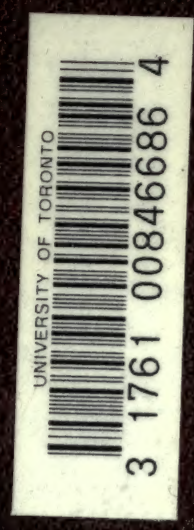




Digitized by the Internet Archive in 2007 with funding from Microsoft Corporation 
A TEXT-BOOK

$\mathrm{OF}$

HUMAN PHYSIOLOGY. 


\section{StaNDARD MeDical Works.}

BY T. M'CALL ANDERSON, M.D.,

Professor of Clinical Medicine, University of Glasgow.

Royal 8vo, with Two Chromo-Lithographs, Steel-Plate, and Numerous Illustrations,

Handsome Cloth. 25s.

SKIN DISEASES (A Treatise on). With Special Reference to Diagnosis and Treatment, including an Analysis of 11,000 Consecutive Cases.

BY Dr: EDOUARD MEYER,

Prof. à l'Ecole Pratique de la Faculté de Médecine de Paris, Chev. of the Legion of Honour, c'c. Royal 8vo, with Three Chromo-Lithographs and Numerous Illustrations. 25s.

DISEASES OF THE EYE (A Practical Treatise on). Translated, with the assistance of the Author, from the Third. French Edition, with additions as contained in the Fourth German Edition, by A. Freeland Fergus, M. B.

BY JOHN THORBURN, M.D., F.R.C.P.,

Late Professor of Obstetric Medicine, Victoria University, Manchester.

Royal 8vo, Illustrated, Handsome Cloth. 21s.

THE DISEASES OF WOMEN (A Practical Treatise on). Prepared with Special Reference to the Wants of the General Practitioner and Advanced Student.

BY A. C. HADDON, M.A.,

Professor of Zoology, Royal College of Science, Dublin.

Royal 8vo, Illustrated, Handsome Cloth. 18s.

EMBRYOLOGY (An Introduction to the Study of). For the use of Students.

By $\mathrm{S}_{I R}$ DYCE DUCKWORTH, M.D. (Edin.), F.R.C.P.

Royal 8vo, Illustrated.

GOUT (A Treatise on). For the use of Practitioners and Students.

Br ALEXANDER MACALISTER, M.D., F.R.S.,

Professor of Anatomy in the University of Cambridge.

Royal 8vo, with Numerous Illustrations.

HUMAN ANATOMY (A Text-Book on). For the use of Students and Practitioners.

BY W. BEVAN LEWIS, L.R.C.P., M.R.C.S.,

Medical Director of the West Riding Asylum, Wakefield.

Royal 8vo, Illustrated.

MENTAL DISEASES (A Text-Book of). With Special Reference to the Pathological Aspects of Insanity.

BY DR RUDOLPH v. JAKSCH,

University of Prague.

Royal 8vo, with Numerous Illustrations.

CLINICAL DIAGNOSIS : A Text-Book of the Chemical, Microseopical, and Bacteriological Evidence of Disease. Translated from the German by James CAGNeY, M.A., M.D., St Mary's Hospital. 


\title{
A TEXT-BOOK OF
}

\section{H U M A PHYSIOLOGY,} INCLUDING

\section{HISTOLOGY AND MICROSCOPICAL ANATOMY;}

\author{
WITH SPECIAL REFERENCE TO THE REQUIREMENTS OF
}

\section{PRACTICAL MEDICINE.}

D R L. L A N D O I S,

PROFESSOR OF PHYSIOLOGY ANI DIRECTOR OF THE PHYSIOLUGICAL INSTITUTE, UNIVERSITY OF GREIFSWALD.

TRANSLATED FROM THE SIXTH GERMAN EDITION.

WITH ADDITIONS BY

W I L L I A M S T I R L I N G, M. D., Sc. D., BRACKENBURY PROFESSOR OF PHYSIOLOGY AND HISTOLOGY IN THE OWENS COLLEGE AND PROFESSOR IN THE VICTORIA UNIVERSITY, MANCHESTER; EXAMINER IN PHYSIOLOGY, UNIVERSITY OF OXFORD.

WITA VERY NUIMEROUS ILIUSTRATIONS.

THIRD EDITION.

L O N D O N :

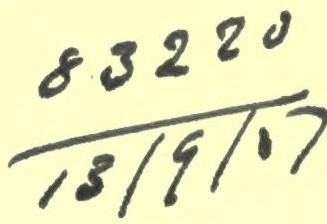

CHARLES GRIFFIN A N C C M A N Y, EXETER STREET, STRAND.

I 888 .

[All Rights Reserved.] 


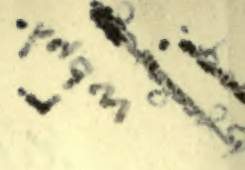

$$
\frac{65358}{1.516}
$$




\section{SIR JOSEPH LISTER, BARONET,}

M.D., D.C.L., LL.D., F.R.SS. (LOND. AND EDIN.),

PROFESSOR OF CLINICAL SURGERY IN KING'S COLLEGE, LONDON, SURGEON-EXTRAORDINARY TO THE QYEEN ; FORMERLY REGIUS PROFESSOR OF CLINICAL SURGERY IN THE UNIVERSITY OF FDINBIRAH,

IN ADMIRATION OF

\section{The Atan of srience,}

WHOSE BRILLIANT DISCOVERIES HAVE REYULUTIONISEI

MEDICAL PRACTICE, AND CONTRIBUTED INCAICULABLY TO THF

WELL-BEING OF MANKINU;

A N I) IN ( \& R A T I T U D E T O

\section{The Teacher,}

WHOSE NOBLE EARNESTNESS IN INCLLCATINA:

THE SACREDNESS OF HUMAN LIFH

STIRREI THE HEARTS OF ALL WHO HEARI HIM :

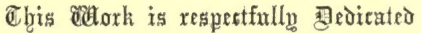

BY HIS FORMER PUPIL,

THE TRANSLATOR. 


\section{PREFATORY NOTE TO THE THIRD) ENGLISH ETITION.}

Is offering to the Profession this Third English Edition, I would only say that the whole work has again been thoroughly revised and in many parts extended. In all respects I have endeavoured to keep it abreast of the latest investigations in Physiology and their bearing on Practical Medicine and Surgery.

I have again to thank my publishers for enabling me to enhance the usefulness of the work by very numerous additions to the Illustrations, which now number 692 as compared with the 494 of the First Edition. Many of these new engravings are original; others are derived from the 6th German edition of the work, from Stöhr's Lehrbuch der Histologie, Quain's Anatomy, Ferrier's Functions of the Brain (2nd Edition), H. Obersteiner's Anleitung beim Studium des Baues der nervösen C'entralorgane, Rollett's Article on "Muscle" in the Real-Encyclopectie, Gowers' Diseases of the Nerrous System, and most of those for the chapter on Reproduction from Haddon's Introduction to Embryology.

In addition, I have to tender special acknowledgments to my colleagues and friends, Professors A. H. Young, James Ross, A. W. Hare, and Dr Aug. D. Waller; as well as to Messrs Carl Reichert of Vienna, W. Petzold of Leipzic, Pothe of Prague, Maw, Cassella, Krohne and Sesemann, Evans \& Wormall of London, and Ferries \& Co. of Bristol.

For the first time the work appears here in one volume-an arrangement adopted both to meet the wishes of Students and to facilitate easy reference. I can but express a hope that the present Edition, in its new form, will meet with the same very kind reception accorded to its predecessors,

WILLIAM STIRLING.

The Owens College, Manchester, September 1888. 
. 


\section{PREFACE TO THE FIRST ENGLISH EDITION.}

The fact that Professor Landois' "Lehrbuch der Physiologie des Menschen" has already passed through Four large Editions since its first appearance in 1880, shows that in some special way it has met the wants of Students and Practitioners in Germany. The characteristic which has thus commended the work will be found mainly to lie in its eminent practicality; and it is this consideration which has induced me to undertake the task of putting it into an English dress for English readers.

Landois' work, in fact, forms a Bridge between Physiology and the Practice of Medicine. It never loses sight of the fact that the Student of to-day is the practising Physician of to-morrow. Thus, to every Section is appended-after a full description of the normal processesa short résumé of the pathological variations, the object of this being to direct the attention of the Student, from the outset, to the field of his future practice, and to show him to what extent pathological processes are a disturbance of the normal activities.

In the same way, the work offers to the busy physician in practice a ready means of refreshing his memory on the theoretical aspects of Medicine. He can pass backwards from the examination of pathological phenomena to the normal processes, and, in the study of these, find new indications and new lights for the appreciation and treatment of the cases under consideration.

With this object in view, all the methods of investigation which may with advantage be used by the Practitioner, are carefully and fully described; and Histology, also, occupies a larger place than is usually assigned to it in Text-books of Physiology.

A word as to my own share in the present version :

(1.) In the task of translating, I have endeavoured throughout to convey the author's meaning accurately, without a too rigid adherence to the original. Those who from experience know something of the difficulties of such an undertaking will be most ready to pardon any shorteomings they may detect.

(2.) Very considerable additions have been made to the Histological, and also (where it has seemed necessary) to the Physiological sections. All such additions are enclosed within square brackets [ ]. I have to 
acknowledge my indebtedness to many valuable Papers in the various Medical Journals-British and Foreign-and also to the Histological Treatises of Cadiat, Ranvier, and Klein; Quain's Anatomy, vol. II., ninth edition; Hermann's Hundbuch der Phlysiolorgie; and the Text-books on l'hysiology by Rutherford, Foster, and Kirkes; C'amgee's Physiological Chemistry; Ewald's 1)igestion; and Loberts's Digestive Ferments.

(3.) The Illustrations have been greatly increased in number, viz., from 275 in the Fourth (ierman Elition to 494 in the English version. These additional Diagrams, with the sources whence derived, are distinguished in the List of Woodcuts by an asterisk.

There only remains for me now to express my thanks to all who lave kindly helped in the progress of the work, either by furnishing Illustrations or otherwise- especially to Drs Byrom Bramwell, Dudgeon, Lauder Brunton, and Knott; Mr Hawksley; Professors Hamilton and I'Kendrick; to my esteemed teacher and friend, Professor Ludwig, of Leipzic; and, finally, to my friend, $\operatorname{Mr}$ A. W. Robertson, M.A., formerly Assistant Lilurarian in the University, and now Librarian of the Aberdeen I'ublic Library, for much valuable assistance while the work was passing through the press.

In conclusion-and forgetting for the moment my own connection with it-I heartily commend the work per se to the attention of Medical Men, and can wish for it no better fate than that it may speedily become as popular in this country as it is in its Fatherland.

WILLIAM STIRLING.

Aberdeen University,

Nivember 1884. 


\section{GENERAL CONTENTS.}

\section{INTRODUCTION.}

The Scope of Physiology and its Relation to the other Branches of Natural Science, . xxxv

Matter,

Forces,

Law of the Conservation of Energy,

Animals and Plants,

Vital Energy and Life,

\section{PHYSIOLOGY OF THE BLOOD.}

SECTION

1. Physical Properties of the Blood,

2. Microscopic Examination of the Blood,

3. Histology of the Human Red Blood-Corpuscles,

4. Effects of Reagents on the Blood-Corpuscles, .

5. Preparation of the Stroma-Making Blood "Take Coloured,"

6. Form and Size of the Blood-Corpuscles of Different Animals, $\quad . \quad$. $\quad 9$

7. Origin of the Red Blood-Corpuscles, . . . . . . . $\quad .10$

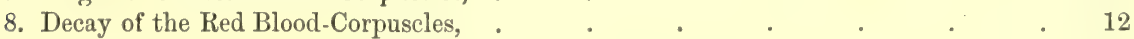

9. The Colourless Corpuscles_Leucocytes-Blood Plates-Granules, . $\quad$. $\quad 13$

10. Abnormal Changes of the Blood-Corpuscles, . . . . . . 17

11. Chemical Constituents of the Red Blood-Corpuscles, . . . . . 18

12. Preparation of Hæmoglobin Crystals, . $\quad$. $\quad . \quad$. $\quad . \quad 19$

13. Quantitative Estimation of Hæmoglobin, . $\quad$ * . . . $\quad . \quad 19$

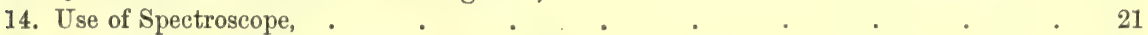

15. Compounds of Hæmoglobin-Methæmoglobin, . . . . $\quad 22$

16. Carbonic Oxide-Hæmoglobin-Poisoning with Carbonic Oxide, . . $\quad .24$

17. Other Compounds of Hæmoglobin, . . . . . . . . . 25

18. Decomposition of Hæmoglobin, . . . . . . . . . . $\quad$. 25

19. Hæmin and Blood Tests, . . . . . . . . . . . $\quad .26$

20. Hæmatoidin, . . . . . . . . . . . 27

21. The Colourless Proteid of Hæmoglobin, . . . . . . . 28

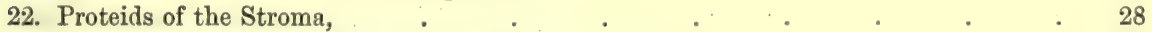

23. The other Constituents of Red Blood-Corpuscles, . . . . . . . $\quad 28$

24. Chemical Composition of the Colourless Corpuscles, . . . . . . . . . $\quad 29$

25. Blood-Plasma, and its Relation to Serum, . $\quad . \quad$. $\quad$. $\quad$. $\quad 29$

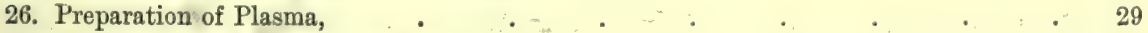

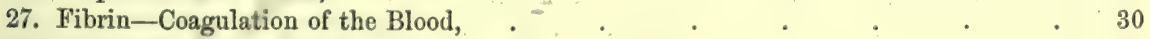

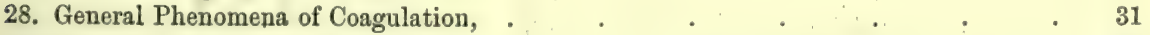

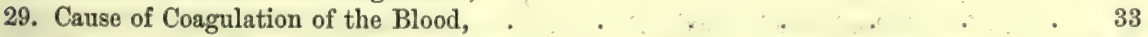

30. Source of the Fibrin-Factors, . $\quad . \quad \ldots \quad \ldots \quad \ldots \quad \ldots \quad \ldots \quad \ldots$ 
SECTION

31. Relation of the Red Blood-Corpuseles to the Formation of Fibrin, . . . $\quad 37$

32. Chemical Composition of the Plasma and Serum, . . . . 37

33. The Gases of the Blood, . . . . . . . . . . 39

34. Extraction of the Blood Gases, . . . . . . . . . 40

35. Quantitative Estimation of the Blood Gases, . . . . . . . 42

36. The Blood Gases, . $\quad . \quad 42$

37. Is Ozone $\left(\mathrm{O}_{3}\right)$ present in Blood ? . . . . . . . . . . . 43

38. Carbon dioxide and Nitrogen in Blood, . . . . . . . . . . 44

39. Arterial and Venous Blood, . . . . . . . . . . . . . 45

40. Quantity of Blood, . . . . . . . . . . . . . . 45

41. Variations from the Normal Conditions of the Blood, . . . . . 46

\section{PHYSIOLOGY OF THE CIRCULATION.}

42. General View of the Circulation,

43. The Heart,

44. Arrangement of the Cardiac Muscular Fibres,

45. Arrangement of the Ventricular Fibres,

46. Pericardium, Endocardium, Valves,

47. Automatic Regulation of the Heart,

48. The Movements of the Heart, .

49. Pathological Disturbances of Cardiac Action, :

50. The Apex-Beat-The Cardiogram,

51. The Time occupied by the Cardiac Movements,

52. Pathological Disturbance of the Cardiac Impulse,

53. The Heart-Sounds,

54. Variations of the Heart-Sounds,

55. The Duration of the Movements of the Heart,

56. Physical Examination of the Heart,

57. Innervation of Heart-Cardiac Nerves,

58. The Automatic Motor-Centres of the Heart,

59. The Cardio-Pneumatic Movements,

60. Influence of the Respiratory Pressure of the Heart,

THE CIRCULATION.

61. The Flow of Fluids throngh Tubes,

62. Propelling Force, Velocity of Current, Lateral Pressure,

63. Currents through Capillary Tubes,

64. Movements of Fluids and Wave-Motion in Elastic Tubes,

65. Structure and Properties of the Blood-Vessels,

66. Investigation of the Pulse,

67. Pulse Tracing or Sphygmogram,

68. Origin of the Dicrotic Wave, .

69. Dicrotic Pulse, .

70. Characters of the Pulse,

71. Variations in the Strength, Tension, and Volume of the Pulse,

72. The Pulse-Curves of various Arteries, .

73. Anacrotism,

74. Influence of the Respiratory Movements on the Pulse-Curve,

75. Influence of Pressure upon the Form of the Pulse-Wave,

76. Rapidity of Transmission of Pulse-Waves,

77. Propagation of the Pulse-Wave in Elastic Tubes,

78. Velocity of the Pulse-Wave in Man, 
80. Vibrations communicated to the Body by the Action of the Heart, . $\quad .116$

81. The Blood-Current, . . . . . . . . . . . . . 116

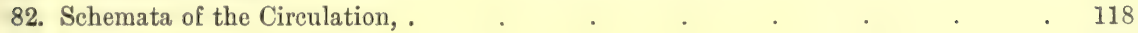

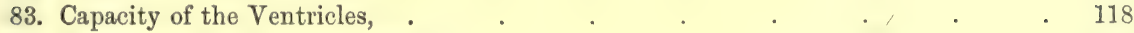

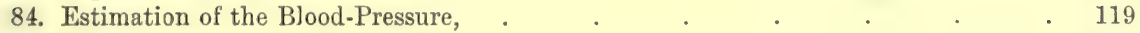

85. Blood-Pressure in the Arteries, . $\quad . \quad$. $\quad . \quad$. $\quad . \quad 122$

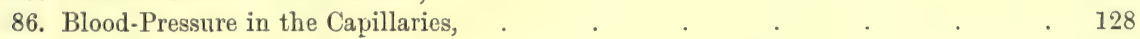

87. Blood-Pressure in the Veins, . $\quad . \quad$. $\quad . \quad$. $\quad . \quad . \quad 128$

88. Blood-Pressure in the Pulmonary Artery, . . . . . . . $\quad . \quad 130$

89. Measurement of the Velocity of the Blood-Stream, . . . . . . $\quad . \quad 132$

90. Velocity of the Blood in Arteries, Capillaries, and Veins, . . . . $\quad . \quad 134$

91. Estimation of the Capacity of the Ventricles, . . . . . . . 135

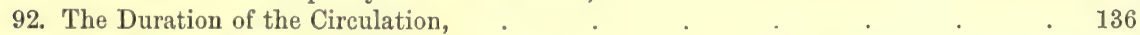

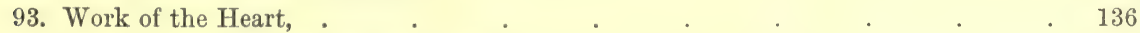

94. Blood-Current in the Smallest Vessels, . . . . . . . . $\quad . \quad 137$

95. Passage of the Blood-Corpuscles out of the Vessels-[Diapedesis], . _ . $\quad 139$

96. Movement of the Blood in the Veins, _ . $\quad . \quad \ldots \quad . \quad$. $\quad . \quad 139$

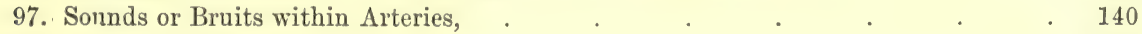

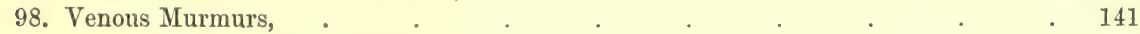

99. The Venous Pulse--Phlebogram, . . . . . . . . . . $\quad$. 142

100. Distribution of the Blood, . . . . . . . . . . . . . 144

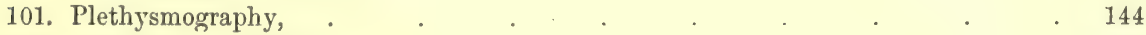

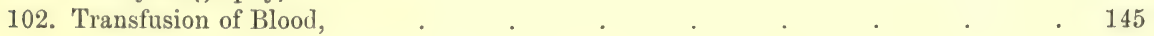

THE BLOOD-GLANDS.

103. The Spleen-Thymus-Thyroid-Supra-Renal Capsules-Hypophysis CerebriCoccygeal and Carotid Glands, . . . . . . . . . 148

104. Comparative,. . . . . . . . . . . . 157

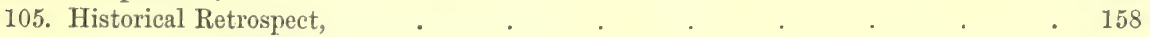

\section{PHYSIOLOGY OF RESPIRATION.}

106. Structure of the Air-Passages and Lungs, . $\quad . \quad$. $\quad . \quad$. $\quad . \quad 159$

107. Mechanism of Respiration, . . . . . . . . $\quad$. 165

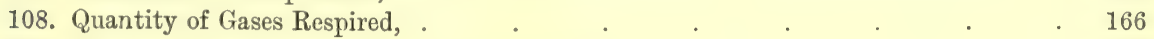

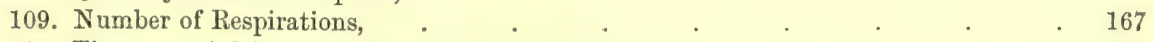

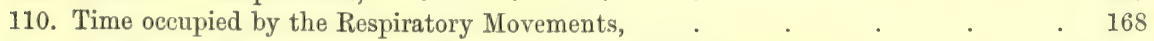

111. Pathological Variations of the Respiratory Movements, . . . . . 171

112. General View of the Respiratory Muscles, . . . . . . $\quad$. 172

113. Action of the Individual Respiratory Muscles, _ . . . . $\quad$. 173

114. Relative Size of the Chest, . . . . . . 176

115. Pathological Variations of the Percussion Sounds, . $\quad . \quad$. $\quad$. 178

116. The Normal Respiratory Sounds, . $\quad . \quad$. $\quad . \quad$. $\quad . \quad 179$

117. Pathological Respiratory Sounds, . . . . . . . $\quad$. 180

118. Pressure in the Air ${ }_{2}$ Passages during Respiration, . . . . . . . 181

119. Appendix to Respiration, . . . . . . . . . $\quad$. 182

120. Peculiarly Modified Respiratory Sounds, . $\quad$. $\quad$. $\quad$. $\quad$. $\quad 182$

121. Quantitative Estimation of $\mathrm{CO}_{2}, \mathrm{O}$, and Watery Vapour, . . . . . 183

122. Methods of Investigation, . . . . . . . . 184

123. Composition and Properties of Atmospheric Air, . . . . . . $\quad 186$

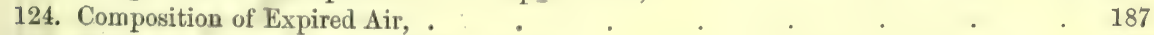

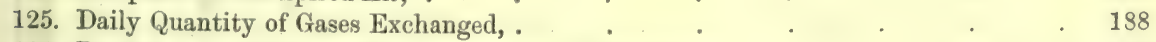

126. Review of the Daily Gaseous Income and Expenditure. ․ . . . $\quad$. 188

127. Conditions influencing the Gaseous Exchanges, . $\quad$. $\quad$. $\quad$. 188 
128. Diffusion of Gases within the Lungs,

129. Exchange of Gases between the Blood aud Air,

130. Dissociation of Gases,

131. Cutaneous Respiration,

132. Internal Respiration, .

133. Respiration in a Closed Space,

134. Dyspnoea and Asphyxia,

135. Respiration of Foreign Gases,

136. Accidental Impurities of the Air,

137. Ventilation of Rooms,

138. Formation of Mucus,

\section{PHYSIOLOGY OF DIGESTION.}

141. The Mouth and its Glands, .

142. The Salivary Glands, .

143. Histological Changes in Salivary Glands,

144. The Nerves of the Salivary Glands,

145. Action of Nerves on the Salivary Secretion,

146. The Saliva of the Individual Glands,

147. The Mixed Saliva in the Mouth,

148. Physiological Action of Saliva,

149. 'Tests for Sugar,

150. Quantitative Estimation of Sugar,

151. Mechanism of the Digestive Apparatus,

152. Introduction of the Food,

153. The Movements of Mastication,

154. Structure and Development of the Teeth,

155. Movements of the Tongue,

156. Deglutition,

157. Movements of the Stomach,

158. Vomiting,

159. Movements of the Intestine,

160. Excretiou of Freal Matter,

161. Conditions intluencing the Movements of the Intestine,

162. Structure of the Stomach,

163. The Gastric Juice,

164. Secretion of Gastric Juice,

165. Methods of obtaining Gastric Juice,

166. Process of Gastric Digestion, .

167. Gases in the Stomach,

168. Structure of the Pancreas,

169. The Pancreatic Juice,

170. Digestive Action of the Pancreatic Juice,

171. The Secretion of the Pancreatic Juice,

172. Preparation of Peptonised Food,

173. Structure of the Liver,

174. Chemical Composition of the Liver-Cells,

175. Diabetes Mellitus,' or Glycosuria,

176. The Functions of the Liver,

177. Constituents of the Bile, 
181. Functions of the Bile,

182. Fate of the Bile in the Intestine, . . . . . . 275

183. The Intestinal Juice, . _ _ _ _ . 276

184. Fermentation Processes in the Intestine, . . . . . . . . 279

185. Processes in the Large Intestine, . . . . . . . . . 283

186. Pathological Variations, . . . . . . . . . . . $\quad 285$

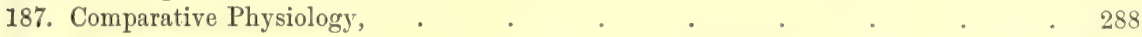

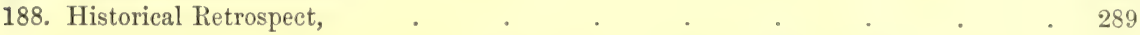

\section{PHYSIOLOGY OF ABSORPTION.}

189. The Organs of Absorption,

190. Structure of the Small and Large Intestines,

191. Absorption of the Digested Food,

192. Absorptive Activity of the Wall of the Intestine, . . . . . 297

193. Influence of the Nervous System, . _ . . . . . . . . 301

194. Feeding with "Nutrient Enemata," . . . . . . . . . . 301

195. Chyle-Vessels and Lymphatics, . . . . . . . . . . $\quad . \quad 301$

196. Origin of the Lymphatics, . $\quad$. $\quad$. $\quad$. $\quad$. $\quad$. $\quad 302$

197. The Lymph-Glands, . . . . . . . . . . . . . 304

198. Properties of Chyle and Lymph, . . . . . . . . . . . 306

199. Quantity of Lymph and Chyle, . . . . . . . . . . . . . $\quad . \quad 308$

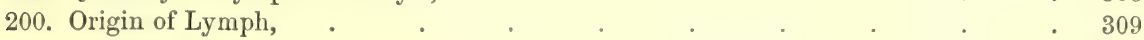

201. Movement of Chyle and Lymph, . . . . . . . . $\quad$. 310

202. Absorption of Parenchymatous Effusions, . . . . . . . . . $\quad 312$

203. Dropsy, Edema, Serous Effusions, . . . . . . $\quad . \quad 312$

204. Comparative Physiology, . $\quad . \quad$. $\quad . \quad$. $\quad$. $\quad$. $\quad$. 314

205. Historical Retrospect, . . . . . . . . . . $\quad$. 314

\section{PHYSIOLOGY OF ANIMAL HEAT.}

206. Sources of Heat,

207. Homoiothermal and Poikilothermal Animals, . $\quad 318$

208. Methods of Estimating Temperature-Thermometry, _ . . . $\quad 319$

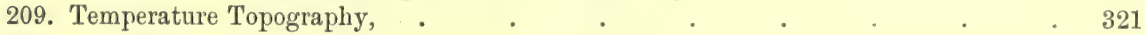

210. Conditions Influencing the Temperature of Organs, . . . . . . 322

211. Estimation of the Amount of Heat-Calorimetry, . . . . . . . 324

212. Thermal Conductivity of Animal Tissues, . . . . . . . . 325

213. Variations of the Mean Temperature, . . . . . . . . . . 325

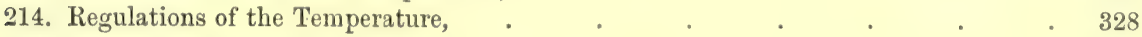

215. Income and Expenditure of Heat, . . . . . . . . . . . . . $\quad . \quad 331$

216. Variations in Heat Production, . . . . . . . . . $\quad 333$

217. Relation of Heat Production to Bodily Work, . . . . . . . . 333

218. Accommodation for Different Temperatures, _ . . . . . . . $\quad$. 334

219. Storage of Heat in the Body, . . . . . . . . . . . . 334

220. Fever, . . . . . . . . . . . . . . . 335

221. Artificial Increase of the Temperature, . . . . . . . . $\quad 336$

222. Employment of Heat, . . . . . . . . . . . . . $\quad 337$

223. Increase of Temperature post mortem, . . . . . . . . . . . . . . . $\quad 337$

224. Action of Cold on the Body, . . . . . . . . . . . . . . . . . . . . $\quad 337$

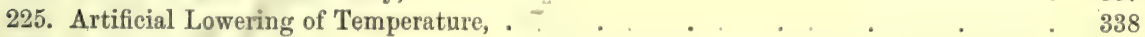

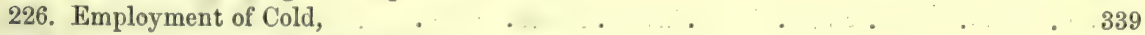

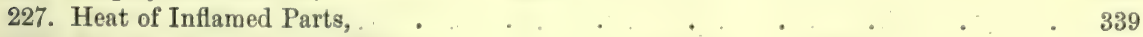

228. Historical and Comparative, . . . . . . . . . . . . . . . . 340 


\section{PHYSIOLOGY OF THE METABOLIC PHENOMENA OF THE BODY. \\ OF THE BODY.}

\section{SECTION}

229. General View of Food-Stuffs,

230. Structure and Secretion of the Mammary (ilands,

231. Milk and its Preparations,

232. Eggs,

233. Flesh and its Preparations, .

234. Vegetable Foods,

235. Condiments-Coffee, Tea, and Alcohol,

236. Equilibrium of the Metabolism,

237. Metabolism during Hunger and Starvation, .

238. Metabolism during a purely Flesh Diet,

239. A Diet of Fat or of Carbohydrates,

240. Mixture of Flesh and Fat,

241. Origin of Fat in the Body,

243. The Metabolisin of the Tissues,

244. Regeneration of Organs and Tissues,

245. Transplantation of the Tissues,

246. Increase in Size and Weight during Growth, THE ORGANISM.

247. Inorganic Constituents,

248. Organic Compounds-Proteids,

249. The Animal and Vegetable Proteids and their Properties,

250. The Albuminoids,

251. The Fats,

252. The Carbohydrates,

253. Historical Retrospect,

\section{THE SECRETION OF URINE.}

254. Structure of the Kidney,

255. The Urine,

256. Organic Constituents of Urine-Urea,

257. Qualitative and Quantitative Estimation of Urea, .

258. Uric Acid,

259. Qualitative and Quantitative Estimation of Uric Acid,

260. Kreatinin and other Substances,

261. Colouring Matters of the Urine,

262. Indigo, Phenol, Kresol, Pyrokatechin,

263. Spontaneous Changes in Urine, Fermentations,

264. Albumin in Urine,

265. Blood in Urine,

266. Bile in Urine,

267. Sugar in Urine,

268. Cystin,

269. Leucin, Tyrosin,

270. Deposits in Urine,

271. General Scheme for Detecting Urinary Deposits, 
275. Passage of Various Substances into the Urine,

276. Influence of Nerves on the Renal Secretion,

277. Uræmia, Ammoniæmia,

278. Structure and Functions of the Ureter,

279. Urinary Bladder and Urethra,

\section{FUNCTIONS OF THE SHIN.}

283. Structure of the Skin, Nails, and Hair,

284. The Glands of the Skin,

285. The Skin as a Protective Covering,

286. Cutaneous Respiration and Secretion-Sweat,

287. Conditions Influencing the Secretion of Sweat,

288. Pathological Variations,

289. Cutaneous Absorption-Galvanic Conduction,

290. Comparative-Historical,

\section{PHYSIOLOGY OF THE MOTOR APPARATUS.}

291. Ciliary Motion, Pigment Cells,

292. Structure and Arrangement of the Muscles,

293. Physical and Chemical Properties of Muscle,

294. Metabolism in Muscle,

295. Rigor mortis,

296. Muscular Excitability,

297. Changes in a Muscle during Contraction,

298. Muscular Contraction,

299. Rapidity of Transmission of a Muscular Contraction,

300. Muscular Work,

301. The Elasticity of Muscle,

302. Formation of Heat in an Active Muscle, .

303. The Muscle-Sound,

304. Fatigue and Recovery of Muscle,

305. The Mechanism of the Joints,

312. Voice and Speech,

313. Arrangements of the Larynx,

314. Organs of Voice-Laryngoscopy,

315. Conditions Modifying the Laryngeal Sounds, 


\section{GENERAL PHYSIOLOGY OF THE NERVES AND ELECTRO-PHYSIOLOGY.}

SECTION

321. Structure and Arrangement of the Nerve-Elements,

325. Diminution of Excitability-Degeneration and Regeneration of Nerves, . . 537

326. The Galvanic Current

327. Action of the Galvanic Current-Galvanometer, . . . 543

328. Electrolysis,

329. Induction-Extra-Current-Magneto-Induction, _ . . . . . . 549

330. Du Bois-Reymond's Inductorium, . _ _ . . . . 551

331. Electrical Currents in Passive Muscle and Nerve, _ _ _ _ . . . 554

332. Currents of Stimulated Muscle and Nerve, . . . . $\quad$. 557

333. Currents in Nerve and Muscle during Electrotonus, _ $\quad 561$

334. Theories of Muscle and Nerve Currents, _ _ $\quad 562$

335. Electrotonic Alteration of the Excitability, . . . . . . . 565

336. Electrotonus-Law of Contraction, . . . . . $\quad 567$

337. Rapidity of Transmission of Nervous Impulses, _. . . . $\quad 570$

338. Double Conduction in Nerves, . . . . 573

339. Therapeatical Lses of Electricity-Reaction of Degencration . . 574

340. Electrical Charging of the Boly, . . . . . $\quad 579$

341. Comparative-Historical, . . . . . . . . $\quad 579$

\section{PHYSIOLOGY OF THE PERIPHERAL NERVES.}

342. Classification of Nerve-Fibres, . . . . . . . . 581

343. Nervus Olfactorius, . $\quad . \quad \ldots \quad \ldots \quad$. $\quad . \quad 584$

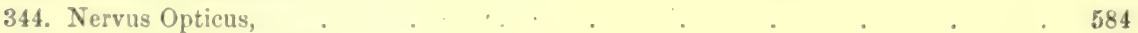

345. Nervus Oculomotorills, _ . . 587

346. Nervus Trochlearis, . . . . . . . $\quad 589$

347. Nervus Trigeminus, . _ _ . . . . $\quad 590$

348. Nervus Abducens, _ _ . . $\quad 599$

349. Nervus Facialis, . $\quad 599$

350. Nervus Acusticus, ?. 603

351. Nervus Glosso-pharyngeuz, . . . . : . . . $\quad 606$

352. Nervus Vagus, . . . . 606

353. Nervus Accessorius, . . . . . . . . 614

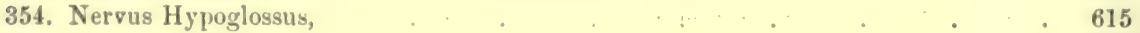

355. The Spiual Nerves, . . . . 615

356. The Sympathetic Nerve, . . . . . . . . . 620

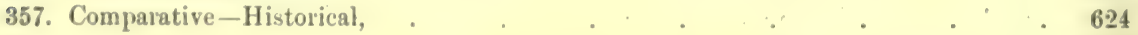

\section{PHYSIOLOGY OF THE NERVE-CENTRES.}

858. General,

359. Structure of the Spinal Cord,

360. Spinal reflexes,

361. Inhibition of the reflexes,

362. Centres in the Spinal Cord, .

363. Excitability of the Spinal Cord,

364. The Conducting Paths in the Spinal Cord,

365. General Schema of the Brain, 
367. Reflex Centres of the Medulla Oblongata, .

368. The Respiratory Centre, . . . . . . . . . 661

369. The Cardio-Inhibitory Centre, . . . . . 667

370. The Accelerans Cordis Centre, . . . . . . . . 669

371. Vaso-motor Centre and Vaso-motor Nerves, _ . . . . . $\quad 672$

372. Vaso-dilator Centre and Vaso-dilator Nerves, . . . . . . 678

373. The Spasm Centre-The Sweat Centre, . . . . . . 680

374. Psychical Functions of the Cerebrum, . . . . . . . 681

375. Structure of the Cerebrum-Motor Cortical Centres, _ . . . . . 686

376. The Sensory Cortical Centres, . . . . . . . 701

377. The Thermal Cortical Centres, . . . . . . 705

378. Topography of the Cortex Cerebri, . . . . . . . . 706

379. The Basal Ganglia-The Mid-brain, . . . . . . 715

380. The Structure and Functions of the Cerebellum, . . . . . . . 723

381. The Protective Apparatus of the Brain, . . . . . . . 726

382. Comparative-Historical, . . . . . . . . 728

\section{PHYSIOLOGY OF THE SENSE ORGANS.}

\section{SIGHT.}

383. Introductory Observations, . . . . . . . . . . . 731

384. Histology of the Eye, . . . . . . . . . . 733

385. Dioptric Observations, . $\quad . \quad$. $\quad . \quad$. $\quad . \quad . \quad . \quad 742$

386. Formation of a Retinal Image, . . . . . . . . . . 747

387. Accommodation of the Eye, . . . . . . . . . . . . 749

388. Normal and Abnormal Refraction, . . . . . . . . . . $\quad$. 753

389. The Power of Accommodation, . . . . . . . $\quad$. 555

390. Spectacles, . . . . . . . . . . . . . 756

391. Chromatic Aberration and Astigmatism, . . . . . . . . 757

392. The Iris, . . . . . . . . . . . . . . 758

393. Entoptical Phenomena, . . . . . . . . . . . 762

394. Illumination of the Eye--The Ophthalmoscope, . . . . . . . $\quad$. 764

395. Aetivity of the Retina in Vision, . . . . . . . . . 768

396. Perception of Colours, . . . . . . . . . 773

397. Colour-blindness, . . . . . . . . 778

398. Stimulation of the Retina, . . . . . . . . . . . $\quad$. $\quad$. 779

399. Movements of the Eyeballs, . . . . . . . 783

400. Binocular Vision, . . . . . . . . . 787

401. Single Vision-Identical Points, . . . . . . . . 787

402. Stereoscopic Vision, . . . . . . . . 789

403. Estimation of Size and Distance, . . . . . . . . . . 791

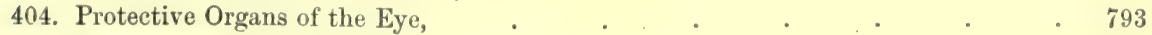

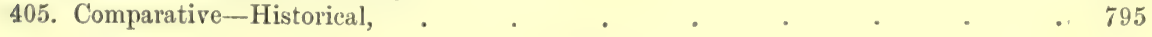

\section{HEARING.}

406. Structure of the Organ of Hearing,

407. Physical Introduction,

408. Ear Muscles,

409. Tympanic Membrane,

410. The Auditory Ossicles and their Muscles,

411. Eustachian Tube-Tympanum,

412. Conduction of Sound in the Labyrinth,

413. Structure of the Labyrinth, 
414. Auditory Perceptions of Pitch,

415. Perception of Quality-Vowels,

416. Action of the Labyrinth,

417. Harmony-Discords-Beats,

418. Perception of Sound,

419. Comparative-Historical,

420. Structure of the Organ of Smell,

421. Olfactory Sensations,

4. TASTE.

422. Position and Structure of the Organs of Taste,

423. Gustatory Seusations,

5. TOUCH.

424. Terminations of Sensory Nerves,

425. Sensory and Tactile Sensations,

426. The Sense of Locality,

427. The Pressure Sense, .

428. The Temperature Sense,

429. Common Sensation-Pain,

\section{PHYSIOLOGY OF REPRODUCTION AND DEVELOPMENT.}

431. Forms of Reproduction,

432. Testis-Seminal Fluid,

433. The Ovary-Ovum-Uterus, .

434. Puberty,

435. Menstruation,

436. Penis-Erection,

437. Ejaculation-Reception of the Semen,

438. Fertilisation of the Ovum,

439. Impregnation and Cleavage of the Ovum,

440. Structures formed from the Epiblast,

441. Structures formed from the Mesollast and Hypoblast,

442. Formation of the Heart and Embryo,

443. Further formation of the Body,

444. Formation of the Amnion and Allantoi.

445. Human Fotal Menibranes-Placenta,

446. Chronology of Human Development,

447. Formation of the Osseous System,

448. Development of the Vascular System,

449. Formation of the Intestinal Canal,

450. Development of Genito-Urinary Organs,

451. Formation of the Central Nervous Systenı,

452. Development of the Sense Organs,

453. Birth,

454. Comparative-Historical, Appendix A.; Bibliography, . 


\section{LIST OF ILLUSTRATIONS.}

FIGURE

1. Human coloured blood-corpuscles, .

2. Apparatus of Abbé and Zeiss for estimating the blood-corpuscles, . 4

3. Mixer,

*4. Gower's hæmacytometer (Hawksley),

*5. Crenation of human blood-corpuscles,

6. Red blood-corpuscles showing various changes of shape,

*7. Effect of reagents on blood-corpuscles (Stirling)

8. Vaso-formative cells, . . . . . . . . . . 11

9. White blood-corpuscles and fibrin, . . . . . . . $\quad$. 13

*10. White blood-corpuscles (Klein), . . . . . . . . . . . 14

11. Amoboid movements of colourless corpuscles, . $\quad . \quad$. $\quad . \quad$. $\quad 15$

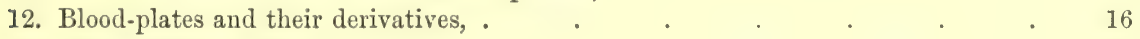

13. Hæmoglobin crystals, . . . . . . . . 18

*14. Gower's hæmoglobinometer (Hawksley), . $\quad$. $\quad$. $\quad$. $\quad$. $\quad$. 20

*15. Fleischl's hæmometer (Reichert), . . . . . . . . 20

16. Scheme of a spectroscope, . . . . . . . . . . 21

17. Various spectra of hæmoglobin and its compounds, . . . . . . 22

18. Hæmin crystals, . . . . . . . . . . 26

19. Hæmin crystals prepared from traces of blood, . $\quad$. $\quad$. $\quad$. $\quad 26$

20. Hæmatoidin crystals, . . . . . . . . 27

*21. Hewson's experiment, . . . . . . . . $\quad$. 33

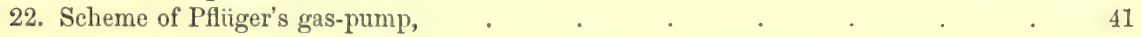

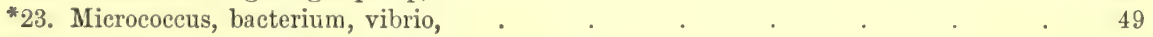

*24. Bacillus anthracis, . . . . . . . . . . . 49

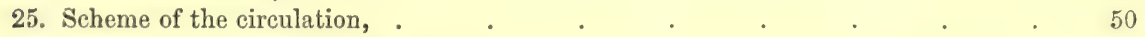

26. Muscular fibres from the heart, . . . . . . . $\quad$. 51

27. Muscular fibres in the left auricle, . $\quad . \quad 5 \quad$. $\quad . \quad$. $\quad . \quad 52$

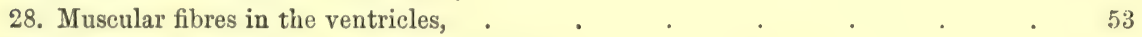

*29. Lymphatic from the pericardium (Cadiat), . . . . . . . . $\quad 54$

*30. Section of the endocardium (Cadiat), . $\quad . \quad$. $\quad . \quad$. $\quad . \quad 54$

*31. Purkinje's fibres (Ranvier), . . . . . . . . . . . 55

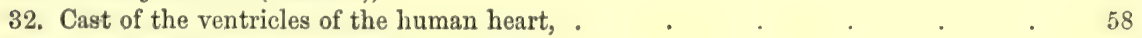

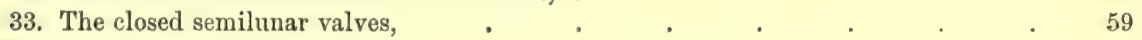

*34. Gaule's maximum and minimum manometer (Gscheidlen), . $\quad$. $\quad$. $\quad$. 59

*35. Manometer of Gaule (Gscheidlen), . $\quad$. $\quad$. $\quad$. $\quad$. $\quad$. $\quad$. 59

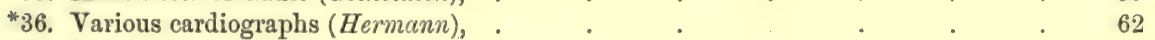

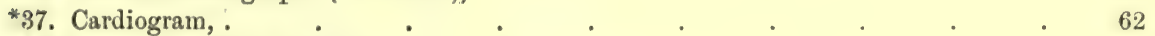

*38. Arteriogram and Cardiogram, . $\quad . \quad$. $\quad . \quad$. $\quad . \quad$. $\quad 62$

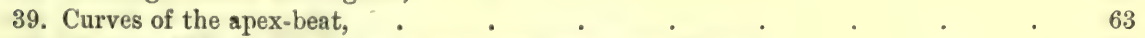

40. Changes of the heart during systole, and sections of thorax, $\quad . \quad$. $\quad . \quad 64$

*41. Dog's heart, posterior surface (Ludvig and Hesse), . . . . . 65

*42. Left lateral surface (Ludwig and Hesse), . . . . . . . . 65

*43. Anterior surface (Ludwig and Hesse), . . . . . . . . 65

*44. Base of heart (Ludwig and Hesse), . . . . . . . . . . . 65 
FIGURE

*45. Base of heart in systole and diastole (Lulvig and Hesse), . . . . $\quad 65$

46. Curves from a rabbit's ventricle, . . . . . . . . . . $\quad .67$

"47. Marey's registering,tambour (Hermann), . . . . . . . . 67

48. Curves obtained with a cardiac sound, . . 68

49. Curves from the cardiac impulse, . . $\quad 70$

"50. Schene of cardiac cycle, . $\quad .71$

"51. Position of the heart in the chest (Luschka und Gairluer), . . $\quad 73$

*52. Curves of excised rabbits' hearts (Stivling, after Waller), . . . . . $\quad 74$

"53. Heart of frog from the front $($ Ecker $)$, . . . . . . . . . . . 77

*54. Heart of frog from behind $(E c k c r)$, . . . . . . . . . . . . . $\quad$. $\quad$.7

*55. Auricular septum (Ecker), . . . . . . . . . . . . . . . 77

*56. Bipolar nerve-cells from a frog's heart, . . . . . . . . . . 77

"57. Scheme of frog's heart (Brunton), . . . . . . . . . . . . $\quad 78$

"58. Stannius's experiment (Brunton), . . . . . . . . . . . . 78

"59. Luciani's groups of cardiac pulsations (Hcrmann), . . . . . . 81

*60. Scheme of a frog-manometer (Stirling), . . . . . . . . . . 82

*61. Perfusion cannula (Kronecker and Stivling), . . . . . . . . 82

*62. Roy's tonometer (Stirling), . . . . . . . . . . . 82

*63. Curves of a frog's heart at different temperatures (Hermamn), . . . $\quad 83$

64. Cardio-pneumograph of Landois, . . . . . . . . . . . . 87

65. Aplaratus for showing the effect of respiration, . . . . . . $\quad 88$

66. Cylindrical vessel filled with water, . . . . . . . 0

67. Cylindrical ressel with manometers, . . . . . . . . . . 90

68. Small artery with its various coats, _ . _ . $\quad . \quad$. $\quad . \quad 92$

69. Capillaries injected with silver nitrate, . . . . . . . . . . . 93

*70. Longitudinal section of a vein at a valve (C'adiat), . . . . . 94

71. Sphymometer of Herisson, . . . . . . . . . . . . $\quad .97$

72. Scheme of Marey's sphygniograph, . . . . . . . . . . . . . 98

*73. Marey's.improved sphygmograph (B. Bramucll), . . . . . . . . 9 98

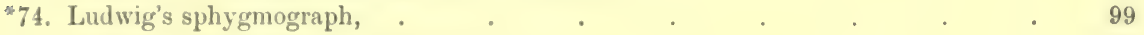

*75. Dudgeon's sphygmograph (Dudgcon), . . . . . . . . . . 99

76. Scheme of Brondgeest's pansphygmograph, . . . . . $\quad .100$

77. Scheme of Landois' angiograph, . $\quad . \quad$. $\quad . \quad . \quad . \quad 100$

78. Pulse-curves of the carotid, radial, and posterior tibial arteries, . $\quad . \quad .101$

*79. S. Mayer's gas-sphy gmoscope, . . . . . . . . . $\quad . \quad 101$

80. Hremautographic curve, . . . . . . . . . . . . . $\quad . \quad 101$

*81. Sphygmogram of raclial artery (Dudgcon), . . . . . . . $\quad$. 102

"82. Irregular pulse, mitral regurgitation, . . . . . . . . $\quad$. 103

83. Sphygmograms of various arteries, . . . . . . . . . . $\quad .104$

*81. P'ulse tracings after amyl nitrite (Slirling, after Murrell), . . . . 105

*85. Aortic regurgitation, . . . . . . . . . 105

86. Pulsus dicrotus, P. caprizans, P. monocrotus, . . . . . . $\quad .106$

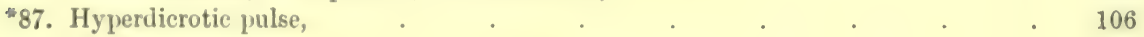

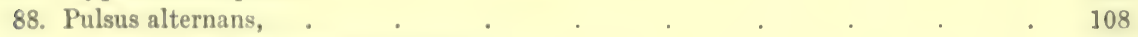

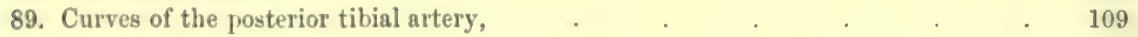

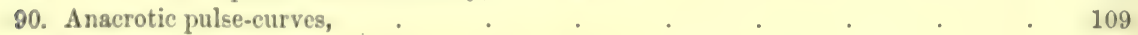

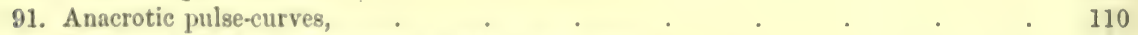

92. Influence of the respiration on the spliygmogram, $\quad$. . . . 111

93. Pulse-curves during Miiller's and Valsalva's experiments, . . . . 112

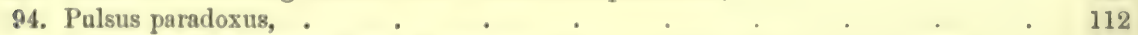

95. Various radial curves altered by pressure, . $\quad . \quad$. $\quad . \quad$. $\quad . \quad 113$

96. Pulse tracings of the radial artery, . $\quad . \quad$. $\quad . \quad$. $\quad . \quad 114$

97. Tracings from the posterior tibial, and carotid arteries, . . . . $\quad .115$

98. Apparatus for registering the molar motions of the body, . . . . $\quad 115$

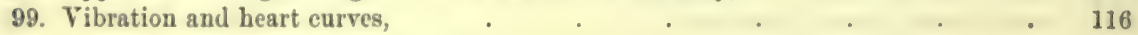


100. Ludwig and Fick's kymographs, . . . . .

*101. Ludwig's improved revolving cylinder (Hermann), _ . . . . . . 119

*102. Blood-pressure tracing of the carotid of a dog (Hermann), _ . . $\quad 120$

*103. Fick's spring manometer, by Hering (Hernann), . . . . . . . 121

104. Fick's flat spring kymograph, . . . . . . . . . . . 121

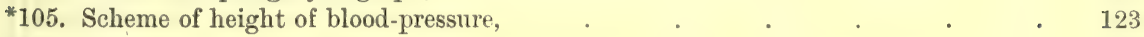

*106. Depressor curve (Stirling),. . . . . . . . . . . . 124

*107. Blood-pressure and respiration tracings taken simultaneously (Stirling), . . 125

*108. Blood-pressure tracing during stimulation of the vagus (Stirling), . . 127

*109. Apparatus of v. Kries for capillary pressure (C. Luduig), . . . . . . 128

*110. Scheme of the blood-pressure, . . . . . . . . . . $\quad$. 129

111. Volkmann's hæmadromometer, . $\quad$. $\quad$. $\quad$. $\quad$. $\quad$. $\quad$. 132

112. Ludwig and Dogiel's rheometer, . . . . . . . 132

113. Vierordt's hæmatachometer-Dronograph, . . . . . . . 133

114. Photohæmatachometer, . . . . . . . 134

*115. Scheme of sectional area $(\operatorname{after} Y e 0), \quad$. $\quad . \quad$. . . . . . . $\quad$. 134

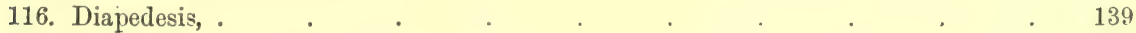

117. Various forms of venous pulse, . $\quad . \quad$. $\quad . \quad$. $\quad . \quad$. 143

118. Mosso's plethysmograph, . . . . . . . . . . 144

*119. Section of spleen $($ Stöhr), . . . . . . . . . . . . . 148

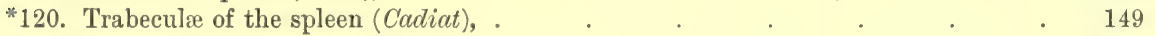

*121. Adenoid tissue of spleen (Cadiat), . . . . . . . . . . . 149

*122. Malpighian corpuscle of the spleen (Cadiat), . . . . . . 149

*123. Elements of splenic pulp (Stöhr), . . . . . . . . . . $\quad 150$

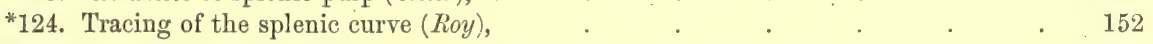

*125. Thymus gland (Cadiat), . . . . . . . . . . 153

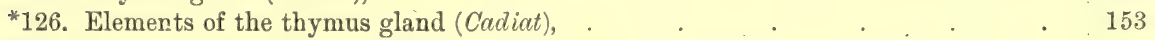

*127. Thyroid gland (Cadiat), . . . . . . . 154

*128. Supra-renal capsule (Cadiat), . . . . . . . . . . $\quad$. 156

129. Schemata of the circulation, . . . . . . . . $\quad{ }^{157}$

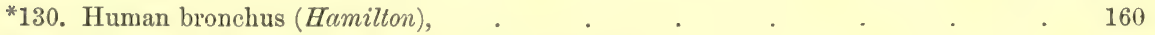

*131. Air-vesicles injected with silver nitrate (Hamilton), . . . . . 162

132. Scheme of the air-vesicles of lung, . . . . . . . . . 163

*133. Interlobular septa of lung (Hamilton), . . . . . . . . . 164

134. Scheme of Hutchinson's spirometer, _ . . . . . . . $\quad$. 167

135. Brondgeest's tambour and curve, . $\quad . \quad$. $\quad . \quad$. . . 168

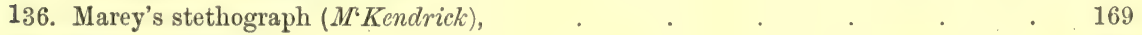

137. Pneumatograms, . . . . . . . . . 170

138. Section through diaphragm (Hermann), . $\quad . \quad$. $\quad . \quad$. $\quad$. 173

139. Action of intercostal muscles, . . . . . . . . . 175

140. Crytometer curve, . . . . . . . . . . 176

141. Sibson's thoracometer, . . . . . . . . . . . . 176

142. Topography of the lungs and heart, . . . . . . . . $\quad$. 178

143. Andral and Gavarret's respiration apparatus, . . . . . . 184

144. Scharling's apparatus, . . . . . . . . . 185

145. Regnault and Reiset's apparatus, . $\quad . \quad$. $\quad . \quad$. $\quad . \quad 185$

146. v. Pettenkofer's apparatus, . . . . . . . . . $\quad$. 186

147. Valentin and Brunner's apparatus, . . . . . . . . $\quad .187$

148. Ciliated epithelium, . . . . . . . . . . . $\quad$. 200

149. Objects found in sputum, . . . . . . 202

*150. Squamous epithelium from the mouth (Stirling), . . . . . $\quad 206$

151. Mucous follicle and salivary corpuseles (Schenik), . . . . . 207

*152. Section of tonsil (Stöhr), . . . . . . . . 207

*153. Scheme of glands, . . . . . . . . . . . . . 208

154. Rodded epithelium of a salivary duct, . . . . . . . . 208 
155. Histology of the salivary glands, .

*156. Human sub-maxillary gland (Heidenhrin),

*157. Parotid gland of rabbit at rest (Heidenhain),

*158. Parotid gland of rabit, active phase (Heidenhain),

*159. Scheme of the nerves of the salivary glands (Stirling),

*160. Diagram of a salivary gland (Stirling),

161. Apparatus for estimation of sugar, .

162. Polarisation apparatus,

163. Vertical section of a tooth,

164. Dentine,

165. Interglobular spaces,

166. Dentine and enamel,

167. Dentine and crusta petrosa,

168.

169. Development of a tooth,

171. Section of œesophagus (Sihenk),

172. Perinæum and its muscles,

173. Levator ani externus and internus,

*175. Auerbach's plexus (Cadiat),

"176. Meissner's plexus (Cadiat),

*177. Vertical section of stomach (Stöhr),

178. Goblet cells,

179. Surface section of gastric mucous membranr,

180. Fundus gland of the stomach, .

181. Pyloric gland,

182. Scheme of the gastric inucous membrane, ,

*183. Pyloric nucous menubrane (Hermann), . . . . . . . . . . 243

"184. Pyloric glands during digestion (Hermann), . . . . . . . . . 243

*185. Scheme of pyloric fistula (Stirling), . . . . . . . . . . . 245

*186. Section of the acini of the pancreas (Hermann), . . . . . $\quad 252$

187. Changes of the pancreatic cells during activity, : $\quad . \quad 253$

*188. Section of human liver $($ Stöhr), . . . . . . . . . . . . 258

189. Scheme of a liver lobule, . $\quad$. . . . . . . . $\quad 259$

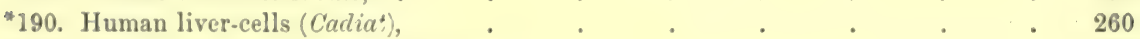

*191. Liver-cells during fasting (Hermann), . . . . . . . . 260

192. Bile ducts, . . . . . . . . . . . . . . $\quad 260$

*193. Liver-cells (Stirling, after Stolnikoff), _ . . . . . . . $\quad .261$

194. Various appearances of the liver-cells, . . . . . . . . $\quad 261$

195. Interlobular bile duct, . . . . . . . 262

*196. Cholesterin (Stirling), . . . . . . . . . . . . . 269

*197. Biliary fistulæ (Stirling), . . . . . . . . . . . 271

*198. Section of duodenum (Stöhr), . . . . . . . . . . . . 276

*199. Lieberkuhn's gland (Hermann), . . . . . . . . . . 277

200. Trausverse section of Lieberkuhn's follicles (Schenk), . . . . $\quad 277$

*201. Schemata of intestinal fistulæ (Stirling), . . . . . . . . . 277

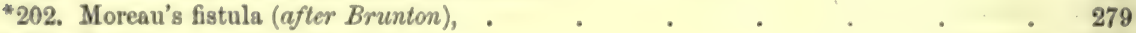

203. Bacterium aceti and B. butyricus, _ . . . . . . . . $\quad 280$

204. Bacillus subtilis, . . . . . . . . . . . . $\quad 281$

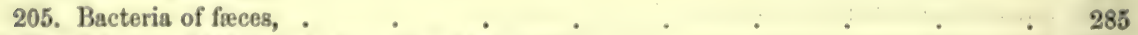

*206. Scheme of intestinal absorption (Beaunis), . . . . . . . 290

*207. Longitudinal section of small intestine (Srlecnk), . . . . . . . $\quad .291$

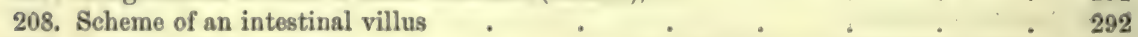

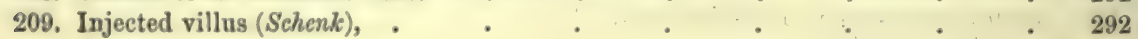


*210. Villi of small intestine injected (Cadiat), .

*211. Duodenum injected (Stöhr),

*212. Section of a solitary follicle (Cadiat),

*213. Section of a Peyer's patch (Cadiat),

214. Section of large intestine (Schenk),

215. Endosmometer,

216. Origin of lymphaties in the tendon of diaphragm,

*217. Lymphatics of disphragm silvered (Ranvier),

218. Perivascular lymphatics, .

219. Stomata from lymph-sac of frog, .

220. Section of two lymph-follicles,

*221. Scheme of a lymphatic gland (Sharpey), . . . . . . . . . . . 305

222. Part of a lymphatic gland, .

*223. Section of the central tendon of diaphragm (Brunton),

*224. Section of fascia lata of a dog (Brunton),

*225. Lymph hearts (Ecker),

226. Water-calorimeter of Favre and Silbermann,

*227. Water-calorimeter of Dulong (Rosenthal), .

*228. Clinical Thermometers,

229. Walferdin's metastatic thermometer,

230. Scheme of thermo-electric arrangements, .

231. Kopp's apparatus for specific heat,

232. Daily variations of temperature,

234. Milk-glands during inaction and secretion,

*235. Milk and colostrum (Stirling),

*236. Section of a grain of wheat (Blyth),

238. Composition of animal and vegetable foods,

*239. Starch grains,

*240. Longitudinal section of the kidney (Henle),

*241. Malpighian pyramid (Tyson, after Ludwig),

*242. Scheme of the uriniferous tubules (Klein and Noble Simith),

243. Scheme of the structure of the kidney,

244. Glomerulus and renal tubules,

*245. Convoluted renal tubule (Heidenhain),

*247. Transverse section of the apex of a Malpighian pyramid (Cadiat),

*248. Development of a glomerulus (Cadicat),

249. Graduated urinary flask,

251. Graduated burette, .

*253. Oxalate of urea (after Beale),

255. Graduated pipette, .

256. Uric acid, .

257. Kreatinin-zinc-chloride,

*258. Oxalate of lime,

259. Hippuric acid,

260. Deposit in urine during the "acid fermentation," .

261. Deposit in ammoniacal urine,

262. Micrococcus ureæ,

263. Esbach's albumimeter, 
265. Peculiar forms of blood-corpuscles,

266. Coloured and colourless corpuscles in urine,

267. Blood-corpuscles and triple phosphate,

268. Spectroscopic examination of urine,

*269. Picio-saccharimeter (G. Johnson), .

*270. Inusit (Bcale, after Funke),

271. Cystin and oxalate of lime,

272. Leucin, tyrosin, and ammonium urate,

273. Fungi in urine,

274. Epithelial casts,

275. Blood casts,

276. Leucocyte cast,

277. Cast of urate of soda,

278. Finely granular casts,

279. Coarsely granular casts,

280. Hyaline casts,

281. Calcic carbonate and phosphate,

282. Triple phosphate,

283. Imperfect forms of triple phosphate,

284. Acid ammonium urate,

285. Basic magnesic phosphate,

*286. Oncometer (Stirling, after Roy),

*287. Oncograph (Stirling, after Roy),

*289. Section of ureter $(S t o ̈ h r)$,

290. Transitional epithelium (Beale),

*292. Nervous mechanism of micturition (Stirling, after Goucrs),

*293. Section of epidermis and its nerves (Ranvier),

294. Scheme of the structure of the skin,

*295. Papillæ of the skin injected,

296. Transverse section of a nail,

297. Transverse section of a hair-follicle,

298. Longitudinal section of a hair-follicle,

299. Sebaceous gland, .

300. Ciliated epithelium,

*301. Pigment and guanin cells of frog (Stirling),

302. Histology of muscular tissue,

*303. Muscular fibre (Quain),

*304. Insect's muscle (Rollett),

*305. Insect's muscle (Rollett),

*306. Network in muscle (Melland),

307. Tendon attached to a muscle,

*308. Injected blood-vessels of muscle (Kölliker),

309. Motorial end-plates, .

*310. Termination of a nerve in a frog's muscle (Kühne),

1. Scheme of nerve-ending in muscle (Rollett, after Kühne),

*313. Non-striped muscle-cell (Stirling),

*314. Nerve-ending in smooth muscle (Cadiat), .

*315. Frog with its sciatic artery ligatured (Stirling),

*316. Scheme of the curara experiment (after Rutherford),

*317. Excitability in a frog's sartorius (Stirling, after Pollitzer),

*318. Excitability in a curarised sartorius (Stirling, after Pollitzer) 
*321. Pendulum myograph,

*322. Scheme of the pendulum myograph (Stivling), . 479

*323. Du Bois-Reymond's spring myograph, . . . . . . . . $\quad .479$

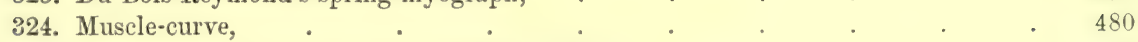

*325. Muscle-curve of pendulum myograph (Stirling), . . . . . . $\quad$. 481

*326. Method of studying a muscular contraction (after Ruticerford), . . $\quad 481$

*327. Effect of make and break induction shocks (Stirling), . . . . 481

328. Muscle-curves, . . . . . . . . 482

329. Muscle-curve, opening and closing shocks, _ . . . . . . . $\quad 482$

*330. Veratrin-curve (Stirling), . . . . . . . . . . . . . . 483

331. Muscle-curves, tetanus, . . . . . . . . . . . . . 484

*332. Staircase contractions (Buckmaster), . . . . . . . . . . . $\quad .485$

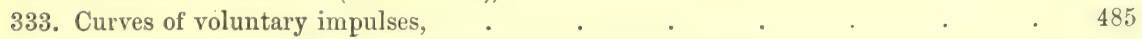

*334. Curves of a red and pale muscle (Kronecker and Stirling), . . . 486

*335. Muscle-curves (Kronecker and Stirling), . . . . . . . . . 487

*336. Tone-inductorium (Kronecker and Stirling), . . . . . . 487

*337. Muscle-curves (Marey), . . . . . . . . . . . . . . . 488

*338. Height of the lift by a muscle, . $\quad . \quad$. $\quad . \quad$. $\quad . \quad$. 489

*339. Dynamometer, . . . . . . . . . . . . . 490

*340. Curve of elasticity (after Marey), . . . . . . . . . . . $\quad$. 491

*341. Curve of elasticity of a muscle (after Marey), . . . . . . . 491

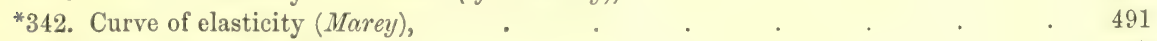

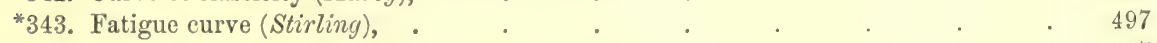

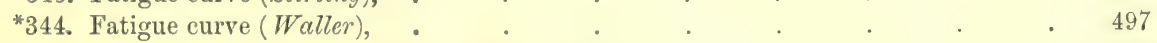

*345. Vertical section of articular cartilage (Stirling), . . . . . . . $\quad 498$

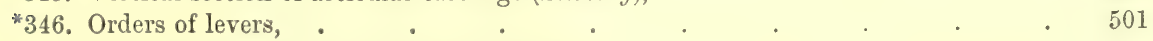

*347. Scheme of the action of muscles on bones, . . . . . . . $\quad$. 501

348. Phases of walking, . . . . . . . . . . . 506

349. Instantaneous photograph of a person walkincr, . $\quad$. $\quad . \quad$. 506

350. Instantaneous photograph of a runner, . . . . . . . . . 507

351. Instantaneous photograph of a person jumping, . . . . . . 508

352. Larynx from the front, . $\quad . \quad$. $\quad . \quad$. $\quad . \quad$. $\quad . \quad 511$

353. Larynx from behind, . $\quad . \quad$. $\quad . \quad$. $\quad . \quad$. $\quad . \quad 511$

354. Larynx from behind, . $\quad . \quad$. $\quad . \quad$. $\quad . \quad 512$

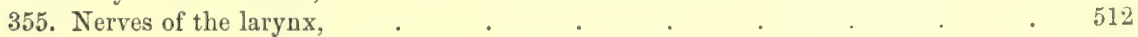

356. Action of the posterior crico-arytenoid muscles, . . . . . . . . . 513

357. Action of the arytenvid muscles, . $\quad . \quad$. $\quad . \quad$. $\quad . \quad 513$

358. Action of the lateral crico-arytenoid muscles, . . . . . . . . $\quad 513$

359. Vertical section of the head and neck, . . . . . . . . . . 515

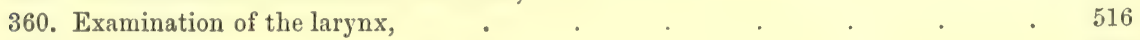

361. Laryngoscopic view of the larynx, . . . . . . . . $\quad .516$

362. View of the larynx during a high note, . . . . . . . . . $\quad .517$

363. View of the larynx during a deep inspiration, . . . . . . . $\quad 517$

364. Rhinoscopy, . . . . . . . . . . . . $\quad . \quad 517$

365. View of the posterior nares, . . . . . . . . . $\quad . \quad 518$

366. Parts concerned in phonation, . . . . . . . . . $\quad .520$

367. Tumours on the vocal cords, . . . . . . . . . . . . . $\quad .523$

368. Histology of nervous tissues, . . . . . . . . . 526

*369. Transverse section of nerve-fibres (Cadiat), . . . . . $\quad .527$

*370. Sympathetic nerve-fibre (Ranvier), . . . . . . . 527

*371. Medullated nerve-fibre (Stirling), ․ . . . . . . . . . . 527

372. Medullated nerve-fibre, . . . . . . . . . . . 528

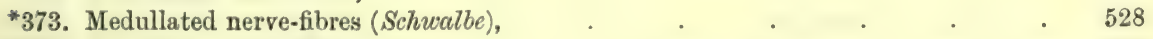

*374. Ranvier's crosses (Ranvier), . . . . . . . . . . . . . $\quad . \quad 529$ 
375. Transverse scction of a nerve,

*376. Cell from the Gasserian ganglion (Schwalbc),

377. Degeneration and regeneration of nerve-fibres,

*378. Waller's experiments (after Dalton),

379. Rheocord of du Bois-Reymond,

380. Scheme of a galvanometer,

"381. Large Grove's battery (Gscheidlen),

*382. Daniell's cell (Stirling),

*383. Grennet's battery (Gscheidlen),

*884. I.eclanché's element (Gscheidlen), .

*385. Non-polarisable electrodes (Elliott Brothers),

*386. Fleischl's non-polarisable electrodes (Petzoldt),

*387. Thomson's galvanometer (Elliott Brothers),

*388. Lamp and scale (Elliott Brothers), .

*389. Galvanometer shunt (Elliout Brothers),

*390. Scheme of the induced currents (Hermann),

*391. Helmholtz's modification (Hermann),

392. Scheme of an induction machine,

*393. Inductorium (Elliott Brothers),

*394. Inductorium (Petzoldt),

395. Stöhrer's apparatus,

*396. Friction key (Elliwtt Brothers), .

*397. Plug key (Elliott Brother's),

*398. Capillary contact (Kronceker and stirling),

399. Scheme of the muscle-current,

400. Capillary electrometer,

*401. Nerve-muscle preparation,.

*402. Kühne's experiment (S'tirling),

* 403. Electrometer' curve, frog's muscle ( $W$ aller $)$,

* 404. Electrometer curve, frog's heart (Waller),

*405. Secondary contraction,

406. Bernstein's differential rheotome, .

407. Nerve-current in electrotonus,

408. Scheme of electrotonic excitability,

409. Method of testing electrotonic excitability,

410. Distribution of an electrical current,

411. Velocity of nerve-energy, .

*412. Scheme for testing velocity of a nerve-impulse,

"413. Curves of a nerve-impulse (Marcy),

*414. Kühne's gracilis experiment,

* 415. Sponge rheophores (Weiss),

"416. Disk rheophore (Weiss), .

*417. Metallic brush (Weiss),

418. Motor points of the arm, .

419. Motor points of the arm, .

420. Motor points of the leg,

421. Motor points of the leg,

*422. Scheme of a reflex act (Stirling),

423. Optic chiasma,

*424. Relation of field of vision, retina, and optic tracts (Gowers),

* 425. Decussation of the optic tracts (Charcot), .

*426. Soheme of images in squinting (Bristonce),

427. Medulla oblongata,

* 428. Under surface of the brain,

429. Connections of the cranial nerves, . 
430. Sensory nerves of the face,

431. Motor points of the face and neck,

*432. Disposition of the semicircular canals (Stirling),

433. Scheme of the branches of vagus and accessorius,

*434. Cardiac nerves of the rabbit (Stirling),

*435. Diagram of a spinal nerve (Ross), .

*436. Spinal ganglion (Cadiat), .

437. Cutaneous nerves of the arm,

438. Cutaneous nerves of the leg (Henle),

*439. Visceral nerves of the dog (Gaskell).

440. Transverse section of the spinal cord,

*441. Transverse section of the white matter (Obersteiner),

601

605

608

610

616

617

618

619

622

626

627

627

*442. Multipolar nerve-cells of the cord (Cadiat),

*443. Relation of white and grey matter of the cord (Schäfer), .

*444. Transverse sections of the spinal cord,

627

628

629

629

*446. Transverse section of the cord (Cadiat),

630

*447. Longitudinal section of the cord (Cadiat), .

*448. Multipolar nerve-cell,

*449. Scheme of fibres in cord (Obersteiner),

*450. Glia cell (Obersteiner),

*451. Glia cells of cord (Obersteiner)

*452. Spinal cord injected (Obersteiner), .

*453. Injected blood-vessels of the cord (Kölliker),

454. Conducting paths in the cord,

*455. Degeneration paths in the cord (Bramwell),

*456. Scheme of a reflex act (Stirling),

*457. Section of a spinal segment (Stirling),

*458. Propagation of reflex movements (Beaunis),

*459. Effect of section of half of the cord $(E r b)$,

*460. Brain, ventricles, and basal ganglia,

630

630

631

631

632

632

633

461. Scheme of the brain,

*462. Connections of the cerebellum,

*463. Diagram of a spinal segment (Bramwell), .

*464. Section across the pyramids (Schusalbe), . . . . 656

*465. Section of the medulla oblongata (Schwalbe), . . . . . . . . . $\quad 657$

*466. Section of the olivary body (Schwalbe), . . . . . . . 657

*467. Scheme of the respiratory centres (Rutherford), . _ . . . . . . . $\quad 662$

*468. Action of vagus on frog's heart (Stirling), . . . . . . . $\quad 668$

*469. Scheme of the accelerans fibres (Stirling), . . . . . . . 670

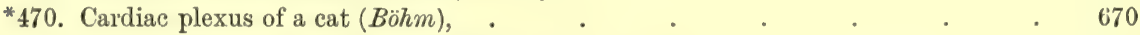

*471. Frog without its cerebrum (Stirling, after Goltz), . $\quad . \quad$. . . . . 683

*472. Frog without its cerebrum (Stirling, after Goltz), . _ . . . . . . . 683

*473. Pigeon with its cerebrum removed (after Dalton), _ . _ . . . . 683

*474. Motor area of cerebral convolution (Ferrier and B. Lewis), . . . . . 687

*475. Cerebral convolution; sensory area (Ferrier and B. Lewis), . . . . 688

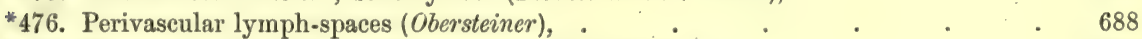

*477. Frontal convolution by Weigert's method (Obersteiner), . . . . . 688

*478. Cerebral convolution injected,

*479. Left side of the human brain (Ecker),

*480. Inner aspect of right hemisphere (Ecker), .

*481. Left frontal lobe and island of Reil (Turner),

*482. Brain from above (Ecker), .

483. Cerebrum of dog, carp, frog, pigeon, and rabbit, .

484. Relation of the cerebral convolutions to the skull, 
"485. Motor areas of a monkey's brain (Horsley and Schïfer), . . . . 698

*486. Motor areas of the marginal convolution (Horsley and Scheifer), . . . 699

*487. Pyscho-optic fibres $(M u n k)$, . . . . . 702

*488. Motor areas (after Gowers),

"489. Motor centres (after Schäfer and Horsley),

*490. Section of a cerebral hemisphere (Horsley),

"491. Innervation of associated muscles (Ross), .

* 492. Secondary degeneration in a crus (Charcot),

*493. Transverse section of the crus cerebri (Churcot),

*494. Scheme of aphasia (Lichthcim),

* 495. Scheme of aphasia (Lichtheim),

"496. Scheme of aphasia (Ross), .

*497. Relation of the convolutions to the skull ( $R . W . R e i d)$,

*498. Relation of motor centres to skull (Hare),

*499. Outline markings on skull (Hare),

*500. Basal ganglia and the ventricles,

*501. Transverse section of the right hemisphere (Gegenbaur),

*502. Transverse section of the crura (Wernicke and Gowers),

*503. Transverse section of the pons (Wernicke),

*504. Course of the fibres in pons $(E r b)$,

*505. Longitudinal section of a human brain (Wiedersheim),

*506. Section of the cerebellum (Sankcy),

"507. Purkinje's cell (Oberstciner),

"508. Pigeon with its cerebellum removed (Dalton),

"509. Cortex cerebri and its membranes (Schuralbe),

*510. Circle of Willis (Charcot), .

"511. Ganglionic arteries (Charcot),

*512. Corneal corpuscles (Ranvier),

*513. Corneal spaces (Ranvier), .

514. Junction of the cornea and sclerotic,

"515. Vertical section of cornea with nerve fibuli (Ranvier),

516. Horizontal section of cornea with nerve hibuli (Ranvicr),

518. Blood-vessels of the eyeball,

"519. Vertical section human retina (Cadiat), .

520. Layers of the retina,

"521. Vertical section of the fovea centralis (Codiat),

*522. Fibres of the lens (Kölliker),

523. Section of the optic nerve,

524. Action of lenses on light, .

525. Refraction of light,

526. Construction of the refracted ray, .

527. Optical cardinal points,

528. Construction of the refracted ray, .

529. Construction of the image,

530. Refracted ray in several media, .

531. Visual angle and retinal iniage, .

532. Scheme of the ophthalmometer,

533. Horizontal section of the eyeball, .

534. Scheme of accommodation,

535. Sanson-Purkinje's images, .

"536. Phakoscope ( $\boldsymbol{M}^{\prime}$ Kendrick),

537. Scheiner's experiment, 
540. Hypermetropic eye,

541. Power of accommodation, .

*542. Diagram of astigmatism (Frost),

543. Cylindrical glasses,

*544. Scheme of the nerves of the iris (Erb),

*545. Pupilometer (Gorham),

*546. Pupilometer (Gorham),

547. Entoptical shadows,

548. Scheme of the original ophthalmoscope, . . . . . . . . . . . $\quad 764$

549. Scheme of the indirect method, . . . . . . . . . . . . 765

550. Action of a divergent lens,

551. Action of a divergent lens,

552. View of the fundus oculi,.

*553. Morton's ophthalmoscope (Pickard and Curry),

*554. Frost's artificial eye (Frost),

*555. Action of the orthoscope,

*556. Mariotte's experiment,

*557. Horizontal section of the right eye,

*558. M 'Hardy's perimeter (Pickard and Curry)

*559. Priestley Smith's perimeter (Pickard and Curry),

560. Perimetric chart,

561. Geometrical colour cone, .

562. Action of light rays on the retina,

*564. Irradiation,

*565. Irradiation,

566. Scheme of the action of the ocular muscles,

567. Identical points of the retina,

568. The horopter,

569. Two stereoscopic drawings,

570. Wheatstone's stereoscope,

790

571. Brewster's stereoscope,

790

572. T'elestereoscope,

791

573. Wheatstone's pseudoscope,

791

792

793

794

797

7.9 
595. Scheme of the canalis cochlearis,

*596. Galton's whistle (Krohne and Sesemann),

597. Curve of a musical note and its overtones,

"598. Konig's manometric capsule (Konig),

*599. Flame-pictures of vowels (Kunig),

*00. Kœnig's analysing apparatus (Koenig),

601. Nasal and pharyngo-nasal cavities, !

"602. Section of the olfactory region (Stöhr),

603. Olfactory cells,

*604. Filiform papillæ (Stöhr),

"605. Fungiform papillæ (Stöhr),

606. Circnmvallate papilla and taste-bulbs,

*607. Papillæ foliatæ (Stöhr),

608. Vertical section of skin,

609. Wagner's touch corpuscle (Ranvier),

610. Pacini's corpuscle,

*611. End-bulb from conjunctiva (Quain),

*612. Tactile corpuscle from clitoris (Quain),

*613. Tactile corpuscles from a duck's bill (Quain),

*614. Bouchon epidermique (Ranvicr),

615. Esthesiometer,

*616. Esthesiometer of Sieveking,

*617. Aristotle's experiment,

618. Pressure-spots,

619. Landois' pressure mercurial balance,

620. Cold- and hot-spots,

621. Cold-and hot-spots,

622. Topography of temperature-spots,

*623. Karyokinesis (Gegenbaur),

*624. Typical nucleated cell (Camoy),

*625. Mitosis or nuclear division (Flemming),

626. Ovum of Tænia solium,

627. Cysticercus,

628. Cysticerci of 'Trnia solium,

629. Scolex,

630. Echinococcus,

631. Tænia solium,

*632. Section of testis (Schenk),

*633. Tubule of testis (Schenk),

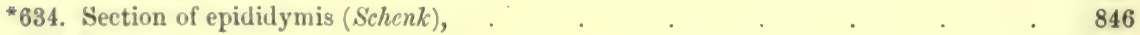

635. Spermatic crystals, . . . . . . . . . . . $\quad .847$

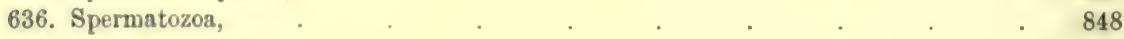

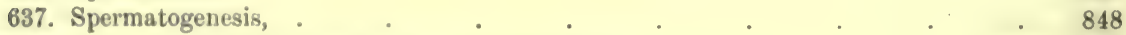

"638. A cat's ovary (Hart and Barbour, after Schrön), . . . . . . $\quad$. 849

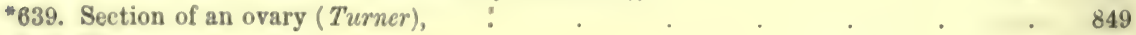

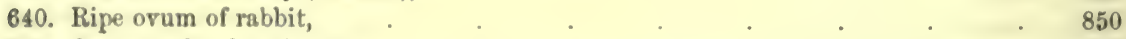

641. Ovary and polar globules,

642. Scheme of a meroblastic ovum,

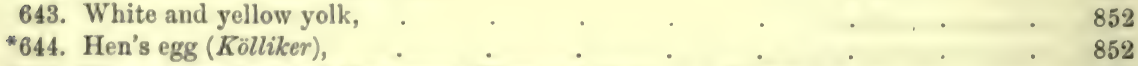

*645. Mucous membrane of the uterus (Hart and Barbour, aftcr Turner), . $\quad .853$

*646. Fallopian tube and its annexes (Henle), . . . . . . . . 854

*647. Section of Fallopian tube (Schenk), . . . . . . . . . $\quad .854$

*648. Uterus before menstruation (J. Williams), _ . . . . . . . $\quad .855$

*649. Uterus after menstruation (J. Williams), 
650. Fresh corpus luteum, . . . . . . . . . $\quad 856$

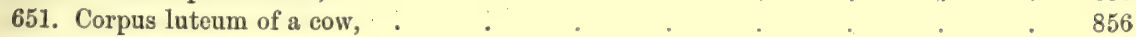

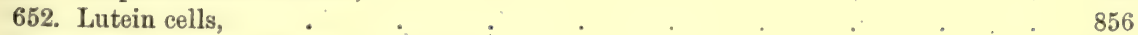

*653. Erectile tissue (Cadiat), . . . . . . . . . . . 857

654. The urethra and adjoining muscles, . . . . . . . . . . . 858

*655. Formation of polar globules, . . . . . . . . . . . 861

656. Extrusion of a polar globule, . . . . . . . . . $\quad . \quad 861$

657. Polar globules, male and female pronucleus, . . $\quad$. . . $\quad . \quad 862$

*658. Segmentation of a rabbit's ovum (Quain, after v. Beneden), . . . $\quad 862$

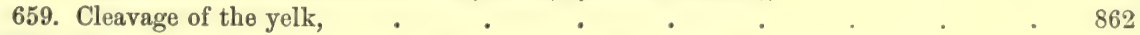

*660. Blastodermic vesicle of rabbit (Quain, after v. Beneden), . _ . . . $\quad .863$

661. The blastoderm, . . . . . . . . . . . . 863

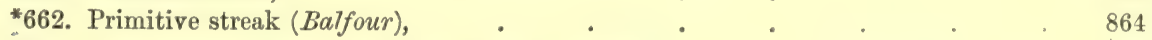

*663. Transverse section of an embryo newt (Hertwig), $\quad$. . . . 864

*664. Vertical section of a blastoderm $($ Klein), . . . . . . . $\quad .865$

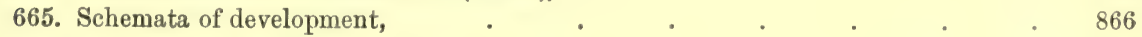

*666. Embryo fowl, 2nd day (Kölliker), ‥ . . . . . . . $\quad 867$

*667. Transverse section of an embryo duck (Balfour), . . . . . 868

*668. Uterine mucous membrane (Coste), . . . . . . . $\quad .873$

*669. Placental villi (Cadiat), . $\quad . \quad$. $\quad . \quad$. $\quad$. $\quad . \quad 874$

*670. Fotal circulation (Cleland), . . . . . . . . . $\quad$. 876

*671. Head of embryo rabbit (Kölliker), $\quad$. $\quad$. $\quad$. $\quad$. $\quad 879$

672. Hare lip, . . . . . . . . . . . . . . $\quad . \quad 879$

*673. Meckel's cartilage ( W. K. Parker), $\quad$. $\quad$. $\quad$. $\quad$. $\quad$. 879

674. Centres of ossification in the innominate bone, . . . . . . $\quad . \quad 881$

675. Development of the heart, . . . . . . . . . . $\quad 883$

676. The aortic arches, $\quad . \quad$. . . . . . . . . . $\quad 884$

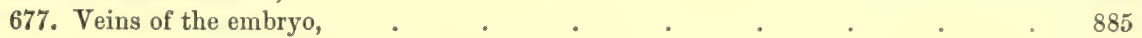

678. Development of the veins and portal system, . $\quad . \quad$. $\quad . \quad 885$

679. Development of the intestine, . . . . . . . . . . . $\quad .886$

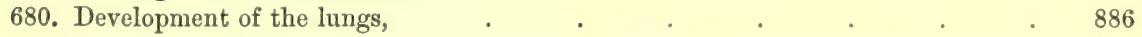

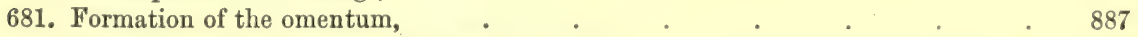

682. Development of the internal generative organs, . . . . . . . 887

*683. Development of ova (Wiedersheim), . . . . . . . . . . $\quad .888$

684. Development of the external genitals, . . . . . . . . 890

*685. ) 890

*686. Changes in the external organs of generation in the female (after Schroeder), $\{890$

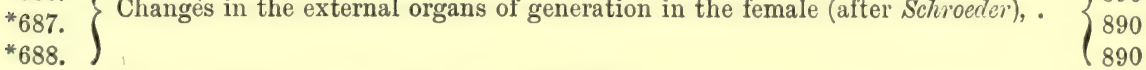

*689. Transverse section of an embryo brain (Kölliker), ․ . . . . 891

*690. Embryo brain of fowl (Quain, after Mihalkowics), $\quad$. . . . $\quad 892$

691. Development of the eye, . . . . . . . . . . . . . $\quad .893$

*692. Development of the vertebrate ear $($ Haddon), . . . . . . . $\quad 893$

[The illustrations indicated by the word Hermann, are from Hermann's Handbuch der Physiologie; by Cadiat, from Cadiat's Traité d'Anatomie Générale; by Ranvier, from Ranvier's Traité Technique d'Histologie; by Brunton, from Brunton's Text-book of Pharmacology, Therapeutics, and Materia Medica; by Schenk, from Schenk's Grundriss der normalen Histologie; by Ecker, from Ecker's Anatomie des Frosches, 2nd ed.; by Quain, from Quain's Anatomy; by Stöhr, from Stöhr's Lehrbuch der Histologie, Jena, 1887 ; by Obcrsteiner, from H. Obersteiner's Anleitung beim Studium des Baues der nervösen Centralorgane, Wien, 1888.] 
- 


\section{Introduction.}

\section{The Scope of Physiology and its Relations to other Branches of Natural Science.}

Physiology is the science of the vital phenomena of organisms, or, broadly, it is the Doctrine of Life. Correspondingly to the divisions of organisms, we distinguish -(1) Animal Physiology; (2) Vegetable Physiology; and (3) the Physiology of the Lowest Living Organisms, which stand on the border line of animals and plants, i.e., the so-called Protistæx of Haeckel, micro-organisms, and those elementary organisms or cells which exist on the same level.

The object of Physiology is to establish these phenomena, to determine their regularity and causes, and to refer them to the general fundamental laws of Natural Science, viz., the Laws of Physics and of Chemistry.

The following Scheme shows the relation of Physiology to the allied branches of Natural Science :-

\section{BIOLOGY.}

The science of organised beings or organisms (animals, plants, protistæ, and elementary organisms).

\section{Morphology.}

The doctrine of the form of organisms.

General

Morphology.

The doctrine of the formed elementary constituents of organisms.

(Histology)-

(a) Histology of Plants.

(b) Histology of Animals.

\section{Special} Morphology.

The doctrine of the parts and organs of organisms.

(Organology -

Anatomy)-

(a) Phytotomy.

(b) Zootomy.

\section{Physiology.}

The doctrine of the vital phenomena of organisms.

\section{General \\ Physiology. \\ Special \\ Physiology.}

The doctrine of vital The doctrine of the phenomena in gene- activities of the inraldividual organs-
(a) Of Plants.
(a) Of Plants.
(b) Of Animals.
(b) Of Animals.

\section{Embryology.}

The doctrine of the generation and development of organisms.

Morphological part of the doctrine of development, i.e., the doctrine of form in its stages of development-

(a) General.

(b) Special.
( 1. History of the development of single beings, of the individual (e.g., of man) from the ovum onwards (Ontogeny)-
(a) In Plants.
(b) In Animals.

2. History of the development of a whole stock of organisms from the lowest forms of the series upwards (Phylogeny) -
(a) In Plants.
(b) In Animals.

Physiological part of the doctrine of development, i.e., the doctrine of the activity during development-
(a) General.
(b) Special. 
Morphology and Physiology are of equal rank in biological science, and a previous acquaintance with Morphology is assumed as a basis for the comprehension of Physiology, since the work of an organ can only be properly understood when its external form and its internal arrangernents are known. Development occupies a middle place between Morphology and Physiology ; it is a morphological discipline in so far as it is concerned with the description of the parts of the developing organism; it is a physiological doctrine in so far as it studies the activities and vital phenomena during the course of development.

MATTER.-The entire visible world, including all organisms, consists of matter, i.e., of substance which occupies space.

We distinguish ponderuble matter which has weight, and imponderable matter which cannot be weighed in a balance. The latter is generally termed ether.

In ponderable materials, again, we distinguish their form, i.e., the nature of their limiting surfaces; further, their volume, i.e., the amount of space which they occupy; and lastly, their "gyreyate condition, i.e., whether they are solid, fluid, or gaseous bodies.

Ether.-The ether fills the space of the universe, certainly as far as the most distant visible stars. This ether, notwithstanding its imponderability, possesses distinct mechanical properties; it is infinitely more attenuated than any known kind of gas, and behaves more like a solid body than a gas, resembling a gelatinous mass rather than the air. It participates in the luminous phenomena due to the vibrations of the atoms of the fixed stars, and hence it is the transmitter of light, which is conducted by means of its vibrations, with inconceivable rapidity $(42,220$ geographical-miles per second) to our visual organs (Tyndall).

Imponderable matter (ether) and ponderable matter are not separated sharply from each other; rather does the ether penetrate into all the spaces existing between the smallest particles of ponderable matter.

Particles.-Supposing that ponderable matter were to be subdivided continuously into smaller and smaller portions, until we reach the last stage of division in which it is possible to recognise the aggregate condition of the matter operated upon, we should call the finely-divided portions of matter in this state particles. Particles of iron would still be recognised as solid, particles of water as fluid, particles of oxygen as gaseous.

Molecules. - Supposing, however, the process of division of the particles to be carried further still, we should at last reach a limit, beyond which, neither by mechanical nor by physical means, could any further division be effected. We should have arrived at the molecules. A molecule, therefore, is the smallest amount of matter which can still exist in a free condition, and which as a unit no longer exhibits the aggregate condition.

Atoms. - But even molecules are not the final units of matter, since every molecule consists of a group of smaller units, called atoms. An atom cannot exist by itself in a free condition, but the atoms unite with other similar or dissimilar atoms to form groups, which are called molecules. Atoms are incapable of further subdivision, hence their name. We assume that the atoms are invariably of the same size, and that they are solid. From a chemical point of view, the atom of 
an elementary body (element) is the smallest amount of the element which can enter into a chemical combination. Just as ponderable matter consists in its ultimate parts of ponderable atoms, so does the ether consist of analogous small ether-atoms.

Ponderable and Imponderable Atoms.-The ponderable atoms within ponderable matter are arranged in a definite relation to the ether-atoms. The ponderable atoms mutually attract each other, and similarly they attract the imponderable ether-atoms; but the ether-atoms repel each other. Hence, in ponderable masses, ether-atoms surround every ponderable atom. These masses, in virtue of the attraction of the ponderable atoms, tend to come together, but only to the extent permitted by the surrounding ether-atoms. Thus the ponderable atoms can never come so close as not to leave interspaces. All matter must, therefore, be regarded as more or less loose and open in texture, a condition due to the interpenetrating ether-atoms, which resist the direct contact of the ponderable atoms.

Aggregate Condition of Atoms.-The relative arrangement of the molecules, i.e., the smallest particles of matter which can be isolated in a free condition, determines the aggregate condition of the body.

Within a solid body, characterised by the permanence of its volume as well as by the independence of its form, the molecules are so arranged that they cannot readily be displaced from their relative positions.

Fluid bodies, although their volume is permanent, readily change their shape, and their molecules are in a condition of continual movement.

When this movement of the molecules takes so wide a range that the individual molecules fly apart, the body becomes gaseous, and as such is characterised by the instability of its form as well as by the changeableness of its volume.

Physics is the study of these molecules and their motions.

\section{Forces.}

1. Gravitation-Work done.-All phenomena appertain to matter. These phenomena are the appreciable expression of the forces inherent in matter. The forces themselves are not appreciable, they are the causes of the phenomena.

Gravitation.-The law of gravitation postulates that every particle of ponderable matter in the universe attracts every other particle with a certain force. This force is inversely as the square of the distance. Further, the attractive force is directly proportional to the amount of the attracting matter, without any reference to the quality of the body. We may estimate the intensity of gravitation by the extent of the movement which it communicates to a body allowed to fall, for one second, through a given distance, in a space free from air. Such a body will fall in vacuo $9 \cdot 809$ metres per second. This fact has been arrived at experimentally.

Let us represent $g=9.809$ metres, the final velocity of the freely falling body at the end of one second. The velocity, $\mathrm{V}$, of the freely falling body is proportional to the time, $t$, so that

$$
\mathrm{V}=g t \ldots . . . . .(1) \text {; }
$$

i.e., at the end of the 1st sec., and $\mathrm{V}=g, 1=g=9 \cdot 809 \mathrm{M}$-the distance traversed-

$$
s=\frac{g}{2} t^{2} .
$$


i.e., the distances are as the square of the times. Hence, from (1) and (2) it follows (by eliminating $t$ ) that-

$$
\mathrm{V}=\sqrt{2 g s}
$$

The velocities are as the square roots of the distances traversed-

Therefore

$$
\frac{\mathrm{V}^{2}}{2 g}=8
$$

The freely falling body, and in fact every freely moving body, possesses kinetic energy, and is in a certain sense a magazine of energy. The kinetic energy of any moring body is always equal to the product of its weight (estimated by the balance), and the height to which it would rise from the earth, if it were thrown from the earth with its own velocity.

Let $\mathrm{W}$ represent the kinetic energy of the moving body, and $\mathrm{P}$ its weight, then $\mathrm{W}=\mathrm{P} . s$, so that from (4) it follows that-

$$
\mathrm{W}=\mathrm{P} \frac{\mathrm{V}}{2 g} .
$$

Hence, the kinetic energy of a body is proportional to the square of its velocity.

Work.-If a force (pressure, strain, tension) be so applied to a body as to move it, a certain amount of rork is performed. The amount of work is equal to the product of the amount of the pressure or strain which moves the body, and of the distance through which it is moved.

Let $\mathrm{K}$ represent the force acting on the boly, and $\mathrm{S}$ the distance, then the work $\mathrm{W}=\mathrm{KS}$. The attraction between the earth and any body raised above it is a source of work.

It is usual to express the value of $\mathrm{K}$ in kilogrammes, and $\mathrm{S}$ in metres, so that the "unit of work" is the kilogramme-metre, i.e., the force which is required to raise 1 kilo. to the height of 1 metre.

2. Potential Energy.-The transformation of Potential into Kinetic Energy, and conversely: Besides kinetic energy, there is also "potential energy," or energy of position. By this term are meant various forms of energy, which are suspended in their action, and which, although they may cause motion, are not in themselves motion. A coiled watch-spring kept in this position, a stone resting upon a tower, are instances of bodies possessing potential energy, or the energy of position. It requires merely a push to develop kinetic from the potential energy, or to transform potential into kinetic energy.

Work, $u$, was performed in raising the stone to rest upon the tower.

$$
w=p, s \text {, where } p=\text { the weight and } s=\text { the height, }
$$

$p=m . g$, is = the prorluct of the mass $(m)$, and the force of gravity $(g)$, so that $v=m g s$.

This is at the same time the expression for the potential energy of the stone. This potential energy may readily be transformed into kinetic energy by merely pushing the stone so that it falls from the tower. The kinetic energy of the stone is equal to the final velocity with which it impinges upon the earth.

$$
\begin{aligned}
\mathrm{V} & =\sqrt{2 g} s \text { (see above (3)). } \\
\mathrm{V}^{2} & =2 g 8 . \\
m \mathrm{~V}^{2} & =2 m g s . \\
\frac{m}{2} \mathrm{~V}^{2} & =m g s .
\end{aligned}
$$

$m$ g 8 was the expression for the potential energy of the stone while it was still 
resting on the height; $\frac{m}{2} V_{2}$ is the kinetic energy corresponding to this potential energy (Brïcke).

Potential energy may be transformed into mechanical energy under the most varied conditions; it may also be transferred from one body to another.

The movement of a pendulum is a striking example of the former. When the pendulum is at the highest point of its excursion, it must be regarded as absolutely at rest for an instant, and as endowed with potential energy, thus corresponding with the raised stone in the previous instance. During the swing of the pendulum this potential energy is changed into kinetic energy, which is greatest when the pendulum is moving most rapidly towards the vertical. As it rises again from the vertical position, it moves more slowly, and the kinetic energy is changed into potential energy, which once more reaches its maximum when the pendulum comes to rest at the utmost limit of its excursion. Were it not for the resistances continually opposed to its movements, such as the resistance of the air and friction, the movement of the pendulum, due to the alternating change of kinetic into potential energy and vice versa , would continue uninterruptedly, as with a mathematical pendulum. Suppose the swinging ball of the pendulum, when exactly in a vertical position, impinged upon a resting but movable sphere, the potential energy of the ball of the pendulum would be transferred directly to the sphere, provided that the elasticity of the ball of the pendulum and the sphere were complete; the pendulum would come to rest, while the sphere would move onward with an equal amount of kinetic energy, provided there were no resistance to its movement. This is an example of the transference of kinetic energy from one body to another. Lastly, suppose that a stretched watch-spring on uncoiling causes another spring to become coiled; and we have another example of the transference of kinetic energy from one body to another.

The following general statement is deducible from the foregoing examples:If, in a system, the individual moving masses approach the final position of equilibrium, then in this system the sum of the kinetic energies increases; if, on the other hand, the particles move away from the final position of equilibrium, then the sum of the potential energies is increased at the expense of the kinetic energies, i.e., the kinetic energies diminish (Brïcke).

The pendulum, which, after swinging from the highest point of its excursion, approaches the vertical position, i.e., the position of equilibrium of a passive pendulum, has in this position the largest amount of potential energy ; as it again ascends to the highest point of its excursion on the other side, it again gradually receives the maximum of potential energy at the expense of the gradually diminishing movement, and therefore of the kinetic energy.

3. Heat.-Its Relation to Potential and Kinetic Energy. - If a lead weight be thrown from a high tower to the earth, and if it strike an unyielding substance, the movement of the mass of lead is not only arrested, but the kinetic energy (which to the eye appears to be lost) is transformed into a lively vibratory movement of the atoms. When the lead meets the earth, heat is produced. The amount of heat produced is proportional to the kinetic energy, which is transformed through the concussion. At the moment when the lead weight reaches the earth, the atoms are thrown into vibrations; they impinge upon each other; then rebound again from each other in consequence of their elasticity, which opposes their direct juxtaposition; they fly asunder to the maximum extent permitted by the attractive force of the ponderable atoms, and thus oscillate to and fro. All the atoms vibrate like a pendulum, until their movement is communicated to the ethereal atoms surrounding them on every side, i.e., until the heat of the heated mass is "radiated." Heat is thus a vibratory movement of the atoms. 
As the amount of heat produced is proportional to the kinetic energy, which is transformed through the concussion, we must find an adequate measure for both forces.

Heat-Unit.-As a standard of measure of heat, we have the "heat-unit" or" calorie. The "heat-unit" or calorie is the amonnt of energy required to raise the temperature of 1 gramme of water $1^{\circ}$ centigrade. The "heat-unit" corresponds to 425.5 gramme-metres, i.e., the same energy required to heat 1 gramme of water $1^{\circ} \mathrm{C}$. would raise a weight of 425.5 grammes to the height of 1 metre; or, a weight of 425.5 grammes, if allowed to fall from the height of 1 metre, would by its concussion produce as much heat as would raise the temperature of 1 gramme of water $1^{\circ} \mathrm{C}$. The "mechanical equivalent" of the heat-unit is, therefore, 425.5 gramme-metres.

It is evident that from the collision of moving masses an immeasurable amount of heat can be produced. Let us apply what has already been said to the earth. Suppose the earth to be disturbed in its orbit, and suppose further that, owing to the attraction of the sun, it were to impinge on the latter (whereby, according to J. R. Mayer, its final velocity would be 85 geographical miles per second), the amount of heat produced by the collision would be equal to that produced by the combustion of a mass of pure charcoal more than 5000 times as heavy (Julius Robert Mayer, Helmholtz).

Thus, the heat of the sun itself can be produced by the collision of masses of cold matter. If the cold matter of the universe were thrown into space, and there left to the attraction of its particles, the collision of these particles would ultimately produce the light of the stars. At the present time, numerous cosmic bodies collide in space, while innumerable small meteors $(94,000$ to 188,000 billions of kilos. per minute) fall into the sun. The force of gravity is perhaps, in fact, the only source of all heat ( $J$. R. Mayer, Tyndall).

We have a homely example of the transformation of kinetic energy into heat in the fact that a blacksmith may make a piece of iron red-hot by hammering it. Of the conversion of heat into kinetic energy we have an example in the hot watery vapour (steam) of the steam-engine raising the piston. An example of the conversion of potential energy into heat occurs in a metallic spring, when it uncoils and is so placed as to rub against a rough surface, producing heat by friction.

4. Chemical Affinity : Relation to heat. - Whilst gravity acts upon the particles of matter without reference to the composition of the body, there is another atomic force which acts between atoms of a chemically different nature; this is chemical affinity. This is the force in virtue of which the atoms of chemically different bodies unite to form a chemical compound. The force itself varies greatly between the atoms of different chemical bodies; thus we speak of strong chemical affinities and weak affinities. Just as we were able to estimate the potential energy of a body in motion from the amount of heat which was produced when it collided with an unyielding body, so we can measure the amount of heat which is formed when the atoms of chemically different bodies unite to form a chemical compound. As a rule, heat is formed when separate chemically-different atoms form a compound body. When, in virtue of chemical affinity, the atoms of 1 kilo. of hydrogen and 8 kilos. of oxygen unite to form the chemical compound water, an amount of heat is thereby evolved which is equal to that produced by a weight of 47,000 kilos. falling and colliding with the earth from a height of 1000 feet above the surface of the earth. If 1 gramme of $\mathrm{H}$ be burned along with the requisite amount of $\mathrm{O}$ to form water, it yields 34,460 heat-units or calories : and 1 gramme carbon burned to carbonic acid (carbon dioxide) yields 8080 heat-units. Wherever, in chemical processes, strong chemical affinities are satisfied, heat is set free, i.e., chemical affinity is 
changed into heat. Chemical affinity is a form of potential energy obtaining between the most different atoms, which during chemical processes is changed into heat. Conversely, in those chemical processes where strong affinities are dissolved, and chemically-united atoms thereby pulled asunder, there must be a diminution of temperature, or, as it is said, heat becomes latent-that is, the energy of the heat which has become latent is changed into chemical energy, and this, after decomposition of the compound chemical body, is again represented by the chemical affinity between its isolated different atoms.

LAW OF THE CONSERVATION OF ENERGY.- Julius Robert Mayer and Helmholtz have established the important law that, in a system which does not receive any influence and impression from without, the sum of all the forces acting within it is always the same. The various forms of energy can be transformed one into the other, so that kinetic energy may be transformed into potential energy and vice versî, but there is never any part of the energy lost. The transformation takes place in such measure that, from a certain definite amount of one form of energy, a definite amount of another can be obtained.

The various forms of energy acting in organisms occur in the following modifications :-

1. Molar motion (ordinary movements), as in the movements of the whole body of the limbs, or of the intestines, and even those observable microscopically in connection with cells.

2. Movements of Atoms as Heat.-We know, in connection with the vibration of atoms, that the number of vibrations in the unit of time determines whether the oscillations appear as heat, light, or chemically-active vibrations. Heat-vibrations have the smallest number, while chemically-active vibrations have the largest number, light-vibrations standing between the two. In the human body we only observe heat-vibrations, but some of the lower animals are capable of exhibiting the phenomena of light.

In the human organism the molar movements in the individual organs are constantly being transformed into heat, e.g., the kinetic energy in the organs of the circulation is transformed by friction into heat. The measure of this is the "unit of work " = 1 gramme-metre, and the " unit of heat " $=425 \cdot 5$ gramme-metres.

3. Potential Energy.-The organism contains many chemical compounds which are characterised by the great complexity of their constitution, by the imperfect saturation of their affinities, and hence by their great tendency to split up into simpler bodies.

The body can transform the potential energy into heat as well as into kinetic energy, the latter always in conjunction with the former, but the former always by itself alone. The simplest measure of the potential energy is the amount of heat which can be obtained by complete combustion of the chemical compounds representing the potential energy. The number of work-units can then be calculated from the amount of heat produced.

4. The phenomena of electricity, magnetism, and diamagnetism may be recognised in two directions, as movements of the smallest particles, which are recognised in the glowing of a thin wire when it is traversed by strong electrical 
currents (against considerable resistance), and also as molar movement, as in the attraction or repulsion of the magnetic needle. Electrical phenomena are manifested in our bodies by muscle, nerve, and glands, but these phenomena are relatively small in amount when compared with the other forms of energy. It is not improbable that the electrical phenomena of our bodies become almost completely transformed into heat. As yet experiment has not determined with accuracy a "unit of electricity" directly comparable with the "heat-unit" and the "work-unit."

It is quite certain that within the organism one form of energy can be transformed into another form, and that a certain amount of one form will yield a definite amount of another form; further, that new energy never arises spontaneously, nor is energy already present ever destroyed, so that in the organism the law of the conservation of energy is continually in action.

ANIMALS AND PLANTS. - The animal body contains a quantity of chemicallypotential energy stored up in its constituents. The total amount of the energy present in the human body might be measured by burning completely an entire human body in a crelorimeter, and thereby determining how many heat-units are produced when it is reduced to ashes (see Animal Heat).

The chemical compounds containing the potential energy are characterised by the complicated relative position of their atoms, by a comparatively imperfect saturation of the affinities of their atoms, by the relatively small amount of oxygen which they contain, by their great tendency to decomposition, and the facility with which they undergo decomposition.

If a man were not supplied with food he would lose 50 grammes of his borlyweight every hour; the material part of his body, which contains the potential energy, is used up, oxygen is absorbed, and a continual process of combustion takes place; by the process of combustion simpler substances are formed from the more complex compounds, whereby potential is converted into kinetic energy. It is immaterial whether the combustion is rapid or slow; the same amount of the same chemical substances always produces the same amount of kinetic energy, i.e., of heat.

A person, when fasting, experiences after a certain time the disagreeable feeling of exhaustion of his reserve of potential energy, hunger sets in, and he takes food. All food for the animal hingdom is obtained, either directly or indirectly, from the vegetable kingdom. Even carnivora, which eat the flesh of other animals, only eat organised matter which has been formed from vegetable food. The existence of the animal kingdom presupposes the existence of the vegetable kingdom.

All substances, therefore, necessary for the food of animals occur in vegetables. Besides water and the inorganic constituents, plants contain, amongst other organic compounds, the following three chief representatives of food-stuff s-fats, carbohydrates, and proteids.

All these contain stores of potential energy, in virtue of their complex chemical coustitution.

The fats contain :- $\left\{\begin{array}{l}\mathrm{CnH}_{2 \mathrm{n}-1} \mathrm{O}(\mathrm{OH})=\text { fatty acids } \\ +\mathrm{C}_{3} \mathrm{H}_{5}(\mathrm{OH})_{3}=\text { glycerin }\end{array}\right\}(\$ 251)$.

The carbohydrates contain :- $\mathrm{C}_{6} \mathrm{H}_{10} \mathrm{O}_{5}$. $\quad$ (\$252). 
The proteids contain per cent.:- $\left\{\begin{array}{l}\text { C. } 51 \cdot 5-54 \cdot 5 \\ \text { H. } 6 \cdot 9-7 \cdot 3 \\ \text { N. } 15 \cdot 2-17 \cdot 0 \\ \text { O. } 20 \cdot 9-23 \cdot 5 \\ \text { S. } 0 \cdot 3-2 \cdot 0\end{array}\right\}$ (\$ 248 and 249).

A man who takes a certain amount of this food adds thereto oxygen from the air in the process of respiration. Combustion or oxidation then takes place, whereby chemically-potential energy is transformed into heat.

It is evident that the products of this combustion must be bodies of simpler constitution-bodies with less complex arrangement of their atoms, with the greatest possible saturation of the affinities of their atoms, of greater stability, partly rich in $\mathrm{O}$, and possessing either no potential energy, or only very little. These bodies are carbon dioxide, $\mathrm{CO}_{2}$; water, $\mathrm{H}_{2} \mathrm{O}$; and as the chief representative of the nitrogenous excreta, urea $\left(\mathrm{CO}\left(\mathrm{NH}_{2}\right)_{2}\right)$, which has still a small amount of potential energy, but which outside the body readily splits into $\mathrm{CO}_{2}$ and ammonia $\left(\mathrm{NH}_{3}\right)$.

The human body is an organism in which, by the phenomena of oxidation, the complex nutritive materials of the vegetable kingdom, which are highly charged with potential energy, are transformed into simple chemical bodies, whereby the potential energy is transformed into the equivalent amount of kinetic energy (heat, work, electrical phenomena).

But how do plants form these complex food-stuffs so rich in potential energy? It is plain that the potential energy of plants must be obtained from some other form of energy. This potential energy is supplied to plants by the rays of the sun, whose chemical light-rays are absorbed by plants. Without the rays of the sun there could be no plants. Plants absorb from the air and the soil $\mathrm{CO}_{2}, \mathrm{H}_{2} \mathrm{O}$, $\mathrm{NH}_{3}$, and $\mathrm{N}$, of which carbon dioxide, water, and ammonia (from urea) are also produced by the excreta of animals. Plants absorb the kinetic energy of light from the sun's rays and transform it into potential energy, which is accumulated during the growth of the plant in its tissues, and in the food-stuffs produced in them during their growth. This formation of complex chemical compounds is accompanied by the simultaneous excretion of $\mathrm{O}$.

Occasionally, kinetic energy, such as we universally meet with in animals, is liberated in plants. Many plants develop considerable quantities of heat in their flowers, e.g., the arum tribe. We must also remember that during the formation of the solid parts of plants, when fluid juices are changed into solid masses, heat is set free. In plants, under certain circumstances, $\mathrm{O}$ is absorbed, and $\mathrm{CO}_{2}$ is excreted, but these processes are so trivial as compared with the typical condition in the vegetable kingdom, that they may be regarded as of small moment.

Plants, therefore, are organisms which, by a reduction process, transform simple stable combinations into complex compounds, whereby potential solar energy is transformed into the chemically-potential energy of vegetable tissues. Animals are living beings, which by oxidation decompose or break up the complex grouping of atoms manufactured by plants, whereby potential is transformed into kinetic energy. Thus, there is a constant circulation of matter and a constant exchange of cnergy between plants and animals. All the energy of animals is derived from plants. All the energy of plants arises from the sun. Thus the sun is the cause, the original source of all energy in the organism, i.e., of the whole of life. 
As the formation of solar heat and solar light is explicable by the gravitation of masses, gravity is perhaps the original form of energy of all life.

We may thus represent the formation of kinetic energy in the animal body from the potential energy of plants. Let us suppose the atoms of the substances formed in organiisms, as simple small bodies, balls, or blocks. As long as these lie in a single layer, or in a few layers, upon the surface, there is a stable arrangement, and they continue to remain at rest. If, however, an artificial tower be built of these blocks, so that an unstable erection is produced, and the same tower be afterwards knocked down, then for this purpose we require-(1) the motor power of the workman who lifts and carries the blocks ; (2) a blow or other impulse from without applied to the unstable structure-when the atoms will fall together, and as they fall collide with each other and produce heat. Thus, the energy employed by the workman is again transformed into the last-named form of energy.

In plants the complex unstable building of the groups of atoms is carried on, the constructer being the sun. In animals, which eat plants, the complex groups of the atoms are tumbled down, with the liberation of kinetic energy.

Vital Energy and Life.-The forces which act in organisms, in plants, and animals are exactly the same as are recognisable as acting in dead matter. A socalled "vital force," as a special force of a peculiar kind, causing and governing the vital phenomena of living beings, does not exist. The forces of all matter, of organised as well as unorganised, exist in connection with their smallest particles or atoms. As, however, the smallest particles of organised matter are, for the most part, arranged in a very complicated way, compared with the much simpler composition of inorganic bodies, so the forces of the organism connected with the smallest particles yield more complicated phenomena and combinations, whereby it is excessively difficult to ascribe the vital phenomena in organisms to the simple fundamental laws of physics and chemistry.

The Exchange of Material, or Metabolism ("Stoffwechsel") as a Sign of Life. -Nevertheless, there appears to be a special exchange of matter and energy peculiar to living beings. This consists in the capacity of organisms to assimilate the matter of their surroundings, and to work it up into their own constitution, so that it forms for a time an integral part of the living being, to be given off again. The whole series of phenomena is called metabolism or "Stoffwechsel," which consists in the introduction, assimilation, integration, and excretion of matter.

We have already shown that the metabolism of plants and that of animals are quite different. The processes, as already described, actually occur in the typical higher plants and animals.

But there is a large group of organisms which, throughout their entire organisation, exhibit so low a degree of development, that by some observers they are considered as undifferentiated "ground-forms." They are regarded as neither plants nor animals, and are the most simple forms of aniniated matter. Haeckel has called these organisms Protistæ, as being the original and primitive forms.

We must assume that, corresponding with their simpler vital conditions, their metabolism is also simpler, but on this point we still require further observations and experiments. 


\section{Physiology of the Blood.}

[THE blood is aptly described by Claude Bernard as an internal medium which acts as a "go-between " or medium of exchange for the outer world and the tissues. Into it are poured those substances which have been subjected to the action of the digestive fluids, and in the lungs or other respiratory organs it receives oxygen. It thus contains new substances, but in its passage through the tissues it gives up some of these new substances, and receives in exchange certain waste products which have to be got rid of. Its composition is thus highly complex. Besides carrying the new nutrient fluids to the tissues, it is also the great oxygen-carrier, as well as the medium by which some of the waste products, r.g., CO.2, urea, are removed from the tissues, and brought to the organs, e.\%, the lungs, kidneys, skin, which eliminate them from the body. It is at once a great pabulum-supplying medium and a channel for getting rid of useless materials. As the composition of the organs through which the blood Hows varies, it is evident that its composition must vary in different parts of the circulatory system; and it also varies in the same individual under different conditions. Still, with slight variations, there are certain general physical, histological, and chemical properties which characterise blood as a whole.]

1. PHYSICAL PROPERTIES.--(1) Colour.--The colour of blood varies from a bright scarlet-red in the arteries to a deep, dark, bluish-red in the veins. Oxygen (and, therefore, the air) makes the blood bright red; want of oxygen makes it dark. Blood free from oxygen (and also venous blood) is dichroic-i.e., by reflected light it appears dark red, while by transmitted light it is green. [Arterial blood is monochroic.]

In thin layers blood is opaque, as is easily shown by shaking blood so as to form bubbles, or by allowing blood to fall upon a plate with a pattern on it, and pouring it off again. [Printed matter cannot be read through a thin layer of blood spread on a glass slide.] Blood behaves, therefore, like an "opaque coluur," as its colouringmatter is suspended in the form of fine particles-the blood-corpuscles.

Hence, it is possible to separate the colouring-matter from the fluid part of the blood by filtration. This is accomplished by mixing the blood with fluids which render the bloodcorpuscles sticky or rough. If mammalian blood be treated with one-seventh of its volume of solution of sollic sulphate, or if frog's blood be mixed with a 2 jer cent. solution of sugar ( $J_{o} /$. Müller ) and filtered, the shrivelled corpuscles, now robbed of part of their water, remain upon the filter.

(2) Reaction.-The reaction is alkaline, owing to the presence of disodic phosphate, $\mathrm{Na}_{2} \mathrm{HPO}_{4}$, and bicarbonate of soda. Alter blood is shed, its alkalinity rapidly diminishes, and this occurs more rapidly the greater the alkalinity of the blood. This is due to the formation of an acid, in which, perhaps, the coloured corpuscles take part, owing to the decomposition of their colouring-matter. A high 
temperature and the addition of an alkali favour the formation of the acid $(N$. Zuntz).

The alkaline reaction of blool is diminished: $(a)$ by great muscular exertion, owing to the formation of a large amount of acill in the muscles; $(\boldsymbol{\beta})$ during coagulation ; $(\gamma)$ in old blood, or bloot dissolved by water from old blood-stains, steh blood being usually acid; fresh cruor las a stronger alkaline reaction than serum; $(\delta)$ after the prolonged use of soda the alkalinity is increased, after the use of acids it is decreased.

Methods. - Owing to the colour of the hloot we cannot employ ordinary litmus paper to test its reaction. One of the following methols may be used:-(1) Moisten a strip of glazed red litmus paper with solution of commou salt, ancl allow a drop of blood to fall on the paper; then rapidly wipe it off hefore its colouring matter has time to penetrate and tinge the paper (Zunt:). (2) Liebreich used thin plite's of plister-of-Paris of a perfectly nentral reaction. 'These are dried, and afterwards moistened with a neutral solution of litmus. When a drop of hood is placed upon the porous plite, the fluid part of the blood passes into it, while the (orpuscles are washerl off with water, and the altered colour of the litmus-stained slab is apparent. [(3) Schiffer uses dry fatintly-redlened glazed litmus paper, and on it is placed a drop of blood, which is wiped iff after a few seennils. The place where the blood rested is indicated by a blue patch upon a red or violet ground.]

Estimation of the Alkalinity.-A very dilute solution of tartaric acid (1 cubic centimetre combines with 3.1 milligrams of sorla, i.t, 1 litre of water contains 7.5 grams of erystallised tartaric arid) is added to hlool until a blue litmus paper is turned red (by Zuntz's method). 100 grams of rabbit's blood have an alkalinity corresponding to 150 milligrams of soda ; the blook of carnivora to about 180 milligrams (Lesser), while 100 c.e. of normal human bloot have an alkalinity equal to $260-300$ milligrams of soda $(v$. Jaksch).

The following method can be used with a few drops of blood :-To nentralise the blood, tartarie acil in the above concentration is used. Prepare the following mixture by mixing it with a concentrated neutral solution of solic sulphate, and then adding sodic sulphate until the mixture is completely saturated. I., 10 parts of solution of tartaric acid to 100 parts of concentraterl solie sulphate solution; II., 20 prarts tartaric acid solution to 90 sodie sulphate solution; III. contains these substances in the proportion of 30 to 80 ; IV., 40 to 70 ; V., 50 to 60 ; VI., 60 to 50 ; VII., 70 to 40 ; VIII., 80 to 30 ; IX., 90 to 20 ; and X., 100 to 10 . Excess of sodic sulphate is present in all the flasks.

A known volume of the blood to be investigated is mixed with an equal volume of each of the mixtures, in a small tube, which is made by drawing out a glass tube 1 millimetre in diameter to a fine point. To calibrate this tube, suck up water, say, to the height of $8 \mathrm{~mm}$., make a mark on the tube with a fine file, then suck up the water until its lower level corresponds with the mark. Again mark the upper limit of the water. To test the blood, suck a irop of the mixture I. up to the level of the first mark on the glass pipette, anul, after wiping its point, suck up an equal yuantity of blood. Again clean the point of the pipette, and blow its contents into a watch-glass; then mix, and test the reaction with sensitive violet-coloured litmus paper. Proceed in the same way with the sereral mixtures, II., to X., until the alkaline reaction disappears or the acid appears. The narrow strips of litmus paper are dipped into each of the mixtures, the corpuseles remain in the wetted part of the paper, while the fluid permeates further and shows the reaction. As a rule, the degree of alkalinity in human blood corresponils to VI. Human blood can be sucked directly from a small wound made by a needle, either by attaching an elastic tube or a small hypodermic syringe to the pipette(Landois).

Pathological.-The alkalinity is increased during persistent vomiting, and decreased in mronounced anemia, cachexia, uritnia, rheumatism, high fever, diabetes, and cholera. [Immediately before death by cholera it may be acid (Cantani).]

(3) Odour.--Blood emits a peculiar odour, the halitus sanguinis, which differs in animals and man.

It depends upon the presence of volatile fatty acils. If concentrated sulphuric acid he adderl to blood, whereby the volatile fatty acids are set free from their combinations with alkalies, the characteristic odour, somewhat similar to that of butyric acid, becomes much more perceptible.

(4) Taste.-Blood has a saline taste, deyending upon the salts dissolved in the fluid of the blood.

(5) Specific Gravity.-The specific gravity is 1056-1059 in man, 1051-1055 in woman; in children less. The specific gravity of the blood-corpuscles is 1105 , that of the plasma 1027 . Hence the corpuscles tend to sink.

Clinical Method.-A thin glass tube is drawn out till it is of small calibre, and then bent at a right angle, and closed above with a caoutchouc eap. Press slightly on the caoutchoue cap, and suck up a drop of the freshly-drawn blood obtained by pricking the finger. The fine capil- 
lary-tube is at once immersed in a solution of sodic sulphate, and a drop of the blood expressed into the saline solution. It is necessary to prepare several solutions of sodic sulphate with specific gravities varying from 1050-1070. The solution in which the corpuseles remain suspended indicates the specific gravity of the blood (Roy, Landois).

The drinking of water and hunger dininish the specific gravity temporarily, while thirst and the digestion of dry food raise it. If blood be passed through an organ artificially, its specific gravity rises in consequence of the absorption of dissolved matters and the giving off of water. It falls after hæmorrhage, and is diminished in badly-nourished individuals. [By working with solutions of glycerine, Jones finds that it is highest at birth, is at a minimum between the second week and the second year; it rises gradually until the 35 th-45th year. It is usually higher in the male than the female, is diminished by pregnaney, the ingestion of solid or liquiil food, and gentle exercise.]

[(6) Temperature.-Blood is viscid, and its temperature varies from $36.5^{\circ} \mathrm{C}$. $\left(97.7^{\circ}\right.$ F.) to $37 \cdot 8^{\circ}\left(100^{\circ}\right.$ F.). The warmest blood in the body is that of the hepatic vein $(\$ 210)$.]

2. MICROSCOPIC EXAMINATION.--[Blood, when examined by the microscope, is seen to consist of an enormous number of corpuscles - coloured and colourless-floating in a transparent fluid, the plasma, or liquor sanguinis.

A

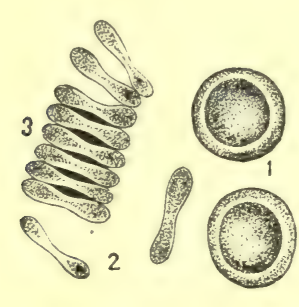

B

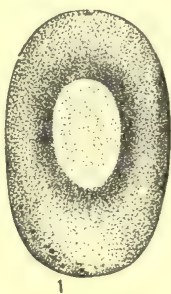

C

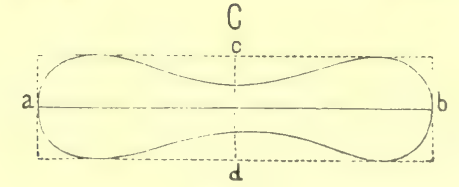

Fig. 1.

A, human coloured blool-corpuscles--1, on the flat; 2, on edge ; 3 , rouleau of coloured corpuscles. $B$, amphibian coloured blood-corpuscles-1, on the flat; 2 , on edge. $\mathrm{C}$, ideal transverse section of a human coloured blood-corpuscle magnified 5000 times linear- $a b$, diameter ; $c d$, thickness.

Human Red Blood-Corpuscles.--(/ ( ) Form.--They are circular, coin-shaped, homogeneous discs, with saucer-like depressions on both surfaces, and with rounded margins ; in other words, they are bi-concave, circular non-nucleated discs.

(b) Size. - The diameter $(a b)$ is $7 \cdot 7 \mu,{ }^{1}(6 \cdot \tau-9 \cdot 3 \mu)$ the greatest thickness $(c d) 1 \cdot 9 \mu$ (fig. 1, C), [i.e., it is $\frac{1}{3.500}$ to $\frac{1}{3200}$ of an inch in diameter, and about one-fourth of that in thickness].

They are slightly diminished in size by septic fever, inanition, morphia, increased bodily temperature, and $\mathrm{CO}_{2}$; and increased by $O$, watery condition of the blood, cold, consumption of alcohol, quinine, and hydrocyanic acid. Compare $\$ 10,3$.

If the total amount of blood in a man be taken at 4400 cubic centimetres, the corpuscles therein contained have a surface of 2816 square metres, which is equal to a square surface with a side of 80 paces ; 176 cubic centimetres of blood pass through the lungs in a second, and the blood-corpuscles in this amount of blood have a superficies of 81 square metres, equal to a square surface with a side of 13 paces (Welcker).

(c) The weight of a blood-corpuscle is 0.00008 milligramme.

${ }^{1}$ The Greek letter $\mu$ represents one-thousandth of a millimetre $(\mu=0.001 \mathrm{~mm}$.), and is the sign of a micro-millimetre, or a micron. 
(d) The number exceeds 5,000,000 per cubic millimetre in the male, and $4,500,000$ in the female; so that, in $10 \mathrm{lbs}$. of blood, there are 25 billions of corpuscles. The number is in inverse ratio to the amount of plasma; hence, the number must vary with the state of contraction of the blood-vessels, the pressure, diffusion currents, and other conditions.

The number of red corpuscles is increased ; in venous blool (especially in the small cutaneons veins), after the use of solid forl, after much sweating, and the excretion of much water by the bowel and kidneys; during inanition, hecause the blood-plasma undergoes decomposition sooner than the blood-corpuscles themselves; in the blood of the newly-born child, especially when the umbilical cord is long in leeing tied $(\$ 40)$, from the 4 th day onward the number is liminished; in persons of robust constitution. and in those who live in the country. The number is diminished, during pregnaney, after copious draughts of water. In the earlier period of furtal life the number is only $\frac{1}{2}-1$ million in 1 cubic millimetre. (For the pathological conditions see \& 10 .

Methods of Counting the Blood Corpuscles. - The pointed ent of a glass pipette (fig. 3), the mixer, is dipled into the blood, and by sucking the elastic tube $f$, blood is drawn into the tube
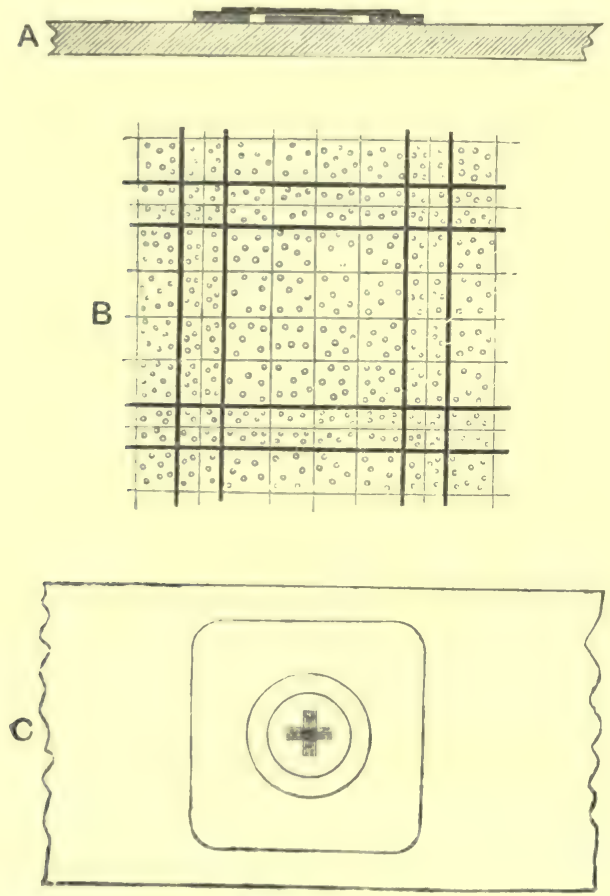

Fig. 2 .

Apparatus of Abbé and \%eiss for counting the corpuscles. A, in section; C, surface view without cover-glass; $\mathrm{B}$, microscopic appearance with the blood-corpuseles.

A

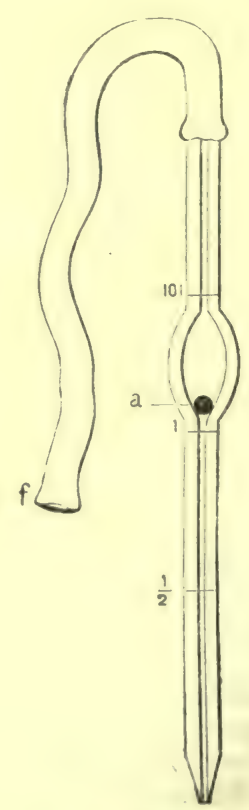

Fig. 3.

The Melangéur, piputte or mixer,

until it reaches the mark $\frac{1}{2}$, on the stem of the pipette, or until the mark 1 is reached. The carefully-cleaned point of the pipette is dipped into the artificial serum, and this is sucked into the pipette until it reaches the mark, 101. The artificial serum consists of 1 vol. of solution of gum arabic (sp. gr. 1020) and 3 vols. of a solution of equal parts of sodic sulphate and sodic chloride (sp. gr. 1020). The process of mixing the two fluids is aided by the presence of a little glass ball (a) in the bulb of the pipette. If blood is sucked up to the mark $\frac{1}{2}$, the strength of the mixture is $1: 200$; if to the mark 1 , it is $1: 100$; a small drop of the mixture is allowel to run into the counting chamber of $A$ bbé and Zeiss (fig. 2). The first portions are not useil, in order to obtain a uniform sample from the hulb of the pipette. This ohamber consists of a glass 
receptacle $0.1 \mathrm{~mm}$. deep, with its base divided into squares, and cemented to a glass slide, the whole being covered with a thin covering-glass. The space over each square $=\frac{1}{4000}$ cubi millimetre. Count, with the aid of a microscope, the number of blood-corpuscles in each square, and the number found, multiplied by 4000 , will give the number of blood-corpuscles in 1 c.mm. This number, again, must be multiplied by 100 or 200 , according as the blood was diluted 100 or 200 times. To ensure greater accuracy, it is well to count the number in serceral squares, and to take the mean of these.

[Gowers' Method. - "The Hæmacytometer (fig. 4) consists of-(1) a small pipette, which, when filled to the mark on its stem, holds exactly 995 cubic millimetres. It is furnished with an india-rubber tube and mouthpiece to facilitate filling and emptying. (2) A capillary tube marked to contain exactly 5 cubic millimetres, with india-rubber tube for filling, \&e. (3) A small glass jar in which the dilution is made. (4) A glass stirrer for mixing the blood anci solution in the glass jar. (5) A brass stage plate, carrying a glass slip, on which is a cell, $\frac{1}{5}$ of a millimetre deep. The bottom of this is divided into $\frac{1}{10}$ millimetre squares. Upon the top of the cell rests the cover-glass, which is kept in its place by the pressure of two springs proceeding from the ends of the stage plate." The diluting solution used is a solution of sodic sulphate in distilled water, sp. gr. 1025, or the following-sodic sulphate, 104 grains ; acetic acid, 1 drachm ; distilled water, 4 oz.

" 995 cubic millimetres of the solution are placed in the mixing jar ; 5 cubic millimetres of blood are drawn into the capillary tube from the puncture in the finger, and then blown into the solution. The two fluids are well mixed by rotating the stirrer between the thumb and finger, and a small drop of this dilution is placed in the centre of the cell, the covering-

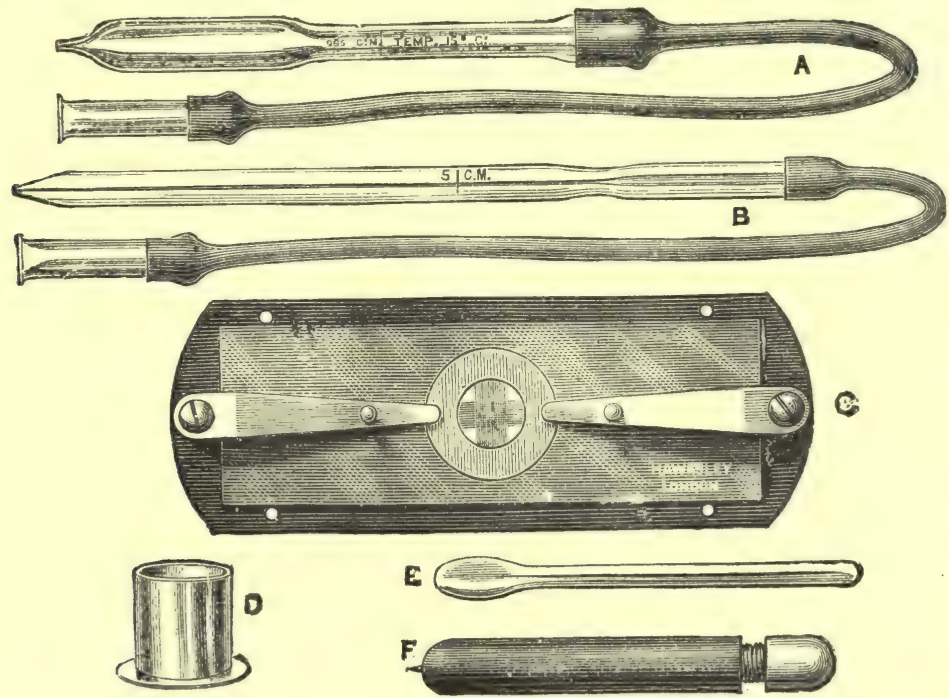

Fig. 4.

Gowers' apparatus. A, pipette for measuring the diluting solution; B, capillary tube for measuring the blood ; C, cell with divisions on the floor, mounted on a slide ; 1, vessel in which the dilution is made; $\mathbf{E}$, glass stirrer ; $F$, guarded spear-pointed needle.

glass gently put upon the cell, and secured by the two springs, and the plate placed upon the stage of the microscope. The lens is then focussed for the squares. In a few minutes the corpuscles have sunk to the bottom of the cell, and are seen at rest on the squares. The number in ten squares is then counted, and this, multiplied by 10,000, gives the number in a cubic millimetre of blood."

To estimate the colourless corpuscles only, mix the blood with 10 parts of 0.5 per cent. solution of acetic acid, which destroys all the red corpuscles (Thoma).

(e) Red blood-corpuscles are characterised by their great elasticity, flexibility, and softness. [The elastic property is shown by the extent to which red corpuscles while circulating may be distorted, and yet resume their original for $\mathrm{m}$ as soon as the pressure is removed.] 
3. HISTOLOGY OF THE HUMAN RED BLOOD-CORPUSCLES.-When observed singly, human red blood-corpuscles are bi-concave circular discs of a yellow colour with a slight tinge of green; they seem to be devoid of an envelope, are certainly non-nucleated, and appear to be homogeneous throughout. Each corpuscle consists (1) of a framework, an exceedingly pale, transparent, soft protoplasm - the stroma ; and (2) of the red pigment, or hremoglobin, which impregnates the stroma, much as fluid passes into and is retained in the interstices of a bath-sponge.

4. EFFECT OF REAGENTS.--(1) On their Vital Phenomena.-The bloodcorpuscles present in shed blood-or even in defibrinatcd blood, when it is reintroduced into the circulation-retain their vitality and functions undiminished. Heat acts powerfully on their vitality, for if blood be heated to $52^{\circ} \mathrm{C}$., the vitality of the red corpuscles is clestroyed. Nammalian blood may be kept for four or tive days in a ressel under iced water, and still retain its functions; but if it be kept longer, and reintroduced into the circulation, the corpuscles rapidly break up

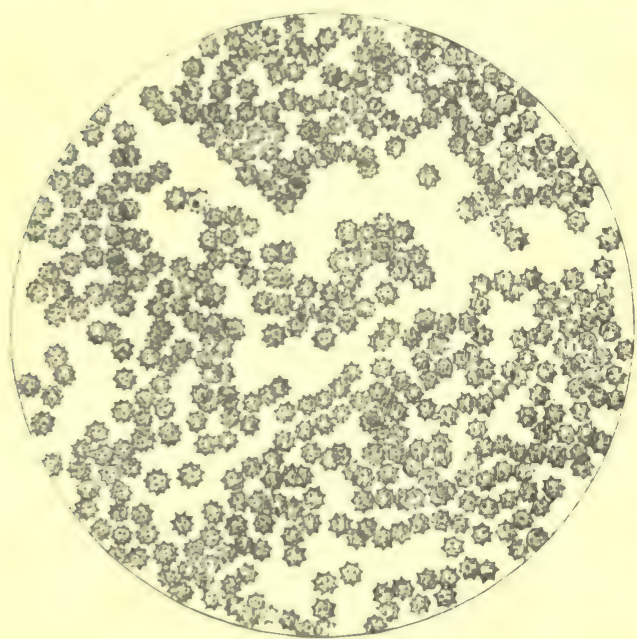

Fig. 5.

Crenation of human red blood-corpuseles. -a proof that they have lost their vitality. The red corpuscles in freshly shed blood sometimes exhibit a peculiar mulberry-like appearance (figs. $5,6, g, h)$. [This is called crenation of the coloured corpuscles. It occurs in cases of poisoning with Calabar bean; and also by the addition of a 2 per cent. solution of common salt.] The blood of many persons crenates spontaneously - a condition ascribed to an active contraction of the stroma, but it is doubtful if this is the cause. The red corpuscles of the embryo-chick undergo active contraction.

(B) On their External Characters.-(u) The colour is changed by many grases. $\mathrm{O}$ makes blood scarlet, want of $\mathrm{O}$ renders it dark bluish-red, CO makes it cherry-red, NO viulet-red. There is no difference between the shape of the corpuscles in arterial and venous blood. All reagents (e.\%,, a concentrated solution of sodic sulphate), which cause great shrinking of the coloured corpuscles, produce a very bright scarlet or brick-red colour. The red colour so" produced is quite different from the scarletred of arterial blood. Rieagents which render blood-corpuscles globular darken the blood, e.g., water.

[The contrast is very striking, if we compare blood to which a 10 per cent. solution of common salt has been added with blowil to which water has been added. With reflected light the one is bright red, and the other a very dark deep crinison, almost black.]

(b) Formation of Rouleaux.-A very common phenomenon in shed blood is the tendency of the corpuscles to run into rouleaux (fig. 1, A, 3).

Conditions that increase the coagulability of the blood favour this phenomenon, which is ascribed by Dogiel to the attraction of the dises and the formation of a sticky substance. [The cause of the formation of rouleaux is by no means clear. The corpuscles may be detached from each other by gently touching the cover-glass, but the rouleaux may re-form. Lister suggesterl that the surfaces of the corpuscles were so altered that they became adhesive. Norris made experiments with corks weighted with tacks or pins, so as to produce partial submersion of the cork 
dises. These dises rapidly cohere, owing to eapillarity, and form rouleaux. If the dises be completely submerged they remain apart, as occurs with unaltered blood-corpuscles within the blood-vessels. If, however, the corpuscles be dipped in petroleum, and then placed in water, rouleaux are formed.] If reagents which cause the corpuscles to swell up be added to the blood, the corpuseles become globular and the rouleaux break up. According to E. Weber and Suchard, the uniting medium is not fibrin (although it may sometimes assume a fibrous form), but belongs to the peripheral layer of the corpuscles.

(c) Changes of Form.-The discharge of a Leyden jar causes the corpuscles to crenate, so that their surfaces are beset with coarse or fine projections (fig. $6, c, d$, $e, g, h)$; it also causes the corpuscles to assume a spherical form $(i, i)$, and they

a

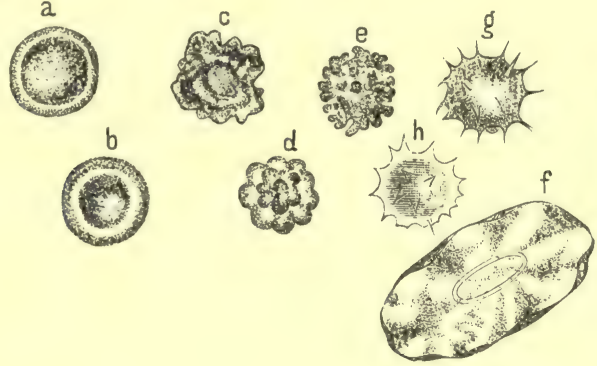

Fig. 6 .
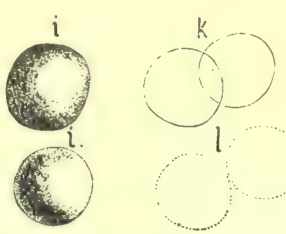

Red blood-corpuscles. $a, b$, normal human red corpuscles, the central depression more or less in focus; $c, d, e$, mulberry, and $g, h$, crenated forms; $k$, pale corpuscles decolorised by water ; $l$, stroma ; $f$, frog's blood-corpuscle acted on by a strong saline solution.

become smaller than normal. The corpuscles so altered are sticky, and run together like drops of oil, forming larger spheres. The prolonged action of the electrical spark causes the hæmoglobin to separate from the stroma $(k)$, whereby the fluid part of the blood is reddened, while the stroma is recognisable only as a faint shadow $(l)$. Similar forms are to be found in decomposing blood, as well as after the action of many other reagents. Heat.-When blood is heated, on a warm stage, to $52^{\circ} \mathrm{C}$. the corpuscles exhibit remarkable changes. Some of them become spherical, others biscuit-shaped; some are perforated, while in others small portions become detached and swim about in the surrounding fluid, a proof that heat destroys the histological individuality of the corpuscles. If the heat be continued, the corpuscles are ultimately dissolved $(\$ 10,3)$.

Heat acts like the addition of a concentrated solution of urea to blood. If strong pressure he exerted upon a microscopic preparation, the blood-corpuseles nay break in pieces. The latter process is called hæmocytotrypsis, in contradistinction to that of solution of the corpuscles or hæmocytolysis.

If a finger moistened with blood be rapilly clrawn across a warm slip of glass, so that the fluid dries rapidly, the corpuscles exhibit very remarkable shapes, showing their great ductility and softness.

Cytozoon-Gaule's Experiment.-A few drops of freshly-shed frocg's blood are mixed with 5 c.c. of 0.6 per cent. solution of common salt, and the mixture defibrinated by shaking it along with a few c.c. of mercury. A drop of the defibrinated blood is examined on a hot stage $\left(30^{\circ}-32^{\circ}\right.$ C. $)$ under a microseope, when a protoplasmic mass, the so-called "Wïrmchen," escapes with a lively movement from many corpuscles, and ultimately dissolves. Similar "cytozoa" were discovered by Gaule in the epithelium of the cornea, of the stomach and intestine, in connective-tissue, in most of the large glands, and in the retina (frog, triton). In mammals also he found similar but smaller structures. Most probably these structures are parasitic in their nature, as suggested by Ray Lankester, who called the parasite Drepanidium ranarum.

[Staining Reagents.--Such reagents as magenta, picro-carmine, carmine, and many of the aniline dyes, stain the nucleus deeply when such is present, and although they must traverse the hæmoglobin to reach the nucleus, the hæmoglobin itself is not stained. When no nucleus is present, therefore, the corpuscles are not 
stained. Magenta causes one or more small spots or maculæe to appear on the edge of the corpuscles (fig. $7, a$ ). What its significance is, is entirely unknown. Normal saline solution $(0.6$ per cent. $\mathrm{NaCl})$, tinged with methyl violet, is a good staining and preservative agent.]

[Agitation with Mercury. - If ox blood be shaken up with mercury for 7 or 8 hours, the corpuscles completely disappear, no trace of stroma or corpuscles being found in the fluid (Meltzer and $W$ elch $)$. The adlition of pyrograllie acid ( 20 per cent.), potassic chlorate (6 per cent.), and silver nitrate $(3$ per cent.), completely prevents dissolution of the corpuscles, even though the shaking be kept up for fourteen clays. ]

If blood be mixed with concentrated gum solution, and if concentrated salt solution be added to it under the microscope, the corpuseles assume elongated forms. Similar forms are obtained by mixing bloor with an eynal volume of gelatine at $36^{\circ} \mathrm{C}$., allowing it to cool, and then making sections of the coagulated mass. The corpuscles may be broken up by pressing firmly on the cover-glass. In all these experiments no trace of an envelope around the corpuscles is observed. [An excellent reagent for "fixing" the blood-corpuscles is either a dilute solution or the vapour of osmic acid.]

Conservation of the Corpuscles. - In investigating blood with the microscope for forensic purposes, it is necessary to have a solvent for the blood when it oecurs as stains on a garment or iustrument. Driel stains are dissolved lyy a concentrated, or a 30 per cent., solution of
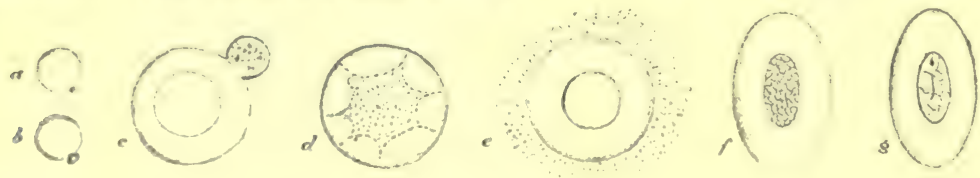

\section{Fig. 7}

", b, luman red blood-corpuscles; ;, actel on by magenta ; b, by tamuic acid. The others are amphibian reel blood-corpuseles; $c, d$, e, effect of tamnic acid ; $f$, of dilute acetic acid ; $g$, of dilute alcohol; $d$, by boracic acid (Stirling).

eaustir protash, or with one of the preserving fluids. If the stain be softened with concentrated tartaric acid, colourless corpuseles are specially distinct (S'truuse). Nevertheless, corpuscles are often nut foumd in such stains. If the corpuseles have become very pale, their colour may be improved hy adhling a solution of iodide of potassium, a saturated solution of picric acid, 20 per cent. pyrogallic acid, or 3 per cent. solution of silver nitrate.

5. STROMA-LAKE-COLOURED BLOOD.-Many reagents cause the hæmoglobin to separate from the stroma. The hæmoglobin dissolves in the serum; the blood becomes dark red and transparent, as it contains its colouring matter in solution, and hence it is called "lake-coloured" (Rollett). The aggregate condition of the hæmoglobin is not altered when the corpuscles are dissolved-it only changes its place, leaving the stroma and plassing into the serum. Hence, the temperature of the blood is not lowered thereby.

Methods. - To obtain a large quantity of the stroma for chemical purposes, add 10 vols. of a solution of common salt (1 vol. coneritraterl solution, and 15 to 20 vols. of water) to 1 vol. of ilefibrinated blood, when the stromata are thrown down as a whitish precipitate.

For microscopical purposes, mix blood with an equal volume of a concentrated solution of sodic sulphate, and cautiously add a 1 per cent. solution of tartaric acid.

The following reagents cause a separation of the stroma from the hrmoglobin, and thus make blood transparent:-

(a) Physical Agents. - 1. Heating the blood to $60^{\circ}$ C. (Schultse); the temperature, however, varies for the blood of different animals. 2. Repeated freezing and thawing of the bloor (Rollett). 3. Sparks from an electrical machine (but not after the addition of salts to the blood) (Rollett) ; the constant and induced currents (Neumann).

(b) Chemically active Substances produced within the Body.--4. Bile (Hünefeld), or bile salts (Plattner, v. Dusch). 5. Serum of other species of animals (Landois); thus dog's serum and frog's serum dissolve the blood-corpuscles of the rabbit in a few minutes. 6. The addition of lake-coloured blood of many species of animals (Landois).

(c) Other Chemical Reagents.-7. Water. 8. The vapour of chloroform (Böttcher); ether ( $v$. Wittich); amyls, small quantities of alcohol (Rollett); thymol (Marchand); nitrobenzol, ethylic ether, aceton, petroleum ether, \&c. (L. Levin). 9. Antimoniuretted hydrogen, arseniuretted hydrogen; carbon bisulphide; boraric acid ( 2 per ceut.), added to amphilian blood, causes the rerl mass (which also encloses the nucleus when such is present), the so-called 
zooid, to seprarate from the œcoid (fig. $7, d$ ). The zooid may shrink from the periphery of the corpuscle, or it may pass out of the corpuscle altogether (Brücke) ; Briicke regarls the stroma in a certain sense as a house, in which the remainder of the substance of the corpuscle, the chief part endowed with vital phenomena, lives. 11. Strong solutions of acids dissolve the corpuscles; more dilute solutions cause precipitates in the hæmoglobin. This is easily seen with carbolic acid (Hüls and Landois, Stirling and Rannie). 12. Alkalies of moderate strength cause sudden solution. A 10 per cent. solution of potash, placed at the edge of a coverglass, shows the process of solution going on under the microscope. At first the corpuscles become globular, and so appear smaller, but afterwards they burst like soap-bubbles. [13. $\mathrm{NH}_{4} \mathrm{Cl}$ injected into the blood causes vacuolation of the red corpuscles (Bobritiky). 14. Soclic salicylate, benzoate, and colchicin, dissolve the red corpuscles (N. Paton).]

[Tannic Acid. - A freshly prepared solution of tannic acid has a remarkable effect on the coloured blood-corpuscles of man and animals-causing a separation of the hemoglobin from the stroma $(W$. Roberts $)$. The usual effect is to produce one or more granular buds of hæmoglobin on the side of the corpuscles (fig. $7, b, c)$; more rarely the hremoglobin collects arounil the nucleus, if such be present (fig. $7, d$ ), or is extruded, as shown in fig. $7, e$.]

[Ammonium or Potassium Sulpho-cyanide removes the hrmoglobin, and reveals a reticular structure-intra-nuclear plexus of fibrils (Stirling and Rannie).]

The Amount of Gases in the blood exercises an important influence on their solubility. The corpuseles of venous blood, which contains much $\mathrm{CO}_{2}$, are more easily dissolved than those of arterial blood; while between both stands blood containing $\mathrm{CO}$. When the gases are completely removed from the blood, it becomes lake-coloured.

Salts increase the resistance of the corpuscles to physical means of solution, while they facilitate the action of chemical solvents.

If certain salts be aulded in substance to blood, they make blood lake-coloured; potassic sulphocyanide, sodic ehloride, \&c. (Kowalewsky).

Resistance to Solvents.-The red blood-corpuscles offer a certain degree of resistance to the action of solvents,

Method.-Mix a small drop of blood with an equal volume of a 3 per cent. solution of sodic chloride, and then add distilled water until all the coloured corpuscles are dissolved. Fill the mixer (fig. 3) up to the mark 1 with blood obtained by pricking the finger, and blow this bloor into an equal volume of a 3 per cent. solution of $\mathrm{NaCl}$ previously placed in a hollow in a glass slide. Mix the fluids, and the corpuscles will remain undissolved. By means of the pipette add distilled water, and go on doing so until all the corpuscles are dissolved ; which is ascertainecl with the microscope. In normal blood, solution of the corpuseles occurs after 30 volumes of distilled water have been added to the blood (Landois).

There are some individuals whose blood is more soluble than that of others; their corpuscles are soft, and readily undergo changes. Many conditions, such as cholæmia, poisoning with substances which dissolve the corpuscles, and a markedly venous condition of the blood, affect the corpuscles. Interesting observations may be made on the blood in infectious diseases, hæmoglobinuria, and in cases of burning. In anæmia and fever, the capacity for resistance seems to be diminished.

6. FORM AND SIZE OF THE BLOOD-CORPUSCLES OF ANIMALS.-All mammals (with the exception of the camel, llama, alpaca, and their allies), and the cyclostomata amongst fishes, e.g., Petromyzon, possess circular bi-concave nonnucleated disc-shaped corpuscles. Elliptical corpuscles without a nucleus are found in the above-named mammals, while all birds, reptiles, amphibians (fig. 1, B, 1, 2), and fishes (except cyclostomata) have nucleated elliptical bi-convex corpuscles.

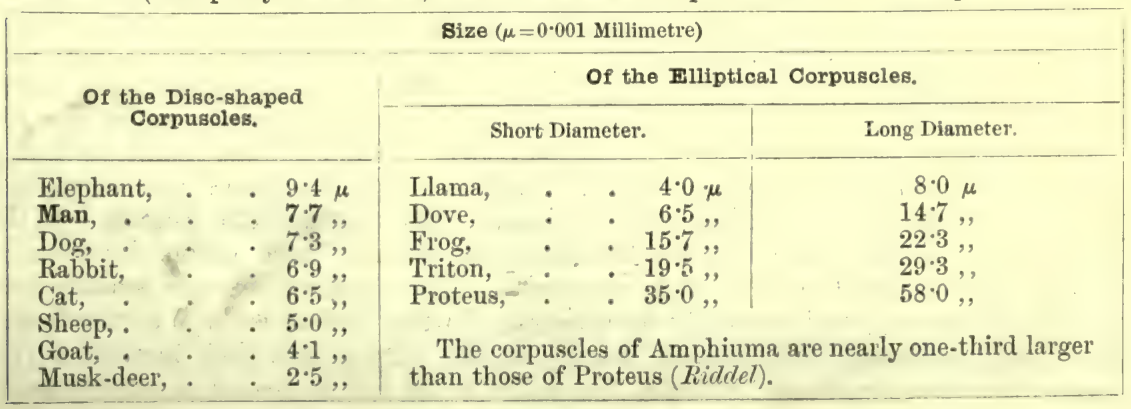


Amongst vertebrates, amphioxus has colourless blood. The large blood-corpuscles of many amphibia, e.g., amphiuma, are visible to the naked eye. The blood-corpuscles of the frog contain, in addition to a nucleus, a nucleolus (Auerbach, Ranvier), [and the same is true of the coloured corpuscles of the newt (Stirling). The nucleolus is revealed by acting on the corpuseles with dilute alcohol (1, alcohol ; 2, water ; Ranvier's "alcool au tiers" (fig. 7, g). ] It is evident that the larger the blood-corpuscles are, the smaller must be the number and total superticies of the corpuscles in a given volume of blood. In birds, however, the number is relatively larger than in other classes of vertebrates, notwithstanding the larger size of their corpuseles; this, doubtless, has a relation to the very energetic metabolism that takes place in birds (Mulusses). Amongst mammals, carnivora have more blood-corpuscles than herbivora. (ioat's blood contains 9.720,000 corpuseles per cubic millimetre ; llama's, 13,000,000; bullfinch's, 3,600,000; lizard's, 1,420,000 ; frog's, 404,000 ; and that of proteus, 36,000 (Welcker). In hybernating animals, the number diminishes from $\bar{\tau}, 000,000$ to $2,000,000$ per cubic millimetre. No relation exists between the size of the animal and that of its bloodcorpuscles.

The invertebrata generally have colomless blood, with colourless corpuscles; but the earthworm, and the larva of the large gnats, \&e., have red blood whose plasma contains hæmoglobin, while the blood-corjuscles themselves are colourless. Many invertebrates possess red, violet, brown, or green opalescent blood with colourless corpuscles (amoboid cells). In cephalopods, and some crabs, the blood is blue, owing to the presence of a colouring matter (hæmocyanin), which contains copper, and combines with $\mathrm{O}$.

\section{ORIGIN OF THE RED BLOOD-CORPUSCLES.-(A) During Embryonic}

Life.-Blood-corpuscles are developed in the fowl during the first days of embryonic life. [They appear in groujs within the large branched cells of the mesoblast, in the vascular area of the blastoderm outside the developing body of the chick, where they form the "blood-islands" of Pander. The mother-cells form an irregular network by the union of the processes of adjoining cells, and meantime the central masses split up, and the nuclei multiply. The small nucleated masses of protoplasm, which represent the blood-corpuscles, acquire a reddish hue, while the surrounding protoplasm, and also that of the processes, becomes vacuolated or hollowed out, constituting a branching system of canals ; the outer part of the cells remaining with their nuclei to form the walls of the future blood-vessels. A fluid appears within this system of branched canals in which the corpuscles lie, and gradually a communication is established with the blood-vessels developed in connection with the heart. According to Klein, the nuclei of the protoplasmic wall also proliferate, and give rise to new cells, which are washed away to form blood-corpuscles.] At first the corpuscles exhibit amceboid movements, are devoid of pigment, nucleated, ylobular, larger, and more irregular than the permanent corpuscles. They becorne. coloured, retain their nucleus, and are capable of undergoing multiplication by division; Remak observed all the stages of the process of division, which is best seen from the 3 rd to the 5 th day of incubation. Increase by division also takes place in the larve of the salamander, triton, and toad (Flemming); and during the intrauterine life of a mammal, in the spleen, bone-marrow, the liver, and the circulating blood (Bizzozero).

Neumann found in the liver of the embryo protoplasmic cells containing red bloodcorpuscles. Cells, some with, others without, hæmoglobin, but with large nuclei, have been found. These cells increase by division, their nucleus shrivels, and they ultimately form blood-corpuscles (Löwit). The spleen is also regarded as a centre of their formation, but this seems to be the case only during embryonic life (Neumann). Here the red corpuscles are said to arise from yellow, round, nucleated cells, which represent transition forms. Foa and Salvioli found red corpuscles forming endogenously within large protoplasmic cells in lymphatic glands. In the later period of embryonic life, the characteristic non-nucleated corpuscles seem to be developed from the nucleated corpuscles. The nucleus becomes smaller and smaller, breaks up, and gradually disappears. In the human embryo at the fourth week, only nucleated corpuscles are found; at the third month their number is still $1-\frac{1}{8}$ of the total corpuscles, while at the end of fotal life nucleated blood-corpuscles 
are very rarely found. Of course, in animals with nucleated blood-corpuscles, the nucleus of the embryonic blood-corpuscles remains.

(B) During Post-Embryonic Life.--Kölliker assumed that in the tail of the tadpole capillaries are formed by the anastomoses of the processes of branched and radiating connective-tissue corpuscles. These corpuscles lose their nuclei and protoplasm, become hollowed out, join with neighbouring capillaries, and thus form new bloodchannels. J. Arnold and Golubew oppose this view, asserting that the bloodcapillaries in the tail of the tadpole give off solid buds at different places, which grow more and more into the surrounding tissues, and anastomose with each other ; after their protoplasm and contents disappear they become hollow, and a branched system of capillaries is formed in the tissues. Ranvier noticed the same mode of growth in the omentum of newly-born kittens.

Young rabbits, a week old, have, in their omentum, small white or milk spots (Ranvier), in which lie "vaso-formative cells," i.e., highly refractive cells of variable shape, with long cylindrical protoplasmic processes (fig. 8). In its refractive power the protoplasm of these cells resembles that of lymphcorpuscles. Long rod-like nuclei lie within these cells $(\mathrm{K}, \mathrm{K})$, and also red blood-corpuscles $(r, r)$, and both are surrounded with protoplasm. These vaso-formative cells give off protoplasmic processes $(a, a)$, some of which end free, while others form a network. Here and there elongated connective-tissue corpuscles lie on the branches, and ultimately form the adventitia of the blood-vessel. The vaso-formative cells have many forms : they may be elongated cylinders ending in points, or more round and oval, resembling lymph cells, or modified connective-tissue corpuscles. These cells are always the sert of origin of nonnucleated red blood-corpuscles, which arise in the protoplasm of vaso-formative cells, as chlorophyll grains or starch granules arise within the cells of plants. The corpuscles escape, and are washed into the circulation when the cells, by means of their processes, form connections with the circulatory system. Probably the vessels so formed in the omentum are only temporary. May it not be that there are many other situations in the body where blood is regenerated?

[The observations of Schäfer also prove the intra-cellular origin of red blood. corpuscles, and although this mode usually ceases before birth, still it is found in the rat at birth. The protoplasm of the subcutaneous connective-tissue corpuscles, which are derived from the mesoblast, has in it small coloured globules about the size of a coloured corpuscle. The mother-cells elongate, become pointed at their ends, and unite with processes from adjoining cells. The cells become vacuolated; fluid or plasma, in which the liberated corpuscles float, appears in their interior, and ultimately a communication is established with the general circulation.]

Neumann observed similar formations in the embryonic liver; Wissotzky in the rabbit's amnion ; Klein in the embryo chick; and Bayerl in ossifying cartilage. All these observations go to show that at a certain early period of development blood-corpuscles are formed within other large cells of the mesoblast, and that part of the protoplasm of these blood-forming cells remains to form the wall of the future blood-vessel. 
(C) Later Formation.-Most observers agree that the red blood-corpuscles are formed from special nucleated cells, which gradually assume the form and colour of the perfect red corpuscle. According to Neumann, however, these corpuscles are pigmented from the first. In the tailed amphibians and fishes, the spleen, in all other vertebrates the red marrow of bone, are the seats of formation of these corpuscles, which subsequently increase by division (Neumann, Rinclfteisch. Bissusero). In the red marrow of bone we can study all the stages of the transformation; especially pale contractile cells similar to colourless corpuscles, and also red nucleated corpuscles, which are similar to the nucleated corpuscles of the embryo, and the progenitors of the red corpuscles. These transition cells are said by Erb to be more numerous after severe hirmorrhace, the number of them occurring in the blood corresponding with the energy of the formative process. After copious hrmorrhage, these transition forms appear in numbers in the blood-stream. The small veins, and, perhajs, the capillaries of the red marrow of bone and the spleen have no proper walls, so that the red corpuscles when formed can pass into the circulation.

Red or blood-forming marrow occurs in the bones of the skull, and in most of the bones of the trunk, while the bones of the extremities either contain yellow marrow (which is essentially fatty in its nature), or, at most, it is only the heads of the long bones that contain red marrow. Where the blood-regeneration process is very active, however, the yellow marrow may be changed into red, even throughout all the bones of the extremities (Neumanu).

8. DECAY OF THE RED BLOOD-CORPUSCLES-The blood-corpuscles undergo decay within a limited time, and the liver is regarded as one of the chief places in which their disintegration occurs, because bile-pigments are formed from hemoglobin, and the blood of the hepatic vein contains fewer red corpuscles than the portal vein.

The splenic pulp contains cells which indicate that coloured corpuscles are lroken up within it. These are the so-called "blood-corpuscle-containing cells" (\$102). Quincke's olservations go to show that the red corpuscles-which may live from three to four weeks - when about to disintegrate, are taken up by the white blood-corpuscles in the hepatic capillaries, by the cells of the spleen and the bonemarrow, and are stored up chiefly in the capillaries of the liver, in the spleen, and in the marrow of bone. They are transformed, partly into coloured, and partly into colourless proteids which contain iron, and are either deposited in a granular form, or are dissolved. Part of the products of decomposition is used for the formation of new blood-corpuscles in the marrow and in the spleen, and also. perhaps in the liver, while a portion of the iron is excreted by the liver in the bile.

That the normal red blood-crifuscles and other particles suspended in the blood-stream are not taken up in this way, may be due to their being smooth and polished. As the corpuscles grow older and become more rigid, they, as it were, are caught by the amceboid cells. As cells containing blool-corpuscles are very rarely found in the general circulation, one nay assume that the occurrence of these cells within the spleen, liver, and marrow of bone is favoured by the slowness of the circulation in these organs (Quincke).

Pathological.- In certain pathological conditions, ferruginous substances derived from the red bloot-rorpuseles are found in masses in the spleen, the marrow of bone, and the capillaries of the liver:-(1) When the disintegration of blool-corpuseles is increased, as in ancmia (Stahel). (2) When the formation of red blood-corpuscles from the old material is diminished. If the excretion from the liver cells be prevented, iron aceumulates within them ; it is also more abundant in the blcod-serum, and it may even accumulate in the secretory cells of the cortex of the kidney and pancreas, in glanil cells, and in the tissue elements of other organs. When the amount of blool in dogs is greatly increased, after four weeks an enormous number of granules containing iron occur in the leucocytes of the liver capillaries, the cells of the spleen, bone-marrow, lymph-glanils, liver cells, and the epithelium of the cortex of the kidney. The iron reaction in the last two situations occurs after the introduction of hrmoglobin, or of salts of iron into the blood (Glaeveck, v. Stark.)

When we reflect how rapidly large quantities of blood are replaced after hamorrhage and after menstruation, it is evident that there must be a brisk manu- 
factory somewhere. As to the number of corpuscles which daily decay, we have in some measure an index in the amount of bile-pigment and urine-pigment resulting from the transformation of the liberated hæemoglobin ( $\$ 20)$.

\section{COLOURLESS CORPUSCLES, BLOOD-PLATES, AND GRANULES.}

White Blood-Corpuscles.-Blood, like many other tissues, contains a number of cells or corpuscles which reach it from without; the corpuscles vary somewhat in form, and are called colourless or white blood-corpuscles, or "leucocytes" (Hewson, 1770). Similar corpuscles are found in lymph, adenoid tissue, marrow of bone, and as wandering cells or leucocytes in connective-tissue, and also between glandular and epithelial cells. So that these corpuscles are by no means peculiar to blood alone. They all consist of more or less spherical masses of protoplasm, which is sticky, highly refractile, soft, capable of movement, and devoid of an envelope (fig. 9). When they are quite fresh (A) it is difficult to detect the nucleus, but after they have been shed for some time, or after the addition of water (B), or acetic acid, the uucleus (which is usually a compound one) appears ; acetic acid clears up the perinuclear protoplasm, and reveals the presence of the nuclei, of which the number varies from one to four, although generally three are found. The subsequent addition of magenta solution causes the nuclei to stain deeply. Water makes the contents more turbid, and causes the corpuscles to swell up. One or more nucleoli may be present in the nucleus. The size of the corpuscles varies from 4-13 $\mu$, and as a rule they are about $\frac{1}{2500}$ of an inch in diameter; in the smallest forms the layer of the protoplasm is extremely thin. They all exhibit amœboid movements which are very apparent in the larger corpuscles, and were discovered by Wharton Jones in
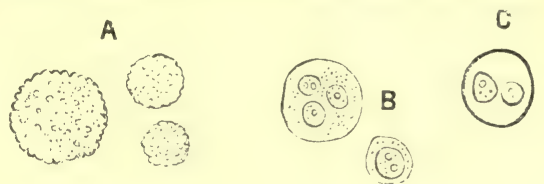

D

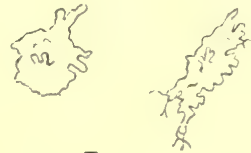

$E$

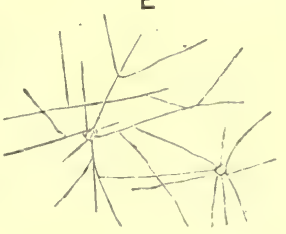

Fig. 9.

A, human white blood-corjuseles, without any reagent ; $\mathrm{B}$, after the action of water; C, after acetic acid ; D, frog's corpuscles, changes of shape due to amceboid movement; $\mathrm{E}$, fibrils of fibrin from coagulated blood; F, elementary granules.

the skate (1846), and by Davine in the corpuscles of man (1850). Max Schultze describes three different forms in human blood :

(1) The smallest, spherical forms, less than the red corpuscles, with one or two nuclei, and a very small amount of protoplasm.

(2) Spherical forms, the same size as the coloured blood-corpuscles.

(3) The large amoboid corpuscles, with much protoplasm and distinctly evident movements.

[On examining human blood microscopically, more especially after the coloured blood-corpuscles have run into rouleaux, the colourless corpuscles may readily be detected, there being usually three or four of them visible in the field at once. They adhere to the glass slide, for if the cover-glass be moved, the coloured corpuscles readily glide over each other, while the colourless can be seen still adhering to the'slide.]

[White Corpuscles of Newt's Blood.-The characters of the colourless corpuscles are best studied in a drop of newt's blood, which contains the following varieties :-

(1) The large finely granular corpuscle, which is about $\frac{1}{1} \frac{1}{00}$ of an inch in liameter, irregular in outline, with fine processes or pseuclopodia, projecting from its surface. It rapidly changes 
its shape at the ordinary temperature, and in its interior a bi- or tri-partite uncleus may be seen, surrounded with fine granular protoplasm, whose outline is continually ehanging. Sometimes vocuotes are seen in the protoplasm.

(2) The coarsely granular variety is less conmmon than the first-mentioned, but when detected its characters are distinct. The protoplasm contains, besides a nucleus, a large number of highly refractive granules, and the corpuscle usually exhibits active amoboid novements; suddenly the granules may be seen to rush from one side of the corpuscle to the other. The processes are usually more blunt than those emitted by (1). The relation between these two kinds of corpuscles has not been ascertained.

(3) The small colourless corpuscles are more like the ordinary human colourless corpuscle, and they, too, exhibit amœboid movements.]

Two kinds of colourless corpuseles like (1) and (2) exist in frog's blood. In the coarsely granular corpuscles the glancing granules may be of a fatty nature, since they dissolve in alcohol and ether, but other granules exist which are insoluble in these fluids. The nature of the latter is unknown. Very large colourless corpuscles exist in the axolotl's blood.

[Action of Reagents.-( ( 1 ) Water, when added slowly, causes the colourless corpuscles to become globular, and the granules within them to exhibit Brownian movements. (l, Pigments, such as magenta or carmine, stain the nuclei very deeply, and the protoplasm to a less extent. (c) Dilute Acetic Acid clears up the surrounding protoplasm and brings clearly into view the composite nucleus, which may be stained thereafter with magenta. (d) Iodine gives a faint port-wine colour, especially in horse's blood, indicating the presence of glycogen. (e) Dilute Alcohol

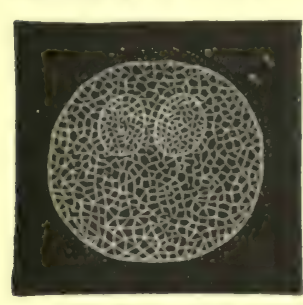

Fig. 10.

Plexus of fibrils in a colourless bloot-corjuscle. causes the formation of clear blebs on the surface of the corpuscles, and brings the nuclei into view (Ranvier, Stirling).] [A delicate plexus of fibrils-intra-nuclear plexus-exists within the nucleus just as in other cells. It is very probable that the protoplasm itself is pervaded by a similar plexus of fibrils, and that it is continuous with the intranuclear plexus (fig. 10).] The colourless corpuscles divide, and in this way reproduce themselves.

The Number of Colourless Corpuscles is very much less than that of the red corpuscles, and is subject to considerable variations. It is certain that the colourless corpuscles are very much fewer in shed blood than in blood still within the circulation. Immediately after blood is shed, an enormous number of white corpuscles disappear (\$31).

Al. Schmilt estimates the number that remain at $\frac{1}{10}$ of the whole originally present in the circulating blood. The proportion is greater in children than in adults. The following table gives the number in shed blood :-

\begin{tabular}{|c|c|c|}
\hline \multicolumn{3}{|c|}{ Nlubeit of Wuite is Proportion to Red Blood-Cohplscles- } \\
\hline In Normul Cunditions. & In I ifferent Places. & In Different Conditions. \\
\hline $\begin{array}{l}1: 335 \text { (Welcher). } \\
1: 357 \text { (Moleschott). }\end{array}$ & $\begin{array}{l}\text { Splenic Vein, } 1: 60 \\
\text { Splenic Artery, } 1: 2,260 \\
\text { Hepatic Vein, } 1: 170 \\
\text { Portal Vein, } 1: 740 \\
\text { Generally more numerous } \\
\quad \text { in Veins than Arteries. }\end{array}$ & $\begin{array}{l}\text { Increased by Digestion, Loss } \\
\text { of Blood, Prolonged Sup- } \\
\text { puration, Parturition, Leuk- } \\
\text { amia, Quinine, Bitters. } \\
\text { Diminished by Hunger, Bad } \\
\text { Nourishment. }\end{array}$ \\
\hline
\end{tabular}

[The number also varies with the Age and Sex :--

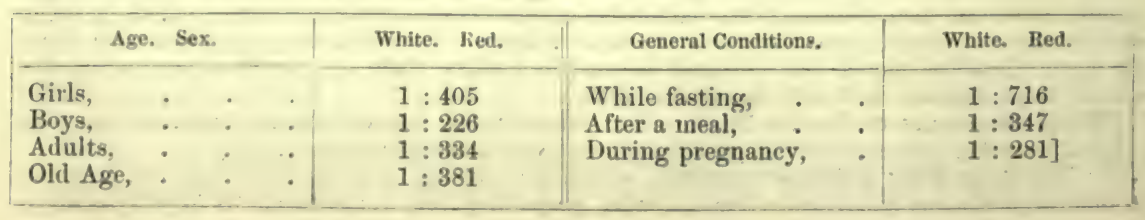


The amœboid movements of the white corpuscles (so called because they resemble the movements of amœba) consist in an alternate contraction and relaxation of the protoplasm surrounding the nucleus. Processes are given off from the surface, and are retracted again. There is an interial current in the protoplasm, and the nucleus has also been observed to change its form [and exhibit contractions without the corpuscle dividing. The karyokinetic aster, and convolution of the intranuclear plexus have been seen]. Two series of phenomena result from these movements :-(1) The "wandering" or locomotion of the corpuscles due to the extension and retraction of their processes ; (2) the absorption of small particles into their interior (fat, pigment, foreign bodiess). The particles adhere to the sticky external surface, are carried into the interior by the internal currents, and may eventu. ally be excreted, just as particles are taken up by amœba and the effete particles excreted. [Max Schultze observed that coloured particles were readily taken up by these corpuscles. Conditions for move-

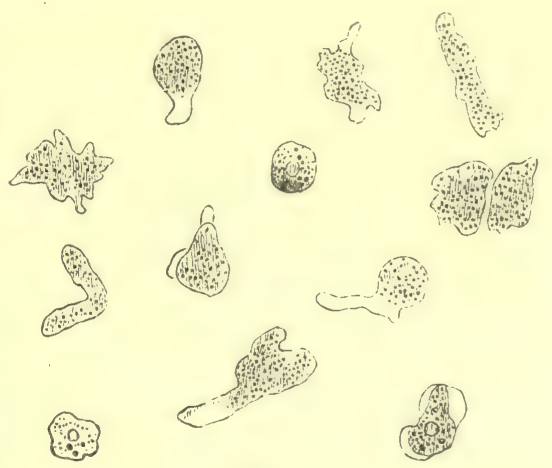

Fig. 11.

Human leucocytes showing amceboid movements.

ment.-In order that the amoeboid movements of the leucocytes may take place, it is necessary that there be-(1) a certain temperature and normal atmospheric pressure ; (2) the surrounding medium, within certain limits, must be "indifferent," and contain a sufficient amount of water and oxygen ; (3) there must be a basis or support to move on.]

Struggle between Microbes and the Organism. - Metschnikoff emphasises the activity of the leucocytes in retrogressive processes, whereby the parts to be removed are taken up by them in fine granules, and, as it were, are "caten." Hence, he calls such cells "phagocytes." They may be found in the atrophied tails of batrachians, the cells containing in their interior whole pieces of nerve-fibre and primitive muscular bundles. Schizomycetes which have fouml their way into the blood ( $\$ 183$ ) have been found to be partly taken up by the colourless corpuscles. [The spores of a kind of yeast are similarly attacked in the transprarent tissues of the waterflea by the leucocytes, and the connective-tissue cells also destroy microbes.]

Effect of Reagents.-On a hot stage $\left(35^{\circ}-40^{\circ}\right.$ C. $)$ the colourless corpuscles of warm-blooded animals retain their movements for a long time ; at $40^{\circ} \mathrm{C}$. for two to three hours ; at $50^{\circ} \mathrm{C}$. the proteids are coagulated and cause "heat rigor" and death, [when their movements no longer recur on lowering the temperature]. In cold-blooded animals (frogs), colourless corpuscles may be seen to crawl out of small coagula, in a moist chamber, and move about in the serum. [Draw a drop of newt's blood into a capillary tube, seal up the ends of the latter and allow the blood to coagulate. After a time, examine the tube in clove oil, when some of the colourless corpuscles will be found to have made their way out of the clot.] Induction shocks cause them to withdraw their processes and become spherical, and, if the shocks be not too strong, their movements recommence. Strong and continued shocks kill them, causing them to swell up, and completely disintegrating them.

Diapedesis.-These amoboid movements are of special interest on account of the "wandering out" (diapedesis) of colourless blood-corpuscles through the walls of the blood-vessels ( $(95)$.

[Effect of Drugs. - Acids and alkalies, if very dilute, at first increase, but afterwards arrest their movements. Sodic chloride in a 1 per cent. solution at first accelerates their movements, but afterwards produces a tetanic contraction, and, it may be, expulsion of any food particles they contain. 'The Cinchona alkaloids-quinine, quinidine, cinchonidine $(1: 1500)$-quickly 
arrest the locomotive movements, as well as the protrusion of pseudopoulia, although the leurnytes of different animals vary somewhat in their resistance to the action of drugs. Quinine not only arrests the movements of the lencocytes when applied to them directly, but when injeeted into the cireulation of a frog the lencocytes no longer pass through the walls of the capillaries (Віяz).

The chyle contains leucorytes, which are more resistant than thos of the blood, but less so than theise of the coarulable transudations. The lencocytes of the lymphatic glamels may also be clissolved (Ranschenbuch).

Relation to Aniline Pigments. - Fihrlich las observed a remarkable relation of the white corpuscles to wid (eosin, picric arill, aurantia), lussic (dahlia, acetate of rosanilin), or neutral (ficeate of rosanilin reactions. The smallest protoplasmic gramules of the cells have different chemical affinities for these pigments. Thus kihrlich listinguishes " eosinophile," "basophile," and "n-utrophile" gramules within the cells Eosinophile granules oceur in the leucoertes which come from bone-marrow. the myelogenie leurocytes. The small leucocytes, i.c., those about the size of a coloume. blowel-orpuscle or slightly larger, are formed in the lymphatic

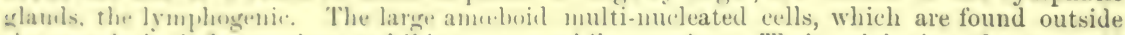
the ressels in inflammations, exhil,it a neutrophile reation. Their origin is unknown, and $\cdots$ is that of the large uni-nmileated cells, and the large cells with ronstrieted nuelei. The "usinophile compuscles are considerahly increased in leukitmia. The besophete granules occur Also in comertive-tissue corpuscles, "sperially in the neighbourhood of epithelium; they are always greatly increased where chronic inflammation occurs.

1II. Blood-Plates.-... - inecial attention lias recently been directed to a third "lement of the Liood, the "blood-plates" or "blood-tablets" of Bizzozero; pale, colourles, oval, roumd, or lenticular discs of variable size (mean, $3 \mu$ ). In a healthy man Fusari found 18,000 to 250,000 in 1 cubic millimetre of blood. These blood-plates may le reconnised in the circulating blood of the mesentery of a
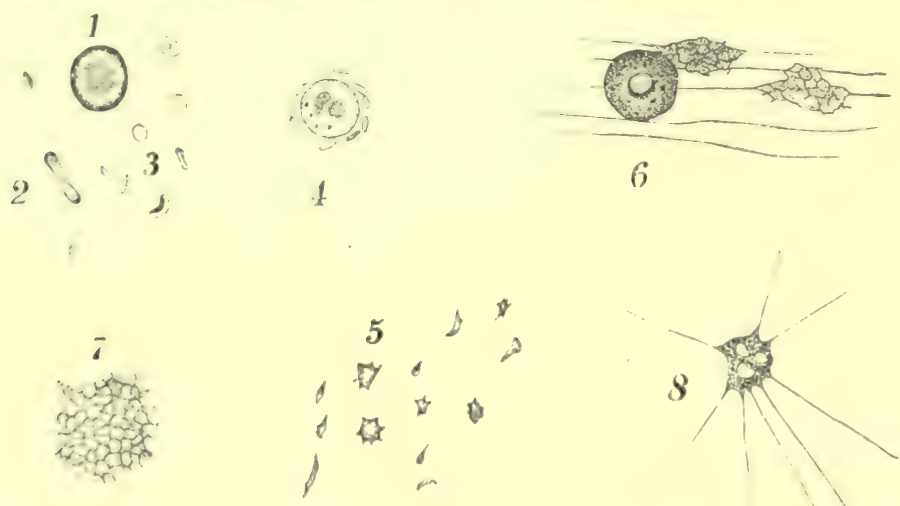

Fig. 12.

- Blood-plates" and their lerivatives. 1 , a real blood-"orpuscle on the flat; 2 , on the side; 3 , unchanged blood-plates ; 4, lymph-corpuscle, surrounded by blood-plates ; 5 , altered blood-

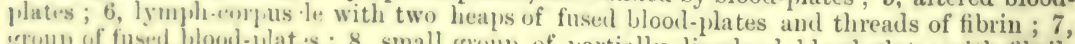
group of fused l, lood-plat:s ; 8 , small group of prartially dissolved blood-plates with fibrils
of fibrin.

chloralised guinea-pig and the wing of the bat. They are precipitated in enormous numbers upon threads susi en led in fresh shed blood. They nay be obtained from blood flowing directly from a blood-vessel, on mixing it with 1 per cent. solution of osmic acid. They rapidly change in shed blood (fig. 12, 5), disintegrating, forming small particles, and ultimately dissolving. When several occur together they rapidly unite, form small groups $(7)$, and collect into finely granular masses. These masses may be associated in coagulated blood with fibrils of fibrin (fig. 12).

[These blowi-plates are best seen in the shed blood of the guinea-pig, especially if it be mixed with a solution of sodic sulphate (sl). gr. 1022) or i jer cent. NaCl tinged with methylviolet. Bizzozero regards them as the agents which immediately iuluce coagulation and take 
part in the formation of fibrin during coagulation of the blood ; Eberth and Schimmelbusch ascribe the initial formation of white thrombi to them. According to Löwit they are formed from partially disintegrated leucocytes, as a consequence of alteration of the blood. Along with the leucosytes they are concerned in the formation of fibrin (Hlava). These structures were known to earlier observers ; but their significance has been variously interpreted. Hayem called them hæmatoblasts. Halla found that they increased in pregnancy, Afanassiew in conditions of regeneration of the blood, and Fusari in febrile anæmia; they are diminished in fever.

[As to the hæmatoblasts, or, as they have also besn called, the "globules of Donné" by Pouchet, there seems to be some confusion, for both coloured and colourless granules are described under these names. As Gibson suggests, the former are, perhaps, parts of disintegrated coloured corpuscles, whilst the latter are the blood-plates. The "invisible blood-corpuscles" described by Norris seem to be simply decolorised red corpuscles (Hart, Gibson).]

IV. Elementary Granules.--Blood contains elementary granules (fig. 9, F), [i.e., the elementary particles of Zimmermann and Beale. They are irregular bodies, much smaller than the ordinary corpuscles, and appear to consist of masses of protoplasm detached from the surface of leucocytes, or derived from the disintegration of these corpuscles, or of the blood-plates. Others, again, are completely spherical granules, either consisting of some proteid substance or fatty in their nature. The protoplasmic and the proteid granules disappear on the addition of acetic acid, while the fatty granules (which are most numerous after a diet rich in fats) dissolve in ether].

V. In coagulated blood, delicate threads of fibrin (figs. 9, E, and 12, 6, 7, 8) are seen, more especially after the corpuscles have run into rouleaux. At the nodes of these fibres are found granules which closely resemble those described under III.

[When the blood-forming process is particularly active, "nucleated coloured corpuscles" or the "corpuscles of Neumann," are sometimes found in the blood. They are identical with the nucleated coloured blool-corpuscles of the foetus, being somewhat larger than the nonnucleated coloured corpuscle $(\S 7)$.]

10. ABNORMAL CHANGES OF THE BLOOD-CORPUSCLES. - (1) Hæmorrhages diminish the number of red corpuseles (at most one-half), and so does menstruation. The loss is partly . covered by the absorption of fluid from the tissues. Menstruation shows us that a moderate loss of red corpuscles is replaced within twenty-eight days. When a large amount of blood is lost, so that all the vital processes are lowered, the time may be extended to five weeks. In acute fevers, as the temperature increases, the number of red corpuscles diminisines, while the white corpuscles increase in number. By greatly cooling peripheral parts of the body, as by keeping the hands in iced water, in some individuals possessing red blood-corpuscles of low resisting power, these corpuscles are dissolved, the blood-plasma is reddened, and even hæmoglobinuria may occur ( $\$ 265)$.

Diminished production of new red corpuscles causes a decrease, since blood-corpuscles are continually being used up. In chlorotic females there seems to be a congenital weakness in the blood-forming and blood-propelling apparatus, the cause of which is to be sought for in some faulty condition of the mesoblast. In them the heart and the blood-vessels are small, and the absolute number of corpuseles may be dininished one-half, although the relative number may be retained, while in the corpuscles themselves the hrmoglobin is diminished almost onethird ; but it rises again after the administration of iron (Hayem). The administration of iron increases the amount of hæmoglobin in the blood. [The action of iron in anæmic persons has been known since the time of Sydenham. Hayem also finds in certain forms of anæmia that there is considerable variation in the size of the red corpuscles, and that in chronic anæmia the mean diameter of the corpuseles is always less than normal $(7 \mu$ to $6 \mu)$. There is, moreover, a persistent alteration in the volume, colouring power, and consistence of the corpuseles, consequently a want of accord between the number of the corpuscles and their colouring power, i.e., the amount of hæmoglobiu which they contain. In pernicious anæmia, in which the continued decrease in the red corpuscles may ultimately produce death, there is undoubtedly a severe affection of the blood-forming apparatus. The corpuscles assume many abnormal and bizarre forms, often being oval or tailed, irregularly shaped, and sometimes very pale; while numerous cells containing blood-corpuscles are found in the marrow of bone. In this disease, although the red blood-corpuscles are diminished in number, some may be larger and contain more hæmoglobin than normal corpuseles. The number of coloured corpuscles is also diminished in chronic poisoning by lead or miasmata, and also by the poison of syphilis.

(2) The size of the corpuscles varies in disease from $2 \cdot 9-12 \cdot 9 \mu$ (mean 6-8 $\mu$ ); " $d$ warf corpuscles" or microcytes ( $6 \mu$ and less) are regarded as young forms, and occur plentifully in 
nearly all cases of anamia. "Giant blood-corpuscles" or macrocytes (10 $\mu$ and more) are constant in pernicious anamia, and sometimes in leukiemia, chlorosis, and liver cirrhosis (Gram).

(3) Abnormal forms of the red corpuscles have been observed after severe burns (Lesser); the corpuscles are inuch smaller, and under the influence of the heat, prarticles seem to be detached from them just as can be seen happening under the microscope as the effect of heat. Disintegration of the corpuscles into fine droplets has been observed in various diseases, as in severe malarial fevers. The dark granules of a pigment closely relatel to hematin are derived from the granules arising from the disintegration of the blood-corpuscles, and these particles float in the f.lool melanmmia. This condition ean he produced artificially by injecting bisulphide of

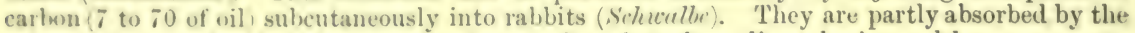
colourless corpuscles, but they are also deposited in the spleen, liver, brain, and bone-marrow.

14 Sometimes the red corpuscles are abnomally soft, and readily yield to pressure.

Parasites of Blood-Corpuscles. - Within the red blood-corpuscles of birds, fishes, and tortoises, parasites are occasionally developed in the form of round "pseudo-vacuoles" from which free parasites are subseguently discharged (Inonilmesky). In malarial conditions in man, protozoonlike organisms have been seen within the red corpuseles, the plasmodium malaria (Marchiutacul).

The white corpuscles are enormously increased in number in leukrmia ( $J$. $H$. Bennett, birhurl. In some case's the hlood looks as if it were mixed with milk. The colourless corpuscles seem to be formed chiefly in bone-marrow ( $E$. Neumann), and also in the spleen and ymphatic glands (myelogenic, splenic, and lymphatic leukrmia).

\section{CHEMICAL CONSTITUENTS OF THE RED BLOOD-CORPUSCLES.-} (1) The colouring matter or hæmoglobin $(\mathrm{Hb})$ is the cause of the red colour of blood; it also occurs in muscle, and in traces in the fluid part of blood, but in the last case only as the result of the solution of some red corpuscles. Its percentage composition is :-C $53 \cdot 85, \mathrm{H} 7 \cdot 3 \cdot 2, \mathrm{~N} 16 \cdot 17$, Fe $0.42, \mathrm{~S} 0.39$, O $21 \cdot 84$ (dog). Its rational formula is unknown, but Preyer gives the empirical formula $\mathrm{C}_{600}, \mathrm{H}_{960}$, $\mathrm{N}_{15}, \mathrm{Fe}, \mathrm{S}_{3}, \mathrm{O}_{1, \ldots}$. Although it is a colloid substance it crystallises in all classes

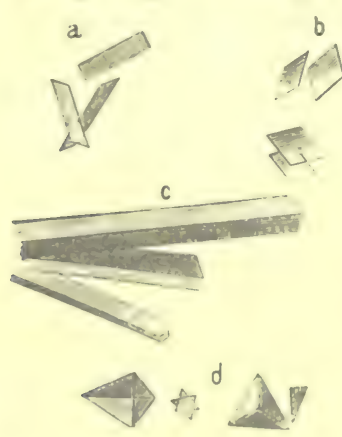
of vertebrates, according to the rhombic system, and chiefly in rhombic plates or prisms; in the gninea-pig in rhombic tetrahedra ; in the squirrel, however, it yields hexagonal plates. The varying forms, perhaps, correspond to slight differences in the chenical composition in different cases. Crystals separate from the blood of all classes of vertebrata during the slow evaporation of lakecoloured blood, but with varying facility (fig. 13).

The colouring matter erystallises very readily from the blood of man, dog, mouse, guinea-pig, rat, cat, hedgehog, horse, rabbit, birds, fishes ; with difficulty from that of the sheep,ox, and pig. Coloured crystals are not obtained from the blood of the frog. More rarely a crystal is formed from a single corpuscle enclosing the stroma. Crystals have been found near the nucleus of the large corpuseles of fishes, and in this class of vertebrates colourless erystals have been observel.

Dichroism.- Hiemoglobin crystals are doubly Fig. 13. refractive and pleo-chromatic; they are bluishHatmoglobin crystals from blood. a, red with transmitted light, scarlet-red by reflected
$b$, human ; $c$, cat; ; $d$, yuinea-pig; light. They contain from 3 to 9 per cent. water $c$, hamster ; $f$, squirrel. of crystallisation, and are soluble in water, but more so in dilute alkalies. They are insoluble in alcohol, ether, chloroform, and fats. The solutions are dichroic: red in reflected light, and green in transmitted light.

In the act of crystallisation the hæmoglobin seems to undergo some internal change. Before it crystallises it does not diffuse like a true colloid, and it also rapidly decomposes bydric peroxide. If it be relissolved after crystallisation, it diffuses, although only to a small extent, but it no longer decomposes hydric peroxide, and is decolorised by it. [The prestnce of $O$
favours crystallisation.] 
12. PREPARATION OF HAMOGLOBIN CRYSTALS. - Method of Rollett. - Put defibrinated blood in a platinum capsule placed on a freezing nixture, freeze the blood, and then thaw it; pour the lake-coloured blood into a plate, until it forms a stratum not more than $1 \frac{1}{2} \mathrm{~mm}$. in thickness, and allow it to evaporate slowly in a cool place, when crystals will separate.

Method of Hoppe-Seyler.-Mix defibrinated blood with 10 volumes of a 20 per cent. salt solution, and allow it to stand for two days. Remove the clear upper fluid with a pipette, wash the thick deposit of blood-corpuscles with water, and afterwards shake it for a long time with an equal volume of ether, which dissolves the blood-corpuscles. Remove the ether, filter the lake-coloured blood, add to it $\frac{1}{4}$ of its volume of cold alcohol $\left(0^{\circ}\right)$, and allow the mixture to stand in the cold for several days. The numerous crystals can be collected on a filter and pressed between folds of blotting-paper.

Method of Gscheidlen. - Take defibrinated blood, which has been exposed for twenty-four hours to the air, and keep it in a closed tube of narrow calibre for several days at $37^{\circ} \mathrm{C}$. When the blood is spread on glass, the crystals form rapidly. [Vaccine tubes answer very well.]

[Method of Stirling and Brito. - It is in many cases sufficient to mix a drop of blood with a few drops of water on a glass slide, and to seal up the preparation. After a few days beautiful crystals are developed. The addition of water to the blood of some animals, such as the rat and the guinea-pig, is rapidly followed by the formation of crystals of hemoglobin." Very large crystals may be obtained from the stomach of the leech several days after it has suckeil blood.]

13. QUANTITATIVE ESTIMATION OF HÆIMOGLOBIN. - $(a)$ From the Amount of Iron. As dry $\left(100^{\circ}\right.$ C. ) hæmoglobin contains 0.42 per cent. of iron, the amount of hæmoglobin may be calculated from the amount of iron. If $m$ represents the percentage arnount of metallic iron, then the percentage of hamoglobin in blood is $=\begin{gathered}100 \mathrm{~m} \\ 0.42\end{gathered}$. The procedure is the following:Calcine a weighed quantity of blood, and exhaust the ash with $\mathrm{HCl}$ to obtain ferric chloride, which is transformed into ferrous chloride. The solution is then titrated with potassic permanganate.

(b) Colorimetric Method.---Prepare a dilute watery solution of hæmoglobin exystals of a known strength. With this compare an aqueous dilution of the blood to be investigated, by adding water to it until the colour of the test solution is obtained. Of course, the solutions must be compared in vessels with parallel sides and of exactly the same width, so as to give the same thickness of fluid (Hoppe-Seyler). [In the vessel with parallel sides, or hæmatinometer, the sides are exactly 1 centimetre apart. Instead of using a standard solution of oxyhæmoglobin, a solution of picro-carminate of ammonia may be used (Rajewsky, Malassez).]

(c) By the Spectroscope. - Preyer found that a 0.8 per cent. watery solution $(1 \mathrm{~cm}$. thick), allowed the red, the yellow, and the first strip of green to be seen (fig. 17, 1). Take the blood to be investigated (about $0.5 \mathrm{c.cm}$.), and dilute it with water until it shows exactly the same optical effects in the spectroscope. If $k$ is the percentage of $\mathrm{Hb}$, which allows green to pass through $(0.8$ per cent.), $b$, the volume of blood investigated (about $0.5 \mathrm{c.cm}$.), $w$, the necessary amount of water added to dilute it, then $x=$ the percentage of $\mathrm{Hb}$ in the blood to be investi. gated-

$$
x=\frac{k(w+b)}{b}
$$
CO.

It is very convenient to add a drop of caustic potash to blood and then to saturate it with

$[(d)$ The Hæmoglobinometer of Gowers is used for the clinical estimation of hæmoglobin (fig. 14). "The tint of the dilution of a given volume of blood with distilled water is taken as the index of the amount of hæmoglobin. The distilled water rapidly dissolves out all the hæmoglobin, as is shown by the fact that the tint of the dilution undergoes no change on standing. The colour of a dilution of average normal blood one hundred times is taken as the standard. The quantity of hæmoglobin is indicated by the amount of distilled water needed to obtain the tint with the same volume of blood under examination as was taken of the standard. On account of the instability of a standard dilution of blood, tinted glycerine-jelly is employed instead. This is perfectly stable, and by means of carmine and picro-carmine the exact tint of diluted blood can be obtained. The apparatus consists of two glass tubes of exactly the same size. One contains (D) a standard of the tint of a dilution of $20 \mathrm{cubic} \mathrm{mm}$. of blood, in 2 cubic centimetres of water (1 in 100). The second tube (C) is graduated, 100 degrees $=2$ centimetres (100 times 20 cubic millimetres). The 20 cubic millimetres of blood are measured by a capillary pipette (B). This quantity of the blood to be tested is ejected into the bottom of the tube, a few drops of distilled water being first placed in the latter. The mixture is rapidly agitated to prevent the coagulation of the blood. The distilled water is then added drop by drop (from the pipette stopper of a bottle (A) supplied for that purpose), until the tint of the dilution is the same as that of the standard, and the amount of water which has been added (i.e., the degree of dilution) indicates the amount of hæmoglobin." 
" Since average normal blood yields the tint of the standard at 100 degrees of dilution, the number of degrees of dilution necessary to obtain the same tint with a given specimen of blood is the percentage proportion of the haemoglobin contained in it, compared to the normal. For

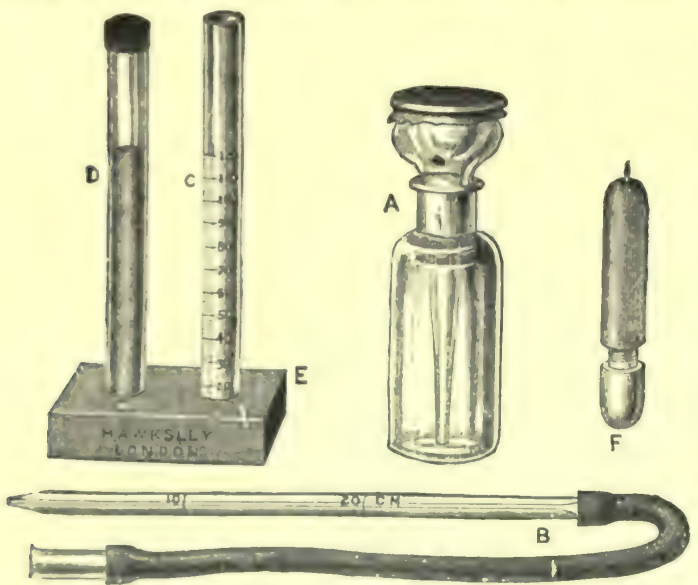

Fig. 14. instance, the 20 cubic millimetres of blood from a patient with anæmia gave the standard tint of 30 degrees of dilution. Hence it contained only 30 per cent. of the normal quantity of hæmoglobin. By ascertaining with the hæmacytometer the corpuscular richness of the blood, we are able to compare the two. A fraction, of which the numerator is the percentage of hremoglobin, and the denominator the percentage of corpuscles, gives at once the average value per corpuscle. Thus the blood mentioned above containing 30 per cent. of hæmoglobin, contained 60 per cent. of corpuscles; hence the average value of each corpuscle was $\frac{3}{6} \frac{0}{6}$ or $\frac{1}{2}$ of the normal. Variations in the amount of hæmoglobin may be recorded on the same chart as that employed for the corpuscles. The instrument is only expected to Ciwwers harmoglobinometer. A, pipette bottle for distilled yield approximate results, accurate water: 3 , capillary pipette: (C, graduated tube: 1). tule within 2 or 3 per cent. It has, with standard dilution ; F, lancet for pricking the finger. however, been found of much utility in clinical observation."']

(c) Fleischl's Hæmometer. - For clinical purposes this instrument (fig. 15) is useful. A

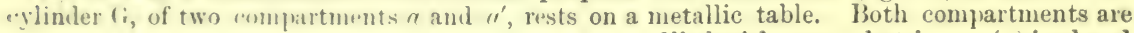

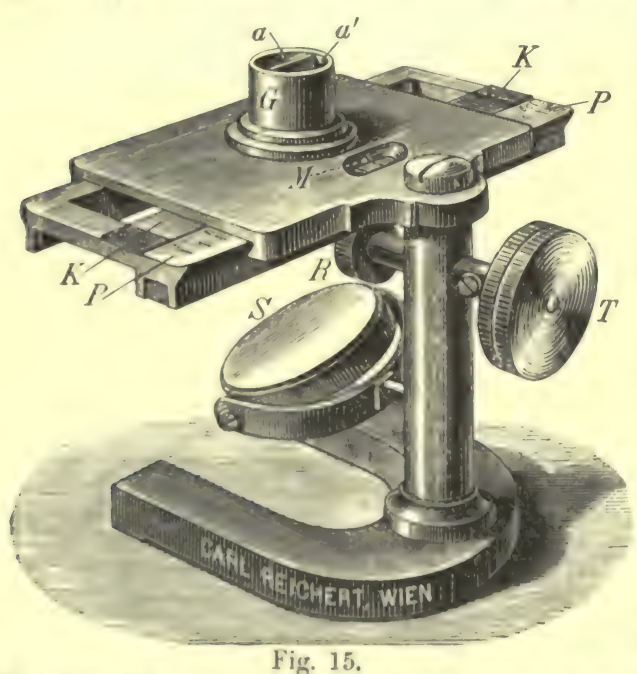

V. Fleischl's hamometer. $K$, red coloured wedge of glass movel by $\mathrm{R} ; \mathrm{G}$, mixing yessel with two compartments $u$ and $u^{\prime} ; \mathrm{M}$, table with hole to read off the percentage of hamoglobin on the scale $\mathrm{P}$; $\mathrm{T}$, tw move $\mathbf{K}$; $\mathrm{S}$, mirror of plaster of Paris. tween six months and five years it is smallest in amount; it reaches its second highest maximum between twenty-one and forty-five, and then sinks again. From filled with water, but in one $(a)$ is placed a measuring-tube of known capacity. 'The red colour of the solution of hremoglobin thus obtained is compared with a red wedge of glass $(K)$, which is moved by means of a wheel ( $R$ and $T$ ) under the other compartment $\left(a^{\prime}\right)$ until the two colours are identical. The illuminaation of the dilute blood solution and the red glass wedge is done from below by lamp light reffected from the white reflecting surface (S). The frame in which the red glass wedge is fixed bears numbers, and when the colour is iden. tical in the two compartments $a$ and $\boldsymbol{a}^{\prime}$, the percentage of hrmoglobin as compared with normal blood can be read off directly. Suppose it to be 80 on the scale, then the blood examined contains 80 per cent. of the hremoglobin of normal blood.

The amount of hæmoglobin in man is 13.77 per cent., in the woman 12.59 per cent., during jregnancy 9 to 12 per cent (Preyer). According to Ieichtenstern, $\mathrm{Hb}$ is in greatest amount in the blood of a newly-born infant, but after ten 
the tenth year onwards, the blood of the female is poorer in $\mathrm{Hb}$. The taking of food causes a temporary decrease of the $\mathrm{Hb}$, owing to the dilution of the blood.

In Animals. - In the dog, $9 \cdot 7$; ox, 9.9 ; sheep, 10.3 ; pig, 12.7 ; horse, 13.1 ; birds, $16-17$ per cent.

Pathological.-A decrease is observable during recovery from febrile conditions, and also during phthisis, cancer, ulcer of the stomach, cardiac disease, chronic diseases, chlorosis, leukæmia, pernicious anæmia, and during the rapid mercurial treatment of syphilitic persons.

14. THE SPECTROSCOPE. - As the spectroscope is frequently used in the investigation of blood and other substances, a short description of the instrument is given here (fig. 16). It

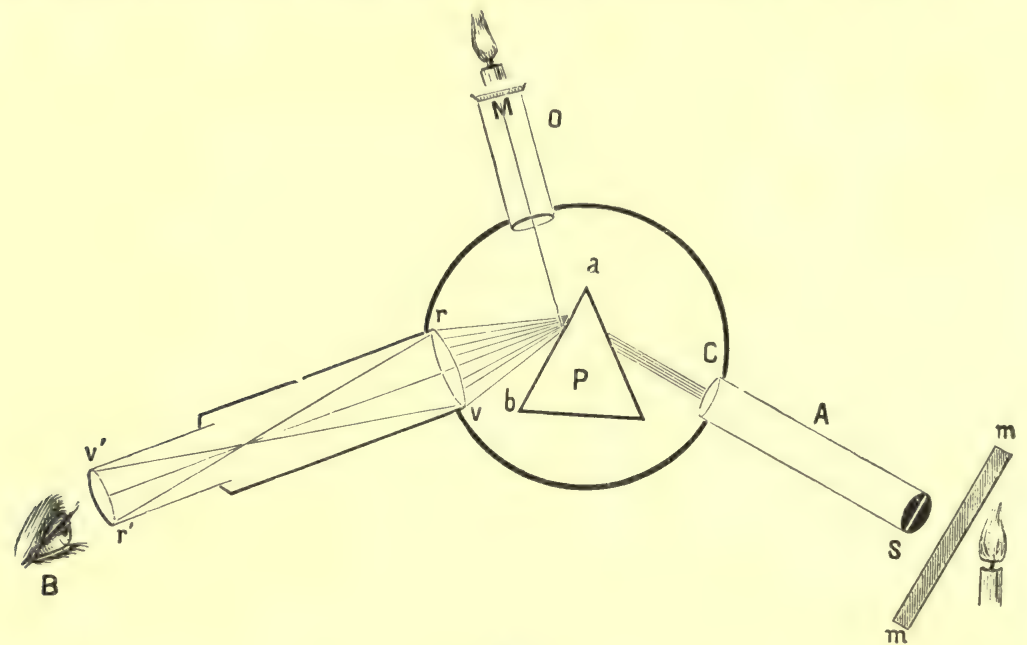

Fig. 16.

Scheme of a spectroscope for observing the spectrum of blood. A, tube; S, slit; $m$, $m$, layer of blood with flame in front of it ; P, prism; M, scale ; B, eye of observer looking through a telescope $; r, v$, spectrum.

consists of-(1) a tube, A, which has at its peripheral end a slit, $\mathbf{S}$ (that can be narrowed or widened). At the other end a collecting lens, C (called a collimator), is placed, so that its focus is in exact line with the slit. Light (from the sun or a lamp) passes through the slit, and thus goes parallel through $\mathrm{C}$ to-(2) the prism, $\mathrm{P}$, which decomposes the parallel rays into a coloured spectrum, $r, v$. (3) An astronomical telescope is directed to the spectrum $r, r$, and the observer, $\mathrm{B}$, with the aid of the telescope, sees the spectrum magnified from six to eight times. (4) A third tube, D, contains a delicate scale, M, on glass, whose image, when illuminated, is reflected from the prism to the eye of the observer, so that he sees the spectrum, and over or above it the scale. To keep out other rays of light the inner ends of the three tubes are covered by metal or by a dark cloth (see also \& 265).

[The micro-spectroscope, e.g., as made by Browning or Zeiss, may be used when small quantities of a solution are to be examined. Every spectroscope ought to give two spectra, so that the position of any absorption-band may be definitely ascertained. The spectroscope is fitted into the ocular end of the tube of a microscope instead of the eye-piece. Small cells for containing the fluid to be examined are made from short pieces of barometer-tubes cemented to a plate of glass.]

Absorption Spectra.-If a coloured medium (e.g., a solution of blood) be placed between the slit and a source of light, all the rays of coloured light do not pass through it-some are absorbed; many yellow rays are absorbed by blood, hence that part of the spectrum appears dark to the observer. On account of this absorption, such a spectrum is called an "absorption spectrum."

Flame spectra. - If mineral substances be burned on a platinum-wire in a non-luminous flame or Bunsen's burner in front of the slit, the elements present in the mineral or ash give special coloured band or bands, which have a definite position. Sodium gives a yellow, potassium a red and violet line. These substances are found on burning the ashes of almost all organs.

If sunlight be allowed to fall upon the slit, the spectrum shows a large number of lines 
(Fraunhofer's lines) which occupy definite positions in the coloured spectrum. These lines are indicated by the letters A, B, C, D, \&c., $a, b, c, \& c$. (fig. 17).

15. COMPOUNDS OF HB WITH O; OXYHAMOGLOBIN AND METHEMOGLOBIN. - 1. Oxyhæmoglobin $\left(\mathrm{O}_{2} \mathrm{Hb}\right)$ behaves as a weak acid, and occurs to the extent of 86.78 to $94 \cdot 30$ per cent. in dry human red corpuscles (Jüdell). It is formed very readily whenever $\mathrm{Hb}$ comes into contact with $\mathrm{O}$ or atmospheric air. According to Bohr, 1 gramme $\mathrm{Hb}$ unites with 1.56 cubic centimetre of $\mathrm{O}$ at $0^{\circ}$ and $760 \mathrm{~mm}$. $\mathrm{Hg}$ pressure, the union being stronger in weak than in concentrated solutions. Oxybamoglolin is a very loose chemical compound, and is slightly less soluble than Hb; its spectrum shows in the yellon and the green two dark

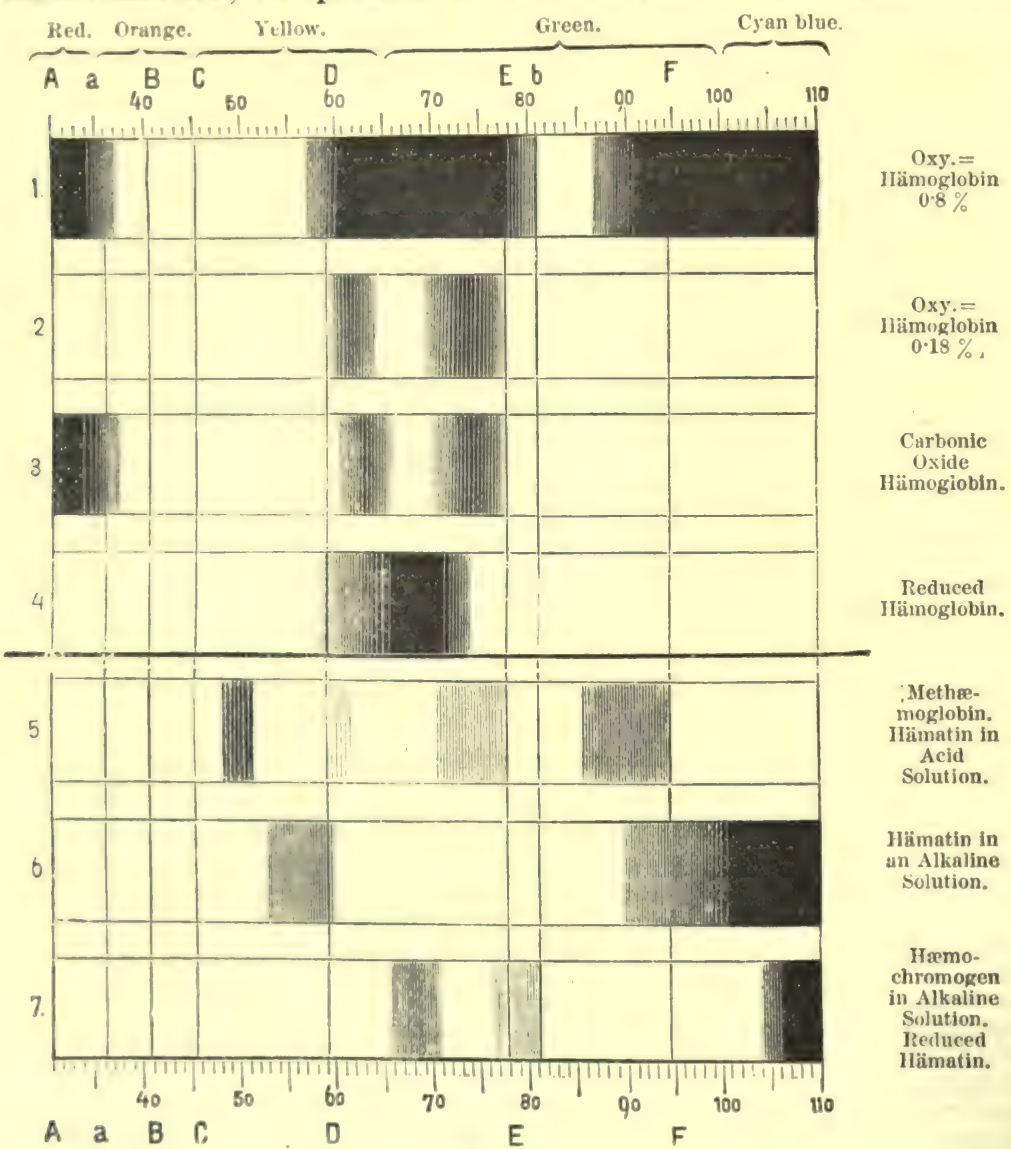

Fig. 17 .

Spectra of hæmoglobin and its compounds.

absorption-bands, whose length and breadth in a 0.18 per cent. solution are given in fig. $17(2)$.

It occurs in the blood-corpuscles circulating in arteries and capillaries, as can be shown by the spectroscopic examination of the ear of a rabbit, of the prepuce, and the web of the fingers (Vierordt).

[Spectrum of 0xyhæmoglobin.-In the spectrum of a dilute solution of hæmoglobin crystals or arterial blood, part of the red and violet rays are absorbed, 
but two well-marked absorption-bands exist between $\mathrm{D}$ and $\mathrm{E}$. The line nearest $\mathrm{D}$, i.e., next the red end of the spectrum, sometimes designated by the letter $(\alpha)$ is narrow, sharply defined, and black at its centre, and its position corresponds to the wave-length 579. The other absorption-band near E, conveniently designated by $(\beta)$, is broader, not so dark, and its edges are less sharply defined. Its centre corresponds to the wave-length $553 \cdot 8$. In very dilute solutions the $\alpha$ band is the only one visible. In a strong solution, as shown in fig. 17, the two bands fuse, but are again made visible as two on dilution of the blood.]

Reduction of $\mathbf{0 x y h æ m o g l o b i n . - I t ~ g i v e s ~ u p ~ i t s ~} \mathrm{O}$ very readily, however, even when means which set free absorbed gases are used. It is reduced by the removal of the gases by the air-pump, by the conduction through its solution of other gases (CO), and by heating to the boiling-point. In the circulating blood its $\mathrm{O}$ is very rapidly given up to the tissues, so that in suffocated animals only reduced hemoglobin is found in the arteries. Some constituents of the serum and sugar remove its $\mathrm{O}$. By adding to a solution of oxyhæmoglobin reducing substances-e.\%., ammonium sulphide, iron filings, or Stokes's fluid [tartaric acid, iron proto-sulphate, and excess of ammonia] - the two absorption-bands of the spectrum disappear, and reduced hrmoglobin (gas-free), with one absorption-band, is formed. The colour changes from a bright red to a purplish or claret tint. The two bands are reproduced by shaking the reduced hæmoglobin with air, whereby $\mathrm{O}_{2} \mathrm{Hb}$ is again formed. Solutions of oxyhæmoglobin are readily distinguished by their scarlet colour from the purplish tint of reduced hæmoglobin.

[The single absorption-band (fig. 17,4) designated by the letter $(\gamma)$, lying about midway between the position of the two previous bands, is broader, fainter, less deeply shaded, and its centre is about, but not quite, intermediate between $\mathrm{D}$ and E. It extends between the wave-lengths 595 and 538 , and is blackest opposite the wave-length 550 , so that it lies nearer $\mathrm{D}$ than $\mathrm{E}$. At the same time more of the blue rays are transmitted. On dilution the band is not resolved into two, but simply becomes fainter and disappears.]

[Hæmoglobin has certain remarkable characters :-(1) Although it is a crystalloid body it diffuses with difficulty through an animal membrane, owing to the large size of its molecule. (2) It readily combines with $\mathrm{O}$ to form an unstable and loose chemical compound, oxyhæmoglobin. (3) This $\mathrm{O}$ it gives up readily to the tissues or other deoxidising reagents. (4) Its composition is very complex, for, in addition to the ordinary elements present in proteids, it contains a remarkable amount of iron $(0.4$ per cent.).]

If a string be tied round the base of two fingers so as to interrupt the circulation, spectroscopic examination shows that the oxyhæmoglobin rapidly passes into reduced $\mathrm{Hb}$ (Vierordt). Cold delays this reduction; it is accelerated in youth, during muscular activity, or by suppressed respiration, and usually also during fever.

The spectroscopic examination of small blood-stains is often of the utmost forensic importance. A minimal drop is sufficient. Dissolve the stain in a few drops of distilled water, and place the solution in a thin glass tube in front of the slit of the spectroscope.

Para-hæmoglobin. - If $\mathrm{O}_{2} \mathrm{Hb}$ be preserved under alcohol it passes into a molified form, which is insoluble in water (Nencki and Sieber).

2. Methæmoglobin is a more stable, crystalline compound (Hoppe-Seyler). It contains the same amount of $\mathrm{O}$ as $\mathrm{O}_{2} \mathrm{Hb}$, but in a different chemical union, while the $\mathrm{O}$ is also more firmly united with it. It shows four absorption-bands like hæmatin in acid solution (fig. 17.,5), of which that between $\mathrm{C}$ and $\mathrm{D}$ is distinct; the second is very indistinct, while the third and fourth readily fuse, so that these last two bands are only well seen with good apparatus.

It is produced spontaneously in old brown blood-stains, in the crusts of bloody wounds, in blood cysts, and in bloody urine. Chemically, it can be prepared from a solution of $\mathrm{Hb}$, by the action of potassic ferri-cyanide (Jäderholm) or potassic chlorate (Marchand), [or by adding to a solution of $\mathrm{Hb}$ a freshly prepared solution of potassic permanganate], and in non-laky blood by alloxantin 
(Knralcueshy). It crystallises if defibrinated blood is shaken with amyl nitrite and the mahogany-brown laky fluill be allowed to evaporate slowly (Halliburton).

If a trace of ammonia he alled to a solution of methamoglobin, it gives an alkaline solution of methamoglolin, which shows two hands like oxyhamoglobin, of which the first one is the broaler, and extenils more towards the red. If ammonium sulphide be added to the methæmo. globin solution, reluced $\mathrm{Hb}$ is formed.

[Action of Nitrites. - The addition of amyl nitrite dissolved in alcohol, or sodic or potassic nitrite to defibrinated blood causes the latter to assume a chocolate colour, which, on the addition of ammonia, changes to red. The chocolate-coloured fluid shows one well-defined band in the red, and less distinctly other three bands like methæmoglobin (Gamgee).]

[The nitrites therefore form a compound with its oxygen more firmly fixed than the 0 in Hho., so that large dose's of nitrites arrest the internal respiration and are poisonous. It is, however, affieterl by the prolucts formed in the blood during asphyxia, while CO-Hb is not, the methirmolobin formed by the nitrites is reduced by these products to $\mathrm{Hb}$, which as it passes through the lungs takes up 0.$]$

16. CARBONIC OXIDE-H \&MOGLOBIN, POISONING WITH CO.--3. COHæmoglobin is a more stable chemical compound than the foregoing, and is produced at once when carbonic oxide is brought into contact with pure $\mathrm{Hb}$ or $\mathrm{O}_{2} \mathrm{Hb}$ (Cl. Bernurd, 18.57). It has an intensely florid or cherry red colour, is not dichroic, and its spectrum shows two absorption-bands, very like those of $\mathrm{O}_{2} \mathrm{Hb}$, but they are slightly closer together and lie more towards the violet (fig. 17, 3). Reducing substances which act upon $\mathrm{HbO}_{.,}$e.\%., ammonium sulphide or Stokes's fluid, do not affect these bands, i.e., they cannot convert the $\mathrm{CO}-\mathrm{Hb}$ into reduced $\mathrm{Hb}$. If a 10 per cent. solution of caustic soda be added to a solution of $\mathrm{CO}-\mathrm{Hb}$, and heated, it gives a cimneluer reel colsur; while, with an $\mathrm{HbO}_{2}$ solution, it gives a dark brown, greenish, greasy mass. Oxidising substances [solutions of potassic permanganate $(0.025$ per cent.), potassic chlorate (5) per cent.), and dilute chlorine solution] make solutions of ( ( ()-Hb cherry red in colour, while they turn solutions of $\mathrm{O}_{2} \mathrm{Hb}$ pale yellow. After this treatment both solutions show the absorption -bands of methrmogollin, but those of the $\mathrm{CO}-\mathrm{Hb}$ appear considerably later. If ammonium sulphide be added, $\mathrm{O}_{2} \mathrm{Hb}$ and $\mathrm{CO}-\mathrm{Hb}$ are re-formed.

()n account of its stability, ( $\mathrm{O}-\mathrm{Hb}$ resists external influences and even putrefaction for a long time, and the two bands of the spectrum may he visible after many months. Landois obtained the soda test and sprestroseopic: bands in the blood of a woman poisoned eighteen months previously hy CO, and after great putrefaction of the boly had taken place. [Stirling has kept $\mathrm{CO}-\mathrm{Hb}$ in a stoppered bottle for five years without putrefaction taking place.]

If ('O or air containing it be inspired, it gradually displaces the $\mathrm{O}$, volume for voluine, out of the red blood-corpuscles, and death soon occurs; $1000 \mathrm{c.cm}$. inspired at once will kill a man. A very small quantity in the air $\left(\frac{1}{400}-\frac{1}{1000}\right)$ suffices, in a relatively short time, to form a large quantity of $\mathrm{CO}-\mathrm{Hb}$. As continued contact with other gases (such as the passing of $O$ through it for a very long time) gradually separates the $\mathrm{C}^{\prime} \mathrm{O}$ from the $\mathrm{IIb}$, with the formation of $\mathrm{O}_{2} \mathrm{Hb}$, it happens that, in very partial poisoning with $\mathrm{CO}$, the blood gradually gets rid of the $\mathrm{CO}$ by the respiratory organs. It is uncertain if any part is excreted as $\mathrm{CO}_{2}$. $[\mathrm{CO}$ Hamoglobin, being a stable compound when once formed, circulates in the bloodvessels ; but it neither gives up oxygen to the tissues, nor takes up oxygen in the lungs, hence its very poisonous properties. The real cause of death in animals poisoned with it is, that the internal respiration is arrested.]

Poisoning with Carbonic 0xide. - Carbonic oxide is formed during the incomplete combustion of coal or coke, and passes into the air of the room, provided there is not a free outlet for the products of combustion. It occurs to the extent of 12-28 per cent. in ordinary gas, which largely owes its poisonous properties to the presence of $\mathrm{CO}$. If the $\mathrm{O}$ be gradually displaced from the blood by the respiration of air containing $\mathrm{CO}$, life can only be maintained as long as suffi. cient $\mathrm{O}$ can be obtained from the blood to support the oxidations necessary for life. Death occurs before all the $\mathrm{O}$ is displaced from the blood. $\mathrm{CO}$ has no effect when directly applied to muscle and nerve. When it is mixed with air, as in coal-gas poisoning, and inhaled, there is first 
stimulation and afterwards paralysis of the nervous system, as shown by the symptoms induced, e.g., violent headache, great restlessness, excitement, increased activity of the heart and respiration, salivation, tremors, and spasms. Later, unconsciousness, weakness, and paralysis occur, laboured respiration, diminished heart-beat, and lastly, complete loss of sensibility, cessation of the respiration and heart-beat, and death. At first the temperature rises several tenths of a degree, but it soon falls $1^{\circ}$ or more. The pulse is also increased at first, but afterwards it becomes very small and frequent. In poisoning with pure CO there is no dyspnœe, but sometimes muscular spasms occur, the coma not being very marked. There is also temporary but pronounced paralysis of the limbs, followed by violent spasms. After death the heart and brain are congested with intensely florid blood. In poisoning with the vapour of charcoal, where $\mathrm{CO}$ and $\mathrm{CO}_{2}$ both occur, there is a varying degree of coma; pronounced dyspncea, muscular spasms which may last several minutes, gradual paralysis and asphyxia, moniliform contractions and subsequent dilatation of the blood-vessels, with congestion of various organs, occur, accompanied by a fall of the blood-pressure $(K l e b s)$, indicating initial stimulation and subsequent paralysis of the vaso-motor centre. This also explains the variations in the temperature and the occasional occurrence of sugar in the urine after poisoning with CO. After death, the blood-vessels are found to be filled with fluid blood of an exquisitely bright eherry red colour, while all the muscles and viscera and exposed parts of the body (such as the lips) have the same colour. The brain is soft and friable; there is catarrh of the respiratory organs and degeneration of the muscles, and great congestion and degeneration of the liver, kidneys, and spleen. The spots of lividity, post-mortem, are bright red. After recovery from poisoning with CO there may be paraplegia and (although more rarely) disturbances of the cerebral activity.

17. OTHER COMPOUNDS OF HAMOGLOBIN.-4. Nitric Oxide-Hæmoglobin $(\mathrm{NO}-\mathrm{Hb})$ is formed when $\mathrm{NO}$ is brought into contact with $\mathrm{Hb}$ ( $L$. Hermann).

As $\mathrm{NO}$ has a great affinity for $\mathrm{O}$, red fumes of nitrogen peroxide $\left(\mathrm{NO}_{2}\right)$ being formed whenever the two gases meet, it is clear that, in order to prepare $\mathrm{NO} \cdot \mathrm{Hb}$, the $\mathrm{O}$ must first be removed. This may be done by passing $\mathrm{H}$ through it, [or ammonia may be added to the blood, and a stream of NO passed through it ; the ammonia combines with all the acid formed by the union of the $\mathrm{NO}$ with the $\mathrm{O}$ of the blood]. $\mathrm{NO}-\mathrm{Hb}$ is a more stable chenical compound than $\mathrm{CO}-\mathrm{Hb}$, which, as we have seen, is again more stable than $\mathrm{O}_{2} \mathrm{Hb}$. It has a bluish-violet tint, and also gives two absorption-bands in the spectrum similar to those of the other two compounds, but not so intense. These bands are not abolished by the action of reducing agents. As NO-Hb cannot be formed in the body, it has no practical siguificance.

The three compounds of $\mathrm{Hb}$, with $\mathrm{O}, \mathrm{CO}$, and $\mathrm{NO}$ are crystalline, like reduced $\mathrm{Hb}$; they are isomorphous, and their solutions are not dichroic. All three gases unite in equal volumes with $\mathrm{Hb}$. If $\mathrm{O}$ be conducted through a concentrated solution of $\mathrm{Hb}$ devoid of gases, a crystalline mass of $\mathrm{O}_{2} \mathrm{Hb}$ is thereby readily formed.

5. Cyanogen, CNH (Hoppe-Seyler), and acetylene, $\mathrm{C}_{2} \mathrm{H}_{4}$ (Bistrow and Licbreich), form easily decomposable compounds with $\mathrm{Hb}$. The former occurs in poisoning with hydrocyanic acid, and has a spectrum nearly identical with that of $\mathrm{O}_{2} \mathrm{Hb}$, and, like $\mathrm{O}_{2} \mathrm{Hb}$, it is reduced, but very slowly, by special reagents. [The existence of these compounds is, however, highly doubtfuil (Gamgee).]

18. DECOMPOSITION OF HAMOGLOBIN.-In solution and in the dry state $\mathrm{Hb}$ gradually becomes decomposed, whereby the iron-containing pigment hæmatin (along with certain bye-products, formic, lactic, and butyric acids), is formed. Hæmoglobin, however, may be decomposed at once into--(1) Hæmatin, a body containing iron, and (2) a colourless proteid closely related to globulin ; by $(a)$ the addition of all acids, even by $\mathrm{CO}_{2}$ in the presence of plenty of water ; $(b)$ strong alkalies; $(c)$ all reagents which coagulate albumin, and by heat at $70^{\circ}-80^{\circ}$ C.; (d) by ozone.

(A) Hæmatin, $\mathrm{C}_{32} \mathrm{H}_{32} \mathrm{~N}_{4} \mathrm{FeO}_{4}$ (Nencki and Sieber), is a bluish-black amorphous body, which forms about 4 per cent. of hæmoglobin (dog). It is insoluble in water, alcohol, and ether ; soluble in dilute alkalies and acids, and in acidulated ether and alcohol.

(1) Acid Hæmatin.-Lecanu extracted it from dry blood-corpuscles by using alcohol containing sulphuric and tartaric acids. [If acetic acid be added to a solution of $\mathrm{Hb}$ and slightly heated, a mahogany-brown fluid is obtained, containing 
hamatin in arid solution, which gives a spectrum with one absorption-band to the red side of D near C (fig. 17,5). There is at the same time a considerable absorption of the blue end of the spectrum. If an ethereal extract of the acidhrmatin be made, the ether is coloured brown and shows four absorption-bands, as in fig. 17,5 .]

(2) Alkali-hæmatin. - If to the above solution ammonia or caustic soda be added, on heating gently, the colcur changes, and the fluid becomes dichroic, showing a greenish tinge. On mixing the solution thoroughly with air the spectrum of oxy-alkali-hrmatin is obtained, i.e., one absorption-band just to the red side of D (fig. 17,6), so that it is much nearer $\mathrm{D}$ than the corresponding band of acidhamatin. IIuch of the blue end of the spectrum is absorbed as well.]

[(3) Reduced Alkali-hæmatin or Hæmochromogen.--If the solution of alkalihermatin be reduced by ammonium sulphide, the spectrum of hæmochromogen is obtained, viz., two absorption-bands between $\mathrm{D}$ and $\mathrm{E}$, but they are nearer the violet end than in the case of $\mathrm{HbO}_{2}$ and $\mathrm{Hb} \cdot \mathrm{CO}$ (fig. 17, 7).]

[(4) Hæmatoporphyrin or Iron-free Hæmatin.-On adding blood to concentrated sulphuric acid a clear purplish-red solution is obtained, which shows two absorption-bands, one close to and on the red side of D, and a second half-way between 1) and $\mathrm{E}$. If water be added a brown precipitate is thrown down. When this precipitate is dissolved in caustic soda, it gives a fluid which shows four absorption-bands.]

Action of $\mathrm{CO}_{0}$. If C $\mathrm{CO}_{2}$, he passed through a solution of oxyhæmoglobin for a considerable time, realuced $\mathrm{Hb}$ is first formed; but if the process be prolonged the $\mathrm{Hb}$ is decomposed, a frecipitate of ghlubulin is thrown down, and an absorption-band, similar to that obtained when Hb is decomposed with acids, is observed (p. 25).

An alkaline solution of hrematin, when reduced by tin and hydrochloric acid, yields urobilin (compare $\$ 261)$.

When hirmoglolin is extravasated into the subcutaneous tissue, it becomes so altered that at first himativilin $\leqslant 20$ ), and ultimately hydrated oxide of iron, appear in its place.

19. HÆMIN AND BLOOD TESTS.-In 1853 Teichmann prepared crystals of hæmin from blood, which Hoppe-Seyler showed to be chloride of hæmatin

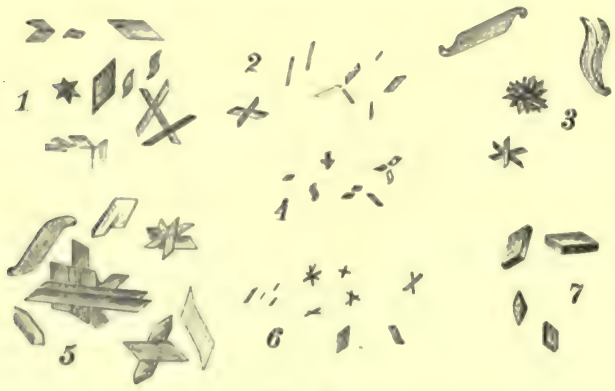

Fig. 18.

Hæmin crystals. 1, human ; 2, seal ; 3, calf ; 4 , pig; 5 , lamb; 6 , pike; 7 , rabbit.

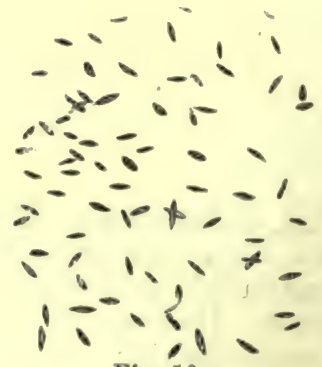

Fig. 19.

Hæmin crystals prepared from traces of blood.

(Hiematin, +2HCl), with the formula $\mathrm{C}_{32} \mathrm{H}_{31} \mathrm{ClN}_{4} \mathrm{FeO}_{3}$ (Nencki and Sieber). The presence of these crystals is used as a test for blood-stains or blood in solution. They (fig. 18) are prepared by adding a small crystal of common salt to dry blood on a glass slide, and then an excess of glacial acetic acid; the whole is gently heated until bubbles of gas are given off. On allowing the preparation to cool, the characteristic hæmin crystals are obtained.

Characters.-When well formed, the crystals are small microscopic rhombic 
plates, or rods; sometimes they are single-at other times they are aggregated in groups, often crossing each other. Some kinds of blood (ox and pig) yield very irregular, scarcely crystalline, masses. The crystalline forms of hæmin are identical in all the different kinds of blood that have been examined. They are doubly refractive; under the polarization microscope they are a glancing yellow, appearing raised on the dark field, with a strong absorption of the light parallel to the long axis of the crystals (Falk and Morache). They are pleochromatic: by transmitted light they are mahogany-brown, and by reflected light bluish-black, glancing like steel.

(1) Preparation from Dry Blood-Stains. - Place a few particles of the blood-stain on a glass slide, add 2 to 3 drops of glacial acetic acid and a small crystal of common salt; cover with a cover-glass, and heat gently over the flame of a spirit lamp until bubbles of gas are given off. On cooling, the crystals appear in the preparation (fig. 19).

(2) From Stains on Porous Bodies. -The stained object (cloth, wood, blotting paper, earth) is extracted with a small quantity of dilute caustic potash, and afterwards with water in a watch-glass. Both solutions are carefully filtered, and tannic acid and glacial acetic acid are added until an acid reaction is obtained. The dark precipitate which is formed is collected on a filter and washed. A small part of it is placed on a microscope slide, a granule of common salt is added, and the whole dried; the dry stain is treated as in (1) (Struwe).

(3) From Fluid Blood. - Dry the blood slowly at a low temperature, and proceed as in (1).

(4) From Dilute Solutions of Hæmoglobin. - (a) Struwe's Method.-Add to the fluid, ammonia, tannic acid, and afterwards glacial acetic acid, until it is acid; a black precipitate of tannate of hæmatin is thrown down. This is isolated, washed, dried, and treatel as in (1), but instead of $\mathrm{NaCl}$ a granule of ammonium chloride is added.

Hæmin crystals may sometimes be prepared from putrefying or lake-coloured blood, but they are very small, and the test often fails. When mixed with ironrust, as on iron weapons, the blood-crystals are generally not formed. In such cases, scrape off the stains and boil them with dilute caustic potash. If blood be present, the dissolved hæmatin forms a fluid, which in a thin layer is green, in a thick layer red (H. Rose).

Hæmin erystals have been prepared from all classes of vertebrates and from the blood of the earth-worm. From the blood of the ox and nig they may he almost amorphous.

Chemical Characters. - They are insoluble in water, alcohol, ether, chloroform; but concentrated $\mathrm{H}_{2} \mathrm{SO}_{4}$ dissolves them, expelling the $\mathrm{HCl}$, and giving a violet-red colour. Ammonia also dissolves them, and if the resulting solution be evaporated, heated to $130^{\circ} \mathrm{C}$., and treated with boiling water (which extracts the ammonium chloride), hæmatoporphyrin-identical with MLulder's iron-free hæmatoin, and with Preyer's hæmatoin, is obtained (Hoppe-Seyler). It is a bluish-black substance, which on being pounded forms a brown and amorphous powder. Its solutions in caustic alkalies are dichroic: in reflected light brownish-red; in transmitted light, in a thick stratum, red-in a thin one, olive-green. The acid solutions are monochromatic and brown.

Preparation in Bulk. - To obtain it in quantity, heat dried horse's blood with 10 parts of formic acid. If the crystals be suspended in methyl alcohol, on adding iodine and heating them they dissolve with a purple colour; after adding bromine, brown; and after passing chlorine gas, green ; all these give a characteristic spectrum (Axenfeld).

The glacial acetic acid may be replaced by oxalic or tartaric acid, the common salt by salts of iodine or bromine; in the latter 'case similar bromine- or iodine-hæmatin is formed (Bikfalvi).

20. HEMATOIDIN. - Virchow discovered this important derivative of hæmoglobin. It occurs in the body wherever blood stagnates outside the circulation, and becomes decomposed-as when blood is extravasated into the tissues-e.g., the brain-in solidified blood-

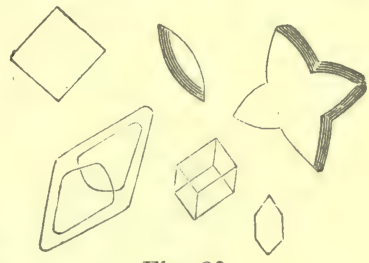

Fig. 20.

Hæmatoidin crystals. plugs or thrombi; especially in veins; invariably in the Graafian follicles. It contains no iron $\left(\mathrm{C}_{32} \mathrm{H}_{36} \mathrm{~N}_{4} \mathrm{O}_{6}\right)$, and crystallises in clino-rhombic prisms (fig. 20) of a yellowish-brown colour. It is soluble in warm alkalies and chloroform. Very probably it is identical with the bile-pigment-bilirubin. [When acted upon 
by impure nitric acid (Gmelin's reaction), it gives the same play of colours as. bile.]

Pathological _- In cases where a large amount of blood has undergone solution within the blood-vessels (as hy injecting foreign blood) hrmatoidin crystals have been found in the urine. For their oceurrence in the urine in jaundice $(\$ 180)$, and in the sputum ( $\$ 138)$.

21. (B.) THE COLOURLESS PROTEID OF HEMOGLOBIN.-It is closely related to globulin; but, while the latter is precipitated by all acids, even by $\mathrm{CO}_{2}$, and re-dissolved on passing () through it, the proteid of hæmoglobin, on the other hand, is not dissolved after precipitation on passing through it a stream of $\mathrm{O}$.

As crystals of hemoglobin can be decolorised under special circumstances, it is probable that there owe their crystalline form to the proteid which they contain. Landois placed crystals of hamoglobin along with alcohol in a dialyser, putting ether acidulated with sulphuric acid outside, and thereby obtained colourless crystals. [If frogs' blood be sealed up on a microscopic slicle along with a few drops of water for several days, long colourless acicular crystals are developell in it (Stirling and Brito).]

22. II. PROTEIDS OF THE STROMA.-Dry red human blood-corpuscles contain from $5 \cdot 10-1 \cdot 2+2$ per cent. of these proteids, but little is known about them (Jiidell). One of them is globulin, which is combined with a body resembling nuclein (Wouldrillg), and traces of a diastatic ferment ( $v$. Wittich). The stroma tends to form masses which resemble fibrin.

I. Brunton found a body resembling mucin in the nuclei of red blood-corpuscles, and Miescher letected nuclein $(\$ 250,2)$.

\section{OTHER CONSTITUENTS OF RED BLOOD-CORPUSCLES.--III. Lecithin} $(0.35-0.72$ per cent.) in dry blood-corpuscles $(\$ 250,2)$. Cholesterin $(0.25$ per cent.) (§ 250 , III.), no Fats.

Lecithin is regarded as a glycero-phosphate of neurin; in which, in the radical of glycerophosphoric acid, two atoms of $\mathrm{H}$ are replaced by two of the radical of stearic acid. By gentlo heat glycero-phosphoric acid is split up into glycerine and phosphoric acid (\$250).

These substances are ohtained by extracting old stromata or isolated blood-corpuscles with ether. "hen the ether evaporates, the characteristic globular forms ("myelin-forms") of lecithin, and erystals of cholesterin are recognised. The amount of lecithin may be determined from the amount of phosphorus in the ethereal extract.

IV. Water (681.63 per 1000-C. Schmidt).

V. Salts (7.28 per 1000), chiefly compounds of potash and phosphoric acid; the phosphoric acid is derived only from the burned lecithin ; while the greater part of the sulphuric acid is derived from the burning of the hrmoglobin in the analysis.

Analysis of Blood. -1000 parts, by weight, of horse's blood contain :-

$344 \cdot 18$ blood-corpuscles (containing about 128 per cent. of solids).

$655 \cdot 82$ plasma (containing about 10 per cent. of solids).

1000 parts, by weight, of moist blood-corpuscles contain :-

$$
\text { Solids, , . } 367 \cdot 9 \text { (pig); } 400 \cdot 1 \text { (ox). }
$$

The solids are :-

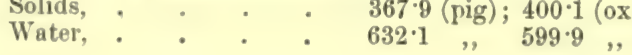

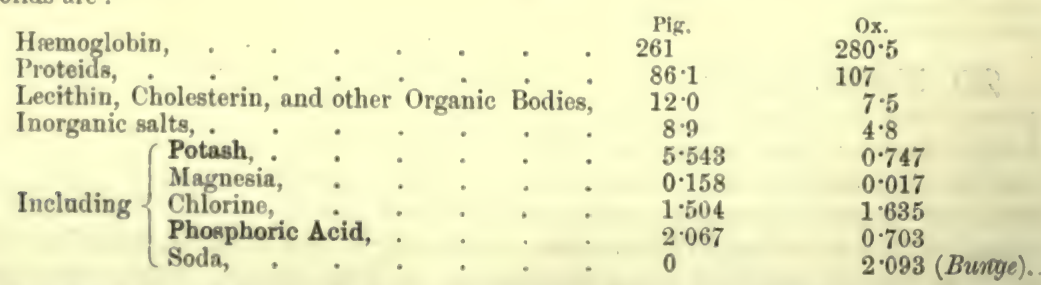

[An approximate estimate of the composition of human blood is given in the following table :- 
Composition of Human Blood as a Whole.

Water,

Solids-of these-

Corpuscles,

Serum-albumin,

Serum-globulin,

Fibrin of Clot (? Fibrinogen),

Inorganic Salts (of serum),

Extractives,

Gases, $\mathrm{O}, \mathrm{CO}_{2}, \mathrm{~N}$.]

Fatty matters,

24. CHEMICAL COMPOSITION OF THE WHITE CORPUSCLES.-Investi:gations have been made on pus cells, which closely resemble colourless bloodcorpuscles. They contain several proteids; alkali-albuminate, a proteid which coagulates at $48^{\circ} \mathrm{C}$, an albuminate resembling myosin, paraglobulin, peptone, and a coagulating ferment; nuclein in the nuclei $(\$ 250,2)$, glycogen $(\$ 252)$, lecithin, cerebrin, cholesterin, and fat.

100 parts, by weight, of dry pus contain the following Salts :-

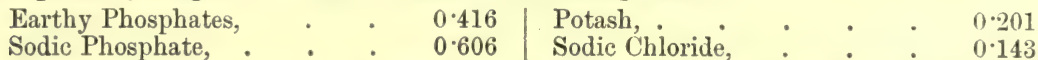

25. BLOOD-PLASMA AND ITS RELATION TO SERUM.-The unaltered fluid in which the blood-corpuscles float is called blood-plasma, or liquor sanguinis. This fluid, however, after blood is withdrawn from the vessels, rapidly undergoes a change, owing to the formation of a solid fibrous substance-fibrin. After this occurs, the new fluid which remains, no longer coagulates spontaneously (it is plasma, minus the fibrin-factors), and is called serum. Apart from the presence of the fibrin-factors, the chemical composition of plasma and serum is the same.

[When blood coagulates, Table I. shows what takes place, while Table II. shows what occurs when it is beaten :-

Coagulation.

BLOOD.

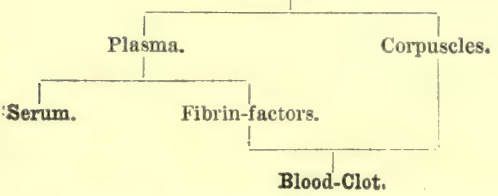

'II.

When beaten.

BLOOD.

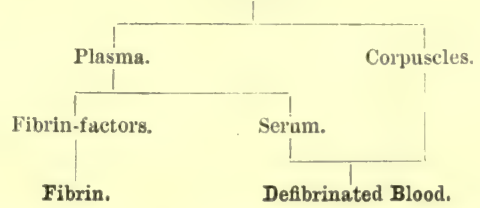

Plasma is a clear, transparent, slightly thickish fluid, which, in most animals (rabbit, ox, cat, dog), is almost colourless; in man it is yellow, and in the horse citron yellow.

26. PREPARATION OF PLASMA.-(A) Without Admixture.-Taking advantage of the fact that plasma, when cooled to $0^{\circ}$ outside the body, does not coagulate for a considerable time, Brücke prepares the plasma thus :-The blood of the horse (because it coagulates slowly, and its corpuscles sink rapidly to the bottom) is received, as it flows from an artery, into a tall narrow glass, placed in a freezingmixture, and cooled to $0^{\circ}$. The blood remains fluid, the coloured corpuscles isubside in a few hours, while the plasma remains above as a clear layer, which can be removed with a cooled pipette. If this plasma be then passed through a cooled filter, it is robbed of all its colourless corpuscles. [Burdon-Sanderson uses a vessel consisting of three compartments-the outer and inner contain ice, while the blood is caught in the central compartment, which does not exceed half an inch in diameter.] The quantity of plasma may be roughly (but only roughly) estimated by using a tall, graduated measuring-glass. If the plasma be warmed, it soon 
coagulates (owing to the formation of the fibrin), and passes into a trembling jelly. If, however, it be beaten with a glass-rod, the fibrin is obtained as a white stringy mass, adhering to the rod. The quantity of fibrin in a given volume of plasma is very small (1. 31), although it varies much in different cases.

(B) With Admixture.-Blood flowing from an artery is caught in a tall vessel containing 1 th of its volume of a concentrated solution of sodic sulphate (Hewson) - or in a 25 per cent. solution of magnesic sulphate ( 1 vol. to 4 vols. bloodSimmer)-or 1 vol. blood with 2 vols. of a 4 per cent. solution of monophosphate of potash (.l/cisil). When the blood is mixed with these fluids and put in a cool place, the corpuscles subside, and the clear stratum of plasma mixed with the salts may be removed with a pipette. [The plasma so obtained is called "salted plasma."] If the salts be removed by dialysis, coagulation occurs ; or it may be caused by the addition of water $(J o h$. Hüller). Blood which is mixed with a 4 per cent. solution of common salt does not coagulate, so that it also may be used for the preparation of plasma. [For frogs' blood Johannes Müller used a $\frac{1}{2}$ per cent. solution of cane-sugar, which permits the corpuscles to be separated from the plasma by filtration. The plasma mixed with the sugar coagulates in a short time.]

\section{FIBRIN-COAGULATION OF THE BLOOD. - General Characters.-} Fibrin is that substance which, becoming solid in shed blood, in plasma and in lymph causes coagulation of these fluids. In these fluids, when left to themselves, fibrin is formed, consisting of innumerable, excessively delicate, closely-packed, microscopic, doubly refractive fibrils (fig. $7, E$ ). These fibrils entangle the bloodcorpuscles as in a spider's web, and form with them a jelly-like solid mass called the blood-clot or placenta sanguinis. At first the clot is very soft, and after the first ¿ to 15 minutes a few fibres may be found on its surface; these may be removed with a needle, while the interior of the clot is still fluid. The fibres ultimately extend throughout the entire mass, which, in this stage, has been called '"um. Ifter from 12 to 15 hours the fibrin contracts, or, at least, shrinks more and more closely round the corpuscles, and a fairly solid, trembling, jelly-like clot, which can be cut with a knife, is formed. During this time the clot takes the shape of the vessel in which the blood coagulates, and expresses from its substance a fluid-the blood-serum. Fibrin may be obtained by washing away the corpuscles from the clot with a stream of water.

Crusta Phlogistica.- If the corpuscles subside very rapidly, and if the blood coagulates slowly, the upper stratum of the clot is not red, but only yellowish, on account of the absence of coloured corpuscles. This is regularly the case in horse's blood, and in human blood it is observed especially in inflammations; hence this layer has been called crusta phlogistica. Such blood contains more fibrin, and so coagulates more slowly.

The crusta is formed under other circumstances, c.g., with increased sp. gr. of the corpuseles, or diminished sp. gr. of the plasma (as in hydremia and chlorosis), whereby the corpuscles sink more rapidly, and also during pregnancy. The taller and narrower the glass, the thicker is the crusta (compare $\$ 41$ ). The upper end of the clot, where there are few corpuscles, shrinks more, and is therefore smaller than the rest of the clot. This upper, lighter-coloured layer is called the "buffy coat"; but it gradually passes both in size and colour into the normal darkcoloureal clot. [Sometimes the upper surface of the clot is concave or "cupped." The older physicians attached great importance to this condition, and also to the occurrence of the buffy coat. ]

Defibrinated Blood. - If freshly-shed blood be beaten or whipped with a glass. rod, or with a bundle of twigs, fibrin is deposited on the rod or twigs in the form of a solid, fibrous, yellowish-white, elastic mass, and the blood which remains is called "defibrinated blood" (p. 29). [The twigs and fibrin must be washed in a stream of water to remove adhering corpuscles.] 
Coagulation of Plasma.-Plasma shows phenomena exactly analogous, save that the clot is not so well marked, owing to the absence of the resisting corpuscles; there is, however, always a soft trembling jelly formed when plasma coagulates. [In Hewson's experiment on the blood of a horse tied in a vein, he found that the plasma coagulated-fibrin being formed, so that he showed coagulation to be due to changes in the plasma itself (\$29).]

Properties of Fibrin.-Although the fibrin appears voluminous, it only occurs to the extent of 0.2 per cent. $(0.1$ to 0.3 per cent.) in the blood. The amount varies considerably in two samples of the same blood. It is insoluble in water and ether; alcohol shrivels it by extracting water; dilute hydrochloric acid $(0.1$ per cent.) causes it to swell up and become clear, and changes it into syntonin or acidalbumin (\$ 249, III.). When fresh, it has a greyish-yellow fibrous appearance, and is elastic ; when dried, it is horny, transparent, brittle, and friable.

When fresh it dissolves in 6-8 per cent. solutions of sodium nitrate or sulphate, in dilute alkalies, and in ammonia, thus forming alkali-albuminate. Heat does not coagulate these solutions. [It is also soluble in, or rather decomposed by, 5-10 per cent. solutions of neutral salts, e.g., NaCl, yielding two fibro-globulins (Green).] Hydric peroxide is rapidly decomposed by fibrin into water and $\mathrm{O}$ (Thenard). Fibrin which has been exposed to the air for a long time is no longer soluble in solution of potassic nitrate, but in neurin (Mauthner). During putrefaction it passes into solution, albumin being formed. Fibrin contains, entangled in it, ferric, calcic, and magnesic phosphates, and calcium sulphate whose origin is unknown.

Time for Coagulation. - The first appearance of a coagulum occurs in man's blood after 3 minutes 45 seconds, in woman's blood after 2 min. 20 sec. (H. Nasse). Age has no effect; withdrawal of food accelerates coagulation (H. Vierordt).

28. GENERAL PHENOMENA OF COAGULATION.-I. Blood in direct contact with living unaltered blood-vessels does not coagulate. [Hewson (1772) found that when he tied the jugular vein of a horse in two places, and excised it, the blood did not coagulate for a long time.] Brïcke filled the heart of a tortoise with blood which had stood 15 minutes exposed to the air at $0^{\circ}$, and kept it in a moist chamber ; at $0^{\circ} \mathrm{C}$. the blood was still uncoagulated in the contracting heart after eight days. Blood in a contracting frog's heart preserved under mercury does not coagulate. If the wall of the vessel be altered by pathological processes (e.g., if the intima becomes rough and uneven, or undergoes inflammatory change), coagulation is apt to occur at these places. Blood rapidly coagulates in a dead heart, or in blood-vessels (but not in capillaries) or other canals (e.g., the ureter). If blood stagnates in a living vessel, coagulation begins in the central axis, because here there is no contact with the wall of the living blood-vessel.

II. Conditions which Hinder or Delay Coagulation.-(a) The addition of small quantities of alkalies, ammonia, or concentrated solutions of neutral salts of the alkalies and earths (alkaline chlorides, sulphates, phosphates, nitrates, carbonates). Magnesic sulphate acts most favourably in delaying coagulation ( 1 vol. solution of 28 per cent. to $3 \frac{1}{2}$ vols. blood of the horse).

(b) Precipitation of the fibrino-plastin by adding weak acids, or $\mathrm{CO}_{2}$.

By the addition of acetic acid until the reaction is acid, coagulation is completely arrested. A large amount of $\mathrm{CO}_{2}$ delays it, hence venous blood coagulates more slowly than arterial, and the blood of suffocited persons remains fluid for the same reason.

(c) The addition of egg-albumin, syrup, glycerine, and much water. If uncoagulated blood be brought into contact with a layer of already-formed fibrin, coagulation occurs later.

(d) By cold $\left(0^{\circ} \mathrm{C}\right.$.) coagulation may be delayed for one hour. If blood is frozen at once, after thawing it is still fluid; and then coagulates (Hewson). When shed blood is under high pressure it coagulates slowly.

(e) Blood of embryo-fowls does not coagulate before the twelfth or fourteenth day of incubation $(B o l l)$; that of the hepatic vein very slightly; menstrual blood shows 
little tendency to coagulate when alkaline mucus from the vagina is mixed with it. If it be rapidly discharged, it coagulates in masses. Fotal blood at the moment of birth coagulates soon.

(f) Bloor rich in fibrin from inflamed parts cuagulates slowly, but the clot so formed is firm.

(g) [Blood coagulates more slowly in a smooth than a rough vessel, and alsu in a shallow vessel than in a deep one.]

Hrmophilia. - A very slight serateh in some persons may cause very free bleeding. These persons are called colloguially "bleeders," and are said to have hæmophilia or the hæmorrhagic diathesis. In "l, leeclers" coagulation seems not to take place, owing to a want of the substances prolucing fibrin; hence, in these cases, wounds of vessels are not plugged with fibrin. [A temlency to hermorrhage occurs in scurvy, purpura, in sorne infectious diseases, such as trphus, plague, yellow fever, and in poisoning with phosphorus. ]

Injection of Peptones. - Alhertoni observed that if tryptic pancreas ferment (dissolved in mlycerine) be injected into the blood of an animal, the blood does not coagulate. SehmidtIfilheim found that after the injection of pure peptone in to the blood $(0.5$ gram per kilo. $)$ of a dog, the blood lost its power of coagulating. ['This oceurs in the dog, but not in the rabbit. Peptonised blood coagulates when it is treated with $\mathrm{CO}_{2}$ or water. It appears, however, that it is not the preptone which prevents the coagulation, but the albumoses adhering to it which (i) so.] A sulstance is formed in the plasma, which prevents coagulation, but which is pre-

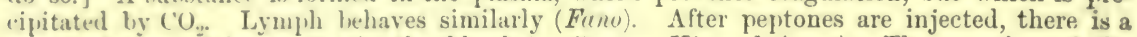
great solution of lencocytes in the blood ( 2 . Sumson-Himmelstjorna). The secretion of the mouth of the medicinal leech, [although its action is not due to a ferment (Haycraft)], and snake proison also prevent coagulation $\left(I^{*}\right.$ all). [Diastatic ferment also prevents coagulatiou [Salvioli).]

III. Coagulation is accelerated-( (I) By contact with foreign Substances of all kinds, but only when the blood adheres to them, hence, threads or needles introduced into arteries are rapidly covered with fibrin. Blood does not coagulate in contact with bodies covered with fat or vaseline (F'reund). Even the introduction of air-lubbles into the circulation or the passage of indifferent gases, $\mathrm{N}$ or $\mathrm{H}$, llirough blood, accelerates it. The pathologically altered wall of a vessel acts like a foreign body. Blood shed from an artery rapidly coagulates on the walls of ressels, on the surfaces exposed freely to air, and on the rods or twigs used to beat it.

(b) The products of the retrogressive metabolism of proteids (uric acid, glycin, leucin, taurin, kreatin, sarkin, but not urea) favour coagulation by increased ferment formation; but if they are added in excess, they retard the process.

From a watery extract of the testis or thymus, on the aldition of acetic acid, is precipitated a substance which is soluble in sodic carbonate. It is a mixture of lecithin and albumin, and when it is injected into the bloud-stream it causes almost instantaneous death by intravascular coagulation (Wooldridge).

(c) During rapid hiemorrhage, the last portions of blood coagulate most rapidly (Holmann).

(d) Heating the blood from $39^{\circ}$ to $55^{\circ} \mathrm{C}$. (Hewson).

(e) Agitation of the blood (Hewson and Hunter).

$[(f)$ The addition of a small quantity of water.

(I) A vectery condition of the blood. The clot is small and soft.

(h) Contact with oxygen.]

IV. Rapidity of Coagulation.-Amongst vertebrates, the blood of birds (especially of the pigeon) coagulates almost momentarily; in cold-blooded animals coagulation occurs much more slowly, while mammals stand midway between the two.

[The blood of a fowl begins to coagulate in $\frac{1}{2}$ to $1 \frac{1}{2}$ minute; pig, sheep, rabbit, in $\frac{1}{2}$ to $1 \frac{1}{2}$ minute; dog, 1 to 3 minutes ; horse and ox, 5 to 13 minutes ; man, 3 to 4 minutes; solidification is completed in 9 to 11 minutes (Nasse).] The blood of invertebrates, which is usually colourless when it is oxidised (\$32), forms a soft whitish clot of fibrin. Even in lymph and chyle, a small solt clot is formed.

V. When coagulation occurs, the aggregate condition of the fibrin-factors is altered, so that heat must be set free (Valentin, 1844). 
VI. In blood shed from an artery, the degree of alkalinity diminishes from the time of its being shed until coagulation is completed (Pfliger and Zuntz). This is probably due to a decomposition in the blood, whereby an acid is developed, which diminishes the alkalinity (p. 1).

VII. During coagulation there is a diminution of the $\mathbf{0}$ in the blood, although a similar decrease also occurs in non-coagulated blood. Traces of ammonia are also given off, which Richardson erroneously supposed to be the cause of the coagulation of the blood.

[This is refuted-(1) by the fact that blood, when collected under mercury (whereby no escape of ammonia is possible), also coagulates ; and (2) by the following experiment of Lister :- $\mathrm{He}$ placed two ligatures on a vein containing blood, moistening one-half of the outer surface of the vein with ammonia, leaving the other half intact. The blood coagulated in the first half, and not in the other, owing to the properties of the wall of the vein of the former being altered. Neither the decrease of $O$ nor the evolution of ammonia seems to have any causal connection with the formation of fibrin.]

Pathological. - When the blood coagulates within the vessels during life, the process is called thrombosis, and the coagulum or plug so formed is termed a thrombus. When a clot of blood or other body is carried by the blood-stream to another part of the vascular system where it blocks up a vessel, the plug is called an embolus, and the result embolism.

29. CAUSE OF THE COAGULATION OF BLOOD.-[Hewson's Experiments (1772.) - Hewson tied the jugular vein of a horse between two ligatures, removed it, and then suspended it by one end (fig. 21). He found that the blood remained fluid for a long time (48 hours), the red corpuscles sank $(\mathrm{RC})$ and left a clear layer of plasma on the surface $(\mathrm{P})$. On drawing off some of this clear plasma it coagulated, thus proving coagulation to be due to changes in the plasma. Lister repeated this experiment, and found that, even if the upper end of the tube be opened and the blood freely exposed to the air, coagulation is but slightly hastened. He showed that the blood might be poured from one vein into another, just as one might pour fluid from one test-tube into another. In this case there were two test-tubes, i.e., the veins-and although the blood, on being poured from the one to the other, came into contact with the air, it did not coagulate. Hewson, however, found that blood poured from the vein into a glass vessel coagulated, so that, in his opinion, the blood-vessels exerted a restraining influence on coagulation. By cooling the blood and preventing it from coagulating, he proved that coagulation was not due to the loss of heat. Nor could it be a vital act, as sodic sulphate or other neutral salt prevented coagulation indefinitely, but coagulation took place when the blood was diluted with water.]

[Buchanan's Researches.-The serous sacs of the body contain a fluid Vein of horse tied which in some respects closely resembles lymph. The pericardial fluid of some animals coagulates spontaneously $(e . g .$, in the rabbit, ox, horse, and sheep) if the fluid be removed immediately after death. If this be not done till several hours after death, the fluid does not coagulate spontaneously. The fluid of the tunica vaginalis of the testis sometimes accumu. lates to a great extent, and constitutes hydrocele, but this fluid shows no

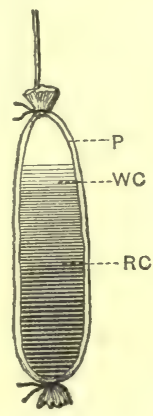

Fig. 21.

between two ligatures. P, plasma; WC, white, and $\mathrm{RC}$, red corpuscles. tendency to coagulate spontaneously. Andrew Buchanan found, however, that if to the fluid of ascites, pleuritic fluid, or hydrocele fluid, there be added clear blood-serum, then coagulation takes place, i.e., two fluids-neither of which shows any tendency by itsclf to coagulate-form a clot when they are mixed (1831). He also found, that if "washed blood-clot" (which consists of a mixture of fibrin and colourless corpuscles) be added to hydrocele fluid, coagulation occurred. He compared the action of washed blood-clot to the action of rennet in coagulating milk, and he imagined the agents which determined the coagulation to be colourless corpuscles. Thus, the buffy coat of horses' blood is a powerful agent, and it contains numerous colourless corpuscles. He finally concluded that some constituent in the plasma, to which he gave the name of a "soluble fibrin," is acted upon by the colourless corpuscles and converted into fibrin. The soluble fibrin of Buchanan is comparable to the fibrinogen in Hammarsten's theory. Buchanan, however, did not separate the substance.]

[Denis's Plasmine (1859). - Denis mixed uncoagulated blood with a saturated solution of sodic sulphate, and allowed the corpuscles to subside. The salted plasma thus obtained he precipitated with sodic chloride. The precipitate, when washed with a saturated solution of sodic chloride, he called plasmine. If plasmine be mixed with water, it coagulates spontaneously, resulting in the formation of fibrin, while another proteid remains in solution. According to the view of Denis, fibrin is produced by the splitting up of plasmine into two bodies-tibrin and a soluble proteid.] 
[A. Schmidt's Researches (1861). - This observer rediscovered the chief facts already knowir to Buchanan, viz., that some fluids which do not coagulate spontaneously, clot when mixed with other fluids which show no teudency to coagulate spontaneously, e.g., hydrocele fluid and blood-serum. He isolated from these fluids the bodies deseribed us fibrinogen and fibrinoplastin. The bodies so obtained were not jure, but Schmidt supposed that the furmation of fibrin was due to the interaction of these two proteids. The reason hydrocele fluid does not coagulafe, he says, is that it contaius filbrinogen and no fibrino-plastin, while blood-serum contains the latter, but not the former. Sehnidt afterwards discovered that these two substances may be present in a fluid, and jet coagulation may not occur (c.g., occasionally in hydrocele fluid1). He supposed, therefore, that blood or blood-serum contained some other constituent necessary for coagulation. This he afterwarls isolated in an impure condition and called fibrin-ferment.]

A. Schmidt's theory is that fibrin is formed by the coming together of tuo proteid substunces which occur dissolved in the plasma, viz:- (1) fibrinoger, i.e., the substance which yields the chief mass of the fibrin, and (2) fibrino-plastic substance or fibrino-plastin (serum-globulin or paraglobulin, §32). In order to determine the coagulation a ferment seems to be necessary, and this is supplied liy (3) the fibrin-ferment.

1. Properties.-Fibrinogen and fibrino-plastin belong to the group of proteids called globulins, i.e, they are insoluble in pure water, but are soluble in dilute solutions of common salt $(\$ 249)$, and are not distinguished from each other by wellmarked chemical characters. Still they differ as follows :-

Fibrino-plastin is more easily precipitated from its solutions than fibrinogen. It is more readily redissolved when once it is precipitated. It forms when precipitated a very light granular powder.

Fibrinogen adheres as a sticky deposit to the side of the vessel. It coagulates at $56^{\circ} \mathrm{C}$.

On account of their great similarity, both substances are not usually prepared from blood-plasma. Fibringyen is prepared from serous transudations (pericardial, abdominal, or pleuritic fluid, or the fluid of hydrocele), which contain no fibrinoplastiu. Fibrino-plustin is most readily prepared from serum, in which there is still plenty of fibrino-plastin, but no fibrinogen.

2. Preparation of Fibrino-plastin, Serum-globulin, or Paraglobulin.- $(a)$ Dilute blood-serum with twelve times its volume of ice-cold water, and almost neutralise it with acetic acid [add 4 drops of a 25 per cent. solution of acetic acid to every 120 c.c. of diluted serum]; or $(b)$ pass a stream of carbon dioxide through the diluted serum, which suon becomes turbid; after a time a fine white powder, copious and granular, is precipitated.

[(c) Method of Hammarsten. - All the fibrino-plastin in serum is not precipitated either by adding acetic acid or by $\mathrm{CO}_{2}$. Hammarsten found, however, that if crystals of magnesium sulphate be added to complete saturation, it precipitates the whole of the serum-globulin, but does not precipitate serum-albumin; serum-globulin is more abundant than serum-albumin in the serum of the $o x$ and horse, while in man and the rabbit the reverse obtains; (compare $\$ 32)$.

Schuilt found that 100 c.c. of the serum of ox blood yielded 0.7 to $0.8 \mathrm{grm}$; horse's serum, 0.3 to $0.56 \mathrm{grm}$. of dry fibrino-plastin. Fibrino-plastin occurs not only in serum, but also in red blood-corpuscles, in the fluids of connective-tissue, and in the juices of the cornea.

3. Preparation of Fibrinogen.-This is best prepared from hydrocele fluid, although it may also be obtained from the fluids of serous cavities, e.g., the pleura, pericardium, or peritoneum. It does not exist in blood-serum, although it does exist in blood-plasma, lymph, and chyle, from which it may be obtained by a stream of $\mathrm{CO}_{2}$, after the paraglobulin is precipitated. (a) Dilute hydrocele fluid with ten to fifteen times its volume of water, and pass a stream of $\mathrm{CO}_{2}$ through it for a long time. (b) Add powdered common salt to saturation to a seruus transudation, when a sticky glutinous (not very abundant) precipitate of fibriuogen is obtained. 
[Hammarsten and Eichwald find that, although paraglobulin and fibrinogen are soluble in solutions of common salt (containing 5 to 8 per cent. of the salt), a saline solution of 12 to 16 per cent. is required to precipitate the fibrinogen, leaving still in solution paraglobulin, which is not precipitated until the amount of salt exceeds 20 per cent.]

Properties of the Fibrin-Factors.-They are insoluble in pure water, but dissolve in water containing $\mathrm{O}$ in solution. Both are soluble in very dilute alkalies, e.g., caustic soda, and are precipitated from this solution by $\mathrm{CO}_{2}$. They are soluble in dilute common salt-like all globulins-but if a certain amount of common salt be added in excess, they are precipitated. Very dilute hydrochloric acid dissolves them, but after several hours they become changed into a body resembling syntonin or acid-albumin (\$249, III.). Fibrinogen held in solution by common salt coagulates at $52^{\circ}$ to $55^{\circ} \mathrm{C}$. [Frédéricq finds that fibrinogen exists as such in the plasma ; it coagulates at $56^{\circ} \mathrm{C}$., and the plasma thereafter is uncoagulable.

4. Preparation of the Fibrin-Ferment.-(a) Mix blood-serum (ox) with twenty times its volume of strong alcohol, and after one month filter off the deposit thereby produced. The deposit on the filter consists of coagulated insoluble albumin and the ferment; dry it carefully over sulphuric acid, and reduce to a powder. Triturate 1 gram of the powder with 65 c.c. of water for ten minutes, and filter. The ferment is dissolved by the water, and passes through the filter, while the coagulated albumin remains behind (Schmidt).

[(b) Gamgee's Method.-Buchanan's "washed blood-clot" (p. 33) is digested in an 8 per cent. solution of common salt. The solution so obtained possesses in an intense degree the properties of Schmidt's fibrin-ferment.]

In the preparation of fibrino-plastin, the ferment is carried down with it mechanically. The ferment seens to be formed first in fluids outside the body, very probably by the solution of the colourless corpuseles. More ferment is formed in the blood the longer the interval between its being shed and its coagulation. It is destroyed at $70^{\circ} \mathrm{C}$. Blood flowing directly from an artery into alcohol contains no ferment. It is also formed in other protoplasnic parts (Rauschenbach), e.g., in dead muscle, brain, suprarenal capsule, spermatozoa, testicle (Foa and Pellacani), and in vegetable micro-organisms [e.g., yeast] and protozoa (Grohmann), [so that it would seem to be a general product of protoplasm. As the ferment does not pre-exist in colourless blood-corpuscles, it seems to be formed from some mother-substance in them, the blood-plasma itself decomposing this substance].

Coagulation Experiments.-According to A. Schmidt, if pure solutions of (1) fibrinogen, (2) fibrino-plastin, and (3) fibrin-ferment le mixed, fibrin is formed. The process goes on best at the temperature of the body ; it is delayed at $0^{\circ}$; and the ferment is destroyed at the boiling-point. The presence of $\mathrm{O}$ seems necessary for coagulation. The amount of the ferment appears to be immaterial ; large quantities produce more rapid coagulation, but the amount of fibrin formed is not greater.

[Foa and Pellacani find that a filtered watery extract of fresh brain, capsule of the kidneys, testes, and some other tissues, when injected into the blood-vessels of a rabbit, causes coagulation of the blood in the pulmonary eirculation and the heart, death being caused by the action of a substance identical with the fibrin-ferment.]

The amount of salts present has a remarkable relation to coagulation. Solutions of the fibrin-factors deprived of salts, and redissolved in very dilute caustic soda, when mixed, do not coagulate until sufficient $\mathrm{NaCl}$ be added to make a 1 per cent. solution of this salt $(S c h m i d t)$. [Green finds that calcium sulphate brings about coagulation in plasma which shows little or no tendency to clot, while coagulation in its absence is almost or quite prevented.]

When blood or blood-plasma coagulates, all the fibrinogen is used up, so that the serum contains only fibrino-plastin and fibrin-ferment; hence, the addition of hydrocele fluid (which contains fibrinogen) to serum causes coagulation.

[Hammarsten's Theory.-Hammarsten's researches led him to believe that fibrino-plastin is quite unnecessary for coagulation. According to him, fibrin is formed from one body, viz., fibrinogen, which is present in plasma when it is acted upon by the fibrin-ferment; the latter, however, has not been obtained in a pure 
state. Neither he nor Schmidt asserts that this body is of the nature of a ferment, although they use the term for convenience. It is quite certain that fibrin may be formed when no fibrino-plastin is present, coagulation being caused by the addition of calcic chloride or casein prepared in a special way. But, whether one or two proteids be required, in all cases it is clear that a certain quantity of salts, especially of $\mathrm{NaCl}$, is necessary.]

[The main drift of the foregoing evidence points to the presence of one proteid - filrinogen-in the plasma, which under certain circumstances yields fibrin. In shed blood this act seems to be determined by a ferment, perhaps derived from the disintegration of colourless corpuscles.]

[Theory of Wooldridge.-Wooldridge attributes great importance to lecithin. In shed blood the coagulation is brought about by the interaction of the plasma and the colourless corpuscles. If lecithin (which is jresent in considerable amount in the colourless corpuseles) diffuses into the blood, coagulation takes place. When peptone is injected into the blood of the $\log$, the blood does not clot; this is due, according to Wooldridge, to the peptone "preventing the interaction of lencocytes and plasma." If, however, the corpuscular elements are removed by the centrifugal machine, the peptone-plasma can be made to clot. He also believes that fibrin-ferment does not pre-exist in normal plasma, but that "it may make its appearance in that plasma in the absence of all cellular elements, and must therefore come from some constituent or constituents of the plasma itself."]

30. SOURCE OF THE FIBRIN-FACTORS. - Al. Schmidt maintains that all the three substances out of which fibrin is said to be formed, arise from the breaking up of colourless blood-corpuscles. In the blood of man and mammals, fibrinogen exists dissolved in the circulating blood as a dissolution-product of the retrogressive changes of the white corpuscles. Plasma contains dissolved fibrinogen and serumalbumin. The circulating blood is very rich in colourless blood-corpuscles, much richer, indeed, than was formerly supposed. As soon as blood is shed from an artery, enormous numbers of the colourless corpuscles are dissolved-according to Al. Schmidt 71.7 per cent. (horse). First the body of the cell disappears, and then the nucleus. The products of their dissolution are dissolved in the plasma, and one of these products is fibrino-plastin. At the same time the fibrin-ferment is also produced, so that it would seem not to exist in the intact blood-corpuscles. Fibrino-plastin and fibrin-ferment are also produced by the "transition forms" of blood-corpuscles, i.e., those forms which are intermediate between the red and the white corpuscles. They seem to break up immediately after blood is shed. The 1,10od-plutes (p. 16) are also probably sources of these substances.

In amplibians and biris the rel nucleatel corpuscles rapidly break up after blood is shed, and yielit the substance or substances which form fibrin. Al. Schmidt convinced himself that in these animals fibrinogen is originally a constituent of tne bloor-corpuscles.

It is clear, therefore, according to Schmidt's view, that as soon as the bloodcorpuscles, white or red, are dissolved, the fibrin-factors pass into solution, and the formation of fibrin by the interaction of the three substances will ensue.

If a large number of leucocytes be introduced into the circulation of an animal, the leucocytes are dissolved in great numbers in the blood, so that death takes place by diffuse coagulation. Should the animal survive the immediate danger of death, the blood, owing to the want of leucocytes, is completely incapable of coagulating (Groth).

[And. Buchanan thought that the potential element of his "washed blood-clot" resided in the colourless corpuscles, "primary cells or vesicles." He, like Schmidt, found that the buffy coat of horses' blood, which is very rich in white corpuscles, produced coagulation rapidly. Buchanan comprared the action of his washed clot to that of rennet in coagulating milk.]

Pathological. - Al. Schmidt and his pupils have shown that some ferment, probably derived from the clissolution of colourless corpuscles, is found in circulating blood, and that it is more abuntant in venous than in arterial hlood, while it is most abundant in shed blood. It is specially remarkal,le that in septic fever the amount of ferment in blood may increase to such an extent as to permit the occurrence of spontancous coagulation (thrombosis), which may even produce ileath (Arn. Köhler). In febrile cases generally, the anount of ferment is somewhat 
more a bundant (Edelberg and Birk). After the injection of ichor into the blood an enormous number of colourless corpuscles are dissolved ( $F$. Hoffmann). The injection of peptone, $\mathrm{Hb}$, and to a less degree of distilled water, is followed by dissolution of numerous leucocytes.

There are changes in the blood, constituting true blood diseases, in which the physiological metabolism of the colourless corpuscles is enormously increased, so that the metabolic products accumulate in the blood (Alex. Schmidt). The result of this is spontaneous coagulation within the circulatory system, and death even may occur; there is always an increase of temperature. After such a condition, the coagulability of the blood is diminished.

31. Formation of Fibrin. - After several observers had shown that the red blood-corpuscles (bird, horse, frog) participate in the production of fibrin, Landois observed, in 1874, under the microscope that the stromata of the red blood-corpuscles of mammals passed into fibrin. If a drop of defibrinated rabbit's blood be placed in serum of frog's blood, without mixing them, the red corpuscles can be seen collecting together ; their surfaces are sticky, and they can only be separated by a certain pressure on the cover-glass, whereby some of the now spherical corpuscles are drawn out into threads. The corpuscles soon become spherical, and those at the margin allow the hæmoglobin to escape, the decolorisation progresses, from the margin inwards, until at last there remain masses of stroma adhering together. The stroma-substance is very sticky, but soon the cell-contours disappear, and the stromata adhere and form fine fibres. Thus (according to Landois) the formation of fibrin from red blood-corpuscles can be traced step by step. The red corpuscles of man and animals, when dissolved in the serum of other animals, show much the same phenomena.

Stroma-Fibrin and Plasma-Fibrin.-Landois ealls fibrin formed direct from stroma, stromafibrin; fibrin formed in the usual way plasma-fibrin. The stroma-fibrin is closely relaterl chemically to stroma itself; as yet, however, the two kinds of fibrin have not been sharply distinguished chemically. Substances which rapidly dissolve red corpuscles cause extensive coagulation, e.g., injection of bile or bile salts, or lake-coloured blood, into arteries. After the injection of foreign blood the newly-injected blood often breaks up in the blood-vessels of the recipient, while the finer vessels are frequently found plugged with small thrombi (\$ 102).

Coagulable Fluids.-With regard to coagulability, fluids containing proteids may be classified thus :-

(1) Those that coagulate spontaneously, i.e., blood, lymph, chyle.

(2) Those capable of coagulating, e.g., fluids secreted pathologically in serous cavities; for example, hydrocele fluid, which, as usually containing fibrinogen only, does not coagulate spontaneously, but it coagulates on the addition of fibrino-plastin and ferment (or of bloodserum in which both occur).

(3) Those which do not coctgulate, e.g., milk or seminal fluid, which do not seem to contain fibrinogen.

32. CHEMICAL COMPOSITION OF PLASMA AND SERUM.-I. Proteids occur to the amount of 8 to 10 per cent. in the plasma. Only $0 \cdot 2$ per cent. of these go to form fibrin. After the formation of the fibrin the plasma is converted into serum. The sp. gr. of human serum is 1027 to 1029 . It contains several proteids. [According to Hammarsten, humen serum contains $9 \cdot 207$ per cent. of solids, - of these, $3 \cdot 103=$ serum-globulin, and $4 \cdot 516=$ serum-albumin, i.e., in the ratio of $1: 1 \cdot 511$. In horse-serum the proportion is $4 \cdot 5: 2 \cdot 6$, in ox-serum $4 \cdot 16: 3 \cdot 29$, and rabbit-serum $6 \cdot 22: 1 \cdot 78$. The total amount of proteids in blood seems to be much more constant than are the relative proportions of serum-albumin and serumglobulin (Salvioli).]

(a) Serum-globulin or Paraglobulin ( 2 to 4 per cent.). If crystals of magnesium sulphate be added to saturation to serum at $35^{\circ} \mathrm{C}$., serum-globulin is precipitated, but not serum-albumin. It is soluble in 10 per cent. solution of common salt, and coagulates at $69-75^{\circ} \mathrm{C}$. Its specific rotatory power is $-47 \cdot 8^{\circ}$ (Frédéricq).

[Serum-globulin was described by Panum under the name of "serum-casein"; by Al. Schmidt, as "fibrino-plastic substance"; and by Kühne, as "paraglobulin."] During hunger the globulin increases and the albumin diminishes.

(b) Serum-albumin (3-4 per cent:). Its solutions begin to be turbid at $60^{\circ} \mathrm{C}$., and coagulation occurs at $73^{\circ} \mathrm{C}$., the fluid becoming slightly more alkaline at the same time. If sodium chloride be cautiously added to serum, the coagulating temperature may be lowered to $50^{\circ} \mathrm{C}$. Its specific rotatory power is from $-62 \cdot 6$ 
to $64.5^{\circ}$ (Starke). It is changed into syntonin or acid-albumin by the action of dilute HCl, and by dilute alkalies into alkali-albuminate.

Serum-albumin is absent from the blood of starving snakes; and reappears atter they are fed (Tiegel).

[Serum-Albumin $v$. Egg-Albumin. -Although serum-albumin is closely related to eggalbumin they differ-( (c) as remards their action upon polarised light ; $(b)$ the precipitate prodnced by adling $\mathrm{HCl}$ or $\mathrm{H} \mathrm{NO}_{3}$ is realily soluble in 4 c.c. of the reagent in the case of serumalbumin, while the precipitate in egg-albumin is dissolved with very great difficulty ; $(c)$ eggalbumin, injected into the veins, is exereted in the urine as a foreign body, while serum-albumin is not; (d) serum-albumin is not coagulated by ether, while egrralbumin is, if the solution is unt alkaline $(\$ 249)$. Serum albumin has never been obtained free from salts, even when it is dialysed for a very long time.]

After all the scrum-globulin in serum is precipitated by magnesium sulphate, serum-albumin still remains in solution. If this solution he heated to 40 or $50^{\circ} \mathrm{C}$. a copious precipitate of non-enamlated serum-albumin is obtained, which is soluble in water. If the serum-albumin be filtered from the fluid, and if the clear fluid he heated to over $60^{\circ} \mathrm{C}$., Fréderieg found that it lwecomes turbid from the precipitation of other proteids; the amount of these other bodies, however, is small.

[Proteids of the Serum. - Halliburton has shown by the method of "fractional heat-coagulation" (i.", ascertaining the temperature at which a proteid is "oagulated, filtering the fluid and again heating the filtrate to a higher temperature), that from the same fluid perhals two or more proteids, all with different temperatures of coagulation, may be obtained. ('are must be taken to keep the reaction c onstant. He finds that serum-globulin coagulates at $75^{\circ} \mathrm{C}$, while serum-albumin in reality consists of three proteids, which coagulate at different temperatures ; $(a)$ at $73^{\circ},(\beta)$ at $77^{\circ}$, and $(\gamma)$ at $84^{\circ} \mathrm{C}$.]

[Precipitation by Salts. - Sulphinte of magnesia not only precipitates serum-globulin but also filminogen. The fluid must be shaken for several hours to get complete saturation. Sodie sulphate, when addecl to serum deprived of its globulin by $\mathrm{MgSO}_{4}$, precipitates serum-albumin, lut it molues no preripitate with pure serum. In this way serum-albumin may be obtained in a pure, uncuagulated, and still soluhle condition. Serum-globulin is thrown down by sodic nitrate, aretate. or carhonate; while all the proteids of the serum are precipitated by potassic anetate or phosphate, and the same result is brought about by adding two salts, e.g., $\mathrm{MgSO}_{4}$ and $\mathrm{Na}_{2} \mathrm{SO}_{4}$ (in this case sollio-magnesic sulphate is formed); $\mathrm{MgSO}_{4}$ and $\mathrm{NaNO}_{3} ; \mathbf{M} \mathrm{NSO}_{4}$ and KI : $\mathrm{XaCl}$ and $\mathrm{Xa}_{2} \mathrm{SO}_{4}$. After serum-globulin is thrown down by $\mathrm{MgSO}_{4}$, the addition of $\mathrm{MaSO}_{4}$ and $\mathrm{Na}_{2} \mathrm{SO}_{4}$ or the donhle-salt, precipitates the serum-albumin, which is still soluble in water. As sulphate of ammonia precipitates all the proteids except peptones, it, may be used (Halliburton).]

(The plasma of Invertebrata (ilecapod erustaceans, some gasteropods, ceplaalopols, \&c.) clots like vertehrate hlool, and contains fibrinogen, but, in addition, there is found in it a substance corresponding to hiemoglolin, and called by Frédéricq, hæmocyanin. It exists like $\mathrm{Hb}$ in two conditions, one reduced and the other oxy-hemocyanin, the former being colourless, the latter whe. In its general characters it resemilles $\mathrm{Hl}$, although it contains copper instead of iron, and gives no absorption-bands (Holliburton). In the blood of some decapod crustaceans there is a reldish pigment, tetronerythrin, which is illentical with that in the exoskeleton and hypolerm. It belongs to the group of lipochromes, like some of the pigments of the retina. The hamocyanin is respiratory in function, and it is remarkable that it is contained in the flasma, and not in the formed elements like the $1 \mathrm{~b}$ of vertebrates. So that, stated broadly, in these invertebrates the pla-ma is both nutritive and respiratory in its functions, while in vertebrates the recl corpuscles chiefly are respiratory and the plasma nutritive.]

II. Fats $(0.1$ to 0.2 per cent.). - Neutral fats (tristearin, tripalmitin, triolein) occur in the blood in the form of small microscopic granules, which, after a meal rich in fat (or milk) render the serum quite milky.

[The anount of fat in the serum of fasting animals is about 0.2 per cent.; during digestion 0.4 to 0.6 per cent.; and in dogs fecl on a diet rich in fat it may be 1.25 per cent. There are also minute traces of fatty ariels (succinic). Rihrig showed that soluble sorps, i.e., alkaline salts of the fatty acids, cannot exist in the blood. Cholesterin may be considered along with the fats. It occurs in considerable amount in nerve-tissues, and, like fats, is extracted by ether from the dry resilue of blood-serum. Hoppe-Seyler found 0.019 to 0.314 per cent. in the serum of the blood of fattened geese. There is no fat in the red blood-corpuscles. Lecithin (its decomposition-products, glycerin-phosphoric acid and protagon) occur in serum and also in the blood-corpuscles.] 
III. Traces of Grape-Sugar [0.1 to 0.15 per cent. (more in the hepatic vein, 0.23 per cent.)] derived from the liver and muscles, and increased after hæmorrhage (\$ 175); some glycogen, and another reducing fermentative substance also increased by hæmorrhage.

The amount of grape-sugar in the blood increases with the absorption of sugar from the intestine, and this increase is most obvious in the blood of the portal and hepatic veins; there is also a slight increase in the arterial blood, but there it is rapidly changed. The presence of sugar is ascertained by coagulating blood by boiling it with sodium sulphate, pressing out the fluid and testing it for sugar with Fehling's solution ( $\mathrm{Cl}$. Bernard). Pavy coagulates the blood with alcohol.

IV. Extractives.-Kreatin, urea (0.016 per cent., increased after nitrogenous food), succinic acid, and uric acid (more abundant in gouty conditions), guanin (?), carbamic acid, sarcolactic acid ; all occur in very small amounts.

V. Salts $(0.85$ per cent.), especially sortic chloride $(0.5$ per cent.) and sodic carbonate. [It is most important to note that the sorla salts are far more abundant in the serum than the potassium salts. The ratio may be as high as $10: 1$.] Animal diet increases the amount of salts, vegetable food diminishes it temporarily.

Salts in human blood-serum (Hoppe-Seyler).

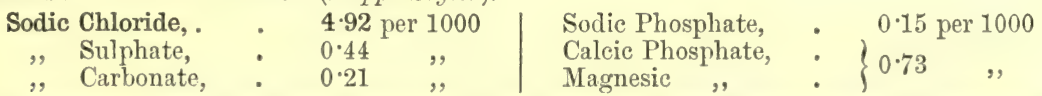

If large quantities of salts are introduced into the blood, they almost entirely disappear from the blood-stream within a few minutes, chiefly by diffusion into the tissues. They are gradually eliminated by the kidneys. The same is true of sugar and peptones (Luduig and Klicouicz).

VI. Water about 90 per cent.

\section{A yellow pigment.}

The pigment may be extracted with methylic alcohol. It shows two absorption-bands of a lipochrome like lutein (Krukenberg). Thudichum regards the pigment of the serum as lutein ; Maly, as hydrobilirubin; and MacMunn as choletelin.

33. THE GASES OF THE BLOOD. - Absorption by Solid Bodies. - A considerable attraction exists between the particles of solid porous bodies and gases, whereby the latter are attracted and condensed within the pores of solid bodies, i.e., the gases are absorbed. Thus, 1 volume of boxwood charcoal (at $12^{\circ} \mathrm{C}$. and ordinary barometric pressure) absorbs 35 volumes $\mathrm{CO} ., 9 \cdot 4$ vol. $\mathrm{O}, 7.5$ vol. $\mathrm{N}, 1.75 \mathrm{vol}$. H. Heat is always formed when gases are absorbed, and the amount of heat evolved bears a relation to the energy with which the absorption takes place. Non-porous bodies are similarly invested by a laver of condensed gases on their surface.

By Fluids. - Fluids can also absorb gases. A known quantity of fluid at different pressurcs always absorbs the same volume of gas. Whether the pressure be great or small, the volume of the gas absorbed is equally great ( $W$. Henry). But according to Boyle (1662) and Mariotte's law (1679) on the compression of gases, when the pressure within the same volume of gas is increased, the volume varies inversely as the pressure. Hence it follows that, with varying pressure, the volume of gas absorbed remains the same, but the quantity of gas (weight) is directly proportional to the pressure. If the firessure $=0$, the weight of the gas absorbed must also $=0$. As a necessary result of this, we see that (1) fluids can be freed of their absorbed gases in a vacuum under an air-pump.

Coefficient of Absorption means the volume of a gas $\left(0^{\circ} \mathrm{C}\right.$.) which is absorbed by a unit of volume of a liquid (at $760 \mathrm{~mm}$. Hg) at a given temperature. The volume of a gas absorbed, and therefore the coefficient of absorption, is quite independent of the pressure, while the weight of the gas is proportional to it. Temperature has an important influence on the coefficient of absorption. With a low temperature it is greatest; it diminishes as the temperature increases ; and at the boiling-point it $=0$. Hence, it follows that-(2) absorlied gases may be expelled from fluids simply by causing, the fluids to boil. The coefficient of absorption diminishes for different fluids and gases, with increasing temperature, in a special, and by no means uniform, manner, which must be determined empirically for each liquid and gas. Thus the coefficient of absorption for $\mathrm{CO}_{2}$ in water diminishes with an increasing temperature, while that for $\mathrm{H}$ in water remains unchanged between $0^{\circ}$ and $20^{\circ} \mathrm{C}$.

Diffusion of Gases. - Gases which do not enter into chemical combinations with each other mix with each other in definite proportions. If the necks of two flasks be placed in communication by means of a glass or other tube, and if the lower flask contain $\mathrm{CO}_{2}$, and the upper one $\mathrm{H}$, the gases mix quite independently of their different specific gravities, both gases forming in 
each flask a perfectly uniforn mixture. The phenomenon is called the diffusion of gases. If a porous mimbiane be previously inserted between the gases, the exchange of gases still goes on through the menbrane. But (as with endosmosis in fluids) the gases pass with unequal rapidity through the pores, so that at the beginning of the experiment a larger amount of gas is found on one side of the membrane than on the other. According to Graham, the rapidity of the diffusion of the gases through the pores is inversely proportional to the square root of their specific gravities. (According to Bunsen, however, this is not quite correct.)

Different Gases in a Gaseous Mixture do not Exert Pressure upon one another. - Gases, therefore, pass into a space filled with another gas, as they would pass into a vacuum. If the surface of a fluid containing absorberl sases be placed in contact with a very large quantity of another gas, the ahsorbel gase's liffuse into the latter. Hence, absorbed yases can be removed hy (3) pussing a stream of another ges thiongh the fluil, of by merely shaking up the fluid with another gas.

Partial Pressure. - If tu or mor' yases are mime in a closel space over a fluid, as the different gases existing in a gaseous mixture exert no pressure upon each other, the several gases are alsoritul. The weight of each absorbed is proportional to the pressure under which each gas woulil he, were it the only gas in the space. This pressure is called the partial pressure of a gas (liunsen). The absorption of gases from their mixtures, therefore, is proportional to the pritial pressur. The partial pressure of a gas in a space is at the same time the expression for the tension of the gas absorbed by a fluid.

The air contains $(1 \cdot 2096$ volume of $\mathrm{O}$, and 0.7904 volume $\mathrm{N}$. If 1 volume of the air be placed under a pressure, $P$, over water, the partial pressure under which $\mathrm{O}$ is absorbed $=0.2096 \mathrm{P}$; that for $X=0.79114$ P'. At $0^{\circ} \mathrm{C}$., and $760 \mathrm{~mm}$. jressure, 1 volume of water absorbs 0.02477 volume of air, consisting of 0.00862 volume 0 , and $0^{\circ}(1) 1615$ volume $\mathrm{N}$. It contains, therefore, 34 per crnt. O and 66 jer cent. N. Therefore, rater alsorls from the air a mixture of gases containing a larger percentage of (1) than the air itself.

34. EXTRACTION OF THE BLOOD GASES. - [The blood to be analysed must be collected over mercury so as to avoil contact with air. This is lone by means of a special apparatus, consistinn of a fraluaterl tube filled with mercury and communicating with a glass globe also filled with merriry, which ran le lowered as the blood flows into the graduated tube.] The extraction of the irases from the blool, and their collection for chemical analysis, are carried out by means of the mremial fump (C. Lul, *ig). Fig. 22 shows in a diagrammatic form the arrangement of Pflüger's gas-pump.

It consists of a reepptarle for the blool, or "blood-bulb" ( $\mathrm{A}$ ), a glass globe capable of containing 250 to 300 c.e., connecterl above and below with tubes, each of which is provided with a stop-cock, " and $b ; b$ is an orelinary stop-cock, while $a$ has through its long axis a perforation which opens at $r$, and is so arranged that, according to the position of the handle, it leads up into the blood-bulb (position r', ${ }^{\prime}$ ), or downwarls through the lower tube (position $x^{\prime}, a^{\prime}$ ). This hlood-lull is first completely emptied of air (by means of a mercurial air-pump), and then care. fully weighed. One ent $\left(e^{\prime}\right)$ of it is tied into an artery or a vein of an animal, and when the lower stop.cock is placel in the position $r$, a blool flows into the receptacle. When the neessary amount of blool is collected, the lower stop-cock is put into the position $x^{\prime}$, $a^{\prime}$, and the blood-hilh, after being cleaned most carefully, is weighed to ascertain the weight of the amount of blool collected. The seconl part of the apparatus consists of the froth-chamber, B, leading upwards and downwards into tubes, each of which is provided with an orlinary stop-cock, $c$ and d. The froth-chamler, as its name denotes, is to catch the froth which is formed during the energetic evolution of the gases from the blool. The lower averture of the froth-chamber is connected by means of a well-ground tube with the blood-bulh, while alove it communicates with the third part of the apparatus, the drying-chamber, G. This consists of a U-shaped tube, provileel below with a small glass bulb, which is half filled with sulphuric acid, while in its limbs are placel pieces of pumice-stone also moistened with sulphuric acid. As the blood gases pass through this apparatus (which may be shut off by the stop-cocks $c$ and $f$ ), they are freel from their walory vapme, hy the sulphuric acil, so that they pass quite dry through the stop-cock, $f$. The short well-ground tube, D, is fixed to $f$, and to the former is attached the small burmetric tule or monometer, y, which indieates the extent of the vacuum. From D we pass to the pump proper. This consists of two large glass bulbs, which are continued above and below into open tubes; the lower tubes, $\mathrm{Z}$ and $w$, being united by a caoutchouc tube, $\mathrm{G}$. Poth the bullos and the caoutchouc tube contain mereury - the bulbs being about half full, and $\mathrm{F}$ being larger than $\mathrm{E}$. The bulb, E, is fixed; but $\mathrm{F}$ can be raised or lowered by means of a pulley with a rack and pinion motion. If $\mathrm{F}$ be raised, $\mathrm{E}$ is filled; if $\mathrm{F}$ be lowered, $\mathrm{E}$ is emptied. The upper end of $\mathrm{E}$ divides into two tubes, $g$ and $h$, of which $g$ is united to $D$. The ascending tube, $h$ (gas-delivery tube), is very narrow, and is bent so that its free end dips into a vessel containing mercury, $v$ (a pmeumatic trough), and the opening is placed exactly under the tube for collecting the gases, the eudiometer, $\mathrm{J}$, which is also filled with mercury. Where $\mathrm{g}$ and $\mathrm{H}$ unite, there is a two-way stop-cock, which in one position, $\mathrm{H}$, places $\mathrm{E}$ in comnunication with $A, B, G, D$, the chambers to be exhausted, and in the position $K$ shuts off $A, B, G, D$, and 
places the bulb, E, in communication with the gas-delivery tube, $h$, and the eudiometer, J. $\mathrm{B}, \mathrm{G}, \mathrm{D}$ are completely emptied of air, thus :-The stop-cock is placed in the position, $\mathrm{K}$; raise $F$ until drops of mercury issue from the fine tube, $i$ (not yet placed under $J$ ); place the stopcock in the position $\mathrm{H}$, lower $\mathrm{F}$; stop-cock in position, $\mathrm{K}$, and so on until the barometer, $y$, indicates a complete vacuum. $J$ is now placed over $i$. Open the cocks, $c$ and $b$, so that the blood-bulb, A, communicates with the rest of the apparatus, and the blood gases froth up in $\mathrm{B}$,

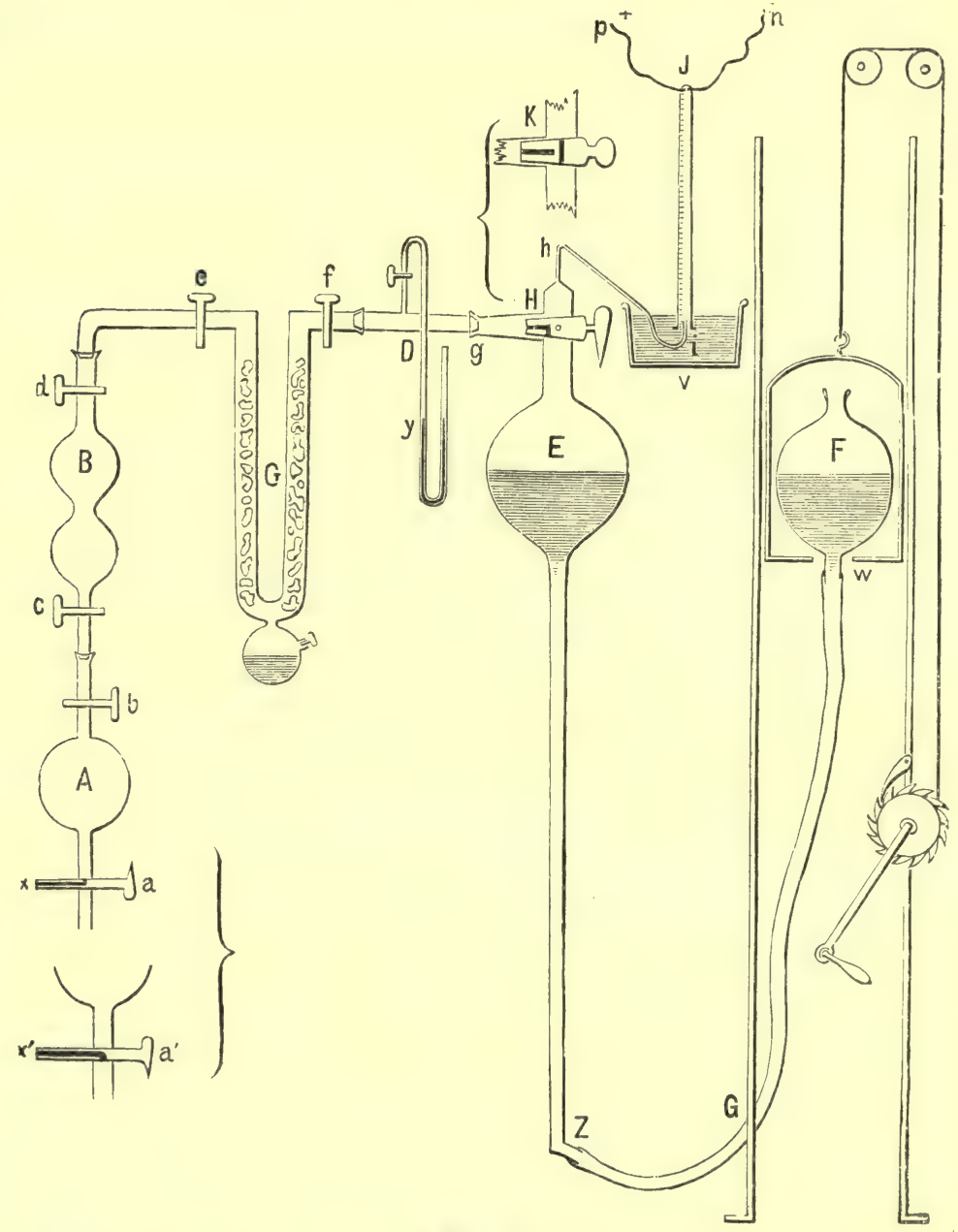

Fig. 22.

Scheme of Pflüger's gas-pump. A, blood-bulb; $a$, stop-cock, with a longitudinal perforation opening upwards; $a^{\prime}$, the same opening downwards; $b$ and $c$, stop-cocks ; B, froth-chamber ; $d, e, f$, stop-cocks; $G$, drying-chambers, containing sulphuric acid and pumice-stone ; $\mathrm{D}$, tube, with manometer, $y$.

and after being dried in $\mathrm{G}$ pass towards $\mathrm{E}$. Lower $\mathrm{F}$, and they pass into $\mathrm{E}$; stop-cock in position, $K$, raise $F$, and the gases are collected in $J$ under mercury. The repeated lowering and raising of $\mathrm{F}$ with the corresponding position of the stop-cocks ultimately drives all the gases into J. The removal of the gases is greatly facilitated by placing the blood-bulb, $\mathbf{A}$, in a vessel containing water at $60^{\circ} \mathrm{C}$.

It is well to remove the gases from the blood immediately after it is collected from a bloodvessel, because the $O$ undergoes a diminution if the blood be kept. Of course, in making several 
analyses it is diffienlt to do this, and the best plan to pursue in that case is to keep the receptacles containing the blood on ice.

Mayow $(1670)$ observed that gases were given off from blood in vacuo. Magnus (1837) investigated the percentage composition of the blood gases. The more important recent investigations have been maile by Lothar Meyer (1857), and by the pupils of C. Ludwig and E. Pfliiger. .

35. QUANTITATIVE ESTIMATION OF THE BLOOD GASES.-The gases ohtrined from blood consist of $\mathrm{O}, \mathrm{CO}_{2}$, and $\mathrm{N}$. Pflïger obtained (at $0^{\circ} \mathrm{C}$. and 1 metre $\mathrm{Hg}$ pressure) $47 \cdot 3$ volumes per cent., consisting of-

$$
\mathbf{0}, 16.9 \text { per cent. ; } \mathbf{C O}_{2}, 29 \text { per cent.; } \mathbf{N}, 1.4 \text { per cent. }
$$

$\Lambda \mathrm{s}$ is shown in fig. $22, \mathrm{~J}$, the gases are obtained in an eudiometer, i.e., in a narrow tube, closed at one end, and with a very exact scale marked on it, and having two fine platinum wires melted into its upper end, with their free ends projecting into the tube $(p$ and $n)$.

(1) Estimation of the $\mathrm{CO}_{\ldots} . \mathrm{A}$ small ball of fused caustic potesh, fixed on a platinum wire, is introduced into the mixture of gases through the lower end of the eudiometer under cover of the mercury. The surface of the potash hall is moistened before it is introduced. The $\mathrm{CO}_{2}$ unites with the jotash to form potassium carlonate. The potash bulb is withdrawn after 24 hours. The diminution in volume inclicates the amount of $\mathrm{CO}_{2}$ absorbed.

(2) Estimation of the 0.-(u) Just as in estimating the $\mathrm{CO}_{2}$, a ball of phosphorus on a platinum wire is introluced into the eudiometer; it absorls the $\mathrm{O}$ and forms phosphoric acid. Another plan is to employ a small papier-mache ball saturated with pyrogallic acid in crustic. potush, which rapidly alsorhis $O$. After the ball is removerl, the diminution in volume indicates the quantity of $O$.

(h) The $O$ is most easily and accurately estimated by cxploding it in the eudiometer. Introduce a sufficient quantity of $\mathrm{H}$ into the euliometer, and accurately ascertain its volume; an electrical spark is now passeel between the wires, $p$ and $n$, through the mixture of gases; the 0 and $H$ unite to form water, which canses a diminution in the volume of the gases in the endiometer, of which $\frac{7}{3}$ is due to the $\mathrm{O}$ user to form water $\left(\mathrm{H}_{2} \mathrm{O}\right)$.

(.) Estimation of the $\mathrm{N}$. - When the $\mathrm{CO}_{2}$ and $\mathrm{O}$ are estimated by the above method, the remainder is pure $\mathrm{N}$.

36. THE BLOOD GASES. - [In human blood the average total gases are estimated to be, at $0^{\circ} \mathrm{C}$. and 1 metre pressure,

$\begin{array}{lccc} & \mathbf{0} & \mathbf{C O}_{2} & \mathrm{~N} \\ \text { Arterial blood, } & 17 & 30^{2} & 1 \text { to } 2 \text { per cent. } \\ \text { Venous blood, } & 6 \text { to } 10 & 35 & 1 \text { to } 2\end{array}$

or, calculated at $0^{\circ} \mathrm{C}$. and $760 \mathrm{~mm}$. pressure,

$\begin{array}{lcccc}\text { Arterial blood, } & 20 & 39 & 1.4 & \text { per cent. } \\ \text { Venous blood, } & 8 \text { to } 12 & 46 & 1.4 \quad, \quad]\end{array}$

I. 0xygen exists in arterial blood ( $\mathrm{dog}$ ) on an average to the extent of 17 volumes per cent. (at $0^{\circ} \mathrm{C}$. and 1 metre $\mathrm{Hg}$ pressure) (Pflüiger). According to Pfliiger, arterial blood $(\mathrm{dog})$ is saturated to $\frac{9}{10}$ with $\mathrm{O}$, while, according to Hüfner, it is saturated to the extent of $\frac{14}{15}$. In venous blood the quantity varies very greatly; in the blood of a passive muscle 6 volumes per cent. have been found; while in the blood after asphyxia it is absent, or occurs only in traces. It is certainly more abundant in the comparatively red blood of active glands (salivary glands, kidney), than in ordinary dark venous blood.

[Modifying Conditions. - The amount of $\mathrm{O}$ obtainable from the blood depends upon the organ from which the lilood comes, or whether the organ be active or at rest. Thus the $O$ present
in the

Carotil artery is .

Renal artery, : 21 per cent.

Bert finls that increase of the atmospheri Renal vein (kidney at rest), 6 ," amount of $\mathrm{O}$ in arterial blood from 20 to ler cent., while the $\mathrm{CO}_{2}$ is but slightly affected.]

The $\mathbf{0}$ in Blood occurs-(a) simply absorbed in the plasma. This is only a 
minimal amount, and does not exceed what distilled water at the temperature of the body would take up at the partial pressure of the $\mathrm{O}$ in the air of the lungs (Lothar Meyer).

(b) Almost the total $O$ of the blood is chemically united, and therefore not subject to the law of absorption. It is loosely united to the hæmoglobin of the red corpuscles, with which it forms oxyhrmoglobin $(\$ 15)$. With regard to the taking up of $\mathrm{O}$, the total quantity of blood behaves exactly like a solution of hæmoglobin free from $\mathrm{O}$ (Preyer). The absorption of $\mathrm{O}$ is more rapid in blood than in a solution of $\mathrm{Hb}$.

The absorption of this quantity of $\mathrm{O}$ is completely independent of pressure; hence, animals confined in a closed space, until they are nearly asphyxiated, can use up almost all the $\mathrm{O}$ from the surrounding atmosphere. The fact of the union being independent of pressure is proved by the following:- The blood only gives off copiously its chemically united $\mathrm{O}$ when the atmospheric pressure is lowered to 20 millimetres, $\mathrm{Hg}$ (Worm Müller); and, conversely, blood only takes up a little more $\mathrm{O}$ when the pressure is increased to 6 atmospheres (Bert).

Physical Methods of obtaining 0 from Blood.-Notwithstanding the chemical union between the $\mathrm{Hb}$ and $\mathrm{O}$, all the $\mathrm{O}$ of the blood can be expelled from its state of combination by those means which set free absorbed gases-. (a) by introducing blood into a Torricellian vacuum; $(b)$ by boiling; $(c)$ by the conduction of other gases $[\mathrm{H}, \mathrm{N}, \mathrm{CO}$, or $\mathrm{NO}]$ through the blood, because the oxyhæmoglobin compound is so loose that it is decomposed even by these physical means.

Reducing Reagents.-Amongst chemical reagents the following reducing substances-ammonium sulphide, sulphuretted hydrogen, alkaline solutions of subsalts or Stokes's fluid, iron filings, \&c., rob blood of its O (\$15).

Relation to Fe.- The amount of iron in the blood $(0.55$ in 1000 parts) stands in direct relation to the amount of $\mathrm{Hb}$; this to the quantity of blood-corpuseles ; and this, in turn, to the specific gravity of the blood. The amount of $\mathrm{O}$ in the blood, therefore, is nearly proportional to the specific gravity of the blood, and it is also in proportion to the anıunt of iron in the blond. Picard affirms that 2:36 grams of iron in the blood can fix chemically 1 gram $\mathrm{O}$; while, according to Hoppe-Seyler, the proportion is 1 atom iron to 2 atoms $\mathrm{O}$.

During morphia nareosis the amount of $\mathrm{O}$ in the blood is diminished (Evald); after hæmorrhage the arterial blood is saturated with $\mathrm{O}(J . G$. Ott $)$.

Disappearance of $\mathbf{0}$ in Shed Blood.-Even immediately after blood is shed, there is a slight disappearance of $\mathrm{O}$, as a physiological index of respiration of the tissues within the living blood itself ( $\$ 132)$. When blood is kept long outside of the blood-vessels, the quantity of O gradually diminishes, and if it be kept for a length of time at a high temperature it may disappear altogether. This depends upon decomposition occurring in the blood, whereby reducing substances are formed which consume the 0 . All kinds of blood, however, do not act with equal energy in consuming $\mathrm{O}$, e.g., venous blood from active muscles acts most energetically, while that from the hepatic vein has very little effect. $\mathrm{CO}_{2}$ appears in the blood in place of the $\mathrm{O}$, and the colour darkens. The amount of $\mathrm{CO}_{2}$ produced is sometimes greater than that of the $\mathrm{O}$ consumed.

Relation to Acids. - If blood (or a solution of oxyhæmoglobin) be acted upon by acids (e.g., tartaric acid) until it is strongly acid, $\mathrm{O}$ can be pumped out in considerably less amount, while the formation of $\mathrm{CO}_{2}$ is not increased. We must, therefore, assume that, during the decomposition of the $\mathrm{Hb}$ caused by the acids ( $\$ 18$ ), a decomposition product becomes more highly oxidised by the intense chemical union of the $\mathrm{O}$ at the moment of its origin (Lothar Meyer, Zuntz, Strassburg). The same phenomenon occurs when oxyhæmoglobin is decomposed by boiling.

37. IS OZONE PRESENT IN BLOOD?-On account of the numerons and energetic oxidations which occur in connection with the blood, the question has often been raised as to whether the $\mathrm{O}$ of the blood exists in the form of ozone $\left(\mathrm{O}_{3}\right)$. Ozone, however, is contained neither in the blood itself (Schönbein) nor in the blood-gases obtained from it. Nevertheless, the red corpuscles (and $\mathrm{Hb}$ ) have a distinct relation to ozone.

(1) Tests for Ozone.-Hæmoglobin acts as a conveyer of ozone, i.e., it is able to remove the active $\mathrm{O}$ of other bodies and to convey or transfer it at once to other easily oxidisable substances. (a) Turpentine which has been exposed to the air for a long time always contains ozone. The tests for the latter are starch and potassium iodide, the ozone decomposing the iodide, when the iodine strikes a blue with the starch. (b) Freshly-prepared tincture of guaiacum is also rendered 
blue by ozone. If some tincture of guaiacum be arlded to turpentine there is no reaction, but on adling a drop of blood a deep blue colour is immediately produced, i.e., blood takes the ozone from the turpentine and converys it at once to the dissolved guaiacum, which becomes blue. It is immaterial whether the $\mathrm{Hb}$ contains $\mathrm{O}$ or not.

(2) It is also assertel that hrmoglobin acts as an ozone-producer, i.e., that it can convert the ordinary .0 of the air into ozone. Hence the reason why red blood-corpuseles alone render guaiacum blue. This reaction succeeds best when the guaiacum solution is allowed to dry on blotting-paper, and a few drops of blood (diluted 5 to 10 times) are poured on it. That the $\mathrm{Hb}$ forms ozone from the surrouming $\mathrm{O}$, is shown by the fact that red blood-corpuseles containing carbonic oxille eanse the blue colour (Kühne and Sikolz). According to PHiiger, however, these reactions only oreur from deromposition of the $\mathrm{Hb}$, so that on this view the blood-corpuscles cannot be regarded as prolucers of ozone.

Sulphuretted hydrogen is decomposed by blood (as by ozone itself) into sulphur and water. Hydric peroxide is decomposed by blool into $\mathrm{O}$ and water [but this reaction is prevented by the adtition of a small amount of hydrocyanic acid (Schünbein)]. Crystallised $\mathrm{Hb}$ does not do this, and $\mathrm{H}_{2} \mathrm{O}_{2}$ may be cautiously injected into the blood-vessels of animals. This would show that unchanged $\mathrm{Hb}$ does not produce ozone.

Various Forms of 0xygen. - There are three forms of oxygen:-(1) The ordinary oxygen $\left(\mathrm{O}_{2}\right)$ in the air. (2) Active or nasient oxygen $(0)$, which never can oceur in the free state, but the moment it is formerl acts as a powerful oxidising agent and produces chemical compounds. It converts water into hydric peroxide - the $\mathrm{N}$ of the air into nitrous and nitric acids, and even $\mathrm{CO}$ into $\mathrm{CO}_{3}$, which ozone does not. It certainly plays an important part in the organism. (3) Ozone $\left(\vec{U}_{3}\right)$, which is formerl by the decomposition of several molecules of ordinary oxygen $\left(\mathrm{O}_{2}\right)$ into two atoms of $\mathrm{O}$, and the appropriation of each of these atoms by a molecule of undecomposed oxygen. It is oxygen conilensed to $\frac{g}{3}$ of its volume.

38. CO AND N IN BL00D.-II. Carbon Dioxide.-In arterial blood there are about 30 volumes per cent. of $\mathrm{CO}_{2}$ at $0^{\circ} \mathrm{C}$. and 1 metre pressure (Setschenonv); but in venous bluod the amount is very variable, e.g., in the venous blood of passive muscles there are 35 volumes per cent. (Scallom'), while in the blood of asphyxia there may be $5 \cdot 6$ volumes per cent. The $\mathrm{CO}_{2}$ in the lymph of asphyxia is less than that in the blood (Buchner, Gaule). The $\mathrm{CO}_{2}$ of the blood may be extracted from it or ismlitetely pumperl sut, but during the process of evacuation, or removal of the gas, a new property of the red blood-corpuscles is produced, whereby they assume the function of an acid, and thus aid in the chemical expulsion of the $\mathrm{CO}_{2}$ This acid-like property of the red corpuscles occurs especially in the presence of $O$ and heat.

(1) The $\mathrm{CO}_{2}$ in the Plasma. - The largest portion of the $\mathrm{CO}_{2}$ belongs to the plasma (or serum), and it all appears to be in a state of chemical combination. Serum takes up $\mathrm{CO}_{2}$ quite independently of pressure, lience it cannot be merely absorbed. $A$ certain part of the $\mathrm{CO}_{2}$ can be removed from the serum (plasma) by the Torricellian vacuum, while another part is obtained only after the addition of an acid. [The latter is called the "fixed" $\mathrm{CO}_{2}$, while the former is known as the "loose" $\mathrm{CO}_{2}$. $]$ The $\mathrm{CO}_{2}$ in the serum exists in the following conditions :-

(1) $\mathrm{CO}_{2}$ is united to the soda of the plasma in the form of "neutral sodic carbonate." This portion of the $\mathrm{CO}_{2}$ can only be displaced from its combination by the addition of an acid. (In depriving blood of its gases the red corpuscles play the rôle of an acid.)

(2) A portion of the $\mathrm{CO}_{2}$ is lorsely united to sodic carbonate in the form of sodic Iricrolumate; the carbonate takes up 1 equivalent of $\mathrm{CO}_{2} ; \mathrm{Na}_{2} \mathrm{CO}_{3}+\mathrm{CO}_{2}+\mathrm{H}_{2} \mathrm{O}=$ $2 \mathrm{NaHCO}_{3}$. This $\mathrm{CO}_{2}$ may be pumped out, as in the process the bicarbonate splits up again into the neutral carbonate and $\mathrm{CO}_{2}$.

Preyer has oljected to this view on the ground that blood is alkaline in reaction, whilst all solutions that contain $\mathrm{CO}_{2}$ in a state of absorption, or loose chemical combination, are always acid. Pfliiger and Zuntz showed that blood, after being completely saturated with $\mathrm{CO}_{2}$, still.
remains alkaline.

As the bicarbonate only gives up its $\mathrm{CO}_{2}$ very slowly in vacu, while blood gives off its $\mathrm{CO}_{3}$ and forms a complex compound, from which the $\mathrm{CO}_{2}$ is rapidly given off in vacuo.

(3) A minimal portion of the $\mathrm{CO}_{2}$ may be chemically united with neutral sodic 
phosphate in the plasma (Fernet). One equivalent of this salt can fix one equivalent of $\mathrm{CO}_{2}$, so acid sodium phosphate and acid sodium carbonate are formed, $\mathrm{Na}_{2} \mathrm{HPO}_{4}+\mathrm{CO}_{2}+\mathrm{H}_{2} \mathrm{O}=\mathrm{NaH}_{2} \mathrm{PO}_{4}+\mathrm{Na}_{2} \mathrm{H} \mathrm{CO}_{3}$ (Hermann). When the gases are removed the $\mathrm{CO}_{2}$ escapes, and neutral sodic phosphate remains.

It is probable, however, that almost all the sodic phosphate found in the blood-ash arises from the burning of lecithin; we have, therefore, to consider only the very small amount of this salt which occurs in the plasma (Hoppe-Seyler and Sertoli).

(B) The $\mathbf{C O}_{2}$ in the Blood-Corpuscles. - The red corpuscles contain $\mathrm{CO}_{2}$ in loose chemical combination; for (1) a volume of blood can fix nearly as much $\mathrm{CO}_{2}$ as an equal volume of serum (Ludwig, Al. Schmidt); and (2) with increasing pressure the absorption of $\mathrm{CO}_{2}$ by blood takes place in a different ratio from what occurs with serum (Pflüger, Zuntz). The red corpuscles can fix more $\mathrm{CO}_{2}$ than their own volume, and the union of the $\mathrm{CO}_{2}$ seems to depend upon the $\mathrm{Hb}$, for Setschenow found that, when $\mathrm{Hb}$ was acted on by $\mathrm{CO}_{2}$, its power of fixing the latter was increased, which is perhaps due to the formation of some substance more suited for fixing $\mathrm{CO}_{2}$. The colourless corpuscles, after the manner of the serum-constituents, also fix $\mathrm{CO}_{2}$, to the extent of $\frac{1}{8}$ to $\frac{1}{12}$ of the absorbing power of serum.

After the use of $\mathrm{I}, \mathrm{Hg}$, sodic oxalate, and nitrite, there is a diminution of $\mathrm{CO}_{2}$ in arterial blood (Feitelberg), and also in fever (Geppert, Minkowski). [In the last case it is perhaps due to the diminished akalinity, and this is in part owing to the acid products formed during the decomposition of the tissues.]

III. Nitrogen exists in the blood to the extent of 1.4 to 1.6 vol. per cent., and it appears to be simply absorbed.

It is doubtful if any part of the $\mathbf{N}$ exists chemically united in the red corpuscles. Blood warmed outside the body, and with a free supply of oxygen, gives off a minute quantity of ammonia, which is perhaps derived from the decomposition of some salt of ammonia as yet unknown (Kühne and Strauch).

39. ARTERIAL AND VENOUS BLO0D. - Arterial blood contains in solution all those substances which are necessary for the nutrition of the tissues, those which are employed in secretion, and it also contains a rich supply of O. Venous blood must contain less of all these, but in addition it holds the used-up or effete substances derived from the tissues, and the products of their retrogressive metabolism are more numerous; there is in venous blood a larger amount of $\mathrm{CO}_{2}$. It is evident also that the blood of certain veins, the portal and hepatic, must have special characters.

The following are the most important points of difference between arterial blood and venous blood:-

$$
\begin{aligned}
& \text { more } \mathrm{O}_{\text {, }} \\
& \text { less } \mathrm{CO}_{2} \text {, } \\
& \text { more water, } \\
& \text { more fibrin, }
\end{aligned}
$$

\section{Arterial Blood contains-}

more extractives, more salts, more sugar,

fewer blood-corpuscles, less urea.

It is bright red and not dichroic.

As a rule it is $1^{\circ} \mathrm{C}$. warmer.

The bright red colour of arterial blood depends on the presence of oxyhæmoglobin, whilst the dark colour of venous blood is due to its smaller proportion of oxyhæmoglobin, and the quantity of reduced hæmoglobin which it contains. The dark change of colour is not to be attributed to the larger quantity of $\mathrm{CO}_{2}^{\prime}$ in venous blood (Marchand); for if equal quantities of $\mathrm{O}$ be added to two portions of blood, and if $\mathrm{CO}_{2}$ be added to one of them, the colour is not changed (Pflüger).

[According to C. Schmidt, the blood of the portal vein contains more water, plasma, salts, and fats, but less extractives and corpuscles than the blood of the hepatic vein; while (when an animal is not digesting) sugar is absent or at least only in traces in the portal vein, and in considerable amount in the hepatic vein (\$ 175).]

40. QUANTITY OF BIOOD. - In the adult the quantity of blood is equal to $\frac{1}{13}$ part of the body-weight (Bischoff), in newly-born children $\frac{1}{19}$ (Welcker).

According to Schiicking, the amount of blood in a newly-born child depends to some extent npon the time at which the umbilical cord is ligatured. The amount $=\frac{1}{15}$ of the body-weight 
when the corl is tied at once, while if it is tied somewhat later it may be 1 . Immediate. ligrature of the cord may, therefore, deprive a newly-born child of 100 grams of blood. Further, the number of corpuscles is less in a child after immediate ligature of the umbilical cord than when it is tied somewhat later (Helot).

The methods of Valentin (1838), and Ed. Wuber (1850), are not now used, as the results obtained are not sufficiently accurate.

Method of Welcker (1854).--Begin by taking the weight of the animal to be experimented on ; place a cannula in the carotid, and allow the blood to run into a flask previously weighed, aud in which small pebbles (or $\dot{H} g$ ) have been placed in order to clefibrinate the blood by shaking. Take a part of this ilefibrinated blood, and make it cherry-red in colour by passing through it a stream of $\mathrm{CO}$ (because ordinary blood varies in colour according to the amount of 0 contained in it-Gschidlen, Heidenhin). Tie a - shaped cannula in the two cut ends of the carotid, and allow a $0^{\circ} 6$ per cent. solution of common salt to flow into the vessel from a pressure bottle; collect the coloured Huid issuing from the jugular veins and inferior vena cava until the fluil is yuite clear. The entire boly is then chopped up (with the exception of the contents of the stomach aml intestines, which are weighed, and their weight deducted from the borly-weight, and extracted with water, and after twenty-four hours the fluid is expressed. This water, as well as the washings with salt solution, are collected and weighed, and part of the mixture is saturated with CO. A sample of this dilute blood is placed in a vessel with parallel siles (1 cm. aparti oplosite the light (the so-ealled hæmatinometer), and in a second vessel of the same dimpusions a sample of the undiluted $\mathrm{CO}$ blood is diluted with water from a burette, until loth fluils give the sume intensity of colour. From the quantity of water required to dilute the lilooul to the tint of the washings of the bloor-vessels, the quantity of blood in the washings is calculaterl. (In chopping up the muscles alone, we obtain the amount of $\mathrm{Hb}$ present in them, which is not taken into calculation.

Quantity of Blood in Various Animals.-The quantity of blood in the mouse

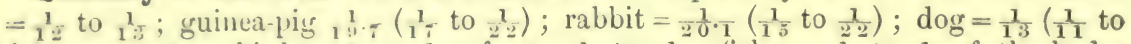
$\left.{ }_{1}^{1}\right)$; cat $=\frac{1}{3} ;$; birds $=\frac{1}{10}$ to $\frac{1}{1: 5} ;$ frog $=\frac{1}{15}$ to $\frac{1}{2}$; fishes $=\frac{1}{14}$ to $\frac{1}{19}$ of the bodyweight (without the contents of the stomach and intestines).

The sprecifie gravity of the blool ought always to be taken when estimating the amount of hlovel. The anomut of hloul is liminished during inanition; fat persons have relatively less hlool; after hirmorrhage the loss is at first replaced by a watery fluid, while the blood-corpuscles are gradually regenerated.

Blood in Organs.- The estimation of the quantity of blocd in different organs is done by suddenly ligaturing their blood-vessels intra vitam. A watery extract of the chopped-up organ is prepared, and the quantity of blood estimated as described above. Koughly it may be said that the lungs, heart, large arteries, and veins contain $\frac{1}{3}$; the muscles of the skeleton, $\frac{1}{4}$; the liver, $\frac{1}{4}$; and other organs, $\frac{1}{4}$ (Ranke).]

41. ABNORMAL CONDITIONS OF THE BL00D. - (A) 1 Polyæmia. - (1) An increase in the entire mass of the blool, unifurmly in all organs, constitutes polyamia or plethora, and in overnourished indiviluals it may approach a pathological condition. A bluish-red colour of the skin, swollen veins, large arteries, hard full pulse, injection of the capillaries and smaller vessels of the visible mucous membranes are signs of this state, and, when accompanied by congestion of the brain, there is vertigo, congestion of the lungs, and breathlessness. After major anputations with little loss of blood a relative but transient increase of blood has been found (?) (plethora apocoptica).

Transfusion. - Polyamia nay be produced artificially by the injection of blood of the same species. If the normal quantity of blood be increased 83 per cent. no abnormal condition occurs, because the hlood-pressure is not permanently raised. The excess of blood is accommodated in the grently disteniled capillaries, which may be stretched beyond their normal elasticity. If it be increased to $150 \mathrm{per}$ cent. there are variations in the blood-pressure, life is endangered, and there may be sudden rupture of blood-vessels (Worm Müller).

Fate of Transfused Blood. - After the transfusion of blood the formation of lymph is greatly increased; but in one or two days the serum is used up, the water is excreted chiefly by the urine, and the albumin is partly changed into urea. Hence, the blood at this time appears to be relatively richer in blool-corjuseles (Panum, Lesser, Worm Müller $)$. The red corpuseles break up much more slowly, and the products thereof are partly excreted as urea and partly (but not constantly) as bile-pigments. Even after a month an increase of coloured blood. corpuscles has been olserved (T'schirjew). That the blood-corpuscles are broken up slowly in the economy is proved liy the fact, that the amount of urea is much larger when the same quan. tity of bloor is swallowed by the animal than when an equal amount is transfused ( $T$ schirjew, Landois). In the latter case there is a moderate increase of the urea, lasting for days, a proof 
of the slow decomposition of the red corpuscles. Pronounced over-filling of the vessels causes loss of appetite and a tendency to hæmorrhage of the mucous membranes.

(2) Polyæmia serosa is that condition in which the amount of serum, i.e., the amount of water in the blood, is increased. This may be produced artificially by the transfusion of bloodserum from the same species. The water is soon given off in the urine, and the albumin is decomposed into urea, without, however, passing into the urine. An animal forms more urea in a short time from a quantity of transfused serum than from the same quantity of blood, a proof that the blood-corpuscles remain longer undecomposed than the serum (Forster, Landois). If serum from another species of animal be used (e.g., dog's serum transfused into a rabbit), the blood-corpuscles of the recipient are dissolved; hæmoglobinuria is produced (Ponfick); and if there be general dissolution of the corpuscles, death may occur (Landois).

(3) Polyæmia aquosa is a simple increase of the water of the blood, and occurs temporarily after copious drinking, but increased diuresis sonn restores the normal condition. Diseases of the kidneys, which destroy their secreting parenchyma, produce this condition, and often general dropsy, owing to the passage of water into the tissues. Ligature of the ureter produces a watery condition of the blood.

(4) Plethora polycythæmica, Hyperglobulie.-An increase of the red corpuscles has been assumed to occur when periodically recurring hemorrhages are interrupted, c.g., menstruation, bleeding from the nose, \&c.; but the increase of corpuscles has not been definitely proved. There is a proved case of temporary polycythæmia, viz., when similar blood is transfused, a part of the fluid being used up, while the corpuseles remain unchanged for a considerable time. There is a remarkable increase in the number of blood-corpuscles (to 8.82 millions per cubic millimetre) in certain severe cardiac affections where there is great congestion, and much water transudes through the vessels. In cases of hemiplegia, for the same reason, the number of corpuseles is greater on the paralysed congested side (Pensoldt). After diarrhoea, which dimin. ishes the water of the blood, there is also an increase (Brouardel), and the same is the case after profuse sweating and polyuria. Drugs (alcohol, chloral, amyl nitrite) which act on the bloodvessels affect the number of corpuscles; during contraction of the blood-vessels their number increases, during dilatation they diminish in number (Andreesen). There is a temporary iucrease in the hæmatoblasts as a reparative process after severe hæmorrhage ( $\$ 7$ ), or after acute cliseases. In cachectic conditions this increase continues, owing to the diminished non-conversion of these corpuscles into red corpuscles. In the last stages of cachexia the number diminishes more and more until the formation of hæmatoblasts ceases (Hayen).

(5) Plethora hyperalbuminosa is a term applied to the increase of albumins in the plasna, such as occurs after taking a large amount of food. A similar condition is producerl by transfusing the serum of the same species, whereby, at the same time, the urea is increased. Injection of egg-albumin produces albuminuria (Stokvis, Lehmann).

[T'he subcutaneous injection of human blood has been practised with good results in anæmia (v. Ziemssen). When defibrinated human blood is injected subcutaneously, while its passage into the circulation is aided by massage, it causes neither pain nor inflammation, but the blood of animals, and a solution of hæmoglobin, always induce abscess (Benczur). Blood is also rapidly absorbed when injected in small amount into the respiratory passages.]

Mellitæmia.-The sugar in the blood is partly given off by the urine, and in "diabetes mellitus" 1 kilo. $(2 \cdot 2$ lbs. $)$ may be given off daily, when the quantity of urine may rise to 25 kilos. To replace this loss of grape-sugar a large amount of food and drink is required, wherehy the urea may be increased threefold. The increased production of sugar causes an increased decomposition of albuminous tissues; hence the urea is always increased, even though the supply of albumin be insufficient. The patient loses flesh ; all the glands, and even the testicles, atrophy or degenerate (pulmonary phthisis is common); the skin and bones become thinner; the nervous system holds out longest. The teeth become carious on account of the acid saliva, the crystalline lens becomes turbid from the amount of sugar in the fluid of the eye which extracts water from the lens, and wounds heal badly because of the abnormal condition of the blood. Absence of all carbohydrates in the food causes a diminution of the sugar in the blood, but does not cause it to disappear entirely. [The sugar in the blood is also increased after the inhalation of chloroform or amyl nitrite, and after the use of curara, nitro-benzole, and chloral (\$ 175).] An excessive amount of inosite has been found in the blood and urine ( $\$ 267$, constituting mellituria inosita ( $V o h l)$.

Lipæmia, or an increase of the Fat in the Blood, occurs after every meal rich in fat (e.g., in sucking kittens), so that the serum may become turbid like milk. Pathologically, this occurs in a high degree in drunkards and in corpulent individuals. When there is great decomposition of albumin in the body (and therefore in very severe diseases), the fat in the blood increases, and this also takes place after a liberal supply of easily decomposable carbohydrates and much fat.

After injuries to bones affecting the marrow, not unfrequently fatty granules pass from the marrow through the imperfect walls of the blood-vessels into the blood-stream. These fatty particles may form fat emboli, e.g., in the liver or lungs, or they may appear in the urine.

If granules of cimnabar or indigo are injected into the blood, they are taken up by the 
leucocytes, and by them are carried outside the blood-stream. The cells of the splenic pulp, marrow of bone, and the liver also take up these particles (Sirbel).

The salts remain very persistently in the blood. 'The withdrawal of common salt produces albuminuria, and, if all salts be withheld, paralytic phenomena occur (Forster). Over-feeding with salted food, such as salt meat, has caused death through fatty degeneration of the tissues, esprecially of the glanils. Withdrawal of lime and phosphoric acill produces atrophy and softening of the bones. In infectious diseases and dropsies the salts of the blood are often increased, and diminished in inflammation and cholera. $[\mathrm{NaCl}$ is absent from the urine in certain stages of pneumonia, and it is a good sign when the chlorides begin to return to the urine.] [In scurvy the corpusicular elements are diminished in amount, but we have not precise information as to the salts, although this disease is prevented, in persons forced to live upon preserved and salted food, by a liberal use of the salts - especially potash salts-of the organic acids, as contained in lime-juice. In gout the blood, during an acute attack, and also in chronic gout, contains an excess of uric acid (Garrod).]

The amount of fibrin is increased in inflammations of the lung and pleura [croupous pmeumonia, erysipelas], hence such blood forms a crusta phlogistica (\$ 27 ). In other diseases, where decomposition of the blood-corpuseles occurs, the fibrin is increased, perhaps because the dissolved red corpuscles yield material for the formation of fibrin. After repeated hromorrhages, sigm. Mayer found an increase of fibrin. Blood rich in fibrin is said to coagulate more slowly than when less fibrin is present-still there are many exceptions.

(B) (I. Diminution of the Quantity of Blood, or its Individual Constituents. - (1) Oligømia vera, Anæmia, or diminution of the guantity of blood as a whole, occurs whenever there is hemorrhage. Life is endangered in newly-born children when they lose a few ounces of blood; in children a year old, on losing half a poumd; and in alults, when one-half of the total blood is lost. Women bear loss of hlood much better than men. The perioulical formation of blood after each menstruation seems to enable blood to be renewed more rapidly in their case. Stout persons, old people, and children do not bear the loss of blood well. The more rapidly blood is lost, the more dangerous it is. [A moderate loss of blood is soon made up, but the fluid part is more quickly restored than are the corpuscles.]

Symptoms of Loss of Blood. - Great loss of blood is accompanied by general paleness and coldness of the cutaneous surface, inereased oppression, twitching of the eyeballs, noises in the ears and vertigo, loss of voice, great breathlessness, stopluage of secretions, coma ; dilatation of the pupils, involuntary vacuations of urine and freces, and lastly, general convulsions, are sure signs of death by hæmorrhage. In the gravest cases recovery is only possible by means of transfusion. Animals can bear the loss of one-fourth of their entire blood without the bloodpressure in the arteries permanently falling, because the blood-vessels contract and accommodate themselves to the smaller yuantity of blood (in conseguenee of the stimulation of the vasomotor centre in the medulla). The loss of one-third of the total blood diminishes the blood-pressure considerably (one-fourth in the carotid of the dog). If the hemorrhage is not such as to cause death, the fluid part of the blool and the dissolved salts are restored by absorption from the tissues, the blood-pressure grallually rises, and then the albumin is restoreal, though a longer time is remuires for the formation of red corpuscles. At first, therefore, the blood is abnormally rich in water (hydræmia), and at last abnormally poor in corpuscles (oligocythæmia, hypoglobulie). With the increased lymph-stream which pours into the blood, the colourless corpuscles are considerably increased above normal, and during the period of restitution fewer red corpuscles seem to be used up (e.g., for bile).

After moderate bleeding from an artery in animals, Buntzen observed that the volume of the hlood was restored in several hours; after more severe hiemorrhage in 24 to 48 hours. The red blood-corfuscles, after a loss of blood equal to $1 \cdot 1$ to $4 \cdot 4$ per cent. of the body-weight, are restored only after 7 to 34 days. The regeneration begins after 24 hours. During the period of regeneration the number of the blood-corpuscles in an early stage of development is increased. The newly-formed corpuscles contain less $\mathrm{Hb}$ than normal (Jac. G. Ott). Even in man the duration of the periou of regeneration depends upon the amount of blood lost (Lyon). The amount of hamoglobin is diminished nearly in proportion to the anount of the hremorrhage
(Bizzozero and Salvioli).

Metabolism in Anæmia. - The condition of the metabolism within the bodies of anæmic persons is important. The decomposition of proteids is increased (the same is the case in hunger), hence the excretion of urea is increased (Bauer). The decomposition of fats, on the contrary, is diminished, which stands in relation with the diminution of $\mathrm{CO}_{2}$ given off. Anemic and chlorotic persons put on fat easily. The fattening of cattle is aided by occasioual bleedings and by intercurrent periods of hunger (Aristolle).

(2) An excessive thickening of the blood through loss of water is called oligæmia sicca. This occurs in man after copious watery evacuations, as in cholera, so that the thick tarry blood stagnates in the vessels. Perhaps a similar condition-though to a less degree-may
exist after very copious perspiration.

(3) If the proteids in blood be abnormally diminished the condition is called oligrmia hypalbuminosa; they may be diminished about one-half. They are usually replaced by an 
excess of water in the blood [so that the blood is watery, constituting hydræmia]. Loss of albumin from the blood is caused directly by albuminuria (25 grams of albumin may be given off by the urine daily), persistent suppuration, great loss of milk, extensive cutaneous ulceration, albuminous diarrhoea (dysentery). Frequent and copious hæmorrhages, however, by increasing the absorption of water into the vessels, at first produce oligæmia hypalbuminosa.

For the abnormal changes of the red and white blood-corpuseles, see $\$ 10$; for Hæmophilia, $\S 28$.

[Organisms in the Blood.-The presence of animal and vegetable parasites in the blood gives rise to certain diseases. Some of these, and especially the vegetable organisms, have the power of multiplying in the blood. The regetable forms belonging to the schizomycetes are frequently spoken of collectively under the title bacteria. They are classified by Cohn into

I. Sphærobacteria.

II. Microbacteria, exhibit movements.

III. Desmobacteria
IV. Spirobacteria exhibit movements.

These forms are shown in fig. 23. The micrococci (A) are examples of I. ; while Bacterium termo (B) is an example of II. In III. the members are short cylindrical rods, straight (Bacillus, D) or wavy (Vibrio, C). Splenic fever of cattle is due to the presence of Bacillus anthracis (fig. 24). These rod-shaped bodies under proper conditions divide transversely and

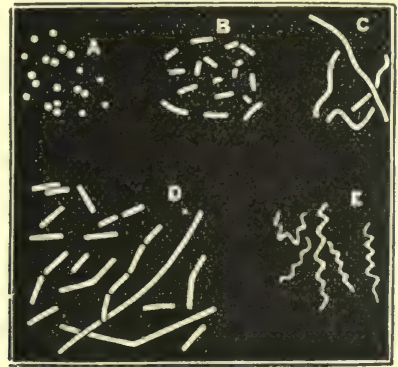

Fig. 23.

$\mathrm{A}$, micrococcus ; B, bacterium ; C, vibrios ; $\mathrm{D}$, bacilli ; E, spirillum.

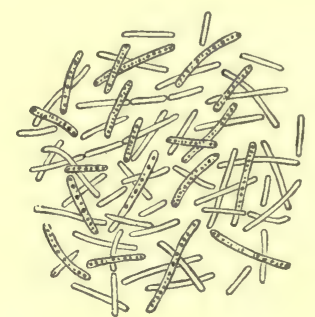

Fig. 24.

Bacillus anthracis from the blood (ox) in splenic fever.

elongate, but they also form spores in their interior, which in turn under appropriate conditions may germinate (fig. 24). Class $I V$. is represented by two genera, Spirochæta and Spirillum (fig. 23), the former with close, and the latter with open spirals. The Spirochrata Obermeieri (often spoken of as "Spirillum") is present in the blood during the paroxysms in persons suffering from relapsing fever. Amongst animal parasites are Filaria sanguinis, and Bilhar\%ia Hrematobia, which occurs in the portal vein and in the veins of the urinary apparatus.] 


\section{Physiology of the Circulation.}

42. GENERAL VIEW.-The blood within the vessels is in a state of continual motion, being carried from the ventricles by the large arteries (aorta and pulmonary)

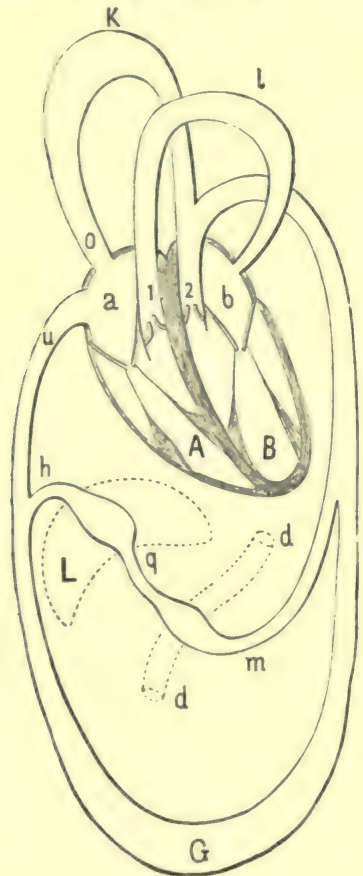

Fig. 25 .

Scheme of the circulation. - $a$, right, $b$, left auricle ; $A$, right, $B$, left ventricle; 1 , pulmouary artery ; 2 , aorta ; $l$, area of pulmonary, $\mathrm{K}$, area of systemic circulation ; $o$, the superior vena cava; G, area supplying the inferior vena cava, $u$; $d, d$, intestine; $m$, mesenteric artery ; $q$, portal vein; $\mathrm{L}$, liver ; $h$, hepatic vein. and their branches to the system of capillary vessels, from which again it passes into the veins that end in the atria of the auricles (W. Harvey, 1628).

The cause of the circulation is the difference of pressure which exists between the blood in the aorta and pulmonary artery on the one hand, and the two venæ cavæ and the four pulmonary veins on the other. The blood, of course, moves continually in its closed tubular system in the direction of least resistance. The greater the difference of pressure, the more rapid the movement will be. The cessation of the difference of pressure (as after death) naturally brings the movement to a standstill (\$ 81). The circulation is usually divided into-

(1) The greater, or systemic circulation, which includes the course of the blood from the left auricle and left ventricle, through the aorta and all its branches, the capillaries of the body and the veins, until the two venæ cavæ terminate in the right auricle.

(2) The lesser, or pulmonic circulation, which includes the course from the right auricle and right ventricle, the pulmonary artery, the pulmonary capillaries, and the four pulmonary veins springing from them, until these open into the left auricle.

(3) The portal circulation is sometimes spoken of as a special circulatory system, although it represents only a second set of capillaries (within the liver) introduced into the course of a venous trunk. It consists of the vena portarum-formed by the union of the intestinal or mesenteric and splenic veins, and it passes into the liver, where it divides into capillaries, from which the hepatic veins arise. The hepatic vein joins the inferior vena cava.

Strictly speaking, however, there is no special portal circulation. Similar arrangements occur in other animals in different organs, e.g., suakes have such a system in their supra-renal 
capsules, and the frog in its kidneys. When an artery splits up into fine branches during its course, and these branches do not form capillaries, but reunite into an arterial trunk, a rete mirabile is formed, such as occurs in apes and the edentata. Microscopic retia mirabilia exist in the human mesentery ( $S c h o ̈ b l)$. Similar arrangements may exist in connection with veins, giving rise to venous retia mirabilia.

43. THE HEART. - The muscular fibres of the mammalian heart consist of short $(50$ to $70 \mu$ in man), very fine, transversely striated fibres, which are actual unicellular elements, devoid of a sarcolemma (15 to $25 \mu$ broad), and usually divided at their blunt ends, by which means they anastomose and form a network (fig. 26, A, B). The individual muscle-cells contain in their
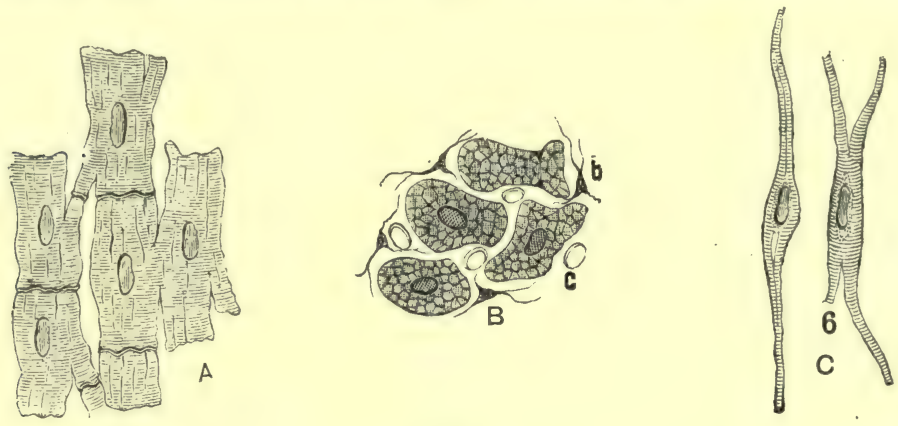

Fig. 26.

$A$, muscular fibres from the heart of a mammal, and $C$ from a frog ; $B$, transverse section of the cardiac fibres ; $b$, connective-tissue corpuscles ; $c$, capillaries.

centre an oval nucleus, and are held together by a cement which is blackened by silver nitrate, and dissolved by a 33 per cent. solution of caustic potash. This cement is also dissolved by a 40 per cent. solution of nitric acid. The transverse striæ are not very distinct, and not unfrequently there is an appearance of longitudinal striation, produced by a number of very small granules arranged in rows within the fibres. The fibres are gathered lengthwise in bundles, or fasciculi, surrounded and separated from each other by delicate processes of the perimysium. When the connective-tissue is dissolved by prolonged boiling, these bundles can be isolated, and constitute the so-called "fibres" of the heart. The transverse sections of the bundles in the auricles are polygonal or rounded, while in the ventricles they are somewhat flattened. [The muscular mass of the heart is called the myocardium, and is invested by fibrous tissue. It is important to notice that the connectivetissue of the visceral pericardium (epicardium) is continuous with that of the endocardium by means of the perimysium surrounding the bundles of muscular fibres.] The fine spaces which exist between these bundles form narrow lacunæ, lined with epithelium, and constituting part of the lymphatic system of the heart.

[The cardiac muscular fibres occupy an intermediate position between striped and plain muscular fibres. Although they are striped, they are involuntary, not being directly under the influence of the will, while they contract more slowly than a voluntary muscle of the skeleton.] In the frog's heart the muscular fibres are in shape elongated spindles, or fusiform, in this respect resembling the plain muscle-cells, but they are transversely striped (fig. $26, \mathrm{C}$ ). They are easily isolated by means of a 33 per cent. solution of potash or dilute alcohol.

44. ARRANGEMENT OF THE CARDIAC MUSCULAR FIBRES.-The study of the embryonic heart is the key to a proper understanding of the complicated arrangement of the fibres in the adult heart. The simple tubular heart of the embryo has an outer circular and an inner longitudinal layer of fibres. The septum is formed later; hence, it is clear that a part, at least, of the fibres must be common to the two auricles, and a part also to the two ventricles, since there is, originally, but one chamber in the heart. The muscular fibres of the auricles are, however, completely separated from those of the ventricles by the fibro-cartilaginous rings. In the auricles the fundamental arrangement of the embryonic fibres partly remains, while in the ventricles it becomes obscured as the cavities undergo a saclike dilatation, and also become twisted in a spiral manner. 
(1) The muscular fibres in the auricles are completely separated from the fibres of the ventricles by the fibrous rings which surround the auriculo-ventricular orifices, and which serve as an attachment for the auriculo-ventricular valves (fig. 27, I). The auricles are much thinner than the ventricles, and their fibres are generally arranged in two layers; the outer transverse layer is continuous over both auricles, whilst the inner one is directed longitudinally. The outer transverse fibres may be traced from the openings of the venous trunks anteriorly and posteriorly over the auricular walls. The longitudinal fibres are specially well marked where they are inserted into the fibro-cartilaginous rings, while in some parts of the anterior auricular wall they are not continuous. In the auricular septum, some fibres, circularly disposed around the fossa ovalis (formerly the embryonic opening of the foramen ovale), are well marked. Circulcer bands of striped muscle exist around the veins where they open into the heart; these are least marked on the inferior vena cava, and are stronger and reach higher $(2.5 \mathrm{~cm}$.) on the superior vena cava (fig. 27, II). Similar fibres exist around the pulmonary veins, where they join the left auricle, and these fibres (which are arranged as an inner circular and an outer longitudinal layer) can be traced to the hilus of the lung in man and some
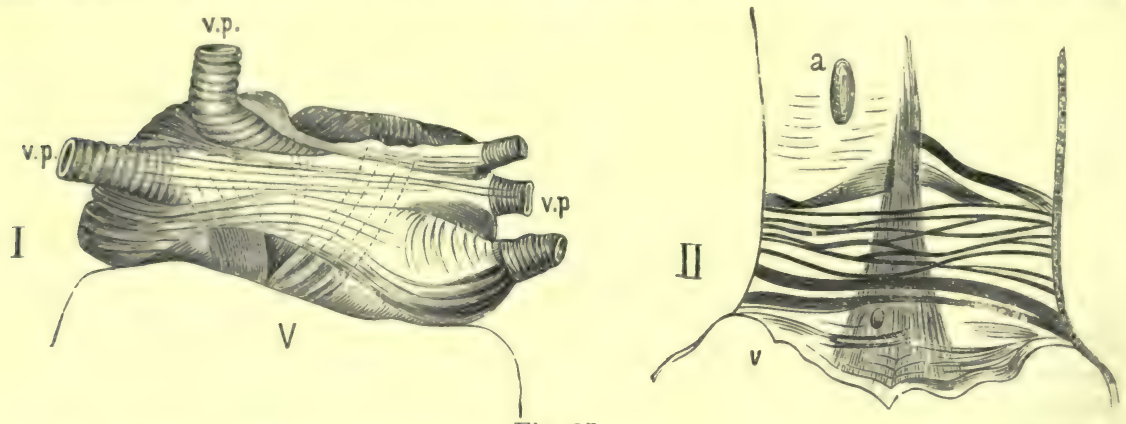

Fig. 27.

1. Course of the muscular fibres on the left auricle with the outer transverse and inner longitudinal fibres, the circular fibres on the pulmonary veins $(v . p.) ; \mathrm{V}$, the left ventricle (John licid). II. Arrangement of the striped muscular fibres on the superior vena cava (Elischer) $-a$, opening of vena azygos; $v$, auricle.

mammals; in the ape and rat they extend on the pulmonary veins right into the lung. In the mouse and bat, again, the striped muscular fibres pass so far into the lungs that the walls of the smaller veins are largely composed of striped muscle (Stieda).

Circular muscular fibres are found where the vena magna cordis enters the heart, and in the Valvula Thebesii which guards it.

Physiological Significance.-(1) The auricles contract independently of the ventricles. This is seen when the heart is about to die; when there may be several auricular contractions for one ventricular, and at last only the auricles pulsate. The auricular portion of the right auricle beats longest; hence it is called the "ultimum moriens." Independent rhythmical contractions of the venæ cavæ and pulmonary veins are often noticed after the heart has ceased to beat. [This beating can also be observed in those veins in a rabbit after the heart is cut out of the body.]

(2) The double arrangement of the fibres (transverse and longitudinal) produces a simultaneous and uniform diminution of the auricular cavity (such as occurs in most of the hollow viscera).

(3) The contraction of the circular muscular fibres around the venous orifices; and the subsequent contraction of the auricle, cause these veins to empty themselves 
into the auricle ; and by their presence and action they prevent any large quantity of blood from passing backward into the veins when the auricle contracts. [No valves are present in the superior and inferior vena cava in the adult heart, or in the pulmonary veins, hence the contraction of these circular muscular fibres plays an important part in preventing any reflux of blood during the contraction of the auricles.]

\section{ARRANGEMENT OF THE VENTRICULAR FIBRES.-(2) The} muscular fibres in the thick wall of the ventricles are arranged in several layers (fig. 28, A) under the pericardium. First, there is an outer longitudinal layer (A) which is in the form of single bundles on the right ventricle, but forms a complete layer on the left ventricle, where it measures about one-eighth of the thickness of the ventricular wall. A second longitudinal layer of fibres lies on the inner surface of the ventricles, distinctly visible at the orifices, and within the vertically placed papillary muscles, whilst elsewhere it is replaced by the irregularly arranged trabeculæ carneæ. Between these two layers there lies the thickest layer, consisting of more or less transversely arranged bundles, which may be broken up into single layers more or less circularly disposed. The deep lymphatic vessels run between the layers, whilst the blood-vessels lie within the substance of the layers, and are surrounded by the primitive bundles of muscular fibres. All three layers are not completely independent of each other; on the contrary, the fibres which run obliquely form a gradual transition between the transverse layers and the inner and outer longitudinal layers. It is not, however, quite correct to assume that the
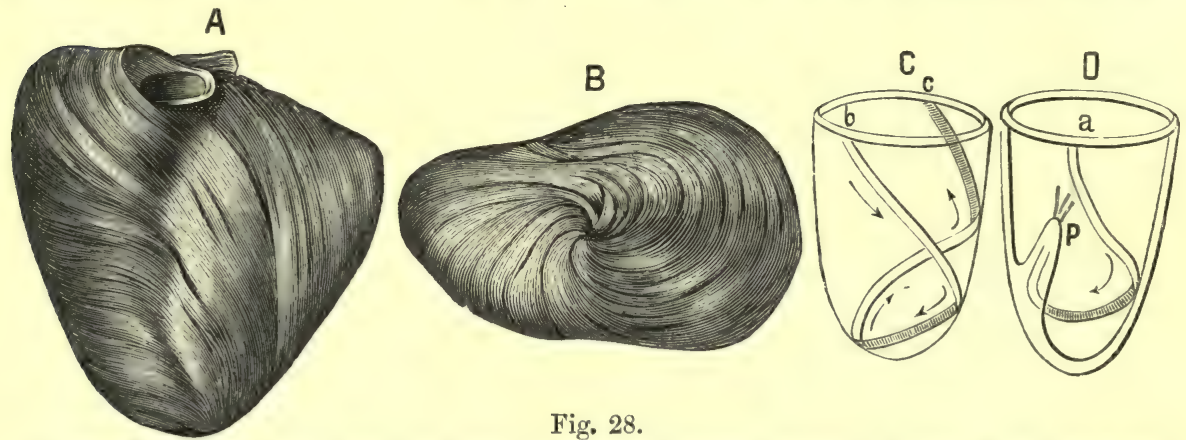

Course of the ventricular muscular fibres. A, on the anterior surface ; B, view of the apex with the vortex; C, course of the fibres within the ventricular wall ; D, fibres passing into a papillary muscle.

outer longitudinal layer gradually passes into the transverse, and this again into the inner longitudinal layer (as is shown schematically in C) ; because, as Henle pointed out, the transverse fibres are relatively far greater in amount. In general, the outer longitudinal fibres are so arranged as to cross the inner longitudinal layer at an acute angle. The transverse layers lying between these two form gradual transitions between these directions. At the apex of the left ventricle, the outer longitudinal fibres bend or curve so as to meet at the so-called vortex B, where they enter the muscular substance, and, taking an upward and inward direction, reach the papillary muscles, $P, D$; although it is a mistake to say that all the bundles which ascend to the papillary muscles arise from the vertical fibres of the outer surface : many seem to arise independently within the ventricular wall. According to Henle, all the external longitudinal fibres do not arise from the fibrous rings or the roots of the arteries. The mitral orifice is surrounded by circular fibres which act like a sphincter (Henle). 
[The assumption that the muscles of the ventricle are arranged so as to form a figure of 8 , or in loops, seems to be incorrect ; thus, fibres are said to arise at the base of the ventricle, to pass over it, and to reach the vortex, where they pass into the interior of the muscular substance, to end either in the papillary muscles or high up on the inner surface of the heart at its base. Figs. C and D give a schematic representation of this view.]

Only the general arrangement of the ventricular muscular fibres has been indicated. According to Pettigrew, there are seven layers in the ventricle, viz., three external, a fourth or central layer, and three internal. These interual layers are continuous with the corresponding external layers at the apex, thus - one and seven, two and six.

46. PERICARDIUM, ENDOCARDIUM, VALVES. - The pericardium encloses within its two layers [risceral and parietal] a lymph space-the pericardial space-which contains a small

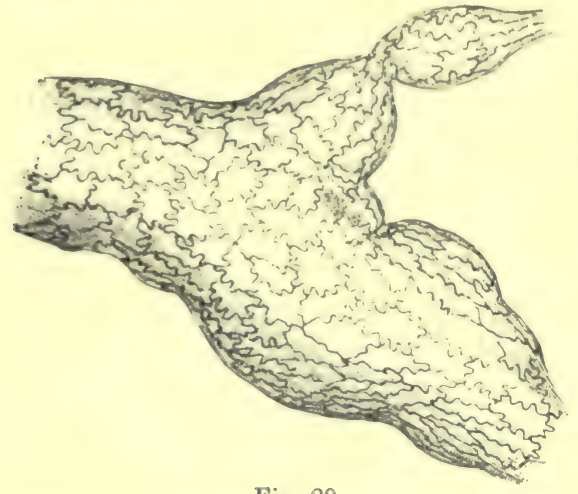

Fig. 29.

Lymphatic of the pericardium, epithelium stained th with nitrate of silver.

aprearance.] Then follow's, as the busis of the memb in the auricles, and in some places thereof assuming the characters of a fenestrated membrane.

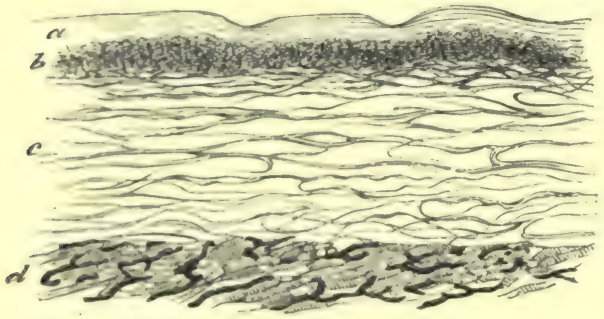

Fig. 30 .

Section of the endocardium. $a$, hyaline layer ; $b$, network of fine elastic fibres; $c$, network of stronger elastic fibres; $d$, myocardium with blood-vessels, which do not pass into the endo. cardium,

artery, which prevent the blood from passing back into the ventricles, and the tricuspid (right auriculo-ventricular) and mitral (left auriculo-ventricular), which protect the auricles from the same result. The lower vertebrata have valves in the orifices of the venæ cavæ, which prevent regurgitation into them; while in birds and some mammals these valves exist in a rudimentary condition. The valves are fixed by their base to resistant fibrous rings, consisting of elastic and fibrous tissue. They are formed of two layers-(1) the fibrous, which is a direet continuation of the fibrous rings, and (2) a layer of elastic elements. The elastic layer of the 
auriculo-ventricular valves is an immediate prolongation of the endocardium of the auricles, and is directed towards the auricles. The semi-lunar valves have a thin elastic layer directed towards the arteries, which is thickest at their base. The connective-tissue layer directed towards the ventricle is about half the thickness of the valve itself.

The auriculo-ventricular valves also contain striped muscular fibres. Radiating fibres proceed from the auricles and pass into the valves, which, when the atria contract, retract the valves towards their base, and thus make a larger opening for the passage of the blood into the ventricles; according to Paladino, they raise the valves after they have been pressed down by the blood-current. This observer also described some longitudinal fibres which proceed from the ventricles to enter these valves. There is also a concentric layer of fibres arranged near their point of attachment, and directed more towards their ventricular surface. These fibres seem to contract sphincter-like when the ventricle contracts, and thus approximate the base of the valves, and so prevent too great tension being put upon them. The larger chordæ tendineæ also contain striped muscle, while a delicate muscular network exists in the valvula Thebesii and valvula Eustachii.

Purkinje's Fibres consist of an anastomosing system of greyish fibres which exist in the subendocardial tissue of the ventricles, especially in the heart of the sheep and ox. The fibres are made up of polyhedral clear cells, containing some granular protoplasm, and usually two nuclei (fig. 31). The margins of the cells are striated. Transition-forms are found between these cells and the ordinary cardiac fibres; in fact, these cells become continuous with the true fully developed cardiac fibres. They represent cells which have been arrested in their development. They are absent in man and the lower vertebrates, but in birds and some mammals they are well marked (Schweigger-Seidel, Ranvier).

Blood-Vessels occur in the auriculoventricular valves only where muscular fibres are present, while the semi-lunar valves are usually devoid of vessels except at their base. The best figures of the blood-vessels of the valves are given by Langer. The network of lymphatics in the endocardium reaches towards the middle of the valves.

Weight of the Heart.-According to W. Muiller the proportion between

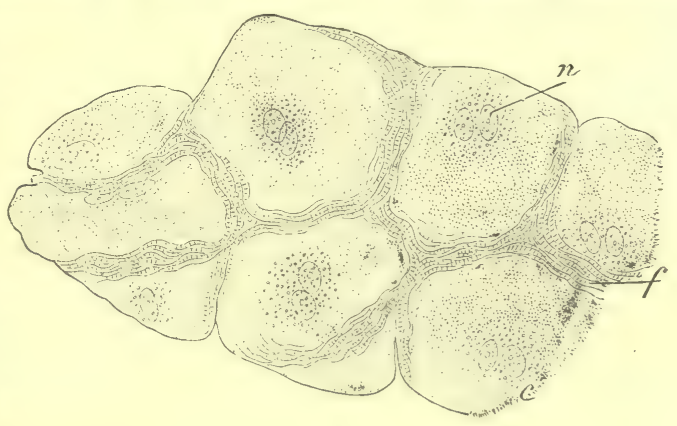

Fig. 31.

Purkinje's fibres isolated with dilute alcohol. $c$, cell ; $f$ : striated substance; $n$, nucleus. $\times 300$. the weight of the body and the heart in the child, and until the body reaches 40 kilos., is 5 grms. of heart-substance to 1 kilo. of body-weight; when the body-weight is from 50 to 90 kilos. the ratio is 1 kilo. to 4 grms. of heart-substance; at 100 kilos. 3.5 grms. As age advances, the auricles become stronger. The right ventricle is half the weight of the left. The weight of the heart of an adult man is about $309 \mathrm{grms}$; ; female, $274 \mathrm{grms}$. [According to Laennec the heart is about the size of the closed fist of the individual.] Blosfeld and Dieberg give 346 grms. for the male, and 310 to 340 grms. for the female heart. The specific gravity of the heartmuscle is 1.069 . The thickness of the left ventricle in the middle in man is $11.4 \mathrm{~mm}$., and in woman $11 \cdot 15$; that of the right is $4 \cdot 1$ and $3 \cdot 6 \mathrm{~mm}$. respectively.

47. AUTOMATIC REGULATION OF THE HEART.-Anatomical Investigations. - The two coronary arteries arise from the first part of the aorta in the region of the sinus of Valsalva. The position of origin varies-(1) either the orifices lie within the sinus, or (2) their openings are only partially reached by the margins of the semi-lunar valves (which is usually the case in the left coronary artery of man and the ox), or (3) their orifices lie clear above the margins of the valves. Post-mortem observations seem to show that during contraction of the ventricle it is very improbable that the semi-lunar valves constantly cover the origin of the coronary arteries.

Antomatic Regulation of the Heart.-Brücke attempted to show that during the systole, or contraction of the ventricle, the semi-lunar valves covered the openings of the coronary arteries, so that these vessels could be filled with blood 
only during the diastole or relaxation of the ventricle. To him it seemed that $(a)$ the diastolic filling of the coronary arteries would help to dilate the ventricles; (b) on the contrary, a systolic filling of these arteries would oppose the contraction, because the systolic filling and expulsion of the blood from the coronary arteries would diminish the force of the ventricular contraction. [To this supposed arrangement Brücke gave the name "Selbststeuerung," which may be rendered as above, or as "self-controlling" action of the heart by the aortic valves.]

Arguments against Brïcke's View. - The following considerations militate against this theory :- (1) Filling the coronary vessels under a high pressure in a dead heart causes a diminution of the ventricular cavity ( $v$. Wittich). (2) The chief trunks of the coronary arteries lie in loose sub-pericardial fatty tissue in the cardiac sulei, hence a dilatation of the ventricle through this agency is most unlikely (Landois). (3) Experiments on animals have shown that a coronary artery spouts, like all arteries, during the systole of the ventricle. Von Ziemssen found that in the case of a woman who had a large part of the anterior wall of the thorax removed by an operation, the heart being covered only by a thin membrane, the pulse in the roronary arteries was synchronous with the pulse in the pulmonary artery. H. N. Martin and Selgwick placed a manometer in connection with the coronary artery, and another with the carotid in a large dog, and they founi that the pulsations occurred simultancously. When a coronary artery is divided, the blood flows out continuously, but undergoes acceleration during the systole of the ventricles (Eindemann, Pcrls). (4) If a strong intermittent current of water he allowed to flow through a sufficiently wille tube into the left auricle of a fresh pig's heart, so that the water passes into the aorta, and if the aorta be provided with a vertical tube, the water flows continuously from the coronary arteries, and is accelerated during the systole. (5) It is exceedingly improbable that the coronary arteries should be filled during the diastole, while all the other arteries are filled lluring systole of the ventricle. (6) There is always a sufficient quantity of blood in the sinus of Valsalva to fill the arteries during the first part of the systole. (7) The valves, when raised, are not applied directly to the aortic wall (Hamberger, Riudlinger) even by the most energetic pressure from the ventricle (Sandborg and W orm Müller). (8) Observations on voluntary museles have shown that the small arteries dilate during contraction of the muscle, and the blood-stream is accelerated. (9) By the systolic filling of the anta the arterial path is elongated - this elastic distention is compensated before the diastole occurs. By the recoil of the aortic walls the layer of blood in them is driven backwards and closes the valves (Cercedini). According to Sandborg and Worm Miiller, the semilunar valves close just after the ventricles have begun to relax, which agrees with the curve obtained from the cardiac impulse (fig. $39, \mathrm{~A}$ ).

During the systole, the small arterial trunks lying next the ventricular cavities have to bear a higher pressure than that borne by the aorta, and their lumen must be compressed during the systole so that their contents are propelled towards the veins.

Peculiarities of the Cardiac Blood-Vessels. - The capillary vessels of the myocardium are very numerous, corresponding to the energetic activity of the heart. Where they pass into veins, several unite at once to form a wide venous trunk, whereby an easy passage is offered to the blood. The veins are provided with valves so that (1) during systole of the right auricle the venous stream is interrupted; (2) during contraction of the ventricles, the blood in the coronary veins is similarly accelerated as in the veins of muscles. The coronary arteries are characterised by their very thick connective-tissue and elastic intima, which perhaps accounts for the frequent occurrence of atheroma of these vessels (Henle). Some observers maintain that the coronary arteries do not anastomose, but this is denied by Langer and Krause. [West has injected the one artery from the other.] Many of the small lower vertebrates have no bloodvessels in their heart-muscle, e.g., frog ( $H$ yrtl).

Ligature of the Coronary Arteries. - The phenomena produced by partial obliteration or ligature of the coronary arteries are most important. In man analogous conditions occur, as in atheroma or calcification of these arteries. Sée and others have ligatured the coronary arteries in dogs, and found that after two minutes the cardiac contractions gave place to twitchings of the muscular fibres, and ultimately the heart ceased to beat. Ligature of the anterior coronary artery alone, or of both its branches, is sufficient to produce this result. If the coronary arteries be compressed or tied in a rabbit in the angle between the bulbus aortæ and the ventricle, the heart's action is soon weakened, owing to the sudden anæmia and to the retention of the decomposition-products of the metabolism in the heart- 
muscle. Ligature of one artery first affects the corresponding ventricle, then the other ventricle, and, last of all, the auricles. Hence, compression of the left coronary artery, (with simultaneous artificial respiration in a curarised animal), causes slowing of the contractions, especially of the left ventricle, whilst the right one at first contracts more quickly, and then, gradually its rhythm is slowed. The contractions of the left ventricle are not only slowed but also weakened, whilst the right pulsates with undiminished force. Hence it follows that, as the left half of the heart cannot expel the blood in sufficient quantity, the left auricle becomes filled, whilst the right ventricle, not being affected, pumps blood into the lungs. CEdema of the lungs is produced by the high pressure in the pulmonary circulation, which is propagated from the right heart through the pulmonary vessels into the left auricle (Samuelson and Grïnhagen). According to Sig. Mayer, protracted dyspnoea causes the left ventricle to beat more feebly sooner than the right, so that the left side of the heart becomes congested. Perhaps this may explain the occurrence of pulmonary œdema during the death-agony.

Cohnheim and v. Schulthess-Rechberg found, after ligature of one of the large branches of a coronary artery in a dog, that at the end of a minute the pulsations become intermittent. This intermittence becomes more pronounced, the two sides of the heart do not contract simultaneously (arhythmia), the heart beats more slowly, and the blood-pressure falls. Suddenly, about 105 seconds after the ligature is applied, both ventricles cease to beat, and there is a great fall of the blood-pressure. After an arrest lasting for 10 to 20 seconds, twitching movements occur in the ventricles, while the auricles pulsate regularly, and may continue to do so for many minutes, but the ventricles cease to beat altogether after 50 seconds. According to Lukjanow, there is a peristaltic condition which operates upwards and downwards, and occurs in the period between the regular contraction and the twitching vibratory movement. Stimulation of the vagus does not arrest these peristaltic movements.

Pathological. - In so-called sclerosis of the coronary arteries in old age, there are attacks of diminished cardiac activity, weakness of the heart, an altered rhythm and frequency, with consequent breathlessness; there may also be loss of consciousness, congestions, and attacks of pulmonary œdema. Death may take place unexpectedly from sudden arrest of the heart's action.

48. MOVEMENTS OF THE HEART.-Cardiac Revolution.-The movement of the heart is characterised by an alternate contraction and relaxation of its walls. The total cardiac movement is called a "cardiac revolution," or a "cardiac cycle," and consists of three acts-the contraction or systole of the auricles, the contraction or systole of the ventricles, and the pause (fig. 50). During the pause, the auricles and ventricles are relaxed; during the contraction of the auricles the ventricles are at rest; whilst during the contraction of the ventricles the auricles are relaxed. The rest during the phase of relaxation is called the diastole. The following is the sequence of events in the heart during a cardiac revolution:-

(A) The blood flows into the auricles. and thus distends them and the auricular appendices. This is caused by-

(1) The pressure of the blood in the venæ cavæ (right side) and the pulmonary veins (left side) being greater than the pressure in the auricles. (2) The elastic traction of the lungs (\$ 68), which, after complete systole of the auricles, pulls asunder the now relaxed and yielding auricular walls. The auricular appendages are also filled at the same time, and they act to a certain extent as accessory reservoirs for the large supply of blood streaming into the auricles.

(B) The auricles contract, and we observe in rapid succession-

(1) The contraction and emptying of the auricular appendix towards the atrium. Simultaneously the mouths of the veins become narrowed, owing to the contraction of their circular muscular fibres (more especially the superior vena cava and the pulmonary veins); (2) the auricular walls contract simultaneously towards the auriculo-ventricular valves and the venous orifices, whereby (3) the blood is driven into the relaxed ventricles, which are considerably distended thereby.

The contraction of the auricles is followed by 
(a) A slight stagnation of the blood in the large venous trunks, as can be observed in a rabbit after division of the pectoral muscles so as to expose the junction of the jugular with the subclavian vein. There is no actual regurgitation of the blood, but only a partial interruption of the inflow into the auricles, because the mouths of the veins are contracted, and the pressure in the superior vena cava and pulmonary veins soon holds in equilibrium any reflux of blood; and lastly, because any reflux into the cardiac veins is prevented by valves. The movement

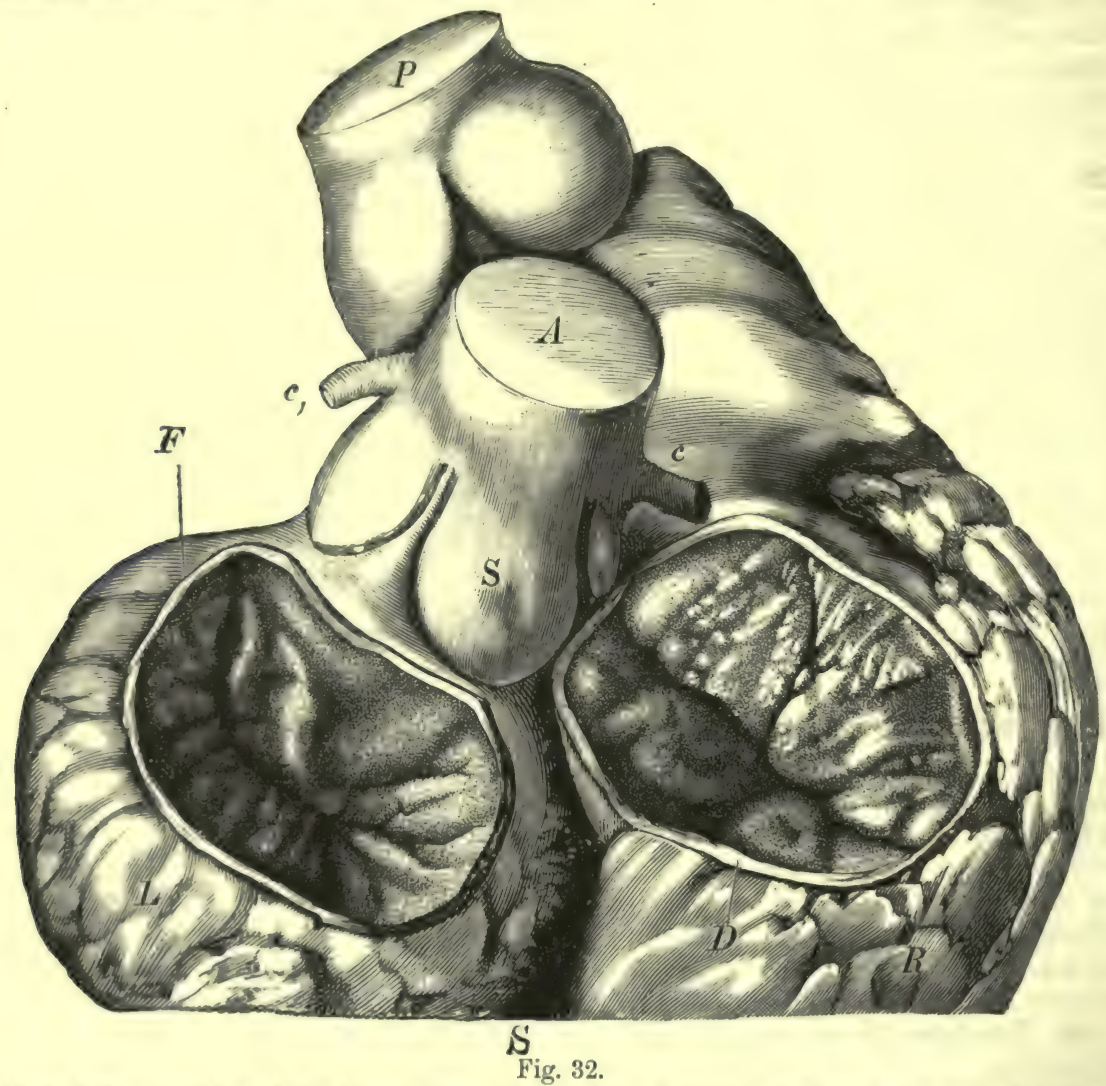

Cast of the ventricles of the human heart viewed from behind and above; the walls have been removed, and only the fibrous rings and the auriculo-ventricular valves are retained. $L$, left, $R$, right ventricle; $\mathrm{S}$, septum ; $\mathrm{F}$, left fibrous ring, with mitral valve closed ; D, right fibrous ring, with tricuspid closed; $A$, aorta, with the left $\left(C_{1}\right)$ and right $(C)$ coronary arteries; $\mathbf{S}$, sinus of Valsalva ; P, pulmonary artery.

of the heart causes a regular pulsatile phenomenon in the blood of the venæ cavæ, which under abnormal circumstances may produce a venous pulse (see $\$ 99$ ).

(b) The chief motor effect of the contraction of the auricles is the dilatation of the relaxed ventricle, which has already been dilated to a slight extent by the elastic traction of the lungs.

Aspiration of the Ventricles. - The dilatation of the ventricles has been ascribed to the elasticity of the muscular walls-the strongly contracted ventricular walls (like a compressed india-rubber bag), in virtue of their elasticity, are supposed, in returning to their normal resting form, to suck in or aspirate the blood under a negative pressure; this power on the part of the ventricle is not great (p. 59).. 
(c) When the ventricles are distended by the inflowing blood, the auriculoventricular valves are floated up, partly by the recoil or reflexion of the blood from the ventricular wall, and partly owing to their lighter specific gravity, whereby they easily float into a more or less horizontal position. The valves are also raised to a slight extent by the longitudinal muscular tibres, which pass from the auricles into the cusps of the valve.

(C) The ventricles now contract, and simultaneously the auricles relax, whereby-

(1) The muscular walls contract forcibly from all sides, and thus diminish the ventricular cavity. (2) The blood is at once pressed against the under surface of the auriculo-ventricular valves, whose curved margins are opposed to each other like teeth, and are pressed hermetically against each other (fig. 32). It is impossible for the blood to push the cusps backwards into the auricle, as the chordæ tendineæ hold fast their margins and surfaces like a taut sail. The margins of the neighbouring cusps are also kept in apposition, as the chordæ tendineæ from one papillary muscle always pass to the adjoining edges of two cusps. The extent to which the ventricular wall is shortened is compensated by the contraction of the papillary muscle, and also of the large muscular chordæ, so that the cusps cannot be pushed into the auricle. When the valves are closed, their surfaces are horizontal, so that, even when the ventricles are contracted to their greatest extent, there remains in the supra-papillary space a small amount of blood which is not expelled (Sandborg and Worm Müller). (3) When the pressure within the ventricles exceeds that in the arteries, the semi-lunar valves are forced open and stretched like a sail across the pocket-like sinus, without, however, being directly applied to the wall of the arteries (pulmonary and aorta), and thus the blood enters the arteries.

(D) Pause.-As soon as the ventricular contraction ends, and the ventricles begin to relax, the semi-lunar valves close (fig. 33). The diastole of the ventricles is followed by the pause. Under normal circumstances, the right and left halves of the heart always contract or relax uniformly and simultaneously.

Negative Pressure in the Ventricle.-Goltz and Gaule found that there was a negative pressure of $23.5 \mathrm{~mm} . \mathrm{Hg}$. $(\mathrm{dog})$ in the interior of the ventricle during a certain phase of the heart's action. This they determined by a maximal and minimal manometer. They surmised that this phase coincided with the diastolic dilatation, for which they assumed a considerable power of aspiration. Moens is of opinion that this negative pressure within the ventricle obtains shortly before the systole has reached its height, i.e., just before the inner surface of the ventricles and the valves, after the blood is expelled, are nearly in apposition. $\mathrm{He}$ explains this aspiration as being due to the formation of an empty space in the ventricle caused by the energetic expulsion of the blood throngh the aorta and pulmonary artery.

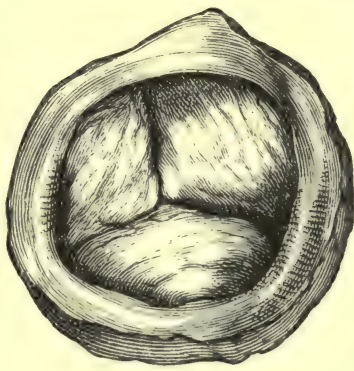

Fig. 33.

The closed semi-lunar valve of the pulmonary artery seen from below.

[Maximum and Minimum Manometer. - Into the tube connecting the interior of the ventricle of the heart with the ordinary $\mathrm{U}$-shaped mercury manometer, is introduced the maximum

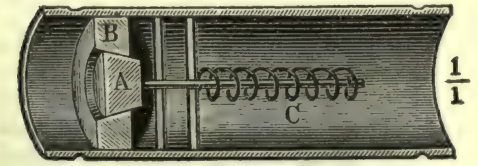

Fig. 34.

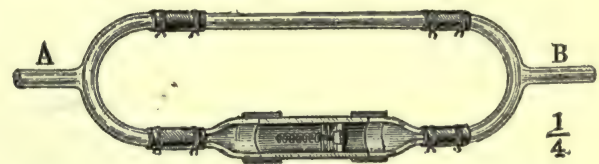

Fig. 35 .

Gaule's maximum and minimum manometer.

manometer, which is constructed on the principle of a ball and cup valve (fig. 34), the ball A being kept closed in B by a spring C. To make it a maximum manometer the end A is connected with the heart, and $B$ with the mercurial manometer (fig. 35). When a clamp is placed 
on the upprer limb the valve is acterl on only at each systole of the heart, blood is driven beyond it, but during diastole it closes and no blood can return. This goes on until the pressure beyond the ralve in the mercury manometer is the same as in the heart. If the valve be reversed, it is converterl into a minimum inanometer.]

49. PATHOLOGICAL CARDIAC ACTION. - Cardiac Hypertrophy.-All resistances to the movement of the blool through the various chambers of the heart, and through the vessels communicating with it, cause a greater amount of work to be thrown upon the portion of the heart specially related to this part of the circulatory system; consequently, there is produced an increase in the thickness of the nuscular walls and dilatation of the heart. If the resistance or obstacle does not act upon me part of the heart alone, but on parts lying in the onvord direction of the blood-stream, these parts also subsequently undergo hypertrophy. If in addition to the museular thickening of a part of the heart, the cavity is simultaneously dilated, it is spoken of as eccentric hypertrophy or hypertrophy with dilatation. The obstacles most likely to occur in the blood-ressels, are narrowing of the lumen or want of elasticity in their walls; in the heurt, narrowing of the arterial or venous orifices or insufficiency or incompetency of the valves. Incompetency of the valves forms an obstruction to the movement of the blood, by allowing part of the hlood to flow back or regurgitate, thus throwing extra work upon the heart.

Thus arisp-1) Hypertrophy of the left ventricle, owing to resistance in the area of the systemic circulation, especially in the arteries and capillaries-not in the veins. Amongst the canses are-constriction of the orifice or other parts of the aorta, calcification, atheroma, and want of elasticity of the large arteries and irregular dilatations or aneurisms in their course ; insufficiency of the aortic valves, in which case the same pressure always obtains within the ventri.le and in the aorta ; and, lastly, cirrhosis of the kidneys, whereby the excretion of water by these organs is diminisheel. Even in mitral insufficiency, compensatory hypertrophy of the left ventric.fe must oceur, owing to the hypertrophy of the left atrium in consequence of the increased blood-pressure in the pulmonary circuit.

(2) Hypertrophy of the left auricle occurs in stenosis or constriction of the left auriculo-ventricular orifice, or in insufficieney of the mitral valve, and it occurs also as a result of aortic insufficiney, lecause the auricle has to overeome the continual aortic pressure within the ventricle.

(3) Hypertrophy of the right ventricle occurs $(a)$ when there is resistance to the bloodstream through the pulmonary circuit. The resistance may be due to $(\alpha)$ obliteration of large rascular areats in consequence of destruction, shrinking or compression of the lungs, and the disappearance of numerous capillaries in emphysematous lungs; $(\boldsymbol{\beta})$ overfilling of the pulmonary cirenit with blool in consequence of stenosis of the left auriculo-ventricular orifice, or mitral insufficiency-consequent upon hypertrophy of the left auricle resulting from aortic insufficiency. (b) When the valves of the pulmonary artery are insufficient, thus permitting the blood to flow bark into the ventricle, so that the pressure within the pulmonary artery prevails within the right ventricle (very rare).

(4) Hypertrophy of the right auricle occur' in consequence of the last-named condition, and also from stenosis of the tricuspid orifice, or insufficiency of the tricuspid valve (rare).

Artificial Injury to the Valves. - If the aortic valves are perforated, with or without simultaneous injury to the mitral or tricuspid valves, the heart does more work; thus the physical defect is overeome for a time, so that the blood-pressure does not fall. The heart seems to have a store of reserve energy which is called into play. Soon, however, dilatation takes place, on account of the regurgitation of the blood into the heart. Hypertrophy then occurs, but the compensation meanwhile must be obtained through the reserve energy of the heart (O. Roscnbach).

Impeded Diastole. - Among causes which hinder the diastole of the heart are-copious effusion into the pericarlium, or the pressure of tumours upon the heart. The systole is greatly interf red with when the heart is united to the pericarlium and to the connective-tissue in the mediastiuum. As a consequence the connective-tissue, and even the thoracic wall, are drawn in during contraction of the heart, so that there is a retraction of the region of the apex-beat luring systole, and a protrusion of this part during the diastole.

[Palpitation is a symptom indicating generally very rapid and quick action of the heart, the pulsations often being unequal in time and intensity, while the person is generally conscious of the irregalarity of the cardiac action. It may be due to some organic condition of the heart itself, especially where the cardiac muscles are weak, in cases of dilatation and hypertrophy of the left ventricle, where the heart is gradually becoming unable to overcome the resistances offered to its work, and especially during exertion when the heart is taxed above its strength. It may also occur where the blond-pressure is low, as in anæmia, so that the heart contracts quickly, there being little resistance opposed to its action. The excitability of the cardiac muscle may he increased as in fatty heart, when very slight exertion may excite it often in a paroxysmal way. In other cases, it is nervous in its origin, being either direct or reflex. In very emotional and excitable people (especially in women) it is easily set up, and in some people it may be 
produced reflexly by gastric or intestinal irritation or dyspepsia. It also frequently results from excesses of all kinds and the over-use of tobacco. The remedies to be used obviously depend on the cause. Where the blood-pressure is low, as in anæmia, digitalis and iron will do good; the former by increasing the blood-pressure, and the latter by improving the general nutrition of the body and the blood in particular. In neurotic cases cardiac sedatives are indicated, while in cases due to indigestion hydrocyanic acid is useful (Brunton)].

[Fainting or Syncope. - In fainting the person loses consciousness, owing to a sudden arrest of the blood-supply to the brain, the face is pallid, the respiration is feeble or ceases, while the heart beats but feebly or not at all. The defective supply of blood to the brain may depend upon sudden arrest of the heart's action, caused, it may be, by a fright, or the heart's action may be arrested reflexly. Any cause which suddenly diminishes the blood-pressure may produce it, or when pressure is suddenly removed from the large vessels, as in tapping the abdomen in ascites, without at the same time giving sufficient support to the abdominal viscera. When a person has been long in the recumbent position, on being rapidly set up in bed he may faint. In some forms of heart disease, sudden exertion or change of posture may produce it.

[Treatment.-The object is to restore consciousness and the action of the heart. Place the person in the horizontal position, keep the head low, even lower than the body, and do not support it with pillows. Dashing cold water on the face, so as to stimulate the fifth nerve, usually succeeds in causing the person to take a deep inspiration. In other cases a sniff of smelling salts or ammonia, acting through the nasal branch of the fifth nerve, will excite the cardiac and respiratory functions $(\$ 368)$.]

50. THE APEX-BEAT, CARDIOGRAM.-Cardiac Impulse.-By the term " apex-beat" or "cardiac impulse" is understood under normal circumstances an elevation (perceptible to touch and sight), in a circumscribed area of the fifth left intercostal space, and caused by the movement of the heart. [The apex-beat is felt in the fifth left intercostal space, 2 inches below the nipple, and 1 inch to its sternal side, or at a point 2 inches to the left of the sternum.] The impulse is more rarely felt in the fourth intercostal space, and it is much less distinct when the heart beats against the fifth rib itself. The position and force of the cardiac impulse vary with changes in the position of the body.

[The cardiac impulse is synchronous with the systole of the heart, but although this name and apex-beat are frequently used as synonymous terms, it is to be remembered that the impulse may be caused by different parts of the heart being in contact with the chest-wall. The cardiac impulse is usually higher than normal in children, while it is lower during inspiration than expiration.]

[Methods. - To obtain a curve of the apex-beat or a cardiogram, we may use one or other of the following cardiographs (fig. 35). Fig. 36, A, is the first form used by Marey, and it consists of an oval wooden capsule applied in an air-tight manner over the apex-beat. The disc, $p$, capable of being regulated by the screw, $s$, presses upon the region of the apex-beat, while $t$ is a tube which may be connected with a recording tambour (fig. 47). B is an improved form of the instrument, consisting essentially of a tambour, while attached to the membrane is a button, $p$, to be applied over the apex-beat. The movements of the air within the capsule are communicated by the tube, $t$, to a recording tambour. Fig. $36, \mathrm{C}$, is the pansphygmograph of Brondgeest, which consists of a Marey's tambour, in an iron horse-shoe frame, and adjustable by means of a screw, $s$. Burdon-Sanderson's cardiograph is shown in D. The button, $p$, carried by the spring, $e$, does not rest upon the caoutchouc membrane, but on an aluminium plate attached to it. The apparatus is adjusted to the chest by three supports. Fig. 36, E, shows a modified instrument on the same principle by Grummach and v. Knoll. In all these figures the $t$ indicates the exit-tube communicating with a recording tambour (fig. 47), D and E may be used for other purposes, e.g., for the pulse, so that they are polygraphs. See also fig. 76.]

Fig. 39, A, shows the cardiogram or the impulse-curve of the heart of a healthy man ; B, that of a dog, obtained by means of a sphygmograph. In both the following points are to be noticed:- $a b$, corresponds to the time of the pause and the contraction of the auricles. As the atria contract in the direction of the axis of the heart from the right and above towards the left and below, the apex of the heart moves towards the intercostal space. The two or three smaller elevations are perhaps caused by the contractions of the ends of the veins, the auricular appendices, and the atria themselves.

The portion $b c$, which communicates the greatest impulse to the instrument, and also to one's hand when it is placed on the apex-beat, is caused by the contraction 
of the ventricles, and during it the first sound of the heart occurs. Frequently,

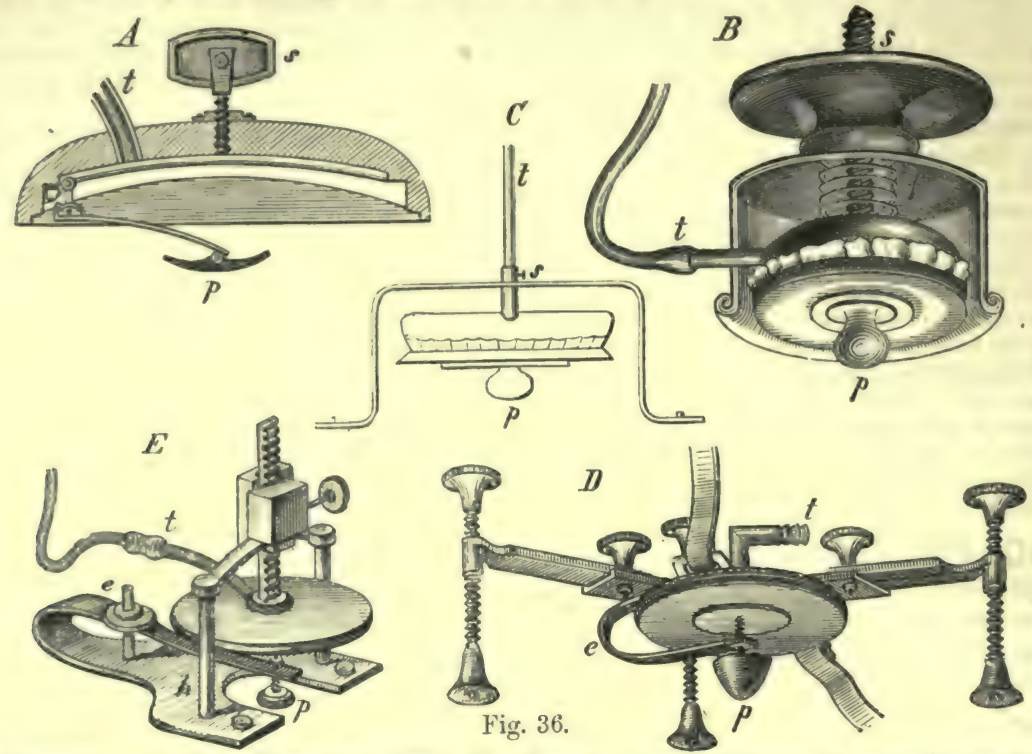

Cardiographs. A, Marey's original form ; B, Marey's improved form ; C, pansphygmograph (Brondgeest); D, cardiograph (Burdon-Sanderson); $\mathrm{E}$, that of $\mathbf{v}$. Knoll.

Lut erroneously, the cardiac impulse has been ascribed to the contraction of the

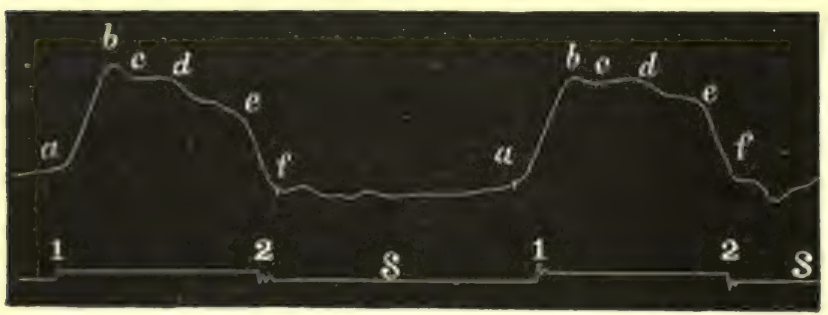

Fig. 37.

Cardiogram. $a-f ; 1$, beginning of 1 st, and 2,2 nd sound. ventricles alone. It, however, is due to all those conditions which cause an elevation in the region of the cardiac impulse.

[Edgren recorded a human cardiogram, and listened at the same time to the heart sounds, recording the latter by means of an electric signal. The curve rises at $a$, with the beginning of the first sound, i.e., with the contraction of the ventricles, and reaches the abscissa at $f$ with the
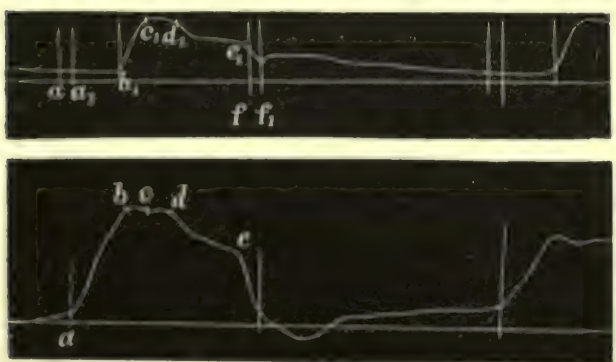

Fig. 38.

The upper curve from the human carotid; the lower a cardiogram taken simultaneously. beginning of the second sound, i.e., when the seni-lunar valves are closed. The relation between $a$ and the points intermediate between it and $f$, and to the pulsocurve of the carotid, is shown in fig. 38. The letters with the dash correspond to the unmarked letters in the cardiogram.]

The cause of the ventricular impulse has been much discussed. It depends upon the following :-

(1) The base of the heart (auriculoventricular groove) represents during diastole a transversely-placed ellipse (fig. 40, I., FG), while during contraction it has a more circular figure, 
$a b$. Thus, the long diameter of the ellipse (FG) is diminished, the small diameter $d c$ is increased, while the base is brought nearer to the chest-wall $e$. This alone
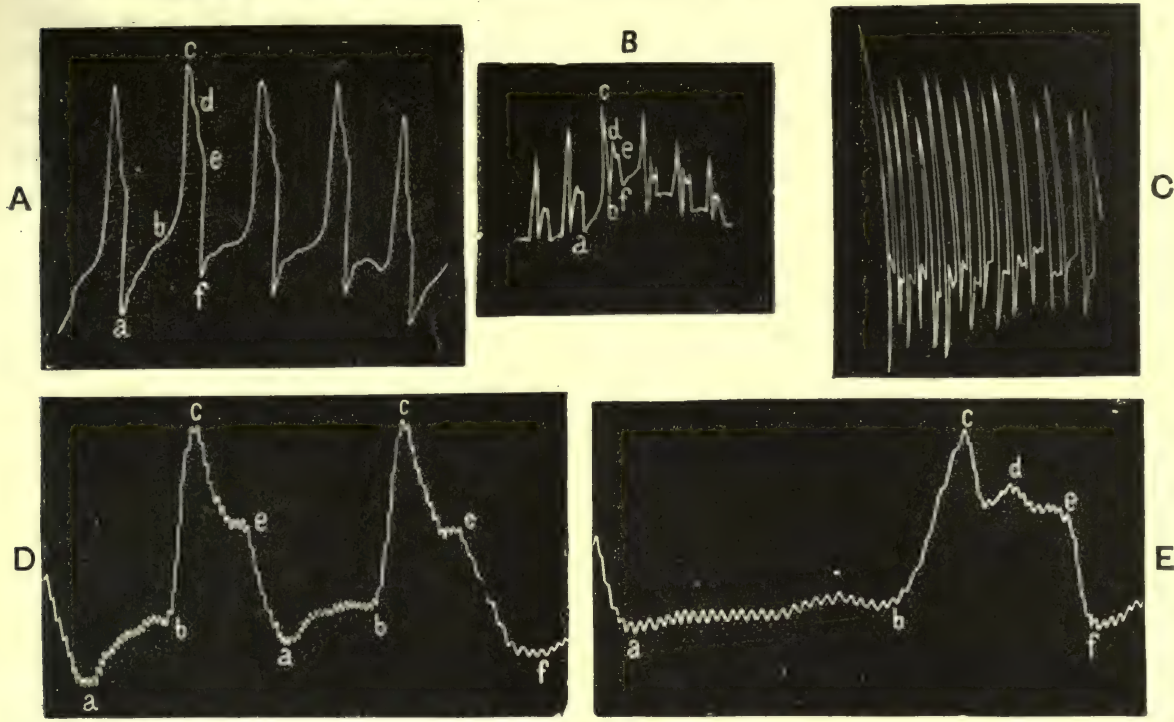

Fig. 39.

Curves from the apex-beat. A, normal curve (man); B, from a dog; C, very rapid curve $(\operatorname{dog}) ; \mathrm{D}$ and $\mathrm{E}$, normal curves (man) registered on a vibrating glass plate where each indentation $=0.01613 \mathrm{sec}$. In all the curves $a b$ means contraction of the auricles, and $b c$ of the ventricles; $d$, closure of the aortic, and $e$, of the pulmonary valves; ef, diastole of the ventricle.

does not cause the impulse, but the basis of the heart, being liardened during the systole and brought nearer to the chest-wall, allows the apex to execute the movement which causes the impulse (p. 61).

(2) During relaxation, the ventricle lies with its apex (fig. 40, II., i) obliquely downwards, and with its long axis in an oblique direction-so that the angles (bci, aci) formed by the axis of the ventricles with the diameter of the base are unequal - during systole it represents a regular cone, with its axis at right angles to its base. Hence, the apex $(i)$ must be erected from below and behind $(p)$, forwards and upwards (Harvey - "cor sese erigere"), and when hardened during systole presses itself into the intercostal space (fig. 40, II.).

(3) The ventricles undergo during systole a slight spiral twisting on their long axis ("lateralem inclinationem"-Harvey), so that the apex is brought from behind more forward, and thus a greater portion of the left ventricle is turned to the front. This rotation is caused by the muscular fibres of the ventricles, which proceed from that part of the fibrous rings between the auricles and ventricles which lies next the anterior thoracic wall. The fibres pass from above obliquely downwards, and to the left, and also run in part upon the posterior surface of the ventricles. When they contract in the axis of their direction, they tend to raise the apex, and also to bring more of the posterior surface of the heart in relation with the anterior thoracic wall. It is favoured by the slightly spiral arrangement of the aorta and pulmonary artery.

These are the most important causes, but the minor causes are,

(4) The "reaction impulse" or "recoil," or that movement which the ventricles are said to undergo, (like an exploded gun or rocket), at the moment when the 
blood is discharged into the aorta and pulmonary artery, whereby the apex goes in the opposite direction, i.e., downwards and slightly outwards. Landois, however, has shown that the mass of blood is discharged into the vessels 0.08 of a second after the beginning of the systole, while the cardiac impulse occurs with the first sound.

(5) When the blood is discharged into the aorta and pulmonary artery, these vessels are slightly elonguted, owing to the increased blood-pressure. As the heart is suspended from above by these vessels, the apex is pressed slightly downwards and forwards towards the intercostal space (?).

As the cardiac impulse is observed in the empty hearts of dead animals, (4) and (5) are certainly of only second-rate importance. Filehne and Pentzoldt maintain that the apex during systole does not move to the left and downwards, as must

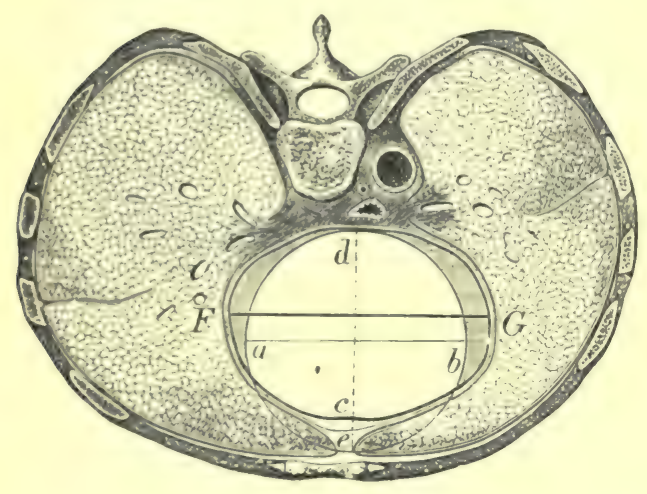

I.

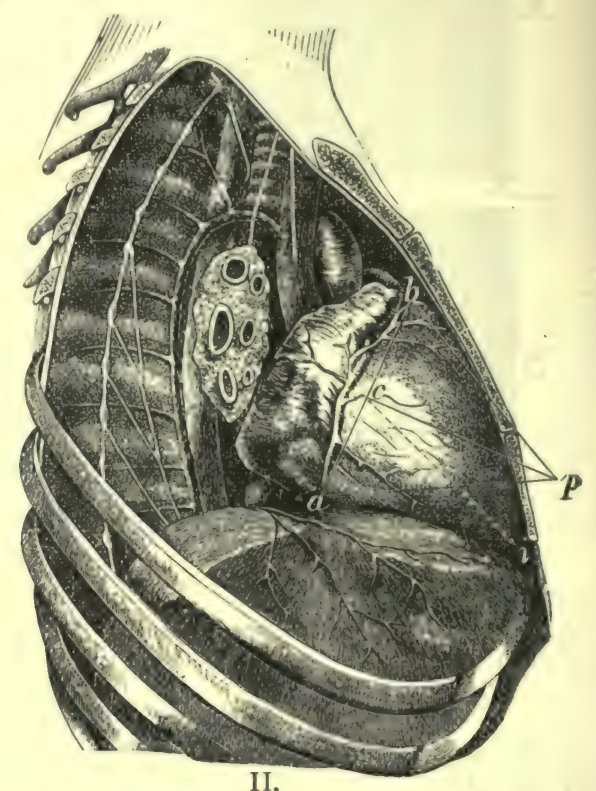

II.

Fig. 40 .

I. Schematic horizontal section through the heart, lungs, and thorax, to show the change of shape which the base of the heart undergoes during contraction of the ventricle $-\mathrm{F}, \mathrm{G}$, transverse diameter of the ventricle during diastole ; $c$, position of the thoracic wall ; $a, b$, transverse diameter of the heart during systole, with $e$, position of the anterior thoracic wall during systole. II. Side-view of the heart-i, apex during diastole; $p$, during systole.

be the case in (4) and (5), but that it moves upward and to the right-a result corroborated by v. Zienıssen. [Barr attributes the cause of the impulse to the rigidity or hardening of the ventricle during systole, to the rotatory movement and lengthening downwards of the bloodcolumn in the aorta and pulmonary artery, while towards the end of the systole the maximum of recoil takes place and also contributes to cause it.]

It is to be remembered that as the apex is always applied to the chest-wall, separated from it merely by the thin margin of the lung, it only presses against the intercostal space during systole (Kiwisch).

After the apex of the curve, $c$, has been reached at the end of the systole, the curve falls rapidly, as the ventricles quickly become relaxed. In the descending part of the curve, at $d$ and $e$, are two elevations, which occur simultaneously with the second sound. These are caused by the sudden closure of the semi-lunar valves, whereby an impulse is propagated through the axis of the ventricle to its apex, and thus causes a vibration of the intercostal space; $d$ corresponds to the closure of the aortic valves, and $e$ to the closure of the pulmonary valves. The closure of the valves in these two vessels is not simultaneous, but is separated by an interval of 0.05 to 0.09 sec. The aortic valves close sooner on account of the 
greater blood-pressure there. Complete diastolic relaxation of the ventricle occurs from $e$ to $f$ in the curve.

It is clear, then, that the cardiac impulse is caused chiefly by the contraction of the ventricles, while the auricular systole and the vibration caused by the closure of the semi-lunar valves are also concerned in its production.

[Change in Shape of Heart.-The experiments of Ludwig and Hesse on the heart of the dog show that the shape of the ventricles varies remarkably in systole and diastole, and that the shape of the heart as found post-mortem is not its natural shape.]

[Method.-Bleed a dog rapidly from the carotids, defibrinate the blood, expose the heart, tie graduated straight tubes into the pulmonary artery and aorta, and ligature the auricular vessels. Pour the blood into the heart until it is dilated under a pressure equal to the mean arterial pressure $(150 \mathrm{~mm}$.). The ventricles are in the diastolic phase, the auricles still pulsate. A plaster cast is now rapidly made of the ventricles. This represents the diastolic phase. To obtain what may be regarded as the systolic phase, a heart, similarly prepared but emptied of blood, is suddenly plunged into a hot $\left(50^{\circ} \mathrm{C}\right.$.) saturated solution of potassic bichromate, when the heart gives one rapid and final contraction and remains permanently contracted owing to the heatrigor, its proteids being coagulated ( $\$ 295)$. This is the systolic phase. Little pins with twisted points are previously inserted in the organ to mark certain parts of both hearts for comparison.]

[In diastole, the shape of the ventricle is hemispheroidal, the apex being rounded, while the posterior surface is flatter than the anterior (fig. 41). In the plane of the ventricular base, the greatest diameter is from right to left, and the shortest from base to apex. The conus arteriosus is above the plane of the base. During

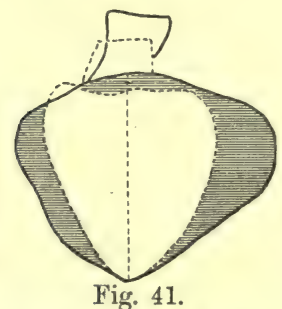

Projection of a dog's heart.

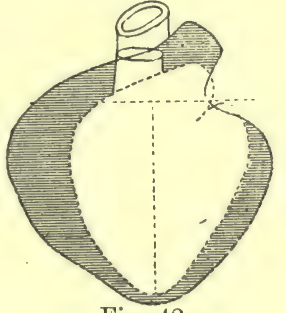

Fig. 42.

Anterior surface.

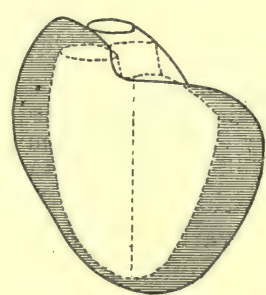

Fig. 43.

Left lateral surface.

systole, the apex is more pointed, the ventricle more conical, while all the diameters in the plane of the base are equally diminished, hence the vertical measurement from base to apex is longer now than either of the diameters at the base (fig. 43). The conus arteriosus sinks towards the plane of the base, while the base of the ventricle becomes more circular, so that the difference of the curvatures of the anterior and posterior surfaces vanishes (fig. 42). In all these figs. the shaded part represents diastole and the clear part systole. The most remarkable point is that the vertical measurement remains unchanged. This refers to the left ventricle, which of course forms the apex; the right is shortened. The plane of the ventricular base in systole is about one-

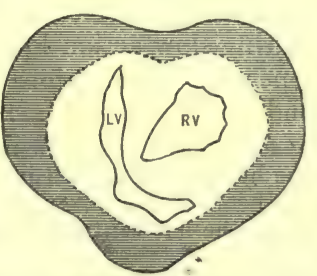

Fig. 44.

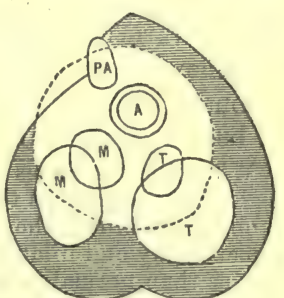

Fig. 45 .

half of what it is in diastole, as is Projection of the base in A, aorta; PA, pulmonshown in fig. 44. Thus the heart is systole and diastole, RV, _. ary artery; M, mitral, diminished in all its diameters except right, and $L V$, left ven- and $T$, tricuspid orione, the arterial orifices are scarcely affected, while the area of the auriculo-ventricular orifices (M, T) is diminished about one-half (fig. 45). This is most important in connection with the closure of 
the auriculo-ventricular valves; as it shows that the muscular fibres of the heart, by diminishing these orifices during systole, greatly aid in the perfect closure of these valves. Thus we explain why defective nutrition of the cardiac muscle may give rise to incompetency of these valves, without the valves themselves being diseased (Macalister).]

[In order to account for the vertical diameter remaining unchanged, we may represent the ventricular fibres as consisting of three layers, viz., an inner and outer set, more or less longitudinal, and a middle set, circular. Both sets will tend, when they contract, to diminish the cavity, but the shortening of the longitudinal layers is compensated for by the contraction, i.e., the elongation produced by the circular set.]

[In order to obtain the shape of the cavities, dogs were taken of the same litter and as nearly alike as possible. One heart was filled with blood, as already described, and placed in a cool solution of potassie bichromate, whereby it was slowly hardened in the diastolic form, while the other was plunged as before into a hot solution. Casts were then made of the cavities.]

51. THE TIME OF THE CARDIAC MOVEMENTS. - Methods. - The time occupied by the various pheses of the moccments of the heart may be determined by studying the apex-beat curve.

(1) If we know at what rate the plate on which the curve was obtained moved during the experiment, of course all that is necessary is to measure the distance, and so calculate the time occupied by any event (see Pulse, § 67).

(2) It is preferable, however, to cause a tuning-fork, whose rate of vibration is known, to write its vibrations under the curve of the apex-beat, or the curve may be written upon a plate attached to a vibrating tuning-fork (fig. $39, \mathrm{D}, \mathrm{E}$ ). Such a curve contains fine teeth caused by the vibrations of the tuning-fork. I) and $\mathrm{E}$ are curves obtained from the cardiac impulse in this way from healthy students. In $\mathrm{D}$ the notch $d$ is not indicated. Each complete vibration of the tuning-fork, reckoned from apex to apex of the teeth $=0.01613$ second, so that it is simply necessary to count the number of teeth and multiply to obtain the time. The values obtained vary within certain limits even in health.

The value of $\iota b=$ pause + contraction of the auricles, is subject to the greatest variation, and depends chiefly upon the number of heart-beats per minute. The more quickly the heart beats, the shorter is the pause, and conversely. In some curves, even when the heart beats slowly, it is scarcely possible to distinguish the auricular contraction (indicated by a rise) from the part of the curve corresponding to the pause (indicated by a horizontal line). In one case (heart-beats 55 per minute) the pause $=0.4$ second, the auricular contraction $=0.177$ second. In fig. $39, \mathrm{~A}$, the time occupied by the pause + the auricular contraction ( 74 beats per minute) $=0.5$ second. In $\mathrm{D}, a b=19$ to 20 vibrations $=0.32$ second; in $\mathrm{E}=$ 26 vibrations $=0.42$ second.

The ventricular systole is calculated from the beginning of the contraction $b$, to $e$ when the semi-lunar valves are closed; it lasts from the first to the second sound. It also. varies somewhat, but is more constant. When the heart beats rapidly, it is somewhat shorter-during slow action longer. In $\mathrm{E}=0.32$ second ; in $\mathrm{D}=0.29$ second; with 55 beats per minute Landois found it $=0.34$, with a very high rate of beating $=0.199$ second.

When the ventricles beat feebiy, they contract more slowly, as can be shown by applying the registering apparatus to the heart of an animal just killed. In fig. 46 , from the ventricle of a rabbit just killed, the slow heart-beats, B, are seen to last longest. In cases of enormous hypertrophy and dilatation of the left ventricle, the duration of the ventricular systole is not longer than normal (Landois).

In calculating the time occupied by the ventricular systole we must remember-(1) The time between the two sounds of the heart, i.e., from the beginning of the first to the end of the second sound $(b$ to $e)$. (2) The time the blood flows into the aortu, which comes to an end at the depression between $c$ and $d$ (in fig. 39, E). Its commencement, however, does not coincide with $b$, as the aortic valves open 0.085 to 0.073 second after the beginning of the ventricular systole. Hence the aortic current lasts 0.08 to 0.09 second. This is calculated in the following way :The time between the first sound of the heart and the pulse in the axillary artery is 0.137 second, and of this time 0.052 second is occupied in the propagation of the pulse-wave along the $30 \mathrm{~cm}$. of artery lying between the root of the aorta and the axilla. Thus the pulse-wave in 
the aorta occurs 0.137 minus $0.052=0.085$ second after the beginning of the first sound. The current in the pulmonary artery is interrupted in the depression betiveen $d$ and $e$. (3) Lastly, the time occupied by the muscular contraction of the ventricle, which begins at $b$, reaches its

A

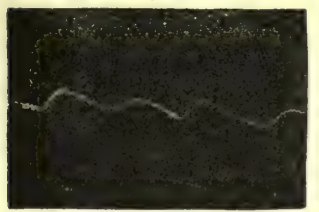

B

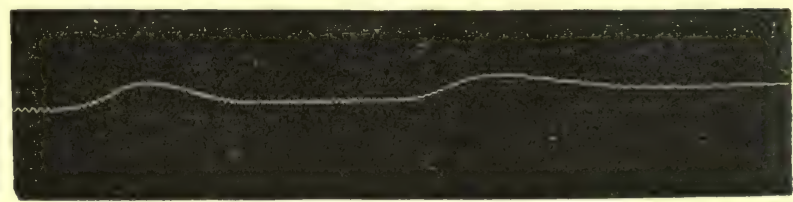

Fig. 46.

Curves recorded by the ventricle of a rabbit, upon a vibrating plate attacher to a tuning-fork (vibration $=0.01613 \mathrm{sec}$.). A, soon after death; $\mathrm{B}$, from the dying ventricle.

greatest extent at $c$, and is completely relaxed at $f$. The apex of the curve, $c$, nay be higher or lower according to the flexibility of the intercostal space, hence the position of $c$ varies. In hypertrophy with dilatation of the left ventricle, the duration of the ventricular contraction does not greatly exceed the normal.

The time which elapses between $d$ and e, i.e., between the complete closure of the aortic and pulmonary valves, is greater the more the pressure in the aorta exceeds that in the pulmonary artery, as the valves are closed by the pressure from above, and the difference in time may be 0.05 second, or even double that time, in which case the second sound appears double (compare $\$ 54$ ). If the aortic pressure diminishes while that in the pulmonary artery rises, $d$ and $e$ may be so near each other that they are no longer marked as distinct elements in the curve.

The time, ef, during which the ventricles relax varies somewhat: 0.1 second may be taken as a mean.

Accelerated Cardiac Action, - When the action of the heart is greatly accelerated, the pause is considerably shortened in the first instance (Donder's), and to a less extent the time of contraction of the auricles and ventricles. When the pulse-rate is very rapid, the systole of the atria coincides with the closure of the arterial valves of the preceding contraction, as is shown in fig. $39, \mathrm{C}(\mathrm{dog})$.

In registering the cardiac impulse, the apparatus is separated by a greater or less depth of soft parts from the heart itself, so that in all cases the intercostal tissues do not follow exactly the movements of the heart, and thus the curve obtained may not coincide mathematically with the movements of the heart. It is desirable that curves be obtained from persons whose hearts are exposed, i.e., in cases of ectopia cordis.

Cleft Sternum. - Gibson inscribed cardiograms from the heart of a man with cleft sternum. The following were the results obtained:-Auricular contraction $=0.115$; ventricular contraction $(b, d)$ $=0.28$; difference between closure of valves $(d, e)$ $=0.09$; ventricular diastole $(e, f)=0.11$; pause $=0.45$ second.

Endocardial Pressure.-In large mammals, such as the horse, Chauveau and Marey (1861) determined the duration of the events that occur within the heart, and also the endocardial pressure by means of a cardiac sound. Small elastic bags attached to tubes were introduced through

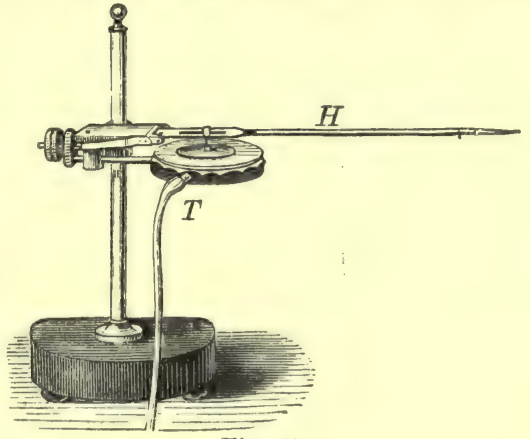

Fig. 47 .

Marey's registering tambour. $\mathrm{T}$, metallic capsule, with thin india-rubber stretched over it, and bearing an aluminium dise, which acts upon the writing lever, $\mathbf{H}$. By means of a thick-walled caoutchoue tube, it may be connected with any system containing.ail, so as to reeord variations of pressure.

the jugular vein into the right auricle and ventricle. Each of these tubes was connected with a registering tambour (fig. 47), and simultaneous tracings of the variations of pressure within the cavities of the heart were obtained by causing the writing-points of the levers of the tambours to write upon a revolving cylinder. 
Fig. 48, A, gires the result obtained when one elastic bag was placed in the right auricle, being introduced through the jugular vein and superior vena cava; $\mathrm{B}$, when the other bag pushed through the tricuspid orifice was in the right ventricle; $D$, in the root of the aorta, pushed in through the carotid ; C, pushed past the semi-lunar valves into the left ventricle; while at E a similar bag has been placed externally between the heart's apex and the inner wall of the chest. In all cases $v=$ auricular contraction; $V$, that of the ventricle; $s$, closure of semi-lunar valves, sooner in $\mathrm{C}$ than $\mathrm{B} ; \mathrm{P}=$ pause.

Methods.--(1) The cardiac sound consists of a tube containing two separate air-passages, and in connection with each of these there is a small elastic bag or ampulla. One of the bags is fixed to the free end of the sound, and communicates with one of the air-passages. The other

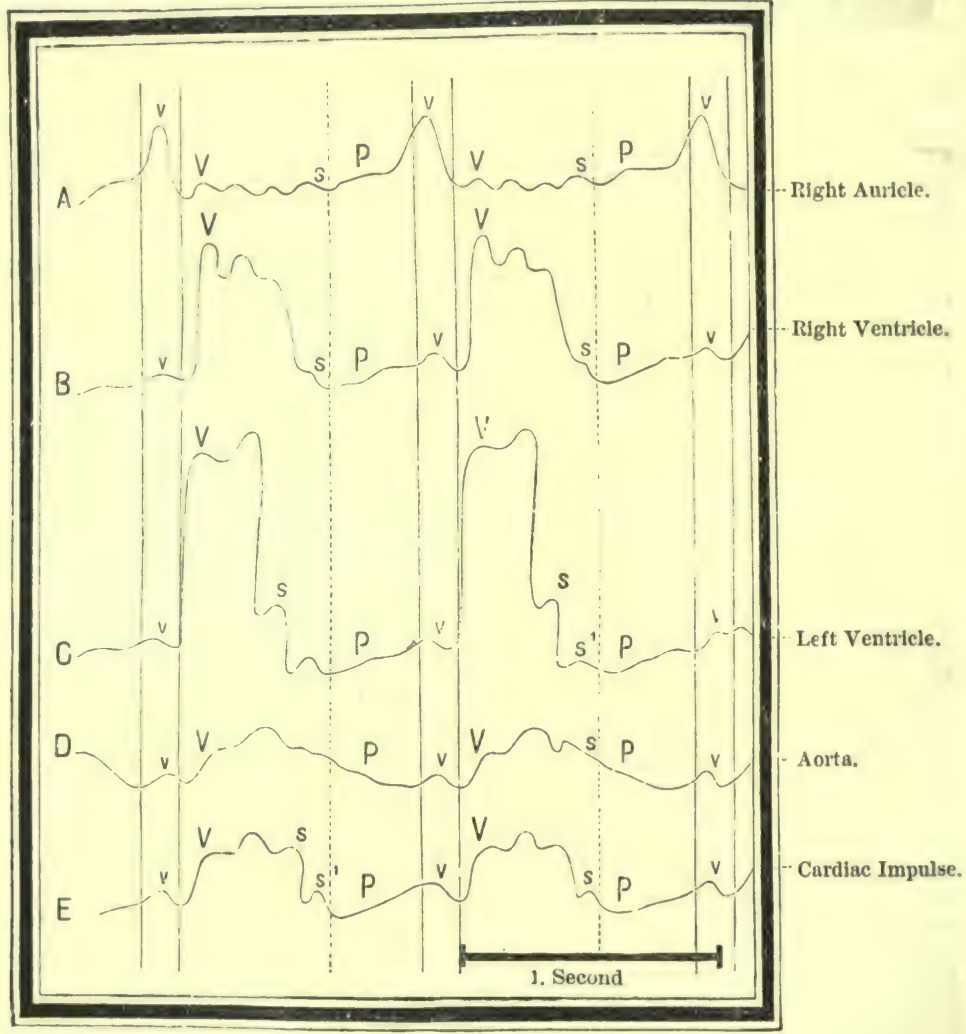

Fig. 48.

Curves obtained from the heart of a horse by the cardiac sound.

bag is placed in connection with the second air-passage in the sound, and at such a distance that, when the former bag lies within the ventricle, the latter is in the auricle. Each bag and air-tube communicating with it is connected with a Marey's tambour (fig. 47), provided with a lever which inscribes its movements upon a revolving cylinder. Any variation of pressure within the auricle or ventricle will affect the elastic ampulle, and thus raise or depress the lever. Care must be taken that the writing-points of the levers are placed exactly above each other. A tracing of the cardiac impulse is taken simultaneously by means of a cardiograph attached to a separate tambour.

It has still to be determined whether the auricles and ventricles act alternately, so that at the moment of the beginning of the ventricular contraction the auricles relax, or whether the ventricles are contracted while the auricles still remain slightly contracted, so that the whole heart is contracted for a short time at least. The latter view was supported by Harvey, Donders, and others, while Haller and many 
of the more recent observers support the view that the action of the auricles and ventricles alternates. In the case of Frau Serafin, whose heart was exposed, v. Ziemssen obtained curves from the auricles, which showed that the contraction of the auricles continued even after the commencement of the ventricular systole. In Marey's curve the contraction of the ventricle is represented as following that of the auricle (fig, 48).

[(2) Rolleston used a special apparatus which was connected with the interior of the heart, and he finds that there is no distinct rise of pressure in the dog within the ventricles corresponding to the auricular systole such as was obtained by Marey in the horse. During the ventricular diastole in certain cases the pressure falls below the atmospheric pressure, and may be equal to $-20 \mathrm{~mm}$. mercury or more in the left ventricle (\$ 48 ). It is probably caused by the elastic expansion of the ventricle continuing after the blood in the auricle at the moment of the cessation of the ventricular systole has entered the ventricle, i.e., the quantity of blood in the auricle is not sufficient in all cases to distend the left ventricle to the point at which its suction action ceases. Magini, operating on dogs with a trocar which perforated the cavities of the heart, found none of the secondary elevations obtained by Marey with his sound.]

A. Fick regards the alternating contraction as a means whereby the pressure in the large venous trunks is kept nearly constant. At the moment of ventricular systole the auricles relax, and the venous blood flows freely into the latter, while if the auricles remained contracted, the blood in the veins would be kept back. Further, at the moment of ventricular diastole the auricles contract, so that there is not an abnormal diminution of the pressure in the veins. Thus the pressure in the auricle is more equable, while the current in the terminal parts of the veins is kept more constant.

52. PATHOLOGICAL CARDIAC IMPULSES. - Change in the Position of the Apex-Beat. The position of the cardiac impulse is changed-(1) by the accumulation of fluids (serum, pus, blood) or gas in one pleural cavity. A copious effusion into the left pleural cavity compresses the lung, and may displace the heart towards the right side, while effusion on the right side may push the heart more to the left. As the right heart must make a greater effort to propel the blood through the compressed lung, the cardiac impulse is usually increased. Advanced emphysema of the lung, causing the diaphragm to be pressed downwards, displaces the heart downwards and inwards, while pushing or pulling up of the diaphragm (by contraction of the lung, or through pressure from below) causes the apex-beat to be displaced upwards, and also slightly to the left. Thickening of the muscular walls with dilatation of the cavities of the left ventricle makes that ventricle longer and broader, while the increased cardiac impulse may be felt in the axillary line in the sixth, seventh, or even eighth intercostal space to the left of the mammary line. Hypertrophy, with dilatation of the right side, increases the breadth of the heart, so that the cardiac impulse is felt more to the right, even to the right of the sternum. In the rare cases where the heart is transposed, the apexbeat is felt on the right side. When the cardiac impulse goes to the left of the left mammary line, or to the right of the parasternal line, the heart is increased in breadth, and there is hypertrophy of the heart. A greatly increased cardiac impulse may extend to several intercostal spaces.

The cardiac impulse is abnormally weakened in cases of atrophy and degeneration of the cardiac muscle, or by weakening of the innervation of the cardiac ganglia. It is also weakened when the heart is separated from the chest-wall owing to the collection of fluids or air in the pericardium, or by a greatly distended left lung; and, indeed, when the left side of the chest is filled with fluid, the cardiac impulse may be extinguished. The same occurs when the left ventricle is very imperfectly filled during its contraction (in consequence of marked narrowing of the mitral orifice), or when it can only empty itself very slowly and gradually, as during marked narrowing of the aortic orifice.

An increase of the cardiac impulse occurs during hypertrophy of the walls, as well as under the influence of various stimuli (psychical, inflammatory, febrile, toxic) which affect the cardiac ganglia. Great hypertrophy of the left ventricle causes the heart to heave, so that a part of the left chest-wall may be raised and also vibrate during systole.

A pulling in of the anterior wall of the chest during the cardiac systole occurs in the third and fourth interspaces, not unfrequently under normal circumstances, sometimes during increased cardiac action, and in eccentric hypertrophy of the ventricles. As the heart's apex is slightly displaced, and the ventricle becomes slightly smaller during its systole, the empty space is filled by the yielding soft parts of the intercostal space. When the heart is united with the pericardium and the surrounding connective-tissue, which renders systolic locomotion of the heart impossible, retraction of the chest-wall during systole takes the place of the cardiac impulse $(S k o d a)$. During the diastole, a diastolic cardiac impulse of the corresponding part of the chest-wall may be said to occur. 
Clinically, changes in the carliac impulse are best ascertained by taking graphic representations of the cardiac impulse, and studying the curves so obtained (fig. 49).

In curve $\mathrm{P}$ (much reduced), from a case of marked hypertrophy with dilatation, the ventricular contraction, $b c$, is usually very great, while the time occupied by the contraction is not much increased. $\mathbf{P}$ and $\mathbf{Q}$ were obtained from a case of marked eccentric hypertrophy of the left ventricle, due to insufficiency of the aortic valves. Curve $Q$ was taken intentionally over the auriculo-ventricular groove, where retraction of the chest-wall occurred during systole; nevertheless the indivilual events oceurring in the heart are indicated.

Fig. $\mathrm{E}$ is from a case of aortic stenosis. The auricular contraction $(a b)$ lasts only a short time: the ventricular systole is obvionsly lengthened, and after a short elevation $(b c)$ shows a
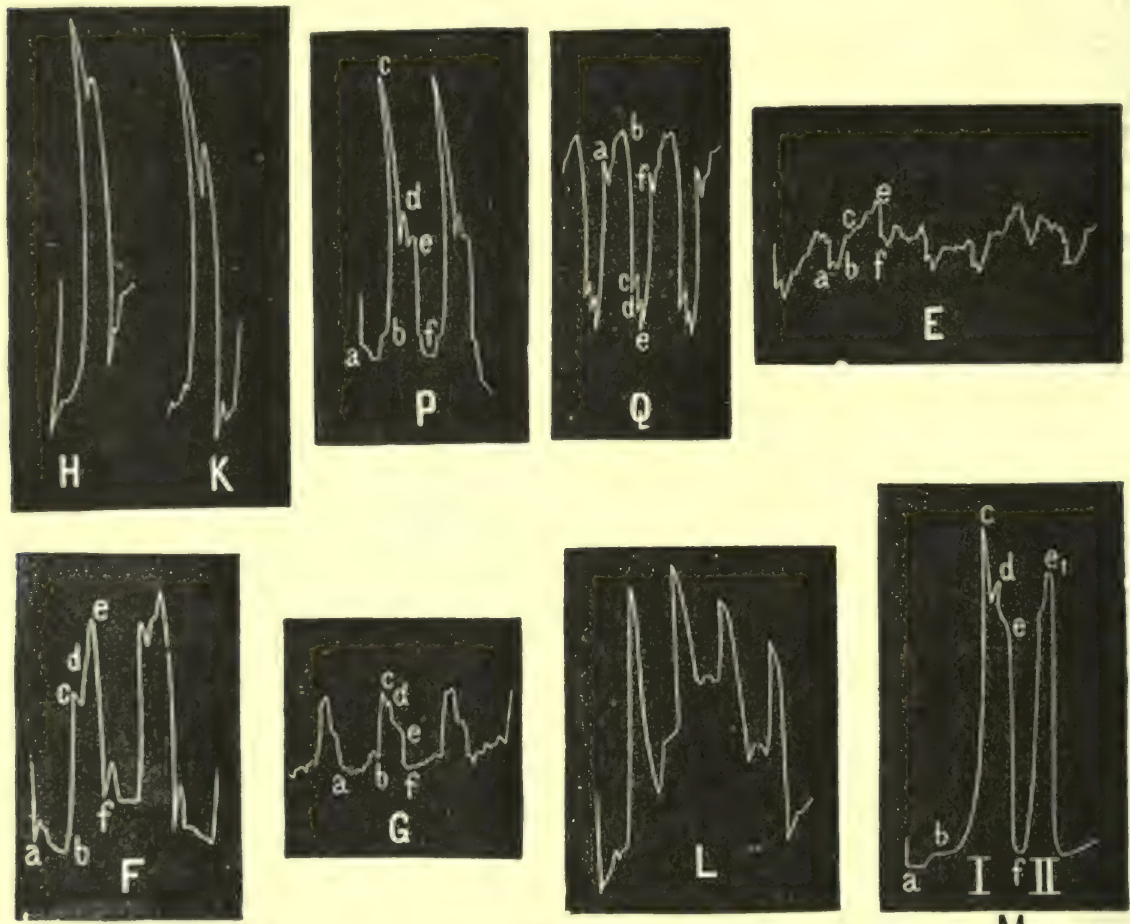

Fig. 49 .

Curves of the carliac impulses. $a b$, contraction of auricles; $b c$, ventricular systole; $d$, closure of aortic, and $e$, of pulmonary valves; $c f$, diastole of ventricle; $\mathrm{P}, \mathrm{Q}$, hypertrophy and dilatation of the left ventricle ; $\mathrm{E}$, stenosis of the aortic orifice; F, mitral insufficiency ; (i, nitral stenosis; L, nervous palpitation in Basedow's disease ; M, so-called hemisystole.

series of fine indentations $(c, c)$ caused by the blood being pressed through the narrowed and roughened aorta.

Fig. F, from a case of insufficiency of the mitral valve, shows (ab) well marked on account of the increased activity of the left auricle, while the shock $(d)$ from the closure of the aortic valves is small, on account of the diminished arterial tension. On the other hand, the shock from the accentuated pulmonary sound $(c)$ is very great, and is in the apex of the curve. On account of the great tension in the pulmonary artery, the second pulmonary tone may be so strong, and succeed the second aortic sound $(d)$ so rapidly, that both almost merge completely into each other $(\mathbf{H}$ and $\mathrm{K})$.

The curve of stenosis of the mitral orifice (G) shows a long, irregular, notched, auricular contraction $(a b)$, caused by the blood being forced through an irregular narrow orifice. The ven. tricular contraction $(b c)$ is feeble because the ventricle is imperfectly filled. The closures of the two valves, $d$ and $\ell$, are relatively far apart, and one can hear distinctly a reduplicated second sonnd. The aortie valves close rapidly, because the aorta is imperfectly supplied with blood, while the more copious inflow of blood into the pulmonary artery causes its valves to close later. 
If the heart beats rapidly and feebly-if the blood-pressure in the aorta and pulmonary artery be low, the signs of closure of the pulmonary valves may be absent-as in curve Ltaken from a girl suffering from nervous palpitation and morbus Basedowii.

In very rare cases of insufficiency of the mitral valve, it has been observed that at certain times both ventricles contract simultaneously, as in a normal heart, but that this alternates with a condition where the right ventricle alone seems to contract. Curve $M$ is such a curve obtained by Malbranc, who called this condition intermittent hemisystole. The first curve (I.) is like a normal curve, during which the whole heart acted as usual. The curve II., however, is caused by the right side of the heart alone ; it wants the closure of the aortic valves, $d$, and there was no pulse in the arteries. Owing to insufficiency of the tricuspid valve, the same person had a venous pulse with every cardiac impulse, so that the arterial and venous pulses first occurred together, and then the venous pulse alone occurred. In these cases the mitral insufficiency leads to the right ventricle being overdistended, while the left is nearly empty, so that the right side requires to contract more energetically than the left. It does not seem that the right ventricle alone contracts in these cases, but rather that the action of the left side is very feeble.

53. THE HEART-SOUNDS.-On listening over the region of the heart in a healthy man, either with the ear applied directly to the chest-wall (Harvey), or by means of a stethoscope (Laennec, 1819), we hear two characteristic sounds, the socalled " heart-sounds." The two sounds are called first and second, and together they correspond to a single cardiac cycle. These sounds are separated by silences. [Fig. 50 shows the relation of the events occurring in the heart during a cardiac cycle to the sounds and silences.]

1. The first sound.

2. The first or short silence.

3. The second sound.

4. The second or long silence.

[Relative Duration.--There is no absolute duration of each phase of a cardiac cycle, but we may take the average duration calculated from the measurements of Gibson, in a case of fissure of the sternum, to be as follows :-

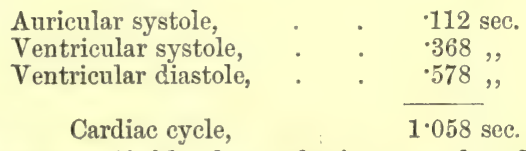

Suppose we divide the cycle into tenths (Walshe), then the first sound will last $\frac{4}{10}$, the first silence $\frac{1}{10}$, the second sound $\frac{2}{10}$, and the long silence $\frac{3}{10}$ of the entire period.]

The first sound [long or systolic] is twice as long as, somewhat duller, and one-third or one-fourth deeper, than the second sound; it is less sharply defined at first, and is synchronous with the systole of the ventricles.

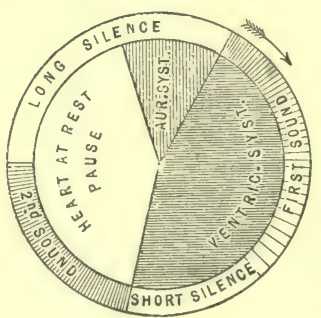

Fig. 50.

Scheme of a cardiac cycle. The inner circle shows what events occur in the heart, and the outer, the relation of the sounds and silences to these events.

The second sound [short or diastolic] is clearer, sharper, shorter, more sudden, and is one-third to one-fourth higher; it is sharply defined and synchronous with the closure of the semi-lunar valves. The sounds emitted during each cardiac cycle have been compared to the pronunciation of the syllables $l \bar{u} b b$, düp. Or the result may be expressed thus-
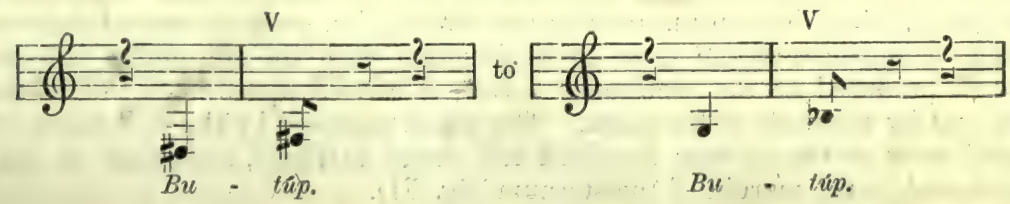
[It is to be remembered that in reality four sounds are produced in the heart, but the two first sounds occur together and the two second, so that only a single first and a single second sound are heard.]

The causes of the first sound are due to two conditions. As the sound is heard, although enfeebled, in an excised heart in which the movements of the valves are arrested, and also when the finger is introduced into the auriculo-ventricular orifices so as to prevent the closure of the valves (C. Ludwig and Dogiel), one of the chief factors lies in the "muscle sound" produced by the contracting muscular fibres of the ventricles. This sound is supported and increased by the sound produced by the tension and vibration of the auriculo-ventricular valves and their chordæ tendineæ, at the moment of the ventricular systole. Wintrich, by means of proper resonators, has analysed the first sound and distinguished the clear, short, valvular part from the deep, long, muscular sound.

The muscle-sound produced by transversely-striped muscle does not occur with a simple con. traction (p. 86), but only when several contractions are superposed to produce tetanus $(\S 303)$. The ventricular contraction is only a simple contraction, but it lasts considerably longer than the contraction of other muscles, and herein lies the cause of the occurrence of the muscle-sound during the ventricular contraction.

Defective Heart-Sounds. - In certain conditions (typhus, fatty degeneration of the heart), where the muscular substance of the heart is much weakened, the first sound may be completely inaudible. In aortic insufficiency, in consequence of the reflux of blood from the aorta into the ventricle, the mitral valve is gradually stretched, and sometimes even before the beginning of the ventricular systole, the first sound may be absent. Such pathological conditions seem to show that, for the production of the first sound, muscle-sound and valve-sound must eventually work together, and that the tone is altered, or may even disappear, when one of these causes is absent. [Yeo and Barrett state that the sound is purely muscular (?).]

The cause of the second sound is undoubtedly due to the prompt closure, and therefore sudden stretching or tension, of the semi-lunar valves of the aorta and pulmonary artery, so that it is purely a valvular sound. Perhaps it is augmented by the sudden vibration of the fluid-particles in the large arterial trunks. [The second sound has all the characters of a valvular sound. That the aortic valves are concerned in its production, is proved by introducing a curved wire through the left carotid artery and hooking up one or more segments of the valve, when the sound is modified, and it may disappear or be replaced by an abnormal sound or "murmur." Again, when these valves are diseased, the sound is altered, and it may be accompanied or even displaced by murmurs.] Although the aortic and pulmonary valves do not close simultaneously, usually the difference in time is so small that both valves make one sound, but the second sound may be double or divided when, through increase of the difference of pressure in the aorta and pulmonary artery, the interval becomes longer. Even in health this may be the case, as occurs at the end of inspiration or the beginning of expiration ( $v$. Dusch).

Where the Sounds are Heard Loudest. - The sound produced by the tricuspid valve is heard loudest at the junction of the lower right costal cartilages with the sternum; as the mitral valve lies more to the left and deeper in the chest, and is covered in front by the arterial orifice, the mitral sound is best heard at the apexbeat, or immediately above it, where a strip of the left ventricle lies next the chestwall. [The sound is conducted to the part nearest the ear of the listener by the muscular substance of the heart.] The aortic and pulmonary orifices lie so close together that it is convenient to listen for the second (aortic) sound in the direction of the aorta, where it comes nearest to the surface, i.e., over the second right costal cartilage or aortic cartilage close to its junction with the sternum. The sound, although produced at the semi-lunar valves, is carried upwards by the column of blood, and by the walls of the aorta. The sound produced by the pulmonary artery is heard most distinctly over the third left costal cartilage, somewhat to the left and external to the margin of the sternum (fig. 51). 
54. VARIATIONS OF THE HEART-SOUNDS. - Increase of the first sound of both ventricles indicates a more energetic contraction of the ventricles and a simultaneously greater and more sudden tension of the auriculo-ventricular valves. Increase of the second sound is a sign of increased tension in the interior of the corresponding large arteries. Hence increase of the

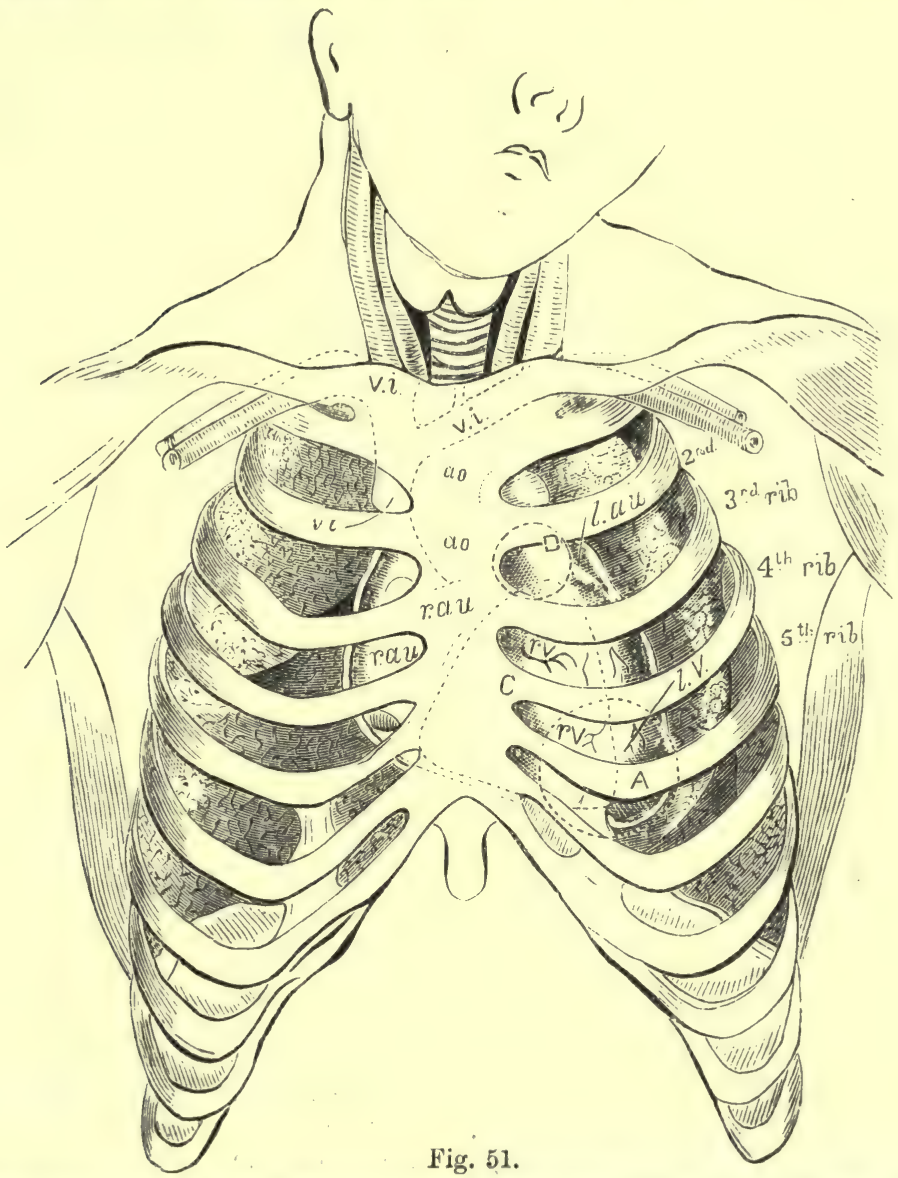

The heart-its several parts and great vessels in relation to the front of the thorax. The lungs are collapsed to their normal extent, as after death, exposing the heart. The outlines of the several parts of the heart are indicated by very fine dotted lines. The area of propagation of valvular murmurs is marked out by more visible dotted lines. A, the circle of mitral murmur, corresponds to the left apex. The broad and somewhat diffused area, roughly triangular, is the region of tricuspid murmurs, and corresponds generally with the right ventricle, where it is least covered by lung. The letter $\mathrm{C}$ is in its centre. The circumscribed circular area, D, is the part over which the pulmonic arterial murmurs are commonly heard loudest. In many cases it is an inch, or even more, lower down, corresponding to the conus arteriosus of the right vêntricle, where it tonches the wall of the thorax. The internal organs and parts of organs are indicated by letters as follows :r.au, right auricle, traced in fine dotting; $a 0$, arch of aorta, seen in the first intercostal space, and traced in fine dotting on the sternum; $v . i$., the two innominate veins; $r . v$, right ventricle;. .v., left ventricle.

second (pulmonary) sound indicates overfilling and excessive tension in the pulmonary circuit. $A$ feeble action of the heart, as well as abnormal want of blood in the heart, causes weak heart-sounds, which is the case in degenerations of the heart-muscle.

Irregularities in structure of the individual valves may cause the heart-sounds 'to become 
" impure." If a pathological cavity, filled with air, be so placed, and of such a form as to act as a resonator to the heart-sounds, they may assume a "metallic" character. The first and second sounds may be "reduplicated" or [although "duplication" is a more accurate term (Barr)] doubled. The reduplication of the first sound is explained by the tension of the tricuspid and that of the mitral valves not occurring simultaneously. Sometimes in disease a sound is produced by a hypertrophied auricle producing an audible presystolic sound, 3.e., a sound or "murmur," precelling the first sound. [This has been questioned quite recently.] As the aortic and pulmonary valves do not close quite simultaneously, a reduplicated second sound is only an increase of a physiological condition. All conditions which cause the aortic valves to close rapidly (diminished amount of blood in the left ventricle) and the pulmonary valves to close later (congestion of the right ventricle-both conditions together in mitral stenosis), favour the production of a reduplicated second sound.

Cardiac Murmurs. - If irregularities occur in the valves, either in cases of stenosis or in insufficiency, so that the blood is subjected to vibratory oscillations and friction, then, instead of the heart-sounds, other sounds-murmurs or bruits-arise or accompany these. A combination of these sounds is always accompanied by disturbances of the circulation. [These murmurs may be produced within the heart, when they are termed endocardial; or outside it, when they are called exocardial murmurs. But other murmurs are due to changes in the quality or amount of the blood, when they are spoken of as hæmic murmurs. In the study of all murmurs, note their rhythm or exact relation to the normal sounds, their point of maximum intensity, and the direction in which the murmur is propagated.] It is rare that tumours or other deposits projecting into the ventricles cause murmurs, unless there be present at the same time lesions of the valves and disturbances of the circulation. The cardiac murmurs are always related to the systole or diastole, and usually the systolic are more accentuated and louder. Sometimes they are so loud that the thorax trembles under their irregular oscillations (fremitus, frémissement cataire).

In cases where diastolic murmurs are heard, there are always anatomical changes in the cardiac mechanism. These are insufficiency of the arterial valves, or stenosis of the auriculo-ventricular orifice (usually the left). Systolic murmurs do not always necessitate a disturbance in the cardiac mechanism. They may occur on the left side, owing to insufficiency of the mitral valve, stenosis of the aorta, and in the calcification and dilatation of the ascending part of the aorta. These murmurs occur very much less frequently on the right side, and are due to insufficiency of the tricuspid and stenosis of the pulmonary orifice.

Functional Murmurs. - Systolic murmurs often occur without any valvular lesion, although they are always less loud, and are caused by abnormal vibrations of the valves or arterial walls. The $y$ oceur most frequently at the orifice of the pulmonary artery [and are generally heard at the hase], less frequently at the mitral, and still less frequently at the aortic or the tricuspid orifice. Anerinia, general malnutrition, acute febrile affections, are the causes of these murmurs. [Some of these are due to an altered condition of the blood, and are called hrmic, and others to defective cardiac muscular nutrition, and are called dynamic (Walshe).]

Sounds may also occur during a certain stage of inflammation of the pericardium (pericarditis) from the roughened surfaces of this membrane rubbing upon each other. Audible friction sounds are thus produced, and the vibration may even be perceptible to touch. ['These are "friction sounds," and quite distinct from sounds produced within the heart itself.]

55. DURATION OF THE MOVEMENTS OF THE HEART.-The heart con-

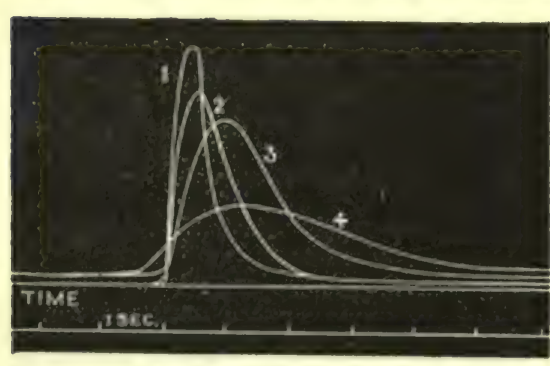

Fig. 52.

Curves of excised rabbit's heart, 1, 6 mins. after excision; 2, 10 mins. ; 3,20 mins. ; 4, 70 mins. (after $W$ aller and Reid). to occur in the right auricle (rabbit) 15 hours after death; in a mouse's heart, 46 hours; in a dog's, 96 hours. An excised frog's heart beats, at the longest, $2 \frac{1}{2}$ days

tinues to beat for some time after it is cut out of the body. The movement lasts longer in cold-blooded animals (frog, turtle)-extending even to days - than in mammals. A rabbit's heart beats from 3 minutes up to 36 minutes after it is cut out of the body. The average of many experiments is about 11 minutes. [Waller and Reid recorded the ventricular contractions of a rabbit's heart 72 minutes after its excision. Fig. 52 shows the prolongation of the ventricular systole in an excised rabbit's heart, the movements being recorded by a lever resting on the heart.] Panum found the last trace of contraction 
(Valentin). In a human embryo (third month) the heart was found beating after 4 hours. In this condition stimulation causes an increase and acceleration of the action. The ventricular contraction weakens, and soon each auricular contraction is not followed by a ventricular contraction, two or more of the former being succeeded by only one of the latter. At the same time the ventricles contract more slowly (fig. 46), and soon stop altogether, while the auricles continue to beat. If the ventricles be stimulated directly, as by pricking them with a pin, they may execute a contraction. The left auricle soon ceases to beat, while the right auricle still continues to contract. The right auricular appendix continues to beat longest, as was observed by Galen and Cardanus (1550), and it is termed "ultimum moriens." Similar observations have been made upon the hearts of persons who have been executed.

If the heart has ceased to beat, it may be excited to contract for a short time ky direct stimulation, more especially by heat (Harvey); even under these circumstances the auricles and their appendices are the last parts to cease contracting. As a general rule, direct stimulation, although it may cause the heart to act more vigorously for a short time, brings it to rest sooner. In such cases, therefore, the regular sequence of events ceases, and there is usually a twitching movement of the muscular fibres of the heart. C. Ludwig found that, even after the excitability is extinguished in the mammalian heart, it may be restored by injecting arterial blood into the coronary arteries: conversely lesion of these vessels is followed by enfeebled action of the heart ( $\$ 47)$. Hammer found that in a man, whose left coronary artery was plugged, the pulse fell from 80 to 8 beats per minute.

[The beats of the excised heart of a rabbit gradually decline in force and frequency, the latent period and contraction become longer and the excitability more obtuse. The duration of a contraction may be 6 sec., the normal being 3 sec. The beats have often a bigeminal character. An excised heart may be frozen quite hard, yet on being thawed it contracts spontaneously. The contraction proceeds in a wave from the spot stimulated in the frog's heart at $8^{\circ}$ to $12^{\circ} \mathrm{C}$. at 30 to $90 \mathrm{~mm}$. per sec.; in the mammalian excised heart about 8 metres per sec. (Waller and Reid).]

Action of Gases on the Heart.-During its activity the heart uses $\mathrm{O}$, and produces $\mathrm{CO}_{2}$, so that it beats longest in pure $\mathrm{O}$ (12 hours), and not so long in $\mathrm{N},-\mathrm{H}$ ( 1 hour) $-\mathrm{CO}_{2}$ (10 minutes) - $\mathrm{CO}$ (42 minutes) - $-\mathrm{Cl}$ (2 minutes), or in a vacuum (20 to 30 minutes), even when there is watery vapour present to prevent evaporation. If the heart be reintroduced into $\mathrm{O}$ it begins to beat again. '[A frog's heart ceases to beat in compressed $\mathrm{O}$ (10 to 12 atmospheres) in about one-third of the time it would do were it simply excised and left to itself. An excised heart suspended in ordinary air beats three to four times as long as a heart which is placed upon a glass-plate.]

[56. PHYSICAL EXAMINATION OF THE HEART.-The physical methods of diagnosis enable us to obtain precise knowledge regarding the actual state of the heart. The methods available are :-
1. Inspection.
2. Palpation.

3. Percussion.

4. Auscultation.

To arrive at a correct'diagnosis all the methods must be employed.]

[Inspection. - The person is supposed to have his chest exposed and to be in the recumbent position. It is important to remember the limits of the heart. The base corresponds to a line joining the upper nargins of the third costal cartilages, the apex to the fifth interspace, while transversely it extends from a little to the right of the sternum to within a little of the left nipple; this area occupied by the heart being called the deep cardiac region. By the eye we can detect any alteration in the configuration of the precordia, bulging or retraction of the region as a whole or of the intercostal spaces, and we may detect.variations in the position, character, extent of the cardiac impulse, or the presence of other visible pulsations.]

[Palpation.--By placing the whole hand flat upon the præcordia, we can ascertain the presence or absence, the situation and extent, and any alterations in the characters of the apexbeat; or we may detect the existence of abnormal pulsations, vibrations, thrills, or friction in this region. In feeling for the apex-beat, if it be at all feeble, it is well to make the patient lean forward. Of course, it must be remembered that the whole heart may be displaced by tumours or accumulations of fluids pressing upon it, i.e., conditions external to itself, or the apex-beat may be displaced from canses within the heart itself, as in hypertrophy of the left ventricle.] 
[Percussion.-As the heart is a solid organ, and is surrounded by the lungs, which contain air, it is evident that the sound enitted by striking the chest over the region of the former must be different from that produced over the latter. Not only is there a difference in the sound or note emitted, but the "sensation of resistance" which one feels on percussing the two organs is different. We may ascertain-

1. The superficial or absolute cardiac dulness.

2. The deep or relative dulness.]

[Superficial Cardiac Dulness. - This theoretically is the part of the heart in direct contact with the chest-wall and uncovered by lung, but obviously as the lungs vary in size during respiration, it must be smaller during inspiration and larger during expiration. It forms a roughly triangular space, whose base cannot be accurately determined, as the heart-dulness merges into that of the liver, situate below it, but it corresponds to a horizontal line $2 \frac{1}{2}$ inches long, extending from the apex-beat to the middle of the sternum. The internal side corresponding to the left edge of the sternum is 2 inches long, and reaches from the junction of the fourth costal cartilage with the sternun-apex of the triangle-to the sternal end of the base line. The superior, outer, or oblique line, 3 inches in length, is somewhat curved, and passes lownwards and outwards from the apex of the triangle to the apex of the heart.]

[Deep Cardiac Dulness. - By this method theoretically we seek to define the exact limits of the heart as a whole, and thus to ascertain its absolute size, and of course percussion has to be done through a certain thickness of lung tissue, and hence one must strike the pleximeter forcibly. It extends vertically from the third rib and ends at the sixth, but owing to the cardiac merging in the hepatic dulness, this lower limit cannot be accurately ascertained; while transtersely at the fourth rib it extends from just within the nipple line to slightly beyond the right of the sternum. By these means we may detect increase in the size of the heart or alterations in the relation of the lungs to the heart, fluid in pericardium, \&c.]

[Auscultation. - This is one of the most valuable methods, for by it we can detect variations and modifieations in the healthy sounds of the heart, the rhythm and frequency of the heartbeat, the existence of abnormal sounds, and their exact relation to the normal sounds, also their characters and relation to the cardiac cycle, and the direction in which these sounds are propagated (\$54).]

\section{INNERVATION OF THE HEART. - [ntra- and Extra-Cardiac Nervous}

Mechanism.- When the heart is removed from the body, or when all the nerves which pass to it are divided, it still beats for some time, so that its movements must depend uyon some mechanism situated within itself. The ordinary rhythmical movements of the heart are undoubtedly associated with the presence of nerve ganglia, which exist in the substance of the heart-the intra-cardiac ganglia. But the movements of the heart are influenced by nervous impulses which reach it from without, so that there falls to be studied an intra-cardiac and an extra-cardiac nervous mechanism.]

The cardiac plexus is composed of the following nerves:-(1) The cardiac branches of the vagus, the branch of the same name from the external branch of the superior laryngeal, a branch from the inferior laryngeal, and sometimes branches from the pulmonary plexus of the vagus (more numerous on the right side); (2) the superior, middle, inferior, and lowest cardiac branches of the three cervical ganglia and the first thoracic ganglia of the sympathetic; $(3)$ the inconstant twig of the descending branch of the hypoglossal nerve, which, according to Luschka, arises from the upper cervical ganglion. From the plexus there proceed-the deep and the superficial nerves (the latter usually at the division of the pulmonary artery under the arch of the aorta, and containing the ganglion of Wrisberg) (§370). The following nerves may be separately traced from the plexus :-

(a) The plexus coronarius dexter and sinister, which contains the vaso-motor nerves for the coronary vessels (physiological proof still wanting) as well as the nerves (sensory ?) proceeding from them (to the pericardium?).

(b) Intra-cardiac nerves and ganglia.-The nerves lying in the grooves of the heart and in its substance contain numerous ganglia (Remak), and are regarded as the automatic motor centres of the heart. A nervous ring containing numerous ganglia corresponds to the margin of the septum atriorum; there is another in the auriculo-ventricular groove. Where the two meet, they exchange fibres. The 
ganglia usually lie near the pericardium. In mammals, the two largest ganglia lie near the orifice of the superior vena cava-in birds, the largest ganglion (containing thousands of ganglionic cells) lies posteriorly where the longitudinal and transverse sulci cross each other. Fine branches, also provided with small ganglia, proceed from these ganglia, and penetrate the muscular walls of the auricles and ventricles.

[Frog's Heart.-The frog's heart consists of the sinus venosus, into which open the single inferior and the two superior venæ cavæ (fig. 54). There are two auricles; the right one communicates with the sinus venosus, and opens into the single ventricle; the left auricle also

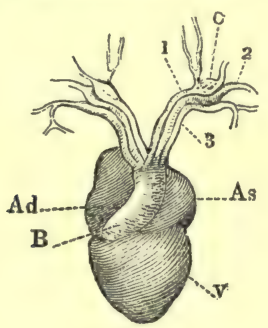

Fig. 53.

Heart of frog from the front. V, single ventricle; $A d, A s$, right and left auricles ; B, bulbus arteriosus ; 1, carotid, 2, aorta, and 3 , pulmo-cutaneous arteries; C, carotid gland.

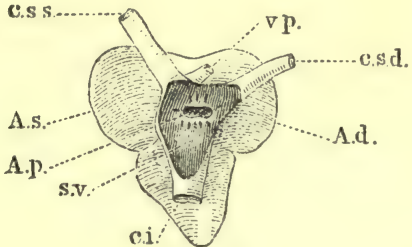

Fig. 54.

Heart of frog from behind. s.v., sinus venosus opened ; $c i$, inferior ; csd, css, right and left superior venæ cavæ; vp., pul. monary vein; $A d$, and $A s$, right and left auricles; $A p$, communication between the right and left auricle.

opens into the single ventricle (fig. 53,v), and in the latter are mixed the venous blood returned by the right auricle and the arterial blood from the left auricle. The aorta with its bulbus arteriosus conducts the blood from the ventricle. The various orifices are giaarded by projec-

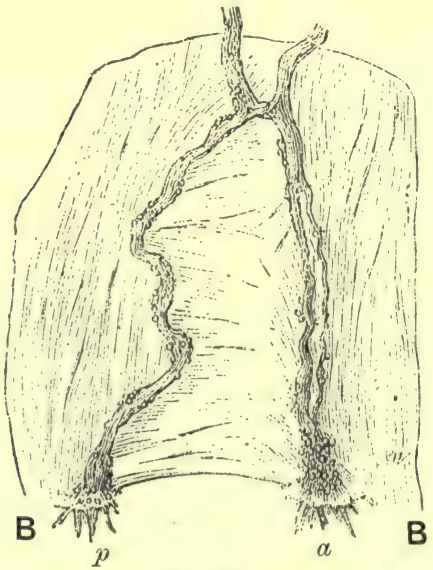

Fig. 55.

Auricular septum of a frog's heart. $a$, anterior, and $p$, posterior branch of the car. diac vagus ; B, Bidder's ganglion.

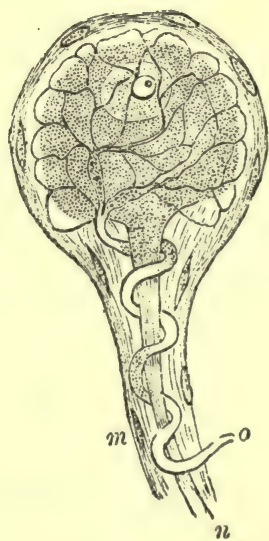

Fig. 56.

Pyriform ganglionic bi-polar nerve-cell from the heart of a frog. $m$, sheath; $n$, straight process ; 0 , spiral process.

tions of tissue, which act like valves. The two auricles are completely separated by a septum. This septum ends posteriorly in a free concave margin so as to divide the auriculo-ventricular orifice into a right and a left orifice. Each orifice is guarded by two thick fleshy valves, which close it.]

[Nerves. - The two cardiac branches of the vagi-the nervi cardiaci--proceed to the posterior surface of the sinus venosus, and where the latter joins the auricle they interlace, and are mixed with a number of ganglion cells (fig. 57). This spot is called Remak's ganglion, is sometimes 
single, at others double, and it can be seen as a white "crescent" when the heart is lifted up and looked at from behind (fig. 54). The cardiac nerves proceod downwards on the auricular septum, exchanging fibres in their course to join two ganglia at the auriculo-ventricular groove, and known as Bidder's ganglia (fig. 57). It has been stated by one observer that the bulbus arteriosus contains ganglionic cells, but this is denied by others.]

Aocording to Openchowsky, every part of the heart (frog, triton, tortoise) contains nervefibres which are connected with every muscular fibre. In the auricles, at the end of the non-

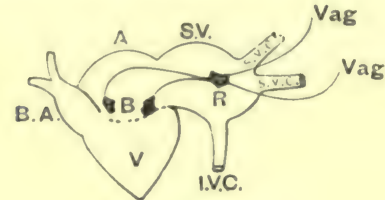

Fig. 57 .

Scheme of nerves of frog's heart. R. Remak's, aud B, Bidder's ganglia; S.V., sinus venosus; A, auricles; V, ventricle ; B.A., bulbus arteriosus ; vag, vagi.
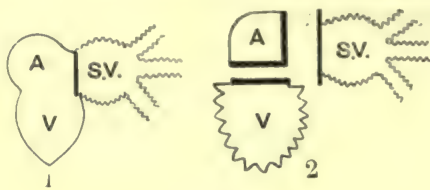

Fig. 58.

Stannius's experiment. A, auricle; $\mathrm{V}$, ventricle ; S.V., sinus venosus. The zig-zay lines indicate which parts continue to beat; in 2 the ventricle beats at a different rate.

melullated fibre, a tri-radiate nucleus exists which gives off fibrils to the muscular bundles. There is a network of fine nerve-fibres distributed immediately under the endocardium-these fibres act partly in a centripetal direction on the cardiac ganglia, and are partly motor for the endocardial muscles. The parietal layer of the pericardium contains (sensory) nerve-fibres. The following kinds of nerve-cells are found-unipolar cells, the single processes of which afterwards divide; bipolar pyriform cells (fig. 56), which in the frog possess a straight $(n)$ and usually also a spiral process $(0)$.

58. THE AUTOMATIC MOTOR CENTRES OF THE HEART.-(1) It is generally assumed that the nervous centres which excite the cardiac movements, and maintain the rhythm of these movements, lie within the heart, and that they are probably represented by the ganglia.

(2) There are-not one, but several of these centres in the heart, which are connected with each other by conducting paths. As long as the heart is intact, all its parts move in rhythmical sequence from a principal central point, an impulse being conducted from this centre through the conducting paths. What the "discharging forces" of these regular progressive movements are, is unknown. If, however, the heart be subjected to the action of diffuse stimuli (e.g., strong electrical currents), all the centres are thrown into action, and a spasm-like action of the heart occurs. The dominating centre lies in the auricles, hence the regular progressive movement usually starts from them. If the excitability is diminished, as by touching the septum with opium, other centres seem to undertake this function, in which case the movement may extend from the ventricles to the auricles. According to Kronecker and Schmey, in the dog's heart there is a spot above the lower limit of the upper third of the ventricular septum, which, when it is injured, e.y., by destroying it with a stout needle, brings the heart to a standstill ; this has been called a co-ordinating centre.

(3) All stimuli of moderate strength applied directly to the heart cause at first an increase of the rhythmical heart-beats ; stronger stimuli cause a diminution, and it may be paralysis, which is often preceded by a convulsive movement. Increased activity exhausts the energy of the heart sooner.

(4) Single very weak stimuli, which have no effect on the heart when applied singly, if repeated sufticiently often, may become active owing to "summation of the stimuli " (v. Basch).

(5) Even the weakest stimulus which can excite a contraction always causes an energetic contraction, i.e., "the minimal stimulus causes a maximal effect" (Bowditch, Kronecker and Stirling). 
(6) After every contraction of the heart there is a short period of "diminished excitability" or Marey's "refractory period," during which the heart is less susceptible to further stimulation.

(7) The non-ganglionic apex of the heart, when it is not stimulated, no longer beats spontaneously, but it responds each time by a single contraction to a single direct stimulus. If, however, a continuous stimulus, e.g., a continuous current of electricity, be applied to it, it executes a series of beats. Such continuous stimuli are obtained through a continuous pressure of fluid, exerted on the interiur of the heart or by moistening the heart with chemical substances.

(8) The auricular centres seem to be more excitable than those of the ventricle; hence, in a heart left to itself the auricles pulsate longest.

(9) The heart may be excited (reflexly) from its inner surface. Weak stimuli applied to the inner surface of the heart greatly accelerate the heart's action, the stimulus required being much feebler than that applied to the external surface of the heart. Strong stimuli, which bring the heart to rest, also act more easily when applied to the inner surface than when they are applied to its outer surface. The ventricle is always the first part to be paralysed.

(10) In order that the heart may continue to contract, it is necessary that it be supplied with a fluid which, in addition to $\mathrm{O}$, must contain the necessary nutritive materials. The most perfect fluid, of course, is blood. Hence the heart after a time ceases to beat in an indifferent fluid ( 0.6 per cent. sodium chloride), but its activity may be revived by supplying it with a proper nutritive fluid.

Cardiac Nutritive Fluids.-These nutritive fluids are such as contain serum-albumin, e.g., blood, serum, or lymph. Serum retains its nutritive properties even after it has been subjecter to diffusion (Martius and Kronecker). Milk and whey (v. Ott), normal saline solution mixed with blood, albumin, or peptone, and 0.3 per cent. sodium carbonate (Kronecker, Merunowicz and Stiénon), a trace of caustic soda (Gaule), or a solution of the salts of serum, are suitable. Alkaline solution of soda revives a feebly beating heart by neutralising the acid formed in the cardiac muscle, or normal saline containing calcic phosphate and potassic chloride (S. Ringer).

(11) The independent pulsations of parts of the heart which are devoid of ganglia, show that the presence of ganglia is not absolutely necessary in order to have rhythmical pulsation. Direct stimulation of the heart may cause these movements. But the ganglia are more excitable than the heart muscle itself, and they conduct the impulses which lead to the regular alternating action of the various parts of the heart, so that, under normal circumstances, we must assume that the action of the heart is governed by the ganglia.

(12) If a heart be cut into pieces, so that the individual pieces still remain connected with each other, the regular peristaltic or wave-like movements proceeding from the auricles to the ventricle may continue for a long time (Donders, Engelmann). If the heart, however, be completely divided into two distinct pieces (auricle and ventricle), the movements of both parts continue, but not in the same sequence-they beat at different rates.

The chief experiments upon which the above statements are based are as follows :-

I. Experiments by cutting and ligaturing the heart. These experiments have been made chiefly upon the heart of the frog. The ligature experiments are performed by tightening and then relaxing a ligature placed around the heart, so that the physiological connection is destroyed, while the anatomical or mechanical connections (continuity of the cardiac wall, intact condition of its cavities) still exist. The most important of these experiments are-

(1) Stannius's Experiment.- - If the sinus venosus of a frog's heart be separated from the auricles, either by an incision or by a ligature, the auricles and ventricle stand still in diastole, whilst the veins and the remainder of the sinus continue to beat (fig. 58, 1). If a second incision be made at the auriculo-ventricular groove, 
as a rule the ventricle begins at once to beat again, whilst the auricles remain in the condition of diastolic rest. [Thus the sinus venosus and ventricle continue to beat, while the auricle stands still, but the two former no longer beat with the same rhythm, the ventricle usually beats more slowly, as is shown in fig. 58,2 , by the large zig-zags.] According to the position of the second ligature or incision, the auricles may also beat along with the ventricles, or the auricles alone may beat, while the ventricles remain at rest.

Theoretical. - Various explanations of these experiments have been given :- $(a)$ Remak's ganglion in the sinus venosus is distinguished by its great excitability, while Bidder's ganglion in the auriculo-ventricular groove is less excitable; in the normal condition of the heart the motor impulse is carried from the former to the latter. If the sinus venosus be separated from the heart, Remak's ganglion has no action on the heart. The heart stops for two reasonsfirst, because Bidder's ganglion alone has not sufficient energy to excite it to action, and because the inhibitory fibres of the vagus going to the heart have been stinulated by being divided at this point (Heidenhain). [That stimulation of the inhibitory fibres of the vagus is not the cause of the standstill, is proved by the fact that the standstill occurs even after the administration of atropine, which paralyses the cardiac inhibitory mechanism.] The passive heart, however, may be made to contract by mechanically stimulating Bidder's ganglion, e.g., by a slight prick with a needle in the auriculo-ventricular groove, or by the action of a constant current of moderate strength (Eckhard), the ventricular pulsation at the same time preceding the auricular ( $v$. Bezold, Bernstein). If the auriculo-ventricular groove be divided, the ventricle pulsates again, because Bidder's ganglion has been stimulated by the act of dividing it ; while, at the same time, the ventricle is withdrawn from the inhibitory influence of the vagus produced by the first division at the sinus venosus. If the line of separation is so made that Bidder's ganglion remains attached to the auricles, these pulsate, and the ventricle rests; if it be divided into halves, the auricles and ventricles pulsate, each half being excited by the portion of the ganglion in relation with it. (b) According to another view, both Remak's $(a)$ and Bidder's ganglia $(b)$ are motor centres, but in the auricles there is in addition an inhibitory ganglionic system (c) (Bezold, Traube). Under normal circumstances $a+b$ is stronger than $c$, while $c$ is stronger than $a$ or $b$ separately. If the sinus venosus be separated it beats in virtue of $a$; on the other hand, the heart rests because $c$ is stronger than $b$. If the section be made at the level of the auriculo-ventricular groove, the auricles stand still owing to $c$, while the ventricle beats owing to $b$.

(2) If the ventricle of a frog's heart be separated from the rest of the heart by means of a ligature, or by an incision carried through it at the level of the auriculo-ventricular groove, the sinus and atria pulsate undisturbed as before (Des'curtes, 1644), but the ventricle stands still in diastole. A single local stimulus applied to the ventricle is responded to by a single contraction. If the incision be so made that the lower margin of the auricular septum remains attached to the ventricle, the latter pulsates. Even the ventricles of a rabbit's heart, when separated with a part of the auricles in connection with them, pulsate (Tigerstedt).

[Gaskell's Clamp. -Gaskell uses a clamp, regulated by a millimetre screw, to compress the heart, and thus to obstruct the passage of impulses from one part of the heart to the other, or to "block" the way, the pulsations of the auricles and ventricles being separately registered. By compressing the heart at the auriculo-ventricular groove, the ratio of auricular and ventricular beats alters, and instead of being $1: 1$, there may be 2,3 , or more auricular beats for each beat of the ventricle, expressed thus $-\frac{A}{V}, \frac{\text { II }}{I}, \frac{\text { III }}{I}, \frac{\text { IV }}{\text { I }}$. After the heart is fixed by the clamp, levers are placed horizontally above and below the heart. These levers are fixed to part of the auricles and to the apex by means of threads. Each part of the heart attached to a lever, as it contracts, pulls upon its own lever, so that the extent and duration of each contraction may be registered. This method is applicable for studying the effect of the vagus and other nerves upon the heart.]

(3) Section.-A. Fick showed that the process of excitement in the contractile tissue of the frog's heart is propagated in all directions (1874), so that to a certain extent the whole frog's heart behaves like one continuous muscular fibre, thus one transverse cut into the ventricle does not prevent contraction from taking place in the separated parts. Engelmann's experiments also show that if the ventricle of a frog's heart be cut up into two or more strips in a zig-zag way, so that the individual parts still remain connected with each other by muscular tissue, the 
strips still beat in a regularly progressive rhythmical manner, provided one strip is caused to contract. The rapidity of the transmission is about 10 to $15 \mathrm{~mm}$. per sec. Hence, it appears that the conducting paths for the impulse causing the contraction are not nervous, but must be the contractile mass itself. It has not been proved that nerve-fibres proceed from the ganglia to all the muscles.

[According to Marchand's experiments, it takes a very long time for the excitement to pass from the auricles to the ventricle-a much longer time, in fact, than it would require to conduct the excitement through muscle-so that it is probable that the propagation of the impulse from the auricles to the ventricle is conducted by nervous channels to the auriculo-ventricular nervous apparatus. In fact, in the mammalian heart the muscular fibres of the auricles are quite distinct from those of the ventricles.]

(4) When the apex of a frog's heart is ligatured off from the rest of the heart, it no longer pulsates (Heidenhain, Goltz), but such an apex, if stimulated directly, e.g., by a prick of a pin, responds with a single contraction. If the "heart-apex" be filled with normal saline solution under pressure, which acts as a stimulus, the heart begins to pulsate, and the same is the case with a solution of delphinin or quinine. If a cannula be tied into the heart over the auriculo-ventricular groove, the ventricle does not beat, but if the ventricle be filled through the cannula with blood containing oxygen, under a constant and sufficient pressure, it also pulsates (Ludwig and Merunowicz).

[(5) Luciani found that a heart ligatured above the auriculo-ventricular groove, when filled with pure serum, produced groups of pulsations with a long diastolic pause between every two groups (fig. 59). The successive beats in each group

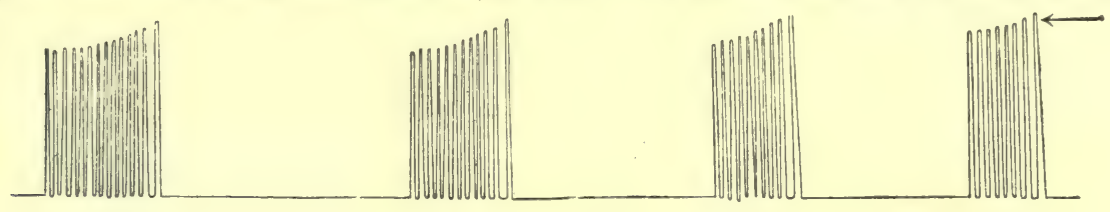

Fig. 59.

Four groups of pulsations with intervening pauses, with their "staircase" character. The points on the abscissa were marked every 10 seconds.

assume a "staircase" character (p. 84). These periodic groups undergo many changes; they occur when the heart is filled with pure serum free from bloodcorpuscles, and they disappear and give place to regular pulsations when defibrinated blood or serum containing hæmoglobin or normal saline solution is used (Rossbach). They also occur when the blood within the heart has become dark-coloured, i.e., when it has been deprived of certain of its constituents, and if a trace of veratrin be added to bright red blood they occur.]

(6) An apex preparation, when stimulated with even a weak induction shock, always gives its maximal contraction, and when a tetanising current is applied, tetanus does not occur (Kronecker and Stirling). When the opening and closing shocks of a sufficiently strong constant current are applied to the heart-apex, it contracts with each closing or opening shock. [When a constant current is applied to the lower two-thirds of the ventricle (heart-apex), under certain conditions the apex contracts rhythmically. This is an important fact in connection with any theory of the cardiac beat.]

(7) If the bulbus aortæ (frog) be ligatured, it still pulsates, provided the internal pressure be moderate. Should it cease to beat, a single stimulus makes it respond by a series of contractions. Increase of temperature to $35^{\circ} \mathrm{C}$, , and raising the pressure within it, increase the number of pulsations (Engelmann).

Action of Fluids. - Haller was of opinion that the venous blood was the natural stimulus which caused the heart to contract. That this is not so, is proved at once 
by the fact that the heart beats rhythmically when it contains no blood. Blood and other fluids which are supplied to an excised heart are not the cause of its rhythmical movements, but only the conditions on which these movements depend.

[Methods. - The study of the action of fluids upon the excised frog's heart has been rendered possible by the invention of Ludwig's "frog-manometer." The apparatus (fig. 60) consists

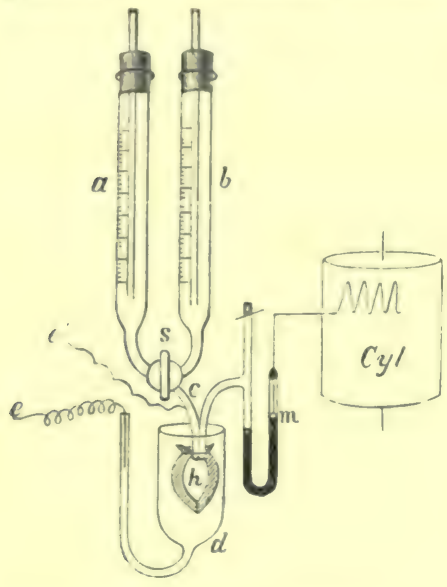

Fig. 60 .

Scheme of a frog-manometer. $a, b$, Mariotte's flasks for the nutrient fluids ; $s$, stop-cock ; $c$, cannula ; $m$, manometer ; $h$, heart ; $l$, glass cup for $h ; e^{\prime}, e$, electrodes ; ml, revolving cylinder.

a ligature is tied over it around the anicle, ic. above the auriculo-ventricular groove. Thus the auriculo-ventricular ganglia and other nervous structures remain in the preparation. This

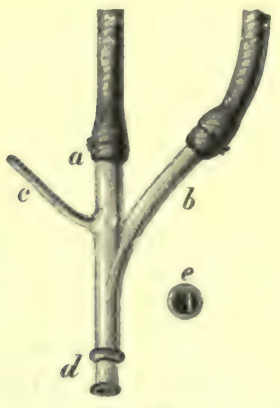

Fig. 61 .

Perfusiou cannula for a frog's heart. c, for fixing an electrode; $d$, the heart is tied over the flanges, preventing it from slipping out ; $e$, section of $d$. of (1) a double-way cannula, $c$, which is tied into the heart, $h ;(2)$ a manometer, $m$, connected with $c$, and registering the movements of its mercury on a revolving cylinder, cyl; (3) two Mariotte's flasks, $a$ and $b$, which are connected with the other linb of the cannula. Either $a$ or $b$ can be placed in communication with the interior of the heart by means of the stop-cock, s. The fluid in one graduated tube may be poisoned, and the other not; $\hat{d}$ is a glass vessel for fluid, in which the heart pulsates, $e^{\prime}$ and $e$ are electrodes, $e$ is inserted into the fluid in $d, e^{\prime}$ is attached to the German silver cannula which is shown in fig. 61.]

[In the tonometer of Roy (fig. 62) the ventricle, $h$, or the whole heart, is placed in an air-tight chamber, $o$, filled with oil. As before, a "perfusion" cannula is tied into the heart. A piston, $p$, works up and down in a cylinder, and is adjusted by means of a thin flexible animal membrane, such as is used by perfumers, Attached to the piston by means of a thread is a writing-lever, $l$, which records the variations of pressure within the chamber, $o$. When the ventricle contracts, it becomes smaller, diminishes the pressure within $o$, and hence the piston and lever rise ; conversely, when the heart dilates, the lever and piston descend. Variations in the volume of the ventricle may be registered, without in any way interfering with the flow of fluids through it.]

[Two preparations of the frog's heart have been used -(1) The "heart," in which case the cannula is introduced into the heart through the sinus venosus, and was the heart preparation employed by Luciani and Rossbach. (2) In the "heart-apex" or apex preparation, the cannula is introduced as before, but the ligature is tied on it over the ventricle, several millimetres below the auriculo-ventricular groove, so that this preparation contains none of the auriculo. ventricular ganglia, and, according to the usual statement, this part of the heart is devoid of nerve ganglia. This is the preparation. which was used by Bowditch, Kronecker and Stirling, Merunowicz, and others.

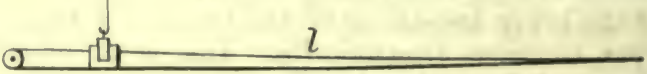

Fig. 62.

Roy's heart tonometer. $h$, heart; 0 , air-tight chamber ; $p$, piston ; $l$, writing-lever ; $e$, outflow tube.

The first effect of the application of the ligature in both cases is, that both preparations cease to beat, but the "heart" usually resumes its rhythmical contractions within several minutes, while the "heart-apex" does not contract spontaneously until after a much longer time (10 to 90 mins.).]

[If the "heart-apex" be filled with a 0.6 per cent. solution of common salt, the contractions are at first of greater extent, but they afterwards cease, and the preparation passes into a con- 
dition of "apparent death," lasting 30-90 mins. ; while, if the action of the fluid be prolonged, the heart may not contract at all, even when it is stimulated electrically or mechanically. It may be made, however, to pulsate again, if it be supplied with saline solution containing blood (1 to 10 per cent.). If the ventricle be nipped with wire forceps at the junction of the upper with its middle third, so as to separate the lower two-thirds of the ventricle, physiologically but not anatomically, from the rest of the heart, then the apex will cease to contract, although it is still supplied with the frog's own blood (Bernstein, Bowditch). 'The physiologically isolated apex may be made to beat by clamping the aortic branches so as to prevent blood passing out of the heart, and thus raising the intracardiac pressure. The rate of the beat of the apex is independent of and slower than that of the rest of the heart. This experiment proves that the amount of pressure within the apex-cavity is an important factor in the causation of the spontaneous beats of the apex. If blood-serum, to which a trace of delphinin is added, be transfused or "perfused" through the heart, it begins to beat within a minute, continues to beat for several seconds, and then stands still in diastole (Bowditch). Quinine and a mixture of atropine and muscarin have a similar action. These experiments show that, provided no nervous apparatus exists within the heart-apex, the cause of the varying contraction is to be sought for in the musculature of the heart, and that the stimulus necessary for the systole of the heart's apex may arise within itself. If there is no nervous apparatus of any kind present, then we must assume that the heart-muscle may execute rhythmical movements independently of the presence of any nervous mechanism, although it is usually assumed that the ganglia excite the heartmuscle to pulsate rhythmically. It is by no means definitely proved that the heart-apex is devoid of all nervous structures, which may act as originators of these rhythmical impulses.]

[Action of Drugs.-If the heart-apex contains no nervous structures, it must form a good object for the study of the action of drugs on the cardiac muscle. Some of these have been mentioned already. Ringer finds that a calcium salt makes the contractions higher and longer. Dilute acids added to saline solution, e.g., lactic, cause complete relaxation of the cardiac musculature, while dilute alkalies produce an opposite effect or tonic contraction, even though the apex be not pulsating. The action of a dilute acid may be set aside by a dilute alkali and vice versâ. Digitalin, antiarin, barium, and veratria act like alkalies, while saponin, muscarin, and pilocarpin have the effect of acids $(\$ 65)$. An isolated frog's heart, fatigued after being

A

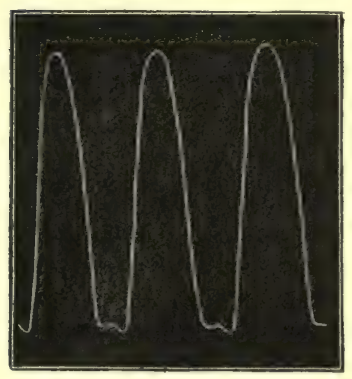
supplied with a solution of blood, is caused to beat more vigorously by a solution of kreatinin, or extract of meat (Mays).]

[T'he "heart" preparation in many respects behaves like the foregoing, i.e., it is exhausted after a time by the continued application of normal saline solution $\left(0^{\circ} 6\right.$ per cent. $\left.\mathrm{NaCl}\right)$, while its activity may be restored by supplying it with albuminous and other fluids (p. 79).]

II. Direct Stimulation of the Heart.-All direct cardiac stimuli act more energetically on the inner than on the outer surface of the heart. If strong stimuli are applied for too long a time, the ventricle is the part first paralysed.

(a) Thermal Stimuli.-[Heat affects the number or frequency C

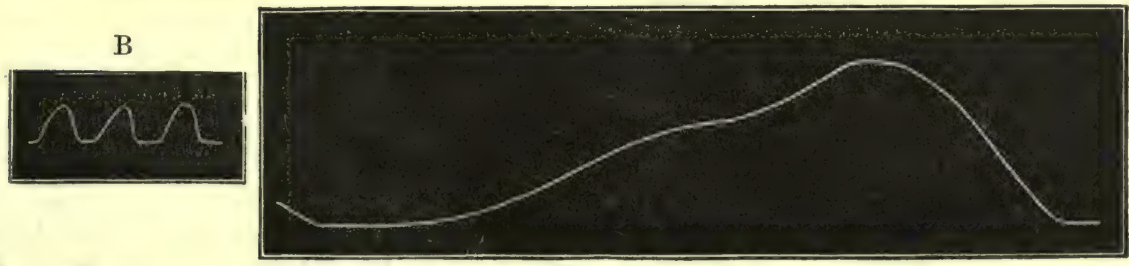

Fig. 63.

$\mathrm{A}$, contractions of a frog's heart at $19^{\circ} \mathrm{C}$.; B, at $34^{\circ} \mathrm{C}$.; C, at $3^{\circ} \mathrm{C}$.

and the amplitude of the pulsations, as well as the duration of the systole and diastole and the excitability of the heart.] Descartes (1644) observed that heat increases the number of pulsations of an eel's heart. As the temperature increases, the number of beats is at first considerably increased, but afterwards the beats again. become fewer, and if the temperature is raised above a certain linit the heart stands still, the myosin of which its fibres consist is coagulated, and "heat-rigor" occurs. Even before this stage is reached, however, the heart may stand still, the muscular fibres appearing to remain contracted. The ventricles usually cease to beat before the auricles (Schelske). The size and extent of the contractions increase up to about $20^{\circ} \mathrm{C}$., but above this point they diminish (fig. 63). The time occupied by any singlo 
contraction at $20^{\circ} \mathrm{C}$. is only about $\frac{1}{10}$ of the time occupied by a contraction occurring at $5^{\circ} \mathrm{C}$. A heart which has been warmed is capable of reacting pretty rapidly to intermittent stimuli, while a heart at a low temperature reacts only to stimuli occurring at a considerable interval (Gaule).

Cold. - When the temperature of the blood is diminished, the heart beats more slowly. A frog's heart, placed between two watch-glasses and laid on ice, beats very much more slowly. The pulsations of a frog's heart stop when the heart is exposed to a temperature of $4^{\circ} \mathrm{C}$. to $0^{\circ}$. If a frog's heart be taken out of warm water, and suddenly placed upon ice, it beats more rapidly, and conversely, if it be taken from ice and placed over warm water, it beats more slowly at first and more rapidly afterwards (Aristow).

[Methods. - The effect of heat on a heart may be studied by the aid of the frog-manometer, the fluid in which the heart is placenl being raised to any temperature required. For demonstra. tion purposes, the heart of a pithed frog is excised and placed on a glass slide under a light lever, such as a straw. The slide is warmed by means of a spirit-lamp. In this way the frequeney and amplitude of the contractions are readily made visible at a distance.]

(b) Mechanical Stimuli. - Pressure applied to the heart from without accelerates its action. In the case of Frau Serafin, v. Ziemssen found that slight pressure on the auriculo-ventricular groove caused a second short contraction of both ventricles after the heart-beat. Strong pressure causes a very irregular action of the cardiac musele. This may readily be produced by compressing the freshly excised heart of a dog between the fingers. The intra-cardiac pressure also affects the heart-beat (p. 83). If the pressure within the heart be increased, the heartbeats are gradually increased, if it be diminished the number of beats dininishes (Ludwig and Thiry). If the intra-cardiac pressure be very greatly increased, the heart's action becomes very irregular and slower. A heart which has ceased to beat may, under certain circumstances, be caused to execute a single contraction if it be stimulated mechanically.

(c) Electrical Stimuli. $-\Lambda$ constant electrical current of moderate strength increases the number of heart-beats. V. Ziemssen found, in the case of Frau Serafin $(\S 47,3)$, that the number of beats was doubleil when a constant uninterrupted strong current was passed through the ventricles. If the constant current be very strong, or if tetanising induction currents be used, the cardiac muscle assumes a condition resembling, but not identical with, tetanus (Ludwig and Hoffa), and of course this results in a fall of the blood-pressure. If the auriculoventricular groove be compressed so as to cause the ventricle of a frog's heart to cease to beat, on placing one electrode of a constant current on the ventricular wall and the other electrode on an indifferent part of the body, we obtain on making the current, a systolic contraction of the ventricle only when the cathode touches the ventricle; and conversely on breaking, only when the anode is on the heart (Biedermann).

When a single induction shock is applied to the ventricle of a frog's heart during systole, it has no apparent effect; but if it is applied during diastole, the succeeding contraction takes place sooner. The auricles and also the apex behave in a similar manner. Whilst they are contracted, an induction shock has no effect; if, however, the stimulus is applied during diastole, it causes a contraction, which is followed by systole of the ventricle. Even when strong tetanising induction shocks are applied to the heart, they do not produce tetanus of the entire cardiac musculature, or as it is said, "the heart knows no tetanus" (Kronecker and Stirling). Small white local weal-like elevations-such as occur when the intestinal musculature is stimulated-appear between the electrodes. They may last several minutes. A frog's heart, which yields weak and irregular contractions, may be made to execute regular rhythmical contractions synchronous with the stimuli, if electrical stimuli are used (Bowditch).

[Break induction shocks, if of sufficient strength, cause the heart to contract, while weak stimuli have no effect; on the other hand, moderate stimuli, when they do cause the heart to contract, always cause a maximal contraction, so that a minimal stimulus acts at the same time like a maximal stimulus. The heart either contracts or it does not contract, and when it contracts the result is always a "maximal" contraction (Kronecker and Stirling). Bowditch found that the excitability of the heart was increased by its own movements, so that after a heart had once contracted, the strength of the stimulus required to excite the next contraction may be greatly diminished, and yet the stimulus be effectual. Usually the amplitude of the first beat so produced is not so great as the second beat, and the second is less than the third, so that a "staircase" ("Trejpe") of beats of successively greater extent was produced (fig. 59). Under certain circumstances, however, a skeletal muscle gives contractions of a "staircase" character. This staircase arrangement occurs even when the strength of the stimulus is kept constant, so that the production of one contraction facilitates the occurrence of the succeeding one. A staircase arrangement of the pulsations is also seen in Luciani's groups (p. 81). The question, whether a stimulus will cause a contraction, depends upon what particular phase the heart is in when the shock is applied. Even comparatively weak stimuli will cause a heart to contract, provided the stimuli are applied at the proper momeut and in the proper tempo, i.e. to say, they become what are called "infallible." If stimuli are applied to the heart, at intervals which are longer than the time the heart takes to execute its contraction, they are effectual or "adequate," but if they are applied before the period of pulsation comes to an end, then they are ineffectual 
(Kronecker). It is quite clear, therefore, that the relation of the strength of the stimulus to the extent of the contraction of the cardiac muscle, is quite different from what occurs in a muscle of the skeleton, where within certain limits the amplitude of the contraction bears a relation to the stimulus, while in the heart the contraction is always maximal.]

Human Heart. - V. Ziemssen found that he could not alter the heart-beats of the human heart (Frau Serafin, $\S 4 \tau, 3$ ), even with strong induction-currents. The ventricular diastole seemed to be less complete, and there were irregularities in its contraction. By opening and closing, or by reversing a strong constant current applied to the heart, the number of beats was increased, and the increase corresponded with the number of electrical stimuli ; thus, when the electrical stimuli were $120,140,180$, the number of heart-beats was the same, the pulse beforehand being 80 . The normal pulse-rate of 80 was reduced to 60 and 50 when the number of shocks was reduced in the same ratio. [In Frau Serafin's case the electrodes were applied to the heart, separated from it merely by the pericardium. Ziemssen found that the Faradic current did not modify the heart's action when the thorax was intact, but that the constant current did; if of sufficient strength. Herbst and Dixon Mann obtained negative results with both kinds of electricity in the normal thorax.]

(d) Chemical Stimuli.-Many chemical substances, when applied in a dilute solution to the inner surface of the heart, increase the heart-beats, while if they are concentrated, or allowed to act too long, they diminish the heart-beats, and paralyse it. Bile, and bile salts, diminish the heart-beats (also when they are absorbed into the blood as in jaundice); in very dilute solutions both increase the heart-beats. A similar result is produced by acetic, tartaric, citric, and phosphoric acids. Chloroform and ether, applied to the inner surface, rapidly diminish the heart-beats, and then paralyse it; but very small quantities of ether ( 1 per cent.) accelerate the heart-beat of the frog (Kronecker and $M^{\prime}$ Gregor-Robertson), while a solution of $1 \frac{1}{2}$ to 2 per cent. passed through the heart arrests it temporarily or completely. Dilute solutions of opium, strychnia, or alcohol applied to the endocardium, increase the heart-beats ; if concentrated they rapidly arrest its action. Chloral-hydrate paralyses the heart.

Action of Gases. - When blood containing different gases was passed through a frog's heart, Klug found that blood containing sulphurous acid rapidly and completely killed the heart; chlorine stimulated the heart at first, and ultimately killed it; and langhing-gas rapidly killed it also. Blood containing sulphuretted hydrogen paralysed the heart without stimulating it. Carbonic oxide also paralysed it, but if fresh blood was transfused the heart recovered. [B]lood containing $\mathrm{O}$ excites the heart (Castell), while the presence of much $\mathrm{CO}_{2}$ paralyses it, and the presence of $\mathrm{CO}_{2}$ is more injurious than the want of $\mathrm{O}$. Blood or serum completely saturated with $\mathrm{CO}_{2}$ exhausts the heart (Saltet and Kronecker), but it recovers itself when the $\mathrm{CO}_{2}$ is removed. $\mathrm{H}$ and $\mathrm{N}$ have no effect.]

Cardiac Poisons are those substances whose action is characterised by special effects upon the movements of the heart. Amongst these are neutral potash salts, which cause the heart to stand still in diastole. An excised frog's heart ceases to beat after one-half to one minute when it is placed in a 2 per cent. solution of potassic chloride.] Even a very dilute solution of yellow prussiate of potash injected into the heart of a frog causes the ventricle to stand still in systole. Antiar (Java arrow-poison) causes the ventricle to stand still in systole and the auricles in diastole. Some heart-poisons, in small doses, diminish the heart's action, and in large doses not unfrequently accelerate it, e.g., digitalis, morphia, nicotin. Others, when given in small doses, accelerate its action, and in large doses slow it-veratria, aconitin, camphor.

Special Actions of Cardiac Poisons. - The complicated actions of various poisons upon the heart have led observers to suppose that there are various intra-cardiac mechanisms on which these substances may act. Besides the muscular fibres of the heart and its automatic ganglia, some toxicologists assume that there are inhibitory ganglia into which the inhibitory fibres of the vagus pass, and accelerator ganglia, which are connected with the accelerating nerve-fibres of the heart. Both the inhibitory and accelerator ganglia are connected with the automatic ganglia by conducting channels.

Muscarin and all other trimethylammonium bases stimulate permanently the inhibitory ganglia, so that the heart stands still (Schmiedeberg and Koppe). [According to Gaskell, however, when the action of the sinus is arrested by muscarin, there is no deflection of the galvanometer similar to that produced by the excitation of the vagus. He infers that muscarin does not cause arrest of the beat by acting as an excitant of inhibitory mechanisms, but as a depressant to motor activity.] As atropin and daturin paralyse thêse ganglia, the standstill of the heart brought about by muscarin may be set aside by atropin. [If a frog's heart be excised and placed in a watch-glass, and a few drops of a very dilute solution of muscarin be placed on it with a pipette, it ceases to beat within a few minutes, and will not beat again. If, however, the muscarin be removed, and a solution of atropine applied to the heart, it will resume its contractions after a short time.] Physostigmin or Calabar bean excites the energy of the cardiac muscle to such an extent, that stimulation of the vagus no longer causes the heart to stand still. Iodine-aldehyd, chloroform, and chloral-hydrate paralyse the automatic ganglia. The heart stands still, and it cannot be made to contract again by atropine. The cardiac muscle itself remains excitable after the action of muscarin and iodine-aldehyd, so that if it be stimulated 
it contracts. [According to Caskell, antiarin and digitalin solutions produce an alteration in the condition of the muscular tissne of the apex of the heart of the same nature as that produced by the action of a very dilute alkali solution, while the action of a blood-solution containing muscarin closely resembles that of a dilute acid solution (p. 95, § 65).]

[Nature of a Cardiac Contraction.-The question as to whether this is a simple contraction or a compound tetanic contraction has been much discussed. So much is certain, that the systolic contraction of the heart is of very much longer duration ( 8 to 10 times) than the contraction of a skeletal muscle produced by stimulation of its motor nerve. When the sciatic nerve of a nerve-muscle preparation is adjusted upon a contracting heart, a simple secondary twitch of the limb, and not a tetanic spasm, is produced when the heart (auricle or ventricle) contracts This of itself is not sufficient proof that the systole is a simple spasm, for tetanus of a muscle does not in all cases give rise to secondary tetanus in the leg of a rheoscopic limb. Thus, a simple "initial" contraction occurs when the nerve is applied to a muscle tetanised by the action of strychnia, and the contracted diaphragm gives a similar result. The question whether the heart can be tetanised has been answered in the negative, and as yet it has not been shown that the heart can be tetanised in the same way that a skeletal muscle is tetanised.]

[MacWilliam finds, when the quadriceps extensor cruris contracts to cause the knee-jerk, that a sound similar to the first sound of the heart is heard. As the former is regarded as a simple contraction, it is argued that a simple contraction can produce a muscle-sound. Frédéricq regards the ventricular systole not as a simple contraction, but as composed of three or more fused contractions corresponding to tetanus. This he concludes from a study of cardiograms as well as from the electro-motive phenomena of the heart.]

The peripheral or extra-cardiac nerves (\$S 369 and 370 ).

59. CARDIO-PNEUMATIC MOVEMENT. - As the heart within the thorax occupies a smaller space during the systole than during the diastole, it follows that, when the glottis is open, air must be drawn into the chest when the heart contracts ; whenever the heart relaxes, i.e., during diastole, air must be expelled through the open glottis. But we must also take into account the degree to which the larger intra-thoracic vessels are filled with blood. These movements of the air within the lungs, although slight, seem to be of importance in hybernating animals. In animals in this condition, the agitation of the gases in the lungs favours the exchange of $\mathrm{CO}_{2}$ and $\mathrm{O}$ in the lungs, and this slow current of air is sufficient to aerate the blood passing through the lungs. [Ceradini called the diminution of the volume of the entire heart which occurs during systole meiocardia, and the subsequent increase of volume, when the heart is distended to its maximum, auxocardia.]

Method. - A manometric flame may be used. Insert one limb of a $\mathrm{Y}$-tube into the opened trachea of an animal, while the other limb passes to a small gas-jet, and connect the other tube with the gas supply. The movements of the heart affect the column of gas, and thus affect the flame. It may also be done in man by inserting the tube into one nostril, while the other nostril and the mouth are closed. [A simpler and less irritating plan is to fill a wide curved glass-tube with tobacco smoke, and insert one end of the tube into one nostril while the other nostril anil the mouth are closed. If the glottis be kept open, and respiration be stopped, then the movements of the column of smoke within the tube are obvious. Or a nianometer containing a drop of a coloured fluid may be used under the same conditions.]

The cardiac pneumograph (fig. 64) consists of a tube (D), about 1 inch in diameter and 6 to 8 inclies in length; the tube is bent at a right angle, and communicates with a small metal capsule about the size of a sancer $(\mathrm{T})$, over which a membrane composed of collodion and castor oil is loosely stretched. To this membrane is attached a glass rou $(\mathrm{H})$ used as a writing-style, which records its movements on a glass-plate $(\mathrm{S})$ movell by clock-work. A small valve $(\mathrm{K})$ is placed on the side of the tube (D), which enables the experimenter to breathe when necessary. The tube (D) is held in an air-tight manner between the lips, the nostrils being closed, the glottis open, and respiration stopyed. In the curves (fig. $64, \mathrm{~A}, \mathrm{~B}$ ) we observe that-

(1) At the moment of the first sound (1) the respiratory gases undergo a sharp expiratory movement, because at the moment of the first part of the ventricular systole the blood of the ventricle has not left the thorax, while venous blood is streaming into the right auricle through the venæ cave, and because the dilating branches of the pulmonary artery compress the 
accompanying bronchi. The blood of the right ventricle has not yet left the thorax, it passes merely into the pulmonary circuit. The expiratory movement is diminished somewhat $(\alpha)$ by the muscular mass of the ventricle occupying slightly less bulk during the contraction, and $(\boldsymbol{\beta})$ owing to the thoracic cavity being slightly increased by the fifth intercostal space being pushed forward by the cardiac impulse.

(2) Immediately after (1) there follows a strong inspiratory current of the respiratory gases. As soon as the blood from the root of the aorta reaches that part of the aorta lying outside the thorax, more blood leaves the chest than passes into it simultaneously through the venæ cavæ.

(3) After the second sound (at 2), indicated sometimes by a slight depression in the apex of

A

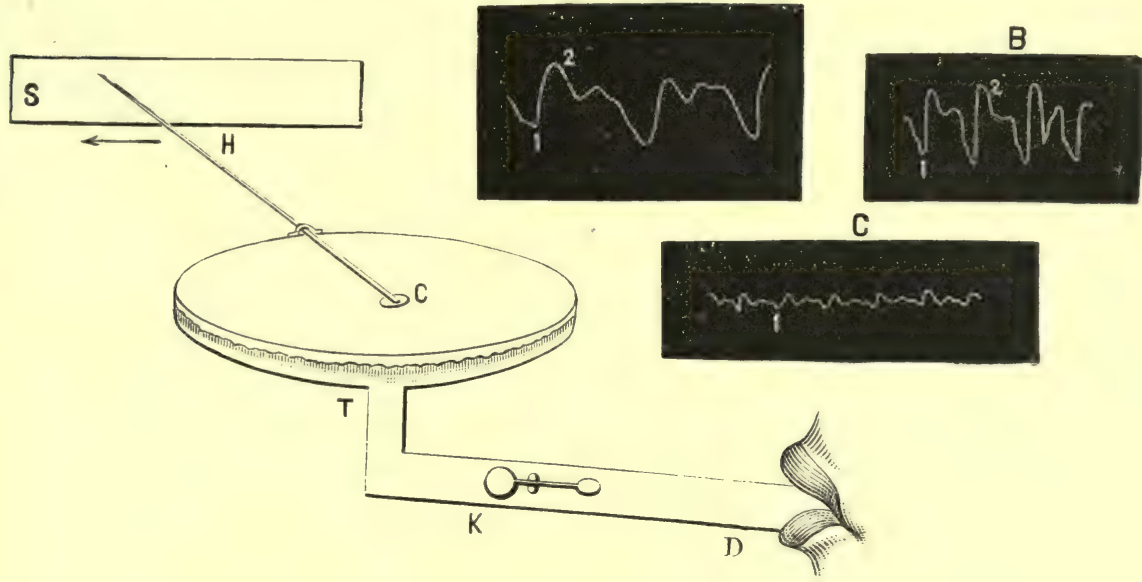

B

Fig. 64 .

Landois' cardio-pneumograph, and the curves obtained therewith. A and B, from man, 1 and 2 correspond to the periods of the first and second heart-sounds; C, from dog; D, method of using the apparatus.

the curve, the arterial blood accumulates, and hence there is another expiratory movement in the curve.

(4) The peripheral wave-movements of the blood from the thorax cause another inspiratory movement of the gases.

(5) More blood flows into the chest through the veins, and the next heart-beat occurs.

60. INFLUENCE OF THE RESPIRATORY PRESSURE ON THE HEART. -The variation in pressure to which all the intra-thoracic organs are subjected, owing to the increase and decrease in the size of the chest caused by the respiratory movements, exerts an influence on the movements of the heart. Examine first the relations in different passive conditions of the thorax, when the glottis is open.

The diastolic dilatation of the cavities of the heart, besides the pressure of the venous blood and the elastic stretching of the relaxed muscle-wall, is fundamentally due to the elastic traction of the lungs. This is stronger the more the lungs are distended (inspiration), and is less active the more the lungs are contracted (expiration). Hence it follows :-

(1) When the greatest possible expiratory effort is made, (of course, with the glottis open), only a small amount of blood flows into the heart; the heart in diastole is small and contains a small amount of blood. Hence the systole must also be small, thus causing a small pulse-beat.

(2) On taking the greatest possible inspiration (with the glottis open), and therefore causing the greatest stretching of the elastic tissue of the lungs, the elastic traction of the lungs is, of course, greatest $=30 \mathrm{~mm}$. Hg (Donders), and may interfere with the contraction of the thin-walled atria and appendices, in consequence of which these cavities do not completely empty themselves into the ventricles. The heart is in a state of great diastolic distension, and filled with 
blood ; nevertheless, in consequence of the limited action of the auricles, only small pulse-beats are observed. In several individuals Donders found the pulse to be smaller and slower; afterwards it became larger and faster.

(3) When the chest is in a position of moderate rest, whereby the elastic traction is moderate $=7.5 \mathrm{~mm}$. $\mathrm{Hg}$, we have the condition most favourable to the action of the heart-sufficient diastolic dilatation of the cavities of the heart, as well as unhindered emptying of them during systole.

Voluntary increase or diminution of the intra-thoracic pressure affects the action of the heart.

(1) Valsalva's Experiment (1740). - If the thorax is fixed in the position of deepest inspiration, and the glottis be then closed, and if a powerful expiratory effort be made by bringing into action all the expiratory muscles, so as to contract
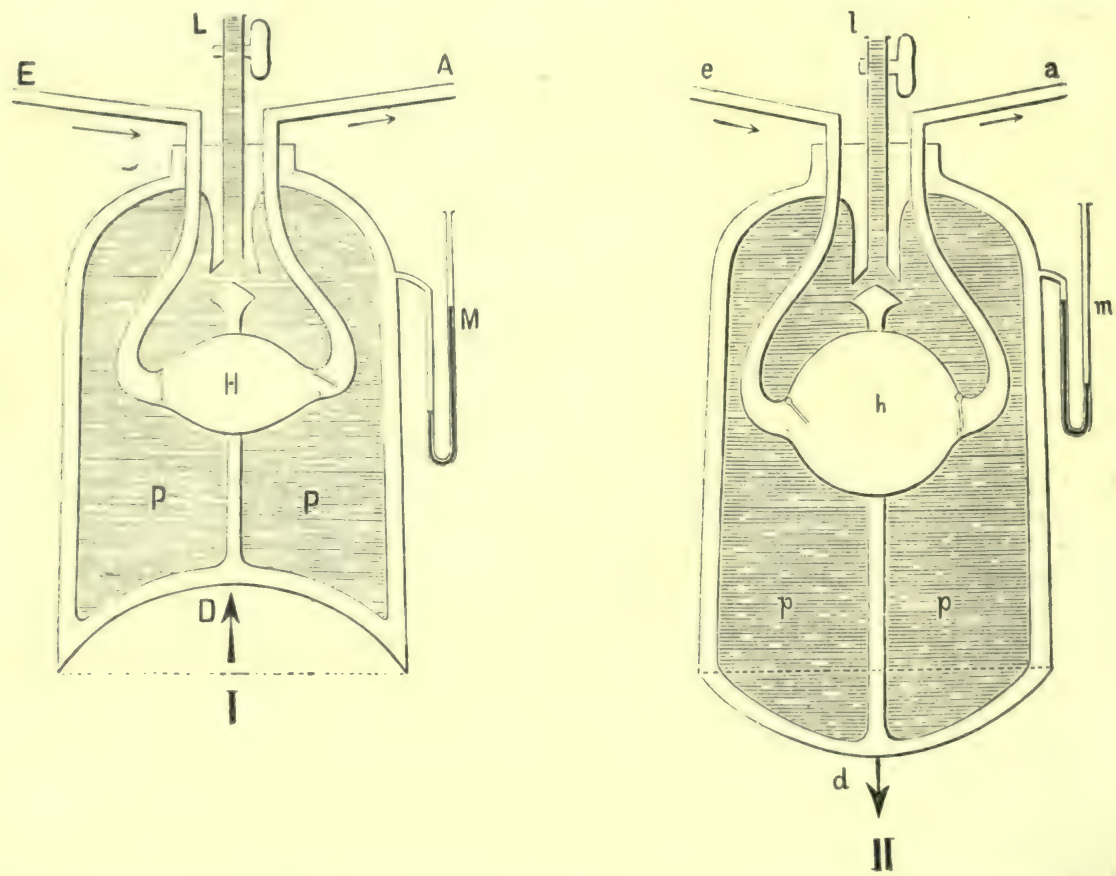

Fig. 65 .

Apparatus for demonstrating the action of inspiration, II., and expiration, I., on the heart and blood-stream. P, $p$, lungs ; H, h, heart ; L, l, closed glottis; M, $m$, manometers ; E, $e$, ingoing hlood-stream, vein ; A, $a$, outgoing blood-stream, artery; $\mathrm{D}$, diaphragm during expiration ; $d$, during inspiration.

the chest, the cavities of the heart are so compressed that the circulation of the blood is temporarily interrupted. In this expiratory phase the elastic traction is very limited, and the air in the lungs being under a high pressure also acts upon the heart and the intra-thoracic great vessels. No blood can pass into the thorax from without; hence the visible veins swell up and become congested, the blood in the lungs is rapidly forced into the left ventricle by the compressed air in the lungs, and the blood soon passes out of the chest, so that the heart and lungs contain little blood, thus leading to a greater supply of blood in the systemic than in the pulmonary circulation and the heart. The heart-sounds disappear, and the pulse is absent $(E . H$. Weher, Donders). 
(2) J. Müller's Experiment (1838).-Conversely, if after the deepest possible expiration the glottis be closed, and the chest be now dilated with a great inspiratory effort, the heart is powerfully dilated, the elastic traction of the lungs, and the very attenuated air in these organs, act so as to dilate the cavities of the heart. More blood flows into the right heart, and, in proportion as the right auricle and ventricle can overcome the traction outwards, the blood-vessels of the lungs become filled with blood, and thus partly occupy the lung space. Much less blood is driven out of the left heart, so that the pulse may disappear. Hence, the heart is distended with blood and the lungs are congested, while the aortic system contains a small amount of blood, i.e., the systemic circulation is comparatively empty, while the heart and the pulmonary vessels are engorged with blood.

In normal respiration, the air in the lungs during inspiration is under slight pressure, while during expiration the pressure is higher, so that these conditions favour the circulation ; inspiration favours the occurrence of diastole, the supply of blood (and lymph) through the venæ cavæ. In operations where the axillary or jugular vein is cut, air may be sucked into the circulation during inspiration, and cause death. Expiration favours the flow of blood in the aorta and its branches, and aids the systolic emptying of the heart.

The elastic traction of the lungs aids the lesser-circulation through the lungs; the blood of the pulmonary capillaries is exposed to the pressure of the air in the lungs, while the blood in the pulmonary veins is exposed to a less pressure, as the elastic traction of the lungs, by dilating the left auricle, favours the outflow from the capillaries into the left auricle. The elastic traction of the lungs acts slightly as a disturbing agent on the right ventricle, and, therefore, on the movement of blood through the pulmonary artery, owing to the overpowering force of the bloodstream through the pulmonary artery, as against the elastic traction of the lungs (Donders).

The above apparatus (fig. 65) shows the effect of the inspiratory and expiratory movements on the dilatation of the heart, and on the blood-stream in the large blood-vessels. The large glass vessel represents the thorax; the elastic membrane, $\mathrm{D}$, the diaphragm ; $\mathrm{P}, p$, the lungs; $\mathrm{L}$, the trachea supplied with a stop-cock to represent the glottis; $\mathrm{H}$, the heart; $\mathrm{E}$, the venæ cavæ; A, the aorta. If the glottis be closed, and the expiratory phase imitated by pushing up $\mathrm{D}$ as in $\mathrm{I}$, the air in $\mathrm{P}, \mathrm{P}$ and the heart $\mathrm{H}$ are compressed, the venous valve closes, the arterial is opened, and the fluid is driven out through A. The manometer, M, indicates the intrathoracic pressure. If the glottis be closed, and the inspiratory phase imitated, as in II., $p, p$ and $h$ are dilated, the venous valve opens, the arterial valve closes; henee, venous blood flows from $e$ into the heart. Thus, inspiration always favours the venous stream, and hinders the arterial ; while expiration hinders the venous, and favours the arterial stream. If the glottis $\mathrm{L}$ and $l$ be open, the air in $\mathrm{P}, \mathrm{P}, p, p$ will be changed during the respiratory movements $\mathrm{D}$ and $d$, so that the action on the heart and blood-vessels will be diminished, but it will still persist, although to a much less extent.

\section{The Circulation of the Blood.}

61. FLOW OF FLUIDS THROUGH TUBES. - Toricelli's Theorem states that the velocity of efflux $(v)$ of a fluid-through an opening at the bottom of a cylindrical vessel-is exactly the same as the velocity which a body falling freely would acquire, were it to fall from the surface of the fluid to the base of the orifice of the outflow. If $-h$ be the height of the propelling force, the velocity of efflux is given by the formula-

$$
v=\sqrt{2 g h} \text { (where } g=9.8 \text { metres). }
$$

The rapidity of outflow increases with increase in the height of the propelling force, $h$. The former occurs in the ratio $1,2,3$, wher $h$ increases in the ratio $1,4,9, i . e$, , the velocity of efflux is as the square root of the height of the propelling force. Hence, it follows that the velocity of efflux depends upon the height of the liquid above the orifice of outflow, and not upon the nature of the fluid.

Resistance.-Toricelli's theorem, however, is only valid when all resistance to the outflow is 
absent ; but in every physical experiment such resistance exists. Hence, the propelling force, $h$, has not only to cause the efflux of the fluid, but has also to overcome resistance. These two forces may be expressed by the heights of two columns of water placed over each other, viz., by the height of the column of water causing the outflow, F, and the height of the column, D, which overcomes the resistance opposed to the outflow of the fluid. So that

$$
h=\mathrm{F}+\mathrm{D} \text {. }
$$

62. VELOCITY OF THE CURRENT. RESISTANCE. - In the case of a fluid flowing through a tube, which it fills completely, we have to consider the propelling force, $h$, causing the fluid to flow through the varions sections of the tube. The amount of the propelling force depends. upon two factors :-

(1) On the velurity of the current, $v ;(2)$ on the pressure (amount of resistance) to which the fluid is subjected at the various parts of the tube, D.

(1) The velocity of the current, $v$, is estimated-(a) from the lumen, $l$, of the tube ; and (b). from the yuantity of fluid, $q$, which flows through the tube in the unit of time. So that $v=q: l$. Both values, " as well as $l$, ean le aceurately measured. (The circumference of a circular tube, whose diameter $=d$ is $3 \cdot 14 . d$. The sectional area (lumen of the tube) is $\left.l=\frac{3 \cdot 14}{4} \cdot d^{2}\right)$. Having in this way determinet $r$, from it we may calculate the height of the column of fluid, $\mathrm{F}$, which will give this velocity, i.e, the height from which a hody must fall in vacuo, in order to attain the velocity $r$. In this case $F=\frac{r^{2}}{4 g}$ (where $y=$ the distance traversed by a falling body in 1 sec. $=4 \cdot 9$ metre).

(2) The pressure, D (amount of resistance), is measured lirectly by placing manometers at different parts of the tube (fig. 67).

The propelling force at any part of the tube is $h=\mathrm{F}+\mathrm{D}$; or, $h=\frac{v^{2}}{4 g}+\mathrm{D}$ (Donders). This is proved experimentally by taking a tall cylindrical vessel, $A$, of sufficient size, which is kept fillerl with water at a constant level, $h$. The rigid outflow tube, $a b$, has in connection with it a number of tubes placed vertically, $1,2,3$, constituting a piezometer. At the end of the

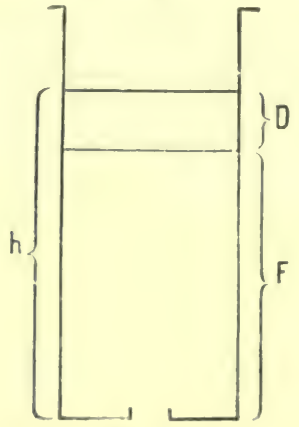

Fig. 66 .

Cylindrical ressel filled with water. $h$, height of the column of fluid; D, height required to overcome the resistance; $\mathrm{F}$, height causing the efflux.

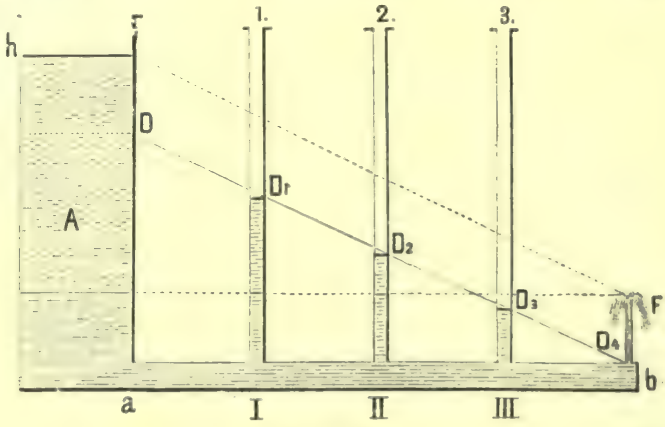

Fig. 67.

A cylindrical vessel filled with water. $a b$, outflow tube, along which are placed at intervals vertical tubes, $1,2,3$, to estimate the pressure.

tube, $b$, there is an opening with a short tube fixed in it, from which the water issues to a constant height, provided the level of $h$ is kept constant. The height to which it rises depends on the height of the column of fluid causing the velocity, F. As the pressure in the manometric tubes, $\mathrm{D}^{1}, \mathrm{D}^{2}, \mathrm{D}^{3}$, can be read off directly, the propelling force of the water at the sections of the tubes, I, II, III, is-

$$
h=\mathrm{F}+\mathrm{D}^{1} ; \mathrm{F}+\mathrm{D}^{2} ; \mathrm{F}+\mathrm{D}^{3} .
$$

At the end of the tube, $b$, where $\mathrm{D}^{4}=\mathrm{O}, h=\mathrm{F}+\mathrm{O}$, i.e., $h=\mathrm{F}$. In the cylinder itself it is the constant pressure, $h$, which causes the movement of the fluid. It is clear that the propelling force of the water gradually diminishes as we pass from the inflow towards the outflow of the tube, $b$. The water in the pressure-cylinder, falling from the height, $h$, only rises as high as $\mathrm{F}$ at $b$. This diminution of the propelling power is due to the presence of resistances, which oppose the current in the tube, i.e., part of the energy is transformed into heat. As the propelling force at $b$ is represented only by $\mathrm{F}$, while in the vessel it is $h$, the difference must be due to the sum of the resistances, $\mathrm{D}=h-\mathbf{F}$; hence it follows that $h=\mathrm{F}+\mathrm{D}$. 
Estimation of the Resistance. - When a fluid flows through a tube of uniform calibre, the propelling force, $h$, diminishes from point to point on account of the uniformly acting resistance, hence the sum of the resistance in the whole tube is directly proportional to its length. In a uniformly wide tube, fluid flows through each sectional area with equal velocity, hence $v$ and also $\mathrm{F}$ are equal in all parts of the tube. The diminution which $h$ (propelling force) undergoes can only occur from a diminution of pressure $\mathrm{D}$, as $\mathrm{F}$ remains the same throughout (and $h=\mathrm{F}+\mathrm{D}$ ). Experiment with the pressure-cylinder shows that the pressure towards the outflow end of the tube gradually diminishes. In a uniformly wide tube, the height of the pressure in the manometers expresses the resistances opposed to the current of fuid, which it has to overcome in its course from the point investigated to the free orifice of efflux.

Nature of the Resistance. - The resistance opposed to the flow of a fluid depends upon the cohesion of the particles of the fluid amongst themselves. During the current, the outer layer of fluid which is next the wall of the tube, and which moistens it, is at rest. All the other layers of fluid, which may be represented as so many cylindrical layers, one inside the other, move more rapidly as we proceed towards the axis of the tube, the axial thread or stream being the most rapidly moving part of the liquid. On account of the movement of the cylindrical layers, one within the other, a part of the propelling energy must be used up. The amount of the resistance greatly depends upon the amount of the cohesive force which the particles of the fluid have for each other; the more firmly the particles cohere the greater will be the resistance, and vice versâ. Hence, the sticky blood-current experiences greater resistance than water or ether.

Heat diminishes the cohesion of the particles, hence it also diminishes the resistance to the onflow. These resistances are first developed by, and result from, the movement of the particles of the fluid, they being, as it were, torn from each other. The more rapid the current, therefore, i.e., the larger the number of particles of fluid which are pulled asunder in the unit of time, the greater will be the sum of the resistance. As the layer of fluid lying next the tube, and moistening it, is at rest, the material which composes the tube exerts no influence on the resistance.

Tubes of Unequal Diameter. - When the velocity of the current is uniform, the resistance depends upon the diameter of the tube-the smaller the diameter the greater the resistance; the greater the diameter the less the resistance. The resistance in narrow tubes, however, increases more rapidly than the diameter of the tube decreases, as has been proved experimentally. In tubes of unequal calibre, at different parts of their course, the velocity of the current varies-it is slower in the wide part of the tube and more rapid in the narrow parts. As a general rule, in tubes of unequal diameter the velocity of the current is inversely proportional to the diameter of the corresponding section of the tube; i.e., if the tube be cylindrical, it is inversely proportional to the square of the diameter of the circular transverse section. In tubes of uniform diameter, the propelling force of the moving fluid diminishes uniformly from point to point, but in tubes of unequal calibre it does not diminish uniformly. As the resistance is greater in narrow tubes, of course the propelling force must diminish more rapidly in them than in wide tubes. Hence, within the wide parts of the tube the pressure is greater than the sum of the resistances still to be overcome, while in the narrow portions it is less than these.

Tortuosities and bending of the vessels add new resistance, and the fluid presses more strongly on the convex side than on the concave side of the bend, and there the resistance to the flow is greater than on the concave side.

Division of a tube into two or more branches is a source of resistance, and diminishes the propelling power. When a tube divides into two smaller tubes, of course some of the particles of the fluid are retarded, while others are accelerated on account of the unequal velocities of the different layers of the fluid. Many particles which had the greatest velocity in the axial layer come to lie more towards the side of the tube where they move more slowly ; and conversely many of those lying in the outer layers reach the centre, where they move more rapidly. Hence, some of the propelling force is used up in this process, and the pulling asunder of the particles where the tube divides acts in a similar manner. If two tubes join to form one tube, new resistance is thereby caused, which must diminish the propelling force. The sum of the mean velocities in both branches is independent of the angle at which the division takes place (Jacobson). If a branch be opened from a tube, the principal current is accelerated to a considerable extent, no matter at what angle the branch may be given off:

63. FLOW IN CAPILLARY TUBES.-Poiseuille proved experimentally that the flow in the capillaries is subject to special conditions-

(1) The quantity of fluid which flows out of the same capillary tube is proportional to the pressure.

(2) The time necessary for a given quantity of fluid to flow out (with the like pressure, diameter of tube and temperature), is proportional to the length of the tubes.

(3) The product of the outflow (other things being equal) is as the fourth power of the diameter. 
(4) The relocity of the current is proportional to the pressure and to the square of the diameter, and inversely proportional to the length of the tube.

(5) The resistance in the capillaries is proportional to the velocity of the current.

64. FLOW IN ELASTIC TUBES. - (1) When an uninterrupted uniform current flows through an elastic tube, it follows exactly the same laurs as if the tube had rigid walls. If the propelling power increases or diminishes, the elastic tubes become wider or narrower, and they behave, as far as the movement of the fluid is concerned, as wider or narrower rigid tubes.

(2) Wave-Motion. - If, however, more fluid be forced in jerks into an elastic tube, i.e., intcrruptedly, the first part of the tube dilates suldenly, corresponding to the quantity of fluid propelled into it. The jerk communicates an oscillatory movement to the particles of the fluid, which is communicated to all the fluid particles from the beginning to the end of the tube; $a$ positive ware is thus rapially propagatal throughout the whole length of the tube. If we imagine the elastic tube to be closed at its neripheral end, the positive wave will be reflected from the point of occlusion, and it may be propagated to and fro through the tube until it finally disappears. In such a closed tube a sudden jet of fluid produces only a wave-movement, i.e., only a vibratory movement, or an alteration in the shape of the liquid, there being no actual translation of the particles along the tube.

(3) If, however, fluid be pumped interruptedly or by jerks into an elastic tube filled with fluid, in which there is already a continuous current, the movement of the current is combined with the wave movement. We must carefully distinguish the movement of the current of the fluirl, i.e., the translation of a mass of fluid through the tube, from the vave-movement, the oscillatory movement, or movement of change of form in the column of fluid. In the former, the particles are actually translated, while in the latter they merely vibrate. The current in elastic tubes is slower than the wave-movement, which is propagated with great rapidity. This last case obtains in the arterial system. The blood in the arteries is already in a state of continual movement, directed from the aorta to the capillaries ; by means of the systole of the left ventricle a quantity of fluid is suddenly pumped into the aorta, and causes a positive wave, the

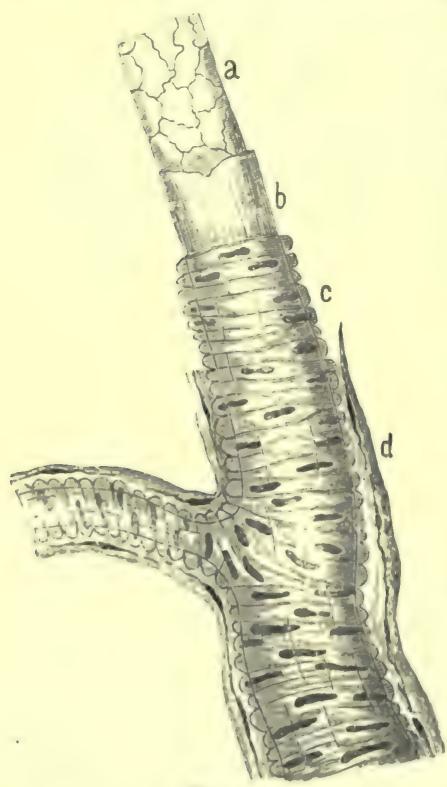

Fig. 68. pulse-wave which is propagated with great rapidity to the terminations of the arteries, while the current of the blood itself moves much more slowly.

Rigid and Elastic Tubes.-If a quantity of fluid be forced into a rigid tube under a certain pressure, the same quantity of fluid will flow out at once at the other end of the tube, provided there be no special resistance. In an elastic tube, immediately after the forcing in of a quantity of fluid, at first only a small quantity flows out, and the remainder flows out only after the propelling force has ceased to act. If an equal quantity of fluid be periodically injected into a rigid tube, with each jerk an equal quantity is forced out at the other end of the tube, and the outflow lasts exactly as long as the jerk or the contraction, and the pause between two periods of outflow is exactly the same as between the two jerks or contractions. In an elastic tube it is different, as the outflow continues for a time after the jerk; hence, it follows that a continuous outflow current will be produced in elastic tubes, when the time between two jerks is made shorter than the duration of the outflow after the jerk has been completed. When fluid is pumped periodically into rigid tubes, it causes a sharp abrupt outflow synchronous with the inflow, and the outflow becomes continuous only when the inflow is continuous and uninterrupted. In elastic tubes, an intermittent current under the above conditions causes a continuous outflow, which is increased with the systole or contraction.

65. STRUCTURE AND PROPERTIES OF Coats of a small artery. $a$, endo. THE BLO0D-VESSELS. - In the body the large thelium; $b$, internal elastic lamina; vessels carry the blood to and from the various $c$, circular muscular fibres of the tissues and organs, while the thin-walled capillaries middle coat ; $d$, the outer coat. bring the blood into intimate relation with the tissues. Through the excessively thin walls of the capillaries the fluid part of the blood transudes, to nourish the tissues outside the capillaries. [At the same time fluids pass from the tissues into the blood. Thus, there is an exchange between the 
blood and the fluids of the tissues. The fluid after it passes into the tissues constitutes the lymph, and acts like a stream irrigating the tissue elements.]

I. The arteries are distinguished from veins by their thicker walls, due to the greater development of smooth muscular and elastic tissues-the middle coat (tunica media) of the arteries is specially thick, while the outer coat ( $t$. adventitia) is relatively thin. [The absence of valves is by no means a characteristic feature.]

A typical artery consists of three coats (fig. 68). (1) The tunica intima, or inner coat, consists of a layer of $(a)$ irregular, long, fusiform nucleated squamous cells forming the excessively thin transparent endothelium, immediately in contact with the blood-stream. [Like other endothelial cells, these cells are held together by a cement substance, which is blackened by the action of silver nitrate.] Outside this lies a very thin, more or less fibrous, layer-sub-epithelial layer-in which numerous spindle or branched protoplasmic cells lie embedded within a corresponaing system of plasma canals. Outside this is an elastic lamina (b), which in the smallest arteries is a structureless or fibrous elastic membrane-in arteries of medium size it is a fenestrated membrane (Henle), while in the largest arteries there may be several layers of elastic laninæ or fenestrated elastic membrane mixed with connective-tissue. [In some arteries the elastic membrane is distinctly fibrous, the fibres being chiefly arranged longitudinally. It can be stripped off, when it forms a brittle elastic membrane, which has a great tendency to curl up at its margins. In a transverse section of a middle-sized artery it appears as a bright wavy line, but the curves are probably produced by the partial collapse of the vessel. It forms an important guide to the pathologist, in enabling him to determine which coat of the artery is diseased.] In middle-sized and large arteries a few non-striped muscular fibres are disposed longitudinally between the elastic plates or laminæ. Along with the circular muscular fibres of the middle coat, they may act so as to narrow the artery, and they may also aid in keeping the lumen of the vessel open and of uniform calibre.

(2) The tunica media, or middle coat, contains much non-striped muscle (c), which in the smallest arteries consists of transversely disposed non-striped muscular fibres lying between the endothelium and the T. adventitia, while a finely granular tissue with few elastic fibres forms the bond of union between them. As we proceed from the very smallest to the small arteries, the number of muscular fibres becomes so great as to form a well-marked fibrous ring of nonstriped muscle, in which there is comparatively little connective-tissue. In the large arteries the amount of connective-tissue is considerably increased, and between the layers of fine connectivetissue numerous (as many as 50) thick, elastic fibrous or fenestrated laminæ are concentrically arranged. A few non-striped fibres lie scattered amongst these, and some of them are arranged transversely, while a few have an oblique or longitudinal direction.

The first part of the aorta and pulmonary artery, and the retinal arteries, are devoid of muscle. The descending aorta, common iliac, and popliteal have longitudinal fibres between the transverse ones. Longitudinal bundles lying inside the media occur in the renal, splenic, and internal spermatic arteries. Longitudinal bundles occur both on the outer and inner surfaces of the umbilical arteries, which are very muscular.

(3) The tunica adventitia, or outer coat, in the smallest arteries consists of a structureless membrane with a few connectivetissue corpuscles attached to it; in somewhat larger arteries there is a layer of fine fibrous elastic tissue mixed with bundles of fibrillar connective-tissue $(d)$. In arteries of middle size, and in the largest arteries, the chief mass consists of bundles of fibrillar connective-tissue containing connective-tissue corpuscles. The bundles cross each other in a variety of directions, and fat cells often

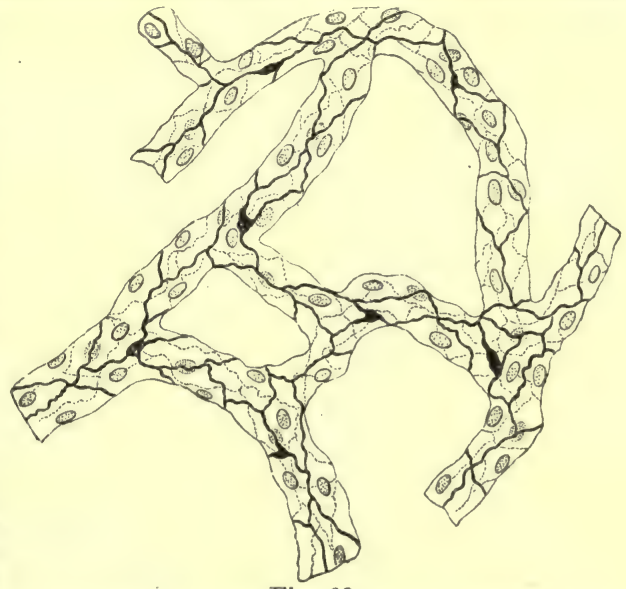

Fig. 69 .

Capillaries. The outlines of the nucleated endo. thelial cells with the cement blackened by the action of silver nitrate.

lie between them. Next the media there are numerous fibrous or fenestrated elastic lamellæ. In medium sized and small arteries the elastie tissue next the media takes the form of an independent elastic membrane (Henle's external elastic membrane). Bundles of non-striped muscle, arranged longitudinally, occur in the adventitia of the arteries of the penis, and in the renal, splenic, spermatic, iliac, hypogastric, and superior mesenteric arteries.

II. The capillaries, while retaining their diameter, divide and reunite so as to form net- 
works, whose shape and arrangenient differ considerably in different tissues. The diameter of the capillaries varies considerably, but as a general rule it is such as to admit freely a single row of blood-corpuscles. In the retina and the muscles the diameter is 5-6 $\mu$, and in bone-marrow, liver, and choroid 10-20 $\mu$. The tubes consist of a single layer of transparent, excessively thin, nucleated, endothelial cells joined to each other by their margins. [The nuclei contain a wellmarked intra-nuclear plexus of fibrils, like other nuclei.] The cells are more fusiform in the smaller capillaries and more polygonal in the larger. The body of the cells presents the characters of very faintly refractive protoplasm, but it is doubtful whether the body of the cell is endowed with the property of contractility (p. 96).

If a dilute solution (1 per cent.) of silver nitrate be injected into the blood-vessels, the cement substance of the endothelium, [and of the muscular fibres as well], is revealed by the presence of the black "silver lines." The blackened cement substance shows little specks and large black slits at different points. It is not certain whether these are actual holes through which colourless corpuseles may pass out of the vessels, or are merely larger accumulations of the cement sulstance. [If a capillary is examined in a perfectly fresh condition (while living) and without the addition of any reagent, it is impossible to make out any line of demarcation between adjacent cells owing to the uniform refractive index of the entire wall of the tube.]

[Arnold ealled these small areas in the black silver lines when they are large stomata, and when small stigmata. They are most numerous after venous congestion, and after the dis-

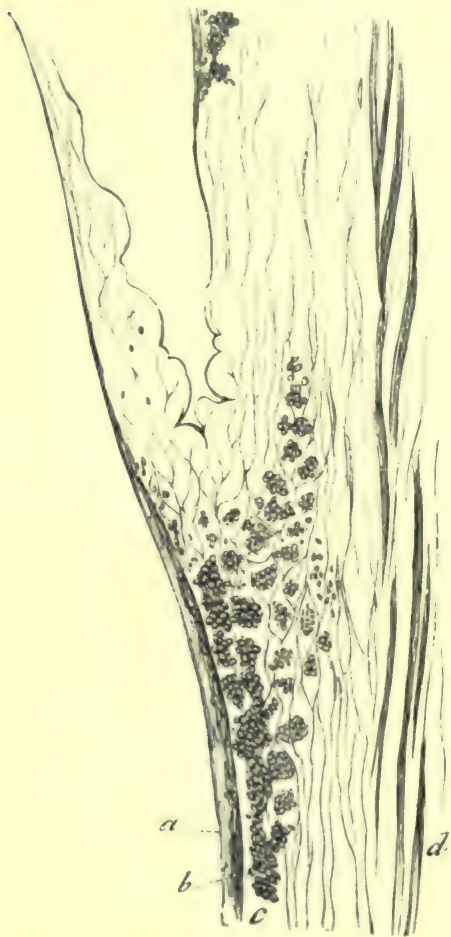

Fig. 70 .

Longitudinal section of a vein at the level of a valve. $a$, hyaline layer of the internal coat ; $b$, elastic lamina ; $c$, groups of smooth muscular fibres divided transversely ; $d$, longitudinal muscular fibres in the adventitia. turbances which follow inflammation of a part. They are not always present. The existence of cement substance between the cells may also be inferred from the fact that indigo-sulphate of soda is deposited in it (Thoma), and particles of cinnabar and China ink are fixed in it, when these substances are injected into the blood (Foa).]

Fine anastomosing fibrils derived from non-medullated nerves terminate in small end-buds in relation with the capillary wall; ganglia in connection with the nerves of capillaries occur only in the region of the sympathetic.

The small vessels next in size to the capillaries, and continuous with them, have a completely structureless covering in addition to the endothelium.

III. The veins are generally distinguished from the arteries by their lumen being wider than the lumen of the corresponding arteries; their walls are thinner on account of the smaller amount of non-striped muscle and elastic tissue (the non-striped muscle is not unfrequently arranged longitudinally in veins). They are also more extensile (with the same strain). The adventitia is usually the thickest coat. The occurrence of valves is limited to the veins of certain areas.

Structure.-(1) The tunica intima consists of a layer of shorter and broader endothelial cells, under which in the smallest veins there is a structureless elastic membrane, sub-epithelial layer, which is fibrous in veins somewhat larger in size, but in all cases is thinner than in the arteries. In large veins it may assume the characters of a fenestrated membrane, which is double in some parts of the crural and iliac veins. Isolated muscular fibres exist in the intima of the femoral and popliteal veins.

(2) The $t$. media of the larger veins consists of alternate layers of elastic and muscular tissue united to each other by a considerable amount of connectivetissue, but this coat is always thinner than in the corresponding arteries. This coat diminishes in the following order in the following vessels :popliteal, veins of the lower extremity, veins of the upper extremity, superior mesenteric, other abdominal veins, hepatic, pulnonary, and coronary veins. The following veins contain no muscle:-veins of bone, central nervous system and its membranes, retina, the superior cava, 
with the large trunks that open into it, the upper part of the inferior cava. Of course, in these cases the media is very thin. In the smallest veins the media is formed of fine connectivetissue, with very few muscular fibres scattered in the inner part.

(3) The t. adventitia is thicker than that of the corresponding arteries; it contains much connective-tissue, usually arranged longitudinally, and not much elastic tissue. Longitudinally arranged muscular fibres occur in some veins (renal, portal, inferior cava near the liver, veins of the lower extremities). The valves consist of fine fibrillar connective-tissue with branched cells. An elastic network exists on their convex surface, and both surfaces are covered by endothelium. The valves contain many muscular fibres (fig. 70). [Ranvier has shown that the shape of the epithelial cells on the side over which the blood passes, are more elongated than on the cardiac side of the valve, where the long axes of the cells are placed transversely.]

The sinuses of the dura mater are spaces covered with endothelium. The spaces are either duplicatures of the membrane, or channels in the substance of the tissue itself.

Cavernous spaces we may imagine to arise by numerous divisions and anastomoses of tolerably large veins of unequal calibre. The vascular wall appears to be much perforated and like a sponge, the internal space being traversed by threads and strands of tissue, which are covered with endothelium on their surfaces, that are in contact with the blood. The surrounding wall consists of connective-tissue, which is often very tough, as in the corpus cavernosum, and it not unfrequently contains non-striped muscle.

Cavernous formations of an analogous nature on arteries are the carotid gland of the frog, and a similar structure on the pulmonary arteries and aorta of the turtle, and the coccygeal gland of man. The last structure is richly supplied with sympathetic nerve-fibres, and is a convoluted mass of ampullated or fusiform dilatations of the middle sacral artery, surrounded and permeated by non-striped muscle.

Vasa Vasorum. - [These are small vessels which nourish the coats of the arteries and veins. They arise from one part of a vessel and enter the walls of the same, or another vessel at a lower level. They break up chiefly in the outer coat, and none enter the inner coat.] In structure they resemble other small blood-vessels. The blood circulating in the arterial or venous wall is returned by small veins.

[Lymphatics. - There are no lymphatics on the inner surface of the muscular coat, or under the intima in large arteries. They are numerous in a gelatinous layer immediately ontside the muscular coat, and the same relation obtains in large muscular veins and lymphatic trunks (Hoggan).]

Intercellular Blood-Channels. - Intercellular blood-channels of narrow calibre, and without walls, occur in the granulation tissue of healing wounds. At first blood-plasma alone is found between the formative cells, but afterwards the blood-current forces blood-corpuscles through the channels. The first blood-vessels in the developing chick are formed in a similar way from the formative cells of the mesoblast.

Properties of the Blood-Vessels.-The larger blood-vessels are cylindrical tubes composed of several layers of various tissues, more especially elastic tissue and smooth muscular fibres, and the whole is lined by a smooth polished layer of endothelium. One of the most important properties is the contractility of the vascular wall, in virtue of which the calibre of the vessel can be varied, and therefore the supply of blood to a part is altered. The contractility is due to the plain muscular fibres, which are, for the most part, arranged circularly. It is most marked in the small arteries, and of course is absent where no muscular tissue occurs. The amount and intensity of the contraction depend upon the development of the muscular tissue ; in fact, the two go hand in hand. [If an artery be exposed in the living body it soon contracts under the stimulus of the atmosphere acting upon the muscular fibres.]

[Action of Drugs on the Vascular System. - Gaskell finds that a very dilute solution of lactic acid ( $1: 10,000$ parts of saline solution), passed through the blood-vessels of a frog, always enlarges the calibre of the blood-vessels, while an alkaline solution ( 1 part sodium hydrate to 10,000 saline solution) always diminishes their size, usually to absolute closure, and indeed the artificial constriction of the blood-vessels may be almost complete. These fluids are antagonistic to each other as far as regards their action on the calibre of the arteries. Dilute alkaline solutions act on the heart in the same way. After a series of beats the ventricle stops beating, the standstill being in a state of contraction. Very dilute lactic acid causes the ventricle to stand still in the phase of complete relaxation. The acid and alkaline saline solutions are antagonistic in their action on the ventricle. Cash and Brunton find that dilute acids have a tendency to increase the transudation through the vessels and produce oedema of the surrounding tissues. They also observed that barium, calcium, strontium, copper, iron, and tin produce contraction of the blood-vessels when solutions of their salts are driven through then, while the same effect is produced by very dilute solutions of potassium. Nicotin, atropin, and 
chloral differ in their action according to the dose. In these experiments the effect was ascertained by the amount of fluid which flowed out of the vessels in a given time.] If blood containing certain drugs be perfused through the blood-vessels of a freshly excised organ, the blood-vessels are dilated; e.g., by amyl nitrite, chloral hydrate, morphia, $\mathrm{CO}$, paraldehyde, kairin, quinine, atropin, ferricyanide of potassium, (urea and sodic chloride in the renal vessels), - they are contracted by digitalin, veratria, helleborin (Kobert). Heat causes contraction of the blood-vessels of the frog's mesentery (Gürtner). According to Roy the blood-vessels shorten when heated.

That the capillaries undergo dilatation and contraction, owing to variations in the size of the protoplasmic elements of their walls, must be admitted.

Stricker has described capillaries as "protoplasm in tubes," and observed that in the tadpole they exhibited movements when stimulated. Golubew described an active state of contraction of the capillary wall, but he regarded the nuclei as the parts which underwent change. Rouget observed the same result in the capillaries of new-born mammals. Tarchanoff found that mechanical or electrical stimulation caused a change in the shape and size of the nuclei, so that he regards these as the actively contractile parts. [Severini also attaches great importance to the contractility of the capillaries and especially of their nuclei as influencing the blood-stream. Oxygen acts on the nuclei of the capillary wall (membrana nictitans of frog) and causes them to swell, while $\mathrm{CO}_{2}$ has an opposite effect. The circulation through a lung suddenly filled with $\mathrm{O}$ or atmospheric air is at first very rapid, but it soon diminishes, while with $\mathrm{CO}_{2}$ the circulation remains constant.] As the capillaries are excessively thin, soft, and delicate, it is obvious that the form of the individual cells must depend to a considerable extent upon the degree to which the vessels are filled with blood. In vessels which are distended with blood the endothelial cells are flattened, but when the capillaries are collapsed they project more or less into the lumen of the vessel (Rencut).

[It is well known that the eapiliaries present great variations in their diameter at different times. As these variations are usually accompanied by a corresponding contraction or dilatation of the arterioles, it is usually assumed that the variations in the diameter of the capillaries are due to differences of the pressure within the capillaries themselves, viz., to the elasticity of their walls. Every one is agreed that the capillaries are very elastic, but the experiments of Roy and Graham Brown show that they are contractile as well as elastic, and these observers conclude that, under normal conditions, it is by the contractility of the capillary wall as a whole that the diameter of these vessels is changed, and to all appearance their contractility is constantly in action. "The individual capillaries (in all probability) contract or expand in accordance with the requirements of the tissues through which they pass. The regulation of the vascular blood-flow is thus more complete than is usually imagined.'

Physical Properties.-Amongst the physical properties of the blood-vessels, elasticity is the most important ; their elasticity is small in amount, i.e., they offer little resistance to any force applied to them so as to distend or elongate them but it is perfect in quality, i.e., the blood-vessels rapidly regain their original size and form after the force distending them is removed.

[Uses of Elasticity. - The elasticity of the arteries is of the utmost importance in aiding the conversion of the unequal movement of the blood in the large arteries into a uniform flow in the capillaries. E. H. Weber comprared the elastic wall of the arteries with the air in the airchamber of a fire-engine. In both cases an elastic medium is acted upon-the air in the one case and the elastic tissue in the other-which in turn presses upon the fluid, propelling it onwards continually, while the action of the pump or the heart, as the case may be, is intermittent. The ordinary spray-producer acts on this principle. A uniform spray or jet is obtained by pumping intermittently, but only when the resistance is such as to bring into action the elasticity of the bag between the pump and the spray-orifice.]

According to E. H. Weber, Volkmann, and Wertheim, the elongation of a blood-vessel and moist tissues generally is not proportional to the weight used to extend it, the elongation being relatively less with a large weight than with a small one, so that the curve of extension is nearly [or, at least, bears a certain relation to] a hyperbola. According to Wundt, we have not only to consider the extension produced at first by the weight, but also the subsequent "elastic after-effect," which occurs gradually. The elongation which takes place during the last few moments occurs so slowly and so gradually that it is well to observe the effect by means of a magnifying lens. Variations from the general law occur to this extent, that if a certain weight is exceeded, less extension, and, it may be, permanent elongation of the artery not unfrequently occur. K. Bardeleben found, especially in veins elongated to 40 or 50 per cent. of their original length, that when the weight employed increased by an equal amount each time, the elongation was proportional to the square-root of the weight. This is apart from any elastic after-effect. Veins may be extended to at least 50 per cent. of their length without passing the limit of their elasticity. 
[Roy experimented upon the elastic properties of the arterial wall. A portion of an artery, so that it could be distended by any desired internal pressure, was enclosed in a small vessel containing olive oil arranged in the same way as in fig. 62 for the heart. The variations of the contents were recorded by means of a lever writing on a revolving cylinder. The instrument is termed a sphygmotonometer. The aorta and other large arteries are most elastic and most distensible at pressures corresponding more or less exactly to their normal blood-pressure, while in veins the relation between internal pressure and the cubic capacity is very different. In them the maximum of distensibility occurs with pressures immediately above zero. Speaking generally, the cubic capacity of an artery is greatly increased by raising the intra-arterial tension, say from zero to about the normal internal pressure which the artery sustains during life. Thus in the rabbit, the capacity of the aorta was quadrupled by raising the intra-arterial pressure from zero to $200 \mathrm{~mm}$. Hg., while that of the carotid was more than six times greater at that pressure than it was in the undistended condition. The pulmonary artery is distinguished by its excessive elastic distensibility. Its capacity (rabbit) was increased more than twelve times on raising the internal pressure from zero to about $36 \mathrm{~mm}$. $\mathrm{Hg}$. Veins, on the other hand, are distinguished by the relatively small increase in their cubic capacity produced by greatly raising the internal pressure, so that the enormous changes in the capacity of the veins during life are due less to differences in the pressure than to the great differences in the quantity of blood which they contain.]

Pathological. - Interference with the nutrition of an artery alters its elasticity, [and that in cases where no structural changes can be found]. Marasmus preceding death causes the arteries to become wider than normal. In some old people they become atheromatous and even calcifierl.

Cohesion.-The cohesion of blood-vessels is very great, and in virtue of this they are able to resist even considerable internal pressure without giving way. The carotid of a sheep is ruptured only when fourteen times the usual pressure it is called upon to bear is put upon it (Volkmann). Given a vein and an artery of the same thickness, a greater pressure is required to rupture the former than the latter. The human carotid or iliac artery resists a pressure of 8 atmospheres, the veins about the half of this.

66. INVESTIGATION OF THE PULSE.-[The characters of the pulse may be investigated by-(1) the eye (inspection); (2) the finger (palpation); (3) instruments.

Two or three fingers are placed over the course of the radial artery, and the various phenomena in connection with the pulse are noted. It takes much practice for the physician to acquire the tactus eruditus, and notwithstanding the value of instruments, every physician should make a careful study of the pulse-beat with his finger. In order to feel the pulse-beat or to take a pulse-tracing, there must be some resistant body, e.g., a bone behind the artery, and a certain degree of pressure must be exerted on the artery.]

The individual phases of the movement of the pulse can only be accurately investigated by the application of instruments to the arteries.

(1) Poiseuille's Box Pulse-Measurer (1829). - An artery is exposed and placed in an oblong box filled with an inaifferent fluid. A vertical tube with a scale attached communicates with the interior of the box. The column of fluid undergoes a variation with every pulse-beat.

(2) Hérisson's Tubular Sphygmometer consists of a glass tube whose lower end is covered with an elastic membrane (fig. 71). The tube is partly filled with $\mathrm{Hg}$. The membrane is placed over the position of a pulsating artery, so that its beat causes a movement in the $\mathrm{Hg}$. Chelius used a similar instrument, and he succeeded with this instrument in showing the existence of the double beat (dicrotism) in the normal pulse (1850).

(3) Vierordt's Sphygmograph (1855). - In this, one of the earliest sphygmo-

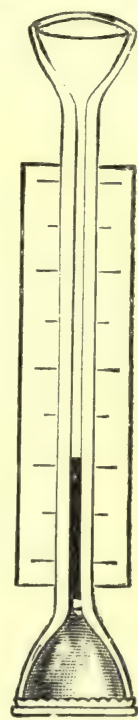

Fig. 71 .

Sphygmometer of Hérisson and Chelius. graphs, Vierordt departed from the principle of a fluctuating fluid column, and adopted the principle of the lever. Upon the artery rested a small pad, which moved a complicated system of levers. At first he used a straw 6 inches long, which rested on the artery. The point of one of the levers inscribed its movements upon a revolving cylinder. This instrument was soon discarded.

(4) Marey's Sphygmograph consists of a combination of a lever with an elastic spring. The 
elastic spring (fig. $72, \Lambda$ ) is fixed at one end, $z$, free at the other end, and provided with an ivory pad, $y$, which is pressed by the spring upon the radial artery. On the upper surface of the pad there is a vertically-placed fine toothed rod, $k$, which is pressed upon by a weak spring, $e$,

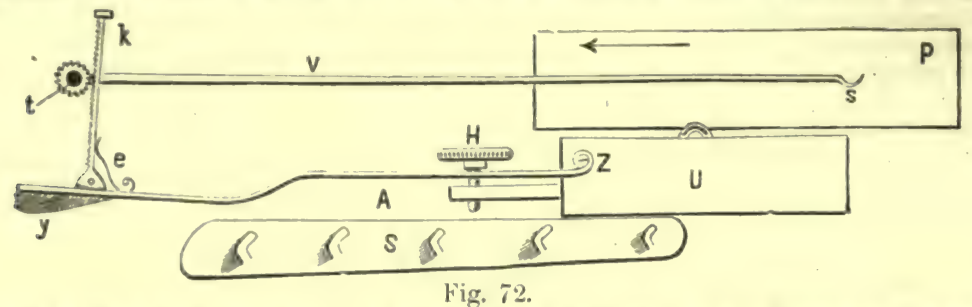

Scheme of Marey's sphygmograph. A, spring with ivory pad, $y$, which rests on the artery; $e$, weak spring pressing $l$ into $t$; $r$, writing lever; $P$, piece of smoked glass or paper moved by clock-work, $\mathrm{U} ; \mathrm{H}$, screw to limit excursion of $\mathrm{A} ; \mathrm{S}$, arrangement for fixing the instrument to the arm of the patient.

so that its teeth dovetail with similar teeth in the small wheel, $t$, from whose axis there projects a long, light, wonden lever, $r$, running nearly parallel with the elastic spring. This lever has a fine style at its free end, s, which writes upon a smoked plate, I, moved by clock-work, $\mathrm{U}$, in front of the style. Marey's instrument, as improved by Nahomed and others, has been very largely used.

[Its more complete form, as in fig. 73, where it is shown applied to the arm, consists of -(1) a steel spring, $A$, whirh is provided with a parl resting on the artery, and moves with each movement of the artery ; $(2)$ the lever, $\mathrm{C}$, which recorls the movement of the artery and spring in a magnified form on the smokesl paper. $\mathrm{G}:(3)$ an arrangement, L, whereby the exact pressure exerted upon the artery is indieated on the dial, $\mathrm{II} ;(4)$ the clock-work, $\mathbf{H}$, which moves the smoked paper, $G$, at a uniform rate; (5) a franiework to which the various parts of the instrument are attathed, and by means of which the instrument is fastened to the arm by straps, K, K (Byrom Bramwell).]

[Application.--Iin applying the sphymograph, cause the patient to seat himself beside a low table, and place his arm on the double-inclined plane (fig. 73). In the newer form of instru-

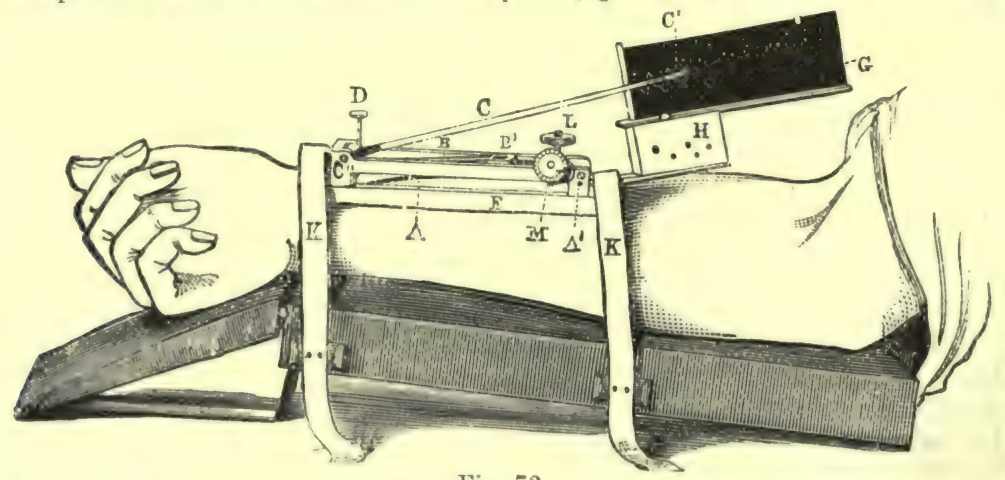

Fig. 73.

Marey's improved sphygmograph. A, steel spring; B, first lever; C, writing lever ; C', its free writing end; $\mathrm{D}$, screw for bringing $\mathrm{B}$ in contact with $\mathrm{C} ; \mathrm{G}$, slide with smoked paper; $\mathrm{H}$, clock-work; L, serew for inereasing the pressure; $\mathbf{M}$, dial indicating the pressure ; $\mathbf{K}, \mathbf{K}$, straps for fixing the instrument to the arm, and the arm to the double-inclined plane or support.

ment, the lid of the box is so arranged as to unfold to make this support. The fingers ought to be semi-flexed. Mark the position of the radial artery with ink. See that the clock-work is wound up, and apply the ivory pad exactly over the radial artery where it lies upon the radius, fixing it to the arm by the non-elastic straps, K, K. Fix the slide holding the smoked paper in position. The best paper to use is that with a very smooth surface, or an enamelled card smoked over the flame of a turpentine lamp, over a piece of burning camphor, or over a fantailed gas-burner. The writing-style is so arranged as to write upon the smoked paper with the least possible friction. It is most important to regulate the pressure exerted upon the 
artery by means of the milled head, L. This must be determined for each pulse, but the rule is to graduate the pressure until the greatest amplitude of movement of the lever is obtained. Set the clock-work going, and a tracing is obtained, which must be "fixed" by dipping it in a rapidly drying varuish, e.g., photographic. In every case scratch on the tracing with a needle the name, date, and amount of pressure employed.]

[(5) Dudgeon's Sphygmograph.-This is a convenient form of sphygmograph, although Broadbent regards its results as untrustworthy. The instrument after being carefully adjusted

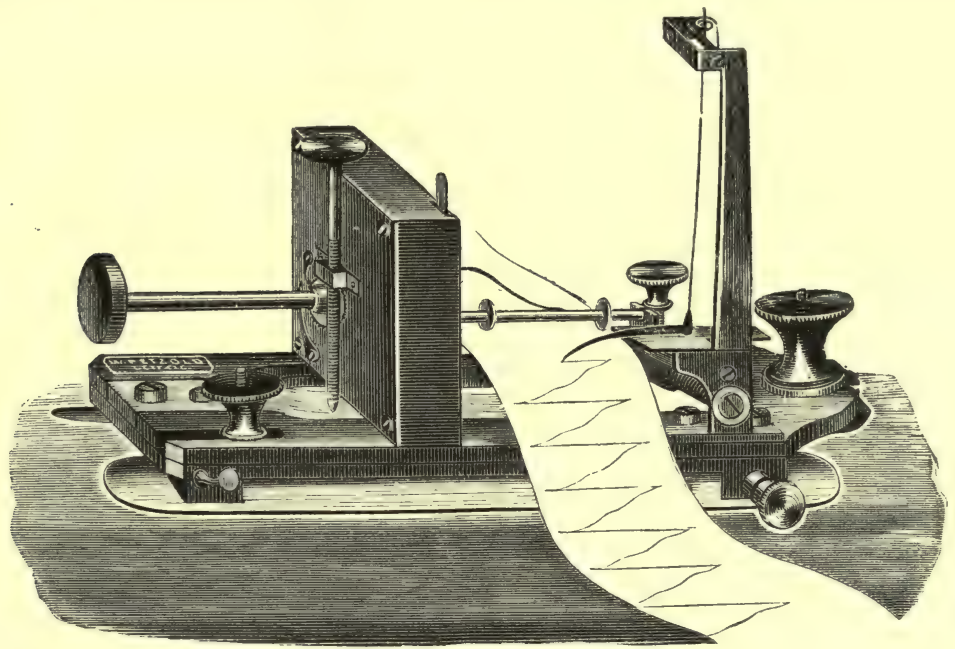

Fig. 74.

Ludwig's sphygmograph.

upon the radial artery is kept in position by an inelastic strap. The pressure of the spring is regulated by the eccentric wheel to any amount from 1 to 5 ounces. As in other instruments

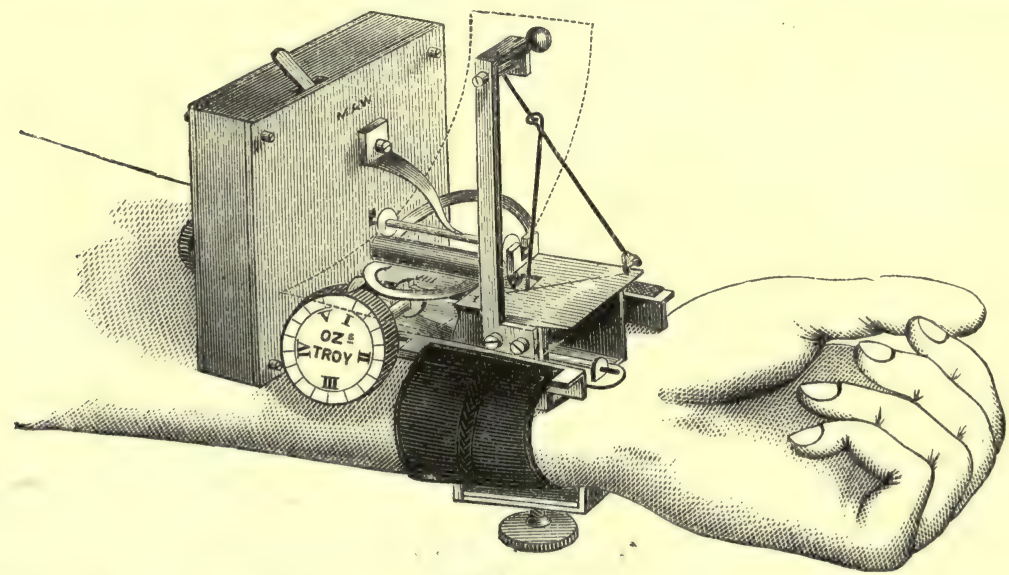

Fig. 75 .

Dudgeon's sphygmograph.

the tracing paper is moved in front of the writing-needle by means of clock-work. The writinglevers are so adjusted that the movements of the artery are magnified fifty times (fig. 75).]

(6) [Ludwig's improved form is a very serviceable instrument (fig. 74).]

(7) Marey's tambours are also employed for registering the movements of the pulse. They are used in the same way as the pansphygmograph. Two pairs of metallic cups (fig. $76, \mathrm{~S}, \mathrm{~S}$, 
and $\mathbf{S}^{\prime}, \mathbf{S}^{\prime}$, Upham's capsules) are pierced in the middle by thin metal tubes, whose free ends are counected with caoutchouc tubes, $\mathrm{K}$ and $\mathrm{K}^{\prime}$. All the four metallic vessels are covered with an elastic membrane. Ou $\mathrm{S}$ and $\mathrm{S}^{\prime}$ are fixed two knob-like pads, $p$ and $p^{\prime}$, which are applied to the pulsating arteries, and the metal ares, $\mathrm{B}$ and $\mathrm{B}^{\prime}$, retain them in position. On the other tambours are arranged the writing-levers, $Z$ and ' $Z$ '. Pressure on the one tambour necessarily compresses the air, and makes the other, with which it is connected, expand, so as to move the writing-lever. This arrangement does not give absolutely exact results ; still, it is very easily

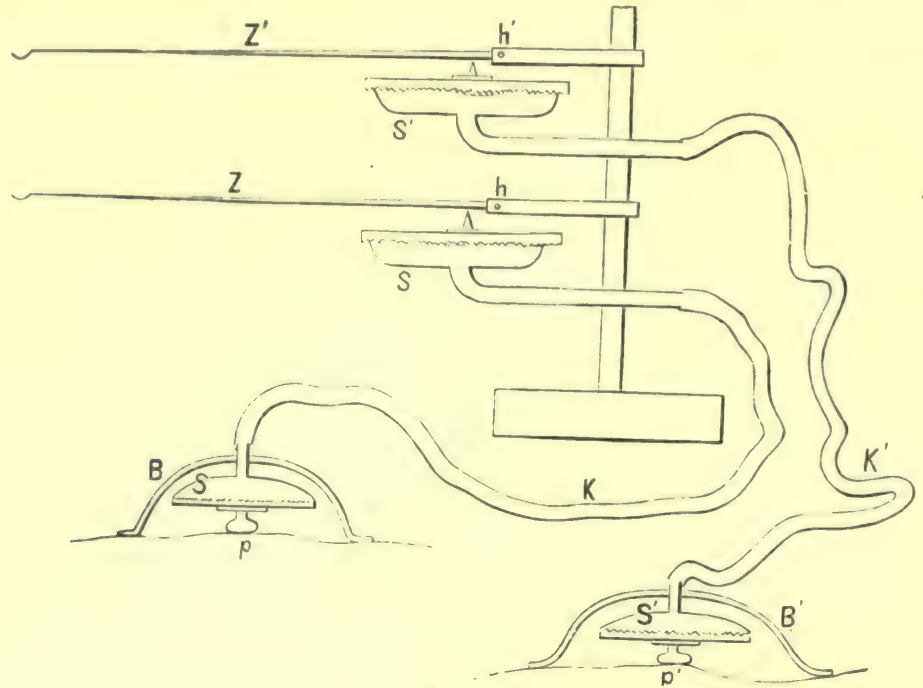

Fig. 76.

Scheme of Brondgeest's sphygmograph. S, $\mathbf{S}^{\prime}$, receiving aud recording $\left(\mathrm{S}, \mathrm{S}^{\prime}\right)$ tambours with writing levers, $Z$ and $Z^{\prime} ; \mathrm{K}, \mathrm{K}^{\prime}$, conducting tubes : $p$, over heart, $p^{\prime}$, over a distant artery.

used, and is convenient. In fig. 76 a double arrangement is shown, whereby one instrument, $\mathrm{B}$, may be placed over the heart, and the other, $\mathrm{B}^{\prime}$, on a distant artery.

(8) Landois' Angiograph. - To a basal plate, G, G', are fixed two upright supports, $p$, which carry between them at their upper part the movable lever, $d, r$, carrying a rod bearing a pad, $r$, directed downwarls, which rests on the pulse. The short arm earries a counterpoise, $d$, so

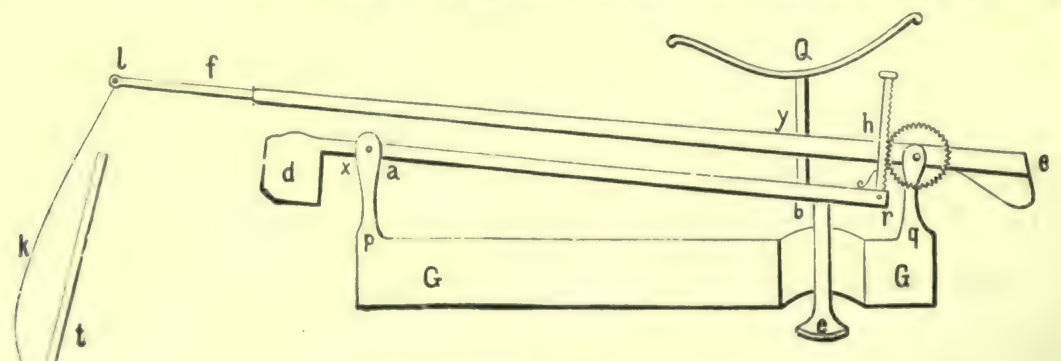

Fig. 77 .

Scheme of Landois' angiograph.

as exactly to balance the long arm. The long arm has fixed to it at $r$ a vertical rod provided with teeth, $h$, which is pressed against a toothed wheel firmly fixed on the axis of the very light writing-lever, $e, f$, which is supported between two uprights, $q$, fixed to the opposite end of the basal plate, G, G. 'The writing-lever is equilibrated by means of a light weight. The writing-needle, $k$, is fixed by a joint to $e$, and it writes on the plate, $t$. The first-mentioned lever, $d, r$, carries a shallow cup, $Q$, just above the pad, into which weights may be put to press on the pulse. In this instrument the weight can be measured 
and varied; the writing-lever moves vertically, and not in a curve as in Narey's apparatus, which greatly facilitates the measuring of the curves (fig. 77 ).

Other sphygmographs are used, both in this country and abroad, including that of Sommerbrodt, which is a complicated form of Marey's sphygmograph, and those of Pond and Mach.

In every pulse-curve - sphygmogram or arteriogram - we can distinguish the ascending part (ascent) of the curve, the apex, and the descending part (descent). Secondary elevations scarcely ever occur in the ascent, which is usually represented by a straight line, while they are always present in the descent. Such elevations occurring in the descent are called catacrotic, and those in the ascent, anacrotic. When the recoil elevation or dicrotic wave occurs in a wellmarked form in the descent, the pulse is said to be dicrotic, and when it occurs twice, tricrotic.

Measuring Pulse-Curves. - If the smoked surface on which the tracing is inscribed is moved at a uni- $\mathrm{N}$ form rate by means of the clock-work, then the height and length of the curve are measured by means of an ordinary rule. If we know the rate at which the paper was moved, then it is easy to calculate the duration of any event in the curve.

The curve may be recorded on a plate of glass fixed to a tuning-fork kept in vibration. Every part of the curve shows little elevations (whose rate of vibration is known beforehand). All that is required is to count the number of vibrations in order to ascertain the duration of any part of the curve (fig. 78).

Gas-Sphygmoscope.-A small metallic or glass capsule (fig. 79), provided with an inlet and an outlet tube, and closed below by a fine membrane, is placed over an artery. The inlet tube is connected to a gas supply, and the outlet to a rat-tailed gas-burner $(b)$. The gas-jet responds to every pulse- $l$ beat. Czermak photographed a beam of light set in motion by the movements of the pulse.

Hæmautography. - Expose a large artery of an animal, and divide it so that the stream of blood issuing from it strikes against a piece of paper drawn in front of the blood-stream.

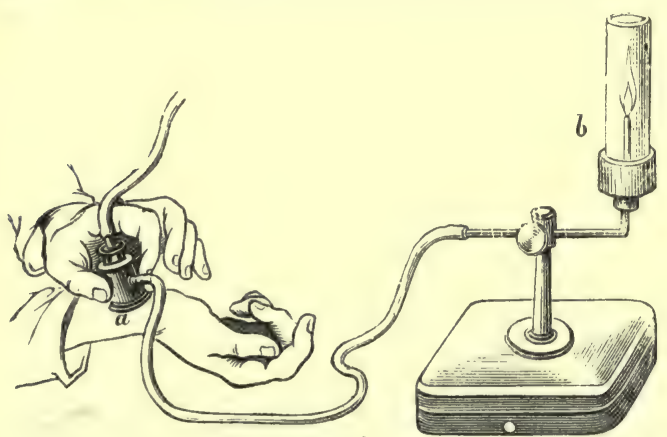

Fig. 79 .

Gas-sphygmoscope of S. Mayer.

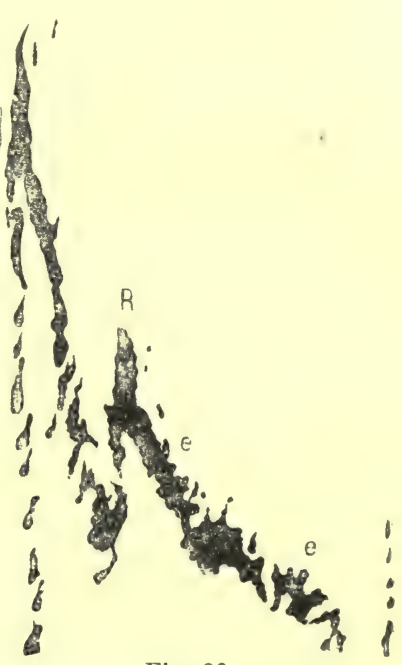

Fig. 80 .

Hæmautographic curve of the posterior tibial artery of a dog. $\mathrm{P}$, primary pulse-wave ; $\mathrm{R}$, dicrotic wave ; $e, e$, elevations due to elasticity.

The curve so obtained (fig. 80 ) shows, in addition to the primary wave, $\mathrm{P}$, a distinct dicrotic wave, $\mathrm{R}$, and slight vibrations, $e, e$, due to the variations in the elasticity of the arterial wall, which shows that the movements occur in the blood itself, and are communicated as waves to the arterial wall. By estimating the amount of blood in the various parts of the curve, we obtain a knowledge of the amount of blood discharged by the divided artery during the systole and diastole (i.e., the narrowing and dilatation) of the artery-the ratio is $7: 10$. Thus in the unit of time, during arterial dilatation, rather more than twice as much blood flows out as compared with what occurs during arterial contraction. 
67. PULSE-TRACING OR SPHYGMOGRAM.- [The Pulse.-With each systole of the heart, a certain quantity of blood is forced into the already filled and partially distended arteries, the resistance in the vessels is lowest between the pulsations, and at this time the arterial tubes are somewhat flattened, but with each systole of the left ventricle the pulse-wave, or rather the liquid pressure within the vessel, is increased, thus forcing the artery back into the circular form. "The

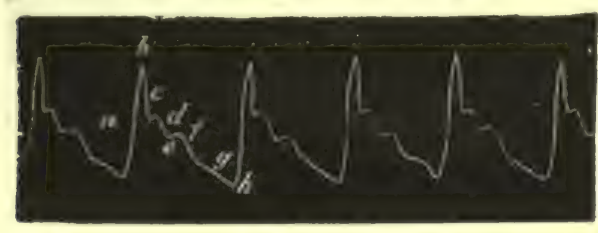

Fig. 81 .

Sphygmogram of radial artery : pressure $2 \%$. Fach part of the curve between the base of one up-stroke anil the base of the next up-stroke corresponds to a beat of the heart, so that this figme shows five heart-beats and part of a sixth. change of shape, from the flattened condition impressed upon the vessel by the finger or the sphygmograph lever, to the round cylindrical shape which it assumes under the distending force of the blood within it, constitutes the pulse," and it indicates the degree and duration of the increased pressure in the arterial system caused by the ventricular systole (Broadbent).]

Analysis.-A sphygmogram or pulse-tracing consists of a series of curres (fig. 81) each of which corresponds with one beat of the heart. Each pulsecurve consists of-

\section{The line of ascent ( $a$ to $b$ in fig. 81). \\ 2. The apex ( $\mathrm{P}$ in fig. 83 , and $b$ in fig. 81$)$. \\ 3. The line of descent ( $b$ to $h)$.}

(1) The line of ascent, up-stroke, or percussion stroke, is nearly vertical, and occurs during the dilatation of the artery produced by the systole of the left ventricle, when the aortic valves are forced open and the ventricular contents are projected into the arterial system. [The ascent is nearly vertical, but in some cases, where the ventricle contracts very suddenly, as occasionally happens in aortic regurgitation, it is quite vertical (fig. 85).]

(2) The apex or percussion wave in a normal pulse is pointed.

(3) The line of descent is gradual, and corresponds to the diminution of diameter or contraction of the artery. It is interrupted by two completely distinct elevations or secondary waves. Such elevations are called "catacrotic." The more distinct of the two occurs as a well-marked elevation about the middle of the descent $(\mathrm{R}$ in fig. 83 and $f$ in fig. 81 ); it is called the dicrotic wave, or, with reference to its mode of origin, the "reroil wuve." [As the descent corresponds to the time when blood is flowing out of the arteries at the periphery into the capillaries, its direction will depend on the rapidity of the outflow. Thus it will be more rapid in paralysis of the arterioles and very rapid in aortic regurgitation, where, of course, much of the blood flows backward into the left ventricle (fig. 85). In this case, the artery will recoil suddenly from under the finger or pad of the instrument, and this constitutes the "pulse of empty arteries."]

The dicrotic wave, or recoil wave, corresponds to the time following the closure of the aortic valves, and is preceded in the descent by a slight depression, the aortic notch

[The tidal wave, or pre-dicrotic, occurs between the apex and the dicrotic wave (fig. 81, $d$ ). It occurs on the descent, and during the contraction of the ventricle. The tidal wave is best marked in a hard pulse, i.e., where the blood-pressure is high, so that it is usually well marked in cirrhotic disease of the kidney, accompanied by hypertrophy of the left ventricle.]

[In some cases, e.g., mitral regurgitation, the pre-dicrotic wave may be present in some pulse-beats and absent in others (fig. 82), where the tidal wave is present in the largest pulse, 
and absent in the others, while the base line is uneren. In mitral stenosis the amount of blood discharged into the left ventricle frequently varies, hence the variations in the characters of the arterial pulse.]

There may be other secondary waves in the lower part of the descent.

[Respiratory or Base Line.- If a line be drawn so as to touch the bases of all

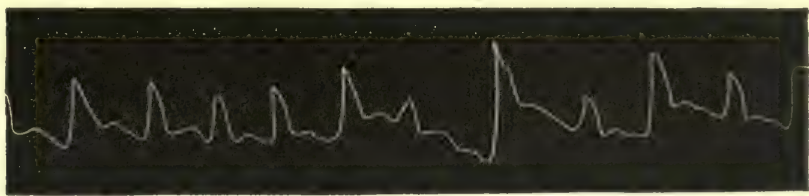

Fig. 82.

Irregular pulse of mitral regurgitation.

the up-strokes, we obtain a. straight line, hence called by this name. The base line is altered in disease and during forced respiration (\$74).]

The pulse-curve indicates the variations of pressure which the blood exerts on the arterial walls, for the lever rises and falls with the pressure, hence v. Kries calls it the "pressure-pulse."

68. ORIGIN OF THE DICROTIC WAVE.-The dicrotic or recoil wave, which is always present in a normal pulse, is caused thus :-During the ventricular systole a mass of blood is propelled into the already full aorta, whereby a positive wave is rapidly transmitted from the aorta throughout the arterial system, even to the smallest arterioles, in which this primary wave is extinguished. As soon as the semi-lunar valves are closed, and no more blood flows into the arterial system, the arteries, which were previously distended by the mass of blood suddenly thrown into them, recoil or contract, so that in virtue of the elasticity (and contractility) of their walls, they exert a counter-pressure upon the column of blood, and thus the blood is forced onwards. There is a free passage for it towards the periphery, but towards the centre (heart) it impinges upon the already closed semi-lunar valves. This develops a new positive wave, which is propagated peripherally through the arteries, where it disappears in their finest branches. In those cases where there is sufficient time for the complete development of the pulse-curve, (as in the short course of the carotids, and in the arteries of the upper arm, but not in those of the lower extremity, on account of their length), a second reflected wave may be caused in exactly the same way as the first. Just as the pulse occurs later in the more peripherally placed arteries than in those near the heart, so the secondary wave reflected from the closed aortic valves must appear later in the peripheral arteries. Both kinds of waves, the primary pulse-wave, the secondary, and eventually even the tertiary reflected wave-arise in the same place, and take the same course, and the longer the course they have to travel to any part of the arterial system, the later they arrive at their destination.

[The conditions which favour dicrotism are low blood-pressure and a rapid sharp cardiac contraction. When the blood-pressure is low, there is less resistance to the inflow of hlood at the aorta from the left ventricle, so that its systole oceurs sharply, forcing on the blood and distending the arterial walls. The elastic eoats rebound on the contained blood, and thus start a wave from the closed semi-lunar valves.]

The following points regarding the dicrotic wave have been ascertained experimentally, chiefly by Landois :-

1. The dicrotic wave occurs later in the descending part of the curve, the further the artery experimented upon is distant from the heart. Compare the curves, fig. 83 .

The shortest accessible course is that of the carotid; where the dicrotic wave reaches its maximum 0.35 to $0.37 \mathrm{sec}$. after the beginning of the pulse. In the upper extremity the apex of the dicrotic wave is 0.36 to 0.38 to 0.40 sec. after the beginning of the pulse-beat. The longest course is that of the arteries of the lower extremity. The apex of the dicrotic wave 
occurs 0.45 to 0.52 to 0.59 sec. after the beginning of the curve. It varies with the height of the individual.

2. The dicrotic elevation in the descent is lower, and is less distinct, the further the artery is situated from the heart, so that the longer the distance which the wave has to travel the less distinct it becomes.

3. It is best marked in a pulse where the primary pulse-wave is short and
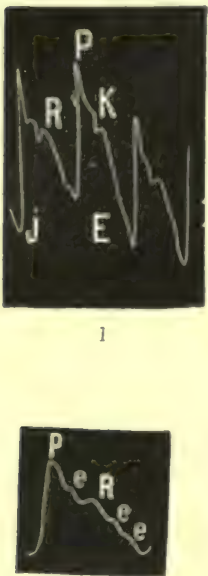

VI

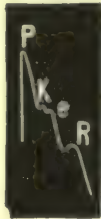

II

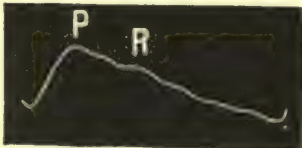

VII

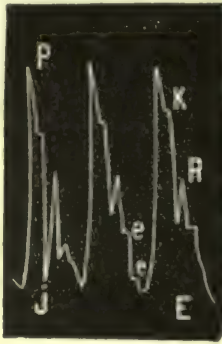

IV

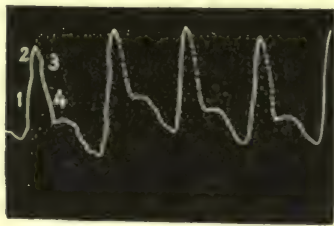

VIII

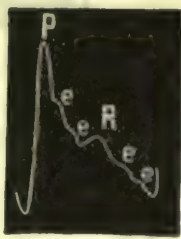

V

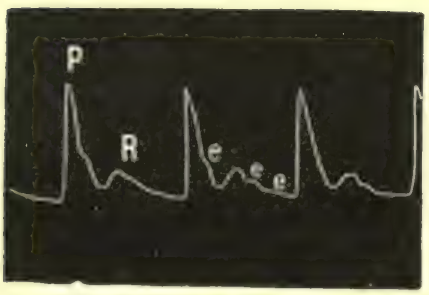

IX

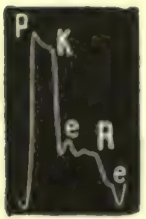

$\mathbf{X} 1$

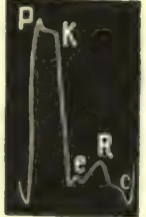

XII

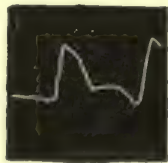

$\mathrm{X} 111$

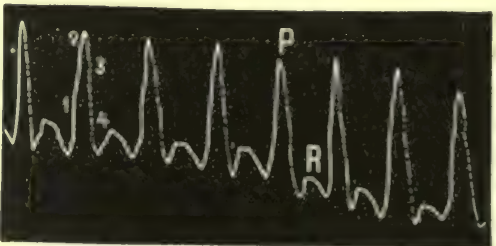

$\mathbf{X}$

Fig. 83.

I. II, III, sphygmograms of carotid artery ; IV, axillary ; V to IX, radial ; X, dicrotic radial pulse; XI, XII, crural ; XIII, posterior tibial; XIV, XV, pedal. In all the curves $P$ indicates apex; $\mathrm{R}$, dicrotic wave ; $e$, $c$, elevations due to elasticity ; $\mathrm{K}$, elevation caused by the closure of the semi-lunar valves of the aorta.

energetic. It is greatest relatively when the systole of the heart is short and energetic.

4. It is better marked the lower the tension of the blood within the arteries, [and 
is best developed in a soft pulse]. In fig. 83, IX and X were obtained when the tension of the arterial was low; V and VI, medium; and VII with high tension.

Conditions influencing Arterial Tension. - It is diminished at the beginning of inspiration (\$74), by hæmorrhage, stoppage of the heart, heat, an elevated position of parts of the body, amyl nitrite, nitro-glycerine, and the nitrites generally. [Both drugs accelerate the pulse-beats and produce marked dicrotism; with amyl nitrite the full effect is obtained in from 15 to 20 sec. after the inhalation of the dose (fig. $84, \mathrm{~A}, \mathrm{~A}^{\prime}$ ), but with nitro-glycerine not until 6 or 7 min. (fig. 84, B, B') and in the latter case the effects last longer.] It is increased at the

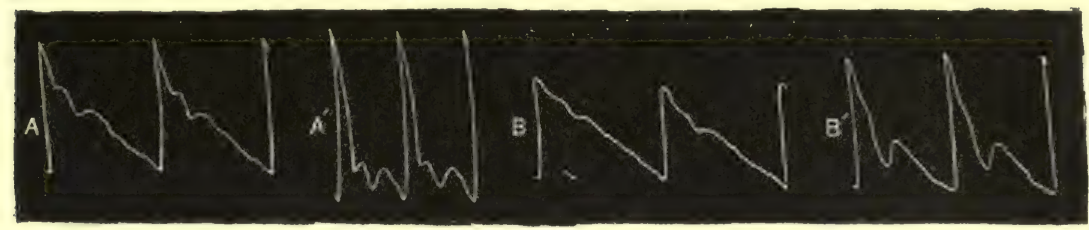

Fig. 84 .

Pulse-tracings. A, normal ; A', one minute after inhalation of amyl nitrite ; $\mathrm{B}$, normal ; $\mathrm{B}^{\prime}$, after a dose of nitro-glycerine (Stirling after Murrell).

heginning of expiration, by accelerated action of the heart, stimulation of vaso-motor nerves, diminished outllow of blood at the periphery, and by inflammatory congestion by certain poisons, as lead ; compression of other large arterial trunks, action of cold and electricity on the small cutaneous vessels, and by impeded outflow of venous blood. When a large arterial trunk is exposed, the stimulation of the air causes it to contract, resulting in an increased tension within the vessel. In many diseased conditions the arterial tension is greatly increased-[e.g., in Bright's disease, where the kidney is contracted ("granular"), and where the left ventricle is hypertrophied].

In all these conditions increased arterial tension is indicated by the dicrotic wave being less high and less distinct, while with diminished arterial tension it is a larger and apparently more independent elevation. Moens has shown that the time between the primary elevation and the dicrotic wave increases with increase in the diameter of the tube, with diminution of its thickness, and when its coefficient of elasticity diminishes.

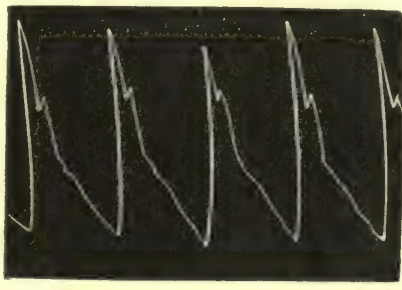

Fig. 85 .

Aortic regurgitation.

[The dicrotic wave is absent or but slightly marked in cases of atheroma and in aortic regurgitation (fig. 85). In this fig. observe also the vertical character of the up-stroke.]

Elastic Elevations.-Besides the dicrotic wave, a number of small less-marked elevations occur in the course of the descent in a sphygmogram (fig. 83,e,e). These elevations are caused by the elastic tube being thrown into vibrations by the rapid energetic pulse-wave, just as an elastic membrane vibrates when it is suddenly stretched. The artery also executes vibratory movements when it passes suddenly from the distended to the relaxed condition. These small elevations in the pulsecurve, caused by the elastic vibrations of the arterial wall, are called "elastic elevations" by Landois.

(1) The elastic vibrations increase in number in one and the same artery with the degree of tension of the elastic arterial wall. A very high tension occurs in the cold stage of intermittent fever, in which case these elevations are well marked.

(2) If the tension of the arterial wall be greatly diminished, these elevations may disappear, so that, while diminished tension favours the production of the dicrotic wave, it acts in the opposite way with reference to the "elastic elevations." (3) In diseases of the arterial walls affecting their elasticity, these elevations are either greatly diminished or entirely abolished. (4) The farther the arteries are distant from the heart, the higher are the elastic elevations. (5) When the mean pressure within the arteries is increased by preventing the outflow of blood from them, the elastic vibrations are higher and nearer the apex of the curve. (6) They vary 
in number and length in the pulse-curves obtained from different arteries of the body.

When the arm is held in an upright position, after five minutes the blood-vessels empty themselves, and collapse, while the elasticity of the arteries is diminished.

69. Dicrotic Pulse.-Sometimes during fever, especially when the temperature is high, at dicrotic pulse may be felt, each pulse-beat, as it were, being composed of two beats (fig. $83, \mathrm{X}$ ), one beat being large and the other small, and more like an after-beat. Both beats correspond to me beat of the heart. The two beats are quite distinguishable by the touch. The phenomenon is only an exagrerated condition of what occurs in a normal pulse. The sensible

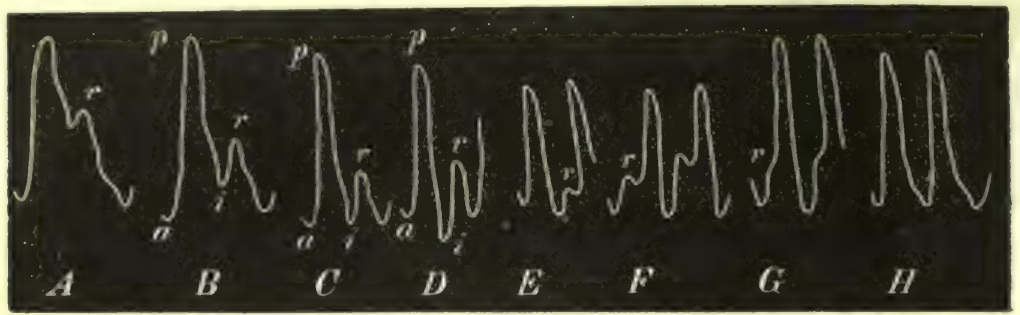

Fig. 86 .

Development of the Pulsus dicrotus--P. caprizans; P. monocrotus.

serond beat is mothing more than the greatly incicased dicrotic elevation, which, under ordinary conditions, is not felt by the finger.

Conditions. - The occurrence of a dicrotic pulse is favoured (1) by a short primary pulse. wave, as in fevers, where the heart beats rapidly.

(2) By diminishel arterial tension. A short systole and diminished arterial blood-pressure are the most farourable conditions for causing a dicrotic pulse. [So that dicrotism is best marked in a soft pulse.] The double beat may be felt only at certain jarts of the arterial system, whilst at other parts only a single beat is felt. A favourite site is the radial artery of one or other side, where conditions favourable to its occurrence appear to exist. This seems to be due to a local diminution of the blood-pressure in this area, owing to the paralysis of its vasomotor nerves (Landois). If the tension be increased by compresisng other large arterial trunks or the veins of the part, the double beat becomes a simple pulse-beat. The dicrotic pulse in fever seems to he due to the increased temperature $\left(39^{\circ}\right.$ to $40^{\circ} \mathrm{C}$. $)$, whereby the artery is more distended, and the heart-beat is shorter and more prompt.

(3) It is absolutely necessary that the elasticity of the arterial wall be normal. The dicrotic pulse does not occur in old persons with atheromatous arteries.

Monocrotic Pulse. - In fig. 86, A, B, C, we observe a gradual passage of the normal radial curve, $\mathrm{A}$, into the dicrotic beat, $\mathrm{B}$, and $\mathrm{C}$, where the dicrotic wave, $r$, appears as an independent elevation. If the frequency of the pulse increases more and more in fever, the next following pulse-beat may oecur in the aseending part of the dicrotic wave, D, E, F, and it may even nceur close to the apex, ( $:$ (P. caprizans). If the next following beat occurs in the

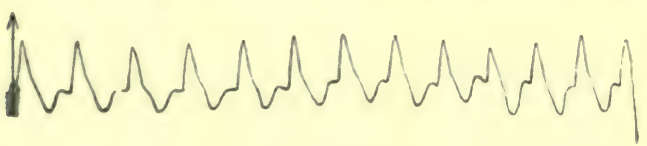

Fig. 87 .

Hyperdicrotic pulse. depression, $i$, between the primary elevation, $p$, and the dicrotic elevation, $r$, the latter entirely disappears, and the curve, $H$, assumes what Lan. dois calls the "monocrotic" type.

[Degrees of Dicrotism. - When the aortic notch reaches the respiratory or base line, the tidal wave having disappeared, the pulse is said to be fully dicrotic. When the aortic notch falls below the hase line, i.e., below where the up-stroke begins, the pulse is said to be hyperdicrotic (fig. 87 ). This form occurs during high fever $\left(104^{\circ}\right.$ F.), and is usually a grave sign, indicating exhaustion and the need for stimulants.]

70. CHARACTERS OF THE PULSE.- [The three factors concerned in the production of the pulse are, (1) the action of the heart, (2) the elasticity of the large vessels, (3) the resistance in the small arteries and capillaries. Any or all or several of these factors may be modified.] (1) Frequency. - Accorling as a greater or less number of beats occurs in a given time, e.g., per minute, the pulse is sail to be frequent or infrequent. The normal rate, in man $=71$ per minute, and somewhat more in the female; in fever it may exceed 120 (250 have been connted by Bowles), while in other diseases it may fall to 40 , and even 10 to 15 ; but such cases are rare, and are probably due to an affection of the cardiac nerves $(\$ 41)$. The frequency 
of the pulse is usually increased when the respirations are deeper, but not nore numerous, i.e., rapid shallow respirations do not affect the frequency of the pulse, but deep respirations do. [The frequency may be regular or irregular with regard to time.]

(2) Celerity or Rapidity. - If the pulse-wave is cleveloped, so that the distension of the artery slowly reaches its height, and the relaxation also takes place gradually, we have the p. tardus or slow or long pulse ; the opposite condition gives rise to the p. celer or quick or short pulse. The rapidity of the pulse is increased by quick action of the heart, power of expansion of the arterial walls, easy efflux of blood owing to the dilatation of the small arteries, and by nearness to the heart. [The quiekness has reference to a single pulse-beat, the frequency to a number of beats.] In a quick pulse, the curve is high and the angle at the apex is acute, while in a slow pulse the ascent is low and the angle at the apex is large.

(3) Conditions affecting the Pulse-Rate.-Frequency in Health. - In man the normal pulserate $=71$ to 72 beats per minute, in the female about 80 . In some individuals the pulse-rate may be higher (90 to 100), in others lower (50), and such a fact must be borne in miud.

(a) Age :-

\begin{tabular}{|c|c|c|c|c|c|c|c|}
\hline & $\begin{array}{l}\text { Beats per } \\
\text { Minute. }\end{array}$ & & & $\begin{array}{l}\text { Beats per } \\
\text { Minute. }\end{array}$ & & & $\begin{array}{l}\text { Beats per } \\
\text { Minute. }\end{array}$ \\
\hline Newly born, & - 130 to 140 & 5 years, & & 94 to 90 & 25 to 50 years, & & 70 \\
\hline year, & - 120 to 130 & 10, & • & about 90 & 60 years, . & - & \\
\hline years, & 105 & 10 to 15 years, & • & - $\quad 78$ & $80,, \quad \cdot$ & - & $\begin{array}{l}79 \\
\cdot \quad 79\end{array}$ \\
\hline , & 100 & 15 to 20 & - & 70 & 80 to 90 years, & & over 80 \\
\hline - & 97 & 20 to 25 & - & 70 & & & \\
\hline
\end{tabular}

(b) The length of the body has a certain relation to the frequency of the pulse. The following results have been obtained by Czarnecki from the formulre of Volkmann and Rameaux :-

Length of Body

in $10 \mathrm{~cm}$.

80 to 90 ,

90 to 100

100 to 110 ,

110 to 120 ,

120 to 130 ,

130 to 140 ,

\begin{tabular}{cc}
\multicolumn{3}{c}{ Pulse. } \\
Calculated. & Observe \\
90 & 103 \\
86 & 91 \\
81 & 87 \\
78 & 84 \\
75 & 78 \\
72 & 76
\end{tabular}

Length of Body in $10 \mathrm{~cm}$.

140 to 150 ,

150 to 160 ,

160 to 170 ,

170 to 180 ,

Above 180,
Pulse. Calculated. Observed $\begin{array}{ll}69 & 74\end{array}$ $67 \quad 68$ $65 \quad 65$ $63 \quad 64$ $60 \quad 60$

(c) The pulse-rate is increased by muscular activity, ly every increase of the arterial bloodpressure, by taking of food, increased temperature, painful sensations, by psychical disturbances, and [in extreme debility]. Increased heat, fever, or pyrexia increases the frequency, and as a rule the increase varies with the height of the temperature. [Dr Aitken states that an increase of the temperature of $1^{\circ} \mathrm{F}$. above $98^{\circ} \mathrm{F}$. corresponds with an increase of ten pulse-beats per minute; thus-

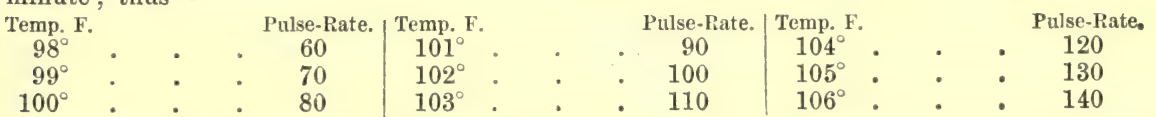

This is merely an approximate estimate.] It is more frequent when a person is standing than when he lies down. Music accelerates the pulse and increases the blood-pressure in dogs and men. Increased barometric pressure diminishes the frequency.

The Variation of the Pulse-Rate during the Day. -3 to 6 A.M. $=61$ beats ; 8 to $11 \frac{1}{2}$ A. M. $=74$. It then falls towards 2 P.M. ; towards 3 (at dinner-time) another increase takes place and goes on until 6 to 8 P.M. $=70$; and it falls until midnight $=54$. It then rises again towards 2 A.M., when it soon falls again, and afterwards rises as before towards 3 to 6 A.M.

[Pulse-Rate in Animals. - $($ Colin $)$.]

\begin{tabular}{|c|c|c|c|c|c|c|c|c|c|}
\hline Elephant, & & & $\begin{array}{c}\text { Per Min. } \\
25-28\end{array}$ & Lioness, & $\begin{array}{c}\text { Per Min. } \\
68\end{array}$ & Rabbit, & . & & $\begin{array}{l}\text { Per Min. } \\
120-150\end{array}$ \\
\hline Camel,. & & & $28-32$ & Tiger, & 74 & Mouse, . & & & 120 \\
\hline Giraffe, & • & . & 66 & Sheep, & $70-80$ & Goose, . & & & 110 \\
\hline Horse, . & . & . & $36-40$ & Goat, & $70-80$ & Pigeon, & . & & 136 \\
\hline $\mathrm{Ox}$ & . & . & $45-50$ & Leopard, . & 60 & Hen, . & & & 140 \\
\hline Tapir, . & . & . & 44 & Wolf (female), & 96 & Snake, . & ${ }^{\circ}$ & $v^{\circ}$ & 24 \\
\hline Ass, . & - & & $46-50$ & Hyæna, . & -55 & Carp, . & & & 20 \\
\hline Pig, . & - & & $70-80$ & Dog, & $90-100$ & Frog, . & - & - & 80 \\
\hline Lion, . & • & • & 40 & Cat, . & $120-140$ & Salamander & - & . & 77 \\
\hline
\end{tabular}

(4) Variations in the Pulse-Rhythm (Allorhythmia). - On applying the fingers to the normal pulse, we feel beat after beat occurring at-apparently equal intervals. Sometimes in a normal series a beat is omitted = pulsus intermittens, or intermittent pulse. [In feeling an intermittent pulse, we imagine or have the impression that a beat is omitted. This may be due to a reflex arrest of the ventricular contraction, caused by digestive derangement, in which case it has no great significance ; but if it be due to failure of the ventricular action, intermittent pulse 
is a serious symptom, being frequently present when the muscular walls are degenerated.] $\Lambda t$ other times the beats become smaller and smaller, and after a certain time begin as large as before $=p$. myurus. When an extra beat is intercalated in a normal series $=\mathbf{p}$. intercurrens.

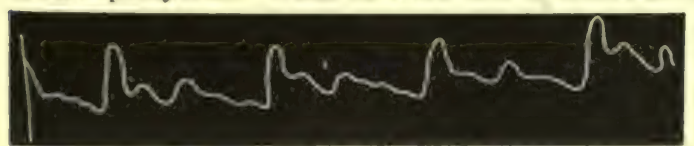

Fig. 88 .

Pulsus alternans.

The regular alternation of a high and a low beat $=$ p. alternans (fig. 88). In the $\mathbf{p}$. bigeminus of Traube the beats occur in pairs, so that there is a longer pause after every two beats. Traube found that he could produce this form of pulse in curarised dogs by stopping the artificial respiration for a long time. The p. trigeminus and quadrigeminus occur in the same way, but the irregularities oceur after every third and fourth beat. Knoll found that in animals such irregularities of the pulse were apt to occur, as well as great irregularity in the rhythm generally, when there is much resistance to the circulation, and consequently the heart has great demands upon its energy. The same occurs in man when an improper relation exists between the force of the 'Ariliac muscle and the work it has to do (Riegel). Complete irregularity of the heart's action is called arhythmia cordis.

71. VARIATIONS IN THE CHARACTERS OF THE PULSE.-Compressibility.-The relative strength or compressibility of the pulse ( $\mathrm{p}$. fortis and debilis), i.e., whether the pulse is strong or vecti, is estimated by the weight which the pulse is able to raise. A sphygmograph, provided with an index indicating the amount of pressure exerted upon the spring pressing upon the artery, may be usel (fig. 73). In this case, as soon as the pressure exerted upon the artery overomes the julse-beat, the lever ceases to move. The weight employal indicates the strength of the pulss. [The finger may be, and generally is used. The finger is pressed upon the artery intil the pulse-beat in the artery loyomel the joint of pressure is obliterated. In health it reyuires a pressure of several ounces to do this. Handfield Jones uses a sphygmometer for this furpose. It is constructed like a cylindrical letter-weight, and the pressure is exerted by means of a spiral spring which has heen carefully graduaterl.] The pulse is haid or soft when the artery, according to the mean blool-juressure, crives a feeling of greater or less resistance to the finger, and this quite independent of the energy of the individual pulse-beats (p. durus and mollis). In estimating the tension of the artery and the pulse, i.e., whether it is hard or soft, it is important to observe whether the artery has this quality only during the pulse-wave, $i . c$., if it is hard during diastole, or whether it is hard or soft during the period of rest of the arterial wall. All arteries are harder and less compressible during the pulse-beat than during the period of rest, but an artery which is very hard during the pulse-beat may be hard also during the fause between the pulse-beats, or it may be very soft, as in insufficiency of the aortic valves. In this case, after the systole of the left ventricle, owing to the incompetency of the aortic semilunar valves, a large amount of blood flows back into the ventricle, so that the arteries are thereby sudienly reniered partially empty. [The suilden collapse of the artery. gives rise to the characteristic " pulse of unfilled arteries" (fig. 85).]

Under similar conditions, the volume of the pulse is obvious from the size of the sphygmogram, so that we speak of a large and a small pulse ( $p$. magnus and parvus). Sometimes the julse is so thready and of such diminished volume that it can scarcely be felt. $\Lambda$ large pulse occurs in disease when, owing to hypertrophy of the left ventricle, a large amount of blood is forced into the aorta. A small pulse orcurs under the opposite condition, when a small amount of blood is forced into the aorta, either from a diminution of the total amount of the blood, or from the aortic orifice being narrowed [aortic stenosis], or from disease of the mitral valve; again, where the ventricle contracts feebly, the pulse becomes small and thready.

Compare the two radials. Sometimes the pulse differs on the two sides, or it may be absent on one sidle. [The pulse-wave in the two radials is often different when an aneurism is present on one side.]

Angiometer. - Waldenburg constructed a "pulse-clock" to register the tension, the diameter of the artery, and the volume of the pulse upon a clial. It does not give a graphic tracing, the results being marked by the position of an indicator.

72. THE PULSE-CURVES OF VARIOUS ARTERIES.-1. Carotid (fig. 83, I, II, III ; fig. $93, \mathrm{C}$ and $\mathrm{C}_{1}$ ). The ascending part is very steep-the apex of the curve (fig. $83, \mathrm{P}$ ) is sharp and high. Below the apex there is a small notch-the "aortic notch" (fig. 83, K) - which depends on a positive wave formed in the root of the aorta, owing to the closure of the aortic valves, and propagated with almost wholly undiminished energy into the carotid artery. Quite close to this notch, if the curve be obtained with minimal friction, the first elastic vibration occurs (fig. 83, II, e). Above the middle of the descending part of the curve is the dicrotic elevation, $R$, produced by the reflection of a positive wave from the already closed semi-lunar valves. The dicrotic wave is relatively small on account of the high tension in the carotid artery. After this the curve falls rapidly, but in its lowest third two small elevations may be seen. Of these the former is due to elastic vibration. The latter represents a second dicrotic 
wave fig. 83 , III, R). Here there is a true tricrotism, which is more easily obtained from the carotid on account of the shortness of the arterial channel.

2. Axillary Artery (fig. 83, IV). In this curve the ascent is very steep, while in the descent near the apex there is a small (aortic) elevation, $\mathbf{K}$, caused by a positive wave, produced by the closure of the aortic valves. Below the middle there is a tolerably high dicrotic elevation, R, higher than in the carotid curve; because in the axillary artery the arterial tension is less, and permits a greater development of the dicrotic wave. Further on, two or three sunall elastic vibrations occur, $e, e$.

3. Radial Artery (fig. 78 ; fig. $83, \mathrm{~V}$ to $\mathrm{X}$; fig. $93, \mathrm{R}$ and $\mathrm{R}_{1}$ ). The line of ascent (fig. 83) is tolerably high and sudden-somewhat in the form of a long $f$. The apex, $\mathrm{P}$, is well marked. Below this, if the tension be high, two elastic vibrations may occur $(\mathrm{V}, e, e)$, but if it be low only one (VI to IX, $e$ ). About the middle of the curve is the well-marked dicrotic elevation, $\mathrm{R}$. This wave is least pronounced in a small hard pulse, and when the artery is much distended (fig. 83, VII, $\mathrm{R}_{1}$ ); it is larger when the tension is low (fig. $83, \mathrm{IX}, \mathrm{R})$, and is greatest of all when the pulse is dicrotic (X, R). Two or three small elastic elevations occur in the lowest part of the curve.

4. Femoral Artery (fig. 83, XI, XII). The ascent is steep and high-the apex of the curve is not unfrequently broad, and in it the closure of the aortic valves $(\boldsymbol{K})$ is indicated. The curve falls rapidly towards its lowest third. The dicrotic elevation, $\mathrm{R}$, occurs late after the beginning of the curve, and there are also small elastic elevations $(e, e)$.

5. Pedal Artery (fig. $83, \mathrm{XIV}, \mathrm{XV}$ ), and Posterior Tibial (fig. 89 and fig. 83, XIII). In pulse-curves obtained from these arteries, there are well-marked indications that the apparatus (heart) producing the waves is placed at a considerable distance. The ascent is oblique and low-the dicrotic elevation occurs late. Two elastic

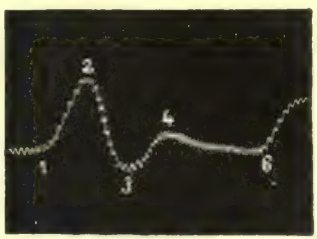

Fig. 89.

Curve of posterior tibial. Written by the angiograph upon a vibrating plate. vibrations (fig. 83, XIV, $e, e$ ) occur in the descent, but they are very close to the apex, while the elastic vibrations at the lower part of the curve are feebly marked. Fig. 89 is from the posterior tibial. When measured, it gives the following result :-

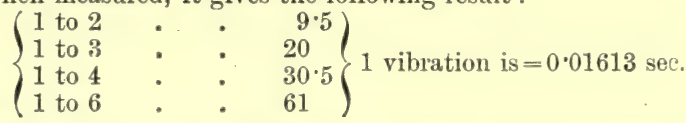

73. ANACROTISM. - As a general rule, the line of ascent of a pulse-curve has the forni of an $f$, and is nearly vertical. The arterial walls are thrown into elastic vibration by the pulse-beat, and the number of vibrations depends greatly upon the tension of the arterial walls. The distension of the artery, or what is the same thing, the ascent of the sphygmogram, usually occurs so rapidly that it is equal to one elastic vibration. The elongated $f$-shape of the ascent is fundamentally just a prolonged elastic vibration. When the number of vibrations causing the elastic variation is small, and when the line of ascent is prolonged, two elevations occasionally occur in the line of ascent. Such a condition may occur normally (fig. 83, VIII, at 1 and $2 ; X$, at 1 and 2 ). When a series of closely-placed elastic vibrations occur in the upper
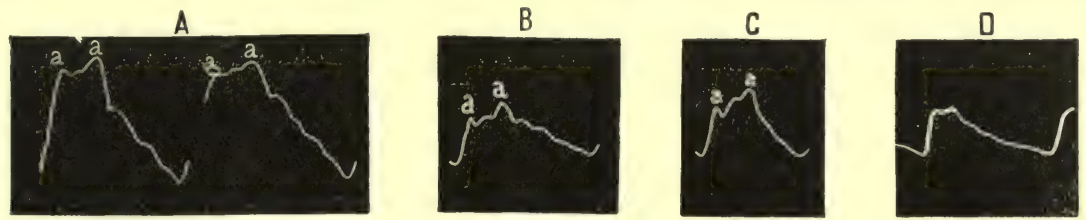

Fig. 90.

Anacrotic radial curves. $\alpha, \alpha$, the anacrotic parts.

part of the line of ascent, so that the apex appears dentate and forms an angle with the line of ascent, then the condition becomes one of anacrotism (fig. 90, $\alpha, \alpha$ ), which, when it is so marked, may be characterised as pathological. Anacrotism of the pulse occurs when the time of the influx of the blood is longer than the time occupied by an elastic vibration. Hence it takes place :-

(1) In dilatation and hypertrophy of the left ventricle, e.g., fig. 90, A, a tracing from the radial artery of a man suffering from contracted kidney. The large volume of blood expelled with each systole requires a long time to dilute the tense arteries.

(2) When the extensibility of the arterial wall is diminished, even the normal amount of blood expelled from the heart at every systole requires a long time to dilate the artery. This occurs in old people where the arteries tend to become rigid, e.g., in atheroma. Cold also 
stimulates the arteries, so that they become less extensile. Within one hour after a tepid bath, the pulse assumes the anacrotic form (fig. 90, D) (G. v. Licbig).

(3) When the blood stagnates in consequence of great diminution in the velocity of the blood-stream, as occurs in paralysed limbs, the volume of blood propelled into the artery at every systole no longer produces the normal distension of the arterial coats, and anacrotic notches oceur (fig. $90, \mathrm{~B}$ ).

(4) After ligature of an artery, when blood slowly reaches the peripheral part of the vessel through a relatively small collateral cireulation, it also occurs. If the brachial artery be compressed so that the blood slowly reaches the radial, the radial pulse may become anacrotic. It often oecurs in stenosis of the aorta, as the blood has difficulty in getting into the aorta (fig. $90, \mathrm{C})$.

Recurrent Pulse.--If the radial artery be compressed at the wrist, the pulsebeat reappears on the distal side of the point of pressure through the arteries of the palm of the hand (Janumd, I'idert). The curve is anacrotic, and the dicrotic wave is diminished, while the elastic elevations are increased.

(5) A special form of anacrotism oecurs in cases of well-marked insufficiency of the aortic valves. Practically, in these cases, the aorta remains permanently open. The contraction of the left auricle causes in the blood a wave-motion, which is at once propagated through the open mouth of the aorta into the large blood-vessels. This wave is followed by the wave caused by the contraction of the liypertrophied left ventricle, but of course the former wave is not so large as the latter. In insuffieiency of the aortic valves, the auricular wave occurs before the ventricular wave in the ascenting part of the curve. The auricular is well marked only in the large vessels, for it soon becomes lost in the peripheral vessels. Fig. 91, I, was obtained from

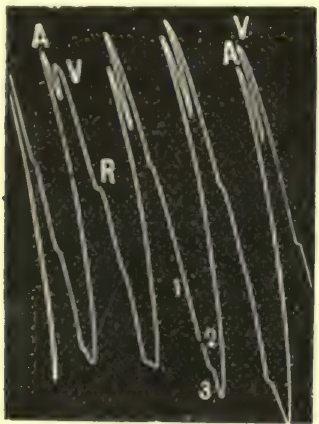

I.

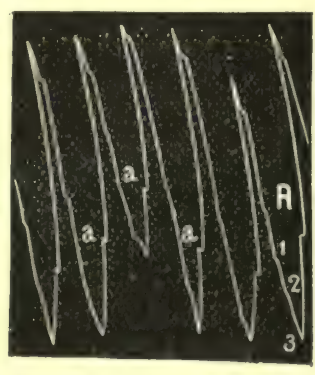

II.
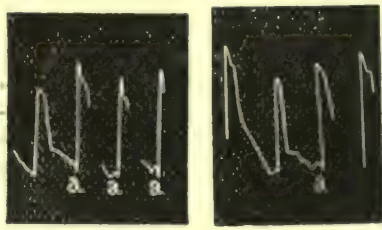

III.

Fig. 91.

I., 11., I1I., curves with anacrotic elevations , in insufficiency on the aortic valves.

the carotid of a man suffering from vell-marked insufficiency of the aortic valres, with con siderable hypertrophy of the left ventricle and left auricle. The ascent is steep, caused by the force of the contracting heart. In the apex of the curve are two projections; $\mathrm{A}$ is the anacrotic auricular wave, and $V$ is the ventricular wave. Fig. 91, II, is a curve obtained from the sub. clavian arfery of the same individual. In the femoral artery the auricular projection is only obtainel when the friction of the writing-style is reduced to the minimum, and when it occurs it immediately precedes the beginning of the ascent (fig. 86 , III, $a$ ). The pulse-curve, in cases of aortic insufficiency, is also characterised by--(1) its considerable height; (2) the rapid fall of the lever from the apex of the curve, because a large part of the blood which is forced into the aorta regurgitates into the left ventricle when the ventricle relaxes; (3) not unfrequently a projection occurs at the apex, due to the elastic vibration of the tense arterial wall; (4) the dierotie wave $(\mathbf{R})$ is small compared with the size of the curve itself, because the pulse-wave, owing to the lesion of the aortic valves, has not a sufficiently large surface to be reflected from (fig. 85). The great height of the curve is explained by the large amount of blood projected into the aortic system by the greatly hypertrophied and dilated ventricle.

74. INFLUENCE OF RESPIRATION ON THE PULSE-CURVE.-The respiratory movements influence the pulse (1) in a purely physical way. Stated broadly, the blood-pressure rises during inspiration and falls during expiration, but when we consider the effect on the pulse-curve, it is found that it varies with the depth, rapidity, and ease of respiration: (2) the respiratory movements are 
accompanied by stimulation of the vasomotor centre, which produces variations of the blood-pressure.

1. Normal Respiration.-Fig. 92 shows what sometimes, but by no means always, happens. During inspiration, owing to the dilatation of the thorax, more arterial blond is retained within the chest, while at the same time venous blood is

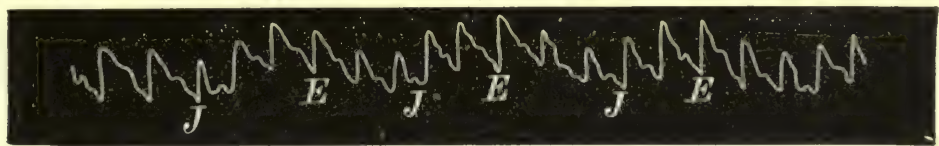

Fig. 92.

Influence of the respiration upon the pulse. J, inspiration; E, expiration.

sucked into the right auricle by the aspiration of the thorax; as a consequence of this, the tension in the arteries during inspiration must be less. The diminution of the chest during expiration favours the flow in the arteries, while it retards the flow of the venous blood in the venæ cavæ, two factors which raise the tension in the arterial system. The difference of pressure explains the difference in the form of the pulse-curve obtained during inspiration and expiration, as in fig. 92 and fig. 83, I, III, IV, in which $J$ indicates the part of the curve which occurred during inspiration, and $\mathrm{E}$ the expiratory portion. The following are the points of difference:-(1) The greater distension of the arteries during expiration causes all the parts of the curve occurring during this phase to be higher; (2) the line of ascent is lengthened during expiration, because the expiratory thoracic movement helps to increase the force of the expiratory wave; (3) owing to the increase of the pressure, the dicrotic wave must be less during expiration; (4) for the same reason the elastic elevations are more distinct and occur higher in the curve near its apex. The frequency of the pulse is slightly greater during expiration than during inspiration.

2. This purely mechanical effect of the respiratory movements is modified by the simultaneous stimulation of the vasomotor centre which accompanies these movements. At the beginning of inspiration the blood-pressure in the arteries is lowest, but it begins to rise during inspiration, and increases until the end of the inspiratory act, reaching its maximum at the beginning of expiration; during the remainder of the expiration the blood-pressure falls until it reaches its lowest level again at the beginning of inspiration (compare $\$ 85, f^{\circ}$ ); the pulse-curves are similarly modified, and exhibit the signs of greater or less tension of the arteries corresponding to the phases of the respiratory movements. [There is, as it were, a displacement of the blood-pressure curve relative to the respiratory curve.]

Forced Respiration.-With regard to the effect produced on the pulse-curve by a powerful expiration and a forced inspiration, observers are by no means agreed.

Valsalva's Experiment.-Strong expiratory pressure is best produced by closing the mouth and nose, and then making a great expiratory effort $(\S 60)$; at first there is increase of the blood-pressure, while the form of the pulse-waves resembles that which occurs in ordinary expiration, the dicrotic wave being less developed; but, when the forced pressure is long continued, the pulse-curves have all the signs of diminished tension. This effect is due to the action of the vasomotor centre, which is affected reflexly from the pulmonary nerves. We must assume that forced expiration, such as occurs in Valsalva's experiment, acts by depressing the activity of the vasomotor centre ( $\$ 371$, II.). Coughing, singing, and declaiming act like Valsalva's experiment, while the frequency of the pulse is increased at the same time. After the cessation of Valsalva's experiment, the blood-pressure rises above the normal state (Sommerbrodt), almost as much as it fell below it; the normal condition being restored within a few minutes (Lenzmann). 
Muller's Experiment.-When the thorax is in the expiratory phase, close the mouth and nose, and take a deep inspiration so as forcibly to expand the chest $(\$ 60)$. At first the pulse-curves have the characteristic signs of diminished tension, viz., a higher and more distinct dicrotic wave; then the tension can, by nervous influencęs, be increased, just as in fig. 93 , where $\mathrm{C}$ and $\mathrm{R}$ are tracings taken from
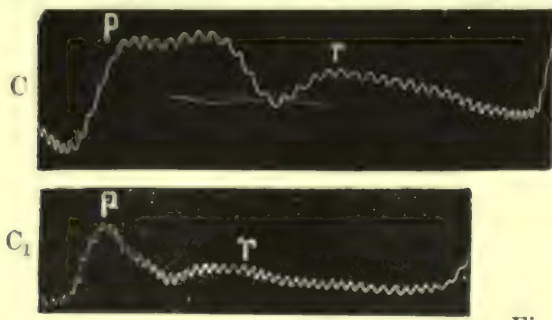
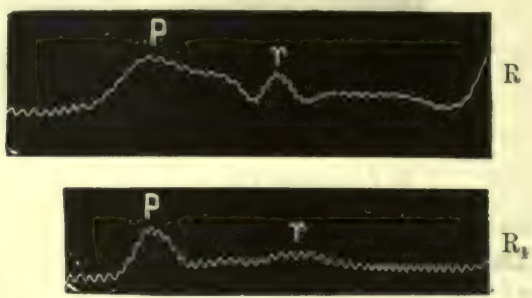

Fig. 93.

( ; curse from the carotid, and $R$, raclial, during Mïller's experiment; $C_{1}$ and $R_{1}$, during Valsalva's experiment. Curves written on a vibrating surface.

the carotid and radial arteries respectively, during Müller's experiment, in which the dicrotic waves, $r, r$, indicate the diminished tension in the vessels. In $\mathrm{C}_{1}$ and $\mathbf{R}_{1}$, taken from the same person during Valsalva's experiment, the opposite condition occurs.

Compressed Air.-On cxpiring into a vessel resembling a spirometer (see Respiration), (Walclenhurg's respiration apparatus), and filled with compressed air, the same result is obtained as in Valsalva's experiment - the blood-pressure falls and the pulse-beats increase; conversely, the inspiretion from this apparatus of air under less pressure acts like Miiller's experiment, i.e., it increases the effect of the inspiration, and afterwards increases the blood-pressure, which may either remain inereased on continuing the experiment, or may fall (Lenzmann).

The inspirution of compressed air diminishes the mean blood-pressure (Zuntz), and the aftereffect cuntinues for some time. The pulse is more frequent both during and after the experiment. Expirmtion in rarefied air increases the blood-pressure. The effects which depend upon the artion of the nervous system do not occur to the same extent in all cases. Exposure to. compressed air in a pneumatic cabinet lowers the pulse-curve, the elastic vibrations become indistinct, and the dicrotic wave diminishes and may disappear ( $v$. Vivenot). The heart's beat is slowed and the blood-pressure raised (Bert). Exposure to rarefied air causes the opposite result, which is a sign of diminished arterial tension.

Pulsus Paradoxus. - Under pathological conditions, especially when there is union of the heart or its large vessels with the surrounding parts, the pulse during inspiration may be

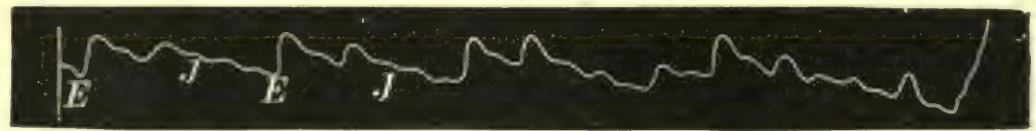

Fig. 94 .

Pulsus paradoxus (after Kussmaul). E, expiration ; J, inspiration.

extremely small and changed, or may even be absent (fig. 94). This condition has been called pulsus paradoxus (Griesinger, Kussincul). It depends upion a diminution of the arterial lumen during the inspiratory movement. Even in health, it is possible by a change of the inspiratory movement to produce the p. paradoxus (Riegel, Sommerbrodt).

75. INFLUENCE OF PRESSURE ON THE PULSE-CURVE.-It is most important to know the actual pressure which is applied to an artery while a sphygmogram is being taken. The changes affect the form of the curve as well as the relation of individual parts thereof. In fig. $95, a, b, c, d, e$ are radial curves ; $a$ was taken with minimal pressure, $b$ with $100, c 200, d 250$, and $e 450$ grams pressure, while A, B, C, D show the relations as to the time of occurrence of the individual phenomena where the weight was successively increased. The study of these curves yields the following results :-(1) When the weight is small, the dicrotic wave is relatively less; the whole curve is high; (2) with a moderate weight (100 to 200 grams) the dicrotic wave is best marked, the whole curve is somewhat lower; (3) on increasing the weight the size of the licrotic wave again diminishes; $(4)$ the fine elastic vibrations preceding the dicrotic wave appear first when a weight of 220 to 300 grams is used; (5) the rapidity of the 
pulse changes with increasing weight, the time occupied by the ascent becoming shorter, the descent becoming longer ; $(6)$ the height of the entire curve decreases as the weight increases. In every sphygmogram the pressure under which it was obtained ought always to be stated. In fig. 95, A, B are curves obtained from the radial artery of a healthy student. The pressure exerted upon the artery for A was $100 ; \mathrm{B}, 220 \mathrm{grms}$. (1 vibration $=0.01613 \mathrm{sec}$.).

If pressure be exerted upon an artery for a long time, the strength of the pulse is gradually increased. If, after subjecting an artery to considerable pressure, a lighter weight be used, not
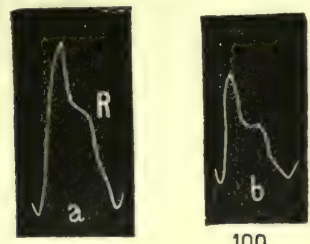

100

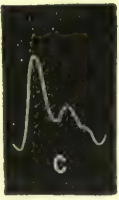

200

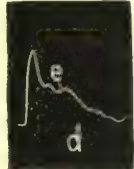

250

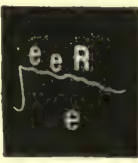

450
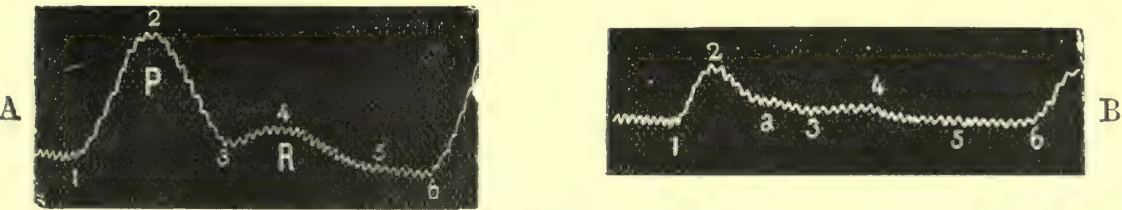

Fig. 95 .

Various forms of curves (radial) obtained by gradually increasing the pressure.

tunfrequently the pulse-curve assumes the form of a dicrotic pulse, owing to the greater development of the dicrotic elevation. When strong pressure is applied, the blood is forced to find its way through collateral channels. When the chief artery ceases to be compressed, the total area is, of course, considerably and suddenly enlarged, which results in the production of a dicrotic elevation. Fig. 83, X, is such a dicrotic curve obtained after considerable pressure had been applied to the artery.

76. TRANSMISSION OF PULSE-WAVES.-The pulse-wave proceeds throughout the arterial system from the root of the aorta, so that the pulse is felt sooner in parts lying near the heart than in the peripheral arteries. E. H. Weber calculated the velocity of the pulse-wave as $9 \cdot 240$ metres [28 $\frac{1}{2}$ feet] per second, from the difference in time between the pulse in the external maxillary artery and the dorsal artery of the foot. Czermak showed that the elasticity was not equal in all the arteries, so that the velocity of the pulse-wave cannot be the same in all. The pulse-wave is propagated more slowly in the arteries with soft extensile walls than in arteries with resistent and thick walls, so that it is transmitted more rapidly in the arteries of the lower extremities than in those of the upper. It is still slower in children.

77. PULSE-WAVE IN ELASTIC TUBES.--Waves similar to the pulse may be produced in elastic tubes. (1) According to E. H. Weber the velocity of propagation of the waves is $11 \cdot 205$ metres per sec.; according to Donders, 11-13 metres (34-42 feet). (2) According to E. H. Weber increased internal tension causes only an inconsiderable decrease ; Rive found a great decrease; Donders found no obvious difference; while Marey found an increased velocity. (3) Donders found the velocity to be the same in tubes $2 \mathrm{~mm}$. in diameter as in wider tubes, but Marey believes that the velocity varies when the diameter of the tube changes. (4) The velocity is less, the smaller the elastic coefficient. (4) The velocity increases with inereased thickness of the wall, while it diminishes when the specific gravity of the fluid increases.

Moens has recently formulated the following laws as to the velocity of propagation of waves in elastic tubes:-(1) It is inversely proportional to the square root of the specific gravity of the fluid; (2) it is as the square root of the thickness of the wall, the lateral pressure being the same; (3) it is inversely as the square root of the diameter of the tube, the lateral pressure being the same; (4) it is as the square root of the elastic coefficient of the wall of the tube, the lateral pressure being the same (Valentin).

(A) The velocity of the wave is 11.809 metres per second.

(B) The intra-vascular pressure has a decided influence on the velocity: thus, in the tube, 
$A$, with $18 \mathrm{~cm}$. ( $\mathrm{Hg}$.) pressure, the velocity per metre $=0.093$ second, while with $21 \mathrm{~cm}$. pressure (Hg.) $=0.095$ second per metre.

(C) The specific grarity of the liquid influences the velocity of the pulse-wave. In mercury the wave is propagated four times more slowly than in water.

(D) The velocity in a tube which is more rigid and not so extensile is greater than in a tube which is easily distended.

78. VELOCITY OF THE PULSE-WAVE IN MAN. - Landois obtained the following results in a student:-Difference between carotid and ralial $=0.074$ second (the distance being takeu as 62 (entimetres); carotid and femoral $=0.068$ second ; femoral (inguinal region) and posterior tibial $=0.097$ second (distance estimated at 91 centimetres). [Waller obtained between the heart and earotid $0 \cdot 10$ second : heart and femoral, 0.18 sec.; heart and dorsalis pedis, 0.22 .]

The velocity of the pulse-wave in the arteries of the upper extremities $=8 \cdot 43$ metres per second, and in those of the lower extremity 9.40 metres per second, [i.e., about 30 feet per second]. The velocity is greater in the less extensile arteries of the lower extremities than in those of the upper limb. For the same reason it is les: in the peripheral arteries and in the yielding arteries of children (Czermak).

E. H. Weber estimated the relocity at 9.24 metres per second; Garrod, 9-10.8 metres; (irashey, $8 \cdot 5$ metres: Noens, $8 \cdot 3$ metres, and with diminished pressure during Valsalva's experiment $7 \cdot 3$ metres $(\$ 60, \S 74)$.

Influencing Conditions. - In animals, hemorrhage, slowing of the heart produced by stimulation of the vagus (1/oens), section of the spinal cord, deep morphia-narcosis, and dilatation of the blowl-vessels by heat, produce sluriniy of the velocity, while stimulation of the spinal cord mecelerefes it (Grunimach).

The wave-length of the pulse-wave is obtained by multiplying the duration of the inflow of blood into the aorta $=0.08$ to 0.09 second $(\$ 51)$, by the velocity of the pulse-wave.

Method. - Place the knolis of two tambours (fig. 76) upon the two arteries to be investigated, or place ouf orer the alex-heat and the other upon an artery. These receiving tambours are connected with two registering tambours, as in Brondgeest's pansphygmograph ( $\$ 67$, fig. 76 ), so that their writing-levers are directly over each other, and so arranged as to write simultanewusly on onc vibrating plate attached to a tuning-fork. [Or they may be made to write upon a revolving cylinder, whose rate of movement is ascertained by causing a tuning-fork of a known

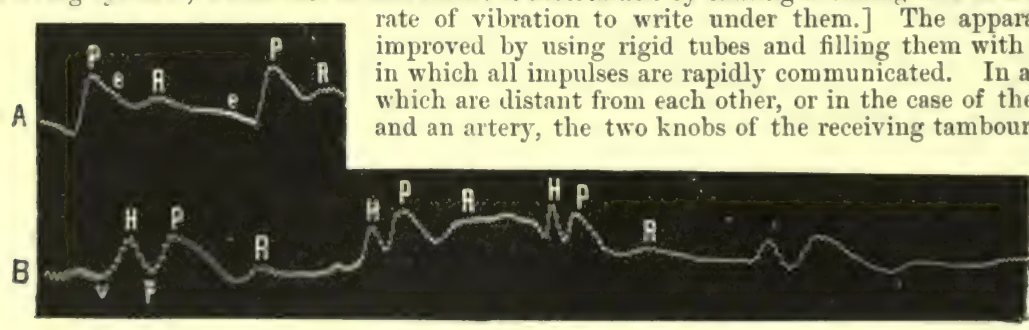

Fig. 96.

A, curve of ralial artery on a vibrating surface $(1$ vil. $=0.01613 \mathrm{sec}$ ) $; \mathrm{P}$, apex of curve $; e, e$, elastic vibrations; $R$, dicrotic wave. $B$, curve of same radiai taken along with the heartbeat ; $r, \mathrm{H}, \mathrm{P}$, contraction of the ventricle.

be connected by means of a $\mathrm{Y}$-tube with one writing-lever. In fig. 96, B is a curve from the radial artery taken in this way. In it $v \mathrm{H}$ P indicates contraction of the ventricle; $\mathrm{H}$, the apex of the ventricular coutraction; $\mathrm{P}$, the primary apex of the radial curve; $v$, the beginning of the ventricular contraction; $p$, of the radial pulse. A is the curve of the radial artery alone. From these curves it is evident that in this instance nine vibrations occur between the beginning of the ventricular contraction and the beginning of the pulse in the radial artery $=$ $0 \cdot 15 \mathrm{sec}$.

In fig. 97 the difference between the carotid and the posterior tibial pulse $=0.137$ sec.

Pathological. - In cases of diminished cotensibility of the arteries, $e . g_{\text {. }}$, in atheroma $(\S 77, \mathrm{D})$, the julse-wave is propagated more rapidly. Local dilatations of the arteries, as in aneurisms, cause a retardation of the wave, and a similar result arises from local constrictions. Relaxation of the walls of the vessels in high fever retards the movement (Hamernjk).

79. OTHER PULSATILE PHENOMENA. - 1. In the mouth and nose, when they are filled with air, and the glottis closed, pulsatile phenomena (due to the arteries in their soft parts), 
may be found communicating a movement to the contained air. The curves obtained are relatively small, and closely resemble the curve of the carotid. A similar pulse is obtained in the tympanum with intact membraua tympani, and when the soft parts of the tympanum are congested (Schwartze, Tröltsch).

2. Entoptical Pulse.-After violent exercise, an illumination, corresponding to each pulse beat, occurs on a dark optical field. When the optical field is bright, an analogous darkening

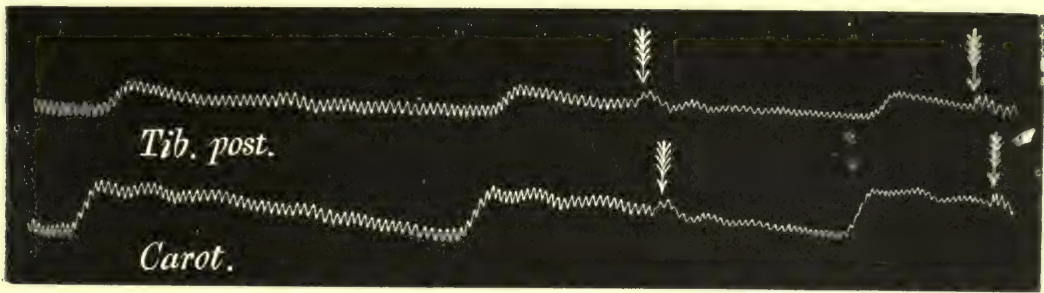

Fig. 97.

Curves of the carotid and posterior tibial taken simultaneously with Brondgeest's pansphygmograph writing upon a vibrating-plate attached to a tuning-fork. The arrows indicate tlet identical moment of time in each curve.

occurs. The ophthalmoscope occasionally reveals pulsation of the retinal arteries (Jïgci), which becomes marked in insufficiency of the aortic valves.

3. Pulsatile Muscular Contraction. - The orbicularis palpebrarum muscle contracts undel similar conditions synchronously with the pulse; and it is perhaps due to the pulse-beat exciting the sensory nerves reflexly. The Brothers Weber found that not unfrequently, while walking, the step and pulse gradually and involuntarily coincide.

4. When the legs are crossed as one sits in a chair, the leg which is supported is raised witl each pulse-beat, and it gives also a second or dicrotic elevation.

5. If, while a person is quite quiet, the incisor teeth of the lower jaw be made just to touch the upper incisors very lightly, we detect a double beat of the lower acrainst the upper teeth, owing to the pulse-beat in the external maxillary artery raising the lower jaw. The second elevation is due to the closure of the semi-lunar valves, and not to a dicrotic wave.

6. Brain and Fontanelles. - The large arteries at the base of the brain communicate a movement to it, while similar movements oceur with respiration-rising during expiration and falling during inspiration. These movements are visible in the fontanelles of infonts. The respiratory movements depend upon variations in the amount of blood in the veins of the cranial cavity, and also upon the respiratory variations of the blood-pressure.

7. Amongst pathological phenomena are $(a)$ the beating in the epigastrium, e.g., in hypertrophy of the right or left ventricle, cansed, it may be, by deep insertion of the diaphragm, and it may be, partly, by the beating of a dilated abdominal aorta or cœliac axis.

(b) Aneurisms or abnomal dilatations of the arteries cause an abnormal pulsation, while they

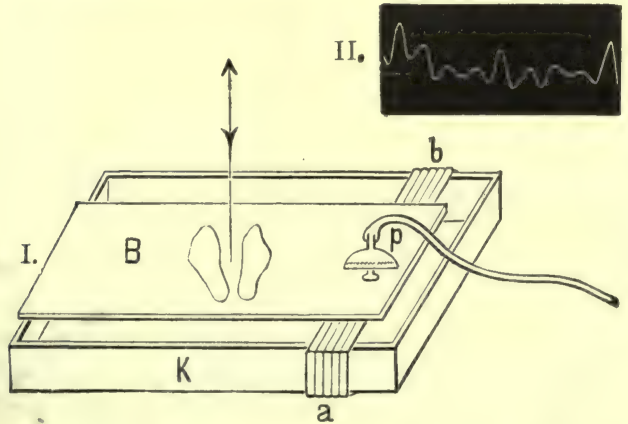

III.

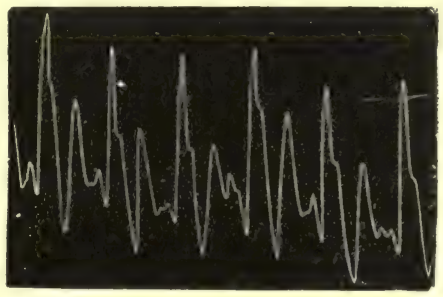

Fig. 98 .

I. Elastic support for registering the molar motions of the body-K, wooden box; B, feet of patient; $p$, cardiograph ; $\alpha, b$, elastic tubing. II. Vibration curves of a healthy person. III. Curve obtained from a patient with insufficiency of the aortic valves and great hypertrophy of the heart.

produce a slowing in the velocity of the pulse-vave in the corresponding artery. Hence the pulse appears later in such an artery than in the artery on the healthy side. Hypertrophy and, 
dilatation of the left ventricle cause the arteries near the heart to pulsate strongly. In the analogous condition of the right ventricle, the beat of the pulmonary artery may be seen and felt in the second left intercostal space.

80. VIBRATIONS OF THE BODY DUE TO THE HEART. - The beating of the heart and large arteries communicates vibrations to the body as a whole; the vibration being not simple but compound. Gordon was the first to represent this pulsatory vibration graphically. If a person be placed in an erect attitude in the scale-pan of a large balance, the index oscillates, and its movements coincide with the heart's movements.

Method. - Take a long four-sided box, $\mathbf{K}$, open at the top, and arrange several coils, $a, b$, of stout caoutchouc tubing round oue end (fig. 98). A wooden board, B, is so placed that it rests with one end on the caoutchouc tubing, and with the other on the narrow end of the box. The person to be experimented upon, A, stands vertically and firmly on this board. A receiving tambour, $p$, is placed against the surface of the board next the elastic tube, which registers the vibrations of the foot-support. Fig. III. is a curve showing such vibrations, each heart-beat being followed in this case by four oscillations. To ascertain the relations and causes of these vibrations, it is necessary to obtain, simultaneously, a tracing of the heart and the vibratory 'urve. For this purpose use the two tambours of Brondgeest's pansphygmograph $(\S 67,76)$, placing one knob or pad over the heart and the other on the foot-support, and allow the writingtambours to inseribe their vibrations on a glass plate attached to a tuning-fork.

In the locer or curdiuc impulse curce (fig. 99), the rapidly-rising part is due to the ventricular

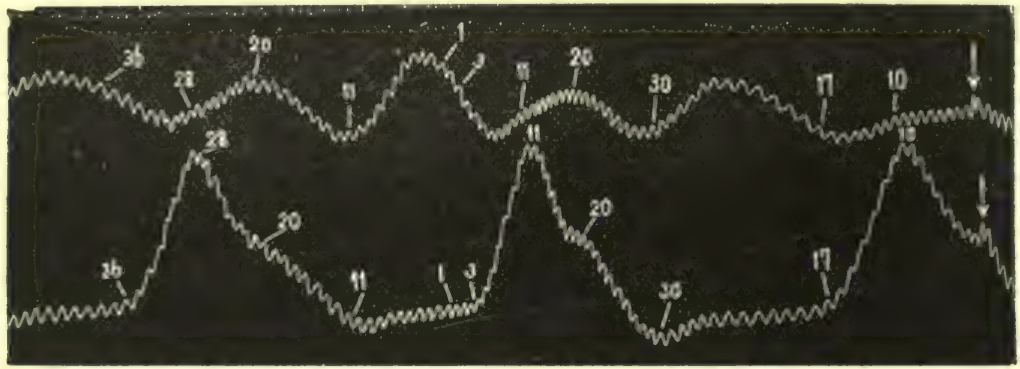

Fig. 99.

The upper curve is the vibration-curve of a healthy person, and the lower one a tracing of the apex-beat.

systole. It contains eight vibrations ( 1 vib. $=0.01613 \mathrm{sec}$.). The beginning of the ventricular systole is indicated in the fig. by $-36,-3,-17$.

If the corresponding numbers in the upper or vibratory curve are studied, it is obvious that "t the moment of ventricular systole the body makes a downward vibration, i.e., it exercises greater pressure upon the foot-support. Gordon interprets his curve as giving exactly the opposite result. This downward motion, however, lasted only during five vibrations of the tuning-fork : during the last three vibrations, corresponding to the systole, there is an ascent of the body corresponding to a less pressure upon the foot-plate. When the ventricle empties itself, it undergoes a movement in a downward and outward direction-Gutbrodt's "reaction impulse."

In the upper curve analogous numbers are employed to indicate the vibrations occurring simultaneously, viz., $-28,-11,-10$. The closure of the semi-lunar valves is well marked in the three heart-beats at $20,-20$. This closure is indicated in analogous points in both curves, after which there is a descent of the foot-support, and this corresponds to the downward propagation of the pulse-wave through the aorta to the vessels of the feet.

Pathological.-In insufficiency of the aortic valves, as shown in fig. 98, III., the vibration communicated to the body is very considerable.

81. THE BLOOD-CURRENT.-Canse.-The closed and much-branched vascular system, whose walls are endowed with elasticity and contractility, is not only completely filled with blood, but it is over-filled. The total volume of the blood is somewhat greater than the capacity of the entire vascular system. Hence it follows that the mass of blood must exert pressure on the walls of the entire system, thus causing a corresponding dilatation of the elastic vascular walls (Brunner). This occurs only during life; after death the muscles of the vessels relax, and fluid passes into the tissues, so that the blood-vessels come to contain less fluid, and some of them may be empty. 
If the blood were uniformly distributed throughout the vascular system and under the same pressure, it would remain in a position of equilibrium (as after death). If, however, the pressure be raised in one section of the tube, the blood will move from the part where the pressure is higher to where it is lower ; so that the bloodcurrent is a result of the difference of pressure within the vascular system. If either the aorta or the venæ cavæ be suddenly ligatured in a living animal, the blood continues to flow, but gradually more slowly, until the difference of pressure is equalised throughout the entire vascular system.

The velocity of the current will be greater the greater the difference of pressure, and the less the resistance opposed to the blood-stream.

The difference of pressure which causes the current is produced by the heart. Both in the systemic and pulmonary circulation the point of greatest pressure is in the root or beginning of the arterial system, while the point of lowest pressure is in the terminal portion of the venous orifices at the heart. Hence the blood flows continually from the arteries through the capillaries into the venous trunks. The heart keeps up the difference of pressure required to produce this result; with each systole of the ventricles a certain quantity of blood is forced into the beginning of the arteries, while at the same time an equal amount flows from the venous orifices into the auricles during their diastole (E. H. Weber).

Donders showed that the action of the heart not only causes the difference of pressure necessary to establish a blood-current, but also raises the mean pressure within the vascular system. The terminations of the veins at the heart are wider and more extensible than the arteries where they arise from the heart (fig. 133), As the heart propels a volume of blood into the arteries equal to that which it receives from the veins, it follows that the arterial pressure must rise more rapidly than the venous pressure diminishes, since the arteries are not so wide nor so extensible as the veins. Thus the total pressure must also increase.

Cause of Continuous Flow. - The volume of blood expelled from the ventricle at every systole would give rise to a jerky or intermittent movement of the bloodstream-(1) if the tubes liad rigid walls, as in such tubes any pressure exerted upon their contents is propagated momentarily throughout the length of the tube, and the motion of the fluid ceases when the propelling force ceases; (2) the flow would also be intermittent in character in elastic tubes if the time between two successive systoles were longer than the duration of the current necessary for the compensation of the difference of pressure caused by the systole. If the time between two successive systoles be shorter than the time necessary to equilibrate the pressure, the current will become continuous, provided the resistance at the periphery of the tube be sufficiently great to bring the elasticity of the tube into action. The more rapidly systole follows systole, the greater the difference of pressure becomes, and the more distended the elastic walls. Although the current thus produced is continuous, a sudden rise of pressure is caused by the forcing in of a mass of blood at every systole, so that with every systole there is a sudden jerk and acceleration of the blood-stream corresponding to the pulse (compare $\$ 64$ ).

This sudden jerk-like acceleration of the blood-current is propagated throughout the arterial system with the velocity of the pulse-wave : both phenonema are due to the same fundamental cause. Every pulse-beat causes a temporary rapid progressive acceleration of the particles of the fluid. - But just as the form-movement of the pulse is not a simple movement, neither is the pulsatile acceleration a simple acceleration. It follows the course of the development of the pulse-wave. The pulse-curve is the graphic representation of the pulsatory acceleration of the bloodstream. Every rise in the curve corresponds to an acceleration, every depression to a retardation of the current.

[Method: Rigid and Elastic Tubes. - These facts are easily demonstrated. Tie a Higginson's syringe to a piece of an ordinary gas-pipe. On forcing water through the tube, by compressing 
the elastic pump, the water will flow ont at the other end of the tube in jets, while during the intervals of pulsation no water will flow ont. As the walls of the tube are rigid, just as much fluid flows out as is forced into the tube. If a similar arrangement be made, and a long elastic. tube he used, a continuous outflow is obtained, provided the pulsations oceur with sufficient rapidity and the length of the tube, or the resistance at its periphery, be sufficient to bring the elasticity of the tube into action. This can be done by putting a narrow cannula in the outflow end of the tube, or by placing a clainp on it so as to diminish the exit aperture. This apparatus converts the intermittent flow into a contimuous current.] The fire-engine is a good example of the conversion of an intermittent inflow into a uniform outflow. The air in the reservoir is in a state of elastic tension, and it represents the elasticity of the vaseular walls. When the pump is worked slowly, the outflow of the water occurs in jets, and is interrupted. If the pumping movement be sufficiently rapid, the compressed air in the reservoir causes a continuous cutflow, which is distinctly accelerated at every movement of the pump. [The ordinary sprayproducer is another good example.]

[Thus, there are two factors - a central one, the heart,-and a peripheral one, the amount of resistance in the arterioles. Either or both may be varied, and as this is done, so will the pressure and velocity vary.]

Current in the Capillaries. - In the capillaries the pulsatile acceleration of the current ceases with the extinction of the pulse-wave. The great resistance which is offered to the current towards the capillary area causes both to disappear. It is only when the capillaries are greatly dilated, and when the arterial blood-pressure is high, that the pulse is propagated through the capillaries into the beginning of the veins. A venous pulse is observed in the veins of the sub-maxillary gland after stimulation of the chorda tympani nerve, which contains the vaso-dilator nerves for the blood-vessels of this gland. If the finger be constricted with an elastic land, so as to hinder the return of the venous blood, and to increase the arterial blood-pressure, while at the same time dilating the capillaries, an intermittent increased redness occurs, which corresponds with the well-known throbbing sensation in the swollen finger. This is due to the capillary pulse. [Roy and (iraham Brown found that pulsatile phenomena were produced in the capillaries by increasing the extra-vascular pressure (\$86). Quincke called attention to the rapillary pulse, which can often be seen under the finger-nails. Extend the fingers completely, when a whitish area appears under the nails. A red area near the free margin of the nail advances and retires with each pulse-beat. It is well marked in some diseased conditions of the heart, especially in incompetence of the aortic valves, and is probably produced by increased extra-vascular pressure.]

82. SCHEMATA OF THE CIRCULATION.-E. H. Weher constructed a scheme of the cirmlation. It consisted of a force-pump with properly arranged valves to represent the heart, portions of gut for the arteries and veins, and a piece of glass tubing containing a piece of sponge to represent the eapillaries. Various schemes have been invented, including the very complicated one of Marey, [the extremely ingenious one of v. Tlianhoffer, and the thoroughly practical one of Rutherford].

83. CAPACITY OF THE VENTRICLES. - Since the right and left ventricles contract simultaneously, and just the same volume of blood passes through the pulmonary as through the systemic circulation, it follows that the right ventricle must be just as capacious as the left. The capacity of the ventricles has been estimated in the following ways:-

Methods. - (1) Directly, by filling the deced relaxed ventricle with blood or an injection mass. This method is unsatisfactory and inaccurate.

(2) Indirectly. Volkmann (1850) estimated it thus :-Estimate the sectional area of the aorta. and the velocity of the blood-stream in it $(\$ 1)$. From this calculate the amount of blood passing through the aorta in the unit of time. As the total quantity of blood in the body is known $\left(=\frac{1}{18}\right.$ of the body-weight), we can easily calculate how long this takes to flow through the aorta. We must also know the number of beats during the time of the circulation. From these data, and from experiments on animals, Volkmann estimated the volume of blond discharged at each systole by the ventricle to be $\frac{1}{5} \delta$ of the body-weight. For a nan weighing 75 kilos. this is $187 \cdot 5$ grams. This estimate still leaves much to be desired.

Place calculates it in the following manner:- $\mathrm{A}$ man uses about 500 litres of $\mathrm{O}$ in 24 hours. To absorb this into the venous blood (which contains about 7 vols per cent. less $O$ than arterial), 
about 7000 litres of blood must pass through the lungs in 24 hours. If one calculates 100,000 heart-beats in 24 hours, then at each systole only 70 cubie centimetres are discharged.
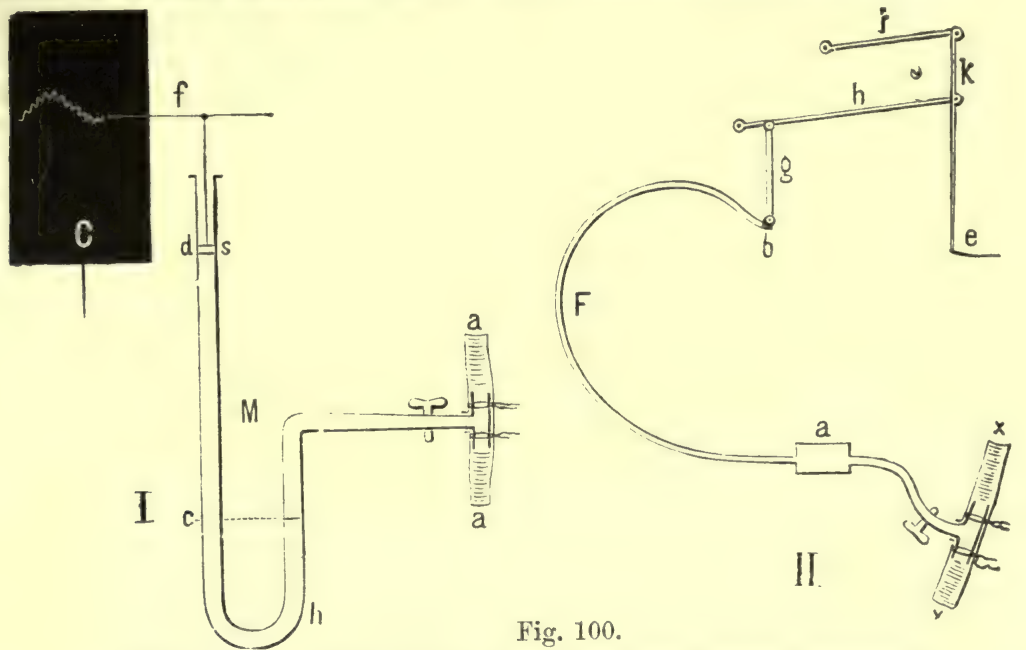

I. Scheme of C. Ludwig's kymograph. II. Fick's spring-kymograph.

84. ESTIMATION OF THE BLOOD-PRESSURE. - (A) In Animals : (1) Methods of Hales.

- The Rev. Stephen Hales (1727) was the first to introduce a long glass tube into a blood-vessel in order to estimate the blood-pressure by measuring the height of the column of blood.

The tube was provided at its lower end with a copper tube bent at a right angle (Pitot's tube). [The tube he used was one-sixth of an inch bore and about 9 feet long, and was inserted into the femoral artery of a horse. The height to which the blood rose in the tube was noted, as well as the oscillations that occurred with every pulsation. From the height of the column of fluid he calculated the force of the heart.]

(2) The Hæmadynamometer of Poiseuille (1828). - This observer used a $\mathrm{U}$-shaped tube partially filled with mercury - a manometer-which was brought into connection with a bloodvessel by means of a rigid tube. [The mercury oscillated with every pulsation, and the extent of the oscillations was read off by means of a scale attached to the bent tube. He called the instrument a hoemadynamometer.]

[(3) Vierordt used a tube 5 or 6 feet long, and filled it with a solution of sodium carbonate, thus preventing much blood from entering the tube, while at the same time the soda solution prevented the coagulation of the blood.]

(4) C. Ludwig's Kymograph. -C. Ludwig employed a $\mathrm{U}_{\text {-shaped }}$ manometer, but he placed a light float (fig. 100, $d, s$ ) upon the surface of the mer-

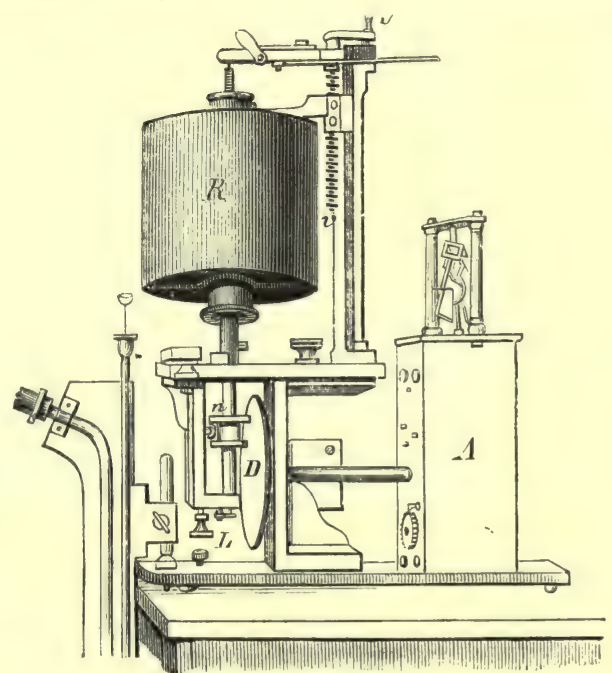

Fig. 101.

Ludwig's improved revolving cylinder, $R$, moved by the clock-work in the box $A$, and regulated by a Foucault's regulator placed on the top of the box. The disc, $D$, moved by the clock-work, presses upon the wheel, n, which can be raised or lowered by the screw, $L$, thus altering the position of $n$ on $D$, so as to cause the cylinbe raised by the handle, $U$. On the left side of the figure is a mercurial manometer. When the cylinder is used, it is covered with smoked smooth paper. der to rotate at different rates. The cylinder itself can 
cury in the open limb of the tube. A writing-style, $f$, placed transversely on the free end of the float, inscribed the movements of the float-and, therefore, of the mercury - upon a cylinder, $c$, caused to revolve at a uniform rate. This apparatus registered the height of the blood-pressure, as well as the pulsatile and other oscillations occurring in the mercury. Volkmann called this instrument a kymograph or "wave-writer." The difference of the height of the column of mercury, $c, d$, in both limbs of the tube indicates the pressure within the vessel. If the height of the column of mercury be multiplied by $13 \cdot 5$, this gives the height of the corresponding column of blood. Setschenow placed a stop-cock in the lower bend, $h$, of the tube. If this be closed so as just to permit a small aperture of communication to remain, the pulsatile vibrations no longer appear, and the apparatus indicates the mean pressure. By the term mean pressure is meant the limit of pressure, above and below which the oscillations occurring in an ordinary blood-pressure-tracing range. [Briefly, it is the averayp elevation of the mercurial column.]

In a blood-pressure-tracing, such as fig. 102, each of the smaller waves corresponds to a heart-beat, the ascent corresponds to the systole and the descent to the diastole. The large

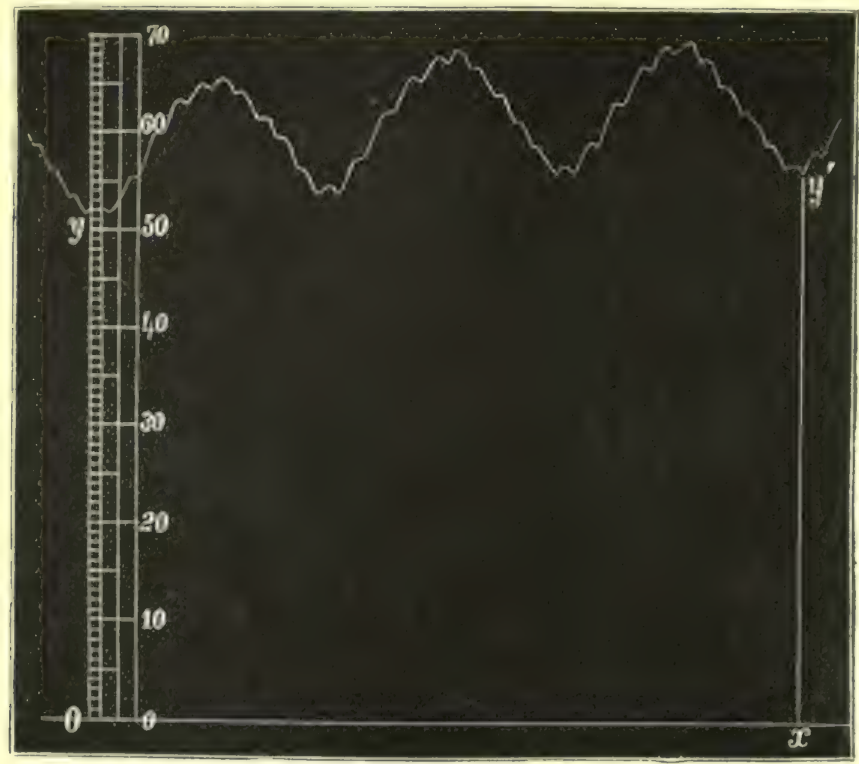

Fig. 102.

Blood-pressure curve of the carotid of a dog obtained with a mercurial manometer. $\quad 0-x=$ line of no pressure, zero line, or abscissa; $y-y^{\prime}$ is the blood-pressure-tracing with small waves, each one caused by a heart-beat, and the large waves due to the respiration. A millimetre scale shows the height of the pressure in millimetres of mercury.

undulations are due to the respiratory movements. It is clear that the heart-beat is expressed as a simple rise and fall (fig. 102), so that the curve of the heart-beat obtained with a mercurial kymograph differs from a sphygmographic curve.

Faults of a Mercurial Manometer. - A perfect recording instrument ought to indicate the height of the blood-pressure, and also the size, form, and duration of any wave-motion communicated to it. The mercurial manometer does not give the true form of the pulse-wave, as the mercury, when once set in motion, executes vibrations of its own, owing to its great inertia, and thus the finer movements of the pulse-wave are lost. Hence a mercurial kymograph is used for registering the blood-pressure, and not for obtaining the exact form of the pulse-wave. Instruments with less inertia, and with no vibrations peculiar to themselves, are required for this purpose.

[Method.-Expose the carotid of a chloralised rabbit, and isolate a portion of the vessel 
between two ligatures, or two spring clamps. With a pair of scissors make an oblique slit in the artery, and into it tie a straight glass cannula, directing the pointed end of the cannula towards the heart. Fill the cannula with a saturated solution of sodium carbonate, taking care that no air-bubbles enter, and connect it with the lead tube which goes to the ascending limb of the manometer. The tube which connects the artery with the manometer must be flexible and yet inelastic, and a lead tube is best. It is usual to connect a pressure-bottle, containing a saturated solution of sodium carbonate, by means of an elastic tube, with the tube attached to the manometer. This bottle can be raised or lowered. Before beginning the experiment, raise the pressure-bottle until there is a positive pressure of mercury in the manometer about equal to the estimated blood-pressure, and then clamp the tube of the pressure-bottle where it joins the lead tube. This positive pressure prevents the escape of blood from the artery into the solution of sodium carbonate. When all is ready, the ligature on the cardiac side of the cannula is removed, and immediately the float begins to oscillate and inscribe its movements upon the recording surface. The fluid within the artery exerts pressure latterly upon the sodium carbonate solution, and this in turn transmits it to the mercury. Peptones, or rather the albumoses, when injected into the blood keep it from coagulating (p. 32). Roy finds that oil may take the place of sodic carbonate.]

[Precautions.-In taking a blood-pressure tracing, after seeing that the apparatus is perfect, care must be taken that the animal is perfectly quiescent, as every movement causes a rise of blood-pressure. This may be secured by giving curara and keeping up artificial respiration, or by the carefully regulated inhalation of ether. When a drug is to be injected to test its action, if it be introduced into the jugular vein it is apt to affect the heart directly. This may be avoided by injecting it into a vein of the leg, or under the skin. The solution of the drug must not contain particles which will block up the capillaries. Care should also be taken that the carbonate of soda does not flow back into the artery.]

[Continuous Tracing. - When we have occasion to take a tracing for any length of time, it must be written upon a strip of paper which is moved at a uniform rate in front of the writingstyle on the float (fig. 100). Various arrangements are employed for this purpose, but it is usual to cause a cylinder to revolve, so as to unfold a roll or riband of paper placed on a movable bobbin. As the cylinder revolves, it gradually winds off the strip of paper, which is kept applied to the revolving surface by ivory frietion-wheels. In Hering's complicated kymograph a long strip of smoked paper is used. The writing-style may consist of a sable birush, or a fine glass pen filled with aniline blue dissolved in water, to which a little alcohol and glycerine are added.]

[In order to measure the height of the pressure, we must know the position of the abscissa or line of no pressure, and it may be recorded at the same time as the blood-pressure, or afterwards. In fig. $102 O-x$ is the zero-line or the abscissa, and the height of the vertical lines or ordinates may be measured by the millimetre scale on the left of the figure. The height of the blood-pressure is obtained by drawing ordinates from the curve to the abscissa, measuring their length, and multiplying by two.]

(5) Spring-Kymograph.-A. Fick (1864) uses a "hollow spring-kymograph" on the principle of Bourdon's manometer (fig. 100, II).

A hollow $\mathbf{C}$-shaped metallic spring, F, is filled with alcohol. One end of the hollow spring is closed, and the other end, covered by a membrane, is brought into connection with a bloodvessel by a junction-piece filled with a solution of sodium carbonate. As soon as the communication with the artery is opened, the pressure rises, and the spring, of course, tends to straighten itself. To the closed end, $b$, there is fixed a vertical rod attached to a series of levers, $h, i, k, \varepsilon$, one of which writes its movements upon a surface moving at a uniform rate. The blood-pressure and the periodic variations of the pulse are both recorded, although the latter is not done with absolute accuracy.

[Hering improved Fick's instrument (fig. 103). $a, b, c$, is the hollow spring filled with alcohol, and communicating at $\alpha$ with the lead tube, $d$, passing to the cannula in the artery. To $c$ is attached a series of light wooden levers with a writing-style, s. The lower part of 4 dips into a vessel, $e$, filled with oil or glycerine, which serves to damp the vibrations of the levers. At $f$ is a syringe communicating with the tube, $d$, filled with solution of sodic carbonate, and used for regulating the amount of fluid in the tube connecting the nanometer with the blood-vessel. The whole apparatus can be raised or lowered on the toothed rod, $h$, by means of the millhead opposite, $g$, to which all the parts of the apparatus are attached.]

(6) Fick's Flat Spring-Kymograph.-The narrow tube $\alpha, a(1 \mathrm{~mm}$. diam.) is placed in con. nection with a blood-vessel by means of the cannula, $c$, and over its vertical expanded end, $A$, is fixed a caoutchoue membrane, with a projecting point, $s$, which presses against a horizontal spring, $\mathrm{F}$, joined to a writing-lever, $\mathrm{H}$, by an intermediate piece, $b$. The whole is held in the metallic frame, R R (fig. 104). In order to estimate the absolute pressure, the instrument must be compared previously with a mercurial manometer. 
(B) In man the blood-pressure may be estimated by means of-(1) a properly graduated sphygmograph $(\$ 67)$. The pressure required to abolish the movement

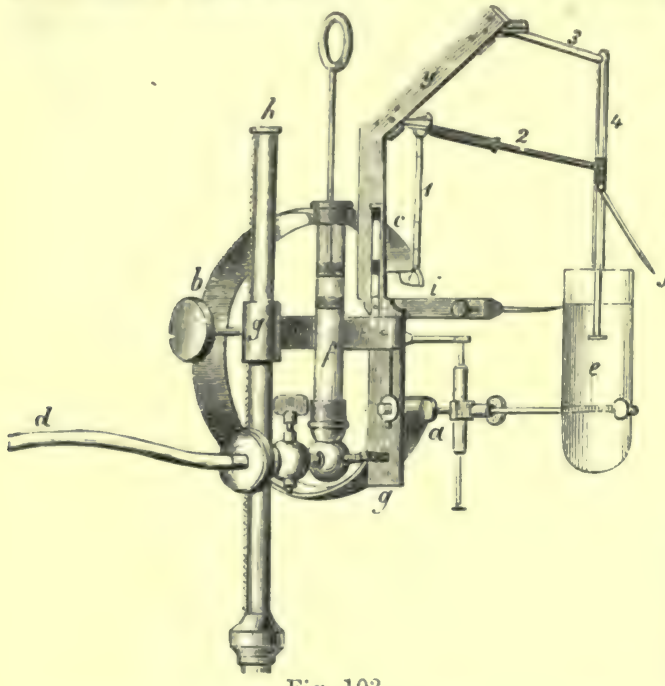

Fig. 103

Fick's spring-manometer, as improved by Hering. of the lever indicates approximately the vascular tension. The mean blood-pressure in the radial artery is equal to 550 grams.

(2) Sphygmomanometer of v. Basch. - A capsule containing fluid was placed upon a pulsating artery, while the capsule itself communicated with a mercurial manometer. As soon as the pressure within the manometer slightly exceeded that within the artery, the artery was compressed so that a sphygmograph placed on a peripheral portion of the vessel ceased to beat. Both arrangements do not give the exact pressure within the artery; they only indicate the pressure which is required to compress the artery and the overlying soft parts. The pressure required to compress the arterial walls, however, is very small compared with the blood-pressure. It is only $4 \mathrm{~mm}$. $\mathrm{Hg}$. $\mathrm{V}$. Pasch estimated the jressure in the radial artery of a healthy man to be 135 to 165 millimetres of mercury.

Variations. - In children the hlood-pressure increases with age, height, and weight. In the superficial temporal artery, at 2 to 3 years, it is $=97 \mathrm{~mm}$.: 12 to 13 years, $113 \mathrm{~mm}$. Hg. (A. Eckert, c. $\S 100$ ). The blood-pressure is raised immediately after bodily movements ; it is higher when a person is in the horizontal position than when sitting, and in sitting than in standing. After a

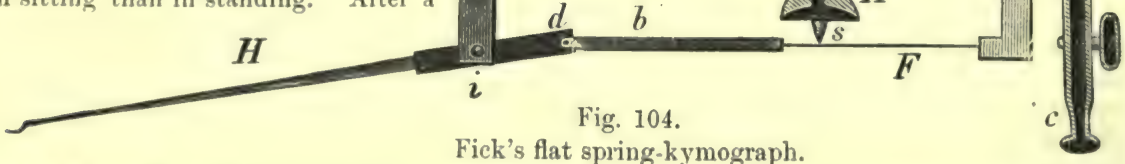

cold as well as after a warm bath, the first effect is an increase of blood-pressure and of the rquantity of urine.

85. BLOOD-PRESSURE IN THE ARTERIES.-The following results have been obtained by experiment on systemic arteries:-

(a) Mean Blood-Pressure.-The blood-pressure is very considerable, varying within pretty wide linits; in the large arteries of large mammals, and perhaps in man, it $=140$ to 160 millimetres [ $5 \cdot 4$ to $6 \cdot 4$ inches] of a mercurial column.

The following results have heen obtained, those marked thus * by Poiseuille, and those + by

* Carotid, Horse, $161 \mathrm{~mm}$.

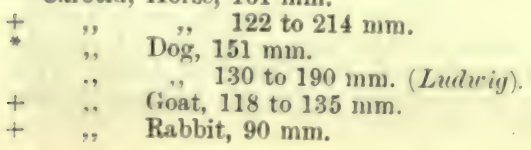

+ Carotid, Fowl, 88 to $171 \mathrm{~mm}$.

+ Aorts of Frog, 22 to $29 \mathrm{~mm}$.

+ Gill artery of Pike, 35 to $84 \mathrm{~mm}$.

Brachial artery of Man during an operation, 110 to $120 \mathrm{~mm}$. (Faivre). Perhaps too low owing to the injury. 
E. Albert estimated the blood-pressure by means of a manometer, placed in connection with the anterior tibial artery of a boy whose leg was to be amputated, to be 100 to $160 \mathrm{~mm}$. Hg. "The elevation with each pulse-beat was 17 to $20 \mathrm{~mm}$. ; coughing raised it to 20 or $30 \mathrm{~mm}$.; tight bandaging of the healthy leg, $15 \mathrm{~mm}$. ; while passive elevation of the body, whereby the hydrostatic action of the column of blood was brought into play, raised it $40 \mathrm{mn}$.

The pressure in the aorta of mammals varies from 200 to $250 \mathrm{~mm}$. Hg. As a general rule, the blood-pressure in large animals is higher than in small animals, because in the former the blood-channel is considerably longer, and there is greater resistance to be overcome. In very young and in very old animals the pressure is lower than in individuals in the prime of life.

The arterial pressure in the fœtus is scarcely half that of the newly-born, while the venous pressure is higher, the difference of pressure between arterial and venous blood being scarcely half so great as in adult animals (Cohnstein and Zuntz).

The arterial blood-pressure is highest in the aorta, and falls towards the smaller vessels, but the fall is very gradual, as shown in fig. 105. A great fall takes place on passing from the area of the arterioles into the capillary area (C), while it is less in the venous area, and negative near the heart, as indicated in the dotted line passing below the abscissa, so that the pressure is lowest in the cardiac ends of the venæ cavæ (compare fig. 111).

(b) Branching of the BloodVessels.-Within the large arteries the blood-pressure diminishes relatively little as we pass towards the periphery, because the difference of

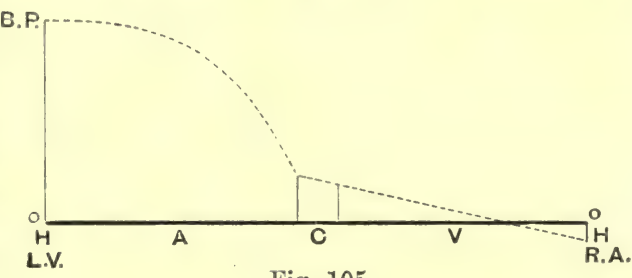

Fig. 105.

the resistance in the different sections of large tubes is very small. As soon, however, as the arteries begin to divide frequently, and undergo a considerable diminution in their lumen, the blood-pressure in them rapidly diminishes, because the propelling energy of the blood is much weakened, owing to the resistance which it has to overcome (\$99).

(c) Amount of Blood.-The blood-pressure is increased with greater filling of the arteries, and vice versa; hence it

\section{Increases.}

1. With increased and accelerated action of the heart;

2. In plethoric persons;

3. After considerable increase of the quantity of blood by direct transfusion, or after a copious meal.

\section{Decreases.}

1. During diminished and enfeebled action of the heart;

2. In anæmic persons;

3. After hæmorrhage or considerable excretions from the blood by sweating, the urine, severe diarrhoea.

The blood-pressure does not vary in the same proportion as the variations in the amount of blood. The vascular system, in virtue of its muscular tissue, has the property, within liberally wide limits, of accommodating itself to larger or smaller quantities of blood (C. Ludvig and Worm Müller, § 102, d). [In fact, a large amount of blood may be transfused without materially raising the blood-pressure.] Small and moderate hæmorrhages (in the dog to 2.8 per cent. of the body-weight) have no obvious effect on the blood-pressure. After a slight loss of blood the pressure may even rise ( Worm Müller). If a large amount of blood be withdrawn, it causes a great fall of the blood-pressure, and when hæmorrhage occurs to 4-6 per cent. of the bodyweight, the blood-pressure $=0$. The transfusion of a modlerate amount of blood does not raise the mean arterial blood-pressure. There are important practical deductions from these experiments, viz., that the arterial blood-pressure cannot be diminished directly by moderate bloodletting, and that the blond-pressure is not necessarily high in plethoric persons. ]

(d) Capacity of the Vessels. - The arterial pressure rises when the capacity of the arterial system is diminished, and conversely. The circularly-disposed smooth muscular fibres of the arteries are the chief agents concerned in this process. When they relax, the arterial blood-pressure falls, and when they contract, it rises. These 
actions of muscular fibres are controlled and regulated by the action of the vasomotor nerves $(\$ 371)$.

(e) Collateral Vessels. - The arterial pressure within a given area of the vascular system must rise or fall according as the neighbouring areas are diminished, whether by the application of pressure, or a ligature, or are rendered impervious, or as these areas dilate. The application of cold or warmth to limited areas of the body-increasing or diminishing the atmospheric pressure on a part-the paralysis or stimulation of certain vaso-motor areas $(\$ 371)$, all produce remarkable variations in the blood-pressure. [The effect of dilatation of a large vascular area on the arterial pressure is well shown by what happens when the blood-vessels of the abdomen are dilated. Divide both vagi in the neck of a rabbit and stimulate the central end of the superior cardiac or depressor nerve; after a few seconds, the blood-vessels of the abdomen dilate, and gradually there is a steady fall of the blood-pressure in the systemic arteries. Fig. 106 is a blood-pressure tracing showing the height of the blood-pressure before stimulation, $a$. The stimulation was continued from $a$ to $b$, and after a certain latent period there is a steady fall of the blood-pressure.]

$(f)$ Respiratory Undulations. - The arterial pressure also undergoes regular variations or undulations owing to the respiratory movements. These undulations

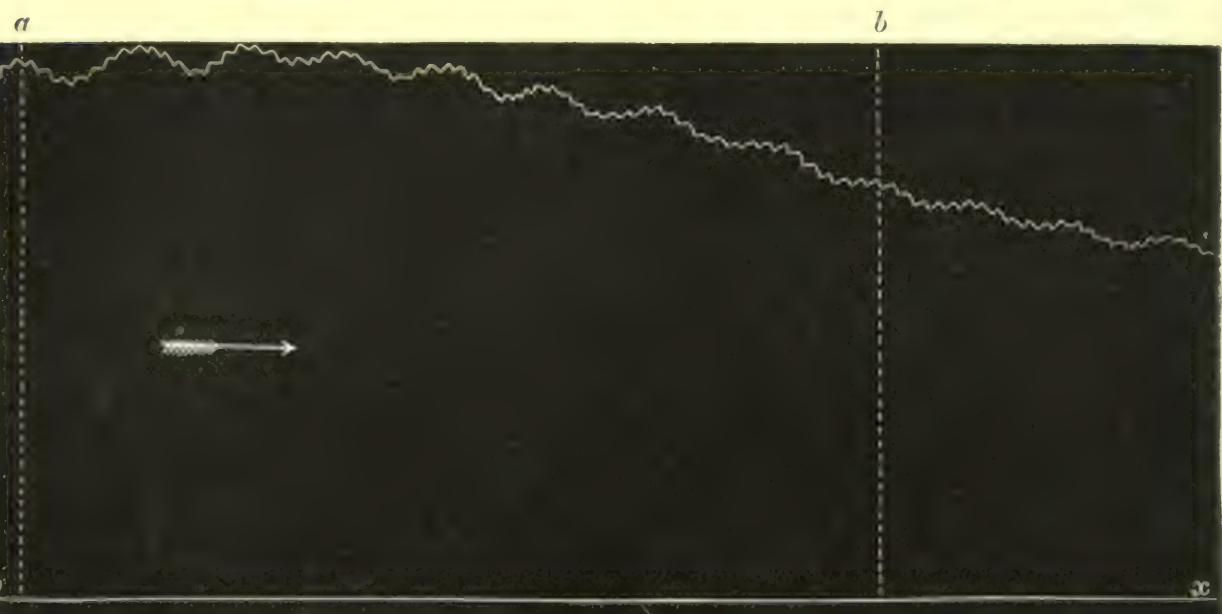

Fig. 106.

Kymographic traeing showing the effect on the blood-pressure of stimulation of the central end of the depressor nerve in the rabbit after section of both vagi in the neck. Stimulation began at $a$ and ended at $b ; 0-x$, the abscissa.

are called respiratory undulations (figs. 102 and 107.) Stated broadly, during every strong inspiration the blood-pressure rises, and during expiration it falls (\$ 74). This is not quite correct. These undulations may be explained by the fact that, with every expiration, the blood in the aorta is subjected to an increase of pressure through the compressed air in the chest; with every inspiration, on the other hand, it is diminished owing to the rarefaction of the air in the lungs acting upon the aorta. Besides, the inspiratory movements of the chest aspirate blood from the venæ cavæ towards the heart, while expiration retards it, and thus influences the blood-pressure. The undulations are most marked in the arteries lying nearest to the heart. The respiratory undulations are due in part to a stimulation or condition of excitement of the vaso-motor centre, which runs parallel with the respiratory movements. This stimulation of the vaso-motor centre causes 
the arteries to contract, and thus the blood-pressure is raised. The variations in the pressure which depend upon a varying activity of the vaso-motor centre are known as the "curves of Traube and Hering." Fig. 107 shows the carotid bloodpressure tracing of a dog. In this curve, when inspiration begins (I) the bloodpressure is still falling slightly, but gradually rises until it reaches its maximum shortly after the beginning of expiration (E). [The maxima and minima of the respiratory and blood-pressure curves do not coincide exactly, but in addition the number of pulse-beats is greater in the ascent than in the descent. This is well marked in a blood-pressure tracing from a dog's carotid (fig. 107), while in a rabbit this difference of the pulse-rate is but slightly marked (fig. 106). The smaller number of pulse-beats during the descent, i.e., during the greater part of expiration

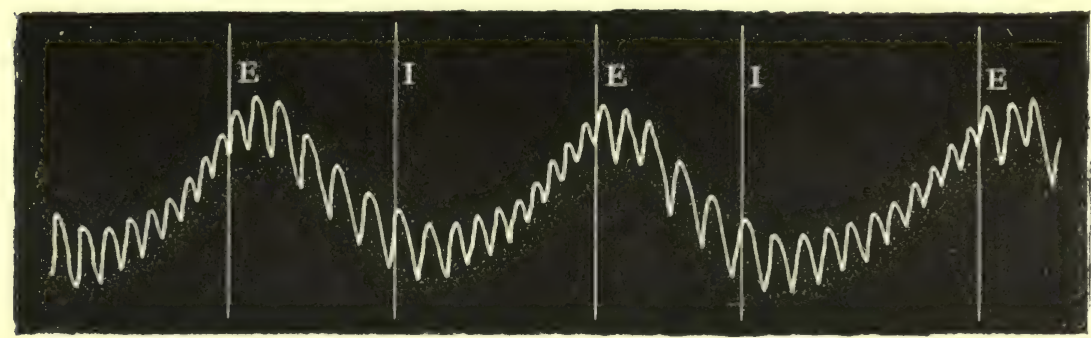

Fig. 107.

Carotid blood-pressure tracing of $\mathrm{dog}$; vagi not divided ; $\mathrm{I}=$ inspiration, $\mathrm{E}=$ expiration. (Stirling).

-is due to the activity of the cardio-inhibitory centre in the medulla oblongata. This is proved by the fact that section of both vagi in the dog causes the difference of pulse-rate to disappear, while other conditions remain the same as before, except that the heart beats more rapidly. It would seem that, during the ascent, the cardio-inhibitory centre is comparatively inactive. It is clear, therefore, that the respiratory and cardio-inhibitory centres in the medulla oblongata act to a certain extent in unison, so that it is reasonable to suppose that other centres situated in close proximity to these may also act in unison with them, or, as it were, "in sympathy." As already stated, the vaso-motor centre is also in action during a particular part of the time.]

[If a dog be curarised and artificial respiration established, the respiratory undulations still occur, although in a modified form. In artificial respiration, the mechanical conditions, as regards the intra-thoracic pressure, are exactly the reverse of those which obtain during ordinary respiration. Air is forced into the chest during artificial respiration, so that the pressure within the chest is increased during inspiration, while in ordinary inspiration the pressure is diminished. Thus, the same mechanical explanation will not suffice for both cases.]

If the artificial respiration be suddenly interrupted in a curarised animal, the bloodpressure rises steadily and rapidly. This rise is due to the stimulation of the vaso-motor centre in the medulla oblongata by the impure blood. This causes contraction of the small arteries throughout the body, which retards the outflow from the large arteries, and thus the pressure within them is raised. [Stated broadly, the arterial pressure depends on the central organ-the heart, and on the condition of the peripheral organs-the small arteries. Both are influenced by the nervous system. If the action of the vaso-motor centre be eliminated by dividing the spinal cord in the cervical region, arrest of the respiration causes a very slight rise of the blood-pressure; hence, it is evident that venous blood acts but slightly on the heart, or on any local peripheral nervous mechanism, or on the 
muscular fibres of the arteries. This experiment shows that it is the vaso-motor centre which is specially acted upon by the venous blood.]

[Traube-Hering Curves.-The following experiment proves that the varying activity of the vaso-motor centre suffices to produce undulations in the bloodpressure tracing. Take a dog, curarise it, expose both vagi and establish artificial respiration; then estimate the blood-pressure in the carotid. After section of the vagi, the heart will continue to beat more rapidly, but it will be undisturbed by the cardio-inhibitory centre. Thus the central factor in the causation of the bloodpressure remains constant. Suddenly interrupt the respiration, and, as already stated, the blood-pressure will rise steadily and uniformly, owing to the stimulation of the vaso-motor centre by the venous blood. In this case the peripheral factor, or state of tension of the small arteries throughout the body, is influenced by the condition of the nerve-centre, which controls their action. After a time, the bloodpressure tracing shows a series of bold curves higher than the original tracing. These can only be due to an alteration in the state of the small arteries, brought about by a condition of rhythmical activity of the vaso-motor centre. These curves were described and figured by Traube, and are called the Traube or TraubeHering curves. As in other conditions, stimulation causes exhaustion, and soon the venous blood paralyses the vaso-motor centre and the small arteries relax, blood flows freely out of the larger arteries, and the blood-pressure rapidly sinks. Variations in the blood-pressure have been observed after a mechanical pump has been substituted for the heart, i.e, after all respiratory movements have been set aside, so that the only factor which would account for the phenomena of the TraubeHering curves is the variation in the peripheral resistance in the small arteries, determined by the condition of the vaso-motor centre.]

Variations. - The respiratory undulations of the blood-pressure become more pronounced the greater the force of the respirations, which produce greater variations of the intra-thoracic pressure. In man, the diminution of the pressure within the trachea is $1 \mathrm{~mm}$. $\mathbf{H g}$. during trancuil inspiration; while during forced respiration, when the respiratory passage is closed, it may be $57 \mathrm{~mm}$. Conversely, during ordinary expiration, the pressure is increased within the trachea $2-3 \mathrm{~mm}$. Hs., while during forced expiration, owing to the compression of the abdominal muscles, it may reach $87 \mathrm{~mm}$. $\mathrm{Hg}$.

Other Factors. - The increase of the blood-pressure during inspiration, as well as the fall during expiration, must in part depend upon the pressure within the abdomen. As the diaphragm descends during inspiration, it presses upon the abdominal contents, including the abdominal vessels, whereby the blood-pressure must be increased. The reverse effect occurs during expiration (Schuceinburg). [Section of both phrenic nerves and opening of the abdominal cavity cause the respiratory undulations almost entirely to disappear. The respiratory undulations, therefore, depend in great part upon the changes of the abdominal pressure and the effect of these changes on the amount of bluod in the abdominal vessels. When making a bloodjressure experiment, pressure upon the abclomen of the animal with the hand causes the bloodpressure to rise rapidly.]

(g) Variations with each Pulse-Beat. - The inean arterial pressure undergoes a variation with each heart-beat or pulse-bent, causing the so-called pulsatory undulations (fig. 107). The mass of blood forced into the arteries with each ventricular systole causes a positive wave and an increase of the pressure corresponding with it, which of course corresponds in its development and in its form with the pulse-curve.

In the large arteries Volkmann found the increase during the heart-beat to be $=\frac{1}{16}$ (horse) and $i_{\text {t }}(\mathrm{dog})$ of the total pressure.

None of the apparatus described in $\$ 84$ gives an exact representation of the pulse-curve. They all show simply a rise and fall, a simple curve. The sphygmograph alone gives a true expression of the undulatious in the blood-pressure which are due to the heart-beat.

(h) Arrest of the Heart's Action.--If the heart's action be arrested or interrupted by continued stimulation of the vagus, or by high positive respiratory pressure, the arterial blood-pressure falls enormously, while it rises in the veins as the blood flows into them from the arteries to equilibrate the difference of pressure in the two 
sets of vessels. This experiment shows that, even when the difference of pressure is almost entirely set aside, the passive blood presses upon the arterial walls, i.e., on account of the overfilling of the blood-vessels a slight pressure is exerted upon the walls, even when there is no circulation. [As already stated, the arterial pressure depends on the condition of the central organ-the heart-and on the peripheral organs - the small arteries. If the action of the heart be arrested, then the blood-pressure rapidly falls. Fig. 108 shows the effect on the blood-pressure

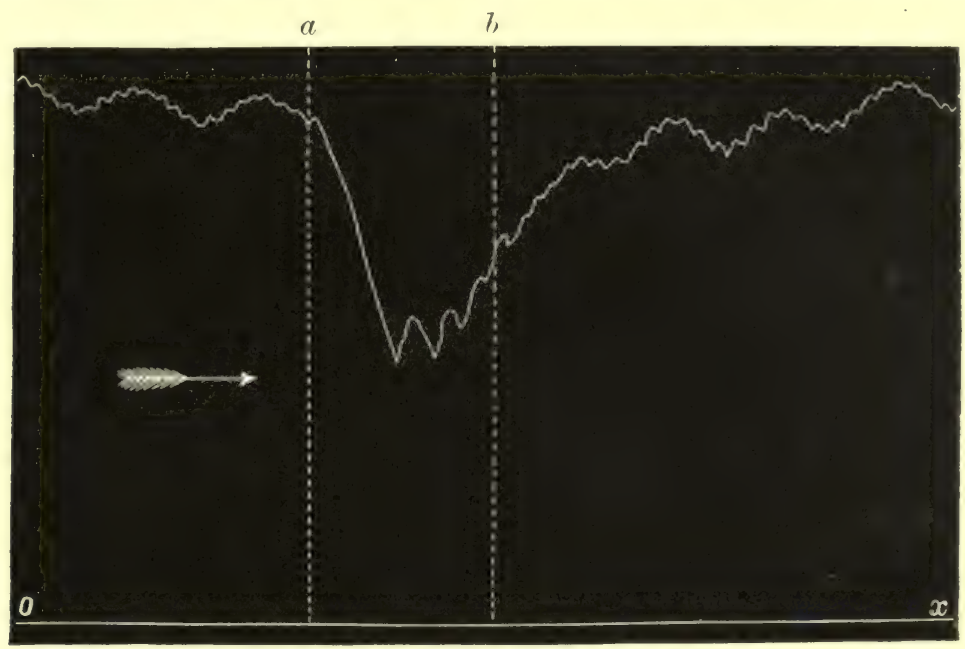

Fig. 108,

Blood-pressure tracing taken with a mercurial kymograph from the carotid of a rabbit. $0-x$, abscissa ; stimulation of vagus begun at $a$ and stopped at $b$.

of arresting the action of the heart by stimulation of the peripheral end of the vagus. There is a sudden fall of the arterial pressure, as shown by the rapid fall of the curve from $a$.]

[Variations in Animals. - The pressure in the arterial system depends upon the balance between the inflow and outflow, i.e., upon the heart and the state of the arterioles. But it is to be noted that the central factor, the heart, varies in different animals. In the rabbit the heart normally beats rapidly, so that section of the vagi does not cause any great increase in the number of beats, nor is the blood-pressure much raised thereby. In the dog, on the other hand, the beats are considerably increased by section of the vagi, while the blood-pressure rises considerably. Atropin paralyses the cardiac terminations of the vagus, and thereby trebles the number of heart-beats in the dog, while it only raises it 25 per cent. in the rabbit ; in man, again, the number may be doubled. As Brunton has shown, this difference of the initial number of heart-beats and the action of the vagus have important relations to the action of drugs on the blood-pressure. For example, if an intact rabbit be caused to inhale amyl nitrite, the blood-pressure falls at once and rapidly, while in the dog the fall may be slight. The pulse of the dog, however, is greatly accelerated, so much so as to be nearly as rapid as that of the rabbit. In both, the vessels are dilated, but in the dog, notwithstanding this dilatation, which per se would cause the pressure to fall, the heart of the dog beats now so rapidly as to compensate for this, and thus keeps the blood-pressure nearly normal ; while the increased rate of beating in the rabbit is not sufficient for this purpose: " If the vagi in the dog be divided, the subsequent inhalation of amyl nitrite causes a fall of blood-pressure like that in the rabbit (Brunton).]

[Relation of Blood-Pressure to Pulse-Rate.-When the blood-pressure rises in an intact animal, as a rule the pulse-rate falls, owing to stimulation of the vagus centre increasing the cardio-inhibitory action, while a fall of blood-pressure is accompanied by an increase of the number of pulse-beats for the opposite reason, the action of the medullary cardio-inhibitory centre being increased. But the 
blood-pressure may be increased either by the action of the heart or the arterioles. If we divide the vagi the pulse beats more quickly, and in some animals the blood-pressure rises; in this case, the rise in the two curves occurs together, and if the vagi be stimulated there is a sudden fall of the blood-pressure, due to arrest of the heart's action, so that again the two curves are parallel. If the arterioles contract the blood-pressure rises, but by and by the pulse-rate falls, owing to the caraio-inhibitory action of the vagus; while, on the other hand, if the arterioles are dilated, the blood-pressure falls, and the heart beats faster. Thus, in both of these cases the pulse-curve and blood-pressure curve run in opposite directions. These results only obtain when the vagi are intact (Brunton).]

[The increase in the pulse-rate and blood-pressure following section of the vagi do not run parallel. Both sooner or later reach a maximum, but the blood-pressure gradually falls to or below the normal, while the pulse-rate remains above the normal (Münzel). J

For the effects of the nervous system upon the blood-pressure see $\S 371$.

Pathological. - In persons suffering from granular or contracted kidney and sclerosis of the arteries, in lead poisoning, and after the injection of ergotin, which causes contraction of the small arteries, it is found, on employing the method of v. Basch, that the blood-pressure is raised. It is also increased in eases of cardiac hypertrophy with dilatation, and by digitalis in cardiac affections, while it falls after the injection of morphia. The blood-pressure falls in fever, a fact also indicated in the sphygmogram ( $\$ 69)$, and it is low in chlorosis and phthisis.

86. BLOOD-PRESSURE IN THE CAPILLARIES. - Methods. - Direct estimation of the capillary pressure is not possible on account of the smallness of the capillary tubes. If a glass plate of known dimensions be placed on a portion of the skin rich in blood-vessels, and if it be weighted

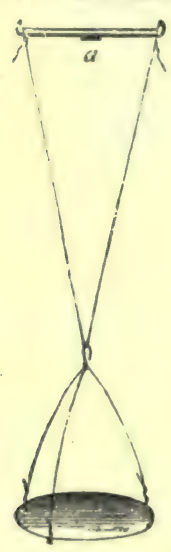

Fig. 109. until the capillaries become pale, we obtain approximately the pressure necessary to overcume the capillary pressure. N. v. Kries placed a small glass plate (fig. 109) 2.5-5 sq. mm., on a suitable part of the skin, e.g., the skin at the root of the nail on the terminal phalanx, or on the ear in man, and on the gum in rabbits. Into a scale-pan attached to this, weights were placed until the skin became pale. The pressure in the capillaries of the hand, when the hand is raised, Kries found to be $24 \mathrm{~mm}$. Hg. ; when the hand hangs down, $54 \mathrm{~mm}$. Hg. : in the ear, $20 \mathrm{~mm}$., and in the gum of a rabbit, $32 \mathrm{~mm}$.

Roy and (iraham Brown compressed from below transparent vascular memhranes against a glass plate by means of an elastic bag connected with a manometer, while the variations in the capillaries were observed from above by a microscope.

Conditions influencing Capillary Pressure.-The capillary blood-pressure in a given area increases-(1) When the afferent small arteries dilate, so that the blood-pressure within the large arteries is propagated more easily into them. (2) By increasing the pressure in the small afferent arteries. (3) By narrowing the diameter of the veins leading from the capillary area. Closure of

V. Kries's apparatus for capillary pressure. square of glass. the veins may quadruple the pressure.

(4) By increasing the pressure in the veins (e.g., by altering the position of a limb). A diminution of the capillary pressure is caused by the opposite conditions.

Changes in the diameter of the capillaries influence the internal pressure. We have to consider the movements of the capillary wall itself as well as the pressure, swelling, and consistence of the surrounding tissues. The resistance to the blood-stream is greatest in the capillary area, and it is evident that the blood in a long capillary must exert more pressure at the commençement than at the end of the capillary; in the middle of the capillary area the blood-pressure is just about one-half of the pressure within the large arteries (Donders). The capillary pressure must also vary in different regions of the body. Thus, the pressure within the intestinal capillaries, in those constituting the glomeruli of the kidney, and in those of lower limbs when the person is in the erect posture, must be greater than in other regions, depending in the former cases partly upon the double resistance caused by two sets of capillaries, and in the latter case partly on purely hydrostatic causes.

87. Blood-Pressure in the Veins.-In the large venous trunks near the heart (innominate, subclavian, jugular) a mean negative pressure of about -0.1 
mm. Hg. prevails ( $H$. Jacobson). Hence, the lymph-stream can flow unhindered. As the distance of the veins from the heart increases, there is a gradual increase of the lateral pressure; in the external facial vein $($ sheep) $=+3 \mathrm{~mm}$.; brachial, $4.1 \mathrm{~mm}$., and in its branches $9 \mathrm{~mm}$. ; crural, $11.4 \mathrm{~mm}$. [The pressure is said to be negative when it is less than that of the atmosphere. The gradual fall of the blood-pressure from the capillary area $(\mathrm{C})$ to the venous area $(\mathrm{V})$ is shown in fig. 108, while within the thorax, where the veins terminate in the right auricle, the pressure is negative.]

Modifying Conditions.-(1) All conditions which diminish the difference of pressure between the arterial and venous systems increase the venous pressure, and vice versâ.

(2) General plethora of blood increases it ; anæmia diminishes it.

(3) Respiration, or the aspiration of the thorax, affects specially the pressure in the veins near the heart; during inspiration, owing to the diminished tension, blood flows towards the chest, while during expiration it is retarded. The effects are greater, the deeper the respiratory movement, and these may be very great when the respiratory passages are closed $(\$ 60)$.

[When a vein is exposed at the root of the neck, it collapses during inspiration, and fills during expiration. The respiratory movements do not affect the venous stream in peripheral veins. The veins of the neck and face become distended with blood during crying, and on making violent expiratory efforts, as in blowing upon a wind instrument. Every surgeon is acquainted with the fact that air is particularly liable to be sucked into the veins, especially in operations near the root of the neek. This is due to the negative intra-thoracic pressure occurring during inspiration.]

(4) Aspiration of the Heart.-Blood is sucked or aspirated into the auricles when they dilate (p. 59), so that there is a double aspiration-one synchronous with inspiration, and the other, which is but slight, synchronous with the heart-beat. There is a corresponding retardation of the blood-stream in the venæ cavæ, caused by the contraction of the auricle (p. 58, a). The respiratory and cardiac undulations are occasionally observable in the jugular vein of a healthy person ( $\$ 99)$.

(5) Change in the position of the limbs or of the body, for hydrostatic reasons, greatly alters the venous pressure. The veins of the lower extremity bear the greatest pressure, while at the same time they contain most muscle ( $K$. Bardeleben, $\S 65)$. Hence, when these muscles from any cause become insufficient, dilatations occur in the veins, giving rise to the production of varicose veins.

[Braune showed that the femoral vein under Poupart's ligament collapsed when the lower limb was rotated outwards and backwards, but filled again when the limb was restored to its former position. All the veins which open into the femoral vein have valves, which permit blood to pass into the femoral vein, hut prevent its reflux. This mechanism acts to a slight degree as a kind of suction and

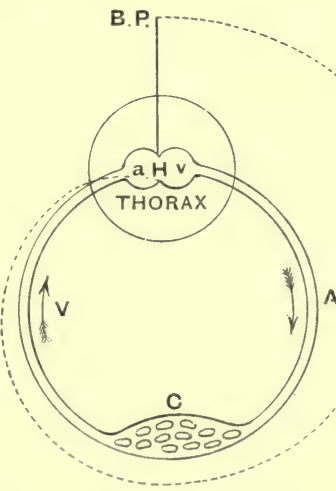

Fig. 110.

Scheme of the blood-pressure. H, heart; $\alpha$, auricle ; $v$, ventricle ; $\mathrm{A}$, arterial ; C, capillary ; and $\mathrm{V}$, venous areas. The circle indicates the parts within the thorax; B.P., pressure in the aorta. pressure apparatus when a person walks, and thus favours the onward movement of the blood.]

[(6) Muscular Movements.--Veins which lie between muscles are compressed when these muscles contract, and as valves exist in the veins, the flow of blood is accelerated towards the heart; if the outflow of the blood be obstructed in any way, then the venous pressure on the distal side of the obstruction may be greatly increased. When a fillet is tied on the upper-arm, and the person moves the muscles of the fore-arm, the superficial veins become turgid, and can be distinctly traced on the surface of the limb.] 
(7) Gravity exercises a greater effect upon the blood-stream in the extensile veins than upon the stream in the arteries. It acts on the distribution of the blood, and thus indirectly on the motion of the blood-stream. It favours the emptying of descending veins, and retards the emptying of ascending veins, so that the pressure becomes less in the former and greater in the latter. If the position of the limb be changed, the conditions of pressure are also altered. If a person be suspended with the head hanging downwards, the face soon becomes turgid, the position of the body favouring the inflow of blood through the arteries and retarding the outflow through the veins. If the hand hangs down it contains more blood in the veins than if it is held for a short time over the head, when it becomes pale and bloodless. [As Lister has shown, the condition of the resiels in the limb is influenced not only by the position of the limb, but also by the fact that a nervous mechanism is called into play.]

[Ligature of the portal vein causes congestion of the rootlets and dilatation of all the bloodverssels in the abilomen: graclually nearly all the blood of the animal accumulates within its belly, so that, paradoxiral as it may seem, an animal may be bled into its own belly. As a conserguene of studen and complete ligature of this vein, the arterial blood-pressure gradually and rapilly falls, and the animal dies very quickly. If the ligature be removed before the hlood-pressure falls ton much, the animal may recover. Schiff and Lautenbach regard the symptoms as due chiefly to the artion of a poison, for when the blood of the portal vein in an animal treated in this way is injerted into a frog, it eauses death within a few hours, while the ordinary blood of the portal vein has no eflect.]

Ligature of the Veins of a Limb. - The effect of ligaturing or compressing all the veins of a limb is well seen in cases where a handage has been applied too tightly. It leads to congestion and increase of pressure within the veins and capillaries, increased transulation of fluid through the eapillaries, and ronserquent wedcme of the parts beyond the obstruction. Ligature of one vein lioes not always proiluce melema, but if several veins of a limb be ligatured, and the vasomotor nerves be divided at the same time, the rapid production of cedema is ensuret. In pathulugical ases the pressure of a tumour upon a large vein may produce similar results $(\$ 20) 3)$.

88. BLOOD-PRESSURE IN THE PULMONARY ARTERY.-Methods.-(1) Direct estimation of the hlood-pressure in the pulmonary artery by opening the ehest was made by C. Ludwig and Beutner (1850). Artificial respiration was kept up, and the manometer was placed in connection with the left branch of the pulmonary artery. The circulation through the left lung of cats and rabbits was thereby completely cut off, and in logs to a great extent interrupted. There was an adlitional disturbing element, vi\%, the removal of the elastic force of the lungs, owing to the opening of the chest, whereby the venous blood no longer flowed normally into the right heart, while the heart itself was under the full pressure of the atmosphere. The estimated pressure in the $\log =29 \cdot 6$; in the eat $=17 \cdot 7$; in the rabbit, $12 \mathrm{~mm}$. Hg., i.e., in the $\log 3$ times, the rabbit 4 times, and the cat 5 times less than the carotid pressure.

(2) Hering (1850) experimented upon a calf with ectopia cordis. He introduced glass tubes directly into the heart, by pushing them through the muscular walls of the ventricles. The 1, lool rose to the height of 21 inches in the right tube, and 33.4 inches in the left.

(3) Faivie (1856) introrlucel a catheter through the jugular vein into the right ventricle, and placed it in connection with a recording tambour.

Indirect measurements have been male by comparing the relative thickness of the walls of the right and left ventricles, or the walls of the pulmonary artery and aorta.

Beutner and Marey estimated the relation of the pulmonary artery to the aortic pressure as 1 to 3 ; Goltz and Gaule as 2 to 5 ; Fick and Badoud found a pressure of $60 \mathrm{~mm}$. in the pulmonary artery of the dog, and in the carotid $111 \mathrm{~mm}$. $\mathrm{Hg}$. The blood-pressure within the pulmonary artery of a child is relatively higher than in the adult.

Elastic Tension of Lungs. - The lungs within the chest are kept in a state of distension, owing to the fact that a negative pressure exists on their outer pleural surface. When the glottis is open, the inner surface of the lung and the walls of the capillaries in the pulmonary air-vesicles are exposed to the full pressure of the air. The heart and large blood-vessels within the chest are not exposed to the full pressure of the atmosphere, but only to the pressure which corresponds to the atmospheric pressure minus the pressure exerted by the elastic traction of the lungs 
(\$60). The trunks of the pulmonary artery and veins are subjected to the same conditions of pressure. The elastic traction of the lungs is greater the more they are distended. The blood of the pulmonary capillaries will, therefore, tend to flow towards the large blood-vessels. As the elastic traction of the lungs acts chiefly on the thin-walled pulmonary veins, while the semi-lunar valves of the pulmonary artery, as well as the systole of the right ventricle, prevent the blood from flowing backwards, it follows that the blood in the capillaries of the lesser circulation must flow towards the pulmonary veins.

If tubes with thin walls be placed in the walls of an elastic distensible bag, the lumen of these tubes changes according to the manner in which the bag enclosing them is distended. If the bag be directly inflated so as to increase the pressure within it, the lumen of the tubes is diminished (Funke and Latschenberger). If the bag be placed within a closed space, and the tension within this space be diminished so that the bag thereby becomes distended, the tubes in its wall dilate. In the latter case-viz., by negative aspiration-the lungs are kept distended within the thorax, hence the blood-vessels of the lungs containing air are wider than those of collapsed lungs (Quincke and Pfeiffer, Bowditch and Gurland, De Jïger). Hence also, more blood flows through the lungs distended within the thorax than through collapsed lungs. The rilatation which takes place durins inspiration acts in a similar manner. The negative pressure that obtains within the lungs during inspiration causes a considerable dilatation of the pulmonary veins, into which the blood of the lungs flows readily, whilst the blood under high pressure in the thick-walled pulmonary artery scarcely undergoes any alteration. The velocity of the blood-stream in the pulmonary vessels is accelerated during inspiration ( $D$ Jäger, Lalesque). The blood-pressure in the pulmonary circuit is raised when the lungs are inflated. Contraction of small arteries, which causes an increase of the blood-pressure in the systemic circulation, also raises the pressure in the pulmonary circuit, because more blood flows to the right side of the heart.

The vessels of the pulmonary circulation are very distensible and their tonus is slight. [Occlusion of one branch of the pulmonary artery does not raise the pressure within the aorta. Even when one pulmonary artery is plugged with an embolon of paraffin, the pressure within the aortic system is not raised (Lichtheim). When a large branch of the pulmonary artery becomes impervious, the obstruction is rapidly compensated for, and this is not due to the action of the nervous system. The vaso-motor system has much less effect upon the pulmonary blood-vessels than upon those of the systemic circulation. The compensation seems to be due chiefly to the great distensibility and dilatation of the pulmonary vessels (Lichtheim).] We know little of the effect of physiological conditions upon the pulmonary artery. According to Lichtheim suspension of the respiration causes an increase of the pressure. [In one experiment he found that the pressure within the pulmonary artery was increased, while it was not increased in the carotid, and he regards this experiment as proving the existence of vaso-motor nerves in the lung.]

During the act of great straining, the blood at first flows rapidly out of the pulmonary veins, and afterwards ceases to flow, because the inflow of blood into the pulmonary vessels is interfered with. As soon as the straining ceases, blood flows rapidly into the pulmonary vessels (Lalesque).

Severini found that the blood-stream through the lungs is greater and more rapid when the lungs are filled with air rich in $\mathrm{CO}_{2}$ than when the air within them is rich in $\mathrm{O}$. He supposes that these gases act upon the vascular ganglia within the lung, and thus affect the diameter of the vessels.

Pathological. - Increase of the pressure within the area of the pulmonary artery occurs frequently in man, in certain cases of heart disease. In these cases the second pulmonary sound is always accentuated, while the elevation caused thereby in the cardiogram is always more marked and occurs earlier (\$52). Electrical and mechanical stimulation of abdominal organs raises the blood-pressure in the pulmonary artery ( $\mathrm{Morel}$ ).

[The action of drugs on the pulmonary circulation may be tested by Holmgren's apparatus 
(\$94), which permits of distension of the lung and retention of the normal circulation in the frog. Cold contracts the pulmonary capillaries to one-third of their diameter, and anasthetics arrest the pulmonary circulation, chloroform leing most and ether least active, while ethidene is intermediate in its effect.]

[Influence of the Nervous System.-The pulmonary circulation is much less dependent on the nervous system than the systemic circulation. Very considerable variations of the blood-pressure within the other parts of the body may occur, while the pressure within the right heart and pulmonary artery is but slightly affected thereby. The pressure is increased by electrical stimulation of the medulla oblongata, and it falls when the medulla is destroyed. Section and stimulation of the central or peripheral ends of the vagi, stimulation of the splanchnics, and of the central end of the sciatic, have but a minimal influence on the pressure of the pulmonary artery $(A u b e r t)$.]

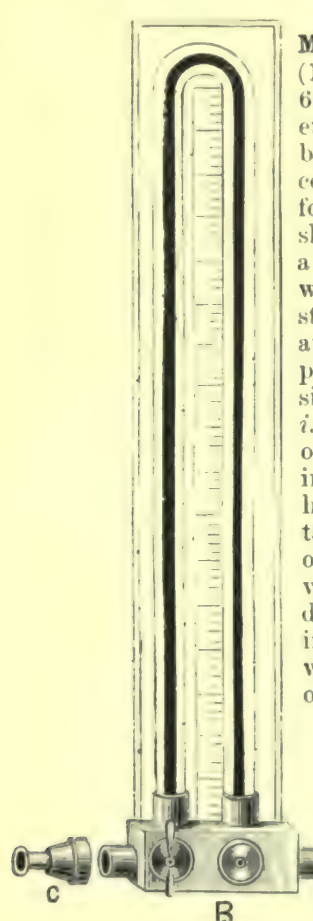

B

89. VELOCITY OF THE BLOOD-STREAM. Methods: (1) A. W. Volkmann's Hrmadromometer (1850). - A glass tube of the shape of a hair-pin, $60-130 \mathrm{~cm}$. long and 2 or $3 \mathrm{~mm}$. broal, with a seale etchel on it, or attached to it, is fixed to a metallic basal plate, $B$, so that each limb passes to a stopcock with three channels. 'The basal plate is perforated along its length, and carries at each end short cammula, $c$, $c$, which are tied into the ends of a clivicled artery. The whole apparatus is first filled with water, [or, better, with salt solution]. The stop-cocks are moverl simultaneously, as they are attached to a toothed wheel, and have at first the position given in fig. $111, \mathrm{I}$, so that the blood simply flows through the hole in the basal piece, i.f., directly from one end of the artery to the other. If at a given moment the stop-cock is turned in the direction indieated in fig. 111, II, the blood has to pass through the glass tube, and the time it takes to make the circuit is noted; and as the length of the tube is known, we can easily calculate the velocity of the blood. The method has very obvious defects arising from the narrowness of the tube ; the introluction of such a tube offers new resistance, while there are no respiratory or pulse-variations observable in the stream in the glass tube.

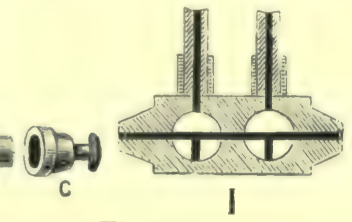

Fig. 111.

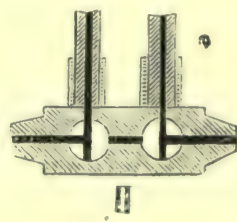

[.

Volkmann's hamalromometer (B). I, blool flows from artery to artery ; II, blool must pass through the glass tube of 13 ; c, c, cammulit for the divided artery.

Volkmann found the velocity to be in the carotid $(\operatorname{dog}):=205$ to $357 \mathrm{~mm}$.; carotid (horse) $=306$; maxillary (horse) $=232$; metatarsal $=56 \mathrm{~mm}$. per second.

(2) C. Ludwig and Dogiel (1867) devised a "stromuhr" or rheometer for measuring the amount of blood which passed through an artery in a given time (fig. 112).

It consists of two glass bulbs, A and B, of exactly the same capacity. These bulbs communicate with each other above, their lower ends being fixed by means of the tubes, $c$ and $d$, to the metal disc, $e, e_{1}$. This disc rotates round the axis, $\mathrm{X}, \mathrm{I}$, so that, after a complete revolution, the tube $c$ communicates with $f$, and $d$ with $g ; f$ and $g$ are proviled with horizontally placed 
cannulre, $h$ and $k$, which are tied into the ends of the diviled artery. The cannula $h$ is fixed in the central end, and $k$ in the peripheral end of the artery (e.g., carotid); the bulb, A, is filled with oil, and $B$ with defibrinated blood; at a certain moment the communication through $h$ is opened, the blood flows in, driving the oil before it, and passes into $\mathrm{B}$, while the defibrinated blood flows through $k$ intu the peripheral part of the artery. As soon as the oil reaches $m-a$ moment which is instantly noted, or, what is better, inscribed upon a revolving cylinder-the bulbs, A, B, are rotated upon the axis $\mathbf{X}, \mathbf{Y}$, so that $\mathbf{B}$ comes to occupy the position of $\mathbf{A}$. The same experiment is repeated, and can be continuel for a long time. The quantity of blook which passes in the unit of time ( 1 sec.) is calculaterl from the time necessary to fill the bulb with blood. Important results are obtained by means of this instrument.

[Suppose $50 \mathrm{c.cm}$. of blool are lelivered in 100 secs., then $1 \mathrm{c.cm}$. flows through in 2 secs. Suppose the sectional area of the artery to be $3 \frac{1}{4} \mathrm{~mm}$. As the velocity is measurel by the ratio of the quantity to the sectional area, then $\frac{5000}{3 \cdot 14}=159 \mathrm{~mm}$. per second.]

[As peptone injected into the blood prevents it from coagulating (dog), this fact has been turned to account in using the rheometer.]

(3) Vierordt's Hæmatuchometer (1858) consists of a small metal box (fig. 113, I) with parallel glass sides. To the narrow sides of the box are fitted an inlet $"$, and an exit cannula, $\alpha$. In its interior is suspended, against the entrance opening, a pendulum, $p$, whose vibrations may be rearl off on a curved scale. [This instrument, as well as Volkmann's apparatus, has only an historical interest.]

(4) Chauveau and Lortet's Dromograph (1860) is constructed on the same principle. A tube A, B (fig. 113), of sufficient liameter, with a sile tube fixed to it, C, which can be placed in connection with a manometer, is introduced into the carotid artery of a horse. At $\alpha$ a small piece is cut out and provided with a covering of gutta-percha which has a small hole in it; through this a light pendulum, $a, b$, with a long imlex, $b$, projects into the tube, i.e., into the blookl-
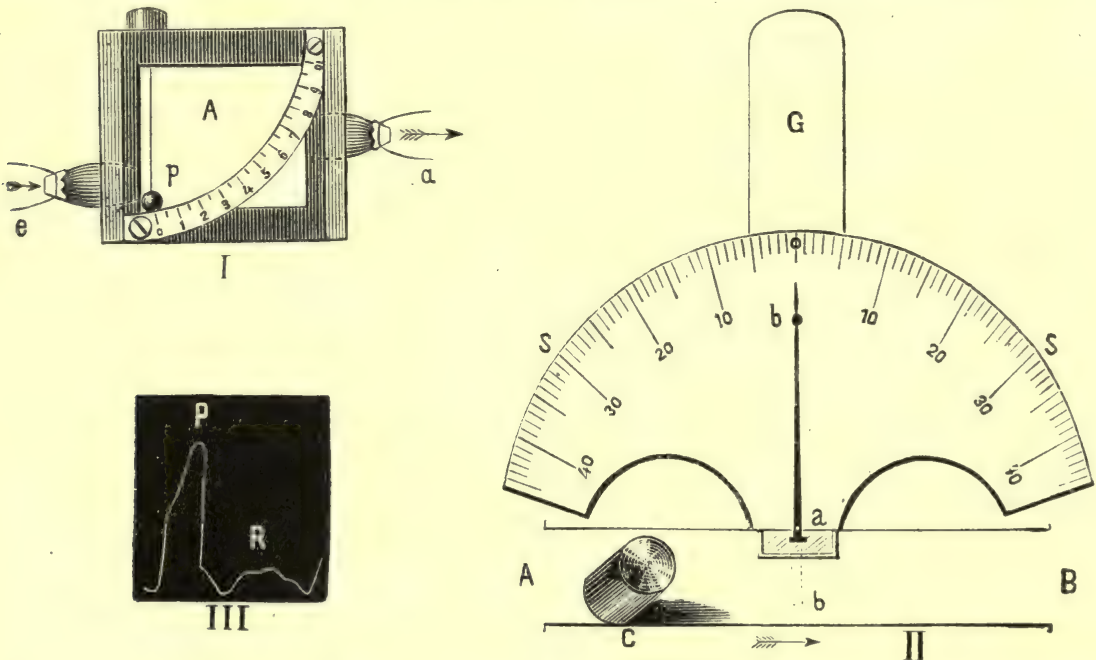

Fig. 113.

I. Vierorlt's hæmatachometer. A, glass; $e$, entrance ; $\iota$, exit camnula ; $p$, pendulum. II. Dromograph. A, B, tube inserted in artery ; C, lateral tube comectel with a manometer; $b$, index moving in a caoutchoue membrane, $a$; $G$, handle. III. Curve obtained by dromograph.

current, which causes the pendulum to vibrate, and the extent of the vibrations can be real off on a scale, S, S. G is an arrangement to permit the instrument to be held. Both this and the former instrument are tested beforehand with a stream of water sent through them with varying velocities.

(5) Cybulski's Photohæmatachometer. - When fluid flows into a tube (fig. 114, II, de) in the direction of the arrow, the fluid stands higher in the manometer $p$ than in $m$. The tube $m y$ indicates the lateral pressure, but $p x$ gives this plus the velocity of the fluid (p. 89). The velocity of the current may be estimated from the difference in the level in the two tubes.

Pitot's tube as used by Cybulski is bent at a right angle $(\mathbf{I}, c p)$, the end $c$ being inserted and 
tied into the central, and $p$ into the peripheral, part of a divided artery. As the blood flows through the tube, the blood rises higher in $\alpha$ than $b$.

To aroid haring the manometers $a$ and $b$ too long, they are connected with each other by a capillary tube filled with air and provided above with a stop-cock $i$. The blood is allowed to

$I$.

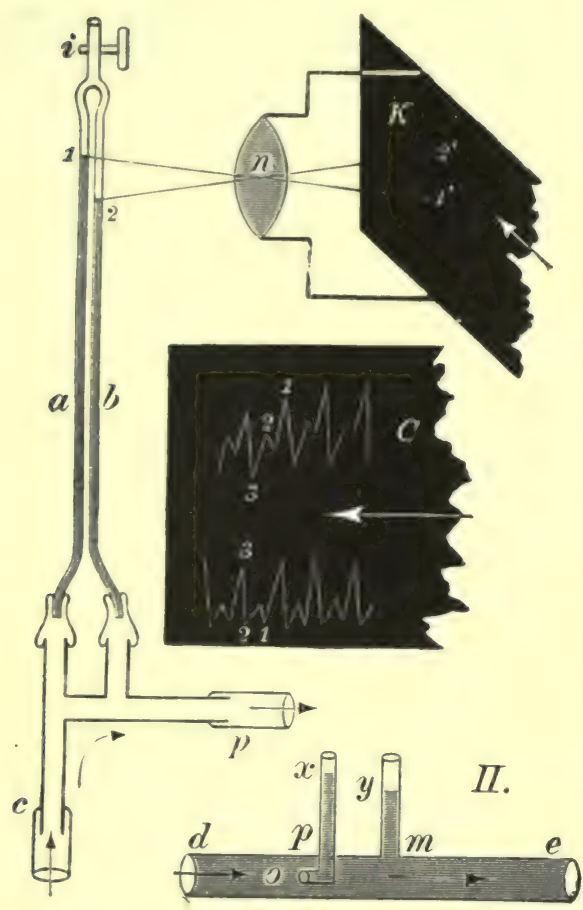

Fig. 114

I. Scheme of the photohematachometer ; 1I. Pitot's tube. rise to the height of 1 and 2, the stop-cock $i$ is closed, and practically an air-manometer is made, which shows a marked difference in the level of the blood of the two tubes. The level of the blood in 1 and 2 is continually changed by the movements of the heart and those of respiration, and these variations are photographed by means of a camera $n$ with a rapidly moving plate $k$.

Fig. C shows a curve obtained from the carotid of a dog. The velocity of the current at $1_{1}-1=238 \mathrm{~mm}$, in the phase $2_{1}-2=225 \mathrm{~mm}$., and at $3_{1}-3=177$ $\mathrm{mm}$. The velocity is greatest at the end of inspiration and the beginning of expiration. Asphyxia increases it at first. Paralysis of the sympathetic increases it. while stimulation of this nerve diminishes it. Section of the vagi increases the velocity, while stimulation diminishes it.

The curve of the velocity may be written off on a smoked glass plate, moving parallel with the index $b$. The dromograph curve, III, shows the primary elevation, $\mathrm{P}$, and the dicrotic elevation $\mathrm{R}$.

\section{VELOCITY OF THE BLOOD.} -(1) Division of Vessels - Arteries. In estimating the velocity of the blood, it is important to remember that the sectional area of all the branches of the aorta becomes greater as we proceed from the aorta towards the capillaries, so that the capillary area is 700 times greater than the sectional area of the aorta. As the veins join and form larger trunks, the venous area gradually becomes smaller, but the sectional area of the

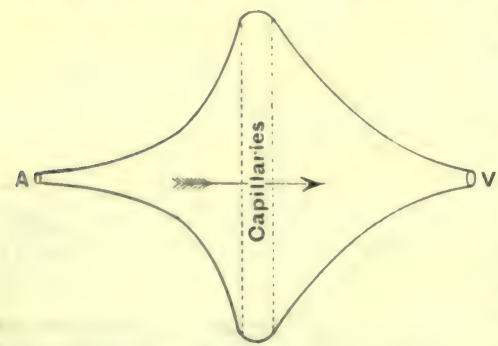

Fig. 115. venous orifices at the heart is greater than that of the corresponding arterial orifices. [We may represent the result as two cones placed base to base (fig. 115), the bases meeting in the capillary area. The sectional area of the venous orifice $(\mathrm{V})$ is represented larger than that of the arterial (A). The increased sectional area influences the velocity of the bloodcurrent, while the resistance affects the pressure.]

The common iliacs are an exception; the sum of Scheme of the sectional area. A, arterial, their sectional areas is less than that of the aorta; and $\mathrm{V}$, venous orifice.

the sections of the four pulmonary veins are together less than that of the pulmonary artery.

(2) Sectional Area. - $\Lambda$ erpunl quentity of blood must pass through every section of the circulatory system, through the pulmonic as well as through the systemic circulation, so that the same amount of blood must pass through the pulmonary 
artery and aorta, notwithstanding the very unequal blood-pressure in these two vessels.

(3) Lumen.--The velocity of the current, therefore, in various sections of the vessels, must be inversely as their lumen.

(4) Capillaries.-Hence, the velocity must diminish very considerably as we pass from the root of the aorta and the pulmonary artery towards the capillaries, so that the velocity in the capillaries of mammals $=0.8$ millimetre per sec.; frog $=0.53 \mathrm{~mm}$. (E. H. Weber $) ; \operatorname{man}=0.6$ to 0.9 (C. Vierordt $)$. According to A. W. Volkmann, the blood in mammalian capillaries flows 500 times slower than the blood in the aorta, so that the total sectional area of all the capillaries must be 500 times greater than that of the aorta. Donders found the velocity of the stream in the small afferent arteries to be 10 times faster than in the capillaries.

Veins. - The current becomes accelerated in the veins, but in the larger trunks it is 0.5 to 0.75 times less than in the corresponding arteries.

(5) Mean Blood-Pressure.--The velocity of the blood does not depend upon the mean blood-pressure, so that it may be the same in congested and in anæmic parts (Volkmann, Hering).

(6) Difference of Pressure.-On the other hand, the velocity in any section of a vessel is dependent on the difference of the pressure which exists at the commencement and at the end of that particular section of a blood-vessel; it depends, therefore, on (1) the vis a tergo (i.e., the action of the heart), and (2) on the amount of the resistance at the periphery (dilatation or contraction of the small vessels).

Corresponding to the smaller difference in the arterial and venous pressure in the foetus $(\$ 85)$, the velocity of the blood is less in this case (Cohnstein and Zuntz).

(7) Pulsatory Acceleration.-With every pulse-bect a corresponding acceleration of the blood-current (as well as of the blood-pressure) takes place in the arteries (pp. 126, 133). In large vessels, Vierordt found the increase of the velocity during the systole to be greater by $\frac{1}{4}$ to $\frac{1}{2}$ than the velocity during the diastole. The variations in the velocity caused by the heart-beat are recorded in fig. 113, obtained by Chauveau's dromograph from the carotid of a horse. The velocity curve corresponds with a sphygmogram- $\mathrm{P}$ represents the primary elevation and $\mathrm{R}$ the dicrotic wave. This acceleration, as well as the pulse, disappears in the capillaries. A pulsatory acceleration, more rapid during its first phase, is observable in the small arteries, although the arteries themselves are not distended thereby.

(8) Respiratory Effect.-Every inspiration retards the velocity in the arteries, every expiration aids it somewhat; but the value of these agencies is very small.

If we compare what has already been said regarding the effect of the respiration on the contraction and dilatation of the heart and on the blood-stream $(\$ 60)$, it is clear that respiration favours the blood-stream, and so does artificial respiration. When artificial respiration is interrupted, the blood-stream becomes slower (Dogiel). If the suspension of respiration lasts sonıwhat longer, the current is again accelerated on account of the dyspncic stimulation of the vaso-motor centre (Heidenhain) (\$371, I.).

(9) Modifying Conditions.-Many circumstances affect the velocity of the blood in the veins. (1) There are regular variations in the large veins near the heart due to the respiration and the movements of the heart (\$5 50 and 60). (2) Irregular variations due to pressure, e.g., from contracting muscles $(\$ 87)$, friction on the skin in the direction or against the direction of the venous current; the position of a limb or of the body. The pump-like action of the veins of the groin on moving the leg has been referred to ( $\$ 87)$. When the lower limb is extended and rotated outwards, the femoral vein in the iliac fossa collapses, owing to an internal negative pressure; when the thigh is flexed and raised, it fills under a positive pressure (Braune). A similar condition obtains in walking.

91. CAPACITY OF THE VENTRICLES. - Vierordt calculated the capacity of the left ventricle from the velocity of the blood-stream, and the amount of blood discharged per second 
by the right earotil, right subelavian, the two coronary arteries, and the aorta below the origin of the innominate artery. He estimated that with every systole of the heart, 172 cubic centimetres (eriual to 180 grammes) of blood were discharged into the aorta ; this, therefore, must be the capacity of the leit ventricle (compare \& 83).

92. THE DURATION OF THE CIRCULATION.-The time required by the blood to make a complete circuit through the course of the circulation was first determined by Hering (1829) in the horse. He injected a 2 per cent. solution of potassium ferrocyanide into a special vein, and ascertained (by means of ferric chloride) when this substance appeared in the blood taken from the corresponding vein on the opposite side of the body. The ferrocyanide may also be injected into the central or cardiac end of the jugular vein, and the time noted at which its presence is detected in the blood of the peripheral end of the same vein. Vierordt (1858) improved this method by placing under the corresponding vein of the opposite side a rotating disc, on which was fixed a number of cups at regular intervals. The first appearance of the potassium ferrocyanide is detected by adding ferric chloride to the serum which separates from the samples of blood after they have stood for a time. The duration of the circulation is as follows :-

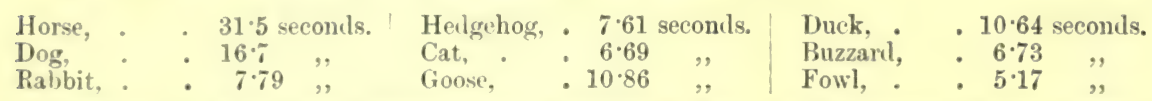

Results. - When these numbers are compared with the frequency of the normal pulse-beat in the corresponding animals, the following deductions are obtained :-

(1) The mean time required for the circulation is accomplished during 27 heartheats, i.r., for $\operatorname{man}=32 \cdot .2$ seconds, supposing the heart to beat 72 times per minute.

(2) (ienerally, the mean time for the circulation in two warm-blooded animals is inversely as the frequency of the pulse-beats.

Modifying Conditions. - The time is influenced by the following factors :-

1. Long vascular channels (.$\%$, from the metatarsal vein of one foot to the other foot) reyuire a longer time than short chamnels (as between the jugulars). The difference may be equal to 10 per cent. of the time required to complete the entire circuit.

2. In young animals (with shorter vascular chamels and higher pulse-rate) the time is shorter than in old animals.

3. Rapid and energetic carliac contractions (as during muscular exercise) diminish the time. Hence rapid and at the same time less energetic eontractions (as after section of both vagi), and sluw but vigorous systoles (6.g., after slight stimulation of the vagus), have no effect.

C. Virrorlt estimates the quantity of blood in a man, in the following mammer :-In all warm-blooderl animals. 27 systoles correspond to the time for completing the circulation. Hence, the total mass of the bloor must he eipual to 27 times the capacity of the ventricle, i.e., in man, $187.5 \mathrm{grm} s . \times 27=5062^{\circ} 5 \mathrm{grms}$. This is equal to $\frac{1}{13}$ of the bolly-weight in a person weighing $65 \cdot 8$ kilos. (compare \$ 49 ).

It is not to be forgotten that the salt used is to some extent poisonous, but Hermann uses the corresponding imnocuous soda salt (25 per cent.).

Pathological. - The lluation of the circulation seems to be increased during septic fever (E. $\left.W o l f f^{\circ}\right)$.

93. WORK OF THE HEART.-The left ventricle expels 0.188 kilo. of blood with each systole, and in doing so it overcomes the pressure in the aorta, which is equal to a column of blood 3.21 metres in height. [The amount of blood expelled from each ventricle during the systole is about $180 \mathrm{grms} .(6 \mathrm{oz}$.$) . It is forced out$ against a pressure of $250 \mathrm{~mm}$. Hg. $=3.21$ metres of blood.] The work of the heart at each systole is $0.188 \times 3.21=0.604$ kilogramme-metre. If the number of beats $=75$ per minute, then the work of the left ventricle in 24 hours $=(0.604 \times$ $75 \times 60 \times 24)=65,230$ kilogramme-metres; while the "work" done by the right. ventricle is about one-third that of the left, and therefore $=21,740$ kilogrammemetres. Both ventricles do work equal to 86,970 kilogramme-metres. A workman during eight hours produces 300,000 kilogramme-metres, i.e., about four times as 
much as the heart. As the whole of the work of the heart is consumed in overcoming the resistance within the circulation, or rather is converted into heat, the body must be partly warmed thereby- 42.5 .5 gramme-metres are equal to 1 heat-unit, i.e., the force required to raise 425.5 grammes to the height of 1 metre may be made to raise the temperature of 1 cubic centimetre of water $1^{\circ}$ C.). So that 204,000 "heat-units" are obtained from the transformation of the kinetic energy of the heart.

One gramme of coal when burned yields 8080 heat-units, so that the heart yields as much energy for heating the body as if about 2.5 grammes of coal were burned within it to produce heat.

94. BLOOD-CURRENT IN THE SMALLER VESSELS.-Methods. - The most important observations for this purpose are made by means of the microscope on transparent parts of living animals. Malpighi was the first to observe the circulation in this way in the lung of a frog (1661).

The following parts have been employed :- The tails of tadpoles and small tishes; the web, tongue, mesentery, and lungs of curarised frogs ; the wing of the bat; the third eyelid of the pigeon or fowl ; the mesentery ; the vessels of the liver of frogs and newts, pia mater of rabbits, the skin on the belly of the frog, the mucous membrane of the inner surface of the human lip (Hüter's Cheilangioscope, 1879); the conjunctiva of the eyeball and eyelids. All these may be examined by reflected light.

[Holmgren's Method. - In studying the circulation in the frog's lung, it must be inflated. A cannula with a bulge on its free end is placed in the larynx, while to the other end is fixed a piece of caoutchouc tubing. The lung is inflated and then the caoutchone tube is closed, after which the lung is placed in a chamber with glass above and below, and examined microscopically.]

[Entoptical appearances of the circulation (Purkinje, 1815). Under certain conditions a person may detect the movement of the blood-corpuscles within the blood-vessels of his own eye. The best method is that of Rool, viz., to look at the sky through a lark blue glass, or through several pieces of cobalt glass placed over each other (Holmholtz).]

Form and Arrangement of Capillaries. - Regarding the form and arrangement of the capillaries, we find that-

1. The diameter which, in the finest, permits only the passage of single corpuscles in a rowone behind the other-may vary from $5 \mu$ to $2 \mu$, so that 2 or more corpuscles may move abreast when the capillary is at its widest.

2. The length is about $0.5 \mathrm{~mm}$. They terminate in small veins.

3. The number is very variable, and the capillaries are most numerous in those tissues where the metabolism is most active, as in lungs, liver, muscles-less numerous in the sclerotic and in the nerve-trunks.

4. They form numerous anastomoses, and give rise to networks, whose form and arrangement are largely determined by the arrangement of the tissue elements themselves. 'They form simple loops in the skin, and polygonal networks in the serous membranes, and on the surface of many gland tubes; they occur in the form of elongated networks, with short commecting branches in muscle and nerve, as well as between the straight tubules of the kiduey; they converge radially towards a central point in the lobules of the liver, and form arches in the free margins of the iris, and on the limit of the selerotic and cornea.

[Direct Termination of Arteries in Veins. - Arteries sometimes terminate lirectly in veins, without the intervention of capillaries, $e_{.}$. ., in the ear of the rabbit, in the terminal phalanges of the fingers and toes in man and some animals, in the cavernous tissue of the penis. They may be regarded as secondary channels which protect the circulation of adjacent parts, and they may also be related to the heat-regulating mechanisms of peripheral parts (Hoyer).]

In connection with the termination of arteries in capillaries, it is important to ascertain if the arterioles are terminal arteries, i.e., if they do not form any further anastomoses with other similar arterioles, but terminate directly in capillaries, and thus only communicate by capillaries with neighbouring arterioles - or the arteries may anastomose with other arteries just before they break up into capillaries. This distinction is important in connection with the nutrition of parts supplied by such arteries (Cohnheim).

Capillary Circulation.-On observing the capillary circulation, we notice that the red corpuscles move only in the axis of the current (axial current), while the lateral transparent plasma-current flowing on each side of this central thread is free from these corpuscles. [The axial current is the more rapid.] This plasma layer or "Poiseuille's space" is seen in the smallest arteries and veins, where $\frac{3}{5}$ are 
taken up with the axial current, and the plasma layer occupies $\frac{1}{5}$ on each side of it. (fig. 116). A great many, but not all, of the colourless corpuscles move in this layer. It is much less distinct in the capillaries. Rud. Wagner stated that it is absent in the finest ressels of the lung and gills, [although Gunning was unable to confirm this statement]. The coloured corpuscles move in the smallest capillaries in single jile one after the other; in the larger vessels, several corpuscles may move abreast, with a gliding motion, and in their course they may turn over and even be twisted if any obstruction is offered to the blood-stream. As a general rule, in these ressels the movement is uniform, but at a sharp bend of the vessel it may partly le retarded and partly accelerated. Where a vessel divides, not unfrequently u corpuscle remains upon the projecting angle of the division, and is doubled over it so that its ends project into the two branches of the tube. There it may remain for a time, until it is dislodged, when it soon regains its original form on account of its elasticity. Not unfrequently we see a red corpuscle becoming bent where two ressels meet, but on all occasions it rapidly regains its original form. This is a good proof of the elasticity of the coloured corpuscles. The motion of the colourless corpuscles is quite different in character; they roll directly on the masulı, mull. moistened on their peripheral zone by the plasma in Poiseuilles space, their other surface being in contact with the thread of coloured corpuscles in the centre of the stream. Sichklarewsky (1868) has shown by physical experimenti, that the particles of least specific gravity in all capillaries (e.!., of glass) are presised toward the wall, while those of greater specific gravity remain in the middle of the strean. [Graphite and particles of carmine were suspended in water, and caused to circulate through capillary tubes placed under a microscope, when the sraphite kept the centre of the stream, and the carmine moved in the layer next the wall of the tube.]

When the colourless corpuscles reach the wall of the vessel, they must roll along, partly on account of their surface being sticky, whereby they readily adhere to the vessel, and partly because one surface is directed towards the axis of the vessel where the movement is most rapid, and where they receive impulses directly from the rapidly moring coloured blood-corpuscles (Donder's). The rolling motion is not always uniform, not unfrequently it is retrograde in direction, which seems to be due to an irresular adhesion to the vascular wall. Their slover movement (10 to 1.2 times slower than the red corpuscles) is partly due to their stickiness, and partly to the fact that, as they are placed near the wall, a large part of their surface lies in the peripheral threads of the fluid, which of course move more slowly (in fact the layer of fluid next the wall is passive-p. 91).

[D. J. Hamilton finds that, when a frog's web is examined in a vertical position, by far the greater proportion of lencocytes float on the upper surface, and only a few on the lower surface, of a small bloot-ressel. In experiments to determine why the coloured corpuscles float or glide "xclusively in the axial stream, while a great many, but not all, of the leucocytes roll in the jwipheral layers. Hanilton ascertained that the nearer the suspended body approaches to the sprecific gravity of the liquicl in which it is immersed, the more it tencls to occupy the centre of the stream. He is of opinion that the phenomenon of the separation of the blood-corpuseles in the circulating fluil is due to the colourless corpuscles being specifically lighter, and the coloured either of the same or of very slightly greater specific gravity, than the blood-plasma. Hamilton controverts the statement of Schklarewsky, and he finds that it is the relative specific gravity of a body which ultimately determines its position in a tube. These experiments point to the immense importance of a due relation subsisting between the specific gravity of the blood-plasma and that of the corpuscles.]

In the vessels first formed in the incubated egg, as well as in young tadpoles, the movement of the blood from the heart occurs in jerks (Spallansani, 1768).

The velocity of the blood-stream is influenced by the diameter of the vessels, which undergo periodic changes of calibre. This change occurs not only in vessels provided with muscular fibres, but also in the capillaries, which vary in diameter, owing to the contraction of the cells composing their walls (p. 96). 
The amount of water in the blood is of importance; when it is increased, the circulation is facilitated and accelerated $(\$ 62)$.

The velocity of the blood is greater in the pulmonary than in the systemic capillaries; so that the total sectional area of the pulmonary capillaries is less than that of all the systemic capillaries.

95. DIAPEDESIS. - If the circulation be studied in the vessels of the mesentery, we may observe colourless corpuscles passing out of the vessels in greater or less numbers (fig. 116). The mere contact with the air suffices to excite slight inflammation. At first, the colourless corpuscles in the plasma-space move more slowly; several accumulate near each other, and adhere to the walls-soon they bore into the wall, ultimately they pass quite through it, and may wander for a distance into the perivaseular tissues. It is doubtful whether they pass through the so-called "stomata" which exist between the endothelial cells, or whether they simply pass through the cement substance between the endothelial cells (p. 94). This process is called diapedesis, and consists of several acts:- $(a)$ The adhesion of lymph-cells or colourless corpuscles to the inner surface of the vessel (after moving more slowly along the wall up to this point). (b) They send processes into and through the vascular wall. (c) The body of the cell is drawn after or follows the processes, whereby the corpuscle appears constricted in the centre (fig. 116, c). (d) The complete passage of the corpuscle through the wall, and its farther motion in virtue of its own amoeboid movements. Hering observed that in large vessels with perivascular lymph-spaces, the corpuscles passed into the spaces, hence cells are found in lymph before it has passed through lymphatic glands. The cause of the diapedesis is partly due to the independent locomotion of the corpuscles, and it is partly a physical act,

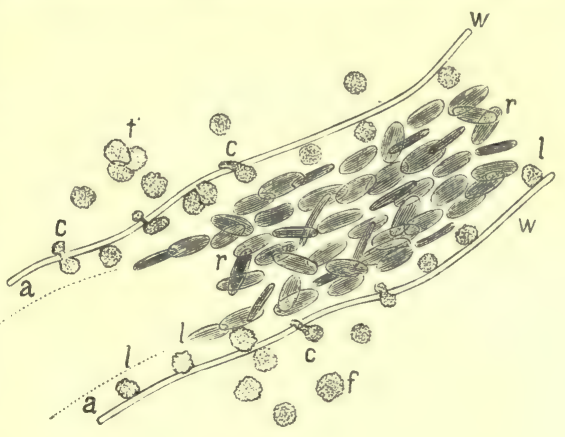

Fig. 116.

Small vessel of a frog's mesentery showing diapedesis. $u, v$, vascular walls ; $a, a$, Poiseuille's space; $r, r$, red corpuscles; $l, l$, colourless corpuscles adhering to the wall, and $c, c$, in various stages of extrusion; $f, f$, extruded corpuscles. viz., a filtration of the colloid mass of the cell under the force of the blood-pressure (Hering) in the latter respect depending upon the intravascular pressure and the velocity of the bloorstream. Hering regards this process, and even the passage of the coloured corpuscles through the vascular wail, as a normal process. The red corpuscles pass ont of the vessels when the venous outflow is obstructed, which also causes the transudation of plasma through the vascular wall. The plasma carries the coloured corpuscles along with it, and at the moment of their passage through the wall they assume extraordinary shapes, owing to the tension put upon them, regaining their shape as soon as they pass out (Cohnheim). This remarkable phenomenon was described by Waller in 1846. It was re-described by Cohnheim, and according to him the out-wandering is a sign of inflammation, and the colourless corpuscles which accumulate in the tissues are to be regarded as true pus-corpuscles, which may undergo further increase by division.

Stasis. - When a strong stimulus acts on a vascular part, hyperæmic redness and swelling occur. Microscopic observation shows, that the capillaries and the small vessels are dilated and overfilled with blood-corpuscles; in some cases, a temporary narrowing precedes the dilatation ; simultaneously the velocity of the strean changes, rarely there is a temporary acceleration, more frequently it becomes slower. If the action of the stimulus or irritant be continued, the retardation becomes considerable, the stream moves in jerks, then follows a to-and-fro movement of the blood-column - a sign that stagnation has taken place in other vascular areas. At last the blood-stream comes completely to a standstill-stasis - and the blood-vessels are plugged with blood-corpuscles. Numerous colourless blood-corpuscles are found in the stationary blood. Whilst these various processes are taking place, the colourless corpuscles-more rarely the red - pass out of the vessels. Under favourable circumstances the stasis nay disappear. The swelling which occurs in the neighbourhood of inflamed parts is chiefly due to the exudation of plasma into the surrounding tissues.

96. MOVEMENT OF THE BLOOD IN THE VEINS. - In the smallest veins coming from the capillaries, the blood-stream is more rapid than in the capillaries themselves, but less so than in the corresponding arteries. The stream is uniform, and if no other conditions interfered with it, the venous stream towards the heart ought to be uniform, but many circumstances affect the stream 
in different parts of its course. Amongst these are:-(1) The relative laxness, great distensibility, and the ready compressibility of the walls, even of the thickest veins. (2) The incomplete filling of the veins, which does not amount to any considerable distension of their walls. (3) The numerous and free anastomoses between adjoining veins, not only between veins lying in the same plane, but also between superficial and deep veins. Hence, if the course of the blood be obstructed in one direction, it readily finds another outlet. (4) The presence of numerous valves which permit the blood-stream to move only in a centripetal direction. They are absent from the smallest veins, and are most numerous in those of middle size.

Position of Valves. - The venous valves always have two pouches, ancl are placed at definite intervals, which correspond to the $1,2,3$, or $\mathbf{n}^{\text {th }}$ power of a certain "fundamental distance," which is $=7 \mathrm{~mm}$. for the lower extremity and $5.5 \mathrm{~mm}$. for the upper. Many of the original valves disappear. On the proximal side of every valve a lateral branch opens into the vein, while on the distal side of each branch lies a valve. The same is true for the lymphaties (K. Baideleben).

Effect of Pressure.-As soon as pressure is applied to the veins, the next lowest valves close, and those immediately above the seat of pressure open and allow the blood to move freely toward the heart. The pressure may be exerted from without, as by anything placed asainst the body ; the thickened contracted muscles, especially the muscles of the limbs, compress the veins. That the blood flows out of a divided vein more rapidly when the muscles contract, is shown during venesection. If the muscles are kept contracted, the venous blood passing out of the muscles collects in the passive parts, e.g., in the cutaneous veins. The pulsatile pressure of the arteries accompanying the veins favours the venous current. From a hydrostatic point of view the valves are of considerable importance, as they serve to divide the column of blood into segments (e.g., in the crural vein in the erect attitude), so that the fine blood-vessels in the foot are not subjected to the whole amount of the hydrostatic pressure in the veins.

The velocity of the venous blood has been measured directly (with the hæmadromometer and the rheometer $-\$ 89$ ). Volkmann found it to be $225 \mathrm{~mm}$. per sec. in the jugular vein. Reil observed that $2 \frac{1}{2}$ times more blood flowel from an arterial orifice than from a venous orifice of the same size. The velocity of the venous current obviously depends upon the sectional area of the vessel. Borelli estimaterl the capacity of the venous system to be 4 times greater than that of the arterial; while, accorling to Haller, the ratio is 9 to 4 .

Large Veins. - Is we proceed from the small veins towards the venæ cavæ, the sectional area of the veins, taken as a whole, becomes less, so that the velocity of the current increases in the same ratio. The velocity of the current in the venæ cavæ may be about half of that in the aorta (Haller). As the pulmonary veins are narrower than the pulmonary artery, the blood moves more rapidly in the former.

97. SOUNDS WITHIN ARTERIES. - The sounds produced within arteries are, speaking strictly from a physical point of view, only noises or bruits. Still, following Skoda's lead, they are spoken of by physicians as "tones." Clinically, there is no sharp distinction between "tones," soumls, noises, or bruits. In four-fifths of all healthy men two sounds-corresponding in duration and other characters to the two heart-sounds - are heard in the carotid (Conrad, $W_{\text {cil }}$. Sometimes only the second heart-sound is distinguishable, as its place of origin is near to the carotill. They are not true arterial sounds, but are simply "propagated heart-sounds." Sometimes the sound of the pulmonaly artery can be heard in this way (Weil, Bettelhein). These murmurs, sounds, or bruits occur either spontaneously, or are produced by the application of cxtrrnal pressicre, whereby the lumen of the vessel is diminished. Hence one distinguishes: (1) Spontaneous Murmurs, and (2) Pressure Murmurs.

Arterial Sounds or murmurs are readily produced by pressing upon a strong artery, e.\%., the crural in the inguinal region, so as to leave only a narrow passage for the blood ("stenosal murmur"). A fine blood-stream passes with great rapidity and force through this narrow part, into a wider portion of the artery lying behind the point of compression. Thus arises the "pressure-stream " (P. Viemeyer), or the "fluid vein" ("veine fluide" of Chauveau). The particles 
of the fluid are thrown into rapid oscillation, and undergo vibratory movements, and by their movements produce the sound within the peripheral dilated portion of the tube. A sound is produced in the fluid by pressure (Corrigan). The sounds are not caused by vibrations of the vascular wall, as supposed by Bouillaud.

A murmur of this sort is the "sub-clavicular murmur" (Roser), occasionally heard during systole in the subclavian artery; it occurs when the two layers of the pleura adhere to the apex of the lung (especially in tubercular diseases of the lungs), whereby the subclavian artery undergoes a local constriction due to its being made tense and slightly curved (Fricdreich). This result is indicated in a diminution or absence of the pulse-wave in the radial artery (Weit).

It is obvious that arterial murmurs will occur in the human body:-(a) When, owing to pathological conditions, the arterial tube is dilated at one part, into which the blood-current is forcibly poured from the normal narrow tube. Dilatations of this sort are called aneurisms, in which murmurs are generally audible. (b) When pressure is exerted upon an artery, e.g., by the pressure of the greatly enlarged arteries during pregnancy, or by a large tumour pressing upon a large artery.

Spontaneous Murmurs. - In cases where no source of external pressure is discoverable, and when no aneurism is present, the spontaneously occurring sounds are favoured, when at the moment of arterial rest (cardiac systole) the arterial walls are distended to the slightest extent, and when during the movement of the pulse (cardiac diastole) the tension is most rapid ( $T$ raube, $W e i l)$, i.e., when the low systolic minimum tension of the arterial wall passes rapidly into the high maximum tension. This is especially the case in insufficiency of the aortic valves, in which ease the sounds in the arteries are audible over a wide area. If the minimum tension of the arterial wall is relatively great, even during diastole, the sounds in the arteries are greatly diminished.

Arterial murmurs are favoured by-(1) Sufficient delicacy and elasticity of the arterial walls. (2) Diminished peripheral resistance, e.g., an easy outflow of the fluid at the end of the stream. (3) Accelerated current in the vascular system generally. (4) A considerable difference of the pressure in the narrow and wide portions of the tube. (5) Large calibre of the arteries.

In normal pulsating arteries, sounds may be heard especially at an acute bend of the artery. Murmurs of this sort are loudest where several large arteries lie together; hence, during pregnancy, we hear the uterine murmur, or placental bruit, or souffle in the greatly dilated uterine arteries. It is much less distinct in the umbilical arteries of the cord (umbilical murmurs). Similar sounds are heard through the thin walls of the head of infants, and a murmur is sometimes heard in the enlarged spleen in ague (Maissurianz).

Auscultation of the Normal Pulse. - On auscultating the radial artery uncler favourable circumstances, and especially in old thin persons with wide arteries and dicrotic pulse, one may hear two sounds corresponding to the primary and dicrotic waves.

In insufficiency of the aortic valves, characteristic sounds may be heard in the crural artery. If pressure be exerted upon the artery, a double blowing murmur is heard ; the tirst one is due to a large mass of blood being propelled into the artery synchronously with the heart-beat, the second to the fact that a large quantity of blood flows back into the heart during diastole. If no pressure be exercised two sounds are heard, and these seem to be due to a wave propagated into the arteries by the auricles and ventricles respectively-compare $\$ 73$, fig. 86 , III. In atheroma a double sound may sometimes be heard $(\$ 73,2)$.

98. VENOUS MURMURS.-I. Bruit de Diable. - This sound is heard above the clavicles in the furrow between the two heads of the sterno-mastoid, most frequently on the right side, and in 40 per cent. of all persons examined. It is either a continuous or a rhythmical murmur, occurring during the diastole of the heart or during inspiration; it has a whistling or rushing character, or even a musical quality, and arises within the bulb of the common jugular vein. When this sound is heard without pressure being exerted by the stethoscope, it is a pathological phenomenon. If, however, pressure be exerted, and if, at the same time, the person examined turn his head to the opposite side, a similar sound is heard in nearly all cases. The pathological bruit de diable occurs especially in anæmic persons, in lead poisoning, in syphilitic and scrofulous persons, sometimes in young persons; and less frequently in elderly people. Sometimes a thrill of the vascular wall may be felt.

Causes.-It is due to the vibration of the blood flowing in from the relatively narrow part of the common jugular vein into the wide bulbous portion of the 
vessel, and seems to occur chietly when the walls of a thin part of the vein lie close to each other, so that the current must purl through it. It is clear that pressure from without, or lateral pressure, as by turning the head to the opposite side, must favour its occurrence. Its intensity will be increased when the velocity of the stream is increased, hence inspiration and the diastolir action of the heart (both of which assist the venous current) increase it. The erect attitude acts in a similar manner. A similar bruit is sometimes, though rarely, heard in the subclavian, axillary, thyroid, facial, innominate and crural veins, and superior cava.

II. Regurgitant Murmurs. - On making a sulden effort, a murmur may be heard in the crural vein during expiration, which is caused hy a centrifugal current of blood, owing to the incompetence or absence of the valves in this region. If the valves at the jugular bulb are not tight, there may be a bruit with expiration (crpiratory jugular vein bruit-Hamernjk), or luring the cardiac systole (systolic jugular vein bruit-v. Bamberger).

III. Valvular Sounds in Veins. - When the trieuspid valve is incompetent, during the ventricular systole, a large volume of blood is propelled backwards into the venæ cavæ. The venous valves are closed suldenly thereby and a sound produced. This occurs at the bulb or dilatation on the jugnlar vein ( $e$. Bimberyrr), and in the crural vein at the groin (N. Fricdreich), i.e., only as long as the valves are competent. Foreed expiration may cause a valvular sound in the crural vein. No sound is heard in the veins uniler perfectly normal circumstances.

99. THE VENOUS PULSE-PHLEBOGRAM.-Methods. - A tracing of the movements of a vein, taken with a lightly weighted sphygmograph, has a characteristic form, and is called a phlebogram (fig. 117). In orler to interpret the various events of the phlebogram it is most important to record simultaneonsly the events that take place in the heart. The auricular con-

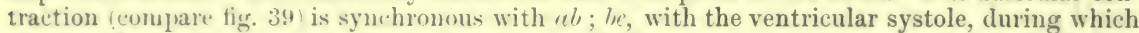
time the first somml oecurs, whilst $" b$ is a fresystolic movement. The carotid pulse coineides nearly with the alm $x$ of the carliogram, i.e, almost simultaneously with the descending limb of the phlebogran (Riegel).

Occasionally in healthy individuals a pulsatile movement, synchronous with the action of the heart, may be observed in the common jugular vein. It is either confined to the lower part of the vein, the so-called bulb, or extends farther up along the trumk of the vein. In the latter case, the valves above the bulb are insufficient, which is by no means rare, even in health. The wave-motion passes from below upwards, and is most obvious when the person is in the passive horizontal position, and it is more frequent on the right side, because the right vein lies nearer the heart than the left. It is propagated more slowly than the arterial pulse-wave. The venous pulse resembles very closely the tracing of the cardiac impulse. Compare fig. 117, 1, with fig. 39.

It is obvious that, as the jugular vein is in direct communication with the right auricle, and as the pressure within it is low, the systole of the right auricle nust cause a positive wave to be propagated towards the peripheral end of the jugular vein. Fig. 117,9 and 10 , are venous pulse-tracings of a healthy person with insufficiency of the valves of the jugular vein. In these curves, the part $a b$ corresponds to the contraction of the auricle. Occasionally this part consists of two elevations, corresponding to the contraction of the atrium and auricle respectively. As the blood in the right auricle receives an impulse from the sudden tension of the tricuspid valve, synchronous with the systole of the right ventricle, there is a positive wave in the jugular vein in fig. 117,9 and 10 , indicated by $b, c$. Lastly, the sudden closure of the pulmonary valves may even be indicated $(e)$. As the aorta lies in direct relation with the pulmonary artery, the sudden closure of its valves may also be indicated (fig. 91, 9, at $d$ ). During the diastole of the auricle and ventricle, blood flows into the heart, so that the vein partly collapses and the lever of the recording instrument descends.

Sinus and Retinal Pulse. - The blood in the sinuses of the brain also undergoes a pulsatile novement, owing to the fact that during cardiae diastole much blood flows into the veins (Mosso). Under favourable circumstances, this movement may be propagated into the veins of the retina, constituting the venous retinal pulse of the older observers (Helfreich).

Pathological Jugular Vein Pulse. - The venous nulse in the jugular vein is far better marked 
in insufficiency of the tricuspid valve, and the vein may pulsate violently, but if its valves be perfect, the pulse is not propagated along the vein, so that a pulse in the jugular vein is not necessarily a sign of insufficiency of the tricuspid valve, but only of insufficiency of the valve of the jugular vein (Friedreich).

Liver Pulse. - The ventricular systole is propagated into the valveless inferior vena cava, and causes the liver pulse. With each systole blood passes into the hepatic veins, so that the liver undergoes a systolic swelling and injection.

Fig. 117, 2-8, are curves of the pulse in the common jugular vein. Although at first sight the curves appear to be very different, they all agree in this, that the various events occurring in the heart during a cardiac revolution are indicated more or less completely. In all the curves, $a b=$ auricular contraction. The auricle, when it contracts, excites a positive wave in the veins. The elevation, $b c$, is eaused by the large blood-wave produced in the veins, owing to the emptying of the ventricle. It is always greater, of course, in insufficiency of the trienspid valves than under normal circumstances (fig. 117, 9 and $10 \%$. In the latter case, the closure of the tricuspid valve causes only a slight wave-motion in the auricle. The apex, $c$, of this wave may be higher or lower, according to the tension in the vein and the pressure exerted by the sphygmograph. As a general rule, at least one notch $(4,5,6, e)$ follows the apex, due to the
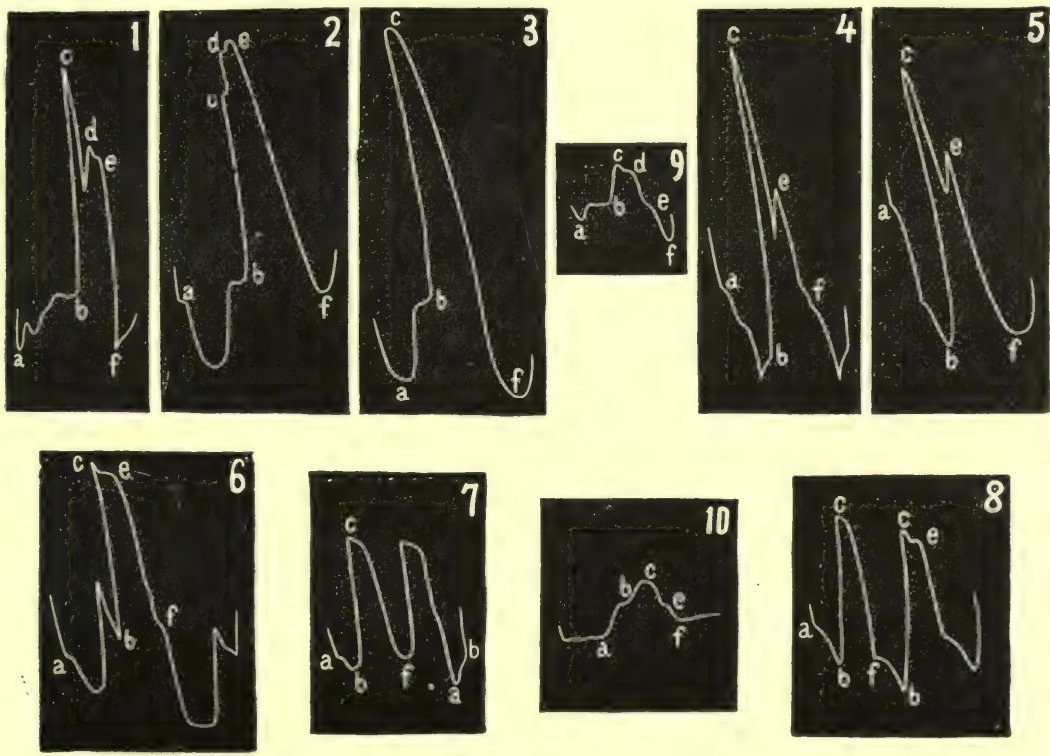

Fig. 117.

Venous pulses (Friedreich). 1-8, from insufficiency of the tricuspid ; 9, 10, pulse of the jugular vein of a healthy person. In all the curves, $a b=$ contraction of the right auricle; $b c$, of the right ventricle; $d$, closure of the aortic valves; $e$, closure of the pulmonary valves; $e, f$, diastole of the right ventricle.

prompt closure of the valves of the pulmonary artery. The closure of the closely adjacent aortic valves may cause a small secondary wave near to $e$ (as in 1 and $2, d$ ). The curve falls towards $f$, corresponding to the diastole of the heart.

A well-marked venous pulse occurs when the right auricle is greatly congested, as in cases of insufficiency of the mitral valve or stenosis of the same orifice. In rare cases, in addition to the pulse in the common jugular vein, the external jugular, the facial, thyroid, external thoracic veins, or even the veins of the upper and lower, extremities may pulsate. A similar pulsation must occur in the pulmonary veins in mitral insufficiency, but of enurse the result is not visible.

On rare occasions, a pulse occurs in the veins on the back of the hand and foot owing to the arterial pulse being propagated through the capillaries into the veins. This may occur under normal circumstances, when the peripheral ends of the arteries become dilated and relaxed (Quincke), or when the blood-pressure within these vessels rises rapidly and falls as suddenly, as in insufficiency of the aortic valves.

In progressive effusion into the pericardium, the carotid pulse at first becomes smaller and the venous pulse larger; beyond a certain stage of pressure the latter ceases (Riegel). 
100. DISTRIBUTION OF THE BLOOD. - In the rabbit, one-fourth of the total amount of the blood is found in each of the following:- $a$, in the passive muscles; $b$, in the liver; $c$, in the organs of the circulation (heart and great vessels); $d$, in all other parts together.

Methods. - The methods adopted do not give exact results. J. Ranke ligatured the parts during life, remosed them, and investigated the amount of blood while the tissues were still warm.

Influencing Conditions. - The amount of blood is influenced by-(1) the anatonical distribution of the vessels (vascularity or the reverse) as a whole ; 2 ) the diameter of the vessels, which deprends upon physiological causes- $(\alpha)$ on the blood-pressure within the vessels ; $(b)$ on the condition of the vasu-motor or vaso-dilator nerves ; $(c)$ on the condition of the tissues themselves, e.g., the vessels of the intestine during absorption; by the vessels of muscle during muscular contraction ; of vessels in inflamed parts.

The most important factor, however, is the state of activity of the organ itself ; hence, the saying, "ubi irritatio, ibi affluxus." We may instance the congestion of the salivary glands and the gastric mucous miembrane during digestion, and the increased vascularity of muscles during contraction. As the activity of organs varies at different times, the amount of blood in the purt or organ goes hand in hand with the veriations in its states of artivity. When some organs are congested, others are at rest ; during digestion, there is muscular relaxation and less mental activity : violent muscular exertion retards digestion-during great congestion of the cutaneous vessels the activity of the kidneys diminishes. Many organs (heart, muscles of respiration, certain nerve-centres) seem always to be in a nearly uniform state of activity and vascularity. During the activity of an organ, the amount of blood in it may be increased 30 per cent., nay, even 47 per cent. The motor organs of young muscular persons are relatively more vascular than those of old and feeble persons $(J$. Ranke). In the condition of increased activity, a more rapind renewal of the blood seems to occur ; after muscular exertion the duration of the circulation diminishes (Vierordt).

During a condition of mental activity, the carotid is dilated, the dicrotic wave in the carotid curve is increased (the radial shows the opposite condition), and the pulse is increased in frequency (Glcy).

Age.--The development of the heart and large ressels determines a different distribution of the blood in the child from that which obtains in the adult. The heart is relatively small from infancy up to puberty, the vessels are relatively large; while after puberty the heart is large,

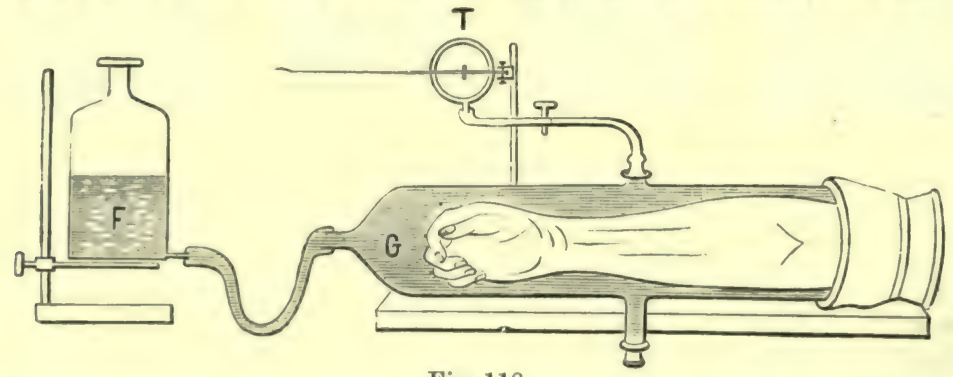

Fig. 118.

Mosso's plethysmograph. G, glass-vessel for holding a limb ; F, flask for varying the waterpressure in $\mathbf{G}$; $\mathrm{T}$, recording apparatus.

and the vessels are relatively smaller. Hence it follows that the blood-pressure in the arteries of the systemic circulation must be lower in the child than in the adult. The pulmonary artery is relatively wide in the child, while the aorta is relatively small ; after puberty both vessels have nearly the same size. Hence, it follows that the blood-pressure in the pulmonary vessels of the child is relatively higher than that in the adult (Bencke).

101. PLETHYSMOGRAPHY.-In order to estimate and register the amount of blood in a limb Mosso devised an instrument (fig, 118), which he termed a. plethysmograph. 
It consists of a long cylindrical glass-vessel, G, suited to accommodate a limb. The opening through which the limb is introduced is closed with caoutchouc, and the vessel is filled with water. There is an opening in the side of the vessel in which a manometer tube, filled to a certain height with water, is fixed. As the arm is enlarged owing to the increased supply of arterial blood passing into it at each pulse-beat, of course the water column in the manometer is raised. Fick placed a float upon the surface of the water, and thus enabled the variations in the volume of the fluid to be inscribed on a revolving cylinder. The curve obtained resembled the pulse-curve ; it was even dicrotic. In fig. 118 the movement of the fluid is represented as conveyed to a Marey's tambour, T, similar to the recording apparatus employed in Brondgeest's pansphygmograph (fig. 76).

The cylinder $\mathrm{C}$ may be filled with air. Kries fills it with gas and connects the tube leading to $\mathrm{T}$ to a gas-burner. The variations in the gas-flame are then photographed.

Results.-(1) Pulsatile Variations in the Volume.-As the venous current is regarded as uniform in the passive limb, every increase of the volume-curve indicates a greater velocity of the arterial current towards the periphery, and vice versa (Fick). The curves registered by the apparatus are volume-pulses, and they resemble the curve of the dromograph (fig. 113, III). The ascent of the curve indicates a greater, the descent a diminished inflow of arterial blood.

At first sight the plethysmograph curve (volume-pulse, $\S 90,7$ ) is very like the pulse-curve (pressure pulse); both are dicrotic. But there are differences; the volume pulse-curve beyond the apex falls more rapidly. This rapid fall, which is not accompanied by a corresponding fall of the pressure, is attributed by v. Kries to peripheral reflexion. The dicrotic wave occurs sooner in the volume-pulse than in the pulse-curve.

(2) The respiratory undulations correspond to similar variations in the bloodpressure tracing $(\$ 85, f)$. Vigorous respiration and cessation of the respiration cause a diminution of the volume. The limb swells during straining and coughing, but diminishes during sighing. (3) Certain periodic undulations occur, due to the regular periodic contractions of the small arteries. (4) Other undulations, due to various accidental causes, affect the blood-pressure: changes of the position of a limb acting hydrostatically, and dilatation or contraction of the vessels in other vascular regions. (5) Movement of the muscles of the limb under observation causes diminution of volume, as the venous current is accelerated, the musculature is also very slightly diminished in volume, even when the intra-muscular vessels are dilated. (6) Mental exercise causes a diminution in the volume of the limb, and so does sleep (Mosso). Music influences the blood-pressure in dogs, the pressure rising or falling under different conditions. The stimulation of the auditory nerve is transmitted to the medulla oblongata, where it acts so as to cause acceleration of the action of the heart (Dogiel). (7) Compression of the afferent artery causes a decrease, and compression of the vein an increase in the volume of the limb (Mosso). (8) Stimulation of the vaso-motor nerves causes a decrease, that of the vasodilators an increase in the volume (Bowditch and Warren).

102. TRANSFUSION OF BLOOD.--Transfusion is the introduction of blood from one animal into the vascular system of another animal.

(a) The red corpuscles are the most important elements in connection with the restorative powers of the blood. They seem to preserve their functions even in blood which has been defibrinated outside the body $(\$ 4, \mathrm{~A})$.

(b) With regard to the gases present in the blood, arterial blood never acts injuriously; but venous blood overcharged with carbonic acid ought only to be transfused when the respiration is sufficient to oxygenate the blood as it passes through the pulmonary capillaries, whereby venous is transformed into arterial blood. If the respiratory movements have ceased, or are imperfectly performed, the blood becomes rapidly richer in carbonic acid, and in this condition reaches the heart ; thence it is propelled into the blood-vessels of the medulla oblongata, where it acts as a powerful stimulus of the respiratory centre, causing dyspnoea, convulsions, and death.

(c) The fibrin, and the substances from which it is formed, do not seem to play 
any part in connection with the restorative powers of the blood ; hence, defibrinated blood performs all the functions of non-defibrinated blood within the body (Panum, Landois).

(d) The investigations of Worm Müller showed that an excess of 83 per cent. of blood may be transfused into the vascular system of an animal $(\mathrm{dog})$ without producing any injurious effects. Hence it follows that the vaseular system has the power of accommodating large quantities of blood within it. That the vascular system can accommodate itself to a diminished amount of blood has been known for a long time $(\$ 85, c)$. [It is very important to observe that the transfusion of a large yuantity of blood does not materially or permanently raise the bloodpressure.]

When Employed.--The transfusion of blood is used-(1) in acute anæmia (\$ 41, I), e.g., after copious hæmorrhage. New blood (150 to 500 c.c.), from the same speries of animal, is introduced directly into the vessels, to supply the place of the blood lost by the hæmorrhage.

(2) In cases of poisoning, where the blood has been rendered useless by being mixed with a poisonous substance, and hence is unable to support life. In such cases remove a considerable quantity of the blood, and replace it by fresh blood. Carbonic oxide is a poison of this kind, and its effects on the body have already been described (\$16). A similar practice is indicated in poisoning with ether, rhloral, chloroform, opium, morphia, strychnine, cobra poison, and such substances as dissolve the blood-corpuscles, e.g., potassic chlorate.

(3) Inder certain pathological conditions, the blood may become so altered in quality as to be unable to support life. The morphological elements of the blood may be altered, and so may the relative proportion of its other constituents. Amongst these conditions may be cited the pathological condition of uræmia, due, it may be, to the accumulation of urea or the products of its decomposition within the blood; accumulation of the biliary constituents in the blood, and great increase of the carbonic acid. All these three conditions, when very pronounced, may cause death. In these cases, part of the impure blood may be replaced by normal human blood.

Amongst conditions where the morphological constituents of the blood are altered qualitatively or quantitatively are : hydræmia (excessive amount of water in the blood, 41, 1); oligocythæmia (abnormal diminution of red blood-corpuscles). When these conditions are highly developed, more especially in pernicious anæmia $(\$ 10,2)$, healthy bluod may be substituted. Transfusion is not suited for persons suffering from leukæmia (compare p. 18).

After-Effects. - A i ularter or half an hour after normal blood has been injected into the blood-vessels of a man, there is a greater or less febrile reaction, according to the amount of blood transfused (Fever, $\S 220$.)

Operation. - The operative procedure to be adopted in the process of transfusion varies according as defibrinated or non-defibrinated blood is used. In order to defibrinate blood, some blood is withdrawn from a vein of a healthy man in the ordinary way, collected in an open vessel, and whippecl or beaten with a glass rod until all the fibrin is completely removed from it. It is then filtered through an atlas filter, heated to the temperature of the body (by placing it in a vessel in warm water), and injected by means of a syringe into an artery opened for the purpose. A vein (e.g., basilic or great saphenous) may be selected for the transfusion, in which ease the blood is driven inward in the direction of the heart; if an artery is selected (radial or posterior tihial) the blood is injected towards the periphery (Hüter), or towards the heart (Landois, Schäfor).

If non-defibrinated human blood is used, the blood may be passed directly from the arm of the giver to the arm of the receiver by means of a flexible tube. The tube used must be filled with normal saline solution to prevent the entrance of air. [J. Duncan collects the blood shed during an operation in a 5 per cent. solution of sodic phosphate (Pavy), and injects the mixture especially where much blood has been lost previously.]

Dangers. - It is most important that no air be allowed to pass into the circulation, for if it be introduced in sufficient quantity it may cause death. When air enters the circulation it reaches 
the right side of the heart, where, owing to the movement of the blood, it forms air-bubbles and makes a froth. The air-bubbles are pumped into the branches of the pulmonary artery, in which they become impacted, arrest the pulmonary circulation, and rapidly cause death.

Peritoneal Transfusion. - Recently, the injection of defibrinated blood into the peritoneal cavity has been recommended. The blood so injected is absorbed (Ponfick). Even after twenty minutes the number of blood-corpuscles in the blood of the recipient (rabbit) is increased, and the number is greatest on the first or second day. The operation, however, may cause death, and one fatal case, owing to peritonitis, is recorded (Mosler). It is evident that this method of transfusion is not applicable in cases where blood must be introduced into the circulation as rapidly as possible (e.g., after severe hæmorrhage or in certain cases of poisoning. [Blood has been injected into the subcutaneous cellular tissue of the abdomen in cases of great debility.]

Heterogeneous Blood. - The blood of animals ought never to be transfused into the blood-vessels of man. It is to be remembered, however, that the blood-corpuscles of the sheep are rapidly dissolved by human blood, so that the active constituents of the blood are rendered useless (Landois). As a general rule, the blood-serum of some mammals dissolves the blood-corpuscles of other mammals $(\$ 5,5)$.

Solution of the Blood-Corpuscles. - The serum of dog's blood is a powerful solvent, while that of the blood of the horse and rabbit dissolves corpuscles relatively slowly. The bloodcorpuscles of mammals vary very greatly with reference to their power to resist the solvent action of the serum of other animals. The red blood-corpuscles of rabbits' blood are rapidly dissolved by the blood-serum of other aninals, whilst those of the cat and dog resist the solvent action much longer. Solution of the corpuscles occurs in defibrinated as well as in ordinary blood. When the blood of a rabbit or lamb is injected into the blood-vessels of a dog, the red blood-corpuscles are dissolved in a few minutes. If blood be withdrawn by pricking the skin with a needle, the partially dissolved corpuscles may be detected.

Liberation of Hæmoglobin and Hæmoglobinuria. - As a result of the solntion of the coloured corpuscles, the blood-plasma is reddened by the liberated hæmoglobin. Part of the dissolved material may be used up in the body of the recipient, some of it for the formation of bile, but if the solution of the corpuscles has been extensive, the hæmoglobin is excreted in the urine (hæmoglobinuria), in less amount in the intestine, the bronchi, and the serous cavities. Bloody urine has been observed in man after the injection of 100 grammes of lamb's blood. Even some of the recipient's blood-corpuscles are dissolved by the serum of the transfused blood, e.g., on transfusing dog's blood into man. In the rabbit, whose corpuscles are readily dissolved, the transfusion of the blood-serum of the dog, man, pig, sheep, or cat produces serious symptoms, and even death. The dog, whose corpuscles are more resistant, bears transfusion of other kinds of blood well.

Dangers. --When foreign or heterogeneous blood (i.e., blood from a different species) is trans. fused, two phenomena, which may be dangerous to life, occur:-

(1) Before the corpuseles are dissolved, they usually run together and form sticky masses, consisting of 10 or 12 corpuseles, which are apt to occlude the capillaries. After a time they give up their hæmoglobin, leaving the stroma, which yields a sticky fibrin-like mass that may occlude fine vessels (\$31).

(2) The presence of a large quantity of dissolved hæmoglobin may cause extensive coagulation within the blood-vessels. The injection of dissolved hæmoglobin causes extensive coagulations (Naunyn and Francken).

The coagulation occurs usually in the venous system and in the larger vessels, and may cause death either suddenly or after a considerable time.

Dissolved hæmoglobin seems greatly to increase the activity of the fibrin-ferment $(\S 30)$, perhaps by accelerating the disintegration of the colourless corpuscles. Hæmoglobin exposed to the air gradually loses this property ; and the fibrin-ferment, when in contact with hæmoglobin, is either destroyed or rendered less active (Sachssendahl).

Vascular Symptoms. - As a result of the above-named causes of occlusion of the vessels, there are often signs of the circulation being impeded in various organs. In man, after transfusion of lamb's blood, the skin is bluish-red, in consequence of the stagnation of blood in the cutaneous vessels. Difficulty of breathing occurs from obstruction in the capillaries of the lung; while there may be rupture of small bronchial vessels, causing sanguineous expectoration. The dyspnoea may increase, especially when the circulation through the medulla oblongata-the seat of the respiratory centre-is interfered with. In the digestive tract, for the same reason, increased peristalsis, evacuation of the contents of the rectum, vomiting, and abdominal pain may occur. These phenomena are explained by the fact that disturbances of the circulation in the intestinal vessels cause increased peristaltic movements. Degeneration of the parenchyma of the kidney occurs as a result of the occlusion of some of the renal vessels. The uriniferous tubules become plugged with cylinders of coagulated albumin (Ponfick). Owing to the occlusion of numerous small muscular branches, the muscles may become stiff, or coagulation of their myosin may occur. Other symptoms, referable to the nervous system, sense-organs, and heart, are all due to the interference with the circulation through them. An important symptom is the occurrence of a considerable amount of fever half an hour or so after the trans- 
fusion of heterogeneous blood $(\S 200)$. When many vessels are occluded, rupture of some small blood-vessels may take place. This explains the oceurrence of slight, yet persistent hæmorrhages, which occur on the free surfaces of the mucous and serous membranes, and in the parenchyma of organs, as well as in wounds. The blood coagulates with difficulty, and imperfectly.

Transfusion of other Fluids, - Other substances have been transfused. Normal saline solution $(0.6$ per cent. $\mathrm{NaCl})$, or serum from the same species, aids the circulation in a purely mechanical way (Goltz), and it even excites the circulation (Kronecker). In severe anæmia this fluid cannot maintain life (Eulenburg and Landois). The injection of peptone, even in moderate amount, is dangerous to life, as it causes paralysis of the vessels (p. 32).

\section{The Blood-Glands.}

103.-I. THE SPLEEN, - Structure. -The spleen is covered by the peritoneum, except at the hilum. Under this serous corering there is a tough, thick, elastic, fibrous capsule, which

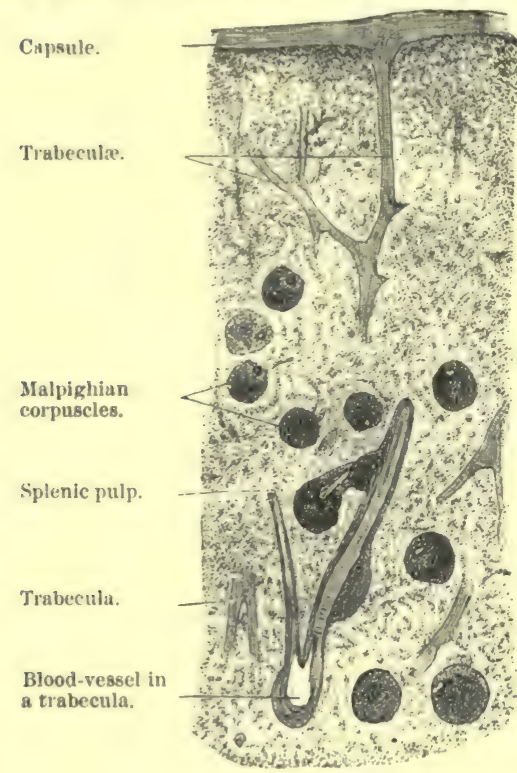

Fig. 119 .

closely invests the organ and gives a covering to the vessels which enter or leave it at the hilum, so that fibrous tissue is carried into the organ along the course of the vessels (lig. 119). [The capsule cannot be separated without tearing the splenic pulp.] Numerous trabeculø pass into the spleen from the deep surface of the capsule, where they branch and anastomose so as to produce a network of sustentacular tissue, which is continuous with the connective-tissue, prolonged inwards and surrounding the blood-vessels (fig. 120). Thus, the connective-tissue in the spleen, as in other viscera, is continuous throughout the organ. In this way an irregular dense network is formed, comparable to the meshes of a bath sponge. [This network is easily demonstrated by washing out the pulp lying in its meshes by means of a stream of water, when a beautiful soft semi-elastic network or framework of rounded and flattened threads is obtained.] The capsule (fig. 119) is composed of interlacing bundles of connective-tissue mixed with numuerous fine fibres of elastic tissue and some non-striped muscular fibres.

Reticulum. - Within the meshes of the trabecular framework there is disposed a very delicate network or reticulum of adenoid tissue, which, with the other coloured elements that fill up the meshes, constitutes the splenic pulp (fig. 121). The reticulum is continuous with the fibres of the trabeculæ. [If a fine section of the spleen be Section of human spleen $\times 10$ "pencilled" in water, so as to remove the cellular times. elements, the preparation presents much the same characters as a section of a lymph-gland similarly treated, viz., a very fine network of adenoid tissue, continuous with, and surrounding the walls of, the blood-vessels. The spaces of this tissue are filled with lymph-and blood-corpuscles.]

The pulp is a dark reddish-coloured, semi-fluid material, which may be squeezed or washed out of the meshes in which it lies. It contains a large number of coloured blood-corpuscles, and becomes brighter when it is exposed to the action of the oxygen of the air.

Blood-Vessels and Malpighian Corpuscles. - The large splenic artery, accompanied by a vein, splits up into several branches before it enters the spleen. Both vessels and their branches are enclosed in a fibrous sheath, which becomes continuous with the trabeculø. The smaller branches of the artery gradually lose this fibrous investment, and each one ultimately divides into a group or pencil of arterioles or penicilli, which do wot anastomose with each other. [Thus each branch is terminal - a condition which is of great importance in connection with the pathology of embolism or infarction of the vessels of the spleen.] At the points of division of the branches of the artery, or scattered along their course, are small oval or globular masses of adenoid tissue ( $\frac{1}{80}$ to $\frac{1}{20}$ inch in diameter), the Malpighian corpuscles. [These bodies are visible to the naked eye as small, round, or oval white structures, about the size of millet seed, in a section of a fresh spleen. They are very numerous-[70,000 in man] -and are readily dotected in the dark reddish pulp. One must be careful not to mistake sections of the trabeculæe 
for them. These corpuscles consist of adenoid tissue, whose meshes are filled with lymphcorpuscles, and they present exactly the same structure as the solitary follicles of the intestine

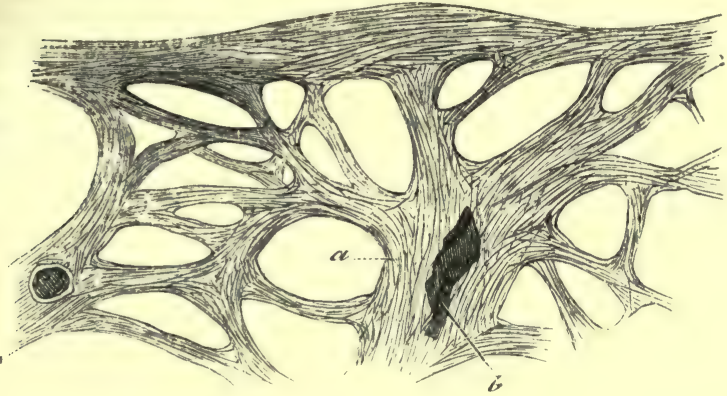

Fig. 120.

Trabeculæ of the spleen of a cat with the splenic pulp washed out. $a$, trabecula; $b$, vein.
(\$ 197). They are small lymphatic accumulations around the arteries - peri-arterial masses of adenoid tissue similar to those masses that occur in a

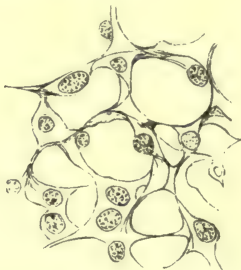

Fig. 121.

Adenoid reticulum of spleen of cat.

slightly different form in other organs, e.g., the lungs. In a section of the spleen the artery may pass through the centre of the mass or through one side of it, and in some cases the tissue is collected unequally on opposite sides of the vessel, so that it is lob-sided. They are not surrounded by any special envelope. In some animals the lymphatic tissue is continued for some distance along the small arteries, so that to some extent it resembles a peri-vascular sheath of adenoid tissue. In a well-injected spleen, a few fine capillaries are to be found within these corpuscles. The capillaries distributed in the substance of the Malpighian corpuscle (fig. 122) form a network, and ultimately pour their bloor into the spaces in the pulp. According to Cadiat, the corpuseles are separated from the splenic pulp by a lymphatic sinus, which is traversed by efferent capillaries passing to the pulp (fig. 122).]

Connection of Arteries and Veins.-It is very difficult to determine what is the exact mode of termination of the arteries within the spleen, more especially as it is extremely difficult to inject the blood-vessels of the spleen. According to Stieda, and others, the fine "capillary arteries" formed by the division of the small arteries do not open directly into the capillary veins, but the connection between the arteries and veins is by means of the "intermediary intercellular spaces" of the reticulum of the spleen, so that, according to this view, there is no continuous channel lined throughout by epithelium connecting these vessels one with another. Thus the blood of the spleen flows into the spaces of the adenoid reticulum just as the lymph-stream flows through the spaces in a lymph-gland. According to Billroth and Kölliker, a closed blood-channel actually does exist between the capillary arteries and the veins, consisting of dilated spaces (similar to those of erectile tissue). These intermediary spaces are said to

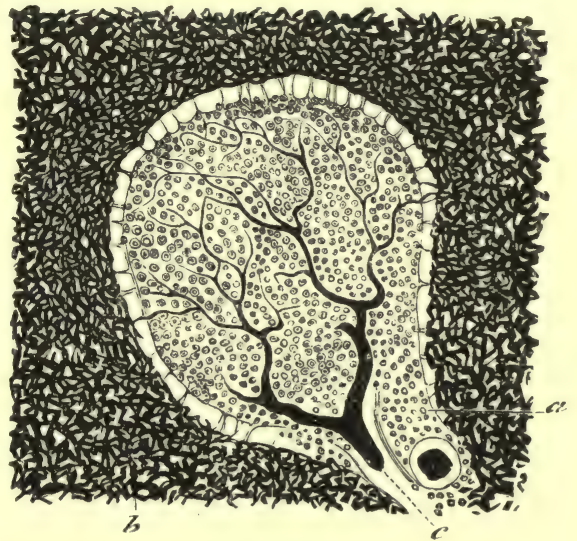

Fig. 122.

Malpighian corpuscle of a cat's spleen injected. $a$, artery ; $b$, meshes of the pulp injected; $c$, the artery of the corpuscle ranifying in the lymphatic tissue composing it.

be completely lined by spindle-shaped epithelium, which abuts externally on the reticulum of the pulp. [According to Frey, owing to the walls of the terminal vessels being incomplete, there being elefts or spaces between the cells composing them, the blood passes freely into spaces of the adenoid tissue of the pulp "in the same way as the water of a river finds its way amongst the pebbles of its bed," these "intermediary passages" being bounded directly by the cells and fibres of the network of the pulp. From these passages the venous radicles arise. At first, their walls are imperfect and cribriform, and they often present peculiar transverse markings, due to the circular disposition of the elastic fibres of the reticulum. The small veins have at first a different course from the arteries. They anastomose freely, but they soon become ensheathed, and accompany the arteries in their course.] 
Elements of the Pulp (fig. 123). - The morphological elements are very various-(1) Lymph-corpuscles of various sizes, sometimes partly swollen, and at other times with granular contents. (2) Red blood-corpuscles. (3) Transition forms between 1 and 2 [although this is

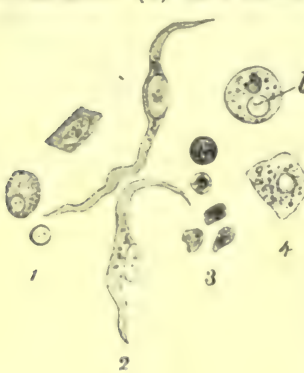

Fig. 123. denied by some observers $(\$ 7, \mathrm{C})]$. corpuscles and pigment granules.

(4) Cells containing red bloodntanules. [These cells exhibit amœeboid

[Lymphatics undoubtedly arise within the spleen, but they are not numerous. There are two systems-a superficial or capsular, and trabecular system; and a peri-vascular set. The superficial lymphaties in the capsule are rather more numerous. Some of them seem to communicate with the lymphaties within the organ (Tomsa, Kolliker). In the horse's spleen they communicate with the lymphatics in the traberula, and with the peri-vascular lymphatics. The exact mode of origin of the peri-vascular system is unknown, but in part at least it begins in the spaces of the adenoid tissue of the Malpighian corpuscles and peri-vascular adenoid tissue, and runs along the arteries towards the hilum. There seem to be no afferent lymphatics in the spleen such as exist in a lymphatic gland.]

Elements of human splenic pulp. 1, colourless cells; 2 , endothelium ; 3 , coloured blood-corpuscles; 4 , cells containing granules, the upper one with a colourless blood-corpuscle, $b$, enclosed.

The nerves of the spleen are composed for the most part of nonmedullated nerve-fibres, and run along with the artery. Their exact mode of termination is unknown, but they probably go to the blood-vessels and to the muscular tissue in the capsule and trabeculæ. [They are well seen in the spleen of the ox, and in their course very small ganglia, placed wide apart, have been found by Remak and W. Stirling. ]

Chemical Composition.--Several of the more highly oxidised stages of albuminous bodies exist in the spleen. Besides the ordinary constituents of the blood, there exist :-leucin, tyrosin, xanthin, hypoxanthin ; lactic, butyric, acetic, formic, succinic, and uric acids, and perhaps glycero-phosphoric acid (Salkowski); cholesterin, a glutin-like body, inosit, a pignent containing iron, and even free iron oxide (Nrsse). The ash is rich in phosphoric acid and iron ( 1.151$)$ - poor in chlorine compounds. The splenic juice is alkaline in reaction ; the specific gravity of the spleen $=1059-1066$.

The functions of the spleen are obscure, but we know some facts on which to form a theory. [The spleen differs from other organs in that no very apparent effect is produced by it, so that we must determine its uses in the economy from a consideration of such facts as the following:-(1) The effects of its removal or extirpation. (2) The changes which the blood undergoes as it passes through it. (3) Its chemical composition. (4) The results of experiments upon it. (5) The effects of diseases.]

(1) Extirpation. - The spleen may be removed from an animal-old or youngwithout the organism suffering any very obvious change (Galen). The human spleen has been successfully removed by Köberle, Péan, and others. As a result (compensatory ?) the lymphatic glands enlarge, but not constantly, while the bloodforming activity of the red marrow of bone is increased. Small brownish-red patches were observed in the intestines of frogs after extirpation of the spleen. These new formations are regarded by some observers as compensatory organs. Tizzoni asserts that new splenic structures are formed in the omentum (horse, dog) after the destruction of the parenchyma and blood-vessels of the spleen. The spleen is absent extremely seldom.

[The weight of the animal ( $\mathrm{dog}$ ) diminishes after the operation, but afterwards increases. The number of red blood-corpuscles is lessened, reaching its minimum about the 150 th to the 200 th day, while the colourless corpuseles are increased in number. The lymphatic glands (especially the internal, and those in the neck, mesentery, and groin) enlarge, while on section the cortical substance of these structures is redder, owing to the great number of red corpuscles, many of them are nucleated in the lymph spaces (Gibson). The marrow of all the long boues (those of the foot excepted), becomes very red and soft, with the characters of embryonic bonemarrow. Such animals withstand hæmorrhage (to $\frac{1}{3}$ of the total amount of blood) without any specially bad results (Tizzoni, Winogradow). Schindeler observed that animals after extirpation of the spleen became very ravenous.]

[Regeneration, - After entire removal of the spleen, nodules of splenic tissue are reproduced 
(fox); while new adenoid tissue is formed in the lymphatic glands, and in Peyer's patches, the parenchyma of the former coming to resemble splenic tissue (Tizzoni, Eternod). ]

(2) According to Gerlach and Funke the spleen is a blood-forming gland. The blood of the splenic vein contains far more colourless corpuscles than the blood of the splenic artery (p. 14). Many of these corpuscles undergo fatty degeneration, and disappear in the blood-stream. That colourless blood-corpuscles are formed within the spleen seems to be proved by the enormous number of these corpuscles which are found in the blood in cases of leukæmia (Bennett (1852), Virchow). Bizzozero and Salvioli found that, several days after severe hæmorrhage, the spleen became enlarged, and its parenchyma contained numerous red nucleated hæmatoblasts.

(3) Other observers (Kölliker and Ecker) regard the spleen as an organ in which coloured blood-corpuscles are destroyed, and they consider the large protoplasmic cells containing pigment granules as a proof of this (p. 12). According to the observations of Kusnetzow, these structures are merely lymph-corpuscles, which, in virtue of their amœboid movements, have entangled coloured blood-corpuscles. [Such corpuscles exhibit similar properties when placed upon a warm stage.] Similar cells occur in extravasations of blood. The coloured blood-corpuscles within the lymph-cells gradually become disintegrated, and give rise to the production of granules of hæmatin and other derivatives of hæmoglobin. [The spleen contains so much free iron that a section of this organ, especially from a young animal, when treated with Tizzoni's fluid, i.e., with potassic ferrocyanide and hydrochloric acid, gives a distinct blue colour $(\$ 174,4)$.] Hence, the spleen contains more iron than corresponds to the amount of blood present in it. When we consider that the spleen contains a large number of extractives derived from the decomposition of proteids, it is very probable that coloured blood-corpuscles are destroyed in the spleen. Further, the juice of the spleen contains salts similar to those that occur in the red blood-corpuscles.

The blood from the spleen is said to have undergone other changes, but the following statement must be accepted with caution :-The blood of the splenic vein contains more water and fibrin, its red blood-corpuscles are smaller, brighter, less flattened, more resistant, and do not form rouleaux; its hæmoglobin crystallises more easily, and there is a large proportion of $\mathrm{O}$ during digestion.

[The spleen has therefore very direct relations to the blood; in it coloured bloodcorpuscles undergo disintegration, it produces colourless corpuscles, and it is said to transform white corpuscles into red.]

(4) Contraction.-In virtue of the plain muscular fibres in its capsule and trabeculæ, the spleen undergoes variations in its volume. Stimulation of the spleen or its nerves, by cold, electricity, quinine, eucalyptus, ergot of rye, and other "splenic reagents" causes it to contract, whereby it becomes paler, and its surface may even appear granular. After a meal, the spleen increases in size, and it is usually largest about five hours after digestion has begun, i.e., at a time when the digestive ergans have almost finished their work, and have again become less vascular. After a time it regains its original volume. For this reason the spleen was formerly regarded as an apparatus for regulating the amount of blood in the digestive organs. [The congestion of the spleen after a meal is more probably related to the formation of new colourless corpuscles than to the destruction of red corpuscles. It may be, however, that some of the products of digestion are partially acted upon in the spleen, and undergo further change in the liver.] There is a relation between the size of the spleen and that of the liver, for it is found that when the spleen contracts-e.g., by stimulation of its nerves-the liver becomes enlarged, as if it were injected with more blood than usual (Drosdow and Botschetschkarow).

[Oncograph.-Botkin, and more recently Roy, have studied various conditions which affect the size of the spleen. Roy enclosed the spleen of a dog in a box with rigid walls, the oncograph (órкos, volume) and filled with oil after the manner 
of the plethysmograph ( $§ 101,276$ ). Any variations in the size of the organ caused a variation in the amount of oil within the box, and these variations were recorded. The blood-pressure was recorded at the same time. The circulation through the spleen is peculiar, and is not due to the blood-pressure within the arteries, but is carried on chiefly by a rhythmical contraction of the muscular fibres of the capsule and trabeculæ. The spleen undergoes very regular rhythmical contractions (systole) and dilatations (diastole). This alternation of systole and diastole may last for hours, and the two events together occupy about one minute (fig. 124). Changes in the arterial blood-pressure have comparatively little in-

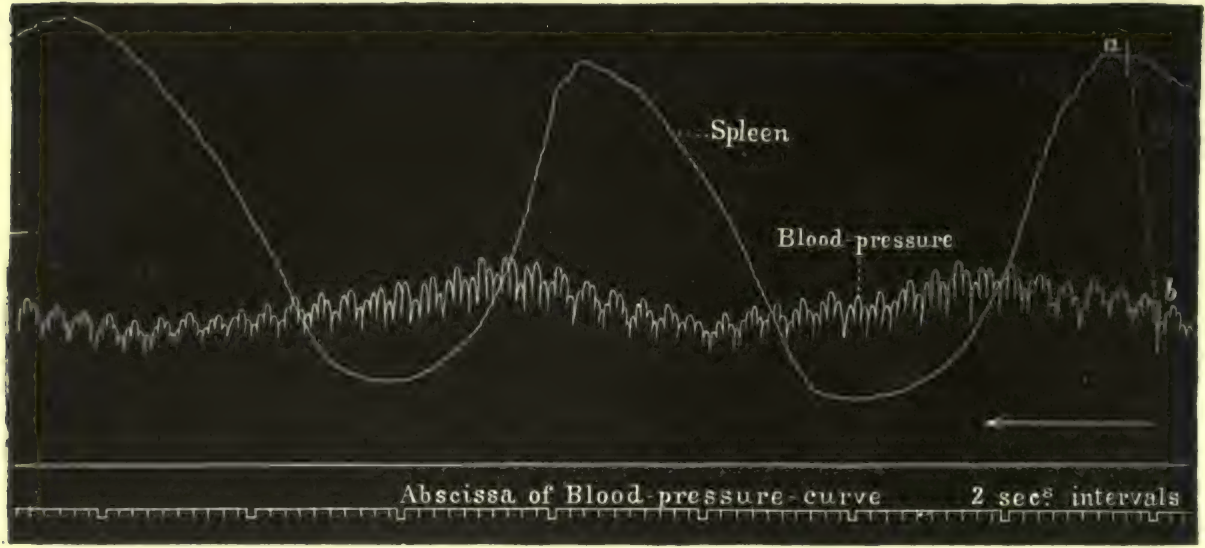

Fig. 124.

Tracing of a splenic curve, reduced one-half, taken with the oncograph. The upper line with large waves is the splenie curve, each ascent corresponds to an increase, and each descent to a diminution in the volume of the spleen. The curve beneath is a blood-pressure tracing from the carotid artery. The lowest line indicates the time, the interruptions of the marker occurring every two seconds. The vertical lines, $a$ and $b$, give the relative positions of the lever-point of the oncograph, and of the point of the recording style of the kymograph respectively (Roy).

fluence on the volume of the spleen. The rhythmical contractions, although modified, still go on after section of the splenic nerves. This would seem to indicate that the spleen has an independent (nervous) mechanism within itself, causing its movements.]

[Influence of Nerves. - Section of the splenic nerves is followed by an increase in the size of the spleen. The nerves have their centre in the medulla oblongata. Stimulation of the medulla oblongata, either directly or by means of asphyxiated blood, causes contraction of the spleen, hence the spleen is "small and contracted" in death from asphyxia. The fibres proceed down the cord, and leaving it in the dorsal region, enter the left splanchnic, pass through the semi-lunar ganglion, and thus reach the splenic plexus. Stimulation of the peripheral ends of these nerves causes contraction of the spleen, and so does cold applied to the spleen directly or over the region of the organ. In the last case the result is brought about reflexly. Botkin found that the application of the induced current to the skin over the spleen, in a case of leukæmia, caused well-marked contraction of the spleen in all its dimensions, and the result lasted some time. After every stimulation the number of colourless corpuscles in the blood increased, and the condition of the patient improved.]

[There is a popular notion that the spleen is influenced by the condition of the nervous system. Botkin found that depressing emotions increased its size, while 
exhilarating ideas diminished it. The causes of these changes are referable not only to changes in the amount of blood in the spleen, but also to the greater or less degree of contraction of its muscular tissue. And it would appear that, like the small arteries, the muscular tissue of the spleen is in a state of tonic contraction. The size of the spleen may be influenced reflexly. Thus, Tarchanoff found that stimulation of the central end of the vagus, when the splanchnics were intact, caused contraction of the spleen, while stimulation of the central end of the sciatic also caused contraction, but to a less degree. It is quite certain that all the phenomena are not due to the action of vaso-motor nerves on the splenic bloodvessels. There is a certain amount of independent action of the muscular fibres of the organ, and it is not improbable that the innervation of the spleen is similar to the innervation of arteries, and that it has a motor centre in the cord capable of being influenced reflexly by afferent nerves, while it also sends out efferent impulses.]

[Stimulation of (1) the central end of a sensory nerve ; $(2)$ of the peripheral ends of both splanchnics; (3) of the peripheral ends of both vagi, causes contraction of the spleen. But even after section of the splanchnics and vagi, stimulation of a sensory nerve still causes contraction, so that there must be some other channel as yet unknown (Roy). Bochefontaine found that electrical stimulation of certain parts of the cortex cerebri produced contraction of the spleen.] Sensory nerves seem to occur only in the peritoneum covering the spleen.

Pressure on the splenic vein causes enlargement of the spleen, hence, increased pressure in this vein (congestion of the portal vein, cessation of hæmorrhoidal and menstrual discharges) also causes its enlargement. With regard to the action of "splenic reagents," such as quinine, on the contraction of the spleen, Binz is of opinion that this drug retards the formation of the colourless blood-corpuseles, so that its chief function is interfered with and the organ becomes less vascular. It is not definitely decided, however, whether it is contraction or dilatation of the spleen that alters the proportion of red and white corpuseles in the blood.

Splenic Tumours. - The increase in size of the spleen in various diseases early attracted the attention of physicians. The healthy spleen undergoes several variations in volume during the course of a day, corresponding with the varying activity of the digestive organs. In this respect the spleen resembles the arteries. In many fevers the spleen becomes greatly enlarged,

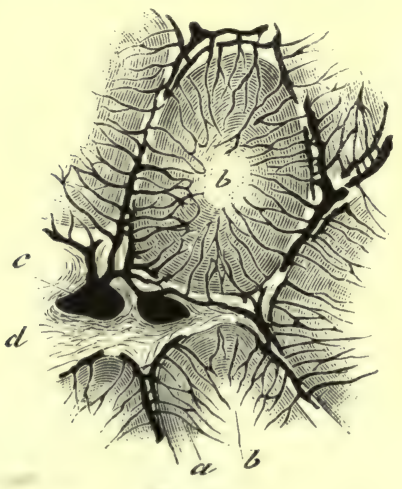

Fig. 125. probably due to paralysis of its nerves. It is greatly increased in intermittent fever or ague, and often during the course of typhus. When it becomes abnormally enlarged, and remains so after repeated attacks of the ague, it is greatly hypertrophied, and constitutes "ague cake."

In cases of splenic leukæmia it is greatly enlarged, and at the same time there is a great increase in the number of colourless corpuscles in the blood and also a decrease of the coloured ones $(\S 10)$.

II. The Thymus. - During foetal life this gland is largely developed, and it increases during the first two or three years of life, remaining stationary until the tenth or fourteenth year, when it begins to atrophy and undergo fatty degeneration. [The degeneration begins at the outer part of each lobule and

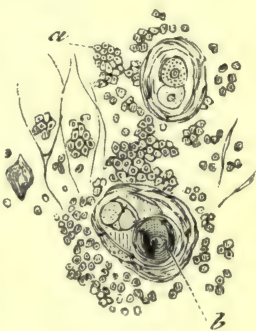

Fig. 126.

Elements of the thymus $(\times 300), a$, lymphcorpuscles ; $b$, concentric corpuscle of Hassall. part $a$, and a centre, $b$. $a$, lymphoid nective-tissue.

\section{progresses inwards (His).]}

Structure._" It consists of an aggregation of lymph-follicles (resembling the glands of Peyer) or masses of adenoid tissue held together by a framework of connective-tissue which contains blood-vessels, lymphatics, and a few nerves (fig. 125). The framework of connectivetissue gives off septa which divide the gland into lobes, these being further subdivided by finer 
septa into lobules, the lobules being separated by fine intra-lobular lamellæ of connective-tissueinto follicles $(0.5-1.5 \mathrm{~mm}$.). These follicles make up the gland-substance, and they are usually polygonal when seen in a section. Each follicle consists of a cortical and a medullary part, and the matrix or framework of both consists of a fine adenoid reticulum whose meshes are filled with lymph-corpuscles" (fig. 126,a).] Many of these corpuscles exhibit various stages of disintegration. In the medulla are found the concentric corpuscles of Hassall. [ "They consist of a central granular part, around which are disposed layers of flattened nucleated endothelial cells arranged concentrically. When seen in a section they resemble the 'cellnests' of epithelioma (fig. 126, b). They have also been compared to similar bodies which occur in the prostate. They are most numerous when the gland undergoes its retrograde metamorphosis." Sig. Mayer finds that the thymus of the frog contains structures, with transverse markings, identical with the stripes of striped muscular fibres. The structures are identieal with those called "sarcoplasts" by Margo and Paneth, and "sarcolytes" by Sig. Mayer. They also occur in large numbers in the tail of the larvæ of batrachians, when the tail is undergoing a retrograde metamorphosis.]

Simon, His, and others described a convoluted blind canal, the "central canal," as occurring within the gland, and on it the follicles were said to be placed. Other observers, Jendrassik and Klein, either deny its existence or regard it merely as a lymphatic or an artificial product. Numerous fine lymphatics penetrate into the interior of the organ, and many are distributed over its surface, but their mode of origin is unknown. [They seem to be channels through which the lymph-corpuscles are conveyed away from the gland.] Numerous blood-vessels are also distributed to the septa and follicles (fig. 125, c).

Chemical Composition. - Besides gelatin, albumin, soda-albumin, there are sugar and fat, leucin, xanthin, hypoxanthin, formic, acetic, butyric, and succinic acids. Potash and phosphoric acid are more abundant in the ash than soda, calcium, magnesium (? ammonium), chlorine, and sulphuric acia.

Function. - As long as it exists, it seems to perform the functions of a true lymph-gland. This view is supported by the fact that in reptiles and amphibians, which do not possess lymph-glands, the thymus remains as a permanently active organ. [Extirpation gave few positive results, but chemical investigation shows that the parenchyma contains a large number of products indicating considerable metabolic activity (Friedleben).]

III. The Thyroid. - Structure. - The gland consists of lobes and lobules held together by connective-tissue rich in cells. Each lobule is made up of numerous completely closed sacs.

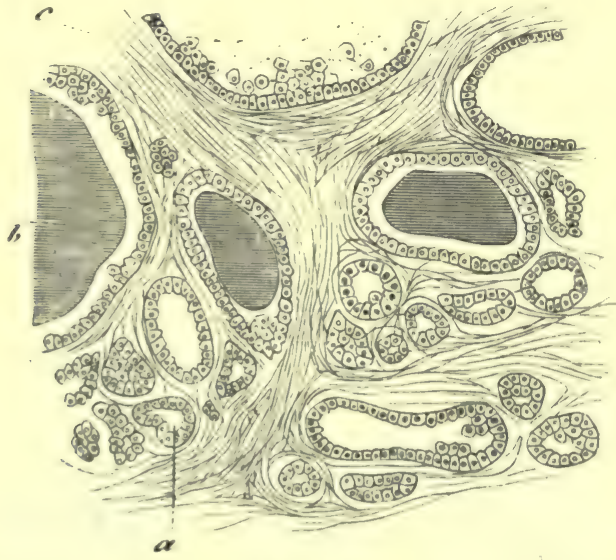

Fig. 127.

$\left(0.04\right.$ to $0^{\circ} 1 \mathrm{~mm}$. in diameter $)$, which in the embryo and the newly-born animal are composed of a membrana propria lined by a single layer of nucleated cubical cells (fig. 127). The sacs contain a transparent, viscid, albuminous fluid. [Not unfrequently the sacs contain many coloured bloodcorpuscles (Baber).] Each sac is surrounded by a plexus of capillaries which do not penetrate the membrana propria. There are also numerous lymphatics. At an early period the sacs dilate, their cellular lining atrophies, and their contents undergo colloid degeneration. When the gland-vesicles are greatly enlarged, "goitre" is produced.

The chemical composition of this gland has not been nuch investigated. In addition to the ordinary constituents, leucin, xanthin, sarkin, lactic, succinic, and volatile fatty acids have been found.

Section of the thyroid gland. $a$, closed vesicles; $b$, distended by colloid masses and lined by low columnar epithelium ; $c$, inter-vesicular connective-tissue.

[Excision. - The effects differ according to the animal operated on.

This gland has been excised in the human subject in cases of goitre. Reverdin pointed out that a peculiar condition results, called cachexia stumipriva, and practically the human being becomes a cretin. This operation therefore is highly questionable when performed on man. Rabbits endure the operation well, and so do the sheep, calf, and horse. Of dogs, cats, and foxes, only a very small number survive, nearly all die. It appears therefore that herbivora bear the operation and suffer fewer after-effects than carnivora (Sanquirico and Orecchia). The inmediate effects are fibrillar contractions, which ultimately influence the gait of the animals, convulsions, anæsthesia, great diminution of sensibility, loss of flesh, redness of the ears and 
intense heat of the skin (which disappear after several days), difficulty in seizing and eating food, kerato-conjunctivitis, and frequently disturbance of the rhythm of respiration with dyspnoea and spasms of the abdominal muscles (Schiff). The arterial blood contains about the same amount of $\mathrm{O}$ as venous blood. Certain parts of the peripheral nerves undergo a kind of degeneration similar to that found after nerve-stretching. There is albuminuria and fall of the blood-pressure. Death usually occurs between the third and fourth day, the animals being comatose (Wagner). Schiff found that if one-half of the gland was excised at once, and the other half a month afterwards, death did not occur; but Wagner denies this, for he asserts that the remaining half hypertrophies, and if it be excised, death occurs with the usual symptoms. In monkeys, five days after the operation, there are symptoms of nervous disturbance. The animals have lost their appetite, there are fibrillar contractions of the muscles of the face, hands, and feet, but the tremors disappear on voluntary effort. The appetite returns and is increased, but notwithstanding, the animal grows thin and pale; while the tremors increase and affect all the muscles of the body. These tremors are of central origin, because they disappear on dividing the nerve. Thus there is profound alteration of the motor powers. Amongst the outward symptoms are puffiness of the eyelids, swelling of the abdomen, increased hebetude, and dyspnœea, while afterwards there is a fall of the temperature and imbecility ; the tremors disappear, there is a palor of the skin, and ultimately after five to seven weeks the animals die comatose. Thus there is a slow onset of hebetude, terminating in imbecility. Very remarkable changes occur in the blood. There is a steady fall of the blood-pressure; a diminution of the red blood-corpuscles, or rather profound anæmia; leucocythæmia, the colourless corpuscles being increased to the ratio of four to fourteen; and lastly mucin is present in the blood, although normally it is not so. The salivary glands are hypertrophied, owing to the presence of mucin, which is found even in the parotid, although this is normally a serous gland ( $\$ 141)$. The swelling of the abdomen is due to hypertrophy of the great omentum. Mucin is found in the peritoneal fluid, and the spleen is also enlarged. Thus these symptoms present many features in common with those of myxœema as described by $\operatorname{Ord}(v$. Horsley).]

[Stages.- Horsley distinguishes three stages. In the first or neurotic stage, the animals exhibit constant tremors, 8 per second, and young animals do not appear to survive this stage. In the second or mucinoid stage, mucin is deposited in the tissues and blood; this change, however, is only seen to perfection in monkeys. If these animals be kept at a high artificial temperature, their life is considerably prolonged. In the third, atrophic, or marasmic period, the animals die of marasmus, while they lose their excess of mucin. Age seems to exert an important influence in thyroidectomy; young dogs survive but a short time, while old dogs merely exhibit symptoms of indolence and incapacity; and, as a matter of fact, the activity of the gland seems to be most active when tissue-metabolism is most active.]

[The following table, after Horsley, indicates the symptoms that follow loss of the function of the thyroid gland.

\begin{tabular}{|c|c|c|c|c|}
\hline & Stages. & Duration. & Symptoms. & Remarks. \\
\hline & Neurotic. & $\begin{array}{l}1 \text { to } 2 \text { weeks in dogs; } \\
1 \text { to } 3 \text { weeks in } \\
\text { monkeys. }\end{array}$ & $\begin{array}{l}\text { Tremors, rigidity, dys- } \\
\text { pnoea. }\end{array}$ & $\begin{array}{l}\text { Young dogs and monkeys } \\
\text { alike die in this stage. }\end{array}$ \\
\hline II. & Mucinoid. & $\begin{array}{l}\frac{1}{2} \text { to } 1 \text { week in dogs; } \\
3 \text { to } 7 \text { weeks in } \\
\text { monkeys. }\end{array}$ & $\begin{array}{l}\text { Commencing hebetude } \\
\text { and mucinoid degen- } \\
\text { eration of the connec- } \\
\text { tive-tissues. }\end{array}$ & $\begin{array}{l}\text { Dogs survive only to the } \\
\text { beginning of this stage; } \\
\text { monkeys die at the end, } \\
\text { if not treated. }\end{array}$ \\
\hline III. & Atrophic. & $\begin{array}{l}5 \text { to } 8 \text { weeks in mon- } \\
\text { keys. }\end{array}$ & $\begin{array}{l}\text { Complete imbecility and } \\
\text { atrophy of all tissues, } \\
\text { especially muscles. }\end{array}$ & $\begin{array}{l}\text { Monkeys survive accord- } \\
\text { ing to the temperature } \\
\text { of the air-bath.] }\end{array}$ \\
\hline
\end{tabular}

Functions.-The functions of the thyroid gland are very obscure. Perhaps it may be an apparatus for regulating the blood-supply to the head (?). It becomes enlarged in Basedow's disease, in which there is great palpitation, as well as protrusion of the eyeball or exophthalmos, which seem to depend upon a simultaneous stimulation of the accelerating nerve of the heart, and the sympathetic fibres of the smooth muscles in the orbital cavity and the eyelids, as well as of the inhibitory fibres of the vessels of the thyroid. In many localities it is common to find swelling of the thyroid constituting goitre, which is sometimes, but far from invariably, associated with idiocy and cretinism. [Horsley finds that its removal is the essential cause of myxodema and cretinism. He regards it (1) as a blood-forming gland, so that it has a hæmapoietic function, but Gibson finds no grounds for supporting this view. During the anæmia resulting from its removal, the blood of the thyroid vein contains 7 per cent. more red bloodcorpuscles than the corresponding artery (Horsley). (2) It seems to regulate the formation of mucin in the body. After its removal the normal metabolism is no longer maintained, and there is a corresponding increasingly defective condition of nutrition.] 
In the Tunicata, this gland, represented by a groove, secretes a digestive fluid. In vertebrates, it is an organ which has undergone a retrograde change (Gegenbaur).

IV. The Suprarenal Capsules. - Structure.-These organs are invested by a thin capsule which sends processes into the substance of the organ. They consist of an outer (broad) or cortical layer and an inner (narrow) or medullary layer. The former is yellowish in colour, firm and striated, while the latter is softer and deeper in tint. In the outermost zone of the cortex (fig. 128, $b$ ), the trabeculæ form polygonal meshes, which contain the cells of the glandsubstance ; in the broader middle zone the meshes are elongated, and the cells filling them are arranged in columns radiating outwards. Here the cells are transparent and nucleated, often

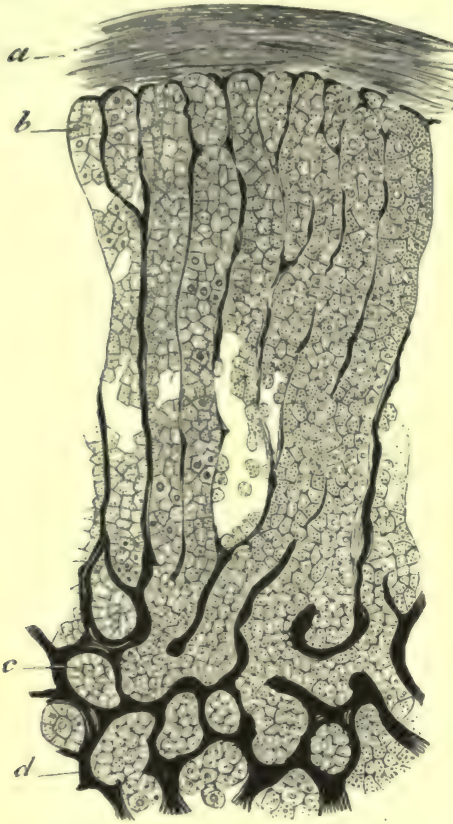

Fig. 128.

Section of a human suprarenal capsule. $a$, capsule ; $b$, gland-cells of the cortex arranged in columns ; $c$, glandular network of the medulla; $d$, blood-vessels containing oil-globules ; in the innermost narrow zone the polygonal arrangement prevails, and the cells often contain yellowish-brown pigment. In the medulla (c), the stroma forms a reticulum containing groups of cells of very irregular shape. Numerous blood-vessels occur in the gland, especially in the cortex. [The nerves are extremely numerous, and are derived from the renal and solar plexuses. Many of the fibres are medullated. After they enter the gland, numerous ganglionic cells occur in the plexuses which they form. Indeed, some observers regard the cells of the medulla as nervous. Undoubtedly, numerous multipolar nervecells exist within the gland.]

Chemical Composition. - The suprarenals contain the constituents of connective- and nerve-tissue ; also leucin, hypoxanthin, benzoic, hippuric, and taurocholic acids, taurin, inosit, fats, and a body which becomes pigmented by oxidation. Amongst inorganic substances potash and phosphoric acid are most abundant.

The function of the suprarenal bodies is very obscure. It is noticeable, however, that in Addison's disease ("bronzed skin"), which is perhaps primarily a nervous affection, these glands have frequently, but not invariably, been found to be diseased. Owing to the injury to adjacent abdominal organs, extirpation of these organs is often, although not always, fatal ; in dogs pigmented patches have been found in the skin near the mouth. Brown Séquard thinks they may be concerned in preventing the over-production of pigment in the blood.

[Spectrum.-MacMunn finds that the medulla of the suprarenal bodies (in man, cat, dog, guinea-pig, rat, \&c.) gives the spectrum of hæmochromogen (\$18), while the cortex shows that of what he calls histohæmatin, the latter being a group of respiratory pigments. He finds that hæmochromogen is only found in excretory organs (the bile, the liver), hence he regards the medulla as excretory, so that part of the function of the adrenals may be "to metamorphose effete hæmoglobin or hæmatin into hæmochromogen," and when they are diseased, the effete pigment is not removed, hence the pigmentation of the skin and mucous membranes. Taurocholic acid has been found in the medulla by Vulpian, and pyro-catechin by Krukenberg. MacMunn believes that "they have a large share in the downward metamorphosis of colouring matter."']

V. Hypophysis Cerebri-Coccygeal and Carotid Glands.-The hypophysis cerebri, or pituitary body, cousists of an anterior lower or larger lobe, partly embracing the posterior lower or smaller lobe. These two lobes are distinct in their structure and development. The posterior lobe is a part of the brain, and belongs to the infundibulum. The nervous elements are displaced by the ingrowth of connective-tissue and blood-vessels. The anterior portion represents an inflected and much altered portion of ectoderm, from which it is developed. It contains gland-like structures, with connective-tissue, lymphatics, and blood-vessels, the whole being surrounded by a capsule. According to Ecker and Mihalkowicz, it resembles the suprarenal capsule in its structure, while, according to other observers, in some animals it is more like the thyroid. Its functions are entirely unknown. [Excision.-Horsley has removed this gland twice successfully in dogs, which lived from five to six months. No nervous or other symptoms were noticed, but when the cortex of the brain was exposed and stimulated, a great increase in the excitability of the motor regions was induced, even slight stimulation being followed by violent tetanus and prolonged epilepsy.] 
Coccygeal and Carotid Glands. - The former, which lies on the tip of the coccyx, is composed to a large extent of plexuses of small, more or less cavernous, arteries, supported and enclosed by septa and a capsule of connective-tissue (Luschka). Between these lie polyhedral granular cells arranged in networks. The carotid gland has a similar, structure (p. 77). Their functions are quite unknown. Perhaps both organs may be regarded as the remains of embryonal blood. vessels (Arnold).

104. COMPARATIVE. - The heart in fishes (fig. 129, I.), as well as in the larvæ of amphibians with gills, is a simple venous heart, consisting of an auricle and a ventricle. The ventricle propels the blood to the gills, where it is oxygenated (arterialised);

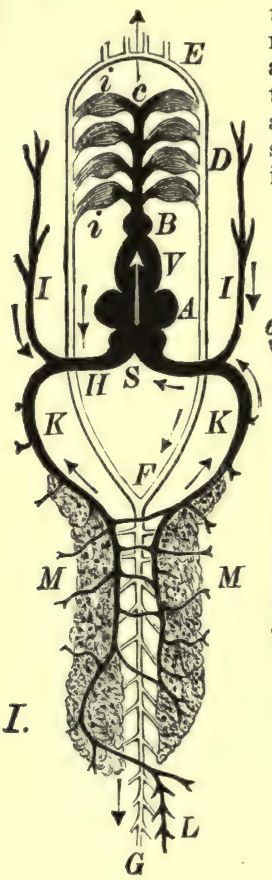
thence it passes into the aorta to be distributed to all parts of the body, and returns through the capillaries of the body and the veins to the heart. The amphibians (frogs) have two auricles and one ventricle (Frog, II.). From the latter there proceeds one vessel which gives off the pulmonary arteries, and as the aorta supplies the rest of the body with blood, the veins of the systemic circulation carry their blood to the right auricle, those of the lung into the left auricle. In fishes and amphibians there is a dilatation at the
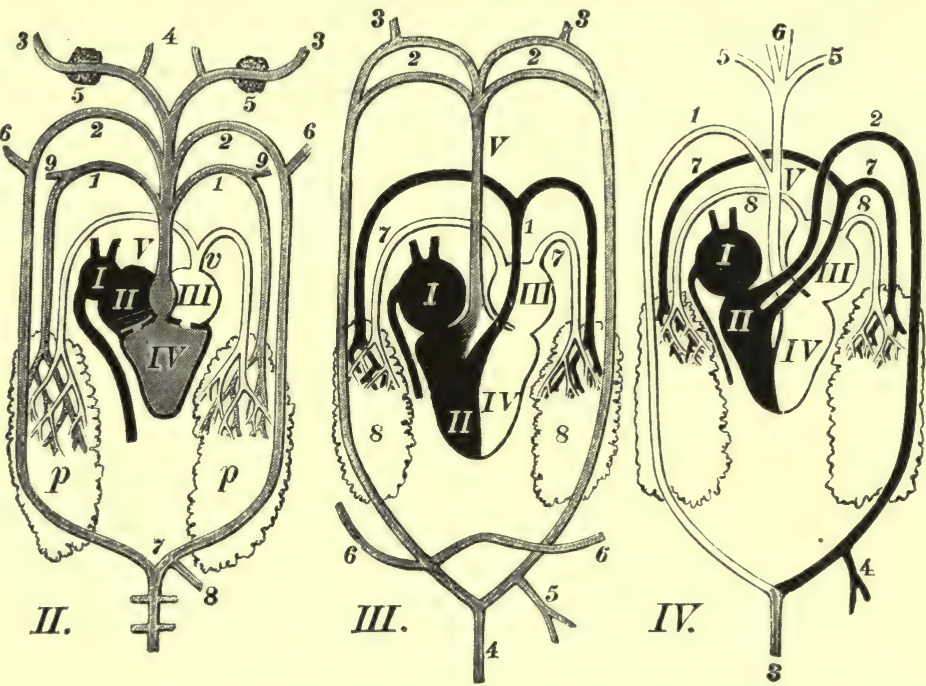

Fig. 129.

Schemata of the circulation. I. Fish $-A$, auricle ; $S$, sinus venosus ; $V$, ventricle ; $B$, bulbus aortæ ; $c$, branchial arteries ; $i$, branchial vessels ; $V v$, branchial veins ; $E$, circulus cephalicus aortæ ; $F$, common aorta ; $G$, caudal artery ; $H$, duct of Cuvier ; $I$, anterior, and $K$, posterior cardinal veins ; $L$, caudal vein ; $M, M$, kidneys. II. Frog.-I, sinus venosus ; II, and III, right and left auricles; IV, ventricle; V, aorta with the bulb; 1 , pulmonary arteries ; 2, arch of the aorta ; 3, carotid ; 4, lingual ; 5, carotid gland, and 6, axillary arteries ; 7, common aorta ; 8 , coeliac artery ; 9 , cutaneous artery ; $V v$, pulmonary veins ; $p$, $p$, lungs. III. Saurians.-I, right auricle, with the venæ cavæ; II, right ventricle ; III, left auricle; IV, left ventricle ; V, anterior common aorta ; 1 , pulmonary artery ; 2 , arch of the aorta; 3 , carotid artery ; 4, posterior common aorta; 5 , coliac, and 6 , subclavian, arteries ; 7, pulmonary veins ; 8, lungs. IV. Tortoise._I, right auricle with the venæ cavæ; II, right, and IV, left ventricles; III, left auricle; 1 and 2 , right and left aortæ; 3, posterior common aorta ; 4, cœliac, 5, subclavian, 6, carotid, and 7, pulmonary arteries ; 8, pulmonary veins.

commencement of the aorta, the bulbus arteriosus, which is partly provided with strong muscles. The reptiles (III.) possess two separate auricles, and two imperfectly separated ventricles. The aorta and pulmonary artery arise separately from the two latter chambers. The venous blood of the systemic and pulmonary circulations flows separately into the right and left auricles, and the two streams are mixed in the ventricle. In some reptiles the opening in the ventricular septum seems capable of being closed. The complete separation of the ventricle into two is seen in fig. IV., in the tortoise. The lower vertebrates have valves at the orifices of the venæ cavæ, which are rudimentary in birds and some mammals. All birds and mammals have two 
completely separate auricles and two separate ventricles. In the halicore the apex of the ventricles is deeply cleft. Some animals have accessory hearts, e.g., the eel in its caudal vein. They are very probably lymph-hearts (Robin). The veins of the wing of the bat pulsate (Schiff). The lowest vertebrate, amphioxus, has no heart, but only a rhythmically-pulsating vessel.

Amongst blood-glands, the thymus and spleen occur throughout the vertebrata, the latter being absent only in amphioxus and a few fishes.

Amongst invertebrata a closed vascular system, with pulsatile movement, occurs here and there, e.g., amongst echinodermata (star-fishes, sea-urchins, holothurians) and the higher worms. The insects have a pulsating "dorsal vessel" as the central organ of the circulation, which is a contractile tube provided with valves and dilated by muscular action; the blood being propelled rhythmically in one direction into the spaces which lie amongst the tissues and organs, so that these animals do not possess a closed vascular system. The mollusca have a heart with a lacunar vascular system. The cephalopods (cuttle-fish) have three hearts - a simple arterial heart, and two venous simple gill-hearts, each placed at the base of the gills. The vessels form a completely closed cireuit. The lowest animals have either a pulsatile vesicle, which propels the colourless juice into the tissues (infusoria), or the vascular apparatus may be entirely absent.

105. HISTORICAL RETROSPECT. - The ancients held various theories regarding the movement of the blood, but they knew nothing of its circulation. According to Aristotle (384 3.C.), the heart, the acropolis of the body, prepared in its cavities the blood, which streamed through the arteries as a nutrient fluid to all parts of the body, but never returned to the heart. With Herophilus and Erasistratus (300 B.c.), the celebrated physicians of the Alexabdrian school, originated the erroneous view that the arteries contain air, which was supplied to them by the respiration (hence the name artery). They were led to adopt this view from the empity condition of the arteries after death. By experiments upon animals, Galen disproved this view (131-201 A.D.)-“" Whenever I injured an artery," he says, "blood always flowed from the womuled vessel. On tying part of an artery between two ligatures, the part of the artery so included is always filled with blood."

Still, the idea of a single centrifugal movement of the blood was retained, and it was assumed that the right and left sides of the heart ecmmunicated directly by means of openings in the septum of the heart, until Vesalius showed that there are no openings in the septum. Michael Servetus (a Spanish monk, burned at Geneva, at Calvin's instigation, in 1553) discovered the pulmonary circulation. Cesalpinus confirmed this observation, and named it "Circulatio." Fabricius ab Aquapendente (Padua, 1574) investigated the valves in the veins more carefully (although they were known in the 5th century to Theodoretus, Bishop in Syria), and he was acquainted with the centripetal movement of the blood in the veins. Up to this time it was imagined that the veins carried blood from the centre to the periphery, although Vesalius was acquainted with the centripetal direction of the blood-stream in the large venous trunks. At length William Harvey, who was a pupil of Fabricius (1604), demonstrated the complete circulation (1616-1619), and published his great discovery in 1628. [For the history of the discovery of the circulation of the blood, see the works of Willis on "W. Harvey," "Servetus and Calvin," those of Kirchner, and the various Harveian orations.]

According to Hippecrates, the heart is the origin of all the vessels; he was acquainted with the large vessels arising from the heart, the valves, the chordie tenclinex, the auricles, and the closure of the semi-lunar valves. Aristotle was the first to apply the terms aorta and venæ cavie; the school of Erasistratus used the term carotid, and indicated the functions of the venous valves. In Cicero a distinction is drawn between arteries and veins. Celsus mentions that if a vein be struck below the spot where a ligature has been applied to a limb, it bleeds, while Aretaeus (50 A.D.) knew that arterial blood was bright and venous dark. Pliny $(+79$ A.D.) described the pulsating fontanelle in the child. Galen (131-203 A.D.) was acquainted with the existence of a bone in the septum of the heart of large animals (ox, deer, elephant). He also surmised that the veins communicated with the arteries by fine tubes. The demonstration of the capillaries, however, was only possible by the use of the microscope, and employing this instrument, Malpighi (1661) was the first to demonstrate the capillary circulation. Leuwenhoek (1674) described the capillary circulation more carefully, as it may be seen in the web of the frog's foot and other transparent membranes. Blancard (1676) proved the existence of capillary passages by means of injections. William Cooper (1697) proved that the same condition exists in warm-blooded animals, and Ruysch made similar injections. Stenson (born 1638) established the muscular nature of the heart, although the Hippocratic and Alexandrian schools had already surmised the fact. Cole proved that the sectional area of the blood-stream became wider towards the capillaries (1681). Joh. Alfons Borelli (1608-1679) was the first to estimate the amount of work done by the heart. 


\section{Physiology of Respiration.}

THE object of respiration is to supply the oxygen necessary for the oxidationprocesses that go on in the body, as well as to remove the carbon dioxide formed within the body. The most important organs for this purpose are the lungs. There is an outer and an inner respiration - the former embraces the exchange of gases between the external air and the blood-gases of the respiratory organs (lungs and skin) - the latter, the exchange of gases between the blood in the capillaries of the systemic circulation and the tissues of the body.

[The pulmonary apparatus consists of (1) an immense number of small sacsthe air-vesicles-filled with air, and covered externally by a very dense plexus of capillaries; (2) air-passages - the nose, pharynx, larynx, trachea, and bronchi communicating with (1); (3) the thorax with its muscles, acting like a pair of bellows, and moving the air within the lungs.]

106. STRUCTURE OF THE AIR-PASSAGES AND LUNGS.-The lungs are compound tubular glands, which separate $\mathrm{CO}_{2}$ from the blood. Each lung is provided with an excretory duct (bronchus) which joins the common respiratory passage of both lungs-the trachea.

Trachea. - The trachea and extra-pulmonary bronchi are similar in structure. The basis of the trachea consists of 16-20 C-shaped incomplete cartilaginous hoops placed over each other. These rings consist of hyaline cartilage, and are united to each other by means of tough fibrous tissue containing much elastic tissue, the latter being arranged chiefly in a longitudinal direction. The function of the cartilages is to keep the tube open under varying conditions of pressure. Pieces of cartilage having a similar function oceur in the bronchi and their branches, but they are absent from the bronchioles, which are less than $1 \mathrm{~mm}$. in diameter. In the smaller bronchi, the cartilages are fewer and scattered more irregularly. [In a transverse section of a large intra-pulmonary bronchus, two, three, or more pieces of eartilage, each invested by its perichondrium, may be found.] At the points where the bronchi subdivide, the cartilages assume the form of irregular plates embedded in the bronchial wall.

An external fibrous layer of connective-tissue and elastic fibres covers the trachea and the extra-pulmonary bronchi externally. Towards the oesophagus, the elastic elements are more numerous, and there are also a few bundles of plain muscular fibres arranged longitudinally. Within this layer there are bundles of non-striped muscular fibres which pass transversely between the cartilages behind, and also in the intervals between the cartilages. [These pale reddish fibres constitute the trachealis muscle, and are attached to the inner surfaces of the eartilages at a little distance from their free ends. The arrangement varies in different animals -thus, in the cat, dog, rabbit, and rat the muscular fibres are attached to the external surfaces of the cartilages, while in the pig, sheep, and ox they are attached to their internal surfaces (Stirling).] Some museular fibres are arranged longitudinally external to the transverse fibres. The function of these muscular fibres is to prevent too great distension when there is great pressure within the air-passages.

The mucous membrane consists of a basis of very fine connective-tissue, contuining much adenoid tissue with numerous lymph-corpuscles. Numerous elastic fibres are arranged chiefly in a longitudinal direction under the basement membrane. They are also abundant in the deep layers of the posterior part of the membrane opposite the intervals between the cartilages. A small quantity of loose sub-mucous connective-tissue containing the large blood-vessels, glands, and lymphatics unites the mucous membrane to the perichondrium of the cartilages. The epithelium consists of a layer of columnar ciliated cells with several layers of immature 
cells under them. [The superficial layer of cells is colunnar and ciliated (fig. 130, b), while those lying under them present a variety of forms, and below all is a layer of somewhat flattened squames, $c$, resting on the basement membrane, $d$. These squames constitute a layer quite distinct from the basement membrane, and they form the layer described as Débove's. membrane. They are active germinating cells, and play a most important part in connection

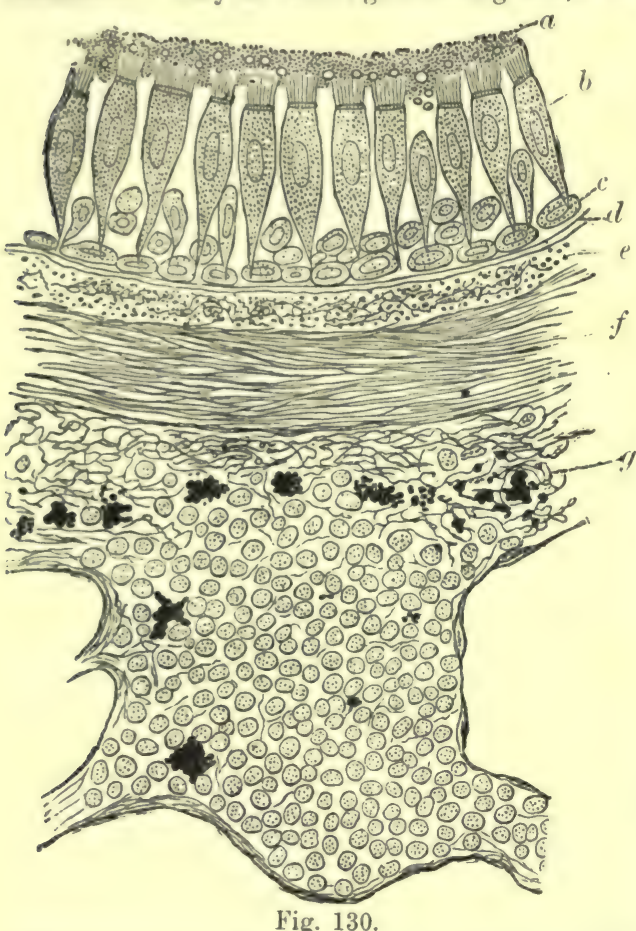

Transverse section of part of a human bronchus $(\times 450)$ $a$, precipitated mucus; $b$, ciliated columnar epithelium ; $c$, deep germinal layer of cells (Débove's membrane); $d$, elastic basement membrane; $e$, elastic fibres divided transversely (inner fibrous layer); $f$, bronchial muscle; $g$, outer fibrous layer with leucocytes and pigment granules (black); below a mass of arlenoid tissue. with the regeneration of the epithelium, after the superficial layers have been shed, in such conditions as bronchitis. Not unfrequently a little viseid mucus (a) lies on the free ends of the cilia. In the intermediate layer, the cells are more or less pyriform or battledore-shaped, with their long tapering process inserted amongst the deepest layer of squames. According to Drasch, this long process is attached to one of these cells and is an outgrowth from it, the whole constituting a "foot-cell."]

Under the epithelium is the homogeneous basement membrane, through which fine canals pass, connecting the cement of the epithelium with spaces in the mucosa. [This membrane is well marked in the human trachea, where it plays an important part in many pathological conditions, $e_{.} g$., bronchitis. It is stained bright red with picrocarmine.] The cilia act so as to carry any secretion towards the larynx. Goblet cells exist between the ciliated columnar cells. Numerous small compound tubular mucous glands occur in the mucous membrane, chiefly between the cartilages. Their ducts open on the surface by means of a slightly funnel-shaped aperture, into which the ciliated epithelium is prolonged for a short distance. [The acini of some of these glands lie outside the trachealis muscle. The acini are lined by cubical or columnar secretory epithelium. In some animals (dog) these cells are clear, and present the usual characters of a mucus-secreting gland; in man, some of the cells may be clear, and others "granular," but the appearance of the cells depends upon the physiological state of activity.] These glands secrete the mucus, which entangles particles inspired

with the air, and is carried towards the larynx by ciliary action. [Numerous lymphatics exist in the mucous and sub-inucous coat, and not unfrequently small aggregations of adenoid tissue occur (especially in the cat) in the mucous coat, usually around the ducts of the glands. They are comparable to the solitary follicles of the alimentary tract. The blood-vessels are not so numerous as in some other mucous membranes. [A plexus of nerves containing numerous ganglionic cells at the nodes exists on the posterior surface of the trachealis muscle. The tibres are derived from the vagus, recurrent laryngeal, and sympathetic (C. Frankenhauser, $W$. Stirling, Kandarazi).]

[The mucous membrane of the trachea and extra-pulmonary bronchi, therefore, consists of the following layers from within outwards :-

(1) Stratified columnar ciliated epithelium.

(2) A layer of flattened cells (Débove's membrane).

(3) A clear homogeneous basement membrane.

(4) A basis of areolar tissue, with adenoid tissue and blood-vessels, and outside this a layer of longitudinal elastic fibres.

Outside this, again, is the sub-mucous coat, consisting of loose areolar tissue, with the larger vessels, lymphatics, nerves, and mucous glands.]

[The Bronchi. - In structure the extra-pulmonary bronchi resemble the trachea. As they 
pass into the lung they divide very frequently, and the branches do not anastomose. In the intra-pulmonary bronchi the subdivisions become finer and finer, the finest branches being called terminal bronchi, or bronchioles, which open separately into clusters of air-vesicles.]

[Eparterial and Hyparterial Bronchi.-As the bronchi proceed, one main trunk passes into the lung, running towards its base, and from it are given off branches dorsally and ventrally, and these branches again subdivide. In man one main branch comes off from the right bronchus and proceeds to the upper right lobe, above the place where the pulmonary artery crosses the bronchus. Such branches are called eparterial, and they are more numerous in birds. In man, all the branches, both on the right and left side, come off below the point where the pulmonary artery crosses the bronchus, and are called hyparterial bronchi $(C$. Aeby).]

[In the middle-sized intra-pulmonary bronchi, the usual characters of the mucous membrane are retained, only it is thinner ; the cartilages assume the form of irregular plates situated in the outer wall of the bronchus; while the muscular fibres are disposed in a complete circle, constituting the bronchial muscle (fig. $130, f$ ). When this muscle is contracted, or when the bronchus as a whole is contracted, the mucous membrane is thrown into longitudinal folds, and opposite these folds the elastic fibres form large elevations. This muscle is particularly well developed in the smaller microscopic bronchi. Numerous elastic fibres, $\varepsilon$, disposed longitudinally, exist under the basement membrane, $d$. They are continuous with those of the trachea, and are prolonged onwards into the lung. The mucous membrane of the larger intrapulmonary bronchi consists of the following layers from within outwards :-

(1) Stratified columnar ciliated epithelium (fig. 130, b).

(2) Débove's membrane (fig. 130,c).

(3) Transparent homogeneous basement membrane (fig. 130, d).

(4) Areolar tissue with longitudinal elastic fibres (fig. 130,e).

(5) A continuous layer of non-striped muscular fibres disposed circularly (bronchial muscle, fig. $130, f)$.

Outside this is the sub-mucous coat, consisting of areolar tissue mixed with much adenoid tissue (fig. $130, g$ ), sometimes arranged in the form of cords, the lymph-follicular cords. It also contains the acini of the numerous mucous glands, blood-vessels, and lymphatics. The ducts of the glands perforate the muscular layer, and open on the free surface of the mucous membrane. The sub-mucous coat is connected by areolar tissue with the perichondrium of the cartilages. Outside the eartilages are the nerves and nerve-ganglia accompanying the bronchial vessels. The branches of the pulmonary artery and of the pulmonary vein usually lie on opposite sides of the bronchus, while there are several branches of the bronchial arteries and veins. Fat cells also occur in the peri-bronchial tissue.]

In the small bronchi the cartilages and glands disappear, but the circular muscular fibres are well developed. They are lined by lower columnar ciliated epithelium, containing goblet cells.

Bronchioles. - After repeated subdivision, the bronchi form the "smallest bronchi" (about 0.5 to $1 \mathrm{~mm}$.) or lobular bronchial tubes. Each tube is lined by a layer of ciliated epithelium, but the glands and cartilages have disappeared. These tubes have a few lateral alveoli or aircells communicating with them. Each smallest bronchus ends in a "respiratory bronchiole" (Kölliker), which gradually becomes beset with more air-cells, and in which squamous epithelium begins to appear between the eiliated epithelial cells. [Each bronchiole opens into several wider alveolar or lobular passages. Each passage is completely surrounded with air-cells, and from it are given off' several similar but wider blind branches, the infundibula, which, in their turn, are beset on all sides with alveoli or air-cells. Several infundibula are connected with each bronchiole, and the former are wider than the latter. Each bronchiole, with its alveolar passages, infundibula, and air-vesicles, is termed a lobule, whose base is directed outwards, and whose apex may be regarded as a terminal bronchus. The lung is made up of an immense number of these lobules, separated from each other by septa of connective-tissue, the interlobular septa (fig. 133, e) which are continuous on the one hand with the sub-plural connectivetissue, and on the other with the peri-bronchial connective-tissue.]

[There is an alteration in the structure of the bronchi, as we proceed from the larger to the smaller tubes. The cartilages and glands are the first structures to disappear. The circular bronchial muscle is well developed in the smaller bronchi and bronchioles, and exists as a continuous thin layer over the alveolar passages, but it is not continued over and between the air-cells. Elastic fibres, continuous, on the one hand, with those in the smaller bronchi, and on the other with those in the walls of the air-cells, lie outside the muscular fibres in the bronchioles and infundibula. In the respiratory bronchioles, the ciliated epithelium is reduced to a single layer, and is mixed with the stratified form of epithelium, while, where the alveolar passages open into the air-cells or alveoli, the epithelium is non-ciliated, low, and polyhedral.]

Alveoli or Air-Cells. - The form of the cells, which are $250 \mu$ ( $\frac{1}{100}$ inch) in diameter, may be more or less spherical, polygonal, or cup-shaped. They are disposed around and in communication with the alveolar passages. Their form is determined by the existence of a nearly structureless membrane, composed of slightly fibrillated connective-tissue containing a few 
corpuscles. This is surrounded by numerous fine elastic fibres, which give to the pulmonary parenchyma its well-marked elastic characters (fig. $132, e, e$ ). These fibres often bifurcate, and are arranged with reference to the alveolar wall. They are very resistant, and in some cases of lung disease may be recognised in the sputum. A few non-striped muscular fibres exist in the delicate connective-tissue between adjoining air-vesicles. These muscular fibres sometimes become greatly developed in certain diseases (Amold, W. Stirling). The air-cells are lined by two kinds of cells-(1) large, transparent, clear polygonal (nucleated ?) squames or placoids $(22-45 \mu)$ lying over and between the capillaries in the alveolar wall (fig. 131,a); (2) smahl irregular "granular" nucleated cells $(7-15 \mu)$ arranged singly or in groups (two or three) in the interstices between the capillaries. They are well seen in a cat's lung (fig. 131, $d$ ). [When acted

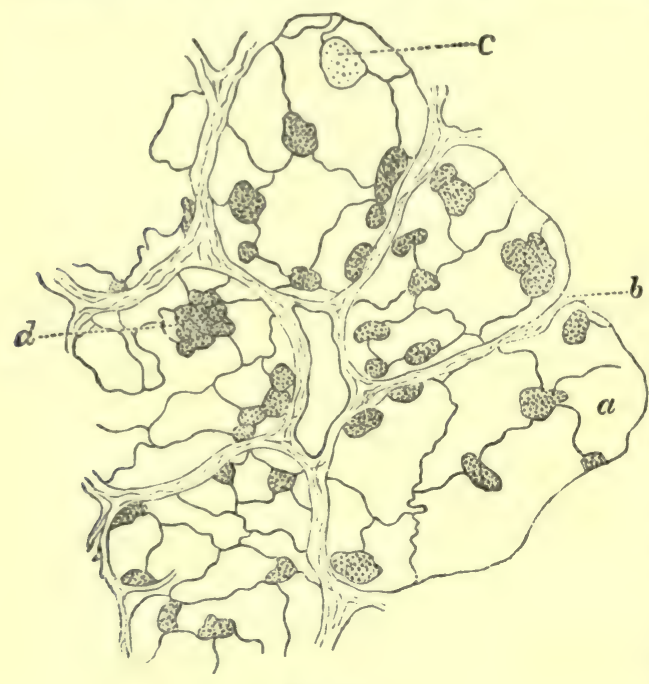

Fig. 131. on with nitrate of silver the cementsubstance bounding the clear cells is stained, but the small cells become of a uniform brown granular appearance, so that they are readily recognised. Small holes or "pseudo-stomata" seem to exist in the cement-substance, and are most obvious in distended alveoli. They open into the lymph-canalicular system of the alveolar wall (Klein), and through them the lymph-corpuscles, which are always to be found on the surface of the air-vesicles, migrate, and carry with them into the lymphatics particles of carbon derived from the air.] In the alveolar walls is a very dense plexus of fine capillaries (fig. 132, c), which lie more towards the cavity of the air-vesicle, being covered only by the epithelial lining of the air-cells. Between two adjacent alveoli there is only a single layer of capillaries (man), and on the boundary-line between two air-cells the course of the capillaries is twisted, thus projecting sometimes into the one alveoIus, sometimes into the other.

[The number of alveoli is stated to Air-vesicles injected with silver nitrate. $a$, outlines be about 725 millions, a result obtained of squamous epithelium ; $b$, alveolar wall ; $c$, young by measuring the size of the air-vesicles epithelium cell ; $d$, aggregation of young epithelial and ascertaining the amount of air in cells germinating.

the lung after an ordinary inspiration, determining how much of this air is in the air-vesicles and bronchi respectively. The superficial area of the air-vesicles is about 90 square metres, or 100 times greater than the surface of the body ( 8 to $\cdot 9$ sq. metre).]

The Blood-vessels of the lung belong to two different systems:-(A) Pulmonary vessels (lesser circulation). The branches of the pulmonary artery accompany the bronchi and are closely applied to them. [As they proceed they branch, but the branches do not anastomose, and ultimately they terminate in small arterioles, which supply several adjacent alveoli, each arteriole splitting up into capillaries for several air-cells (fig. 132, v, c). An efferent vein usually arises at the opposite side of the air-cells, and carries away the purified blood from the capillaries. In their course these veins unite to form the pulmonary veins, which, again, are joined in their course by a few small bronchial veins. The veins usually anastomose in the earlier part of their course, whilst the corresponding arteries do not.] Although the capillary plexus is very fine and dense, its sectional area is less than the sectional area of the systemic capillaries, so that the blood-stream in the pulmonary capillaries must be more rapid than that in the capillaries of the body generally. The pulmonary veins, unlike veins generally, are collectively narrower than the pulmonary artery (water is given off in the lung), and they have no valves. [The pulmonary artery contains venous blood, and the pulmonary veins pure or arterial blood].

(B) The bronchial vessels represent the nutrient system of the lungs. They (1-3) arise from the aorta (or intereostal arteries) and accompany the bronchi without anastomosing with the branches of the pulmonary artery. In their course they give branches to the lymphatic glands at the hilum of the lung, to the walls of the large blood-vessels (vasa vasorum), the pulmonary pleura, the bronchial walls, and the interlobular septa. The blood which issues from their capillaries is returned-partly by the pulmonary veins-hence, any considerable interference with the pulmonary circulation causes congestion of the bronchial mucous membrane, resulting in a catarrhal condition of that membrane. The greater part of the blood is returned by the bronchial veins, which open into the vena azygos, intercostal vein, or superior vena cava. The 
veins of the smaller bronchi (fourth order onwards) open into the pulmonary veins, and the anterior bronchial also communicate with the pulmonary vein (Zuckerkandl).

[The Pleura. - Each pleural cavity is distinct, and is a large serous sac, which really belongs to the lymphatic system of the lung. The pleura consists of two layers, visceral and parietal. The visceral pleura covers the lung; the parietal portion lines the wall of the chest, and the two layers of the corresponding pleura are continuous with one another at the root of the lung. The visceral pleura is the thicker, and may readily be separated from the inner surface of the chest. Structurally, the pleura resembles a serous membrane, and consists of a thin layer of fibrous tissue covered by a layer of endothelium. Under this layer, or the pleura proper, is a deep or sub-serous layer of looser areolar tissue, containing many elastic fibres. The layer of the pleura pulmonalis of some animals, as the guinea-pig, contains a network of non-striped muscular fibres (Klein). Over the lung it is also continuous with the interlobular septa. 'The interlobular septa (fig. 133, e) consist of bands of fibrous tissue separating adjoining lobules,

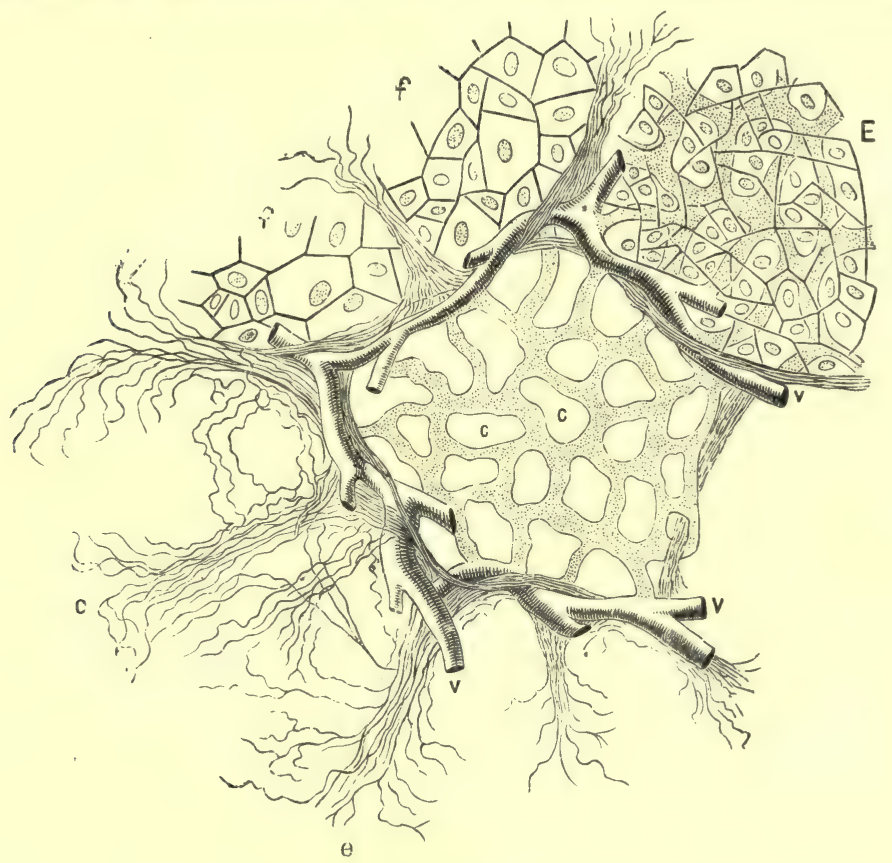

Fig. 132.

Semi-diagrammatic representation of the air-vesicles of the lung. $v, v$, blood-vessels at the margins of an alveolus ; $c, c$, its blood-capillaries ; $\mathrm{E}$, relation of the squamous epithelium of an alveolus to the capillaries in its wall ; $f$, alveolar epithelium shown alone; $e$, $e$, elastic tissue of the lung.

and they become continuous with the peri-bronchial connective-tissue entering the lung at its hilum. Thus the fibrous framework of the lung is continuous throughout the lung, just as in other organs. The connection of the sub-pleural fibrous tissue with the connective-tissue within the substance of the lung has most important pathological bearings. The interlobular septa contain lymphatics and blood-vessels. The endothelium covering the parietal layer is of the ordinary squamous type, but on the pleura pulmonalis- the cells are less flattened, more polvhedral, and granular. They must necessarily vary in shape with changes in the volume of the lung, so that they are more flattened when the lung is distended, as during inspiration. The pleura contains many lymphatics, which communicate by means of stomata with the pleural cavity.]

[The Lymphatics of the lung are numerous, and are arranged in several systems. The various air-cells are connected with each other by very delicate connective-tissue, and, according to J. Arnold, in some parts this interstitial tissue presents characters like those of adenoid tissue ; so that the lung is traversed by a system of juice-canals or "Saft-canälehen."] [In the deep layer of the pleura there is a $(a)$ sub-pleural plexus of lymphatics partly derived from the pleura, 
but chiefly from the lymph-canalicular system of the pleural alveoli. Some of these branches proceed to the bronchial glands, but others pass into the interlobular septa, where they join (b) the peri-vascular lymphatics which arise in the lymph-canalieular system of the alveoli. These trunks, provided with valves, run alongside the pulmonary artery and vein, and in their course they form frequent anastomoses. Snecial vessels arise within the walls of the bronchi, and occur chiefly in the outer coat of the latter, constituting $(c)$ the peri-bronchial lymphaties, which anastomose with $b$. The branches of these two sets run towards the bronchial glands. Not unfrequently (cat) masses of adenoid tissue are found in the course of these lymphatics.] The lymph-canalicular system and the lymphaties besome injected when fine-coloured particles are inspired, or are introduced into the air-cells artificially. The pigment particles pass through the semi-fluid cement-substance into the lymph-canalicular system and thence into the lym-

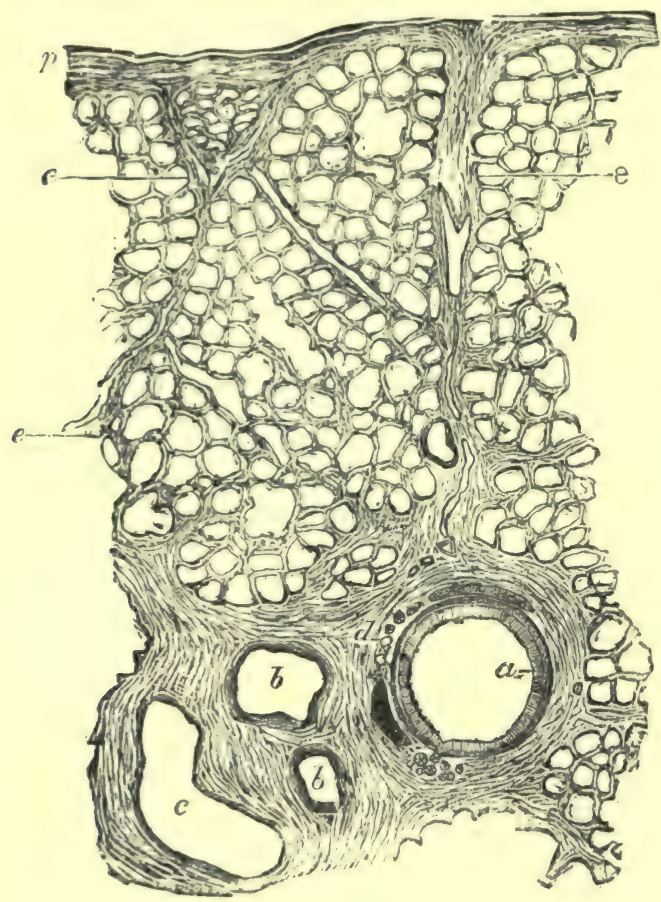

Fig. 133. phatics; or, according to Klein, they pass through actual holes or pores in the cement (p. 162).] [This pigmentation is well seen in coal-miner's lung or anthracosis, where the particles of carbon pass into and are found in the lymphatics. Sikorski and Küttner showed that pigment reached the lymphatics in this way during life. If pigment, China ink, or indigo-carmine be introduced into a frog's lung, it is found in the lymphatic system of the lung. Ruppert, and also Schotielius, showed that the same result occurred in dogs after the inhalation of charcoal, cinnabar, or precipitated Berlin blue, and von Ins after the inhalation of silica. Schestopal used China ink and cinnabar suspended in $\frac{3}{4}$ per cent. salt solution.] Excessively fine lymphcauals lie in the wall of the alveoli in the interspaces of the capillaries, and there are slight dilatations at the points of crossing. According to Pierret and Renaut every air-cell of the lung of the $o x$ is surrounded by a large lymph. space, such as occurs in the salivary glands. When a large quantity of fluid is injected into the lung, it is absorbed with great rapidity; even blood-corpuscles rapidly pass into the lymphatics.

The superficial lymphatics of the pulmonary pleura communicate with the pleural cavity by means of free

Human lung $\left(\times 50\right.$ and reduced $\left.\frac{1}{4}\right)$. $a$, small bronchus; openings or stomata, and the same is
$b, b$, pulmonary artery ; $c, j$ lmonary vein ; $e$, inter- true of the lymphatics of the parietal lobular septa, continuous with the deep layer of the pleura, but these stomata are confiued pleura, $p$.

to linited areas over the diaphragmatic pleura. [The lymphatics in the eostal pleura occur over the intercostal spaces and not over the ribs (Inblowski).] The large arteries of the lung are provided with lymphatics which lie between the middle and outer coats. [The movements of the lung during respiration are most important factors in moving the lymph onwards in the pulmonary lymphatics. The reflux of the lymph is prevented by the presence of valves.]

[The nerves of the lung are derived from the anterior and posterior pulmonary plexuses, and consist of branches from the vagus and sympathetic. They enter the lungs and follow the distribution of the brunchi, several sections of nerve-trunks being usually found in a transverse section of a large bronchial tube. The nerves lie outside the cartilages, and are in close relation with the branches of the bronchial arteries. Medullated and non-medullated nerve-fibres occur in the nerves, which also contain numerous small ganglia (Remak, Klein, Stirling). In the lung of the calf the ganglia are large. The exact mode of termination of the nerve-fibres within the lung has yet to be ascertained in mammals, but some fibres pass to the bronchial muscle, others to the large blood-vessels of the lung, and it is highly probable that the mucous glands are also supplied with nerve-filaments. In the comparatively simple lungs of the frog, nerves with numerous nerve-cells in their course are found (Arnold, Stirling), and in the very 
simple lung of the newt, there are also numerous nerve-cells disposed along the course of the intra-pulmonary nerves. Some of these fibres terminate in the uniform layer of non-striped muscle which forms part of the pulmonary wall in the frog and newt, and others end in the muscular coat of the pulmonary blood-vessels (Stirling). The functions of these ganglia are unknown, but they may be compared to the nerve-plexuses existing in the walls of the digestive tract.]

The Function of the non-striped muscle of the entire bronchial system seems to be to offer a sufficient amount of resistance to increased pressure within the airpassages ; as in forced expiration, speaking, singing, blowing, \&c. The vagus is the motor nerve for these fibres, and according to Longet, the "lung-tonus" during increased tension depends upon these muscles.

[Effect of Nerves. - By connecting the interior of a small bronchus with an oncograph ( $\$ 103)$ in curarised dogs (the thorax being opened), Graham Brown and Roy found that section of one vagus causes a marked expansion of the bronchi of the corresponding lung, while stimulation of the peripheral end of a divided vagus causes a powerfui contraction of the bronchi of both lungs. Stimulation of the central end of one vagus, the other being intact, also causes a contraction (feebler) under the same circumstances. Especially in etherised dogs, expansion and not contraction results. If both vagi be divided, no effect is produced by stimulation of the central end of either vagus. It seems plain that the vagi contain centripetal or afferent fibres, which can cause both expansion and contraction of the bronchi. Asphyxia causes contraction provided the vagi are intact, but none if they are divided, although in etherised dogs expansion frequently occurs, while stimulation of the central end of other sensory nerves has very rarely any, or, if any, but a slight, effect on the calibre of the bronchi, so that in the log the only connection between the cerebro-spinal centres and the bronchi is through the vagi.]

Pathological. - Stimulation of the smooth muscles, whereby a spasmodic narrowing of the smaller bronchi is produced, may excite asthmatic attacks. If the expiratory blast be interfered with, acute emphysema may take place (Biernier).

Chemistry. - In addition to connective, elastic, and muscular tissue, the lungs contain lecithin, inosit, uric acid (taurin and leucin in the ox), guanin, xanthin (?), hypoxanthin (dog) - soda, potash, magnesium, oxide of iron, much phosphoric acid, also chlorine, sulphuric, and silicic acids-in diabetes sugar occurs--in purulent infiltration glycogen and sugar-in renal degeneration urea, oxalic acid, and ammonia salts; and in diseases where decomposition takes place, leucin and tyrosin.

[Physical Properties of the Lungs. - The ]ungs, in virtue of the large amount of elastic tissue which they contain, are endowed with elasticity; and when the chest is opened they collapse. If a cannula with a small lateral opening be tied into the trachea of a rabbit's or sheep's lungs, the lungs may be inflated with a pair of bellows, or elastic pump. After the artificial inflation, the lungs, owing to their elasticity, collapse and expel the greater part of the air. As much air remains within the light spongy tissue of the lungs, even after they are removed from the body, a healthy lung floats in water. If the air-cells are filled with pathological fluids or blood, as in certain diseased conditions of the lung (pneumonia), then the lungs or parts thereof may sink in water. The lungs of the foetus, before respiration has taken place, sink in water, but after respiration has been thoroughly established in the child, the lungs float. Hence, this hydrostatic test is largely used in medico-legal cases, as a test of the child's having breathed. If a healthy lung be squeezed between the fingers, it emits a peculiar and characteristic fine crackling sound, owing to the air within the air-cells. A similar sound is heard on cutting the vesicular tissue of the lung. The colour of the lungs varies much; in a young child it is rose-pink, but afterwards it becomes darker, especially in persons living in towns or a smoky atmosphere, owing to the deposition of granules of carbon. In coal-miners the lungs may become quite black.]

[Excision of the Lung.-Dogs recover after the excision of one entire lung, and they even survive the removal of portions of lung infected with tubercle (Biondi).]

107. MECHANISM OF RESPIRATION:-The mechanism of respiration consists in an alternate dilatation and contraction of the chest. The dilatation is called inspiration, the contraction expiration. As the whole external surfaces of both elastic lungs are applied directly, and in an air-tight manner, by their smooth moist pleural investment to the inner wall of the chest, which is covered by 
the parietal pleura, it is clear that the lungs must be distended with every dilatation of the chest, and diminished by every contraction thereof. The movements of the lungs, therefore, are entirely passive, and are dependent on the thoracic movements.

On account of their complete elasticity and their great extensibility, the lungs are able to accommodate themselves to any variation in the size of the thoracic cavity, without the two layers of the pleura becoming separated from each other. As the capacity of the non-distended chest is greater than the volume of the collapsed lungs after their removal from the body, it is clear that the lungs, even in their natural position within the chest, are distended, i.e., they are in a certain state of elastic tension $(\$ 60)$. The tension is greater the more distended the thoracic cavity, and vice versa. As soon as the pleural cavity is opened by perforation from without, the lungs, in virtue of their elasticity, collapse, and a space filled with air is formed between the surface of the lungs and the inner surface of the thoracic wall (pneumo-thorax). The lungs so affected are rendered useless for respiration; hence a double pneumo-thorax causes death.

Pneumo-thorax. - It is also clear that, if the pulmonary pleura be perforated from within the lung, air will pass from the respiratory passages into the pleural sac, and also give rise to pneumo-thorax. [Not unfrequently the surgeon is called on to open the chest, say by removing a portion of a rib to allow of the free exit of pus from the pleural cavity. If this be done with proper precautions, and if the external wound be allowed to heal, after a time the air in the pleural cavity becomes absorbed, the collapsed lung tends to regain its original form, and again becomes functionally active.]

Estimation of Elastic Tension. - If a manometer be introduced through an intercostal space into the pleural cavity, in a lead subject, we can measure, by means of a column of mercury, the amount of the elastic tension required to keep the lung in its position. This is equal to $6 \mathrm{~mm}$. in the rlead subject, as well as in the conctition of expiration. If, however, the thorax be brought into the position of inspiration by the application of traction from without, the elastic tension may be increased to $30 \mathrm{~mm}$. Hg. (Donders).

If the glottis be closed and a deep inspiration taken, the air within the lungs must become rarefied, because it has to fill a greater space. If the glottis be suddenly opened, the atmospheric air passes into the lungs until the air within the lungs has the same density as the atmosphere. Conversely, if the glottis be closed, and if an expiratory effort be made, the air within the chest must be compressed. If the glottis be suddenly opened, air passes out of the lungs until the pressure outside and inside the lung is equal. As the glottis remains open during ordinary respiration, the equilibration of the pressure within and without the lungs will take place gradually. During tranquil inspiration there is a slight negative pressure; during expiration a slight positive pressure, in the lungs ; the former $=1 \mathrm{~mm}$., the latter $2-3$ $\mathrm{mm}$. Hg. in the human trachea (measured in cases of wounds of the trachea).

108. QUANTITY OF GASES RESPIRED.-As the lungs within the chest never give out all the air they contain, it follows that only a part of the air of the lungs is changed during inspiration and expiration. The volume of this air will depend upon the depth of the respirations.

\begin{tabular}{|c}
\hline COMPLEMENTAI \\
AIR \\
110 \\
\hline TIDAL AIR \\
20 \\
\hline RESERVE AIR \\
100 \\
\hline RESIDUAL AIR \\
100 \\
\hline
\end{tabular}

(5) Vital Capacity is
Hutchinson defined the following :-

(1) Residual air is the volume of air which remains in the chest after the most complete expiration. It is $=1230-1640$ c.c. [100-130 cubic inches].

(2) Reserve or supplemental air is the volume of air which can be expelled from the chest after a normal quiet expiration. It is $-1240-1800$ c.c. [100 cubic inches].

(3) Tidal air is the volume of air which is taken in and given out at each respiration. It is -500 cubic centimetres [20 cubic inches].

(4) Complemental air is the volume of air that can be forcibly inspired over and above what is taken in at a normal respiration. It amounts to about 1500 c.c. [100-130 cubic inches]. 
forcibly expelled from the chest after the deepest possible inspiration. It is equal to 3772 c.c. (or 230 cubic inches) for an Englishman (Hutchinson), and 3222 for a German (Haeser).

Hence, after every quiet inspiration, both lungs contain $(1+2+3)=3000$ to 3900 c.cm. [220 cubic inches]; after a quiet expiration $(1+2)=2500$ to 3400 c.cm. [ 200 cubic inches]. So that about $\frac{1}{6}$ to $\frac{1}{7}$ of the air in the lungs is subject to renewal at each ordinary respiration. air.

Donders calculated that the entire bronchial system and the trachea contain about 500 c.c. of

Estimation of Vital Capacity.-This was formerly thought to be of great utility, but at the present time not much importance is attached to it, nor is it frequently measured in cases of disease. It is estimated by means of the spirometer of Hutchinson (fig. 134), which consists of a graduated cylinder filled with water and inverted like a gasometer over water, and balanced by means of a counterpoise. Into the cylinder a tube projects, and this tube is connected with a mouthpiece. The person to be experimented upon takes the deepest possible inspiration, closes his nostrils, and breathes forcibly into the mouthpiece of the tube: After doing so the tube is closed. The cylinder is raised by the air forced into it, and after the water inside and outside the cylinder is equalised, the height to which the cylinder is raised indicates the amount of air expired, or the vital or respiratory capacity. In a man of average height, 5 feet 8 inches, it is equal to 230 cubic inches.

The following circumstances affect the vital capacity :-

(1) The Height. - Every inch added to the height of persons between 5 and 6 feet gives an increase of the vital capacity $=130$ c.c. [8 cubic inches.]

(2) The Body-weight.-When the body-weight exceeds the normal by 7 per cent. there is a diminution of 37 c.c. of the vital capacity for every kilo. of increase.

(3) Age. - The vital capacity is at its maximum at 35 ; there is an annual decrease of 23.4 c.c., from this age onwards to 65 , and backwards to 15 years of age.

(4) Sex.-It is less in women than men, and even where there is the same circumference of chest, and the same height in a man and a woman, the ratio is $10: 7$.

(5) Position and Occupation.-More air is respired in the erect than in the recumbent position.

(6) Disease. - Abdominal and thoracic diseases diminish it.

109. NUMBER OF RESPIRATIONS.-In the adult, the number of respirations varies from 16 to 24 per minute, so that about 4 pulse-beats occur during each respiration. The number of respirations is influenced by many conditions :-

(1) The Position of the Body.-In the adult, in the horizontal position, Guy counted 13 , while sitting 19 , while standing 22 , respirations per minute.

(2) Age.-Quetelet found the mean number of respiratiqns in 300 individuals to be :-

$\left.\left.\begin{array}{rr|c|c}\text { Year. } & \text { Respirations. } \\ 0 \text { to } 1, & 44 \\ 5, & 26 \\ 15 & \text { to } 20, & 20\end{array}\right\} \quad \begin{array}{ccc}\text { Year. } & \text { Respirations. } \\ \text { Average } & \text { to } 25, & 18 \cdot 7 \\ \text { Number per } & \text { to } 30, & 16 \\ 30 \text { to } 50, & 18 \cdot 1\end{array}\right\} \begin{gathered}\text { Average } \\ \text { Number per } \\ \text { Minute. }\end{gathered}$

(3) The State of Activity.-Gorham counted in children of 2 to 4 years of age during standing 32 , in sleep 24, respirations per minute. During bodily exertion the number of respirations increases before the heart-beats. [Very slight muscular exertion suffices to increase the frequency of the respirations.]

[(4) The Temperature of the surrounding medium.-The respirations become more numerous 
the higher the surrounding temperature, but this result only occurs when the actual temperature of the blood is increased, as in fever.

(5) Digestion. - There is a slight variation during the course of the day, the increase boing most marked after mid-day dinner (Vierordt).

(6) The Will can to a certain extent modify the number and also the depth of the respirations, but after a short time the impulse to respire overcomes the voluntary impulse.

(7) The Gases of the Blood have a marked effeet, and so has the heat of the blood in fever.]

[(8) In Animals-

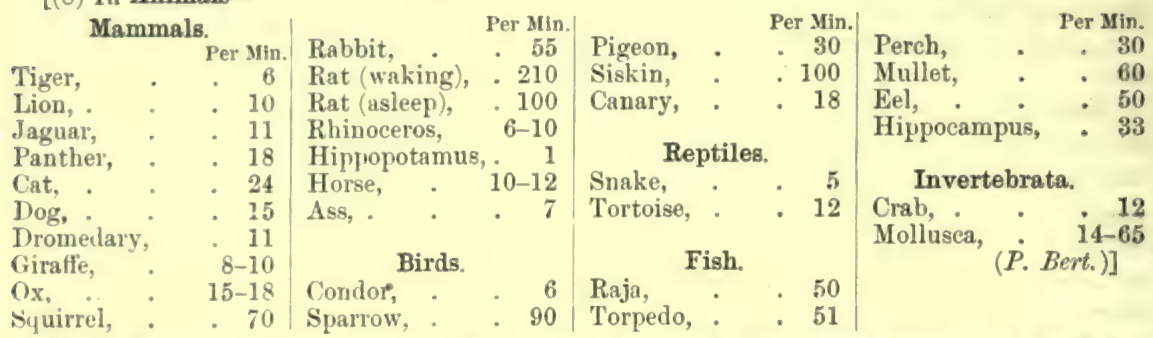

[(9) In Disease.-The number may he greatly increased from many causes, e.g., in fever, pleurisy and pucumonia, some heart diseases, or in certain cases of alteration of the blood, as
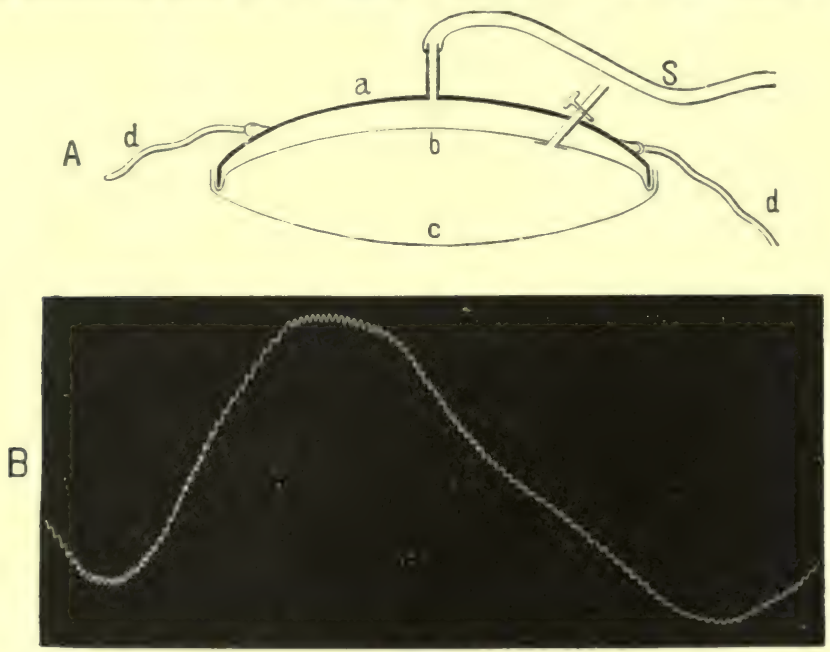

Fig. 135.

A, Brondgeest's tambour for registering the respiratory movements. $b$, $c$, inner and outer caoutchouc membranes; $a$, the capsule; $d, d$, cords for fastening the instrument to the chest; $S$, tube to the recording tambour. B, normal respiratory curve obtained on a vibrating plate (each vibration $=0.01613 \mathrm{sec}$.).

in anæmia ; and diminished where there is pressure on the respiratory centre in the medulla, in coma. It is important to note the ratio of pulse-beats to respirations.]

110. TIME OCCUPIED BY THE RESPIRATORY MOVEMENTS.-The time occupied in the various phases of a respiration can only be accurately ascertained by obtaining a curve or pneumatogram of the respiratory movements by means of recording apparatus.

Methods.-The graphic method can be employed in three directions:-(1) To record the movements of individual parts of the chest-wall.

(1) Vierordt and C. Ludwig transferred the movements of a part of the chest-wall to a lever which inscribed its movements upon a revolving cylinder. Riegel (1873) constructed a "double stethograph" on the same principle. This instrument is so arranged that one arm of the lever may be applied in connection with the healthy side of a person's chest, and the 
other on the diseased side. In the case of animals placed on their backs, Snellon introduced a long needle vertically through the abdominal walls into the liver. Rosenthal opened the abdomen and applied a lever to the under surface of the diaphragm, and thus registered its movements (Phrenograph).

(2) An air-tambour, such as is used in Brondgeest's pansphygmograph (fig. 135, A), may be employed. It consists of a brass vessel, $a$, shaped like a small saucer. The mouth of the brass vessel is covered with a double layer of caoutchonc membrane, $b, c$, and air is forced in between the two layers until the external membrane bulges outwards. This is placed on the chest, and the apparatus is fixed in position by means of the bands, $d, d$. The cavity of the tambour communicates by means of a caoutchouc tube, $s$, with a recording tambour, which inscribes its movements upon a revolving cylinder. Every dilatation of the chest compresses the membrane, and thus the air within the tambour is also compressed. [A somewhat similar apparatus is used by Burdon-Sanderson, and called a "recording-stethograph." By it movements of the corresponding points on opposite sides of the chest can be investigated.] A cannula or nesophageal sound may be introduced into that portion of the resophagus which lies in the chest, and a connection established with Marey's tambour (Rosenthal). [This method also enables one to measure the intrathoracic pressure.]

Marey's Stethograph or Pneumograph.-[There are two forms of this instrument, one modified by P. Bert and the more modern form (fig. 136). A tambour $(h)$ is fixed at right angles to a thin elastic plate of steel $(f)$. The aluminium disc on the caoutchouc of the tambour is attached to an upright $(b)$, whose end lies in contact with a horizontal screw $(g)$. Two arms $(d, c)$ are attached to opposite sides of the steel plate, and to them the belt $(e)$ which fastens the instrument to the chest is attached. When the chest expands, these two arms are pulled asunder, the steel plate is bent, and the tambour is affected, and any movement of the tambour is transmitted to a registering tambour by the air in the tube $(a)$ ].

(2) To record variation in volume of the thorax or of the respired gases.

For this purpose E. Hering secures the animal, and places it

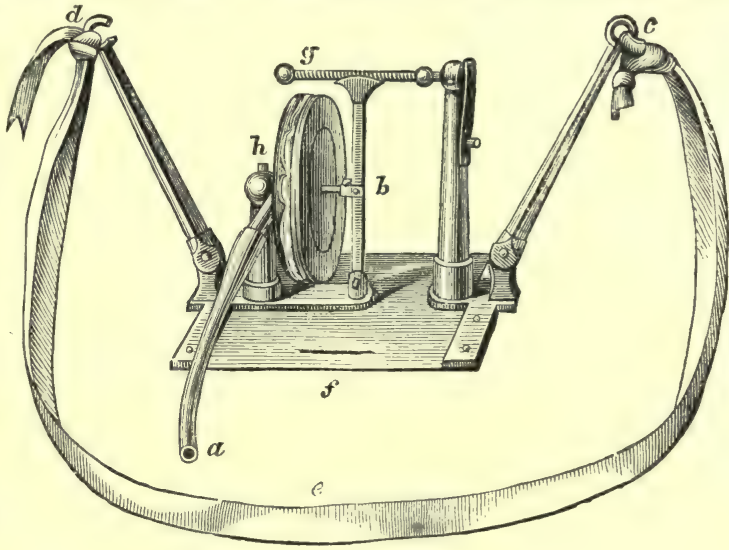

Fig. 136.

Marey's stethograph. in a tight box provided with two openings in its side; one hole contains a tube, which is connected to a cannula tied into the transversely divided trachea of the animal, so that respiration can go on undisturbed. In the other orifice is fixed a water-manometer provided with a swimmer arranged to write on a recording surface. Gad registered graphically the respired air by means of a special apparatus; the expired air raised a very light and carefully equipoised box placed over water. As it was raised, it moved a writing-style. During inspiration the box sank.

\section{(3) To record the rate at which the respiratory gases are exchanged.}

If the trachea of an animal, or the mouth of a man (the nostrils being closed), be connected with a tube like that of the dromograph (fig. 113), then during inspiration and expiration the pendulum will be moved to and fro by the air, and the movements of the pendulum can be registered. [Some years ago, an instrument, called the "Anapnograph," was constructed on this principle.]

The curve (fig. 135, B) was obtained by placing the tambour of a Brondgeest's pansphygmograph upon the xiphoid process, and recording the movement upon a plate attached to a vibrating tuning-fork. The inspiration (ascending limb) begins with moderate rapidity, is accelerated in the middle, and towards the end again becomes slower. The expiration also begins with moderate rapidity, is then accelerated, and becomes much slower at the latter part, so that the curve falls very gradually.

Inspiration is slightly shorter than Expiration.-According to Sibson, the 
ratio for an adult is as 6 to 7 ; in women, children, and old people, 6 to 8 or 6 to 9. Vierordt found the ratio to be 10 to $14 \cdot 1$ (to $24 \cdot 1$ ); J. R. Ewald, 11 to 12. It is only occasionally that cases occur where inspiration and expiration are equally long, or where expiration is shorter than inspiration. When respiration proceeds quietly and regularly, there is usually no pause (complete rest of the chest-walls) between the inspiration and expiration. The very flat part of the expiratory curve has been wrongly regarded as due to a pause. Of course, we may make a voluntary pause between two respirations, or at any part of a respiratory act.

Some observers, however, have described a pause as occurring between the end of expiration and the beginning of the next inspiration (expiration pause), and also another pause at the end of inspiration (inspiration pause). The latter is always of very short duration, and considerably shorter than the former. During very deep and slow respiration, there is usually an

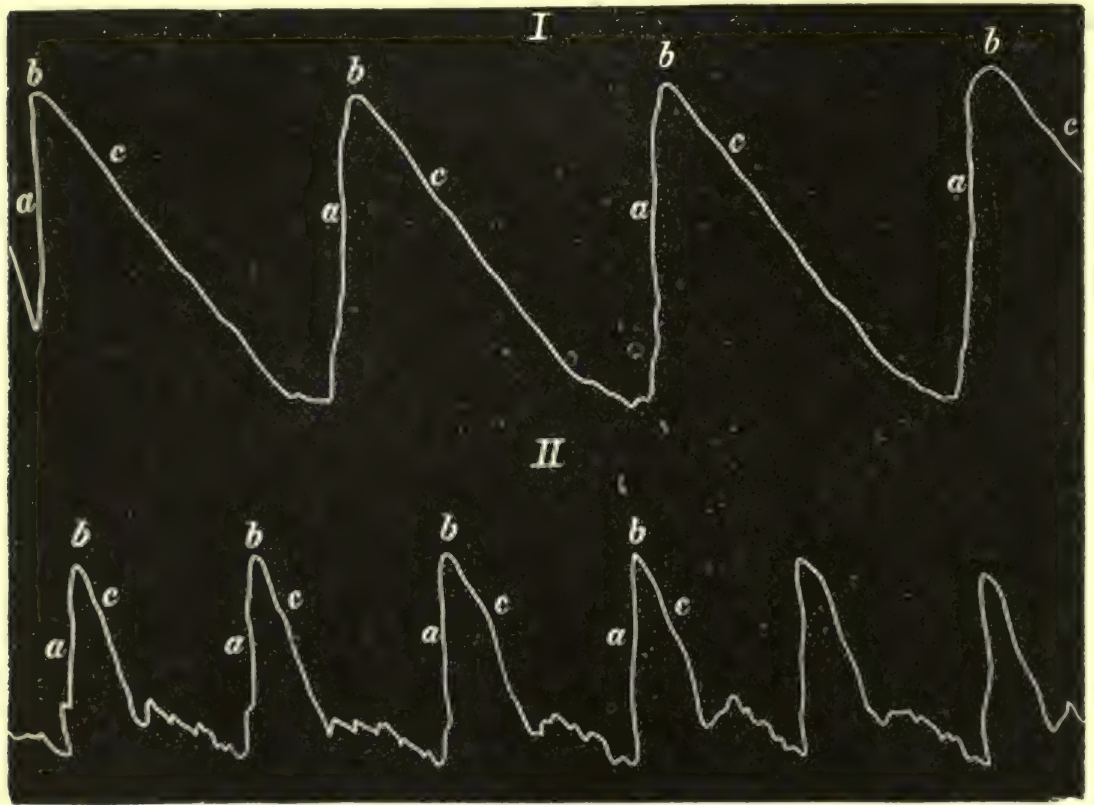

Fig. 137.

Pneumatograms obtained by means of Riegel's stethograph. I, normal curves; II, curve from a case of emplysema ; $a$, ascending limb ; $b$, apex ; $c$, descending limb of the curve. The small elevations are due to the cardiac impulse.

expiration pause, while it is almost invariably absent during rapid breathing. An inspiration pause is always absent under normal circumstances, but it may occur under pathological conditions.

In certain parts of the respiratory curve slight irregularities may appear, which are sometimes due to vibrations communicated to the thoracic walls by vigorous heart-beats (fig. 137).

The "type" of respiration may be ascertained by taking curves from various parts during the respiratory movements. Hutchinson showed that, in the female, the thorax is dilated chiefly by raising the sternum and the ribs (Respiratio costalis), while in man it is caused chiefly by a descent of the diaphragm (Respiratio diaphragmatica or abdominalis). In the former, there is the so-called "costal type," in the latter the "abdominal or diaphragmatic type."

This difference in the type of respiration in the sexes occurs only during normal quiet respiration. During deep and forced respiration, in both sexes the dilatation of the chest is caused chiefly by raising the chest and the ribs. In man, the epigastrium may be pulled in sooner than it is protruded. During sleep, the type of respiration in both sexes is thoracic, 
while at the same time the inspiratory dilatation of the chest precedes the elevation of the abdominal wall (Mosso). It is not determined whether the costal type of respiration in the female depends upon the constriction of the chest by corsets or other causes (Sibson), or whether it is a natural adaptation to the child-bearing function in women (Hutchinson). Some observers maintain that the difference of type is quite distinct, even in sleep, when all constrictions are removed, and that similar differences are noticeable in young children. This is denied by others, while a third class of observers hold that the costal type occurs in children of both sexes, and they ascribe as a cause the greater flexibility of the ribs of children and women, which permits the muscles of the chest to act more efficiently upon the ribs.

111. PATHOLOGICAL. - Examination of the Lungs. - T'he same methods that are applicable to the heart, viz., I., Inspection; II., Palpation; III., Percussion; and IV., Auscultation, apply here also.]

[By inspection we may determine the presence of symmetrical or unilateral alterations in the shape of the chest, the presence of bulging or flattening at one part, and variations in the movement of the chest-walls. By palpation, the presence or absence, character, seat, and extent of any movements are more carefully examined. But we may also study what is called vocal fremitus (\$117). Percussion ( $\$ 114)$, Auscultation (\$ 116).]

[In investigating the respiratory movements, we should observe (1), the frequency ( $\$ 109)$; (2), the type $(\S 110) ;(3)$, the nature, character, and extent of the movements, noting also whether they are accompanied by pain or not (\$110); (4), the rhythm.]

I. Changes in the mode of Movement.-In persons suffering from disease of the respiratory organs, the dilatation of the chest may be diminished (to the extent of 5 or $6 \mathrm{~cm}$.) on both sides or only on one side. In affections of the apex of the lung (in phthisis), the sub-normal expansion of the upper part of the wall of the chest may be considerable. Retraction of the soft parts of the thoracic wall, the xiphoid process, and the parts where the lower ribs are inserted, occurs in cases where air cannot freely enter the chest during inspiration, e.g., in narrowing of the larynx; when this retraction is confined to the upper part of the thoracic wall, it indicates that the portion of the lung lying under the part so affected is less extensile and diseased.

Harrison's Groove.-In persons suffering from chronic difficulty of breathing, and in whom, at the same time, the diaphragm acts energetically, there is a slight groove, which passes horizontally outwards from the xiphoid cartilage, caused by the pulling in of the soft parts and corresponding to the insertion of the diaphragm.

The duration of inspiration is lengthened in persons suffering from narrowing of the trachea or larynx ; expiration is lengthened in cases of dilatation of the lung, as in emphysema, where all the expiratory muscles must be brought into action (fig. 137, II).

II. Variations in the Rhythm. - When the respiratory apparatus is much affected, there is either an increase or a deepening of the respirations, or both. When there is great difficulty of breathing, this is called dyspnoea.

Causes of Dyspnœa. - (1) Limitation of the exchange of the respiratory gases in the blood due to- $(a)$ diminution of the respiratory surface (as in some diseases of the lungs); $(b)$ narrowing of the respiratory passages; $(c)$ diminution of the red blood-corpuseles; $(d)$ disturbances of the respiratory mechanism (e.g., due to affections of the respiratory muscles or nerves, or painful affections of the chest-wall); (e) impeded circulation through the lungs due to various forms of heart-disease. (2) Heat-dyspncea. - The frequency of the respirations is increased in febrile conditions. The warm blood acts as a direct irritant of the respiratory centre in the medulla oblongata, and raises the number of respirations to $30-60$ per minute ("Heat-dyspnoea"). If the carotids be placed in warm tubes, so as to heat the blood going to the medulla oblongata, the same phenomena are produced ( $\$ 368)$. [When a child sucks, it breathes exclusively through the nose, hence catarrhal conditions of the nasal mucous membrane are fraught with danger to the child.]

[Orthopnœa. - Sometimes the difficulty of breathing is so great that the person can only respire in the erect position, i.e., when he sits or is propped up in bed. This occurs frequently towards the close of some heart affections, notably in mitral lesions; dropsical conditions, especially of the cavities, may be present.]

Cheyne-Stokes' Phenomenon. - This remarkable phenomenon occurs in certain diseases, where the normal supply of blood to the brain is altered, or where the quality of the blood itself is altered, e.g., in certain affections of the brain and heart, and in uræmic poisoning. Respiratory pauses of one-half to three-quarters of a minute alternate with a short period ( $\frac{1}{2}-\frac{3}{4} \mathrm{~min}$.) of increased respiratory activity, and during this time $20-30$ respirations occur. The respirations constituting this "series" are shallow at first ; gradually they become deeper and more dyspnœic, and finally become shallow or superficial again. Then follows the pause, and thus there is an alternation of pauses and series (or groups) of modified respirations. During the pause, the pupils are contracted and inactive; and when the respirations begin, they dilate and become sensible to light; the eyeball is moved as a whole at the same time. Hein observed that consciousness was abolished during the pause, and that it returned when respiration commenced. 
Canses. - Luciani and Rosenbach regard variations in the excitability of the respiratory centre as the cause of the phenomenon, which they compare with the periodic contraction of the heart (§58). The excitability of the respiratory centre is lowest during the pause. They observed this phenomenon after injury to the medulla oblongata above the respiratory centre, and after apncea produced in animals deeply narcotised with opium, and in the last stages of asphyxia, during respiration in a closed space. During hybernation, this mode of respiration is normal in Myoxus, the hedgehog, and the caiman.

Periodic Respiration.-If frogs be kept under water, or if the aorta be clamped, after several hours, they hecome passive. If they be taken out of the water, or if the clamp be removed from the aorta, they gradually recover and always exhibit the Cheyne-Stokes' phenomenon. In such frogs the blool-current may be arrested temporarily, while the phenomenon itself remains (Sokolow and Luchsinger). If the blood-current be arrested by ligature of the aorta, or if the frogs be bled, the respirations occur in groups. This is followed by a few single respirations, and then the respiration ceases completely. During the pause between the periods, mechanical stimulation of the skin causes the discharge of a group of respirations (Sicbert and Langendorff).

Action of Drugs. - Muscarin, digitalin, curara, chloral, sulphuretted hydrogen, and the poison of many infectious diseases (typhus, diphtheria, scarlet fever) may also cause periodic respiration in frogs [which is not due to the action of these drugs on the heart].

Periodic respiration without any variation in the size of the individual respirations-the socalled "Biot's respiration"-occurs normally during sleep. While the nervous system as it were strives to rest, and thus forgets the respiration, the organism does not observe the short pauses (Mosso). [There is a periodic increase or decrease in the depth of the respiration, especially in old people and children, even to the extent of the respiration becoming "remittent," or even "intermittent," for a period of 30 sec. during sleep. During periodic-respiration the action of the several respiratory muscles does not coincide. As a rule, one respires more than is required by the organism. Mosso calls this "luxus-respiration."] Periodic irregularitics in the respiration are often of reflex origin ( $K$ noll).

\section{GENERAL VIEW OF THE RESPIRATORY MUSCLES.}

\section{(A) Inspiration.}

\section{During Ordinary Inspiration.}

1. The diaphragm (Nervus phrenicus).

2. The $\mathrm{VIm}$. levatores costarum longi et breves (Rami posteriores $\mathrm{Nn}$. dorsalium).

3. The $\mathrm{Mm}$. intercostales externi et intercartilaginei $(\mathrm{N} n$. intercostales).

\section{During Forced Respiration.}

(a) Muscles of the Trunk.

1. The three $\mathrm{Mm}$. scaleni (Rrmi musculares of the plexus cervicalis et brachialis).

2. M. sternocleidomastoideus (Ram. externus $N$. accessorii).

3. M. trapezius ( $R$. externus $N$. accessorii et Ram. musculares plexus cervicalis).

4. I. pectoralis minor ( $\mathrm{Nn}$. thoracici anteriores).

5. M. serratus posticus superior ( $N$. dorsalis scapulx).

6. Mm. rhomboidei ( $N$. dorsalis scapulx).

7. $\mathrm{Mm}$. extensores columnæ vertebralis (Ram. posteriores nervorum dorsalium)

[8. $\mathrm{Mm}$. serratus anticus major ( $N$. thoracicus longus). ? ?]

(b) Muscles of the Larynx.

1. M. sternohyoideus (Ram. descendens hypoglossi).

2. M. sternothyreoideus (Ram. descendens hypoglossi).

3. M. crico-arytaenoideus posticus ( $N$. laryngeus inferior vagi).

4. M. thyrec-arytaenoideus (N. laryngeus inferior vagi).

(c) Muscles of the Face.

1. M. dilatator narium anterior et posterior ( $N$. facialis).

2. M. levator alæ nasi $(N$. facialis).

3. The dilators of the mouth and nares, during forced respiration, [ "gasping for breath "] ( $N$. facialis).

1. M. levator veli palatini ( $N$. facialis).

(d) Muscles of the Pharynx.

2. M. azygos uvulæ ( $N$. facialis).

3. According to Garland, the pharynx is always narrowed. 
(B) Expiration.

\section{During Ordinary Respiration.}

The thoracic cavity is diminished by the weight of the chest, the elasticity of the lungs, costal cartilages, and abdominal muscles.

\section{During Forced Expiration. \\ The Abdominal Muscles.}

1. The abdominal muscles [including the obliquus externus and internus, and transversalis abdominis] ( $N n$. abdominis internis anteriores e nervis intercostalibus, $8-12)$.

2. Mm. intercostales interni, so far as they lie between the osseous parts of the ribs, and the $\mathrm{Mm}$. infracostales ( $\mathrm{Nn}$. intercosiales).

3. M. triangularis sterni $(\mathrm{Nn}$. intercostales $)$.

4. M. serratus posticus inferior (Ram. externi nerv. dorsalium).

5. M. quadratus lumborum (Ram. muscular e plexu lumbali).

113. ACTION OF THE INDIVIDUAL RESPIRATORY MUSCLES.-(A) Inspiration. - (1) The Diaphragm arises from the cartilages and the adjoining osseous parts of the lower six ribs (costal portion), by two thick processes or crura, from the upper three or four lumbar vertebræ, and a sternal portion from the back of the ensiform process. It represents an arched double cupola or dome-shaped partition, directed towards the chest; in the larger concavity on the right side lies the liver, while the smaller arch on the left side is occupied by the spleen and stomach. During the passive condition, these viscera are pressed against the under surface of the diaphragm, by the elasticity of the abdominal walls, and by the intra-abdominal pressure, so that the arch of the diaphragm is pressed upwards into the chest. The elastic traction of the lungs also aids in producing this result. The greater part of the upper surface of the central tendon of the diaphragm is united to the pericardium. The part on which the heart rests, and which is perforated by the inferior vena cava (foramen quadrilaterum) is the deepest part of the middle portion of the diaphragm during the passive condition.

Action of the Diaphragm.-When the diaphragm contracts, both arched portions become flatter, and the chest is thereby elongated from above downwards. In this act, the lateral muscular parts of the diaphragm pass from an arched condition into a flatter form (fig. 138), and during a forced inspiration the lowest lateral portions, which during rest are in contact with the chest-wall, become separated from it. The middle of the central tendon where the heart rests (fixed by means of the pericardium and inferior vena cava) takes no share in this movement, especially in ordinary quiet breathing, but during the deepest in- Sa spiration it sinks somewhat.

Undoubtedly, the diaphragm is the most powerful agent in increasing the cavity of the chest. Bruicke believes that in addition to increasing the length of the thoracic cavity from above downwards, it also

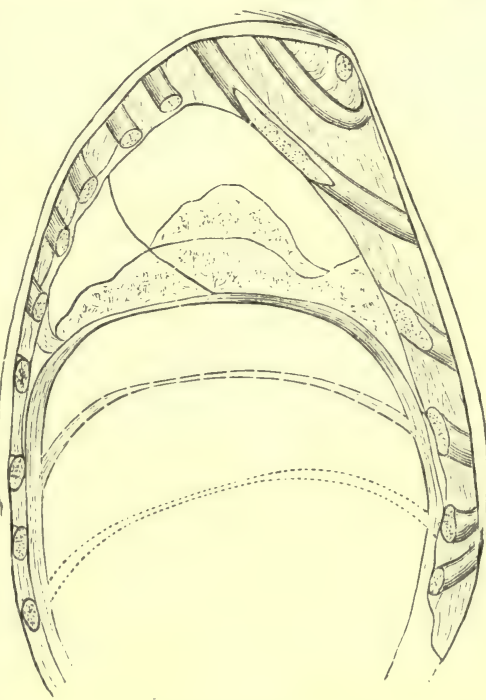

Fig. 138.

Sagittal section through the second rib on the right side. When the arched muscular part of the diaphragn contracts, a wedge-shaped space, with its apex downwards, is formed around the circumference of the lower part of the chest. increases the transverse diameter of the lower part of the chest. It presses upon the abdominal viscera from above, and strives to press these outwards, thus tending to push out the adjoining thoracic wall. If the contents of the abdomen are removed from a living animal, every time the diaphragm contracts the ribs are drawn inwards. This, of course, hinders the chest from becoming wider below, hence the presence of the abdominal viscera seems to be necessary for the normal activity of the diaphragm. Every contraction of the diaphragm, by increasing the 
intra-abdominal pressure, favours the venous blood-current in the abdomen towards the vena cava inferior.

Phrenic Nerve.-The immense importance of the diaphragm as the great inspiratory muscle is proved by the fact that, after both phrenic nerves (third and fourth cervical nerves) are divided, death occurs. The phrenic nerve contains some sensory fibres for the pleura, pericardium, and a portion of the diaphragm. The contraction of the diaphragm is not to be regarded as a " simple nuscular contraction," since it lasts 4 to 8 times longer than a simple contraction; it is rather a short tetanic contraction, which we may arrest in any stage of its activity, without bringing in to action any antagonistic muscles (Kronecker and Marckwald).

(2) The Elevators of the Ribs. - The ribs at their vertebral ends (which lie nuch higher than their sternal ends) are united by means of joints by their heads and tubercles to the bodies and transverse processes of the vertebræ. A horizontal axis can be drawn through both joints, around which the ribe can rotate upwards and downwards. If the axis of rotation of each pair of ribs be prolonged on both sides until they meet in the middle line, the angles so formed are greatest above $\left(125^{\circ}\right)$, and smallest below $\left(88^{\circ}\right)$. Owing to the ribs being curved, we can imagine a plane which, in the passive (expiratory) condition of the chest, has a slope from behind and inwards to the front and outwards. If the ribs move on their axis of rotation, this plane becomes more horizontal, and the thoracic cavity is increased in its transverse diameter. As the axis of rotation of the upper ribs runs in a more frontal, and that of the lower ribs in a more sagittal direction, the elevation of the upper ribs causes a greater increase from before backwards, and the lower ribs from within outwards (as the movements of ribs which are directed downwards are vertical to the axis). The costal cartilages undergo a slight tension at the same time, which brings their elasticity into play.

Changes in the Chest.-All "inspirutory muscles" which act directly upon the chest-wall do so by raising the ribs:-(a) When the ribs are raised, the intercostal spaces are widened. (b) When the upper ribs are raised, all the lower ribs and the sternum must be elevated at the same time, because all the ribs are connected with each other by means of the soft parts of the intercostal spaces. (c) During inspiration, there is an elevation of the ribs and a dilatation of the intercostal spaces. (The lowest rib is an exception : during forced respiration, at least, it is drawn downwards.) (ll) If, on a preparation of the chest, the ribs be raised as in inspiration, we may regard all those muscles as elevators of the ribs, whose origin and insertion become approximated. Every one is agreed that the scaleni and levatores costarum longi et breves, the serratus posticus superior, are inspiratory muscles. These are the most important inspiratory muscles which act upon the ribs.

Intercostal Muscles. - With regard to the action of the intercostal muscles, there is a great difference of opinion. According to the above experiment, the external intercostals and the intercartilaginous parts of the internal intercostals act as inspiratory muscles, whilst the remaining portions of the internal intercostals (as far as they are covered by the external) are elongated when the ribs are raised, while they shorten when the chest-wall descends. A muscle shortens only during its activity. The internal intercostals were regarded by Hamberger as depressors of the ribs or expiratory muscles.

In fig. $139, \mathrm{I}$, when the rods, $a$ and $b$ (which represent the ribs), are raised, the intercostal space nust be widened $(e f>c d)$. On the opposite side of the figure, it is evident that when the rods are raised, the line, $g h$, is shortened $(i k<g h$, direction of the external intercostals) $l m$ is lengthened ( $l m<0 n$, direction of internal intercostals). Fig. 139, II, shows, that when the ribs are raised, the inter-cartilaginei, indicated by $g h$, and the external intercostals indicated by $l k$, are shortened. When the ribs are raised, the position of the muscular fibres is indicated by the diagonal of the rhomb becoming shorter.

The mode of action of the intercostal muscles is an old story, Galen (131-203 A.D.) regarding the exteruals as inspiratory, the internals as expiratory. Hamberger (1727) accepted this proposition, and considered the intercartilaginei also as inspiratory. Haller looked upon both the external and internal intercostals as inspiratory, while Vesalius (1540) regarded both as expiratory. Landerer, observing that the upper two or three intercostal spaces became narrower duriug inspiration, regarded both as active during inspiration and expiration. They keep one rib attached to the other, so that their action is to transmit any strain put upon them to the wall of the ehest. On this view they will be in action, even when the distance between their points of attachment becomes greater. Landois regards the external intercostals and intercartilaginei as active only during inspiration, the internal intercostals only during expiration. Martin and Hartwell exposed the internal intercostals, und observed whether they contracted 
along with the diaphragm, or whether the contractions of these two muscles alternate. As the result of their experiments, they conclude that " the internal intercostal muscles are expiratory throughout their whole extent, at least in the dog and cat; and that in the former animal they are almost 'ordinary' muscles of respiration, while in the latter they are "extraordinary" respiratory muscles."] Landois is of opinion that the chief action of these muscles is not to raise or depress the ribs, but rather that the external intercostals and the intereartilaginei offer resistance to the inspiratory dilatation of the intercostal spaces, and to the simultaneously increased elastic tension of the lungs. The internal intercostals act during powerful expiratory efforts, (e.g., coughing), and oppose the distension of the lungs and chest caused by this act. Unless muscles were present to resist the uninterrupted tension and pressure, the intercostal substance would become so distended that respiration would be impossible. [According to Rutherford, the internal intercostals are probably muscles of inspiration.]

The Pectoralis minor and (? Serratus anticus major) can only act as elevators of the ribs when the shoulders are fixed, partly by the rhomboidei, and partly by fixing the shoulder-joint and supporting the arms, as is done instinctively by persons suffering from breathlessness.

(3) Muscles acting on the Sternum, Clavicle, and Vertebral
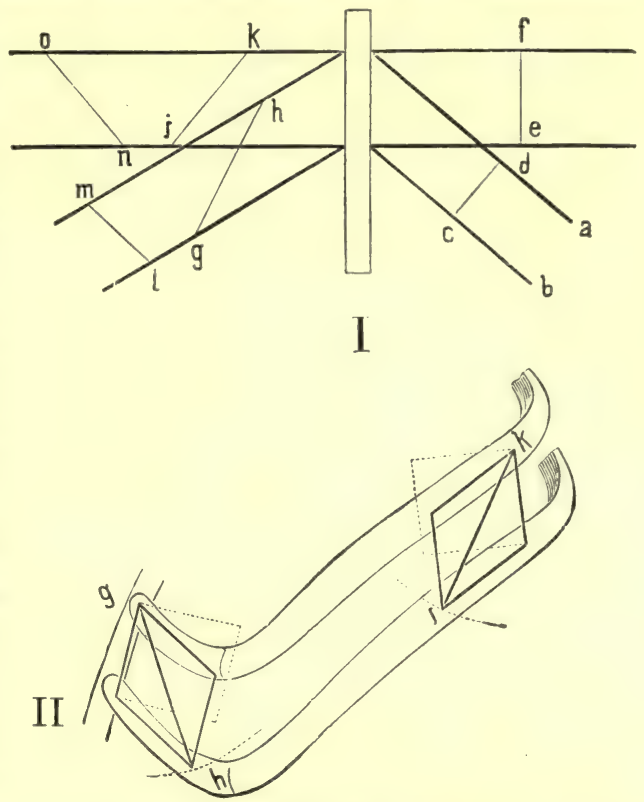

Fig. 139.

Scheme of the action of the intercostal muscles.

Column. - When the head is fixed by the muscles of the neck, the sternocleidomastoid raises the manubrium sterni and the sternal end of the clavicle, so that the thorax is raised and thereby dilated. The scaleni also aid in this act. The clavicular portion of the trapezius may act in a similar although less energetic manner. When the vertebral column is straightened, it causes an elevation of the upper ribs, and a dilatation of the intercostal spaces which aid inspiration. During deep respiration, the straightening of the vertebral column takes place involuntarily.

(4) Laryngeal Movements:-During laboured respiration, with every inspiration, the larynx descends and the glottis is opened. At the same time the palate is raised, so as to permit a free passage to the air entering through the mouth.

(5) Facial Movements.-During laboured respiration, the facial muscles are involved; there is an inspiratory dilatation of the nostrils (well marked in the horse and rabbit). When the need for respiration is very great, the mouth is gradually widened, and the person as it were gasps for breath. During expiration, the muscles that are active during (4) and (5) relax, so that a position of equilibrium is established without there being any active expiratory movement to counteract the inspiratory movement. During inspiration the pharynx becomes narrow (Garland).

(B) Expiration.-Ordinary expiration occurs without the aid of muscles, owing to the weight of the chest, which tends to fall into its normal position from the position to which it was raised during inspiration. This is aided by the elasticity of the various parts of the chest. When the costal cartilages are raised, which is accompanied by a slight rotation of their lower margins from below forwards and upwards, their elasticity is called into play. As soon, therefore, as the inspiratory forces cease, the costal cartilages return to their normal position, i.e., the position 
of expiration, and tend to untwist themselves; at the same time, the elasticity of the distended lungs draws upon the thoracic walls and the diaphragm. Lastly, the tense and elastic abdominal walls, which, in man chiefly, are stretched and pushed forward, tend to return to their non-distended passive condition when the abdominal viscera are relieved from the pressure of the contracted diaphragm. (When the position of the body is reversed, the action of the weight of the chest is removed, but in place of it there is the weight of the viscera, which press upon the diaphragm.)

The abdominal muscles [obliquus internus and externus, transversalis abdominis and levator ani] are always active during laboured respiration. They act by diminishing the abdominal cavity, and they press the abdominal contents upwards against the diaphragm. When they act simultaneously, the abdominal cavity is diminished throughout its whole extent. The triangularis sterni depresses the sternal ends of the united cartilages and bones, from the third to sixth ribs downwards; and the serratus posticus inferior depresses the lowest four ribs, causing the others to follow. It is aided by the quadratus lumborum, which depresses the last rib. According to Henle, the serratus posticus inferior fixes the lower ribs for the action of the slips of the diaphragm inserted into them, so that it acts during inspiration. According to Landerer, the downward movement of the ribs in the lower part of the thorax dilates the chest.

In the erect position, when the vertebral column is fixed, deep inspiration and expiration naturally alter the position of the centre of gravity, so that during inspiration, owing to the protrusion of the thoracic and abdominal walls, the centre of gravity lies somewhat more to the front. Hence, with each respiration there is an involurtary balancing of the body. During very derp inspiration, the accompanying straightening of the vertebral column and the throwing backwards of the head compensate for the protrusion of the anterior walls of the trunk.

114. RELATIVE DIMENSIONS OF THE CHEST. - The diameter of the chest is ascertained by means of callipers; the circumference with a flexible centimetre or other measure.

In strong men, the circumference of the upper part of the chest (immediately under the arms) is 88 centi-

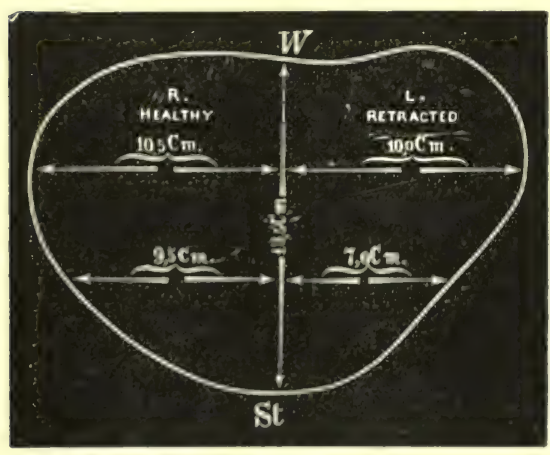

Fig. 140.

Cyrtometer curve. Left side of the chest retracted in a girl aged twelve.

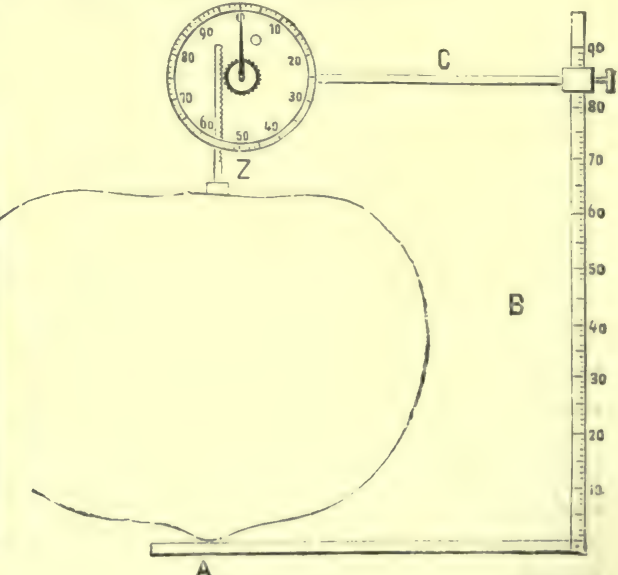

Fig. 141.

Sibson's thoracometer.

metres $(34 \cdot 3$ inches), in females 82 centimetres ( 32 inches); at the level of the ensiform process 82 centimetres $(32$ inches) and 78 centimetres $(30.4$ inches) respectively. When the arms are placed horizontally, during moderate expiration, the circumference immediately under the nipple and the angles of the scapulæ is equal to half the length of the body; in man 82 , and during deep inspiration 89 centimetres. The circumference at the level of the ensiform cartilage is 6 centimetres less. In old people, the circumference of the upper part of the chest is diminished, 
so that the lower part becomes the wider of the two. The right half of the chest is usually slightly larger than the left half, owing to the greater development of the muscles on that side. The long diameter of the chest-from the clavicle to the margin of the lowest rib-varies very much.

The transverse diameter in man, above and below, is 25 to 26 centimetres $(9 \cdot 7$ to $10 \cdot 1$ inches), in females 23 to 24 centimetres $(8.9$ to $9 \cdot 2$ inches); above the nipple it is 1 centimetre more. The antero-posterior diameter (distance of anterior chest-wall from the tip of a spinous process) in the upper part of the chest is $=17(6.6$ inches $)$, in the lower 19 centimetres $(7 \cdot 4$ inches). Valentin found that in a man, during the deepest inspiration, the chest on a level with the groove in the heart was increased about $\frac{1}{12}$ to $\frac{1}{7}$; while Sibson estimates the increase at the level of the nipple to be $\frac{1}{10}$.

Thoracometer.-In order to obtain a knowledge of the degree of movement-rising or falling - of the chest-wall during respiration, varions instruments have been invented. The thoracometer (fig. 141) measures the elevation in different parts of the sternum. It consists of two metallic bars placed at right angles to each other; one of them, $\mathrm{A}$, is placed on the vertebral column. On B there is placed a movable transverse bar, C, which carries on its free end a toothed rod, Z, directed downwards. The lower end of this rod is provided with a pad which rests on the sternum, while its toothed edge drives a small wheel, which moves an index, whose excursions are indicated on a circle with a scale attached to it.

The Cyrtometer of Woillez consists of a brass chain of movable links, to be applied in a definite direction to part of the chest-wall, e.g., transversely on a level with the nipple, or vertically upon the mammillary or axillary lines anteriorly. There are freely movable links at two parts, which permit the chain to be easily removed, so that as a whole it still retains its form. The chain is laid upon a sheet of paper, and a line drawn with a pencil around its inner margin gives the form of the thorax (fig. 140). [A lead wire answers the same purpose.]

Limits of the Lungs. - The extent and boundaries of the lungs are ascertained in the living subject by means of percussion, which consists in lightly tapping the chest-wall by means of a hammer (percussion-hammer). A small ivory or bone plate or pleximeter, held in the left hand, is laid on the chest, and the hammer is made to strike this plate, whereby a sound is emitted, which sound varies with the condition of the subjacent lung-tissue. Whenever the lung-substance in contact with the chest-wall contains air, a clear resonant tone or sound-such as is obtained by striking a vessel containing air, a clear percussion-sound-is obtained. Where the lung does not contain air, a dull sound-like striking a limb-is obtained. If the parts containing air be very thin, or only partially filled with air, the sound is " muffled."

Fig. 142 indicates the relation of the lungs to the anterior surface of the chest. The apices of the lungs reach 3 to 7 centimetres $(1 \cdot 1$ to $2 \cdot 7$ inches) above the clavicles anteriorly, while posteriorly they extend from the spines of the scapulæ as high as the seventh spinous process. The lower margin of the right lung in the passive position (moderate expiration) of the chest, commences at the right margin of the sternum at the insertion of the sixth rib, runs under the right nipple, nearly parallel to the upper border of the sixth rib, and descends a little in the axillary line, to the upper margin of the seventh rib. On the left side (apart from the position of the heart), the lower limit reaches as far down anteriorly as the right. In fig. 142 the line $a, t, b$ shows the lowest limit of the passive lungs. Posteriorly both lungs reach as far down as the tenth rib. During the deepest inspiration, the lungs descend anteriorly as far as between the sixth-and seventh ribs, and posteriorly to the eleventh rib-whereby the diaphragm is separated from the thoracic wall (fig. 143). During the deepest expiration, the lower margins of the lungs are elevated almost as much as they descend during inspiration. In fig. $142, m, n$ indicates the margin of the right lung during deep inspiration; $h, l$, during deep expiration. [The part of the chest-wall covered by the costal pleura is considerably larger than the circumference of the lung. This is specially marked at the lower margin of the lung, and where the left lung is incised over the heart. In these 
regions, during expiration, the surfaces of the visceral and parietal pleuræe are in contact, but during inspiration they are separated, and allow the thin margins of the lung to be insinuated between them. This available space is called complemental space (Gerhurdt), or "disposable" or reserve-pleural space by Luschka (Eichhorst).]

It is important to observe the relation of the margin of the left lung to the heart. In fig. 142 a somewhat triangular space, reaching from the middle of the point of insertion of the fourth rib to the sixth rib on the left side of the sternum, is indicated. In the passive chest, the heart lies in contact with the thoracic wall in this triangular area $(\$ 56)$. This area is represented by the triangle $t, t^{\prime}, t^{\prime \prime}$, and percussion over it gives a dull sound (superficial dulness).

In the area of the larger triangle, $d, d^{\prime}, d^{\prime \prime}$, where the heart is separated from the chest-wall by the thin anterior margins of the lung, percussion gives a muffled sound, while further outwards a clear lung percussion-sound is obtained. During deep inspiration, the inner margin of the left lung reaches over the heart as far as the

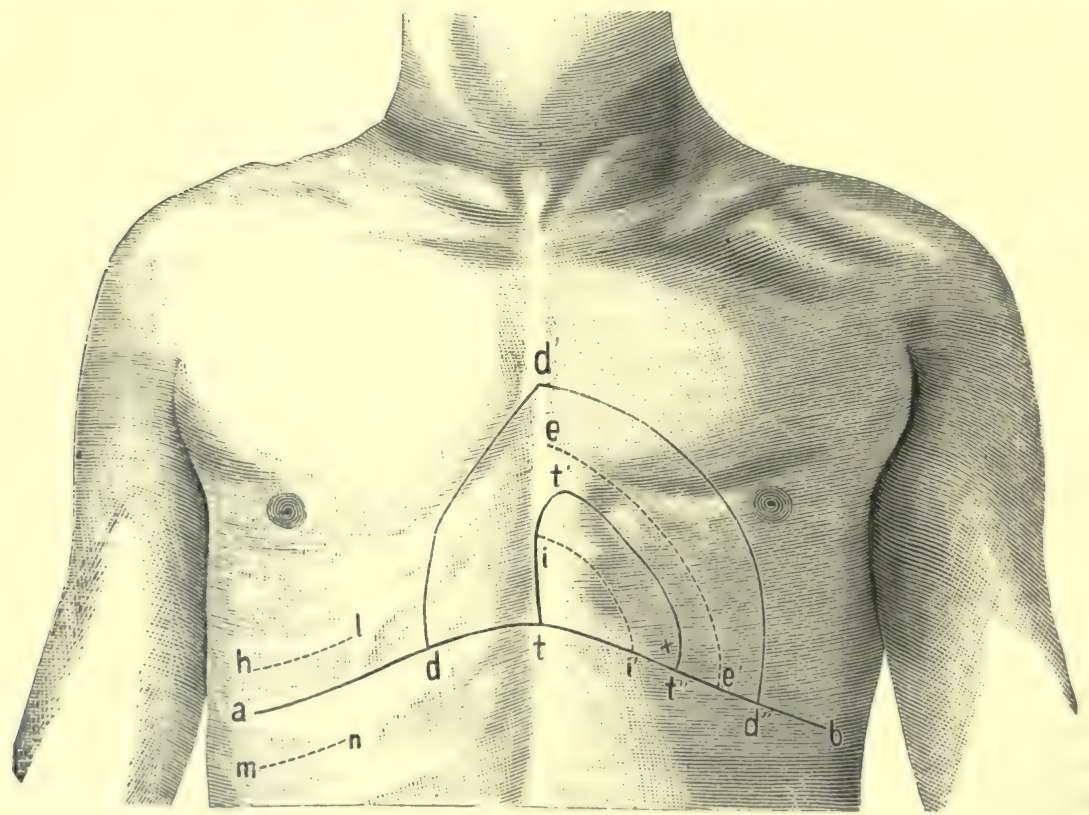

Fig. 142

Topography of the lungs and heart. $h, l$, upward limit of margin of lung during deepest expiration; $m, n$, lower limit during deepest inspiration; $t, t^{\prime}, t^{\prime \prime}$, triangular area where the heart is uncovered by lung, dull percussion-sound ; $d, d^{\prime}, d^{\prime \prime}$, muffled percussion-sound ; $i$, $i^{\prime}$, anterior margin of left lung reaches this line during deep inspiration, and during deep expiration it recedes as far as $e, e^{\prime}$.

insertion of the mediastinum, whereby the dull sound is limited to the smallest triangle, $t, i, i^{\prime}$. Conversely, during very complete expiration, the margin of the lung recedes so far that the cardiac dulness embraces the space, $t, e, e^{\prime}$.

115. PATHOLOGICAL PERCUSSION-SOUNDS.-Abnormal Dulness.-The normal clear resonant percussion-sound of the lungs becomes muffled when infiltration takes place into the lungs, so us to diminish the normal amount of air within them, or when the lungs are compressed from without, c.g., by effusion of fluid into the pleura. The percussion-sound becomes clearer when the chest-wall is very thin, as in spare individuals, during very deep inspiration, 
and especially in emphysema, where the air-vesicles of certain parts of the lung (apices and margins) become greatly dilated.

The pitch of the percussion-sound ought also to be noted. It depends upon the greater or less tension of the elastic pulmonary tissue, and on the elasticity of the thoracic wall. The tension of the elastic tissue is increased during inspiration and diminished during expiration, so that even under physiological conditions, the pitch of the sound varies.

The sound is said to be tympanitic when it has a musical quality resembling in its timbre the sound produced on drums, and when it has a slight variation in pitch. If a caoutchouc ball be placed near the ear, on tapping it gently; a well-marked tympanitic sound is heard, and the sound is of higher pitch the smaller the diameter of the ball. A tympanitic sound is always produced on tapping the trachea in the neck. A tympanitic sound produced over the chest is always indicative of a diseased condition. It occurs in cases of cavities or vomicæ within the substance of the lung, (the sound becomes deeper when the mouth, or better, the mouth and nose, are closed), when air is present in one pleural cavity, as well as in conditions where the tension of the pulmonary tissues is diminished. The tympanitic sound resembles the metallic tinkling which is heard in large pathological cavities in the lungs, or which occurs when the pleural cavity contains air, and when the conditions which permit a more uniform reflection of the sound-waves within the cavity are present.

[When a cavity, freely communicating with a large bronchus, exists in the upper and anterior part of the lung, a peculiar "cracked-pot sound" is heard on percussing over the part. Some notion of this sound may be obtained by clasping the two hands so as to bring the palms nearly together, leaving an air-space between, and then striking them on the knee. When percussion is made over a large cavity communicating with a bronchus, some of the air is expelled, and the sound thereby emitted is blended with the fundamental note of the air in the cavity itself, the combination of these two sounds thus producing the "cracked-pot" sound.]

Resistance.--When percussing a chest, we may determine whether the substance lying under the portion of the chest under examination presents great or small resistance to the blow, either of the percussion-hammer or of the tips of the fingers, as the case may be, [e.g., in great pleuritic effusion exerting much pressure on, and so distending, the thoracic walls].

Phonometry. - If the stem of a vibrating tuning-fork be placed on the chest-wall over a part containing air, its sound is intensified; but if it be placed over a portion of the lung which contains little or no air, its sound is enfeebled (von Baas).

116. THE NORMAL RESPIRATORY SOUNDS.-If the ear directly, or through the medium of a stethoscope, be placed in connection with the chest-wall, we hear over the entire area, where the lung is in contact with the chest, the so-called "normal vesicular sound," which is audible during inspiration, and its typical characters may be studied by listening in the infra-scapular region in an adult. It is a fine sighing or breezy sound, [which gradually increases in intensity until it reaches a maximum, and falls away before expiration begins]. It is said to be caused by the sudden dilatation of the air-vesicles (hence "vesicular ") during inspiration, and it is also ascribed to the friction of the current of air entering the alveoli. The sound has, at one time, a soft, at another, a sharper character ; the latter occurs constantly in children up to 12 years of age. In their case, the sound is sharper, because the air, in entering vesicles one-third narrower, is subjected to greater friction. This is followed by an expiratory sound, which may be absent during quiet breathing. It is a feeble sighing sound, of an indistinct soft character, caused by the air passing out of the air-vesicles, is three or four times shorter than the inspiratory, is loudest at first, and soon disappears, the latter part of the expiratory act giving rise to no audible sound. Its absence is not a sign of disease, but when it is prolonged and loud, suspicion is aroused.]

Bronchial Respiration. - Within the larger air-passages-larynx, trachea, bronchi -during inspiration and expiration, there are loud, rough, harsh sounds like a sharp h or ch-the "bronchial" - the laryngeal, tracheal, or "tubular" sound, or breathing. [In normal bronchial breathing, as heard over the trachea, there is a pause between the inspiratory and expiratory sounds, which are of nearly equal duration and of about the same intensity throughout. These sounds are also heard between the scapulæ, at the level of the fourth dorsal vertebra (bifurcation of trachea), and they occur also during expiration, being slightly louder on the right side, owing to the slightly greater calibre of the right bronchus. At all other parts 
of the chest, the vesicular sound obscures the tubular or bronchial sound. If the air-vesicles are deprived of their air, the tubular breathing becomes distinct.

Bronchial respiration is produced chiefly in the larynx, owing to the formation of air-eddies in consequence of the narrowing of the respiratory part of the glottis. This "laryngeal stenosis sound " excites resonance of the tracheo-bronchial column of air, and communicates to it the specific character of bronchial breathing which is heard over the large tubes of the bronchial system (Dehio).

It is asserted that, when lungs containing air are placed over the trachea, the tubular sound there produced becomes vesicular. In this case, we must suppose that the vesicular sound arises from the tubular breathing becoming weakened, and acoustically altered by being conducted through the lung alveoli. A sighing sound is often produced at the apertures of the nose and mouth during forced inspiration.

117. PATHOLOGICAL RESPIRATORY SOUNDS. - [The breath-sounds heard in disease may be merely modifications of the normal vesicular or bronchial sounds, or new sounds, such as friction sounds, râles, or rhonchi.]

[Puerile Breathing is merely an exaggerated vesicular sound, so called because it resembles the louder vesicular sound heard in children. It occurs when some part of the lung is unable to act, and there is, as it were, extra work of the other parts to compensate, and thus the sound is exaggerated.]

(1) Bronchial or Tubular Breathing occurs over the entire area of the lung, either when the air-vesicles are devoill of air, which may be caused by the exudation of fluid or solid constituents, or when the lungs are compressed from without. In both cases vesicular sounds disappear, and the condensed or solidified lung-tissue conducts the tubular sound of the large bronchi to the surface of the chest. [The sound heard over a hepatised lobe of the lung in pneumonia is a typical example.] It also occurs in large cavities, with resistent walls near the surface of the lung, proviled these cavities communicate with a large bronchus. [In this case it is termed cavernous breathing.]

(2) The amphoric sound is compared to that produced by blowing over the mouth of an empty bottle. It occurs either when a cavity - at least the size of the fist-exists in the lung, which is so blown into during respiration that a peculiar amphoric-like sound, with a metallic timbre, called metallic tinkling, is produced; or when the lung still contains air, and is capable of expansion; as there is still air in the pleural cavity, it acts as a resonator, and causes an amphoric sound, sinultaneous with the change of air in the lungs. [The amphoric sound or echo and metallic tinkling are the only certain signs of the existence of a cavity in the lung.]

(3) If obstruction occurs in the course of the air-passages of the lungs, various results may accrue, according to the nature of the resistance :- $(a)$ owing to various causes, e.g., in the apices of the lungs, there may be partial swelling of the walls of the air-tubes, or infiltration into the air-cells which hinders the regular supply of air. In these cases, parts of the lung are not supplied with air continuously; it only reaches them periodically, when a cogwheel sound occurs. A similar sound may be heard occasionally in a normal lung, when the muscles of the chest contract in a periodic spasmodic manner. (b) When the air entering large bronchi causes the formation of bubbles in the mucus which may have accumulated there, "mucous râles" are produced. They also occur in small spaces when the walls are separated from their fluid contents by the air entering during inspiration, or when the walls, being adherent to each other, are suddenly pulled asunder. The râles are distinguished as moist (when the contents are fluid), or as dry (when the contents are sticky); they may be inspiratory, expiratory, or continuous, or they may be coarse or fine; further, there is the very fine crepitation, or crackling sound, and, lastly, the metallic tinkling caused in large cavities through resonance. [Crepitation or vesicular râles are fine crepitating sounds like those produced by rubbing a lock of hair between the fingers near one's ear ; they occur only during inspiration, and are a proof that some air is entering the air-vesicles. It is heard in its typical form during the first stage of pneumonia, and seems to be produced by the bursting of minute bubbles of air in a fluid.] (c) When the mucous membrane of the bronchi is greatly swollen, or is so covered with viscid mucus that the air must force its way through, deep sonorous rhonchi (rhonshi sonori) may occur in the large air-passages, and clear shrill sibilant sounds (rhonchi sibilantes) in the smaller ones. [Rhonchi are usually due to catarrh or to affections of the bronchial mucous membrane or bronchitis.] When there is extensive bronchial catarrh, not unfrequently we feel the chestwall vibrating with the râle sounds (bronchial fremitus).

(4) If fluid and air occur together in one pleural cavity in which the lung is collapsed, on shaking the person's thorax vigorously we hear a sound such as is produced when air and water are shaken together in a bottle. This is the succussion sound of Hippocrates. Much more rarely this sound is heard under similar conditions in large pulmonary cavities.

(5) Pleural Friction.-- When the two opposed surfaces of the pleura are inflamed, have become soft, and are covered with exudation; they move over each other during respiration, and 
in doing so give rise to friction-sounds, which can be felt (often by the patient himself), and can also be heard. The sound is comparable to the sound produced by bending new leather.

(6) Pectoral Fremitus. - When we speak or sing in a loud tone, the walls of the chest vibrate, because the vibration of the vocal cords is propagated throughout the entire bronchial ramifications. The vibration is, of course, greatest near the trachea and large bronchi. The ear cannot detect the sounds distinctly. If there be much exudation or air in the pleura, or great accumulation of mucus in the bronchi, the pectoral fremitus is diminished or altogether absent. [ $\mathrm{In}$ health, when a person speaks, the vocal resonance over the trachea, although loud, may be inarticulate; and on listening over the sternum the sound is diminished and quite inarticulate ; while over the chest-wall generally the sound, though distinct, is feeble.

All conditions which cause bronchial breathing increase the pectoral fremitus. Under normal circumstances, therefore, it is louder where bronchial breathing is heard normally. The ear hears an intensified sound, called bronchophony, [which is a sound like that heard normally over the trachea or bronchi, but audible over the vesicular lung-tissue. The conditions that cause it are the same as those on which bronchial breathing depends, so that it is heard in pneumonia and phthisis. If, through effusion into the pleura or inflammatory processes in the lung-tissue, the bronchi are pressed flat, a peculiar bleating sound (ægophony) may be heard.]

\section{PRESSURE IN THE AIR-PASSAGES DURING RESPIRATION.-} Respiratory Pressure.--If a manometer be tied into the trachea of an animal, so that the respiration goes on completely undisturbed, i.e., normal respiration, during every inspiration there is a negative pressure $(-3 \mathrm{~mm} . \mathrm{Hg})$ and during expiration a positive pressure. Donders placed the $\mathrm{U}$-shaped manometer tube in one nostril, closed his mouth, leaving the other nostril open, and respired quietly. During every quiet inspiration the mercury showed a negative pressure of $-1 \mathrm{~mm}$., and during expiration a positive pressure of $2-3 \mathrm{~mm}$. $(\mathrm{Hg})$.

Forced Respiration.-As soon as the air was inspired or expired with greater force, the variations in pressure became very much greater, e.g., during speaking, singing, and coughing. The inspiratory pressure was $=-57 \mathrm{~mm}$. (36-74), the greatest expiratory pressure $+87(82-100) \mathrm{mm}$. Hg. The pressure of forced expiration, therefore, is $30 \mathrm{~mm}$. greater than the inspiratory pressure (Donders).

Resistance to Inspiration. - Notwithstanding this, we must not conclude that the expiratory muscles act more powerfully than the inspiratory; for during inspiration a variety of resistances have to be overcome, so that after these have been met, there is only a residue of the force for the aspiration of the mercury. The resistances to be overcome by the inspiratory muscles are:-(1) The elastic tension of the lungs, which during the deepest expirations $=6 \mathrm{~mm}$. ; during the deepest inspirations $=30$ $\mathrm{mm}$. Hg (\$ 107). (2) The raising of the weight of the chest. (3) The elastic torsion of the costal cartilages. (4) The depression of the abdominal contents, and the elastic distension of the abdominal walls. All these not inconsiderable resistances, which the inspiratory muscles have to overcome, act during expiration, and aid the expiratory muscles. The forces concerned in inspiration are decidedly much greater than those of expiration.

Intra-thoracic Pressure.-As the lungs within the chest, in virtue of their elasticity, continually strive to collapse, necessarily they must cause a negative pressure within the chest. This amounts in dogs, during inspiration, to $-7 \cdot 1$ to $-7 \cdot 5 \mathrm{~mm}$. $\mathrm{Hg}$, and during expiration to $-4 \mathrm{~mm}$. $\mathrm{Hg}$. The corresponding values for man have been estimated at $-4.5 \mathrm{~mm}$. $\mathrm{Hg}$ and $-3 \mathrm{~mm}$. $\mathrm{Hg}$, by Hutchinson.

[We must distinguish between the respiratory pressure of the air within the respiratory passages, and the intra-thoracic pressure. The former is the same as the atmospheric pressure when the chest is passive, but less than it as the chest is being enlarged, and greater than it when it is being diminished in size. The intra-thoracic pressure is the pressure within the chest, but outside the lungs, i.e., in the pleura, mediastinum, \&c. It is negative, i.e., less than the atmospheric pressure, and must vary with the degree of distension of the lungs.]

[Methods.-A direct estimation was made by Adamkiewiez and Jacobson. A trocar with its stylette was forced into the fourth left intercostal space near the sternum and pushed into the pericardium (sheep). The stylette was then withdrawn, and the trocar connected with a manometer, and the negative pressure of -3 to $-5 \mathrm{~mm}$. $\mathrm{Hg}$ was obtained. During severe dyspnœa it was $-9 \mathrm{~mm}$. $\mathrm{Hg}$. Rosenthal introduced an cesophageal sound with an elastic 
ampulla on its lower end into the cesophagus, so that the ampulla came to lie opposite the posterior mediastinum. The sound was connected with a registering tambour or manometer. During inspiration the manometer fell, and during inspiration it rose.]

Even the greatest inspiratory or expiratory pressure is always much less than the bloodpressure in the large arteries; but if the pressure be calculated upon the entire respiratory surface of the thorax, very considerable results are obtained.

Pneumatometer. - This instrument of Waldenburg is merely a mercurial manometer fixed to a stand, and connected to an elastic tube with a suitable monthpiece, which is fitted over the mouth and nose, while the variations of the $\mathrm{Hg}$ can be read off on a scale. [In the male, the expiratory pressure is $90-120 \mathrm{~mm}$. Hg, and the respiratory $70-100$. The relation of the pressures luring expiration and inspiration is more important than the absolute pressure.] The inspiratory pressure is liminished in nearly all diseases where the expansion of the lung is impaired [phthisis], or the expiratory pressure is diminished, as in emphysema and asthma.

Effects of the first Respiration on the Thorax. - Until birth, the airless lungs are completely collapsed (atelectic) within the chest, and fill it, so that on opening the chest in a dead fotus, jneumo-thorax does not occur (Bernstein). Supposing, however, respiration to have been fully established after birth, and air to have freely entered the lungs, if a manometer be placed in connection with the trachea, and the chest be opened, the manometer will register a pressure of $6 \mathrm{~mm} . \mathrm{Hg}$, due to the collapse of the elastic lungs. Bernstein supposes that the thorax assumes a new permanent form, due to the first respiratory distension; it is as if, owing to the respiratory elevation of the ribs, the thorax had become permanently too large for the lungs, which are, therefore, kept permanently distended, but collapse as soon as air passes into the pleura. When a lung has once been filled with air, it cannot be emptied by pressure from without, as the small bronchi are compressed before the air can pass out of the alveoli. The expiratory muscles cannot possibly expel all the air from the lungs, while the inspiratory muscular force is sufficient to distend the lungs beyond their elastic equilibrium. Inspiration listends the lungs, increasing their elastic tension, while expiration diminishes the tension without abolishing it.

\section{APPENDIX TO RESPIRATION.-Nasal Breathing.-During quiet} respiration we usually. breathe-or ought to breathe-through the nostrils, the mouth being closed. The current of air passes through the pharyngo-nasal cavity - so that, in its course during inspiration, it is (1) warmed and rendered moist, and thus irritation of the mucous memurane of the air-passages by the cold air is prevented; (2) small particles of sont, or other foreign substances in the air, adhere to, and become embedded in the mucus covering the somewhat tortuous walls of the respiratory passages, and are carried outwards by the agency of the ciliated epithelium of the respiratory passages ; (3) disagreeable odours and certain impurities are detected by the sense of smell.

If a lung be inflated, air constantly passes through the walls of the alveoli and trachea. This also occur's during violent expiratory efforts (cutaneous emphysema in whooping-cough), so that pneumo-thorax may occur $(J . R$. Ewald and Kobert).

Pulmonary Edema, or the exudation of lymph into the pulmonary alveoli, occurs-(1) When there is very great resistance to the blood-stream in the aorta or its branches, e.g., by ligaturing all the arteries going to the head or the arch of the aorta, so that only one carotid remains pervious. (2) When the pulmonary veins are occluded. (3) When the left ventricle, owing to mechanical injury, ceases to beat, while the right ventricle goes on contracting $(\$ 47)$. These conditions produce at the same time anæmia of the vaso-motor centre, which results in stimulation of that centre, and consequent contraction of all the small arteries. Thus the blood-stream through the veins to the right heart is favoured, and this in its turn favours the production of relema of the lungs. [The injection of muscarin rapidly causes pulmonary cedema, due to the increase of pressure and slowing of the blood-stream in the pulmonary capillaries. It is set aside by atropin (W.einzweig, Grossmann).]

120. MODIFIED RESPIRATORY MOVEMENTS.-(1) Coughing consists in a sudden violent expiratory explosion after a previous deep inspiration and closure of the glottis, whereby the glottis is forced open, and any substance, fluid, gaseous, or solid, in contact with the respiratory mucous membrane is violently ejected through the open mouth. It is produced voluntarily or reflexly; in the latter case, it can be controlled by the will only to a limited extent.

[Causes. - A cough may be discharged reflexly from a large number of surfaces:-(1) A draught of cold air striking the skin, especially of the upper part of the body. This may cause congestion of blood in the air-passages, this in turn exciting the cough. (2) More frequently it is discharged from the respiratory mucous membrane, especially of the larynx, the sensory branches of the vagus and the superior laryngeal nerve being the afferent nerves. A cough cannot be discharged from every part of the larynx : thus there is none from the true vocal cords, 
but only from the glottis respiratoria. All other parts of the larynx are inactive, and so is the trachea as far as the bifurcation, where stimulation excites cough (Kohts). (3) Sometimes an offending body, such as a pea or inspissated cerumen in the external auditory meatus, gives rise to coughing, the afferent nerve being the auricular branch of the vagus. (4) There seems to be no doubt that there may be a "gastric or stomach cough," produced by stimulation of the gastrie branches of the vagus, especially in cases of indigestion, accompanied by irritation of the larynx and trachea. (5) Irritation of the costal pleura and even of the œesophagus (Kohts). (6) Irritation of some parts of the nose. (7) Sometimes also from irritation of the pharynx, as by an elongated uvula. (8) In some diseases of the liver, spleen, and generative organs, when pressure is exerted on these parts.]

(2) Hawking, or clearing the throat.-An expiratory current is forced in a continuous stream through the narrow space between the root of the tongue and the depressed soft palate, in order to assist in the removal of foreign bodies. When the act is carried out periodically, the closed glottis is suddenly forced open, and it is comparable to a voluntary gentle cough. This act can only be produced voluntarily.

(3) Sneezing consists in a sudden violent expiratory blast through the nose, for the removal of mucus or foreign bodies (the mouth being rarely open) after a simple or repeated spasm-like inspiration-the glottis remaining open. It is usually caused reflexly by stimulation of sensory nerve-fibres of the nose [nasal branch of the fifth nerve], or by sudden exposure to a bright light [the afferent nerve is the optic]. This reflex act may be interfered with to a certain extent, or even prevented, by stimulation of sensory nerves, or firmly compressing the nose where the nasal nerve issues. The continued use of sternutatories, as in persons who take snuff, dulls the sensory nerves, so that they no longer act when stimulated reflexly.

[Sternutatories or Errhines, such as powered ipecacuanha, snuff, and euphorbium, also increase the secretion from the nasal glands. The afferent impulses sent to the respiratory centre also affect the vaso-motor centre, so that, even when sneezing does not occur, the blood-pressure throughout the body is raised.]

(4) Snoring occurs during respiration through the open mouth, whereby the inspiratory and expiratory stream of air throws the uvula and soft palate into vibration. It is involuntary, and usually occurs during sleep, but it may be produced voluntarily.

(5) Gargling consists in the slow passage of the expiratory air-current in the form of bubbles through a fluid lying between the tongue and the soft palate, when the head is held backwards. It is a voluntary act.

(6) Crying, caused by emotional conditions, consists in short, deep inspirations, long expirations with the glottis narrowed, relaxed facial and jaw muscles, secretion of tears, often combined with plaintive inarticulate expressions. When crying is long continued, sudden and spasmodic involuntary contractions of the diaphragm occur, which cause the inspiratory sounds in the pharynx and larynx known as sobbing. This is an involuntary act.

(7) Sighing is a prolonged inspiration, usually combined with a plaintive sound, often caused involuntarily, owing to painful or unpleasant recollections.

(8) Laughing is due to short rapid expiratory blasts through the tense vocal cords, which cause a clear tone, and there are characteristic inarticulate sounds in the larynx, with vibrations of the soft palate. The mouth is usually open, and the countenance has a characteristic expression, owing to the action of the M. zygomaticus major. It is usually involuntary, and can only be suppressed, to a certain degree, by the will (by forcibly closing the mouth and stopping respiration).

(9) Yawning is a prolonged deep inspiration occurring after successive attempts at numerous inspirations - the mouth, fauces, and glottis being wide open; expiration shorter-both acts often accompanied by prolonged characteristic sounds. It is quite involuntary, and is usually excited by drowsiness or ennui.

$[(10)$ Hiccough is due to a spasmodic involuntary contraction of the diaphragm, causing an inspiration, which is arrested by the sudden closure of the glottis, so that a characteristic sound is emitted. Not unfrequently it is due to irritation of the gastric mucous membrane, and sometimes it is a very troublesome symptom in uræmic poisoning.]

121. CHEMISTRY OF RESPIRATION-CARBON DIOXIDE, OXYGEN, and WATERY VAPOUR GIVEN OFF. - I. Estimation of $\mathrm{CO}_{2},-1$. The volume of $\mathrm{CO}_{2}$ is estimated by means of the anthracometer (fig. 143, II). The volume of gas.is collected in a graduated tube, $r r$, provided with a bulb at one end (previously filled with water and carefully calibrated, i.e., the exact amount which each part of the tube contains is accurately measured), and the tube is closed. The lower end has a stop-cock, $h$, and to this is screwed a flask, $n$, completely filled with a solution of caustie potash ; the stop-eock is then opened, the potash solution is allowed to ascend into the tube, which is moved about until all the $\mathrm{CO}_{2}$ unites with the potash to form potassium carbonate. Hold the tube vertically and allow the potash to run back into the flask, close the stop-cock, and remove the bottle with the potash. Place the stop-cock under water, open it, and allow the water to ascend in the tube, when the space in the tube occupied by the fluid indicates the volume of $\mathrm{CO}_{2}$ which is combined with the potash. 
2. By Weight. - A large quantity of the mixture of gases which has to be investigated is made to pass through a Liebig's bulb filled with caustic potash. The potash apparatus having been carefully weighed beforehand, the increase of weight indicates the amount of $\mathrm{CO}_{2}$ which has been taken up by the potash from the air passed through it.

3. By Titration.-A large volume of the air to be investigated is conducted through a known volume of a solution of barium hydrate. The $\mathrm{CO}_{2}$ unites with the barium and forms barium carbonate. The fluid is neutralised with a standard solution of oxalic acid, and the more barium that has united with the $\mathrm{CO}_{2}$ the smaller will be the amount of oxalic acid used, and vice versa.

II. Estimation of Oxygen.-According to volume-(a) By the union of the $\mathrm{O}$ with potassium

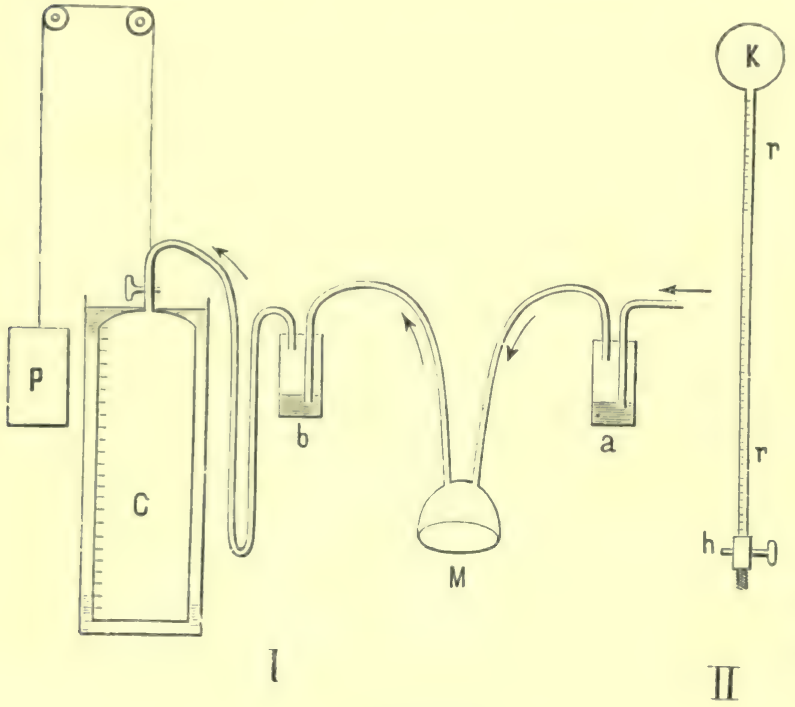

Fig. 143. pyrogallate. The same procedure is adopted as for the estimation of $\mathrm{CO}_{2}$, only the flask, $n$, is filled with the pyrogallate solution instead of potash. (b) By explosion in an eudiometer (see BloodGases, § 35).

III. Estimation of Watery Vapour. - The air to be investigated is passed through a bulb containing concentrated sulphuric acid, or through a tube filled with pieces of calcium chloride. The amount of water is directly indicated by the increase of weight.

122. METHODS OF INVESTIGATION.-I. Collecting the Expired Air. - (1) The air expired may be collected in the cylinder of the

I. Apparatus of Andral and Gavarret for collecting the expired air. C, spirometer, which is large cylinder to collect the air expired; $P$, weight to balance cylin- suspended in concender; $a, b$, two Miiller's valves; M, mouthpiece. II. Anthracometer trated salt solution to of Vierordt. avoid the absorption of $\mathrm{CO}_{2}(\S 108)$.

Andral and Gavarret's Apparatus.-The operator breathed several times into a capacious cylinder (fig. 143). A mouthpiece (MI) was placed air-tight over the mouth while the nostrils were closed. The direction of the respiratory current was regulated by two so-called "Müller's Valves" (mercurial), $(a$ and $b$ ). With every inspiration the bottle or valve, $a$ (filled below with $\mathrm{Hg}$ and hermetically closed above), permits the air inspired to pass to the lungs-during every expiration the expired air can pass only through $b$ to the collecting-cylinder C.

(2) If the gases given off by the skin are to be collected, a limb, or whatever part is to be investigated, is secured in a closed vessel, and the gases so obtained are analysed.

II. The most important apparatus for this purpose are those of-(a) Scharling (fig. 144), which consists of a closed box, $\mathrm{A}$, of sufficient size to contain a man. It is provided with an inlet $z$ and outlet $b$. The latter is connected with an aspirator, $\mathrm{C}$, a large barrel filled with water. When the stop-cock, $h$, is opened and the water flows out of the barrel, fresh air will rush in continuously into the box, $\mathrm{A}$, and the air mixed with the expired gases will be drawn towards C. A Liebig's bulb, $d$, filled with caustic potash, is connected with the entrance tube, $\approx$, through which the in-going air must pass, whereby it is completely deprived of $\mathrm{CO}_{2}$, so that the person experimented on is supplied with air free from $\mathrm{CO}_{2}$. The air passing out by the exit tube, $b$, has to pass first through $e$, where it gives up its watery vapour to sulphurie aciả, whereby the amount of watery vapour is estimated by the increase of the weight of the apparatus, e. Afterwards the air passes through a bulb, $f$, containing caustic potash, which absorbs all the $\mathrm{CO}_{2}$, while the tube, $g$, filled with sulphuric acid, absorbs any watery vapour that may come from $f$. The increase in weight of $f$ and $g$ indicates the amount of $\mathrm{CO}_{2}$. The total volume of air used is known from the capacity of $\mathrm{C}$.

(b) Regnault and Reiset's Apparatus is more complicated, and is used when it is necessary to keep animals for some time under observation in a bell-jar. It consists of a globe, $\mathrm{R}$, in 
hich is placed the dog to be experimented on (fig. 145). Around this is placed a cylinder, $g, g$, (provided with a thermometer, $t$ ), which may be used for calorimetric experiments. A tube, $c$, leads into the globe, $\mathrm{R}$; through this tube passes a known quantity of pure oxygen (fig. 145, O). To absorb any trace of $\mathrm{CO}_{2}$, a vessel containing potash (fig. 145, $\mathrm{CO}_{2}$ ) is placed

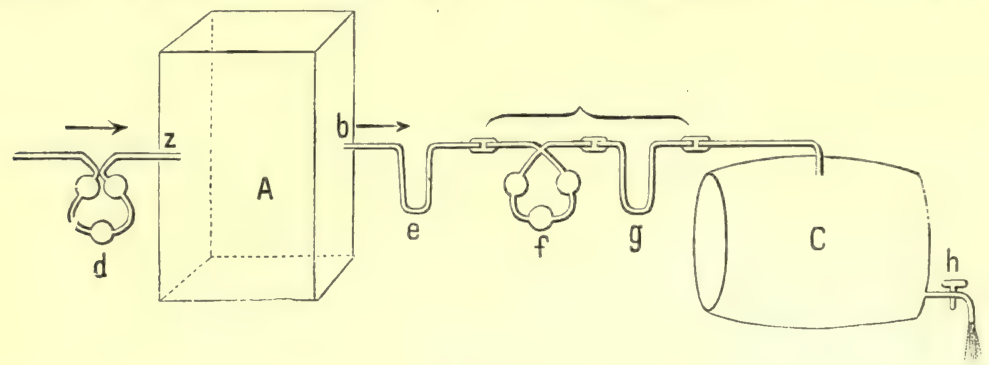

Fig. 144 .

Scharling's apparatus. $d$, bulb containing caustic potash to absorb $\mathrm{CO}_{2}$ from in-going air ; $\mathrm{A}$, box for animal experimented on ; $e$ and $g$, tubes containing sulphuric acid to absorb watery vapour; $f$, potash bulb to absorb $\mathrm{CO}_{2}$ given off; $\mathrm{C}$, vessel filled with water to aspirate air ; $h$, stop-cock.

in the course of the tube. The vessel for measuring the $\mathrm{O}$ is emptied towards $\mathrm{R}$, through a solution of calcium chloride from a large pan $\left(\mathrm{CaCl}_{2}\right)$ provided with large flasks. Two tubes, $d$ and $e$, lead from $\mathrm{R}$, and are united by caoutchouc tubes with the potash bulbs (KOH, Koh), which can be raised or depressed alternately by means of the beam, W. In this way they

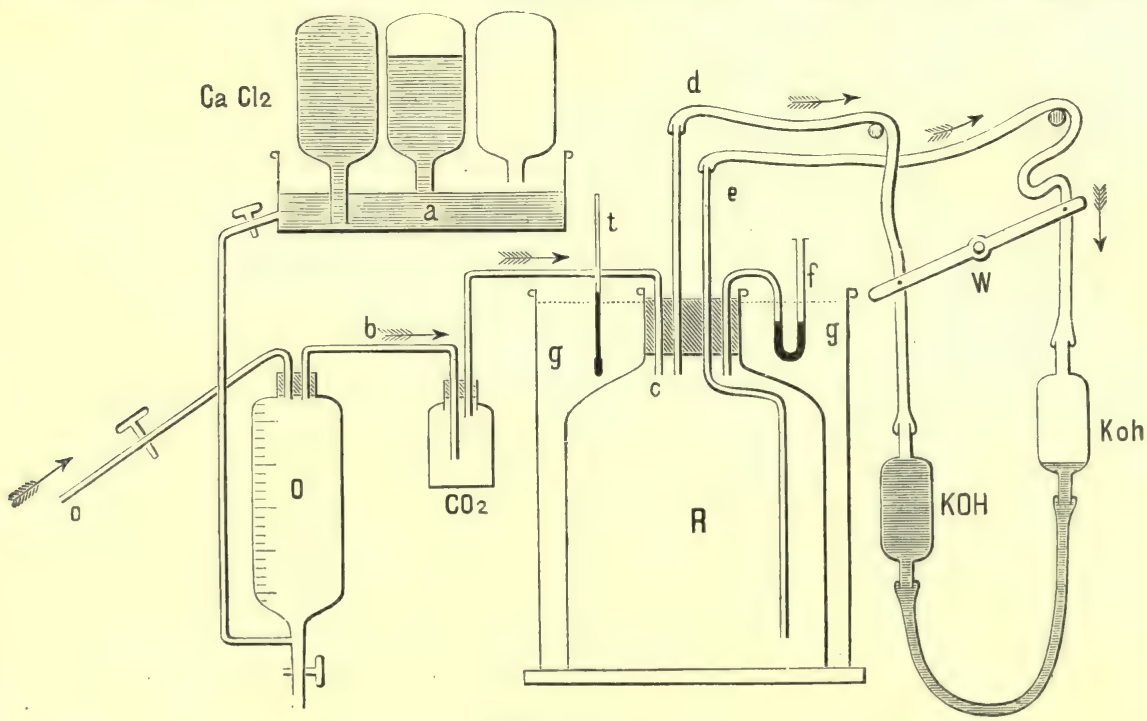

Fig. 145 .

Scheme of the respiration apparatus of Regnault and Reiset. R, globe for animal ; $g, g$, outer casing for $\mathrm{R}$, provided with a thermometer, $t ; d$ and $\grave{e}$, exit tubes to movable potash bulbs, $\mathrm{KOH}$ and $\mathrm{K} o h ; \mathrm{O}$, in-going oxygen; $\mathrm{CO}_{2}$, vessel to absorb any carbonic acid; $\mathrm{CaCl}_{2}$, apparatus for estimating the amount of $O$ supplied ; $f$, manometer.

aspirate alternately the air from $\mathrm{R}$, and the caustic potash absorbs the $\mathrm{CO}_{2}$. The increase in weight of these flasks after the experiment-indicates the amount of $\mathrm{CO}_{2}$ expired. The manometer, $f$, shows whether there is a difference of the pressure outside and inside the globe, $R$.

(c) V. Pettenkofer has invented the most complete apparatus (fig. 146). It consists of a chamber, Z, with metallic walls, and provided with a door and a window. At $\alpha$ is an opening for the admission of air, while a large double suction-pump, $\mathrm{PP}_{1}$ (driven by means of a steam- 
engine) continually renews the air within the chamber. The air passes into a vessel, $b$, filled with pumice-stone saturated with sulphuric acid, in which it is dried; it then passes through a large gas-meter, $c$, which measures the total amount of the air passing through it. After the air is measured, it is emptied outwards by means of the pump, $\mathrm{PP}_{1}$. From the chief exit tube, $x$, of the chamber provided with a small manometer, $q$, a narrow laterally placed tube, $n$, passes conducting a small secondary stream, which is chemically investigated. This current passes through the suction-apparutus, $\mathrm{MM}_{1}$ (constructed on the principle of Miiller's mercurial valve, and (iriven by a steam-engine). Before reaching this apparatus, the air passes through the bulb, $\mathrm{K}$, filled with sulphuric acid, whose increase in weight indicates the amount of watery vapour. After passing through $\mathrm{MMI}_{1}$, it goes through the tube, $\mathrm{R}$, filled with baryta solution, which takes up $\mathrm{CO}_{2}$. The quantity of air whioh passes through the accessory current, $n$, is

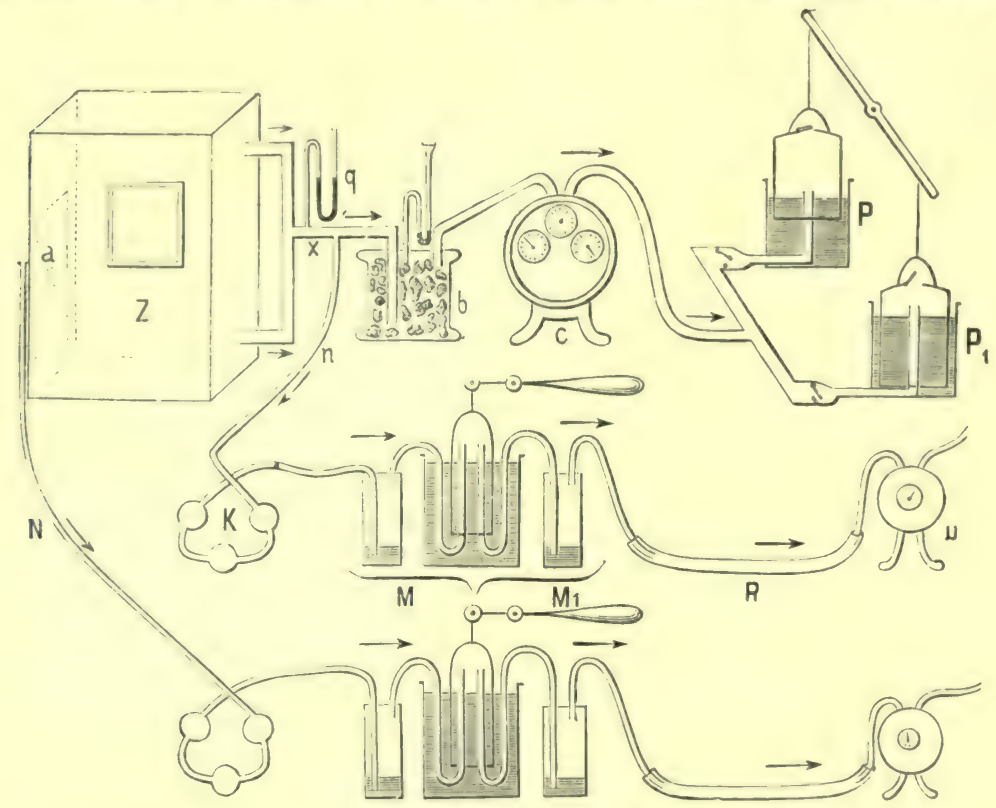

Fig. 146.

Respiration apluaratus of $\mathrm{v}$. Pettenkofer. Z, chamber for person experimentel on; $x$, exit tube with manometer, $q$; $b$, vessel with sulphuric acid; C, gas-meter; $\mathrm{PP}_{1}$, pump; $n$ secondary current, with, $k$, bulb; $\mathbf{M N}_{1}$, suction apparatus; $u$, gas-meter; $\mathrm{N}$, stream for investigating air before it enters $\mathrm{Z}$.

measured by the small gas-meter, $u$, from which it passes outwarils. The second accessory stream, $\mathrm{N}$, enables us to investigate the air before it enters the chamber, and it is arranged in ractly the same way as $n$. The increase of $\mathrm{CO}_{2}$ and $\mathrm{H}_{2} \mathrm{O}$ in the accessory strean, $n$ (i.e., more than in $\mathrm{N}$ ), inclicates the amount of $\mathrm{CO}_{2}$ given off by the person in the chamber, $\mathrm{Z}$.

\section{COMPOSITION OF ATMOSPHERIC AIR.-1. Dry Air contains:- \\ Gas. \\ $\stackrel{\mathrm{O}}{\mathrm{N}}$ \\ $\mathrm{CO}_{3}$ \\ By Weight. \\ $23 \cdot 015$ \\ $76 \cdot 985$}

2. Aqueous vapour is always present in the air, but it varies greatly in amount, and generally increases with the increase of the temperature of the air. We distinguish $(a)$ the absolute moisture, i.e., the quantity of watery vapour which a volume of air contains in the form of vapour ; and (b) the relative moisture, i.e., the amount of watery vapour which a volume of air contains with respect to its temperature.

Experience shows that people generally can breathe most comfortably in an atmosphere which not completely saturated with aqueous vapour according to its temperature, but is only 
saturated to the extent of 70 per cent. If the air be too dry, it irritates the respiratory mucous membrane ; if too moist, there is a disagreeable sensation, and if it be too warm, a feeling of eloseness. Hence, it is important to see that the proper amount of watery vapour is present in the air of our sitting-rooms, bedrooms, and hospital wards.

The absolute amount of moisture varies:- In towns during the day it increases with increase of temperature, and diminishes when the temperature falls; it also varies with the direction of the wind, season of the year, and the height above sea-level.

The relative amount of moisture is greatest at sunrise, least at midday; small on high mountains ; greater in winter than in summer ; larger with a south or a west wind than with a north or an east wind.

The air in midsummer contains absolutely three times as much watery vapour as in midwinter, nevertheless the air in summer is relatively drier than the air in winter.

3. The air expands by heat. Rudberg found that 1000 volumes of air, at $0^{\circ}$, expanded to 1365 when heated to $100^{\circ} \mathrm{C}$.

4. The density of the air diminishes with increase of the height above the sea-level.

124. COMPOSITION OF EXPIRED AIR.-1. The expired air contains more $\mathbf{C O}_{2}$-in normal respiration $=4 \cdot 38$ vols. per cent. $(3 \cdot 3$ to 5.5 per cent.), so that it contains nearly 100 times more $\mathrm{CO}_{2}$ than the atmospheric air.

2. It contains less $\mathbf{0}(4 \cdot 782$ vols. per cent. less $)$ than the atmospheric air, i.e., it contains only 16.033 vols. per cent. of $\mathrm{O}$.

3. Respiratory Quotient.-Hence, during respiration, more $\mathrm{O}$ is taken into the body from the air than $\mathrm{CO}_{2}$ is given off; so that the volume of the expired air is $\left(\frac{1}{40}\right.$ to $\left.\frac{1}{50}\right)$ smaller than the volume of the air inspired, both being calculated as dry, at the same temperature, and at the same barometric pressure. The relation of the $\mathrm{O}$ absorbed to the $\mathrm{CO}_{2}$ given off is $4 \cdot 38: 4 \cdot 782$. This is expressed by the "respiratory quotient" -

$$
\frac{\mathrm{CO}_{2}}{\mathrm{O}}\left(=\frac{4 \cdot 38}{4 \cdot 782}\right)=0.906
$$

4. An excessively small quantity of $\mathbf{N}$ is added to the expired air (Regnault and Reiset). Segen found that all the $\mathrm{N}$ taken in with the food did not reappear in the excreta (urine and fæces), and he assumed that a small part of it was given off by the lungs.

5. During ordinary respiration the expired air is saturated with watery vapour. It is evident, therefore, that when the watery vapour in the air varies, the lungs give off different quantities of water from the body. The percentage of watery vapour falls during rapid respiration (Moleschott).

6. The expired air is warmer $\left(36.3^{\circ} \mathrm{C}.\right)$. It is very near the temperature of the body, and although the temperature of the sur-

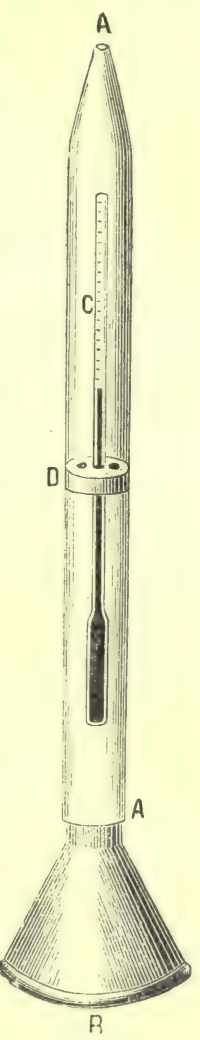

Fig. 147. rounding atmosphere be very'variable, the temperature of the expired air still remains nearly the same.

Fig. 147 shows the instrument used by Valentin and Brunner to determine the temperature of the expired air. It consists of a glass tube, A, A, with a mouthpiece, B, and in it is a fine thermometer, C. The operator breathes through the nose and expires slowly through the mouthpiece into the tube.
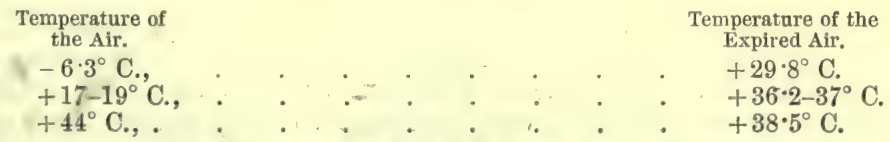

7. The diminution of the volume of the expired air mentioned under (3) is far more than compensated by the warming which the inspired air undergoes in the 
respiratory passages, so that the volume of the expired air is one-ninth greater than the air inspired.

8. A very small quantity of ammonia is found in the expired air $=0.0204$ grammes in 24 hours; it is probably derived from the blood.

9. Small quantities of $\mathbf{H}$ and $\mathbf{C H}_{4}$ are expired, both being absorbed from the intestine. In herbivora, Reiset found that 30 litres of $\mathrm{CH}_{4}$ were expired in 24 hours:

125. QUANTITY OF GASES EXCHANGED.-As under normal circumstances more $\mathrm{O}$ is absorbed than there is $\mathrm{CO}_{2}$ given off (equal volumes of $\mathrm{O}$ and $\mathrm{CO}_{2}$ contain equal quantities of $\mathrm{O}$ ), a part of the $\mathrm{O}$ must be used for other oxidation-processes in the body. According to the extent of these latter processes, the ratio of the $\mathrm{O}$ taken in to the $\mathrm{CO}_{2}$ given out-

$$
\left(\frac{\mathrm{CO}_{2}}{\mathrm{O}}=0.906 \text { normally }\right) \text { must vary. }
$$

The amount of $\mathrm{CO}_{2}$ given off may be less than the "mean" above stated. The quantity of $\mathrm{CO}_{2}$ alone is not a reliable indication of the entire exchange of gases during respiration; we must estimate simultaneously the amount of $\mathrm{O}$ absorbed and the $\mathrm{CO}_{2}$ given off.

\section{DAILY GASEOUS INCOME AND EXPENDITURE:-}

\section{0xygen- \\ 744 grms. $=516: 500 \mathrm{c} . \mathrm{cm}$ tr \\ Income in 24 hours.}

(Vierordt).

(At $0^{\circ} \mathrm{C}$. and mean barometric pressure.)

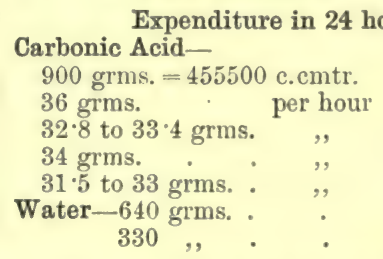

(Vierordt). (Scharling). (Liebermeister). (Panum). (Ranke).

(Valentin). (Vierordt).

127. CONDITIONS INFLUENCING THE GASEOUS EXCHANGES. - The formation of $\mathrm{CO}_{2}$, in all probability, consists of two distinct processes. First, compounds containing $\mathrm{CO}_{2}$, which are oxidation-products of substances containing carbon, seem to be formed in the tissues. The second process consists in the separation of this $\mathrm{CO}_{2}$, which, however, takes place without the absorption of $\mathrm{O}$. Both processes do not always occur simultaneously, and the one process may exceed the other in extent. The formation of $\mathrm{CO}_{2}$ is affected by :-

1. Age.- Until the body is fully developed, the $\mathrm{CO}_{2}$ given off increases, but it diminishes as the bodily energies decay. Hence, in young persons the $\mathrm{O}$ absorbed is relatively greater than the $\mathrm{CO}_{2}$ given off ; at other periods both values are pretty constant. Example :-

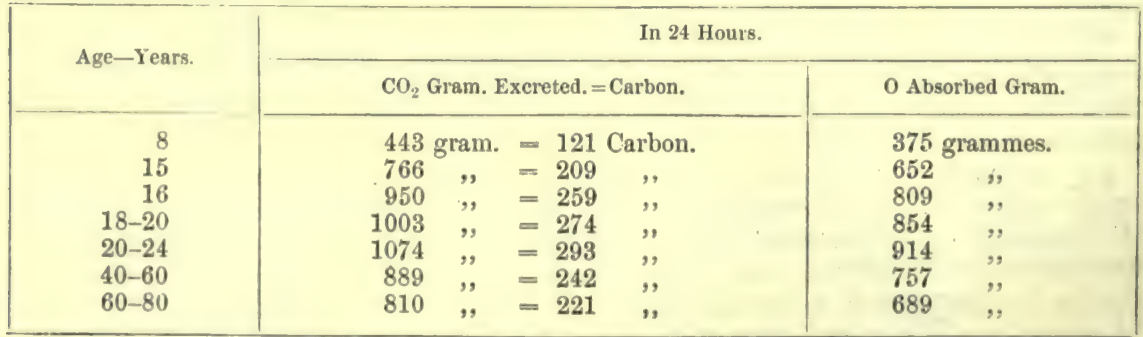

The absolute amount of $\mathrm{CO}_{2}$ given off is less in children than in adults; but if the $\mathrm{CO}_{2}$ given off be calculated with reference to body-weight, then, weight for weight, a child gives off twice as much $\mathrm{CO}_{2}$ as an adult.

2. Sex.-Males, from the eighth year onward to old age, give off about one- 
third more $\mathrm{CO}_{2}$ than females. This difference is more marked at puberty, when the difference may rise to one-half. After cessation of the menses, there is an increase, and in old age the amount of $\mathrm{CO}_{2}$ given off diminishes. Pregnancy increases the amount, owing to causes which are easily understood (Andral and Gavarret).

3. Constitution. - In general, muscular energetic persons use more $\mathrm{O}$ and excrete more $\mathrm{CO}_{2}$ than less active persons of the same weight.

4. Alternation of Day and Night. - The CO., given off is diminished about onefourth during sleep, due to the constant heat of the surroundings (bed), darkness, absence of muscular activity, and the non-taking of food (see 5, 6, 7, 9). $\mathrm{O}$ is not stored up during sleep (S. Lewin). After awaking in the morning, the respirations are deeper and more rapid, while the amount of $\mathrm{CO}_{2}$ given off is increased. It decreases during the forenoon, until dinner at mid-day causes another increase. It falls during the afternoon, and increases again after supper.

During hybernation, when no food is taken, and when the respirations cease, or are greatly diminished, the respiratory exchange of gases is carried out by diffusion and the cardiopneumatic movements $(\S 59)$. The $\mathrm{CO}_{2}$ given off falls to $\frac{1}{75}$, the $\mathrm{O}$ taken in to $\frac{1}{4}$, of what they are in the waking condition. Much less $\mathrm{CO}_{2}$ is given off than $\mathrm{O}$ taken in, so that the bodyweight may increase through the excess of 0 .

5. Temperature of the Surroundings. - Cold-blooded animals become warmer when the temperature of their environment is raised, and they give off more $\mathrm{CO}_{2}$ in this condition than when they are cooler; e.g., a frog with the temperature of the surroundings at $39^{\circ} \mathrm{C}$. excreted three times as much $\mathrm{CO}_{2}$ as when the temperature was $6^{\circ} \mathrm{C}$. Warm-blooded animals behave quite differently when the temperature of the surrounding medium is changed. When the temperature of the animal is lowered thereby, there is a considerable decrease in the amount of $\mathrm{CO}_{2}$ given off, as in cold-blooded animals, but if the temperature of the animal be increased (and also in fever), the $\mathrm{CO}_{2}$ is increased (C. Ludwig and Sanders-Ezn). Exactly the reverse obtains when the temperature of the surroundings varies, and the bodily temperature remains constant. As the cold of the surrounding medium increases, the processes of oxidation within the body are increased through some as yet unknown reflex inechanism; the number and depth of the respirations increase, whereby more $\mathrm{O}$ is taken in and more $\mathrm{CO}_{2}$ is given out. A man in January uses $32 \cdot 2$ grammes $\mathrm{O}$ per hour; in July only $31 \cdot 7$ grammes. In animals, with the temperature of the surroundings at $8^{\circ} \mathrm{C}$., the $\mathrm{CO}_{2}$ given off was one-third greater than with a temperature of $38^{\circ} \mathrm{C}$. When the temperature of the air increases-the body temperature remaining the same-the respiratory activity and the $\mathrm{CO}_{2}$ given off diminish, while the pulse remains nearly constant. On passing suddenly from a cold to a warm medium the amount of $\mathrm{CO}_{2}$ is considerably diminished ; and conversely, on passing from a warm to a cold medium, the amount is considerably increased (\$ 214).

6. Muscular exercise causes a considerable increase in the $\mathrm{CO}_{2}$ given out, which may be three times greater during walking than during rest (Ed. Smith). Ludwig and Sezelkow estimated the $\mathrm{O}$ taken in and the $\mathrm{CO}_{2}$ given off by a rabbit during rest, and when the muscles of the hind limbs were tetanised. During tetanus the $\mathrm{O}$ and $\mathrm{CO}_{2}$ were increased considerably, but in tetanised animals more $\mathrm{O}$ was given off in the $\mathrm{CO}_{2}$ expired than was taken up simultaneously during respiration. The passive animal absorbed nearly twice as much $\mathrm{O}$ as the amount of $\mathrm{CO}_{2}$ given off $(\$ 294)$.

7. Taking of food causes a not inconsiderable increase in the $\mathrm{CO}_{2}$ given off, which depends upon the quantity taken; the increase generally occurs about an hour after the chief meal-dinner. During inanition, the exchange of gases diminishes eonsiderably until death occurs. At first the $\mathrm{CO}_{2}$ given off diminishes more quickly than the $\mathrm{O}$ is taken up. The quality of the food influences the $\mathrm{CO}_{2}$ given off to this extent, that substances rich in carbon (carbohydrates and fats) cause a greater excretion of $\mathrm{CO}_{2}$ than substances which contain less $\mathrm{C}$ (albumins). 
Regnault and Reiset found that a dog gave off 79 per cent. of the $\mathrm{O}$ inspired after a flesh diet, and 91 per cent. after a diet of starch. If easily oxidisable substances (glycerin or lactate of soda) are injected into the blood, the $\mathrm{O}$ taken in, and the $\mathrm{CO}_{2}$ given off, undergo a considerable increase (Ludwig and Scheremetjewsky). Alcohols, tea, and ethereal oils diminish the $\mathrm{CO}_{2}$ (Prout, Vierordt). [Ed. Smith divided foods, with reference to the excretion of $\mathrm{CO}_{2}$, into two classes. The respiratory excitants include nitrogenous foods, rum, beer, sugar, stout, \&c.; the nonexciters starch, fat, some alcoholic mixtures. The most powerful respiratory excitants, however, are tea, sugar, coffee, and rum, and the maximum effect is usually experienced within an hour. He also found that the effects produced by alcoholic drinks varied with the nature of the spirituous liquor. Thus brandy, whisky, and gin diminish the amount; while pure alcohol, rum, ale, and porter tend to increase it.]

8. The number and depth of the respirations have practically no influence on the formation of $\mathrm{CO}_{2}$ or the oxidation-processes within the body, these being regulated by the tissues themselves, by some mechanism as yet unknown (Pflüger). They have a marked effect, however, upon the removal of the already formed $\mathrm{CO}_{2}$ from the body. An increase in the number of respirations (their depth remaining the same), as well as an increase of their depth (the number remaining the same), causes an absolute increase in the amount of $\mathrm{CO}_{2}$ given off, which, with reference to the total amount of gases exchanged, is relatively diminished. The following example from Vierordt illustrates this:-

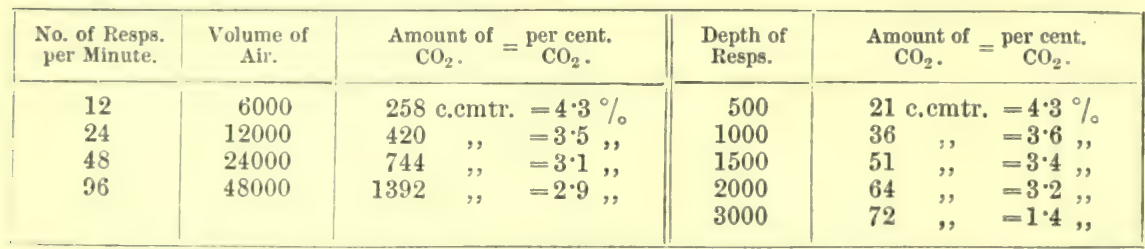

9. Exposure to a bright light causes an increase in the $\mathrm{CO}_{2}$ given off in frogs, in mammals and birds, even in frogs deprived of their lungs, or in those whose spinal cord has been divided high up. The consumption of $\mathrm{O}$ is increased at the same time. The same results occur in blind persons, although to a less degree. Bluish-violet light is almost as active as white light, while red light is less active.

10. The experiments of Gréhant, on dogs, seem to show that intense inflammation of the bronchial mucous membrane influences the $\mathrm{CO}_{2}$ given off.

11. Amongst poisons, thebaia increases the $\mathrm{CO}_{2}$ given off, while morphia, codeia, narcein, narcotin, papaverin, diminish it (Fübini).

128. DIFFUSION OF GASES WITHIN THE LUNGS. The air within the airvesicles contains most $\mathrm{CO}_{2}$ and least $\mathrm{O}$, and as we pass from the small to the large bronchi and onwards to the trachea, the composition of the air gradually approaches more closely to that of the atmosphere. Hence, if the air expired be collected in two portions, the first half (i.e., the air from the larger air-passages) contains less $\mathrm{CO}_{2}(3 \cdot 7$ vols. per cent.) than the second half $(5 \cdot 4$ vols. per ceut.). The difference in the percentage of gases gives rise to a diffusion of the gases within the air-passages; the $\mathrm{CO}_{2}$ must diffuse from the air-vesicles outwards, and the $\mathrm{O}$ from the atmosphere and nostrils inwards ( $\$ 33$ ). This movement is aided by the cardio-pneumatic movement $(\$ 59)$. In hybernating animals and in persons apparently but not actually dead, the exchange of gases within the lungs can only occur in the abovementioned ways. For ordinary purposes this mechanism is insufficient, and there are added the respiratory movements whereby atmospheric air is introduced into the larger air-passages, from which and into which the diffusion currents of $\mathrm{O}$ and $\mathrm{CO}_{2}$ pass, on account of the difference of tension of the gases. 
129. EXCHANGE OF GASES IN THE AIR-VESICLES.--The exchange of gases between the gases of the blood and those in the air-vesicles occurs almost exclusively through the agency of chemical processes, and therefore independently of the diffusion of gases.

Method.-It is important to ascertain the tension of the $\mathrm{O}$ and $\mathrm{CO}_{2}$ in the venous blood of the pulmonary capillaries. Pfliiger and Wolfberg estimated the tension by "catheterising the lungs." An elastic catheter was introduced through an opening in the trachea of a dog into the bronchus leading to the lowest lobe of the left lung. An elastic sac was placed round the catheter, and when the latter was introduced into the bronchus, the sac around the catheter was distended so as to plug the bronchus. No air could escape between the catheter and the wall of the bronchus. The outer end of the catheter was closed at first, and the dog was allowed to respire quietly. After four minutes the air in the air-vesicles was completely in equilibrium with the blood-gases. The air of the lung was sucked out of the catheter by means of an airpump, and afterwards analysed.

Thus we may measure indirectly the tension of the $\mathrm{O}$ and $\mathrm{CO}_{2}$ in the venous blood of the pulmonary capillaries. The direct estimation of the gases in different kinds of blood is made by shaking up the blood with another gas. The gases so removed indicate directly the proportion of blood-gases.

The following statement shows the tension and percentage of $\mathrm{O}$ and $\mathrm{CO}_{2}$ in arterial and venous blood, in the atmosphere, and in the air of the alveoli :-

\section{I.}

0.Tension in arterial blood $=29.6 \mathrm{~mm} . \mathrm{Hg}$ (corresponding to a mixture containing $3 \cdot 9$ vol. per cent. of $\mathrm{O}$ ).

II.

$\mathrm{CO}_{2}$-Tension in arterial blood $=21 \mathrm{~mm} . \mathrm{Hg}$ (corresponding to $2 \cdot 8$ vol. per cent.).

III.

O-Tension in venous blood $=22 \mathrm{~mm} . \mathrm{Hg}$ (corresponding to $2 \cdot 9$ vol. per eent.).

IV.

$\mathrm{CO}_{2}$-Tension in venous blood $=41 \mathrm{~mm}$. $\mathrm{Hg}$ (corresponding to $5 \cdot 4$ vol. per cent.).
V.

O-Tension in the air of the alveoli of the catheterised lung $=27 \cdot 44 \mathrm{~mm}$. $\mathrm{Hg}$ (corresponding to $3 \cdot 6$ vol. per cent.).

VI.

$\mathrm{CO}_{2}$-Tension in the air of the alveoli of the catheterised lung $=27 \mathrm{~mm}$. $\mathrm{Hg}$ (corresponding to $3 \cdot 56$ vol. per cent.).

VII.

O-Tension in the atmosphere $=158 \mathrm{~mm} . \mathrm{Hg}$ (corresponding to 20.8 vol. per cent.).

VIII.

CO. - Tension in the atmosphere $=0.38 \mathrm{~mm}$. $\mathrm{Hg}$ (corresponding to $0^{\circ} 03-0.05$ vol. per cent.).

When we compare the tension of the $\mathrm{O}$ in the air (VII. $=158 \mathrm{~mm} . \mathrm{Hg}$ ) with the tension of the $\mathrm{O}$ in venous blood (III. $=22 \mathrm{~mm}$. $\mathrm{Hg}$, or $\mathrm{V} .=27 \cdot 44 \mathrm{~mm}$. $\mathrm{Hg}$ ), we might be inclined to assume that the passage of the $O$ from the air of the airvesicles into the blood was due solely to diffusion of the gases ; and similarly, we might assume that the $\mathrm{CO}_{2}$ of the venous blood (IV. or VI.) diffused into the airvesicles, because the tension of the $\mathrm{CO}_{2}$ in the air is much less (VIII.). There are a number of facts, however, which prove that the exchange of the gases in the lungs is chiefly due to chemical forces.

[V. Fleischl finds that fluids yield up their gases very much more easily when they receive a shock, and he regards the shock communicated to the blood, by the contraction of the heart, as an important factor in preparing the blood for the diffusion of $\mathrm{CO}_{2}$ from the blood-plasmia into the lungs.]

[Changes produced in the Blood by Respiration.-The blood of the pulmonary artery is changed from venous into arterial blood (\$39), the most obvious alterations being (1) the change in colour from dark crimson to bright scarlet.

It loses $\mathrm{CO}_{2}$. (3) It gains $\mathrm{O}$. (4) The reduced $\mathrm{Hb}$ of the venous blood is converted into $\mathrm{HbO}_{2}$. (5) As to a supposed difference of temperature, see $\S 209,3$. (6) Pawlow finds that blood which passes several times through the lungs loses its power of coagulation. Are we to assume that the pulmonary tissues have the property of destroying the tibrin-ferment?]

1. Absorption of 0 .-Concerning the absorption of $\mathrm{O}$ from the air in the alveoli into the venous blood of the lung-capillaries, whereby the blood is arterialised, it is 
proved that this is a chemical process. The gas-free (reduced) hæmoglobin takes up $\mathrm{O}$ to form oxyhæmoglobin $(\S 15,1)$. That this absorption has nothing to do directly with the diffusion of gases, but is due to a chemical combination of the atomic compounds, is shown by the fact that, when pure $\mathrm{O}$ is respired, the blood does not take up more $\mathrm{O}$ than when atmospheric air is respired ; further, that animals made to breathe in a limited closed space can absorb almost all the $\mathrm{O}$-even to traces-into their blood before suffocation occurs. Of course, if the absorption of $\mathrm{O}$ were due to diffusion, in the former case more $\mathrm{O}$ would be absorbed, while in the latter case the absorption of $\mathrm{O}$ could not possibly occur to such an extent as it does. The law of diffusion comes into play in connection with the absorption of $\mathrm{O}$ to this extent, viz., that the $\mathrm{O}$ diffuses from the air-cells of the lung into the blood-plasma, where it reaches the blood-corpuscles floating in the plasma. The hæmoglobin of the blood-corpuscles forms at once a chemical compound (oxyhæmoglobin) with the $\mathrm{O}$.

Even in very rarefied air, such as is met with in the upper regions of the atmosphere during a balloon ascent, the absorption of $\mathrm{O}$ still remains independent of the partial pressure. But a much longer time is required for this process at the ordinary temperature of the body, so that in rarefied air, the absorption of $\mathrm{O}$ is greatly delayed, but it is not diminished. This is the cause of death in aeronauts who have ascended so high that the atmospheric pressure is diminished to one-third (Setschenow).

2. Excretion of $\mathrm{CO}_{2}$ - With regard to the excretion of $\mathrm{CO}_{2}$ from the blood, we must remember that the $\mathrm{CO}_{2}$ in the blood exists in two conditions. Part of the $\mathrm{CO}_{2}$ forms a loose or feeble chemical compound, while another portion is more firmly combined. The former is obtained by those means which remove gases from fluids containing them in a state of absorption, so that in removing the $\mathrm{CO}_{2}$ from the blood it is difficult to determine whether the $\mathrm{CO}_{2}$, so removed, obeyed the law of diffusion, or if it was expelled by chemical means.

Although it is convenient to represent the excretion of $\mathrm{CO}_{2}$ from the blood into the air-vesicles of the lung, as due to equilibration of the tension of the $\mathrm{CO}_{2}$ on opposite sides of the alveolar membrane, i.e., to diffusion-nevertheless, chemical processes play an important part in this act. The absorption of $\mathrm{O}$ by the coloured corpuscles acts, at the same time, in expelling $\mathrm{CO}_{2}$. This is proved by the fact that the expulsion of $\mathrm{CO}_{2}$ from the blood takes place more readily when $\mathrm{O}$ is simultaneously adınitted. The free supply of $\mathrm{O}$ not only favours the removal of the $\mathrm{CO}_{2}$, which is loosely combined, but it also favours the expulsion of that portion of the $\mathrm{CO}_{2}$ which is more firmly combined, and which can only be expelled by the addition of acids to the blood. That the oxygenated blood-corpuscles (i.e., their oxyhæmoglobin) are concerned in the removal of $\mathrm{CO}_{2}$ is proved by the fact that $\mathrm{CO}_{2}$ is more easily removed from serum which contains oxygenated blood-corpuscles than from serum charged with $\mathrm{O}$.

[The following scheme may serve to illustrate the extent to which diffusion comes into play. The $\mathrm{O}$ must pass through the alveolar membrane, $\mathrm{AB}$-including the alveolar epithelium and the wall of the capillaries-as well as the blood-plasma, to reach the hæmoglobin of the blood-corpuscles. Similarly, the $\mathrm{CO}_{2}$ must leave the salts of the plasma with which it is in combination, and diffuse in the opposite direction, through the wall of the capillaries, the alveolar membrane, and epithelium, to reach the air-vesicles. Let $\mathrm{AB}$ represent the alveolar membrane; on the one side of it is represented the partial pressure of the $\mathrm{CO}_{2}$ and $\mathrm{O}$ in the air-vesicles ; and on the other, the partial pressure of the $\mathrm{CO}_{2}$ and $\mathrm{O}$ in the venous blood entering the lung. The arrows indicate the direction of diffusion.]

$\begin{gathered}\text { Partial preasure of air in } \\ \text { alveoli of lung. }\end{gathered}$
$\begin{gathered}\text { Tension of gases in venous } \\ \text { blood of lung. }\end{gathered}$$\left\{\begin{array}{l}\mathrm{CO}_{\mathrm{a}} \\ 27\end{array}\right\}$


Nature of the Process.-The exchange of gases between the blood and the air in the lungs has been represented by Donders as due to the process of dissociation.

[Bohr used a modified rheometer of Ludwig's, whereby living arterial blood was brought into direct contact with a volume of air containing a greater or less percentage of $\mathrm{CO}_{2}$. Even when the amount of $\mathrm{CO}_{2}$ in the air in direct contact with the blood was very small, it was found that very little $\mathrm{CO}_{2}$ diffused from the blood into the air-space. Bohr therefore concludes that the separation of $\mathrm{CO}_{2}$ from the venous blood in the lungs, and its passage into the air-vesicles, are not explicable on the hypothesis of diffusion, but we must rather regard the $\mathrm{CO}_{2}$ as removed from the blood by the pulmonary tissue by means of a kind of secretory process, analogous to the excretion-processes in glands.]

130. DISSOCIATION OF GASES.-Many gases form true chemical compounds with other bodies (i.e., they combine according to their equivalents), when the contact of these bodies is effected under conditions such that the partial pressure of the gases is high. The chemical compound formed under these conditions is broken up, whenever the partial pressure is diminished, or when it reaches a certain minimum level, which varies with the nature of the bodies forming the compound. Thus, by increasing' and diminishing the partial pressure alternately, a chemical compound of the gas may be formed and again broken up. This process is called dissociation of the gases. The minimal partial pressure is constant for each of the different substances and gases, but temperature, as in the case of the absorption of gases, has a great effect on the partial pressure ; with increase of temperature the partial pressure, under which dissociation occurs, diminishes.

As an example of the dissociation of a gas, take the case of calcium carbonate. When it is heated in the air to $440^{\circ} \mathrm{C}$., $\mathrm{CO}_{2}$ is given off from its state of chemical combination, but is taken up again and a chemical compound formed, which is changed into chalk when it cools.

Dissociation in the Blood.-The chemical combinations containing $\mathrm{CO}_{2}$ and those containing $\mathrm{O}$ within the blood-stream, viz., the salts of the plasma, which are combined with $\mathrm{CO}_{2}$, and the oxyhæmoglobin, behave in a similar manner. If these compounds of $\mathrm{O}$ and $\mathrm{CO}_{2}$ are placed under conditions where the partial pressure of these gases is very low-i.e., in a medium containing a very small amount of these gases, the compounds are dissociated, i.e., they give off $\mathrm{CO}_{2}$ or $\mathrm{O}$. If after being dissociated they are placed under conditions where, owing to the large amount of these gases, the partial pressure of $\mathrm{O}$ or of $\mathrm{CO}_{2}$ is high, these gases are taken up again, and enter into a condition of chemical combination.

The hæmoglobin of the blood in the pulmonary capillaries finds plenty of $\mathrm{O}$ in the alveoli ; hence, it unites with the $\mathrm{O}$ owing to the high partial pressure of the $\mathrm{O}$ in the lung, and sa forms the compound oxyhæmoglobin. On its course through the capillaries of the systemic circulation, the oxyhæmoglobin of the blood comes into relation with tissues poor in $\mathrm{O}$; the oxyhæmoglobin is dissociated, the $\mathrm{O}$ is supplied to the tissues, and the blood freed from this $\mathrm{O}$ returns to the right heart, and passes to the lungs, where it takes up the new $\mathrm{O}$.

The blood whilst circulating meets with most $\mathrm{CO}_{2}$ in the tissues; the high partial pressure of the $\mathrm{CO}_{2}$ in the tissues causes the $\mathrm{CO}_{2}$ to unite with certain constituents in the blood so as to form chemical compounds, which carry the $\mathrm{CO}_{2}$ from the tissues to the lungs. In the air of the lungs, however, the partial pressure of the $\mathrm{CO}_{2}$ is very low, dissociation of these chemical compounds occurs under the low partial pressure, and the $\mathrm{CO}_{2}$ passes into the air-cells of the lung, from which it is expelled during expiration. It is evident that the giving up of $\mathrm{O}$ from the blood to the tissues, and the absorption of $\mathrm{CO}_{2}$ from the tissues, go on side by side and take place simultaneously, while in the lungs the reverse processes occur almost simultaneously.

131. CUTANEOUS RESPIRATION.-Methods. - If a man or an animal be placed in the chamber of the respiratory apparatus ( $\$ 122)$, and if tubes be so arranged that the respiratory gases do not enter the chamber, of course we obtain only the "perspiration" of the skin in the 
chamber. It is less satisfactory to leave the head of the person outside the chamber, while the neck is fixed air-tight in the wall of the chamber. The extent of the cutaneous respiration of a limb may be ascertained by enclosing it in an air-tight vessel (Röhrig) similar to that used for the arm in the plethysmograph $(\S 101)$.

Loss by Skin.-A healthy man loses by the skin, in 24 hours, $\frac{2}{2}$ of his bodyweight, which is greater than the loss by the lungs, in the ratio of $3: 2$. Only 10 grammes - 150 grains, - or it may be 3.9 grammes 60 grains, - of the entire loss are due to the $\mathrm{CO}_{2}$ given off by the skin. The remainder of the excretion from the skin is due to water $\left[1 \frac{1}{2}-2 \mathrm{tb}\right.$ daily $]$ containing a few salts in solution. When the surrounding temperature is raised, the $\mathrm{CO}_{2}$ is increased, in fact it may be doubled ; violent muscular exercise has the same effect.

0 Absorbed.-The $\mathrm{O}$ taken up by the skin is either equal to, or slightly less than, the $\mathrm{CO}_{2}$ given off. As the $\mathrm{CO}_{2}$ excreted by the skin is only $\frac{1}{220}$ of that excreted by the lungs, while the $\mathrm{O}$ taken in $=\frac{1}{180}$ of that taken in by the lungs, it is evident that the respiratory activity of the skin is very slight. Animals whose skin has been covered by an impermeable varnish die, not from suffocation, but from other causes $(\$ 225)$.

In animals with a thin moist epidermis (frog) the exchange of gases is nuch greater, and in them the skin so far supports the lungs in their function, and may even partly replace them funetionally. In mammals with thick dry cutaneous appendages, the exchange of gases is, again, much less than in man.

132. INTERNAL RESPIRATION.-Where $\mathrm{CO}_{2}$ is formed.-By the term "internal respiration" is understood the exchange of gases between the capillaries of the systemic circulation and the tissues of the organs of the body. As organic constituents of the tissues, during their activity, undergo gradual oxidation, and form, amongst other products, $\mathrm{CO}_{2}$; we may assume-(1) that the chief focus for the absorption of $\mathrm{O}$ and the formation of $\mathrm{CO}_{2}$ is to be sought for within the tissues themselves. That the $\mathrm{O}$ from the blood in the capillaries rapidly penetrates or diffuses into the tissues, is shown by the fact that the blood in the capillaries rapidly loses $\mathrm{O}$ and gains $\mathrm{CO}_{2}$, while blood containing $\mathrm{O}$, and kept warm outside the body, changes very slowly and incompletely. If portions of fresh tissues be placed in defibrinated blood containing $\mathrm{O}$, then the $\mathrm{O}$ rapidly disappears. Frogs deprived of their blood exhibit an exchange of gases almost as great as normal. This shows that the exchange of gases must take place in the tissues themselves. If the chief oxidations took place in the blood and not in the tissues, then, during suffocation, when $\mathrm{O}$ is excluded, the substances which use up $\mathrm{O}$, i.e., those substances which act as reducing agents, ought to accumulate in the blood. But this is not the case, for the blood of asphyxiated animals contains mere traces of reducing materials (Pfliger). It is difficult to say how the $\mathrm{O}$ is absorbed by the tissues, and what becomes of it immediately it comes in contact with the living elements of the tissues. Perhaps it is temporarily stored up, or it may form certain intermediate less oxidised compounds. This may be followed by a period of rapid formation and excretion of $\mathrm{CO}_{2}$. On this supposition, it is evident that the absorp: tion of $\mathrm{O}$ and the excretion of $\mathrm{CO}_{2}$ need not occur to the same extent, so that the amount of $\mathrm{CO}_{2}$ given off at any period is not necessarily an index of the amount of $\mathrm{O}$ absorbed during the same period $(\$ 127)$.

[There are two views as to where the $\mathrm{CO}_{2}$ is formed as the blood passes through the tissues. One view is that the seat of oxidation is in the blood itself, and the other is that it is formed in the tissues. If we knew the tension of the gases in the tissues, the problem would be easily solved, but we can only arrive at a know. ledge of this subject indirectly, in the following ways]:-

$\mathrm{CO}_{2}$ in Cavities. - That the $\mathrm{CO}_{2}$ is formed in the tissues, is supported by the fact that the a mount of $\mathrm{CO}_{2}$ in the fluids of the cavities of the body is greater than the $\mathrm{CO}_{2}$ in the blood of the capillaries. The tension of $\mathrm{CO}_{2}$ in - 


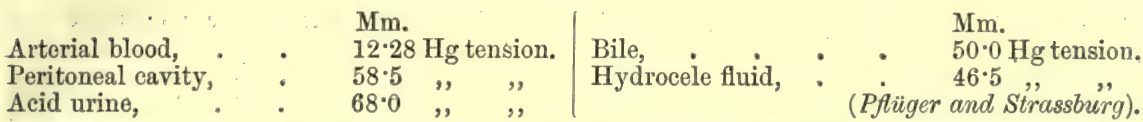

The large amount of $\mathrm{CO}_{2}$ in these fluids can only arise from the $\mathrm{CO}_{2}$ of the tissues passing into them.

Gases of Lymph. - In the lymph of the ductus thoracicus the tension of $\mathrm{CO}_{2}=33 \cdot 4$ to $37 \cdot 2$ $\mathrm{mm} . \mathrm{Hg}$, which is greater than in arterial blood, but considerably less than in venous blood (41.0 mm. Hg). [Ludwig and Hammarsten, Tschirjerv.] This does not entitle us to conclude that in the tissues from which the lymph comes only a small quantity of $\mathrm{CO}_{2}$ is formed, but rather that in the lymph there is less attraction for the $\mathrm{CO}_{2}$ formed in the tissues than in the blood of the capillaries, where chemical forces are active in causing it to combine, or that in the course of the long lymph-current, the $\mathrm{CO}_{2}$ is partly given back to the tissues, or that $\mathrm{CO}_{2}$ is formed in the blood itself. Further, the nuseles, which are by far the largest producers of $\mathrm{CO}_{2}$, contain few lymphaties, nevertheless they supply much $\mathrm{CO}_{2}$ to the blood. The amount of free "non-fixed" $\mathrm{CO}_{2}$ contained in the juices and tissues indicates that the $\mathrm{CO}_{2}$ passes from the tissues into the blood; still, Preyer believes that in venous blood $\mathrm{CO}_{2}$ undergoes chemical combination. The exchange of $\mathrm{O}$ and $\mathrm{CO}_{2}$ varies much in the different tissues. The muscles are the most important organs, for in their active condition they excrete a large amount of $\mathrm{CO}_{2}$, and use up much $\mathrm{O}$. The $\mathrm{O}$ is so rapidly used up by them that no free $\mathrm{O}$ can be pumped out of muscular tissue (L. Hermann). The exchange of gases is more vigorous during the activity of the tissues. Nor are the salivary glands, kidneys, and pancreas any exception, for although, when these organs are actively secreting, the blood flows out of the dilated veins in a bright red stream, still the relative diminution of $\mathrm{CO}_{2}$ is more than compensated by the increased volume of blood which passes through these organs.

Reductions by the Tissues. - The researches of Ehrlich have shown that in most tissues very energetic reductions take place. If colouring-matters, such as alizarin blue, indophenol blue, or methyl blue, be introduced into the blood-stream, the tissues are coloured by them. Those tissues or organs which have a particular affinity for $\mathrm{O}(e .9$. , liver, cortex of the kidney, and lungs), absorb $O$ from these pigments, and render them colourless. The pancreas and submaxillary gland scarcely reduce them at all.

(2) In the blood itself, as in all tissues, $\mathrm{O}$ is used up and $\mathrm{CO}_{2}$ is formed. This is proved by the following facts:- That blood withdrawn from the body becomes poorer in $\mathrm{O}$ and richer in $\mathrm{CO}_{2}$; that in the blood of asphyxia, free from $\mathrm{O}$, and in the blood-corpuscles, there are slight traces of reducing agents, which become oxidised on the addition of $\mathrm{O}$. Still, this process is comparatively insignificant as against that which occurs in all the other tissues. That the walls of the vessels-more especially the muscular fibres in the walls of the small arteries-use $\mathrm{O}$ and produce $\mathrm{CO}_{2}$ is unquestionable, although the exchange is so slight that the blood in its whole arterial course undergoes no visible change.

Ludwig and his pupils have proved that $\mathrm{CO}_{2}$ is actually formed in the blood. If the easily oxidisable lactate of soda be mixed with blood, and this blood be caused to circulate in an excised but still living organ, such as a lung or kidney, more $\mathrm{O}$ is used up and more $\mathrm{CO}_{2}$ is formed than in unmixed blood similarly transfused.

(3) That the tissues of the living lungs use $\mathrm{O}$ and give off $\mathrm{CO}_{2}$ is probable. When C. Ludwig and Müller passed arterial blood through the blood-vessels of a lung deprived of air, the $\mathrm{O}$ was diminished and the $\mathrm{CO}_{2}$ increased. As the total amount of $\mathrm{CO}_{2}$ and $\mathrm{O}$ found in the entire blood, at any one time, is only 4 grammes, and as the daily excretion of $\mathrm{CO}_{2}=900$ grammes, and the $\mathrm{O}$ absorbed daily $=744$ grammes, it is clear that exchange of gases must go on with great rapidity, that the $\mathrm{O}$ absorbed must be used quickly, and the $\mathrm{CO}_{2}$ must be rapidly excreted.

Still, it is a striking fact that oxidation-processes of such magnitude, as $e . g_{\text {. }}$, the union of $\mathrm{C}$ to form $\mathrm{CO}_{2}$, occur at the relatively low temperature of the blood and the tissues. It has been surmised that the blood acts as an ozone-producer, and transfers this active form of $\mathrm{O}$ to the tissues. Liebig showed that the alkaline reaction of most of the juices and tissues favours the processes of oxidation. Numerous organic substances, which are not altered by $\mathrm{O}$ alone, become rapidly oxidised in the presence of free alkalies, e.g., gallic acid, pyrogallic acid, and sugar; while many organic acids, which are unaffected by ozone alone, are changed into carbonates when in the form of alkaline salts (Gorup-Besanez); and, in the same way, when they are introduced into the body in the form of acids, they are partly or wholly excreted in 
the urine, but when they are administered as alkaline compounds they are changed into earbonates.

\section{RESPIRATION IN A LIMITED SPACE.-Respiration in a limited} space causes-(1) a gradual diminution of $\mathrm{O}$; (2) a simultaneous increase of $\mathrm{CO}_{2}$; (3) a diminution in the volume of the gases. If the space be of moderate dimensions, the animal uses up almost all the $\mathrm{O}$ contained therein, and dies ultimately from spasms caused by the asphyxia. The $\mathrm{O}$ is absorbed, therefore-independently of the laws of absorption-by chemical means. The $\mathrm{O}$ in the blood is almost completely used up (\$129). In a larger space, the $\mathrm{CO}_{2}$ accumulates rapidly, before the diminution of $\mathrm{O}$ is such as to affect the life of the animal. As $\mathrm{CO}_{2}$ can only be excreted from the blood when the tension of the $\mathrm{CO}_{2}$ in the blood is greater than the tension of $\mathrm{CO}_{2}$ in the air, as soon as the $\mathrm{CO}_{2}$ in the surrounding air in the closed space becomes the same as in the blood, the $\mathrm{CO}_{2}$ will be retained in the blood, and finally $\mathrm{CO}_{2}$ may pass back into the body. This occurs in a large closed space, when the amount of $\mathrm{O}$ is still sufficient to support life, so that death occurs under these circumstances (in rabbits) through poisoning with $\mathrm{CO}_{2}$ causing diminished excitability, loss of consciousness, and lowering of temperature, but no spasms (Worm Müller). In pure $\mathbf{0}$ animals breathe in a normal way; the quantity of $\mathrm{O}$ absorbed and the $\mathrm{CO}$, excreted is quite independent of the percentage of $\mathrm{O}$, so that the former occurs through chemical agency independent of pressure. In limited spaces filled with $\mathrm{O}$, animals died by absorption of the $\mathrm{CO}_{2}$ excreted. Worm Müller found that rabbits died after absorbing $\mathrm{CO}_{2}$ equal to half the volume of their body, although the air still contained 50 per cent. O. Animals can breathe quite quietly a mixture of air containing 14.8 per cent. (20.9 per cent. normal) ; with 7 per cent. they breathe with difficulty ; with 4.5 per cent. there is marked dyspnoea; with 3 per cent. O there is tolerably rapid asphyxia. The air expired by man normally contains 14 to 18 per cent. O. According to Hempner, mammals placed in a mixture of gases poor in $\mathrm{O}$ use slightly less $\mathrm{O}$.

Dyspncea occurs when the respired air is deficient in $\mathrm{O}$, as well as when it is overcharged with $\mathrm{CO}_{2}$, but the dyspnœa in the former case is prolonged and severe; in the latter, the respiratory activity soon ceases. The want of $O$ causes a greater and more prolonged increase of the blood-pressure than is caused by excess of $\mathrm{CO}_{2}$. Lastly, the consumption of $\mathrm{O}$ in the body is less affected when the $\mathrm{O}$ in the air is diminished than when there is excess of $\mathrm{CO}_{2}$. If air containing a diminished amount of $\mathrm{O}$ be respired, death is preceded by violent phenomena of excitement and spasms, which are absent in cases of death caused by breathing air overcharged with $\mathrm{CO}_{2}$. In poisoning with $\mathrm{CO}_{2}$, the excretion of $\mathrm{CO}_{2}$ is greatly diminished, while with diminution of $\mathrm{O}$ it is almost unchanged.

If animals be supplied with a mixture of gases similar to the atmosphere, in which $\mathrm{N}$ is replaced by $\mathrm{H}$, they breathe quite normally (Lavoisier and Seguin); the $\mathrm{H}$ undergoes no great change.

Cl. Bernard found that, when an animal breatherl in a limited space, it became partially accustomed to the condition. On placing a bird under a bell-jar, it lived several hours ; but if several hours before its death, another bird fresh from the outer air were placed under the same bell-jar, the second bird died at once, with convulsions.

Frogs, when placed for several hours in air devoid of $\mathrm{O}$, give off just as much $\mathrm{CO}_{2}$ as in air containing $\mathrm{O}$, and they do this without any obvious disturbance. Hence, it appears that the formation of $\mathrm{CO}_{2}$ is independent of the absorption of $\mathrm{O}$, and the $\mathrm{CO}_{2}$ must be formed from the decomposition of other compounds. Ultimately, however, complete motor paralysis occurs, whilst the circulation remains undisturbed (Aubert).

[134. DYSPNEA AND ASPHYXIA.-For the causes of dyspnœa see $\$ 111$, and those of asphyxia see $\S 368$. If from any cause an animal be not supplied with a due amount of air, normal respiration becomes greatly altered, passing through the phases of hyperpnca, or increased respiration, dyspncea, or difficulty of breathing, to the final condition of suffocation or asphyxia. The phenomena of asphyxia may be developed by closing the trachea of an animal with a clamp, or by any means which prevents the entrance of air or blood into the lungs. 
The phenomena of asphyxia are usually divided into several stages :-1. During the first stage there is hyperpnœa, the respirations being deeper, more frequent, and laboured. The extraordinary muscles of respiration-both those of inspiration and expiration ( $\S 118$ ) - are called into action, dyspnœa is rapidly produced, and the struggle for air becomes more and more severe. At the same time the oxygen of the blood is being used up, while the blood itself becomes more and more venous. The venous blood circulating in the medulla oblongata and spinal cord stimulates the respiratory centres, and causes the violent respirations. This stage usually lasts about a minute, and gradually gives places to-

2. The second stage, when the inspiratory muscles become less active, while those concerned in laboured expiration contract energetically, and indeed almost every muscle in the body may contract; so that this stage of violent expiratory efforts ends in general convulsions. The convulsions are due to stimulation of the respiratory centres by the venous blood. The convulsive stage is short, and is usually reached in a little over one minute. This storm is succeeded by-

3. The third stage, or stage of exhaustion, the transition being usually somewhat sudden. It is brought about by the venous blood acting on and paralysing the respiratory centres. The pupils are widely dilated, consciousness is abolished, and the activity of the reflex centres is so depressed that it is impossible to discharge a reflex act, even from the cornea. The animal lies almost motionless, with flaccid muscles, and to all appearance dead, but every now and again, at long intervals, it makes a few deep inspiratory efforts, showing that the respiratory centres are not quite, but almost paralysed. Gradually the pauses become longer and the inspirations feebler and of a gasping character. As the venous blood circulates in the spinal cord, it causes a large number of muscles to contract, so that the animal extends its trunk and limbs. It makes one great inspiratory spasm, the mouth being widely opened and the nostrils dilated, and ceases to breathe. After this stage, which is the longest and most variable, the heart becomes paralysed, partly from being overdistended with venous blood, and partly, perhaps, from the action of the venous blood on the cardiac tissues, so that the pulse can hardly be felt. To this pulseless condition the term "asphyxia" ought properly to be applied. In connection with the resuscitation of asphyxiated persons, it is important to note that the heart continues to beat for a few seconds after the respiratory movements have ceased.

The whole series of phenomena occupies from 3 to 5 minutes, according to the animal operated on, and depending also upon the suddenness with which the trachea was closed. If the causes of suffocation act more slowly, the phenomena are the same, only they are developed more slowly.

The Circulation.-The post-mortem appearances in man or in an animal are generally well marked. The right side of the heart, the pulmonary artery, the venæ cavæ, and the veins of the neck are engorged with dark venous blood. The left side is comparatively empty, because the rigor mortis of the left side of the heart, and the elastic recoil of the systemic arteries, force the blood towards the systemic veins. The blood itself is almost black, and is deprived of almost all its oxygen, its hæmoglobin being nearly all in the condition of reduced hæmoglobin, while ordinary venous blood contains a considerable amount of oxyhæmoglobin as well as reduced $\mathrm{Hb}$. The blood of an asphyxiated animal practically contains none of the former and much of the latter. It is important to study the changes in the circulation in relation to phenomena exhibited by an animal during suffocation.

We may measure the blood-pressure in any artery of an animal while it is being asphyxiated, or we may open its chest, maintain artificial respiration, and place a manometer in a systemic artery, e.g., the carotid, and another in a branch of the pulmonary artery. In the latter case, we can watch the order of events in the heart itself, when the artificial respiration is interrupted. It is well to study the events in both cases. 
If the blood-pressure be measured in a systemic artery, e.g., the carotid, it is found that the blood-pressure rises very rapidly, and to a great extent during the first and second stages; the pulse-beats at first are quicker, but soon become slower and more vigorous. During the third stage it falls rapidly to zero. The great rise of the blood-pressure, during the first and second stages, is chiefly due to the action of the venous blood on the general vaso-motor centre, causing constriction of the small systemic arteries. The peripheral resistance is thus greatly increased, and it tends to cause the heart to contract more vigorously, but the slower and more vigorous beats of the heart are also partly due to the action of the venous blood on the cardio-inhibitory centre in the medulla.

If the second method be adopted, viz., to open the chest, keep up artificial respiration, and measure the blood-pressure in a branch of the pulmonary artery, as well as in a systemic artery,-e.\%., the carotid,-we find that when the artificial respiration is stopyed, in addition to the rise of the blood-pressure indicated in the carotid manometer, the cavities of the heart and the large veins near it are engorged with venous blood. There is, however, but a slight comparative rise in the bloodpressure in the pulmonary artery. This may be accounted for, either by the pulmonary artery not being influenced to the same extent as other arteries by the vaso-motor centre, or by its greater distensibility (\$88). But, as the heart itself is supplied through the coronary arteries with venous blood, its action soon becomes weakened, each beat becomes feebler, so that soon the left ventricle ceases to contract, and is unable to overcome the great peripheral resistance in the systemic arteries, although the right ventricle may still be contracting. As the blood becomes more venous, the vaso-motor centre becomes paralysed, the small systemic arteries relax, and the blood flows from them into the veins, while the blood-pressure in the carotid manometer rapidly falls. The left ventricle, now relieved from the great internal pressure, may execute a few feeble beats, but they can only be feeble, as its tissues have been subjected to the action of the very impure blood. More and more blood accumulates in the right side from the causes already mentioned. The violent inspiratory efforts in the early stages aspirate blood from the veins towards the right side of the heart, but of course this factor is absent when the chest is opened.]

[Convulsions during asphyxia occur only in warm-blooded animals, and not in frogs. If a drug when injected into a mammal excites convulsions, but does not do so in the frog, then it is usually concluded that the convulsions are due to its action on the circulation and respiration, and not to any direct stimulating effect upon the motor centres. But if the drug excites convulsions both in the mammal and frog, then it probably acts directly on the motor centres (Brunton).]

[Recovery from the condition of Asphyxia. - If the trachea of a dog be closed suddenly and completely, the average duration of the respiratory movements is 4 minutes $\mathbf{5}$ seconds, while the heart continues to beat for about 7 minutes. Recovery may be obtained if proper means be adopted before the heart ceases to beat; but after this, never. If a dog be drowned, the result is different. After complete submersion for $1 \frac{1}{2}$ minute, recovery did not take place. In drowning, air passes out of the chest, and water is inspired into and fills the air-vesicles. It is rare for recovery to take place in a person deprived of air for more than five minutes. If the statements of sponge-divers are to be trusted, a person may become accustomed to the deprival of air for a longer time thain usual. In cases where recovery takes place after a much longer period of submersion, it has been suggested that, in these cases, syncope occurs, the heart beats but feebly or not at all, so that the oxygen in the blood is not used up with the same rapidity. It is a well-known fact that newly-born and young puppies can be submerged for a long time before they are suffocated.]

Artificial Respiration in Asphyxia.-In cases of suspended animation, artificial respiration must be performed. The first thing to be done is to remove auy foreign substance from the respiratory passages (mucus or cedematons fluids) in the newly-born or asphyxiated. In doubtful cases, open the trachea and suck out any fluid by means of an elastic catheter ( $v$. Hüter). Recourse must in all cases be had to artificial respiration. There are several methods of dilating and compressing the chest so as to cause an exchange of gases. One method is to compress the chest rhythmically with the hands. 
[Marshall Hall's Method. - "After clearing the mouth and throat, place the patient on the face, raising and supporting the chest well on a folded coat or other article of dress. Turn the body very gently on the side and a little beyond, and then briskly on the face, back again, repeating these measures cautiously, efficiently, and perseveringly, about fifteen times in the minute, or once every four or five seconds, occasionally varying the side. By placing the patient on the chest, the weight of the body forces the air out ; when turned on the side,. this pressure is removed, and air enters the chest. On each occasion that the body is replaced on the face, make uniform but efficient pressure with brisk movement on the back between and below the shoulder-blades or bones on each side, removing the pressure immediately before turning the body on the side. During the whole of the operations let one person attend solely to the movements of the head and of the arm placed under it."]

[Sylvester's Method.-." Place the patient on the back on the flat surface, inclined a little upwards from the feet; raise and support the head and shoulders on a small firm cushion or folded article of dress placed under the shoulder-blades. Draw forward the patient's tongue, and keep it projecting beyond the lips; an elastic band over the tongue and under the chin will answer this purpose, or a piece of string or tape may be tied round them, or by raising the lower jaw the teeth may be made to retain the tongue in that position. Remove all tight clothing from about the neck and chest, especially the braces." "To Imitate the Movements of Breathing.-Standing at the patient's head grasp the arms just above the elbows, and draw the arms gently and steadily upwards, above the head, and keep them stretched upwards for two seconds. '.. By this means air is drawn into the lungs. Then turn down the patient's arms, and press them gently and firmly for two seconds against the sides of the chest. By this means air is pressed out of the lungs. Repeat these measures alternately, deliberately, and perseveringly about fifteen times in a minute, until a spontaneous effort to respire is perceived, immediately upon which cease to imitate the movements of breathing, and proceed to induce circulation and warmeth:"]

Howard advises rhythmical compression of the chest and abdomen by sitting like a rider astride of the body, while Schiiller advises that the lower ribs be seized from above with both hands and raised, whereby the chest is dilated, especially when the thigh is pressed against the abdomen to compress the abdominal walls. The chest is compressed by laying the hands flat upon the hypochondria. Artificial respiration acts favourably by supplying $\mathrm{O}$ to, as well as removing $\mathrm{CO}_{2}$ from, the blood ; further, it aids the movement of the blood within the heart and in the large vessels of the thorax. If the action of the heart has ceased, recovery' is impossible. In asphyxiated newly-born children, we must not cease too soon to perform artificial respiration. Even when the result appears hopeless, we ought to persevere. Pfliger and Zuntz observed that the reflex excitability of the fotal heart continued for several hours after the death of the mother.

Resuscitation by compressing the heart.-Böhm found that in the case of cats poisoned with potash salts or chloroform, or asphyxiated, so as to arrest respiration and the action of the heart,- - even for a period of forty minutes, - and even when the pressure within the carotid had fallen to zero, he could restore animation by rhythmical compression of the heart, combined with artificial respiration. The compression of the heart causes a slight movement of the blood, while it acts at the same time as a rhythmical cardiac stimulus. After recovery of the respiration, the reflex excitability and gradually also voluntary movements are restored. The animals are blind for several days, the brain acts slowly, and the urine contains sugar. These experiments show how important it is in cases of asphyxia to act at the same time upon the heart.

For physiological purposes, artificial respiration is often made use of, especially after poisoning with curara. Air is forced into the lungs by means of an elastic bag or bellows, attached to a cannula tied in the trachea. The cannula has a small opening in the side of it to allow the expired air to escape.

Pathological. - After the lungs have once been properly distended with air, it is impossible by any amount of direct compression of them to get rid of all the air. This is probably due to the pressure acting on the small bronchi, so as to squeeze them, before the air can be forced out of the air-vesicles. If, however, a lung be filled with $\mathrm{CO}_{2}$, and be suspended in water, the $\mathrm{CO}_{2}$ is absorbed by the water, and the lungs become quite free from air and are atelectic (Hermann and Keller). The atelectasis, which sometimes occurs in the lung, may thus be explained :- If a bronchus is stopped with mucus or exudation, $\mathrm{CO}_{2}$ accumulates in the air-vesicles belonging to this bronchus. If the $\mathrm{CO}_{3}$ is absorbed by the blood or lymph, the corresponding area of the lung will become atelectic. Sometimes there is spasm of the respiratory muscles, brought about by direct or reflex stimulation of the respiratory centre.

135. RESPIRATION OF FOREIGN GASES. - No gas without a sufficient admixture of $O$ can support life. Even with completely innocuous and indifferent gases, if no $O$ be mixed with them, they cause suffocation in 2 to 3 minutes.

I. Completely indifferent Gases are $\mathrm{N}, \mathrm{H}, \mathrm{CH}_{4}$. The living blood of an animal breathing these gases yields no $\mathrm{O}$ to them (Pflïger).

II. Poisonous Gases, - 0-displacing Gases. - $(a)$ Those that displace 0 , and form a stable com. 
pound with the hrmoglobin-(1) CO (\$ 16 and 17). (2) CNH (hydrocyanie acid) displaces (?) $\mathrm{O}$ from hæmoglobin, forming a more stable compound, and kills exceedingly rapidly. Bloodcorpuscles charged with hydrocyanic acid lose the property of decomposing hydric peroxide into water and $\mathrm{O}(\$ 17,5)$.

(b) Narcotic Gases. - (1) $\mathrm{CO}_{2} .-\mathrm{V}$. Pettenkofer characterises atmospheric air containing '1 per cent. $\mathrm{CO}_{2}$ as " bad air"; still, air in a room containing this amount of $\mathrm{CO}_{2}$ produces a disagreeable feeling, rather from the impurities mixed with it than from the actual amount of $\mathrm{CO}_{2}$ itself. Air containing 1 per cent. $\mathrm{CO}_{2}$ produces decided discomfort, and with 10 per cent. it endangers life, while larger amounts cause death, with symptoms of coma. (2) $\mathrm{N}_{2} \mathrm{O}$ (nitrous oxide), respired, mixed with $\frac{1}{5}$ volume $\mathrm{O}$, causes, after 1 to 2 minutes, a short temporary stage of excitement ("Laughing gas" of H. Davy), which is succeeded by unconsciousness, and afterwards by an increased excretion of $\mathrm{CO}_{2}$. (3) Ozonised air causes similar effects (Binz).

(c) Reducing Gases.-(1) $\mathrm{H}_{2} \mathrm{~S}$ (sulphuretted hydrogen) rapidly robs blood-corpuscles of $\mathbf{O}$, $\mathrm{S}$ and $\mathrm{H}_{2} \mathrm{O}$ being formed, and death occurs rapidly before the gas can decompose the hæmoglobin to form a sulphur-methæmoglobin compound.

(2) $\mathrm{PH}_{3}$ (phosphuretted hydrogen) is oxidised in the blood to form phosphoric acid and water, with decomposition of the hæmoglobin.

(3) $\mathrm{AsH}_{3}$ (arseniuretted hydrogen) and $\mathrm{SbH}_{3}$ (antimoniuretted hydrogen) act like $\mathrm{PH}_{3}$, but the hæmoglobin passes out of the stroma and appears in the urine.

(4) $\mathrm{C}_{2} \mathrm{~N}_{2}$ (cyanogen) absorbs $\mathrm{O}$, and decomposes the blood.

III. Irrespirable gases, $i . e_{\text {, }}$ gases which, on entering the larynx, cause reflex spasm of the glottis. When introduced into the trachea, they cause inflammation and death. Under this category come hydrochloric, hydrofluoric, sulphurous, nitrous, and nitric acids, ammonia, chlorine, fluorine, and ozone.

136. ACCIDENTAL IMPURITIES OF THE AIR.-Amongst these are dust-particles, which occur in enormous amount suspended in the air, and thereby act injuriously upon the respiratory organs. The ciliated epithelium of the respiratory passages eliminates a large number of them (fig. 148). Some of them, however, reach the air-vesicles of the lung, where they penetrate the

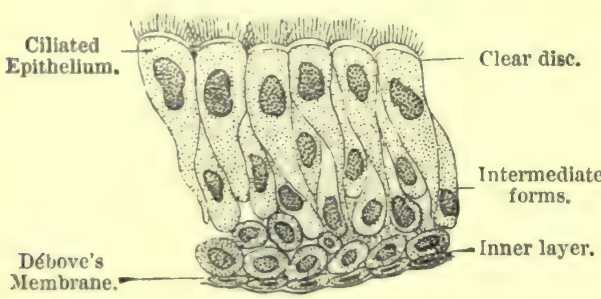

Fig. 148. epithelium, reach the interstitial lung-tissue and lymphatics, and so pass with the lymphstream into the bronchial glands. Particles of coal or charcoal are found in the lungs of all elderly individuals, and blacken the alveoli. In moderate amount, these black particles do not seem to do any harm in the tissues, but when there are large accumnlations they give rise to lung-affections, which lead to disintegration of these organs. [In coal-miners, for example, the lungtissues along the track of the lymphatics and in the bronchial glands are quite black, constituting "coal-miners' lung."] In many trades various particles occur in the

Ciliated epithelium.

air ; miners, grinders, stone-masons, file-makers, weavers, spinners, tobacco manufacturers, millers, and bakers suffer from lung affections caused by the introduction of particles of various kinds inhaled during the time they are at work.

Germs. - There seems no doubt that the seeds of some contagious diseases may be inhaled. Diphtheritic bacteria (Bacillus diphtheriæ) become localised in the pharynx and in the larynxglanders in the nose-measles in the bronchi-whooping-cough in the bronchi-hay-monads in the nose-the Bacillus pneumoniæ of pneumonia in the pulmonary alveoli. Tuberculosis, according to $\mathrm{R}$. Koch, is due to the introduction and development of the Bacillus tuberculosis in the lungs, the bacillus being derived from the dust of tuberculous sputa. The same seems to be the case with the Bacillus of leprosy and with Bacillus malariæ, which is the cause of malaria. The latter organism thus reaches the blood; it changes the $\mathrm{Hb}$ within the red blood-corpuscles into melanin $(\$ 10,3)$, and causes them to break up. The Micrococcus vaccinæ of smallpox gains access to the blood in the same way, also the Spirillum of remittent fever (fig. 23), the microbe of scarlet fever, \&c.

Seeds of disease passing into the mouth along with air, and also with the food, are swallowed, and undergo development in the intestinal tract, as is probably the case in cholera (Comma. bacillus of $R$. Koch), dysentery, typhoid, and anthrax which is due to Bacterium anthracis (fig. 24).

137. VENTILATION OF ROOMS, - Fresh air is as necessary for the healthy as for the sick. Every healthy person ought to have a cubic space of at the very least 800 cubic feet, and every sick person at the very least 1000 cubic feet of space. [The cubic space allowed per individual varies greatly, but 1000 cubic feet is a fair average. If the air in this space is to be kept sweet, 
so that the $\mathrm{CO}_{2}$ does not exceed 06 per cent., 3000 cubic feet of air per hour must be supplied, i.e., the air in the space must be renewed three times per hour.]

[Floor-Space. - It is equally important to secure sufficient floor-space, and this is especially the case in hospitals. If possible, 100-120 square feet of floor-space ought to be provided for each patient in a hospital-ward, and if it is obtainable a cubic space of 1500 cubic feet (Parkes). In all cases the minimum floor-space should not be less than $\frac{1}{1^{2}}$ of the cubic space.]

Overcrowding. - When there is overcrowding in a room, the amount of $\mathrm{CO}_{2}$ increases. $\mathrm{V}$. Pettenkofer found the normal amount of $\mathrm{CO}_{2}$ (.04 to .05 per 1000) increased in comfortable rooms to $0.54-0.7$ per 1000 ; in badly ventilated sick chambers $=2.4$; in overcrowded auditoriums, $3 \cdot 2$; in pits $=4 \cdot 9$; in schoolrooms, $7 \cdot 2$ per 1000 . Although it is not the quantity of $\mathrm{CO}_{2}$ which makes the air of an overerowded room injurious, but the excretions from the outer and inner surfaces of the body, which give a distinct odour to the air, quite recognisable by the sense of smell, still the amount of $\mathrm{CO}_{2}$ is taken as an index of the presence and amount of these other deleterious substances. Whether or not the ventilation of a room or ward occupied by persons is sufficient, is ascertained by estimating the amount of $\mathrm{CO}_{2} . \mathrm{A}$ room which does not give a disagreeable, somewhat stuffy, odour has less than 0.7 per 1000 of $\mathrm{CO}_{2}$, while the ventilation is certainly insufficient if the $\mathrm{CO}_{2}=1$ per 1000. As the air contains only 0.0005 cubic metre $\mathrm{CO}_{2}$ in 1 cubic metre of air, and as an adult produces hourly 0.0226 cubic metres $\mathrm{CO}_{2}$, calculation shows that every person requires 113 cubic metres of fresh air per hour, if the $\mathrm{CO}_{2}$ is not to exceed 0.7 per $1000:$ for $0.7: 1000=(0.0226+x \times 0.0005): x, i . e_{.}, x=113$.

[Vitiating Products.-In a state of repose, an adult man gives off from 12 to 16 cubic feet of $\mathrm{CO}_{2}$ in twenty-four hours, or on an average 6 cubic feet per hour. To this must be added a certain quantity of organic matter, which is extremely deleterious to health. While the $\mathrm{CO}_{2}$ diffuses readily and is easily disposed of by opening the windows, this is not the case with the organic matter, which adheres to clothing, curtains, and furniture; hence to get rid of it, a room, and especially a sleeping apartment, requires to be well aired for a long time, together with the free admission of sunlight. We must also remember that an adult gives off from 25 to $40 \mathrm{oz}$. of water by the skin and lungs. The nature of the organic matters is not precisely known, but some of it is particulate, consisting of epithelium, fatty matters, and organic vapours from the lungs and mouth. It blackens sulphuric acid, and decolourises a weak solution of potassic permanganate. As a test, if we expire through distilled water, and this water be set aside for some time in a warm place, it will soon become fœetid. We must also take into consideration the products of combustion; thus 1 cubic foot of coal-gas, when burned, destroys all the $\mathrm{O}$ in 8 cubic feet of air (Parkes).]

Methods. - In ordinary rooms, where every person is allowed the necessary cubic space (1000 cubic feet), the air is sufficiently renewed by means of the pores in the walls of the room, by the opening and shutting of doors, and by the fireplace, provided the damper is kept open. It is most important to notice that the natural ventilation be not interfered with by dampness of the walls, for this influences the pores very greatly. At the same time, damp walls are injurious to health by conducting away heat, and in them the germs of infectious diseases may develop.

[Natural Ventilation.-By this term is meant the ventilation brought about by the ordinary forces acting in nature; such as diffusion of gases, the action of winds, and the movements excited owing to the different densities of air at unequal temperatures.]

[Artificial Ventilation. - Various methods are in use for ventilating public buildings and dwelling-houses. Two principles are adopted for the former, viz., extraction and propulsion of air. In the former method, the air is sucked out of the rooms by a fan or other apparatus, while in the latter, air is forced into the rooms, the air being previously heated to the necessary temperature.]

[A very convenient method of introducing air into a room is by means of Tobin's tubes, placed in the walls. The air enters through these tubes from the outside near the floor, and is carried up six or more feet, to an opening in the wall ; the cool air thus descends slowly. For a sitting-room, a convenient plan of window ventilation is $\mathbf{H}$. Bird's Method :-Raise the lower sash and place under it, so as to fill up the opening, a piece of wood 3 or 4 inches high. Air will then pass in, in an upward direction, between the upper part of the lower sash-frame and the lower part of the upper one.]

138. FORMATION OF MUCUS, SPUTUM.-The respiratory mucous membrane is covered normally with a thin layer of mucus (fig. 130,a). It so far inhibits the formation of new mucus by protecting the mucous glands from the action of cold or other irritative agents. New mucus is secreted as that already formed is removed. An increased secretion accompanies congestion of the respiratory mucous membrane [or any local irritation]. - Division of the nerves on one side of the trachea (cat) causes redness of the tracheal mucous membrane and increased secretion (Rossbach), [but the two processes do not stand in the relation of cause and effect]. The secretion cannot be excited by stimulating the nerves going to the 
mucous membrane. This merely causes anæmia of the mucous membrane, while the secretion continues.]

Modifying Conditions. - If ice be placed on the belly of an animal so as to cause the animal "to take a cold," the respiratory mucous membrane first becomes pale, and afterwards there is a copious mucous secretion, the membrane beconing deeply congested. The injection of sodium carbonate and ammonium chloride into the blood limits the secretion. The local application of alum, silver nitrate, or tannic acid, makes the mucous membrane turbid, and the epithelium is shed. The secretion is excited by apomorphin, emetin, pilocarpin, and ipecacuanha when given internally, while it is limited by atropin and morphia (Rosshach).

[Expectorants favour the removal of the secretions from the air-passages. This they may do either by $(a)$ altering the character and qualities of the secretion itself, or $(b)$ by affecting the expulsive mechanism. Some of the drugs already mentioned are examples of the first class. The second class act chiefly by influencing the respiratory centre, e.g., ipecacuanha, strychnia, ammonia, senega ; emeties also act energetically as expectorants, as in some cases of chronic bronchitis ; warmth and moisture in the air are also powerful adjuncts.]

Sputum.-Under normal circumstances, some mucus - mixed with a little salivamay be coughed up from the back of the throat. In catarrhal conditions of the
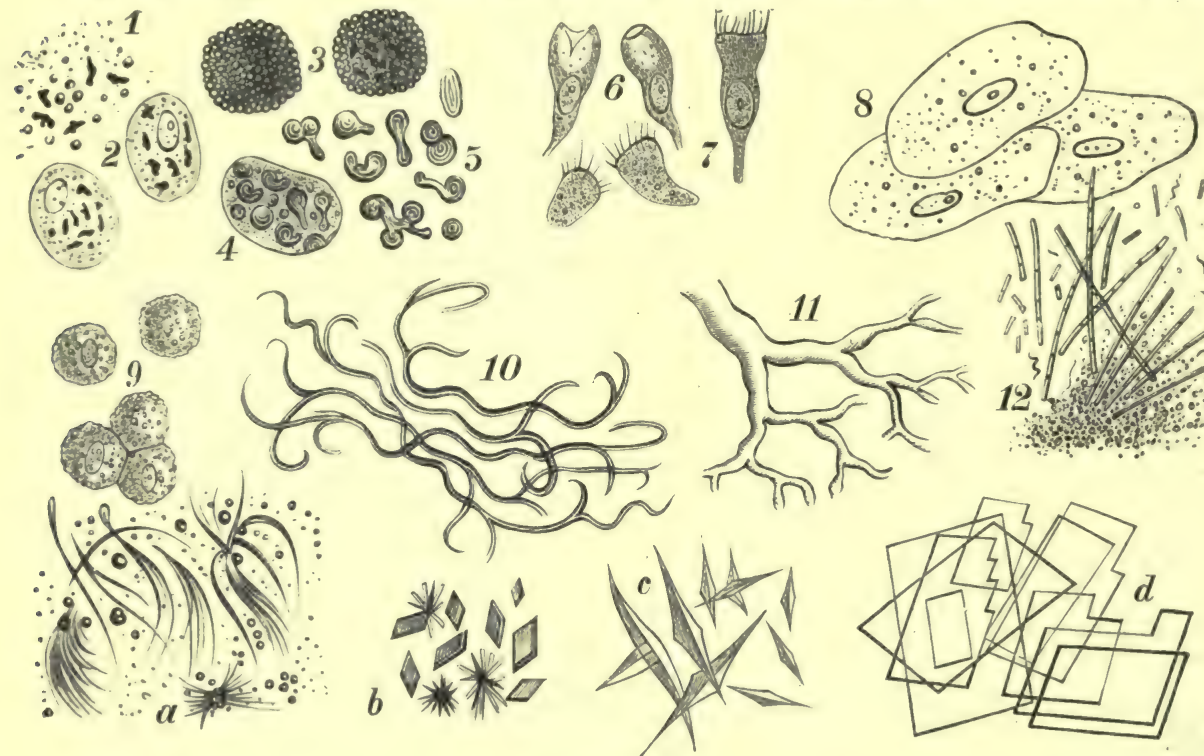

Fig. 149 .

Various objects found in sputum. 1, detritus and particles of dust; 2, alveolar epitheliun with pigment ; 3 , fatty and pigmented alveolar epithelium ; 4, alveolar epithelium with myelin-forms ; 5 , free myelin-forms ; 6,7 , ciliated epithelium, some without cilia ; 8 , squamous epithelium from the mouth; 9 , leucocytes ; 10 , elastic fibres ; 11 , fibrin-cast of a small bronchus ; 12 , leptothrix buccalis with cocci, bacteria, and spirochaetae ; $\alpha$, fatty acid crystals and free fatty granules; $b$, hæmatoidin ; $c$, Charcot's crystals ; $d$, cholesterin.

respiratory mucous membrane, the sputum is greatly increased in amount, and is often mixed with other characteristic products. Microscopically, sputum contains-

1. Epithelial Cells, chiefly squames from the mouth and pharynx (fig. 149), more rarely alveolar epithelium and ciliated epithelium (7) from the respiratory passages. They are often altered owing to maceration or other changes. Thus some cells may have lost their cilia (6).

The epithelium of the alveoli (2) is squamous epithelium, the cells being two to four times the breadth of a colourless blood-corpuscle. These cells occur chiefly in the morning sputum in individuals over 30 years of age. In younger persons their presence indicates a pathological condition of the pulmonary parenchyma. 
They often undergo fatty degeneration, and they may contain pigment-granules (3); or they may present the appearance of what Buhl has called "myelin degenerated cells," i.e., cells filled with clear refractive drops of various sizes, some colourless, others with coloured particles, the latter having been absorbed (4). Mucin in the form of myelin drops (5) is always present in sputum.

2. Lymphoid cells (9) are colourless blood-corpuscles which have wandered out of the blood-vessels ; they are most numerous in yellow sputum, and less numerous in the clear mucus-like excretion. The lymph-cells often present alterations in their characters ; they may be shrivelled up, fatty, or present a granular appearance.

The fluid substance of the sputum contains much mucus, arising from the mucous glands and goblet cells, together with nuclein, and lecithin, and the constituents of saliva, according to the amount of the latter mixed with the secretion. Albumin occurs only during the inflammation of the respiratory passages, and its amount increases with the degree of inflammation. Urea has been found in cases of nephritis.

In cases of catarrh, the sputum is at first usually sticky and clear (sputa cruda), but later it becomes more firm and yellow (sputa cocta). Under pathological conditions, there may be found in the sputum-(a) red blood-corpuscles from rupture of a blood-vessel. (b) Elastic-fibres (10) from disintegration of the alveoli of the lung; usually the bundles are fine, curved, and the fibres branched. [In certain cases it is well to add a solution of caustic potash, which dissolves most of the other elements, leaving the elastic fibres untouched.] Their presence always indicates destruction of the lung-tissue. (c) Colourless plugs of fibrin (11), casts of the smaller or larger bronchi, occur in some cases of fibrinous exudation into the finer air-passages. $(d)$ Crystals of various kinds-crystals of fatty acids in bundles of fine needles (fig. 149, a). They indicate great decomposition of the stagnant secretion. Leucin and tyrosin crystals are rare (\$ 269). Tyrosin occurs in considerable amount when an old abscess breaks into the lungs. Colourless, sharp-pointed, octagonal or rhombic plates-Charcot's crystals $(c)$-have been found in the expectoration in asthma, and exudative affections of the bronchi. Hæmatoidin $(b)$ and cholesterin crystals $(d)$ occur much more rarely.

Fungi and other lowly organisms are taken in during inspiration (\$ 136). The threads of Leptothrix buccalis (12), detached from the teeth, are frequently found.(\$147). Mycelium and spores are found in thrush (Oidium albicans), especially in the mouths of sucking infants. In malodorous expectoration rod-shaped bacteria are present. In pulmonary gangrene are found monads, and cercomonads; in pulmonary phthisis the tubercle bacillus ; very rarely sarcina, which, however, is often found in gastric catarrh in the stomach and also in the urine $(\$ 270)$.

Physical Characters. - Sputum, with reference to its physical characters, is described as mucous, muco-purulent, or purulent.

Abnormal coloration of the sputum-red from blood; when the blood remains long in the lung it undergoes a regular series of changes, and tinges the sputum dark-red, bluish-brown, brownish-yellow, deep yellow, yellowish-green, or grass-green. The sputum is sometimes yellow in jaundice. The sputum may be tinged by what is inspired as in the case of the "black-spit" of miners].

The odour of the sputum is more or less unpleasant. It becomes very disagreeable when it has remained long in pathological lung-cavities, and it is stinking in gangrene of the lung.

139. ACTION OF THE ATMOSPHERIC PRESSURE.-At the normal pressure of the atmosphere (height of the barometer, 760 millimetres $\mathrm{Hg}$ ), pressure is exerted upon the entire surface of the body $=15,000$ to 20,000 kilos,, according to the extent of the superficial area. This pressure acts equally on all sides upon the body, and also occurs in all internal cavities containing air, both those that are constantly filled with air (the respiratory passages and the spaces in the superior maxillary, frontal, and ethmoid bones), and those that are temporarily in direct communication with the outer air (the digestive tract and tympanum). As the fluids of the body (blood, lymph, secretions, parenchymatous juices) are practically incompressible, their volume remains unchanged under the pressure; but they absorb gases from the air corresponding to the prevailing pressure (i.e., the partial pressure of the individual gases), and according to their temperature ( $\$ 33)$. The solids consist of elementary parts (cells and fibres), each of which presents only a microscopic surface to the pressure, so that for each cell the prevailing pressure of the air can only be calculated at a few millimetres-a pressure under which the most delicate histological tissues undergo development. As an example 
of the action of the pressure of the atmospheric pressure upon large masses, take that brought about by the adhesion of the smooth, sticky, moist, articular surfaces of the shoulder and hip joints; the arm and the leg are supported without the action of muscles. The thigh-bone remains in its socket after section of all the muscles and its capsule. Even when the cotyloid cavity is perforated, the head of the femur does not fall out of its socket. The ordinary barometric variations affect the respiration-a rise of the barometric pressure excites, while a fall diminishes, the respirations. The absolute amount of $\mathrm{CO}_{2}$ remains the same $(\S 127,8)$.

Great diminution of the atmospheric pressure, such as occurs in ballooning (highest ascent, 8600 metres), or in ascending mountains, causes a series of characteristic phenomena :-1) In consequence of the diminution of the pressure upon the parts directly in contact with the air, they become greatly congested, hence there is redness and swelling of the skin and free mucous membranes ; there may be hæmorrhage from the nose, lungs, gams; turgidity of the cutaneous veins; copious secretion of sweat; great secretion of mucus. (2) A feeling of weight in the limbs, a pressing outwards of the tympanic membrane (until the tension is equilibrated by opening the Eustachian tube), and as a consequence noises in the ears and difficulty of hearing. (3) In consequence of the diminished tension of the $O$ in the air (\$129), there is difficulty of breathing, pain in the chest, whereby the respirations (and pulse) become more rapid, deeper, and irregular. When the atmospheric pressure is diminished $\frac{1}{2}-\frac{1}{3}$, the amount of $\mathrm{O}$ in the blood is diminished, the $\mathrm{CO}_{2}$ is imperfectly removed from the blood, and in consequence there is dininished oxidation within the body. When the atmospheric pressure is diminished to onehalf, the amount of $\mathrm{CO}_{2}$ in arterial blood is lessened ; and the amount of $\mathrm{N}$ diminishes proportionally with the decrease of the atmospheric pressure. The diminished tension of the air prevents the vibrations of the vocal cords from occurring so forcibly, and hence the voice is feeble. (5) In consequence of the amount of blood in the skin, the internal organs are relatively anæmic ; hence, there is diminished secretion of urine, muscular weakness, disturbances of digestion, dulness of the senses, and it may be unconsciousness, and all these phenomena are intensified by the conditions mentioned under (3). Some of these phenomena are modified by usage. The highest limit at which a man may still retain his senses is placed by Tissandier at an elevation of 8000 metres $(280 \mathrm{~mm}$. $\mathrm{Hg})$. In dogs the blood-pressure falls, and the pulse becomes small and diminished in frequency, when the atmospheric pressure falls to $200 \mathrm{~mm}$. $\mathrm{Hg}$.

Those who live upon high mountains suffer from a disease " mal de montagne," which consists essentially in the above symptoms, although it is sometimes complicated with anæmia of the internal organs. Al. v. Humboldt found that in those who lived on the Andes the thorax was capacious. At 6000 to 8000 feet above sea-level, water contains only one-third of the absorbed gases, so that fishes cannot live in it. Animals may be subjected to a further diminution of the atmospheric pressure by being placed under the receiver of an air-pump. Birds die when the pressure is reduced to $120 \mathrm{~mm}$. $\mathrm{Hg}$; mammals at $40 \mathrm{~mm}$. $\mathrm{Hg}$; frogs endure repeated evacuations of the receiver, whereby they are much distended, owing to the escape of gases and water, but after the entrance of air they become greatly compressed. The cause of death in mammals is ascribed hy Hoppe-Seyler to the evolution of bubbles of gas in the blood; these bubbles stop up the capillaries, and the circulation is arrested. Local diminution of the atmospheric pressure causes marked congestion and swelling of the part, as occurs when a cupping-glass is used.

Great increase of the atmospheric pressure causes phenomena, for the most part, the reverse of the foregoing, as in pneumatic cabinets and in diving-bells, where men may work even under $4 \frac{1}{2}$ atmospheres pressure. (1) Paleness and dryness of the external surfaces, collapse of the cutaneous veins, diminution of perspiration, and mucous secretions. (2) The tympanic membrane is pressed inwards (until the air escapes through the Eustachian tube, after causing a sharp sound), acute sounds are heard, pain in the ears, and difficulty of hearing. (3) A feeling of lightness and freshness during respiration, the respiration becomes slower (by 2-4 per minute), inspiration easier and shorter, expiration lengthened, the pause distinct. The capacity of the lungs increases, owing to the freer movement of the diaphragm, in consequence of the diminu. tion of the intestinal gases. Owing to the more rapid oxidations in the body, muscular movement is easier and more active. The $\mathrm{O}$ absorbed and the $\mathrm{CO}_{2}$ excreted are increased. The venous blood is reddened. (4) Difficulty of speaking, alteration of the tone of the voice, inability to whistle. (5) Increase of the urinary secretion, more muscular energy, more rapid metabolism, increased appetite, subjective feeling of warmth, pulse beats slower, and pulse-curve is lower (compare \& 74). In animals subjected to excessively high atmospheric pressure, P. Bert found that the arterial blood contained 30 vols. per cent. $\mathrm{O}$ (at $760 \mathrm{~mm}$. $\mathrm{Hg}$ ); when the amount rose to 35 vol. per cent., death occurred with convulsions. Compressed air has been used for therapeutical purposes, but in doing so a too rapid increase of the pressure is to be avoidecl. Waldenburg has constructed such an apparatus, which may be used for the respiration of air under a greater or less pressure.

Frogs, when placed in compressed $O$ (at 14 atmospheres), exhibit the same phenomena as if 
they were in a vacuum, or pure $\mathrm{N}$. There is paralysis of the central nervous system, sometimes preceded by convulsions. The heart ceases to beat (not the lymph hearts), while the excitability of the motor nerves is lost at the same time, and lastly the direct muscular excitability disappears. An excised frog's heart placed in 0 under a very high pressure (13 atmospheres), scarcely beats one-fourth of the time during which it pulsates in air. If the heart be exposed to the air again, it begins to beat, so that compressed $\mathrm{O}$ renders the vitality of the heart latent before abolishing it.

Phosphorus retains its luminosity under a high pressure in $\mathrm{O}$, but this is not the case with the luminous organisms, e.g., Lampyris, and luıninous bacteria. High atmospheric pressure is also injurious to plants.

140. COMPARATIVE AND HISTORICAL. -Mammals have lungs similar to those of man. The lungs of birds are spongy, and united to the chest-wall, while there are openings on their surface communicating with thin-walled "air-sacs," which are placed amongst the viscera. The air-sacs communicate with cavities in the bones, which give the latter great lightness. The diaphragm is absent. In reptiles the lungs are divided into greater and smaller compartments ; in snakes one lung is abortive, while the other has the elongated form of the body. The amphibians (frog) possess two simple lungs, each of which represents an enormous infundibulum with its alveoli. The frog pumps air into its lungs by the contraction of its throat, the nostrils being closed and the glottis opened. When young-until their metamorphosis-frogs breathe like fishes by means of gills. The perennibranchiate amphibians (Proteus) retain their gills throughout life. Amongst fishes, which breathe by gills and use the $\mathrm{O}$ absorbed by the water, the Dipnoi have in addition to gills a swim-bladder provided with afferent and efferent vessels, which is comparable to the lung. The Cobitis respires also with its intestine. Insects and centipedes respire by " tracheæ," which are branched canals distributed throughout the body ; they open on the surface of the body by openings (stigmata) which can be closed. Spiders respire by means of tracheæ and tracheal sacs, erabs by gills. The molluses and cephalopods have gills ; some gasteropods have gills and others lungs. Amongst the lower invertebrata some breathe by gills, others by mearss of a special "water-vascular system," and others again by no special organs.

Historical. -Aristotle (384 B.c.) regarded the object of respiration to be the cooling of the body, so as to moderate the internal warmth. He observed correctly that the warmest animals breathe most actively, but in interpreting the fact he reversed the cause and effect. Galen (131-203 A.D.) thought that the "soot" was removed from the body along with the expired water. The most important experiments on the mechanics of respiration date from Galen; he observed that the lungs passively follow the movements of the chest; that the diaphragm is the most important muscle of inspiration; that the external intercostals are inspiratory ; and the internal, expiratory. He divided the intercostal nerves and muscles, and observed that loss of voice occurred. On dividing the spinal cord higher and higher, he found that as he did so the muscles of the thorax lying higher up became paralysed. Oribasius $(360$ A.D. $)$ observed that in double pneumothorax both lungs collapsed. Vesalius (1540) first described artificial respiration as a means of restoring the beat of the heart. Malpighi (1661) described the structure of the lungs. J. A. Borelli $(+1679)$ gave the first fundamental description of the mechanism of the respiratory movements. The chemical processes of respiration could only be known after the discovery of the individual gases therein concerned. Van Helmont $(+1644)$ detected $\mathrm{CO}_{2}$. [Joseph Black (1757) discovered that $\mathrm{CO}_{2}$, or "fixed air," is given out during expiration.] In 1774 Priestley discovered 0 . Lavoisier detected N (1775), and ascertained the composition of atmospheric air, and he regarded the formation of $\mathrm{CO}_{2}$ and $\mathrm{H}_{2} \mathrm{O}$ of the breath as a result of a combustion within the lungs themselves. J. Ingen-Houss (1730-1799) discovered the respiration of plants. Vogel and others proved the existence of $\mathrm{CO}_{2}$ in venous blood, and Hotfmann and others that of $\mathrm{O}$ in arterial blood. The more complete conception of the exchange of gases was, however, only possible after Magnus had extracted and analysed the gases of arterial and venous blood (\$36). 


\section{Physiology of Digestion.}

141. THE MOUTH AND ITS GLANDS. - The mucous membrane of the cavity of the mouth, which becomes continuous with the skin at the red nıargin of the lips, has a number of sebaceous glands in the region of the red part of the lip. The buccal mucous membrane consists of bundles of fine fibrous tissue mixed with elastic fibres, which traverse it in every direction. Papillæ-simple or compound-occur near the free surfaces. The sub-mucous tissue, which is directly continuous with the fibrous tissue of the mucous membrane itself, is thickest where the mucous membrane is thickest, and densest where it is firmly fixed to the periosteum of the

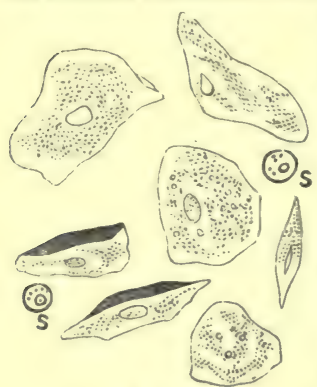

Fig. 150. bone and to the gum; it is thinnest where the mucous membrane is most movable, and where there are most folds. The cavity of the mouth is lined by stratified squamous epithelium (fig. 150), which is thickest, as a rule, where the longest papillæ occur.

All the glands of the mouth, including the salivary glands, may be divided into different classes according to the nature of their secretions.

1. The serous or albuminous glands [true salivary], whose secretion contains a certain amount of albumin, e.g., the human parotid.

2. The mucous glands, whose secretion, in addition to some albumin contains the characteristic constituent mucin.

3. The mixed [or muco-salivary] glands, some of the Cells of stratified squamous acini secreting an albuminous fluid and others mucin, e.g., the epithelium detachedfrom human maxillary gland.

the mouth. $s$, salivary Numerous mucous glands (labial, buccal, palatine, lingual, molar) corpuscles.

have the appearance of small macroscopic bodies lying in the submucosa. They are branched tubular glands, and the contents of their secretory cells consist partly of mucin, which is expelled from them during secretion. The excretory ducts of these glands, which are lined by cylindrical epithelium, are constricted where they enter the mouth. Tot unfrequently one duct receives the secretion of a neighbouring gland.

The glands of the tongue form two groups, which differ morphologically and physiologically. (1) The mucous glands ( $W$ eber's glands), occurring chiefly near the root of the tongue, are branched tubular glands lined with clear transparent secretory cells whose nuclei are placed near the attached end of the cells. The acini have a distinct membrana propria. (2) The serous glands (Ebner's) are acinous glands occurring in the region of the circumvallate papillæx (and in animals near the papillæ foliatæ). They are lined with turbid granular epithelium with a central nucleus, and secrete saliva. (3) The glands of Blandin and Nuhn are placed near the tip of the tongue, and consist of mucous and serous acini, so that they are mixed glands (Podvisotzky).

The blood-vessels are moderately abundant, and the larger trunks lie in the sub-mucosa, whilst the finer twigs penetrate into the papillæ, where they form either a capillary network or simple loops.

The larger lymphatics lie in the sub-mucosa, whilst the finer branches form a fine network placed in the mucosa. The lymph-follicles also belong to the lymphatic system (\$197). On the dorsum of the posterior part of the tongue they form an almost continuous layer. They are round or oval (1-1.5 mm. in diameter), lying in the sub-mucosa, and consist of adenoid tissue loaded with lymph-corpuseles. The outer part of the adenoid reticulum is compressed so as to 
form a kind of capsule for each follicle. Similar follicles occur in the intestine as solitary follicles; in the small intestine they are collected together into Peyer's patches, and in the spleen they occur as Malpighian corpuscles. On the dorsum of the tongue several of these follicles form a slightly oval elevation, which is surrounded by connective-tissue. In the centre of this elevation there is a depression, into which a mucous gland opens, which fills the small crater with mucus (fig. 151).

The tonsils have fundamentally the same structure. On their surface are a number of depressions into which the ducts of small mucous glands open. These depressions are surrounded by groups (10-20) of lymphfollicles, and the whole is environed by a capsule of connective-tissue (fig. 152). Large lymph-spaces, communicating with lymphatics, occur in the neighbourhood of the tonsils, but as yet a direct connection between the spaces in the follicles and the lymphvessels has not been proved to exist. Similar structures occur in the tubal and pharyngeal tonsils. [Stöhr asserts that an enormous number of leucocytes wander out of the tonsils, solitary and Peyer's glands, and the adenoid tissue of the bronchial mucous membrane. The cells pass out between the epithelial cells, but do not pass into the interior of the latter.]

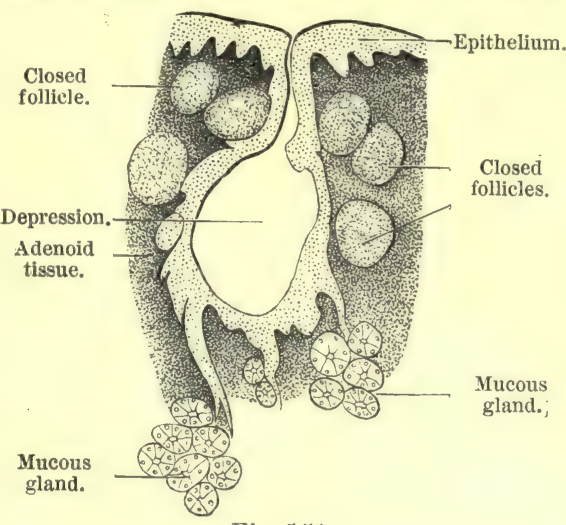

Fig. 151.

Section of a mucous follicle from the tongue.

Nerves. - Numerous medullated nerve-fibres occur in the sub-mucosa, pass into the mucosa, and terminate partly in the individual papillæ in Krause's end-bulbs, which are most abundant in the lips and soft palate, and not so numerous in the cheeks and floor of the mouth. The nerves administer not only to common sensation, but they are also the organs of transmissions for tactile (heat and pressure) impressions. It is highly probable, however, that some nerve-fibres end in fine terminal fibrils, between the epithelial cells, as in the cornea and elsewhere.

[Secretory glands may be simple (fig. 153, B, C, D) or compound $(\mathbf{E})$. In the latter case the duct is branched. In the process of development, a solid process of the epithelium sinks into the subjacent fibrous tissue, and, to form a simple gland, a cavity appears in this bud, but for a compound gland, other epithelial buds sprout from its blind end. Each bud acquires a central cavity, these elongate and increase in number, thus forming a much branched system, the terminal blind ends forming the acini, alveoli or true secretory part. If the alveoli are tubular in shape, the gland is called a compound

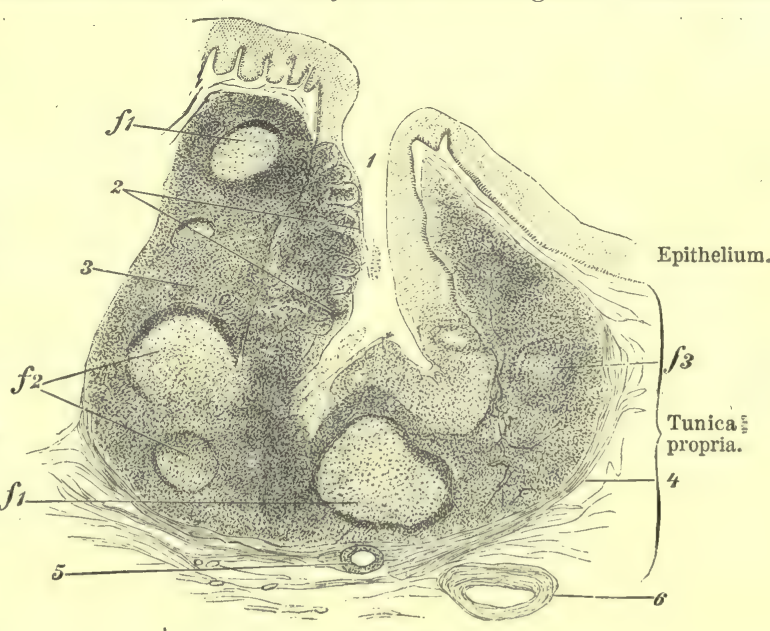

Fig. 152.

Vertical section of a human tonsil, $\times 20$. 1, cavity ; 2, epithelium infiltrated with leucocytes below and on the left, but free on the right ; 3 , adenoid tissue with sections $\alpha, b, c$, of masses of it ; 4 , fibrous sheath ; 5 , section of a gland-duct; $d$, blood-vessel.

tubular gland. Thus in the compound glands some parts are secretory, and others act as ducts, while in the simple glands, all the parts may be secretory. All the glands opening on the surface of the body are of epiblastic origin. The secretory cells lining the acini rest on a basement membrane, and outside this are the lymph-spaces and capillary blood-vessels.]

142. THE SALIVARY GLANDS. - The three pairs of salivary glands, sub-maxillary, sublingual, and parotid, are compound tubular glands. Fig. 155, A, shows a fine duct, terminating in the more or less flask-shaped alveoli or acini.. . E Each gland consists of a number of lobes, and 
each lobe in turn of a number of lobules, which, again, are composed of acini. All these are held together by a framework of counective-tissue. The larger branches of the duct lie between the lobules, and constitute the interlobular ducts, giving branches to each lobule which they enter,

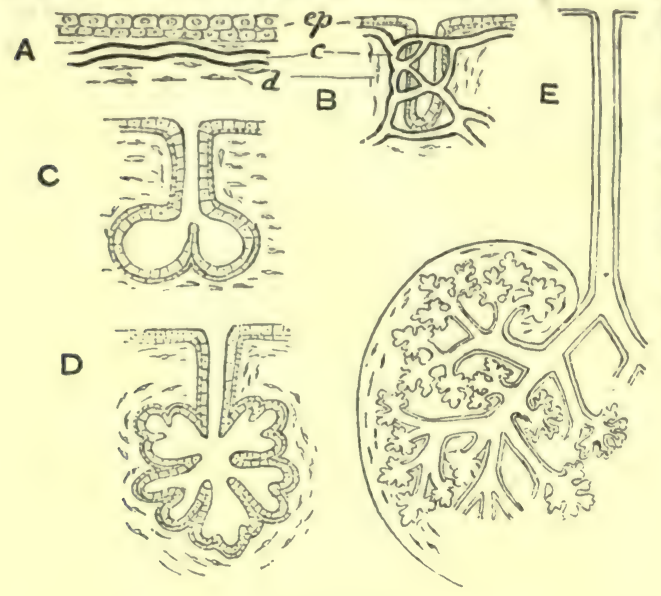

Fig. 153. constituting the intralobular ducts, which branch and finally terminate in connection with the alveoli, by means of an intermediary or intercalary part. The larger interlobar and interlobular ducts consist of a membrana propria, strengthened outside with fibrous and elastic tissue, and in some places also by non-striped muscle, while the ducts are lined by columnar epithelial cells. In the largest branches, there is a second row of smaller cells, lying between the large cells and the membrana propria.

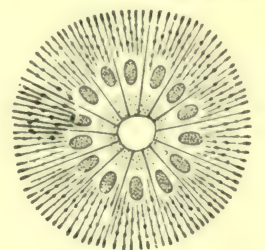

Fig. 154.

Fig. 153. - Evolution of glands. A, schema of skin ; ep, epidermis; $d$, cutis with a capillary $c$; B, simple gland with its blood-vessels; C, D, more complex glauds ; E, compound gland, blood-vessels omitted.

Fig. 154.--Rodded epithelium lining the duct of a salivary gland.
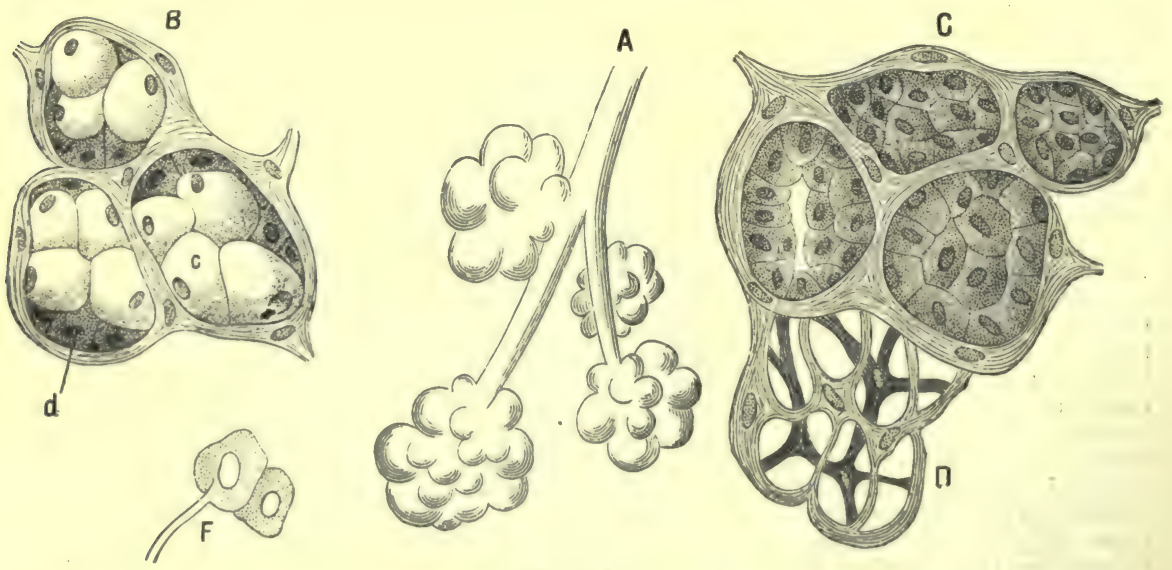

Fig. 155.

$A$, duct and acini of the parotid gland of a dog ; B, acini of the sub-maxillary gland of a dog ; $c$, refractive mucous cells ; $d$, granular half-moons of Gianuzzi ; C, similar alveoli after prolonged secretion ; D, basket-shaped tissue-investment of an acinus ; F, entrance of a non-medullated nerve-fibre into a secretory cell.

The intralobular ducts are lined by a single layer of large cylindrical epithelium with the nucleus about the middle of the cell, while the outer half of the cell is finely striated longitudinally, or "rodded," which is due to fibrillse (fig. 154); the inner half next the lumen is granular. The intermediary part is narrow, and is lined by a single layer of flattened cells, each with an elongated oval nucleus. There is usually a narrow "neck," where the intralobular duct becomes continuous with the intermediary part, and here the cells are polyhedral.]

The acini, or alveoli, are the parts where the actual process of secretion takes place. They vary somewhat in shape-some are tubular, others branched, some are dilated and resemble a 
Florence flask, and several of them usually open into one intermediary part of a duct. Each alveolus is bounded by a basement membrane, with a reticulate structure made up of nucleatel, branched, and anastomosing cells so as to resemble a basket (D). There is a homogeneous membrane bounding the alveoli in addition to this basket-shaped structure. Immediately outside this membrane is a lymph-space, and outside this again the network of capillaries is distributed. [The extent to which this lymph-space is filled with lymph determines the distance of the capillaries from the membrana propria. The interalveolar lymph-spaces communicate with large lymph-spaces between the lobules, which in turn communicate with perivascular lymphatics around the arteries and veins.] The lymphatics emerge from the gland at the hilum.

The secretory cells vary in structure, according as the salivary gland is a mucous [sub-maxillary and sub-lingual of the dog and cat'], a serous [parotid of man and mammals, and sub-maxillary of rabbit], or a mixed gland [human sub-maxillary and sub-lingual].

Mucous Acini, - The secretory cells of mucous glands, and the mucous acini of mixed glands (fig. 156), are lined by a single layer of "mucin cells" (fig. 155, B, c), which are large cells distended with mucin, or with a hypothetical substance, mucigen, which yields mucin. The mucin cells are more or less spheroidal in shape, clear, shining, highly refractive, and nearly fill the acinus. The flattened nucleus is near the wall of the acinus. Each cell has a fine process which overlaps the fixed parts of the cells next to it. Owing to the body of each cell being infiltrated. with mucin, these cells do not stain with carmine, although the nucleus and its immediately investing protoplasm do. Another kind of cell occurs in the sub-maxillary gland of the dog. It forms a half-moon-shaped structure lying in direct contact with the wall of the acinus (Gianuzzi). Each "half-moon" or " crescent" consists of a number of small, closely packed, angular, highly albuminous cells with small oval nuclei, which, however, are separated only with difficulty. Hence, Heidenhain has called them "composite marginal cells" $(\mathrm{B}, d)$. They are granular, darker, devoid of mucin, and stain readily with pigments. [In the sub-maxillary gland of the cat, there is a complete layer of these "'marginal " carminestaining cells lying between the mucous cells and the membrana propria.]

[Serous Acini. - In true serous glands (parotid of man and mammals) and in the

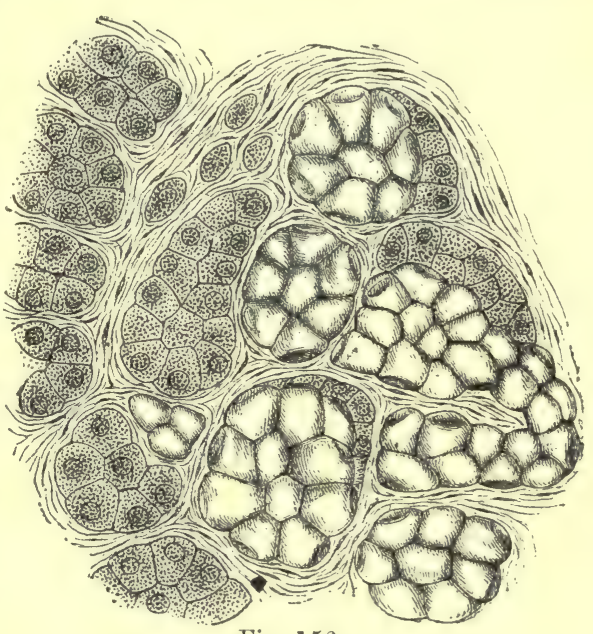

Fig. 156.

Section of a human sub-maxillary gland. On the left is a group of serous alveoli, and on the right a group of mucous alveoli. serous acini of mixed glands, the acini are lined by a single layer of secretory columnar finely. granular cells, which in the quiescent condition completely fill the acinus, so that scarcely any lumen is left. Just before secretion, or when these cells are quiescent, Langley has shown that they are large and filled with numerous granules, which obscure the presence of the nucleus. As secretion takes place, these granules seem to be used up or discharged into the lumen; at least, the outer part of each cell gradually becomes clear and more transparent, and this condition spreads towards the inner part of the cell.]

[In the mixed or muco-salivary glands (e.g., human sub-maxillary) some of the alveoli are mucous and others serous in their characters, but the latter are always far more numerons, and the one kind of acinus is directly continuous with the others (fig. 156).]

\section{HISTOLOGICAL CHANGES DURING THE ACTIVITY OF THE} SALIVARY GLANDS. - [The condition of physiological activity of the gland-cells is accompanied by changes in the histological characters of the secretory celis. Changes in serous glands have been carefully studied in the parotid of the rabbit, but the appearances vary somewhat, according as the glands are examined in the fresh condition or after hardening in various reagents, such as absolute alcohol. When the gland is at rest, in a preparation hardened in alcohol, and stained with carmine, the cells consist of a pale, almost uncoloured substance, with a few fine 
granules, and a small, irregular, red-stained, shrivelled nucleus devoid of a nucleolus. The appearance of the nucleus suggests the idea of its being shrivelled by the action of the hardening reagent (fig. 157.]

[During activity, if the gland be caused to secrete by stimulating the sympathetic, all parts of the cells undergo a change (figs. 157, 158). In preparations hardened in alcohol-(1) the cells diminish somewhat in size; (2) the nuclei are no longer irregular, but round, with a sharp contour and nucleoli; (3) the substance of the cell itself is turbid owing to the diminution of the clear substance, and the increase of the granules, especially near the nuclei ; $(4)$ at the same time, the whole cell stains more deeply with carmine (Heidenhain).]

[On studying the changes which occur in a living serous gland, Langley found that the substance of the cells of the parotid is pervaded by fine granules, which

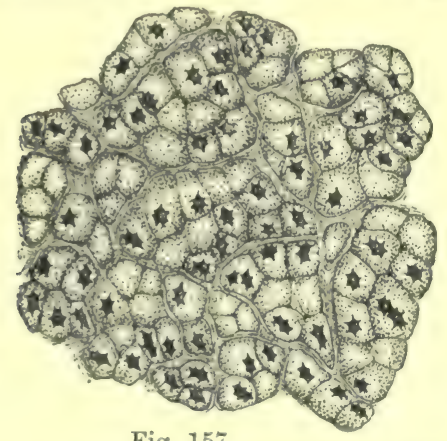

Fig. 157 .

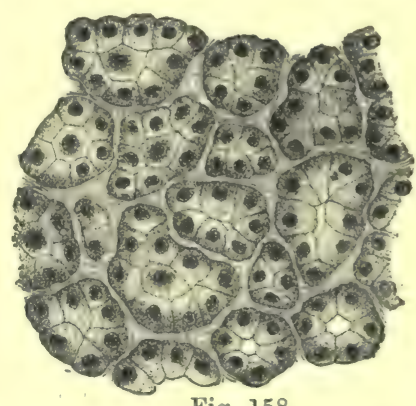

Fig. 158.

Sections of a "serous" gland. The parotid of a rabbit, fig. 157, at rest; fig. 158, alter stimulation of the cervical sympathetic.

are so numerous as to obscure the nucleus, while the outlines of the cells are indistinct. No lumen is visible in the acini during activity, the granules disappear from the outer zone of the cells, the cells themselves becoming smaller and more distinct. After prolonged secretion, the granules largely disappear from the cellsubstance except quite near the inner margin. The cells are smaller, their outlines more distinct, their spherical nuclei apparent, and the lumen of the acini is wide and distinct. Thus, it is evident that, during rest, granules are manufactured, which disappear during the activity of the cells, the disappearance taking place from without inwards. Similar changes occur in the cells of the pancreas.]

[More complex changes occur in the mucous glands, such as the sub-maxillary or orbital glands of the dog (Lavdovsky). The appearances vary according to the intensity and duration of the secretory activity. The mucous cells at rest are large, clear, and refractive, containing a flattened nucleus (fig. 155, C), surrounded with a small amount of protoplasm, and placed near the basement membrane. The clear substance does not stain with carmine, and consists of mucigen lying in the wide spaces of an intracellular plexus of fibrils. After prolonged secretion, produced, it may be, by strong and continued stimulation of the chorda, the mucous cells of the sub-naxillary gland of the dog undergo a great change.] . The distended, refractive, and "mucous cells," which occur in the quiescent gland, and which do not stain with carmine, do not appear after the gland has been in a state of activity. Their place is taken by small dark protoplasmic cells devoid of mucin (fig. 155, C). These cells readily stain with carmine, whilst their nucleus is scarcely, if at all, coloured by the dye. The researches of $R$. Heidenhain (1868) have shed much light on the secretory activity of the salivary glands.

The change may be produced in two ways. Either it is due to the "mucous cells" during secretion becoming broken up, so that they yield their mucin directly to the saliva ; in saliva rich 
in mucin, small microscopic pieces of mucin are found, and sometimes mucous cells themselves are present. Or, we must assume that the mucous cells simply eliminate the mucin from their bodies (Ewald, Stöhr); while, after a period of rest, new mucin is formed. According to this view, the dark granular cells of the glands, after active secretion, are simply mucous cells, which have given out their mucin. If we assume, with Heidenhain, that the mucous cells break up, then these granular non-mucous cells must be regarded as new formations produced by the proliferation and growth of the composite marginal cells, i.e., the crescents, or half-moons of Gianuzzi.

[During rest, the protoplasm seems to manufacture mucigen, which is changed into and discharged as mucin in the secretion, when the gland is actively secreting. Thus, the cells become smaller, but the protoplasm of the cell seems to increase, new mucigen is manufactured during rest, and the cycle is repeated.]

144. THE NERVES OF THE SALIVARY GLANDS.-The nerves are for the most part medullated, and enter at the hilum of the gland, where they form a rich plexus provided with ganglia between the lobules. [There are no ganglia in the parotid gland (Klein).]

All the salivary glands are supplied by branches from two different nerves-from the sympathetic and from a cranial nerve.

1. The sympathetic nerve gives branches $(a)$ to the sub-maxillary and the sublingual glands, derived from the plexus on the external maxillary artery; $(b)$ to the parotid gland from the carotid plexus (fig. 159).

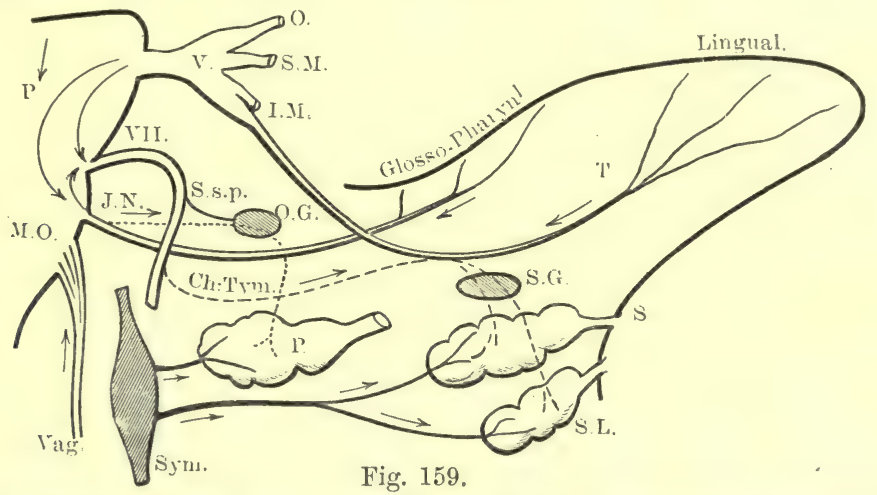

Scheme of the nerves of the salivary glands. P., pons; M.O., medulla oblongata; J.N., nerve of Jacobson; O., S.M., I.M., ophthalmic, superior, and inferior maxillary divisions of fifth nerve, V.; VII., seventh nerve; S.s.p., small superficial petrosal nerve; Vag., vagus; Sym., sympathetic ; O.G., otic, and S.G., submaxillary ganglia; P., S., and S.L., parotid, submaxillary, and sublingual glands ; T., tongue,

2. The facial nerve gives branches to the sub-maxillary and sub-lingual glands from the chorda tympani, which accompanies the lingual branch of the fifth nerve (fig. 159). The branches to the parotid arise from the tympanic branch of the glosso-pharyngeal nerve (dog). The tympanic plexus sends fibres to the small superficial petrosal nerve, and with it these fibres run to the anterior surface of the pyramid in the temporal bone, emerging from the skull through a fissure between the petrous and great wing of the sphenoid, and then joining the otic ganglion. This ganglion sends branches to the auriculo-temporal nerve (itself derived from the third branch of the trigeminus), which, as it passes upwards to the temporal region under cover of the parotid, gives branches to this gland.

The sub-maxillary ganglion, which gives branches to the sub-maxillary and sublingual glands, receives fibres from the tympanico-lingual nerve (chorda tympani) as well as sympathetic fibres from the plexus on the external maxillary artery.

Termination of the Nerve-Fibres. - With regard to the ultimate distribution of these nerves we can distinguish (1) the vaso-motor nerves, which give branches to the walls of the blood-vessels, and (2) the secretory nerves proper. 
Pfliger states, with regard to the latter, that $(a)$ medullated nerve-fibres penetrate the acini; the sheath of Schwann unites with the membrana propria of the acinus; the medullated fibro - still medullated-passes between the secretory cells, where it divides and becomes nonmedullated, and its axial cylinder terminates in connection with the nucleus of a secretory cell. [This, however, is not proved] (fig. 155, F). (b) According to Pfliger, some of the nerve-fibres end in multipolar ganglion cells, which lie outside the wall of the acinus, and these cells send branches to the secretory cells of the acini. [These cells probably correspond to the branched cells of the basket-shaped structure.] (c) Again, he describes medullated tibres which enter the attached end of the cylindrical epithelium liuing the excretory ducts of the glands (E). Pfliiger thinks that those fibres entering the acini directly are cerebral, while those with ganglia in their course are derived from the sympathetic system. $[(d)$ The direct termination of nerve-fibres has been observed in the salivary glands of the cockroach by Kupffer.]

\section{ACTION OF THE NERVOUS SYSTEM ON THE SECRETION OF} SALIVA.-A. Sub-maxillary Gland.-Stimulation of the facial nerve at its origin causes a profuse secretion of a thin watery saliva, which contains a very small amount of specific constituents. Simultaneously with the act of secretion, the blood-vessels of the glands dilate, and the capillaries are so distended that the pulsatile movement in the arteries is propagated into the veins. Nearly four times as much blood flows out of the veins ( $\mathrm{Cl}$. Bermurd), the blood being of a bright red colour, and containing one-third more $\mathrm{O}$ than the venous blood of the non-stimulated gland. Notwithstanding this relatively high percentage of $\mathrm{O}$, the secreting gland uses more O than the passive gland $(\S 131,1)$.

[I. Stimulation of Chorda.-If a cannula be placed in Wharton's duct, e.g., in a dog, and the chorda tympani be divided, no secretion flows from the cannula. On stimulating the peripheral end of the chorda tympani with an interrupted current of electricity, the same results-copious secretion of saliva and vascular dilatation, with increased flow of blood through the gland-occur as when the origin of the seventh nerve itself is stimulated. - The watery saliva is called chorda saliva.]

Two functionally different kinds of nerve-fibres occur in the facial nerve--(1) true secretory fibres, (2) vaso-dilator fibres.

II. Stimulation of the sympathetic nerve causes a scanty amount of a very thick, sticky, mucous secretion, in which the specific salivary constituents, mucin, and the salivary corpuscles are very abundant. The specific gravity of the saliva is raised from 1007 to 1010 . Simultaneously the blood-vessels become contracted, so that the blood flows more slowly from the veins, and has a dark bluish colour.

The sympathetic also contains two kinds of nerve-fibres-(1) true secretory fibres, and (2) vaso-constrictor fibres.

[Electrical Variations during Secretion.-That changes in the electromotive properties of glands occur during secretion was shown in the frog's skin. Bayliss and Brarford find that the same is true of the sub-maxillary gland $(\mathrm{dog})$. During secretion, the excitatory change on stimulating the chorda is a positive variation of the current of rest (the hilum of the gland becoming more positive), but it is frequently followed by a second phase of opposite sign. The latent period is always very short, about $0.37^{\prime \prime}$. Atropin abolishes the chorda variation. On stimulating the sympathetic, the excitatory change is of an opposite sign to that of the chorda, and the hilum becomes less positive, so that there is a negative variation. It requires a more powerful stimulus, is less in amount, and its latent period is longer $\left(2^{\prime \prime}-4^{\prime \prime}\right)$, while atropin lessens but does not abolish it.]

Relation to Stimulus._-On stimulating the cerebral nerves, at first with a weak and gradually with a stronger stimulus, there is a gradual development of the secretion in which the solid constituents-occasionally the organic-are increased (Heidenhain). If a strong stimulus be applied for a long time, the secretion diminishes, becomes watery, and is poor in specific constituents, especially in the organic elements, which are more affected than the inorganic $(C$. Ludwig and Becher). After prolonged stimulation of the sympathetic, the secretion resembles the chorda saliva. It would seem, therefore, that the chorda and sympathetic saliva are not specifically distinct, but vary only in degree. On continuing the stimulation of the nerves up to a certain maximal limit, the rapidity of secretion becomes greater, and the percentage of salts also increases to a certain maximum, and this independently of the former condition of the glands. The percentage of organic constituents also depends on the strength of the nervous stimulation, but not on this alone, as it is essentially contingent upon the condition of the gland 
before the secretion took place, and it also depends upon the duration and intensity of the previous secretory activity. Very strong stimulation of the gland leaves an "after-effect," which predisposes it to give off organic constituents into the secretion (Heidenhain). A latent period of $1 \cdot 2$ sec. to 24 sec. may elapse between the nerve-stimulation and the beginning of the secretion.

[Langley has shown that in the cat the sympathetic saliva of the sub-maxillary gland is less viscid than the chorda saliva.]

Relation to Blood-Supply. - The secretion of saliva is not simply the result of the amount of blood in the glands; that there is a factor independent of the changes in the state of the vessels is shown by the following facts :-

(1) The secretory activity of the glands when their nerves are stimulated continues for some time after the blood-vessels of the gland have been ligatured. [If the head of a rabbit be cut off, stimulation of the seventh nerve, above where the chorda leaves it, causes a flow of saliva, which cannot be accounted for on the supposition that the saliva already present in the salivary glands is forced out of them. Thus we may have secretion without a blood-stream. The saliva is really secreted from the lymph present in the lymph-spaces of the gland (Ludwig).]

(2) Atropin and daturin abolish the activity of the secretory fibres in the chorda tympani, but do not affect"the vaso-dilator fibres (Heidenhain). The same results occur after the injection of acids and alkalies into the excretory duct (Gianuzzi).

[Action of Atropin.-The vascular dilatation and the increased flow of saliva, due to the activity of the secretory cells, produced by stimulation of the chorda tympani, although they occur simultaneously, do not stand in the relation of cause and effect. We may cause vascular dilatation without an increased flow of saliva, as already stated (2). If atropin be given to an animal, stimulation of the chorda produces dilatation of the blood-vessels, but no secretion of saliva. Atropin paralyses the secretory fibres, but not the vaso-dilator fibres (fig. 160). The increased supply of blood, while not causing, yet favours the act of secretion, by placing a larger amount of pabulum at the disposal of the secretory elements, the cells.]

(3) The pressure in the excretory duct of the salivary gland-measured by means of a manometer tied into it-may be nearly twice as great as the pressure within the arteries of the glands, or even in the carotid itself (Ludwig). The pressure in Wharton's duct may reach $200 \mathrm{~mm}$. Hg.

[Secretory Pressure.- The experiment described under (3) proves, in a definite manner, that the passage of the water from the blood-vessels, or at least from the lymph into the acini of the gland, cannot be due to the blood-pressure; that, in fact, it is not a mere process of filtration, such as occurs in the glomeruli of the kidney. In the case of the salivary gland, where the pressure within the gland may be double that of the arterial pressure, the water actually moves from the lymph-spaces against very great resistance. We can only account for this result by ascribing it to the secretory activity of the gland-cells themselves. Whether the activities of the gland-cells, as suggested by Heidenhain, are governed directly by two distinct kinds of nerve-fibres, a set of solid-secreting fibres, and a set of water-secreting fibres, remains to be proved.]

(4) Just as in the case of muscles and nerves, the salivary glands become fatigued or exhausted after prolonged action. This result may also be brought about by injecting acids or alkalies into the duct, which shows that the secretory activity of the gland is independent of the circulation (Gianuzzi).

All these facts lead us to conclude that the nerves exercise a direct effect upon the secretory cells, apart from their action on the blood-vessels.

Extirpation of Salivary Glands. - When the chorda tympani is extirpated on one side in young dogs, the sub-maxillary gland on that side does not develop so much-its weight is 50 per cent. less-while the mucous cells and the "crescents" are smaller than on the sound side (Bufalini).

During secretion, the temperature of the gland rises $1.5^{\circ} \mathrm{C}$. (Ludwig), and the blood flowing from the veins is often warmer than the arterial blood. [The electromotive changes are referred to at p. 212.] 
"Paralytic Secretion" of Saliva.-By this term is meant the continued secretion of a thin watery saliva from the sub-maxillary gland, which occurs twentyfour hours after the section of the cerebral nerves (chorda of the seventh), i.e., those branches of them that go to this gland, whether the sympathetic be divided or not (Cl. Bernard). It increases until the eighth day, after which it gradually diminishes, while the gland-tissue degenerates. The injection of a small quantity of curara into the artery of the gland also causes it.

[Heidenhain showed that section of one chorda is followed by a continuous secretion of saliva from both sub-maxillary glands. The term "paralytic" secretion is applied to that which takes place on the side on which the nerve is cut, and Langley proposes to call the secretion on the opposite side the antilytic. Apnoea (\$368) stops both the paralytic and antilytic secretion, while dyspnea increases the flow in both cases ; and as section of the sympathetic fibres to the gland (where the chorda is cut) arrests the paralytic secretion excited by dyspnoa, it is evident that both the paralytic secretion and the secretion following dyspnoea are cansed by stimuli travelling down the sympathetic fibres. In the later stages of the paralytic secretion, the cause is in the gland itself, for it goes on even if all the nerves passing to the gland be divided, and is probably due to a local nerve-centre. In this stage the secretion is arrested by a large dose of chloroform. The paralytic secretion, in the first stage, may be owing to a venous condition of the blood acting on a central secretory centre whose excitability is increased; and in the latter stages probably on local nerve-centres within the gland. The fibres of the chorda in the cat are only partially degenerated thirteen days after section (Langley).]

[Histological Changes. - In the gland during paralytic secretion, the gland-cells of the alveoli (serous, mucous, and demilunes) diminish in size and show the typical "resting" appearance, even to a greater extent than the normal resting gland (Langley).]

B. Sub-lingual Gland.-Very probably the same relations obtain as in the submaxillary gland.

C. Parotid Gland.-In the dog; stimulation of the sympathetic alone causes no secretion; it occurs when the glosso-pharyngeal branch to the parotid is simultaneously excited. This branch may be reached within the tympanum in the tympanic plexus. A thick secretion containing much organic matter is thereby obtained. Stimulation of the cerebral branch alone yields a clear thin watery secretion, containing a very small amount of organic substances, but a considerable amount of the salts of the saliva.

[Stimulation of Jacobson's Nerve (Parotid of Dog) -

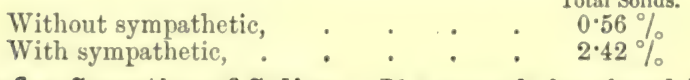

$\begin{array}{cc}\text { Sults. } & \text { Organic Matter. } \\ 0.31 & 0.24 \\ 0.36 & 2 \cdot 06]\end{array}$

[Reflex Secretion of Saliva.-If a cannula be placed in Wharton's duct, e.g., in a dog, during fasting, no saliva will flow out, but on applying a sapid substance to the

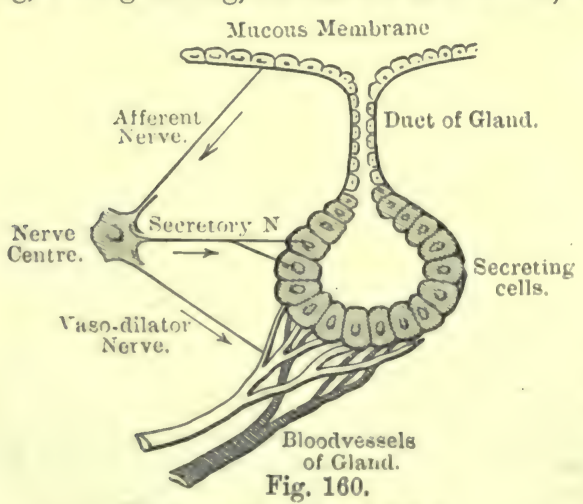

Diagram of a salivary gland. mucous membrane of the mouth or the tongue, there is a copious flow of saliva. If the sympathetic nerve be divided, secretion still takes place when the mouth is stimulated, but if the chorda tympani be cut, secretion no longer takes place. Hence, the secretion is due to a reflex act; in this case, the lingual is the afferent, and the chorda the efferent nerve carrying impulses from a centre situated in the medulla oblongata (fig. 160).] In the intact body, the secretion of saliva occurs through a reflex stimulation of the nerves concerned, whereby, under normal circumstances, the secretion is always watery (chorda or facial saliva). The centripetal or afferent nerve-fibres concerned are:-(1) The nerves of taste. (2) The sensory 
branches of the trigeminus of the entire cavity of the mouth and the glossopharyngeal (which appear to be capable of being stimulated by mechanical stimuli, pressure, tension, displacement). The movements of mastication also cause a secretion of saliva. Pflüger found that one-third more saliva was secreted on the side where mastication took place; and $\mathrm{Cl}$. Bernard observed that the secretion ceased in horses during the act of drinking. (3) The nerves of smell, excited by certain odours. (4) The gastric branches of the vagus. A rush of saliva into the mouth usually precedes the act of vomiting ( $\$ 158)$.

(5) The stimulation of distant sensory nerves, e.g., the central end of the sciatic-certainly through a complicated reflex mechanism-causes a secretion of saliva (Owsjannikow and T'schierjew). Stimulation of the conjunctiva, e.g., by applying an irritating fluid to the eye of carnivorous animals, eauses a reflex secretion of saliva (Aschenbrandt). Perhaps the secretion of saliva which sometimes occurs during pregnancy is caused in a similar reflex manner.

(b) The movements of mastication excite secretion, but although, during the act of rumination; this is the case in ruminants, in whom the process of mastication is very thorough, there is no secretion from the sub-maxillary gland, although the parotid secretes (Colin, Ellenberger and Hofmeister).

The reflex centre for the secretion of saliva lies in the medulla oblongata, at the origin of the seventh and ninth cranial nerves. The centre for the sympathetic fibres is also placed here. 'This region is connected by nerve-fibres with the cerebrum; hence, the thought of a savoury morsel, sometimes, when one is hungry, causes a rapid secretion of a thin watery fluid-[or, as we say, "makes the mouth water"]. If the centre be stimulated directly by a mechanical stimulus (puncture), salivation occurs, while asphyxia has the saine effect. The reflex secretion of saliva may be inhibited by stimulation of certain sensory nerves, e.g., by pulling out a loop of the intestine. Stimulation of the cortex cerebri of a dog, near the sulcus cruciatus, is often followed by secretion of saliva. Disease of the brain in man sometimes causes a secretion of saliva, owing to the effects produced on the intracranial centre.

So long as there is no stimulation of the nerves, there is no secretion of saliva, as in sleep. Immediately after the section of all nerves, secretion stops, for a time at least.

Pathological Conditions and Poisons.-Certain affections, as inflammation of tne mouth, neuralgia ; ulcers of the mucous membrane, and affections of the gums, due to teething or the prolonged administration of mercury, often produce a copious secretion of saliva or ptyalism. Certain poisons cause the same effect by direct stimulation of the nerves, as Calabar bean (physostigmin), digitalin, and especially pilocarpin. Many poisons, especially the narcoticsabove all, atropin-paralyse the secretory nerves, so that there is a cessation of the secretion and the mouth becomes dry; while the administration of muscarin in this condition causes secretion. Pilocarpin acts on the chorda tympani, causing a profuse secretion, and if atropin be given, the secretion is again arrested. Conversely, if the secretion be arrested by atropin, it may be restored by the action of pilocarpin or physostigmin. Nicotin, in small. doses, excites the secretory nerves, but in large doses paralyses them. Daturin, cicutin, and iodide of æthylstrychnin, paralyse the chorda. The saliva is diminished in amount in man, in cases of paralysis of the facial or sympathetic nerves, as is observed in unilateral paralysis of these nerves.

[Sialogogues are those drugs which increase the secretion of saliva. Some are topical, and take effect when applied to the mouth. They excite secretion reflexly by acting on the sensory nerves of the mouth. They include acids, and various pungent bodies, such as mustard, ginger, pyrethrum, tobacco, ether, and chloroform; but they do not all produce the same effect on the amount or quality of the saliva ; others, the general sialogogues, cause salivation when introduced into the blood; physostigmin, nicotin, pilocarpin, muscarin. The drugs named act after all the nerves going to the gland are divided, so that they stimulate the peripheral ends of the nerves in the glands. The two former also excite the central ends of the secretory nerves.]

[Anti-sialics are those substances which diminish the secretion of saliva, and they may take effect upon any part of the reflex arc, i.e., on the mouth, the afferent nerves, the nerve-centre and afferent nerves, or upon the blood-stream through the glands, or on the glands themselves. Opium and morphia affect the centre, large doses of physostigmin affect the blood-supply, but atropin is the most powerful of all, as it paralyses the terminations of the secretory nerves in the glands, e.g., the chorda tympani, and even the sympathetic in the cat (but not in the $\operatorname{dog})$. 
[Excretion by the Saliva. - Some drugs are excreted by the saliva. Iodide of potassium is rapidly eliminated by the kidneys, and by the salivary glands, and so also is iodide of iron. ]

Theory of Salivary Secretion.-Heidenhain has recently formulated the following theory regarding the secretion of saliva:- "During the passive or quiescent condition of the gland, the organic materials of the secretion are formed from and by the activity of the protoplasm of the secretory cells. A quiescent cell, which has been inactive for some time, therefore contains little protoplasm, and a large amount of these secretory substances. In an actively secreting gland, there are two processes occurring together, but independent of each other, and regulated by two different classes of nerve-fibres; secretory fibres cause the act of secretion, while trophic fibres cause chemical processes within the cells, partly resulting in the formation of the soluble constituents of the seeretion, and partly in the growth of the protoplasm. According to the number of both kinds of fibres present in a nerve passing to a gland, such nerve being stimu. lated, the secretion takes place more rapidly (cerebral nerve) or more slowly (sympathetic), while the secretion contains less or more solid constituents. The cercbral nerves contain many secretory fibres and few trophic fibres, while the sympathetic contain more trophic but few secretory fibres. The rapidity and chemical composition of the secretion vary according to the strength of the stimulus. During continued secretion, the supply of secretory materials in the gland-cells is used up more rapidly than it is replaced by the activity of the protoplasm; hence, the amount of organic constituents diminishes, and the microscopic characters of the cells are altered. The microscopic characters of the cells are altered also by the increase of the protoplasm, which takes place in an active gland. The mucous cells disappear, and seem to be dissolved after prolonged secretion, and their place is taken by other cells derived from the jurliferation of the marginal cells. The energy which causes the current of fluid depends upon the protoplasm of the gland-cells."

146. THE SALIVA OF THE INDIVIdUAL GLANDS.-(a) Parotid saliva is obtained by placing a fine cannula in Steno's duct; it has an alkaline reaction, but during fasting, the first few drops may be neutral or even acid on account of free $\mathrm{CO}_{2}$; its specific gravity is 1003 to 1004. After standing it becomes turbid, and deposits, in addition to albuminous matter, calcium carbonate, which is present in the fresh saliva in the form of bicarbonate. It contains small quantities (more abundant in the horse) of a globulin-like body, and never seems to be without CNKS, i.e., sulphocyanide of potassium (or sodium), -which, however, is absent in the sheep and dog.

[The sulphocyanide gives a dark red colour (ferric sulphocyanide) with ferric chloride, and the colour is discharged by mercuric chloride, but this is not the case with meconic acid, which gives a similar colour-reaction.] It also reduces iodic acid when added to saliva, causing a yellow colour from the liberation of iodine, which may be detected at once by starch (Solera).

Amongst the organic substances the most important are ptyalin, a small amount of urea, and traces of a volatile acid. Mucin is absent, hence the parotid saliva is not sticky, and can readily be poured from one vessel into another. It contains 1.5 to 1.6 per cent. of solids in man, of which 0.3 to 1.0 per cent. is inorganic.

Of the inorganic constituents--the most abundant are potassium and sodium chlorides; then potassium, sodium, and calcium carbonates, some phosphates, and a trace of an alkaline sulphate.

Salivary calculi are formed in the ducts of the salivary glands owing to the deposition of lime-salts, and they contain only traces of the other salivary constituents; in the same way is formed the tartar of the teeth, which contains many threads of leptothrix, and the remains of low organisms which live in decomposing saliva in carious cavities between the teeth.

(b) Sub-maxillary saliva is obtained by placing a cannula in Wharton's duct ; it is alkaline, and may be strongly so. After standing for a time, fine crystals of calcium carbonate are deposited, together with an amorphous albuminous body. It always contains mucin (which is precipitated by acetic acid); hence, it is usually somewhat tenacious. It contains ptyalin, but in less amount than in parotid saliva ; and, according to Oehl, only 0.0036 per cent. of potassium sulphocyanide.

Chemical Composition,-Sub-maxillary saliva (dog) :

$$
\begin{aligned}
& \text { Water, . } 991.45 \text { per } 1000 \text {, }
\end{aligned}
$$

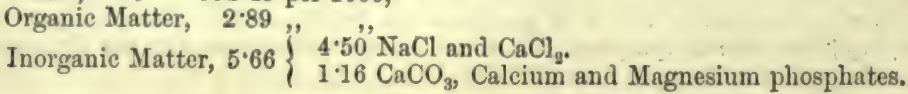




Mixed Saliva
(Human)
(Jacubovitsch).
$99 \cdot 51$
$0 \cdot 49$

$\begin{array}{cc}\begin{array}{c}\text { Parotid } \\ \text { (Human) }\end{array} & \begin{array}{c}\text { Submaxillary } \\ (\text { Dog) }\end{array} \\ \text { (Hoppe-Seyler). } & (\text { Herter }) . \\ 99.32 & 99 \cdot 44 \\ 0.68 & 0.56\end{array}$

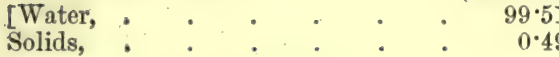

$\left.\begin{array}{l}0.13 \\ 0.16 \\ 0.102 \\ 0.006 \\ 0.084\end{array}\right\}$
$\begin{aligned} & 0 \\ & 0\end{aligned}$

0.34

Soluble organic bodies (ptyalin),

Epithelium, mucin,

Inorganic salts,

Potassic sulphocyanide,

Potassic and sodic chlorides,

0.084

$0 \cdot 34$

0.03

$\left\{\begin{array}{l}0.066 \\ 0.17 \\ 0.43 \\ \cdots \\ \cdots\end{array}\right]$

Gases. - Pflüger found that 100 cubic centimetres of the saliva contained $0.6 \mathrm{O} ; 64 \cdot 7 \mathrm{CO}_{2}$ (part could be pumped out, and part required the addition of phosphoric acid); $0.8 \mathrm{~N}$. ; or, in 100 vol. gas, $0.91 \mathrm{O} ; 97.88 \mathrm{CO}_{2} ; 1.21 \mathrm{~N}$. [It therefore contains much more $\mathrm{CO}_{2}$ than venous blood. Külz obtained from 100 c.c. of human saliva 7 c.c. of gas- $\mathrm{O}=1$ c.c., $\mathrm{N}=2 \cdot 5$ c.c. and $\mathrm{CO}_{2}=3.5$ c.c. Besides this there is 40-60 c.c. of fixed $\mathrm{CO}_{2}$ in the form of carbonates.]

(c) Sub-lingual saliva is obtained by placing a very fine cannula in the ductus Rivinianus; it is strongly alkaline in reaction, very sticky and cohesive, contains much mucin, numerous salivary corpuscles, and some potassium sulphocyanide.

147. THE MIXED SALIVA IN THE MOUTH.-The mixed saliva in the mouth is a mixture of the secretions from the salivary, mucous, and other glands of the mouth.

(1) Physical Characters.-It is an opalescent, tasteless, odourless, slightly glairy fluid, with a specific gravity of 1004 to 1009, and an alkaline reaction. The amount secreted in twenty-four hours $=200$ to 1500 grammes ( 7 to 50 oz.); according to Bidder and Schmidt, however, $=1000$ to 2000 grammes. The solid constituents $=5 \cdot 8$ per 1000 .

Composition. - The solids are :-Epithelium and mucus, $2 \cdot 2$; ptyalin and albumin, 1.4 ; salts, $2 \cdot 2$; potassium sulphocyanide, 0.04 per 1000 . The ash contains chiefly potash, phosphoric acid, and chlorine (Hammerbacher).

Decomposition-products of epithelium, salivary corpuscles, or the remains of food, may render it acid temporarily, as after long fasting, and after much speaking; the reaction is acid in some cases of dyspepsia and in fever, owing to the stagnation and insufficient secretion.

(2) Microscopic Constituents.-(a) The salivary corpuscles are slightly larger than the white blood-corpuscles $(8$ to $11 \mu$ ), and are nucleated protoplasmic globular cells without an envelope (fig. 150,s). During their living condition, the particles in their interior exhibit molecular or Brownian movement. The dark granules lying in the protoplasm are thrown into a trembling movement, from the motion of the fluid in which they are suspended. This dancing motion stops when the cell dies.

[The Brownian movements of these suspended granules are purely physical, and are exhibited by all fine microscopic particles suspended in a limpid fluid, e.g., gamboge rubbed up in water, particles of carmine, charcoal, \&c.]

(b) Pavement epithelial cells from the mucous membrane of the mouth and tongue; they are very abundant in catarrh of the mouth (fig. 150).

(c) Living organisms, which live and thrive in the cavities of teeth, nourished by the remains of food. Amongst these are Leptothrix buccalis (fig. 149, 12) and small bacteria-like organisms. The threads of the leptothrix penetrate into the canals of the dentine, and produce dental caries. [Miller has found twenty-five varieties of micro-organisms, including cocci, bacteria, vibrios, spirilla, and spirochætæ, eight of them present in the stomach and twelve in the intestines.]

(3) Chemical Properties.-_(a) Organic Constituents.-Serum-albumin is precipitated by heat and by the addition of alcohol. In saliva, mixed with much water and shaken up with $\mathrm{CO}_{2}$, a globulin-like body is precipitated ; mucin occurs in small amount. Amongst the extractives, the most important is ptyalin; fats and urea occur only in traces. In twenty-four hours 130 milligrammes of potassium or sodium sulphocyanide are secreted.

(b) Inorganic Constituents.-Sodium and potassium chlorides, potassium sulphate, alkaline and earthy phosphates, ferric phosphate. 
According to Schünbein, the saliva contains traces of nitrites, (detected by adding dilute sulphuric acid and diamido-benzol to dilute saliva), which give a yellow colour (Gries). There are also traces of ammonia (Brïcke).

Abnormal Constituents. - In diabetes mellitus, lactic acid, derived from a further decomposition of grape-sugar, is found. It dissolves the lime in the teeth, giving rise to diabetic dental caries. Frerichs found leucin, and Vulpian increase of albumin in albuminuria. Of foreign substances taken into the body, the following appear in the saliva:-Mercury, potassium, iodine, and bromine.

Saliva of New-Born Children.-In new-born children, the parotid alone contains ptyalin. The diastatic ferment seems to be developed in the sub-maxillary gland and pancreas, at the earliest after two months. Hence, it is not advisable to give starchy food to infants. No ptyalin has been found in the saliva of infants suffering from thrush (Oidium albicans-Zweifel). The diastatic action of saliva is not absolutely necessary for the suckling, feeding as it does upon milk. The mouth during the first two months is not moist, but at a later period saliva is copiously secreted (Korowin); after the first six months, the salivary glands increase considerably. The eruption of the teeth-owing to the irritation of the mucous membrane-produces a copious secretion of saliva.

148. PHYSIOLOGICAL ACTION OF SALIVA.-I. Diastatic Action.-The most important chemical action exerted by saliva in digestion is its diastatic or amylolytic action (Leuchs, 1831), i.e., the transformation of starch into dextrin and some form of sugar. This is due to the ptyalin-a hydrolytic ferment or enzym - which, even when it is present in very minute quantity, causes starch to take up water and become soluble, the ferment itself undergoing no essential change in the process. [Ptyalin belongs to the group of unorganised ferments $(\$ 250,9)$. Like all other ferments it acts only within a certain range of temperature, being most active about $40^{\circ} \mathrm{C}$. Its energy is permanently destroyed by boiling. Its acts best in a slightly alkaline or neutral medium.]

[Action on Starch. - Starch-grains consist of granulose or starch enclosed by coats of cellulose. Cellulose does not appear to be affected by saliva, so that saliva acts but slowly on raw unboiled starch. If the starch be boiled, so as to swell up the starch-grains, and rupture the cellulose envelopes, the amylolytic action takes place rapidly. If starch-paste or starch-mucilage, made by boiling starch in water, be acted upon by saliva, especially at the temperature of the body, the first physical change observable is the liquefaction of the paste, the mixture becoming more fluid and transparent. The change takes place in a few minutes. When the action is continued, important chemical changes occur.]

According to O'Sullivan, Musculus, and v. Mering, the diastatic ferment of saliva (and of the pancreas), by acting upon starch or glycogen, forms dextrin and maltose (both soluble in water). Several closely allied varieties of dextrin, distinguishable by their colour-reactions, seem to be produced (Brïcke). Erythrodextrin is formed first, it gives a red colour with iodine; then a reducing dextrin-achroodextrin, which gives no colour-reaction with iodine. The sugar formed by the action of ptyalin upon starch is maltose $\left(\mathrm{C}_{12} \mathrm{H}_{22} \mathrm{O}_{11}+\mathrm{H}_{2} \mathrm{O}\right)$, which is distinguished from grape-sugar $\left(\mathrm{C}_{12} \mathrm{H}_{24} \mathrm{O}_{12}\right)$ by containing one molecule less of water, which, however, it holds as a molecule of water of hydration. [Maltose also differs from grapesugar in its greater rotatory power on polarised light, the former $=+150^{\circ}$, the:latter $+56^{\circ}$, the ratio being $61: 100$; and in its smaller power of reducing cupric oxide. Thus, between the original starch and the final product, maltose, several intermediate bodies are formed. The starch gives a blue with iodine, but after it has been acted on for a time it gives a red or violet colour, indicating the presence of erythrodextrin, there being a simultaneous production of sugar; but ultimately no colour is obtained on adding iodine-achroodextrin, which gives no colour with iodine, maltose being formed. The presence of the maltose is easily determined by testing with Fehling's solution.] 
[Brown and Heron suggest that the final result of the transformation may be represented by the equation-

$$
\left.\underset{\text { Soluble starch. }}{10}\left(\mathrm{C}_{12} \mathrm{H}_{20} \mathrm{O}_{10}\right)+\underset{\text { Water. }}{8 \mathrm{H}_{2} \mathrm{O}}=\underset{\text { Maltose. }}{8}\left(\mathrm{C}_{12} \mathrm{H}_{22} \mathrm{O}_{11}\right)+\underset{\text { Achroodextrin. }}{2\left(\mathrm{C}_{12} \mathrm{H}_{20} \mathrm{O}_{10}\right.}\right)
$$

The ferment slowly changes maltose into grape-sugar or dextrose. This result may be brought about much more rapidly by boiling maltose with dilute sulphuric or hydrochloric acid.] Achroodextrin ultimately passes into maltose, and this again into dextrose ; the other form of dextrin does not seem to undergo this change (Seegen's Dystropodextrin). For the further changes that maltose undergoes in the intestine, see $\S 183$, II. 2.

[The formula of starch is usually expressed as $\mathrm{C}_{6} \mathrm{H}_{10} \mathrm{O}_{5}$, but the researches already mentioned, and those of Brown and Heron, make it probable that it is more complex, which we may provisionally represent by $n\left(\mathrm{C}_{12} \mathrm{H}_{20} \mathrm{O}_{10}\right)$. According to Musculus and Meyer, erythrodextrin is a mixture of dextrin and soluble starch.]

Preparation of Ptyalin. - (1) Like all other hydrolytic ferments, it is carried down with any copious precipitate that is produced in the fluid which contains it, and it can be isolated from the precipitate. The saliva is acidulated with phosphoric acid, lime-water is added until the reaction becomes alkaline, when a precipitate of the basic calcium phosphate occurs, which carries the ptyalin along with it. This precipitate is collected on a filter, washed with water, which dissolves the ptyalin, and from its watery solution it is precipitated by alcohol as a white powder. It is redissolved in water and reprecipitated, and is obtained pure (Cohnheim).

(2) Glycerine or v. Wittich's Method. - The salivary glands [rat] are chopped up, placed in absolute alcohol for twenty-four hours, taken out and dried, and afterwards placed in glycerine for several days, which extracts the ptyalin. It is precipitated by alcohol from the glycerine extract.

(3) William Roberts recommends the following solutions for extracting ferments from organs which contain them :-(1) A 3 to 4 per cent. solution of a mixture of 2 parts of boracic acid and 1 part borax. (2) Water, with 12 to 15 per cent. of alcohol. (3) 1 part chloroform to 200 of water.

Diastatic Action of Saliva. - (a) The diastatic or sugar-forming action is known by-(1) The disappearance of the starch. When a small quantity of starch is boiled with several hundred times its volume of water, starch-mucilage is obtained, which strikes a blue colour with iodine. If to a small quantity of this starch a sufficient amount of saliva be added, and the mixture kept for some time at the temperature of the body, the blue colour disappears. (2) The presence of sugar is proved directly by using the tests for sugar ( $\$ 149)$.

(b) The action takes place more slowly in the cold than at the temperature of the body-its action is enfeebled at $55^{\circ} \mathrm{C}$, , and destroyed at $75^{\circ} \mathrm{C}$. (Paschutin). The most favourable temperature is $35^{\circ}$ to $39^{\circ} \mathrm{C}$.

(c) The ptyalin itself does not seem to be changed during its action, but ptyalin which has been used for one experiment is less active when used the second time (Paschutin).

Ptyalin differs from diastase-the ferment in germinating grains-in so far that the latter first begins to act at $+66^{\circ}$ C. Ptyalin decomposes salicin into saligenin and grape-sugar (Frerichs and Städler).

(d) Saliva acts best in an exactly neutral medium, but it also acts in an alkaline and even in a slightly acid fluid; strong acidity prevents its action. The ptyalin is only active in the stomach when the acidity is due to organic acids (lactic or butyric), and not when free hydrochloric acid is present (van de Velde). In both cases, however, dextrin is formed. Ptyalin is destroyed by hydrochloric acid or digestion by pepsin (Chittenden and Griswold, Langley). Even butyric and lactic acids formed from grape-sugar in the stomach may prevent its action; but if the acidity be neutralised, the action is resumed $(\mathrm{Cl}$. Bernard).

(e) The addition of common salt, ammonium chloride, or sodium sulphate (4 per cent. solution), increases the activity of the ptyalin, and $\mathrm{CO}_{2}$, acetate of quinine, strychnia, morphia, curara, 0.025 per cent. sulphuric acid, have the same effect.

(f) Much alcohol and caustic potash destroy the ptyalin; long exposure to the air weakens its action, sodium carbonate and magnesium sulphate delay the action (Pfeiffer). Salicylic acid and much atropin arrest the formation of sugar.

(g) Ptyalin acts very feebly and very gradually upon raw starch, only after 2 to 3 hours (Schiff); while upon boiled starch it acts rapidly. [Hence the necessity for boiling thoroughly all starchy foods.]

$(h)$ The various kinds of starch are changed more or less rapidly according to the amount of cellulose which they contain; raw potato starch after 2 to 3 hours, raw maize starch after 2 to 3 minutes (Hammarsten); wheat starch more quickly than that of rice. When the starches are powdered and boiled, they are changed with equal rapidity.

(i) A mixture of the saliva from all the glands is more active than the saliva from any single gland (Jakubowitsch), while mucin is inactive. 
[Effect of Tea.-Tea has an intensely inhibitory effect on salivary digestion, which is due to the large quantity of tannin contained in the tea-leaf. Coffee and cocoa have only a slight effect on salivary digestion. The only way to mitigate the inhibitory effect of tea on salivary digestion is "not to sip the beverage with the meal, but to eat first and drink afterwards" (Roberts).]

II. Saliva dissolves those substances which are soluble in water; while the alkaline reaction enables it to dissolve some substances which are not soluble in water alone, but require the presence of an alkali.

III. Saliva moistens dry food and aids the formation of the "bolus," while by its mucin it helps the act of swallowing, the mucin being given off unchanged in the fæces. The ultimate fate of ptyalin is unknown.

[IV. Saliva also aids articulation, while according to Liebig it carries down into the stomach small quantities of O.]

[ $\mathrm{Y}$. It is necessary to the sense of taste to dissolve sapid substances, and bring them into relation with the end-organs of the nerves of taste.]

Saliva has no action on proteids or on fats.

The presence of a peptone-forming ferment has recently been detected in saliva (Hüfner, Munk, Kühne). [Perfectly healthy human saliva has no poisonous properties.]

149. TESTS FOR SUGAR.-1. Trommer's test depends upon the fact that, in alkaline solutions, sugar acts as a reducing agent; in this case a metallic oxide is changed into a suboxide. To the fluid to be investigated, add $\frac{1}{2}$ of its volume of a solution of caustic potash (soda), specific gravity $1 \cdot 25$, and a few drops of a weak solution of cupric sulphate, which causes at first a bluish precipitate, consisting of hydrated cupric oxide, but it is redissolved, giving a clear blue fluid, if sugar be present. Heat the upper stratum of the fluid, and a yellow or red ring of cuprous oxide is obtained, which indicates the presence of sagar; $2 \mathrm{CuO}-\mathrm{O}=\mathrm{Cu}_{2} \mathrm{O}$.

The solution of hydrated cupric oxide is caused by other organic substances; but the final stage, or the production of cuprous oxide, is obtained only with certain sugars-grape-, fruit-, and milk- (but not cane-) sugar. Fluids which are turbid must be previously filtered, and if they are highly coloured, they must be treated with basic lead acetate; the lead acetate is afterwards removed by the addition of sodium phosphate and subsequent filtration. If very small quantities of sugar are present along with compounds of ammonia, a yellow colour instead of a yellow precipitate may be obtained. In doing the test, care must be taken not to add too much cupric sulphate.

[2. Fehling's Solution is an alkaline solution of potassio-tartarate of copper. Boil a small quantity of the deep-blue-coloured Fehling's solution in a test-tube, and add to the boiling test a few drops of the fluid supposed to contain the sugar. If sugar be present, the copper solution is reduced, giving a yellow or reddish precipitate. The reason for boiling the test itself is, that the solution is apt to decompose when kept for some time, when it is precipitated by heat alone. This is one of the best and most reliable tests for the presence of sugar. In Pavy's modification of this test, ammonia is used instead of a caustic alkali ( $\$ 267)$.]

(3) Böttger's Test. - Alkaline bismuth oxide solution is best prepared, according to Nylander, as follows:-2 grms. bismuth subnitrate, 4 grms. potassic and sodic tartarate, $100 \mathrm{grms}$. caustic soda of 8 per cent. Add 1 c.c. to every 10 c.c. of the fluid to be investigated. When boiled for several minutes, the sugar causes the reduction and deposits a black precipitate of metallic bismuth. [According to Salkowski the urine of a person taking rhubarb gives the same reaction with this test.]

(4) Moore and Heller's Test. - Canstic potash or soda is added until the mixture is strongly alkaline; it is afterwards boiled. If sugar be present, a yellow, brown, or brownish-black coloration is obtained. If nitric acid be added, the odour of burned sugar (caramel) and formic acid is obtained.

(5) Mulder and Neubauer's Test. - A solution of indigo-carmine, rendered alkaline with sodic carbonate, is added to the sugar solution until a slight bluish colour is obtained. When the mixture is heated, the colour passes into purple, red, and yellow. When shaken with atmospheric air, the fluid again becomes blue.

Molisch's Test.-To 5 c.cm. of the fluid add 2 drops of a 17 per cent. alcoholic solution of $a$-naphthol, or a solution of thymol. Add 1 to $2 \mathrm{c.cm}$. of concentrated sulphuric acid, and shake the mixture. The presence of sugar colours the a-naphthol mixture deep violet, 
the thymol deep red. The subsequent addition of water causes a precipitate of similar colour, which is insoluble in concentrated hydrochloric acid. Albumin, casein, and peptone give the same reaction (Seegen), but the deposit on the addition of water is soluble in concentrated hydrochloric acid.

Other tests are described in $\$ 266$.

In all cases where albumin is present it must be removed-in urine by acidulating with acetic acid and boiling; in blood, by adding four times its volume of alcohol and afterwards filtering, while the alcohol is expelled by heat,

150. QUANTITATIVE ESTIMATION OF SUGAR.-I. By Fermentation. - In the glass vessel (fig. 161, a) a measured quantity (20 c.cm.) of the fluid (sugar) is placed along with some yeast, while $b$ contains concentrated sulphuric acid. The whole apparatus is then weighed. When exposed to a sufficient temperature $\left(10^{\circ}\right.$ to $40^{\circ} \mathrm{C}$.), the sugar splits into 2 molecules of alcohol and 2 of carbon dioxide,

$$
\begin{aligned}
\mathrm{C}_{6} \mathrm{H}_{12} \mathrm{O}_{6} & =2\left(\mathrm{C}_{2} \mathrm{H}_{6} \mathrm{O}\right)+\underset{2}{2\left(\mathrm{CO}_{2}\right),} \\
\text { Grape-sugar } & =2 \text { alcohol }+2 \text { carbon dioxide; }
\end{aligned}
$$

and in addition there are formed traces of glycerine and succinic acid. The $\mathrm{CO}_{2}$ escapes from $b$, and as it passes through the $\mathrm{H}_{2} \mathrm{SO}_{4}$, the $\mathrm{CO}_{2}$ yields to the latter its water. The apparatus is weighed after two days, when the reaction is ended, and the amount of sugar is calculated from the loss of weight in the $20 \mathrm{c.cm}$. of fluid. 100 parts of water-free sugar $=48.89$ parts $\mathrm{CO}_{2}$, or 100 parts $\mathrm{CO}_{2}$ correspond to 204.54 parts of sugar.

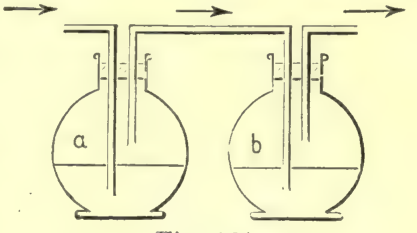

Fig. 161.

Apparatus for the quantitative estimation of sugar by fermentation.

II. Titration. - By means of Fehling's solution, which is made of such a strength that all the copper in 10 cubic centimetres of the solution is reduced by 0.05 grammes of grape-sugar (\$267).

III. Circumpolarisation, - The saccharimeter of Soleil-Ventzke may be used to determine the amount of sugar present. It may also be used for the quantitative estimation of albumin. Sugar rotates the ray of polarised light to the right and albumin to the left. The amount of rotation, or "specific rotatory power," is directly proportional to the amount of the rotating substance present in the solution, so that the amount of rotation of the ray indicates the amount of the substance present. By the term "specific rotatory power" is meant the degree of rotation which is produced by $1 \mathrm{grm}$, of the substance dissolved in $1 \mathrm{c.cm}$. of water, wheu examined in a layer 1 decimeter thick. For yellow light the specific rotation of grape-sugar is $+56^{\circ}$.

In fig. 162 the light from the lamp falls upon a crystal of calc-spar. Two Nicol's prisms are placed at $v$ and $s, v$ is movable round the axis of vision, while $s$ is fixed. In $m$ Soleil's double plate of quartz is placed, so that one-half of it rotates the ray of polarised light as much to the right as the other rotates it to the left. In $n$ the field of vision is covered by a plate of leftrotatory quartz. At $b c$ is the compensator, composed of two right-rotatory prisms of quartz, which can be displaced laterally by the nilled head, $g$, so that the polarised light passing through the apparatus can be made to pass through a thicker or thinner layer of quartz. When these right-rotatory prisms are placed in a certain position, the rotation of the left-rotatory quartz at $n$ is exactly neutralised. In this position the scale on the compensator has its nonius exactly at $o$, and both halves of the double plate at $m$ appear to have the same colour to the observer, who from $v$ looks through the telescope placed at $e$. Rotate the Nicol's prism at $v$ until a bright rose-coloured field is obtained. In this position the telescope must be so adjusted that the vertical line bounding the two halves shall be distinctly visible. The apparatus is now ready for use.

Fill a tube, 1 decimetre in length, with urine containing sugar or albumin, the urine being perfectly clear. The tube is placed between $m$ and $n$. By rotating the Nicol's prisms, $v$, the rose-colour is again obtained. The compensator at $g$ is then rotated until both halves of the field of vision have exactly the same colour. When this is obtained, read off on the scale the number of degrees the nonius is displaced to the right (sugar) or to the left (albumin) from zero. The number of degrees indicates directly the number of grammes of the rotating substance present in 100 c.c. of the fluid. If the fluid is very dark coloured, it must be decolourised by filtering it through animal charcoal (Seegen), [or the colouring matter may be preciptated by the addition of lead acetate.] If the sugary urine contains albumin, the latter must be removed by boiling and filtration. A turbidity not removed by filtration may be got rid of by adding a drop of acetic acid or several drops of sodic carbonate or milk of lime, and afterwards filtering. [One may also employ the apparatus of Mitscherlich, or the "half-shadow apparatus" of Laurent.]

151. MECHANISM OF THE DIGESTIVE APPARATUS-This embraces the following acts :- 
1. The introduction and mastication of the food; the movements of the tongue ; insalivation; formation of the bolus of food.

2. Deglutition.

3. The movements of the stomach, small and large intestine.

4. The excretion of frcal matters.

152. INTRODUCTION OF THE FOOD.-Fluids are taken into the mouth in three ways :-(1) By suction, the lips are applied air-tight to the vessel containing the fluid, while the tongue is retracted (the lower jaw being often depressed) and

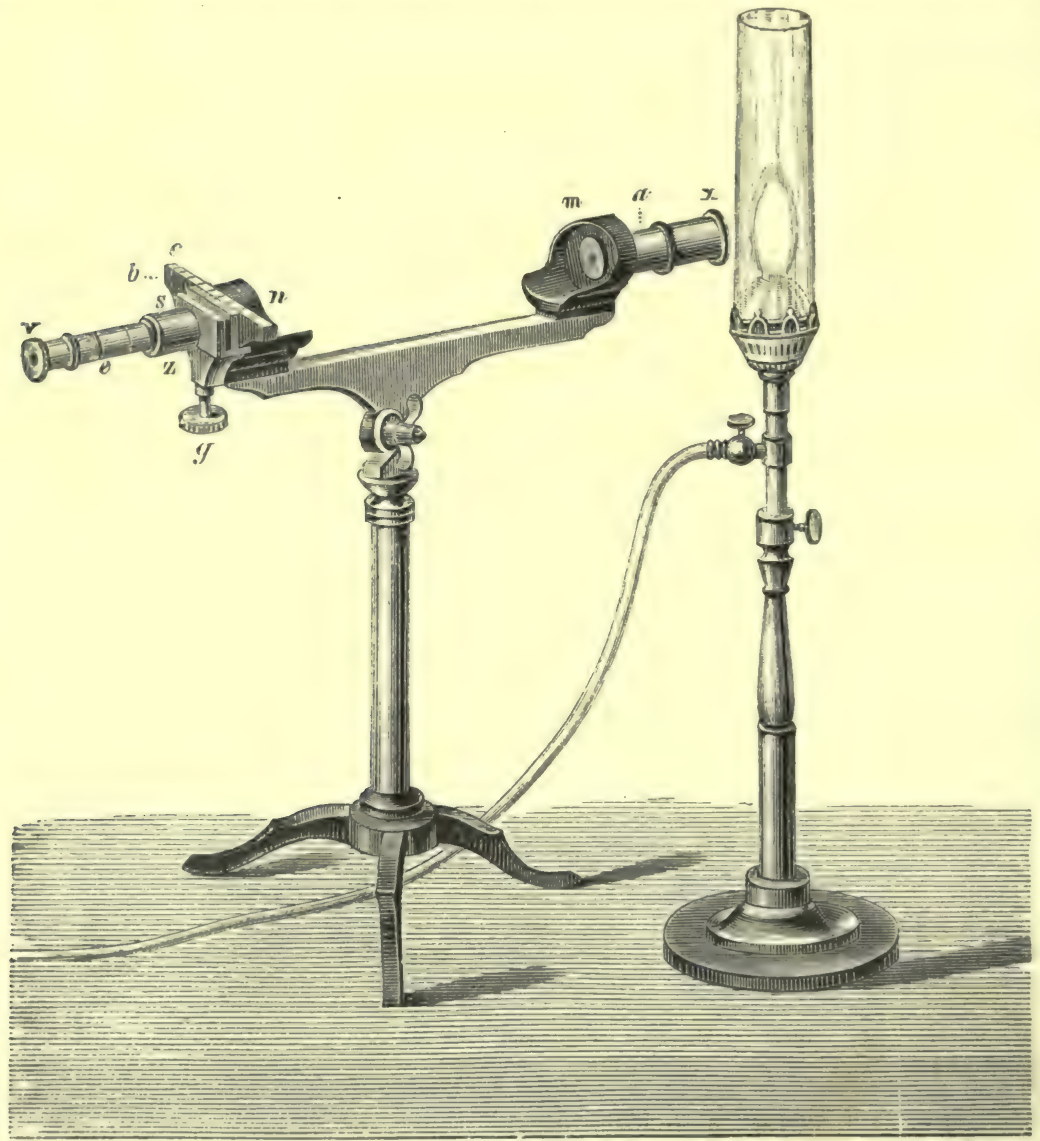

Fig. 162.

Soleil-Ventzke's polarisation apparatus.

acts like the piston in a suction-pump, thus causing the fluid to enter the mouth. Herz found that the negative pressure caused by an infant while sucking $=3$ to 10 $\mathrm{mm}$. Hg. (2) The fluid is lapped when it is brought into direct contact with the lips, and is raised by aspiration and mixed with air so as to produce a characteristic sound in the mouth. (3) Fluid may be poured into the mouth, and as a general rule the lips are applied closely to the vessel containing the fluid.

Solids, when they consist of small particles, are licked up with the lips, aided by the movements of the tongue. In the case of large masses, a part is bitten off with 
the incisor teeth, and is afterwards brought under the action of the molar teeth by means of the lips, cheeks; and tongue.

153. MASTICATION. - The articulation of the jaw is provided with an interarticular cartilage - the meniscus - which prevents direct pressure being made upon the articular surface when the jaws are energetically closed, and which also divides the joint into two cavities, one lying over the other. The capsule is so lax that, in addition to the raising and depressing of the lower jaw, it permits of the lower jaw being displaced forwards, whereby the meniscus moves with it, and covers the articular surface.

The process of mastication embraces:- $(a)$ The elevation of the jaw, accomplished by the combined action of the Temporal, Masseter, and Internal Pterygoid Muscles. If the lower jaw was previously so far depressed that its articular surface rested upon the tubercle, it now passes backwards upon the articular surface.

(b) The depression of the lower jaw is caused by its own weight, aided by the action of the anterior bellies of the Digastrics, the Mylo- and Genio-hyoid and Platysma. The muscles act especially during forcible opening of the mouth. The necessary fixation of the hyoid bone is obtained through the action of the Omo- and Sterno-hyoid, and by the Sterno-thyroid and Thyro-hyoid.

When the articular surface of the lower jaw passes forwards on to the tubercle, the External Pterygoids actively aid in producing this (Bérard).

(c) Displacement of the Articular Surfaces.-During rest, when the mouth is closed, the incisor teeth of the lower jaw are within the arch of the upper incisors. When in this position the jaw is protruded by the External Pterygoids, whereby the articular surface passes on to the tubercle (and, therefore, downwards), while the lateral teeth are thereby separated from each other. The jaw is retracted by the Internal Pterygoids without any aid from the posterior fibres of the Temporals. When one articular surface is carried forwards, the jaw is protruded and retracted by the External and Internal Pterygoid of the same side. At the same time, there is a transverse movement, whereby the back teeth of the protruded side are separated from each other.

During mastication, the individual movements of the lower jaw are variously combined, and especially with lateral grinding movements, while the food to be masticated is kept from passing outwards by the action of the muscles of the lips (Orbicularis oris) and the Buccinators, while the tongue continually pushes the particles between the molar teeth. The energy of the muscles of mastication is regulated by the sensibility of the teeth, and the muscular sensibility of the muscles of mastication, as well as by the general sensibility of the mucous membrane of the mouth and lips. At the same time, the mass is mixed with saliva, the divided particles cohere, and are formed into a mass or bolus, of a long, oval shape, by the muscles of the tongue. The bolus then rests on the back of the tongue, ready to be swallowed.

Nerves of Mastication. - The muscles of mastication receive their motor nerves from the third branch of the trigeminus, the mylo-hyoid and the anterior belly of the digastric being supplied from the same source. The genio-, omo-, and sterno-hyoid, sterno-thyroid, and thyro-hyoid are supplied by the hypoglossal, while the facial supplies the posterior belly of the digastric, the stylo-hyoid, the platysma, the buccinator, and the muscles of the lips. The general centre for the muscles of mastication lies in the medulla oblongata ( $\$ 367$ ).

When the mouth is closed, the jaws are kept in contact by the pressure of the air, as the cavity of the mouth is rendered free from air, and the entrance of air is prevented anteriorly by the lips, and posteriorly by the soft palate. The pressure exerted by the air is from 2 to 4 nim. Hg. (Metzger and Donders).

[Effect on the Circulation. - Marey found that mastication trebled the velocity of the bloodcurrent in the carotid (horse), while François Frank observed that the circulation of the brain (in man) is increased; hence it is evident that mastication implies an increased supply of blood to the nerve-centres.]

154. STRUCTURE AND DEVELOPMENT OF THE TEETH. -A tooth is just a papilla of the mucous membrane of the gum, which has undergone a characteristic development. In its simplest form, as in the teeth of the lamprey, the connective-tissue basis of the papilla is covered with many layers of corneous epithelium. In human teeth, part of the papilla is transformed into a layer of calcified dentine, while the epithelium of the papilla produces the enamel, the fang of the tooth being covered by a thin accessory layer of bone, the crusta petrosa or cement. 
The dentine or ivory which surrounds the pulp-cavity and the canal of the fang (fig. 163) is

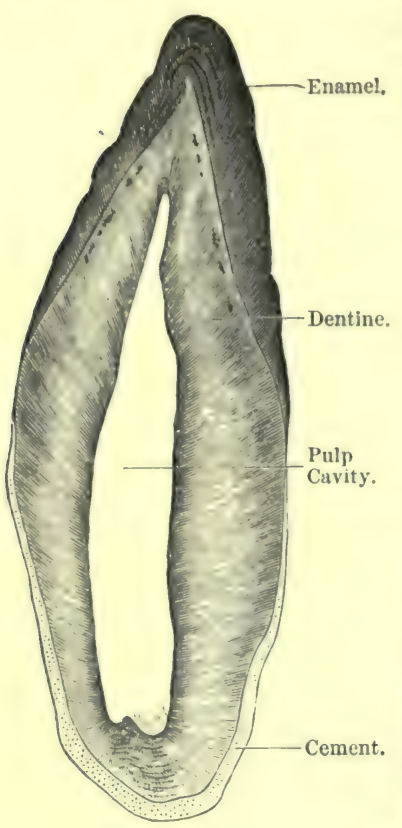

Fig, 163, very firm, elastic, and brittle. Dentine, like the matrix of bone, when treated in a certain way, presents a fibrillar structure. It is permeated by innumerable long, tortuous,

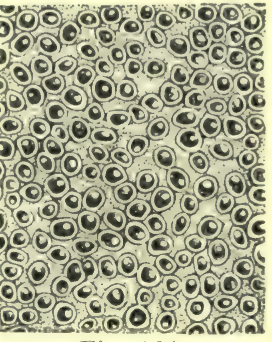

Fig, 164.

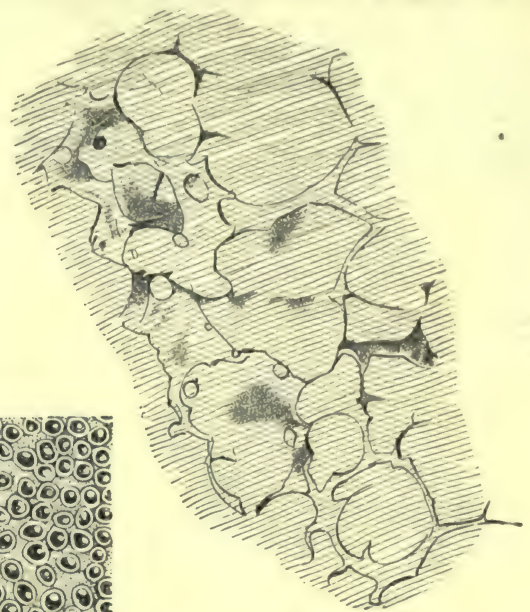

Fig. 165 .

Fig. 163. - Longitudinal section of an incisor tooth. Fig. 164.- Transverse section of dentine. The light rings are the walls of the dentinal tubules; the dark centres with the light points are the fibres of Tomes lying in the tubules. Fig. 165. - Interglobular spaces in dentine.

wavy tubes-the dentinal tubules-each of which communicates with the pulp-cavity by means of a fine opening, and passes more or less horizontally outwards as far as the outer layers of the

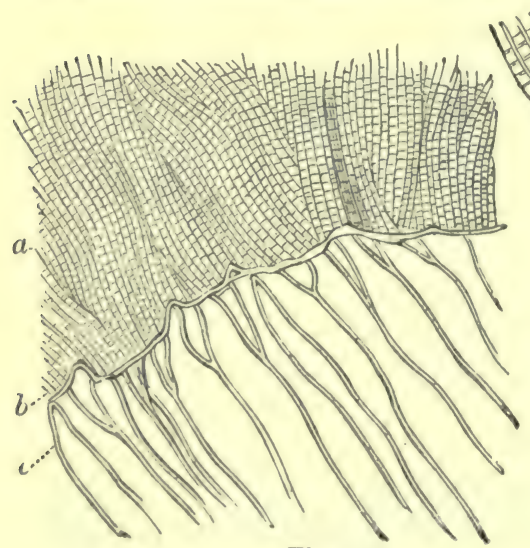

Fig. 166. dentine. The tubules are bounded by an extremely resistant, thin, cuticular membrane, which strongly resists the action of chemical reagents. These

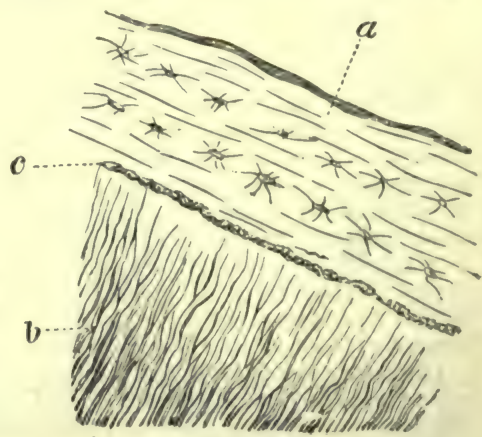

Fig. 167.

Fig. 166. - Section of a tooth between the dentine and enamel. $a$, enamel; $c$, dentinal tubules ; $B$, enamel prisms highly magnified; $C$, transverse sections of enamel prisms. Fig. 167.Transverse section of the fang. $a$, cement with bone-corpuscles; $b$, dentine with tubules; $c$, boundary between both,

tubules are filled completely by soft fibres, the "fibres of Tomes," which are merely greatly elongated and branched processes of the odontoblasts of the pulp. 
The dentinal tubules, as well as the fibres of Tomes, anastomose throughout their entire extent by means of fine processes. As the fibres approach the enamel, which they do not penetrate, some of them bend on themselves, and form a loop (fig. 166, c), whilst others pass into the "interglobular spaces" (fig. 165) which are so abundant in the outer part of the dentine. The interglobular spaces are small spaces bounded by curved surfaces. Certain curved lines, "Schreger's lines," may be detected with the naked eye in the dentine (e.g., of the elephant's tusk) running parallel with the contour of the tooth. They are caused by the fact that at these parts all the chief curves in the dentinal tubules follow a similar course.

The enamel, the hardest substance in the body (resembling apatite), covers the crown of the teeth. It consists of hexagonal flattened prisms arranged side by side like a palisade (fig. 166, $B$ and $C$ ). They are 3 to $5 \mu$ ( $\frac{1}{5000}$ inch) broad, not quite uniform in thickness, curved slightly in different directions, and, owing to inequalities of thickness, they exhibit transverse markings. They are elongated, calcified, cylindrical, epithelial cells. Retzius described dark brown lines running parallel with the outer boundary of the enamel, due to the presence of pigment (fig. 163). The fully formed enamel is negatively doubly refractive and uniaxial, whilethe developing enamel is positively doubly refractive (Hoppe-Seyler).

The cuticula or Nasmyth's membrane covers the free surface of the enamel as a completely structureless membrane 1 to $2 \mu$ thick, but in quite young teeth it exhibits an epithelial structure, and is derived from the outer epithelial layer of the enamel organ.

The cement or crusta petrosa is a thin layer of bone covering the fang (fig. 167, $a$ ). The bone lacunæ communicate directly with the dental tubules of the fang. Haversian canals and lamellæ are only found where the layer of cement is thick, and the former may communicate with the pulp-cavity. Very thin layers of cement may be devoid of bone-corpuscles. Sharpey's fibres occur in the cement of the dog's tooth; while in the horse's tooth single bone-corpuscles are developed by a capsule. In the periodontal membrane, which is just the periosteum of the alveolus, coils of blood-vessels similar to the renal glomeruli occur. They anastomose with each other, and are surrounded by a delicate capsule of connective-tissue.

Chemistry of a Tooth. - The teeth consist of a gelatine-yielding matrix infiltrated with cal cium phosphate and carbonate (like bone). (1) The dentine contains-organic matter, 27·70 ; calcium phosphate and carbonate, 72.06 ; magnesium phosphate, 0.75 ; with traces of iron, fluorine, and sulphuric acid.

(2) The enamel contains an organic proteid matrix allied to the substance of epithelium. It consists of 3.60 organic matter and 96.00 of calcium phosphate and carbonate, 1.05 magnesium. phosphate, with traces of calcium fluoride and an insoluble chlorine compound.

(3) The cement is identical with bone.

The pulp in a fully-grown tooth represents the remainder of the dental papilla around which the dentine was deposited. It consists of a very vascular indistinctly fibrillar connective-tissue, laden with cells. The layers of cells, resembling epithelium, which lie in direct contact with the dentine, are called odontoblasts, i.e., those cells which build up the dentine. These cells send off long branched processes into the dentinal tubules, whilst their nucleated bodies lie on the surface of the pulp, and form connections by processes with other cells of the pulp and with neighbouring odontoblasts. Numerous non-medullated nerve-fibres (sensory from the trigeminus), whose mode of termination is unknown, occur in the pulp.

The periosteum or periodontal membrane of the fang is, at the same time, the alveolar periosteum, and consists of connective-tissue with elastic fibres and many nerves.

The gums are devoid of mucous glands, very vascular, and often provided with long vascular papillæ, which are sometimes compound.

Development of a Tooth. - It begins at the end of the second month of foetal life. Along the whole length of the foetal gum is a thick projecting bridge (fig. 168, $\alpha$ ) composed of many layers of epithelium. A depression, the dental groove, also filled with epithelium, occurs in the gum, and runs along under the ridge. The dental groove becomes deeper throughout its entire length, and on transverse section presents the appearance of a dilated flask $(b)$, while at the same time it is filled with elongated epithelial cells, which form the "enamel organ." A conical papilla, the "dentine germ," grows up from the mucous tissue, of which the gum consists, towards the enamel organ (fig. 169, c), so that the apex of the papilla comes to have the enamel organ resting. upon it like a double cap. Afterwards, owing to the development of connective-tissue, the parts of the enamel organ lying between and uniting the individual dentine germs, disappear, and gradually the connective-tissue forms a tooth-sac enclosing the papilla and its enamel organ $(d)$.

Those epithelial cells (fig. 169,3) of the enamel organ, which lie next the top of the papilla, are cylindrical, and become calcified to form enamel prisms. The layer of cells of the double cap, which is directed towards the tooth-sac (1), becomes flattened, fuses, undergoes a horny transformation, and becomes the cuticula, whilst the cells which lie between both layers undergo an intermediate metamorphosis, so that they come to resemble the branched stellate cells of the mucous tissue (2), and gradually disappear altogether.

The dentine is formed in the most superficial layer of the projecting connective-tissue of the dental papilla, owing to the calcification of the continuous layer of odontoblasts which occur there (figs. 169 and $170, k$ ). During the process, fibres or branches of these cells are left 
unaffected, and remain as the fibres of Tomes. Exactly the same process occurs as in the formation of bone, the odontoblasts forming around themselves a calcified matrix. The cement is formed from the soft connective-tissue of the dental alveolus.

Dentition.-During the development of the first temporary or milk-teeth a special enamel organ (fig. $169, c$ ) is formed near these, but it does not undergo development until the milk. teeth are shed; even the papilla is wanting at first. When the permanent tooth begins to

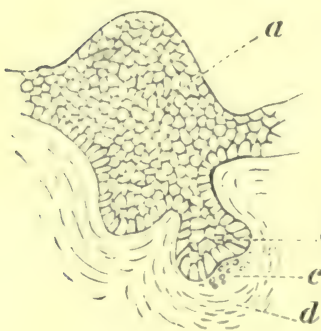

Fig. 168 .

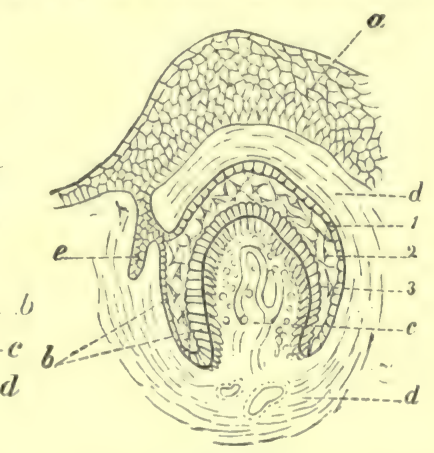

Fig. 169.

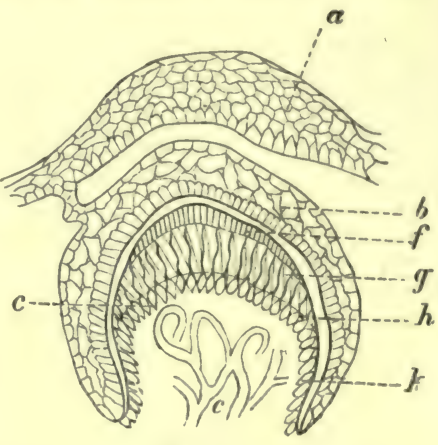

Fig. 170 .

Fig. 168. - $a$, Dental ridge ; $b$, enamel organ ; $c$, beginning of the dentine germ; $d$, first indication of the tooth-sac. Fig. 169. - a, Dental ridge ; $b$, enamel organ with (1) outer epithelium, (2) middle stellate layer, (3) enamel prism-cell layer; $c$, dentine germ with blood-vessels and the long osteoblasts on the surface; $d$, tooth-sac; $e$, secondary enamel germ. Fig. $170 .-a$, Dental ridge ; $b$, enamel organ ; $c$, dentine germ ; $f$, enamel ; $g$, dentine; $h$, interval between enamel organ and the position of the tooth ; $k$, layer of odontoblasts.

develop, it opens into the alveolar wall of the milk-teeth from below. The tissue of this dental sac causes erosion, or eating away of the fang and even of the body of the milk-teeth, without its blood-vessels undergoing atrophy. The chief agents in the absorption are the amœboid cells of the granulation tissue. [Multinuclear giant-cells also erode the fangs of the teeth.]

Eruption of the Milk-Teeth. - The following is the order in which the twenty milk-teeth cut the gum, i.e., from the seventh month to the second year:-Lower central incisors, upper central incisors, upper lateral incisors, lower lateral incisors, first molar, canine, the second molars.

[The figures indicate in months the period of eruption of each tooth.]

\begin{tabular}{|c|c|c|c|c|}
\hline Molars. & Canines. & Incisors. & Canines. & Molars. \\
\hline 2412 & 18 & 9779 & 18 & 2412 \\
\hline
\end{tabular}

[The permanent teeth succeed the milk-teeth, the process beginning about the scventh year. Ten teeth in each jaw take the place of the milk-teeth, while six teeth appear further back in ench jaw. Thus the total number of permanent teeth is thirty-two. As the sacs, from which the permanent teeth are developed, are formed before birth, they merely undergo the same process of development as the temporary teeth, only at a much later period. The last of the permanent molars-the wisdom-tooth - may not cut the jaw until the seventeenth to the twentyfifth year. At the sixth year the jaw contains the largest number of teeth, as all the temporary teeth are present, and, in addition, the crowns of all the permanent teeth, except the wisdomteeth, making forty-eight in all.]

[Eruption of Permanent Teeth. - The age at which each tooth cuts the gum is given in years in the following table:-

\begin{tabular}{|c|c|c|c|c|c|c|c|}
\hline Molars. & Bicuspld. & Canines. & Incisors. & Canines. & Bicuspid. & & Molars. \\
\hline $\begin{array}{lll}17 & 12 & \\
\text { to } & \text { to } & 6 \\
25 & 13 & \end{array}$ & 109 & 11 to 12 & 8778 & 11 to 12 & $\begin{array}{ll}9 & 10\end{array}$ & 6 & $\begin{array}{ll}12 & 17 \\
\text { to } & \text { to } \\
13 & 25\end{array}$ \\
\hline
\end{tabular}

[Action of Drugs on the Teeth. - All the conditions for putrefaction are present in the mouth; and wben putrefaction occurs, the products (often acid) attack the dentine and hasten its decay. Hence, the necessity for thorough daily cleansing of the teeth and mouth. The teeth may be cleaned by means of a soft tooth-brush and water, with or without the use of any of the 
numerous dentifrices, such as powdered chalk or charcoal. Astringents such as catechu and areca-nut are sometimes used. Mineral acids attack the teeth, and ought, when taken, to be sucked through a tube.]

155. MOVEMENTS OF THE TONGUE. - The tongue, being a muscular organ, and extremely mobile, plays an important part in the process of mastication :-(1) It keeps the food from passing from between the molar teeth. (2) It forms into a bolus the finely-divided food after it is mixed with saliva. (3) When the tongue is raised, the bolus lying on its dorsum is pushed backwards into the pharynx and osophagus.

The course of the fibres is threefold-longitudinally, from base to tip ; transversely, the fibres for the most part proceeding outwards from the verticallyplaced septum linguæ; vertically, from below upwards. Some of the muscles are confined to the tongue (intrinsic), while others (extrinsic) are attached beyond it to the hyoid bone, lower jaw, the styloid process, and the palate.

Microscopically, the fibres are transversely striated, with a delicate sarcolemma, and very often they are branched where they are inserted intot he mucous membrane. The muscular bundles cross each other in various directions, and in the interspaces fat-cells and glands occur.

Changes in form and position :-

(1) Shortening and broadening by the longitudinal muscle, aided by the hyoglossus.

(2) Elongation and narrowing, by the transversus linguæ.

(3) The dorsum is rendered concave by the transversus and the simultaneous action of the median vertical fibres.

(4) Arching of the dorsum:-(a) Transversely, by the lowest transverse bundles ; (b) longitudinally, by the lowest longitudinal muscles.

(5) Protrusion, by the genio-glossus, while at the same time the tongue usually becomes narrower and longer (2).

(6) Retraction, by the hyo-glossus and stylo-glossus, and (1) usually occurring at the same time.

(7) Depression into the floor of the mouth, by the hyo-glossus. The floor of the mouth may be made deeper by depressing the hyoid bone.

(8) Elevation of the tongue towards the palate:-(a) At the tip by the anterior part of the longitudinal fibres; $(b)$ in the middle by elevating the entire hyoid bone by the mylo-hyoid ( $N$. trigeminus); $(c)$ at the root by the stylo-glossus and palatoglossus, as well as indirectly by the stylo-hyoid ( $N$. facialis).

(9) Lateral movements, the tip of the tongue passing to the right or left ; these are caused by the longitudinal fibres of one side.

Motor Nerves.-The motor nerve of the tongue is the hypoglossal. When this nerve is divided or paralysed on one side, the tip of the tongue lying in the floor of the mouth is directed towards the sound side, because the tonus of the non-paralysed longitudinal fibres shortens the sound side slightly. If the tongue be protruded, however, the tip passes towards the paralysed side. This arises from the direction of the genio-glossus (from the middle downwards and outwards), and the tongue follows the direction of its action. The tongues of animals which have been killed exhibit fibrillar contractions of the muscles, sometimes lasting for a whole day. [Stirling has frequently found nerve-ganglia in the nerves of the tongue].

156. DEGLUTITION.-The onward movements of the contents of the digestive canal are effected by a special kind of action whereby the tube or canal contracts upon its contents, and as this contraction proceeds along the tube, the contents are thereby carried along. This is the "peristaltic movement," or peristalsis.

In the first and most complicated part of the act of deglutition, we distinguish in order the following individual movements :-

(1) The aperture of the mouth is closed by the orbicularis oris ( $N$. facialis).

(2) The jaws are pressed against each other by the muscles of mastication ( $N$. trigeminus), while at the same time the lower jaw affords a fixed point for the action of the muscles attached to it and the hyoid bone. 
(3) The tip, middle, and root of the tongue, one after the other, are pressed against the hard palate, whereby the contents of the mouth are propelled towards the pharynx.

(4) When the bolus has passed the anterior palatine arch (the mucus of the tonsillar glands making it slippery again), it is prevented from returning to the mouth by the palato-glossi muscles which lie in the anterior pillars of the fauces, coming together like two side-screens or curtains, meeting the raised dorsum of the tongue (Stylo-glossus).

(5) The morsel is now behind the anterior palatine arch and the root of the tongue, and has reached the pharynx, where it is subjected to the successive action of the three pharyngeal constrictor muscles which propel it onwards. The action of the superior constrictor of the pharynx is always combined with a horizontal elevation (Levator veli palatini; $N$. facialis) and tension (Tensor veli palatini; $N$. trigeminus, otic ganglion) of the soft palate. The upper constrictor presses (through the pterygo-pharyngeus) the posterior and lateral walls of the pharynx tightly against the posterior margin of the horizontal, tense, soft palate, whereby the margins of the posterior palatine arches (palato-pharyngeus) are approximated. The pharyngo-nasal cavity is thus completely shut off, so that the bolus cannot be pressed backwards into the nasal cavity.

In persons with congenital or acquired defects of the soft palate, or cleft-palate, during swallowing, food passes into the nose.

(6) Falk and Kronecker assert, that by the energetic contraction of the muscles which diminish the cavity of the mouth, especially the mylo-hyoid, the bolus is "projected" through the pharynx and cesophagus. [They even assert that the bolus reaches the cardia before even the musculature of the pharynx or osophagus can contract, and further that the pharyngeal muscles of a dog may be divided without making swallowing impossible.] If we make a series of efforts to swallow, one after the other, as in drinking, contraction of the pharynx and osophagus takes place only after the last effort. Thus each new act of deglutition in the mouth inhibits (by stimulation of the glosso-pharyngeal nerve) the movements in the parts of the esophageal tube situated below it.

(7) The bolus is propelled onwards by the successive contraction of the upper, middle, and lower constrictors of the pharynx until it passes into the œesophagus. At the same time the entrance to the glottis is closed, else the morsel would pass into the larynx, or, as is generally said, would "pass the wrong way."

Duration. -According to Meltzer and Kronecker, the duration of deglutition in the mouth is $0.3 \mathrm{sec}$; then the constrictors of the pharynx contract $0.9 \mathrm{sec}$; afterwards, the upper part of the oesophagus; then after $1.8 \mathrm{sec}$. the middle; and after another $3 \mathrm{sec}$. the lower constrictor. The closure of the cardia, after the entrance of the bolus into the stomach, is the final act in the total series of movements.

Sounds during Deglutition. - If the region of the stomach be auscultated during the act of swallowing, two sounds may be heard; the first one is produced when the bolus is projected into the stomach; the second occurs when the peristalsis, which takes place at the end of swallow. ing, squeezes the contents of the osophagus through the cardia (Meltzer, Zenker, Ewald). [The latter occurs 4-5 mins. afterwards. In man, when water alone is swallowed, there is no sound, but when it is mixed with air there is, and it is generally heard because air is usually swallowed with the food or drink (Quincke).]

The closure of the glottis is effected in the following manner:-(a) The whole larynx - the lower jaw being fixed-is raised upwards and forwards, while at the same time the root of the tongue hangs over it. The byoid bone is raised forwards and upwards by the genio-hyoid, anterior belly of the digastric, and mylo-hyoid; the larynx is approximated close to the hyoid bone by the thyro-hyoid. (b) When the larynx is raised, so that it comes to lie below the overhanging root of the tongue, the epiglottis is pressed downwards over the entrance to the glottis, and the bolus passes over it. The epiglottis is also pulled down by the special muscular 
fibres of the reflector epiglottidis and aryepiglotticus. (c) The closure of the glottis by the constrictors of the larynx also prevents the entrance of substances into the larynx (\$ 313, II. 2).

Injury to the Epiglottis. - Intentional injury of the epiglottis in animals, or its destruction in man, may cause fluids to "go the wrong way," $i . e$, into the glottis, whilst solid food can be swallowed without disturbance. In dogs, coloured fluids placed on the root of the tongue have been observed to pass directly into the pharynx without coming into contact with it, so as to tinge the upper surface of the epiglottis (Magendie). [The basis of the epiglottis is yellow elastic cartilage, so that it shows no tendency to ossify, and always retains its elasticity (§313).

In order that the descending bolus may be prevented from carrying the pharynx with it, the stylo-pharyngeus, salpingo-pharyngeus, and baseo-pharyngeus contract upwards when the constrictors act.

Nervous Mechanism.-Deglutition is voluntary only during the time the bolus is in the mouth. When the food passes through the palatine arch into the gullet the act becomes involuntary, and is, in fact, a well-regulated reflex action. When there is no bolus to be swallowed, voluntary movements of deglutition can be accomplished only within the mouth; the pharynx only takes up the movement, provided a bolus (food or saliva) mechanically excites the reflex act. The afferent nerves, which, when mechanically stimulated, excite the involuntary act of deglutition, are, the palatine branches of the trigeminus (from the spheno-palatine ganglion) and the pharyngeal branches of the vagus. The centre for the nerves concerned (for the striped muscles) lies in the superior olives of the medulla oblongata. Swallowing can be carried out when a person is unconscious, or after destruction of the cerebrum, cerebellum, and pons $(\$ 367,6)$. [Even in the deep coma of alcoholism, the tube of a stomach-pump is carried into the stomach reflexly, provided the surgeon passes it back into the pharynx.] The nerves of the pharynx are derived from the pharyngeal plexus, which receives branches from the vagus, glossopharyngeal, and sympathetic $(\S 352,4)$.

Within the œsophagus, whose stratified epithelium is moistened with the mucus derived from the mucous glands in its walls, the downward movement is involuntary, and depends upon a complicated reflex movement discharged from the centre for deglutition. There is a peristaltic movement of the outer longitudinal and inner circular non-striped muscular fibres.

In the upper part of the esophagus, which contains striped muscular fibres, the peristalsis takes place more quickly than in the lower part. The movements of the oesophagus never occur independently, but are always the continuation of a foregoing act of deglutition. If food be introduced into the osophagus through a hole in its wall, there it lies; and it is only carried downwards when a movement to swallow is made. The peristalsis extends along the whole length of the osophagus, even when it is ligatured or when a part of it is removed (Mosso). If a dog be allowed to swallow a piece of flesh tied to a string, so that the flesh goes halfway down the œsophagus, and if the flesh be withdrawn, the peristalsis still passes downwards (C. Ludwig and Wild).

The motor nerve of the œsophagus is the vagus (not the accessory fibres) [œsophageal, whose branches have numerous small ganglia in their course]. After it is divided, the food lodges in the lower part of the oesophagus. Very large and very small masses are swallowed with more difficulty than those of moderate size. Dogs can swallow an olive-shaped body weighted with a counterpoise of 450 grammes (Mosso). When the thorax is greatly distended, as in Miiller's experiment, or greatly diminished, as in Valsalva's experiment ( $\$ 60)$, deglutition is rendered more difficult.

Goltz's Experiments. - The œsophagus and stomach of-the frog become more excitable, i.e., the excitability of the ganglionic plexuses in their walls is increased, when the brain and spinal cord or both vagi are destroyed. These organs contract energetically after slight stimulation, while frogs, whose central nervous system is intact, swallow fluids simply by peristalsis. Females, and sometimes men also, suffering from hysteria, not unfrequently have similar spasmodic contractions of the œesophageal region (globus hystericus). After section of both vagi in the dog, Schiff observed spasmodic contraction of the œesophagus.

Effect on Circulation. - Every time one swallows, the heart's action is accelerated, the bloodpressure falls, the necessity for respiration diminishes, while many movements (labour pains, erection) are inhibited. These effects are brought about reflexly (Kronccker and Meltzer, § 369). 
[Structure of the Esophagus. - The walls of the cesophagus are composed of four coatsmucous, sub-mucous, muscular, and fibrous (fig, 171).

(1) The mucous coat is firm, and is thrown into longitudinal folds, which disappear when the tube is distended. It is lined by several layers of stratified squamous epithelium. The membrane itself is composed, especially at its inner part, of dense fibrous tissue, which projects, in the form of papillø, into the stratified epithelium. The papillæ are present in the child, but are largest in old people. At its outer part is a continuous longitudinal layer of non-striped muscle, the muscularis mucosø. The layer consists of small bundles of non-striped muscle separate from each other.

(2) The sub-mucous coat is thicker than the foregoing, and consists of loose connectivetissue, with the acini of small mucous glands imbedded in it. The ducts pierce the muscularis mucose to open on the inner surface of the tube.

(3) The muscular coat consists of an inner, thicker, circular, and an outer, thinner, longitudinal layer of non-striped muscle, commencing on a level with the cricoid cartilage. In man the upper third of the gullet consists of striped muscular fibres. (4) Outside the nuscular coat

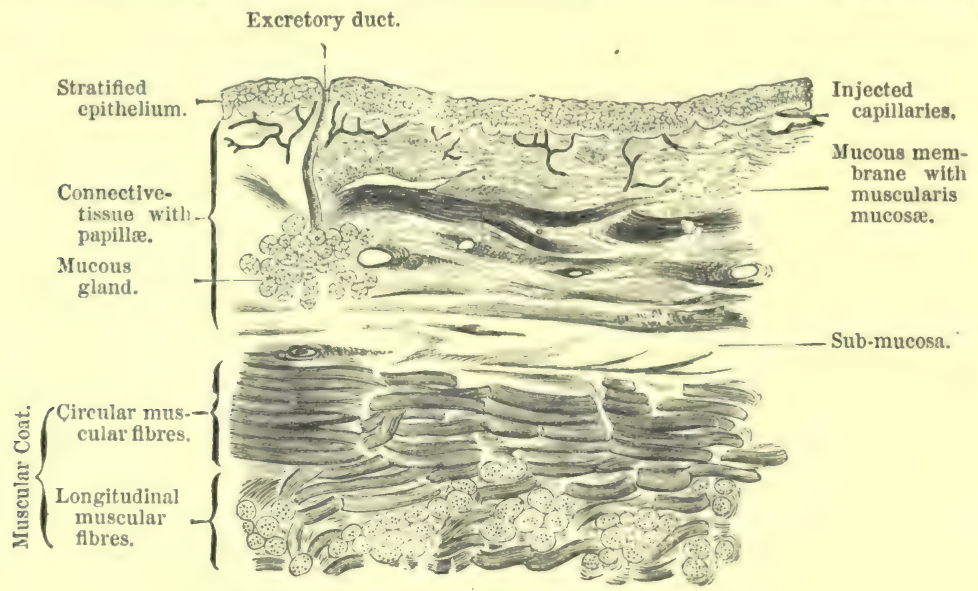

Fig. 171 .

Transverse section of part of the nesophagus.

is a layer of fibrous tissue with elastic fibres. The structure of the muscular coat of the cesophagus varies much in different animals. In the rabbit, in the first quarter of its length, it has two layers, but below this there are three layers, i.e., a circular between an outer and an inner longitudinal layer, while the non-striped fibres are confined to the lowest quarter of the tube.]

[Nerve-Plexuses. - As in the intestine, there are two plexuses of nerves with ganglia; one in the sub-mucous coat (Meissner's) and the other between the two museular coats (Auerbach's), which are continuous with those in the stomach and intestine. Blood-vessels and numerous lymphatics lie in the mucous and sub-mucous coats.]

157. MOVEMENTS OF THE STOMACH.-Position.-When the stomach is empty, the great curvature is directed downwards and the lesser upwards ; but when the organ is full, it rotates on an axis running horizontally through the pylorus and cardia, so that the great curvature appears to be directed to the front and the lesser backwards.

Arrangement of the Muscular Fibres. - The non-striped muscular fibres of the stomach are arranged in three directions or layers, an outer longitudinal continuous with those of the œesophagus. This layer is best developed along the curvatures, especially the lesser. At the pylorus the fibres form a thick layer, and become continuous with the longitudinal fibres of the duodenum. The circular fibres form a conplete layer; at the pylorus they are more numerous, and constitute the sphincter-muscle or pyloric valve; whilst at the cardia (inlet), such a muscular ring is absent. The innermost oblique or diagonal layer is complete.

The Movements of the Stomach are of two kinds:-(1) The rotatory or churning movements, whereby the parts of the wall of the stomach in contact with the 
contents glide to and fro with a slow rubbing movement. Such movements seem to occur periodically, every period lasting several minutes (Beaumont). By these movements the contents are moistened with the gastric juice, while the masses of food are partly broken down. The formation of hair-balls in the stomach of dogs and oxen indicates that such rotatory movements of the contents of the stomach take place. (2) The other kind of movement consists in a periodically occurring peristalsis, whereby, as with a push, the first dissolved portions of the contents of the stomach are forced into the duodenum. They begin after a quarter of an hour, and recur until about five hours after a meal. This peristalsis is most pronounced towards the pyloric end, and the muscles of the pyloric sphincter relax to allow the contents to pass into the duodenum. According to Rüdinger, the longitudinal muscular fibres, when they contract, especially when the pyloric end is filled, may act so as to dilate the pylorus.

Gizzard.-The strongly muscular walls of the stomach of grain-eating birds effect a trituration of the food. The older physiologists found that glass balls and lead tubes, which could be compressed only by a weight of 40 kilos., were broken or compressed in the stomach of a turkey.

Influence of Nerves.- [The stomach is supplied by the vagi and by the sympathetic, the right vagus being distributed to the posterior surface, and the left to the anterior surface, of the organ.] Auerbach's ganglionic plexus of nerve-fibres and nerve-cells, which lies between the muscular coats of the stomach, must be regarded as its proper motor centre, and to it motor impulses are conducted by the vagi. Section of both vagi does not abolish, but it diminishes the movements of the stomach. The muscular fibres of the cardia may be excited to action, or their action inhibited by fibres which run in the vagus $(\mathrm{Nn}$. constrictores, et dilatator cardiæ). [If the vagi be divided in the neck, there is a short temporary spasmodic contraction of the cardiac aperture. On stimulating the peripheral end of the vagus with electricity, after a latent period of a few seconds, the cardiac end contracts, more especially if the stomach be distended, but the movements are slight if the stomach be empty. In curarised dogs, the pylorus contracts with varying intensity, and irregularly whether the vagi and splanchnics be intact or divided. Stimulation of the vagi in the neck causes contraction of the pylorus, when the latent period may be seven seconds. Stimulation of the splanchnics in the thorax arrests the spontaneous pyloric contractions, the left splanchnic being more active than the right $(O s e r)$.]

Local electrical stimulation of the surface of the stomach causes circular constrictions of the organ, which disappear very gradually, while the movement is often propagated to other parts of the gastric wall. When heated to $25^{\circ} \mathrm{C}$., the excised empty stomach exhibits movements. Injury to the pedunculi cerebri, optic thalamus, medulla oblongata, and even to the cervical part of the spinal cord, according to Schiff, causes paralysis of the vessels of certain areas of the stomach, resulting in congestion and subsequent hæmorrhage into the mucous membrane. [It is no uncommon occurrence to find hænorrhage into the gastric mucous membrane of rabbits, after they have been killed by a violent blow on the head.]

[Action of Drugs. - The automatic centres are excited by emetin, apomorphin, tartar emetic, while muscarin causes general contraction of the stomach. The activity of the automatic centres is diminished by chloral, urethan, morphin, and nicotin, while atropin causes paralysis of the nerve-endings ( $E$. Schütz).]

158. VOMITING.-Mechanism.-Vomiting is caused by contraction of the walls of the stomach, the pyloric sphincter being = closed. It occurs most readily when the stomach is distended-(dogs usually greatly distend the stomach by swallowing air before they vomit); it readily occurs in infants, in whom the cul-de-sac at the cardia is not developed. It is quite certain that in children vomiting occurs through contraction of the walls of the stomach, without the spasmodic action of the abdominal walls. When vomiting is violent, the abdominal muscles act energetically. [The act of vomiting is generally preceded by a feeling of nausea, and usually there is a rush of saliva into the mouth, 
caused by a reflex stimulation of afferent fibres in the gastric branches of the vagus, the efferent nerve for the secretion of saliva being the chorda tympani. After this a deep inspiration is taken, and the glottis closed, so that the diaphragm is firmly pressed downwards against the abdominal contents, and it is kept contracted; the lower ribs are pulled in. The diaphragm being kept contracted and the glottis closed, a violent expiratory effort is made, so that the contraction of the abdominal muscles acts upon the abdominal contents, the stomach being forcibly compressed. The cardiac orifice is opened at the same time, and the contents of the stomach are ejected. The chief agent seems to be the abdominal compression, but the walls of the stomach also help, though only to a slight extent.]

The contraction of the walls of the stomach, which causes a general diminution of the gastric cavity, is not a true anti-peristalsis, as can be seen in the stomach when it is exposed. The cardia is opened by the longitudinal muscular fibres, which pull towards the lower orifice of the resophagus, so that when the stomach is full they must act as dilators. The act of romiting is preceded by a ructus-like dilating movement of the intra-thoracic part of the œsophagus, which is caused thus :-The glottis is closed, inspiration occurs suddenly and violently, whereby the cesophagus is distended by gases proceeding from the stomach. The larynx and hyoid bone, by the combined action of the genio-hyoid, sterno-hyoid, sterno-thyroid, and thyro-hyoid muscles, are forcibly pulled forwards, so that the air passes from the pharynx downwards into the upper section of the oesophagus. If the abdominal walls contract suddenly, and if this sudden impulse be aided by the movements of the stomach itself, the contents of the stomach are forced outwards. During continued vomiting, antiperistalsis of the duodenum may occur, whereby bile passes into the stomach, and becomes mixed with its contents.

Children, in whom the fundus is absent, vomit more easily than adults. [In them also the nervous system is more excitable.]

Influence of Nerves.-The centre for the movements concerned in vomiting lies in the medulla oblongata, and is in relation with the respiratory centre, as is shown by the fact that nausea may be overcome by rapid and deep respirations. In animals, vomiting may be inhibited by vigorous artificial respiration. On the other hand, the administration of certain emetics prevents the occurrence of apnœea.

In vomiting, the afferent impulses may be discharged from (1) the mucous membrane of the soft palate, pharynx, root of the tongue (glosso-pharyngeal nerve), as in tickling the fauces with the finger; (2) the nerves of the stomach (vagus and sympathetic); (3) stimulation of the uterine nerves (pregnancy); (4) the mesenteric nerves (inflammation of the abdomen and hernia); (5) nerves of the urinary apparatus (passing a renal calculus); (6) nerves to the liver and gall-duct (vagus); (7) nerves to the lungs in phthisis (vagus). Vomiting is also produced by direct stimulation of the vomiting centre. [The efferent impulses are carried by the phrenics (diaphragm), vagus (œsophagus and stomach), and intercostals (abdominal muscles).]

Vomiting, produced by the thought of something disagreeable, appears to be caused by the conduction of the excitement from the cerebrum to the vomiting centre. [It may also be excited through the brain by a disagreeable smell, shocking sight, or by other impressions on the nerves of special sense.] Vomiting is very common in diseases of the brain [tubercle, inflammation, hæmorrhage]. Section of both vagi generally, but not always, prevents vomiting.

Emetics act (1) partly by mechanically or chemically stimulating the ends of the centripeta (afferent) nerves of the mucous membrane. [These are local emetics.] Tickling the fauces, touching the surface of the exposed stomach (dog); and many chemical emetics, e.g., mustard, cupric and zinc sulphate, and other metallic salts, act in this way. (2) Other substances cause vomiting when they are introduced into the blood (without being first introduced into the stomach), and act directly upon the vomiting centre, e.g., apomorphin. [These are general emetics.] (3) Lastly, there are some substances which act in both ways, e.g., tartar emetic. Emetics may also remove mucus from the lungs, and in this case it is probable that the emetic acts upon the respiratory centre, and so favours the respirations. The general emetics usually create considerable depression, while the vomiting lasts longer than with local emetics. The former increase the salivary, gastric, and respiratory secretions.

[Uses of Emetics. - Emetics are useful not only for removing from the stomach any offending body, be it a poison or the products of imperfect or perverted gastric digestion, or bile which has passed back into the stomach, but foreign bodies impacted in the oesophagus may be got 
xid of on exciting vomiting by the subcutaneous injection of apomorphin. As the diaphragm contracts vigorously during vomiting, it compresses the liver, and thus bile is expelled into the duodenum, or the passage of a small calculus along the bile-duct may be aided. They also are useful in removing mucus or false membranes from the respiratory passages.]

[Anti-Emetics. - Vomiting may be allayed by local anti-emetics such as ice, and many chemical substances such as bismuth, hydrocyanic acid, opium, and morphia, as well as by general remedies which act on the vomiting centre. Some of the foregoing drugs perhaps act both locally and generally.]

Vomiting is analogous to the process of rumination in animals that chew the cud ( $\$ 187$ ). Some persons can empty their stomach in this way.

159. MOVEMENTS OF THE INTESTINE._Peristalsis.—The best example of peristaltic movements is afforded by the small intestine; the progressive narrowing of the tube proceeds from above downwards, thus propelling the contents before it. Frequently after death, or when air acts freely upon the gut, the peristalsis develops at various parts of the intestine simultaneously, whereby the loops of intestine present the appearance of a heap of worms creeping amongst each other. The advance of new intestinal contents again increases the movement. In the large intestine, the movements are more sluggish and less extensive. The peristaltic movements may be seen and felt when the abdominal walls are very thin, and also in hernial sacs. They are more lively in vegetable feeders than in carnivora. The peristalsis is perbaps conducted directly through the muscular substance itself, as in the heart and ureter. The movements of the stomach and intestine cease during sleep (Busch).

[Rate of Motion.-In a Thiry-Velly fistula ( $\$ 183$, II.) Fubini estimated the rate of motion of

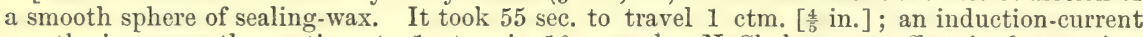
greatly increases the motion, to $1 \mathrm{ctm}$. in 10 seconds; $\mathrm{NaCl}$ does not affect it, but excites secretion ; laudanum paralyses it.]

Method of Observation. - Open the abdomen of an animal under a 6 per cent. saline solution to prevent the exposure of the gut to air (Sanders and Braam-Houckgeest).

The ileo-colic valve, as a rule, prevents the contents of the large intestine from passing backwards into the small intestine.

When fluid is slowly introduced into the rectum through a tube, it passes upwards into the intestine, and even goes through the ileo-colic valve into the small intestine. Muscarin excites very lively peristalsis of the intestines, which may be set aside by atropin (Schmiedeberg and Koppe).

Pathological.-When any condition excites an acute inflammation of the intestinal mucous membrane, catarrh is rapidly produced, and very strong contractions of the inflamed parts filled with food take place. When these parts of the gut become empty, the movements are not stronger than normal. If new material passes into the inflamed part, the peristalsis recurs, becomes more lively than normal, and the result is diarrhœa (Nothnagel). Sometimes a greatly contracted part of the small intestine is pushed into the piece of gut directly continuous with it, giving rise to invagination or intussusception.

Anti-peristalsis, i.e., a movement which travels in an upward direction towards the stomach, does not occur normally. This has been inferred from the fact, that in cases where the intestine is occluded, called ileus, fæcal matter is vomited. Nothnagel's experiments throw doubts upon this view, as he failed to observe anti-peristalsis in cases where the intestine was occluded artificially. The frecal odour of the ejecta may result from the prolonged retention of the material within the small intestine.

160. EXCRETION OF FECAL MATTER.-The contents of the small intestine remain in it about three hours, and about twelve hours in the large intestine, where they become less watery, and they assume the characters of fæces, become "formed" in the lower part of the great intestine. The fæces are gradually carried along by the peristaltic movement, until they reach a point a little above that part of the rectum which is surrounded by both sphincters, the internal sphincter consisting of non-striped, and the external of striped muscle.

Immediately after the expulsion of the fæces the external sphincter (fig. 172, S, and fig. 173) usually contracts vigorously, and remains so for some time. Afterwards it relaxes, when the elasticity of the parts surrounding the anal opening, 
particularly of the two sphincters, suffices to keep the anus closed. In the interval between two evacuations, there does not seem to be a continued tonic contraction of the sphincters. As long as the fæces lie above the rectum, they do not excite any conscious sensations, but the sensation of requiring to go to stool occurs when the freces pass into the rectum. At the same time, the stimulation of the sensory nerves of the rectum causes a reflex excitement of the sphincters. The centre for these movements (Budge's centrum anospinale) lies in the lumbar region of the spinal cord $(\$ 362)$; in the rabbit between the sixth and seventh, and in the dog at the fifth lumbar vertebra (Masius).

In animals whose spinal cord is divided above the centre, a slight touch in the region of the anus causes this orifice to contract, but after this lively reflex contraction, the sphineters relax again, and the anus may remain open for a time. This occurs because the voluntary impulses which proceed from the brain to cause the contraction of the external sphincter are absent.

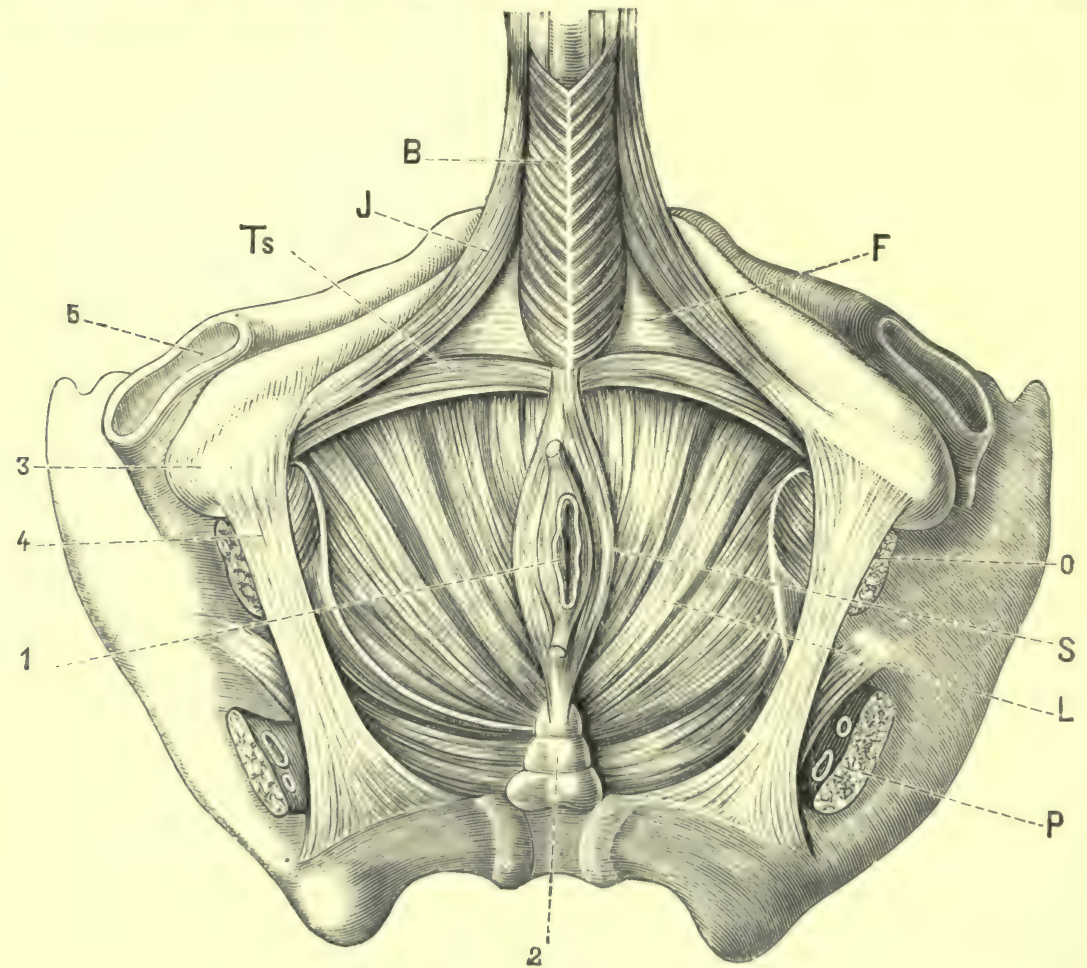

Fig. 172.

The perinæum and its muscles. 1 , anus; 2 , сосеуx; 3 , tuberosity ; 4 , sciatic ligament ; 5 , cotyloid cavity ; B, lulbo-cavernosus muscle ; Ts, superficial transverse perineal musele; $\mathbf{F}$, fascia of the deep transverse perineal muscle ; J, ischio-cavernosus nuscle ; M, obturator internus; $\mathrm{S}$, external anal sphincter; L, levator ani; $\mathrm{P}$, pyriformis.

Landois observed that in dogs with the posterior roots of their lower lumbar and sacral nerves divided, the anus remained open, and not unfrequently a mass of fæces remained half ejected. As the sensibility of the rectum and anus was abolished in these animals, the sphincters could not contract reflexly, nor could there be any voluntary contraction of the sphincters, the result of sensory impulses from the rectum.

The external sphincter can be contracted voluntarily, like any voluntary muscle, but the closure of the anus can only be effected up to a certain degree. When the pressure from above is very great, the energetic peristalsis at last overcomes 
the strongest voluntary impulses. Stimulation of the peduncles of the cerebrum and of the spinal cord below this point causes contraction of the external sphincter.

Defæcation.-The evacuation of the fæces, which in man usually occurs at certain times, begins with a lively peristalsis of the large intestine, which passes downwards to the rectum. In order that the mass of fæces may not excite reflexly the sphincter-muscles, in consequence of mechanical stimulation of the sensory nerves of the rectum, there seems to be a centre which inhibits the reflex action of the sphincters, which is called into play, owing, as it appears, to voluntary impulses. Its seat is in the brain, perhaps in the optic thalami. When this

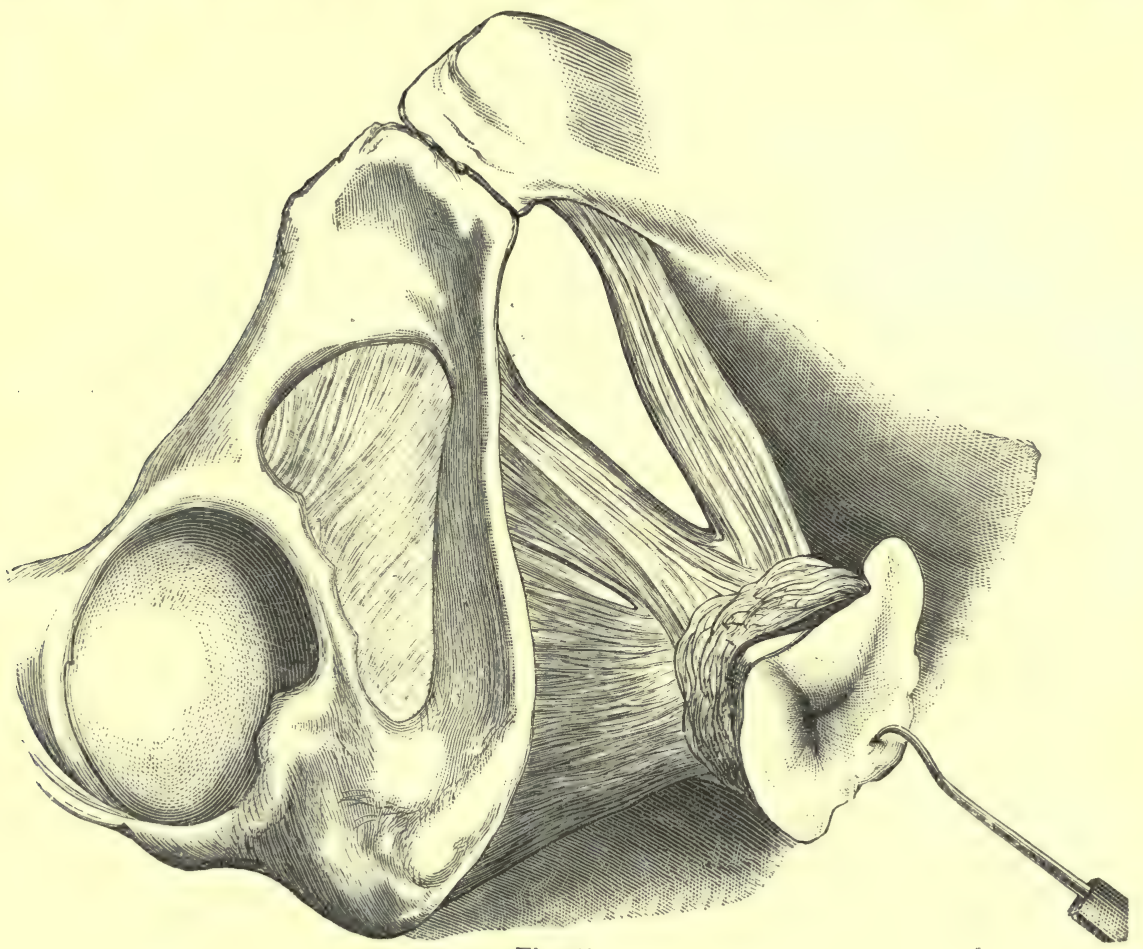

Fig. 173.

Levator ani and sphincter ani externus.

inhibitory apparatus is in action, the fæcal mass passes through the anus, without causing it to close reflexly. The strong peristalsis which precedes defæcation can be aided, and to a certain degree excited, by rapid voluntary movements of the external sphincter and levator ani, whereby the plexus myentericus of the large intestine is stimulated mechanically, thus causing lively peristaltic movements in the large intestine. The expulsion of the fæces is also aided by the pressure of the abdominal muscles, and most efficiently when a deep respiration is taken, so as to fix the diaphragm, whereby the abdominal cavity is diminished to the greatest extent. The soft parts of the floor of the pelvis, during a strong effort at stool, are driven downwards in the form of a cone, causing the mucous membrane of the anus, which contains much venous blood, to be everted. The function of the levator ani (figs. 172, 173) is to raise voluntarily the soft parts of the floor of the pelvis, and to pull the anus to a certain extent upwards over the descending fæcal mass. At the same time, it prevents the distension of the pelvic fascia. As 
the fibres of both levatores converge below, and become united with the fibres of the external sphincter, they aid the latter, during energetic contraction of the sphincter; or, as Hyrtl put it, the levatores are related to the anus, like the two cords of a tobacco pouch. During the periods between the evacuation of the gut, the fæces appear only to reach the lower end of the sigmoid flexure. As a rule, from thence downwards, the rectum is normally devoid of fæces. It seems that the strong circular fibres of the muscular coat, which Nélaton has called sphincter ani tertius, when they are well developed, contract and prevent the entrance of the freces. When the tendency to the evacuation of the rectum is very pressing,

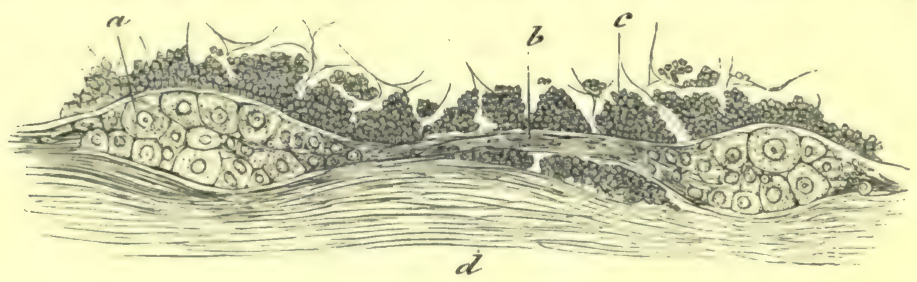

Fig. 174.

Anerbach's plexus shown in section (human). $a$, ganglionic cells; $b$, nerve fibres ; $c$, section of the circular muscular fibres; $d$, longitudinal muscular fibres.

the anus may be closed more firmly from without, by energetically rotating the thigh outwards, and contracting the muscles of the gluteal region.

161. CONDITIONS INFLUENCING THE INTESTINAL MOVEMENTS.The intestinal canal contains an automatic motor centre within its walls, - the

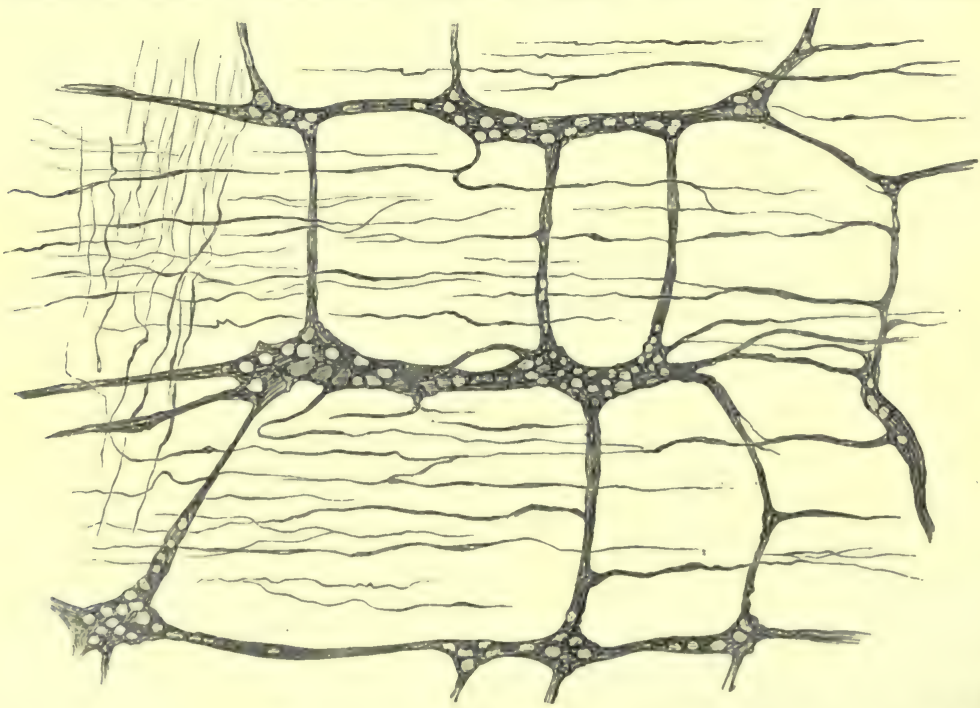

Fig. 175.

Plexus of Auerbach, prepared from the small intestine of a dog, by the action of gold chloride. The nerve-cells are shown at the nodes, while the fibrils proceeding from the ganglia, and the anastomosing fibres, lie between the muscular bundles.

plexns myentericus of Auerbach — which lies between the longitudinal and circular muscular fibres of the gut. It is this plexus which enables the intestine, when cut out of the body, to execute, apparently spontaneously, movements for some time. 
[Structure.-Auerbach's Plexus consists of non-medullated nerve-fibres which form a dense network, groups of ganglion cells occurring at the nodes (fig. 175, and when seen in vertical sections between the muscular coats it is like fig. 174). A similar plexus extends throughout the whole intestine between the longitudinal and circular muscular coats from the osophagus to the rectum. Branches are given off to the muscular bundles. A similar, but not so rich a plexus lies in the submucous coat, Meissner's plexus, which gives branches to supply the muscularis mucosæ, the smooth muscular fibres of the villi, and the glands of the intestine (fig. 176).

1. If this centre is not affected by any stimulus, the movements of the intestine cease-comparable to the condition of the medulla oblongata in apnœa. The same is true-just as in the case of the respiration-during intra-uterine life, in consequence of the fotal blood being well supplied with $\mathrm{O}$. This condition may be termed aperistalsis. It also occurs during sleep, perhaps on account of the greater amount of $\mathrm{O}$ in the blood during that state.

2. When blood containing the normal amount of blood-gases passes through the intestinal bloodvessels, the quiet peristaltic movements of health occur (euperistalsis) provided no other stimulus be applied to the intestine.

3. All stimuli applied to the plexus myentericus increase the peristalsis, which may become so very violent as to cause evacuation of the contents of the large gut, and may even produce spasmodic

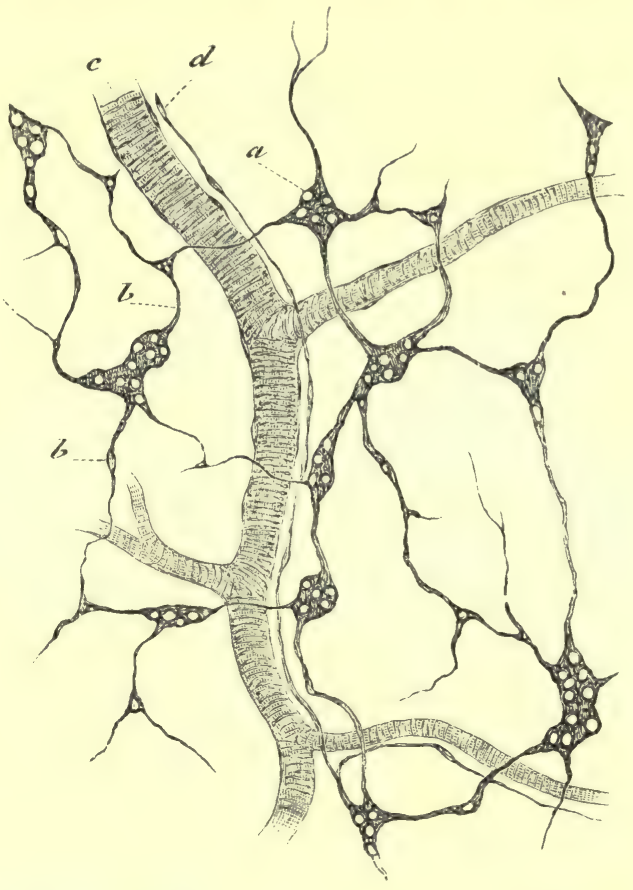

Fig. 176.

Plexus of Meissner. $a$, ganglia ; $b$, anastomosing fibres; $c$, artery ; $d$, vaso-motor nerve-fibres accompanying $c$.

contraction of the musculature of the intestine. This condition may be termed dysperistalsis, corresponding to dyspnœa. The condition of the blood flowing through the intestinal vessels affects the peristalsis.

Condition of the Blood.-Dysperistalsis may be produced by $(a)$ interrupting the circulation of the blood in the intestines, no matter whether anæmia (as after compressing the aortaSchiff) or venous hyperæmia be produced. The stimulating condition is the want of $\mathrm{O}$, i.e., the increase of $\mathrm{CO}_{2}$. Very slight disturbance in the intestinal blood-vessels, e.g., venous congestion after copious transfusion into the veins, whereby the abdominal and portal veins become congested, causes increased peristalsis. The intestines become nodulated at one part and narrow at another, and involuntary evacuation of the fæces takes place when there is congestion, owing to the plugging of the intestinal blood-vessels when blood from another species of animal is used for transfusion ( $\$ 102$ ). The marked peristalsis which occurs on the approach of death is undoubtedly due to the derangements of the circulation, and the consequent alteration of the amount of gases in the blood of the intestine. The same is true of the increased movements of the intestines which occur as a result of psychical excitement, e.g., grief. The stimulus, in this case, passes from the cerebrum through the medulla oblongata (vaso-motor centre) to the intestinal nerves, and causes anæmia of the intestine (corresponding to the palor occurring elsewhere). When the normal condition of the circulation is restored, the peristalsis diminishes. (b) Direct stimulation of the intestine, conducted to the plexus myentericus, causes dysperistalsis; direct exposure of the intestines to the air (stronger when $\mathrm{CO}_{2}$ or $\mathrm{Cl}$ is present)-introduction of various irritating substances into the intestine-increased filling of the intestine when there is any difficulty in emptying the gut (often in man)-direct stimulation of various kinds (also 
inflammation), - all act upon the intestive, either from without or from within. Induction. shocks applied to a loop of intestine in a hernial sac cause lively peristalsis in the hernia. The intestinal movements are favoured by heat.

4. The continued application of strong stimuli causes the dysperistalsis to give place to rest, owing to over-stimulation, which may be called "intestinal paresis," or exhaustion.

This condition is absolutely different from the passive condition of the intestine in aperistalsis. Continued congestion of the intestinal blood-vessels ultimately causes intestinal paralysis, e.g., when transfusion of foreign blood causes coagulation within these vessels. Filling the bloodvessels with "indifferent" fluids, after the peristalsis has been previously brought about by compressing the aorta, also causes cessation of the movements (O. Nasse). The movements cease when the intestines are cooled to $19^{\circ} \mathrm{C}$. (Horwath), while severe inflammation of the intestine has a similar effect. Under favourable circumstances, the intestine may recover from this condition. Arterial blood admitted into the vessels of the exhausted intestine causes peristalsis, which at first is more vigorous than normal.

5. The continued application of strong stimuli causes complete paralysis of the intestine, such as occurs after violent peritonitis, or inflammation of the musculature or mucous coat in man. In this condition, the intestine is greatly distended, as the paralysed musculature does not offer sufficient resistance to the intestinal gases which are expanded by the heat. This constitutes the condition of meteorism.

Influence of Nerves.-With regard to the nerves of the intestine, stimulation of the vagus increases the movements (of the small intestine), either by conducting impressions to the plexus myentericus, or by causing contraction of the stomach, which stimulates the intestine in a purely mechanical manner (Braam-Houckgeest). The splanchnic is (1) the inhibitory nerve of the small intestine (Pflüger), but only as long as the circulation in the intestinal blood-vessels is undisturbed, and the blood in the capillaries does not become venous; when the latter condition occurs, stimulation of the splanchnic increases the peristalsis. If arterial blood be freely supplied, the inhibitory action continues for some time. Stimulation of the origin of the splanchnics, of the spinal cord in the dorsal region (under the same conditions), and even when general tetanus has been produced by the administration of strychnia, causes an inhibitory effect. It is inferred that the splanchnic contains - (2) inhibitory fibres, which are easily exhausted by a venous condition of the blood, and also motor fibres, which remain excitable for a longer time, because after death, stimulation of the splanchnics always causes peristalsis, just like stimulation of the vagus. (3) It is the vaso-motor nerve of the intestinal blood-vessels, so that it governs the largest vascular area in the body. When it is stimulated, all the vessels of the intestine which contain muscular fibres in their walls contract; when it is divided, they dilate. In the latter case, a large amount of blood accumulates within the blood-vessels of the abdomen, so that there is anæmia of the other parts of the body, which may be so great as to cause death-owing to the deficient supply of blood to the medulla oblongata. (4) It is the sensory nerve of the intestine, and, under certain circumstances, it may give rise to very painful sensations.

As stimulation of the splanchnic contracts the blood-vessels, von Basch has raised the question whether the intestine does not come to rest, owing to the want of the blood, which acts as a stimulus. But, when a weak stimulus is applied to the splanchnic, the intestine ceases to move before the blood-vessels contract (van Braam-Houckgeest); it would therefore seem that the stimulation diminishes the excitability of the plexus myentericus. According to Engelmann and $\mathrm{v}$. Brakel, the peristaltic movement is chiefly propagated by direct muscular conduction, as in the heart and ureter, without the intervention of any nerve-fibres.

[Effect of Nerves on the Rectum.-The nervi erigentes, when stimulated, cause the longitudinal muscular fibres of the rectum to contract, while the circular muscular fibres are supplied by the hypogastric nerves. Stimulation of the latter nerves also exerts an inhibitory effect on the longitudinal muscles. Stimulation of the nervi erigentes inhibits not only the spontaneous movements of the circular fibres of the rectum, but also those movements excited by stimulation of the hypogastric nerves (Fellner).]

[Artificial Circulation in the Intestine. - Ludwig and Salvioli excised a loop of intestine 
from an animal, tied a cannula into an artery and another into a vein, and kept it in a warm moist atmosphere. The arterial cannula was connected with a vessel containing defibrinated blood, to which different drugs could be added. A lever rested on the intestine, and registered its movements on a recording surface. As long as arterial blood was tranfused, the intestine was nearly quiescent, but when it was arrested, so that the blood became venous, a series of contractions occurred. Nicotin diminished the flow of blood and quickened the intestinal movements, while at the same time the circular muscular fibres remained contracted or tetanic. Tincture of opium, in the proportion of 01 to $\cdot 04$ in the blood, causes at first contraction of the vessels, and lessens the amount of blood circulating in the intestine; but it very rapidly increases - even to six times - the amount of blood which transfuses, while at the same time the movements of the intestine cease, the walls of the intestine being contracted. Peptone caused first strong and then irregular contractions.]

Effect of Drugs. - Amongst the reagents which act upon the intestinal movements are :-(1) Such as diminish the excitability of the plexus myentericus, $i . e$, which lessen or even abolish intestinal peristalsis, e.g., belladonna. (2) Such as stimulate the inhibitory fibres of the splanchnic, and in large doses paralyse them-opium, morphia; 1 and 2 produce constipation. (3) Other agents excite the motor apparatus-nicotin (even causing spasm of the intestine), muscarin, caffein, and many laxatives, which act as purgatives. The movements produced by muscarin are abolished by atropin. These substances accelerate the evacuation of the intestine, and, owing to the rapid movement of the intestinal contents, only a small amount of water is absorbed; so that the evacuations are frequently fluid. (4) Amongst purgatives, colocynth and croton oil act as direct irritants. With regard to drugs of this sort, they seem to cause a watery transudation into the intestine, just as croton oil causes vesicles when applied to the skin. (5) Calomel is said to limit the absorptive activity of the intestinal wall, and to control the decompositions in the intestine. The stools are thin and greenish, from the admixture of biliverdin. (6) Certain saline purgatives-sodium sulphate, magnesium sulphate-cause fluid evacuations by retaining the water in the intestine ; and it is said that if they be injected into the bloodvessels of animals, they cause constipation. [When a crystal of a potash salt is applied to the peritoneal surface of the intestine of an animal, it causes merely a local constriction of the muscular fibres of the gut, while a sodium salt excites a contraction which passes upwards towards the stomach, and never towards the rectum. In any case it may serve as a useful guide to the surgeon, in determining which is the upper end of a piece of intestine during an operation on the intestines (Nothnagel).]

[Saline Cathartics. - A salt exerts a genuine excito-secretory action on the glands of the intestines, whilst at the same time, in virtue of its low diffusibility, it impedes absorption. Thus, between stimulated secretion and impeded absorption there is an accumulation of fluid within the canal, which reaches the rectum and results in purgation. Purgation does not ensue when water is withheld from the diet for one or two days previous to the administration of the salt in a concentrated form. When a concentrated solution of a salt is administered to an animal whose alimentary canal is empty, but whose blood is in a natural state of dilution, the blood becomes rapidly very concentrated, and reaches the maximum of its concentration in from half an hour to an hour and a half; within four hours the blood has gradually returned to its normal state of concentration, without having reabsorbed fluid from the intestine. It apparently recoups itself from the tissue-fluids. The salt-sulphate of magnesia or sulphate of soda -becomes split up in the small intestine, and the acid is more rapidly absorbed than the base. A portion of the absorbed acid shortly afterwards returns to the intestines, evidently through the intestinal glands. The salt does not purge when injected into the blood, and excites no intestinal secretion; nor does it purge when injected subcutaneously, unless on account of its causing local irritation of the abdominal subcutaneous tissue, which acts reflexly on the intestines, dilating their blood-vessels, and perhaps stimulating their muscular movements $(M$. Hay).

162. STRUCTURE OF THE STOMACH.-[The stomach receives the bolus, and secretes a juice which acts on certain constituents of the food, while by its muscular walls it moves the latter within its own cavity, and after a time expels the partially digested products towards the duodenum.]

Structure.- [The walls of the stomach consist of four coats, which are from without inwards (fig. 177) -

(1) The serous layer, from the peritoneum.

(2) The muscular layer, composed of three layers of non-striped muscular fibres- $(a)$ longitudinal, (b) circular, (c) oblique (\$15).

(3) The sub-mucous layer of loose connective-tissue, with the larger blood-vessels, lymphatics, and nerves.

(4) The mucous layer.]

The well-developed mucous membrane of the stomach is thrown into a series of folds or rugæ, in a contracted condition of the organ. With the aid of a hand-lens, it is seen to be 
beset with small irregular depressions or pits (fig. 179). Throughout its entire extent it is covered by a single layer of moderately tall, narrow, cylindrical epithelium, which seems to

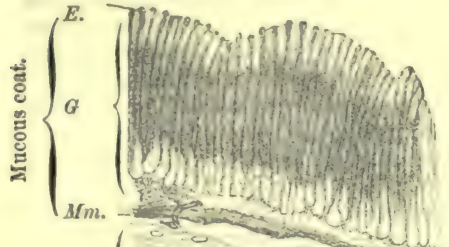
consist of mucus-secreting goblet-cells (fig. 178). The epithelium is sharply defined at the cardin from the stratified epithelium of the resophagus, and also at the pylorus, from the true cylindrical epithelium with the striated dise in the duodenum. [The cells contain a plexus of fibrils, and in the passive condition seem to

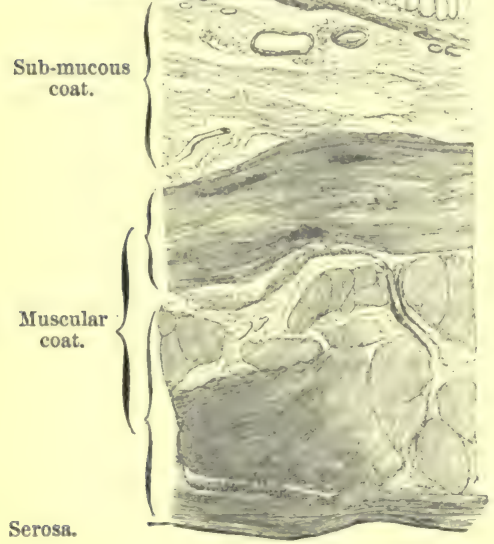

Fig. 177.

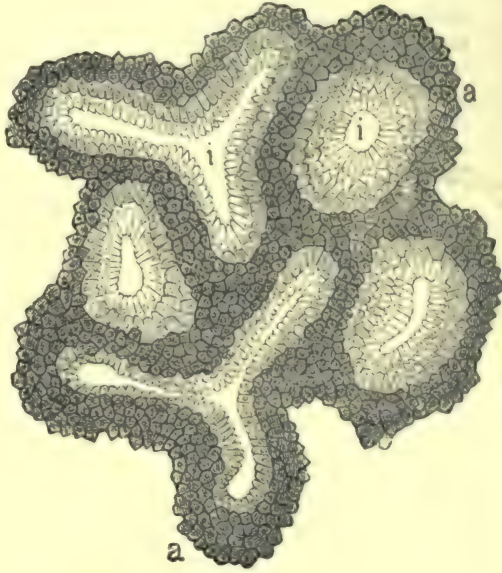

Fig. 179.

Fig. 177. - Vertical section of the wall of the human stomach, $\times 15 . \quad E$., epithelium; $G l$, glands; Mm., muscularis mucosæ. Fig. 178. - Goblet-cells of the stomach. Fig. 179. - Surface section of the dog's gastric mucous membrane, showing pits, $i, i$; $a$, the elevations round $i, i$.

consist of two zones, an outer clear part, next the lumen of the organ, consisting of a substance (mucigen) which yields mucus, the attached end of the cell being granular.] The oval nucleus lies about the centre of the cells. Spindle-shaped, nucleated cells, probably for re-

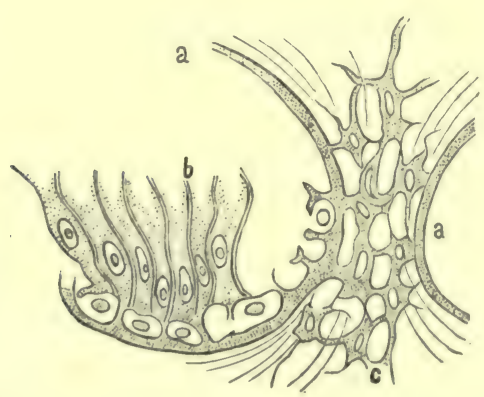

I.

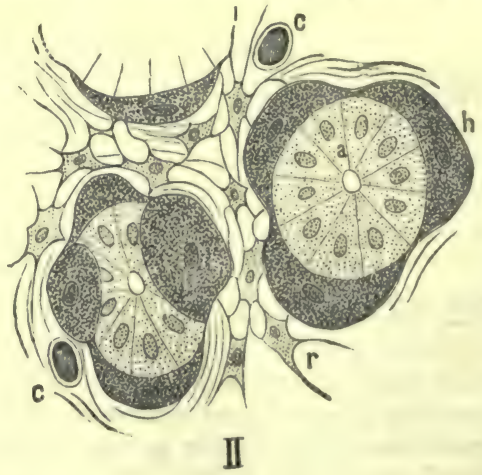

II

Fig. 180.

I, Transverse section of a duct of a fundus gland $-\alpha$, membrana propria ; $b$, mucus-secreting goblet-cells ; $c$, adenoid interstitial substance. II. Transverse section of a fundus-gland$a$, chief, $h$, parietal-cells ; $r$, adenoid tissue ; $c$, capillaries.

placing the others, are said by Ebstein to occur at their bases. All the cells are open at their free ends, so that the mucus is readily discharged, leaving the cells empty. Numerous tubular glands of two distinct kinds are placed vertically, like rows of test-tubes, in the mucous membrane. 
The cardiac portion of the gastric mucous membrane consists of a number of microscopic tubular glands placed side by side, the fundus-glands of Heidenhain, otherwise called peptic, or cardiac. Several gland-tubes, which are wider below, usually open into the duct (fig. 182). Each gland consists of a structureless membrana propria with anastomosing branched cells in relation with it. The duct is short, about one-fifth of the whole tube, and is lined by a layer of cells like those lining the stomach, while the secretory part of the tubes is lined throughout by a layer of granular, short, small, polyhedral, or columnar nucleated cells. These cells border the very narrow lumen, and are called principal (Heidenhain), central (fig. 180, II,

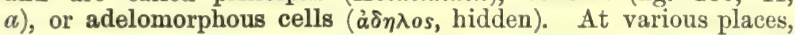
between these cells and the membrana propria, are large, oval, or angular, well-defined, granular, densely reticulated, nucleated cells, the parietal cells of Heidenhain, the delomorphous cells of Rollett, or the oxyntic (acid-forming) cells of Langley (fig. 180, II, $h$ ). They are most numerous in the neck of the glands, and least so in the deep blind end of the tubes. These cells are stained deeply by osmic acid and aniline blue, so that they are readily distinguished from the other cells. They bulge out the membrana propria of the gland opposite where they are placed. The parietal cells in man are said to reach to the lumen of the gland-tubes (Stöhr). Isolated cells are sometimes found under the epithelium of the surface of the stomach, and occasionally in individual pyloric glands. The fundus-glands are most numerous (about five millions), and are of considerable size in the fundus.

2. The pyloric glands occur only in the region of the pylorus, where the mucous membrane is more yellowish-white in colour (fig. 181, A). These glands are generally branched at their lower ends, so that several tubes open into a single duct [which, in contradistinction to the duct of the other glands, is wide and long, extending often to half the depth of the nucous membrane. The duct is lined by epithelium like that lining the stomach, while the secretory part is lined by a single layer of short, finely granular, columnar cells, whose secretion is quite different from that of the cells lining the duct. The lumen is well defined. Nussbaum has occasionally found other cells, which stain deeply with osmic acid, between the bases of these. The appearance of the cells differs according to their state of physiological activity (figs. 183, 184). When they are exhausted they are smaller and more granular, owing to the denser reticulation of their network; at any rate they are granular in preparations hardened in alcohol (fig. 184). There are no parietal cells.]

The glands are supported by very delicate connective-tissue mixed with adenoid tissue (fig. 180). Below this are two layers,

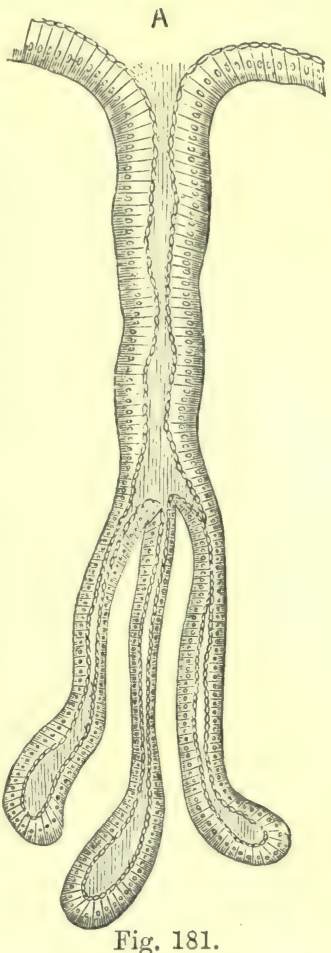

A, Isolated pyloric gland. circular and longitudinal, of non-striped muscle, the muscularis mucosæ (fig. 177, MIm.), and from it fine processes of smooth muscular fibres pass up between groups of the glands towards the free epithelial surface of the mucous membrane. Perhaps these processes are concerned in emptying the glands. [In the gastric mucous membrane of the cat, there is a clear homogeneous layer, which is stained red by picro-carmine, and placed immediately internal to the muscularis mucosæ. It is pierced by the processes passing from the muscularis mucosæ.]

Masses of adenoid tissue occur in the mucous membrane, especially near the pylorus, constituting lymph-follicles, which are comparable to the solitary glands of the small intestine. The lymphatics are numerous, and begin close under the cpithelium by dilated extremities or loops (fig. 182, $d$ ) ; they run vertically, and anastomose in the mucosa between the gland-tubes, which they envelop in sinus-like spaces. They join large trunks in the mucosa ; another plexus of large vessels exists in the sub-mucosa (Loven).

[The Nerves. - A plexus of non-medullated nerve-fibres and a few ganglion cells exist in the muscular coat [Auerbach's], and another [Meissner's] in the sub-mucosa.]

The blood-vessels are very numerous. Small arterial branches, $\alpha$, run in the sub-mucosa, and ascend between the glands to form a longitudinal capillary network, $c, c$, under the epithelium, and between its meshes the gland-ducts open, $g$. The veins gradually collect from this hurizontal capillary network, and run towards the large veins of the sub-mucosa, $v$.

163. THE GASTRIC JUICE.-Properties.-The gastric juice is a tolerably clear colourless fluid, with a strong acid reaction, sour taste, and peculiar characteristic odour; it rotates the plane of polarised light to the left. It is not rendered turbid by boiling, and resists putrefaction for a long time. Its specific gravity = 
1002.5 (dog, 1005), and it contains only $\frac{1}{2}$ per cent. of solid constituents. The quantity secreted in 24 hours was estimated by Beaumont, from observations upon Alexis St Martin, who had a gastric fistula (1834) - at only $180 \mathrm{grms}$. daily (!); by Grunewald (1853), in a similar case, as equal to 26.4 per cent. of the body-weight; while Bidder and Schmidt (from corresponding observations on dogs) estimated it as equal to $6 \frac{1}{2}$ kilos. daily, corresponding to $\frac{1}{10}$ of the body-weight. It contains:-

(1) Pepsin, the characteristic hydrolytic ferment or enzym, which dissolves

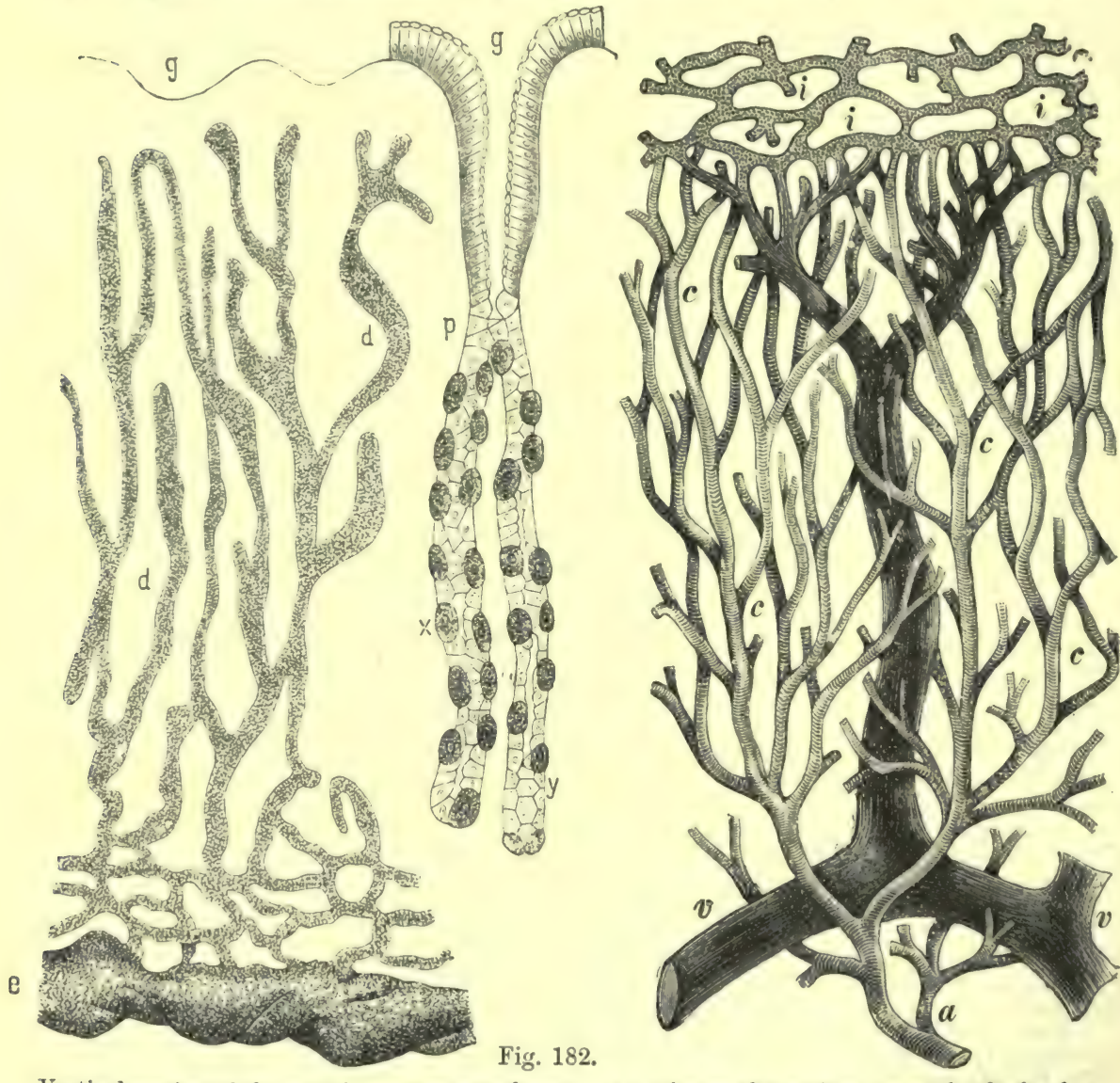

Vertical section of the gastric mucous membrane. $g, g$, pits on the surface; $p$, neck of a fundusgland opening into a duct, $g ; x$, parietal, and $y$, chief cells ; $a, v$, $c$, artery, vein, capillaries; $d, d$, lymphatics, emptying into a large trunk, $e$.

proteids. E. Schütz obtained 0.41 to 1.17 per cent. from a fasting person by means of the œsophageal sound.

(2) Free hydrochloric acid (Prout, 1824), 0.2 to 0.3 (Richet, 0.8 to $2 \cdot 1$ ) per 1000; (in the dog, 0.52 per cent.). It occurs free, as the gastric juice always contains more free chlorine than bases, to which it can be united (C.Schmidt). Lactic acid is usually met with, but it arises from the fermentation of the carbohydrates of the food.

Tests. - Free hydrochloric acid is detected by the following reactions :- 0.025 per ceut. solution of mythyl-violet becomes blue; or alkaline solution of oo-tropæolin becomes lilac; or, red 
Bordeaux wine, treated with amylic alcohol until its colour almost disappears, becomes rosecoloured. [Giinzburg recommends an alcoholic solution of phloroglucin-vanillin. 2 grammes of phloroglucin are mixed with 1 gramme of vanillin in 30 grammes of absolute alcohol, which gives a yellowish-red solution. Concentrated and even very weak mineral acids cause, with this solution, a bright red colour with the formation of bright red crystals, while concentrated organic acids do not affect it. For gastric juice mix equal quantities of the filtered gastric juice and the above solution in a wateh-glass, and evaporate carefully, not allowing it to boil; a red pellicle with red crystals indicates the presence of minute traces of hydrochloric acid. Congo-red, either in solution or as congo-red papers, becomes blue, but the reaction is interfered with by the presence of ammonia, or ammoniacal salts.]

Lactic Acid.-The freshly-prepared blue solution of 10 c.c. of a 4 per cent. solution of carbolic acid, with 20 c.c. of distilled water, and 1 drop of liquor ferri perchloride, is changed to yellow by lactic acid (Uffelmann).

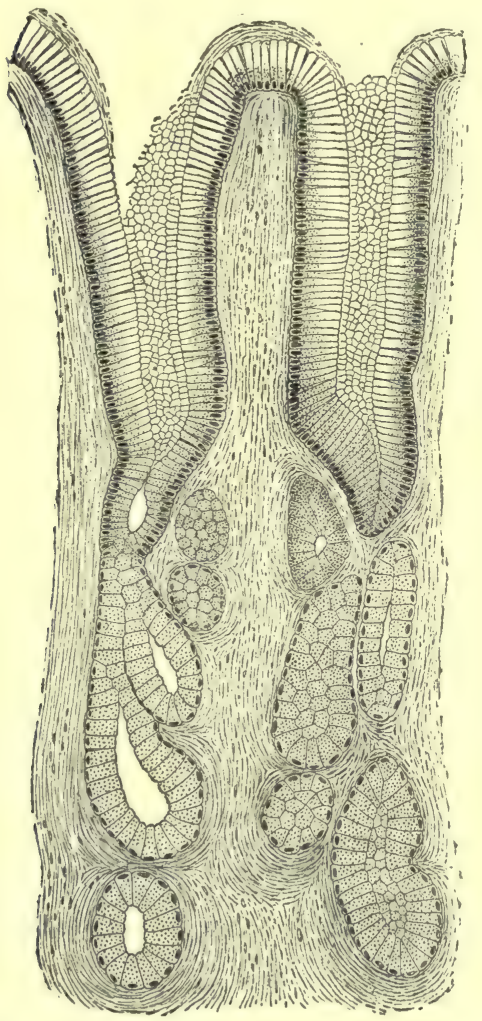

Fig. 183.

Section of the pyloric mucous membrane.

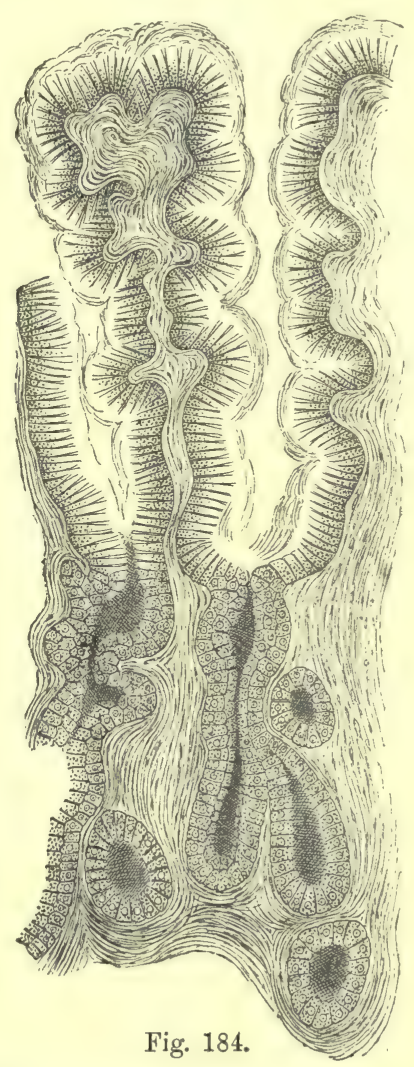

Pyloric glands showing changes of the cells during digestion.

(3) The large amount of mucus covering the surface of the mucous membrane is secreted by the goblet-cells of the mucous membrane ( $\$ 162)$, ( $(136$, II.).

(4) Mineral salts (2 per 1000), and a milk-curdling ferment.

They are chiefly sodium and potassium chlörides, less calcic chloride (ammonium chloride, also in animals), and the compounds of phosphoric acid with lime, magnesium, and iron.

Amongst foreign substances, which may be introduced into the body, the following appear in the gastric juice-HI, after the use of potassium iodide-potassium sulphocyanide, ferric lactate, and sugar ; and ammonium carbonate in uræmia. 
[Composition of Gastric Juice (Hoppe-Seyler after C. Schmidt).

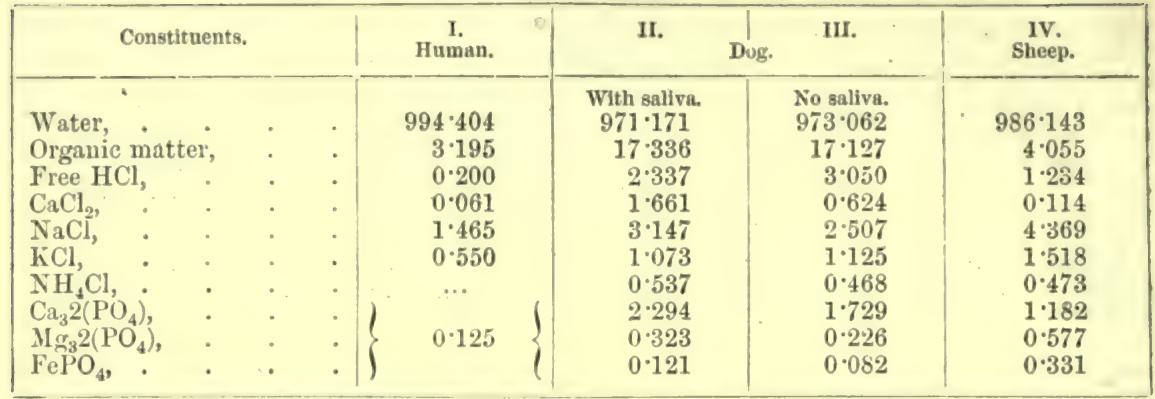

Good human saliva is not so dilute or so poor in $\mathrm{HCl}$ as I. Szabo has found even 3 of $\mathrm{HCl}$ per 1000 in man.]

164. SECRETION OF GASTRIC JUICE.-After the discovery of the two kinds of glands in the stomach and the two kinds of cells in the fundus-glands, the question arose as to whether the different constituents of gastric juice were formed by different histological elements.

Changes of the Cells during Digestion.-During the course of digestion, the cells of the fundus (and pyloric glands, dog) undergo important changes (Heidcnhain). During bunger, the chief cells are clear and large, the parietal investing-cells are small, the pyloric cells clear and of moderate size. During the first six hours of digestion, the chief cells become enlarged and molerately turbid or granular, the parietal cells also cnlarge, while the pyloric cells remain unchanged. The chief cells become diminished and more turbid or granular until the ninth hour, the parietal cells are still swollen, and the pyloric cells enlarge. During the last hours of digestion, the chief cells again become larger and clearer, the parietal cells dimiuish, the pyloric cells decrease in size and become turbid (figs. 183 and 184).

[Langley gives a different description of the appearances presented by these cells. The results may be reconciled by remembering that the gland-cells were examined under different ('onditions. The secretory cells consist of a cell-substance composed of $(a)$ a framework of living protoplasm, either in the form of an intracellular fibrillar network, or in flattened bands. The meshes of this framework enclose at least two chemical substances, viz., $(b)$ a hyaline substance in contact with the framework, and $(c)$ spherical granules which are embedded in the hyaline substance. During active secretion, the granules decrease in number and size, the hyaline substance increases in amount, the network grows. This is the reverse of what is stated above as the observation of Heidenhain, but the granular appearance described by Heidenhain after secretion is very probably due to the action of the hardening agent, alcohol. Langley found that in the living condition, or after the use of osmic acid, in some animals at least, the chief cells are granular during rest, but during a state of activity two zones are differentiated, an outer one, which is clear, owing to the disappearance of the granules, and an inner more or less granular one. Granules reappear in the outer part after rest. During digestion, the parietal cells increase in size, but do not become granular. In all cells containing much pepsinogen, distinct granules are present, and the quantity of pepsinogen varies directly with the number and size of the granules. In the glands of some animals there is little difference between the resting and active phases. Compare Serous Glands, \& 143, and Pancreas, § 168.]

The pepsin is formed in the chief cells (Heidenhain). When these are clear and large, they contain much pepsin; when they are contracted and turbid, the amount is small. The pyloric glands are also said to secrete pepsin, but only to a small extent. Pepsin accumulates during the first stage of hunger, and it is eliminated during digestion and also during prolonged hunger. Pepsin as suck, is not present within the cells, but only as a "mother-substance," a pepsinogen-substance (zymogen), or propepsin, which occurs in the granules of the chief cells. This zymogen, or mothersubstance, by itself, has no effect upon proteids; but if it be treated with hydrochloric acid or sodium chloride, it is changed into pepsin. Pepsin and pepsinogen may be extracted from the gastric mucous membrane by means of water free from acids.

[Pepsinogen and Pepsin.-Glycerine extracts very little pepsin from the perfectly fresh gastric mucous membrane, but a large amount is afterwards obtained by extracting it with 
dilute hydrochloric acid, or with this acid and glycerine. The relative amount of pensinogen and pepsin in a fluid may be determined approximately by the method of Langley and Edkins, A 1 per cent. solution of sodic carbonate exerts a greater destructive action on pepsin than on pepsinogen, while a current of $\mathrm{CO}_{2}$ destroys pepsinogen to a greater extent than pepsin. Both substances are unaffected by $\mathrm{CO}$, but are destroyed at $54^{\circ}$ to $57^{\circ} \mathrm{C}$.]

The pyloric glands secrete pepsin, but no acid. Klemensiewicz excised in a living dog the pyloric portion of the stomach, and afterwards stitched together the duodenum and the remaining part of the stomach. The excised pyloric part, with its vessels intact, he stitched to the abdominal wall, after sewing its lower end. The animals experimented on died, at the latest, after six days. The secretion of this part was thin, alkaline, and contained 2 per cent. of solids, including pepsin.

[Pyloric Fistula.-In fig. $185 \mathrm{P}$ represents the excised pyloric portion, C the cardiac. The parts $\alpha, \alpha$, and $\alpha^{\prime} \alpha^{\prime}$ were then stitched together, and the continuity of the organ established. The lower end $(d)$ of $\mathrm{P}$ was closed by sutures, while the edges of $\mathrm{P}$ at $\mathrm{O}$ were stitched to the abdominal walls, thus making a pyloric fistula.]

In the frog the alkaline glands of the osophagus contain only chief cells which produce pepsin; while the stomach has glands which secrete acid (and perhaps some pepsin), and are lined by parietal cells.

Amongst fishes the carps have no fundus-glands in the stomach (Luchau). [The secreting portions of glands of the cardiac sac (crop) of the herring are lined by a single layer of polygonal cells ( $W$. Stirling).]

The hydrochloric acid is formed, according to Heidenhain, by the parietal cells. It occurs on the

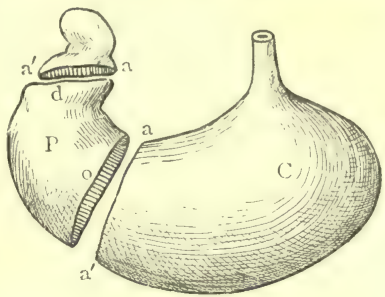

Fig. 185.

Diagram of Klemensiewicz's experiment (Stirling). free surface of the gastric mucous membrane as well as in the ducts of the fundusglands. The deep parts of the glands are usually alkaline. Free $\mathrm{HCl}$ is detected in human gastric juice, within 45 minutes to 1 to 2 hours after a moderate meal, but in 10 to 15 minutes in a fasting condition after drinking water; the amount gradually increases during the process of digestion. Lactic acid, perhaps derived from the food, is found in the stomach immediately after taking food, after half an hour along with $\mathrm{HCl}$, while after an hour only $\mathrm{HCl}$ is found (Ewald and Bocls).

Cl. Bernard injected potassium ferrocyanide and afterwards lactate of iron into the veins of a dog. After death, blue coloration occurred only in the upper acid layers of the mucous membrane. Nevertheless, we must assume that the hydrochloric acid is secreted in the parietal cells of the fundus of the glands, and that it is rapidly carried to the surface along with the pepsin. Brïcke neutralised the surface of the gastric mucous membrane with magnesia usta, chopped up the mucous membrane with water, and left it for some time, when the fluid had again an acid reaction.

As to the formation of a free acid, the following statements may be noted:The parietal cells form the hydrochloric acid from the chlorides which the mucous membrane takes up from the blood. According to Voit, the formation of acid ceases, if chlorides be withheld from the food. Maly suggests that the active agent is lactic acid, which splits up sodium chloride and forms free $\mathrm{HCl}$. The base set free is excreted by the urine, rendering it at the same time less acid. The formation of acid is arrested during hunger. According to $\mathrm{H}$. Schultz, watery solutions of alkaline and earthy chlorides are decomposed, even at a low temperature, by $\mathrm{CO}_{2}$, free hydrochloric acid being formed.

[The source of the $\mathrm{HCl}$ is undoubtedly the sodic chloride in the blood and lymph, but what other acid displaces the $\mathrm{HCl}$ is a matter of conjecture. In this connection, it is important to remember that Jul. Thomsen has shown that every acid can displace a part of another acid from its combination with its base, and the weaker acid may even combine with the greater part of the base. Thomsen calls this " avidity." Even strong mineral acids may be displaced by weak organic ones. Thus the free $\mathrm{CO}_{2}$ in the alkaline blood may set free a small quantity of $\mathrm{HCl}$ from the sodic chloride. What is still more remarkable is, that the free $\mathrm{HCl}$ should be transferred by the cells towards the gland-duct, while the sodic carbonate diffuses towards the blood and lymph.]

Secretion.-When the stomach is empty, there is usually no secretion of gastric 
juice; this takes place only after appropriate (mechanical, thermal, or chemical) stimulation. In the normal condition, it takes place immediately on the introduction of food, but also of indigestible substances, such as pebbles. The mucous membrane becomes red, and the circulation more active, so that the venous blood becomes brighter. [That the vagi are concerned in this vascular dilatation, is proved by the fact, that if both nerves be divided during digestion, the gastric mucous membrane becomes pale (Rutherford).] The secretion is probably caused reflexly, and the centre perhaps lies in the wall of the stomach itself (Meissner's plexus in the sub-mucous coat). It is asserted that the idea of food, especially during hunger, excites secretion. As yet we do not know the effect produced upon the secretion by stimulation or destruction of other nerves, e.g., vagus, sympathetic. [There is no nerve passing to the stomach, whose stimulation causes a secretion of gastric juice, as the chorda tympani does in the submaxillary gland. If the vagi be divided sufficiently low down not to interfere with respiration, the introduction of food still causes a secretion of gastric juice; even if the sympathetic branches be divided at the same time, secretion still goes on (Heidenhain). This experiment points to the existence of local secretory centres in the stomach. But there is evidence to show that there is some connection, perhaps indirect, between the central nervous system and the gastric glands. Richet observed a case of complete occlusion of the œesophagus in a woman, produced by swallowing a caustic alkali. A gastric fistula was made, through which the person could be nourished. On placing sugar or lemon juice in the person's mouth, Richet observed a secretion of gastric juice. In this case no saliva could be swallowed to excite secretion, so that it must have taken place through some nervous channels. Even the sight or smell of food caused secretion. Emotional states also are known to interfere with gastric digestion.]

Effect of Absorption. - Heidenhain isolated a part of the mucous membrane of the fundus so as to form a blind sac of it, and he found that mechanical stimulation caused merely a scanty local secretion at the spots irritated. If, however, at the same time, absorption of digested matter also occurred, secretion took place over larger surfaces. [He distinguishes a primary and merely local secretion excited by the mechanical stimulus of the ingesta, and a secondary depending on absorption, and extending to the whole of the mucous membrane.

The statement of Schiff, that active gastric juice is secreted only after absorption of the so. called peptogenic substances (especially dextrin), is denied.

The acid contents of the stomach called chyme, which pass into the duodenum after gastric digestion is completed, are neutralised by the alkali of the intestinal mucous membrane and the pancreatic juice, [at the same time, a precipitate is formed and deposited on the walls of the duodenum, and it carries the pepsin down with it]. Part of the pepsin is reabsorbed as such, and is found in traces in the urine and muscle juice (Brücke). If the gastric juice be completely discharged externally through a gastric fistula, the alkalinity of the intestine is so strong that the urine becomes alkaline (Maly).

The acid gastric juice of the new-born child is already fairly active; casein is most easily digested by it, then fibrin and the other proteids (Zweifel). When the amount of acid is too great in the stomach of sucklings, large firm indigestible masses of casein are apt to be formed, especially after the use of cow's milk (\$ 230).

[Action of Drugs on Gastric Secretion. - Dilute alkalies, if given before food; saliva ; some substances called peptogens by Schiff, such as dextrin and peptones, alcohol and ether, all excite secretion, the last being very powerful. When the secretion is excessively acid, antacids are given, some diminishing the acidity in the stomach, as the carbonates and bicarbonates of the alkalies, liquor potassæ, and the carbonate of magnesia ; while the citrates and tartrates of the alkalies, becoming converted into carbonates in their passage through the organism, diminish the acidity of the urine.] Small doses of alcohol, introduced into the stomach, increase the secretion of gastric juice; large doses arrest it. Artificial digestion is affected by 10 per cent. of alcohol, is retarded by 20 per cent., and is arrested by stronger doses. Beer and wine hinder digestion, and in an undiluted form interfere with artificial digestion. 
165. METHODS OF OBTAINING GASTRIC JUICE.-Historical-Spallanzani caused starving animals to swallow small pieces of sponge enclosed in perforated lead capsules, and after a time, when the sponges had become saturated with gastric juice, he removed them from the stomach. To avoid the admixture of saliva, the sponges are best introduced through an opening in the œsophagus. Dr Beaumont (1825), an American physician, was the first to obtain human gastric juice, from a Canadian named Alexis St Martin, who was injured by a gun-shot wound, whereby a permanent gastric fistula was established. Various substances were introduced through the external opening, which was partially covered with a fold of skin, and the time requirer for their solution was noted. Bassow (1842), Blondlot (1843), and Bardeleben (1849) were thereby led to make artificial gastric fistulæ.

Gastric Fistula. - The anterior abdominal wall is opened by a median incision just below the ensiform cartilage, the stomach is exposed, and its anterior wall opened and afterwards stitched to the margins of the abdominal walls. A strong cannula is placed in the fistula thus formed. The tube is kept corked. If the ducts of the salivary glands be tied, a perfectly uncomplicated object for investigation is obtained.

According to Leube, dilute human gastric juice may be obtained by means of a syphon-like tube introduced into the stomach. Water is introduced first, and after a time it is withdrawn.

An important advance was made when Eberle (1834) prepared artificial gastric juice, by extracting the pepsin from the gastric mucous membrane with dilute hydrochloric acid. Four litres of solution of hydrochloric acid, containing 4 to $8 \mathrm{c.c}$. $\mathrm{HCl}$ per 1000, are sufficient to extract the chopped-up mucous membrane of a pig's stomach. Half a litre is infused with the stomach and renewed every six hours. The collected fluid is afterwards filtered. The substance to be digested is placed in this fluid, and the whole is kept at the temperature of the body, but it is necessary to add a little $\mathrm{HCl}$ from time to time (Schwann). The $\mathrm{HCl}$ may be replaced by ten times its volume of lactic acid and also by nitric acid; while oxalic, sulphuric, phosphoric, acetic, formic, succinic, tartaric, and citric acids are much less active; butyric and salicyclic acids are inactive.

Von Wittich's Method. - (a) Glycerine extracts pepsin in a very pure form. The mucous membrane is rubbed up with powdered glass until it forms a pulp, mixed with glycerine, and allowed to stand for eight days. The fluid is pressed through cloth, and the filtrate mixed with alcohol, thus precipitating the pepsin, which is washed with alcohol and afterwards dissolved in the dilute $\mathrm{HCl}$, to form an artificial digestive fluid. (b) Or the mucous membrane may be placed for twenty-four hours in alcohol, and afterwards dried and extracted for eight days with glycerine. (c) Wm. Roberts has used other agents for extracting enzymes (§ 148).

Preparation of Pure Pepsin.-Briicke pour's on the pounded mucous membrane of the pig's stomach a 5 per cent. solution of phosphoric acid, and afterwards adds lime-water until the acid reaction is scarcely distinguishable. A copious precipitate, which carries the pepsin with it, is produced. This precipitate is collected on cloth, repeatedly washed with water, and afterwards dissolved in very dilute $\mathrm{HCl}$. A copious precipitation is caused in this fluid, by gradually adding to it a mixture of cholesterin in four parts of alcohol and one of ether. The cholesterin pulp is collected on a filter, washed with water containing acetic acid, and afterwards with pure water. The cholesterin pulp is placed in ether to dissolve the cholesterin, and the ether is then removed. The small watery deposit contains the pepsin in solution.

Pepsin so prepared is a colloid substance; it does not react like albumin with the following tests, viz.:- It does not give the xanthroprotein reaction ( $\$ 248)$, is not precipitated by acetic acid and potassium ferrocyanide, nor by tannic acid, mercuric chloride, silver nitrate, or iodine. In other respects it belongs to the group of albuminoids. It is rendered inactive in an acid fluid by heating it to $55^{\circ}$ to $60^{\circ} \mathrm{C}$.

166. PROCESS OF GASTRIC DIGESTION.-[In the process of gastric digestion we have to consider-

1. The secretion of gastric juice and its action on food.

2. The absorption of the products of this digestion.

3. The movements of the stomach itself.]

Chyme.-The finely divided mixture of food and gastric juice is called chyme The gastric juice acts upon certain constituents of chyme.

I. Action on Proteids.-Pepsin and the dilute hydrochloric acid, at the temperature of the body, transform proteids into a soluble form, to which Lehmann (1850) gave the name of "peptone" (\$ 249, III.). Fibrin (or coagulated proteids) first becomes clear and swollen up.

[It is commonly stated that the first product formed during the gastric digestion of proteids is syntonin or para-peptone, then hemi-albumose or pro-peptone, and 
finally peptone. The products vary, however, with the proteid digested. Kühne has shown that the proteid molecule is split up, and yields two groups, which 'he calls anti-peptone and hemi-peptone; the former can be split up into leucin and tyrosin by trypsin, while the latter does not undergo this change. A mixture of the two he calls ampho-peptone.-The intermediate body or pro-peptone, is really a mixture of several bodies. Kühne called it hemi-albumose. These intermediate bodies from albumin are called albumoses, from globulins globuloses, from casein caseoses. Halliburton calls all these intermediate bodies "proteoses."]

Properties. - Hemi-albumose, although a composite body, gives the following reactions :-It is highly soluhle in water; when heated to $50^{\circ}$ to $60^{\circ}$ it becomes somewhat turbid, but when boiled it becomes clear, and gets turbid again on cooling. This effect is most marked when it is treated with acetic acid and sodic chloride, or the latter alone. It is precipitated by acetic acid and potassic ferrocyanide, but the precipitate disappears on heating and reappears on cooling. It gives the biuret rosy tint reaction like peptones. It is precipitated by nitric acid, and the precipitate adheres to the glass, but is soluble in the acid with the aid of heat, yielding a yellow fluid, but is precipitated on cooling. It is precipitated by boiling with acetic acid and a strong solution of sodic sulphate, metaphosphoric acid, and pyrogallic acid (Kühne). It is said to be present in all animal tissues except inuscle and nerve (\$ 293).

[Albumoses are the first products of the splitting up of proteids by enzymes, and from them peptones are ultiniately formed. They may be made from Witte's peptone, or by the peptic digestion of fibrin. Such a mixture, on being neutralised with sodic carbonate, gives a copious precipitate of para-peptones, which can be filtered off, leaving a clear solution of albumoses. On saturating the clear fluid with $\mathrm{NaCl}$, a dense white precipitate, consisting of three albumoses, called proto-, dys-, and hetero-albumose is obtained ; a fourth, deutero-albumose, remains in solution, but can be precipitated by adding acetic acid. If the albumose precipitate be treated with 10 per cent. $\mathrm{NaCl}$ solution, proto- and hetero-albumose are dissolved, leaving dys-albumose undissolved. Dialysis of the saline solution precipitates hetero-albumose, leaving proto-albumose in solution. It is probable, however, that hetero- and dys-albumose are identical, or that the former is merely an insoluble form of the latter. The albumoses are bodies intermediate between albumins and peptones, and of the three, deutero-albumose is nearest to peptones.]

[Properties.-Proto-albumose is soluble in distilled water, is not changed by heat, but is precipitated by saturation of the solution with sodic chloride, by $\mathrm{HNO}_{3}$, acetic acid and potassie ferrocyanide, copper sulphate, mercuric chloride. Deutero-albumose is very like the foregoing, but it is not precipitated by $\mathrm{HNO}_{3}$ or on adding sodic chloride to saturation, but precipitation occurs when 20 to 30 per rent. of acetic acid is addecl. Hetero-albumose resembles a globulin in its properties; it is insoluble in distilled water, but is soluble in saline solutions (10 to 15 per cent.), and is partly precipitated from its solution by saturation with $\mathrm{NaCl}$ or dialysis. It is coagulated by heat. All give the rosy-pink colour with the biuret-reaction, and they are all precipitated by saturation with neutral ammonia sulphate, which peptones are not (Kühne and Chittenden).]

[Globuloses from the globulin of ox-serum are obtained in the same way, although the ferment has much less action on globulin than on albumin. Speaking generally, they resemble the albumoses].

By the continued action of the gastric juice, the pro-peptone passes into a true soluble peptone. The unchanged albumin behaves like an anhydride with respect to the peptone. The formation of peptone is due to the taking up of a molecule of water, under the influence of the hydrolytic ferment pepsin, and the action takes place most readily at the temperature of the body. Gelatin is changed into a gelatin-peptone.

According to Kiihne, the proteid molecule contains two substances preformed : anti-albumin and hemi-albumin. Gastric juice at first converts them into anti-albumose and hemi-albumose, and both ultimately into anti-peptone and hemi-peptone (\$170, II.). Only the latter is split up by trypsin into leucin and tyrosin.

The greater the amount of pepsin (within certain limits), the more rapidly does the solution take place. The pepsin suffers scarcely any change, and if care be taken to renew the hydrochloric acid, so as to keep it at a uniform amount, the 
pepsin can dissolve new quantities of albumin. Still, it seems that some pepsin is used up in the process of digestion (Grützner). Proteids are introduced into the stomach either in a solid (coagulated) or fluid condition. Casein alone of the fluid forms is precipitated or coagulated, and afterwards dissolved. The noncoagulated proteids are transformed into syntonin, without being previously coagulated, and are then changed into pro-peptone and directly peptonised, i.e., actually dissolved.

When albumin is digested by pepsin at the temperature of the body, a not inconsiderable amount of heat disappears, as can be proved by calorimetric experiment (Maly). Hence, the temperature of the chyme in the stomach falls $0^{\circ} \cdot 2$ to $0^{\circ} \cdot 6 \mathrm{C}$. in two to three hours $(v$. Vintschgau and Dietl).

Coagulated albumin may be regarded as the anhyairide of the fluid form, and the latter again as the anhydride of peptone. The peptones, therefore, represent the highest degree of hydration of the proteids.

Hence, peptones may be formed from proteids by those reagents which usually cause hydration, viz., treatment with strong acids (from fibrin, with $0.2 \mathrm{HCl}$ ), caustic alkalies, putrefactive and various other ferments, and ozone.

The anhydride proteid has been prepared from the hydrated form. Henniger and Hofmeister, by boiling pure peptone with dehydrating substances (anhydrous acetic acid at $80^{\circ}$ C.), have succeeded in decomposing it into a body resembling syntonin.

Peptones.-(1) They are completely soluble in water. (2) They diffuse very easily through membranes. (3) They filter quite easily through the pores of animal membranes. (4) They are not precipitated by boiling, nitric acid, acetic acid and potassium ferrocyanide, acetic acid and saturation with common salt. (5) They are precipitated from neutral or feebly acid solutions by mercuric chloride, tannic acid, bile acids, and phosphoro-molybdic acid. (6) With Millon's reagent they react like proteids, and give a red colour, and with nitric acid give the yellow xanthoprotein reaction. (7) With caustic potash or soda and a small quantity of cupric sulphate, [or Fehling's solution], they give a beautiful rosy-red colour, the biuretreaction. (8) They rotate the plane of polarised light to the left.

[Kühne and Chittenden, making use of the fact that ammonium sulphate to saturation precipitates all proteids from solution except peptone, have reinvestigated the subject, and they find that many of the peptones of commerce contain albumoses. Pure peptone has remarkable properties. When dissolved in water, it hisses and froths like phosphoric anhydride, heat is evolved, and a brown solution is formed. It is difficult to preserve it. It is not precipitated by $\mathrm{NaCl}$, or $\mathrm{NaCl}$ and acetic acid, but is completely precipitated by phospho-tungstic and phospho-molybdic acids, tannin, iodo-mercuric iodide, picric acid. Peptones have a cheesy taste, while albumin and albumoses are tasteless.]

The biuret-reaction is obtained with pro-peptone, as well as with a form of albumin, which is formed during artificial digestion and is soluble in alcohol. It is called "alkophyr" by Briicke. [Darby's fluid-meat gives all the above reactions, and is very useful for studying the tests for peptones.]

The rapidity of solution of fibrin is tested by placing fibrin, which is swollen up by the action of 0.2 per cent. $\mathrm{HCl}$ in a glass funnel, and adding the digestive fluid, observing the rapidity with which the fluid, the altered fibrin, drops from: the funnel, and the fibrin disappears (Grünhagen). Or the fibrin may be coloured with carmine, swollen up in 0.1 per cent. $\mathrm{HCl}$, and placed in the digestive fluid. The more rapidly the fluid is coloured red, the more energetic is the digestion.

Preparation.-Pure peptones are prepared by taking fluid which contains them and neutralising it with barium carbonate, evaporating upon a water-bath, and filtering. The barium is removed from the filtrate by the careful addition of sulphuric acid, and subsequent filtration.

Ptomaines. - Brieger extracted from gastric peptones by amylic alcohol a peptone-free poison, with actions like those of curara. It belongs to the group of ptomaines, i.e., alkaloids obtained from dead bodies or decomposing proteids. [Ptomaines are identical with the alkaloids in plants, and many have been isolated. The term leucomaine has been applied by Gautier to 
alkaloids formed by the decomposition of albuminous bodies during the normal metabolic processes taking place in the tissues. They are not formed by the activity of micro-organisms. Some seem to be formed in muscle, and are closely allied to creatin and xanthin.]

Peptones are undoubtedly those modifications of albumin or proteids which, after their absorption from the intestinal canal into the blood, are destined to make good the proteids used up in the human organism. By giving peptones (instead of albumin) as food, life can not only be maintained, but there may even be an increase of the body-weight (Plosz and Maly, Adamkiewicz). Very probably, before being absorbed into the blood-stream, peptones are retransformed into serum-albumin (\$192).

Conditions affecting Gastric Digestion. - The presence of already-formed peptones interferes with the action of the gastric juice, in so far as the greater concentration of the fluid interferes with and limits the mobility of the fluid-particles. Boiling, concentrated acids, alum, and tannic acid, alkalinity of the gastric juice (e.g., by the admixture of much saliva), abolish the action; also sulphurous and arsenious acids and potassic iodide. The salts of the heavy metals, which cause precipitates with pepsin, peptone, and mucin, interfere with gastric digestion, and so do concentrated solutions of alkaline salts, common salt, magnesium and sodium sulphates. A small quanity of $\mathrm{NaCl}$ increases the secretion (Griutzner) and favours the action of pepsin. Alkalies rapidly destroy pepsin, but less rapidly pro-pepsin (Langley). Alcohol precipitates the pepsin, but by the subsequent addition of water it is redissolved, so that digestion goes on as before. Any means that prevent the proteid bodies from swelling up, as by binding them firmly, impede digestion. Slightly over half a pint of cold water does not seem to disturb healthy digestion, but it does so in cases of disease of the stomach. Copious draughts of water, and violent muscular exercise, disturb digestion; while warm clothing, especially over the pit of the stomach, aids it. Menstruation retards gastric digestion. [Oddi finds that the presence of large quantities of ox bile, or even of its own bile in the stomach of a dog, does not affect the activity of the gastric juice, does not precipitate peptones, and does not excite vomiting.]

[Artificial Digestion. - The action of gastric juice on proteids may be observed outside the body, and we can prove, as is shown in the following table, after Rutherford, that pepsin and an acid-e.g., hydrochloric, along with water-are essential to the formation of gastric peptones:-

\begin{tabular}{|l|l|l|}
\hline \multicolumn{1}{|c|}{ Beaker A. } & \multicolumn{1}{|c|}{ Beaker B. } & \multicolumn{1}{|c|}{ Beaker C. } \\
\cline { 1 - 3 } $\begin{array}{l}\text { Water. } \\
\begin{array}{l}\text { Pepsin, } 0.3 \text { per cent. } \\
\text { Fibrin. }\end{array}\end{array}$ & $\begin{array}{l}\text { Water. } \\
\mathrm{HCl} 0.2 \text { per cent. } \\
\text { Fibrin. }\end{array}$ & $\begin{array}{l}\text { Wepsin, 0.3 per cent. } \\
\text { Hel, 0.2 } \\
\text { Fibrin. }\end{array}$ \\
\hline \multicolumn{1}{|c|}{ Keep all in water-bath at $38^{\circ} \mathrm{C}}$. \\
\hline Unchanged. & $\begin{array}{l}\text { Fibrin swells up, becomes clear, and is } \\
\text { changed into acid-albumin or syntonin. }\end{array}$ & $\begin{array}{l}\text { Fibrin ultimately changed } \\
\text { into peptone. }\end{array}$ \\
\hline
\end{tabular}

[In all animals, gastric digestion is essentially an acid digestion, and between the native proteid, fibrin, albumin, or any other form of proteid, and the end-product peptone, there are numerous intermediate substances, many of whose properties and characters have still to be investigated.

[Exclusion of the Stomach. - Ogata finds that if the stomach be divided at the pyloric end so as to exclude the stomach from the digestive apparatus, a dog can be nourished for a long time by introducing food through the pylorus into the duodenum. A dog has lived several years after excision of its stomach $($ Czerny). Raw flesh so introduced is digested more rapidly in the small intestine than in the stomach. The stomach not only digests, but it acts on the connective. tissue of flesh so as to prepare the latter for intestinal digestion.]

II. Action on other Constituents of Food.-Milk coagulates when it enters the stomach, owing to the precipitation of the casein, and in doing so it entangles some of the milk-globules. During the process of coagulation, heat is given off. The free hydrochloric acid of the gastric juice is itself sufficient to precipitate it; the acid removes from the alkali-albuminate or casein the alkali which keeps it in solution. Hammarsten separated a special ferment from the gastric 
juice-quite distinct from pepsin-the milk-curdling ferment which, quite independently of the acid, precipitates the casein either in neutral or alkaline solutions. It is this ferment or rennet which is used to coagulate casein in the making of cheese. [Rennet is an infusion of the fourth stomach of the calf in brine ( $\$ 231)$. The ferment which coagulates milk is quite distinct from pepsin. If magnesic carbonate be added to an infusion of calf's stomach, a precipitate is obtained. The clear fluid has strongly coagulating properties, while the precipitate is strongly peptic.]

The action of the milk-curdling ferment is perhaps, like the action of all ferments, a hydration of casein ; it is greater in the presence of $0.2 \mathrm{HCl}$.

One part of the rennet-ferment can precipitate 800,000 parts of casein. When casein coagulates, two new proteids seem to be formed-the coagulated proteid which constitutes cheese, and a body resembling peptone dissolved in the whey. The addition of calcium chloride accelerates, while water retards the coagulation (\$ 231) (Hammarsten). [A ferment similar to rennet is contained in the seeds of Withania coagulans (S. Lea).]

Casein is first precipitated in the stomach, then a body like syntonin is formerl, and finally peptone. During the process, a substance containing phosphorus and resembling nuclein appears (Lubavin).

There is a "lactic acid ferment" also present, which changes milk-sugar into lactic acid (Hammarsten). Part of the milk-sugar is changed in the stomach and intestine into grape-sugar.

Action on Carbohydrates.-Gastric juice does not act as a solvent of starch, inulin, or gums. Cane-sugar is slowly changed into grape-sugar. According to Uffelmann, the gastric mucus, and according to Leube, the gastric acid, are the chief agents in this process. On albumenoids.-During the digestion of true cartilage, there is formed a chondrin-peptone, and a body which gives the sugarreaction with Trommer's test. Perfectly pure elastin yields an elastin-peptone, similar to albumin-peptone, and hemi-elastin similar to hemi-albumose. A very minute quantity of fat is broken up into glycerine and fatty acids. [On neutral olive-oil being injected into the stomach of a dog, after several hours-the pylorus being plugged with an elastic bag-it partly splits up and yields oleic acid (Cash and Ogata).]

[We still require further observations on the gastric digestion of fats. Richet observed in his case of fistula, that fatty matters remained a long time in the stomach, and Ludwig found the same result in the dog. In some dyspeptics, rancid eructations often take place towards the end of gastric digestion.]

III. Action on the various Tissues. - (1) The gelatin-yielding substance (collagen) of all the connective-tissues (connective-tissue, white fibro-cartilage, and the matrix of bone), as well as glutin, is dissolved and peptonised by the gastric juice. [Gelatin, when acted on by gastric juice, no longer solidifies in the cold, but a gelatin-peptone is formed, which is soluble and diffusible, although it differs from true peptone. In the dog, connective-tissues are specially acted on in the stomach, while the other parts of organs used as food are prepared for digestion in the small intestine, where the cellular and nuclear elements are digested by the pancreatic juice (Bikfalvi).] (2) The structureless membranes (membranæ propriæ) of glands, sarcolemma, Schwann's sheath of nerve-fibres, capsule of the lens, the elastic laminæ of the cornea, the membranes of fat-cells are dissolved, but the true elastic (fenestrated) membranes and fibres are not affected. (3) Striped muscle, after solution of the sarcolemma, breaks up transversely into dises, and, like non-striped muscle, is dissolved, and forms a true soluble peptone, but parts of the muscle always pass into the intestine. (4) The albuminous constituents of the soft cellular elements of glands, stratified epithelium, endothelium, and lymph-cells, form peptones, but the nuclein of the nuclei does not seem to be dissolved. (5) The horny parts of the epidermis, nails, hair, as well as chitin, silk, conchiolin, and spongin of the lower animals are indigestible, and so are amyloid-substance and wax. (6) The red blood-corpuscles are dissolved, the hæmoglobin decomposed into hæmatin and a globulin-like substance; the latter is peptonised, while the former remains unchanged, and is partly absorbed and transformed into bile-pigment. Fibrin is easily dissolved to form hemi- and anti-peptone. (7) Mucin, which is also secreted by the goblet-cells of the stomach, passes through the intestines unchanged. (8) Vegetable fats are not affected by the gastric juice ; these cells yield their protoplasmic contents to form peptones, while the cellulose of the cell-wall, in the case of man at least, remains undigested ( $\$ 184)$.

Why the Stomach does not digest itself.-That the stomach can digest living things is shown by the following facts:-Bernard introduced the leg of a living frog through a gastric 
fistula into the stomach of a dog. Pavy did the same with the ear of a rabbit, and in both the objects introduced were digested. [Frenzel has modified this experiment, and shown that the legs of a living frog are digested by artificial gastric juice, the tissues being first killed and then digested. His experiments go to show that the alkalinity of the blood is not the protective medium.] The nargins of a gastric ulcer and of gastric fistulæ in man are attacked by the gastric juice. John Hunter (1772) discussed the question why the stomach does not digest itself. Not unfrequently after death the posterior wall of the stomach is found digested, [more especially if the person die after a full meal and the body be kept in a warm place, whereby the contents of the stomach may escape into the peritoneum. Cl. Bernard showed, that if a rabbit be killed and placed in an oven at the temperature of the body, the walls of the stomach are attacked by its own gastric jnice. Fishes also are frequently found with their stomach partially digested after death]. It would seem, therefore, that, so long as the circulation continues, the tissues are protected from the action of the acid by the alkaline blood; this action cannot take place if the reaction be alkaline (Pavy). [This, however, does not explain why the pancreatic juice does not digest the pancreas.] Ligature of the arteries of the stomach, causes digestive softening of the gastric mucous membrane. The thick layer of mucus may also aid in protecting the stomach from the action of its own gastric juice $(\mathrm{Cl}$. Bernard).

167. GASES IN THE STOMACH.-The stomach always contains a certain quantity of gas, derived partly from the gases swallowed with the saliva, partly from gases which pass backwards from the duodenum.

The air in the stomach is constantly undergoing changes, whereby its $\mathrm{O}$ is absorbed by the blood, and for 1 vol. of $\mathrm{O}$ absorbed 2 vols. of $\mathrm{CO}_{2}$ are returned to the stomach from the blood. Hence, the amount of $\mathrm{O}$ in the stomach is very small, the $\mathrm{CO}_{2}$ very considerable (Planer).

Gases in the Stomach, -Vol. per cent. (Planer).

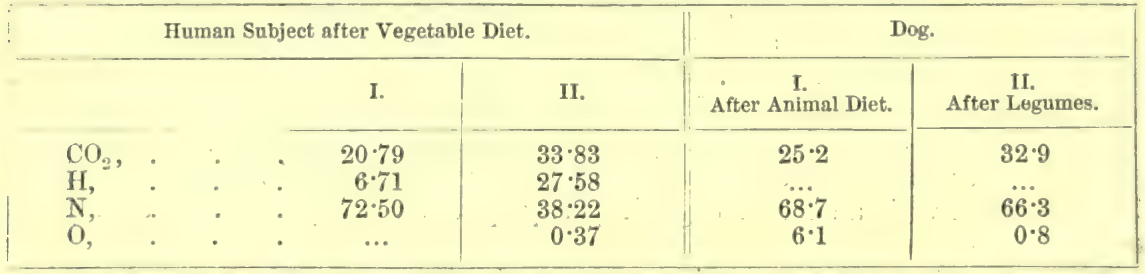

By the acid of the stomach a part of the $\mathrm{CO}_{2}$ is set free from the saliva, which contains much $\mathrm{CO}_{2}(\$ 146)$. The $\mathrm{N}$ acts as an indifferent substance.

Abnormal development of gases in persons suffering from gastric catarrh, occurs when the gastric contents are neutral in reaction ; during the butyric acid fermentation $\mathrm{H}$ and $\mathrm{CO}_{2}$ are

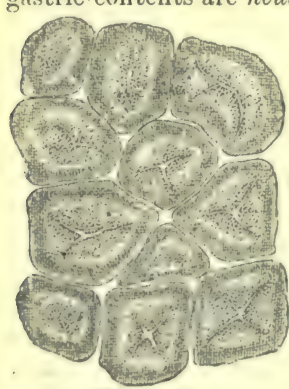

Fig. 186.

Section of the fresh pancreas. formed; the acetic acid and lactic acid fermentations do not cause the formation of gases. Marsh-gas $\left(\mathrm{CH}_{4}\right)$ has been found, but it comes from the intestine, as it can only be formed when no $\mathrm{O}$ is present ( $\$ 184$ ).

168. STRUCTURE OF THE PANCREAS. - The pancreas is a com. pound tubular gland, and in its general arrangement into lobes, lobules, and system of ducts and acini, it corresponds exactly to the taine salivary glands. The epithelium lining the ducts is not at all, or only faintly, striated. The acini are tubular or flasked-shaped, and often convoluted. They consist of a membrana propria, resembling that of the salivary glands, lined by a single layer of somewhat cylindrical cells, with a more or less conical apex, directed towards the very narrow lumen of the acini. [As in the salivary glands, there is a narrow intermediary part of the ducts opening into the acini, and lined by flattened epithelium.] The cells lining the acini consist of two zones (fig. 186):-

(1) The smaller outer or parietal layer is transparent, homogeneous, sometimes faintly striated, and readily stained with carmine and log. wood; and (2) the inner layer (Bernard's granular layer) is granular, and stains but slightly with carmine (fig. 186). It undoubtedly contributes to the secretion by giving off material, the granules being dissolved, while the zone itself becomes smaller. The spherical nucleus lies between the two zones. [The lumen of the acini is very small, and spindle-shaped or branched cells (centro-acinar cells) lie in it, and send their processes between the secretory cells, thus acting as supporting cells for the elements of the wall of the acini. During secretion, there is a 
continuous change in the appearance of the cell-substance; the granules of the inner zone dis. solve to form part of the secretion; new granules are formed in the homogeneous substance of the outer zone, and pass towards the inner zone (Heidenhain, Kühne and Lea).

Changes in the Cells during Digestion.-During the first stage (6 to 10 hours) the granular inner zone diminishes in size, the granules disappear, while the striated outer zone increases in size (fig. 187, 2). In the second stage (10 to 20 hours) the inner zone is greatly enlarged and granular, while the outer zone is small (fig. 187, 3). During hunger the outer zone again enlarges (fig. 187, 1). In a gland, where paralytic secretion takes place, the gland is much diminished in size, the cells are shrivelled (fig. 187, 4) and greatly changed. According to Ogata, some cells actually disappear during secretion.

The axially-placed excretory duct consists of an inner thick and an outer loose wall of connective and elastic tissues, lined by a single layer of columnar epithelium. Small mucous glands lie in the largest trunks. Non-medallated nerves, with ganglia in their course, pass to the acini, but their mode of termination is unknown. The blood-vessels form a rich capillary plexus round some acini, while round others there are very few. Kühne and Lea found peculiar small cells in groups between the alveoli, and supplied with convoluted capillaries like glomeruli. Their significance is entirely unknown. ['They are probably lymphatic in their nature.] The lymphatics resemble those of the salivary glands. When a coloured injection is forced into the ducts under a high pressure, fine intercellular passages between the secreting cells are formed (Saviotti's canals), but they are artificial Changes of the pancreatic cells in various stages of activity. 1 , products.]

[Number of Ducts. - In

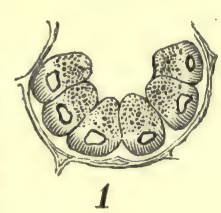

1

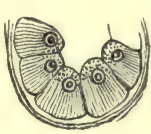

2

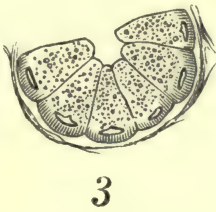

3

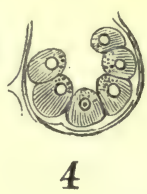

4

Fig. 187.

During hunger; 2 , in the first stage of digestion; 3 , in the second stage; 4, during paralytic secretion.

pancreatic secretion, it is important to remember that the number of pancreatic ducts varies in different animals. In man there is one duct opening along with the common bile-duct at Vater's ampulla, at the junction of the middle and lower third of the duodenum. The rabbit has two ducts, the larger opening separately about 14 inches (30 to $35 \mathrm{~cm}$.) below the entrance of the bile-duct. The dog and cat have each two ducts opening separately.]

Chemistry. - The fresh pancreas contains : water, proteids, ferments; fats, and salts. In a gland which has been exposed for some time, much leucin, isoleucin, butalin, tyrosin, often xanthin and guanin, are found : lactic and fatty acids seem to be formed from chemical decom. positions taking place.

169. THE PANCREATIC JUICE. - Method. - Regner de Graaf (1664) tied a cannula in the pancreatic duct of a dog, and collected the juice in a small bag. Other experimenters made a temporary fistula. To make a permanent fistula, the abdomen is opened (dog), the pancreatic duct pulled forward, and stitched to the abdominal wall, with which it unites. Heidenhain cuts out the part of the duodenum where the duct opens into it, from its continuity with the intestine, and fixes it outside the abdominal wound.

The secretion obtained from a permanent fistula is a copious, slightly active, watery secretion, containing much sodium carbonate ; while the thick fluid obtained from the fistula before inflammation sets in, or that from a temporary fistula, acts far more energetically. This thick secretion, which is small in amount, is the normal secretion. The copious watery secretion is perhaps caused by the increased. transudation from the dilated blood-vessels (possibly in consequence of the paralysis of the vaso-motor nerves). It is, therefore, in a certain sense, a "paralytic secretion" (\$145). The quantity varies : much, according as the fluid is thick or thin. During digestion, a large dog secretes 1 to 1.5 gramme of a thick secretion ( $\mathrm{Cl}$. Bernard). Bidder and Schmidt obtained in twenty-four hours 35 to 117 grammes of a watery secretion per kilo. of a dog. When the gland is not secreting, and is at rest, it is soft, and of a pale yellowish-red colour, but during secretion it is red and turgid with blood, owing to the dilatation of the blood-vessels.

The normal secretion is transparent, colourless, odourless, saltish to the taste, and has a strong alkaline reaction, owing to the presence of sodium carbonate, so that when an acid is added, $\mathrm{CO}_{2}$ is given off. It contains albumin and alkalialbuminate; it is sticky, somewhat viscid, flows with difficulty, and is coagulated 
by heat into a white mass. In the cold, there separates a jelly-like albuminous coagulum. Nitric, hydrochloric, and sulphuric acids cause a precipitate; while the precipitate caused by alcohol is redissolved by water. $\mathrm{Cl}$. Bernard found in the pancreatic juice of a dog 8.2 per cent. of organic substances, and 0.8 per cent. of ash. The juice (dog) analysed by Carl Schmidt contained in 1000 parts :-

$$
\begin{gathered}
\text { Solids, } 90.38 \text { in } \\
1000 \text { parts. }
\end{gathered}\left\{\begin{array}{c}
\text { Organic, }: \\
\text { Inorganic, } \\
\text { (like those of } \\
\text { blood-serum). }
\end{array}\right.
$$

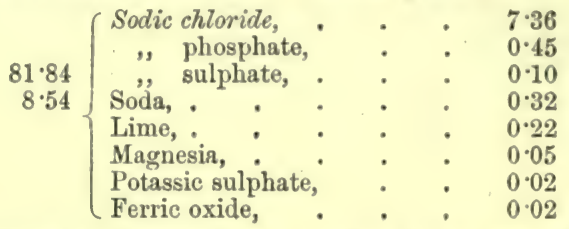

The more rapid and more profuse the secretion, the poorer it is in organic substances, while the inorganic remain almost the same ; nevertheless, the total quantity of solids is greater than when the quantity secreted is small (Bcrnstcin). Traces of leucin and soaps are present in the fresh juice. [It usually contains few or no structural elements. Any structural elements present in the fresh juice, as well as its proteids, are digested by the peptone-forming ferment of the juice, especially if the latter be kept for some time. If the fresh juice is allowed to stand for some time, and then mixed with chlorine water, a red colour is obtained.]

Concretions are rarely formed in the pancreatic ducts; they usually consist of calcic carbonate. Dextrose has been found in the juice in diabetes, and urea in jaundice. Schiff's statement that the pancreas secretes only after the absorption of dextrin, has not been confirmed. The secretory activity of the pancreas is not dependent on the presence of the spleen.

170. ACTION OF THE PANCREATIC JUICE.-The presence of at least four enzymes, or hydrolytic ferments, makes the pancreatic juice one of the most important digestive fluids in the body.

I. Diastatic action is due to the diastatic ferment, amylopsin, a substance which seems to be identical with the saliva ferment; but it acts much more energetically than the ptyalin on saliva, on raw starch as well as upon boiled starch; at the temperature of the body the change is effected almost at once, while it takes place more slowly at a low temperature. Glycogen is changed into dextrin and grape-sugar; and achroodextrin into sugar. Even cellulose is said to be dissolved, and gum changed into sugar by it, but inulin remains unchanged.

According to v. Mering and Musculus, the starch (as in the case of the saliva, § 148) is changed into maltose, and a reducing-dextrin; so also is glycogen. Amylopsin changes achroodextrin into maltose ; at $40^{\circ} \mathrm{C}$. ualtose is slowly changed into dextrose, but cane-sugar is not changed into invert-sugar. The ferment is precipitated by alcohol, while it is extracted by glycerine without undergoing any essential change. All conditions which destroy the diastatic action of saliva (\$148) similarly affect its action, but the admixture with acid gastric juice (its acid being neutralised) or bile does not seem to have any injurious influence. This ferment is absent from the pancreas of new-born children (Korowin).

Preparation. - The ferment is isolated by the same methods as obtain for ptyalin (\$ 148); but the tryptic ferment is precipitated at the same time. The addition of neutral salts (4 per cent. solution), e.g., potassium nitrate, common salt, ammonium chloride, increases the diastatic action.

II. Tryptic action, or the action on proteids, depends upon the presence of a hydrolytic ferment which is now termed trypsin (Kühne). Trypsin acts upon proteids at the temperature of the body, when the reaction is alkaline, and changes them first into a globulin-like substance, then into pro-peptone or albumose, and lastly into a true peptone, sometimes called tryptone. The albumoses are not so abundant or so easily separated as in gastric digestion (see also p. 248). The proteids do not swell up before they are changed into peptone, [but they are eroded or eaten away by the action of the juice]. When the proteid has been previously swollen up by the action of an acid, or when the reaction of the medium is acid, the transformation is interfered with.

Substances yielding gelatin, nuclein, and $\mathrm{Hb}$, resist trypsin ; glutin and swollen-up gelatinyielding substances are changed into gelatin-peptone, but the latter undergoes no further 
change. Hb- $\mathrm{O}_{2}$ is split up into albumin and hæmochromogen. In other respects, trypsin acts on tissues containing albumins just like pepsin (\$ 166, III.).

Trypsin is never absent from the pancreas of new-born children (Zweifel), and it may be extracted by water, which, however, also dissolves the albumin. Kühne has carefully separated the albumin and obtained the ferment in a pure state. It is soluble in water, insoluble in alcohol. Pepsin and hydrochloric acid together act upon trypsin and destroy it; hence it is not advisable to administer trypsin by the mouth, as it would be destroyed in the stomach. When dried it may be heated to $160^{\circ}$ without injury.

Trypsin is formed within the pancreas by a "mother-substance," or zymogen, taking up oxygen. The zymogen is found in small amount, 6 to 10 hours after a meal, in the inner zone of the secretory cells, but after 16 hours it is very abundant in the inner zone of the cells. It is soluble in water and glycerine. Trypsin is formed in the watery solution from the zymogen, and the same result occurs when the pancreas is chopped up and treated with strong alcohol (Kühne). The addition of sodium chloride, carbonate, and glycocholate, favours the activity of the tryptic ferment (Heidenhain). [The following facts show that zymogen ('v́r ${ }^{\prime}$, ferment), or, as it has been called, trypsinogen, is the precursor of trypsin, that it exists in the gland-cells, and requires to be acted upon before trypsin is formed. If a glycerine extract be made of a pancreas taken from an animal just killed, and if another extract be made from a similar pancreas which has been kept for 24 hours, it will be found that an alkaline solution of the former has practically no effect on fibrin, while the latter is powerfully proteolytic. If a fresh, and still warm, pancreas be rubbed up with an equal volume of a 1 per cent. solution of acetic acid, and then extracted with glycerine, a powerfully proteolytic extract is at once obtained. Trypsin is formed from zymogen by the action of acetic acid. There is reason to believe that trypsin is formed from zymogen by oxidation, and that the former loses its proteolytic power after removal of its oxygen. The amount of zymogen present in the gland-cells seems to depend upon the number and size of the granules present in the inner granular zone of the secretory cells.]

Further Effects. - When trypsin is allowed to act upon the hemi-peptone formed by its own action, the latter is partly changed into the amido-acid, leucin, or amido-caproic acid $\left(\mathrm{C}_{6} \mathrm{H}_{13} \mathrm{NO}_{2}\right)$, and tyrosin $\left(\mathrm{C}_{9} \mathrm{H}_{11} \mathrm{NO}_{3}\right)$, which belongs to the aromatic series $(\$ 252, \mathrm{IV} .3)$. Hypoxanthin, xanthin, and aspartic or amido-succinic acid $\left(\mathrm{C}_{4} \mathrm{H}_{7} \mathrm{NO}_{4}\right)$, are also formed during the digestion of fibrin and gluten, and so are glutamic $\left(\mathrm{C}_{5} \mathrm{H}_{9} \mathrm{NO}_{4}\right)$ and amido-valerianic acid $\left(\mathrm{C}_{5} \mathrm{H}_{11} \mathrm{NO}_{2}\right)$. Gelatin is first changed into a geletin-peptone, and afterwards is decomposed into glycin and ammonia.

Putrefactive Phenomena.-If the action of the pancreatic juice be still further prolonged, especially if the reaction be alkaline, a body with a strong, stinking, disagreeable fæcal odour, indol $\left(\mathrm{C}_{8} \mathrm{H}_{7} \mathrm{~N}\right)$, skatol $\left(\mathrm{C}_{9} \mathrm{H}_{9} \mathrm{~N}\right)$, and phenol $\left(\mathrm{C}_{6} \mathrm{H}_{6} \mathrm{O}\right)$, and a substance which becomes red on the addition of chlorine-water (Bernard), [or it gives with bromine-water first a pale red and then a violet tint (Kühne)], volatile fatty acids are formed, while, at the same time, $\mathrm{H}, \mathrm{CO}_{2}, \mathrm{H}_{2} \mathrm{~S}, \mathrm{CH}_{4}$, and $\mathrm{N}$ are given off. The formation of indol and the other substances just mentioned depends upon putrefaction ( $\$ 184$, III.). Their formation is prevented by the addition of salicylic acid, or thymol, which kills the organisms upon which putrefaction depends (Kühne).

[Artificial Digestion.-From fibrin placed in pancreatic juice, or in a 1 per cent. solution of sodium carbonate containing the ferment trypsin, peptones are rapidly formed at $40^{\circ} \mathrm{C}$. When we compare gastric with pancreatic digestion, we find that the fibrin in pancreatic digestion is eroded, or eaten away, and never swells up. The process takes place in an alkaline medium, and never in an acid one. In fact, a 1 per cent. solution of sodic carbonate seems to play the same part in assisting trypsin, that a 2 per cent. solution of $\mathrm{HCl}$ does for pepsin, in gastric digestion. In gastric digestion acid-albumin or syntonin is formed in addition to the true peptones. 
In pancreatic digestion a body resembling alkali-albumin, which passes into a globulin-like body, and ultimately into a peptone, is formed. Of the peptones so produced, one is called anti-peptone, and it is not further changed, but part of the proteid is changed into hemi-peptone. This body, when acted upon, yields leucin and tyrosin. When putrefaction takes place, the bodies above-mentioned are also formed. We might represent the action of trypsin thus :- Proteid + trypsin +1 per cent. sodium carbonate, kept at $38^{\circ} \mathrm{C}$. $=$ formation of a globulin-like body, and then anti-peptone and hemi-peptone are formed.

\begin{tabular}{|c|c|c|c|}
\hline \multirow[t]{2}{*}{ ANTI-PEPTONE } & \multicolumn{3}{|c|}{ HEMI-PEPTONE } \\
\hline & Normal Digestive Products. & \multicolumn{2}{|c|}{ Putrefactive Products. } \\
\hline $\begin{array}{l}\text { undergoes no } \\
\text { further change. }\end{array}$ & $\begin{array}{l}\text { Leucin, } \\
\text { Tyrosin, } \\
\text { Hypoxanthin, } \\
\text { Aspartic Acid. }\end{array}$ & $\begin{array}{l}\text { Indol, } \\
\text { Skatol, } \\
\text { Phenol. }\end{array}$ & $\begin{array}{l}\text { Volatile Fatty Acids, } \\
\mathrm{H}, \mathrm{CO}_{2}, \mathrm{H}_{2} \mathrm{~S}, \\
\mathrm{CH}_{4}, \mathrm{~N} .\end{array}$ \\
\hline
\end{tabular}

It seems that trypsin in pure water can act slowly upon fibrin to produce peptone. Pepsin cannot do this without the aid of an acid.]

[Kuihne's Pancreas Powder.-This is prepared by the prolonged extraction of fresh pancreas of ox with alcohol and then with ether. If the dry powder be extracted for several hours with a 1 per cent. solution of salicylic acid, and filtered, a fluid with powerful proteolytic, but no diastatic, properties is obtained. Several hours afterwards much tyrosin may separate out, which, of course, must be removed by filtration. The clear fluid, when mixed with fibrin and a 1 per cent. solution of sodic carbonate, rapidly digests fibrin. If it be desired to obtain a true pancreatic digestion, with none of the products of putrefaction, the mixture must be strongly "thymolised" with a 25 per cent. alcoholic solution of thymol (Kühne).]

[Setschenow finds that egg-albumin, boiled in a vacuum at $35^{\circ}-40^{\circ} \mathrm{C}$., is more rapidly digested than fibrin by a specially prepared trypsin.] When proteids are boiled for a long time with dilute $\mathrm{H}_{2} \mathrm{SO}_{4}$, we obtain peptone, then leucin and tyrosin ; gelatin yields glycin. Hypoxanthin and xanthin are obtained in the same way by similarly boiling fibrin, and the former may even be obtained by boiling fibrin with water (Chittenden).

It is very remarkable that the juice of the green fruit of the papaya tree, or Carica papaya, possesses digestive properties (Roy, Wittmack), and that the action is due to peptonising ferment, closely related to trypsin, and called caricin or papain. [It forms a true peptone, an intermediate body, and leucin and tyrosin. It also contains a milk-coagulating ferment (Martin).] The milky juice of the fig-tree has a similar action. Sprouting malt, vetch, hop, hemp during sprouting, and the receptacle of the artichoke contain a peptonising ferment. Leucin, tyrosin, glutamic and aspartic acils, and xanthin are formed in the seeds of some plants; hence we may assume that the processes of decomposition in some seeds are closely allied to the fermentative actions that occur in the intestine.

III. The action on neutral fats is twofold:-(1) It acts upon fats so as to form a fine permanent emulsion. (2) It causes neutral fats to take up a molecule of water and split into glycerine and their corresponding fatty acids :-

$$
\left.\left.\left.\left.\underset{\text { Tristearin. }}{\left(\mathrm{C}_{57} \mathrm{H}_{110} \mathrm{O}_{6}\right.}\right)+\underset{\text { Water. }}{3\left(\mathrm{H}_{2} \mathrm{O}\right.}\right)=\underset{\text { Glycerine. }}{\left(\mathrm{C}_{3} \mathrm{H}_{8} \mathrm{O}_{3}\right.}\right)+\underset{\text { Stearic Acid. }}{3\left(\mathrm{C}_{18} \mathrm{H}_{36} \mathrm{O}_{2}\right.}\right) .
$$

The latter result is due to the action of an easily-decomposable fat-splitting ferment $(\mathrm{Cl}$. Bernard), also called steapsin. Lecithin is decomposed by it into glycero-phosporic acid, neurin and fatty acids. The fatty acids thus liberated are partly suponified by the alkali of the pancreatic and intestinal juices, and partly emulsionised by the alkaline intestinal juice. Both the soaps and emulsions are capable of being absorbed ( $\$ 191)$.

Emulsification.-The most important change effected on fats in the small intestine is the production of an emulsion, or their subdivision into exceedingly minute particles (\$191). This is necessary in order that the fats may be taken up by the lacteals. If the fat to be emulsified contain a free fatty acid, i.e., if it be slightly rancid, and if the fluid with which it is mixed 
be alkaline, emulsification takes place extremely rapidly (Brïcke). A drop of cod-liver oil, which in its unpurified condition always contains fatty acids, on being placed in a drop of 0.3 per cent. solution of soda, instantly gives rise to an emulsion $(\mathrm{Gad})$. The excessively minute oil-globules that compose the emulsion are first covered with a layer of soap, which soon dissolves, and in the process small globules are detached from the original oil-globules. The fresh surface is again covered by a soap film, and the process is repeated over and over again until an excessively fine emulsion is obtained. If the fat contain much fatty acid, and the solution of soda be more concentrated, "myelin forms" are obtained similar to those which are formed when fresh nerve-fibres are teased in water. Animal oils enulsionise more readily than vegetable oils ; castor oil does not emulsionise $(G a d)$. [It is extremely difficult to obtain a perfectly neutral oil, as most oils contain a trace of a fatty acid. In fact, if on adding a weak solution of sodic carbonate to oil or fatty matters, fluid at the temperature of the body, an emulsion is obtained, one may be sure that the oil contained a fatty acid, so that Bernard's view about an " emulsive ferment" being necessary is not endorsed. The fatty acid set free by the fat-splitting ferment enables the alkaline pancreatic juice at once to produce an emulsion.]

Fat-Splitting Ferment. - This is a very unstable body, and must be prepared from the perfectly fresh gland by rubbing it up with powdered glass, glycerine, and a 1 per cent. solution of sodic carbonate, and allowing it to stand for a day or two (Grützner). [This ferment is said to cause an emulsion of oil and mucilage tinged blue with litmus at $40^{\circ} \mathrm{C}$. to become red (Gamgee). In performing this experiment notice that the mucilage is perfectly neutral, as gum-arabic is frequently acid.]

[Pancreatic Extracts. - The action of the pancreas may be tested by making a watery extract of a perfectly fresh gland. Such an extract always acts upon starch and generally upon fats, but this extract and also the glycerine extract vary in their action upon proteids at different times. If the extract-watery or glycerine-be made from the pancreas of a fasting animal, the tryptic action is slight or absent, but is active if it be prepared from a gland 4 to 10 hours after a meal. The pancreatic preparations of Benger of Manchester, Savory and Moore, or Burroughs and Welcome, all possess active diastatic and proteolytic properties.]

[Pancreas Salt.-Prosser-James proposes to employ common salt mixed with pepsin, which he calls peptic salt; and he advocates the use of another preparation composed of the pancreatic ferments and common salt, pancreatic salt.]

The pancreas of new-born children contains trypsin and the fat-decomposing ferment, but not the diastatic one (Zweifel). A slight diastatic action is obtained after two months, but the full effect is not obtained until after the first year (Korowin).

IV. The pancreas contains a milk-curdling ferment, which may be extracted by means of a concentrated solution of common salt.

\section{THE SECRETION OF THE PANCREATIC JUICE-Rest and} Activity.-As in other glands, we distinguish a quiescent state, during which the gland is soft and pale, and a state of secretory activity, during which the organ swells up and appears pale red. The latter condition only occurs after a meal, and is caused probably reflexly owing to stimulation of the nerves of the stomach and duodenum. Kühne and Lea found that all the lobules of the gland were not active at the same time. The pancreas of the herbivora secretes uninterruptedly, [but in the dog secretion is not constant].

Time of Secretion.-According to Bernstein and Heidenhain the secretion begins to flow when food is introduced into the stomach, and reaches its maximum 2 to 3 hours thereafter. 'The amount falls towards the 5th or 7 th hour, and rises ragain (owing to the entrance of the chyme into the duodenum) towards the 9 th and 11th hour, gradually falling towards the 17th to 24 th hour until it ceases completely. When more food is taken, the same process is repeated. As a general rule, a rapidly-formed secretion contains less solids than one formed slowly.

Condition of Blood-Vessels.-During secretion, the blood-vessels behave like the blood-vessels of the salivary glands after stimulation of the chorda-they dilate, and the venous blood is bright red-thus, it is probable that a similar nervous mechanism exists, [but as yet no such mechanism has been discovered]. The secretion is excreted at a pressure of more than $17 \mathrm{~mm}$. Hg. (rabbit).

Effect of Nerves.-The nerves arise from the hepatic, splenic, and superior mesenteric plexuses, together with branches from the vagus and sympathetic. The secretion is excited by stimulation of the medulla oblongata, as well as by direct stimulation of the gland itself by induction-shocks. [It is not arrested by 
section of the cervical spinal cord.] The secretion is suppressed by atropin [in the dog, but not the rabbit], by producing vomiting, by stimulation of the central end of the vagus, as well as by stimulation of other sensory nerves, e.g., the crural and sciatic. Extirpation of the nerves accompanying the blood-vessels prevents the above-named stimuli from acting. Under these circumstances, a thin "paralytic secretion," with feeble digestive powers, is formed, but its amount is not influenced by the taking of food. [Secretion is excited by the injection of ether into the stomach.]

Extirpation of the gland may be performed, or the duct ligatured in animals, without causing any very great change in their nutrition; the absorption of fat from the intestine does not cease. After the duct is ligatured it may be again restored. Ligature of the duct may cause the formation of cysts in the duct and atrophy of the gland-substance. Pigeons soon die after this operation.

[172. PREPARATION OF PEPTONISED FOOD.-Peptonised food may be given to patients whose digestion is feeble (Roberts). Food may be peptonised either by peptic or tryptic digestion, but the former is not so suitable as the latter, because in peptic digestion the grateful odour and taste of the food are destroyed, while bitter bye-products are formed, so that pancreatic digestion yields a more palatable anà agreeable product. As trypsin is destroyed by gastric digestion, obviously it is useless to give extract of the pancreas to a patient along with his food. $]$

[Peptonised Milk. - " A pint of milk is diluted with a quarter of a pint of water and heated to $60^{\circ} \mathrm{C}$. Two or three tea-spoonfuls of Benger's liquor pancreaticus, together with 10 or 20 grains of bicarbonate of sola, are then mixed therewith." Keep the mixture at $38^{\circ} \mathrm{C}$. for about two hours, and then boil it for two or three minutes, which arrests the ferment action.]

[Peptonised Gruel, prepared from oatmeal, or any farinaceous food, is more agreeable than

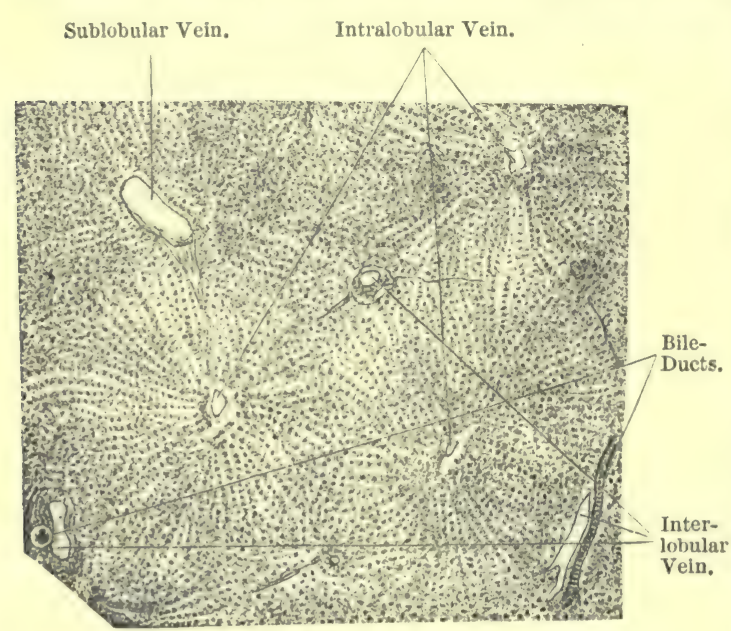

Fig. 188. peptonised milk, as the bitter flavour does not appear to be developed in the pancreatic digestion of vegetable proteids.]

[Peptonised Milk-Gruel yielded Roberts the most satisfactory results, as a complete and highly nutritious food for weak digestions. Make a thick gruel from any farinaceous food, e.g., oatmeal, and while still hot add to it an equal volume of cold milk, when the mixture will have a temperature of $52^{\circ} \mathrm{C}$. $\left(125^{\circ} \mathrm{F}\right.$.). To each pint of this mixture add two or three tea-spoonfuls of liquor pancreaticus and 20 grains of bicarbonate of soda. It is kept warm for two hours under a "cosey." It is then boiled for a few minutes and strained. The bitterness of the digested milk is almost com. pletely covered by the sugar pro. duced during the process.]

[Peptonised soups and beef-tea

Section of human liver, $\times 20$, showing the liver-lobules and have also been made and used the radiate arraugement of their cells from the central or with success, and have been adintralobular vein. ministered both by the mouth and rectum.]

[Peptonising powders containing the proper proportions of ferment and sodic bicarbonate are prepared by Benger, and Burroughs and Welcome.]

173. STRUCTURE OF THE LIVER - The liver, the largest gland in the body, consists of innumerable small lobules or acini, 1 to 2 millimetres $\left(\frac{1}{24}\right.$ to $\frac{1}{12}$ inch) in diameter. These lobules are visible to the naked eye. All the lobules have the same structure. 
1. The Capsule. - The liver is covered by a thin, fibrous, firmly-adherent capsule, which has on its free surface a layer of endothelium derived from the peritoneum. The capsule sends fine septa into the organ between the lobules, but it is also continued into the interior at the trans. verse fissure, where it surrounds the portal vein, hepatic artery, and bile-duct, and accompanies these structures as the capsule of Glisson, or interlobular connective-tissue. The spaces in which these three structures lie are known as portal canals. In some animals (pig, camel, polar bear) the lobules are separated from each other by the somewhat lamellated connective-tissue of Glisson's capsule, but in man this is but slightly developed, so that adjoining lobules are more or less fused. Very delicate connective-tissue, but small in amount, is also found within the lobules. Leucocytes are sometimes found in the tissue of Glisson's capsule.

2. Blood-Vessels. - (a) Branches of the Venous System. - The portal vein, after its entrance into the liver at the portal fissure, gives off numerous branches lying between the lobules, and ultimately forming small trunks which reach the periphery of the lobules, where they form a rich plexus. These are the interlobular veins (figs. 188, 189, V. $i$ ). From these veins numerous

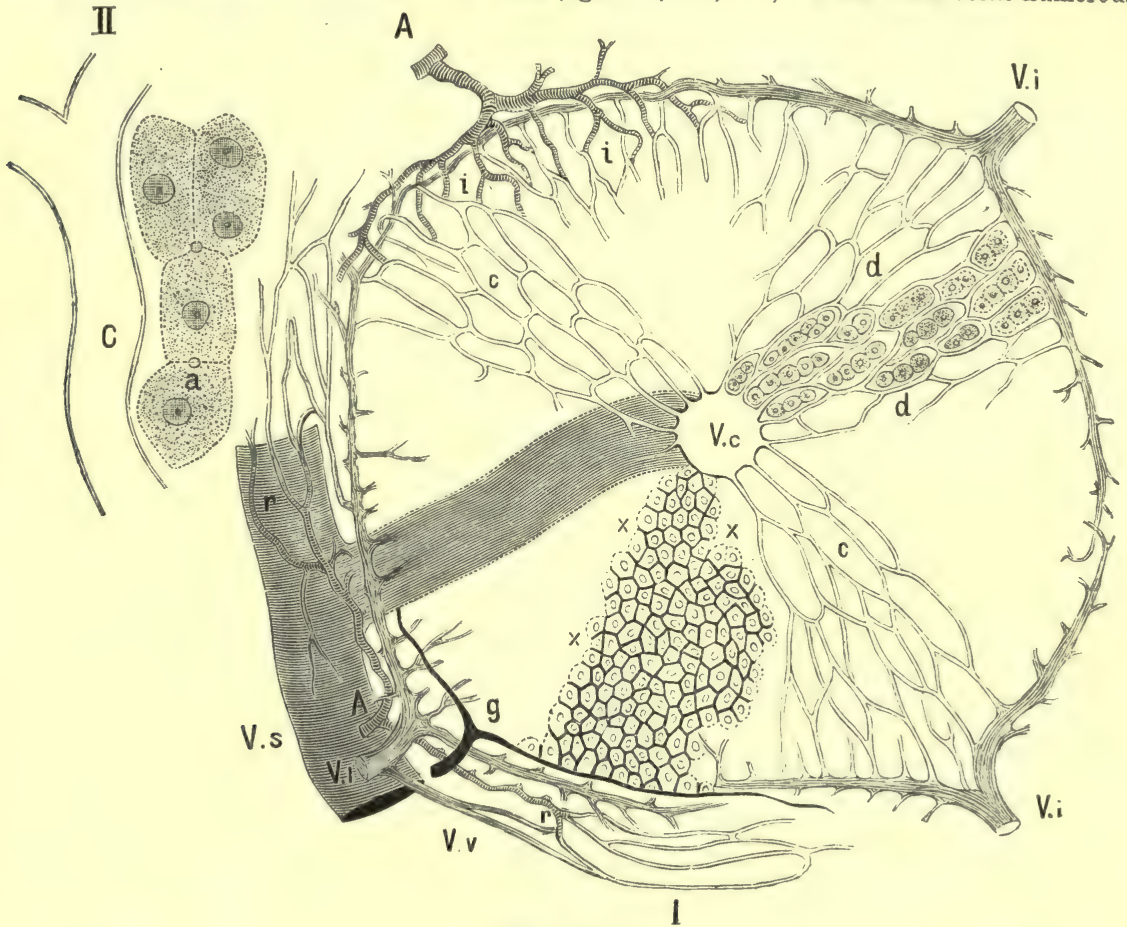

Fig. 189.

I, Scheme of a liver-lobule-V.i, V.i, interlobular veins (portal); $V . c$, central or intralobular vein (hepatic); $c, c$, capillaries between both; $V . s$, sublobular vein ; $V . v$, vena vaseularis ; $A, A$, hepatic artery, giving branches, $r, r$, to Glisson's capsule and the larger vessels, and ultimately forming the venæ vasculares at $i$, opening into the intralobular capillaries; $g$, bile-ducts ; $x, x$, intralobular biliary channels between the liver-cells; $d, d$, position of the liver-cells between the meshes of the blood-capillaries. II, Isolated livercells- $c$, a blood-capillary; $a$, fine bile-capillary channel.

capillaries $(c, c)$ are given off to the entire periphery of the lobule. The capillaries converge towards the centre of the lobule. As they proceed inwards, they form elongated meshes, and between the capillaries lie rows or columns of liver-cells $(d, d)$. The capillaries are relatively wide, and are so disposed as to lie between the edges of the columns of cells, and never between the surfaces of two neighbouring cells. The capillaries converge towards the centre of each lobule, where they join to form one large vein, the intralobular, hepatic, or central vein $(V . c)$, which traverses each lobule, reaches its surface at one point, passes out, and joins similar veins from other lobules to form the sublobular veins $(V . s)$. These in turn unite to form wide veins, the origins of the hepatic vein, which opens into the vena cava inferior.

(b) The branches of the hepatic artery accompany the branches of the portal vein and bile. 
duct in the portal canals between the lobules, and in their course give off capillaries to supply the walls of the portal vein and larger bile-ducts. The branches of the hepatic artery anastomose frequently where they lie between the lobules. On reaching the periphery of the lobules, a certain number of capillaries are given off, which penetrate the lobule and terminate in the eapillaries of the portal vein $(i, i)$. These capillaries, however, which supply the walls of the portal vein and large bile-ducts $(r, r)$, terminate in veins which end in the portal vein $(V \cdot v)$. Several branches-capsular-pass to the surface of the liver, where they form a wide-meshed plexus under the peritoneum. The blood is returned by veins, which open into branches of the portal vein.

[Hepatic Zones.-Pathologists draw a sharp distinction between different zones within a hepatic lobule. Thus the central area, capillaries, and cells form the hepatic vein zone, which is specially liable to cyanotic changes; the area next the periphery of the lobule is the portal

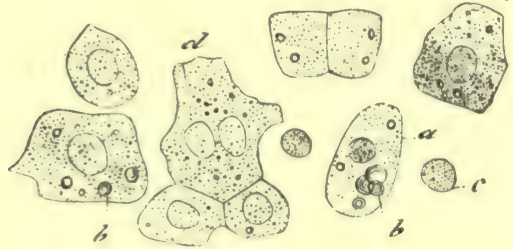

Fig. 190.

Human liver-cells containing oil-globules, $b$; $d$, has two nuclei.

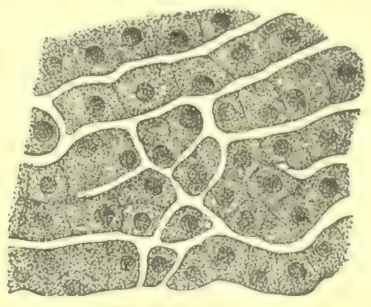

Fig. 191.

Liver-cells after withholding food for 36 hours.

vein zone, whose cells under certain circumstances are particularly apt to undergo fatty degeneration; while there is an area lying midway between the two foregoing-the hepatic artery zone-which is specially liable to amyloid or waxy degeneration.]

3. The hepatic cells (fig. 189, II, $a$ ) are irregular polygonal cells of about $\frac{1}{1000}^{\text {th }}$ th of an inch $(34$ to $45 \mu$ ) in diameter (fig. 190). The arrangement of the capillaries withiu a lobule deter-

Finest bile-duct. Finest bile-duct divided.

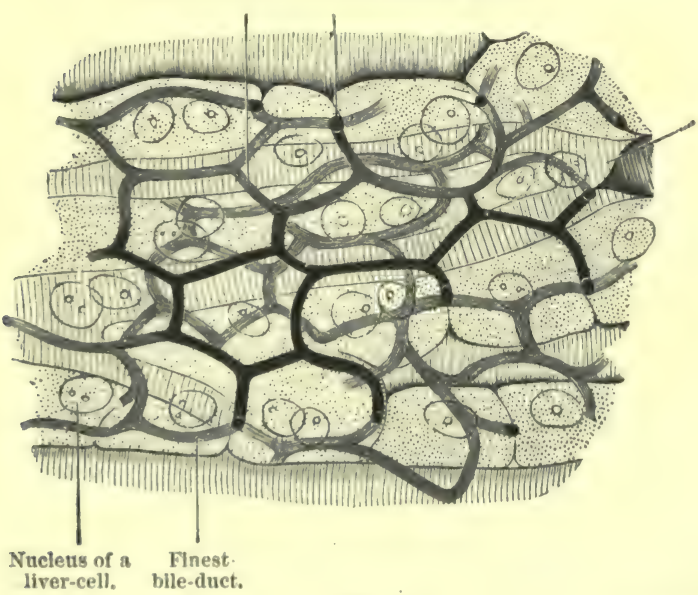

Fig. 192. mines the arrangement of the liver-cells. The liver-cells form anastomosing columns which radiate from the centre to the periphery of each lobule Blood- (fig. 191). [The liver-cells vessel. are usually stated to be devoid of an envelope, although Haycraft states that they possess one. They usually contain a single nucleus, with one or more nucleoli, but sometimes two nuclei occur. The protoplasm and nucleus of each cell contain a plexus of fibrils just like other epithelial cells. In someanimals, globules of oil and pigmentgranules are found in the cell-protoplasm (fig. 190). Each cell is in relation with the wide-meshed blood-capillaries $(d, d)$, and also with the much narrower meshwork of bile-ducts (I, $x$ ).

Blood-capillaries : finest bile-ducts in their relative position in a $\begin{gathered}\text { Changes in Liver-Cells. } \\ \text { The appearance of the vells }\end{gathered}$ rabbit's liver.

varies with the period of di-

gestion. During hunger, the liver-cells are finely granular and very cloudy (fig. 191), [and contain little glycogen, but many pigment-granules, and the nucleus is more frequently absent. Often free nucleoli and pale nuclei are found (Ellenberger and Baum). During activity, i.e., after a full meal, especially of starchy food, the cells are larger and more distinct, stain more deeply with eosin, and contain fewer granules]. The protoplasm contains coarse, glancing masses of glycogen (fig. 194, 2), and near the surface of the cell it is condensed, and a fine network stretches 
toward the centre of the cell, and in it is suspended the nucleus. All the hepatic cells are not in the same phase of activity at the same time. Afanassiew finds that if the formation of bile in the liver be increased (e.g., by section of the hepatic nerves, or feeding with proteids), the cells are moderately enlarged in size, and contain numerous granules, which are proteid in their nature ; such cells resist the action of caustic potash. When there is a great formation of glycogen (as after feeding with potatoes and sugar), all the cells are very large and sharply defined, and contain many granules of glycogen, the cells being so large as to compress the capillaries. These cells dissolve quickly in caustic potash.

Action of Drugs. - Some substances excite the cells to activity, and cause them to present the appearance of cells in activity, e.g., pilocarpin, muscarin, aloes, less so salicylate and benzoate of soda and rhubarb, while atropin and lead acetate inhibit the signs of activity. These results were obtained in the horse by Ellenberger and Baum. [Stolnikow, by using the quadruplestaining method of Gaule, finds that the hepatic cells of the frog undergo remarkable changes in poisoning by phosphorus. It is well known that this drug produces fatty degeneration of the liver-cells, but a deeper study shows that the changes are both histological and chemical. Besides producing remarkable changes in the protoplasm of the cell, the protoplasm of the
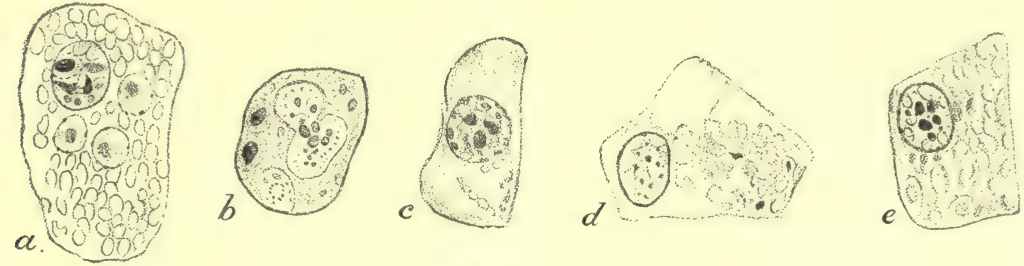

Fig. 193.

Liver-cells of frog. $a$, early, and $b$, late stage in poisoning by phosphorus ; $c$, liver-cell of frog getting water only, $d$, getting sugar, and $e$, peptone (Stirling after Stolnikow).

nucleus, in the form of small masses called plasmosoma, passes out into the cell-body, perhaps to renew the latter. The cells are increased in size, both after poisoning with phosphorus and after excision of the fat bodies in the frog (fig. 193). The fat present in the liver in phosphoruspoisoning is not present as droplets of oil, but probably in a loose combination, $\epsilon . g$., lecithin, and as a matter of fact the amount of liver-lecithin is extracrdinarily increased. There is also an increase of the nuclein; while glycogen is absent. The season of the year also affects them. There is a period of growth from July to November, and one of decay from December to May (A. Leonard). Antipyrin also produces profound changes, especially in the nuclei.]

4. The Bile-Ducts. - The finest bile-capillaries, channels, or canaliculi arise from the centre of the lobule, and indeed throughout the whole lobule they form a regular anastomosing network of very fine tubes or channels. Each cell is surrounded by a polygonal-usually hexagonalmesh (fig. 194, 3). The bile-capillaries always lie in the middle of the surface between two adjoining cells (II, $\alpha$ ), where they form actual intercellular passages (fig. 192). [According to some observers, they are merely excessively narrow channels ( 1 to $2 \mu$ wide) in the cement substance between the cells, while according to others they have a distinct delicate wall. The bile-capillary network is much closer than the blood-capillary network. [Thus, there are three networks within each lobule-

(1) A network of blood-capillaries;

(2) " " hepatic cells ;

(3) $\quad, \quad$ bile-capillaries; (fig. 192).]

Excessively minute intracellular passages are said to pass from the bile-capillaries into the interior of the liver-cells, where they communicate with certain small cavities or vacuoles (Asp, Kupffer) (fig. 194, 3). As the blood-capillaries run along the edge of the liver-cells, and the bile-capillaries between the opposed surfaces of adjacent cells, the two systems of canals within the lobule are kept separate. Some bile-capillaries run along the edges of the liver-cells in the human liver, especially during embryonic life. Towards the peripheral part of the lobule, the bile-capillaries are
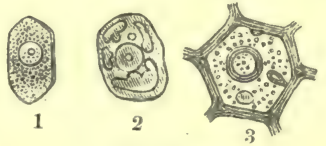

Fig. 194 .

1, Liver-cell during fasting; 2 , containing masses of glycogen ; 3 , a liver-cell surrounded with bile-channels, from which fine twigs proceed into the cell-substance to end in vacuoles.

larger, while adjoining channels anastomose, and leave the lobule, where they become interlobular ducts $(g)$, which join with other similar ducts to form larger interlobular bile-duets. These accompany the hepatic artery and portal vein, and leave the liver at the transverse fissure. The finer interlobular ducts frequently anastomose in Glisson's capsule, possess a structureless basement membrane, and are lined by a single layer of low polyhedral epithelial cells. The larger interlobular ducts have a distinct wall, consisting of connective and elastic tissue, 
mixed with circularly-disposed smooth muscular fibres (fig. 195). Capillaries are supplied to the wall, which is lined by a single layer of columnar epithelium. A sub-mucosa occurs only in the largest bile-ducts, and in the gall-bladder. Smooth muscular fibres, arranged in single

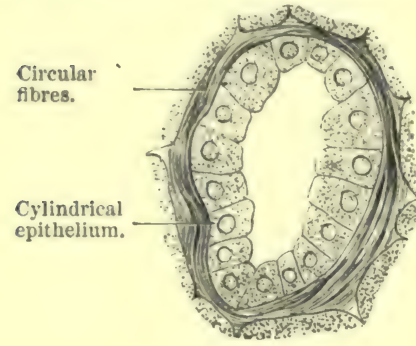

Fig. 195. bundles, occur in the largest ducts, and as longitudinal and circular layers in the gall-bladder, whose mucous membrane is provided with numerous folds and depressions. The epithelium lining the gall-bladder is cylindrical, with a distinct clear disc, and between these cells are goblet-cells. Small branched tubular mucous glands occur in the larger bile-ducts and in the gall-bladder.

Vasa aberrantia are isolated bile-ducts which occur on the surface of the liver, but have no relation to any system of liver-lobules. They occur at the sharp margin of the liver, in the region of the inferior vena cava, of the gallbladder, and of the parts near the portal fissure. It seems that the liver-lobules to which they originally belonged have atrophied and disappeared (Zuckerkandl and Toldt).

5. The lymphatics begin as pericapillary tubes around

Interlobular bile-duct (human), the capillaries within the lobules, emerge from the lobule, and run within the wall of the branches of the hepatic and portal veins, and afterwards surround the venous trunks, thus forming the interlobular lymphatics. These unite to form larger trunks, which leave the liver partly at the portal fissure, partly along with the hepatic veins, and partly at different points on the surface of the organ. There is a narrow superficial meshwork of lymphatics under the peritoneum-sub-peritonealwhich communicate with the thoracic lymphatics through the triangular ligament and suspensorium, while on the under surface they communicate with the lyniphatics of the interlobular connective-tissue.

6. The nerves consist partly of medullated and partly of non-medullated fibres from branches of the sympathetic and left vagus to the hepatic plexus. They accompany the branches of the hepatic artery, and ganglia oceur on their branches within the liver. Some of the nerve-fibres are vaso-motor in function, and, according to Pfliiger, other nerve-fibres terminate directly in connection with liver-cells. [MacCallum describes an interlobular plexus of non-medullated fibres in man and menobranchus, from which a peri-vascular and intercellular plexus proceeds. From the latter fibrils pass to terminate within the cells near the nucleus.]

Pathological. - The connective-tissue between the lobules may undergo great increase in amount, especially in alcohol-and gin-drinkers, and thus the substance of the lobules may be greatly compressed, owing to the cicatricial contraction of the newly-formed connective-tissue (cirrhosis of the liver). In such interlobular connective-tissue, newly-formed bile-ducts are found.

Ligature of the ductus choledochus [causes enlargement of the spleen (rabbit), and a diminution in the number of the blood-corpuscles], and, after a time, interstitial inflammation of the liver. In rabbits and guinea-pigs the liver-parenchyma disappears, and its place is taken by newly-formed connective-tissue and bile-ducts (Charcot and Gombault). In all these cases of interstitial inflammation, there is proliferation of the epithelium of the bile-ducts.

[Regeneration of the Liver.-Tizzoni finds that there may be partial regeneration and new formation of liver-lobules in the dog, the process being the same as that which occurs in the embryonic development of the organ, i.e., the growth of solid cylinders of liver-cells, formed by the pre-existing liver-cells, which penetrate into the connective-tissue uniting the edges of the wound. These cells ultimately differentiate into hepatic cells and bile-ducts. Other observers attribute the new formation to outgrowths of the epithelial cells of the bile-cells.]

174. CHEMICAL COMPOSITION OF THE LIVER-CELLS.-(1) Proteids. - The fresh, soft, parenchyma of the liver is alkaline in reaction; after death, coagulation occurs, the cell-contents appear turbid, the tissue becomes friable, and gradually an acid reaction is developed. This process closely resembles what occurs in muscle, and is due to the coagulation of a myosin-like body, which is soluble during life, but after death undergoes spontaneous coagulation (Ploss). The liver contains other proteids; one coagulating at $45^{\circ} \mathrm{C}$., another at $70^{\circ} \mathrm{C}$, , and one which is slightly soluble in dilute acids and alkalies. The nuclei contain nuclein. The connective-tissue yields gelatin.

(2) Glycogen or Animal Starch-1.2 to $2 \cdot 6$ per cent.-is a true carbohydrate most closely related to inulin, soluble in water, but diffuses with difficulty, and has the formula $6\left(\mathrm{C}_{6} \mathrm{H}_{10} \mathrm{O}_{5}\right)+\mathrm{H}_{2} \mathrm{O}$. It is stored up in the liver-cells in amorphous granules around the nuclei (fig. 194, 2), but is not uniformly distributed in all 
parts of the liver. Like inulin, it gives a deep red colour with solution of iodine in iodide of potassium. It is changed into dextrin and sugar by diastatic ferments, and when boiled with dilute mineral acids, it yields grape-sugar ( $\$ 148$, I.; $\S 170$, I.; § 252, III.).

Preparation of Glycogen. - [Feed a rabbit on carrots or boiled rice, and kill it three or four hours thereafter. Remove the liver immediately after death, cut it into fine pieces, and place these in boiling water, and boil it for some time in order to obtain a watery extract of the liver. The boiling water destroys the ferment supposed to be present in the liver, which would transform the glycogen into grape-sugar. To the cold filtrate are added alternately dilute hydrochloric acid and potassio-mercuric iodide, which precipitates the proteids. Filter, when a clear opalescent fluid, containing the glycogen in solution, is obtained. The glycogen is precipitated from the filtrate, as a white amorphous powder, on adding an excess of 70 to 80 per cent. alcohol. The precipitate is washed with 60 per cent. and afterwards with 95 per cent. alcohol, then with ether, and lastly, with absolute alcohol ; it is dried over sulphuric acid and weighed (Brïcke). Kiilz modifies the method somewhat. After boiling the liver for half an hour, it is rubbed up with liquor potassæ (100 grm. liver, $4 \mathrm{grm}$. KHO). Evaporate in the water-bath until all is dissolved, which occurs in about 3 hours. After cooling, neutralise with $\mathrm{HCl}$ and precipitate the proteids as above. F. Eves asserts that the post-mortem conversion of sugar in the liver is not attributable to a ferment action, and the rapid appearance of sugar in the liver after death is due to the specific metabolic activity of the dying cells.]

Sources. -The "mother-substance" of the glycogen of the liver has been variously stated to be the carbohydrates of the food (Pavy); fats (olive oil, Salomon); glycerine, taurin, and glycin (the latter splitting into glycogen and urea), the proteids ( $\mathrm{Cl}$. Bernard); and gelatin (Salomon). If it is derived from the albumins, it must be formed from a non-nitrogenous derivative thereof.

Rohmann found that the use of ammonia carbonate and asparagin or glycin, along with a carbohydrate diet, in rabbits considerably increased the formation of glycogen. The excessive formation of acid observed by Stadelmann in diabetes unites with the ammonia and diminishes considerably the formation of glycogen.

Effects of Food. - Rabbits, whose livers have been rendered free from glycogen by starvation, yield new glycogen from their livers when they are fed with canesugar, grape-sugar, maltose, or starch. Forced muscular movements soon make the liver of dogs free from glycogen, exposure to cold diminishes its amount. Dextrin and grape-sugar occur in the dead liver, but, in addition, some glycogen is found for a considerable time after death in the liver and in the muscles.

If glycogen is injected into the blood, achroodextrin appears in the urine, and also hæmoglobin, as glycogen dissolves red blood-corpuscles. Ligature of the bile-duct causes decrease of the glycogen in the liver.

Other Situations. - Glycogen is not confined to the liver-cells; it occurs during foctal life in all the tissues of the body of the embyro [including the embryonic skeleton], in young animals (Kühne), the placenta (Bernard). [It occurs in large amount in the liver during intrauterine life.] In the adult it occurs in the testicle, in the muscles (MacDonnel, O. Nasse), in numerous pathological products, in inflamed lungs (Kühne), and also in the corresponding tissues of the lower animals. [It also occurs in the chorionic villi, in colourless bloodcorpuscles, in fresh pus cells which still exhibit amœeboid movements, and in fact in all developing animal cells, with amœboid movement; it is a never-failing constituent in eartilage, and in the muscles and liver of invertebrata, such as the oyster. There is none in the fresh brain of the dog or rabbit, but it is found in the brain in diabetic coma (Abeles).]

Modifying Conditions.-If large quantities of starch, milk-, fruit-, or cane-sugar, or glycerine, but not mannite, or glycol, or inosite, be added to the proteids of the food, the amount of glycogen in the liver is very greatly increased (to 12 per cent. in the fowl), while a purely albuminous or purely fatty diet diminishes it enormously. During hunger it almost disappears. "The injection of dissolved carbohydrates into a mesenteric vein of a starving rabbit causes the liver, previously free from glycogen, to contain glycogen.

[Effect of Drugs.-Arsenic, phosphorus, and antimony destroy the glycogenic function of the liver, no glycogen being present in the liver in animals poisoned with these drugs, so that puncture of the floor of the fourth ventricle no longer causes glycosuria in them. In animals poisoned by strychnia or curara, it is greatly diminished, both in the liver and in the muscles. Sugar is always present in the urine in the latter case but not in the former.] 
During life, under normal conditions, the glycogen in the liver is either not transformed into grape-sugar (Pavy), or, what is more probable, only a very small amount of it is so changed. The normal amount of sugar in blood is 0.5 to 1 per 1000 , although the blood of the hepatic vein contains somewhat more. A considerable amount is transformed into sugar only when there is a decided derangement of the hepatic circulation, and in these circumstances the blood of the hepatic vein contains more sugar. The glycogen undergoes this change very rapidly after death, so that a liver which has been dead for some time always contains more sugar and less glycogen.

The diastatic ferment in the liver is small in amount, and can be obtained from the extract of the liver-cells by the same means as are applicable for obtaining other similar ferments, such as ptyalin; but it does not seem to be formed within the liver-cells, but only passes very rapidly from the blood into them. The ferment seems to be rapidly formed when the blood-stream undergoes considerable derangement. A similar ferment is formed when red blood-corpuscles are dissolved (Tiegel), and, as red blood-corpuscles are continually destroyed within the liver, there is one source from which the ferment may be formed, whereby minute quantities of sugar would be continually formed in the liver.

According to Seegen, the blood of the hepatic vein contains twice as much sugar $(0.23$ per cent.) as that in the portal vein $(0.119$ per cent.); observations on dogs showed that the blood flowing through the liver gives up over 400 grms. sugar in 24 hrs. Hence, in carnivora, the greatest part of the $\mathrm{C}$ of the animal food must pass into sugar, so that the formation of sugar in the liver, and its decomposition in the blood, or in the organs traversed by the blood, must be a very important function of the metabolism. Seegen is also of opinion that the liver. glycogen takes no part in the formation of sugar in the liver.

[Blood when perfused through a freshly excised liver, (or through the kidneys, lungs, or muscles), gains lactic acid (G. Aglio and Wissokowitsch).]

(3) Fats, in the form of highly refractive granules, occur in the liver-cells, as well as free in the bile-ducts; sometimes, when the food contains much fat (more abundant in drunkards and the phthisical), olein, palmatin, stearin, volatile fatty acids, and sarcolactic acid are found.

There are also found traces of cholesterin, minute quantities of urea, uric acid, and the littleknown body jecorin. [Jecorin, discovered by Drechsel, contains S and P, and reduces alkaline solutions of copper like grape-sugar. It is also found in the spleen, muscles, and blood (Baldi). The liver of birds contains a relatively large amount of uric acid, even 6 to 14 times as much as the boor $(v$. Schredler $)$.$] [Leucin (? guanin), sarkin, xanthin, cystin, and tyrosin$ occur pathologically in certain diseases where marked chemical decompositions occur.]

[Fatty Dengeneration and Infiltration.-Fatty granules are of common occurrence within the cells of the liver, constituting fatty infiltration, and when not too numerous do not seem to interfere greatly with the functions of the liver-cells. Fatty particles occur if too much fatty food be taken, and they are commonly found in the livers of stall-fed animals; the well-known paté-de-foie gras is largely composed of the livers of geese, which have been fed on large amounts of farinaceous food, and which have been subjected to other unfavourable hygienic conditions. Fatty granules are recognised by their highly refractive appearance, by their solubility in ether, and by heing blackened by osmic acid.]

(4) The inorganic substances in the human liver are-potassium, sodium, calcium, magnesium, iron, manganese, chlorine, and phosphoric, sulphuric, carbonic, and silicic acids; while copper, zinc, lead, mercury, and arsenic may be accidentally deposited in the hepatic tissue.

[Tizzoni's Reaction. - If a section of a liver (especially of a young animal) hardened in alcohol be treated with a solution of potassic ferrocyanide, and then with dilute hydroehloric acid, as a general rule the preparation becomes blue, even to the naked eye; but failing that, one can usually see with the microscope granules of Prussian blue in the protoplasm of the cells, indicating the presence of free iron oxide.]

175. DIABETES MELLITUS AND GLYCOSURIA.-[Glycosuria is characterised by the presence of grape-sugar in the urine. According to Brücke a trace of sugar exists normally in urine, and when this amount is increased we have glycosuria. When the normal amount of grape-sugar in the blood is 
increased, grape-sugar appears in the urine. In diabetes mellitus, grape-sugar also appears in the urine, but this is really a serious disease, involving the alteration of many tissues, and distinguished by profound disturbance of the whole metabolic activity, which leads to numerous pathological, changes and often to death. The appearance of grape-sugar in urine does not necessarily mean that a person is suffering from this disease.]

The formation of large quantities of grape-sugar by the liver, and its passage into the blood, and from the blood into the urine, constitute glycosuria. Extirpation of the liver in frogs, or destruction of the hepatic cells, as by fatty degeneration from poisoning with phosphorus or arsenic, does not cause this condition. It occurs for several hours, after the injury of a certain part-the centre for the hepatic vaso-motor nerves-of the floor of the lower part of the fourth ventricle $(\mathrm{Cl}$. Bernard's "piqûre"); also after section of the vaso-motor channels in the spinal cord, from above down as far as the exit of the nerves for the liver, viz., to the lumbar region, and in the frog to the fourth vertebra (Schiff). When the vasomotor nerves, which proceed from this centre to the liver, are cut or paralysed in any part of their course, mellituria or glycosuria is produced. All the nerve channels do not run through the spinal cord alone. A number of vaso-motor nerves leave the spinal cord higher up, pass into the sympathetic, and thus reach the liver; so that destruction of the superior (Pavy), as well as of the inferior cervical sympathetic ganglion, and the first thoracic ganglion (Eckhard) of the abdominal sympathetic, and often of the splanchnic itself produces it. The paralysis of the blood-vessels causes the liver to contain much blood, and the intrahepatic bloodstream is slowed. The disturbance of the circulation causes a great accumulation of sugar in the liver, as the blood-ferment has time to act upon the glycogen and transform it into sugar. By stimulation of the sympathetic at the lowest cervical and first thoracic ganglion, the hepatic vessels at the periphery of the liver-lobules become contracted and pale (Cyon). It is remarkable that glycosuria when present may be set aside by section of the splanchnic nerves. This is explained by supposing that the enormous dilatation and congestion, or the hyperæmia of the abdominal blood-vessels thereby produced, renders the liver anæmic.

Continued stimulation of peripheral nerves may act reflexly upon the centre for the vasomotor nerves of the liver. Diabetes has been observed to occur after stimulation of the central end of the vagus ( $\mathrm{Cl}$. Bernard), and also after stimulation of the central end of the depressor nerve (Filehne). Even section and subsequent stimulation of the central end of the sciatic nerve causes diabetes. This may explain the occurrence of diabetes in people who suffer from sciatica. [It may occur also after perverted nervous activity, as psychical excitement, neuralgias (sciatica, trigeminal or occipital), concussion of the brain, as well as after certain injuries to the skull and vertebral column and some cerebral diseases.]

According to Schiff, the stagnation of blood in other vascular regions of the body may cause the ferment to accumulate in the blood to such an extent that diabetes occurs. The glycosuria that occurs after compression of the aorta or portal vein may perhaps be ascribed to this cause, but perhaps the pressure caused by these procedures may paralyse certain nerves. According to Eckhard, injury to the vermiform process of the cerebellum of the rabbit causes diabetes. In man, affections of the above-named nervous regions cause diabetes.

[In most individuals the use of a large quantity of sugar in the food is not followed by the appearance of sugar in the urine; but in some exceptional cases it is often present, e.g., in persons suffering from gastric catarrh, especially if they are gouty.]

A number of poisons which paralyse the hepatic vaso-motor nerves produce diabetes ; curara (when artificial respiration is not maintained), $\mathrm{CO}$, amyl nitrite, ortho-nitro-propionic acid, and methyl-delphinin; less certainly morphia, chloral hydrate, $\mathrm{HCN}$, and some other drugs; [phlorizin (v. Mering)]; and some infectious diseases. But congestion of the liver produced in other ways appears to cause diabetes, e.g., after mechanical stimulation of the liver. To this class belongs the injection of dilute saline solutions into the blood (Bock, Hoffimann), whereby either the change in form or the solution of the coloured blood-corpuscles causes the congestion. The circumstance that repeated blood-letting makes the blood richer in sugar, may perhaps be explained by the slowing of the circulation.

[Most of the means which produce glycosuria in other animals fail to do so in birds; even the piqûre rarely produces it. This Thiel and Minkowski attribute to the intensely active 
oxidation-processes in birds. Phlorizin causes glycosuria, even after extirpation of the liver, which shows that in these cases there are other causes at work that obtain in the forms of glycosuria.] Phlorizin makes animals, which are free from carbohydrates, diabetic. In this case the sugar must be derived from proteids ( $v$. Mering).

Theoretical. - In order to explain the more immediate cause of these phenomena several hypotheses have been advanced :-

(a) The liver-glycogen may be transformed unhindered into sugar, as the blood in its passage through the liver deposits or gives up the ferment to the liver-cells. So that the normal function of the vaso-motor system of the liver, and its centre in the floor of the fourth ventricle, may be regarded as, in a certain sense, an "inhibitory system" for the formation of sugar.

(b) If we assume that, normally there is continually a small quantity of sugar passing from the liver into the hepatic vein, we might explain the diabetes as due to the disappearance of these decompositions-diminished burning-up of the sugar in the blood, which are constantly removing the sugar from the blood. In fact, diabetic persons have been found to consume less $\mathrm{O}$ and to have an increased formation of urea.

[Injection of Grape-Sugar into the Blood. - When grape-sugar is injected into the jugular vein of a dog, only 33 per cent. at most is given off in the urine; within 2 to 5 hours the urine is free from sugar. Even within a few minutes after the injection, only a certain proportion $\left(\frac{1}{2}-\frac{1}{4}\right)$ of the sugar is found in the blood; part of the sugar has been detected in the muscles, liver, and kidneys, but the fate of the remainder is not known. Immediately after the injection, the amount of hemoglobin and also of serum-albumin is diminished (50 per cent.), which is due to increase of the quantity of water within the vessels; but within two hours the normal state is restored (Brasol). In a curarised dog the injection of grape-sugar into a vein increases the blood-pressure, but this effect is not observed after the injection of morphia and chloral.]

Persons suffering from diabetes require a large amount of .food; they suffer greatly from thirst, and drink much fluid. They exhibit signs of marked emaciation, when the loss of the body is greater than the supply. [In advanced diabetes the glycogenic function of the liver is almost abolished, as was proved by removing with a trocar a small part of the liver from man, when almost no glyeogen was found (Ehrlich). The absorbed sugar in the portal vein passes directly into the general circulation without being submitted to the action of the liver $(v$. Frerichs).] In severe cases, towards death, not unfrequently a peculiar comatose conditiondiabetic coma-occurs, when the breath often has the odour of aceton, which is also found in the urine. But neither aceton nor its precursor, aceto-acetic acid, nor æthyl-diacetic acid, nor the unknown substance, in diabetic urine, which gives the red colour with ferric chloride $(v$. Jaksch), is the cause of the coma (Frerichs and Brieger).

176. THE FUNCTIONS OF THE LIVER.-[To understand the functions of the liver, we must remember its unique relation to the vascular and digestive systems, whereby many of the products of gastric and intestinal digestion have to traverse it before they reach the blood, and some of them as they traverse the liver are altered. We have still much to learn regarding the liver. It has several distinct functions-some obvious, others not. (1) The liver secretes bile, which is formed by the hepatic cells, and leaves the organ by the bile-ducts, to pass into the duodenum. (2) The liver-cells also form glycogen, which does not pass into the ducts, but in some altered and diffusible form passes into the blood-stream, and leaves the liver by the hepatic veins. Hence, the study of the liver materially influences our conception of a secreting organ. In this case, we have the products of its secretory activity leaving it by two different channels-the one by the ducts, and the other by the blood-stream. The liver, therefore, is a great storehouse of carbohydrates, and it serves them out to the economy as they are required. All this points to the liver as being an organ intimately related to the general metabolism of the body. (3) In a certain period of development it is concerned in the formation of blood-corpuscles $(\$ 7)$. (4) It has some relation to the breaking up of blood-corpuscles and the formation of urea and other metabolic products $(\S 20, \S 177,3)$. (5) Brunton attributes some importance to the liver in connection with the arrest of certain substances absorbed from the alimentary canal, whereby they are either destroyed, stored up in the liver, or, it may be, prevented from entering the general circulation in too large amount. It is possible that ptomaines may be arrested in this way $(\$ 166)$.]

[The liver has no special action on certain mineral substances which traverse it in the blood, 
e.g., potassic chloride, but it retains the vegetable alkaloids, provided they are not present in too large an amount in the blood. The ptomaines are similarly retained in the liver. The liver possesses this property only as long as it contains glycogen (H. Rogers).]

177. CONSTITUENTS OF THE BILE.-Bile is a yellowish-brown or dark green coloured transparent fluid, with a sweetish, strongly bitter taste, feeble musklike odour, and neutral reaction. The specific gravity of human bile from the gall bladder $=1026$ to 1032 , while that from a fistula $=1020$ to 1011 . It contains :-

(1) Mucus, which gives bile its sticky character, and not unfrequently makes it alkaline; it is the product of the mucous glands and the goblet-cells of the mucous membrane of the larger bile-ducts. When bile is exposed to the air, the mucus causes it to putrefy rapidly. It is precipitated by acetic acid, or alcohol.

[The bile formed in the ultimate bile-ducts does not seem to contain mucin or mucus, but bile from the gall-bladder always does. It is formed by the mucous glands in the larger bileducts (\$ 173).]

(2) The Bile-Acids. - Glycocholic and taurocholic acids, so-called conjugate acids, are united with soda (in traces with potash) to form glycocholate and taurocholate of soda, which have a bitter taste, and rotate the plane of polarised light to the right. In human bile (as well as in that of birds, many mammals, and amphibians) taurocholic acid is most abundant; in other animals (pig, ox) glycocholic acid is most abundant but is absent in sucklings.

(a) Glycocholic acid, $\mathrm{C}_{26} \mathrm{H}_{43} \mathrm{NO}_{6}$; when boiled with caustic potash, or baryta water, or with dilute mineral acids, it takes up $\mathrm{H}_{2} \mathrm{O}$ and splits into-

Glycin $(=$ Glycocoll $=$ Gelatin Sugar $=$ Amido-acetic acid $)=\mathrm{C}_{2} \mathrm{H}_{5} \mathrm{NO}_{2}$.

+ Cholalic acid (also called Cholic acid) . . $=\mathrm{C}_{24} \mathrm{H}_{40} \mathrm{O}_{5}$.

$$
=\text { Glycocholic acid + Water } \cdot \cdot \quad=\mathrm{C}_{26} \mathrm{H}_{43} \mathrm{NO}_{6}+\mathrm{H}_{2} \mathrm{O} \text {. }
$$

(b) Taurocholic acid, $\mathrm{C}_{26} \mathrm{H}_{45} \mathrm{NSO}_{7}$, when similarly treated, takes up water and splits into-

$$
\begin{aligned}
& \text { Taurin (=Amidoæthyl-Sulphuric acid) }=\mathrm{C}_{2} \mathrm{H}_{7} \mathrm{NSO}_{3} \text {. } \\
& \text { + Cholalic acid . . . . }=\mathrm{C}_{24} \mathrm{H}_{40} \mathrm{O}_{5} \text {. } \\
& =\text { Taurocholic acid + Water. } \quad \cdot \quad=\mathrm{C}_{26} \mathrm{H}_{45} \mathrm{NSO}_{7}+\mathrm{H}_{2} \mathrm{O} \text { (Strecker). }
\end{aligned}
$$

[Solutions of taurocholic acid are antiseptic, and if sufficiently strong interfere with the development of bacteria, and prevent the alcoholic and lactic fermentations, as well as the tryptic and diastatic action of the pancreas (Emich).]

Preparation of the Bile-Acids. - Evaporate bile to $\frac{1}{4}$ of its volume, rub it up into a paste with excess of animal charcoal, and dry at $100^{\circ} \mathrm{C}$. Extract the black mass with absolute alcohol, and filter. After a part of the alcohol has been removed by distillation, the bile-salts are precipitated in a resinous form, and on the addition of excess of ether, there is formed immediately a crystalline máss of glancing needles (Platner's "crystallised bile"). The alkaline salts of the bile-acids are freely soluble in water or alcohol, and insoluble in ether. Neutral lead acetate precipitates the glycocholic acid-as lead glycocholate-from the solution of both salts; the precipitate is collected on a filter, dissolved in hot alcohol, and the lead is precipitated as lead sulphide by $\mathrm{H}_{2} \mathrm{~S}$; after removal of the lead sulphide, the addition of water precipitates the isolated glycocholic acid. If, after precipitating the lead glycocholate, the filtrate be treated with basic lead acetate, a precipitate of lead taurocholate is formed, from which the acid may be obtained in the same way as described above (Strecker).

With regard to the decomposition products of the bile-acids, glycin, as such, does not occur in the body, but only in the bile in combination with cholic acid, in urine in combination with benzoic acid, as hippuric acid, and lastly, in gelatin in complex combination.

Cholalic acid rotates the ray of polarised light to the right, and its chemical composition is unknown. It is insoluble in water, soluble in alcohol, but soluble with difficulty in ether, from which it separates in prisms. Its crystalline alkaline salts are readily soluble in water. It is coloured blue by iodine, and occurs free only in the intestine.

Cholalic acid is replaced in the bile of many animals by a nearly related acid, e.g., in pig's bile, by hyo-cholalic acid (Strecker); in the bile of the goose, cheno-cholalic acid is present (Marsson, Otto), 
When cholalic acid is boiled with concentrated $\mathrm{HCl}$, or heated dry at $200^{\circ} \mathrm{C}$., it becomes an anhydride, thus :-

$$
\begin{array}{ll}
\text { Cholalic acid. } & =\mathrm{C}_{24} \mathrm{H}_{40} \mathrm{O}_{5} \text {, produces } \\
\text { Choloidinic acid. } & =\mathrm{C}_{24} \mathrm{H}_{38} \mathrm{O}_{4}+\mathrm{H}_{2} \mathrm{O} \text {, and this again yields } \\
\text { Dyslysin : } & =\mathrm{C}_{24} \mathrm{H}_{36} \mathrm{O}_{3}=\mathrm{H}_{2} \mathrm{O} \text {. }
\end{array}
$$

Choloidinic acid is, however, not improbably a mixture of cholalic acid and dyslysin ; dyslysin, when fused with caustic potash, is changed into cholalate of potash. By oxidation cholalic acid yields a tribasic acid, as yet uninvestigated, and a fair amount of oxalic acid, but no fatty acids (Clève).

Pettenkoffer's Test.-The bile-acids, cholic acid, and their anhydrides, when dissolved in water, yield on the addition of $\frac{2}{3}$ concentrated sulphuric acid (added in drops so as not to heat the fluid above $70^{\circ} \mathrm{C}$.), and several drops of a 10 per cent. solution of cane-sugar, a reddish-purple transparent fluid, which shows two absorption-bands at $\mathrm{E}$ and $\mathrm{F}$ (Schenk). [A very good method is to mix a few drops of the cane-sugar solution with the bile, and to shake the mixture until a copious froth is obtained. Pour the sulphuric acid down the side of the test-tube, and then the characteristic colour is seen in the froth. Any albumin present must be removed before applying the test.]

According to Drechsel, it is better to add phosphoric acid, instead of sulphuric acid, until the fluid is syrupy, then add the cane-sugar, and afterwards place the whole in boiling water. When investigating the amount of bile-acids in a liquid, the albumin must be removed beforehand, as it gives a reaction similar to the bile-acids, but in that case the red fluid has only one absorption-band. If only small quantities of bile-acids are present, the fluid must in the first place be concentrated by evaporation.

[Hay's Test. - The bile-acids or their soluble salts lower the surface-tension of fluids in which they are dissolved. Throw a small quantity of sulphur (sublimed or precipitated) on the surface of the fluid containing bile-acids, and if the bile-acids be present, the sulphur will at once begin to sink, and will be wholly precipitated within a few minutes. (Privately communicated.)]

The bile-acids are formed in the liver. After its extirpation, there is no accumulation of biliary matters in the blood.

How the formation of the nitrogenous bile-acids is effected, is quite unknown. They must be obtained from the decomposition of albuminous materials, and it is important to note that the amount of bile-acids is increased by albuminous food. Taurin contains part of the sulphur of albumin; bile-salts contain 4 to 4.6 per cent., which may perhaps be derived from dissolved red blood-corpuscles.

(3) The Bile-Pigments.-The freshly secreted bile of man and many animals has a yellowish-brown colour, due to the presence of bilirubin. When it remains for a considerable time in the gall-bladder, or when alkaline bile is exposed to the air, the bilirubin absorbs $\mathrm{O}$ and becomes changed into a green pigment, biliverdin. This substance is present naturally, and is the chief pigment in the bile of herbivora and cold-blooded animals.

(a) Bilirubin $\left(\mathrm{C}_{32} \mathrm{H}_{36} \mathrm{~N}_{4} \mathrm{O}_{6}\right)$ is perhaps united with an alkali ; it crystallises in transparent fox-red clinorhombic prisms. It is insoluble in water, soluble in chloroform, by which substance it may be separated from biliverdin, which is insoluble in chloroform. It unites as a monobasic acid with alkalies, and as such is soluble. It is identical with Virchow's hæmatoidin (\$20).

Preparation.- It is most easily prepared from the red (bilirubin-chalk) gall-stones of man or the ox. The stones are pounded, and their chalk dissolved by hydrochloric acid; the pigment is then extracted with chloroform. That bilirubin is derived from hæmoglobin is very probablè, considering its identity with hæmatoidin. Very probably red blood-corpuscles are dissolved in the liver, and their hremoglobin changed into bilirubin.

(b) Biliverdin, $\mathrm{C}_{32} \mathrm{H}_{36} \mathrm{~N}_{4} \mathrm{O}_{8}$, is an oxidised derivative of the former, from which it can be obtained by various oxidation-processes. It is readily soluble in alcohol, very slightly so in ether, and not at all soluble in chloroform. It occurs in the placenta of the bitch. As yet it has not been retransformed by reducing agents into bilirubin.

Tests for Bile-Pigments,-Bilirubin and biliverdin may occur in other fluids, 
e.g., the urine, and are detected by the Gmelin-Heintz' reaction. When nitric acid containing some nitrous acid is added to a liquid containing these pigments, a play of colours is obtained beginning with green (biliverdin), blue, violet, red, ending with yellow. [This reaction. is best done by placing a drop of the liquid on a white porcelain plate, and adding a drop of the impure nitric acid.]

(c) If, when the blue colour is reached, the oxidation process is arrested, bilicyanin (Heynsius, Campbell), in acid solution blue (in alkaline violet), is obtained, which shows two ill-defined absorption-bands near D (Jaffé).

$(d)$ Bilifuscin occurs in small amount in decomposing bile and in gall-stones =bilirubin $+\mathrm{H}_{2} \mathrm{O}$.

(e) Biliprasin (Städler) also occurs $=$ Bilirubin $+\mathrm{H}_{2} \mathrm{O}+\mathrm{O}$.

$(f)$ The yellow pigment, which ultimately results from the prolonged action of the oxidising reagent, is the choletelin $\left(\mathrm{C}_{16} \mathrm{H}_{18} \mathrm{~N}_{2} \mathrm{O}_{6}\right)$ of Maly; it is amorphous, and soluble in water, alcohol, acids, and alkalies.

[Spectrum of Bile.-The bile of carnivorous animals is generally free from absorption-bands, except when acids are added to it, in which case the band of bilirubin is revealed. Bilirubin and biliverdin yield characteristic spectra only when they are treated with nitric acid. The bile of some animals yields bands, but when this is the case they are due to the presence of a derivative of hæmatin, and MacMunn calls this body cholohæmatin, which gives a three- or four-banded spectrum (ox, sheep).]

(g) Bilirubin absorbs $\mathrm{H}+\mathrm{H}_{2} \mathrm{O}$ (by putrefaction, or by the treatment of alkaline watery solutions with the powerfully reducing sodium amalgam), and becomes converted into Maly's hydrobilirubin $\left(\mathrm{C}_{32} \mathrm{H}_{40} \mathrm{~N}_{4} \mathrm{O}_{7}\right)$, which is slightly soluble in water, and more easily soluble in solutions of salts, or alkalies, alcohol, ether, chloroform, and shows an absorption-band at $b, \mathrm{~F}$. This substance, which, according to Hammarsten, occurs in normal bile, is a constant colouring-matter of fæces, and was called stercobilin by Vaulair and Masius, but is identical with hydrobilirubin (Maly). It is, however, probably identical with the urinary pigment urobilin of Jaffé (Stokvis, $\S 20)$.

[The bile of invertebrates contains none of the bile-pigments present in vertebrates, although hæmochromogen is found in the cray-fish and pulmonate molluses. In some organs, and in bile, a pigment like vegetable chlorophyll-entero-chlorophyll-is found, but whether it is derived from without, or formed within the organism, is not certain (MacMunn).]

(4) Cholesterin, $\mathrm{C}_{26} \mathrm{H}_{44} \mathrm{O}\left(\mathrm{H}_{2} \mathrm{O}\right)$, is a monatomic alcohol which rotates the ray of polarised light to the left, it occurs also in blood, yelk, nervous matter [and gallstones]. It forms transparent rhombic plates, which usually have a small oblong piece cut out of the corner (fig. 196). It is insoluble in water, soluble in hot alcohol, ether, or chloroform. It is kept in solution in the bile by the bile-salts.

Preparation.-It is most easily prepared from so-called white gall-stones, which not unfrequently consist entirely of cholesterin, by extracting them with hot alcohol after they are pulverised. Crystals are excreted after evaporation of the alcohol. Tests. They give a red colour with sulphuric acid ( 5 vol. to 1 vol. $\mathrm{H}_{2} \mathrm{O}$ ), while they give a blue $\rightarrow$ as cellulose does-with sulphuric acid and

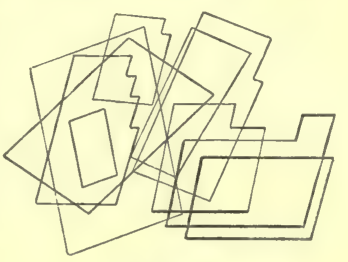

Fig. 196.

Crystals of cholesterin. iodine. When dissolved in chloroform, one drop of concentrated sulphuric acid causes a deep red colour (H. Schiff).

(5) Amongst the other organic constituents :-Lecithin ( $\$ 23)$, or its decomposition-product, neurin (cholin), and glycero-phosphoric acid (into which lecithin may be artifically transformed by boiling with baryta); palmatin, stearin, olein, as well as their soda soaps; diastatic ferment; traces of urea; (in ox bile, acetic acid and propionic acid, united with glycerine and metals, Dogiel).

(6) Inorganic constituents of bile $(0.6$ to 1 per cent.) :-

They are-sodic and potassic chloride, calcic and magnesic phosphate, and much iron, which in fresh bile gives the ordinary reactions for iron, so that iron must occur in one of its oxidised compounds in bile; manganese and silica. Gases. - Freshly-secreted bile contains in the dog more than 50 vol., and in the rabbit 109 vol. per cent. $\mathrm{CO}_{2}$, partly united to alkalies, partly absorbed, the latter, however, being almost completely absorbed within the gall-bladder. 
The mean composition of human bile is :-

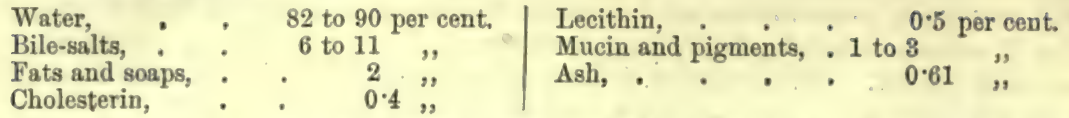

Further, unchanged fat probably'always passes into the bile, but it is again absorbed therefrom (Virchow). The amount of $\mathrm{S}$ in dry dog's bile $=2 \cdot 8$ to $3 \cdot 1$ per cent., the $\mathrm{N}=7$ to 10 per cent. (Spiro) ; the sulphur of the bile is not oxidised into sulphuric acid, but it appears as a sulphurcompound in the urine (Kunkel, v. Voit).

178. SECRETION OF BILE-(1) The secretion of bile is not a mere filtration of substances already existing in the blood of the liver, but it is a chemical production of the characteristic biliary constituents, accompanied by oxidation, within the hepatic cells, to which the blood of the gland only supplies the raw material. The liver-cells themselves undergo histological changes during the process of digestion. It is secreted continually; but part is stored up in the gall-bladder, and is poured out copiously during digestion. The higher temperature of the blood of the hepatic vein, as well as the large amount of $\mathrm{CO}_{2}$ in the bile, indicates that oxidations occur within the liver. The water of the bile is not merely filtered through the blood-capillaries, as the pressure within the bile-ducts may exceed that in the portal vein.

(2) The quantity of bile was estimated by v. Wittich, from a biliary fistula, at 533 cubic centimetres in twenty-four hours (some bile passed into the intestine); by Westphalen, at 453 to 566 grms. [by Murchison, at 40 oz.] ; by Joh. Ranke, on a biliary-pulmonary fistula, at 652 cubic centimetres. The last observation gives 14 grms. (with 0.44 grms. solids) per kilo. of man in twenty-four hours.

Analogous values for animals are -1 kilo. dog, $32 \mathrm{grm}$. (1·2 solids) ; 1 kilo. rabbit, $137 \mathrm{grm}$. (2.5 solids); 1 kilo. guinea-pig, 176 grms. (2.5 solids).

(3) The excretion of bile into the intestine shows two maxima during one period of digestion; the first from 3 to 5 hours, and the second from 13 to 15 hours, after food. The cause is due to simultaneous reflex excitement of the hepatic bloodvessels, which become greatly dilated.

(4) The influence of food is very marked. The largest amount is secreted after a flesh diet, with some fat added; less after vegetable food; a very small amount with a pure fat diet; it stops during hunger. Draughts of water increase the amount, with a corresponding relative diminution of the solid constituents. [The biliary solids are increased by food, reaching their maximum about one hour after feeding.]

(5) The influence of blood-supply is variable:-

(a) Secretion is greatly favoured by a copious and rapid blood-supply. The blood-pressure is not the prime factor, as ligature of the eava above the diaphragm, whereby the greatest blood-pressure occurs in the liver, arrests the secretion.

(b) Simultaneous ligature of the hepatic artery (diameter $5 \frac{1}{2} \mathrm{~mm}$.) and the portal vein (diameter, $16 \mathrm{~mm}$.) abolishes the secretion (Röhrig). These two vessels supply the raw material for the secretion of bile.

(c) If the hepatic artery be ligatured, the portal vein alone supports the secretion. Ligature of the artery or one of its branches ultimately causes necrosis of the parts supplied by that branch, and eventually of the entire liver, as this artery is the nutrient vessel of the liver.

(d) If the branch of the portal vein to one lobe be ligatured, there is only a slight secretion in that lobe, so that the bile must be formed from the arterial blood. Complete ligature of the portal vein rapilly causes death $(\$ 87)$. Neither ligature of the hepatic artery by itself, nor gradual obliteration of the portal vein by itself, causes cessation of the secretion, but it is diminished. That sudden ligature of the portal vein causes cessation is due to the fact that, in addition to diminution of the secretion, the enormous stagnation of blood in the rootlets of the portal vein in the abdominal orgais makes the liver very anæmic, and thus prevents it from secreting.

(e) If the blood of the hepatic artery is allowed to pass into the portal vein (which has been ligatured on the peripheral side), secretion continues (Schiff).

$(f)$ Profuse loss of blood arrests the secretion of bile, before the muscular and nervous apparatus become paralysed. A more copious supply of blood to other organs-e.g., to the 
muscles of the trunk-during vigorous exercise, diminishes the secretion, while the transfusion of large quantities of blood increases it, but if too high a pressure is caused in the portal vein, by introducing blood from the carotid of another animal, it is diminished.

(g) Influence of Nerves. - All conditions which cause contraction of the abdominal blood. vessels, e.g., stimulation of the ansa Vieussenii, of the inferior cervical ganglion, of the hepatic nerves, of the splanchnics, of the spinal cord (either directly by strychnia, or reflexly through stimulation of sensory'nerves), affect the secretion; and so do all conditions which cause stagnation or congestion of the blood in the hepatic vessels (seetion of the splanchnic nerves, diabetic puncture, §175), section of the cervical spinal cord. Paralysis (ligature) of the hepatic nerves causes at first an increase of the biliary secretion.

(h) Portal and Hepatic Veins. - With regard to the raw material supplied to the liver by its blood-vessels, it is important to note the difference in the composition of the blood of the hepatic and portal veins. The blood of the hepatic vein contains more sugar (?), lecithin, cholesterin (Drosdoff), and blood-corpuscles, but less albumin, fibrin, hæmoglobin, fat, water, and salts.

$[(i)$ Uffelmann observed that the flow of bile from a person with a biliary fistula was arrested during fever.]

(6) The formation of bile is largely dependent upon the decomposition of red blood-corpuscles, as they supply the material necessary for the formation of some of its constituents.

Hence, all conditions which cause solution of the coloured blood-corpuscles are accompanied by an increased formation of bile ( $\$ 180)$.

(7) Of course a normal condition of the hepatic cells is required for a normal secretion of bile.

Biliary Fistulæ. - The mechanism of the biliary secretion is studied in animals by means of biliary fistulæ. Schwann opened the belly by a vertical incision a little to the right of the ensiform process, cut into the fundus of the gall-bladder, and sewed its margins to the edges of the wound in the abdomen, and afterwards introduced a cannula into the wound (fig. 197). To secure that all the bile is discharged externally, tie the common bile-duct in two places and divideit between the twoligatures. Aftera fistula is freshly made the secretion falls. This depends upon the removal of the bile from the body. If bile be supplied, the secretion is increased. Regeneration of the divided bile-duct may occur in dogs. V. Wittich observed a biliary fistula in man. [A temporary biliary fistula may also be made. The abdomen is opened in the same way as described above. A long bent glass cannula is introduced and tied into the common bile-duct, and the cystic duct is ligatured or clamped (fig. 197). The tube is brought out through the wound in the abdomen.]
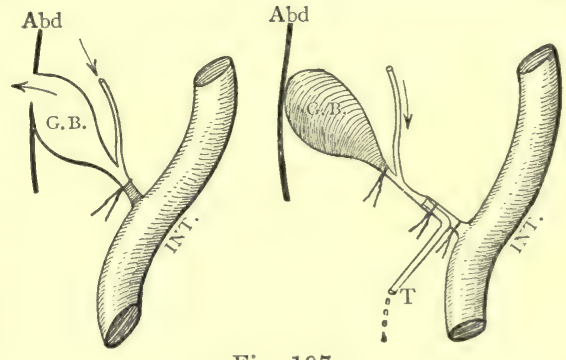

Fig. 197.

Schwann's permanent fistula, and a temporary fistula. Abd, abdominal wall ; G.B., gallbladder; INT., intestine; T, tube in temporary fistula (Stirling).

[Influence of the Liver on Metabolism. - If the liver be excluded from the circulation, im. portant changes must necessarily occur in the metabolism. In birds (the goose) there is an anastomosis between the portal system of the liver and that of the kidneys, so that, when the portal circulation is interrupted in these animals, there is never any great congestion in the abdominal organs. The goose dies generally eight to ten hours after the operation. The uric acid in the urine rapidly falls to a minimum ( $\frac{1}{20}$ to $\frac{1}{30}$ of normal); the chief constituent of the urine is then sarcolactic acid, while in normal urine there is none ; the ammonia is increased (Minkowski). This experiment goes to indicate that uric acid is formed in the liver. Dog. If the liver be excluded from the portal circulation, by connecting the portal vein with the inferior vena cava, and ligaturing the hepatic artery, a dog will live in the former case three to six days and in the latter one to two. The liver does not undergo necrosis, nor does bile cease to be secreted. The liver is nourished by the blood in the hepatic vein, the reflux in this vein being probably caused by the respiratory movements (Stolnikow). Noël Paton finds that in dogs, in a condition of nitrogenous balance, some drugs which increase the flow of bile (e.g., salicylate and benzoate of soda, colchicum, perchloride of mercury, and euonymin) also increase the production of urea ; hence, he concludes that the formation of urea in the liver bears a very direct relationship to the secretion of bile (\$256).]

179. EXCRETION OF BILE.- - In this connection we must keep in view two distinct mechanisms. (1) The bile-secreting mechanism dependent upon the 
liver-cells, which are always in a greater or less degree of activity ; (2) the bileexpelling mechanism, which is specially active at certain periods of digestion (\$ 178)].

Excretion of bile is due to (1) the continual pressure of the newly-formed bile within the interlobular bile-ducts forcing onward the bile in the excretory ducts.

(2) The interrupted periodic compression of the liver from above, by the diaphragm, at every inspiration. Further, every inspiration assists the flow of, blood in the hepatic veins, and every respiratory increase of pressure within the abdomen favours the current in the portal vein.

It is probable that the diminution of the secretion of bile, which occurs after bilateral division of the vagi, is to be explained in this way ; still it is to be remembered, that the vagus sends branches to the hepatic plexus. It is not decided whether the biliary excretion is diminished after section of the phrenic nerves and paralysis of the abdominal muscles.

(3) The contraction of the smooth muscles of the larger bile-ducts and the gallbladder. Stimulation of the spinal cord, from which the motor nerves for these structures pass, causes acceleration of the outflow, which is afterwards followed by a diminished outflow. Under normal conditions, this stimulation seems to occur reflexly, and is caused by the passage of the ingesta into the duodenum, which, at the same time, excites movement of this part of the intestine.

(4) Direct stimulation of the liver, and reflex stimulation of the spinal cord, diminish the excretion; while extirpation of the hepatic plexus and injury to the floor of the fourth ventricle do not exert any disturbing influence.

(5) A relatively small amount of resistance causes bile to stagnate in the bileducts.

Secretion Pressure. - A manometer, tied into the gall-bladder of a guinea-pig, supports a column of 200 millimetres of water; and secretion can take place under this pressure. If this pressure be increased, or too long sustained, the watery bile passes from the liver into the blood, even to the amount of four times the weight of the liver, thus causing solution of the red blood-corpuscles by the absorbed bile; and very soon thereafter hæmoglobin appears in the urine. [This fact is of practical importance, as duodenitis may give rise to symptoms of jaundice, the resistance of the inflamed mucous membrane being sufficient to arrest the outHow of bile.]

Passage of Substances into the Bile.-Some substances which enter the blood pass into the bile; especially the metals, copper, arsenic, iron. \&c. ; potassium iodide, bromide, and sulphocyanide, and turpentine ; to a less degree, cane-sugar and grape-sugar ; sodium salicylate, and carbolic acid. If a large amount of water be injected into the blood, the bile becomes albuminous; mercuric and mercurous chlorides cause an increase of the water of the bile. Sugar has been found in the bile in diabetes; leucin and tyrosin in typhus, lactic acid and albumin in other pathological conditions of this fluid.

180. REABSORPTION OF BILE; JAUNDICE.--I. Absorption-Jaundice.-When resistance is offered to the outflow of bile into the intestine, e.g., by a plug of mucus, or a gall-stone which occludes the bile-duct, or where a tumour or pressure from without makes it impervious -the bile-ducts become filled with bile and cause an enlargement of the liver. The pressure within the bile-ducts is increased. As soon as the pressure has reached a certain amount, which it soon does when the bile-duct is occluded (in the dog $275 \mathrm{~mm}$. of a column of bile), reabsorption of bile from the distended larger bile-ducts takes place into the lymphatics (not the blood-vessels) of the liver, the bile-acids pass into the lymphatics of the liver. [The lymphatics can be seen at the portal fissure filled with yellow-coloured lymph.] The lymph passes into the thoracic duct, and so into the blood (Fleischl). Even when the pressure is very low within the portal vein, bile may pass into the blood without any obstruction to the bile-duct being present. This is the case in Icterus neonatorum, as after ligature of the umbilical cord no more blood passes through the umbilical vein ; further, in the icterus of hunger, "hungerjaundice" as the portal vein is relatively empty, owing to the feeble absorption from the intestinal canal (Cl. Bernard).

II. Cholæmia may also occur, owing to the excessive production of bile (hypercholia), the bile not being all excreted into the intestine, so that part of it is reabsorbed. This takes place when there is solution of a great number of blood-corpuscles ( $\$ 178,6)$, which yield material for the formation of bile. Thick inspissated bile accumulates in the bile-ducts, so that stagnation, with subsequent reabsorption of the bile, takes place. The transfusion of hetero- 
geneous blood obtained by dissolving coloured blood-corpuseles acts in this direction. Icterus is a common phenomenon after too copious transfusion of the same blood. The blood-corpuscles are dissolved by the injection into the blood of heterogeneous blood-serum, by the injection of bile-acids into the vessels, and by other salts, by phosphoric acid, water, chloral, inhalation of chloroform and ether; the injection of dissolved hæmoglobin into the arteries or into a loop of the small intestine acts in the same way.

Icterus Neonatorum. - When, owing to compression of the placenta within the uterus, too much blood is forced into the blood-vessels of the newly-born infant, a part of the surplus blood during the first few days becomes dissolved, part of the hæmoglobin is converted into bilirubin, thus causing jaundice (Virchow, Violet).

Absorption-Jaundice.-When the jaundice is caused by the absorption of bile already formed in the liver, it is called hepatogenic or absorption-jaundice. The following are the symptoms :-

(1) Bile-pigments and bile-acids pass into the tissues of the body; hence, the most pronounced external symptom is the yellowish tint or jaundice. The skin and the sclerotic become deeply coloured yellow. In pregnancy the foetus is also tinged.

(2) Bile-pigments and bile-acids pass into the urine (not into the saliva, tears, or mucus), (\$177). When there is much bile-pigment, the urine is coloured a deep yellowish-brown, and its froth is citron-yellow; while strips of gelatin or paper dipped into it also become coloured. Occasionally bilirubin ( $=$ hæmatoidin) crystals occur in the urine ( $\$ 266)$.

(3) The fæces are "clay-coloured" (because the hydrobilirubin of the bile is absent from the fæcal matter)-very hard (because the fluid of the bile does not pass into the intestine); contain much fat (in globules and crystals), because the fat is not sufficiently digested in the intestine without bile, so that 78 per cent. of the fat taken with the food reappears in the fæces (v. Voit); they have a very disagreeable odour, because the bile normally greatly limits the putrefaction in the intestine. [V. Voit finds that putrefaction does not take place if fats be withheld from the food.] The evacuation of the fæces occurs slowly, partly owing to the hardness of the fæces, partly because of the absence of the peristaltic movements of the intestine, owing to the want of the stimulating action of the bile.

(4) The heart-beats are greatly diminished, e.g., to 40 per minute. This is due to the action of the bile-salts, which at first stimulate the cardiac ganglia, and then weaken them. Bile-salts injected into the heart produce at first a temporary acceleration of the pulse, and afterwards slowing (Röhrig). The same occurs when they are injected into the blood, but in this case the stage of excitement is very short. The phenomenon is not affected by section of the vagi. It is probable, that when the action of the bile-salts is long continued, they act upon the heart-muscle. In addition to the action on the heart, there is slowing of the respiration and diminution of temperature.

(5) That the nervous system, and perhaps also the muscles, are affected, either by the bilesalts or by the accumulation of cholesterin in the blood, is shown by the very general relax. ation, sensation of fatigue, weakness, drowsiness, and lastly deep coma-sometimes there is sleeplessness, itchiness of the skin, even mania, and spasms. Löwit, after injecting bile into animals, observed phenomena referable to stimulation of the respiratory, cardio-inhibitory, and vasomotor nerve-centres.

(6) In very pronounced jaundice there may be "yellow vision," owing to the impregnation of the retina and macula lutea with the bile-pigment.

(7) The bile-acids in the blood dissolve the red blood-corpuscles. The hæmoglobin is changed into new bile-pigment, and the globulin-like body of the hæmoglobin may form urinary cylinders or casts in the urinary tubules, which are ultimately washed out of the tubules by the urine.

[Influence of Drugs on the Secretion of Bile.-On animals one may make either a permanent or a temporary fistula. The latter is the more satisfactory method, and the experiments are usually made on fasting curarised dogs. A suitable cannula is introduced into the common bile-duct (fig. 197), the animal is curarised, artificial respiration being kept up, while the drug is injected into the stomach or intestine. Röhrig used this method, which was improved by Rutherford and Vignal. Röhrig found that some purgatives, croton oil, colocynth, jalap, aloes, rhubarb, senna, and other substances, increased the secretion of bile. Rutherford and Vignal investigated the action of a large number of drugs on the bile-secreting mechanism. They found that croton oil is a feeble hepatic stimulant, while podophyllin, aloes, colchicum, euonymin, iridin, sanguinarin, ipecacuan, colocynth, sodium phosphate, phytolaccin, sodium benzoate, sodium salicylate, dilute nitro-hydrochloric acid, anmonium phosphate, mercuric chloride (corrosive sublimate), are all powerful, or very considerable, hepatic stimulants. Some substances stimulate the intestinal glands, but not the liver, e.g., magnesium sulphate, castor oil, gamboge, ammonium chloride, manganese sulphate, calomel. Other substances stimulate the liver as well as the intestinal glands, although not to the same extent, e.g., scammony (powerful intestinal, feeble hepatic stimulant); colocynth excites both powerfully; jalap, sodium sulphate, 
and baptisin, act with considerable power both on the liver and the intestinal glands. Calabar bean stimulates the liver, and the increased secretion caused thereby may be reduced by sulphate of atropin, although the latter drug, when given alone, does not notably affect the secretion of bile. The injection of water or bile slightly increases the secretion. In all cases where purgation was produced by purely intestinal stimulants, such as magnesium sulphate, gamboge, and castor oil, the secretion of bile was diminished. In all such experiments it is most important that the temperature of the animal be kept up, else the secretion of bile diminishes. Paschkis's results on dogs differ considerably from those of Rutherford. He asserts that only the bile-acids (salts) of all the substances he investigated excite a prompt and distinct cholagogue action.]

[As yet we cannot say definitely whether, or not, these substances stimulate the secretion of bile, by exciting the mucous membrane of the small intestine, and thereby inducing reflex excitement of the liver. Their action does not seem to be due to increase of the blood-stream through the liver. More probably, as Rutherford suggests, these drugs act directly on the hepatic-cells or their nerves. Acetate of lead directly depresses the biliary secretion, while some substances affect it indirectly.]

[Cholesteræmia.-Flint ascribes great importance to the excretion of cholesterin by the bile, with reference to the metabolism of the nervous system. Cholesterin, which is a normal ingredient of nervous tissue, is excreted by the bile ; and if it be retained in the blood "cholesteræmia," with grave nervous symptoms, is said to occur. This, however, is problematical, and the phenomena described are probably referable to the retention of the bile-acids in the blood.]

181. FUNCTIONS OF THE BILE.-[(1) Bile is concerned in the digestion of certain food-stuffs; (2) part is absorbed ; (3) part is excreted.]

(A) Bile plays an important part in the absorption of fats :-

(1) It emulsionises neutral fats, whereby the fatty granules pass more readily through or between the cylindrical epithelium of the small intestine into the lacteals. It does not decompose neutral fats into glycerine and a fatty acid, as the pancreas does (§ 170, III.).

When, however, fatty acids are dissolved in the bile, the bile-salts are decomposed, the bileacids being set free, while the soda of the decomposed bile-salts readily forms a soluble soap with the fatty acids. These soaps are soluble in the bile, and increase considerably the emulsifying power of this fluid. Bile can dissolve fatty acids to form an acid fluid, which has high emulsionising properties (Steiner). Emulsification is influenced by a 1 per cent. solution of $\mathrm{NaCl}$, or $\mathrm{Na}_{2} \mathrm{SO}_{4}$.

(2) As fluid fat flows more easily through capillary tubes moistened with bile, it is concluded that, when the pores of the wall of the small intestine are moistened with bile, the fatty particles pass more easily through them.

(3) Filtration of fat takes place through a membrane moistened with bile or bilesalts under less pressure than when it is moistened with water or salt solutions $(v$. Wistinghausen).

(4) As bile, like a solution of soap, has a certain relation to watery solutions, as well as to fats, it permits diffusion to take place between these two fluids, as the membrane is moistened by both fluids.

It is clear, therefore, that the bile is of great importance in the absorption of fats. This is strikingly illustrated by experiments on animals, in which the bile is entirely discharged externally through a fistula. Dogs under these conditions, absorbed at most 40 per cent. of the fat taken with the food [ 60 per cent. being given off by the fæces, while a normal dog absorbs 99 per cent. of the fat]. The chyle of such animals is very poor in fat, is not white but trans. parent; the fæces, however, contain much fat, and are oily; the animals have a ravenous appetite; the tissues of the body contain little fat, even when the nutrition of the animals has not been much interfered with. Persons suffering from disturbances of the biliary secretion, or from liver affections, ought, therefore, to abstain from fatty food. [The digestion of flesh and gelatin is not interfered with in dogs by the removal of the bile (v. Voit).]

(B) Fresh bile contains a diastatic ferment, which transforms starch into sugar, and also glycogen into sugar.

(C) Bile excites contractions of the muscular coats of the intestine, and contributes thereby to absorption.

(1) The bile-acids act as a stimulus to the muscles of the villi, which contract from time to time, so that the contents of the origins of the lacteals are emptied towards the larger lym. 
phatics, and the villi are thus in a position to absorb more. [The villi act like numerous small pumps, and expel their contents, which are prevented from returning by the presence of valves in the larger lymphatics.]

(2) The musculature of the intestine itself seems to be excited, perhaps through the agency of the plexus myentericus. In animals with a biliary fistula, and in which the bile-duct is obstructed, the intestinal peristalsis is greatly diminished, while the salts of the bile-acids administered by the mouth cause diarrhoea and vomiting. As contraction of the intestine aids absorption, bile is also necessary in this way for the absorption of the dissolved food-stuffs.

(D) The presence of bile seems to be necessary to the vital activity of the intestinal epithelium in its supposed function of being concerned in the absorption of fatty particles (\$190).

(E) Bile moistens the wall of the intestines, and gives to the fæces their normal amount of water, so that they can be readily evacuated. Animals with biliary fistula, or persons with obstruction of the bile-ducts, are very costive. The mucus aids the forward movement of the ingesta through the intestinal canal. [Thus, in a certain sense, bile is a natural purgative.]

(F) The bile diminishes putrefactive decomposition of the intestinal contents, especially with a fatty diet, $\S 190$. [Thus, it is an antiseptic, although this is doubted by $\mathbf{v}$. Voit.]

(G) When the strongly acid contents of the stomach pass into the duodenum, the glycocholic acid is precipitated by the gastric acid, and carries the pepsin with it (Burkart). Some of the albumin, which has been simply dissolved (but not peptone or propeptone), is also precipitated, by the taurocholic acid (Maly and Emich). The bile-salts are decomposed by the acid of the gastric juice. When the mixture is rendered alkaline by the pancreatic juice and the alkali derived from the decomposition of the bile-salts, the pancreatic juice acts energetically in this alkaline medium (Moleschott).

[Taurocholic acid and its soda salts precipitate albumin, but not peptone; glycocholic acid does not precipitate albumin, so that in the intestine the peptone is separated from the albumin (and syntonin), and may therefore be more readily absorbed, while the precipitate adhering to the intestinal wall can be further digested (Maly and Emich). Taurocholic acid behaves in the same way towards gelatin peptone.]

Bilious Vomit. - When bile passes into the stomach, as in vomiting, the acid of the gastric juice unites with the bases of the bile-salts ; sodium chloride and free bile-acids are formed, and the acid-reaction is thereby somewhat diminished. The bile-acids cannot carry on gastric digestion; the neutralisation also causes a precipitation of the pepsin and mucin. As soon, however, as the walls of the stomach secrete more acid, the pepsin is redissolved. The bile which passes into the stomach deranges gastric digestion, by shrivelling the proteids, which can only be peptonised when they are swollen up (p. 250):

182. FATE OF THE BILE.-Some of the biliary constituents are completely evacuated with the fæces, while others are reabsorbed by the intestinal walls.

(1) Mucin passes unchanged into the fæces.

(2) The bile-pigments are reduced, and are partly excreted with the fæces as hydrobilirubin, and partly as the identical end-product urobilin by the urine $(\S 177,3 g)$.

From meconium hydrobilirubin is absent, while crystalline bilirubin and biliverdin, and an unknown red oxidation-product of them, are present [bile-acids, even taurocholic, and small trace of fatty acids] (Zweifel), so that it gives Gmelin's reaction. Heuce, no reduction-but rather oxidation-processes occur in the foetal intestine. [Composition.-Dary gives 727 per cent. water, 23.6 mucus and epithelium, 1 per cent. fat and cholesterin, and 3 per cent. bile. pigments. Zweifel gives 79.78 per cent. water, and solids 20.22 per cent. It does not contain fecithin, but so much bilirubin that Hoppe-Seyler uses it as a good source whence to obtain this pigment. It gives a spectrum of a body related to urobilin.]

(3) Cholesterin is given off with the fæces.

(4) The bile-salts are for the most part reabsorbed by the walls of the jejunum and ileum, to be re-employed in the animal's economy. Tappeiner found them in the chyle of the thoracic duct-minute quantities pass normally from the blood into the urine. Only a very small amount of glycocholic acid appears unchanged 
in the fæces. The taurocholic acid, as far as it is not absorbed, is easily decomposed in the intestine, by the putrefactive processes, into cholalic acid and taurin; the former of these is found in the fæces, but the taurin at least seems not to be constantly present. Part of the cholalic acid is absorbed, and may unite in the liver either with glycin or taurin (Weiss).

(5) The fæces contain mere traces of lecithin.

Impaired Nutrition.-The greatest part of the most important biliary constituents, the bileacids, re-enter the blood, and thus is explained why animals with a biliary fistula, where all the bile is removed (without the animal being allowed to lick the bile), rapidly lose weight. This depends partly upon the digestion of the fats being interfered with, and also upon the direct loss of the bile-salts. If such dogs are to maintain their weight, they must eat twice as much food. In such cases, carbohydrates most beneficially replace the fats. If the digestive apparatus is otherwise intact, the animals, on account of their voracity, may even increase in weight, but the flesh and not the fat is increased.

Bile partly an Excretion.-The fact that bile is secreted during the foetal period, whilst none of the other digestive fluids is, proves that it is an excretion.

The cholalic acid which is reabsorbed by the intestinal walls passes into the body, and seems ultimately to be burned to form $\mathrm{CO}_{2}$ and $\mathrm{H}_{2} \mathrm{O}$. The glycin (with hippuric acid) forms urea, as the urea is increased after the injection of glycin. The fate of taurin is unknown. When large quantities are introduced into the human stomach, it reappears in the urine as tauro-carbamic

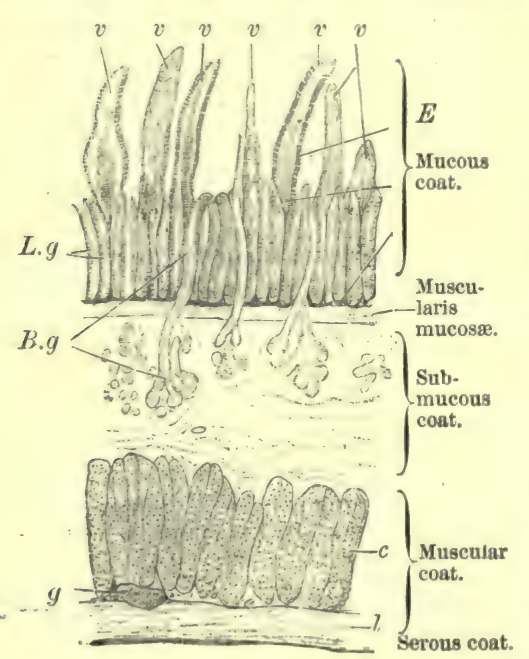

Fig. 198.

Vertical section of duodenum (cat), $\times 30$. $E$, epithelium ; $c$ and $l$, circular and longitudinal muscular fibres; $L . g$, Lieberkühn's glands; B. $g$, Brunner's glands, $g$, ganglion cells ; $v$, villi.

Brunner's glands are small, branched, tubular glands, lying in the sub-mucosa of the duodenum. Their fine ducts run inwards, pierce the mucous membrane, and open at the bases of the villi (fig. 198). The acini are lined by cylindrical cells, like those lining the pyloric glands. In faet Brunner's glands are structurally and anatomically identical with the pyloric glands of the stomach. During hunger, the cells are turbid and small, while during digestion they are large and clear. The glands receive nerve-fibres from Meissner's plexus (Drasch).

I. The Secretion of Brunner's Glands. - The granular contents of the secretory cells of these glands, which occur singly in man, but form a continuous layer in the duodenum of the sheep, besides proteids consist of mucin and a fermentsubstance. of unknown constitution. The watery extract of the glands causes-(1) 
Solution of proteids at the temperature of the body (Krolow). (2) It also has a diastatic action. It converts maltose into glucose (Brown and Heron). It does not appear to act upon fats.

On account of the smallness of the objects, such experiments are only made with great difficulty, and, therefore, there is a considerable uncertainty with regard to the action of the secretion.

Lieberkiihn's glands are simple tubular glands resembling the finger of a glove [or a testtube], which lie closely packed, vertically near each other, in the mucous membrane (fig. 199); they are most numerous in the large intestine, owing to the absence of villi in this region. They consist of a structureless membrana propria lined by a single layer of low cylindrical epithelium, between which numerous goblet-cells occur, the goblet-cells being fewer in the small intestine and much more numerous in the large (fig. 199). The glands of the small intestine yield a thin secretion, while those of the large intestine yield a large amount of sticky mucus from their gobletcells (Klose and Heidenhain). [In a vertical section of the small intestine they lie at the base of the villi (fig. 198). In transverse section they are shown in fig. 200.]

\section{The Secretion of} Lieberkühn's Glands, from the duodenum onwards, is the chief source of the intestinal juice.

Intestinal Fistula.-The intestinal juice is obtained by making a Thiry's Fistula (1864). A loop of the intestine of a dog is pulled forward (fig. 201, 1), and a piece about 4 inches in length is cut out, so that the continuity of the intestinal tube is broken, but the mesentery and its blood-vessels are not divided. One end of this tube is closed, and the other end is left open and stitched to the abdominal wall (fig. 201, 3). The two ends of the intestine, from which this piece was taken, are brought together with sutures, so as to establish the continuity of the intestinal canal (fig. 201,2). The excised piece of intestine yields a secretion which isuncontaminated with any other digestive secretion. [Thiry's method is very unsatisfactory, as judged from the action of the separated loop in relation to medicaments, prob-

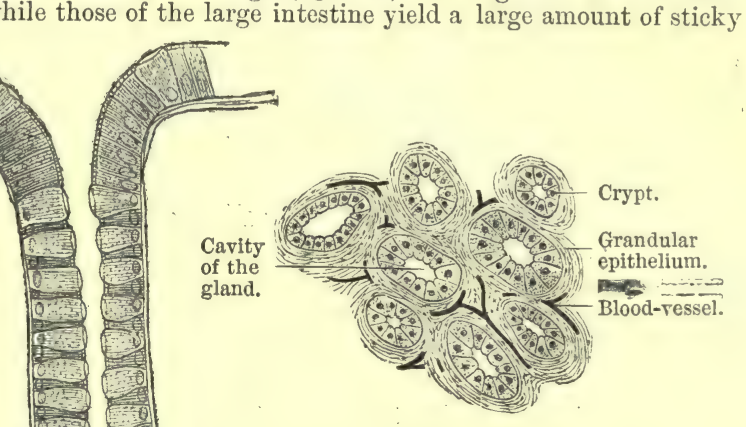

Fig. 200.

Transverse section of Lieberkühn's follicles.

ably owing to its mucous membrane becoming atrophied from disuse, or injured by inflammation.]

[Meade Smith makes a small opening in the intestine, through which he introduces two small collapsed india-rubber balls, one above and the other below the opening, which are then distended by inflation until they completely block a certain length of the intestine. The loop thus blocked off, having been previously well washed out, is allowed to become filled with succus, which is secreted on the application of various stimuli. By means of Bernard's gastric cannula ( $\$ 165$ ) inserted into the fistula in the loop, the secretion can be removed when desired.]

[Vella's Fistula.-Open the belly of a dog, and pull out a loop (30 to 50 ctm.) [1 to $1 \frac{1}{2}$ feet] of small intestine, and ligature it; divide it above and below, re-establish the continuity of the rest of the intestine. Stitch both ends of the loop of intestine into the wound in the linea alba 
(fig. 201, 4), so that there is a loop of intestine supplied by its blood-vessels and nerves, isolated and with an upper and lower aperture.]

The intestinal juice of such fistulæ flows spontaneously in very small amount, and is increased during digestion; it is increased-especially its mucus-by mechanical, chemical, and electrical stimuli; at the same time, the mucous membrane becomes red, so that 100 centimetres yield 13 to 18 grammes of this juice in an hour (Thiry). The juice is light yellow, opalescent, thin, strongly alkaline, specific gravity 1011, evolves $\mathrm{CO}_{2}$ when an acid is added; it contains albumin, ferments, and mucin-especially the juice of the large intestine. Its composition is-water, $97 \cdot 59$; proteids, 0.80 ; other organic substances $=0 \cdot 73$; salts, 0.88 per cent. ; amongst these-sodium carbonate, 0.32 to 0.34 per cent.

[The intestinal juice obtained by Meade Smith's method contained only 0.39 per cent. of organic matter, and in this respect agreed closely with the juice which $\mathrm{A}$. Moreau procured by dividing the mesenteric nerves of a ligatured loop of intestine. The secretion of the large intestine is much more viscid than that of the small intestine.]

Actions of Succus Entericus.-It is most active in the dog, and in other animals it is more or less inactive.

(1) It is less diastatic than the saliva and the pancreatic juice, but it does not form maltose; while the juice of the large intestine does not possess this property (Eichhorst).

(2) It converts maltose into grape-sugar. It seems, therefore, to continue the diastatic action of saliva $(\S 148)$ and pancreatic juice $(\S 170)$, which usually form only maltose.

According to Bourquelot this action is due to the intestinal schizomycetes and not to the intestiual juice as such, the saliva, gastric juice, or invertin. The greater part of the maltose appears, however, to be absorbed unchanged.

(3) Fibrin is slowly (by the trypsin and pepsin-Kühne) peptonised (Thiry, Leube); less easily albumin (Masloff), fresh casein, flesh raw or cooked, vegetable albumin; probably gelatin also is changed by a special ferment into a solution which does not gelatinise (Eichhorst).

(4) Fats are only partly emulsionised (Schiff), and afterwards decomposed (Vella).

(5) According to $\mathrm{Cl}$. Bernard, invertin occurs in intestinal juice (this ferment can also be extracted from yeast). It causes cane-sugar $\left(\mathrm{C}_{12} \mathrm{H}_{22} \mathrm{O}_{11}\right)$ to take up water $\left(+\mathrm{H}_{2} \mathrm{O}\right)$, and converts it into invert-sugar, which is a mixture of left rotating sugar (lævulose, $\mathrm{C}_{6} \mathrm{H}_{12} \mathrm{O}_{6}$ ) and of grape-sugar (dextrose, $\mathrm{C}_{6} \mathrm{H}_{12} \mathrm{O}_{6}$ ). Heat seems to be absorbed during the process.

[Hoppe-Seyler has suggested that this ferment is not a natural product of the body, but is introduced from without with the food. Matthew Hay, however, finds it to be invariably present in the small intestine of the foetus.]

[Effect of Drugs. - The subcutaneous injection of pilocarpin causes the mucous membrane of a Vella's fistula to be congested, when a strongly alkaline, opalescent, watery, and slightly albuminous secretion is obtained. This secretion produces a reducing sugar, converts cane-sugar into invert-sugar, emulsifies neutral fats, ultimately splitting them up, peptonises proteids, and coagulates milk, even although the milk be alkaline. The juice attacks the sarcous substance of muscle before the connective-tissues - the reverse of the gastric-juice. The mucous membrane in a Vella's fistula does not atrophy. K. B. Lehmann finds that the succus entericus obtained from the intestine of a goat by a Vella fistula has no digestive action.]

The Action of Nerves on the secretion of the intestinal juice is not well determined. Section or stimulation of the vagi has no apparent effect; while extirpation of the large sympathetie abdominal ganglia causes the intestinal canal to be filled with a watery fluid, and gives rise to diarrhea. This may be explained by the paralysis of the vaso-motor nerves, and also by the section of large lymphatic vessels during the operation, whereby absorption is interfered with and transudation is favoured. Moreau's Experiment. - Moreau placed four ligatures on a loop of intestine at equal distances from each other (fig. 202). The ligatures were tied so that three loops of intestine were shut off. The nerves $(\mathrm{N})$ to the middle loop were divided, and the intestine was replaced in the abdominal cavity. After a time, a very small amount of secretion, or none at all, was found in two of the ligatured compartments of the gut, i.e., in those with the nerves and blood-ressels intact $(1,3)$, but the compartment (2) whose nerres had been 
divided contained a watery secretion. Perhaps the secretion which occurs after section of the mesenteric nerves is a paralytic secretion. The secretion of the intestinal and gastric juices is diminished in man in certain nervous affections (hysteria, hypochondriasis, and various cerebral diseases); while in other conditions these secretions are increased.

Excretion of Drugs. - If an isolated intestinal fistula be made, and various drugs administered, the mucous membrane excretes iodine, bromine, lithium, sulphocyanides, but not potassium ferrocyanide, arsenious or boracic acid, or iron salts.

In sucklings, 'not unfrequently a large amount of acid is formed, when the fungi in the intestine split up milk-sugar or grape-sugar into lactic acid (Leube). Starch changed into grape-sugar may undergo the same abnormal process; hence, infants ought not to be fed with starchy food.

[Fate of the Ferments. - Langley is of opinion that the digestive ferments are destroyed in the intestinal canal; the diastatic fer-

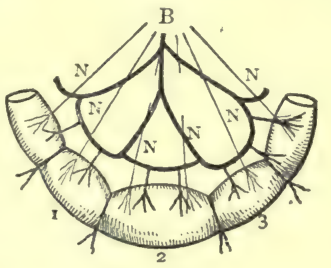

Fig. 202.

Scheme of Moreau's experiment (Stirling). ment of saliva is destroyed by the free $\mathrm{HCl}$ of the gastric juice ; pepsin and rennet are acted upon by the alkaline salts of the pancreatic and intestinal juices, and by trypsin; while the diastatic and peptic ferments of the pancreas disappear under the influence of the acid fermentation in the large intestine.]

184. FERMENTATION IN THE INTESTINE.-Those proeesses, which are to be regarded as fermentations or putrefactive processes, are quite different from those caused by the digestive enzymes or ferments just considered. The putrefactive changes are connected with the presence of lower organisms, so-called fermentation- or putrefaction-producers: and they may develop in suitable media outside the body. The lowly organisms which cause the intestinal fermentation are swallowed with the food and drink, and also with the saliva. When they are introduced, fermentation and putrefaction begin, and gases are evolved.

Intestinal Gases.-During the whole of the foetal period, until birth, fermentation cannot occur; hence gases are never present in the intestine of the newly-born. The first air-bubbles pass into the intestine with the saliva which is swallowed, even before food has been taken. The germs of organisms are thus introduced into the intestine, and give rise to the formation of gases. The evolution of intestinal gases goes hand-in-hand with the fermentations. Air is also swallowed, and an exchange of gases take place in the intestine, so that the composition of the intestinal gases depends upon various conditions. Kolbe and Ruge collected the gases from the anus of a man, and found in 100 vols. :-

\begin{tabular}{|c|c|c|c|c|c|c|c|}
\hline \multicolumn{3}{|c|}{ Food. } & $\mathrm{CO}_{2}$. & H. & $\mathrm{CH}_{4}$. & N. & $\mathrm{H}_{2} \mathrm{~S}$. \\
\hline $\begin{array}{l}\text { Milk, } \\
\text { Flesh, } \\
\text { Peas, }\end{array}$ & : & & $\begin{array}{l}16 \cdot 8 \\
12 \cdot 4 \\
21 \cdot 0\end{array}$ & $\begin{array}{r}43 \cdot 3 \\
2 \cdot 1 \\
4 \cdot 0\end{array}$ & $\begin{array}{r}0 \cdot 9 \\
27 \cdot 5 \\
55 \cdot 9\end{array}$ & $\begin{array}{l}38 \cdot 3 \\
57 \cdot 8 \\
18 \cdot 9\end{array}$ & $\begin{array}{l}\text { Quantity not } \\
\text { estimated. }\end{array}$ \\
\hline
\end{tabular}

1. Air-bubbles are swallowed with the food. The $O$ is rapidly absorbed in the intestinal tract, so that in the lower part of the large intestine, even traces of $\mathrm{O}$ are absent. In exchange, the blood-vessels in the intestinal wall give off $\mathrm{CO}_{2}$ into the intestine, so that part of the $\mathrm{CO}_{2}$ in the intestine is derived by diffusion from the blood.

2. $\mathrm{H}, \mathrm{CO}_{2}, \mathrm{NH}_{3}$, and $\mathrm{CH}_{4}$ are also formed from the intestinal contents by fermentation, which takes place even in the small, intestine.

Fungi. - The chief agents in the production of fermentations, putrefaction, and other similar decompositions are undoubtedly the group of fungi called schizomycetes. They are small unicellular organisms of various forms--globular, micrococcus; short rods, bacterium; long rods, bacillus; or spiral threads, vibrio, spirillum, spirochæta (fig. 23). The mode of reproduction is by division, and they may either remain single or unite to form colonies. Each organism is nsually capable of some degree of motion. They produce profound chemical changes in the fluids or media in which they grow and multiply, and these changes depend upon the vital activity of their protoplasm. These minute microscopic organisms take certain constituents from the "nutrient fluids" in which they live, and use them partly for building up their own 
tissues and partly for their own metabolism. In these processes, some of the substances so absorbed and assimilated undergo chemical changes, some ferments seem thereby to be produced, which in their turn may act upon material present in the nutritive fluid.

These fungi consist of a capsule enclosing protoplasmic contents. Many of them are provided with excessively delicate cilia, by means of which they move about. The new organisms, produced by the division of pre-existing ones, sometimes form large colonies visible to the naked eye, the individual fungi being united by a jelly-like mass, the whole constituting zoogloea. In some fungi, reproduction takes place by spores; more especially when the nutrient fluids are poor in nutritive materials. The bacteria form longer rods or threads, which are jointed, and in each joint or segment small $(1-2 \mu)$ highly refractive globules or spores are developed (fig. $203,7)$. In some cases, as in the butyric acid fermentation, the rods become fusiform before spores are formed. When the envelope of the mother-cell is ruptured or destroyed, the spores are liberated, and if they fall upon or into a suitable medium, they germinate and reproduce organisms similar to those from which they sprang. The process of spore-production is illustrated in fig. $203,7,8,9$, and in $1,2,3,4$ is shown the process of germination in the butyric acid fungus. The spores are very tenacious of life; they may be dried, when they resist death for a very long time; some of them are killed by being boiled. Some fungi exhibit their vital activities only in the presence of $O$ (ærobes), while others require the exclusion of $O$ (anærobes, Pasteur). According to the products of their action, they are classified as follows :Those that produce fermentations (zymogenic schizomycetes); those that produce pigments (chromogenic); those that produce disagreeable odours, as during putrefaction (bromogenic);

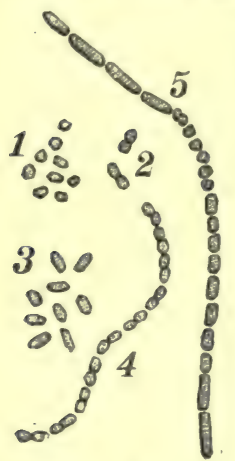

$A$

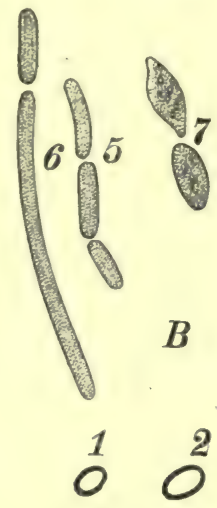

Fig. 203.
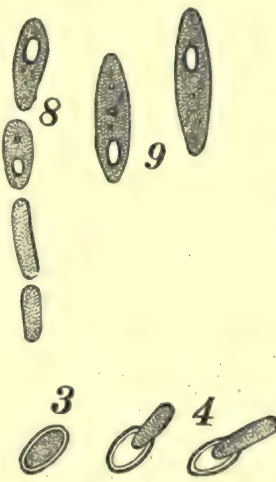

A, Bacterium aceti in the form of-cocci (1); diplococci (2); short rods (3); and jointed threads $(4,5)$. Bacillus butyricus -(1) isolated spore; $(2,3,4)$ germinating condition of the spores; $(5,6)$ short and long rods ; $(7,8,9)$ formation of spores within a cellular fungus.

in length, arranged in groups or isolated. acid;

$$
1 \text { grape-sugar }=\mathrm{C}_{6} \mathrm{H}_{12} \mathrm{O}_{6}=2\left(\mathrm{C}_{3} \mathrm{H}_{6} \mathrm{O}_{3}\right)=2 \text { lactic acid. }
$$

Milk-sugar $\left(\mathrm{C}_{12} \mathrm{H}_{22} \mathrm{O}_{11}\right)$ can be split up by the same ferment causing it to take up $\mathrm{H}_{2} \mathrm{O}$, and forming 2 molecules of grape-sugar, $2\left(\mathrm{C}_{6} \mathrm{H}_{12} \mathrm{O}_{6}\right)$, which are again split into 4 molecules of lactic acid $4\left(\mathrm{C}_{3} \mathrm{H}_{6} \mathrm{O}_{3}\right)$.

This fungus and its spores occur everywhere in the atmosphere, and are the cause of the spontaneous acidification and subsequent coagulation of milk (\$230).

(2) Bacillus butyricus, which in the presence of starch is often coloured blue by iodine, changes lactic acid into butyric acid, together with $\mathrm{CO}_{2}$ and $\mathrm{H}$ (Prazmowski).

$$
2\left(\mathrm{C}_{3} \mathrm{H}_{6} \mathrm{O}_{3}\right) \text { lactic acid }=\left\{\begin{array}{c}
\mathrm{C}_{4} \mathrm{H}_{8} \mathrm{O}_{3}=1 \text { butyric acid. } \\
2\left(\mathrm{CO}_{2}\right)=2 \text { carbon dioxide. } \\
4 \mathrm{H}=4 \text { hydrogen. }
\end{array}\right.
$$


This fungus (fig. 203, B) is a true anærobe; and grows only in the absence of O. The lactic acid fungus uses $\mathrm{O}$ very largely, and is, therefore, its natural precursor. The butyric acid fermentation is the last change undergone by many carbohydrates, especially by starch and inulin. It takes place constantly in the fæces.

(3) Certain micrococci cause alcohol to be formed from carbohydrates. The presence of yeast may cause the formation of alcohol in the intestine, and in both cases also from milk-sugar, which first becomes changed into dextrose.

(4) Bacterium aceti (fig. 203, A) converts alcohol into acetic acid outside the body. Alcohol $\left(\mathrm{C}_{2} \mathrm{H}_{6} \mathrm{O}\right)+\mathrm{O}=\mathrm{C}_{2} \mathrm{H}_{4} \mathrm{O}$ (Aldehyd) $+\mathrm{H}_{2} \mathrm{O}$. Acetic acid $\left(\mathrm{C}_{2} \mathrm{H}_{4} \mathrm{O}_{2}\right)$ is formed from aldehyd by oxidation. According to Nägeli, the same fungus causes the formation of a small amount of $\mathrm{CO}_{2}$ and $\mathrm{H}_{2} \mathrm{O}$. As the acetic fermentation is arrested at $35^{\circ} \mathrm{C}$., this fermentation cannot occur in the intestine, and the acetic acid, which is constantly found in the fæces, must be derived from another source. During putrefaction of the proteids, with exclusion of air, acetic acid is produced (Nencki).

(5) Starch and cellulose are partly dissolved by the schizomycetes (Bac. butyricus and Vibrio rugula) of the intestine. If cellulose be mixed with cloacal-mucus, or with the contents of the intestine, it passes into a saccharine carbohydrate which decomposes into equal volumes of $\mathrm{CO}_{2}$ and $\mathrm{CH}_{4}$ (Hoppe-Seyler).

(6) Fungi, whose nature is unknown, can partly transform starch (? and cellulose) into sugar.

(7) Others produce invertin. Invertin changes cane-sugar into invert-sugar (\$ 183, II., 5). Cane-sugar, $\mathrm{C}_{12} \mathrm{H}_{22} \mathrm{C}_{11}+\mathrm{H}_{2} \mathrm{O}=\mathrm{C}_{6} \mathrm{H}_{12} \mathrm{O}_{6}$ (Dextrose) $+\mathrm{C}_{6} \mathrm{H}_{12} \mathrm{O}_{6}$ (Lævulose).

II. Fermentation of Fats ( $\S 251$ ).-During putrefaction, organisms of an unknown nature cause neutral fats to take up water and split into glycerine and their corresponding fatty acid ( $\$ 170)$. Glycerine is capable of undergoing several fermentations, according to 1 the fungus which acts upon it (\$251). With a neutral reaction, in addition to succinic acid, a number of fatty acids, $\mathrm{H}$ and $\mathrm{CO}_{2}$, are formed.

Fitz found that the

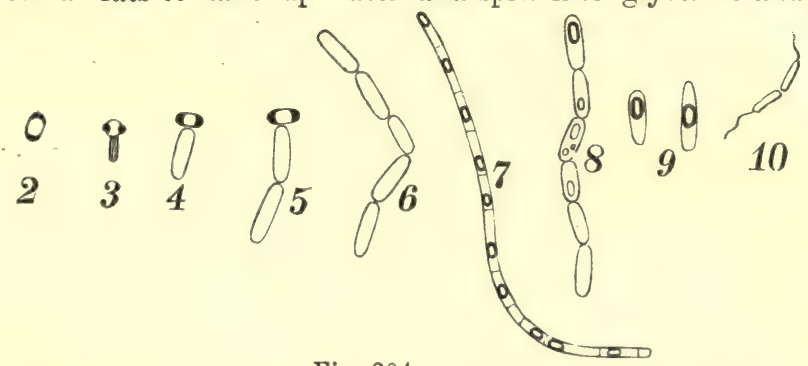

Fig. 204.

Bacillus subtilis. 1, spore ; 2, 3, 4, its germination ; 5, 6, short rods ; 7 , jointed thread, with the formation of spores in each segment; 8 , short rods, some of them containing spores; 9 , spores in single short rods ; 10 fungus with a cilium.

hay-bacillus (Bacillus subtilis, fig. 204) formed alcohol with caproic, butyric, and acetic acids ; in other cases, especially butylic alcohol, van de Velde found butyric, lactic, and traces of succinic acid with $\mathrm{CO}_{2}, \mathrm{H}_{2} \mathrm{O}, \mathrm{N}$.

The fatty acids, especially as chalk soaps, form an excellent material for fermentation. Calcium formiate mixed with cloacal-mucus ferments and yields calcium carbonate, $\mathrm{CO}_{2}$ and $\mathrm{H}$; calcium acetate, under the same conditions, produces calcium carbonate, $\mathrm{CO}_{2}$ and $\mathrm{CH}_{4}$. Amongst the oxy-acids, we are acquainted with the fermentations of lactic, glycerinic, malic, tartaric, and citric acids.

According to Fitz, lactic acid (in combination with chalk) produces propionic and acetic acids, $\mathrm{CO}_{2}, \mathrm{H}_{2} \mathrm{O}$. Other ferments cause the formation of valerianic acid. Glycerinic acid, in addition to alcohol and succinic acid, yields chiefly acetic acid; malic acid forms succinic and acetic acid. The other acids above enumerated yield somewhat similar products.

III. Fermentation of Proteids ( $\$ 249)$. - The undigested proteids and their derivatives appear to be acted upon by fungi. Many schizomycetes (hay bacillus and Bac. subtilis), however, can produce a peptonising ferment. We have already 
seen that pancreatic digestion acts upon the proteids, forming, among other products, amido-acids, leucin, tyrosin, and other bodies ( $\$ 170, \mathrm{II}$.). Under normal conditions, this is the greatest decomposition produced by the pancreatic juice. The putrefactive fermentation of the large intestine causes further and more profound decompositions. Leucin $\left(\mathrm{C}_{6} \mathrm{H}_{13} \mathrm{NO}_{2}\right)$ takes up two molecules of water and yields valerianic acid $\left(\mathrm{C}_{5} \mathrm{H}_{10} \mathrm{O}_{2}\right)$, ammonia, $\mathrm{CO}_{2}$ and $2\left(\mathrm{H}_{2}\right)$; glycin behaves in a similar manner. Tyrosin $\left(\mathrm{C}_{9} \mathrm{H}_{11} \mathrm{NO}_{3}\right)$ is decomposed into indol $\left(\mathrm{C}_{8} \mathrm{H}_{7} \mathrm{~N}\right)$, which is constantly present in the intestine along with $\mathrm{CO}_{2}, \mathrm{H}_{2} \mathrm{O}, \mathrm{H}$. If $\mathrm{O}$ be present, other decompositions take place. These putrefactive products are absent from the intestinal canal of the fotus and the newly-born. During the putrefactive decomposition of proteids, $\mathrm{CO}_{2}, \mathrm{H}_{2} \mathrm{~S}, \mathrm{H}$, and $\mathrm{CH}_{4}$, are formed; the same result is obtained by boiling them with alkalies. Gelatin, under the same conditions, yields much leucin and ammonia, $\mathrm{CO}_{2}$, acetic, butyric, and valerianic acids, and glycin. Mucin and nuclein undergo no change. Artificial pancreatic digestion-experiments rapidly tend to undergo putrefaction.

The substance which causes the peculiar fæcal odour is produced by putrefaction, but its nature is not known. It clings so firmly to indol and skatol that these substances were formerly regarded as the odorous bodies, but when they are prepared pure they are odourless (Bayer).

Amongst the solid substances in the large intestine formed only by putrefaction is indol $\left(\mathrm{C}_{8} \mathrm{H}_{7} \mathrm{~N}\right)$, a substance which is also formed when proteids are heated with alkalies, or by superheating them with water to $200^{\circ} \mathrm{C}$. It is the stage preceding the indican in the urine. If the products of the digestion of the proteids-the peptones-are rapidly absorbed, there is only a slight formation of indol; but when absorption is slight, and putrefaction of the products of pancreatic digestion occurs, much indol is formed, and indican appears in the urine.

Jaffé found much indican in the urine in strangulated hernia, and when the small intestine was obstructed.

Reactions for Indol. - Acidulate strongly with $\mathrm{HCl}$, and shake vigorously after adding a few drops of turpentine. If there be an intense red colour, the pigment is removed by ether. The substance which is formed after the digestion of fibrin by trypsin, and which gives a violet colour with bromine water $(\$ 170,2)$, can be removed by chloroform. In addition to the last pigment, there is a second one, which passes over during distillation, and which can be extracted from the distillate by ether. Both substances seem to belong to the indigo group (Krukenberg).

A. Bayer prepared indigo-blue artificially from ortho-phenyl-propionic acid, by boiling it with dilute caustic soda, after the addition of a little grape-sugar. $\mathrm{He}$ obtained indol and skatol from indigo-blue. Hoppe-Seyler found that on feeding rabbits with ortho-nitrophenylpropionic acid, much indican was present in the urine.

Phenol $\left(\mathrm{C}_{6} \mathrm{H}_{6} \mathrm{O}\right)$ is formed during putrefaction in the intestine, and it is also formed when fibrin and pancreatic juice putrefy outside the body, while Brieger found it constantly in the fæces. It seems to be increased by the same circumstances that increase indol, as an excess of indican in the urine is accompanied by an increase of phenylsulphonic acid in that fluid (\$262).

From putrefying flesh and fibrin, amido-phenylpropionic acid is obtained, as a decomposition. product of tryrosin. A part of this is transformed by putrefactive ferments into hydrocinnamic acid (phenylpropionic acid). The latter is completely oxidised in the body into benzoic acid, and appears as hippuric acid in the urine. Thus is explained the formation of hippuric acid from a purely albuminous diet.

Skatol $\left(\mathrm{C}_{9} \mathrm{H}_{9} \mathrm{~N}=\right.$ methylindol $)$ is a constant human fæcal substance, and has been prepared artificially by Nencki and Secretan from egg-albumin, by allowing it to putrefy for a long time under water. It also appears in the urine as a sulphur compound. The excretin of human fæces, described by Marcet, is related to cholesterin, but its history and constitution are unknown.

According to Salkowski, skatol and indol are both formed from a common substance which exists preformed in albumin, and which, when it is decomposed, at one time yields more indol, at another skatol, according as the hypothetical "indol-fungus," or "skatol-fungus," is the more abundant. 
It is of the utmost importance, in connection with the processes of putrefaction, to determine whether they take place when oxygen is excluded, or not. When $\mathrm{O}$ is absent, reductions take place; oxy-acids are reduced to fatty acids, and $\mathrm{H}, \mathrm{CH}_{4}$ and $\mathrm{H}_{2} \mathrm{~S}$ are formed; while the $\mathrm{H}$ may produce further reductions. If $\mathrm{O}$ be present, the nascent $\mathrm{H}$ separates the molecule of free ordinary oxygen $\left(=\mathrm{O}_{2}\right)$ into two atoms of active oxygen $(=\mathrm{O})$. Water is formed on the one hand, while the second atom of $\mathrm{O}$ is a powerful oxidising agent (Hoppe-Seyler).

It is remarkable that the putrefactive processes, after the development of phenol, indol, skatol, cresol, phenylpropionic and phenylacetic acids are subsequently limited, and after a certain concentration is reached, they cease altogether. The putrefactive process produces antiseptic substances which kill the micro-organisms, so we may assume that these substances limit to a certain extent the putrefactive processes in the intestine.

The reaction of the intestine immediately below the stomach is acid, but the pancreatic and intestinal juices cause a neutral and afterwards an alkaline reaction, which obtains along the whole small intestine. In the large intestine, the reaction is generally acid, on account of the acid fermentation and the decomposition of the ingesta and the fæces.

185. PROCESSES IN THE IARGE INTESTINE.-Within the large intestine, the fermentative and putrefactive processes are certainly more prominent than the digestive processes proper, as only a very small amount of the intestinal juice is found in it. The absorptive function of the large intestine is greater than its secretory function, for at the beginning of the colon its contents are thin and watery, but in the further course of the intestine they become more solid. Water and the products of digestion in the solution are not the only substances absorbed, but under certain circumstances, unchanged fluid egg-albumin, milk and its proteids, flesh-juice, solution of gelatin, myosin with common salt, may also be absorbed. Experiments with acid-albumin, syntonin, or blood-serum gave no result. Toxic substances are certainly absorbed more rapidly than from the stomach. [In the dog the secretion of the large intestine has no digestive properties, but fats are absorbed in it. Klug and Koreck regard its Lieberkiibnian glands not as secretingbut as absorbing-structures.] The fæcal matters are formed or rather shaped in the lower part of the gut. The cæcum of many animals, e:g., rabbit, is of considerable size, and in it fermentation seems to occur with considerable energy, giving rise to an acid-reaction. In man, the chief function of the cæcum is absorption, as is shown by the great number of lymphatics in its walls. From the lower part of the small intestine and the cæcum onwards, the ingesta assume the fæcal odour.

The amount of fæces is about [5 oz. or] 170 grms. (60 to 250 grms.) in twentyfour hours; but if much indigestible food be taken, it may be as much as 500 grms. The amount is less, and the absolute amount of solids is less, after a diet of flesh and albumin, than after a vegetable diet. The fæces are rendered lighter by the evolution of gases, and hence they float in water.

The consistence depends on the amount of water present-usually about 75 per cent. The amount of water depends partly on the food-pure flesh diet causes relatively dry fæces, while substances rich in sugar yield fæces with a relatively large amount of water. The quantity of water taken has no effect upon the amount of water in the fæces. But the energy of the peristalsis has. The more energetic the peristalsis is, the more watery the fæces are, becaúse sufficient time is not allowed for absorption of the fluid from the ingesta. Paralysis of the blood-and lymphvessels, or section of the nerves, leads to a watery condition of the fæces ( $\$ 183)$.

The reaction is often acid, in consequence of lactic acid being developed from the carbohydrates of the food. Numerous other acids produced by putrefaction are also present (\$184). If much ammonia be formed in the lower part of the intestine, a neutral or even alkaline reaction may obtain. A copious secretion of mucus favours the occurrence of a neutral reaction. 
The odour, which is stronger after a flesh diet than after a vegetable diet, is caused by some fæcal products of putrefaction, which have not yet been isolated; also by volatile fatty acids and by sulphuretted hydrogen, when it is present.

The colour of the fæces depends upon the amount of altered bile-pigments mixed with them, whereby a bright yellow to a dark brown colour is obtained.

The colour of the food is also of importance. If much blood be present in the food, the freces are almost brownish-black from hæmatin; green vegetables = brownish-green from chlorophyll ; bones $(\mathrm{dog})=$ white from the amount of lime; preparations of iron = black from the formation of sulphide of iron.

The fæces contain-

(1) The unchanged residue of animal or vegetable tissues used as food; hairs, horny and elastic tissues; most of the cellulose, woody fibres, spiral vessels of vegetable cells, gums.

(2) Portions of digestible substances, especially when these have been taken in too large amount, or when they have not been sufficiently broken up by chewing. Portions of muscular fibres, ham, tendon, cartilage, particles of fat, coagulated albumin - vegetable cells from potatoes, and other vegetables, raw starch, \&c.

All fool yields a certain amount of residue-white bread, $3 \cdot 7$ per cent.; rice, $4^{\cdot 1}$ per cent. ; flesh, 4.7 per cent.; potatoes, 9.4 per cent. ; cabbage, 14.9 per cent. ; black bread, 15 per cent. ; yellow turnip, 20.7 per cent. (Rubner).

(3) The decomposition-products of the bile-pigments, which do not now give Gmelin's reaction; as well as the altered bile-acids $(\S 177,2)$. This reaction, however, may be obtained in pathological stools, especially in those of a green colour; unaltered bilirubin, biliverdin, glycocholic and taurocholic acids occur in meconium (\$182).

[Mac)Iunn found no unchanged bile-pigments in the frees. A substance called stercobilin is obtained from the fæces, and it closely resembles what has been called "febrile" urobilin, but it is certainly different from normal urobilin.]

(4) Unchanged mucin and nuclein-the latter occasionally after a diet of bread, together with partially disintegrated cylindrical epithelium from the intestinal canal, and occasionally drops of oil. Cholesterin is very rare. [Ten grains of a substance, stercorin, said to be a modification of cholesterin, occur in the fæces, $(F$ lint).] The less the mucus is mixed with the fæces, the lower the part of the intestine from which it was derived (Nothnagel).

(5) After a milk diet, and also after a fatty diet, crystalline needles of lime combined with fatty acids and chalk soaps constantly occur, even in sucklings ( Wegscheider). Even unchanged masses of casein and fat occur during the milk cure. Compounds of ammonia, with the acids mentioned as the result of putrefaction ( 184 , III.), belong to the fæcal matters (Brieger).

(6) Amongst inorganic residues, soluble salts rarely occur in the fæces because they diffuse readily, e.g., common salt, and the other alkaline chlorides, the compounds of phosphoric acid, and some of those of sulphuric acid. The insoluble compounds of which ammoniaco-magnesic or triple phosphate, neutral calcic phosphate, yellow coloured lime salts, calcium carbonate, and magnesium phosphate are the chief, form 70 per cent. of the ash. Some of these insoluble substances are derived from the food, as lime from bones, and in part they are excreted after the food has been digested, as ashes are eliminated from food which has been burned.

Concretions. - The excretion of inorganic substances is sometimes so great, that they form incrustations around other fæcal matters. Usually ammoniaco-magnesic phosphate occurs in large crystals by itself, or it may be mixed with magnesium phosphate.

(7) Micro-organisms. - A considerable portion of normal fæcal matter consists of micrococci and microbacteria, yeast is seldom absent (Frerichs, Nothnagel).

To isolate the individual fungi, Escherich has made pure cultivations from the intestinal contents of sucklings, and Bienstock from adults. In the intestine of sucklings which have been nourished entirely on their mother's milk, the Bacterium lactis aërogenes (fig. 205, 2) 
causes the lactic acid fermentation with the evolution of $\mathrm{CO}_{2}$ and $\mathrm{H}$, in the upper part of the canal where some milk-sugar is still unabsorbed. In the evacuations is the characteristic slender Bacterium coli commune (fig. 205, 1). In addition, occasionally there are other bacilli, cocci, spores of yeast, and a mould.

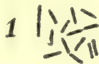

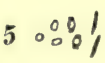

$a$
2

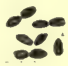

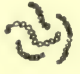

$c$
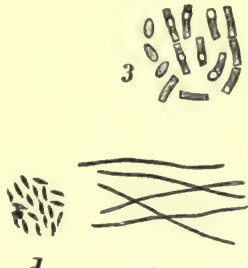

$d$

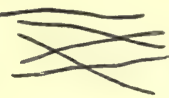

$\boldsymbol{e}$
4

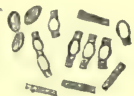

Fig. 205.

1, Bacterium coli commune; 2, bacterium lactis aërogenes; 3 and 4 , the large bacilli of LEEA Bienstock, with partial endogenous spore-formation ; 5, the various stages in the development of the bacillus which causes the fermentation of albumin.

In the fæces of an adult, Bienstock detected two large forms of bacilli (fig. 205, 3, 4), closely resembling Bacillus subtllis in form and size, but distinguished from it only by the form of its pure cultivation, by the mode of growth of its spores, and by the absence of movements. These two forms can be distinguished microscopically by the mode of their cultivation, which is either in the form of a grape or a flat membrane. These two do not excite a fermentative action. A third micrococcus-like, small, very slowly-developing bacillus occurs in three-fourths of all stools. A fourth kind (absent in sucklings) is the specific bacillus ( $\$ 184$, III.), causing the decomposition of albumin, resulting in the products of putrefaction and a fæcal odour. This is the only bacillus that excites these processes in the intestine ; but it does not decompose casein and alkali-albumin. In fig. 205, 5, $a-g$, the stages in the development of this bacillus are represented, but the stages from $c$ and $g$ are absent in the fæces, and are found only in artificial cultivations.

If the fæces are simply investigated microscopically and without special precautions, there are other fungi, some of which may be introduced through the anus. In stools that contain much starch, the bacillus butyricus, which is tinged blue with iodine, occurs (\$184), and other small globular or rod-like fungi, which give a similar reaction (Nothnagel, Uffelmann).

The changes of the intestinal contents have been studied on persons with an accidental intestinal fistula, or an artificial anus.

[The following scheme from Krukenberg shows graphically the reaction of the contents of the various parts of the alimentary canal, and also the distribution of the ferments.]
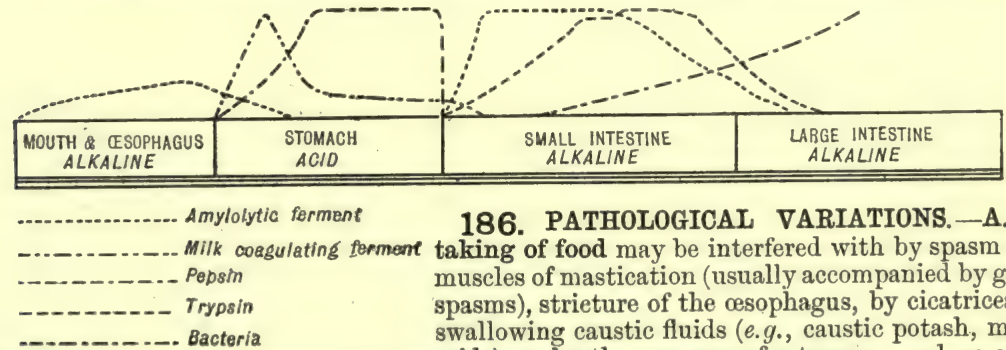

186. PATHOLOGICAL VARIATIONS. - A. The taking of food may be interfered with by spasm of the muscles of mastication (usually accompanied by general spasms), strieture of the cesophagus, by cicatrices after swallowing caustic fluids (e.g., caustic potash, mineral acids), or by the presence of a tumour, such as cancer. Inflammation of all kinds in the mouth or pharynx interferes with the taking of food. Inability to swallow occurs as part of the general phenomena in disease of the medulla oblongata, in consequence of paralysis of the motor centre (superior olives), for the facial, vagus, and hypoglossal nerves, and also for the afferent or sensory fibres of the glosso-pharyngeal, vagus, and trigeminus. Stimulation or abnormal excitation of these parts causes spasmodic swallowing, and the disagreeable feeling of a constriction in the neck (globus hystericus).

B. The secretion of saliva is diminished during inflammation of the salivary glands; occlusion of their ducts by concretions (salivary calculi); also by the use of atropin, daturin, and during fever, whereby the secretory (not the vaso-motor) fibres of the chorda appear to be paralysed (\$145). When the fever is very high, no saliva is secreted. The saliva secreted during moderate fever is turbid and thick, and usually acid. As the fever increases, the diastatic action of the saliva diminishes. The secretion is increased by-stimulation of the buccal 
nerves (inflammation, ulceration, trigeminal neuralgia), so that the saliva is secreted in great quantity. Mercury and jaborandi cause secretion of saliva, the former causing stomatitis, which excites the secretion of saliva reflexly. Even diseases of the stomach accompanied by vomiting cause secretion of saliva. A very thick tenacious sympathetic saliva occurs when there is violent stimulation of the vascular system during sexual excitement, and also during certain psychical conditions. The reaction of the saliva is acid in catarrh of the mouth, in fever in consequence of decomposition of the buccal epithelium, and in diabetes mellitus in consequence of acid fermentation of the saliva which contains sugar. Hence, diabetic persons often suffer from carious teeth. Unless the mouth of an infant be kept scrupulously clean, the saliva is apt to become acid.

C. Disturbances in the activity of the musculature of the stomach may be due to paralysis of the muscular layers, whereby the stomach becomes distended, and the ingesta remain a long time in it. A special form of paralysis of the stomach is due to non-closure of the pylorus (Ebstein). This may be due to disturbances of innervation of a central or peripheral nature, or there may be actual paralysis of the pyloric sphincter, or anæethesia of the pyloric mucous membrane, which acts reflexly upon the sphincter muscle; and lastly, it may be due to the reflex impulse not being transferred to the efferent fibre within the nerve-centre. Abnormal activity of the gastric musculature hastens the passage of the ingesta into the intestine ; vomit. ing often occurs.

D. Gastric digestion is delayed by violent bodily or mental exercise, and sometimes it is arrested altogether. Sulden mental excitement may have the same effect. These efforts are very probably caused through the vaso-motor nerves of the stomach. Feeble and imperfect digestion may be of a purely nervous nature (Dyspepsia nervosa-Leube; Neurasthenia gastricaBurkart). An excessive formation of acid may be due to nervous disturbance, and is called "nervous gastroxynsis," by Rossbach.

[Action of Alcohol, Tea, \&c., in Digestion.-According to J. W. Fraser, all infused beverages, tea, coffee, cocoa, retard the peptic digestion of proteids, with few exceptions. The retarding action is less with coffee than with tea. The tannic acid and volatile oil seem to be the retarding ingredients in teas. Distilled Spirits-brandy, whisky, gin-have but a trifling retarding effect on the digestive processes; and when one considers their action on the secretory glands, it follows that in moderate dietetic doses they promote digestion. Wines are highly inimical to salivary digestion, but this is due to their acidity ; and this effect can be removed by the addition of au alkali. Wines retard peptic digestion, the sparkling less than the still wines. Tea has an intensely inhibitory action on salivary digestion, in fact a small quantity paralyses the action of saliva, while coffee has only a slight effect. This action of tea is due to the tannin. Tea, coffee, and cocoa all retard peptic digestion, when they form 20 per cent. of the digestive mixture ( $W$. Roberts).]

Inflammatory or catarrhal affections of the stomach, as well as ulceration and new formations, interfere with digestion, and the same result is caused by eating too much food which is difficult of digestion, or taking too much highly spiced sauce or alcohol. In the case of a dog suffering from chronic gastric catarrh, Griitzner observed that the secretion took place continuously, and that the gastric-juice contained little pepsin, was turbid, sticky, feebly acid, and even alkaliue. The introduction of food did not alter the secretion, so that in this condition the stomach really obtains no rest. The chief cells of the gastric-glands were turbid. Hence, in gastric catarrh, we ought to eat frequently, but take little at a time, while at the same time dilute hydrochloric acid ought to be administered $(0 \cdot 4$ per cent. $)$. Small doses of common salt seem to aid digestion.

[Absence of $\mathbf{H C l} .-\mathrm{HCl}$ is almost always absent in carcinoma of the stomach (van de Velde), amyloid degeneration of the gastric mucous membrane (Edinger), and sometimes in fever. In all these cases the acid-reaction is due to lactic or butyric acid. The absence of $\mathrm{HCl}$ in cancer of the stomach is an important diagnostic and prognostic symptom. It is not absent in simple dilatation of the stomach. Test the contents of the stomach for free $\mathrm{HCl}$ with tropæolin (red colour), methylviolet (blue), and with ferric chloride and carbolic acid (Uffelmann). $\frac{1}{20}$ per cent. of free $\mathrm{HCl}$ causes the amethyst-blue of the last to become steel-grey, while somewhat more discharges the colour altogether. [In testing for the presence of free lactic acid in the gastric contents use Uffelmann's reaction (\$ 163). The lactic acid is easily extracted by ether from the gastric contents, and the reaction can then be performed with the residue obtained after evaporating the ether. A solution of 1 drop of the liquor perchloride in 50 c.c. of water is made yellow by lactic acid.]

Feeble digestion may be caused either by imperfect formation of acid or pepsin, so that both substances may be administered in such a condition. [It may also be due to deficient muscular power in the wall of the stomach.] In other cases, lactic, butyric, and acetic acids are formed, owing to the presence of lowly organisms. In such cases, small doses of salicylic acid, together with some hydrochloric acid, are useful. Pepsin need not be given often, as it is rarely absent, even from the diseased gastric mucous membrane. Albumin has been found in the gastricjuice in cases of gastric catarrh and cholera.

E. Digestion during Fever and Anæmia. - Beaumont found that in the case of Alexis St 
Martin, when fever occurred, a small amount of gastric-juice was secreted; the mucous membrane was dry, red, and irritable. Dogs suffering from septicæmic fever, or rendered anæmic by great loss of blood, secrete gastric-juice of feeble digestive power and containing little acid (Manasseïn). [In acute diseases accompanied by fever, the inner cells of the fundusglands of the human stomach may disappear (C. Kuptfer).] Hoppe-Seyler investigated the gastric-juice of a typhus patient in which van de Velde found no free acid. Usually no free hydrochloric acid is found in cancer of the stomach. The gastric-juice of the typhus patient did not digest artificially, even after the addition of hydrochloric acid. The diminution of acid, under these circumstances, favours the occurrence of a neutral reaction, so that, on the one hand, digestion cannot proceed, and on the other, fermentative processes (lactic and butyric acid fermentations with the evolution of gases) occur. These results are associated with the presence of micro-organisms and Sarcina ventriculi (Goodsir). Uffelmann found that the secretion of a peptone-forming gastric-juice ceased in fever, when the fever is severe at the outset, when a feeble condition occurs, or when the temperature is very high. The amount of juice secreted is certainly diminished during fever. The excitability of the mucous membrane is increased, so that vomiting readily occurs. The increased excitability of the vaso-motor nerves during fever is disadvantageous for the secretion of the digestive fluids (Heidenhain). Beaumont observed that fluids are rapidly absorbed from the stomach during fever, but the absorption of peptones is diminished on account of the accompanying catarrhal condition of the stomach, and the altered functional activity of the muscularis mucosæ (Leube).

Many salts, when given in large amount, disturb gastric digestion, e.g., the sulphates. While the alkaloids, morphia, strychnia, digitalin, narcotin, veratria have a similar action, quinine favours it (Wolberg). In some nervous individuals "peristaltic unrest of the stomach," conjoined with a dyspeptic condition, occurs (Kussmaul). [Prosser James directs attention to the value of peptic and pancreatic salts, which are preparations of common salt mixed with pepsin and the ferments of the pancreas respectively.]

[Artificial Digestion is affected by various salts according to their nature and dilution. The digestion of fibrin by pepsin goes on best without the addition of salts, being diminished by magnesic sulphate, sodic carbonate, and sulphate. The digestion of fibrin by pancreatic extract is accelerated by sodic carbonate (Heidenhain), and retarded by $\mathrm{MgSO}_{4}$ and $\mathrm{Na}_{2} \mathrm{SO}_{4}$. The diastatic action of the saliva and pancreas on starch is greatly accelerated by $\mathrm{NaCl}$ ( 2 per cent.), while $\mathrm{Na}_{2} \mathrm{CO}_{3}, \mathrm{Na}_{2} \mathrm{SO}_{4}$, and $\mathrm{MgSO}_{4}$ hinder it (Pfeiffer).] According to Schütz, artificial gastric digestion is retarded by a 2 per cent. solution of alcohol, and also by a solution of salicylic acid (.06 to 1 per cent.). Buchner, however, finds that 10 per cent. of alcohol does not affect artificial gastric digestion, while above 20 per cent. arrests it. Beer hinders digestion.

F. In acute diseases, the secretion of bile is affected; it becomes less in amount and more watery, i.e., it contains fewer specific constituents. If the liver undergoes great structural change, the secretion may be arrested.

G. Gall-Stones.-When decomposition of the bile occurs, gall-stones are formed in the gallbladder or in the bile-ducts. Some are white, and consist almost entirely of stratified layers of crystals of cholesterin. The brown forms consist of bilirubin-lime, and calcium carbonate, often mixed with iron, copper, and manganese. The gall-stones in the gall-bladder become facetted by rubbing against each other. The nucleus of the white stones often consist of chalk and bilecolouring matters, together with nitrogenous residues, derived from shed epithelium, mucin, bile-salts, and fats. Gall-stones may occlude the bile-duct and cause cholæmia. When a small stone becomes impacted in a duct, it gives rise to excessive pain, constituting hepatic colic, and may even cause rupture of the bile-duct with its sharp edges.

H. Nothing certain has been determined regarding the pancreatic secretion in disease, but in fever it appears to be diminished in amount and digestive activity. The suppression of the pancreatic secretion, [as by a cancerous tumour of the head of the pancreas], is often accompanied by the appearance of fat, in the form of globules or groups of crystals in the fæces.

I. Constipation is a most important derangement of the digestive tract. It may be caused by-(1) Conditions which obstruct the normal channel, e.g., constriction of the gut from stricture -in the large gut after dysentery, tumours, rotation on its axis of a loop of intestine (volvulus), or invagination, occlusion of a coil of gut in a hernial sac, or by the pressure of tumours or exudations from without, or congenital absence of the anus. (2) Too great dryness of the contents, caused by too little water in the articles of diet, diminution of the amount of the digestive secretions, e.g., of bile in icterus; or in consequence of much fluid being given off by other organs, as after copious secretion of saliva, milk, or in fever. (3) Variations in the functional activity of the muscles and motor-nervous apparatus of the gut may cause constipation, owing to imperfect peristalsis. This condition occurs in inflammations, degenerations, chronic catarrh, and diaphragmatic inflammation. Affections of the spinal cord, and sometimes also of the brain, are usually accompanied by slow evacuation of the intestine. Whether diminished mental activity and hypochondriasis are the cause of, or are caused by, constipation is not proved. Spasmodic contraction of a part of the intestine may cause temporary retention of the intestinal contents, and, at the same time, give rise to great pain or colic; the same is true of spasm of the anal sphinicter, which may be excited reflexly from the lower part of the gut. The fæcal 
masses in constipation are usually hard and dry, owing to the water being absorbed; hence they form large masses or scybala within the large intestine, and these again give rise to new resistance. Amongst the reagents which prevent evacuation of the bowels, some paralyse the -motor apparatus temporarily, e.g., opium, morphia; some diminish the secretion of the intestinal mucous membrane, and cause constriction of the blood-vessels, as tannic acid, vegetables containing tannin, alum, chalk, lead acetate, silver nitrate, bismuth nitrate.

$\mathbf{J}$. Increased evacuation of the intestinal contents is usually accompanied by a watery condition of the fæces, constituting diarrhœea. The causes are:-

1. A too rapid movement of the contents through the intestine, chiefly through the large intestine, so that there is not time for the normal amount of absorption to take place. The increased peristalsis depends upon stimulation of the motor-nervous apparatus of the intestine, usually of a reflex nature. Rapid transit of the contents through the intestine causes the evacuation of certain substances, which cannot be digested in so short a time.

2. The stools become thinner from the presence of much water, mucus, and the admixture with fat, and by eating fruit and vegetables. In rare cases, when the evacuations contain much mucin, Charcot's crystals occur (fig. 144, c). In ulceration of the intestine, leucocytes (pus) are present (Nothnagel).

3. Diarrhoe: may occur as a consequence of disturbance of the diffusion-processes through the intestinal walls, as in affections of the epithelium, when it becomes swollen in inflammatory or catarrhal conditions of the intestina mucous membrane. [Irritation over the abdomen, as from the subcutaneous injection of small quantities of saline solutions, causes diarrhoea.]

4. It may also be due to increased secretion into the intestine, as in capillary diffusion, when magnesium sulphate in the intestine attracts water from the blood.

The same occurs in cholera, when the stools are copious and of a rice-water character, and are loaded with epithelial cells from the villi. The transudation into the intestine is so great that the blood in the arteries becomes very thick, and may even on this account cease to circulate.

Transudation into the intestine also takes place as a consequence of paralysis of the vasomotor nerves of the intestine. This is perhaps the case in diarrhoea following upon a cold. Certain substances seem directly to excite the secretory organs of the intestines or their nerves, such as the drastic purgatives (\$ 180). Pilocarpin injected into the blood causes great secretion (Masloff).

During febrile conditions, the secretion of the intestinal glands seems to be altered quantitatively and qualitatively, with simultaneous alteration of the functional activity of the musculature and the organs of absorption, while the excitability of the mucous membrane is increased (Iffelmann). It is important to note that in many acute febrile diseases the amount of common salt in the urine diminishes, and increases again as the fever subsides.

187. COMPARATIVE. - Salivary Glands.-Amongst mammals, the herbivora have larger salivary glands than the carnivora ; while midway between both are the omnivora. The whale has no salivary glands. The pinnipedia have a small parotid, which is absent in echidna. The log and many carnivora have a special gland lying in the orbit, the orbital or zygomatic gland. In birds the salivary glands open at the angle of the mouth, but the parotid is absent. Imongst reptiles the parotid of some species is so changed as to form poison-glands; the tortoise has sublingual glands; reptiles have labial glands. The amphibia and fishes have merely small glands scattered over the mouth. The salivary glands are large in insects ; some of them secrete formic acid. The salivary glands are well developed in molluscs, and the saliva of Dolium galea contains more than 3 per cent. of free sulphuric acid (?). The cephalopods have double glands.

A crop is not present in any mammal ; the stomach is either simple, as in man, or, as in many rodents, it is divided into two halves, into a cardiac and a pyloric portion. The intestine is short in flesh-eating animals and long in herbivora. The stomach of ruminants is compound, and consists of four cavities. The first and largest is the paunch or rumen, then the reticulum. In these two cavities, especially the former, the ingesta are softened and undergo fermentation. They are then returned to the mouth by the action of the voluntary muscular fibres, which reach to the stomach. This is the process of rumination. The ingesta are chewed again in the mouth, and are again swallowed, but this time they enter the third cavity or psalterium(which is absent in the camel)-and thence into the fourth stomach or abomasum, in which the fermentative digestion takes place. The cæcum is a very large and important digestive organ in herbivora and in most rodents; it is small in man, and absent in carnivora. The cesophagus in grain-eating birds not unfrequently has a blind diverticulum or crop for softening the food. In the crop of pigeons during the breeding season, there is formed a peculiar secretion- "pigeon's milk," which is used to feed the young (J. Hunter). The stomach consists of a glandular proventriculus and a strong muscular stomach which is covered with horny epithelium and triturates the food. There are usually two fluid diverticula on the small intestine near where it joins the large gut. In fishes the intestinal canal is generally simple; the stomach is merely a dilatation of the tube; and at the pylorus there may be one, but usually many, blind glandular appendages (the appendices pyloricæ). They are generally longitudinal folds in 
the intestinal mucous membrane, but in some fishes, e.g., the shark, there is a spiral valve. [The inversive (cane-sugar) ferment is wanting in the herbivora, as the cow, horse, and sheep, but is present in the dog and cat. It is also met with in birds and reptiles, and in many of the invertebrates, as the ordinary earth-worm (M. Hay).]

In amphibia and reptiles the stomach is a simple dilatation; the gut is larger in vegetable feeders than in flesh feeders. The liver is never absent in vertebrates, although the gall-bladder frequently is. The pancreas is absent in some fishes.

Digestion in Plants. - The observations on the albumin-digesting power of some plants are extremely interesting (Canby, $1869 ; C h$. Darwin, 1875). The sundew or drosera has a series of tentacles on the surface of its leaves, and the tentacles are provided with glands. When an insect alights upon a leaf, it is suddenly seized by the tentacles; the glands pour out an acid juice over the prey, which is gradually digested, all except the chitinous structures. The secretion, as well as the subsequent absorption of the products of digestion, are accomplished by the activity of the protoplasm of the cells of the leaves. The digestive juice contains a pepsin-like ferment and formic acid. Similar phenomena are manifested by the Venus flytrap (Dionæa), by pinguicula, as well as by the cavity of the altered leaves of nepenthes. About fifteen species of these "insectivorous" or carnivorous plants are known. [Papain, and other ferments analogous in their action to trypsin, are referred to in $\$ 170$.

188. HISTORICAL, -Digestion in the Mouth. - The older observers regarded the saliva as a solvent, and in addition, many bad qualities, especially in starving animals, were ascribed to it. This arose from the knowledge of the saliva of mad animals, and the parotid saliva of poisonous snakes. The salivary glands have been known for a long time. Galen (131-203 A.D.) was acquainted with Wharton's duct, and Aëtius $(270$ A.D. ) with the sub-maxillary and sub-lingual glands. Hapel de la Chenaye (1780) obtained large quantities of saliva from a horse, in which he was the first to make a salivary fistula. Spallanzani (1786) asserted that food mixed with saliva was more easily digested than food moistened with water. Hamberger and Siebold investigated the reaction, consistence, and specific gravity of saliva, and found in it mucus, albumin, common salt, calcium and sodium phosphates. Berzelius gave the name ptyalin to the characteristic organic constituent of saliva, but Leuchs (1831) was the first to detect its diastatic action.

Gastric Digestion.-Digestion was formerly compared to "coction," whereby solution was effected. According to Galen, only substances that have been dissolved passed through the pylorus into the intestine. He described the movements of the stomach and the peristalsis of the intestines. Aelian gave names to the four stomachs of the ruminants. Vidius $(+1567)$ noticed the numerous small apertures of the gastric glands. Van Helmont $(\uparrow 1644)$ expressly notices the acidity of the stomach. Reaumur (1752) knew that a juice was secreted by the stomach, which effected solution, and with which he and Spallanzani performed experiments on digestion outside the body. Carminati (1785) found that the stomachs of carnivora during digestion secreted a very acid juice. Prout (1824) discovered the hydrochloric acid of the gastric-juice, Sprott and Boyd (1836) the glands of the gastric mucous membrane, while Wasmann and Bischoff noted the two kinds of gastric-glands. After Beaumont (1834) had made his observations upon Alexis St Martin, who had a gastric fistula caused by a gunshot wound, Bassow (1842) and Blondlot (1843) made the first artificial gastric-fistulæ upon animals. Eberle (1834) prepared artificial gastric-juice. Mialhe called albumin, when altered by gastric digestion, albuminose; Lehmann, who investigated this substance more carefully, gave it the name peptone. Schwann isolated pepsin (1836), and established the fact of its activity in the presence of hydrochloric acid.

Pancreas, Bile, Intestinal Digestion. - The pancreas was known to the Hippocratic School; Maur. Hoffmann (1642) demonstrated its duct (fowl), and Wirsung described it in man. Regner de Graaf (1664) collected the pancreatic juice from a fistula, and Tiedemann and Gmelin found it to be alkaline, while Lauret and Lassaigne found that it resembled saliva. Valentin dis. covered its diastatic action, Eberle its emulsionising power, and $\mathrm{Cl}$. Bernard (1846) its tryptic and fat-splitting properties. The last-mentioned function was referred to by Purkinje and Pappenheim (1836). Aristotle characterised the bile as a useless secretion; according to Erasistratus (304 B.c.), fine invisible channels conduct the bile from the liver into the gallbladder. Arëtaeus ascribed icterus to obstruction of the bile-duct. Benedetti (1493) described gall-stones. According to Jasolinus (1573), the gall-bladder is emptied by its own contractions. Sylvius noticed the lymphatics of the liver (1640); Walaeus, the connective-tissue of the socalled capsule of Glisson (1641). Haller indicated the uses of bile in the digestion of fats. The liver-cells were described by Henle, Purkinje, and Dutrochet (1838). Heynsius discovered the nrea and Cl. Bernard (1853) the sugar in the liver, and he and Hensen (1857) found glycogen in the liver. Kiernan gave a more exact description of the hepatic blood-vessels (1834). Beale injected the lymphaties, and Gerlach the finest bile-ducts. Schwann (1844) made the first biliary fistula ; Demareay particularly referred to the combination of the bile-acids with soda (1838); Strecker discovered the soda compounds of both acids, and isolated them. Celsus mentions nutrient enemata (3-5 A.D.). Fallopius (1561) described the valvulæ conniventes and villi of the intestinal mucous membrane, and the nervous plexus of the mesentery. The agminated glands or patches of Peyer were known to Severinus (1645). 


\section{Physiology of Absorption.}

189. THE ORGANS OF ABSORPTION.-[As most substances in the state in which they are used for food are either insoluble, or diffuse but imperfectly through membranes, the whole drift of the complicated digestive processes is to render these substances soluble and diffusible, and thus fit them for absorption; while most of the fats are emulsionised.]

The mucous membrane of the whole intestinal tract, as far as it is covered by a

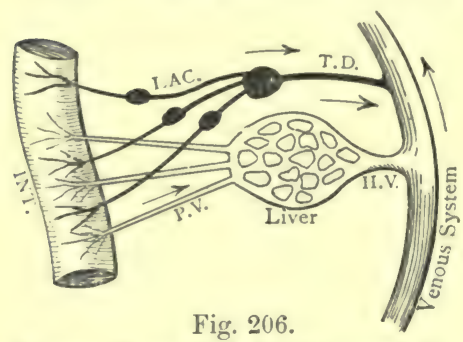

Scheme of intestinal absorption. LAC, lacteals ; T.D., thoracic duct; P. V. and H.V., portal and hepatic veins; INT., intestine. single layer of columnar epithelium, i.e., from the cardiac orifice of the stomach to the anus-is adapted for absorption. The mouth and œesophagus, lined as they are by stratified squamous epithelium, are much less adapted for this pur pose. Still, poisoning is caused by placing potassium cyanide in the mouth. The channels of absorption in the intestinal tract are (fig. 206) (1) the capillaries [direct], and (2) the lacteals [indirect] of the mucous membrane. Almost the whole of the substances absorbed by the former pass into the rootlets of the portal vein, and traverse the liver, while those that enter the lacteals really pass into lymphatics, so that the chyle passes through the thoracic duct and is poured by it into the blood, where the thoracic duct joins the subclavian vein.

Watery solutions of salts, grape-sugar, peptone, poisons, and in a still higher degree alcoholic solutions of poisons, are absorbed in the stomach. The empty stomach absorbs more rapidly than one filled with food; gastric catarrh delays absorption. After a copious diet of milk, fatty granules have been found in the protoplasm of the goblet-cells; so that according to this view, the goblet-cells have a double function, to secrete mucus and to absorb nutriments.

The greatest area of absorption is undoubtedly the small intestine, especially its upper half, owing to the presence of the valvulæ conniventes and the villi.

190. STRUCTURE OF THE SMALL AND LARGE INTESTINES.-[The wall of the small intestine consists of four coats ; which, from without inward, are named serous, muscular, sub-mucous, and mucous (fig. 207).

(1) The serous coat has the same structure as the peritoneum, i.e., a thin basis of fibrous tissue covered on its outer surface by endothelium.

(2) The muscular coat consists of a thin outer longitudinal and an inner thicker circular layer of non-striped muscular fibres (fig. 207).

(3) The sub-mucous coat consists of loose connective-tissue containing large blood-vessels and nerves, and it connects the muscular with the mucous coat.

(4) The mucous coat is the most internal coat, and its absorbing surface is largely increased by the presence of the valvulæ conniventes and villi. [The valvulæ conniventes are permanent folds of the mucous membrane of the small intestine, arranged across the long axis of the gut. They pass round a half or more of the inner surface of the gut. They begin a little below the commencement of the duodenum, and are large and well marked in the duodenum, and remain so as far as the upper half of the jejunum, where they begin to become smaller, and finally disappear about the lower part of the ileum.] The villi are characteristic of the small intestine, and are confined to it; they occur everywhere as closely-set projections over and between the valvulæ conniventes (fig. 207). When the inner surface of the mucous membrane is examined in water, it has a velvety appearance owing to their presence. [They vary in length from $\frac{1}{60}$ to $\frac{1}{30}$ of an inch, are largest and most numerous in the upper part of the intestine, duodenum, and jejunum, where absorption is most active, but they are less abundant in the ileum. Their total number has been calculated at four millions by Krause.] Each villus is a projection of the entire mucous membrane, so that it contains within itself representatives of all the tissue- 
elements of the mucosa. The orifices of the glands of Lieberkühn open between the bases of villi (fig. 207).

Each villus, be it cylindrical or conical in shape, is covered by a single layer of columnar epithelium, whose protoplasm is reticulated, and contains a well-defined nucleus with an intranuclear plexus of fibrils. The ends of the epithelial cells directed towards the gut are polygonal, and present the appearance of a mosaic (fig. 208, D). When looked at from the side, their free surface is seen to be covered with a clear, highly refractive disc or "cuticula," which is marked with vertical striæ. These striæ were supposed by Kölliker to represent pores for the absorption of fatty particles, but this has not been confirmed, while Brettauer and Steinach regarded them as produced by prisms placed side by side.

According to v. Thanhoffer, however, this clear disc is comparable to the thickened flange around the bottom of a vessel, such as is used for collecting gases. On this supposition, the upper end of each cell is open, and from it there project pseudopodia-like bundles of protoplasmic processes (fig. 208, B). These processes are supposed to be extended beyond the margin of the cell, and again rapidly retracted, and in so acting they are said to carry the fatty particles into the interior of the cells, much as the pseudopodia of an amoba entangle its food. [This view has not been confirmed by a sufficient number of observers.] Between the epithelial cells are the so-called goblet-cells (fig. 208, C). [Each goblet-cell is more or less like a chalice, narrower above and below, and broad in the middle, with a tapering fixed extremity. The outer part of these cells is filled with a clear substance or mucigen, which, on the addition of water, yields mucus. The mucigen lies in the intervals of a fine network of fibrils, which pervades the cell-protoplasm, while the protoplasm, containing a globular or triangular nucleus, is pushed into the lower part of the cell. Those goblet-cells are simply altered columnar epithelial cells, which secrete mucus in their interior. They are more numerous under certain conditions. Not unfrequently in a section of the mucous membrane of the gut, after it is stained with logwood, we may see a deep blue plug of mucus partly exuded from these cells. When looked at from above they give the appearance seen in fig. 208, D.] The epithelial cells are shed in enormous numbers in cholera, and in poisoning with arsenic and muscarin (Böhm).

[The epithelial cells covering the villus are placed upon a layer of squamous epithelium (basement membrane) - the sub-epithelial membrane of Débove. This basement membrane is said to be connected by processes with the so-called branched cells of theadenoid tissue of the villus, while it also sends up processes between the epithelial covering.]

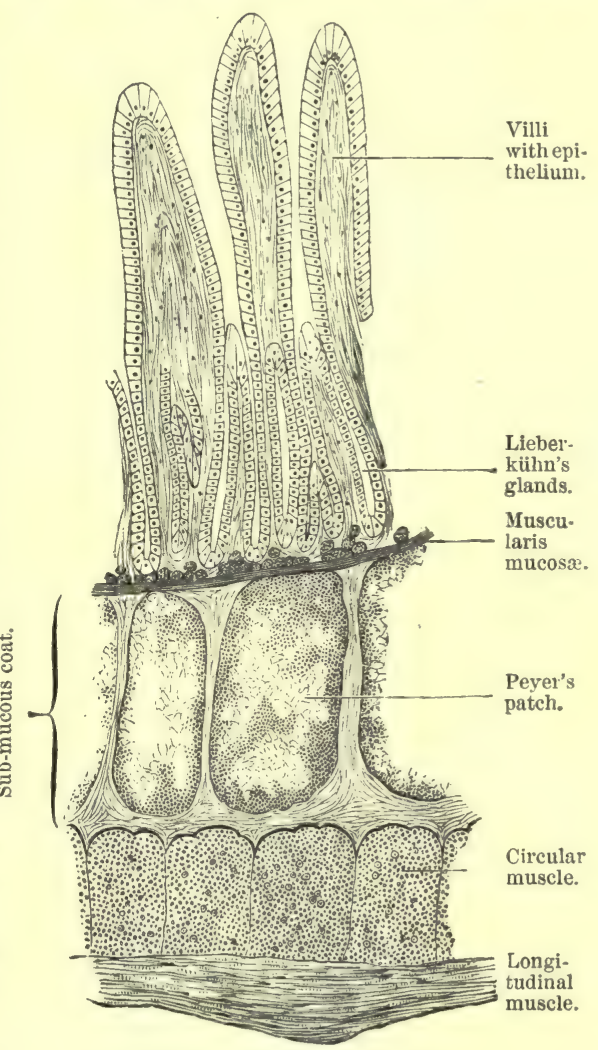

Fig. 207.

Longitudinal section of the small intestine of a dog through a Peyer's patch.

The villus itself consists of a basis of adenoid tissue, containing in its centre one or more lacteals, closely invested with bundles of longitudinal smooth muscular fibres, derived from the muscularis mucosæ, and a plexus of blood-vessels. The adenoid tissue of the villus consists of a reticulum of fibrils with endothelial plates at its nodes. The spaces of the adenoid tissue form a spongy network of inter-communicating channels containing stroma-cells or leucocytes (fig. $208, \mathrm{~A}, e, e)$ These leucocytes or lymph-corpuscles have been seen to contain fatty granules, and they are perhaps concerned in the absorption of fatty particles.

The lymphatic or lacteal lies in the axis of the villus (fig. $210, d$ ). Some regard the lacteal merely as a space in the centre of the villus, but more probably it has a distinct wall composed of endothelial cells, with apertures or stomata here and there between the cell-plates. These stomata place the interior of the lacteal in direct communication with the spaces of the adenoid tissue. Perhaps, white blood-corpuscles wander out of the blood-vessels of the villi into the 
spaces of the adenoid tissue, where they become loaded with fatty granules, and pass into the

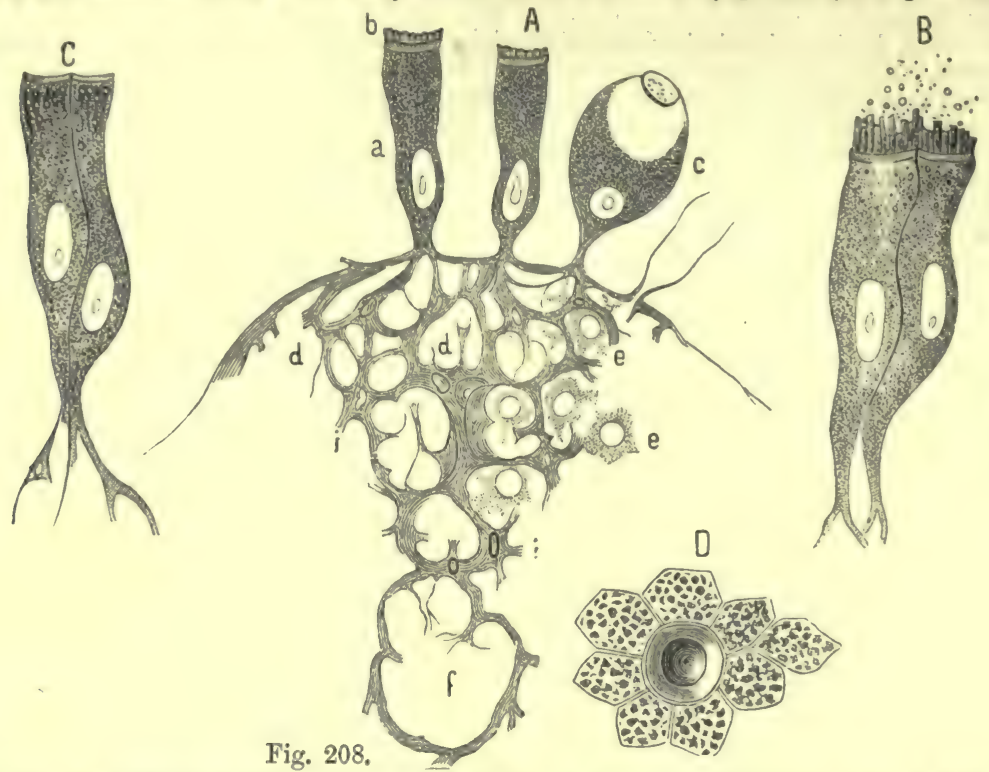

A, scheme of a transverse section of part of a villus ; $a$, columnar epithelium with, $b$, clear dise ; $c$, goblet-cell ; $i$, $i$, adenoid reticulum ; $d, d$, spaces containing leucocytes, $e, e ; f$, section of the central lacteal. B, scheme of a cell with processes projected from its interior. C, columnar epithelium after the absorption of fatty granules. D, columnar epithelium of a villus seen from above with a goblet-cell in the centre.

central lacteal. Zuwarykin and Wiedersheim suppose that the leucocytes pass from the

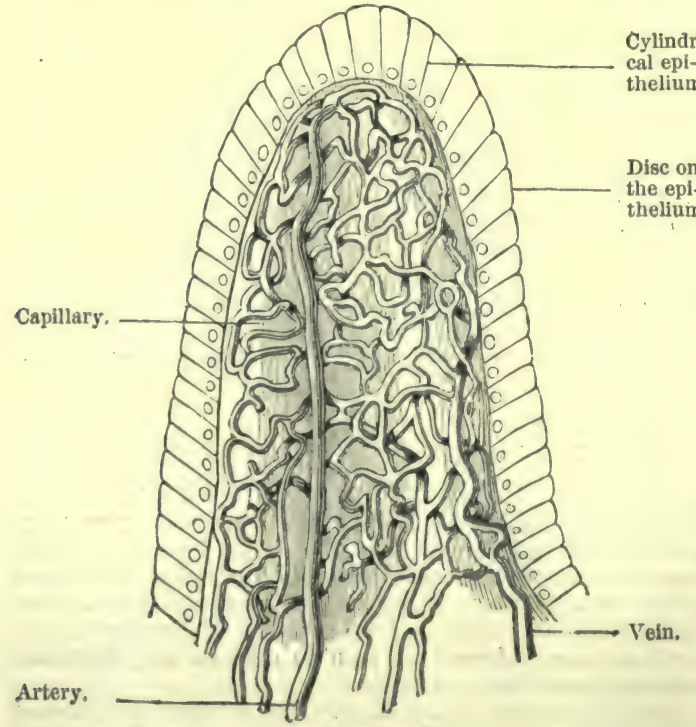

Fig. 209.

Injected blood-vessels of a villus. parenchyma of the villus towards the epithelial layer, and even beCylindri- tween the epithelial cells, from thelium. which they return towards the axis of the villus, laden with substances which they have taken into their interior (\$ 192, II.).

A small artery placed eccentrically passes into each villus (fig. 209). In man it begins to divide about the middle of the villus, but in animals it usually runs to the apex before it divides. The capillaries resulting from the division of the artery form a fine dense network placed superficially, immediately under the epithelium of the surface. The blood is carried out of a villus by one or two veins (figs. 207, 209).

Non-striped muscular fibres are present in villi. They are arranged longitudinally in several bundles from base to apex, immediately outside the central lacteal. When they contract they tend to empty the lacteal. A few muscular fibres are placed more superficially, and run in a more transverse direction. [The longitudinal bundles of non-striped muscle in the villi are connected together by oblique strands; while the longitudinal bundles shorten the villus, the oblique 
fibres keep the lacteal open ; thus the parenchyma of the villus is also compressed transversely, whereby the products of absorption are forced into the lacteal. The muscles are fixed by cement to the sub-epithelial basal membrane. The muscular fibres of the villi are direct prolongations of the muscularis mucosæ.]

Nerves pass into the villi from Meissner's plexus lying in the sub-mucous coat. The nerves to the villi are said to have small granular ganglionic cells in their course, and they terminate partly in the muscular fibres and partly in the arteries of the villi.

[On making a vertical section of the mucous membrane of the small intestine, it is seen to consist of a network of adenoid tissue loaded with leucocytes. This tissue forms its basis, and in it are placed vertically side by side, like test-tubes in a stand, immense numbers of simple tubular glands - the crypts of Lieberkiihn (fig. 207).] [Kultschitzki finds that the connective-tissue framework of the mucous membrane of the small intestine is not true adenoid tissue, but a transition form between the latter and loose fibrous tissue.] Lieberkuihn's glands open above at the bases of the villi, while their closed lower extremity reaches almost to the muscularis mucosæ. Each tube consists of a basement membrane lined by a single layer of columnar epithelium, leaving a wide lumen, the cells lining them being

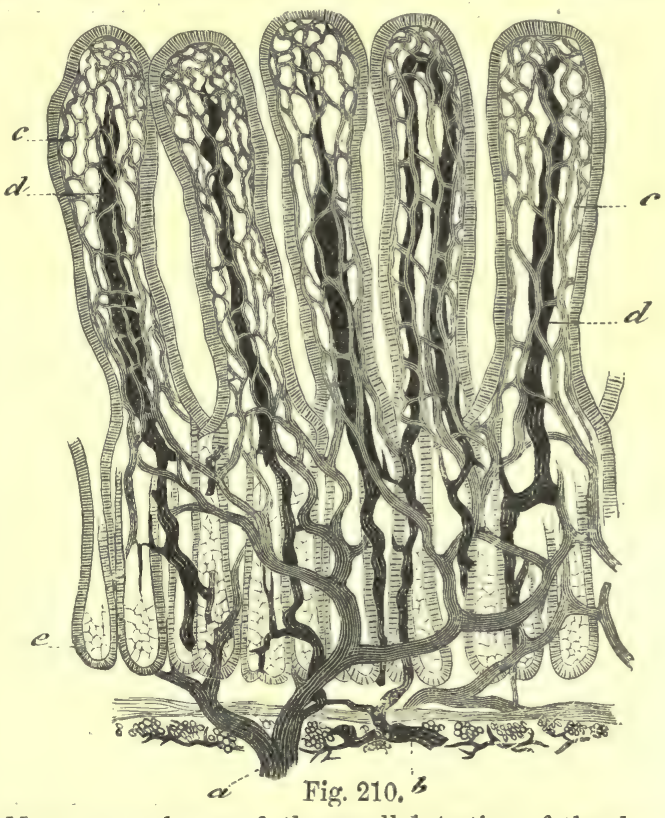

Mucous membrane of the small intestine of the $\mathrm{dog}$; the lacteals are black, and the blood-vessels lighter. $a$, artery; $b$, lymphatic; $c$, plexus of capillaries in the villi; $d$, lacteal; $e$, Lieberkühn's glands. continuous with those that cover the mucous membrane. Some goblet-cells are often found between the columnar epi- Villi with blood-vessels injected. Solitary follicle.

thelium. Immediately below the bases of the follicles of Lieberkuihn is the muscularis mucosø, consisting of two or three narrow layers of non-striped muscular fibres arranged circularly and longitudinally. [It is continuous with the muscularis mucosæ of the stomach, and extends throughout the whole intestine, not as a continuous layer, but as a close network of bundles of smooth muscle. It sends fibres upwards into the villi (fig. 212, e).]

[Brunner's Glands are compound tubular glands lying in and confined to the sub-mucous coat of the duodenum (fig. 198). Their ducts perforate the muscularis mucosæ to open on the surface. They seem to be the homologues of the py. loric glands of the stomach.]

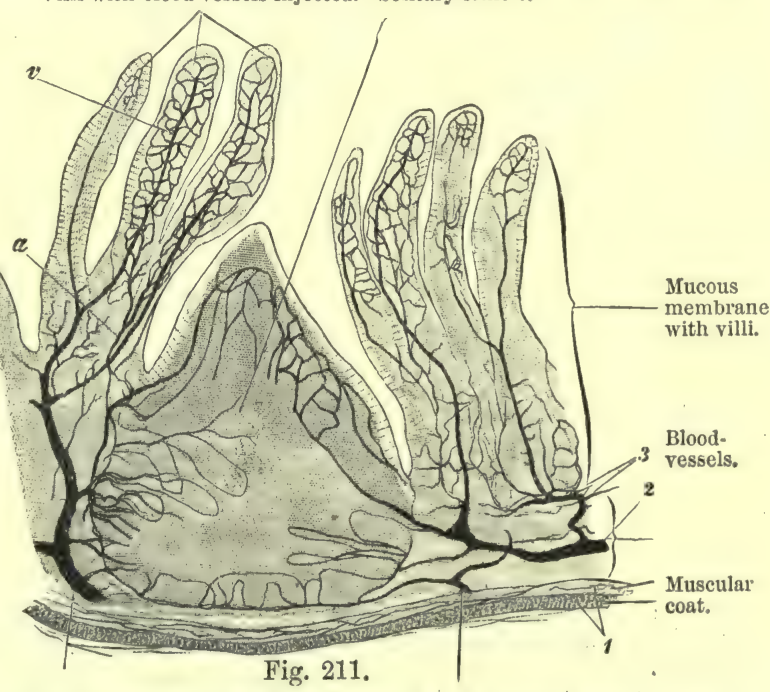

Transverse section of duodenum of a rabbit injected, $x=50$,

[Solitary Follicles are small round or oval white masses of adenoid tissue, with their deeper parts embedded in the sub-muçosa, and their apices projecting into the mucosa of the 
intestine. - They begin at the pyloric end of the stomach and are found throughout the whole intestine. They consist of small masses of adenoid tissue loaded with leucocytes (fig. 214).

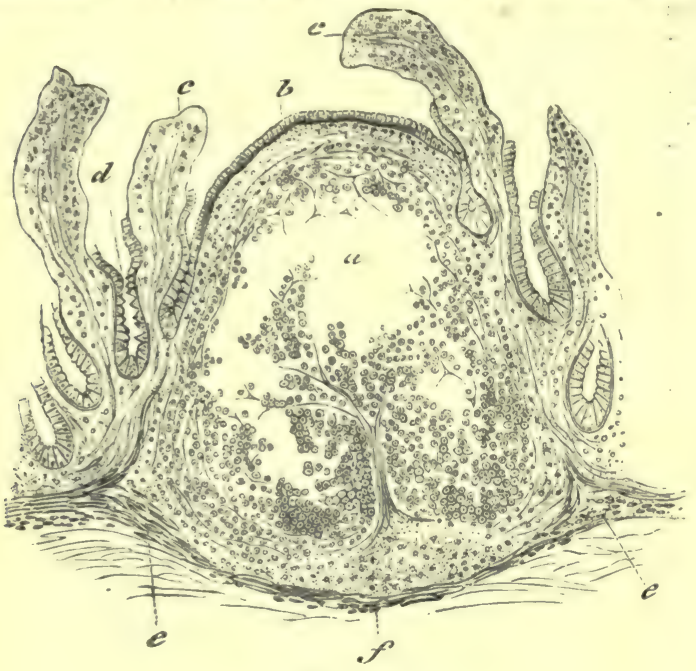

Fig. 212. They are, well supplied with blood-vessels (\$197), although no lymphatic vessels enter them. They are surrounded by lymphatics, and, in fact, they may be said to hang into a lymph-stream. The distribution of solitary follicles is fairly uniform in the small intestine; their number generally increases from the stomach to the large intestine; although there are considerable variations in different individuals, there seems to be the same number of solitary follicles and Peyer's patches in the infant as in the adult (Passov).]

[Peyer's glands, or agminated glands, consist of groups of lymph-follicles like the foregoing (figs. 207, 213). The masses are often more or less fused together, their bases lie in the sub-mucosa, while their summits project into the mucosa, where they are covered merely by the columnar

Section of a solitary follicle of the small intestine (human). epithelium of the intestine. The $a$, lymph-follicle covered with epithelium $(b)$ which has lymph-corpuscles often pass befallen from the villi, $c ; d$, Lieberkiihn's follicle $; e$, mus- tween the epithelial cells. The cularis mucosæ ; $f$, sub-mucous tissue.

patches so formed have their long axis in the axis of the intestine, and they are always placed opposite the attachment of the mesentery. Like the solitary glands, they are well supplied with blood-vessels, while around them is a dense plexus of lymphatics

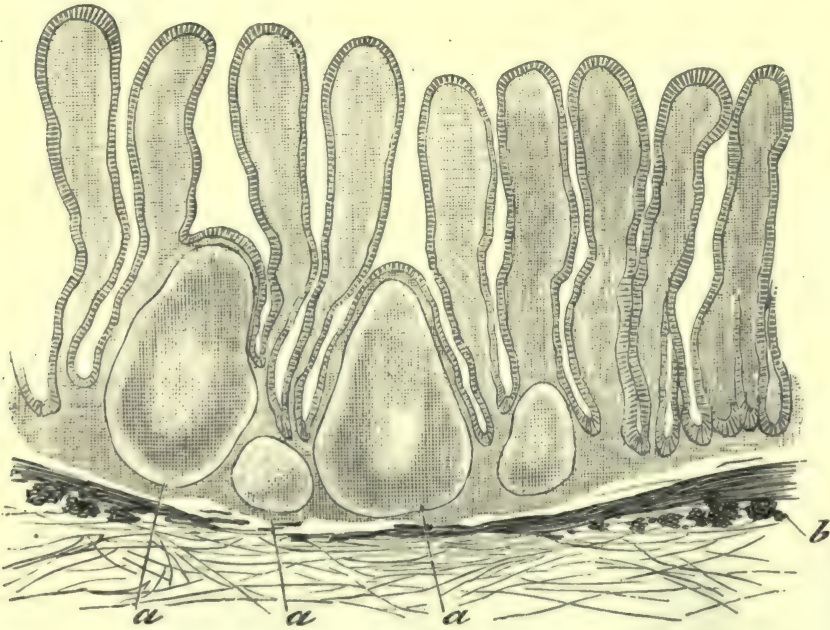

Fig. 213. or lacteals. They are most abundant in the lower part of the ileum. These glands are specially affected in typhoid fever.]

Nerves of the Intestine. - Throughout the whole intestinal tract there exists the plexus of Auerbach, lying between the longitudinal and circular muscular coats (figs. 174, 175). This plexus consists of non-medullated nerves with groups of ganglionic cells at the nodes. Fibres are given off by it to the muscular coats. Connected by branches with the foregoing, and lying in the sub-mucosa, is the plexus of Meissner, which is

Diagram of a vertical section of the mucous membrane of the small much finer, the meshes intestine of a dog showing the closed follicles, $a a ; b$, muscularis being wider, the nodes mucose. smaller, but alsso provided with ganglionic cells: It supplies the muscular fibres and arteries of the mucosa, including those of the villi. It also sends branches to Lieberkühn's glands (fig. 176).

[Structure of the Large Intestine. - It has four coats, like those of the small intestine. The 
serous coat has the same structure as that of the small intestine. The muscular coat has external longitudinal fibres occurring all round the gut, but they form three flat ribbon-like longitudinal bands in the cæcum and colon (fig. 214). Inside this coat are the circular fibres. The sub-mucosa is practically the same as that of the small intestine. The mucosa is distinguished by negative characters. It has no villi and no Peyer's patches, but otherwise it resembles structurally the small intestine, consisting of a basis of adenoid tissue with the simple tubular glands of Lieberkühn (fig. 199). These glands are very numerous and somewhat longer than those of the small intestine, and they always contain far more goblet-cells-about ten times as many. The cells lining them are devoid of a clear disc. Solitary glands occur throughout the entire length of the large intestine. At the bases of Lieberkiihn's glands is the muscularis mucosæ. The blood-vessels and nerves have a similar arrangement to those in the stomach.]

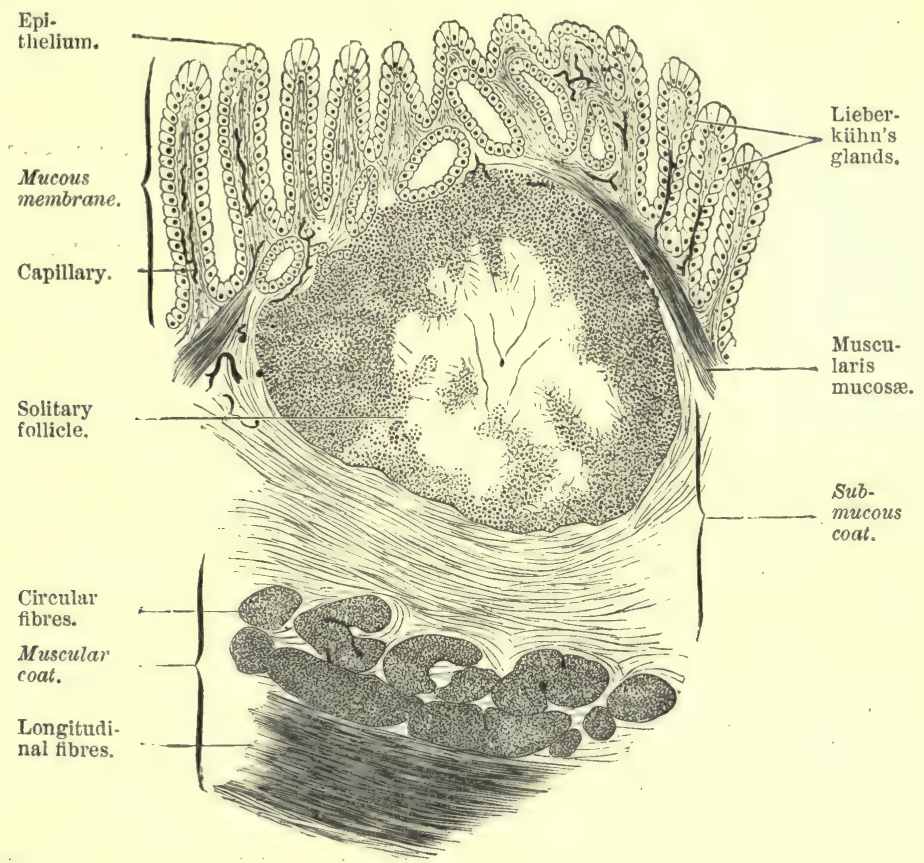

Fig. 214.

Langitudinal section of the large intestine.

[Blood-Vessels. - On looking down on an opaque injection of the mucous membrane of the stomach, one sees a dense meshwork of polygonal areas of unequal size, with depressions here and there. The orifices are the orifices of the gastric glands, each surrounded by a capillary. $\Lambda$ somewhat similar appearance is seen in an opaque injection of the mucous membrane of the large intestine, but in the latter the meshwork is uniform, all the orifices (of Lieberkühn's glands) being of the same size.]

\section{ABSORPTION OF THE DIGESTED FOOD. - The physical forces con- cerned are :-endosmosis, diffusion, and filtration.}

All the constituents of the food, with the exception of the fats, which in part are changed into a fine emulsion, are brought into a state of solution by the digestive processes. These substances pass through the walls of the intestinal tract, either into the blood-vessels of the mucous membrane or into the beginning of the lymphatics. In this passage of the fluids two physical processes come into play-endosmosis and diffusion as well as filtration.

I. Endosmosis and diffusion occur between two fluids which are capable of forming an intimate mixture with each other, e.g., hydrochloric acid and water, but never between two fluids which do not form a perfect mixture, such as oil and water. If two fluids capable of mixing with eaeh other, but of different compositions, be separated from each other by means of a septum with physical pores (which occur even in a homogeneous membrane), an exchange of the constitu- 
ents in the fluids occurs until both fluids have the same composition. This exchange of fluids is termed endosmosis or diosmosis.

Diffusion. - If the two mixible fluids are placed in a vessel, the one fluid over the other, but withont being separated by a porous septum, an exchange of the particles of the fluids also occurs, until the whole mixture is of uniform composition. This process is called diffusion.

Conditions Influencing Diffusion.-Graham's investigations showed that the rapidity of diffusion is influenced by-(1) The nature of the fluids themselves; acids diffuse most rapidly; the alkaline salts more slowly ; and most slowly, fluid albumin, gelatin, gum, dextrin. These

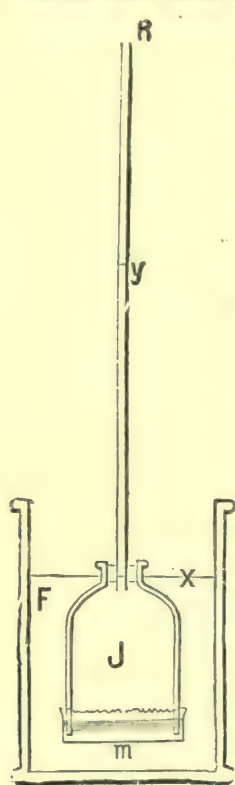

Fig. 215 .

Endosmometer. last do not crystallise, and perhans do not form true solutions. (2) The more concentrated the solutions, the greater the diffusion. (3) Heat accelerates, while cold retards, the process. (4) If a solution of a body which diffuses with difficulty be mixed with an easily diffusible one, the former diffuses with still greater difficulty. (5) Dilute solutions of several substauces diffuse into each other without any difficulty, but if concentrated solutions are employed, the process is retarded. (6) Double salts, one constituent of which diffuses more readily than the other, may be chemically separated by diffusion.

Endosmometer.-The exchange of the fluid-particles takes place independently of the hydrostatic pressure. An endosmometer (fig. 215) consists of a glass cylinder filled with distilled water, and into this is placed a flask, J, without a bottom, instead of which a membrane, $m$, is tied on. A glass tube, $\mathrm{R}$, is fixed firmly by means of a cork into the neck of the flask. The flask is filled up to the lower end of the tube with a concentrated salt solution, and is then placed in the cylindrical vessel until both fluids are on the same level, $x$. The fluid in the tube, $\mathrm{R}$, soon begins to rise, because water passes through the membrane into the concentrated solution in the flask, and this independently of the hydrostatic pressure. Particles of the concentrated salt solution pass into the cylinder and mix with the water, F. These outgoing and ingoing currents continue until the fluids without and within $J$ are of uniform composition, whereby the fluid in $\mathrm{R}$ always stands higher (e.g., at $y$ ), while it is lowered in the cylinder. The circumstance of the level of the fluid within the tube being so high, and remaining so, is due to the fact that the pores in the membrane are too fine to allow the hydrostatic pressure to act through them.

Endosmotic Equivalent. - Experiment has shown that equal weights of different soluble substances attract different amounts of distilled water through the membrane, i.e., a known weight of a soluble substance (in the flask) can be exchanged by endosmosis for a definite weight of water. The term "endosmotic equivalent" indicates the weight of distilled water that passes into the flask of the endosmometer, in exchange for a known weight of the soluble substance (Jolly). For $1 \mathrm{grm}$. alcohol $4^{*} 2 \mathrm{grms}$. water were exchanged; while for $1 \mathrm{grm}$. NaCl, 4.3 grms. water passed into the endosmometer. The following numbers give the endosmotic equivalent of

$$
\begin{aligned}
& \begin{array}{l|l|l}
\text { Acid potassium sulphate, }+=2.3 & \text { Magnesium sulphate, }, \quad=11 \cdot 7
\end{array} \\
& \text { Common salt, . . }=4.3 \text { Potassium sulphate, } .=12.0 \\
& \text { Sugar, . }: \therefore:^{\circ}=7 \cdot 1 \text { Sulphuric acid, . }:{ }^{\circ}=0.39 \\
& \text { Sodium sulphate, } . \quad .=11.6 \text { Potassium hydrate, }{ }^{\circ}=215{ }^{\circ} 0
\end{aligned}
$$

The amount of the substance which passes through the membrane into the water of the cylinder is proportional to the concentration of the solution. If the water in the cylinder, therefore, be repeatedly renewed, the endosmosis takes place more rapidly and the process of equilibration is accelerated. The larger the pores of the membrane, and the smaller the molecules of the substance in solution, the more rapid is the endosmosis. Hence, the rapidity of endosmosis of different substances varies, e.g., the rapidity of sugar, sodium sulphate, common salt, and urea is in the ratio of $1: 1 \cdot 1: 5: 9 \cdot 5$.

The endosmotic equivalent is not constant for each substance. It is influenced by-(1) The temperature, which, as it increases, generally increases the endosmotic equivalent. (2) It also varies with the degree of concentration of the osmotic solutions, being greater for dilute solutions of the substances.

If a substance other than water be placed in the cylinder, an endosmotic current occurs on both sides until complete equality is obtained. In this case, the currents in opposite directions disturb each other. If two substances be dissolved in the water in the flask at the same time, they diffuse into water without affecting each other. (3) It also varies with membranes of varying porosity. Common salt, which gives an endosmotic equivalent with a pig's bladder $=4 \cdot 3$, gives 6.4 when an ox bladder is used; 2.9 with a swimming bladder; and 20.2 with a collodion membrane.

Colloids. - There are many fluid-substances which, on account of the great size of their 
molecules, do not pass, or pass only with difficulty, through the pores of a membrane impregnated with gelatinous bodies, which diffuse slowly. These substances are not actually in a true state of solution, but exist in a very dilute condition of imbibition. Such substances are the fluid proteids, starches, dextrin, gum, and gelatin. These diffuse when no septum is present, but diffuse with difficulty or not at all through a porous septum. Graham called these substances colloids, because, when concentrated, they present a glue-like or gelatinous appearance; further, they do not crystallise, while those substances which diffuse readily are crystalline, and are called crystalloids. Crystallisable substances may be separated from non-crystallisable by this process, which Graham called dialysis. Mineral salts favour the passage of colloids through membranes.

That endosmosis takes place in the intestinal tract, through the mucous membrane and the delicate membranes of the blood and lymph-capillaries, cannot be denied. On the one side of the membrane, within the intestine, are relatively concentrated solutions of highly diffusible salts, peptones, sugar, and soaps, and within the blood-vessels are the colloids which are scarcely diffusible, e.g., the proteids of blood and lymph.

II. Filtration is the passage of fluids through the coarse intermolecular pores of a membrane owing to pressure. The greater the pressure, and the larger and more numerous the pores, the more rapidly does the fluid pass through the membrane ; increase of temperature also accelerates it. Those substances which are imbibed by the membrane filter most rapidly, so that the same substance filters through different membranes with varying rapidity. The filtration is usually slower, the greater the concentration of the fluid. The filter has the property of retaining some of the substances from the solution passing through it, e.g., colloid substances-or water (in dilute solutions of nitre). In the former case, the filtrate is more dilute, in the latter more concentrated, than before filtration. Other substances filter without undergoing any change of concentration. Many membranes behave differently, according to which surface is placed next the fluid; thus the shell-membrane of an egg permits filtration only from without inwards; [and the same is true to a much less extent with filter-paper; the smooth side of the filterpaper ought always to be placed next the fluid to be filtered. The intact skin of the grape prevents the entrance of fungi into the fruit]. There is a similar difference with the gastric and intestinal mucous membrane.

[By using numerous layers of filter-paper, many colloids and crystalloids are retained in the filter, e.g., hæmoglobin, albumin, and many colouring matters, especially aniline colours, the last being arrested by glass-wool (Krysinski).]

[Filtration of Albumin.-Runeberg finds that the amount of albumin in pathological transudations varies with (1) the capillary area, being least in œedema of the subcutaneous tissue. (2) The presence or absence of inflammatory processes in the vascular wall, non-inflammatory pleuritic effusion containing 2 per cent., and inflammatory 6 per cent., of albumin. (3) The condition and amount of albumin in the bbood. The amount of albumin in the transudate never reaches, although it sometimes approaches, that in blood. In ascites in general dropsy the amount is 03 to 04 per cent. (4) The duration of the transudation. (5) Perhaps the bloodpressure and the condition of the circulation.]

Filtration of the soluble substance may take place from the canal of the digestive tract when :-(1) The intestine contracts and thus exerts pressure upon its contents. This is possible when the tube is narrowed at two points, and the musculature between these two points contracts upon the fluid-contents. (2) Filtration, under negative pressure, may be caused by the villi (Brïcke). When the villi contract energetically, they empty their contents towards the blood- and lymph-vessels. The lacteal remains empty, as the chyle is prevented from passing backwards into the origin of the lacteal within the villi, owing to the presence of numerous valves in the lymphatics. When the villi relax, they are refilled with fluids from the intestine.

192. ABSORPTION BY THE INTESTINAL WALL.-I. True solutions undoubtedly pass by endosmosis into the blood-vessels and lymphatics of the intestinal walls, but numerous facts indicate that the protoplasm of the cells takes an active part in the process of absorption. The forces concerned have not as yet been proved to be purely physical and chemical in their nature.

(1) Inorganic Substances.-Water and the soluble salts necessary for nutrition are easily absorbed, the latter especially by the blood-and lymph-vessels. When saline solutions pass by endosmosis into the vessels, water must pass from 
the intestinal vessels into the intestine. The amount of water, however, is small, owing to the small endosmotic equivalent of the salts to be absorbed. More salts are absorbed from concentrated than from dilute solutions. If large quantities of salt, with a high endosmotic equivalent, e.g., magnesium or sodium sulphate, are introduced into the intestine, these salts retain the water necessary for their solution, and may thus cause diarrhœa. Conversely, when these substances are injected into the blood, a large quantity of water passes from the intestine into the blood, so that constipation occurs, owing to dryness of the intestinal contents (Aubert).

[M. Hay concludes from his experiments ( $\$ 161)$, that salts, when placed in the intestines, do not abstact water from the blood, or are themselves absorbed, in virtue of an endosmotic relation being establisher between the blood and the saline solution in the intestines. Absorption is probably due to the filtration and diffusion, or processes of inhibition other than endosmosis, as yet little understood. The result obtained by Aubert, which is not constant, is mostly caused by the great diuresis which the injected salt excites.] The absorption of fluids takes place best at a medium pressure of 80 to $140 \mathrm{c.cm}$. of water within the intestine; higher pressure compresses the blood-vessels and diminishes the absorption. During digestion, owing to the dilatation of the vessels, absorption is more rapid. The fact that 0.5 per cent. solution of $\mathrm{NaCl}$ is ahsorbed better than water, and soda solution than potash solution, seems to show that physical forces are not the only factors concerned.

Numerous inorganic substances, which do not occur in the body, are absorbed by endosmosis from the intestine, e.g., dilute sulphuric acid, potassium iodide, chlorate, and bromide, and many other salts.

(2) The soluble carbohydrates, such as the sugars, of which the chief representatives are dextrose and maltose, with a relatively high endosmotic equivalent. Cane-sugar is changed by a special ferment into invert-sugar $(\S 183,5)$. Absorption appears to take place somewhat slowly, as only very small quantities of grape-sugar are found in the chyle vessels, or the portal vein, at any time. According to v. Mering, the sugar passes from the intestine into the rootlets of the portal vein; dextrin also occurs in the portal vein. When the blood of the portal vein is boiled with dilute sulphuric acid, the amount of sugar is increased. The amount of sugar absorbed depends upon the concentration of its solution in the intestine ; hence the amount of sugar in the blood is increased after a diet containing much of this substance, so that it may appear in the urine; in which case the blood must contain at least $0^{\circ} 6$ per cent. of sugar. A small amount of cane-sugar has also been found in the blood ( $\mathrm{Cl}$. Bernard). The sugar is used up in the bodily metabolism; some of it is perhaps oxidised in the muscles $(\$ 176)$.

(3) The peptones have a small endosmotic equivalent, a 2 to 9 per cent. solution $=7$ to 10 . Owing to their great diffusibility they are readily absorbed, and they are the chief representatives of the proteids which are absorbed. The amount absorbed depends upon the concentration of their solution in the intestine. When animals are fed on peptones (with the necessary fat or sugar), they serve to maintain the body-weight. [According to Plósz and Györgyai, Drosdorff and SchmidtMulheim, peptones occur only in traces in the blood of the portal vein. Neumeister, however, using the best methods, finds that although peptones are abundant in the intestine, not a trace of peptone or of the albumoses is found either in the blood or lymph. This coincides with Hofmeister's researches, and is of course opposed to the results of the above-named observers. As no peptones or albumoses have been found in the blood, and as they can compensate for the total metabolism of the proteids within the body, we must assume that they are rapidly converted into true albuminous bodies.] Hofmeister supposes that the leucocytes absorb the peptones and act as their carriers, much as the red corpuscles are oxygen carriers. They carry the peptones into the mucous membrane of the stomach and small intestine, which are very rich in peptone at the fourth hour of digestion. [The number of leucocytes is greatly increased in the mucous membrane, especially in the stomach and upper part of the duodenum, during digestion, and diminished during fasting in dogs and cats. The same is the case with the lymph-follicles, the 
cells of which are formed by the division of the pre-existing cells.] It is asserted by Salvioli that the mucous membrane possesses the property of changing peptone into albumin.

[Injection of Peptone into Blood.-When peptone is injected into the blood of an animal, within twenty minutes thereafter no trace of the peptone is to be found in the blood, although it has not been excreted by any of the organs. Peptones so injected prevent the blood of the dog (not of the rabbit or pig) from coagulating. In large quantity they are fatal. Fano asserts that the peptone is taken up by the red blood-corpuscles, which thus become of ' greater specific gravity, and change it into globulin. After three or more hours the corpuscles return the globulin to the blood, so that the corpuscles represent a reserve store of proteid. The peptones used in these experiments were really a mixture of peptones and albumoses. Neumeister finds that in the dog, when albumoses are injected into the blood they reappear in the urine, but somewhere in the body they undergo hydration in the sense in which peptic digestion causes hydration. The two primary albumoses reappear almost completely as deutero-albumose, and deutero-albumose, when introduced, reappears as peptone. Peptone, however, reappears unchanged. In rabbits, albumose reappears unchanged in the urine.]

(4) Unchanged true proteids filter with great difficulty, and much albumin remains upon the filter. On account of their high endosmotic equivalent they pass with extreme slowness, and only in traces, through membranes. Nevertheless, it has been conclusively proved that unchanged proteids can be absorbed (Brücke), e.g., casein, soluble myosin, alkali-albuminate, albumin mixed with common salt, gelatin (Voit, Bauer, Eichhorst). They are absorbed even from the large intestine (Czerny and Latschenberger), although the human large intestine cannot absorb more than 6 grms. daily. But the amount of unchanged proteids absorbed is always very much less than the amount of peptone.

Egg-albumin without common salt, syntonin, serum-albumin, and fibrin are not absorbed (Eichhorst). Landois observed, in the case of a young man who took the whites of 14 to 20 eggs along with $\mathrm{NaCl}$, that albumin was given off by the urine for 4 to 10 hours thereafter. The amount of albumin given off rose until the third day, and ceased on the fifth day. The more albumin taken, the sooner the albuminuria appeared, and the longer it lasted. The unchanged egg-albumin reappeared in the urine. If egg-albumin be injected into the blood, part of it reappears in the urine $(\$ 41,2)$ (Stokvis, Lehmann).

(5) The soluble fat-soaps represent only a fraction of the fats of the food which are absorbed ; the greater part of the neutral fats being absorbed in the form of very fine particles-as an emulsion (\$192, II.). The absorbed soaps have been found in the chyle, and as the blood of the portal vein contains more soaps during digestion than during hunger, it has been assumed that the soaps pass into the intestinal blood-capillaries. The investigations of Lenz, Bidder, and Schmidt, render it probable that the organism can absorb only a limited amount of fat within a given period; the amount perhaps bears a relation to the amount of bile and pancreatic juice. The maximum per kilo. (cat) was $0 \cdot 6$ grms. of fat per hour.

Perhaps the soaps reunite with glycerine in the parenchyma of the villi, to form neutral fats, as Perewoznikoff and Will found neutral fats, after injecting these two ingredients into the intestinal canal, while Ewald found that fat was formed when soaps and glycerine were brought into contact with the fresh intestinal mucous membrane. Perhaps this is the explanation of the observation of Bruch, who found fatty particles within the blood-vessels of the villi. No fatty-acids are found in blood, or chyle.

Absorption of other Substances. - Of soluble substances which are introduced into the intestinal canal, some are absorbed and others are not. The following are absorbed :-alcohol, part of which appears in the urine (not in the expired air), viz., that part which is not changed into $\mathrm{CO}_{2}$ and $\mathrm{H}_{2} \mathrm{O}$, within the body; tartaric, citric, malic, and lactic acids ; glycerine, inulin ; gum and vegetable mucin, which give rise to the formation of glycogen in the liver.

Amongst colouring matters, alizarin (from madder), alkannet, indigo-sulphuric acid, and its soda-salt are absorbed; hæmatin is partly absorbed, while chlorophyll is not. Metallic salts seem to be kept in solution by proteids, are perhaps absorbed along with them, and are partly carried by the blood of the portal vein to the liver (ferric sulphate has been found in chyle). Numerous poisons are very rapidly absorbed, e.g., hydrocyanic acid after a few seconds ; potas- 
sium cyanide has been found in the chyle. [If salts (KI, sulphocyanide of ammonium) be injected into a ligatured loop of intestine (dog, cat, rabbit), these substances are absorbed both by the blood- and lymph-vessels, and in both nearly simultaneously.] Even for the absorption of completely fluid substances, endosmosis and filtration seem to be scarcely sufficient. An active participation of the protoplasm of the cells seems here also-in part at least-to be necessary, else it is difficult to explain how very slight disturbances in the activity of these cells, e.g., from intestinal catarrh, cause sudden variations of absorption, and even the passage of fluids into the intestine.

If absorption were due to diffusion alone, when alcohol is injected into the intestine, water ought to pass into the intestine, but this does not occur. Brieger found that the injection of a 0.5 to 1 per cent. solution of salts into a ligatured loop of intestine did not cause water to pass into the intestine; but it appeared when a 20 per cent. solution was injected.

II. Absorption of the Smallest Particles.-The largest amount of the fats is absorbed in the form of a milk-like emulsion, formed by the action of the bile and the pancreatic juice, and consisting of excessively small granules of uniform size (\$170, III.; $\$ 181)$. The fats themselves are not chemically changed, but remain as undecomposed neutral fats. The particles seem to be surrounded by a delicate albuminous envelope, or haptogen membrane, partly derived from the pancreatic juice [probably from its alkali-albuminate]. The villi of the small intestine are the chief organs concerned in the absorption of the fatty emulsion, but the epithelium of the stomach and that of the large intestine also take a part. The fatty granules are recognised in the villi-(1) Within the delicate canals ? (\$190) in the clear band of the epithelium (Kölliker). [It is highly doubtful if the vertical lines seen in the clear disc of the epithelium of the intestine are due to pores.] (2) The protoplasm of the epithelial cells is loaded with fatty granules of various sizes during the time of absorption, while the nuclei of the cells remain free, although, from the amount of fat within the cells, it is often difficult to distinguish them. (3) The granules pass into the spaces of the parenchyma of the villi; these spaces communicate freely with each other. (4) The origin of the lacteal in the axis of the villus is found to be filled with fatty granules. The amount of fat in the chyle of a dog, after a fatty meal, is 8 to 10 per cent., while the fat disappears from the blood within thirty hours.

With regard to the forces concerned in the absorption of fats, v. Wistinghausen proved, that when a porous membrane is moistened with bile, the passage of fatty particles through it is thereby facilitated, but this fact alone does not explain the copious and rapid absorption of fats. It is possible that the protoplasm of the epithelial cells is actively concerned in the process, and that it takes the particles into its interior. Perhaps a fine protoplasmic process is thrown out by these cells, just as pseudopodia are thrown out and retracted by lower organisms. It is possible that absorption may take place through the open mouths of the goblet-cells. The protoplasm of the epithelial cells is in direct communication with the numerous protoplasmic lymph-cells within the reticulum of the villi, so that the particles may pass into these, and from them through the stomata (?) between the endothelial cells into the central lacteal of the villus. According to this view, the absorption of fatty particles, and perhaps also the absorption of true proteids, is due to an active vital process, as indicated by the observations of Brücke and $\nabla$. Thanhoffer. This view is supported by the observation of Grünhagen, that the absorption of fatty particles in the frog is most active at the temperature at which the motor phenomena of protoplasm are most lively. That it is due to simple filtration alone is not a satisfactory explanation, for the amount of fatty particles in the chyle is independent of the amount of water in it. If absorption were chiefly due to filtration, we would expect that there would most probably be a direct relation between the amount of water and fat (Ludwig and Zawilsky). [The observations of Watney have led him to suppose that the fatty particles do not pass through the cell protoplasm to reach the lacteal, but that they pass through the cement-substance between the epithelial cells covering a villus. If this view be 
correct, the absorbing surface is thereby greatly diminished. Zuwarykin and Schäfer suggest that the leucocytes, which have been observed between the columnar cells of the villi of the small intestine, are carriers of at least part of the fat from the lumen of the gut to the lacteal ; they also, perhaps, alter it for further use in the economy. According to Zuwarykin, Peyer's patches in the rabbit seem to be especially active in the absorption of fat, so that he attaches great importance to the leucocytes of the adenoid tissue in the absorption of fat.]

[According to Grünhagen there are several channels for the absorption of fats, but they are different in different animals. Some are absorbed by the columnar epithelium cells themselves, and some passes between them.]

The activity of the cells of the intestine with pseudopodial processes may be studied in the intestinal canal of Distomum hepaticum. Sommer has figured these pseudopodial processes actively engaged in the absorption of particles from the intestine.

193. INFLUENCE OF THE NERVOUS SYSTEM.-With regard to the influence of the nervous system upon intestinal absorption, we know very little. After extirpation of the semi-lunar ganglion, as well as after section of the mesenteric nerves (Moreau), the intestinal contents become more fluid, and are increased in amount (\$183). This may be partly due to diminished absorption. V. Thanhoffer states that he observed the protrusion of threads from the epithelial cells of the small intestine only after the spinal cord, or the dorsal nerves, had been divided for some time.

194. "NUTRIENT ENEMATA."-In cases where food cannot be taken by the mouth, e.g., in stricture of the œsophagus, continued vomiting, \&c., food is given per rectum. As the digestive activity of the large intestine is very slight, fluid food ought to be given in a condition ready to be absorbed, and this is best done by introducing it into the rectum through a tube with a funnel attached, and allowing the food to pass in slowly by its own weight. The patient must endeavour to retain the enema as long as possible. When the fluid is slowly and gradually introduced, it may pass above the ileo-cæcal valve.

Solutions of grape-sugar, and perhaps a small amount of soap solution, are useful; and arnongst nitrogenous substances the commercial flesh-, bread-, or milk-peptones of Sanders-Ezn, Adamkiewicz, in Germany, and Darby's fluid meat, and Carnrick's beef-peptonoids in this country, are to be recommended. The amount of peptone required is $1.11 \mathrm{grm}$. per kilo. of body-weight (Catillon); less useful are butter-milk, egg-albumin with common salt. Leube uses a mixture of 150 grms. flesh, with 50 grms. pancreas and 100 grms. water, which he slowly injects into the rectum, where the proteids are peptonised and absorbed. [Peptonised food prepared after the method of Roberts is very useful (\$ 172).] The method of nutrient enemata only permits imperfect nutrition, and at most only $\frac{1}{4}$ of the proteids necessary for maintaining the metabolism of the body is absorbed ( $v$. Voit, Bauer).

195. CHYLE-VESSELS AND LYMPHATICS.-Lymphatics.-Within the tissues of the body, and even in those tissues which do not contain blood-vessels, e.g., the cornea, or in those which contain few blood-vessels, there exists a system of vessels or channels which contain the juices of the tissues, and within these vessels the fluid always moves in a centripetal direction. These canals arise within the tissues in a variety of ways, and unite in their course to form delicate and afterwards thicker tubes, which ultimately terminate in two large trunks which open at the junction of the jugular and subclavian veins; that on the left side is the thoracic duct, and that on the right, the right lymphatic trunk.

With regard to the lymph and its movements in different organs, it is to be noticed that this occurs in different ways in different places. (1) In many tissues, the lymphatics represent the nutrient channels, by which the fluid that transudes through the neighbouring vessels is distributed, as in the cornea and in many connective-tissues. (2) In many tissues, as in glands, e.g., the salivary glands and the testis, the lymphspaces are the chief reservoirs for fluid, from which the cells during the act of secretion derive the fluid necessary for that process. (3) The lymphatics have the general function of collecting the fluid which saturates the tissues, and carrying it back again to the blood. The capillary blood-system may be regarded as an irrigation system, which supplies the tissues with nutrient fluids, while the lymphatic system may be regarded as a drainage apparatus, which conducts away the fluids that have transuded through the capillary walls. Some of the decomposition pro- 
ducts of the tissues, proofs of their retrogressive metabolism, become mixed with the lymph-stream, so that the lymphatics are at the same time absorbing vessels. Substances introduced into the parenchyma of the tissues in other ways, e.g., by subcutaneous injection, are partly absorbed by the lymphatics. A study of these conditions shows that the lymphatic system represents an appendix to the bloodvascular system, and further that there can be no lymph system when the bloodstream is completely arrested; it acts only as a part of the whole, and with the whole.

Lacteals. - When we speak of the lymphatics proper as against the chyle-vessels or lacteals, we do so from anatomical reasons, because the important and considerable lymphatic channels coming from the whole of the intestinal tract are, in a certain sense, a fairly independent province of the lymphatic vascular area, and are endowed with a high absorptive activity, which, from ancient times, has attracted the notice of observers. The contents of the chyle-vessels or lacteals are mixed with a large amount of fatty granules, giving the chyle a white colour, which distinguishes them at once from the true lymphatics with their clear watery contents. From a physiological point of view, however, the lacteals must be classified with the lymphatics, for, as regards their structure and function, they are true lymphatics, and their contents consist of true lymph mixed with a large amount of absorbed substances, chiefly fatty granules. [The contents of the lacteals are white only during digestion, at other times they are clear like lymph.]

196. ORIGIN OF THE LYMPHATICS.-(1) Origin in Spaces. - Within the connectivetissues (connective-tissue proper, bone) are numerous stellate, irregular, or branched spaces,

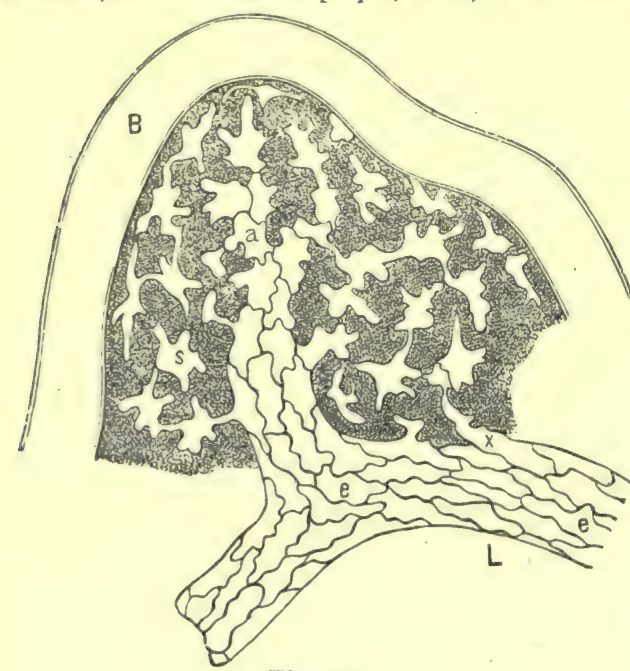

Fig. 216. which communicate with each other by numerons tubular processes (fig. 216, $s$ ); in these communicating spaces lie the cellular elements of these tissues. These spaces, however, are not completely filled. by the cells, but an interval exists between the body of the cell and the wall of the space, which is greater or less according to the condition of movement of the protoplasmic cell. These spaces are the socalled "juice canals" or "Saft-canälchen," and they represent the origin of the lymphatic vessels ( $v$. Recklinghausen). As they communicate with neighbouring spaces, the movement of the lymph is provided for. The cells which lie in the spaces exhibit amoboid movements. Some of these cells remain permanently each in its own space, within which, however, it may change its form-these are the so-called "fixed connectivetissue corpuscles," and bone corpuscleswhile others merely wander or pass into these spaces, and are called "wandering cells," or "leucocytes"; but the latter are merely lymph-corpuscles, or colourless

Origin of lymphatics from the central tendon of the blood-corpuscles which have passed out of diaphragm stained with nitrate of silver. $s$, the the blood-vessels into the origin of the juice-canals, communicating at $x$ with the lym-lymphatics. These cells exhibit amœboid phatics; $a$, origin of the lymphatics by the conflu- movements. These spaces communicate ence of several juice-canals. with the small tubular lymphaties-the so-called lymph-capillaries (L). The spaces lie close together, where they pass into a lymphcapillary $(a)$. The lymph-capillary, which is usually of greater diameter than the bloodcapillary, generally lies in the middle of the space within the capillary arch (B). The finest lymphatics are lined by a layer of delicate, nucleated, endothelial cells $(e, e)$, with characteristic sinuous margins, whose characters are easily revealed by the action of silver nitrate (fig. $217, \mathrm{~L}$ ). This substance blackens the cement-substance which holds the endothelial cells together. Between the endothelial cells are small holes, or stomata, by means of which the lymph-capil. laries communicate (at $x$ ) with the juice-canals. 
It is assumed by Arnold that the blood-vessels communicate with the juice-canals, and that fluid passes out of the thin-walled capillaries through their stomata into these spaces $(\$ 65)$. This fluid nourishes the tissues, the tissues take up the substances appropriate to each, while the effete materials pass back into the spaces, and from these reach the lymphatics, which ultimately discharge them into the venous blood.

Whether the cells within these spaces are actively concerned in the pouring out of the blood. plasma, or take part in its movement, is matter of conjecture. We can imagine that by contracting their body, after it has been impregnated with fluid, this fluid may be propelled from space to space towards the lymphatics. The leucocytes wander through these spaces until they pass into the lymphatics. Fine particles which are contained in these spaces - e.g., after tattooing the skin, and even fatty particles after inunction - are absorbed by the leucocytes, and carried by them to other parts of the body.

pigment particles used to tattoo the finger are usually found within the first lymphatic gland at the elbow.

The migration of cellular elements from the blood-vessels into the origin of the lymphatics is to be considered as a normal process. Granular colouring-matter passes from the blood into the protoplasmic body of the cells within the lymph-spaces; and only when the granular pigment is in large amount, does it appear as a granular injection in the branches of the juice-spaces.

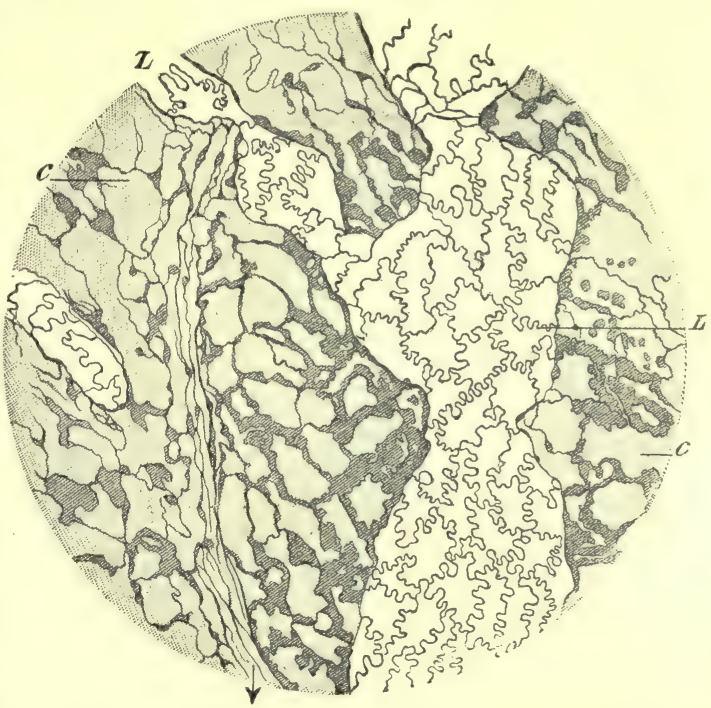

Fig. 217.

Pleural surface of the central tendon of the diaphragm of the rabbit stained with silver nitrate. L, lymphatic with its sinuous endothelium; $c$, cells of the connective-tissue brought into view by the silver nitrate.

(2) Origin within villi-i.e., of the chyle vessel or lacteal-has been described (\$ 190).

(3) Origin in perivascular spaces (fig. 218). - The smallest blood-vessels of bone, the central nervous system, retina and the liver, are completely surrounded by wide lymphatic tubes, so that the blood-vessels are completely bathed by a lymph-stream. In the brain these lymphatics are partly composed of delicate connective-tissue fibres, which traverse the lymph-space and become attached to the wall of the included blood-vessel. Fig. 218, B, represents a transverse section of a small blood-vessel, B, from the brain; $p$ is the divided perivascular space. This space is called the perivascular space of His, but in addition to it the blood-vessels of the brain have a lymph-space within the adventitia of the blood-vessels (Virchow-Robin's space). It is partly lined by well-defined endothelium. Where the blood-vessels begin to increase considerably in diameter, they pass through the wall of the lymphatics, and the two vessels afterwards take separate courses. In all cases, where there is a perivascular space, the passage of lymphand blood-corpuscles into the lymphatics is greatly facilitated. In the tortoise the large bloodvessels are often surrounded with perivascular lymphatics. Fig. 218, A, gives a representation of the aorta surrounded by a perivascular space which is visible to the unaided eye. In mammals the perivascular spaces are microseopic.

(4) Origin in the form of interstitial slits within organs.-Within the testis the lymphatics begin simply in the form of numerous slits, which occur between the coils and twists of the seminal tubules. They take the form of elongated spaces bounded by the curved cylindrical surfaces of the tubules. The surfaces, however, are covered with endothelium. The lymphatics of the testis get independent walls after they leave the parenchyma of the organ. In many other glands the gland-substance is similarly surrounded by a lymph-space. The blood-vessels pour the lymph into these spaces, and from them the secreting cells obtain the materials necessary for the formation of their secretion.

(5) Origin by means of free stomata on the walls of the larger serous cavities, which (fig. $219, a)$ communicate freely with the lymphatics. The investigation of the serous surfaces is most easily accomplished on the septum of the great abdominal lymph-sac of the frog. Silver 
nitrate reveals the presence of relatively large free openings or stomata lying between the endothelium. Each stoma is bounded by several germinating cells, which have a granular appearance, and undergo a change of shape, so that the size of the stoma depends upon the degree of contraction of these cells; thus the stoma may be open $(a)$, half-open $(b)$, or completely closed $(c)$. These stomata are the origin of the lymphatics. The serous cavities belong therefore to the lymphatic systern, and fluids placed in the serous cavities readily pass

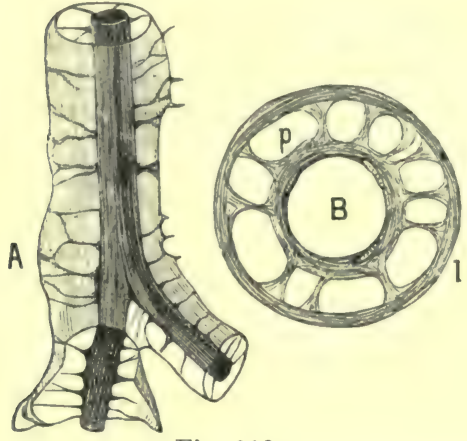

Fig. 218.

Perivascular lymphatics. A, aorta of tortoise ; $\mathrm{B}$, artery from the brain.

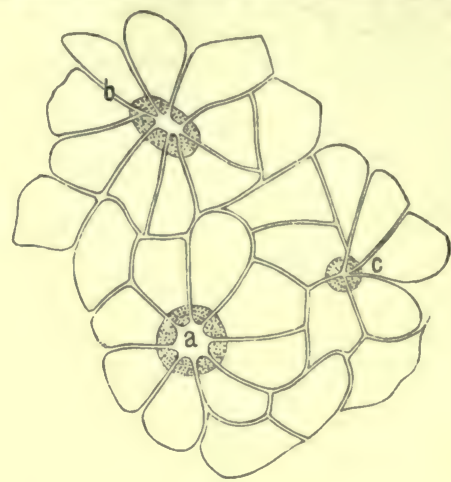

Fig. 219.

Stomata in the great lymph-sac (frog). $a$, open ; $b$, half-closed ; $c$, closed.

into the lymphatics. The cavities of the peritoneum, pleura, pericardium, tunica vaginalis, testis, arachnoid space, aqueous chambers of the eye, and the labyrinth of the ear, are true lymph. cavities, and the fluid they contain is to be regarded as lymph. [Hoffmann has found that a nerve-fibre surrounds the stomata in the frog and sends branches between the germinal epithelium.]

(6) Free open pores have been observed on some mucous membranes, which are regarded as the origin of lymphatics, e.g., in the bronchi, nasal mucous membrane, trachea, and larynx.

Structure. - The larger lymphatics resemble in structure the veins of corresponding size. The valves are particularly numerous in the lymphatics, so that a distended lymphatic resembles a chain of pearls. [Lymphatics have dilations here and there in their course (fig. 217).]

197. THE LYMPH-GLANDS.-The lymphatic glands belong to the lymph apparatus. They are incorrectly termed glands, as they are merely much branched

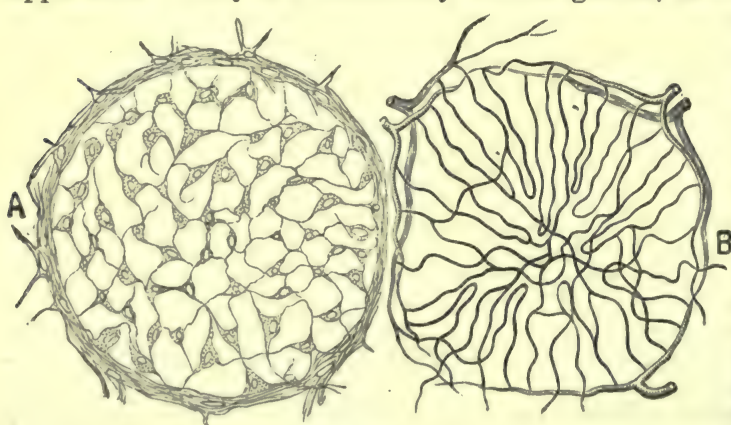

Fig. 220. lacunar labyrinthine spaces composed of adenoid tissue, and intercalated in the course of the lymphatic vessels. There are simple and compound lymph-glands.

(1) The simple lymph-glands, or, more correctly, lymph-follicles, are small rounded bodies, about the size of a pin-head. They consist of a mass of adenoid tissue (fig. $220, A$ ), i.e., of a very delicate network of fine reticular fibres with nuclei at their points of intersection, and in the spaces of the meshwork lie the lymph and

Two lymph-follicles. A, a small follicle highly magnified, showing the adenoid reticulum ; $B$, a follicle less highly magnified, showing injected blood-vessels.

which is not however a true capsule, as.it is penser, where it forms a capsule, Small is spermeated with numerous small sponge-like spaces. Sull these lymph-follicles, and often cover their surface in the form of a close network. The surface of the lymph-follicles is not unfrequently placed in the wall of a lymph-vessel, so that it is directly bathed by the lymph-stream. Although no direct canal-like opening leads from the follicle into the lymphatic stream in 
relation with it, a communication must exist, and this is obtained by the numerous spaces in the follicle itself, so that a lymph-follicle is a true lymphatic apparatus whose juices and lymphcorpuscles can pass into the nearest lymphatic. The follicles are surrounded by a network of blood-vessels which sends loops of capillaries into their interior (fig. 220, B). We may assume that lymph-corpuscles pass from these capillaries into the follicle.

In connection with these follicles, including those of the back of the tongue, the solitary glands of the intestine and the adenoid tissue in the bronchial tract, the tonsils, and Peyer's patches, it is important to remember that enormous numbers of leucocytes pass out between the epithelial cells covering these follicles. The extruded leucocytes undergo disintegration subsequently.

(2) The compound lymph-glands-the lymphatic glands-represent a collection of lymphfollicles, whose form is somewhat altered. Every lymph-gland is covered externally with a connective-tissue capsule (fig. 221,c), which contains numerous non-striped muscular fibres. From its inner surface, numerous septa and trabeculæ $(t r$.$) pass into the$ interior, so that the gland-substance is divided into a large number of compartments. These compartments in the cortical portion of the gland have a somewhat rounded form, and constitute the alveoli, while in the medullary portion they have a more elongated and irregular form. [On making a section of a lymph-gland we can readily distinguish the cortical from the medullary portion of the gland.] All the compartments are of equal dignity, and they all communicate with each other by means of openings, so that the septa bound a rich network of spaces within the gland, which communicate on all sides with each other.

These spaces are traversed by the follicular threads (fig. 222, $f, f$ ). These represent the contents of the spaces, but are smaller than the

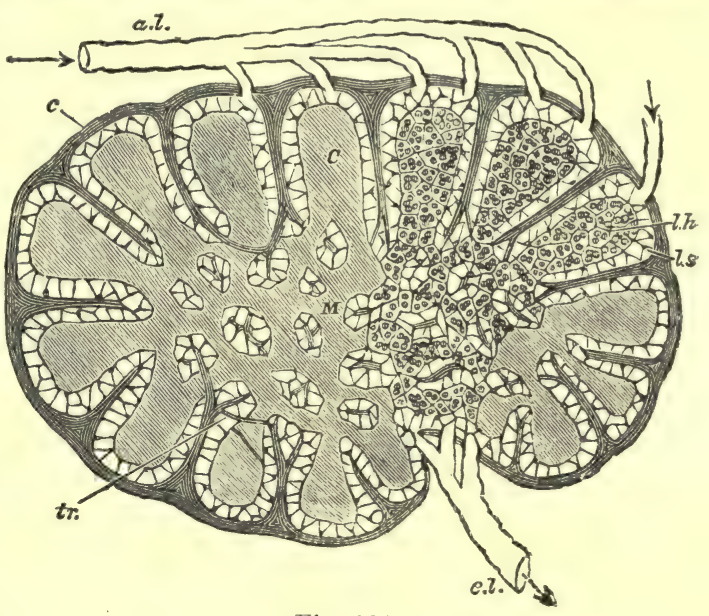

Fig. 221. spaces in which they lie, and do not Diagrammatic section of a lymphatic gland. a.l., afferent, come into contact anywhere with e.l., efferent, lymphatics; C, cortical substance; $\mathrm{M}$, the walls of the spaces. If we reticular cords of medulla; $l . s$, lymph-sinus ; $c$, capsule, imagine the spaces to be injected with a mass, which ultimately shrinks to one-half of its original volume, we obtain a conception of the relation of these follicular threads to the spaces of the gland. The blood-vessels of the gland $(b)$ lie within these follicular threads. They are surrounded by a tolerably thick crust of adenoid tissue, with very fine meshes $(x, x)$ filled with lymph-corpuscles, and with its surface $(0,0)$ covered by the cells of the adenoid reticulum, in such a way as to leave free communications through the narrow meshes.

Between the surface of the follicular threads and the inner wall of all the spaces of the gland, lies the lymph-channel or lymph-path $(\mathrm{B}, \mathrm{B})$, which is traversed by a reticulum of adenoid tissue, containing relatively few lymph-corpuscles. It is very probable that these lymph-paths are lined by endothelium.

The vasa afferentia (fig. $221, a . l$.), of which there are usually several, expand upon the surface of the gland, perforate the outer capsule, and pour their contents into the lymph-paths of the gland $(\mathrm{C})$. The vasa efferentia, which are less numerous than the afferentia, and come out at the hilum, form large, wide, almost cavernous dilatations, and they anastomose near the gland $(e . l$.$) . Through them the lymph passes out at the opposite surface of the gland.$ The lymph percolates through the gland, and passes along the lymph-paths, which represent a kind of rete mirabile interposed between the afferent and efferent lymph-vessels.

During its passage through this complicated branched system of spaces, the movement of the lymph through the gland is retarded, and, owing to the numerous resistances which occur in its path, it has very little propulsive energy. The lymph-corpuseles which lie in the meshes of the adenoid reticulum are washed out of the gland by the lymph-stream. The lymph-corpuscles lying within the follicular threads pass through the narrow meshes $(0)$ into the lymph-paths. The formation of lymph-corpuscles either occurs locally, from division of the pre-existing cells, or new leucocytes wander out into the follicular threads. The movement of the lymph through the gland is favoured by the muscular action of the capsule. When the 
capsule contracts energetically, it must compress the gland like a sponge, and the direction in which the fluil moves is regulated by the position and arrangement of the valves.

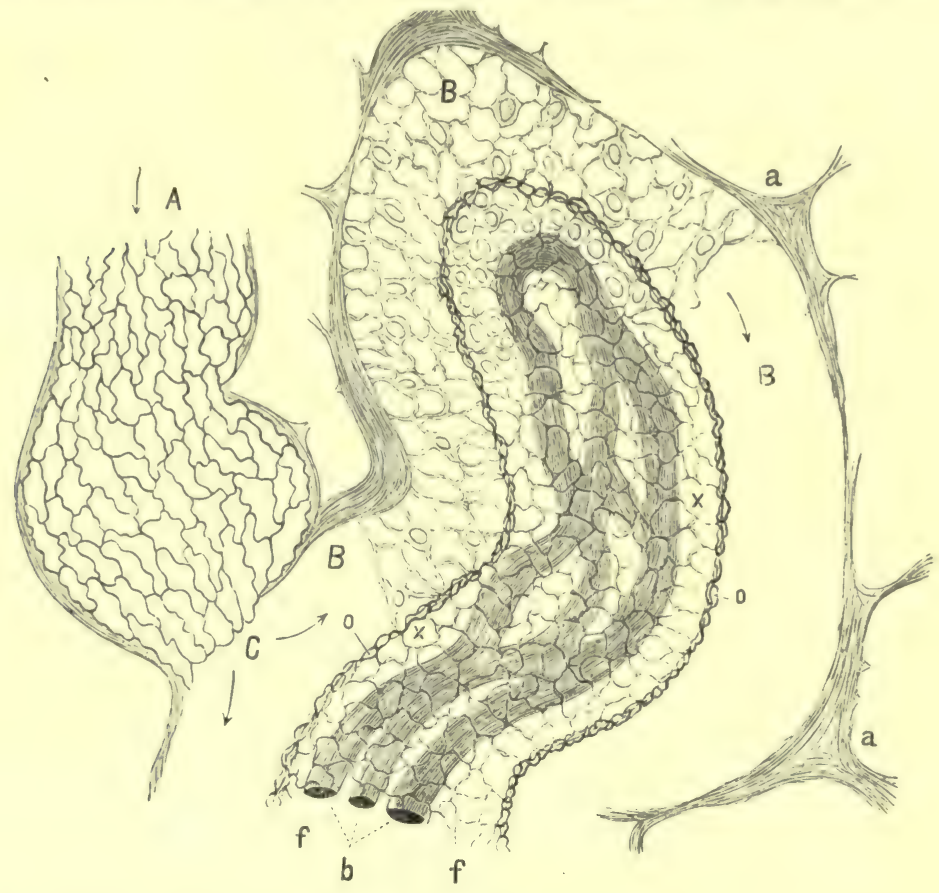

Fig. 222.

Part of a lymphatic gland. A, vas afferens; B, B, lymph-paths; $a$, $a$, trabeculie seen on edge; $f, f$, follicular strand from the medulla ; $x, x$, its adenoid reticulum; $b$, its bloodvessels; 0,0 , narrow-meshed part limiting the follicular strands from the lymph-path.

Chemistry.- In addition to the constituents of lymph, the following chemical substances have been found in lymphatic glands:-leucin and xanthin.

198. PROPERTIES OF CHYLE AND LYMPH.-Chyle and lymph are albuminous, colourless, clear juices, containing lymph-corpuscles, which are identical with the colourless blood-corpuscles $(\$ 9)$. In some places, e.g., in the lymphatics of the spleen, especially in starving animals, and in the thoracic duct, a few coloured blood-corpuscles have been found. The lymph-corpuscles are supplied to the lymph and chyle from the lymphatic glands and the adenoid tissue. As to their source see $\$ 200,2$. They also pass out of the blood-vessels and wander into the lymphatics. $\Lambda$ s red blood-corpuscles have also been seen to pass out of the bloodvessels, this explains the occasional presence of these corpuscles in some lymphatics; but when the pressure within the veins is high, near the central orifice of the thoracic duct, red blood-corpuscles may pass into the thoracic duct. But we are not entitled to conclude from their pressure that lymph-cells form red bloodcorpuscles. In addition, the chyle contains numerous fatty granules, each surrounded with an albuminous envelope. [Thus the chyle, in addition to the constituents of the lymph, contains, especially during digestion, a very large amount of fat, in the form of the finely-emulsionised fat of the food, which gives it its characteristic white or milky appearance. During hunger, the fluid in the lacteals resembles ordinary lymph. The fine fat-granules constitute the so-called "molecular basis" of the chyle.] 
Composition of Lymph.-The lymph consists of lymph-plasma with lymphcorpuscles suspended in it. The corpuscles or leucocytes are described in $\$ 24$. The lymph-plasma contains the three so-called fibrin-factors, derived very probably from the breaking up of lymph-corpuscles (\$29). When lymph is withdrawn from the body, these substances cause it to coagulate. Coagulation occurs slowly, owing to the formation of a soft, jelly-like, small "lymph-clot," which contains most of the lymph-corpuscles. The exuded fluid or lymph-serum contains alkalialbuminate (precipitated by acids), serum-albumin (coagulated by heat), and paraglobulin - the two latter occurring in the same proportion as in blood-serum ; 37 per cent. of the coagulable proteids is paraglobulin.

(1) Chyle, which occurs within the lacteals of the intestinal tract, can only be obtained in very small amount before it is mixed with lymph, and hence the difficulty of investigating it. A few lymph-corpuscles occur even in the origin of lacteals within the villi, but their number increases in the vessels beyond the intestine, more especially after the chyle has passed through the mesenteric glands. The amount of solids, which undergoes a great increase during digestion, on the contrary, diminishes when chyle mixes with lymph. After a diet rich in fatty matters, the chyle contains innumerable fatty granules (2-4 $\mu$ in size). [This is the so-called "molecular basis" of the chyle.] The amount of fibrin-fuctors increases with the increase of lymph-corpuscles, as they are formed from the breaking up of the lymph-corpuscles; a diastatic ferment absorbed from the intestine; occasionally sugar (to 2 per cent.); after much starchy food, lactates; peptone in the leucocytes (§ 192, I., 3), and traces of urea and leucin.

The Chyle of a person who was executed contained $90^{\circ} 5$ per cent. of water.

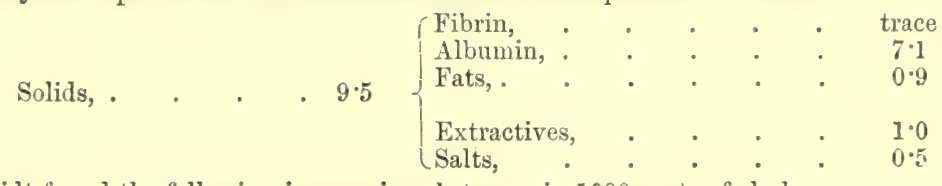

Schmidt found the following inorganic substances in 1000 parts of chyle.

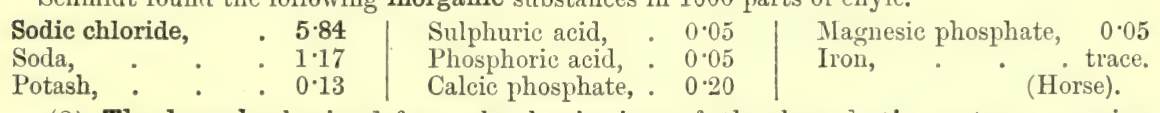

(2) The lymph obtained from the beginning of the lymphatic system contains very few lymph-corpuscles; it is clear, transparent, and colourless, and closely resembles the fluids of serous cavities. That the lymph coming from different tissues varies somewhat, is highly probable, but this has not been proved. After lymph has passed through lymphatic glands, it contains more corpuscles, and also more solids, especially albumin and fat. Ritter counted 8200 lymph-corpuscles in 1 cubic centimetre of the lymph of a dog.

Pure lymph obtained from a lymphatic fistula in the leg of a man has an alkaline reaction and a saline taste, and the following composition :-

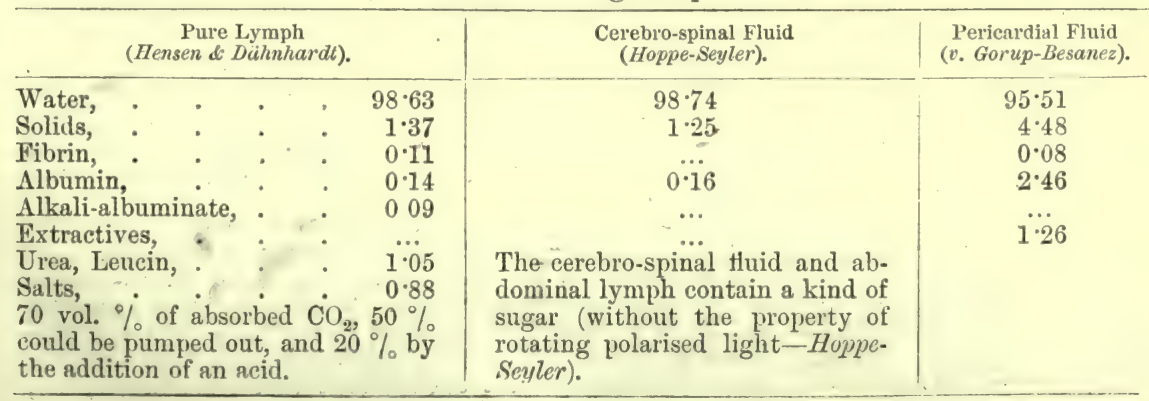


100 parts of the ash of lymph contained the following substances :-

\begin{tabular}{l|l|l|l} 
Sodium chloride, . 74.48 & Lime, . . . 0.98 & Sulphuric acid, . 1.28
\end{tabular}

Soda, . . 10:36 Magnesia, . . 0.27 Carbonic acid, . . 8*21 Potash, . . 3.26 Phosphoric acid, . 1.09 Iron oxide, . 0.06

Just as in blood, potash and phosphoric acid are most abundant in the corpuscles; while soda (chiefly sodium chloride) is most abundant in the lymphserum. The potash and phosphoric acid compounds are most abundant in cerebrospinal fluid, according to C. Schmidt. The amount of water in the lymph rises and falls with that of the blood. Gases.-Dog's lymph contains much CO, - more than 40 vols. per cent., of which 17 per cent. can be pumped out, and 23 per cent. expelled by acids, while there are only traces of $\mathrm{O}$ and 1.2 vols. per cent, $\mathrm{N}$ (Ludwig, Hammersten).

[The cerebro-spinal fluid contains a substance which reduces an alkaline solution of cupric hyilrate. The potassic are in excess of the soda-salts, while the fluid of meningoceles and chronic hychrocephalus contains proto-albumose, some serum-globulin, no serum-albumin, but the last is present in acute bydrocephalus thuid. No albumose is found in pericarlial or pleuritic fluids (Halliburton).]

199. QUANTITY OF LYMPH AND CHYLE. - When it is stated that the total amount of the lymph and chyle passing through the large vessels in twentyfour hours is equal to the amount of the blood, it must be remembered that this is merely a conjecture. Of this amount one-half may be lymph and the other half chyle. The formation of lymph in the tissues takes place continually, and without interruption. Nearly 6 kilos. of lympl were collected in twenty-four hours from a lymphatic fistula in the arm of a woman, by Gubler and Quevenne; 70 to 100 grms. were collected in $1 \frac{1}{2}$ to 2 hours from the large lymph-trunk in the neck of a young horse. The following conditions affect the amount of chyle and lymph :-

(1) The amount of chyle undergoes very considerable increase during digestion, more especially after a full meal, so that the lacteals of the mesentery and intestine are distended with white or milky chyle. During hunger the lymph-vessels are collapsed, so that it is difficult to see the large trunks.

(2) The amount of lymph increases especially with the activity of the organ from which it proceeds. Active or passive muscular movements greatly increase its amount. Lesser obtained in this way 300 cubic centimetres of lymph from a fasting dog, whereby its blood became so inspissated as to cause death.

(3) All conditions which increase the pressure upon the juices of the tissues increase the amount of lymph, and vice ver'sit. These conditions are :-

(a) $A_{n}$ increase of the blood-pressure, not only in the whole vascular system, but also in the vessels of the corresponding organ, augments the amount of lymph and vice versit (Ludwig, Tumsa). This however is doubtful, as has been shown by Paschutin and Emminghaus. [In order to increase the amount of lymph depending upon pressure within the vessels, what must happen is increased pressure within the capillaries and veins.]

(b) Ligature or obstruction of the efferent veins greatly increases the amount of lymph which flows from the corresponding parts (Bidder, Emminghaus). It may be doubled in amount. Tight bandages cause a swelling of the parts on the peripheral side of the bandage, owing to a copious effusion of lymph into the tissue (congestive oedema).

(c) An increased supply of arterial blood acts in the same way, but to a less degree. Paralysis of the vaso-motor nerves, or stimulation of vaso-dilator fibres, by increasing the supply of blood increases the amount of lymph; while diminution of the blood-supply, owing; to stimulation of vaso-motor fibres or other causes, diminishes the amount. Even after ligature of both caroticls, as the heal is still supplied with blood by the vertebrals, the lymph-stream in the large cervical lymphatic does not cease.

(4) When the total amount of the blood is increased, by the injection of blood or serum into the arteries, much fluid passes into the tissues and increases the formation of lymph.

(5) The formation of lymph still goes on for a short time after death, and after complete cessation of the action of the heart, but only to a slight extent. If fresh blood be caused to circulate in the body of an animal, while it is still warm, more lymph flows from the lymphatics. It appears as if the tissues obtained plasma from the blood for a time after the stoppage of the circulation. This perhaps explains the circumstance that some tissues, e.g., 
connective-tissues, contain more fluid after death than during life, while the blood-vessels have given out a considerable amount of their plasma after death.

(6) The amount of lymph is increased under the influence of curara, and so is the amount of solids in the lymph (Lesser). A large amount of lymph collects in the lymph-sacs [especially the sub-lingual] of frogs poisoned with curara, which is partly explained by the fact that the lymph-hearts are paralysed by curara. The amount of lymph is also increased in inflamed parts.

200. ORIGIN OF LYMPH.-(1) Source of the Iymph-Plasma.-The lymph-plasma may be regarded as fluid which has been pressed through the walls of the blood-vessels by the blood-pressure, i.e., by filtration into the tissues. The salts which pass most readily through membranes, go through nearly in the same proportion as they exist in blood-plasma-the fibrin-factors to about two-thirds, and albumin to about one-half of that in the blood. As in the case of other filtrationprocesses, the amount of lymph must increase with increasing pressure.

This was proved by Ludwig and Tomsa, who found that when they passed blood-serum under varying pressures through the blood-vessels of an excised testis, the amount of transuded fluid which flowed from the lymphatics varied with the pressure. This "artificial-lymph" had a composition similar to that of the natural lymph. Even the amount of albumin increased with increasing pressure. The lymph-plasma is mixed in the different tissues with the decomposition products, the results of the metabolism of the tissues.

When the muscles act, not only is the lymph poured out more rapidly, but more lymph is formed. The tendons and fasciæ of the muscles of the skeleton, which are provided with numerous small stomata, absorb the lymph from the muscles. By the alternate contraction and relaxation of these fibrous structures, they act like suction-pumps, whereby the lymphatics are alternately filled and emptied, while the lymph is propelled onwards. Even passive-movements act in the same way. If solutions be injected under the fascia lata, they may be propelled onwards to the thoracic duct by passive movements of the limb (Lucluvig, SchweiggerSeidel).

(2) The source of the lymph-corpuscles varies.-(1) A very considerable number of lymph-corpuscles are derived from the lymphatic glands; they are washed out of these glands into the vas efferens by the lymph-stream, hence, the lymph always contains more corpuscles after it has passed through a lymph-gland. Small isolated lymph-follicles permit corpuscles to pass through their limiting layer into the lymph-stream. (2) Those organs whose basis consists of adenoid tissue, and in whose meshes numerous lymph-corpuscles occur, e.g., the mucous membrane of the entire intestinal tract, red marrow of bone, and the spleen (\$103). The cells reach the origin of the lymph-stream by their own amœboid movements. (3) As lymph-corpuscles are returned to the blood-stream, where they appear as colourless blood-corpuscles, so they again pass out of the blood-capillaries into the tissues, partly owing to their amœboid movements, and they are partly expelled by the blood-pressure. In rare cases lymph-corpuscles wander from lymphatic spaces back again into the blood-vessels.

Fine particles of cinnabar or milk-globules introduced into the blood soon pass into the lymphatics. The extrusion of particles is greater during venous congestion than when the circulation is undisturbed, just as with diapedesis $(\$ 95)$; inflammatory affections of the vascular wall also favour their passage. The vessels of the portal system are especially pervious.

(4) By division of the lymph-corpuscles, and also by proliferation of the fixed connective-tissue corpuscles. This process certainly occurs during inflammation of many organs. This has been proved for the excised cornea kept in a moist chamber; the nuclei of the cornea-corpuscles also proliferate.

That the connective-tissue corpuscles proliferate is shown by the enormous production of lymph-corpuscles in acute inflammations (with the formation of pus), e.g., in extensive erysipelas, and inflammatory purulent effusions into serous cavities, where the number of corpuscles is too great to be explained by the wandering of blood-corpuscles out of the bloocl-vessels.

Decay of Lymph-Corpuscles.-The lymph-corpuscles disappear partly where the 
lymphatics arise. The presence of the fibrin-factors in the lymph-formed as they are from the breaking-up of lymph-corpuscles-seems to indicate this. In inflammation of connective-tissue, in addition to the formation of numerous new lymphcorpuscles, a considerable number seems to be dissolved; hence the lymph, and also the blood, in this case contains more fibrin. Lymph-corpuscles are also dissolved within the blood-stream, and help to form the fibrin-factors.

\section{MOVEMENT OF CHYLE AND LYMPH.-The ultimate cause of the} movement of the chyle and lymph depends upon the difference of the pressure at the origin of the lymphatics, and the pressure where the thoracic duct opens into the venous system.

(1) The forces which are active at the origin of the lymphatics are concerned in moving the lymph, but these must vary according to the place of origin-(a) The lacteals receive the first impulse towards the movements of their contentsthe chyle-from the contraction of the muscular fibres of the villi (pp. 292, 297). When these contract and shorten, the axial lacteal is compressed, and its contents are forced in a centripetal direction towards the large lymphatic trunks. When the villi relax, the numerous valves prevent the return of the chyle into the villi. (b) Within those lymphatics which take the form of perivascular spaces, every time the contained blocid-ressel is dilated the surrounding lymph will be pressed onwards. (c) In the case of the pleural lymphatics with open mouths, every inspiratory movement acts like a suction-pump upon the lymph, and the same is the case with the openings or stomata of the lymphatics on the abdominal side of the diapliaragm. (d) In the case of those vessels which begin by means of fine juice canals, the movement of the lymph must largely depend upon the tension of the juices of

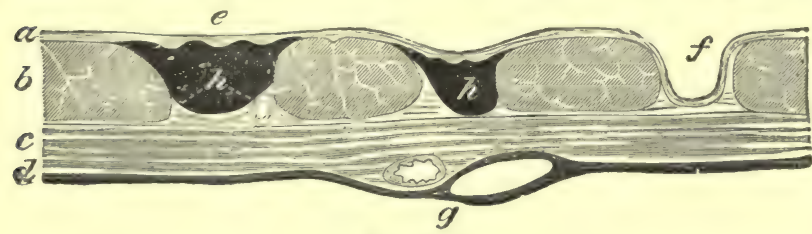

Fig. 223. the parenchyma, and this again must depend upon the tension or pressure in the bloodcapillaries, so that the blood-pressure acts like a vis a tergo in the rootlets of the lym-

Section of central tendon of diaphragm. The injected lymph-spaces, phatics.

$h$ and $h$, are black. At $f$ the walls of the space have collapsed.

[In some organs peculiar r umping arrangements are brought into action. The abdominal surface of the central tendon of the diaphragm is provided with stomata, or open communications between the peritoneal

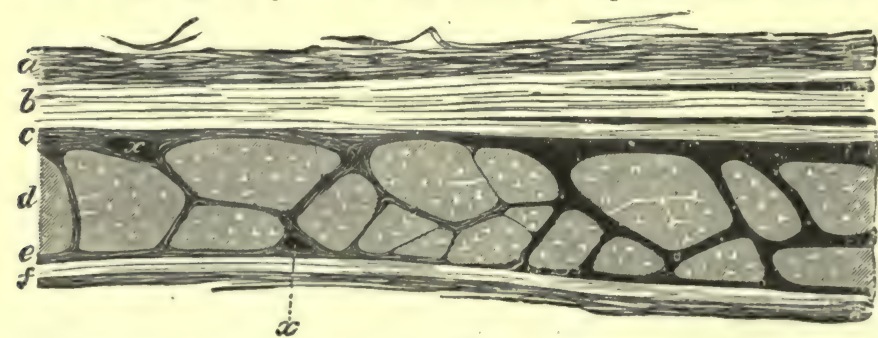

Fig. 224.

Injected lymph-spaces (black) from the fascia lata of the dog. cavity and the lym. phatics in the sub. stance of the tendon. Von Recklinghausen found that milk put upon the peritoneal surface of the central tendon showed little eddies, caused by the milk-globules passing through the stomata and entering the lym. phatics. The central tendon consists of two lavers of fibrous tissue arranged in different directions (fig. 223, $b, c$ ). When the diaphragm moves during respiration, these layers are alternately pressed together and pulled apart. Thus the spaces are alternately dilated and contracted, lymph being drawn into the lymphatics through the stomata (fig. $223, h$ ). The same kind of pumping mechanism exists over the costal pleura. The fascia covering the muscles is another similar mechanism. The fascia consists of two layers of fibrous tissue, with intervening lymphatics (fig. 224). When a muscle contracts, lymph is forced out from between the layers of the fascia, while when it relaxes, the lymph from the 
muscle, carrying with it some of the waste products of muscular action, passes out of the muscle into the fascia, between the now partially separated layers.]

[Ludwig's Experiment. - Tie a respiration cannula in the trachea of a dead rabbit; cut across the body of the animal immediately below the diaphragm; remove the viscera, and ligature the vessels passing between the thorax and abdomen; tie the thorax to an iron ring, and hang it up with the head downwards; pour a solution of Berlin blue upon the peritoneal surface of the diaphragm; connect the respiration cannula either with a pair of bellows or an apparatus for artificial respiration, and imitate the respiratory movements. After a few minutes the lymphatics are filled with a blue injection showing a beautiful plexus.]

(2) Within the lymph-trunks themselves, the independent contraction of their muscular fibres partly aids the lymph-stream. Heller observed in the mesentery of the guinea-pig that the peristaltic movement of the lymphatic wall passed in a centripetal direction. The numerous valves prevent any reflux. The contraction of the surrounding muscles, and pressure upon the vessels and the tissues, aid the current. If the outflow of blood from the veins is interfered with, lymph flows copiously from the corresponding tissues. [If a cannula be tied in a lymphatic of a dog, a few drops of lymph flow out at long intervals. But if even passive movements of the limb be made, e.g., simply flexing and extending the limb, the outflow becomes very considerable and continuous.]

(3) The lymph-glands, which occur in the course of the lymphatics, offer very considerable resistance to the lymph-stream, which must pass through the lymphpaths, whose spaces are traversed by adenoid tissue, and contain a few lymphcorpuscles. But this is, to a certain extent, compensated for by the non-striped muscle which exists in the capsule and trabeculæ of the glands. When they contract they force on the lymph, while the valves prevent its reflux. Enlarged lymphatic glands have been seen to contract when stimulated electrically. [Botkin has stimulated enlarged lymphatic glands with electricity in cases of leukæmia.]

(4) The lymph-vessels gradually join to form larger vessels, and finally end in one trunk. 'Thus the sectional area diminishes, so that the velocity of the current and the pressure are increased. Nevertheless, the velocity is always small; it varied from 230 to 300 millimetres per minute in the large lymphatic in the neck of a horse, a fact which enables us to conclude that the movement must be very slow in small vessels. The lateral pressure at the same place was 10 to $20 \mathrm{~mm}$., and in the $\operatorname{dog} 5$ to $10 \mathrm{~mm}$. of a weak solution of soda, although it was $=12 \mathrm{~mm}$. $\mathrm{Hg}$ in the thoracic duct of a horse.

(5) The respiratory movements exercise a considerable influence upon the lymphstream in the thoracic duct, and in the right lymphatic duct; every inspiration favours the passage of the venous blood, and also of the lymph towards the heart, whereby the tension in the thoracic duct may even become negative. [The diastolic suction of the heart, by diminishing the pressure in the subclavian vein, also favours the inflow of lymph 'into the thorax.]

(6) Lymph-hearts exist in eertain cold-bloodeả animals. The frog has two axillary hearts (above the shoulder near the vertebral column), and two sacral hearts, one on each side of the coccyx near the anus (fig. 225, L). They beat, but not synchronously, about sixty times per minute, and contain 10 cubic centimetres of lymph. 'They have transversely-striped muscular fibres in their walls, and are also provided with nerve-ganglia. The posterior pair pump the lymph

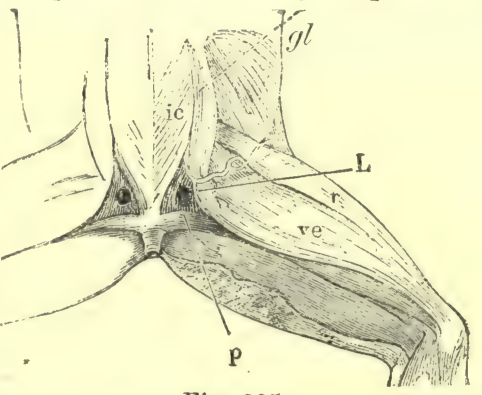

Fig. 225.

Posterior pair of lymph-hearts (L) of the frog.

into the branch of the vena iliaca communicans, and the anterior pair into the vena subscapularis. Their pulsation depends partly, but not exclusively, upon the spinal cord, for if the cord be rapidly destroyed, they may cease to pulsate, but not unfrequently they continue to pulsate after removal of the cord. [And if the cord be destroyed gradually, they continue to beat $(K a b r h e l)$.] A second source of their pulsatile movements is to be sought for in 
Waldever's ganglia. Stimulation of the skin, intestine, or blood-heart influences them reflexly - partly accelerating and partly retarding them, [most frequently arresting them in diastole, so that there seems to be an inhibitory mechanism in the cord, but it is not affected by atropine (Kulrikel).] If the coceygeal nerve, which connects the sacral hearts to the spinal cord, be divided, these effects do not oceur. Strychnia accelerates their movements, and so does heating of the spinal cord; but if the cord be cooled, they are retarded. A lymph-heart arristed by being exposed, or after the action of muscarin, can be caused to beat by filling it nnder pressure, but this is not the ease when the arrest is caused by clestruction of its nerves. Antiurin paralyses the lrmph-heart and the blood-heart at the same time, while curara garalyses the former alone. In other amphibians. there are two lymph-hearts; in the ostrich ant cassowary and some swimming birls, and in the embryo chick 1 or 2 . They occur in sone fishes, e.g., near the caudal vein of the eel.

(7) The nervous system has a direct effect upon the lymph-stream, on account of its connection with the muscles of the lymphatics and lymph-glands, and with the lymph-hearts where these exist. Kiilne observed that the cornea corpuscles contracted when the corneal nerves were stimulated, [and Hoffman has described the t:rmination of nerves in connective-tissue corpuscles]. Goltz also observed that, when a dilute solution of common salt was injected under the skin of a frog, it was rapidly absorbed, but if the central nervous system had been destroyed, it was not absorbed.

If inflammation be produced in the hind legs of a dog, and if the sciatic nerve be divided on one side, welema and a simultaneous increase of the lymph-stream occur on that side. IA combination of congestion and inflammation greatly increases the lymph-stream, and this is still more the case when the nerves are divided at the same time.]

Ligature the leg of a frog, except the nerves, so as to arrest the circulation, and place the leg in water; it swells up very rapilly, but a dead limb does not swell up. So that absorption is inderemlent of the continuance of the circulation. Section of the sciatic nerve, or destruction of the spinal cord (but not section of the brain), arrests absorption.

202. ABSORPTION OF PARENCHYMATOUS EFFUSIONS. - Fluids which pass from the bloml-vessels into the spaces in the tissues, or those injected subcutaneously, are absorbed chiefly hy the blood-vessels, but also by the lymphatics. Small particles, as after tattooing with cinnalar or China ink, may jass from the tissue-spaces into the lymphatics-and so do hlood-corpuscles from extravasations of blood, and fat-granules from the marrow of a broken hone. If all the lymphatics of a part are ligatured, absorption takes place quite as rapidly as before; hence, alsorbed fluid must pass through the thin membranes of the blood-vessels. The correspondling experiment of ligaturing all the blood-vessels, when no absorption of the paren- hymatous juices takes place, does not prove that the lymphatics are not concerned in absorption, for, after ligaturing the blood-vessels of a part, of course the formation of lymph, anil also the lymph-stream, must cease. When fluids are injected under the skin, absorption takes place very rapidly-more rapidly than when the substance is given by the mouth. The subcutaneous injection of drugs is extensively used, but of course the substances used must not corrode, irritate, or coagulate the tissues. Some substances do not act when given by the mouth, as snake poison, joisons from dead bodies, or putrid things, although they act rapilly when introduced subcutaneously. If emulsin be given by the month, and amygdalin be injecterl into the veins of an animal, hydrocyanic acid is not formed, as the emulsin seems to be destroyed in the alimentary canal. If the emulsin, however, be injected into the blood, and the amygdalin be given by the mouth, the animal is rapidly poisoned, owing to the formation of hydrocyanic acid, as the amygdalin is rapidly absorbed from the intestinal canal. The amyglalin, a glucoside $\left(\mathrm{C}_{20} \mathrm{H}_{27} \mathrm{NO}_{11}\right)$, is acted upon by fresh emulsin like a ferment; it takes* nip $2\left(\mathrm{H}_{2} \mathrm{O}\right)$ and yields hydrocyanic acid $(\mathrm{CHN})$, + oil of bitter almonds $\left(\mathrm{C}_{7} \mathrm{H}_{6} \mathrm{O}\right)$, + sugar $2\left(\mathrm{C}_{6} \mathrm{H}_{12} \mathrm{O}_{6}\right)$. Serum injected subcutaneously is rapidly absorbed; it is decomposed within the blood-stream, and increases the amount of urea. Albuminous solutions, oil, peptones, and sugars are also absorbed.

203. EDEMA, DROPSY, AND SEROUS EFFUSIONS.-[Dropsy.-As aptly illustrated by Lauler Brunton, the lymph-spaces may be represented by cisterns, each of which is provided with supply pipes-the arteries and capillaries; while there are two exit pipes-the veins and lymphatics. In health, the balance between the inflow and outflow is such that the spaces are merely moistened with fluid. When a cannula is placed in a lymphatic vessel in a dog, only a few dropis of lymph flow out at long intervals, but if the veins of the limb be ligatured, the lymph flows much more quickly. This is in part due to the increased transudation of fluid from the small blood-vessels, but it may also be due to fluid passing away by the lymphatics when it can no longer be carried away by the veins. We cannot say what is the relative share of the veins and the lymphatics, nor in the above experiment do we know how much is due to 
increased transudation or diminished absorption. When there is an undue accumulation of fluid more or less like serum in the lymph-spaces, we have the condition termed dropsy. When there is general dropsy it is called anasarca.]

Edema. - If the efferent veins and lymphatics of an organ be ligatured, or if resistance be offered to the outflow of their contents, congestion and a copious transudation of lymph into the tissue take place. These are most marked in the skin and subcutaneous cellular tissue. The soft parts swell up, without pain or redness, and a doughy swelling, which pits on pressure with the finger, results. These are the signs of lymph-congestion, which is called œdema when the fluid is watery and localised.

Under similar circumstances lymph is effused in the serous cavities. [In the peritoneum it is ascites-thorax, hydro-thorax-pericardium, hydro-pericardium-cranium, hydrocephalus -tunica vaginalis, hydrocele-joints, hydrarthrosis, \&c.] If, at the same time, a large number of colourless blood-corpuscles pass ont of the blood-vessels into the cavity, the fluid becomes more and more like pus. In order that these corpuscles may proliferate, a considerable percentage of albumin is necessary. When the pressure within the serous cavity rises above that in the small blood-vessels, water may pass into the blood. These sero-purulent effusions not unfrequently undergo changes, and yield decomposition-products, such as leucin, tyrosin, xanthin, kreatin, kreatinin (?), uric acid (?), urea. Endothelium from the serous eavity, sugar in pleuritic effusions and in œedemas with little albumin, cholesterin frequently in hydrocele fluid, and succinic acid in the fluid of echinococci have all been found in these effusions. The effusion of lymph may arise not only from pressure upon the lymphatics, but also from inflammation and thrombosis of the lymphatics themselves, in which cases not unfrequently new lymphatics are formed, so that the communication is re-established. Sometimes the ductus thoracicus bursts, and lymph is poured directly into the abdomen or thorax. [Ligature of the thoracic duct results in rupture of the receptaculum chyli and escape of chyle and lymph into the large serous cavities (Ludwig).]

When dropsy or effusion of fluids occurs into serous cavities, there is always a greater transudation of fluid through the blood-vessels. The abdominal blood-vessels, and those which yield a watery effusion under normal circumstances, are those most liable to be affected.

Transudation is favoured by-(1) Venous congestion, so as to raise the blood-pressure, in which case the effusion usually contains little albumin and few lymph-corpuscles, while the coloured corpuscles, on the contrary, are more numerous the greater the venous obstruction. Ranvier produced cedema artificially by ligaturing the vena cava in a dog, and at the same time dividing the sciatic nerve. The paralytic dilatation of the blood-vessels thereby produced caused an increased amount of blood to pass to the limb, while the blood-pressure was raised, and both factors favoured the transudation of fluid. [Ranvier's experiment proves that mere ligature of the venous trunk of a limb by itself is not sufficient to cause oedema. The cedema is due to the concomitant paralysis of the vaso-motor nerves. If the motor roots of the sciatic nerve alone be divided along with ligature of the vena cava, no œdema occurs, but if the rasomotor fibres are divided at the same time, the limb rapidly becomes cedematous. There is such an increased transudation through the vascular walls that the veins and lymphatics cannot remove it with sufficient rapidity, and oelema occurs. If there be weakness of the vaso-motor nerves, slight obstruction is sufficient to produce œedema.] When the leg-veins are oceluded with an injection of gypsum, œdema occurs. (2) Some unknown physical changes occur in the protoplasm of the endothelium of the capillaries and blood-vessels, which favour the transudation of albumin, hæmoglobin, and even blood-corpuscles. This occurs when abnormal substances accumulate in the blood-e.g., dissolved hæmoglobin-and when the blood contains little $\mathrm{O}$ or albumin. The same has been observed after exposure to too high temperatures, and the swelling of soft parts in the neighbourhood of an inflammatory focus seems due to the transudation of fluid through the altered vascular wall. It is probable that a nervous influence may affect particular areas through its action on the blood-vessels of the part (it may be upon the protoplasm of the blood-capillaries). The transudations of this nature usually contain much albumin and many lymph-corpuscles. (3) When the blood contains a very large amount of water, the tendency to transudation of fluid is increased. After a time it may produce the changes indicated in (2), and when long continued may increase the permeability of the vascular wall. Watery lym. phatic effusions from watery blood-" cachectic oedema"-occur in feeble and badly-nourished individuals. [One of the commonest forms of dropsy is the slight œedema of the legs in anæmic persons, in whom the heart and lungs are healthy. Mány factors are involved-the bloodpressure, watery condition of the blood, the condition of nutrition of the capillaries, and probably a tendency to vaso-motor paresis (Brunton).]

[The fluid poured ont varies according to the rapidity with which this occurs. In acute inflammations effusion or exudation takes place rapidly, and the fluid contains the fibrin-factors, so that it tends to coagulate spontaneously. There is every gradation between the non-coagulable hydrocele fluid and the coagulable exudation in inflammation. The fluids in different dropsies vary in composition, and some have more cells in them, depending on local causes, as in some situations absorption is more active than in others. The pleural fluid contains most solids, then ascitic, cerebro-spinal, and lastly that in the subcutaneous tissue. Transudation cor- 
responds to the process of filtration through animal membranes, i.e., the transudation contains only those substances alrealy present in the blood-plasma. The filtrate may even contain more salts than the original fluid, as is often the case with fluids containing crystalloid and colloid bodies. Senator finds, in cases of cedema of the leg, that increase of the venous pressure increases the proteids in the transudation, but causes no essential change in the amount of the salts.]

[(t) Ostroumoff found that stimulation of the lingual nerve not only causes the blood-vessels of the tongue to dilate, but that the corresponding side of the tongue becomes oedematous. If a solution of lilute hyilrochloric acid or quinine (\$145) be injected into the duct of the submaxillary gland, anil the chorla tympani stimulated, there is no secretion of saliva, but the gland becomes inlematous. In an animal poisoned with atropin, stimulation of the chorda canses dilatation of the blool-vessels, although there is no secretion of saliva, nevertheless the gland der:s not berme cilematous (Heidenhrin). As Brunton suggests, this experiment points to some action of atropin on the blood-vessels which has hitherto been entirely overlooked.]

204. COMPARATIVE PHYSIOLOGY.-In the frog large lymph-saes, lined with endothelimm, exist under the skin, while large lymph-sacs lie in relation with the vertebral column -one on each sile-separated from the alidominal cavity by a thin membrane, perforated with stuniata. This is the cysterna lymphatica magna of Panizza. Some amphilians and many reptiles have under the skin large lymph-spaces, which occupy the whole of the dorsal region of the boly. All reptiles and the tailed amphibians have large elongated reservoirs for lymph along the course of the aorta. The lymph-applaratus of the tortoise (fig. 218) is very extensive. The osseous fishes have in the lateral parts of their backs an elongated lymph-trunk, which reaches from the tail to the anterior fins, and is connected with the dilated lymphatic rootlets in the base of the tail aml in the fins. The largest internal lymph-sinus is in the region of the (M) Mhagus. Many birds pussess a sinus-like dilatation or lymph-space in the region of the tail. The lymph-spaces communicate with the venous system-with valves properly arrangedusually in comnertion with the uper vena cava. Lymph-hearts have alreacly been referred to $(52(1), 6)$. In carnivora the lymph-glands of the mesentery are united into one large compact mass, the so-called "pancreas Asellii."

205. HISTORICAL. - Althongh the Hippocratic School was acquainted with the lymph. glands from their hecoming swollen from time to time, and although Herophilus and Erasistratus hail seen the mesenteric glands, yet Aselli (1662) was the first who accurately dieseribed the lactrals of the mesentery with their valves. Pecquet (1648) discovered the jenplatulum chyli; Rullheck and Thom. Bartholinus the lymphatic vessels (1650-52); Eustarhius (1563) was aequainted with the thoracic duct, which Gassendus (1654) maintained that he was the first to see; Lister noticed that the chyle became blue when indigo was injected into the intestine (16i1); Simmering observel the separation of fibrin when lymph coagulated; lieuss and Emmert discovered the lymph-corpuscles. The chemical investigations date from the first yuarter of this century ; they were carried out by Lassaigne, Tiedemann, Gmelin, and others. The two last-named oliservers noticel that the white colour of chyle was due to the presence of fatty granules. 


\section{Physiology of Animal Heat.}

206. SOURCES OF HEAT.-The heat of the body is an uninterrupted evolution of kinetic energy, which we must represent to ourselves as due to vibrations of the corporeal atoms. The ultimate source of the heat is contained in the potential energy taken into the body with the food, and with the $\mathrm{O}$ of the air absorbed during respiration. The amount of heat formed depends upon the amount of energy liberated.

The energy of the food-stuffs may be called "latent heat," if we assume that when they are used up in the body, chiefly by a process of combustion, kinetic energy is liberated only in the form of heat. As a matter of fact, however, mechanical energy and electrical energy are developed from the potential energy. In order to obtain a unit-measure for the energy liberated, it is advisable to express all the potential energy as heat-units.

The Calorimeter. - This instrument enables us to transform the potential energy of the food into heat, and, at the same time, to measure the number of heat-units produced.

Favre and Silbermann used a watercalorimeter (fig. 226). The substance to be burned is placed in a large cylindrical combustion-chamber (K), suspended in a large cylindrical vessel (L) filled with water $(w)$, so that the combustion-chamber is completely surrounded by the water. Three tubes open into the upper part of the chamber; one of them (O) supplies the air which is necessary for combustion,

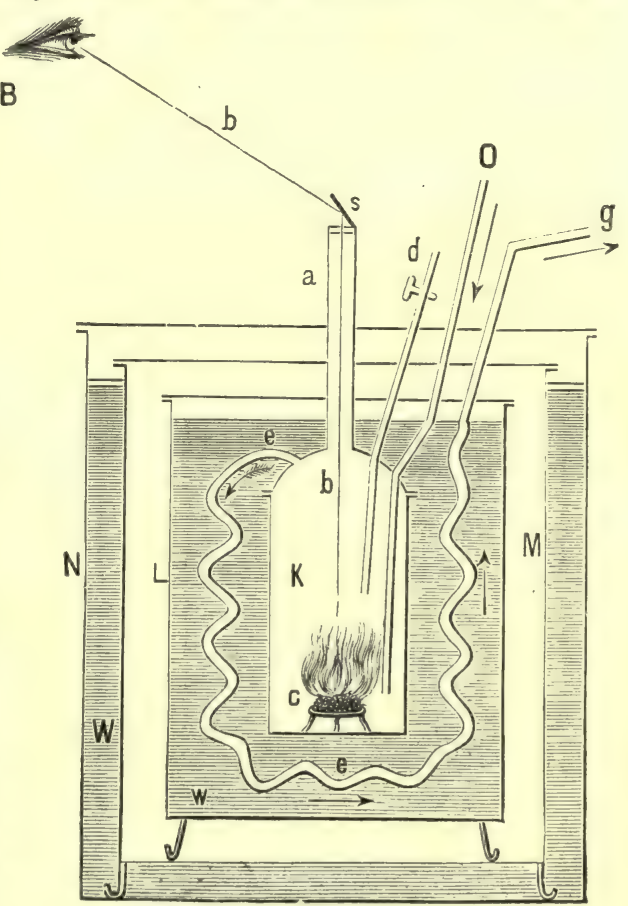

Fig. 226 .

Water-calorimeter of Favre and Silbermann. it reaches almost to the bottom of the chamber; the second $(a)$ is fixed in the middle of the lid, and is closed above with a thick glass plate, and on this is placed, at an angle, a small mirror $(s)$, which enables an observer to look into the chamber, and observe the process of combustion at $c$. The third tube $(d)$ is used only when combustible gases are to be burned in the chamber. It can be closed by means of a stop-cock. A lead tube $(e, e)$, with many twists, passes from the upper part of the chamber through the water, and finally opens at $g$. 
The gaseous prolucts of combustion pass out through this tube, and in doing so help to heat the water. The cylindrical ressel with the water is closed with a lid which transmits the four tubes. The water-erlimler stanils on four feet within a large cylinder (M), which is filled with some cooul non-conilucter of heat, and this again is placed in a large vessel filled with water (W). This is to prevent any lieat reaching the inner cylinder from without. A weighed quantity of the sulstance (o he investigated is placed in the combustion-chamber. When eomhustion is culdel, duriug which the inner water must be repeatedly stirred, the temperature of the water is ascertainel hy means of a delicate thermometer. If the increase of the temperature and the amount of water are known, then it is easy to calculate the number of heat-units producel by the combustion of a known weight of the substance (see Introduction).

The ice calorimeter may also be used. The imner cylinder is filled with ice and not with water. anul ice is also placed in the nuter cylinder to prevent any heat from without from

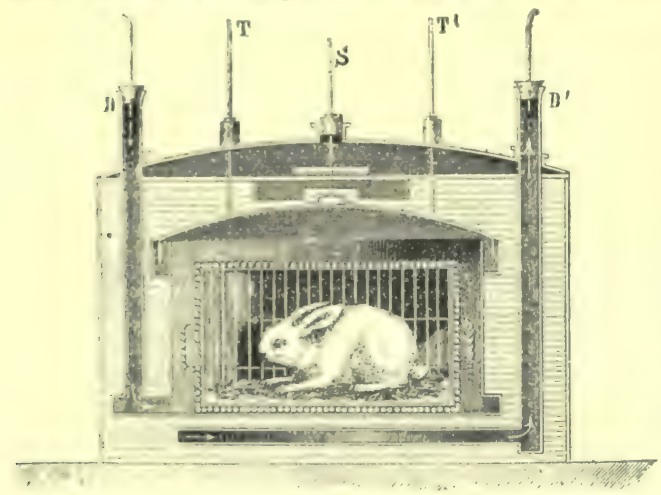

Fig. 227 .

Water-calorimeter of Dulong. acting upon the inner ice. The heat given off from the combustion-chamber causes a certain amount of the ice to melt, and the water thereby produced is collected and measured. It requires 79 heat-units to melt 1 grm. of ice to 1 grm. of water at $0^{\circ} \mathrm{C}$.

[The amount of heat produced by a living animal is similarly measured. The animal (fig. 227), in a cage, is placed in a large vessel, which is placed within another vessel, and the interspace filled with water. The whole should be enclosed in a large box packed with fur, shavings, feathers, or other bad conductor of heat. A tube, D, opens into the inner space, and from it there is an exit-tube, D, which winds many times in the water-space beneath. Air passes in through $D$ and out by $\mathrm{D}^{\prime}$. The temprerature of the water is ascertained hy thermometers $\mathrm{T}$ and $\mathrm{T}$ ', while the water is moved by a stirrer (S) placed between the two.]

Just as in a calorimeter, although much more slouly, the food-stuffs within our body are bumed up, oxygen being supplied, and thus potential energy is transformed into kinetic energy, which, in the case of a person at rest, almost completely appears in the form of heat.

Heat-Units.-Favre, Silhermaun, Frankland, Rechenberg, B. Danilewsky, and others have mal. calmimetric experiments on the heat produced by food. Accorling to Danilewsky, 1 gramme of the following dry substances yields heat-units :-

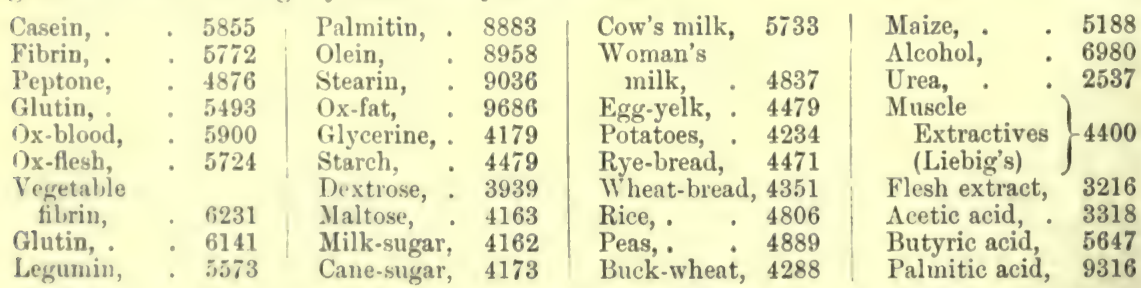

As albumin is only oxillised to the stage of urea, we must deduct the heat-units obtainable from urea from those of allumin, and as 1 part of albumin yields in round numbers about $\hat{s}$ of urea, we obtain about 5100 calories [ $=2170$ kilogram-metres] for $1 \mathrm{grm}$. of albumin. :

Isodynamic foods, i.c., those that produce an equal amount of heat; 100 grms. animal albumin (after leclucting the heat-mnits of urea) $=52$ fat $=114$ starch $=129$ dextrose; 100 grms. fat are isolynamic with 243 dry flesh or 225 of dry syntonin (Rubner); 100 grms. of vegetable allumin $=55 \mathrm{fat}=121$ starch $=137$. dextrose $($ Danilewsky). Rubner calculated that in man, with a mixed diet, the arcilable heat-units for $1 \mathrm{grm}$. of albumin $=4100 ; 1 \mathrm{grm}$. fat $=9300$; and for $1 \mathrm{grm}$. carbohydrate $=4100$ calories.

When we know the weight of any of the above-named substances consumed by a man in twenty-four hours, a simple calculation enables us to determine how many 
heat-units are formed in the body by oxidation, i.e., provided the substance is completely oxidised.

[Several sources of heat-production or thermogenesis are to be found in all tissues wherever oxidation is going on. The metabolism of protoplasm is always associated with the evolution of heat.]

(1) In the transformation of the chemical constituents of the food, endowed with 4 large amount of potential energy, into such substances us have little or no energy. The organic substances used as food consist of $\mathrm{C}, \mathrm{H}, \mathrm{O}, \mathrm{N}$, so that there takes place- (a) Combustion of $\mathrm{C}$ into $\mathrm{CO}_{2}$, of $\mathrm{H}$ into $\mathrm{H}_{2} \mathrm{O}$, whereby heat is produced ; $1 \mathrm{grm}$. $\mathrm{C}$ burned to produce $\mathrm{CO}_{2}$ yields 8080 heat-units, while $1 \mathrm{grm}$. $\mathrm{H}$ oxidised to $\mathrm{H}_{2} \mathrm{O}$ yields 34,460 heat-units. The $\mathrm{O}$ necessary for these purposes is absorbed during respiration, so that, to a certain extent at least, the amount of heat produced may be estimated from the amount of $\mathrm{O}$ consumed. The same consumption of $\mathrm{O}$ gives rise to the same anount of heat whether it is used to oxidise $\mathrm{H}$ or $\mathrm{C}$ (Pflüger). There is a relation, amounting to cause and effect, between the amount of heat produced in the body and the $\mathrm{O}$ consumed. The cold-blooded animals, which consume little $\mathrm{O}$, have a low temperature; amongst warm-blooded animals, 1 kilo. of a living rabbit takes up within an hour $0.914 \mathrm{grm}$. $\mathrm{O}$, and its body is heated to a mean of $38^{\circ} \mathrm{C}$. 1 kilo. of a living fowl uses 1.186 grms. $\mathrm{O}$, and gives a mean temperature of $43.9^{\circ} \mathrm{C}$. The amount of heat produced is the same whether the combustion occurs slowly or quickly; the rapidity of the metabolism, therefore, affects the rapidity, but not the absolute amount of heat-production. The combustion of inorganic substances in the body, e.f., of the sulphur into sulphuric acid, the phosphorus into phosphoric acid, is another, although very small, source of heat.

[The muscles form about the half of the whole mass of the body and the bones nearly the other half. In the latter, oxidation does not go on actively, so that the muscles must be the great seats of heat-production or thermogenesis in the body. This view is supported by the fact that the blood leaving a muscle at rest contains more $\mathrm{CO}_{2}$ than the blood in the right ventricle. Muscular exercise greatly increases the metabolism and the $\mathrm{CO}_{2}$ excreted $(\$ 127)$, but at the same time, there is a great increase in heat-production. The muscles, therefore, are the great thermogenic tissues, and they yield $\frac{4}{5}$ ths of the heat in health. The several secreting glands, especially the liver and the alimentary canal, during digestion, are also foci of heat-formation.]

(b) In addition to the processes of combustion or oxidation, all those chemical processes in our body, by which the amount of the available potential energy which is present is diminished, in consequence of a greater satisfaction of atomic affinities, lead to the production of heat. In all cases where the atoms assume more stable positions with their affinities satisfied, chemical energy passes into kinetic thermal energy, as in the alcoholic fermentation of grape-sugar, and other similar processes.

Heat is also developed during the following chemical processes :-

(a) During the union of bases with acids. The nature of the base determines the amount of heat produced, while the nature of the acid is without effect. Only in those cases where the acid, e.g., $\mathrm{CO}_{2}$, is unable to set aside the alkaline reaction, the amount of heat produced is less. The formation of compounds of chlorine (e.g., in the stomach) produces heat.

$(\boldsymbol{\beta})$ When a neutral salt is changed into a basic one. In the blood the sulphuric and phosphoric acids derived from the combustion of $\mathbf{S}$ and $\mathbf{P}$ are united with the alkalies of the blood to form basic salts. The decomposition of the carbonates of the blood by lactic and phosphoric acids forms a double source of heat, on the one hand, by the formation of a new salt, and, on the other, by the liberation of $\mathrm{CO}_{2}$, which is partly absorbed by the blood.

$(\gamma)$ The combination of hæmoglobin with $\mathrm{O}(\$ 36)$.

During those chemical processes, whereby the heat of the body is produced, heat-absorbing intermediate compounds are not unfrequently formed. Thus, in 
order that the final stage of more complete saturation of the affinities be reached, intermediary atomic groups are formed, whereby heat is absorbed. Heat is also absorbed when the solid aggregate condition is dissolved during retrogressive processes. But these intermediary processes, whereby heat is lost, are very small compared with the amount of heat liberated when the end-products are formed.

(2) C'ertain physical processes are a second source of heat.-(a) The transformation of the kinetic mechanical energy of internal organs, when the work done is not transferred outside the body, produces heat. Thus the whole of the kinetic enercy of the heart is changed into heat, owing to the resistance opposed to the blood-stream (\$93). The same is true of the mechanical energy evolved by many muscular viscera. The torsion of the costal cartilages, the friction of the current of air in the respiratory organs, and the ingesta in the digestive tract, all yield heat.

An excessively minute amount of the mechanical energy of the heart is transferred to surroumding bodies by the carliac impulse and the superficial pulse-beats, but this is infinifesimally small. During respiration, when the respiratory gases and other substauces are cxpired, a very small amount of energy disappears externally, which does not become changed into heat. If we assume that the daily work of the circulation exceeds 86,000 kilogram-metres, the heat evolved is equal to 204,000 calories, in twenty-four hours (\$93), which is sufficieut to raise the temperature of a person of medium size $2^{\circ} \mathrm{C}$.

(l) When, owing to muscular activity, the body produces work which is transferred to external objects, e.\%., when a man ascends a tower or mountain, or throws a heavy weight, a portion of the kinetic energy passes into heat, owing to friction of the muscles, tendons, and the articular surfaces, as well as to the shock and pressure of the ends of the bones against each other.

(r) The electrical currents which occur in muscles, nerves, and glands very probably are clianged into heat. The chemical processes which produce heat evolve electricity, which is also changed into heat. This source of heat, however, is very small.

(d) Other processes are the formation of heat from the alsointion of $\mathrm{C}\left(\mathrm{O}_{2}\right.$, by the concentration uf "ruter as it passes through membranes, in imbilition, anul the formation of the solicls, e.g., of ihalk in the lones. After death, aud in some pathological processes during life, the coagulation of blood and the production of rigor mortis are sources of heat.

207. HOMOIOTHERMAL AND POIKILOTHERMAL ANIMALS.-In place of the uld classification of animals into "cold-blooded " and "warm-blooded," another basis of classification seems desirable, viz., the relation of the temperature of the body to the temperature of the surrounding medium. Bergmann introduced the word homoiothermal for the warm-blooded animals (mammals and birds), because these animals can maintain a.very uniform temperature, even although the surrounding temperature be subject to considerable variations. The so-called coldblooded animals are called poikilothermal, because the temperature of their bodies rises or falls, within wide limits, with the heat of the surrounding medium.

When homoiothermal animals are kept for a long time in a cold medium, their heat-production is increased, and when they are kept for a long time in a warm medium it is diminished.

Forlyce gave a proof of the nearly uniform temperature in man. A man remained ten minutes in an oven containing very dry hot air (\$218), and yet the temperature of the palm of his hanci, mouth, and urine was increased only a few tenths of a degree. Becquerel and Brechet investigated the temperature of the human biceps (by means of thermo-electric needles), when the arm had been one hour in iced water, and yet the temperature of the muscular tissue was cooleil only $0 \cdot 2^{\circ} \mathrm{C}$. 'The same musele did not unclergo any inerease in temperature, or at most $0.2^{\circ} \mathrm{C}$, when the man's arm was placed for a guarter of an hour in water at $42^{\circ} \mathrm{C}$.

If heat be rapidly abstracted $(\$ 225)$ or rapidly supplied $(\$ 221)$ to the body, so as to produce rajid variation of the temperature, life is endangered. 
Poikilothermal animals behave very differently; the temperature of their bodies generally follows, although with considerable variations, the temperature of the surroundings. When the temperature of the surroundings is increased, the amount of heat produced is increased, and when the surrounding temperature falls, the amount of heat evolved within the body also falls.

The following table shows very clearly the characters of poikilothermal animals, e.g., frogs, which were placed in air and water of varying temperatures. They were immersed up to the mouth. The temperature was measured by neans of a thermometer introduced through the mouth into the stomach.

\begin{tabular}{|c|c|c|c|}
\hline \multicolumn{2}{|c|}{ In Water. } & \multicolumn{2}{c|}{ In Air. } \\
\hline $\begin{array}{c}\text { Temperature of the } \\
\text { Water. }\end{array}$ & $\begin{array}{c}\text { Temperature of Frog's } \\
\text { Stomach. }\end{array}$ & $\begin{array}{c}\text { Temperature of the } \\
\text { Air. }\end{array}$ & $\begin{array}{c}\text { Temperature of Frog's } \\
\text { Stornach. }\end{array}$ \\
\hline $41 \cdot 0^{\circ} \mathrm{C}$. & $38 \cdot 0^{\circ} \mathrm{C}$. & $40 \cdot 4^{\circ} \mathrm{C}$ & $31 \cdot 7^{\circ} \mathrm{C}$. \\
$30 \cdot 0$ & $29 \cdot 6$ & $27 \cdot 4$ & $19 \cdot 7$ \\
$20 \cdot 6$ & $20 \cdot 7$ & $16 \cdot 4$ & $14 \cdot 6$ \\
$5 \cdot 9$ & $8 \cdot 0$ & $6 \cdot 2$ & $7 \cdot 6$ \\
$2 \cdot 8$ & $5 \cdot 3$ & $5 \cdot 9$ & $8 \cdot 6$ \\
\hline
\end{tabular}

[Temperature of Different Animals.

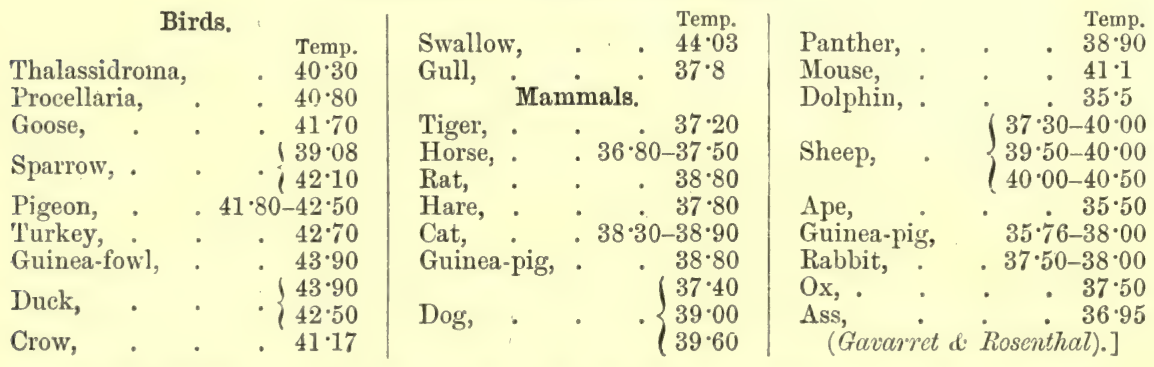

Reptiles-Snakes, $10^{\circ}-12^{\circ}$, but higher when incubating. Amphitians and fishes- $0.5^{\circ}-3^{\circ}$ above the temperature of the surroundings. Arthropoda- $0 \cdot 1^{\circ}-5 \cdot 8^{\circ}$ above the surroundings. Bees in a hive, $30^{\circ}-32^{\circ}$, and when swarming, $40^{\circ}$. The following animals have a temperature higher than the surrounding temperature :-Cephalopods, $0.57^{\circ}$; molluses, $0.46^{\circ}$; echinoderms, $0.40^{\circ}$; medusæ, $0.27^{\circ}$; polyps, $0.21^{\circ} \mathrm{C}$.

208. ESTIMATION OF TEMPERATURE.-By using thermometric apparatus, we are enabled to obtain information regarding the degree of heat of the body to be investigated. For this purpose the following methods are employed:-

A. The Thermometer. - Celsius (1701-1744) divided his thermometer into 100 parts, and each part was again divided into 10 parts, so that $\frac{1}{10}^{\circ} \mathrm{C}$. could be easily real off. All thermometer's which have been used for a loug time give too high readings, hence they should be compared, from time to time, with a normal thermometer. When taking the temperature, the bulb ought to be surrounded for fifteen minutes, and during the last five minutes the mercury column ought not to vary. A very sensitive thermometer will indicate the temperature after seven seconds if the urine stream be directed upou its bulb. Minimal and maximal thermometers are often of use to the physician.

[Clinically, one of the thermometers shown in fig. 228 may be used. They are self-registering maximum thermometers, i.e., a portion of the mercury is separated from the mercurial column, to form the index, the top of which indicates the temperature. Before being used, the index must be well below the normal temperature. Various forms of surface thermometers have been used.]

Walferdin's metastatic thermometer (fig. 229) is specially useful for comparative observation. The tube is very narrow in comparison with the bulb, and in order that the stem be not too long, it is constructed so that the amount of mercury can be varied. A quantity of mercury is taken, so that with the temperature expected the thread of mercury will stand about the middle of the stem. A small bulb at the upper part of the stem receives the excess of $\mathrm{Hg}$. Suppose a temperature between $37^{\circ}-40^{\circ} \mathrm{C}$. is to be measured, the bulb is first heated a little over $40^{\circ} \mathrm{C}$., 
it is then suddenly coolel, and shaken at the same time, so that the thread of mercury is

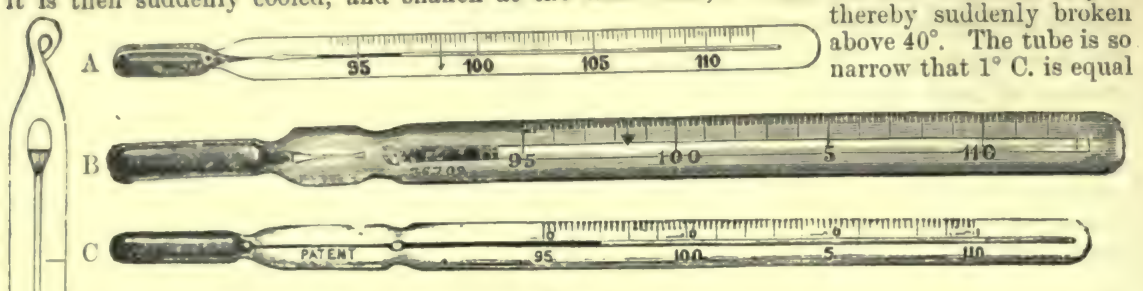

Fig. 228.

A, ('assella's "infallible," B, "Ferris' perfect," and C, Evans' and Wormull's "standard" clinical thermometers.

to ahout 10 centimetres of the length of the tube, so that $\frac{1}{10} 0^{\circ} \mathrm{C}$. is still 1 millimetre in length. The scale is divided empirically, but the value of the divisions must be com.
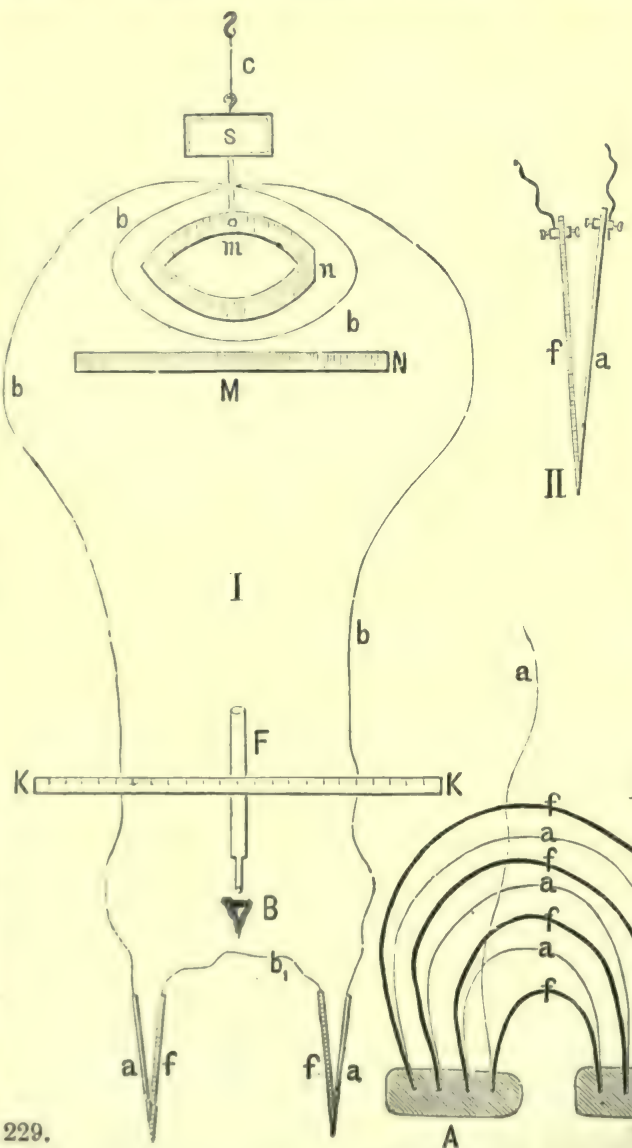

a

Fig. 229.

Walferdin's metastatic

thermo-

meter. pared with a normal thermometer.

Kronecker and Meyer used very small maximal "outflow thermometers," and caused them to pass through the intestinal canal, or through large blood-vessels. The mercury flows out of the short open tube, and of course more flows out the higher the temperature. After these small bulbs have passed throngh the animal, a comparison is instituted with a normal thermo. meter, to determine at what temperature the mercury reaches the free margin of the tube.

B. Thermo-electric Method. - This method enables us to determine the temperature accurately and rapidly (fig. 230, I). The thermo-electric galvanometer of Meissner and Meyerstein consists of a circular magnet $(m)$, suspended by a thread of silk (c), to which a small mirror $(\mathbf{S})$ is attached. $\mathbf{A}$ large stationary bar magnet $(\mathrm{M})$ is placed near the magnet $(m)$, so that the north poles ( $n$ and $\mathrm{N}$ ) of both magnets point in the same direction, and it is so arranged that the suspended magnet is caused to point to the north by a minimal action of M. A thick copper wire $(b, b)$ is coiled several times round $m$ (although in the fig. it is represented as a single coil), and the ends of the wire are soldered to two thermo-elements, each composed of two different metals-iron and 
Terman silver, the two similar free elements being united by a wire $\left(b_{1}\right)$, so that the two therrno-elements form part of a closed circuit. A horizontal scale ( $K, K$ ) is placed at a distance of 3 metres from the mirror, so that the divisions of the scale are seen in the mirror. The scale itself rests upon a telescope $(\mathrm{F})$ directed towards the mirror. The observer (B), who looks through the telescope, can see the divisions of the scale in the mirror. When the magnet, and with it the mirror, swing out of the magnetic meridian, the observer notices other divisions of the scale in the mirror. When one of the thermo-elements is heated, an electrical current is produced, which passes from the iron to the German silver in the heated couple, and causes a deviation of the suspended magnet. Suppose a person were swimming in the direction of the current in the conducting wire, then the north pole of the magnet goes to the north (Ampere). The tangent of the angle $\phi$, through which the freely movable magnet is diverted by a galvanic current, from its position of rest or zero, in the magnetic meridian, is the same as the galvanic stream; $G$ is proportional to the magnetic energy D, i.e., tang. $\phi=\frac{G}{D}$. If $G$ is to remain the same, and the tang. $\phi$ to be as large as possible, the magnetic energy must be diminished as much as possible. If the magnetism of the suspended magnet be indicated by $m$, and that of the earth by $\mathrm{T}$, the magnetic directing energy $\mathrm{D}=\mathrm{T} m$, so that $\mathrm{D}$ can be distinguished in two ways : (1) by diminishing the magnetic moment of the suspended magnet, as may be done by using a pair of astatic needles, such as are used in Nobili's galvanometer; (2) and also by weakening the magnetism of the earth, by placing an accessory stationary magnet (Hauy's rod) in the same direction, and near the suspended magnet. An important arrangement for rapidly getting the magnet to zero is the dead-beat arrangement of Gauss (not figured in the scheme). It consists of a thick copper cylinder, on which the wire of the coil is wound. This mass of copper may be regarded as a closed multiplicator with a very large transverse section. The vibrating magnet induces in this closed circuit a current of electricity, whose intensity is greatest when the velocity of the excursion of the magnet is greatest, and which takes the opposite direction as soon as the magnet returns towards zero. These induced currents cause a diminution of the vibrations of the magnet in this way, that the arc of vibration of the magnet diminishes very rapidly, almost in a geometrical progression. The induced damping-current is stronger, the less the resistance in the closed circuit, and in the damper or dead-beat arrangement itself, the greater the section of the copper ring. This damping arrangement limits the oscillations of the magnet, and it comes to rest rapidly and promptly after 3 or 4 small vibrations, so that much time is saved. The angle of deviation is so small that the angle itself may be taken instead of the tangent.

The thermo-electric needles of Dutrochet (II) may be placed in the circuit. They consist of iron and German silver soldered at their points; or the needles of Beequerel (III) may be used. They consist of the same metals soldered in a straight line, one behind the other. The needles must always be covered by a varnish, which will prevent the parenchymatous juices from acting upon them, and so causing a current. Before the experiment we must cietermine what extent of excursion on the scale is obtained with a certain temperature. In order to determine this, a delicate thermometer is fixed to each of the thermo-couples, and both are placed in oil baths, which differ in temperature-say by $1^{\circ} \mathrm{C}$. - as can be determined by the thermometer. When the current is closed, the excursion on the scale will indicate $1^{\circ} \mathrm{C}$. Suppose that the excursion was $150 \mathrm{~mm}$., then each $\mathrm{mm}$. of the scale would be equal to $\frac{1}{15} 0^{\circ} \mathrm{C}$. When this is determined, the two thermo-needles may be placed in the different tissues or organs of animals, and, of course, we obtain the difference of temperature in these places. Or one thermo-couple may be placed in a bath of constant temperature (nearly that of the body), in which is placed a delicate thermometer, while the other needle is introduced into the organ to be investigated. In this case, we obtain the difference of temperature between the tissue and the source of the constant heat. The electric current passes in the warmer needle from the iron to the German silver, and thus through the wires of the apparatus. For small differences of temperature, such as occur in the body, the thermo-electric energy is always proportional to the difference of temperature of the two needles or couples. In place of a single pair of needles several may be used, whereby the sensitiveness of the apparatus. is greatly increased. Helmholtz found that by using sixteen antimony-bismuth couples, he could detect an increase of $\frac{1}{4000}{ }^{\circ} \mathrm{C}$. Schiffer prepared a simple thermopile (IV) by soldering together alternately four pairs of wires of iron $(f)$ and German silver $(a)$. These are placed in the two organs (A and B) which are to be investigated, whereby a very high degree of exactness is obtained.

209. TEMPERATURE TOPOGRAPHY.-Although the blood, in virtue of its continual motion (completing, as it does, the circulation in twenty-three seconds), must exercise a very considerable influence on the equilibration of the temperature in different organs, nevertheless, a completely uniform temperature does not exist, and the temperature varies in different parts :- 
1. Skin $(J$. Davy).

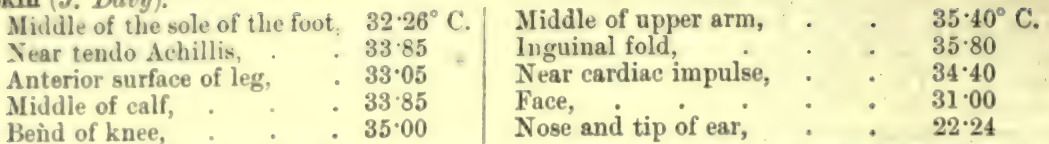

In the closed axilla, 36.49 (mean, of 505 individuals); -36.5 to $37 \cdot 25$ (Wunderlich); $-36.89^{\circ} \mathrm{C}$.

(Liclurmeistr). The skin over muscles is warmer than that over bone (Kunkel).

The temprature of the skin of the head is higher in the forehead and parietal region than in the ocipital region; the skin on the left side of the head is warmer than on the right. Drspnnea increases the temperature of the skin.

Method. - Liehermeister determines the temperature of free cutaneous surfaces thus:-The bulh of the thermometer is heated slightly above the temperature expected; after the mereury heegins to fall, the bulb is placed on the skin, and if the bulb has the same temperature as the skin, the mereury remains stationary. This experiment must be repeated several times.

2. Cavities.

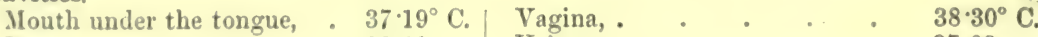

Rectum, . . 38.01 Urine, . . . . 37.03

Uterine cavity somewhat warmer ; cervical canal of the uterus somewhat cooler.

The temperature falls in the stomach during digestion $(\S 166,1)$. Cold injections $\left(11^{\circ}\right.$ (') into the rectum rapidly lower the temperature in the stomach $1^{\circ} \mathrm{C}$. (Winternitz).

3. The temperature of the blood is, as a mean, $39^{\circ} \mathrm{C}$. The venous blood in internal viscera is warmer than the arterial, but it is cooler in peripheral parts :-

Blood of the right heart, . . $38 \cdot 8^{\circ}$

left heart, . $\quad 38 \cdot 6$

aorta,

hepatic vein,

38.7

$39 \cdot 7$

Blood of the superior vena cava, .

$36.78^{\circ}$

,

The lower temperature of the blood in the Fin. According to Heidenhain and hirner, the right heart is slightly warmer because it lies in relation with the warm liver, whilst the left heart is surrounderl by the lung, which contains air. This observation is disputed by others, who say that the left heart is slightly warmer because the combustion-processes are more active in arterial blood, and heat is evolved during the formation of oxyhæmoglobin. The blood in the veins is usually cooler than in the corresponding arteries, owing to the superficial position of the former, whereby they give off heat during their long course ; thus the blood of the ingulue vein is $\frac{1}{2}$ to $2^{\circ} \mathrm{C}$. lower than the blood in the carotid; the crural vein $\frac{3}{4}$ to $1^{\circ}$ cooler than in the crucal cirtery. Superficial veins, more especially those of the skin, give off much heat, and their blood is, therefore, somewhat cooler. The warmest blood is that of the hepatic vein, 39.7 C., partly owing to the great chemical changes which occur within the liver, from its secretory activity $(\$ 210, a)$, and partly to its protected situation.

4. The individual tissues are warmer: (1) the greater the transformation of kinetic energy into heat, i.e., the greater the tissue-metabolism; (2) the more blood they contain; (3) and the more protected their situation. According to Heidenhain and Körner, the cerebrum is the warmest organ in the body.

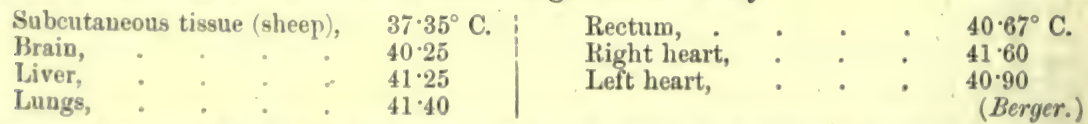

Becyuerel and Brechet found the temperature of the human subeutaneous tissue to be $2 \cdot 1^{\circ} \mathrm{C}$. lower than that of the neighbouring inuscles. The horny tissues do not produce heat, and their low temperature is due to the conduction of heat from the parts on which they grow. The temperature of the cornea partly depends on that of the iris, and the more contracted the pupil is, the more heat it receives from the blood-vessels of the iris.

210. CONDITIONS AFFECTING THE TEMPERATURE OF ORGANS.The temperature of the individual organs is by no means constant; it is influenced by many conditions ; amongst these are the following :-

(1) The more heat produced independently within a pait, the higher is its temperature. As the amount of heat produced within a part depends upon its metabolism, 
therefore, when the metabolism is increased, the amount of heat produced is similarly increased.

(a) Glands produce more heat during the act of secretion, as is proved by the higher temperature of their secretion, or by the higher temperature of the venous blood flowing out of their veins.

Ludwig found that when he stimulated the chorda tympani, the saliva of the submaxillary gland was $1.5^{\circ} \mathrm{C}$. warmer than the blood in the carotid, which supplied the gland with blood (p. 213). The blood in the renal vein in a kidney which is secreting is warmer than the blood in the renal artery. The secreting liver produces much heat $(\$ 178) . \quad \mathrm{Cl}$. Bernard investigated the temperature of the blood of the portal and hepatic veins during hunger, at the beginning of digestion, and when digestion was most active, and he found :-

Temperature of portal vein, . $37.8^{\circ} \mathrm{C}$. After 4 days

" hepatic vein, : 38.4$\}$ starvation.

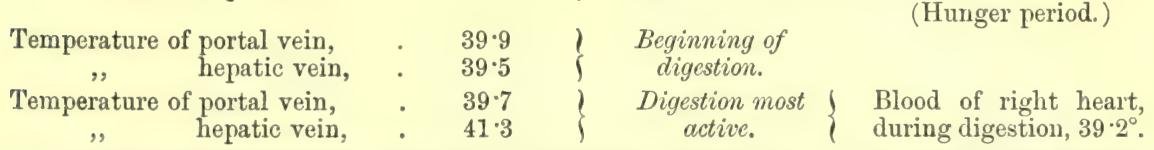

In the dog a moderate diet, chemical or mechanical stimulation of the gastric mucous membrane, or even the sight of food, raises the temperature in the stomach and intestine.

(b) When the muscles contract, they evolve heat. Davy. found that an active muscle became $0.7^{\circ} \mathrm{C}$. warmer; while Becquerel, by means of a thermo-galvanometer, found that human muscles, when kept contracted for five minutes, became $1^{\circ} \mathrm{C}$. warmer (\$ 302).

This is one of the reasons why the temperature may rise above $40^{\circ}$ during rapid running. A temperature obtained by energetic muscular action usually does not fall to the normal until after resting for $1 \frac{1}{2}$ hour. The low temperature of paralysed limbs depends partly upon the absence of the muscular contractions.

(c) With regard to the effect of sensory nerves upon the temperature, some of the chief points to ascertain are-whether the circulation is accelerated or retarded by their stimulation, or whether the respiration is increased or diminished $(\$ 214$, II., 3), and whether the muscles of the skelcton are relaxed or contracted reflexly $(\$ 214, \mathrm{I}, \mathrm{,} 3)$. In the former case the temperature of the interior and rectum is increased; in the latter, diminished.

(d) The temperature of the body rises during mental exertion. Davy observed an increase of $0.3^{\circ} \mathrm{C}$. after vigorous mental exertion.

(e) The parenchymatous fluids, serous fluids, and lymph produce little heat, owing to their feeble metabolism, hence they have the same temperature as their surroundings; the epidermal and horny tissues do not produce heat, they merely conduct it from subjacent structures.

(2) The temperature depends, to a large extent, upon the amount of blood in an organ, and also upon the rapidity with which the blood is renewed by the circulation. This is best observed in the difference of the temperature between a cold, pale, bloodless hand, and a warm, red, congested one.

Becquerel and Brechet found that the temperature of the human biceps fell several tenths of a degree, when the axillary artery was compressed. Ligature of the crural artery and vein in a dog causes a fall of several degrees. If the extremities be kept suspended in the air, they become bloodless and cold.

Liebermeister has pointed out a difference with regard to the external and internal parts of the body. The external parts give off more heat than they produce, so that they become cooler the more slowly new blood flows into them, and warmer the greater the rapidity of the bloodstream through them. Acceleration of the blood-stream, therefore, causes the temperature of peripheral parts to approximate more and more to the temperature of internal organs, while retardation of the blood-stream causes them to approach the temperature of the surrounding medium. Exactly the reverse is the case with internal parts, where a large amount of heat is produced, and heat is given up almost alone to the blood which flows through them. Their temperature must fall when the blood-stream through them is accelerated, and it is raised when the blood-stream is retarded. Hence it follows, that the greater the difference of the temperature between peripheral and internal parts, the slower must be the velocity of the circulation. 
(3) If the position of an organ be such, or if other conditions cause it to give off heat by conduction or radiation, then its temperature falls.

A good example of this is the skin, which varies greatly in temperature according to the temperature of the surrounding medium, whether it is covered or uncovered, whether it is dry or moist with sweat (which abstracts heat when it evaporates). When much cold food or drink is taken, the stomach is cooled, and when ice-cold air is breathed, the respiratory passages as far as the bronchi are cooled.

211. ESTIMATION OF HEAT.-Calorimetry is the method of determining the amount of heat possessed by any body, or what amount of heat it is capable of producing. The unit of measurement is the "heat-unit," i.e., the amount of heat (or potential energy) required to raise the temperature of 1 gramme of water $1^{\circ} \mathrm{C}$. (see Introduction).

Expreriment has shown that equal quentitics of different substances require very unequal "menunts of heut to raise them to the same temperature, e.g., 1 kilo. water requires nine times as much heat as 1 kilo. iron to raise it to the same temperature. In the human body, therefore, which is composed of very different substances, unequal amounts of heat will be required to raise them all to the same temperature. The same amount of heat transferred to two different substances will raise them to different temperatures. Hence, bodies of different temperatures may contain equal amounts of heat. The amount of heat required to raise a definite quantity $(c \%, 1$ grm. $)$ of a sulstance to a certain higher degree (e.g., $1^{\circ} \mathrm{C}$.) is called "specific heat." The sprecific heat of water (which of all bodies has the highest specific heat) is taken as $=1$. By "heat-capacity" is meant, that property of bodies in virtue of which they must absorb a given amount of heat in order to have a certain temperature.

Calorimetry is employed:-I. To letermine the specific heat of the different organs of the burly.-Only a few observations have been made. The mean specific heat of the following animal parts (water $=1$ ) is :-

\begin{tabular}{ll|l|l|l|} 
Human blood $=1.02(?)$ & Human muscle $=0.741$ & Fat tissue & $=0.712$ \\
Arterial blood $=1.031(?)$ & Ox muscle & $=0.787$ & Striped muscle $=0.825$ \\
Venous blood $=0.892(?)$ & Compact bone $=0.3$ & Defibrinated blood = $=0.927$ \\
Cow's milk $=0.992$ & Spongy bone $=0.71$ & & (J. Rosenthal.)
\end{tabular}

The specific heat of the human body, as a whole, is about that of an equal volume of water (?).

Kopp's Method. - The soliel to be investigated is broken in pieces about the size of a pea, and placed in a test-tube, A, with thin walls, which is closed above with a cork, from which a

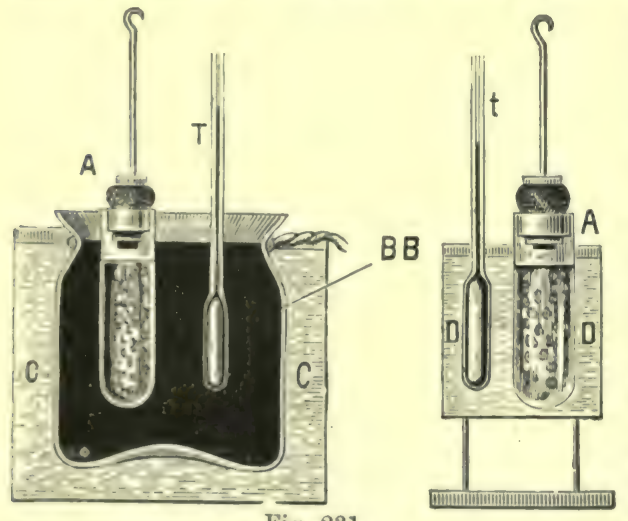

Fig. 231. copper wire with a hook on it projects (fig. 231). The test-tube contains a certain quantity of fluid which does not dissolve the substance, but which lies between its pieces and covers it. It is weighed three times to ascertain the weight (1) of the empty glass, (2) after it is filled with the solid substance, (3) after the fluid is added, so that we obtain the weight of the solid substance, $m$, and that of the fluid, $f$. The test-tube and its contents are placed in a mercury bath, BB, and this again in an oil bath, CC, and the whole is raised to a high temperature. Into BB there is introduced a fine thermometer, $\mathrm{T}$. When the tube, A, has reached the necessary tem. perature (say $40^{\circ}$ ) it is rapidly placed in the water of the accompanying calorimeter-box, DD. The water in this box, which also contains a thermometer, D, is kept in motion until it has completely

T represent the temperature to which $\mathbf{A}$ and its contents were raised in the mercury bath, and

Kopl's apparatus for estimating specific heat. $\mathrm{T}_{1}$ the temperature to which it fell in the calorimeter; let $s$ be the specific heat, and $m$ the weight of the solid substance in the test-tube, while $\sigma$ and $\mu$ represent the specific heat of the weight of the interstitial fluid in the test-tube; and lastly, let $w$ equal the amount of water in contact with $\mathrm{A}$, which absorbs and gives off heat; then W represents the amount of heat which the test-tube and its contents give off during cooling. 


$$
\mathrm{W}=(s . m+w+\sigma \mu)\left(\mathrm{T}-\mathrm{T}_{1}\right) .
$$

The amount of heat, $\mathrm{W}_{1}$, absorbed by the calorimeter is

$$
\mathrm{W}_{1}=\mathbf{M}\left(t_{1}-t\right) \text {, }
$$

where $\mathrm{N}$ represents the amount of water in the calorimeter, $t$ the original temperature of the water in the calorimeter, and $t_{1}$ the temperature to which it is raised by placing $\mathrm{A}$ in it. If $\mathrm{W}$ and $\mathrm{W}_{1}$ are equal, then

$$
\text { the specific heat, } s=\frac{\mathrm{M}\left(t_{1}-t\right)-(w+\sigma \cdot \mu)\left(\mathrm{T}-\mathrm{T}_{1}\right)}{m\left(\mathrm{~T}-\mathrm{T}_{1}\right)} .
$$

If a fluid substance is placed in the test-tube, and its weight $=m$, and its specific heat $=s$, the formula for the specific heat of the fluid to be investigated is

$$
s=\frac{\mathrm{M}\left(t_{1}-t\right)-w\left(\mathrm{~T}-\mathrm{T}_{1}\right)}{m\left(\mathrm{~T}-\mathrm{T}_{1}\right)} .
$$

II. Calorimetry is more important for determining the amount of heat produced in a given time by the body as a whole, or by its individual parts.

Lavoisier and Laplace made the first calorimetric observations on animals in 1783, by means of an ice-calorimeter ; a guinea-pig melted $13 \mathrm{oz}$. of ice in ten hours. Crawford, and afterwards Dulong and Despretz, used Rumford's water-calorimeter, which is similar to Favre and Silbermann's. Small animals are placed in the inner thin-walled copper chamber $(\mathrm{K})$, which is placed in a water-bath surrounded on all sides by some non-conducting material. We require to know the amount of water, and its original temperature. The number of calories is obtained from the increase of the temperature at the end of the experiment, which lasts several hours. The air is supplied to the animal through a special apparatus, resembling a gasometer. The amount of $\mathrm{CO}_{2}$ in the gases evolved is estimated.

According to Despretz, a bitch forms 14,610 heat-units per hour-i.e., 393,000 in twenty-four hours. Other things being equal, a man seven times heavier than this would produce in twenty-four hours about 2,750,000 calories. Senator found that a dog weighing 6330 grms. produced 15,370 calories, and excreted at the same time 367 grms. $\mathrm{CO}_{2}$. The first calorimetric experiments on man were made by Scharling (1849). Liebermeister estimated the amount of heat given off by a man placed in a cold bath, which was surrounded with a woollen covering. Leyden placed a lower limb in the calorimeter, whereby 6000 grms. water were raised $1^{\circ}$ C. in an hour. If we assume that the total superficial area of the body is fifteen times greater than that of the leg, the human body would produce $2,376,000$ calories in twenty-four hours.

212. THERMAL CONDUCTIVITY OF TISSUES. - The thermal conductivity of animal tissues is of special interest in connection with the skin and subcutaneous fatty tissue. The fatty layer under the skin, more especially in the whale, walrus, and seal, forms a protective covering, whereby the conduction of heat from internal organs is rendered almost impossible. Investigations upon this subject, however, are few. Griess attempted to estimate the thermal conductivity by heating one part of the tissue, and determining when and in what direction pieces of wax placed on the tissue to be investigated began to melt. He investigated the stomach of the sheep, the bladder, skin, hoof, horn, and bones of an ox, deer's horn, ivory, mother-of-pearl, shell of haliotis. He found that fibrous tissues conducted heat more readily in the direction of their fibres than at right angles to the course of the fibres. Hence, the figures obtained from the melted wax were usually elliptical. Landois has made similar observations, and he finds that tissues conduct better in the direction of their fibres. After bones, blood-clot was the best conductor, then followed spleen, liver, cartilage, tendon, muscle, elastic tissue, nail and hair, bloodless skin, gastric mucous membrane, washed fibrin. It is specially interesting to note how much better skin containing blood in its blood-vessels conducts than does bloodless skin. Hence little heat is given off from a bloodless skin, while congested skin conducts and gives off much more heat.

Like all other substances, the human body is enlarged by heat. A man weighing 60 kilos., and whose temperature is raised from $37^{\circ} \mathrm{C}$. to $40^{\circ} \mathrm{C}$., is enlarged about 62 cubic centimetres. Connective-tissue (tendon) is extended by heat, while elastic tissue and the skin, like caoutchouc, are contracted.

213. VARIATIONS OF THE MEAN TEMPERATURE-(1) General Climatic and Somatic Influences.-In the tropics the mean temperature of the body is about $\frac{1}{2}$ C. higher than in temperate climates, where again it is several tenths of a degree warmer than in cold climates; but this has recently been 
denied. The difference is comparatively trivial, when we remember that a man is subjected to a rariation of over $40^{\circ} \mathrm{C}$. in passing from the equator to the poles. Observations on more than 4000 persons show that when a person goes from a warm to a cold climate, his temperature is but slightly diminished, but when he goes from a cold to a warm climate his temperature rises relatively considerably more. In the temprerate zone, the temperature of the body during a cold winter is usually $0 \cdot 1$ to $0 \cdot 3^{\circ} \mathrm{C}$. lower than it is on a warm summer day. The elevation of a place above sea-level has no obvious effect on the temperature of the body. There seems to be no difference in different races, nor in the sexes, other conditions heing the same. Persons of powerful physique and constitution are said to have generally a slightly higher temperature than feeble, weak, anæmic persons.

() Influence of the General Metabolism.-As the formation of heat depends upon the transformation of chemical compounds, whose chief final products, in addition to $\mathrm{H}_{2} \mathrm{O}$, are $\mathrm{CO}_{2}$ and urea, the amount of heat formed must go pari passu with the amount of these excreta. The more rapid metabolism which sets in after a full meal causes a rise of temperature to several tenths of a degree ("Digestionfever "). As the metabolism is much diminished during hunger, this explains why the mean temperature in a fasting man is $36.6^{\circ}$, while it is $37.17^{\circ}$ on ordinary days $(\$ 237)$.

Jurgensen also found that the temperature fell on the first day of inanition (although there was a temporary rise on the second day). In experiments made upon starving animals, the temperature at first fell rapidly, then remained constant for a considerable time, while during the last dilys it fell considerably. Schmidt starved a cat-on the 15 th day the temperature was $39 \cdot 6^{\circ}$; on the 16 th, $38 \cdot 3^{\circ} ; 17$ th, $37 \cdot 64^{\circ} ; 18 \mathrm{th}, 35 \cdot 8^{\circ} ; 19$ th $($ death $)=33 \cdot 0^{\circ}$. Chossat found that starving mammals and birds had a temperature $16^{\circ} \mathrm{C}$. below normal on the day of their death.

(3) Age has a decided effect upon the temperature of the body. The extent of the general metabolism is in part an index of the heat of the body at different ages, but it is possible that other, as yet unknown, influences are also active.

\begin{tabular}{|c|c|c|c|}
\hline Age. & $\begin{array}{l}\text { Mean Temperature at the } \\
\text { Ordinary Temperature. }\end{array}$ & Normal Limits. & Where Measured. \\
\hline $\begin{array}{r}\text { Newly-born, } \\
5-9 \text { year, } \\
15-20 \quad, " \\
21-30 \quad, \\
25-30 \quad, \\
31-40 \quad, \\
41-50, \\
51-60 \quad, \\
80, ",\end{array}$ & $\begin{array}{l}37 \cdot 45^{\circ} \mathrm{C} . \\
37 \cdot 72 \\
37 \cdot 37 \\
37 \cdot 22 \\
36 \cdot 91 \\
37 \cdot 1 \\
36 \cdot 87 \\
36 \cdot 83 \\
37 \cdot 46\end{array}$ & $\begin{array}{c}37 \cdot 35-37 \cdot 55^{\circ} \mathrm{C} . \\
36 \cdot 87-37 \cdot 62 \\
36 \cdot 12-38 \cdot 1 \\
\ldots \\
36 \cdot 25-37 \cdot 5 \\
\ldots \\
\ldots\end{array}$ & $\begin{array}{c}\text { Rectum. } \\
\text { Mouth and Rectum. } \\
\text { Axilla. } \\
\text {," } \\
\text {," } \\
\text { ", } \\
\text { Mouth. }\end{array}$ \\
\hline
\end{tabular}

Newly-born animals exhibit peculiarities owing to the sudden change in their conditions of existence. Immediately after birth, the infant is $0.3^{\circ}$ warmer than the vagina of the mother, viz., $37 \cdot 86^{\circ}$. A short time after birth, the temperature falls $0.9^{\circ}$, while twelve to twenty-four hours afterwards, it has risen to the normal temperature of an infant, which is $37 \cdot 45^{\circ}$. Several irregular variations occur during the first weeks of life. During sleep, the temperature of an infant falls $0.34^{\circ}$ to $0.56^{\circ}$, while continued crying may raise it several tenths of a degree. Old people, on account of their feeble metabolism, produce little heat; they become cold sooner, and hence ought to wear warm clothing to keep up their temperature.

(4) Periodical Daily Variations.-In the course of twenty-four hours there are regular periodic variations in the mean temperature, and these occur at all ages. As a general rule, the temperature continues to rise during the day (maximum at 5 to 8 P.M.), while it continues to fall during the night (minimum 2 to 6 A.M.). The mean temperature occurs at the third hour after breakfast. 
The mean height of all the temperatures taken during a day in a patient is called the "daily mean," and according to Jaeger it is $37.13^{\circ}$ in the rectum in

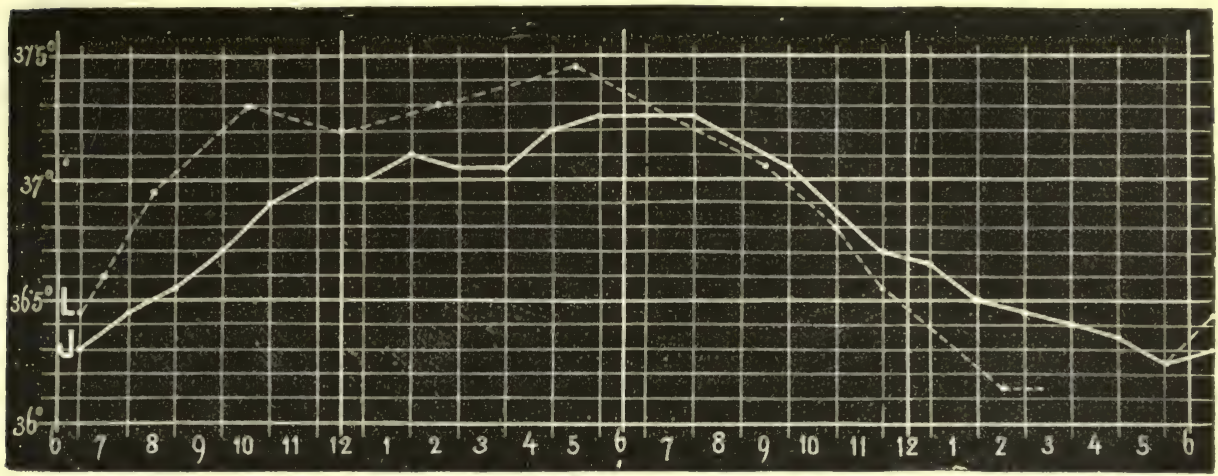

Fig. 232.

Variations of the daily temperature in health during twenty-four hours. Lafter Liebermeister; J___, after Jürgensen.

health. A daily mean of more than $37 \cdot 8^{\circ}$ is a "fever temperature," while a mean under $37 \cdot 0^{\circ} \mathrm{C}$. is regarded as a "collapse temperature."

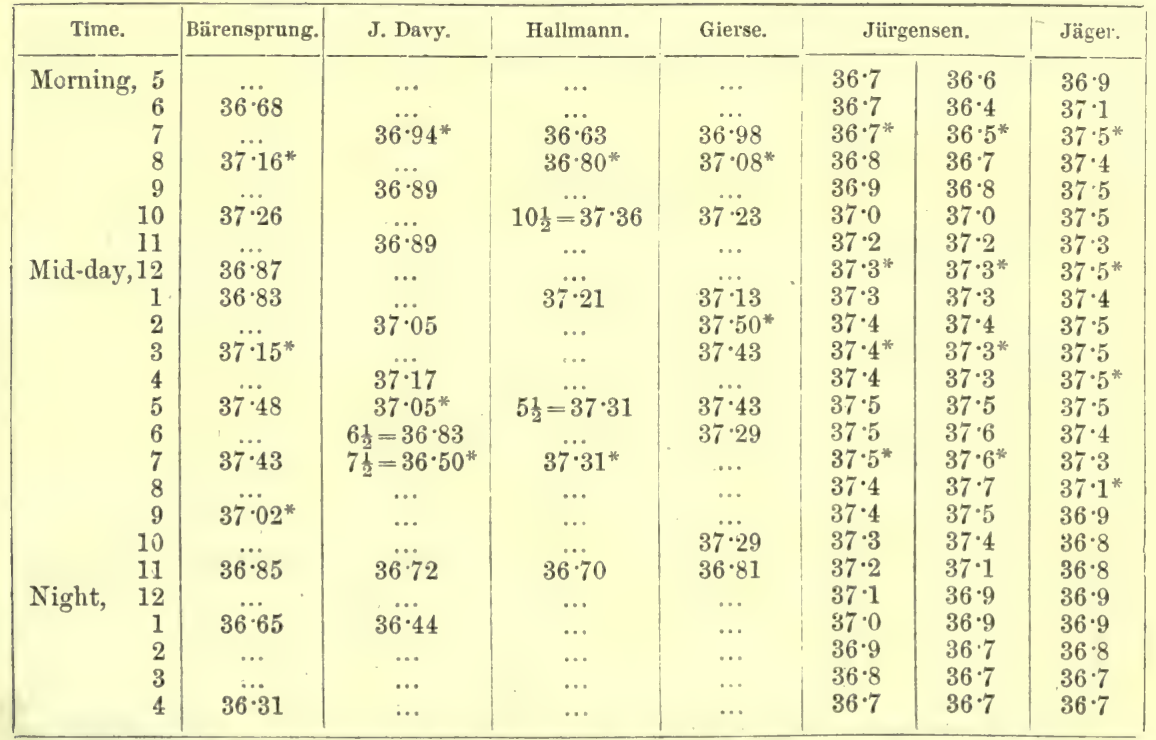

As the variations occur when a person is starved for a day-although those that occur at the periods at which food ought to have been taken are less-it is obvious that the variations are not due entirely to the taking of food. [The * indicatés taking of food.]

The daily variation in the frequency of the pulse often coincides with variation of the temperature. Bärensprung found that the mid-day temperature maximum slightly preceded the pulse maximum $(\$ 70,3, \mathrm{C})$.

If we sleep during the day, and do all our daily duties during the night, the above described typical course of the temperature is reversed. With regard to the effect of activity or rest, it appears that the activity of the muscles during the day tends to increase the mean temperature slightly, while at night the mean temperature is less than in the case of a person at rest. 
The peripheral parts of the body exhibit more or less regular variations of their temperature. In the palm of the hand, the progress of events is the following:-After a relatively high night temperature there is a rapid fall at $6 \mathrm{~A} . \mathrm{M}$., which reaches its minimum at 9 to $10 \mathrm{A.M}$. This is followed by a slow rise, which reaches a high maximum after dinner; it falls between 1 to 3 P.M., and after two or three hours reaches a minimum. It rises from 6 to 8 P.M., and falls again towards morning. A rapid fall of the temperature in a peripheral part corresponds to a rise of temperature in internal parts.

(5) Many operations upon the body affect the temperature. After hæmorrhage the temperature falls at first, but it rises again several tenths of a degree, and is neually accompanied by a shiver or slight rigor; several days thereafter it falls to normal, and may even fall somewhat below it. The sudden loss of a large amount of bloo 3 causes a fall of the temperature of $\frac{1}{2}$ to $2^{\circ} \mathrm{C}$. Very long-continued hæmorrhage (dog) causes it to fall to $31^{\circ}$ or $29^{\circ} \mathrm{C}$.

This is obriously lue to the diminution of the processes of oxidation in the ansemic body, and to the enfeebled circulation. Similar conditions causing diminished metabolism effect the same result. Continued stimulation of the peripheral end of the vagus, so that the heart's action is enormously slowed, diminishes the temperature several degrees in rabbits (Lanulois and Ammon).

The transfusion of a considerable quantity of blood raises the temperature ahout half an hour after the operation. This gradually passes into a febrile attack, which disappears within several hours. When blood is transfused from an artery to a vein of the same animal, a similar result occurs (\$102).

(6) Many poisons diminish the temperature, e./., chloroform and the anæsthetics, alcohol (\$235), digitalis, quinine, aconitin, muscarin. These appear to act partly by rendering the tisiues less liable to undergo molecular transformations for the prodluction of heat. In the case of the anresthetics, this effect perhaps occurs, and is due possibly to a semi-coagulation of the nervous substance (?). They may also act partly by influencing the giving off of heat (§214, II.). Other poisons increase the temperature for opposite reasons.

The temprerature is increased by strychnin, nicotil, picrotoxin, veratrin, laudanin.

(7) Various diseases diminish the temperature, which may be due either to lessened production of heat (liminution of the metabolism), or to increased expenditure of heat. Loewenharit found that in paralyties and in insane persons, several weeks before their death, the rectal temperature was $30^{\circ}$ to $31^{\circ} \mathrm{C}$., in diabetes $30^{\circ} \mathrm{C}$. or less ; the lowest temperature observed and life retained, in a drunk person was $24^{\circ} \mathrm{C}$.

The temperature is increased in fever, and the highest point reached just before death, and recorded by Wunderlich, was $44 \cdot 65^{\circ} \mathrm{C}$. (compare $\$ 220$ ).

214. REGULATION OF THE TEMPERATURE.-As the bodily temperature of man and similar animals is nearly constant, notwithstanding great variations in the temperature of their surroundings, it is clear that some mechanism must exist in the body, whereby the heat economy is constantly regulated. This may be brought about in two ways; either by controlling the transformation of potential energy into heat, or by affecting the amount of heat given off according to the amount produced, or to the action of external agencies.

[The constancy or thermostatic condition of the temperature is brought about by three co-operant factors, the thermogenic or heat-producing, the thermolytic or heat-discharging, and the thermotaxic or mechanism by which heat-production and heat-loss are balanced, and it is obvious that the last must be in relation with the other two. The thermotaxic mechanisns is developed last, is least pronounced in the lower vertebrata, and is most easily liable to fail under injury or disease (MacAlister).]

I. Regulatory Arrangements governing the Production of Heat.-Liebermeister estimated the amount of heat produced by a healthy man at 1.8 calorie per minute. It is highly probable that, within the body, there exist mechanisms which determine the molecular transformations, upon which the evolution of heat depends. This is accomplished chiefly in a reflex manner. The peripheral ends of 
cutaneous nerves (by thermal stimulation), or the nerves of the intestine and the digestive glands (by mechanical or chemical stimulation during digestion or inanition), may be irritated, whereby impressions are conveyed to the heat-centre, which sends out impulses through efferent fibres to the depôts of potential energy, either to increase or diminish the extent of the transformations occurring in them. The nerve channels herein concerned are entirely unknown. Many considerations, however, go to support such an hypothesis ( $\$ 377$ ).

[Thermotaxic Mechanism, Thermal Nerves and Centres.-Just as the respiration and the state of the blood-vessels are regulated from a central focus, so the question arises, does the same obtain with regard to temperature. Studying this question, however, it must be borne in mind that thermometric observations alone are not sufficient; the true test must be calorimetric. Sir Benjamin Brodie observed that in a case of injury of the spinal cord in the neck the temperature in the thigh rose very high. In some cases the temperature falls. Wood has shown that section of cord above the origin of the splanchnics leads to decided increase in the amount of heat dissipated, but to a decided diminution of heat-production. The vaso-motor paralysis has much to do in these cases with the loss of heat. In warm-blooded animals, exposed to a high temperature, the heat-production is diminished, but when they are exposed to a low temperature it is increased. If a warm-blooded animal's medulla oblongata be divided, there is a fall of temperature, chiefly due to its vaso-motor paralysis, and such an animal behaves, as regards the effect of heat and cold, exactly like a poikilothermal animal, i.e., its metabolism and heat-production are increased by cold and diminished by heat. If, however, the incision be made above the pons, so as to leave the vaso-motor centre intact in the dog, there is a rise of the temperature and increased heat-production for 24 hours afterwards. This suggests the idea that this region is traversed by inhibitory nerves, so that when they are cut off from their centres situate above, the augmentor nerves can act more vigorously. This suggests the existence of thermo-inhibitory centres situate higher up in the brain. If an animal be curarised, not only is there paralysis of voluntary motor acts, but on stimulating an ordinary motor nerve, not only is there no muscular contraction, but there is no rise of temperature of the muscles supplied by that nerve. In such an animal the temperature rises and falls with the temperature of the surrounding medium. Even although the respirations be kept constant and the vaso-motor nerves intact, the thermogenic activity of muscles, therefore, seems to be dependent on their innervation.]

[Cerebral Centres. - Apart from the cortical heat centres (\$ 377), Ott, Aronsohn, Sachs, Richet and others have shown that if a needle be thrust through the skull and brain, so as to injure certain deeper-seated parts, there is a rise of temperature and increased heat-production for several hours. The experiment may be repeated several times in the same rabbit. Ott gives three areas which, when so injured, cause these effects-(1) a part of the brain in the inedian side of the corpus striatum, and near the nodus cursorius ; (2) a part between the corpus striatum and the optic thalamus; and (3) the anterior end of the optic thalamus itself. From the effect of atropin, Ott suggests the existence of spinal centres as well.]

The following phenomena indicate the existence of mechanisms regulating the production of heat :-

(1) The temporary application of moderate cold raises the bodily temperature, while heat, similarly applied to the external surface, lowers it ( $\$ 222$ and 224).

(2) Cooling of the surroundings increases the amount of $\mathrm{CO}_{2}$ excreted, by increasing the production of heat, while the $\mathrm{O}$ consumed is also increased simultaneously; heating the surrounding medium diminishes the $\mathrm{CO}_{2}(\S 127,5)$.

D. Finkler found, from experiments upon guinea-pigs, that the production of heat was more than doubled when the surrounding temperature was diminished $24^{\circ} \mathrm{C}$. The metabolism of the guinea-pig is increased in winter 23 per cent. as compared with summer, so that the same relation obtains as in the case of a diminution of the surrounding temperature of short duration.

C. Ludwig and Sanders-Ezn found that in a rabbit there was a rapid increase in the amount of $\mathrm{CO}_{2}$ given off, when the surroundings were cooled from $38^{\circ}$ to $6^{\circ}$ or $7^{\circ} \mathrm{C}$.; while the excretion was diminished when the surrounding temperature was raised from $4^{\circ}-9^{\circ}$ to $35^{\circ}-37^{\circ}$, so that the thermal stimulation, due to the temperature of the surrounding medium, acted upon the combustion within the body. Pfliger found that a rabbit which was dipped in cold water used more $\mathrm{O}$ and excreted more $\mathrm{CO}_{2}$.

If the cooling action was so great as to reduce the bodily temperature to $30^{\circ}$, the exchange of gases diminished, and where the temperature fell to $20^{\circ}$, the exchange of gases was diminished one-half. It is to be remembered, however, that the excretion of $\mathrm{CO}_{2}$ does not go hand in hand with the formation of $\mathrm{CO}_{2}$. If mammals be placed in a warm bath, which is $2^{\circ}$ to $3^{\circ}$ higher than their own temperature, the excretion of $\mathrm{CO}_{2}$ and the consumption of $\mathrm{O}$ are increased, 
owing to the stimulation of their metabolism, while the excretion of urea is also increased in animals and in $\operatorname{man}(\$ 133,5)$.

(3) Cold acting upon the skin causes involuntary muscular movements (shivering, rigors), and also voluntary movements, both of which produce heat.

The cold excites the action of the muscles, which is connected with processes of oxidation (Pflür. After poisoning with curara, which paralyses voluntary motion, this regulation of the heat falls to a minimum (livilrig and $Z$ unt $z$ ), [ while the bodily temperature rises and falls with a rise or fall in the temperature of the surrounding medium].

(4) Variations in the temperature of the surroundings affect the appetite for food: in winter, and in cold regions, the sensation of hunger and the appetite for the fats. or such substances as yield much heat when they are oxidised, are increased: in summer, and in hot climates, they are diminished. Thus the mean temperature of the surroundings, to a certain extent, determines the amount of the heat-producing substances to be taken in the food.

II. Regulatory Mechanisms governing the Excretion of Heat or Thermolysis. - The mean amount of heat given off by the human skin in twenty-four hours, by a man weighing 8.2 kilos., is 2092 to 2592 calories, i.e., 1.36 to 1.60 per minute.

(1) Heat causes dilatation of the cutaneous vessels; the skin becomes red, congested, and soft; it contains more fluids, and becomes a better conductor of heat; the epithelium is moistened, and sweat appears upon the surface. Thus increased excretion of heat is provided for, while the evaporation of the sweat also abstracts heat.

The amount of heat necessary to convert into vapour $1 \mathrm{grm}$. of water at $100^{\circ} \mathrm{C}$., is equal to that recpuired to heat 10 grms. from $0^{\circ}$ to $53.67^{\circ} \mathrm{C}$. The sweat as secreted is at the temperature of the body; if it were completely changed into vapour, it would require the heat necessary to raise it to the boiling point, and also that necessary to convert it into vapour.

Cold causes contraction of the cutaneous vessels; the skin becomes pale, less soft, poorer in juices, and collapsed; the epithelium becomes dry, and does not permit fluids to yass through it to be evaporated, so that the excretion of heat is diminished. The exretion. of heat from the periphery, and the transverse thermal comluction through the skin, are diminished by the contraction of the vessels and muscles of the skin, and by the expulsion of the well-conducting blood from the cutaneous and subcutaneous vessels. The cooling of the body is very much affected, owing to the diminution of the cutaneous blood-stream, just as occurs when the current through a coil or worm of a distillation apparatus is greatly diminished. If the blood-vessels dilate, the temperature of the surface of the body rises, the difference of temperature between it and the surrounding cooler medium is increased, and thus the excretion of heat is increased. Tomsa has shown that the fibres of the skin are so arranged anatomically, that the tension of the fibres produced by the erector pili muscles causes a diminution in the thickness of the skin, this result being bronght about at the expense of the easily expelled blood.

By the systematic application of stimuli, e.g., cold baths, and washing with cold water, the muscles of the skin and its blood-vessels may be caused to contract, and become so vigorous and excital,le, that when cold is suddenly applied to the body, or to a part of it, the excretion of heat is energetically prevented, so that cold baths and washing with cold water are, to a certain extent, "gymnasties of the cutaneous muscles," which, under the above circumstances, protect the body from cold.

(2) Increased temperature causes increased heart-beats, while diminished temperature diminishes the number of contractions of the heart ( $\$ 58$, II., $a)$. The relatively warm blood is pumped by the action of the heart from the internal organs of the body to the surface of the skin, where it readily gives off heat. The more frequently the same volume of blood passes through the skin-twenty-seven heart-beats being necessary for the complete circuit of the blood-the greater will be the amount of heat given off, and conversely. Hence, the frequency of the heart-beat is in direct relation to the rapidity of cooling. In very hot air (over 
$100^{\circ} \mathrm{C}$.) the pulse rises to over 160 per minute. The same is true in fever $(\$ 70$, $3, c)$. Liebermeister gives the following numbers in an adult:-

$\begin{array}{lcccccc}\text { Pulse-beats, per min., } & 78 \cdot 6 & 91 \cdot 2 & 99 \cdot 8 & 108 \cdot 5 & 110 & 137 \cdot 5 \\ \text { Temperature in } \mathbf{C}^{\circ} ., & 37^{\circ} & 38^{\circ} & 39^{\circ} & 40^{\circ} & 41^{\circ} & 42^{\circ}\end{array}$

(3) Increased Temperature increases the Number of Respirations.-Under ordinary circumstances, a much larger volume of air passes through the lungs when it is warmed almost to the temperature of the body. Further, a certain amount of watery vapour is given off with each expiration, which must be evaporated, thus abstracting heat. Energetic respiration aids the circulation, so that respiration acts indirectly in the same way as (2). According to other observers, the increased consumption of $\mathrm{O}$ favours the combustion in the body, whereby the increased respiration must act in producing an amount of heat greater than normal $(\S 127,8)$. This excess is more than compensated for by the cooling factors above mentioned. Forced respiration produces cooling, even when the air breathed is heated to $54^{\circ} \mathrm{C}$., and saturated with watery vapour.

(4) Covering of the Body.-Animals become clothed in winter with a winter fur or covering, while in summer their covering is lighter, so that the excretion of heat in surroundings of different temperatures is thereby rendered more constant.

Many animals which live in very cold air or water (whale) are protected from too rapid excretion of heat by a thick layer of fat under the skin. Man provides for a similar result by adopting summer and winter clothing.

(5) The position of the body is also important; pulling the parts of the body together, approximation of the head and limbs, keep in the heat; spreading out the limbs, erection of the hairs, pluming the feathers, allow more heat to be evolved. If a rabbit be kept exposed to the air with its legs extended for three hours, the rectal temperature will fall from $39^{\circ}$ C. to $37^{\circ}$ C. Man may influence his temperature by remaining in a warm or a cold room-by taking hot or cold drinks, hot or cold baths-remaining in air at rest or air in motion, e.g., by using a fan.

CLOTHING. - Warm Clothing is the Equivalent of Food.-As clothes are intended to keep in the heat of the body, and heat is produced by the combustion and oxidation of the food, we may say the body takes in heat directly in the food, while clothing prevents it from giving off too much heat. Summer clothes weigh 3 to 4 kilos., and winter ones 6 to 7 kilos.

In connection with clothes, the following considerations are of importance :-

(1) Their capacity for conduction. - Those substances which conduct heat badly keep us warmest. Hare-skin, down, beaver-skin, raw silk, taffeta, sheep's wool, cotton wool, flax, spun-silk, are given in order, from the worst to the best conductors. (2) The capacity for radiation. - Coarse materials radiate more heat than smooth, but colour has no effect. (3) Relation to the sun's rays. - Dark materials absorb more heat than light-coloured ones. (4) Their hygroscopic properties are important, whether they can absorb nuch moisture from the skin and gradually give it off by evaporation, or the reverse. The same weight of wool takes up twice as much as linen; hence, the latter gives it off in evaporation more rapidly. Flannel next the skin is not so easily moistened, nor does it so rapidly become cold by evaporation; hence it protects against the action of cold. (5) The permeability for air is of importance, but does not stand in relation with the heat-conducting capacity. The following substances are arranged in order from the most to the least permeable-flannel, buck-skin, linen, silk, leather, waxcloth.

215. HEAT-BALANCE.-As the temperature of the body is maintained within narrow limits, the amount of heat taken in must balance the heat given off, i.e., exactly the same amount of potential energy must be transformed in a given time into heat, as heat is given off from the body. An adult produces as much heat in half an hour as will raise the-temperature of his body $1^{\circ} \mathrm{C}$. If no heat were given off, the body would become very hot in a short time; it would reach the boiling point in thirty-six hours, supposing the production of heat continued uninterruptedly. The following are the most important calculations on the subject :- 
A. Helmholtz was the first to estimate numerically the amount of heat produced by a man :- Heat-income. - (a) A healthy adult, weighing 82 kilos., expires in twenty-four hours 878.4 grms. $\mathrm{CO}_{2}$ (Scharling). The combustion of

the $\mathrm{C}$ therein into $\mathrm{CO}_{2}$ produces
(b) But he takes in more $\mathrm{O}$ than reappears in the $\mathrm{CO}_{2}^{\cdot}$; the excess is
used in oxidation-processes, e.g., for the formation of $\mathrm{H}_{2} \mathrm{O}$, by union with $\mathrm{H}$, so that 13,615 grms. $\mathrm{H}$ will be oxidised by the excess of $O$, which gives .

(c) About 25 per cent. of the heat must be referred to sources other $1,730,760 \mathrm{cal}$. than combustion (Dulong), so that the total . . . . . . $=2,732,000$,,

$2,732,000$ calories are actually sufficient to raise the temperature of an adult, weighing 80 to 90 kilos., from $10^{\circ}$ to $38^{\circ}$ or $39^{\circ} \mathrm{C}$, i.e., to a normal temperature.

(2) Heat-expenditure. - ( II) Heating the food and drink, which have a mean temperature of $12^{\circ} \mathrm{C}$.

(b) Heating the air respired $=16,400$ grms. with an initial temperature of $20^{\circ} \mathrm{C}$.

( When the temperature of the eir is $0^{\circ}, 140,064 \mathrm{cal} .=5 \cdot 2 \mathrm{per}$ cent.)

(c) Evaporation of 656 grmis. water by the lungs, ${ }^{\text {(d) }}$ The remainder given off by radiation and evaporation of water by the skin, . . . . . $(77 \cdot 5$ per cent. to $)=80 \cdot 1$ 70,157 cal. $=2 \cdot 6$ per cent. $70,032,=2 \cdot 6 \quad$, $397,536 \mathrm{cal} .=14 \cdot 7 \quad$,

B. Dulong. - (1) Heat-income.-Dulong and others sought to estimate the amount of heat from the $\mathrm{C}^{\prime}$ and $\mathrm{H}$ contained in the food. As we know that the combustion of $1 \mathrm{grm} . \mathrm{C}=8040$ heat-units, and $1 \mathrm{grm} . \mathrm{H}=34,460$ heat-units, it would be easy to determine the amount of heat were the $\mathrm{C}$ simply converted into $\mathrm{CO}_{\text {, }}$ and the $\mathrm{H}$ into $\mathrm{H}_{2} \mathrm{O}$. But Dulong omitted the $\mathrm{H}$ in the carbohyldrates $\left(e . g_{0}\right.$, crape-sugar $\left.=\mathrm{C}_{6} \mathrm{H}_{12} \mathrm{O}_{6}\right)$ as producing heat, because the $\mathrm{H}$ is already combined with $\mathrm{O}$, or at least is the proportion in which it exists in water. This assumption is hypothetical, for the atoms of $\mathrm{C}$ in a carbohydrate may be so firmly united to the other atorns, that before oxidation can take place their relations must be altered, so that potential energy is used up, i.e., heat must be rendered latent; so that these considerations rendered the following example of Dulong's method given by Vierordt very problematical :-

An alult eats in twenty-four hours, 120 grms. proteids, 90 grms. fat, and 340 grms. starch (carbohydrates). These contain :-

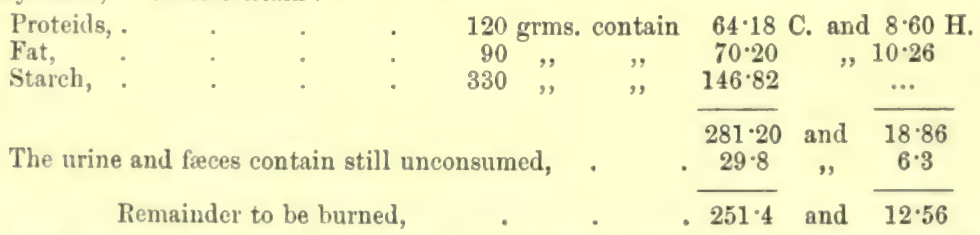

As $1 \mathrm{grm} . \mathrm{C}=8040$ heat-units and $1 \mathrm{grm} . \mathrm{H}=34,460$ heat-units, we have the following calculation:-

$$
\begin{aligned}
251.4 \times 8,040 & =2,031,312 \text { (from combustion of C). } \\
12.56 \times 34,460 & =432,818(,, \quad, \quad H) .
\end{aligned}
$$

(2) Heat-expenditure :-

$2,464,130$ heat-units.

1. 1900 grms. are excreted daily by the urine and freces, and they are $25^{\circ}$ warmer than the food, .

2. $13,000 \mathrm{grms}$. air are heatel (from $12^{\circ}$ to $37^{\circ} \mathrm{C}$.) (heat-capacity of the air $=0.26$ ),

3. 330 grms. water are evaporated by the respiration ( 1 grm. $=58 \dot{2}$ heat-units),

4. 660 grms. water are evaporated from the skin, : : Per cent. of
Heat-units.
the excreta.

Total,

Remainder radiated and conducted from the skin,

$$
47,500
$$
$1 \cdot 9$

C. Heat-income. - Frankland burned the food directly in a calorimeter, and found that $1 \mathrm{grm}$. of the following substances yielded :- 
Albumin, 1 grm.,
Grape-sugar, 1 grm.,
0x fat, 1 grm.,
4998 heat-units.

3217

9069

The albumin, however, is only oxidised to the stage of urea, hence the heat-units of urea must be deducted from 4998 , which gives 4263 heat-units obtainable from $1 \mathrm{grm}$. albumin. When we know the number of grammes consumed, a simple multiplication gives the number of heat-units.

The heat-units will vary, of course, with the nature of the food. J. Ranke gives the following :-

With animal diet,
,,$\quad$ food free from $\mathrm{N}$,
,, mixed diet,
,, during hunger,

$2,779,524$ heat-units.

$2,059,506 \quad$, ,

$2,200,000 \quad$,

$2,012,816$

216. VARIATIONS IN HEAT-PRODUCTION - (1) Influence of Bodily Surface. - Rubner found that the production of heat depended more upon the size of the body and its superficial area than upon the body-weight. Small or young animals have a relatively larger surface than large or older ones, and as the removal of heat takes place chiefly from the external surface, animals with a larger surface must produce more heat. Small animals used relatively more O. Rubner's investigations on dogs of different size gave a heat-production of 1,143,000 calories for every square metre of cutaneous surface. On comparing the body-weight with the cutaneous surface in different animals, he found that for every 1 kilo. of body-weight there was in the rat 1650 , rabbit 946 , man 287 square centimetres of surface.

(2) Age and Sex. - The heat-production is less in infancy and in old age, and it is less in proportion in the female than in the male.

(3) Daily Variation. - The heat-production shows variations in twenty-four hours corresponding with the temperature of the body $(\$ 213,4)$.

(4) The heat-production is greater in the waking condition, during physical and mental exertion, and luring digestion, than in the opposite conditions.

217. RELATION OF HEAT-PRODUCTION TO WORK. - The potential energy supplied to the body may be transformed into heat and kinetic energy (see Introduction). In the resting condition, almost all the potential energy is changed into heat; the workman, however, transforms potential energy into work -mechanical work-in addition to heat. These two may be compared by using an equivalent measurement, thus, 1 heat-unit (energy required to raise 1 gramme of water $1^{\circ}$ C.) $=425.5$ gramme-metres.

Relation of Heat to Work. - The following example may serve to illustrate the relation between heat-production and the production of work:-Suppose a small steam-engine to be placed within a capacious calorimeter, and a certain quantity of coal to be burned, then as long as the engine does not perform any mechanical work, heat alone is produced by the burning of the coal. Let this amount of heat be estimated, and a second experiment made by burning the same amount of coal, but allow the engine to do a certain amount of work-say, raise a weight - by a suitable arrangement. This work must, of course, be accomplished by the potential energy of the heating material. At the end of this experiment, the temperature of the water will be inuch less than in the first experiment, i.e., fewer heat-units have been transferred to the calorimeter when the engine was heated than when it did no work. Comparative experiments of this nature have shown that in the second experiment, the useful work is very nearly proportional to the decrease of the heat (Hirn).

Compare this with what happens within the body:-A man in a passive condition forms from the potential energy of the food between $2 \frac{1}{2}$ to $2 \frac{3}{4}$ million calories. The work done by a workman is reckoned at 300,000 kilogrammemetres $(\S 300)$.

If the organism were precisely similar to a machine, a smaller amount of heat, corresponding to the work done, would be formed in the body. As a matter of fact, the organism produces less heat from the same amount of potential energy when mechanical work is done. There is one point of difference between a workman and a working machine. The workman consumes much more potential energy in the same time than a passive person; much more is transformed in his body; and hence the increased consumption is not only covered, but even overcompensated. Hence, the workman is warmer than the passive person, owing to the increased muscular activity $(\$ 210,1, b)$. Take an example:-Hirn remained 
passive, and absorbed $30 \mathrm{grm}$. O per hour in a calorimeter, and produced 155 calories. When in the calorimeter he did work equal to 27,450 kilogrammemetres, which was transferred beyond it; he absorbed $132 \mathrm{grm}$. O, and produced only 251 calories.

In estimating the work done, we must include only the heat-equivalent of the work transferred beyond the body; lifting weights, pushing anything, throwing a weight, and lifting the body, are examples. In ordinary walking we must take into account that we overcome the resistance of the air and the activity of the muscles.

The organism is superior to a machine in as far as it can, from the same amount of potential energy, produce more work in proportion to heat. Whilst the very best steam-engine gives $\frac{1}{8}$ of the potential energy in the form of work, and $\frac{7}{8}$ as heat, the body produces $\frac{1}{5}$ as work and $\frac{4}{5}$ as heat. Chemical energy can never do work alone, in a living or dead motor, without heat being formed at the same time.

218. ACCOMMODATION FOR VARYING TEMPERATURES-All substances which possess high conductivity for heat, when brought into contact with the skin, ajpear to be very much colder or hotter than bad conductors of heat. The reason of this is that these bodies abstract far more heat, or conduct more heat than other bodies. Thus the water of a cool bath, being a better conductor of heat, is always thought to be colder than air at the same temperature. In our climate it appears to us that-

Air, at $18^{\circ} \mathrm{C}$. is moderately warm ; at $25^{\circ}-28^{\circ} \mathrm{C}$., hot ; above $28^{\circ}$, very hot.

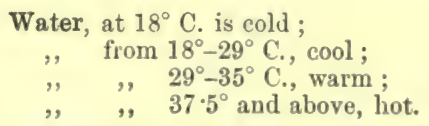

Warm Media. - As long as the temperature of the body is higher than that of the surrounding medium, heat is given off, and that the more rapidly the better the conducting power of the surrounding melium. As soon as the tenperature of the surrounding nedium rises higher than the temperature of the body, the latter absorbs heat, and it does so the more rapidly the better the conducting power of the medium. Hence, hot water appears to be warmer than air at the same temperature. A person may remain eight minutes in a bath at $45.5^{\circ} \mathrm{C}$. (dangerous to life!); the hands may be plunged into water at $50.5^{\circ} \mathrm{C}$., but not at $51.65^{\circ} \mathrm{C}$., while at $60^{\circ}$ violent pain is produced.

A person may remain for eight minutes in hot air at $127^{\circ} \mathrm{C}$, and a temperature of $132^{\circ} \mathrm{C}$. has been borne for ten minutes, and yet the body temperature rises only to $38.6^{\circ}$ or $38.9^{\circ}$. This depends upon the air being a bad conducter, and thus it gives less heat to the body than water would do. Further, and what is more important, the skin becomes covered with sweat, which evaporates and abstracts heat, while the lungs also give off more watery vapour. The enormously increased heart-beatsover 160-and the diluted blood-vessels, enable the skin to obtain an ample supply of blood for the formation and evaporation of sweat. In proportion as the secretion of sweat diminishes, the body becomes unable to endure a hot atmosphere; hence it is that in air containing much watery vapour a person cannot endure nearly so high a temperature as in dry air, so that heat must accumulate in the body. In a Turkish-vapour bath of $53^{\circ}$ to $60^{\circ} \mathrm{C}$, the rectal temperature rises to $40.7^{\circ}$ or $41.6^{\circ}$ C. A person may work continuously in air at $31^{\circ} \mathrm{C}$. which is almost saturated with moisture.

If a person be placed in water at the temperature of the body, the normal temperature rises $1^{\circ} \mathrm{C}$. in one hour, and in $1 \frac{1}{2}$ hour about $2^{\circ} \mathrm{C}$. A gradual increase of the temperature from $38 \cdot 6^{\circ}$ to $40 \cdot 2^{\circ} \mathrm{C}$. causes the axillary temperature to rise to $39 \cdot 0^{\circ}$ within fifteen minutes.

219. STORAGE OF HEAT.-As the uniform temperature of the body, under normal circumstances, is due to the reciprocal relation between the amount of heat 
aciul is also increased; the urine pigment ( $\$ 19)$, derived from the hrmoglobin, may be increased twenty times, while the excretion of potush may be seven-fold. It is important to observe that the oxidation or combustion processes within the body of the fever-patient are greatly increased when he is placed in a warmer atmosphere. The oxidation processes in fever, however, are also increased under the influence of cooler surroundings $(\$ 214,1 ., 2)$, but the increase of the oxidation in a warm medium is very much greater than in the cold (D. Finkler). The amount of $\mathrm{CO}_{3}$ in the blood is diminished, but not at once after the onset even of a very severe fever (Geppert).

(4) The diminished excretion of heat varies in different stages of a fever. We distinguish several stages in a fever-(a) The cold stage, when the loss of heat is greatly diminished, owing to the pale hloolless skin, but at the same time the heat-production is increased $1 \frac{1}{2}$ to $2 \frac{1}{2}$ times. The sudhen and considerable rise of the temperature during this stage shows that the diminished excretion of heat is not the only cause of the rise of the temperature. (b) During the hot stage the huit giren off from the congested red skin is greatly increased, but at the same time more heat is producril. Liebermeister assumes that a rise of $1,2,3,4^{\circ} \mathrm{C}$. corresponds to an increased prolution of heat of $6,12,18,24$ per cent. (c) In the sweating stage the excretion of heat through the red moist skin and evaporation are greatest, more than two or three times the normal. 'The heat-production is either increased, normal, or sub-normal, so that under these conditions the temperature may also be sub-nornal $\left(36^{\circ} \mathrm{C}\right.$. $)$.

5) The heat-regulating mechanism is injured. $-\mathrm{A}$ warm temperature of the surroundings raises the temperature of the ferer-patient more than it does that of a non-febrile person. The depression of the heat-production, which enables normal animals to maintain their normal temperature in a warm medium (\$214), is much less in fever (D. Finkler).

The accessory phenomena of ferer are very important:- Increase in the intensity and number of the heart-beats ( $\$ 214$, II., 2) and respirations (in adults 40 , and children 60 per mitu. ), both being compensatory phenomena of the increased temperature; further, diminished digestive activity and intestinal movements $(\$ 186, D)$; disturbances of the cerebral activities; of secretion; of muscular activity; slower excretion, e.g., of potassium iodide through the urine. In severe fever, molecular degenerations of the tissues are very common. For the condition of the blood-corpuscles in fever, see $\$ 10$, the vaseular tension, $\$ 69$, the saliva, $\$ 146$, ligestion, $\$ 186$.

Quinine, the most important fehrifuge, causes a decrease of the temperature by limiting the production of heat $(\$ 213,6)$. Toxic closes of the metallic salts act in the same way, while there is at the same time climinished formation of $\mathrm{CO}_{2}$. CAntipyretics or Febrifuges. - All methods which diminish abnormal temperature belong to this group. As the constant temperature of the borly deprenls on (1) the amount of heat-production, and (2) the loss of heat, we may lower the temperature either in the one way or the other. When cold water is applied to the boily, it alstracts heat, i.e, it affects the results of fever, so that Liebermeister calls such methols antithermic. But those remedies which diminish the actual heat-production are true antipyretic. In practice, however, both methods are usually employed, and spoken of collectively as antipyretics.]

[Amongst the methouls which are used to abstract heat from the body, are the application of colder fluids, such as the cold bath, affusion, douche, spray, ice, or cold mixtures, \&c. A person suffering from high fever requires to be repeatedly placed in a cold bath to produce any permanent reduction of the temperature. Some remedies act by favouring the radiation of heat, by dilating the cutaneous ressels (alcohol), while others excite the sweat-glands-i.e., are sulorifis - so that the water by its evaporation removes some heat. Amongst the drugs which influence tissue changes and oxidation, and thereby lessen heat-production, are quinine, salicylic acid, some of the salicylates, digitalis, and veratrin. Blood-letting was formerly used to diminish abnormal temperature. Amongst the newer antipyretic remedies are hydrochlorate of kairin and antipyrin, both of which belong to the aromatic group (derivatives of benzol), which includes also many of our best antiseptics.]

221. ARTIFICIAL INCREASE OF THE BODILY TEMPERATURE.-If mammals are kept constantly in air at $40^{\circ} \mathrm{C}$., the excretion of heat from the body ceases, so that the heat produced is stored up. At first the temperature falls somewhat for a very short time, but soon a decided increase occurs. The respirations and pulse are increased, while the latter becomes irregular and weaker. The $\mathrm{O}$ absorbed and $\mathrm{CO}_{2}$ given off are diminished after six to eight hours, and death occurs after great fatigue, feebleness, spasms, secretion of saliva, and loss of consciousness, when the bodily temperature has been increased $4^{\circ}$ or at most $6^{\circ} \mathrm{C}$. Death does not take place owing to rigidity of the muscles, for the coagulation of the myosin of mammals' muscles occurs at $49^{\circ}$ to $50^{\circ} \mathrm{C}$., in birds at $53^{\circ} \mathrm{C}$, and in frogs at $40^{\circ} \mathrm{C}$. If mammals are suddenly placed in air at $100^{\circ} \mathrm{C}$, death occurs (in 15 to 
20 min.) very rapidly, and with the same phenomena, while the bodily temperature rises $4^{\circ}$ to $5^{\circ} \mathrm{C}$. In rabbits the body-weight diminishes 1 grm. per min. Birds bear a high temperature somewhat longer; they die when their blood reaches $48^{\circ}$ to $50^{\circ} \mathrm{C}$.

Even man may remain for some time in air at $100-110-132^{\circ} \mathrm{C}$, , but in ten to fifteen minutes there is danger to life. The skin is burning to the touch, and red; a copious secretion of sweat bursts forth, and the cutaneous veins are fuller and redder. The pulse and respirations are greatly accelerated. Violent headache, vertigo, feebleness, and stupefaction, indicate great danger to life. The rectal temperature is only $1^{\circ}$ to $2^{\circ} \mathrm{C}$. higher. The high temperature of fever may even be dangerous to human life. If the temperature remains for any length of time at $42.5^{\circ}$ C., death is almost certain to occur. Coagulation of the blood in the arteries is said to occur at $42 \cdot 6^{\circ} \mathrm{C}$. If the artificial heating does not produce death, fatty infiltration and degeneration of the liver, heart, kidneys, and muscles begin after thirty-six to forty-eight hours.

Cold-blooded animals, if placed in hot air or warm water, soon have their temperature raised 6 to $10^{\circ} \mathrm{C}$. The highest temperature compatible with life in a frog must be below $40^{\circ} \mathrm{C}$, , as the frog's heart and muscles begin to coagulate at this temperature. Death is preceded by a stage resembling death, during which life may be saved.

Most of the juicy plants die in half an hour in air at $52^{\circ} \mathrm{C}$., or in water at $46^{\circ} \mathrm{C}$. (Sachs). Dried seeds of corn may still germinate after long exposure to air at $120^{\circ} \mathrm{C}$. Lowly organised plants, such as algæ, may live in water at $60^{\circ} \mathrm{C}$. (Hoppe-Seyler). Several bacteria withstand a boiling temperature (Tyndall).

222. EMPLOYMENT OF HEAT.-Action of Heat.-The short, but not intense, action of heat on the surface causes, in the first place, a transient slight decrease of the bodily temperature, partly because it retards reflexly the production of heat, and partly because, owing to the dilatation of the cutaneous vessels and the stretching of the skin, more heat is given off. A warm bath above the temperature of the blood at once increases the bodily temperature.

Therapeutic Uses.- The application of heat to the entire body is used where the bodily temperature has fallen, or is likely to fall, very low, as in the algid stage of cholera, and in infants born prematurely. The general application of heat is obtained by use of warm baths, packing, vapour baths, and the copious use of hot drinks. The local application of heat is obtained by the use of warm wrappings, partial baths, plunging the parts in warm earth or sand, or placing wounded parts in chambers filled with heated air. After removal of the heating agent, care must be taken to prevent the great escape of heat due to the dilatation of the blood-vessels.

223. INCREASE OF TEMPERATURE POST-MORTEM.-Phenomena.-Heidenhain found that in a dead dog, before the body cooled, there was a constant temporary rise of the temperature, which slightly exceeded the normal. The same observation had been occasionally miade on human bodies immediately after death, especially when death was preceded by muscular spasms [also in yellow fever]. Thus, Wunderlich measured the temperature fifty-seven minutes after death in a case of tetanus, and found it to be $45 \cdot 375^{\circ} \mathrm{C}$.

Causes. - (1) A temporary increascd production of heat after death, due chiefly to the change of the semi-solid myosin of the muscles into a solid form (rigor mortis). As the muscle coagulates, heat is produced. All conditions which cause rapid and intense coagulation of the muscles-e.g., spasms, favour a post-mortem rise of temperature (see \$295); a rapid coagulation of the blood has a similar result $(\$ 28,5)$.

(2) Immediately after death a series of chemical processes occur within the body, whereby heat is produced. Valentin placed a dead rabbit in a chamber, so that no heat could be given off from the body, and he found that the internal temperature of the animal's body was increased. The processes which cause a rise of temperature post-mortem are more active during the first than the second hour; and the higher the temperature at the moment of death, the greater is the amount of heat evolved after death.

(3) Another cause is the diminished excretion of heat post-mortem. After the circulation is abolished, within a few minutes little heat is given off from the surface of the body, as rapid excretion implies that the cutaneous vessels must be continually filled with warm blood.

224. ACTION OF COLD ON THE BODY.-Phenomena.-A short temporary slight cooling of the skin (removing one's clothes in a cool room, a cool bath for a short time, or a cool douche) causes either no change or a slight rise in the bodily temperature. The slight rise, when it occurs, is due to the stimulation of the skin 
causing reflexly a more rapid molecular transformation, and therefore a greater production of heat, while the amount of heat given off is diminished owing to contraction of the small cutaneous vessels and the skin itself (Liebermeister). The continuous and Intense application of cold causes a decrease of the temperature, chiefly by conduction, notwithstanding that at the same time there is a greater production of heat. After a cold bath the temperature may be $34^{\circ}, 32^{\circ}$, and even $30^{\circ} \mathrm{C}$.

As an after-effect of the great abstraction of heat, the temperature of the body after a time remains lower than it was before ("primary after-effect"-Liebermeister); thus after an hour it was $0.22^{\circ} \mathrm{C}$. in the rectum. There is a "secondary "itter-effert" which occurs after the first after-effect is over, when the temperature rises (Jïrgensen). This effect begins five to eight hours after a cold bath, and is equal to $+0.2^{\circ} \mathrm{C}$. in the rectum. Hoppe-Seyler found that some time after the application of heat there was a corresponding lowering of the temperature.

Taking Cold.-If a rabbit be taken from a surrounding temperature of $35^{\circ} \mathrm{C}$., and suddenly cooled, it shivers, and there may be diarrhoa. After two days the temperature rises $1.5^{\circ} \mathrm{C}$., and albuminuria oceurs. There are microscopic traces of interstitial inflammation in the kidners, liver, lungs, heart, and nerve-sheaths, the dilated arteries of the liver and lung contain thrombi, and in the neighbourhood of the veins are accumulations of leucocytes. In pregnant animals the futus shows the same conditions. Perhaps the greatly cooled blood acts as an irritant causing inflammation.

Action of Frost. - The continued application of a high degree of cold causes at first contraction of the blood-vessels of the skin and its muscles, so that it becomes pale. If continued paralysis of the cutaneous vessels occurs, the skin becomes red owing to congestion of its vessels. As the passage of fluids through the capillaries is rendered more difficult by the cold, the blood stagnates, and the skin assumes a livid appearance, as the $\mathrm{O}$ is almost completely used up. Thus the peripheral circulation is slowed. If the action of the cold be still more intense, the peripheral circulation stops completely, especially in the thinnest and most exposed organs-ears, nose, toes, and fingers. The sensory nerves are paralysed, so that there is numbness with loss of sensibility, and the parts may even be frozen through and through. As the slowing of the circulation in the superficial vessels gradually affects other areas of the circulation, the pulmonary circulation is enfeebled, and diminished oxidation of the blood occurs, notwithstanding the greater amount of $\mathrm{O}$ in the cold air, so that the nerve centres are affected. Hence arise great dislike to making movements or any muscular effort, a painful sensation of fatigue, a peculiar and almost irresistible desire to sleep, cerebral inactivity, blunting of the sense-organs, and lastly, coma. The blood freezes at $-3.9^{\circ} \mathrm{C}$, while the juices of the superficial parts freeze sooner. Too rapid movements of the frost-bitten parts ought to be avoided. Rubfing with snow, and the very gradual application of heat, produce the best results. Partial death of a part is not unfrequently produced by the prolonged action of cold.

\section{ARTIFICIAL LOWERING OF THE TEMPERATURE.-Phenomena.} -The artificial cooling of warm-blooded animals, by placing them in cold air or in a freezing mixture, gives rise to a series of characteristic phenomena. If the animals (rabbits) are cooled so that the temperature (rectum) falls to $18^{\circ}$, they suffer great depression, without, however, the voluntary or reflex movements being abolished. The pulse falls from 100 or 150 to 20 beats per minute, and the bloodpressure falls to several millimetres of $\mathrm{Hg}$. The respirations are few and shallow. Suffocation does not cause spasms, the secretion of urine stops, and the liver is congested. The animal may remain for twelve hours in this condition, and when the muscles and nerves show signs of paralysis, coagulation of the blood occurs after numerous blood-corpuscles have been destroyed. The retina becomes pale, and death occurs with spasms and the signs of asphyxia. If the bodily temperature be reduced to $17^{\circ}$ and under, the voluntary movements cease before the reflex acts. An animal cooled to $18^{\circ} \mathrm{C}$, and left to itself, at the same temperature as the surroundings, does not recover of itself, but if artificial respiration be employed, the temperature rises $10^{\circ} \mathrm{C}$. If this be combined with the application of external warmth, the animals may recover completely, even when they have been apparently dead for forty minutes. Walther cooled adult animals to $9^{\circ} \mathrm{C}$., and recovered them by artificial respiration and external warmth; while Horvath cooled young animals 
to $5^{\circ} \mathrm{C}$. Mammals, which are born blind, and birds which come out of the egg devoid of feathers, cool more rapidly than others. Morphia, and more so, alcohol, accelerate the cooling of mammals, at the same time the exchange of gases falls considerably; hence, drunk men are more liable to die when exposed to cold.

Artificial Cold-Blooded Condition.-Cl. Bernard made the important observation, that the muscles of animals that had been cooled remained irritable for a long time, to direct stimuli as well as to stimuli applied to their nerves; and the same is the case when the animals are asphyxiated for want of $\mathrm{O}$. An " artificial cold-blooded condition," i.e., a condition in which warm-blooded animals have a lower temperature, and retain muscular and nervous excitability, may also be caused in warm-blooded animals, by dividing the cervical spinal cord and keeping up artificial respiration; further, by moistening the peritoneum with a cool solution of common salt.

Hybernation presents a series of similar phenomena. Valentin found that hybernating animals become half-awake when their bodily temperature is $28^{\circ} \mathrm{C}$.; at $18^{\circ} \mathrm{C}$. they are in a somnolent condition, at $6^{\circ}$ they are in a gentle sleep, and at $1.6^{\circ} \mathrm{C}$. in a deep sleep. The heart-beats and the blood-pressure fall, the former to 8 to 10 per minute. The respiratory, urinary, and intestinal movements cease completely, and the cardio-pneumatic movement alone sustains the slight exchange of gases in the lungs ( $\$ 59)$. They cannot endure cooling to $0^{\circ} \mathrm{C}$.; and awake before the temperature falls so low. Hybernating animals may be cooled to a greater degree than other mammals ; they give off heat rapidly, and they become warm again rapidly, and even spontaneously. New-born mammals resemble hybernating animals more closely in this respect than do adults.

Cold-blooded animals may be cooled to $0^{\circ}$. Even when the blood has been frozen and ice formed in the lymph of the peritoneal cavity, frogs may recover. In this condition they appear to be dead, but when placed in a warm medium they soon recover. A frog's muscle so cooled will contract again. The germs and ova of lower animals, e.g., insects' eggs, survive continued frost; and if the cold be moderate, it merely retards development. Bacteria, e.g., Bacillus anthracis, survive a temperature of $-130^{\circ} \mathrm{C}$.; yeast, even $-100^{\circ} \mathrm{C}$.

Varnishing the skin causes a series of similar phenomena. The varnished skin gives off a large amount of heat by radiation, and sometimes the cutaneous vessels are greatly dilated. Hence the animals cool rapidly and die, although the consumption of $\mathrm{O}$ is not diminished. If cooling be prevented by warming them and keeping them in warm wool, the animals live for a longer time. The blood post-mortem does not contain any poisonous substances, nor even are any materials retained in the blood which can cause death, for if the blood be injected into other animals, these remain healthy.

226. EMPLOYMENT OF COLD. - Cold may be applied to the whole or part of the surface of the body in the following conditions:-

(a) By placing the body for a time in a cold bath to abstract as much heat as possible, when the bodily temperature in fever rises so high as to be dangerous to life. This result is best accomplished and lasts longest when the bath is gradually cooled from a moderate temperature. If the body be placed at once in cold water, the cutaneous vessels contract, the skin becomes bloodless, and thus obstacles are placed in the way of the excretion of heat. A bath gradually cooled in this way is borne longer. The addition of stimulating substances, e.g., salts, which cause dilatation of the cutaneous vessels, facilitates the excretion of heat; even salt water conducts heat better. If alcohol be given internally at the same time, it lowers the temperature.

(b) Cold may be applied locally by means of ice in a bag, which causes contraction of the cutaneous vessels and contraction of the tissues (as in inflammation), while at the same time heat is abstracted locally.

(c) Heat may be abstracted locally by the rapid evaporation of volatile substances (ether, carbon disulphide), which causes numbness of the sensory nerves. The introduction of media of low temperature into the body, respiring cool air, taking cold drinks, and the injection of cold fluids into the intestine act locally, and also produce a more general action. In applying cold it is important to notice that the initial contraction of the vessels and the contraction of the tissues are followed by a greater dilatation and turgescence, i.e., by a healthy reaction.

227. HEAT OF INFLAMED PARTS.- "Cälor," or heat, is reckoned one of the fundamental phenomena of inflammation, in addition to rubor (redness), tumor (swelling), and dolor (pain). But the apparent increase in the heat of the inflamed parts is not above the temperature of the blood. Simon, in 1860, asserted that the arterial blood flowing to an inflamed part was cooler than the part itself, but this has been contradicted. The outer parts of the skin in an inflamed part are warmer than usual, owing to the dilatation of the vessels (rubor) and the consequent 
acceleration of the blood-stream in the inflamed part, and owing to the swelling (tumor) from the presence of good heat-conducting fluids; but the heat is not greater than the heat of the blood. It is not proved that an increased amount of heat is produced owing to increased molecular decompositions within an inflamed part.

228. HISTORICAL AND COMPARATIVE-According to Aristotle, the heart prepares the heat within itself, and sends it along with the blood to all parts of the body. This doctrine prevailet in the time of Hippocrates and Galen, and occurs even in Cartesius and Bartholinus (1667, "flammula cordis"). The iatro-mechanical school (Bocrhave, van Swieten) ascribed the heat to the friction of the blood on the walls of the vessels. The iatro-chemical school, on the other hand, sought the source of heat in the fermentations that arose from the jassage of the alisorbed substances into the blood (ran Helmont, Sylvius, Ettmüller). Lavoisier (1777) was the first to ascribe the heat to the combustion of carbon in the lungs. After the construction of the thermometer by Galileo, Sanctorius (1626) made the first thermometric observations on sick persons, while the first calorimetric observations were made by Lavoisier and Laplate. Comparative observations are given at § 207, and also under Hybernation (\$ $225)$. 


\section{Physiology of the Metabolic Phenomena.}

By the term metabolism we mean those phenomena, whereby all-even the most lowly-living organisms are capable of incorporating the substances obtained from their food into their tissues, and making them an integral part of their own bodies. This part of the process is known as assimilation. Further, the organism in virtue of its metabolism forms a store of potential energy, which it can transform into kinetic energy, and which, in the higher animals at least, appears most obvious in the form of muscular work and heat. The changes of the constituents of the tissues, by which these transformations of the potential energy are accompanied, result in the formation of excretory products, which is another part of the process of metabolism. The normal metabolism requires the supply of food quantitatively and qualitatively of the proper kind, the laying up of this food within the body, a regular chemical transformation of the tissues, and the formation of the effete products which have to be given out through the excretory organs. [Synthetic or constructive metabolism is spoken of as anabolic, and destructive or analytical metabolism as katabolic, metabolism.]

229. THE MOST IMPORTANT SUBSTANCES USED AS FOOD.-Water. -When we remember that 58.5 per cent. of the body consists of water, that water is being continually given off by the urine and fæces, as well as through the skin and lungs, that the processes of digestion and absorption require water for the solution of most of the substances used as food, and that numerous substances excreted from the body require water for their solution, especially in the urine, the great importance of water and its continual renewal within the organism are at once apparent. As put by Hoppe-Seyler, all organisms live in water, and even in running water, a saying which ranks with the old saying- "Corpora non agunt nisi fluida."

Water-as far as it is not a constituent of all fluid foods-occurs in different forms as drink :(1) Rain water, which most closely resembles distilled or chemically pure water, always contains minute quantities of $\mathrm{CO}_{2}, \mathrm{NH}_{3}$, nitrous and nitric acids. (2) Spring water usually contains much mineral substance. It is formed from the deposition of watery vapour or rain from the air, which permeates the soil, containing much $\mathrm{CO}_{2}$; the $\mathrm{CO}_{2}$ is dissolved by the water, and aids in dissolving the alkalies, alkaline earths, and metals, which appear in solution as bicarbonates, e.g., of lime or iron oxide. The water is removed from the spring by proper mechanical appliances, or it bubbles up on the surface in the form of a "spring." (3) River water usually contains much less mineral matter than spring water. Spring water floating on the surface rapidly gives off its $\mathrm{CO}_{2}$ whereby many substances-e.g., lime-are thrown out of solution, and deposited as insoluble precipitates.

Gases. - Spring water contains little $\mathrm{O}$, but much $\mathrm{CO}_{2}$, the latter giving to it its fresh taste. Hence, vegetable organisms flourish in spring water, while animals requiring, as they do, much $\mathrm{O}$, are but poorly represented in such water. Water flowing freely gives up $\mathrm{CO}_{\mathrm{g}}$, and absorbs $O$ from the air, and thus affords the necessary conditions for the existence of fishes and other marine animals. River water contains $\frac{1}{30}$ to $\frac{1}{20}$ of its volume of absorbed gases, which may be expelled by boiling or freezing. 
Drinking water is chiefly obtained from springs. River water, if used for this purpose, must be filtered to get rid of mechanically suspended impurities. For household purposes a charcoal filter may be usecl, as the charcoal acts as a disinfectant. Alum has a remarkable action. When adiled to give a dilution containing 0.0001 per cent., it makes turbid water clear.

Investigation of Drinking Water.-Drinking water, even in a thick layer, ought to be completely colourless, not turbid, and without odour. Any odour is best recognised by heating it to $50^{\circ} \mathrm{C}$., and adding a little caustic soda. It ought not to be ton hurd, i.e., it ought not to contain too much lime (and magnesia) salts.

By the term "degree of hardness" of a water is meant the unit amount of lime (and macnesia) in 100,000 jarts of water; a water of 20 degrees of hardness contains 20 parts of lime (calcium oxide) combined with $\mathrm{CO}_{2}$, sulphuric, or hydrochloric acids (the small amount of magnesia may be neglected). A good drinking vater ouyht not to exceed 20 degrees of hardness. The hardness is determined by titrating the water with a standard soap solution, the result being the formation of a scum of lime-soap on the surface. The hardness of unboiled water is called its tutal hardness, while that of boilal water is called permanent hardness. Boiling drives off the $\mathrm{CO}_{2}$, and precipitates the calcium carbonate, so that the water at the same time becomes softer.

The presence of sulphuric acid, or sulphates, is determined by the water becoming turbid on adding a solution of barium chloride and hydrochloric acid.

Chlorine occurs in small amount in pure spring water, but when it occurs there in large amount-apart from its being derived from saline springs, near the sea or manufactories-we may conclude that the water is contaminated from water-closets or dunghills, so that the estimation of chlorine is of importance. For this purpose use a solution, A, of 17 grms. of crystallised silver nitrate in 1 litre of distilled water; 1 cubic centimetre of this solution precipitates 3.55 milligrammes of chlorine as silver chloride. Use also B, a cold saturated solution of neutral potassium chromate. Take 50 cubic centimetres of the water to be investigated, and place it in a beaker, add to it 2 to 3 drops of $B$, and allow the fluid $A$ to run into it from a burette until the white precipitate first formed remains red, even after the fluid has been stirred. Multiply the number of cubic centimetres of A used by $7 \cdot 1$, and this will give the amount of chlorine in 100,000 parts of the water. Example-50 c.cmtr. requires $2.9 \mathrm{c}$.cmtr. of the silver solution, so that 100,000 parts of the water contain $2.9 \times 7 \cdot 1=20.59$ parts chlorine (Kubel Tiemenn). Good water ought not to contain more than 15 milligrammes of chlorine per litre.

The presence of lime may be ascertained by acidulating 50 cubic centimetres of the water witlı $\mathrm{HCl}$, addling ammonia in excess, and afterwards adding ammonia oxalate; the white precipitate is lime oxalate. According to the degree of turbidity, we judge whether the water is "soft" (poor in lime), or "hard" (rich in lime).

Magnesia is determinel by taking the elear fluid of the above operation, after removing the precipitate of lime, and adding to it a solution of sodium phosphate and some ammonia; the crystalline precipitate which occurs is magnesia.

The more fecble all these reactions which indicate the presence of sulphuric acid, chlorine, lime, and magnesia, are, the better is the water. In addition, good water ought not to contain more than traces of nitrates, nitrites, or compounds of ammonia, as their presence indicates the decomposition of nitrogenous organic substances.

For nitric acid, take 100 cubic centimetres of water acidulated with two or three drops of concentrated sulphuric acil, add several pieces of zinc together with a solution of potassium iodide, and starch solution-a blue colour indicates nitric acid. The following test is very delicate:-Aild to half a drop of water in a capsule two drops of a watery solution of Brucinum sulphuricum, and afterwarls several drops of concentrated sulphuric acid ; a rose-red coloration indicates the presence of nitric acid.

The presence of nitrous acid is ascertained by the blue coloration which results from the addition of a solution of potassium iodide, and solution of starch, after the water has been acidulated with sulphuric acid.

Compounds of ammonia are detected by Nessler's reagent, which gives a yellow or reddish coloration when a trace of ammonia is present in water; while a large amount of these compounds gives a brown precipitate of the iodide of mercury and ammonia.

The contamination of water by decomposing animal substance is determined by the amount of $\mathrm{N}$ it contains. In most cases it is sufficient to determine the amount of nitric acid present. For this purpose we require $(\Lambda)$ a solution of 1.871 grms. potassium nitrate in 1 litre distilled water-1 cubic centimetre contains 1 milligramme nitric acid; (B) a dilute solution of indigo, which is prepared by rubbing together one part of pulverised indigotin with six parts $\mathrm{H}_{2} \mathrm{SO}_{4}$, and allowing the deposit to subside, when the blue fluid is poured into forty times its volume of distilled water and filtered. This fluid is diluted with distilled water until a layer, 12 to 15 mm. in thickness, begins to be transparent.

To test the activity of $\mathrm{B}$, place 1 cubic centimetre of $\mathrm{A}$ in 24 cubic centimetres water, add 
some common salt and 50 cubic centimetres concentrated sulphuric acid, and allow B to flow from a burette into this mixture until a faint green colour is obtained. The number of cubic centimetres of $\mathrm{B}$ used correspond to 1 milligramme of nitric acid.

Twenty-five cubic centimetres of the water to be investigated are mixed with 50 cubic centimetres of concentrated $\mathrm{H}_{2} \mathrm{SO}_{4}$, and titrated with $\mathrm{B}$ until a green colour is obtained. This process must be repeated, and on the second occasion the solution B must be allowed to flow in at once, when usually somewhat more indigo solution is required to obtain the green solution. The number of cubic centimetres of $\mathrm{B}$ (corresponding to the strength of $\mathrm{B}$, as determined above) indicates the amount of nitric acid present in $25 \mathrm{c.cmtr}$. of the water investigated. As much as 10 milligrammes nitric acid have been found in spring water (Marx, Trommsdorff).

Sulphuretted Hydrogen is recognised by its odour ; also by a piece of blotting-paper moistened with alkaline solution of lead becoming brown, when it is held over the boiling water. If it occurs as a compound in the water, sodium nitro-prusside gives a reddish-violet colour.

It is of the greatest importance that drinking water should be free from the presence of organic matter in a state of decomposition. Organic matter in a state of decomposition, and the organisms therewith associated, when introduced into the body, may give rise to fatal maladies, e.g., cholera and typhoid fever. This is the case when the water supply has been contaminated from water which has percolated from water-closets, privies, and dung-pits. The presence of organic matter may be detected thus-(1) A considerable amount of the water is evaporated to dryness in a porcelain vessel, if the residue be heated again a brown or black colour indicates the presence of a considerable amount of organic matter; and if it contain $\mathrm{N}$, there is an odour of ammonia. Good water treated in this way gives only a light brown stain. The presence of micro-organisms may be determined microscopically after evaporating a small quantity of the water on a glass slide. (2) The addition of potassio-gold chloride to the water gives a black frothy precipitate after long standing. (3) A solution of potassium permanganate, added to the water in a covered jar, gradually becomes decolorised, and a brownish precipitate is formed.

Water containing much organic matter should never be used as drinking water, and this is especially the case when there is an epidemic of typhoid fever, cholera, or diarrhœa. In all such circumstances, the water ought to be boiled for a long time, whereby the organic germs are killed. The insipid taste of the water after boiling may be corrected by adding a little sugar or lime juice.

230. THE MAMMARY GLANDS AND MILK. - Milk Duct. - A bout 20 galactoferous ducts open singly upon the surface of the nipple. Each of these, just before it opens on the surface, is provided with an oval dilatation-the sinus lacteus. When traced into the gland, the galactoferous ducts divide like the branches of a tree, and a large branch of the duct passes to each lobe of the gland, all the lobes being held together by loose connective-tissue. Only during lactation do all the fine terminations of the ducts communicate with the globular glandular acini. Every gland acinus consists of a membrana propria, surrounded externally with a network of branched connective-tissue corpuscles, and lined internally with a somewhat flattened polyhedral layer of nucleated secretory cells (fig. 233). The size of the lumen of the acini depends upon the secretory activity of the glands; when it is large, it is filled with milk containing numerous refractive fatty granules. The walls of the milk ducts consist of fibrillar connective-tissue, some fibres are arranged longitudinally, but the chief mass are disposed circularly, and are permeated externally with elastic fibres, while in the finer ducts there is a membrana propria continuous with that of the gland acini. The ducts are lined by cylindrical epithelium.

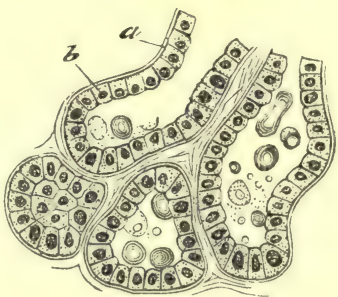

Fig. 233.

Acini of the mammary gland of a sheep during lactation. $a$, membrana propria; $b$, secretory epithelium.

During the first few days after delivery, the breasts secrete a small amount of milk of greater consistence, and of a yellow colour - the colostrum -in which large cells filled with fatty granules occur - the colostrum-corpuscles (fig. 235). Sometimes a nucleus is observable within them, and rarely they exhibit amœboid movements (fig. 234, $c, d, e$ ). The regular secretion of milk begins after three to four days. It was formerly supposed that the cells of the acini underwent a fatty degeneration, and thus produced the fatty granules of the milk. It is more probable, from recent observations, that the cells of the acini manufacture the fatty granules, and their protoplasm eliminates them, at the same time forming the clear fluid part of the milk.

Changes during Secretion.-Pratsch and Heidenhain found that the secretory cells in the non-secreting gland (fig. 234, I), were flat, polyhedral, and uninucleated, whilst the secreting cells (fig. 234, II) often contained several nuclei, were more albuminous, higher, and cylindrical in form. The edge of the cell directed towards the lumen of the acinus undergoes characteristic changes during secretion. 
Fatty granules are formed in this part of the cell, and are afterwards extruded. The decomposed portion of the cell is dissolved in the milk, and the fatty granules become free as milk-globules (fig. 234, II, a). If nuclei are present in that part of the cell which is broken up, they also pass into the milk and give rise to the presence of nuclein in the secretion.

Besides the milk-globules and colostrum cornuscles, Rauber has found leucocytes undergoing fatty degreneration and single pale cells $(f)$. Occasionally milk-globules are found with traces of the cell-substance adhering to their surface $(b)$.

Formation of Milk. - Concerning the formation of the individual constituents of milk, $\mathbf{H}$. Thierfeliler, who digested fresh manmary glands directly after death, found that during the digestion of the grlands, at the temperature of the bolly, a reducing substance, probably lactose, was formed by a process of fermentation. The mother substance (saccharogen) is soluble in water, lut not in alcohol or ether, is not ilestroyed by boiling, and is not identical with glycogen. The ferment which forms the lactose is connected with the gland-cells-it does not pass into the milk, nor into a watery extract of the gland. During the digestion of the mammary glands at the temperature of the boly, casein is formed, probably from serum-albumin, by a process of fermentation. This ferment occurs in the milk.

The nipple and its areola are characterised by the presence of pigment-more abundant Anring fremancy-in the rete Malpighii of the skin, and by large papille in the cutis vera. some of the papille contain tonch-corpuscles. Numerous non-striped muscular fibres surround the milk-luets in the deep layers of the skin and in the subcutaneous tissue, which contains no tit. These muscular fibres can be traced, following a longitulinal course, to the termination of the incts on the surface. The small glands of Montgomery, which occur on the areola during lactation, are just small milk-glands, each with a special duct opening on the surface of the elevation.

Arteries proceed from several sources to supply the mamma, but their branches do not accomprany the milk-lucts; each gland acinus is surrounded by a network of capillaries, which

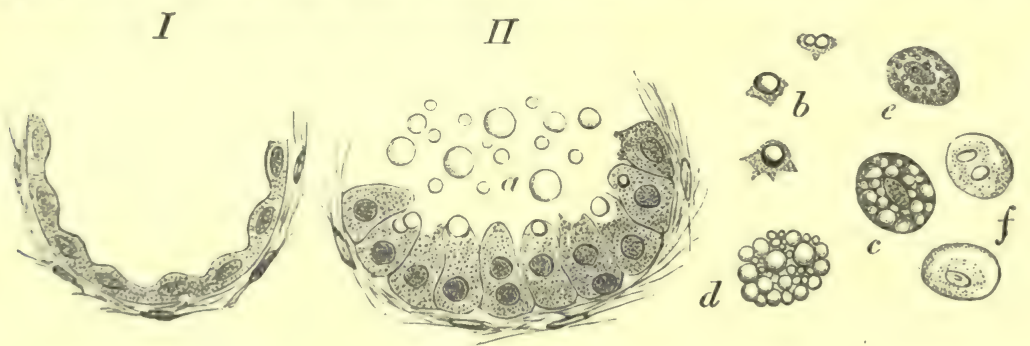

Fig. 234.

I. Inactive acinus of the mamma. II. During the secretion of milk $-a, b$, milk-globules ; $c, d, e$, colostrum corpuscles; $f$, pale cells (bitch).

communicate with those of adjoining acini by small arteries and veins. The veins of the areola are arranged in a circle (circulus Halleri). The nerves are derived from the supraclavieular, and the II $-I V-V I$ intercostals; they proceed to the skin over the gland, to the very sensitive nipple, to the bloor-vessels anil non-striped muscle of the nipple, and to the gland acini, where their mode of termination is still unknown. Lymphatics surround the alveoli, and they are often full. The milk appears to be prepared from the lymph contained in the lymphatics surrounding the acini.

The comparative anatomy of the mamma.-The rolents, insectivora, and carnivora have 10 to 12 teats, while some of them have only 4. The pachydermata and ruminantia have 2 to 4 abdominal teats, the whale has 2 near the vulva. The apes, bats, vegetable-feeding whales, elephants, and sloths have 2, like man. In the marsupials the tubes are arranged in groups, which open on a patch of skin devoid of hair without any nipple. The young animals remain within the mother's pouch, and the milk is expelled into their mouths by the action of a inuscle-the compressor mammæ.

The development of the human mamma begins in both sexes during the third month; at the fourth and fifth months a few simple tubular gland-ducts are arranged radially around the position of the future nipple, which is devoid of hair. In the new-born child the ducts are branched twice or thrice, and are provided with dilated extremities, the future acini. Ip to the twelfth year, in both sexes, the ducts continue to divide dendritically, but without any proper acini being formed. In the girl at puberty, the ducts branch rapidly; but the acini are formed only at the periphery of the gland; during pregnancy, acini are also formed in the 
centre of the gland, while the connective-tissue at the same time becomes somewhat more opened out. At the climacteric period, or menopause, all the acini and numerous fine milkducts degenerate. In the adult male, the gland remains in the non-developed infantile condition. Accessory or supernumerary glands upon the breast and abdomen are not uncommon, sometimes the mamma occurs in the axilla, on the back, over the acromion process, or on the leg. A slight secretion of milk in a newly-born infant is normal.

During the evacuation of the milk (500-1500 cubic centimetres daily), there is not only the mechanical action of sucking, but also the activity of the gland itself (\$152). This consists in the erection of the nipple, whereby its non-striped muscular fibres compress the sinuses on the milk-ducts, and empty them, so that the milk may flow out in streams. The gland acini are also excited to secretion reflexly by the stimulation of the sensory nerves of the nipple. The vessels of the gland are dilated, and there is a copious transudation into the gland-the transuded fluid being manufactured into milk under the influence of the secretory protoplasm. The amount of secretion depends upon the blood-pressure (Röhrig). During sucking, not only is the milk in the gland extracted, but new milk is formed, owing to the accelerated secretion. Emotional disturbances-anger, fear, \&c. - arrest the secretion. Laffont found that stimulation of the mammary nerve (bitch) caused erection of the teat, dilatation of the vessels, and secretion of milk. After section of the cerebro-spinal nerves going to the mamma, Eckhard observed that erection of the teat ceased, although the secretion of milk in a goat was not interrupted. The rarely observed galactorrhœa is perhaps to be regarded as a paralytic secretion analogous to the paralytic secretion of saliva. Heidenhain and Pratsch found that the secretion (bitch) was increased by injecting strychnine or curara after section of the nerves of the gland. The " milk-fever," which accompanies the first secretion of milk, probably depends on stimulation of the vaso-motor nerves, but this condition must be studied in relation with the other changes which occur within the pelvic cavity after birth. [Some substances, such as atropin, arrest the secretion of milk.]

231. MILK AND ITS PREPARATIONS.-Milk represents a complete or typical food in which are present all the constituents necessary for maintaining the life and growth of the body of an infant (\$236). [If an adult were to live on milk alone, to get the $23 \mathrm{oz}$. of dry solids necessary, he would have to take 9 pints of milk daily, which would give far too much water, fat, and proteids.] To every 10 parts of proteids there are 10 parts fat and 20 parts sugar. Relatively more of the fat than the albumin of the milk is absorbed (Rubner); while a part of both is excreted in the fæces.

Characters.-Milk is an opaque, bluish-white fluid with a sweetish taste and a characteristic odour, probably due to the peculiar volatile substances derived from the cutaneous secretions of the glands, and it has a specific gravity of 1026 to 1035 . When it stands for a time, numerous milk globules, butter globules, or cream, collect on its surface, under which there is a bluish watery fluid. Human milk is always alkaline, cow's milk may be alkaline, acid, or amphoteric; while the milk of carnivora is always acid.

Milk-Globules.-When milk is examined microscopically, it is seen to contain numerous small highly refractive oil-globules, floating in a clear fluid-the milk plasma (figs. 234, $a, b, 235)$; while colostrum corpuscles and epithelium from the milk-ducts are not so numerous. The white colour and opacity of the milk are due to the presence of the milkglobules, which reflect the light; the globules consist of a fat, or butter, and are said by

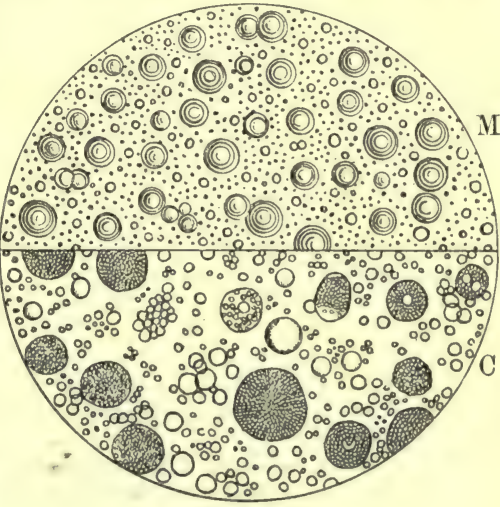

Fig. 235.

Microscopic appearance of milk, (M) upper half, and colostrum (C) lower half. some to be surrounded with a very thin envelope of casein or haptogen membrane.

If acetic acid be added to a microscopic preparation of milk, the fatty granules run together to form irregular masses. If cow's milk be shaken with caustic potash, the casein envelopes 
are dissolved, and if ether be added, the milk becomes clear and transparent, as the ether dissolves out all the fatty particles in the solution. Ether cannot extract the fat from cow's milk until acetic acid or caustic potash is alded to liberate the fats from their envelopes; but shaking with ether is sufficient to extract the fats from human milk. Some observers deny that an envelope of casein exists, and according to them milk is a simple emulsion, kept emulsionised owing to the colloid swollen-up casein in the milk plasma. The treatment of milk with potash and ether makes the casein unable any longer to preserve the emulsion (Soxhlet).

The fats of the milk-globules are the triglycerides of stearic, palmitic, oleic (very little), myristic, arachinic (butinic), capric, caprylic, caproic, and butyric acid, with traces of acetic and formic acids and cholesterin.

Butter. - When milk is beaten or stirred for a long time (i.e., churned), the fat of the milkglobules is ultimately obtained in the form of butter, owing to the rupture of the envelopes of casein. Butter is soluble in alcohol and ether, and it is clarified by heat $\left(60^{\circ} \mathrm{C}_{\text {. }}\right)$, or by washing in water at $40^{\circ} \mathrm{C}$. When allowed to stand exposed to the air, it first becomes sour, owing to the formation of lactic-acid, and afterward̀s rancid, owing to the glycerine of the neutral fats being decomposed by fungi into acrolein and formic acid, while the volatile fatty acids give it its rancid odour.

The milk plasma, obtained by filtration through a clay filter or membranes, is a clear, slightly opalescent fluid, and contains casein ( $\$ 249$, III., 3 ), some serumalbumin $(\$ 32)$, peptone $(0.13$ per cent.), nuclein, and a trace of diastatic ferment (in human milk).

The presence of other peculiar chemical bodies, e.g., lactoprotein, globulin, albumose, galactin, $\& c_{.}$, is disputed by some chemists.

When milk is boiled the albumin coagulates, while the surface also becomes covered with a thin scum or layer of casein, which has become insoluble [the rest of the milk remaining fluid].

Casein. - When milk is filtered through fresh animal membranes or through a clay filter, the casrin loes not pass through. Precipitation. - It is precipitated by adding crystals of $\mathbf{M g S O}_{4}$ to saturation. [If to nilk twice its volume of a saturated solution of $\mathrm{NaCl}$ and crystals of $\mathrm{NaCl}$ be alderl, and the whole shaken thoroughly, casein is precipitated, and carries down with it fat, so that the clear filtrate contains the lactose, salts, and coagulable proteids.]

The plasma contains milk-sugar (\$ 252); a carbohydrate resembling dextrin, (? lactic acid), lecithin, urea, extractives, kreatin, sarkin, (potassic sulphocyanide in cow's milk), sodic and potassic chlorides, alkaline phosphates, calcium and magnesium sulphates, alkaline carbonates, traces of iron, fluorine, and silica, $\mathrm{CO}_{2}, \mathrm{~N}$, and $\mathrm{O}$.

The coagulation of milk depends upon the coagulation of its casein. In milk, casein is combined with calcium phosphate, which keeps it in solution; acids which act on the calcium phosphate cause coagulation of the casein (acetic and tartaric acids in excess redissolve it). All acids do not coagulate human milk. It is coagulated by two or more drops of hydrochloric acid $(0.1$ per cent.) or acetic acid $(0.2$ per cent. $)$. The spontancons coagulation of milk after it has stood for a time, especially in a warm place, is due to the production of lactic acid, which is formed from the milk-sugar in the milk by the action of bacillus acidi lactici [which is introduced from without] ( $\$ 184$, I.). It changes the neutral alkaline phosphate into the acid phosphate, takes the casein from the calcium phosphate, and precipitates the casein. The sugar is decomposed into lactic acid and $\mathrm{CO}_{2}$.

Rennet (\$250,9, $d, \S 166$, II.) coagulates milk with an alkaline reaction (sweet whey). This ferment decomposes the casein into the precipitated cheese and also into the slightly soluble whey-albumin, so that the coagulation by rennet is a process quite distinct from the coagulation of milk by the gastric and pancreatic juices, [and also from the precipitation produced by acids. The presence of calcium phosphate seems to be necessary for the complete action of the rennet (Hammarsten)].

[Experiments. - Warm a little milk to $40^{\circ} \mathrm{C}$., and add a few drops of commercial rennet, setting aside the mixture in a warm place; a solid coagulum is soon formed, and by and by the whey separates from it. If the milk be previously diluted with water, no coagulum is formed; and if the rennet be boiled before, it like other ferments is destroyed. A solution of rennet may be prepared by extracting the fourth stomach of the calf with glycerine. [When the milk is coagulated we obtain the curd, consisting of casein with some milk-globules entangled in it ; the whey contains some soluble albumin and fat, and the great proportion of the salts and milksugar, together with lactic acid.]

[A milk-coagulating ferment is found in certain plants (artichokes, figs, Carica papaya), and causes milk to coagulate in neutral or alkaline solutions. It is also found in the small intestine 
of the calf, while a 5 per cent. $\mathrm{NaCl}$ solution of the seeds of Withania coagulans coagulates milk in an alkaline medium.]

Boiling (by killing all the lower organisms), sodium bicarbonate $\left(\frac{1}{1000}\right)$, ammonia, salicylic acid $\left(\frac{1}{5000}\right)$, glycerine, and ethereal oil of mustard prevent the spontaneous coagulation. Fresh milk makes tincture of guaiacum blue, but boiled milk does not do so. When milk is exposed to the air for a long time, it gives off $\mathrm{CO}_{2}$ and absorbs $\mathrm{O}$; the fats are increased (? owing to the development of fungi in the milk), and so are the alcoholic and ethereal extracts, from the decomposition of the casein. According to Schmidt-Mülheim, some of the casein becomes converted into peptone, but this occurs only in unboiled milk.

Composition. - 100 parts of milk contain-

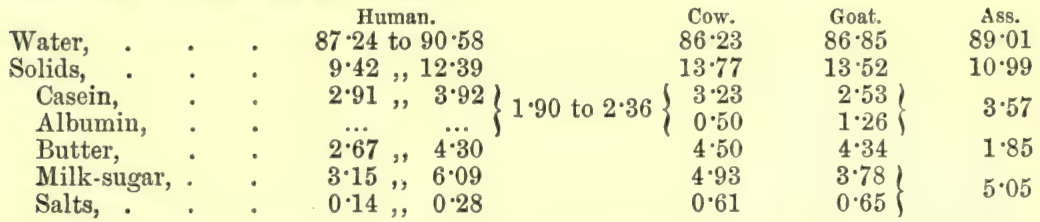

Human milk contains less albumin, which is more soluble than the albumin in the milk of animals.

Colostrum contains much serum-albumin, and very little casein, while all the other substances, and especially the fats, are more abundant.

Gases.-Pfliiger and Setschenow found in 100 vols. of milk 5.01 to $7.60 \mathrm{CO}_{2} ; 0.09$ to $0.32 \mathrm{O}$; 0.70 to $1.41 \mathrm{~N}$, according to volume. Only part of the $\mathrm{CO}_{2}$ is expelled by phosphoric acid.

Salts. - The potash salts (as in blood and muscle) are more abundant than the soda compounds, while there is a considerable amount of calcium phosphate, which is necessary for forming the bones of the infant. Wildenstein found in 100 parts of the ash of human milk-sodium chloride, 10.73 ; potassium chloride, 26.33 ; potash, 21.44 ; lime, 18.78 ; magnesia, 0.87 ; phosphoric acid, 19 ; ferric phosphate, $0 \cdot 21$; sulphuric acid, $2 \cdot 64$; silica, traces. The amount of salts present is affected by the salts of the food.

Conditions Influencing the Composition.- The oftener the breasts are emptied, the richer the milk becomes in casein. The last milk obtained at any time is always richer in butter, as it comes from the most distant part of the gland-viz., the acini. Some substances are diminished and others increased in amount, according to the time after delivery. The following are increased:-Until the 2nd month after delivery, casein and fat; until the 5 th month, the salts (which diminish progressively from this time onwards); from the 8th to the 10th month, the sugar. The following are diminished:-From 10th to 24 th month, easein; from 5 th to 6 th and 10 th to 11 th month, fat; during 1 st month, the sugar; from the 5 th month, the salts.

The greater the amount of milk that is secreted (woman), the more casein and sugar, and the less butter it contains. The milk of a primipara is less watery. Rich feeding, especially proteids (small amount of vegetable food), increases the amount of milk and the casein, sugar, and fat in it; a large amount of carbohydrates (not fats) increases the amount of sugar.

[Modifying Conditions.- That cow's milk is influenced by the pasture and food is well known. Turnip as food gives a peculiar odour, taste, and flavour to milk, and so do the fragrant grasses. The mental state of the nurse influences the quantity and quality of the milk. Jaborandi is the nearest approach to a galactagogue, but its action is temporary. Atropin is a true antigalactagogue. The composition of the milk may be affected by using fatty food, by the use of salts, and above all by the diet (Dolan).]

[Milk may be a vehicle for communicating disease-by direct contamination, from the water used for adulterating it or cleansing the vessels in which it is kept; by the milk absorbing deleterious gases; by the secretion being altered in diseased animals.] Milk ought not to be kept in zine vessels, owing to the formation of zinc lactate.

Substitutes. - If other than human milk has to be used, ass's milk most closely resembles human milk. Cow's milk is best when it contains plenty of fatty matters - it must be diluted with its own volume of water at first, and a little milk-sugar added. The casein of cow's milk differs qualitatively from that of human milk; its coagulated flocculi or curd are much coarser than the fine curd of human milk, and they are only $\frac{3}{4}$ dissolved by the digestive juices, while human milk is completely dissolved. Cow's milk when boiled is less digestible than unboiled.

Tests for Milk.-The amount of cream is estimated by placing the milk for twenty-four hours in a tall cylindrical glass graduated into a hundred parts or creamometer; the cream collects on the surface, and ought to form from 10 to 24 vols. per cent. [The cream is generally about $\frac{8}{100 .]}$ The specific gravity (fresh cow's milk, 1029 to 1034 ; when creamed, 1032 to 1040) is estimated with the lactometer at $15^{\circ} \mathrm{C}$. The sugar is estimated by titration with Fehling's solution ( $\$ 150$, II.), but in this case 1 cubic centimetre of the solution corresponds to $0.0067 \mathrm{grm}$. of milk-sugar; or its amount may be estimated with the polariscopic apparatus 
(\$ 150). The proteids are precipitated and the fats extracted with ether. The fats in fresh milk form ahout 3 per cent., and in skimmed milk $1 \frac{1}{2}$ per cent. The amount of water in relation to the milk-globules is estimated by the lactoscope or the diaphanometer of Donné (modified by Vogel and Hoppe-Seyler), which consists of a glass vessel with plane parallel sides placed 1 centimetre apart. A measured quantity of milk is taken, and water is added to it from a burette until the outline of a canclle flame placed at a distance of 1 metre can be distinctly seen through the diluted milk. This is done in a dark room. For 1 cubic centimetre of good cow's milk, 70 to 85 centimetres water are required. [Other forms of lactoscope are used, all depending on the same principle of an optical test, viz., that the opacity of milk varies with and is proportional to the amount of butter-fats present, i.e., the oil-globules. Bond uses a shallow cylinirical vessel with the bottom covered by black lines on a white surface. A measured yuantity of water is placed in this vessel, and milk is added, drop by drop, until the parallel lines on the pattern at the bottom of the dish cease to be visible. On counting the number of dropls, a table accompanying the appliance gives the percentage of fats. This method gives approximate results. In all cases it is well to use fresh milk.]

Various substances pass into the milk when they are administered to the mother-many odoriferous vegretable bodies, e.g., anise, vermuth, garlic, \&c. ; chloral, rhubarb, opium, indigo, salicylic acid, iodine, iron, zinc, mercury, lead, bismuth, antimony. In osteomalacia the amount of lime in the milk is increased (Gusserow). Potassium iodide diminishes the secretion of milk hy affecting the secretory function. Amongst abnormal constituents are-hæmoglobin, bilepigments, mucin, blood-corpuscles, pus, fibrin. Numerous fungi and other low organisms levelop in evacuated milk, and the rare blue milk is due to the development of bacillus cyanogeneum. The milk-serum is blue, not the fungus. Blue milk is unhealthy, and causes diarrhwa. There are fungi which make milk bluish-black or green. Red and yellow milk are proluced by a similar action of chromogenic fungi (\$ 184). The former is produced by Microroceus prodicriosus, which is colourless. The colour seems to be due to fuchsin. The yellow colour is produred by bacillus synxanthus. Some of the pigments seem to be related to the aniline-, and others to the phenol-colouring matters (Hüppc).

The rennet-like action of bacteria is a willely diffused property of these organisms; they "oagulate and peptonise casein and may ultimately produce further decompositions. The lutyric acil bacillus ( $\$ 184)$ first coagulates casein, then peptonises it, and fiully splits it up, with the evolution of ammonia (Hüppe).

Milk becomes stringy owing to the action of cocci which form a stringy substance $[=$ dextran, $\mathrm{C}_{12} \mathrm{H}_{10} \mathrm{O}_{10}$ (S'schibler)], just as beer or wine undergoes a similar or ropy change. [The milk of diseased animals may contain or transmit directly infectious matter.]

Preparations of Milk.-(1) Condensed milk- 80 grms. cane-sugar are added to 1 litre of milk; the whole is evaporated to $\frac{1}{5}$; and while hot sealed up in tin cans. For children one teaspoonful is dissolved in a pint of cold water, and then boiled.

(2) Koumiss is prepared by the Tartars from mare's milk. After the addition of koumiss and sour milk, the whole is violently stirred, and it undergoes the alcoholic fermentation, wherehy the milk-sugar is first changel into galactose, and then into alcohol ; so that koumiss contains 2 to 3 per cent. of alcohol; while the casein is at first precipitated, but is afterwards partly redissolved and changed into acid-albumin and peptone. Tartar koumiss seems to be produced by the action of a special bacterium (Diaspora caucasia).

(3) Cheese is preprared by coagulating milk with rennet, allowing the whey to separate, and alding salt to the curd. When kept for a long time cheese "ripens," the casein again becomes soluble in water, probably from the formation of soda albuminate; in many cases it becomes semi-fluil, when it takes the characters of peptones. When further decomposition occurs, leucin and tyrosin are formed. The fats increase at the expense of the casein, and they again undergo further change, the volatile fatty acils giving the characteristic odour. The formation of peptone, leucin, tyrosin, and the decomposition of fat recall the digestive processes. [Cheese is coagulated casein entangling more or less fat, so that the richness of the cheese will depend upon the kind of milk from which it is made. There are, in this sense, three kinds of cheese, whole milk, shim milk, and cream cheese, the last being represented by Stilton, Roquefort, Cheshire, \&c. The composition is shown in the following table after Bauer:-

\begin{tabular}{|c|c|c|c|c|c|c|}
\hline & & Water. & $\begin{array}{l}\text { Nitrogenous } \\
\text { Matter. }\end{array}$ & Fat. & Extractives. & Ash. \\
\hline $\begin{array}{l}\text { Cream cheese, } \\
\text { Whole milk, } \\
\text { Skim milk, }\end{array}$ & $\begin{array}{l}\dot{.} \\
\dot{.}\end{array}$ & $\begin{array}{l}35 \cdot 75 \\
46 \cdot 82 \\
48 \cdot 02\end{array}$ & $\begin{array}{r}7 \cdot 16 \\
27 \cdot 62 \\
32 \cdot 65\end{array}$ & $\begin{array}{r}30 \cdot 43 \\
20 \cdot 54 \\
8 \cdot 41\end{array}$ & $\begin{array}{l}2 \cdot 53 \\
2 \cdot 97 \\
6 \cdot 80\end{array}$ & $\begin{array}{l}4 \cdot 13 \\
3 \cdot 05 \\
4 \cdot 12\end{array}$ \\
\hline
\end{tabular}

Cream cheese, especially if it be made from goat's milk, aequires a very high odour and strong flavour when it is kept and "ripens"; the casein is partly decomposed to yield ammonia and ammonium sulphide, while the fats yield butyric, caproic, and other acids.] 
232. EGGS must be regarded as a complete food, as the organism of the young chick is developed from them. The yolk contains a characteristic proteid bodyvitellin (\$249), and an albuminate in the envelopes of the yellow yolk spheresnuclein, from the white yolk; fats in the yellow yolk (palmitin, olein), cholesterin, much lecithin; and as its decomposition-product, glycerin-phosphoric acid-grapesugar, pigments (lutein), and a body containing iron and related to hæmoglobin; lastly, salts qualitatively the same as in blood-quantitatively as in the blood-corpuscles-and gases. The chief constituent of the white of egg is egg-albumin (§249), together with a small amount of palmitin and olein partly saponified with soda ; grape-sugar, extractives ; lastly salts, qualitatively resembling those of blood, but quantitatively like those of serum, and a trace of fluorine. Relatively more of the nitrogenous constituents than of the fatty constituents of eggs are absorbed (Rubner).

[The shell is composed chiefly of mineral matter (91 per cent. of calcic carbonate, 6 per cent. of calcic phosphate, and 3 per cent. of organic matter. A hen's egg weighs about 13. which the shell forms about $\frac{1}{10}$. Note the amount of fats in the yolk.]

Composition :-

$\begin{array}{lccc} & & \text { White of Egg. Yolk. } \\ \text { Water, . } & \text {. } & 84.8 & 51.5 \\ \text { Proteids, } & \text {. } & 12.0 & 15.0 \\ \text { Fats, \&c., } & \text {. } & 2.0 & 30.0\end{array}$

$\begin{array}{lcc} & \text { White of Egg. Yolk. } \\ \text { Mineral matter, } & 1 \cdot 2 & 1 \cdot 4 \\ \text { Pigment extractives, } & \ldots & 2 \cdot 1\end{array}$

233. FLESH AND ITS PREPARATIONS.-Flesh, in the form in which it is eaten, contains, in addition to the muscle-substance proper, more or less of the elements of fat, connective- and elastic-tissue mixed with it (\$293). The following results refer to flesh freed as much as possible from those constituents. The chief proteid constituent of the contractile muscular substance is myosin ; serum-albumin occurs in the fluid of the fibres, in the lymph and blood of muscle. The fats are for the most part derived from the interfascicular fat-cells, while lecithin and cholesterin come from the nerves of the muscles; the gelatin is derived from the connective-tissue of the perimysium, perineurium, and the walls of blood-vessels and tendons. The red colour of the flesh is due to the hæmoglobin present in the sarcous substance, but in some muscles, e.g., the heart, there is a special pigment, myohæmatin (MacMunn). Elastin occurs in the sarcolemma, neurilemma, and in the elastic fibres of the perimysium and walls of the vessels; the small amount of keratin is derived from the endothelium of the vessels. The chief muscular substance, the result of the retrogressive metabolism of the sarcous substance, is kreatin (-0.05 per cent.) ; kreatinin, the inconstant inosinic acid, then lactic, or rather sarcolactic acid (\$293). Further, taurin, sarkin, xanthin, uric acid, carnin, inosit (most abundant in the muscles of drunkards), urea $(0 \cdot 1$ per cent.) dextrin (in horse and rabbit, not constant); grape-sugar, but this is very probably derived post-mortem from glycogen $(0.43$ per cent.), which occurs in considerable amount in fœetal muscles; lastly volatile fatty acids. Amongst the salts, potash and phosphoric acid compounds are most abundant; magnesium phosphate exceeds calcium phosphate in amount. [The composition varies somewhat even in different muscles of the same animal.]

In 100 parts Flesh there are, according to Schlossberger and v. Bibra-

\begin{tabular}{|c|c|c|c|c|c|c|c|c|}
\hline & $0 x$. & Calf. & Deer. & Pig. & Man. & Fowl. & Carp. & Frog. \\
\hline Water, . & $77 \cdot 50$ & $78 \cdot 20$ & $74 \cdot 63$ & $78 \cdot 30$ & $74 \cdot 45$ & $77 \cdot 30$ & $79 \cdot 78$ & $80 \cdot 43$ \\
\hline Solids, : : . & $22 \cdot 50$ & $21 \cdot 80$ & $25 \cdot 37$ & $21 \cdot 70$ & $25 \cdot 55$ & $22 \cdot 7$ & $20 \cdot 22$ & $19 \cdot 57$ \\
\hline Soluble albumin, & $2 \cdot 20$ & $2 \cdot 60$ & $1 \cdot 94$ & $2 \cdot 40$ & 1.93 & $3 \cdot 0\{$ & $2 \cdot 35$ & $1 \cdot 86$ \\
\hline Glutin, . & $1: 30$ & $1 \cdot 60$ & 0.50 & 0.80 & $2 \cdot 07$ & $1 \cdot 2$ & $1 \cdot 98$ & $2 \cdot 48$ \\
\hline Alcoholic extract, . & $1 \cdot 50$ & $1 \cdot 40$ & $4 \cdot 75$ & $1 \cdot 70$ & $3 \cdot 71$ & $1 \cdot 4$ & $3 \cdot 47$ & $3 \cdot 46$ \\
\hline Fats, & $\cdots$ & $\ldots$ & $1 \cdot 30$ & $\ldots$ & $2 \cdot 30$ & $\ldots$ & $1 \cdot 1]$ & $0 \cdot 10$ \\
\hline Blood-vessels, \&c., & $17 \cdot 50$ & $16 \cdot 2$ & $16 \cdot 81$ & $16 \cdot 81$ & $15 \cdot 54$ & $16 \cdot 5$ & $11 \cdot 31$ & $11 \cdot 67$ \\
\hline
\end{tabular}


In 100 parts Ash there are-

\begin{tabular}{|c|c|c|c|c|c|c|c|c|c|}
\hline & & & & & & Horse. & $0 x$. & Calf. & Pig. \\
\hline Potash, . & . & . & - & - & . & $39 \cdot 40$ & $35 \cdot 94$ & $34 \cdot 40$ & $37 \cdot 79$ \\
\hline Soula, $\because$ & . & . & . & . & . & $4 \cdot 86$ & & $2 \cdot 35$ & 4.02 \\
\hline Magnesia, & . & . & . & - & - & $3 \cdot 88$ & $3 \cdot 31$ & 1.45 & $4 \cdot 81$ \\
\hline Chalk, . & . & . & . & - & - & $1 \cdot 80$ & $1 \cdot 73$ & $1 \cdot 99$ & $7 \cdot 54$ \\
\hline Potassium, & . & & . & . & - & $\cdots$ & $5 \cdot 36$ & $\cdots$ & 范 \\
\hline Sodium, & - & - & . & . & - & $1 \cdot 47$ & $\dddot{4} \cdot 86$ & 10.59 & $\begin{array}{l}0.40 \\
0.62\end{array}$ \\
\hline Chlorine, & : & · & . & . & : & $1 \cdot 0$ & $\begin{array}{l}4.80 \\
0.98\end{array}$ & 0.27 & 0.35 \\
\hline $\begin{array}{l}\text { Iron oxide, } \\
\text { Phosphoric }\end{array}$ & Acid, & : & . & . & i. & $46 \cdot 74$ & $34 \cdot 36$ & $48 \cdot 13$ & $44 \cdot 47$ \\
\hline Sulphuric &, & : & . & . & . & $0 \cdot 30$ & $3 \cdot 37$ & & ... \\
\hline Silicic & ," & . & . & . & . & $\ldots$ & $2 \cdot 07$ & 0.81 & $\cdots$ \\
\hline Carbonic & ," & . & & . & . & $\cdots$ & $8 \cdot 02$ & $\cdots$ & $\cdots$ \\
\hline Ammonia, & . & . & . & . & . & $\cdots$ & 0.15 & $\cdots$ & $\cdots$ \\
\hline
\end{tabular}

The amount of fat in flesh varies very much according to the condition of the animal. After removal of the visible fat, human flesh contains $7 \cdot 15$; ox, $11 \cdot 12$; calf, 10.4 ; sheep, 3.9 ; wild goose, $8 \cdot 8$; fowl, $2 \cdot 5$ per cent.

The amount of extractives is most abundant in those animals which exhibit energetic muscular action; hence it is largest in wild animals. The extract is increased after vigorous muscular action, whereby sarcolactic acid is developed, and the flesh becomes more tender and is more palatable. Some of the extractives excite the nervous system, e.g., kreatin and kreatinin ; and others give to flesh its characteristic agreeable flavour ["osmasome,"] but this is also partly due to the different fats of the flesh, and is best developed when the flesh is cooked. The extractives in 100 parts of flesh are in man and pigeon, 3 ; deer and duck, 4; swallow, 7 per cent.

Cooking of Flesh. $-A \mathrm{~s}$ a general rule, the flesh of young animals, owing to the sarcolemma, connective-tissue, and elastic constituents being less tough, is more tender and more easily digesterl than the flesh of old animals; after flesh has been kept for a time it is more friable and tender, as the inosit becomes changed into sarcolactic acid and the glycogen into sugar, and this again into lactic acid, whereby the elements of the flesh undergo a kind of maceration. Finely diviled flesh is more digestible than when it is eaten in large pieces. In cooking meat, the heat ought not to be too intense, and ought not to be continued too long, as the muscular fibres thereby become hard and shrink very much. Those parts are most digestible which are obtained from the centre of a roast where they have been heated to $60^{\circ}$ to $70^{\circ} \mathrm{C}$., as this temperature is sufficient, with the aid of the acids of the flesh, to change the connective-tissue into gelatin, whereby the fibres are loosened, so that the gastric juice readily attacks them. In roasting beef, apply heat suddenly at first, to coagulate a layer on the surface, which prevents the escape of the juice.

Meat Soup is best prepared by cutting the flesh into pieces and placing them for several hours in cold water, and afterwards boiling. Liebig found that 6 parts per 100 of ox flesh were dissolved by cold water. When this cold extract was boiled, 2.95 parts were precipitated as coagulated albunin, which is chiefly removed by "skimming," so that only 3.05 parts remain in solution. From 100 parts of flesh of fowl, 8 parts were extracted, and of these 4.7 was coagulated and $3 \cdot 3$ remained dissolved in the soup. By boiling for a very long time, part of the albumin may be relissolved. The dissolved substances are:-(1) Inorganic salts of the meat, of which $82 \cdot 27$ per cent. jass into the soup; the earthy phosphates chiefly remain in the cooked meat. (2) Kreatin, kreatinin, the inosinates and lactates which give to broth or beef-tea their stimulating 'qualities, and a small amount of aromatic extractives. (3) Gelatin, more abundantly extracted from the flesh of young animals. According to these facts, therefore, flesh broth or beef-tea is a powerful stimulant, supplying muscle with restoratives, but is not a food in the ordinary sense of the term, as kreatin in general leaves the body unchanged ( $v$. Voit). The flesh, especially if it be cooked in a large mass, after the extraction of the broth is still available as a food.

Liebig's Extract of Meat is an extract of flesh evaporated to a thick syrupy consistence. It contains no fat or gelatin, and is chiefly a solution of the extractives and salts of flesh.

[Extract of Fish. - A similar extract is now prepared from fish, and such extract has no fishy flavour, but presents much the same appearance, odour, and properties as extract of flesh.]

234. VEGETABLE FOODS.-The nitrogenous constituents of plants are not so easily absorbed as animal food (Rubner). Carbohydrates, starch, and sugar are very completely absorbed, and even a not inconsiderable proportion of cellulose may be digested. The more fats that are contained in the vegetable food, the less are the carbohydrates digested and absorbed. 
1. The cereals are most important vegetable foods ; they contain proteids, starch, salts, and water about 14 per cent. The nitrogenous body glutin is most abundant under the husk (fig. 236, c). The use of whole meal containing the outer layers of the grain is highly nutritive, but bread containing much bran is somewhat indigestible (Rubner). Their composition is the following :-

\begin{tabular}{|c|c|c|c|c|c|}
\hline \multicolumn{3}{|c|}{100 Parts of the Dry Meal contain } & \multicolumn{3}{|c|}{100 Parts of Ash contain } \\
\hline 0 & Albumin. & Starch. & Red Wheat. & & White Wheat. \\
\hline $\begin{array}{l}\text { Wheat, : } \\
\text { Rye, } \\
\text { Barley, : } \\
\text { Maize, : } \\
\text { Rice, } \\
\text { Buckwheat }\end{array}$ & $\begin{array}{l}16 \cdot 52 \% \\
11 \cdot 92 \\
17 \cdot 70 \\
13.65 \\
7 \cdot 40 \\
6 \cdot 8-10 \cdot 5\end{array}$ & $\begin{array}{l}56 \cdot 25 \% \\
60 \cdot 91 \\
38 \cdot 31 \\
77 \cdot 74 \\
86 \cdot 21 \\
65 \cdot 05\end{array}$ & $\begin{array}{r}27 \cdot 87 \\
15 \cdot 75 \\
1 \cdot 93 \\
9 \cdot 60 \\
1 \cdot 36 \\
49 \cdot 36 \\
0 \cdot 15\end{array}$ & $\begin{array}{l}\text { Potash, : } \\
\text { Soda, : } \\
\text { Lime, : } \\
\text { Magnesia, } \\
\text { Iron oxide, } \\
\text { Phosphoric Acid, } \\
\text { Silica, . }\end{array}$ & $\begin{array}{r}33 \cdot 84 \\
\ldots \\
3 \cdot 09 \\
13 \cdot 54 \\
0 \cdot 31 \\
59 \cdot 21 \\
\quad \ldots\end{array}$ \\
\hline
\end{tabular}

It is curious to observe that soda is absent from white wheat, its place being taken by other alkalies. Rye contains more cellulose and dextrin than wheat, but less sugar; rye-bread is usually less porous.

[0atmeal contains more nitrogenous substances (gliadin and glutin-casein) than wheaten flour, but owing to the want of adhesive properties it cannot be made into bread. The amount of fat and salts is large.]

In the preparation of bread the meal is kneaded with water until dough is formed, and to it is added salt and yeast (Saccharomycetes cerevisiæ). When placed in a warm oven, the proteids of the meal begin to decompose and act as a ferment upon the swollen-up starch, which becomes in part changed into sugar. The sugar is further decomposed into $\mathrm{CO}_{2}$ and alcohol, the $\mathrm{CO}_{2}$ forms bubbles, which cause the bread to "rise" and thus become spongy and porous. The alcohol is driven off by the baking $\left(200^{\circ}\right)$, while much soluble dextrin is formed in the crust of the bread. [But $\mathrm{CO}_{2}$ may be set free within the dough by chemical means without yeast or leaven, thus forming unfermented bread. This is done by mixing with the dough an alkaline carbonate and then adding an acid. Baking powders consist of carbonate of Micr soda and tartaric acid. In Dauglish's process for aerated bread, the $\mathrm{CO}_{2}$ is forced into water, and a dough is made with this

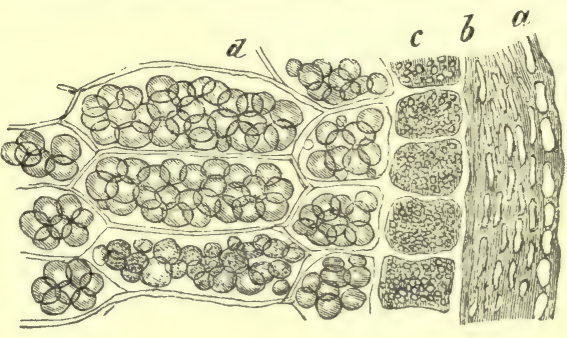

Fig. 236.

Microscopic characters of wheat $(\times 200)$. $\quad a$, cells of the bran; $b$, cells of thin cuticle; $c$, glutin cells ; $d$, starch cells. water under pressure, and when the dough is heated, the $\mathrm{CO}_{2}$ expands and forms the spongy bread. Bread as an article of food is deficient in $\mathrm{N}$, while it is poor in fats and some salts. Hence the necessity for using some form of fat with it (butter or bacon).

2. The pulses contain much albumin, especially legumin : together with starch, lecithin, cholesterin, and 9 to 19 per cent. water. Peas contain 18.02 proteids, and $34 \cdot 81$ starch : beans $28 \cdot 54$, and $37 \cdot 50$ : lentils, $29 \cdot 31$, and 40 , and more cellulose. Owing to the absence of glutin they do not form dough, and bread cannot be prepared from them. On account of the large amount of proteids which they contain, they are admirably adapted as food for the poorer classes.

[3. The whole group of farinaceous substances used as "pudding stuffs," such as corn-flour, arrow-root, rice, hominy, are really very largely composed of starchy substances.]

4. Potatoes contain 70 to 81 per cent. water. In the fresh juicy cellular tissue, which has an acid reaction, from the presence of phosphoric, malic, and hydrochloric acids, there is 16 to 23 per cent. of starch, 2.5 soluble albumin, globulin, and a trace of asparagin. The envelopes of the cells swell up by boiling, and are changed into sugar and gums by dilute acids. The poisonous solanin occurs in the sprouts. In 100 parts of potato ash, May found 49.96 potash, 2.41 sodium chloride, 


\section{$8 \cdot 11$ potassium chloride, $6 \cdot 50$ sulphuric acid derived from burned proteids, $7 \cdot 17$} silica.

5. In fruits the chief nutrient ingredients are sugar and salts; the organic acids give them their characteristic taste, the gelatinising substance is the soluble so-called pectin $\left(\mathrm{C}_{3.2} \mathrm{H}_{45}\left(\mathrm{O}_{32}\right)\right.$, which can be prepared artificially by boiling the very insoluble pectose of unripe fruits and mulberries.

6. Green Vegetables are especially rich in salts, which resemble the salts of the blood: thus, dry salad contains 23 per cent. of salts, which closely resemble the salts of the blood. Of much less importance are the starch, cell-substance, dextrin, sugar, and the small amount of albumin which they contain.

[lemetaliles are chiefly useful for the salts they contain, while many of them are antiscorbutic. Their value is attested by the serious defects of nutrition, such as scurvy, which result when they are not supplied in the food. In Arctic expeditions and in the navy, lime juice is served out as an antiscorbutic.]

[Preserved Vegetables. - The dried and compressed vegetables of Messrs Chollet \& Company are an excellent substitute for fresh vegetables, and are used largely in naval and military expeditions.]

[Utilisation of Food.--As regards what percentage of the food swallowed is actually absorbed, we know that, stated broadly, vegetable food is assimilated to a much less extent than animal food in man. Fr. Hofmann gives the following table as showing this:-

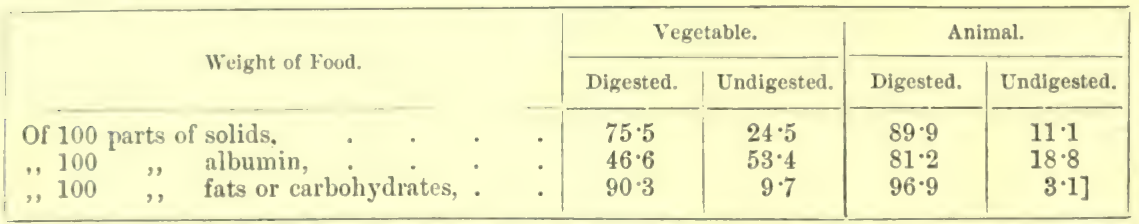

[The following table, abrilged from Parkes, shows the composition of the chief articles of diet, and is also used for calculating diet tables :-

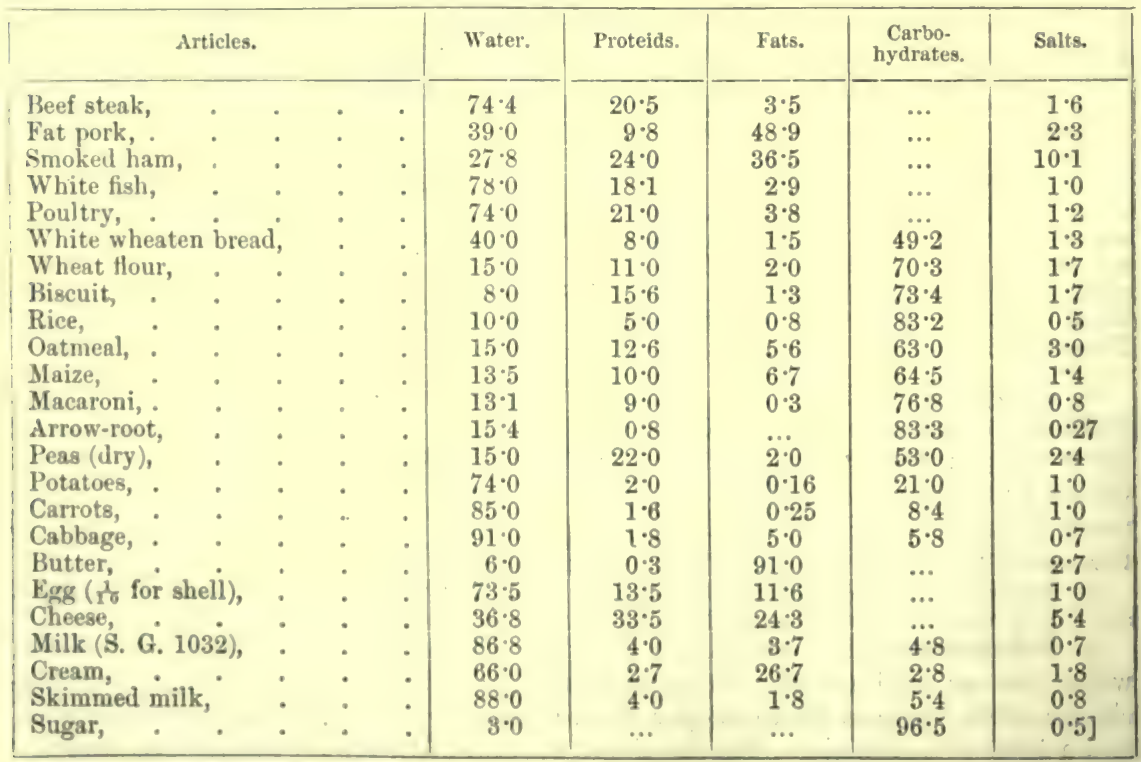

235. CONDIMENTS, COFFEE, TEA, ALCOHOL. - Some substances are used along with food, not so much on account of their nutritive properties as on account of their stimulating 
effects and agreeable qualities, which are exerted partly upon the organ of taste and partly upon the nervous system. These are called condiments.

Coffee, Tea, and Chocolate are prepared as infusions of certain vegetables [the first of the roasted. berry, the second of the leaves, and the third of the seeds]. Their chief active ingredients are respectively caffein, thein $\left(\mathrm{C}_{8} \mathrm{H}_{10} \mathrm{~N}_{4} \mathrm{O}_{2}+\mathrm{H}_{2} \mathrm{O}\right)$, and theobromin $\left(\mathrm{C}_{7} \mathrm{H}_{3} \mathrm{~N}_{4} \mathrm{O}_{2}\right)$, which are regarded as alkaloids of the vegetable bases, and which have recently been prepared artificially from xanthin (E. Fischer). [Guarana, or Brazilian cocoa, is made of the seeds ground into a paste in the form of a sausage. Maté or Paraguay tea (the leaves of a species of holly) is used in South America, and so also is the coca of the Andes (Erythroxylon C'oca).] These "alkaloids" occur as such in the plants containing them; they behave like ammonia; they have an alkaline reaction, and form erystalline salts with acids. All these vegetable bases act upon the nervous system; some more feebly (as the above), others more powerfully (quinine); some stimulate powerfully, or completely paralyse (morphia, atropin, strychnin, curarin, nicotin).

Effects.-All these substances act on the nervous system; they quicken thought, accelerate movement, and stir one to greater activity. In these respects they resemble the stimulating extractives of beef-tea. Coffee contains about $\frac{1}{3}$ per cent. of caffein, part of which only is liberated by the act of roasting. Tea has 6 per cent. of thein; whilst green tea contains 1 per cent. ethereal oil, and black tea $\frac{1}{2}$ per cent.; in green tea there is 18 per cent., in black 15 per cent. tannin; green tea yields about 46 per cent., and the black scarcely 30 per cent. of extract. The inorganic salts present are also of importance; tea contains 3.03 per cent. of salts, and amongst these are soluble compounds of iron, manganese, and sodasalts. In coffee, which yields 3.41 per cent. of ash, potash salts are most abundant; in all three substances the other salts which occur in the blood are also present.

Alcoholic drinks owe their action chiefly to the alcohol which they contain. Alcohol, when taken into the body, undergoes certain changes and produces certain effects:-(1) About 95 per cent. of it is oxidised chiefly into $\mathrm{CO}_{2}$ and $\mathrm{H}_{2} \mathrm{O}$, so that it is so far a source of heat. As it undergoes this change very readily, when taken to a certain extent, it may act as a substitute for the consumption of the tissues of the body, especially when the amount of food is insufficient. [Hammond found that when he lived on an insufficient amount of food, alcohol, if given in a certain quantity, supplied the place of the deficiency of food, and he even gained in weight. If, however, sufficient food was taken, alcohol was unnecessary. As it interferes with oxidation, and where there is a sufficient amount of other food, in health, it is unnecessary for dietetic reasons.] Small doses diminish the decomposition of the proteids to the extent of 6 to 7 per cent. Only a very small part of the alcohol is excreted in the urine; the odour of the breath is not due to alcohol, but to other volatile substances mixed with it, e.g., fusel oil, \&c. (2) In small doses it excites, while in large doses it paralyses the nervous system. By its stimulating qualities it excites to greater action, which, however, is followed by depression. (3) It diminishes the sensation of hunger. (4) It excites the vascular system, accelerates the circulation, so that the muscles and nerves are more active, owing to the greater supply of blood. It also gives rise to a subjective feeling of warmth. In large doses, however, it paralyses the vessels, so that they dilate, and thus much heat is given off $(\$ 213,7 ; \S 227)$. The action of the heart also becomes affected, the pulse becomes smaller, feebler, and more rapid. In high altitudes the action of alcohol is greatly diminished, owing to the diminished atmospheric pressure, whereby it is rapidly given off from the blood.

Alcohol in small doses is of great use in conditions of temporary want, and where the food taken is insufficient in quantity. When alcohol is taken regularly, more especially in large doses, it affects the nervous system, and undermines the psychical and corporeal faculties, partly from the action of the impurities which it may contain, such as fusel oil, which has a poisonous effect upon the nervous system, partly by the direct effects, such as catarrh and inflammation of the digestive organs, which it produces, and lastly, by its effect upon the normal metabolism. 
[The action of alcohol in lowering the temperature, even in moderate doses, is most important. By dilating the cutaneous vessels, it thus permits of the radiating of much heat from the blood. When the action of alcohol is pushed too far, and especially when this is combiued with the action of great cold, its use is to be condemned. Brunton has pointed out that, as regards its action on the nervous system, it seems to induce progressive paralysis, affecting the nervous tissues "in the inverse orler of their development, the highest centres being affected first and the lowest last." The julgment is affected first, although the imagination and "emotions may be more than unusually active." The motor centres and speech are affected, then the cerehellum is influenced, and afterwards the cord, while by and by the centres essential to life are paralysed, provided the dose be sufficiently large.]

Preparation.-Alcoholic drinks are prepared by the fermentation of various carbohydrates, such as sugar lerived from starch. The alcoholic fermentation, such as occurs in the manufacture of beer, is caused by the development of the yeast plant, Saccharomycetes cerevisiæ ; while in the fermentation of the grape (wine), S. Ellipsoideus is the species present (fig. 237). The yeast takes the substances necessary for the maintenance of its organic processes directly from the mixture of the sugar, viz., carbohydrates, proteids, and salts, especially calcium and potassium phosphates and magnesium sulphate. These substances undergo decomposition
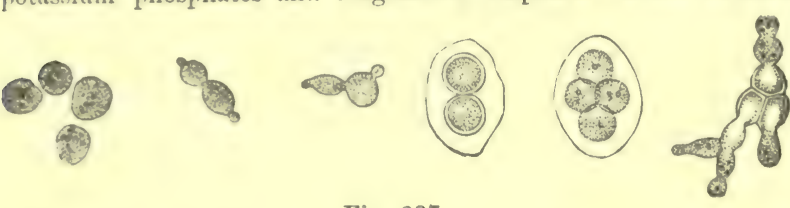

within the cells of the yeast plant, which multiply during the process, and there are produced alcohol and $\mathrm{CO}_{2}(\S 150)$, together with glycerine $(3 \cdot 2$ to $3 \cdot 6$ per cent.) and succinic acid $\left(0^{\circ} 6\right.$

Fig. 237. to 0.7 per cent.). Yeast is either added intentionally

1, Isolated yeast cells ; 2, 3, yeast cells bulding; 4, 5, so-called or it reaches the mixture endogenous formation of cells; 6 , sprouting and formation of buds. from the air, which always contains its spores. When yeast is completely excluded, or if it be killed by boiling [or if its action be prevented by the presence of some germicide], the fermentation does not occur. The alcoholic fermentation is due to the vital activity of a low organism.

In the preparation of brandy, the starch of the grain or potatoes is first changed into sugar by the action of diastase or maltin. Yeast is adled, and fermentation thereby produced; the mixture is distilled at $78.3^{\circ} \mathrm{C}$. The fusel oil is prevented from mixing with the alcohol by passing the vapour through heated charcoal. The distillate contains 50 to 55 per cent. of alcohol.

In the preparation of wine, the saccharine juice of the grape-the must-after being expressed. from the grapes, is exposed to the air at $10^{\circ}$ to $15^{\circ} \mathrm{C}$., and the yeast cells, which are floating about, drop into it and excite fermentation, which lasts 10 to 14 days, when the yeast sinks to the bottom. The clear wine is drawn off into casks, where it becomes turbid by undergoing an after-fermentation, until the sugar is converted into alcohol and $\mathrm{CO}_{2}$, which is accompanied by the deposition of some yeast and tartar. If all the sugar is not decomposed-which occurs when there is not sufficient nitrogenous matter present to nourish the yeast-a sweet wine is obtained. W'ine contains 89 to 90 per cent. water, 7 to 8 per cent. alcohol, together with athylic, propylic, and butylic alcohol. The red colour of some wines is due to the colouring matter of the skin of the grapes, but if the skins be removed before fermentation, red grapes yield white wine. When wine is stored, it clevelops a fine flavour or bouquet. The characteristic vinous odour is due to œnanthic ether. The salts of wine closely resemble the salts of the blood.

In the preparation of beer the grain is moistened, and allowed to germinate, when the temperature rises, and the starch ( 68 per cent. in barley) is changed into sugar. Thus "malt" is formed, which is dried, and afterwards pulverised, and extracted with water at $70^{\circ}$ to $75^{\circ}$, the watery extract being the "wort." Hops are added to wort, and the whole is evapurated, when the proteils are coagulated. Hops give beer its bitter taste, and make it keep, while their tannic acid precipitates any starch that may be present, and clarifies the wort. After being boiled, it is cooled rapidly $\left(12^{\circ} \mathrm{C}\right.$. $)$; yeast is added, and fermentation goes on rapidly and with considerable effervescence at $10^{\circ}$ to $14^{\circ}$. Beer contains 75 to 95 per cent. water; alcohol, 2 to 5 per cent. (porter and ale, to 8 per cent.); $\mathrm{CO}_{2,} 0.1$ to 0.8 per cent.; sugar, 2 to 8 per cent.; gum, lextrin, 2 to 10 per cent.; the hops yield traces of protein, fat, lactic acid, ammonia compounds, the salts of the grain and of the hops. In the ash there is a great preponderance of phosphoric acid and potash, both of which are of great importance for the formation of blood. In 100 parts of ash there are 40.8 potash, $20^{\circ} 0$ phosphorus, magnesium phosphate 20 , calcium phosphate $2 \cdot 6$, silica $16 \cdot 6$ per cent. The formation of blood, muscle, and other tissues from the consumption of beer is due to the phosphoric acid and potash, while if too much be taken, the potash produces fatigue.

Condiments are taken with food, partly on account of their taste, and partly because they excite secretion. Common salt, in a certain sense, is a condiment. 
We may also include many substances of unknown constitution which act upon the gustatory organs, e.g., dextrin, and sulostances in the crust of bread and in meat which has been roasted.

236. EQUILIBRIUM OF THE METABOLISM.-By this term is meant that, under normal physiological conditions, just as much material is absorbed and assimilated from the food as is removed from the body by the excretory organs in the form of effete or end-products, the result of the retrogressive tissue-changes. The income must always balance the expenditure; wherever a tissue is used up, it must be replaced by the formation of new tissue. During the period of growth, the increase of the body corresponds to a certain increase of formation, whereby the metabolism of the growing parts of the body is 2.5 to 6.3 times greater than that of the parts already formed. Conversely, during senile decay, there is an excess of expenditure from the body.

Methods. - The normal equilibrium of the metabolism of the body is investigated-(1) By determining chemically that the sum of all the substances passing into the body is equal to the sum of all the substances given off from it. Thus the $\mathrm{C}, \mathrm{N}, \mathrm{H}, \mathrm{O}$, salts and water of the food, and the $\mathrm{O}$ inspired, must be equal to the $\mathrm{C}, \mathrm{N}, \mathrm{H}, \mathrm{O}$, salts and water given off in the excreta (urine, fæces, air expired, water excreted). (2) 'The physiological equilibrium is determined empirically by observing that the body retains its normal weight with a given diet; so that, by simply weighing a person, a physician is enabled to determine exactly the state of convalescence of his patient. The tedious process of making an elementary analysis of the metabolic substances was first undertaken in the Munich School by v. Bischoff, v. Voit, v. Pettenkofer, and others.

Circulation of $\mathbf{C}$.- In the circulation of materials the total amount of $\mathrm{C}$ taken in the food, if the metabolism be in a condition of physiological equilibrium, must be equalled by the $\mathrm{C}$ in the $\mathrm{CO}_{2}$ given off by the lungs and skin (90 per cent.), together with the relatively small amount of $\mathrm{C}$ in the organic excreta of the urine and fæces (10 per cent.).

Circulation of $\mathbf{N}$. - Nearly all the $N$ taken in with the food is excreted within twenty-four hours in the form of urea. A very small amount of nitrogenous matter is excreted in the fæces, while the other nitrogenous urinary constituents (uric acid, kreatinin, \&c.) represent about 2 per cent. of $\mathrm{N}$. A trace of the $\mathrm{N}$ is given off by the breath (\$124), and a minute proportion in combination, in the epidermal scales (50 milligrammes daily in the hair and nails), and in the sweat.

Deficit of $\mathbf{N}$.- That nearly all the $\mathrm{N}$ taken in the food reappears in the urine and fæces, as was stated by v. Voit to be the case in the carnivora and in the herbivora, and by $\mathrm{v}$. Ranke in man, is contradicted partly by old and partly by new observations, which go to show that the whole of the $\mathrm{N}$ cannot be recovered from these excretions, but that on the contrary there is a considerable deficit.

According to Leo, only 0.55 per cent. of the albumin transformed within the body (assuming 15 per cent. $\mathrm{N}$ in albumin) gives off its $\mathrm{N}$ in the form of gaseous $\mathrm{N}$ (according to Seegen and Nowak 12 times more). In every exact analysis of the metabolism of $\mathrm{N}$ this gaseous excretion of $\mathrm{N}$ must be taken into account.

The excretion of $\mathbf{N}$ after food does not take place regularly from hour to hour, but it increases at once and distinctly, reaches its maximum in five to six hours, and then gradually falls. The same is true of the excretion of $\mathbf{S}$ and $\mathbf{P}$; but in these cases the maximum of excretion is reached at the fourth hour. When fat is added to a diet of flesh, the excretion of $\mathrm{N}$ and $\mathrm{S}$ is uniformly distributed over the individual hours of the day ( $v$. Voit and Feder).

The nitrogenous constituents in the body during metabolism become poorer in $\mathrm{C}$, and richer in $\mathrm{N}$ and $\mathrm{O}$. Thus in albumin to 1 atom of $\mathrm{N}$ there are 4 atoms $\mathrm{C}$; in gelatin, $3 \frac{1}{2} \mathrm{C}$; in glycocoll, $2 \mathrm{C}$; in kreatin, $1 \frac{1}{3} \mathrm{C}$; in uric acid, $1 \frac{1}{4} \mathrm{C}$; in allantoin, $1 \mathrm{C}$; in urea, only $\frac{1}{2}$ atom of $\mathrm{C}$.

The $\mathbf{H}$ leaves the body chiefly in the form of water-a part, however, is in combination in other excreta; the $\mathbf{0}$ is chiefly excreted as $\mathrm{CO}_{2}$ and water; a little is given off in combination in other excreta; water is given off by evaporation from the lungs and skin, and also in the urine and frecs. As $H$ is oxidised to $\mathrm{H}_{2} \mathrm{O}$, more water is excreted than is taken in. Most of the readily soluble salts 
are given off by the urine; the less soluble salts, especially those of potash, and the insoluble salts, in the fixces; while others are given off in the sweat. Of the sulphur of albumin, about one-half is excreted in the sulphur compounds in the urine, and the other half in the freces (taurin) and in the epidermal tissues.

Every organism has a minimum and a maximum limit of metabolism, according to the amount of work done by the body, and its weight. If less food be given than is necesiary to maintain the former, the body loses weight; while, if more be given after the maximum limit is reached, the food so given is not absorbed, but remains as a floating balance, and is given off with the freces. When food is liberally supplied and the weight increases, of course the minimum limit rises; hence, during the process of "feeding" or "fattening," the amount of food necessary is very much sreater than in poorly fed animals, for the same increase of the bodyweight. liy continuing the process, a condition is at last reached, in which the digestive orcans are just sufficient to maintain the existing condition, but cannot act $s u$ as to admit of new additions being made to the body-weight $(v$. Bischoff, $v$. Voit, v. Pettenkotier).

By the term "Iuxus consumption" is meant the direct combustion or oxidation of the superfluous food-stuffs absorbed into the blood. This, however, does not exist; on the contrary, the material in the juices is always being used for building up the tissues. The albumin found in the fluids, which everywhere permeate the tissues, has been called "circulating albumin," and according to v. Voit it undergoes decomposition sooner than the organised or "organic albumin" which forms an interral part of the tissue. According to v. Voit, in 24 hours 1 per cent. of the organic and 70 per cent. of the circulating albumin is used up.

[Lichig taught that the nitrogenous metabolism of the body depended on a corresponding deennposition of the proteids of the organs, so that the proteids in the fool supplied the place of the juteidls of the organs thus used up. He called the proteids "plastic foods" or "tissue formers," while he reirarled the fats and carbohydrates as "respiratory foods," as he supposed that they alone were concerned in the evolution of heat. As a matter of fact, experiment provel that the $\mathrm{N}$ metabolism is to a large extent independent of the proteids of the food. The luxus-consumption theory was invented to explain this. It simply means, that proteids taken with the food not only replace the amount of proteids which have been decomposed during the activity of organs and tissues, but that any excess is immediately consumed without being converterl into tissue, aud thus this surplus amount giving rise to heat, by being oxidised, to a certain extent replaces the fats and carbohydrates. But Voit showed that nitrogenous metabolism is not influenced ly the activity of the organism, and he proved that in ordinary conditions, only a small amount of the organic albumin, i.c., that composing tissues and organs, undergoes decomposition, while, owing to the action of the cellular elements of the tissue, a large amount of the circulating albumin is split up, so that under ordinary conditions, the organic albumin is comparatively stalile. This he demonstrated from a comparison of the urea excreted, for the urea tuay he taken as an index of the $\mathrm{N}$ metabolism in well-fed, fasting, and starving animals. But in certain pathological conditions the organic albumin may undergo rapid change, having hecome less stable, as in fevers, and poisoning with phosphorus.]

Quality and Quantity of the Diet.-As far as his organisation is concerned, man belongs to the omnivorous animals, i.e., those that can live upon a mixed diet. For an adequate diet man requires for his existence and to maintain health a mixture of the following four chief groups of food-stuffs along with the necessary relishes; none of them must be absent from the food for any length of time. They are :-

1. Water-for an adult in his food and drink, 2700 to 2800 grms. [70 to 90 oz.] daily ( $\$ 229$ and $\$ 247,1)$.

[Thirst. - The neels of the economy for water are expressed by the sensation of thirst. The sensation of heat and dryness may be confined to the tongue, mouth and fauces, and indeed may be excited by inhaling dry air. This local thirst may be allayed by swallowing water or by eating substances which excite the secretion of saliva. More frequently, however, the sensation is the expression of a general condition indicating the diminution of water in the tissues; or it may be due to excess of saline matters in the blood. In some diseases this sensation is very intense, e.g., diabetes. If water be injected into the blood-vessels, or stomach, both the general and local thirst are abolished, even although no water enters the mouth.] 
2. Inorganic substances or Salts are an integral part of all tissues, and without them the tissues cannot be formed. They occur in ordinary food. The addition of too much salt increases the consumption of water, and this in turn increases the transformation of $\mathrm{N}$ in the body. If an animal be deprived of salts, nutrition is interfered with; food deprived of its lime affects the formation of the bones; deprival of common salt causes albuminuria (247, A, III). The alkaline salts serve to neutralise the sulphuric acid formed by the oxidation of the sulphur of the proteids. Iron, which is so essential for the formation of blood, exists in animals and plants in combination with complex organic bodies.

Only in times of famine is man driven to eat large quantities of inorganic substances, to extract the organic matter mixed therewith. A. v. Humboldt states, in regard to the inhabitants of the Orinoceo, that they eat a kind of earth which contains innumerable infusoria.

3. At least one animal or vegetable albuminons body or proteid (\$ $\$ 248,250$ ). The proteids are required to replace the used-up nitrogenous tissues, e.g., for muscles. They contain $15 \cdot 4$ to 16.5 per cent. $\mathrm{N}$.

The proteids are in blood $=20.56$ per cent.; muscles, 19.9 per cent.; liver, $11 \cdot 74$ per cent.; brain, 8.63 per cent.; blood-plasma, $7 \cdot 5$ per cent.; milk, 3.94 per cent.; lymph, $2 \cdot 46$ per cent. According to Pfliuger and Bohland, a youth of full stature, and 62 kilos. [136 lbs.] weight, decomposes $89 \cdot 9$ grms. of albumin daily.

Asparagin, in combination with gelatin, can replace albumin in the food (Weiske), while asparagin alone limits the decomposition of albumin in herbivora but not in carnivora $(J$. Munk). Ammoniacal salts, glycocoll, sarkosin, and benzamid increase with the amount of albumin in the body.

4. At least one fat (\$251), or a digestible carbohydrate $(\$ 252)$. These chiefly serve to replace the transformed fats and non-nitrogenous constituents. Owing to the large amount of $\mathrm{C}$ which they contain, when they undergo oxidation, they form the chief source of the heat of the body (\$206). Fats and carbohydrates may replace each other in the food, and in inverse proportion too, corresponding to the amount of $\mathrm{C}$ which each contains. As far as the mere evolution of heat is concerned, 100 parts of fat $=256$ of grape-sugar $=234$ of cane-sugar $=221$ of dry starch (Rubner). A man consumes 210 grms. fat daily.

[5. Every proper diet ought to have'a certain degree of sapidity or flavour. The suhstances which give this are not useful in the evolution of energy or building up the tissues, but they stimulate the nervous system and excite secretion. They are called "Genussmittel" (means of enjoying food) by the Germans, but we have no exact equivalent for this word in English, though the articles themselves are included under" our expression "condiments." These substances are the aromatic matter in roast meat (osmasome), tea, vinegar, salt, mustard, pepper, \&c.]

[Condition of Diet for Health.-In an adequate diet, not only (1) should the total quantity be sufficient and not more than sufficient, but (2) the constituents should exist in proper proportions, (3) be digestible, and (4) the whole should be in good condition, wholesome, and not adulterated with any substance prejudicial to health.]

With regard to the relative proportions of the various kinds of food which ought to be taken, experience has shown that the diet best suited for the body must contain 1 part of nitrogenous foods to $3 \frac{1}{2}$ or, at most, $4 \frac{1}{2}$ of the non-nitrogenous. Looking at ordinary foods from this point of view, we see how far they correspond to this requirement, and how several substances may be combined to produce a satisfactory diet.

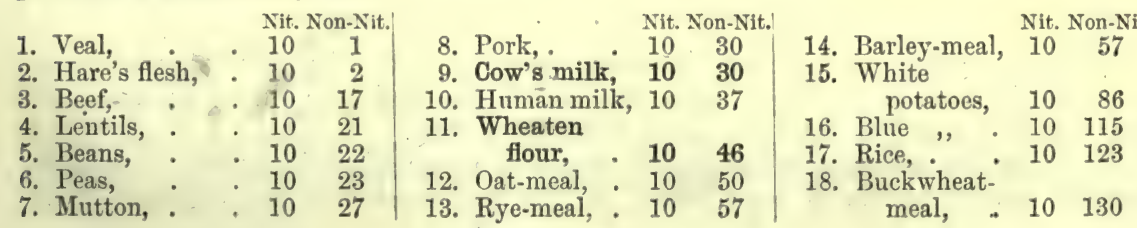


An examination of this table shows that, in addition to human milk, wheat-flour has the right proportion of nitrogrenous to non-nitrogenous substances. A man who tries to nourish himself on heef alone, commits as great a mistake as the one who would feed himself on potatoes alone. Exprerience has tanght people that man may live upon milk and eggs, but that in addition to flesh we must eat breal or potatoes, while pulses require fat or bacon.

The diet varies with the climate and with the season of the year. As the organism must produce more heat in cold latitules, the inhabitants of northern elimates must eat more nonnitrogenous fooxls, stch as fats and sugars or starches, which, on account of the large amount of $\mathrm{C}$ they coutain, are admirably adapted for producing heat $(\S 214, \mathrm{I} ., 4)$.

Animal Foods.

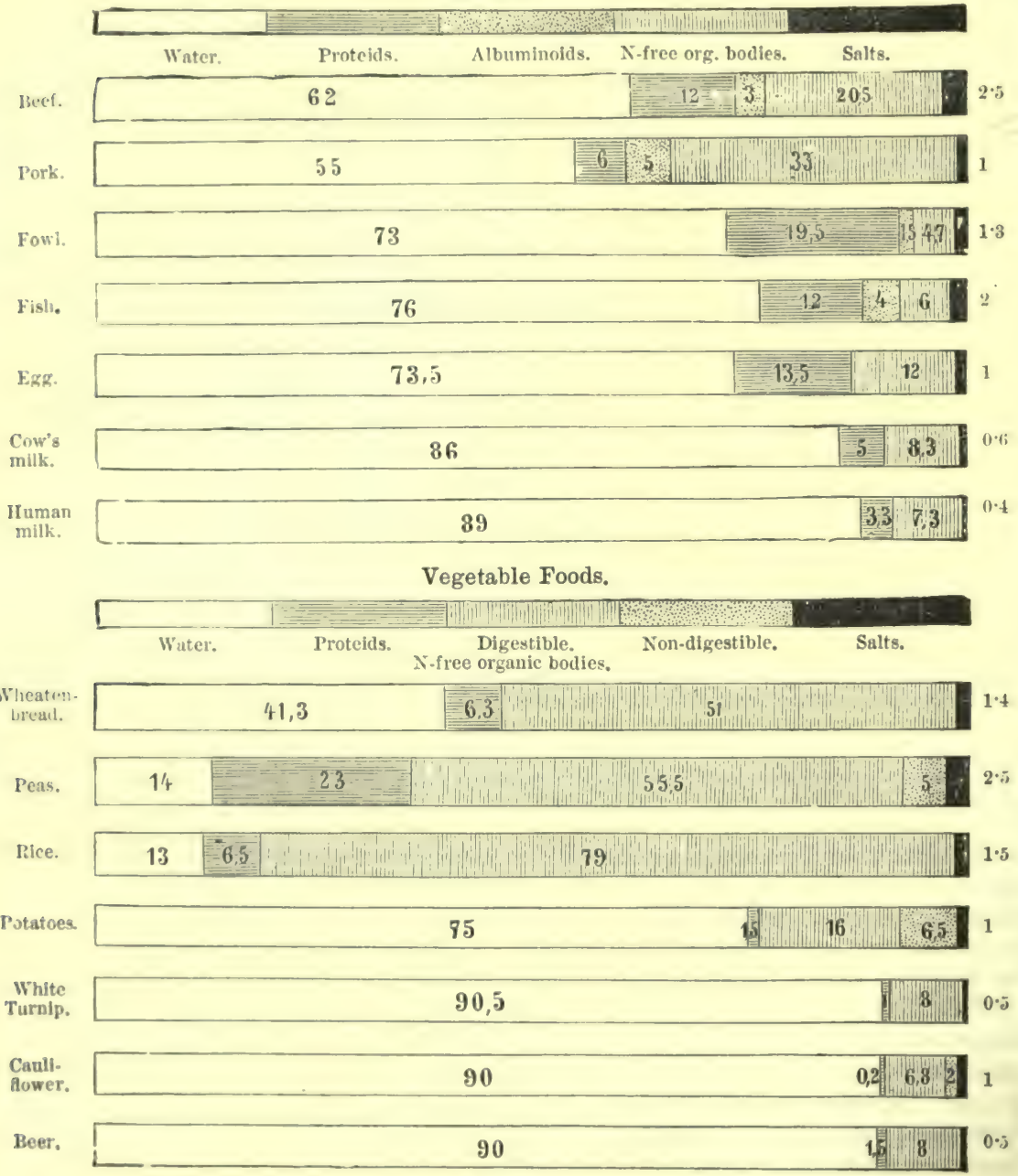

Fig. 238.

The graphic representation of the composition of foods (fig. 238) shows the relative proportions of the most important food-stuffs, and how they vary from the standard of 1 nitrogenous to $3 \frac{1}{2}$ or $4 \frac{1}{2}$ non-nitrogenous.

The absolute amount of food-stuffs required by an adult in twenty-four hours depends upon a variety of conditions. As the food represents the chemical reser- 
voir of potential energy, from which the kinetic energy (in its various forms) and the heat of the body are obtained, the absolute amount of food must be increased when the body loses more heat, as in winter, and when more muscular activity (work) is accomplished. As a general rule an adult requires daily 130 grammes proteids, 84 grammes fats, 404 grammes carbohydrates, and 30 grammes salts.

A Healthy Adult requires in 24 Hours of water-free solids-

\begin{tabular}{|c|c|c|c|c|}
\hline \multirow[b]{2}{*}{ Food in Grammes. } & \multirow{2}{*}{$\begin{array}{l}\text { At Rest } \\
\text { (Playfair). }\end{array}$} & \multirow{2}{*}{$\begin{array}{c}\text { Moderate } \\
\text { Wurk } \\
\text { (Moleschott). }\end{array}$} & \multicolumn{2}{|c|}{ Laborious Work. } \\
\hline & & & (Playfair.) & $\begin{array}{l}\text { (v. Pettenkofer } \\
\text { and } v \text {. Voit.) }\end{array}$ \\
\hline $\begin{array}{l}\text { Proteids, } \quad: \\
\text { Fats, } \\
\text { Carbohydrates (Sugar, Starch, \&c.), } \\
\text { Salts, }\end{array}$ & $\begin{array}{r}70 \cdot 87 \\
28 \cdot 35 \\
310 \cdot 20 \\
14 \cdot 00\end{array}$ & $\begin{array}{r}130 \\
84 \\
404 \\
30\end{array}$ & $\begin{array}{r}155 \cdot 92 \\
70 \cdot 87 \\
567 \cdot 50 \\
40 \cdot 00\end{array}$ & $\begin{array}{r}137 \\
117 \\
352 \\
40\end{array}$ \\
\hline
\end{tabular}

[When we record these numbers in ounces we get the following results as waterfree solids required by an average man (Parkes) :-

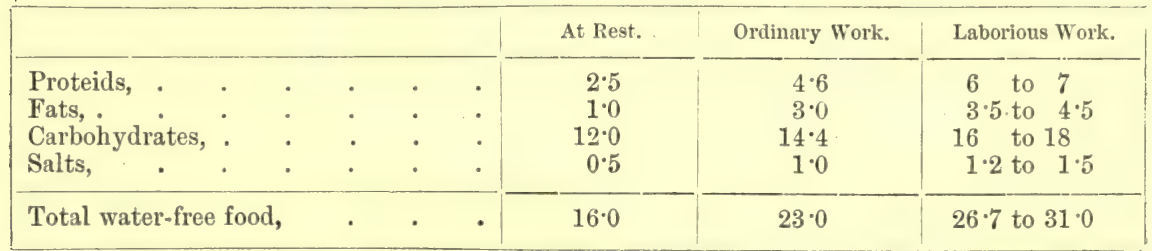

During ordinary work the proportion is about :-

Proteids 1 : fats $0 \cdot 6$ : carbohydrates $3 \cdot 0$,

i.e., 1 nitrogenous to 3.6 non-nitrogenous.]

[In a diet for ordinary work ( $23 \mathrm{oz}$. of $d r y$ solids) a man takes about $\frac{1}{100}$ part of his own weight daily; ordinary food, however, as it is consumed, contains between 50 and 60 per cent. of water; if we add this proportion of water to the actually dry food, we get 48 to $60 \mathrm{oz}$. of ordinary food (exclusive of liquids). But we consume 50 to 80 oz. of water in some liquid form, making the total amount of water 70 to 90 oz. (Parkes).]

The following tables show the elementary composition of the income and expenditure :-

An Adult doing a Moderate Amount of Work takes in:-

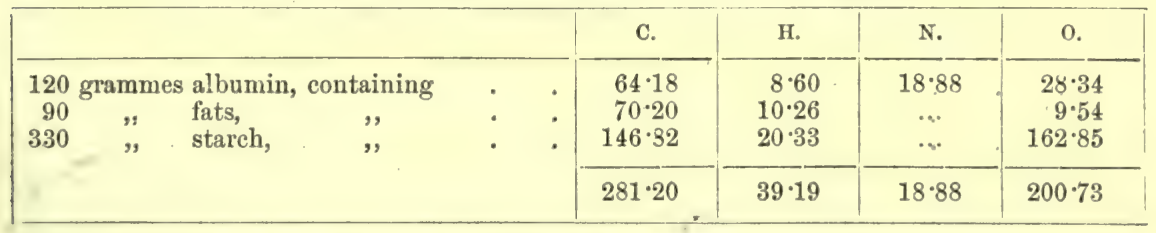

Add $744 \cdot 11 \mathrm{grm}$. $O$ from the air by respiration.

$\begin{array}{lrll}, 2 & 2818 \quad, \quad & \mathrm{H}_{2} \mathrm{O} . \\ , & 32 \quad, & \text { Inorganic compounds (salts). }\end{array}$

The whole is equal to $3 \frac{1}{2}$ kilos. $[7 \mathrm{lbs}$.$] , i.e., about \frac{1}{20}$ of the body-weight; so that about 6 per cent. of the water, about 6 per cent. of the fat, about 1 per cent. albumin, and about 0.4 per cent. of the salts of the body are daily transformed within the organism. 
As Adrlt nolsg a Monerate Amotert of Work gives off in grammes:-

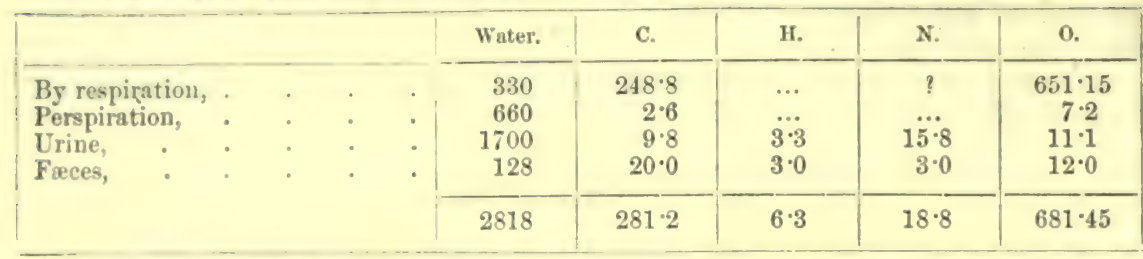

Add to this (besiles 2818 grammes water as drink) 296 grammes water formed in the body by the oxilation of $H$. These 296 grammes of water contain $34.89 \mathrm{grms}$. II, and $263.41 \mathrm{grms}$. 0 ; 26 grms. of salts are given off in the urine, and 6 by the frees. $96.5 \mathrm{grms}$. of proteic $(=1.40 \mathrm{grm}$. per kilo.) are used $u$ by a resting adult in 24 hours ; but while working 107.6 grms. are used. Nominally $2: 3$ times as much fat as albumin are used up.

The investigations of the Munich school have shown that the following numbers represent the minimum amount of food necessary for different ages :-

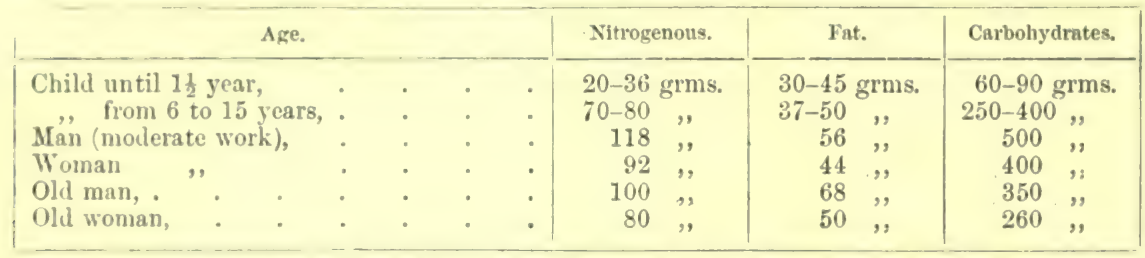

Small animals have a more lively metabolism than large ones. In small animals the decomposition of albumin per unit weight of hody is greater than in large animals $(v$. Voit). Small animals as a rule consume more proteid than larger ones, because they generally have less bodily fat (Rubuer).

Relation of $\mathbf{N}$ to $\mathbf{C}$. - In most of the ordinary articles of diet, nitrogenous and non-nitrugenous substances are present, but in very varying proportion, in the different foods. Man requires that these shall lbe in the proportion of $1: 3 \frac{1}{2}$ to $1: 4 \frac{1}{2}$. If food be taken in which this proportion is not observed, in order to obtain the necessary amount of that substance which is contained in too small proportion in his food, he must consume far too much food. In order to obtain the 130 grammes of proteids necessary a person must use

\begin{tabular}{|c|c|c|c|c|c|c|}
\hline $\begin{array}{l}\text { Cheese, } \\
\text { Lentils, } \\
\text { Peas, . }\end{array}$ & : & $\begin{array}{l}388 \text { grms. } \\
491 \text { ", } \\
582,\end{array}$ & $\begin{array}{l}\text { Beef, } \\
\text { Eggs, : } \\
\text { Wheat-bread, }\end{array}$ & $\begin{array}{r}614 \text { grms. } \\
. \quad 968 ~ ", \\
.1444, "\end{array}$ & $\begin{array}{l}\text { Rice, } \\
\text { Rye-bread, } \\
\text { Potatoes, }\end{array}$ & $\begin{array}{rr} & 2562 \\
\cdot & 2875 \\
\cdot & 10,000, ",\end{array}$ \\
\hline
\end{tabular}

provided he were to take only one of these substances as food; so that if a workman were to live on potatoes alone, in order to get the necessary amount of $\mathrm{N}$ he would have to consume an altogether excessive amount of this kind of food.

To obtain the $\mathbf{4} 48$ grammes of carbohydrates, or the equivalent amount of fat necessary to support him, a man must eat

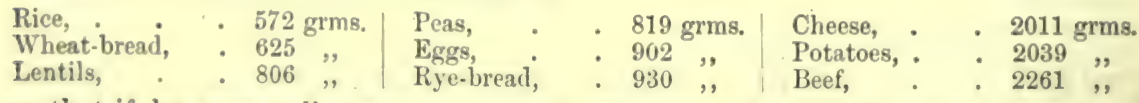

so that if he were to live upon cheese or flesh alone, he would require to eat an enormous amount of these substances.

In the case of herbivora, the proportion of nitrogenous to non-nitrogenous food necessary is 1 of the former to 8 or 9 parts of the latter.

237. HUNGER AND STARVATION.-If a warm-blooded animal be deprived of all food, it must, in order to maintain the temperature of its body and to produce the necessary amount of mechanical work, transform and utilise the potential energy of the constituents of its own body. The result is that its bodyweight diminishes from day to day, until death occurs from starvation. 
The following table, from Bidder and Schmidt, shows the amounts of the different excreta in the case of a starved cat:-

\begin{tabular}{|c|c|c|c|c|c|c|c|c|}
\hline Day. & $\begin{array}{c}\text { Body- } \\
\text { weight. }\end{array}$ & $\begin{array}{l}\text { Water } \\
\text { taken. }\end{array}$ & Urine. & Urea. & $\begin{array}{l}\text { Inorganic } \\
\text { Substances } \\
\text { in Urine. }\end{array}$ & $\begin{array}{c}\text { Dry } \\
\text { Fæces. }\end{array}$ & Expired C. & $\begin{array}{l}\text { Water in } \\
\text { Crine } \\
\text { and Fæces. }\end{array}$ \\
\hline 1. & 2464 & & 98 & $7 \cdot 9$ & $1 \cdot 3$ & $1 \cdot 2$ & $13 \cdot 9$ & $91 \cdot 4$ \\
\hline 2. & 2297 & $11: 5$ & 54 & $5 \cdot 3$ & 0.8 & $1 \cdot 2$ & $12 \cdot 9$ & $50 \cdot 5$ \\
\hline 3. & 2210 & & 45 & $4 \cdot 2$ & 0.7 & $1 \cdot 1$ & 13 & $42 \cdot 9$ \\
\hline 4. & 2172 & $68 \cdot 2$ & 45 & $3 \cdot \overline{8}$ & 0.7 & $1 \cdot 1$ & $12 \cdot 3$ & 43 \\
\hline 5. & 2129 & $\ldots$ & 55 & $4 \cdot 7$ & 0.7 & $1 \cdot 7$ & $11 \cdot 9$ & $54 \cdot 1$ \\
\hline 6. & 2024 & $\ldots$ & 44 & $4 \cdot 3$ & 0.6 & 0.6 & $11 \cdot 6$ & $41 \cdot 1$ \\
\hline 7 & 1946 & $\ldots$ & 40 & 3.8 & 0.5 & 0.7 & 11 & $37 \cdot 5$ \\
\hline 8. & 1873 & & 42 & 3.9 & 0.6 & $1 \cdot 1$ & $10 \cdot 6$ & 40 \\
\hline 9. & 1782 & $15 \cdot 2$ & 42 & 4 & 0.5 & $1 \cdot 7$ & 10.6 & $41 \cdot 4$ \\
\hline 10. & 1717 & 102 & 35 & 3.3 & 0.4 & $1 \cdot 3$ & 10.5 & 34 \\
\hline 11. & 1695 & 4 & 32 & $2 \cdot 9$ & 0.5 & $1 \cdot 1$ & $10 \cdot 2$ & $30 \cdot 9$ \\
\hline 12. & 1634 & $22 \cdot 5$ & 30 & $2 \cdot 7$ & 0.4 & $1 \cdot 1$ & $10 \cdot 3$ & $29: 6$ \\
\hline 13. & 1570 & $7 \cdot 1$ & 40 & $3 \cdot 4$ & 0.5 & 0.4 & $10 \cdot 1$ & $36 \cdot 6$ \\
\hline 14. & 1518 & 3 & 41 & $3 \cdot 4$ & 0.5 & 0.3 & $9 \cdot \overline{7}$ & 38 \\
\hline 15. & 1434 & $\ldots$ & 41 & 2.9 & 0.4 & 0.3 & $9 \cdot 4$ & $38 \cdot 4$ \\
\hline 16. & 1389 & $\ldots$ & 48 & 3 & 0.4 & 0.2 & 8.8 & 45.5 \\
\hline 17. & 1335 & $\cdots$ & 28 & $1 \cdot 6$ & $0 \cdot 2$ & 0.3 & $7 \cdot 8$ & $26 \cdot 6$ \\
\hline \multirow[t]{2}{*}{$18 .+$} & 1267 & ... & 13 & 0.7 & $0 \cdot 1$ & 0.3 & $6 \cdot 1$ & $12 \cdot 9$ \\
\hline & -1197 & $131 \cdot 5$ & 773 & $65 \cdot 8$ & $9 \cdot 8$ & $15 \cdot 7$ & $190 \cdot 7$ & $734 \cdot 4$ \\
\hline
\end{tabular}

The cat lost 1197 grms. in weight before it died, and this amount is apportioned in the following way :-204.43 grms. (=17.01 per cent.) loss of albumin; 132.75 grms. (=11.05 per cent.) loss of fat; 863.82 grms. loss of water $(=71.91$ per cent. of the total body-weight).

Methods. - In order to investigate the condition of inanition it is necessary-(1) to weigh the animal daily; (2) to estimate daily all the $\mathrm{C}$ and $\mathrm{N}$ given off from the body in the frecs, urine, and expired air. The $\mathrm{N}$ and $\mathrm{C}$, of course, can only be obtained from the decomposition of tissues containing them.

Amongst the general phenomena of inanition, it is found that strong well-nourished dogs die after 4 weeks, man after 21 to 24 days - 6 melancholics who took water died after 41 days $)$; small mammals and birds 9 days, and frogs 9 months. Vigorous adults die when they lose $\frac{4}{10}$ of their body-weight, but young individuals die much sooner than adults. The symptoms are obvious :- The mouth is dry, the walls of the alimentary canal become thin, and the digestive secretions cease to be formed ; pulse-beats and respirations are fewer ; urine very acid from the presence of an increased amount of sulphuric and phosphoric acids, whilst the chlorine compounds rapidly diminish and almost disappear. The blood contains less water and the plasma less albumin, the gall-bladder is distended, which indicates a continuous decomposition of blood-corpuseles within the liver. The liver is small and very dark coloured, the museles are very brittle and dry, so that there is great muscular weakness, and death occurs with the sigus of great depression and coma.

The relations of the metabolism are given in the foregoing table, the diminution in the excretion of urea is much greater than that of $\mathrm{CO}_{2}$, which is due to a larger amount of fats than proteids being decomposed. According to the calculation, there is daily a tolerably constant amount of fat used up, while, as starvation continues, the proteids are decomposed in much smaller amounts from day to day, although the drinking of water accelerates their decomposition.

Loss of Weight of Organs.-It is of importance to determine to what extent the individual organs and tissues lose weight; some undergo simple loss of weight, e.g., the bones, the fat undergoes very considerable and rapid decomposition, while other organs, as the heart, undergo little change, because they seem to be able to nourish themselves from the transformation products of other tissues. 
A starving cat, according to $\mathrm{v}$. Voit, lost-

\begin{tabular}{|c|c|c|c|c|c|c|}
\hline & & $\begin{array}{l}\text { Per cent. } \\
\text { originally } \\
\text { present. }\end{array}$ & $\begin{array}{l}\text { Per cent. of } \\
\text { the total loss of } \\
\text { body-weight. }\end{array}$ & & $\begin{array}{l}\text { Per cent. } \\
\text { originally } \\
\text { present. }\end{array}$ & $\begin{array}{l}\text { Per cent. of } \\
\text { the total loss of } \\
\text { body-weights. }\end{array}$ \\
\hline 1. Fat, & . & 97 & $26 \cdot 2$ & 10. Lungs, . & $17 \cdot 7$ & 0.3 \\
\hline Spleen, & & $66 \cdot 7$ & 0.6 & 11. Pancreas, & $17 \cdot 0$ & 0.1 \\
\hline 3. Liver, & . & $53 \cdot 7$ & $4 \cdot 8$ & 12. Bones, & $13 \cdot 9$ & $5 \cdot 4$ \\
\hline 4. Testicles, & . & $40 \cdot 0$ & 0.1 & 13. Central Nerv- & & \\
\hline 5. Muscles, & . & $30 \cdot 5$ & $42 \cdot 2$ & ous System, & $3 \cdot 2$ & 0.1 \\
\hline 6. Blood, & & $27 \cdot 0$ & $3 \cdot 7$ & 14. Heart, & $2 \cdot 6$ & 0.02 \\
\hline 7. Kidneys, & . & $25 \cdot 9$ & $\begin{array}{l}0.6 \\
8 \cdot 8\end{array}$ & 15. Total loss of & & \\
\hline $\begin{array}{l}\text { 8. Skin, } \\
\text { 9. Intestine, }\end{array}$ & . & $\begin{array}{l}20 \cdot 6 \\
18 \cdot 0\end{array}$ & $2 \cdot 0$ & the body, . & $36 \cdot 8$ & $5 \cdot 0$ \\
\hline
\end{tabular}

There is a very important difference according as the animals before inanition have been fed freely on flesh and fat [i.e., if they have a surplus store of food within themselves], or as they have merely had a subsistence diet. Well-fed animals lose weight much more rapidly during the first few days than on the later days. V. Voit thinks that the albumin derived from the excess of food occurs in a state of lonse combination in the body as "circulating" or "storage-albumin," so that during hunger it must decompose more rapidly and to a greater extent than the "organic albumin," which forms an integral part of the tissues $(\S 236$. Further, in fat individuals, the decomposition of fat is much greater than in slender persons.

238. METABOLISM ON A PURELY FLESH DIET.-A man is not able to maintain his metabolism in equilibrium on a purely flesh diet; if he were compelled to live on such a diet, he would succumb. The reason is obvious. In beef the proportion of nitrogenous to non-nitrogenous elementary constituents of food is $1: 1 \cdot \tau(\mathrm{p} .35 \pi)$. A healthy person excretes 280 grammes [ 8 to $9 \mathrm{oz}$.] of carbon in the form of $\mathrm{CO}_{2}$, in the expired air, and in the urine and fæces. If a man is to obtain 280 grammes C from a flesh diet he must consume-digest and assimilate-more than 2 kilos. [ $4.4 \mathrm{lbs}$.] of beef in twenty-four hours. But our digestive organs are unequal to this task for any length of time. The person is soon obliged to take less beef, which would necessitate the using of his own tissues, at first the fatty parts and afterwards the proteid substances.

A carnivorous animal (dog), whose digestive apparatus, being specially adapted for the ligestion of flesh, has a short intestine and powerfully active digestive fluids, can only maintain its metabolism in a state of equilibrium when fed on a flesh diet free from fat, provided its boly is already well supplied with fat, and is muscular. It consumes $\frac{1}{20}$ to $\frac{1}{2 \sigma}$ part of the weight of its bouly in flesh, so that the excretion of urea increases enormously. If it eats a larger amount, it may " put on flesh," when, of course, it requires to eat more to maintain itself in this conclition, until the limit of its digestive activity is reached. If a well-nourished dog is fed on less than $\frac{1}{25}$ to $\frac{1}{2} 0$ of its body-weight of flesh, it uses part of its own fat and muscle, gralually diminishes in weight, and ultimately succumbs. Poorly fed, non-muscular dogs are unable from the very beginning to maintain their metabolism in equilibrium for any length of time on a purely flesh diet, as they must eat so large a quantity of flesh that their digestive organs caunot ligest it. The herbivora cannot live upon flesh food, as their digestive apparatus is adapted solely for the digestion of vegetable food.

[The proteid metabolism depends (1) on the amount of proteids ingested, for the great mass of these becomes changed into circulating albumin; $(2)$ upon the previous condition of nutrition of the organism, for we know that a certain amount of proteid may produce very different results in the same individual when he is in good health, and when he has suffered from some exhausting disease. (3) The use of other foods, e.g., fats and carbohydrates. If a certain amount of fat be added to a diet of flesh, much less flesh is required, so that the $\mathrm{N}$ metabolism is reduced by fat. This is spoken of as the "albumin-sparing action" of fats.]

Exactly the same result occurs with other forms of proteids, as with flesh. It has been proved that gelatin may to a certain extent replace proteids in the food, in the proportion of 2 of gelatin to 1 of albumin. The carnivora, which can 
maintain their metabolism in equilibrium by, eating a large amount of flesh, can do so with less flesh when gelatin is added to their food. A diet of gelatin alone, which produces much urea, is not sufficient for this purpose, and animals soon lose their appetite for this kind of food.

[Gelatin.-Voit has shown that gelatin readily undergoes metabolism in the body and forms urea, and if a small quantity be taken, it is completely and rapidly metabolised. When administered it acts just like fats and carbohydrates as an "albumin-sparing" substance. It seems that gelatin is not available directly for the growth and repair of tissues.] Owing to the great solubility of gelatin, its value as a food used to be greatly discussed. The addition of gelatin in the form of calf's-foot jelly is recommended to invalids. [When a large amount of gelatin is given as food, owing to the large and rapid excretion of urea, the latter excites diuresis.] When chondrin is given along with flesh for a time, grape-sugar is found in the urine.

[The Metabolism of Peptones.-Most of the proteids absorbed into the blood are previously converted into peptones by the digestive juices. It has been asserted, more especially by Bricke, that some albumin is absorbed unchanged (\$192,4), and that only this is capable of forming organic albumin, while the peptones, after undergoing a reconversion into albumin, undergo decomposition as such. This view is opposed by many observers, who maintain that peptones perform all the func. tions of proteids, so that peptones, with the other necessary constituents of an adequate diet, suffice to maintain a proper standard of health.]

239. A DIET OF FAT OR OF CARBOHYDRATES. - If fat alone be given as a food, the animal lives but a short time. The animal so fed excretes even less urea than when it is starving; so that the consumption of fat limits the decomposition of the animal's own proteids. As fat is an easily oxidised body, it yields heat chiefly, and becomes sooner oxidised than the nitrogenous proteids which are oxidised with more difficulty. If the amount of fat taken be very large, all the $\mathrm{C}$ of the fat does not reappear, e.g., in the $\mathrm{CO}_{2}$ of the expired air ; so that the body must acquire fat, whilst at the same time it decomposes proteids. The animal thus becomes poorer in proteids and richer in fats at the same time.

[The metabolism of fats is not dependent on the amount of fats taken with the food. 1. It is largely influenced by work, i.e., by the activity of the tissues, and in fact with muscular work $\mathrm{CO}_{2}$ is excreted in greatly increased amount $(\$ 127,6)$. 2. By the temperature of the surroundings, as more $\mathrm{CO}_{2}$ is produced in the cold $(\$ 214,2)$, and far more fatty foods are required in high latitudes. In their action on the organism, proteids and fats so far oppose each other, as the former increase the waste, and therefore oxidation, while the latter diminish it, probably by affecting the metabolic activity of the cells themselves (Bauer). As a matter of fact, fat animals or persons bear starvation better than spare individuals. In the latter, the small store of fat is soon used up, and then the albumin is rapidly decomposed. For the same reason corpulent persons are very apt to become still more so, even on a very moderate diet.]

When carbohydrates alone are given, they must first be converted by digestion into sugar. The result of such feeding coincides pretty nearly with feeding with fat alone. But the sugar is more easily burned or oxidised within the body than the fat, and 17 parts of carbohydrate are equal to 10 parts of fat. Thus the diet of carbohydrates limits the excretion of urea more readily than a purely fat diet. The animals lose flesh, and appear even to use up part of their own fat.

[The metabolism of carbohydrates also serves to diminish the proteid metabolism, as they are rapidly burned up and thus "spare" the circulating albumin. But Pettenkofer and Voit assert that they are rapidly destroyed in the body, even when given in large amount, so that they differ from fats in this respect. They are more easily oxidised than fats, so that they are always consumed first in a diet of carbohydrates and fat. By being consumed they protect the proteids and fats from consumption.] 
The direct introduction of grape- and cane-sugar into the blood does not increase the amount of $\mathrm{O}$ used, but the amount of $\mathrm{C}^{\mathrm{O}} \mathrm{O}_{2}$ is increased. [The cloctrine of Liebig, that the oxygen taken in is a measure of the metabolic processes, is refuted by these and other experiments. It would seem that fat is not directly oxidised by $O$, but that it is split up into other simpler compoumls which are slowly and gradually oxilised; in fact, fat may lessen the amount of $\mathrm{O}$ taken in, as it diminishes waste.]

240. FLESH AND FAT, OR FLESH AND CARBOHYDRATES.-An amount of Hesh equal to $\frac{x}{5}$ to $\frac{x}{20}$ of the weight of the body is required to nourish a dog, which is fed on a purely flesh diet; if the necessary amount of fat or carbohydrates be added to the diet, a smaller quantity of flesh is required (v. Voit). For I00 parts of fat added to the flesh diet, 245 parts of dry flesh or 227 of syntonin can be disirensed with. If instead of fats carbohydrates are added, then 100 parts of fat $=2.30$ to 2.50 of the latter $($ Rulmer $)$. When the amount of flesh is insufficient, the addition of fat or carbohydrates to the food always limits the decomposition of the animal's own substance. Lastly, when too much flesh is given along with these substances, the weight of the body increases more with them than without them. Inder these circumstances, the animal's body puts on more fat than flesh. The consumption of $\mathbf{0}$ in the body is regulated by the mixture of flesh and nonnitrogenous substances, rising and falling with the amount of flesh consumed. It is remarkable that more $\mathrm{O}$ is consumed when a given amount of flesh is taken, than when the same amount of flesh is taken with the addition of fat.

It seems that, insteal of fat, the corresponding amount of fatty acids has the same effect on the metabolism. [If a dog be fed with fatty acids and a sufficient amount of proteid, no fatty acils are foumd in the chyle, while fat is formed synthetically, the glycerin for the latter probahly bing moluced in the boly.] They are absorbed as an emulsion just like the fats. When so absorlued, they seem to be reconverted into fats in their passage from the intestine to the thoracie duct probalily hy the action of the leucocytes $(J$. Munk, Will). [Glycerin in small doses has no effect on the metabolism of proteid, but in large doses it increases it. It is consumed in the hody, as shown by experiments on the respiratory products, and it prevents a certain amount of fat from being used up. About 20 per cent. is excreted in the urine (Amschink).]

241. ORIGIN OF FAT IN THE BODY.-I. Part of the fat of the body is derived directly from the fat of the food, i.e., it is absorbed and deposited in the tissues. This is shown by the fact that, with a diet containing a small amount of albumin, the addition of more fat causes the deposition of a larger amount of fat in the body (v. Voit, IIofmann).

[Hof mann starverl a fat dog for 30 days until all its fat was used up. He fed it on lard and a little albumin for 5 days anil then killed it. In 5 days it absorbed 1854 grms. of fat and 254 grms. of albumin. It adderl to its body 1353 grms. of fat; but this anount could not be formed from the proteils of the fool, and therefore the fat must have come from the fat of the fool. l'ettenkofer and Yoit arrived at the same result in another way. They ferl dogs on fish and much fat, and hy their respiration apparatus estimated the gaseous income and expenditure (\$122). All the $Y$ triten in rectppecered in the cxcrete, but not all the $C$. The amount of $\mathrm{C}$ retained was very large, therefore a non-nitrogenous residue must have been laid up in the body, and it could only be fat, as this was the only substance found in large amount in the body. They estimated the possible amount of fat that could be formed from the proteids, and found that the amount stored up was far greater than this; so that the fat of the food must have been stored up in the tissues.]

Lebeileff found that dogs, which were starved for a month, so as to get rid of all their own fat, on being fed with linseed oil, or mutton suet and flesh, had these fats restored to their tissues. These fats, therefore, must have been absorbed and deposited. J. Munk found the same on feerling animals with rape-seed oil. Fatty acids may also contribute to the formation of fats, as glycerin when formed in the body must be stored up during metabolism (J. Munk).

Fatty acils may contribute to the formation of fats by union with the glycerin of the body during the metabolism.

II. A second source of the fats is albuminous bodies. In the case of the formation of fat from proteids, which may yield 11 per cent. of fat (according to Henneberg 100 parts of dry albumin can form 51.5 parts of fat), these proteids split up into a non-nitrogenous and a nitrogenous atomic compound. The former, during 
a diet containing much albumin, when it is not completely oxidised into $\mathrm{CO}_{2}$, and $\mathrm{H}_{2} \mathrm{O}$, is the substance from which the fat is formed-the latter leaves the body oxidised chiefly to the stage of urea.

Examples. - That fats are formed from proteids is shown by the following:-1. A cow which produces $1 \mathrm{lb}$. of butter daily does not take nearly this amount of fatty matter in its food, so that the fat would appear to be formed from vegetable proteids. 2. Carnivora giving suck, when fed on plenty of flesh and soine fat, yield milk rich in fat. 3. Dogs fed with plenty of flesh and some fat, add more fat to their bodies than the fat contained in the food. 4. Fatty degeneration, e.g., of nerve and muscle, is due to a decomposition of proteids. 5. The transformation of entire bodies, e.g., such as have lain for a long time surrounded with water, into a mass consisting almost entirely of palmitic acid or adipocere is also a proof of the transformation of part of the proteids into fats. 6. Fungi are also able to form fat from albumin cluring their growth. [7. In starving dogs, Bauer estimated the $\mathrm{N}$ and $\mathrm{CO}_{2}$ given off, and $\mathrm{O}$ taken in, and then slowly poisoned them with phosphorus, and he found that the excretion of $\mathrm{N}$ was increased twofold, while the exeretion of $\mathrm{CO}_{2}$ and the absorption of $\mathrm{O}$ were diminished one-half. Therefore from a large amount of nitrogenous tissue, a nitrogenous body and a small amount of a carbonaceous compound were excreted, while a large amount of a non-nitrogenous residue was retained unconsumed. There was fatty degeneration of all the organs, the fat being derived from the non-nitrogenous part of the proteid. The same obtains with arsenic and antimony.]

Fats not merely absorbed.-Experiments which go to show that the fat of animals, during the fattening process, is not absorbed as such, from the food, are:-1. Fattening occurs with flesh and soaps; it is most improbable that the soaps are transformed into neutral fats by taking up glycerin and giving up alkali. 2. If a lean dog be fed with flesh and palmitin- and stearinsoda-soap, the fat of its body contains, in addition to palmitin and stearin, olein fat, so that the last must be formed by the organism from the proteids of the flesh. Further, Ssubotin found that, when a lean dog was fed on lean meat and spermaceti-fat, a very small amount of the latter was found in the fat of the animal. Although these experiments show that the fat of the body must be formed from the decomposition of proteids, they do not prove that all the fat arises in this way, and that none of it is absorbed and redeposited ( $\$ 241, \mathrm{I}$.).

III. According to v. Voit, no fat is formed in the body directly from carbohydrates, e.g., by reduction. As fattening occurs on a diet of pure flesh with the addition of carbohydrates, it is assumed that the carbohydrates are consumed or oxidised in the body, and that thereby a non-nitrogenous body derived from the proteids is prevented from being burned up, and that it is changed into fat, and stored up as such. No doubt fat is formed indirectly in the blood in this way (\$ 240).

From experiments upon fattening animals, however, Lawes and Gilbert, Lehmann, Heiden, v. Wolff, and others, think they are entitled to conclude that the carbohydrates absorbed are directly concerned in the formation of fats, a view which is supported by Henneberg, B. Schulze, and Soxhlet. According to Pasteur, glycerin (the basis of neutral fats) may be formed from carbohydrates.

[Tscherwinsky fed two similar pigs from the same litter; No. I. weighed $7300 \mathrm{grms}$.; No. II. 7290 grms. No. I. was killed and its fat and proteids estimated. No. II. was fed for four months on grain and then killed, the grain and excreta and the undigested fat and proteids were analysed, so that the amount of fat and proteid absorbed in four months was estimated. The pig then weighed 24 kilos., it was killed and its fat and proteids estimated.

No. II. contained $2 \cdot 50$ kilos. albumin and $9 \cdot 25$ kilos. fat.

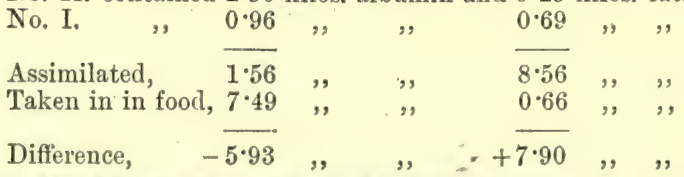

There were therefore 7.90 kilos. of fat in the body which could not be accounted for in the fat of the food. The 5.93 kilos. of albumin of the food which were not assimilated as albumin could yield only a small part of the 7.90 kilos. of fat, so that at least 5 kilos. of fat must have been formed from carbohydrate. Lawes and Gilbert calculated that 40 per cent. of the fat in pigs was derived from carbohydrates. How the carbohydrates are changed into fat in the body is entirely unknown.]

Formerly it was believed that bees could prepare wax from honey alone; this is a mistakean equivalent of albumin is required in addition-the necessary amount is found in the raw honey itself. 
242. CORPULENCE. - The addition of too much fat to the body is a pathological phenomenon which is attemled with disagreeable consequences. With regard to the causes of obesity, without doubt there is an inherited tendency (in 33 to 56 per cent. of the cases) in many families-and in some breeds of cattle, to lay up fat in the body, while other families may be richly supplied with fat, and yet remain lean. The chief cause, however, is taking too much food, i.e., more than the amount required for the normal metabolism; corpulent people, in order to maintain their bodies, must eat absolutely and relatively more than persons of spare liabit, under analogous conditions of nutrition $(\$ 236)$.

Conditions favouring Corpulence.-(1) A diet rich in proteids, with a corresponding addition of fut or curlubluglicutes. As tlesh or muscle is formeil from proteids, and part of the fat of the body is also formed from albumin; the assumption that fats and carbohydrates fatten, or, when taken alone, act as fattening agents, is completely without foundation. (2) Diminished disintegratime of materials withm the body, e.y., (u) diminished muscular activity (much sleep and little ('xercise); (b) rebrugution of the sexull functions (as is shown by the rapid fattening of castrated animals, as well as by the fact that some women, after cessation of the menses, readily become corpulent); (c) diminished mentul actirity (the obesity of dementia), phlegmatic temperament. On the contrary, vigorous mental work, excitable temperament, care and sorrow, counteract the deposit of fat ; (d) diminisher catent of the respiratory activity, as oceurs when there is a great deposition of fat in the abdomen, limiting the action of the diaphragm (breathlessness of corpulent people), whereby the combustion of the fatty matters iwhich become deposited in the borly, is limited : $(r)$ a corpulent person requires to use relatively less heat-giving substances in his bouly, partly because he gives off relctively less heat from his compact body, than is done by a slender long-hodied individual, and partly because the thick layer of fat retards the conduction of heat $(\$ 214,4)$. Thus, corresponding to the relatively diminished production of heat, more fat may be stored up; $(f)$ a diminution of the red blood-corpuscles, which are the great exciters of oxidation in the hody, is generally followed by an increase of fat-fat people, as a rule, are fat because they have relatively less blood ( $\$ 41$ ) - women with fewer red blood. corpuseles are usually fatter than men; $(g)$ the consumption of alcohol favours the conservation of fat in the body, the alcohol is easily oxidised, and thus prevents the fat from being burned uip (\$235).

Disadvantages.--Besiles the inconvenience of the great size and weight of the body, corpulent people sulfir from lireathlessness - they are easily fatigued, are liable to intertrigo between the folds of the skin, the heart becomes loaded with fat, and they not unfrequently are subject to apoplexy.

In orler to counteract corpulence we ought to-(1) lialuce uniformly all articles of diet. The diet and bouly ought to be weighed from week to week, and as long as there is no diminution in the hody-weight the amount of food ought to be gradually and uniformly reduced (notwithstanling the alyetite). This must be done very gradually and not snddenly. A moderate reiluction o, fat and carbohydrates in a normal diet, at the same time leads to a diminution of the fat of the body itself. Let a person; who is capable of muscular exertion take 156 grms. proteisl, 43 grms. fat, and 114 grms. carbohydrates; but in those where congestions, hydrimia, hreathlessness have taken place, take $170 \mathrm{grms}$. proteid, $25 \mathrm{grms}$. fat, and 70 grms. carboliydrates (Oertel). It is not advisable to limit the amount of fat and carbohydrates alone, as is done in the Banting-cure or Bantingism. A part altogether from the fact that fat is formed from proteils, if too little non-nitrogenous food be taken, severe disturbance of the bodily metalsolism is apt to occur. (2) It is advisable during the chief meal to limit the consumption of fluids of all sorts (even until three-yuarters of an hour thereafter), and thus render the absorption and digestive activity of the intestine less active (Oertel). (3) The muscular activity onght to be greatly developed by doing plenty of muscular work, or taking plenty of exercise, both physical and mental. (4) Furver the evolution of heat by taking cold baths of considerable duration, and afterwards rubbing the skin strongly so as to cause it to become red ; further, dresslightly, and at night use light bed-clothing; tea and coffee are useful, as they excite the circulation. (5) Use gentle laxatives; acid fruits, cider; alkaline carbonates (of Marienbad, Carlshad, Vichy, Neuenahr, Ems, \&c.) act by increasing the intestinal evacuations and diminishing absorption. (6) If from accumulation of fat there is danger of failure of the heart's action, Oertel recommends hill-climbing, whereby the cardiac muscle is exercised and strengthened. At the same time the circulation becomes more lively and the metabolism is increased.

[Oertel's Method goes on the idea of strengthening the cardiac musculature, which is sought to be accomplished by (1) limiting the amount of fluids consumed, and (2) carefully regulated muscular exertion. The amount of food is first reduced one-half, and the water to a still lower amount, while the nitrogenous elements in food are increased, the non-nitrogenous are decreased. The person is then instructed to take exercise under certain medical precautions, first, on level ground, and theu on gradually increasing gradients. ]

Fatty Degeneration. - The process of fattening consists in the deposition of drops of fat within the fat-cells of the panniculus and around the viscera, as well as in the marrow of bone (but they are never deposited in the subcutaneous tissue of the eyelids, of the penis, of the red part of the lips, in the ears and nose). This is quite different from the fatty atrophy or fatty 
degeneration which occurs in the form of fatty globules or granules in albuminous tissues, e.g., in muscular fibres (heart), gland-cells (liver, kidney), cartilage-cells, lymph- and pus-corpuscles, as well as in nerve-fibres separated from their nerve centres. The fat in these cases is derived from albumin, much in the same way as fat is formed in the gland-cells of the mammary and sebaceous glands. Marked fatty degeneration not unfrequently occurs after severe fevers, and after artificial heating of the tissues; when a too small amount of $\mathrm{O}$ is supplied to the tissues, as occurs in cases of phosphorus poisoning (Bauer); in drunkards ; after poisoning with arsenic and other substances, and after some disturbances of the circulation and inuervation. Some organs are especially prone to undergo fatty degeneration during the course of certain diseases.

243. METABOLISM OF THE TISSUES.-The blood-stream is the chief anedium whereby new material is supplied to the tissues and the effete products removed from them. The lymph which passes through the thin capillaries comes into actual contact with the tissue elements. Those tissues which are devoid of blood-vessels in their own substance, such as the cornea and cartilage, receive nutrient fluid or lymph from the adjacent capillaries, by means of their cellular elements, which act as juice-conducting media. Hence, when the normal circulation is interfered with, by atheroma or calcification of the walls of the blood-vessels, these tissues are secondarily affected [this, for example, is the case in arcus senilis of the cornea, due to a fatty degeneration of the corneal tissue, owing to some affection of the blood-vessels on which the cornea depends for its nutrition]. Total compression or ligature of all the blood-vessels results in necrosis of the parts supplied by the ligatured blood-vessels.

Atrophies caused by diminution of the normal supply of blood, gradually, in the course of time become less and less (Samuel).

Hence, there must be a double current of the tissue juices; the afferent or supply current, which supplies the new material, and the efferent stream which removes the effete products. The former brings to the tissues the proteids, fats, carbohydrates, and salts from which the tissues are formed. It is evident that any interruption of the arterial supply to the tissues will diminish this supply.

That such a current exists is proved by injecting an indifferent, easily recognisable substance into the blood, e.g., potassium ferrocyanide, when its presence may be cletected in the tissues, to which it has been earried by the outgoing current.

The efferent stream carries away the decomposition products from the various tissues, more especially urea, $\mathrm{CO}_{2}, \mathrm{H}_{2} \mathrm{O}$, and salts, and these are transferred as quickly as possible to the organs through which they are excreted.

That such a current exists is proved by injecting such a substance as potassium ferrocyanide into the tissues, e.g., subcutaneously, when its presence may be detected in the urine within two to five minutes.

If the current from the tissues to the blood is so active that the excretory organs cannot eliminate all the effete products from the blood, then these products are found in the tissues. When certain poisons are injected subcutaneously, they pass rapidly into the blood and are carried in great quantity to other tissues, e.g., to the nervous system, on which they act with fatal effect, before they are eliminated to any great extent from the blood by the action of the excretory organs. The effete materials are carried away from the tissues by two channels, viz., by the veins and by the lymphatics, so that if these be interfered with, the metabolism of the tissues must also suffer. When a limb is ligatured so as to compress the veins and the lymphatics, the efferent stream stagnates to such an extent that considerable swelling of the tissues or oedema may occur ( $\$ 203)$. The action of the muscles and fasciæ are very important in removing these effete matters.

$\mathrm{H}$. Nasse found that the blood of the jugular vein is 0.225 per 1000 specifically heavier than the blood of the carotid, and contains $0^{\circ} 9$ parts per 1000 more solids; 1000 eubic centimetres of blood circulating through the head yield about 5 cubic centimetres of transudation into the tissues,

The extent and intensity of the metabolism of the tissues depend upon a variety of factors. 
1. Upon their activity.-The increased activity of an organ is indicated by the increased amount of blood going to it, and by the more active circulation through it $(\$ 100)$. When an organ is completely inactive, such as a paralysed muscle, or the peripheral end of a divided nerve, the amount of blood and the nutritive exchange of fluids diminish within these parts. The parts thus thrown out of activity become pale, relaxed, and ultimately undergo fatty degeneration. The increased metabolism of an organ during its activity has been proved experimentally in the case of muscle, and $[(\$ 26: 3)$ also in the brain (Speck)]. Langley and Sewell have recently observed directly the metabolic changes within sufficiently thin lobules of glands during life. The cells of serous glands ( $\$ 143)$, and those of mucous, and pepsin-forming glands ( $\$ 164)$, during quiescence, become filled with coarse granules, which are dark in transmitted light and white in reflected light, which granules are consumed or disappear during granular activity. During sleep, when most organs are at rest, the metabolism is limited, darkness also diminishes it; while light excites it, obviously owing to nervous influences. The variations in the total metabolism of the body are reflected in the excretion of $\mathrm{CO}_{2}(\$ 12 \pi, 9)$ and urea $(\$ 257)$, which may be expressed graphically in the form of a curve corresponding with the activity of the organism; this curve corresponds very closely with the daily variations in the respirations, pulse, and temperature (p. 327).

2. The composition of the blood has a marked effect upon the current on which the metaholism of the tissues depends. Tery concentrated blood, which contains a small amount of water, as after profuse sweating, severe diarrhwea, cholera, makes the tissues dry, while if much water be absorbed into the blood, the tissues become more succulent and even (edema may occur. When much common salt is present in the blood, and when the red blood-corpuscles contain a diminished amount of $\mathrm{O}$, and especially if the latter condition be accompanied by muscular exertion causing dyspnua, a large amount of albumin is decomposed, and there is a great formation of urea. Hence, exposure to a rarefied atmosphere is accompanied by increased excretion of urea. Certain abnormal conditions of the blood produce remarkable results; blood charged with crerlomic oxide cannot absorb $\mathrm{O}$ from the air, and does not remove $\mathrm{CO}$. from the tissues $(\$ 16)$. The presence of hydrocyanic acid in the blood (\$16) is said to interrupt at once the chemical oxidation processes in the blood, so that rapid asphyxia, owing to cessation of the internal respiration, occurs. Fermentation is interrupted by the same substance in a similar way. A diminution "f the total amont of the blood causes more fluid to pass from the tissues into the blood, but the absorption of substances-such as poisons or pathological effusions, from the tissues or intestines is delayed. If the substances which pass from the tissues into the blood be rapidly eliminated from it, absorption takes place more rapidly.

3. The blood-pressure, when it is greatly increased, causes the tissues to contain more fluid, while the blood itself becomes more concentrated, to the extent of 3 to 5 per 1000. We may convince ourselves that blood-plasma easily passes through the capillary wall, by pressing upon the efferent vessel coming from the chorium deprived of its epidermis, e.y., by a burn or a blister, when the surface of the wound becomes rapidly suffused with plasma. Diminution of the blood-pressure produces the opposite result. The oxidation processes in the body are diminished after the use of $\mathrm{P}, \mathrm{Cu}$, ether, chloroform, and chloral.

4. Increased temperature of the tissues (several hours daily) does not increase the breaking up of albumin and fats. (See $\$ 221,220,225$.

5. The influence of the nervous system on the metabolism is twofold. On the one hand, it acts indirectly through its effect upon the blood-vessels, by causing them to contract or dilate through the agency of vaso-motor nerves, whereby it influences the amount of blood supplied, and also affects the blood-pressure. But quite independently of the blood-vessels, it is probable that certain special nerves- 
the so-called trophic nerves, influence the metabolism or nutrition of the tissues $(\S 342, c)$. That nerves do influence directly the transformation of matter within the tissues is shown by the secretion of saliva resulting from the stimulation of certain nerves, after cessation of the circulation ( $\$ 145$ ), and by the metabolism during the contraction of bloodless muscles. Increased respiration and apnœa are not followed by increased oxidation (Pflüger) (\$127, 8).

[Gaskell has raised the question as to the existence of katabolic and anabolic nerves con. trolling respectively the analytic and synthetic metabolism of the tissues.]

244. REGENERATION. - The extent to which lost parts are replaced varies greatly in different organs. Amongst the lower animals, the parts of organs are replaced to a far greater extent than amongst warm-blooded animals. When a hydra is divided into two parts, each part forms a new individual-nay, if the body of the animal be divided into several parts in a particular way, then each part gives rise to a new individual (Spallanzani). The Planarians also show a great capability of reproducing lost parts (Dugès). Spiders and crabs can reproduce lost feelers, limbs, and claws; snails, part of the head, feelers, and eyes, provided the central nervous system is not injured. Nany fishes reproduce fins, even the tail fin. Salamanclers and lizards can produce an entire tail, including bones, muscles, and even the posterior part of the spinal cord; while the triton reproduces an amputated limb, the lower jaw, and the eye. This reproduction necessitates that a small stump be left, while total extirpation of the parts prevents reproduction. In amphibians and reptiles the regeneration of organs and tissues, as a whole, takes place after the type of the embryonic development, and the same is true as re. gards the histological processes which occur in the regenerated tail and other parts of the body of the earth-worm.

The extent to which regeneration can take place in mammals and in man is very slight, and even in these cases it is chiefly confined to young individuals. A true regeneration occurs in-

1. The blood, including the plasma, the colourless and coloured corpuscles. $(\$ 7$ and $\$ 41$.

2. The epidermal appendages $(\$ 283)$ and the epithelium of the mucous membranes are reproduced by a proliferation of the cells of the deeper layers of the epithelium, with simultaneous division of their nuclei. Epithelial cells are reproduced as long as the matrix on which they rest and the lowest layer of cells are intact. When these are destroyed cell-regeneration from below ceases, and the cells at the margins are concerned in filling up the deficiency. Regeneration, therefore, either takes place from below or from the margins of the wound in the epithelial covering; leucocytes also wander into the part, while the deepest layer of cells forms large multi-nucleated cells, which reproduce by division polygonal flat nucleated cells. [In the process of division of the cells, the nucleus plays an important part, and in so doing it shows the usual karyokinetic figures (\$431).] The nails grow from the root forwards; those of the fingers in four to five months, and that of the great toe in about twelve months, although growth is slower in the case of fracture of the bones. The matrix is co-extensive with the lunule, and if it be destroyed the nail is not reproduced (\$284). The eyelashes are changed in 100 to 150 days, the other hairs of the body somewhat more slowly. If the papilla of the hair follicle be destroyed, the hair is not reproduced. Cutting the hair favours its growth, but hair which has been cut does not grow longer than uncut hair. After hair has grown to a certain length, it falls out. The hair never grows at its apex. The epithelial cells of mucous membranes and secretory glands seem to undergo a regular series of changes and renewal.. The presence of secretory cells in the milk ( $(231)$ and in the sebaceous secretion $(\$ 285)$ proves this; the spermatozoa are replaced by the action of spermatoblasts. In catarrhal conditions of mucous membranes, there is a great increase in the formation and excretion of new epithelium, while many cells are but indifferently formed and constitute mucous corpuscles. The crystalline lens, which is just modified epithelium, is reorganised like epithelium; its matrix is the anterior wall of its capsule, with the single layer of cells covering it. If the lens be removed and this layer of cells retained, 
these cells proliferate and elongate to form lens fibres, so that the whole cavity of the empty lens capsule is refilled. If much water be withdrawn from the body, the lens fibres become turbid. [A turbid or opaque condition of the lens may oceur in diabetes, or after the transfusion of strong common salt or sugar solution into a frog.

3. The blood-vessels undergo extensive regeneration, and they are regenerated in the same way as they are formed $(\$ 7, B)$. Capillaries are always the first stage, and around them the characteristic coats are added to form an artery or a vein. When an artery is injured and permanently occluded, as a general rule the part of the ressel up to the nearest collateral branch becomes obliterated, whereby the derivatives of the endothelial lining, the connective-tissue corpuscles of the wall, and the leucocytes change into spindle-shaped cells, and form a kind of cicatricial tissue. Blind and solid outshoots are always found on the blood-vessels of young and adult animals, and are a sign of the continual degeneration and regeneration of these vessels. Lymphatics behave in the same way as blood-vessels; after removal of a lymphatic sland, a new one may be formed (Bayer).

4. The contractile substance of muscle may undergo regeneration after it has become partially degenerated. This takes place after amyloid or wax-like degeneration, such as occurs not unfrequently after typhus and other severe fevers. This is chiefly accomplished by an increase of the muscle corpuscles. After being compressed, the muscular nuclei disappear, and at the same time the contractile contents degenerate. After several days, the sarcolemma contains numerous nuclei which reproduce new muscular nuclei and the contractile substance. In fibres injured by a subcutaneous wound, Neumann found that, after five to seven days, there was a bud-like elongation of the cut ends of the fibres, at first without transverse striation, but with striation ultimately. If a large extent of a muscle be removed, it is replaced by cicatricial connective-tissue. Non-striped muscular fibres are also reproduced; the nuclei of the injured fibres divide after becoming enlarged, and exhibit a well-marked intra-nuclear plexus of fibrils. The nuclei divide into two, and from each of these a new fibre is formed, probably by the differentiation of the peri-nuclear protoplasm.

5. After a nerve is divided, the two ends do not join at once so as to permit the function of the nerve to be established. On the contrary, marked changes occur. If a piece be cut out of a nerve-trunk, the peripheral end of the divided nerve degenerates, the axial cylinder and the white substance of Scliwann disappear. The interval is filled up at first with juicy cellular tissue. The subsequent changes are fully described in $\$ 325,4$. There seems to be in peripheral nerves a continual disappearance of fibres by fatty degeneration, accompanied by a consecutive formation of new fibres (Sigm. Mayer). The regeneration of peripheral ganglionic cells is unknown. V. Voit, however, observed that a pigeon, part of whose brain was removed, had within five months reproduced a nervous mass within the skull, consisting of medullated nerve-fibres and nerve-cells. Eichhorst and Naunyn found that in young dogs, whose spinal cord was divided between the dorsal and lumbar regions, there was an anatomical and physiological regeneration, to such an extent that voluntary movements could be executed $(\$ 338,3)$. Vaulair, in the case of frogs, and Masius in dogs, found that mobility or motion was first restored, and afterwards sensibility. Regeneration of the spinal ganglia did not occur.

6. In many glands, the regeneration of their cells during normal activity is very active-sebaceous, mucous, Lieberkihnian, uterine, mammary glands during pregnancy - in others less. If a large portion of a secretory gland be removed, as a general rule, it is not reproduced. A gland, if injured, and if suppuration follows, is not regenerated. But the bile ducts $(\$ 173)$ and the pancreatic duct may be reproduced (\$171). According to Philippeaux and Griffini, if part of the spleen be removed it is reproduced $(\S 103)$. Tizzoni and Collucci observed the formation 
of new liver-cells and bile-ducts after injury to the liver (\$173), and Pisenti makes the same statement as regards the kidney. After mechanical injury to the secretory cells of glands (liver, kidney, salivary, Meibomian), neighbouring cells undergo proliferation and aid in the restoration of the cells.

7. Amongst connective tissues, cartilage, provided its perichondrium be not injured, reproduces itself by division of its cartilage cells; but usually when a part of a cartilage is removed, it is replaced by connective-tissue.

8. When a tendon is divided, proliferation of the tendon cells occurs, and the cut ends are united by connective-tissue.

9. The reproduction of bone takes place to a great extent under certain conditions. If the articular end be removed by excision, it may be reproduced, although there is a considerable degree of shortening. Pieces of bone which have been broken off or sawn off heal again, and become united with the original bone. A tooth may be removed, replanted in the alveolus, and become fixed there. If a piece of periosteum be transplanted to another region of the body, it eventually gives rise to the formation of new bone in that locality. If part of a bone be removed, provided the periosteum be left, new bone is rapidly reproduced; hence, the surgeon takes great care to preserve the periosteum intact in all operations where he wishes new bone to be reproduced. Even the marrow of bone, when it is transplanted, gives rise to the formation of bone. This is due to the osteoblasts adhering to the osseous tissue.

In fracture of a long bone, the periosteum deposits on the surface of the ends of the broken bones a ring of substance which forms a temporary support, the external callus. At first this callus is jelly-like, soft, and contains many corpuscles, but afterwards it becomes more solid and somewhat like cartilage. A similar condition occurs within the bone, where an internal callus is formed. The formation of this temporary callus is due to an inflammatory proliferation of the connective-tissue corpuscles, and partly to the osteoblasts of the periosteum and marrow. According to Rigal and Vignal, the internal callus is always osseous, and is derived from the marrow of the bone. The outer and inner callus become calcified and ultimately ossified, whereby the broken ends are reunited. Towards the fortieth day, a thin layer of bone is formed (intermediary callus) between the ends of the bone. Where this begins to be definitely ossified, the outer and inner callus begin to be absorbed, and ultimately the intermediary callus has the same structure as the rest of the bone.

There are many interesting observations connected with the growth and metabolism of bones. 1. The addition of a very small amount of phosphorus or arsenious acid to the food causes considerable thickening of the bones. This seems to be lue to the non-absorption of those parts of the bones which are usually absorbed, while new growth is continually taking place. 2 . When food devoid of lime-salts is given to an animal, the growth of the bones is not arrested, but the bones become thinner, whereby all parts, even the organic basis of the bone, undergo a uniform diminution. 3. Feeding with madder makes the bones red, as the colouring matter is deposited with the bone-salts in the bone, especially in the growing and last formed parts. In birds the shell of the egg becomes coloured. 4. The continued use of lactic acid dissolves the bones. The ash of bone is thereby diminished. If lime-salts be withheld at the same time, the effect is greatly increased, so that the bones come to resemble rachitic bones. (Development of Bone, \$ 447.)

When a lost tissue is not replaced by the same kind of tissue, its place is always taken by cicatricial connective-tissue.

When this is the case, the part becomes inflamed and swollen, owing to an exudation of plasma. The blood-vessels become dilated and congested, and, notwithstanding the slower circulation, the amount of blood is greater. The blood-vessels are increased, owing to the formation of new ones. Colourless blood-corpuscles pass out of the vessels and reproduce themselves, and many of them undergo fatty degeneration, whilst others take up nutriment and become converted into large uninucleated protoplasma cells, from which giant cells are developed. The newly formed blood-vessels supply all these elements with blood.

245. TRANSPLANTATION OF TISSUES. - The nose, ear, and even a finger, after having been severed from the body by a clean cut, have, under certain circumstances, become united to the part from which they were removed. The skin is frequently transplanted by surgeons, as, for example, to form a new nose. The piece of skin is cut from the forehead or arm, to which it is left attached by a bridge of skin, is then stitched to the part which it is desired to cover in, and when it has become attached in its new situation, the bridge of skin is severed. 
Reverdin cut a piece of skin into pieces about the size of a pea and fixed them on an ulcerated surface, where they, as it were, took root, grew, and sent off from their margins epithelial outgrewths, so that ultimately the whole surface was covered with epithelium. [White skin transplanted to a negro ultimately becomes pigmented, and black skin transplanted to a white person becomes white.] The excised smur of a cock was transplanted and fixed in the comb of the same animal, where it grew (John Hunter). P. Bert cut off the tail and legs of rats and transplanted them under the skin of the back of other rats, where they united with the adjoining parts. Ollier foum that, when periosteum was transplanted, it grew and reproduced bone in its new situation. Even blood and lymph may be transfused (Transfusion, § 102). [Small portions $(15 \mathrm{~mm}$.) of epiphyses, costal cartilage, of a rabbit or kitten, when transplanted quite fresh into the anterior chamber of the eye, testis, submaxillary gland, kidney, and under the skin of a rahl,it, attach themselves and grow, and the growth is more rapid the more vascular the site on which the tissue is transplanted. The cartilage is not essentially different from hyaline cartilage, but the cells are fewer in the centre, while the matrix tends to become fibrous. simall pieces of epiphysial cartilage introduced into the jugular vein were found as cartilaginous fori in the lungs. Tissues transplanted from embryonic structures grow far better than adult tissues. If a portion of the cornea of a rabbit be transplanted to a human eve, provided Descemet's membrane be clear, it will grow and remain clear ( $v$. Hippell). A rablit's nerve has been transplanted to the human subject, but without success.]

Many of these results seem only to be possible between individuals of the same specics, although Helferich has revently found that a piece of a dog's muscle, when substituted for human muscle, united to the arljoining musele, and became functionally active. [J. R. Wolfe has transplanted the conjunctiva of the rabbit to the human eye.] Most tissues, however, do not admit of transplantation, c.g., glands and the sense-organs. They may be removed to other jarts of the loxly, or into the peritoneal cavity, without exciting any inflammatory reaction ; they, in fact, behave like inert foreign matter.

246. INCREASE IN SIZE AND WEIGHT. - The length of the body, which at birth is usually : $1 \frac{1}{5}$ of the arlult borly, undergoes the greatest elongation at an early period :-in the first year, 20 ; in the secund, 10 ; in the thirl, about 7 centimetres; whilst from five to sixteen years the annual increase is about $5 \frac{1}{2}$ centimetres. In the twentieth year the increase is very slight. From fifty onwarls the size of the boly diminishes, owing to the intervertebral discs becoming thinner, and the loss may be 6 to 7 centinetres about the eightieth year. The weight of the body ( $\frac{1}{20}$ of an alult) sinks during the first five to seven days, owing to the evacuation of the meconium aml the small amount of food which is taken at first. Only on the tenth day is the weight the same as at birth.

The increase of weight is greater in the same time than the increase in length. Within the first year a child trebles its weight. The greatest weight is usually reached about forty, while towards sixty a decrease begius, which at eighty may amount even to 6 kilos. The results of measurements, chiefly by Quetelet, are given in the following table :-

\begin{tabular}{|c|c|c|c|c|c|c|c|c|c|}
\hline \multirow{2}{*}{ Age. } & \multicolumn{2}{|c|}{ Length (Cmtr.). } & \multicolumn{2}{|c|}{ Weight (Kilo.). } & \multirow{2}{*}{ Age. } & \multicolumn{2}{|c|}{ Length (Cmtr.). } & \multicolumn{2}{|c|}{ Weight (Kllo.). } \\
\hline & Man. & Woman. & Man. & Woman. & & Man. & Woman. & Man. & Woman. \\
\hline 0 & $49 \cdot 6$ & $48 \cdot 3$ & $3 \cdot 29$ & $2 \cdot 91$ & 15 & $155 \cdot 9$ & $147 \cdot 5$ & $46 \cdot 41$ & $41 \cdot 30$ \\
\hline 1 & $69 \cdot 6$ & $69^{\circ} 0$ & 10.00 & $9 \cdot 30$ & 16 & $161^{\circ} 0$ & $150^{\circ} 0$ & $53 \cdot 39$ & $44 \cdot 44$ \\
\hline 2 & $79 \cdot 6$ & $78 \cdot 0$ & $12 \cdot 00$ & $11 \cdot 40$ & 17 & $167 \cdot 0$ & $154 \cdot 4$ & $57 \cdot 40$ & $49 \cdot 08$ \\
\hline 3 & $86^{\circ} 0$ & $85^{\circ} 0$ & $13 \cdot 21$ & $12 \cdot 45$ & 18 & $170 \cdot 0$ & $156 \cdot 2$ & $61 \cdot 26$ & $53 \cdot 10$ \\
\hline 4 & $93 \cdot 2$ & $91 \cdot 0$ & $15 \cdot 07$ & $14 \cdot 18$ & 19 & $170 \cdot 6$ & & $63 \cdot 32$ & \\
\hline 5 & $99 \cdot 0$ & $97 \cdot 0$ & $16 \cdot 70$ & $15 \cdot 50$ & 20 . & $171 \cdot 1$ & $157 \cdot 0$ & $65 \cdot 00$ & $54 \cdot 46$ \\
\hline 6 & $104 \cdot 6$ & $103 \cdot 2$ & $18 \cdot 04$ & $16 \cdot 74$ & 25 & $172 \cdot 2$ & $157 \cdot 7$ & $68 \cdot 29$ & $55 \cdot 08$ \\
\hline 7 & $111 \cdot 2$ & $109 \cdot 6$ & $20 \cdot 16$ & $18 \cdot 45$ & 80 & $172 \cdot 2$ & $157 \cdot 9$ & $68 \cdot 90$ & $55 \cdot 14$ \\
\hline 8 & $117 \cdot 0$ & $113 \cdot 9$ & $22 \cdot 26$ & $19 \cdot 82$ & 40 & $171 \cdot 3$ & $155 \cdot 5$ & $68 \cdot 81$ & $56 \cdot 65$ \\
\hline 9 & $122 \cdot 7$ & $120 \cdot 0$ & $24 \cdot 09$ & $22 \cdot 44$ & 50 & $167 \cdot 4$ & $153 \cdot 6$ & $67 \cdot 45$ & $58 \cdot 45$ \\
\hline 10 & $128 \cdot 2$ & $124: 8$ & $26 \cdot 12$ & $24 \cdot 24$ & 60 & $163 \cdot 9$ & $151 \cdot 6$ & $65 \cdot 50$ & $56 \cdot 73$ \\
\hline 11 & $132 \cdot 7$ & $127 \cdot 5$ & $27 \cdot 85$ & $26 \cdot 25$ & 70 & $162 \cdot 3$ & $15 \mathrm{i} \cdot 4$ & $63 \cdot 03$ & $53 \cdot 72$ \\
\hline 12 & $135 \cdot 9$ & $132 \cdot 7$ & $31 \cdot 08$ & 30.54 & 80 & $161 \cdot 3$ & $150 \cdot 6$ & $61 \cdot 22$ & $51 \cdot 52$ \\
\hline 13 & $140: 3$ & $138 \cdot 6$ & $35 \cdot 32$ & $34 \cdot 65$ & 90 & & & $57 \cdot 83$ & $49 \cdot 34$ \\
\hline 14 & $148 \cdot 7$ & $144 \cdot 7$ & $40 \cdot 50$ & $38 \cdot 10$ & & (Chie & $y$ from 6 & telet.) & \\
\hline
\end{tabular}

Between the twelfth and fifteenth years the weight and size of the girl are greater than of the boy. Growth is most active in the last months of fetal life, and afterwards from the sixth to the ninth year until the thirteenth to the sixteenth. The full stature is reached about thirty, but not the greatest weight. 


\section{General View of the Chemical Constituents of the Organism.}

247. (A) INORGANIC CONSTITUENTS. - I. Water forms 58.5 per cent. of the whole body, but it occurs in different quantity in the different tissues. The kilneys contain the most water, 82.7 per cent.; bones, 22 per cent.; teeth, 10 per cent.; while enamel contains the least, $0^{\cdot 2}$ per cent. ( $\left.\$ 229\right)$. According to some observers, peroxide of hydrogen $\left(\mathrm{H}_{2} \mathrm{O}_{2}\right)$ is also present in the body.

[Approximately, water forms about two-thirds of the weight of the body, so that a body weighing $\cdot 75$ kilos. (165 lbs.) contains 50 kilos. (110 lbs.) of water. The following table, modified from Beaunis, shows the percentage of water in several tissues and organs :-

Solids.

\begin{tabular}{|c|c|c|c|c|c|c|c|c|}
\hline $\begin{array}{l}\text { Tissue or Organ. } \\
\text { Enamel, }\end{array}$ & $\begin{array}{c}\text { Water. } \\
\quad \cdot 2\end{array}$ & $\begin{array}{l}\text { Solids. } \\
99 \cdot 8\end{array}$ & $\begin{array}{l}\text { Tissue or Organ. } \\
\text { Spinal cord, . }\end{array}$ & $\begin{array}{l}\text { Water: } \\
69 \cdot 7\end{array}$ & $\begin{array}{r}\text { Solids. } \\
30.3\end{array}$ & $\begin{array}{l}\text { Tissue or Organ. } \\
\text { Thymus, }\end{array}$ & $\begin{array}{r}\text { Water. } \\
77 \cdot 0\end{array}$ & $\begin{array}{r}\text { Solids. } \\
23.0\end{array}$ \\
\hline Dentine, & 10.0 & $90 \cdot 0$ & White matter & & & Connective- & & \\
\hline e, & $48^{\circ} 6$ & $51 \cdot 4$ & of brain, . & & $30^{\circ} 0$ & & & \\
\hline Fat, : & $29 \cdot 9$ & $70 \cdot 1$ & Skin, · & $72 \cdot 0$ & $28 \cdot 0$ & Kidney, . & $82 \cdot 7$ & \\
\hline $\begin{array}{l}\text { Elastic tissue, } \\
\text { Cartilage, }\end{array}$ & $\begin{array}{l}49 \cdot 6 \\
55 \cdot 0\end{array}$ & $\begin{array}{l}50 \cdot 4 \\
45 \cdot 0\end{array}$ & $\begin{array}{l}\text { Brain, . } \\
\text { Muscles, }\end{array}$ & & $\begin{array}{l}25 \cdot 0 \\
24 \cdot 3\end{array}$ & $\begin{array}{l}\text { Grey matter o } \\
\text { brain,. }\end{array}$ & & \\
\hline iver, & $69 \cdot 3$ & $30 \cdot 7$ & Spleen, & $75 \cdot 8$ & $24 \cdot 2$ & Vitreous hun & $98 \cdot 7$ & \\
\hline
\end{tabular}

Liquids.

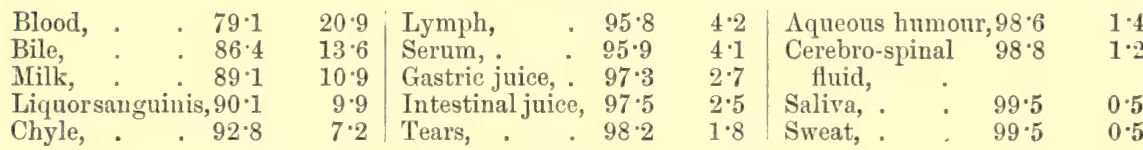

II. Gases. - O, - ozone (§ 37) - H, $-\mathrm{N}-\mathrm{CO}_{2}(\S 38)$. Marsh gas $\mathrm{CH}_{4}(\S 124), \mathrm{NH}_{3}(\S 30$, $\S 124, \S 184), \mathrm{H}_{2} \mathrm{~S}(\S 184)$.

III. Salts. - Sodium chloride [is one of the most important inorganic substances present in the body. It occurs in all the tissues and fluids of the body, and plays a most prominent part in connection with the diffusion of fluids through membranes, ancl its presence is necessary for the solution of the globulins (p. 376). Sometimes it exists in a state of combination with proteid bodies, as in the blood-plasma. Common salt is absolutely necessary for one's existence ; if it be withdrawn entirely, life soon comes to an end. About 15 grammes are given off in twenty-four hours, chiefly by the urine. Boussingault showed that the addition of common salt to the food of cattle greatly improved their condition].

[Calcium phosphate $\left(\mathrm{Ca}_{3} \mathrm{P}_{2} \mathrm{O}_{8}\right)$ is the most abundant salt in the body, as it forms more than one-half of our bones, but it also occurs in dentine, enamel, and to a much less extent in the other solids and fluids of the body. Amongst secretions, milk contains relatively the largest amount ( 2.72 per cent.). In milk it is necessary for forming the calcareous matter of the bones of the infant. It gives bones their hardness and rigidity. It is chiefly derived from the food, and, as only a small quantity is given off in the excretions, it seems not to undergo rapid removal from the body.]

[Sodium phosphate $\left(\mathrm{PNa}_{3} \mathrm{O}_{4}\right)$, acid sodium phosphate $\left(\mathrm{PNa}_{2} \mathrm{HO}_{4}\right)$, acid potassium phosphate $\left(\mathrm{PK}_{2} \mathrm{HO}_{4}\right)$. The sodium phosphate and the corresponding potash salt give most of the fluirls of the body their alkaline reaction. The alkaline reaction of the blood-plasma is partly due to alkaline phosphates, which are çhiefly derived from the food. The acid sodium phosphate is the chief cause of the acid reaction of the urine. A small quantity of phosphoric acid is formed in the body owing to the oxidation of lecithin, which contains phosphorus.]

[Sodium carbonate $\left(\mathrm{Na}_{2} \mathrm{CO}_{3}\right)$ and sodium bicarbonate $\left(\mathrm{NaHCO}_{3}\right)$ exist in small quantities in the food, and are formed in the body from the decomposition of the salts of the vegetable acids. They occur in the blood-plasma, where they play an inportant part in carrying the $\mathrm{CO}_{2}$ from the tissues to the lungs.]

[Sodium and potassium sulphates $\left(\mathrm{Na}_{2} \mathrm{SO}_{4}\right.$ and $\left.\mathrm{K}_{2} \mathrm{SO}_{4}\right)$ exist in very small quantity in the body, and are introduced with the food, but part is formed in the body from the oxidation of organic bodies containing sulphur.]

Potassium chloride $(\mathrm{KCl})$ is pretty widely distributed, and occurs specially in muscle, coloured blood-corpuscles, and milk. Calcium fluoride $\left(\mathrm{CaFl}_{2}\right)$ occurs in small quantity in 
bones and teeth. Calcium carbonate $\left(\mathrm{CaCO}_{3}\right)$ is associated with calcium phosphate in bone, tooth, and in some fluids, but it oceurs in relatively much smaller amount. It is kept in solution by alkaline chlorides, or by the presence of free carbonic acid. Ammonium chloride $\left(\mathrm{NH}_{4} \mathrm{Cl}\right.$ ). - Minute traces occur in the gastric juice and the urine. Magnesium phosphate $\left(\mathrm{Mg}_{3} \mathrm{PO}_{4}\right.$ ) occurs along with ealcium phosphate, but in very much smaller quantity.]

Table by Beaunis of the relative proportions of Salts.

\begin{tabular}{|c|c|c|c|c|c|c|c|}
\hline & Heintz. & Staffel. & Breed. & Oidtmann. & C. Schmidt. & Oidtmann, \\
\hline & & Bone. & $\begin{array}{l}\text { Muscle of } \\
\text { culf. }\end{array}$ & Brain. & Liver. & Lungs. & Spleen. \\
\hline \multirow{2}{*}{$\begin{array}{l}\text { Sorlic chloride, } \\
\text { Potassic chlorile, }\end{array}$} & . & $\cdots$ & $10 \cdot 59$ & $4 \cdot 74$ & $\ldots$ & $13 \cdot 0$ & $\cdots$ \\
\hline & $\therefore \quad:$ & $\begin{array}{l}\ldots \\
\ldots\end{array}$ & $\dddot{2} 35$ & $10 \cdot 69$ & $14 \cdot 51$ & $19 \cdot 5$ & $44: 33$ \\
\hline Potash, : & . & $\cdots$ & $34 \cdot 40$ & $34 \cdot 42$ & $25 \cdot 23$ & i.3 & $9 \cdot 60$ \\
\hline Lime, . . & . & $37 \cdot 58$ & 1.99 & 0.72 & $3 \cdot 61$ & $1 \cdot 9$ & $7 \cdot 48$ \\
\hline Magnesia, & . & $1 \cdot 22$ & $1 \cdot 45$ & $1 \cdot 23$ & $0 \cdot 20$ & $1 \cdot 9$ & 0.49 \\
\hline Ferric oxide, . & . & $\ldots$ & $\ldots$ & $\ldots$ & $2 \cdot 74$ & $3 \cdot 2$ & $7 \cdot 28$ \\
\hline Chlorine, . & - & & $\ldots$ & $\cdots$ & $2 \cdot 58$ & $\ldots$ & 0.54 \\
\hline $\begin{array}{l}\text { Fluorine, } \\
\text { Phosphoric acid (fr }\end{array}$ & & $1 \cdot 66$ & $\cdots$ & $\dddot{0} \cdot 15$ & $\cdots$ & $\cdots$ & $\cdots$ \\
\hline $\begin{array}{l}\text { Phosphoric acid (fr } \\
\text { Phosphoric arid (co }\end{array}$ & nbinedi), & $53 \cdot 31$ & $48 \cdot 13$ & $39 \cdot 02$ & $50 \cdot 18$ & $48 \cdot 5$ & $27 \cdot 10$ \\
\hline Sulphuric acid, & . $\quad$. & & $\ldots$ & 0.75 & 0.92 & $1 \cdot 4$ & $2 \cdot 54$ \\
\hline Carbon dioxide, & . & $5 \cdot 47$ & & & & $\ldots$ & \\
\hline $\begin{array}{l}\text { Silicic acid, : } \\
\text { Ferric phosphate, }\end{array}$ & $\cdot$ & $\cdots$ & $0 \cdot 81$ & 0.12 & 0.27 & $\ldots$ & $0 \cdot 17$ \\
\hline Ferric phosphate, & & $\cdots$ & $\cdots$ & 1.23 & $\cdots$ & $\cdots$ & $\cdots$ \\
\hline
\end{tabular}

Table by Beaunis of the Mineral Matter in Animal Fluids.

\begin{tabular}{|c|c|c|c|c|c|c|c|c|}
\hline & Verdell. & Weber. & Weber. & $\begin{array}{l}\text { Dahn- } \\
\text { hardt. }\end{array}$ & Porter. & $\begin{array}{l}\text { Wilder- } \\
\text { stein. }\end{array}$ & Rose. & Porter. \\
\hline & Blood. & $\begin{array}{l}\text { Blood- } \\
\text { serum. }\end{array}$ & Blood. & Lymph. & Urine. & Bulk. & Bile. & Freces. \\
\hline Sodic chloride, & $58 \cdot 81$ & $72 \cdot 88$ & $17: 36$ & $74 \cdot 48$ & $67 \cdot 28$ & $10 \cdot 73$ & $27 \cdot 70$ & $4 \cdot 33$ \\
\hline $\begin{array}{l}\text { Potassic chloride, } \\
\text { Soda, }\end{array}$ & $\ddot{4} \cdot 15$ & $12 \cdot 93$ & $\begin{array}{r}29 \cdot 87 \\
3.55\end{array}$ & $10 \cdot 35$ & $1 \cdot 33$ & & $36 \cdot 73$ & $5 \cdot 07$ \\
\hline Potash, : & $\begin{array}{r}4.10 \\
11 \cdot 97\end{array}$ & 2.95 & $22 \cdot 36$ & $\begin{array}{r}10 \cdot 05 \\
3 \cdot 25\end{array}$ & $\begin{array}{r}1.00 \\
13.64\end{array}$ & $21 \cdot 44$ & 4.80 & $6 \cdot 10$ \\
\hline Lime, : & $1 \cdot 76$ & $2 \cdot 28$ & $2 \cdot 58$ & 0.97 & $1 \cdot 15$ & $18 \cdot 78$ & $1 \cdot 43$ & $26 \cdot 40$ \\
\hline Magnesia, . & $1 \cdot 12$ & 0.27 & 0.53 & $0 \cdot 26$ & $1 \cdot 34$ & 0.87 & 0.53 & $10 \cdot 54$ \\
\hline Ferric oxide, . & $8 \cdot 37$ & 0.26 & $10 \cdot 48$ & 0.50 & & $0 \cdot 10$ & 0.33 & $2 \cdot 50$ \\
\hline Phosplioric acisl, & $10 \cdot 23$ & $1 \cdot 73$ & $10 \cdot 64$ & $1 \cdot 09$ & $11 \cdot 21$ & $19 \cdot 00$ & $10 \cdot 45$ & $36 \cdot 03$ \\
\hline Sulphuric acid, & $1 \cdot 67$ & $2 \cdot 10$ & 0.09 & & $\ldots$ & $2 \cdot 64$ & $6 \cdot 39$ & $\ldots$ \\
\hline Carbon dioxide, & $1 \cdot 19$ & $4 \cdot 40$ & $2 \cdot 17$ & $8 \cdot 20$ & $\ldots$ & $\ldots$ & $11 \cdot 26$ & \\
\hline Silicic acid, . & $\ldots$ & $0 \cdot 20$ & 0.42 & $1 \cdot 27$ & $4 \cdot 06$ & $\ldots$ & 0.36 & $3 \cdot 13$ \\
\hline
\end{tabular}

IV. Free Acids. - Hydrochloric acid $(\mathrm{HCl})$ [occurs free in the gastric juice, but in combination with the alkalies it is widely distributed as chlorides]. Sulphuric acid $\left(\mathrm{H}_{2} \mathrm{SO}_{4}\right)$ [is said to occur free in the saliva of certain gasteropods, as Dolium galea. In the body it forms sulphates, chiefly in combination with soda and potash].

v. Bases, - Silicon as silicic acid $\left(\mathrm{SiO}_{2}\right)$; manganese, iron, the last forms an integral constituent of hæmoglobin ; copper (?), (\$174).

248. (B) ORganic COMPounds. - I. The Albuminous or Protein Substances.-(1) True Proteids and their Allies are composed of $\mathbf{C}, \mathbf{H}, \mathbf{0}, \mathbf{N}$, and $\mathbf{S}$, and are derived from plants (see Introduction). [The formation of albumin from the elements is accomplished only by plants. What the chemical processes are is quite unknown. We only know that the $\mathrm{N}$ is in the first instance obtained from the nitric acid or ammonia of the soil. The former is probal,ly not used directly as such, but serves, perhaps, for the formation of amides or amido-acids, from which, by the action of non-nitrogenous bodies, proteids are formed.] 
[According to Hoppe-Seyler their general percentage composition is-

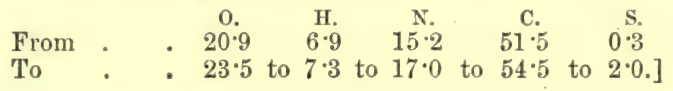

They exist in almost all animal fluids and tissues partly in the fluid form, although Briicke maintains that the molecule of albumin exists in a condition midway between a state of imbibition and a true solution-and partly in a more concentrated condition. Besides forming the chief part of muscle, nerve, and gland, they occur in nearly all the fluids of the body, including the blood, lymph, and serous fluids, but in health mere trases occur in the sweat, while they are absent from the bile and the urine. Unboiled white of egg is the type. In the alimentary canal they are changed into peptones. The chief products derived from their oxidation within the body are $\mathrm{CO}_{2}, \mathrm{H}_{2} \mathrm{O}$, and especially urea, which contains nearly all the $\mathrm{N}$ of the proteids.

Constitution.-Their chemical constitution is quite unknown. The $\mathrm{N}$ seems to exist in two distinct conditions, partly loosely combined, so as to yield ammonia readily when they are decomposed, and partly in a more fixed condition. According to Pfliiger, part of the $\mathrm{N}$ in living proteid bodies exists in the form of cyanogen. [Loew supports Pfliiger's view that the molecule of living (active) albumin differs from that of dead albumin, as he finds that the living protoplasm of certain algre can reduce silver in very dilute alkaline solutions, which dead protoplasm cannot do.] The proteid molecule is very large, and is a very complex one; a small part of the molecule is composed of substances from the group of aromatic bodies (which become conspicuous during putrefaction), the larger part of the molecule belongs to the fatty bodies; during the oxidation of albumin fatty acids especially are developed. Carbohydrates may also appear as decomposition-products. For the decompositions during digestion see $\S 170$, and during putrefaction $\$ 184$. The proteids form a large group of closely related substances, all of which are perhaps modifications of the same body. When we remember that the infant manufactures most of the proteids of its ever-growing body from the casein in milk, this last view seems not improbable.

Characters.-Proteids, the anhydrides of peptones ( $\$ 166)$ are colloids ( $(191)$, and therefore do not diffuse easily through animal membranes; they are amorphous and do not crystallise, and hence are isolated with difficulty ; some are soluble, others are insoluble in water ; insoluble in alcohol and ether; rotate the ray of polarised light to the left; when burned they give the odour of burned horn. Various metallic salts and alcohol precipitate them from their solution; they are coagulated by heat, mineral acids, and the prolonged action of alcohol. Caustic alkalies dissolve them (yellow), and from this solution they are precipitated by acids. By powerful oxidising agents they yield carbamic acid, guanidin, and volatile fatty acids.

Decomposition.-[The number and varieties of these products are exceedingly great, so that it is not easy to separate the several products. In the first place, there is great difficulty in getting in sufficient quantity a perfectly pure proteid, wherewith to institute the necessary experiments. The decomposition-products of albumin when acted on by barium hydrate have been most fully investigated. The action of concentrated $\mathrm{HCl}$, potassic permanganate, and bromine has also been studied. The action of the animal or vegetable digestive ferments is very important $(\S 170)$, and specially that of bacteria causing putrefaction (§ 184).] When acted upon in a suitable manner by acids and alkalies, they give rise to the decomposition-products-leucin (10 to 18 per cent.), tyrosin $(0.25$ to 2 per cent.), aspartic acid, glutamic acid, and also volatile fatty acids, benzoic and hydrocyanic acids, and aldehydes of benzoic and fatty acids; also indol (Hlasiwetz, Hebermann). Similar products are formed during pancreatic digestion $(\$ 170)$ and during putrefaction (§ 184). [Although it is assumed that the proteids have the closest relation to urea, no one, so far, has succeeded in preparing urea by the direct decomposition of albumin. Both by the action of acids and barium hydrate, the splitting up into simpler compounds does not take place at once, but by successive stages, one to the formation of different bodies. Proteids, when fully decomposed, either by acids or alkalies, yield as the final products ammonia, and amido-acids; by alkalies also carbonic, acetic, and oxalic acids. The amido-acids contain several series including leucin, tyrosirt, and glutamic acid. But all proteids do not yield these three bodies, for tyrosin may be absent, while leucin, so far, has been always found. It has therefore been attempted to classify proteids into those that yield tyrosin (i.e., aromatic compounds) and those that do not. Classes I.-VII., p. 376, yield when decomposed aromatic bodies (tyrosin, indol, phenol), while gelatin-yielding bodies and spongin yield no aromatic bodies.]

General Reactions. - (1) Xanthoproteic Reaction.-Heated with strong nitric acid they give a yellow, the addition of ammonia gives a deep orange colour.

(2) With Millon's reagent they give a precipitate, and when heated with this reagent above $60^{\circ} \mathrm{C}$. they give a red one, probably owing to the formation of tyrosin. [If the proteids are 
jresent in large amount, a red preeipitate occurs, but if mere traces are present only the fluid hecomes rect.]

(3) The aldition of a few drops of a dilute solution of cupric sulphate, and the subsequent addition of caustic potash or solla, give a violet colour, which deepens on boiling; [the same colour is obtainet hy alding a few drops of Fehling's solution (biuret-reaction)].

(4) They are precipitated after strong acilulation by acetic acid and by potassium ferrocyanide.

(5) When boiled with concentrated hydrochloric acid, they give a violet-red colour (Liebermann's reaction).

(6) Sulphuric acid containing molybdic acil gives a blue colour (Fröhde).

(7) Their solution in acetic acid is coloured violet with concentrated sulphuric acid, and shows the absorption-band of hydrobilirubin (Adamkievicz).

(8) Iodine is a goot nicrosropic reacent, which strikes a brownish-yellow, while sulphuric acid and cane-sugar give a purplish-violet (E. Schultze).

[?: Wh'n rentered strongly acid with acetic acid and boiled with an equal volume of a con"pntrated sulution of solic sulphate, they are precipitated. This method is used for removing froteids from other liquils, as it does not interfere with the presence of other substances. saturation with solio-magnesic sulphate precipitates the proteids, but not peptones, and the same is the case with saturation with neutral ammonia sulphate (\$249).]

$[(10)$ The precipitation of albumin by aciels is more delicate when the acid is dissolved in alcohol containing 10 per cent. of ether; the precipitate is not clissolved by an excess of the reagent.]

[11) ILost of them are preripitutel by strong mineral acids, and metaphosphoric acid, tannic acid (in an acid solution), phospho-wolframic and phospho-molybilic acids (in acid solution); jotassio-mercuric iorlide (in acisl solutions); many metallic salts, e.y., of $\mathrm{C} u, \mathrm{P}$ 'b, $\mathrm{Ag}, \mathrm{Hg}$; chloral, phenol, trichloracetic acil, picrie acid, alcohol. Taurocholic acid precipitates albumin and syntonin, but not peptone or hemi-albumose (\$275).]

249. THE ANIMAL PROTEIDS AND THEIR CHARACTERS. - Class I. - Native Albumins occur in a natural conclition in animal solids and fluids. They are soluble in water, and are not jrecipitated ly alkaline earhonates, $\mathrm{NaCl}$, or by very dilute acids. Their solutions are "oagulater by heating at 65 to $73^{\circ} \mathrm{C}$. Dried at $40^{\circ} \mathrm{C}$., they yield a clear, yellow, amber-coloured, friahle mass, "soluble albumin," which is soluble in water.

(1) Serum-albumin $(\$ 32$ and $\$ 41)$. - [Its specific rotatory power is $-56^{\circ}$.] Almost all its salts may he removed from it hy dialysis, when it is no longer coagulated by heat. It is coagulated by strong alcohol; and not very readily precipitated by hydrochloric acid, while the precipitate so formed is easily dissolved on adding more acid. When precipitated, it is readily soluble in strong nitric acid. It is not coagulated when shaken up with ether. The addition of water to the hydrochloric solution precipitates acid-albumin. For its presence in urine, $\$ 264$.

(2) Egg-albumin. - When injected into the blood-vessels or unler the skin, or even when introlucesl in large quantity into the intestine, part of it appears unchanged in the urine $(\$ 192$, 4 , and $\$ 264)$. When shaken with ether it is precipitated. These two reactions serve to distingnish it from (1). The specific rotation is $-355^{\circ}, i . c$, for yellow light. Amount of $\mathrm{S}, 1.6$ per cent.

(Metalbumin and Paralbumin have been found by Scherer in ropy solutions in ovarian cysts; they are only prartially precipitated by heat. The precipitate thrown down by the action of strong alcohol is soluble in water.)

Class II. - Globulins are native proteils, insoluble in distilled water, but soluble in dilute neutral saline solutions, i.r, neutral solutions of the alkalies and alkaline earths, e.g., NaCl, $\mathrm{KCl}, \mathrm{XH}_{4} \mathrm{Cl}, \mathrm{MkSO}_{4}$, (hut not $\mathrm{Na}_{2} \mathrm{Cl}_{3}, \mathrm{Na}_{2} \mathrm{HPO}_{4}$ ), sodium chloride of 1 per cent., and in magnesium sulphate. These solutions are coagulated by heat, and are precipitated by the addition of a large quantity of water. Most of them are frecipituted from their sodium chloride solution by the mldition of crystals of sodium chloride, and also by saturating their neutral solution at $30^{\circ}$ with crystals of magnesinm sulphate. When acted upon by dilute acids they yield acid-albumin, and by dilute alkalies, alkali-albumin.

(1) Globulin (Crystallin) is obtained by passing a stream of $\mathrm{CO}_{2}$ through a watery extract of the crystalline lens.

(2) Vitellin is the chief proteid in the yolk of egg. It is also said to occur in the chyle (?) and in the amniotic fluid $(W \cdot y /)$. Both the foregoing are not precipitated from their neutral solutions by saturation with sodium chloride.

(3) Para-globulin or Serum-globulin (\$ 29), and in urine (\$264).

(4) Fibrinogen (829). - In the clear jelly-like secretion of the vesicule seminales of the guinea-pis, there is a globulin-like body closely resembling fibrinogen. It contains 29 per cent. of albumin, with scarcely any ash. If it be touched with a trace of blood-serum, without mixing them, it gradually and completely forms a solid mass quite like fibrin.

(5) Myosin is the chief proteid in dead muscle. Its coagulation in muscle post-mortem constitutes rigor mortis. If muscle be repeatedly washed, and afterwards treated with a 10 per 
cent. solution of sodium or ammonium chloride, it yields a viscid fluid which, when dropped into a large quantity of distilled water, gives a white flocculent precipitate of myosin. It is also precipitated from its $\mathrm{NaCl}$ solution by crystals of $\mathrm{NaCl}$. For Kühne's and other methods see $\S 293$.

(6) Globin (Preyer), the proteid residue of hæmoglobin (§ 18).

Class III.-Derived Albumins (Albuminates).-(1) Acid-albumin or Syntonin. - When proteids are dissolved in the stronger acids, e.g., hydrochloric, they become changed into acidalbumins. They are precipitated from soluticn by the addition of many salts, sodic chloride, acetate or phosphate, or by neutralisation with an alkali, e.g., sodic carbonate, but they are not precipitated by heat. The concentrated solution gelatinises in the cold, and is redissolved by heat. Syntonin, which is obtained by the prolonged action of dilute hydrochloric acid ( 2 per 1000) upon minced muscle, is also an acid-albumin. It is formed also in the stomach during digestion $(\$ 166, \mathrm{I}$.). According to Soyka, the alkali- and acid-albumins differ from each other only in so far as the proteid in the one case is united with the base (metal) and in the other with the acid.

(2) Alkali-albumin.--If egg- or serum-albumin be acted upon for some time by dilute alkalies, a solution of alkali-albumin is obtained. Strong caustic potash acts upon white of egg, and yields a thick jelly, Iieberkühn's jelly. The solution is not precipitated by heat, but it is precipitated by the addition of an acid. [Although alkali-albumin is precipitated on neutralisation, this is not the case in the presence of alkaline phosphates, e.g., sodic phosphate.]

(3) Casein is the chief proteid in milk $(\$ 231)$. It is precipitated by acids and by rennet at $40^{\circ} \mathrm{C}$. In its characters it is closely related to alkali-albuminate, but it contains more $\mathrm{N}$. It contains a large amount of phosphorus $(0.83$ per cent.). It may be precipitated from milk by diluting it with several times its volume of water and adding dilute acetic acid, or by adding magnesium sulphate crystals to milk and shaking vigorously. Owing to the large amount of phosphorus which it contains, it is sometimes referred to the nucleo-albumins. When it is digested with dilute $\mathrm{HCl}\left(0^{\cdot} 1\right.$ per cent. $)$ and pepsin at the temperature of the body, it gradually yields nuclein.

Class IV.-Fibrin. - $(\S 27)$ and for the fibrin-factors $(\$ 29)$.

Class V.-Peptones. - For peptones and propeptone or the albumoses $(\S 166, I)$; in urine $(\S 264)$.

Class VI.-Lardacein and Other Bodies. -There fall to be mentioned the "yelk-plates," which occur in the yelk:- Ichthin (cartilaginous fishes, frog); Ichthidin (osseous fishes); Ichthulin (salmon); Emydin (tortoise); also the indigestible amyloid substance or lardacein, which occurs chiefly as a pathological infiltration into various organs, as the liver, spleen, kidneys, and blood-vessels. It gives a blue with iodine and sulphuric acil (like cellulose), and a mahogany-brown with iodine. It is difficult to change it into an albuminate by the action of acids and alkalies.

Class VII. - Coagulated Proteids. - When any native albumins or globulins are coagulated, e.g., at $70^{\circ} \mathrm{C}$., they yield bodies with altered characters, insoluble in water and saline solutions, but soluble in boiling strong acids and alkalies, when they are apt to split up. They are dissolved during gastric and pancreatic digestion to produce peptones.

Appendix: Vegetable Proteid Bodies.-Plants, like animals, contain proteid bodies, although in less amount. They occur either in solution in the juices of living plants or in the solid form. In composition and reaction they resemble animal proteids.

[The characters of vegetable proteids have a great resemblance to animal proteids. They have frequently been obtained in a crystalline form, e.g., from the seeds of the gourd and various oleaginous seeds. They occur in greatest bulk in the seeds of plants, aleurone grains being for the most part composed of them. In seeds, globulins and "vegetable peptone" form the greater proportion of the proteid constituents.]

[Globulins,-These varieties have been described as occurring in the seeds of plants:vegetable myosin, vitellin, and paraglobulin (Martin). They have practically the same properties as those found in the animal kingdom: vegetable vitellin has, however, not been sufficiently studied. Paraglobulin has been found in papaw juice (Martin). Myosin occurs in the seed of leguminosie, in flour, and in the potato.]

[Albumin.-The existence of a body corresponding to egg- or serum-albumin in the vegetable kingdom is doubtful (Ritthausen). Such a body has been described in papaw juice [Martin).]

[Vegetable Peptone: Albumoses. - A true peptone has not yet been recognised in plants: what has been described as such is hemi-albumose (Vines). Albumoses have been found in the seeds of leguminosæ, in flour, and in papaw juice. In the last, two forms occur, called respectively $\alpha$ - and $\beta$-phytalbumose. The former, $\alpha$-phytalbumose, agrees with the hemi-albunnose lescribed by Vines, being soluble in cold and boiling water; giving also a biuret-reaction, and a precipitate by saturation with sodium chloride only in an acid solution. The latter, $\beta$-phytalbumose, is soluble in cold, but not in bqiling, distilled water; hence it is precipitated by heat. 
It is also readily thrown down by saturation with sodium chloride, and gives a faint biuretreaction (Martin).]

[Vegetable Casein is said to occur in the seeds of leguminosa ; and it is slightly soluble in water, but readily so in weak alkalies and in solutions of basic calcic phosphate. A solution of this body is precipitated by acids and rennet. Two varieties have been described, $-(\alpha)$ legumin, in peas, beans, lentils; acid in reaction, soluble in weak alkalies and very dilute $\mathrm{HCl}$ or acetic acii ; $(\boldsymbol{\beta})$ conglutin, a very similar body occurring in hops and almonds. The existence of vegretable casein is denied. Vines states that both legumin and conglutin are artificial products, being formed from the globulins present by the dilute alkali used in extraction of the proteids. This is denied by Ritthausen.]

[Gluten and Glutin.-Gluten is readily prepared from flour by washing and kneading it in a muslin bag under a stream of water. So prepared it is yellowish-brown in colour, very sticky, and capable of being drawn out into long shreds. It is insoluble in water, soluble (but not completely) by prolonged action in dilute acids and alkalies ( 2 per cent. $\mathrm{KHO}$ and $\mathrm{HCl}$ ). The prolonged action of alcohol ( 80 to 85 per cent.) dissolves part of the substance of gluten, leaving a residue, called by Liebig plant-fibrin and by Ritthausen gluten-casein. The alcohol contains gliadin (glutin), gluten-fibrin, and mucedin. Gluten-casein is readily soluble in dilute alkalies, almost insoluble in dilute acetic acid, and quite insoluble in cold and boiling water ; the products of its decomposition, by heating with $\mathrm{H}_{2} \mathrm{SO}_{2}$, are leucin, tyrosin, glutamic, and asparaginie acils. The three bodies dissolved from glutin by alcohol differ chietly in their solubility in alcohol and water. Gluten-fibrin, the least soluble, is coagulated by the action of absolute alcohol; it is readily soluble in dilute acids and alkalies, being precipitated by neutralisation. Gliadin (glutin, plant-gelatin) may be prepared by boiling gluten with water : it deposits on cooling the solution. Though soluble in water at $100^{\circ} \mathrm{C}$. at first, it becomes insoluble by the prolongerl action of water at that temperature. It is, like gluten-fibrin, soluble in dilute acids and alkalies. Mucedin differs from gliadin in being less soluble in strong alcohol. The water used in washing the flour in the preparation of gluten contains hemi-albumose (Vines) and a globulin $(W c y l)$. Rye-Hour, as well as wheaten, yields gluten under similar treatment with water.]

[Nitrogenous Crystalline Principles.-Leucin, tyrosin, asparagin, and glutamic acid have been found in the seeds of plants.]

250. (2) THE ALBUMINOIDS. --.These substances closely resemble true proteids in their composition and origin, and are amorphous non-crystalline colloids; some of them do not contain S, but the most of them have not been prepared free from ash. Their reactions and decomposition-products closely resemble those of the proteids; some of them produce, in addition to leucin and tyrosin, glycin and alanin (amido-propionic acid). They occur as organised constituents of the tissues and also in fluid form. It is unknown whether they are formed by oxidation from proteid bodies or by synthesis.

1. Mucin is the characteristic substance present in mucus. That obtained from the submaxillary gland contains-C $52 \cdot 31, \mathrm{H} 7 \cdot 22, \mathrm{~N} 11 \cdot 84, \mathrm{O} 28 \cdot 63$. According to Hammarsten it contains $S 1.79$ and $N 13.5$ per cent. It dissolves in water, making it sticky or slimy, and can be filtered. It is precipitated by acetic acid and alcohol ; and the alcohol precipitate is again soluble in water. It is not precipitated by acetic acid and ferrocyanide of potassium, but $\mathrm{HNO}_{3}$ and other mineral acids precipitate it. It occurs in saliva (\$146), in bile, in mucous glands, secretions of mucous membranes, in mucous tissue, in synovia, and in tendons. Pathologically it occurs not unfrequently in eysts; in the animal kingdom, especially in snails and in the skin of holothurians. It yields leucin and 7 per cent. of tyrosin when it is decomposed by prolonged boiling with sulphuric acid. [The precipitate called mucin has not always the same characters, and, in fact, it differs according to the animal from which it is obtained (Landwehr).]

2. Nuclein (Niescher, § 198)-(C 29, H 49, N 9, P 3, O 22)-contains phosphoric acid, and is slightly soluble in water, ensily in ammonia, alkaline carbonates, strong $\mathrm{HNO}_{3}$; it gives the biuret-reaction; no reaction with Millon's reagent; when decomposel it yields phosphorus. It occurs in the nuclei of pus and blood-corpuscles (\$22), in spermatozoids, yelk-spheres, liver, brain, and milk, yeast, fungi, and many seeds. It has resemblances to mucin, and is perhaps an intermediate product between albumin and lecithin (Hoppe-Seyler). It is prepared by the artificial digestion of pus, when it remains as an indigestible residue; acids precipitate it from an alkaline solution. It gives a feeble xantho-proteic reaction; after the prolonged action of alkalies and acid, substances similar to albumin and syntonin are formed. Hypoxanthin and guanin have been obtained as decomposition-products from it (Kossel).

3. Keratin occeurs in all horny and epidermic tissues (epidermic scales, hairs, nails, feathers) - C 50.3-52.5, H 6.4-7, N $16 \cdot 2-17,020 \cdot 8-25$, S $0 \cdot 7-5$ per cent. -is soluble in boiling caustic alkalies, but swells up in cold concentrated acetic acid. When decomposed by $\mathrm{H}_{2} \mathrm{SO}_{4}$ it yields 10 per cent. leucin and $3 \cdot 6$ per cent. tyrosin. Neuro-keratin (\$321).

4. Fibroin is soluble in strong alkalies and mineral acids, in ammonio-sulphate of copper ; when boiled with $\mathrm{H}_{2} \mathrm{SO}_{4}$ it yields 5 per cent. tyrosin, leucin, and glycin. It is the chief constituent of the cocoons of insects and threads of spiders. 
5. Spongin, allied to fibroin, occurs in the bath-sponge, and yields, as decomposition prolucts, leucin and glycin (Stadeler).

6. Elastin, the fundamental substance in elastic tissue, is soluble only when boiled in concentrated caustic potash $-\mathrm{C} 55-55 \cdot 6, \mathrm{H} 7 \cdot 1-7 \cdot 7, \mathrm{~N} 16 \cdot 1-17 \cdot 7, \mathrm{O} 19 \cdot 2-21^{\circ} 1$ per cent. It yields 36 to 45 per cent. of leucin and $\frac{1}{2}$ per cent. of tyrosin.

7. Gelatin (Glutin), obtained from connective-tissues by prolonged boiling with water; it gelatinises in the cold - C $52 \cdot 2-50 \cdot 7, \mathrm{H} 6 \cdot 6-7 \cdot 2, \mathrm{~N} 17 \cdot 9-18 \cdot 8, \mathrm{~S}+\mathrm{O} 23 \cdot 5-25$ (S $0 \cdot 7$ per cent.). [The ordinary connective-tissues are supposed to contain the hypothetical anhydride collagen, while the organic basis of bone is called ossein.] It rotates the ray of polarised light strongly to the left $=-130^{\circ}$. By prolonged boiling and digestion, it is converted into a peptone-like body (gelatin-peptone), which does not gelatinise (\$161, I.). [It swells up, but does not dissolve in cold water; when dissolved in warm water, and tinged with Berlin blue or carmine, it forms the usual coloured mass which is employed by histologists for making fine transparent injections of blood-vessels.] A body resembling gelatin is found in leukæmic blood and in the juice of the spleen $(\S 103$, I.). When decomposed with sulphuric acid it yields glycin, ammonia, leucin, but no tyrosin. [It is precipitated from its solution by alcohol, mercuric chloride, metaphosphoric acid, phospho-wolframic acid, taurocholic acid, tannic acid, but the precipitate with the last does not occur when salts are absent. It is readily soluble in dilute acids, even in acetic acid. When boiled with Millon's reagent, it is not coloured red. With cupric sulphate and caustic soda it gives a violet colour which, on boiling, becomes light red. It gives no colour with concentrated $\mathrm{H}_{2} \mathrm{SO}_{4}$ and acetic acid.]

8. Chondrin occurs in the matrix of hyaline cartilage and between the fibres in fibro-cartilage. It is obtained from hyaline cartilage and the cornea by boiling. [Its solutions gelatinise on cooling.] It occurs also in the mantle of molluses-C $49 \cdot 5-50 \cdot 9, \mathrm{H} 6 \cdot 6-7 \cdot 1, \mathrm{~N} 14 \cdot 4-14 \cdot 9, \mathrm{~S}+\mathrm{O}$ $27 \cdot 2-29$ (S 0.4 per cent.). When boiled with sulphuric acid it yields leucin; with hydrochloric acid, and when digested, chondro-glucose (Meissner); it belongs to the glucosides, which contain N. When acted upon by oxidising reagents it is converted into gelatin (Bramc). The substance which yields chondrin is called chondrogen, which is perhaps an anhydride of chondrin. The following properties of gelatin and chondrin are to be noted :-Gelatin is precipitated by tannic acid, mercuric chloride, chlorine water, platinic chloride, and alcohol, but not by acids, alum, or salts of silver, iron, copper, or lead; its specific rotation is $=-130^{\circ}$. [Compare these precipitants with those of albumin.] Chondrin is precipitated by acetic acid and dilute sulphuric and hydrochloric acids, by alum, and by salts of silver, iron, and lead; its specific rotation $=-213^{\circ}$.

9. The hydrolytic ferments have recently been called enzymes by W. Kiihne, in order to distinguish them from organised ferments, such as yeast. The enzymes, hydrolytic or organic ferments, act only in the presence of water. They act upon certain bodies, causing them to take up a molecule of water. They all decompose hydric peroxide into water and $O$. They are most active between $30^{\circ}$ to $35^{\circ} \mathrm{C}$., and are destroyed by boiling, but when dry they may be subjected to a temperature of $100^{\circ}$ without being destroyed. Their solutions, if kept for a long time, gradually lose their properties and undergo more or less decomposition.

(a) Sugar-forming or diastatic-ferment occurs in saliva (\$ 148), pancreatic juice ( $\$ 170)$, intestinal juice (\$183), bile ( $(180)$, blood (\$ 22$)$, chyle (\$189), liver (\$174), in human milk (\$231). Invertin in intestinal juice (\$183). Almost all dead tissues, organic fluids, and even proteids, although only to a slight degree, may act diastatically. Diastatic ferments are very generally distributed in the vegctable kingdom.

(b) Proteolytic, or ferments which act upon proteids. - Pepsin in gastric juice and in muscles (\$166), in vetches, myxomycetes (Krukenberg), trypsin in the pancreatic juice (\$170), a similar ferment in the intestinal juice ( $\$ 183)$, and urine (\$264).

(c) Fat-decomposing in pancreatic juice (\$170), in the stomach ( $\$ 166)$.

(d) Milk-coagulating in the stornach ( $\$ 166)$, pancreatic juice $(\S 170)$, and perhaps also in the intestinal juice (?)-( W. Roberts).

[The importance of fermentative processes has already been referred to in detail under "Digestion." Ferments are bodies which excite chemical changes in other matter with which they are brought into contact. They are divided into two classes :-

(1) Unorganised ; soluble or non-living.

(2) Organised, or living.]

[(1) The Unorganised Ferments are those mentioned in the following table. They seem to be nitrogenous bodies, although their exact composition is unknown, and it is doubtful if they have ever been obtained perfectly pure. They are present in many secretions, and are produced within the body by the vital activity of the protoplasm of cells. They are termed soluble because they are soluble in water, glycerin, and some other substances (\$148), while they can be precipitated by alcohol and some other reagents. They do not multiply during their activity, nor is their activity prevented by a certain proportion of salicylic acid. They are not affected by oxygen subjected to the compression of many atmospheres $(P$. Bert). They are non-living: Their other properties are referred to above.] 
[The unorganised ferments present in the body, and their actions ( $W$. Robcrts) :-

\begin{tabular}{|c|c|c|c|}
\hline Fluid or Tissues. & Ferment. & $*$ & Actions: \\
\hline Saliva, . . & 1. Ptyalin (\$ 148), & . & Converts starch chiefly into maltose. \\
\hline Gastric juice, & $\begin{array}{l}\text { 1. Pepsin, . } \\
\text { 2. Milk-curdling, } \\
\text { 3. Lactic acid fermen } \\
\text { 4. Fat-splitting, . }\end{array}$ & $\begin{array}{l}\cdot \\
\cdot \\
\cdot \\
\cdot\end{array}$ & $\begin{array}{l}\text { Converts proteids into peptones in an acid } \\
\text { medium, certain bye-products being } \\
\text { formed (\$ 166). } \\
\text { Curdles casein of milk. } \\
\text { Splits up milk-sugar into lactic acid. } \\
\text { Splits up fats into glycerin and fatty acids. }\end{array}$ \\
\hline $\begin{array}{l}\text { Pancreatic } \\
\text { juice, }\end{array}$ & $\begin{array}{l}\text { 1. Diastatic or amylo } \\
\text { 2. Trypsin, } \\
\text { 3. Emulsive (?), . } \\
\text { 4. Fat-splitting or st } \\
\text { 5. Milk-curdling, }\end{array}$ & $\begin{array}{l}\text { psin, } \cdot\{ \\
\cdot \text { eapsin, }^{\cdot}\end{array}$ & $\begin{array}{l}\text { Converts starch chiefly into maltose. } \\
\text { Changes proteids into peptones in an } \\
\text { alkaline medium, certain bye-products } \\
\text { being formed }(\$ 170) \text {. } \\
\text { Emulsifies fats. } \\
\text { Splits fats into glycerin and fatty acids. } \\
\text { Curdles casein of milk. }\end{array}$ \\
\hline $\begin{array}{l}\text { Intestinal } \\
\text { juice, }\end{array}$ & $\begin{array}{l}\text { 1. Diastatic, . } \\
\text { 2. Proteolytic, . } \\
\text { 3. Invertin, : } \\
\text { 4. Milk-curdling, }\end{array}$ & 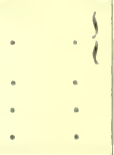 & $\begin{array}{l}\text { Does not form maltose, but maltose is } \\
\text { changed into glucose ( } \$ 183) \text {. } \\
\text { Fibrin into peptone (?). } \\
\text { Changes cane- into grape-sugar. } \\
\text { (? in small intestine). }\end{array}$ \\
\hline $\begin{array}{l}\text { Blood, : } \\
\text { Chyle, } \\
\text { Liver (?), } \\
\text { Milk, } \\
\text { Most tissues, }\end{array}$ & Diastatic ferments. & & $\ldots$ \\
\hline $\begin{array}{l}\text { Muscle, : } \\
\text { Urine, }\end{array}$ & \} Pepsin and other fer & ments. & $\ldots$ \\
\hline Blood, & Fibrin-forming ferme & & $\ldots$ \\
\hline
\end{tabular}

[(2) The Organised or living ferments are represented by yeast ( $\$ 235)$. Other living ferments belonging to the schizomycetes, occurring in the intestinal canal, are referred to in $\$ 184$. Yeast causes fermentation by splitting up sugar into $\mathrm{CO}_{2}$ and alcohol $(\$ 156)$, but this result only occurs so long as the yeast is living. Hence, its activity is coupled with the vitality of the cells of the yeast. If yeast be boiled, or if it be mixed with carbolic or salicylic arill, or chloroform, all of which destroy its activity, it cannot produce the alcoholic fermentation. As yet no one has succeeded in extracting from yeast a substance which will excite the alcoholic fermentation. All the organised ferments grow and multiply during their activity at the expense of the substances in which they occur. Thus the alcoholic fermentation depends upen the "life" of the yeast. They are said to be killed by oxygen subjected to the compression of many atmospheres $(P$. Bert). But it is important to note that Hoppe-Seyler has extracterl from decul yeast (killed by ether) an unorganised ferment which can change canesugar into grape-sugar.]

10. Hrmoglobin, the colouring matter of blood, which, in addition to $\mathrm{C}, \mathrm{H}, \mathrm{O}, \mathrm{N}$, and $\mathbf{S}$, rontains irvil, may be taken with the albuminoids (\$ 11). [Hæmocyanin (§32).]

\section{(3) Glucosides containing Nitrogen.}

In adlition to chondrin, the following glucosides containing nitrogen, when subjected to hydrolytic processes, may combine with water, and form sugar and other substances:-

Cerebrin $(\$ 322)=\mathrm{C}_{57} \mathrm{H}_{110} \mathrm{~N}_{2} \mathrm{O}_{25}$ (Geoghegan). [Parcus has shown that cerebrin as originally prepared by W. Müller is a mixture of three bodies, viz., cerebrin, homocerebrin, and encephalin.]

Protagon-C $66 \cdot 29$, H $10 \cdot 69$, N 2.39, P 1.068 , per cent.-occurs in nerves, and contains phosphorus (\$ 322).

Chitin, $2\left(\mathrm{C}_{15} \mathrm{H}_{28} \mathrm{~N}_{2} \mathrm{O}_{10}\right)$, is a glucoside containing nitrogen, and occurs in the cutaneous coverings of arthropoda, and also in their intestine and tracheæ; ; it is soluble in concentrated aciils, c.g., bydrochloric or nitric acid, but iusoluble in other rengents. According to Sandwick, ehitin is an amin-derivative of a carbohylrate with the general formula $n\left(\mathrm{C}_{12} \mathrm{H}_{20} \mathrm{O}_{10}\right)$. The 
hyalin of worms is closely related to chitin. (Solanin, amygdalin (§ 202), and salicin, \&c., are glucosides of the regetable kingdom.)

\section{(4) Colouring Matters containing Nitrogen.}

Their constitution is unknown, and they occur only in animals. They are in all probability derivatives of hæmoglobin. They are-(1) hæmatin ( $\$ 18, A)$, myohæmatin ( $\$ 232, \S 292, a)$, histo-hæmatin (\$ 103, IV.), and hæmatoidin (\$20). (2) Bile-pigments $(\S 177,3)$. (3) Urinepigments (except Indican). (4) Melanin- $\mathrm{C}_{44^{\prime} \cdot 2}, \mathrm{H}_{3}, \mathrm{~N}_{9 \cdot 9}, \mathrm{O}_{42 \cdot 6}$-or the black pigment, which occurs partly in epithelium (choroid, retina, iris, and in the deep layers of epidermis in coloured races) and partly in connective-tissue corpuscles (Lamina fusca of the choroid). [Turacin occurs in the red feathers of Corythaix Buffoni or Plantain-Eater. Its ash contains nearly 6 per cent. of copper (Church). The reddish spots or parts of feathers burn with a green flame.]

\section{Organic Acids free from Nitrogen.}

(1) The fatty acids, with the formula $\mathrm{C}_{n} \mathrm{H}_{2 n-1} \mathrm{O}(\mathrm{OH})$, occur in the body partly free and partly in combination. Free volatile fatty acids occur in decomposing cutaneous secretions (sweat). In combination, acetic acid and caproic acid occur as amido-compounds in glycin (=amido-acetic acid) and leucin (=amido-caproic acid). More especially do they occur united with glycerin to form neutral fats, from which the fatty acid is again set free by pancreatic digestion ( $\$ 170$, III.).

(2) The acids of the acrylic acid series, with the formula $\mathrm{C}_{n} \mathrm{H}_{2-3} \mathrm{O}(\mathrm{HO})$, are represented in the body by one acid, oleic acid, which in combination with glycerin yields the neutral fat olein.

251. Fats. - (1) Neutral fats occur very abundantly in animals, but they also occur in all plants; in the latter more especially in the seeds (nuts, almonds, cocoa nut, poppy), more rarely in the pericarp (olive) or in the root. They are obtained by pressure, melting, or by extracting them with ether or boiling alcohol. They [e.g., tristearin, $\mathrm{C}_{57} \mathrm{H}_{110} \mathrm{O}_{6}$ ] contain much less $\mathrm{O}$ than the carbohydrates, such as sugar and starch; they give a greasy spot on paper, and when shaken with colloid substances, such as albumin, they yield an emulsion. When treated with superheated steam or with certain ferments (p. 256, c), they take up water and yield glycerin and fatty acids, and if the latter be volatile they have a rancid odour. Treated with caustic alkalies they also take up water, and are decomposed into glycerin and fatty acids; the fatty acid unites with the alkali and forms a soap, while glycerin is set free. The soapsolution dissolves fats.

Glycerin is a tri-atomic alcohol, $\mathrm{C}_{3} \mathrm{H}_{5}(\mathrm{OH})_{3}$, and unites with (1) the following monobasic fatty acids (those occurring in the body are printed in italics):-

\begin{tabular}{|c|c|c|c|c|c|}
\hline $\begin{array}{l}\text { Acids. } \\
\text { 1. Formic, } \\
\text { 2. Acetic, } \\
\text { 3. Propionic, . } \\
\text { 4. Butyric, } \\
\text { [Isobutyric, } \\
\text { 5. Valerianic, } \\
\text { 6. Caproic, . }\end{array}$ & $\begin{array}{ll}\cdot & \mathrm{CH}_{2} \mathrm{O}_{2} \\
\cdot \cdot & \mathrm{C}_{2} \mathrm{H}_{4} \mathrm{O}_{2} \\
\cdot & \mathrm{C}_{3} \mathrm{H}_{6} \mathrm{O}_{2} \\
\cdot & \mathrm{C}_{4} \mathrm{H}_{8} \mathrm{O}_{2} \\
\cdot & \mathrm{C}_{4} \mathrm{H}_{8} \mathrm{O}_{2} \\
\cdot & \mathrm{C}_{5} \mathrm{H}_{10} \mathrm{O}_{2} \\
\cdot & \mathrm{C}_{6} \mathrm{H}_{12} \mathrm{O}_{2}\end{array}$ & $\begin{array}{l}\text { Acids. } \\
\text { 7. AEnanthylic, . } \\
\text { 8. Caprylic, } \\
\text { 9. Pelargonic, } \\
\text { 10. Capric, } \\
\text { 11. Laurostearic, } \\
\text { 12. Myristic, } \\
\text { 13. Palmitic, }\end{array}$ & $\begin{array}{l}\mathrm{C}_{7} \mathrm{H}_{14} \mathrm{O}_{2} \\
\mathrm{C}_{8} \mathrm{H}_{16} \mathrm{O}_{2} \\
\mathrm{C}_{9} \mathrm{H}_{18} \mathrm{O}_{2} \\
\mathrm{C}_{10} \mathrm{H}_{20} \mathrm{O}_{2} \\
\mathrm{C}_{12} \mathrm{H}_{24} \mathrm{O}_{2} \\
\mathrm{C}_{14} \mathrm{H}_{28} \mathrm{O}_{2} \\
\mathrm{C}_{16} \mathrm{H}_{32} \mathrm{O}_{2}\end{array}$ & $\begin{array}{l}\text { Acids. } \\
\text { [Margaric, } \\
\text { is a mixture } \\
\text { of } 13 \text { and 14.] } \\
\text { 14. Stearic, } \\
\text { 15. Arachinic, } \\
\text { 16. Hyänic, } \\
\text { 17. Cerotinic, }\end{array}$ & $\begin{array}{ll}\cdot \mathrm{C}_{17} \mathrm{H}_{34} \mathrm{O}_{2}, \\
.] \\
. & \mathrm{C}_{18} \mathrm{H}_{36} \mathrm{O}_{2} \\
\cdot & \mathrm{C}_{20} \mathrm{H}_{40} \mathrm{O}_{2} \\
\cdot & \mathrm{C}_{25} \mathrm{H}_{50} \mathrm{O}_{2} \\
& \mathrm{C}_{27} \mathrm{H}_{54} \mathrm{O}_{2}\end{array}$ \\
\hline
\end{tabular}

The acids form a homologous series with the formula $\mathrm{C}_{n} \mathrm{H}_{2 n-1} \mathrm{O}(\mathrm{OH})$. With every $\mathrm{CH}_{2}$ added their boiling point rises $19^{\circ}$. Those containing most carbon are solid, and non-volatile; those containing less $\mathrm{C}$ (up to and including 10 ) are fluid like oil, have a burning acid taste, and a rancid odour. The earlier members of the series may be obtained by oxidation from the later, by $\mathrm{CH}_{2}$ being removed, while $\mathrm{CO}_{2}$ and $\mathrm{H}_{2} \mathrm{O}$ are formed ; thus, butyric acid is obtained from propionic acid. Nos. 13 and 14 are found in human and animal fat, less abundant and more inconstant are $12,11,6,8,10,4$. Some occur in sweat $(\$ 287)$ and in milk (\$231). Many of them are developed during the decomposition of albumin and gelatin. Most of the above (except 15 to 17 ) occur in the contents of the large intestine ( $\$ 185$ ).

(2) Glycerin also unites with the monobasic oleic acid, which also forms a series, whose general formula is $\mathrm{C}_{n} \mathrm{H}_{2 \mathrm{n}-3} \mathrm{O}(\mathrm{OH})$; and they all contain $2 \mathrm{H}$ less than the corresponding members of the fatty acid series. The corresponding fatty acids can be obtained from the oleic acid series and vice versâ. Oleic acid (olein-elainic acid), $\mathrm{C}_{18} \mathrm{H}_{34} \mathrm{O}_{2}$, is the only one found in the organism; united with glycerin, it forms the fluid fat, olein. The fat of new-borm children contains more glyceride of palmitic and stearic acid than that of adults, which contains more glyceride of oleic acid. Oleic acid also occurs united with alkalies (in soaps) and (like some fatty acids) in the lecithins (\$23). If lecithin be acted on with barium hydrate, we obtain insoluble stearic, or oleic, or palmitie acids and barium oleate, together with dissolved neurin $(\S 322, b)$ and baric glycerin phosphate. It appears as if there were several lecithins, of which the most abundant are the one with stearic acid and that with palmitin + oleic acid radicle (Diakonow). Lecithin occurs in the blood-corpuscles (\$23), semen, and nerves, while neurin is constantly present in fungi. 
The neutral fats [palmitin, stearin (both solid), and olein (fluid)], the glycerides of fatty acids, and of oleic acid, are triple ethers of triatomic alcohol glycerin. With the neutral fats may be associated glycerin-phosphoric acid, an acid glycerin ether, formed by the union of glycerin and phosphoric acid, with the giving off of a molecule of water $\left(\mathrm{C}_{3} \mathrm{H}_{9} \mathrm{PO}_{6}\right)$; it is a decomposition-product of lecithin ( $\$ 23)$.

(3) The glycolic acids (acids of the lactic acid series) have the formula $\mathrm{C}_{n} \mathrm{H}_{2 n-2} \mathrm{O}(\mathrm{OH})_{2}$. They are formed by oxidation from the fatty acid series by substituting $\mathrm{OH}$ (hydroxyl) for one atom of $\mathrm{H}$ of the fatty acids. Conversely, fatty acids may be obtained from the glycolic acids. The following acids of this series occur in the body:-

(i) Carbonic Acid (oxy-formic acid), $\mathrm{CO}(\mathrm{OH})_{2}$; in this form, however, it only makes salts. Free carbonic acicl or carbon dioxide is an anhydride of the same $=\mathrm{CO}_{2}$.

(b) Glycolic Acid (oxy-acetic acid), $\mathrm{C}_{2} \mathrm{H}_{2} \mathrm{O}(\mathrm{OH})_{2}$, does not occur free in the body. One of its comprumls, glycin (glycocoll, amido-acetic acid, or gelatin-sugar), occurs as a conjugate acid, viz., as glycocholic acid in the bile $(\$ 177,2)$, and as hippurie acid in the urine (\$260). Glycin exists in complex combination in the gelatin.

(c) Lactic Acid (oxy-propionic acid), $\mathrm{C}_{3} \mathrm{H}_{4} \mathrm{O}(\mathrm{OH})_{2}$, occurs in the body in two isomeric forms -1. The chylidene-lactic acill, which occurs in two modifications-as the right rotatory sarculactic acid (paralactic), a metabolic product of muscle; and as the ordinary optically inactive product of "lactic fermentation," which occurs in gastric juice, in sour milk (sauerkraut, aril cucumber), and can be obtained by fermentation from sugar ( $\$ 184)$. 2. The isomer, ethylenc-lactic acid, occurs in the watery extract of muscles (\$293).

(d) Leucic Acid (oxy-eaproie acid), $\mathrm{C}_{6} \mathrm{H}_{12} \mathrm{O}_{3}$, does not oceur as such, but only in the form of one of its derivatives, leucin (anido-caproic acid), as a product of the metabolism in many tissues, and is formed during pancreatic digestion (\$170, II.). Leucic acid may be prepared from leucin, and glycolic acid from glycin, by the action of nitrous acid.

(4) Acids of the 0xalic Acid or Succinic Acid Series, having the formula $\mathrm{C}_{11} \mathrm{H}_{2 n-4} \mathrm{O}_{2}(\mathrm{OH})_{2}$, are bi-hasic acids, which are formed as completely oxidised products by the oxidation of fatty acids and glycolic acid, water being removed. It is important to note their origin from sub. stances rich in earbon, e.g., fats, carbohydrates, and proteids.

(i) Oxalic Acid, $\left.\mathrm{C}_{2} \mathrm{O}_{2}(\mathrm{O}) \mathrm{H}\right)_{2}$, arises from the oxidation of glycol, glycin, cellulose, sugar, stareh, glycerin, and many vegetable acils-it occurs in the urine as calcium oxalate $(\$ 260)$.

(li) Succinic Acid, $\mathrm{C}_{4} \mathrm{H}_{4} \mathrm{O}_{3}\left(\mathrm{OH}_{2,2}\right.$, has been found in small amount in animal solids and fluids : spleen, liver, thymus, thyroid; in the fluids of echinococeus, hydrocephalus, and hydrocele, and more abunlantly in dog's urine after fatty and flesh food ; in rabbit's urine after feeding with yellow turnips. It is also formed in small amount during alcoholic fermentation (\$ 150$)$.

(5) Cholalic Acid in the bile $(\S 177)$ and in the intestine $(\$ 182)$.

(6) Aromatic Acids contain the raulicle of benzol. Benzoic acid (=phenyl-formic acid) occurs in urine united with glycin, as hippuric acid (\$260).

\section{Alcohols.}

Alcohols are bodies which originate from earbohydrates, in which the radical hydroxyl (HO) is substituted for one or more atoms of $\mathrm{H}$. They may be regarded as water, $\left.\mathrm{H}_{\mathrm{H}}^{\mathrm{H}}\right\} \mathrm{O}$, in which the half of the $\mathrm{H}$ is replaced by a $\mathrm{CH}$ compound. Thus, $\mathrm{C}_{2} \mathrm{H}_{6}$ (ethyl-hydrogen) passes into $\left.\mathrm{C}_{\mathrm{g}} \mathrm{H}_{5}\right\} \mathrm{O}$ (ethylic alcohol).

( bile $(\$ 177,4)$, and generally in vegetable cells, and it is the only solid monatomic alcohol in the body.

(b) Glycerin, $\mathrm{C}_{3} \mathrm{H}_{5}\left\{\begin{array}{l}\mathrm{OH} \\ \mathrm{OH} \\ \mathrm{OH}\end{array}\right.$ is a triatomic alcohol. It occurs in neutral fats united with fatty acids and oleic acid; it is formed by the splitting-up of neutral fats during pancreatic digestion (\$170, III.), and during the alcoholic fermentation $(\$ 150)$.

(c) Phenol (= phenylic acid, carbolic acid, oxybenzol) (\$184, III.).

(d) Pyrokatechin (- dioxybenzol) (\$ 252).

(e) The Sugars are closely related to the alcohols, and they may be regarded as polyatomic alcohols. Their constitution is unknown. Together with a series of closely-related bodies they form the great group of the carbohydrates, some of which occur in the animal body, while others are widely distributed in the vegetable kingdom.

252. THE CARBOHYDRATES.-Oecur in plants and animals, and received their name, because in addition to $\mathrm{C}$ (at least 6 atoms), they contain $\mathrm{H}$ and $\mathrm{O}$, in the proportion in which these occur in water. They are all solid, chemically indifferent, and without odour. They have either a sweet taste (sugars), or can be readily changed into sugars by the action of dilute acids; they rotate the ray of polarised light either to the right or left; as far as their 
constitution is concerned, they may be regarded as fatty bodies, as hexatomic alcohols, in which $2 \mathrm{H}$ are wanting.

They are divided into the following groups :-

I. Division. - Glucoses $\left(\mathrm{C}_{6} \mathrm{H}_{12} \mathrm{O}_{6}\right)$. - (1) Grape-sugar (glucose, dextrose, or diabetic sugar) occurs in minute quantities in the blood, chyle, muscle, liver (?), urine, and in large amount in the urine in diabetes mellitus ( $\$ 175)$. It is formed by the action of diastatic ferments upon other carbohydrates, during digestion. In the vegetable kingdom, it is extensively distributed in the sweet juices of many fruits and flowers (and thus it gets into honey). It is formed from cane-sugar, maltose, dextrin, glycogen, and starch, by boiling with dilute acids. It crystallises in warty masses with one molecule of water of crystallisation; unites with bases, salts, acids, and alcohols, but is easily decomposed by bases; it reduces many metallic oxides ( $\$ 149)$. Fresh solutions have a rotatory power of $+106^{\circ}$. By fermentation with yeast it splits up into alcohol and $\mathrm{CO}_{2}(\S 150)$; with decomposing proteids it splits into 2 molecules of lactic acid (\$184, I.); the lactic acid splits up under the same conditions in alkaline solutions, into butyric acid, $\mathrm{CO}_{2}$, and $\mathrm{H}$. For the qualitative and quantitative estimation of glucose, see $\$ 149$ and $\S 150$. In alcoholic solution, it forms very insoluble compounds with chalk, barium, and potassium, and it also forms a crystalline compound with common salt (Estimation, $\$ 150$ ).

(2) Galactose, obtained by boiling milk-sugar (lactose) with dilute mineral acids; it crystallises readily, is very fermentable, and gives all the reactions of glucose. When oxidised with nitric acid it becomes transformed into mucic acid. Its specific rotatory power $=+88^{\circ} 08^{\circ}$.

(3) Laevulose (left-fruit-, invert-, or mucin-sugar) occurs as a colourless syrup in the acid juices of some fruits and in honey; is non-crystallisable, and insoluble in alcohol ; specitic rotatory power $=-106^{\circ}$. It is formed normally in the intestine (\$183), and occurs rarely as a pathological product in urine.

II. Division. - This contains carbohydrates with the formula $\mathrm{C}_{12} \mathrm{H}_{22} \mathrm{O}_{11}$, and its members may be regarded as anhydrides of the first division-1. Milk-sugar or lactose occurs only in milk, crystallises in cakes (with 1 molecule of water) from the syrupy concentrated whey ; it rotates polarised light to the right $=+59 \cdot 3$, and is much less soluble in water and alcohol than grape-sugar. When boiled with dilute mineral acids it passes into galactose, and can be directly transformed into lactic acid only by fermentation; the galactose, however, is capable of undergoing the alcoholic fermentation with yeast (Koumiss preparation, \& 232). For its quantitative estimation ( $\$ 231)$. Rare in urine (\$267).

2. Maltose $\left(\mathrm{C}_{12} \mathrm{H}_{22} \mathrm{O}_{11}\right)+\mathrm{H}_{2} \mathrm{O}$ (O'Sullivan) has 1 molecule of water less than grape-sugar $\left(\mathrm{C}_{12} \mathrm{H}_{24} \mathrm{O}_{12}\right)$, is formed during the action of a diastatic ferment, such as saliva upon starch (\$ 148); is soluble in alcohol, right-rotatory power $=+150^{\circ}$; it is crystalline, while its reducing power is only two-thirds that of dextrose. [The ratio of the reducing power of maltose to that of glucose is 100 to 66.$]$

(3. Saccharose (cane-sugar) occurs in sugar-cane and some plants, it does not reduce a solution of copper, is insoluble in alcohol, is right-rotatory, and not capable of fermentation. When boiled with dilute acids, it becomes changed into a mixture of easily fermentable glucose (right-rotatory) and laevulose (invert sugar, $\$ 183,5$, and $\S 184$, I., 6), which ferments with difficulty and is left-rotatory $(\$ 183)$. When oxidised with nitric acid, it passes into glucic acid and oxalic acid.)

(4. Melitose, from Eucalyptus-manna; Meleztose, from Larch-manna; Trehalose (Mycose), from Ergot : are all right-rotatory, and do not reduce alkaline cupric solutions).

III. Division. - This contains carbohydrates, with the formula, $\mathrm{C}_{6} \mathrm{H}_{10} \mathrm{O}_{5}$, which may be regarded as anhydrides of the second division.

1. Glycogen, with a dextro-rotatory power of $211^{\circ}$, does not reduce cupric oxide. It occurs in the liver ( $(174)$, muscles, many embryonic tissues, the embryonic area of the chick (Külz), in normal and pathological epithelium; in diabetic persons it is widely distributed ; brain, pancreas, and cartilage; and in the spleen, pancreas, kidney, ovum, brain, and blood, together with a small amount of glucose (Pavy). It also occurs in the oyster and some of the molluses (Bizio), and indeed in all tissues and classes of the animal kingdom.

2. Dextrin was discovered by Limpricht in the muscles of the horse. It is right-rotatory = $+138^{\circ}$, soluble in water, and forms a very sticky solution, from which it is precipitated by alcohol or acetic acid; it is tinged slightly red with iodine. It is formed in roasted starch. (hence it occurs in large quantity in the crust of bread-see Bread, § 234), by dilute acids, and in the body by the action of ferments ( $\$ 148$ ). It is formed from cellulose by the action of dilute sulphuric acid. It occurs in beer, and is found in the juices of most plants.

3. Amylum or Starch occurs in the "mealy" parts of many plants, is formed within vegetable cells, and consists of concentric layers with an excentric nucleus (fig. 239). The diameter and characters of starch-grains vary greatly with the plant from which they are derived. At $72^{\circ} \mathrm{C}$. it swells up in water and forms a mucilage; in the cold, iodine colours it blue. Starch-grains always contain more or less cellulose and a substance, erythrogranulose, which is coloured red with iodine (\$ 148) It and glycogen are transformed into dextrose by certain digestive ferments in the saliva, pancreatic, and intestinal juices, and artificially by boiling with dilute sulphuric acid. 
(4. Gum, $\mathrm{C}_{10} \mathrm{H}_{20} \mathrm{O}_{10}$, occurs in vegetable juices (especially in acacie and mimosæ), also in the salivary glands, mucous tissue, lungs, and urine; is partly soluble in water (arabin), partly swells up like mucin (bassorin). Alcohol precipitates it. It is fermentable, and when boiled with dilute acids yields a reducing sugar.)

(5. Inulin, a crystalline powder oceurring in the root of chicory, dandelion, and specially in the bulbs'of the dahlia ; it is not coloured blue by iodine.)

(6. Lichenin occurs in the intercellular substance of Iceland moss (Cetraria islandica) and algae; is transformed into glucose by dilute sulphuric acid.)

(7. Paramylum oecurs in the form of grauules resernbling stareh, in the infusorian, Euglena viridis.)

(s. Cellulose vecurs in the cell-walls of all plants (in the exo-skeleton of arthropoda, and the skin of snakes); soluble only in ammonio-cupric oxide; rendered blue by sulphuric acid

(I

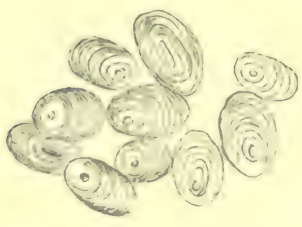

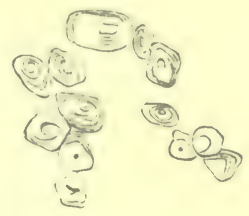

Fig. 239.

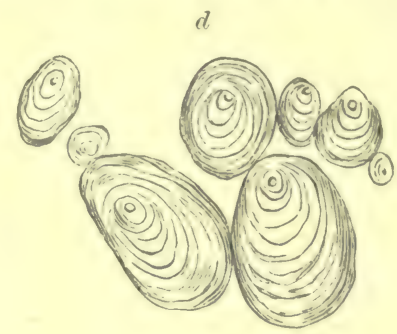

$a$, West Indian arrow-root ; $c$, Tahiti arrow-root ; $d$, Potato starch.

and iodine. Boiled with dilute sulphuric acil, it yields dextrin and glucose. Concentrated nitric acid mixed with sulphuric acid changes it (cotton) into nitro-cellulose (gun-cotton) $\mathrm{C}_{6} \mathrm{H}_{7}\left(\mathrm{NO}_{2}\right)_{3}()_{5}$, which dissolves in a mixture of ether and alcohol and forms collodion.)

(9. Tunicin is a substance resembling cellulose, and occurs in the integument of the Tunicata or Ascidians.)

IV. Division. - This contains the carbohydrates which do not ferment.

1. Inosit (phaseo-mannit, muscle-sugar) occurs in muscle (Scherer), lung, liver, spleen, kilney, hrain of ox, human kilney; pathologically in urine and the fluid of echinococcus. In the vegetable kingdom, in beans (leguminose), and the juice of the grape. It is an isomer of grape-sugar; optically it is inactive, crystallises in warts with 2 nolecules of water, in long monoclinic crystals; it has a sweet taste, is insoluble in water, does not give Trommer's reaction, is capable of undergoing only the sarcolactic acid fermentation. (Nearly allied are Sorbin, from sorbic acid-Scyllit, from the intestines of the hag-fish and skate-and Eukalyn, arising from the fermentation of melitose.)

\section{Derivatives of Ammonia and their Compounds.}

The ammonia derivatives are obtained from the proteils, and are decomposition-products of their metabolism.

(1) Amines, i.e., compound ammonias which can be obtained from ammonia $\left(\mathrm{NH}_{3}\right)$, or from ammonium-hydroxide $\left(\mathrm{NH}_{4}-\mathrm{OH}\right)$, by replacing one or all the atoms of $\mathrm{H}$ by groups of carbohyclrates (alcohol radicals). The amine derived from one molecule of ammonia is called monamine. We are only ucquainted with

$$
\left.\left.\begin{array}{c}
\mathrm{H} \\
\mathrm{H} \\
\mathrm{CH}_{3}
\end{array}\right\} \mathrm{~N} \quad \text { Methylamine and Tri-Methylamine } \quad \begin{array}{l}
\mathrm{CH}_{3} \\
\mathrm{CH}_{3}
\end{array}\right\} \quad \mathrm{N} \text {, }
$$

as decomprosition-products of cholin (neurin) and of kreatin. Neurin occurs in lecithin in a very complex combination (see Lecithin, p. 381, and also \& 23).

(2) Amides, i.e., derivatives of acids, which have exchanged the hydroxyl $(\mathrm{HO})$ of the acids for $\mathrm{NH}_{2}$. Urea, $\mathrm{CO}\left(\mathrm{NH}_{2}\right)_{2}$, the biamid of $\mathrm{CO}_{2}$, is the chief end-product of the metabolism of the nitrogenous constituents of our bodies (see Urine, \$256). Carbon dioxide containing water $=\mathrm{CO}(\mathrm{OH})_{2}$, where both $\mathrm{OH}$ are replaced by $\mathrm{NH}_{2}$-thus we get $\mathrm{CO}\left(\mathrm{NH}_{2}\right)_{2}$, urea.

(3) Amido-acids, i.e., nitrogenous compounds, which show partly the character of an acid and partly that of a weak base, in which the atoms of $\mathrm{H}$ of the acid-radical are replaced by $\mathrm{NH}_{2}$, or by the substituted ammonia groups.

(a) Glycin (or amido-acetic acid, glycocoll, gelatin-sugar, $\$ 177,2$ ) is formed by boiling gelatin with dilute sulphuric acid. It has a sweet taste (gelatin-sugar), behaves as a weak acid, but also unites with acids as an amine-base. It occurs as glycin + benzoic acid=hippuric aciel in urine (\$ 260); and also as glycin + cholalic acid=glycocholic acid in bile (\$ 177). (b) Leucin 
- $(\$ 170)=$ amido-caproic acid. (c) Serin - (= ? amido-lactic acid) obtained from silk-gelatin. (d) Aspartic acid-(amido-succinic acid); and (e) Glutamic acid, obtained by the splitting up, of proteids ( $\$ 170)$. Other amido-acids are- $(f)$ Cystin=amido-lactic acid, in which 0 is replaced by $\mathbf{S}(\$ 268)$. (g) Taurin-(\$ 177), amido-ethyl-sulphonic acid occurs (except in certain glands) chiefly in combination with cholalic acid, as taurocholic acid in bile. Tyrosin (parahydro-oxyphenyl-amido-propionic acid), an amido-acid of unknown constitution, occurs along with leucin during pancreatic digestion ( $\$ 170$ ), is a decomposition-product of proteids, and occurs plentifully in the urine in acute yellow atrophy of the liver $(\$ 269)$.

To the amido-acids are related-(a) Kreatin in muscle, brain, blood, urine, regarded as methyl-uramido-acetic acid $\left(\mathrm{C}_{4} \mathrm{H}_{9} \mathrm{~N}_{3} \mathrm{O}_{2}\right)$. It has been prepared artificially. When boiled with baryta-water, it takes up $\mathrm{H}_{2} \mathrm{O}$, and splits into urea-and (b) Sarkosin $\left(\mathrm{C}_{3} \mathrm{H}_{7} \mathrm{NO}_{2}\right)$, methylamido-acetic acid. When boiled with water, heated with strong acids, in the presence of putrefying substances, kreatin gives off water, and is changed into kreatinin $\left(\mathrm{C}_{4} \mathrm{H}_{7} \mathrm{~N}_{3} \mathrm{O}\right)$. This: strong base can be rechanged by alkalies into kreatin.

(4) Ammonia Derivatives of Unknown Constitution. - Uric acid (\$ 258); allantoin (\$ 260), is formed by the oxidation of uric acid by means of potassium permanganate; cyanuric acid in dog's urine; inosinic acid in muscle; guanin in traces in the liver and pancreas, in guano, the excrements of spiders, in the skin of amphibia and reptiles, in the silver sheen of many fishes (A. Ewald and Krukenberg); by oxidation it yields urea (p. 439); hypoxanthin or sarkin oceurs along with xanthin in many organs and in urine. Kossel prepared hypoxanthin from nuclein by prolonged boiling of the latter. It may be obtained from fibrin by putrefaction, by gastric and pancreatic digestion, and by dilute acids (Salomon, H. Krause, Chittenden); xanthin is prepared by oxidation from hypoxanthin. It occurs very rarely in the form of a urinary calculus. Paraxanthin in urine, and a similar body carnin in flesh ( $\$ 233)$. [Adenin $\left(\mathrm{C}_{5} \mathrm{H}_{5} \mathrm{~N}_{5}\right)$, discovered by Kossel in the pancreas, yeast, and tea-leaves, has also been isolated from the spleen, lymphatic glands, and kidney ; it appears to be present in all highly cellular animal and vegetable tissues. Like the allied bases-xanthin and guanin, it is a derivative of the nuclein of the nuclei.]

\section{Aromatic Substances,}

1. Monatomic phenols- $(a)$ Phenol (hydroxyl of benzol) in the intestine ( $\$ 184)$. Phenylsulphonic acid in urine $(\$ 262)$. (b) Kresol, in the form of orthokresol and parakresol, united with sulphonic acid, occur in urine $(\$ 262)$. 2. Diatomic phenols- $(a)$ pyrokatechin united with sulphonic acid in urine (\$262). 3. Aromatic oxyacids- $(a)$ Hydroparacumaric acid; (b) Paraoxyphenylacetic acid in urine (\$262). 4. Indol and skatol in the intestine (\$184), conjoined with sulphonic acid in urine (\$262).

253. HISTORICAL. - According to Aristotle, the organism requires food for three purposes - for growth, for the production of heat, and to compensate for the loss of the boaily excreta. The formation of heat takes place in the heart by a process of concoction, the heat so formed being distributed to all parts of the body by means of the blood, while the respiration is regarded as an act whereby the body is cooled. Galen accepted this view in a somewhat modified form ; according to him, the metabolic processes may be compared to the processes going on in a lamp; the blood represents the oil ; the heart, the wick; the lungs, the fanning apparatus. According to the view of the iatrochemical school (van Helmont), the metabolic processes of the body are fermentations, whereby the food is mixed with the juices of the body. Since the middle of the seventeenth century (Boyle), the knowledge of the metabolic processes has followed the development of chemistry. A. v. Haller regarded heat as due to chemical processes - the food continually supplying the waste which is excreted from the body. After the discovery of oxygen (1774, by Priestley and Scheele), Lavoisier formulated the theory of combustion in the lungs, whereby carbonic acid and water were formed. Mitscherlich compared the decomposition-processes in the living body with putrefactive processes. Magendie was the first to emphasise the difference between nitrogenous and non-nitrogenous foods, and he showed that the latter alone were not able to support life. Even gelatin alone is not sufficient for this purpose. The greatest advance in the theory of nutrition was made by J. v. Liebig, who laid the foundation of our present knowledge of this subject. According to Liebig, foods may be divided into two classes, viz., the "plastic," suitable for the construction of the organism, and the "respiratory" for the maintenance of the temperature; to the former class he referred the albuminates or proteids, to the latter, the non-nitrogenous carbohydrates and fats (p. 356). Amongst recent observers, the Munich School, as represented by v. Bischoff, v. Pettenkofer, and v. Voit, has done most to give us an exact knowledge of this department of physiology. 


\section{The Secretion of Urine.}

254. STRUCTURE OF THE KIDNEY.-[Capsule. - The kidney is a compound tubular gland, and is investel by a thin, tough, fibrous capsule, easily stripped off fron the substance of the organ, to which it is attached by fine processes of connective-tissue and blood-vessels.]

[Naked Eye Appearances. - On dividing the kidney longitudinally from the hilum to its outer borter, and examiniug the cut surface with the naked eye, we observe the parenchyma

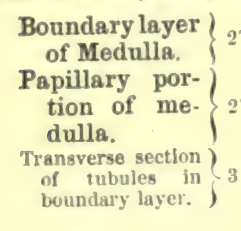

Fat of renal sinus, 4 .

Artery. 5.

Artery. 8.

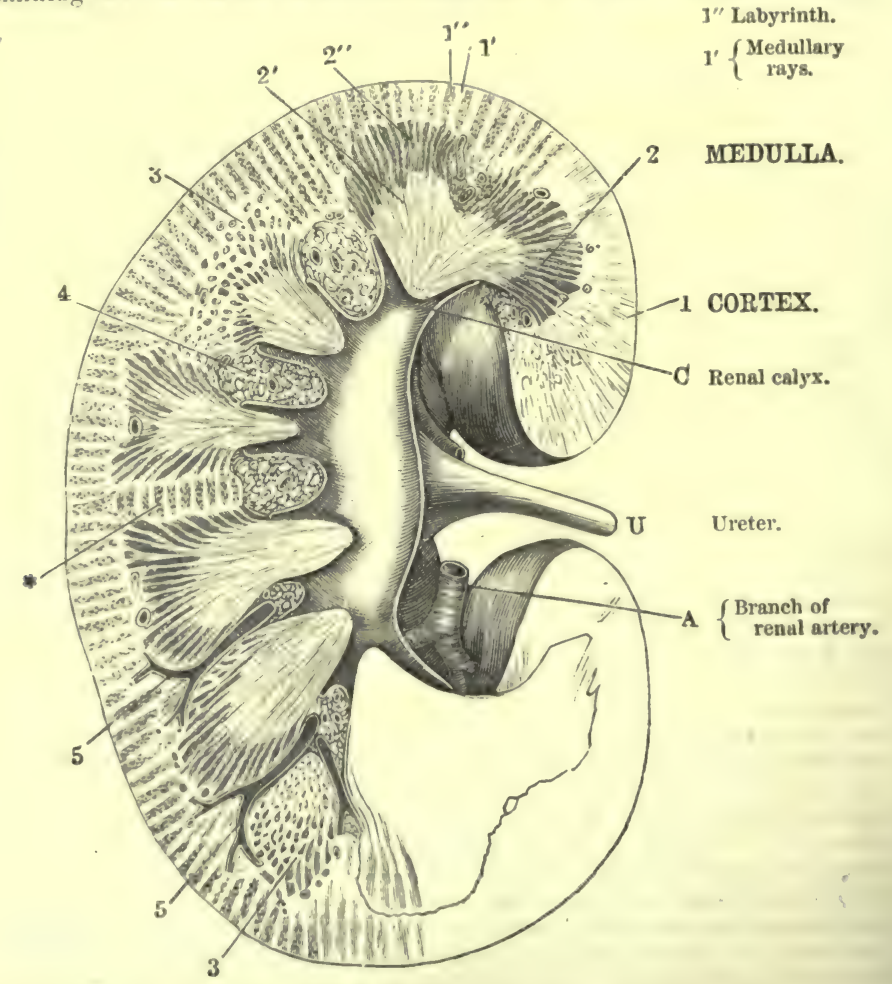

Fig 240.

Longitudinal section through the kidney (Tyson, after Henle).

of the kidney, consisting of an outer cortical and an inner medullary, or pyramidal portion, the latter composed of about twelve conical papillæ, or pyramids of Malpighi, with their apices directed towards and embracel by the calices of the pelvis of the kidney (fig. 240). The medullary portion is further subdivided into the boundary layer of Ludwig and the papillary 
portion. According to Klein, the relative proportions of these three parts are-cortex, 3.5 ; boundary layer, $2 \cdot 5$; and papillary portion, 4 . The cortex has a light brown colour, and when torn presents a slightly granular aspect, with radiating lines running at regular distances. The granules are due to the presence of the Malpighian corpuscles, and the strix to the medullary rays. The boundary zone is darker, and often purplish in colour. It is striated with clear and red lines alternating with opaque ones, the former being blood-vessels and the latter uriniferous tubules. The papillary zone is nearly white and uniformly striated, the striæ converging to the apex of the pyramid. The medulla is much denser and less friable than the cortex, owing to the presence of a large amount of connective-tissue between the tubules. The bundles of straight tubes of the medulla may be traced at regular intervals ruuning outwards in to the cortex, constituting medullary rays, which become smaller as they pass outwards in the cortical zone, so that they are conical and form the pyramids of Ferrein (fig. 241, PF). The portion of the cortex lying between the medullary rays is known as the labyrinth, from the complicated arrangement of its tubules.]

[Size, Weight. - The adult kidney is about 11 centimetres ( 4.4 inches) in length, 5 centimetres ( 2 inches) wide, and 3 centimetres (1 inch) in thickness. It weighs in the male 113.5 to $170 \mathrm{grms}$. (4 to $6 \mathrm{oz}$.), in the female 113.5 to 156 grms. ( 4 to $5 \frac{1}{2}$ oz.). The width of the cortex is usually 5 to 6 millimetres ( $\frac{1}{5}$ to $\frac{1}{4}$ inch).]

I. The uriniferous tubules all arise within the labyrinth of the cortex by means of a globular enlargement, 200 to $300 \mu\left[\frac{1}{160}\right.$ to $\frac{1}{125}$ inch] in diameter, called Bowman's capsule (figs. 242, 243). After pursuing a complicated course, altering their direction, diameter, and structure, and being joined by other tubules, they ultimately form large collecting tubes, which terminate by minute apertures, visible with the aid of a haud-lens, on the apices of the papillæ projecting into the calices of the kidney. Each urinary tubule is composed of a homogeneous membrana propria, lined by epithelial cells, so as to leave a lumen for the passage of the urine from the Malpighian corpuscles to the pelvis of the kidney. The diameter and direction of the tubules vary, and the epithelium differs

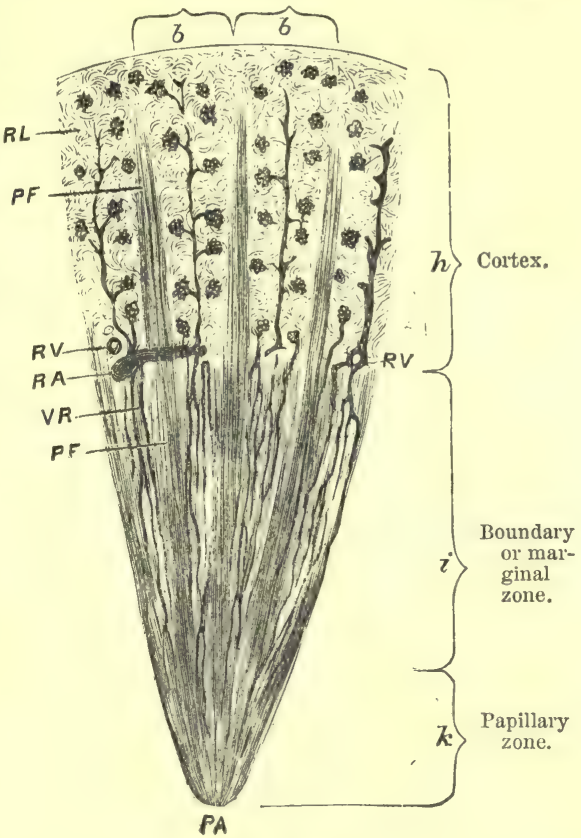

Fig. 241.

Longitudinal section of a Malpighian pyramid. $\mathrm{PF}$, pyramids of Ferrein ; $\mathrm{RA}$, branch of renal artery ; RV, lumen of a renal vein receiving an interlobular vein; VR, vasa recta; PA, apex of a renal papilla: $b, b$, embrace the bases of the renal lobules.

in its characters at different parts of the tube, while the lumen also undergoes alterations in its diameter.

Course and Structure of the Tubules. - In the labyrinth of the cortex, tubules arise in the spherical enlargement known as Bowman's capsule (fig. 242,1 ), which invests (in the manner presently to be described) the tuft of capillary blood-vessels called a glomerulus or Malpighian corpuscle. By means of a short and narrow neck (2) the capsule becomes continuous with a convoluted tubule, $\mathrm{X}$ in fig. 243 . This tubule is of considerable length, forming many windings in the cortex (fig. 242, 3); the first part of it is $45 \mu$ wide, constituting the proximal or first convoluted tubule. It becomes continuous with a spiral tubule of Schachowa (4), which lies in a medullary ray where it pursues a slightly wavy or spiral course. On the boundary line between the cortical and boundary zone, the spiral tubule suddenly becomes smaller and passes into the descending portion of Henle's loop (5), which is $14 \mu$ in breadth, and is continued downwards through the boundary zone into the medulla, where it forms the narrow loop of Henle (6), which runs backwards in the medullary part to the boundary zone. Here it becomes wider $(20-26 \mu)$, and as it continues its undulating course, it enters a medullary ray, where it constitutes the ascending looped tube (7), which becomes narrower in the cortex. Leaving the medullary ray again, it passes into the labyrinth, where it forms a tube with irregular angular outlines - the irregular tubule (10); which is continuous with (fig. 243, $n, n$ ) the second or distal convoluted tubule (11), which resembles the proximal tubule of the same name. Its diameter is $40 \mu$. A short, narrow, wavy junctional or curved collecting tubule 
(12) connects the latter with one of the straight collecting tubes (13) of a medullary ray. As the collecting tubule proceeds through the bounlary zone, it receives numerous junctional tubes, and when it reaches the boundary zone, it forms one of the collecting tubes (fig. 243, 0 ),

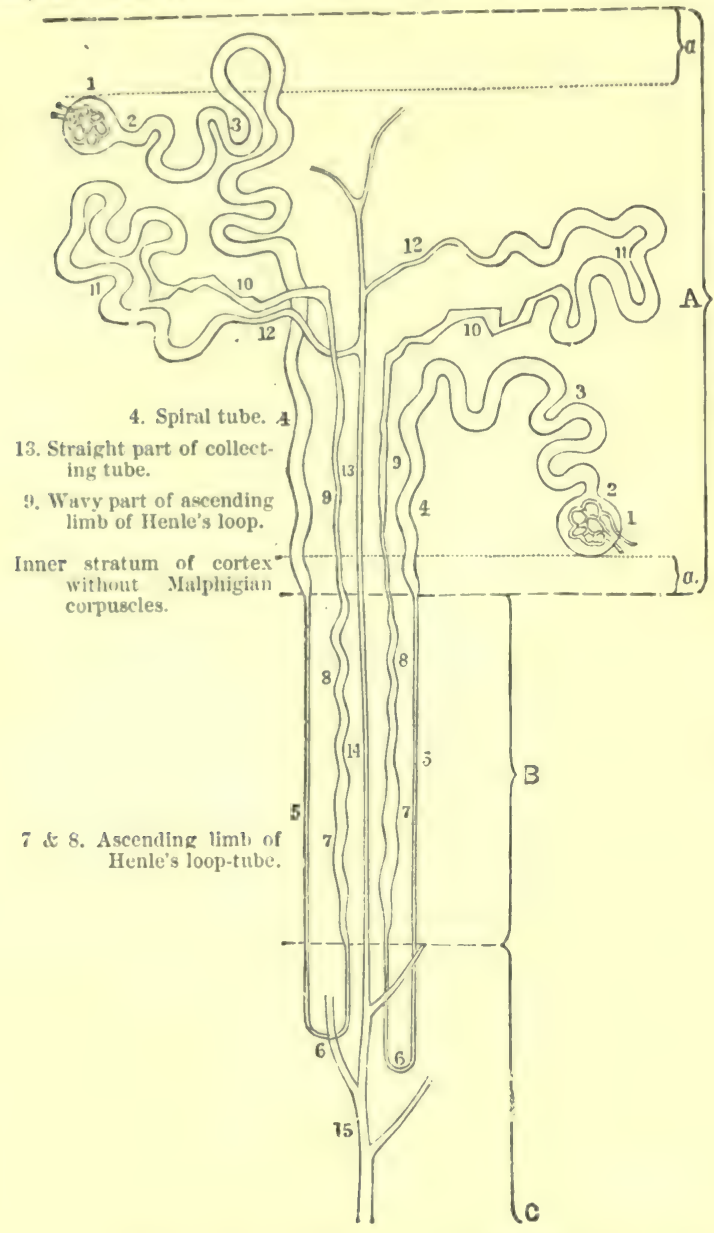

Sub-capsular layer without Malpighian corpuscles.

12. First part of collecting tube.

11. Distal convoluted tubule.

A. CORTEX.

10. Irregular tubule.

i. Proximal convoluted tubule.

9. Wary part of ascending limb.

2 . Constriction or neck.

4. Spiral tubule.

1. Malpighian tuft surrounded by Bowman's capsule.

8. Spiral part of ascending liml of Henle's loop.

B. BOUNDARY ZONE.

5. Descending limb of Henle's loop-tube.

6. Henle's loop.

Fig. 242.

Diagram of the course of two uriniferous tubules (Klein and Noble-Smith).

which unite with one another at acnte angles to form the larger straight excretory tubes or ducts of Bellini (15), which open on the summit of the Malpighian pyramids into a calyx of the pelvis of the kidney. In the cortex the collecting tubules are $45 \mu$ in diameter, but where they have formed an excretory tube (O), their diameter is 200 to $300 \mu ; 24$ to 80 of these tubes open on the ajpex of each of the 12 to 15 Malpighian pyramids. In the lowest and broadest part, the membrana propria is strengthened by the presence of a thick supporting framework of connective-tissue.

Structure of the Tubules.-[Below the neck, the tubules are lined everywhere by a single layer of nucleated epithelium. ] Bowman's capsule, which is about $\frac{1}{2 b}$ inch in diameter (fig. 244 , II), consists of a homogeneous basement membrane lined internally by a single continuous layer of flattened cells $(k)$. According to Roth, the basement membrane itself is composed of endothelial cells. [In the foetus the lining cells are more polyhedral.] Within the capsule lies the glomerulus or tuft of blood-vessels. The cells lining the capsule are reflected over and between the lobules of which the glomerulus consists. The glomerulus may not completely fill the capsule, so that, according to the activity of the kidney, there may be a larger or smaller 
space between the glomerulus and the capsule into which the filtered urine passes. The neck is lined by cubical cells. These cells, in some animals, e.g., the rabbit, sheep, mouse, and frog, are ciliated.

The proximal convoluted tubule is lined by characteristic epithelium. The cells, which are short or polyhedral, contain a turbid or cloudy protoplasm (fig. 244, III, 1 and 2), which not unfrequently contains oil-globules, and they form a single layer. Each cell consist of two parts ; the inner, containing the spherical nucleus, is next the lumen, and granular (III, 2, g), while the outer part, next the membrana propria, appear's fibrillated, or "rodded," from the presence of rods or fibrils placed vertically to the basement-membrane (fig. 245). These appear like the hairs of a brush pressed upon a plate of glass (III, 2). The cells are not easily separated from each other, as neighbouring cells interlock by means of the branched ridges on their surfaces (III, 1)-(Heidenhain, Schachowa). The lumen is well lefined, but its size seems to lepend upon the state of imbibition of the cells bounding it.

The spiral tubule has similar epithelium and a corresponding lumen, although the epithelium becomes lower and somewhat altered in its characters at the lower part of the tube.

The descending limb of Henle's loop, and the loop itself with a relatively wide lumen, are bounded by clear, flattened, epithelial cells, with a bulging nucleus (IV, S); the cells lying on one side of the tube being so placed that the bulging part of the bodies of the cells is opposite the thin part of the cells on the opposite side of the tube. [These tubes might be mistaken for blood-capillaries, but in addition to their squamous lining, they have a basement-membrane, which capillaries have not.] In the ascending limb, the lumen is relatively wide, while its epithelium agrees generally with that in the convoluted tubule, excepting that the "rods" are shorter. Sometimes the cells are arranged in an "imbricate" manner.

In the irregular tubule, which has a very small lumen, the polyhedral cells lining it contain oval nuclei, and are shorter than

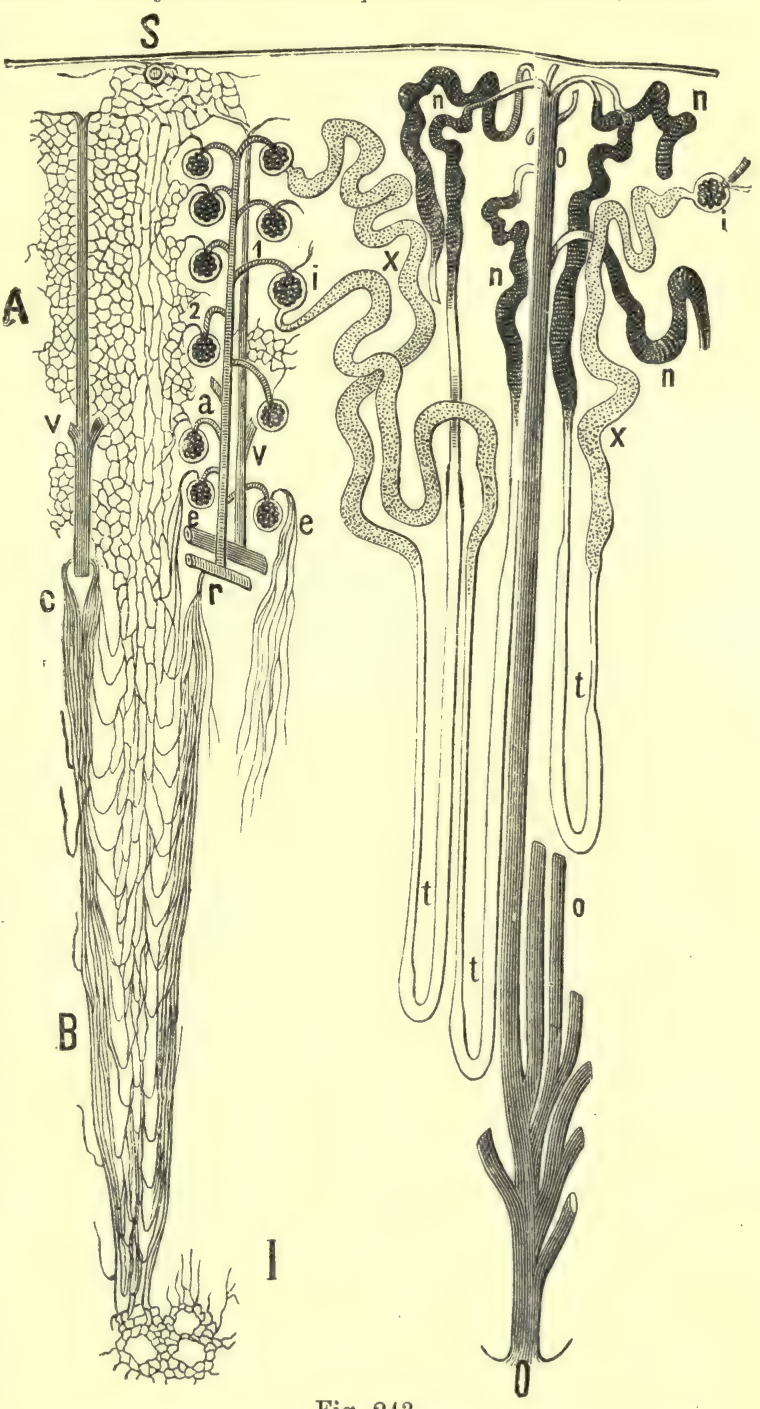

Fig. 243.

I, Blood-vessels and uriniferous tubules of the kidney (semidiagrammatic); A, capillaries of the cortex, $\mathrm{B}$, of the medulla ; $a$, interlobular artery ; 1 , vas afferens; 2 , vas efferens ; $r, e$, vasa recta; $c$, venæ rectæ; $v, v$, interlobular vein; $\mathbf{S}$, origin of ä vena stellata; $i$, Bowman's capsule and glomerulus; $\mathrm{X}, \mathrm{X}$, convoluted tubules; $t, t$, Henle's loop ; $n, n$, junctional piece; $o, o$, collecting tubes; $\mathrm{O}$, excretory tube.

those of the convoluted tubules. The cells, again, are very irregular in size, while their "rodded" character is much coarser and more defined (fig. 246).

The distal convoluted tubule closely resembles in its structure the proximal convoluted 
tubule, and is linet by similar cells. The curved collecting, or junctional tubule, although narrow, has a relatively wide lumen, as it is lined by clear, somewhat flattened cells.

The collecting tubes have a distinct lumen, and are lined by clear, somewhat irregular, cubical cells (fig. 244, V), which in the larger excretory tubes are distinctly columnar (VI). The basement-membrane is said to be absent in the larger tubes. [Klein describes a thin, delicate, nucleated centro-tubular membrane lining the surface of the epithelium next the lumen.]

II. The Blood-Vessels. - The renal artery (fig. 250) divides into four or five branches, which pass into the kidney at the hilum. These branches, surrounded by connective-tissue continuous with that of the capsule, continue to divide, and pass between the papillæ, to reach the bases of the pyramids on the limits between the cortical and boundary zones, where they form incomplete arches. From these horizontal trunks, the interlobular arteries (fig. 243,
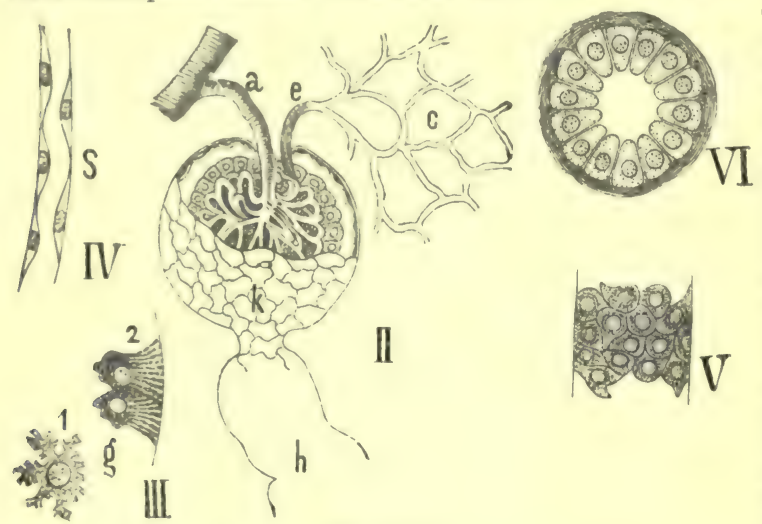

a) run vertically and singly into the cortex, between each two medullary rays, and in their course they give off on all sides the short undivided vasa afferentia (1), each of which enters a Malpighian capsule at the opposite pole from which the urinary tubule is given off. Within the capsule, each afferent artery breaks up into capillaries arranged in lobules and supported by connective-tissue, the whole forming a tuft of capillary blood-vessels, or a glomerulus. Each glomerulus is covered on its surface, directed towards the wall of the capsule by a layer of flat, nucleated, epithelial cells (fig. 229, II), which also dip down between the capillaries.

Fig. 244.

II, Bowman's capsule and glomerulus. $a$, vas afferens; $e$, vas efferens; $c$, capillary network of the cortex; $k$, endothelium of the capsule; $h$, origin of a convoluted tubule. III, "rodded" cells from a convoluterl tubule-2, seen from the side, with $g$, inner granular zone; 1 , from the surface. IV, cell lining Henle's looped tubule. 'V, cells of a col-

lecting tube. VI, section of an excretory tube. vessel enters it (fig. 244, II).
In their structure and distribution all the efferent vessels resemble arteries, as they divide into branches to form a dense, narrow-meshed, capillary network (fig. 243, A, and fig. 244, II, c), which ramifies over and between the convoluted tubules. The meshes are elongated around

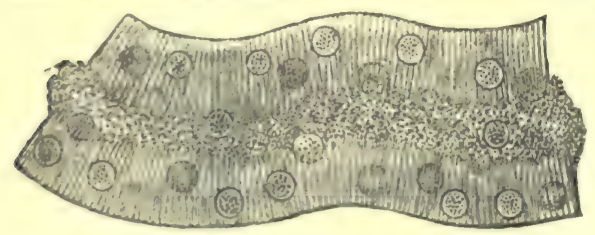

Fig. 245.

Convoluted tubule (after ammonium chromate) showing "rodded" epithelium.

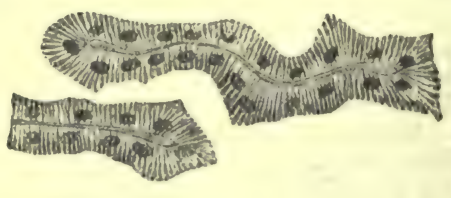

Fig. 246.

Epithelium of an irregular tubule of the kidney of a dog.

the tubules of the medullary rays, and more polygonal around the convoluted tubules (fig. 243). Some of the lowest efferent vessels split up into vasa recta, which run towards the medulla. The interlobular arteries become smaller as they pass towards the surface of the kidney, and some of their terminal capillaries communicate with the capillaries of the external capsule itself. Venous trunks proceed from the capillary network, to terminate in the interlobular veins (V), which begin close under the capsule by venous radicles arranged in a stellate manner (constituting the stellulæ Verheynii, or venæ stellatæ), and accompany the corresponding artery to the limit between the cortex and boundary zone, where they communicate with the large venous trunks in that situation.

The blood-vessels of the medulla arise from the vasa recta (fig. $243, r$ ), which begin on the limit of the cortex and medulla, either as single, direct, muscular branches $(v)$ of the large 
arterial trunks, or from those efferent vessels $(e)$ which lie next to the medulla. The latter are said to be devoid of muscle. According to Huschke, a few vasa recta are formed by the union of the capillaries of the medullary rays. All the vasa recta enter the boundary layer, where they split up into a leash or pencil of small arterioles, which pass between the straight tubules towards the pelvis, and form in their course a capillary network with elongated meshes. From these capillaries there arise venous radicles, which, as they proceed towards the limit between the cortex and medulla, form the venæ rectæ $(c)$, and open into the concave side of the venous trunks in this region. At the apex of the papillæ, the capillaries of the medulla form connections with the rosette-like capillaries surrounding the excretory ducts (at I).

[The circulation through the vasa recta is most important. The cortical system of bloodvessels communicates with the medullary, but as most of the vasa recta are derived from the same vessel as the interlobular arteries, it is evident that they may form a side stream through which much of the blood may pass without traversing the vessels of the cortex. Very probably the "short-cut" is useful in congestions of the kidney. The amount of distention of these vessels also will influence the size of the tubules lying between them. There are two other channels by which blood can pass throngh the renal arteries without traversing the glomeruli(1) The anastomoses between the terminal twigs of the renal artery and the subcapsular venous plexus; (2) small branches given off, either by the interlobular arteries or by the afferent vessels before entering the glomeruli (Brunton).]

The blood-vessels of the external capsule are derived partly from the terminal twigs of the interlobular arteries, partly from branches of the supra-renal, phrenic, and lumbar arteries, which anastomose with each other. The capillary network has simple meshes. The venous radicles pass partly into the venæ stellatex, and partly into the veins of the same name as the arteries. The connection of the area of the renal artery with the other arteries of the capsule explains why, after ligature of the renal artery within the kidney, the blood still circulates in the external capsule (C. Ludwig, M. Herrmann); in fact, these blood-vessels still supply the kidney with a small amount of blood, which may suffice to permit a slight secretion of urine to take place (Litten, Pautynski).

III. The lymphatics form a wide-meshed plexus in the capsule of the kidney, while under it they form large spaces (Heidenhain). In the parenchyma of the kidney, the lymphatics are said to be represented by large slits devoid of a wall in the tissues, and are more numerous around the convoluted than the straight tubules. The slits pass to the surface of the kidney, and expand under the capsule. When the lymphatics are greatly distended, they tend to compress the uriniferous tubules and the blood-vessels (C. Ludwig and Zawarykin). According to Ryndowsky, the uriniferous tubules are surrounded by true lymphatics with an endothelial lining, and they even penetrate into the capsule of Bowman along with the vas afferens. [The large bloodvessels are also surrounded by lymphatics.] Large lymphatics, provided with valves, pass out of the kidneys at the hilum, while others emerge through the capsule; both sets are connected with the lymph-spaces of the capsule of the kidney (A. Budge).

IV. The nerves form small trunks provided with ganglia, and accompany the blood-vessels. [They are derived from the renal plexus and the lesser splanchnic nerve.] They contain medullated and non-medullated fibres, and the latter have been traced by W. Krause as far as the apices of the papillæ. Their mode of termination is unknown. Physiologically, we are certain that they contain both vaso-motor and sensory fibres; perhaps there may be also vaso-dilator and secretory tibres.

v. The connective-tissue, or interlobular stroma, forms in the papillæ, especially at their apices, fibrous, concentric layers of considerable thickness between the

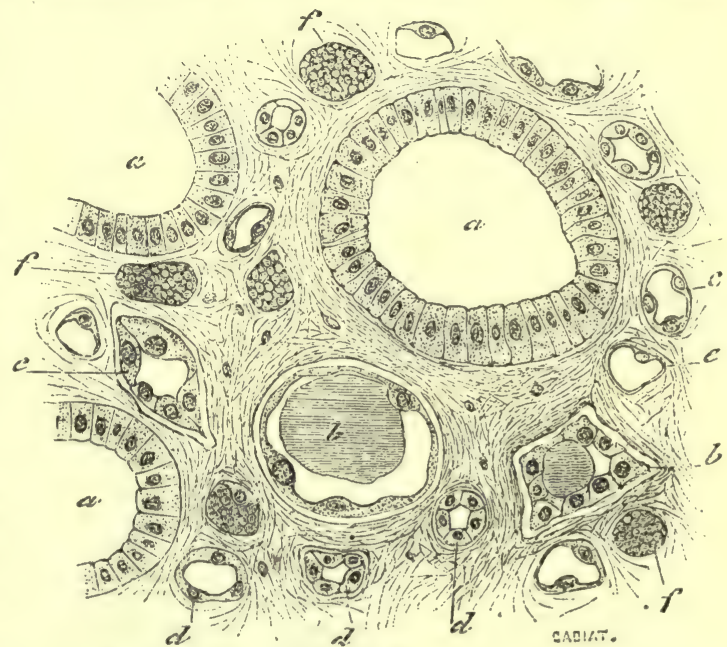

Fig. 247.

Transverse section of apex of Malpighian pyramid. $a$, large collecting tubes; $b, c, d$, tubules of Henle; $e, f$, bloodcapillaries.

excretory tubules (fig. 247). Farther outwards, the fibrillar character becomes less distinct, while at the same time branched connective-tissue corpuscles occur in greater numbers. In the 
cortex, the interstitial strona consists almost entirely of branched corpuscles, which anastomose with each other. [There is also a small quantity of delicate fibrous tissue around Bowman's capsule, and along the course of the arteries. The connective-tissue often plays an important rôle in pathological conditions of the kidney, as interstitial nephritis.] The outer layers of the capsule of the kidney are composed of dense bundles of tibrous tissue, while the deeper layers are more loose, and send processes into the cortical layers. The capsule is easily stripped off. None of the secretory substance is removed with it. Under the capsule in the human kidney, there is a thin plexus of non-striped muscular fibres. At the hilum it becomes continuous with the outer fibrous coat of the dilated upper end of the ureter. Smooth muscular fibres also oscur in a sphincter-like arrangement round the apex of each papilla, while others proceed from the pelvis hetween the pyramids along the blood-vessels (Jardet). The fat surrounding the kilney is united to the latter partly by blood-vessels and partly by bands of connective-

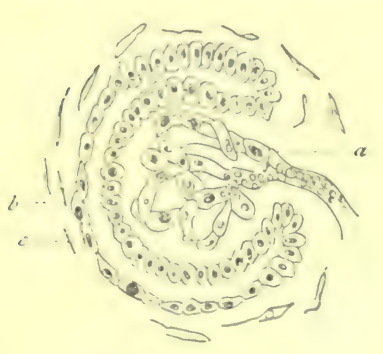

Fire. 248 .

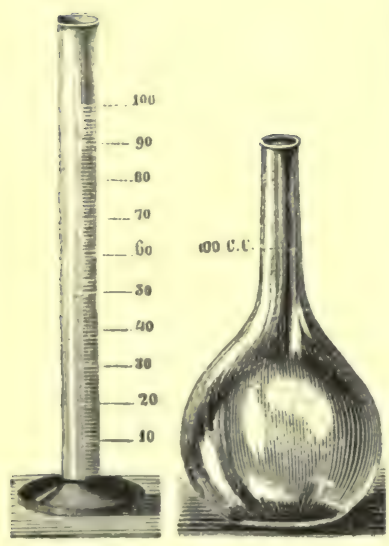

Fig. 249.

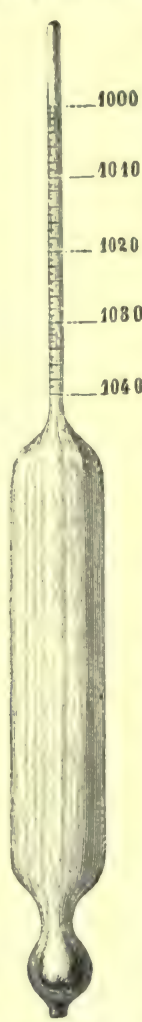

Fig. 250 . tissue. [The subcapsular layer of the cortex, and a thin layer next the boundary zone (fig. $242, a, a)$, are devoid of Malpighian corpuscles.

[Development of a Malpighian Capsule. - The upper end of the urinary tubule is dilated and closed, and into it there grows a tuft of bloodvessels $(a)$ pushing one layer of the tube before it (b), hence the capillaries become invested by it, just as an organ is surrounded by a serous sac, so that one layer - the reflected one $(b)$ - of the tubule is closely applied to the blood-vessels, while the other $(c)$ lies loosely over it with a space between the two (fig. 248).]

\section{THE URINE. - Physical} Characters. - A knowledge of the composition of this secretion is of the greatest value to the physician and surgeon.

1. The quantity of urine passed by an adult man in twenty-four hours is between 1000 and 1500 cubic centimetres, or about $50 \mathrm{ozs}$. , and in the female 900 to 1200 c.c. The minimum is secreted between 2 to 4 A.M., and the maximum between 2 to 4 P.M. (Weigelin).

The amount is diminished by profuse sweating, diarrhoea, thirst, non-nitrogenous food, diminution of the general blood-pressure, after severe hrmorrhage, and in some diseases of the kidneys. The minimum, which may be normal, is 400 to 500 c.c. It is increased by increase of the general blood-pressure, or of the pressure within the area of the renal artery, by copious drinking, contraction of the cutaneous vessels through the action of cold, the passage of a large amount of soluble substances (urea, salts, Fig. 248,-Development of a glomerulus and and sugar) into the urine, a large amount of Malpighian capsule. $a$, capillary; $b$, vis- nitrogenous food, as well as by various drugs, ceral; $c$, parietal layer of capsule.

Fig. 249. -Graduated cylinder and flask for measuring the amount of urine.

Fig. 250.-Uirinometer. such as digitalis, alcohol, squills. After taking fluids charged with $\mathrm{CO}_{2}$, the amount of urine is increased during the following hours (Quincke).

The secretion is influenced directly by the nervous system, as in the sudden polyuria fol.

lowing nervous excitement, such as hysteria, [when the person usually passes a large amount of rery pale-coloured urine]; after an epileptic attack, and also after pleasurable excitement (Bencke). We may have polyuria unaccompanied by the presence of sugar in the urine, which follows injury to a certain part of the floor of the fourth ventricle $(C l$. Bernard $)$. The urine is measured in tall graduated cylindrical vessels (fig. 249). [In estimating the quantity of urine jassed, the patient must, of course, be directed always to empty his bladder at a particular hour, and collect the urine passed during the next twenty-four hours.]

2. The specific gravity varies, as a mean, between 1015 and 1025 ; the mini- 
mum, after copious draughts of water, may be 1002 ; while the maximum, after profuse perspiration and great thirst, may be 1040. The mean specific gravity is about 1020. In newly-born children, the specific gravity falls very considerably during the first three days, which is due to the amount of food taken (Martin and Ruge). [The specific gravity of the urine in infants is about 1003 to 1006.] A

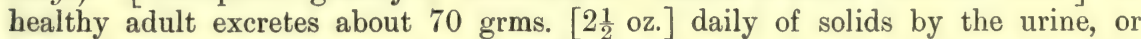
about $1 \mathrm{grm}$. of solids per 1 kilo. of body-weight.

The specific gravity is estimated by means of a urinometer (fig. 250), the urine being at the temperature of $16^{\circ} \mathrm{C}$. [The urinometer, when placed in distilled water, ought to float at the mark $0^{\circ}$ or zero, which is conventionally spoken of as 1000 . Place the urine to be tested in a tall cylindrical glass, of such width that the urinometer, when placed in it, may float freely and not touch the sides. Take care that no air-bubbles adhere to the instrument. When reading off the mark on the stem, raise the vessel to the eye and bring the eye on a level with the surface of the water, noting the number which corresponds to this. This rule is adopted, because the water rises on the stem in virtue of capillarity. It is essential that a sample of the mixed urine of the twenty-four hours be used for ascertaining the mean specific gravity.]

Christison's Formula. - To estimate the amount of solids in the urine. This may be done approximately by means of the formula of Trapp or Haeser, or, as it is called in this country, "Christison's formula," viz., "Multiply the two last figures of a specific gravity expressed in four figures by $2 \cdot 33$ " (Christison and Haeser), or by 2 (Trapp), or $2 \cdot 2$ (Loebisch). 'I'his gives the amount of solids in every 1000 parts. [Suppose a person passes 1200 c.c. urine in twentyfour hours, and the specific gravity is 1022, then

$22 \times 2 \cdot 33=51 \cdot 26$ grms. in 1000 c.c.

To ascertain the amount in 1200 c.c.

$$
\left.1000: 1200:: 51 \cdot 26: \times=\frac{51 \cdot 26 \times 1200}{1000}=61 \cdot 51 \mathrm{grms} .\right]
$$

Direct Estimation of Solids.-Place 15 c.c. of urine in a capsule of known weight, and evaporate it over a water-bath, afterwards completely dry the residue in an air-bath at $100^{\circ} \mathrm{C}$, and then cool it over concentrated sulphuric acid. During the process, a small amount of urea is decomposed, so that the value obtained is slightly too small. Of course the specific gravity varies with the amount of water in the urine. The most concentrated (highest specific gravity) urine is the morning urine (Urina noctis), especially after being retained in the bladder, e.g., in prolonged sleep a certain amount of water is absorbed, so that the urine becomes more concentrated. The most dilute urine is secreted after copious drinking (Urina potus). Under pathological conditions, as in diabetes mellitus ( $\$ 175)$, the urine is, at the same time, very copious (as much as 10,000 c.c.), and very concentrated, while the specific gravity varies from 1030 to 1060 , [due to the presence of a large amount of grape-sugar]. In fever the urine is concentrated, and small in amount. In polyuria, due to certain nervous conditions, the urine is very dilute and copious, while the specific gravity may be as low as 1001 .

3. The colour of the urine depends on the colouring-matters present in it, and varies greatly, but the differences in colour are due chiefly to variations in the amount of water. Normally it has a pale straw colour, but if it contains more water than usual it has a very pale tint, and in certain cases (as in the sudden polyuria occurring after an attack of hysteria) it may be as clear as water. Concentrated urine, as after meals, or the first urine passed in the morning, has a darker colour; it is a dark yellow or brownish-red; while it is usually dark coloured in fever.

Fœtal urine, and also the urine first passed after birth, are as clear and colourless as water. The admixture of various substances with the urine alters its colour. When mixed with blood, aceording to the degree of decomposition of the hæmoglobin, the urine is red or dark brownishred [more frequently it is smoky], especially if the blood comes from the kidneys and the urine is acid. When mixed with bile pigments, it is of a deep yellowish-brown, with an intense yellow froth; senna taken internally makes it intensely red, rhubarb brownish-yellow, and carbolic acid black. Urine undergoing the ammoniacal fermentation may present a dirty bluish appearance owing to the formation of indigo. The colour of urine is estimated by Neubauer and Vogel by means of an empirical "colour-scale."

Urine, but especially ammoniacal urine, exhibits fluorescence, which disappears on the addition of an acid, and reappears after the addition of an alkali.

Normal urine, after standing for several hours, deposits a fine cloud of vesical mucus [like delicate cotton wool]. The froth of normal urine is white, and disappears pretty rapidly, while that on an albuminous urine persists much longer. The urine not unfrequently contains some epithelial cells from the bladder and urethra. 


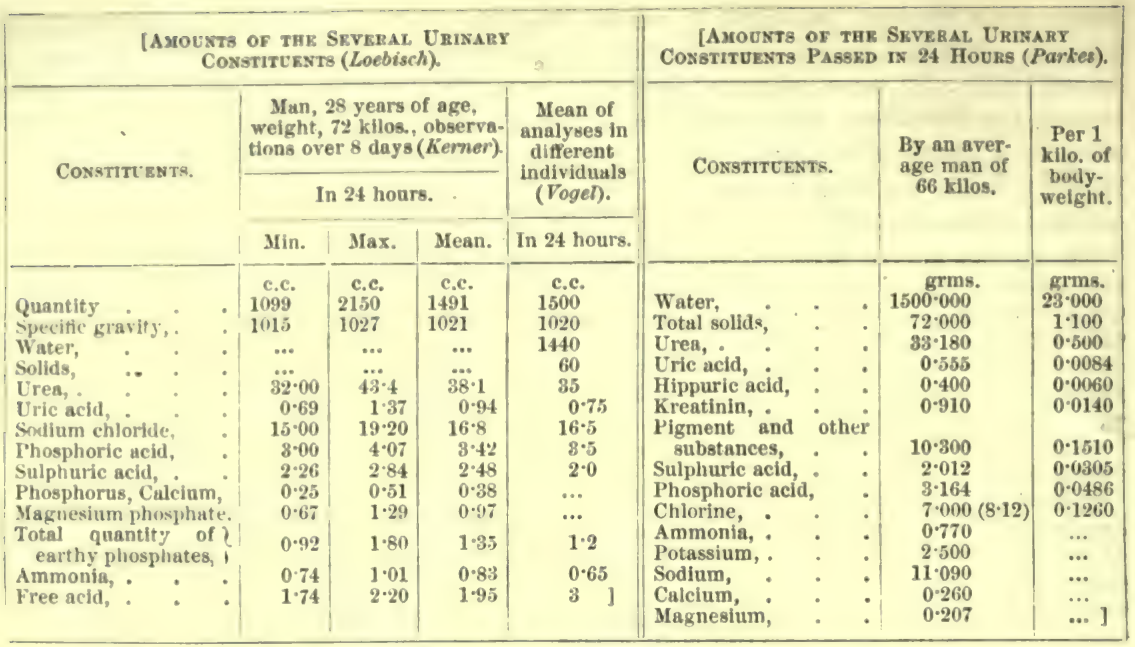

4. Consistence.-Normal urine, like water, is a freely mobile fluid.

Large quantities of sugar, albumin, or mucus make it less mobile; while the so-called chylous urine of warm climates may be like a white jelly.

5. The taste is a saline bitter, the odour is characteristic and aromatic.

Ammoniacal urine has the odour of ammonia. Turpentine taken internally gives rise to the orlour of violets, copaiba and cubebs a strongly aromatic, and asparagus an unpleasant odour. Valerian, assafortida, and castoreum [but not camphor] also produce a characteristic odour. [The odour of diabetic urine is described as "sweet."]

6. The reaction of normal urine is acid, owing to the presence of acid salts, chiefly acid sodic phosphate, which seems to be derived from basic sodic phosphate, owing to the uric acid, hippuric acid, sulphuric acid, and $\mathrm{CO}_{2}$ taking to themselves part of the soda, so that the phosphoric acid forms an acid salt. After a diet of flesh, acid potassic phosphate is the cause of the acidity. That the urine contains no free acid is proved by the fact that it gives no precipitate with sodic hyposulphite (v. Voit, Huppert).

The acid reaction is increased after the use of acids, e.g., hydrochloric and phosphoric, also by ammoniacal salts, which are changed within the body into nitric acid; lastly, after prolonged muscular exertion. The morning urine is strongly acid.

The urine becomes less acid or alkaline-(1) By the use of caustic alkalies, alkaline carbonates, or alkaline salts of the vegetable acids, the last being oxidised within the body into carbonates. (2) By the presence of calcic, or magnesic carbonate. (3) By admixture with alkaline blood, or pus. (4) By removing the gastric juice through a gastric fistula (p. 246, Maly); further, from one to three hours after a meal. [The reaction of urine passed during digestion may be neutral, or even alkaline. This is due either to the furmation of acid in the stomach (Bence Jones), or to a fixed alkali derived from the basic alkaline phosphates taken with the food ( $W$. Roberts).] (5) The urine is rarely alkaline in anæmia, owing to a deficiency of phosphoric and sulphuric acids. [(6) The nature of the food-vegetable food makes it alkaline. (7) By profuse sweating. (8) By absorption of alkaline transudations (blood, serum).]

[Method. - The reaction of urine is tested by means of litmus paper. Normal urine turns blue litmus paper red, and does not affect red litmus. An alkaline urine makes red litmus paper blue, while a neutral urine does not alter either blue or red litmus paper.] Sometimes violet litmus paper is userl, which becomes red in acid, and blue in alkaline urine.

Estimation of the Acidity. - This is done by determining the amount of caustic soda necessary to proluce a neutral reaction in 100 c.c. of urine. $\mathbf{A}$ soda solution, containing 0.0031 grm. of soda in each c.c. is used; 1 c.c. of this solution exactly neutralises 0.0063 grm. oxalic acid. To the 100 c.c. of urine in a beaker, soda solution is added, drop by drop, from a graduated burette (fig. 251), until violet litmus paper becomes neither red nor blue. The number of c.c. of soda solution is now read off on the burette, and as each c.c. corresponds to 
0.0063 grm. oxalic acid, we can easily calculate the amount of oxalic acid which is equivalent to the degree of acidity in 100 c.c. of urine. So that the degree of acidity of the urine is expressed by the equivalent amount of oxalic acid, which is completely neutralised by the same amount of caustic soda.

Urine of Mammals. - The urine of carnivora is pale, passing into a golden-yellow ; its specific gravity is high, and its reaction strongly acid. The urine of herbivora is alkaline ; it shows a precipitate of earthy carbonates (hence, it effervesces on the addition of an acid), and of basic earthy phosphates. During hunger, the urine presents the character of that of carnivora, as the animal in this case practically lives upon its own flesh and tissues.

256.-I. THE ORGANIC CONSTITUENTS OF URINE.-Urea, $\mathrm{CO}\left(\mathrm{NH}_{2}\right)_{2}$, the diamide of $\mathrm{CO}_{2}$, or carbamid, is the chief end-product of the oxidation of the nitrogenous constituents of the body. Its composition is comparatively simple: 1 carbonic acid +2 ammonia - 1 water. It crystallises in silky foursided prisms with oblique ends (rhombic system), without water of crystallisation (fig. 252, 1), if it crystallises rapidly it forms delicate white needles. It has no action on litmus, is odourless, and has a weak, bitter, cooling taste, like saltpetre ; is readily soluble in water and alcohol, but insoluble in ether. It is an isomer of anmonium cyanate, from which it may be prepared by evaporation, whereby the atoms rearrange themselves (Wöhler, 1828). It can be prepared artificially in many other ways.

Decomposition.--When heated above $120^{\circ}$, it gives off ammonia vapour, while a glassy mass of biuret and cyanic acid is left. When urine undergoes the alkaline fermentation ( $\$ 263$ ), or when urea is treated with strong mineral acids, or boiled with the hydrates of the alkalies, or superheated with water $\left(240^{\circ} \mathrm{C}\right.$.), it takes up two molecules of water and produces ammonium carbonate, thus-

$$
\mathrm{CO}\left(\mathrm{NH}_{2}\right)_{2}+2 \mathrm{H}_{2} \mathrm{O}=\mathrm{CO}\left(\mathrm{NH}_{4} \mathrm{O}\right)_{2} \text {. }
$$

When brought into relation with nitrous acid, it splits up into water, $\mathrm{CO}_{2}$, and $\mathrm{N}$. The two last decompositions are made the basis of methods for the quantitative estimation of urea $(\$ 257)$.

Quantity.-In normal urine, urea occurs to the extent of 2.5 to 3.2 per cent. An adult man excretes daily from 30 to 40 grms. [500 grains, or a little over $1 \mathrm{oz}$.$] ; women less, children relatively$ more ; owing to the relatively greater metabolism in children, the unit weight of body produces more urea than the unit weight of an adult, in the proportion of $1.7: 1$. If the metabolism of the body is in a condition of equilibrium ( $\$ 236)$, the urea excreted contains almost as much $\mathrm{N}$ as is taken in with the nitrogenous constituents of the food.

Variations in the Quantity.-The amount of urea increases when the amount of pro-

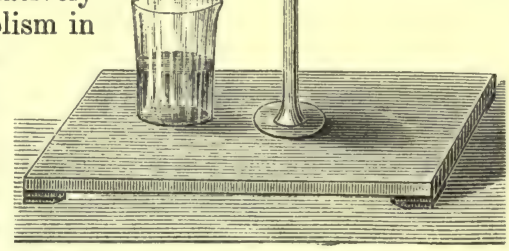

Fig. 251.

Graduated burette. teids in the food is increased; and also when there is a more rapid breaking up of the nitrogenous tissues of the body itself. As this breaking up is increased by diminution of $\mathrm{O}$, and by loss of blood, so these conditions also increase the urea ( $\S 41)$. It is also increased by drinking large draughts of water, by various salts, by frequent urination, and by exposure to compressed air. In diabetic persons, who eat very large quantities of food, it may exceed 100 grms. [over 3 
oz.] per day ; during hunger it sinks to $6 \cdot 1$ grms. [90 grains] per day. During inanition, the maximum amount is excreted towards mid-day, and the minimum in the morning. The daily amount of urea varies with the quantity of urine; three to five hqurs after a meal, the formation of urea is at a maximum, when it sinks and reaches its minimum during the night. Muscular exercise, as a rule, does not increase it (v. Voit, Fick and Wislicenus- $\$ 295)$, but only when deficiency of

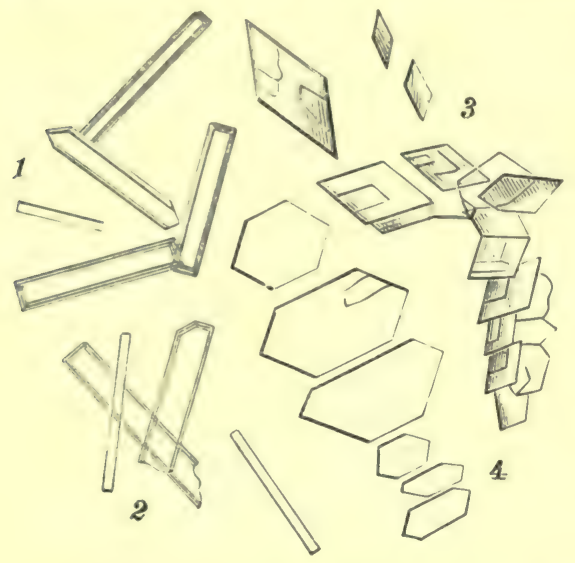

$\mathrm{O}$, causing dyspnœa, occurs at the same time (Oppenheim).

Fig. 252.

1, 2, Prisms of pure urea ; 3 , rhomboilal plates ; 4 , hexagonal tablets ; 5,6 , irregular scales and plates of urea nitrate.

Pathological.--In acute febrile inflammations, and in fevers generally ( $\$ 22,3$ ), the urea increases until the crisis is reachell, and afterwards it diminishes. After the fever has passed off, the amount excreted is often under the normal. In some cases of high fever, although the amount of urea formed is increased, it may not be excreted; there is a retention of the urea, which, later on, may lead to an increased excretion (Naunyn). In chronic discases, the amount depends largely upon the state of the nutrition, the metabolism, and also upon the degree of fever present. Degenerative changes in the liver, e.g., due to poisoning with phosphorus, may be accompanied by diminished excretion of urea and increased excretion of ammonia (Stadelmunn). It is increased in man by morphia, narcotin, narcein, papaverin, codein, thebain (Fubini), arsenic (Güthgens), compounds of antimony, and small doses of phosphorus (Boner), which favour the decomposition of proteids, and by substances which increase the bile formation in the liver ( $N$. Paton). Quinine, which "spares" the proteids, diminishes it.

Occurrence.-Urea occurs in the blood $(1: 10,000)$, lymph, chyle $(2: 1000)$, liver, lymphglands, spleen, lungs, brain, eye, bile, saliva, amniotic fluid, and pathologically in sweat, e.g., in cholera, in the vomit and sweat of uræmic patients, and in dropsical fluids.

Formation.- It is certain that it is the chief end-product of the metabolism of the proteids. Less oxidised products are uric acid, guanin, xanthin, hypoxanthin, alloxan, allantoin. Uric acid administered internally appears in the urine as urea ; alloxan and hypoxanthin can be changed directly into urea. The urea excretion is increased by the administration of leucin, glycin, aspartic acid, or ammonia salts (Schulzen, Nencki). As yet it has not been definitely determined where urea is formed, but the liver and, perhaps, the lymph glands, are organs where it is produced (\$ 178).

In birds, the liver forms uric acid from ammonia. The liver can be readily excluded from the circulation in birds, and Minkowski found that after this operation the uric acid was diminished and the ammoniacal salts were increased (\$178).

Antecedents.-During digestion, the proteids are converted into leucin, tyrosin, glycin, and aspartic acid. If the amido-acids, glycin, leucin, or aspartic acid, or ammoniacal salts, be given to an animal, the amount of urea excreted is increased. As the molecule of the amido-acids contains only one atom of $\mathrm{N}$, and the molecule of urea contains two of $\mathrm{N}$, it is probable that urea may be formed synthetically from these acids. It is possible that the amido-acids meet 
with nitrogenous residues in the juices of the body, e.g., carbamic acid or cyanic acid. The union of these may produce urea. According to Salkowski, feeding with these substances causes the breaking up of the proper proteids of the body so as to provide the necessary components. Schmiedeberg is of opinion that urea is formed in the body from ammonia carbonate by the removal of water; and $\mathbf{v}$. Schröder found that, when he passed blood containing ammonia carbonate through a fresh liver, the urea in the blood was greatly increased. Drechsel succeeded in producing urea at ordinary temperatures by the rapid alternating oxidation and reduction of a watery solution of ammonia carbonate. [We know that the greater part of the urea exists in the blood, and that the renal epithelium removes it from the blood. Although it is surmised that some of the nitrogenous bodies named above, more especially leucin, and perhaps also kreatin, are the precursors of urea, yet we cannot say definitely how or where the transformation takes place. Perhaps this is effected in the liver, and, it may be, also in the spleen (\$ 193).]

Preparation.-Urea may be prepared from dog's urine (especially after a diet of flesh) by evaporating it to a syrupy consistence, extracting it with alcohol, and again evaporating the filtrate to a syrupy consistence. The crystals which separate are washed with water to remove any extractives that may be mixed with them, and dissolved in absolute alcohol. It is then filtered and allowed to crystallise slowly. Or, human urine may be evaporated to one-sixth of its volume and cooled to $0^{\circ}$, and excess of strong nitric acid added, which precipitates urea nitrate mixed with colouring-matter. This precipitate is pressed in blottingpaper, then dissolved in boiling water containing animal charcoal, and filtered while hot. When it cools, colourless crystals of urea nitrate separate (fig. 252). These crystals are redissolved in warm water, and barium carbonate added until effervescence ceases; urea and barium carbonate are formed. Evaporate to dryness, extract with absolute alcohol, filter, and allow evaporation to take place, when urea separates.

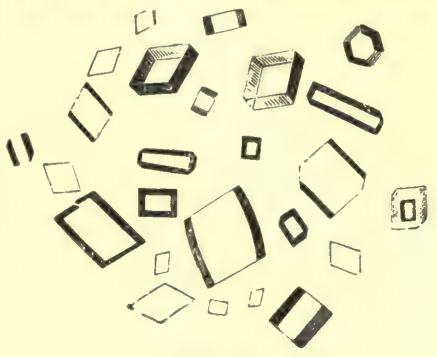

Fig. 253.

Perfect crystals of oxalate of urea.

Compounds of Urea.-Urea combines with acids, bases, and salts. The following are the most important combinations :-

1. Urea nitrate $\left(\mathrm{CH}_{4} \mathrm{~N}_{2} \mathrm{O}, \mathrm{HNO}_{3}\right)$ is easily soluble in water, and not so soluble in water containing nitric acid. It forms characteristic rhombic crystals (fig. 252, 3, 4, 5, 6). Sometimes the formation of these crystals is used to determine microscopically the presence of urea in a fluid. If a fluid is suspected to contain minute traces of urea, it is concentrated and a drop of the fluid is put on a microscopic slide. A thread is placed in the fluid, and the whole is covered with a cover-glass. A drop of concentrated nitric acid is allowed to flow under the cover-glass, and after a time crystals of urea nitrate adhering to the thread may be detected with the microscope.

2. Urea oxalate $\left(\mathrm{CH}_{4} \mathrm{~N}_{2} \mathrm{O}\right)_{2}, \mathrm{C}_{2} \mathrm{H}_{2} \mathrm{O}_{4}+\mathrm{H}_{2} \mathrm{O}$, is made by mixing a concentrated solution of urea with oxalic acid. The crystals form groups of rhombic tables, often of irregular shape. It is only slightly soluble in cold water, and still less so in alcohol (fig. 253).

3. Urea phosphate $\left(\mathrm{CH}_{4} \mathrm{~N}_{2} \mathrm{O}, \mathrm{H}_{3} \mathrm{PO}_{4}\right)$, forms large, glancing, rhombic erystals, very easily soluble in water. It is obtained by evaporating the urine of pigs fed on dough.

4. Sodic chloride + urea $\left(\mathrm{CH}_{4} \mathrm{~N}_{2} \mathrm{O}, \mathrm{NaCl}+\mathrm{H}_{2} \mathrm{O}\right)$ forms rhombic, shining prisms, which are sometimes deposited in evaporated human urine.

5. Urea + mercuric nitrate is obtained as a white cheesy precipitate, when mercuric nitrate is added to a solution of urea. Liebig's titration method for urea depends on this reaction (\$ 257, II.).

257. QUALITATIVE AND QUANTITATIVE ESTIMATION OF UREA.-I. The qualitative Estimation of Urea. - (1) It may be isolated as such. If albumin be present, add to the fluid three or four times its volume of alcohol, and, after several hours, filter. Evaporate the filtrate over a water-bath, and dissolve the residue in a few drops of water.

(2) The crystals of urea nitrate may be detected microseopically (fig. 252).

II. Quantitative Estimation.-(1) Sodic hypobromite decomposes urea into $\mathrm{CO}_{3}, \mathrm{H}_{2} \mathrm{O}$, and N. On this reaction depends the Knop-Huifner method of quantitative estimation. The $\mathrm{N}$ rises in the form of small bubbles in the mixed fluid, while the $\mathrm{CO}_{2}$ is absorbed by the caustic soda. [The reaction is the following :-

$$
\mathrm{N}_{2} \mathrm{H}_{4} \mathrm{CO}+3 \mathrm{NaBrO}=3 \mathrm{NaBr}+\mathrm{CO}_{2}+2 \mathrm{H}_{2} \mathrm{O}+\mathrm{N} \text {. }
$$

The nitrogen is collected and estimated in a graduated tube, and the amount of urea calculated from the volume of nitrogen. The uric acid is also decomposed, but that can be estimated separately and a correction made. We may use the apparatus of Russell and West, or Dupré, or that of Charteris (fig. 254).]

[Ureameter.-Make a solution of hypobromite of soda by mixing 100 grammes $\mathrm{NaHO}$ in 250 c.c. of water, and adding 25 c.c. of bromine. It is better to be made fresh, as it decomposes by 
keeping. The graduated tube is placed in a cylindrical vessel, filled with water, and depressed until the zero on the tubes coincides with the level of the water. Introduce 15 c.c. of the hypobromite solution into the pyramidal-shaped bottle, while into a short test-tube are placed 5 c.c. of urine. The test-tube with the urine is introduced into the bottle by means of a pair of forceps in such a way that it does not spill. Close the bottle tightly with the caoutchouc stopner, through which passes a glass tube to connect it with the graduated burette. Incline the bottle so as to allow the urine to mix with the hypobromite solution when the gases are given off, and pass into the collecting tube, which is gradually raised until the surfaces of the liquids, outside and in, coincide. Time should be allowed to permit the whole apparatus to have the same temperature. Read off the amount of gas $\mathrm{N}$ evolved, for the $\mathrm{CO}_{2}$ is absorbed by the caustic soda. The collecting tube is usually graduated beforehand, so that each division of the tube is $=0^{\circ} 1$ per cent. of urea, or $0^{\circ} 44 \mathrm{gr}$. per fluid oz. Thus, suppose that $50 \mathrm{oz}$. of urine are passed in twenty-four hours, and that 5 c.c. of urine evolve 18 measures of $\mathrm{N}$, then $0.44 \times 18 \times 50=396$ grs. of urea. If, however, the tube be graduated into
c.c., then $30.3 \mathrm{c} . \mathrm{c}$ of $\mathrm{N}=0^{\circ} \mathrm{grm}$. of urea at the ordinary temperature and
pressure. $]$ (fig. 255), 40 cubic centimetres of the urine are placed in a beaker; add 20 enbic centimetres of barium mixture to precipitate the sulphuric and phosphoric acids. The barium mixture consists of 1 vol. of a cold saturated solution of barium nitrate and 2 vols. of a cold saturated solution of barium hydrate. Filter through a $d r y$ filter, and take 15 cubic centimetres of the filtrate, which correspond to $10 \mathrm{c.c}$. of urine, and place in a beaker. Allow a titrated standard solution of mercuric nitrate to drop from a burette into the urine until a precipitate no longer occurs. The mercuric nitrate is made of such a strength that 1 cubic centimetre of it will combine with 10 milligrammes of urea. Test a drop of the mixture from time to time in a watch-glass or piece of glass blackened on its under surface, with a solution of sodic carbonate, which is called the indicator. Whenever the slightest excess of mercuric nitrate is added, the mixture strikes a yellow colour with the soda. The standard solution must

Fig. 254. be added drop by drop until this result is obtained. Read off the Ureameter of Charteris.

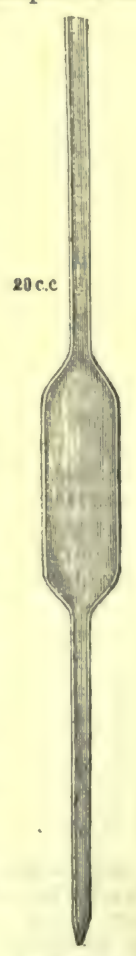

Fig. 255.

Graduated pipette.

number of cubic centimetres of the standard solution used; as each centimetre corresponils to 10 milligrammes of urea, multiply by ten, and the amount of urea in 10 cubic centimetres of urine is obtained.

This method does not give quite accurate results even in normal urine. To urine containing much phosphates is added an equal volume of the barium nixture. Very acid urines may require several volumes to be added. Urine containing albumin or blood must be boiled, after the addition of a few drops of acetic acid, to remove the albunin. The sodic chloride in the urine also interferes with the accuracy of the process, as on adding mercuric nitrate to urine, mercuric ehloride and solic nitrate are formed, so that the urea does not combine until the sodic chloride is decomposed. When the urine contains, as is usually the case, 1 to $1 \frac{1}{2}$ per cent. $\mathrm{NaCl}$, deduct 2 c.c. from the number of c.c. of the S.S. added to 10 c.c. of urine.

Estimation of the total $\mathrm{N}$ in Urine.-Pfliger and Bohland recommend the following modification of the method of Kjeldahl. Five c.c. of a urine of medium concentration are allowed to flow from a burette into Erlenmeyer's flask, capable of containing about 300 c.c., and to it are added 20 c.c. of concentrated sulphuric acid. The whole is hoiled until all the water and gases are driven off. The fluid at first becomes black from the action of the sulphuric acid, but when it has become of brownish tone lessen the heat of the Bunsen burner. About half an hour suffices to heat it, when the fluid at last becomes bright yellow. Allow it to cool, dilute it with water to 200 c.c., and place the whole in a flask, add 80 c.c. of caustic soda (S.9.1.3), cork the flask as quickly as possible, and distil its contents. The distillate must pass over into sulphuric acid, which must be titrated beforehand. The quantity of sulphuric acid not combined with ammonia must be estimated by titration with caustic soda.

The $\mathbf{N}$ in the Urine may be estimated approximately thus. To 10 c.c. of the urine add from 
a burette Liebig's mercuric nitrate solution, and test the mixture in a black glass plate with dry sodic bicarbonate until a yellow speck remains. Multiply the number of c.c. of the burette fluid used by 0.04 (Pflïger and Bohland).

258. URIC $\mathbf{A C I D}=\mathbf{C}_{5} \mathbf{H}_{4} \mathbf{N}_{4} \mathbf{O}_{3}$ is the nitrogenous substance which, next to urea, carries off most of the $\mathrm{N}$ from the body; in twenty-four hours $0.5 \mathrm{grm}$. ( 7 to 10 grains); during hunger, 0.24 grm. (4 grains); after a strongly animal diet, 2.11 grm. (30 to 35 grains) are excreted. The proportion of urea to uric acid is $45: 1$. If a mammal be fed with uric acid, part of it becomes more highly oxidised into urea, while the oxalic acid in the urine is also increased ( $\$ 260)$; in fowls, feeding with leucin, glycin, or aspartic acid (v. Knieriem), or ammonia carbonate (Schroeder), increases the amount of uric acid. When urea is administered to fowls, it is reduced chiefly to uric acid.

It is the chief nitrogenous product in the urine of birds, reptiles, and insects, while it is absent from herbivorous urine.

Properties. - It is dibasic, colourless, and crystallises in various forms (figs. 256 and 257), belonging to the rhombic system (1). When the angles are rounded, the whetstone form (2) is produced, and if the long surfaces be flattened, six-sided tables occur. Not unfrequently diabetic urine deposits spontaneously, large, yellow, transparent rosettes $(6,8)$. If 20 c.c. of $\mathrm{HCl}$, or acetic acid, be added to 1 litre of urine, crystals (9) are deposited, like cayenne pepper, on the surface and sides of the glass, after several hours. [The $\mathrm{HCl}$ decomposes the urates, and liberates the acid, which does not crystallise at once, owing to the presence of the phosphates in the urine. Crystals of uric acid are usually yellowish in colour from the pigment of the urine, and they are soluble in caustic potash.]

Solubility. - It is tasteless and odourless; reddens litmus; is soluble in 6 18,000 parts of cold and in 15,000 of boiling water, and insoluble in alcohol and ether. Horbaczewski prepared it synthetically by melting together glycin, or, as it is also called, glycocin, and urea. It is freely soluble in alkaline carbonates, borates, phosphates, lactates, and aceFig. 256.
tates, these salts at
the same time re-Forms of uric acid. 1, Rhombic plates; 2 , whetstone forms; 3 , quadrate moving a part of forms ; 4,5 , prolonged into points ; 6,8 , rosettes ; 7 , pointed bundles; the base ; thus, there 9 , barrel forms precipitated by adding hydrochloric acid to urine.

are formed acid urates and acid salts from the neutral salts. It is soluble in concentrated sulphuric acid, from which it may be precipitated by the addition of water.

Decomposition.--During dry distillation it decomposes into urea, cyanuric acid, hydrocyanic acid, and ammonium carbonate. Superoxide of lead converts it into urea, allantoin, oxalic acid, and $\mathrm{CO}_{2}$; while ozone forms the same substances, with the addition of alloxan. When it is reduced by $\mathrm{H}$ in statu nascendi, as by sodium amalgam, it forms xanthin and sarkin. It is a less oxidised metabolic product than urea, but it is by no means proved that uric acid is a precursor of urea.

Occurrence.-Uric acid occurs dissolved in the urine in the form of acid urates of soda and potash. These salts occur also in urinary calculi, gravel, and in gouty 
deposits. Ammonium urate occurs in very small quantity in a deposit of "urates," but is formed in considerable amount when urine becomes ammoniacal from decomposition (fig. 261). Free uric acid occurs in normal urine only in the very smallest amount. It is sometimes deposited after a time (fig. 260). It frequently forms urinary calculi and gravel.

The urine of newly-born children contains much uric acid. Uric acid and its salts are increased after severe muscular exertion, accompanied by perspiration, in catarrhal and rheumatic fevers, and such conditions as are accompanied by disturbance of the respiration; in leuk:emia and tumours of the spleen, cirrhotic liver, and generally in cases of catarrh of the stomach and intestinal tract, following the excessive use of alcohol. [It is also increased during agne and fevers, and perhaps this has some relation to the congestion of the spleen which accompanies these conditions.]. It is diminished after copious draughts of water, after large doses of yuinine, caffein, potassic iodide, common salt, sodic and lithic carbonates, sodic sulphate, inhalation of $\mathrm{O}$, slight muscular exertion. In gout, the amount excreted in the urine is small. In chronic tumours of the spleen, anemia, and chlorosis, when the respiration is not at the same time embarrassed, it is also diminished.

Urates. - Cric acid forms salts-chiefly acid urates-with several bases, which dissolve with difficulty in cold water, but are easily soluble in warm water. Neutral urates are changed by $\mathrm{CO}_{2}$ into acid salts. Hydrochloric and acetic acids break up the compounds, and crystals of uric acid separate.

(1) Acid sorlic urate usually appears as a brick-red deposit in urine; more rarely grey or white (lateritious deposit), tinged with uroërythrin, in catarrhal conditions of the digestive organs, and in rheumatic and felorile affections. Microscopically, it is completely amorphous, consisting of granules, sometimes lisposed in groups (fig. 260, $b$ )-sometimes the granules have spines on them. The corresponding potash salt occurs not unfrequently under the same con. ditions, and presents the same characters.

(2) Acid ammonium urate (fig. 261, a) always occurs as a sediment in ammoniacal urine, either with (1), or mixed with free uric acid, accompanied by triple phosphate. Microscopically, it is the same as (1). (1) and (2) are distinguished by the sediment dissolving when the urine is heuted. If a drop of hydrochloric acid be added to a microscopic preparation of the sediment, crystals of uric acid separate.

(3) Acid calcic urate occurs sometimes in calculi, and is a white, amorphous powder slightly soluble in watel. When heated on platinum it leaves an ash of calcium carbonate. Magnesium urate rarely occurs in urinary calculi.

\section{ESTIMATION OF URIC ACID. - I. Qualitative.-1. Microscopic Char-} acters. - The appearances presented by uric acid and its salts under the microscope. It is deposited from urine after several hours, on adding acetic or hydrochloric acid.

2. Murexide Test._-Gently heat a urate or uric acid in a porcelain vessel along with nitric acid. Decomposition takes place and the colour changes to yellow. N and $\mathrm{CO}_{2}$ are giren off; urea and alloxan $\left(\mathrm{C}_{4} \mathrm{H}_{2} \mathrm{~N}_{2} \mathrm{O}_{4}\right)$ remain. Evaporate slowly and allow the yellowish-red stain to cool; on adding a drop of dilute ammonia a purplish-red colour of murexide is obtained, it becomes blue on the addition of caustic potash. If potash or soda be added instead of ammonia, a violet colour is obtained.

3. Schiff's Test.-Dissolve uric acid or a urate in a solution of an alkaline carbonate, and drop it upon hotting-paper saturated with a solution of silver nitrate, reduction of the silver takes place at once, and a black spot is formed.

4. On boiling a solution of uric acid or a urate in an alkali, with Fehling's solution $(\S 149,2)$, at first white urate of the suboxide of copper is deposited, while later, red copper suboxide is formed.

II. Quantitative Estimation.-Add 5 cubic centimetres of concentrated $\mathrm{HCl}$ to 100 c.c. of urine, and allow it to stand for forty-eight hours in the dark, when the uric acid is precipitated like fine cayenne pepper crystals. All the uric acid is not precipitated by the $\mathrm{HCl}$, even after standing for a time. [E. A. Cook uses sulphate of zinc to precipitate the uric acid as urate of zinc. Caustic solla is added to precipitate the phosphates, and then to the clear fluid zinc sulphate solution, which precipitates urate of zinc as a white gelatinous deposit.]

Fokker-Salkowski Method.-Make 200 c.c. of urine strongly alkaline with sodic carbonate, and after an hour ald 200 c.c. of a concentrated solution of ammonium chloride, whereby acid urate of ammonium is precipitated. After forty-eight hours filter, through a small weighed filter, and wash it several times. Fill the filter with dilute $\mathrm{HCl}$ and collect the filtrate. Do this until all the acid urate is dissolved. From the total filtrate after a time all the uric acid separates. It is collected in the same filter, washed with water and alcohol until the acid reaction disappears, dried at $100^{\circ} \mathrm{C}$. and weighed. To the weight in excess of the filter add $0.030 \mathrm{grm}$. 
260. KREATININ AND OTHER SUBSTANCES.-Kreatinin $\mathrm{C}_{4} \mathrm{H}_{9} \mathrm{~N}_{3} \mathrm{O}_{2}$ is derived from the kreatin of muscle by the removal of a molecule of water, and partly from flesh food. The quantity excreted daily is 0.6 to 1.3 gramme (8 to 18 grains).

It is diminished in progressive muscular atrophy, tetanus, anæmia, marasmus, chlorosis, consumption, paralysis; and is increased in typhus, inflammation of the lung; it is absent from the urine of sucklings.

Properties. - Kreatinin is alkaline, easily soluble in water and hot alcohol. It forms colourless oblique rhombic columns; unites with acids and salts, silver nitrate, mercuric chloride, and especially with zinc chloride. Kreatinin-zinc chloride (fig. 257) is used to detect its presence. Weyl's Test. - Add to urine a few drops of a slightly brownish solution of nitro-prusside of soda, and then weak caustic soda solution, producing a Burgundy-red colour, which soon disappears. When heated with glacial acetic acid, the colour changes to green, which after a time changes to blue (Salkowski). [The blue colour-Berlin blue-is due to the formation of an iron-salt ferro-cyanide of sodium from the decomposition of the nitro-prusside. The reaction also succeeds with formic acid-instead of glacial acetic acid-if some time be allowed to elapse after Weyl's reaction.]

Xanthin $=\mathrm{C}_{5} \mathrm{H}_{4} \mathrm{~N}_{4} \mathrm{O}_{2}$ occurs only to the amount of 1 gramme in 300 kilos. of urine. It is a substance intermediate between sarkin and uric acid. Guanin and hypoxanthin may be changed into xanthin; in contact with water and ferments it passes into uric acid. When evaporated with nitric acid, it gives a yellow stain, which becomes yellowish-red on adding potash, and violet-red on applying more heat. It is an amorphous, yellowish-white powder, fairly soluble in boiling water. It has also been found in traces in muscles, brain, liver, spleen, pancreas, and thymus. The crystalline body paraxanthin (dimethylxanthin) and the amorphous heteroxanthin (methylxanthin) occur in traces in the urine ( $S a l o$ mon).

Sarkin or Hypoxanthin, $\mathrm{C}_{5} \mathrm{H}_{4} \mathrm{~N}_{4} \mathrm{O}$.As yet this substance has been found only in the urine of leukæmic patients (Jakubasch), and it has been prepared in the form of needles or flattened scales from muscle, spleen, thymus, biain, bone, liver, and kidney. In normal urine a body nearly related to, and possibly identical with, hypoxanthin occurs

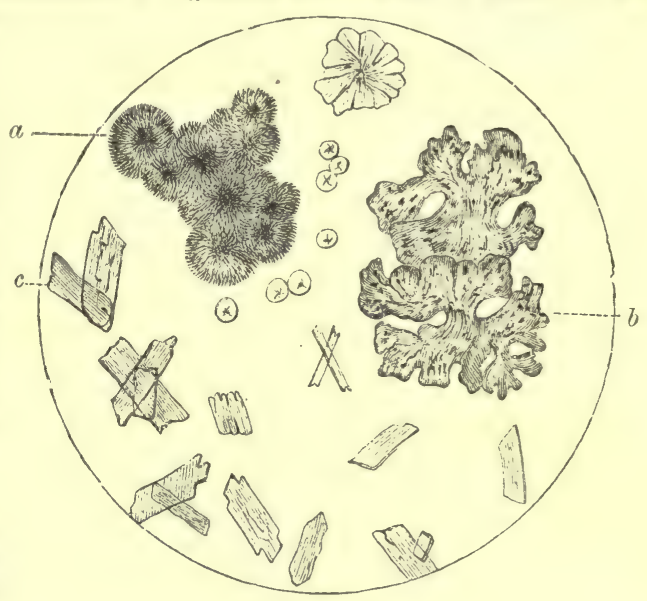

Fig. 257.

Kreatinin-zinc chloride. $\alpha$, balls with radiating marks : $b$, crystallised from water; $c$, from alcohol.

(E. Salkowski). Hypoxanthin closely resembles xanthin, and can be changed into it by oxidation. Nascent hydrogen, on the other hand, reduces uric acid to xanthin and hypoxanthin. When evaporated with nitric acid it gives a light yellow stain, which becomes deeper, jut not reddish-yellow, on adding caustic soda. It is more easily soluble in water than xanthin, and by this means the two substances can be separated from each other. Guanin is insoluble in water.

Oxaluric acid $\left(\mathrm{C}_{3} \mathrm{H}_{4} \mathrm{~N}_{2} \mathrm{O}_{4}\right)$ occurs in very small quantity combined with ammonia in urine. Physiologically, it is interesting on account of its relation to uric acid. It is a white powder slightly soluble in water. Ammonia oxalurate can be prepared from uric acid.

Oxalic Acid $\left(\mathrm{C}_{2} \mathrm{H}_{2} \mathrm{O}_{4}\right)$ occurs, but not constantly, to the amount of 20 milligrammes daily as oxalate of lime, which is known by the "envelope" shape of the crystals (fig. 258); insoluble in acetic acid, and forming transparent octahedra. More rarely it assumes a biscuit or sand-glass form. The genetic relation of oxalic acid to uric acid is shown by the fact, that dogs fed with uric acid excrete much oxalate of lime. Oxalic acid may also be produced by the oxidation of products derived from the fatty acid series (p. 381).

Oxaluria. - The eating of substances containing oxalate of lime (rhubarb) increases the excretion. Increased excretion is called oxaluria ; it is regarded as a sign of retarded metabolism (Beneke), and it may give rise to the formation of a calculus. In oxaluria the uric acid is also often in. 
creased in amount. Perhaps, in the first instance, there is an increased formation of uric acid, from which oxalic acid, urea, and $\mathrm{CO}_{2}$ may be formed. The amount of oxalic acid is increased after the nse of wine and sodic bicarbonate.

Hippuric Acid $=\mathrm{C}_{3} \mathrm{H}_{9} \mathrm{NO}_{3}$ (Benzoylamidoacetic acid) occurs in large amount in the urine of herbivora, and in them is the chief end-product of the metabolism of nitrogenous substances; in human urine the daily quantity is small, 0.3 to 3.8

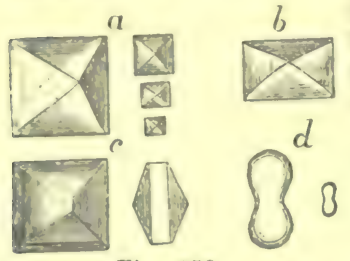

Fig. 258 grms. (5 to 50 grains). It is an odourless monobasic acid with a bitter taste, crystallising in colourless foursided prisms (fig. 259). Readily soluble in alcohol, and soluble in 600 parts of water. Oxalate of lime. $4, b$, octa- benzoic acid, or some nearly related chemical body, such hedra; c, compound forms; as the cuticular substance of plants, or from oil of bitter d, dumb-bells. almonds, cinnamic or chinic acid, which easily pass by reduction (chinic acid) or by oxidation (cinnamic acid) into benzoic acid; glycin uniting with it, with the formation of water-

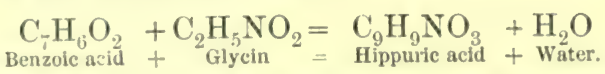

Formation. - When benzoic acid is introduced into the alimentary canal of an animal (rabbit or dog), it appears in the urine as hippuric acid; while nitro-benzoic

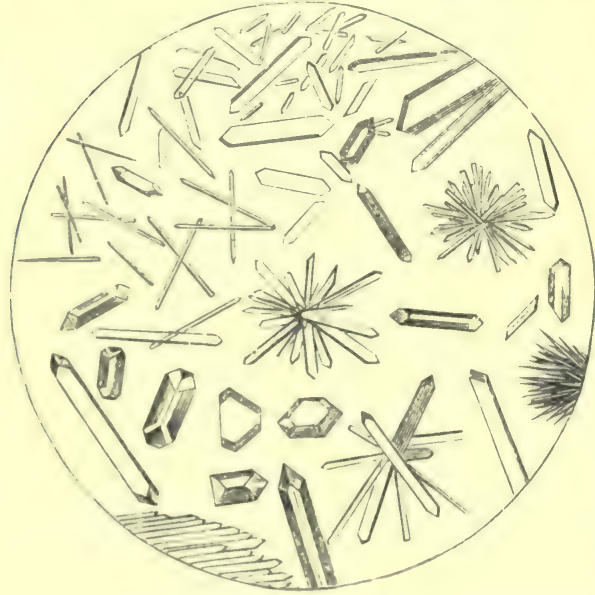

Fig. 259 .

Hippuric Acid. acid appears as nitro-hippuric acid. As the benzoic acid passes through the body, it becomes conjugated with glycin or glycocin, chiefly in the kidneys. The hippuric acid in the urine of herbivora is chiefly derived from some substance with a benzoic acid residue present in the cuticular coverings of the food. That hippuric acid, in part at least, is formed in the kidneys is shown by the following considerations :- If arterialised blood, containing benzoic acid and glycin, or even benzoic acid alone, be passed through the blood-vessels of a fresh, living, excised kidney, hippuric acid is found in the blood after it is perfused. Even after forty-eight hours, if the kidney be kept cool, the synthesis takes place. If the kidney be kept too long, the conjugation does not take place. If the fresh kidney be chopped up, and kept at the temperature of the body with benzoic acid and glycin, hippuric acid is formed. Oxygen seems to be necessary for the process, for, if blood or serum containing carbonic oxide be used, there is no formation of hippuric acid.]

According to this view, it is derived chiefly from the food of herbivorous animals, and hence it is absent from the urine of sucking calves, as well as after feeding with grain devoid of husk. But it is also formed in the body from the proteids. In the dog, the formation of hippuric acid occurs in the kidney (Schmialcberg and Bunge); and in the froy also outside the kidney. Kühne and Hallwachs thought it was formed in the liver, and Jaarsveld and Stockvis in the kidney, liver, and intestine. The observation of Salomon that, after excision of the kidneys in rabbits, 
and injection of benzoic acid into the blood, hippuric acid was found in the muscles, blood, and liver, goes to show that it must be formed in other organs beside the kidneys. The power of changing benzoic acid introduced into the human body, into hippuric acid, may even be abolished in disease of the kidney. Under certain circunstances it seems that hippuric acid, already formed, may be again decomposed in the tissues.

It is greatly increased after eating pears, plums, and cranberries; in icterus, some liver affections, and in diabetes. When boiled with strong acid or alkalies, or with putrid substances, it takes up $\mathrm{H}_{2} \mathrm{O}$ and splits into benzoic acid and glycin.

Preparation. - Add milk of lime to the fresh urine of horses or cows to form calcic hippurate. Filter, evaporate the filtrate to a small bulk, and precipitate the hippuric acid with excess of hydrochloric acid. To purify the hippuric acid, crystallise it several times from a hot watery solution.

Cynuric Acid. $-\mathrm{C}_{20} \mathrm{H}_{14} \mathrm{~N}_{2} \mathrm{O}_{6}+\mathrm{H}_{2} \mathrm{O}$ occurs in the urine of dogs (J. v. Liebig).

Allantoin, $\mathrm{C}_{4} \mathrm{H}_{6} \mathrm{~N}_{4} \mathrm{O}_{3}$, which occurs in the amniotic fluid of the cow, is found in minute traces in normal urine after flesh food, and is more abundant during the first weeks of life, and during pregnancy.

After large doses of tannic acid, the amount is increased (Schottin), while in dogs feeding with uric acid also increases it (Salkowski).

Properties. - It forms shining, prismatic crystals; from the urine of sucking calves it crystallises in transparent prisms. It is decomposed by ferments into urea, ammonium oxalate, and carbonate, and another as yet unknown body. Preparation- $(a)$ the urine is precipitated with basic lead acetate, the lead in the filtrate is removed by sulphuretted hydrogen, and the filtrate itself is then evaporated to a syrup, from which the crystals separate, after standing for several days. They are then washed with water, and recrystallised from the water (Salkowski).

261. COLOURING-MATTERS OF THE URINE.-1. Urobilin is most abundant in the highly coloured urine of fevers, but it also occurs in normal urine (Jaffé). It is identical with the hydrobilirubin of Maly $(\$ 117,3, g)$. It is a derivative of hæmatin, which also yields the bile-pigments (\$177). It gives a red, or reddish-yellow colour to urine, which becomes yellow on the addition of ammonia.

[MacMunn, chiefly from spectroscopic observations, finds that two entirely different substances have been included under the name of " urobilin," viz., that of normal and that of pathological urine, and that hydrobilirubin is not identical with either. The pathological urobilin seems to be closely connected with stercobilin (\$ 185).]

Preparation.- Prepare a chloroform extract of urine containing urobilin-add iodine to the extract, and remove the ioline by shaking the mixture with dilute caustic potash, which forms potassic iodide. This potash solution becomes yellow or brownish-yellow, and exhibits beautiful green fluorescence (Gerhardt).

Urobilin may be extracted from many urines by ether (Salkoveski). When subjected to the action of reducing agents, e.g., sodium amalgam, a colourless product is obtained, which on exposure to the air absorbs $\mathrm{O}$, and becomes retransformed into urobilin. This colourless body is identical with the chromogen which Jaffé found in urine.

If urine is treated with soda or potash, the characteristic absorption-band lying between $b$ and F, passes nearer to $b$, becomes darker and more sharply defined. According to Hoppe-Seyler, urobilin is formed in urine after it is voided, from another urobilin-forming body (Jaffé's chromogen) absorbing oxygen. If urine containing urobilin be made alkaline with ammonia, and zine chloride be added, it exhibits marked fuorescence; it has a green shimmer by reflected light. When urobilin is isolated, it fluoresces without the addition of zinc chloride. In cases of jaundice $(\$ 180)$, where Gmelin's test sometimes fails to reveal the presence of bile-pigments, nrobilin occurs. This "urobilin-icterus" (Gerhardt) occurs chiefly after the absorption of large extravasations of blood. According to Cazeneuve, the urobilin is increased in all diseases where there is increased disintegration of coloured blood-corpuscles.

2. Urochrome (Thudichum) is regarded as the chief colouring-matter of urine. It may be isolated in the form of yellow scales, soluble in water, and in dilute acids and alkalies. The watery solution oxidises, and when exposed to air becomess red owing to the formation of uroerythrin. When acted on by acids, new decomposition-products are formed, e.g., uromelanin. Uroerythrin gives the red colour to deposits of urates (\$258).

3. A brown pigment containing iron is carried down with uric acid, which is precipitated on the addition of hydrochloric acid ( $\$ 258$ ). By repeatedly adding sodic urate to the urine, and precipitating the uric acid by hydrochloric acid, a considerable amount may be obtained (Kunkel).

4. Urine boiled with $\mathrm{HCl}$ yields a garnet-red crystalline pigment, urorubin, to ether.

In eases of melanotic tumours, there has been occasionally observed urine, which becomes dark, owing to melanin $(\S 250,4)$, or to a colouring-matter containing iron (Kunkel). 
262. INDIGO, PHENOL, KRESOL, PYROKATECHIN, AND SKATOLFORMING SUBSTANCES.-1. Indican $\left[\mathrm{C}_{8} \mathrm{H}_{7} \mathrm{NSO}_{4}\right]$, or indigo-forming substance (Schunck), is derived from indol, $\mathrm{C}_{8} \mathrm{H}_{7} \mathrm{~N}$, the basis of indigo, which is formed in the intestine by the pancreatic digestion of proteids $(\S 170, \mathrm{II}$.$) , but it$ also arises as a putrefactive product $(\S 184,6)$. Indol, when united with the radical of sulphuric acid, $\mathrm{HSO}_{3}$, and combined with potassium, forms the so-called imligoryen or indican of urine (Brieger, Baumann). This substance $\left(\mathrm{C}_{8} \mathrm{H}_{6} \mathrm{NSO}_{4} \mathrm{~K}\right.$ = potassium indoxyl-sulphate) forms white glancing tablets and plates; readily soluble in water and less so in alcohol. By oxidation it forms indigo-blue; 2 indican $+\mathrm{O}_{2}=\mathrm{C}_{16} \mathrm{H}_{10} \mathrm{~N}_{2} \mathrm{O}_{2}$ (indigo-blue) $+2 \mathrm{HKSO}_{4}$ (acid potassic sulphate). It is more abundant in the urine in the tropics, and it is absent from the urine of the newly-born (Senator).

Tests.-(1) Adil to 40 drops of urine, 3 to 4 c.c. of strong fuming hydrochloric acid, and 2 to 3 drops of nitric acill. Boil, a riulet-red colour (with the deposition of true crystalline imlig-l, lu (rhombic) and indigo-red attests its presence. Putrefaction causes a similar decomposition in inclican; hence, we not unfrequently observe a bluish-red pellicle of microscopic crystals of indigo-blue, or even a precipitate of the same. (2) Mix in a beaker equal quantities of urine and hydrochloric acil, and aild two drops of solution of chlorinated lime; the mixture at first becomes clear, then blue (Juffe). Add chloroform, and shake the mixture vigorously for some time; the chloroform dissolves the blue colouring matter, which is obtained as a deposit, when the chloroform evaporates (Scnator, Salkou'ski). (3) Heat to $70^{\circ}$ one part of urine with two parts of nitric acil, and shake up with chloroform; the chloroform dissolves the indigo which is formed, assumes a violet colour, and gives an absorption band between $\mathrm{C}$ and $\mathrm{D}$, slightly nearer 1) (H"In'-sicyler). Quantity. -Jaflé fomd in 1500 c.c. of normal human urine, 4.5 to 19.5 millimrammes of indigo; horse's urine contains 23 times as much. The subcutaneous injection of inlol increases the inclican in the urine (Jaffé). E. Ludwig obtained indiean by heating hermatin or urobilin with a caustic alkali and zinc dust. It has also been found in the sweat (Bizio).

Pathological. - The indican in the urine is increased when much indol is formed in the intestine $(\$ 172,11),. c . \%$, in typhus, lear colic, trichinosis, catarrh, and hemorrhage of the stomach, cholera, carcinoma of the liver and stomach; obstruction of the bowel or ileus, peritonitis, and diseases of the small intestine.

2. Phenol, $\mathrm{C}_{t:} \mathrm{H}_{t i} \mathrm{O}$ (carbolic acid, $\$ 252$ ), was discovered by Städeler in human urine (more abundant in horse's urine). It does not occur as carbolic acid, but in combination with a substance from which it is separated by distillation with dilute mineral acids. The "phenol-forming substance" is, according to Baumann, "phenolsulphonic acid" $\left(\mathrm{C}_{6} \mathrm{H}_{5} \mathrm{O}, \mathrm{SO}_{3} \mathrm{H}\right)$, which in urine is united with potash.

Thenol is derivel from the decomposition of proteids by pancreatic digestion ( $\$ 17 \cdot 2$, II.), and also from jutrefartion $(\$ 184,6)$, the mother-substance being tyrosin. Hence, the formation of phenolsulphonic acid is analogous to the formation of indican.

If in the employment of carbolic acid it be absorber, the phenolsulphonic acid becomes greatly increased in amount, so that sulphuric acid must be united with it; hence, alkaline sulphates are lecomposed in the looly, so that the latter may be absent from the urine (Baumann). Living muscle or liver, when digested in a stream of air for several hours with blood to which phenol and sodic sulphate are added, yields phenolsulphonic acid; while, under the same circumstances, pyrokatechin forms ethersulphonic acid.

Carboluria. - When carbolic acid is used externally or internally, and it is absorbed, it causes a derp drik-coloured urine due to the oxidation of phenol into hydrochinon (orthobioxybenzol $=\mathrm{C}_{6} \mathrm{H}_{6} \mathrm{O}_{2}$ ), which for the most part appears in the urine as ethersulphonic acid (Baumann and others).

3. Parakresol (hydroxyltoluol, $\mathrm{C}_{-} \mathrm{H}_{5} \mathrm{O}$ ), with its isomers ortho- and meta-kresol (the latter in traces), is more abundant in urine (Baumann, Preusse). It also occurs in combination with sulphonic acid.

Test for phenol (and also kresol) :-Distil 150 c.c. urine with dilute sulphuric acid. The distillate gives a brown crystalline deposit of tribromophenol with bromine water, as well as a red colour with Millon's reagent.

Hydroxybenzol (pyrokatechin, hydrochinon) is obtained from urine, when it is heated for a long time with hydrochloric acid.

Resorcin, which is an isomer of hylrochinon, when administered internally, also appears in the urine as ethersulphonic acid. Toluol and naphthalin behave similarly. Benzol is oxidised to phenol. 
4. Pyrokatechin $=\mathrm{C}_{6} \mathrm{H}_{6} \mathrm{O}_{2}$ (metadihydroxylbenzol), is formed along with hydrochinon from phenol, and is an isomer of the former. It behaves like indol and phenol, for when united with sulphonic acid, it yields the pyrokatechin-forming substance. Small quantities sometimes occur in human urine; it is more abundant in the urine of children; it becomes darker when the urine putrefies.

5. Skatol, which is crystalline, and is formed during putrefaction in the intestine, also appears in the urine as a compound of sulphonic acid (\$252). On feeding a dog with skatol, Brieger found much potassic skatol-oxy-sulphate.

Test. - Skatol compounds are recognised by adding dilute nitric acid, which causes a violet colour, or fuming nitric acid, which precipitates red flakes (Nencki). Its quantity is regulated by the same conditions as indican.

The aromatic oxyacids, hydroparacumaric acid, and paraoxyphenylacetic acid (the former a putrefactive product of flesh, the latter obtained by . E. and H. Salkowski from putrid albumin) occur in the urine (Baumann, § 252). Shake the urine treated with a mineral acid with ether, evaporate the latter, and dissolve the resilue in water. If aromatic oxyacids are present, they give a red colour with Millon's reagent.

Baumann gives the following series of bodies, which are formed from tyrosin by decomposition and oxidation; most of the substances are formed both during the decomposition of albumin. and also in the intestine, whence they pass into the urine:-Tyrosin, $\mathrm{C}_{9} \mathrm{H}_{11} \mathrm{NO}_{3}+\mathrm{H}_{2}$ $=\mathrm{C}_{9} \mathrm{H}_{10} \mathrm{O}_{3}$ (hydroparacumaric acid) $+\mathrm{NH}_{3} . \quad \mathrm{C}_{9} \mathrm{H}_{10} \mathrm{O}_{3}=\mathrm{C}_{8} \mathrm{H}_{10} \mathrm{O}$ (paraethylphenol, not yet proved) $+\mathrm{CO}_{2} \cdot \quad \mathrm{C}_{8} \mathrm{H}_{10} \mathrm{O}+\mathrm{O}_{3}=\mathrm{C}_{8} \mathrm{H}_{8} \mathrm{O}_{3}$ (paraoxyphenylacetic acid) $+\mathrm{H}_{2} \mathrm{O} . \quad \mathrm{C}_{8} \mathrm{H}_{8} \mathrm{O}_{3}=\mathrm{C}_{7} \mathrm{H}_{8} \mathrm{O}$ (parakresol) $+\mathrm{CO}_{2} \cdot \mathrm{C}_{7} \mathrm{H}_{8} \mathrm{O}+\mathrm{O}_{3}=\mathrm{C}_{7} \mathrm{H}_{6} \mathrm{O}_{3}$ (paroxybenzoic acid, not yet proved) $+\mathrm{H}_{2} \mathrm{O}$. $\mathrm{C}_{7} \mathrm{H}_{6} \mathrm{O}=\mathrm{C}_{6} \mathrm{H}_{6} \mathrm{O}$ (phenol) $+\mathrm{CO}_{2}$.

Potassium sulphocyanide, derived from the saliva, also occurs in urine. After acidulation with hydrochloric acid, its presence may be detected by the ferric chloride test (\$146 - Gscheidlen and J.Munk). One litre of human urine contains 0.02 to 0.08 gramme combined with an alkali.

Succinic acid $\left(\mathrm{C}_{4} \mathrm{H}_{6} \mathrm{O}_{4}\right)$ occurs chiefly after a diet of flesh and fat, and almost disappears after a vegetable diet. It is a decomposition-product of asparagin, and occurs in considerable amount in the urine after eating asparagus. It is also a product of the alcoholic fermentation $(\$ 150)$, and as it passes out of the body unchanged, it occurs in the urine of those who imbibe spirituous liquors. It passes unchanged into the urine (Naubauer).

Lactic acid $\left(\mathrm{C}_{3} \mathrm{H}_{6} \mathrm{O}_{3}\right)$ is a constant constituent of urine. Other observers have found fermentable lactic acid in diabetic urine; sarcolactic acid after poisoning with phosphorus and in trichinosis. Occasionally traces of volatile fatty acids are present. Some animal gum occurs in urine (p. 384), and Bechamp's "nephrozymose" consists for the most part of gum (Landwehr). 'This substance is precipitated from urine by adding to it three times its volume of 90 per cent. alcohol. It is not a simple body, but at $60^{\circ}$ to $70^{\circ} \mathrm{C}$. it transforms starch into sugar (v. Vintschgau).

Ferments. - Traces of diastatic, peptic, and rennet ferment have been found, especially in urine of high specific gravity. Trypsin is said not to occur normally (Leo).

Traces of sugar (Brïcke, Bence Jones), to the amount of 0.05 to 0.01 per cent., occur in normal urine. After the ingestion of milk-, cane-, or grape-sugar (50 grms.) these varieties of sugar appear in small quantity in the urine (Worm-Müller-\$267,7).

Kryptophanic acid $\left(\mathrm{C}_{3} \mathrm{H}_{9} \mathrm{NO}_{5}\right)$, according to Thudichum, occurs as a free acid in urine, but Landwehr regards it as an animal gum.

Aceton $\left(\mathrm{C}_{3} \mathrm{H}_{6} \mathrm{O}\right)$ is formed when normal urine is oxidised with potassic bichromate and sulphuric acid, and it is formed from a reducing substance present in normal urine (apparently derived from the grape-sugar of the blood). Aceton occurs in traces as a normal urinary constituent, which is increased during increased decomposition of the tissues, e.g., carcinoma, inanition. It has also been found in the blood in fever ( $v$. Jacksch). Lieben's Test. -Acidulate half a litre of urine with $\mathrm{HCl}$ and distil; when treated with tincture of iodine and ammonia there is a turbidity due to iodoform.

II. THE INORGANIC CONSTITUENTS OF THE URINE.-The inorganic constituents are either taken into the body as such with the food and pass off unchanged in the urine, or they are formed in the body, owing to the sulphur and phosphorus of the food being oxidised and the products uniting with bases to form salts. The quantity of salts excreted daily in the urine is 9 to 25 grammes $\left[\frac{1}{4}\right.$ to $\frac{3}{4}$ oz.].

1. Sodic chloride - to the amount of 12 (10 to 13$)$ grammes [180 grains]-is excreted daily. It is increased, after a meal, by muscular exercise, drinking of water, and generally, when the quantity of urine is increased, by the free use of 
large quantities of common salt, but by potash salts also; it is diminished under the opposite conditions.

In disease it is greatly diminished ; in pneumonia and other inflammations accompanied by effusions, in continued diarrhea and profuse sweating, constantly in albuminuria and in drupisies. [In cases of pneumonia, sodic chloride may at a certain stage almost disappear from the urine, and it is a grood sign when the chlorides begin to reappear.] In other chronic diseases, the amount of $\mathrm{NaCl}$ exereted runs nearly parallel with the amount of urine passed. In conditions of excitement the amount of sodic chloride is diminished, and potassic chloride increased; in conditions of depression the reverse is the case (Zeulzer).

Test. - A hil to the urine nitric acid and then nitrate of silver solution, which gives a white curly jurifitate of chloride of silver. In alhuminous urine the albumin must first be removed.

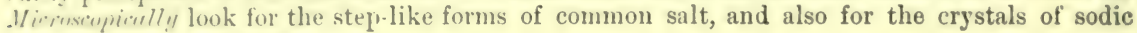
chloride and urea $(\S 256,4)$.

2. Phosphoric acid occurs in urine as acid sodic phosphate and acid calcic and magnesic phosphates to the amount of about 2 grammes daily [ 30 grains]; it is more abundant after an animal than after a vegetable diet. The amount increases after a mid-day meal until evening, and falls during the night until next day at noon. It is partly derived from the alkaline and earthy phosphates of the food, and partly as a decomposition-product of lecithin and nuclein. As phosphorus is an important constituent of the nervous system, the relative increase of phosphoric acid is due to increased metabolism of the nervous substance.

Pathological. - In fevers, the increased excretion of potassic phosphate is due to a consumption of hlowl and muscle $(\$ 220,3)$. It is also increased in inflammation of the brain, softening of the hones, liabetes, and oxaluria ; after the administration of lactic acid, morphia, ehloral, or chloroform. It is diminished cluring pregnaney, owing to the formation of the fortal bones; also after the use of ether and alcohol, and in inflammation of the kidney.

Tests. - To urine add nitric acid and solution of ammonium molyblate and boil, a canaryyellow precipitate of ammonium phosphomolybdate indicates the presence of phosphoric acic. ()r, ald half its volume of caustic potash to urine, and boil. The earthy phosphates are precipitated, but not the alkaline phospliates.]

Earthy phosphates are precipitated by heat in some pathological urines. This precipitate is distinguished from albumin, which is also precipitated by heat, by being soluble in nitric acid, which precipitated albumin is not. [The earthy phosphates are not precipitated until near the boiling point.]

Quantitative. - The amount of phosphoric acid is estimated by tritation with a standard solution of uranium acctate; ferrocyanide of potassium being the indicator. The indicator gives a brownish-red colour when there is an excess of free uranium acetate.

In addition to phosphoric acid, phosphorus occurs in an incompletely oxidised form in the urine, e.g., glycerinphosphoric acid $(\$ 251,2)$, which occurs to the anount of 15 milligrammes in a litre of urine; it is increased in nervous diseases and after chloroform narcosis.

3. Sulphuric acid occurs in the urine, the greater part in combination with the alkaties, and the remainder united with indol, skatol, and pyrokatechin, in the form of cromatic ethersulphonic compounds, the ratio being 1:0.1045. All conditions which favour the formation of indol, skatol, or pyrokatechin, increase the amount of combined sulphuric acid. The total daily amount of sulphuric acid is 2.5 to 3.5 grammes [37 to 5: grains]. It is increased by the administration of sulphur, (Krause). The sulphuric acid is chiefly derived from the decomposition of proteids, and hence its amount runs parallel with the amount of urea excreted. The amount of alkaline sulphates in the food is, as a rule, very small.

An increased excretion of sulphuric acid in fevers indicates an increased metabolism of the tissues of the body. In renal inflammation it has been observed to be diminished, and in eczema it is greatly increased. Feeding with taurin (which contains sulphur), in the case of rabbits, (but not in carnivora or man), increases the sulphuric acid in the urine (Salkowski). According to Ziulzer, a copious secretion of bile lessens the relative amount of sulphuric acid in the urine.

Test.-Barium chloride gives a copious white heavy precipitate of barium sulphate, insoluble in nitric acid.

In addition to sulphuric acid, sulphur (b) occurs in an incompletely oxidised form in the urine (potassium sulphocyanide, cystin, and sulphur-bearing compounds derived from the bile) 
(Kunkel, v. Voit-\$177,6). Hyposulphurous acid, as an alkaline salt, is an abnormal con. stituent in typhus; and so is sulphuretted hydrogen, which is recognised by the blackening of a piece of paper moistened with lead acetate and ammonia, held over the urine.

4. Excessively minute traces of silicic acid and nitric acid derived from drinking water have been found in urine. Organic acids, e.g., citric and tartaric, when taken internally, increase the amount of carbonates in the urine. The urine may effervesce on the addition of an acid.

The sodium in the urine is chiefly combined with chlorine, but a small part of it is united with phosphoric and uric acids; potassium (which is about $\frac{1}{3}$ of the sodium) is chiefly combined with chlorine. In fevers, more potash is excreted than soda, and during convalescence, the reverse is the case; calcium and magnesium exist in normal acid urine as chlorides or acid phosphates. If the urine is neutral, neutral calcium phosphate and magnesium phosphate are precipitated. Ebstein found the latter in alkaline urine, as large clear four-sided prisms, in diseases of the stomach. If the urine is alkaline, calcium carbonate (fig. 281) and tribasic calcic phosphate are deposited as such, while the magnesium is precipitated in the form of armmonio-magnesium phosphate, or triple phosphate. The calcium is derived from the food, and depends upon the amount of lime salts absorbed from the intestine. Free ammonia is said to occur $(0.72$ gramme, or 7 grains daily) in perfectly fresh urine (Neubauer, Brïcke), and the amount is greater with an animal than with a vegetable diet (Coranda). The amount of fixed ammonia is increased by the administration of mineral acids (Walter, Schmiedeberg, Gï̈thgens). Iron (1 to 11 milligrammes per litre) is never absent. There is a trace of hydric peroxide (Schonbein), which is detected by its decolorising indigo-solution on the addition of iron sulphate.

Gases. $-24 \cdot 4$ c.c. of gas was obtained from one litre of urine-100 volumes of the gases pumped out consisted of $65 \cdot 40$ vol. $\mathrm{CO}_{2}, 2 \cdot 74 \mathrm{O}, 13 \cdot 86 \mathrm{~N}$. After severe muscular action, the amount of $\mathrm{CO}_{2}$ may be doubled; digestion also increases it, copious drinking diminishes it.

263. FERMENTATIONS OF URINE.-Acid Fermentation.-When perfectly fresh urine is set aside, it gradually becomes more acid from day to day. This is called the "acid fermentation." It seems to be due to the development of special fungi (fig. 260,a), and the process is accompanied by the deposition of uric acid $(c)$, acid sodium urate, in amorphous grains (b), and calcium oxalate $(d)$. According to Scherer, the fungus and the mucus from the bladder decompose part of the urinary pigment into lactic and acetic acids. The latter sets free uric acid from neutral sodium urate, so that free uric acid and sodium urate must be formed. Butyric and formic acids have been found as abnormal decompositionproducts of other urinary constitu- D ents. When the acid fermentation begins, the urine absorbs oxygen

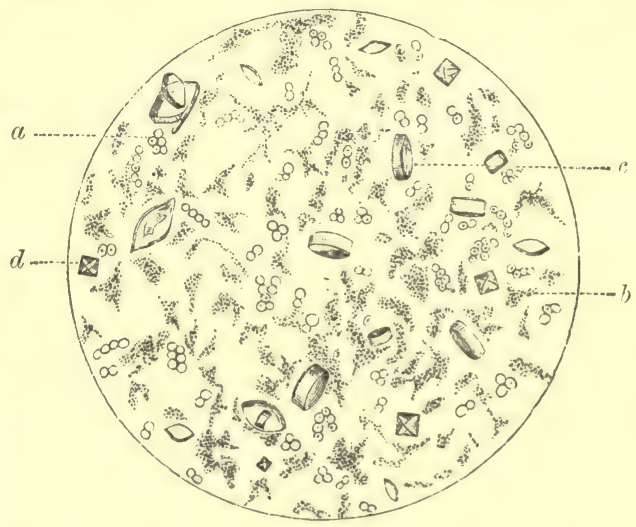

Fig. 260.

Deposit in "acid fermentation" of urine. a, fungus ; $b$, amorphous sodium urate ; $c$, uric acid; $d$, calcium oxalate. (Pasteur). According to Bricke, it is the lactic acid, formed from the minute traces of sugar present in urine, which causes the acidity. According to Röhmann, who recognises the acid fermentation as an exceptional phenomenon, the acids are formed from the decomposition of sugar, and from alcohol which may be present 
accidentally. While the urine is still acid, it becomes turbid and contains nitrous acid, whose source is entirely unknown. According to v. Voit and Hofmann, phos-

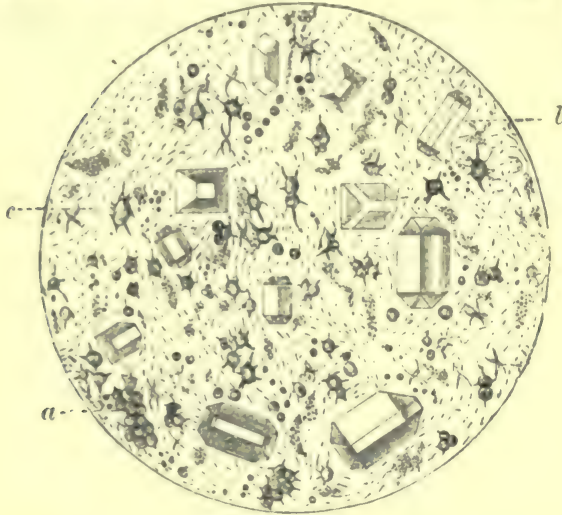

Fig. 261. phoric acid and a basic salt are formed from acid sodium phosphate, whereby part of the uric acid is displaced from sodium urate, thus causing the formation of an acid urate.

Alkaline Fermentation. - When urine is exposed for a still longer time, more especially in a warm place, it becomes neutral and ultimately ammoniacal, i.e., it undergoes the alkaline fermentation (fig. 261).

This condition is accompanied by the formation of the micrococcus ureæ (fig. 262) (Pasteur, Cohn) and Bacterium ureæ (fig. 261), which causes the urea to take up water, and decompose into $\mathrm{CO}_{2}$ and ammonia.

Deposit in ammoniacal urine (alkaline fermentation). $a$, acil ammonium urate ; $b$, ammonio magnesium phosphate; $c$, bacterium ureæ.

Urea $\left[\mathrm{CO}\left(\mathrm{HN}_{2}\right)_{2}+2\left(\mathrm{H}_{2} \mathrm{O}\right)=\right.$ ammonium carbonate $\left[\left(\mathrm{NH}_{4}\right)_{2} \mathrm{CO}_{3}\right]$.

The property of decomposing urea belongs to many different kinds of bacteria, including even the sarcina of the lungs-whose germs seem to be universally diffused in the air. These organisms produce a soluble ferment (Musculus), which, however, only passes from the body of the cells into the fluid after the cell or organism has been killed by alcohol (Lea).

The presence of ammonia causes the urine to become turbid, and those substances which are insoluble in an alkaline urine are precipitated-earthy phosphates, con-

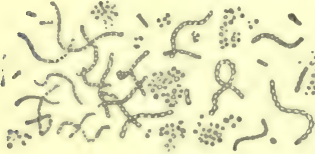

Fig. 262.

Micrococcus nreæ. sisting of the amorphous calcic phosphate, acid ammonium urate (fig. 261, a), in the form of small dark granules covered with spines; and, lastly, the large clear knife-rest or "coffin-lid" form of ammonio-magnesic phosphate, or triple phosphate (fig. 282). [The last substance does not exist as such in normal urine; but it is formed when ammonia is set free by the decomposition of urea, the ammonia uniting with the magnesium phosphate. Its presence therefore always indicates ammoniacal fermentation of the urine.] In cases of catarrh or inflammation of the bladder, this decomposition may take place within the bladder, when the urine always contains pus-cells (fig. 267) and detached epithelium. When much pus is present, the urine contains albumin. Ammoniacal urine forms white fumes of ammonium chloride, when a glass rod dipped in hydrochloric acid is brought near it. [When ammonia is added to normal urine, triple phosphate is precipitated in a feathery form (fig. 283).]

[Significance of Triple Phosphate.--If urine be alkaline when it is passed, and the alkalinity be due to a volatile alkali, i.e., to $\mathrm{NH}_{3}$, then decomposition of the urine has taken place, and this kind of urine is a sure sign that there is disease of the genito-urinary mucous membrane.]

264. ALBUMIN IN URINE OR ALBUMINURIA.-Serum-albumin is the most important abnormal constituent in urine which engages the attention of the physician. It occurs in blood ( $\$ 32)$, and its characters are described in $\S 249$.

Causes of Albuminuria.-1. Serum-albumin may appear in urine withont any apparent anatomical or structural change of the renal tissues. This condition has been called by $\mathbf{v}$. Bamberger "Homatogenous albuminuria." It occurs but rarely, however, and sometimes in healthy individuals when there is an excess of albumin in the blood-plasma (e.g., after suppression of the secretion of milk), and after too free use of albuminous food. 2. As a result of increased blood-pressure in the renal vessels, e.g., after copious drinking. It may be temporary, 
or it may be persistent, as in cases of congestion following heart discase, emphysema, chronic pleuritic effusions, infiltrations of the lungs, and after compression of the chest, causing congestion in the pulmonary circuit, which extends even into the renal veins, \&c. 3. After section or paralysis of the vaso-motor nerves of the kidneys, which causes great congestion of these organs. The albuminuria, which accompanies intense and long-continued abdominal pain, is brought about owing to a reflex paralysis of the renal vessels. 4. After violent muscular exercise. [Senator found that forced marches in young recruits were very frequently followed by the appearance of albumin in the urine, which persisted for several days.] Convulsive disorders, e.g., epilepsy, the spasms of dyspnœea after strychnin poisoning, in shock of the brain, apoplexy, spinal paralysis, and violent emotions; the excessive use of morphia, which perhaps acts on the vaso-motor centres. 5. It may accompany many acute febrile diseases, e.g., the exanthemata (scarlet fever), typhus, pneumonia, and pyæmia. In these cases, it may be due to the increase of temperature paralysing the vessels, but more probably the secretory apparatus of the kidney is so changed (e.g., cloudy swelling of the renal epithelium) that the albumin can pass through the renal membrane. 6. Certain degenerations and inflammations of the kidneys at several of their stages. 7. Inflammation or suppuration in the ureter or urinary passages. 8. Certain chemical substances which irritate the renal parenchyma, e.g., cantharides, carbolic acid. 9. The complete withdrawal of common salt from the food. The albumin disappears when the common salt is given again. 10. The epithelium may be in such a condition that it cannot retain the albumin within the vessels, due to imperfect nourishment and functional weak. ness of the excretory elements. This includes the albuminuria of ischrmia, and that after hæmorrhage, in anæmia, scorbutus, icterus, diabetes. [Grainger Stewart finds that albuminuria is more common among presumably healthy people than was formerly supposed.]

[Besides being derived from the secreting parenchyma of the kidney, albumin may be present owing to admixture with the secretions from any part of the urinary tract, including the vagina and uterus in the female. In some cases the transudation of albumin is favoured by changes in the capillary walls, the albumin being forced through by the intravascular pressure. Sometimes albuminuria occurs during the course of severe typhoid fever, and in acute fevers generally, where the temperature is persistently above $40^{\circ} \mathrm{C}$. (104 ${ }^{\circ} \mathrm{F}$.). The high temperature alters the filtering membrane and permits the filtration of albumin.]

[Physiological Albuminuria. - This term has been applied to that condition of the urine, where traces of albumin are found in individuals apparently in perfect health. Johnson and Pavy cite such cases, while Posner asserts that all urine--even healthy urine-contains traces of proteids, whose presence is ascertained after concentrating the urine. It is safe to assume that normal urine should give no reaction with the usual tests for albumin. Posner precipitated the urine with alcohol, washed the precipitate, dissolved it in acetic acid, and tested it with the ferrocyanide test for albumin. He finds that minute traces of proteid are detected by the following modification of the biuret test:-Make the urine alkaline, and by the "contact method" bring a layer of very dilute cupric sulphate over it; when the two fluids touch, a reddish-violet ring is obtained.]

The tests for albumin in urine depend upon the facts that it is coagulated by heat in neutral or acid solutions, and it is precipitated by various reagents.

[(1) Heller's Test.-Place 10 c.c. of the urine in a test-glass, and pour in pure colourless $\mathrm{HNO}_{3}$ so as to run down the side of the glass, forming a layer beneath the urine. A white zone of coagulated albumin indicates the presence of albumin. In this test it is important to wait a certain time for the development of the reaction. In urines of high specific gravity, a haziness due to acid urates may be formed above where the two fluids meet, but its upper edge is not circumscribed. The acid decomposes the neutral urates and forms a more insoluble acid salt. This cloud of acid urates is readily dissolved by heat, while the albumin is not; the latter is always a sharply defined zone between the two fluids. In very concentrated urine (rare), nitric acid may gradually precipitate crystalline urea nitrate. In patients taking copaiba, nitric acid, by acting on the resin, causes a slight milkiness.]

(2) Boiling and Nitric Acid.-Place 10 c.c. of urine in a test-tube and boil. If albumin be present in small quantity, a faint haziness, which may be detected in a proper light, will be produced. Add 10 to 12 drops of $\mathrm{HNO}_{3}$. If the turbility disappears it is due to phosphates, while if any remains it is due to albumin. If albumin be present in large quantity, a copious whitish coagulum is obtained. Precautions.-- (a) In all "cases, if the urine be turbid, filter it before applying any test. (b) How to boil.-Boil the upper strata of the liquid, and take care, if any coagulum be formed, that it does not adhere to the side of the tube, else the tube is liable to break. (c) In performing this test with a neutral solution, note when the precipitate falls, for albumin is precipitated about $70^{\circ} \mathrm{C}$, phosphates not till about the boiling point. (d) Amount of Acid. - If too little (2 or 3 drops) $\mathrm{HNO}_{3}$ be added, or too much (30 or 40 drops), we may fail to detect albumin, although it is present.]

(3) Ferrocyanide Test. - By the addition of acetic acid and potassium ferrocyanide. [If albumin be present, a white flocculent precipitate separates in the cold. Dr Pavy has introduced pellets, consisting of a mixture of citric acid and sodic ferrocyanide. All that is required 
is to add a pellet to the suspected urine. Oliver's papers. - Dr Oliver uses papers, one saturated with citric acid and another with ferrocyunide of potassium. The two papers are added to the clear filtered urine. Other precipitants of albumin, such as small pieces of paper impregnated with potassio-mercuric jodide, are used by Oliver.]

(4) Boiling Acid Urine. - If the urine be alkaline, although albumin may be present, it is not preeipitated by heat alone. We require to add acetic acid until a slightly acid reaction is obtained. Boiling may give a precipitate of earthy phosphates in an alkaline urine, owing jerhaps to the $\mathrm{CO}_{2}$ being driven off. This precipitate might be mistaken for albumin, but on adling acetic or nitric acid, the earthy precipitate is dissolved, while the precipitate of albumin is not dissolved. In testing for albumin, always use clear urine. If it is turbid, filter it.

[(5) Metaphosphoric acid is clissolved in water just before it is to be used and added to clear urine (Himlenleny). Graham pointed out that metaphosphoric acid precipitated albumin. A 20 per cent. solution of the ordinary glacial phosphoric acid is a good test for albumin, but it also precipitates peptones. It, however, changes into ordinary phosphoric acid by keeping, and then it no longer precipitates albumin.]

[(6) Sodic Sulphate and Acetic Acid.-Acidulate 10 c.c. of urine with acetic acid, and add $\frac{1}{6}$ of its volume of a concentrated solution of sulphate of soda or magnesia. On heating, if albumin be present, $\mathrm{a}$ distinct cloudiness is obtained.]

[( 7 ) In picric acid, according to Dr Johnson, we have a more delicate test for minute traces of allumin than either heat or nitric acid, or than both these tests combined. It is used either in the form of crystals or powder, or as a saturated arpueous solution. Take a four-inch column

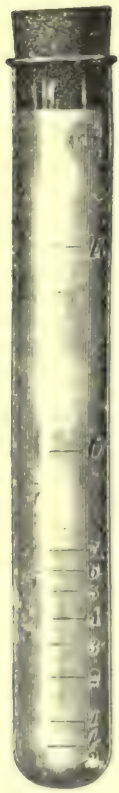

Fig. 263.

Esbach's

albumiof urine in a test-tube, hold the tube in a slanting direction, and pour an inch of the picric acid solution on the surface of the urine, where in consequence of its low specific gravity (1005) it mixes only with the upper layer of the urine. It coagulates any albumin present. The precipitate occurs at once, and is increased by heat, while the urate of soda, which is sometimes precipitated, is soluble on leating.]

[Dr Roberts regards any test for albumin which requires strong acidulation with an organic acid, citric, acetic, or lactic, as unsatisfactory, since it precipitates mucin. For this reason he rejects the tungstate, mercuric iodide, and potassic ferrocyanide tests. Dr Roberts regards the heat test, with the addition of a small definite (juantity of acetic acid, as the best test for the detection of small quantities of albumin.]

1. Quantitative Estimation of albumin. - 100 c.c. of urne are boiled in a capsule, some acetic acid being ultimately added, whereby the albumin is precipitated in flakes. The precipitate is collected on a weighed, dried $\left(110^{\circ}\right)$, ash-free filter, and repreatenly washed with hot water, then with alcohol, and dried in an air-bath at $110^{\circ}$. The weight of the filter is deducted, and finally the dried filter with the albumin is lumed in a weighed platinum capsule, and the weight of the ash also deducted. [This method is not available for the busy practitioner on account of the time it takes. Practically, it is sufficient to compare from day to day the froportion that the precipitated albumin bears to the bulk of the urine tested. A graduated tube may be used, so that after the precipitate has subsided, the physician may see what proportion of the whole the precipitate occupies.]

Esbach's Albumimeter (fig. 263). - A glass cylinder is filled with the urine up to the mark $U$, and to $R$ with the precipitant ( 20 citric acid, 10 picric acid, 970 water). The vessel is corked and then shaken. After twenty-four hours the coagulated albumin subsides, when the graduation on the tube indicates the number of grms. of albumin per 1000 c.c. of urine. Very albuminous urine must be previously diluted.

2. Globulin occurs only in albuminous urine, and is frequently present. Its presence is ascertained by adding powdered magnesium sulphate in excess to the urine; when it is present it is precipitated $(\$ 32)$. The more globulin there is in the presence of albumin, the more difficult it is to precipitate it. [Sometimes, when an albuminous urine is dropper into a large cylinder of water, each drop as it sinks is follower by a milky train, and when a sufficient number of drops has been added, the water becomes opalescent, the oplescence disappearing on adding an acid. The globulin is kept in solution by cominon salt and other neutral salts, but when these are largely ililuted, the globulin is precipitated (Roberts).]

3. Peptone occurs in some specimens of albuninous urine, but also in non-albuminous urine. Maixner found it constantly in the urine in all cases where suppuration is present, and even in phthisis, constituting pyogenic peptonuria. Peptone occurs in pus, and the peptonuria in these cases is a sign of the breaking up of the pus-cells (Hofmeister). Also when many leucocytes are broken up in the blood (hømatogenic). It occurs in cases where there is great disintegration of albuminous tissues, e.g., in cancer. It is frequently found after child-birth. Ammonium sulphate precipitates all proteids except peptones (p. 249). 
Test. - Separate the albumin by boiling and the addition of acetic acid. Treat the filtrate with three volumes of alcohol; this precipitates the peptone, which, when dissolved in water, gives the characteristic reactions for peptone $(\$ 166, \mathrm{I}$.).

4. Propeptone, or Hemialbumose, occurs very rarely, e.g., in osteomalacia and intestinal tuberculosis (Bence Jones). The urine is treated to saturation with $\mathrm{NaCl}$ and a large quantity of acetic acid added, and filtered while hot, to separate the albumin and globulin. In the cold filtrate propeptone forms a turbidity, which is redissolved by heat. The precipitate thrown down by $\mathrm{HCl}$ and $\mathrm{HNO}_{3}$ is soluble by heat (Kühne). The precipitate is isolated by filtration, and dissolved in a little warm water, when it gives with $\mathrm{HNO}_{3}$ a yellow reaction; like peptone the solution gives the biuret-reaction ( $p .248$ ).

5. Egg-albumin appears in the urine when much egg-albumin is taken in the food, and also when it is injected into the blood-vessels (\$ 192, 4). According to Semmola, the albumin present in the urine in Bright's disease has undergone a molecular change (similar to eggalbumin), and hence it is excreted.

6. Mucus is present in large amount, especially in catarrh of the bladder. It contains numerous mucus corpuscles, which are scarcely distinguishable from pus corpuscles. They contain albumin, so that urine containing much mucus is albuminous; mucin is not precipitated by heat, but acetic acid gives a flocculent precipitate in clear urine. [Minute traces of mucin nccur normally in urine. If clear normal urine be set aside for a short time, a floceulent haziness, like a cloud of cotton wool, is seen floating in the urine. This is mucus entangling a few epithelial cells from the genito-urinary tract. Mucin Reaction.-According to W. Roberts, the addition of a concentrated solution of citric acid to urine, as in Heller's test $(\$ 264, a)$, where the two fluids meet, causes an opalescent zone gradually to be formed above the layer of acid.]

265. BLOOD IN URINE (HAMATURIA)--HAMOGLOBINURIA.-I. Source of the Blood.--(1) In hæmaturia, the blood may come from any part of the urinary apparatus. 1. In hæmorrhage from the kidney, the amount of blood is usually small and well mixed with the urine. The presence of "blood-cylinders," long microscopic blood coagula, casts of the uriniferous tubules, washed out of them by the urine, is characteristic when they are found in the urine (fig. 275). The urine usually has a smoky appearance. [The urine slowly dissolves out the colouring matter, the stroma of the corpuscles after a time being deposited as a brownish sediment. The smoky hue occurs only in acid urine; if the urine becomes alkaline, the hue becomes brighter red.] The blood-corpuscles show peculiar changes of form, [they become crenated] (fig. 264), and exhibit evidence of division, due to the action of urea on them (\$5). Large coagula are never found in urine mixed with blood derived from the kidney. 2. In hæmorrhage from the ureter, we occasionally find worm-like masses of clotted blood, casts of the canal of the ureter. 3. The relatively largest coagula occur in hæmorrhage from the bladder. In all cases where blood is present, we must examine microscopically for the blood-corpuscles, and it may be for coagula of fibrin. In acid urine, blood-corpuscles, but never arranged in rouleaux, may be found after two to three days. The blood-corpuseles settle as a red sediment at the bottom. If the hæmorrhage is copious many retain their original shape, but if the urine is very concentrated, they may become crenated.

When there is a small and slow hremorrhage from ruptured small capillaries, the red bloodcorpuscles are of unequal size, many $\frac{1}{2}$ to $\frac{1}{3}$ the size of normal, while the pigment has become brownish-yellow (fig. 265).

If a hæmorrhage of this kind be accompanied by catarrhal inflammation of the bladder, there is found between the red, numerous shrivelled leucocytes (fig. 265), which in freshly passed urine often exhibit lively amoboid movements. If the urine be alkaline, as it usually is, crystals of triple phosphate also occur.

If the remains of the red blood-corpuscles become very pale, their presence may be frequently ascertained by adding iodine in a solution of KI (fig. 265). Blood is constantly present in the urine during menstruation.

II. Hæmoglobinuria is quite distinct from hæmaturia. It depends upon the excretion of hæmoglobin as such through the kidneys, and it is produced when hæmoglobin occurs free within the blood-vessels, as in cases where the coloured blood-corpuscles have been dissolved inside the blood-vessels (hæmocytolysis). It occurs when foreign blood is transfused, e.g., when lamb's blood is transfused into man. The foreign blood-corpuscles are dissolved in the blood of the recipient, and the hæmoglobin appears in the urine (\$102). In addition, microscopic 
"cylinders," or "casts," consisting of a globulin-like body tinged yellow with hæmoglobin, may likewise be found in the urine. It also occurs in cases of severe burns $(\$ 10,3)$; after decomposition of the blood in pyæmia, scorbutus, purpura, severe typhus, after respiring arseniuretted hydrogen, and after the passage of azobenzol, naphtol, pyrogallic acid, potassic chlorate, chloral, phosphorus, or carbolic acid into the circulation. [The injection of laky blood, water ether; glycerin (Adams), or toluylendiamin (Afanassiew), also causes it, and in such

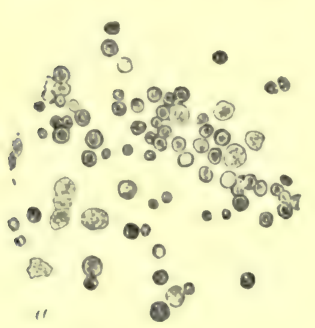

Fig. 266.

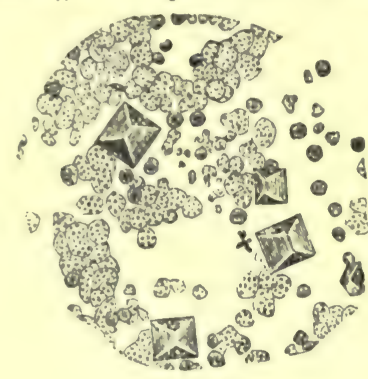

Fig. 267 . cases Afanassiew asserts that the $\mathrm{Hb}$ passes out through the glomeruli, while brown degeneration-products of the red blood-corpuscles, which are dissolved by these agents, were found in the convoluted tubules.]

- These substances dissolve the red blood-corpuscles. Sometimes it occurs periodically from causes and conditions as yet but little understood, e.g., the application of cold to the skin.

Tests for Blood in Urine. -1 . The colour of bloody urine shows every tint, from a faint red to a dark Fig. 266-Coloured and (a) colourless blood-corpuscles blackish-brown, according to the of various forms. Fig. 267.-Shrivelled blood-corpuscles amount of blood present. The urine in urine (catarrh of the bladder), with numerous lymph. is often turbid.

corpuscles, and crystals of triple phosphate, $\times 350$.

2. Urine containing blood or bloodpigment contains albumin.

3. Heller's Blood Test. - Aild to urine half its volume of solution of caustic potash, and heat gently. The earthy phosphates are precipitated, and they carry the hænatin with them, falling as garnet-red flocculi. [This is not a reliable test.]

4. Hæmin Test. - The coloured earthy phosphates may be collected on a filter, and from them hremin may be prepared as directed in $\$ 19$.

5. Almen's Test.-Add to urine, freshly prepared tincture of guaiacum and ozonised ether ; a blue colour indicates the presence of blood (\$37).

6. Spectroscope (see $§ 14$ ). Fig. 268 shows the arrangement of the apparatus. The urine

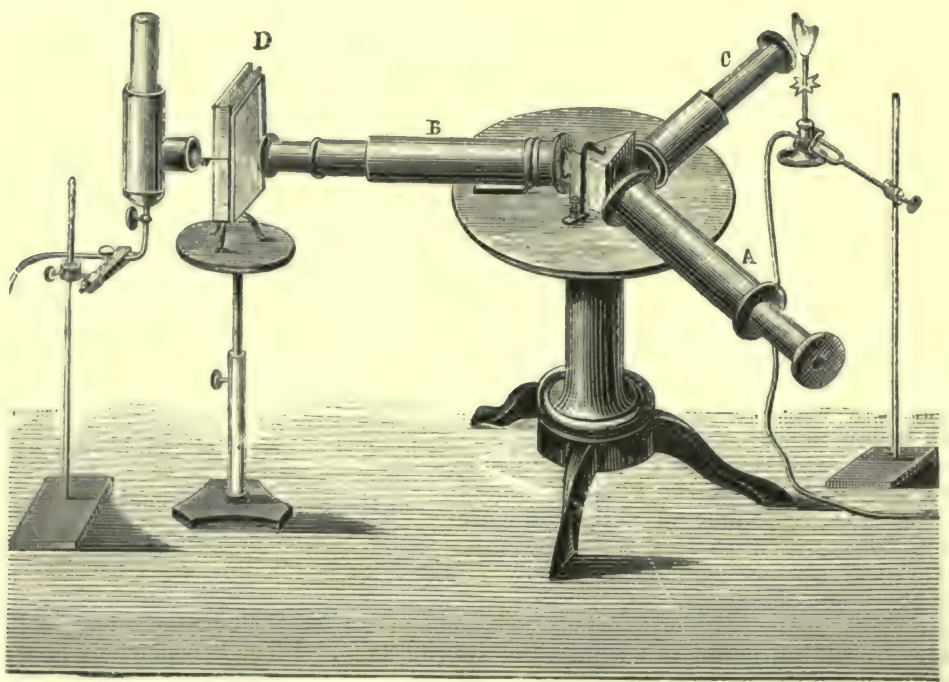

Fig. 268.

Spectroscope for investigating the presence of hæmıglobin in urine.

is placed in a glass vessel, $\mathrm{D}$, with parallel sides, 1 centimetre apart (hæmatinometer). Light from a lamp, $\mathbf{E}$, passes through the fluid. The lamp, $\mathbf{F}$, illuminates the scale which is seen by the observer through the telescope, A. (a) Fresh urine containing blood gives the spectrum 
of oxyhæmoglobin (fig. 17). (b) When bloody urine is exposed for some time, especially in a warm place, it becomes more acid, and assumes a dark brownish-black colour. The hæmoglobin becomes changed into methæmoglobin $(\$ 15)$. It is precipitated by lead acetate, which does not precipitate oxyhæmoglobin; the spectrum of methæmoglobin resembles that of hæmatin in an acid solution (\$15, fig. 17). The spectra may be combined. (c) The microscopic investigation must never be omitted. The shape of the corpuscles may vary considerably (figs. 264 to 266).

266. BILE IN URINE (CHOLURIA). - The physiological conditions which cause the bileconstituents to appear in the urine are mentioned in part at $\$ 180$.

Hæmatogenic, or Anhepatogenic Icterus (Quincke), occurs when bilirubin $(\$ 20)$ is formed from extravasated blood by the action of the connective-tissue corpuscles, so that bile pigments, in addition to colouring the tissues, pass into the urine.

I. Bile Pigments. - Their presence is ascertained by Gmelin-Heintz's test. Green (Biliverdin) is the characteristic hue in the play of colours obtained with this test, which is fully described in $\$ 177$.

Modifications of the Test.-1. If icteric urine be filtered through filtering or blotting paper, a drop of nitric acid containing nitrous acid, when applied to the inner surface of the spreadout filter, gives a yellowish coloured ring (Rosenbach). 2. In order that the reaction may not take place too rapidly, add a concentrated solution of sodic nitrate, and then slowly pour in sulphuric acid (Fleischl). 3. On shaking 50 c.c. of icteric urine with 10 c.c. of chloroform, the bilirubin is dissolved by the latter. On adding bromide water, a beautiful ring of colours is obtained (Maly). If the chloroform extract be treated with ozonised turpentine and dilute caustic potash, a green colour, due to biliverdin, occurs in the watery fluid (Gerhardt).

In slight degrees of jaundice, urobilin alone may be found ( $\$ 261,1)$ (Quincke).

In persistent high fever, the urine contains especially biliprasin (Huppert). If it contains choletelin alone, add to the urine some hydrochloric acid, and examine it with the spectroscope, which gives a pale absorption-band between $b$ and $\mathrm{F}(\S 177,3, f)$.

Hæmatoidin. - Sometimes crystals of hæmatoidin (\$20, fig. 14) appear in the urine, especially when blood-corpuscles are dissolved within the blood-stream; occasionally in scarlet fever and typhus, and sometimes in cases of periodic hæmoglobinuria. The breaking up of old bloodclots in the urinary passages, as in pyonephrosis (Ebstein), or the dissolution of necrotic areas (Hofinann and Ultzmann) produces them, and similar crystals occur in analogous cases in the sputum (\$138). In jaundice due to congestion ( $\$ 180)$, the identical crystalline substance, bilirubin, is found.

II. Bile acids occur in largest amount in absorption jaundice, but they are never present to any extent. The test is described at $\$ 177,2$, the cane-sugar solution consisting of $0.5 \mathrm{grm}$. to 1 litre of water. If the urine be dilute, it is advisable to concentrate it on a water-bath. [It is rare to get a satisfactory result with Pettenkoffer's test in ordinary icteric urine:] V. Pettenkofer's test may be used with the alcoholic extract of the nearly dry residue, but no albumin must be present. Dragendorff found $0.8 \mathrm{grm}$. in 100 litres of normal urine.

Strassburg's Modification. - Dip filter paper into the urine, to which a little cane-sugar has been added; dry the paper and apply to it a drop of sulphuric acid. A violet-red colour is obtained after a short time. [Hay's Reaction (\$ 177).]

267. SUGAR IN URINE (GLYCOSURIA).-Diabetes Mellitus.-The excessively minute trace of grape-sugar or dextrose, which is constantly present in normal urine, sometimes becomes greatly increased and constitutes the conditions of diabetes mellitus and glycosuria. The physiological conditions which determine this result are given at $\$ 175$. In this condition, the quantity of urine is greatly increased, it may reach 10 or more litres. Many pints may be passed daily. [The usual abnormal amount of sugar is from 1 to 8 per cent., although 15 per eent. has been found, i.e., found from 5 to 50 grs. per fluid oz., or 300 to 4000 grs. in twentyfour hours.] The specific gravity is also increased (1030 to 1040). [In a case where a large amount of urine is passed of a pale colour and a specific gravity above 1030, always suspect sugar.] A diabetic person gives off relatively more water by the kidneys and less by the skin (and lungs?) than a healthy person. The colour is very pale yellow, although the amount of pigment is by no means diminished-it is only diluted [the depth of the colour being inversely as the quantity passed]. The amount of the nitrogenbus urinary excreta is increased. The sugar is increased by a diet of carbohydrates and diminished by an albuminous diet. The uric acid and oxalate of lime are often increased at the commencement of the disease, while yeast cells are constantly present after the urine has been exposed to the air for some time.

Sugar has been found occasionally after poisoning with or after the use of morphia, CO, chloral, chloroform, curara; after the injection of ether and amyl nitrite into the blood; and in gout, intermittent fever, cholera, cerebro-spinal meningitis, hepatic cirrhosis, and cardiac and pulmonary affections.

[There is no doubt that normal healthy human urine contains a reducing agent, which reduces cupric oxide to the same extent as if the urine on an average contained 6 grains of glucose in 
every 10 fluid ounces of urine, or 1.34 grms. per litre. As this substance does not cause alcoholic fermentation in its solutions, its identity with glucose appears to be doubtful. The most active rellucing agent is probably kreatinin (G.S. Johnson).]

Tests. - Any of the tests described at $\$ 149$ may be used, but the urine must be free from albumin. The quantitative estimation by fermentation and the titration methods are described in \$ 149. [The tests for grape-sugar described in \$ 149 are (1) Trommer's ; (2) Fehling's ; (3) Moore \& Heller's ; (4) Bittgrer's; (5) Mulder \& Neubauer's; (6) Fermentation test.]

7 . Worm-Muiller recommends the following molification of Fehling's test. Use a 2.5 per cent. solution of cupric sulphate solution, and a nother of 10 parts of sodio-potassic tartarate in 100 parts of 4 per eent. solution of soda. Boil $5 \mathrm{c} . \mathrm{cm}$. of urine in a test-tube, while in a second test-tube is boileal 1 to $3 \mathrm{c} . \mathrm{cm}$. of the copper solution and $2.5 \mathrm{c.cm}$. of the potassio-tartarate solution. The boiling of both fluids is stopped simultaneously, und after 20 to 25 seconds the contents of one test-tube are added to those of the other, but without shaking the mixture, the reduction taking place spontaneously.

8. Nylander's modification of Böttger's test is also good (\$149).

[9. Picric Acid and Potash Test. - Bram showed that grape-sugar, when boiled with picric aciil amil potash, reduces the yellow picrie acid to the deep red picramic acid, the depth of the colour ilepending on the amount of sugar present. Dr Johnson uses this test for detecting the presence of sugar in urine, and also for estimating the amount of sugar present, the depth of the ret colour obtainesl in boiling being compared with a standard dilution of ferric acetate. In doing the test, use 1 drachm of urine, $\frac{1}{2}$ a drachm of liquor potasse, and 10 minims of picric acid solution: make up to 2 drachms with distilled water, and boil the mixture for one minute. This test indicates the presence of 0.6 grain of sugar per fluid ounce of normal urine. Dr Johnson claims for this test that it possesses all the advantages of the other tests, while it is not aflected by uric acid or any other normal ingredient of urine; neither does the presence of albumin interfere with the action of the test as it does with all the forms of copper testing.]

[10. Indigo-carmine Test. $-A$ blue solution of this substance, when boiled with diabetic urine containing sodic carbonate, changes from a blue to a violet, purple, red, yellow, and finally, straw-yellow colour. After cooling and exposure to the air, the various colours are obtained in the reverse order until the mixture becomes blue again. Dr Oliver uses this test in the form of test-papers. One bibulous paper is impregnated with the indigo-carmine and the other with sodic carbonate. Drop one of the test-papers and a sollic carbonate paper into a test-tube containing $1 \frac{1}{2}$ inches of water, heat gently, when a blue solution is obtained. Add the urine slowly, one drop at a time, and boil the mixture, observing any change of colour by holling the tube against a white surface below the level of the eye. Uric acid and urates, which reduce Fehling's solution, do not affect the earmine test, nor does kreatinin, although it reacts with the picric acid test. ]

[Quantitative Estimation. --( ( ) Fermentation Test (\$150). Take 4 oz. (120 c.c.) of the urine ; alli a lump of German yeast, about the size of a walnut, lightly cork the bottle, and place it aside for twenty-four hours in a molerately warm place, e.g., on the mantelpiece. Take the specific. gravity before and after the fermentation. Thus, if the specific gravity be 1038 before and 1013 afterwards, the difference or "density lost" is 25, which gives 25 grs. of sugar per Huill $0 \%$. (lioherts). If it be desirel to get the percentage, multiply the density lost by $0 \cdot 23$, thus $25 \times 0 \cdot 23=5 \cdot 69$ in 100 parts.]

$[(b)$ Volumetric Analysis. -10 c.e. of Fehling's solution $=\cdot 05$ gramme of sugar.

1. Ascertain the quantity of urine passed in twenty-four hours. 2. Filter the urine, and remove any albumin present by boiling and filtration. 3. Dilute 10 c.c. of Fehling's solution with alout twenty times its volume of distilled water, and place it in a white porcelain capsule on a wire gauze support under a burette. (It is diluted because any change of colour is more easily observed.) 4. Take 5 c.c. of the urine, and 95 c.c. of distilled water, and place the diluted urine in a burette. 5. Gradually boil the diluted Fehling's solution, and whilst it is boiling gradually adl the diluted urine from the burette, until all the cuprous oxide is precipitated as a redlish powder, and the supernatent fluid has a straw-yellow colour, not a trace of blue remaining. Read off the number of c.c. of dilute urine employed. Say 36 c.c. were used-that, of cour'se, represents 1.8 c.c. of the original urine. Suppose the patient passes 1550 c.c., as 1.8 c.c. of urine reduced all the cupric oxide in the 10 c.c. of Fehling's solution, it must contain 05 gramme sugar, hence,

$$
1 \cdot 8: 1550:: \cdot 05: \frac{1550 \times \cdot 05}{1 \cdot 8}=237 \cdot 5 \text { grammes of sugar passed in } 24 \text { hours. ] }
$$

[Preparation of Fehling's Solution. - 34.64 grammes of pure crystalline cupric sulphate are powdered and dissolved in 200 c.c. of distilled water; in another vessel dissolve 173 grammes of Rochelle salts in 480 c.c. of pure caustic sola, specific gravity 1.14 . Mix the two solutions, and dilute the deep coloured fluid which results to 1 litre. N.B.-Fehling's ought not to be kept too long; it is apt to decompose, and should therefore be preserved from the light, or protected with oparue paper pasted on the bottle. Some other substances in urine, e.g., urates and uric acid, reduce cupric oxide.] 
(c) According to Worm-Müller, the polarization method is almost valueless for diabetic urine.

[Picro-Saccharimeter.-G. Johnson uses a stoppered bottle 12 inches long and $\frac{3}{4}$ inch wide, graduated in $\frac{1}{10}$ ths and $\frac{1}{10}$ ths (fig. 269). To it is fixed a shorter bottle containing the standard iron-solution for comparison, a standard solution, composed of liquor ferri perchloride $3 \mathrm{j}$, liq. ammon. acetatis $3 \mathrm{iv}$, glacial acetic acid $3 \mathrm{iv}$, liq. ammoniæ $3 \mathrm{i}$, and water to make up 3 iv. All B. P. preparations give a colour identical with a solution containing $1 \mathrm{gr}$. of grape-sugar per oz., reduced by picric acid and afterwards diluted four times, so that this tint $=\frac{1}{4}$ gr. of sugar per oz. After reducing the sugar with the picric acid, pour into the tall tube the dark saccharine liquid produced by boiling to occupy ten divisions of the tube, and add distilled water cautiously until the colour approaches that of the standard; read off the level of the fluid. The amount of sugar present is determined from the amount of water added. In making the test, the picric acid must be added in proportion to the amount of sugar present.]

If large quantities of dextrose are taken in the food, a part of it (and more in diabetic persons) appears in the urine. Lævulose, when taken internally, does not increase the amount of sugar in diabetes. The free use of starch does not cause glycosuria in health, but in diabetes it increases the amount of sugar. A large consumption of cane- or milk-sugar causes the passage of small quantities of both of these sugars into the urine in health, while in diabetes the amount

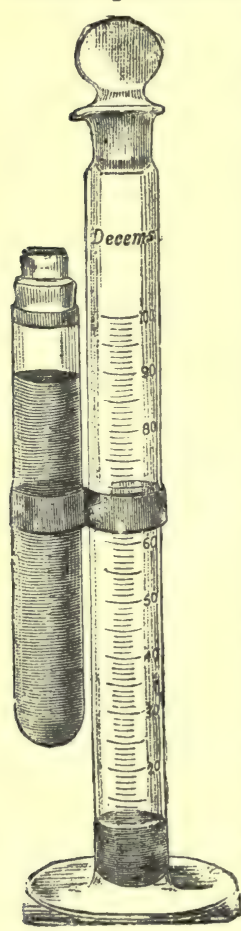

Fig. 269. of dextrose is increased (Worm-Müller). According to Külz, in diabetic persons cane-sugar splits up into grape- and fruit-sugar, the latter being used up in the body, the former partly excreted ; and the same is the case with milk-sugar.

In severe cases of diabetes mellitus, Külz found the left-rotatory $\beta$-oxybutyric acid (the next highest analogue of lactic acid) in the urine, from which acetic acid is formed by oxidation $(\$ 175)$, which in its turn readily yields $\mathrm{CO}_{2}$ and aceton. $\alpha$-crotonic acid is formed in urine by the removal of water from oxybutyric acid in the urine in diabetes (Stadelmann). The administration of aceton causes albuminuria, and this may in part explain in some cases the complication of albuminuria in diabetes (Albertoni and Pisenti).

Aceton, or Aceton-yielding substance, probably aceto-acetic acid, is sometimes found in diabetic urine. It has a peculiar vinous odour, and it has been detected in the urine during fever. Gerhardt described a peculiar substance in diabetic urine, which gave a deep red colour with perchloride of iron. This substance is probably diacetic ether, and he considered it to be the source of aceton; but it is more probably derived from aceto-acetic acid. Tests for Aceton.-(1) Perchloride of iron=Burgundy-red colour; but this is not reliable. (2) Lieben suggested an iodoform test. Dissolve 20 grains of KI in a fluid drachm of liq. potassæ, and boil the fluid. Pour the suspected urine on the surface, when a ring of phosphates is deposited from the urine by the hot alkaline solution. If aceton be present, after a time the deposit becomes yellow, and yellow granules of indoform ap. pear and sink to the bottom of the test-tube. The only other substance which may be met with in the urine giving this reaction is lactic acid.

Milk-sugar is sometimes found in the urine of women who are nursing; when the secretion of Picro-saccharimeter mulk is arrested, absorption takof $\mathrm{G}$. Johnson.

sten, Spiegelberg). Lævulose is sometimes found in diabetic urine (\$252). Dextrin has also been found in diabetic nrine. Fig. 270 .
Inosit, or muscle-sugar ( $\$ 252)$, is sometimes found Inosit crystallised partly from alcohol and in diabetes, in polyuria, and albuminuria. It is found in traces, even in normal urine. Occasionally,

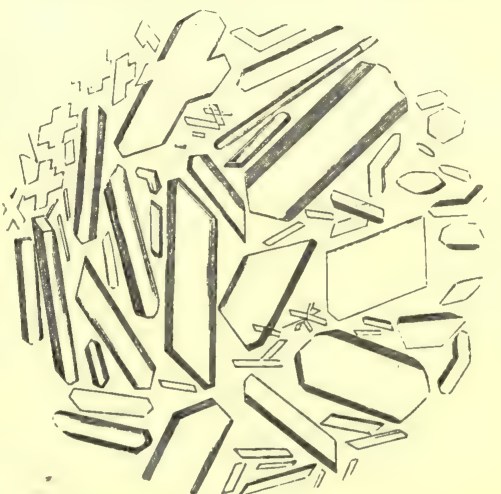

Fig. 270.

after the piqûre in animals ( $\$ 175$ ), inosit, instead of grape-sugar, appears in the urine (fig. 270 ). In testing for inosit, remove the grape-sugar by fermentation, and the albumin by heat after the addition of a few drops of acetic acid and sodic sulphate. Some of the filtrate is evaporated nearly to dryness on a capsule. To the residue add two drops of nercuric nitrate (Liebig's titration fluid for urea), which gives a yellow precipitate. When this coloured residue is spread 
out and carefully heated, a dark red colour, which disappears on cooling, is obtained (Gallois, Külz). [Inosit gives a green when boiled with Fehling's solution.]

268. CYSTIN. - This left-rotatory body $\mathrm{C}_{6} \mathrm{H}_{12} \mathrm{~N}_{2} \mathrm{~S}_{2} \mathrm{O}_{4}$, occurs very seldom in large amount in urine, although it seems to be a constituent of normal urine. It may be in solution or in the form of hexagonal crystals (fig. $271, \mathrm{~A}$ ). It is insoluble in water, alcohol, and ether, but easily soluble in ammonia, from which solution it may be crystallised. According to Baumann and Preusse, there are intermediate products of the metabolism, from which are furnished the materials necessary for the formation of cystin. During normal metabolism these materials undergo further changes, and the sulphur appears oxidised in the urine as sulphuric acid. In rare cases these oxiclations do not take place, and then the sulphur appears in the cystin of the urine (Skadthagen).

269. LEUCIN $=\mathrm{C}_{6} \mathrm{H}_{13} \mathrm{NO}_{2}$. TYROSIN $=\mathrm{C}_{5} \mathrm{H}_{11} \mathrm{NO}_{3}$. - Both bodies occur in the urine in acute yellow atrophy of the liver, and in poisoning by phosphorus. (Their formation during pancreatic digrestion has been referred to in $\$ 170$, II.) As the urea excreted is usually diminished at the same time, it is assumed that, in these diseases, the further oxidation of the

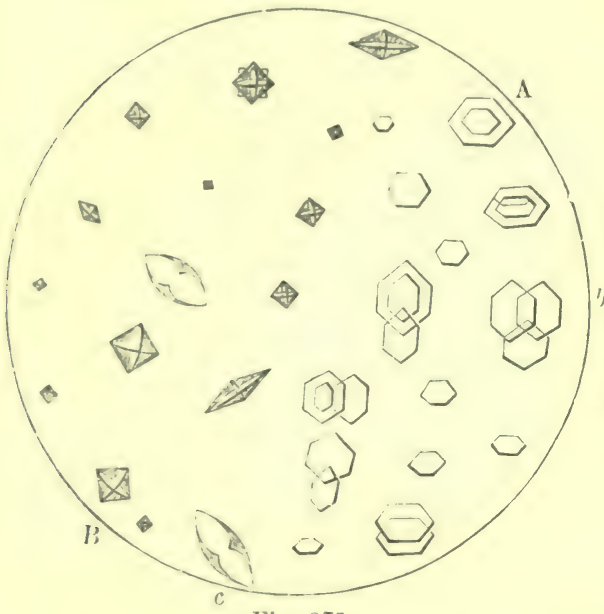

Fig. 271.

$A$, crystals of cystin ; B, oxalate of lime ; $c$, hour-glass forms of $\mathrm{B}$.

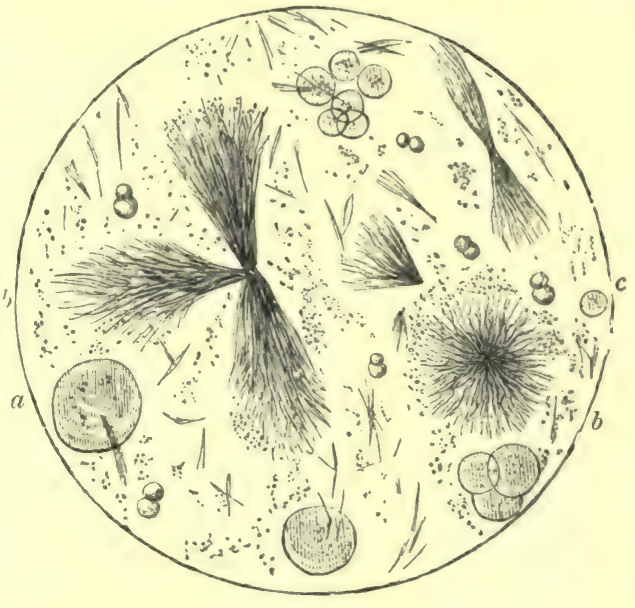

Fig. 272.

$a, a$, leucin balls; $b, b$, tyrosin sheaves; $c$, double balls of ammonium urate.

derivatives of the proteids is interfered with. Leucin, which is either precipitated spontaneonsly or obtained after evaporating an alcoholic extract of the concentrated urine, occurs in the form of yellowish-brown balls (fig. 272,a,a), often with concentric markings, or with fine spines on their surface. When heated, it sublimes without fusing.

Tyrosin forms silky colourless sheaves of needles (fig. $272, b, b$ ). When boiled with mercuric nitrate and nitric acid it gives a red colour, and afterwards a brownish-red precipitate. Piria's Test. - When slightly heated with a few drops of concentrated sulphuric acid, it dissolves with a temporary deep, red colour. On diluting with water, adding barium carbonate until it is nentralised, boiling, filtering, and adding dilute ferric chloride, a violet colour is obtained (Piria, Stiideler).

270. DEPOSITS IN URINE.-Deposits may occur in normal and in pathological urine, and they may be either "organised" or " unorganised."

\section{Organised Deposits.}

A. Blood : red and white blood-corpuscles and sometimes fibrin (figs. 264-266).

B. Pus, in greater or less amount in catarrh or inflammation of the urinary passages. Pus cells exactly resemble colourless blood-corpuscles (figs. 9, 267). Donné's Test.-Pour off the supernatent fluid and add a piece of caustic potash to the deposit; if it be pus it becomes gelatinous, ropy, and more viscid (alkali-albuminate). Mucus, when so acted on, becomes more fluid and mixed with floceuli.

C. Epithelium of various forms occurs, but it is not always possible to say whence it is derived. 
D. Spermatozoa may be present.

E. Lower organisms occur in the urinary passages very seldom, but they may be present, e.g., in the bladder, when germs are introduced from without by means of a dirty catheter. [Before introducing a catheter into the bladder one ought always to make sure that the instrument is perfectly aseptic.] Micrococci are found in the urine in certain diseases, e.g., diphtheria. The following forms are distinguished :-

1. Schizomycetes (\$ 184). Normal human urine contains neither schizomycetes nor their spores. In pathological conditions, however, fungi may pass from the blood into the urinary tubules and thus reach the urine (Leube). During the alkaline fermentation of urine, micrococci, rod-shaped bacteria or bacilli (fig. 273) appear. Sarcinæ belong to the group ( $\$ 186)$.

2. Saccharomycetes (fermentation fungi): $(a)$ The fungus of the acid urine fermentation ( $S$. urinæ) consists of small bladder-like cells arranged either in chains or in groups (figs. 260,a;273, f). (b) Yeast (S. fermentum) occurs in diabetic urine, as oval cells with a dotted eccentrically-placed nucleus (fig. 237).

3. Phytomycetes (moulds) occur in putrid urine (fig. 273,e). They are without clinical significance.

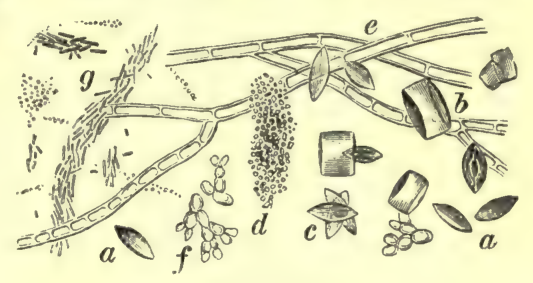

Fig. 273.

Fungi in urine. $e$, mould ; $f$, yeast ; $d, g$, micrococci and bacilli ; $a, b, c$, uric acid.

F. Tube Casts. -The occurrence of tube casts, i.e., casts of the uriniferons tubules (Henle, 1837), is of great importance in the diagnosis of renal diseases. If these structures are relatively thick and straight, they probably come from the collecting tubules, but if they are smaller and twisted, they probably come from the convoluted tubules. There are various forms of tube

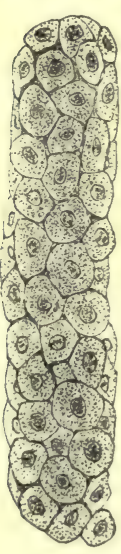

Fig. 274 . casts :-1. Epithelial casts, consisting of the actual cells of the uriniferous tubules. They indicate that there is no very great change going on, but only that, as in catarrhal inflammation of any mucous membrane, the epithelium is in process of desquamation. 2. Hyaline casts (fig. 280) are quite clear and homogeneous, usu-

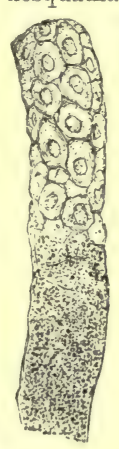
ally long and small; sometimes they are "finely granular," from the presence of fat or other particles. They are best seen after the addition of a solution of iodine. They are probably formed from albumin, which

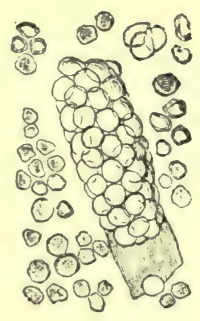

Fig. 275.

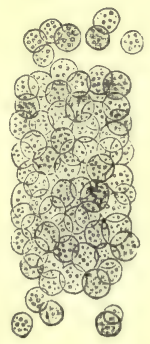

Fig. 276.

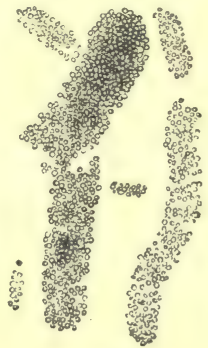

Fig. 277.
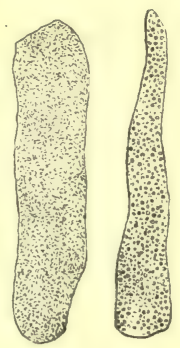

Fig. 278.

Fig. 274.-Epithelial casts. Fig. 275. -Blood cast. Fig. 276. - Leucocyte cast. Fig. 277.Acid sodic urate in cylinders. Fig. 278. - Finely granular cast.

passes into the uriniferous tubules. They are dissolved in alkaline urine, while acid urine favours their formation. They usually occur in the late stages of renal disease, after the tubular epithelium has been shed. 3. Coarsely granular casts (fig. 279) are brownishyellow, opaque, and granular, usually broader than 2. There are various forms. Not unfrequently there are fatty granules, and, it may be, epithelial cells in them. 4. Amyloid casts oceur in amyloid degeneration of the kidneys (fig. 280). They are refractive and completely homogeneous, and give a blue colour (amyloid reaction) with sulphuric acid and iodine. 5. Blood casts occur in capillary hrmorrhage of the kidney, and consist of coagulated blood entangling blood-corpuseles (fig. 275). When tube casts are present, the urine is always albuminous.

Leucocyte casts oecur in suppurating conditions of the urinary tubules (fig. 280). The urates in the form of casts (fig. 277) are without significance.

\section{Unorganised Deposits.}

Some of these are crystalline and others are amorphous, and they have been referred to in treating of the urinary constituents. 
271. SCHEME FOR DETECTING URINARY DEPOSITS. - I. In acid urine there may occur-

1. An amorphous granular deposit:

(a) Which is lissolved by heat and reappears in the cold; the deposit is often reddish in - colour = urates (fig. 260).

(11) Which is not dissolved by heat, but is dissolved by acetic acid, but without efferves. cence $=$ probably tribasic calcic phosphate.

(a) Small bright refractive granules, soluble in ether $=$ fat or oil granules (§ 41), (Lipæmia). Fat vecurs in the urine, especially when the round worm, Filaria sanguinis hominis, is present in the blood; sometimes, along with sugar, in phthisis, poisoning with phosphorus, yellow fever, premia, after long-continued suppuration, and lastly, after the injection of fat or milk into the blood (\$102). It occurs also in fatty degeneratiun of the urinary apraratus, almixture with pus from old abscesses, and after severe injuries to bones. In these cases attention ought to be directed to the presence of cholesterin and lecithin. Tery rarely is the fat present in such amount in the urine as to form a cream on the surface (chyluria).

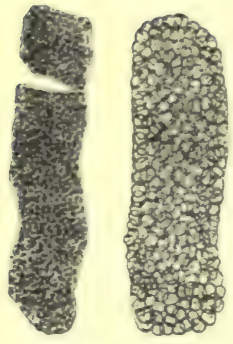

Fig. 279
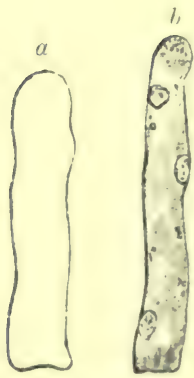

Fig. 280 .

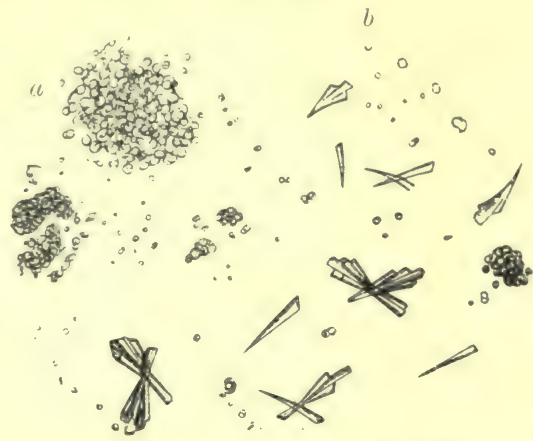

Fig. 281.

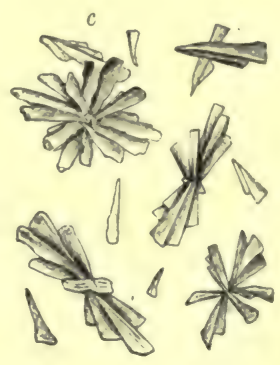

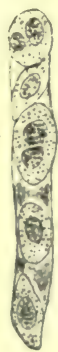

Fig. 279. - Coarsely granular casts. Fig. 280.-Hyaline casts, $a$; $b$, with leucocytes ; $c$, with renal epithelium. Fig 281. - a, Granules of calcic carbonate of lime; $b$, $c$, crystalline neutral calcic phosphate. Fig. 282. - Ammonio-magnesic phosphate. Fig. 283.-Imperfect forms of the same.

2. A crystalline deposit may be-
(a) Uric acid (fig. 256).
(b) Calcium oxalate (fig. 258)-octahedra insoluble in acetic acid.
(c) Cystin (fig. 271).
(d) Leucin and tyrosin-very rare (fig. 272).

II. In alkaline urine there may occur-

1. A completcly amorphous granular deposit, soluble in acids without effervescence= tribasic calcic phosphate. 
2. Sediment crystalline, or with a characteristic form.

(a) Triple phosphate (figs. 282, 283), soluble at once in acids.

(b) Acid ammonium urate-dark yellowish small balls often beset with spines, also amorphous (fig. 284).

(c) Calcium carbonate-small whitish balls or biscuit-shaped bodies. Acids dissolve them with effervescence (fig. 281).

(d) Leucin and tyrosin (fig. 272) - very rare.

(e) Neutral calcic phosphate and long plates of tribasic magnesic phosphate (fig. 285).

Organised deposits may occur both in alkaline and in acid urine; pus-cells are more abundant in alkaline urine, and so are the lower vegetable organisms.

272. URINARY CALCULI.-Urinary concretions may occur in granules the size of sand, or in masses as large as the fist. According to their size they are spoken. of as sand, gravel,

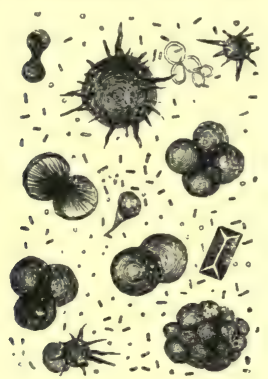

Fig. 284.

Acid ammonium urate. stone, or calculi. They occur in the pelvis of the kidney, ureters, bladder, and sinus prestaticus.

We may classify them as follows (Ultzmann) :-

1. Calculi, whose nucleus consists of the sedimentary deposits that occur in acid urine (primary formation of calculi). They are all formed in the kidney, and pass into the bladder, where they enlarge by the deposition of matter on their surface.

2. Calculi, which are either sedimentary forms from alkaline urine, or whose nucleus consists of a forcign body (secondary formation of calculi). They are formed in the bladder.

The primary formation of calculi begins with free uric acid in the form of sheaves (fig. 256)

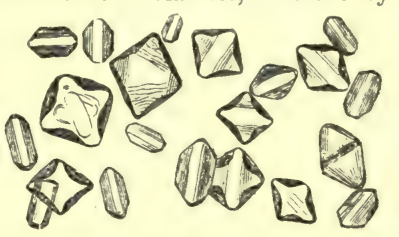

Fig. 285 .

Basic magnesic phosphate.

which form a nucleus, with concentric layers of oxalate of lime. The secondary formation occurs in neutral urine by the deposition of calcic carbonate and crystalline calcic phosphate; in alkaline urine, by the deposition of acid ammonium urate, triple phosphate, and amorphous calcic phosphate.

Chemical Investigation. - Scrape the calculus, burn the scrapings on platinum foil to ascertain if they are burned or not.

I. Combustible concretions can consist only of organic substances.

(a) Apply the murexide test $(\$ 259,2)$, and, if it succeeds, uric acid is present. Uric acid ealculi are very common, often of considerable size, smooth, fairly hard, and yellow to reddishbrown in colour.

(b) If another portion, on being boiled with caustic potash, gives the odour of ammonia (or when the vapour makes damp turmeric paper brown, or if a glass rod dipped in $\mathrm{HCl}$ and held over it gives white fumes of ammonium chloride), the concretion contains ammonium urate. If $b$ gives no result, pure uric acid is present. Calculi of ammonium urate are rare, usually small, of an earthy consistence, i.e., soft and pale yellow or whitish in colour.

(c) If the xanthin reaction succeeds ( $\$ 260)$, this substance is present (rare). Indigo has been found on one occasion in a calculus (Ord).

(d) If, after solution in ammonia, hexagonal plates (fig. 271, A) are found, cystin is present.

(e) Concretions of coagulated blood or fibrin, without any crystals, are rare. When burned they give the odour of singed hair. They are insoluble in water, alcohol, and ether; but are soluble in caustic potash, and are precipitated therefrom by acids.

$(f)$ Urostealith is applied to a caoutchouc-like soft elastic substance, and is very rare. When dry it is brittle and hard, brown or black. When warm it softens, and if more heat be applied it melts. It is soluble in ether, and the residue after evaporation becomes violet on being heated. It is soluble in warm caustic potash, with the formation of a soap.

II. If the concretions are only partly combustible, thus leaving a residue, they contain organic and inorganic constituents.

(a) Pulverise a part of the stone, boil it in water, and filter while hot. The urates are dissolved. To test if the uric acid is united with soda, potash, lime, or magnesia, the filtrate is evaporated and burned. The ash is investigated with the spectroscope $(\S 14)$, when the characteristic bands of sodium or potash are observed. Magnesic urate and calcic urate are changed into carbonate by burning. To separate ther, dissolve the ash in dilute hydrochloric acid, and filter. The filtrate is neutralised with ammonia, and again redissolved by a few drops of acetic acid. The addition of ammonium oxalate precipitates calcic oxalate. Filter, and add to the filtrate sodic phosphate and ammonia, when the magnesia is precipitated as ammonio-magnesic phosphate.

(b) Calcic oxalate (especially in children, either as small smooth pale stones, or in dark, 
warty, hard "mulberry calculi") is not affected by acetic acid, is dissolved by mineral acids without effervescence, and again precipitated by anmonia. Heated on platinum foil it chars and blackens, then it becomes white, owing to the formation of calcic carbonate, which effervesces on the addition of an acid.

(c) Calcic carbonate (chiefly in whitish-grey, earthy, chalk-like calculi, somewhat rare) dissolves with effervescence in hydrochloric acid. When burned it first becomes black, owing to admixture with mucus, and then white.

(d) Ammonio-magnesic phosphate and basic calcic phosphate usually occur together in soft, white, earthy stones, which occusionally are very large. These stones show that the urine has heen ammoniacal for a very long time. The first substance when heated gives the odour of ammonia, which is more distinct when heated with caustic potash; is soluble in acetic acid without effervescence, and is again precipitated in a crystalline form from this solution on the addition of ammonia. When heated it fuses into a white enamel-like mass : [hence, it is called "fusible calculus"]. Basic calcic phosphate does not effervesce with acids. The solution in hydrochloric acid is precipitated by ammonia. When ammonium oxalate is added to the acetic acid solution, it yields calcic oxalate.

(c) Neutral calcic phosphate is rare in calculi, while it is frequent in the form of gravel. l'hysically and chemically, these concretions resemble the earthy phosphates, only they do not contain magnesia.

\section{THE SECRETION OF URINE.-[The functions of the kidney are-}

1. To excrete waste products, chiefly nitrogenous bodies and salts;

2. To excrete water ;

3. And perhaps also to reabsorb water from the uriniferous tubules, after it has washed out the waste products from the renal epithelium.

The chief parts of the organs concerned in 1, are the epithelial cells of the convoluted tubules; the glomeruli permit water and some solids to pass through them, while the constrictions of the tubules may prevent the too rapid outflow of water, and thus enable part of it to be reabsorbed.]

Theories. - The two chief older theories regarding the secretion of urine are the following :-1. According to Bowman (184\%), through the glomeruli are filtered only the verter and some of the highly diffusible and soluble salts present in the hood, while the specinic urinary constituents are secreted by the activity of the epithelium of the urinary tubules, and are extracted or removed from the epithelium hy the water flowing along the tubules. This has been called the "vital " theory. 2. C. Ludwig (1844) assumes that very dilute urine is secreted or filtered through the glomerulus. As it passes along the urinary tubules it becomes more concentrated, owing to endosmosis. It gives back some of its water to the blood and lymph of the kidney, thus becoming more concentrated, and assuming its normal character. [This is commonly known as the "mechanical " theory.]

The secretion of urine in the kidneys does not depend upon definite physical forces only. A great number of facts force us to conclude that the vital activity of certain secretory cells plays a foremost part in the process of secretion $(R$. Heidenhain).

The secretion of urine embraces-(1) The water, and (2) the urinary constituents therein dissolved; both together form the urinary secretion. The amount of urine depends chiefly upon the amount of water which is filtered through or secreted by the glomeruli; the amount of solids dissolved in the urine determines its concentration.

(A) The amount of urine, which is secreted chiefly within the Malpighian capsules, depends mimarily upon the blood-pressure in the area of the renal artery, and follows, therefore, the laws of filtration ( $\$ 191$, II.) (Ludwig and Goll). [In this respect the secretion of urine differs markedly from that of saliva, gastric juice, or bile. We may state it more accurately thus, that the amount of urine depends very closely upon the differences of pressure between the blood in the glomeruli and the pressure within the renal tubules. If the ureter be ligatured, the secretion of urine is ultimately arrested, even although the blood-pressure be high. The secretion may also be arrested by ligature of the renal vein; and in some cases of 
cardiac or pulmonary disease the venous congestion thereby produced may bring about the same result.]

Glomerular Epithelium.-The amount of urine secreted does not depend upon the hydrostatic pressure alone, but it seems that the epithelial cells covering the glomerulus also participate actively in the process of secretion. Besides the water, a certain amount of the salts present in the urine is excreted through the glomeruli. The serum-albumin of the bood, however, is prevented from passing through. With regard to the secretory activity of these cells, the quantity of water must also depend upon the amount of the urinary constituents and water present in the blood (R. Heidenhain).

Only when the vitality of the secretory cells is intact, is there independent activity of these secretory cells (Heidenhain). When the renal artery is closed temporarily, their activity is paralysed, so that the kidneys cease to secrete, and even after the compression is removed and the circulation re-established, secretion does not take place for some time (Overbeck).

That the secretion depends in part upon the blood-pressure is proved by the following considerations :-

1. Increase of the total contents of the vascular system, so as to increase the bloodpressure, increases the amount of water which filters through the glomeruli. The injection of water into the blood-vessels, or drinking copious draughts of water, acts partly in this way. If the blood-pressure rises above a certain height, albumin may pass into the urine. The active participation of the cells of the glomeruli is rendered probable by the fact that, after very copious drinking, the blood-pressure is not always raised (Pawlow) ; further, after copious transfusion, the quantity of urine is not increased. Conversely, the loss of water owing to profuse sweating or diarrhœa, copious hæmorrhage, or prolonged thirst, diminishes the secretion of urine.

2. Diminution of the capacity of the vascular system, provided the pressure within the renal area be thereby increased, acts in a similar manner. This may be produced by contraction of the cutaneous vessels, owing to the action of cold, stimulation of the vaso-motor centre, or large vaso-motor nerves, ligature, or compression of large arteries $(\$ 85, e)$, or enveloping the extremities in tight bandages. All these conditions cause an increase in the amount of urine, and of course the opposite conditions bring about a diminution of urine, e.g., the action of heat on the skin causing redness and dilatation of the cutaneous vessels, weakening of the vaso-motor centre, or paralysis of a large number of vaso-motor nerves.

3. Increased action of the heart, whereby the tension and rapidity of the blood in the arteries are increased $(\S 85, c)$, augments the amount of urine; conversely, feeble action of the heart (paralysis of motor cardiac nerves, disease of the cardiac musculature, certain valvular lesions) diminishes the amount. Artificial stimulation of the vagi in animals, so as to slow the action of the heart, and thus diminish the mean blood-pressure from 130 to $100 \mathrm{~mm}$. Hg, causes a diminution in the amount of urine to the extent of one-fifth ( $\mathrm{Goll}, \mathrm{Cl}$. Bernard); when the pressure in the aorta falls to $40 \mathrm{~mm}$. the secretion of urine ceases. [If the medulla oblongata be divided (dog), there is an immediate fall of the general blood-pressure, and although, as a general rule, the secretion of urine is arrested when the pressure falls to 40 to $50 \mathrm{~mm}$. Hg, yet secretion has been observed to takke place with a lower pressure than this.]

4. The amount of urine secreted rises or falls according to the degree of fulness of the renal artery (Ludwig, Max Hermann); even when this artery is moderately constricted in animals, there is a decided diminution in the amount of urine.

Pathological. - In fever the renal vessels are less full and there is consecutive diminution of urine (Mendelson). It is most important, in connection with certain renal diseases, to note that ligature of the reual artery, even when it is obliterated for only two hours, causes necrosis 
of the epithelium of the uriniferous tubules. When the arterial anæmia is kept up for a long time, the whole renal tissue dits (Litten). After long-continued ligation of the renal artery, the epithelium of the glomeruli becomes greatly changed (Ribbert).

5. Most diuretics act in one or other of the above mentioned ways.

[Some iliuretics act hy increasing the general blool-pressure (digitalis and the action of cold on the skin, others may increase the blool-pressure locully within the kidney, and this they may do in sereral ways. The nitrites are sail to paralyse the muscular fibres in the vasa afferentia, and thus raise the blood-pressure within the glomeruli. But some also act on the suretury prithitium, such as urea and caffein. Brunton recommends the combination of diuretirs in appropriate cases, and the diuretics must be chosen according to the end in viewas we wish to renove excess of fluids from the tissues and serous cavities, or as we wish to remove injurious waste products, or merely to dilute the urine.]

[6. The amount of urine also depends upon the composition of the blood. Drinking a large quantity of water, whereby the blood becomes more watery, increases the amount of urine, but this is true only within certain limits. It is not merely the increase of volume of the blood acting mechanically which causes this increase, as we know that large quantities of fluid may be transfused without the general blood-pressure being materially raised thereby.]

[Heidenhain argues, that it is not so much the pressure of the blood in the slomeruli as its velocity, which determines the process of the secretion of water in the kidney. He contends that, while increase of the pressure in the renal artery causes an increased flow of urine, ligature of the renal vein, whereby the pressure in the glomeruli is also increased, arrests the secretion altogether. In both cases the pressure is increased within the glomeruli, and the two cases differ essentially in the velacity of the blood-current through the glomeruli.]

Pressure in the Vas Afferens. - The pressure in each vas afferens must be relatively great, because (1) the double set of capillaries in the kidney offers consillerable resistance, and (2) the lumen of the vas efferens is narrower than that of the vas afferens. Hence, owing to the high blood-pressure in the capillaries of the renal glomeruli, filtration must take place from the blood into the Malpighian capsules. When the vasa afferentia are dilated, the filtration-pressure is increased, while, when they are contracted, the secretion is lessened. When the pressure becomes so diminished as to retard greatly the blood-stream in the renal vein, the secretion of urine begins to be arrested. Occlusion of the renal vein completely. suppresses the secretion (II. Meyer, v. Frerichs). Ludwig concluded from this observation, that the filtration or excretion of fluid could not take place through the renal capillaries jiroper, as, owing to occlusion of the renal vein, the blood-pressure in these capillaries must rise, which ought to lead to increased filtration. Such an experiment joints to the conclusion that the filtration must take place through the rapilluries of the glomeruli. The venous stasis distends the vas efferens, which surings from the centre of the glomerulus, and compresses the capillary loops against the wall of the Malpighian capsule, so that filtration cannot take place through them. It is not decided whether any fluid is given off through the convoluted urinary tubules.

Venous congestion in the kilneys diminishes the quantity of urine and the urea. The $\mathrm{NaCl}$ remains constant, but pathological albumin is increased (Senator and Munk).

Pressure in Ureter.-As the blood-pressure in the renal artery is about 120 to $140 \mathrm{~mm}$. $\mathrm{Hg}$, and the urine in the ureter is moved along by a very slight propelling force, so that a counterpressure of from 10 ( $L i j / l l$ ) to $40 \mathrm{~mm}$. of $\mathrm{Hg}$ is sufficient to arrest its flow, it is clear that the blood-pressure can also act as a vis a tergo to propel the urine through the ureter. The pressure in the ureter is measured by dividing the ureter transversely and inserting a manometer in it.

(B) Secretory Activity of the Renal Epithelium.-The degree of concentration of the urine depends upon the quantity of the dissolved constituents which has passed from the blood into the urine. The secretory cells of the convoluted tubules, by their own proper vital activity, seem to be able to take up, or secrete, 
some at least of these substances from the blood (Bowman, Heidenhain). The watery part of the urine, containing only easily diffusible salts, as it flows along the tubules from the glomeruli, extracts or washes out these substances from the secretory epithelium of the convoluted tubules.

Experiments.-1. Sulphindigotate of soda and sodium urate, when injected into the blood, pass into the urine, and are found within the protoplasm of the cells of the convoluted tubules [only in those parts lined by "rodded" epithelium], but not in the Malpighian capsules (Heidenhain). A little later these substances are found in the lumen of the urinary tubules, from which they are washed out by the watery part of the urine coming from the glomeruli. If, however, two days before the injection of these substances into the blood, the cortical part of the kidney containing the Malpighian capsules be cauterised, [e.g., by nitrate of silver], or sliced off, the blue pigment remains within the convoluted tubules. It cannot be carried onward, as the water which should carry it along has ceased to be secreted, owing to the destruction of the glomeruli. This experiment also goes to show that, through the glomeruli the watery part of the urine is chiefly excreterl, while through the convoluted tubules the specific urinary constituents are excreted. Uric acid, salts, injected into the blood, were observed by Heidenhain to be excreted by the convoluted tubules. Von Wittich had previously observed that in birds, crystals of uric acid were excreted by the epithelium of the convoluted tubules. [The presence of crystals of uric acid in the renal epithelium was observed by Bowman, and used as an argument to support his theory.] Nussbaum, in 1878, stated that urea is secreted by the urinary tubules and not by the glomeruli.

The same is true for the bile-pigments, for the iron salts of the vegetable acids when injected subcutaneously, and for hæmoglobin. After injection of mith into the blood-vessels, numerous fatty granules occur within the epithelium of the urinary tubules (\$ 102).

[Nussbaum's Experiments. - In the frog and newt, the kidney is supplied with blood in a manner different from that obtaining in mammals. The glomeruli are supplied by branches of the renal artery. The tubules are supplied by the renalportal vein. The vein coming from the posterior extremities divides at the upper end of the thigh into two branches, one of which enters the kidney, and breaks up to form a capillary plexus which surrounds the uriniferous tubules, but this plexus is also joined by the efferent vessels of the glomeruli. These two systems are partly independent of each other. After ligaturing the renal artery, Nussbaum asserted that the circulation in the glomeruli was cut off, while ligature of the renalportal vein excluded the functional activity of the tubules. By injecting a substance into the blood, after ligaturing either the artery or renal-portal vein, and observing whether it occurs in the urine, he infers that it is given off either by the glomeruli or the tubules. Sugar, peptones, and egg-albumin rapidly pass through an intact kidney, but if the renal artery be tied they are not excreted. Urea when injected into the circulation is excreted after the artery is tied, so that it is excreted through the tubules, but at the same time it takes with it a considerable quantity of water. Thus, water is excreted in two ways from the kidney, by the glomeruli and also from the venous plexus around the tubules along with the urea. Indigo-carmine merely passes into the tubular epithelium of the convoluted tubules, but it does not cause a secretion of urine. Albumin passes through the glomeruli, but only after their membranes have been altered in some way, as by clamping the renal artery for a time.]

[Adami's Experiments on the kidney of the frog tend to show that Nussbaum's conclusions are not justified, for Adami found that if the renal arteries in the frog be ligatured, within a few hours a collateral circulation is established, and a certain amount of blood flows through the kidney. He proved this by injecting into the blood, carmine or painter's vermilion, in a state of fine suspension, and after ligature of the renal arteries, he found it in many of the glomeruli, while laky blood similarly injected revealed its presence as menisci of $\mathrm{Hb}$ in the Malpighian corpuscles. Even secretion of some urine may go on after ligature of the renal 
arteries. It is evident, then, that Nussbaum's method is not a reliable one for locating the parts of the kidney through which certain substances are excreted. Adami's experiments also give some support to Heidenhain's view, that the glomerular epithelium "possesses powers of a selective secretory nature," for he finds that in frogs, after ligature of the renal arteries, where, of course, the pressure in the glomeruli is just nearly that in the veins, and in the dog after section of the spinal cord, so that the blood-pressure has fallen below $40 \mathrm{~mm}$. $\mathrm{Hg}$, whereby the secretion of urine is arrested, the injection of laky blood causes $\mathrm{Hb}$ to appear in the capsules, although there is no simultaneous excretion of water.]

Excretion of Pigments. - Only during very copious excretion does the capsule participate. After the introduction of a large amount of sodic sulphindigotate, and when the experiment has lasted for a long time, the epithelium of the capsule becomes blue. In albuminuria, the abnormal excretion of urine takes place first in the urinary tubules, and afterwards in the capsules, $\mathrm{Hb}$ is partly found in the capsules. According to Nussbaum, egg-albumin passes out through the capsule.

2. Even when the secretion of the watery part of the urine is completely arrested, either by ligature of the ureter, or after a very great fall of the blood-pressure in the renal artery, [as after section of the cervical spinal cord], the before-mentioned substances, when injected into the blood, are found in the cells of the convoluted tubules. The injection of urea under these circumstances causes renewed secretion. These facts show that, independently of the filtration pressure, the secretory activity of these cells is still maintained.

The independent vital activity of the secretory cells of the urinary tubules, which as yet we are unable to explain on purely physical grounds, renders it probable that the tubules are not to be compared to an apparatus provided with physical membranes. This is proved by the following experiment: $-\Lambda$ beles caused arterial blood to circulate through freshly excised living kidneys. A pale urine-like fluid dropped from the ureter. On adding some urea or sugar to the blood, the secretion became more concentrated. Thus, the excised living kidney also excretes substances in a more concentrated form than those supplied to it in the diluted blood streaming through it. J. Mfunk obtained similar results in excised kidneys with common salt, nitre, caffein, grape-sugar, glycerin, with increase in the amount of urine secreted. The addition of caffein or theobromin to the perfused blood increases the secretion, exciting the secretory cells to greater activity (v. Schroeder).

Salts and Gases. - The vital activity explains why the serum-albumin of the blood does not pass into the urine, while egg-albumin and dissolved hæmoglobin readily do so. Among the salts which occur in the blood and blood-corpuscles, of course only those in solution can pass into the urine. Those which are united with proteid bodies, or are fixed in the cellular elements, cannot pass out, or at least only after they been split up. Thus, we may explain the difference between the salts of the urine and those of the blood. Similarly, the urine can only contain the absorbed and not the chemically-united gases.

Ligature of the Ureter. - If the secretion be arrested by compression or by ligature of the ureter, the lymph-spaces of the kidney become filled with fluid, which may pass into the blood, so that the organ becomes nedematous, owing to the passage of fluid into its lymph-spaces. The secretion undergoes a change, as first water passes back into the blood, then the sodic chloride, sulphuric, and phosphoric acids diminish, and lastly the urea (C. Ludwig, Max Herrmann). Kreatinin is still present in considerable amount. There is no longer secretion of proper urine (Löbell).

Non-Symmetrical Renal Activity.-It is remarkable that both kidneys do not secrete symmetrically - there is an alternate condition of hyperæmia and secretory activity on opposite sides $(\$ 100)$. One kidney secretes a more watery urine, which at the same time contains more $\mathrm{NaCl}$ and urea. Von Wittich observed that the secretion of uric acid was not uniform in all the urinary tubules of the same bird. Extirpation of one kidney, or disease of one kidney in man, does not seem to diminish the secretion (Rosenstein). The remaining kidney becomes more active and larger.

Reabsorption in the Kidney. - In discussing the secretion of the kidney, we must attach considerable importance to the variations in the calibre of the renal tubules in their course. Perhaps in the narrowing of the descending part of the looped tubule of Henle there may be either a reabsorption of water, so that the urine becomes more concentrated, or there may be absorption even of albumin, which may perhaps pass through the glomeruli in small amount. ['That reabsorption of fluid takes place within the kidney was part of Ludwig's theory, which is practically a process of filtration and reabsorption. Hifner pointed out that the structure of the kidneys of various classes of vertebrates corresponded closely with the requirements for reabsorption of water. The experiments of Ribbert show that the urine actually secreted in the cortex of the kidney is more watery than that secreted normally by the entire organ. He extirpated the medullary portion in rabbits, leaving the cortical part intact, and in this way 
collected the dilute urine from the Malpighian corpuscles before it passed through Henle's loops.]

274. FORMATION OF THE URINARY CONSTITUENTS.-The question

has often been discussed, whether all the urinary constituents are merely excreted through the kidneys, i.e., that they exist preformed in the blood; or whether some of them do not exist preformed in the blood, but are formed within the kidneys, as a result of the activity of the renal epithelium.

Urea is formed Outside the Kidney.-Urea exists preformed in the blood, from which it is separated by the activity of the kidney. This is proved by the following considerations :-

1. The blood contains one part of urea in 3000 to 5000 parts, but the renal vein contains less urea than the blood of the corresponding artery.

2. After extirpation of the kidneys, or nephrotomy, or after ligature of the renal vessels, the amount of urea accumulates in the blood, and increases with the duration of the experiment to $\frac{1}{300}$ to $\frac{1}{400}$. At the same time there is vomiting and diarrhoea, and the fluids so voided contain urea $(\mathrm{Cl}$. Bernard). Animals die in from one to three days after the operation.

3. After ligature of the ureters, the secretion of urine is soon arrested. Urea accumulates in the blood, but not to a greater extent than after nephrotomy. It is possible, however, that the kidneys, like other organs, may form a small amount of urea, due to the metabolism of their own tissues.

[Urea exists in the blood, whence does the blood derive it? It can only obtain it from one or more of several organs-(1) muscle, (2) nervous system, and (3) glands, of which the liver is the most prominent. This is best stated by the method of exclusion.]

[1. That urea is not formed in muscle is shown, among other considerations, by the fact that only a trace of urea occurs in muscle ( $\$ 293)$, and that the amount is not increased by exercise. Blood which has been transfused through a muscle, or the blood after circulating in a muscle during violent exercise, does not contain an increase of urea, nor does the addition of ammonia carbonate to blood circulating through muscle show any increase of urea. Again, muscular exertion does not (as a rule) increase the amount of urea in the urine, as shown by the experiments of Fick and Wislicenus $(\S 294)$, Parkes, and others. The excretion chiefly increased by muscular exertion is the pulmonary $\mathrm{CO}_{2}(\$ 127)$.]

[2. From what we know of the nervous system, it is not formed there. We are therefore forced to consider the evidence as to the liver, as the organ, or, at least, the chief organ in which it is formed. This evidence is in some respects contradictory, but it is partly experimental and partly clinical. Although Hoppe-Seyler denies the existence of urea in the liver, its existence there was proved by Gscheidlen; and Cyon, on passing blood through an exeised liver by the "perfusion" method of Ludwig, found that blood, after being passed several times through the organ, contained an increased amount of urea. The objection to these experiments is that Cyon's method of estimating the urea was unreliable. But von Schroeder, using a similar method, finds that if blood be perfused through the liver of a dog in full digestion, there is a great increase in the amount of urea, while there is none in the liver of a fasting dog. If ammonia carbonate be added to the blood, there is a very much greater amount of urea in the blood of the hepatic vein. This last fact is confirmed by Salomon. The experiments of Minkowski on the liver of the goose $(\$ 386)$ show that, when the liver is excluded from the circulation, lactic acid takes the place of uric acid in this bird. Brouardel further states, that if the region of the liver be so beaten as to cause congestion of that organ, there is an increase of the urea in the urine.]

[The clinical evidence points strongly to the formation of urea in the liver. Parkes pointed ont that in hepatic abscess, during the early congestive stage, the urea in the urine is increased, while it is diminished in the suppurative stage, when the hepatic parenchyma is destroyed. The urea is also diminished in cancer of the liver, phthisis, and some forms of hepatic cirrhosis, while it is increased during hepatic congestion, and specially so in some cases of diabetes mellitus. The most striking fact of all is that, in acute yellow atrophy of the liver, the urea is enormously diminished in the urine, and may even disappear from it while its place is taken by the intermediate products, leucin and tyrosin (v. Frerichs). In poisoning by phosphorus, coincident with the atrophy of the liver, there is a fall in the urea excretion. Noell-Paton finds that some drugs which inerease the quantity of bile in dogs in a state of N-equilibrium ( $\$ 178)$, e.g., sodic salicylate and benzoate, colchicum, mercuric chloride and euonymin also increase the urea in the urine, he therefore concludes "that the formation of urea in the liver bears a very direct relationship to the secretion of bile by that organ."]

As to the antecedents of urea there is the greatest doubt (\$256). 
Uric Acid is formed Outside the Kidneys. - 1. Birds' blood normally contains uric acil (Meissac $)^{\circ}$ ). [The liver of the pigeon contains 6 to 14 times as much uric acid as the blood.] Ligature of the ureters or renal blood-vessels (Puxlinoff), or gradual destruction of the renal secretory parenchyma hy the suheutaneous injection of neutral potassium chromate (Ebstcin), is followed by the deposition of uric acid in the joints and tissues, and it may even form a white incrustation on the serons membranes. The brain remains free $(\boldsymbol{Z}$ aleshy, Oppler $)$. Acid urates of ammonia, solla, anil magnesia are also similarly deposited. Extirpation of a snake's kidneys gives the same result, but to a less degree.

[Minkowsti foum that, after excluding the liver from the circulation, lactic acid took the place of uric acill in the urine (p. 425). Some uric acid still appears in the urine, which cannot fue derivel from the small amount in the hlood, so that, according to v. Schroeder, there are perhaps other foci of formation of uric acill.]

[The latter experiment points to the formation of uric acid in the liver in birds, and this is supposed to be strengthened by the appearance of the deposition of urates in the urine in certain disorders of digestion.] Von Schroeder and Colasanti, however, as the result of their experiments upon snakes, come to the conclusion that there is no special organ concerned in the formation of uric acid.

Hippuric acid is partly formel in the kidney, for the blood of herbivora does not contain a trace of it (Meissner and Shrpurd). In rabbits, perhaps it is formed synthetically, in other tissues as well as in the killney. If blool containing sodic benzoate and glycin be passed through the lulond-vessels of a fresh kidney, hipuuric acid is formed $(\$ 260)$ (Bunge, Schmiedeberg, Kochs). [The other evilence is given in $\$ 260$.]

Kreatinin has intimate relations to kreatin of musele, but where it is formed is not known. If phrmil and pyrokatechin are digested along with fresh renal substance, a compound of sulpleuriu acie! similar to that necurring in urine is formed (\$ 262). The latter substance, however, is also formed hy similarly digesting liver, pancreas, and muscle. It is concluded from these experiments that these substances are formed in the body within the kidneys and the other organs mentioned (Kochs).

Chemistry of the Kidney. - The kidneys contain a very large amount of water. Besides serum-alluumin, glolulin, alhumin soluble in sodium carbonate (Gottucalt), gelatin-yielding sulistances, fat in the epithelium, elastic substance derived from the membrana propria of the tubules, the kilneys contain leucin, xanthin, hypoxanthin, kreatin, taurin, inosit, cystin (the last in no other tissur), but only in very small amount. The occurrence of these substances frints to a lively metaholism in the kilneys, which is also proved by the liberal supply of blood they receive.

Blood-Vessels. - The kidneys receive a very large supply of blood, and during secretion the blood of the renal vein is bright red. [In the dog, the diameter of the renal artery may be diminished to $5 \mathrm{~mm}$. without the amount of blood flowing through the kidney being thereby greatly interfered with. Hence, within wide limits, the amount of blood is independent of the size of the arterial lumen, and is therefore dependent on the blood-pressure in the aorta, and the resistance to the blood-current within and beyond the kidney (Heidenhain).]

The reaction of the killneys is acil, even in those animals whose urine is alkaline. Perhaps this fact is conmected with the retention of the albumin in the vessels.

275. PASSAGE OF VARIOUS SUBSTANCES INTO THE URINE.-1. The following substances jlass unchanged into the urine :-Sulphate, borate, silicate, nitrate, and carbonate of the alkalies; alkaline chlorides, bromicles, iodicles; potassium sulphocyanide and ferrocyanicle ; bile salts, urea, kreatinin ; cumaric, oxalic, camphoric, pyrogallic, and carbolic acids. Many alkuloirls, e.g., morphia, strychnia, curara, quinine, caffein ; pigments, sulphindigotate of soda, carmine, madder, logwood, colouring matter of cranberries, cherries, rhubarb; santonin ; lastly, salts of gold, silver, mercury, antimony, arsenic, bismuth, iron (but not lead), although the greatest part of these is excreted by the bile and the frecs.

2. Inorganic acirls reappear in man and carnivora as neutral salts of ammonia, in herbivora, as nentral salts of the alkalies.

3. Certain sulistances which, when injected in small amount, seem to be decomposed in the blood, prass in lart into the urine, when they occur in such large amount in the blood that they cannot be completely decomposed-sugar, hæmoglobin, egg-albumin, alkaline salts of the vegetable acids, alcohol, chloroform.

4. Many sulistances appear in an oxidised form in the urine-moderate quantities of organic alkaline salts as alkaline carbonates (Wöhler), uric acid in part as allantoin (Salkoveski), sulphicles and sulphites of soda, in part as sodium sulphate, potassium sulphide as potassium sulphate, some oxyduls as oxides, benzol as phenol (Naumyn and Schulzen). 
5. Those bodies which are completely decomposed, as glycerin, resins, give rise to no special derivatives in the urine.

6. Many substances combine and appear as conjugated compounds in the urine, e.g., the origin of the hippuric acid by conjugation ( $\$ 260$ ), the conjugation of sulphonic acid (\$262), and the formation of urea by synthesis from carbamic acid and ammonia (Drechsel) (\$256). After the use of camphor, chloral, or butylchloral, a conjugated compound with glycuronic acid (an acid nearly related to sugar) appears in the urine. Taurin and sarcosin unite with sulphaminic acid. When bromophenol is given, it unites with mercapturic acid, a body nearly related to cystin ( $\$ 268$ ).

7. Tannic acid, $\mathrm{C}_{14} \mathrm{H}_{10} \mathrm{O}_{9}$, takes up $\mathrm{H}_{2} \mathrm{O}$, and is decomposed into two molecules of gallic acid $=2\left(\mathrm{C}_{7} \mathrm{H}_{6} \mathrm{O}_{5}\right)$.

8. The iodates of potash and soda are reduced to iodides; malic acid $\left(\mathrm{C}_{4} \mathrm{H}_{6} \mathrm{O}_{5}\right)$ partly to succinic acid $\left(\mathrm{C}_{4} \mathrm{H}_{6} \mathrm{O}_{4}\right)$; indigo-blue $\left(\mathrm{C}_{16} \mathrm{H}_{10} \mathrm{~N}_{2} \mathrm{O}_{2}\right)$ takes up hydrogen and becomes indigo-white $\left(\mathrm{C}_{16} \mathrm{H}_{12} \mathrm{~N}_{2} \mathrm{O}_{2}\right)$.

9 . Some substances do not pass into the urine at all, e.g., oils, insoluble metallic salts and metals.

276. INFLUENCE OF NERVES AND OTHER CONDITIONS.-At present we are acquainted merely with the influence of the vaso-motor nerves on the filtration of the urine through the renal vessels. Each kidney seems to be supplied with vaso-motor nerves, which spring from both halves of the spinal cord (Nicolaides). As a general rule, dilatation of the branches of the renal artery, chiefly the vasa afferentia, must raise the pressure within the glomeruli, and thus increase the amount of water filtered through them. The more the dilatation is confined to the area of the renal artery alone, the greater is the amount of the urine. [As yet we know the nervous system influences the secretion of urine only in so far as it modifies the pressure and velocity of the blood-current in the kidney. We have no satisfactory evidence of the existence of direct secretory nerves in the kidney.]

1. Renal Plexus and its Centre.-Section of the nerves of the renal plexusthe nerves around the renal artery-generally causes a considerable increase in the secretion of urine, hydruria or polyuria; sometimes, on account of the great rise of the pressure within the glomeruli, albumin passes into the urine, and there may be rupture of the vessels of the glomeruli, leading to the passage of blood into the urine. The nerve-centre for the renal nerves lies in the floor of the fourth ventricle, in front of the origin of the vagus. Injury to this part of the floor of the fourth ventricle, e.g., by puncture (piqûre), may increase the amount of urine (diabetes insipidus), which is sometimes accompanied by the simultaneous appearance of albumin and blood in the urine $(\mathrm{Cl}$. Bernard $)$. Section of the parts which lie directly in the course of these fibres, as they pass from their centre to the kidney, produces the same effects. Close to this centre in the medulla, lies the centre for the vaso-motor nerves of the liver, whose injury causes diabetes mellitus $(\S 175)$. Eckhard found that stimulation of the vermiform process of the cerebellum produced hydruria. In man, stimulation of these parts by tumours or inflammation, drc., produces similar results.

2. Paralysis of Limited Vascular Areas.-If, simultaneously with the paralysis of the nerves of the renal artery, the nerves of a neighbouring large vascular area be paralysed, necessarily the blood-pressure in the renal artery area will not be so high, as more blood flows into the other paralysed province. Under these circumstances, there may be only a temporary, or, indeed, no increase of urine, provided the paralysed area be sufficiently large. There is a moderate increase of urine for several hours after section of the splanchnic nerve. This nerve contains the renal vaso-motor nerves (which, in part, at least, leave the spinal cord at the first dorsal nerve and pass into the sympathetic nerve), but it also contains the vaso-motor nerves for the large area of the intestinal and abdominal viscera. Stimulation of this nerve has the opposite effect $(\mathrm{Cl}$. Bernard, Eckhard). [The polyuria thus produced is not so great as after section of the renal nerves, because the splanchnic supplies such a large vascular area, that much blood accumulates in that area, and also because all the renal nerves do not run in the splanchnics.] 
3. Paralysis of Large Areas. - If, simultaneously with paralysis of the renal nerves, the great majority of the vaso-motor nerves of the body be paralysed [as by section of the medulla oblongata], then, owing to the great dilatation of all these vessels, the blood-pressure falls at once throughout the arterial system. The result of this may be, provided the pressure is sufficiently low, that there is a great decrease, or, it may be, entire cessation of the secretion of urine. The secretion is arrested when the cervical cord is completely divided, down even as far as the seventh cervical vertebra $(E$ cklhard $)$. The polyuria caused by injury to the floor of the fourth ventricle at once disappears when the spinal cord (even down to the twelfth dorsal nerve) is divided.

[4. Other Conditions - As already stated, section of the renal nerves is followed by polyuria, owing to the increased pressure in the glomeruli, but this polyuria may be increased by stimulating the spinal cord below the medulla oblongata, because the contraction of the blond-vessels throughout the body still further raises the blood-pressure within the glomeruli. If, however, the spinal cord be divided below the medalla oblongata - the renal nerve being also divided-the polyuria ceases, because of the fall of the general blood-pressure thereby produced. Division of the spinal cord in the dorsal region also diminishes or arrests the secretion of urine, owing to the fall of the blood-pressure; but animals recover from this operation, the general blood-pressure rises, and with it the secretion of urine. Stimulation of the cord below the medulla arrests the secretion, as it causes contraction of the renal arteries along with the other arteries of the body.]

[Volume of the Kidney-Oncometer.-By means of the plethysmograph (\$101) we can measure the variations in the size of a limb, while by the oncograph (ó $\gamma \kappa$ s, volume) similar variations in the volume of the spleen are measured (\$ 103). Roy and Cohnheim have measured the variations in the volume of the kidney by means of an instrument which consists of two parts, one termed the oncometer or renal plethysmometer, in which the organ is enclosed, while the other part is the registering portion or oncograph. The kidney is enclosed in a kidney-shaped metallic capsule (fig. 286), composed of two halves which move on the hinge, $h$, to

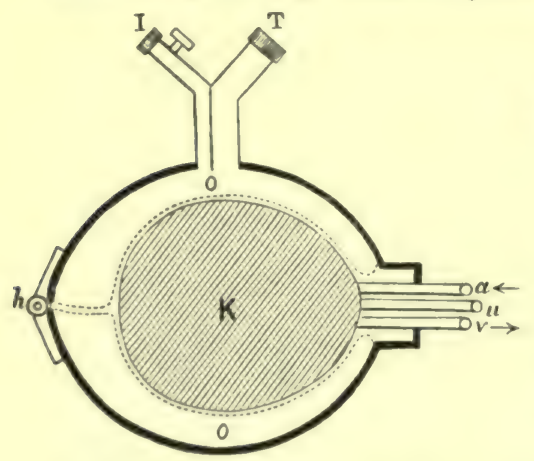

Fig. 286 .

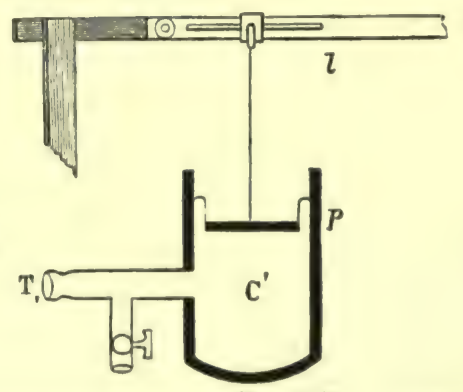

Fig. 287.

Fig. 286.-Oncometer. K, kidney; the thick line is the metallic capsule; $h$, hinge ; $I$, tube for filling apparatus ; $\mathrm{T}$, tube to connect with $\mathrm{T}_{1} ; a, v, u$, artery, vein, ureter (Stirling, after Royj). Fig. 287.-Oncograph. $\mathrm{C}^{\prime}$, chamber filled with oil, communicating by $\mathrm{T}_{1}$ with T ; $p$, piston ; $q$, writing-lever (Stirling, after Roy).

introduce the organ. The renal vessels pass out at $a, v$. The kidney is surrounded with a thin membrane, and between this membrane and the inner surface of the capsule is a space filled with warm oil through the tube, I, which is closed by means of a stop-cock after the space is filled with oil. The tube, $\mathrm{T}$, can be made to communicate with another tube, $\mathrm{T}_{1}$, leading into a metallic chamber, $\mathrm{C}^{\prime}$, of the 
oncograph (fig. 287), which is provided with a movable piston, $p$, attached by a thread to the writing-lever, $l$. Any increase in the size of the organ expels oil from the chamber, $\mathrm{O}$, into $\mathrm{C}^{\prime}$, and thus the piston is raised, while a diminution in the size of the kidney diminishes the fluid in $\mathrm{C}^{\prime}$, and the lever falls. The actual volume of the living kidney depends upon the state of distension of its structural elements, upon the amount of lymph in its lymph-spaces, but chiefly upon the amount of blood in its blood-vessels, and this again must depend upon the condition of the non-striped muscles in the renal arteries. When the vessels dilate, the kidney increases in size, and when they contract it contracts, so that we can register on the same revolving cylinder the variations of the volume at the same time that we record the general arterial blood-pressure.]

[In the normal circulation through the kidney, the kidney curve, i.e., the curve of the volume of the kidney, runs parallel with the blood-pressure curve, and shows the large respiratory undulations, as well as the smaller elevations due to the systole of the heart (fig. 288). Usually, when the blood-pressure falls, the kidney curve sinks, and when the blood-pressure rises the volume of the kidney increases. When the blood-pressure curve is complicated by Traube-Hering waves $(\$ 85)$ the opposite effect is produced on the kidney curve; the highest blood-pressure corresponds to the smallest size of the kidney, and conversely. This is due to the fact that, when these curves occur, all the small arterioles, including those in the kidney, are contracted. A kidney placed in an oncometer secretes urine like a kidney under natural conditions.]

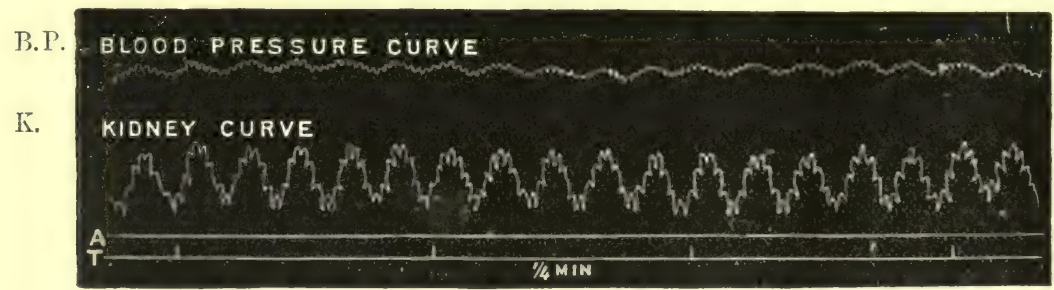

Fig. 288.

B.P., Blood-pressure curve ; K., curve of the volume of the kidney ; T, time curve, intervals indicate a quarter of a minute; A, abscissa (Stirling, after Roy).

[Arrest of the respiration in a curarised animal produces a rapid and great diminution of the volume of the kidney, caused by the venous blood stimulating the vaso-motor centres, and thus contracting the small arterioles, including those of the kidney. This result occurs whether one or both splanchnics are divided, proving that all the vaso-motor nerves of the kidney do not reach it through the splanchnics. When all the renal nerves at the hilum are divided, arrest of the respiration causes dilatation of the organ, which condition runs parallel with the rise of the blood-pressure. Stimulation of a sensory nerve, e.g., the central end of the sciatic nerve, while causing an increase of the blood-pressure, makes the kidney shrink.]

[In poisoning with strychnin, the kidney shrinks while the blood-pressure rises. Stimulation of the central or peripheral end of the splanchnics, divided at the diaphragm, causes contraction of the renal vessels of both sides; the former is a reflex, the latter a direct effect. Stimulation of the peripheral end of one splanchnic sometimes affects both kidneys. Stimulation of the peripheral end of the renal nerves always causes a diminution in the volume of the kidney, so that Cohnhein and Roy inferred that, although there was evidence of the existence of vaso-motor and sensory nerves to the kidney, they found none of vaso-dilators. Each kidney acts independently of the other. Sudden compression of one rental artery has not the slightest effect upon the blood-current of the other kidney. If a kidney be 
exposed in an animal, by making an incision in the lumbar region, on stimulating the medulla oblongata directly with electricity, we may observe the kidney itself becoming paler, the palor appearing in a great many small spots on the surface of the organ, corresponding to the distribution of the interlobular arteries.]

[Cohnheim showed that the composition of the blood has a remarkable effect on the renal circulation. Some substances (water and urea), when injected into the blood, cause the kidney first to shrink and then to expand, while sodic acetate dilates the kidney, even after all the renal nerves are divided-an operation which is very difficult indeed. Provided all the renal nerves be divided, these effects would indicate the existence of some local intra-renal vaso-motor mechanism governing the renal blood-vessels. The general blood-pressure is not thereby modified; nor need we wonder at this, as ligature of one renal artery does not increase the pressure in the aorta.]

[The reciprocal relation between the skin and the kidneys is known to every one. On a cold day, when the skin is pallid, owing to contraction of the cutaneous vessels, the amount of urine secreted is great, and conversely, in summer less urine is passed than in winter. Washing the skin of a dog for two minutes with ice-cold water causes a great contraction of the kidney.]

The perfusion of blood through a living excised kidney is materially influenced by the substances mixed with the blood perfused. This effect may in part be due to the action of these chemical ingredients upon the nuclei of the endothelial lining of the blood-vessels, especially the capillaries, or the effects upon the muscular fibres of the blood-vessels.

[Strvchnin seems to cause contraction of the renal vessels, independently of its action on the general vaso-motor centre. Brunton and Power found that digitalis caused an increase of the blool-pressure (dog), but the secretion of urine was either at the same time diminished, or it eeased altogether. The latter result was due to contraction of the renal blood-vessels, but when the aortic blood-pressure began to fall, the amount of urine secreted rose much above normal, i.e., when the arteries had begun to relax.]

During fever, the renal vessels are probably contracted in consequence of the stimulation of the renal centre by the abnormally warm blood (Mendelson).

The repeated respiration of $\mathrm{CO}$ is said to produce polyuria, perhaps in consequence of paralysis of the renal vaso-motor centre.

Action of the Vagus. - According to $\mathrm{Cl}$. Bernard, stimulation of the vagus at the cardia increases the urinary secretion, while at the same time the blood of the renal vein becomes red. This nerve may contain vaso-dilator nerve-fibres corresponding to the fibres in the facial nerve for the salivary glands ( $\$ 145$ ).

277. UR丨MIA-AMMONIÆMIA. - Symptoms of Uræmia.-After excision of the kidneys, nephrotomy, or ligature of the ureter ; in man, also, as a result of certain diseased conditions of the kidney, leading to the suppression of the secretion of urine, there is developed a series of characteristic symptoms which are followed by death. The condition is called uræmic intoxication or uracmia. There are marked cerebral phenomena, drowsiness, and deep coma, and occasionally local or more general spasins. Sometimes there is delirium; Cheyne-Stokes phenomenon is often observed ( $\$ 111$, II.), and there nay be vomiting and diarrhoea, while in the fluids voided, as well as in the expired air, ammonia may sometimes be detected.

The cause of these phenomena has been ascribed to the retention in the blood of those substances which normally are excreted by the urine, but as yet it has not been definitely ascer. tained which of these substances canses the phenomena:-

1. The first thought is to ascribe them to the retention of the urea. V. Voit found that dogs exhibited uremic symptoms if they were fed for a long time on food containing urea and little water. Meissner found that in nephrotomised animals, the uremic symptoms were hastened by the injection of urea into the blood. The injection of a moderate amount of urea in perfectly healthy animals is not followed by uræmic symptoms, probably because the urea is rapidly excreted by the kidneys ; 1 to 2 grms. [15 to 30 grains] so injected produce comatose symptoms in rabbits.

2. The injection of ammonium carbonate produces symptoms resembling those of uræmia, so that $\mathrm{v}$. Frerichs thought that the urea was decomposed in the blood, yiclding ammonium carbonate-ammoniæmia. Demjankow observed uræmic phenomena after nephrotomy, if at the time he injected urea-ferment into the blood (\$ 263). Feltz and Ritter obtained uræmic symptoms in dogs by injecting salts of ammonia. 
3. As ligature of the ureters produces a comatose condition in those animals which excrete chiefly uric acid in the urine-e.g., birds and snakes (Zalesky) - it is possible that other substances may produce the poisonous symptoms. The injection of kreatinin causes feebleness and contraction of the museles in dogs (Meissner). Bernard, Traube, and more recently Feltz and Ritter ascribe the symptoms to an accumulation of the neutral potassium salts in the blood $(\$ 54)$. The injection of kreatin, succinic acid (Meissner), uric acid, and sodic urate (Ranke), is without effect. Schottin and Oppler ascribe the results to an accumulation of normal or abnormal extractives. It is possible that several substances and their decomposition-products contribute to produce the result, so that there is a combined action of several factors, but perhaps the retention of the potash salts plays the most important part.

The direct application of some urinary substances (kreatinin, kreatin, acid potassic phosphate, urates) to the surface of the cerebrum causes all the symptoms of uræmia. Urea is inactive, and slightly active are ammonium and sodic carbonate, leucin, $\mathrm{NaCl}, \mathrm{KCl}$ (Landois).

[Alkaloids in Urine. - Human urine, and especially febrile urine, when injected under the skin of frogs or rabbits, acts as a poison, and even causes death, by arresting the respiration. The alkaloids seem to be formed by the action of vegetable organisms in the intestine, whence they are absorbed into the blood and pass into the urine (\$116). Urine rendered colourless by charcoal loses half its toxic power, and the poisonous substance is not volatile, and even resists boiling. These alkaloids are increased in the urine in typhoid fever, pneumonia, but not in diabetes.]

Ammoniæmia. - When urine undergoes the alkaline fermentation within the bladder, and ammonium carbonate is formed, the ammonia may be absorbed and produce this condition. The breath and excretions smell strongly of ammonia; the mouth, pharynx, and skin are very dry ; there is vomiting, with diarrhoea or constipation, while ulcers may form in the intestine. The patient rapidly loses flesh, and death occurs without any disturbance of the mental faculties.

Uric Acid Diathesis. - When too much nitrogenous food, too much of any alcoholic fluid is persistently used, and little muscular exercise taken, especially if the respiratory organs are interfered with, uric acid may not unfrequently accumulate in the blood (Garrod). It may be deposited in the joints and their ligaments, especially in the foot and hand, giving rise to painful inflammation, and forming gout-stones or chalk-stones. The heart, liver, and kidneys are rarely affected. The tissues near these deposits undergo necrosis.

278. STRUCTURE AND FUNCTIONS OF THE URETER.-Mucous Membrane.-The pelvis of the kidney and the ureter are lined by a mucous membrane, consisting of connectivetissue, and covered with several layers of stratified "transitional" epithelium (fig. 290). The cells are of various shapes, those of the lowest layer being usually more or less spherical and small, while many of the cells in the upper layers are irregular in shape, often with long processes passing into the deeper layers.

Sub-mucosa.-Under the epithelium there is a layer of adenoid tissue (Hamburger, Chiari), which may contain small lymph-follicles [embedded in loose connectivetissue]. In the pelvis of the kidney and ureter there are a few small mucous glands lined by a single layer of columnar epithelium (Unruh, Egli).

The muscular coat consists of an inner somewhat stronger layer of longitudinal non-striped fibres, and an outer circular layer (fig. 289).

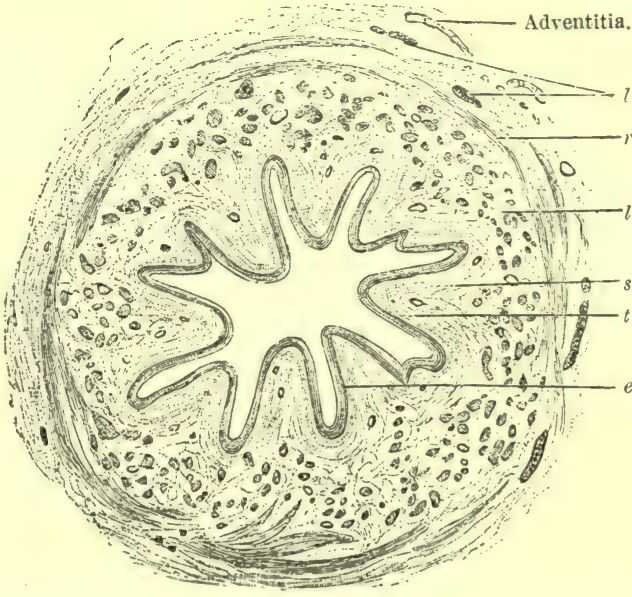

Fig. 289.

In the lowest third of the ureter Transverse section of the lower part of human ureter, $\times 15$. there are in addition a number of $e$, epithelium ; $t$, tunica propria; $s$, sub-mucosa; $l$ and $r$, scattered muscular fibres. All these layers are surrounded and supported by connective-tissue. The outer layers of the connective-tisste form an outer coat or adventitia, which contains the large vessels and nerves. The various coats of the ureter can be followed up to the pelvis of the kidney, and to its calices. The papillæ are covered only by the mucous membrane, while the muscular layer ceases at the apex of the pyramids, where they are disposed circularly, to form a kind of sphincter muscle for each papilla (Henle). 
The blood-vessels supply the various coats, and form a capillary plexus under the epithelium. The nerves are not very numerous, but they contain medullated (few) and non-medullated fibres, with numerous ganglia scattered in their course. They are partly motor and supply the muscular layers, and some pass towards the epithelium, and are sensory and excito-reflex in function. These nerves are excited when a calculus passes along the ureter, and thus give rise to severe pain. The ureter perforates the wall of the bladder obliqucly. The inner opening is a narrow slip in the mucous membrane, directed downiwards and inwards, and provided with a pointed valve-like process (fig. 291).

Movement of the Urine.-The urine is propelled along the ureter thus:-(1) The secretion, which is continually being formed under a high pressure in the

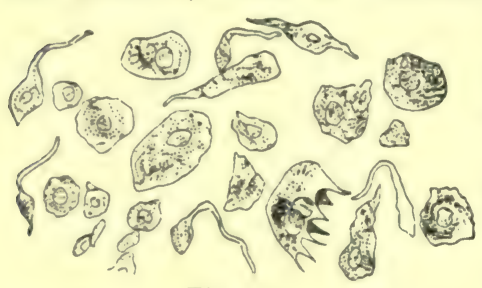

Fig. 290. kidney, propels the urine onwards in front of it, as the urine is under a low pressure in the ureter. (2) Gravity aids the passage of the urine when the person is in the erect posture. (3) The muscles of the ureter contract rhythmically and peristaltically, and so propel it towards the bladder. This movement is reflex, and is due to the presence of the urine in the ureter. Every three-quarters of a minute several drops Transitional epithelium from the bladder. of urine pass into the bladder. But the fibres IIany of the large cells lie upon the may also be excited directly. The contraction summit of the columnar and caudate passes along the tube at the rate of 20 to 30 cells, and depressions are seen on their $\mathrm{mm}$. per second, always from above downwards. under surface.

the urine, the more rapid is the peristaltic movement.

Local Stimulation.-On applying a stimulus to the ureter directly, the contraction passes both upwards and downwarls. Engelmam observed that the movements occur in parts of the

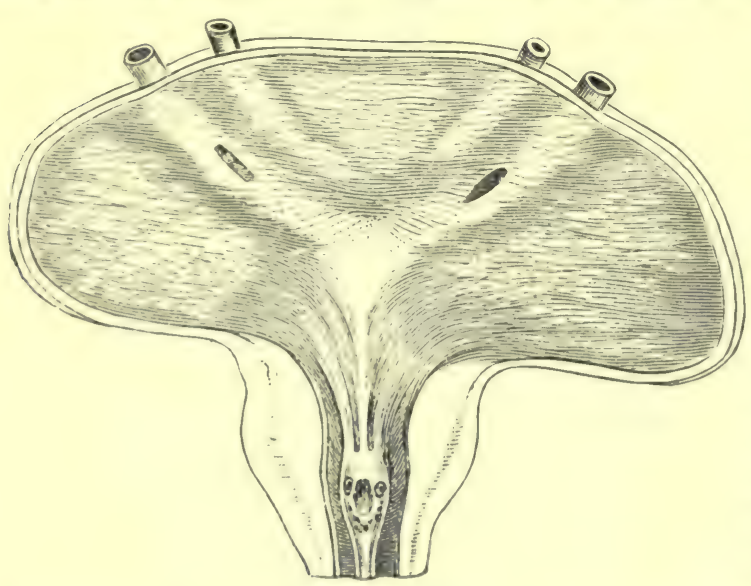

Fig. 291. ureter where neither nerves nor ganglia were to be found, and he concluded that the movement was propagated by "muscular conduction." If this be so, then an impulse may be propagated from one non-striped muscular cell to anotherwithout the intervention of nerves (see Heart, \&58, I., 3).

\section{Prevention of Reflux.} - The urine is prevented from exerting a backward pressure towards the kidneys:-(1) The urine which collects in the pelvis of the kidney is under a high pressure, and thus tends uniformly to compress the pyramids, so that the urine Lower part of the human bladder laid open, showing clear part, cannot pass into the minute
or trigone, the slit-like openings of the ureters, the divided orifices of the urinary tubureters, and vesiculie seminales; the sinus prostaticus, and ules. (2) When there is a on each side of it, the openings of the ejaculatory ducts, and considerable accumulation below both numerous small apertures of the prostate ducts. of urine in a ureter, e.g., from the presence of an impacted calculus or other cause, there is also more energetic peristalsis, and, at the same time, the circular muscular tibres round the apices of pyramids compress the pyramids and prevent the reflux of urine through the collecting tubules. The urine is prevented from passing back from the bladder into the ureter, the wall of the bladder itself, and the part of the ureter which 
passes through it, are compressed, so that the edges of the slit-like opening of the ureter are rendered more tense, and are thus approximated towards each other (fig. 291).

279. URINARY BLADDER AND URETHRA. - Structure. - The mucous membrane of the bladder resembles that of the ureter; the upper layers of the stratified transitional epithelium are flattened. It is obvious that the form of the cells must vary with the state of distention or contraction of the bladder. [The mucous membrane and muscular coats are thicker than in the ureter. There are mucous glands in the mucous membrane, especially near the neck of the bladder.]

Sub-mucous Coat.-There is a layer of delicate fibrillar connective-tissue mixed with elastic fibres between the mucous and muscular layers.

[The serous coat is continuous with, and has the same structure as the peritoneum, and it covers only the posterior and upper half of the organ.]

Musculature. - The non-striped muscular fibres are arranged in bundles in several layers, an external longitudinal layer, best developed on the anterior and posterior surfaces, and an inner circular layer. [Between these two is an oblique layer.] There are other bundles of muscular fibres arranged in different directions. Physiologically, the musculature of the bladder represents a single or common hollow muscle, whose function when it contracts is to diminish uniformly the size of the bladder, and thus to expel its contents (\$306).

The blood-vessels resemble those of the ureter. The nerves form a plexus, and are placed partly in the mucous membrane and partly in the muscular coat, and, like all the extra-renal parts of the urinary apparatus, are provided with ganglia, lying in the mucosa, sub-mucosa, and connected to each other by fibres (Maier). Ganglia occur in the course of the motor nervefibres in the bladder $(W . W o l f f)$. Their functions are motor, sensory, excito-motor, and vasomotor. [Sympathetic nerve-ganglia also exist underneath the serous coat (F.Darwin).]

A too minute dissection of the several layers and bundles of the musculature of the bladder has given rise to erroneous inferences. Thus, we speak of a detrusor urinæ, which, however, consists chiefly of fibres running on the anterior and posterior surfaces, from the vertex to the fundus. There does not seem to be a special sphincter vesicæ internus; it is mercly a thicker circular (6 to $12 \mathrm{~mm}$.) layer of non-striped muscle which surrounds the beginning of the urethra, and which, from its shape, helps to form the funnel-like exit of the bladder. Numerous muscular bundles, connected partly with the longitudinal and partly with the circular fibres of the bladder, exist, especially in the trigone, between the orifice of the ureters.

In the female, the urethra serves merely for the passage of urine. The mucous membrane consists of connective-tissue with many elastic fibres, and provided with papillæ. It is covered by stratified epithelium and contains several mucous glands (Littré). Outside this is a layer of longitudinal, smooth, muscular fibres, and outside this again a layer of circular fibres. Many elastic fibres exist in all the layers, which are traversed by numerous wide venous channels.

The proper sphincter urethræ is a transversely striped muscle subject to the will, and consists of completely circular fibres which extend downwards as far as the miadle of the urethra, and partly of longitudinal fibres, which extend only on the posterior surface towards the base of the bladder, where they become lost between the fibres of the circular layer.

In the male urethra, the epithelium of the prostatic part is the same as that in the bladder ; in the membranous portion it is stratified, and in the cavernous part the simple cylindrical form. The nucous membrane, under the epithelium itself, is beset with papillce, chiefly in the posterior part of the urethra, and contains the mucous glands of Littré.

Non-striped muscle occurs in the prostatic part arranged longitudinally, chiefly at the colliculus seminalis ; in the membranous portion the direction of the fibres is chiefly circular, with a few longitudinal fibres intercalated; the cavernous part has a few circular fibres posteriorly, but anteriorly the muscular fibres are single and placed obliquely and longitudinally.

Closure of the Bladder.-The so-called internal vesical sphincter of the anatomists, which consists of non-striped muscle, is in reality an integral part of the muscular coat of the bladder and surrounds the orifice of the urethra as far down as the prostatic portion, just above the colliculus seminalis. It is, however, not the sphincter muscle. The proper sphincter urethræ (sph. vesicæ externus) lies below the latter. It is a completely circular muscle disposed around the urethra, close above the entrance of the urethra into the septum urogenitale at the apex of the prostate, where it exchanges fibres with the deep transverse muscle of the perinæum which lies under it. 
Some longitudinal fibres, which run along the upper margin of the prostate from the bladder, belong to this sphincter muscle. Single transverse bundles passing forward from the surface of the neck of the blachler, the transverse bands which lie within the prostate, the apex of the colliculus seminalis, and a strong transverse bundle passing in front of the origin of the urethra into the substance of the prostate-all belong to the sphincter muscle (Henle). In the male urethra, the blood-ressels form a rich capillary plexus under the epithelium, below which is a wide-meshed lymphatic plexus.

[Tonus of Sphincter Urethræ. - Open the abdomen of a rabbit, ligature oue ureter, tie a cannula in the other, and pour water into the bladder until it runs out through the urethra, which is usually under a pressure of 16 to 20 inches. If the spinal cord be divided between the tifth and seventh lumbar vertebrex, a column of 6 inches is sufficient to overcome the resistance of the sphincter, while section at the fourth lumbar vertebra has no effect on the height of the pressure. In such an animal the bladder becomes distended, but in one with its cord divided between the fifth and seventh lumbar vertebrx, there is incontinence of urinein the former case because the excito-motor impulses are cut off from the centre $(5$ to 7 vert.), and in the latter because the tonus of the sphincter is destroyed (Kupressow). This tonus is denied by Landois and others. ]

280.-ACCUMULATION OF URINE-MICTURITION.-After emptying the bladder, the urine slowly collects again, the bladder being thereby gradually distended. [A healthy bladder may be said to be full when it contains 20 oz.] As long as there is a moderate amount of urine in the bladder, the elasticity of the elastic fibres surrounding the urethra, and that of the sphincter of the urethra (and in the male of the prostate), suffice to retain the urine in the bladder. This is shown by the fact that the urine does not escape from the bladder after death. If the bladder be greatly distended ( 1.5 to 1.8 litre), so that its apex projects above the pubes, the sensory nerves in its walls are stimulated and cause a feeling of a full bladder, while at the same time the urethral opening is dilated, so that a few drops of urine pass into the beginning of the urethra. Besides the subjective feeling of a full bladder, this tension of the walls of the bladder causes a reflex effect, so that the urinary bladder contracts periodically upon its fluid contents, and so do the sphincter of the urethra and the muscular fibres of the urethra, and thus the urethra is closed against the passage of these drops of urine. As long as the pressure within the bladder is not very high, the reflex activity of the transversely striped sphincter overcomes the other (as during sleep); but, as the pressure rises and the distension increases, the contraction of the walls of the bladder overcomes the closure produced by the sphincter, and the bladder is emptied, as occurs normally in young children.

As age advances, the sphincter urethræ comes under the control of the will, so that it can be contracted voluntarily, as occurs in man when he forcibly contracts the bulbo-cavernosus muscle to retain urine in the bladder. The sphincter ani usually contracts at the same time. The reflex activity of the sphincter may also be inhibited voluntarily, so that it may be completely relaxed. This is the condition when the bladder is emptied voluntarily.

Slight movements, confined to the bladder, occur during psychical or emotional disturbances (e.g., anger, fear), [the bladder may be emptied involuntarily during a fright], after stimulation of sensory nerves, auditory impressions, restraining the respiration, and by arrest of the heart's action. There are slight periodic variations coincident with variations in the blood-pressure. The contractions of the bladder cease after deep inspiration, and also during apnoea (Mosso and Pellucani). The excised bladder of the frog, and even portions free from ganglia, exhibit rhythmical contractions, which are increased by heat (Pfalz). [Ashdown found in dogs that the bladder exhibits regular rhythmical contractions, which were influenced by the degree of distension of the bladder, being most marked with moderate dilatation and least when the bladder was feebly or over-distended. The contractions could be registered by means of a water-manometer communicating with the interior of the bladder.]

Nerves. - The nerves concerned in the retention and evacuation of the urine are :1. The motor nerves of the sphincter urethræ, which lie in the pudendal nerve (anterior roots of the third and fourth sacral nerves). When these nerves are divided, as soon as the bladder becomes so distended as to dilate the urethral opening, the 
urine begins to trickle away (incontinence of urine).

2. The sensory nerves of the urethra, which excite these reflexes, leave the spinal cord by the posterior roots of the third, fourth, and fifth sacral nerves. Section of these nerves causes incontinence of urine. The centre in dogs lies opposite the fifth, and in rabbits, opposite the seventh, lumbar vertebra (Budge). 3. Fibres pass from the cerebrumthose that convey voluntary impulses through the peduncles, and the anterior columns of the spinal cord (according to Mosso and Pellacani, through the posterior columns and the posterior part of the lateral columns), to the motor fibres of the sphincter urethræ. 4. The inhibitory fibres concerned in the reflex-inhibition of the sphincter urethræ, take the same course (perhaps from the optic thalamus?) downwards through the cord to where the third, fourth, and fifth sacral nerves leave it. 5. Sensory nerves proceed from the urethra and bladder to the brain, but their course is not known. Some of the motor and sensory fibres lie for a part of their course in the sympathetic.

Transverse section of the spinal cord above where the nerves leave it, is always followed in the first instance by retention of urine, so that the bladder becomes distended. This occurs because-(1) the section of the spinal cord increases the reflex activity of the urethral sphincter; and (2), because the inhibition of this reflex can no longer take place. As soon, however, as the bladder becomes so distended, as in a purely mechanical manner to cause dilatation of the urethral orifice, then the urine trickles away, but the amount of urine which trickles out in drops is small. Thus the bladder becomes more and more distended, as the continuously distended walls of the organ yield to the increased tension, so that the bladder may become distended to an enormous extent. The urine very frequently becomes ammoniacal, accompanied by catarrh and inflammation of the bladder (\$263).

Voluntary Micturition.-Observers are not agreed as to the mechanism concerned in emptying the bladder when it is only partially full. It is stated by some that a voluntary impulse passes from the brain along a cerebral peduncle, and the cord, to the anterior roots of the 3rd and 4th sacral nerves, and partly through motor fibres from the 2nd to the 5th lumbar nerves (especially the 3rd), to act directly upon the smooth muscular fibres of the bladder. This is assumed, because electrical stimulation of any part of this nervous channel causes contraction of the bladder. This view, however, does not seem to be the true one. It is to be remembered that Budge showed that the sensory nerves of the wall of the bladder are contained in the first, second, third, and fourth sacral nerves, and also in part in the course of the hypogastric plexus, whence they ultimately pass by the rami communicantes into the spinal cord.

According to Landois, the smooth musculature of the bladder cannot be excited directly by a voluntary impulse, but it is always caused to contract reflexly. If we wish to micturate when the urinary bladder contains a small quantity of urine, we first excite the sensory nerves of the opening of the urethra, either by causing contraction or relaxation of the sphincter urethræ, or by means of slight abdominal pressure, and thus force a little urine into the urethral orifice. This sensory stimulation causes a reflex contraction of the walls of the urinary bladder. At the same time, this condition is maintained voluntarily, by the action of the intracranial reflex-inhibitory centre of the sphincter urethræ. The centre for the reflex stimulation of the movements of the walls of the urinary bladder is placed somewhat higher in the spinal cord than that for the sphincter urethræ. In dogs, it is opposite the 4th lumbar vertebra (Gianuzzi, Budge).

[Two centres are assumed to exist in the cord, fig. 292, one the automatic (A.C.) at the segment corresponding to the $2 \mathrm{nd}$, 3rd, and 4 th sacral nerves, which maintains the tonic action of the sphincter; the other, a reflex centre (R.C.), is situated higher, and through it the detrusor urinæ is excited to contraction. Both centres are connected to and governed or controlled by a cerebral centre (C.). The automatic centre is connected with the sphincter, and the other with the urineexpelling fibres. They are also connected with afferent fibres from the bladder and elsewhere. 
The afferent or sensory fibres are also connected with the brain. The automatic centre maintains the closure of the bladder, but if the latter be distended, different impulses proceeding from it reach the spinal centre, and it may be the cerebrum. The impulses reaching the automatic centre inhibit its action and those to the reflex centre excite it, so that the detrusor urinæe contracts. If the afferent impulses be powerful, a desire to urinate is excited, and voluntary

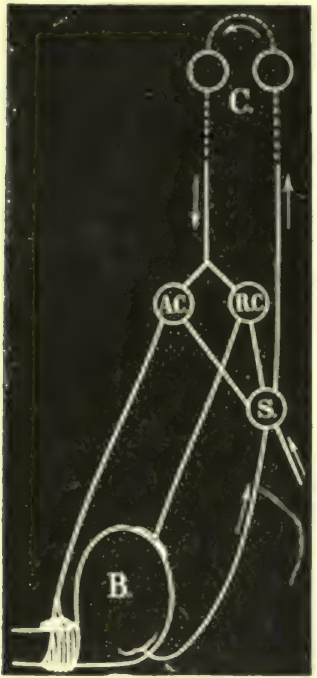

Fig. 292. impulses are excited which act upon the spiual centres as the afferent impulses do, and thus the act of urination is more easily accomplished.]

We may conceive a voluntary impulse to pass down special fibres to an inhibitory centre, which may either act directly on the motor centre, or possibly may send branches directly to the sphineter muscles.

Painful stimulation of sensory nerves causes reflex contraction of the bladder and evacuation of the urine (in children during teething). Reflex contraction of the bladder can be brought about in cats, by stimulation of the inferior mesenteric ganglion. After suction of all the nerves going to the bladder, hæmorrhage and asphyxia cause contraction by a direct effect upon the structures in the wall of the bladder. As yet no one has succeeded in exciting artificially the iuhibitory centre in the brain for the sphincter muscle (Sokowin and Kowalesky).

It seems probable that, as in the case of the anal sphincter (\$160), there is not a continuous tonic reflex stimulation of the sphincter urethræ; the reflex is excited each time by the contents. The sphincter vesicæ of the anatonists, which consists of smooth muscular tissue, does not seem to take part in closing the bladder. Budge and Landois found that, after removal of the transversely striped sphincter urethræ, stimulation of the smooth sphincter did not cause occlusion of the bladder, nor could L. Rosenthal or $\mathrm{v}$. Wittich convince themselves of the presence of tonus in this muscle. Indeed, its very existence is questioned by Henle.

Scheme of micturition:-A.C., Changes of the Urine in the Bladder. - When the urine is R.C., C., automatic, reflex, retained in the bladder for a considerable time, according to and cerebral centres;B.,blad- Kaupp, there is an increase in the sodium chloride and a decrease. der; S., sensory centre acted in the urea and water. Urine which remains for a long time in on by afferent impulses. the bladder is prone to undergo ammoniacal decomposition.

Absorption.-Many observers have shown that the mucous membrane of the bladder is raprable of absorbing substances - potassium iodide and other soluble salts. [Ashdown has shown that poisons, such as watery solutions of strychnin, curare, eserin, emulsions of chloroform and - ther, are absorbed when injected into the bladder of rabbits. In rabbits, KI injected into the bladder through a catheter was found in the urine obtained from the divided ureters. Water and urea are also absorbed-the latter in larger proportion than the former.]

As the ureters enter near the base of the bladder, the last secreted urine is always lowest. If a person remain perfectly quiet, strata of urine are thus formed, and the urine may be voided so as to prove this (Edlefsen).

The pressure within the bladder, when in the supine position $=13$ to 15 centimetres of water. Increase of the intra-abdominal pressure (by inspiration, forced expiration, coughing, beariug-(own) increases the pressure within the bladder. The erect posture also increases it, owing to the pressure of the viscera from above (Schatz, Dubois). [James obtained 4 to $4 \cdot 5$ inches $\mathrm{Hg}$ as the highest expulsive power of the bladder including the abdominal pressure, voluntary and involuntary. In praraplegia, where there is merely. the expulsive power of the bladder, he found 20 to 30 inches of water.]

[Hydronephrosis occurs when the ureters and pelvis of the kidney become dilated, owing to partial and gradual obstruction of the outflow of urine from the ureters: if the obstruction become complete, there is cessation of the urinary secretion. James has shown that the bladder remains contracted for several seconds after it is emptied, and this is specially the case in irritable bladder; so that this condition may also give rise to hydronephrosis by damming up the urine in the ureters.]

Rapidity of Micturition. - The amount of urine voided at first is small, but it increases with the time, and towards the end of the act it again diminishes. In men, the last drops of urine. are ejected from the urethra by voluntary contractious of the bulbo-cavernosus muscle. Adult dogs increase the stream rhythmically by the action of this muscle.

281.-RETENTION AND INCONTINENCE OF URINE.-Retention of urine or ischuria occurs:-1. When there is obstruction of the urethra, from foreign bodies, concretions, stricture, swelling of the prostate. 2. Paralysis or exhaustion of the musculature of the 
bladder ; the latter sometimes occurs after delivery, in consequence of the pressure of the child against the bladder. 3. After section of the spinal cord (p.435), 4. Where the voluntary impulses are uuable to act upon the inhibitory apparatus of the sphincter urethræ reflex, as well as when the sphincter urethræ reflex is increased.

Incontinence of urine (stillicidium urinæ) occurs in consequence of-1. Paralysis of the sphincter urethræ. 2. Loss of sensibility of the urethra, which of course abolishes the reflex of the sphincter. 3. Trickling of the urine is a secondary consequence of section of the spinal cord, or of its degeneration.

Strangury is an excessive reflex contraction of the walls of the bladder and sphincter, due to stimulation of the bladder and urethra; it is observed in inflammation, neuralgia [and after the use of some poisons, e.g., cantharides].

Enuresis nocturna, or involuntary emptying of the bladder at night, may be due to an increased reflex excitability of the wall of the bladder, or weakness of the sphincter.

282. COMPARATIVE AND HISTORICAL.-Amongst vertebrates, the urinary and genital organs are frequently combined, except in the osseous fishes. The Wolffian bodies which act as organs of excretion during the embryonic period, remain thronghout life in fishes and amphibians and continue to act as such. Fishes. - The myxinoids (cyclostomata) have the simplest kidneys; on each side is a long ureter with a series of short-stalked glomeruli with capsules arranged along it. Both ureters open at the genital pore. In the other fishes, the kidneys lie often as elongated compact masses along both sides of the vertebral column. The two ureters unite to form a urethra, which always opens behind the anus, either united with the opening of the genital organs, or behind this. In the sturgeon and hag-fish, the anus and orifice of the urethra together form a cloaca. Bladder-like formations, which, however, are morphologically homologous with the urinary bladder of mammals, oceur in fishes, either on each ureter (ray, hag-fish), or where both join. In amphibians, the vasa efferentia of the testicles are united with the urinary tubules; the duct in the frog unites with the one on the other side, and both conjoined opens into the cloaca, whilst the capacious urinary bladder opens through the anterior wall of the cloaca. From reptiles upwards, the kidney is no longer a persistent Wolffian body, but a new organ. In reptiles, it is usually flattened and elongated; the ureters open singly into the cloaca. Saurians and tortoises have a urinary bladder. In birds, the isolated ureters open into the urogenital sinus, which opens into the cloaca, internal to the excretory ducts of the genital apparatus. The urinary bladder is always absent. In mammals, the kidneys often cousist of many lobules, e.g., dolphin, ox.

Amongst invertebrates, the mollusca have excretory organs in the form of canals, which are provided with an outer and an inner opening. In the mussel, this canal is provided with a sponge-like organ, often with a central cavity, and consisting of ciliated secretory cells, placed at the base of the gills (organ of Bojanus). In gasteropods, with analogous organs, uric acid has been found. Insects, spiders, and centipedes have the so-called Malpighian vessels, which are excretory organs partly for uric acid and partly for bile. These vessels are long tubes, which open into the first part of the large intestine. In crabs, blind tubes connected with the intestinal tube, perhaps have the same functions. The vermes also have renal organs.

Historical. - Aristotle directed attention to the relatively large size of the human bladderhe named the ureters. Massa (1552) found lymphatics in the kidney. Eustachius $(+1580)$ ligatured the ureters and found the bladder empty. Cusanus (1565) investigated the colour and weight of the urine. Pousset (1581) described the muscular nature of the walls of the bladder. Vesling described the trigone (1753). The first important chemical investigations on the urine date from the time of van Helmont (1644). He isolated the solids of the urine and found among them common salt; he ascertained the higher specific gravity of fever-urine, and ascribed the origin of urinary calculi to the solids of the urine. Scheele (1766) discovered uric acid and calcium phosphate; Arand and Kunckel, phosphorus; Rouelle (1773), urea ; and it got its name from Fourcroy and Vauquelin (1799). Berzelius found lactic acid; Seguin, albumin in pathological urine : Liebig, hippuric acid ; Heintz and v. Pettenkofer, kreatin and kreatinin ; Wollaston (1810), cystin. Marcet found xanthin ; and Lindbergson, magnesic carbonate.

\section{Functions of the Skin.}

283. STRUCTURE OF THE SKIN, HAIRS, AND NAIL.-The skin $(3.3$ to $2.7 \mathrm{~mm}$. thick ; specific gravity, 1057) consists of -

[1. The epidermis ;

2. The chorium, or cutis vera, with the papillæ (fig. 294).]

The epidermis $(0.08$ to $0.12 \mathrm{~mm}$. thick) consists of many layers of stratified epithelial cells united to each other by cement substance (figs. 293, 291). The superficial layers-stratum 
corneum-consist of several layers of dry horny non-nucleated squames, which swell up in solution of caustic solla (fig. $294, \mathrm{E}$ ). [It is always thickest where intermittent pressure is applied, as on the sole of the foot and palm of the hand.] The next layer is the stratum lucidum, which is clear and transparent in a section of skin, hence the name, and consists of compact layers of clear cells with vestiges of nuclei. Under this is the rete mucosum or rete Malpighii (fig. 294, $d$ ), consisting of many layers of nucleated protoplasmic epithelial cells which contain pigment in the dark races, and in the skin of the scrotun, and around the anus. [The superficial cells are more fusiform and contain granules which stain deeply with carmine. They constitute, 3, the stratum granulosum. In these cells the formation of keratin is about to begin, and the granules have been called eleidin granules by Ranvier. They are chemically on the way to the transformed into keratin. All corneous structures contain similar granules in the area where

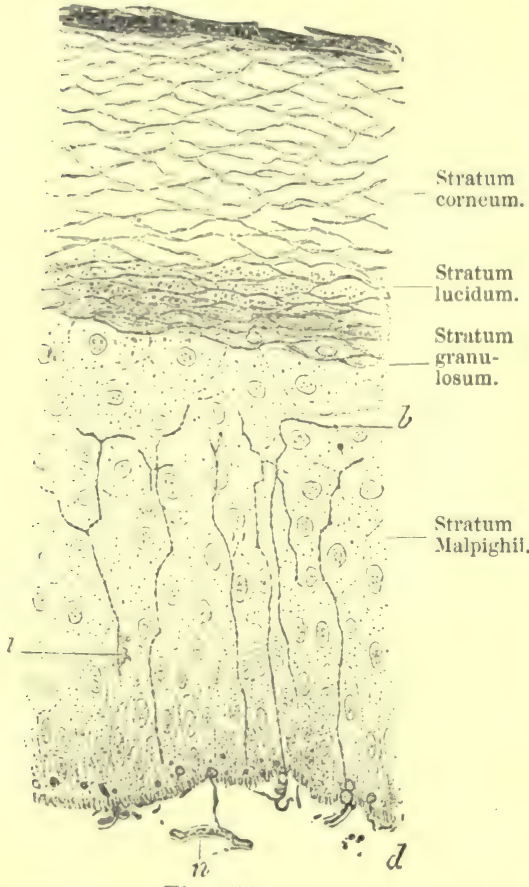

Fig. 293.

Vertical section of the human epidermis ; the nerve-fibrils, $n, b$, stained with gold chloride. papills (fise.294), the larcest beincr upon the volar surface of the hand and foot, on the nipple (Iost of the papilla contain a looped capillary $(g)$, while in certain regions some of them contain a touch-corpuscle (fig. 295, $\iota$ ). The papilla are disposed in groups, whose arrangement varies in different parts of the body. In the palm of the hand and sole of the foot they occur in rows, which are marked out by the existence of delicate furrows on the surface visible to the naked eye. The chorium consists of a dense network of bundles of white fibrous tissue mixed with a network of elastic fibres, which are more delicate in the papillæ. In silversmiths the elastic fibres are blackened by the partial deposition of reduced silver, and the same obtains in those who take silver nitrate in such quantity as to produce argyria. The connective-tissue contains many connective-tissue corpuscles and numerous lencocytes. The deeper connective-tissue layers of the choriun gradually pass into the subcutaneous tissue, where they form a trabecular arrangement of bundles, leaving between them elongated rhomboidal spaces filled for the most part with groups of fat cells (fig. 294, a, a). [In microscopic sections, after the action of alcohol, the fat cells not unfrequently contain crystals of margarin.] The long axis of the rhomb corresponds to the greater tension of the skin at that part (C. Langer $)$. In some situations the subcutaneous tissue is devoid of fat [penis, evelids]. In many situations, the skin is fixed by solid fibrous bands to subjacent structures, as fascire, ligaments or bones 
(tenacula cutis); in other parts, as over bony prominences, bursæ partially lined with endothelium and filled with synovia-like fluid, occur.

Smooth muscular fibres occur in the chorium in certain situations on extensor surfaces

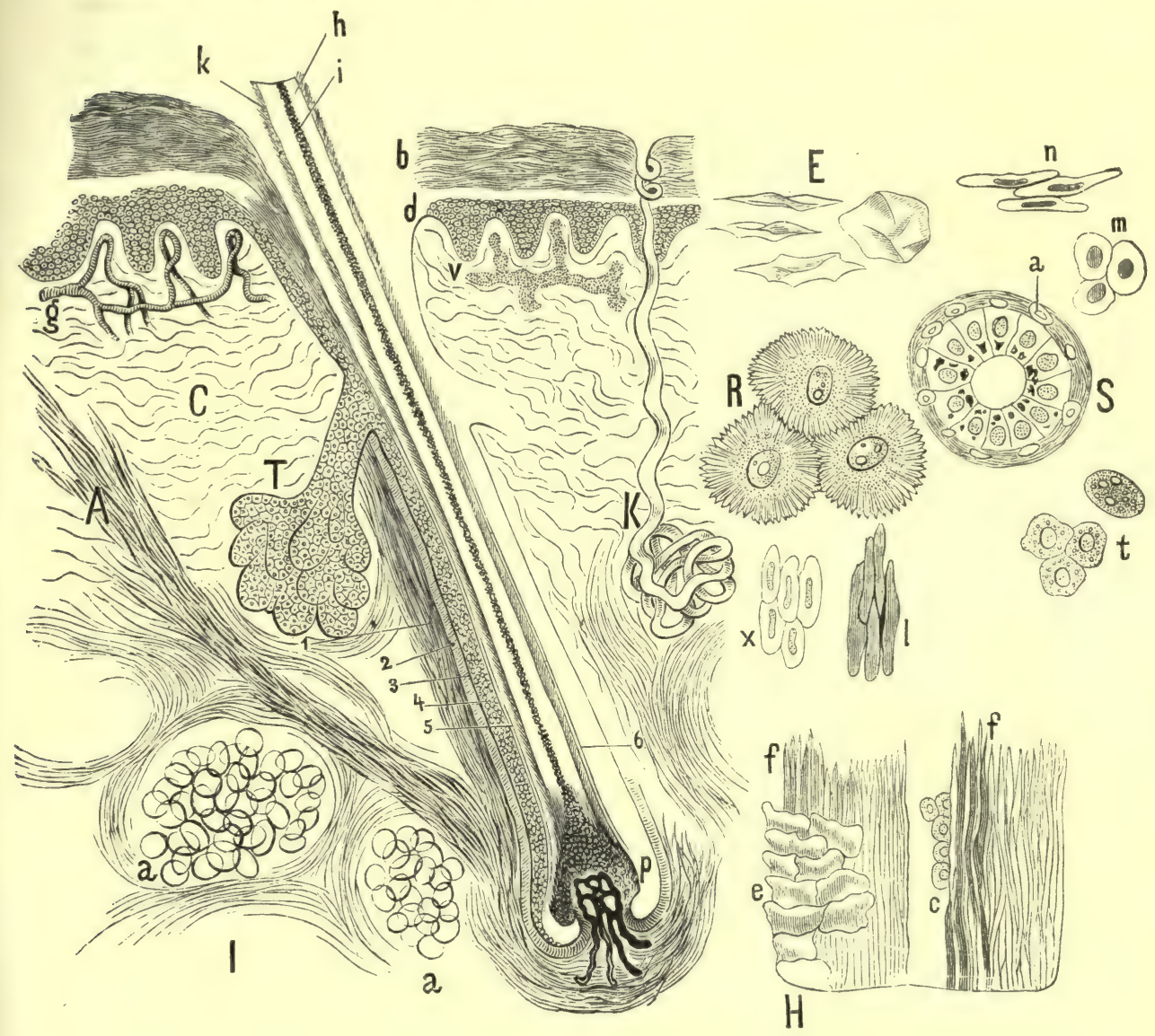

Fig. 294.

I, Vertical section of the skin, with a hair and sebaceous gland, T. Epidermis and chorium shortened-1, outer ; 2 , inner fibrous layer of the hair-follicle; 3 , its byaline layer ; 4 , outer root sheath; 5, Huxley's layer of the inner root sheath; 6, Henle's layer of the same ; $p$, root of the hair, with its papilla; $\mathrm{A}$, arrector pili muscle ; $\mathrm{C}$, chorium ; $a$, subcutaneous fatty tissue ; $b$, epidermis (horny layer); $d$, rete Malpighii ; $g$, blood-vessels of papillæ; $v$, lymphatics of the same; $h$, horny or corneous substance; $i$, medulla or pith ; $k$, epidermis or cuticle of hair ; K, coil of sweet-gland; $\mathrm{E}$, epidermal scales (seen from above and en face) from the stratum cornem ; $\mathrm{R}$, prickle cells from the rete Malpighii ; $n$, superficial, and $m$, deep cells from the nail ; $\mathrm{H}$, hair magnified ; $e$, cuticle ; $c$, medulla, with cells ; $f, f$, fusiform fibrous cells of the substance of the hair ; $x$, cells of Huxley's layer ; $l$, those of Henle's layer; S, transverse section of a sweat-gland from the axilla ; $a$, smooth muscular fibres surrounding it; $t$, cells from a sebaceous gland, some of them containing granules of oil.

(Neumann); nipple, areola mammæ, prepuce, perinæum, and in special abundance in the tunica dartos of the scrotum.

[Guanin in the Skin.-The skin of many amphibians and reptiles contains brown or black pigment-granules, and other granules of a white, silvery, or chalky appearance. Ewald and Krukenberg have shown that the latter consists of guanin, and that this substance is very widely diffused in the skin of fishes, amphibians, and reptiles. Test:-Select a piece of skin 
from the belly of a frog; place it in a porcelain capsule as for the murexide test; add concentrated nitric acid, and heat to dryness, when a yellow resilue is obtained; on adding a drop of caustic soda a red colour is struck. The yellow residue gives no reaction with ammonia. If to the fluid more water be added, and it be then heated, distributed over the surface of the capsule, ant cooled by blowing upon it, various shades of purple and violet are obtained.]

The nails (specific gravity 1.19) consist of numerous layers of solid, horny, homogeneous, epilermal, or nail-eells, which may be isolated with a solution of caustic alkali, when they swell up and exhibit the remains of an elongated nucleus (fig. $294, n, m$ ). The whole under surface of the nail rests upon the nail-bed; the lateral and posterior edges lie in a deep groove, the nail-groove (fig. 296, c). The chorium under the nail is covered throughout its entire extent by longitulinal rows of papillø (fig. 296, d). Above this there lies, as in the skin, many

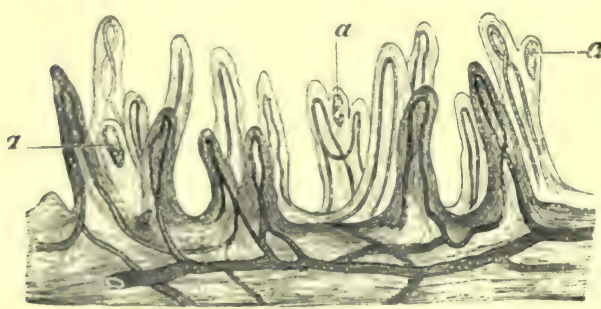

Fig. 295.

l'apillie of the skin, epiclermis removed, blood. ressels injerterl ; some contain a Wagner's touch. corpuscle, ", the others a capillary loop. layers of prickle cells like those in the rete Malpighii (fig. 294, d), and above this again is the substance of the nail (fig. 296, $\alpha$ ). [The stratum granulosum is rudimentary in the nail-bed. The substance of the nail represents the stratum lucidum, there being no stratum corneum (Klein).] The posterior part of the nail-groove and the half moon, brighter part or Iunule, form the root of the nail. They are, at the same time, the matrix, from which growth of the nail takes place. The lunule is present in an isolated nail, and is due to diminished transparency of the posterior part of the nail, owing to the special thickness and uniform distribution of the cells of the rete Malpighii (Toldt).

Growth of the Nail. - According to Lnna, the matrix extends to the front part of the lunule. The nail grows continually from hehind forwarls, and is formed by layers secreted or formed ly the mitrix. These layers run parallel to the surface of the matrix. They run obliquely

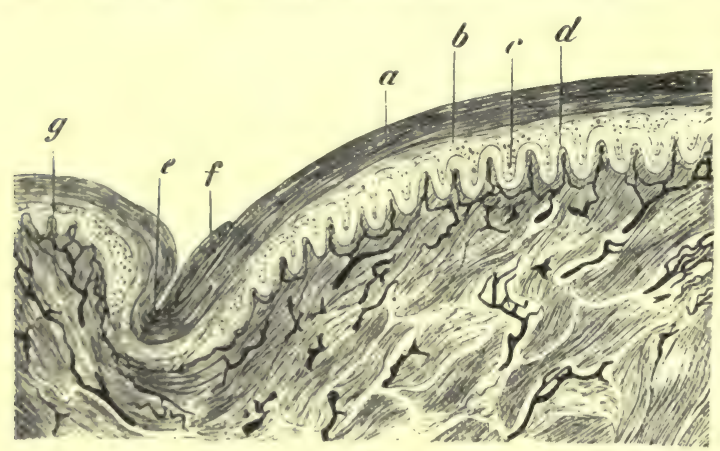

Fig. 296. from above and behind, downwards and forwards, through the thickness of the substance of the nail. The nail is of the same thickness from the anterior margin of the lunule forwards to its free margin. Thus the nail does not grow in thickness in this region. In the course of a year the fingers produce about 2 grms. of nail substance, and relatively more in summer than in winter.

Development, - 1. From the second to the eighth month of fœtal life, the position of the nail is indicated by a partial but marked horny condition of the epidernis on the back of the first phalanx, the "eponychium." The remainder of this substance is represented during life by the normally former epidermal layer, which separates the future nail from the surface of the furrow. 2. The future nail is

formed under the eponychium, with its first nail-cells still in front of the nail-groove; then the ransverse section of one-half of a nail. $\quad \alpha$, nail-subst $b$, more open layer of cells of the nail-bed; $c$, stratum Malpighii of the nail-bed; $d$, transversely divided papille ; $e$, nail-groove ; $f$, horny layer of $c$ projecting over the nail ; g, papille of the skin on the back of the finger. nail grows and pushes forward towards the groove. At the seventh month, the nail (itself covered by the eponychium) covers the whole extent of the nail-bed. 3. When, at a later period, the eponychium splits off, the nail is uncovered. After birth the papilla are formed on the bed of the nail, while simultaneously the matrix passes backwards to the most posterior part of the groove (Inna).

Absence of Hairs. - The whole of the skin, with the exception of the palmar surface of the hand, sole of the foot, dorsal surface of the third phalanx of the fingers and toes, outer surface of the eyelids, glans penis, inner surface of the prepuce, and part of the labia is covered with hairs, which may be strong or fine (lanugo).

A Hair (specific gravity $1 \cdot 26$ ) is fixed by its lower extremity (root) in a depression of the skin or a hair-follicle (fig. 294, I, $p$ ) which passes obliquely through the thickness of the skin, 
sometimes as far as the subcutaneous tissue. The structure of a hair-follicle is the following:1. The outer fibrous layer (figs. 294, 1, 293), composed of interwoven bundles of connectivetissue, arranged for the most part longitudinally, and provided with numerous blood-vessels and nerves. [It is just the connective-tissue of the surrounding chorium.] 2. The inner fibrous layer (figs. 294, 2, 297) consists of a layer of fusiform cells (? smooth muscular fibres) arranged circularly. [It does not extend throughout the whole length of the follicle.] 3. Inside this layer is a transparent, hyaline, glass-like basement membrane (figs. 292, 3, 297), which ends at the neck of the hair-follicle; while above it is continued as the basement membrane which exists between the epidermis and chorium. In addition to these coverings, a hair-follicle has epithelial coverings which must be regarded in relation to the layers of the epidermis. Immediately within the glass-like membrane is the outer root-sheath (figs. 294, 4, 297,298 ), which consists of so many layers of epithelial cells that it forms a conspicuous covering. It is, in fact, a direct continuation of the stratum Malpighii, and consists of many layers of soft cells, the cells of the outer layer being cylindrical. Towards the base of the hairfollicle it becomes narrower, and is united to, and continues with the cells of the root of the hair itself, at least in fully developed hairs. The horny layer of the epidermis continues to retain its properties as far down as the orifice of the sebaceous follicle; below this point, however, it is continued as the inner root-sheath. This consists of (1) a single layer of elongated, flat, homogeneous, non-nucleated cells (figs. $294,6,297, f-H e n l e ' s$ layer placed next and within the outer root-sheath. Within this lies (2) Huxley's layer (figs. $294,5,297, g$ ), consisting of nucleated elongated polygonal cells (fig. 294, $x$, and 3 ), while the cuticle of the hair-follicle is composed of cells analogous to those of the surface of the hair itself. Towards the bulb of the hair these three layers become fused together.

[Coverings of a hair-follicle arranged from without inwards-

1. Fibrous layers, $\{(\alpha)$ Longitudinally arranged fibrous tissue.

2. Glass-like (hyaline) membrane.

3. Epithelial
layers, $\quad\left\{\begin{array}{l}(a) \text { Outer root-sheath. } \\ (b) \text { Inner root-sheath. } \\ (c) \text { Cuticle of the hair. }\end{array}\left\{\begin{array}{l}\text { Henle's layer. } \\ \text { Huxley's layer. }\end{array}\right.\right.$

4. The hair itself.

The arrector pili muscle (fig. 294, A) is a fan-like arrangement of a layer of smooth muscular fibres, attached below to the side of a hair-follicle and extending towards the surface of the chorium; as it stretches obliquely upwards, it subtends the obtuse angle formed by the hair-follicle and the surface of the skin, [or, in other words, it forms an acute angle with the hair-follicle, and between it and the follicle lies the sebaceous gland]. When these muscles contract, they raise and erect. the hair-follicles, producing the condition of cutis anserina or gooseskin. As the sebaceous gland lies in the angle between the muscle and the hairfollicle, contraction of the muscle compresses the gland and favours the evacuation of the sebaceous secretion. It also compresses the blood-vessels of the papilla (Unna).

The hair with its large bulbous extremity-hair-bulbsits upon, or rather embraces, the papilla. It consists of (1)

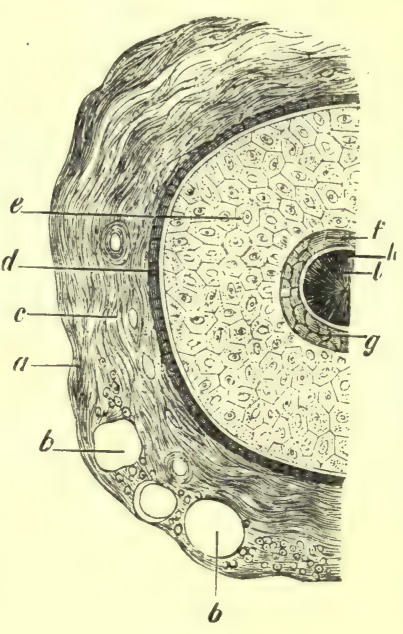

Fig. 297.

Transverse section of a hair and its follicle. $a$, outer fibrous coat with $b$, blood-vessels ; $c$, inner circularly disposed layer; $d$, glass-like layer; $e$, outer, $f, g$, inner, root-sheath; $f$, outer layer of the same 'Henle's sheath); $g$, inner layer of the same (Huxley's sheath); $h$, cuticle ; $l$, hair.

the narrow or medulla (fig. 294, $i$ ), which is absent in woolly hair and in the hairs formed during the first year of life. It is composed of two or three rows of cubical cells $(H, c)$. (2) Outside this lies the thicker cortex $(h)$, which consists of elongated, rigid, horny, fibrous cells $(\mathbf{H}, f, f)$, while in and between these cells lie the pigment granules of the hair. (3) The surface of the hair is covered with a cuticle $(k)$, and consists of imbricated layers of non-nucleated squames.

Grey Hair. - When the hair becomes grey, as in old age, this is due to defective formation of pigment in the cortical part. The silvery appearance of white hair is increased when small aircavities are developed, especially in the medulla and to a less extent in the cortex, where they reflect the light. Landois records a case of the hair becoming suddenly grey, in a man whose hair became grey during a single night, in the course of an attack of delirium tremens. Numerous air-spaces were found throughout the entire marrow of the (blond) hairs, while the hair-pigment still remained.

[Blood-Pigment in Hairs. - The feelers of albino rabbits contain in some part of their substances blood-pigment (Sig. Mayer).] 
Development of Hair. - According to Kölliker, from the 12th to 13th week of intra-nterine life, solid finger-like processes of the epidermis are pushed down into the chorium. The process becomes flask-shaped, while the central cells of the cylinder become elongated and form a conical body, arising as it were from the depth of the recess. It soon differentiates into an inner darker part, which becomes the hair, and a thinner, clearer layer covering the former,

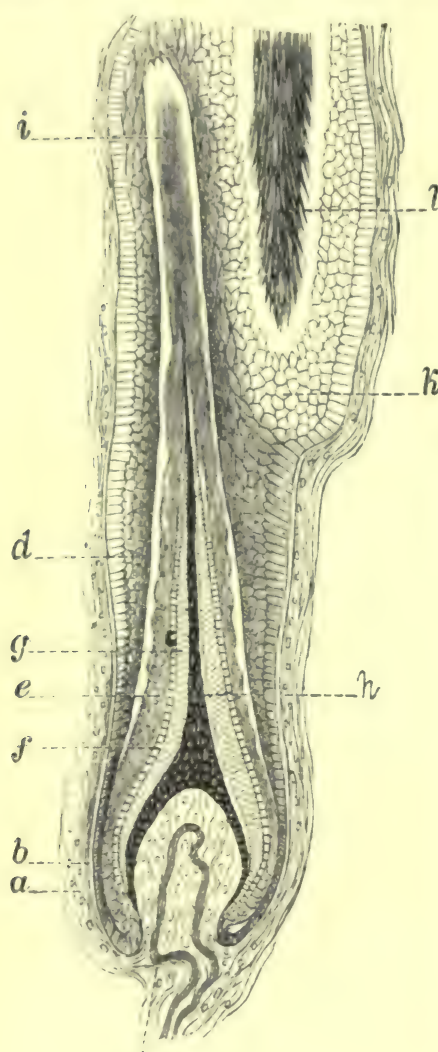

$c^{\prime}$

Fig. 298. the inner root-sheath. The outer cells, i.e., those lying next the wall of the sac, form the outer root-sheath. Outside this, again, the fibrous tissue of the chorium forms a rudimentary hair-follicle, while one of the papillre grows up against it, indents it, and becomes embraced by the bulb of the hair. This is the hair papilla, which contains a loop of blood-vessels. The cells of the bulb of the hair proliferate rapidly and thus the hair grows in length. The point of the hair is thereby gradually pushed upwards, pierces the inner root-sheath, and passes obliquely through the epidermis. The hairs appear upon the forehead at the 19th week; at the 23rd to 25th week the lanugo hairs appear free, and they have a characteristic arrangement on different parts of the body.

Physical Properties. - Hair has very considerable elasticity (stretching to 0.33 of its length), considerable cohesion (carrying 3 to $5 \mathrm{lbs}$.), resists putrefaction for a long time, and is highly hygroscopic. The last property is also possessed by epidermal scales, as is proved by the pains that occur in old wounds and scars during damp weather.

Growth of a hair occurs by proliferation of the cells on the surface of the hair papilla, these cells representing the matrix of the hair. Layer after layer is formed, and gradually the hair is raised higher within its follicle.

Change of the Hair.-According to one view, when the hair has reached its full length, the process of formation on the surface of the hair papilla is interrupted; the root of the hair is raised from the papilla, becomes horny, remains almost devoid of pigment, and is gradually more and more lifted upwards from the surface of the papilla, while its lower bulbous end becomes split up like a brush. The lower empty part of the hairfollicle becomes smaller, while on the old papilla a new formation of a hair begins, the old hair at the same time falling out (Unna). According to Stieda, the old papilla disappears, while a new one is formed in the hair-follicle, and from it the new hair is developed. According to Kölliker, again, both processes obtain.

284. THE GLANDS OF THE SKIN. - The sebaceous glands (fig. 294, I, T) are simple acinous glands, which Section of a hair-follicle while a hair open by a duct into the hair-follicles of large hairs near is being shed. a, outer and midlle their upper part; in the case of small hairs, they may sheaths of liair-follicle; $b$, hyaline project from the duct of the gland (fig. 299). In some membrane; $c$, papilla, with a capil- situations, the ducts of the glands open free upon the lary ; $l$, outer, $e$, inner root-sheath ; surface, e.g., the glands of labia minora, glans, prepuce $f$, cuticle of the latter ; $g$, cuticle of (Tyson's glands), and the red margins of the lips. The the hair; $h$, young non-medullated largest glands occur in the nose and in the labia; they hair ; $i$, tip of new hair; $l$, hair- are absent only from the vola manus and planta pedis. knob of the shell hair, with $k$, the The oblong alveoli of the gland consist of a basement remainder of the cast-off outer root- membrane lined with small polyhedral nucleated granusheath.

lar secretory cells (fig. 294, $t$ ). Within this are other polyhedral cells, whose substance contains numerous oil-globules; the cells become more fatty as we proceed towards the centre of the alveolus. The cells lining the duct are continuous with those of the outer root-sheath. The detritus formed by the fatty metamorphosis of the cells constitutes the sebum or sebaceous secretion. [If the "oil or coceygeal-gland" of a duck be removed, it is found that, when the animal is submerged, it takes up between its feathers about the same amount of water as an intact duck; but it retains 2 to $2 \frac{1}{2}$ times as much water in its feathers (Max Joseph).]

The sweat-glands (fig. 294, I, $k$ ), sometimes called sudoriparous glands, consist of a long 
blind tube, whose lower end is arranged in the form of a coil placed in the areolar tissue under the skin, while the somewhat smaller upper end or excretory portion winds in a vertical, slightly wave-like manner, through the chorium, and in a cork-screw or spiral manner through the epidermis, where it opens with a free, somewhat trumpet-shaped, mouth. The glands are very numerous and large in the palm of the hand, sole of the foot, axilla, forehead, and around the nipple; few on the back of the trunk, and are absent on the glans, prepuce, and margin of the lips. The circumanal glands and the ceruminous glands of the external auditory meatus, and Moll's glands, which open into the hair-follicles of the eyelashes, are modifications of the sweat-glands.

Each gland-tube consists of a basement membrane lined by cells; the excretory part or sweat-canal of the tube is lined by several layers of cubical cells, whose surface is covered by a delicate cuticular layer, a small central lumen being left. Within the coil the structure is different. The first part' of the coil resembles the above, but as the coil is the true secretory part of the gland, its structure differs from the sweat-canal. This, the so-called distal portion of the tube, is lined by a single layer of moderately tall clear nucleated cylindrical epithelium (fig. $294, \mathrm{~S})$, of ten containing oil-globules. Smooth museular fibres are arranged longitudinally along the tube in the large glands (fig. 294, S, a). There is a distinct lumen present in the tube. $\Lambda$ s the duct passes through the epidermis, it winds its way between the epidermal cells without any independent membrane lining it (Heynold). A network of capillaries surrounds the coil. Before the arteries split up into capillaries, they form a true rete mirabile around the coil (Brïcke). This is comparable to the glomerulus of the kidney, which may also be regarded as a rete mirabile. Numerous nerves pass to form a plexus, and terminate in the glands ( $T o m s a)$.

The total number of sweat-glands is estimated by Krause at $2 \frac{1}{2}$ millions, which gives a secretory surface of nearly 1800 square metres. These glands secrete sweat. Nevertheless, an oily or fatty substance is often mixed with the sweat. In some animals (glands in the sole of the foot of the dog, and in birds) this oily secretion is very marked.

Numerous lymphatics occur in the cutis, some arise by a blind end, and others from loops within the papilla on a plane lower than the vascular capillary. [These open into more or less horizontal networks of tubular lymphatics in the cutis, and these again into the wide lymphatics of the subcutaneous tissue, which are well provided with valves.] Special lymphatic spaces are disposed in relation with the hair-follicles and their glands (Neumann), [and also with the fat (Klein). The lymphatics of the skin are readily injected with Berlin blue by the puncture method].

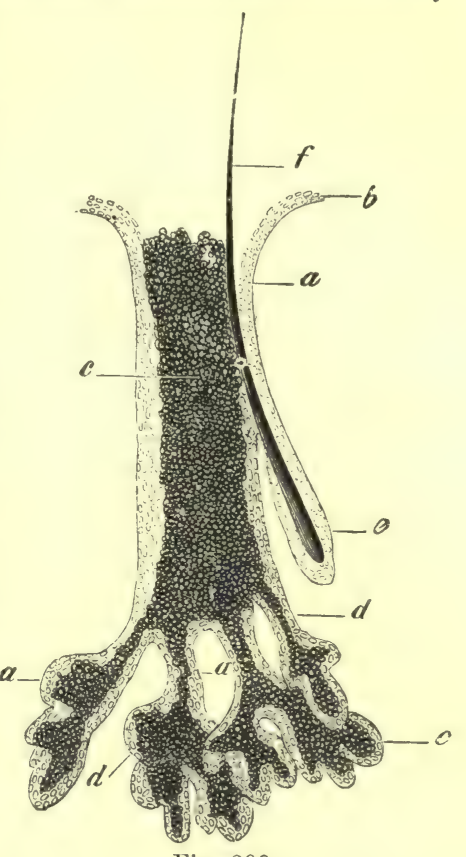

Fig. 299.

Sebaceous gland, with a lanngo hair. $a$, granular epithelium ; $b$, rete Malpighii continuous with $a ; c$, fatty cells and free fat; $d$, acini ; $e$, hair-follicle, with a small hair, $f$.

The blood-vessels of the skin are arranged in several systems. There is a superficial system, from which proceed the capillaries for the papillæ. There is a deeper system of vessels which supplies special blood-vessels to $(a)$ the fatty tissue; $(b)$ the hair-follicles, each of which has a special vascular arrangement of its own, and in connection with this each sebaceous gland receives a special artery; $(c)$ an artery goes also to each coil of a sweat-gland, where it forms a dense plexus of capillaries ( $T$ omsa).

285. THE SKIN AS A PROTECTIVE COVERING.--The subcutaneous fatty tissue fills up the depressions between adjoining parts of the body and covers projecting parts, so that a more rounded appearance of the body is thereby obtained. It also acts as a soft elastic pad and protects delicate parts from external pressure (sole of the foot, palm of the hand), and it often surrounds and protects blood-vessels, nerves, \&c. It is a bad conductor of heat, and thus acts as one of the factors regulating the radiation of heat ( $\$ 214$, II., 4), and, therefore, the temperature of the body. The epidermis and cutis vera also act in the same manner $(\S 212)$. Klug found that the heat-conduction is less through the skin and sub- 
cutaneous fatty tissue than through the skin alone; the epidermis conducts heat less easily than the fat and the chorium.

The solid, elastic, easily movable cutis affords a good protection against external, mechanical injuries; while the dry, impermeable, horny epidermis, devoid of nerves and blood-vessels, affords a further protection against the absorption of poisons, and at the same time it is capable of resisting, to a certain degree, thermal and even chemical actions. A thin layer of fatty matter protects the free surface of the epidermis from the macerating action of fluids, and from the disintegrating action of the air. The epidermis is important in connection with the fluids of the borly. It exerts pressure upon the cutaneous capillaries, and, to a limited extent, prevents too great diffusion of fluid from the cutaneous vessels. Parts of the skin devoid of epidermis are red and always moist. When dry, the epidermis and the epidermal appendages are bad conductors of electricity (\$ 326). Lastly, the existence of uninjured epidermis prevents adjoining parts from growing together.

As the epidermis is but slightly extensile it is stretched over the folds and papillæ of the cutis vera, which becomes level when the skin is stretched, and the papillæ may even disappear with strong tension (Lewinski).

286. CUTANEOUS RESPIRATION: SEBUM-SWEAT.-The skin, witb a surface of more than $1 \frac{1}{2}$ square metre, has the following secretory functions:-

1. The respiratory excretion ;

2 . The secretion of sebaceous matter; and

3 . The secretion of sweat.

[Besides this the skin is protective, contains sense-organs, is largely concerned in regulating the temperature, and may be concerned in absorption.]

1. Respiration by the skin has been referred to (\$131). The organs concerned are the tubes of the sweat-glands, moistened as they are with fluids, and surrounded by a rich network of capillaries. It is uncertain whether or not the skin gives off a small amount of $\mathrm{N}$ or ammonia. Rihrig made experiments upon an arm placed in an air-tight metal box. According to him the amount of $\mathrm{CO}_{2}$ and $\mathrm{H}_{2} \mathrm{O}$ excreted is subject to certain daily variations; it is increased by digrestion, increased temperature of the surroundings, the application of cutaneous stimuli, ancl by impeding the pulmonary respiration. The exchange of gases also depends upon the vascularity of certain parts of the skin, while the cutaneous absorption of $\mathrm{O}$ also depends upon the number of coloured corpuscles in the blood.

In frogs and other amphibians, with a thin, always moist epidermis, the cutaneous respiration is more considerable than in warm-blooded animals. In winter, in frogs, the skin alone yields $\frac{3}{4}$ of the total amount of $\mathrm{CO}_{2}$ excreterl; in summer, $\frac{2}{3}$ of the same (Bidder); thus, in these animals it is a more important respiratory organ than the lungs themselves.

Suppression of the cutaneous activity by varnishing or dipping the skin in oil, causes death by asphyxia (frogs) sooner than ligature of the lungs. Varnishing the Skin.-When the skin of a warm-blooded animal is covered with an impermeable varnish [such as gelatin] (Fourcault, Becquercl, Brechet), death occurs after a time, probably owing to the loss of too much heat. The formation of crystalline ammonio-magnesic phosphate in the cutaneous tissue of such animals (Ellenhuizen) is not sufficient to account for death, nor are congestion of internal organs and serous effusions satisfactory explanations. The retention of the volatile substances (acids) present in the sweat is not sufficient. Strong animals live longer than feeble ones; horses die after several days (Gerlach); they shiver and lose flesh. The larger the cutaneons surface left unvarnished, the later does death take place. Rabbits die when $\frac{7}{8}$ of their surface is varnished. When the entire surface of the animal is varnished, the temperature rapidly falls $\left(\right.$ to $\left.19^{\circ}\right)$, the pulse and respirations vary; usually they fall when the varnishing process is limited; increased frequency of respiration has been observed (\$ 225). Pigs, dogs, horses, when one-half of the body is varnished, exhibit only a temporary fall of the temperature, and show signs of weakness, but do not die (Ellenberger and Hofmeister). [In extensive burns of the skin, not only is there disintegration of the coloured blood-corpuscles (v. Lesser), but in some cases ulcers occur in the duodenum. The cause of the ulceration, however, has not been ascertained satisfactorily (Curling).]

2. Sebaceous Secretion.-The fatty matter as it is excreted from the acini of the sebaceous glands is fluid, but even within the excretory duct of the gland it stagnates and forms a white fat-like mass, which may sometimes be expressed (at the side of the nose) as a worm-like white body, the so-called comedo. The 
sebaceous matter keeps the skin supple, and prevents the hair from becoming too dry. Microscopically, the secretion is seen to contain innumerable fatty granules, a few gland-cells filled with fat, visible after the addition of caustic soda, crystals of cholesterin, and in some men a microscopic mite-like animal (Demodex folliculorum).

Chemical Composition. - The constituents are for the most part fatty ; chiefly olcin (fluid) and palmitin (solid) fat, soaps, and some cholesterin ; a small amount of albumin and unknown extractives. Amongst the inorganic constituents, the insoluble earthy phosphates are most abundant; while the alkaline chlorides and phosphates are less abundant.

The vernix caseosa, which covers the skin of a new-born child, is a greasy mixture of sebaceous matter and macerated epidermal cells (containing 47.5 per cent. fat). A similar product is the smegma præputialis (52.8 per cent. fat), in which an ammonia soap is present.

The cerumen or ear-wax is a mixture of the secretions of the ceruminous glands of the ear (similar in structure to the sweat-glands) and the sebaceous glands of the auditory canal. Besides the constituents of sebum, it contains yellow or brownish particles, a bitter yellow extractive substance derived from the ceruminous glands, potash soaps, and a special fat. The secretion of the Meibomian glands is sebum.

[Lanoline.-Liebreich finds in feathers, hairs, wool, and keratin-tissnes generally, a cholesterin fat, which however is not a true fat, although it sayonifies, but an ethereal compound of certain fatty acids with cholesterin. In commerce it is obtained from wool, and is known by the above name; it forms an admirable basis for ointments, and it is very readily absorbed by the skin.] Thus, the fat-like substance for protecting the epidermis is nartly formed along with keratin in the epidermis itself.

3. The Sweat. - The sweat is secreted in the coil of the sweat-glands. As long as the secretion is small in amount, the water secreted is evaporated at once from the skin along with the volatile constituents of sweat; as soon, however, as the secretion is increased, or evaporation is prevented, drops of sweat appear on the surface of the skin. The former is called insensible perspiration, and the latter sensible perspiration. [Broadly, the quantity is about 2 lbs. in twenty-four hours.]

The sensible perspiration varies greatly; as a rule, the right side of the body perspires more freely than the left. The palms of the hands secrete most, then follow the soles of the feet, cheek, breast, upper arm, and fore-arm (Peiper). It falls from morning to mid-day, and rises again towards evening, reaching its maximum before midnight. Much moisture and cold in the surrounding atmosphere diminish it, and so does diuresis. In children, the insensible perspiration is relatively great. The drinking of water favours it, alcohol diminishes it (H. Schmid).

Method. - Sweat is obtained from a man by placing him in a metallic vessel in a warm bath; the sweat is rapidly secreted and collected in the vessel. In this way Favre collected 2560 grammes of sweat in $1 \frac{1}{2}$ hour. An arm may be inclosed in a cylindrical vessel, which is fixed air-tight round the arm with an elastic bandage (Schottin).

Amongst animals, the horse sweats, so does the ox, but to a less extent ; the vola and planta of apes, cats, and the hedgehog secrete sweat ; the snout of the pig sweats (?), while the goat, rabbit, rat, mouse, and dog are said not to siveat (Luchsinger). [The skin over the body and the pad on the dog's foot contain numerous sweat-glands, which open free on the surface of the pad and into the hair-follicles on the general surface of the skin (W. Stirling).]

Microscopically. - The sweat contains only a few epidermal scales accidentally mixed with it, and fine fatty granules from the sebaceous glands.

Chemical Composition.-Its reaction is alkaline, although it frequently is acid, owing to the admixture of fatty acids from decomposed sebum. During profuse secretion it becomes neutral, and lastly alkaline again (Trümpy and Luchsinger). The sweat is colourless, slightly turbid, of a saltish taste, and has a characteristic odour varying in different parts of the body; the odour is due to the presence of volatile fatty acids. The constituents are water, which is increased by copious draughts of that fluid, and solids, which amount to $1 \cdot 180$ per cent. $(0 \cdot 70$ to 2.66 per cent.-Funke), and of these 0.96 per cent. is organic and 0.33 inorganic. Amongst the organic constituents are neutral fats (palmitin, stearin), also present in the sweat of the palm of the hand, which contains no sebaceous glands, cholesterin, volatile fatty acids (chiefly formic, acetic, butyric, propionic, caproic, capric acids), varying qualitatively and quantitatively in different parts of the body.-These acids are most abundant in the sweat first (acid) secreted. There are also traces 
of albumin (similar to casein) and urea, about 0.1 per cent. In uræmic conditions (anuria in cholera) urea has been found crystallised on the skin. When the secretion of sweat is greatly increased, the amount of urea in the urine is diminished, both in health and in uriemia (Leube). The nature of the reddish-yellow pigment, which is extracted from the residue of sweat by alcohol, and coloured green by oxalic acid, is unknown. Amongst inorganic constituents, those that are easily soluble are more abundant than those that are soluble with difficulty, in the proportion of 17 to 1 ; sodium chloride, 0.2 ; potassium chloride, 0.2 ; sulphates, 0.01 per 1000, together with traces of earthy phosphates and sodium phosphate. Sweat contains $\mathrm{CO}_{2}$ in a state of absorption and some $\mathrm{N}$. When decomposed with free access of air, it yields ammonia salts (Gorup-Besanez).

Excretion of Substances. - Some substances when introduced into the body reappear in the sweat-benzoic, cinnamic, tartaric, and succinic acids are readily excreted ; quinine and potassic iodide with more difficulty. Mercuric chloride, arsenious and arsenic acids, sodium and potassium arseniate have also been found. After taking arseniate of iron, arsenious acid has been found in the sweat, and iron in the urine. Mercury iodide reappears as a chloride in the sweat, while the iodine occurs in the saliva.

Formation of Pigments. - The leucocytes furnish the material, and the pigment is deposited in granules in the deeper layers, and, to a less extent, in the upper layers, of the rete Malpighii. This occurs in the folds around the anus, scrotum, nipple, [especially during pregnancy], and everywhere in the coloured races. There is a diffuse, whitish-yellow pigment in the stratum corneum, which becomes darker in old age. The pigmentation depends on chemical processes, reduction taking place, and these processes are aided by light. Granular pigment lies also in the layers of prickle cells. The dark coloration of the skin may be arrested by free $\mathrm{O}$ [hydric peroxide], while the corneous change is prevented at the same time (Unna).

Pathological. - To this belongs the formation of liver spots or cholasma, freckles, and the pigmentation of Addison's disease, [prigmentation round old ulcers, \&c.] (\$ 103, IV.). [The curious cases of pigmentation, especially in neurotic women, e.g., in the eyelids, deserve further study in relation to the part played by the nervous system in this process. ]

287. INFLUENCE OF NERVES ON THE SECRETION OF SWEAT.The secretion of the skin, which averages about $\frac{1}{6 i}$ of the body-weight, i.e., about double the amount of water excreted by the lungs, may be increased or diminished. The liability to perspire varies much in different individuals. The following conditions influence the secretion-1. Increased temperature of the surroundings causes the skin to become red, while there is a profuse secretion of sweat $(\$ 214$, II., 1). Cold, as well as a temperature of the skin about $50^{\circ} \mathrm{C}$., arrests the secretion. 2. A very watery condition of the blood, e.g., after copious draughts of warm water, increases the secretion. Increased cardiac and vascular activity, whereby the blood-pressure within the cutaneous capillaries is increased, have a similar effect; increased sweating follows increased muscular activity. 3. Certain drugs favour sweating, e.g., pilocarpin, Calabar bean, strychnin, picrotoxin, muscarin, nicotin, camphor, ammonia compounds; while others, as atropin and morphia, in large doses, diminish or paralyse the secretion. [Drugs which excite copious perspiration, so that it stands as beads of sweat on the skin, are called sudorifics, while those that excite the secretion gently are diaphoretics, the difference being one of degree. Those drugs which lessen the secretion are called antihydrotics.] 4. It is important to notice the antagonism which exists, probably upon mechanical grounds, between the secretion of sweat, the urinary secretion, and the evacuation of the intestine. Thus copious secretion of urine (e.g., in diabetes) and watery stools coincide with dryness of the skin. If the secretion of sweat be increased, the percentage of salts, urea, and albumin is also increased, whilst the other organic substances are diminished. The more saturated the air is with watery vapour, the sooner does the secretion appear in drops upon the skin, while in dry air or air in 
motion, owing to the rapid evaporation, the formation of drops of sweat is prevented, or at least retarded. [The complementary relation between the skin and kidneys is well known. In summer, when the skin is active, the kidneys separate less water; in winter, when the skin is less active, it is cold and comparatively bloodless, while the kidneys excrete more water, so that the action of these two organs is in inverse ratio.]

The influence of nerves upon the secretion of sweat is very marked.

I. Just as in the secretion of saliva (\$ 145), vaso-motor nerves are usually in action at the same time as the proper secretory nerves; the vaso-dilator nerves (sweating with a red congested skin) are most frequently involverl. The fact that secretion of sweat does occasionally take place when the skin is pale (fear, deathagrony) shows that, when the vaso-motor nerves are excited, so as to constrict the cutaneous blood-vessels, the sweat-secretory nerve-fibres may also be active.

Under certain circumstances, the amount of blood in the skin seems to determine the occurrence of sweating; thus, Dupuy found that section of the cervical sympathetic caused secretion on that side of the neck of a horse; while Nitzelnadel found that percutaneous electrical stimulation of the cervical sympathetic in man, limited the sweating.

[We may draw a parallel between the secretion of saliva and that of sweat. Both are formed in glands derived from the outer layer of the embryo. Both secretions are formed from lymph supplied by the blood-stream, and if the lymph be in sufficient quantity, secretion may take place when there is no circulation, although in both cases secretion is most lively when the circulation is most active and the secretory nerves of both are excited simultaneously ; both glands have secretory nerves distinct from the nerves of the blood-vessels; both glands may be paralysed by the action of the nervous system, or in disease (fever), or conversely, both are paralysed by atropine and excited by other drugs, e.g., pilocarpin. In the gland cells of both, histological changes accompany the secretory act, and no doubt similar electromotor phenomena occur in both glands.]

II. Secretory nerves, altogether independent of the circulation, control the secretion of sweat. Stimulation of these nerves, even in a limb which has been amputated in a kitten, causes a temporary secretion of sweat, i.e., after complete arrest of the circulation (Goltz, Kendall and Luchsinger, Ostroumow). In the intact condition of the body, however, profuse perspiration, at all events, is always associated with simultaneous dilatation of the blood-vessels (just as, in stimulation of the facial nerve, an increased secretion of saliva is associated with an increased blood-stream- $\_145$, A, I.). The secretory nerves and those for the blood-vessels seem to lie in the same nerve-trunks.

The secretory nerves for the hind limbs (cat) lie in the sciatic nerve. Luchsinger found that stimulation of the peripheral end of this nerve caused renewed secretion of sweat for a period of half an hour, provided the foot was always wiped to remove the sweat already formed. If a kitten, whose sciatic nerve is divided on one side, be placed in a chamber filled with heated air, all the three intact limbs soon begin to sweat, but the limb whose nerve is divided does not, nor does it do so when the veins of the limb are ligatured so as to produce congestion of its blood-vessels. [The cat sweats only on the hairless soles of the feet.] As to the course of the secretory fibres to the sciatic nerve, some pass directly from the spinal cord (Vulpian), some pass into the abdominal sympathetic (Luchsinger, Nawrocki, Ostroumow), through the rami communicantes and the anterior spinal roots from the upper lumbar and lower dorsal spinal cord (9th to 13 th dorsal vertebræ-cat), where the sweat-centre for the lower limbs is situated.

The sweat-centre may be excited directly:-(1) By a highly venous condition of the blood, as during dyspnœea, e.g., the secretion of sweat that sometimes precedes death; (2) by overheated blood $\left(45^{\circ}\right.$ C.) streaming through the centre; (3) by certain poisons (see p. 446). The centre may be also excited reflexly, although the results are variable, e.g., stimulation of the crural and peroneal nerves, as well as the central end of the opposite sciatic nerve, excites it. [The pungency of mustard in the mouth may excite free perspiration on the face.] 
Anterior Extremity.-The secretory fibres lie in the ulnar and median nerves, for the fore-limbs of the cat ; most of them, or indeed all of them (Nawrocki), pass into the thoracic sympathetic (Ggl. stellatum), and part (?) run in the nerve-roots direct from the spinal cord (Luchsinger, Vulpian, Ott). A similar sweat-centre for the upper limbs lies in the lower part of the cervical spinal cord. Stimulation of the central ends of the brachial plexus causes a reflex secretion of sweat upon the foot of the other side (Adamkiewicz). At the same time the hind feet also perspire.

Pathological. - Degeneration of the motor ganglia of the anterior horns of the spinal cord canses loss of the secretion of sweat, in addition to paralysis of the voluntary muscles of the trunk. The perspiration is increased in paralysed as well as in cedematous limbs. In nephritis, there are great variations in the amount of water given off by the skin.

Head. - The secretory fibres for this part (horse, man, snout of pig) lie in the thoracic sympathetic, pass into the ganglion stellatum, and ascend in the cervical sympathetic. Percutaneous electrical stimulation of the cervical sympathetic in man, causes sweating of that side of the face and of the arm (M. Meyer). In the cephalic portion of the sympathetic, some of the fibres pass into, or become applied to, the branches of the trigeminus, which explains why stimulation of the infraorbital nerve causes secretion of sweat. Some fibres, however, arise directly from the roots of the trigeminus (Luchsinger), and the facial (Vulpian, Adamkiewicz). Undoubtedly the cerebrum has a direct effect either upon the vaso-motor nerves (p. 447, I.) or upon the sweat-secretory fibres (II.), as in the sweating produced by psychical excitement (pain, fear, \&c.).

$\Lambda$ damkiewicz and Senator found that, in a man suffering from abscess of the motor region of the cortex cerebri for the arm, there were spasms and perspiration in the arm.

Sweat-Centre.-According to Adankiewicz, the medulla oblongata contains the dominating sweat-centre (\$373). When this centre is stimulated in a cat, all the four feet sweat, even three-quarters of an hour after death (Adamkiewicz).

III. The nerve-fibres which terminate in the smooth muscular fibres of the sweatglands act upon the excretion of the secretion.

[Changes in the Cells during Secretion.-In the resting glands of the horse, the cylindrical cells are clear with the nucleus near their attached ends, but after free perspiration they become granular, and their nucleus is more central (Renaut).]

If the sweat-nerves be divided (cat), injection of pilocarpin causes a secretion of sweat, even at the end of three days. After a longer period than six days, there may be no secretion at all. This observation coinciles with the phenomenon of dryness of the skin in paralysed limbs. Dieffenbach found that transplanted portions of skin first began to sweat when their sensibility was restored. If a motor nerve (tibial, median, facial) of a man be stimulated, sweat appears on the skin over the muscular area supplied by the nerve, and also upon the corresponding area of the opposite non-stimulated side of the body. This result occurs when the circulation is arrested as well as when it is active. Sensory and thermal stimulation of the skin always cause a bilateral reflex secretion independently of the circulation. The area of sweating is independent of the part of the skin stimulated.

288. PATHOLOGICAL VARIATIONS.-1. Anidrosis or diminution of the secretion of sweat occurs in diabetes and the cancerous cachexia, and along with other disturbances of nutrition of the skin in some nervous diseases, e.go, in dementia paralytica ; in some limited regions of the skin, it has occurred in certain tropho-neuroses, e.g., in unilateral atrophy of the face and in pralysed parts. In many of these cases it depends upon paralysis of the corresponding nerves or their spinal sweat-centres.

2. Hyperidrosis, or increase of the secretion of sweat, occurs in easily excitable persons, in consequence of the irritation of the nerves concerned (\$288), e.g., the sweating which occurs in debilitated conditions and in the hysterical (sometimes on the head and hands), and the socalled epileptoid sweats (Eulenburg). Sometimes the increase is confined to one side of the head (H. unilateralis). This condition is often accompanied with other nervous phenomena, partly with the symptoms of paralysis of the cervical sympathetic (redness of the face, narrow pupil), partly with symptoms of stimulation of the sympathetic (dilated pupil, exophthalmos). It may occur without these phenomena, and is due perhaps to stimulation of the proper secretory fibres alone. [Inereased sweating is very marked in certain fevers, both during their course and at the crisis in some; while the sweat is not only copious but acid in acute rheumatism. The "night-sweats" of phthisis are very marked and disagreeable.] 
3. Paridrosis or qualitative changes in the secretion of sweat, e.g., the rare case of "sweating of blood" (hæmatohidrosis), is sometimes unilateral. According to Hebra, in some cases this condition represents a vicarious form of menstruation. It is, however, usually one of many phenomena of nervous affections. Bloody sweat sometimes occurs in yellow fever. Bilepigments have been found in the sweat in jaundice; blue sweat from indigo (Bizio), from pyocyanin (the rare blue colouring-matter of pus), or from phosphate of the oxide of iron (Osc. Kollmann) is extremely rare. Such coloured sweats are called chromidrosis. Numerous microorganisms (which, however, are innocuous) live between the epidermal seales and on the hairs, two varieties of Saccharomycetes; in cutaneous folds Ieptothrix epidermalis, various Schizomycetes, and five kinds of Micrococci ; and between the toes-Bacterium graveoleus (BordoniUffreduzzi), which causes the odour of the sweat of the feet. Micro-organisms are also the cause of yellow, blue, and red sweat; the last is due to Micrococcus hæmatodes.

Grape-sugar occurs in the sweat in diabetes mellitus; uric acid and cystin very rarely ; and in the sweat of stinking feet, leucin, tyrosin, valerianic acid, and ammonia. Stinking sweat (bromidrosis) is due to the decomposition of the sweat, from the presence of a special microorganism (Bacterium foetidum-Thin). In the sweating stage of ague butyrate of lime has been found, while in the sticky sweat of acute articular rheumatism there is more albumin (Anselmino), and the same is the case in artificial sweating (Leube); lactic acid is present in the sweat in puerperal fever.

The sebaceous secretion is sometimes increased, constituting seborrhœa, which may be local or general. It may be diminished (Asteatosis cutis). The sebaceous glands degenerate in old people, and hence the glancing of the skin (Rémy). If the ducts of the glands are occluded the sebum accumulates. Sometimes the duct is oceluded by black particles or ultramarine (Unna) from the blue used in colouring the linen. When pressed out, the fatty worm-shaped secretion is called "comedo."

289. CUTANEOUS ABSORPTION_GALVANIC CONDUCTION.-After long immersion in water the superficial layers of the epidermis become moist and swell up. The skin is unable to absorb any substances, either salts or vegetable poisons, from watery solutions of these. This is due to the fat normally present on the epidermis and in the pores of the skin. If the fat be removed from the skin by alcohol, ether, or chloroform, absorption may occur' in a few minutes (Parisot). According to Röhrig, all volatile substances, e.g., carbolic acid and others, which act upon and corrode the epidermis, are capable of absorption. While according to Juhl, such watery solutions as impinge on the skin, in a finely divided spray, are also capable of absorption, which very probably takes place through the interstices of the epidermis.

[Inunction.-When ointments are rubbed into the skin so as to press the substance into the pores, absorption occurs, e.g., potassium iodide in an ointment so rubbed in is absorbed, so is mercurial ointment. V. Voit found globules of mercury between the layers of the epiclermis, and even in the chorium of a person who was executed, into whose skin mercurial ointment had been previously rubbed. The mercury globules, in cases of mercurial inunction, pass into the hair-follicles and ducts of the glands, where they are affected by the secretion of the glands and transformed into a compound capable of absorption. An abraded or inflamed surface (e.g., after a blister), where the epidermis is removed, absorbs very rapidly, just like the surface of a wound (Endermic method).]

[Drugs may be applied locally where the epidermis is intact-epidermic method-as when drugs which affect the sensory nerves of a part are painted over a painful area to diminish the pain. Another method, the hypodermic, now largely used, is that of injecting, by means of a hypodermic syringe, a non-corrosive, non-irritant drug, in solution, into the subcutaneous tissue, where it practically passes into the lymph spaces and comes into direct relation with the lymph-and blood-stream; absorption takes place with great rapidity, even more so than from the stomach.]

Gases. - Under normal conditions, minute traces of $\mathrm{O}$ are absorbed from the air; hydrocyanic acid, sulphuretted hydrogen $-\mathrm{CO}, \mathrm{CO}_{2}$, the vapour of chloroform and ether may be absorbed (Chaussier, Gerlach, Röhrig). In a bath containing sulphuretted hydrogen, this gas is absorbed, while $\mathrm{CO}_{2}$ is given off into the water (Röhrig).

Absorption of watery solutions takes place rapidly through the skin of the frog (Guttmann, W. Stirling, $v$. Wittich). Even after the circulation is excluded and the central nervous system destroyed, much water is absorbed through the skin of the frog, but not to such an extent as when the circulation is intact (Spina).

Galvanic Conduction through the Skin.-If the two electrodes of a constant current be impregnated with a watery solution of certain substances and applied to the skin, and if the direction of the current be changed from time to tirne, strychnin may be caused to pass through the skin of a rabbit in a few minutes, and that in sufficient amount to kill the animal ( $H$. Munk). In man, quinine and potassium iodide have been introduced into the body in this way, and their presence detected in the urine. This process is called the cataphoric action of the constant current ( $\$ 328)$.

290. COMPARATIVE-HISTORICAL. - In all vertebrates, the skin consists of chorium 
and epidermis. In some reptiles, the epidermis becomes horny, and forms large plates or seales. Similar structures occur in the edentata among mammals. The epidermal appendages assumie various forms-such as hair, nail, spines, bristles, feathers, claws, hoof, horns, spurs, \&c. The scales of some fishes are partly osseous structures. Many glands occur in the skin ; in some amphibia they secrete mucus, in others the secretion is poisonous. Snakes and tortoises are levoil of cutaneous glands; in lizards the "leg-glands" extend from the anus to the bend of the knee. In the crocodile, the glands open under the margins of the cutaneo-osseous scales. In birds, the entaneous glands are absent; the "coccygeal glands" form an oily secretion for lubricating the feathers. [This is denied by 0 . Liebreich, as he finds no cholesterin-fats in their secretion.] The ciret ylunds, at the anus of the civet cat, the preputial glands of the musk leer, the glands of the hare, and the pedal glands of ruminants, are really greatly developed sebaceous glands. In some invertebrata, the skin, consisting of epidermis and chorium, is intimately united with the subjacent muscles, forming a musculo-cutaneous tube for the body of the animal. The cephalopoda have chromatophores in their skin, i.e., round or irregular spaces filled with coloured granules. Muscular fibres are arranged radially around these spaces, so that when these muscles contract the coloured surface is increased. The change of colour in these animals is due to the play or contraction of these muscles (Brücke). Special glands are encerned in the production of the shell of the snail. The annulosa are covered with a chitinous investment, which is continued for a certain distance along the digestive tract and the trache:e. It is thrown off when the animal sheds its covering. It not only protects the animal, but it forms a structure for the attachment of muscles. In echinodermata, the cutaneous covering contains calcareous masses; in the holothurians, the calcareous structures assume the form of ealcareous spicules.

Historical.-Hiplocrates (born 460 B.c) and Theophrastus (born 371 B. c.) distinguished the perspiration from the sweat; and, accorling to the latter, the secretion of sweat stands in a certain antagonistic relation to the urinary secretion and to the water in the freces. According to Cassius Felix (97 A.D.), a person placed in a bath absorbs water through the skin ; Sanctorius (1614) measured the amount of sweat given off; Alberti (1581) was acquainted with the hairbulb; Donatus (1588) deseribed hair becoming grey suddenly; Riolan (1626) showed that the colour of the skin of the negro was due to the epidermis. 


\section{Physiology of the Motor Apparatus.}

291. [CILIARY MOTION-PIGMENT CELLS]-_[(a) Muscular Movement.-By far the greatest number of the movements occurring in our bodies is " accomplished through the agency of muscular fibre, which, when it is excited by a stimulus, contracts, i.e., it forcibly shortens, and thus brings its two ends nearer together, while it bulges to a corresponding extent laterally. In muscle, the contraction takes place in a definite direction.]

[(b) Amœboid Movement.-Motion is also exhibited by colourless blood-corpuscles, lymph-corpuscles, leucocytes, and some other corpuscles. In these structures we have examples of amœboid movement $(\$ 9)$, which is movement in an indefinite direction.]

[(c) Ciliary Movement.-There is also a peculiar form of movement, known as ciliary movement. There is a gradual transition between these different forms of movement. The cilia which are attached to the ciliated epithelium are the motor agents (fig. 300).]

[Ciliated epithelium-where found, -In the nasal mucous membrane, except the olfactory region; the cavities accessory to the nose; the upper half of the pharynx, Eustachian tube, larynx, trachea, and bronchi; in the uterus, except the lower half of the cervix; Fallopian tubes ; vasa efferentia to the lower end of epilidymis ; ventricles of brain (child); and the central canal of the spinal cord.]

[The cilia are flattened blade-like or hair-like appendages attached to the free end of the cells. They are about $\frac{1}{3000}$ inch in length, and are apparently homogeneous and structureless. They are planted upon a clear non-contractile disc on the free end of the cell, and some observers state that they pass through the disc to become continuous with the protoplasm of the cell, or with the plexus of fibrils which pervades the protoplasm, so that by some observers they

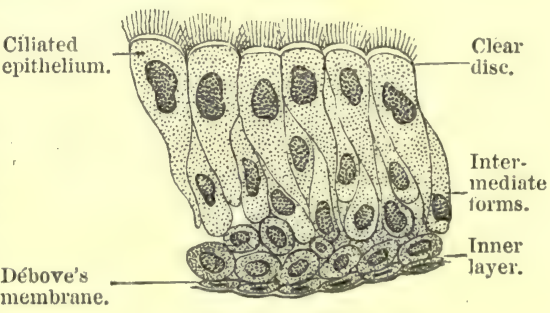

Fig. 300.

Ciliated epithelium. are regarded as prolongations of the intra-epithelial plexus of fibrils. They are specially modified parts of an epithelial cell, and are contractile and elastic. They are colourless, tolerably strong, not coloured by staining reagents, and are possessed of considerable rigidity and flexibility. They are always connected with the protoplasm of cells, and are never outgrowths of the solid cell membranes. There may be 10 to 20 cilia distributed uniformly on the free surface of a cell (fig. 300).]

[In the large ciliated cells in the intestine of some molluscs (mussel), the cilia perforate the clear refractile disc, which appears to consist of small globules-basal pieces-united by their edge, so that a cilium seems to spring from each of these, while continued downwards into the protoplasm of the cell, but not attached to the nucleus, there is a single varicose fibril-rootlet, and the leash of these fibrils passes through the substance of the cell and may unite towards its lower tailed extremity (Engelmann).]

[Ciliary motion may be studied in the gill of a mussel, a small part of the gill being teased 
in sea water; or the hard palate of a frog, newly killed, may be scraped and the scraping ex-

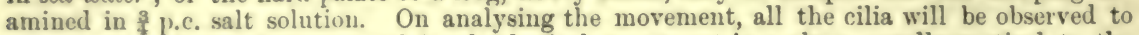
execute a regular, periotic, to and fro rhythmical movement in a plane usually vertical to the surface of the cells, the direction of the movement being parallel to the long axis of the organ. The appearance presented by the movements of the cilia is sometimes described as a lashing movement, or like a field of corn moved by the wind. Each vibration of a cilium consists of a rapid forward movement or flexion, the tip moving more than the base, and a slower backward movement, the cilium again straightening itself. The forward movement is about twice as rapid as the lackward movement. The amplitude of the movement varies according to the kind of cell and other conditions, being less when the cells are about to die, but it is the same for all the cilia attached to one cell, and is seldom more than $20^{\circ}$ to $50^{\circ}$. There is a certain periodicity in their movement-in the frog they contract about 12 times per second. The result of the rapid forward movement is that the surrounling fluid, and any particles it may contain, are moved in the direction in which the cilia bend. All the eilia of adjoining cells do not move at once, but in regular succession, the movement travelling from one cell to the other, but how this co-ordination is brought about we do not know. At least it is quite independent of the nervous system, as eiliary movement goes on, in isolated cells, and in man it has been observed in the trachea two days after leath. Conditions for Movement. - In order that ciliary movement may go on, it is essential that (1) the cilia be connected with part of a cell ; (2) moisture ; (3) oxygen be present; and (4) the temperature be within certain limits.]

[A ciliated epithelial cell is a good example of the physiological division of labour. It is derived from a cell which originally held motor, antomatic, and nutritive functions all com. bined in one mass of protoplasm, but in the fully developed cell, the nutritive and regulative functions are confined to the protoplasm, while the cilia-alone are contractile. If the cilia be separated from the cell, they no longer move. If, however, a cell be divided so that part of it remains attached to the cilia, the latter still move. The nucleus is not essential for this act. It would seem, therefore, that though the cilia are contractile, the motor impulse probably proceels from the cell. Each cell can regulate its own nutrition, for during life they resist the entrance of certain coloured fluids.]

[Effect of Reagents. - Gentle heat accelerates the number and intensity of the movements, cold retards them. A temperature of $45^{\circ} \mathrm{C}$. causes coagulation of their proteids, makes them permanently rigid, aud kills them, just in the same way as it acts on muscle, causing heatstiffening ( 295,1$)$. Weak alkalies may cause them to contract after their movement is arrested or nearly so (Virchouc), and any current of fluid in fact may do so. Lister showed that the vapour of ether and chloroform arrests the movements as long as the narcosis lasts, but if the vapour be not applied for too long a time, the cilia may begin to move again. The prolonged action of the vapour kills them. As yet we do not know any specific poison for cilia-atropin, veratrin, and curara acting like other substances with the same endosmotic equivalent (Engelmann).]

[Functions of Cilia.-The moving cilia propel fluids or particles along the passages which they line. By carrying secretions along the tubes which they line towards where these tubes open on the surface, they aid in excretion. In the respiratory passages, they carry outwards along the bronchi and trachea, the mucus formed by the mucous glands in these regions. When the mucus reaches the larynx it is either swallowed or coughed up. That the cilia carry particles upwards in a spiral direction in the trachea has been proved by actual laryngoscopic investigation, and also by excising a trachea and sprinkling a coloured powder on its mucous membrane, when the coloured particles (Berlin blue or charcoal) are slowly carried towards the upper end of the trachea. In bronchitis the ciliated epithelium is shed, and hence the mucus tends to accumulate in the bronchi. They remove mucus from cavities accessory to the nose, and from the tympanum, while the ova are carried partly by their agency from the ovary along the Fallopian tube to the uterus. In some of the lower animals they act as organs of locomotion, and in others as adjuvants to respiration, by creating currents of water in the region of the organs of respiration.]

[The Force of Ciliary Movement.-Wyman and Bowditch found that the amount of work that can be done by cilia is very considerable. The work was estimated by the weight which a measured surface of the mucous membrane of the frog's hard palate was able to carry up an inclined plane of a definite slope in a given time.]

[Pigment-cells belong to the group of contractile tissues, and are well developed in the frog, and many other animals where their characters have been carefully studied. They are generally regarded as comparable to branched connective-tissue corpuscles, loaded with pigmented granules 
of melanin. The pigment-granules may be diffused in the cell, or aggregated around the nucleus ; in the former case, the skin of the frog appears dark in colour, in the latter, it is but slightly pigmented (fig. 301). The question has been raised whether they are actual cells or merely spaces, branched, and containing a fluid with granules in suspension. In any case, they undergo marked changes of shape under various influences. If the motor-nerve to one leg of a frog be divided, the skin of the leg on that side becomes gradually darker in colour than the intact leg. $\Lambda$ similar result is seen in the curara experiment, when all parts are ligatured except the nerve. Local applications affect the state of diffusion of the pigment, as v. Wittich found that turpentine or electricity caused the cells of the tree-frog to contract, and the same effect is produced by light. In Rana temporaria local irritation has little effect, but light, on the contrary, has, although the effect of light seems to be brought about through the eye, probably by a reflex mechanism (Lister). A pale-coloured frog, put in a dark place, assumes, after

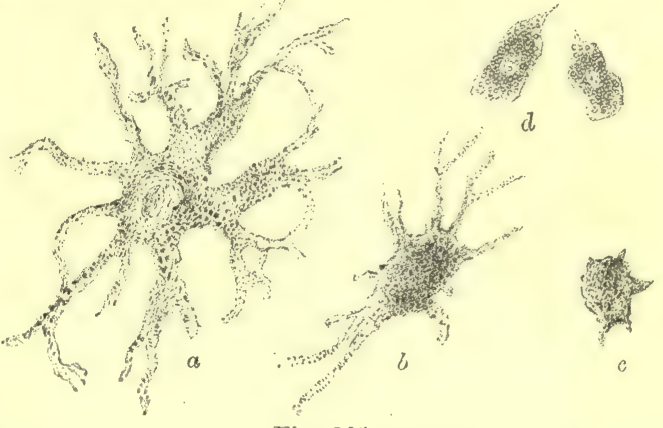

Fig. 301.

Pigment-cells from the web of frog's foot; $\alpha$, cell with pigment-granules diffused; $b$, granules more concentrated; $c$, more concentrated still; $d$, cells with guaningranules (Stirling).

a time, a different colour, as the pigment is diffused in the dark; but if it be exposed to a bright light it soon becomes nale again. The same phenomenon may be seen on studying the web of a frog's leg under the microscope. The marked variations of colour-within a certain range-in the chameleon is due to the condition of the pigment-cells in its skin, covered as they are by epidermis, containing a thin stratum of air (Brïcke). When it is poisoned with strychnin, its whole body turns pale; if it be ill, its body becomes spotted in a dendritic fashion, and if its cutaneous nerves be divided, the area supplied by the nerve changes to black. The condition of its skin, therefore, is readily affected by the condition of its nervous system, for psychical excitement also alters its colour. If the sympathetic nerve in the neck of a turbot be divided, the skin on the dorsal part of the head becomes black. It is notorious that the colour of fishes is adapterl to the colour of their environment. If the nerve proceeding from the stellate ganglion in the mantle of a cuttle-fish be divided, the skin on one half of the body becomes pale.]

[Guanin in Cells.-Besides the pigment-cells in the web of a frog's foot (especially in Rana temporaria) there are other cells which contain granules of guanin (fig. 301, d). If the web of a frog's foot be mounted in Canada balsam and examined microscopically between crossed Nicol's prisms, each guanin-cell is seen to contain numerous very strongly doubly refractive granules of guanin (\$283).]

292. STRUCTURE AND ARRANGEMENT OF MUSCLES.-[Muscular Tissue is endowed with contractility, so that when it is acted upon by certain forms of energy or stimuli, it contracts. There are two varieties of this tissue-

(1) Striped, striated (or voluntary);

(2) Non-striped, smooth, organic (or involuntary).

Some muscles are completely under the control of the will, and are hence called "voluntary," and others are not directly subject to the control of the will, and are hence called "involuntary;" the former are for the most part striped, and the latter non-striped; but the heart-muscle, although striped, is an involuntary muscle.]

1. Striped Muscles. - The surface of a muscle is covered with a connective-tissue envelope or perimysium externum, from which septa, carrying blood-vessels and nerves, the perimysium internum, pass into the substance of the muscle, so as to divide it into bundles of fibres or fasciculi, which are fine in the eye-muscles and coarse in the glutei. In each such compartment or mesh, there lie a number of maiscular fibres arranged more or less parallel to each other. [The fibres are held together by delicate connective-tissue or endomysium, which surrounds groups of the fibres; each fibre being, as it were, separated from its neighbour by delicate fibrillar connective-tissue.] Each muscular fibre is surrounded with a rich plexus of capillaries [which form an elongated meshwork, lying between adjacent fibres, but never pene- 
trating the fibres, which, however, they cross (fig. 307). In a contracted muscle, the capil. laries may be slightly sinuous in their course, but when a muscle is on the stretch these curves disappear. The eapillaries lie in the endouysium, and near them are lymphatics]. Each muscular fibre receives a nerve-fibre. [Where found.-Striped muscular fibres occur in the skeletal muscles, heart, diaphragm, pharynx, upper part of cesophagus, muscles of the middle ear and pinna, the true sphincter of the urethra, and external anal sphincter.]

A muscular fibre (fic. 302,1 ) is a more or less cylindrical or polygonal fibre, 11 to $67 \mu$ [ $\frac{1}{100}$ to $\pi \frac{1}{0}$ in.] in diameter, and never longer than 3 to 4 centimetres [1 to $1 \frac{1}{2}$ in.]. Within short muscles, $e . \%$, stapedius, tensor tympani, or the short muscles of a frog, the fibres are as long as the muscle itself; within longer museles, however, the individual fibres are pointed,
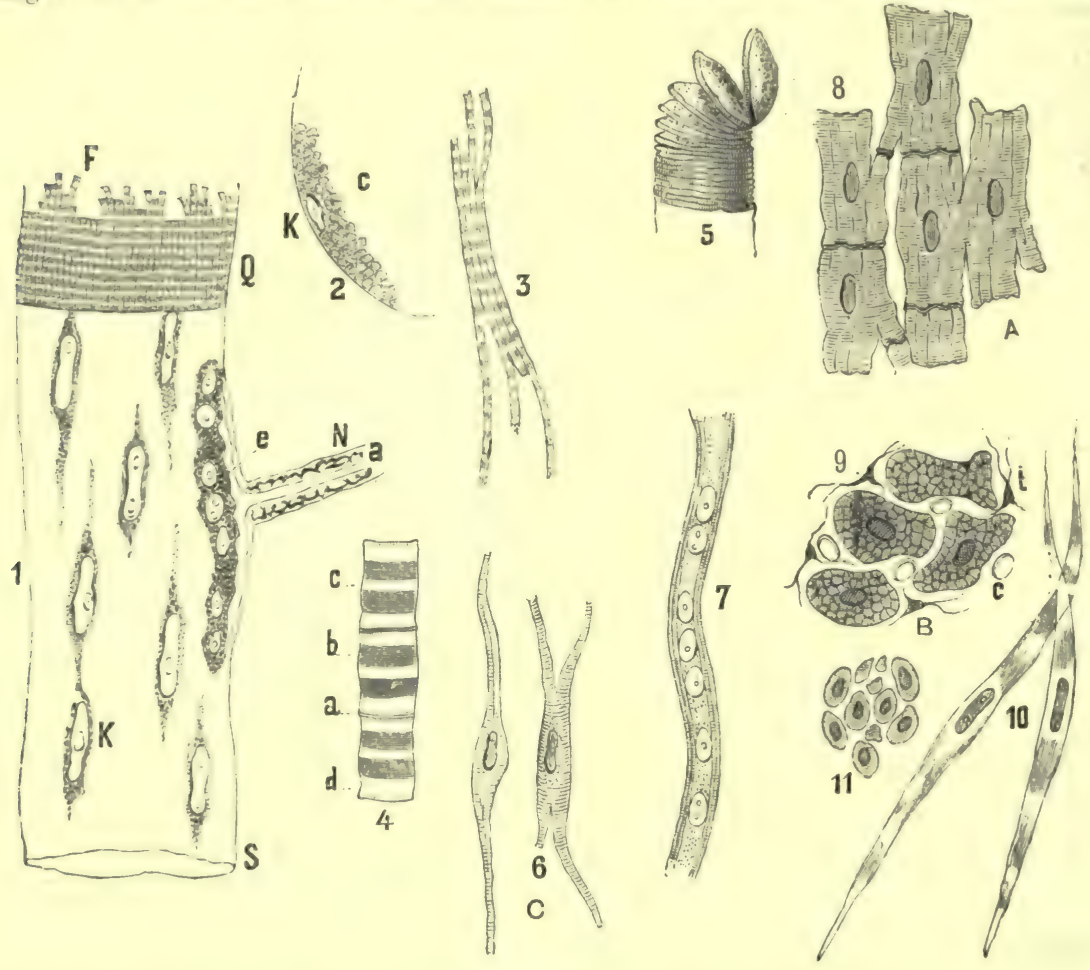

Fig. 302.

Histology of muscular tissue. 1, Diagram of part of a striped muscular fibre ; S, sarcolemma ; Q, transverse stripes; $\mathrm{F}$, fibrilla; $\mathrm{K}$, the muscle nuclei ; $\mathrm{N}$, a nerve-fibre entering it with $x$, its axis cylinder and Kiihne's motorial end-plate, $c$, seen in profile; 2 , transverse section of part of a muscular fibre, showing Cohnhein's areas, $c$; 3 , isolated muscular fibrillæ; 4, part of an insect's muscle crreatly magnified; $a$ Krause-Amici's line limiting the muscular cases ; $b$, the doubly-refractive substance ; $c$, Hensen's disc ; $d$, the singly-refractive substance ; 5 , fibres cleaving transversely into dises ; 6 , muscular fibre from the heart of a frog; 7 , development of a striped muscle from a human foetus at the third month ; 8,9 , muscular fibres of the heart ; $c$, capillaries ; $b$, connective-tissue corpuscles; 10 , smooth muscular fibres ; 11, transverse section of smooth muscular fibres.

and are united obliquely by cement-substance with a similar bevelled or pointed end of another fibre lying in the same direction. Muscular fibres may be isolated by maceration in nitric acid with excess of potassic chlorate or by a 36 per cent. solution of caustic potash.

[Each muscular fibre consists of the following parts :-

1. Sarcolemma, an elastic sheath, with transverse partitions, stretching across the fibre at regular intervals - the membrane of Krause;

2. The included sarcous substance ;

3. The nuclei or muscle corpuscles.] 
Sarcolemma. - Each muscular fibre is completely enclosed by a thin colourless, structureless, transparent elastic sheath (fig. 302, 1, S), which, chemically, is midway between connective and elastic tissue, and within it is the contractile substance of the muscle. [When a muscular fibre is being digested by trypsin, Chittenden observed, at the beginning, the sarcolemma raised from its sarcous contents as a folded tube, but it is ultimately digested by trypsin. It is thus distinguished from the collagen substance of connective-tissue, which is not digested by trypsin. It is not dissolved by boiling, and it resists the action of acids and dilute alkalies, while it is dissolved by concentrated alkalies. Thus, it differs from elastic fibres, and on the whole, chemically, it seems to be most closely related to the membrana propria of glands. It has much more cohesion than the sarcous substance which it encloses, so that sometimes, when teasing fresh muscular tissue under the microscope, one may observe the sarcous substance torn across, with the unruptured sarcolemma stretching between the ends of the ruptured sarcous substance. If muscular fibres be teased in distilled water, sometimes fine clear blebs are seen along the course of the fibre, due to the sarcolemma being raised by the fluid diffusing under it. The sarcous substance, but not the sarcolemma, may be torn across by plunging a muscle in water at $55^{\circ} \mathrm{C}$., and keeping it there for some time (Ranvier).]

Sarcous Substance. - The sarcous substance is marked transversely by alternate light and dim layers, bands, stripes or dises (fig. $302,1, \mathrm{Q}$ ), so that each fibre is said to be "transversely striped." [The stripes do not occur in the sarcolemma, but are confined to the sarcous substance, and they involve its whole thickness.]

[The animals most suited for studying the structure of the sarcous substance are some of the insects. The muscles of the water-beetle, Dytiscus marginalis, and the Hydrophilus piceus are well suited for this purpose. So is the crab's muscle. In examining a living muscle microscopically, no fluid except the musele-juice should be added to the preparation, and very high powers of the microscope are required to make out the finer details.]

Bowman's Discs. - If a muscular fibre be subjected to the action of hydrochloric acid (1 per 1000), or if it be digested by gastric juice, or if it be frozen, it tends to cleave transversely into discs (Bowmon), which are artificial products, and resemble a pile of coins which has been knocked over (fig. 302, 5).

Fibrillæ.-Under certain circumstances, a fibre may exhibit longitudinal striation. This is due to the fact that it may be split up longitudinally into an immense number of ( 1 to $1.7 \mu$ in diameter) fine, contractile threads, the primitive fibrillø (fig. 302, 1, F), placed side by side, each of which is also transversely striped, and they are so united to each other by semi-fluid cement-substance, that the transverse markings of all the fibrillæ lie at the same level. Several of these fibrils are united together owing to the mutual pressure, and prismatic in form, so that when a transverse section of a perfectly fresh muscular fibre is observed after it is frozen, the end of each fibre is mapped out into a number of small polygonal areas called Cohnheim's areas (fig. 302, 2). [Each bundle of fibrils or polygonal area represents what Kölliker called a "MuscleColumn."]

Fibrillæ are easily obtained from insects' muscles, while those from a mammal's muscle are readily isolated by the action of dilute alcohol, Miiller's fluid [or, best of all, $\frac{1}{6}$ per cent. solution of chromic acid] (fig. 302, 3).

[In studying the structure of muscle, it is well to remember that there are considerable differences between the muscles of Vertebrates and those of Arthropoda. ]

[When a living unaltered vertebrate muscular fibre is examined microscopically, in its own juice, we observe the alternate dim and light transverse dises. Amici, Krause, and Dobie showed that a fine dark line runs across the light disc, and divides it into two (fig. 303). Amici resolved it into a row of granules, and by others (e.g., Krause) it is regarded as due to the existence of a membrane,-hence it is called Krause's membrane, - which runs transversely

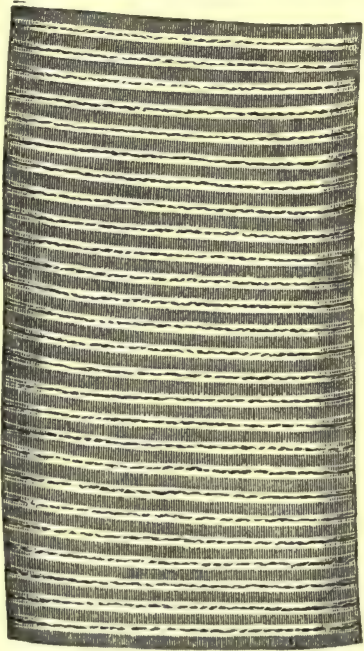

Fig. 303.

Human muscular fibre, $\times 300$. across the fibre, being attached all round to the sarcolemma, thus dividing each fibre into a series of compartments placed end to end. Hensen described a dise or stripe in the centre of the dim dise. $]$

[On Krause's theory, each muscular compartment contains (1) a broad dim disc, which is the contractile part of the sarcous substance. It is doubly refractive (anisotropous), and is composed of Bowman's sareous elements. (2) On each end of this disc, and between it and Krause's membranes, is a narrower, clear, homogeneons, and but singly refractile (isotropous), soft or fluid substance, which forms the lateral disc of Engelmann. In some insects it contains a row of refractive granules, constituting the granular layer of Floggel. If a muscular fibre be 
stretched and stained with logwood, the central part of the dim dise appears lighter in colour than the two enils of the same disc. This has been described as a separate disc, and is called the median dise of Hensen (fig. 302, 4, c).]

[In an unalterel fibre, the dim broad stripe may appear homogeneous, but after a time it cleaves throughout its entire extent in the long axis of the fibre into a number of prismatic elements or fibrils, the sarcous elements of Bowman (fig. 302). These at first are prismatic, but as they solidify they shrink and seem to squeeze out of them a fluid, becoming at the same time more constricted in the centre. This separation into bundles of fibrils with an interstitial matter gives rise to the appearance seen on transverse section of a frozen muscle, and known as Cohnheim's areas (fig. $302,2, c$ ). In all probability the cleavage also extends through the lateral dises, and thus fibrils are formed by longitudinal cleavage of the fibre.]

[Muscles of Arthropoda. - Engelmann showed that the muscles of these animals have a large number of dises. In a musele of an animal killed by being plunged into alcohol, according to the position of the lens of the microscope, one sees :-

1. The broad dim dise, composed of two darker lateral portions or discs, and a lighter disethat of Hensen, between them. In fig. 304 the whole disc is marked Q, and Hensen's dise is distinguished as $h$.

2. On both sicles of this is a small, clear, slightly refractive stripe, $\mathrm{J}$, corresponding to one of Engelmann's isotropons stripes.

3. On hoth sides there follows symmetrically a dark strongly refractive stripe, $\mathbf{N}$, corresponding to Engelmann's accessory stripe and Flögel's granular layer.

4. Then on both sides there is a clear, feebly refractive disc, $\mathbf{E}$.

5. Beyond $\mathrm{E}$ is a small, dark, highly refractive stripe, $\mathrm{Z}$ - usually the darkest-corresponding to the Amici-Krause line.]

[From Z, the stripes are repeated in the inverse order to $Q$, then in the same order to $Z$, and so $\mathrm{om}$. This is the appearance with a low position of the lens. Many muscles do not show all these stripes, thus $h$ is often absent.]

[If the lens of the microscope be raised, to get a more superficial view of the fibre, the distribution of the light is reversed (fig. 304 , II), as all strongly refractive sections become

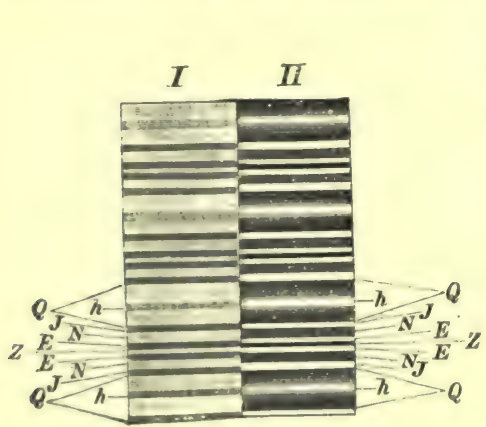

Fig. 304 .

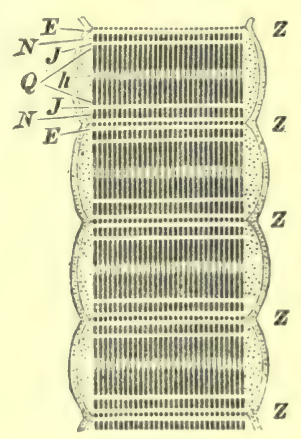

Fig. 305 .

Fig. 304.-Insect's muscle; I, with a high position of the lens, and II, with a deeper position. Fig. 305.-Muscular fibre of Carabus cancellatus. are thicker at their ends, and thinner and lighter at their middle. Rollett regards the clear light, and all feebly refractive appear darker, while with a deep position of the lens, the reverse is the case.]

[Experiment shows that the dim dise rapidly swells up in dilute acids, and also that the dim discs $(Q)$, the accessory dises (N), and the Amici-Krause line (K), stain more deeply with logwood than the other discs, and $h$ less than the rest of Q.]

[If a muscle which has been some time in alcohol be examined as to its longitudinal striation, it will be seen to consist of rods with light intervals between them (fig. 305). The rods intervals between these rods as consisting of sarcoplasma, a body closely related to protoplasm, and the rods as bundles of fibrillæ or "muscle columns."]

[If a muscle be acted upon by certain acids the relative appearance of the muscle-columns and the sarcoplasma is altered; and the latter may appear in these and in gold preparations as a plexus of fibrils with regular longitudinal and transverse meshes (Melland, Marshull, fig. 306).]

[Muscle Rods. - Schitfer describes the appearance differently :--Double rows of granules are seen lying in or at the boundaries of the light streaks (discs), and very fine longitudinal lines may be detected running through the dark streak (dim disc) and uniting the minute granules. These fine lines, with their enlarged extremities, are "muscle rods." They are most conspicuous in insects. During the contraction of a living muscular fibre, Schäfer describes the "reversal of the stripes" ( $(\$ 297)$ as follows:- "When the fibres contract, the light stripes are seen, as the fibre shortens and thickens, to become dark, an apparent reversal being thereby produced in the strix. This reversal is due to the enlargement of the rows of dark dots and the formation by their juxtaposition and blending of dark discs, whilst the muscular substances between these discs has by contrast a bright appearance." With polarised light in a living muscular fibre, 
all the sarcous substance, except the muscle rod, is doubly refractive or anisotropous, so that it appears bright on a dark field when the Nicol's prisms are crossed, while under the same conditions contracted muscle and dead muscle show alternate dark and light bands (Schäfer).]

The nuclei or muscle-corpuscles are found immediately under the sarcolemma in all mammals, and their long axis lies in the long axis of the fibre ( 8 to $13 \mu$ long, 3 to $4 \mu$ broad).

[In the muscles of the frog, reptiles, and some other animals, e.g., the red muscles of the rabbit and hare and in some muscles of birds, they lie in the substance of the fibre surrounded by a small amount of protoplasm.] When they occur immediately under the sarcolemma they are more or less flattened, and lie embedded in a small amount of protoplasm (fig. 302, 1 and 2, K). They contain one or two nucleoli, and it is said that the protoplasm sends ont fine processes which unite with similar processes from adjoining corpuscles, so that, according to this view, a branched protoplasmic network exists under the sarcolemma. [Each nucleus has a reticulated appearance due to the presence of a plexus of fibrils, consisting of chromatin ; in its meshes lies an achromatic substance. The nuclei are specially large in Otiorrhynchus planatus, one of the beetles. Mitotic figures indicating division of the nuclei have been observed. The nuclei are not seen in a perfectly fresh muscle, because, until they have undergone some change, their refractive index is the same as that of the sarcous substance.] They become specially evident after the addition of acetic acid. Histogenetically, they are the remainder of the cells from which the muscular fibres were developed (fig. 302, 7). According to M. Schultze, the sarcous substance is an intercellular substance differentiated and formed by their activity. Perhaps they are the centres of nutrition for the muscular fibres. In amphibians, birds, fishes, and reptiles, they lie in the axis of the fibres between the fibrils.

It is said that the protoplasm of the muscle-corpuscles forms a fine network throughout the whole muscular fibre, the transverse branches taking the course of the lines of Krause or Dobie, and the longitudinal branches running in the interstices between Cohnheim's area (Retzius, Bremer, Melland, fig. 306).

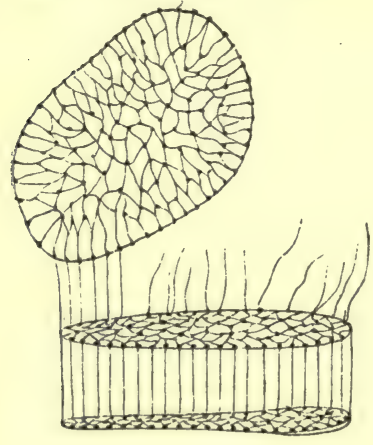

Fig. 306.

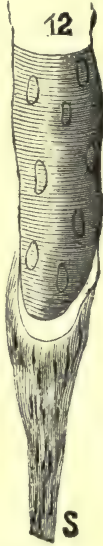

Fig. 307.

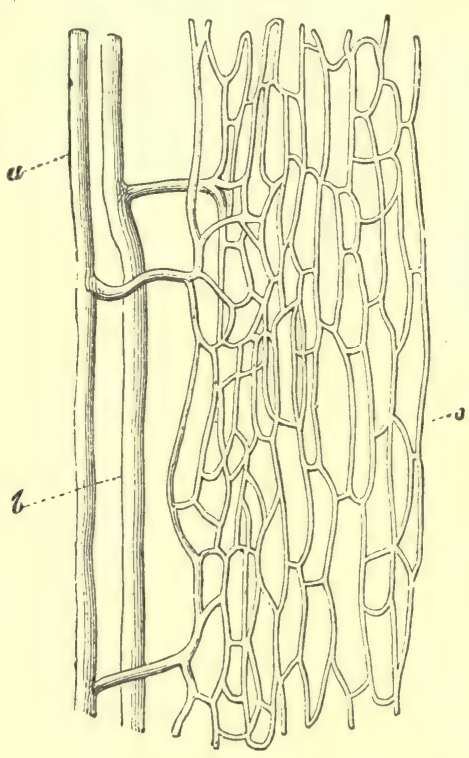

Fig. 308 .

Fig. 306. - Network in a muscular fibre. Fig. 307. - Relation of a tendon, S, to its muscular fibre. Fig. 308. - Injected blood-vessels of a human muscle. $a$, small artery; $b$, vein; $c$, capillaries. $\times 250$.

Relation to Tendons. -According to Toldt, the delicate connective-tissue elements, which cover the several muscular fibres, pass from the ends of the latter directly into the connectivetissue elements of the tendon. The end of the muscular fibre is perhaps united to the smooth surface or hollow end of the tendon by means of a special cement (Weismann-fig. 307, S). In arthropoda, the sarcolemma passes directly into and becomes continuous with the tendon (Leydig). The tendon itself consists of longitudinally arranged bundles of white fibrous tissue with cellstendon cells-embracing them. There is a loose capsule or sheath of connective-tissue-the peritendineum of Kollman-surrounding the whole and carrying the blood-vessels, lymphatics, and nerves. The tendons move in the tendon-sheaths, which are moistened by a mucous fluid. In most situations, muscular fibres are attached by means of tendons to some fixed point, but in other situations (face) the ends terminate between the connective-tissue elements of the skin.

[Blood-Vessels. - Muscles, being very active organs, are richly supplied with blood. The blood-supply of a muscle differs from some organs in not constituting an actual vascular unit, 
supplied ouly by one artery and one vein, thus being unlike the kidney, spleen, \&c. Each muscle usually receives screral branches from different arteries, and branches enter it at certain distances along its whole length. The artery and vein usually lie together in the connectivetissue of the perimysium, while the capillaries lie in the endomysium. The capillaries lie between the muscular fibres, but outside the sarcolemma, where they form an elongated rich plexus with numerous transverse branches (fig. 308). The lymph to nourish the sarcous substance must traverse the sarcolemina to reach the former. In the red muscles of the rabbit (e.g., semitendinosus) the capillaries are more wavy, while on the transverse branches of some of the capillaries, and on the veins, there are small, oval, saccular dilatations, which act as reservoirs for blood (Ranvier).]

[Lymphatics. - We know very little of the lymphatics of muscle, although the lymphatics of tendon and fascia have been carefully studied by Ludwig and Schweigger-Seidel. There are lymphatics in the endomysium of the heart, which are continuous with those under the pericarlium. This subject still requires further investigation. Compare the lymphatics of the fascia lata of the dog (fig. $227, \S 201$.]

Entrance of the Nerve. - The trunk of the motor nerve, as a rule, enters the muscle at its geometrical centre (Schuralbe); hence, the point of entrance in muscles with long, parallel, or spindle-shaped fibres lies near its middle. If the muscle with parallel fibres is more than 2 to 8 centimetres [1-3 inches] in length, several branches enter its middle. In triangular muscles, the point of entrance of the nerve is displaced more towards the strong tendinous point of convergence of the muscular fibres. A nerve-fibre usually enters a muscle at the point where there is the least displacement of the muscular substance during contraction.

Motor Nerve.-Every muscle-fibre receives a motor nerve-fibre (fig. 302, 1, N). Each nerve does not contain originally as many motor nerve-fibres as there are

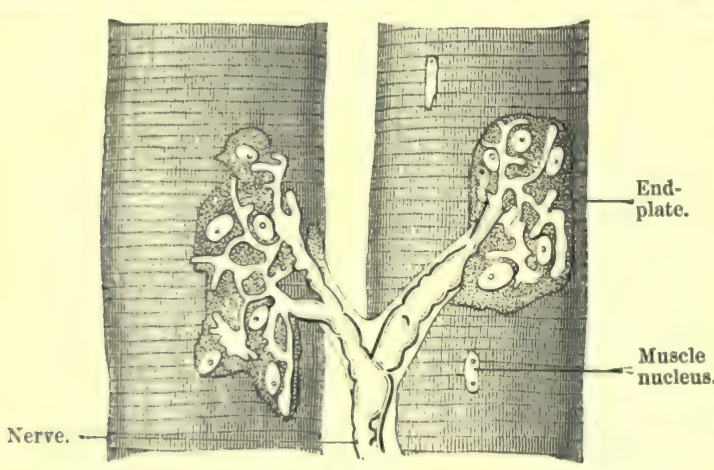

Fig. 309 .

Jluscular fibres with motorial end-plates. muscular fibres in the muscle it enters; in the human eyemuscles, there are only 3 nerve-fibres to 7 muscular fibres; in other muscles (dog), 1 nerve-fibre to 40 or 80 (Tergast). Hence, when a nerve enters a muscle it must divide, which occurs dichotomously [at.Ranvier's nodes], the structure undergoing no change until there are exactly as many nervefibres as muscular fibres. In warm-blooded animals each muscular fibre has only one, while cold-blooded animals

have several points of insertion of the nerve-fibre (Sandmann). A nerve-fibre enters each muscular fibre, and where it enters it forms an eminence (Doyère, 1840), the "motorial end-plate" (figs. 302, 1, e, 309, 310, 311).

[The elaborate investigations of $\mathrm{K}$. Mays on the exact distribution of nerve-fibres in the muscles of the frog have conclusively proved - apart from experimental reasons - that parts of muscles receive no nerve-fibres at all, large portions being free from nerves. This has been proved for all classes of vertebrates except osseous fishes.]

[The mode of termination of a motor nerve in a muscular fibre is not the same in all animals, but in every case it pierces the sarcolemma, and its ultimate distribution has a distinct hypolemmal character. The Doyère's eminence is present in most mammals and reptiles, but in amphibians and birds, the ending is flat on the muscle-fibre. Most of the results known to us have been worked out by Kiihne. The nerve-endings, then, are confined to very small spots or areas on the nuscular filres, termed by Kiihne "fields of innervation." Most nerve-fibres have only one such field, but very long fibres may have, at most, eight. One or more medullated nerve-fibres pass-as preterminal or epilemmal fibres - from the point of division of the nerve-fibre to the nuscular fibre, to pass into the nerve-endings. The nerve-endings consist of divisions of the axial cylinder, which are distributed over the sarcons substance without (so far as is known) forming any direct connection with it. The endings, however, lie in direet 
contact with it. This branched arrangement of the axis-cylinder under the sarcolemma, Kiihne has called a "motor-spray" ("motorisches Geweih"), and the mode of distribution of the branches varies in different classes of animals. In the frog (fig. 310), tailed amphibians, and birds, the hypolemmal branches of the axis-cylinder form bayonet-like and branched endings. In the lizard, snakes, and mammals, the branches are often curved or twisted, and possessed of lobes, and as the division is very variable, there is every form from a simple hook-like bend to a highly arborescent termination.]

[Where a motor nerve enters a muscular fibre at the eminence of Doyère, the sheath of the

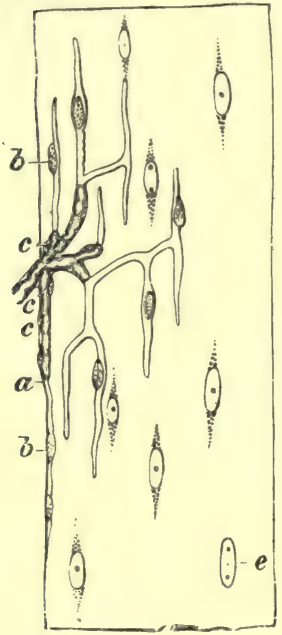

Fig. 310 . nerve-fibre, known as the perineural or Henle's sheath ( $\$ 321$ ), becomes continuous with the sarcolemma. The eminence itself consists of a mass of protoplasm - or sarcoplasm - called by Kühne sarcoglia - which contains granules and nuclei, the latter with a membrane and peculiar nucleoli; the nuclei themselves are the fundamental or basal nuclei of the sarcoglia. The outer surface of the eminence is covered by a membrane called telolemma by Kiihne, but which in reality consists of two membranes, an outer one, the epilemma, continuous with the perineural or Henle's sheath, and an inner one, the endolemma, the continuation of the sheath of Schwann of the nerve-fibre, both ultimately being connected with the sarcolemma. As the nerve pierces Fig. 311.

Fig. 310.-Motor nerve-ending in the frog (Kühne). $\quad \alpha$, Profile. view of entrance of the nerve; $b, b$, nuclei of the branches of the axial cylinder ; $c, c, c$, nuclei of Henle's sheath ; $e$, muscle nuclei. Fig. 311.-Motor nerve-ending in lizards, mammals, and man. Schematic after Kiihne. A, axis-cylinder; $\mathrm{A}^{\prime} \mathrm{A}^{\prime}$, terminal branches of $\mathrm{A} ; a, a$, myelin of nerves; $b$, perineural or Henle's sheath, and its nuclei $(c) ; d$, nuclei of telolemma ;

$\mathrm{B}$, bed ; D, large granule in B ; C, nuclei of the bed ; E, muscle nuclei ;

F, contractile substance.

the muscular fibre, it loses its myeline, and with it disappears the keratin sheath or axilemma of the axis-cylinder, so that the spraylike ending is accompanied only by the telolemma (fig. 311). The telolemma contains nuclei which are derived from Henle's sheath (Kühne).]

[In some animals, such as the lizard, in order to see the nerve terminations, it is sufficient to stain portions of fresh muscles with Delafield's logwood.]

[Nerve-endings, then, are sublemmar, and the terminations of the nerves never penetrate into the depth of the muscular fibre, but come into direct contact with the contractile prism or cylinder moistened by the fluids of the muscle. In many cases the striped substance is separated from the blunt nerve-endings by some of the sarcoglia, which in some cases penetrate and traverse the other constituent of the fibre. The latter Kühne has called "rhabdia." The antler-like division of the axis-cylinder or spray, in contact with the muscular substance, serves to conduct the excitation from the former to the latter, but excitation of the muscular substance is never transmitted in the reverse order to the nerve-ending (Kühne).]

Each muscular fibre of the cray fish is supplied by two nerve-fibrils arising from separate axis-cylinders (Biedermann).

Sensory fibres also occur in muscles, and they are the channels for muscular sensibility. They seem to be distributed on the outer surface of the sarcolemma, where they form a branched plexus and wind round the muscular fibres (Arndt, Sachs); but, according to Tschirjew, the sensory nerves traverse the substance of the muscle, and after dividing dichotomously, end only in the aponeurosis, either 
suddenly or by means of a small swelling - a view confirmed by Rauber. The existence of sensory nerves in muscles is also proved by the fact that stimulation of the central end of a motor nerve, e.g., the phrenic, causes increase of the bloodpressure and dilatation of the pupil (Asp, Kowalewsky, Nawrocki), as well as by the fact that when they are inflamed they are painful. They of course do not degenerate after section of the anterior root of the spinal nerves.

Red and Pale Muscles.--In many fishes, (skate, plaice, herring, mackerel) ( $W$. Stirling), birds, and mammals (rabbits), there are two kinds of striped muscle (Krause), differing in colour, histolosical structure (lanvier), and physiological properties (Kronecker and Stirling). some are "red," e.g., the soleus and semitenlinosus of the rabbit, and others "pale," e.g., the adhluctor magnus. In the pale muscles the transverse striation is less regular, and their nuclei fewer than in the rell muscles (Ranvier); they contain less glycogen and myosin. [W. Stirling finds that the red muscles in many fishes, e.g., the mackerel, contain granules of oil, and present all the apjearance of. muscle in a state of fatty degeneration, while the pale muscles, lying side by side, contain no fatty granules.]

Julius Arnold found in human nuscles an extensive distribution of pale fibres amongst the red ones, and indeed in the same muscle in the frog and mammals, red and pale fibres occur together, in fact this is the case in almost every muscle (Grïtzner).

[Spectrum. - The red colour of the ordinary skeletal muscle is due to hæmoglobin in the sarcous substance $(K$ iuhne). This is proved by the fact that the colour is retained after all the blood is washed out of the vessels, when a thin muscle still shows the absorption-bands of hæmoglobin when examined with the spectroscope. 7

[Myo-hæmatin.-Mac-Munn points out that although most voluntary muscles owe their colour to hemoglobin, it is accompanied by myo-hamatin in most cases, and sometimes entirely replaced by it. Myo-hiematin is found in the heart of vertebrates, in the papillary muscles of the human heart, and in abundance in the pectoral muscles of pigeons, and in some muscles of vertebrates and invertebrates, c.I., certain beetles (Hydrophilus, Dytiscus), the common fly, and other insects, spiders, crustaceans, and molluses.]

Muscular Fibres of the Heart. - The mammalian cardiac muscle has certain peculiarities alrealy mentioned (\$43):-(1) It is striped, but it is involuntary ; (2) it has no sarcolemma ; (3) its fibres branch and anastomose; (4) the transverse striation is not so distinct, and it is sometimes striated longitudinally; (5) the nucleus is placed in the centre of each cell (see $\$ 43)$. [The cardiac muscle, - viewed from a physiological point of view, stands midway between striped and unstriped muscle. Its contraction occurs slowly and lasts for a long time (p. 86), while, although it is transversely striped, it is involuntary.]

[Purkinje's Fibres. - These fibres, which form a plexus of greyish fibres under the endo. carlium of the heart of ruminants, have been described already (fig. 28); the cells have, as it were, advanced only to a certain stage of development $(\$ 46)$.]

Development. - Each muscular fibre is developed from a uni-nucleated cell of the mesoblast, which elongates into the form of a spindle. As the cell elongates, the nuclei multiply. The superficial or parietal part of the cell-substance shows transverse markings (fig. 302, $\boldsymbol{\tau}$ ), while the nuclei with a small amount of protoplasm are continuous along the axis of the fibre, where they remain in some animals, but in man they pass to the surface where they come to lie under the sarcolemma. The muscles of the young are smaller and have fewer fibres than those of adults (Budlyr). In developing muscle, the number of fibres is increased by the proliferation of the muscle-corpuscles, which form new fibres.

Striped muscle, besides occurring in the corresponding organs of vertebrata, occurs in the iris and choroid of birds. The arthropoda have only striped muscle, the molluscs, worms, and echinoderms chiefly smooth muscles; in the latter are muscles with double oblique striation (Schucclbe). Accorling to Paneth, in old individuals separate cells with aggregation of contractile substance-so-called Sarcoplasts-unite to form new muscular fibres. Sig. Mayer regards these structures as retrogressive structures, and he calls them Sarcolytes (\$103, II.).

2. Non-Striped Muscle. - [Distribution. - It occurs very widely distributed in the body, in the muscular coat of the lower half of the human œesophagus, stomach, small and large intestine, muscularis muscosæ of the intestinal tract, in the arteries, veins, and lymphatics, posterior part of the trachea, bronchi, infundibula of the lung, muscular coat of the ureter, bladder, urethra, vas deferens, vesicule seminales, and prostate; corpora cavernosa and spongiosa penis, ovary, Fallopian tube, uterus, skin, ciliary muscle, iris, upper eyelid, spleen and capsule of lymphatic glands, tunica dartos of the scrotum, gall-bladder, in ducts of glands, and in some other situations.]

Structure. - Smooth muscular fibres consist of fusiform or spindle-shaped elongated cells, with their ends either tapering to fine points or divided (fig. 312). These contractile fibre-cells may be isolated by steeping a piece of the tissue in a 30 per cent. solution of caustic potash, or a strong solution of nitric acid. They are 45 to $30 \mu\left[\frac{1}{d \sigma}\right.$ to $-\frac{1}{2} \frac{1}{2}$ in. $]$ in length, and 4 to $10 \mu$

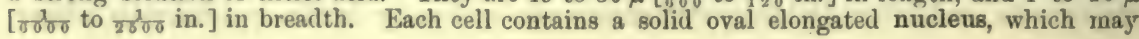


contain one or more nucleoli. It is brought into view by the action of dilute acetic acid, or by staining reagents. The mass of the cell appears more or less homogeneous, [and is surrounded by a thin elastic envelope]. In some places it shows longitudinal fibrillation. [Method.-This fibrillation is revealed more distinctly thus:-Place the mesentery of a newt (Klein) or the bladder of the salamandra maculata (Flemming) in a 5 per cent. solution of arnmonium chromate, and afterwards stain it with picro-carmine. Each cell consists of a thin elastic sheath (sarcolemma of Krause) enclosing a bundle of fibrils (F) which run in a longitudinal direction within the fibre (fig. 313). They are continuous at the poles of the nucleus with the plexus of fibrils which lies within the nucleus, and, according to Klein, they are the contractile part, and when they contract the sheath becomes shrivelled transversely and exhibits what looks like thickenings (S). These fibrils have been observed by Flemming in the cells while living. Sometimes the cells are branched, while in the frog's bladder they are triradiate.

[Arrangement. - Sometimes the fibres occur singly, but usually they are arranged in groups, forming lamellæ, sheets, or bundles, or in a plexiform manner, the bundles being surrounded by connective-tissue.] A very delicate elastic cement-substance unites the individual cells to each other. [This cement may be demonstrated by the action of nitrate of silver. In transverse section (fig. 312, 11) they appear oval or polygonal, with the delicate homogeneous cement between them; but, as the fibres are cut at various levels, the areas are unequal in size, and all of them, of course, are not divided at the position of the nucleus.]

They vary in length from $\frac{1}{100}$ to $\frac{1}{20}$ of an inch; those in the middle coat of the arteries are short, while they are long in the intestinal tract, and-especially in the pregnant uterus. According to Engelmann, the separation of the smooth muscular substance into its individual spindle-like elements is a post-mortem change of the tissue. Sometimes transverse thickenings are seen, which are not due to transverse striation, but to a partial contraction. Occasionally they have a tendinous insertion.

Blood-Vessels. - Non-striped muscle is richly supplied with blood-vessels, and the capillaries

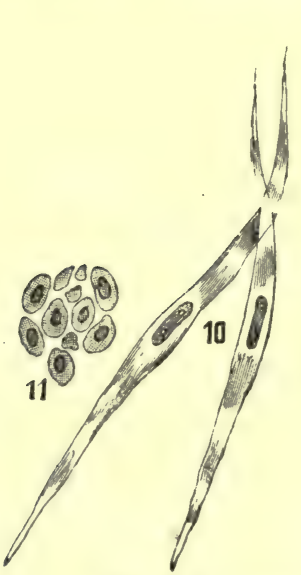

Fig. 312.

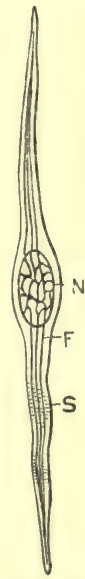

Fig. 313.

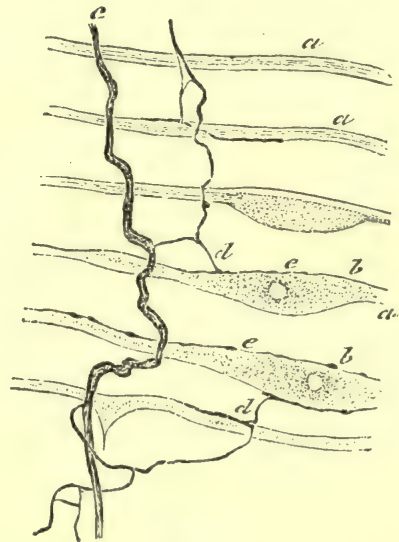

Fig. 314 .

Fig. 312. - Smooth muscular fibres (10); (11) transverse section. Fig. 313. -Smooth muscular fibre from the mesentery of a newt (ammonium chromate). N, nucleus ; F, fibrils ; $\mathrm{S}$, markings in the sheath. F'ig. 314.-Termination of nerve in non-striped muscle.

form elongated meshes between the fibres, [although it is not so vascular as striped muscle]. Iymphatics also occur between the fibres.

Motor Nerves.-According to J. Arnold, they consist of medullated and non-medullated fibres [derived from the sympathetic system] which form a plexus-ground plexus - partly provided with ganglionic cells, and lying in the connective-tissue of the perimysium. [The fibres are surrounded with an endothelial sheath.] Small branches [composed of bundles of fibrils] are given off from this plexus, forming the intermediary plexus with angular nuclei at the noda] points. It lies either immediately upon the musculature or in the connective-tissue between the individual bundles. From the intermediary plexus, the finest fibrillæ $(0.3$ to $0.5 \mu)$ pass off, either singly or in groups, and reunite te form the intermuscular plexus (fig. $314, d$ ), which lies in the cement substance between the muscle-cells, to end, according to Frankenhäuser, in the nucleoli of the nucleus, or in the neighbourhood of the nucleus (Lustig). According to J. Arnold, the fibrils traverse the fibre and the nucleus, so that the fibres appear to be strung upon a fibril passing through their nuclei. According to Löwit, the fibrils reach ouly the interstitial 
substance, while Gscheillen also observed that the finest terminal fibrils, one of which goes to each muscular fibre, ran along the margins of the latter (fig. 314). The course of these fibrils can only be tracel after the action of gold chloride. [Ranvier has traced their terminations in the stomach of the leech.]

Nerves of Tendon. - Within the tendons of the frog, there is a plexus of medullated nervefibres, from which brush-like livided fibres proceed, which ultimately end with a point in nucleated plates, the nerve-flakes of Rollett. According to Sachs, bodies like end-bulbs occur in tendons, while Rauber found Vater's corpuscles in their sheaths ; Golgi found, in addition, spindle-shaped terminal corpuscles, which he regards as a specific apparatus for estimating tension.

\section{PHYSICAL AND CHEMICAL PROPERTIES OF MUSCLE.-1.}

The consistence of the sarcous substance is the same as that of living protoplasm, e.g., of lymph-cells; it is semi-solid, i.e., it is not fluid to such a degree as to flow like a fluid, nor is it so solid that, when its parts are separated, these parts are unable to come together to form a continuous whole. The consistence may be comprared to a jelly at the moment when it is dissolved (e.g., by heat). The power of imbilition is increased in a contracted muscle (Ranke).

Proofs. - The following facts corroborate the view expressed above :--(a) The analogy between the function of the sarcous substance and the contractile protoplasm of cells $(\$ 9)$. (b) The so-called Porret's phenomenon, which consists in this, that when a galvanic current is conducted through the living, fresh, sarcous substance, the contents of the muscular fibre exhibit a stream. ing movement from the positive to the negative pole (as in all other fluids), so that the fibre swells at the negative pole (Kïhne). (c) By the fact that wave-movements have been observed to pass along the muscular fibre. (d) Direct observation has shown that a small parasitic round worm (Myoryctes Weismanni) moved freely in the sarcous substance within the sarcolemma, while the semi-solid mass closed up in the tract behind it (Kühne, Eberth).

2. Polarised Light. - The contractile substance doubly refracts light, and is said to be anisotropous, while the ground substance causes single refraction, and is isotropous. According to Briicke, muscle behaves like a loubly refractive, positively uniaxial body, whose optical axis lies in the long axis of the fibre. When a muscular fibre is examined under the polarisation microseope, the doubly refractive substance is recognised by its appearing bright in the dark field of the microscople when the Nicols are crossed $(\$ 297)$. During contraction of the muscular fibre, the contractile part of the fibre becomes narrower, and at the same time broader, whilst the optical constants do not thereby undergo any change. Hence, Briicke concludes that the contractile dises are not simple bodies like crystals, but must consist of a whole series of small, cloubly refractive elements arranged in groups, which change their position during contraction and relaxation. These small elements Bricke called disdiaclasts. According to Schipiloff, Danielewsky, and O. Nasse, the contractile anisotropous substance consists of myosin, which occurs in a crystalline condition and represents the disdiaclasts. According to Engelmann, however, all contractile elements are donbly refractive, and the direction of contraction always coincides with the optical axis.

The investigations of $\mathbf{v}$. Ebner have shown that during the process of growth of the tissue, tension is produced-the tension of bodies subjected to imbibition-which results in double refraction, and so gives rise to the condition called anisotropous. During a sustained contraction, the index of refraction of the muscular fibre increases (Exner).

[Reaction. - If a transverse section of a living excised muscle be pressed upon a strip of blue litmus paper, the latter may assume a reddish tinge, and if upon a red litmus paper the latter may assume a bluish tinge, but it will not alter violet litmus paper. This is the amphochromatic or amphoteric reaction, indicating that the muscle is neutral. It may, however, give only an alkaline reaction. A living muscle plunged into boiling water still retains its neutral or alkaline reaction; but a muscle, which has been tetanised, or is in rigor mortis, is decidedly acid.]

The chemical composition of muscle undergoes a great change after death, owing to the spontaneous coagulation of a proteid within the muscular fibres. As 'frog's muscles may be frozen and thawed, and still remain contractile, they cannot, therefore, be greatly changed by the process of freezing. Kühne bled frogs, cooled their muscles to $10^{\circ}$ or $7^{\circ} \mathrm{C}$., pounding them in an iced mortar, and expressed their juice through linen. The juice so expressed, when filtered in the cold, forms a neutral, or alkaline, slightly yellowish, opalescent fluid, the so-called "muscle-plasma." Like blood-plasma, it coagulates spontaneously; at first it is like a uniform soft jelly, but soon becomes opaque; doubly refractive fibres and 
specks, similar to the fibrin of blood, appear in the jelly, and as these begin to contract, they squeeze out of the jelly an acid "muscle-serum." [Halliburton finds that the muscles of warm-blooded animals yield a similar muscle-plasma.] Cold prevents or delays the coagulation of the muscle-plasma; above $0^{\circ}$, coagulation occurs very slowly, and the rapidity of coagulation increases rapidly as the temperature rises, while coagulation takes place very rapidly at $40^{\circ} \mathrm{C}$. in cold-blooded animals, or at $48^{\circ}$ to $50^{\circ} \mathrm{C}$. in warm-blooded muscles. The addition of distilled water or an acid to muscle-plasma causes coagulation at once. The coagulated proteid, most abundant in muscle, and which arises from the doubly refractive substance, is called "myosin" (W. Kühne).

Myosin.--It is a globulin ( $\$ 245$ ), and is soluble in strong (10 per cent.) solution of conmon salt, and is again precipitated from such a solution by dilution with water, or by the addition of very small quantities of acids $(0.1$ to 0.2 per cent. lactic or hydrochloric acid). It is soluble in dilute alkalies or slightly stronger acids $(0.5$ per cent. lactic or hydrochloric acid), and also in 13 per cent. ammonium chloride solution. ['The more myosin is freed from salts (especially of calcium) by washing, the more insoluble does it become, both in saline solutions and weak liydrochloric acid. When once precipitated from its solution, it can be redissolved, reprecipitated, and again undergo coagulation a second or even a third time (Halliburton).] Like fibrin, myosin rapidly decomposes hydric peroxide. When treated with dilute hydrochloric acid and heat, it is very rapidly changed into syntonin (\$ 245). Myosin may be extracted from muscle by a 10 to 15 per cent. solution of $\mathrm{NH}_{4} \mathrm{Cl}$, and if it be heated to $65^{\circ}$, it is precipitated again (Danielewsky). Danielewsky succeeded in partly changing syntonin into myosin by the action of milk of lime and ammonium chloride. Myosin occurs in other animal structures (cornea), nay, even in some vegetables ( 0 . Nasse).

Muscle-serum, according to Kühne, still contains three proteids ( $2 \cdot 3$ to 3 per cent.), viz. :-1. Alkali-albuminate, which is precipitated on adding an acid, even at $20^{\circ}$ to $24^{\circ} \mathrm{C}$. 2. Ordinary serum-albumin, 1.4 to 1.7 per cent. $(\$ 32$, a), which cuagulates at $73^{\circ} \mathrm{C}$. 3. An albuminate which coagulates at $47^{\circ} \mathrm{C}$.

[Halliburton finds, however, the following proteids in muscle-plasma.-

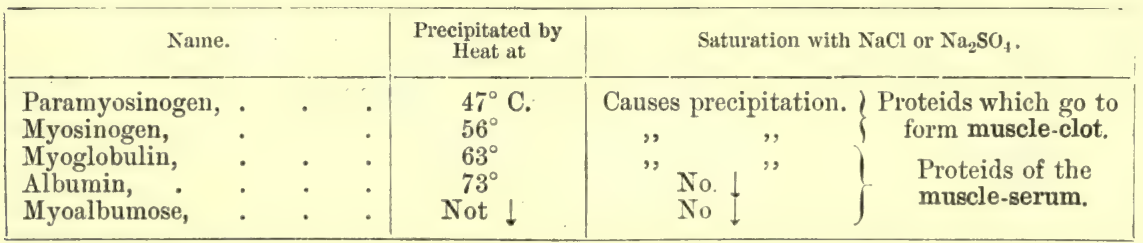

Although the first two go to form the clot of muscle or myosin, paramyosinogen is not essential for coagulation. Besides these bodies there are hæmoglobin and also myo-hæmatin, which is not identical with the blood-pigment. It can be extracted by ether from muscle (e.g., the breast muscle of a pigeon), whereby the ether becomes red. It can exist in an oxidised and reduced condition (MacMunn).]

The other chemical constituents of muscle have been referred to in treating of flesh (§ 233). 1. Muscle-ferments.-Brücke found traces of pepsin and peptone in muscle-juice, [the latter is denied by Halliburton]; Piotrowsky, a trace of a diastatic ferment. [When muscle becomes acid, as in rigor mortis, the pepsin at a suitable temperature $\left(35^{\circ}\right.$ to $40^{\circ} \mathrm{C}$.) acts on the proteids, and albumoses and peptones are formed. Halliburton found a myosin-ferment which has the characters of an albumose. 2. In addition to volatile fatty acids (formic, acetic, butyric), there are two isomeric forms of lactic acid $\left(\mathrm{C}_{3} \mathrm{H}_{6} \mathrm{O}_{3}\right)$ present in muscle with an acid reaction : -(a) Ethylidene-lactic acid, in the modification known as right rotatory sarcolactic or paralactic acid, which occurs only in muscles, and some other animal structures. (b) Ethylene-lactic acid in small amount $(\$ 251,3, c)$. It was formerly assumed that lactic acid is formed by fermentation from the carbohydrates of the muscle (glycogen, dextrin, sugar), and Maly has observed that paralactic acid is occasionally formed when these bodies undergo fermentation. According to Böhm, 
however, the glycogen of muscle does not pass into lactic acid, as during rigor mortis, if putrefaction be prevented, the amount of glycogen does not diminish. If muscle be suddenly boiled or treated with strong alcohol, the ferment is destroyed, aud hence the aciditication of the muscular tissue is prevented (Du Bois-Reymond). Acil potassium phosphute also contributes to the acid reaction. 3. Carnin $\left(\mathrm{C}_{7} \mathrm{H}_{5} \mathrm{~N}_{4} \mathrm{O}_{3}\right)$ which is changed by bromine or nitric acid into sarkin, occurs to the extent of 1 per cent. in Liebig's extract of meat (Weidel). 4. Urea, 0.01 per cent. (Haycreft). [There is much urea in the muscles of the skate.] 5. Glycogen occurs to the amount of over 1 per cent. after copious flesh feeding, and to 0.5 per cent. during fasting. It is stored up in the muscles, as well as in the liver, during digestion, but it disappears during hunger. It is perhaps formed in the muscles from proteids $(\S 174,2)$. 6. Lecithin, derived in part from the motur nerve-endings (\$23 and $\$ 251)$. 7. The gases are $\mathrm{CO}_{2}$ (15 to 18 vol. per cent.), partly absorbed, partly chemically united; some absorbed $\mathrm{N}$, but no $\mathrm{O}$, although muscle continually absorbs $\mathrm{O}$ from the blood passing through it (L. Hermann). The muscles contain a substance whose decomposition yields $\mathrm{CO}_{2}$. When muscles are exercised, this substance is used up, so that severely fatigued muscles yield less $\mathrm{CO}_{2}$ (Stinzing). [All muscles have not the same chemical composition.]

294. METABOLISM IN MUSCLE.-[In living muscle we have to study the transformations of energy, and the chemical changes on which these depend. But as we cannot examine the chemical changes which occur during a contraction, we are confined to a study of (1) the composition of a muscle before and after contraction, and (2) the effect of contraction on the medium surrounding or passing through a muscle. We may observe the effect produced by a muscle upon air or other gases to which an excised muscle is exposed, or we may investigate the changes which the blood undergoes in passing through a muscle, and if the muscle be still in situ, the effect upon the general excreta. These methods may be applied to muscle in various conditions, passive or active, dead or dying, to excised muscles or those still under normal circumstances.]

I. A passive muscle continually absorbs a certain amount of $\mathrm{O}$ from the blood flowing through its capillaries, and returns a certain amount of $\mathrm{CO}_{2}$ to the bloodstream. The amount of $\mathrm{CO}_{2}$ given off is less than corresponds to the amount of $\mathrm{O}$ absorbed. Excised muscles freed from blood exhibit an analogous but diminished gaseous exchange. As an excised muscle remains longer excitable in $\mathrm{O}$ or in air than in an atmosphere free from $\mathrm{O}$, or in indifferent gases, we must conclude that the above-named gaseous exchange is connected with the normal metabolism, and is a condition on which the life and activity of the muscle depend. [Resting living muscles also exhale $\mathrm{CO}_{2}$ ]

If a living muscle be excised, and if blood be perfused through its blood-vessels, the amount of $\mathrm{O}$ used uy is, within pretty wide limits, almost independent of temperature ; if the variations of temperature be great, it rises and falls with the temperature. The $\mathrm{CO}_{2}$ given off by muscular tissue (less than the $\mathrm{O}$ used up) falls when the muscle is cooled, but it is not increased when the muscle is subsequently warmed (Rubner).

This exchange of gases must be distinguished from the putrefactive phenomena due to the development of living organisms in the muscle. These putrefactive phenomena are also connected with the consumption of $\mathrm{O}$ and the excretion of $\mathrm{CO}_{2}$, and occur soon after death $(L$. Hermann).

II. In an active muscle the blood-vessels are always dilated (Ludwig and Sczelkow, Gaskell) - a condition pointing to a more lively material exchange in the organ. [The dilatation of the blood-vessels can be observed microscopically in the contracting mylo-hyoid muscle of the frog.] Hence, the active muscle is distinguished from the passive one by a series of chemical transformations.

1. Reaction. - The neutral or feebly alkaline reaction of a passive muscle (also of the non-striped variety) passes into an acid reaction during the activity of the muscle, owing to the formation of paralactic acid (Du Bois-Reymond, 1859); the 
degree of acidity increases up to a certain extent, according to the amount of work performed by the muscle ( $R$. Heidenhain). The acidification is due, according to Weyl and Zeitler, to the phosphoric acid produced by the decomposition of lecithin and (? nuclein).

It is doubtful if the acidity is due to lactic acid, as Warren and Astaschewsky find that there is less lactic acid in the active than in the passive miscle. Marcuse, however, supports the lactic acid theory, while Moleschott and Battistini, agree that the passive musele contains acid, but the fatigued muscle contains more, especially of phosphoric acid and $\mathrm{CO}_{2}$.

2. Production of $\mathbf{C O}_{2}$ - An active muscle excretes considerably more $\mathrm{CO}_{2}$ than a passive one :- $(a)$ active muscular exertion on the part of a man or of animals increases the amount of $\mathrm{CO}_{2}$ given off by the lungs $(\$ 127) ;(b)$ venous blood flowing from a tetanised muscle of a limb contains more $\mathrm{CO}_{2}$, more $\mathrm{CO}_{2}$ being formed than corresponds to the O, which has simultaneously been absorbed (Ludvig and Sczelkow). The same result is obtained when blood is passed through an excised muscle artificially; $(c)$ an excised muscle caused to contract excretes more $\mathrm{CO}_{2}$. (Compare $\$ 368$.)

3. Consumption of 0xygen.-An active muscle uses up more $\mathrm{O}-(a)$ when more muscular work is done, the body absorbs much more O $(\$ 217)$-even 4 to 5 times as much (Regnault and Reiset); (b) venous blood flowing from an active muscle of a limb contains less O (Ludwig, Sczelkow, and Al. Schmidt). Nevertheless, the increase of $\mathrm{O}$ used up by the active muscle is not so great as the amount of $\mathrm{CO}_{2}$ given off ( $v$. Pettenkofer and $v$. Voit). The increase of $\mathrm{O}$ used up may be ascertained even during the period of rest directly following the period of activity, and the same is the case with the $\mathrm{CO}_{2}$ excreted (v. Frey).

As yet, it is not possible to prove by gasometric methods, that $\mathrm{O}$ is used up in an excised muscle free from blood. Indeed, the presence of $\mathrm{O}$ does not seem to be absolutely necessary for the activity of muscle during short periods, as an excised muscle may continue to contract in a vacuum, or in a mixture of gases free from $\mathrm{O}$, and no $\mathrm{O}$ can be obtained from muscular tissue (L. Hermann). A frog's muscles rob easily reducible substances of their $\mathrm{O}$; they discharge the colour of a solution of indigo; muscles which have rested for a time, acting less energetically than those which have been kept in a state of continued activity (Grützner, Gscheidlen).

4. Glycogen.-The amount of glycogen $(0.43$ per cent. in the muscles of a frog or rabbit) and grape-sugar is diminished in an active muscle (O. Nasse, Weiss), but muscles devoid of glycogen do not lose their excitability and contractility. Hence, glycogen is certainly not the direct source of the energy in an active muscle. Perhaps it is to be sought for in an as yet unknown decomposition-product of glycogen (Luchsinger). [There is more glycogen in the red than in the pale muscles of a rabbit.]

5. Extractives. - An active muscle contains less extractive substances soluble in water, but more extractives soluble in alcohol (v. Helmholtz, 1845); it also contains less of the substances which form $\mathrm{CO}_{2}$ (Ranke); less fatty acids (Sczelkow); less kreatin and kreatinin (v. Voit).

6. During contraction, the amount of water in the muscular tissue increases, while that of the blood is correspondingly diminished (J.Ranke). The solid substances of the blood are increased, while they (albumin) are diminished in the lymph (Fano).

7. Urea.-The amount of urea excreted from the body is not materially increased during muscular exertion (v. Voit, Fick and Wislicenus). According to Parkes, however, although the excretion of urea is not increased immediately, yet after 1 to $1 \frac{1}{2}$ day there is a slight increase. The amount of work done cannot be determined from the amount of albumin which is changed into urea.

[Relation of Muscular Work to Urea. - Ed. Smith, Parkes, and others have made numerous 
investigations on this subject. Fick and Wislicenus (1866) ascended the Faulhorn, and for seventeen hours before and for six hours after the ascent no proteid food was taken-the diet consisting of cakes made of fat, sugar, and starch. The urine was collected in three periods, as follows :-

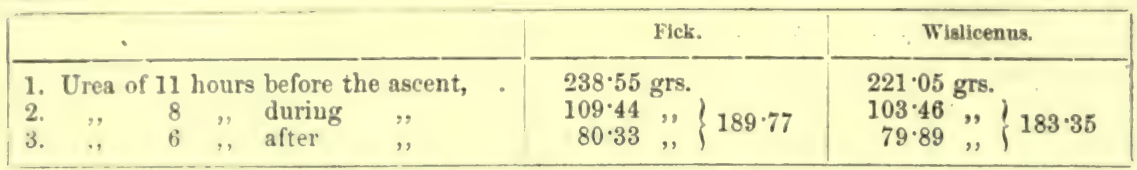

A hearty meal was taken after this period, and the urine of the next eleven hours after the period of rest contained $159 \cdot 15$ grains of urea (Fick), and $176 \cdot 71$ (Wislicenus). All the experiments go to show that the amount of urea excreted in the urine is far more dependent upon the nitrogen ingested, i.c., the nature of the food, than upon the decomposition of the muscular substauce. $A$ regetable diet diminishes, while an animal diet greatly increases, the amount of urea in the urine. North's researches confirm those of Parkes, but he finds that the disturbance procuced by severe muscular labour is considerable. The elimination of phosphates is not affected, while the sulphates in the urine are increased.]

During the activity of a muscle, all the groups of the chemical substances present in muscle undergo more rapid transformations ( $J$. Ranke). It is still a matter of doubt, therefore, whether we may assume that the kinetic energy of a muscle is chiefly due to the transformation of the chemical energy of the carbohydrates which are decomposed or used up in the process of contraction. As yet we do not know whether the glycogen is supplied by the blood-stream to the muscles, perhaps from the liver, or whether it is formed within the muscles themselves from some unknown derivative of the proteids. The normal circulation is certainly one of the conditions for the formation of glycogen in unuscle, as glycogen diminishes after ligature of the blood-vessels (Chanclelon). A muscle in which the blood circulates freely is capable of doing more work than one devoid of blood, and even in the intact body, more blood is always supplied to the contracted muscles.

[Source of Muscular Energy.-The experiment of Fick and Wislicenus definitely proved that the jroteids are not the exclusive, or by any means the chief source of muscular energy. As it is conclusively proved, that during muscular work, there is a great increase in the amount of $\mathrm{O}$ absorbed, and $\mathrm{CO}_{2}$ given off, it is evident that the non-nitrogenous substances of the food must be the chief sources of this energy. We turn naturally to the carbohydrates, and as the latter are chiefly stored up in the form of glycogen in the muscles, it is assumed that glycogen is the chief source of the energy. Glycogen in muscle diminishes during muscular work, and is stored up during rest (Bernard). Külz also found that in dogs the glycogen disappears from the liver during work, and Voit found that the muscle-glycogen disappears before that in the liver. It appears, therefore, that the carboliydrates are a source of muscular energy. But they, again, are not the only source. It is highly probable that glycogen can be formed from proteids, and it is allowable, therefore, to assume that proteids may also serve as a source of muscular energy. If this be not so, it is difficult to understand how carnivora can be fed and maintained in good health for long periods ou lean flesh. The fats are probably also another source. Hence, it would appear that all three of the chief groups of food-stuffs-carbohydrates, proteids, and fats-may serve as the source of muscular energy; but that, so long as nonnitrogenous elements are supplied in the food in sufticient quantity, or are stored up in the body, the muscles do their work chiefly on these. After they are used up, the proteids are, as it were, called up.]

295. RIGOR MORTIS.-Cause.-Excised striped, or smooth muscles, and also the muscles of an intact body, at a certain time after death, pass into a condition of rigidity-cadaveric rigidity or rigor mortis. When all the muscles 
of a corpse are thus affected, the whole cadaver becomes completely stiff or rigid. The cause of this phenomenon depends upon the spontaneous coagulation of a proteid, viz., the myosin of the muscular fibres (Kiihne). Under certain circumstances, the coagulation of the other proteids of the muscle may increase the rigidity. During the process of coagulation, an acid is formed, heat is set free ( $v$. Walther, Fick- $-\$ 223$ ), owing to the passage of the fluid myosin into the solid condition, and also to the simultaneous and subsequently increased density of the tissue.

Properties of a Muscle in Rigor Mortis. - It is shorter, thicker, and somewhat denser (Schmulewitsch); stiff, compact, and solid; turbid and opaque (owing to the coagulation of the myosin); incompletely elastic, less extensible, and more easily torn or ruptured; it is completely inexcitable to stimuli; the muscular electrical current is abolished, (or there is a slight current in the opposite direction); its reaction is acid, owing to the formation of both forms of lactic acid ( $\$ 293$ ), glycero-phosphoric acid (Diakanow); while it also develops free $\mathrm{CO}_{2}$. When an incision is made into a rigid muscle, a fluid, the muscle-serum, appears spontaneously in the wound (\$293).

The first formed lactic acid converts the salts of the muscle into acid salts ; thus, potassium lactate and acid potassium phosphate are formed from potassium phosphate. The lactic acid, which is formed thereafter, remains free and ununited in the muscle.

Amount of Glycogen. - The newest observations of Bohm are against the view that, during rigor mortis, a jartial or complete transformation of the glycogen into sugar and then into lactic acid takes place. During digestion, a temporary storage of glycogen oceurs in the muscles as well as in the liver, so that about as much is found in the muscles as in the liver. There is no diminution of the glycogen when rigidity takes place, provided putrefaction be prevented; so that the lactic acid of rigid muscles cannot be formed from glycogen, but more probably it is formed from the decomposition of the albuminates (Demant, Böhm).

The amount of acid does not vary, whether the rigidity occurs rapidly or slowly (J. Ranke) ; when acidification begins, the rigidity becomes more marked, owing to the coagulation of the alkali-albuminate of the inuscle. Less $\mathrm{CO}_{2}$ is formed from a rigid muscle, the more $\mathrm{CO}_{2}$ it has given off previously, during muscular exertion. A rigid muscle gives off $\mathrm{N}$, and absorbs $\mathrm{O}$. In a cadaveric rigid muscle, fibrin-ferment is present (Al. Schmidt and others). It seems to be a product of protoplasm, and is never absent where this occurs (Ranschenbach). [The myosinferment seems not to be identical with the fibrin-ferment (p. 463).]

[Rigor Mortis and Coagulation of Blood.-Thus, there is a marked analogy between the coagulation of the blood and that of muscle. In both cases, a fluid body yields a solid body, fibrin from blood, and myosin from muscle; the coagula. tion of blood is prevented by neutral salts, and so is the coagulation of myosin; dilution of the salted plasma produces coagulation in both cases; and perhaps the coagulation in both is due to the action of a ferment, the one the fibrin-ferment the other the myosin-ferment. There are, however, points of difference, for myosin can be dissolved, reprecipitated, and coagulated several times, while fibrir. does not undergo recoagulation; the formation of myosin from myosinogen, again, is accompanied by the development of an acid, whereas that of fibrin from fibrinogen is not; further, the formation of myosin is not accompanied by the formation of another globulin, whereas that of fibrin from fibrinogen is.]

Stages of Rigidity. - Two stages are recognisable in cadaveric muscles:- In the first stage, the muscle is rigid, but still excitable; in this stage the myosin seems to be in a jelly-like condition. Restitution is still possible during this stage. In the second stage, the rigidity is well pronounced, with all the phenomena above mentioned.

The onset of the rigidity varies in man from ten minutes to seven hours [but as a rule it is complete within four to six hours after death. The muscles of the jaws are first affected, then those of the neck and trunk, afterwards (as a rule) the lower limbs, and finally the upper limbs]. Its duration is equally variable-one to six days. After the cadaveric rigidity has disappeared, the muscles, owing to 
further decompositions and an alkaline reaction, become soft, and the rigidity disappears (Nysten). The onset of the rigidity is always preceded by a loss of nervous activity. Hence, the muscles of the head and neck are tirst affected, and the other muscles in a descending series (\$352). Disappearance of the rigidity occurs first in the muscles first affected (Nysten). Great muscular activity before death (e.g., spasms of tetanus, cholera, strychnin, or opium poisoning) causes rapid and intense rigidity; hence, the heart becomes rigid relatively rapidly, and strongly. Hunted animals may become affected within a few minutes after death. Usually the rigidity lasts longer the later it occurs. Rigidity does not occur in a foetus before the seventh month. A frog's muscle cooled to $0^{\circ} \mathrm{C}$. does not begin to exhibit cadaveric rigidity for four to seven days.

Stenson's Experiment.- The amount of blood in a muscle has a marked effect upon the onset of the rigidity. Ligature of the muscular arteries causes at first in all mammals an increase of the muscular excitability, and then a rapid fall of the excitability (schmulevitsch); thereafter stiffness occurs, the one stage following closely upon the other (Swammerdam, Nic. Stenson, 1667). [If the ligature be removed in the first stage, the muscle recovers, but in the later stages the rigidity is permanent.] If the artery going to a muscle be ligatured, Stannius observed that the excitability of the motor nerves disappeared after an hour, that of the muscular substance after four to five hours, and then cadaveric rigidity set in.

Pathological. - When the blood-vessels of a muscle are occluded, by coagulation taking place within them, rigidity of the muscles is produced (\$102). True cadaveric rigidity may be produced by too tight bandaging; the muscles are paralysed, rigid, and break up into flakes, while the contents of the fibre are afterwards absorbed ( $R$. Volkmann). Occlusion of the bloodvessels of muscles by infarcts, especially iu persons with atheromatous arteries, may even cause necrosis of the muscles implicated (Finch, Gircendeau).

If the circulation be re-established during the first stage of the rigidity, the muscle soon recovers its excitability (Stannius). When the second stage has set in, restitution is impossible (Kühne). In cold-blooded animals, cadaveric rigidity does not occur for several days after ligaturing the blood-vessels. Brown-Séquard, by injecting fresh oxygenated blood into the blood-vessels, succeeded in restoring the excitability of the muscles of a human cadaver four hours after death, i.e., during the first stage of cadaveric rigidity. Ludwig and Al. Schmidt found that the onset of cadaveric rigidity was greatly retarded in excised muscles, when arterial blood was passed through their blood-vessels. Blood deprived of its $\mathrm{O}$ did not produce this effect. Cadaveric rigidity occurs relatively early after severe hæmorrhage. If a weak alkaline fluid be perfused through the blond-vessels of the dead muscles of a frog, cadaveric rigidity is prevented (Schipilotf).

Section of Nerves.-Preliminary section of the motor nerves causes a later onset of the rigidity in the corresponding muscles (Brown-Séquard, Heineke). [The same result occurs after a hemi-section of the spinal cord or after removal of one cerebral hemisphere (Bierfreund).] In fishes, whose medulla oblongata is suddenly destroyed, cadaveric rigidity occurs much more slowly than in those animals that die slowly (Blane).

[Other Influences. - Rigidity begins much later in the red (11 to 15 hours) than in pale muscles ( 1 to 3 hours post-mortem); the rigor is complete in the white muscles in 10 to 14 hours, in the red in 52 to 58 hours. The extent of shortening due to the rigor is 2 to $2 \frac{1}{2}$ times as great as in the white. In both muscles the resolution of the rigor begins 12 to 15 hours after the completion of the rigidity, so that the red muscles are not completely rigid before the other muscles appear to have passed from a state of rigidity. Temperature has a markerl effect, but it acts more on the resolution than on the onset of the rigor. At $60^{\circ} \mathrm{C}$. the onset begins almost at once, and is complete in a few minutes (Bierfreund). Ether and ehloroform injected into the blood-vessels cause almost instantaneous rigor (Kussmaul).]

Rigidity may be produced artificially by various reagents :-

1. Heat ["Heat-stiffening "] causes the myosin to coagulate at $40^{\circ} \mathrm{C}$. in coldblooded animals, in birds about $53^{\circ} \mathrm{C}$., and in mammals at $48^{\circ}$ to $50^{\circ} \mathrm{C}$. The 
protoplasm of plants and animals, e.g., of the amœba, is coagulated by heat, giving rise to heat rigor.

Schmulewitsch found that the longer a muscle had been excised from the body, the greater was the heat required to produce stiffening. Heat-stiffening differs from cadaveric rigidity thus :-a 13 per cent. solution of ammonium chloride dissolves out the myosin from a cadaveric rigid muscle, but not from one rendered rigid by heat (Schipiloff). If the rigid cadaveric muscles of a frog be heated, another proteid coagulates at $45^{\circ}$, and lastly at $75^{\circ}$ the serumalbumin itself. Hence, both processes together make the muscle more rigid (\$295).

2. When a muscle is saturated with distilled water, it produces "waterstiffening "- an acid reaction being developed at the same time.

Muscles rendered stiff by water still exhibit electromotive phenomena, while muscles rendered rigid by other means do not (Biedermann). If the upper limb of a frog be ligatured, deprived of its skin, and dipped in warm water, it becomes rigid. If the ligature be removed and the circulation re-established, the rigidity may be partially set aside. If there be wellmarked rigidity, it can only be set aside by placing the limb in a 10 per cent. solution of common salt, which dissolves the coagulum of myosin (Preyer).

3. Acids, even $\mathrm{CO}_{2}$, rapidly produce "acid-stiffening," which is probably different from ordinary stiffening, as such muscles do not evolve any free $\mathrm{CO}_{2}$ (L. Hermann). The injection of 0.1 to 0.2 per cent. solutions of lactic or hydrochloric acid into the muscles of a frog produces stiffening at once, which may be set aside by injecting 0.5 per cent. solution of an acid, or by a solution of soda, or by 15 per cent. solution of ammonium chloride. The acids form a compound with myosin (Schipiloft).

4. Freezing and thawing a part alternately, rapidly produce stiffening; and it is aided by mechanical injuries.

Poisons. - Rigor mortis is favoured by quinine, caffein, digitalin, [a concentrated solution of caffein or digitalin, applied to the muscle of a frog, produces rigor mortis], veratrin, hydrocyanic acid, ether, chloroform, the oils of mustard, fennel, and aniseed ; direct contact of muscular tissue with potassium sulphocyanide (Bernard, Setschenow), ammonia, alcohol, and metallic salts.

Position of the Body. - The attitude of the body during cadaveric rigidity is generally that occupied at death ; the position of the limbs is the result of the varying tensions of the different muscles. During the occurrence of rigor mortis, a limb, or more frequently the arm and fingers, may move (Sommer). Thus, if stiffening occurs rapidly and firmly in certain groups of muscles, this may produce movements, as is sometimes seen in cholera. If cadaveric rigillity occurs very rapidly, the body may occupy the same position which it did at the moment of death, as sometimes happens on the battle-field. In these cases it does not seem that a contracted condition of the muscle passes at once into rigor mortis; but between these two conditions, according to Brücke, there is always a very short relaxation.

Muscles which have been plunged into boiling water do not undergo rigor mortis, neither do they become acid (Du Bois-Reymond), nor evolve free $\mathrm{CO}_{2}$ (L. Hermann).

Work done during Rigidity. $-\mathrm{A}$ muscle in the act of becoming stiff will lift a weight, but the height to which it is lifted is greater with small weights, less with heavier weights, than when a living muscle is stimulated with a maximal stimulus.

Analogy between Contraction and Rigidity.-L. Hermann has drawn attention to the analogy which exists between a muscle in a state of contraction and one in a state of cadaveric rigidity - both evolve $\mathrm{CO}_{2}$ and the other acids from the same source; [both acts take place without the consumption of $\mathrm{O}$ ]. The form of the contracted and of the stiffened muscles is shorter and thicker; both are denser, less elastic, and evolve heat; in both cases, the muscular contents behave negatively as regards their electromotive force, in reference to the unaltered, living, resting substance. Hence, he is inclined to regard a muscular contraction as a temporary, physiological, rapidly disappearing rigor. Rigor mortis is in a certain sense the last flickering act of a living muscle, [and he regards contraction as partial death of a muscle. But this is no explanation, and moreover there are important points of difference. We have no proof of a coagulum being formed during contraction, while the extensibility is increased during contraction and much diminished during rigor.]

Disappearance of Rigidity.-When rigor mortis passes off, there is a consider- 
able amount of acid formed in the muscle, which dissolves the coagulated myosin. After a time putrefaction sets in, accomplanied by the presence of micro-organisms and the evolution of ammonia and putrefactive gases $\left(\mathrm{H}_{2} \mathrm{~S}, \mathrm{~N}, \mathrm{CO}_{2}-\$ 184\right)$. [Hermann and Birerfreund attach much importance to the resolution of rigor mortis independently of putrefaction.]

Accorling to Onimus, the loss of excitability which precedes the ouset of rigor mortis occurs in the following order in man:- left ventricle, stomach, intestine (55 minutes); urinary bladder, right ventricle (60 min.); iris (105 min.); muscles of face and tongue (180 min.); the extensors of the extremities (ai)out one hour before the flexors): the muscles of the trunk (five to six hours). The oesophagus remains excitable for a long time (\$ 325).

296. MUSCULAR EXCITABILITY.-By the term excitability or irritability of a muscle, is meant that property of a muscle in virtue of which it responds to stimuli, at the same time becoming shorter and correspondingly thicker. The condition of excitement is the active condition of a muscle produced by the application of stimuli, and is usually indicated by the act of contraction. Stimuli are simply various forms of energy, and they throw the muscle into a state of excitement, while at the moment of activity the chemical energy of the muscle is transformed into work and heat, so that stimuli act as "liberating" or "discharging forces." [These "discharging forces" may themselves be very feeble, but they are capable of causing the manifestation of the transformation of a large amount of energy.] The normal temperature of the body is most favourable for maintaining the normal muscular excitability; the excitability varies as the temperature rises or falls.

As long as the blood-stream within a muscle is uninterrupted, the first effect of stimulation of a muscle is to increase its energising power, partly because the circulation is more lively and the blood-vessels are dilated, but after a time, the energising power is diminished. Even in excised muscles, especially when the large nerve-trunks have already lost their excitability, the excitability is increased after a stimulus, so that the application of a series of stimuli of the same strength causes a series of contractions which are greater than at first (Wundt). Hence, we account for the fact that, although the first feeble stimulus may be unable to discharge a contraction, the second may, because the first one has increased the muscular excitability (Fick).

Effects of Cold.-If the muscles of a frog or tortoise be kept in a cool place, they may remain excitable for ten days, while the muscles of warm-blooded animals cease to be excitable after one and a half to two and a half hours. (For the heart see \$55.) A muscle, when stimulated directly, always remains excitable for a longer time when its motor nerve is already dead.

[Independent Muscular Excitability.-Since the time of Albrecht v. Haller, and R. Whytt, physiologists have ascribed to muscle a condition of excitability which is entirely independent of the existence of motor nerves, but is dependent on certain constituents of the sarcous substance. Excitability, or the property of responding to a stimulus, is a widely distributed function of protoplasm or its modifications. A colourless blood-corpuscle or an amoba is excitable, and so are secretory and nerve-cells. In the first case, the application of a stimulus results in motion in an indefinite direction, in the second in the formation of a secretion, and in the third in the discharge of nerve-energy. In the case of muscle, a stimulus causes movement in a definite direction, called a contraction, and depending on the contractility of the sarcous substance. There are many considerations which show, that excitability is independent of the nervous system, although in the higher animals, nerves are the usual medium through which the excitability is brought into action. Plants however are excitable, and they contain no nerves.]

Numerous experiments attest the "independent excitability" of muscle :-1. There are chemical stimuli, which do not cause movement when applied to motor nerves, but do so when they are applied directly to muscle; ammonia, lime water, 
carbolic acid. 2. The ends of the sartorius of the frog, in which no nerve-terminations are observable by means of the microscope, contract when they are stimulated directly (Kühne). 3. Curara paralyses the extremities of the motor nerves, while the muscles themselves remain excitable ( $\mathrm{Cl}$. Bernard, Kölliker). The action of cold, or arrest of the blood supply in an animal, abolishes the excitability of the nerves, but not of the muscles at the same time. 4. After section of its nerve, a muscle still remains excitable, even after the nerves have undergone fatty degeneration (Brown-Séquard, Bidder). 5. Sometimes electrical stimuli act only upon the nerves and not upon the muscle itself (Brïcke). [6. The foetal heart contracts rhythmically before any nervous structures are discoverable in it.]

[The Action of Curara. - Curara, woorali, urari, or Indian arrow poison of South America, is the inspissated juice of the Strychnos crevauxi. A watery extract of the drug, when injected under the skin or into the blood of an animal, acts chiefly upon the motor nerve-endings, and does not affect the muscular contractility. An active substance, curarin, has been isolated from it (p. 474). Poison a frog by injecting a few milligramınes into the dorsal lymph-sac. In a few minutes after the poison is absorbed, the animal ceases to support itself on its fore-limbs; it lies flat on the table, its limbs are paralysed, and so are the respiratory movements in the throat. When completely uncler the action of the poison, the frog lies in any position, limp and motionless, neither exhibiting voluntary nor reflex movements. If the brain be destroyed and the skin removed, on faradising the sciatic nerve, no contraction of the muscles of the hindlimb occurs, but if the electrical stimulus be applied directly to the muscles, they contract, thus proving that curara poisons the motor connections and not the muscles. If the dose be not too large, the heart still continues to beat, and the vaso-motor nerves remain active.]

[Methods.-(1) Local Application.-Bernard took two nerve-muscle preparations, put some solution of curara into two watch-glasses, and dipped the nerve into one glass and the muscle of the other preparation into the other glass. The curara penetrated into both preparations, and he found, on stimulating the nerve which had been steeped in curara, that its muscle still contracted, so that the curara had not acted on the motor nerve-fibres; while stimulation of the nerve of the other preparation produced no contraction, although the corresponding muscle contracted. In the latter case, the curara had penetrated into the muscle and affected the intramuscular portions of the nerve.]

$[(2)$ But it is the terminal or intra-muscular portions of the nerves, not the nerve-trunk, which are paralysed. Ligature the sciatic artery, or, better still, tie all the parts of the hind-limb of a frog, except the sciatic nerve, at the upper part of a thigh (fig. 315). Inject curara into the dorsal lymph-sac. The poisoned blood will, of course, circulate in every part of the body except the ligatured limb. The shaded parts are traversed by the poison. The animal can still, at a certain stage of the poisoning, pull up the non-poisoned limb, while it cannot move the poisoned one. At this time, although poisoned blood has circulated in the sacral and intra-abdominal parts of the nerves, yet they are not paralysed, so that the poison does not act on this part of the trunk of the

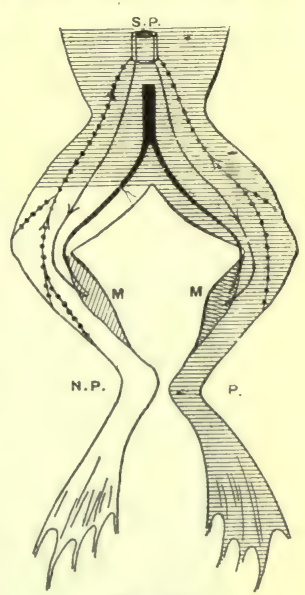

Fig. 315.

Frog with sciatic artery ligatured. S.P., spinal cord with afferent and efferent nerves; P., poisoned, N.P., non-poisoned leg; M, gastrocnemius muscles.

nerve. But we can show that it does not act on any part of the extra-muscular trunk of the nerve. This is done by ligaturing the arteries going to the gastrocnemius muscle, and then poisoning the animal. On stimulating the nerve on the ligatured side, the gastrocnemius of that side contracts, although the whole length of the nerve-trunk was supplied by poisoned blood. Therefore, it is the intramuscular terminations of the nerves which are acted on.] 
[By means of the following arrangement we may prove that the terminal parts of the nerve are paralysed. Ligature the sciatic artery of one leg of a frog, and

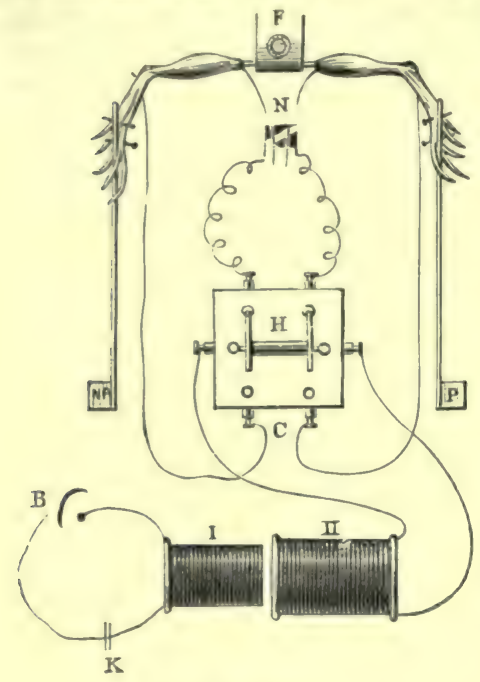

Fig. 316. then inject curara into a lymph-sac. After the animal is fully poisoned, expose the sciatic nerve in both legs, leaving all the muscles below the knee-joint, then clean and divide the femur at its middle. Pin a straw flag to each limb, and fix both femora in a clamp, with the gastrocnemii uppermost, as in fig. 316. Place the two nerves, $\mathbf{N}$, on electrodes attached to two wires coming from a commutator, C (fig. 316). From the opposite binding screws of the commutator, two wires pass to the gastrocnemii. The other two binding screws of the commutator are connected with the secondary coil of an induction machine ( $\S 330)$. The bridge of the commutator can be turned so as to pass the current either through both muscles or both nervesthe latter is the case in the diagram $(\mathrm{H})$. When both nerves are stimulated, only the nompoisoned leg (NP) contracts. Reverse the commutator, and pass the current through both muscles, when both contract.]

Scheme of the curara experiment. $\mathrm{B}$, battery ; I, primary, II, secondary spiral ; $N$, nerves; $F$, clamp; NP, non-poisoned leg; $\mathrm{P}$, poisoned leg; C, commutator ; K, key.

[Rosenthal's Modification. - Pull the secondary coil far away from the primary, and pass the current through both muscles. Gradually approximate the secondary to the primary coil, and in doing so, it will be found that the non-poisoned leg contracts first, but on continuing to push up the secondary coil, both limbs contract. Thus, the poisoned limb

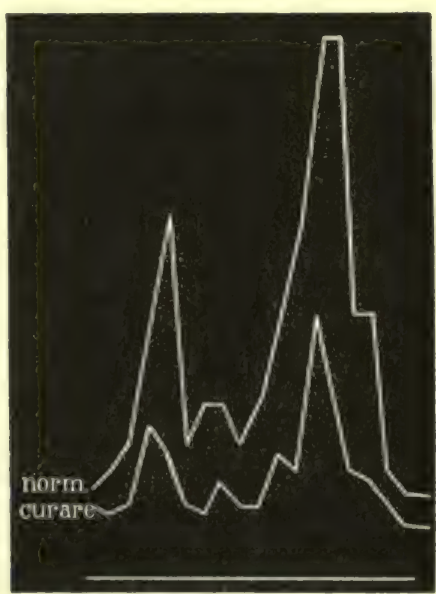

Fig. 317.

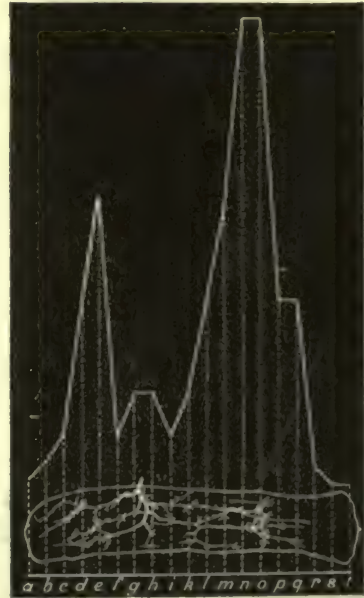

Fig. 318.

Fig. 317. - Curve showing the excitahility in the sartorius of a frog in a normal and curarised muscle. Fig. 318. - Distribution of nerves in the sartorius of a frog and the curve of excitability in different parts of the muscle, i.e., the excitability is greatest where there are most nerve-endings.

does not respond to so feeble a faradic stimulus as the non-poisoned one, a result which is not due? to the action of the curara on the excitability of the muscle. The non-poisoned limb 
responds to a feebler stimnlus because its motor nerve terminations are not paralysed, while the poisoned leg does not do so, because the motor terminations are paralysed. A feebler induced shock suffices to cause a muscle to contract when it is applied to the nerve, than when it is applied to the muscle itself directly. In large doses, curara also affects the spinal cord (p. 474).]

[On what structures does curara act?-These experiments prove that curara does not paralyse the motor nerve-trunks, nor the muscular fibres, and that it acts on the motor terminations within the muscles, but they do not enable us to state the precise part of the nerve-ending so affected. It may act on (1) the nerve just before it pierces the sarcolemma, (2) the sub-lemmar axis-cylinder, (3) the end-plates, (4) the terminal branches or spray. Kiihne and Pollitzer have made it probable that, even when a muscle is thoroughly impregnated with curara, some of the nervous apparatus is unaffected. The sartorius is most excitable where there are most nerves (fig. 318), and even in a muscle profoundly poisoned with curara, the distribution of excitability varies with the number of nerves in the several parts of the musele (fig. 317 ) just as in a normal muscle, with this difference, that the excitability of all the parts of the muscle containing nerves is less than normal. That this variation in excitability is due to nervous structures, is shown by using a polarising anelectrotonic current $(\$ 335)$, which depresses the excitability of nerve-fibres, and then this difference of excitability disappears, the curve of excitability running parallel with the abscissa, so that the difference does not seem to be due to purely muscular causes.]

[Pollitzer, speculating as to which part of the terminal nerve is affected, supposes that all parts beyond the last node of Ranvier retain their functions, and he supposes that it is not the axis-cylinders themselves, but the cement at the nodes, on which the drug exerts its specific action.]

Neuro-Muscular Cells. - Even in the lower animals, e.g., Hydra and Medusæ, there are unicellular structures called "neuro-muscular cells," in which the nervous and muscular substances are represented in the same cell (Kleinenberg and Eimer). [The outer part of these cells is adapted for the action of stimuli, and corresponds to the nervous receptive organ, while the inner deeper part is contractile, and is the representative of the muscular part.]

Muscular Stimuli.-Various stimuli cause a muscle to contract, either by acting upon its motor nerve, or upon the muscular substance itself (\$324). [The former is called indirect stimulation, the latter direct stimulation.]

1. Under ordinary circumstances, the normal stimulus exciting a muscle to contract is the nerve impulse which passes along a nerve, but its exact nature is unknown.

2. Chemical Stimuli.-All chemical substances which alter the chemical composition of a muscle with sufficient rapidity, act as muscular stimuli. Mineral acids ( $\mathrm{HCl} 0 \cdot 1$ per cent.), acetic and oxalic acids, the salts of iron, zinc, copper, silver, and lead, bile, all act in weak solutions as muscular stimuli; they act upon the motor nerve only when they are more concentrated. Lactic acid and glycerin, when concentrated, excite only the nerve; when dilute, only the muscle. [The lower end of the sartorius, which contains no nerves, may be dipped into glycerin, and it will not contract, but if it be dipped deeper to where there are nerveendings, it will contract at once.] Neutral alkaline salts act equally upon nerve and muscle; alcohol and ether act on both very feebly. When water is injected into the blood-vessels, it causes fibrillar muscular contractions ( $v$. Wittich), while a 0.6 per cent. solution of $\mathrm{NaCl}$ may be passed through a muscle for days without causing contraction (Kölliker, O. Nasse). [Carslaw, under Ludwig's direction, however, found that solutions containing 0.5 to 0.2 per cent. $\mathrm{NaCl}$, when perfused through the muscles of a frog, excite many short, powerful attacks of tetanus, separated from each other by periods of rest. Solutions containing 0.5 to 0.7 per cent. $\mathrm{NaCl}$, i.e., so-called "indifferent fluids" or "normal saline," are not without influence, but of all known saline solutions, they injure a nerve-muscle preparation least. Solutions of 1 to 2 per cent. rapidly kill the muscle.] Acids, alkalies, and extract of flesh diminish the muscular excitability, while the muscular stimuli, in small doses, increase it (Ranke). Gases and vapours stimulate muscle; they cause either a simple contraction $(e . g ., \mathrm{HCl})$, or at once permanent contraction or contracture (e.g., Cl). Long exposure to the gas causes rigidity. The vapour of bisulphide of carbon stimulates only the nerves, while most vapours (e.g., $\mathrm{HCl}$ ) kill without exciting then (Kühne and Jani). 
Method. - In making experiments upon the chemical stimulation of muscle, it is inadvisablo to dip the transverse section of the muscle into the solution of the chemical reagent (Hering). The chemical stimulus ought to be applied in solution to a limited portion of the uninjured surface of the muscle; after a few secouds, we obtain a contraction or fibrillar twitchings of the superficial muscular layers (Hering).

Rhythrnical Contraction. - While rhythmical contractions are very marked in smooth muscle, (especially if it is stretched or subjected to considerable internal pressure, as in the hollow viscera), e.g., the intestine, uterus, ureter, blood-vessels, and also in the striped but involuntary cardiac nusculature (\$ \$8), they are not, as a rule, very common in striped voluntary muscle. Chemical stimuli are particularly effective in producing them.] If the sartorius of a eurarised frog be dipved into a solution composed of 5 grms. $\mathrm{NaCl}, 2$ grms. alkaline sodium phosphate, and $0.5 \mathrm{grm}$. solium carbonate in 1 litre of water, at $10^{\circ} \mathrm{C}$., the muscle contracts rhythmically, and may do so for several days, especially with a low temperature (Biedermann). This recalls the rhythmical contraction of the heart. [Kiihne found a similar result. The rhythm is arrester by lactic acid and restored by an alkaline solution of $\mathrm{NaCl}$.] Rhythmical movements may also be induced in the sartorius (frog), by the combined action of a dilute solution of sodic carbonate and an ascending constant electrical current. Compare also the action of a constant current on the heart ( $\$ 58)$.

3. Thermal Stimuli. - If an excised frog's muscle be rapidly heated to $28^{\circ} \mathrm{C}$., a gradually increasing contraction occurs, which, at $30^{\circ} \mathrm{C}$., is more pronounced, reaching its maximum at $45^{\circ} \mathrm{C}$. If the temperature be raised, "heat-stiffening" rapidly ensues. The smorth muscles of warm-blooded animals also contract when they are warmed, but those of cold-blooded animals are elongated by heat (Grïnhagen). If a frog's muscle be cooled to $0^{\circ}$, it is very excitable to mechanical stimuli (Griühagen); it is even excited by a temperature under $0^{\circ}$ (Eckhard).

Cl. Bernari observed that the muscles of animals, artificially cooled, remained excitable many hours after death $(\$ 225)$. Heat causes the excitability to disappear rapidly, but increases it temporarily.

4. Mechanical Stimuli.-Every kind of sudden mechanical stimulus, provided it be applied with sufficient rapidity to a muscle (and also to a nerve), causes a contraition. If stimuli of sufficient intensity be repeated with sufficient rapidity, tetanus is produced. Strong loral stimulation causes a weal-like, long-continued contraction at the part stimulated $(\S 297,3, a)$. Moderate tension of a muscle increases its excitability.

5. Electrical Stimuli will be referred to when treating of the stimulation of nerve (\$324).

Other Actions of Curara. - When it is injected into a frog, either into the blood or subcutaneously, it causes at first paralysis of the intra-muscular cnds of the motor nerves (p. 471), while the muscles themselves remain excitable. The sensory nerves, the central nervous system, viscera, heart, intestine, and the blood-vessels are not affected at first (Cl. Bernard, Kölliker $)$. [If the skin be stimulated, the frog pulls up the ligatured leg reflexly, although the other leg remains quiescent; this shows that the sensory nerve and nerve-centres are still intact; but when the action of the drug is fully developed, no amount of stimulation of the skin or the posterior roots of the nerves will give rise to a reflex act, although the motor nerve of the ligatured limb is known to be excitable; hence, it is probable that the nerve-centres in the cord themselves are ultimately affected. If the dose be very large, the heart and blood-vessels are affected.] In warm-blooded animals, death takes place by asphyxia, owing to paralysis of the diajhragm, but of course there are no spasms. In frogs, where the skin is the most important respiratory organ, if a suitable dose be injected subcutaneously, the animal may remain motion. less for days and yet recover, the poison being eliminated by the urine $(K \ddot{u} h n)$. If the dose be large, the inhibitory fibres of the vagus may be paralysed. In electrical fishes, the sensory nerves, and in frogs, the lymph-hearts are paralysed. A dose sufficient to kill a frog, when injected under its skin, will not do so if administered by the mouth, because the poison seems to be eliminated as rapidly by the kidneys as it is absorbed from the gastric mucous nembrane. For the same reason the flesh of an animal killed by curara is not poisonous when eaten. If, however, the ureters be tied, the poison collects in the blood, and poisoning takes place (L. Hermann). [In this case the manimal may exhibit convulsions. Why? Curara paralyses the respiratory nerves, so that asphyxia is prouluced from the venosity of the blood. It affects the respiratory nerve-endings before those in the muscles generally, so that when the venous blood stimulates the nerve-centres, the partially affected muscles respond by convulsions. Other narcotics may excite convulsions indirectly by inducing a venous condition of the blood, while the motor centres, nerves, and muscles are still unaffected.] Large doses, however, poison un- 
injured animals even when given by the mouth. The nerves and muscles of poisoned animals exhibit considerable electromotive force. [For the effect of curara on lymph-formation $(\S 199,6)$.]

Atropin appears to be a specific poison for smooth muscular tissue, but different muscles are differently affected (Szpilmann, Luchsinger). [This is doubtful. A small quantity of atropin seems to affect the motor nerves of smooth muscle in the same way that curara does those of striped muscle; we must remember, however, that there are no end-plates proper in the former, so that the link between the nerve-fibrils and the contractile substance is probably different in the two cases. It is well known that the amount of striped and smooth muscle varies in the œesophagus in different animals. Szpilmann and Luchsinger found that, after the action of atropin, stimulation of the peripheral end of the vagus will still cause contraction of the striped muscular fibres in the œsophagus, but not of the smooth fibres, although both forms of muscular tissue respond to direct stimulation.]

After section of the motor nerve of a muscle, the excitability undergoes remarkable changes; after three to four days the excitability of the paralysed muscle is diminished, both for direct and indirect stimuli (p. 473); this condition is followed by a stage, during which a constant current is more active than normal, while induced currents are scarcely or not at all effective $(\S 339$, I. $)$. The excitability to mechanical stimuli is also increased. The increased excitability occurs until about the seventh week; it gradually diminishes until it is abolished towards the sixth to the seventh month. Fatty degeneration begins in the second week after section of the motor nerve, and goes on until there is complete muscular atrophy. Immediately after section of the sciatic nerve, Schmulewitsch found that the excitability of the muscles supplied by it was increased.

\section{CHANGES IN A MUSCLE DURING CONTRACTION.-I. Macro-} scopic Phenomena.-1. When a muscle contracts, it becomes shorter and at the same time correspondingly thicker.

The degree of contraction, which in very excitable frogs may be 65 to 85 per cent. (72 per cent. mean) of the total length of the muscle, depends upon various conditions :- $(\alpha)$ Up to a certain point, increasing the strength of the stimulus causes a greater degree of contraction ; (b) as the muscular fatigue increases, i.e., after continued vigorous exertion, the stimulus remaining the same, the extent of contraction is diminished; $(c)$ the temperature of the surroundings has a certain effect. The extent of the contraction is increased in a frog's musclethe strength of stimulus and degree of fatigue remaining the same-when it is heated to $33^{\circ} \mathrm{C}$. If the temperature be increased above this point, the degree of contraction is diminished (Schmulewitsch).

2. The volume of a contracted muscle is slightly diminished (Swammerdam, † 1680). Hence, the specific gravity of a contracted muscle is slightly increased, the ratio to the non-contracted muscle being 1062:1061 (Valentin); the diminution in volume is, however, only $\frac{1}{137}$, although this has recently been denied by J. Ewald.

Methods. - (a) Erman placed portions of the body of a live eel in a glass-vessel filled with an indifferent fluid. A narrow tube communicated with the glass-vessel, and the fluid rose in the tube to a certain level. As soon as the muscles of the eel were caused to contract, the fluid in the index-tube sank. (b) Landois denıonstrates the decrease in volume by means of a manometric flame. The cylindrical vessel containing the muscle is provided with two electrodes fixed into it in an air-tight manner. The interior of the vessel communicates with the gas supply, while there is a small narrow exit-tube for the gas, which is lighted. Every time the muscle contracts, the flame diminishes. The same experiment may be performed with a contracting heart.

3. Total and Partial Contraction.-Normally, all stimuli applied to a muscle or its motor nerve cause contraction in all its muscular fibres. Thus, the muscle conducts the state of excitement to all its parts. Under certain circumstances, however, this is not the case, viz. :- (a) when the muscle is greatly fatigued, or when it is about to die, violent mechanical stimuli, as a vigorous tap with the finger or a percussion hammer (and also chemical or electrical stimuli), cause a localised contraction of the muscular fibres. This is Schiff's "idio-muscular contraction." The same phenomenon is exhibited by the muscles of a healthy man, when the blunt edge of an instrument is drawn transversely over the direction of the muscular fibres. (b) Under certain as yet but imperfectly known conditions, a muscle exhibits so-called fibrillar contractions, i.e., short contractions occur alternately in 
different bundles of muscular fibres. This is the case in the muscles of the tongue, after section of the hypoglossal nerve; and in the muscles of the face, after section of the facial nerve.

[In some phthisical patients there is marked muscular excitability, so that if the pectoral muscle be percussed, a local contraction-idio-muscular-occurs, either confined to the spot, or two waves may proceed outwards and return to the spot struck.]

Cause of Fibrillar Contraction.-According to Bleuler and Lehmann, section of the hypoglossal nerve in rabbits is followed by fibrillar contractions after sixty to eighty hours; these contractions may continue for months, even when the divided nerve has healed and is stimu. lated above the cicatrix so as to produce movements in the corresponding half of the tongue. Stimulation of the lingual nerve increases the fibrillar contractions or arrests them. This nerve contains vaso-dilator fibres derived from the chorda tympani. Schiff is of opinion that the increased blood-stream through the organ is the cause of the contractions. Sig. Mayer found that, by compressing the carotids and subclavian, and again removing the pressure so as to permit free circulation, the muscles of the face contracted. Section of the motor nerves of the face did not abolish the phenomenon, but compression of the arteries did. The cause of the phenomenon, therefore, seems to lie within the muscles themselves. This phenomenon may be compared to the paralytic secretion of saliva and pancreatic juice which follows section of the nerves going to these glands ( $\mu$ p. 214,258). Similar fibrillar contractions occur in man under pathological conditions, but they may also occur without any signs of pathological disturbance. [Fibrillar contractions, due to a central cause, occur in monkeys after excision of the thyroid gland (\$ 103, III.). Some drugs cause fibrillar muscular contractions, e.g., aconitin, guanidin, nicotin, pilocarpin, but physostigmin produces them in warm-blooded animals (not in frogs). According to Brunton these drugs probably act by irritating the motor nerve-endings, as the contractions are gradually abolished by curara.]

II. Microscopic Phenomena.-1. Single muscular fibrilla exhibit the same phenomena as an entire muscle, in that they contract and become thicker. 2. There is great difficulty in observing the changes that occur in the individual parts of a muscular fibre during the act of contraction. This much is certain, that the muscular elements become shorter and broader during contraction, and that the transverse strix approach nearer to each other (Bowman, 1840). 3. There is great difference of opinion as to the behaviour of the doubly refractive (anisotropous) and the singly refractive media.

Engelmann's View. - Fig. 319, 1, on the left represents a passive mus. cular element-from $c$ to $d$ is the doubly refractive contractile substance, with the median dise, $a, b$, in it $h$ and $g$ are the lateral discs. Besides these, in each of the singly refractive dises there is a clear disc- "secondary lise " $f$ and $e$, which is only slightly doubly refractive. This occurs only in the muscles of insects. Fig. 1, on the right, shows the same element in polarised light, whereby the middle area of the element, as far as the contractile substance proper extends, is, owing to its double refraction, bright; while the other part of the muscular element, owing to its being singly refractive, is black. Fig. 319,2 , is the transition stage, and 3 the proper stage of contraction of the muscular element. In both cases, the figures on the left are viewed in ordinary light, and on the right, in polarised light. During contraction (fig. 319,3 ), the singly refractive dise becomes as a whole more refractive, the doubly refractive less so. Consequently, a fibre at a

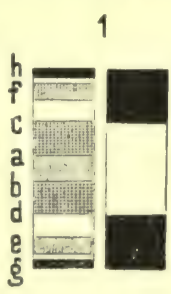
1

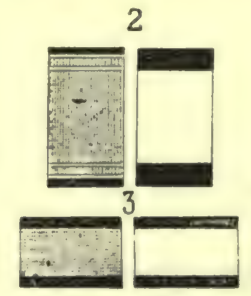

Fig. 319.

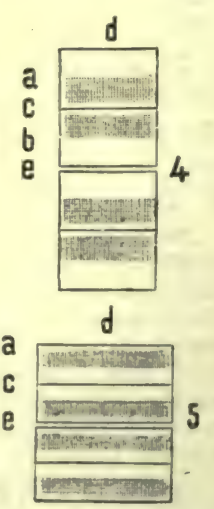

The microscopic appearances during a muscular contraction in the individual elements of the fibrilla. 1,2,3 (after Engelmann); 4, 5 (after Merkel). certain degree of contraction (2), when viewed in ordinary light, may appear homogeneous and but slightly striped transversely = the homogencous or transition stage. During a greater degree of contraction (3), very dark transverse stripes reappear, corresponding to the singly refractive discs. At every stage of the contraction, as well as in the transition stage, the singly and doubly refractive dises are sharply defined, and are recognised by the polariscope as regular alternating layers (in 1,2, and 3 on the right). These do not change places during the contraction. The height of both discs is diminished during contraction, but the singly refractive 
do so more rapidly than the doubly refractive discs. The total volume of each element does not undergo any appreciable alteration in volume during the contraction. Hence, the doubly refractive dises increase in volume at the expense of the singly refractive. From this it is concluded that, during the contraction, fluid passes from the singly refractive into the doubly refractive dises; the former shrink, the latter swell.

Merkel's view is partially diff'erent. In fig. 319, 4, are two muscular elements at rest ; in (5), two in a state of contraction, after Merkel. The grey punctuated areas are the dotibly refractive substance, $c$, the median disc. According to Merkel, during contraction the dark substance lying in the middle of the element changes its position-either in part or as a whole; it leaves the iniddle of the element (the two surfaces of Hensen's median discs, 4,c), and places itself at the lateral dises, 5 at $e$ and $d$, while the clear substance leaves the lateral disc, $4, e$ and $d$, and applies itself to both surfaces of the median disc, 5, c. The clear substance of the isotropous dises is fiuid, and plays a more passive rôle ; during contraction it is in part absorbed by the dark substance which thus swells up. This mutual exchange of place of the substances is accompanied by an intermediate "stage of dissolution," in which the whole contents of the element appear equally homogeneous, in which, therefore, the fluid singly refractive substance has uniformly penetrated the doubly refractive substance. At this moment only the lateral dises are still visible.

[If a living portion of an insect's muscle be examined in its own juice, contraction-waves may be seeu to pass over the fibres. When a contraction-wave passes over part of the fibres, the dises become shorter and broader; at the same time, in the fully contracted part, the dim disc appears lighter than the centre of the light disc. There is said to be a "reversal of the stripes" from what obtains in a passive muscle. Before this stage is reached, there is an intermediate stage where the two bands are almost uniform in appearance.]

Methods. - These phenomena are best observed by "fixing" the different stages of rest or contraction, by suddenly plunging the muscular fibrillæ of insect's muscles into alcohol or osmic acid, which coagulates the muscle-substance. The actual contraction may be observed under the microscope in the transparent parts of the larvæ of insects.

Spectrum. - A thin nuscle, e.g., the sartorius of the frog, when placed directly behind a narrow slit running at right angles to the course of the fibres, yields a diffraction-spectrum. When the muscle contracts, as by mechanical stimulation, the spectrum broadens, a proof that the interspaces of the transverse stripes become narrower (Ranvier).

\section{MUSCULAR CON-} TRACTION. - Methods. - In order to determine the duration of each phase of a muscular contraction, myographs of various forms are used.

V. Helmholtz's Myograph is shown in fig. 320. A muscle, M-say the gastrocnemius of a frog attached to the femur-is fixed by the femur in a clamp, $\mathrm{K}$; its lower end is attached to a movable lever carrying a scale-pan and weight, $\mathrm{W}$, the weight being varied at pleasure. When the muscle contracts, necessarily it raises the lever. At the free end of the lever is a movable style, $\mathrm{F}$, which inscribes its movements on a revolving cylinder caused to rotate at a uniform rate by means of clock-work. The cylinder is covered with smoked

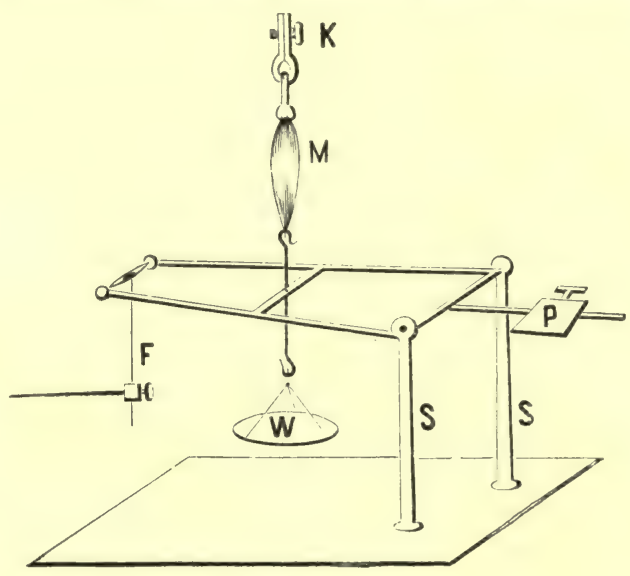

Fig. 320 .

Scheme of v. Helmholtz's myograph. M, muscle fixed in a clamp, $\mathrm{K} ; \mathrm{F}$, writing style ; $\mathrm{P}$, weight or counterpoise for the lever; W, scale-pan for weights; S, S, supports for the lever.

enamelled paper in the flame of a turpentine lamp. When the muscle contracts, it inscribes a curve-the "muscle-curve," or "myogram." The abscissa of the curve indicates the duration of the contraction, but of course the rate at which the cylinder is moving must be known. The ordinates represent the height of contraction at any particular part of the curve.

The muscle-curve may be inscribed upon a smoked glass plate attached to one limb of a vibrating tuning-fork. Such a curve registers the time-units in all its parts. Suppose each vibration of the tuning-fork $=0.01613$ second, then the duration of any part of such a curve is obtained by counting the number of vibrations and multiplying by 0.01613 second.

[Fick's Pendulum Myograph. - A board fixed to the wall carries a heavy iron pendulum, P, 
whose axis, A, A, moves on friction rollers (fig. 321). At the lower swinging end are two glass plates, $G$ and $G^{\prime}$, fixed to a bearer, $T$. 'The plates can be adjusted by meaus of the screw, $s$, so that several curves can be written one above the other. The plate, $\mathrm{G}^{\prime}$, on the posterior surface is merely a compensator, so that, when $\mathrm{G}$ is elevated, $\mathrm{G}^{\prime}$ is lowered, and thus the duration of the oscillation is not altered. The spring catches, $\mathrm{H}, \mathrm{H}$, which can be turned inwards or outwards, are used to fix the pendulum by the teeth, $a$, $a$, when it is drawn to one side. The pendulum is drawn to one sile and fixed, $a$, in $\mathrm{H}$, so that when $\mathrm{H}$ is pulled down, it is liberated

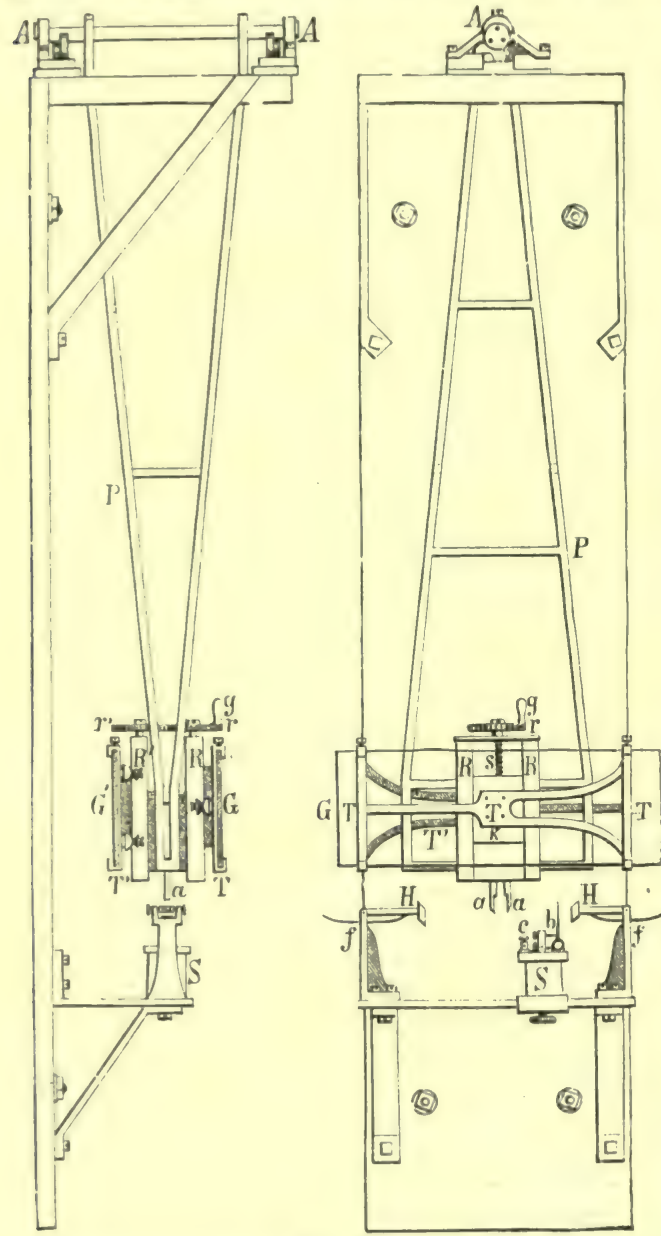

Fig. 321.

Fick's pendulum myograph, as improved by v. Helmholtz ( $\frac{1}{12}$ natural size), side and front view. and swings to the other side, where it is caught by $\mathrm{H}$ at the opposite side. In the improved form, the catches, $\mathrm{H}$, are made to slide along a rod like the arc of a circle, so that the length of the swing can be varied. As the pen. dulum swings from one side to the other, the projecting points, $a, a$, knock over the contact key, $b$, and the current is opened and a shock transmitted to the muscle. The writing-lever to which the muscle is attached is usually a heavy one, and a style writes upon the smoked surface of the glass. Of course, when the pendulum swings, it moves with unequal velocities at diferent parts of its course.]

[When using the pendulum myograph to study a muscular contraction, arrange it as in fig. 322 . The frog's muscle is attached to a writing-lever, which is very like the lever in fig. 321, while the style inscribes its movements on the blackened plate.]

[The pendulum is fixed in the catch, $\mathrm{C}$, as shown in the figure; the key, $\mathrm{K}^{\prime}$, is closed and placed in the primary circuit, while two wires from the secondary coil of an induction machine are attached to the muscle. Wheu the pendulum swings, the projecting tooth, S, knocks over the contact at $\mathrm{K}^{\prime}$, and breaks the primary circuit, when a shock is instantly transmitted through the muscle. Before stimulating, allow the pendulum to swing to obtain an abscissa. The time is recorded by a vibrating tuning-fork, of known rate of vibration, connected with a Dupré's electric ehronograph. Duprés chronograph is merely a sniall electro-magnet with a fine writing style attached, which vibrates when it is introduced in an electrical circuit, in which is placed a vibrating tuningfork. The signal vibrates just as ofteu as the tuning-fork.]

[Du Bois' Spring Myograph. -It consists of a glass plate tixed in a frame, and moving on two polished steel wires, stretched between the supports A and B (fig. 323). At $b$ is a spring which, when it is compressed between the upright, $\mathrm{B}$, and the knob, $b$, drives the glass plate from $\mathrm{B}$ to $\mathrm{A}$. As the plate moves from one side to the other, a small tooth, $d$, on its under surface, opens the $\mathrm{key}, h$, and thus a shock is transmitted to the muscle. The arrangement otherwise is the same as for the pendulum myograph. The smoked glass plate is liberated by the projecting fingerplate attached to the upright, A.]

[Marey's Simple Myograph. - The gastrocnemins is attached to a horizontal lever, which inscribes its movements on a revolving cylinder. This form of myograph, when provided with two levers, is very useful for comparing the action of a poison on one limb, the other being unpoisoned. ]

[Pflüger's stationary form is simply a Helmholtz's myograph (fig. 320) arranged to record 
its movements on a stationary glass plate, so that the muscle merely makes a vertical line or ordinate instead of a curve ; it thus merely indicates the height or extent of the contraction, not its duration.]

A rapidly rotating disc was used by Valentin and Rosenthal for registering the musclecurve, while Harless used a plate which was allowed to fall rapidly, the so-called "Fallmyograph." In all these experiments it is necessary to indicate at the same time the moment of stimulation.

\section{Contraction Curve of Human Muscle.} - In man, another principle is adopted, viz., to measure the increase in thickness during the contraction, either by means of a lever or a compressible tambour, such as is used in Brondgeest's pansphygmograph (fig. 36). [The thickening of the adductor muscles of the thumb may be registered by means of Marey's pince myographique.]

I. Simple Contraction.-If a single shock or stimulus of momentary duration be applied to a muscle, a "simple muscular contraction" [or shortly, a contraction or twitch] is the result, i.e., the muscle rapidly shortens and quickly returns again to its original relaxed condition.

Myogram or Muscle-Curve.-Suppose a single stimulus be applied to a muscle attached to a light writing-lever, which is not "overweighted" with any weight

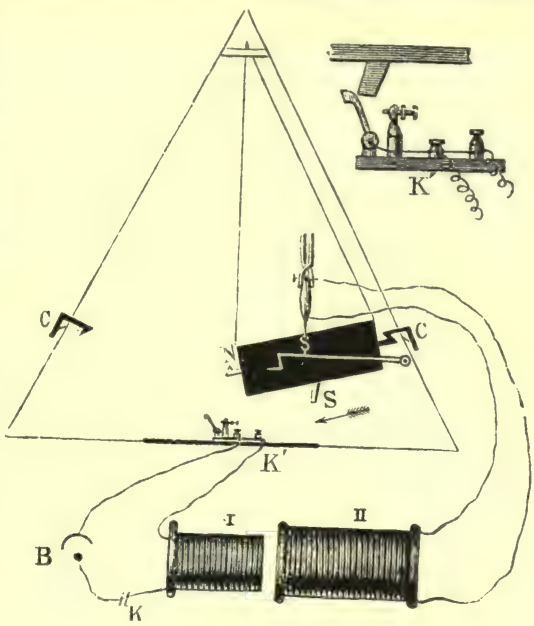

Fig. 322.

Scheme of the arrangement of the pendulum myograph. B, battery ; I, primary, II, secondary spiral of the induction machine; $\mathrm{S}$, tooth ; $\mathrm{K}^{\prime}$, key; $\mathrm{C}, \mathrm{C}$, eatches ; $\mathrm{K}^{\prime}$ in the corner, scheme of $\mathbf{K}^{\prime}$; $\mathbf{K}$, key in primary circuit. attached to it, then, when the muscle contracts, the following events take place :-

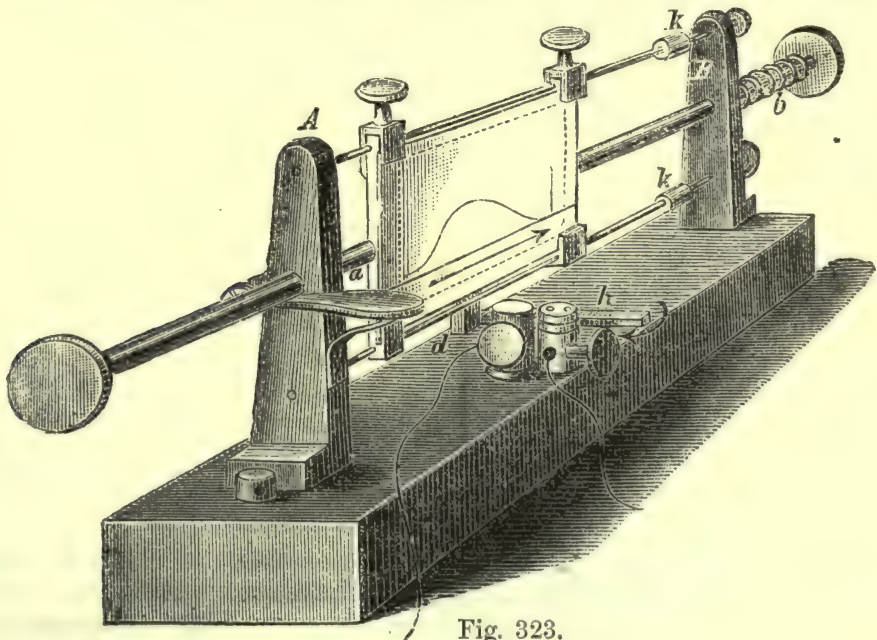

Spring myograph or "shooter."

[(1) A period or stage of latent stimulation (figs. 324, 325).

(2) A period of increasing energy or contraction.

(3) A period of decreasing energy or more rapid relaxation.

(4) A period of slow relaxation, or the elastic after-vibration.] 
The muscle-curve proper is composed of 2,3 , and 4 .

1. The latent period (fig. $324, a, b$ ) consists in this, that the muscle does not begin to contract precisely at the moment the stimulus is applied to it, but the contraction occurs somewhat later, i.e., a short, but measurable interval, elapses

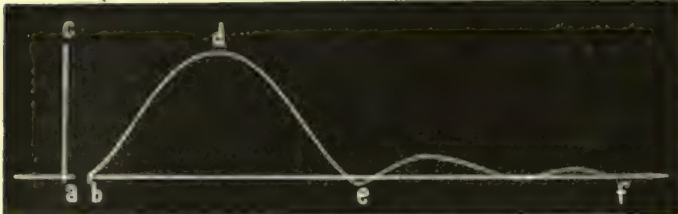

Fig. 324 . between the application of a momentary stimulus and the contraction ( $v$. Helmholtz). If the entire muscle be stimulated by 'a momentary stimulus, e.g., a single break induction shock, the duration of the latent period is about 0.01 second. In smooth muscle, the latent period may last for several seconds.

Muscle-curve produced by a single induction shock applied to a muscle. $a-f$, abscissa ; $a-c$, ordinate ; $a b$, period of latent stimulation : $b d$, period of increasing energy ; $d e$, period of decreasing energy ; $e f$, elastic after-vibrations.

[Although no change be visible in a muscle during the latent period, nevertheless we have proof that some change does take place within the muscle-substance, for we know that the electrical current of the muscle is diminished during this period, or we have what is known as the negative variation of the muscle-current (Bernstein$\$ 333)$.]

In man, the latent periol varies between 0.004 and 0.01 second. If the experiment be so arranged that the muscle can contract as soon as the stimulus is applied to it, i.e., before time is lost in making the musele tense ; or to put it otherwise, if the muscle has not " to take in slack," as it were, the latent period may fall below 0.004 second $(\mathrm{Gad})$. If the muscle be still attached to the body, protected as much as possible from external influences and properly supplied with blood, the latent period may be reduced to 0.0033 or even 0.0025 .

Modifying Influences. - The latent period is shortened by an increased strength of the stimulus and by heat; while fatigue, cooling, and increasing weight lengthen it (Lauterbach, Memulelssohn, Yeo, Cush). The latent period of a break-contraction is longer than that of a make-contraction. The red muscles have a longer latent period than the white. Before the muscle contracts as a whole, the individual fibres within it must have contracted. We must, therefore, conclude that the latency of the individual muscular elements is shorter than that of the entire muscle ( $\mathrm{Gad}$, Tigerstedt).

2. The contraction or stage of increasing energy, i.e., from the moment the muscle begins to shorten until it reaches its greatest degree of contraction $(b d)$. At first the muscle contracts slowly, then more rapidly, and again more slowly, so that the ascending limb of the curve has somewhat the form of an $f$. This stage lasts 0.03 to 0.4 second. It is shorter when the contraction is shorter (weak stimulus) and the less the weight the muscle has to lift. It also varies with the excitability of the muscle, being shorter in a fresh, non-fatigued muscle.

3. Elongation or stage of decreasing energy.-After the muscle has contracted up to its maximum for any particular stimulus, it begins to relax - at first slowly, then rapidly - and lastly more slowly, so that an inverse of an $f$ is obtained $(d e)$. This stage is usually of shorter duration than 2. The duration varies with the strength of the stimulus, being shorter than 2 with a weak stimulus, and longer with a strong stimulus. It also depends upon the extent to which the muscle is loaded during contraction.

4. The fourth stage has received various names-stage of elastic after-vibration [residual contraction or contraction-remainder (Hermann). The aftervibrations $(e f)$, which disappear gradually, depend upon the elasticity of the muscle. The duration of this stage is longest with a powerful contraction, and when the weight attached to the muscle is small].

If the stimulus be applied to the motor nerve instead of to the muscle itself, the contraction is greater (PHüger), and lasts longer (Wundt) the nearer to the spinal cord the stimulus is applied to the nerve. 
[In studying a muscle-curve, the more or less vertical character of the ascent will indicate the rapidity of the contraction, the height above the base line, itsextent, the length of the curve, the duration, and the line of descent, the rate of its extensibility. The form of the muscle-curve will vary with the kind of myograph

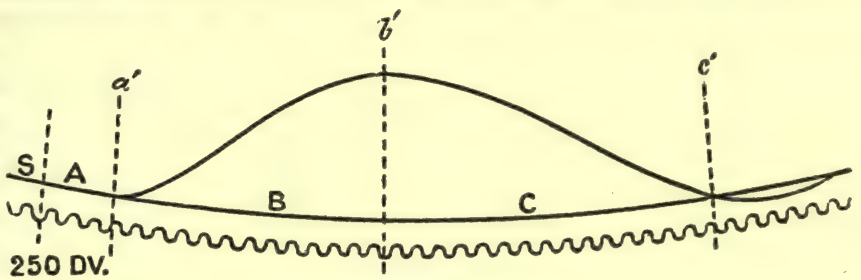

Fig. 325.

Pendulum myograph curve of a frog's gastrocnemius. S, point of stimulation; A, latent period; $\mathrm{B}$, period of shortening, and $\mathrm{C}$, of relaxation. $250 \mathrm{DV}$., tuning-fork vibrating 250 double vibrations per sec. The dotted vertical lines are ordinates (Stirling).

used; if it be stationary, then the muscle will merely record a vertical line ; if the recording surface move quickly, the two parts of the curve will form an acute angle (fig. 327); and if it move with great rapidity, they will have the form of fig. 325 , that obtained with a pendulum myograph. A vibrating tuningfork records time directly under the tracing; whereby the duration of each part of the curve is readily determined.]

[In measuring the myogram, all that is required is to know the moment at which the stimulus was applied, and to note when the curve begins to leave the base line or abscissa. Raise a vertical line or ordinate from each of these points, and the interval between these lines, as measured by the chronograph, indicates the time (fig. 325).]

[The time-relations of a simple muscular contraction caused by a single induction shock may be studied by means of the following arrangement:-Attach a frog's gastrocnemius to a lever, as in fig. 326, and through the frog's muscle place two wires from the secondary coil of an induction machine. A scale-pan with a weight is attached to the lever. On the same support adjust an electro-magnet with a writingstyle in the primary circuit, and in this circuit

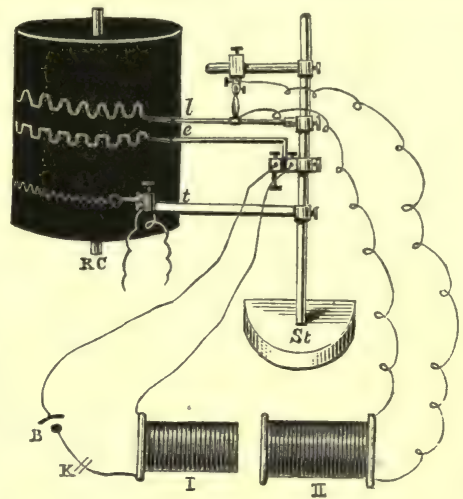

Fig. 326.

Arrangement for estimating the timerelations during contraction of a muscle produced by a faradic shock. $\mathrm{B}$, battery ; K, key in primary circuit; I, primary, II, secondary spiral ; $l$, muscle lever; $e$, electro-magnet in primary circuit ; $t$, electric signal ; $S t$, support; RC, revolving cylinder (after Rutherford).

also place a key $(\mathrm{K})$ to make and break the current. Fix also a Dupré's chrono. graph to the same support, and make it vibrate by connecting it in circuit with a tuning-fork of known rate of vibration, and driven by a galvanic battery. See that the points of all three levers write exactly over each other on the revolving cylinder. 'The upper lever registers the contraction, the electro-magnet the moment the stimulus is applied to the muscle, and the electrical chronograph the time.]

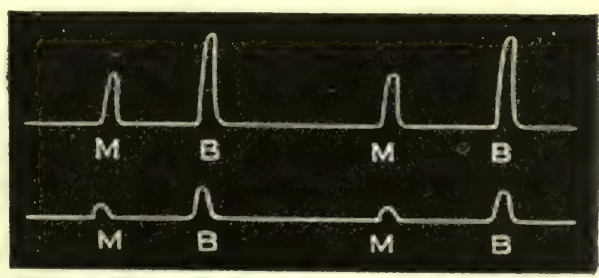

Fig. 327.

[Single make (closing) or break shows the same, but with the muscle fatigued. (opening) induction shocks. A muscle or nerve may be stimulated either with a "make" or "break" induction shock, but it is important to notice that the break 
shock is strunger than the make. In fig. $327, \mathrm{~B}$ shows the effect produced by a single break induction shock, and $\mathrm{M}$ that of a single make shock.]

Overweighted Muscles. - The foregoing remarks apply to curves obtained by a light lever connected with the muscle. If the muscle lever be "overweighted," or overloaded, i.e., if the lever be loaded, so that when the muscle contracts it has to lift these weights, the course of the curve varies according to the weight to be lifted. It is necessary, however, to support the lever in the intervals when the muscle is at rest. As the weights are increased, the occurrence of the contraction is delayed. This is due to the fact that the muscle, at the moment of stimulation, must accumulate as much energy as is necessary to lift the weight. The greater the weight, the longer is the time before it is raised. Lastly il c muscle n ay be so "loaded," or "overloaded," that it camnot contract at all ; this is the limit of the miscular or mechanical energy of the muscle (v. Helmholtz).

Fatigue.-If a muscle be caused to contract so frequently that it becomes "rictijued," the latent period is longer, the curve is not so high, because the

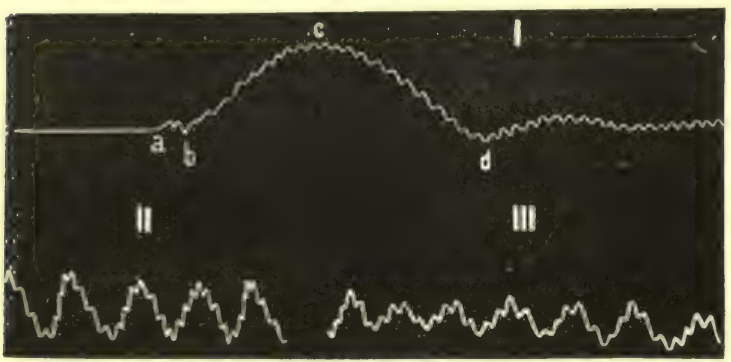

Fig. 328. muscular contraction is less, and the abscissa is longer, i.e., the contraction is slower and lasts longer (fig. 328). Cooling a muscle has the same effect. Soltmann finds that, the fresh muscles of new-born animals behave in a similar manner. The myogram has a flat apex and considerable elongation in the descending limb of the curve.

Constant Current. - If the

I, Contraction of a futiguel frog's muscle writing its contraction on a vibrating plate attached to a tuning-fork. Each vibration $=0.01613$ second ; $a b$, =latent period ; $b c$, stage of increasing energy ; $c d$, of decreasing energy. II, The most rapid writing movements of the right hand inseribed on a vibrating plate. III, The most rapid trembling tetanic the resulting muscular conmovements of the right fore-arm inscribed on the same traction corresponds exactly plate.

to that already described. If, however, the current be made or broken with the muscle itself directly in the circuit, during the make shock, there is a certain degree of contraction which lasts

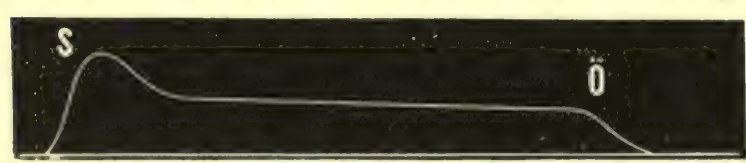

Fig. 329.

for a time, so that the curve assumes the form of fig. 329 , where $\mathrm{S}$ represents the moment of closing or making the current, and $\ddot{O}$ the moment of opening or breaking it $(\S 336, \mathrm{D})$.

The investigations of Cash and Effect on a muscle of closing and opening a constant current. $\mathrm{S}$, closing ; $\ddot{\mathrm{O}}$, opening shock $(\boldsymbol{W} u n d t)$.

Kronecker show that individual muscles have a special form of muscle-curve; the omohyoid of the tortoise contracts more rapidly than the pectoralis. Similar differences occur in the muscles of frogs and mammals. The flexors of the frog contract more rapidly than the extensors (Griit:ner) Sometimes within one and the same muscle there are "red" (rich in glycogen) and "pale" fibres (\$292). The red fibres contract more slowly, are less excitable, and less easily fatigued (Grützner). The muscles of flying insects contract very rapidly, even more than 100 times per second.

Poisons. - Very small doses of curara or quinine increase the height of the contraction (excited by stimulation of the motor nerve), while larger doses diminish it, and finally abolish it altogether. Guanidin has a similar action in large doses, but the maximum of contraction lasts for a longer time. Suitable doses of veratrin also increase the contractions, but the stage of relaxation is greatly strengthenel (Rossbach and Clustermeyer). Veratrin, antiarin, and digitalin, in large doses, act upon the sarcous substance in such a way that the contractions become very prolonged, not uulike a condition of prolonged tetanus (Harless, 1862). The latent period of muscl is poisoned with veratrin and strychnin is shortened at first, and after- 
wards lengthened. The gastrocnemius of a frog supplied by blood containing soda contracts more rapidly (Grütznci). Kunkel is of opinion that muscular poisons act by controlling the imbibition of water by the sarcous substance. As muscular contraction depends on imbibition ( $\$ 297$, II.), the form of the contraction of the poisoned muscle will depeud upon the altered condition of imbibition produced by the drug.

[Veratrin.-If a frog be poisoned with veratrin, and then be made to spring, it does so rapidly, but when it alights again the hind legs are extended, and they are only drawn up after a time. Thus, rapid and powerful contraction, with slow and prolonged relaxation, are the character of the movements. In a muscle poisoned with veratrin, the ascent is quick enough, but it remains contracted for a long time, so that this condition has been called "contracture." A single stimulation may cause a contraction lasting five to fifteen seconds, according to circumstances (fig. 330). Brunton and Cash find that cold has a marked effect on the action of veratrin -in fact, its effect may be permanently destroyed by exposure to extremes of heat or cold. The muscle-curve of a brainless frog cooled artificially, and then poisoned by veratrin, occasionally gives no indications of the action of the poison until its temperature is raised, and

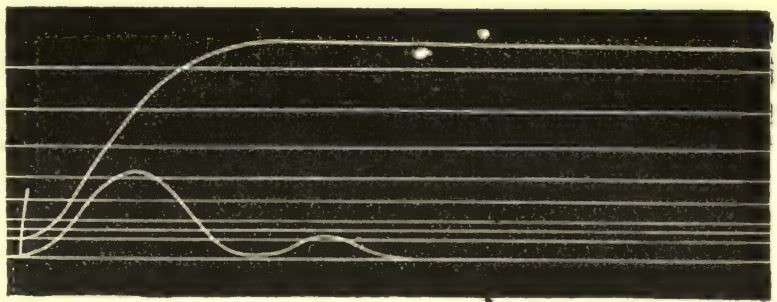

Fig. 330 .

Lower curve is the normal muscle-curve (frog), upper one of the same muscle with veratrin (Stirling).

this is not due to non-absorption of the poison. Cold, therefore, abolishes or lessens the contracture peculiar to a veratrin-curve. Similar results are obtained with salts of barium, and to a less degree by those of strontium and calcium (Brinton and Cash).]

Smooth Muscles. - The muscle-curve of smooth or non-striped muscles is similar to that of the striped muscles, but the duration of the contraction is visibly much longer, and there are other points of difference. Some muscles stand midway between these two, at least as far as the duration of their contractions is concerned.

The "red" muscles of rabbits, the muscles of the tortoise, the adductors of the common mussel, and the heart, all react in a similar manuer.

Contraction-Remainder. - A contracted muscle assumes its original length only when it is extended by sufficient traction, e.g., by means of a weight. Otherwise, the muscle may remain partially shortened for a long time. This condition has been called "contracture" (Tiegel), or, better, contraction-remainder (Hermann). This condition is most marked in muscles that have been previously subjected to strong, direct stimulation, and are greatly fatigued, which are distinctly acid, and ready to pass into rigor mortis, or in muscles excised from animals poisoned with veratrin (fig. 330 ).

Rapidity of Muscular Contraction.-In man, single muscular movements can be executed with great rapidity. The time-relations of such movements can be ascertained by inscribing the movements upon a smoked glass plate attached to a tuning-fork. Fig. 328, II, represents the most rapid voluntary movements that Landois could execute, as, e.g., in writing the letters $n, n$, and every contraction is equal to about 3.5 vibravions ( 1 vibration $=0.01613$ second $)=0.0564$ second. In III, the right arm was tetanised, in which case $2^{\circ}$ to 2.5 vibrations occur $=0.0323$ to 0.0403 second.

V. Kries found that a simple muscular twitch, caused by a single induction shock, is shorter than a momentary voluntary single movement. If the thickening caused by a single voluntary contraction of a muscle be registered directly, the curve shows that the contraction within the muscle lasts longer than the duration of the movement produced in the passive motor apparatus itself. This paradoxical phenomenon is due to the fact that, shortly after the primary voluntary muscular 
contraction, there is a contraction of the antagonistic muscles, whereby a part of the intended movement is, as it were, cut off. During the most rapid voluntary movement in human muscles, v. Kries found that 4 stimuli per second were active, so that a voluntary contraction is really a short tetanus.

Pathological. - In secondary degeneration of the spinal cord after apoplexy, atrophic muscular anchylosis of the linbs, muscular atrophy, progressive ataxia, and paralysis agitans of long standing, the latent period is lengthened; while it is shortened in the contracture of senile chorea and spastic tabes (Ifendelssohn). The whole curve is lengthened in jaundice and diabetes (Eilinger). In cerebral hemiplegia, during the stage of contracture, the muscle-curve resembles the curre of a muscle poisoned with veratrin, and the same is the case in spastic spinal paralysis and amyotrophic lateral sclerosis; in pseudo-hypertrophy of the muscles the ascent is short and the descent very elongated. In muscular atrophy, after cerebral hemiplegia, and in tabes, the latent preriod increases, while the height of the curve dininishes. In chorea, the curve is short (Reaction of Degeneration, \$339). In rare cases in man, it has been observed that the execution of spontaneous movements results in a very prolonged contraction (Thomsen's disease). such cases the muscular fibres are very broad, and the nuclei increased $(E r b)$.

II. Action of Two Successive Stimuli.-Let two momentary stimuli be applied successively to a muscle:-(A) If each stimulus or shock be of itself sufficient to cause a maximal contraction, i.e., the greatest possible contraction which the muscle can accomplish, then the effect will vary according to the time which elapses between the application of the two stimuli. (a) If the second stimulus is applied to the muscle after the relaxation of the muscle following upon the first stimulus, we obtain merely two maximal contractions. (b) If, however, the second stimulus be applied to the muscle during the time that the effect of the first is present, i.e., while the muscle is in the phase of contraction or of relaxation; in this case the second stimulus causes a new maximal contraction, according to the time of the particular phase of the contraction. (c) When, lastly, the second stimulus follows the first so rapidly that both occur during the latent period, we obtain only one maximal contraction $(v$. Helmholtz). It is to be specially noted

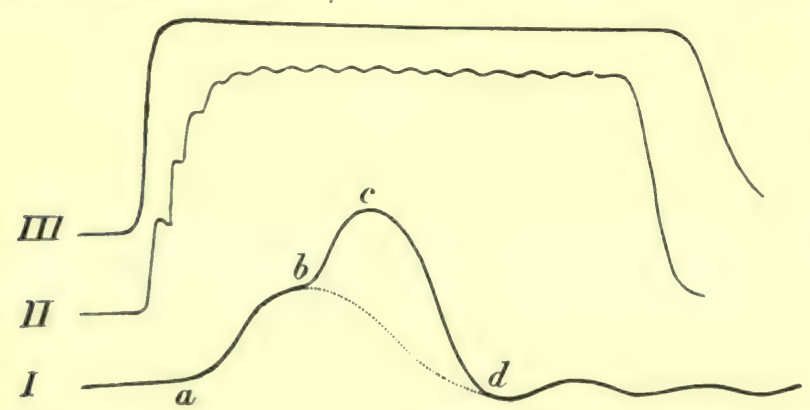

Fig. 331. that a single maximal stimulus never excites the same degree of shortening as tetanic stimulation (III), but only about $\frac{1}{3}$ of the height of the contraction in tetanus.

(B) If the stimuli be not maximal, but only such as cause a medium or sub-maximal contraction, the

I, two successive sub-maximal contractions; II, successive contrac. effects of both stimuli
tions produced by stimulating a muscle with 12 induction shocks are superposed, or per second; III, curve produced with very rapid induction shocks there is a summation (complete tetanus).

331). It is of no consequence at what particular phase of the primary contraction the second shock is applied. In all cases, the second stimulus causes a contraction, just as if the phase of contraction caused by the first shock was the natural passive form of the muscle, i.e., the new contraction $(b, c)$ starts from that point as from an abscissa (fig. 331, I, $\ell$ ). Thus, under favourable conditions, the contraction may be twice as great as that caused by the first stimulus. The most favourable time for the application of the second stimulus is $\frac{1}{20}$ th second after the application of the first (Sewcall). The effects of both stimuli are obtained even when the second stimulus is applied during the latent period ( $v$. Helmholtz). 
III. Tetanus-Summation of Stimuli.-If stimuli, each capable of causing a contraction, and following each other with medium rapidity, be applied to a muscle, the muscle has not sufficient time to elongate or relax in the intervals of stimulation. Therefore, according to the rapidity of the successive stimuli, it remains in a condition of continued vibratory contraction, or in a state of tetanus. Tetanus is, however, not a continuous uniform condition of contraction, but it is a discontinuous condition or form of the muscle, depending upon the summation or accumulation of contractions. If the stimuli are applied with moderate rapidity, the individual contractions appear in the curve (fig. 331, II) ; if they occur rapidly, and thus become superposed and fused, the curve appears continuous and unbroken by elevations and depressions (fig. 331, III). As a fatigued muscle contracts slowly, it is evident that such a muscle will become tetanic by a smaller number of stimuli per second than will suffice for a fresh muscle (Marey). All muscular movements of long duration occurring in our bodies are probably tetanic in their nature $(E d$. Weber).

[Summation of Stimuli.-If a stimulus, insufficient in itself to cause contraction of a muscle, be repeatedly applied to a muscle in proper tempo and of sufficient strength, at first a slight and then a stronger or maximal contraction may be produced. This process of summation occurs also in nervous tissue (\$360).]

[Staircase or "Treppe." Bowditch showed that the cardiac contractions exhibited a "staircase" character, i.e., the height of the second beat is greater than that of the first; and the third than that of the second (p. 84). The same occurs in the case of the muscles of the frog (Ticgel, Minot) and in mammals (Rossbach). Bohr showed that the successive ascending apices in a tetanus-curve have really a stairease character, and that its exact form is that of a hyperbola. Bohr found that (1) this form-the muscle not being fatigued - is independent of the strength and frequency of the stimuli. (2) The height of the series of contractions in tetanus is independent of the frequency of the stimuli, increase of frequency merely causing the staircase to reach its maximum more rapidly. (3) The height of the staircase increaseswithin certain limits - with the

Minimal, Maximal. Sub-maximal. Maximal.

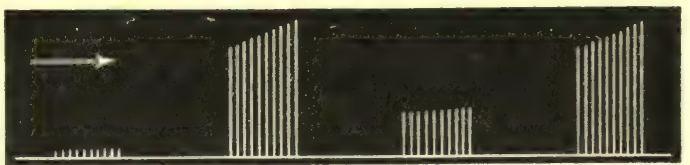

Fig. 332.

Four groups of contractions, interval of simulation 2 seconds, and 5 minutes pause between two groups (Buckmaster). strength of the stimulus. Buckmaster has confirmed this for simple contractions, but as shown in fig. 332, when the stimuli are minimal or sub-maximal, there is usually no staircase character of the contractions, but maximal stimuli always cause it.]

A continued voluntary contraction in man, consists of a series of single contractions rapidly following each other. Every such movement, on being carefully analysed, consists of intermittent vibrations, which reach their maximum when a person shivers $(E d$. Weber $)$.

I.

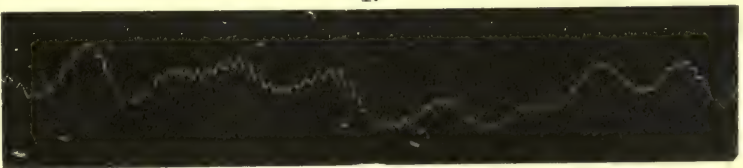

II.

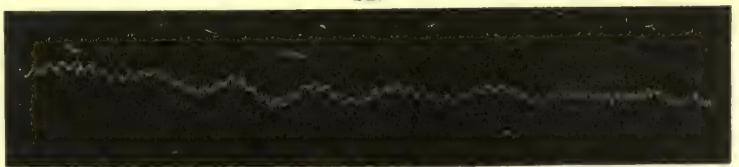

Fig. 333.

- [Baxt found that the I., vibration obtained from the flexor brevis follicis; II., from simplest possible voluntary

the extensor digiti tertii.

contractions, e.g., striking with the index finger, occupies on an average nearly twice as long a time as a similar movement discharged by a single induction shock.]

The number of single impulses sent to our muscles during a voluntary movement is tolerably variable, during a slow contraction $=8$ to 12 , and during a rapid 
contraction $=18$ to 20 impulses per second. Fig. 333 , I., represents a myogram of a sustained contraction of the flexor brevis pollicis and abductor pollicis, recorded on a vibrating plate. The wave-like elevations indicate the single impulses, each tooth $=0.01613$ second. II. is a similar curve registered by the extensor digiti tertii (Landris). "Sichifer finds that a prolonged voluntary contraction in man is an incomplete tetanus produced by 8 to 13 successive nervous impulses per second. About 10 per second may be taken as the average.]

The requisite degree of shortening is obtained, by the summation of single stimuli applied to the slowly contracting muscle, until the desired degree of shortening is obtained. In estimating exactly the amount of movement, we generally oppose some resistance by contracting antagonistic muscles, as is shown by observations on spare individuals (Briucke).

The tetanic contractions, which occur normally in an intact body, are proverl to consist of a series of successive contractions, because they can give rise to sccondary tetanus (\$ 332), which ma also be caused ly muscles thrown into tetanus by strychnin poisoning (Lorèn). The muscle-sound cannot be regarded as a proof of the oscillatory movement in tetanus, [as Helmholtz has shown that this sound coincides with the resonance sound of the ear (Hering and Friedrich)].

If a muscle be connected with a telephone, whose wires are brought into connection with two needles, one placed in the tendon, and the other in the substance of the muscle, we hear a sound when the muscle is thrown into tetanus, which proves that periodic vibratory processes, i.e., successive contractions, occur in the muscle (Bernstein and Schönlein). The sound is most distinct when the tetanising Neef's hammer of an induction machine vibrates about 50 times per second ( $W$ edenski and Kronecher).

The number of stimuli requisite to produce tetanus varies in different animals, and in different muscles of the same animal. About 15 stimuli per second are recuired to produce tetanus in the muscles of the from (hyoglossus only 10, gastrocnemius 27); very feeble stimuli (more than 20 per second) cause tetanus (Kronecker); the museles of the tortoise become tetanic with 2 to 3 shocks per second; the ral muscles of the rabbit by 10, the pale by over 20 (Kronecker and Stirling); nuscles of birds not even with 70 (Narey); muscles of insects 330 to 340 per second (Marey). Tetanic stimulation of the muscles of the crayfish (Astacus) and also in hydrophilus, may cause rhythmical contractions (Richet), or rhythmically interrupted tetanus (Schönlein).

Curarised muscles sometimes pass into tetanus on the application of a momentary stimulus (Kïhne, Hering).

O. Soltmann found that the pale muscles of new-born rabbits were rendered tetanic with 16 stimuli per second, so that tetanus was produced in them with the same number of shocks as in

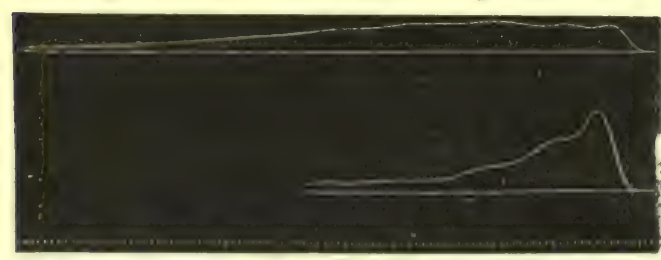

Fig. 334.

Curves obtained from red (upper) and pale (lower) muscles of a rabhit, by stimulating the sciatic nerve with a single induction shock. The lowest line (Kronecker and Stirling).

muscle. Four stimuli per second cause an inconplete plete tetauus in the red muscles of a rabbit, while the pale muscles require 20 to 30 stimuli per second to be completely tetanised. Fig. 335 shows the results produced by induction shocks applied to both muscles at intervals of $\frac{1}{4}$ second.]

The extent of shortening in a tetanically contracted muscle, within certain limits, is dependent upon the strength of the individual stimuli-but not upon their frequency. The contractionremainder after tetanus is greater the stronger the stimuli, the longer they are applied, and the feebler the muscle used (Buhr). For an unweighted muscle, the height of a contraction and that of tetanus are the same $(v$. Frey $)$. Only when a muscle is weighted is the height of a single contraction less than by a tetanic contraction. Sometimes a stimulus applied to a muscle immediately after tetanus produces a greater effect than it dill before the tetanus (Rossbach, Bohr). 
Duration of Tetanus. - A tetanised muscle cannot remain contracted to the same extent for an indefinite period, even if the stimuli are kept constant. It gradually begins to elongate, at first somewhat rapidly, and then more slowly, owing to the occurrence of fatigue. If the

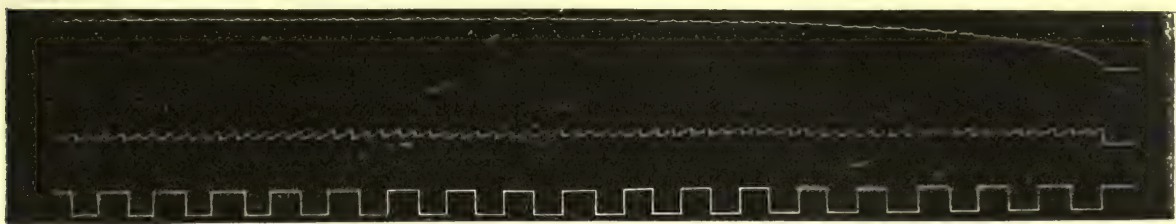

Fig. 335 .

Make and break induction shocks of 300 units, applied at intervals of a $\frac{1}{4}$ second to the pale (lower) and red (upper) muscles of a rabbit. The lowest line marks $\frac{1}{4}$ second (Kronecker and stirling).

tetanic stimulation is arrested, the muscle does not regain its original position and shape at once, but a contraction-remainder exists for a certain time, this being more evident after stimulation with induction shocks.

IV. If very rapid induction shocks (224 to 360 per second) be applied to a muscle, the tetanus after a so-called "initial contraction" (Bernstein) may cease (Harless, Heidenhain). This occurs most readily when the nerves are cooled. Kronecker and Stirling, however, found that stimuli following each other at greater rapidity than 24,000 per second produced tetanus.

[Tone-inductorium of Kronecker and Stirling. - This apparatus (fig. 336), consists of a rod of iron, $d$, fixed in an iron upright at $a$. The primary, $s_{f}$, and secondary spiral, $s_{n}$, rest on wooden supports, which can be pushed over both ends of the rod. One end of the rod lies

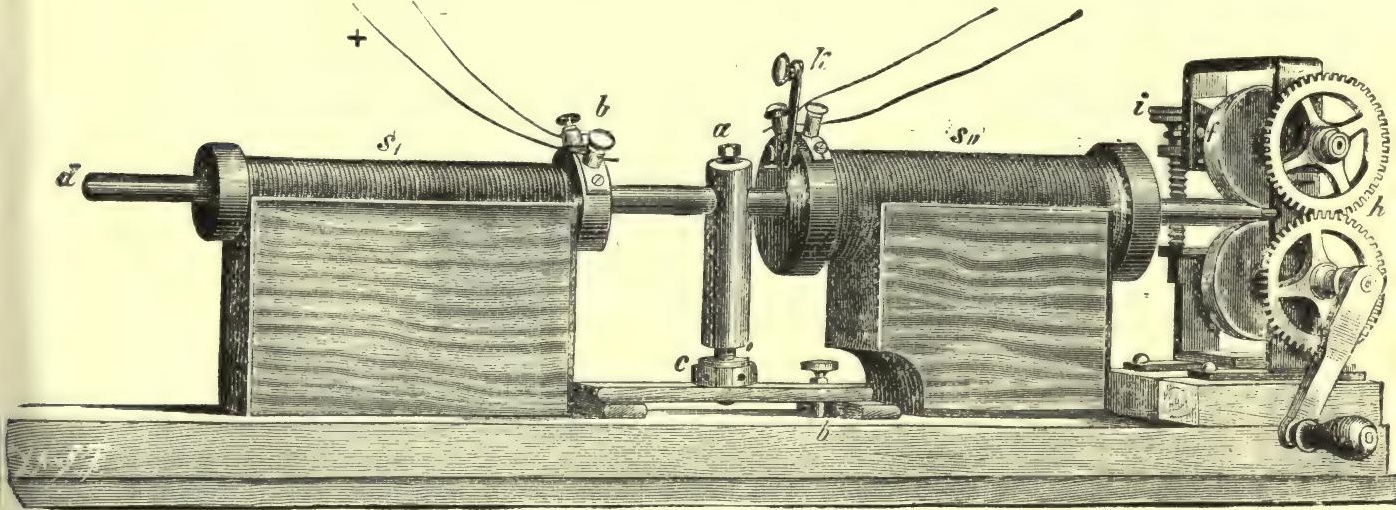

Fig. 336.

Tone-indnctorium of Kronecker and Stirling. $d$, iron rod, clamped at $\alpha$; $s_{t}$, primary, $s_{\|,}$ secondary spiral, with a key, $k$; leather rollers, $f$ and $g$, driven by wheels, $h$.

between leather rollers, $f$ and $g$, which can be made to rub on the rod by moving the toothed wheels, $h$. In this way a tone is produced by the longitudinal vibrations of the rod, the number of vibrations being proportional to the length of the rod, so that by means of this instrument we can produce from 1000 to 24,000 alternating induction shocks per second.]

Fick has recently investigated the changes-tension-undergone by a muscle when it is stimulated, and when its length remains constant, and he calls this process an "isometrical muscular act." He finds that a voluntary contraction in an isometrical act in man causes a higher tension than a contraction excited electrically. In the frog, the tension is nearly twice as great during tetanus as during a single maximal muscular contraction; in human muscles, it may be ten times as great.

299. RAPIDITY OF TRANSMISSION OF A CONTRACTION.-1. If a long muscle be stimulated at one end, a contraction occurs at that point, and is rapidly 
propagated in a wave-like manner through the whole length of the muscle, until it reaches its other end. The condition of excitement or molecular disturbance is communicated to each successive part of the muscle, in virtue of a special conductive capacity of the muscle. The mean velocity of the contraction-wave is 3 to 4 metres per second in the frog (Bernstein, 3.869 metres); rabbit, 4 to 5 metres (Bernstein and Steiner); lobster, 1 metre (Frédéricq and van de Velde); in smooth muscle and in the heart, only 10 to 15 millimetres per second $(\S 58,4)$. These results have reference only to excised muscles, the velocity of transmission being much greater in the voluntary muscles of a living man, viz., 10 to 13 metres (Her$\operatorname{mann}, \S 334$, II.).

Methods. - Aeby placed writing-levers upon both ends of a muscle, the levers resting transversely to the direction of the muscular fibres. The muscle was stimulated, and both levers registered their movements, the one directly over the other on a revolving cylinder. On stimulating one end of the muscle, the lever nearest to this point is raised by the contraction-wave, and a little later the other lever. When we know the rate at which the cylinder is moving, and the distance between the two elevations, it is easy to calculate the rapidity of transmission of the contraction-wave.

Duration and Wave-Length.-The time, corresponding to the length of the abscissa of the muscle-curve inscribed by each writing-lever, is equal to the duration of the contraction of this jart of the muscle (according to Bernstein, 0.053 to 0.098 second). If this value be multiplied by the rapidity of transmission of the muscular contraction-wave, we obtain the wave-length of the contraction-wave (=206 to 380 millimetres).

Modifying Influences.-Cold (fig. 337), fatigue, approaching death, and many poisons [veratrin, $\mathrm{KCy}$ ] diminish the velocity and the height of the contraction-

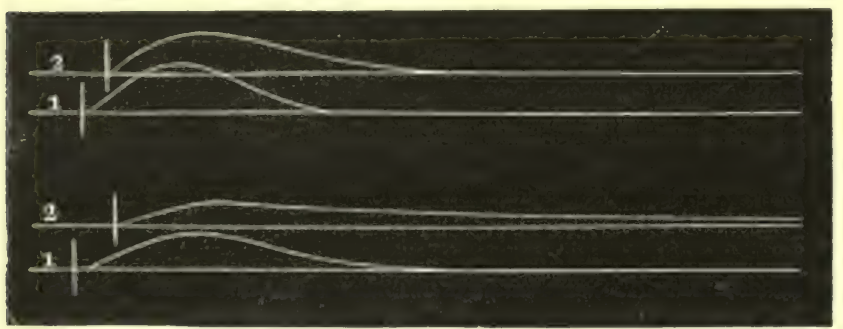

Fig. 337.

Upper two curves, 2 and 1, obtained from a rabbit's muscle by the above arrangement; the lower two curves from the same muscle, when it was cooled by ice.

and animals. The contraction-wave never passes from one neighbouring fibre.

[Fig. 337 shows the effect of cold on the muscles of a rabbit, in delaying the contractionwave. There is a longer distance between 1 and 2 in the lower than in the upper curves.]

2. If a long musele be stimulated locally near its middle, a contraction-wave is propagated towards both ends of the muscle. If several points be stimulated simultaneously, a wave movement sets out from each, the waves passing over each other in their course (Schiff).

3. If a stimulus be applied to the motor nerve of a muscle, an impulse is communicated to every muscular fibre; a contraction-wave begins at the end-organ [motorial end-plate], and must be propagated in both directions along the muscular fibres, whose length is only 3 to 4 centimetres. As the length of the motor fibres from the nerve-trunk to where they terminate in the motorial end-plates is unequal, contraction of all the muscular fibres cannot take place absolutely at the same moment, as the nerve impulse takes a certain time to travel along a nerve. 
Nevertheless, the difference is so small that, when a muscle is caused to contract by stimulation of its motor nerve, practically the whole muscle appears to contract simultaneously and at once.

4. A complete, uniform, momentary contraction of all the fibres of a muscle can only take place when all the fibres are excited at the same moment. This occurs when the electrodes are placed at both ends of the muscle, and an electrical stimulus of momentary duration passes through the whole length of the muscle.

300. MUSCULAR WORK.-Muscles are most perfect machines, not only because they make the most thorough use of the substances on which their activity depends (\$217), but they are distinguished from all machines of human manufacture by the fact that, by frequent exercise they become stronger, and are thereby capable of accomplishing more work (Du Bois-Reymond).

The amount of work (W) which a muscle can perform is equal to the product of the weight lifted $(p)$ and the height to which it is lifted $(h)$, i.e., $\mathrm{W}=p h$ (Introduction). Hence, it follows that when a muscle is not loaded (where $p=0$ ), then $w$ must be $=0$, i.e., no work is performed. If, again, it be overloaded with too great a load, so that it is unable to contract $(h=0)$, here also the work is nil. Between these two extremes an active muscle is capable of doing a certain amount of " work."

I. Work with Maximal Stimulation.-When the strongest possible, or maximal stimulus is applied-i.e, when the strength of the stimulus is such as to cause a muscle to contract to the greatest possible extent of which it is capable, the amount of work done increases more and more as the weight is increased, but only up to a certain maximum. If the weight be gradually increased, so that it is lifted to a less height, the amount of work diminishes more and more, and gradually falls to $\mathrm{be}=0$, when the weight is not lifted at all.

Example of the work done by a frog's muscle $(E d$. Weber $)$ :-

\begin{tabular}{|c|c|c|}
\hline Weight lifted in Grammes. & Height in Millimetres. & Work done in Gramme-Millimetres. \\
\hline 5 & $27 \cdot 6$ & 138 \\
15 & $25 \cdot 1$ & 376 \\
25 & $11 \cdot 45$ & 286 \\
30 & $7 \cdot 3$ & 220 \\
\hline
\end{tabular}

[Suppose a muscle be loaded with a certain number of grammes, and then caused to contract, we get a certain height of contraction. Fig. 338 shows the result of an experiment of this kind. The vertical lines represent the height to which the weights (in grammes) noted under them were raised, so that, as a rule, as the weight increases the height to which it is raised decreases.]

Laws of Muscular Work.-1. A muscle can lift a greater load the larger its transverse section, i.e., the more fibres it contains arranged parallel ? to each other.

2. The longer the muscle, the higher it can lift a weight.

3. When a muscle begins to contract, it can lift the largest load; as the contraction proceeds, it . can only lift a less and less load, and when it is at its maximum of shortening, only relatively very light loads.

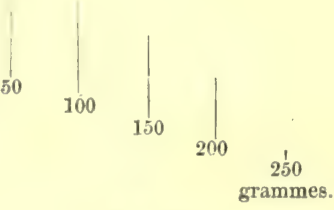

Fig. 338.

Height to which each of the weights is raised.

4. By the term "absolute muscular force" is meant, according to Ed. Weber, just the weight which a muscle undergoing maximal stimulation is no longer able to lift (the muscle being in its normal resting phase), and without the muscle at the moment of stimulation being elongated by the weight. 
Comparative.-Comparing the absolnte muscular force of different muscles, even in different animals, it is usuil to calculate it with reference to that of a square centimetre. The mean transverse section of a muscle is obtained by dividing its volume by its length. The volume is equal to the absolute weight of the muscles diviled by its specific gravity $=1058$. The absolute muscular force for $1 \square$ centimetre of a frog's muscle $=2 \cdot 8$ to 3 kilos. [ $6.6 \mathrm{lbs}$.] ( $J$. Rosenthal) ; for $1 \square$ centimetre of human muscle $=7$ to 8 (Henke and $K$ nor $z$ ), or even 9 to 10 kilos. [20 to 23 lbs.] (Kinster, Haughton). Insects can perform an extraordinary amount of workan insect can drag along sixty-seven times its body-weight; a horse scarcely three times its own weight.

5. During tetanus, when a weight is kept suspended, no work is done as long as the weight is suspended, but of course work is done in the act of lifting the load. To produce tetanus, successive stimuli are required, the muscular metabolism is increased, and fatigue rapidly occurs. The potential energy in this case is converted into heat $(\$ 302)$. When a muscle is stimulated with a maximal stimulus, it cannot lift so great a weight with one contraction as when it is stimulated tetanically (Hemann). The energy evolved, even during tetanus, is greater the more frequent the stimulation, at least up to 100 stimuli per second (Bernstein).

II. Medium Stimuli. - If a muscle be caused to contract by stimuli of maderate strength, i.e., such as do not cause a maximal contraction, there are two possibilities: -Either the feeble stimulus is kept constant whilst the load is varied, in which case the amount of work done follows the same law as obtains for maximal stimulation; or, the load may be kept the same, whilst the strength of the stimulus is varied. In the latter case, Fick observed that the height to which the load was lifted increased in a direct ratio with the strength of the stimulus.

The stimulus which causes a muscle to contract must reach a certain strength or intensity hefore it becomes effective, i.e., the "liminal intensity" of the stimulus, but this is independent of the weight applied to the muscle. With minimal stimuli, a small weight is raised higher than a large one, but as the stimulus is increased, the contractions also increase in a larger ratio with an increased load (v. Kries).

The blood-stream within the muscles of an intact body is increased during muscular activity. The blood-vessels of the muscle dilate, so that the amount of blood flowing through them is increased (Ludwig and Sczellow). At the time that the motor fibres are excited, so also are the vaso-dilator fibres, which lie in the same nervous channels ( $\$ 294$, II.). [Gaskell found that faradisation of the nerve of the mylohyoid muscle of the frog not only caused tetanus of the muscle, but also dilatation of its blood-vessels.]

Testing Individual Muscles. - In estimating the absolute force of the individual muscles or groups of muscles in man, we must always pay particular attention to the physical relations,

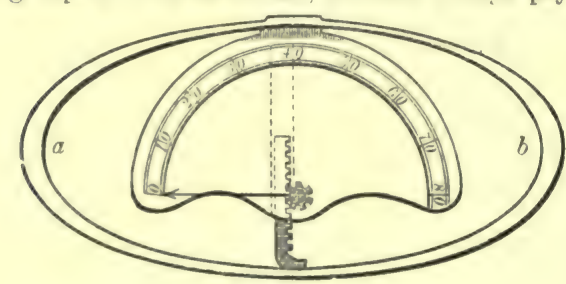

Fig. 339 .

Dynamometer of Mathieu. $i . e .$, to the arrangement of the levers, direction of the traction, degree of shortening, \&c. (\$306). Dynamometer. - The absolute force of certain groups of muscles is very conveniently and practically ascertained by means of a dynamometer (fig. 339). This instrument is very useful for testing the difference between the power of the two arms in cases of paralysis. The patient grasps the instrument in his hand and an index registers the force exerted. Quetelet has estimated the force of certain muscles-the pressure of both hands of a man to be $=70$ kilos.; while by pulling he can move double this weight. The force of the female hand is one-third less. A man can carry more than double his own weight; a woman about the half of this. Boys can carry about one-third more than girls. [Very convenient dynamometers are made by Salter of Birminghan, both for testing the strength of pull and squeeze ; in testing the former, the instrument is held as an archer holds his bow when in the act of drawing it, and the strength of pull is given by an index; in the latter another form of the instrument is used. Large numbers of observations were marle by means of these instruments by Francis Galton at the Health Exhibition, 1885. ]

Amount of Work Daily. - In estimating the work done by a man, we have to consider, not only the amount of work done at any one moment, but how often, time after time, he can 
succeed in doing work. The mean value of the daily work of a man working eight hours a day is $10(10.5$ to 11 at most) kilogramme-metres per second, i.e., a daily amount of work= $288,000(300,000)$ kilogramme-metres.

[Ergostat.-Sometimes it is desirable that patients-especially those who suffer from excessive corpulence-should do a certain amount of work daily; this can be carried ont by Gaertner's Ergostat, which resembles a winch, driven by a handle. The pressure upon the wheel can be regulated by means of a strap, lever, and weights, and according to the weight and number of revolutions of the wheel, can the amount of mechanical work be accurately regulated. This instrument is recommended for therapeutical purposes.]

Modifying Conditions.-Many substances, after being introduced into the body, diminish, and ultimately paralyse the production of work-mercury, digitalin, helleborin, potash salts, \&c. Others increase the muscular activity-veratrin (Rossbach), glycogen, [caffein, and allied alkaloids], muscarin (Klug and Fr. Högyes), kreatin and hypoxanthin ; extract of meat rapidly restores the muscles after fatigue $($ Kobert). [Those drugs which excite muscular tissue restore it after fatigue. Kreatin is a waste product of muscle, and beef-tea and Liebig's extract of meat perhaps owe their restorative qualities partly to these extractives.]

301. THE ELASTICITY OF MUSCLE. - Physical. - Every elastic boly has its " natural shape," i.e., its shape when no external force (tension or pressure) acts upon it so as to listort it. Thus, the passive muscle has a "natural form." If, however, a muscle be extended in the course of its fibres, the parts of the muscle are evidently pulled asunder. If the stretching be carried only to a certain degree, the muscle, in virtue of its elasticity, will regain its natural form. Such a body is said to possess "complete elasticity," i.e., after being stretched it regains exactly its original shape. By the term "amount of elasticity" (modulus) is meant the weight (expressed in kilogrammes) necessary to extend an elastic body $1 \square$ millimetre in diameter, its own length, without the body breaking. Of course many bodies are ruptured before this occurs. For a passive muscle it is $=0.2734$ ( $\mathrm{Wundt}$ ) [that of bone $=2264$ ( $I V$ ertheim), tendon $=1 \cdot 6693$, nerve $=1.0905$, the arterial walls $=0.0726(W u n d t)]$. Thus, the amount of elasticity of a passive muscle is small, as it requires only a slight stretching force to extend it to its own length. It has, therefore, no great amount of elasticity. The term "coefficient of elasticity" is applied to the fraction of the length of an elastic bouy, to which it is elongated by the unit of weight applied to stretch it. It is large in a passive muscle. If the tension be sufficiently great, the elastic body ruptures at last. "The "camrying capacity" of muscular tissue, until it ruptures, is in the following ratios for youth, middle, and old age, nearly $7: 3: 2$. [Instead of the word "elasticity," Brunton suggests the use of extensibility and retractibility, terms suggested by Marey, the one referable to the elongation on the application of a weight, and the other to the shortening after its removal.]

Curve of Elasticity.-In inorganic elastic bodies, the line of elongation, or the extension, is directly proportional to the extending weight; in organic bodies, and therefore in muscle, this is not the case, as the weight is continually increased by equal increments - the muscle is less extended than at the beginning, so that the extension is not proportional to the weight. If equal weights be added to a scale-

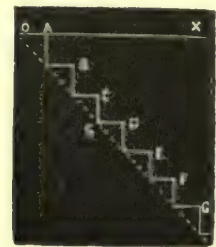

Fig. 340 .

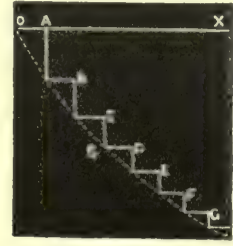

Fig. 341.

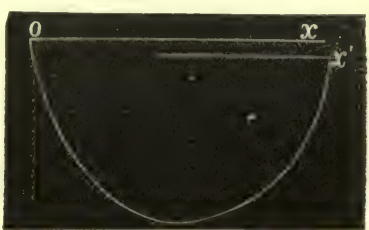

Fig. 342.

Fig. 340.-Curve of elasticity from an inorganic body (india-rubber). Fig. 341.-Curve of elasticity from the sartorius of a frog, obtained by adding equal increments of weight at A, B, C, \&c. Fig. 342.-Curve of elasticity produced by continuous extension and recoil of a frog's muscle; $a x$, abscissa before, $x^{\prime}$ after extension.

pan attached to a piece of india-rubber, with a writing-lever connected with it, and writing its movements on a plate of glass that can be moved with the hand, we get such a curve as in fig. 340 , while, if the same be done with the sartorius of a frog, we get a result similar to fig. 341. A straight line joins the apices of the former, while the curve of elasticity is a hyperbola, or something near it, in the latter case. 
Elastic After-Effect. - At the same time, after the first elongation, corresponding to the extending weight, is reached, the muscle may remain for days, and even weeks, somewhat elongated. This is called the "elastic after-effect" (\$ 65). [Marey attached a lever to a frog's muscle, and allowed the latter to record its movements on a slowly revolving cylinder. To the lever was fixed a vessel into which mercury slowly flowed. This extended the muscle, and when it had ceased to elongate, the mercury was allowed slowly to run out again. The curve obtained is shown in fig. 342 . The abscissæ, $o x$ and $x^{\prime}$, indicate the position of the writingstyle before and after the experiment, and we observe that $x^{\prime}$ is lower than $0 x$, so that the recoil is imperfect. There has been an actual elongation of the muscle, so that the limit of its elasticity is exceeded. Although a frog's gastrocnemius may be loaded with 1500 grammes without rupturing it, 100 grammes will prevent its regaining its original length.]

Method.-In order to test the elasticity of a muscle, fix it to a support provided with a graduated seale, and to the lower end of the muscle attach a scale-pan, in which are placed various weights, measuring on each occasion the corresponding elongation of the muscle thereby obtained $(E d$. $W$ c ber $)$. In orler to obtain the curve of elongation or extensibility take as abscisse the successive units of weight added, and the elongation corresponding to each weight as ordinates. Example from the hyoglossus of the frog:-

\begin{tabular}{|c|c|c|c|}
\hline \multirow{2}{*}{ Weight in Grammes. } & \multirow{2}{*}{$\begin{array}{l}\text { Length of the Muscle } \\
\text { in Jillimetres. }\end{array}$} & \multicolumn{2}{|c|}{ Extension. } \\
\hline & & In Millimetres. & Percentage. \\
\hline 0.3 & $24 \cdot 9$ & $\ldots$ & $\ldots$ \\
\hline $1 \because 3$ & $30 \cdot 0$ & $5 \cdot 1$ & 20 \\
\hline $2 \cdot 3$ & $32 \cdot 3$ & $2 \cdot 3$ & 7 \\
\hline $3 \cdot 3$ & $33 \cdot 4$ & $1 \cdot 1$ & 3 \\
\hline $4 \cdot 3$ & $34 \cdot 2$ & 0.8 & 2 \\
\hline $5 \cdot 3$ & $34 \cdot 6$ & 0.4 & 1 \\
\hline
\end{tabular}

The elasticity of passive muscle is small, but very complete, and is comparable to that of a caoutchouc fibre. Small weights greatly elongate the muscle. If the weights be uniformly increased, there is not a uniform elongation; with equal increments of weight, the greater the load, the increase in elongation always becomes less; or, to express it in another way, the amount of elasticity of the passive muscle increases with its increased extension ( $E d$. Weber).

In inorganic bodies, the curve of extension is a straight line, but in organic bodies, it more closely resembles a hyperbola (Wertheim). The elasticity of a passive fatigued muscle does not differ essentially from that of a non-fatigued muscle.

Muscles in the living body, and still in connection with their nerves and blood-vessels, are more extensible than excised ones. Muscles, when quite fresh, are elongated (within certain small limits as regards the weight) at first with a uniformly increasing weight, to an extent proportional to the latter, just as with an inorganic body. When heavy weights are used, we must be careful to take into consideration the "elastic after-effect" (\$65).

The volume of a stretched muscle is slightly less than an unstretched one, similar to the contracted $(\$ 297,2)$ and stiffened muscle $(\$ 295)$.

Dead muscles and muscles in rigor mortis have greater elasticity, i.e., they require a heavier weight to stretch them than fresh muscles; but, on the other hand, the elasticity of dead muscles is less complete, i.e., after they are stretched, they only recover their original form within certain limits.

Elasticity of Intact Muscles.-Normally, within the body, the muscles are stretched to a very slight extent, as can be shown by the slight degree of retraction which occurs when the insertion of a muscle is divided. This slight degree of extension, or stretching, is important. If this were not so, when a muscle is about to contract, and before it could act upon a bone as a lever, it would have to "take in so much slack." The elasticity of muscles is manifested during the contraction 
of antagonistic muscles. The position of a passive limb depends upon the resultant of the elastic tension of the different muscle groups.

The elasticity of an active muscle is less than that of a passive muscle, i.e., it is elongated by the same weight to a greater extent than a passive muscle. For this reason, the active muscle, as can be shown in an excised contracted muscle, is softer; the apparently great hardness manifested by stretched contracted muscles depends upon their tension. When the active muscle becomes fatigued, its elasticity is diminished ( $\$ 304)$.

Method.-Ed. Weber took the hyoglossus muscle of a frog and suspended it vertically, noticing its length when it was passive. It was then tetanised with induction shocks and its height again noted. One after the other heavier weights were attached to it, and the length of the passive and tetanised muscle observed for each weight. The extent to which the active loaded muscle shortened from the position of the passive loaded muscle he called the "height of the lift" (or "Hubhöhe"). The latter becomes less as the weight increases, and lastly, the tetanised muscle may be so loaded that it cannot contract, i.e., the height of the lift is $=0$.

Weber's Paradox.- The case may occur where, when a muscle is so loaded that it cannot contract when it is stimulated, it may even elongate. According to Wundt, even in this condition the elasticity is not changed. [The usual explanation given is that, as the elasticity of a muscle is diminished during contraction, it is more extended with the same weight in the contracted as compared with the passive or uncontracted state, so that a heavily weighted muscle, when stimulated, may elongate instead of shorten.] According to Wundt, however, as stated, there is no change in the elasticity of the nuscle. In these experiments, the length of the active loaded muscle is equal to the length of the passive muscle when similiarly loaded, minus the "height of the lift."

Poisons. - Potash causes shortening of a muscle with simultaneous increase of its elasticity. Digitalin produces other changes with increased elasticity. Physostigmin increases it, while veratrin diminishes it, and interferes with its completeness (Rossbach and v. Anrep), and tannin makes a muscle less extensible, but more elastic (Lewin). Ligature of the blood-vessels produces at first a decrease, and then an increase, of the elasticity ; section of the motor nerve diminishes the elasticity ( $v$. Anrep); heat increases it.

Eduard Weber concluded from his experiments that a muscle assumes two forms, the active and the passive form. Each of these corresponds to a special natural form. The passive muscle is longer and thinner-the active is shorter and thicker in form. The passive as well as the active muscle strives to retain its form. If the passive muscle be set into activity, the passive rapidly changes into the active form, in virtue of its elastic force. The latter is the energy which causes muscular work. Schwann compared the force of an active muscle to a long, elastic, tense spiral spring. Both can lift the greatest weight, only from that form in which they are most stretched. The more they shorten, the less the weight which they can lift.

[Uses of Elasticity.-As already pointed out, all muscles are slightly on the stretch, so that no time is lost nor energy wasted, in "taking in slack," as it were; but the elasticity also lessens the shock of the contraction, so that it is developed gradually, and muscles are not liable to be torn from their attachments. The muscular energy is transmitted to the mass to be moved through an elastic and easily extensible body (muscle), whereby the shock due to the contraction is lessened, but, as Marey has shown, the amount of work is thereby considerably increased.]

[Tonicity of Muscle ( $\$ 362)$ - Sensibility of Muscle. - That muscles contain sensory fibres is certain $(\$ 430)$. Section of inflamed muscles is painful, and during muscular cramp intense pain is felt. Sachs discharged a reflex action by stimulating the central end of an intra-museular nerve-filament in a frog, while stimulatiou of the central end of the phrenic nerve raises the blood-pressure (Muscular Sense, § 430). ]

302. Formation of Heat in an Active Muscle.-After Bunzen, in 1805 $(\S 209,1, b)$, showed that during muscular activity heat is evolved, v. Helmholtz proved that an excised frog's muscle, when tetanised for two to three minutes, exhibited an increase of its temperature of $0.14^{\circ}$ to $0 \cdot 18^{\circ} \mathrm{C}$. R. Heidenhain succeeded in showing an increase of $0.001^{\circ}$ to $0.005^{\circ} \mathrm{C}$. for each single contraction. The heart is warmer during every systole (Marey).

[Method. - The rise in temperature of a frog's muscle may be estimated by placing the two gastrocnemii of a frog's muscle on the two junctions of a thermo-electric pile, connected with a 
heat galvanometer. Of course, when the two muscles are at the same temperature, the needle of the galvanometer is statiouary ; but, if one muscle is made to contract, or is tetanised, then an electrical current is set up which deflects the needle $(\$ 208, \mathrm{~B})$. Lujankow has, by means of a delicate thermometer placed between the thigh muscles of a dog, estinated the rise of temperature under different conditions of the muscle, while the latter was still in situ and intact.]

The following facts have been ascertained with regard to the development of heat :-

1. Relation to Work. - It bears a relation to the amount of work.

(a) If a muscle during contraction carries a weight which extends it again during rest, no work is transferred beyond the muscle (§300). In this case all the chemical potential energy during this movement is converted into heat. Under these circumstances, the amount of heat evolved runs parallel with the amount of work done, i.e., it increases as the load and the height increase up to a maximum point, and afterwards diminishes as the load is increased. The heat-maximum is reached with a less load sooner than the work-maximum (Heilenhain).

(b) If, when the muscle is at the height of its contraction, the load be removed, then the muscle has produced work referable to something outside itself; in this case the amount of leat produced is less (A. Fick). The amount of work produced, and the diminished amount of heat formed, when taken together, represent the same amount of energy, corresponding to the law of the conservation of energy.

(c) If the same amount of work is performed in one case by many but small contractions, and in another by fewer but larger contractions, then, in the latter case, the amount of heat is greater (Heidenhain and Nawalichin). This shows that larger contractions are accompanied by a relatively greater metabolism of the muscular substance than small contractions, which is in harmony with practical experience; thus the ascent of a tower with steep high steps causes fatigue more rapidly (metabolism greater) than the ascent of a more gentle slope with lower steps.

(d) If the weighted muscle executes a series of contractions one after the other, and at the same time does work, then the amount of heat it produces is greater than when it is tetanic, and keeps a weight suspended. Thus, the transition of the muscle into a shortened form causes a greater production of heat than the maintenance of this form.

2. Relation to Tension. - The amount of heat evolved depends upon the tension of the muscle; it also increases as the muscular tension increases (Heidenhain). If the ends of a muscle be so fixed that it cannot contract, the maximum of heat is obtained (Béclard), and this the more quickly the more rapidly the stimuli follow each other (Fick). Such a condition occurs during tetanus, in which condition the violently contracted muscles oppose each other, and very high temperatures have been registered by Wunderlich $(\$ 213,7)$, while the same is true of animals that are tetanised (Leyden). Dogs kept in a state of tetanus by electrical stimulation die, because their temperature rises so high $\left(44^{\circ}\right.$ to $45^{\circ} \mathrm{C}$.) that life no longer can be maintained (Ricket). In addition to the formation of heat, there is a considerable amount of acid, and of alcoholic extractives produced in the muscular tissue.

3. Relation to Stretching.-Heat is also evolved during the elongation or relaxation of a contracted muscle, e.g., by causing a muscle to contract without the addition of any weight, and loading it when it begins to relax, whereby heat is produced (Steiner, Schmulewitsch, and Westerman). If weights be attached to a muscle by means of an inextensible medium, and the weights be allowed to fall from a height so as to give a jerk to the muscle, then an amount of heat equivalent to the work done by the drop, is set free in the muscle (Fick and Danilewsky).

4. The formation of heat diminishes as the inuscular fatigue increases.

5. In a muscle duly supplied with blood, the production of heat (as well as the mechanical work) is far more active than in a muscle whose blood-vessels are ligatured or its blood-stream cut off. Recovery takes place more rapidly and com- 
pletely after fatigue, while, at the same time, there is a new increase in the production of heat (Meade Smith).

The amount of work and heat in a muscle must always correspond to the transformation of an equivalent amount of chemical energy. A greater part of this energy is manifested as work, the greater the resistance that is offered to the muscular contraction. When the resistance is great, $\frac{1}{4}$ of the chemical energy may be manifested as work, but when it is small, only a small part of it is so converted.

It was stated that a nerve in action is $\frac{1}{30}^{\circ} \mathrm{C}$. warmer (Valentin), but this is denied by v. Helmholtz and Heidenhain.

In man, if the muscles be stimulated with electricity or contracted voluntarily, the production of heat may be detected through the skin (v. Ziemssen). The venous blood flowing from an actively contracting muscle is $0.6^{\circ} \mathrm{C}$. warmer than the arterial blood (Mcade Smith).

303. THE MUSCLE SOUND.-Muscle Sound.-When a muscle contracts, and is at the same time kept in a state of tension by the application of sufficient resistance, it emits a distinct sound or tone with a semi-musical quality, depending upon the intermittent variations of tension occurring within it (Wollaston).

Methods. - The muscle sound may be heard by placing the ear over the tetanically contracted and tense biceps of another person; or we may insert the tips of our index fingers into our ears, and foreibly contract the museles of our arm; or the sound of the museles that close the jaw may be heard by forcibly contracting them, especially at night when all is still, and when the outer ears are closed. V. Helmholtz found that this tone coincides with the resonance tone of the ear, and he thought that the vibrations of the muscles caused this resonance tone. The sound of an isolated frog's muscle may be heard by placing one end of a rod in the ear, the other ear being closed. To the other end of the rod is attached a loaded frog's musele kept in a tetanic condition. The pitch of the note, i.e., the number of vibrations, may be estimated by comparing the muscle sound with that produced by elastic springs vibrating at a known rate.

When a muscle contracts voluntarily, i.e., through the will, it makes 19.5 vibrations per second. [Schäfer and others give the number as 10 successive nervous impulses per second, p.486.] We do not hear this very low tone, owing to the number of vibrations per second being too few, but what we actually hear is the first overtone, with double the number of vibrations. The muscle sound has 19.5 vibrations, when the muscles of an animal are caused to contract, by stimulating its spinal cord (v. Helmholtz), and also when the motor nerve-trunk is excited by chemical means (Bernstein). If, however, tetanising induction shocks be applied to a muscle, then the number of vibrations of the muscle sound corresponds exactly with the number of vibrations of the vibrating spring or hammer of the induction apparatus. Thus, the tone may be raised or lowered by altering the tension of the spring.

Lovèn found that the muscle sound was loudest, when the weakest currents capable of producing tetanus were employed. The sound corresponded to the number of vibrations of the octave just below it in the scale. With stronger currents the muscle-sound disappears, but it reappears with the same number of vibrations as that of the interrupter of the induction apparatus, if still stronger currents are used.

If the induction shocks be applied to the nerve, the sound is not so loud, but it has the same number of vibrations as the interrupter. With rapid induction shocks, tones caused by 704 (Lovèn) and 1000 vibrations per second have been produced (Bernstein).

The first heart-sound is partly muscular (\$53).

A single induction shock is said to cause the muscle-sound in a contracting muscle. If this be so, it is doubtful if the muscle-sound can be regarled as a sign that tetanus is due to a series of single variations of the muscle (\$ 298 , III.).

304. FATIGUE AND RECOVERY OF MUSCLE.-Fatigue.-By the term fatigue is meant that condition of diminished capacity for work which is produced in a muscle by prolonged activity. This condition is accompanied in the living person with a peculiar feeling of lassitude, which is referred to the muscles. A fatigued muscle rapidly recovers in a living animal, but an excised muscle recovers only to a slight extent (Ed. Weber, Valentin).

[Waller recognises a certain resemblance between experimental fatigne and the natural decline of excitability at death, in disease, and in poisoning.] 
The cause of fatigue is probably partly due to the accumulation of decompositionproducts-" fatigue stuffs" - in the muscular tissue, these products being formed within the muscle itself during its activity. They are phosphoric acid, either free or in the form of acid phosphates, acid potassium phosphate (\$ 294), glycerinphosphoric acid (?) and $\mathrm{CO}_{2}$. If these substances be removed from a muscle, by passing through its blood-vessels an indifferent solution of common salt $(0.6$ per cent.), or a weak solution of sodium carbonate [or a dilute solution of permanganate of potash (Kronecker)], the muscle again becomes capable of energising (J. Ranke, 1863). The using up of $\mathrm{O}$ by an active muscle favours fatigue (v. Pettenkofer and v. Voit). The transfusion of arterial blood (not of venous-Bichat) removes the fatigue (Ranke, Kronecker), probably by replacing the substances that have been used up in the muscle. Conversely, an actively energising muscle may be rapidly fatigued by injecting into its blood-vessels a dilute solution of phosphoric acid, of acid potassium phosphate, or dissolved extract of meat (Kemmerich). A muscle fatigued in this way absorbs less $\mathrm{O}$, and when so fatigued, it evolves only a small amount of acids and $\mathrm{CO}_{2}$. The conditions which lead up to fatigue are connected with considerable metabolism in the muscular tissue.

[Zabludowski found that if a frog's muscles be systematically stimulated by maximum induction shocks until they cease to contract, massage or kneading them rapidly restored their excitability, while simple rest had little effect. Massage acts on the nerves, but chiefly by favouring the blood-and lymph-streams which wash out the waste products from the muscle. A similar result obtains in man, so that the ancient Roman practice of "rubbing" after a bath and after exercise was one conducive to restoration of the power of the muscles.]

Modifying Conditions. - In order to obtain the same amount of work from a fatigued muscle, a much more powerful stimulus must be applied to it than to a fresh one. A fatigued muscle is incapable of lifting a considerable load, so that its absolute muscular force is diminished. If, during the course of an experiment, an excised muscle be loaded with the same weight, and if the muscle be stimulated at regular intervals with maximal stimuli (strong induction shocks), contraction after contraction gradually and regularly diminishes in height, the decrease being a constant fraction of the total shortening. Thus the fatigue-curve is represented by a straight line [i.e., a straight line will touch the apices of all the contractions]. 'The more rapidly the contractions succeed each other, the greater is the fall in the height of the contraction [i.e., if the interval between the contractions be short, the fatiguecurve falls rapidly towards the abscissa], and conversely. After a certain number of contractions, an excised muscle becomes exhausted.

This result occurs whether the stimuli are applied at short or long intervals (Kronecker), and a similar result is obtained with sub-maximal stimuli (Tiegel). A fatigued muscle contracts more slowly than a fresh one, while the latent period is also longer during fatigue (p. 480). The fatigued muscle is said to be more extensible (Donders and van Mansvelt). If a muscle be so loaded that, when it contracts, it cannot lift the load, fatigue occurs even to a greater extent than when the load is such that the muscle can lift it (Leber). The metabolism and the formation of acid are greater in a contracted muscle kept on the stretch, than in a contracted muscle allowed to shorten (Heidenhain). If a muscle contract, but be not required to lift any load, it becomes fatigued only very gradually. If a muscle be loaded only during contraction, and not during relaxation, it is fatigued more slowly than when it is loaded during both phases; and the same is true when a muscle has to lift its load only during the course of its contraction, instead of at the beginning of the contraction. Loads may be suspencled to perfectly passive muscles without fatiguing them (Harless, Leber).

[Signs of Fatigue (fig. 343). - In the record of the series of contractions; (1) the contractions become more prolonged; (2) they decrease in height; (3) the latent period becomes longer; (4) if maximal shocks be used, the beginuing of the 
series exhibits a "staircase" character of its contractions just like the heart $(\S 57)$.

[While an excised frog's muscle is fairly rapidly exhausted by single opening induction shocks, at intervals of one second, human muscle in its normal relations may be almost indefinitely so treated, and there is no change in the record or any sensation of fatigue. Waller regards this as favouring the view that the "fatigue consequent upon prolonged muscular exertion is normally central rather than peripheral." Such results, however, do not harmonise with those of Zabludowski on the kneading of muscles, or massage. Probably there are two factors, one central, the other peripheral.]

Blood Supply.-If the arteries of a mammal be ligatured, stimulation of the motor nerves produces complete fatigue after 120 Fatigue-curve of a frog's muscle. The sciatic nerve to 240 contractions (in two to four minutes), but direct muscular stimulation still causes the muscles to contract. In both cases the fatigue-curve is in the form of a straight

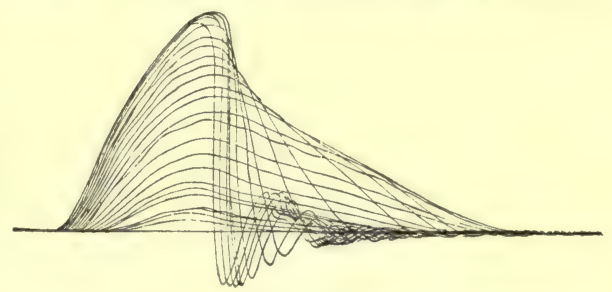

Fig. 343. was stimulated with maximal induction shocks and every fifteenth contraction recorded (Stirling).

line. If the blood supply to a mammalian muscle be normal, on stimulating the motor nerve, the muscular contractions at first increase in height and then fall, their apices forming a straight line (Rossbach and Harteneck). In persons who have used their muscles until fatigue sets in, it is found that at the beginning the nerves and muscles react better to galvanic and faradic stimulation, but afterwards always to a less degree (Orschanski). According to v. Kries, a muscle tetanised and fatigued with maximal stimuli behaves like a fresh muscle tetanised with sub-maxinal stimuli ; both show an incomplete transition from the passive to the active condition.

[Relation of End-Plates, - Muscle is fatigued far more rapidly than nerve, and the fatigue begins in the muscle and not in the nerve, and it seems to be the weakest link in the chain between nerve and muscle which is affected during excessive action, viz., the motor end-plate (Waller). In a nerve its conductivity is sooner affected by fatigue than its direct excitability. Waller finds that after death "the excitability of a nerve persists when its action upon muscle has ceased, such muscle being still excitable by direct stimulation." Some link in the chain is obviously affected, and it is perhaps the end-plates.]

[Action of Drugs on Fatigue.-Waller finds, in a frog poisoned with veratrin, that if the muscles be stimulated electrically, the characteristic elongation of the descent ( $\$ 298$ ) gradually disappears, but reappears after a period of rest. In this respect, strychnin in its action on the spinal cord behaves precisely the same as veratrin on muscle, viz., its effect is dissipated by action and restored by rest.] Curara and the ptomaines cause an irregular course of the fatigue-curve (Guareschi and Mosso). [If strychnin be injected into a frog, and the sciatic nerve on one side divided after the

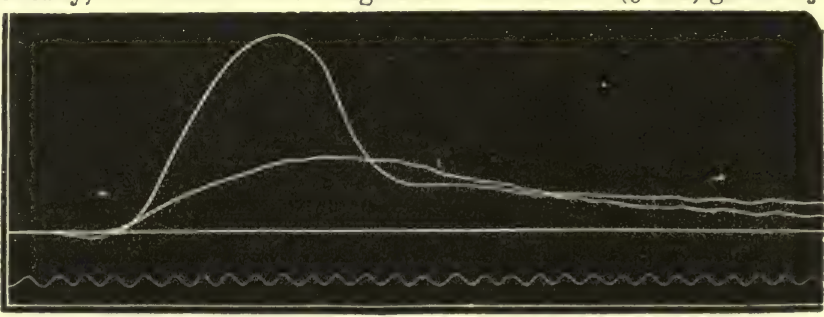

Fig. 344 .

Curves obtained by direct stimulation of the gastrocnemius of a frog poisoned with strychnin, the sciatic nerve divided on one side (upper curve) and not on the other (lower or fatigue-curve).

strychnin tetanus has lasted for a time, the leg muscles of the side with the nerve undivided exhibit signs of fatigue, as shown by direct stimulation of the muscles of both legs, when a curve similar to fig. 344 is obtained. The higher one is the non-fatigued, the lower that of the side with the nerve undivided (Waller).]

Recovery from the condition of fatigue is promoted by passing a constant electrical current through the entire length of the muscle (Heidenhain), also by injecting fresh arterial blood into its blood-vessel, or by very small doses of veratrin, [or permanganate of potash], and by rest.

If the muscle of an intact animal be stimulated continuously (fourteen days or so), until complete fatigue occurs, the muscular fibres become granular and exhibit a wax-like degenera- 
tion. The transverse striation is still visible as long as the sarcous substance is in large masses, but as soon as it breaks up into small pieces the transverse striation disappears completely (0. Roth).

305. MECHANISM OF THE BONES AND JOINTS.-Bones exhibit in the inner architecture of their spongiosa an arrangement of their lamellæ and spicules which represents the static result of those forces-pressure and traction-which act on the developing bone (Structure of Bone, $\$ 447$ ). They are so arranged that, with the minimum of material, they afford the greatest resistance as a supporting structure or framework (H. v. Meyer, Culmann, Jul. Wolft').

I. The joints permit the freest movements of one bone upon another, [such as exist between the extremities of the bones of the limbs. In other cases, sutures are formed, which, while permitting no movement, allow the contents of the cavity which they surround to enlarge, as in the case of the cranium]. The articular end of a fresh bone is covered with a thin layer or plate of hyaline eartilage, which in virtue of its elasticity moderates any shocks or impulses communicated to the bones. The surface of the articular cartilage is perfectly smooth, and facilitates an easy gliding movement of the one surface upon the other. At the outer boundary line of the cartilage, there is fixed the capsule of the joint, which encloses the articular ends of the bones like a sac. The inner surface of the capsule is lined by a synovial membrane, which secretes the sticky, semi-fluid, synovia, moistening the joint. The outer surface of the capsule is provided at various parts with bands of fibrous tissue, some of which strengthen it, whilst others restrain or limit the morement of the joint. Some osseous processes limit the movements

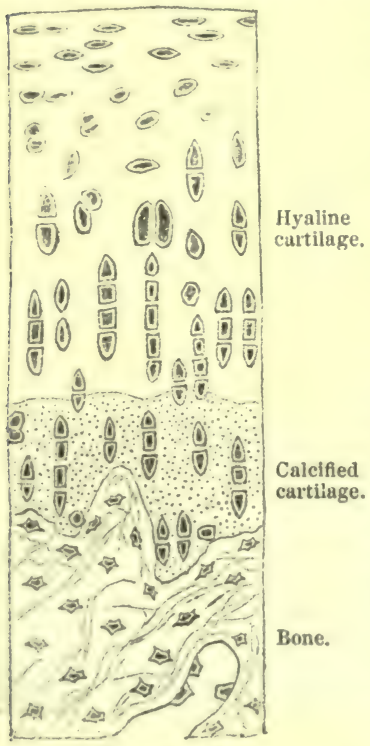

Fig. 345 . of particular joints, $c_{.}$. , the coronoid process of tine ulna, which permits the fore-arm to be flexed on the upper arm only to a certain extent; the olecranon, which prevents over-extension at the elbow-joint. The joint surfaces are kept in apposition -(1) by the adhesion of the synovia-covered smooth articular surface ; (2) by the capsule and its fibrous bands; and (3) by the elastic tension and contraction of the muscles.

[Structure of Articular Cartilage. - The thin layer of hyaline encrusting cartilage is fixed by an irregular surface upon the corresponding surface of the head of the bone (fig. 345). In a vertical section through the articular cartilage of a bone which has been softened in chromic or other suitable acid, we observe that the cartilage cells are flattened near the free surface of the cartilage, and their long axes are parallel to the surface of the joint; lower down, the cells are arrauged in irregular groups, and farther down still, nearer the bone, in columns or rows, whose long axis is in the long axis of the bone. These rows are produced by transverse cleavage of pre-existing cells. In the upper two-thirds or thereby the matrix of the cartilage is hyaline, but in the lower third, near the bone, the matrix is granular and sometimes fibrillated. This is the calcified zone, which is impregnated with lime salts, and sharply defined by a nearly straight line from the hyaline zone above it, and by a very bold wavy line from the osseous head of the bone.]

Synovial Membrane. - Synovial membrane consists of bundles of delicate connective-tissue mixed with elastic tissue, while on its inner surface it is provided with folds, some of which contain fat, and others blood-vessels (synovial villi). The inner surface is lined with endothelium. The intra-capsular ligaments and cartilages are not covered by the synovial membrane, nor are they covered by endothelium. The synovia is a colour. less, stringy, alkaline fluid, with a chemical composition closely

Vertical section of articular car tilage (Stirling).

allied to that of transudations, with this difference, that it contains much mucin, together with albumin and traces of fat. 'Excessive movement diminishes its amount, makes it more inspissated, and increases the mucin, but diminishes the salts.

Joints may be divided into several classes, according to the kind of movement which they permit :-

1. Joints with movement around one axis: (a) The Ginglymus, or Hinge-Joint. -The one articular surface represents a portion of a cylinder or sphere, to which the other surface is adapted by a corresponding depression, so that, when flexion or extension of the joint takes place, it moves only on one axis of the cylinder or sphere. The joints of the fingers and toes are hinge-joirts of this description. Lateral ligaments, which prevent a lateral displacement of the articular surfaces, are always present. 
The Screw-hinge Joint is a modification of the simple hinge form (Langer, Henke), e.g., the humero-ulnar articulation. Strictly speaking, simple flexion and extension do not take place at the elbow-joint, but the ulua moves on the capitellum of the humerus like a nut on a bolt; in the right humerus, the screw is a right spiral, in the left, a left spiral. The ankle-joint is another example ; the nut or female screw is the tibial surface, the right joint is like a lefthanded screw, the left the reverse. (b) The Pivot-Joint (rotatoria), with a cylindrical surface, e.g., the joint between the atlas and the axis, the axis of rotation being around the odontoid process of the axis. In the acts of pronation and supination of the fore-arm at the elbowjoint, the axis of rotation is from the middle of the cotyloid cavity of the head of the radius to the styloid process of the ulna. The other joints which assist in these movements are above the joint, between the circumferential part of the head of the radius and the sigmoid cavity of the ulna, and below the joint, between the sigmoid cavity of the radius which moves over the rounded lower end of the ulna.

2. Joints with movements around two axes.-(a) Such joints have two unequally curved surfaces which intersect each other, but which lie in the same direction, e.g., the atlantooccipital joint, or the wrist-joint, at which lateral movements, as well as flexion and extension, take vlace. (b) Joints with curved surfaces, which intersect each other, but which do not lie in the same direction. To this group belong the saddle-shaped articulations, whose surface is concave in one direction, but convex in the other, e.g., the joint between the metacarpal bone of the thumb and the trapezium. The chief movements are-(1) flexion and extension, (2) abduction and adduction. Further, to a limited degree, movement is possible in all other directions ; and, lastly, a pyramidal movernent can be described by the thumb.

3. Joints with movement on a spiral articular surface (spiral joints), e.g., the knee-joint (Goodsir). The condyle of the femur, curved from before backwards, in the antero-posterior section of its articular surface, represents a spiral $(E d$. Weber), whose centre lies nearer the posterior part of the condyle, and whose radius vector increases from behind, downwards and forwards. Flexion and extension are the chief movements. The strong lateral ligaments arise from the condyles of the femur corresponding to the centre of the spiral, and are inserted into the head of the fibula and internal condyle of the tibia. When the knee-joint is strongly flexed, the lateral ligaments are relaxed-they become tense as the extension increases; and when the knee-joint is fully extended, they act quite like tense bands which secure the lateral fixation of the joint. Corresponding to the spiral form of the articular surface, flexion and extension do not take place around one axis, but the axis moves continually with the point of contact; the axis moves also in a spiral direction. The greatest flexion and extension cover an angle of about $145^{\circ}$. The anterior crucial ligament is more tense during extension, and acts as a check ligament for too great extension, while the posterior is more tense during flexion, and is a check ligament for too great flexion. The movements of extension and flexion at the knee are further complicated by the fact that the joint has a screw-like movement, in that during the greater extension the leg moves outwards. Hence, the thigh, when the leg is fixed, must be rotated outwards during flexion. Pronation and supination take place during the greatest flexion to the extent of $41^{\circ}$ (Albert) at the knee-joint, while with the greatest extension it is nil. It occur's because the external condyle of the tibia rotates on the internal. In all positions during flexion, the crucial ligaments are fairly and uniformly tense, whereby the articular surfaces are against each other. Owing to their arrangement, during increasing tension of the anterior ligament (extension), the condyles of the femur must roll more on to the anterior part of the articular surface of the tibia, while by increasing tension of the posterior ligament (flexion), they must pass more backwards.

4. Joints with the axis of rotation round one fixed point.-These are the freely movable arthrodial joints. The movements can take place around innumerable axes, which all intersect each other in the centre of rotation. One articular surface is nearly spherical, the other is cup-shaped. The shoulder- and hip-joints are tvpical "ball-and-socket-joints." We may represent the movements as taking place around three axes, intersecting each other at right angles. The movements which can be performed at these joints may be grouped as :-(1) pendulum-like movements in any plane, (2) rotation round the long axis of the limb, and (3) circumscribing movements [circumduction], such as are made round the circumference of a sphere; the centre is in the point of rotation of the joint, while the circumference is described by the limb itself.

Limited arthrodial joints are ball joints with limited movements, and where rotation on the long axis is wanting, e.g., the metacarpo-phalangeal joints.

5. Rigid joints or amphiarthroses are characterised by the fact that movement may occur in all directions, but only to a very limited extent, in consequence of the tough and unyielding external ligaments. Both articular surfaces are usually about the same size, and are nearly plane surfaces, e.g., the articulations of the carpal and the tarsal bones.

II. Symphyses, synchondroses, and syndesmoses unite bones without the formation of a proper articular cavity, are movable in all directions, but only to the slightest extent. Physiologically they are closely related to amphiarthrodial joints.

III. Sutures unite bones without permitting any movement. The physiological importance 
of the suture is that the bones can still grow at their edges, which thus renders possible the distension of the cavity enclosed by the bones (Herm. v. Meyer).

306. ARRANGEMENT AND USES OF MUSCLES. - The muscles form 45 per cent. of the total mass of the body, those of the right side being heavier than those on the left. Muscles may be arranged in the following groups, as far as their mechanical actions are concerned :-

\section{A. Muscles without a definite origin and insertion :-}

1. The hollow muscles surrounding globular, oval, or irregular cavities, such as the urinary bladder, gall-bladder, uterus, and heart; or the walls of more or less cylindrical canals (intestinal tract, muscular gland ducts, ureters, Fallopian tubes, vasa deferentia, blood-vessels, lymphatics). In all these cases the muscular fibres are arrangred in several layers, $e_{.} g$., in a longitudinal and a circular layer, and sometimes also in an oblique layer. All these layers act together and thus diminish the cavity. It is inadmissible to ascribe different mechanical effects to the different layers, e.y., that the circular fibres of the intestine narrow it, while the longitudinal dilate it. Both sets of fibres rather seem to act simultaneousty, and diminish the cavity by making it narrower and shorter at the same time. The only case where muscular fibres may act in partially dilating the cavity is when, owing to pressure from without, or from partial contraction of some fibres, a fold, projecting into the lumen, has been formed. When the fibres, necessarily stretching across the depression thereby produced, contract, they must tend to undo it, i.e., enlarge the cavity. The various layers are all innervated from the same motor source, which supports the view of their conjoint action.

2. The sphincters surround an opening or a short canal, and by their action they either constrict or close it, e.g., sphincter pupillie, palpebrarum, oris, pylori, ani, cunni, urethræ.

B. Muscles with a definite origin and insertion:-

1. The origin is completely fixed when the muscle is in action. The course of the muscular fibres, as they pass to where they are inserted, permits of the insertion being approximated in a straight line towards their origin during contraction, $\because g$. , the attolens, attrahens, and retrahentes of the outer ear, and the rhomboidei. Some of these muscles are inserted into soft parts which necessarily must follow the line of traction, e.g., the azygos uvulæ, levator palati mollis, and most of the muscles which arise from bone and are inserted into the skin, such as the muscles of the face, styloglossus, stylopharyngeus, drc.

2. Both Origin and Insertion movable. - In this case the movements of both points are inversely as the resistance to be overcome. The resistance is often voluntary, which may be increased either at the origin or insertion of the muscle. Thus, the sternocleidomastoid may act either as a depressor of the head or as an elevator of the chest; the pectoralis minor may act as an abductor and depressor of the shoulder, or as an elevator of the 3rd to 5th ribs (when the shoulder-girdle is fixed).

3. Angular Course.-Many muscles having a fixed origin are diverted from their straight course; either their fibres or their tendons may be bent out of the straight course. Sometimes the curving is slight, as in the occipito-frontalis and levator palpebre superioris, or the tendon may form an angle round some bony process, whereby the muscular traction acts in quite a different direction, i.e., as if the muscle acted directly from this process upon its point of insertion, e.g, the obliquus oculi superior, tensor tympani, tensor veli palatini, obturator internus.

4. Nany of the muscles of the extremities act upon the long bones as upon levers:- (a) Some act upon a lever with one arm, in which case the insertion of the muscle (power) and the weight lie upon one side of the fulcrum or point of support, e.g., biceps, deltoid. The insertion (or power) often lies very close to the fulcrum. In such a case, the rapidity of the movement at the end of the lever 
is greatly increased, but force is lost [i.e., what is gained in rapidity is lost in power]. This arrangement has this advantage, that, owing to the slight contraction of the muscle, little energy is evolved, which would be the case had the muscular contraction been more considerable (\$300, I., 3). (b) The muscles act upon the bones as upon a lever with two arms, in which case the power (insertion of the muscle) lies on the other side of the fulcrum opposite to the weight, e.g., the triceps and muscles of the calf. In both cases, the muscular force necessary to overcome the resistance is estimated by the principles of the lever : equilibrium is established when the static moments (= product of the power in its vertical distance from the fulcrum) are equal ; or when the power and weight are inversely proportional, as their vertical distance from the fulcrum.

[The Bony Lever. - All the three orders of levers are met with in the body. Indeed, in the elbow-joint all the three orders are represented. The annexed scheme shows the relative positions of P, W, and F (fig. 346). The first order represented by such a movement as notding the head, the second by raising the body on the tiptoes by the muscles of the calf, and the third by the action of the biceps in raising the fore-arm. At the elbow-joint, the first order is illustrated by extending the flexed fore-arm on the upper arm, as in striking a blow on the table, where the triceps attached to the olecranon is the power, the trochlea the fulcrum, and the hand the weight. If the hand rest on the table and the body be raised on it, then the hand is the fulcrum, while the triceps is the power raising the humerus and the parts resting on it (W). The third order has already been referred to, e.g., flexing the fore-arm.]

Direction of Action. - It is most important to observe the direction in which the muscular force and weight act upon the lever-arm.

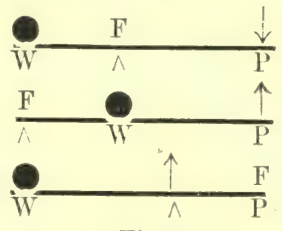

Fig. 346.

The three orders of levers. Thus, the direction may be vertical to the lever in one position, while after flexion it may act obliquely upon the lever. The static moment of a power acting obliqucly on the lever-arm is obtained by multiplying the power with the power acting in a direction vertical to the point of rotation.

Examples :-In fig. 347 , I., $\mathrm{B} x$ represents the humerus, and $x \mathrm{Z}$ the radius; $\mathrm{A} y$, the direction of the traction of the biceps. If the biceps acts at a right angle only, as by lifting horizontally a weight (P) lying on the fore-arm or in the hand, then the power of the biceps $(=\mathrm{A})$ is obtained from the formula, $\mathrm{A} y x=\mathrm{P} x \mathrm{Z}$, i.e., $\mathrm{A}=(\mathrm{P} x \mathrm{Z}): y x$. It is evident
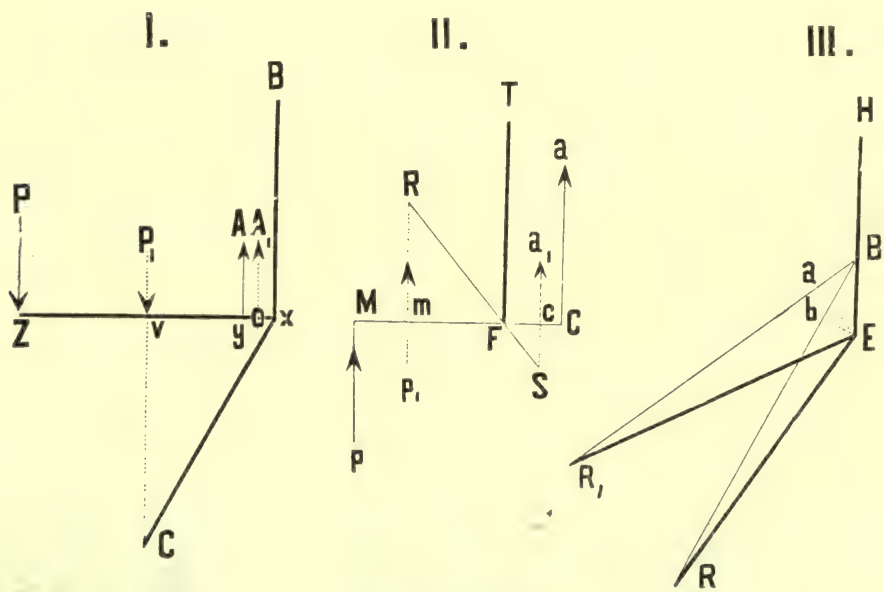

Fig. 347 .

Scheme of the action of the muscles on bones.

that, when the radius is depressed to the position $x \mathrm{C}$, the result is different ; then the force of the biceps $=\mathrm{A}_{1}=\left(\mathrm{P}_{1} v x\right): o x$. In fig. 347, II., TF is the tibia, F, the ankle-joint, MC, the foot in a horizontal position. The power of the muscles of the calf $(=a)$ necessary to equalise a force, $p$, directed from below against the anterior part of the foot, would be 
of the suture is that the bones can still grow at their edges, which thus renders possible the distension of the cavity enclosed by the bones (Herm. v. Meyer).

306. ARRANGEMENT AND USES OF MUSCLES. - The muscles form 45 per cent. of the total mass of the body, those of the right side being heavier than those on the left. Iruscles may be arranged in the following groups, as far as their mechanical actions are concerned :-

\section{A. Muscles without a definite origin and insertion :-}

1. The hollow muscles surrounding globular, oval, or irregular cavities, such as the urinary bladder, gall-bladder, uterus, and heart; or the walls of more or less cylindrical canals (intestinal tract, muscular gland ducts, ureters, Fallopian tubes, vasa deferentia, blood-vessels, lymphatics). In all these cases the muscular fibres are arranged in several layers, $e . g$., in a longitudinal and a circular layer, and sometimes also in an oblique layer. All these layers act together and thus diminish the cavity. It is inadmissible to ascribe different mechanical effects to the different layers, e.y., that the circular fibres of the intestine narrow it, while the longitudinal dilate it. Both sets of fibres rather seem to act simultaneousty, and diminish the eavity by making it narrower and shorter at the same time. The only case where muscular fibres may act in partially dilating the cavity is when, owing to pressure from without, or from partial contraction of some fibres, a fold, projecting into the lumen, has been formed. When the fibres, necessarily stretching across the depression thereby produced, contract, they must tend to undo it, i.e., enlarge the cavity. The various layers are all innervated from the same motor source, which supports the view of their conjoint action.

2. The sphincters surround an opening or a short canal, and by their action they either constrict or close it, e.g., sphincter pupillix, palpebrarum, oris, pylori, ani, cunni, urethræ.

B. Muscles with a definite origin and insertion:-

1. The origin is completely fixed when the muscle is in action. The course of the muscular fibres, as they pass to where they are inserted, permits of the insertion being approximated in a straight line towards their origin during contraction, \%.g., the attolens, attrahens, and retrahentes of the outer ear, and the rhomboidei. Some of these muscles are inserted into soft parts which necessarily must follow the line of traction, e.g., the azygos uvulæ, levator palati mollis, and most of the muscles which arise from bone and are inserted into the skin, such as the muscles of the face, styloglossus, stylopharyngeus, dc.

2. Both Origin and Insertion movable. - In this case the movements of both points are inversely as the resistance to be overcome. The resistance is often voluntary, which may be increased either at the origin or insertion of the muscle. Thus, the sternocleidomastoid may act either as a depressor of the head or as an elevator of the chest; the pectoralis minor may act as an abductor and depressor of the shoulder, or as an elevator of the 3rd to 5th ribs (when the shoulder-girdle is fixed).

3. Angular Course.-Many muscles having a fixed origin are diverted from their straight course; either their fibres or their tendons may be bent out of the straight course. Sometimes the curving is slight, as in the occipito-frontalis and levator palpebrie superioris, or the tendon may form an angle round some bony process, whereby the muscular traction acts in quite a different direction, i.e., as if the muscle acted directly from this process upon its point of insertion, e.g, the obliquus oculi superior, tensor tympani, tensor veli palatini, obturator internus.

4. Many of the muscles of the extremities act upon the long bones as upon levers:- (a) Some act upon a lever with one arm, in which case the insertion of the muscle (power) and the weight lie upon one side of the fulcrum or point of support, e.g., biceps, deltoid. The insertion (or power) often lies very close to the fulcrum. In such a case, the rapidity of the movement at the end of the lever 
is greatly increased, but force is lost [i.e., what is gained in rapidity is lost in power]. This arrangement has this advantage, that, owing to the slight contraction of the muscle, little energy is evolved, which would be the case had the muscular contraction been more considerable (\$300, I., 3). (b) The muscles act upon the bones as upon a lever with two arms, in which case the power (insertion of the muscle) lies on the other side of the fulcrum opposite to the weight, e.g., the triceps and muscles of the calf. In both cases, the muscular force necessary to overcome the resistance is estimated by the principles of the lever: equilibrium is established when the static moments (= product of the power in its vertical distance from the fulcrum) are equal ; or when the power and weight are inversely proportional, as their vertical distance from the fulcrum.

[The Bony Lever.-All the three orders of levers are met with in the body. Indeed, in the elbow-joint all the three orders are represented. The annexed scheme shows the relative positions of P, W, and F (fig. 346). The first order represented by such a movement as nodding the head, the second by raising the body on the tiptoes by the muscles of the calf, and the third by the action of the biceps in raising the fore-arm. At the elbow-joint, the first order is illustrated by extending the flexed fore-arm on the upper arm, as in striking a blow on the table, where the triceps attached to the olecranon is the power, the trochlea the fulcrum, and the hand the weight. If the hand rest on the table and the body be raised on it, then the hand is the fulcrum, while the triceps is the power raising the humerus and the parts resting on it (W). The third order has already been referred to, e.g., flexing the fure-arm.]

Direction of Action. - It is most important to observe the direction in which the muscular force and weight act upon the lever-arm.

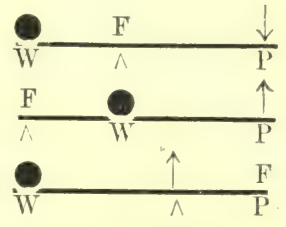

Fig. 346.

The three orders of levers. Thus, the direction may be vertical to the lever in one position, while after flexion it may act obliquely upon the lever. The static moment of a power acting obliqucly on the lever-arm is obtained by multiplying the power with the power acting in a direction vertical to the point of rotation.

Examples :-In fig. 347 , I., $\mathrm{B} x$ represents the humerus, and $x \mathrm{Z}$ the radius; $\mathrm{A} y$, the direction of the traction of the biceps. If the biceps acts at a right angle only, as by lifting horizontally a weight (P) lying on the fore-arm or in the hand, then the power of the biceps $(=\mathrm{A})$ is obtained from the formula, $\mathrm{A} y x=\mathrm{P} x \mathrm{Z}$, i.e., $\mathrm{A}=(\mathrm{P} x \mathrm{Z}): y x$. It is evident
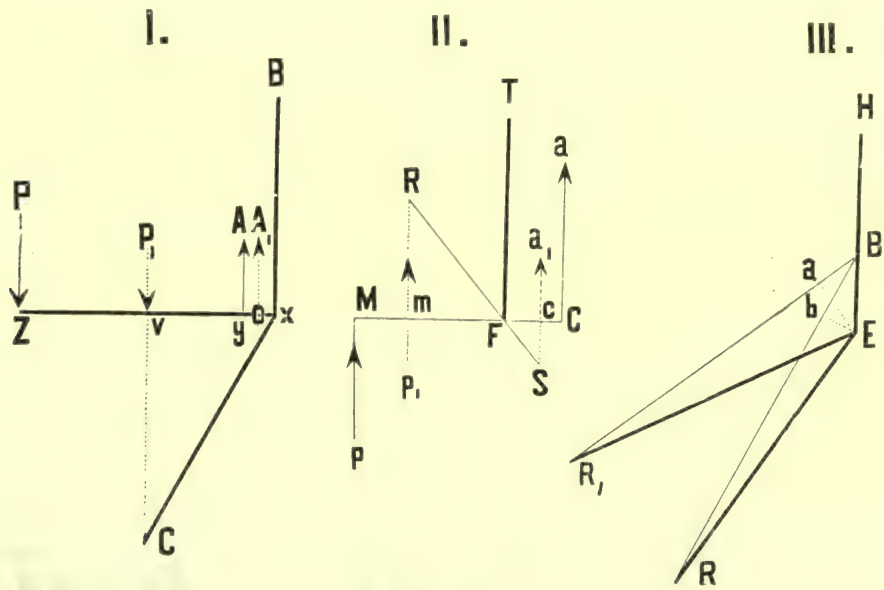

Fig. 347 .

Scheme of the action of the muscles on bones.

that, when the radius is depressed to the position $x \mathrm{C}$, the result is different ; then the force of the biceps $=A_{1}=\left(P_{1} v x\right): o x$. In fig. 347, II., TF is the tibia, F, the ankle-joint, MC, the foot in a horizontal position. The power of the muscles of the calf $(=\alpha)$ necessary to equalise a force, $p$, directed from below against the anterior part of the foot, would be 
$a=(p \mathrm{M} \mathrm{F}):$ F C. If the foot be altered to the position $\mathrm{R} \mathrm{S}$, the force of the muscles of the calf would then be $\sigma_{1}=\left(p_{1} \mathrm{M}\right.$ F $): \mathrm{F} \mathrm{C}$.

In muscles also, which, like the coraco-brachialis, are stretched over the angle of a hinge, the same result obtains.

In fig. 347 , III., $\mathrm{H} \mathrm{E}$ is the humerus, $\mathrm{E}$, the elbow-joint, $\mathrm{E} \mathrm{R}$, the radius, $\mathrm{B} \mathbf{R}$, the coracobrachialis. Its moment in this position is $=\mathrm{A}, a \mathrm{E}$. When the radius is raised to $\mathrm{E} \mathbf{R}_{1}$, then it is $=\mathrm{A}, a \mathrm{E}$. We must notice, however, that $\mathrm{B} \mathrm{R}_{1}<\mathrm{B} \mathrm{R}$. Hence, the absolute muscular force must be less in the flexed position, because every muscle, as it becomes shorter, lifts less weight. What is lost in power is gained by the elongation of the lever-arm.

5. Many muscles have a double action; when contracted in the ordinary way they execute a combined movement, e.g., the biceps is a flexor and supinator of the fore-arm. If one of these movements be prevented by the action of other muscles, the muscle takes no part in the execution of the other movement.

If the fore-arm be strongly pronated and flexed in this position, the biceps takes no part therein; or, when the elbow-joint is rigidly supinated, only the supinator brevis acts, not the biceps. The muscles of mastication are another example. The masseter elevates the lower jaw, and at the same time pulls it forward. If the depressed jaw, however, be strougly pulled back. wards when the jaw is raised, the masseter is not concerned. The temporal muscle raises the jaw, and at the same time pulls it backwards. If the depressed jaw be raised after being pushed forward, then the temporal is not concerned in its elevation.

6. Muscles acting on two or more joints are those which, in their course from their origin to their insertion, jass over two or more joints. Either the tendons may deviate from a straight course, e.g., the extensors and flexors of the fingers and toes, as when the latter are flexed; or the direction is always straight, e.g., the gastrocnemius. The muscles of this group present the following points of interest( ( ) The phenomenon of so-called "active insufficiency." If the position of the joints over which the muscle passes be so altered that its origin and insertion come too near each other, the muscle may require to contract so much before it can act on the bones attached to it, that it cannot contract actively any further than to the extent of the shortening from which it begins to be active; e.g., when the knee-joint is bent, the gastrocnemius can no longer produce plantar flexion of the foot, but the traction on the tendo Achilles is produced by the soleus. (b) "Passive insufficiency" is shown by many-jointed muscles under the following circumstances:- In certain positions of the joint, a muscle may be so stretched that it may act like a rigid strap, and thus limit or prevent the action of other muscles, e.g., the gastrocnemius is too short to permit complete dorsal flexion of the foot when the knee is extended. The long flexors of the leg, arising from the tuber ischii, are too short to permit complete extension of the knee-joint when the hip-joint is flexed at an acute angle. The extensor tendons of the fingers are too short to permit of complete flexion of the joints of the fingers when the hand is completely flexed.

7. Synergetic muscles are those which together subserve a certain kind of movement, e.g., the flexors of the leg, the muscles of the calf, and others. The abdominal muscles act along with the diaphragm in diminishing the abdomen during straining, while the muscles of inspiration or expiration, even the different origins of one muscle, or the two bellies of a biventral muscle, may be regarded from the same point of view.

Antagonistic muscles are those which, during their action, lave exactly the opposite effect of other muscles, e.g., flexors and extensors-pronators and supinators-adductors and abductors-elevators and depressors - sphincters and dilatorsinspiratory and expiratory.

When it is necessary to bring the full power of our muscles into action, we quite involuntarily bring them beforehand into a condition of the greatest tension, as a muscle in this condition is in the most favourable position for doing work $(\S 300, \mathrm{I},, 3)$. Conversely, when we execute delicate movements requiring 
little energy, we select a position in which the corresponding muscle is already shortened.

All the fasciæ of the body are connected with muscles, which, when they contract, alter the tension of the former, so that they are in a certain sense aponeuroses or tendons of the latter (K. Bardeleben). [For the importance of muscular movements and those of fasciæ in connection with the movements of the lymph, see $\S 201$.]

307. GYMNASTICS; MOTOR PATHOLOGICAL VARIATIONS.-Gymnastic exercise is most inportant for the proper development of the muscles and motor power, and it ought to be commenced in both sexes at an early age. Systematic muscular activity increases the volume of the muscles, and enables them to do more work. The amount of blood is increased with increase in the muscular development, while at the same time the bones and ligaments become more resistant. As the circulation is more lively in an active muscle, gymnastics favour the circulation, and ought to be practised, especially by persons of sedentary habits, who are apt to suffer from congestion of blood in abdominal organs (e.g., hæmorrhoids), as it favours the movement of the tissue juices [\$201]. An active muscle also uses more $O$ and produces more $\mathrm{CO}_{2}$, so that respiration is also excited. The total increase of the metabolism gives rise to the feeling of well-being and vigour, diminishes abnormal irritability, and dispels the tendency to fatigue. The whole body becomes firmer, and specifically heavier (Jäger).

By Ling's, or the Swedish system, a systematic attempt is made to strengthen certain weak muscles, or groups of muscles, whose weakness might lead to the production of deformities. These muscles are exercised systematically by opposing to them resistances, which must either be overcome, or against which the patient must strive by muscular action.

Massage, which consists in kneading, pressing, or rubbing the muscles, favours the bloodstream ; hence, this system may be advantageously used for such muscles as are so weakened by disease that an independent treatment by means of gymnastics cannot be adopted. [The importance of massage as a restorative practice in getting rid of the waste products of muscular activity has been already referred to $(\$ 304)$.]

Disturbances of the normal movenents may partly affect the passive motor organs (e.g., the bones, joints, ligaments, and aponeuroses), or the active organs (muscles with their tendons, and motor nerves).

Passive Organs. - Fractures, caries and necrosis, and inflammation of the bones, which make movements painful, influence or even make movement impossible. Similarly, dislocations, relaxation of the ligaments, arthritis, or anchylosis interfere with movement. Also curvature of bones, hyperostosis or exostnsis; lateral curvature of the vertebral column (Scoliosis), backward angular curvature (Kyphosis), or forward curvature (Lordosis). The latter interfere with respiration. In the lower extremities, which have to carry the weight of the body, genu valgum may occur in flabby, tall, rapidly-growing individuals, especially in some trades, e.g., in bakers. The opposite form, genu varum, is generally a result of rickets. Flat foot depends upon a depression of the arch of the foot, which then no longer rests upon its three points of support. Its causes seem to be similiar to those of genu valgum. The ligaments of the small tarsal joints are stretched, and the long axis of the foot is usually directed outwards; the inner margin of the foot is more turned to the ground, while pain in the foot and malleoli make walking and standing impossible. Club-foot (Talipes varus), in which the inner margin of the foot is raised, and the point of the toes is directed inwards and downwards, depends upon imperfect development during foetal life. All children are born with a certain very slight degree of bending of the foot in this direction. Talipes equinus, in which the toes, and T. calcaneus, in which the heel touches the ground, usually depend upon contracture of the muscles causing these positions of the foot, or upon paralysis of the antagonistic muscles.

Rickets and Osteomalacia. - If the earthy salts be withheld from the food, the bones gradually undergo a change; they become thin, translucent, and may even bend under pressure. In certain persistent defects of nutrition, the lime and other salts of the food are not absorbed, giving rise to rachitis, or rickets, in children. If fully formed bones lose their limesalts to the extent of $\frac{1}{2}$ to $\frac{1}{3}$ (halisterisis), they become brittle and soft (osteomalacia). This occurs to a limited extent in old age.

Muscles. - The normal nutrition of muscle is intimately dependent on a proper supply of sodium chloride and potash salts in the food, as these form integral parts of the muscular tissue (Kemmerich, Forester). Besides the atrophic changes which occur in the muscles when these substances are withheld, there are disturbances of the central nervous system and digestive apparatus, and the animals ultimately die. The condition of the muscles during inanition is given in $\$ 237$. If muscles and bones be kept inactive, they tend to atrophy (\$244). In atrophic muscles, and in cases of anchylosis, there is an enormous increase, or " atrophic proliferation," of the muscle-corpuscles, which takes place at the expense of the contractile contents (Cohnheim). A certain degree of muscular atrophy takes place in old age. The uterus, after delivery, undergoes a great decrease in size and weight-from 1000 to 350 grammes-due chiefly to the diminished blood supply to the organ. In chronic lead poisoning, the extensors 
and interossei chiefly undergo atrophy. Atrophy and degeneration of the museles are followed by shortening aud thinning of the bones to which the muscles are attached.

Section and paralysis of the motor nerves cause palsy of the muscle, thus rendering them inactive, and they ultimately degenerate. Atrophy also occurs after inflammation or softening of the multipolar nerve-cells in the anterior horn of the grey matter of the spinal cord, or the motor nuilei (facial, spinal aceessory, and hypoglossal of Stilling in the medulla oblongata), in the muscles connected with these parts. Kapid atrophy takes place in certain forms of spinal paralysis and in acute bulbar praralysis (paralysis of the medulla oblongata), and in a chronic form in progressive muscular atrophy and progressive bulbar paralysis. The muscles and their nerves berome small and soft. The muscles show many nuclei, the sarcous substance becomes fatty, and ultimately disappears. According to Charcot, these areas are at the same time the trophic centres for the nerves proceeding from them, as well as for the muscles belonging to them. Acrorling to Friedreich, the primary lesion in progressive muscular atrophy is in the muscles, and is due to a primary interstitial inflammation of the muscle, resulting in atrophy and degenerative changes, while the nerve-centres are affected secondarily, just as after amputation of a limb, the corresponding part of the spinal cord degenerates.

In pseudo-hypertrophic muscular atrophy the muscular fibres atrophy completely, with copious development of fat and connective-tissue between the fibres, without the nerves or spinal corrl undergoing degeneration. The muscular substance may also undergo amylloil or wax-7ike degeneration, whereby the amyloid substance infiltrates the tissue (\$249, VI.). Sometimes atrophic muscles have a drip bionen colour, due to a change of the hemoglobin of the muscle. When muscles are nuch used they hypertrophy, as the heart in certain cases of valvular lesion or obstruction $(\$ 40)$, the bladder, and intestiue. [In true hypertrophy there is an increased number or increase in the size of its tissue elements, throughout the entire tissue or organ, without any deposit of a fureign boly. Perhaps, in hypertrophy of the bladder, the thickened muscular coat not only serves to overcome resistance, but it offers greater resistance to bursting under the increased intra-vesical pressure. Mere enlargement is not hypertrophy, for this may be bronght ahout ly foreign elements. In atrophy there is a diminution in size or bulk, even when the blood-stream is kept up, the decrease being due to pressure. An atrophied organ may be even enlarget, as seen in pseudo-hypertrophic paralysis, where the muscles are larger, owing to the interstitial growth of fatty and connective-tissue, while the true muscular tissue is diminished and truly atrophied.]

308. STANDING.- The act of standing is accomplished by muscular action, and is the vertical position of equilibrium of the body, in which a line drawn from the centre of gravity of the body falls within the area of both feet placed upon the ground. In the military attitude, the muscles act in two directions-(1) to fix the jointed body, as it were, into one unbending column; and (2) in case of a variation of the equilibrium, to compensate by muscular action for the disturbance of the equilibrium.

The following individual motor acts occur in standing :-

1. Fixation of the head upon the vertebral column. The occiput may be moved in various directions upon the atlas, as in the acts of noddiny. As the long arm of the lever lies in front of the atlas, necessarily when the muscles of the back of the neck relax, as in sleep or death, the chin falls upon the breast. The strong neck muscles, which pull from the vertebral column upon the occiput, fix the head in a firm position on the vertebral column. The chiei rotatory movement of the heal on a vertical axis occurs round the odontoid process of the axis. The articular surfaces on the pedicles, and part of the bodies of the 1st and 2nd vertebre, are convex towards each other in the middle, becoming somewhat lower in front and behind, so that the head is highest in the erect posture. Hence, when the head is greatly rotated, compression of the medulla oblongata is prevented (Henke). In standing, these muscles do not require to be fixed by muscular action, as no rotation can take place when the neck muscles are at rest.

2. Fixed Vertebral Column. - The vertebral column itself must be fixed, especially where it is most mobile, i.e., in the cervical and lumbar regions. This is bronght about by the strong muscles situate in these regions, .9. , the cervical spinal muscles, Extensor dorsi communis and Quadratus lumborum.

Mobility of the Vertebræ. - The least movable vertebræ are the 3rd to the 6th dorsal; the sacrum is quite immovable. For a certain length of the column, the mobility depends on $(a)$ the number and height of the interarticular fibro-cartilages. They are most numerous in the neck, thickest in the lumbar region, and relatively also in the lower cervical region. They permit movement to take place in every direction. Collectively the interarticular discs form one-fourth of the height of the whole vertebral column. They are compressed somewhat by the pressure of the body; hence, the body is longest in the morning and after lying in the horizontal position. The smaller periphery of the bodies of the cervical vertebræ favours the mobility of these vertebræ compared with the larger lower ones. (b) The 
position of the processes also influences greatly the mobility. The strongly depressed spines of the dorsal region hinder hyperextension. The articular processes on the cervical vertebræ are so placed that their surfaces look obliquely from before and upwards, backwards, and downwards; this permits relatively free movement, rotation, lateral and nodding movements. In the dorsal region, the articular surfaces are directed vertically and directly to the front, the lower directly backwards ; in the lumbar region, the position of the articular processes is almost completely vertical and antero-posterior. In bending backwards as far as possible, the most mobile parts of the column are the lower cervical vertebræ, the 11 th dorsal to the 2nd lumbar and the lower two lumbar vertebre $(E . H . W e b e r)$.

3. The centre of gravity of the head, trunk, and arms when fixed as above, lies in front of the 10th dorsal vertebra. It lies further forward, in a horizontal plane, passing through the xiphoid process, the greater the distension of the abdomen by food, fat, or pregnancy. A line drawn vertically downwards from the centre of gravity passes behind the line uniting both hipjoints. Hence, the trunk would fall backwards on the hip-joint, were it not prevented partly by ligaments and partly by muscles. The former are represented by the ileo-femoral band and the anterior tense layer of the fascia lata. As ligaments alone, however, never resist permanent traction, they are aided, especially by the ileo-psoas muscle inserted into the small trochanter, and in part, also, by the rectus femoris. Lateral movement at the hip-joint, whereby the one limb must be abducted and the other adducted, is prevented especially by the large mass of the glutei. When the leg is extended, the ileo-femoral ligament, aided by the fascia lata, prevents adduction.

4. The rigid part of the body, head, and trunk, with the arms and legs, whose centre of gravity lies lower and only a little in front, so that the vertical line drawn downwards intersects a line connecting the posterior surfaces of the knee-joints, must now be fixed at the kneejoint. Falling backwards is prevented by a slight action of the quadriceps femoris, aided br the tension of the fascia lata. Indirectly it is aided also by the ileo-femoral ligament. Lateral movement of the knee is prevented by the disposition of the strong lateral ligaments. Rotation cannot take place at the knee-joint in the extended position ( $\$ 305, \mathrm{I}, \mathrm{s}$ ).

5 . A line drawn downwards from the centre of gravity of the whole body, which lies in the promontory, falls slightly in front of a line between the two ankle-joints. Hence, the body would fall forvard on the latter joint. This is prevented especially by the muscles of the calf, aided by the muscles of the deep layer of the leg (tibialis posticus, flexors of the toes, peroneus longus et brevis).

Other Factors :- $(\alpha)$ As the long axis of the foot forms with the leg an angle of $50^{\circ}$, falling forward can only occur after the feet are in a position more nearly parallel with their long axis. (b) The form of the articular surfaces helps, as the anterior broad part of the astragalus must be pressed between the two malleoli. The latter mechanism cannot be of much importance.

6. 'The metatarsus and phalanges are united by tense ligaments to form the arch of the foot, which tonches the ground at three points-tuber calcanei (heel), the head of the first metatarsal bone (ball of the great toe), and of the fifth toe. Between the latter two points, the heads of the metatarsal bones also form points of supports. The weight of the body is transmitted to the highest part of the arch of the foot, the caput tali. The arching of the foot is fixed only by ligaments. The toes play no part in standing, although, when moved by their muscles, they greatly aid the balancing of the body. The maintenance of the erect attitude fatigues one more rapidly than walking.

309. SITTING.-Sitting is that position of equilibrium whereby the body is supported on the tubera ischii, on which a to and fro movement may take place (H. v. Meyer). The head and trunk together are made rigid to form an immovable column, as in standing. We may distinguish-(1) the forward posture, in which the line of gravity passes in front of the tubera ischii ; the body being supported either against a fixed object, e.g., by means of the arm on a table, or against the upper surface of the thigh. (2) The backward posture, in which the line of gravity falls behind the tubera. A person is prevented from falling backward either by leaning on a support, or by the counter-weight of the legs kept extended by muscular action, whereby the sacrum forms an additional point of support, while the trunk is fixed on the thigh by the ileo-psoas and rectus femoris, the leg being kept exteuded by the extensor quadriceps. Usually the centre of gravity is so placed that the heel also acts as a point of support. The latter sitting posture is of course not suited for resting the muscles of the lower limbs. (3) When "sitting erect" the line of gravity falls between the tubera themselves. The muscles of the legs are relaxed, the rigid trunk only requires to be balanced by slight museular action. Usually the balancing of the head is sufficient to maintain the equilibrium.

310. WALKING, RUNNING, AND SPRINGING.-By the term walking is understood progression in a forward horizontal direction with the least possible muscular exertion, due to the alternate activity of the two legs.

Methods. - The Brothers Weber were the first to analyse the various positions of the body in walking, running, and springing, and they represented them in a continuous series, which 
represents the successive phases of locomotion. These phases may be examined with the zoetrope $(\$ 398,3)$. Marey estimated the time-rclations of the individual acts, by transferring the movements by menns of his air-tambours to a recorling surface. Recently, by means of a revolving camera, he has succeeled in photographing, in instantaneous pictures ( $\frac{2}{1000}$ second),

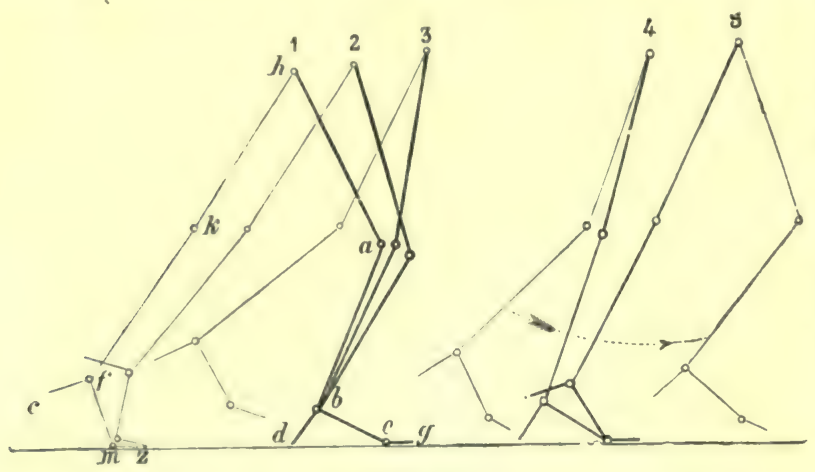

Fig. 348. the whole series of acts. Of course this series, when placed in the zoetrope, represents the natural movements. Figs. 349, 350,351 represent these acts.

In walking, the legs are active alternately; while one-the "supporting" or "active" leg-carries the trunk, the other is "inactive" or "passive." Each leg is alternately in an active and a passive phase. Walk-

Phases of walking. The thick lines represent the active, the thin the ing may be divided passive leg; $h$, the lip-joint ; $k, a$, knee; $f, b$, ankle ; $c, d$, heel; into the following $i n, e$, ball of the tarso-metatarsal joint; $z, g$, point of great toe. movements :-

I. Act (fig. 348, 2). - The actire leg is vertical, slightly flexed at the knee, and it alone supports the centre of gravity of the body. The passive leg is completely extended, and touches the ground only with the tip of the great toe $(z)$. This position of the leg corresponds to a right-angled triangle, in which the active leg and the ground form two sides, while the passive leg is the hypothenuse.

II. Act. - For the forward movement of the trunk, the active leg is inclined slightly from its vertical position (eathetus) to an oblique and more forward (hypothenuse) position (3). In order that the trunk may remain at the same height, it is necessary that the active leg be lengthened. This is accomplished by completely extending the knee $(3,4,5)$, as well as by lifting the heel from the ground $(4,5)$, so that the foot rests on the balls or the heads of the

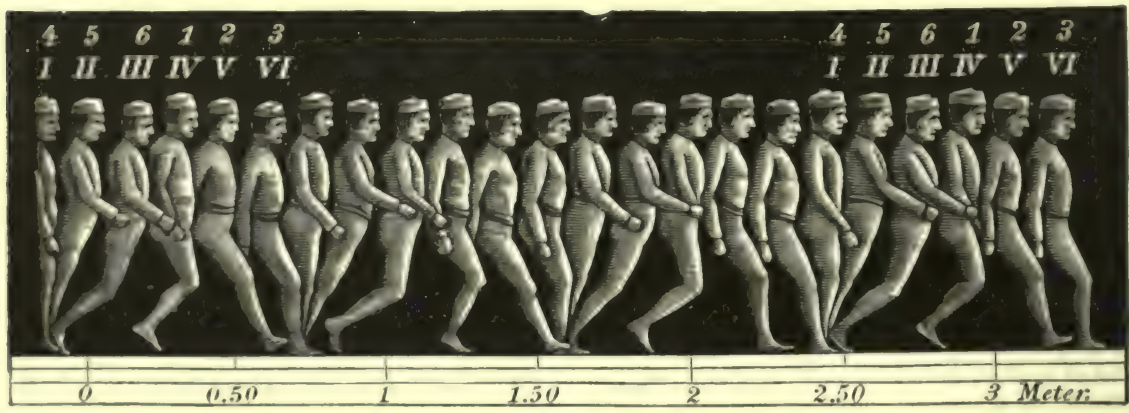

Fig. 349 .

Phases of slow walking. Instantaneous photograph (Marcy), only the side directed to the observer is shown. From the vertical position of the right, active leg; (I.), all the phases of this leg are represented in six pictures (I. to VI.), while after VI. the vertical position is regained. The Arabic numerals indicate the simultaneous position of the corresponding left leg; thus $1=$ I., $2=$ II., \&c., so that during the position IV. of the right leg, at the same time the left leg has the position as 1.

metatarsal bones, and, lastly, by elevating it on the point of the great toe (2, thin line). During the extension and forward movement of the active leg, the tips of the toes of the passive leg have left the ground (3). It is slightly flexed at the knee-joint (owing to the shortening), it performs a "pendulum-like movement" $(4,5)$, whereby its foot is moved as far in front of the active leg as it was formerly behind it. The foot is then placed flat upon the ground $(1,2$, 
thick lines) ; the centre of gravity is now transferred to this active leg, which, at the same time, is slightly flexed at the knee, and placed vertically. The first act is then repeated.

Simultaneous Movements of the Trunk,-During walking, the trunk performs certain characteristic movements. (1) It leans every time towards the active leg, owing to the traction of the glutei and the tensor fasciæ latæ, so that the centre of gravity is moved, which in short heavy persons with a broad pelvis leads to their "waddling" gait. (2) The trunk, especially during rapid walking, is inclined slightly forward to overcome the resistance of the air. (3) During the "pendulum-like action," the trunk rotates slightly on the head of the active femur. This rotation is compensated, especially in rapid walking, by the arm of the same side as the oscillating leg swinging in the opposite direction, while that on the other side at the same time swings in the same direction as the oscillating limb.

Modifying Conditions: 1 . The Duration of the Step.-As the rapidity of the vibration of a pendulum (leg) depends upon its length, it is evident that each individual, according to the length of his legs, must have a certain natural rate of walking. The "duration of a step" depends also upon the time during which both feet touch the ground simultaneously, which, of course, can be altered voluntarily. When "walking rapidly" the time $=0$, i.e., at the same inoment in which the active leg reaches the ground, the passive leg is raised. 2. The length of the step is usually about 6 to 7 decimetres [ 23 to 27 inches], and it must be greater, the more the length of the hypothenuse of the passive leg exceeds the cathetus of the active one. Hence, during a long step, the active leg is greatly shortened (by flexion of the knee), so that the trunk is pulled downwards. Similarly, long legs can make longer steps.

According to Marey and others, the pendulum movement of the passive leg is not a true pendulum movement, because its moveinent, owing to muscular action, is of more uniform rapidity. During the pendulum movement of the whole limb, the leg vibrates by itself at the knee-joint (Lucae, H. Vierordt).

Fixation of the Femur. - According to Ed. and W. Weber, the head of the femur of the passive leg is fixed in its socket chiefly by the atmospheric pressure, so that no muscular action is necessary for carrying the whole limb. If all the muscles and the capsule be divided, the head of the femur still remains in the cotyloid cavity. Rose refers this condition not to the action of the atmospheric pressure, but to two adhesion surfaces united by means of synovia.

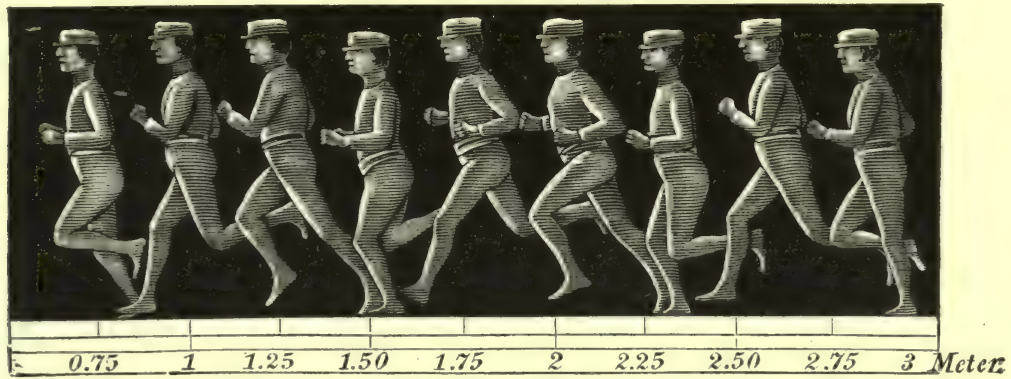

Fig. 350 .

Instantaneous photograph of a runner (Marcy). Ten pictures per second. The abscissa indicates the length of the step in metres.

The experiments of Aeby show that not only the weight of the limb is supported by the atmospheric pressure, but that the latter can support several times this weight. When traction is exerted on the limb, the margins of the cotyloid ligament of the cotyloid cavity are applied like a valve tightly to the margin of the cartilage of the head of the femur. According to the Brothers Weber, the leg falls from its socket as soon as air is admitted by making a perforation into the articular cavity.

Work done during Walking.-Marey and Demery estimate the amount done by a man weighing 64 kilos. [10 stones], when walking slowly, as $=6$ kilogrammetres per second; rapid running $=56$ kilogrammetres. The work done is due to the raising of the entire body and extremities, to the velocity communicated to the body, as well as to the maintenance of the centre of gravity.

In springing or leaping, the body is rapidly projected upwards by the greatest possible and most rapid contraction of the muscles, while at the same time the centre of gravity is maintained by other muscular acts (fig. 351 ).

The pressure upon the sole of the foot in walking is distributed in the following manner:The supporting leg always presses more strongly on the ground than the other; the longer the step the greater the pressure. The heel receives the maximum amount of pressure sooner than the point of the foot (Carlet). 
the cords rapidly return to their former position, and are again pushed asunder, and caused to vibrate.

1. Thus, when a membrane vibrates, the air must be alternately condensed and rarefied. The condensation and rarefaction are the chief cause of the tone or note (as in the siren), not so much the membranes themselves ( $v$. Helmholtz).

2. The air-tube or "porte vente," conducting the air to the membranes in man is the lower portion of the laryux, the trachea, and the whole bronchial system; the bellows are represented by the chest and lungs, which are forcibly diminished in size by the expiratory muscles.

3. The cavities which lie above the membranes constitute "resonators," and consist of the upper part of the larynx, pharynx, and also of the cavities of the nose and mouth, arranged, as it were, in two stories, the one over the other, which can be closed alternately.

The pitch of the tone produced by a membranous apparatus depends upon the following factors :-

(i) On the length of the elastic nembranes or plates. The pitch is inversely proportional to the length of the elastic membrane, i.e., the shorter the membrane the higher the pitch, or the greater the number of vibrations per second. Hence, the pitch of a child's vocal cords (shorter) is higher than that of an adult.

(b) The pitch of the tone is directly proportional to the square root of the amount of the elasticity of the elastic membrane. In membranous reeds, and also with silk, it is directly proportional to the square root of the extending weight, which in the case of the larynx is the force of the muscles rendering the cords tense.

(c) The tone of membranous reeds is not only strengthened by a more powerful blast, as the amplitude of the vibrations is increased, but the pitch of the tone may also be raised at the same time, hecause, owing to the great amplitude of the vibration, the mean tension of the elastic membrane is increased.

(d) The supra-laryngeal cavities, which act as resonators, are also inflated when the larynx is in action, so that the tone producec by these cavities is added to and blended with the sound of the elastic membranes, whereby certain partial tones of the latter are strengthened $(\$ 415)$. The characteristic timbre of the voice largely depends upon the form of the resonators.

(e) When vocalising, the strongest resonance takes place in the air-tubes, as they contain compressed air. It causes the vocal fremitus which is audible on placing the ear over the chest $(\$ 117,6)$.

( $f$ ) Nurrouing or diluting the glottis has no effect on the pitch of the tone, only with a wide glottis much more air must be driven through it, which, of course, greatly increases the work of the thorax.

\section{ARRANGEMENT OF THE LARYNX. - I. Cartilages and Ligaments.}

-The fundamental part of the larynx consists of the cricoid cartilage, whose small narrow portion is directed forwards and the broad plate backwards. The thyroid cartilage articulates by its inferior cornu with the posterior lateral portion of the cricoid. This permits of the thyroid cartilage rotating upon a horizontal axis directed through both of the articular surfaces, so that the upper margin of the thyroid passes forward and downward, while the joint is so constructed as to permit also of a slight upward, downward, forward, and backward movement of the thyroid upon the cricoid cartilage. The triangular arytenoid cartilages articulate at some distance from the middle line, with oval, saddle-like, articular surfaces placed upon the upper margin of the plate of the cricoid cartilage. The articular surfaces permit two kinds of movements on the part of the arytenoid cartilages; first, rotation on their base around their vertical long axis, whereby either the anterior angle or processus vocalis, which is directed forwards, is rotated outwards; while the processus muscularis, which is directed outwards and projects over the margin of the cricoid cartilage, is rotated backwards and inwards, or conversely. Further, the arytenoids may be slightly displaced upon their bases either outwards or inwards.

The true vocal cords, or thyro-arytenoid ligaments, are in man about 15 millimetres, and in woman 11 millimetres in length, and consist of numerous elastic fibres. They arise close to each other from near the middle of the inner angle of the thyroid cartilage, and are inserted, each into the anterior angle or processus vocalis of the arytenoid cartilages. The ventricles of Morgagni permit free vibration of the true vocal cords, and separate them from the upper or false cords, which consist of folds of mucous membrane. The false vocal cords are not concerned in 
phonation, but the secretion of their numerous mucous glands moistens the true vocal cords.

The obliquely directed under-surface of the vocal cords causes the cords to come together very easily when the glottis is narrow during respiration (e.g., in sobbing), while the closure may be made more secure by respiration. The opposite is the condition of the false vocal cords, which, when they touch, are easily separated during inspiration; while during expiration, owing to the dilatation of the ventricles of Morgagni, they easily come together and close (Wyllie, L. Brinnton and Cash).

II. Action of the Laryngeal Muscles.-These muscles have a double function : -1. One connected with respiration, in as far as the glottis is widened and

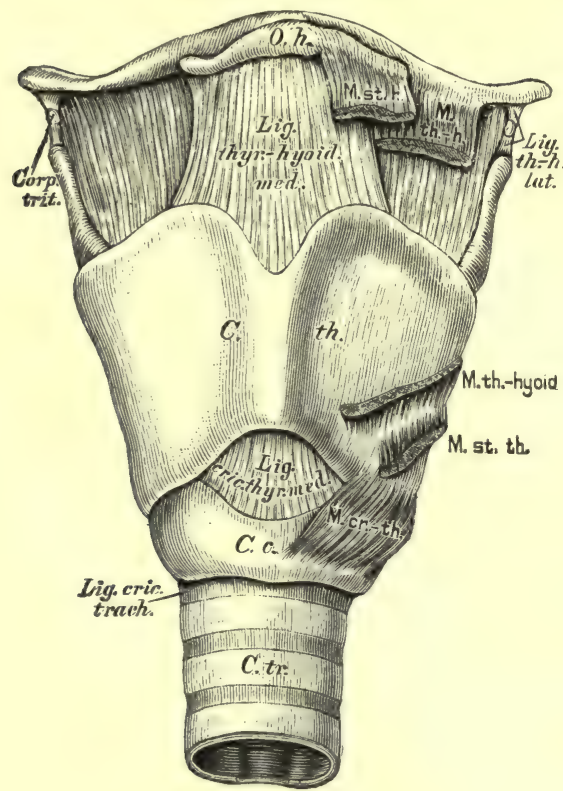

Fig. 352.

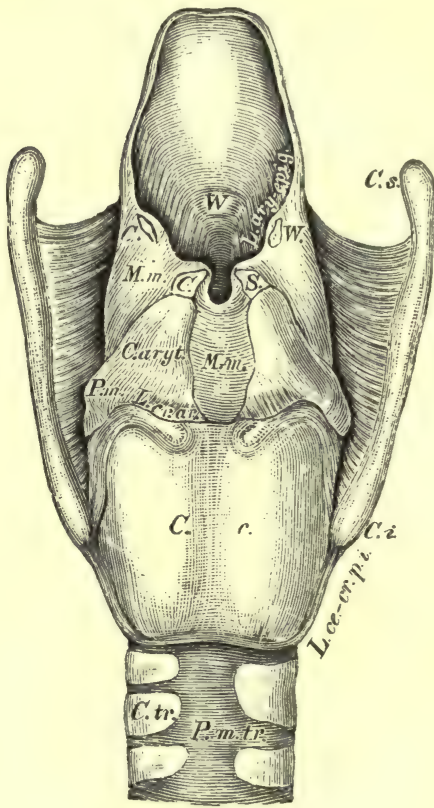

Fig. 353.

Fig. 352.-Larynx from the front, with the ligaments and the insertions of the nuscles. O.h., Os hyoideum; C.th., Cart. thyreoidea; Corp. trit., Corpus triticeum; C.c., Cart. cricoidea; C.tr., Cart. tracheales ; Lig. thyr.-hyoid. med., Ligamentum thyreo-hyoideum medium ; Lig. th.-h. lat., Ligam. thyreo-hyoideum laterale; Lig., cric. thyr. med., Ligam. crico-thyreoideum medium; Lig. cric.-trach., Ligam. crico-tracheale; M. St.-h., Musc. sterno-hyoideus ; $M$. th.-hyoid., Muse. thyreo-hyoideus ; $M$. st.-th., Musc. sterno-thyreoideus; $M . c r$.-th., Musc. crico-thyreoideus. Fig. 353.-Larynx from behind after removal of the muscles. E., Epiglottis cushion (W.) ; L. ar.-ep., Lig. ary-epiglotticum ; M.m., Membrana mucosa ; C.W., Cart. Wrisbergii ; C.S., Cart. Santorini ; C. aryt., Cart. arytænoidea ; C.c., Cart. cricoidea ; P.m., Processus muscularis of Cart. arytæn.; L. cr.-ar., Ligam. crico-arytæan.; C.s., Cornu superius; C.i., Cornu inferius Cart. thyreoidea. $L$. ce.-cr. p. $i$., Lig. kerato-cricoideum. post. inf.; C.tr., Cart. tracheales ; P.m.tr., Pars membranacea tracheæ.

narrowed alternately during respiration; further, when the glottis is firmly closed by these muscles, the entrance of foreign substances into the larynx is prevented. The glottis is closed immediately before the act of coughing $(\$ 120)$. 2. The larnyngeal muscles give the vocal cords the proper tension and other conditions for phonation.

1. The glottis is dilated by the action of the posterior crico-arytenoid muscles. When they contract they pull both processus musculares of the arytenoid cartilages 
backwards, dowuwards, and towards the middle line (fig. 356), so that the processus vocales (I, I) must gro apart and upwards (II, II). Thus, between the vocal cords (glottis vocalis), as well as between the inner margins of the arytenoid cartilages, a large triangular space is formed (glottis respiratoria), and these spaces are so arranged that their bases come together, so that the aperture between the cords and the arytenoid cartilacres has a rhomboidal form. Fig. 356 shows the action of the muscles. The vocal cords, represented by lines converging in front, arise from the anterior angle of the arytenoid cartilages (I, I). When these cartilages are rotated

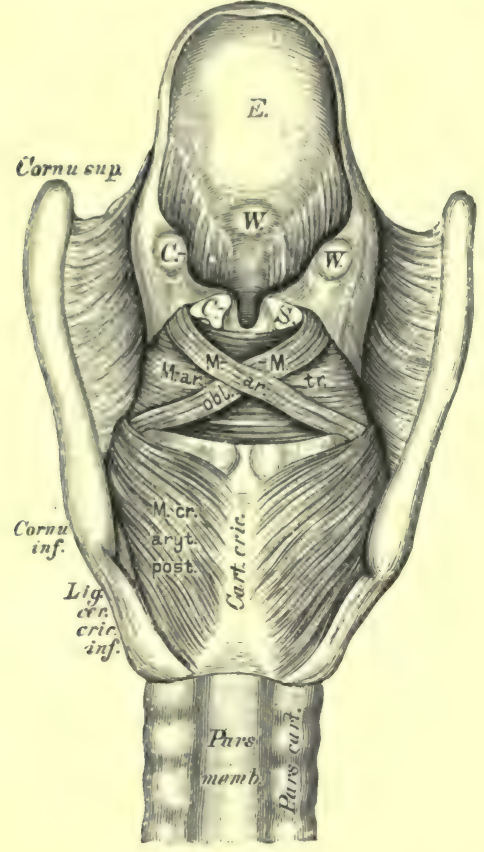

Fig. 354

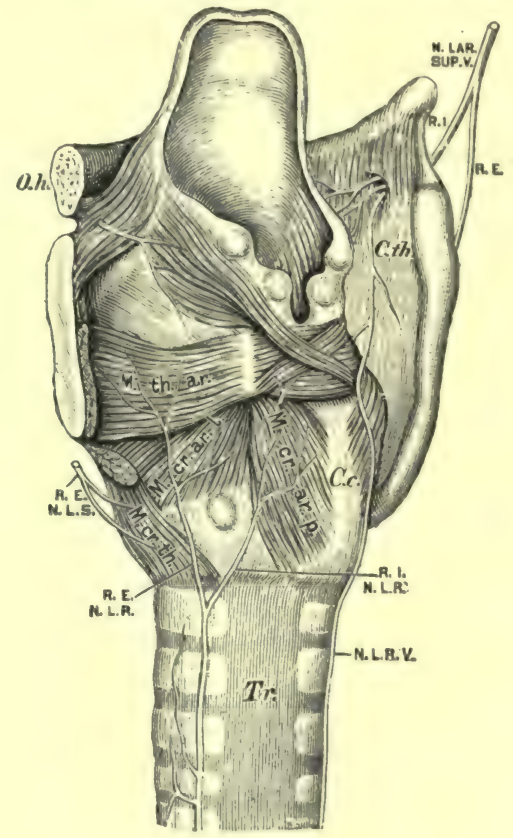

Fig. 355.

Fig. !354.-Larynx from behind with its muscles. E., Epiglottis, with the cushion (W.); C.W., Cart. Wrisbergii ; C.S., Cart. Santorini; C.c., Cart. cricoidea. Cornu sup.Cormu inf., Cart. thyreoidea ; $M_{\text {. }} a r$. $t r$. . Musc. arytienoideus transversus; $M m$. ar. obl., Musculi arytenoidei obliquui ; M. cr.-aryt. post., Musculus crico-arytænoideus posticus ; Pars cert., Pars cartilaginea; Pars memb., Pars membranacea trachee. Fig. 355. -Nerves of the larynx. O.h., Os hyoideum; C.th., Cart. thyreoidea; C.c., Cart. cricoidea; Tr., Trachea; M. th.oat., M. thyreo-arytienoideus; $M$. cr.-ar. $p_{.}$, M. crico-arytæenoideus posticus; M. $c r .-a r$ l., M. crico-arytan. lateralis; M. $c r_{0}-t h$. , M. crico-thyreoideus; I. Tar. sicp. v., N. laryngeus sup.; R.I., Ramus internus ; R.E., Ramus ext.; N. lar. rec. v., N. laryngeus recurrens; R.I.N.L.R., Ramus int.; R.E.N.L.R., Ramus ext. nervi laryngei recurrentis vagi.

into the position (II, II), the cords take the position indicated by the dotted lines. The widening of the respiratory portion of the glottis between the arytenoid cartilages is also indicated in the diagram.

Pathological. - When these muscles are paralysed, the widening of the glottis does not take place, and there may be severe dyspnoea during inspiration, although the voice is unaffected (Riegel, $L$. Weber). In a larynx just excised, the dilators are the first to lose their excitability (Semon and Horsley).

2. The entrance to the glottis is constricted by the arytenoid muscle (transverse), which extends transversely between both outer surfaces of the arytenoids along their whole length (fig. 357). On the posterior surface of this muscle is 
placed the cross bundles (fig. 354) of the thyro-aryepiglotticus (or arytænoidei obliqui); they act like the foregoing. The action of these muscles is indicated in fig. 357 ; the arrows point to the line of traction.

Pathological. - Paralysis of this muscle enfeebles the voice and makes it hoarse, as much air escapes between the arytenoid cartilages during phonation.

3. In order that the vocal cords be approximated to each other, which occurs during phonation, the processus vocales of the arytenoid cartilages must be closely apposed, whereby they must be rotated inwards and downwards. This result is brought about by the processus musculares being moved in a forward and upward direction by the thyro-arytenoid muscles. These muscles are applied to, and in fact are imbedded in, the substance of the elastic vocal cords, and their fibres reach to the external surface of the arytenoid cartilages. When they contract, they rotate these cartilages so that the processus vocales must rotate inwards. The glottis vocalis is thereby narrowed to a mere slit (fig. 358), whilst the glottis respiratoria remains as a broad triangular opening. The action of these muscles is indicated in fig. 358.

The lateral crico-arytenoid muscle is inserted into the anterior margin of the articular surface of the arytenoid cartilage; hence, it can only pull the cartilage

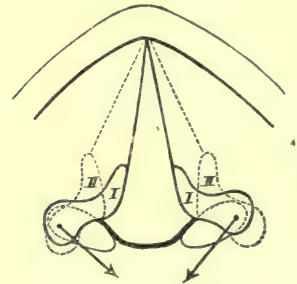

Fig. 356.

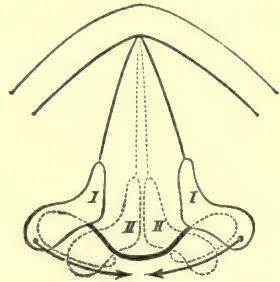

Fig. 357 .

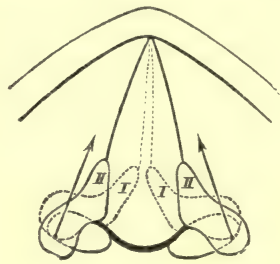

Fig. 358.

Fig. 356. - Schematic horizontal section of the larynx. I, Position of the horizontally divided arytenoid cartilages during respiration; from their anterior processes run the converging vocal cords. The arrows show the line of traction of the posterior crico-arytenoid muscles; II, II, the position of the arytenoid muscles as a result of this action. Fig. 357.Schematic horizontal section of the larynx, to illustrate the action of the crytenoid muscle. I, I, position of the arytenoid cartilages during quiet respiration. The arrows indicate the direction of the contraction of the muscle; II, II, the position of the arytenoid cartilages after the arytenoideus contracts. Fig. 358.- Scheme of the closure of the glottis by the thyro-arytenoid muscles. II, II, position of the arytenoid cartilages during quiet respiration. The arrows indicate the direction of the muscular traction.-I, I, position of the arytenoid cartilages after the muscles contract.

forwards; but some have supposed that it can also rotate the arytenoid cartilage in a manner similar to the thyro-arytenoid (?), with this difference, that the processus vocales do not come so close to each other.

Pathological._Paralysis of both thyro-arytenoid muscles causes loss of voice.

4. The vocal cords are rendered tense by their points of attachment being removed from each other by the action of muscles. The chief agents in this action are the crico-thyroid muscles, which pull the thyroid cartilage forwards and downwards. At the same time, however, the posterior crico-arytenoids must pull the arytenoid cartilages slightly backwards, and also keep them fixed.

The genio-hyoid and thyro-hyoid, when they contract, pull the thyroid upwards and forwards towards the chin, and also tend to increase the tension of the vocal cords (C. Mayer, Griutzner).

Pathological.-Paralysis of the crico-thyroid causes the voice to become harsh and deep, owing to the vocal cords not being sufficiently tense.

Position during Phonation.-The tension of the vocal cords brought about in this way is not of itself sufficient for phonation. The triangular aperture of the glottis respiratoria between the arytenoid cartilages, produced by the unaided action 
of the internal thyro-arytenoid muscles (see 3) must be closed by the action of the transverse and oblique arytenoid muscles. The vocal cords themselves must have a concave margin, which is obtained through the action of the crico-thyroids and posterior crico-arytenoids, so that the glottis vocalis presents the appearance of a myrtle leaf (Henle), while the rima glottidis has the form of a linear slit (fig. 362). The contraction of the internal thyro-arytenoid converts the concave margin of the vocal cords into a straight margin. This muscle adjusts the delicate variations of tension of the vocal cords themselves, causing more especially such variations as are necessary for the production of tones of slightly different pitch. As these muscles come close to the margin of the cords, and are securely woven, as it were, amongst the elastic fibres of which the cords consist, they are specially adapted for the abovementioned purpose. When the muscles contract, they give the necessary resistance to the cords, thus favouring their vibration. As some of the muscular fibres end in the elastic fibres of the cords, these fibres, when they contract, can render certain parts of the cords more tense than others, and thus favour the modifications in the formation of the tones. The coarser variations in the tension of the vocal cords are produced by the separation of the thyroid from the arytenoid cartilages, while the finer variations of tension are produced by the thyro-arytenoid muscles. The value of the elastic-tissue of the cords does not depend so much upon its extensibility, as upon its property of shortening without forming folds and creases.

Pathological. - In paralysis of these muscles, the voice can only be produced by forcible expiration, as nuch air escapes through the glottis; the tones are at the same time deep and impure. laralysis of the muscle of one side causes flapping of the vocal cord on that side (Gerluerdt).

5. The relaxation of the vocal cords occurs spontaneously when the stretching forces cease to act; the elasticity of the displaced thyroid and arytenoid cartilages comes into play, and restores them to their original position. The vocal cords are also relaxed by the action of the thyro-arytenoid and lateral crico-arytenoid muscles.

It is evident, from the above statements, that tension of the vocal cords and narrowing of the glottis are necessary for phonation. The tension is produced by the crico-thyroids and posterior crico-arytenoids; the narrowing of the glottis respiratoria by the arytenoids, transverse and oblique, the glottis vocalis being narrowed by the thyro-arytenoids and (? lateral crico-arytenoids), the former muscles causing the cords themselves to become tense.

Nerves $(352,5)$. - The crico-thyroid is supplied by the superior laryngeal branch of the vagus, which at the same time is the sensory nerve of the mucous membrane of the larynx. All the other intrinsic muscles of the larynx are supplied by the inferior laryngeal.

The mucous membrane of the larynx is richly supplied with elastic fibres, und so is the sub-mucosa. The sub-mucosa is more lax near the entrance to the glottis and in the ventricles of Morgagni, which explains the enormous swelling that sometimes occurs in these parts in wdena glottidis. A thin clear limiting membrane lies under the epithelium. The epithelium is stratitied, cylindrical, and ciliated with intervening goblet cells. On the true vocal cords and the anterior surface of the epiglottis, however, this is replaced by stratified squamous epithelium, which covers the small papillæ of the mucous membrane. Numerous branched mucons glands occur over the cartilages of Wrisberg, the cushion of the epiglottis, and in the ventricles of Morgagni ; in other situations, as on the posterior surface of the larynx, the glands are more scattered. The blood-vessels form a dense capillary plexus under the membrana propria of the mucous membrane; under this, however, there are other two strata of bloodvessels. The lymphatics form a superficial narrow mesh-work under the blood-capillaries, with a deeper, coarser plexus. The medullated nerves have ganglia in their branches, but their mode of termination is unknown. [W. Stirling has described a rich sub-epithelial plexus of medullated nerve-fibres on the anterior surface of the epiglottis, while he finds that there are ganglionic cells in the course of the superior laryngeal nerve.]

Cartilages. - The thyroid, cricoid, and nearly the whole of the arytenoid cartilages consist of hyaline cartilage. The two former are prone to ossify. The apex and processus vocalis of the 
arytenoid cartilages consist of yellow fibro-cartilage, and so do all the other cartilages of the larynx.

The larynx grows until about the sixth year, when it rests for a time, but it becomes again much larger at puberty $(\$ 434)$.

314. LARYNGOSCOPY.-Historical. -After Bozzini (1807) gave the first impulse towards the investigation of the internal cavities of the body, by illuminating them with the aid of mirrors, Babington (1829) actually observed the glottis in this way. The famous singer, Manuel Garcia (1854), made investigations both on himself and other singers, regarding the movements of the vocal cords, during respiration and phonation. The examination of the larynx by means of the laryngoscope was rendered practicable chiefly by T'irck (1857) and
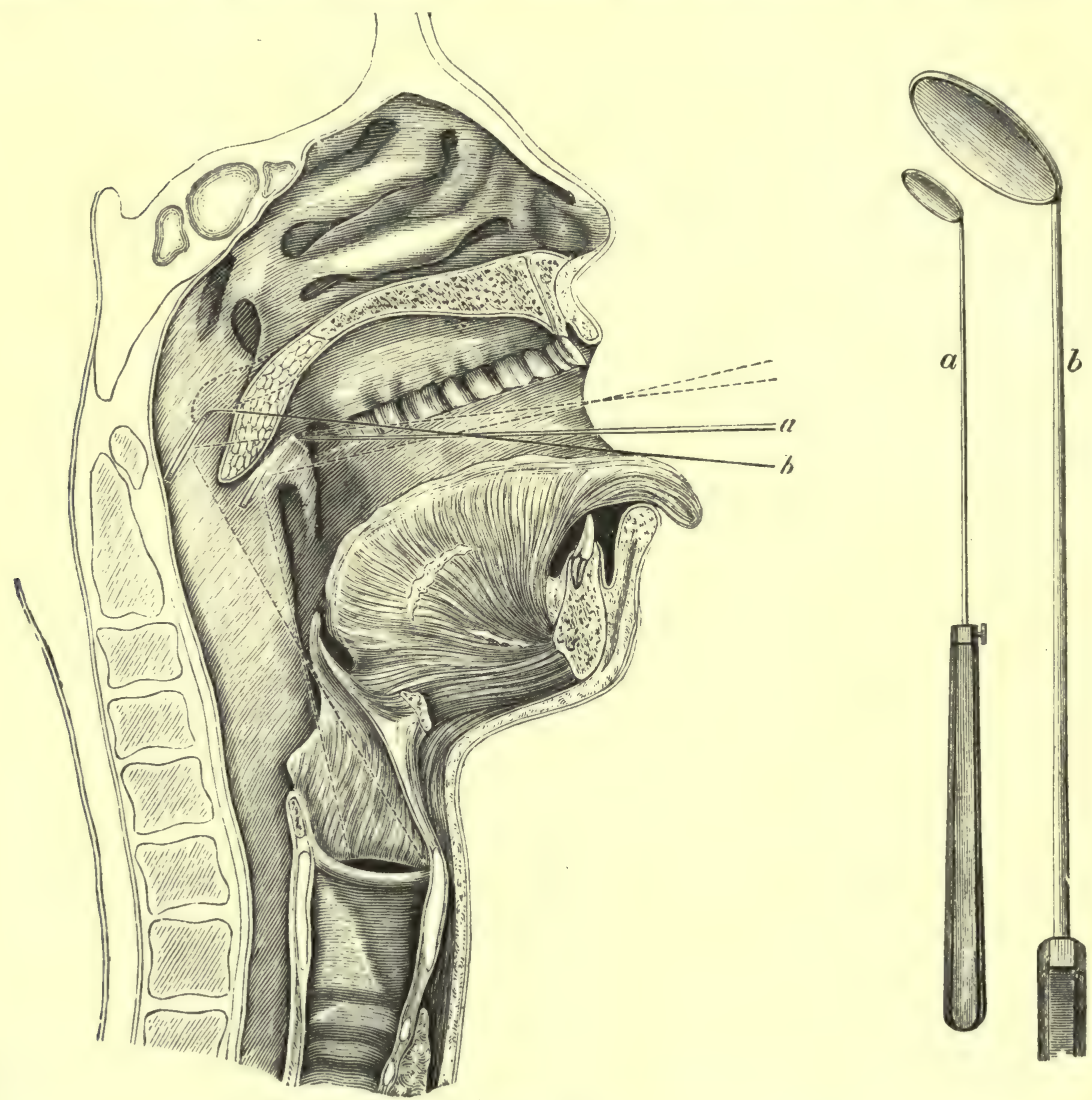

Fig. 359.

Vertical section through the head and neck, to the 1st dorsal vertebra, $a$, position of the laryngoscope on observing the posterior part of the glottis, arytenoid cartilages, and upper surface of the posterior wall of the larynx; $b$, its position on observing the anterior angle of the glottis. Large, $a$, and $b$, small laryngoscopic mirrors.

Czermak, the latter observer being the first to use the light of a lamp for the illumination of the larynx. Rhinoseopy was actually first practised by Baumè (1838), but Czermak was the first person who investigated this subject systematically.

The Laryngoscope consists of a small mirror fixed to a long handle, at an angle of $125^{\circ}$ to $130^{\circ}$ (fig. $\left.359, a, b\right)$. When the mouth is opened, and the tongue drawn forward, the mirror is introduced, as is shown in fig. 360. The position of the mirror must be varied, according to the position of the larynx we wish to examine; in some cases, the soft palate has to be raised by the back of the mirror, as in the position $b$. A picture of the part of the larynx examined is formed in the small mirror, the rays of light passing in the direction indicated by the dotted 
lines from the mirror ; they are reflected at the same angle through the mouth into the eye of the observer, who must place himself in the direction of the reflected rays.

The illumination of the larynx is accomplished either by means of direct sunlight or by light from an artificial source, e.g., an ordinary lamp, an oxyhydrogen lime-light, or the electrie light. The beam of light impinges upon a concave mirror of 15 to 20 centimetres focus, and 10 centimetres in width, and from its surface the concentrated beam of light is reflected through the mouth of the patient, and directed upon the small mirror held in the back part of the throat. The beam of light is reflected at the same angle towards the larynx by the small throat mirror, so that the larynx is brightly illuminated. The observer has now to direct his eye in the same

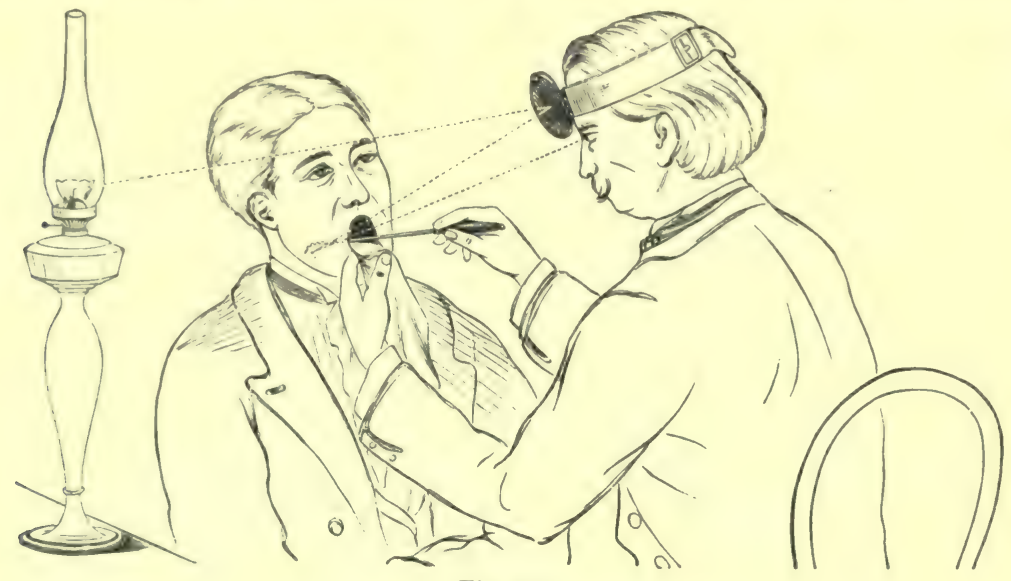

Fig. 360.

Method of examining the larynx.

lirection as the illuminating rays, which can be accomplished by having a hole in the centre of the concave mirror, through which the observer looks. Practically, however, this is unnecessary; all that is necessary is to fix the concave mirror to the forehead by means of a broad elastic band, so that the observer, by looking just under the margin of the concave mirror, can see the

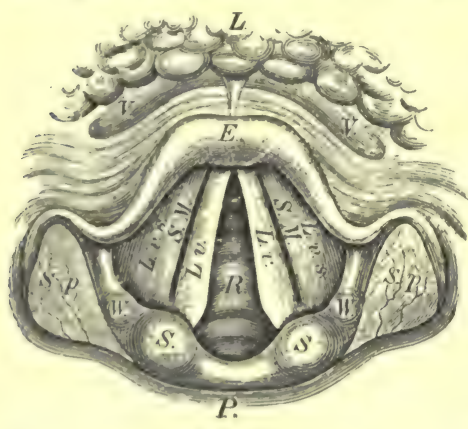

Fig. 361.

The laryux, as seen with the laryngoscope. $L$., tongue ; $E$., epiglottis; $V$., valleculla; $R$., glottis; $L . v$., true vocal rords; S.M., sinus Morgagni ; L.v.s., false vocal cords; $P$., position of pharynx; $S$., cartilage of Santorini ; $W$., of Wrisberg; S.p., sinus piriformes, picture of the larynx in the small throat mirror(fig. 360).

In order to examine the larynx, place the patient immediately in front of you, and cause him to open his mouth and protrude his tongue. A lamp is placed at the side of the head of the patient, and light from this source is reflected from the concave mirror on the observer's forehead, and concentrated upon the laryngoscopic mirror introduced into the back part of the throat of the patient (fig. 360).

Oertel was able by means of a rapid intermittent illumination of the larynx through a stroboscopic dise, to study the movements of the vocal cords directly with the eye. Simanowsky put a photographic camera in the position of the eye, and photographed the movements of the vocal cords of an artificial larynx. [Brown and Behnke have photographed the human vocal cords.]

Laryngeal Electrodes. - V. Ziemssen introduces long narrow electrodes into the larynx, to stimulate the muscles and study their actions. Rossbach finds that the muscles and nerves of the interior of the larynx may be stinulated by stimulating the skin, i.e., percutaneously. Those methods are used both for physiological and therapeutical purposes.

Picture of the Larynx.-Fig. 361 shows the following structures:- $L$., the root of the tongue, with the ligamentum glosso-epiglotticum continued from its middle; on each side of the latter are $V . V$., the so-called valleculce. The epiglottis $(E$.) appears 
like an arched upper lip; under it, during normal respiration, are the lancet-shaped glottis $(R$.$) and on each side of it the true vocal cords (L . v)$. The length of the vocal cord in a child is 6 to $8 \mathrm{~mm}$., in the female 10 to $15 \mathrm{~mm}$. when they are relaxed, and 15 to $20 \mathrm{~mm}$. when tense. In man, the lengths under the same conditions are 15 to $20 \mathrm{~mm}$. and 20 to $25 \mathrm{~mm}$. The breadth varies from 2 to $5 \mathrm{~mm}$. On the external side of each vocal cord is the entrance to the sinus of Morgagni

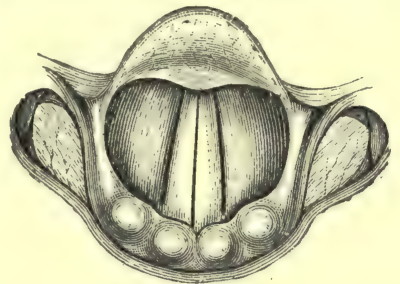

Fig. 362.

Position of the vocal cords on uttering a high note.

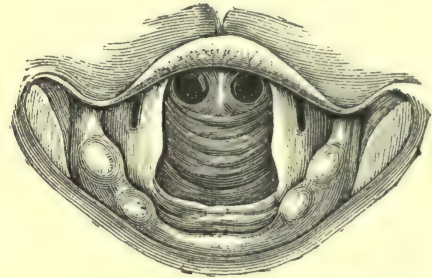

Fig. 363 .

View of the rings and bifurcation of trachea.

(S.M.), represented as a dark line. Further upwards and more external are (L.v.s.) the upper or false vocal cords. [The upper or false vocal cords are red, the lower or true, white.] On each side of $P$. are $(S . S$.$) , the apices of the cartilages of San-$ torini, placed upon the apices of the arytenoid cartilages, while immediately behind is the wall of the pharynx, $P$. In the arytenoepiglottidean fold are $(W . W$.$) the$ cartilages of Wrisberg, while outside these are the depressions $(S . p$. constituting the sinus piriformes.

During normal respiration, the glottis has the form of a lancetshaped slit between the bright, yellowish-white, vocal cords (fig. 362 ). If a deep inspiration be taken, the glottis is considerably widened (fig. 363), and if the mirror be favourably adjusted we may see the rings of the trachea, and even the birfurcation of the trachea.

If a high note be uttered, the glottis is contracted to a very narrow slit (fig. 362).

Rhinoscopy.-If a small mirror, fixed to a handle at an angle of $100^{\circ}$ to $110^{\circ}$, be introduced into the pharynx, as shown in fig. 364, and if the mirror be directed upwards, certain structures are with difficulty rendered visible (fig. 365 ).

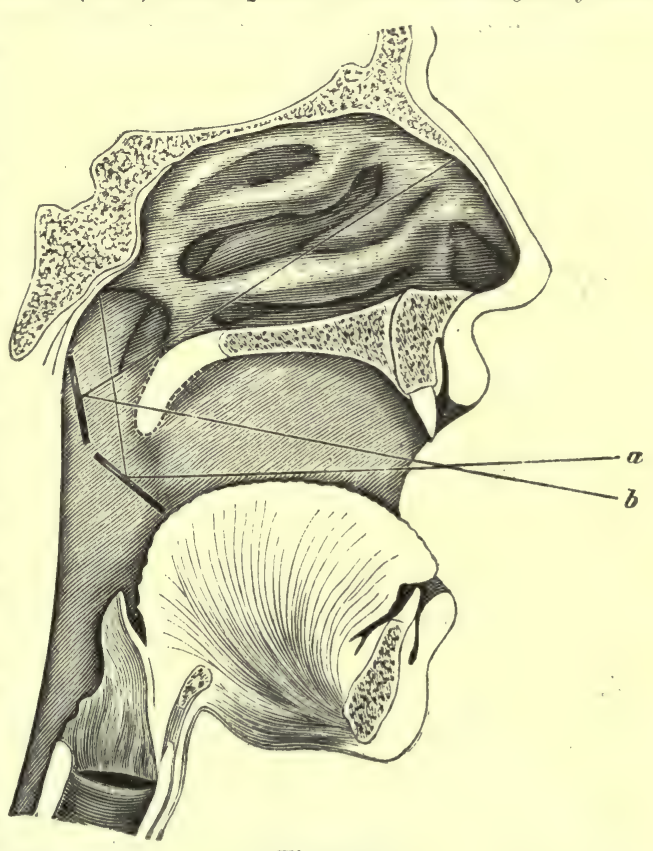

Fig. 364.

Position of the laryngoscopic mirror in rhinoscopy. In the middle is the septum narium $(S . n$.$) , and on each side of it the long oval large posterior$ nares $(C h$.$) , below this the soft palate \left(P . m_{.}\right)$, with the pendant uvula (U.). In the posterior nares are the posterior extremities of the lower $(C . i$.$) , middle (C . m$.$) , and upper turbinated bones$ (C.s.). At the upper part, a portion of the roof of the pharynx $\left(O . R_{0}\right)$ is seen, with the arched masses of adenoid tissue lying between the openings of the Eustachian tubes (T.T.), and called by Luschka the pharyngeal tonsils. External to the openings of the Eustachian tube is the tubular eminence $(W$.$) , and outside this is the groove of Rosenmiller (R$.$) .$ 
Experiments on the Larynx. - Ferrein (\$741) and Joh. Niuller made experiments upon the excised larynx. A tracheal tube was tied into the excised human larynx, and air was blown through it, the pressure being measured by means of a mercurial manometer, while various

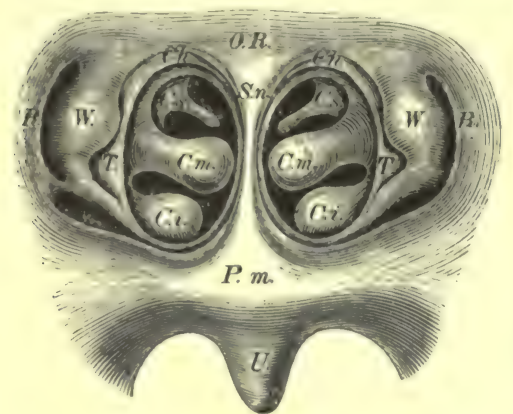

Fig. 365. arrangements were adopted for putting the vocal cords on the stretch and for opening or closing the glottis.

315. CONDITIONS INFLUENCING THE LARYNGEAL SOUNDS. - The pitch of the note emitted by the larynx depends upon:-

1. The Tension of the Vocal Cords, i.e., upon the degree of contraction of the cricothyroid and posterior crico-arytenoid muscles, and also of the internal thyro-arytenoids (\$313, II., 4).

2. The Length of the Vocal Cords.(a) Children and females with short vocal cords produce high notes. (b) If the ary-

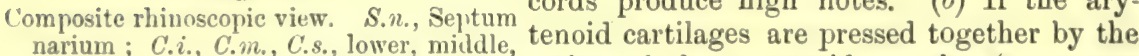
narium; ; C.i., C.m., C.s., lower, milde, turbinated bones; $T$., Eusta- action of the arytenoid muscles (transverse chian tube ; $W$., tubular eminence; $R$., and oblique), so that the vocal cords alone groove of Rosenmiiller; $P$.m., soft can vibrate, while their intercartilaginous palate; $O . R$., roof of pharynx; $U$., uvula. portions lying between the processus vocales do not, the tone thereby produced is higher (Garcia). In the production of low notes, the vocal cords, as well as the margins of the arytenoid cartilages, vibrate. At the same time the space above the entrance to the glottis is enlarged and the larynx becomes more prominent. (c) Every individual has a certain medium pitch of his voice, which corresponds to the smallest possible tension of the intrinsic muscles of the larynx.

3. The Strength of the Blast.-That the strength of the blast from below raises the pitch of the tones of the human larynx is shown by the fact, that tones of the highest pitch can only be uttered by powerful expiratory efforts. With tones of medium pitch, the pressure of the air in the trachea is $160 \mathrm{~mm}$., with high pitch $200 \mathrm{~mm}$., and with very high notes $945 \mathrm{~mm}$., and in whispering $30 \mathrm{~mm}$., of water (Cagniard-Latour). These results were obtained in a case of tracheal fistula.

Accessory Phenomena. - The following as yet but partially explained phenomena are observed in connection with the production of high notes :- $(a)$ As the pitch of the note rises, the larynx is elevated, partly because the muscles raising it are active, partly because the increased intratracheal pressure so lengthens the trachea, that the larynx is thereby raised; the uvula is raised more and more (Labus). (b) The upper vocal cords approximate to each other more and more, without, however, coming into contact, or participating in the vibrations. (c) The epiglottis inclines more and more backwards over the glottis.

4. The falsetto voice with its soft timbre and the absence of resonance or pectoral fremitus in the air-tubes is particularly interesting. Oertel observed that, during the falsetto voice, the vocal cords vibrated so as to form nodes across them, but sometimes there was only one node, so that the free margin of the cord and the basal margin vibrated, being separated from each other by a nodal line (parallel to the margins of the vocal cord). During a high falsetto note, there may be three such nodal lines parallel to each other. The nodal lines are produced probably by a partial contraction of the fibres of the thyro-arytenoid muscle (p. 513), while at the same time the vocal cords must be reduced to as thin plates as possible by the action of the crico-thyroid, posterior arytenoid, thyro- and genio-hyoid muscles (Oertel). The form of the glottis is elliptical, while with the chest-voice the vocal cords are limited by straight surfaces; the air also passes more freely through the larynx. 
Oertel also found that during the falsetto voice the epiglottis is erect. The apices of the arytenoid cartilages are slightly inclined backwards, the whole larynx is larger from before backwards, and narrower from side to side, the aryepiglottidean folds are tense with sharp margins, and the entrance to the ventricles of Morgagni is narrowed. The vocal cords are narrower, the processus vocales touch each other. The rotation of the arytenoid cartilages necessary for this is brought about by the action of the crico-arytenoid alone, while the thyroarytenoid is to be regarded only as an accessory aid. The pitch of the note is increased solely by increased tension of the vocal cords. In addition, there are a number of transverse and longitudinal partial vibrations. During the chest-voice, a smaller part of the margin vibrates than in the falsetto voice, so that in the production of the latter we are conscious of less muscular exertion in the larynx. The uvula is raised to the horizontal position.

Production of Voice. - In order that voice be produced, the following conditions are necessary :-(1) The necessary amount of air is collected in the chest; (2) the larynx and its parts are fixed in the proper position; (3) air is then forced by an expiratory effort either through the linear chink of the closed glottis, so that the latter is forced open, or at first some air is allowed to pass through the glottis without producing a sound, but as the blast of air is strengthened the vocal cords are thrown into vibration.

316. RANGE OF THE VOICE.-The range of the human voice for chest notes is given in the following scheme:-

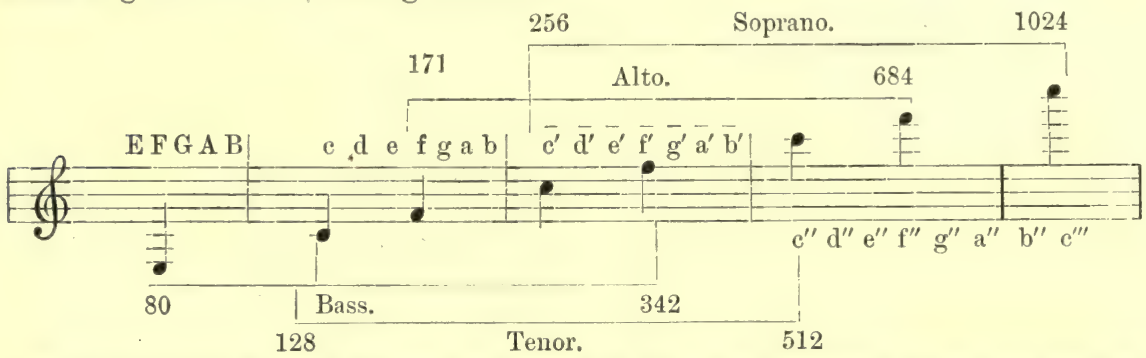

The accompanying figures indicate the number of vibrations per second in the corresponding tone. It is evident that from $c^{\prime}$ to $f^{\prime}$ is common to all voices, nevertheless, they have a different timbre. The lowest note or tone, which, however, is only occasionally sung by bass singers, is the contra-F, with 42 vibrations-the highest note of the soprano voice is $a^{\prime \prime \prime}$, with 1708 vibrations.

Timbre.-The voice of every individual has a peculiar quality, clang, or timbre, which depends upon the shape of all the cavities connected with the larynx. In the production of nasal tones, the air in the nose is caused to vibrate strongly, so that the entrance to the nares must necessarily be open.

317. SPEECH - THE VOWELS. - The motor processes connected with the production of speech occur in the resonating cavities, the pharynx, mouth, and nose, and are directed towards the production of musical tones and noises.

Whispering and Audible Speech.-When sounds or noises are produced in the resonating chambers, the larynx being passive, the vox clandestina, or whispering is produced; when the vocal cords, however, vibrate at the same time, "audible speech" is produced. [Whispering, therefore, is speech without voice.]. Whispering may be fairly loud, but it requires great exertion, i.e., a great expiratory blast, for its production; hence it is very fatiguing. It may be performed both with inspiration and expiration, while audible speech is but temporary and indistinct, if it is produced during inspiration. Whispering is caused by the sound produced by the air passing over the obtuse margins of the cords. During the production of audible sounds, however, the sharp margins of the vocal cords are directed towards the air by the position of the processus vocales.

During speech the soft palate is in action; at each word it is raised, while at the same time, Passavant's transverse band is formed in the pharynx (\$ 156). The soft palate is raised highest 
when $\mathbf{u}$ and $\mathbf{i}$ are sounded, then with $o$ and $e$, and least with $a$. When sounding $\mathbf{m}$ and $\mathbf{n}$ it does not move ; it is high (like $\mathbf{n}$ ) during the utterance of the explosives. With $\mathbf{1}, \mathbf{8}$, and especially with the gutteral r, it exhibits a trembling movement (Gentzen, Falkson).

Speech is composed of vowels and consonants.

A. Vowels (analysis and artificial formation, $\$ 415$ ).-A. During whispering, a vowel is the musical tone produced, either during expiration or inspiration, by the inflated characteristic form of the mouth, which not only has a definite pitch, but also a particular and characteristic timblre. The characteristic form of the mouth may be called "vowel-cavity."

I. The pitch of the vowels may be estimated musically. It is remarkable that the fundamental tone of the "vowel-cavity" is nearly constant at different ages and in the sexes. The different capacities of the mouth can be compensated for by different sizes of the oral aperture. The pitch of the vowel-cavity may be estimatel by placing a number of vibrating tuning-forks of different pitch in front of the mouth, and testing them until we find the one which corresponds with the fundamental tone of the vowel-cavity. This is known by the fact, that the tone of the tuning-fork is intensified by the resonance of the air in the mouth, or the vibrations may be transferred to a vibrating membrane and recorded on a smoked surface, as in the phonautograph of Donders.

According to König, the fundamental tones of the vowel-cavity are for

$$
\mathrm{U}=\mathrm{b}, \mathrm{O}=\mathrm{b}^{\prime}, \mathrm{A}=\mathrm{b}^{\prime \prime}, \mathrm{E}=\mathrm{b}^{\prime \prime \prime}, \mathrm{I}=\mathrm{b}^{\prime \prime \prime \prime} \text {. }
$$

If the vowels be whispered in this series, we find at once that their pitch rises. The fundamental tone in the production of a vowel may vary within certain limits. This may be shown by giving the mouth the characteristic position and then percussing the cheeks (Auerbach); the sound emitted is that of the vowel, whose pitch will vary accordingly to the position of the mouth.

When sounding $\mathbf{A}$, the mouth has the form of a funnel widening in front (fig. 366, A). The tongue lies in the floor of the mouth, and the lips are widle open. The soft palate is moderately

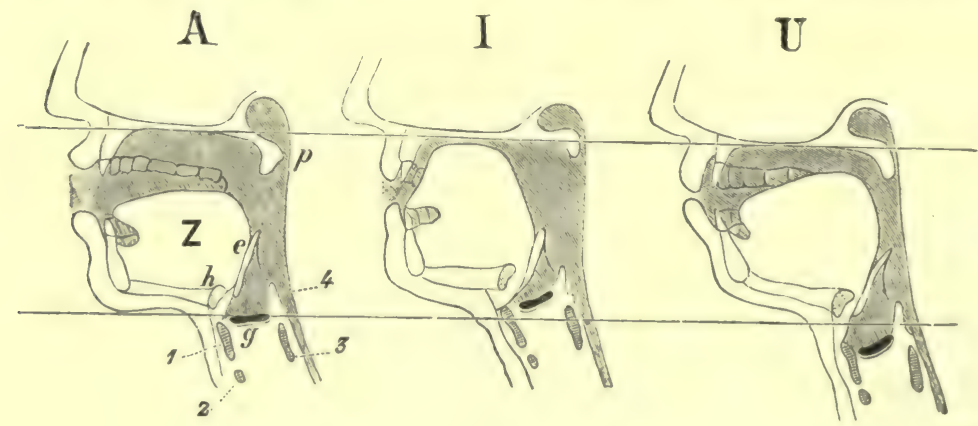

Fig. 366 .

Section of the parts concerned in phonation. Z, tongue ; $p$, soft palate ; $e$, epiglottis ; $g$, glottis; $h$, hyoid bone ; 1 , thyroid, 2, 3 cricoid, 4, arytenoid cartilage.

raised ( $C$ zermak). It is more elevated successively with $\mathrm{O}, \mathrm{E}, \mathrm{U}, \mathrm{I}$. The hyoid bone appears as if at rest, but the larynx is slightly raised. It is higher than with $\mathrm{U}$, but lower than with $\mathrm{I}$.

If we sound $\mathrm{A}$ to $\mathrm{I}$, the larynx and the hyoid bone retain their relative position, but both are raised. In passing from $A$ to $U$, the larynx is depressed as far as possible. The hyoid bone passes slightly forward (Brïcke). When sounding A, the space between the larynx, posterior wall of the pharynx, soft palate, and the root of the tongue, is only moderately wide; it becomes wider with E, and especially with I (Purkinje), but it is smallest with U.

When sounding $\mathbf{U}$ (fig. 366), the form of the cavity of the mouth is like that of a capacious flask with a short, narrow neck. The whole resonance apparatus is then longest. The lips are protruded as far as possible, are arranged in folds and closed, leaving only a small opening. The larynx is depressed as far as possible, while the root of the tongue is approximated to the posterior margin of the palatine arch.

When sounding 0 , the mouth, as in $\mathrm{U}$, is like a wide-bellied flask with a short neck, but the 
latter is shorter and wider as the lips are nearer to the teeth. The larynx is slightly higher than with $\mathrm{U}$, while the resonance chambers also are shorter (fig. 366).

When sounding I, the cavity of the mouth, at the posterior part, is in the form of a smallbellied flask with a long narrow neck, of which the belly has the fundamental tone, $f$, the neck that of $\mathrm{d}^{\prime \prime \prime}$. The resonating chambers are shortest, as the larynx is raised as much as possible, while the mouth, owing to the retraction of the lips, is bounded in front by the teeth. The cavity between the hard palate and the back of the tongue is exceedingly narrow, there being only a median narrow slit. Hence, the air can only enter with a clear piping noise, which sets even the vertex of the skull in vibration, and when the ears are stopped the sounds seem very shrill. When the larynx is depressed and the lips protruded, as for sounding U, I cannot be sounded.

When sounding $\mathbf{E}$, which stands next to I, the cavity hàs also the form of a flask with a small belly (fundamental tone, $\mathrm{f}^{\prime}$ ), and with a long, narrow neck (fundamental tone, $\mathrm{b}^{\prime \prime \prime}$ ). The neck is wider, so that it does not give rise to a piping noise. The larynx is slightly lower than for I, but not so high as for A.

Fundamentally, there are only three primary vowels-I, A, U, the others and the so-called diphthongs standing between them (Brïcke).

Diphthongs occur when, during vocalisation, we pass from the position of one vowel into that of another. Distinct diphthongs are sounded only on passing from one vowel with the mouth wide open to one with the mouth narrow; during the converse process, the vowels appear to our ear to be separate (Brücke).

II. Timbre or Clang-Tint.-Besides its pitch, every vowel has a special timbre, quality, or clang-tint.

The vocal timbre of $U$ (whispering) has, in addition to its fundamental tone, $b$, a deep, piping timbre. The timbre depends upon the number and pitch of the partials or overtones of the vowel sound ( $\$ 415)$.

Nasal Timbre. - The timbre is modified in a special manner when the vowels are spoken with a "nasal" twang, which is largely the case in the French language. The nasal timbre is produced by the soft palate not cutting off the nasal cavity completely, which happens every time a pure vowel is sounded, so that the air in the nasal cavity is thrown into sympathetic vibration. When a vowel is spoken with a nasal timbre, air passes out of the nose and mouth simultaneously, while with a pure vowel sound, it passes out only through the mouth.

When sounding a pure vowel (non-nasal), the shutting off of the nasal cavity from the mouth is so complete, that it requires an artificial pressure of 30 to $100 \mathrm{~mm}$. of mercury to overcome it (Hartmann).

The vowels, a, $\ddot{a}(æ), \ddot{o}(œ), 0$, e, are used with a nasal timbre-a nasal i does not occur in any language. Certainly it is very difficult to sound it thus, because when sounding $i$, the mouth is so narrow that when the passage to the nose is open, the air passes almost completely through the latter, whilst the small amount passing through the mouth scarcely suffices to produce a sound.

In sounding vowels, we must observe if they are sounded through a previously closed glottis, as is done in the German language in all words beginning with a vowel (spiritus lenis). The glottis, however, may be previously opened with a preliminary breath, followed by the vowel sound; we obtain the aspirate vowel (spiritus asper of the Greeks).

B. If the vowels are sounded in an audible tone, i.e., along with the sound from the larynx, the fundamental tone of the vocal cavity strengthens in a characteristic manner the corresponding partial tones present in the laryngeal sound (Wheatstone, v. Helmholtz).

318. CONSONANTS. - The consonants are noises which are produced at certain parts of the resonance chamber. [As their name denotes, they can only be sounded in conjunction with a vowel.]

Classification. - The most obvious classification is according to-(I.) Their acoustic properties, so that they are divided into-(1) liquid consonants, i.e., such as are appreciable without a vowel $(\mathrm{m}, \mathrm{n}, \mathrm{l}, \mathrm{r}, \mathrm{s}) ;(2)$ mutes, including all the others, which cannot be distinctly heard without an accompanying vowel. (II.) According to their mechanism of formation, as well as the type of the organ of speech, by which they are produced. They are divided into-

1. Explosives. - Their enunciation is accompanied by a kind of bursting open of an obstacle, or an explosion, occasioned by the confined and compressed air which causes a stronger or weaker noise; or, conversely, the current of air is suddenly interrupted, while, at the same time, the nasal cavities are cut off by the soft palate.

2. Aspirates, in which one part of the canal is constricted or stopped, so that the air rushes out through the constriction, causing a faint whistling noise. (The nasal cavity is cut off.) In 
uttering $\mathrm{L}$, which is closely related to the aspirates, but differs from them in that the narrow jassage for the rush of air is not in the middle, but at both sides of the middle of the closed part. (The nasal cavity is shut off.)

3. Vibratives, which are froduced by air being forced through a narrow portion of the canal, so that the marcrins of the narrow tube are set in vibration. (The nasal cavity is shut off.)

4. Resonants (also called nasals or semi-vowels). The nasal cavity is completely free, while the vocal canal is completely closed in the front part of the oral channel. According to the position of the obstruction in the oral cavity, the air in a larger or smaller portion of the mouth is thrown into sympathetic vibration.

We may also classify them according to the position in which they are produced -the "articulation positions" of Bricke. These are :-

A. Between both lips; B, between the tongue and the hard palate; C, between the tongue and the soft palate; $\mathrm{D}$, between the true vocal cords.

\section{A. Consonants of the First Articulation Position.}

1. Explosive Labials. - b, the voice is sounded before the slight explosion occurs; $\mathbf{p}$, the voice is souncled after the much stronger explosion has taken place (Kempelen). [The former is spoken of as "voiced" and the latter as "breathed."]

2. Aspirate Labials. - $\mathbf{f}$, between the upper incisor teeth and the lower lip (labiodental). It is absent in all true Slavic words (Purkiñc); $\mathbf{v}$, between both lips (labial); w is formed when the mouth is in the position for $\mathbf{f}$, but instead of merely forcing in the air, the voice is sounded at the same time. Really there are two different $\mathbf{w}$-one corresponding to the labial $\mathbf{f}$, as in wiirde, and the labiodental, e.g., quelle (Brïcke).

3. Vibrative Labials. - The burring sound, emitted by grooms, but not used in civilised language.

4. Resonant Labials. - $\mathrm{m}$ is formed essentially by sounding the voice whereby the air, in the mouth and nose, is thrown into sympathetic vibration [" voiced"].

\section{B. Consonants of the Second Articulation Position.}

1. The explosives, when enunciated sharply and without the voice, are $\mathbf{T}$ hard (also dt and th); when they are feeble and produced along with simultaneous laryngeal sounds (voice), we have $\mathbf{D}$ soft.

2. The aspirates embrace $\mathbf{S}$, including $\mathbf{s}$ sharp, written $\mathbf{8} \mathbf{s}$ or $\mathbf{s} \mathbf{z}$, which is produced without any audible laryngeal vibration; or soft, which requires the voice. Then, also, there are modifications according to the position where the noises are produced. The sharp aspirates include Sch, and the hard English Th; to the soft belong the French J soft, and the English Th soft. $\mathbf{L}$, which occurs in many modifications, appears here, e.g., the $\mathbf{L}$ soft of the French. $\mathbf{L}$ may be sounded soft with the voice, or sharp without it.

3. The vibrative, or $\mathbf{R}$, which is generally voiced, but it can be formed without the larynx.

The resonants are $\mathbf{N}$-sounds, which also occur in several modifications.

\section{Consonants of the Third Articulation Position.}

1. The explosives are the $\mathbf{K}$-sounds, which are hard and breathed and not voiced; $\mathbf{G}$-sounds, which are voiced.

2. The aspirates, when hard and breathed but not voiced, the $\mathbf{C h}$, and when sounded softly and not voiced, $\mathbf{J}$ is formed.

3. The vibrative is the palatal $\mathbf{R}$, which is produced by vibration of the uvula (Briicke).

4. The resonant is the palatal $\mathbf{N}$.

\section{Consonants of the Fourth Articulation Position.}

1. An explosive sound aloes not occur when the glottis is forced open, if a vowel is loudly sounded with the glottis previously closed. If this occurs during whispering, a feeble short noise, due to the sudden opening of the glottis, may be heard.

2. The aspirates of the glottis are the $\mathbf{H}$-sounds, which are produced when the glottis is moderately wide.

3. A glottis-vibrative occurs in the so-called laryngeal $\mathbf{R}$ of lower Saxon (Brücke).

4. A laryngeal resonant cannot exist.

The combination of different consonants is accomplished by the successive movements neces. sary for each being rapidly executed. Compound consonants, however, are such as are formed when the oral parts are adjusted simultaneously for two different cousonants, so that a mixed sound is formed from two. Exampies: Sch-tsch, tz, ts $-P_{B}(\psi)-K_{s}(X \Xi)$.

319. PATHOLOGICAL VARIATIONS OF VOICE AND SPEECH. - Aphonia.-Paralysis of the motor nerves (vagus) of the larynx by injury, or the pressure of tumours, causes aphonia or loss of voice (Galen). In aneurism of the aortic arch, the left recurrent nerve may be paralysed from pressure. The laryngeal nerves may be temporarily paralysed by rheumatism, over-exer- 
tion, and hysteria, or by serous effusions into the laryngeal muscles. If the tensors are paralysed, monotonia is the chief result: the disturbances of respiration in paralysis of the larynx are important. As long as the respiration is tranquil, there may be no disturbance, but as soon as increased respiration occurs, great dyspnœa sets in, owing to the inability of the glottis to dilate.

If only one vocal cord is paralysed, the voice becomes impure and falsetto-like, while we may feel from without that there is less vibration on the paralysed side (Gerhardt). Sometimes the vocal cords are only so far paralysed that they do not move during phonation, but do so during forced respiration and during coughing (phonetic paralysis).

Diphthongia. - Incomplete unilateral paralysis of the recurrent nerve is sometimes followed by a double tone, owing to the unequal tension of the two vocal cords. According to Tiirck and Schnitzler, however, the double tone occurs when the two vocal cords touch at some part of their course (e.g., from the presence of a tumour, fig. 367), so that the glottis is divided into two unequal portions, each of which produces its own sound.

Hoarseness is caused by mucus upon the vocal cords, by roughness, swelling, or laxness of the cords. If, while speaking, the cords are approximated, and suddenly touch each other, the "speech is broken," owing to the fornation of nodal points (\$352). Disease of the pharynx, naso-pharyngeal cavity, and uvula may produce a change in the voice reflexly.

Paralysis of the soft palate (as well as congenital perforation or cleft palate) causes a nasal timbre of all vowels; the former renders difficult the normal formation of consonants of the third articulation position; resonance is imperfect, while the explosives are weak, owing to the escape of the air through the nose.

Paralysis of the tongue weakens I ; E and A ( $\mathbb{E})$ are less easily pronounced, while the formation of consonants of the second and third articulation position is affected. The term aphthongia is applied to a condition in which every attempt to speak is followed by spasmodic movements of the tongue (Fleury).

In paralysis of the lips (facial nerve), and in hare-lip, regard must be had to the formation of consonants of the first articulation position. When the nose is closed, the speech has a characteristic sound. The normal formation of resonants is of course at an end. After excision of the larynx, a netal reed, enclosed in a tube, and acting like an artificial larynx, is introduced between the trachea aud the cavity of the mouth (Czerny).

Stammering is a disturbance of the formation of sounds. [Stammering is due to long-continued spasmodic contraction of the diaphragm, just as hiccough is $(\S 120)$, and, therefore, it is essentially a spasmodic inspiration. As speech depends upon the expiratory blast, the spasm prevents expiration. It may be brought about by mental excitement or emotional conditions. Hence, the treatment of stammering is to regulate the respirations. In stuttering, which is defective speech due to inability to form the proper sounds, the breathing is normal.]

320. COMPARATIVE-HISTORICAL. - Speech may be classified with the "expression of the emotions" (Darwin). Psychical excitement causes in man characteristic movements, in which certain groups of muscles are always concerned, e.g., laughing, weeping, the facial expression in anger, pain, shame, \&c. These movements afford a means whereby one creature can communicate with another. Primarily in their origin, the movements of expression are reflex motor phenomena; when they are produced for purposes of explanation, they are voluntary imitations of this reflex. Besides the emotional movements, impressions upon the sense-organs produce characteristic reflex movements, which may be used for purposes of expression (Geiger), e.g., stroking or painful stimulation of the skin, movements after smelling pleasant or unpleasant or disagreeable odours, the action of sound and light, and the perception of all kinds of objects.

The expression of the emotions occurs in its simplest form in what is known as expression by means of signs or pantomime or mimicry. Another means is the imitation of sounds by the organ of speech, constituting onamatopoesy, e.g., the hissing of a stream, the roll of thunder, the tumult of a storm, whistling, \&c. The expression of speech is, of course, dependent upon the process of ideation and perception.

The occurrence of different sounds in different languages is very interesting. Some languages (e.g., of the Hurons) have no labials; in some South Sea Islands, no laryngeal sounds are spoken; $f$ is absent in Sanscrit and Finnish ; the short $e, o$, and the soft sibilants in Sanscrit ; $d$, in Chinese and Mexican; $s$, in many Polynesian languages; $r$, in Chinese, \&c.

Voice in Animals. - Animals, more especially the higher forms, can express their emotions by facial and other gestures. The vocal organs of mammals are essentially the same as those of man. Special resonance organs occur in the orang-outang, mandril, macacus, and mycetes monkeys, in the form of large cheek pouches, which can be inflated with air, and open between the larynx and the hyoid bone. 
Birds have an upper (larynx) and a lower laryux (syrinx); the latter is placed at the bifurcation of the trachea, and is the true vocal organ. Two folds of mucous membrane (three in singing birds) project into each bronchus, and are rendered tense by muscles, and are thus adapted to serve for the production of voice.

Amongst reptiles, the tortoises produce merely a snifting sound, which in the Emys has a peculiar piping character. The blind snakes are voiceless, the chameleon and the lizards have a very feeble voice; the cayman and crocodile emit a feeble roaring sound, which is lost in some adults owing to changes in the larynx. The snakes have no special vocal organs, but by forcing out air from their capacious lung, they make a peculiar hissing sound, which in some species is loud. Amongst amphibians, the frog has a larynx provided with muscles. The sound emitterl without any muscular action is a deep intermittent tone, while more forcible expiration, with contraction of the laryngeal constrictors, causes a clearer continuous sound. The nale, in liana esculenta, has at each side of the angle of the mouth a sound-bag, which can be inflated with air and acts as a resonance chamber. The "croaking" of the male frog is quite eharacteristic. In Pipa, the larynx is provided with two cartilaginous rods, which are thrown into vibration by the blast of air, and act like vibrating rods or the limbs of a tuning. fork. Some fishes emit sounds, either by rubbing together the upper and lower pharyngeal bones, or by the expulsion of air from the swimming bladder, mouth, or anus.

Some insects cause sounds partly by forcing the expired air through their stigmata provided. with muscular reeds, which are thus thrown into vibration (bees and many diptera). The wings, owing to the rapid contraction of their muscles, may also cause sounds (flies, cockroach, bees). The Sphinx atropos (death-head moth) forces air from its sucking stomach. In others, sounds are produced by rubling their legs on the wing-cases (Acridium), or the wing-cases on each other (Gryllus, locust), or on the thorax (Cerambyx), on the leg (Geotrupes), on the abdomen or the margin of the wing (Nekrophorus). In Cicalacia, membranes are pulled upon by museles, and are thus caused to vibrate. Friction sounds are proluced between the cephalothorax and the ablomen in some spiders (Theridium), and in some crabs (Palinurus). Some mollusca (Pecten) emit a sound on separating their shells.

Historical. - The Hipyocratic School was aware of the fact that division of the trachea abolished the voice, and that the epiglottis prevented the entrance of food into the larynx. Aristotle made numerous observations on the voice of animals. The true cause of the voice escaped him as well as Galen. Galen observed complete loss of voice after double pneumothorax, after section of the intercostal muscles or their nerves, as well as after destruction of part of the spinal cord, even although the diaphragm still contracted. He gave the cartilages of the larynx the names that still distinguish them; he knew some of the laryngeal muscles, and asserted that voice was produced only when the glottis was narrowed. He compared the larynx to a flute. The weakening of the voice, in feeble conditions, especially after loss of hlood, was known to the ancients. Dodart $(1700)$ was the first to explain voice as due to the vibration of the vocal cords by the air passing between them.

The production of vocal sounds attracted much attention amongst the ancient Asiatics and Arabians-less amongst the Greeks. Pietro Ponce $(+1584)$ was the first to advocate instruction in the art of speaking in cases of dumbness. Bacon (1638) studied the shape of the mouth for the pronunciation of the various sounds. Kratzenstein (1781) made an artificial apparatus for the production of vowel sounds, by placing resonators of various forms over vibrating reeds. Von heinpelen (1769 to 1791) constructed the first speaking-machine. Rob. Willis (1828) found that an elastic vilbrating spring gives the vowels in the series-U, O, A, E, I-according to the depth or height of its tone; further, that by lengthening or shortening an artificial resonator on an artificial vocal apparatus, the vowels may be obtained in the same series. The newest and most important investigations on speech are by Wheatstone, v. Helmholtz, Donders, Briicke, \&c., and are mentioned in the context. Hensen succeeded in showing exactly the pitch of vocal tone, thus:-The tone is sung against a Kinnig's capsule with a gas flame. Opposite the flame is placed a tuning-fork vibrating horizontally, and in front of one of its limbs is a mirror, in which the image of the flame is reflected. When the vocal tone is of the same number of vibrations as the tuning-fork, the flame in the mirror shows orfe elevation, if double, i.e., the octave, 2 , and with the double octave, 4 elevations. 


\title{
General Physiology of the Nerves and Electro-Physiology.
}

\section{STRUCTURE OF THE NERVE ELEMENTS.-The nervous elements} present two distinct forms :-

\author{
I. Nerve-Fibres. $\left\{\begin{array}{l}\text { Non-medullated. } \\ \text { Medullated. }\end{array}\right.$ \\ II. Nerve-Cells. $\left\{\begin{array}{r}\text { Of various forms } \\ \text { and functions. }\end{array}\right.$
}

An aggregation of nerve-cells constitutes a nerve-ganglion. The fibres represent a conducting apparatus, and serve to place the central nervous organs in connection with peripheral end-organs. The nerve-cells, however, besides transmitting impulses, act as physiological centres for automatic or reflex movements, and also for the sensory, perceptive, trophic, and secretory functions.

I. (1) The non-medullated nerve-fibres occur in several forms :-

1. Primitive Fibrils. - The simplest form of nerve-fibre, which is visible with a magnifying power of 500 to 800 diameters linear, consists of primitive nerve-fibrils. They are very delicate tibres (fig. 368, 1), often with small varicose swellings here and there in their course, which, however, are due to changes post-nortem. They are stained of a brown or purplish colour by the gold-chloride method, and they occur when a nerve-fibre is near its termination, being formed by the splitting up of the axis-cylinder of the nerve-fibre, e.g., in the terminations of the corneal nerves, the optic nerve-layer in the retina, the terminations of the olfactory fibres, and in a plexiform arrangement in non-striped muscle (p. 461). Similar fine fibrils occur in the grey matter of the brain and spinal cord, and in the finely divided processes of nerve-cells.

2. Naked or simple axial cylinders (fig. 368,2 ), which represent bundles of primitive fibrils held together by a slightly granular cement, so that they exhibit very delicate longitudinal striation with fine granules scattered in their course. The best example is the axial cylinder process of nerve-cells (fig. 368, I, $z$ ). [The thickness of the axis-cylinder depends upon the number of fibrils entering into its composition.]

3. Axis-cylinders surrounded with Schwann's sheath, or Remak's fibres ( 3.8 to $6.8 \mu$ broad), the latter name being given to them from their discoverer (fig. 368, 3). [These fibres are also called pale or non-medullated, and from their abundance in the sympathetic nervous system, sympathetic.] They consist of a sheath, corresponding to Schwann's sheath [neurilemma, or primitive sheath, which encloses an axial cylinder; while lying here and there under the sheath, and between it and the axial cylinder are nerve-corpuscles. These fibres are always fibrillated longitudinally]. The sheath is delicate, structureless, and elastic. Dilute acids clear the fibres without causing them to swell up, while gold chloride makes them brownish-red. They are widely distributed in the sympathetic nerves, [e.g., splenic], and in the branches of the olfactory nerves. All nerves in the embryo, as well as the nerves of many invertebrata, are of this kind. [According to Ranvier, these fibres do not possess a sheath, but the nuclei are merely applied to the surface, or slightly embedded in the superficial parts of the fibre, so that they belong to the fibre itself. These fibres also branch and form an anastomosing net-work (fig. 370). This the medullated fibres never do. These fibres, when acted on by silver nitrate, never show any crosses. The branched forms occur in the ordinary nerves of distribution, and they are numerous in the vagus, but the olfactory nerves have a distinct sheath which is nucleated.]

(2) Medullated fibres occur also in several forms:-

4. Axis-cylinders, or nerve-fibrils, covered only by a medullary sheath, or white substance of Schwann, are met with in the white and grey matter of the central nervous system, in the 
optic and auditory nerves. These medullated nerve-fibres, without any neurilemma, often show after death, varicose swellings in their course [due to the accumulation of fluid between the medulla of myelin and the axis-cylinder]. Hence they are called varicose fibres. [The raricose appearance is easily produced by squeezing a small piece of the white matter of the spinal cord between a slide anil a cover-glass. Nitrate of silver does not reveal any crosses, and there are no nodes of Rauvier. When acted upon by coagulating reagents, e.g., chromic acid, the

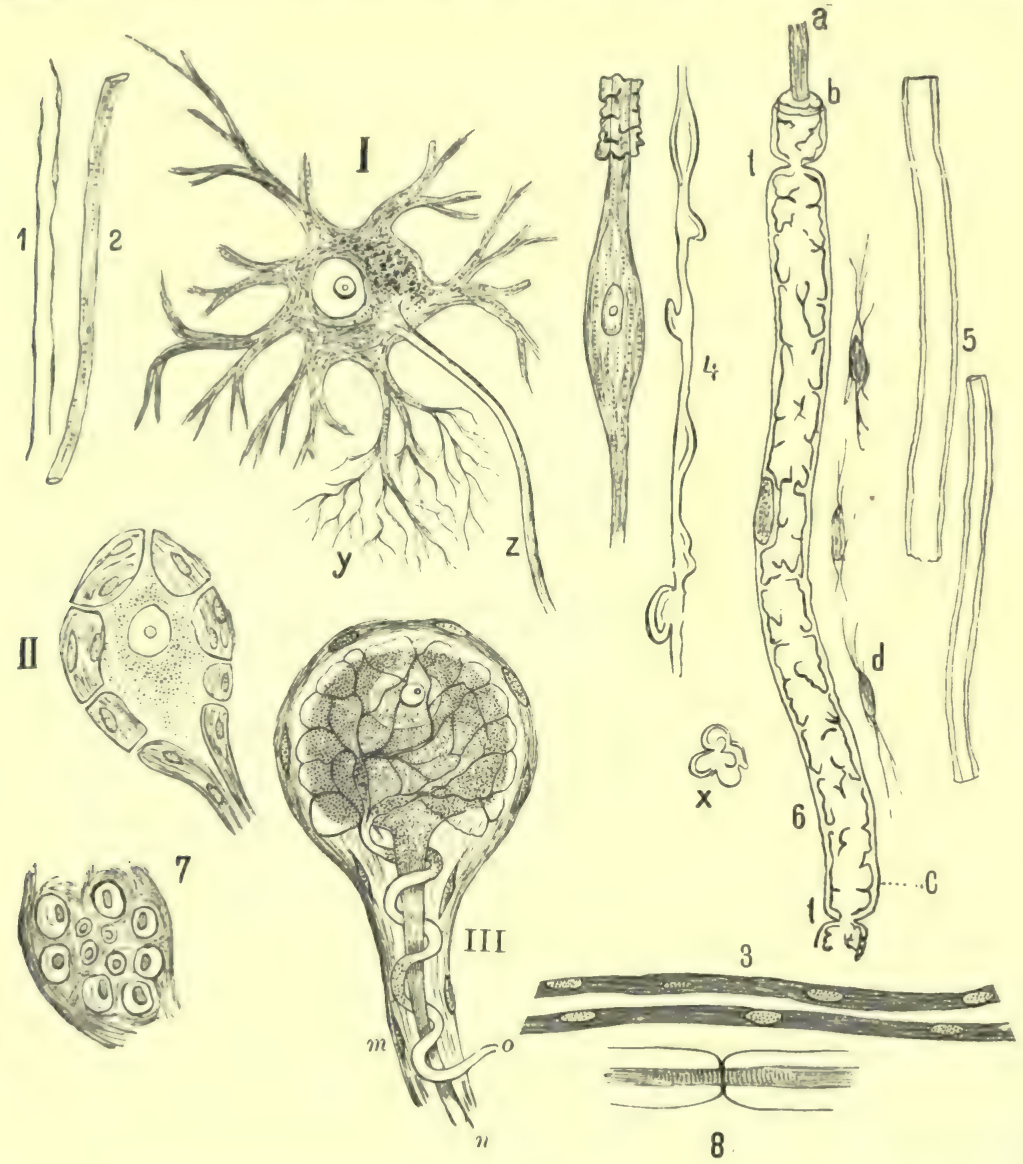

Fig. 368 .

1, Primitive fibrillæ; 2 , axis-cylinder; 3 , Remak's fibres; 4, medullated varicose fibre ; 5,6 , medullated fibre, with Schwann's sheath; $c$, neurilemma ; $t, t$, Ranvier's nodes; $b$, white substance of Schwann; $d$, cells of the endoneurium ; $a$, axis-cylinder ; $x$, myelin drops ; 7 , transverse section of nerve-fibres; 8 , nerve-fibre acted on with silver nitrate and show. ing Fromann's lines. I, multipolar nerve-cell from the spinal cord; $z$, axial cylinder process; $y$, protoplasmic processes-to the right of it a bipolar cell. II, peripheral ganglionic cell, with a connective-tissue capsule. III, ganglionic cell, with 0 , a spiral, and $n$, straight process ; $m$, sheath.

medullary sheath appears laminated, so that on transverse section, when the axis-cylinder is stained, it is surrounded by concentric circles (fig. 369).

5. Medullated Nerve-Fibres with Schwann's Sheath (fig. 368, 5, 6). - These are the most

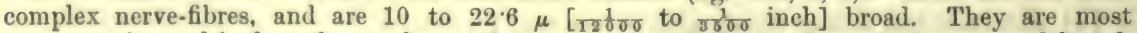
numerous in, and in fact they make up the great mass of, the cerebro-spinal nerves, although they are also present in the sympathetic nerves. [When examined in the fresh and living condition in situ, they appear refractive and homogeneous (Ranvier, Stirling); but if acted upon 
by reagents, they are not only refractive, but exhibit a double contour, the margins being dark and well defined.] Each fibre consists of--

[1. Schwann's sheath, neurilemma, or primitive sheath;

2. White substance of Schwann, medullary sheath, or myelin;

3. Axis-cylinder composed of fibrils and surrounded by a sheath called the axilemma;

4. Nerve-corpuscles.]

A. The axis-cylinder, which occupies $\frac{1}{4}$ to $\frac{1}{5}$ of the breadth of the fibre, is the essential part of the nerve, and lies in the centre of the fibre, like the wick in the centre of a candle (fig. 368, 6, a). Its usual shape is cylindrical, but sometimes it is flattened or placed eccentrically - [this is most probably due to the hardening process employed]. It is composed of fibrils [united by cement or stroma; they become more obvious near the terminations of the nerve, or after the action of reagents, which sometimes cause the fibrils to appear beaded. It is quite transparent, and stains deeply with carmine or logwood], while during life, its consistence is semi-fluid. According to Kupffer, a fluid-"neuroplasma"-lies between the fibrils [while, according to other observers, the whole cylinder is enclosed in an elastic sheath peculiar to itself and composed of neuro-keratin. This sheath is called by Kühne, the axilemma. Each axis-cylinder is'an enormously long process of a ganglionic cell].

Fromann's Lines,-Chloroform and collodion render it visible, while it is most easily isolated as a solid rod, bý the action of nitric acid with excess of potassium chlorate. When acted on by silver nitrate, Fromann observed transverse markings on it, but their significance is unknown (fig. 368,8 ).

B. The white substance of Schwann, medullary sheath or myelin, surrounds the axis-cylinder, like an insulating

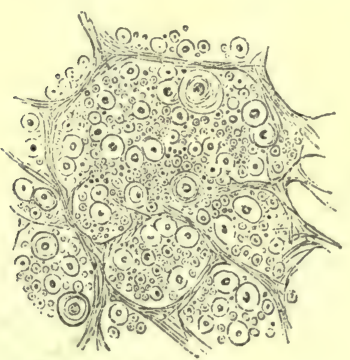

Fig. 369.

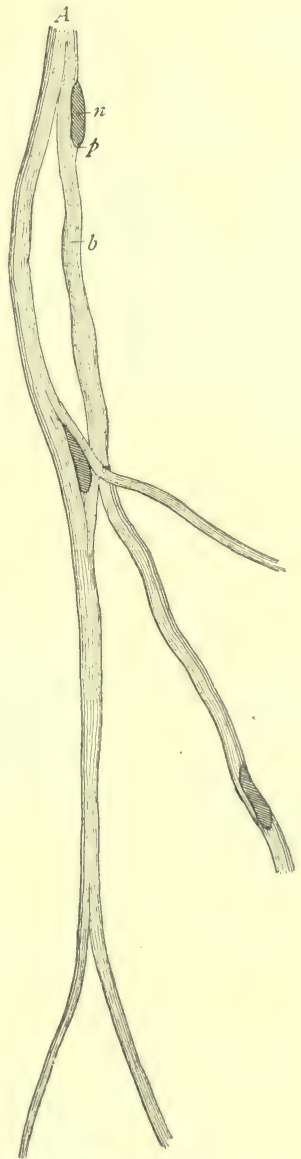

Fig. 370 .

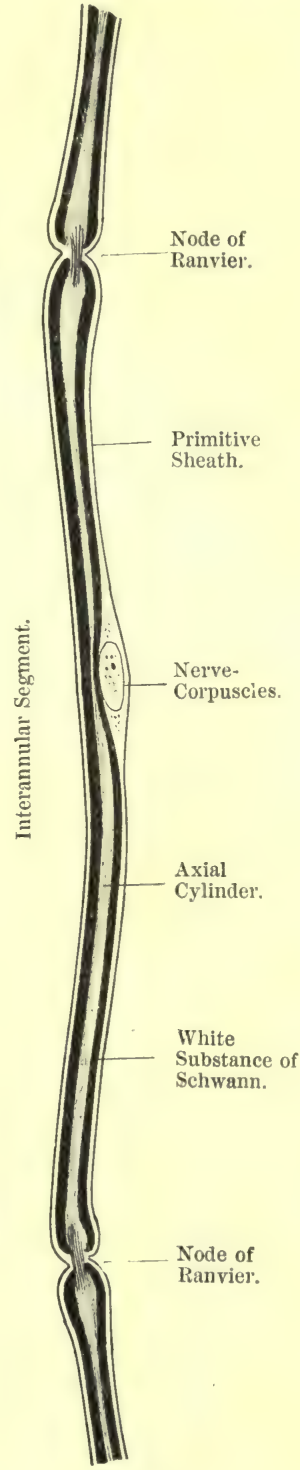

Fig. 371.

Fig. 369. -Transverse section of the nerve-fibres of the spinal cord, the axis-cylinders like dots surrounded by a clear space (myelin). Fig. 370.-Remak's fibre from vagus of dog. $b$, fibrils ; $n$, nucleus; $p$, protoplasm surrounding it. Fig. $371 .-$ Medullated nerve-fibre of a rabbit acted on by osmic acid, $\times 400$.

medium around an electric wire. In the perfectly fresh condition it is quite homogeneous, highly glistening, bright, and refractive; its consistence is fluid, so that it oozes out of the cut ends of the fibres in spherical drops (fig. 368, x), [myelin drops, which are always marked by concentric lines, are highly refractive, and best seen when a fresh nerve is teased in salt solution]. After death, or after the action of reagents, it shrinks slightly from the sheath, so 
that the fibres have a double contour, while the substance itself breaks up into smaller or larger droplets, due not to coagulation (Pertik), but, according to Toldt, to a process like emulsification, the drops pressing against each other. Thus, the fibre is broken up into masses, so that it has a characteristic appearance (fig. 368,6 ). It contains a large amount of cerebrin and lecithin, which swell up to form myelin-like forms in warm water. It also contains fatty matter, so that these fibres are blackened by osmic acid, [while boiling ether extracts cholesterin from them]. Chloroform, ether, and benzin, by dissolving the fatty and some other constituents of the fibres, make them very transparent. [Some observers describe a fluid lying between the medulla and the axis-cylinder.]

C. The Sheath of Schwann, or the neurilemma, lies immecliately outside of and invests the white sheath (fig. $368,6, c$ ), and is a delicate structureless membrane, comparable to the sarcolemma of a muscular fibre.

D. Nerve-Corpuscles. - At fairly wide intervals under the neurilemma, and lying in depressions between it and the medullary sheath, are the nucleated nerve-corpuscles, which are readily stained by pigments (fig. 371). [They may be compared to the muscle-corpuscles, the nuclei being surrounded by a small amount of protoplasm which sometimes contains pigment. They are not so numerous as in muscle.] [Adamkiewicz describes nerve-corpuscles, or "demilunes" under the neurilemma, quite distinct from the ordinary nerve-corpuscles. They are stained yellow by safranin, while the ordinary nerve-corpuscles are

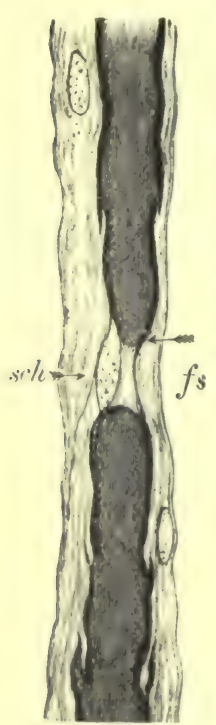

Fig. 372. stained by methylanilin.]

Ranvier's Nodes or Constrictions.-The neurilemma forms in broad fibres at longer, and in narrower ones at shorter intervals, the nodcs or constrictions of Ranvier (fig. $368,6, t, t$; fig. 371 ; fig. $372, f s)$. They are constrictions which occur at regular intervals along a nervefibre; at them the white substance of Schwann is interrupted, so that the sheath of Schwann lies upon the axis-cylinder [or its elastic sheath] at the nodes. The part of the nerve lying between any two nodes is called an interannular or inter-nodal segment, and each such segment contains one or more nuclei, so that some observers look upon the whole segment as equivalent to one cell.

The function of the nodes seems to be to permit the diffusion of plasma through the outer sheath into the axis-cylinder, while the decomposition-products are similarly given off. [A colouring-matter like picro-carmine diffuses into the fibre only at the nodes, and stains the axis-cylinder red, although it does not diffuse through the white substance of Schwann.

Incisures (of Schmidt and Lantermann).Each interannular segment in a stretched nerve shows, rumning across the white substance, a number of oblique lines, which are called I e d ull a t e d incisures (figs. 372,373 ). They indicate that nerve-fibres the segment is built up of a series of conical blackened by sections, each of which is bevelled at its ends, osmic acid. $f s$, and the bevels are arranged in an imbricate
Ranvier's node; interval between them appears as an incisure. sch, Schwann's interval between them appears as an incisure.
sheath. a cylinder cone (Kuhnt).

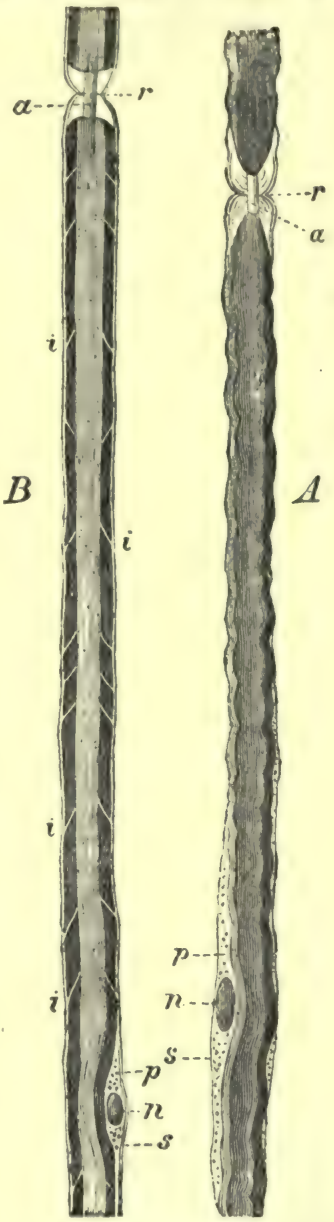

Fig. 373.

Medullated nerve-fibres with osmic acid. $a$, axis-cylinder; $s$, sheath of Schwann; $n$, nucleus; $p, p$, granular substance at the poles of the nucleus; $r, r$, Ranvier's nodes where the medullary sheath is interrupted and the axis-cylinder appears ; $i, i$, incisures of Schmidt.

Neuro-Keratin Sheath.-According to Ewald and Kiihne, the axis-cylinder, as well as the white substance of Schwann, is covered with an excessively delicate sheath, consisting of neurokicrctin, and the two sheaths are connected by numerous transverse and oblique fibrils, which permeate the white substance. [The myelin seems to lie in the interstices of this mesh-work.]

[Rod-like Structures in Myelin.-If a nerve be hardened in ammonium chromate (or picric acid), $M^{\prime}$ Carthy has shown that the myelin exhibits rod-like structures, radiating from the 
axis-cylinder outwards, which are stained with logwood and carmine. The rods are probably not distinct from each other, but are perhaps part of the neuro-keratin network already described.]

Action of Nitrate of Silver.-When a small nerve, e.g., the intercostal nerve of a mouse, is acted on by silver nitrate, it is seen to be covered by an endothelial sheath composed of flattened endothelial cells (fig. 374 ), while the nerve-fibres themselves exhibit crosses along their course. These crosses are due to the penetration of the silver solution at the nodes, where it stains the cement-substance and also part of the axis-cylinder, so that the latter sometimes exhibits transverse markings called Fromann's lines (fig. 368, 8).]

[New Methods. - Much progress has recently been made in tracing the course of medullated nerve-fibres by the action of new staining reagents; thus acid fuchsin stains the myelin deeply, leaving the other parts unstained, at least it can be so manipulated as to yield this result. Weigert's Method and its modifications have yielded most important results, and proved that medullated nerve-fibres exist in many parts of the central nervous system where they cannot be seen in the ordinary way. The nerve-tissue is hardened in a solution of a chromium salt, and placed in a half-saturated solution of cupric acetate; it is then stained with logwood, and afterwards the elements are differentiated by steeping the sections in a solution of ferricyanide of potash and borax. The myelin is coloured a logwood tint.]

In the spinal nerves, those fibres are thickest which have the longest course before they reach their end-organ (Schwalbe), while those ganglion-cells are largest which send out the longest nerve-fibres (Pierret). [Gaskell finds that the longest nerves are not necessarily the thickest, for the visceral nerves in the vagus are small nerves, and yet run a very long course.]

Division of Nerves. - Nerve-fibres run in the nerve-trunks without dividing; but when they approach their termination they often divide dichotomously [at a node], giving rise to two similar fibres, but there may be several branches at a node (fig. $376, t$ ). [The divisions are numerous in motor

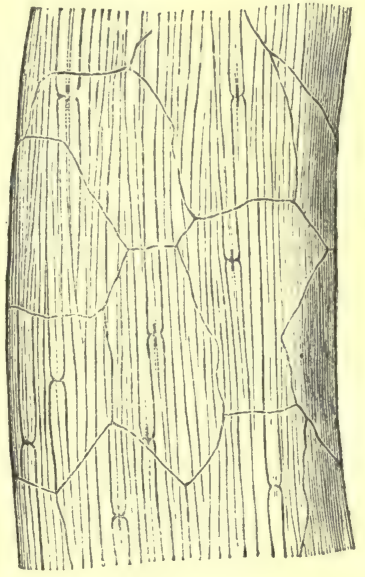

Fig. 374.

Intercostal nerve of a mouse (single fasciculus of nerve-fibres) stained with silver nitrate. Endothelial sheath stained, and some nodes of Ranvier indicated by erosses. nerves to striped muscles.] In the electrical nerves of the malapterurus and gymnotus, there is a great accumulation of Schwann's sheaths round a nerve, so that a nerve-fibre is as thick as a sewing-needle. Such a fibre, when it divides, breaks up into a bundle of smaller fibres.

[Nerve-Sheaths. A nerve-trunk consists of bundles of nerve - fibres. The bundles are held together by a common connective - tissue sheath (fig. 375, ep ), the epineurium which contains the larger blood-vessels, lymphatics, and sometimes fat and plasma cells. Each bundle is surrounded with its own sheath or perineurium $(p e)$, which consists of lamellated

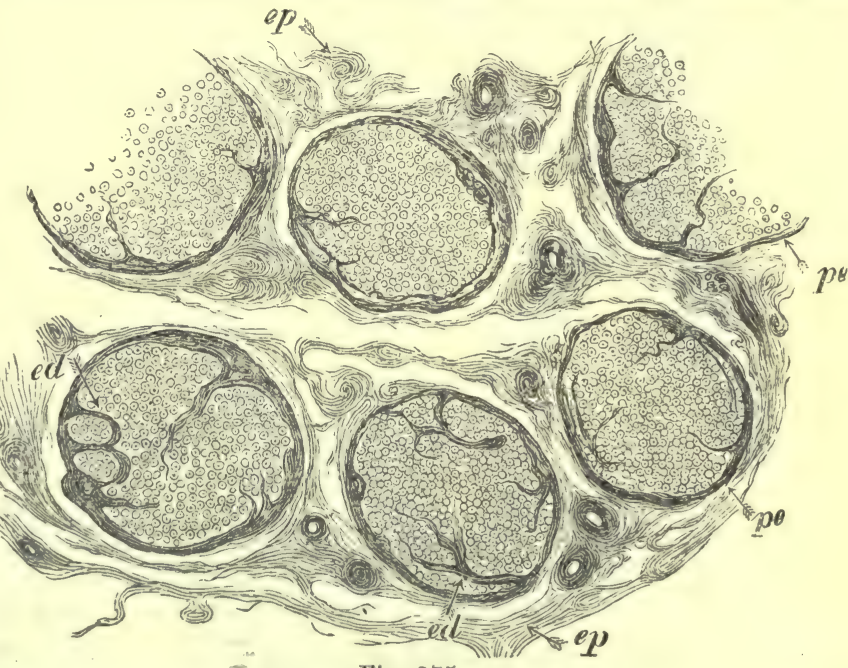

Fig. 375 .

Trans. section of a nerve (median). ep, epineurium; $p e$, perineurium ; $e d$, endoneurium.

connective-tissue disposed circularly, and between the lamellæ are lymph spaces lined by flattened endothelial plates. These lymph spaces may be injected from and communicate with 
the lymphaties (Axel Key and lietzius).] The nerve-fibres within any bundle are held together by delicate connective-tissue, which penetrates between the adjoining tibres, constituting the endoneurium $(e d)$. It consists of delicate fibres with branched connective-tissue corpuscles (fig. $368,6, d$ ), and in it lie the capillaries, which are not very numerous, and are arranged to form elongated open meshes.

[Henle's Sheath. - When a nerve is traced to its distribution, it branches and becomes smaller, until it may consist only of a few bundles or even a single bundle of nerve-fibres. As the bundle branches, it has to give off part of its lamellated sheath or perineurium to each branch, so that, as we pass to the periphery, the smaller bundles are surrounded by few lamellæ. In a bundle containing only a few fibres, this sheath may be much reduced, or may consist only of thin, flattened, connective-tissue corpuscles with a few fibres. A sheath surrounding a few nervefibres is called Henle's Shcath by Ranvier.]

[Nervi Nervorum. - Ilarshall and v. Horsley have shown that the nerve-sheaths are provided with special nerve-fibres, in virtue of which they are endowed with sensibility.]

Development. - At first nerve-fibres consist only of fibrils, i.e., of axis-cylinders, which hecome covered with connective substance, and ultimately the white substance of Schwann is

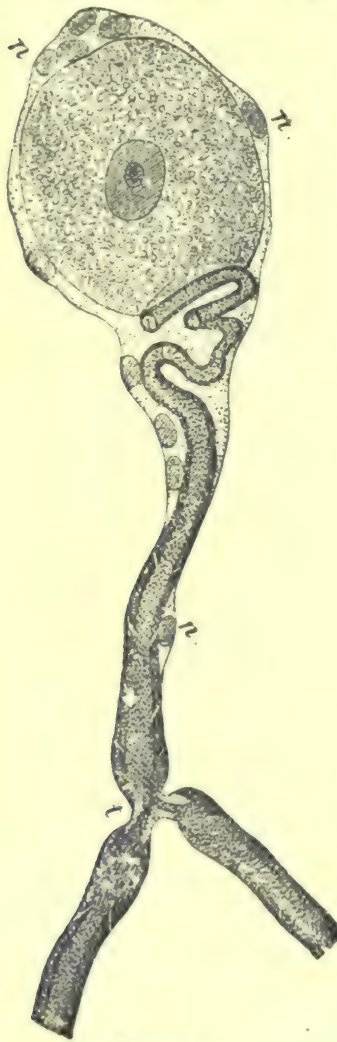

Fig. 376. developed in some of them. The growth in length of the fibres takes place by elongation of the individual "interannular" segments, and also by the new formation of these ( Vignal).

II. Ganglionic or Nerve-Cells. - 1. Multipolar nerve-cells (fig. $368, \mathrm{I})$ occur partly as large cells $(100 \mu$ ), and are visible to the unaided eye as in the anterior horn of the spinal cord, and in a different form in the cerebellum, and partly in a smaller form ( 20 to $10 \mu$ ) in the posterior horns of the spinal cord, niany parts of the cerebrum and cerebellum, and in the retina. They may be spherical, ovoid, pyramidal [cerebrum], pear- or flask-shaped [cerebellum], and are provided with numerous branched processes which give the cells a characteristic appearance. [Deiters isolated such cells from the anterior horn of the grey matter of the spinal cord, so that this special form of cell is sometimes called "Deiters' cell" (fig. 368, I).] They are devoid of a cell envelope, are of soft consistence, and exhibit a fibrillated structure, which may extend even into the processes. Fine granules lie scattered throughout the cell-substance between the fibrils. Not unfrequently yellow or brown granules of pigment are also found, either collected at certain parts in the cell or scattered throughout it. The relatively large nucleus consists of a clear envelope enclosing a resistant substance. It does not appear to have a membrane in youth (Schwalbe). Within the nucleus lies the nucleolus, which in the recent condition is angular, provided with processes and capable of motion, but after death is highly refructive and spherical. There is always one unbranched process, constituting the axial cylinder process $(I, z)$ which remains unbranched, but it soon becomes covered with the white substance of Schwann, and the other sheaths of a medullated nerve, so that it becomes the axial cylinder of a nerve-fibre. [T'hus a nerve-fibre is merely an excessively long, tinbranched process of a nerve-cell pushed outwards towards the periphery.] It is not definitely ascertained that the cerebral cells have such processes. All the other processes divide very frequently until they form a branched, root-like, complex arrangement of the finest primitive fibrils. These are called protoplasmic processes $(\mathbf{I}, y)$. By means of these processes, adjoining cells are brought into communication with each C'ell from the Gasserian ganglion. other, so that impulses can be conducted from one cell to $n$, nuclei of the sheath; $t$, fibre another. Further, many of these fibrils approximate to each dividing at a node of Ranvier. other and join together to form axis-cylinders of other nervefibres. [ $V$. Thanhoffer states that he has traced the axis-cylinder process to the nucleus and nucleolus.]

2. Bipolar cells are best developed in fishes, e.g., in the spinal ganglia of the skate, and in the (rasserian ganglion of the pike. They appear to be nucleated, fusiform enlargements of the axis-cylinder (fig. 368, on the right of I). The white substance often stops short on each side of the enlargement, but sometimes the white substance and the sheath of Schwann pass over the enlargement.

3. Nerve-cells with connective-tissue capsules occur in the peripheral ganglia of man (fig. 
$368, \mathrm{II})$, e.g., in the spinal ganglia. The soft body of the cell, which is provided with several processes, is covered by a thick, tough capsule composed of several layers of connective-tissue corpuscles; while the inner surface of the composite capsule is lined by a layer of delicate endothelial cells (fig. 376). The body of the cells in the spinal ganglia is traversed by a network of fine fibrils (Flemming). The capsule is continuous with the sheath of the nerve-fibre.

Rawitz and G. Retzius find that the cells of the spinal ganglia are unipolar, the outgoing fibre taking a half-turn within the capsule before it leaves the cell (fig. 376). Retzius [and Ranvier] observed the process to divide like a T. . Perhaps this division corresponds to the two processes of a bipolar cell. The jugular ganglion and plexus gangliiformis vagi in man contain ouly unipolar cells, so that, in this respect, they may be compared to spinal ganglia. The same is the case in the Gasserian ganglion; while the ciliary, spheno-palatine, otic, and submaxillary ganglia structurally resemble the ganglia of the sympathetic.

4. Ganglionic cells with spiral fibres oceur chiefly in the abdominal sympathetic of the frog (Beale, J. Arnold). The body of the cell is usually pyriform in shape, and from it proceeds a straight unbranched process (fig. 368 , III, $n$ ), which ultimately becomes the axis-cylinder of a nerve. A spiral fibre springs from the cell (? a network), emerges from it, and curves in a spiral direction round the former $(0)$. The whole cell is surrounded by a nucleated capsule $(m)$. We know nothing of the significance of the different fibres.

\section{CHEMICAL AND MECHANICAL PROPERTIES OF NERVOUS} SUBSTANCE.-1. Proteids.-Albumin occurs chiefly in the axis-cylinder and in the substance of the ganglionic cells. Some of this proteid substance presents characters not unlike those of myosin (\$293). Dilute solution of common salt extracts a proteid from nervous matter, which is precipitated by the addition of much water and also by a concentrated solution of common salt (Petrowsky). Potash-albumin and a globulin-like substance are also present. Nuclein occurs especially in the grey matter $(\S 250,2)$, while neuro-keratin, a body containing much sulphur and closely related to keratin, occurs in the corneous sheath of nervefibres (p. 528). If grey nervous matter be subjected to artificial digestion with trypsin, both of these substances remain undigested (Kühne and Ewaldl). Pure neuro-keratin is obtained by treating the residue with caustic potash. The sheath of Schwann does not yield gelatin, but a substance closely related to elastin $(\$ 250,6)$, from which it differs, however, in being more soluble in alkalies. The connective-tissue of nerves yields gelatin.

2. Fats and other allied substances soluble in ether, more especially in the white matter:- $(a)$ Cerebrin, free from phosphorus $(\S 250,3)$.

Cerebrin is a white powder composed of spherical granules soluble in hot alcohol and ether, but insoluble in cold water. It is decomposed at $80^{\circ} \mathrm{C}$, and its solutions are neutral. When boiled for a long time with acids, it splits up into a left-rotatory body like sugar, and another unknown product. Preparation. - Rub up the brain into a thin fluid with baryta water. Extract the separated coagulum with boiling alcohol. The extract is frequently treated with cold ether to remove the cholesterin ( $W$. Müller $)$. Parkus separated from cerebrin its homologue, homocerebrin, which is slightly more soluble in alcohol, and the clyster-like boily, encephalin, which is soluble in hot water.

(b) Lecithin and its decomposition-products-glycero-phosphoric acid and oleophosphoric acid (\$251).

Neurin (or Cholin $=\mathrm{C}_{5} \mathrm{H}_{15} \mathrm{NO}_{2}$ ) is a strongly alakaline, colourless fluid, forming crystalline salts with acids. It is soluble in water and alcohol, and has been formed synthetically from glycol and trimethylamin. Lecithin is a salt of the base neurin.

(c) Protagon, which contains $\mathrm{N}$ and $\mathrm{P}$, is similar to cerebrin, and is, according to its discoverer, the chief constituent of the brain (Liebreich).

According to Hoppe-Seyler and Diaconow, it is a mixture of lecithin and cerebrin. [The investigations of Gamgee and Blankenhorn have shown, however, that protagon is a definite chemical body. They find that, instead of being unstable, it is a very stable body.] It is a glucoside, and crystalline, and can be extracted from the brain by warm alcohol, and when boiled with baryta yields the decomposition-products of lecithin.

3. The following substances are extracted by water:-Xanthin and hypoxanthin (Scherer), kreatin (Lerch), inosit (W. Müller), ordinary lactic acid (Gscheidlen), acetic and formic acids, uric acid (?), and volatile fatty acids ; leucin (in disease), urea (in uræmia), and a substance like starch in the human brain (Jaffé). All these substances are for the most part products of the regressive metabolism of the tissues. 
Reaction.-Nerrous substance, when passive, is neutral or feebly alkaline in reaction, while active (? and dead) it is acid (Funke). The grey matter of the brain, when quite fresh, is alkaline (Liebreich), but death rapidly causes it to become acid (Gscheidlen).

The reaction of nerve-fibres varies during life. After introducing methyl-blue into the boly of a living animal, Ehrlich found that the axis-cylinder became blue, i.e., in those nerves which have an alkaline reaction (cortex cerebri, cardiac, sensory, motor (nou-striped), gustatory and olfactory fibres), while the termination of motor (voluntary) nerves remained uncoloured. The latter he regards as acid.

The nerves after death have a more solid consistence, so that in all probability some coagrulation or change, comparable to the stiffening of muscle, occurs in them after death, while at the same time a free acid is liberated (\$295). If a fresh Irain be rapidly "broiled" at $100^{\circ} \mathrm{C}$., it, like a muscle similarly treated, remains alkaline $(\$ 295)$.

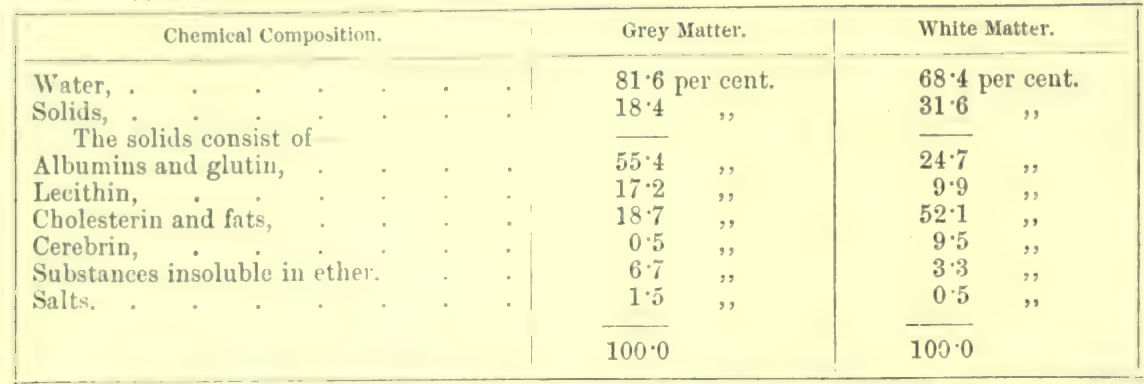

In 100 parts of ash, Breed found potash 32, soda 11, magnesia 2, lime 0.7, $\mathrm{NaCl} 5$, iron phosphate $1 \cdot 2$, fixed phosphoric acid 39 , sulphuric acid $0 \cdot 1$, silicic acid $0 \cdot 4$.

[1'tomaines $(\$ 166)$ are ohtained from putrefying brain. They have an effect on the motor nerves like curara, but in much less degree, while the phenomena last for a much shorter time (Guareschi and Mosso).]

Mechanical Properties.-One of the most remarkable mechanical properties of nerve-fibres is the absence of elastic tension according to the varying positions of the body. Divided nerves do not retract; such nerves exhibit delicate, microscopic, transverse folds, [like watered silk] or Fontana's transverse markings.

The cohesion of a nerve is very considerable. When a limb is forcibly torn from the body, as sometimes happens from its becoming entangled in machinery, then erve not unfrequently remains unsevered, while the other soft parts are ruptured. [Tillaux found that a weight of 110 to $120 \mathrm{lbs}$. was required to rupture the sciatic nerve at the popliteal space, while to break the median or ulnar nerve of a fresh boly, a force equal to 40 to $50 \mathrm{lbs}$. was required. The toughness and elasticity of nerves are often well shown in cases of injury or gun-shot wounds. The median or ulnar nerve will gain 15 to 20 centimetres (6 to 8 inches) before breaking. Weir Mitchell has shown that a healthy nerve will bear a very considerable amount of pressure and handling, and, in fact, the method of nerve-stretching depends upon this property of a nerve-trunk.]

323. METABOLISM OF NERVES.-Influence of Blood-Supply.-We know very little regarding the metabolic processes that occur in nerve-tissue. Some extractives are obtained from nerve-tissue, and they may, perhaps, be regarded as decomposition-products (p. 531). It has not been proved satisfactorily that during the activity of nerves there is an exchange of $\mathrm{O}$ and $\mathrm{CO}_{2}$. That there is an exchange of materials within the nerves is proved by the fact that, after compression of the blood-vessels of the nerves, the excitability of the nerves falls, and is restored again when the circulation is re-established. Compression of the abdominal aorta causes paralysis and numbness of the lower half of the body, 
while occlusion of the cerebral vessels causes almost instantaneously cessation of the cerebral functions. The metabolism of the central nervous organs is much more active than that of the nerves themselves. [If the abdominal aorta of a rabbit be compressed for a few minutes, the hind limbs are quickly paralysed, the animal crawls forward on its fore-legs, drawing the hind limbs in an extended position after it.] The ganglia form much lymph.

324. EXCITABILITY OF THE NERVES-STIMULI.-Nerves possess the property of being thrown into a state of excitement by stimuli, and are, therefore, said to be excitable or irritable. The stimuli may be applied to, and may act upon, any part of the nerve. [The following are the various kinds of stimuli, i.e., modes of motion, which act upon nerves] :-

1. Mechanical stimuli act upon nerves when they are applied with sufficient rapidity to produce a change in the form of the nerve-particles, e.g., a blow, pressure, pinching, tension, puncture, and section. In the case of sensory nerves, when they are stimulated, pain is produced, as is felt when a limb "sleeps," or when pressure is exerted upon the ulnar nerve at the bend of the elbow. When a motor nerve is stimulated, motion results in the muscle attached to the nerve. If the continuity of the nerve-fibres be destroyed, or, what is the same thing, if the continuity of the axial cylinder be interrupted by the mechanical stimulus, the conduction of the impulse across the injured part is interrupted. If the molecular arrangements of the nerves be permanently deranged, e.g., by a violent shock, the excitability of the nerves may be thereby extinguished.

A slight blow applied to the radial nerve in the fore-arm, or to the axillary nerves in the supraclavicular groove, is followed by a contraction of the muscles supplied by these nerves. Under pathological conditions, the excitability of a nerve for mechanical stimuli may be increased enormously.

Tigerstedt ascertained that the minimal mechanical stimulus is represented by 900 milligrammillimetres, and the maximum by 7000 to 8000 . Strong stimuli cause fatigue, but the fatigue does not extend beyond the part stimulated. A nerve when stimulated mechanically does not become acid. Slight pressure without tension increases the excitability, which diminishes after a short time. The mechanical work produced by an excited muscle in consequence of a stimulus was 100 times greater than the mechanical energy of the mechanical nerve-stimulus.

Continued pressure upon a mixed nerve paralyses the motor sooner than the sensory fibres. If the stimulus be applied very gradually, the nerve may be rendered inexcitable without manifesting any signs of its being stimulated (Fontana, 1758). Paralysis, due to continuous pressure gradually applied, may occur in the region supplied by the brachial nerves; the left recurrent laryngeal nerve also may be similarly paralysed from the pressure of an aneurism of the arch of the aorta.

By increasing the pressure on a nerve by using a gradually increasing weight, there is at first an increase and then a decrease of the excitability. Pressure on a mixed nerve abolishes reflex conduction sooner than motor conduction (Kronecker and Zederbaum).

Nerve-stretching is employed for therapeutical purposes. If a nerve be exposed and stretched, or if it be made sufficiently tense, the nerve is stimulated. Slight tension increases the reflex excitability (Schleich), while violent extension produces a temporary diminution or abolition of the excitability (Valentin). The centripetal or sensory fibres of the sciatic nerve are sooner paralysed thereby than the centrifugal or motor (Conrad). During the process of extension, mechanical changes are produced, either in the nerve itself or in its end-organs, causing an alteration of the excitability, but it may also affect the central organs. The paralysis, which sometimes occurs after forcible stretching, usually rapidly disappears. Therefore, when a nerve is in an excessively excitable condition, or when this is due to an inflammatory fixation or constriction of the nerve at some part of its course, nerve-stretching may be useful, partly by diminishing the excitability, partly by breaking up the inflammatory adhesions. In cases where stimulation of an afferent nerve gives rise to cpileptic or tetanic spasms, nerve-stretching may be useful by diminishing the excitability at the periphery, in addition to the other effects already described. It has also been employed in some spinal affections, which may not as yet have resulted in marked degenerative changes.

For physiological purposes, a nerve may be stimulated mechanically by means of Heiden- 
hain's tetanomotor, which is simply an ivory hammer attached to the prolonged spring of a Neefs hammer of an inluction machine. [A more delicate form of this instrument was used by Tigersteclt $(\$ 335)$.] The rapid vibration of the hammer communicates a series of mechanical shocks to the nerve upon which it is caused to beat. Rhythmic extension of a nerve causes contractions and even tetanus.

2. Thermal Stimuli.--If a frog's nerve be heated to $45^{\circ} \mathrm{C}$., its excitalility is first increased and then diminished. The higher the temperature, the greater is the excitability, and the shorter its duration (Afinasieff'). If a nerve be heated to 50 C. for a short time, its excitability and conductivity are abolished. The frog's nerve alone regains its excitability on being cooled (Pickford). If the temperature be raised to $65 \mathrm{C}$., the excitability is abolished without the occurrence of a contraction, while its medulla is broken up (Eckhart). Sudden cooling of a nerve to 5 C. acts as a stimulus, causing contraction in a muscle, while sudden heating to 40 or 45 C. produces the same result. If the temperature be increased still more, instead of a single contraction a tetanic condition is produced. All such rapid variations of temperature quickly exhaust the nerve and kill it. If a nerve be frozen gradually, it retains its excitability on being thawed. The excitability lasts long in a coulect nerve; in fact, it is increased in a motor nerve, but the contractions are not so high and more prolonged, while the conduction in the nerve takes place more slowly. Amongst ntammuliun nerves, the afferent and vaso-dilator nerves at 45 to 50 C. exhibit the results of stimulation, while the others only show a change in their excitability. When cooled to $+5^{\circ} \mathrm{C}$., the excitability of all the fibres is diminished (G'ritzner).

3. Chemical Stimuli excite nerves when they act with a certain rapidity, and thereby alter the condition of the nerve (p. 473). Most chemical stimuli act by first inereasing the nervous excitability, and then diminishing or paralysing it. Chemical stimuli, as a rule, have less effect upon sensory than upon motor fibres (Erlikred). According to Griitzner, the inactivity of chemical stimuli, so often observed when they are applied to sensory nerves, depends in great part upon the non-simultaneous stimulation of all the nerve-fibres. Amongst chemical stimuli are-(n) ropicl alstraction of water by dry air, blotting paper, exposure in a chamber containing sulphuric acid, or by the action of solutions which absorb fluids, e.g., concentrated solutions of neutral alkaline salts $(\mathrm{NaCl}$, excites only motor fibres in mammals-Grietzner), sugar, urea, concentrated glycerin (and ? some metallic salts). 'The subsequent addition of water may abolish the contractions, while the nerve may still remain excitable. The abstraction of water first increases and afterwards diminishes the excitability. The imbibition of water diminishes the excitability. (b) Free alkalies, mineral acids (not phosphoric), many organic acids (acetic, oxalic, tartaric, lactic), and most salts of the heavy metals. While the acids act as stimuli, only when they are somewhat concentrated, the caustic alkalies act in solutions of 0.8 to 0.1 per cent. (Kïiline). Neutral potash salts, in a concentrated form, rapidly kill a nerve, but they do not excite it nearly so strongly as the soda compounds. Dilute solutions of the neutral potash salts first increase and afterwards diminish it (Ranke), as can be shown by stimulation with an induction shock (Biedermann). (c) Various substances, e.g., dilute alcohol, ether, chloroform, bile, bile-salts, and sugar. These substances usually excite contractions, and afterwards rapidly kill the nerve. Ammonia, lime-water, some metallic salts, carbon bisulphide, and ethereal oils kill the nerve without exciting it-at least without producing any contraction in a frog's nerve-muscle preparation. [The nerve of a nerve-muscle preparation may be dipped into ammonia, but no contraction results, while the slightest traces of ammonia applied to a muscle cause contraction.] Carbolic acid does the same, although when applied directly to the spinal cord it produces spasms. These substances excite the muscles when they are directly applied to them. Tannic acid does not act as a stimulus either to nerve or muscle. 
As a general rule, the stimulating solutions must be more concentrated when applied to a nerve than to muscle, in order that a contraction may be produced.

[Methods. - If a nerve-muscle preparation of a frog's limb be made, and a straw flag (p. 472) attached to the toes while the femur is fixed in a clamp, and its nerve be then dipped in a saturated solution of common salt, the toes soon begin to twitch, and by and by the whole limb becomes tetanic, and thus keeps the straw flag extended. The effect of fluid on a muscle or nerve is easily tested by fixing the muscle in a clamp, while a drop of the fluid is placed on a greased surface, which gives it a convex form. The end of the muscle or nerve is then brought into contact with the cupola of the drop (Kühne).]

4. The Physiological or normal stimulus excites the nerves in the normal intact body. Its nature is entirely unknown. The "nerve-motion" thereby set up travels either in a "centrifugal" or outgoing direction from the central nervous system, giving rise to motion, inhibition of motion, or secretion; or in a "centripetal " or ingoing direction from the specific end-organs of the nerves of the special senses or the sensory nerves. In the latter case, the impulse reaches the central organs, where it may excite sensation or perception, or it may be transferred to the motor areas and be conducted in a centrifugal direction, constituting a "reflex" stimulation (\$360). A single physiological nerve-impulse travels more slowly than that excited by the momentary application of an induction shock (Lovin, v. Kries). It is not a uniform process excited by varying intensity and greater or less frequency of stimulation, but it is essentially a process varying considerably in duration, and it may even last as long as $\frac{1}{3}$ second ( $v$. Kries).

5. Electrical Stimuli.-[The following forms of electrical stimuli may be used :-

(1) A constant current, which may be made or broken ( $\$ 328)$.

(2) Induction shocks, either make or break shocks (\$329).

(3) An interrupted current (\$329).

The electrical current acts most powerfully upon the nerves at the moment when it is applied, and at the moment when it ceases (\$336); in a similar way, any increase or decrease in the strength of a constant current acts as a stimulus. If an electrical current be applied to a nerve, and its strength be very gradually increased or diminished, then the visible signs of stimulation of the nerve are very slight. As a general rule, the stimulation is more energetic the more rapid the variations of the strength of the current applied to the nerve, i.e., the more suddenly the intensity of the stimulating current is increased or diminished (du Bois-Reymond).

An electrical current must have a certain strength or liminal intensity before it is effective. By uniformly increasing the strength of the current, the size of the contraction increases rapidly at first, then more slowly (Tigerstedt and Willhard).

An electrical current, in order to stimulate a nerve, must have a certain duration, it must act at least during 0.0015 second (Fick, 1863); even with currents of slightly longer duration, the opening shock may have no effect. If the duration of the closing shock of a constant current be so arranged that it is just too short to be active, then it merely requires to last 1.3 to 2 times longer to produce the most complete effect (Grünhagen).

The electrical current is most active when it flows in the long axis of the nerve ; it is inactive when applied vertically to the axis of the nerve (Galvani). Similarly, muscles are incomparably less excited by transverse than by longitudinal currents (Giuffrè).

The greater the length of nerve traversed by the current, the less the stimulus that is required (Pfaff).

Constant Current.- If the constant current be used as a nervous stimulus, the stimulating effect on the sensory nerves is most marked at the moment of making and breaking the current; during the time the current passes, only slight excitement is perceived, but, even under these circumstances, very strong currents may cause very considerable, and even unbearable, sensations. If a constant current be 
applied to a motor nerve, the greatest effect is produced when the current is made or closed [closing or make contraction], and when it is broken or opened [opening or break contraction]. But while the current is passing, the stimulation does not cease completely, for, with a certain strength of stimulus, the muscle remains in a state of tenanus (galvanotonus or "closing tetanus") (Pflüger). For the same effect on muscles, see p. 482. With strong currents this tetanus does not appear, chiefly because the current diminishes the excitability of the nerves, and thus develops resistance, which prevents the stimulus from reaching the muscle. According to Hermann, a descending current applied to the nerve, at a distance from the muscles, causes this tetanus more readily, while an ascending current causes it more readily when the current is closed near the muscle. The constant current is said by Grïtzner to have no effect on vaso-motor and secretory fibres.

Over-maximal Contraction.-By gralually increasing the strength of the electrical stimulus alpilied to a motor nerve, Fick observed that the muscular contractions (height of the lift) at first increased proportionally to the increase of the stimulus, until a maximal contraction was chitained. If the strength of the stimulus be increased still further, another increase of the contraction above the first reached maximum is obtained. This is called an "over-maximal contraction." Occasionally hetween the first maximum and the second there is a diminution, or indeed absence of, or gap or hiatus, in the contractions. The cause of this lies in the positive pole, which with a certain strength of current is sufficient to prevent the further transmission of the excitement ( $\$ 335)$. On continuing to increase the induction current, ultimately a stage is reached where the stimulation at the negative pole again becomes stronger than the inhibition at the positive, and this overcomes the latter. The contractions before the gap are caused by the occurrence of the induction current (their latent period is short); the contractions (long

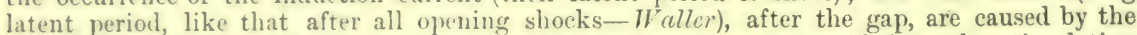
disappearance of the induction current, i.e., by polarisation: this is added to the stimulation proceeling from the negative pole, which after the gap overcomes the inhibition at the positive pole, and excites the over-maximal contractions (Tigerstedt and Willhard).

Tetanus.-If single shocks of short duration be rapidly applied after each other to a nerve, tetanus in the corresponding muscle is produced ( $\$ 298$, III.).

A motor nerve has a greater specitic excitability for electrical stimuli than the muscle-substance. This is proved by the fact that, a feebler stimulus suffices to excite a muscle when applied to the nerve than when it is applied to the muscle directly, as occurs when the terminations of the motor nerves are paralysed by curara (Rosenthal).

Soltmann found that the excitability of the motor nerves of new-born animals for electrical stimuli is less than in adults. The excitability increases until the 5 th to 10 th month.

Unequal Excitability.-Under certain circumstances, the nearer the part of the motor nerve stimulated lies to the central nervous system, the greater is the effect produced (contraction); [or what is the same thing, the further the point of a nerve which is stimulated is from the muscle, the stimulus being the same, the greater is the contraction. This led Pfluiger to his "avalanche-theory," i.e., that the "nervemotion" increases in the nerve as it passes towards the muscles. This effect is explained, however, by the unequal excitability of different parts of the same nerve]. According to Fleischl, all parts of the nerve are equally excitable for chemical stimuli. Further, it is said that the higher placed parts of a nerve are more excitable only when the stimulating current passes in a descending direction; the reverse is the case when the current ascends (Hermann). On stimulating a sensory nerve, Rutberford and Hälsten found that the reflex contraction was greater, the nearer the stimulated point was to the central nervous system.

Unequal Excitability in the same Nerve.-Nerve-fibres, even when functionally the same and included in the same nerve-trunk, are not all equally excitable. Thus, feeble stimulation of the sciatic nerve of a frog causes contraction of the flexor muscles, while it requires a stronger stimulus to produce contraction of the extensors (Ritter, 1805, Rollett). According to Ritter, the nerves for the flexors die first. 
Direct stimulation of the muscles in curarised animals shows that the flexors contract with a feebler stimulus (but also fatigue sooner) than the extensors; the pale muscles of the rabbit are also more excitable than the red. As a rule, poisons affect the flexors sooner than the extensors. In some muscles some pale fibres are present, and they are more excitable than the red (Grützner) (\$298). If a frog's nerve-muscle preparation be exposed to the action of ether, on strong stimulation of the sciatic nerve, flexion occurs (Grützner, Boveditch), but, if the current be made stronger, extension takes place. During deep ether-narcosis, strong stimulation of the recurrent nerve causes dilatation, and with slight narcosis, narrowing of the glottis takes place; dilatation occurs on slight stimulation (Bowditch). The adductor muscle of the claw of a crayfish is relaxed under a weak stimulus, but it contracts when a strong stimulus is applied to it. The reverse is the case with the muscle which opens the claw (Biedermann).

Unipolar Stimulation.-If one electrode of an induction apparatus be applied to a nerve, it may act as a stimulus. Du Bois-Reymond has called this "unipolar induction action." It is due to the movement of the electrical current to and from the free ends of the open induction current at the moment of induction. [Unipolar induction is more apt to occur with the opening than the closing shock, because the former is more intense.]

Upon muscle, electrical stimuli act quite as they do upon nerves. Electrical currents of very short duration have no effect upon muscles whose nerves are paralysed by curara (Brïcke), and the same is true of greatly fatigued muscles, or muscles about to die or greatly weakened by diseased conditions (\$399).

\section{DIMINUTION OF THE EXCITABILITY-DEGENERATION AND} REGENERATION OF NERVES.-1. Normal Nutrition.-The continuance of the normal excitability in the nerves of the body depends upon the maintenance of the normal nutrition of the nerves themselves and a due supply of blood. Insufficient nutrition causes, in the first instance, increased excitability, and if the condition be continued the excitability is diminished (\$339, I.).

When the physician meets with the signs of increased excitability of the nerves, under bad or abnormal conditions of nutrition, this is to be regarded as the beginning of the stage of decrease of the nerve-energy. Invigorating measures are required.

If the terminal nervous apparatus be subjected to a temporary disturbance of its nutrition, the return of the normal nutritive process is heralded by a more or less marked stage of excitement. The more excitable the nervous apparatus, the shorter must be the duration of the disturbance of nutrition, e.g., cutting off the arterial blood supply or interfering with the respiration.

2. Fatigue.-Continued excessive stimulation of a nerve, without sufficient intervals of repose, causes fatigue of the nerve, and by exhaustion rapidly diminishes the excitability. A nerve is more slowly fatigued than a muscle (Bernstein), but it recovers more slowly $(\$ 304)$. [Nerves of cold-blooded animals (Widenskii) and mammals (Bowditch) may be tetanised for hours without becoming fatigued.]

[To show that a muscle is much more rapidly fatigued than a nerve, Bernstein arranged two nerve-muscle preparations so that both nerves were tetanised simultaneously, but through one of the nerves, a polarising constant current was passed by means of non-polarisable electrodes $(\S 327)$, so that the condition of anelectrotonus ( $\$ 335$ ) was set up in this nerve, and thus "blocked" the propagation of impulses to the corresponding muscle. Only one muscle, therefore, was tetanised. Both nerves were continuously stimulated until fatigue of the contracting muscle took place, and on breaking the polarising current applied to the other nerve, the corresponding muscle at once became tetanic. Now, as both nerves were equally stimulated, and the muscle in connection with one nerve was fatigued, while the other muscle at once contracted, it is evident that a muscle is much more rapidly fatigued than a motor nerve. In sensory nerves, fatigue and recovery are analogous to the corresponding processes in motor nerves (Bernstein).]

Recovery.-When a nerve recovers, at first it does so slowly, then more rapidly, and afterwards again more slowly. If recovery does not occur within half an hour after a frog's nerve has been subjected to very long and intense stimulation, it will not take place at all. 
3. Continued inaction of a nerve diminishes, and may ultimately abolish the excitability.

Thus, the central ends of divided sensory nerves, after amputation of a limb, lose their excitability, although the nerves are still connected with the central nervous system, becatise the end-organs through which they were normally excited have been removed.

4. Separation from their Nerve-Centres.-The nerve-fibres remain in a condition of normal nutrition, only when they are directly connected with their centre, which governs the nutritive processes within the nerve. If a nerve within the body be separated from its "nutritive centre"--either by section of the nerve or compressing it-within a short time it loses its excitability, and the peripherel end undergoes fatty degeneration, which hegins in four to six days in warm-blooded animals, and after a long time in cold-blonded ones $(J, h . M /$ iller $)$. See also the changes of the excitability during this condition, the so-called "Reaction of degeneration" (\$339). If the sensomy nerve-fibres of the root of a spinal nerve be divided on the central side of the ganglion, the fibres on the peripheral side do not degenerate, for the ganglion is the trophic or nutritive centre
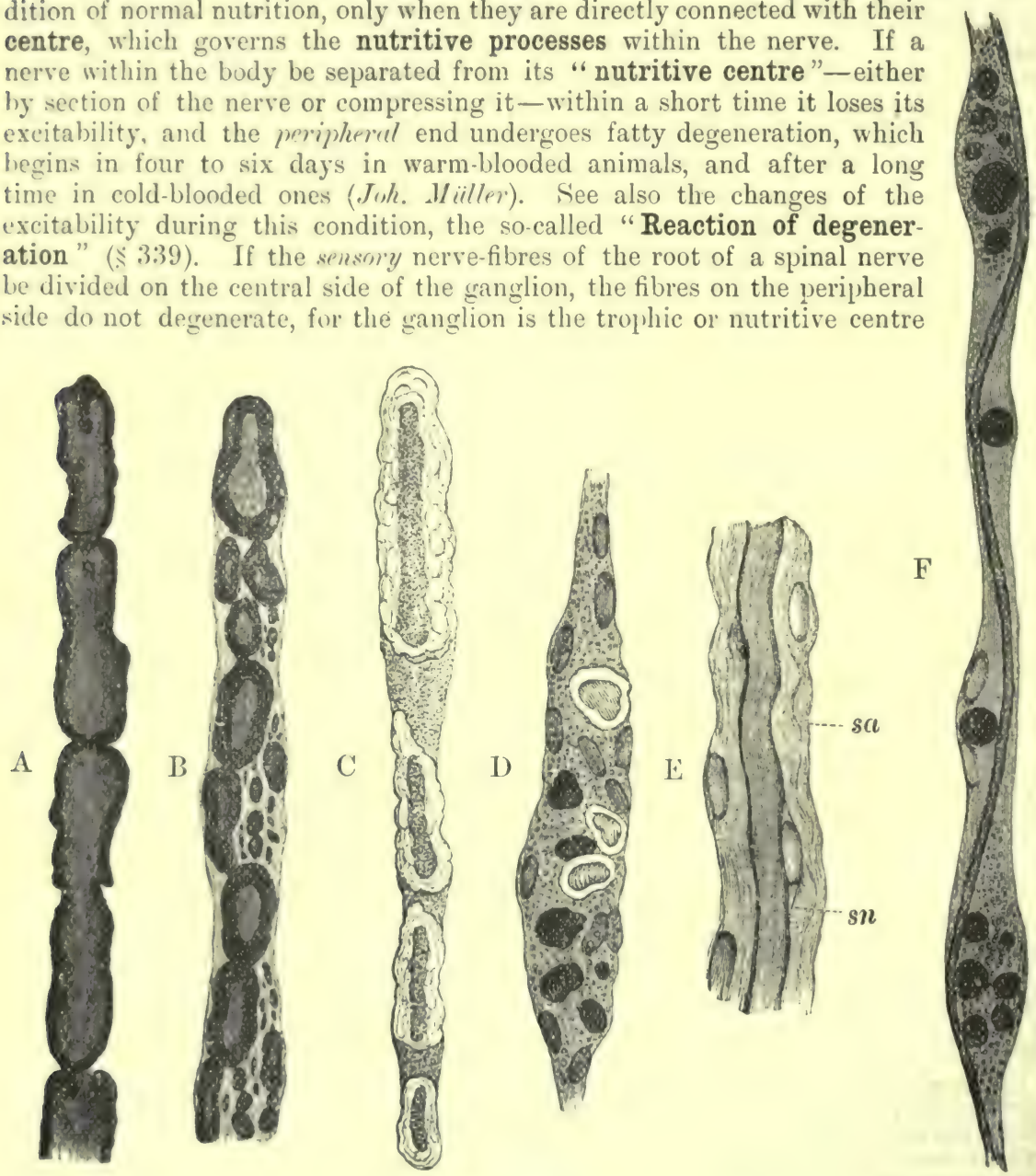

Fig. 377 .

Degeneration and regeneration of nerves. A, sub-division of the myelin ; B, further disintegration thereof (osmic acid staining); C, interruption of the axial cylinder, which is surrounded with the broken-up myelin; D, accumulation of nuclei, with the remainder of the myelin in a spindle-shaped fibre; $\mathrm{E}$, a new nerve-fibre, with a new sheath of Schwann, $s n$, within the old sheath of Schwann, $s a ; \mathrm{F}$, a new nerve-fibre passing in a curved course through an old nerve-fibre sheath.

for the sensory nerves; but the fibres still in connection with the cord degenerate (Waller).

[Wallerian Law of Degeneration. - If a spinal nerve be divided, the peripheral 
part of the nerve and its branches, including the sensory and motor fibres, degenerate completely (fig. 378 , A), while the central parts of the nerve remain unaltered. If the anterior root of a spinal nerve alone be divided before it joins the posterior root, all the peripheral nerve-fibres connected with the anterior root degenerate (fig. $378, \mathrm{~B}$ ), so that in the nerve of distribution only the motor fibres degenerate. The portion of the nerve-root which remains attached to the cord does nut degenerate. If the posterior root alone be divided, between the spinal cord and the ganglion, the effect is reversed, the part of the nerve-root lying between the section and the spinal cord degenerates, while the part of the nerve connected with the ganglion does not degenerate (fig. $378, \mathrm{C}$ ). The central fibres degenerate because

A

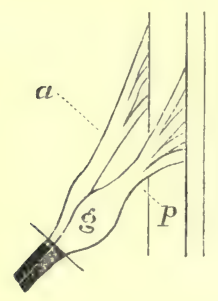

B

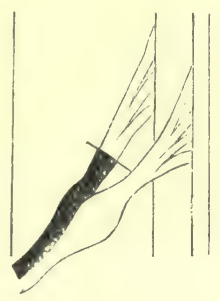

$\mathrm{C}$

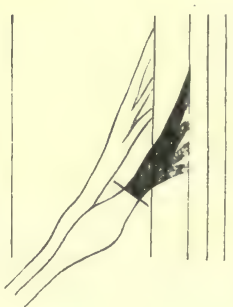

D

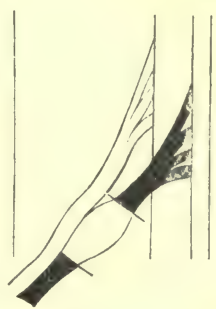

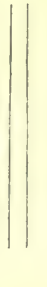

Fig. 378.

Diagram of the roots of a spinal nerve, showing the effect of section (the black arts represent the degenerated parts). A, section of the nerve-trunk beyond the ganglic $n$; $B$, of the anterior root, and C, of the posterior; D, excision of the ganglion; $a$, anterior, $p$, posterior root ; $g$, ganglion.

they are separated from the ganglion. If the ganglion be excised, or if separated, as in fig. 378, D, both the central and peripheral parts of the posterior root degenerate. These experiments of Waller show that the fibres of the anterior and posterior roots are governed by different centres of nutrition or "trophic centres." As the anterior root degenerates when it is separated from the cord, and the posterior when it is separated from its own ganglion, it is assumed that the trophic centre for the fibres of the anterior root lies in the multipolar nerve-cells of the anterior horn of the grey matter of the spinal cord, while that for the fibres of the posterior root lies in the cells of the ganglion placed on it. The nature of this supposed trophic influence is entirely unknown.]

Traumatic and Fatty Degeneration.-Both ends of the nerve at the point of section immediately begin to undergo "traumatic degeneration." (In the frog on the 1st and 2 nd day.) After a time neither the myelin nor axis-cylinder is distinguishable (Schitf'). According to Engelmann, this condition extends only to the nearest node of Ranvier, and afterwards the socalled "fatty degeneration" begins. The process of "fatty" degeneration begins simultaneously in the whole peripheral portion; the white substance of Schwann breaks up into masses (fig. 377, A), just as it does after death, in microscopic preparations; afterwards, the myelin forms globules and round masses (B), the axial-cylinder is compressed or constricted, and is ultimately broken across $(C)$ in many places (7th day). The nerve-fibre seems to break up into two substances-one fatty, the other proteid in constitution, the fat being absorbed (S. Mayer). The nuclei of Schwann's sheath swell up and proliferate (D-until the 10th day). According to Ranvier, the nuclei of the interannular segments and their surrounding protoplasm proliferate, and ultimately interrupt the continuity of the axis-cylinder and the myelin. They then undergo considerable development with simultaneous disappearance of the medulla and axis-cylinder, or at least fatty substances formed by their degeneration, so that the nerve-fibres look like fibres of connective-tissue. [According to this view, the process is in part an active one, due to the growth of the nerve-corpascles breaking up the contents of the neurilemma, which then ultimately undergo chemical degenerative changes.] According to Ranvier, Tizzoni, and others, leucocytes wander into the cut ends of the nerves, and also at Ranvier's nodes, insinuating themselves into the nerve-fibres, where they take myelin into their bodies, and subject it to certain changes. [These cells are best revealed by the action of osmic acid, which blackens any myelin particles in their interior.] Degeneration also takes place in the 
motorial end-plates, beginning first in the non-medullated branches, then in the terminal fibrils, and lastly in the nerve-trunks (Gessler).

Regeneration of Nerves. - In order that regeneration of a divided nerve may take place (Cruickshunk, 1795), the divided ends of the nerve must be brought into contact (\$244). In man this is done by means of sutures. About the middle of the fourth week, small clear bands apjiear within the neurilemma, winding between the nuclei and the remains of the myelin $(\mathrm{E})$. They soon become wider, and receive myelin with incisures, and nodes, and a sheath of schwann (2nd to $3 \mathrm{rd}$ month-F). The regeneration process takes place in each interannular segment, while the individual segments unite end to end at the nodes of Ranvier $(\$ 321,1,5)$. On this view, each nerve-segment of the fibre corresponds to a "cell-unit" (E. Veuminn, Eichhorst). The same process oceurs in nerves ligatured in their course. Several new fibres may be formed within one old nerve-sheath. The divided axis-cylinders of the antiul end of the nerve begin to grow about the 14th day, until they meet the newly formed ones, with which they unite.

[Primary and Secondary Nerve Suture.-Numerous experiments on animals and man have stahlished the fact that, immediate or primary suture of a nerve, after it is divided, either awilentally, or intentionally, hastens reunion and regeneration, and accelerates the restoration of function. Secondary suture, i.e., bringing the ends together long after the nerve has been divided, has been practised with success. Surgeons have recorded cases where the function was restored after division had taken place for 3 to 16 months, and even longer, and in most citses the sensibility was restored first, the average time being 2 to 4 weeks. Motion is rinovered much later. The ends of the nerve should be stitched to each other with catgut, the muscles at the same time being kent from becoming atrophied by electrical stimulation and the systematic use of massage $(\$ 307)$. After suture of a nerve, conductivity is restored in the rablit in 40 days, on the $31 \mathrm{st}$ in dogs, and 25 th in fowls, but after simple division without suture, not till the 6uth day in the rabbit. Trausplantation of nerve does not succeed (John. son).].

Union of Nerves. - The central end of a divided motor nerve may unite with the peripheral end of another, and still conduct impulses (Rara). [It is stated that sensory fibres will reunite with sensory fibres, and motor fibres with motor fibres, and the regenerated nerve will, in the former case, conduct sensory impulses, and the latter motor impulses. There is very considerable diversity of opinion, however, as to the regeneration or union of sensory with motor fibres. Paul Bert made the following experiment:- He stitched the tail of a rat into the animal's back, and after union had taken place, he cut the tail from the borly at the root, so that the tail, as it were, grew out of the animal's back, broad end uppermust. On irritating the end of the tail, which was formerly the root, the animal gave signs of pain. This experiment was devised by Bert to try to show that nerve-fibres can conduct imprulses in both directions. One of two things must have occurred. Either the motor fibres, which normally carried impulses down the tail, now convey them in the opposite direction, and convey them to sensory fibres with which they have united; or the sensory fibres, which mirmally conducted inpulses from the tip upwards, now carry them in the opposite direction. If the former were actually what happened, it would show that nerve-fibres of different function do unite (\$349). Reichert asserts that he has succeeded in uniting the hypoglossal with the vagus in the dog. According to Gessler the end-plate is the first to regenerate.]

Trophic Centres. - The regeneration of the nerves seems to take place under the influence of the nerve-centres, which act as their nutritive, or trophic centres. Nerves permanently separated from these centres never regenerate.

I) uring the regeneration of a mixed nerve, sensibility is restored first, subsequently voluntury motion, and lastly, the movements of the muscles, when their motor nerves are stimulated directly (Schiff, Erb, v. Ziemssen).

Wallerian Method of Investigation. - As the peripheral end of a nerve undergoes degeneration after section, we use this method for determining the course of nerve-fibres in a complex arrangement of nerves. The course of speeial nerve-fibres may be ascertained by tracing the degeneration tract $(W a l l e r)$. If after section, reunion or regeneration of a motor nerve does not take place, the muscle supplied by this nerve ultimately undergoes fatty degeneration.

5. Modifying Conditions.-Under the action of various operations, e.g., compressing a nerve [so as not absolutely to sever the physiological continuity], it has been found that voluntary impulses or stimuli applied above the compressed spot, give rise to impulses which are conducted through the nerve, and in the case of a motor nerve, cause contraction of the muscles, whilst the excitability of the parts below the injured spot is greatly diminished (Schiff). In a similar manner, it is found that the nerves of animals poisoned with $\mathrm{CO}_{2}$, curara or coniin, sometimes even the nerves of paralysed limbs in man, are not excitable to direct stimuli, while they 
are capable of conducting impressions coming from the central nervous system (Duchenne). The injured part of the nerve, therefore, loses its excitability sooner than its power of conducting an impulse.

6. Certain poisons, such as veratrin, at first increase the excitability of the nerves, and afterwards abolish it; with some other poisons, the abolition of the excitability passes off very rapidly, e.g., curara. Conium, cynoglossum, iodide of methylstrychnin, and iodide of æthylstrychnin have a similar action.

If the nerve or muscle of a frog be placed in a solution of the poison, we obtain a different effect from that which results when the poison is injected into the body of the animal. Atropin diminishes the excitability of a nerve-muscle preparation of the frog without causing any previous increase, while alcohol, ether, and chloroform increase and then dininish the excitability (Mommsen).

7. Ritter-Valli Law.-If a nerve be separated from its centre, or if the centre die, the excitability of the nerve is increased; the increase begins at the central end, and travels towards the periphery - the excitability then falls until it disappears entirely. This process takes place more rapidly in the central than in the peripheral part of the nerve, so that the peripheral end of a nerve separated from its centre remains excitable for a longer time than the central end.

The rapidity of the transmission of impulses in a nerve is increased when the excitability is increased, but it is lessened when the excitability is diminished. In the latter condition, an electrical stimulus must last longer in order to be effective; hence rapid induction shocks may not produce any effect.

The law of contraction also undergoes some modification in the different stages of the changes of excitability (\$336, II.).

8. Excitable Points. - Many nerves are more excitable at certain parts of their course than at others, and the excitability may last longer at these parts. One of these parts is the upper third of the sciatic nerve of a frog, just where a branch is given off (Budge).

The motor and sensory fibres of the upper third of the sciatic nerve of a frog are more excitable for all stimuli than the lower parts (Grützner and Elpon). Whether this arises from injury during preparation, (a branch is given off there), or is due to anatomical conditions, e.g., more connective-tissue and more nodes in the lower part of the sciatic, is undetermined (Clarce Halperson).

This increased excitability may be due to injury to the nerve in preparing it for experiment. After section or compression of a nerve, all electrical currents employed to stimulate the nerve are far more active when the direction of the current passes away from the point of injury, than when they pass in the opposite direction. This is due to the fact, that the current produced in the nerve after the lesion is added to the stimulation current $(\S 331,5)$. Even in intact nerves - sciatic of a frog - where the nerve ends at the periphery or at the centre, or where large branches are given off, there are points which behave in the same way as those points where a lesion has taken place (Grützner and Moschner).

Death of a Nerve.--In a deal nerve the excitability is entirely abolished, death taking place according to the Ritter-Valli Law, from the centre towards the periphery. The reaction of a dead nerve has been found by some observers to be acid (\$322).

The functions of the brain cease immediately death takes place, while the vital functions of the spinal cord, especially of the white matter, last for a short time; the large nerve-trunks gradually die, then the nerves of the extensor muscles, those of the flexors atter three to four hours; while the sympathetic fibres retain their excitability longest, those of the intestine even for ten hours (Onimus). Compare $\$ 295$. The nerves of a dead frog may remain excitable for several days, provided the animal be kept in a cool place.

Electro-Physiology.-Before beginning the study of electro-physiology, the student ought to read and study carefully the following short preliminary remarks on the physics of this question :-

326. PHYSICAL_THE GALVANIC CURRENT-RHEOCORD.-1. Electro-motive Force -If two of the under-mentioned bodies be brought into direct contact, in one of them positive electricity, and, in the other, negative electricity can be detected. The cause of this phenomenon is the electro-motive force. The electro-motive substances may be arranged in a series of the first class, so that if the first-mentioned substance be brought into contact with any of the 
other bodies, the first substance is negatively, the last positively, electrified. 'This series is : - carbon, platinum, gold, silver, copper, iron, tin, lead, zinc + .

The amount of the electro-motive force produced by the contact of two of these bodies is greater, the further the bodies are apart in the series. The contact of the bodies may take place at one or more points. If several of the bodies of this series be arranged in a pile, the electrical tension thereby proluced is just as great as if the two extreme bodies were brought into contact, the intermediate ones being left out.

2 . The nature of the two electricities is readily determined by placing one of the bodies of the series in cuntact with a Aluid. If zinc be placed in pure or acidulated water, the zinc is + (nositive) and the water-(negative). If copner be taken instead of zinc, the copper is + but the fluid - Experiment shows that those metals, in contact with fluid, are negatively electrifien most strongly which are most acted on chemically by the fluid in which they are placed. Each such combination affords a constant difference of tension or potential. The tension [or power of overcoming resistance] of the amount of electricity obtained from both bodies depends iunon the size of the surfaces in contact. The fluids, e.g., the solutions of acids, alkalies, or salts are called exciters of electricity of the second class. They do not form among themselves a lefinite series with different tensions. When placed in these fluids, the metals lying next the $\therefore$ and of the above series, especially zinc, are most strongly electrified negatively, and to a less "xtent those lying nearer the - end of the series.

3. Galvanic Battery. - If two different exciters of the first class be placed in fluid, without the bollies coming into contact, e.y., zinc and copper, the projecting end of the (negative) zine shows free negative electricity, while the free end of the (positive) copper shows free positive * letricity. Such a combination of two electro-motors of the first class with an electro-motor of the second class is called a galuanic battery. As long as the two metals in this fluid are kept separate, the circuit is said to be broken or open, but as soon as the free projecting ends of the metals are connected outside the fluid, e.\%, by a copper wire, the circuit or current is made (1) cluserl, and a galvenic or constant current of electricity is obtained. The galvanic current has resistance to encounter in its course, which is called "conduction resistance" (W). It is directly proportional-(1) to the length (7) of the circuit; (2) and with the same length of rircuit, inversely as the section $(q)$ of the same; and $(3)$ it also depends on the molecular properties of the conducting material (specific conduction resistance $=s$ ), so that the conduction resistance, $W=(s . l): q$. The resistance to conduction increases with the increase of the ten perature of the metals, but diminishes under similar conditions with fluids.

Ohm's Law. - The strength of a galvanic current (S), or the amount of electricity passing through the rlosed circuit, is proportional to the electro-motive force (E)--or the electrical tension, but inversely proportional to the total resistance to conduction (L)-

\section{So that $\mathbf{S}=\mathbf{E}: \mathrm{L}$ (Ohm's Law, 1827).}

The total resistance to conduction, however, in a elosed circuit is composel of (1) the resistance outside the battery ("extraordinary resistance"); and (2) the resistance within the battery itself ("essential resistance"). The specific resistance to conduction is very variable in lifferent sulistances : it is relatively small in metals (e.g., for copper $=1$, iron $=6.4$, German silver $=12$ ), lut very great in fluids (e.g., for a concentrated solution of common salt $6,515,000$, for a concentrated solution of copper sulphate 10,963,600).

Conduction in Animal Tissues.-It is also very great in animal tissues, almost a million times greater than in metals. When a constant current is applied to the skin so as to traverse the body, the resistance liminishes because of the conduction of water in the epiclermis under the action of the constant current $(\$ 290)$, and the congestion of the cutaneous blood-vessels in conseguence of the stimulation. But the resistance varies in different parts of the skin, the least being in the jalm of the hand and sole of the foot. The chief seat of the resistance is the "pidermis, for after its removal by means of a blister, the resistance is greatly diminished. Dear tissue, as a rule, is a worse conductor than living tissues (Jolly). When the current is passed firensecrsely to the direction of the fibres of a muscle, the resistance is nearly nine times as great as when the eurrent prasses in the direction of the fibres-a condition which disappears in rigor mortis (Ir,manu). In nerves, the resistance longitudinally is two and a half million times greater than in mercury, transversely about twelve million times greater (Hermann). Tetanus and rigor mortis diminish the resistance in muscle (du Bois-lieymond).

Deductions. - It follows from Ohm's law that-I. If there is very great resistance to the current outside the battery [i.e., between the electrorles], as is the case when a nerve or a muscle lies on the electrodes, the strength of the current can only be increased by increasing the number of the electro-motive elements. II. When, however, the extraordinary resistance is very small compared with that within the battery itself, the strength of the current camnot be increased by increasing the number of the elements, but only by increasing the surfaces of the plates in the battery.

Strength and Density. - We must carefully distinguish the strength (intensity) of the current from its density. As the same amount of electricity always flows through any given transverse section of the circuit, then, if the size of the transverse section of the circuit varies, the elec- 
tricity must be of greater density in the narrower parts, and it is evident that the density will be less where the transverse section is greater. Let $\mathbf{S}=$ the strength of the current, and $q$ the transverse section of the given part of the circuit, then the density $(d)$ at the latter part is $d=\mathrm{S}: q$.

If the galvanic current passing from the positive pole of a battery is divided into two or more streams, which are again reunited at the other pole, then the sum of the strength of all the streams is equal to the strength of the undivided stream. If, however, the different streams are different as regards length, section, and material, then the strength of the current passing in each of the streams is inversely proportional to the resistance to the conduction.

Du Bois-Reymond's Rheocord.-This instrument, constructed on the principle of the "secondary" or "short circuit," enables us to graduate the strength of a galvanic current to any required degree, for the stimulation of nerve and muscle. From the two poles (fig. $379, a, b$ ) of a constant battery, there are two conducting wires $(a, c$ and $d, b)$, which go to the nerve of a frog's nerve-muscle preparation $(\mathrm{F})$. The portion of nerve $(c, d)$ introduced into this circuit $(\alpha, c, d, b)$ offers very great resistance. The second stream or secondary circuit ( $a \mathrm{~A}, b \mathrm{~B}$ ) conducted from $a$ and $b$ passes through a thick brass plate (A, B), consisting of seven pieces of brass ( 1 to 7 ) placed end to end, but not in contact. They can all, with the exception of 1 and 2 , be made to form a continuous conductor by placing in the spaces between them the brass plugs $\left(\mathbf{S}_{1}\right.$ to $\left.\mathbf{S}_{5}\right)$. Evidently, with the arrangement shown in fig. 379 , only a minimal part of the current will pass through the nerve $(c, d)$, owing to the very great resistance in it, while by far the greatest part will pass through the good conducting medium of brass $(A, L, B)$. If new resistance be introduced into this circuit, then the $a, c, d, b$ stream will be strengthened." This resistance can be introduced into the latter circuit by means of the thin wires marked I $a$, I $b$, I $c$, II, V, X. Suppose all the brass plugs from $S_{1}$ to $S_{5}$ to be removed, then the current entering at A must traverse the whole system of thin wires. Thus, there is more resistance to the passage of this current, so that the current through the nerve must be strengthened. If only one brass plug be taken out, then the current passes through only the corresponding length of wire. The resistances offered by the different lengths of wire from I $a$ to $\mathrm{X}$ are so arranged that $\mathrm{I} \alpha, \mathrm{I} b$, and $\mathrm{I} c$ each represents a unit of resistance; II, double; V, five times; and $\mathrm{X}$, ten times the resistance. The length of wire, $\mathrm{I} a$, can also be shortened by the movable bridge (L) [composed of a small tube filled with mercury, through which the wires pass], the scale $(x, y)$ indicating the length of the resistance wires. It is evident that, by means of the bridge, and by the method of using the brass plugs, the apparatus can be graduated to yield very variable currents for stimulating

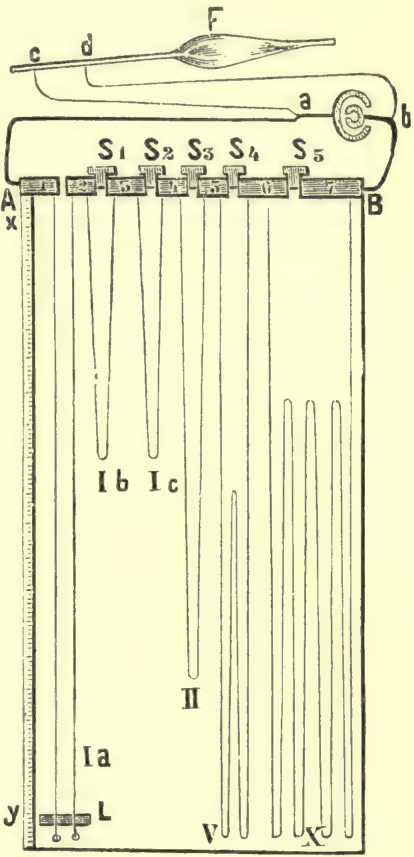

Fig. 379 .

Scheme of du Bois-Reymond's rheocord. nerve or muscle. When the bridge $(\mathrm{L})$ is pushed hard up to 1 and 2 , the current passes directly from $\mathrm{A}$ to $\mathrm{B}$, and not through the thin wires (I $a$ ).

The rheostat is another instrument used to vary the resistance of a galvanic current (Wheatstone).

327. ACTION OF THE GALVANIC CURRENT ON A MAGNETIC NEEDLE-GALVANOMETER. - In 1820, Oerstedt of Copenhagen found that a magnetic needle, suspended in the magnetic meridian, was deflected by a constant current of electricity passed along a wire parallel to it. [The side to which the north pole is deflected depends upon the direction of the current, and whether it passes above or below the needle.]

Ampère's Rule.-Ampère has given a simple rule for determining the direction. If an observer be placed parallel to and facing the needle, and if the current be passing from his feet to his head, then the north pole of the needle will always be deflected to the lcft, and the south pole in the opposite direction. The effect exerted by the constant current acts always in a lirection towards the so-called electro-magnetic plane. The latter is the plane passing through the north pole of the needle, and two points in the straight wire running parallel with the needle. The force of the constant current, which causes the deflection of the masnetic needle, is proportional to the sine of the angle between the electro-magnetic plane and the plane of vibration of the needle.

Multiplicator. - The deflection of the needle caused by the constant current may be increased 
by coiling the conducting wire many times in the same direction on a rectangular frame, or merely around and in the same direction as the needle, [provided that each turn of the wire be properly insulated from the other]. An instrument constructed on this principle is called a multiplicator or multiplier. The greater the number of turns of the wire, the greater is the angle of deflection of the needle, although the deflection is not directly proportional, as the several turns or coils are not at the same distance from, or in the same position as, the needle. By means of the multiplier we may detect the presence [and also the amount and direction] of fecble currents. [The instrument is now termed a galvanometer.] Experience has shown that, when great resistance, (as in animal tissues), is opposed to the weak galvanic currents, we must use a very large number of turns of thin wire round the needle. If, however, the resistance in the circuit is but small, e.g., in thermo-electrical arrangements, a few turns of a thick wire round the needle are sufficient. The multiplier may be made more sensitive by wrukening the mugnetic directive force of the ncedle, which keeps it pointing to the north.

Galvanometer and Astatic Needles. - In the multiplier of Schweigger, used for physiological furpuses, the tenden'y of the neelle to point to the north is greatly weakened by using the

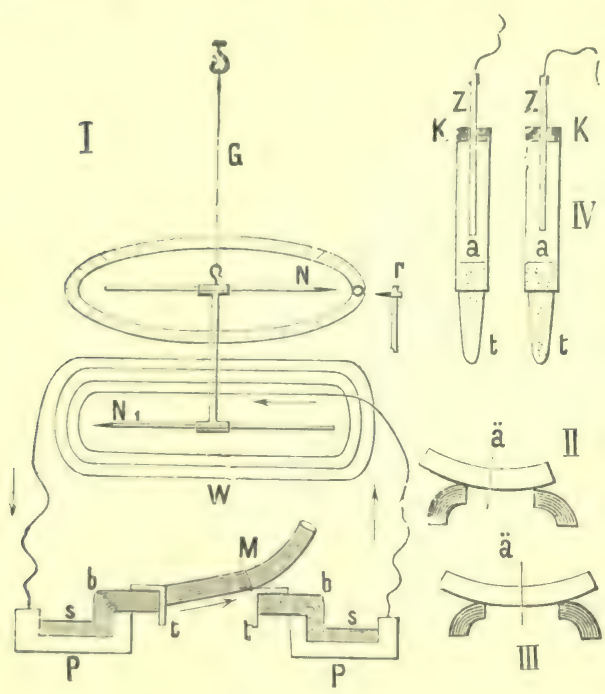

Fig. 380 .

Scheme of the galvanometer. N, N, astatic needles suspented by the silk fibre, G; P, P, non-polarisable clectrodes, containing zinc sulphate solution, $s$, and pauls of blotting paper, $b$, covered with clay, $t, t$, on which the muscle, M, is placed; II and III, arrangement of the muscle on the electrodes; IV, non-pularisable electrodes; ' $\mathrm{l}$, zine wire; $\mathrm{K}$, cork ; ", zinc sulphate sulution; $t, t$, clay points. astatic needles of Nobili. [A multiplier or galvanometer with a single magnetic needle always requires comparatively strong currents to deflect the needle. The needle is continually acted upon by the directive magnetic influence of the earth, which tends to keep it in the maguetic meridian, and, as soon as it is moved out of the magnetic meridian, the directive action of the earth tends to bring it back. Hence, such a simple form of galvanometer is not sufficiently sensitive for detecting feeble currents. In 1827, Nobili devised an astatic combination of needles, whereby the action of the earth's magnetism was diminished.] Two similar magnetic needles are united by a solid light piece of horn [or tortoise shell], and are so arranged that the north pole of the one is placed over or opposite to the south pole of the other (fig. 380). [If both needles are equally magnetised, then the earth's influence on the needle is neutralised, so that the needles no longer adjust themselves in the magnetic meridian; hence, such a system is called astatic.] As it is impossible to make both needles of absolutely equal magnetic strength, one needle is always stronger than the other. The difference, however, must not be so great that the stronger needle points to the north, but only that the freely suspended system of needles forms a certain angle with the magnetic meridian, into which position the system always swings after it is deflected from this position. This angular deviation of the astatic system towards the magnetic meridian is called the "free deviation." The more perfectly an astatic condition is reached, the nearer does the angle formerl hy the direction of the free deviation with the magnetic meridian become a right angle. The greater, therefore, the astatic condition, the fewer vibrations will the astatic system make in a given time, after it has been deflected from its position. The duration of each single vibration is also very great. [Hence, when using a galvanometer, and adjusting its needle to zero, if the magnets dance about or move quickly, then the system is not sensitive, but a sensitive condition of the needles is indicated by a slow period of oscillation.]

In making a galvanometer, the turns of the wire must have the same direction as the needles. In Nobili's galvanometer, as improved by du Bois-Reymond, the upper needle swings above a card divided into degrees (fig. 380), on which the extent of its deflection may be read off. Even the purest copper wire used for the coils round the needles always contains a trace of iron, which exerts an influence upon the needles. Hence, a small fixed directive or compensatory magnet $(r)$ is placed near one of the poles of the upper needle to compensate for the action of the iron on the needles. 
328. ELECTROLYSIS, POLARISATION, BATTERIES.-Electrolysis.-Every galvanic current which traverses a fluid conductor causes decomposition or electrolysis of the fluid. The decomposition-products, called "ions," accumulate at the poles (electrodes) in the fluid, the

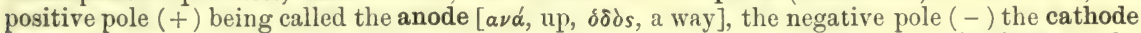
( $\alpha \tau \tau \dot{\alpha}$, down, $\delta \delta \delta s$, a way). The anions accumulate at the anode and the kations at the eathode.

Transition Resistance. - When the decomposition-products accumulate upon the electrodes, by their presence they either increase or diminish the resistance to the electrical current. This is called transition resistance. If the resistance within the battery is thereby increased, the transition resistance is said to be positive; if diminished, negative.

Galvanic Polarisation. - The ions accumulated on the electrodes may also vary the strength of the current, by developing between the anions and kations a new galvanic current, just as occurs between two different bodies connected by a fluid medium. This phenomenon is called galvanic polarisation. Thus, when water is decomposed, the electrodes being of platinum, the oxygen (negative) accumulates at the + pole, and the hydrogen (positive) at the - pole. Usually the polarisation current has a direction opposite to the original current; hence, we speak of negative polarisation. When the two currents have the same direction, positive polarisation obtains. Of course, transition resistance and polarisation may occur together during electrolysis.

Test.-Polarisation, when present, may be so slight as not to be visible to the eye, but it may be detected thus:-After a time exclude the primary source of the current, especially the element connected with the electrodes, and place the free projecting end of the electrodes in connection with a galvanometer, which will at once indicate, by the deflection of its needle, the presence of even the slightest polarisation.

Secondary Decompositions. - The ions excreted during electrolysis cause, especially at their moment of formation, secondary decompositions. With platinum electrodes in a solution of common salt, chlorine accumulates at the anode and sodium at the cathode, but the latter at once decomposes the water, and uses the oxygen of the water to oxidise itself, while the hydrogen is deposited secondarily upon the cathode. The amount of polarisation increases, although only to a slight extent, with the strength of the current, while it is nearly proportional to the increase of the temperature. The attempts to get rid of polarisation, which obviously must very soon alter the strength of the galvanic current, have led to the discovery of two important arrangements, viz., the construction of constant galvanic batteries, and non-polarisable electrodes $(d u$ Bois-Reymond).

Constant Batteries, Elements, or Cells. - A perfectly constant element produces a constant current, $i . e_{\text {, }}$ one remaining of equal strength, by the ions produced by the electrodes being got rid of the moment they are formed, so that they cannot give rise to polarisation.

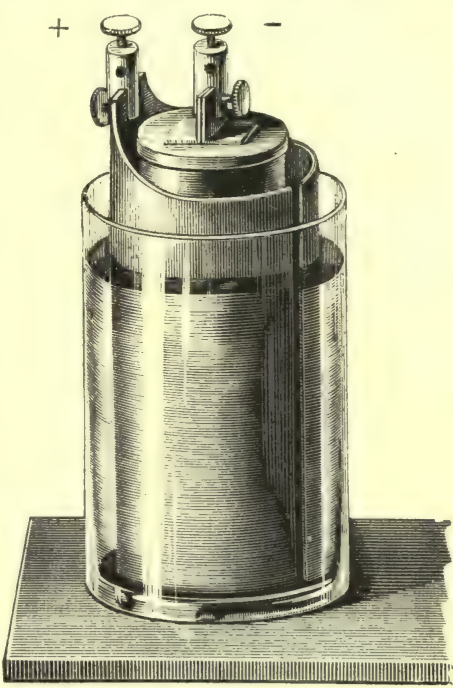

Fig. 381.

Large Grove's cell. For this purpose, each of the substances from the tension series used is placed in a special fluid ( $\$ 326)$, both fluids being separated by a porous septum (porcelain cylinder).

Grove's cell has two metals and two fluids (fig. 381). The zine is in the form of a roll placed in dilute sulphuric acid [ 1 acid to 7 of water, which is contained in a glass, porcelain, or ebonite vessel]. The platinum is in contact with strong nitric acid, [which is contained in a porous cell placed inside the roll of zinc]. The $O$, formed by the electrolysis and deposited on the zinc plate, forms zinc oxide, which is at once dissolved by the sulphuric acid. The hydrogen on the platinum unites at once with the nitric acid, which gives up $\mathrm{O}$ and forms nitrous acid and water, thus-

$$
\left[\mathrm{H}_{2}+\mathrm{HNO}_{3}=\mathrm{HNO}_{2}+\mathrm{H}_{2} \mathrm{O} .\right]
$$

[Platinum is the + pole, and zine the - .]

[Grove's battery is very powerful, but the nitrous fumes are very disagreeable and irritating; hence these elements should be kept in a special well-ventilated recess in the laboratory, in an evaporating chamber, or under glass. The fumies also attack instruments. ]

Bunsen's cell is similar to Grove's, only a piece of compressed carbon is substituted for the platinum in contact with the nitric acid.

[The carbon is the + pole, the zinc the - .]

[Daniell's cell consists of an outer vessel of glass or earthenware, and sometimes of metallic 
copper, filled with a saturated solution of cupric sulphate (fig. 382). A roll of copper, perforated with a few holes, is placed in the copper solution, and in order that the latter be kept saturated,

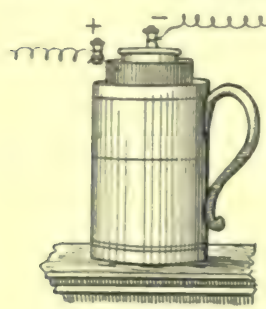

Fig. 382.

Daniell's cell. and to supply the place of the copper used up by the battery when in action, there is a small shelf on the copper roll, on which are placed crystals of cupric sulphate. A porous earthenware vessel containing zinc in contact with dilute sulphuric acid $(1: 7)$ is placed within the copper cylinder. When the circuit is completed, the zinc is acted on, zinc sulphate being formed, and hydrogen liberated. The hydrogen in statu nascendi passes through the porous cell, reduces the cupric sulphate to metallic copper, which is preeipitated on the copper cylinder, so that the latter is always kept bright and clean. The liberated sulphuric acid replaces that in contact with the zinc. Owing to the absence of polarisation, the Daniell is one of the most constant batteries, and is generally taken as the standard of comparison.]

[The copper is the + pole, zinc the - .]

[Smee's cell contains only one fluid, viz., dilute sulphuric acid $(1: 7)$, in which the two metals, zine and platinum, or zinc and platinised silver, are placed.

The platinum is the + pole, and zinc the - .]

[Grennet's or the Bichromate cell consists of one plate of zinc and two plates of compressed carbon in a fluid, containing bichromate of potash, sulphuric acid, and water. The fluid consists of 1 part of potassium bichromate dissolved in 8 parts of water, to which one part of sulphuric acid is added. Measure by weight. The cell consists of a wide-mouthed glass bottle (fig.

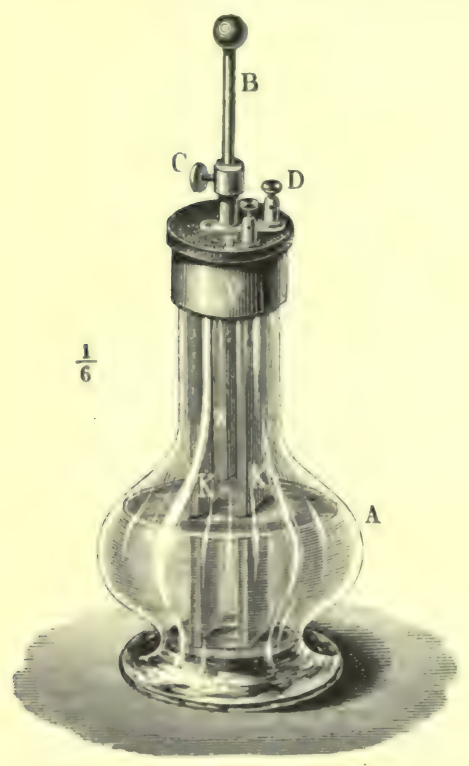

Fig. 383.

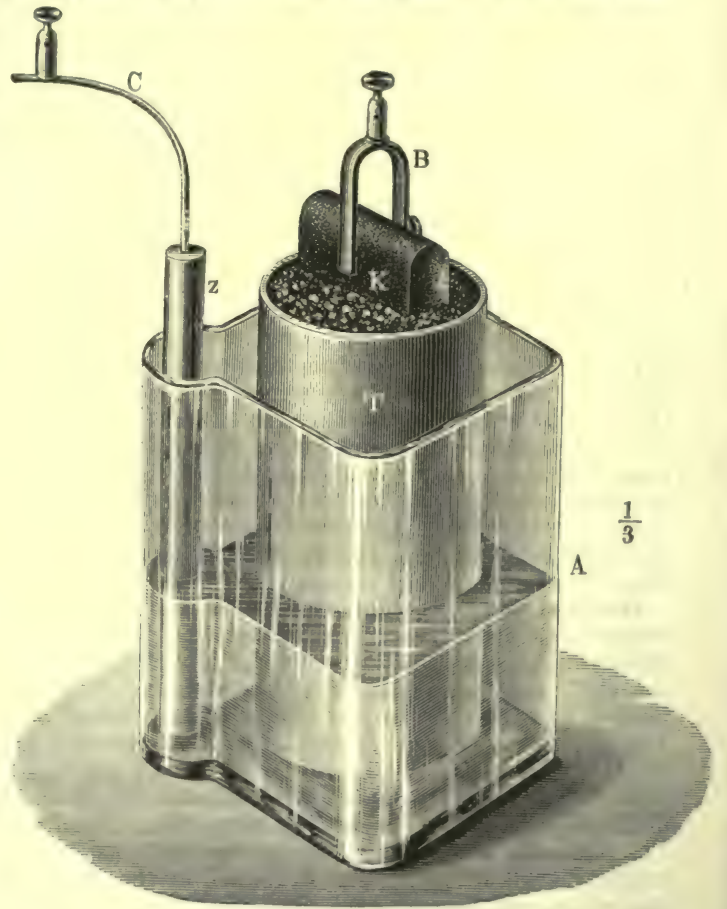

Fig. 384 .

Fig. 383.-Grennet's cell. A, glass vessel; $\mathbf{K}, \mathrm{K}$, carbon; $\mathrm{Z}$, zinc; $\mathrm{D}, \mathrm{E}$, binding screws for the wires; $B$, rod to raise the zinc from the fluid; C, screw to fix $B$. Fig. 384.Leclanché's cell. A, outer vessel; T, porous cylinder, containing $\mathrm{K}$, carbon; $\mathrm{B}$, binding screw ; $\mathrm{Z}$, zine; C, binding screw of negative pole.

383); the carbons remain in the fluid, while the ziuc can be raised or depressed. When not in action, the zinc, which is attached to a rod $(\mathrm{B})$, is lifted out of the fluid. It is not a very constant battery. When in action, the zinc is acted on by the sulphuric acid, hydrogen being liberated, which reduces the bichromate of potash.

The carbon is the + pole, and the zinc the - .]

[Leclanche's cell (fig. 384) consists of an outer glass vessel containing zinc in a solution of 
ammonium chloride, while the porous cell contains compressed carbon in a fluid mixture of black oxide of manganese and carbon. It is most frequently used for electric bells, as its feeble current lasts for a long time.

The carbon is the + pole, and the zinc the - .]

Non-polarisable Electrodes. - If a constant current be applied to moist animal tissues, c.g.,

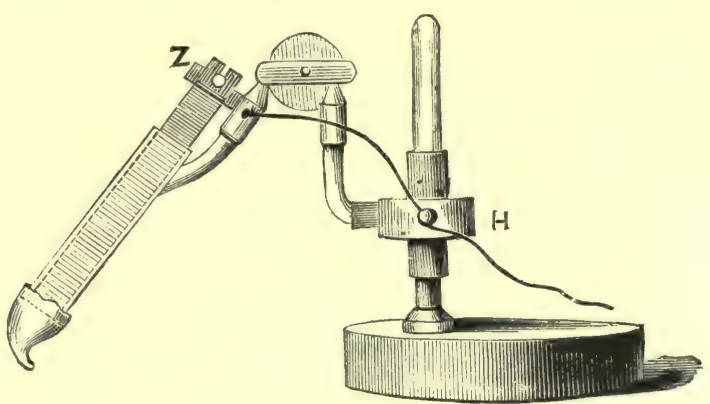

Fig. 385

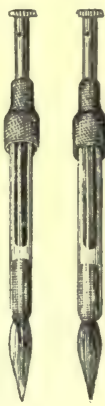

Fig. 386 .

Fig. 385. - Non-polarisable electrode of du Bois-Reymond, Z, zine; H, movable support ; C, clay point-the whole on a universal joint. Fig. 386.-Brush electrodes of v. Fleischl.

nerve or muscle, by means of ordinary electrodes composed either of copper or platinum, of course electrolysis must occur, and in consequence thereof polarisation takes place. In order to avoid this, non-polarisable electrodes (figs. 380,385 ) are used. Such electrodes are made by taking two pieces of carefully amalgamated, pure, zinc wire $(z, z)$, and dipping these in a saturated solution of zinc sulphate contained in tubes $(a, a)$, whose lower ends are closed by means of modeller's clay $(t, t)$, moistened with 0.6 per cent. normal saline solution. The contact of the tissues with these electrodes does not give rise to polarity. [The brush electrodes of v. Fleischl are very serviceable (fig. 386). The lower end of the glass tube is plugged with a camel-hair pencil, moistened with modeller's clay, otherwise the arrangement is the same as shown in fig. 380, IV.]

Arrangement for the Muscle- or Nerve-Current. - In order to investigate the electrical currents of nerve or muscle, the tissue must be placed on non-polarisable electrodes, which may either be one of the forms described above, or the original form used by du Bois-Reymond (fig. 380). The last consists of two zinc troughs $(p, p)$ thoroughly amalgamated inside, insulated on vulcanite, and filled with a saturated solution of zinc sulphate $(s, s)$. In each trough is placed a thick pad or cushion of white blotting paper $(b, b)$ saturated with the same fluid [deriving cushions]. [The cushion consists of many layers, almost sufficient to fill the trough, and they are kept together by a thread. To prevent the action of the zinc sulphate upon the tissue, each cushion is covered with a thin layer of modeller's clay $(t, t)$, moistened with $0^{\circ} 6$ per cent. saline solution, which is a good conductor [clay guard]. The clay guard prevents the action of the solution upon the tissue. Connected with the electrodes are a pair of binding screws, whereby the apparatus is conuected with the galvanometer (fig. 380).

[Reflecting Galvanometer.-The form of galvanometer, used in this country for physiological purposes, is that of Sir William Thomson (fig. 387). In Gerınany, Wiedemann's form is more commonly used. In Thomson's instrument, the astatic needles are very light, and connected to each other by a piece of aluminium, and each set of needles is surrounded by a separate coil of wire, the lower coil $(l)$ winding in a direction opposite to that of the upper $(u)$. A small, round,

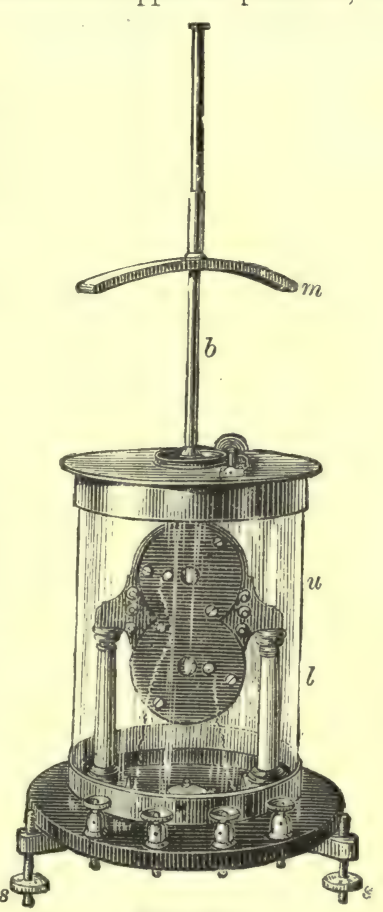

Fig. 387.

Thomson's reflecting galvanoineter. $u$, upper, $l$, lower coil ; $s, s$, levelling screws; $m$, magnet, on a brass support, $b$. light, slightly concave mirror is fixed to the upper set of needles. The needles are suspended by a delicate silk fibril, and they san be raised or lowered as required by means of a 
small milled head. When the milled head is raised, the system of needles swings freely. The coils are protected by a glass shade, and the whole stands on a vulcanite base, which is levelled by three serews $(s, s)$. On a brass rod $(b)$ is a feeble magnet $(m)$, which is used to give an artificial meridian. The magnet $(m)$ can be raised or lowered by means of a milled head.]

[Lamp and Scale. - When the instrument is to be used, place it so that the coils face east and west. At 3 feet distant from the front of the galvanometer, facing west, is placed the lamp and sial. (fic. 35. There is a small vertical slit in front of the lamp, and the image of this slit is

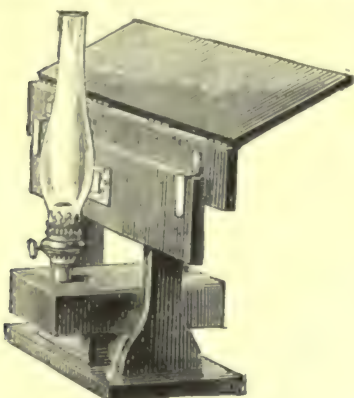

Fir. 388. projected on the mirror attached to the upper needles, and by it is reflected on to the paper scale fixed just above the slit. 'The spot of light is focussed at zero by means of the magnet, $m$. The needles are most sensitive when the oscillations occur slowly. The sensitiveness of the needles can be regulated by means of the magnet. In every case the instrument must be quite level, and for this purpose there is a small spirit-level in the base of the galvanometer.]

[Shunt. - As the galvanometer is very delicate, it is convenient to have a shunt to regulate to a certain extent the amount of electricity transmitted through the galvanometer. The shunt (fig. 389) consists of a brass box containing coils of German silver wire, and is constructed on the same principle as resistance coils or the rheocord (\$326). On the upper surface of the box are several plates of brass separated from each other, like those of the rheocord, hut which ean be united by brass plugs. The two wires coming from the electrodes are connected lamp and scale for Thomson's with the two binding screws, and from the latter two wires are galvanometer. led to the outer two binding screws of the galvanometer. By Jlacing a plug between the brass plates attached to the two binding screws in the figure, the current is short-circuitenl. On removing both plugs, the whole of the current must pass through the galvanometer. If one plug be placed between the central disc of brass and the plate marked $\frac{1}{1}$, (the other being left out), then ${ }_{1}^{1}$ th of the current goes through the galvano-

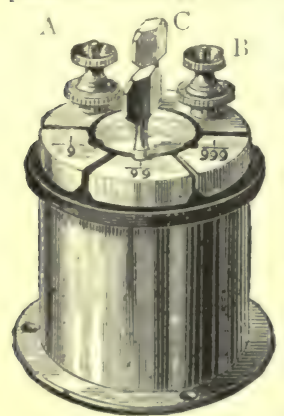

Fig. 389.

Shunt for galvanometer. meter and $\frac{0}{10}$ ths to the electrodes. If the plug be placed as shown in the figure opposite $\frac{1}{90}$, then $\frac{1}{100}$ th part of the current goes to the galvanometer, while $\frac{99}{100}$ ths are short-circuited. If the plug be placed opposite , 1, only $\pi_{1 \pi}^{1}$ th part goes through the galvanometer.]

Internal Polarisation of Moist Bodies.-Nerves and muscular fibres, the juicy parts of vegetables and animals, fibrin, and other similar bodies jossessing a porous structure filled with fluid, exhibit the phenomena of polarisation when subjected to strong currents-a condition termed internal polarisation of moist bodies by du Bois-Reymond. It is assumed that the solid parts in the interior of these bodies which are better conductors, produce electrolysis of the adjoining fluid, just like metals in contact with fluid. The ions produced by the decomposition of the internal fluids give rise to differences of potential, and thus cause intermal polarisation ( $\$ 333$ ).

Cataphoric Action. - If the two electrodes from a galvanic battery be placed in the two compartments of a fluid, separated from each other by a porous septum, we observe that the fluid particles pass in the direction of the galvanic current, from the + to the - pole, so that after some time, the fluid in the one half of the vessel increases, while it diminishes in the other. The phenomena of direct transference were called by du Bois-Reymond the cataphoric action of the constant current. The introduction of dissolved substances through the skin by means of a constant current depends upon this action $(\S 290)$, and so does the so-called Porret's phenomenon in living muscle (\$ 293, I., b).

External Secondary Resistance. - This condition also depends on cataphoric action. If the copper electrodes of a constant battery be placed in a vessel filled with a solution of cupric sulphate, and from each electrode there project a cushion saturated with this fluid, then, on placing a piece of muscle, cartilage, vegetable tissue, or even a prismatic strip of coagulated albumin across these cushions, we observe that, very soon after the circuit is closed, there is a considerable variation of the current. If the direction of the current be reversed, it first becomes stronger, but afterwards diminishes. By constantly altering the direction of the current we cause the same changes in the intensity. If a prismatic strip of coagulated albumin be used for the experiment, we observe that, simultaneously with the enfeeblement of the current in the neighbourhood of the + pole, the albumin loses water and becomes more shrivelled, while at the - pole the albumin is swollen up and contains more water. If the direction of the current be altered, the phenomena are also changed. The shrivelling and removal of water in the albumin at the positive pole must be the cause of the resistance in the 
circuit, which explains the enfeeblement of the galvanic current. This phenomenon is called "external secondary resistance" (du Bois-Reymond).

329. INDUCTION-EXTRA-CURRENT-MAGNETIC-INDUCTION.-Induction of the Extra-Current. - If a galvanic element is closed by means of a short are of wire, at the moment the circuit is again opened or broken, a slight spark is observed. If, however, the circuit is made or closed by means of a very long wire rolled in a coil, then on breaking the circuit there is a strong spark. If the wires be connected with two electrodes, so that a person can hold one in each hand, the current at the moment it is opened must pass through the person's body, then there is a violent shock communicated to the hand. This phenomenon is due to a current induced in the long spiral of wire which Faraday called the extra-current. It is caused thus : -When the circuit is closed by means of the spiral wire, the galvanic current passing along it excites an electric current in the adjoining coils of the same spiral. At the moment of closing or making the circuit in the spiral, the induced current is in the opposite direction to the galvanic current in the circuit; hence its strength is lessened, and it causes no shock. At the moment of opening, however, the induced current has the same direction as the galvanic stream, and hence its action is strengthened.

Magnetisation of Iron. - If a rod of soft iron be placed in the cavity of a spiral of copper wire, then the soft iron remains magnetic as long as a galvanic current circulates in the spiral. If one end of the iron rod be directed towards the observer, and the other away from him, and if, further, the positive current traverse the spiral in the same direction as the hands of a clock, then the end of the magnet directed towards the person is the negative pole of the magnet. The power of the magnet depends upon the number of spiral windings, and on the thickness of the iron bar. As soon as the current is opened, the magnetism of the iron rod disappears.

Induced or Faradic Current. - If a very long, insulated, wire be coiled into the form of a spiral roll, which we may call the secondary spiral, and if a similar spiral, the primary spiral, be placed near the former, and the ends of the wire of the primary spiral be connected with the poles of a constant battery, every time the current in the primary circuit is made (closed), or broken (opened), a current takes place, or, as it is said, is induced in the secondary spiral. If the primary circuit be kept closed, and if the secondary spiral be brought nearer to, or removed further from, the primary spiral, a current is also induced in the secondary spiral (Furculay, 1832). The current in the secondary circuit is called the induced or Faradic current. When the primary circuit is closed, or when the two spirals are brought nearer to each other, the current in the secondary spiral has a direction opposite to that in the primary spiral, while the current produced by opening the primary circuit, or by removing the spirals further apart, has the same direction as the primary. During the time the primary cireuit is closed, or when both spirals remain at the same distance from each other, there is no current in the secondary spiral.

Difference between the Opening [break] and Closing [make] Shocks. - The opening and closing shocks in the secondary spiral are distinguished from each other in the following respects (fig. $390)$ :- The amount of electricity is the same, during the opening, as during the closing shock, but during the opening shock, the electricity rapidly reaches its maximum of intensity and lasts but a short time, while during the closing shock, it gradually increases, but does not reach the high maximum, and this occurs more slowly. [In fig. $390, \mathbf{P}_{1}$ and $\mathbf{S}_{0}$ are the abscissæ of the primary (inducing) and induced currents respectively. The vertical lines or ordinates represent the intensity of the current, while the length of the abscisse indicates its duration. Curve 1 indicates the course of the primary current, and 2, that in the secondary spiral (induced) when the current is closed, while at I the primary current is suddenly opened, when it gives rise to the induced current, 4 , in the secondary spiral.] The cause of the difference is the following:- When the primary circuit is closed, there is developed in it the extra-current, which is opposite in direction to the primary current. Hence, it opposes considerable resistance to the complete development of the strength of the primary current, so that the current induced in the secondary spiral must also develop slowly. But when the primary spiral is opened, the extra-current in the latter has the same direction as the primary current-there is no extra resistance. The rapid and intense action of the opening induction shock is of great physiological importance.

Break or Opening Shock.--[On applying a single induction shock to a nerve or a muscle, the effect is greater with the break or opening shock. If the secondary spiral be separated from the primary, so that the induced currents are not sufficient to cause contraction of a muscle when applied to its motor nerve, then, on gradually approximating the secondary to the primary spiral, the break or opening shock will cause a contraction before the closing one does so.]

Helmholtz's Modification. - Under certain circumstances, it is desirable to equalise the make and break shocks. This may be done by greatly weakening the extra-current, which may be accomplished by making the primary spiral of only a few coils of wire. V. Helmholtr. accomplishes the same result by introducing a secondary circuit into the primary current. By this arrangement the current in the primary spiral never completely disappears, but by 
alternately making and breaking this secondary circuit where the resistance is much less, it is alternately weakened and strengthened.

[In fig. 391 a wire is introduced between $a$ and $f$, while the binding screw, $f$, is separated from the platinum contact, $c$, of Neef's hammer, but, at the same time the screw, $d$, is raised so that it touches Neef's hammer. The current passes from the battery, $K$, through the pillar, $a$,

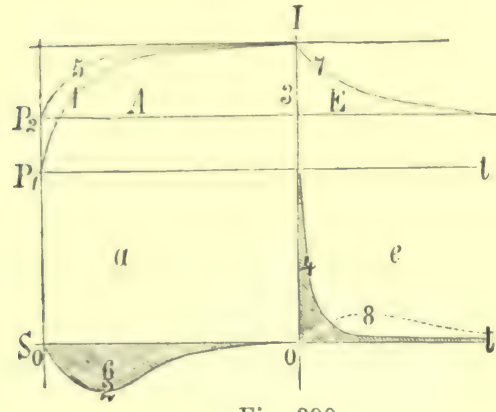

Fig. 390 .

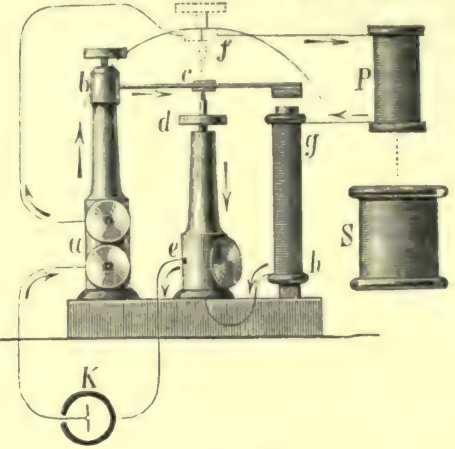

Fig. 391.

Fig. 390. - Scheme of the induced eurrents. $\mathbf{P}_{1}$, abscissa of the primary, and $\mathbf{S}_{0}$, of the secondary current; $\Lambda$, beginning, and $E$, end of the inducing current; 1 , curve of the primary current weakened by the extra-current; 3 , where the primary current is opened; 2 and 4 . corresponding currents induced in the secondary spiral; $\mathrm{P}_{2}$, height, i.e., the strength of the constant inducing current; 5 and 7 , the curve of the inducing current when it is opened and closerl during Helmholtz's modification; 6 and 8, the corresponding currents indureel in the secondary cireuit. Fig. 391.-Helmholtz's modification of Neef's hammer. As lonif as $c$ is not in contact with $d, g h$ remains magnetic ; thus $c$ is attracted to $d$ and a secontary circuit, $a, b, c, d, r$ is formed; $c$ then springs back again, and thus the process goes on. A new wire is introduced to connect $\alpha$ with $f$. $K$, battery.

to $f$ in the direction of the arrow, through the primary spiral, $\mathrm{P}$, to the coil of soft wire, $g$, and back to the battery, through $h$ and $e$. Put $g$ is magnetised thereby, and when it is so, it attracts $c$ and makes it touch the screw $\%$. Thus a secondary circuit, or short circuit, is formed through $a, b, c, d$, $r$, which weakens the current passing through the electro-magnet, $g$, so that the elastic metallic spring flies up again and the current through the primary spiral is long-cireuited, and thus the process is repeated. In fig. 390 the lines 1 and 7 indicate the course of the current in the primary circuit at closing $(a)$, and opening $(e)$. It must be remembered that in this arrangement there is always a current passing through the primary spiral, $\mathrm{P}$ (fig. 391). The dotted lines, 6 and 8 , above and below $\mathrm{S}_{0}$, represent the course of the opening $(a)$ and closing shocks $(c)$ in the secondary spiral. Even with this arrangement the opening is still slightly stronger than the closing shock.] The two shocks, however, may be completely equalised by placing a resistance coil or rheostat in the short circuit, which increases the resistance, and thus increases the current through the primary spiral when the short circuit is closed.

Unipolar Induction. - When there is a very rapid current in the primary spiral, not only is there a current induced in the secondary spiral, when its free ends are closed, e.g., by being connected with an animal tissue, but there is also a current when one wire is attached to a binding screw connected with one end of the wire of the secondary spiral (p. 537). A muscle of a frog's leg, when connected with this wire, contracts, and this is called a unipolar induced contraction. It usually occurs when the primary circuit is opened. The occurrence of these contractions is favoured, when the other end of the spiral is placed in connection with the ground, and when the frog's muscle prepraration is not completely insulated.

Magneto-Induction. - If a magnet be brought near to, or thrust into the interior of, a coil of wire, it excites a current, and also when a piece of soft iron is suddenly rendered magnetic or suldenly demagnetised. The direction of the current so induced in the spiral is exactly the same as that with Faradic electricity, i.e., the occurrence of the magnetism, on approximating the spiral to a inagnet, excites an induced current in a direction opposite to that supposed to circulate in the magnet. Conversely, the demagnetisation, or the removal of the spiral from the magnet, causes a current in the same direction.

Acoustic Tetanus. - If a magnet be rapidly moved to and fro near a spiral, which can easily be done by fixing a vibrating magnetic rod at one end and allowing the other end to swing freely near the spiral, then the pitch of the note of the vibrating rod gives us the rapidity of the induction shoeks. If a frog's nerve-muscle preparation be stimulated, we get what Grossmann called "acoustic tetanus." 
330. DU BOIS-REYMOND'S INDUCTORIUM-MAGNETO-INDUCTION APPARATUS. The inductorium of du Bois-Reymond, which is used for physiological purposes, is a modification of the magneto-electromotor apparatus of Wagner and Neef. A scheme of the apparatus is given in fig. 392. D represents the galvanic battery. The wire from the positive pole, $a$, passes to a metallic column, $\mathrm{S}$, which has a horizontal vibrating spring, $\mathrm{F}$, attached to its upper end. To the outer end of the spring a square piece of iron, $e$, is attached. The middle point of the upper surface of the spring [covered with a little piece of platinum] is in contact with a movable screw, $b$. A moderately thick copper wire, $c$, passes from the screw, $b$, to the primary spiral or coil, $x, x$, which contains in its interior a number of pieces of soft iron wire, $i, i$, covered with an insulating varnish. The copper wire which surrounds the primary spiral is covered with silk. The wire, $d$, is continued from the primary spiral to a horse-shoe piece of soft iron, $\mathbf{H}$, around which it is coiled spirally, and from thence it proceeds, at $f$, back to the negative pole of the battery, $g$. When the current in this circuit-called the primary circuit-is closed, the following effects are produced:-The horse-shoe, H, becomes magnetic, in consequence of which it attracts the movable spring or Neef's hammer, $e$, whereby the contact of the spring, $\mathbf{F}$, with the screw, $b$, is broken. Thus the current is broken, the horse-shoe is demagnetised, the spring, $e$, is liberated, and, being elastic, it springs upwarcis again to its original position in contact with $b$, and thus the current is re-established. The new contact causes $H$ to be remagnetised, so that it must alternately rapidly attract and liberate the spring, $e$, whereby the primary current is rapidly made and broken between $\mathrm{F}$ and $b$.

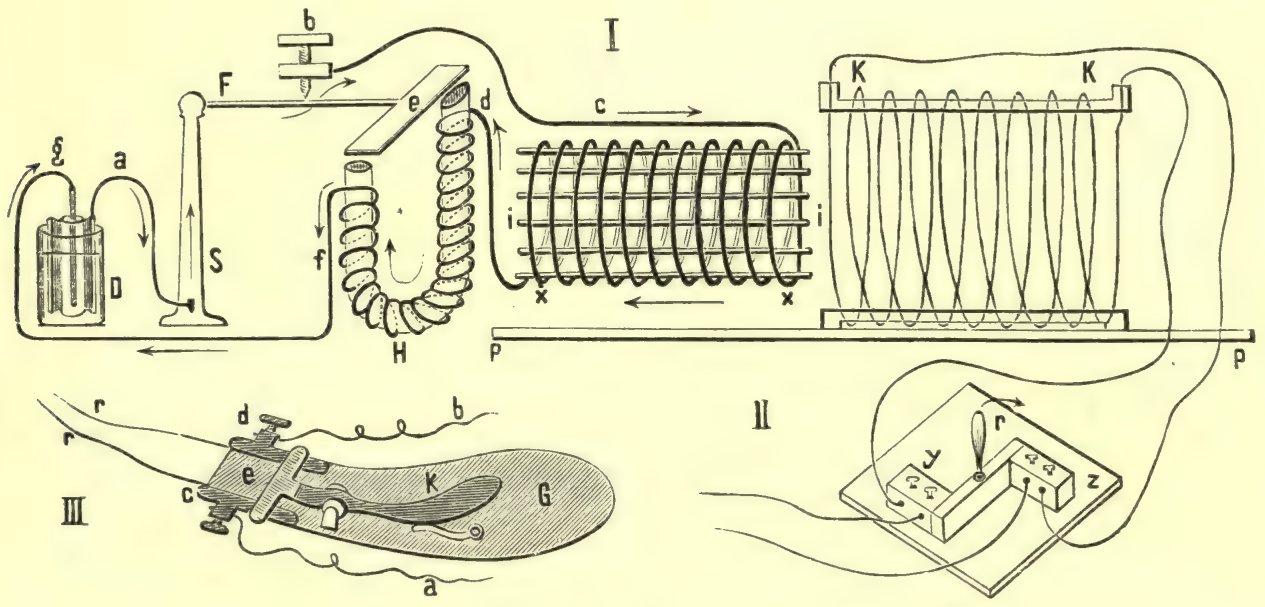

Fig. 392

I, Scheme of du Bois-Reymond's sledge induction machine. D, galvanic battery; $\alpha$, wire from + pole, $(g)$ - pole ; $\mathrm{S}$, brass upright; $\mathrm{F}$, elastic spring; $b$, binding screw ; $c$, wire round primary spiral $(x, x)$, containing $(i, i)$ soft iron wire; $\mathbf{K}, \mathbf{K}$, secondary spiral, with board $(p, p)$ on which it can be moved; $\mathbf{H}$, soft iron magnetised by current $(d, f)$ passing round it. II, key for secondary circuit, as shown it is short-circuited. III, electrodes $(r, r)$, with a key $(\mathrm{K})$ for breaking the circuit.

A secondary spiral or coil $(K, K)$ is placed in the same direction as the primary $(x, x)$, but having no connection with it. It moves in grooves upon a long piece of wood $(p, p)$. The secondary spiral consists of a hollow cylinder of wood covered with numerous coils of thin silkcovered wire. The secondary spiral, moving in slots, can be approximated to or even pushed entirely over the primary spiral, or can be removed from it to any distance desired.

[Fig. 393 shows the actual arrangement of du Bois-Reymond's inductorium. The primary coil $\left(\mathrm{R}^{\prime}\right)$ consists of about 150 coils of thick insulated copper wire, the wire being thick to offer slight resistance to the galvanic current. The secondary coil ( $\left.R^{\prime \prime}\right)$ consists of 6000 turns of thin insulated copper wire arranged on a wooden bobbin ; the whole spiral can be moved along the board (B) to which a millimetre scale (I) is attached, so that the distance of the secondary from the primary spiral may be ascertained. At the left end of the apparatus is Wagner's hammer, as adapted by Neef, which is just an automatic arrangement for opening and breaking the primary circuit. When Neef's hammer is used, the wires from the battery are connected as in the figure; but when single shocks are required, the wires from the battery are connected with a key, and this again with the two terminals of the primary spiral, $\mathbf{S}^{\prime \prime}$ and $\mathbf{S}^{\prime \prime \prime}$. In the 
improved form of this apparatus (fig. 394) the secondary spiral is equiposed over a pulley with a back weight, so that it can move easily in a vertical direction to and from the primary spiral. A. de Watteville has used a form similar to this for a long time.]

According to the law of induction (\$329), when the primary circuit is closed, a current is induced in the secondary circuit in a direction the reverse of that in the primary, while, when it is opened; the induced current has the same direction. Further, according to the laws of magneto-induction, the magnetisation of the iron rods $(i, i)$ within the primary spiral $(x, x)$, canses a werse current in the secondary spiral $(K, K)$, while the demagnetisation of the iron rods, on opening the primary circuit, causes an induced current in the same direction. Thus, we explain the much more powerful action of the opening or break shock as compared with the closing or make shock (p. 481). [The direction of the inducing current remains the same, while the induced currents are constantly reversed. ]

The magneto-induction $(\mathrm{R})$ apparatus of Pixii, as improved by Stohrer, consists of a very powerful horse-shoe steel magnet (fig. 395). Opposite its two poles ( $\mathbf{N}$ and $\mathbf{S}$ ) is a horse-shoeshaped piece of iron $(\mathbf{H})$, which rotates on a horizontal axis $(a, b)$. On the ends of the horse-shoe are fixed wooden bobbins $(c, d)$, with an insulated wire coiled round them. When the horseshoe is at rest, as in the figure, it becomes magnetised by the

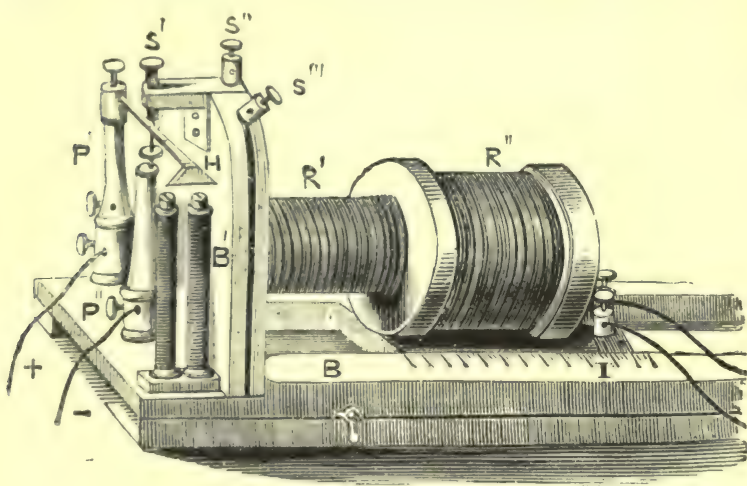

Fig. 393.

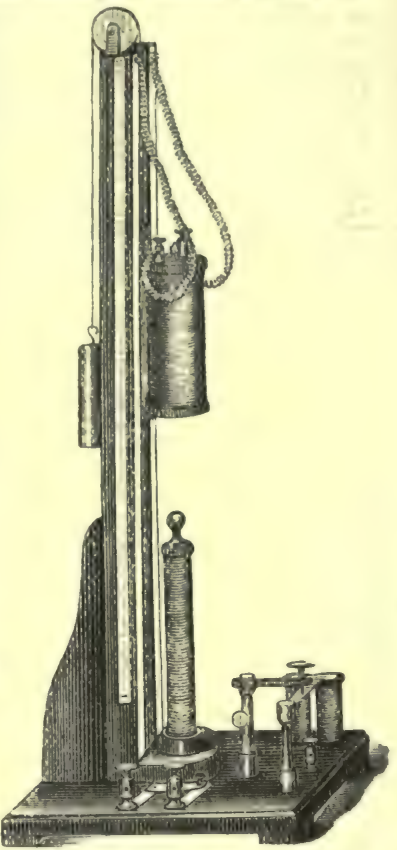

Fig. 394.

Fig. 393. - Induction apparatus of du Bois-Reymond. R', primary, $\mathrm{R}^{\prime \prime}$, secondary spiral ; $\mathrm{B}$, hoarl on which $\mathrm{R}^{\prime \prime}$ moves; $\mathrm{I}$, scale; + - wires from battery; $\mathrm{P}^{\prime}, \mathrm{P}^{\prime \prime}$, pillars; $\mathrm{H}$, Neef's hammer; $\mathrm{B}^{\prime}$, electro-magnet; $\mathrm{S}^{\prime}$, binding screw touching the steel spring $(\mathrm{H}) ; \mathrm{S}^{\prime \prime}$ and $\mathrm{s}^{\prime \prime \prime}$, binding serews to which to attach wires where Neef's hammer is not required. Fig. 394. - New form of du Bois-Reymond's inductorium.

steel magnet, while in the wires of both bobbins ( $c$ and $d)$ an electric current is developed every time the horse-shoe is demagnetised, and again magnetised. When the bobbins rotate in front of the magnet, as each coil approaches one pole, a current is induced, and similarly when it is carried past the pole of the magnet, so that four currents are induced in each coil by a single rotation. By means of Stiihrer's commutator $(m, n)$ attached to the spindle $(a, b)$, and the dividerl metal plates $(y, z)$ which pass to the electrodes, the two currents induced in the bobbins are obtained in the same direction.

Keys, or arrangements for opening or closing a circuit, are of great use. Fig. 392, II, shows a scheme of the friction key of du Bois-Reymond, introduced into the secondary circuit. It consists of two brass bars ( $z$ and $y$ ) fixed to a plate of ebonite, and as long as the key is down on the metal bridge $(y, r, z)$ it is "short-circuited," i.e., the conduction is so good through the thick brass bars that none of the current goes through the wires learling from the left of the key. When the bridge $(r)$ is lifted the current is opened. [Fig. 396 shows the form of the key, $v$ being a screw wherewith to clamp it to the table.] Similarly the key electrodes (III) may be used, the current heing made as soon as the spring connecting-plate $(e)$ is raised by pressing upon $k$. This instrument is opened by the hand; $a, b$ are the wires from the battery or induction machine;; $r, r$, those going to the tissue; $G$, the handle of the instrument.

[Plug Key. - Other forms of keys are in use, c.g., fig. 397, the plug key, the two brass plates to which the wires are attached heing fixed on a plate of ebonite. The brass plug is used to connect the two lurass plates. All these are dry contacts, but sometimes a fluid contact is used 
as in the mercury key, which merely consists of a block of wood with a cup of mercury in its centre. The ends of the wires from the battery dip into the mercury; when both wires dip into the mercury the circuit is made, and when one is out it is broken.]

[Capillary Contact Key.-Where an ordinary mercury key is used to open and close the

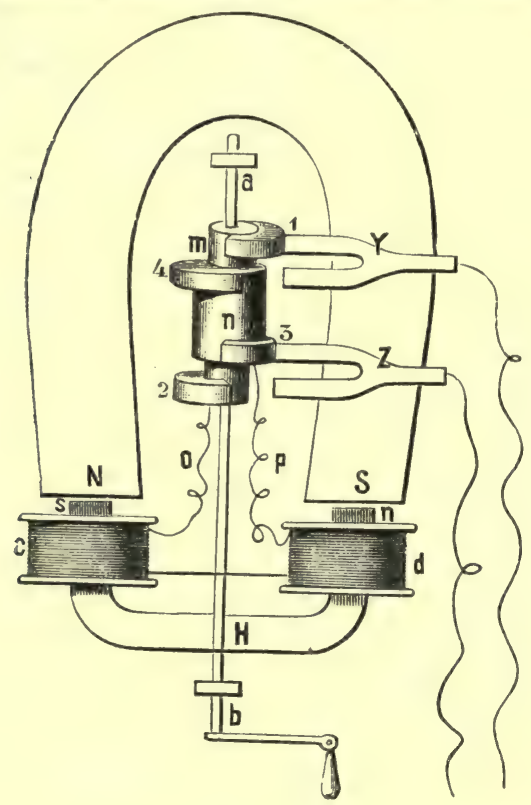

Fig. 395 .

Magneto-induction apparatus, with Stöhrer's commutator.

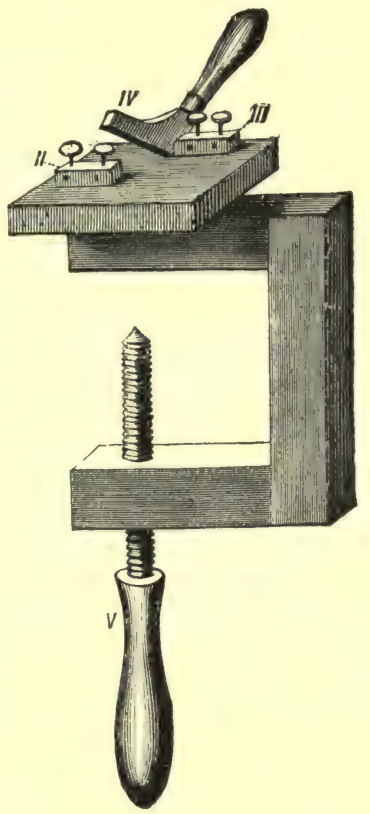

Fig. 396.

Du Bois-Reymond's friction key.

primary circuit, the layer of oxide formed on the surface by the opening spark disturbs the conduction after a short time; hence, it is advisable to wash the surface of the mercury with a dilute solution of alcohol and water ( $W$. Stirling). A handy form of "capillary contact" is shown in fig. 398 , such as was used by Kronecker and Stirling in their experiments on the heart. "A glass $\mathrm{T}$-tube is provided at the crossing point with a small opening $(a)$. The vertical tube $(b)$

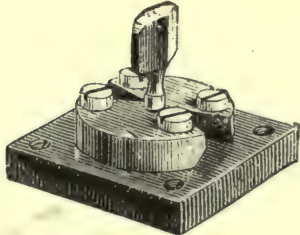

Fig. 397.

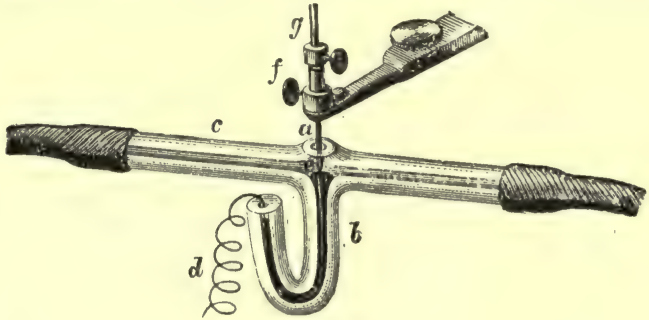

Fig. 398.

Fig. 397.-Plug key. Fig. 398. - Capillary contact. $e$, vibrating platinum style adjustable by $f$ and $g$ and dipping into mercury at $a ; b$, bent tube filled with mercury, into which dips a wire $(\dot{d}) ; \alpha$, opening in cross tube $(c)$.

is bent in the form of a $\mathrm{U}$, and filled so full with mercury that the convex surface of the latter projects within the lumen of the transverse tube $(c)$. One end of $c$ is connected with a Mariotte's flask containing diluted alcohol, and the supply of the latter can be regulated by means of a stop-cock. The fluid flows over the apex of the mercury and keeps it clean. The vibrating platinum style $(e)$ is attached to the end of a rod, which in turn is connected with the positive 
pole of the battery, while the platinum wire $(d)$ is connected with the negative pole of the "battery."]

331. ELECTRICAL CURRENTS in PASSIVE MUSCLE and NERVE - SKIN CURRENTS. - Methods. - In orter to investigate the laws of the muscle-current, we must use a muscle composed of parallel fibres, and with a simple arrangement of its fibres in the form of a prism or eylinder (fig. 399, I and II). The sartorius muscle of the frog supplies these conditions. In such a muscle, we distinguish the surface or the natural longitudinal section, its tendinous eads or the natural transverse section ; further, when the latter is clivided transversely to the long axis, the artificial transverse section (fig. $399, \mathrm{I}, c, d)$; lastly, the term equator $(a, b-m, n)$ is applied to a line so drawn as exactly to divide the length of the muscle into halves. As the currents are very feeble, it is necessary to use a calvanometer with a periodic damped magnet (figs. 380, I, and 387), or a tangent mirror-boussole similar to that used for thermo-electric purposes (fig. 230). The wires leading from the tissue are connected with non-polarisable electroiles (fig. $380, \mathrm{P}, \mathrm{P}$ ).

The capillary-electrometer of Lippmann may be usel for detecting the current (fig. 400). A thread of mercury enclosed in a capillary tube and touching a conducting fluid, e.g., dilute
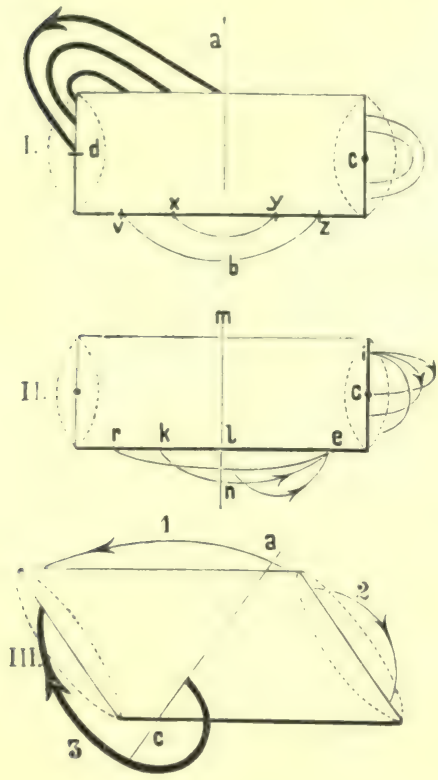

Fig. 399.

Scheme of the muscle-current.

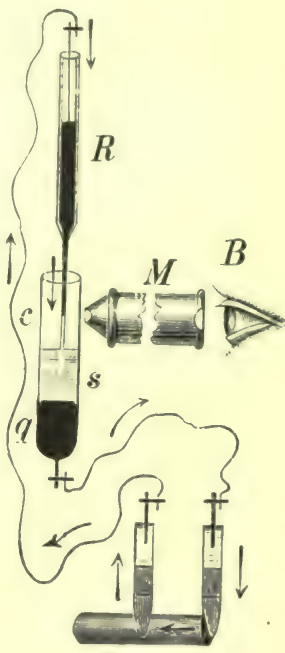

Fig. 400 . sulphuric acid, is displaced by the constant current, in consequence of the polarisation taking place at the point of contact altering the constancy of the capillarity of the mercury. The displacement of the mercury which the observer (B) detects by the aid of the microscope (M) is in the direction of the positive current. $\mathrm{R}$ is a capillary glass tube, filled from above with mercury, and from below with dilute sulphuric acid. Its lower narrow end opens into a wide glass tube, provided below with a platinum wire fused into it and filled with $\mathrm{Hg}(q)$, and this again is covered with dilute sulphuric acid $(s)$. The wires are connected with noy-polarisable electrodes applied to the + and - surfaces of the muscle. On closing the circuit, the thread of mercury passes downwards from $c$ in the direction of the Capillary electrometer. arrow.

$\mathrm{R}$, mercury in tube; Compensation. $-\mathrm{T}$ he capillary tube ; $s$, sul- strength of the current in aniphuric acid; $q, \mathrm{Hg}$; mal tissues is best measured by $\mathrm{B}$, observer; M, mi- the compensation method of croscope.

Poggendorf and du Bois-Reymond. A current of known strength, or which can be accurately graluated, is passed in an opposite direction through the same galvanometer or boussole, until the current from the animal tissue is just neutralised or compensated. [When this occurs, the needle deflected by the tissue-current returns to zero. The principle is exactly the same as that of weighing a body in terms of some standard weights placed in the opposite scale-pan of the balance.]

[Hermann calls the current obtained from an injured muscle, i.e., one on which an artificial transverse or other section has been made, a demarcation-current, while the currents obtained when such a muscle contracts, he calls action-currents. This section deals with demarcation-currents, or the muscle-current of du BoisReymond.]

1. Perfectly fresh uninjured muscles yield no current, and the same is true of dead muscle (L. Hermann, 1867).

2. Strong electrical currents are observed when the transverse section of a muscle is placed on one of the cushions of the non-polarisable electrodes (fig. 380, I, M), 
while the surface is in connection with the other (Nobili, Matteucci, du BoisReymond). The direction of the current is from the (positive) longitudinal section to the (negative) transverse section in the conducting wires (i.e., within the muscle itself from the transverse to the longitudinal section (figs. 380, I, and 399, I)). This current is stronger the nearer one electrode is to the equator, and the other to the centre of the transverse section; while the strength diminishes, the nearer the one electrode is to the end of the surface, and the other to the margin of the transverse section.

Smooth muscles also yield similar currents between their transverse and longitudinal sturfaces (§334, II.).

3. Weak electrical currents are obtained when-(a) two points at unequal distances from the equator are connected; the current then passes from the point nearer the equator $(+)$ to the point lying further from it $(-)$, but of course this direction is reversed within the muscle itself (fig. 399, II, ke and le). (b) Similarly weak currents are obtained by connecting points of the transverse section at unequal distances from the centre, in which case the current outside the muscle passes from the point lying nearer the edge of the muscle to that nearer the centre of the transverse section (fig. 399, II, $i, c$ ).

4. When two points on the surface are equidistant from the equator (fig. 399, $\mathrm{I}, x, y, v, z,-\mathrm{II}, r, e)$, or two equidistant from the centre of the transverse section (II, $c$ ) are connected, no current is obtained. [Because the points are iso-electrical, that is of equal potential.]

5. If the transverse section of the muscle be oblique (fig. 399, III), so that the muscle forms a rhomb, the conditions obtaining under III are disturbed. The point lying nearer to the obtuse angle of the transverse section or surface is positive to the one lying near to the acute angle. The equator is oblique $(a, c)$. These currents are called "deviation currents or inclination currents" by du Bois-Reymond, and their course is indicated by the lines 1,2 , and 3 .

The electro-motive force of a strong muscle-current (frog) is equal to 0.05 to 0.08 of a Daniell's element; while the strongest deviation current may be 0.1 Daniell. The muscles of a curarised animal at first yield stronger currents ; fatigue of the muscle dininishes the strength of the current (Roeber), while it is completely abolished when the muscle dies. Heuting a muscle increases the current ; but above $40^{\circ} \mathrm{C}$. it is diminished (Steiner). Cooling diminishes the electro-motive force. The warmed living muscular and nervous substance is positive to the cooler portions (Her$\operatorname{mann}$ ); while, if the dead tissues be heated, they behave practically as indifferent bodies as regards the tissues that are not heated.

6. The passive nerve behaves like muscle, as far as 2, 3, and 4 are concerned.

The electro-motive force of the strongest nerve-current, according to du Bois-Reymond, is 0:02 of a Daniell. Heating a nerve from $15^{\circ}$ to $25^{\circ} \mathrm{C}$. increases the nerve-current, while high temperatures diminish it (Steiner).

7. If the two transversely divided ends of an excised nerve, or two points on the surface equidistant from the equator, be tested, a current-the axial current--flows in the nerve-fibre in the opposite direction to the direction of the normal impulse in the nerve; so that in centrifugal nerves it flows in a centripetal direction, and in centripetal nerves in a centrifugal direction (Mendelssohn and Christiani).

The electro-motive force increases with the length of the nerve and with the area of its trausverse section. Fatigue (e.g., tetanic stimulation) weakens it, especially in motor nerves, and to a less extent in centripetal nerves.

[Nerve-Muscle Preparation. - This term has been used on several occasions. It is simply the sciatic nerve with the gastrocnemius of the frog attached to it (fig. 401). 
The sciatic nerve is dissected out entire from the vertebral column to the knee; the muscles of the thigh separated from the femur, and the latter divided about its middle, so that the preparation can be fixed in a clamp by the remaining portion of the femur; while the tendon of the gastrocnemius is divided near to the foot. If a straw flag is to be attached to the foot, do not divide the tendo Achilles.]

Rheoscopic Limb.-The existence of a muscle-current may be proved without the aid of a galvanometer :-1. By means of a sensitive nerve-muscle preparation of a frog, or the so-called "physiologicul rheoscope." Place a moist conductor on the transverse and another on the longitudinal surface of the gastrocnemius of a frog. On placing the sciatic nerve of a nerve-muscle preparation of a frog on these conductors, so as to bridge over or connect their two surfaces, contraction of the muscle connected with the nerve occurs at once; and the same occurs when the nerve is removed.

Make a transverse section of the gastrocnemius muscle of a frog's nerve-muscle preparation, and allow the sciatic nerve to fall upon this transverse section; the limb will contract as the muscle-current from the longitudinal to the transverse surface now traverses the nerve (ficlerni, Al.v. Humboldt). These experiments have long been known as "contraction without metals."

[ 'se a nerve-muscle preparation, or, as it is called, a physiological limb. Hold the prepara. tion by the femur, and allow its own nerve to fall upon the gastrocnemius, and the muscle will "ontract, hut it is better to allow the nerve to fall suddenly upon the eross section of the muscle. The nerve then enmpletes the cirenit between the longitulinal and transverse section of the muscle, so that it is stimulated by the current from the latter, the nerve is stimulated, and through it the muscle. That it is so, is proved by tying a thread round the nerve near the muscle, when the latter no longer contracts.]

2. Self-Stimulation of the Muscle.-We may use the muscle-current of an isolated muscle to stimulate the latter directly and cause it to contract. If the transverse and longitudinal surfaces of a curarised frog's nerve-muscle preparation he placed on non-polarisable electrodes, and the circuit be closed by dipping the wires coming from the electrodes in mercury, then the muscle contracts. Similarly a nerve may be stimulated with its own demarcation-current (du Bois-Reymond and otheis). If the lower end of a muscle with its transverse section be dipped into normal saline solution $(0.6$ per cent. $\mathrm{NaCl})$, which is quite an indifferent fluid, this fluid forms an accessory circuit between the transverse and adjoining longitudinal surface of the muscle, so that the muscle contracts. Other indifferent fluids used in the same way produce a similar result.

[Kuhne's Experiment (fig. 402).-The demarcation-current of the nerve of a

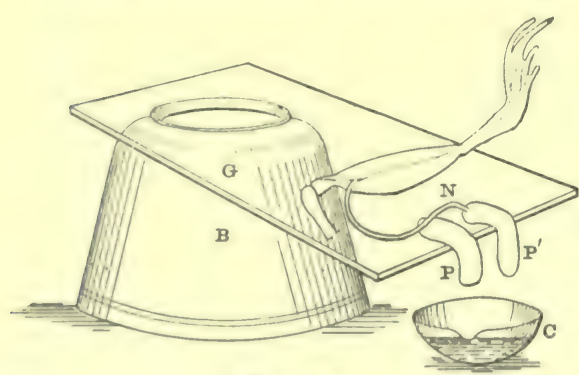

Fig. 402.

Kïhne's nerve demarcation-curreut experiment. nerve-muscle preparation may be used as the stimulus to that nerve on completing the circuit. On an earthenware bowl (B) is fixed a glass plate (G), and thin rolls of modeller's clay $\left(\mathrm{P}^{\mathrm{P}^{\prime}}\right)$ are bent over G. A nerve-muscle preparation is placed with its nerve (N) on the clay, touching the latter with its transverse and longitudinal surfaces. On dipping the clay into a vessel containing normal saline (C), the muscle contracts, and on withdrawing the normal saline, it again contracts. In this case the nerve is stimulated by the completion of the circuit of its own demarcation-current.]

3. Electrolysis. - If the muscle-current be conducted through starch mixed with potassic iorlide, then the iodine is deposited at the + pole, where it makes the starch blue. 
Frog Current.-It is asserted that the total current in the body is the sum of the electrical currents of the several muscles and nerves which, in a frog deprived of its skin, pass from the tip of the toes toward the trunk, and in the trunk from the anus to the head. This is the "corrente propria della rana" of Leopoldo Nobili (1827), or the "frog current" of du Bois: Reymond. In mammals, the corresponding current passes in the opposite direction.

After death, the currents disappear sooner than the excitability (Valentin); they remain longer in the muscle than the nerves, and in the latter they disappear sooner in the central portions. If the nerve-current after a time become feeble, it may be strengthened by making a new transverse section of the nerve. A motor nerve completely paralysed by curara gives a current (Funke), and so does a nerve beginning to undergo degeneration, even two weeks after it has lost its excitability. Muscles in a state of rigor mortis give currents in the opposite direction, owing to inequalities, which take place during decomposition. The nerve-current is reversed by the action of boiling water or drying.

Currents from Skin and Mucous Membranes.-In the skin of the frog the outer surface is + , the inner is - (du Bois-Reymond $)$, and the same is true of the mucous membrane of the intestinal tract (Rosenthal), the cornea (Grünhagen), as well as the non-glandular skin of fishes (Hermann) and molluses (Oehler). Currents are also manifested by glands.

332. CURRENTS OF STIMULATED MUSCLE AND NERVE-ACTIONCURRENTS. - 1. Negative Variation of the Muscle-Current.--If a muscle, which yields a strong electrical current, be thrown into a state of tetanic contraction by stimulating its motor nerve, then, when the muscle contracts, there is a diminution of the muscle-current, and occasionally the needle of the galvanometer may swing almost to zero. This is the "negative variation of the muscle-current" (du BoisReymond). It is larger, the greater the primary deflection of the galvanometer needle and the more energetic the contraction.

After tetanus, the muscle-current is weaker than it was before. If the muscle was so placed upon the electrodes that the current was "feeble," equally during tetanus there is a diminution of this current. In the inactive arrangement, the contraction of the muscle has no effect on the needle. If the muscle be prevented from shortening, as by keeping it teuse, the negative variation still takes place.

2. Current during Tetanus.-An excised frog's muscle tetanised through its nerve shows electro-motive force-the so-called "action-current." In a tetanised frog's gastrocnemius, there is a descending current. In completely uninjured human muscles, however, thrown into tetanus by acting on their nerves, there is no such current (L. Hermann); similarly, in quite uninjured frog's muscles, as well as when these muscles are directly and completely tetanised, there is no current.

3. Current during the Contraction-Wave.-If one end of a muscle be directly excited with a momentary stimulus, so that the contraction-wave (§ 299) rapidly passes along the whole length of the muscular fibres, then each part of the muscle, successively and immediately before it contracts, shows the negative variation. Thus, the "contraction-wave" is preceded by a "negative wave" of the musclecurrent, the latter occurring during the latent period. Both waves have the same velocity, about 3 metres per second. The negative wave, which first increases and then diminishes, lasts at each point only 0.003 second (Bernstein).

4. During a Single Contraction.-A single contraction also shows a musclecurrent. [The electrical variation takes place during the latent period of the muscular contraction, so that it precedes the latter. The variation begins ${ }^{\circ} 01^{\prime \prime}$ to ' $04^{\prime \prime}$ after excitation, while the contraction does not begin until ' $11^{\prime \prime}$ to $' 33^{\prime \prime}$ (Waller). A frog's muscle may be made to record its contraction, and simultaneously the variation of the electrical current, as ascertained by the capillary electrometer, may be photographed (fig. 403), and the same may be done in the case of the heart (fig. 404). The capillary electrometer may with advantage be employed to measure this time-difference, the electrical and the mechanical events being simultaneously recorded.]

The diphasic variation-1st phase middle negative to end; 2 ud phase and negative to middle begins about ' $01^{\prime \prime}$ before the commencement of muscular contraction (Waller). 
The variation is diphasic-1st phase base negative to apex ; 2nd phase apex negative to base ( $\left(\mathrm{V}\right.$ alle $\left.{ }^{\prime}\right)$. The first phase begins $\frac{1}{10}{ }^{\prime \prime}$ before the commencement of contraction.

One of the best objects for this purpose is the contracting heart, which is placed upon the non-polarisable electrodes connected with a sensitive galvanometer. Each

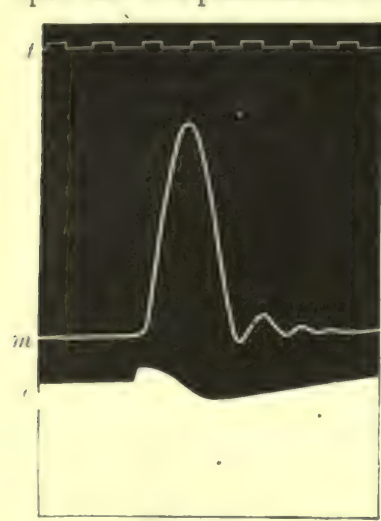

Fig. 403 . beat of the heart causes a deflection of the needle, which occurs before the contraction of the cardiac muscle (Kölliker and H. Müller). The electrical disturbance in the muscle causing the negative variation always precedes the actual contraction ( $v$. Helmholtz, 1845). Still it lasts throughout the whole duration of the contraction (Lee). When the completely uninjured frog's gastrocnemius contracts by stimulating the nerve, there is at first a descending and then an ascending current (Sig. Mayer, \$334, II.).

More exact observations on the electrical processes of the pulsating heart show that complicated phenomena oceur. The apex of the dog's heart is negative to the base during systole. In many cases this is preceded, and in some it is followed, by an opposite condition (Frédérieq), i.e., a diphasic variation. If the heart be arrested in diastole by stimulation of the vagus ( $\$ 369)$, there is a positive variation of the muscle-current (Gaskell, Fano). Waller has demonstrated a true electrical variation of Frog. Gastrocnemius led off the human intact heart.

to "lectrometer from the millle of the musele and [Heart.-Gaskell has shown that, when the vagus of from the tendon. Contrac- a tortoise is stimulated so as to arrest its heart in diastole, tion excited by a single the action of the inhibitory nerve is accompanied by a break inluction shock ap- positive electrical variation of the heart-current, while plied to the sciatic nerve. stimulation of the sympathetic (augmentor) nerve causes $\therefore$ time in 1 th sec. (muscle an electrical variation of the same sign as that caused by to $\mathrm{H}_{2} \mathrm{SO}_{4}$; tendon to $\mathrm{Hg}$ ) a contraction in the non-beating tissue of the ventricle $\left(W\right.$ alle $\left.r^{\circ}\right)$ of the toad. In both cases, the respective nerves can produce their electrical effect after the heart has been brought to standstill by the application of muscarin to the sinus. These experiments are of the utmost

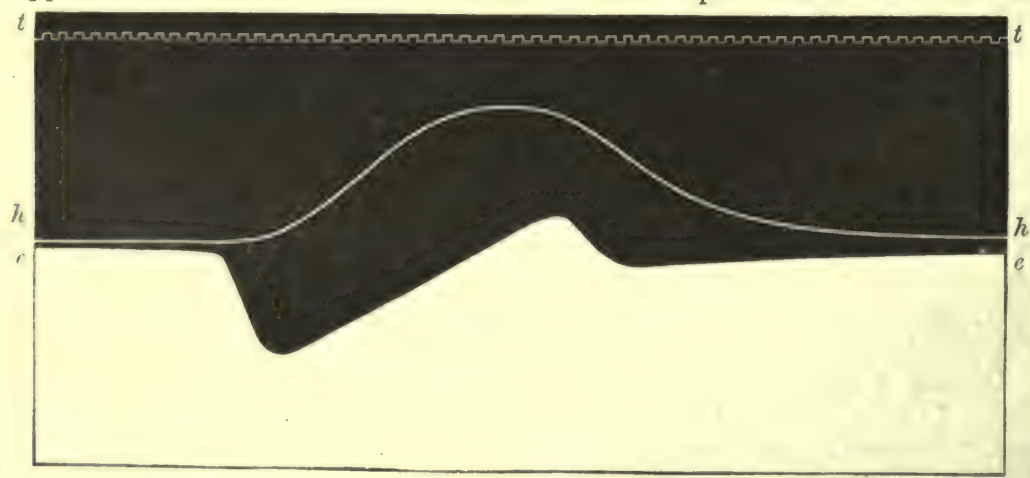

Fig. 404.

Frog's heart. Spontaneous contraction. $e, e$, electrometer; $h$, $h$, heart's contraction ; $t, t$, time in $\frac{1}{2}$ th sec. (apex to $\mathrm{H}_{2} \mathrm{SO}_{4}$, base to $\mathrm{Hg}$ ) (Waller).

importance in connection with the theory of the action of these nerves on the heart ( $\$ 370)$, and the mode of action of poisons on the heart itself.]

Secondary Contraction. - A nerve-muscle preparation may be used to demonstrate the electrical changes that occur during a single contraction. If the sciatic nerve, A, of such a preparation be placed upon another muscle, B, as in fig. 405 , then 
every time the latter, B, contracts, the frog's muscle, A, connected with the nerve also contracts.

If the nerve of a frog's nerve-muscle preparation be placed on a contracting mammalian heart, then a contraction of the muscle occurs with every beat of the heart (Matteucci, 1842). The diaphragm, even after section of the phrenic nerve, especially the left, also contracts during the heart-beat (Schiff). This is the "secondary contraction" of Galvani.

Secondary Tetanus.- Similarly, if a nerve of a nervemuscle preparation be placed on a muscle which is tetanised, then the former also contracts, showing "secondary tetanus" (du Bois-Reymond). The latter experiment is regarded as a proof that, during the process of negative variation in the muscle, many successive variations of the current must take place, as only rapid variations of this kind can produce tetanus by acting on a nerve-continuous variations being unable to do so.

Usually, there is no secondary tetanus in a frog's nervemuscle preparation when it is laid upon a muscle which is tetanised voluntarily, or by chemical stimuli, or by poisoning with strychnin (Hering, Kühne); still, Lovèn has observed secondary strychnin tetanus composed of six to nine shocks per

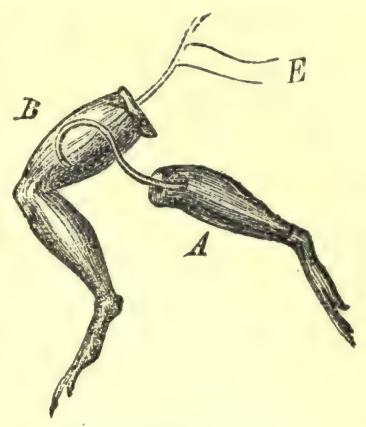

Fig. 405.

Secondary contraction. The sciatic nerve of $\mathrm{A}$ lies on $\mathrm{B}$; E, electrodes applied to the sciatic nerve of $B$. second. Observations with a sensitive galvanometer, or Lippmann's capillary electrometer (fig. 400 ), show that the spasms of strychnin poisoning, as well as a voluntary contraction, are discontinuous processes (Lovèn, p. 485).

Biedermann observed that striped muscle, under the influence of the vapour of ether, passes into a condition in which it shows no obvious change of form or movement when it is stimulated, whilst at the spot stimulated, there are galvanometric variations of the same strength as occurred during stimulation before the action of the ether. Owing to the abolition of the power of conductivity, they can only manifest themselves locally.

[Secondary Contraction from Muscle to Muscle (Kühne).-If $5 \mathrm{~mm}$. of one end of the sartorius of a curarised frog be laid upon a corresponding $5 \mathrm{~mm}$. of the other sartorius, so that both muscles are in line, and if the surfaces of contact be pressed together, either by an ebonite press or other means, on stimulating the free end of one of the muscles-either electrically, mechanically, or chemically-the other muscle also contracts, and if the first one be tetanised, the second one also is thrown into tetanus. The experiment may be repeated with five or six muscles in line. The conduction is interrupted at once by ligature of the muscle. The second muscle contracts, because it is stimulated directly by the action-currents of the contracting muscular fibres. The effect is prevented by introducing, between the overlapping ends of the muscle, a thin plate of gutta-percha, tinfoil, or any insulator. This experiment of Kühne's shows us how important a rôle electrical phenomena play in connection with muscular contraction. Secondary contraction from nerve has long been known.]

Negative Variation in Nerve. - If a nerve be placed with its transverse section on one non-polarisable electrode, and its longitudinal surface on the other, and if it be stimulated electrically, chemically, or mechanically, the nerve-current is also diminished (du Bois-Reymond). This negative variation is propagated towards both ends of a nerve, and is composed of very rapid, successive, periodic, interruptions of the original current, just as in a contracted muscle (Bernstein). Hering succeeded in obtaining from a nerve, as from a muscle, a secondary contraction or secondary tetanus. The amount of the negative variation depends upon the extent of the primary deflection, also upon the degree of nervous excitability, and on the strength of the stimulus employed. The negative variation occurs on stimulating with tetanic as well as with single shocks. The negative variation is not observed in completely uninjured nerves. 
Hering found that the negative variation of the nerve-current caused by tetanic stimulation is followed by a positive variation, which occurs immediately after the former, i.e., it is diphasic. It increases to a certain degree with the duration of the stimulation, as well as with the strength of the stimulus, and with the drying of the nerve (Head). (Effect of Electrotonus, \$ 335, I.).

Negative Variation of the Spinal Cord. - This is the same as in nerves generally. If a current be conducted from the transverse aud longitudinal surfaces of the upper part of the medulla oblongata, we observe spontaneons, intermittent, negative variations, perhaps due to the intermittent excitement of the nerve-centres, more especially of the respiratory centre. Similar variations are obtained reflexly by single stimuli applied to the sciatic nerve, while strong stimulation by common salt or induction shocks inhibits them.

Velocity. - The process of negative variation is propagaterl at a measurable velocity along the nerve, most rapidly at $15^{\circ}$ to $25^{\circ} \mathrm{C}$. (Steiner), and at the same rate as the velocity of the nervous impulse itself, about 27 to 28 metres per second. The duration of a single variation (of which the process of negative variation is composed) is only 0.0005 to 0.0008 second, while the wave-length in the nerve is calculated by Pernstein at $18 \mathrm{~mm}$.

$\because 7$

Differential Rheotome. -J. Bernstein estimated the velocity of the negative variation in a nerve by means of a differential rheotome thus (fig. 406):-A long stretch of a nerve $(\mathrm{N} n)$ is so arranged that at one end of it $(\mathrm{N})$ its transverse and longitudinal surfaces are connected with

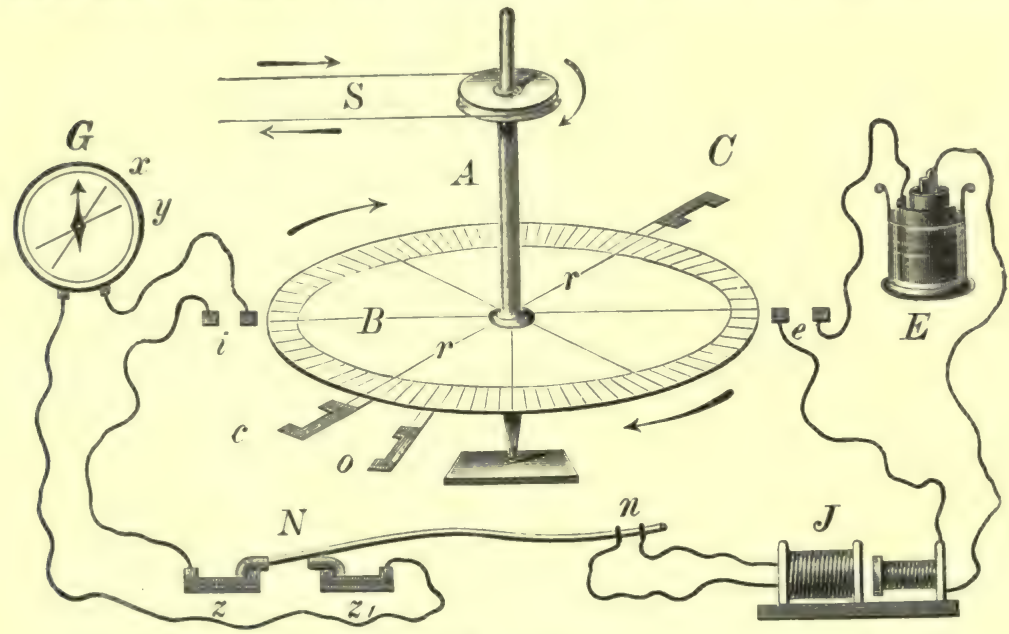

Fig. 406.

Scheme of Bernstein's differential rheotome ; $\mathrm{N} n$, nerve ; $\mathrm{J}$, induction machine ; G, galvanometer, $x, y$, deflection of needle ; $\mathrm{E}$, battery and primary circuit with $\mathrm{C}$ for opening it at 0 ; $c$, for closing galvanometer circuit; $z \approx$, electrodes in galvanometer circuit ; $\mathbf{S}$, motor.

a galvanometer $(\mathrm{G})$, while at the other end $(n)$ are placed the electrodes of an induction machine $(\mathrm{J}) . \quad \mathrm{A}$ disc (B) rapidly rotating on its vertical axis (A) has an arrangement (C) at one point of its circumference, by means of which the current of the primary circuit (E) is rapidly opened and closed during each revolution. This causes, with each rotation of the disc, an opening and a closing shock to be applied to the end of the nerve. At the diametrically opposite part of the circumference is an arrangement $(c)$ by which the galvanometer circuit is closed and opened during each revolution. Thus, the stimulation and the closing of the galvanometer circuit occur at the same moment. On rapidly rotating the disc, the galvanometer indicates a strong nerve-current, an excursion of the magnetic needle to $y$. At the moment of stimulation, the negative variation has not yet reached the other end of the nerve. If, however, the arrangement which closes the galvanometer circuit be so displaced (to $o$ ) along the circumference, that the galvanometer circuit is closed somewhat later than the nerve is stimulated, then the current is weakened by the negative variation (the needle passing backward to $x$ ). When we know the velocity of rotation of the disc, it is easy to calculate the rate at which the impulse causing the negative variation passes along a given distance of nerve from $\mathrm{N}$ to $n$.

The negative variation is absent in degenerated nerves as soon as they lose their excita. bility. 
Rectinal and Eye Currents. - If a freshly-excised eyeball be placed on the non-polarisable electrodes connected with a galvanometer, and if light fall upon the eye, then the normal eyecurrent from the cornea $(+)$ to the transverse section of the optic nerve $(-)$ is at first increased. Yellow light is most powerful, and less so the other colours (Holmgren, M'Kendrick and Dewar). The inner surface of the passive retina is positive to the posterior. When the retina is illuminated there is a double variation, a negative variation with a preliminary positive increase; while, when the light ceases, there is a simple positive variation. Retinæ, in which the visual purple has disappeared owing to the action of light, show smaller variations (Kühne and Steiner).

Stimulation of the secretory nerves of the glandular membranes, besides causing secretion, affects the current of rest (Roeber). This secretion-current passes in the same direction in the skin of the frog and warm-blooded animals as the current of rest, although in the frog it is occasionally in the opposite direction (Hermann). If the current be conducted uniformly from both hind feet of a cat, on stimulating the sciatic nerve of one side, not only is there a secretion of sweat (§ 288), but a secretion-current is developed (Luchsinger and Hermann). If two symmetrical parts of the skin in the leg or arm of a man be similarly tested, and the muscle of one side be contracted, a similar current is developed. Destruction or atrophy of the glands abolishes both the power of secretion and the secretion-current. There is no secretion-current from skin covered with hairs, but devoid of glands (Bubnoff). [The secretion-current from the submaxillary gland is referred to in $\$ 145$ (Bayliss and Bradford).]

333. ELECTROTONIC CURRENTS IN NERVE AND MUSCLE.-[When a constant current called the "polarising current" is passed though a stretch of nerve, the nerve is thrown into a peculiar condition, called the "electrotonic condition," or briefly electrotonus. In this condition, the vital properties of the nerve are modified, i.e.,

(1) Its electromotivity ( $(333)$.

(2) Its excitability (\$335).

The former is considered in this section, and the latter in a subsequent section.]

1. Positive Phase of Electrotonus. - If a nerve be so arranged upon the electrodes (fig. 407, I) that its transverse section lies on one, and its longitudinal on the other electrode, then the galvanometer indicates a strong current. If now a constant current be transmitted through the end of the nerve projecting beyond the electrodes (the so-called "polarising" end of the nerve), and if the direction of this current coincide with that in the nerve, then the magnetic needle gives a greater deflection, indicating an increase of the nerve-current- "the positive phase of electrotonus." The increase is greater the longer the stretch of nerve traversed by the current, the stronger the galvanic current, and the less the distance between the part of the nerve traversed by the constant current and that on the electrodes.

2. Negative Phase of Electrotonus.-If in the same length of nerve, the constant current passes in the opposite direction to the nerve-current (fig. 407 , II), there is a diminution of the electromotive force of the latter-"negative phase of $\mathrm{N}$ electrotonus."

3. Equator.-If two points of the nerve equi-

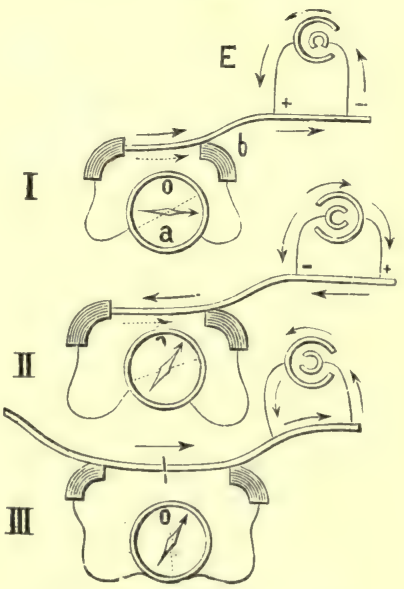

Fig. 407.

distant from the equator be placed on the electrodes (III), there is no deflection of the galvanometer needle (p. 555, 4). If a constant current be passed through one free projecting end of the nerve, then the galvanometer indicates an electro-motive effect in the same direction as the constant current.

Electrotonus.-These experiments show that a constant current causes a change of the electro-motive force of the part of the nerve directly traversed by the constant 
current, i.e., in the intrapolar area, and also in the part of the nerve outside the electrodes, i.e., in the extrapolar area. This condition is called electrotonus (du Bois-Reymond, 1843).

The eleotrotonic current is strongest not far from the electroles, and it may be twenty-five times as strong as the nerve-current of rest $(\$ 331,5)$; it is greater ou the anode than on the cathole sicle; it undergoes a negative variation like the resting nerve-current during tetanus; it oceurs at once on closing the constant current, although it diminishes uninterruptedly at the cathode (du Bois-lirymond). On the contrary, between the electrodes, besides the polarising 'urrent itself, there is no obvious electrotonic increase of the current to be observed (Hermann). These phenomena take place only as long as the nerve is excitable. If the nerve be ligatured in the projecting part in the galvanometer circuit, the phenomena cease in the ligatured part. The alwe-described gaivanic electrotonic changes of the extra-polar part are absent in nonmedullated nerve-fibres, whilst, on the contrary, the physiological electrotonus is present. The phrsiological electrotonus of medullated nerves can be set aside by treating medullated nerves with ether, whilst the physical phenomena remain (Biedermann).

The negative variation ( $\$ 332$ ) occurs more rapilly than the electrotonic increase of the current, so that the former is over before the electro-motive increase occurs. The velocity of the electrotonic change in the current is less than the rapidity of propagation of the excitement in the nerves-being only 8 to 10 metres per second (Tschirjew, Bernstein).

"The secondary contraction from a nerve" depends upon the electrotonic state. If the s.iatic nerve of a frog's nerve-muscle preparation be placed on an excised nerve, and if a constant current he passed through the free end of the latter-non-electrical stimuli being inactive - the muscles contract. This occur's because the electrotonising current in the excised nerve stimulates the nerve lying on it. By rapidly closing and opening the current, we obtain "secondamy tetanus from a nerve" (p. 55்).

[Paradoxical Contraction.-Exactly the same occurs when the current is applied to one of the two branches into which the sciatic nerve of the frog divides. The sciatic nerve of the frog divides at the lower end of the thigh into the peroneal and tibial branches. If the sciatic nerve be divided above, and the peroneal branch be also divided and stimulated with interrupted induction shocks, the muscles supplied by the tibial branch will contract. There is no contraction of the muscle if the leroneal nerve be ligatured.]

Polarising After-Currents. - When the constant current is opened, there are after-currents depending upon internal polarisation (\$ 328). In living nerves, muscle, and electrical organs this internal polarisation current, when a strong primary current of very short duration is used, is always positive, i.e., has the same direction as the primary current. Prolonged duration of the primary current ultimately causes negative polarisation. Between these two is a stage when there is no polarisation. Positive polarisation is especially strong in nerves when the primary current has the direstion of the impulse in the nerve; in muscle, when the primary current is directed from the point of entrance of the nerve into the muscle towards the end of the muscle (\$334, II.).

4. Muscle-Current during Electrotonus.-The constant current also produces an electrotonic condition in muscle; a constant current in the same direction increases the muscle-current, while one in an opposite direction weakens it, but the action is relatively feeble.

[Electrotonic Phenomena in Conductors. - Matteucei found that a metallic wire surrounded by a moist conductor, when traversed by a galvanic current, exhibits currents possessing the froperties of electrotonic currents of nerves. He also found that the currents ceased if the wire was of zinc, and the envelope a saturated solution of zine sulphate. This shows that these currents were due to polarisation between the core and the fluid. Hermann fiuds that the currents only obtain when a polarisable core is present. A straw without joints, if filled with a saturated solution of common salt, or the tentacles of a lobster when moistened with saline solution, and traversed by a constant current, exhibit similar electrotonic currents (Hering).]

334. THEORIES OF MUSCLE- AND NERVE-CURRENTS.-I. Molecular or pre-existence Theory.-To explain the currents in muscle and nerve, du BoisReymond proposed the so-called molecular theory. According to this theory, a nerve- or muscle-fibre is composed of a series of small electro-motive molecules arranged one behind the other, and surrounded by a conducting indifferent fluid. The molecules are supposed to have a positive equatorial zone directed towards the 
surface, and two negative polar surfaces directed towards the transverse section. Every fresh transverse section exposes new negative surfaces, and every artificial longitudinal section new positive areas.

'This scheme explains the strong currents, - when the + longitudinal surface is connected with the - transverse surface, a current is obtained from the former to the latter, - but it does not explain the feeble currents. To explain their occurrence we must assume that, on the one hand, the electro-motive force of the molecules is weakened with varying rapidity at unequal distances from the equator; on the other, at unequal distances from the transverse section. Then, of course, differences of electrical tension obtain between the stronger and the feebler molecules.

Parelectronomy. - But the natural transverse section of a muscle, i.e., the end of the tendon, is not negative, but more or less positive electrically. To explain this condition, du BoisReymond assumes that on the end of the tendon there is a layer of electro-positive musclesubstance. He supposes that each of the peripolar elements of muscle consists of two bipolar elements, and that a layer of this half element lies at the end of the tendon, so that its positive side is turned towards the free surface of the tendon. This layer he calls the "parelectronomic layer." It is never completely absent. Sometimes it is so marked as to make the end of the tendon + in relation to the surface. Cauterisation destroys it. [It is supposed to be favoured by cold.]

The negative variation is explained by supposing that, during the action of a muscle and nerve, the electro-motive force of all the molecules is diminished. During partial contraction of a muscle, the contracted part assumes more the characters of an indifferent conductor, which now becomes connected with the negative zone of the passive contents of the muscular fibres.

The electrotonic currents beyond the electrodes in nerves must be explained. To explain the electrotonic condition, it is assumed that the bipolar molecules are capable of rotation. The polarising current acts upon the direction of the molecules, so that they turn their negative surfaces towards the anode, and their positive surfaces to the cathode, whereby the molecules of the intrapolar region have the arrangement of a Volta's pile. In the part of the nerve outside the electrodes, the further removed it is, the less precisely are the molecules arranged. Hence, the swing of the needle is less, the further the extrapolar portion is from the electrodes.

II. Difference or Alteration Theory.-The difference theory was proposed by L. Hermann, and, according to him, the four following considerations are sufficient to explain the occurrence of the galvanic phenomena in living tissues :-(1) Protoplasm, by undergoing partial death in its continuity, whether by injury or by (horny or mucous) metamorphosis, becomes negative towards the uninjured part. (2) Protoplasm, by being partially excited in its continuity, becomes negative to the uninjured part. (3) Protoplasm, when partially heated in its continuity, becomes positive, and by cooling negative, to the unchanged part. (4) Protoplasm is strongly polarisable on its surface (muscle, nerve), the polarisation constants diminishing with excitement and in the process of dying.

Streamless Fresh Muscles.-It seems that passive, uninjured, and absolutely fresh nerves, and muscles, are completely devoid of a current, e.g., the heart (Engelmann), also the musculature of fishes while still covered by the skin.

[According to Hermann, the currents obtained from muscle are due to injury of the muscle-substance, whereby a difference of potential is set up, the injured part being negative to the uninjured. In fact, it is impossible to isolate a muscle without injuring it, owing to its connections. Frogs exhibit skin-currents after the skin is destroyed; the muscles still exhibit currents, but Hermann explains this by the action of the irritant, used to destroy the skin, also affecting the muscle. In fishes, however, there are no skin-currents, and if they be curarised, absolutely no current is obtained from their uninjured muscles (Hermann). The heart also when passive and uninjured gives no current, although it exhibits an action-current when it contracts, and every injured part in it possesses a negative electrical potential with reference to the rest.]

L. Hermann also finds that the muscle-current is always developed after a time, which is very short, when a new transverse section is made. [By means of his "Fall-rheotom," an arrangement whereby a weight, covered with shagreen, injured a muscle, and at the same time, closed and opened a galvanometer circuit, Hermann was able to show that the current-demarcationcurrent-took a certain time to develop. Had it been pre-existent, as supposed by du BoisReymond, this ought not to have been the case.] 
Demarcation-Current.-Every injury of a muscle or nerve causes at the point of injury (demarcation surfuce) a dying substance, which behaves negatively to the positive intact substance. The current thus produced is called by Hermann the "demarcation-current." If individual parts of a muscle be moistened with potash salts or muscle-juice, they become negatively electrical; if these substances be removed these parts cease to be negative (Bicdermann).

It appears that all living protoplasmic substance has a special property, whereby injury of a part of it makes it, when dying, negative, while the intact parts remain positively electrical. Thus, all transverse sections of living parts of plants are negative to their surface (Buff); and the same occurs in animal parts, e.g., glands and bones. Engelmann made the remarkable observation that the heart and smooth muscle again lose the negative condition of their transverse section. When the muscle-cells are completely dead, as far as the cement-substance of the nearest cells; in nerves, when the divided portion dies, as far as the first node of Ranvier. When all these organs are again completely streamless, then the absolutely dead substance behaves essentially as an indifferent moist conductor. Muscles divided subcutaneously and healed do not exhibit a negative reaction of the surface of their section.

All these considerations go to show that the pre-existence of a current in living uninjured tissues can no longer be maintained.

Theoretical. - Griinhagen and L. Hermann explain the electrotonic currents as being due to internal polarisation in the nerve-fibre between the conducting core of the nerve and the enclosing sheaths. Matteucei found that, when a wire is surrounded with a moist conductor, and the covering placed in comnection with the electrodes of a constant current, currents similar to the rlectrotonic currents in nerves, and due to polarisation, are developed. If either the wire or the moist covering be interrupted at any part, then the polarisation current does not extend beyond the rupture (1).562). The polarisation developed on the surface of the wire by its transition-resistance causes the conlucted current to extend much beyond the electrodes.

Muscles and nerves consist of fibres surrounded by indifferent conductors. As soon as a constant current is closed, on their surface, internal polarisation is developed, which produces the electrotonic variation; it disappears again on opening or breaking the current. Polarisation is letected by the fact that, in living nerve, the galvanic resistance to conduction across a fibre is about five times, and in muscles about seven times greater than in the longitudinal direction.

Action-Currents. - The term "action-current" is applied by L. Hermann to the currents obtainel during the activity of a muscle or nerve. When a single stimulation-wave (contraction) passes along muscular fibres, which are connected at two points with a galvanometer, then that point through which the wave is just passing is negative to the other. Occasionally, in exirised museles, local contractions occur, and these points are negative to the other passive parts of the muscle (Biedemann). In orler, therefore, to explain the currents obtained from a frog's lerg during tetanus, we must assume that the end of the fibre which is negative participates less in the excitement than the middle of the fibre. But this is the case only in dying or fatigued muscles.

According to $\$ 336$, D, the direct application of a constant current to a muscle causes contraction first at the cathode, when the current is closed, and when it is opened, at the anode. This is explained by assuming that, during the closing contraction, the muscle is negative at the cathode, while with the opening contraction the negative condition is at the anode.

If a muscle be thrown into contraction by stimulating its nerve, then the wave of excitement travels from the entrance of the nerve to both ends of the muscle, which also behave negatively to the passive parts of the muscle. Accorling to the point at which the nerve enters the muscle, the ascending or descending wave of excitement will reach the end (origin or insertion) of the muscle sooner than the other. On placing such a muscle in the galvanometer circuit, then at first that end of the muscle will be negative which lies nearest to the point of entrance of the nerve (e.g., the upver end of the gastrocnemius), and afterwards the lower end. Thus, there appears rapilly after each other, at first a descending, and then an ascending, current in the galvanometer circuit, of course reversed within the muscle itself (Sig. Mayer) (§332, 4).

The same occurs in the muscles of the human fore-arm. When these were caused to contract through their nerves, at first the point of entrance of the nerve $(10 \mathrm{~cm}$. above the elbow-joint) was negative, and then followed the ends of the muscles when the contraction-wave, with a velocity of 10 to 13 metres per second, reached them $(L$. Hermann) $(\S 399,1)$.

If a completely uninjured, streamless muscle be made to contract directly and in toto, then neither during a single contraction, nor in tetanus, is there a current, because the whole of the muscle passes at the same moment into a condition of contraction.

Nerve-Currents. - Hermann also supposes that the contents of dying or active nerves behave negatively to the passive normal portions.

Imbibition Currents. - When water flows through capillary spaces, this is accompanied by an electrical movement in the same direction (Quincke, Zöllner). Similarly, the forward movement of water in the capillary interspaces of non-living parts (pores of a porcelain plate) is also connected with electrical movements, which have the same direction as the current of water. 
The same effect occurs in the movement of water, which results in that condition known as imbibition of a body. We must remember, that at the demarcation surface of an injured nerve or muscle, imbibition takes place ; that also at the contracted parts of a muscle imbibition of fluid occurs ( $\$ 227$, II.) ; and that during secretion there is a movement of the fluid particles.

In plants, electrical phenomena have been observed during the passive bending of vegetable parts (leaves or stalks), as well as during the active movements which are associated with the bending of certain parts, e.g., as in the mimosa and dionæa (Burdon-Sanderson). These phenomena are perhaps explicable by the movement of water which must take place in the interior of the vegetable parts $(A . G$. Kunkel). The root cap of a sprouting plant is negative to the seed coverings (Hermann); the cotyledons positive to the other parts of the seedling (Müller-Hettlingen). In the incubated hen's egg, the embryo is + , the yelk - (Hermann and v. Gendre).

335. ELECTRONIC ALTERATION OF THE EXCITABILITY.--Cause of Electrotonus. - If a certain stretch of a living nerve be traversed by a constant electrical ("polarising") current, it passes into a condition of altered excitability (Ritter, 1802, and others), which du Bois-Reymond called the electrotonic condition, or simply electrotonus. This condition of altered excitability extends not only over the part actually traversed by the current, intrapolar portion, but it is communicated to the entire nerve, i.e., to the extrapolar portions. Pfliiger (1859) discovered the following laws of electrotonus :-

At the positive pole or anode (fig. 408, $A$ ) the excitability is diminished-this is the region of anelectrotonus ; at the negative pole or cathode $(K)$ it is increased - this is the region of cathelectrotonus. The changes of excitability are most marked in the regions of the poles themselves.

Indifferent Point.- In the intrapolar region a point must exist where the anelectrotonic and cathelectrotonic regions meet, where therefore the excitability is unchanged; this is called the indifference or neutral point. This point lies nearer the anode $(i)$ with a weak current, but with a strong current nearer the cathode $\left(i_{l}\right)$; hence, in the first case, almost the whole intrapolar portion is more excitable; in the latter, less excitable. [Expressed otherwise, a weak current increases the area over which the negative pole prevails, while the reverse is the case with a strong current. Or in the intrapolar region, the diminution of excitability extends as the strength of the current increases, or to put it otherwise, with an increasing strength of current, the indifferent point moves from the positive to the negative pole.] Very strong currents greatly diminish the conductivity at the anode, and indeed may make the nerve completely incapable of conduction at this part.

At the cathode also, but only after the polarising current has passed for some time through the nerve (Werigo), the excitability is diminished, and the nerve in this area is rendered incapable of conduction (Grünhagen).

Extrapolar Region.-The extrapolar area, or that lying outside the electrodes, is greater the stronger the current. Further, with the weakest currents, the extrapolar anelectrotonic area is greater than the extrapolar cathelectrotonic. With strong currents this relation is reversed.

Fig. 408 shows the excitability of a nerve $(N, n)$ traversed by a constant current in the direction of the arrow. The curve shows the degree of increased excitability in the neighbourhood of the cathode $(K)$ as an elevation above the nerve, diminution at the anode $(A)$ as a depression. The curve $m, o, i_{n}, p, r$, shows the degree of excitability with a strong current; $e, f, i, h, k$, with a medium current ; lastly, $a, b, i, c, d$, with a weak current.

The electrotonic effect increases with the length of the nerve traversed by the current. The changes of the excitability in electrotonus occur instantly when the circuit is closed, while anelectrotonus develops and extends more slowly. Cold diminishes electrotonus (Hermann and v. Gendre).

When the polarising current is opened or broken, at first there is a reversal of the relations of the excitability, and then there follows a transition to the normal condition of excitability of the passive nerve (Pflüger). At the very first moment of closing, Wundt observed that the excitability of the whole nerve was increased. 
I. Proof of Electrotonus in Motor Nerves.-To test the laws of electrotonus, take a frog's nerve-muscle preparation (fig. 401). A constant current (p. 542) is applied to a limited part of the nerve by means of non-polarisable electrodes. A stimulus, electrical, chemical (saturated solution of common salt), or mechanical is applied either in the region of the anode or cathode ; and we olserve whether the contraction which results is greater when the polarising current is opened or closed. We shall consider the following eases (fig. 409).

(a) Descending extrapolar anelectrotonus. With a descending current we have to test the excitability of the extrapolar region at the anode. If the stimulus (common salt) applied at $\mathrm{R}$ (while the circuit was open) causes in this case $(\mathrm{A})$ molerately strong contractions in the

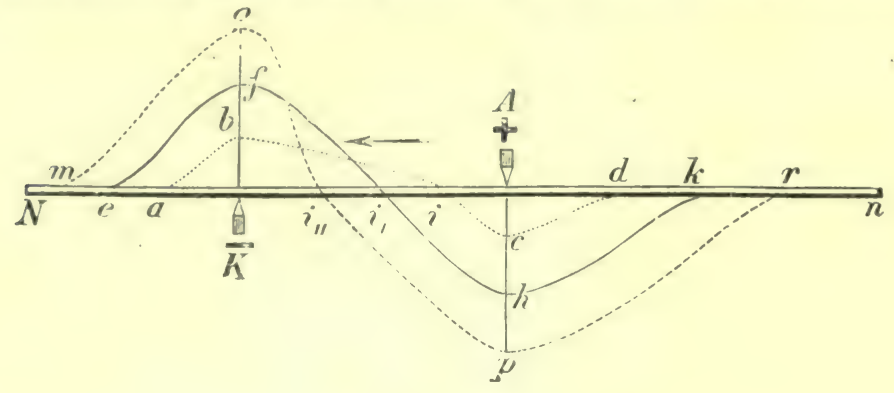

Fig. 408 .

Scheme of the electrotonic excitability.

limb, then these at once become weaker, or disappear as soon as the constant current is transmitted through the nerve. After the circuit is opened, the contractions produced by the salt again occur of the original strength.

(b) Descending extrapolar cathelectrotonous $(\Lambda)$. The stimulus (salt) is at $R_{1}$, and the rontractions thereby produced are at once incrased after elosing the polarising current. On opening it they are again weakened.

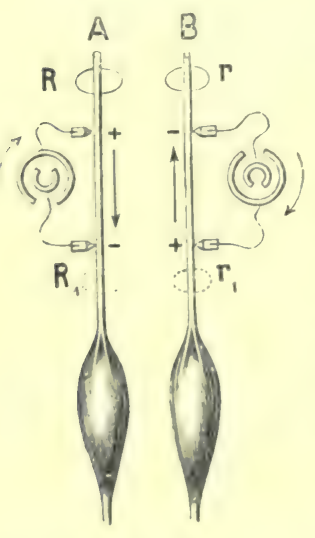

Fig. 409 .

(c) Ascending extrapolar anelectrotonus (B). The salt lies at $r_{1}$; the moderately strong contractions excited by the salt hefore the current is matle, become feebler after the current is made.

(d) Ascending extrapolar cathelectrotonus (B). The salt lies at $r$. In this case we must distinguish according to the strength of the polarising current:-(1) When the current is very weak, which can be obtained with the aid of the rheocord (fig. 379), on closing the polarising current, there is an increase of the contraction produced by salt. (2) If, however, the current is stronger, the contractions become either smaller or cease. This is due to the fact that with strong currents the conductivity of the nodes is diminished or even abolisher (p. 565). Although the salt acts on the excitable nerve, there is no contraction of the muscle, as the conduction of an impulse is prevented by the resistance in the nerve.

The law of electrotonus may also be demonstrated on a completely isolated nerve. The end of the nerve is properly disposed upon electrodes connected with a galvanometer, so as to obtain a strong current. If the nerve, when the constant current is closed, is stimulated in the anelectrotonic area, e.g., by an induction shock, then the negative variation is weaker than when the polarising circuit was open. Conversely, it is stronger when

Method of testing the excita- it is stimulated in the cathelectrotonic area. The currents fron bility in electrotonus. $\mathrm{R}$, the extrapolar areas of a nerve in a condition of electrotonus, $r, \mathbf{R}_{1}, r_{1}$, where the com- exhibit the negative variation when the nerve is stimulated mon salt (stimulus) is ap. (Bernstein).

plied.

[Tigerstedt, instead of employing an electrical or chemical stimulus to excite the electrotonic nerve, used an apparatus like Heidenhain's tetanometer, whereby the nerve was beaten gently with a small ivory hammer. He fully confirms Pfliiger's results.]

Proof in Man. - In performing this experiment it is important to remember the distribution of the current in the body. If both electrodes, for example, be placed over the course of the ulnar nerve (fig. 410), the currents entering the nerve at the anode $(+a a)$ must diminish the 
excitability; only above and below the anode (at $c c$ ) the positive current emerges from the nerve and excites cathelectrotonus at these points. Similarly, where the cathode is applied $(-c c)$ there is increased excitability, but in higher and lower parts of the nerve, where (at $a a)$ the positive current (coming from +) enters the nerve, the excitability is diminished (anelectrotonus) ( $v$. Helmholtz, Erb). If we desire to stimulate in the neighbourhood of an electrode, then we cannot act upon that part of the nerve whose excitability is influenced by the electrode. In order, therefore, to stimulate directly the same point on which the electrode acts, it is necessary to apply the stimulus at the same time by the electrode itself, e.g., either mechanically or by conducting the stimulating current through the polarising circuit (Waller and de Watteville).

II. Proof of Electrotonus in Sensory Nerves. - Isolate the sciatic nerve of a decapitated frog. When this nerve is stimulated in its course with a saturated solution of common salt, reflex movements are excited in the other leg, the spinal cord being intact. These disappear as soon as a constant current is applied to the nerve, provided the salt lies in the anelectrotonic area (Pflüger and Zurhelle, Hallstén).

III. Proof of Electrotonus in Inhibitory Nerves. - To show this, proceed thus :-On causing dyspncea in a rabbit, the number of heart-beats is diminished, owing to the action of the dyspnœic blood on the cardio-inhibitory centre in the medulla oblongata. If, after dividing the vagus on one side, a constant descending current be passed through the other intact vagus, the number of pulse-beats is again increased (descending extrapolar anelectrotonus). If, however, the current through the nerve be an ascending one, then with weak currents the number

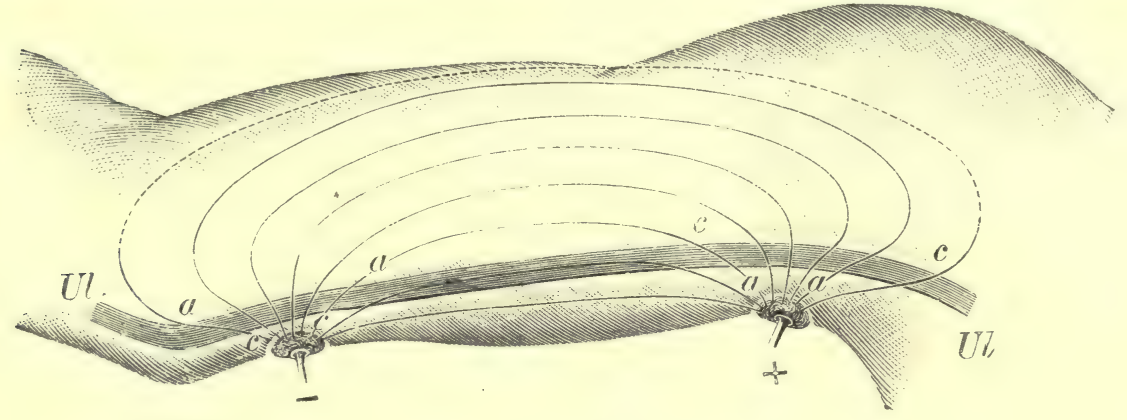

Fig. 410.

Scheme of the distribution of an electrical current in the nerve on galvanising the ulnar nerve.

of heart-beats increases still more (ascending extrapolar cathelectrotonus). Hence, the action of inhibitory nerves in electrotonus is the opposite of that in motor nerves.

During the electrotonus of muscle, the excitability of the intrapolar portion is altered. The delay in the conduction is confined to this area alone $(v$. Bezold $)$ compare $\S 337,1$.

336. ELECTROTONUS-LAW OF CONTRACTION.-Opening and Closing Shocks. - A nerve is stimulated both at the moment of the occurrence and that of disappearance of electrotonus (i.e., by closing and opening the current-Ritter):(1) When the current is closed, the stimulation occurs only at the cathode, i.e., at the moment when the electrotonus takes place. (2) When the current is opened, stimulation occurs only at the anode, i.e., at the moment when the electrotonus disappears. '[This is Pflüger's well-known principle-" A given tract of nerve is stimulated by the appearance of cathelectrotonus and the disappearance of anelectrotonus - not, however, by the disappearance of cathelectrotonus nor by the appearance of anelectrotonus." From this principle can be deduced the law of contraction.] (3) The stimulation at the occurrence of cathelectrotonus is stronger than that at the disappearance of anelectrotonus (Pflüger).

Ritter's Opening Tetanus. - That stimulation occurs only at the anode, when the current is opened, was proved by Pflüger by means of "Ritter's opening tetanus." Ritter's tetanus consists in this, that when a constant current is passed for a long time through a long stretch of nerve, on opening the current, tetanus. lasting for a considerable time results. If the current 
was a descending one, then this tetanus ceases at once after section of the intrapolar area, a proof that the tetanus resulted from the now separated anode. If the current was an ascending one, section of the nerve has no effect on the tetanus.

Pfliiger and v. Bezold found a further proof that the closing or make contraction proceeds from the cathole, and the opening or break contraction from the anode, by showing that with a descending current, the closing contraction in the muscle, at the moment of closing occurred earlier, while the opening contraction at the moment of opening occurred later; and, conversely, with an ascending current the closing contraction oceurred later, and the opening contraction sooner. The difference in time corresponds to the time required for the propagation of the impulse in the intrapolar region $(\$ 337)$. If a large part of the intrapolar region in a frog's nerve be rendered inexcitalle by applying ammonia to it, then only the electrode next the muscle stimulates, i.e., always on closing or making a descending current and on opening or breaking an ascending one (Biedermann).

A. The law of contraction is valid for all kinds of nerves-I. The contraction nccurring at the closing or opening of a constant current varies with $(a)$ the direction (I'fuff'), and (b) the strength of the current (Heidenhain).

(1) Very feeble currents, in conformity with the third of the above statements, (ause only a closing contraction, both with an ascending and a descending current. The disappearance of electrotonus is so feeble a stimulus as not to excite the nerve.

(2) Medium currents cause opening or closing contractions both with an ascending and descending current.

(3) Very strong currents cause only a closing contraction with a descending current: the opening shock does not occur, because, with very strong currents, almost the whole of the intrapolar portion of the electrotonic nerve is incapable of conducting an impulse (p. 565). Ascending currents cause only an opening contraction for the same reason. With a certain strength of current, the muscle remains tetanic while the current is closed ("closing tetanus").

[The lan of contraction is formulated:- $\mathrm{R}=$ rest ; $\mathrm{C}=$ contraction.]

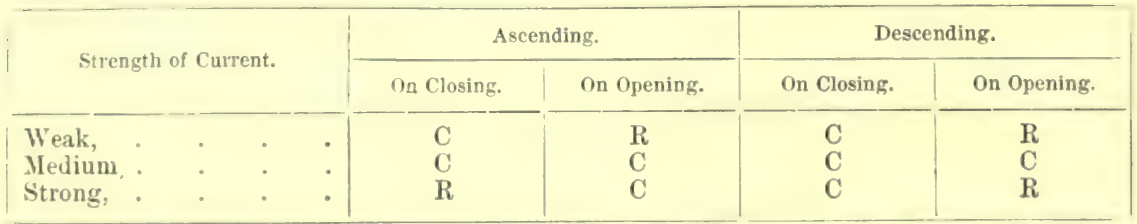

II. In a dying nerve, losing its excitability, according to the Ritter-Valli law $(\$ 325,7)$, the law of contraction is modified. In the stage of increased excitability, weak currents cause only closing contractions with both directions of the current. In the following stage, when the excitability begins to diminish, weak currents cause opening and closing contractions with both currents. Lastly, when the excitability is very greatly diminished, the descending current is followed only by a closing contraction, and the ascending by an opening contraction (Ritter, 1829).

III. As the various changes in excitability occur in a centrifugal direction along the nerve, we may detect the various stages simultaneously at different parts along the course of the nerve. According to Valentin and Fick, the living intact nerve shows only a closing contraction with both directions of the current, and opening contractions only with very strong currents.

Fleischl's Law of Contraction. - V. Fleischl and Stricker have stated a different law, in respect to the fact, that the excitability varies at certain points in the course of a nerve. The sciatic nerve is divided into three areas:-(1) Stretches from the muscle to the place where the branches for the thigh muscles are given off; (2) from here to the intervertebral ganglion; (3) from here into the sjinal cord. Each of these three areas consists of two parts (" upper and lower pole"), which adjoin each other at an equator. In each upper pole, the excitability of the nerve is greater for descending currents, and in each lower pole for ascending ones. At each equator the excitability of the nerve is the same for ascending and descending currents. The difference in the activity, due to the direction of the current, is greater for each stretch of nerve the greater this stretch is distant from the equator. The excitability is less at those points of the nerve where the three areas join each other. 
Eckhard observed that, on opening an ascending medium current applied to the hypoglossal nerve of a rabbit, one-half of the tongue exhibited a trembling movement instead of a contraction, while on closing a descending current, the same result occurred ( $\$ 297,3)$. According to Pfluiger, the molecules of the passive nerve are in a certain state of medium mobility. In cathelectrotonus the mobility of the molecules is increased, in anelectrotonus diminished.

B. The law for inhibitory nerves is similar. Moleschott, v. Bezold, and Donders have found similar results for the vagus, with this difference, that, instead of the contraction of a muscle, there is inhibition of the heart.

C. For sensory nerves also the result is the same, but we must remember that the perceptive organ lies at the central end of the nerve, while in a motor nerve it is at the periphery (muscle). Pflïger studied the effect of closing and opening a current on sensory nerves by observing the reflex movement which resulted. Weak currents cause only closing contractions ; medium currents both opening and closing contractions; strong descending currents only opening contractions; and ascending only closing contractions. Weak currents applied to the human skin cause a sensation with both directions of the current only at closing ; strong descending currents a sensation only at opening; strong ascending currents a sensation only at closing (Marianini, Matteucci). When the current is closed, there is prickly feeling, which increases with the strength of the current (Volta). Analogous phenomena have been observed in the sense organs (sensations of light and sound) by Volta and Ritter.

D. In muscle, the law of contraction is proved thus-by fixing one end of the muscle, keeping it tense, so that it cannot shorten, and opening and closing the current at this end. The end of the muscle, which is free to move, shows the same law of contraction as if the motor nerve were stimulated ( $v$. Bezold ). On closing the current, the contraction begins at the cathode; on opening, at the anode (Engelmann). E. Hering and Biedermann showed more clearly that both the closing and opening contractions are purely polar effects; when a weak current applied to a muscle is closed, the first effect is a small contraction limited to the cathodic surface of the muscle. Increase of the current causes increased contraction which extends to the anode, but which is weaker there than at the cathode; at the same time, the muscle remains contracted during the time the current is closed. On opening, the contraction begins at the anode; even after opening, the muscle for a time may remain contracted, which ceases on closing the current in the same direction.

By killing the end of a muscle in various ways, the excitability is diminished near this part. Hence, at such a place the polar action is feeble (van Loon and Engelmann, Biedermann). Touching a part with extract of flesh, potash, or alcohol diminishes locally the polar action, while soda salts and veratrin increase it (Biedermann).

Closing Continued Contraction. - The moderate continued contraction, which is sometimes observed in a muscle while the current is closed (fig. 329, $\ddot{0}$ ), depends upon the abnormal prolongation of the closing contraction at the cathode when a strong stimulus is used, or during the stage of dying, or in cooled winter frogs; sometimes the opening of the current is accompanied by a similar contraction proceeding from the anode (Biedermann). This tetanus is also due to the summation of a series of simple contractions (\$298, III.). By acting on a muscle with a 2 per cent. saline solution containing sodic carbonate, the duration of the contraction is increased considerably, and occasionally the muscle shortens rhythmically ( $\$ 296)$ (Biedermann).

If the whole muscle is placed in the circuit, the closing contraction is strongest with both directions of the current; during the time the current is closed, a continued contraction is strongest when the current is ascending ( $W$ undt).

Inhibitory Action.-The constant current, when applied to a muscle in a condition of continued and sustained contraction, has exactly the opposite effect to that on a relaxed muscle. If a constant current be applied by means of nonpolarisable electrodes to a muscle in a state of continued contraction, e.g., after poisoning with veratrin or through the contracted ventricle, when the current is closed, there is a relaxation beginning at the anode and extending to the other 
parts; on opening the current applied to muscle in continued contraction, the relaxation proceeds from the cathode.

Corresponding to this remarkable phenomenon, Biedermann found as regards the currents in the muscle-substance following the ordinary law, that every contracted part is negative to every passive section of the musele. Perhaps the experiment of Pawlow, who found nerve-fibres in the alductor muscle of the mussel, whose stimulation caused relaxation of the muscular contraction, may throw some light on this question.

Ritter's Opening Tetanus.-If a nerve or muscle be traversed by a constant current for some time, we often obtain a prolonged tetanus, after opening the current (Ritter's opening tetanus, 1798). It is set aside by closing the original current, while closing a current in the opposite direction increases it ("Volta's alternative "). The continued passage of the current increases the excitability for the opening of the current in the same direction, and for the closing of the reverse current: conversely, it diminishes it for the closing of the current in the same direstion, and for the opening of the reverse current (Volta).

Aceording to (iritzner and Tigerstedt, the cause of the opening contraction is partly due to the oceurrence of polarising after-currents ( $\$ 333$ ), and according to Hermann to a diminution of the anodic positive polarisation.

Engelmann and Grimhagen explain the occurrence of opening and closing tetanus, thus, as due to latent stimulations, drying, variations of the temperature of the prepared nerve, which of themselves are ton feeble to cause tetanus, but which become effective if an increased excitability obtiins at the cathode after closure, and at the anode after opening the current.

Biedermann showed that, under certain conditions, two successive opening contractions can be olstaines in a frog s nerve-musele preparation, the second and later one corresponding to Ritter's tetanus. The first of these contractions is due to the disappearance of anelectrotonus in l'fliger's sense; the second is explained, like Ritter's opening tetanus, in Engelmann and Griinhagen's sense.

Simultaneous action of the constant current and the nerve-current. - Action of two currents. In a nerve-muscle preparation used to prove the law of contraction, of course a demarcationcurrent is developed in the nerve $(\$ 334$, II.). If an artificial weak stimulating-current be applien to such a nerve, we obtain an interference effect clue to these two currents; closing a weak constant current causes a contraction, which, however, is not properly a closing contraction, but depends upon the opening (or derivation) of a branch of the demarcation-current; conversely, the opening of a weak constant current may excite a contraction, which is really due to the closing of a side branch of the nerve-eurrent, in a secondary circuit through the electrodes (Hering, Biedermann, Grützner).

If two induction shocks be simultaneously applied to a motor nerve, two cases are possible. Either the one shock is so feeble that the nerve is not thereby sufficiently excited to cause a contraction, while the other shock causes only a feeble contraction. In this case, the submaximal shock plays the part of a weak constant current, and the size of the contraction depenils only upon whether the effective stimulus was applied in the area of the anode or the (athode of the submaximal shock (Sewall, Grïnhagen, Werigo). If, however, unequal, strong, inluwtion shocks, wch of which is effective-but separated from each other on account of the electrotonic action-be applied to a nerve, then the result is as if the stronger alone was active. The feebler wave of excitation passes completely into the stronger one (Grünhagen, Werigo).

337. TRANSMISSION OF NERVOUS IMPULSES.-1. If a motor nerve be stimulated at its central end (1) a condition of excitation is set up, and (2) an impulse is transmitted along the nerve to the muscle with a certain velocity. The latter depends on the former and represents the function of conductivity. The velocity is about $27 \frac{1}{4}$ metres [about 90 feet] per second ( $v$. Helmholtz), and for the human motor nerves 33.9 [100 to 120 feet per second] (v. Helmholtz and Baxt).

The velocity is less in the visceral nerves, e.g., in the pharyngeal branches of the vagus 8.2 metres [ 26 feet] (Chauveau); in the motor nerves of the lobster 6 metres [18 feet] (Frédéricq and van de Velde).

Modifying Conditions. - The velocity is influenced by various conditions :Temperature.-It is lessened considerably by cold ( $v$. Helmholtz), but both high and low temperatures of the nerve (above or below $15^{\circ}$ to $25^{\circ} \mathrm{C}$.) lessen it (Steiner and Trojtzky) ; also curara, the electrotonic condition (v. Bezold) ; or only anelectrotonus, while cathelectrotonus increases it (Rutherford, Wundt). It varies also with 
the length of the conducting nerve, but it increases with the strength of the stimulus (v. Helmholtz and Baxt), although not at first ( $v$. Vintschgau).

Methods. - 1. V. Helmholtz (1850) estimated the velocity of the nerve-impulse in a frog's motor nerve after the method of Pouillet. The method depends unon the fact that, the needle of a galvanometer is deflected by a current of very short duration, the extent of the deflection being proportional to the duration and strength of the current. The apparatus is so arranged that the "timemarking current" is closed at the moment the nerve is stimulated, and opened again when the muscle contracts. If the nerve attached to a muscle be now stimulated at the further point from the muscle, and a second time near its entrance to the muscle, then in the latter case the time between the application of the stimulus and the beginning of the contraction of the muscle, i.e., the deflection of the galvanometer, will be less than in the former case, as the impulse has to traverse the whole length of the nerve to reach the muscle. The difference between the two times is the time required by the impulse to traverse a given distance of nerve. Fig. 411 shows in a diagrammatic manner the arrangement of the ex-

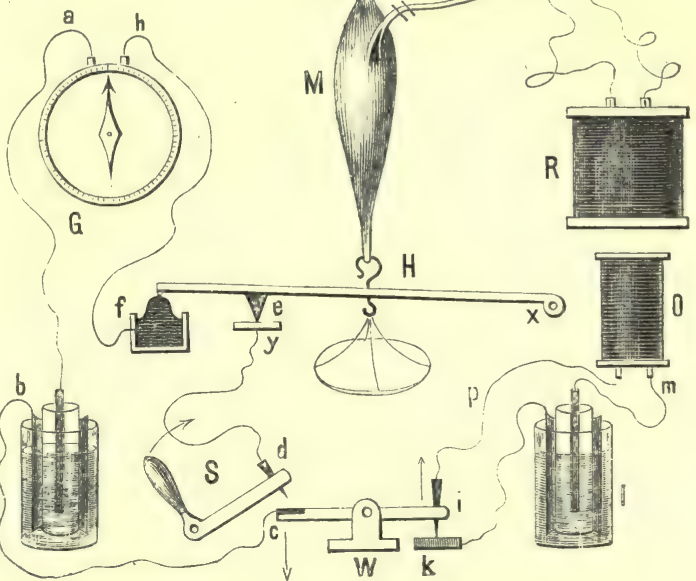

Fig. 411.

V. Helmholtz's method of estimating the velocity of a nerve-pulse.

periment. The galvanometer, $\mathrm{G}$, is placed in the time-marking circuit (open at first), $a, b$ (element), $c$ (piece of platinum on a key, W), introdnced into the time-marking circuit, $d, e$, $f, h$. The circuit is made by closing the key, $\mathbf{S}$, when $d$ depresses the platinum plate of the key, W. At once when the current is closed, the magnetic needle is deflected, and its extent noted. At the same moment in which the current between $c$ and $d$ is closed, the primary circuit of the induction machine is opened, the circuit being $i, k, l$ (element), $m, \mathrm{O}$ (primary spiral), $p$. Thereby an opening shock is induced in the secondary spiral, $\mathrm{R}$, which stimulates the nerve of the frog's leg at $n$. Thus, the closing of the galvanometer circuit exactly coincides with the stimulation of the nerve. The impulse is propagated through the nerve to the muscle, M, and the latter contracts when the impulse reaches it, at the same time opening the time-measuring circuit at the double contact, $e$ and $f$, by raising the lever, $\mathrm{H}$, which rotates on $x$. At the moment of opening, the further deflection of the magnetic needle ceases. The contact at $f$ is made by a pointed cupola of mercury.

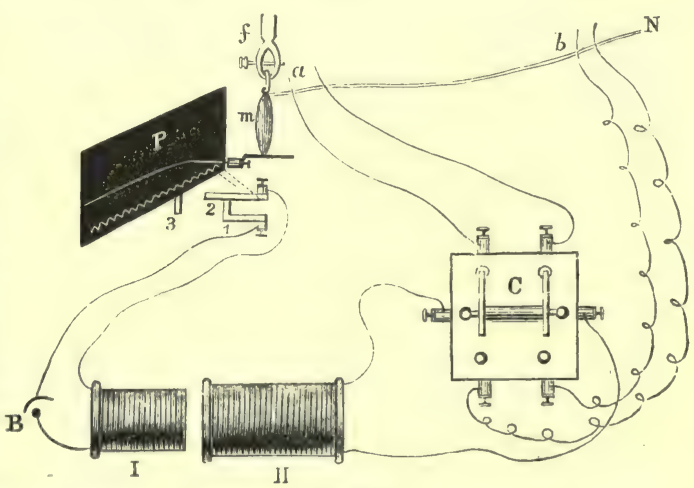

Fig. 412.

Scheme for measuring the velocity of nerve energy. $f$, clamp for femur; $m$, muscle; $\mathrm{N}$, nerve; $a$, near, $b$, removed from, C, commutator; II, secondary; I, primary spiral of induction machine ; B, battery; 1, 2, key; 3 , tooth on the smoked plate $\mathrm{P}$.

When the lever, $\mathrm{H}$, falls after the contact of the muscle, so that the point, $e$, comes into contact with the underlying solid plate, $y$, the contact at $f$ still remains open, i.e., through the galvanometer circuit. If the nerve be stimulated with the opening shock, first at $n$, and then 
at $\mathrm{N}$, the deflection of the needle is greater in the former than in the latter case. From the difference, we calculate the time for the conduction of the impulse in the stretch of the nerve between $n$ and $\mathrm{N}$.

[2. A simpler method is that shown in the scheme, fig. 412. Use a pendulum or spring myograph (fig. 323), and suspend in a suitable manner a frog's gastrocnemius $(m)$, with a long portion of the sciatic nerve $(\mathrm{N})$ dissected out, by fixing the femur in a clamp $(f)$, while the tendo Achilles is fixed to a lever, which inscribes its movements on the smoked glass plate $(P)$ of the myograph; place the key of the myograph (2) in the circuit with the battery (B), and the primary circuit of the induction machine (I). To the secondary coil (II) attach two wires, and connect them with a commutator without cross-bars (C). Connect the other binding serews of the commutator with two pairs of wires, arranged so that one pair can stimulate the nerve near the muscle $(a)$, and the other at a distance from it (b). When the glass plate flies from one side to the other, the tooth (3) on its framework opens the key (:2) in the primary circuit, and if the commutator be in the position indicated, then the induced current will stimulate the nerve at $a$, and a curve will be obtained on the glass plate. Rearrange the pendulum as before, but turn the handle of the commutator, and allow the glass plate to fly again. This time the induced current will stimulate the nerve at $b$, and a second contraction, a little later than the first one, will be obtained. Register the velocity of the glass plate by means of a tuning-fork, and the curve obtained will be something like fig. 413 , although this curve was obtained on a cylinder travelling at a uniform

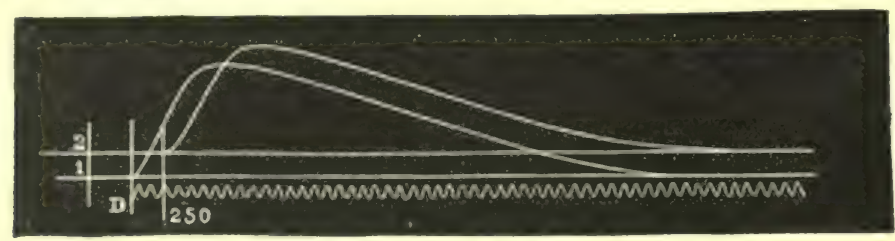

Fig. 413.

1, curve obtained on stimulating a nerve (man) near the muscle; 2 , when the stimulus was applied to the nerve at a distance from the muscle; D, vibrations of a tuning-fork (250 per second).

rate. The difference between the beginning of the $a$ and $b$ curves indicates the time that the nerve-impulse took to travel from $b$ to $a$. This time is measured by the tuning-fork, and if the distance between the points $a$ and $b$ is known, then the calculation is a simple one. Suppose the stretch of nerve between $a$ and $b$ to be 2 inches, and the time required by the impulse to travel from $a$ to $b$ to be $\frac{1}{4} \frac{1}{80}$ second, then we have the simple calculation -2 inches : 12 inches : : $\frac{1}{4} \frac{1}{80}: \frac{1}{80}{ }^{\prime \prime}$, or 80 feet per second. In fig. 413 the experiment was made on man; the curve 1 was obtained by stimulating the nerve near the muscle, and 2 when the nerve was stimulated at a distance of 30 centimetres. The interval between the vertical lines corresponds to $\frac{1}{100}$ second, i.e., the time required by the nerve-impulse to pass along 30 centimetres of nerve, which is equal to a velocity of 30 metres (90 feet) per second.]

In man, v. Helmholtz and Baxt estimated the velocity of the impulse in the median nerve by causing the muscles of the ball of the thumb to write off their contractions on a rapidly revolving cylinder. [In this case the "pince myographique" of Marey may be used ( $\$ 708)$. The ends of the pince are applied so as to embrace the ball of the thumb, so that when the muscles contract, the increase in thickncss of the muscles expands the pince, which acts on a Marey's tambour, by which the movement is transmitted to another tambour provided with a writing-style, and inscribing its movements upon a rapidly moving surface, either rotatory or swinging.] The nerve is stimulated at one time in the axilla and again at the wrist. Two curves are obtained, which, of course, do not begin at the same time. The difference in time between the beginning of the two curves is the time taken by the impulse to traverse the above- 
mentioned length of nerve. [The time is easily ascertained by causing a tuning-fork of a known rate of vibration to write its movements under the curves.]

3. In the sensory nerves of man, the velocity of the impulse is probably about the same as in motor nerves. The rates given vary between 94 to 30 metres [ 280 to 90 feet] per second ( $v$. Helmholtz).

Method.-Two points are chosen as far apart as possible, and at unequal distances from the brain, and they are successively excited by a momentary stimulus, e.g., an opening induction shock applied successively to the tip of the ear and the great toe. The moment of the application of the stimulus is indicated on the registering surface. The person experimented on is provided with a key attached to an electric arrangement, by which he can mark on the registering surface the moment he feels the sensation in each case.

Reaction Time. - The time which elapses between the application of the stimulus and the reaction is called the "reaction time." It is made up of the time necessary for conduction in the sensory nerve, that for the process of perception in the brain, for the conduction in the motor nerves to the muscles, by which the signs on the registering surface were made, and lastly by the latent period (p. 480). The reaction time is usually about 0.125 to 0.2 second $(\S 374)$.

Pathological. - The conduction in the cutaneous nerves is sometimes greatly delayed, in alterations of the cutaneous sensibility, in certain diseases of the spinal cord $(\$ 364)$. The sensation itself may be unchanged. Sometimes only the conduction for painful impressions is retarded, so that a painful impression on the skin is first perceived as a tactile sensation, and afterwards as pain, or conversely. When the interval of time between these two sensations is long, then there is a distinctly double sensation (Naunyn). It is rarely that voluntary movements are executed much more slowly from causes depending on the motor nerves, but occasionally the time between the voluntary impulse and the contraction is lengthened, but there may be in addition slower or longer continued contraction of the muscle. In tabes dorsalis or locomotor ataxia, the discharge of reflex movements is delayed; it is slower with thermal stimuli $\left(60^{\circ}\right)$ than with cold ones $\left(0.52^{\circ} \mathrm{C}\right.$., Ewald $)$.

338. DOUBLE CONDUCTION IN NERVES.-Conductivity is that property of a living nerve in virtue of which, on the application of a stimulus, it transmits an impulse. [The nature of a nerve-impulse is entirely unknown; we may conveniently term the process nerve-motion, but there is some reason to believe that nerve energy is transmitted by some sort of molecular vibration.] The conductivity is destroyed by all influences or conditions which injure the nerve in its continuity (section, ligature, compression, destruction by chemical agents); or which abolish the excitability at any part of its course (absolute deprival of blood; certain poisons, e.g., curara for motor nerves ; also strong anelectrotonus, $§ 335$ ).

Law of Isolated Conduction.-Conduction always takes place only in the continuity of fibres, the impulse never being transferred to adjoining nerve-fibres.

Double Conduction. - Although apparently conduction in motor nerves takes place only in a centrifugal direction towards the muscles, and in sensory nerves in a centripetal direction, i.e., towards the centre; nevertheless, experiment has proved that a nerve conducts an impulse in both directions, just as in a non-living conductor. If a pure motor or sensory nerve be stimulated in its course, an impulse is propagated at the same time in a centrifugal and in a centripetal direction. This is the phenomenon of "double conduction."

Proofs.- 1. If a nerve be stimulated, its electro-motive properties are affected both above and below the point of stimulation (see Negative Variation in Nerves, $\S 332)$.

2. Electrical Nerves.-If the posterior free-end of the electrical centrifugal nerves of the malapterurus be stimulated, the branches given off above the point of stimulation are also excited, so that the whole electrical organ discharges its electricity (Babuchin, Mantey).

3. Kuihne's Experiments.-The sartorius of the frog has no nerve-fibres at its upper and lower ends. If the lower end be cut off, and if the lower third of the muscle be suspended and divided vertically, on stimulating mechanically one apex of the muscle, then the impulse passes in the motor nerves centripetally to the place 
where the nerve-fibre bifurcates in the muscle, and from thence centrifugally into the other or non-stimulated apex, and causes it to contract.

[The Gracilis of the frog is divided into a larger and smaller portion (L) by a tendinous inscription ( $\mathrm{K}$ ) running across it (fig. 414). The nerve (N) enters at the hilum in the larger portion, bifurcates, and gives a branch $(k)$ to

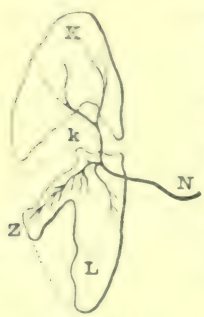

Fig. 414 . the smaller portion and another to the larger portion of the muscle. Let the muscle be cut as shown in fig. 414, avoiding injury to the nerves, so that only the nerve-twig $(k)$ connects the larger and smaller portions of the muscle. If the tongue or tip of muscle $(\mathrm{Z})$ with its nerves be stimulated, contraction occurs both in L and M, which is due to centripetal conduction in the motor nerve. The nerve-fibres divide dichotomously above where the nerves are given off to the portions $\mathrm{L}$ and $\mathrm{M}$.]

[If the inscription be left, and the lower tip of the muscle (which is devoid of nerves) be stimulated, only the lower and not the upper Kuhne's Gracilis prart twitches; but if a part of the muscle containing nerves common

experiment. to both parts be stimulated, then both parts of the muscle contract. This also proves that pure muscular excitation does not travel backwards from the muscle to the nerves. How this comes about, we are entirely ignorant.]

The following experiments used to be cited as proofs, but they do not stand the test of criticism.

4. Union of Motor and Sensory Nerves. - If the hypoglossal and lingual nerves be divided in a dog, and if the peripheral end of the hypoglossal be stitched, so as to unite with the central

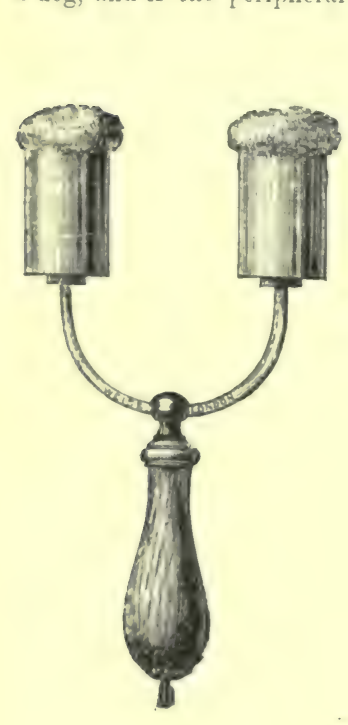

Fig. 415.

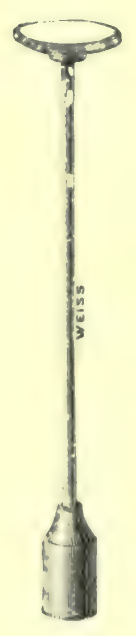

Fig. 416.

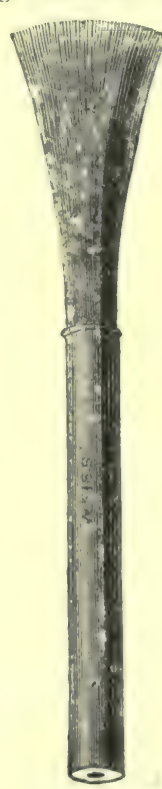

Fig 417.

Double sponge rheophore. Dise rheophore. Metallic brush. end of the lingual (Bidder), then, several months after the union and restitution of the nerves, stimulation of the central end of the lingual causes contraction in the corresponding half of the tongue. Hence, it has been assumed that the lingual, which is the sensory nerve of the tongue, must conduct the impulse in a peripheral direction to the end of the hypoglossal. This experiment is not conclusive, as the trunk of the lingual receives high up the centrifugal fibres from the seventh, viz., the chorda tympani, which may unite with those of the hypoglossal. Further, if the chorda be divided and allowed to degenerate before the above described experiment is made, then no contractions occur on stimu. lating the lingual above the point of union (\$349).

5. Bert's Experiment. - Paul Bert removed the skin from the tip of the tail of a rat, and stitched it into the skin of the back of the animal, where it united with the tissues. After the first union had taken place, the tail was then divided at its base, so that the tail, as it were, grew out of the skin on the back of the animal. On stimulating the tail, the animal exhibited signs of seusation. For the explanation of this experiment, see \& 325 .

339. ELECTRO-THERAPEUTICS-REACTION OF DEGENERATION. - Electricity is frequently employed for therapeutical purposes, the rapidly interrupted current of the induction machine, or Faradic current, being frequently used (especially since Duchenne, 1847), the 
magneto-electrical apparatus, and the extra-current apparatus. The constant or galvanic current is also used, especially since Remak's time, $1855(\S 330)$.

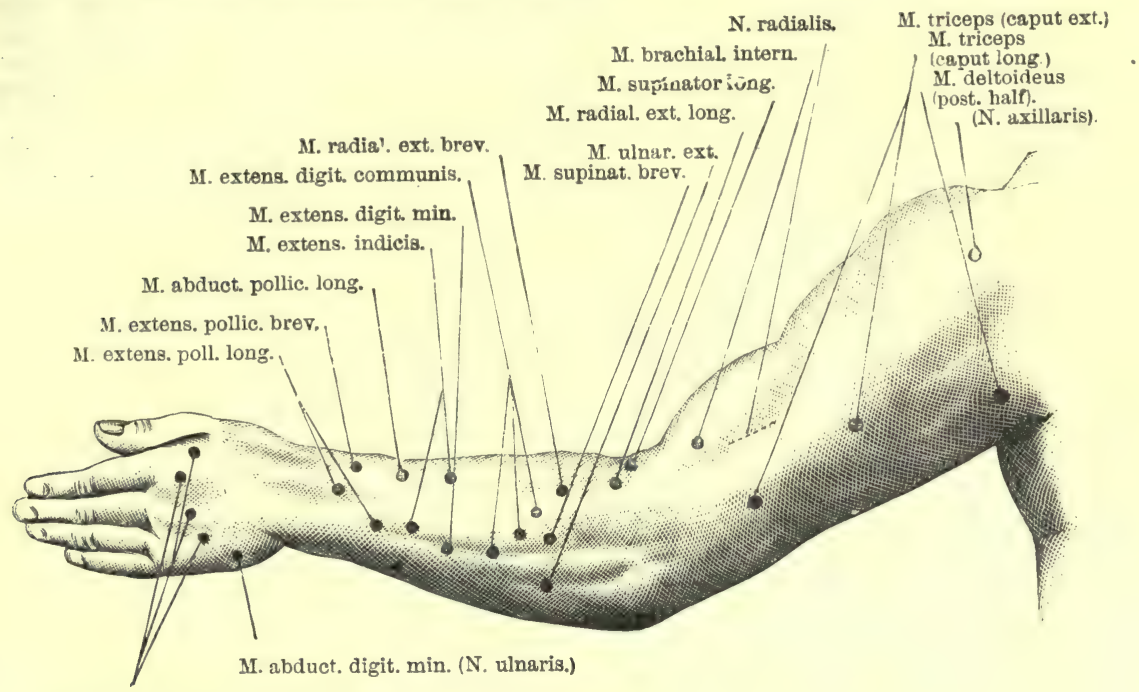

IIm. inteross. dorsal. I, II, III, et IV.

(N. ulnaris.)

Fig. 418.

Motor points of the radial nerve and the muscles supplied by it; dorsal surface.

1. In paralysis, Faradic currents are applied, either to the muscles themselves (Duchenne), or the points of entrance of the motor nerves, by means of suitable electrodes, or rheophores covered with sponge, \&c., and moistened (v. Ziemssen).

M. deltoideus (ant. half) N. axillaris.
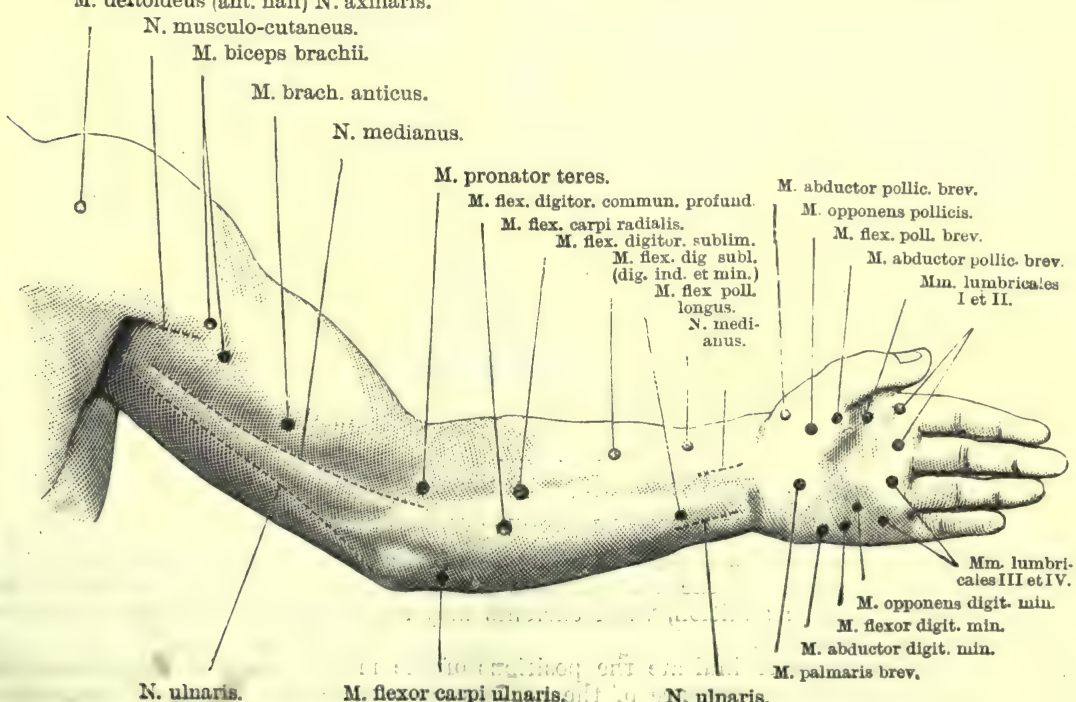

Fig. 419.

Motor points of the median and ulnar nerves, with the muscles supplied by them.

[Rheophores. - Many different forms are used, according to the organ or part to be stimu- 
lated, or the effect desirecl. When electricity is applied to the skin to remove anæsthesia, hyperesthesia, or altered sensibility, and we desire to limit the effect to the skin alone, then the rheophores are applied dry, and are usually made of metal. If, however, deeper-seated structures, as museles or nerve-trunks, are to be affected, the skin must be well moistened and softened by sponging with warm water, while the rheophores are fitted with sponges moistened with continon salt and water, which diminishes the resistance of the skin to the passage of electricity (figs. 415-417). ]

In faradising the paralysed muscle, the object is to cause artificial movements in it, and thus prevent the degeneration which it would otherwise undergo, merely from inaction. If, in atdition to the motor nerres, its trophic nerves are also paralysed, then a muscle atrophies,

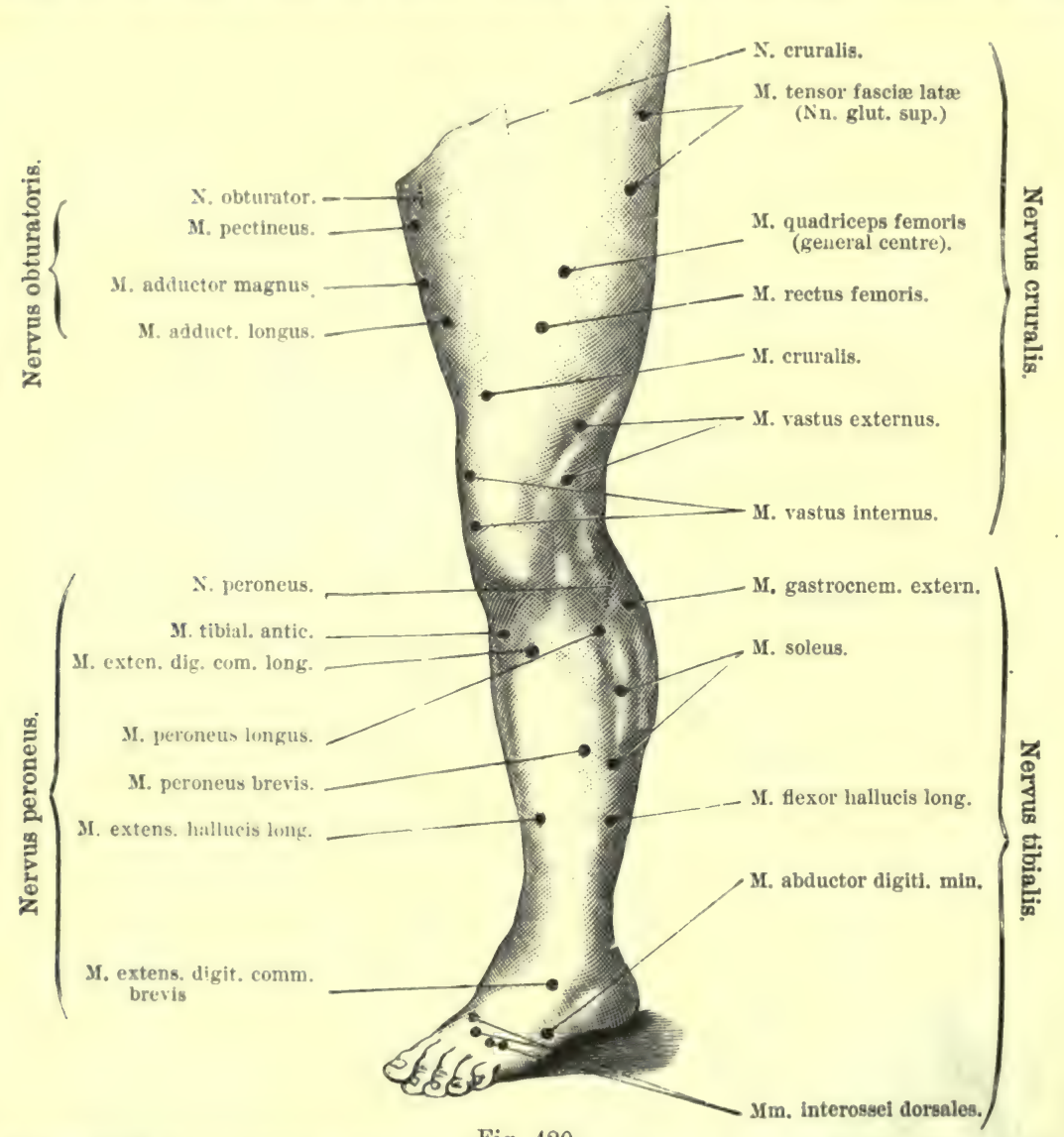

Fig. 420 ,

Motor points of the peroneal and tibial nerves on the front of the leg; the peroneal on the left, the tibial on the right (after Eichhorst).

notwithstanding the faradisation ' $(\$ 325,4)$. The use of the induced current also improves a paralysed muscle, as it increases the blood-stream through it, while it affects the metabolism of the muscle reflexly. In addition, weak currents may restore the excitability of enfeebled nerves (v. Bezold, Engelmann).

The figs. 418, 419, 420, 421 indicate the positions of the motor points of the extremities, where, by stimulating at the entrance of the nerve, each muscle may be caused to contract singly. In $\$ 349$ the motor points of the face, and in $\$ 347$ those of the neck, are indicated.

The constant current may be employed as a stimulus, when it is closed and opened, in the form of an interrupted current, by altering its direction and increasing or diminishing its intensity, but it also causes a polar action. On closing the current, the nerve at the cathode is 
stimulated ; similarly, on opening the current, at the anode ( $\$ 336)$. Thus, when the current is closed, the excitability of the nerve is increased at the cathode ( $\$ 335$ ), which may act favourably upon the nerve. Increased excitability in electrotonus at the anode, although feebler, has been observed during percutaneous galvanisation in man. This is especially the case by repeatedly reversing the current, sometimes also by opening and closing, or even with a uniform current. If the increase of the excitability is obtained, then the direction of the current increases the excitability on closing the reverse current, and on opening the one in the same direction.

Restorative Effect.-Further, in using the constant current, we have to consider its restorative effects, especially when it is ascending. R. Heidenhain found that feeble and fatigued muscles recover after the passage of a constant current through them.

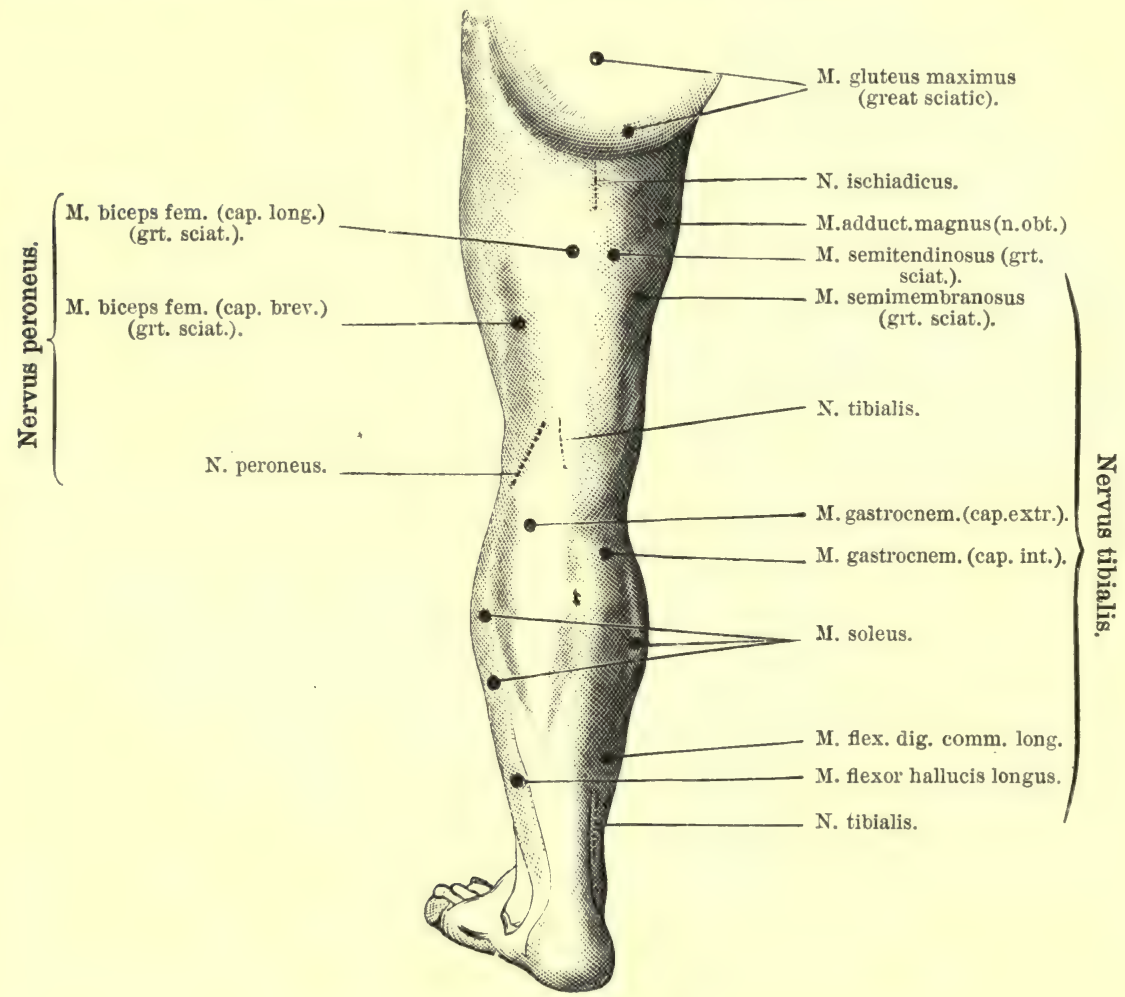

Fig. 421.

Motor points of the sciatic nerve and its branches; the peroneal and tibial nerves.

Lastly, the constant current may be useful from its catalytic or cataphoric action (\$328). The effect is directly upon the tissue elements. It may also act directly or reflexly upon the blood-and lymph-vessels.

Faradisation in Paralysis. - If the primary cause of the paralysis is in the muscles themselves, then the induced current is generally applied directly to the inuscles themselves by means of sponge electrodes (fig. 415); while, if the motor nerves are the primary seat, then the electrodes are applied over them. The current used must be only of very moderate strength; strong tetanie contractions are injurious, and so is too prolonged application (Eulenburg).

The galvanic current nay also be applied to the muscles or to their motor nerves, or to the centres of the latter, or to both muscle and nerve simultaneously. As a rule, the cathode is placed nearer the centre, as it increases the excitability. When the electrode is moved along the course of the nerve, or when the strength of the current is varied, the action is favoured. If the seat of the lesion is in the central nervous system, then the electrodes are applied along the vertebral column, or on the vertebral column, and the course of the nerves at the same 
time, or one on the head and the other on a point as near as possible to the supposed seat of the lesion. The current must not be too strong nor applied too long.

Induced $v$. Constant Current : Reaction of Degeneration.-Paralysed nerves and muscles behave quite differently as regards the induced (rapidly interrupted) and the constant current. This is called the "reaction of degeneration." We must remember the physiological fact that a dying nerve attached to a muscle (\$ $\$ 25)$, and also the muscles of a curarised animal, react much less strongly to rapidly interrupted currents than fresh nou-curarised muscles. Baierlacher, in 1859 , found that, in a case of facial paralysis, the facial muscles contracted but feebly to the induced current, but very energetically on the constant current being used. The excitability for the constant current may be abnormally increased, but may disappear on recovery taking place. Acrording to Neumann, it is the longer duration of the constant current as opposed to the momentary closing and opening of the induced current which makes the contraction of the muscle possible. If the constant current be broken as rapidly as the Faradic current is broken, then the constant current does not cause contraction. Conversely, the induced current may be remlered effective by causing it to last longer. We may also keep the primary circuit of the induction machine closed, and move the secondary spiral to and fro along the slots. Thus we whtain slow gradations of the induced current which act energetically upon curarised muscles (Iricick). Hence, in stimulating a muscle or nerve, we have to consider not only the strength, lut also the duration, of the current, just as the deflection of the magnetic needle depends upon these two factors.

[Galvanic excitability is the term applied to the condition of a nerve or muscle, whereby it responds to the opening or closing of a continuous current. The effects differ according as the current is opened or closed, and according to its strength. As a rule, the cathode causes a contraction chiefly at closure, the anode at opening the current, while the cathode is the stronger stimulus. With a weck current, the cathode produces a simple contraction on closing the current, but no contraction from the anode. With a medium current, we get with the eathode a strong closing contraction but no opening contraction, while the anode excites feeble opening and closing contrations. With a strong current, we get with the cathode a tetanic contraction at ('losure, and a prerceptible contraction at opening, while with the anode there is contraction both at opening and closing.]

[The law of contraction is usually expressed by the following formula $(E r b):-A n=$ anode, $\mathrm{C}_{\mathrm{i}}=$ cathole, $\mathrm{C}=$ contraction, $c=$ feeble contraction, $\mathrm{C}^{\prime}=$ strong contraction, $\mathrm{S}=$ closure of "urrent, $\mathrm{O}=$ opening of current, $\mathrm{Te}=$ tetanic contraction-so that, expressing the above statements briefly, we have-
Weak currents produce $\mathrm{Ca} \mathrm{S} \mathrm{C}$;
Medium ," , Ca S C', An S $c$, An O $c$;
Strong ," $\quad, \quad$ Ca S Te, An S C, An O C. Ca Oc.]

[Typical Reaction of Degeneration.-When the reaction of the nerve and muscle to electrical stimulation is altered both qualitatively and quantitatively, we have the reaction of degeneration, which is characterised essentially by the following conditions]:-The excitability of the muscles is diminished or abolished for the Faradic current, while it is increased for the galvanic current from the 3rd to 58 th day ; it again diminishes, however, with variations, from the 72 nd to 80 th day ; the anode closing contraction is stronger than the cathode closing contraction. The contractions in the affected muscles occur slowly in a peristaltic manner, and are local, in contrast with the rapid contraction of normal muscle. The diminution of the excitability of the nerves is similar for the galvanic and Faradic currents. If the reaction of the nerves be normal, while the muscle during direct stimulation with the constant current exhibits the reaction of degeneration, we speak of "partial reaction of degeneration," which is constantly present in progressive muscular atrophy $(E r b)$.

[The "reaction of degeneration" may occur before there is actual paralysis, as in lead poisoning. When it occurs we have to deal with some affection of the nerve-fibres, or of the trophic nerve-cells. When it is established, (1) stimulation of the nerve with Faradic and galvanic electricity does not cause contraction of the muscle; (2) direct Faradic stimulation of the wuscles does not cause contraction; (3) the galvanic current usually excites contraction more readily than in a normal muscle, so that the muscle responds to much feebler currents than act on healthy muscles, but the contraction is longer and more of a tonic character, and shows a tendency to become tetanic. The electrical excitability is generally unaffected in paralysis of cerebral origin, and in some forms of spinal paralysis, as primary lateral sclerosis and transverse myelitis, but the "reaction of degeneration" oceurs in traumatic paralysis, due to injury of the nerve-trunks, neuritis, rheumatic facial paralysis, lead palsy, and in affections 
of the nerve-cells in the anterior cornu of the grey matter of the spinal cord.] In rare cases the contraction of the muscles, caused by applying a Faradic current to the nerve, follows a slow peristaltic-like course- "Faradic reaction of degeneration" (E. Remak, Erb).

II. In Various Forms of Spasm (spasins, contracture, muscular tremor) the constant current is most effective (Remak). By the action of anelectrotonus, a pathological increase of the excitability is subdued. Hence, the anode ought to be applied to the part with increased excitability, and if it be a case of reflex spasm, to the points which are the origin or seat of the increased excitability. Weak currents of uniform intensity are most effective. The constant current may also be useful from its cataphoric action, whereby it favours the removal of irritants from the seat of the irritation. Further, the constant current increases the voluntary control over the affected muscles. In spasms of central origin, the constant current may be applied to the central organ itself. Faradisation is used in spasmodic affections to increase the vigour of enfeebled antagonistic muscles. Muscles in a condition of contracture are said to become more extensible under the influence of the Faradic current (Remak), as a normal muscle is more excitable during active contraction (\$301).

In Cutaneous Anæsthesia, the Faradic current applied to the skin by means of hair-brush electrodes is frequently used (fig. 417). When using the constant current, the cathode must be applied to the parts with diminished sensibility. The constant current alone is applied to the central seat of the lesion, and care must be taken to what extent the occurrence of cathelectrotonus in the centre affects the occurrence of sensation.

III. In Hyperæsthesia and Neuralgias, Faradic currents are applied with the object of over-stimulating the hyper-sensitive parts, and thus to benumb them. Besides these powerful currents, weak currents act reflexly and accelerate the bloorl-stream, increase the heart's action, and constrict the blood-vessels, while strong currents cause the opposite effects (O. Naumann). Both may be useful. In employing the constant current in neuralgia (Remak), one object is by exciting anelectrotonus in the hyper-sensitive nerves, to cause a diminution of the excitability. According to the nature of the case, the anode is placed either on the nerve-trunk, or even on the centre itself, and the cathode on an indifferent part of the body. The catalytic and cataphoric effects also are most important, for by means of them, especially in recent rheumatic neuralgias, the irritating inflammatory products are distributed and conducted away from the part. A descending current is transmitted continuously for a time through the nerve-trunk, and in recent cases its effects are sometimes very striking. Lastly, of course, the constant current may be used as a cutaneous stimulus, while the Faradic current also acts reflexly on the cardiac and vascular activity.

Recently, Charcot and Ballet have used the electric spark from an electrical machine in cases of anæsthesia, facial paralysis, and paralysis agitans. In some cases of spinal paralysis, muscles can be made to contract with the electric spark, which do not contract to a Faradic current. [Electricity is sometimes used to distinguish real from feigned disease, or to distinguish death from a condition of trance.]

Galvano-Cautery.-The electrical current is used for thermal purposes, as in the galvanocantery.

Galvano-Puncture. - The electrolytic properties of electrical currents are employed to cause coagulation in aneurisms or varix. [If the electrodes from a constant battery in action be inserted in an aneurismal sac, after a time the fibrin of the blood is deposited in the sac, whereby the cavity of the aneurism is gradually filled up. A galvanic current passed through defibrinated blood causes the formation of a coagulum of proteid matter at the positive pole and bubbles of gas at the negative.]

340. ELECTRICAL CHARGING OF THE BODY. - Saussure investigated by means of the electroscope the "charge" of a person standing on an insulated stool. The phenomena observed by him, which were always inconstant, were due to the friction of the clothes upou the skin. Gardini, Hemmer, Ahrens (1817), and Nasse regarded the body as normally eharged with positive electricity, while Sjosten and others regarded it as negatively charged. Most probably all these phenomena are due to friction, and are modified effects of the air in contact with the heterogeneous clothing (Hankel). A strong charge resulting in an actual spark has frequently been described. Cardanus (1553) obtained sparks from the tips of the hair of the head. According to Horsford (1837), long sparks were obtained from the tips of the fingers of a nervous woman in Oxford, when she stood upon an insulated carpet. Sparks have often been observed on combing the hair or stroking the back of a cat in the dark. Freshly voided urine is negatively electrical (Vasalli-Eandi, Volta); so is the freshly formed web of a spider, while the blood is positive.

341. COMPARATIVE-HISTORICAL.-Electrical Fishes. - Some of the most interesting phenomena connected with animal electricity are obtained in electrical fishes, of which there are about fifty species, including the electrical eel, or Gymnotus electricus, of the lagoons of the region of the Orinoco in South America-it may measure over 7 feet in length - the Torpedo marmoratco and some allied species, 30 to 70 centimetres [1 to $2 \frac{1}{2}$ feet], in the Adriatic and Mediterranean, the Malapternirus electricus of the Nile, and the Mormyrus also of the same river. By means of 
special electrical organs (Redi, 1666), these animals can in part voluntarily (gymnotus and malapterurus), and in part reflexly (torpedo), give a very powerful electrical shock. The electrical organ consists of "compartments" of various forms, separated from each other by connective-tissue, and filled with a jelly-like substance, which the nerves enter on one surface and ramify to produce a plexus. From this plexus there proceed branches of the axial cylinder, which end in a nucleated plate, the "electrical plate" (Billharz, M. Schulze). When the "electrical nerves" proceeding to the organ are stimulated, an electrical discharge is the result.

In Gymnotus, the electrical organ consists of several rows of columns arranged along both sides of the spinal column of the animal, under the skin as far as the tail. It receives on the anterior surface several branches from the intercostal nerves. Besides this large organ there is a smaller one lying on both sides above the anal fins. Here the plates are vertical, and the direction of the electrical current in the fish is ascending, so that of course it is descending in the surrounding water (Faraday, du Bois-Reymond).

In Malapterurus, the organ surrounds the body like a mantle, and receives only one nervefibre (1. 529), whose axis cylinder arises near the nedulla oblongata from one gigantic ganglionic cell (Billhar $\approx$ ), and is composed of protoplasmic processes (Fritsch). The plates are also vertical, and receive their nerves from the posterior surface. The direction of the current is descending in the fish during the discharge (du Bois-Reymond).

In the Torpedo, the organ lies immediately under the skin laterally on each side of the head, reaching as far as the pectoral fins. It receives several nerves which arise from the lobus clectricus, between the corpora quadrigemina and the medulla oblongata. The plates, which do not increase in number with the growth of the animal (Delle Chiaje, Babuchin), lie horizontally, while the nerve-fibres enter them on their dorsal surfaces, the current in the fish being from the abdominal to the dorsal surface (Galvani).

It is extremely probable that the electric organs are modified muscles, in which the nerve terminations are highly developed, the electrical plates corresponding to the motorial end-plates of the muscular fibres, the contractile substance having disappeared, so that during physiological activity the chemical energy is changed into electricity alone, while there is no "work" done. This view is supported by the observation of Babuchin, that during development the organs are originally formed like nuscles; further, that the organs when at rest are neutral, but when active or dead, acid; and lastly, they contain a substance related to myosin which coagulates after death (\$295- $-W c y l)$. The organs manifest fatigue; they have a "latent period" of 0.016 second, while one shock of the organ (comparable to the current in an active muscle) lasts 0.07 second. About twenty-five of these shocks go to make a discharge, which lasts about 0.23 second. The discharge, like tetanus, is a discontinuous process (Marey). Mechanical, chemical, thermal, and electrical stimuli cause a discharge; a single induction shock is not effective (Sachs). During the electrical discharge the current traverses the inuscles of the animal itself; the latter contract in the torpedo, while they do not do so in the gymnotus and malapterurus during the discharge (Steiner). A torpedo can give about fifty shocks per minute; it then becomes fatigued, and requires some time to recover itself. It may only partially discharge its organ (Al. v. Humbolut, Sachs). Cooling makes the organ less active, while heating it to $22^{\circ} \mathrm{C}$. makes it more so. The organ becomes tetanic with strychnin (Becquerel), while curara paralyses it (Sachs). Stimulation of the electrical organ of the torpedo causes a discharge (Matteucci); cold retards it, while section of the electrical nerves paralyses the organ. The electrical fishes themselves are but slightly affected by very strong induction shocks transmitted through the water in which they are swimming (du Bois-Ricymond). The sulstance of the electrical organs is singly refractive; excised portions give a current during rest, which has the same direction as the shock; tetanus of the organ weakens the current (Sachs, du Bois-Reymond). Perhaps the electrical organs of malapterurus is evolved from modified cutaneous glands (Fritsch).

Historical.- Richer (1672) made the first communication about the gymnotus. Walsh (1772) made investigations on the torpedo, on its discharge, and its power of communicating a shock. J. Davy magnetised particles of steel, caused a deflection of the magnetic needle, and obtained electrolysis with the electrical discharge. Becquerel, Brechet, and Matteucci studied the direction of the discharge. Al, v Humboldt described the habits and actions of the gymnotus of South America. Hausen (1743) and de Sauvages (1744) supposed that electricity was the active force in nerves. The actual investigations into animal electricity began with $G$. Aloisio Galvani (1791), who observed that frogs' legs connected with an electrical machine contracted, and also when they were touched with two different metals. He believed that nerves and muscles generated electricity. Alessandro Volta ascribed the second experiment to the electrical current prorluced by the contact of dissimilar metals, and therefore outside the tissues of the frog. The contraction without metals described by Galvani was confirmed by Alex. v. Humboldt (1798). Pfaff (1793) first observed the effect of the direction of the current upon the contraction of a frog's leg obtained by stimulating its nerve. Bunzen made a galvanic pile of frogs' legs. The whole subject entered on a new phase with the construction of the galvanometer and since the introduction of the classical methods devised by du Bois-Reymond, i.e., from 1843 onwards. 


\section{Physiology of the Peripheral Nerves.}

342. FUNCTIONAL CLASSIFICATION OF NERVE-FIBRES.--As nervefibres, on being stimulated, are capable of conducting impulses in both directions ( $\$ 338$ ), it is obvious that the physiological position of a nerve-fibre must depend essentially upon its relations to the peripheral end-organ on the one hand, and its central connection on the other. Thus, each nerve is distributed to a special area within which, under normal circumstances, in the intact body, it performs its functions. This function of the individual nerves, determined by their anatomical connections, is called their "specific energy."

I. Centrifugal or Efferent Nerves.-(a) Motor.-Those nerve-fibres whose peripheral end-organ consists of a muscle, the central ends of the fibres being connected with nerve-cells :-

1. Motor fibres of striped muscle ( $\$ 292$ to 320 ).

2. Motor nerves of the heart $(\$ 57)$.

3. Motor nerves of smooth muscle, e.g., the intestine (\$171). The vaso-motor nerves are specially treated of in $\S 371$.

(b) Secretory.-Those nerve-fibres whose peripheral end-organ consists of a secretory cell, the central ends of the fibres being connected with nerve-cells.

Examples of secretory nerves are the secretory nerves for saliva ( $\$ 145)$ and those for sweating (\$289, II.). It is to be remembered, however, that these fibres not unfrequently lie in the same sheath with other nerve-fibres, so that stimulation of a nerve may give rise to several results, according to the kind of nerve-fibres present in the nerve. Thus, the secretory and vaso-motor nerves of glands may be excited simultaneously.

(c) Trophic.-The end-organs of these nerve-fibres lie in the tissues themselves, and are as yet unknown. These nerves are called trophic, because they are supposed to govern or control the normal metabolism of the tissues.

In some tissues, we know of a direct connection of their elements with nerve-fibres, which may influence their nutrition. Nerves are connected with the corneal corpuscles $(\S 201,7)$, with the pigment-cells of the frog's skin (Ehrmann), the connective-tissue corpuscles of the serous membrane of the stomach of the frog, and the cells around the stomata of lymphatic surfaces $(\$ 196,5)(E . F \cdot$ Hoffmann $)$.

Trophic Influence of Nerves. - The trophic functions of certain nerves are referred to as under:-On the influence of the trigeminus on the eye, the mucous membrane of the mouth and nose, the face ( $\$ 347)$; the influence of the vagus on the lungs ( $\$ 352)$; motor nerves on muscle ( $\$ 307)$; nerve-centres on nerve-fibres $(\$ 325,4)$; certain central organs upon certain viscera $(\$ 379)$.

Section of certain nerves influences the growth of the bones. H. Nasse found that, after section of their nerves, the bones showed an absolute diminution of all their individual constituents, while there was an increase of the fat. Section of the spermatic nerve is followed by degeneration of the testicle (Nelaton, Obolensky). After extirpation of their secretory nerves, there is degeneration of the sub-maxillary glands (p. 213). Section of the nerves of the cock'scomb interferes with the nutrition of that organ (Legros, Schiff). After section of the 2 nd cervical nerve in rabbits and cats, the hair falls off the ear on that side (Joseph). Section of the 
cervical sympathetic nerve in young, growing animals is followed by a more rapid growth of the ear upon that side (Bidder, Stirling, Stricker), also of the hair on that side (Schiff, Stirling); while it is said that the corresponding half of the brain is smaller, which, perhaps, is due to the pressure from the dilated blood-vessels (Browon-Séquard).

Blood-Vessels. - Lewaschew found that prolonged uninterrupted stimulation of the sciatic nerve of docs, by means of chemical stimuli [threads dipped in sulphuric acid], eaused hypertrophy of the lower limb and foot, together with the formation of aneurismal dilatations upon the blood-vessels.

Skin and Cutaneous Appendages. - In man, stimulation or paralysis of nerves, or degeneration of the grey matter of the spinal corl, is not unfrequently followed by changes in the pigmentation of the skin, in the nails, in the hair and its mode of growth and colour (Jarisch). [Injury to the lrain, as by a fall, sometimes results in paralysis of the hair follicles, so that, after sucl, an injury, the hair is lost over nearly the whole of the body.] Sometimes there may be eruptims upon the shin, apparently traumatic in their origin ( $v$. Bürcnsprung). Sometimes there is a temlency to decubitus $(\$ 379)$, and in some rare cases of tabes, there is a peculiar degeneration if the joints (Charcot's disease). The changes which take place in a nerve separated from its centre are described in $\$ 325$.

[Trophoneuroses. - Some of the chief data on which the existence of trophic nerves is assumed are indieated above. There are many pathological conditions referable to diseases or injuries of nerves.]

[Muscles,-As is well known, paralysis of a motor nerve leads to simple atrophy of the corresponding muscle, provided it he not exercised; but when the motor ganglionic cells of the anterior horn of grey matter, or the corresponding cells in the crus, pons, and medulla, are paralysed, there is an active condition of atropliy with proliferation of the muscular nuclei. Progressive muscular atrophy, or wasting lalsy, is another trophic change in muscle, whereby either individual muscles, or crouls of muscles, are one after the other paralysed and become atropliterl. In pseudo-hypertrophic paralysis, there is cirrhosis or increased development of the connective-tissue, with a climinution of the true muscular elements, so that although the muscles increase in bulk their power is dininished.]

Cutaneous Trophic Affections.-Amongst these may be mentioned the occurrence of red jatches or erythema, urticaria or nettle-rash, some forms of lichen, eczema, the bulle or blebs of pemplingus, and some forms of ichthyosis, each of which may occur in limited areas after injury to a nerve or its spinal or cerebral centre. The relation between the cutameous eruption and the distribution of a nerve is sometimes very marked in herpes zoster, which frequently follows the distribution of the intercostal and supraorbital nerves. Glossy skin (Paget, Weiv Mitchell) is a condition lepending upon impaired nutrition and circulation, and due to injuries of nerves. The skin is smooth and glossy in the area of distribution of certain nerves, while the wrinkles and folds have disappeared. In myxœdema, the subeutaneous tissue and other oruans are infiltrated with, while the blood contains, mucin. The subcutaneous tissue is swollen, and the pratient looks as if suffering from renal dropsy. There is marked alteration of the cerebral faculties, and a condition resembling a "cretinoid state" occurs after the excision of the thyroid gland. Victor Horsley has shown that a similar condition occurs in monkeys after excision of the thyroid gland (\$ 103, III.). [Laycock described a condition of nervous codema which occurs in some cases of hemiplegia, and apparently it is independent of renal or cardiac disease.]

[There are alterations in the colour of the skin depending on nervous affections, including localised leucoderma, where circumscribed patches of the skin are devoid of pigment. The ligmentatiou of the skin in Addison's disease or bronzed skin, which occurs in some cases of disease of the suprarenal capsules, may le partly nervous in its origin, more especially when we consider the remarkable pigmentation that occurs around the nipple and some other parts of the body lluring pregnancy, and in some uterine and ovarian affections.

In anmsthetic leprosy, the anresthesia is due to the disease of the nervous structure, which results in listurbance of motion and uutrition. Amongst other remarkable changes in the skin, perhajss due to trophic conditions, are those of symmetrical and local gangrene, and acute decubitus or bed-sores:]

[Bed-Sores. - Besides the simple chronic form, which results from over-pressure, bad nursing, and inattention to cleanliness, combined with some defect of the nervous conditions, there is another form, acute decubitus, which is due directly to nerve influence (Charcot). The latter usually aprears within a few hours or days of the cerebral or spinal lesion, and the whole cycle of changes-from the appearance of the erythematous dusky patch to inflammation, ulceration, and gangrene of the buttock-is completed in a few days. An acute bed-sore may form when every attention is paid to the avoidance of pressure and other unfavourable conditions. When it depends on cerebral affections, it begins and develops rapidly in the centre of the gluteal region on the paralysed side, but when it is due to disease of the spinal cord, it forms more in the midlle line in the sacral region; while in unilateral spinal lesions it oceurs not on the paralysed, but on the anasthetic side, a fact which seems to show that the trophic, like the sensory fibres, decussate in the cord (Ross) 1 
[There are other forms due to nervous disease, including symmetrical gangrene and local asphyxia of the terminal parts of the body, such as toes, nose, and external ear, caused perhaps by spasm of the small arterioles (Raynaud's disease); and the still more curious condition of perforating ulcer of the foot. Hrmorrhage of nervous origin sometimes occurs in the skin, including those that occur in locomotor ataxia after severe attacks of pain, and hæmatoma aurium, or the insane ear, which is specially common in general paralytics.]

(d) [Inhibitory nerves are those nerves which modify, inhibit, or suppress a motor or secretory act already in progress.]

Take as an example the effect of the vagus upon the action of the heart. Stimulation of the peripheral end of the vagus causes the heart to stand still in diastole $(\S 85)$; see also the effect of the splanchnic upon the intestinal movements (\$161). The vaso-dilator nerves, or those whose stimulation, is followed by dilatation of the blood-vessels of the area which they supply, are referred to especially in $\S 237$.

[There is the greatest uncertainty as to the nature and mode of action of inhibitory nerves, but take as a type the vagus, which depresses the function of the heart, as shown by the slower rhythm, diminution of the contractions, relaxation of the muscular tissue, lowering of the excitability and conduction. These phenomena are not due to exhaustion. Gaskell points out that the action is beneficial in its after effects, so that this nerve, although it causes diminished acitivity, is followed by repair; of function; hence, he groups it as anabolic nerve, the outward symptoms of cessation of function indicating that constructive chemical changes are going on in the tissue.]

(e) Thermic and electrical nerves have also been surmised to exist.

[Gaskell classifies the efferent nerves differently. Besides motor nerves to striperl muscle, he groups them as follows:-

1. Nerves to vascular muscles.

(a) Vaso-motor, i.e., vaso-constrictor, accelerators and augmentors of the heart.

(b) Vaso-inhibitory, i.e., vaso-dilators and inhibitors of the heart.

2. Nerves of the visceral muscles.

(a) Viscero-motor.

(b) Viscero-inhibitory.

3. Glandular nerves.]

[Other terms are applied to nerves with reference to the chemical changes they excite in a tissue in which they terminate. The ordinary metabolism is the resultant of two processes-one constructive, the other destructive, or of assimilation and dissimilation respectively. The former process is anabolism, the latter katabolism. A motor nerve excites chemical destructive changes in a muscle, and is so far the katabolic nerve of that tissue; in the same way the sympathetic to the heart, by causing more rapid contraction, is also a katabolic nerve, while the vagus, as it arrests the heart's action, and brings about a constructive metabolism of the cardiac tissue, is an anabolic nerve (Gaskell).]

II. Centripetal or Afferent Nerves.-(a) Sensory Nerves (sensory in the narrower sense), which by means of special end-organs conduct sensory impulses to the central nervous system.

(b) Nerves of Special Sense.

(c) Reflex or Excito-motor Nerves.-When the periphery of one of these nerves is stimulated, an impulse is set up which is conducted by them to a nervecentre, from whence it is transferred to a centrifuyal or efferent fibre, and the mechanism (I, a, b, c, d) in connection with the peripheral end of this efferent fibre is set in action; thus, there are--Reflex motor, Reflex secretory, and Reflex inhibitory fibres. [Fig. 422 shows the simplest mechanism necessary for a reflex Scheme of a reflex motor act. $\mathrm{S}$, motor act. The impulse starts from the skin, $\mathrm{S}$, travels up the nerve, af, to the nerve-centre or nerve-cell, $\mathrm{N}$,

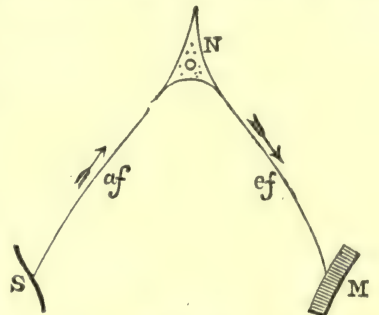

Fig. 422. skin ; af, afferent nerve; $\mathbf{N}$, nerve-cell ; ef, efferent fibre. situate in the spinal cord, where it is modified and transferred to the outgoing fibre, ef, and conveyed by it to the muscle, M.]

III. Intercentral Nerves.-These fibres serve to connect ganglionic centres 
with each other, as, for example, in co-ordinated movements, and in extensive reflex acts.

\section{THE CRANIAL NERVES.}

343. I. NERVUS OLFACTORIUS.-Anatomical. - The three-siled prismatic tractus olfactorius, lying in a groove on the uncler surface of the frontal lobe, arises by means of an inner, outer, and millle root, from the tuber olfactorium (fig. 428, I). The tractus swells out upon the cribriform plate of the ethmoid bone, and becomes the bulbus olfactorius, which is the analorue of the special portion of the brain, existing in different mammals with a well-developed sense of smell (frrutiolet). From twelve to tifteen olfactory filaments pass through the foramina in the cribriform plate of the ethmoid bone. At first they lie between the periosteum and the mucous membrane, but in the lower third of their course they enter the mucous membrane of the regio olfactoria. The bulb consists of white matter below, and above of grey matter mixed with small spinille-shaped ganglionic cells. Henle describes six, and Meynert eight layers, of nervous matter seen on transverse section. [The centre for smell lies in the tip of the uncinate grrus on the inner surface of the cerebral hemisphere (Ferricr).] According to Gudden, removal of the olfactory bulh is followed by atrophy of the gyrus uncinatus on the same side. According to Hill, the three roots of the olfactory bulb stream backwards, the inner one is small, the mildle one is a thick bundle, which grooves the head of the caudate nucleus, curves inwards to the anterior commissure, and crosses ria this commissure where it decussates, and passes to the extremity of the temporo-sphenoidal lobe. The outer roots pass transversely into the pvriform lobe, thence via the fornix, corpora albicantia, the bundle of Vicq d'Azyr into the anterior end of the optic thalamus. Hill also points out that the elements contained in the olfactory bulb are identical with those contained in the four outer layers of the retina. Flechsig traces its origin (1) to the gyrus fornicatus, (2) through the lamia perforata anterior to the internal capsule (sensory part), and to the gyrus uncinatus (sensory area of the cerebrum) ( $\$ 378$, IV.). Proliably the fibres at their origin cross to the cerebrum. There is a connection between the olfactory bulbs in the anterior commissure. [Each nerve is related to both hemispheres.]

Function. - It is the only nerve of smell. Physiologically, it is excited only by gaseous odorous bodies-(Sense of Smell, $\$ 420)$. Stimulation of the nerve, by any other form of stimulus, in any part of its course, causes a sensation of smell. [It also conveys those impressions which we call flavours, but in this case the sensation is combined with impressions from the organs of taste. In this case also the stimulus reaches the nerve by the posterior nares.] Congenital absence or section of both olfactory nerves abolishes the sense of smell (easily performed on young animalsBiffi).

Pathological.-The term hyperosmia is applied to cases where the sense of smell is excessively and abnomally acute, as in some hysterical persons, and in cases where there is a purely subjective sense of smell, as in some insane persons. The latter is perhaps due to an abnornal stimulation of the cortical centre ( $\$ 378$, IV.). Hyposmia and anosmia (i.e., diminution and abolition of the sense of smell) may be due to mechanical causes, or to overstimulation. Strychnin sometimes increases, while morphia diminishes, the sense of smell. [Method of Testing, $\S 421$.

344. II. NERVUS OPTICUS. - Anatomical. - The tractus opticus (fig. 428, II) arises from the anterior corpora quadrigemina, the corpus geniculatum externum, and the thalamus opticus (fig. 428), as well as from the grey matter which lines the third ventricle (Tartuferi). A broad bundle of fibres passes from the origin of the optic tract to the cortical visual centre, at the apex of the occipital lohe on the same side (Wernicke- $\$ 379, I V$.$) . Fibres pass from$ the cerebellum through the crura.

The optic tract bends round the pedunculus cerebri, where it unites with its fellow of the opposite side to form the chiasma, and from the opposite side of this the two optic nerves spring.

[Connections of Optic Tract.-There is very considerable difficulty in ascertaining the exact origin of all the fibres of the optic tract. Although as yet the statement of Gratiolet is not provel that the optic tract is directly connected with every part of the cerebral hemisphere in man, from the frontal to the occipital lobe, still the researches of $\mathrm{D}$. J. Hamilton have shown that its connections are very extensive. It is certain that some of them are ganglionic, i.e., connected with the ganglia at the base of the brain, while others are cortical, and form connections with the cortex cerebri. The ganglionic fibres arise from the corpora geniculata, pulvinar, and anterior corpora quadrigemina, and probably also from the substance of the thalamus. The cortical fibres join the ganglionic to form the optic tract. According to D. J. Hamilton, the connection with the cortex in the frontal region is brought about by "Meynert's commissure." The latter arises directly from the lenticular-nucleus-loop, decussates in the lamina cinerea, and 
passes into the optic nerve of the opposite side. The lenticular-nucleus-loop is formed below the lenticular nucleus by the junctior of the striæ medullares; the striæ medullares form part of the fibres of the internal capsule, and the inner capsule is largely composed of fibres descending from the cortex. Hamilton also asserts that other cortical connections join the tract as it winds round the pedunculus cerebri, and they include $(a)$ a large mass of fibres coming from the motor areas of the opposite cerebral hemisphere, crossing in the corpus callosum, entering the outer capsule, and joining the tract directly ; $(b)$ fibres uniting it to the temporo-sphenoidal lobe of the same side, especially the first and second temporo-sphenoidal convolutions; $(c)$ fibres to the gyrus hippocampi of the same side; $(d)$ a large leash of fibres forming the "optic radiation" of Gratiolet, which connect it directly with the tip of the occipital lobe. There are probably also indirect connections with the occipital region through some of the basal ganglia. Although some observers do not admit the connections with the frontal and sphenoidal lobes, all are agreed as to its connection with the occipital by means of the "optic radiation."]

[The optic radiation of Gratiolet is a wide strand of fibres expanding and terminating in the occipital lobes. It is composed of, or, stated otherwise, gives branches to $(a)$ the optic tract directly, $(b)$ the corpus geniculatum internum and externum, $(c)$ to the pulvinar and substance of the thalamus, $(d)$ a direct sensitive band (Meynert's "Sensitive band") to the posterior third of the posterior limb of the inner capsule, $(e)$ fibres which run between the island of Reil and the tip of the occipital lobe (D.J. Hamilton).]

Chiasma. - The extent of the decussation of the optic fibres in the chiasma is subject to variations. As a rule, rather more than half of the fibres of one tract cross to the optic nerve of the opposite side (fig. 423), so that the left optic tract

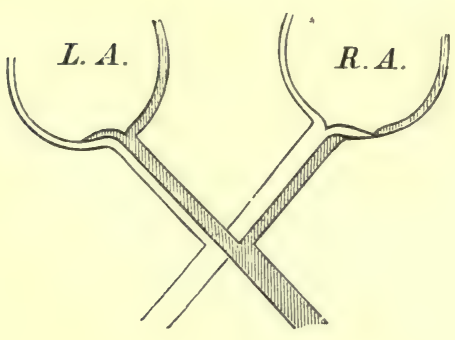

Fig. 423.

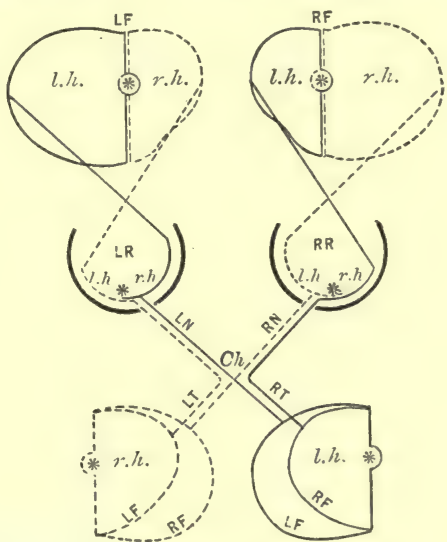

Fig. 424.

Fig. 423. - Scheme of the semi-decussation of the optic nerves. L.A., left eye ; R.A., right eye. Fig. 424.-Diagram of the relation of the field of vision, retina, and optic tracts. $\mathrm{RF}, \mathrm{LF}$, right and left fields of vision-the asterisk is at the fixing point ; RK, LR, right and left retina-the asterisk is at the macula lutea; $l . h ., r . h$. , left half and right half of each retina, receiving rays from the opposite half of the field; RN, LN, right and left optic nerves; Ch, chiasma; RT, LT, right and left optic tracts; below, the halves of the fields from which impressions pass by each optic tract are superimposed (Govers).

sends fibres to the left half of both eyes, while the right tract supplies the right half of both eyes ( $\$ 378$, IV.). [Thus, the corresponding regions of each retina are brought into relation with one bemisphere. The fibres which cross are from the nasal half of each retina (fig. 424).]

Hence, in man, destruction of one optic tract (and its central continuation in the occipital lobe of the cerebrum) produces "equilateral or homonymous hemianopia." In the cat there is a semi-decussation; hence, in this animal extirpation of one eyeball causes atrophy and degeneration of half of the nerve-fibres in both optic tracts (Gudden). Baumgarten and Mohr have observed a similar result in man. A sagittal section of the chiasma in the cat produces partial blindness of both eyes (Nicati). According to Gudden, the fibres which decussate are more numerous than those which do not, although J. Stilling maintains that they are only slightly more numerous. According to J. Stilling, the decussating fibres lie in the central axis of the nerve, while those which do not decussate form a layer around the former. 
Other observers maintain that there is complete decussation of all the fibres in the chiasma. Hence, section of one optic nerve causes dilatation of the pupil and blindness on the same side, while section of one opitic tract causes dilatation of the pupil and blindness of the opposite eye (Knoll). In osseous fishes, both optic nerves are isolated and merely cross over each other, while in the cyclostomata they do not cross at all. [Total decussation occurs in those animals where the eyes do not act together.]

Injury of the external geniculate body and section of the anterior brachium have the sume effect as section of the optic tract of the same side ( $\$ 359$-Bechtercw).

In very rare cases the decussation is absent in man, so that the right tract passes directly into the right eveliall, and the left into the left eyeball (Vesalius, Caldani), the sight not being interfered with.

It is quite certain that the individual fibres do not divide in the chiasma. Two commissures, the inferior commissure (Gudden) and Meynert's commissure, unite both optic tracts further back.

A special commissure (C. inferior) extends in a curved form across the posterior angle of the chiasma (Gudden). It does not degenerate after enucleation of the eyeballs, so that it is regarlerl as an intercentral connection. After excision of an eye, there is central degeneration of the fibres of the optic nerve entering the eyeball (Gudden), and in man about the half of the fibres in the corresponding optic tract (Baumgarten, Mohr). After section of both optic nerves, or enucleation of both eyeballs, there is a degeneration, proceeding centrally, of the whole optic

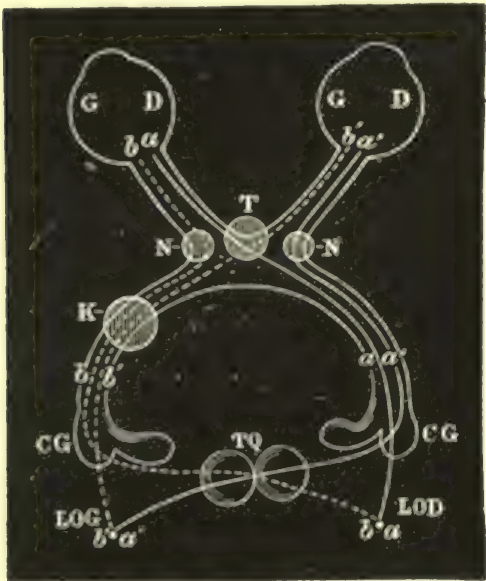

Fig. 425. tract. The degeneration extends to the origins in the corpora quadrigemina, corpora geniculata, and pulvinar, but not into the conducting paths leading to the cortical visual centre $(v$. Monakow) (\$ 378 , IV. I.)

[Hemianopia and Hemianopsia. - When one optic tract is interfered with or divided, there is interference with or loss of sight in the lateral halves of both retinæ, the blind part being separated from the other half of the field of vision by a vertical line. When it is spoken of as paralysis of one-half of the retina, the term hemiopia, or preferably hemianopia, is applied to it ; when with reference to the field of vision, the term hemianopsia is used (see Eye). Suppose the left optic tract to be divided or pressed upon by a tumour at K (fig. 425), then the outer half of the left and the inner half of the right eye are blind, causing right lateral hemianopsia, i.e., the two halves are affected which correspond in ordinary vision, so that the condition is spoken of homonymous hemianopsia. Suppose the lesion to be at $\mathrm{T}$ (fig. 425), then there is paralysis of the inner halves of both eyes, causing double temporal

Diagram of the decussation of the optic tracts.

$T$, semi-decussation in the chiasma; TQ, decussation of fibres behind the ext. geniculate bodies (CG); $a^{\prime} b$, fibres which do not decussate in the chiasma; $b^{\prime} a^{\prime}$, fibres proceeding from the right eye, and coming together in the left hemisphere (LOG); $\mathrm{LOG}, \mathrm{K}$, lesion of the left optic tract producing right lateral hemianopsia; $A$, lesion in the left hemisphere producing crossed amblyopia (right eye); $T$, lesion producing temporal hemianopsia; NN, lesion producing nasal hemianopsia.

hemianopsia. When there are two lesions at NN, which is very rare, the outer halves of both retinæ are paralysed, so that there is double nasal hemianopsia. In order to explain some of the eye symptoms that occasionally occur in cerebral disease, Chareot has supposed that some of the fibres which pass from the external geniculate body to the visual centres in the occipital lobe cross behind the corpora quadrigemina, and this is represented in the diagram as occurring at $\mathrm{TQ}$, in the corpora quadrigemina. On this view, all the occipital cortical fibres from one eye would ultimately pass to the cortex of the oceipital lobe of the opposite hemisphere. This view, however, by no means explaing all the facts, for in cases of homonymous henianopsia the point of central vision on both sides, i.e., both maculæ lutere are always unaffected, so that it is assumed that each macula lutea is connected with both hemispheres. The second crossing suggested by Charcot probably does not occur. Affections of the optic nerve, e.g., between the eyeball and the chiasma, i.e., in the orbit, optic foramen, or within the skull, affect one eye only; of the middle of the chiasma, cause temporal hemiopia; of the optic tract, between the chiasma and occipital cortex, hemiopia, which is always symmetrical (Gowers).

Fig. 424, reduced from that of Gowers, shows the relation of the fields of vision of the retina, tracts, and the cerebral optic centre. 
Function.-The optic nerve is the nerve of sight; physiologically, it is excited only by the transference of the vibrations of the ether to the rods and cones of the retina (\$ 383). Every other form of stimulus, when applied to the nerve in its course or at its centre, causes the sensation of light. Section or degeneration of the nerve is followed by blindness. Stimulation of the optic nerve causes a reflex contraction of the pupils, the efferent nerve being the oculomotorius or third cranial nerve. If the stimulus be very strong, the eyelids are closed and there is a secretion of tears. The influence of light upon the general metabolism is stated at $\S 127,9$.

As the optic nerve has special and independent connections with the so-called visual centre (\$378, IV.), as well as with the centre for narrowing the pupil (\$345), it is evident that, under pathological circumstances, there may be, on the one hand, blindness with retention of the action of the iris, and on the other loss of the movements of the iris, the sense of vision being retained (Wernicke).

Pathological. - Stimulation of almost the whole of the nervous apparatus may cause excessive sensibility of the visual apparatus (hyperæsthesia optica), or even visual impressions of the most varied kinds (photopsia, chromatopsia), which in cases of stimulation of the visual centre may become actual visual hallucinations (\$ 378, IV.). Material change in, and inflammation of, the nervous apparatus are often followed by a nervous weakness of vision (amblyopia), or even by blindness (amaurosis). Both conditions, however, may be the signs of disturbances of other organs, i.e., they are "sympathetic" signs, due it may be to changes in the movement of the blood-stream, depending upon stimulation of the vaso-motor nerves. The discovery of the partial origin of the optic nerve from the spinal cord explains the occurrence of amblyopia with partial atrophy of the optic nerve, in disease of the spinal cord, especially in tabes. Many poisons, such as lead and alcohol, disturb vision. There are remarkable intermittent forms of amaurosis known as day-blindness or hemeralopia, which occurs in some diseases of the liver and is sometimes associated with incipient cataract. [The person can see better in a dim light than during the day or in a bright light. In nightblindness or nyctalopia, the person cannot see at night or in a dim light, while vision is good during the day or in a bright light. It depends upon disorder of the eye itself, and is usially associated with imperfect conditions of nutrition.]

345. III. NERVUS OCULOMOTORIUS. - Anatomical. - It springs from the oculomotorius nucleus (united with that of the trochlearis), which is a direct continuation of the anterior horn of the spinal cord, and lies under the aqueduct of Sylvius (fig. 428). [The motor nucleus (fig. 427) gives origin to three sets of fibres, for (1) the most of the muscles of the eyeballs, (2) the sphincter pupillæ, (3) ciliary muscle. The nucleus of the 3rd and 4th nerves is also connected with that of the 6 th under the iter, so that all the nerves to the ocular muscles are thus co-related at their centres.]

The origin is connected with the corpora quadrigemina, to which the intraocular fibres may be traced, and also with the opposite half of the brain to the angular gyrus $(\S 378, \mathrm{I}$. ) through the pedunculus cerebri. Beyond the pons, it appears on the inner side of the cerebral peduncle, between the superior cerebellar and posterior cerebral arteries (fig. 428, III).

Function.-It contains-(1) the voluntary motor fibres for all the external muscles of the eyeballs - except the external rectus and superior oblique-and for the levator palpebræ superioris. The co-ordination of the movements of both eyeballs, however, is independent of the will. (2) The fibres for the sphincter pupillow, which are excited reflexly from the retina. (3) The voluntary fibres for the muscle of accommodation, the tensor choroideæ or ciliary muscle. The intrabulbar fibres of 2 and 3 proceed from the branch for the inferior oblique muscle, as the short root of the ciliary ganglion (fig. 429). They reach the eyeball through the short ciliary nerves of the ganglion. V. Trautvetter and others observed that stimulation of the nerve caused changes in the eye similar to those which accompany near vision. The three centres for the muscle of accommodation, the sphincter pupillæ, and the internal rectus muscle, lie directly in relation with each other, in the most posterior part of the floor of the third ventricle (Hensen and Völckers).

The centre for the reflex stimulation of the sphincter fibres by light was said to be in the corpora quadrigemina, but newer researches locate it in the medulla oblongata (\$3 379,392$)$. The narrowing of the pupil, which accompanies the act 
of accommodation for a near object, is to be regarded as an associated movement (§ 392, 5).

Anastomoses. - In man, the nerve anastomoses on the sinus cavernosus with the ophthalmic branch of the trigeminus, whereby it receives sensory fibres for the muscles to which it is distributed (Valentin, Adamiik), with the sympathetic through the carotid plexus, and (?) indirectly through the abducens, whereby it receives vaso-motor fibres (?).

Varieties, - In some rare cases, the pupillary fibres for the sphincter run in the abducens (Adamük), or even in the trigeminus (Schiff, v. Oräfe).

Atropin paralyses the intrabulbar fibres of the oculomotorius, while Calabar bean stimulates them (or paralyses the sympathetic, or both-compare $§ 392$ ).

Stimulation of the nerve, which causes contraction of the pupil, is best demonstrated on the decapitated and opened head of a bird. The pupil is dilated in paralysis of the oculomotorius, in asphyxia, sudden cerebral anæmia (e.g., by ligature of the carotids, or beheading), sudden venous congestion, and at death.

Pathological. - Complete paralysis of the oculomotorius is followed by-(1) drooping of the uprer evelid (ptosis paralytica); (2) immobility of the eyelall; (3) squinting (strabismus) outwards and downwards, and consequently there is double vision (diplopia); (4) slight protrusion of the eychall, because the action of the superior oblique muscle in pulling the eyeball forward is no longer compensated by the action of three paralysed recti muscles. In animals provided with a retractor bulbi muscle, the protrusion of the eyeball is more pronounced; (5) moderate dilatation of the pupil (mydriasis paralytica); (6) the pupil does not contract to light; (7) inability to accommorlate for a near object. It is to be noted, however, that the paralysis may be confined to individual branches of the nerve, i.c., there may be incomplete paralysis.

[Squinting. - In paralysis of the superior rectus, the eye cannot be moved upwards, and especially upwards and outwards. There is diplopia on looking upwards, the false image being above the true, and turned to the right when the left eye is affected (fig. 426, 3). Inferior Rectus.-Defect of downwarl, and especially downward and outward movement, the eye being directed upwards and outwards. Diplopia with crossed images, the false one is below the true image and placed obliquely, being turned to the left when the left eye is affected. Diplopia is
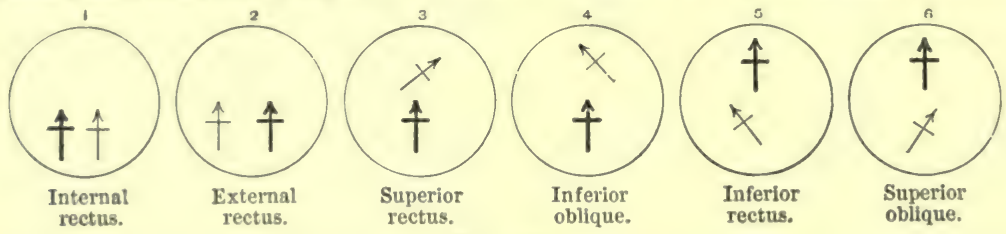

Fig. 426.

The black cross represents the true image, the thin cross the false image. The left eye is represented as affected in all cases (Bristow).

most troublesome when the ohject is below the line of vision (fig. 426, 5). Internal Rectus.Defective inward movement, divergent squint, and diplopia, the images being on the same plane, the false one to the patient's right when the left eye is affected. The head is turned to the healthy side, when looking at an object, while there is secondary deviation of the healthy eye outwards (fig. 426, 1). Inferior oblique is rare, the eye is turned slightly downwards and inwards, and defective movement upwards. Diplopia with the false image above the true one, especially on looking upwards; the false image is oblique, and directed to the patient's left when the left eye is affected (fig. 426, 4).]

Stimulation of the branch supplying the levator palpebræ in man causes lagophthalmus spasticus, while stimulation of the other motor fibres causes a corresponding strabismus spas. ticus. The latter form of squinting may be caused also reflexly-e.g., in teething, or in cases of diarrhoea in children; [the presence of worms or other source of irritation in the intestines of children is a frequent cause of squinting]. Clonic spasms occur in both eyes, and also as involuntary movements of the eyeballs constituting nystagmus, which may be produced by stimulation of the corpora quadrigemina, as well as by other means. Tonic contraction of the sphincter pupillæ is called myosis spastica, and clonic eontraction, hippus. Spasm of the muscle of accommodation (ciliary muscle) is sometimes observed ; owing to the imperfect judgment of distance, this condition is not unfrequently associated with macropia.

[Conjugate Deviation. - Some movements are produced by non-corresponding museles ; thus, on looking to the right, we use the right external rectus and left internal rectus, and the same is the case in turning the head to the right, e.g., the inferior oblique, some muscles of the right side act along with the left sterno-mastoid. In hemiplegia, the muscles on one side are paralysed, so that the head and often the eyes are turned away from the paralysed side, i.e., to the side of 
the brain on which the lesion occurs. This is called "conjugate deviation" of the eyes, with rotation of the head and neck. If the right external rectus be paralysed from an affection of the sixth nerve, on telling the patient to look to the right it will be found that the left eye will squint more inwards even than the right eye, i.e., owing to the strong voluntary effort, the muscle, the left internal rectus which usually acts along with the right external rectus, contracts vigorously, and so we get secondary deviation of the sound eye. Similar results occur in connection with paralysis of other ocular muscles.]

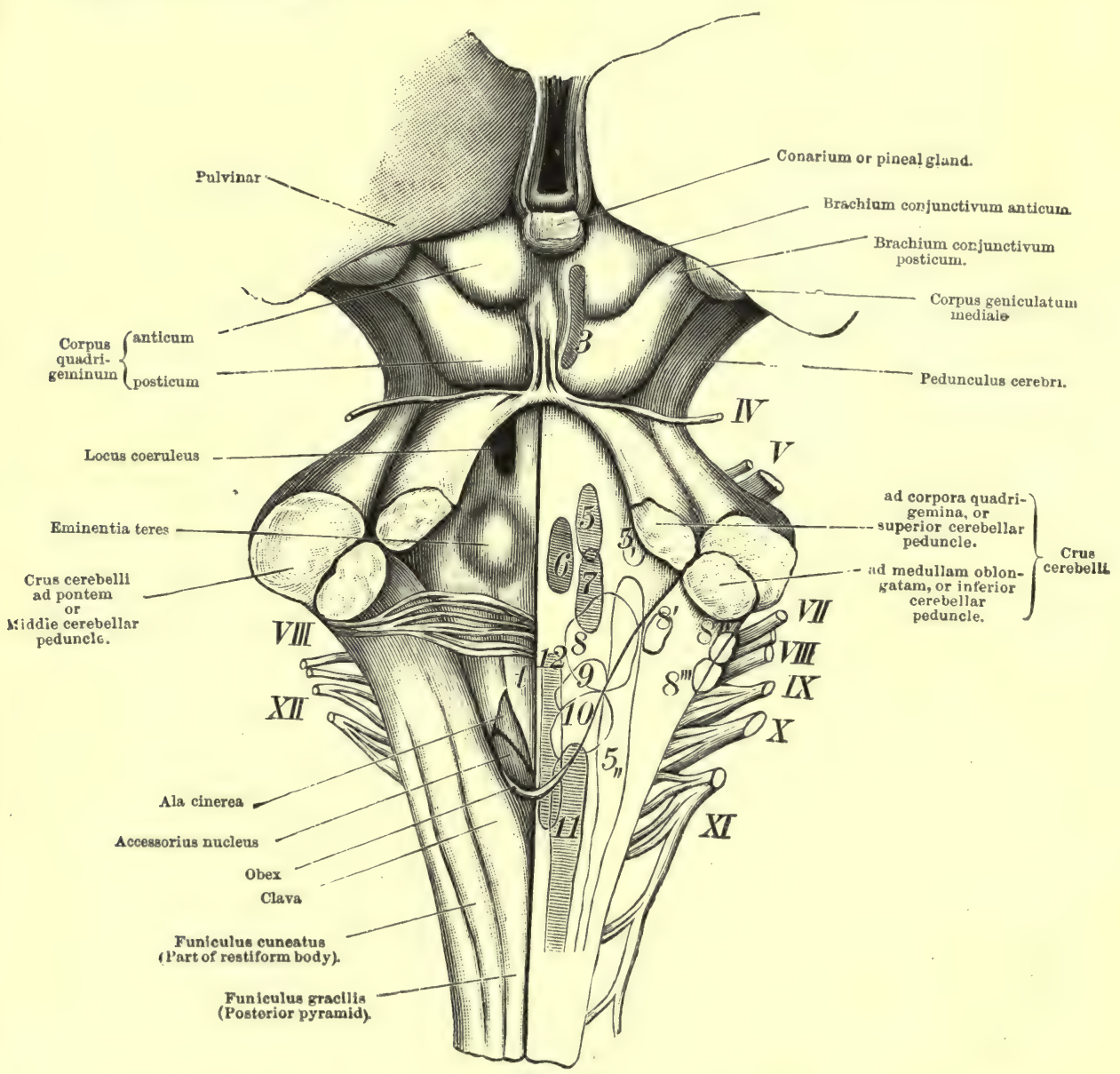

Fig. 427.

Medulla oblongata, with the corpora quadrigemina. The numbers $I V-X I I$ indicate the superficial origins of the cranial nerves, while those (3-12) indicate their deep origin, i.e., the position of their central nuclei; $t$, funiculus teres.

346. IV. NERVUS TROCHLEARIS. - Anatomical. - It arises from the valve of Vieussens, i.e., behind the fourth ventricle, but its fibres pass to the oculomotorius from the trochlearis nucleus, which is to a certain extent a continuation of the anterior horn of the spinal cord (fig. 427). It passes to the lower margin of the corpora quadrigemina, pierces the roof of the aqueduct of Sylvius, then into the velum medullare superius, and after decussating with the root of the opposite side behind the iter, it pierces the crus at the superior and external border (fig. 428). Its fibres cross between its nucleus and its distribution. It has also an origin from the locus cœruleus. The root of the nerve receives some fibres from the nucleus of the abducens of the opposite side. Physiologically, there is a necessity for a connection between the centre and the cortical motor centre for the eye muscles. 
Function.-It is the voluntary motor nerve of the superior oblique muscle." (In

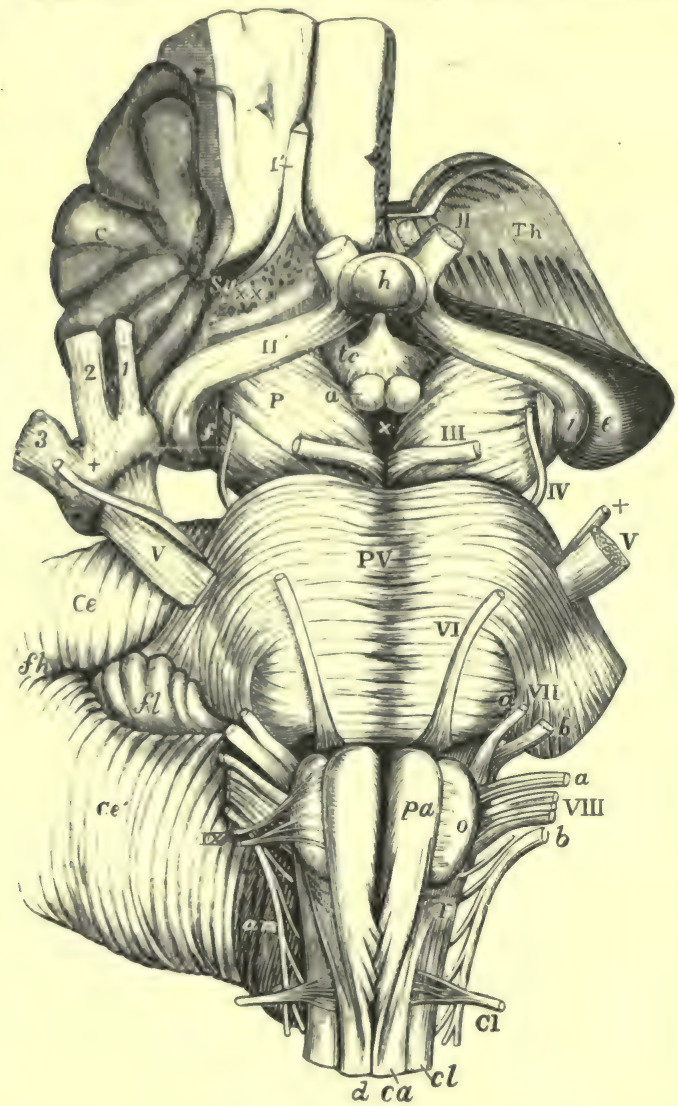

Fig. 428 .

Part of the base of the brain, with the origins of the cranial nerves; the convolutions of the island of Reil on the right side, but removed on the left. I', olfactory tract cut short ; II, left optic nerve ; II', right optic tract ; Th, cut surface of the left optic thalamus; C, central lobe, or island of Reil ; Sy, fissure of Sylvius; XX, the locus perforatus anticus; $e$, the external, and $i$, the interual corpus geniculatum ; $h$, hypophysis cerebri ; $t c$, tuber cinereum, with the infundibulum ; $a$, points to one of the corpora albicantia; $\mathrm{P}$, the cerebral peduncle; $f$, the fillet; III, left oculomotor nerve ; $\mathrm{X}$, the locus perforatus posticus ; PV, pons Varolii ; V, the greater part of the fifth nerve; + , the lesser root (on the right side this mark is placed on the Gasserian ganglion and points to the lesser root); 1, ophthalmic division of the fifth ; VII $\alpha$, facial, VII $b$, auditory ; VIII, vagus ; VIII $a$, glosso-pharyngeal ; VIII $b$, spinal accessory ; IX, hypoglossal ; $f$, flocculus ; $f h$, horizontal fissure of the cerebellum $(C e)$; am, amygdala ; $p \alpha$, anterior pyramid; 0 , olivary body ; $\varepsilon$, restiform body; $d$, anterior median fissure ; $c l$, the lateral column of the spinal cord ; CI, the sub-oecipital or first cervical nerve. the cerebellum, throngh the crura cerebelli. The origins of the sensory root anastomose with co-ordinated movements, however, it is involuntary.)

Anastomoses. - Its connections with the plexus caroticus sympathici, and with the first branch of the trigeninus, have the same significance as similar branches of the oculomotorius.

Pathological,-Paralysis of the trochlearis nerve causes a very slight loss of the mobility of the eyeball outwards and downwards. There is slight squinting inwards and upwards, with diplopia or double vision. The images are placed obliquely over each other [the false image being the lower, and directed to the patient's right when the left eye is affected (fig. 426,6)] ; they approach each other when the head is turned towards the sound side, and are separated when the head is turned towards the other side. The patient at first directs his head forwards, later he rotates it round a vertical axis towards the sound side. In rotating his head (whereby the sound eye may retain the primary position), the eye rotates with it. Spasm of the trochlearis causes squinting outwards and downwards.

347. V. NERVUS TRIGEMINUS. - Anatomical. - The trigeminus (fig. 429,5), arises like a spinal nerve by two roots (fig. 428, V.) The smaller, anterior, motor root proceeds from the "motor trigeminal nucleus" (5), which is provided with many multipolar nerve-cells, and lies in the floor of the medulla oblongata, not far from the middle line. Fibres connect this nucleus with the cortical motor centres on the opposite side of the cerebrum. Besides this the "descending root" also supplies motor fibres. It extends laterally from the corpora quadrigemina along the aqueduct of Sylvius downwards to the exit of the nerve (Henle, Forel). The large posterior sensory root receives fibres :-(1) From the small cells of the "sensory trigeminal nucleus " which lies at the level of the pons, and is the analogue of the posterior horn of the grey matter of the spinal cord. (2) From the grey matter of the posterior horn of the spinal cord, downwards as far as the second cervical vertebra. These fibres run into the posterior column of the cord and then appear as the "ascending root" in the trige- 
the metor nuclei of all the nerves arising from the medulla oblongata, with the exception of the abducens. This explains the vast number of reflex relations of the fifth nerve. The thick trunk appears on each side of the pons (fig. 428), when its posterior root (perhaps in connection with some fibres from the anterior) forms the Gasserian ganglion, upon the tip of the petrous part of the temporal bone (fig. 429). Fibres from the sympathetic proceed from the plexus cavernosus to the ganglion. The nerve divides into three large branches.

I. The ophthalmic division (fig. $429, d$ ) receives sympathetic fibres (vaso-motor nerves) from the plexus cavernosus ; it passes through the superior orbital fissure [sphenoidal] into the orbit. Its branches are :-

1. The small recurrent nerve which gives sensory branches to the tentorium cerebelli. Fibres-the vaso-motor nerves for the dura mater-proceed along with it from the carotid plexus of the sympathetic.

2. The lachrymal nerve gives off- $(a)$ Sensory branches to the conjunctiva, the upper eyelid, and the neighbouring part of the skin over the temple (fig. 429, a); (b) true sensory fibres to the lachrymal gland (?). Stimulation of this nerve is said to cause a secretion of tears, while its section prevents the reflex secretion excited through the sensory nerves of the eye. After a time, section of the nerve is followed by a paralytic secretion of tears (Herzenstein and Wolferz), although the statement is contested by Reich. The secretion of tears may be excited reflexly, by strong stimulation of the retina by light, by stimulation of the first and second branches of the trigeminus, and through all the sensory cranial nerves (Demtschenko) $(\$ 356$, A, 6).

3. The frontal $(f)$ gives off the supratrochlear, which supplies sensory fibres to the upper eyelids, brow, "glabella, and those which excite the secretion of tears reflexly; and by its supraorbital branch $(b)$, analogous branches to the upper eyelid, skin of the forehead, and the adjoining skin over the temple as far as the vertex.

4. The naso-ciliary nerve $(n c)$, by its infratrochlear branch supplies fibres, similar to those of 3 , to the conjunctiva, caruncula, and saccus lacrimalis, the upper eyelid, brow, and root of the nose. Its ethmoidal branch supplies the tip and alæ of the nose, outside and inside, with sensory branches, as well as the upper part of the septum and the turbinated bones with sensory fibres, which can act as afferent nerves in the reflex secretion of tears; while it is probable that vaso-motor fibres are supplied to these parts through the same channel. (These fibres may be derived from the anastomosis with the sympathetic (?).) The naso-ciliary nerve gives off the long root $(l)$ of the ciliary ganglion $(c)$, and 1 to 3 long ciliary nerves.

The ciliary ganglion (fig. 429, c), which, according to Schwalbe, perhaps belongs rather to the third than the fifth nerve, has three roots - (a) the short or oculomotorius $(3$ - see $\S 345)$; $(b)$ the long $(l)$, from the naso-ciliary; and $(c)$ the sympathetic $(s)$ sometimes united with $b$, from the carotid plexus. The short ciliary nerves $(t)$, six to ten in number, proceed from the ganglion, along. with the long ciliary nerves, to near the entrance of the optic nerve, where they perforate the sclerotic coat and run forwards between it and the choroid.

Ciliary Nerves.-Physiologically, these nerves contain :-

1. The motor fibres for the sphincter pupillæ and the tensor choroideæ from the root of the oculomotorius $(\S 345,2,3)$.

2. Sensory fibres for the cornea, which are distributed as excessively fine fibrils between the epithelium of the conjunctiva bulbi; they perforate the sclerotic. These fibres cause a reflex secretion of tears (N. lacrimalis) and closure of the eyelids (N. facialis). Sensory fibres are supplied to the iris (pain in iritis and in operations on the iris), the choroid (painful tension when the ciliary muscle is strained), and the sclerotic.

3. Vaso-motor nerves for the blood-vessels of the iris, choroid, and retina. They arise in part from the sympathetic root, and the anastomosis of the sympathetic 


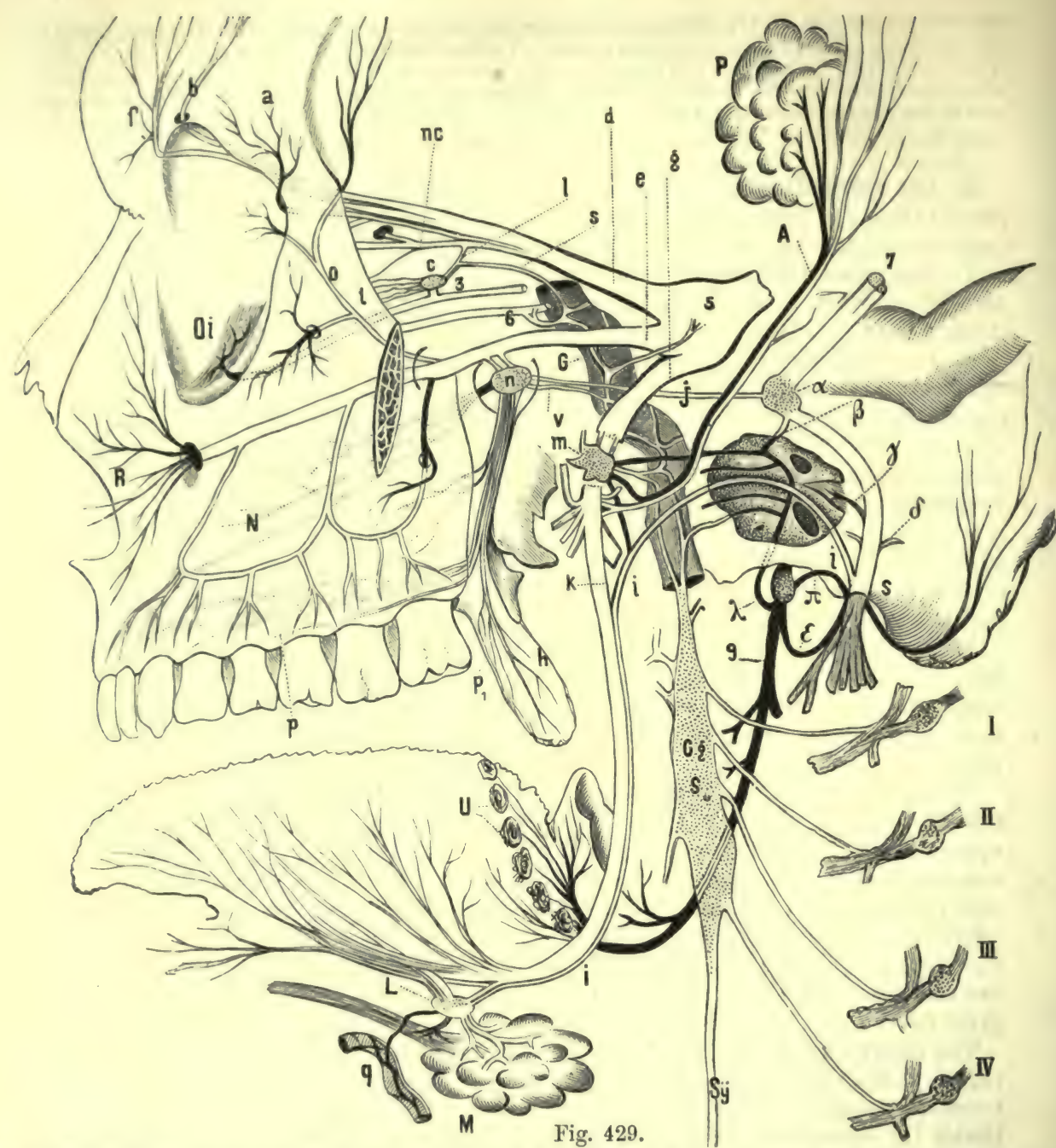

Semi-diagrammatic representation of the nerves of the eyeball, the connections of the trigeminus and its ganglia, together with the facial and glosso-pharyngeal nerves. 3 , Branch to the inferior oblique muscle from the oculomotorius, with the thick short root, to the ciliary ganglion $(c)$; $t$, ciliary nerves; $l$, long root to the ganglion from the naso-ciliary $(n c)$; $s$, sympathetic root from the sympathetic plexus $(S y)$ surrounding the internal earotid $(\mathbf{G}) ; d$, first or ophthalmic division of the trigeminus (5), with the naso-ciliary $(n c)$, and the terminal branches of the lachrymal $(a)$, supraorbital $(b)$, and frontal $(f)$; $c$, second or superior maxillary division of the trigeminus ; $\mathbf{R}$, infraorbital ; $n$, spheno-palatine (Meckel's) ganglion with its roots; $j$, from the facial, and $v$, from the sympathetic ; $\mathbf{N}$, the nasal branches, and $p p_{1}$, the palatine branches of the ganglion; $g$, third or inferior maxillary division of the trigeminus; $k$, lingual ; $i i$, chorda tympani ; $m$, otic ganglion, with the roots from the tympanic plexus, the carotid plexus, and from the 3rd branch, and with its branches to the auriculo-temporal $(\mathbf{A})$, and to the chorda $(i i) ; \mathrm{L}$, sub-maxillary ganglion with its roots from the tympanico-lingual, and the sympathetic plexus ou the external artery $(q) .7$, Facial nerve $-j$, its great superficial petrosal branch; $a$, gang. geniculatum; $\beta$, branch to the tympanic plexus ; $\gamma$, branch to the stapedius ; $\delta$, anastomatic twig to the auricular branch of the vagus ; $i i$, chorda tympani; $\mathrm{S}$, stylo-mastoid foramen. 9. Glosso-pharyngeal $-\lambda$, its tympanic branch; $\pi$ and $\epsilon$, connections with the facial; $U$, terminations of the gustatory fibres of 9 in the circumyallate papille; $S y$, sympathetic with $G g, s$, the superior cervical ganglion; $I, I I, I I I, I V$, the four upper cervical nerves ; $\mathrm{P}$, parotid, $\mathrm{M}$, sub-maxillary gland. 
with the ophthalmic division of the trigeminus (Wegner). The iris and retina receive most of their vaso-motor nerves from the trigeminus itself (Rogow), and few from the sympathetic; according to Klein and Svetlin, the retinal vessels are not influenced either by stimulation or division of the sympathetic.

4. Motor fibres for the dilator pupillæ, which for the most part are derived from the sympathetic (Petit, 1727), through the sympathetic root of the ganglion, and the anastomosis of the sympathetic with the trigeminus (Balogh, Oehl). Some observers deny altogether the existence of a dilator pupillæ muscle (\$384). The ophthalmic division contains independent fibres for the dilatation of the pupil (Schiff), which arise in the medulla oblongata and proceed directly into the ophthalmic (? or arise from the Gasserian ganglion-Oehl).

It is not conclusively determined whether in man dilator fibres also proceed throtigh the sympathetic root of the ciliary ganglion, and reach the iris through the ciliary nerves. In the dog and cat these fibres do not pass through the ciliary ganglion, but go directly along the optic nerve to the eye (Hensen and Völekers) through the Gasserian ganglion, to its ophthalmic branch and through the long ciliary nerves (Jegorow). In birds, the dilator fibres run only in the fifth (Zeglinski). For the centre $(\$ 367,8)$.

After section of the trigeminus, the pupil becomes contracted after a short period of dilatation (rabbit, frog), but this effect is not permanent. After excision of the superior cervical ganglion of the sympathetic, the power of dilatation of the pupil is not completely abolished. The narrowing of the pupil which follows section of the trigeminus in the rabbit, and which rarely lasts more than half an hour, may be regarded as due to a reflex stimulation of the oculomotorius fibres of the sphincter, in consequence of the painful stimulation caused by section of the trigeminus.

Stimulation of the Sympathetic.-Either in the neck, or in its course to the eye, when the peripheral end of the cervical sympathetic is stimulated, besides the effect on the blood-vessels, there is dilatation of the pupil, as well as contraction of the smooth muscular fibres in the orbit and eyelids. The membrana orbitalis, which separates the orbit from the temporal fossa in animals, contains numerous smooth muscular fibres (muscular orbitalis). The corresponding membrane of the inferior orbital fissure [spheno-maxillary fissure] in man has a layer of smooth muscle, one millimetre thick, and arranged for the most part longitudinally. Both eyelids contain smooth muscular fibres which serve to close them; in the upper lid they lie as if they were a continuation of the levator palpebræ superioris, in the lower lid they lie close under the conjunctiva. Tenon's capsule also contains smooth muscular fibres. The sympathetic nerve supplies all these muscles (Heinr. Muiller)-(the orbital muscle is partly supplied from the spheno-palatine ganglion); in animals, the retractor of the third eyelid at the inner angle of the eye is sinilarly supplied. Hence, stimulation of the sympathetic causes dilatation of the pupil and of the palpebral fissure, with protrusion of the eyeball. This result may be caused reflexly by strong stimulation of sensory nerves. Strong stimulation of the nerves of the sexual organs is followed by similar phenomena in the eye. The dilatation of the pupil, which occurs in children affected with intestinal worms, is perhaps an analogous phenomenon. The pupil is dilated when the spinal cord is stimulated (at the origin of the sympathetic), as in tetanus.

Section of the sympathetic, besides other effects, causes narrowing of the fissure between the eyelids, the eyeball sinks in its socket (and in animals, the third eyelid is relaxed and protruded). In dogs, section causes internal squint, as the external rectus receives some motor fibres from the sympathetic. (Origin of these fibres from the cilio-spinal region. Spinal Cord, $\S 362,1$.

5. It is probable that trophic fibres occur in the trigeminus, and pass through the ciliary nerves to reach the eye. If the trigeminus be divided within the cranium, after six to eight days, inflammation, necrosis of the cornea, and ultimately complete destruction of the eyeball take place, constituting panophthalmia (Fodera, 1823 ; Magendie).

Trophic Fibres. - In weighing the evidence for and against the existence of trophic fibres, we must bear in mind the following considerations:-1. Section of the trigeminus makes the whole eye insensible ; the animal is therefore unconscious of direct injury to its eye, and cannot therefore remove any offending body. Dust or mucus, which may adhere to the eye, is no longer removed by the reflex closing of the eyelids; while, owing to the absence of the reflex, the eye is more open and is therefore subject to more injuries; the reflex secretion of tears is also arrested. Snellen (1857) tixed the ear of a rabbit in front of its eye so as to protect the 
latter and shield it from injuries, and he found that the inflammation and other events occurred at a later date, while, according to Mleissner and Bittner, if the eye be protected by means of a complete capsule, the inflammation does not occur at all. There can be no doubt that the loss of the sensibility of the eye favours the occurrence of inflammation. But Meissner, Bittner, and Schiff observed that inflammation of the eye occurred when the trophic (most internal) fibres alone wete divided, the eye at the same time retaining its sensibility; this would seem to indieate the existence of trophic fibres, but Cohnheim and Senftleben dispute the statement. Conversely, the sensibility of the eye may be abolished by partial section of the nerve, yet the eye does not hecome inflamed (Sichiff). Ranvier, who denies the existence of trophic nerves, male a circular incision round the margin of the cornea through its superficial layers, so as to divile all the corneal nerves. Insensibility of the cornea was thereby produced, but never keratitis. Further, in man and animals, when they are unable to close their eyelids, there is redness with secretion of tears, or slight dryness and opacity of the surface of the eyeball (rerosis), liut never the inflammation alrealy described (Samucl). 2. We must also take into ronsideration the following:- Section of the trigeminus paralyses the vaso-motor nerves in the interior of the eyeball, which must undoulitedly cause a disturbance in the intraocular circulation. According to Jesner and Grinhagen, the trigeminus also contains vaso-dilator fibres, whose stimulation is followed hy increased flow of blood to the eye, with consecutive excretion of the fibrin-factors and increase in the amount of albumin of the aqueous humour. 3. After section of the nerve, the intraocular tension is diminished (while stimulation of the nerve is followed by increase of the intraocular pressure) (Hippell, Grïnhagen). This diminution of the normal tension necessarily must alter the normal relation of the filling of the blood-and lymphvessels, and also the movement of the fluids, upon which the normal nutrition is largely dependent. 4. Kiihne olserved that stimulation of the corneal nerves was followed by contraction of the so-called corneal-corpuscles. Perhaps the movements of these corpuscles may influence the normal movement of the lymph in the canalicular system of the cornea $(\$ 384)$; these movements, however, would seem to dejend upon the nervous system, so that its destruction is likely to produce disturbance of nutrition.

[There are three conilitions on which the changes may depend-(1) mere loss of sensibility, which alone is not sufficient to explain the pheuomena; (2) vaso-motor disturbance, which is excluded by the above facts, and also by the other consideration that, if the fifth nerve be livided and the superior cervical ganglion excised simultaneously, ophthalmia does not occur, and, in fact, excision of this sympathetic ganglion may modify the results of section of the fifth (Sinitzin). Thus, we are forced to (3) the theory of trophic fibres, whose centre is the Gasserian ganglion.]

Pathological. - In cases of anresthesia of the trigeminus in man, and, more rarely, in severe irritation of this nerve, inflammation of the conjunctiva, ulceration and perforation of the cornea, and finally panophthalmia, have been observed (Charles Bell). This condition has been called ophthalmia neuroparalytica. Samuel found that a similar result was produced by electrical stimulation of the Gasserian ganglion in animals.

There are other affections of the eye depending upon disease of the vaso-motor nerves, which are quite different from the foregoing, as they never lead to degenerative changes. Such is ophthalmia intermittens (lue to malaria), a unilateral, intermittent, excessive filling of the blood-vessels of the eye, accompanied by the secretion of tears, photophobia, often accompanied by iritis and effusion of pus into the chambers of the eye. This condition is regarded as a vasoneurotic affection of the ocular blood-vessels by Eulenburg. Pathological observations, as well as experiments upon animals, have shown that there is an intimate physiological connection between the vascular areas of both eyes, so that affections of the vascular area of one eye are apt to induce similar disturbances of the opposite eye. This serves to explain the fact that inflammatory processes in the interior of one eyeball are apt to produce a similar condition in the other eye. This is the so-called "sympathetic ophthalmia." Thus, stimulation of the ciliary nerves, or the fifth on one side, causes dilatation of the blood-vessels not only on its own side but also on the other side as well (Jesner and Grïnhagen). The pathological condition of glaucoma simplex, where the intraocular tension is greatly increased, is ascribed by Donders to irritation of the trigeminus. [Increased intraocular tension nay be produced by irritation of the secretory fibres contained in the fifth nerve (Donders), by stimulating the nucleus of the trigeminus in the medulla oblongata (Hippell and Grünhagen), and also reflexly by irritation of the peripheral branches of the fifth, as by nicotin placed in the eye. It is possible, however, that sone forms of glaucoma are produced by diminished removal of the aqueous humour from the eye.] Unilateral secretion of tears, due to irritation of the ophthalmic division of the fifth, has been repeatedly observed, but unilateral cessation of tears, due to paralytic conditions, very rarely.

II. Superior Maxillary Division (fig. 429, e).-It gives off-

1. The delicate recurrent nerve, a sensory branch to the dura mater, which accompanies the vaso-motor nerves, derived from the superior cervical ganglion of the sympathetic, and is distributed to the area of the middle meningeal artery. 
2. The subcutaneous malar or orbital (o) supplies by its temporal and orbital branches sensibility to the lateral angle of the eye and the adjoining area of skin of the temple and cheek. Certain fibres are said to be the true secretory nerves for tears. Compare N. lacrimalis, p. 591.

3. The dental, anterior, posterior, and median, and with them the anterior fibres from the infraorbital nerve, supply sensory fibres to the teeth in the upper jaw, the gum, periosteum, and the cavities of the jaw (p. 592). The vaso-motor nerves of all these parts are supplied from the upper cervical ganglion of the sympathetic.

4. The infraorbital $(R)$, after its exit from the infraorbital foramen, supplies sensory nerves to the lower eyelid, the bridge and sides of the nose, and the upper lip as far as the angle of the mouth. The accompanying artery receives its vasomotor fibres from the superior cervical ganglion of the sympathetic. For the sweat-secreting fibres which occur in it (pig) see $\S 288$.

The spheno-palatine ganglion (Meckel's $-n$ ) forms connections with the second division. To it pass two short sensory root-fibres from the second division itself, which are called spheno-palatine. Motor fibres enter the ganglion from behind, through the large superficial petrosal branch of the facial $(j)$; and grey vasomotor fibres $(v)$ from the sympathetic plexus on the carotid (the deep large petrosal nerve). The motor and vaso-motor fibres from the Vidian nerve, which reach the ganglion through the canal of the same name.

Branches of the Ganglion.-(1) The sensory fibres (N) which supply the roof, lateral walls, and septum of the nose (posterior and superior nasal); the terminal fibres of the naso-palatine pass through the canalis incisivus to the hard palate, behind the incisor teeth. The sensory inferior and posterior nasals for the lower and middle turbinated bones, and both lower nasal ducts, are derived from the anterior palatine branch of the ganglion, which descends in the palato-maxillary canal. Lastly, the sensory branches for the hard $(p)$ and soft palate $\left(p_{1}\right)$, and the tonsils arise from the posterior palatine nerve. All the sensory fibres of the nose (see also the Ethmoidal nerve), when stimulated, cause the reflex act of sneezing (\$120). Preparatory to the act of sneezing, there is always a peculiar feeling of tickling in the nose, which is perhaps due to dilatation of the nasal blood-vessels. This dilatation is rapidly caused by cold, more especially when it is applied directly to the skin. The dilatation of the vessels is followed by an increased secretion of watery fluid from the nasal mucous membrane. Stimulation of the nasal nerves also causes a reflex secretion of tears, and it may also cause stand-still of the respiratory movements in the expiratory phase (Hering and Kratschmer)-(compare Respiratory centre, § 368). (2) The motor branches descend in the posterior palatine nerve through the small palatine canal, and give off $(h)$ motor branches to the elevator of the soft palate and azygos uvulæ $(N u h n)$. The sensory fibres for these muscles are supplied by the trigeminus. According to Politzer, spasmodic contraction of these muscles occasionally causes crackling noises in the ears. (3) The vaso-motor nerves of this entire area arise from the sympathetic root, i.e., from the upper cervical ganglion. (4) The root of the trigeminus supplies the secretory nerves of the mucous glands of the nasal mucous membrane. Stimulation excites secretion, while section of the trigeminus diminishes it with simultaneous atrophic degeneration of the mucous membrane. Thus, trophic functions for the mucosa have been ascribed to the trigeminus (Aschenbrandt).

Stimulation of the Ganglion. - Feeble electrical stimulation of the exposed ganglion causes a copious secretion of mucus and an increase of the temperature in the nose (Prévost), with dilation of the vessels (Aschenbrandt). [Meckel's ganglion has been excised in certain cases of neuralgia ( $W$ alsham).]

III. Inferior Maxillary (g).-It contains all the motor fibres of the fifth, along with a number of sensory fibres; it gives off-

1. The recurrent, which springs by itself from the sensory root, enters the skull 
through the foramen spinosum, and, along with the nerve of the same name from the II. division, supplies sensory fibres to the dura mater. Fibres proceed from it through the petroso-squamosal fissure to the mucous membrane of the cells of the mastoid process.

2. Motor fibres for the muscles of mastication, viz., the masseteric, the two deep temporal nerves, and the internal and external pterygoid nerves. The sensory fibres for the muscles are supplied by the sensory fibres.

3. The buccinator is a sensory nerve for the mucous membrane of the cheek, and the angle of the mouth as far as the lips.

Accorling to Jolyet and Laffont, it contains, in addition, vaso-motor fibres for the mucous membrane of the check, lower lip, and their mucous glands; but these fibres are probably derived from the sympathetic.

Trophic Fibres. - As this region of the mucous membrane of the mouth ulcerates after section of the trigeminus, some have supposed that the buccinator nerve contains trophic fibres. But, as Rollett pointed out, section of the inferior maxillary nerve paralyses the muscles of mastication on the same side, and hence the teeth do not act vertically upon each other, but press against the cheek. Owing to the loss of the sensibility of the mouth, food passes between the gum and the cheek, where it may remain attached, undergo decomposition, and perhaps chemically irritate the mucuus membrane. At a later stage, owing to the wearing away of the teeth in an oblique manner, ulcers begin to form on the sound side. Hence, there is no necessity for assuming the existence of trophic fibres in this nerve. After section of the trigeminus, the nasal mucons membrane on the same side becomes red and congested. This is due to the fact that dust or mucus, not being removed from the nose by the usual reflex acts, remains there, irritates, and ultimately causes inflammation.

4. The lingual $(k)$ receives at an acute angle the chorda tympani $(i \quad i)$, a branch of the facial coming from the tympanic cavity. The lingual does not contain any motor fibres; it is the sensory and tactile nerve of the anterior two-thirds of the tongue, of the anterior palatine arch, the tonsil, and the floor of the mouth. These, as well as all the other sensory fibres of the mouth, when stimulated, cause a reflex secretion of saliva (compare $\$ 145)$. The lingual is accompanied by the nerve of taste (chorda) for the tip and margins of the tongue (i.e., the parts not supplied by the glosso-pharyngeal). After section of the lingual nerve in man, Busch, Inzani, and Lusanna found that the tactile sensibility was lost in the half of the tongue, and there was loss of taste in the anterior part [two-thirds] of the tongue. The fibres which administer to the sense of taste do not as a rule belong to the lingual itself, but are derived from the chorda tympani (p. 600). According to Schiff, the lingual nerve is the gustatory nerve, and some cases of Erb and Senator support this view. Such cases, however, seem to be exceptions to the general rule. The lingual nerve in the substance of the tongue is provided with small ganglia (Remuk, Stirling). Schiff observed that section of the lingual (and also of the hypoglossal) caused redness of the tongue, so that vaso-motor fibres are present in its course. It is unknown whether these are derived from the anastomoses of the Gasserian ganglion with the sympathetic. The lingual appears to receive vasodilator fibres from the chorda for the tongue and gum ( $\$ 349)$.

After section of the trigeminus, animals frequently bite their tongue, as they cannot feel the position and movements of this organ in the mouth.

5. The inferior dental is the sensory branch to the teeth and gum; the vasomotor fibres reach it from the superior cervical ganglion. Before it passes into the canal in the lower jaw, it gives off the mylo-hyoid nerve, which supplies motor fibres to the mylo-hyoid and the anterior belly of the digastric, and also some fibres to the triangularis menti and the platysma; the muscular sensory nerves also lie in these branches. The mental nerve, which issues from the mental foramen, is the sensory nerve for the chin, lower lip, and the skin at the margin of the jaw.

6. The auriculo-temporal gives sensory branches to the anterior wall of the external auditory meatus, the tympanic membrane, the anterior part of the ear, the adjoining region of the temple, and to the maxillary articulation. 
Fig. 430 shows the distribution of the branches of the trigeminus on the head, and the cervical nerves, so that the distribution of anæsthetic and hyperæsthetic areas may easily be made out.

The otic ganglion $(m)$ lies beneath the foramen ovale on the inner side of the third division. Its roots are-(1) short motor fibres from the third division; (2) vaso-motor from the plexus around the middle meningeal artery (ultimately derived from the cervical ganglion of the sympathetic); (3) fibres $(\lambda)$ run from the tympanic branch of the glosso-pharyngeal to the tympanic plexus, and from thence through the canaliculus petrosus in the small superficial petrosal in the cranium, then through a small canal between the apex of the petrous bone and the sphenoid,

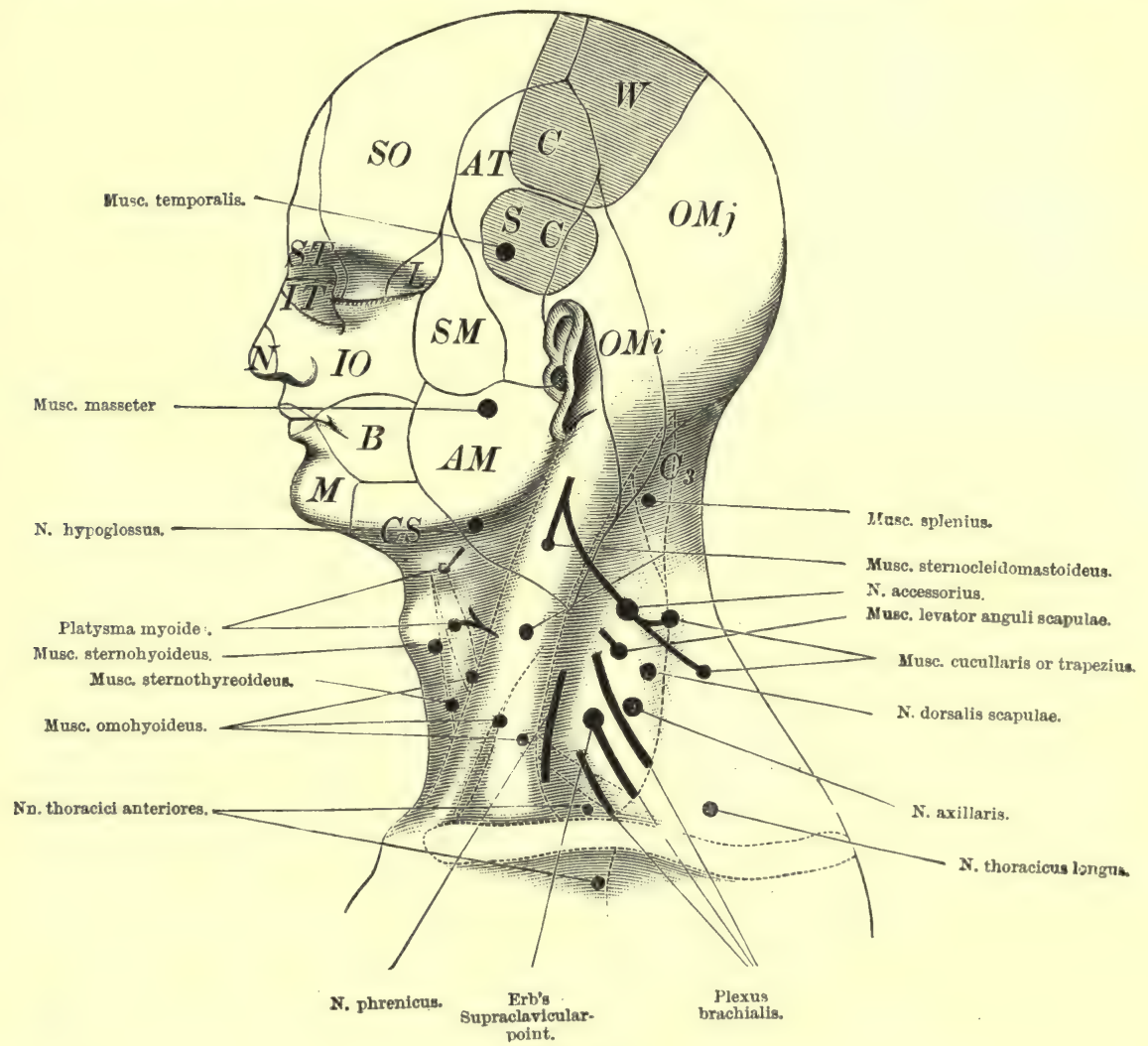

Fig. 430.

Distribution of the sensory nerves on the head as well as the position of the motor points on the neck. SO, area of distribution of the supraorbital nerve; $S T$, supratrochlear; $I T$, infratrochlear; $L$, lachrymal ; $N$, ethmoidal ; $I O$, infraorbital ; $B$, buccinator ; $S M$, subcutaneus malæ; $A T$, auriculo-temporal ; $A M$, great auricular ; $O M j$, great occipital ; $O M i$, lesser occipital; $C_{3}$, three cervical nerves; $C S$, cutaneous branches of the cervical nerves ; $C W$, region of the central convolutions of the brain ; $S C$, region of the speechcentre (third left frontal convolution).

to reach the otic ganglion. Through the chorda tympani the facial nerve is constantly connected with the ganglion (fig. 430).

The branches of the otic ganglion are-(1) motor twigs for the tensor tympani and tensor of the soft palate (these fibres are mixed with muscular sensory fibresIndwig and Politzer); (2) one or more branches connecting the ganglion with the 
auriculo-temporal are carried by the roots 2 and 3 from the sympathetic and glossopharyngeal, which the auriculo-temporal nerve (A), as it passes through the parotid gland $(\mathrm{P})$, gives off to the gland. These are the secretory fibres for the parotid; their functions are stated in $\$ 145$.

Section of the trigeminus is followed by inflammatory changes in the tympanic cavity (rabbit); the degree of inflammation varies much (Berthold and Grïnhagen). Section of the sympathetic or glosso-pharyngeal has no effect.

The sub-maxillary ganglion (fig. 429 , L) lies close to the convex arch of the tymunico-lingual nerve and the excretory duct of the sub-maxillary gland (M). Its roots are-(1) branches of the chorda tympani, $i i$, which undergo fatty degeneration after section of facial nerve. This root supplies secretory fibres to the sub-maxillary and sub-lingual glands, but it also supplies vaso-dilator fibres for the blood-vessels of the same glands (\$ 145). In addition, fibres are supplied to the smooth muscular fibres in Wharton's duct. All the fibres of the chorda do not pass into the gland; some pass along with the lingual nerve into the tongue (see Chorla, under Facial Nerve). (2) The sympathetic root of the ganglion arises from the plexus around the submental branch of the external maxillary artery (y), i.e., ultimately from the superior cervical ganglion; it passes to the gland, and contains secretory fibres, whose stimulation is followed by the secretion of thick concentrated saliva (trophic nerve of the gland). It also carries the vaso-constrictor nerves to the gland (p. 212). (3) The sensory root springs from the lingrual. Some of the fibres, after passing through the ganglion, supply the gland and its excretory ducts, while a few issue from the ganglion, and again join the tympanico-lingual nerve to reach the tongue.

Pathological. - Trismus, or spesm of the muscles of mastication, supplied by the third division, is ustally lilateral; it may be clonic in its nature (ehattering of the teeth), or tonic, when it constitutes the condition of lock-jaw or trismus. The spasms are usually individual symptoms of more extensive convulsions; more rarely when they occur alone, they are symptomatic of dise:ase of the cerebrum, medulla, pons, and cortex of the motor convolutions (Eulenburg). The sprasms may be caused reflexly, e.g., by stimulation of the sensory nerves of the head.

Paralysis. - Degeneration of the motor nuclei, or an affection of the intracranial root of the nerve, causes paralysis of the muscles of mastication, which is very rarely bilateral. Paralysis of the tensor tymprani is said to cause difticulty of hearing (Romberg), or buzzing in the ears (Benclict). We require further olsservations upon this point, as well as upon paralysis of the tensor of the soft palate.

Neuralgia may occur in all the branches of the fifth. It consists of severe attacks of pain shooting into the expansions of the nerves. It is usually unilateral, and in fact is often confined to one branch, or even to a few twigs of one branch. The point from which the pain proceeds is frequently the bony canal through which the branch issues. The ear, dura mater, and tongue are rarely attacked. The attack is not unfrequently accompanied by contractions or twitchings of the corresponding group of the facial muscles. The twitchings are either reflex, or are due to direct peripheral irritation of the fibres of the facial nerve, which are mixed with the terminal branches of the trigeminus. The reflex twitchings may be extensively distributed, involving even the muscles of the arm and trunk.

Redness or congestion of the affected part of the face is not an unfrequent symptom in neuralgia, and it may be accompanied by increased.or diminished secretion from the nasal and buccal mucous membranes. This is a reflex phenomenon, the sympathetic being affected. Reflex stimulation of the vaso-motor nerves frequently gives rise to disturbance of the cerebral activities, owing to changes in the distribution of the blood in the head. Ludwig and Dittmar found that stimulation of sensory nerves caused a reflex contraction of the arterial blood-vessels, and increase of the blood-pressure in the cerebral vessels. Sometimes there is melancholy or hypochondriasis, and in one case of violent pain in the inferior maxillary nerve, the attack was accompanied by hallucinations of vision.

The trophic disturbances which sometimes accompany affections of the trigeminus are particularly interesting. They are : a brittle character of the hair, which frequently becomes grey, or may fall out; circumscribed areas of inflammation of the skin, and the appearance of a vesicular eruption upon the face [often following the distribution of certain nerves], and constituting herpes, which may also occur on the cornea, constituting the neuralgic herpes cornero of Schmidt-Rimpler. Lastly, there is the progressive atrophy of the face which is usually confined to one side, but may occur on both sides (Eulenburg). It is caused very probably by a trophic affection of the trigeminus, although the vaso-motor nerves may also be affected reflexly. 
Landois found that in the famous case of Romberg, a man named Schwahn, the sphygmographic tracing of the carotid pulse of the atrophied side was distinctly smaller than on the sound side.

Urbantschitsch made the remarkable observation that stimulation of the branches of the trigeminus, especially those going to the ear, caused an increase of the sensation of light in the person so stimulated. Blowing upon the cheeks or nasal mucous membrane, electrical stimulation, the use of snuff, smelling strong perfumes-all temporarily increase the sensation of light. The senses of taste and smell, as well as the sensibility of certain areas of the skin, can all be exalted reflexly by gentle stimulation of the trigeminus. In intense affections of the ear, whereby the fibres of the trigeminus are often affected sympathetically, these sensory functions may be diminished. As the ear-malady begins to improve, the excitability of these sense organs also again begins to improve.

[Complete section of the trigeminus results in loss of sensibility in all the parts supplied by it (fig. 430), including one side of the face, temple, part of the ear, the fore part of the head, conjunctiva, cornea, mouth, gums, Schneiderian mucous membrane, anterior two-thirds of the tongue, and part of pharynx. In drinking from a vessel, the patient feels as if one side of it were cut away. The muscles of mastication are paralysed on that side, food is not chewed on one side, and fur accumulates on the tongue on that side. The mucous membranes tend to ulcerate, that of the mouth being chafed by the teeth, the gums get spongy, the nasal mucous membrane tends to ulcerate, so that smelling is interfered with, and ammonia excites no reflex acts, while the eye undergoes panophthalmia.]

[Gowers is of opinion that the sensation of taste on the posterior part of the tongue, soft palate, and palatine arch depends on the fifth nerve and not on the glosso-pharyngeal nerve.]

348. VI. NERVUS ABDUCENS. - Anatomical.-It arises slightly in front of and partly from the nucleus of the facial nerve (which corresponds to the anterior horn of the spinal cord), from large-celled ganglia in the deeper part of the anterior region of the fourth ventricle, (emenentia teres, fig. 427). [Its nucleus is connected with the nucleus of the third nerve of the opposite side. It appears at the posterior margin of the pons (fig. 428, VI.). This nerve has a very long course before it enters the orbit, and as it bends over the posterior margin of the pons, it is liable to be compressed there or from pressure upon the tentorium cerebelli, so that both nerves are very liable to paralysis.]

Function.-It is the voluntary nerve of the external rectus muscle. In coordinated movements of the eyeballs, however, it is involuntary.

Anastomoses. - Branches reach it from the sympathetic upon the cavernous sinus (fig. 429). A few come from the trigeminus, and their function is analogous to similar fibres supplied to the trochlearis and oculomotorius.

Pathological. - Complete paralysis causes squinting inwards [or convergent squint] and consequent diplopia. [The eye cannot be rotated outwards beyond the middle line, the double images are in the same horizontal plane and vertical, the false one is to the left of the patient's eye when the left eye is affected (fig. 426, 2). The feeling of giddiness is often severe. There is secondary deviation to the inner side, and the head is turned towards the affected side.] In dogs, section of the cervical sympathetic causes a slight deviation of the eyeball inwards (Petit). This is explained by the fact that the abducens receives a few motor fibres from the cervical sympathetic. Spasm of the abducens causes external squint.

Squint. - In addition to paralysis or stimulation of certain nerves producing squint, it is to be remembered that it may also be caused by a primary affection of the muscles themselves, e.g., congenital shortness, contracture, or injuries of these muscles. It may also be brought about owing to opacities of the transparent media of the eye; a person with, say an opacity of the cornea, rotates the affected eye involuntarily, so that the rays of light may enter the eye through a clear part of the media.

349. VII. NERVUS FACIALIS.-Anatomical. - This nerve consists entirely of efferent fibres, and arises from the floor of the fourth ventricle from the "facial nucleus" (fig. 427, 7), which lies behind the origin of the abducens, and also by some fibres from the nucleus of the abducens [although Gowers' observations do not confirm this (\$.366)]. Other fibres arise from the cerebrum of the opposite side ( $\$ 378, \mathrm{I}$.). It consists of two roots, the smaller-portio intermedia of Wrisberg - forms a connection with the auditory nerve (see § 350 ). The original fibres of the portio intermedia are developed from the glosso-pharyngeal nucleus (Sapolini). It would thus appear that the sensory and gustatory fibres which are present in the chorda tympani enter it through these fibres (Duval, Schultze, Vulpian), so that the portio intermedia is a special part of the nerve of taste, which becomes conjoined with the facial, and runs to the tongue in the chorda. Along with the auditory nerve, it traverses the porus acusticus internus, where it 
passes into the facial or Fallopian canal. At first it has a transverse direction as far as the hiatus of this canal; it then bends at an acute angle at the "knee" (a) above the tympanic cavity, to descend in an osseous canal in the posterior wall of this space (fig. 429). It emerges from the stylo-mastoid foramen, pierces the parotid gland, and is distributed in a fan-shaped manner (pes anserinus major). ['The superficial origin is at the lower margin of the pons, in the depression between the olivary body and the restiform body, as indicated in fig. 428, VII $a_{0}$ ]

Its branches are:-1. The motor large superficial petrosal (j). It arises from the "knee" or geniculate ganglion within the Fallopian canal, in the cavity of the skull, runs upon the anterior surface of the temporal bone, traverses the foramen lacerum medium on the under surface of the base of the skull, and passes through the Vidian canal to reach the spheno-palatine ganglion (p. 595). It is uncertain whether this nerve conveys sensory branches from the second division of the trigeminus to the facial.

2. Connecting branches $(\beta)$ pass from the geniculate ganglion to the otic ganglion. For their course and function, see Otic ganglion (p. 597).

3. The motor branch to the stapedius muscle $(\gamma)$.

4. The chorda tympani $(i i)$ arises from the facial before it emerges at the stylomastoid foramen $(s)$, runs through the tympanic cavity (above the tendon of the tensor tympani, between the handle of the malleus and the long process of the incus), passes out of the skull through the petro-tympanic fissure, and then joins the lingual nerve at an acute angle (p. 596, 4). Before it unites with this nerve, it exchanges fibres with the otic ganglion $(m)$. Thus, sensory fibres can enter the chorda from the third division of the trigeminus, which may run centripetally to the facial to be distributed along with it. In the same way, sensory fibres may pass from the lingual nerve through the chorda into the facial (Longet). Stimulation of the chorda-which even in man may be done in cases where the tympanic membrane is destroyed-causes a prickling feeling in the anterior margins and tip of the tongue (Trülsch). O. Wolfe found that the section of the chorda in man abolished the sensibility for tactile and thermal stimuli upon the tip of the tongue; and the same was true of the sense of taste in this region. It is supposed by Calori, that these fibres enter the facial nerve at its periphery (especially through the auriculo-temporal into the branches of the facial), that they run in a centripetal direction in the facial, and afterwards pursue a centrifugal course in the chorda. [It is possible that sensory fibres pass from the spheno-palatine ganglion of the fifth through the Vidian nerve and large superficial petrosal to enter the facial. These fibres may be those that appear in the seventh as the chorda fibres which administer to taste. Bigelow asserts that the chorda tympani is not a branch of the facial, but the continuation of the nervus intermedius of Wrisberg.] The chorda also contains secretory and vaso-dilator fibres for the sub-maxillary and sub-lingual glands (\$ 145).

Gustatory Fibres. - The chorda also contains fibres administering to the sense of taste, for the margin and tip of the tongue (anterior two-thirds), which are conveyed to the tongue along the course of the lingual. Urbantschitsch made observations upon a man whose chorda was freely exposed, and in whom its stimulation in the tympanic cavity caused a sensation of taste (and also of touch) in the margins and tip of the tongue.

It would seem, therefore, that the gustatory fibres of the chorda have their origin in the glosso-pharyngeal nerve. They may reach the chorda:-1. Through the portio intermedia of Wrisberg, as already mentioned.

2. There is a channel beyond the stylomastoid foramen, viz., through the ramus communicans cum glosso-pharyngeo (fig. 429), which passes from the last mentioned nerve in that branch of the facial which contains the motor fibres for the stylohyoid and posterior belly of the digastric muscle (Henle's N. styloideus). This nerve also supplies muscular sensibility to the stylohyoid and posterior belly of the digastric muscles. It is also assunied that, by means of these anastomoses, motor fibres are supplied by the facial to the glosso-pharyngeal nerve. 3. A union of the 
glosso-pharyngeal and facial nerves occurs in the tympanic cavity. The tympanic branch of the glosso-pharyngeal $(\lambda)$ passes into this cavity, where it unites in the tympanic plexus with the small superficial petrosal nerve $(\boldsymbol{\beta})$, which springs from the knee on the facial. The gustatory fibres may first pass into the otic ganglion, which is always connected with the chorda (Otic ganglion, p. 597,3). Lastly, a connection is described through a twig $(\pi)$ from the petrous ganglion of the glosso-pharyngeal, direct to the facial trunk within the Fallopian canal (Garibaldi).

According to some observers, the chorda contains vaso-dilator fibres for the anterior two-thirds of the tongue (Vulpian).

Pseudo-motor Action.-From one to three weeks after the section of the hypoglossal nerve, stimulation of the chorda causes movements in the tongue (Philippeaux and Vulpian). These movements are not so energetic as, and occur more slowly than, those caused by stimulation of the hypoglossal. Nicotin first excites, then paralyses, the motor effect of the chorda. Even after cessation of the circulation, stimulation of the chorda causes movements. Heidenhain supposes

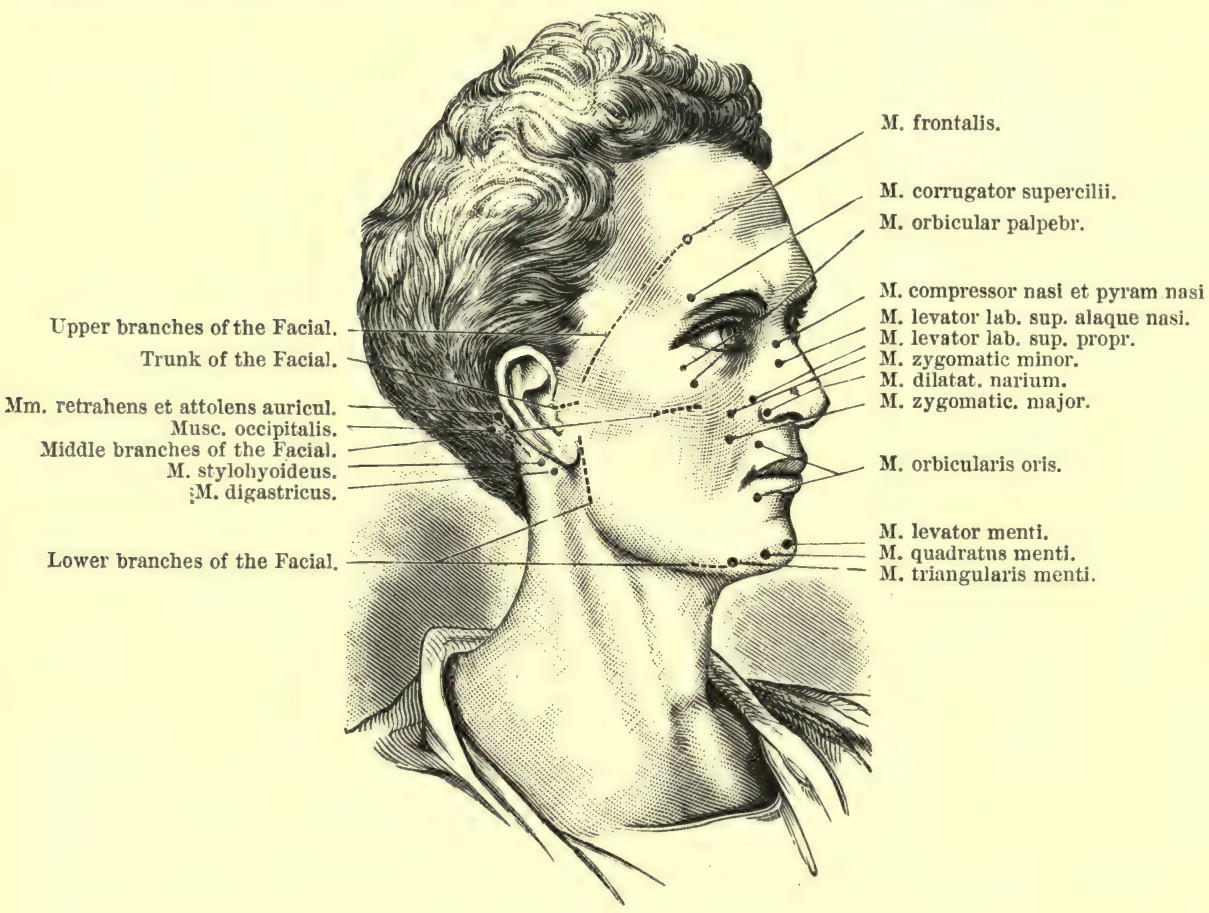

Fig. 431.

Motor points of the facial nerve and the facial muscles supplied by it.

that, owing to the stimulation of the chorda, there is an increased secretion of lymph within the musculature, which acts as the cause of the muscular contraction. He called this action "pseudo-motor."

[If, after the union of the central end of the lingualis and the peripheral end of the hypoglossal nerve, the lingualis be stimulated, there is a genuine contraction of the musculature of the tongue, on that side. A pseudo-motor contraction is easily distinguished from a true contraction, for when a telephone is connected with the tongue, on stimulating the hypoglossal the tone of the tetanus thereby produced is heard, but on stimulating the lingual, although the pseudo-motor contractions occur, no sound is heard (Rogowicz).]

5. Connection with Vagus.-Before the chorda is given off, the trunk of the facial comes into direct relation with the auricular branch of the vagus $(\delta)$, which crosses it in the mastoid canal, and supplies it with sensory nerves (see $V$ agus).

6. Peripheral Branches.-After the facial issues from its canal, it supplies 
motor fibres to the stylohyoid and posterior belly of the digastric, occipitalis, all the muscles of the external ear, the muscles of expression, buccinator and platysma. The facial also contains secretory fibres for the face (compare $\$ 288$ ).

Although most of the branches of the facial are under the influence of the will, yet most men cannot voluntarily move the muscles of the nose and ear.

Anastomoses. - The branches of the seventh nerve on the face anastomose with those of the trigeminus, whereby sensory fibres are conveyed to the muscles of expression. The sensory branches of the auricular branch of the vagus, and the great auricular, enter the peripheral ends of the facial, and supply sensibility to the muscles of the ear; while the sensory fibres of the third cervical nerve similarly supply the platysma with sensibility. Section of the facial at the stylomastoid foramen is painful, but it is still more so if the peripheral branches on the face are divided (Recurrent sensibility, §355).

Pathological. - In all cases of paralysis of the facial, the most important point to determine is whether the seat of the affection is in the periphery, in the region of the stylomastoid foramen, or in the course of the long Fallopian canal, or is central (cerebral) in its origin. This point must be determined by an analysis of the symptoms. Paralysis at the stylomastoid foramen is very frequently rheumatic, and probably depends upon an exudation compressing the nerve; the exulation probably oceupying the lymph-space described by Riidinger on the inner side of the Fallopian canal, between the periosteum and the nerve, and which is a continuation of the arachnoil space. Other causes are-inflammation of the parotid gland, direct injury, and pressure from the forceps during delivery. In the course of the canal, the causes are-fratcture of the temporal bone, effusion of the blood into the canal, syphilitic effusions, and caries of the temporal bone; the last sometimes occurs in inflammation of the ear. Anongst intracranial causes are--affections of the membranes of the brain, and of the base of the skull in the region of the nerve, disease of the "facial nucleus"; lastly, affection of the cortical centre of the nerve and its connections with the nucleus. [No nerve is so liable as the seventh to be paralysed independently.]

Symptoms of Unilateral Paralysis of the Facial [or Bell's Paralysis].-1. Paralysis of the muscles of expression: The forehead is smooth, without folds, the eyelids remain open (lagophthalmus paralyticus), the outer augle being slightly lower. The anterior surface of the eye rapidly becomes dry, the cornea is dull, as, owing to the paralysis of the orbicularis, the tears are not properly distributed over the conjunctiva, and, in fact, in consequence of the dryness of the eyeball, there may be temporary inflammation (keratitis xerotica). In order to protect the eyeball from the light, the patient turns it upwards under the upper eyelid (Bell), relaxes the levator palpebre, which allows the lid to fall somewhat (Hasse). The nose is immovable, while the naso-labial fold is obliterated. As the nostrils cannot be dilated, the sense of smell is interfered with. The impairment of the sense of smell depends more, however, upon the imperfect conduction of the tears, owing to paralysis of the orbicularis palpebrarum and Horner's muscle, thus causing dryness of the corresponding side of the nasal cavity. Horses, which distend the nostrils widely during respiration, after section of both facial nerves, are said by $\mathrm{Cl}$. Bernard to die from interference with the respiration, or at least they suffer from severe dyspncea (Ellenberger). The face is drawn towards the sound side, so that the nose, mouth, and chin are oblique. Paralysis of the buccinator interferes with the proper formation of the bolus of food; the food collects between the cheek and the gum, from which it is usually removed by the patient with his fingers; saliva and fluids. escape from the angle of the mouth. During vigorous expiration, the cheeks are puffed outwards like a sail. The speech may be affected owing to the difficulty of sounding the labial consonants, (especially in double paralysis), and the vowels, $u$, ii (ue), $\ddot{i}$ (oe); while the speech, in paralysis of the branches to both sides of the palate, becomes nasal ( $\$ 628$ ). The acts of whistling, sucking, blowing, and spitting are interfered with. In double paralysis, many of these symptoms are greatly in. tensified, while others, such as the oblique position of the features, disappear. The features are completely relaxed; there is no inimetic play of the features, the patients weep and laugh, "as it were, behind a mask" (Romberg). 2. In paralysis of the palate, when the uvula is directed towards the sound side, and the paralysed half of the palate hangs down and cannot be raised (large superficial petrosal nerve), it is not determined to what extent this condition influences the act of deglutition and the formation of the consonants. 3. Taste is interfered with; either it is absent on the anterior two-thirds of the tongue, or the sensation is delayed and altered. This is due to an affection of the chorda. 4. Diminution of saliva on the affected side was first described by Arnold; still, we must determine to what extent a simultaneous affection of the sense of taste may cause a reflex interference with the secretion of saliva, or whether rapid removal of the saliva through the opened lips and angle of the mouth may cause the dryness on the affected side of the mouth. 5. Roux pointed out that hearing is affected, 
the sensibility to sounds being increased (oxyakoia, hyperakusis willisiana). The paralysis of the stapedius muscle makes the stapes loose in the fenestra ovalis, so that all impulses from the tympanum act vigorously upon the stapes, which consequently excites considerable vibrations in the Hluid of the inner ear. More rarely, in paralysis of the stapedius, it has been observed that low notes are heard at a greater distance than on the sound side (Lucae, Movn). 6. As the facial in man appears to contain fibres for the secretion of sweat, this explains the loss of the power of sweating in the face when the nerve begins to atrophy (Strauss, Bloch).

Section of the facial in young animals causes atrophy of the corresponding muscles. The facial bones are also imperfectly developed; they remain smaller, and hence the bones of the sound side of the face grow towards, and ultimately across, the middle line towards the affected side (Brown-Séquard). The salivary glands also remain smaller.

Stimulation - or irritation in the area of the facial-causes partial or extensive, either direct or reflex, tonic or clonic spasms. The extensive forms are known as " mimetic facial spasm." Amongst the partial forms are tonic contraction of the eyelid (blepharospasm), which is most common; and is caused reflexly by stimulation of the sensory nerves of the eye, e.g., in scrofulous ophthalmia, or from excessive sensibility of the retina (photophobia). More rarely, the excitement proceeds from some more distant part, e.g., in one cause recorded by v. Gräfe, from inflammatory stimulation of the anterior palatine arch. The centre for the reflex is the facial nucleus. The clonic form of spasm-spasmodic winking (spasmus nictitans) - is usually of reflex origin, due to irritation of the eye, the dental nerves, or even of more distant nerves. In severe cases, the affection may be bilateral, and the spasms may extend to the muscles of the neck, trunk, and upper extremities. Contraction of the muscles of the lip may be excited by emotions (rage, grief), or reflexly. Fibrillar contractions occur after section of the facial as a "degeneration-phenomenon" (p. 476). [If the facial be torn out at the stylomastoid foramen, there is paralytic oscillation of the lip muscles (Schiff). If, in such an animal, the posterior root of the annulus of Vieussens be stimulated electrically, as it contains vaso-dilator fibres (Dastre and Morat), not only do the blood-vessels of the cheek and lips dilate, but the veins pulsate and florid blood escapes from the veins, just as occurs in the sub-maxillary gland when the chorda is stimulated. On stimulating the ansa, after section of the seventh, there is a pseudo-motor effect on the muscles of the cheek and lips, so that there is an analogy between the chorda and the ansa (Rogowicz).] Intracranial stimulation of the most varied description may cause spasms. Lastly, facial spasm may be part of a general spasmodic condition, as in epilepsy, chorea, hysteria, tetanus. Aretaeus (81 A.D.) made the interesting observation that the muscles of the ear contracted during tetanus. Very rarely have spasmodic elevation of the palate and increased salivation been described as the result of irritation of the facial (Leube). Moos observed a profuse secretion of saliva on stimulating the chorda during an operation on the tympanic cavity.

350. VIII. NERVUS ACUSTICUS.-Arises by two roots (Stieda); a larger anterior and a smaller posterior one. From the former proceeds the vestibular nerve, and from the latter the cochlear nerve; these are separated in the sheep and horse (Horbaczewski). Each root springs from a median and a lateral nucleus, so that there are four nuclei. Some fibres come from the cerebellum, and these may be connected with equilibration. The ehief mass of the posterior ganglion fibres of the cochlear nerve cross and pass to the corpora quadrigemina, the internal geniculate, and finally to the temporo-sphenoidal lobe $(\$ 378, I V ., 2)$. After extirpation of the ternporo-sphenoidal lobe, these fibres atrophy into the internal capsule and internal geniculate body (v. Monakow). The strix acusticæ form a second decussating projection system. The origin of both acoustic nerves are connected by commissures in the brain (Flechsig).

In the course of the internal auditory meatus, the auditory and portio intermedia of the facial exchange fibres, but the physiological significance of this is unknown.

Function.-The acusticus or auditory nerve has a double function :- 1 . It is the nerve of hearing; when stimulated, either at its origin, in its course, or at its peripheral terminations, it gives rise to sensations of sound. Every injury, according to its intensity and extent, causes hardness of hearing or even deafness.

2. Quite distinct from the foregoing is the other function, which depends upon the semicircular canals, viz., that stimulation of the peripheral expansions in the ampullæ influences the movements necessary for maintaining the equilibrium of the body.

Brenner's Formula. - The relation of the auditory nerve to the galvanic current is very important. In healthy persons, when there is closure at the cathode, there is the sensation of a clang (or tone) in the ear, which continues with variations while the current is closed. When the anode is opened, there is a feebler tone (Brenner's Normal Acoustic Formula). This clang coincides exactly with the resonance fundamental tone of the sound-conducting apparatus of the ear itself. 
Pathological. - Increascd sensibility of the auditory nerve in any part of its course, its centre, or feripheral expansions causes the condition known as hyperakusis, which usually is a sign of greatly increased nervous excitability, as in hysteria. When excessive, it may give rise to distinctly painful impressions, which condition is known as acoustic hyperalgia (Eulenburg). Stimulation of the parts above-named causes sensations of sound, the most common being the sensation of singing in the cars, or tinnitus. This condition is often due to changes in the amount of blool in the blood-vessels of the ear-either anæmic or hyperæmic stimulation. There is well-marked tinnitus after large doses of quinine or salicin, due to the vaso-motor effect of these drugs upon the vessels of the labyrinth (Kirchner). Not unfrequently, in cases of timnitus, the reaction due to the galvanic current is often increased. More rarely there is the so-called "priculorical raction"-i.e., on applying the galvanic current to one ear, in addition to the reaction in this ear, there is the opposite result in the non-stimulated ear. In ther cases of disease of the auditory nerve, noises rather than musical notes are produced by the current; stimulation, especially of the cortical centre of the auditory nerve, chiefly in lunatics, may cause auditory delusions (\$ 378, IV.). According as the excitability of the auditory nerve is diminished or abolished, there is the condition of nervous hardness of hearing hypakusis), or nervous deafness (anakusis).

The Semicircular Canals of the Labyrinth.-Section or injury to these canals does not interfere with hearing, but other important symptoms follow their injury, such as disturbances of equilibrium due to a feeling of giddiness, especially when the injury is bilateral (Flourens). This does not occur in fishes (Kieselbach). The pendulum-like movement of the head, in the direction of the plane of the injured canal, is very characteristic. If the horizontal canal be divided, the head (of the pigeon) is turned alternately to the right and left. The rotation takes place, especially when the animal is about to execute a movement : when it is at rest, the movement is less pronounced. The phenomenon may last for months, and injury to the posterior vertical canals causes a well-marked up and down movement or nodding of the head, the animal itself not unfrequently falling forwards or backwards. Injury to the superion vertical canals also causes pendulum-like vertical movements of the head, while the animal often falls forwards. When all the canals are destroyed, various pendulum-like movenents are performed, while standing is often impossible. Breuer found that electrical stimulation of the canals caused rotation of the head, while Landois, on applying a solution of salt to the canals, observed pendulumlike movements, which, however, disappeared after a time. A 25 per cent. solution of chloral dropped into the ear of a rabbit causes, after fifteen minutes, a similar destruction of the canals (Vulpian). Section of the acoustic nerves within the cranium has the same result (Bechterew).

Explanation. - Goltz regards the canals as organs of sense for ascertaining the equilibrium or prosition of the head in space; Mach, as an organ for ascertaining the movements of the head. According to C'oltz's statical theory, every position of the head causes the endolymph to exert the greatest pressure upon a certain part of the canals, and thus excites in a varying degree the nerve-terninations in the ampullæ. According to Breuer, when the head is rotated, currents are produced in the endolymph of the canals, which must have a fixed relation to the direction and extent of the movements of the head, and these currents, therefore, when they are perceived, afford a means of determining the movement of the head. The nervous end-organs of the ampulla are arranged for ascertaining this perception. If the semicircular canals are an apparatus-in fact, "sense-organs"-for the sensation of the equilibrium, and if their function is to determine the position or movements of the head, necessarily their destruction or stimulation must alter these perceptions, and so give rise to abnormal movements of the head. Vulpian regards the rotation of the head as due to strong auditory perceptions (?) in consequence of affections of the canals. Böttcher, Tomaszewicz, and Baginsky regard the injury to the cerehellum as the cause of the phenomena. The pendulum-like movements, however, are so eharacteristic that they cannot be confounded with disturbances of the equilibrium which result from injury to the cerebellum.

[Kinetic Theory.-In 1875 Crum Brown pointed out that, if a person be rotated passively, his eyes being handaged, he can, up to a certain point, indicate pretty accurately the amount of movement, but after a time, this cannot be done, and if the rotation, as on a potter's wheel, be stopped, the sense of rotation continues. Crum Brown suggested that currents were produced in the endolymph, while the terminal hair-cells lagged behind, and were, in fact, dragged through the fluid. He pointed out that the right posterior canal is in line with the left superior, and the left posterior with the right superior, a fact which is readily observed by looking from 
behind at a skull, with the semicircular canals exposed (fig. 432). He assumes that the canals are paired organs, and that each pair is connected with rotation or movement of the head in a particular direction.]

Giddiness. - This feeling of false impressions as to the relations of the surroundings and consequent movements of the body, occurs especially during acquired changes in the normal movements of the eyes, whether due to involuntary to and fro movements of the eyeballs (nystagmus), or to paralysis of some eye muscle.

Active or passive movements of the head or of the body are normally accompanied by simultaneous movements of both eyeballs, which are characteristic for every position of the body. The general character of these "compensatory" bilateral movements of the eyes consists in this, that during the various changes in the position of the head and body, the eyes strive to maintain their primary passive position. Section of the aqueduct of Sylvius at the level of the corpora quadrigemina, of the floor of the fourth ventricle, of the auditory nucleus, both acustici, as well as destruction of both membranous labyrinths, causes disappearance of these movements; while, conversely,
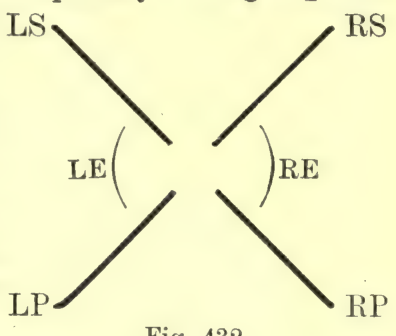

Fig. 432.

Diagram of the disposition of the semicircular canals. RS and LS, right and left superior ; $\mathrm{LP}$ and $\mathrm{RP}$, right and left posterior; $\mathrm{LE}$ and $\mathrm{RE}$, right and left external. stimulation of these parts is followed by bilateral associated movements of the eyeballs.

Compensatory movements of the eyeballs, under normal circumstances, may be caused reflexly from the membranous labyrinth. Nerve channels, capable of exciting reflex movements of both eyes, proceed from both labyrinths, and, indeed, both eyes are affected from both labyrinths. These channels pass through the auditory nerve to the centre (nuclei of the $3 \mathrm{rd}, 4 \mathrm{th}, 6 \mathrm{th}$, and 8 th cranial nerves), and from the latter efferent fibres pass to the muscles of the eye (Högyes).

Cyon found that stimulation of the horizontal semicircular canal was followed by horizontal nystagmus; of the posterior, by vertical, and of the anterior canal, by diagonal nystagmus. Stimulation of one auditory nerve is followed by rotating nystagmus, and rotation of the body of the animal on its axis towards the stimulated side.

Poisons.-Chloroform and other poisons enfeeble the compensatory movements of the eyeballs, while nicotin and asphyxia suppress them, owing to their action on their nerve-centre.

It is probable that the disturbances of equilibrium and the feeling of giddiness which follow the passage of a galvanic current through the head between the mastoid processes, are also due to an action upon the semicircular canals of the labyrinth $(\$ 300)$. Deviation of the eyeballs is produced by such a galvanic current (Hitzig). The same result is produced when the two electrodes are placed in the external auditory meatuses.

Pathological.-Menière's Disease.-The feeling of giddiness, not unfrequently accompanied by tinnitus, which occurs in Menière's disease, must be referred to an affection of the nerves of the ampullæ or their central organs, or of the semicircular canals themselves. By injecting fluid violently into the ear of a rabbit, giddiness, with nystagmus and rotation of the head towards the side operated on, are produced (Baginsky). In cases in man, where the tympanic membrane was defective, Lucæ, when employing the so-called ear-air-douche at 0.1 atmosphere, observed abduction of the eyeball with diplopia, giddiness, darkness in front of the eyes, while the respiration was deeper and accelerated. These phenomena must be due to stimulation or exhaustion of the vestibular branch of the auditory nerve (Högyes). In chronic gastric catarrh, a tendency to giddiness is an occasional symptom (Trousseau's gastric giddiness). This may, perhaps, be caused by stimulation of the gastric nerves exciting the vaso-motor nerves of the labyrinth, which must affect the pressure of the endolymph. Analogous giddiness is excited from the larynx (Charcot), and from the urethra (Erlenmeyer).

[Vertigo or giddiness is a very common symptom in disease, and may be produced by a great many different conditions. It literally means "a turning." As Gowers points out, the most common symptom is that the patient himself has a sense of movement in one or other direction; or objects may appear to move before him; and more rarely there is actual movement "com- 
monly in the same direction as the subjective sense of movement." It is sometimes due to a want of harmony between the impressions derived from different sense-organs or "contradictoriness of sensory impressions (Grainger Stewart), as is sometimes felt on ascending or descending a stair, or by some persons while standing on a high tower, constituting tower or cliff gidliuess. One of the most remarkable conditions is that called "agoraphobia" (Benedikt, $W$ esplial). The person can walk quite well in a narrow lane or street, but when he attempts to cross a wide square, he experiences a feeling closely allied to giddiness. The giddiness of sea-sickness is proverbial, while some persons get giddy with waltzing or swinging. Besides occurring in Meniere's disease, it sometimes occurs in locomotor ataxia, and some cerebral and cerebellar affections, including cerebral anemia. Very distressing giddiness and headache are often proulued by paralysis of some of the ocular muscles, c.g., the external rectus. Defective or perverted ocular impressions, as well as similar auditory impressions, may give rise to vertigo; in the latter or labyrinthine form the vertigo may be very severe. Severe vertigo is often accompranied by vomiting. A hard plug of ear-wax may press on the membrana tympani and eause severe gridliness. The forms of dyspeptic giddiness and the toxic forms due to the abuse of alcohol, tohacco, and some other drugs are familiar examples of this condition.]

[Tinnitus Aurium, or subjective noises in the ear, is a very common symptom in disease of the ear; the noise may be continuous or discontinuous, be buzzing, singing, or rumbling in character.]

351. IX. NERVUS GLOSSO-PHARYNGEUS. - Anatomical.-This nerve (fig. 429, 9) arises from the nucleus of the same name, which consists partly of large cells (motor) and partly of small cells (belonging to the gustatory fibres). The nucleus lies in the lower half of the fourth ventricle, deep in the medulla oblongata, near the olive (fig. 427), and posteriorly it abuts on that of the vagus. The anterior part of the central nucleus is regarded as the root of the portio intermetia of the facial ( $\$ 349$ ). The nerve also receives fibres from the vagal centres. The fibres collect into two trunks, which afterwards unite and leave the medulla oblongata in front of the vagus. In the fossula petrosa it has on it the petrous ganglion, from which, occasionally, a special part on the prosterior twig is separated within the skull as the ganglion of Ehrenritter. Communicating branches are sent from the petrous ganglion to the trigeminus, facial $(\epsilon$ and $\pi$ ), vagus and carotid plexus. From this ganglion also the tympanic nerve $(\lambda)$ ascends vertically in the tympanic cavity, where it unites with the tympanic plexus. This branch $(\$ 349,4)$ gives sensury fibres to the tympanic cavity and the Eustachian tube; while, in the log, it also carries secretory fibres for the parotid into the small superficial petrosal nerve (Heidenhain-\$145).

Function.-1. It is the nerve of taste for the posterior third of the tongue, the lateral part of the soft palate, and the glosso-palatine arch (compare $\$ 422$ ). [This is denied by Gowers (p. 600).]

The nerve of taste for the anterior two-thirds of the tongue is referred to under the lingual (\$ 347 , III., 4) and (horda tympani nerves $(\$ 349,4)$. The glossal branches are provided with ganglia, especially where the nerve divides at the base of the circumvallate papillæ (Remak, Kiolliker, stirling). The nerve ends in the circumvallate papillæ (fig. 429, U), and the endorgans are represented by the taste bulbs $(\$ 422)$.

2. It is the sensory nerve for the posterior third of the tongue, the anterior surface of the epiglottis, the tonsils, the anterior palatine arch, the soft palate, and a part of the pharynx. From this nerve there may be discharged reflexly, movements of deglutition, of the palate and pharynx, which may pass into those of vomiting $(\$ 158)$. These fibres, like the gustatory fibres, can excite a reflex secretion of saliva (\$145).

3. It is motor for the stylo-pharyngeus and middle constrictor of the pharynx (Volkmann); and, according to other observers, to the (?) glosso-palatinus (Hein) and the (??) levator veli palatini and azygos uvulæ (compare Spheno-palatine ganglion, $\$ 347$, II.). It is doubtful whether the glosso-pharyngeal nerve is really a motor nerve at its origin-although Meynert and others have described a motor nucleus-or whether the motor fibres reach the nerve at the petrous ganglion, through the communicating branch from the facial. 4. A twig accompanies the lingual artery ; this nerve, perhaps, is vaso-dilator for the lingual
blood-vessels.

Pathological. - There are no satisfactory observations on man of uncomplicated affections of the glosso-pharyngeal nerve.

352. X. NERVUS VAGUS.-Anatomical,--The nucleus from which the vagus arises along with the 9 th and 11th nerve is in (1) the ala cinerea in the lower half of the calamus 
scriptorius (fig. 427, 10) [and it is very probably the representative of the cells of the vesicular column of Clarke (\$ 366)]. (2) Other fibres come from the "longitudinal bundle" or "respiratory bundle" lying outside the nucleus, and reaching down into the cervical enlargement. (3) A motor nucleus - the nucleus ambiguus - a prolongation of some of the cells of the anterior horn of the spinal cord, gives some motor fibres. It leaves the medulla oblongata by 10 to 15 threads behind the 9 th nerve, between the divisions of the lateral column, and has a ganglion (jugular) upon it in the jugular foramen (fig. 428, VIII). Its branches contain fibres which subserve different functions.

1. The sensory meningeal branch from the jugular ganglion accompanies the vaso-motor fibres of the sympathetic on the middle meningeal artery, and sends fibres to the occipital and transverse sinus.

When it is irritated, as in congestion of the head and inflammation of the dura mater, it gives rise to vomiting.

2. The auricular branch (fig. 433 , au.) from the jugular ganglion receives a communicating branch from the petrous ganglion of the 9 th nerve, traverses the canaliculus mastoideus, crossing the course of the facial, with which it exchanges fibres whose function is unknown. On its course, it gives sensory branches to the posterior part of the auditory meatus, and the adjoining part of the outer ear. A branch runs along with posterior auricular branch of the facial, and confers sensibility on the muscles.

When this nerve is irritated, either through inflammation or by the presence of foreign bodies in the outer ear passage, it may give rise to vomiting. Stimulation of the deep part of the external auditory meatus in the region supplied by the auricular branch causes coughing reflexly [e.g., from the presence of a pea in the ear]. Similarly, contraction of the blood-vessels of the ear may be caused reflexly (Snellen, Lovèn).

The nerve is the remainder of a considerable branch of the vagus which exists in fishes and the larvæ of frogs, and runs under the skin along the side of the body.

3. The connecting branches of the vagus are:-(1) A branch which directly connects the petrous ganglion of the 9 th with the jugular ganglion of the 10th ; its function is unknown. (2) Directly above the plexus gangliiformis vagi, the vagus is joined by the whole inner half of the spinal accessory. This nerve conveys to the vagus the motor fibres for the larynx, and the cervical part of the oesophagus (which according to Steiner lie in the inner part of the nerve-trunk), as well as the inhibitory fibres for the heart ( $\mathrm{Cl}$. Bernard). (3) The plexus gangliiformis fibres, whose function is unknown, join the trunk of the vagus from the hypoglossal, superior cervical ganglion of the sympathetic, and the cervical plexus.

4. Pharyngeal Plexus.-The vagus sends one or two branches (fig. 433, 2) from the upper part of the plexus gangliiformis to the pharyngeal plexus, where at the level of the middle constrictor of the pharynx, it is joined by the pharyngeal branches of the 9 th nerve and those of the upper cervical sympathetic ganglion, near the ascending pharyngeal artery, to form the pharyngeal plexus. The vagal fibres in this plexus supply the three constrictors of the pharynx with motor fibres, while the tensor palati (Otic ganglion, § 347, III.) and levator of the soft palate (compare Spheno-palatine ganglion, $\S 347$, II.) also receive motor (? sensory) fibres. Sensory fibres of the vagus from the pharyngeal plexus supply the pharynx from the part beneath the soft palate downwards. These fibres excite the pharyngeal constrictors reflexly, during the act of swallowing ( $\$ 156)$. If stimulated very strongly, they may cause vomiting. (The sympathetic fibres of the œsophageal plexus give vaso-motor nerves to the œsophageal vessels; for the osophageal branches of the 9th nerve see above.)

5. The vagus supplies two branches to the larynx, the superior and inferior laryngeal.

(a) The superior laryngeal (fig. 433,3 ) receives vaso-motor fibres from the superior cervical ganglion of the sympathetic. It divides into two branches, external and internal :-(1) The external branch receives vaso-motor fibres from the same source (they, accompany the superior thyroid artery), and supply the crico-thyroid 
608

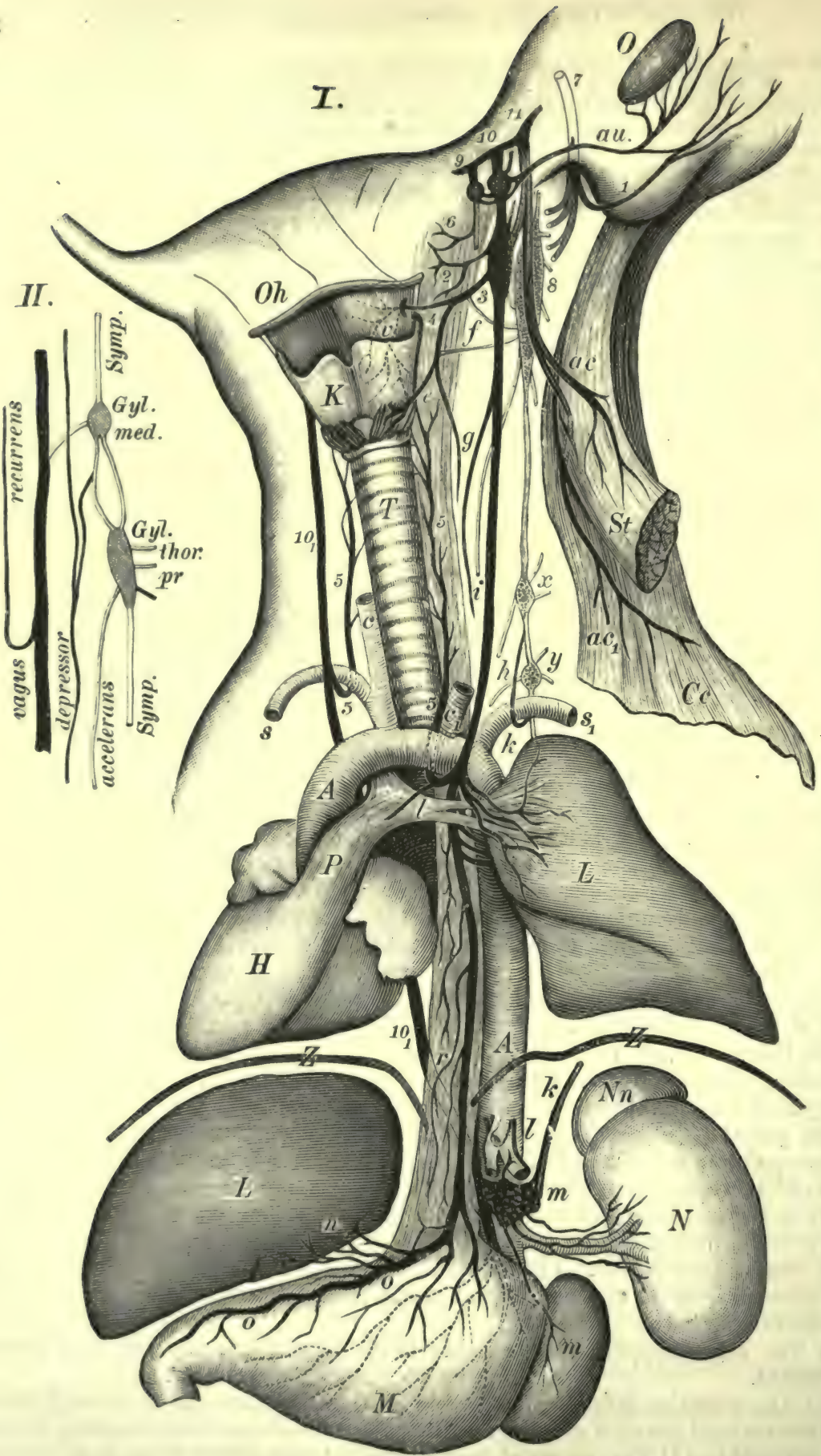

Fig. 433.

I. Scheme of the distribution of the vagus and accessorius. - 10, Exit of left vagus from the skull ; $10_{1}$, right vagus ; 9 , glosso-pharyngeal ; 7 , facial ; 1 , deep post-auricular from the facial ; 
muscle with motor fibres, and sensory fibres to the lower lateral portion of the laryngeal mucous membrane. (2) The internal branch gives off sensory branches only to the glosso-epiglottidean fold, and the adjoining lateral region of the root of the tongue, the aryepiglottidean fold, and to the whole anterior part of the larynx, except the part supplied by the external branch (Longet). Stimulation of any of these sensory fibres causes coughing reflexly. Coughing is produced by stimulation of the boundaries of the glottis respiratoria, but not of the vocal cords, and by stimulation of the sensory branches of the vagus to the tracheal mucous membrane, especially at the bifurcation, and also from the bronchial mucous membrane (Kohts). Coughing is also caused by stimulation of the auricular branch of the vagus, especially in the deep part of the external auditory meatus, of the pulmonary tissue, especially when altered pathologically; in pathological conditions (inflammation) of the pleura (? certain changes in the stomach [stomach-cough]), of the liver and spleen (Naunyn). The coughing centre is said to lie on each side of the raphe, in the neighbourhood of the ala cinerea (Kohts). Cases of violent coughing may, owing to stimulation of the pharynx, be accompanied by vomiting as an associated movement ( $\$ 120)$.

In many individuals, coughing can be excited by stimulation of distant sensory nerves $(\$ 120,1)$, e.g., from the outer ear (auricular nerve), nasil mucous membrane, liver, spleen, stomach, intestine, uterus, mammæ, ovaries, and even from certain cutaneous areas (Ebstein). It is uncertain if these conditions act directly upon the coughing centre, or first of all affect the vascularisation and secretion of the respiratory organs, which in their turn affect the coughing centre.

The cough (dog, cat) caused by stimulation of the trachea and bronchi occurs at once, and lasts as long as the stimulus lasts ; in stimulation of the larynx, the first effect is inhibition of the respiration accompanied by movements of deglutition, while the cough occurs after the cessation of the stimulation (Kandarazky).

The superior laryngeal contains afferent fibres which, when stimulated, cause arrest of the respiration and closure of the rima glottidis (Rosenthal)—(see Respiratory centre, § 368). Lastly, fibres which are efferent and serve to excite the vaso-motor centre, and are in fact "pressor fibres"--(see Vaso-motor centre, § 371 , II.).

(b) The inferior laryngeal or recurrent bends on the left side around the arch of the aorta, and on the right around the subclavian, and ascends in the groove between the trachea and cesophagus, giving motor fibres to these organs, and the lower constrictors of the pharynx, and passes to the larynx, to supply motor fibres to all its muscles, except the cricothyroid. It also has an inhibitory action upon the respiratory centre (see \$ 368).

A connecting branch runs from the superior laryngeal to the inferior (the anastomosis of Galen), which occasionally gives off sensory branches to the upper half of the trachea (sometimes to the larynx ?) ; perhaps also to the osophagus (Longet), and sensory fibres (?) for the muscles of the larynx supplied by the recurrent laryngeal. According to François Franck, seusory fibres pass by this anastomosis from the recurrent into the superior laryngeal. According to Waller and Burckhard, the motor fibres of both laryugeal nerves are all derived from the accessorius ; while Chauveau maintains that the cricothyroid is an exception.

Stimulation of the superior laryngeal is painful, and causes contraction of

2, pharyngeal branches of vagus; 6 , pharyngeal branch of the glosso-pharyngeal; 3 , superior laryngeal, with its anastomoses, $f$, with the sympathetic and its division, 4 , into its internal, $v$, and external branches, $e ; 5$, inferior or recurrent laryngeal; au., auricular branch of vagus. Cardiac nerves: $-g$, cardiac branches from the vagus and superior laryngeal ; $i, h$, the three cardiac branches from the upper, $g$, middle, $x$, and lower, $y$, cervical ganglion of the sympathetic; $k$, ring of Vieusses; $l$, cardiac branch from the recurrent laryngeal; $L$, lung with the anterior and posterior pulmonary plexuses; $r$, œsophageal plexus ; 00 , gastric branches, and near them the hepatic branches, $n$; $m$, cœliac plexus ; $k$, splanchnic entering former; 11 , accessory nerve sending its inner branch into. the gangliform plexus of the vagus-its outer branch, $\alpha c$, supplies the sternomastoid, St and $a c_{1}$, and the trapezius, $C c$; $O$, external auditory meatus; Oh, hyoid bone ; $K$, thyroid cartilage ; $T$, trachea ; $H$, heart ; $P$, pulmonary artery ; $A A$, aorta ; $c$, right carotid ; $c_{1}$, left carotid; $s$ and $s_{1}$, right and left subclavian artery; $Z Z$, diaphragm; $N$, kidney ; $N n$, suprarenal capsule; $M$, stomach; $m$, spleen; $L L$, lung and liver. II. Scheme of the course of the depressor and accelerans in the cat. 
the cricothyroid muscle (while the other laryngeal muscles contract reflexly). Section of both nerves, owing to paralysis of the cricothyroids, causes slight slowing of the respirations (sklarek). In dogs, the voice becomes, deeper and hoarser, owing to diminished tension of the vocal cords (Longet). The larynx becomes insensible, so that saliva and particles of food pass into the trachea and lungs, without causing reflex contraction of the glottis or coughing. This excites "traumatic pneumonia," which results in death (Friedländer).

Stimulation of the recurrent nerves causes spasm of the glottis. Section of these nerves paraly'ses the laryngeal muscles supplied by them, the voice becomes husky and hoarse (in the pig-Galen, Riolan, 1618) in man, dog, and cat; while rabbits retain their shrill cry. The glottis is small, with every inspiration, the

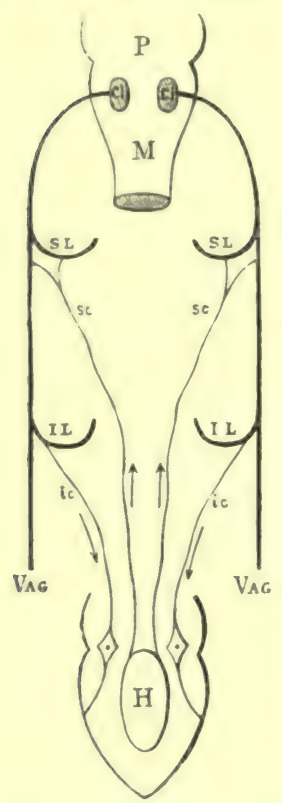

Fig. 434.

Sheme of the cardiac nerves in the rabbit. $P$, pons ; M, medulla oblongata ; VAG, vagus ; SL, superior, iL, inferior laryngeal, s', superior cardiac or depressor ; $i c$, inferior cardiac or cardio-in. hibitory ; H, heart. vocal cords approximate considerably at their anterior parts, while, during expiration, they are relaxed and are separated from each other. Hence, the inspiration, especially in young individuals whose glottis respiratoria is narrow, is difficult and noisy (Legallois); while the expiration takes place easily. After a few days, the animal (carnivore) becomes more quiet, it respires with less effort, and the passive vibratory movements of the vocal cords become less. Even after a considerable interval, if the animal be excited, it is attacked with severe dyspnoea, which disappears only when the animal has become quiet again. Owing to paralysis of the laryngeal muscles, foreign bodies are apt to enter the trachea, while the paralysis renders difficult the first part of the process of swallowing in the oesophageal region. Broncho-pneumonia may be produced (Arnsperger).

6. The depressor nerve, which in the rabbit arises by one branch from the superior laryngeal, and usually also by a second root from the trunk of the vagus itself [runs down the neck in close relation with the vagus, sympathetic, and carotid artery, enters the thorax], and joins the cardiac plexus (fig. $434, s c$ ). It is an afferent nerve, and when its central end is stimulated [provided both vagi be divided], it diminishes the energy of the vaso-motor centre, and thus causes a fall of the blood-pressure (hence the name given to it by Cyon and Ludwig, § 371 , II.). At the same time, [if the vagus on the opposite side be intact], its stimulation affects the cardio-inhibitory centre, and thus reflexly diminishes the number of heart-beats. [Its stimulation also gives rise to pain, so that it is the sensory nerve of the heart. If in a rabbit the vagi be divided in the middle of the neck, and the central end of the depressor nerve, which is the smallest of the three nerves near the carotid, be stimulated, after a short time there is no alteration of the heart-beats, but there is steady fall of the blood-pressure (fig. 106), which is due to a reflex inhibition of the vaso-motor centre, resulting in a dilatation of the blood-vessels of the abdomen. Of course, if the vagi be intact, there is a reflex inhibitory effect on the heart. It is doubtful if the depressor comes into action when the heart is overdistended. If it did, of course the blood-pressure would be reduced by the reflex dilatation of the abdominal blood-vessels.]

The depressor nerve is present in the cat ( $\$ 370$ ), hedgehog (Aubert, Röver), rat and mouse ; in the horse and in man, fibres analogous to the depressor re-enter the trunk of the vagus (Bernhardt, Kreidmann). Depressor fibres are also found in the rabbit in the trunk of the vagus (Dreschfeldt, Stelling). 
7. The cardiac branches (fig. $433, g, l$ ), as well as the cardiac plexus, have been described in $\$ 57$. These nerves contain the inhibitory fibres for the heart (fig. 434, ic-cardio-inhibitory-Edward Weber, November, 1845; Budge, independently in May 1846), also sensory fibres for the heart 「in the frog (Budge), and partly in mammals $(G o l t z)]$. Lastly, in some animals the heart receives some of the accelerating fibres through the trunk of the vagus. Feeble stimulation of the vagus occasionally causes acceleration of the beats of the heart (Schiff). [This occurs when the vagus contains accelerator fibres.] In an animal poisoned with nicotin, or atropin, which paralyses the inhibitory fibres of the vagus, stimulation of the vagus is followed by acceleration of the heart-beats (Schiff, Schmiedeberg) [owing to the unopposed action of any accelerated fibres that may be present in the nerve, e.g., of the frog].

8. The pulmonary branches of the vagus join the anterior and posterior pulmonary plexuses. The anterior pulmonary plexus gives sensory and motor fibres to the trachea, and runs on the anterior surface of the branches of the bronchi into the lungs $(L)$. The posterior plexus is formed by three to five large branches from the vagus, near the bifurcation of the trachea, together with branches from the lowest cervical ganglion of the sympathetic and fibres from the cardiac plexus. The plexuses of opposite sides exchange fibres, and branches are given off which accompany the bronchi in the lungs. Ganglia occur in the course of the pulmonary branches in the frog (Arnold, W. Stirling) [newt-W. Stirling; and in mammals (Remak, Egorow, W. Stirling)], in the larynx [Cock, W. Stirling], in the trachea and bronchi $[W$. Stirling, Kandarazki]. Branches proceed from the pulmonary plexus to the pericardium and the superior vena cava (Luschka, Zuckerkandl).

The functions of the pulmonary branches of the vagus are-(1) they supply motor branches to the smooth muscles of the whole bronchial system $\left(\S 106^{\circ}\right)$; (2) they supply a small part of the vaso-motor nerves of the pulmonary vessels (Schiff), but by far the largest number of these nerves (? all) is supplied from the connection with the sympathetic (in animals from the first dorsal ganglion)-(BrownSéquard, A. Fick, Badoud, Lichtheim); (3) they supply sensory (cough-exciting) fibres to the whole bronchial system, and to the lungs; (4) they give afferent fibres, which, when stimulated, diminish the activity of the vaso-motor centre, and thus cause a fall of the blood-pressure during forced expiration; (5) similar fibres which act upon the inhibitory centre of the heart, and so influence it as to accelerate the pulse-beats (\$369, II.). Simultaneous stimulation of 4 and 5 alters the pulse rhythm (Sommerbrodt); (6) they also contain afferent fibres from the pulmonary parenchyma to the medulla oblongata, which stimulate the respiratory centre. [These fibres are continually in action], and consequently section of both vagi is followed by diminution of the number of respirations; the respirations become at the same time deeper, while the same volume of air is changed (Valentin). Stimulation of the central end of the vagus again accelerates the respirations (Traube, J. Rosenthal). Thus, laboured and difficult respiration is explained by the fact that the influences conveyed by these fibres which excite the respiratory centre reflexly are cut off ; so it is evident, that centripetal or afferent impulses proceeding upwards in the vagus are intimately concerned in maintaining normal reflex respiration; after these nerves are divided, conditions exciting the respiratory movements must originate directly, especially in the medulla oblongata itself ( $(368)$.

Pneumonia after Section of both Vagi.-The inflammation which follows section of both vagi has attracted the attention of many observers since the time of Valsalva, Morgagni (1740), and Legallois (1812). In attempting to explain this phenomenon, we must bear in mind the following considerations :-(a) Section of both vagi is followed by loss of motor power in the muscles of the larynx, as well as the sensibility of the larynx, trachea, bronchi, and the lungs, provided the section be made above the origin of the superior laryngeal nerves. Hence, the glottis is not closed during swallowing, nor is it closed reflexly when foreign bodies (saliva, 
particles of foot, irrespirable gases) enter the respiratory passages. Even the reflex act of coughing, whicl. under orlinary circumstances, would get rid of the offending bodies, is abolished. Thus, foreigu bolies may readily enter the lungs, and this is favoured by the fact that, owing to the simultaneous paralysis of the oesophagus, the food remains in the latter for a time, and may therefore easily enter the larynx. That this constitutes one important factor was proved by Traulve, who foumd that the pueumonia was prevented when he caused the animals to respire by means of a tube inserted into the trachea through an ajerture in the neck. If, on the contrary, only the motor recurrent nerves were divided and the cesophagus ligatured, so that in the juress of attempting to swallow, fool must necessarily euter the respiratory passages, "traumatic pneumonia" was the invariable result (Tr(ube, O. Frey). (b) A second factor depends on the circumstance that, owing to the laboured and difficult respiration, the lungs becmin surchurgal with blond, because during the long time that the thorax is distended, the pressure of the air within the lungs is abmormally low. This condition of congestion, or abnormal filling of the julmonary vessels with blood, is followed by serous exudation (pulmonary oedema), and even ly exulation of blood and the formation of pus in the air-vesicles (Frey). This same circumstance favours the entrance of fluids through the glottis $(\$ 352, b)$. The introduction of a tracheal cannula will prevent the entrance of fluids and the occurrence of inflammation. It is probalile that a partial paralysis of the pulmonary caso-motor nerves may be concerned in the inflammation, as this conduces to an engorgement of the pulmonary capillaries. (c) Lastly, it is of conseyuence to determine whether tiophic fibres are present in the vagus, which may influence the normal condition of the pulmonary tissues. According to Michaelson, the pueumonia which takes place immediately after section of the vagi occurs especially in the lower and midule lolses; the pneumonia which follows section of the recurrents occurs more slowly, and causes catarrhal inflammation, especially in the upper lobes. Rabbits, as a rule, die within twenty-four hours, with all the symptoms of pneumonia; when the above-mentioned precautions are taken, they may live for several days. Dogs may live for a long time. If the 9th, 10th, and 12th nerves lie torn out on one side in a rabbit, death takes place from pneumonia (Grünhagen). In birds, bilateral section of the vagi is not followed by pneumonia (Blainville, Billroth), because the upver laryn remains capable of closing firmly-death takes place in eight to ten days with the symptoms of inanition (Einbrodt, Zander, $v$. Anrep), while the heart undergoes fatty degeneration (Eichlurst), aml so do the liver, stomach, and muscles (v. Anrep). According to Wassilieff, the heart shows eloudy swelling and slight wax-like degeneration. Frogs, which at every respiration open the glottis, and close it during the pause, die of asphyxia. Section of the julmonary hranches has no injurious effect (Bidder). [Unilateral section of the vagus in rabbits is followed within forty-eight hours by the appearauce of yellowish-white spots on the myocardium, especially near the interventricular septum, on the papillary muscles, and along the furrows for the coronary arteries. The muscular fibres exhibit retrogressive changes, whereby their stria disappear; they become swollen up and filled with albuninous granules. After eight to ten days, the interstitial tissue of these foci becomes infiltrated with small round granular cells, especially near the blood-vessels. At a later stage, the interstitial connective. tissue increases in amount, and the muscle atrophies. No effect is produced by section of the depressor or sympathetic, and Fantino concludes that some of the fibres of the vagus exert a trophic action on the myocardium.]

9. The œsophageal plexus (fig. $434, r$ ) is formed principally by branches from the vagus above the inferior laryngeal, from the pulmonary plexus, and below from the trunk itself. This plexus supplies the osophagus with motor power $(\$ 156)$, the sensibility which is present only in the upper part, and it also supplies fibres capable of exciting reflex actions.

10. The gastric plexus $(\omega \circ)$ consists of $(a)$ the anterior (left) termination of the vagus, which supplies fibres to the oesophagus and courses along the small curvature, and sends a few fibres through the portal fissure into the liver; $(b)$ the posterior (right) vagus, after giving off a few fibres to the œesophagus, takes part in the formation of the gastric plexus to which (c) sympathetic fibres are added at the pylorus. Section of the vagi is followed by hyperæmia of the gastric mucous membrane (Panum, Pincus), but it does not interfere with digestion (Bidder and Schmidt), even when it is performed at the cardia (Kritzler, Schiff).

11. About two-thirds of the right vagus on the stomach joins the cœliac plexus, and from it branches accompany the arteries to the liver, spleen, pancreas, duodenum, kidney, and suprarenal eapsules. The vagus supplies motor fibres to the stomach, which belong to the root of the vagus itself and not to the accessorius (Stilling, Bischoff). The gastric branches also contain afferent fibres, which, when stimulated, cause reflexly a secretion of salive (\$145). It is undetermined whether 
they also cause vomiting. For the effect of the vagus upon the movements of the intestine (see $\S 161$ ). According to some observers, stimulation of the vagus is followed by movement of the large as well as of the small intestine (Stilling, Kupffer, C. Ludwig, Remak). Stimulation of the peripheral end of the vagus causes contraction of the smooth muscular fibres in the capsule and trabeculæ of the spleen (in the rabbit and dog, §103). Stimulation of the vagus at the cardia causes increase in the secretion of urine with dilatation of the renal vessels, while the blood of the renal vein becomes more arterial $(\mathrm{Cl}$. Bernard). According to Rossbach and Quellhorst, a few vaso-motor fibres are supplied by the vagus to the abdominal organs, whilst the greatest number comes from the splanchnic.

12. Reflex Effects.-The vagus and its branches contain fibres, some of which have been referred to already, which act reflexly (afferent) upon certain nervous mechanisms.

(a) On the vaso-motor centre there act (a) pressor fibres (especially in both laryngeal nerves), whose stimulation is followed by a reflex contraction of the arterial blood-channels, and thus cause a rise of the blood-pressure; $(\boldsymbol{\beta})$ depressor fibres (in the depressor or the vagus itself), which have exactly an opposite effect. (This subject is specially referred to under the head of the Vaso-motor nerve-centre, \$ 371.$)$

(b) On the respiratory centre there act ( $\alpha$ ) fibres (pulmonary branches) whose stimulation is followed by acceleration of the respiration; and $(\boldsymbol{\beta})$ inhibitory fibres (in both laryngeals), whose stimulation is followed by slowing or arrest of the respiration. (See Respiratory centre, § 368.)

(c) On the cardio-inhibitory system. - [When the central end of one vagus is stimulated, provided the other vagus is intact, the heart may be arrested reflexly in the diastolic phase.] Mayer and Pribram observed that sudden distension of the stomach caused slowing and even arrest of the heart, while, at the same time, there was contraction of the arteries of the medulla oblongata and increase of the blood-pressure.

$(d)$ On the vomiting centre. - This centre may be affected by stimulation of the central end of the vagus, and, as already mentioned, by stimulation of many afferent fibres in the vagus $(\S 158)$.

(e) On the pancreatic secretion.- Stimulation of the central end of the vagus is followed by arrest of this secretion $(\$ 171)$.

( $f$ ) According to $\mathrm{Cl}$. Bernard, there are fibres present in the pulmonary nerves, which, when they are stimulated, increase reflexly the formation of sugar in the liver, perhaps through the hepatic branches of the vagus.

Unequal Excitability. - The various branches of the vagus are not all endowed with the same degree of excitability. If the peripheral end of the vagus be stimulated, first of all with a weak stimulus, the laryngeal muscles are first affected, and afterwards the heart is slowed (Rutherford). If the central end be stimulated with feeble stimuli, the "excito-respiratory" fibres are exhausted before the "inhibito-respiratory" (Burkart). According to Steiner, the varion's fibres are so arranged in the vagus that the afferent fibres lie in the outer, and the efferent in the inner, half of the trunk, in the cervical region.

Pathological. - Stimulation or paralysis in the area of the vagus must necessarily present a very different picture according as the affection is referred to the whole trunk or only to some of its branches, or whether the affection is unilateral or bilateral. Paralysis of the pharynx and œsophagus, which is usually of central or intracranial origin, interferes with or abolishes deglutition, so that when the oesophagus becomes filled with food there is difficulty of breathing, and the food may even pass into the nasal cavities. A peculiar sonorous gurgling is occasionally heard in the relaxed canal (deglutatio sunora). In incomplete paralysis, the act of deglutition is delayed and rendered more difficult, while large masses are swallowed more easily than small ones. Increased contraction and spasmodic stricture of the œsophagus are referred to under the phenomena of general nervous excitability ( $\$ 186)$.

Spasm of the laryngeal muscles causes spasmodic closure of the glottis (Spasmus glottidis). This condition is most apt to occur in children, and takes place in paroxysms, with symptoms of dyspnoea and crowing inspiration; if the case be very severe, there may be muscular contractions (of the eye, jaw, digits, \&c.). The symptoms are very probably due to the reflex spasms which may be discharged from the sensory nerves of several areas (teeth, intestine, skin). The impulse is conducted along the sensory nerves proceeding from these areas to the medulla oblongata, where it causes the discharge of the reflex mechanism which produces the abovementioned results. There may be spasm- of the dilators of the glottis and other laryngeal muscles (Fräntzel).

Stimulation of the sensory nerves of the larynx, as is well known, produces coughing. If the stimulation be very intense, as in whooping-cough, the fibres lying in the laryngeal nerves, which inhibit the respiratory centre, may also be stimulated; the number of respirations is 
diminished, and ultimately the respiration ceases, the diaphragm being relaxed: while, with the most intense stimulation, there may be spasmodic expiratory arrest of the respiration with closure of the glottis, which may last for fifteen seconds. Paralysis of the laryngeal nerves, which causes disturbances of specech, has been referred to in $\$ 313$. In bilateral paralysis of the recurrent nerves, in consequence of tension upon them due to dilatation of the aorta and the subclavian artery, a considerable amount of air is breathed out, owing to the futile efforts which the patient makes in trying to speak; expectoration is more difficult, while violent coughing is impossible ( $v$. Ziemssen). Attacks of dyspncea occur just as in animals, if the person make violent efforts. Some observers (Salter, Beryson) have referred the paroxysms of nervous asthma, which last for a quarter of an hour or more, and constitute asthma bronchiale, to stimulation of the pulmonary plexus, causing spasmodic contraction of the bronchial muscle ( $\S$ 106). Plysical investigatiou during the proxysms reveals nothing but the existence of some rhonchi $(\$ 117)$. If this condition is really spasmodic in its nature (? of the vessels), it must be usually of a reflex character ; the afferent nerves may be those of the lung, skin, or genitals (in hysteria). Perhaps, however, it is due to a temporary paralysis of the pulmonary nerves (afferent), which excite the respiratory centre (excito-respiratory).

Stimulation of the cardiac branches of the ragus may cause attacks of temporary suspension of the cardiac contractions, which are accompanied by a feeling of great depression and of impending dissolution, with occasionally pain in the region of the heart. Attacks of this sort may be produced refferly, c.g., by stimulation or irritation of the abdominal organs (as in the experiment of Goltz of tapping the intestine's). Hennoch and Silbermann observed slowing of the action of the heart in children suffering from gastric irritation. Similarly, the respiration may be affected reflexly through the vagus, a condition deseribed by Hennoch as asthma dyspepticum. In cases of intermittent paralysis of the cardiac branches of the vagus, we rarely find acceleration of the pulse above 160 (Riegel), 200 (Tuczck, L. Langer); even 240 pulse-beats per minute have been recorded ( $\boldsymbol{K}^{\prime}$ uppert), and in such cases, the beats vary much in rhythm and force, and they are very irregular. These cases require to be more minutely analysed, as it is not clear how much is due to paralysis of the vagus and how much to the action of the accelerating mechanism of the heart. Little is known of affections of the intra-abdominal fibres of the vagus. It seems that the sensory branches of the stomach do not come from the vagus. If the trunk of the vagus or its centre be paralysed, there are laboured, deep, slow respirations, such as follow the section of both vagi (Guttrann).

353. XI. NERVUS ACCESSORIUS WILLISII.-Anatomical.-This nerve arises by two completely separate roots; one from the accessorius nucleus of the medulla oblongata (fig. 427,11 ), which is connected with the vagus nucleus; while the other root arises between the anterior and posterior nerve-roots from the spinal cord, usually between the 5th and 6th rervical vertebre. In the spinal cord, its tibres can be traced to an elongated nucleus lying on the outer side of the anterior cornu, as far downwards as the 5th cervical vertebra. Near the jugular foramen both portions come together, but do not exchange fibres $(\boldsymbol{H o l l})$; both roots afterwards separate from each other to form two distinct branches, the anterior (inner), which arises from the medulla oblongata, passing en masse into the plexus gangliiformis vagi. This branch supplies the vagus with most of its motor fibres (compare $\$ 352,3$ ), and also its cardioinhibitory fibres (fig. 433). [The upper cervical metameres or segments give origin not only to the anterior and posterior roots of the corresponding nerve-roots, but between these roots arise the roots of the spinal accessory nerve. This nerve contains large medullated nerve-fibres, and fine medullated fibres such as characterise the visceral branches of the thoracic and sacral regions $(\S 356)$. The nerve passes by the jugular ganglion of the vagus, then divides into the external and internal branch. All the large fibres pass into the external branch, which, along with branches from the cervical plexus, supply the sternomastoid and trapezius. The internal branch, composed of small fibres, passes into the ganglion of the trunk of the vagus. Gaskell therefore regards the internal branch "as formed by the rami viscerales of the upper cervical and vagus nerves." These fine medullated nerve-fibres probably arise from the cells of the posterior vesicular column of Clarke. The motor fibres to the trapezius and sternomastoid arise from the cells of the lateral horn of grey matter.]

If the accessorius be pulled out by the root in animals, the cardio-inhibitory fibres undergo degeneration. If the trunk of the vagus be stimulated in the neck four to five days after the operation, the action of the heart is no longer arrested thereby [owing to the degeneration of the cardio-inhibitory fibres] (Waller, Schiff, Daszkiewicz, Heidenhain); according to Heidenhain, the heart-beats are accelerated immediately after pulling out the nerve.

The external branch arises from the spinal roots. This nerve communicates with the sensory branches of the posterior root of the 1st, more rarely of the 2 nd cervical nerve, and these fibres supply sensibility to the muscles; it then turns 
backwards above the transverse process of the atlas, and terminates as a motor nerve in the sternomastoid and trapezius (fig. 433). The latter muscle usually receives motor fibres also from the cervical plexus (fig. 429).

The external branch communicates with several cervical nerves. These fibres either participate in the innervation of the above-named muscles, or the accessorius returns part of the sensory fibres supplied by the posterior roots of the two upper cervical nerves.

Pathological. - Stimulation of the outer branch causes tonic or clonic spasn of the abovenamed muscles, usually on one side. If the branch to the sternomastoid be affected alone, the head is moved with each clonic spasm. If the affection be bilateral, the spasm usually takes place on opposite siles alternately, while it is rare to have it on both sides simultaneously. In spasm of the trapezius the head is drawn backwards and to the side. Tonic contraction of the flexors of the head causes the characteristic position of the head known as caput obstipum (spasticum) or wryneck. In paralysis of one of these muscles, the head is drawn towards the sound side (torticollis paralyticus). Paralysis of the trapezius is usually only partial.

Paralysis of the whole trunk of the spinal accessory (usually caused by central conditions), besides causing paralysis of the sternomastoid and trapezius, also paralyses the motor branches of the vagus already referred to (Erb, Fränkel).

354. XII. NERVUS HYPOGLOSSUS.-Anatomical.-It arises from two large-celled nuclei within the lowest part of the calamus scriptorius, and one adjoining small-celled nucleus (Roller), while additional fibres come from the brain ( $\$ 378)$, and also perhaps from the olive (fig. 427, 12). It springs by 10 to 15 twigs in a line with the anterior roots of the spinal nerve (fig. 420, IX.). In its development part of the hypoglossal behaves as a spinal nerve (Froriep).

Function.-It is motor to all the muscles of the tongue, including the geniohyoid and thyrohyoid.

Connections.-The trunk of the hypogiossal is connected with-(1) the superior cervical ganglion of the sympathetic, which supplies it with vaso-motor fibres for the blood-vessels of the tongue. After section of the hypoglossal and lingual nerves, the corresponding half of the tongue becomes red and congested (Schiff). (2) There is also a branch from the plexus gangliiformis vagi, its small lingual branch to the commencement of the hypoglossal arch. These fibres supply the hypoglossal with sensory fibres for the muscles of the tongue, for even after section of the lingual the tongue still possesses dull sensibility. It is uncertain whether fibres with a similar function are partly derived from the cervical nerves or from the anastomosis which takes place with the lingual. (3) It is united with the upper cervical nerves by means of the loops known as the ansa hypoglossi. These connecting fibres rum in the descendens noni to the sternohyoid, omohyoid, and sternothyroid. Cervical fibres do not, as a rule, enter the tongue; stimulation of the root of the hypoglossal acts upon the above-named muscles only very rarely and to a very slight extent (Volkmann). (Compare § 297, 3, and § 336, III.).

Bilateral section of the nerve causes complete motor paralysis of the tongue. Dogs can no longer lap, they bite the flaccid tongue. F'rogs, which seize their prey with the tongue, must starve; when the tongue hangs from the mouth, it must prevent the closure of the mouth, so that these animals must die from asphyxia, as air is pumped into the lungs only when the mouth is closed.

Pathological.-Paralysis of the hypoglossal (glossoplegia), which is usually central in its origin, causes disturbance of speech $(\$ 319)$. [In unilateral palsy, the tongue lies in the mouth in its normal position, but the base is more prominent on the paralysed side. When the tongue is protruded, it passes to the sound side by the genio-hyoglossus (\$155).] Paralysis of the tongue also interferes with mastication, the formation of the bolus in the mouth, and deglutition in the mouth. Owing to the imperfect movements of the tongue, taste is imperfect, and the singing of high notes and the falsetto voice, which require certain positions of the tongue, appear to be impossible (Bennati).

Spasm of the tongue, which causes aphthongia (\$ 318$)$, is usually reflex in its origin, and is extremely rare. Idiopathic cases of spasm of the tongue have been described; the seat of the irritation lay either in the cortex cerebri or in the oblongata (Berger, E. Remak). For Pseudomotor Action, p. 601.

355. THE SPINAL NERVES. - Anatomical. - The thirty-one pairs of spinal nerves arise by means of a posterior [superior, gangliated] root (consisting of a few large rounded bundles), from the suleus between the posterior and lateral columns of the spinal cord, and by means of an anterior [inferior, non-gangliated] root (consisting of numerous fine flat strands), from the furrow between the anterior and lateral columns, fig. 435. The posterior roots, with the exception of the 1st cervical nerve, are the larger. Occasionally the roots on opposite sides are not symmetrical; one or other root, or even a whole nerve, may be absent from the dorsal region (Adamkiewicz): On the posterior root is the spindle-shaped spinal ganglion (\$ 321 , II., 
3), which is occasionally double on the lumbar and sacral nerves. Beyond the ganglion, the two roots unite to form within the spinal canal the mixed trunk of a spinal nerve. The branches of the nerve-trunk invariably contain fibres coming from both roots. The number of fibres in the nerve-trunk is exactly the same as in the two roots; hence, we must conclude that the nerve-cells in the spinal ganglion are intercalated in the course of the fibres (Gaule and Birge).

Varieties.-The spinal ganglion is sometimes double, and according to Hyrtl, isolated ganglionic cells frequently oceur in the posterior root, between the ganglion and the cord. Occasionally the roots are somewhat unsymmetrical on opposite sides; in the dorsal part one or other, or hoth roots of a spinal nerve are sometimes absent (Adamkiewicz).

[Morphology of the Spinal Nerves and Limb-Plexuses.-A typical segmental spinal nerve (fig. 435 ) divides, after its formation, into three parts, a dorsal branch, or superior primary

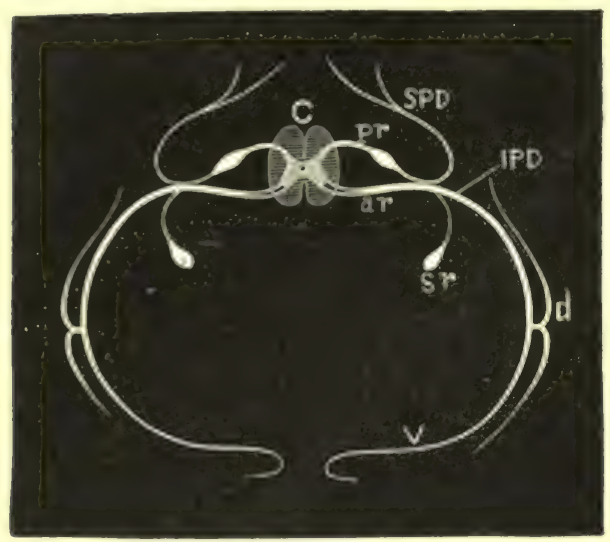

Fig. 435.

Diagram of a spinal nerve; C, spinal cord; $p r$, ar, posterior and anterior roots; SPD, IPD, superior and inferior prinary divisions; $d, v$, dorsal and ventral branches; sr, sympathetic root (Ross). division, distributed to the back, a somatic branch, or inferior primary division, supplying the body-wall or limbs; and a splanchnic or visceral branch, or ramus communicans, connected with the sympathetic gangliated cord, and distributed to the large vessels and viscera. The somatic branch is the largest, and is generally, by human anatomists, spoken of as the "anterior primary division." In the thoracic and upper lumbar regions, the distribution of this nerve is simple. It divides into an external (or lateral) branch, and an internal (or anterior) branch, which supply respectively the lateral and anterior portions of the thoracic and abdominal walls.]

[In the region of the neck, and in relation to the limbs, the arrangement of the somatic branches becomes complicated by the formation of the plexuses. In the embryo, however, the distribution of the nerves is simpler, and a comparison can be made both with the adult arrangement, and with the typical nerve as seen in the thoracic region. In the embryo, the neck as such does not exist, and the upper limb sprouts out directly beyond the segmented visceral arches. In this state, the somatic branch is distributed as in the thoracic region; the nerve divides into an external and an internal branch, distributed to the side and front of the corresponding part of the arches in the neck, in the regions where the limbs are appearing as two flattened buds from the ventrolateral aspect of the body. The somatic branch sweeps round into the blastema forming the limb, and divides into its two branches, external and internal, or dorsal and ventral, which are distributed to the outer (dorsal) and inner (ventral) surfaces, respectively, of the primitive limb. At this time, the cartilaginous and muscular elements of the limb have not become differentiated. While this is occurring, the dorsal and ventral parts of the somatic branches of the uerves entering the limb unite with adjacent dorsal and ventral branches, in various comhinations, so as to produce the limb-plexuses. The nerves resulting from these combinations are distributed to the primitive, dorsal, and ventral surfaces of the limbs. Thus, the plexuses are formed, and the peripheral distribution of the nerves has taken place before the period of flexion and angulation of the limbs. These processes mark the conditions in the adult; but even then it is easy to make out that the nerves in the upper limb derived from the posterior (dorsal) cords of the brachial plexus supply the scapular region, extensor surface of the arm and fore-arm, and the back of the hand, - parts which are derived from the dorsal surface of the primitive limb; while the nerves produced from the anterior (ventral) cords supply the pectoral region, front of the arm, fore-arm, and hand, - parts representing the primitive ventral surface.]

[In the lower limb, the nerves derived from a union of the posterior branches are the external cutaneous, anterior crural, gluteal, and external popliteal. These supply the iliac surfaces, the front of the thigh, leg, and foot,-belonging to the primitive dorsal surface of the limb. The nerves formed by the union of anterior branches, - genitocrural, obturator, and internal popliteal, in like manner supply the parts of the limb corresponding to the ventral surface, the inner side and back of the thigh, the back of the leg, and the sole of the foot $(A . M$. Paterson).]

[Structure of a Spinal Ganglion. - The ganglion is invested by a thin, firmly adherent, sheath 
of connective-tissue, which sends processes into the swelling, and is continuous with the sheaths of the nerve entering and leaving the ganglion (fig. $436, c$ ). In mammals, e.g., rabbit, a longitudinal section of such a ganglion exhibits the cells arranged in groups, with strands of nerve-fibres coursing longitudinally between them (fig. $436, a, b)$. The nervecells are usually globular in form, with a distinct capsule lined with epithelium, and the cell-substance itself contains a well-defined nucleus with a nuclear envelope and a nucleolus. The capsule of the cell is continuous with the sheath of Schwann of a nerve-fibre. The exact relation between the nerve-fibres and the nerve-cells is difficult to establish, but it is probable that each nerve-cell is connected with one nerve-fibre, i.e., they are unipolar. In the spinal ganglia of the vertebrates above fishes, and also in the Gasserian ganglion, cells are found with a single process or fibre attached to them, the nerve-fibre process not unfrequently coiling a few times within the capsule. This process, after emerging from the capsule, becomes coated with myelin, and usually soon divides at a node of Ranvier (fig. 341, t). Ranvier, who first observed this arrangement, describes it as a $\mathrm{T}$-shaped fibre. These nerve-cells with $T$-shaped fibres have been observed in the spinal ganglia of all vertebrates above fishes, in the Gasserian and geniculate ganglia, as well as in the jugular and cervical ganglia of the vagus. In fishes, the nervecells of the spinal ganglia are bipolar (fig. 368, 4). There is a rich plexus of capillaries in these ganglia, and each cell is surrounded by a mesh-work of capillaries, which never penetrate the cell capsules. ]

Bell's Law.- Sir Charles Bell discovered (1811) that the anterior roots of the spinal nerves are motor, the posterior are sensory.

Recurrent Sensibility.-Magendie discovered (1822) the remarkable fact that sensory fibres are also present in the anterior roots, so that their stimulation causes pain. This is due to the fact that sensory fibres pass into the anterior root after the two roots have joined, and these fibres run in

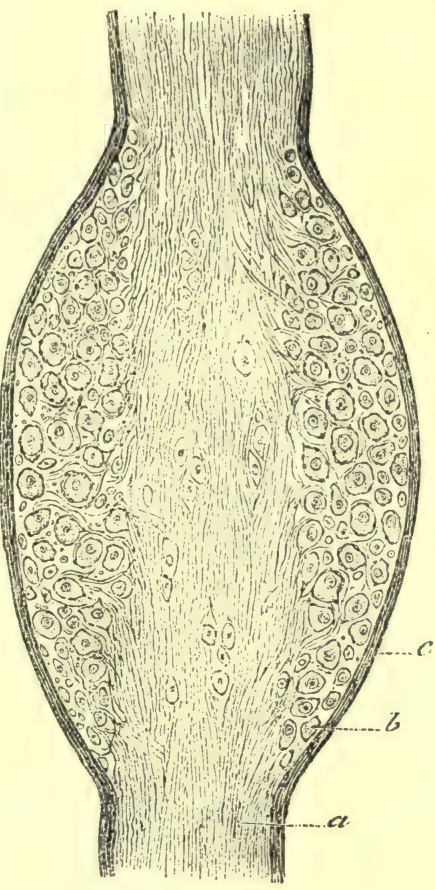

Fig. 436.

Longitudinal section of a spinal ganglion. $a$, nerve-fibre; $b$, nerve-cells; $c$, capsule.

the anterior root in a centripetal direction (Schiff, Cl. Bernard). The sensibility of the anterior root is abolished at once by section of the posterior root. This condition is called "recurrent sensibility" of the anterior root. When the sensibility of the anterior root is abolished, so is the sensibility of the surface of the spinal cord in the neighbourhood of the root. A long time after section of the anterior, and when the degeneration phenomena have had time to develop ( $\$ 325)$, a few non-degenerated sensory fibres are always to be found in the central stump (Schiff, Vulpian). Schiff found that, in cases where the motor fibres had undergone degeneration, there were always non-degenerated fibres to be found in the anterior root, which passed into the membranes of the spinal cord. The sensory fibres pass into the motor root, either at the angle of union of the roots, or in the plexus, or in the region of the peripheral terminations. Sensory fibres enter many of the branches of the motor cranial nerves at their periphery, and afterwards run in a centripetal direction (p.602). Even into the trunks of sensory nerves, sensory branches of other sensory nerves may enter. This explains the remarkable observation, that after section of a nerve trunk (e.g., the median), its peripheral terminations still retain their sensibility (Arloing and Tripier). The tissue of the motor and sensory nerves, like most other tissues of the body, is provided with sensory nerves (Nervi nervorum, p. 530).

[It does not follow that section of a peripheral cutaneous nerve will cause anæsthesia in the part to which it is distributed; in fact, one of the principal nerve trunks of the brachial plexus 
may be divided without giving rise to complete anæsthesia in any part of the area of distribution of the sensory branches of the nerve, and even if there be partial or complete cutaneous anesthesia, it is much less in extent than corresponds to the anatomical area of distribution. The anasthetic area tends to become smaller in extent (Ross). Thus, there is not complete independence in the distrilution of these nerves. These results are explained by the anastomosis leetween branches of nerves, the exchange of fibres in the terminal networks, while some sensory fibres enter the peripheral parts of a nerve and run centripetally, perhaps being distributel to the skin and conferring recurrent sensibility on the peripheral part of the nerve.]

Relative Position of Motor and Sensory Fibres. - In embryos (rabbit) the motor fibres stain more deeply with carmine than the sensory fibres, so that their position in the peripheral nerves

A

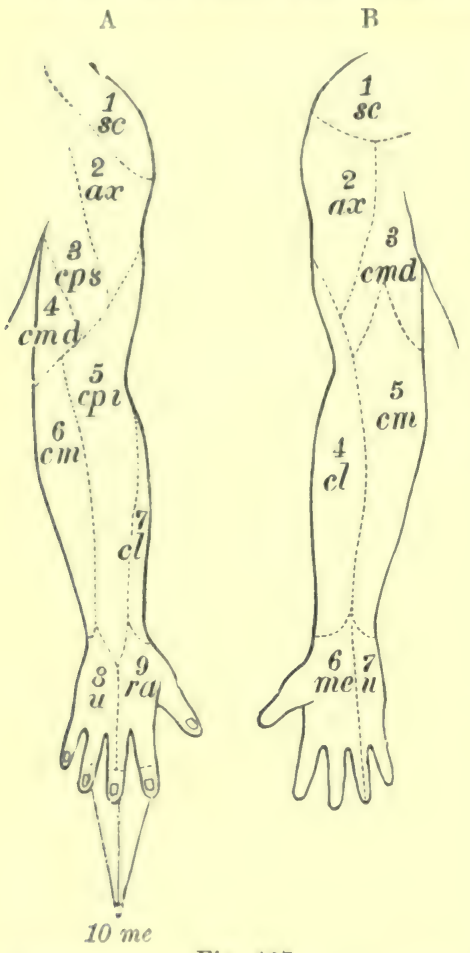

Fig. 437 .

Distribution of the cutaneous nerves of the arm. A, Dorsal surface-1 sc, supraclavicular ; $2 \mathrm{c} x$, axillary ; $3 \mathrm{cps}$, superior posterior cutaneous ; $4 \mathrm{cmd}$, median cutaneous ; 5 cpi, inferior posterior cutaneous ; $6 \mathrm{~cm}$, median cutaneous; 7 $c l$, lateral cutaneous ; $8 u$, ulnar; $9 \mathrm{ra}$, radial ; 10 me, median. $\mathrm{B}$, volar surface-1 $s c$, supra-clavicular; $2 a x$, axillary ; $3 \mathrm{cmd}$, internal cutaneous ; 4 $c l$, lateral cutaneous; $5 \mathrm{~cm}$, cutaneous medius ; 6 me, median; $7 u$, ulnar. of distribution may thereby be made out. In the anterior branch of a spinal nerve, the sensory fibres lie in the outer part of the branch, the motor in the inner part; while this relation is reversed in the posterior root (L. Löwe).

Deduction from Bell's Law.-Careful observations of the effects of section of the roots of the spinal nerves (Magendie, 1822), as well as the discovery of the reflex relation of the stimulation of the sensory roots to the anterior, constituting reflex movements (Marshall Hall, Johannes Miiller, 1832), enable us to deduce the following conclusions from Bell's law:-1. At the moment of section of the anterior root, there is a contraction in the muscles supplied by this root. 2. There is at the same time a sensation of pain due to the "recurrent sensibility." 3. After the section, the corresponding muscles are paralysed. 4. Stimulation of the peripheral trunk of the anterior root (immediately after the operation) causes ccntraction of the muscles, and eventually pain, owing to the recurrent sensibility. 5. Stimulation of the central end is without effect. 6. The sensibility of the paralysed parts is retained completely. 7. At the moment of section of the posterior root, there is severe pain. 8. At the same time movements are discharged reflexly. 9. After the section, all parts supplied by the divided roots are devoid of sensibility. 10. Stimulation of the peripheral trunk of the divided nerve is without effect. 11. Stimulation of the central end causes pain and reflex movements. 12. The ceutral end ultimately degenerates. 13. Movement is retained completely in the paralysed parts, e.g., in the extremities.

The ultimate effect, known as Wallerian degeneration, which follows section of the nerve or its roots, is referred to in $\S 325$. Recently, Joseph has slightly modified the statements of Waller on the degeneration in the posterior roots. According to him, the spinal ganglion is the nutritive centre for by far the largest number of the fibres of this root; but individual fibres traverse the ganglion without forming connections with its cells, so that the nutritive or trophic centre for this small number of nerve-fibres is in the spinal cord. 
Inco-ordinated Movements of Insensible Limbs. - After section of the posterior roots, e.g., of the nerves for the posterior extremities, the muscles retain their movements, nevertheless there are characteristic disturbances of their motor power. This is expressed in the awkward manner in which the animal executes its movement-it has lost to a large extent its harmony and elegance of motion. This is due to the fact that, owing to the absence of the sensibility of the muscles and skin, the animal is no longer conscious of the resistance which is opposed to its movements. Hence, the degree of muscular energy necessary for any particular effort cannot be accurately graduated. Animals which have lost the sensibility of their extremities often allow their limbs to lie in abnormal positions, such as a healthy animal would not tolerate. In man also, when the peripheral ends of the cutaneous nerves are degenerated, there are ataxic phenomena $(\S 364,3)$.

Increased Excitability.-Harless (1858), Ludwig, and Cyon (controverted by v. Bezold, Uspensky, Griinhagen, and G. Heidenhain) observed that the anterior root is more excitable as long as the posterior roots remain intact and are sensitive, and that their excitability is diminished as soon as the posterior roots are divided. In order to explain this phenomenon, we must assume that, in the intact body, a series of gentle impulses (impressions of touch, temperature, position of limbs, \&c.) are continuously streaming through the posterior roots to the spinal cord, where they are transferred to the motor roots, so that a less stimulus is required to excite the anterior roots than when these reflex impulses of the posterior root, which increase the excitability, are absent. Clearly, a less stimulus will be required to excite a nerve already in a gentle state of excitement than in the case of a fibre which is not so excited. In the former case, the discharging stimulus becomes as it were superposed on the excitement already present. (Compare § 362.)

The anterior roots of the spinal nerves supply efferent fibres to-

1. All the voluntary muscles of the trunk and extremities.

Every muscle always receives its motor fibres from several anterior roots (not from a single nerve-root). Hence, every root supplies branches to a particular group of muscles (Preyer, P. Bert, Gad). The experiments of Ferrier and Yeo show that stimulation of each of the anterior roots in apes (brachial and lumbo-sacral plexuses) caused a complex co-ordinated movement. Section of one root did not cause complete paralysis of the muscles concerned in these co-ordinated movements, although the force of the movement was impaired. These experiments confirm the results of clinical observation on man. The fibres for groups of muscles of different functions (e.g., for flexors, extensors) arise from special limited areas of the spinal cord. The cervical and lumbar enlargements of the spinal cord are great centres for highly co-ordinated muscular movements.

2. The anterior roots also supply motor

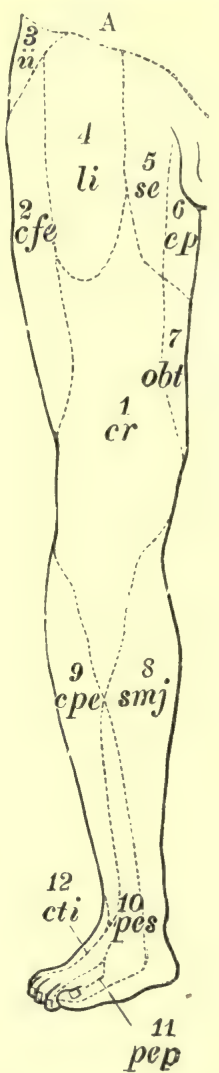

Fig. 438.

Distribution of the cutaneous nerves of the leg (after Henle). A, Anterior surface -1, crural nerve; 2, external lateral cutaneous ; 3 , ilio-inguinal ; 4 , lumboinguinal ; 5 , external spermatic ; 6 , posterior cutaneous ; 7 , obturator ; 8 , great saphenous; 9 , communicating peroneal ; 10, superficial peroneal; 11, deep peroneal; 12, communicating tibial. B, Posterior surface-1, posterior cutaneous ; 2 , external femoral cutaneous; 3 , obturator ; 4 , median posterior femoral cutaneous ; 5 , communicating peroneal ; 6 , great saphenous ; 7 , communicating tibial ; 8 , plantar cutaneous ; 9, median plantar; 10, lateral plantar.

fibres for a number of organs provided with smooth muscular fibres, e.g., the bladder $(\S 280)$, ureter, uterus. [These are the viscero-motor nerves of Gaskell, and from them come also viscero-inhibitory nerves.]

3. Motor fibres for the smooth muscular fibres of the blood-vessels, the vaso- 
motor, vaso-constrictor, or vaso-hypertonic nerves [also accelerator or augmentor nerves of the beart]. They run in the sympathetic for a part of their course (\$ 371).

4. Inhibitory fibres for the blood-vessels. These are but imperfectly known. They are also called vaso-dilator or vaso-hypotonic nerves (\$372). nerves for the heart, which leave the spinal axis in the vagus.]

5. Secretory fibres for the sweat-glands of the skin $(\$ 289)$. For a part of their course they run in the sympathetic.

6. Trophic fibres of the tissues $(\$ 342$, I., c).

The posterior roots contain all the sensory nerves of the whole of the skin and the internal tissues, except the front part of the head, face, and the internal part of the head. They also contain the tactile nerves for the areas of the skin already mentioned. Stimuli which discharge reflex movements are conducted to the spinal cord through the posterior roots. The sensory fibres of a mixed nerve-trunk supply the cutaneous area, which is moved by those muscles (or which covers those muscles) to which the same branch supplies the motor fibres. The special distribution of the motor and sensory nerves of the body belongs to anatomy (figs. 429, 430, 437,438 ).

[Physiology of the Limb-Plexuses. - The idea that the nerve-strands become rearranged in the limb-plexuses so as to comnect nerves derived from different parts of the spinal cord with particular groups of muscles, in order to allow of "co-ordination of muscular action," does not seem to be borne out by more extended observation. Herringham has shown by dissection (and the same is seen in cases of paralysis of motion and sensation) that a given muscle or part of a muscle, and a given spot of skin, are supplied by particular branches of individual spinal nerves proceeding directly from the spinal cord. The reason that the plexuses exist is, apparently, not a phissiologrical one. C'o-ordination cannot be effected in the plexus, where the axis-cylinders of the nerves do not divide; but only in the spinal cord and central nervous system, and through the intervention of nerve-cells. The existence of the plexuses is due to the fact that embryologically the limb consists of a flattened lappet, or bud, derived from certain somites, but at first presenting no sigus of segmentation, with a preaxial and a postaxial border, and outer (dorsal) and inner (ventral) surfaces of skin, covering a double layer of muscle on each surface. The dorsal and ventral branches of the nerves supply these respective surfaces; and after the nerves have grown out, the simple muscular strata become split up into individual muscles, which contain elements derived from one or more segments represented in the primitive limb. Each nerve is segmental, and, therefore, supplies a muscle derived, for example, from the plements of two segments; the nerve of distribution must contain corresponding parts of two segmental nerves. The plexuses appear, therefore, from an embryological cause, and have no direct physiological significance (A.M. Paterson).]

356. THE SYMPATHETIC NERVE.-[Anatomical.-The sympathetic nervous system contains a large number of non-medullated or Remak's fibres, and consists of a series of ganglia lying on each side of the vertebral column and connected with each other by inter-ganglionic fibres. The typical distribution obtains in the thoracic region, where the lateral or vertebral ganglia lie close on the vertebre. In front of this is a second series of ganglia, which do not form a double line, but are connected with the former and with each other. They are the prevertebral or collateral ganglia, e.g., semilunar, inferior mesenteric, \&c., the nerves connecting them with the former being called rami efferentes. From these, fibres proceed to connect them with ganglia lying in or about tissues or organs-the terminal ganglia (Gaskell).]

[Each spinal nerve in this region is connected with its corresponding sympathetic ganglion by the ramus communicans, which is formed by fibres both from the anterior and posterior roots of a spinal nerve. It corresponds to the visceral nerve of the morphologist, and is composed of two parts - a white and a grey ramus. The white ramus is composed entirely of medullated fibres, and coming from the anterior and posterior roots of a spinal nerve, passes into the lateral and collateral ganglia. These white rani occur in the dog only from the 2 nd thoracic to the 2nd lumbar nerve (fig. 439). Above and below this, the rami are all grey and composed of nonmedullated nerve-fibres (Gaskell).]

[In man, the four upper rami communicantes from the four upper cervical nerves all join the superior cervical ganglion (fig. $428, G g, s$ ), the 5 th and 6 th join the middle cervical, the 7 th and 8 th the inferior cervical ganglion. The lowest pair of ganglia are generally united by a loop on the front of the first coccygeal vertebra, and they lie in relation with the coccygeal ganglion.]

[Cephalic Portion.-As the sympathetic ascends to the head it forms connections with many 
of the cranial nerves, and there is a free exchange of fibres between these nerves. (The function and significance of these exchanges are referred to under the physiology of the cranial nerves).]

[Dorsal and Abdominal Portion. - Numerous fibres pass from these parts chiefly to the thoracic and abdominal cavities, where they form large gangliated plexuses, from which functionally different fibres proceed to the different organs.]

[In the dog, the 2nd, 3rd, 4th, and 5th thoracic pass upwards into the cervical sympathetic, those in the dorsal region being directed downwards from the lateral ganglia to form the splanchnics (fig. 439). The grey non-medullated nerve-fibres of each grey ramus are connected with the cells of its ganglion (lateral) ; the fibres do not go beyond the ganglion, but really run to the corresponding spinal nerve to ramify in the sheaths of the nerves, the connective-tissue on the vertebræ and the dura mater, and perhaps the other spinal membranes ; so that, according to Gaskell, no non-medullated nerves leave the central nervous system by the spinal nerveroots. Thus, the white rami communicantes alone constitute the rami viscerales of the morphologist, and all the visceral nerves passing out from the central nervous system into the sympathetic system pass out by them alone. All the nerves in the white ramus are of small calibre $(1.8 \mu$ to $2.7 \mu)$ and medullated, while the true motor fibres are much larger $(14.4 \mu$ to $19 \mu)$. The small, white fibres can be traced upwards as medullated fibres into the superior cervical ganglion, and in the thorax over the lateral ganglia to form the splanchnics into the collateral ganglia, beyond which they cease to be medullated. By the 2nd and 3rd sacral nerves some fibres of smallest calibre issue to form the nervi erigentes, which pass over and do not communicate with the lateral ganglia, but enter the hypogastric plexus, whence they send branches upwards to the inferior mesenteric plexus and downwards to the bladder, rectum, and generative organs. Gaskell proposes to call them the pelvic splanchnic nerves (fig. 439).]

[In the cervical region, there is no white ramus, and the nerve-roots contain no nerve-fibres of small calibre. But in this region rises the spinal accessory nerve, between the anterior and posterior roots. It contains small and large nerve-fibres; the former pass into the internal division of this nerve and join the ganglion of the trunk of the vagus, while the large motor fibres form its external branch and supply the sternomastoid and trapezius muscles.]

[All the vaso-motor nerves arise in the central nervous system, and they leave the spinal cord as the finest medullated fibres in the anterior roots of all the spinal nerves between the 2 nd thoracic and 2nd lumbar (dog) "along the corresponding ramus visceralis, enter the lateral or" main sympathetic chain of ganglia, where they become non-medullated, and are thence distributed either directly or after communication with other ganglia" (Gaskell).]

[The vaso-dilator nerves leave the central nervous system among the fine medullated fibres, which help to form the cervico-cranial and sacral rami viscerales, and pass without altering their character into the distal ganglia" (Gaskell).]

["The viscero-motor nerves upon which the peristaltic contraction of the thoracic portion of the oesophagus, stomach, and intestines depends, leave the central nervous system in the outflow of fine medullated nerves which occurs in the upper part of the cervical region, and pass by way of the rami viscerales of the accessory and vagus nerves to the ganglion trunci vagi, where they become non-medullated" (Gaskell).]

["The inhibitory nerves of the circular muscles of the alimentary canal and its appendages leave the central nervous system in the anterior roots, and pass out among the fine medullated fibres of the rami viscerales into the distal ganglia without communication with the proximal ganglia" (Gaskell).]

[Structure of a Ganglion.-The structure of the sympathetic nerve-fibres and nerve-cells has already been deseribed in $\$ 321$. On making a section of a sympathetic ganglion, e.g., the human superior cervical, we observe groups of cells with bundles of nerve-fibres-chietly nonmedullated-running between them, and the whole surrounded by a laminated capsule of connective-tissue, which sends septa into the ganglion. The nerve-cells have many processes, and are, therefore, multipolar, and each cell is surrounded by a capsule with nuclei on its inner surface (fig. 368, II). The processes pierce the capsule, and one of them certainly-and perhaps all the processes-are connected with a nerve-fibre. Ranvier states that each cell has a fibrillated outer portion and a more granular inner part. Each of the processes becomes continuous with a fibre of Remak. Not unfrequently yellowish-brown pigment is found in the cell-substance. Sinilar cells have been found in the ophthalmic, sub-maxillary, otic, and spheno-palatine ganglia. The number of medullated nerve-fibres diminishes as the sympathetic nerves are traced towards their distribution. Ranvier states that it is possible in the rabbit to trace the conversion of a medullated fibre into a branched fibre of Remak. The blood-vessels of the sympathetic ganglia in mammals are peculiar. The arteries are small, and after subdivision form a capillary network, each mesh of which encloses several ganglionic cells. The veins on the contrary are very large, tortuous, varicose, and often terminate in culs-de-sac, into which several capillaries open. The arrangement of the veins is spoken of as the venous sinuses of these ganglia, being compared by Ranvier to the sinuses of the dura mater and venous plexuses of the spinal canal.]

Functions. - The following is merely a general summary :- 
I. Independent Functions of the sympathetic are those of certain nerve plexuses which remain after all the nervous connections with the cerebro-spinal branches have been divided. The activities of these plexuses may be influenced-either in

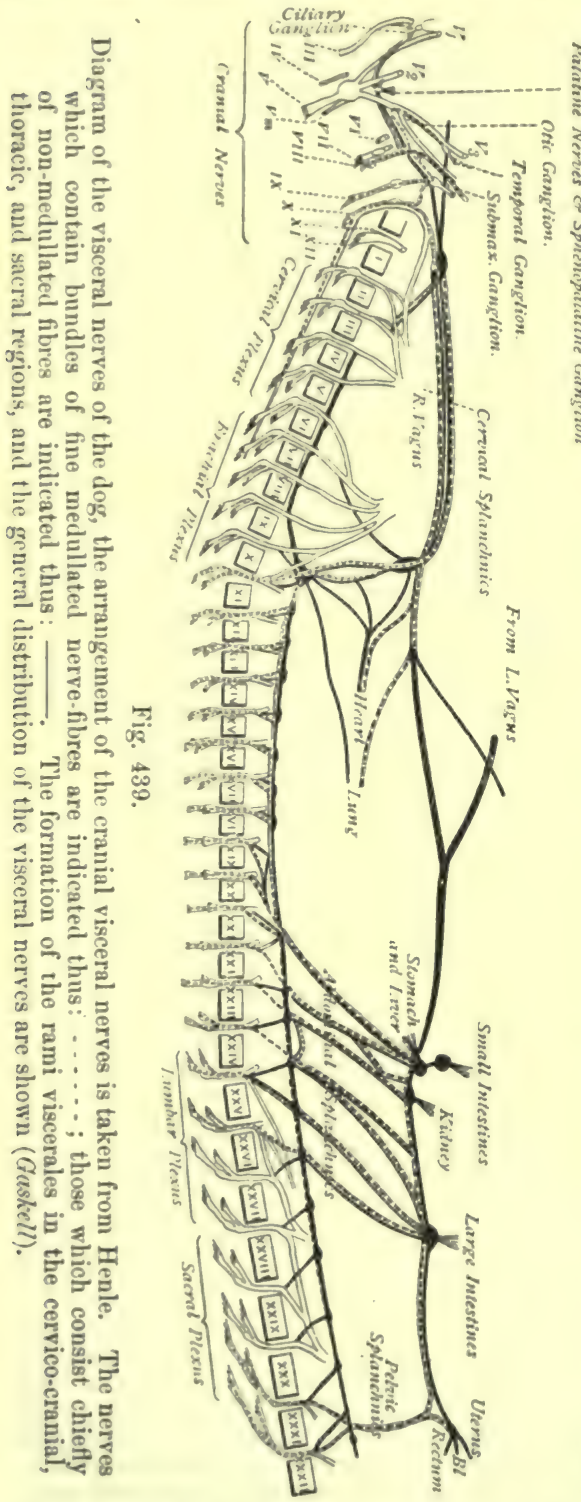
the direction of inhibition or stimulation-through fibres reaching them from the cerebro-spinal nerves.

To these belong:-

1. The automatic ganglia of the heart ( $\$ 58)$.

2. The mesenteric plexus of the intestine (§161).

3 . The plexuses of the uterus, Fallopian tubes, ureters (also of the bloodand lymph-vessels).

II. Dependent Functions.-Fibres run in the sympathetic, which (like the peripheral nerves) are active only when their connection with the central nervous system is maintained, e.g., the sensory fibres of the splanchnic. Others again convey impulses from the central nervous system to the ganglia, while the ganglia in turn modify the impulses which inhibit or excite the movements of the corresponding organs.

The following statement is a resume of the functions of the sympathetic, according to the anatomical arrangement:-

\section{A. Cervical Part of the Sympa-} thetic. - 1.Pupil-dilating fibres (compare Ciliary ganglion, § 347, I., and Iris, § 392). According to Budge, these tibres arise from the spinal cord, and run through the upper two dorsal and lowest two cervical nerves into the cervical sympathetic, which conveys them to the head. Section of the cervical sympathetic or its rami communicantes causes contraction of the pupil. (The central origin of these fibres is referred to in $\S 362,1$, and $\S 367,8$.)

2. Motor fibres for Müller's smooth muscle of the orbit, and partly for the external rectus muscle (\$348).

3. Vaso-motor branches for the outer ear and the side of the face $(\mathrm{Cl}$. Bernard), tympanum (Prussak), conjunctiva, iris, choroid, retina (only in part-see Ciliary ganglion, 3347 , I.), for the vessels of the œsophagus, larynx, thyroid gland-fibres for the vessels of the brain and its membranes (Donders and Callenfels).

4. In the cervical portion are afferent fibres which excite the vaso-motor centre in the medulla (Aubert).

5. Secretory (trophic) and vaso-motor fibres for the salivary glands ( $\$ 145)$. 
6. Sweat-secretory fibres (see $\S 288$, II.).

7. According to Wolferz and Demtschenko the lachrymal glands receive sympathetic secretory fibres (?).

B. Thoracic and Abdominal Sympathetic.-First of all there is-

1. The sympathetic portion of the cardiac plexus $(\S 57,2)$, which receives accelerating or augmentor fibres for the heart from the lower cervical and 1st thoracic ganglion ( $\mathrm{Cl}$. Bernard, $v$. Bezold, Cyon, Schmiedeber.g). The fibres arise partly from the sympathetic and partly from the plexus around the vertebral artery (v. Bezold, Bever). (Compare § 370.)

2. For the vaso-motor fibres passing through the sympathetic to the extremities, skin of the trunk, and lungs (see $\$ 371)$. For vaso-dilators (\$472).

3. The cervical sympathetic and the splanchnic contain fibres which, when their central ends are stimulated, excite the cardio-inhibitory system in the medulla oblongata (Bernstein).

4. The functions of the splanchnic are referred to in $\$ \S 164,175,276$, and 371.

5. The functions of the cœliac and mesenteric plexuses are referred to in $\$ \S 183$ and 192. After extirpation of the cœliac ganglion, Lamansky observed temporary disturbance of digestion, undigested food being passed per anum.

6. For the secretory fibres for sweating, see $\S 289$, II.

7. Lastly, the abdominal portion of the sympathetic contains motor and vasomotor fibres for the spleen, the large intestine (accompanying its arteries), bladder (§ 280), ureters, uterus (running in the hypogastric plexus), vas deferens, and vesiculæ seminales. Stimulation of all of these nerve channels causes increased movement of the organs, but it must be remembered that the diminished supply of blood thereby produced also acts as a stimulus (\$161). Section of these nerves is followed by dilatation of the blood-vessels, with subsequent derangement of the circulation, and ultimately of the nutrition. The relation of the suprarenal bodies to the sympathetic is referred to in $\S 103$, IV. The renal plexus is referred to in $\S 276$, while the cavernous plexus is treated of in $\S 436$.

Pathological.-Considering the numerous connections of the sympathetic, we would naturally suppose that it offers an extensive area for pathological changes. Affections involving the vasomotor system are referred to in $\S 371$.

The cervical sympathetic is most frequently paralysed or stimulated by traumatic conditions, wounds by bullets or knives, tumours, enlarged lymph-glands, aneurisms, inflammation of the apices of the lungs and the adjacent pleuræ, while exostoses of the vertebræ may stimulate it in part or paralyse it in part. The phenomena so produced have been partly analysed in treating of the ciliary ganglion $(\S 347, \mathrm{I}$.). Stimulation of the cervical sympathetic in man causes dilatation of the pupil (mydriasis spastica), palor of the face, and occasionally hyperidrosis or profuse sweating ( $\$ 289,2$, and $\S 288$ ); disturbance of vision for near objects, as the pupil cannot be contracted (see Accommodation), and hence the spherical aberration of the lens (§ 391) must also interfere with vision; protrusion of the eyeball with widening of the palpebral fissure. Paralysis or section of the cervical sympathetic causes increased fulness of the bloodvessels of the side of the head, with occasional anidrosis; contraction of the pupil (myosis paralytica), which undergoes changes in its diameter during accommodation, but not as the effect of the stimulation of light-atropin dilates it slightly. The slit between the eyelids is narrowed, the eyeball retracted and sunk in the orbit, the cornea somewhat flattened, and the consistence of the eyeball diminished. Stimulation of the sympathetic is followed by an increased secretion of saliva ( $\$ 145)$. The above described symptoms have been occasionally accompanied by unilateral atrophy of the face.

[Section of the Cervical Sympathetic.-This experiment is easily done on a rabbit, preferably an albino one. Divide the nerve in the neck, and immediately thereafter (1) the ear and adjoining parts on that side become greatly congested with blood, blood-vessels appear that were formerly not visible, and as a result of the increased quantity of blood in the ear (hyperæmia), there is (2) a rise of the temperature amounting to even $4^{\circ}$ to $6^{\circ} \mathrm{C}$. $(\mathrm{Cl}$. Bernard $)$. These are the vasomotor changes. (3) The pupil is contracted, the cornea flattened, and there is retraction of the eyeball and consequent narrowing of the palpebral fissure. These 
are the oculo-pupillary symptoms. Stimulation (electrical) of the peripheral end produces the opposite results, - pallor of the ears, owing to contraction of the bloodvessels, with consequent fall of the temperature; dilatation of the pupil, bulging of the cornea, protrusion of the eyeball (exophthalmos), and widening of the palpebral fissure. At the same time, the blood-vessels to the salivary glands are contracted, and there is a secretion of thick saliva. The last results are due to the vasoconstrictur and secretory fibres. The vaso-motor and oculo-pupillary fibres, although they lie in the same trunk in the neck, do not issue from the cord by the same nerve-roots; the latter come out of the cord with the anterior roots of the 1 st and 2nd dorsal nerves (dog), while section of the cord between the 2 nd and 4 th dorsal vertebrie produces the vaso-motor changes only. The nasal mucous membrane and lachrymal gland are influenced by the sympathetic.]

[Division of the cervical sympathetic in young, growing, animals results in hypertrophy of the ear, and increased growth of the hair on that side (Bidder, W. Stirling).]

[The vago-sympathetic nerve (dog) in the neck contains vaso-dilator fibres (really in the sympathetic) for the skin and mueous membranes of that side of the head. Weak stimulation of the central end of the sympathetic causes dilatation of the blood-vessels of these parts. The vaso-dilator fibres of the superior maxillary nerve probably come from the same source. The centre for these nerves is in the dorsal region of the cord between the 1st and 5th dorsal vertebrat, where the fibres pass out with the rami communicantes to enter the cervical sympathetic (Distre und Murut). The vasu-dilator fibres occur in the posterior segment of the ring of Vieussens, and when they are stimulated after section of the 7 th cranial nerve, there is a "pseudo-motor" effect on the muscles of the cheek and lip (\$349).]

Irritation in the area of the splanchnic, as occurs oceasionally in lead poisoning, is characterised by violent pain (lead colic), inhibition of the intestinal movements, (hence the persistent constipation), slowing of the heart's action, brought about reflexly, just as in Goltz's "tapping" experiment $(\$ 369)$. Irritation in the area of the sensory nerves of the sympathetic may give rise to that condition which is called by Romberg neuralgia hypogastrica, a painful affection of the lower abdomiual and sacral regions, hysteralgia, neuralgia testis, which are localised in the plexuses of the sympathetic. In affections of the abdominal sympethetic, there may be severe constipation, with diminished or increased secretion of the intestinal glands $\left(\S 18 \mathrm{~b}^{\circ}\right)$.

357. COMPARATIVE-HISTORICAL -Comparative.-Some of the cranial nerves may be absent, others, again, may be abortive, or exist as branches of other nerves. The facial nerve, which supplies the muscles of expression in man, and is, at the same time, the nerve for facial respiratory movements, diminishes more and more in the lower classes of the vertebrata, pari passu, with the diminution of the facial muscles. In birds and reptiles, it supplies the muscles of the hyoid bone, or the superficial cervical muscles of the nape of the neck. In amphibians (frog), the facial no longer exists as a separate nerve, the nerve which corresponds to it springing from the trigeminus. In fishes, the 5th and 7 th nerves form a joint complex nerve. The part corresponding to the facial (also called ramus opercularis trigemini) is the chief motor nerve of the muscles of the gill-cover, and is, therefore, the respiratory uerve. In the cyclostomata (lamprey) there is an independent facial. The vagus is present in all vertebrata; in fishes it gives off a large nerve, the lateral nerve of the body (N. lateralis), which runs along each side of the body close to the lateral canal. It is also present in the tadpole. Its rudimentary representative in man is the auricular branch. In the frog the 9th, 10th, and 11 th arise together from one trunk, and the 7 th and 8 th from another. In fishes and amphibia, the hypoglossal is the first cervical nerve. In amphioxus, the cerebral and spinal nerves are not distinct from each other. The spinal nerves are remarkably similar in all classes of the vertebrata. The sympathetic is absent in the cyclostomata, where it is represented by the vagus. Its course is along the vertebral column, where it receives the rami communicantes of the spiual nerves. In the region of the head its connections with the 5th and 10th nerves are specially developed. In frogs, and still more so in birds, the number of connections with the cranial nerves increases.

Historical. - The vagus and sympathetic were known to the Hippocratic School. According to Erasistratus, all the nerves proceed from the brain and spinal cord ; Herophilus was the first to distinguish the nerves from the tendons, which Aristotle confounded with each other. Marianus ( 80 A.D.) recognised seven pairs of cranial nerves. Galen was in possession of a wide range of important facts in the physiology of the nervous system $(\$ 140)$; he observed that loss of voice followed ligature of the recurrent nerves; and he was acquainted with the accessorius, and the ganglia on the abdominal nerves. The cauda equina is referred to in the Talmud; Coiter (1573) described exactly the anterior and posterior spinal nerve-roots. Van Helmont $(+1644)$ states that the peripheral motor nerves also give rise to impressions of pain, and 
Cesalpinus (1571) remarks that interruption of the blood-stream makes the parts insensible. Thomas Willis described the chief ganglia (1664). In Des Cartes there is the first indication of reflex movements; Stephen Hales and Robert Whytt showed that the spinal cord was necessary for such acts. Prochaska described the reflex channels, [while Marshall Hall established the doctrine of reflex, or, as he called them, "diastaltic" actions]. Duverney (1761) discovered the ciliary ganglion. Gall traced more carefully the course of the 3rd and 6th nerves, and also the spinal nerves into the grey matter. Hitherto only nine nerves of the brain had been enumerated; Sömmerring (1791) separated the facial from the auditory nerve, Andersch (1797) the 9th, 10th, and 11th nerves.

\section{Physiology of the Nerve-Centres.}

358. GENERAL. - [The nerve-fibres and nerve-cells constitute the elements out of which nerve-centres are formed, being held together by connective-tissue. In the process of evolution, groups of nerve-cells with connecting fibres are arranged to constitute nervous masses, whereby there is a corresponding integration of function. Thus, with structural integration there is a functional integration. When the structure suffers so also does the function, and those parts which are most evolved, as well as those actions which have to be learned by practice, are the first to suffer during the dissolution of the nervous system.]

General Functions. - The central organs of the nervous system are in general characterised by the following properties :-

1. They contain nervescells, which are either arranged in groups in the interior of the central organs of the nervous system, or embedded in the peripheral branches of the nerves. [Nerve-cells are centres of activity, originate impulses and conduct impulses as well, while nerve-fibres are chiefly conductors. ]

2. The nerve-centres are capable of discharging reflexes, e.g., reflex-motor, reflexsecretory, and reflex-inhibitory acts.

3. The centres may be the seat of automatic excitement, i.e., they may manifest phenomena, without the application of any apparent external stimulus. The energy so liberated may be transferred to act upon other organs. This automatic state of excitement or stimulation may be continuous, i.e., may be continued without interruption, when it is called tonic automatic or tonus; or it may be intermittent, and occur with a certain rhythm (rhythmical automatic).

4. The central organs are trophic centres for the nerves proceeding from them; they may also perform sinilar functions for the tissues innervated by them.

5. The psychical activities are dependentupen an intact condition of the ganglionic central organs. These various functions are distributed over different centres.

[The term "centre" is merely applied to an aggregation of nerve-cells so related to each other as to subserve a certain function, but, inasmuch as these cells are connected to each other and with other cells in many ways, various combinations of them may result; again, we have also to take into account the greater or less resistance in some paths than in others, so that the variety of combinations which these cells may subserve is enormous. These cells give off processes which branch, and anastomose with processes from other cells. Thus, innumerable ways are opened up to nervous impulses by these combinations, so that in a certain. way we may regard a cell as a junction of these conducting fibres, or a "shunt" whereby an impulse may be shunted on to one or other branch in the direction of least resistance, or in the best beaten path as it were, while there may be a "block" in other directions.]

[In connection with the histology of the central nervous system we have to study:-

A. The nervous constituents.

(1) Nerve-fibres.-

(2) Nerve-cells.

\section{B. Non-nervous constituents.}

(1) Vessels (blood and lymph).

(2) Epithelium.

(3) Suistentacular tissue.

(a) Connective-tissue.

(b) Neuroglia.] 


\section{The Spinal Cord.}

359. STRUCTURE OF THE SPINAL CORD. - [The key to the study of the central nervous system is to remember that it begins as an involution of the epiblast, and is originally tubular, with a central canal, dilated in the brain-end into ventricles. In the spinal cord there are three concentric parts: first, the columnar ciliated epithelium, outside this the central grey tube, and covering in all, the outer white conducting fibres (Hill).]

Structure.-The spinal cord consists of white matter exterually and grey matter internally. IIt is invested by membranes-the pia mater, composed of two layers and consisting of conne-tive-tissue with blood-vessels, heing firmly adherent to the white matter and sending septa into the sulnstance of the cord. Both layers dip into the anterior median fissure, and only the inner one into the posterior median groove. The arachnoid is a more delicate memlirane and non-vaseular, while the dura mater is a tough membrane lining the vertebral canal, and forming a theca or protective coat for the cord (\$ 381).] The grey mater has the form of two (rescents) - placed back to back [or a capital H], in which we can distinguish an anterior (' $)$ and a posterior horn (p), a middle part, and a grey commissure connecting the two crescents. In the centre of this grey commissure is a canal-central canal-which runs from the calamus seriptorius downwards; it is lined throughout by a single layer of ciliated cylindrical epithelium, [in the futus, the cilia not being visible in the adult], and the canal itself is the representative of the embryonal "medullary tube" (figs. 440, 446). [The part of

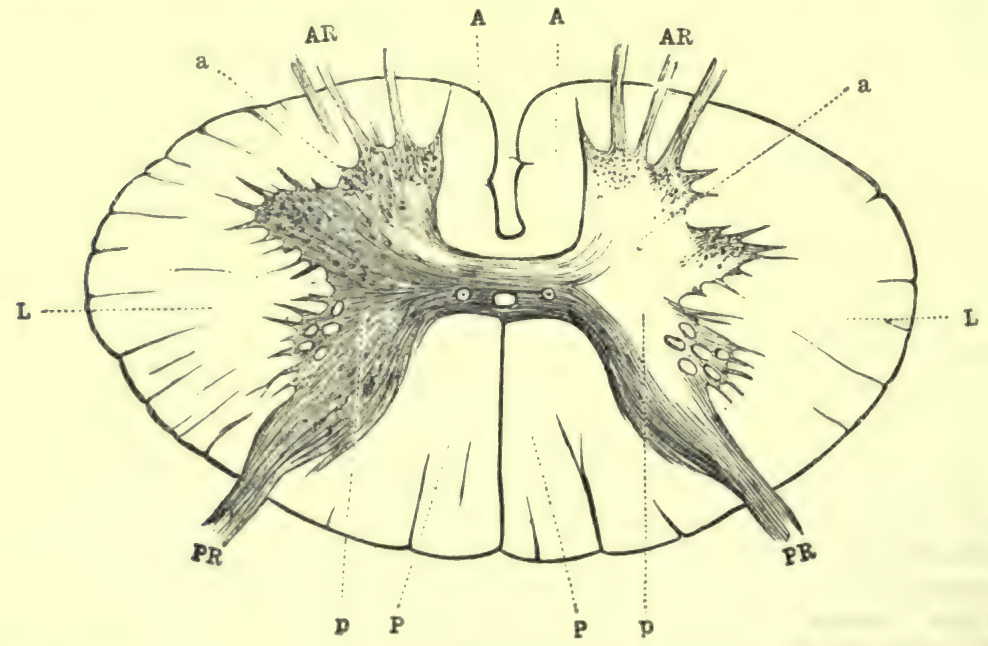

Fig. 440 .

Transverse section of the spinal cord; in the centre is the butterfly form of the grey matter surrounded by white matter. $p$, posterior, and $a$, anterior, horns of the grey matter; PR, posterior roots ; $\mathrm{AR}$, anterior roots of a spinal nerve ; $\mathrm{A}, \mathrm{A}$, the white anterior ; $\mathrm{L}, \mathrm{L}$, the lateral ; P, P, the posterior columns.

the grey commissure in front of this canal is called the anterior, and the part behind, the posterior grey commissure. In front of the grey commissure, and between it and the base of the anterior median fissure, are bundles of white nerve-fibres passing in a horizontal or oblique direction from the anterior column of one side to the grey matter of the anterior cornu of the opposite side (fig. 440). These decussating fibres constitute the white commissure.]

The white matter surrounds the grey, and is arranged in several columns [anterior, lateral, and posterior-by the passage of the nerve-roots to the cornua (figs. 440, 446)]. Along the anterior surface of the cord there runs a well-marked fissure, which dips into the cord itself, but does not reach the grey matter, as a mass of white matter-the white commissure-runs from one side of the cord to the other. Between this fissure, known as the anterior median fissure, and the line of exit of the anterior roots of the spinal nerves, lies the anterior column 
(A) ; the white matter lying laterally between the origin of the anterior and posterior roots of the spinal nerves is the lateral column $(\mathrm{L})$, while the white matter lying between the line of origin of the posterior roots and the so-called posterior median fissure, is the posterior column $(\mathrm{P})$. [The posterior median fissure is not a real fissure, but is filled up with the inner layer of the pia mater, which dips down from the under surface of this membrane quite to the grey matter of the posterior commissure.] Each posterior column, in certain regions of the cord, may be subdivided into an inner part lying next the fissure, the postero-median or Goll's column, or the inner root-zone (Charcot, fig. $454, c)$; and an outer larger part next the posterior root, known as the postero-external or Burdach's column, or the outer rootzone (Charcot, fig. 454, d).

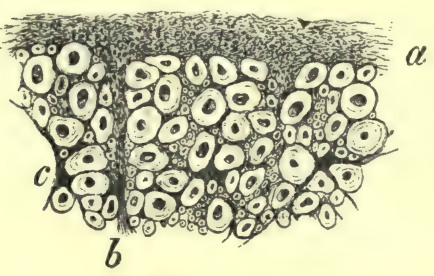

Fig. 441.

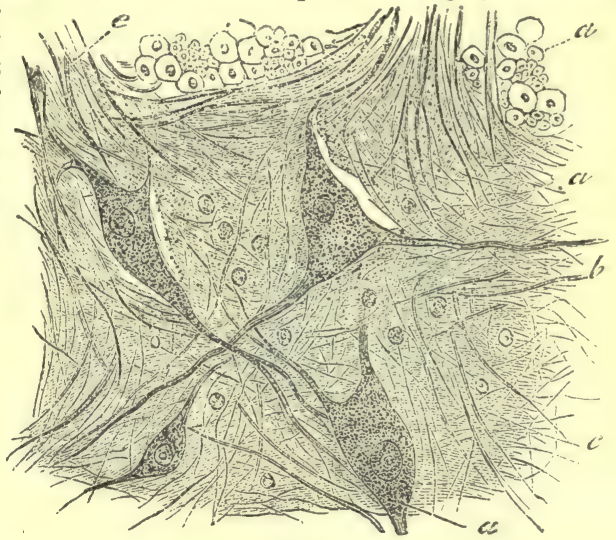

Fig. 442.

Fig. 441. -Transverse section of the white matter of the cord ; $\times 150$. a, peripheral layer. Besides the transverse sections of the nerve-fibres, large and fine, there are three branched connective-tissue corpuscles (c). Fig. 442. - Multipolar nerve-cells from the grey matter of the anterior horn of the spinal cord (ox). $a$, nerve-cell ; $b$, axis-cylinder ; $c$, grey matter ; $d$, white matter of column ; $e, e$, branches of cells.

The white matter consists chiefly of medullated fibres without the sheath of Schwann and Ranvier's nodes, but provided with the neuro-keratin sheaths of Kühne and Ewald (\$ 321), the fibres themselves being chiefly arranged longitudinally. [The incisures of Schmidt exist in these fibres, and can be demonstrated by the interstitial injection of osmic acid (Ranvier).] The nerve-fibres of the nerve-roots, as well as those that pass from the grey matter into the columns, have a transverse or oblique course. There are also decussating fibres in the anterior or white commissure. [In a transverse section of the white matter of the spinal cord, the nerve-fibres are of different sizes, and appear like small circles with a rounded dot in their centre-the axiscylinder-the latter may be stained with carmine or other dye (fig. 441). They are smallest in the postero-median or Goll's column, and largest in the crossed and direct pyramidal tracts, which are motor. The white substance of Schwann, especially in preparations hardened in salts of chromium, often presents the appearance of concentric lines. Fine septa of connectivetissue carrying blood-vessels lie between groups of the nerve-fibres, while here and there between the nerve-fibres may be seen branched neuroglia corpuscles. Immediately underneath the pia mater there is a pretty thick layer of neuroglia, which invests the prolongations of the pia into the cord.]

[The grey matter differs in shape in the different regions of the cord, and so does the grey commissure (fig.

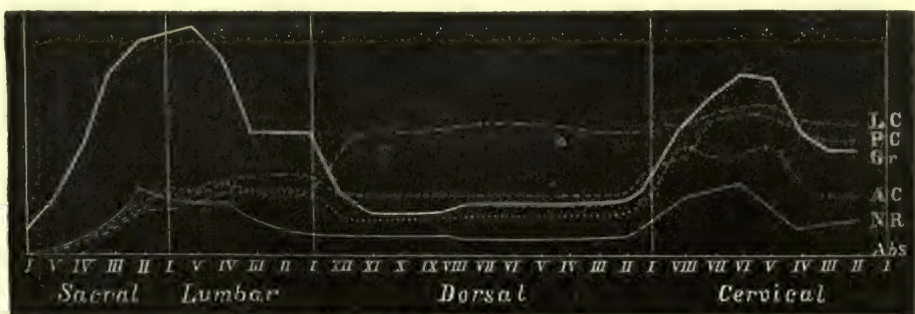

Fig. 443.

444). The latter Diagram of the absolute and relative extenc of the grey matter, and of the is thicker and shorter in the cervical than in the dorsal rewhite columns in successive sectional areas of the spinal cord, as well as the sectional areas of the several entering nerve-roots. NR, nerve-roots; AC LC, PC, anterior, lateral, and posterior columns ; Gr, grey matter.

gion, while it is very narrow in the lumbar region. The amount of grey matter undergoes a great increase opposite the origins of the large nerves, the increase being most marked opposite 
the cervical and lumbar enlargements. Ludwig and Worschiloff constructed a series of curves from measurements by Stilling of the sectional areas of the grey and white matter of the cord, as well as of the several nerve-roots. These curves have been arranged in the annexed convenient form by Schäfer after Woroschiloff (fig. 443)] :-

[In the cervical region, the lateral white columus are large, the anterior cornu of the grey matter is wide and large, while the posterior cormu is narrow: Goll's column is marked off by a depression and a prolongation of the pia mater; the cord itself is broadest from side to side. In the dorsal region, the grey matter is small in animals, and both cornua are narrow and of nearly equal breadth, while the cord itself is smaller and cylindrical. In it the intermedio-lateral and posterior vesicular groups of cells are distinct. They have probably relations to viscera. The commissure lies well forward between the crescents. In the lumbar region, the grey
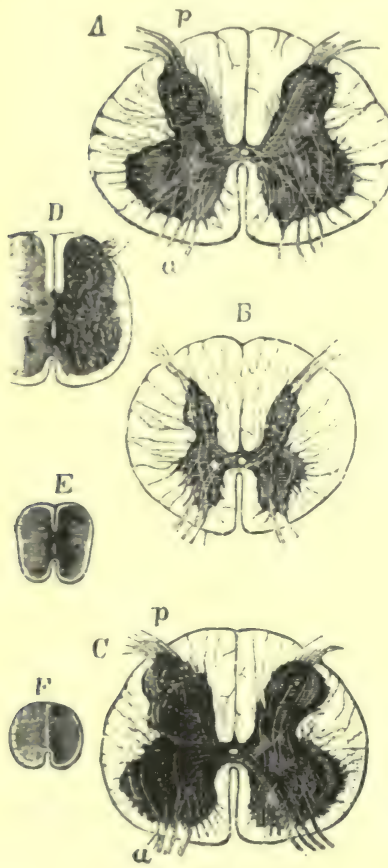

Fig. 444.

Transverse sections of the spinal cord in different regions. A, through the middle of the cervical ; $\mathrm{B}$, the dorsal; $\mathrm{C}$, the lumbar enlargement; $\mathrm{D}$, upper part of the conus medullaris ; $\mathbf{E}$, at the 5 th sacral vertebra; $\mathrm{F}$, at coccyx; A, B, C, enlarged twice; D, E, F, thrice; $a$, anterior, $p$, posterior root. natter is relatively and absolutely greatest, while the white lateral columns are sinall, the central canal in the commissure being nearly in the middle of the cord. In the conus medullaris, the grey matter makes up the great mass of it, with a few white fibres externally (fig. 444).]

The anterior cornu of the grey matter is shorter and broader, and does not reach so near to the surface as the posterior; moreover, each anterior nerve-root arises from it by several bundles-it contains several groups of large multipolar gauglionic cells (fig. 442); the posterior cornu is more pointed, longer, and narrower, and reaches nearer to the surface, the posterior root arising by a single bundle at the postero-lateral fissure; while the cornu itself contains a few fusiform nerve-cells, and is covered by the substantia gelatinosa of Rolando, which is in part an accumulation of neuroglia.

[The substantia gelatinosa on the posterior cornu is marked by striation where the posterior root-fibres traverse it. It contains some connective-tissue cells and some fusiform nerve-cells, especially near the margins. The substance itself stains deeply with carmine.]

[The outer margin of the grey matter near its middle is not so sharply defined from the white matter as elsewhere; and, in fact, a kind of anastomosis of the grey matter projects into the lateral column, especially in the cervical region, constituting the processus reticularis (fig. 446).]

[Arrangement of Nerve-Cells. - The nerve-cells are arranged in four groups, forming columns more or less continuous. There are those of the anterior and posterior horns, those of the lateral column (intermedio-lateral), and the posterior vesicular column of Clarke. The anterior and posterior groups exist as continuous columns along the entire cord. The cells in the anterior cornu are subdivided into smaller groups, which vary in the different regions of the coid. There is an inner or median group near the anterior angle of the cornu. It is the smallest group, and is absent in the lumbar region. Near the anterior edge is the anterior group, and in the external part of the cornu is the antero-lateral group. These two groups are often united, as in the mid-cervical region. There is usually a third large group-the external or postero-lateral in the posterior outer angle of the anterior cornu-the cells of the anterior horn being very large $(67$ to $135 \mu)$, while the fusiform cells of the posterior lorn are $18 \mu$ in diameter. Those of the lateral column are distinct, except in the lumbar and cervical enlargements, where they blend with the anterior horn. The column of Clarke (cells 40 to $90 \mu$ ) is discontinued, and is limited to (1) the thoracic region, (2) cervico-eranial region, (3) sacral region, being nost conspicuous in (1), where it corresponds absolutely to the outflow of visceral nerves (Gaskell). In the sacral region it corresponds to the "sacral nucleus of Stilling," while in the cervical region it begins in the dog at the 2 nd cervical nerve, forming the cervical nucleus, being continued above into the nuclei of the vagus and glosso-pharyngeal nerves. The cells of this column give rise to small medullated nerve-fibres or the leucenteric
fibres of Gaskell.]

The multipolar ganglion cells are largest, and arranged in groups in the anterior horns of the grey matter (fig. 446-" motor ganglionic cells"). [They also occur in the lateral process and in the processus reticularis. It is to be noted that the cells become more branched as we 
proceed upwards amongst the vertebrata. These cells usually contain pigment-granules, and, according to Pierret, their size has a direct relation to the length of the nerve-fibre proceeding from them ; so that they are largest in the lumbar enlargement, smaller in the cervical enlargement, and smallest in the dorsal region. Smaller spindle-shaped ("sensory") cells occur in much smaller numbers in the grey matter of the posterior horn. The cells of Clarke's column (fig. 445) are smaller $(30-60 \mu$ ), and are usually arranged with their long axis in the long axis of the cord. The processes are fewer, but one is generally directed towards the head, and some towards the caudal end of the body. They usually contain much pigment, which is generally disposed towards the cerebral pole of the cell.]

[In a longitudinal section of the cord (fig. 447), these cells are seen to be arranged in columns, the large multipolar cells in the anterior horn $(m)$; in the same section are shown, the longitudinal direction of the nerve-fibres in the anterior $(a)$ and posterior white columns $(c)$, the horizontal direction of the fibres of the anterior and posterior nerve-roots $(b$ and $f)$.

The grey matter contains an exceedingly delicate fibrous network of the finest nerve-fibrils (Gerlach), which is produced by the repeated division of the protoplasmic processes of the

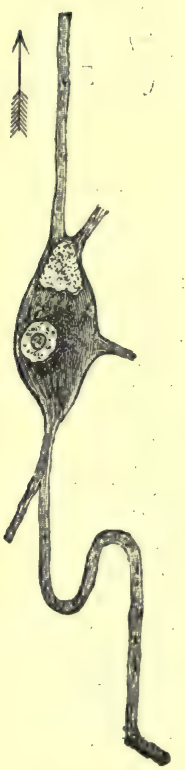

Fig. 445 .

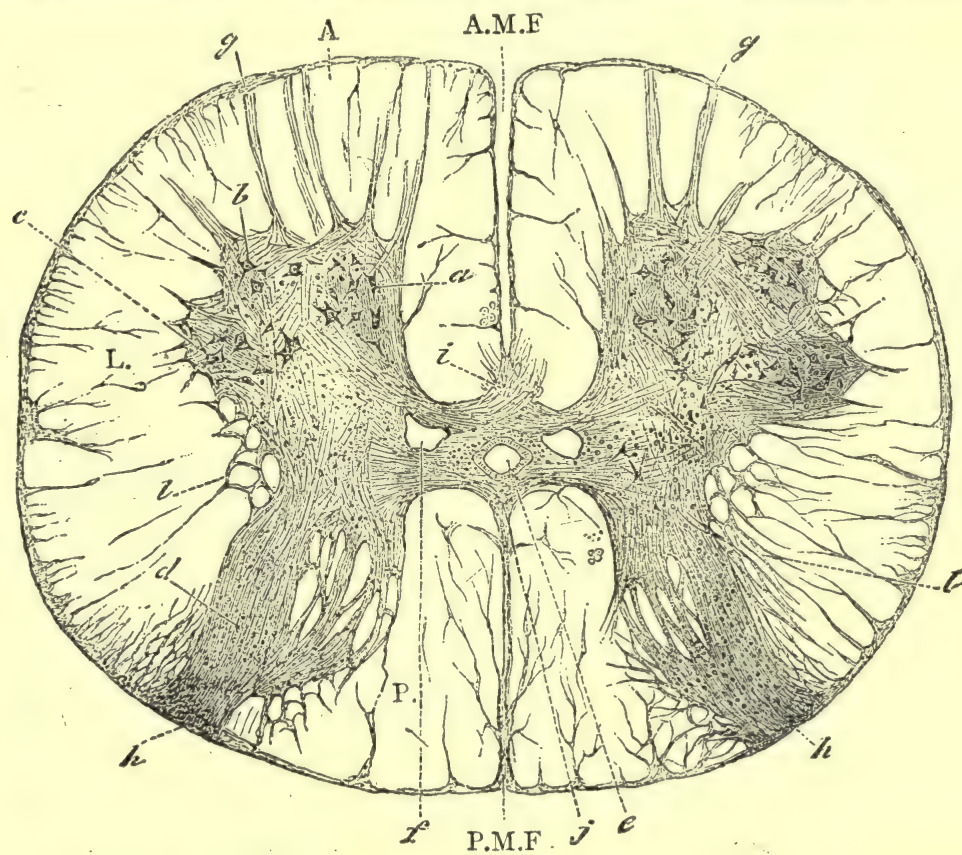

Fig. 446.

Fig. 445. - Nerve-cell from Clarke's column (horse). The arrow indicates the cerebral end. Fig. 446.-Transverse section of the spinal cord (lower dorsal). A, L, P, anterior, lateral, and posterior columns; A.M.F., P.M.F., anterior and posterior median fissures; $a, b$, $c$, ceils of the anterior horn; $d$, posterior cornu and substantia gelatinosa; $e$, central canal ; $f$, veins ; $g$, anterior root bundles; $h$, posterior root bundles ; $i$, white commissure ; $j$, grey commissure ; $l$, reticular formation.

multipolar ganglionic cells. Medullated nerve-fibres traverse and divide in the grey matter and become non-medullated; some of them merely pass through the grey matter of the nonmedullated fibres and terminate in Gerlach's network. Fibres pass from the grey matter of one side to that of the other through the commissures in front of and behind the central canal.

[By means of Weigert's method of staining medullated nerve-fibres (p. 529), it has been proved that numerous, fine, medullated nerve-fibres exist in the grey substance.]

Gerlach's Theory. - According to Gerlach, the connection of the fibres and cells is as follows : -The fibres of the anterior root proceed directly to the ganglionic cells of the anterior horn, with which they form direct communications by means of the unbranched axial cylinder pro. eesses (fig. $443, z$ ). The grey network of protoplasmic processes, produced by the repeated branchings of the fibres of these cells, gives origin to broad fibres. A part of the latter (the median bundle) passes through the anterior white commissure to the other side, and then ascends 
in the anterior column of the opposite side. Other fibres (the lateral bundle) pass into the

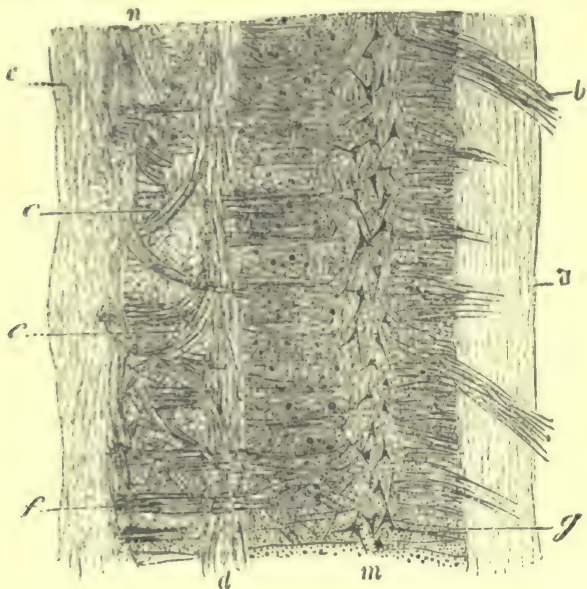

Fig. 447 .

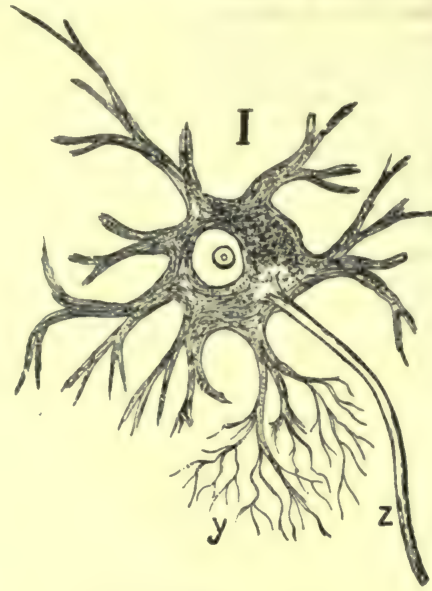

Fig. 448.

Fir. ${ }^{447}$. - Longitudinal section of the human spinal cord. $a$, anterior, $c$, posterior, $d$, lateral white columns ; $b$, anterior, $c$, josterior nerve-roots ; $f$, horizontal (pyramidal) fibres passing t. 1, . cells of anterior cornu ; $n$, oblinue fibres of posterior root. Fig. 448. - Multipolar nerve-cell, from the anterior horn of the spinal cord. $z$, axis-cylinder process ; $y$, branched processes.

latural column of the same side, and ascend in it as far as the decussation of the pyramids, 16. 13 where they cross in the medulla to the other

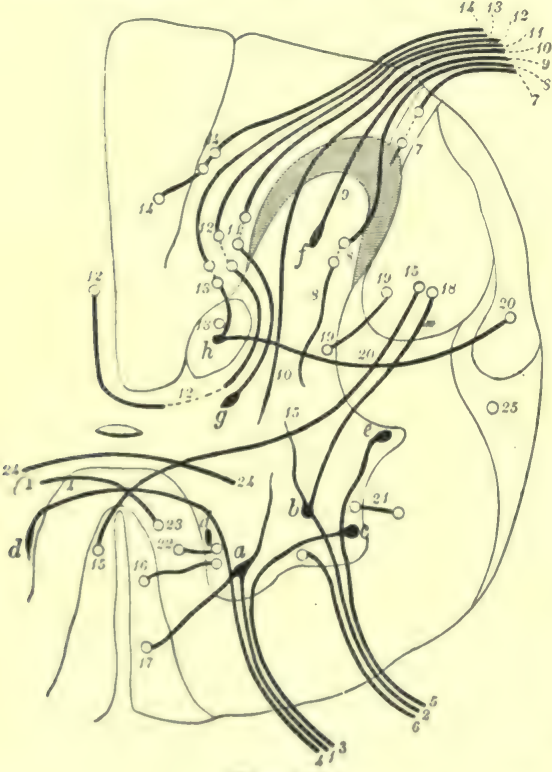

Fig. 449 . posterior horn, and, after dividing, terminate in the nervous protoplasmic network of the grey matter. By means of this network they are connected indirectly with the ganglionic cells of the posterior horn, which are said not to have an axial cylinder process. The grey network, which connects the ganglia of the anterior and posterior horns with each other, also sends fibres, which pass to the other side of the cord in front of and behind the central canal. They then take a backward course, to ascend partly in the posterior horns and partly in the lateral columns.

[The anterior root enters in several bundles of coarse fibres which diverge before they reach the grey matter. Most, or perhaps all, the fibres end in the large motor nerve-cells in the anterior cornu or its lateral process (fig. 449, $a, b, c, d, e)$. But the fibres diverge in all directions, some of the fibres of the bundle nearest the middle line (3) end in the laterally placed cells $(c)$; a part (4) crosses the anterior commissure to end in cells on the opposite side $(d)$. Some of them (6) run upwards to become connected with motor cells lying further up the cord.]

[The posterior root enters as a single bundle, composed of finer fibres with bundles of

Scheme of the course of the fibres in the spinal cord. The longitudinal fibres are indicated by small circles; while the nerve-cells are black. thicker ones. The finest fibres, which are usu. ally placed most laterally (7), or outer radicular fibres, curve into the longitudinal fibres, so that they are cut across in a transverse section, but they again take a horizontal course and enter the substantia gelatinosa. The remainder 
of the fibres split into an outer and inner part. The lateral smaller part or central fibres ( 8 to 10) passes into the substantia gelatinosa, where it divides into several strands, some of which pass into the central part of the grey matter (10), while others (8) pass upwards and downwards in a longitudinal direction. Some of the fibres (9) perhaps end in the nerve-cells $(f)$ in the posterior cornu. The median, inner or internal radicular fasciculus (11 to 14), sweeps through the postero-external column, and, after running a longitudinal course in the white matter, enters the grey substance of the posterior cornu. Some fibres (11) pass to the small fusiform cells $(g)$; and others (13) pass to be connected with the cells of Clarke's column $(h)$, when it is present. From the cells of Clarke's column, fibres seem to pass to the direct cerebellar tract (20). Some of the fibres (12) pass into the posterior grey commissure, to reach the opposite side. This so far only accounts for a part of the fibres. Some of them (8 to 10) are concerned in the formation of the fine nerve-plexus in the grey matter, whereby, perhaps, they become connected with the cells in the anterior cornu. It is asserted that some of the fibres (14) ultimately pass into Goll's column. Many of the fibres in the posterior root have been proved to be directly connected with nerve-cells, e.g., in Petromyzon by Freund, and in the Proteus by Klaussner, so that it is very doubtful if, in the higher animals, the fibres of the posterior root are directly connected with the plexus of grey fibres as suggested by Gerlach (p. 629).]

Neuroglia.-The connective-tissue of the spinal cord arises in part from the pia mater and passes into the white matter, carrying with it blood-vessels, and forming septa, which separate the nerve-fibres into bundles. [The connective-tissue of the central nervous system is so far peculiar, that the intercellular substance is reduced to a minimum. It consists of a reticulated connective-tissue composed of fine fibres, which form a network. Fig. 450 shows one of the

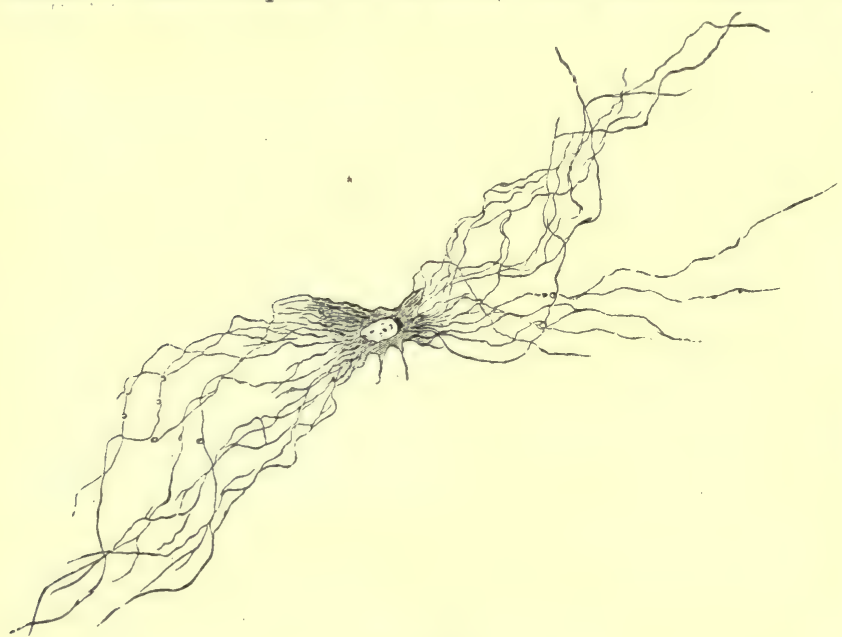

Fig. 450. cells, "glia-cells," isolated. It consists of a small, granular, nucleated body, with numerous excessively fine, slightly branched, stiff processes. The processes form a sustenta-

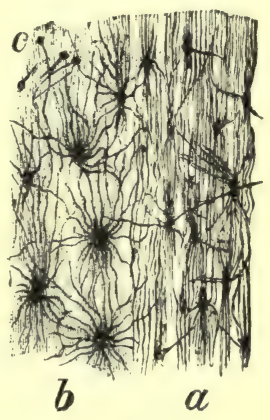

Fig. 451.

Fig. 450.-Isolated connective-tissue corpuscle or "glia-cell" from the human spinal cord ; $\times 800$. Fig. 451 . - Longitudinal section of the spinal cord. $a$, white, $b$, grey matter; $c$, crystals of mercuric chloride. Prepared by Golgi's mercuric chloride method; $\times 80$.

cular tissue for the nerve-fibres and blood-ressels. The arrangement and distribution of these cells is best seen in sections of a cord hardened by Golgi's method in corrosive sublimate solution (fig. 451). In some situations, e.g., the white matter of the cerebrum and cerebellum, the cells are smaller and more angular, and the processes are often connected with the outer coat of the blood-vessels. On the whole, the connective-tissue is much finer. in the brain than in the cord. The central canal is surrounded with a denser layer of this tissue, known as the "central ependyma," which stains deeply with carmine, and is very like the substantia gelatinosa in its structure (p. 628). We must distinguish from this form of connective-tissue that special form in the grey matter to which Virchow gave the name of neuroglia. It is specially adapted to fill up the spaces left by the other elements, and without interfering with the exchange of fluids serves to hold the elements together. It is an excessively finely granular ground-substance in the grey matter. It is also an intercellular substance, but in the adult the cells to which it owes its origin are no longer to be found. It is doubtful, from its chemical nature, if it is really to be reckoned along with the connective-tissues. It seems to be rather a tissue sui generis, belonging to the nervous system, and it is present in very small amount.] 
The neuroglia is also abundant on the sides and apex of the posterior horns, where it is called the gelatinous zubstance of Rolando.

[Blood-Vessels. - The spinal cord is partly supplied with blood by arteries from the vertebrals, and partly by branches of the intercostal, lumbar, and sacral arteries; which reach it through the intervertebral foramina, and pass to the cord along the anterior and posterior roots.]

[Blood-Vessels. - The anterior median (or anterior spinal) (fig. 452) artery gives off branches. which dip into the fissure of the same name, pass to its base, and, after perforating the anterior commissure, divide into two branches, one for each mass of grey matter, and each branch in turn splits into three, which supply part of the anterior, median, and posterior grey matter. The arteries lying in the sulci are called arterim sulci $(s)$ by Adamkiewicz. In the grey matter, there is usually a special branch to Clarke's column $(c l)$. The vaso-coronary arteries include all those arterial branches which proceed

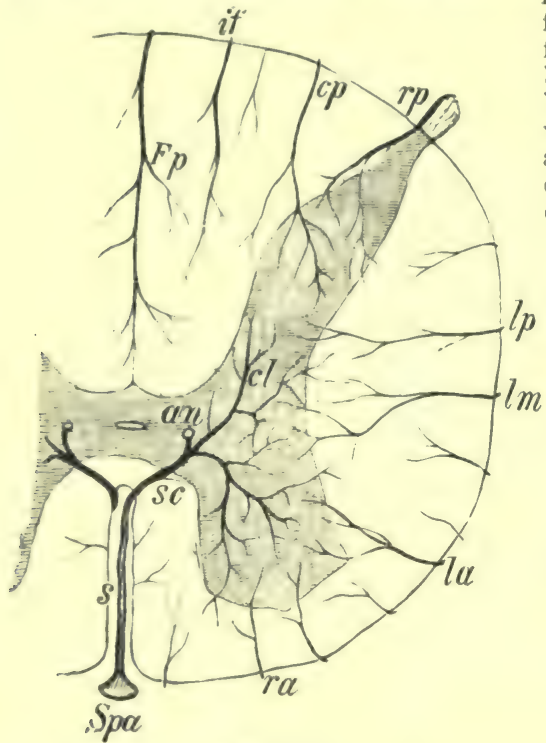

Fig. 452. from the periphery into the white matter; the finer branches pass only into the white matter, but the larger into the grey substance. The largest branch is the artery of the posterior fissure $(F p)$, which passes along the posterior septum and reaches almost to the commissure, giving branches in its course. There is a large artery between the column of Goll and the postero-external column, viz., the

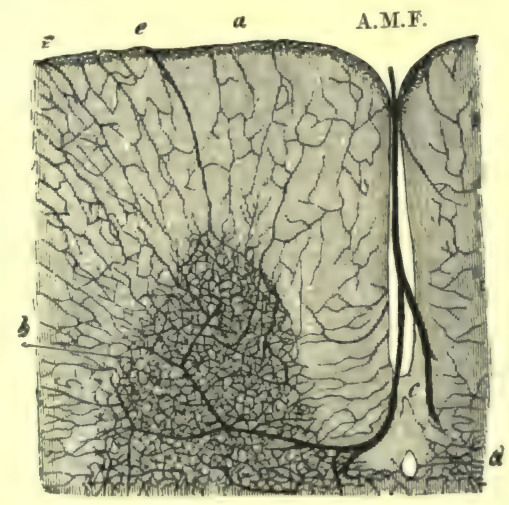

Fig. 453.

Fig. 452. - Semi-diagrammatic arrangement of the arteries in the spinal cord. Spa, anterior spinal ; $s$, sulcine artery ; $s c$, sulco-commissural ; $a n$, its anastomosing branch; $c l$, to Clarke's column ; $F_{p}$, posterior fissure; $r a, r p$, branches along anterior and posterior roots; $c p$, for post. cornu ; if, interfunicular ; $l a, l m, l p$, anterior, median, and posterior lateral. Fig. 453. - Injected blood-vessels of the spinal cord.

interfunicular artery (if). Arteries enter along the anterior and posterior roots $(r a, r p)$. There are also a median lateral artery $(l m)$, and an anterior and posterior lateral $(l p, l a)$, which enter the lateral column. The general result is that the grey matter is much more vascular than the white, as is shown in fig. 453. Some small vessels come from the pia and send branches to the white matter, and unbranched arteries to the grey matter, where they form a capillary plexus. The blood-vessels are surrounded by perivascular lymph-spaces (His).] [With regard to the blood-vessels supplying the cord as a whole, Moxon has pointed out that, owing to the cord not being as long as the vertebral canal, the lower nerves have to run down within the vertebral canal, before they emerge from the appropriate intervertebral foramina. As re-enforcing arteries enter the cord along the course of these nerves, necessarily the branches entering along the course of the lumbar and lower dorsal nerves are long, and this, together with their small size, offers considerable resistance to the blood-stream. Hence, perhaps, the reason why the lower part of the cord is so apt to be affected by various pathological conditions.]

[Functions of the Spinal Cord.-(1) It is a great conducting medium, conducting impulses upwards and downwards, and within itself from side to side; (2) the great reflex centre, or rather series of so-called centres; (3) impulses originate within it.] 
Conducting Systems. - The whole of the longitudinal fibres of the spinal cord may be arranged systematically in special bundles, according to their function.

[Methods.-The course fof the fibres and their division into so-called systems has been ascertained partly by anatomical and embryological, partly by physiological and pathological means. Apart from experimental methods, such as dividing one column of the cord and observing the results, we have the following methods of investigation :-(1) Türck found that injury or disease of certain parts of the brain was followed by a degeneration downwards, or secondary descending degeneration of certain of the nerve-fibres connected with the seat of injury, i.e., they were separated from their trophic centres and underwent degeneration. (2) P. Schieferdecker found also, after section of the cord, that above and below the level of the section, certain definite tracts of white matter underwent degeneration [thus showing that certain tracts had their trophic centre below; this constitutes secondary ascending degeneration]. [(3) Gudden's Method.-He showed, as regards the brain, that excision of a senseorgan in a young growing animal was followed by atrophy of the nerve-fibres and some other parts connected with it. Thus, the optic nerve and anterior corpora quadrigemina atrophy after excision of the eyeball in young rabbits.] (4) Embryological. - Flechsig showed that the fibres of the cord [and the brain also] during development became covered with myelin at different periods, those fibres becoming medullated latest which had the longest course. In this way he mapped out the following systems :-

Flechsig's Systems of Fibres.-1. In the anterior column lie $(a)$ the uncrossed, anterior, or direct pyramidal tract [also called the Column of Türck]; and external to it is $(b)$ the anterior ground bundle, or anterion radicular zone (fig. 454). [The direct pyramidal tract varies in size, and it generally extends downwards in the cord to about the middle of the dorsal region, diminishing steadily in its course; so that it would seem that this tract contains chiefly fibres for the arm. We do not know, exactly, how these fibres end, whether they cross to the opposite side, or remain on the same side, but most probably most of them pass through the anterior commissure to the grey matter of the opposite side.]

2. In the posterior column he distinguishes (c) Goll's column, or the postero-median (postero-internal) column; and $(d)$ the funiculus cuneatus, Burdach's column, or the posterior radicular zone, or the postero-external column.

3. In the lateral column are $(e)$ the anterolateral tract and $(f)$ the lateral mixed paths, or lateral limiting tract, $(g)$ the lateral or crossed pyramidal tract, and $(h)$ the direct cerebellar tract.

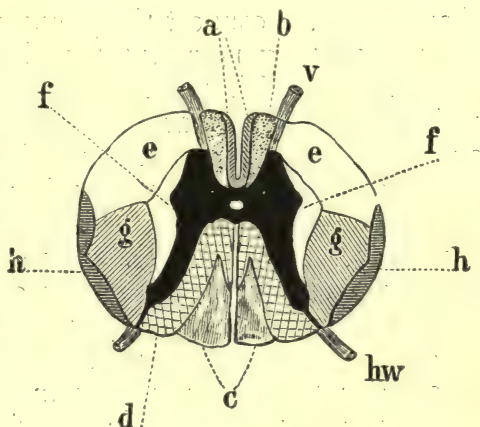

Fig, 454.

Scheme of the conducting paths in the spinal cord at the 3rd dorsal nerve. The black part is the grey matter. $v$, anterior, hw, posterior, root; $\alpha$, direct, and $g$, crossed, pyramidal tracts; $b$, anterior column ground bundle; $c$, Goll's column ; $d$, postero-external column ; $e$ and $f$, mixed lateral paths; $h$, direct cerebellar tracts.

[All the impulses from the central convolutions or motor areas of the cerebrum, by means of which voluntary movements are executed, are conducted by the pyramidal tracts $a$ and $g(\$ 365)$. The fibres in these tracts descend from the central convolutions, i.e., the motor areas pass through the white matter of the cerebrum, converging like the rays of a fan to the internal capsule, where they lie in the knee and anterior two-thirds of its posterior segment (the fibres for the face at the knee, and behind this in order those for the arm and leg), they then enter the middle-third of the crusta (fig. 502, Py), pass through the pons into the anterior pyramids of the medulla oblongata, where the great mass crosses over to the lateral column of the opposite side of the cord (crossed pyramidal tract), a small part descending in the cord on the same side as the antero-median tract (direct pyramidal tract, a). The crossed pyramidal tract lies external to the posterior half of the grey matter in the lateral column (fig. 454,g), and it extends throughout the length of the cord. In 
the greater part of its course, it is separated from the surface by the direct cerebellar tract, but where the latter lies further forward, as at the third cervical segment and lower dorsal region, its posterior surface reaches the surface, while from the last dorsal segment, throughout the lumbar region, it comes quite to the surface, as the direct cerebellar tract ceases at the first lumbar vertebra. The pyramidal tract diminishes from above downwards, and its fibres pass into the grey matter of the anterior cornu, and in all probability they subdivide to form fine fibrils, which become connected with the dense plexus of fine fibrils produced by the subdivision of the processes of the multipolar nerve-cells. From each multipolar nerve-cell, a nervefibre proceeds and passes into the anterior root. The direct cerebellar tract $(h)$ begins ahout the first lumbar nerve, and increases somewhat in thickness from below upwards, but most of its fibres enter it at the first lumbar and lowest dorsal nerves. It forms a thin layer on the surface of the cord. Its fibres very probably arise in the cells of Clarke's column. As Clarke's column is connected with some of the fibres of the posterior root (for the trunk of the body), it follows that this tract connects certain parts of the posterior roots with the cerebellum. The fibres pass up through the cord and restiform body to the cerebellum. When it is divided, it degenerates upwards, so that it conducts impulses in a centripetal direction.] The anterior $(e)$ and lateral paths $(f)$ and the anterior ground bundle $(b)$ represent the channels which connect the grey matter of the spinal cord and that of the medulla oblongata; they represent the channels for reflex effects, and they also contain those fibres which are the direct continuation of the anterior spinal nerve-roots, which enter the cord at different levels and penetrate into the grey matter. In $e$ and $f$ there are some sensory paths. Lastly, $c$ unites the posterior roots with the grey nuclei of the funiculi graciles of the medulla oblongata; $d$ connects some of the posterior nerve-roots through the restiform body with the vermiform process of the cerebellum (Flechsig). The direction of conduction in the posterior columns, which are continnations of some of the fibres of the posterior roots, is upwards, as part of them degenerates upwards after section of the posterior root. Of the fibres of each posterior root, some pass directly into the posterior horn, another part ascends in the posterior column of the same side, and gradually as it ascends, it comes nearer the posterior median fissure. Some of these fibres enter the grey matter of the posterior horn at a higher level. The fibres of the posterior columns run upwards as far as the interolivary layer and the decussation of the pyramids, where they seem to end, or at least form connections with the nerve-cells of the funiculi graciles [clava] and cuneati [triangular nucleus]. A small part as arcuate fibres join the restiform body, and thus the cerebellum is connected with the posterior columns.

Further, the transverse sectional area of the direct and crossed pyramidal tracts $(a$ and $g$ ), the lateral cerebellar tract $(h)$, and Goll's column $(c)$ gradually diminish from above downwards; they serve to connect intracranial central parts with the ganglionic centres distributed along the spinal cord. The anterior root bundle $(b)$, the funiculus cuneatus $(d)$, and the anterior mixed lateral tracts (e) vary in diameter at different parts of the cord, corresponding to the number of nerve-roots. It has been concluded from this that these tracts serve to connect the grey matter at different levels in the cord with each other, and ultimately with the medulla oblongata, so that they do not pass directly to the higher parts of the brain (fig. 443).

Nutritive Centres of the Conducting Paths.-Türck observed that the destruction of certain parts of the brain caused a secondary degeneration of certain parts of the cord, corresponding to the parts called pyramidal tracts by Fleschig (fig. 455). P. Schieferdecker found the same effects below where he divided the spinal cord in a dog. Hence, it is concluded that the nutritive or trophic centre of the pyramidal tracts lies in the cerebrum. [Section of the cord, or an injury compressing the cord, besides giving rise to loss of certain functions (p. 648), results in structural changes in certain limited areas of the cord itself. Below the section 
after a time, the direct and crossed pyramidal tracts (fig. 45. , 1, 1', 2, 2') degenerate downwards, i.e., they undergo descending secondary degeneration, because they are cut off from their nutritive or trophic centres, which are situated above in the pyramidal cells of the motor areas of the brain (\$ 378). The trophic centre for the fibres of the anterior root lies in the multipolar nerve-cells of the anterior cornu of the grey matter of the cord. After section of the spinal cord, Goll's column and the direct cerebellar tracts degenerate upwards, i.e., they undergo ascending secondary degeneration. If the posterior columns even be divided, Goll's column degenerates upwards towards the medulla oblongata, and the degeneration ends in the posterior pyramidal nucleus or clava. The same, result occurs if the posterior nerveroots of the cauda equina be

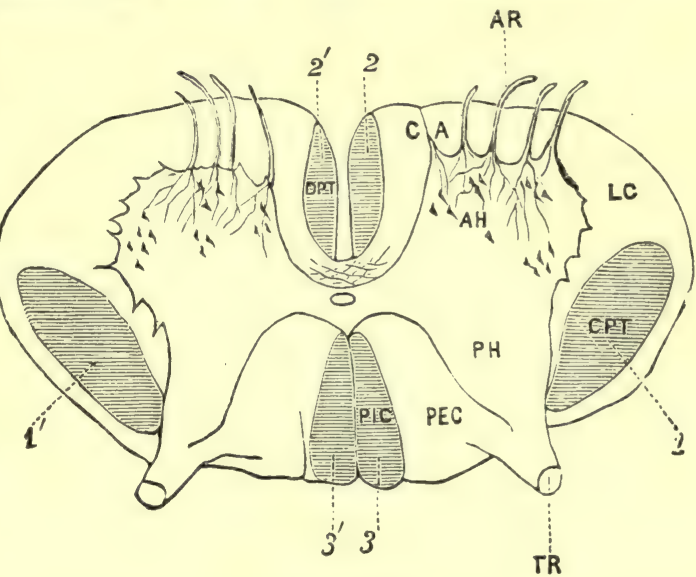

Fig. 455 .

Transverse section of the spinal cord, showing the secondary degeneration tracts. AR, anterior, TR, posterior root; $1, I^{\prime}$ (CPT), region of the crossed pyramidal tract ; 2, 2' (DPT), direct pyramidal tract; PEC, postero-external column ; LC, lateral column.

injured. Hence, fibres seem to pass from the posterior root into these columns, and the nerve-cells in the clava must also have an important relation to these nerve-fibres and the parts whence they are derived. The postero-external column remains undegenerated, so that there is a very sharp distinction between the two parts of the posterior column. As Goll's column degenerates upwards, it points to its fibres conducting impulses in a centripetal direction, and to the nutritive centre for its nerve-fibres being below. The trophic centre is probably in the spinal ganglion of the posterior root.]

[If the cord be divided above the junction of the dorsal and lumbar regions, the direct cerebellar tract undergoes ascending degeneration, which extends through the restiform body to the cerebellum. Its trophic centre is probably in the cells of Clarke's column.] Those fibres of the spinal cord which do not degenerate after section of the cord, especially numerous in the lateral and anterior columns [anterior ground bundle, the anterior and lateral mixed zones of the lateral column, and the postero-external part of the posterior column], are commissural in function, connecting ganglionic cells with each other, and are, therefore, provided with a trophic centre at both ends.

Time of Development. - With regard to the time of development of the individual systems, Flechsig finds that the first formed paths are those between the periphery and the central grey matter, especially the nerve-roots, i.e., they are the first to be covered with the myelin. Then fibres which connect the grey matter at different levels are formed-the fibres which connect the grey matter of the cord with the cerebellum, and also the former with the tegmentum of the cerebral peduncle. At last the fibres which connect the ganglia of the pedunculus cerebri, and perhaps also the grey matter of the cortex cerebri with the grey matter of the cord are formed. In cases of anencephalous foctuses, i.e., where the cerebrum is absent, neither the pyramidal tracts nor the pyramids are developed. In the brain before birth, medullated nerve-fibres are formed in the paracentral, central, and oceipital convolutions, and in the island of Reil, and last of all in the frontal convolutions (Tuczek).

360. SPINAL REFLEXES.-By the term reflex movement is meant a movement caused by the stimulation of an afferent (sensory) nerve. The stimulus, 
on being applied to an afferent nerve, sets up a state of excitement (nervous impulse) in that nerve, which state of excitement is transmitted or conducted in a centripetal direction along the nerve to the centre (spinal cord in this case); where the nerve-cells represent the nerve-centre in the cord, the impulse is transferred to the motor, efferent or centrifugal channel. Three factors, therefore, are essential for a reflex motor act-a centripetal or afferent filre, a transferring centre, a centrifugal or efferent filre; these together constitute a reflex arc (fig. 456). In a purely reflex act, all voluntary activity is excluded.

Reflex movements may be divided into the three following groups:-

I. The simple or partial reflexes, which are characterised by the fact that stimulation of a sensory area discharges movement in one muscle only, or at least

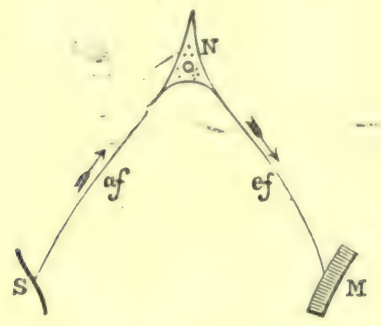

Fig. 456.

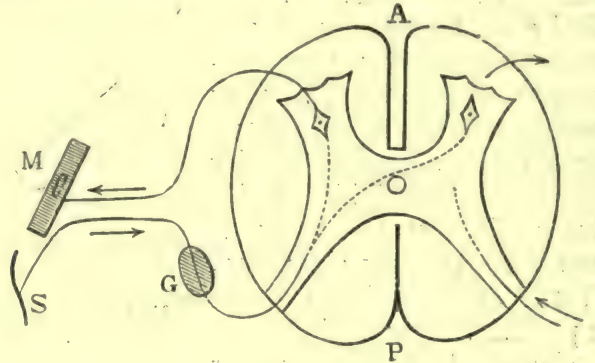

Fig. 457.

Fig. 456. - Scheme of a reflex arc. S, skin; M, muscle; $\mathbf{N}$, nerve-cell, with af, afferent, and $c f$, efferent fibres. Fig. 457. - Section of a spinal segment, showing a unilateral and crossed reflex act. A, anterior, and $\mathrm{P}$, posterior surface; $\mathrm{M}$, muscle; $\mathrm{S}$, skin ; $\mathrm{G}$, ganglion.

in one limited group of muscles. Examples:-A blow upon the knee causes a contraction in the quadriceps extensor cruris; contact with the conjunctiva causes closure of the eyelids. In the former case, the afferent channels arise in the tendon of the quadriceps, and the efferent channels lie in the nerve which supplies the quadriceps; in the latter case, the afferent nerve is the 5 th and the efferent the 7 th cranial nerve. In the former case the centre is in the lumbar region of the cord; in the latter, in the grey matter of the medulla oblongata.

II. The extensive inco-ordinate reflexes, or reflex spasms.-These movements occur in the form of clonic or tetanic contractions; individual groups of muscles, or all the muscles of the body may be implicated. Causes:-A reflex spasm depends upon a double cause-(a) Either the grey matter or the spinal cord is in a condition of exalted excitability, so that the nervous impulse, after having reached the centre, is easily transferred to the neighbouring centres. This excessive excitability is produced by certain poisons, more especially by strychnin, brucia, caffein, atropin, nicotin, carbolic acid, \&c. The slightest touch applied to an animal poisoned with strychnin is sufficient to throw the animal at once into spasms. Pathological conditions may cause a similar result; thus, there is excessive excitability in hydrophobia and tetanus. On the other hand, the central organ may be in such a condition that extensive reflexes cannot take place; thus, in the condition of apnoea, the spasms that occur in poisoning with strychnin do not take place $(J$. Rosenthal and Leube), and the same result is brought about by passive artificial respiratory movements $(\$ 361,3)$. The performance of other passive periodic movements in various parts of the body also produces a similar condition (Buchheim). If the spinal cord be cooled very considerably, reflex spasms may not occur (Kunde). (b) Extensive reflex movements may also take place when the discharging stimulus is very strong. Examples of this condition occur in man, thus-intense neuralgia may be accompanied by extensive spasmodic movements. 
[Fig. 458 shows the mechanism of simple and complex reflex movements. Suppose the skin to be stimulated at $\mathrm{P}$, an impulse is sent to $\mathrm{A}$ and from it to a muscle 1 on the same side, resulting in a unilateral simple reflex movement-the resistance being less in this direction than in the other channels. If the impulse be stronger, or the transverse resistance in the cord dimin. ished, the impulse may pass to $\mathrm{B}$, thence to 2 , resulting in a symmetrical reflex movement on both sides. But if a very strong impulse reach the cord, or if the excitability of the grey matter be increased, e.g., by strychnin, the resistance to the diffusion of the impulse is diminished, and it passes upward to $\mathrm{C}$ and $\mathrm{D}$, resulting in more complex movements--thus there is irradiation-or it may even affect the centres in the medulla oblongata, E, giving rise to general convulsive movements.]

General spasms usually manifest themselves as "extensor tetanus," because the extensors overcome the flexor muscles. Nerves which arise from the medulla oblongata may be excited through the stimulation of distant afferent nerves, without general spasms being produced.

Strychnin is the most powerful reflex-producing poison we possess, and it acts upon the grey matter of the spinal cord. [An animal poisoned with strychnin exhibits tetanic spasms on the application of the slightest stimulus. All the tnuscles become rigid, but the extensors overcome the flexors.] If the heart of a frog be ligatured, and the poison afterwards applied directly to the spinal cord, reflex spasms are produced, proving that strychnin acts upon the spinal cord. During the spasm the heart is arrested in diastole, owing to the stimulation of the vagus, while the arterial blood-pressure is greatly increased, owing to stimulation of the central vaso-motor centres of the medulla oblongata and spinal cord. Mammals may die from asphyxia during the attack; and, after large doses, death may occur, owing to paralysis of the spinal cord, due to the frequently recurring spasms. Fowls are unaffected by comparatively large doses. [We can prove that strychnin does not produce spasms by acting on the brain, muscle, or nerve. Destroy the brain of a frog, divide one sciatic nerve high up, and inject a small dose of strychnin into the dorsal lymph-sac; in a few minutes all the muscles of the body, except those supplied by the divided nerve, will be in spasms, showing that, although the poisoned blood has circulated in the nerves and muscles of the leg, it does not act on them. Destroy the spinal cord, and the spasms cease at once.]

Summation of Stimuli.-By this term is meant, that a single weak stimulus, which in itself is incapable of discharging a reflex act, may, if repeated sufficiently often, produce this act. The single im-

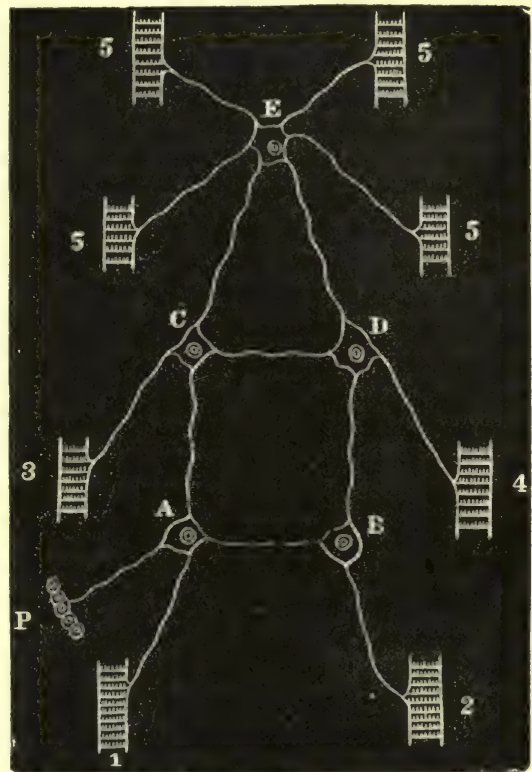

Fig. 458.

Scheme of mode of propagation of reflex movements. P, skin ; A, B, C, D, motor cells in spinal cord ; $1,2,3,4,5$, muscles. pulses are conducted to the spinal cord, in which the process of "summation" takes place. According to J. Rosenthal, 3 feeble stimuli per second are capable of producing this effect, although 16 stimuli per second are most effective. On increasing the number of stimuli per second, no further increase of the reflex act is possible. Other observers (Stirling, Ward) have found that stimuli, such as induction shocks, are active within much wider limits; e.g., from 0.05 to 0.4 second interval. W. Stirling has shown it to be extremely probable that all reflex acts are due to the repetition of impulses in the nerve-centres.

[Strychnin interferes with the summation of stimuli, but the reflex excitability is so greatly exalted that a minimal stimulus is at the same time a maximal one.]

Pfliiger's Law of Reflex Actions. - (1) The reflex movement occur's on the same side on which the sensory nerve is stimulated; while only those muscles contract whose nerves arise from the same segment of the spinal cord. (2) If the reflex occur on the other side, only the corresponding muscles contract. (3) If the contractions be unequal upon the two sides, then the most vigorous contractions always occur on the side which is stimulated. (4) If the reflex excitement extend to other motor nerves, those nerves are always aftected which lie in the 
direction of the medulla oblongata. Lastly, all the muscles of the body may be thrown into contraction.

Crossed Reflexes. - There are exceptions to these rules. If the region of the eye be irritatecl in a frog whose cerebrum is removed, there is frequently a reflex contraction in the hind limb of the opposite side (Luchsinger, Langendorff). In beheaded tritons and tortoises, and in deeply narcotised dogs and cats, tickling one fore limb is frequently followed by a movement of the lint limb of the opposite side (Luchsinger). This phenomenon is called a "crossed reflex" (fig. 45i). If the spinal cord be divided along the middle line throughout its entire extent, then of course the reflexes are confined to one side only (Schiff).

III. Extensive co-ordinated reflexes are due to stimulation of a sensory nerve, causing the discharge of complicated reflex movements in whole groups of different muscles, the movements being "purposive" in character, i.e., as if they were intended for a particular purpose.

Methods. - The experiments are made upon coll-lilvoded animals (decapitated or pithed frogs, tortoises, or eels) or upon mammals. In the latter, artificial respiration is kept up, and the four arteries going to the head are ligatured, in order to eliminate the action of the brain (Sig. Mum, Luchsinger). The reflexes of the lower part of the spinal cord may be studied on animals (or men), in cases where the spinal cord is divided transversely in the upper dorsal region. In such cases, some time must elapse in order that the primary effect of the lesion (the so-called shock), which usually causes a diminution of the reflexes, may pass off. Very young mammals exhibit reflexes for a considerable time after they are beheaded.

Examples:-1. The protective morements of pithed or decapitated frogs. [If a drop of a dilute acid be applied to the skin of such a frog, immediately it strives to get rid of the offending body, and it generally succeeds in doing so.] Similarly, it kicks against any fixed body pushed against it. These movements are so purposive in their character, and the actions of groups of muscles are so adjusted to perform a particular act, that Pflïger regarded them as directed by, and due to "consciousness of the spinal cord." If a flame be applied to the side or part of the body of an eel, the body is moved away from the flame. The tail of a decapitated triton, tortoise, newt, eel, or snake is directed towards a gentle stimulus, but if a violent stimulus is used, it is directed away from it (Luchsinger).

2. Goltz's Croaking Experiment. - A pithed (male) frog, i.e., one with its cerebral lobes alone removed (or one with its eyes or ears destroyed-Langendorff), croaks every time the skin of its back or flanks is gently stroked. [Some male frogs, when held up by the finger and thumb immediately behind the fore legs, croak every time gentle pressure is made on their flank.]

3. Goltz's "Embrace Experiment."-During the breeding season in spring, the part of the body of the male frog between the skull and the fourth vertebra, embraces every rigid object, which is brought into contact with, and gently stimulates, the skin over the sternum.

In the intact animal, the exciting stimulus lies in the degree of filling of the male seminal organ (Turchanoff). The reflex ceases at once on gently stimulating the optic lobes (Albertoni).

4. In mammals (dogs), the following reflex acts are performed by the posterior part of the spinal cord, even after it is separated from the rest of the cord:Scratching with the hind feet a part of the skin which has been tickled (just as in intact animals); the movements necessary for emptying the bladder and for defæcation, as well as those necessary for erection; the movements necessary for parturition (Goltz, Freusberg and Gergens). Co-ordinated movements do not, as a rule, occur simultaneously in portions of the spinal cord lying widely apart after removal of the medulla oblongata. According to Ludwig and Owsjannikow, the medulla oblongata perhaps contains a reflex organ of a higher order, which forms, as it were, a centre for combining, through the medium of the nerve-fibres, the various reflex provinces in the spinal cord.

5. Co-ordinated reflexes may occur in man during sleep, and during pathological comatose conditions.

Most of the movements which we perform while we are awake, and which we execute uncon- 
sciously - or even when our psychical activities are concentrated upon some other object-really belong to the eategory of co-ordinated reflexes. Many complicated motor acts must first be learned-e.g., dancing, skating, riding, walking-before unconscious harmonious co-ordinated reflexes can again be discharged. The co-ordinated reflex movements of coughing, sneezing, and vomiting depend upon the spinal cord, together with the medulla oblongata.

The following facts are also important :-

1. Reflexes are more easily and more completely discharged, when the specific end-organ of the afferent nerve is stimulated, than when the trunk of the nerve is stimulated in its course (Marshall Hall, 1837). [Thus, by gently tickling the skin, it is easy to discharge a reflex act, while it requires a strong stimulus to be applied to an exposed sensory nerve in order to do so.]

2. A stronger stimulus is required to discharge a reflex movement than for the direct stimulation of motor nerves.

3. A movement produced reflexly is of shorter duration than the corresponding movement executed voluntarily. Further, the occurrence of the movement after the moment of stimulation is distinctly delayed. In the frog, a period nearly twelve times as long elapses before the occurrence of the contraction, than is occupied in the transmission of the impulse in the sensory and motor nerves (Helmholtz, 1854). Thus, the spinal cord offers resistance to the transmission of impulses through it.

The term "reflex time" is applied to the time necessary for transferring the impulse from the afferent fibre to the nerve-cells of the cord, and from them to the efferent fibre. In the frog it is equal to 0.008 to 0.015 second. The time, however, is increased by almost one-third, if the impulse pass to the other side of the cord, or if it pass along the cord, e.g., from the sensory nerves of the anterior extremity to the motor roots of the posterior limb. Heat diminishes the reflex time and increases the reflex excitability. Lowering the temperature (winter frogs), as well as the reflex-exciting poisons already mentioned, lengthens the reflex time, whilst the reflex excitability is simultaneously increased. Conversely, the reflex time diminishes as the strength of the stimulus increases, and it may even become of minimal duration ( $J$. Rosenthal). The reflex time is determined by ascertaining the moment at which the sensory nerve is stimulated, and the subsequent contraction occurs. Deduct from this the time of latent stimulation ( $\$ 298$, I.), and the time necessary for the conduction of the impulse ( $\$ 298)$ in the afferent and efferent nerves ( $v$. Helmholtz, J. Rosenthal, Exner, Wundt).

[Influence of Poisons. - The latent period and reflex time are influenced by a large number of conditions. In a research as yet unpublished, W. Stirling finds that the latent period may remain nearly constant in a pithed frog for nearly two days, when tested by Türck's method. Sodic chloride does not influence the time, nor does sodic bromide or iodide. Potassic chloride, however, lengthens it enormously, or even abolishes reflex action after a very short time, and so do potassic bromide, ammonium chloride and bromide, chloral and croton-chloral. The lithia salts also lengthen the reflex time, or abolish the reflex act after a time.]

361. INHIBITION OF THE REFLEXES.-Within the body there are mechanisms which can suppress or inhibit the discharge of reflexes, and they may therefore be termed mechanisms inhibiting the reflexes. These are:-

1. Voluntary Inhibition.-Reflexes may be inhibited voluntarily, both in the region of the spinal cord and brain. Examples :-Keeping the eyelids open when the eyeball is touched; arrest of movement when the skin is tickled. We must cbserve, however, that the suppression of reflexes is possible only up to a certain point. . If the stimulus be strong, and repeated with sufficient frequency, the reflex impulse ultimately overcomes the voluntary effort. It is impossible to suppress those reflex movements which cannot at any time be performed voluntarily. Thus, erection, ejaculation, parturition, and the movements of the iris, are neither direct voluntary acts, nor can they, when they are excited reflexly, be suppressed by the will.

2. Setschenow's inhibitory centre is another cerebral apparatus, which in the frog is placed in the optic lobes. If the optic lobes be separated from the rest of the brain and spinal cord, by a section made below it, the reflex excitability is increased. If the lower divided surface of the optic lobes be stimulated with a 
crystal of common salt or blood, the reflex movements are suppressed. The same results obtain when only one side is operated on. Similar organs are supposed to be present in the corpora quadrigemina and medulla oblongata of the higher vertebrates. From 1 and 2 we may explain why reflex movements occur more regularly and more readily after separation of the brain from the spinal cord.

[Quinine greatly diminishes the reflex excitability in the frog, but if the medulla oblongata be clivided, the reflex excitability of the cord is restored. The depression is ascribed by Chaperon to the action of the quinine on Setschenow's centres.]

3. Strong stimulation of a sensory nerve inhibits reflex movements. The reflex does not take place if an afferent nerve be stimulated very powerfully (Goltz, Levisson). Examples :-Suppressing a sneeze by friction of the nose, [compressing the skin of the nose over the exit of the nasal nerve]; suppression of the movements produced by tickling, by biting the tongue. Very violent stimulation may even suppress the co-ordinated reflex movements usually controlled by voluntary impulses. Violent pain of the abdominal organs (intestine, uterus, kidneys, bladder, or liver) may prevent a person from walking or even from standing. To the same category belongs the fact that persons fall down when internal organs richiy supplied with nerves are injured, there being neither injury of the motor nerves nor loss of blood to account for the phenomenon. Excitement of the central organs through other centripetal channels (nerves of special sense, and those of the generative organs) diminishes the reflexes in other channels.

4. It is important to note that in the suppression of reflexes, antagonistic muscles are often thrown into action, whether voluntarily or by the stimulation of sensory nerves, i.e., reflexly. In some cases, in order to cause suppression of the reflex, it appears to be sufficient to direct our attention to the execution of such a complicated reflex act. Thus, some persons cannot sneeze when they think intently upon this act itself (Darwin). The voluntary impulse rapidly reaches the reflex centre, and begins to influence it so that the normal course of the reflex stimulation, due to an impulse from the periphery, is interfered with (Schlösser).

5. Poisons. - Chloroform diminishes the reflex excitability by acting upon the centre, and a similar effect is produced by picrotoxin, morphia, narcotin, thebain, aconitin, quinine, hydrocyanic acid. [W. Stirling finds that chloral, potassic bromide and chloride, ammonium chloride, but not sodium chloride, greatly diminish the reflex excitability. Nicotin increases it in frogs (Freusberg).]

A constant current of electricity passed longitudinally through the cord diminishes the reflexes (Ranke), especially if the direction of the current is from above downwards (Legros and Onimus, Uspensky).

[Some drugs affect the reflex excitability directly by acting on the spinal cord, e.g., methylconine, but other drugs may produce the same result indirectly by affecting the heart and the blood-supply to the cord. If the abdominal aorta of a rabbit be compressed for a few minutes to cut off the supply of blood to the cord and lower limbs, temporary paraplegia is produced.]

If frogs be asphyxiated in air deprived of all its $\mathrm{O}$, the brain and spinal cord become completely unexcitable, and can no longer discharge reflex acts. The motor nerves and the muscles, however, suffer very little, and may retain their excitability for many days (Aubcrt).

[Nature of Inhibition. - The foregoing view assumes the existence of inhibitory centres, but it is important to point out that it has been attempted to explair this phenomenon without postulating the existence of inhibitory centres. During inhibition the funetion-of an organ is restrained -during paralysis it is abolished, sa that there is a sharp distinction between the two conditions. The analogy between inhibitory phenomena and the effects of interference of waves of light or sound has beeu pointed out by Bernard and Romanes, while Lander Bruntou has tried to explain the question on a physical basis, indicating that inhibition is not dependent on the existenee of special inlibitory centres, but that stimulation and inhibition are different phases of excitement, the two terms being relative conditions depending on the length of the path along which the impulse has to travel and the rate of its transmission. Brunton points out that the known facts are more consistent with an hypothesis of the interference of waves, one with another, than with the supposition that there are inhibitory centres for erery so-called inhibitory act in the body. In discussing this question great regard must be had to the action of the vagus on the heart (\$369).] 
Tuirck's method of testing the reflex excitability of a frog is the following:-A frog is pithed, and after it has recovered from the shock, its foot is dipped into dilute sulphuric acid [2 per 1000]. The time which elapses between the leg being dipped in and the moment it is withdrawn is noted. [The time may be estimated by means of a metronome, or the movements may be inscribed upon a recording surface. The time which elapses is known as the "period of latent stimulation."]

This time is greatly prolonged after the optic lobes have been stimulated with a crystal of common salt or blood, or after the stimulation of a sensory nerve.

Setschenow distinguished tactile reflexes, which are discharged by stimulation of the nerves of touch; and pathic, which are due to stimulation of sensory (pain-conducting) fibres. He and Paschutin suppose that the tactile reflexes are suppressed by voluntary impulses, and the pathic by the centre in the optic lobes.

Theory of Reflex Movements. - The following theory has been propounded to account for the phenomena already described :- It is assumed that the afferent fibre within the grey matter of the spinal cord joins one or more nerve-cells, and thus is placed in communication in all directions with the network of fibres in the grey substance. Any inpulse reaching the grey matter of the cord has to overcome considerable resistance. The least resistance lies in the direction of those efferent fibres which emerge in the same plane aud upon the same side as the entering fibre. Thus, the feeblest stimulus gives rise to a simple reflex, which generally is merely a simple protective movement for the part of the skin which is stimulated. Still greater resistance is opposed in the direction of other motor ganglia. If the reflex impulse is to pass to these ganglia, either the discharging stimulus must be considerably increased, or the resistance within the connections of the ganglia of the grey matter must be diminished. The latter condition is produced by the action of the above-named poisons, as well as during general increased nervous excitability (hysteria, nervousness). Thus, extensive reflex spasms may be produced either by increasing the stimulus, or by dininishing the resistance to conduction in the spinal cord. Those conditions which render the occurrence of reflexes more difficult, or abolish them altogether, must be regarded as increasing the resistance in the reflex arc in the cord. The action of the reflex inhibitory mechanism may be viewed in a similar manner.

The fibres of the reflex arc must have a connection with the reflex inhibitory paths; we must assume that equally by the reflex inhibitory stimulation resistance is introduced into the reflex arc. The explanation of extensive co-ordinated movements is accompanied with difficulties. It is assumed, that by use and also by heredity, those ganglionic cells which are the first to receive the impulse are placed in the path of least resistance in connection with those cells which transfer the impulse to the groups of muscles, whose contraction, resulting in a co-ordinated purposive movement, prevents the body or the limb from being affected by any injurious influences.

Pathological.-Anomalies of reflex activity afford an important field to the physician in the investigation of nervous diseases. Enfeeblement, or even complete abolition of the reflexes may occur:-(1) Owing to diminished sensibility or complete insensibility of the afferent fibres ; (2) in analogous affections of the central organ; (3) or, lastly, of the efferent fibres. Where there is general depression of the nervous activity (as after shocks, compression or inflammation of the central nervous organs; in asphyxia, in deep conra, and in consequence of the action of many poisons), the reflexes may be greatly diminished or even abolished.

[Reflexes.-The physician, by studying the condition of the reflexes, can form an idea as to the condition of practically every inch of the spinal cord. There are three groups of reflexes, $(a)$ the superficial, $(b)$ the deep or tendon, $(c)$ the organic reflexes.]

[The superficial or skin reflexes are excited by stimulating the skin, e.g., by tickling, pricking, scratching, \&c. We can obtain a series of reflexes from below as far up as the lower part of the cervical region. The plantar reflex is obtained by tickling the soles of the feet, when the leg on that side, or, it may be, both legs are drawn up. It is always present in health, and its centre is in the lumbar enlargement of the cord. The cremasteric reflex is well marked in boys, and is easily produced by exciting the skin on the inner side of the thigh, when the testicle on that side is retracted. The gluteal reflex consists in a contraction of the gluteal muscles, when the skin over the buttock is stimulated. The abdominal reflex consists in a similar contraction of the abdominal muscles, when the skin over the abdomen in the mammary line is stimulated. The epigastric reflex is obtained by stimulating the skin in front between the fourth and sixth ribs. The interscapular reflex results in a contraction of the muscles attached to the scapula, 
when the skin between the scapulæ is stimulated. Its centre corresponds to the lower cervical and upper dorsal region.]

[The following table, after Gowers, shows the relation of each reflex to the spinal segment or segments on which it depends:-

\begin{tabular}{|c|c|c|c|c|c|c|}
\hline Cervical, & . & . & - & 6 ) & & Lumbar \\
\hline ", & • & . & - & 7 & Interscapular. & $\therefore$ \\
\hline & . & . & - & 8 & & ", \\
\hline orsal, & & . & - & 1) & & , \\
\hline$\therefore$ & & . & - & $\begin{array}{l}5 \\
6\end{array}$ & Enigastric & Sacral. \\
\hline$\because$ & " & . & - & 7 & & , , \\
\hline$"$, & & & . & 8 & &,, \\
\hline , & . & . & - & 9 & Ah hominol & , , \\
\hline ", & - & . & - & 10 & - Abdominal. & , \\
\hline ", & - & - & - & 11 & & \\
\hline , & - & . & . & 12 & & \\
\hline
\end{tabular}

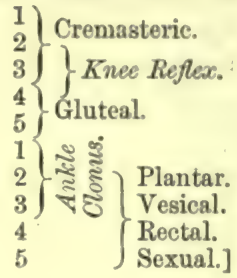

Another important diagnostic reflex is the "abdominal reflex," which consists in this, that when the skin of the abdomen is stroked, e.g., with the handle of a percussion-hammer, the abdominal muscles contract. When this reflex is absent on both sides in a cerebral affection, it indicates a diffuse disease of the brain; its absence on one side indicates a local affection of the opposite half of the brain. The cremasteric, conjunctival, mammillary, pupillary, and nasal reflexes may also be specially investigated. In hemiplegia complicated with cerebral lesions, the reflexes on the paralysed side are diminished, whilst not unfrequently the patellar reflex may be increased. In extensive cerebral affections accompanied by coma the reflexes are absent on both sides, including of course those of the anus and bladder (O. Rasenbach).

[Horsley finds that in the deepest narcosis produced by nitrous oxide gas the superficial reflexes (e.\%., Jlantar, conjunctival) are abolished, while the deep (knee-jerk) remain. Anæmia of the lumbar enlargenent (compression of the abdominal aorta) causes disappearances of both reflexes (Prevost). Chloroform and asphyxia abolish the deep as well as the superficial reflexes. Horsley regards the so-called deep reflex or knee-jerk not as depending on a centre in the cord, but the contraction of the rectus femoris is due to local irritation of the muscle from sudden elongation.]

Deep or Tendon Reflexes.-Under pathological conditions, special attention is directed to the so-called tendon reflexes, which depend upon the fact, that a blow upon a tendon (e.g., the quadriceps femoris, tendo Achilles, \&c.) discharges a contraction of the corresponding muscle (Westphal, Erb, 1875). The patellar tendon reflex (also called "knee phenomenon") or simply "knee-reflex," or "kneejerk," is invariably absent in cases of ataxic tabes dorsalis, while in spastic spinal paralysis it is abnormally strong and extensive (Erb). [The "knee-jerk" is elicited by percussing the ligamentum patellæ, and is due to a single spasm of the rectus. The latent period is 0.03 to 0.04 second, and it is argued by Waller and others that it is doubtful if this tendon-reflex is subserved by a spinal nervous arc, while admitting the effect of the spinal cord in modifying the response of the muscle.] Section of the motor nerves abolishes the patellar phenomenon in rabbits (Schultz), and so does section of the cord opposite the 5th and 6th lumbar vertebræ (Tschirjew). Landois finds that in his own person the contraction occurs 0.048 second after the blow upon the ligamentum patellæ. According to Waller, the patellar reflex and the tendo Achilles reflex occur 0.03 to 0.04 second, and according to Eulenburg, 0.032 second after the blow. According to Westphal, these phenomena are not simple reflex processes, but complex conditions intimately dependent upon the muscle tonus, so that when the tonus of the quadriceps femoris is diminished, the phenomenon is abolished. In order that the phenomenon may take place, it is necessary that the outer part of the posterior column of the spinal cord remain intact (Westphal). [The knee-jerk can be increased or reinforced 
by volitional acts directed to other parts of the body, e.g., by exercising voluntary pressure with the hand (Jendrássik).] [A "jaw-jerk" is obtained by suddenly depressing the lower jaw (Gowers, Beevor, and De Watteville), and the last observer finds that the latent period is 0.02 second, and if this be the case, it is an argument against these so-called "tendon reflexes" being true reflexes, and that they are direct contractions of the muscles due to sudden stimulation by extension.]

[Method.-The knee-jerk is easily elicited by striking the patellar tendon with the edge of the hand or a percussion-hammer when the leg is semi-flexed, as when the legs are hanging over the edge of a table or when one leg is crossed over the other. It is almost invariably present in health, but it becomes greatly exaggerated in descending degeneration of the lateral columns and lateral sclerosis.]

[Ankle clonus is another tendon reflex, and it is never present in health. If the leg be nearly extended, and pressure made upon the sole of the foot so as suddenly to flex the foot at the ankle, a series of ( 5 to 7 per second) rhythmical contractions of the muscles of the calf takes place. Gowers describes a modification elicited by tapping the muscles of the front of the leg, the "front-tap contraction." Ankle clonus is excessive in sclerosis of the lateral columns and spastic paralysis.]

[In "ankle clonus" excited by sudden passive flexion of the foot, there is a multiple spasm of the gastrocnemius. Here also the latent period is about 0.03 to 0.04 second, and the rhythm 8 to 10 per second. This short latent period has led some observers to doubt the essentially reflex nature of this act.]

When we are about to sleep ( $\$ 374$ ), there is first of all a temporary increase of the reflexes ; in the first sleep the reflexes are diminished, and the pupils are contracted. In deep sleep the abdominal, cremasteric, and patellar reflexes are absent; while tickling the soles of the feet and the nose onlyacts when the stimulus is of a certain intensity. In narcosis, e.g., chloroform or morphia, the abdominal, then the conjunctival and patellar reflexes disappear; lastly, the pupils contract (O. Rosenbach).

Abnormal increase of the reflex activity usually indicates an increase of the excitability of the reflex centre, although an abnormal sensibility of the afferent nerve may be the cause. As the harmonious equilibrium of the voluntary movements is largely dependent upon and regulated by the reflexes, it is evident that in affections of the spinal cord, there are frequent disturbances of the voluntary movements, e.g., the characteristic disturbance of motion in attempting to walk, and in grasping movements exhibited by persons suffering from ataxic tabes dorsalis [or, as it is more generally called, locomotor ataxia].

[The organic reflexes include a consideration of the acts of micturition, erection, ejaculation, defæcation, and those connected with the motor and secretory digestive processes, respiration, and circulation.]

362. CENTRES IN THE SPINAL CORD.-Centres capable of being excited reflexly, and which can bring about the discharge of certain complicated, yet well-co-ordinated, motor acts exist in various parts of the spinal cord. They still retain their activity after the spinal cord is separated from the medulla oblongata ; further, those centres lying in the lower part of the spinal cord still retain their activity after being separated from the higher centres, but in the normal intact body, they are subjected to the control of higher reflex centres in the medulla oblongata. Hence, we may speak of them as subordinate spinal centres. The cerebrum also, partly by the production of perceptions, and partly as the organ of volition, can excite or suppress the action of certain of these subordinate spinal centres. [For the significance of the term "Centre," see p. 625.]

1. The cilio-spinal centre connected with the dilatation of the pupil lies in the lower cervical part of the cord, and extends downwards to the region of the 1st to the 3rd dorsal vertebra. It is excited by diminution of light; both pupils always react simultaneously, when one retina is shaded. Unilateral extirpation of this part of the spinal cord causes contraction of the pupil on the same side. The motor fibres pass out by the anterior roots of the two lower cervical and two upper dorsal nerves, into the cervical sympathetic (§ 392). Even the idea of darkness may sometimes, though rarely, cause dilatation of the pupil (Budge). 
In goats and cats, this centre, even after being separated from the medulla oblongata, can be exeited directly by dyspnceic blood, and also reflexly by the stimulation of sensory nerves, e.g., the median, especially when the reflex excitability of the cord is increased by the action of strychnin or atropin (Luchsinger). For the dilator centre in the medulla oblongata, see \& 367,8 .

2. The ano-spinal centre, or centre controlling the act of defæcation. The afferent nerves lie in the hæmorrhoidal and inferior mesenteric plexuses, the centre at the 5 th $(\mathrm{dog})$ or 6 th to 7 th (rabbit) lumbar vertebra; the efferent fibres arise from the pudendal plexus and pass to the sphincter muscles. For the relation of this centre to the cerebrum see $\$ 160$. After section of the spinal cord [in dogs], Goltz observed that the sphincter contracted rhythmically upon the finger introduced into the anus; the co-ordinated activity of the centre therefore would seem to be possible only when the centre remains in connection with the brain.

3. The vesico-spinal centre for regulating micturition, or Budge's vesico-spinal centre. The centre for the sphincter muscle lies at the 5 th $(\mathrm{dog})$ or the 7 th (rabbit) lumbar vertebra, and that for the muscles of the bladder somewhat higher. The centre acts only in a properly co-ordinated way in connection with the brain $(\$ 280)$.

4. The erection centre also lies in the lumbar region (\$436). The afferent nerves are the sensory nerves of the penis; the efferent nerves for the deep artery of the penis are the vaso-dilator nerves, arising from the 1st to 3rd sacral nerves, or Eckhard's nervi erigentes - while the motor nerves for the ischio-cavernosus and deep transverse perineal muscles arise from the 3 rd to 4 th sacral nerves $(\$ 356)$. The latter may also be excited voluntarily, the former also partly by the brain, by directing the attention to the sexual activity. Eckhard observed erection to take place after stimulation of the higher regions of the spinal cord, as well as of the pons and crura cerebri.

5. The ejaculation centre. The afferent nerve is the dorsal of the penis, the centre (Budge's genito-spinal centre) lies at the 4 th lumbar vertebra (rabbit); the motor fibres of the vas deferens arise from the 4th and 5th lumbar nerves, which jlass into the sympathetic, and from thence to the vas deferens. The motor fibres for the bulbo-cavernosus muscle, which ejects the semen from the bulb of the urethra, lie in the 3 rd and 4 th sacral nerves (perineal).

6. The parturition centre lies at the 1 st and 2 nd lumbar vertebræ $(\S 453)$; the afferent fibres come from the uterine plexus, to which also the motor fibres proceed (Körner). Goltz and Freusberg observed that a bitch became pregnant after its spinal cord was divided at the lst lumbar vertebra.

7. Vaso-motor Centres.-Both vaso-motor and vaso-dilator centres are distributed throughout the whole spinal axis. To them belongs the centre for the spleen, which in the dog is opposite the 1 st to 4 th cervical vertebræ (Bulgak). They can be excited reflexly, but they are also controlled by the dominating centre in the medulla oblongata ( $\$ 371)$. Psychical disturbance (cerebrum) influences them (\$377).

[8. Perhaps there are vaso-dilator centres (\$ 372).]

9. The sweat centre is perhaps distributed similarly to the vaso-motor centre (\$288).

The reflex movements discharged from these centres are orderly co-ordinated reflexes, and may thus he compared to the orderly reflexes of the trunk and extremities.

Muscle Tonus. - Formerly automatic functions were ascribed to the spinal cord, one of these being that it caused a moderate active tension of the muscles-a condition that was termed muscle tone, or tonus. The existence of tonus in a striped muscle was thought to be proved hy the faet that, when such a muscle was divided, its ends retracted. This is due merely to the fact that all the museles are stretched slightly beyond their normal longth (§301). Even paralysed muscles, which have lost their muscular tone, show the same phenomenon. Formerly, the stronger contraction of certain muscles, after paralysis of their antagonists, and the retraction of the facial muscles to the sound side, after paralysis of the facial nerve, were 
also regarded as due to tonus. This result is due to the fact that, during the activity of the intact muscles, the other ones have not sufficient power to restore the parts to their normal median position. The following experiment of Auerbach and Heidenhain is against the assumption of a tonic contraction :- If the muscles of the leg of a decapitated frog be stretched, it is found that they do not elongate after section of the sciatic nerve, or after it is paralysed by touching it with ammonia or carbolic acid.

Reflex Tonus.-If, however, a decapitated frog be suspended in an abnormal position, we observe, after section of the sciatic nerve, or the posterior nerve-roots on one side, that the leg on that side hangs limp, while the leg of the sound side is slightly retracted. The sensory nerves of the latter are slightly and continually stimulated by the weight of the limb, so that a slight reflex retraction of the leg takes place, which disappears as soon as the sensory nerves of the leg are divided. If we choose to call this slight retraction tonus, then it is a reflex tonus (Brondgeest). (See the experiments of Harless, C. Ludwig, and Cyon-§355.)

363. EXCITABILITY OF THE SPINAL CORD.-Even at the present time observers are by no means agreed whether the spinal cord, like peripheral nerves, is excitable, or whether it is distinguished by the remarkable peculiarity that most of its conducting paths and ganglia do not react to direct electrical and mechanical stimuli.

It is contended by some observers that if stimuli be cautiously applied either to white or grey matter, there is neither movement nor sensation (Van Deen (1841), Brown-Séquard). Care must be taken not to stimulate the roots of the spinal nerves, as these respond at once to stimuli, and thus may give rise to movements or sensations. As the spinal cord conducts to the brain impulses communicated to it from the stimulated posterior roots, but does not itself respond to stimuli which produce sensations, Schiff has applied to it the term " æasthesodic." Further, as the cord can conduct both voluntary and reflex motor impulses, without, however, itself being affected by motor impulses applied to it directly, he calls it "kinesodic."

Schiff's views are as follows:-

1. In the posterior columns the sensory root-fibres of the posterior root which traverse these columns give rise to painful impressions, but the proper paths of the posterior columns themselves do not do so. The proof that stimulation of the posterior column produces sensory impressions, he finds in the fact that dilatation of the pupil occurred with every stimulation (\$292). Removal of the posterior column produces anæsthesia (loss of tactile sensation). Algesia [or the sensation of pain] remains intact, although at first there may even be hyperalgesia.

2. The anterior columns are non-excitable, both for striped and non-striped muscle, as long as the stimuli are applied only to the proper paths of this column. But movements may follow, either when the anterior nerve-roots are stimulated, or when, by the escape of the current, the posterior columns are affected, whereby reflex movements are produced.

According to Schiff, therefore, all the phenomena of irritation, which oceur when an uninjured cord is stimulated (spasms, contracture), are caused either by simultaneous stimulation of the anterior roots, or are reflexes from the posterior columns alone, or simultaneously from the posterior columns and the posterior roots. Diseases affecting only the anterior and lateral columns alone never produce symptoms of irritation, but always of paralysis. In complete anæsthesia and apnœa, every form of stimulus is quite inactive. According to Schiff's view, all centres, both spinal and cerebral, are inexcitable by artificial means.

Direct Excitability. - Many observers, however, oppose these views, and contend that the spinal cord is excitable to direct stimulation. Fick observed movements to take place when he stimulated the white columns of the cord of a frog, isolated for a long distance so as to avoid the escape of the stimulating currents. Sirotinin, also, who stimulated the transverse section of the frog's cord from point to point, obtained contraction of the muscles both by mechanical and electrical stimuli. Biedermann comes to the following conclusions:- The transverse section of a motor nerve is most excitable. Weak stimuli (descending opening shocks) excite the cut surface of the transversely divided spinal cord, but do not act when applied further down. Luchsinger asserts that, after dipping the anterior part of a beheaded snake into warm water, the reflex movements of the upper part of the cord are abolished, while the direct excitability remains.

3. Excitability of the Vaso-motors. - The vaso-constrictor nerves, which proceed from the vaso-motor centre and run downwards in the lateral columns of the cord, are excitable by all stimuli along their whole course; direct stimulation of 
any transverse section of the cord constricts all the blood-vessels below the point of section (C. Ludurig and Thiry). In the same way, the fibres which ascend in the cord, and increase the action of the vaso-motor centre-pressor fibres, are also excitable (C. Ludurig and Dittmar- $\$ 364,10)$. Stimulation of these fibres, although it affects the vaso-motor centre reflexly, does not cause sensation.

4. Chemical stimuli such as the application of common salt, or wetting the cut surface with blood, appear to excite the spinal cord.

5. The motor centres are directly excited by blood heated above $40^{\circ} \mathrm{C}$., or by asphyxiated blood, or by sudden and complete anrmia of the cord produced by ligature of the aorta (Sigm. Mayer); and also by certain poisons-picrotoxin, vicotin, and compounds of barium (Luchsinger).

Action of Blood and Poisons. - In experiments of this kind, the spinal cord ought to be diviled at the lst lumbar vertebra, at least twenty hours before the experiment is begun. It is well to divile the posterior roots beforehand to avoid reflex movements. If, in a cat thus operateml on, dyspmera be produced, or its blood orerheuted, then spasms, contraction of the vessels, and srcretion of swcut occur in the hind limbs, together with evacuation of the contents of the bluder and rectum, while there are movements of the uterus and the ras deferens. Some poisons act in a similar manuer. In animals with the medulla oblongata divided, rhythmical respiratory movements may be produced if the spinal cord has been previously rendered very sensitive by strychnin or overheated blood (P.v. Rokitansky, v. Schroff-\$368).

The ganglion-cells of the anterior cornu can be excited mechanically (Birge), and, according to Biedermann, the grey matter also responds to electrical stimuli.

Hyperæsthesia.-After unilateral section of the cord, or even only of the posterior or lateral columns, there is hyperæsthesia on the same side below the point of section (Fodira, 1823, and others), so that rabbits shriek on the slightest touch. The phenomenon may last for three weeks, and then give place to normal or sub-normal excitability. On the sound side the sensibility remains permanently diminished. A similar result has been observed in cases of injury in man. An analogrous phenomenon, or a tendency to contraction in the muscles below the section (hyperkinesia), has been observed by Brown-Séquard after section of the anterior columns.

The excitability of the cord is intimately dependent on the continuance of the circulation, for lisature of the abdominal aorta rapidly paralyses the lower extremities (Stenson, 1667), due to anæmia of the cord (Schiffer). Later, the anterior roots of the spinal nerves, and the anæmic part of the grey matter of the cord, undergo degeneration.

364. THE CONDUCTING PATHS IN THE SPINAL CORD.-[Posterior Root.-(a) The inner part, or internal radicular fasciculus is supposed to convey the impressions from tendons and those for touch and locality. When the posteroexternal column is diseased, as in locomotor ataxia, the deep reflexes, especially the patellar tendon reflex, are enfeebled, or it may be abolished, while the implication of the fibres of the internal fasciculus gives rise to severe pain. (b) The outer radicular fibres enter the grey matter of the posterior horn, and are supposed to convey the impressions for cutaneous reflexes and temperature. (c) The central flbres pass directly into the grey matter, and are supposed to conduct painful impressions into the grey matter (fig. 449).]

1. Localised tactile sensations (temperature, pressure, and the muscular sense impressions) are conducted upwards through the posterior roots to the ganglia of the posterior cornu, and lastly into the posterior column of the same side.

In man, the conducting path from the legs runs in Goll's column, while those for the arms run in the ground-bundle (fig. 454) (Flechsig). In rabbits, the path of localised tactile impressions lies in the lower dorsal region in the lateral columns (Ludwig and Woroschiloff, Ott and Meade-Smith).

Anæathesia. - Section of individual parts of the lateral columns abolishes the sensibility for the parts of the skin connected with the part destroyed, while total section produces the same 
result for the whole of the opposite side of the body below the section. The condition where tactile and muscular sensibility is lost is known as anoesthesia.

2. Localised voluntary movements in man are conducted on the same side through the anterior and lateral columns ( $\$ 358$ and 365$)$, in the parts known as the pyramidal tracts. The impulses then pass into the cells of the anterior cornu, and thence to the corresponding anterior nerve-roots to the muscles. The exact section experiments of Ludwig and Woroschiloff showed that, in the lower dorsal region of the rabbit, these paths were confined to the lateral columns. Every motor nervefibre is connected with a nerve-cell in the anterior horn of the frog's spinal cord (Gaule and Birge). Section of one lateral column abolishes voluntary movement in the corresponding individual muscles below the point of section. It is obvious, from the conduction in 1 and 2 , that the lateral columns must increase in thickness and number of fibres from below upwards (Stilling, Woroschiloff) [see fig. 443].

3. Tactile reflexes (extensive and co-ordinated).-The fibres enter by the posterior root, and proceed to the posterior cornu. The groups of ganglionic cells, which control the co-ordinated reflexes, are connected together by fibres which run in the anterior tracts, the anterior ground bundle and (?) the direct cerebellar tracts (p. 633). The fibres for the muscles which are contracted pass from the motor ganglia outwards through the anterior roots.

In ataxic tabes dorsalis, or locomotor ataxia, there is a degeneration of the posterior columns, characterised by a peculiar motor disturbance. The voluntary movements can be executed with full and normal vigour, but the finer harmonious adjustments are wanting or impaired, both in intensity and extent. These depend in part upon the normal existence of tactile and muscular impressions, whose channels.lie in the posterior columns. After degeneration of the latter, there is not only anæsthesia, but also a disturbance in the discharge of tactile reflexes, for which the centripetal arc is interrupted. But a simultaneous lesion of the sensory nerves alone may in a similar manner materially influence the harmony of the movements, owing to the analgesia and the disappearance of the pathic reflexes $(\$ 355)$. As the fibres of the posterior root traverse the white posterior columns, we can account for the disturbances of sensation which characterise the degenerations of these parts (Charcot and Pierret). But even the posterior roots themselves may undergo degeneration, and this may also give rise to disturbances of sensation (p. 618). The sensory disturbances usually consist in an abnormal increase of the tactile or painful sensations, with lightning pains shooting down the limbs, and this condition may lead to one where the tactile and painful sensations are abolished. At the same time, owing to stimulation of the posterior columns, the tactile sensibility is altered, giving rise to the sensation of formication, or a feeling of constriction ["girdle sensation"]. The conduction of sensory impressions is often slowed ( $(337)$. The sensibility of the muscles, joints, and internal parts is altered.

The maintenance of the equilibrium is largely guided by the impulses which travel inwards to the co-ordinating centres through the sensory nerves, special and general, deep and superficial. In many cases of locomotor ataxia, if the patient place his feet close together and close his eyes, he sways from side to side and may fall over, because by cutting off the guiding sensations obtained through the optic nerve, the other enfeebled impulses obtained from the skin and the deeper structures are too feeble to excite proper co-ordination.

4. The inhibition of tactile reflexes occurs through the anterior columns; the impulses pass from the anterior column at the corresponding level into the grey matter, where they form connections with the reflex conducting apparatus.

5. The conduction of painful impressions occurs through the posterior roots, and thence through the whole of the grey matter. There is a partial decussation of these impulses in the cord, the conducting fibres passing from one side to the other. The further course of these fibres to the brain is given in $\S 365$.

If all the grey matter be divided, except a small connecting portion, this is sufficient to conduct painful impressions. In this case, however, the conduction is slower (Schiff). Only when the grey matter is completely divided, is the conduction of painful impressions from below completely interrupted. This gives rise to the condition of analgesia, in which, when the posterior columns are still intact, tactile impressions are still conducted. This condition is sometimes observed in man during incomplete narcosis from chloroform and morphia (Thiersch). Those poisons act sooner on the nerves which administer to painful sensations than on those for tactile impressions, so that the person operated on is conscious of the contact of a knife, but 
not of the painful sensations caused by the knife dividing the parts. As painful impressions are conducted by the whole of the grey matter, and as the impressions are more powerful the stronger the painful impression, we may thus explain the so-called irradiation of painful impressions. During violent pain, the pain seems to extend to wide areas; thus, in violent toothache, proceeding from a particular tooth, the pain may be felt in the whole jaw, or it may be over one side of the head.

According to Bechterew, the paths for the conduction of painful impressions lie in the anterior part of the lateral column (dog, rabbit).

The experiments of Weiss on dogs, ly dividing the lateral column at the limit of the dorsal and lumbar regions, showed that each lateral column contains sensory fibres for both sides. The chief mass of the motor fibres remains on the same side. Section of both lateral columns abolishes completely sensibility and motility on both sides. The anterior columns and the grey matter are not sufficient to maintain these.

6. The conduction of spasmodic, involuntary, inco-ordinated movements takes place through the grey matter, and from the latter through the anterior roots.

It oceurs in epilepsy, poisoning with strychnin, uræmic poisoning, and tetanus $(\S 360$, II. $)$. The ansmic and dyspneie spasms are excited in and conducted from the medulla oblongata, and communicated through the whole of the grey matter.

7. The conduction of extensive reflex spasms takes place from the posterior roots, perhaps to the cells of the posterior cornu and then to the cells of the anterior cornu, above and below the plane of the entering impulse (fig. 458), and, lastly, into the anterior roots, under the conditions already referred to in $\$ 360$, II.

8. The inhibition of pathic reflexes occurs through the anterior columns downwards, and then into the grey matter to the connecting channels of the reflex organ, into which it introduces resistance.

9. The vaso-motor fibres run in the lateral columns (Dittmar), and, after they have passed into the ganglia of the grey matter at the corresponding level, they leave the spinal cord by the anterior roots. They reach the muscles of the bloodvessels either through the paths of the spinal nerves, or they pass through the rami communicantes into the sympathetic, and thence into the visceral plexuses (\$ 356).

Section of the spinal cord paralyses all the vaso-motor nerves below the point of section; while stimulution of the peripheral end of the spinal cord causes contraction of all these vessels. [Ott's experiments on cats show that the vaso-motor fibres run in the lateral columns, and that they as well as the sudorific nerves decussate in the cord.]

10. Pressor fibres enter in the posterior roots, run upwards in the lateral columns, and undergo an incomplete decussation (Ludwig and Miescher).

They ultimately terminate in the dominating vaso-motor centre in the medulla oblongata, which they excite reflexly. Similarly, depressor fibres must pass upwards in the spinal cord, but we know nothing as to their course.

11. From the respiratory centre in the medulla oblongata, respiratory nerves run downwards in the lateral columns on the same side, and after forming connections with the ganglia of the grey matter pass through the anterior roots into the motor nerves of the respiratory muscles (Schiff).

Unilateral, or total destruction of the spinal cord, the higher up it is done, accordingly paralyses more and more of the respiratory nerves, on the same or on both sides. Section of the eord alove the origin of the phrenic nerves causes death, owing to the paralysis of these nerves of the diaphragm (\& 113).

In pathological cases, in degeneration of, or direct injury to, the spinal cord or its individual parts, we must be careful to observe whether there may not be present simultaneously paralytic and irritative phenomena, whereby the symptoms are obscured.

[Complete transverse section of the cord results immediately in complete paralysis of motion and sensation in all the parts supplied by nerves below the seat of the injury, although the muscles below the injury retain their normal trophic and electrical conditions. There is a narrow hyperæsthetic area at the upper limit of the paralysed area, and when this occurs in the dorsal region, it gives rise to the feeling of a belt tightly drawn round the waist, or the "girdle sensation." There is also vaso-motor paralysis below the lesion, but the blood-vessels soon regain their 
tone, owing to the subsidiary vaso-motor centres in the cord. The remote effects come on much later, and are secondary descending degeneration in the crossed and direct pyramidal tracts and ascending degeneration in the postero-internal columns (fig. 455). According to the seat of the lesion, the functions of the bladder and rectum may be interfered with. Injury to the upper cervical region sometimes causes hyperpyrexia.]

[Unilateral section results in paralysis of voluntary motion in the muscles supplied by nerves given off below the seat of the injury, although the muscles do not atrophy, but when secondary descending degeneration occurs they become rigid, and exhibit the ordinary signs of contracture. There is vaso-motor paralysis on the same side, although this passes off below the injury, while the ordinary and muscular sensibility are diminished on both sides (fig. 459). There is bilateral anæsthesia. On the opposite side there is total anæsthesia and analgesia below the lesion, but on the same side in the dorsal region there is a narrow circular anæsthetic zone (fig. $459, b$ ), corresponding to the sensory nerve-fibres destroyed at the level of the section. The sensory nerves decussate shortly after they enter the cord, hence the anæsthesia on the opposite side, but they do not cross at once, but run obliquely upwards before they enter the grey matter of the opposite side, so that a unilateral section will involve some fibres coming from the same side, and hence the slightly diminished sensibility in a circular area on the same side. There is a narrow hyperæsthetic area on the same side as the lesion, at the upper limit of the paralysed cutaneous area (fig. 459, c), due perhaps to stimulation of the cut ends of the sensory fibres on that side. In man there is hyperæsthesia (to touch, tickling, pain, heat, and cold) on the parts below the lesion on the same side, but the cause of this is not known. The remote effects are due to the usual descending and ascending degeneration which set in.]

[In monkeys, after hemi-section of the cord in the dorsal region, there is paralysis of voluntary motion and retention of sensibility with vaso-motor paralysis of the same side, and retention of voluntary motion with anæsthesia and analgesia on the opposite side. The existence of hyperæsthesia on the side of the lesion is not certain in these animals, but there is no doubt of it in man. Ferrier also finds (in opposition to

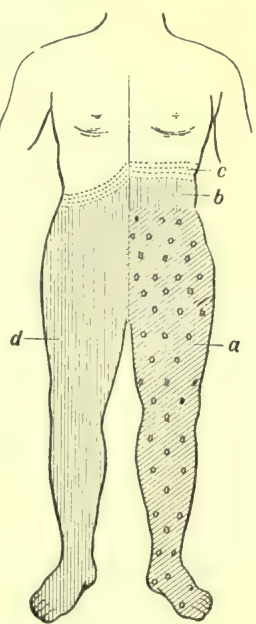

Fig. 459 .

Diagrammatic representation of a lesion of the left half of the spinal cord in the dorsal region. (a) obliqua lines, motor and vaso-motor paralysis ; $(b, d)$, com plete anæsthesia ; $(a$, c), hyperæsthesia of the skin.

Brown-Séquard) that the muscular sense is paralysed as well as all other forms of sensibility, on the side opposite to the lesion, but unimpaired on the side of the lesion. The muscular sense, in fact, is entirely separable from the motor innervation of muscle (Ferrier). The power of emptying the bladder and rectum was not affected.]

\section{The Brain.}

365. GENERAL SCHEMA OF THE BRAIN. - In an organ so complicated in its structure as the brain, it is necessary to have a general view of the chief arrangements of its individual parts. Meynert gave a plan of the general arrangement of this organ, and although this plan may not be quite correct, still it is useful in the study of brain function. The weight of the brain is in man about 1358 grammes, and in woman 1220 grammes (Bischoff).

[A special layer of grey matter of the cerebrum is placed externally and spread as a thin coating over the white matter or centrum ovale - which lies internally, and consists of nervefibres or the white matter. That part lying in each hemisphere is the centrum semi-ovale. The grey matter is folded into gyri or convolutions separated from each other by fissures or sulci. Some of the latter are very marked, and serve to separate adjacent lobes, while the lobes themselves are further subdivided by sulci into convolutions. For a description of the lobes see $\S 375$. Some masses of grey matter are disposed at the base of the brain, forming the 
corpus striatum (projecting into the lateral ventricles), which in reality is composed of two parts, the nucleus caudatus and lenticular nucleus (fig. $460, b$ ), the optic thalamus which lies behind the former, and bounds the $3 \mathrm{rd}$ ventricle (fig. $460, d$ ), the corpora quadrigemina lying on the upper surface of the crura cerebri (fig. 480, hi); within the tegmentum of the crura cerebri are the red nucleus and locus niger (fig. 502). Lastly, there is the continuation of the grey matter of the cord up through the medulla, pons, and around the iter, forming the central grey tube and terminating anteriorly at the tuber cinereum. These various parts are connected in a variety of ways with each other, some by transverse fibres stretching between the two sides

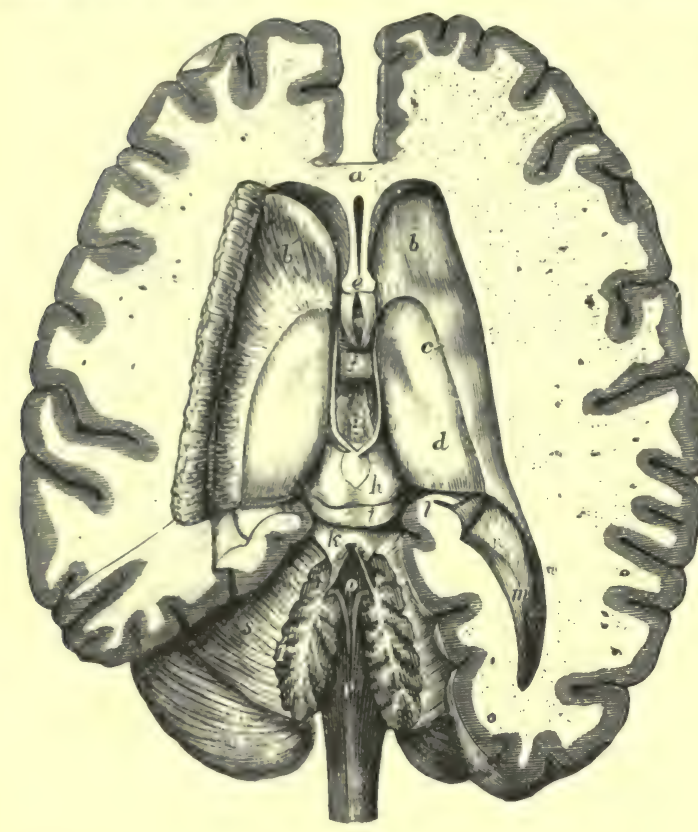

Fig. 460 . of the brain, while other longitudinal fibres bring the hinder and lower parts into relation with the fore parts.]

[Under cover of the occipital lobes, but connected with the cerebrum in front, and the spinal cord below, is the cerebellum, which has its grey matter externally and its white core inter. nally. Thus we have to consider cerebro-spinal and cerebello-spinal connections.]

Meynert's Projection Systems. - The cortex of the cerebrim consists of convolutions and sulci, the "peripheral grey matter" (fig. $461, \mathrm{C}$ ), which is recognised as a nervous structure, from the presence in it of numerous gan. glionic cells $(\$ 358,1)$. From it proceed all the motor fibres which are excited by the will, and to it proceed all the fibres coming from the organs of special sense and sensory organs, which give rise to the psychical perception of external impressions. [In fig. 461 the decussation of the sensory fibres is represented as occurring near the medulla oblongata. It is more probable that a large number of the sensory fibres decussate shortly after they enter

Dissection of the brain from above, showing the lateral, 3rd, and 4 th ventricles, with the basal ganglia, and surrounding parts. $a$, knee of the corpus callosum; $b$, anterior part of the right corpus striatum; $b^{\prime}$, grey matter dissected off to show white fibres; $c$, points to trenia semicircularis; $d$, optic thalamus ; $e$, anterior pillars of fornix, with 5 th ventricle in front of them, between the two laminæe of the septum lucidum ; $f$, niddle or soft commissure; $g$, 3rd ventricle; $h, i$, corpora quadrigemina ; $k$, superior cerebellar peduncle; $l$, hippocampus major ; $m$, posterior cornı of lateral ventricle; $n$, eminentia collateralis; 0,4 th ventricle; $p$, medulla oblongata ; $s$, cerebellum, with $r$, arbor vite.

the cord, as is represented in fig. 463. Some observers assert that some of the sensory fibres decussate in the medulla oblongata.]

First Projection System.-The channels lead to and from the cortex cerebri, some of them traversing the basal ganglia, or ganglia of the cerebrum-the corpus striatum (C.s) (composed of the caudate nucleus and lenticular nucleus (N.l), ) optic thalamus (T.o), and corpora quadrigemina -some fibres form connections with cells within this central grey matter. The fibres which proceed from the cortex through the corona radiata in a radiate direction constitute Meynert's first projection system. Besides these, the white substance also contains two other systems of fibres:- $(a)$ Commissural fibres, such as the corpus callosum and the anterior commissure $(c, c)$, which are supposed to counect the two hemispheres with each other; and $(b)$ a connecting or association system, whereby two different areas of the same side are connected together $(\alpha, \alpha)$. The ganglionic grey matter of the basal ganglia forms the first stage in the course of a large number of the fibres. When they enter the central grey matter, they are interrupted in their course. According to Meynert, the corona radiata contains bundles of fibres from the corpus striatum $(1,1)$, lenticular nucleus $(2,2)$, optic thalamus $(3,3)$, and corpora quadrigemina $(4,4)$.

The second projection system consists of longitudinal bundles of fibres, which proceed downwards and reach the so-called "central grey tube," which is the ganglionic grey matter reaching 
from the 3rd ventricle through the aqueduct of Sylvins, and the medulla oblongata, to the lowest part of the grey matter of the spinal cord. It lines the inner surface of the medullary tube. It is the second stage in the course of the fibres extending from the basal ganglia to the

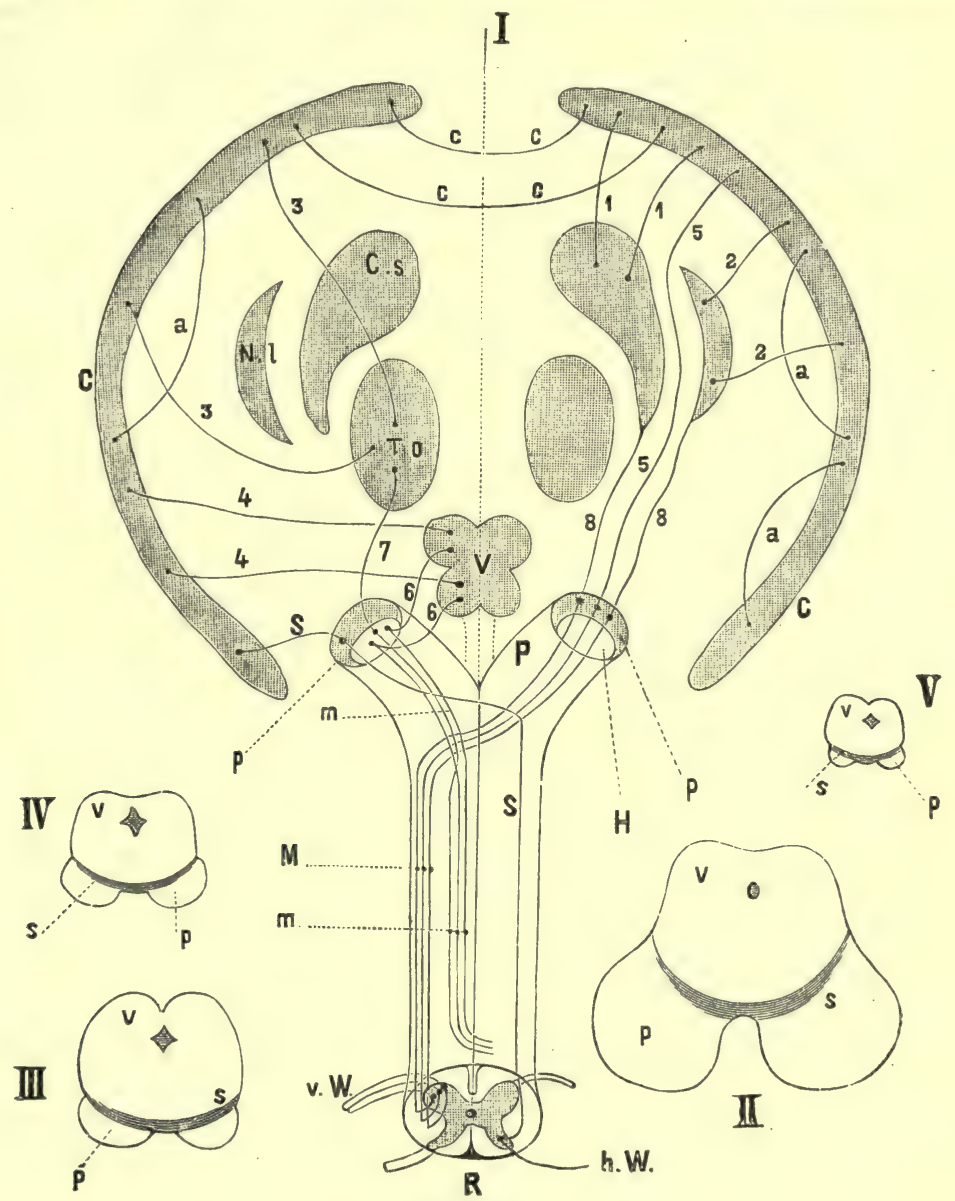

Fig. 461 .

I, Scheme of the brain.-C, C, cortex cerebri ; C.s, corpus striatum; N.l, nucleus lenticularis; T.o, optic thalamus; $v$, corpora quadrigemina; $\mathrm{P}$, pedunculus cerebri; $\mathrm{H}$, tegmentum ; and $p$, crusta ; 1,1 , corona radiata of the corpus striatum ; 2,2 , of the lenticular nucleus ; 3,3 , of the optic thalamus ; 4,4 , of the corpora quadrigemina ; 5 , pyramidal fibres from the cortex cerebri (Flechsig) ; 6, 6, fibres from the corpora quadrigemina to the tegmentum; $m$, further course of these fibres; 8,8 , fibres from the corpus striatum and lenticular nucleus to the crusta of the pedunculus cerebri ; M, further course of these; S, S, course of the sensory fibres; $\mathrm{R}$, transverse section of the spinal cord ; $v . \mathrm{W}$, anterior, and $h$.W, posterior roots ; $a, a$, association system of fibres; $c, c$, commissural fibres. II, Transverse section through the posterior pair of the corpora quadrigemina and the pedunculi cerebri of man. $-p$, crusta of the peduncle ; $s$, substantia nigra ; $v$, corpora quadrigemina, with a section of the aqueduct. III, The same of the dog; IV, of an ape; V, of the guinea-pig. [See p. 650.]

central tubular grey matter. The fibres of this system must obviously vary greatly in length ; some fibres end in the central grey matter above the medulla oblongata, e.g., in the oculomotor nucleus, while others reach to the level of the last spinal nerves. In the central grey matter, 
not only is the course of the fibres interrupted, but there is in it an increase in the number of fibres, for far more fibres proceed peripherally from the grey matter of the medulla and spinal cord, than are sent to it from the central grey matter of the brain.

$\mathrm{As}$ to the arrangement of the fibres in this second system, the fibres descending from the candate and lenticular nucleus $(8,8)$ are grouped into a special channel, which descends through the crusta of the cerebral peduncle, and enters the medulla oblongata, or (according to Flechsig) the pons. In the same way there proceeds from the thalamus (S) and corpora quadrigemina $(6,6)$ a bundle which descends through the tegmentum $(\mathrm{H})$ of the cerebral peduncle. Both sets of fibres--those in the crusta and in the tegmentum-come together in the cord.

Accorling to Wernicke, the lenticular nucleus and caudate nucleus are not the parts of the brain into which, from the cerebral cortex and through the corona, radiate fibres enter; but they are independent parts, analogous to the cortex, and from them fibres proceed. These filures pass into the crusta and run along with those fibres proceeding from the thalamus and corpora quadrigemina.

Acerling to Meynert, the fibres which pass from the thalamus and corpora quadrigemina, through the tegmentum of the cerebral peduncle, are reflex channels; so that these portions of the lirain are centres for certain extensive, co-ordinated reflexes. This is shown by the fact that, after destruction of the voluntary motor paths, in animals, the technical completeness of

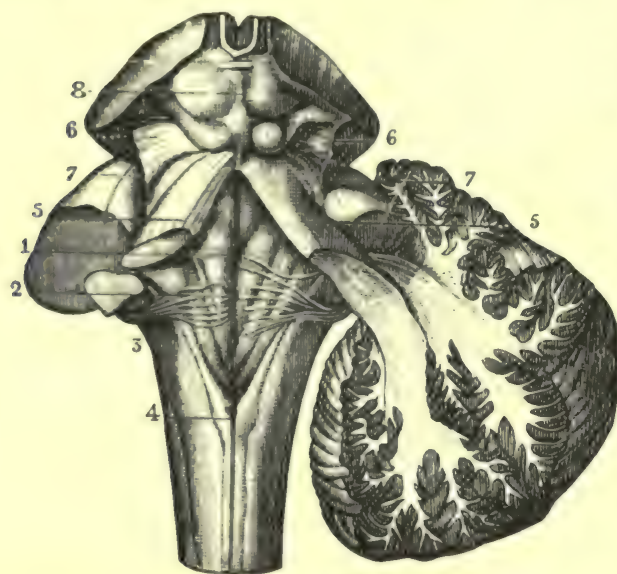

Fig. 462.

Floor of the 4 th ventricle and the connections of the cerebellum. On the left side the three cerebellar peduncles are cut short ; on the right the connections of the superior and inferior peduncles have been preserved, while the middle one has been cut short. 1, median groove of the 4 th ventricle with the fasciculi teretes; 2 , the striæ of the auditory nerve on each side emerging from it; 3 , inferior peduncle; 4 , posterior pyramid and clava, with the calamus scriptorius above it; 5 , superior peduncle; 6 , fillet to the side of the crura cerebri ; 8 , corpora quadrigemina.

nucleus, and a number of these fibres decussate in part of the cerebellum and from its dentate tum, some of then becoming connected with the red nucleus in the tegmentum of the opposite side. Some of the fibres seem to connect the cerebellum with the frontal lobes, constituting a fronto-cerebellar tract, and they are also crossed (Gowers). When the cerebellum is congenitally absent, these fibres are absent (Flechsig). By the two inferior peduncles or restiform bodies, it is connected with all the columns of the spinal cord, and it is to be noted that some of the fibres forming these peduncles are connected with the olivary body of the opposite side, so that they decussate. The middle peduncle is formed by the transverse fibres of the pons (figs. $462,503)$. It is evident that there is a cerebello-spinal, as well as a cerehro-spinal connection to be considered.]

[The grey matter is external and the white internal, and on section the foliated branched appearance of the cerebellum constitutes the arbor vitoe. Within each lateral lobe is a folded mass of grey matter like that in the olivary body, called the corpus dentatum, and from its 
interior white fibres proceed. Stilling describes in the front part of the middle lobe roof-nuclei - so called because they lie in the roof of the 4th ventricle. As is shown in fig. 462, the white fibres of the superior peduncle pass to the grey matter on the inferior surface of the cerebellum, while the inferior peduncular fibres pass to the superior surface, chiefly of the median part; but both are said to form connections with the corpus dentatum; the middle peduncle is connected with the grey matter of the lateral lobes. The minute structure is described in $\$ 380$.]

The distribution of the blood-vessels of the brain is of much practical importance. The middle cerebral artery of the Sylvian fissure supplies the motor areas of the brain in animals; in man, the paracentral lobule is supplied by the anterior cerebral artery (Duret). The region of the third left frontal convolution, which is the speech-centre, is supplied by a special branch of the middle cerebral. According to Ferrier, that part of the brain, any injury to which causes disturbance of intelligence, is supplied by the anterior cerebral; while those regions, where injury is followed by hemi-anæsthesia, are supplied by the posterior cerebral. It is stated that anæmia of isolated parts of this area of the brain is associated with melancholia in man.

Conduction to and from cerebrum-Voluntary motor fibres.-The course of the fibres which convey impulses for voluntary motion-the pyramidal tracts - proceeds from the motor regions of the cerebrum (\$§ 375,378, I.), passing into and through the white matter of the cerebrum through the corona radiata, and converges to the internal capsule, which lies between the nucleus caudatus and opticus thalamus internally and the lenticular nucleus externally (fig. 500). [The motor fibres for the face and tongue occupy the knee of the capsule (F), those for the arm the anterior third of the posterior segment or limb (A), and those for the leg the middle third (L). They pass beneath the optic thalamus, enter the crusta of the cerebral peduncle, and occupy its middle third, or two-fifths, extending almost to the substantia nigra, the fibres for the face being next the middle line, and those for the leg most external, the fibres for the arm lying between the two. They pass into the pons on the same side, where the fibres for the face (and tongue) cross to the opposite side, to become connected with the nuclei from which the facial and hypoglossal nerves arise. The fibres for the arm, and leg (and trunk) continue their course to the medulla oblongata, where they form the anterior pyramids. In the pons, the pyramidal tracts are broken up into bundles lying between its superficial and deep transverse fibres, and surrounded by grey matter (fig. 503); but they have no connection with the grey matter of the pons. By far the greater proportion of the fibres cross at the decussation of the pyramids to form the crossed pyramidal tracts, or lateral pyramidal tracts, of the lateral column of the opposite side. The small uncrossed portion is continued as the direct pyramidal tract on the same side. The latter fibres, perhaps, supply those muscles of the trunk (e.g., respiratory, abdominal, and perineal), which always act together on both sides. According to other observers, however, they cross to the other side of the cord through the anterior white commissure, and descend in the crossed pyramidal tract or pyramidal tract of the lateral column. The fibres of the pyramidal tracts split up into fine fibrils, which form connections with the fibrils produced by the subdivision of the processes of the multipolar nerve-cells. Thus, fibres form connections with the multipolar ganglionic cells of the anterior cornu of the grey matter of the spinal cord at successively lower levels, and from each multipolar cell is directed peripherally a single unbranched process, which ultimately becomes a nerve-fibre. The pyramidal tracts thus end in the multipolar nerve-cells of the grey matter of the spinal cord, from which the anterior roots of the spinal nerves arise.

[The course of the pyramidal tracts and the decussation of these fibres in the medulla oblongata, explain why a hæmorrhage involving the cerebral motor centres, or affecting these fibres in any part of their course above the decussation, results in paralysis of the muscles supplied by the fibres so involved on the opposite side of the body. In their passage through the brain, the paths for direct motor impulses are not interrupted anywhere in their course by ganglion cells, not even in the corpus striatum or pons. They pass in a direct uninterrupted line, until each fibre 
becomes connected with a multipolar nerve-cell in the anterior horn of the grey matter of the spinal cord, so that they have the longest course of any fibres in the central nervous system.]

Variation in Decussation. - There are variations as to the number of fibres which cross at the pyramils (Flechsig). In some cases the usual arrangement is reversed, and in some rare instances there is no decussation, so that the pyramidal tracts from the brain remain on the same side, In this way we may explain the very rare cases where paralysis of the voluntary movements takes place on the same side as the lesion of the cerebrum (Morgagni, Pierret). This is direct paralysis. [Usually about 90 per cent. of the fibres decussate.]

The motor cranial nerves have the centres through which they are excited voluntarily in the cortex cerebri (\$378). The paths for such voluntary impulses also pass through the internal capsule and the crusta of the cerebral peduncle. [In the internal capsule, the fibres for the face (and tongue) lie in the knee, while they occupy the part of the middle of the crusta next the middle line. Their course is then directed across the middle line to their respective nuclei, from which fibres proceed to the muscles supplied by these nuclei.] The exact course of many of the fibres is still unknown. The hypoglossal nerve runs with the pyramidal tracts, and behaves like the anterior root of a spinal nerve (\$354,357).

[Sensory Paths.-Our knowledge is by no means precise. Sensory impulses, passing into the cord, enter it by the posterior nerve-roots, and may pass to the cerebrum or cerebellum. If to the cerebellum, the course, probably, is partly to the direct cerebellar tract and posterior column to the restiform body, thence to the cerebellum. If to the cerebrum, they cross the middle line in the cord not far above where they enter and pass to the lateral column, in front of the pyramidal tract. Some enter the posterior column, and others ascend in the grey matter to pass upwards. As the two subdivisions of the posterior column terminate above in the nuclei of the funiculus gracilis and funiculus cuneatus, and this column contains fibres from the posterior root, it is suggested that above the clava and cuneate nucleus the fibres cross in the superior pyramidal decussation to reach the pons and tegmentum. In the medulla, it is probable that those fibres which do not decussate there do so in the pons, the impulses perhaps travelling upwards in the formatio reticularis, thence, into the posterior half of the pons, into the tegmentum of the crus under the corpora quadrigemina, to enter the posterior third of the posterior limb of the internal capsule (fig. 500, S). But, of course, the sensory fibres from the face have to be connected with the sensory centres in the cerebrum, so that the sensory paths from the cord, i.e., from the trunk and limbs, are joined by those from the face in the pons, and they also occupy part of the posterior third of the posterior segment of the internal capsule, so that this important part of the internal capsule conducts sensory impulses from the opposite half of the body. Some of the fibres pass into the optic thalamus, and others enter the white matter of the cerebrum, but their exact course is very uncertain. The sensory fibres derived from the organs of special sense, e.g., the ear, go to the superior temporosphenoidal convolution, but whether directly or indirectly we do not know ; perhaps some of those for vision traverse the optic thalamus. Some of the afferent fibres perhaps go to the occipital region, and Gowers asserts that some of them go to the parietal and central regions, i.e., to the "motor" regions, for he holds "that disease of the motor cortex often causes impairment of the tactile sensibility."]

[Charcot has called the posterior third of the posterior segment of the internal capsule, lying between the posterior part of the lenticular nucleus and the optic thalamus, the "carefour sensitiv" or "sensory crossway" (fig. 500, S). If it be divided there is hemi-anæsthesia of the opposite side.]

Sensory Decussation in Cord.-As the greater part of the sensory fibres from the skin decussate in the spinal cord, and thus pass to the opposite side of the cord (fig. 463), unilateral section of the spinal cord in man (and monkey-Ferrier) 
abolishes sensibility on the opposite side below the lesion. There is hyperæsthesia of the parts below the seat of the section on the side of the injury ( $\$ 363$ ). From experiments on mammals, Brown-Séquard concludes that the decussating sensory nerve-fibres pass to the opposite side within the cord at different levels, the lowest being the fibres for touch, then those for tickling and pain, and, highest of all, those which administer to sensations of temperature.

All the fibres, therefore, which connect the spinal cord with the grey matter of the brain, undergo a complete decussation in their course. Hence, in man a destructive affection of one hemisphere usually causes complete motor paralysis and loss of sensibility on the opposite side of the body. The fibres proceeding from the nuclei of origin of the cranial nerves also cross within the cranium.

Not unfrequently the motor paralysis and anæsthesia occur on the same side of the head, in which case the lesion (due to pressure or inflammation) involves the cranial nerves lying at the base of the brain.

The positions of decussation are (1) in the spinal cord, (2) in the medulla oblongata, and lastly (3) in the pons. The decussation is complete in the peduncle.

Alternate Paralysis. - Gubler observed that unilateral injury to the pons caused paralysis of the facial nerve on the same side, but paralysis of the opposite half of the body. $\mathrm{He}$ concluded that the nerves of the trunk decussate before they reach the pons, while the facial fibres decussate within the pons. To these rare cases the name "alternate hemiplegia" is given. [When hæmorrhage takes place into the lower part of the lateral half of the pons, there may be alternate paralysis, but when the upper part of the lateral half is injured, the facial is paralysed on the same side as the body, § 379.]

The olfactory nerve is said not to decussate (?), while the optic nerve undergoes a partial decussation at the chiasma (\$344). Some observers assert that the fibres of the trochlearis decussate at their origin.

366. THE MEDULLA OBLONGATA.-[Structure.-In the medulla oblongata, the fibres from the cord are rearranged, the grey matter is also much changed, while new grey matter is added. Each half of the medulla oblongata consists of the following parts, from before backwards :- The anterior pyramid, olivary body, restiform body, and posterior pyramid, or funiculus gracilis (figs. 464, 465, 466). By the divergence of the posterior pyramids and the restiform bodies, the floor of the 4 th ventricle is exposed. As the central canal of the cord gradually comes nearer to the posterior surface of the medulla, it opens into the 4 th ventricle. At the lower end of the medulla oblongata, on separating the anterior pyra-

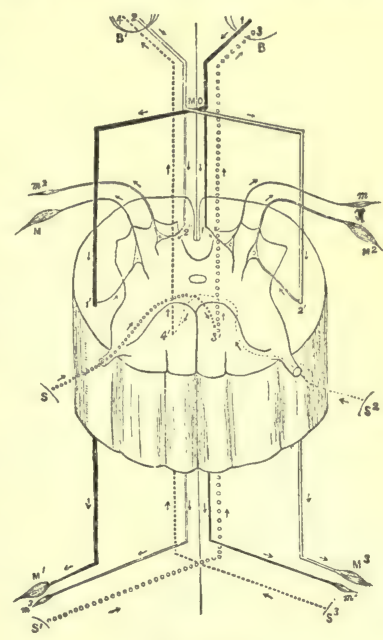

Fig. 463.

Diagram of a spinal segment as a spinal centre and conducting medium. $\mathrm{B}$, right, $\mathrm{B}$, left cerebral hemisphere; MO, lower end of medulla oblongata; 1 , motor tract from the right hemisphere, the larger part decussating at $\mathrm{MO}$, and passing down the lateral column of the cord on the opposite side to the muscles $\mathrm{M}$ and $\mathrm{M}^{\prime} ; 2$, motor tract from the left hemisphere; S, S', sensitive areas on the left side of the body; 3 , 3, the main sensory tract from the left side of the body -it decussates shortly after entering the cord ; $\mathrm{S}^{2}, \mathrm{~S}^{3}$, sensitive areas, and $4^{\prime}, 4$, tracts from the right side of the body. The arrows indicate the direction of theimpulses (Bramwell). [Here all the sensory fibres are shown as erossing in the cord.] mids, we may see the decussation of the pyramids, where the fibres cross over to the lateral columns of the cord. The anterior pyramid receives the direct pyramidal tract of the anterior column of the cord from its own side, and the crossed pyramidal tract from the lateral column of the cord of the opposite side (fig. 464). The decussating fibres (crossed pyramidal tract) of the lateral column pass across in bundles to form the decussation of the pyramids. Most of the pyramidal fibres pass through the pons directly to the cerebrum, a few fibres pass to the cerebellum, while some join fibres proceeding from the olivary body to form the olivary fasciculus or fillet.]

[Thus, only a part of the anterior column of the cord-direct pyramidal tract-is continued into the anterior pyramid, where it lies external to the fibres which pass to the lateral column of the opposite side. The remainder of the anterior column - the antero-external fibres-are continued upwards, but lie deeper under cover of the anterior-pyramid, where they serve to form part of the formatio reticularis (p. 658).] 
[Of the fibres of the latcral column of the cord, some, the direct cerebellar tract, pass backwards to join the restiform bolly and go to the cerebellum. These fibres lie as a thin layer on the surface of the restiform body. The crossed pyramidal fibres cross obliquely, at the lower end of the medulla, to the anterior pyramid of the opposite side, and in their course they traverse the grey matter of the anterior cornu (fig. 464, py). These fibres form the larger and mesial portion of the anterior pyramid. The remaining fibres of the lateral columns are continued upwards, and pass beneath the olivary body, where they are concealed by this structure and also by the arcuate fibres, but they appear in the floor of the medulla oblongata and are here known as the fusciculus teres, which goes to the cerebrum. As they pass upwards, they help to form the lateral part of the formatio reticularis.]

[The posterior pyramid of the oblongata is merely the upwarl continuation of the posteromedian column, or funiculus gracilis of the cord. As it passes upwards at the medulla it broadens

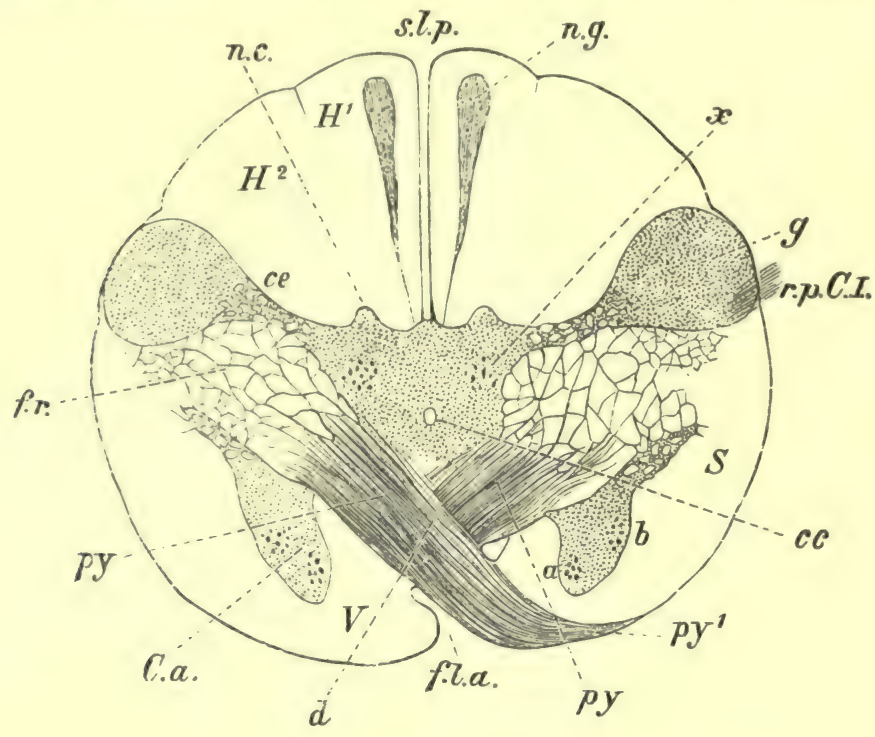

Fig. 464

Section of the decussation of the pyramids. $f a$, anterior median fissure, displaced laterally by the fibres decussating at $d ; V$, anterior column ; $C a$, anterior cornu, with its nerve-cells, $a, b ; c c$, central canal ; $S$, lateral column; $f r$, formatio reticularis ; $c e$, neck, and $g$, head of the posterior cornu ; rpCl, posterior root of the 1st cervical nerve; $n c$, first indication of the nucleus of the funiculus cuneatus; $n g$, nucleus (clava) of the funiculus gracilis ; $H^{1}$, funiculus gracilis; $H^{2}$, funiculus cuneatus; $s l p$, posterior median fissure; $x$, groups of ganglionic cells in the base of the posterior cornu. $\times 6$.

out, forming the clava, which tapers away above. The clava contains a mass of grey matterthe clavate nucleus.]

[The restiform body consists chiefly of the upward continuation of the postero-external column or funiculus cuneatus of the cord. It contains a mass of grey matter, called the cuneate or triangular nucleus. A bove the level of the clava, the funiculus cuneatus forms part of the lateral boundary of the 4 th ventricle. Immediately outside this, i.e., between it and the continuation of the posterior nerve-roots, is a longitudinal prominence, which Schwalbe has called the funiculus of Rolando. It is formed by the head of the posterior cornu of grey matter coning nearer the surface. It also forms part of the restiform body. Some arcuate fibres issue from the anterior median fissure, turn transversely outwards over the anterior pyramids and olivary body, and pass along with the funiculus cuneatus, the funiculus of Rolando, and the direct cerebellar fibres, to enter the corresponding lateral lobe of the cerebellum, all these structures forming its inferior peduncle. Some observers suggest that the funiculus cuneatus and funiculus of Rolando do not pass into the cerebellum.]

[The olivary body forms a well-marked oval or olive-shaped body, which does not extend the whole length of the medulla (fig. $466, o$ ). Above, it is separated from the pons by a gronve from which the 6th nerve emerges. In the groove between it and the anterior pyramid arise 
the strands of the hypoglossal nerve, while in a corresponding groove along its outer surface is the line of exit of the vagus, glosso-pharyngeal, and spinal accessory nerves. It is covered on its surface by longitudinal and arcuate fibres, while in its interior it contains the dentate nucleus. ]

[The functions of the olivary bodies are quite unknown, but it is important to remember that they are connected by fibres with the dentate nuclei of the cerebellum. Fibres pass into the olivary body from the posterior column of the cord of the opposite side, and it is also connected with the dentate body of the opposite side, while, as we know, the dentate body is connected with the tegmentum, so that through the left dentate body of the opposite side, the tegmentum of, say, the right crus, is connected with the right olivary body (Gowers).]

[Decussation of the pyramids is the term given to those fibres which cross obliquely in several bundles, at the lower part of the medulla, from the anterior pyramid of the medulla

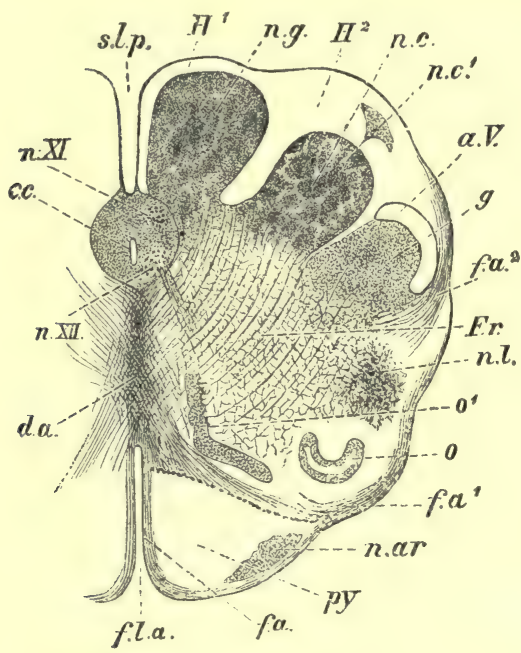

Fig. 465 .

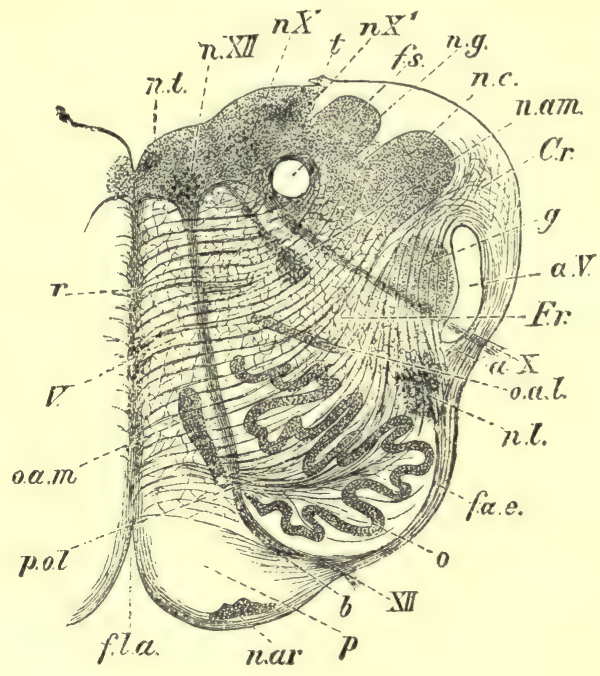

Fig. 466.

Fig. 465. -Section of the medulla oblongata at the so-called upper decussation of the pyramids. $f l a$, anterior, $s l p$, posterior median fissure ; $n X I$, nucleus of the accessorius vagi ; $n X I I$, nucleus of the hypoglossal ; $d a$, the so-called superior or anterior decussation of the pyramids; $p y$, anterior pyramid ; n.ar, nucleus arciformis; $O^{1}$, median parolivary body ; $O$, beginning of the nucleus of the olivary body; $n l$, nucleus of the lateral column ; Fr, formatio reticularis; $g$, substantia gelatinosa, with $(a V)$ the ascending root of the trigeminus; $n c$, nucleus of the funiculus cuneatus; $n c^{1}$, external nucleus of the funiculus cuneatus; $n g$, nucleus of the funiculus gracilis (or clava); $H^{1}$, funiculus gracilis ; $H^{2}$, funiculus cuneatus ; $c c$, central canal ; $f a, f a^{1}, f a^{2}$, external arciform fibres $\times 4$. Fig. 466.Section of the medulla oblongata through the olivary body. nXII, nucleus of the hypoglossal ; $n X, n \mathrm{X}^{1}$, more or less cellular parts of the nucleus of the vagus ; $X I 1$, hypoglossal nerve; $X$, vagus; $n$.am, nucleus ambiguus; $n l$, nucleus lateralis; $o$, olivary nucleus ; oal, external, and oam, internal parolivary body ; $f s$, the round bundle, or funiculus solitarius ; $C r$, restiform body ; $p$, anterior pyramid, surrounded by arciform fibres ; fae, pol, fibres proceeding from the olive to the raphe (pedunculus olivæ); $r$, raphe. $\times 4$.

into the lateral column of the cord of the opposite side (fig. 464, d) to form its lateral pyramid tracts, or crossed pyramidal tracts. The number of fibres which decussate varies, and in some cases all the fibres may cross.]

[The grey matter of the medulla is largely a continuation of that of the cord, although it is arranged differently. As the fibres from the lateral column of the cord pass over to form part of the anterior pyramid of the medulla on the opposite side, they traverse the grey matter, and thus cut off the tip of the anterior cornu, which is also pushed backwards by the olivary body, and exists as a distinct mass, the nucleus lateralis (fig. 465, nl). Part of the anterior grev matter also appears in the floor of the 4th ventricle as the eminence of the fasciculus teres, and from part of it springs the hypoglossal nerve (fig. 466, XII). The neck joining the modified anterior and posterior cornua is much broken up by the passage of longitudinal and transverse 
fibres through it, so that it forms a formatio reticularis, separating the two cornua (fig. $465, f r$ ). The caput cornu posterioris comes to be covered higher up by the ascending root of the 5 th nerve (fig. 465 , $a V$ ), and arcuate fibres jassing to the restiform body. The posterior cornu is also broken up and is thrown outwards, its caput giving rise to part of the elevation seen on the surface and described as the funiculus of Rolando, while part of the base now greatly enlarged forms the grey matter in the funiculus gracilis [elavate nucleus] (fig. 464 , ng) and funiculus cuneatus [cuncate or triangular nucleus] (fig. $464, n c$ ). Nearer the middle line, the grey matter of the pusteriur grey cornu appears in the floor of the 4th ventricle, above the point where the central caual opens into it, as the nuclei of the spinal accessory, vagus, and glosso-pharyngeal nerves.]

[I the floor of the th ventricle near the raphe, and quite superficial, is a longitudinal mass of lare. multipular nerve-cells, lerived from the base of the anterior cornu from which spring the s.vial bumlles forming the hypoglossal nerve; it is the hypoglossal nucleus (fig. $466, n X I 1$ ), the nerve-tibres passing obliquely outwards to apjear between the anterior pyramid and the wlivary body. Internal to it, and next the median groove, is a small mass of cells continuous with these in the raphe, and called the nucleus of the funiculus teres (fig. 466, nt). Around the central canal at the lower part of the medulla is a group of cells (fig. $466, n X 1$ ), which beromes displaced laterally as it comes nearer the surface in the floor of the medulla oblongata, where it lies outside the hypoglossal nucleus, and corresponds to the prominence of the ala (1urea fig. f(it, $n \boldsymbol{X}$; ; and from it and its continuation upwards arise from below upwards part of the sinal accessury $(11 \mathrm{th})$, and the vagus (10th, corresponding to the position of the "Hinentia cincrea-fig. $\left.466, I^{\prime}\right)$, so that this column of cells forms the vago-accessorius nucleus. External to and in front of this is the nucleus for the glosso-pharyngeal nerve. Further up in the medulla, on a level with the auditory strie and outside the previous column, is a tract of cells from which the auditory nerve $(8 t h)$ in great part arises; it is the principal auditory nucleus, and lies just under the commencement of the inferiot cerebellar peduncle fi: $\left.4_{-7}, s^{\prime}, s^{\prime \prime}, s^{\prime \prime \prime}\right)$. It consist of an outer and inner nucleus, which extend to the middle line. It forms connetions with the cerebellum, and some fibres are sail to enter the inferior cerebellar jecluncle. 'This is an inportant relationship, as we know that the vestibular branch of the anditury nerve comes jartly from the semicircular canals, so that in this way these organs may be connected with the cerebellum.]

Superadded Grey Matter.-There is a superadded mass of grey matter not represented in the (ord, that of the olivary body, enclosing a nucleus, the corpus dentatum, with its wavy strip of grey matter containing many small multipolar nerve-cells embedded in neuroglia. The grey matter is coverel on the surface by longitudinal and transverse fibres. It is open towards the middle line hilum), and into it run white fibres forming its peduncle (fig. $466, p, 0, l$ ). These fibres diverge like a fan, some of them ending in connection with the small multipolar (..lls of the deutite body, while others traverse the lamina of grey matter and pass backwards (1) appear as arcuate fibies which join the restiform body ; others, again, jass directly through to the surface of the olivary holy, which they help to cover as the superficial arcuate fibres. The accessory olivary nuclei (fig. $465, o^{\prime}, o^{\prime \prime}$ ) are two small masses of grey matter similar to the last, and looking as if they were detached from it, one lying above and external, sometimes callet the parolivary body, and the other slightly below and internal to the olivary nucleus, the latter heing separated from the dentate body by the roots of the hypoglossal nerve. The latter is sometumes called the internal parolivary body, or nucleus of the pyramid.]

[The formatio reticularis occupies the greater part of the central and lateral parts of the medulla, and is produced by the intercrossing of bundles of fibres running longitudinally and more or less trausversely in the medulla (fig. $465, f r$ ). In the more lateral portions are large multipolar nerve-cells, perhays continued upwards from part of the anterior cornu, while the part next the raphe has no such cells. The longitudinal fibres consist of the upward prolongation of the antero-external columns of the cord, while some seem to arise from the clavate nuclei and olives as arcuate fibres passing upwards. In the lateral portions, the longitudinal fibres are the direct continuation upwards of Flechsig's antero-lateral mixed tracts of the lateral colunus (1. 633). The horizontal fibres are formed by arcuate fibres, some of which run more or less trausversely outwards from the raphe. The superficial arcuate fibres (fig. $466, f, a, e$ ) appear in the anterior median fissure, and perhajs come through the raphe from the opposite side of the medulla, curve round the anterior pyramids, form a kind of capsule for the olives, and join the restiform body (p. 656), but they are reinforced by some of the deep arcuate fibres which traverse the olivary body (p. 656). The deep arcuate fibres run from the clavate and triangular nuclei horizontally inwards to the raphe, and cross to the other side; others pass from the raphe to the olivary body, and through it to the restiform body. In the raphe, which contains nerve-cells, soine fibres run transversely, others longitudinally, and others from before backwards. ]

[Other Nerve Nuclei-Sixth Nerve.-Under the elevation called eminentia teres (fig. 427) in front of the auditory stria, close to the middle line, is a tract of large multipolar nerve-cells. It was ouce thought to be the common nucleus of the 6 th and 7 th facial nerves, but Gowers has shown that "the facial ascends to this nuclous, forms a loop round it (some fibres indeed 
go through it), and then passes downwards, forwards, and outwards, to a column of cells more deeply placed in the medulla than any other nucleus in the lower part." But the 7 th has no real origin from this nucleus. Facial Nerve. - The nucleus lies deep in the formatio reticularis of the pons under the floor of the 4 th ventricle, but outside the position of the nucleus of the 6th (fig. 427,7 ). It extends downwards about as far as the auditory striæ, or a little lower. The fifth nerve arises from its motor nucleus (with large multipolar cells), which lies more superficially above and external to the 6 th (fig. 427, 5). T'he fibres run backwards, where they are joined by fibres from the upper sensory nucleus, but another sensory nucleus extends down nearly to the lower end of the medulla $\left(5^{\prime \prime}\right)$. Doubtless this extensive origin brings this nerve into intimate relation with the other cranial nerves, and accounts for the numerous reflex acts which can be discharged through the fifth nerve. Some sensory fibres are said to pass up beneath the corpora quadrigernina (Gowers). The fourth nerve arises from the valve of Vieussens, i.e., the lamina of white and grey matter which stretches between the superior cerebellar peduncles. It arises, therefore, behind the 4 th ventricle, but some of the fibres spring from nerve-cells at the lower part of the nucleus of the 3rd nerve. Some fibres also descend in the pons to form a connection with the nucleus of the 6 th nerve. The fibres decussate behind the aqueduct, so that in it alone, of all the cranial nerves, decussation occurs between its nucleus and its superficial origin (Gowers). The third nerve arises from a tract of cells beneath the aqueduct and near the middle line, and the fibres descend through the tegmentum to appear at the inner side of the crus cerebri. Gowers points out that, in reality, there are three distinct functional centres, (1) for accommodation (ciliary muscle), (2) for the light reflex of the iris, and (3) most of the external muscles of the eyeball. It is important to notice the connection between the nuclei of the $3 \mathrm{rd}, 4$ th, and 6 th nerves, in relation to the innervation of the ocular muscles.]

Functions.-The medulla oblongata, which connects the spinal cord with the brain, has many points of resemblance with the former. [Like the cord it is concerned (1) in the conduction of impulses.] (2) In it, numerous reflex centres are present, e.g., for simple reflexes similar to the nerve-centres in the spinal cord, e.g., closure of the eyelids, [so that they subserve the transference of afferent into efferent impulses]. There are other centres present which seem to dominate or control similar centres placed in the cord, e.g., the great vaso-motor centre, the sweat-secreting, pupil-dilating centres, and the centre for combining the reflex movements of the body. Some of the centres are capable of being excited reflexly $(\$ 358,2)$. (3) It is also said to contain automatic centres $(\$ 358,3)$. The normal functions of the centres depend upon the exchanges of blood-gases, effected by the circulation of the blood through the medulla. If this gaseous exchange be interrupted or interfered with, as by asphyxia, sudden anæmia, or venous congestion, these centres are first excited, and exhibit a condition of increased excitability, and at last, if they are over-stimulated, they are paralysed. An excessive temperature also acts as a stimulus. All the centres, however, are not active at the same time, and they do not all exhibit the same degree of excitability. Normally, the respiratory centre and the vaso-motor centre are continually in a state of rhythmical activity. In some animals, the inhibitory centre of the heart remains continually non-excited; in others, it is stimulated very slightly under normal conditions, simultaneously with the stimulation of the respiratory centre, and only during inspiration. The spasm centre is not stimulated under normal conditions; and during intra-uterine life, the respiratory centre remains quiescent. The medulla oblongata, therefore, contains a collocation of nerve-centres which are essential for the maintenance of life, as well as various conducting paths of the utmost importance. We shall treat of the reflex, and afterwards of the automatic centres.

367. REFLEX CENTRES OF THE MEDULLA OBLONGATA.-The medulla oblongata contains a number of reflex centres, which minister to the discharge of a large number of co-ordinated movements.

1. Centre for closure of the eyelids. The sensory branches of the 5th cranial nerve to the cornea, conjunctiva, and the skin in the region of the eye, are the afferent nerves. They conduct impulses to the medulla oblongata, where they are transferred to, and excite part of, the centre of the facial nerve, whence, through 
branches of the facial, the efferent impulses are conveyed to the orbicularis palpebrarum. The centre extends from about the middle of the ala cinerea upwards to the posterior margin of the pons (Nickell).

The reflex closure of the eyelids al ways occurs on both sides, but closure may be produced voluntarily on one side (winking). When the stimulation is strong, the corrugator and other groups of muscles which raise the cheek and nose towards the eye may also contract, and so form a more perfect protection and closure of the eye. Intense stimulation of the retina causes closure of the eyelids [and in this case the shortest reflex known, the latent period, is 0.05 second $($ Waller $)$ ].

2. Sneezing centre.-The afferent channels are the internal nasal branches of the trigeminus and the olfactory, the latter in the case of intense odours. The efferent or motor paths lie in the nerves for the muscles of expiration (\$ 120,3, and 347 , II.). Sneezing cannot be performed voluntarily, [but it may be inhibited by compressing the nasal nerve at its exit on the nose].

3. Coughing centre.-According to Kohts, it is placed a little above the inspiratory centre; the afferent paths are the sensory branches of the vagus $(\$ 3.5,5,5)$. The efferent paths lie in the nerves of expiration and those that close the glottis $(\$ 120,1)$.

4. Centre for sucking and mastication.-The afferent paths lie in the sensory branches of the nerves of the mouth and lips ( 2 nd and 3rd branches of the trigeminus and glosso-pharyngeal). The efferent nerves for sucking are (\$152):Facial for the lips, hypoglossal for the tongue, the inferior maxillary division of the trigeminus for the muscles which elevate and depress the jaw. For the movements of mustication, the same nerves are in action (\$153); but when food passes within the dental arch, the bypoglossal is concerned in the movements of the tongue, and the facial for the buccinator.

5. Centre for the secretion of saliva (p. 215) lies in the floor of the 4th ventricle. Stimulation of the medulla oblongata causes a profuse secretion of saliva when the chorda tympani and glosso-pharyngeal nerves are intact, a much feebler secretion when the nerves are divided, and no secretion at all when the cervical sympathetic is extirpated at the same time (Griitzner).

6. Swallowing centre lies in the floor of the 4 th ventricle $(156)$. - The afferent paths lie in the sensory branches of the nerves of the mouth, palate, and pharynx ( 2 nd and 3rd branches of the trigeminus, glosso-pharyngeal, and vagus); the efferent channels, in the motor branches of the pharyngeal plexus $(\$ 352,4)$. Stimulation of the glosso-pharyngeal nerve does not cause deglutition; on the contrary, this act is inhibited (p. 228).

According to steiner, every time we swallow there is a slight stimulation of the respiratory centre, resulting in a contraction of the diaphragm. [Kronecker has shown that if a glass of water be sipued slowly, the action of the cardio-inhibitory centre is interfered with reflexly, so that the heart beats much more rapidly, whereby the circulation is accelerated, hence probably the reason why sipping an alcoholic driuk intoxicates more rapidly than when it is quickly swaliuwed (p. 668).]

7. Vomiting centre ( $\$ 158)$. - The relation of certain branches of the vagus to this act are given at $\$ 352,2$, and $12, d .1$

8. The upper centre for the dilator pupillæ muscle, the smooth muscles of the orbit, and the eyelids lies in the medulla oblongata. The fibres pass out partly in the trigeminus (\$347, I., 3), partly in the lateral columns of the spinal cord as far down as the cilio-spinal region, and proceed by the two lowest cervical and the two upper dorsal nerves into the cervical sympathetic $(\$ 356, \mathrm{~A}, 1)$. The centre is normally excited reflexly by shading the retina, i.e., by diminishing the amount of light admitted into the eye. It is directly excited by the circulation of dyspnœic blood in the medulla. (The centre for contracting the pupil is referred to at $\$ 345$ and 392.)

The centre may be excited reflexly by stimulation of sensory nerve, e.g., the sciatic. These afferent fibres pass upwards through both lateral columns to their centre (Kowalcusky). 
9. There is a subordinate centre in the medulla oblongata, which seems to be concerned in bringing the various reflex centres of the cord into relation with each other. Owsjannikow found that, on dividing the medulla $6 \mathrm{~mm}$. above the calamus scriptorius (rabbit), the general reflex movements of the body still occurred, and the anterior and posterior extremities participated in such general movements. If, however, the section was made $1 \mathrm{~mm}$. nearer the calamus, only local partial reflex actions occurred ( $\$ 360$, III., 4); [thus, on stimulating the hind-leg, the fore-legs did not react-the transference of the reflex was interfered with]. The centre reaches upwards to slightly above the lowest third of the oblongata.

The medulla in the frog also contains the general centre for movements from place to place. Section of this region abolishes the power to move from place to place; when external stimuli are applied, there remains only simple reflex movements (Steiner).

Pathological. - The medulla oblongata is sometimes the seat of a typical disease, known as bulbar paralysis, or glosso-pharyngo-labial paralysis (Duchenne, 1860), in which there is a progressive invasion of the different nerve-nuclei (centres) of the cranial nerves which arise within the medulla, these centres being the motor portions of an important reflex apparatus. Usually, the disease begins with paralysis of the tongue, accompanied by fibrillar contractions, whereby speech, formation of the food into a bolus, and swallowing are interfered with $(\$ 354)$. The secretion of thick, viscid saliva points to the impossibility of secreting a thin watery facial saliva ( $\$ 145, \mathrm{~A})$, owing to paralysis of this nerve-nucleus. Swallowing may be impossible, owing to paralysis of the pharynx and palate. This interferes with the formation of consonants [especially the linguals, $l, t, s, r$, and, by and by, the labial explosives $\mathrm{b}, \mathrm{p}$ ] $(\$ 318, \mathrm{C})$; the speech becomes nasal, while fluids and solid food often pass into the nose. Then follows paralysis of the branches of the facial to the lips, and there is a characteristic expression of the mouth "as if it were frozen." All the muscles of the face may be paralysed; sometimes the laryngeal muscles are paralysed, leading to loss of voice and the entrance of food into the windpipe. The heart-beats are often retarded, pointing to stimulation of the cardio-inhibitory fibres (arising from the accessorius). Attacks of dyspnoea, like those following paralysis of the recurrent nerves ( $\$ 313$, II., 1 , and $\S 352,5, b$ ), and death may occur. Paralysis of the muscles of mastication, contraction of the pupil, and paralysis of the abducens are rare. [This disease is always bilateral, and it is important to note that it affects the nuclei of those muscles that guard the orifices of the mouth, including the tongue, the posterior nares including the soft palate, and the rima glottidis with the vocal cords.]

368. RESPIRATORY CENTRE. INNERVATION OF THE RESPIRATORY ORGANS. - The respiratory centre lies in the medulla oblongata (Legallois, 1811), behind the superficial origin of the vagi, on both sides of the posterior aspect of the apex of the calamus scriptorius, between the nuclei of the vagus and accessorius, and was named by Flourens the vital point, or nœud vital. The centre is double, one for each side, and it may be separated by means of a longitudinal incision (Longet, 1847), whereby the respiratory movements continue symmetrically on both sides. Section of Vagi.-If one vagus be divided, respiration on that side is slowed. If both vagi be divided, the respirations become much slower and deeper, but the respiratory movements are symmetrical on both sides. Stimulation of the central end of one vagus, both being divided, causes an arrest of the respiration only on the same side, the other side continues to breathe. The same result is obtained by stimulation of the trigeminus on one side (Langendorff). When the centre is divided transversely on one side, the respiratory movements on the same side cease (Schiff). Most probably the dominating respiratory centre lies in the medulla oblongata, and upon it depend the rhythm and symmetry of the respiratory movements ; but, in addition, other and subordinate centres are placed in the spinal cord, and these are governed by the oblongata centre. If the spinal cord be divided in newly-born animals (dog, cat) below the medulla oblongata, respiratory movements of the thorax are sometimes observed (Brachet, 1835).

[If the cord be divided below the medulla, or the cranial arteries ligatured (rabbit), there may still be respiratory movements, which become more distinct if strychnin be previously administered, so that Langendorff assumes the existence of a spinal respiratory centre, which he finds is also influenced by reflex stimulation of sensory nerves.]

Nitschmann, by means of a vertical incision into the cervical cord, divided the spinal centre 
into two equal halves, each of which acted on both sides of the diaphragm after the medulla was divided just below the calamus scriptorius. The spinal centres must, therefore, be consected with each other in the cord. The spinal respiratory centre can be excited or inhibited reflexly (Wertheimer).

Anstomical. - S.hiff locates the respiratory centre near the lateral margins of the grey matter in the flour of the 4 th ventricle, but not reaching so far backwards as the ala "cinerea. According to (iierke, Heilenhain, and Langendorff, those parts of the medulla oblongata whose destruction rauses cessation of the respiratory movements are single or double strands of nervous matter, containing grey nervous substance with small ganglion cells, and running dowuwarls in the substance of the medulla oblongata. These strands are said to arise partly from the roots of the vagus, trigeminus, spinal accessory, and glosso-pharyngeal (Meynert), forming connections by means of fibres with the other side, and descending as far downwards as the rervical enlargement of the spinal cord (Goll). According to this view, this strand represents an inter-central band connecting the spinal cord (the place of origin of the motor respiratory nerves) with the nuclei of the above-named cranial nerves.

Cerebral Inspiratory Centre.-According to Christiani, there is a cerebral insfirutory centre in the optic thalamus in the floor of the 3rd ventricle, which is

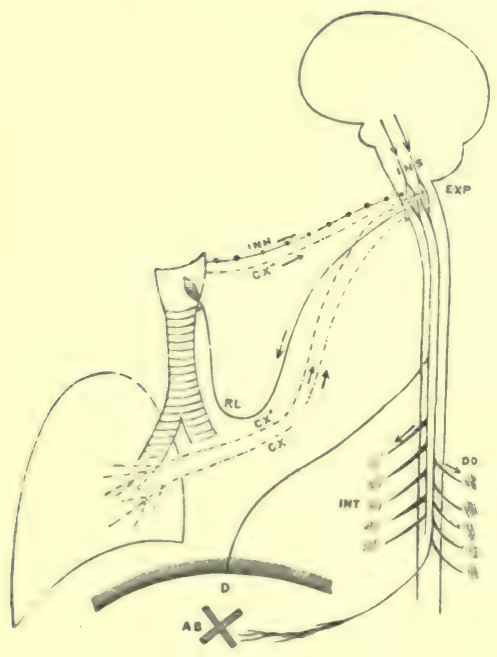

Fig. 467. stimulated through the optic and auditory nerves, even after extirpation of the cerebrum and corpora striata; when it is stimulated directly, it deepens and accelerates the inspiratory movements, and may even cause a standstill of the respiration in the inspiratory phase. This inspiratory centre may be extirpated. After this operation, an expiratory centre is active in the substance of the anterior pair of the corpora quadrigemina, not far from the aqueduct of Sylvius. Martin and Booker describe a second cerebral inspiratory centre in the posterior pair of the corpora quadrigemina. These three centres are connected with the centres in the medulla oblongata.

The respiratory centre consists of two centres, which are in a state of activity alternately - an inspiratory and an expiratory centre (fig. 467), each one forming the motor central point for the acts of inspiration and expiration (\$112). The centre is automatic, for, after section of all the sensory nerves which can act reflexly upon the centre, it still retains its activity. The degree of excitability and the stimulation of the centre depend upon the state of the blood, and chiefly upon the amount of the blood-gases, the $\mathrm{O}$ and $\mathrm{CO}_{2}$ (J. Rosenthal). According to the condition of the centre, there are several well-recognised respiratory conditions :-

1. Apnœa.-Complete cessation of the the inspiratory centre.

respiration constitutes apncea, i.e., cessation of the respiratory movements, owing to the absence of the proper stimulus, due to the blood being saturated with $\mathrm{O}$ and poor in $\mathrm{CO}_{2}$. Such blood saturated with $\mathrm{O}$ fails to stimulate the centre, and hence the respiratory muscles are quiescent. This seems to be the condition in the foetus during intrauterine life. If air be vigorously and rapidly forced into the lungs of an animal by artificial respiration, the animal will cease to breathe for a time, after 
cessation of the artificial respiration (Hook, 1667), the blood being so arterialised that it no longer stimulates the respiratory centre. If a person takes a series of rapid, deep respirations his blood becomes surcharged with oxygen, and long " apnœic pauses" occur.

Apnoeic Blood.-A. Ewald found that the arterial blood of apnoic animals was completely saturated with $\mathrm{O}$, while the $\mathrm{CO}_{2}$ was diminished ; the venous blood contained less $\mathrm{O}$ than normal - this latter condition being due to the apnœic blood causing a considerable fall of the bloodpressure and consequent slowing of the blood-strean, so that the $\mathrm{O}$ can be more completely taken from the blood in the capillaries (Pfiuger). The amount of $\mathrm{O}$ used in apnoea on the whole is not increased (\$127). Gad remarks that during forced artificial respiration, the pulmonary alveoli contain a very large amount of atmospheric air; hence, they are able to arterialise the blood for a longer time, thus diminishing the necessity for respiration. According to Gad and Knoll, the excitability of the respiratory centre is reduced during apnœa, and this is caused reflexly during artificial respiration by the distension of the lungs stimulating the branches of the vagus. In quite young mammals apnoea cannot be produced (Runge).

[Drugs. - If the excitability of the respiratory centre be diminished by chloral, apnœa is readily induced, while, if the centre be excited, as by apomorphine, it is difficult to produce it.]

2. Eupnœa.-The normal stimulation of the respiratory centre, eupnoea, is caused by the blood, in which the amount of $\mathrm{O}$ and $\mathrm{CO}_{2}$ does not exceed the normal limits (\$ 35 and 36 ).

3. Dyspnœa.-All conditions which diminish the $\mathrm{O}$ and increase the $\mathrm{CO}_{2}$ in the blood circulating through the medulla and respiratory centre cause acceleration and deepening of the respirations, which may ultimately pass into vigorous and laboured activity of all the respiratory muscles, constituting dyspnoea, when the difficulty of breathing is very great ( $\$ 134)$. [Changes in the rhythm, $\S 111$. ]

During normal respiration, and with the commencement of the need for more air, according to Gad, the gases of the blood excite only the inspiratory centre; while the expiration follows owing to reflex stimulation of the pulmonary vagus by the distension of the lungs (p. 666). $\mathrm{He}$ is also of opinion that the normal respiratory movements are excited by the $\mathrm{CO}_{2}$.

[Muscular work, as is well-known, increases the respirations and may even cause dyspnøea. This is not due to the nervous connections of the muscles or other organs with the respiratory centre, but to changes in the blood. Geppert and Zuntz have shown, however, that the result cannot be explained by changes in the blood caused either by diminution of $\mathrm{O}$ or increase of $\mathrm{CO}_{2}$. It seems to be due to the blood taking up some as yet unknown products from the contracting muscle, and carrying them to the respiratory centre, which is directly excited by them. The nature of these substances is unknown. It has been shown that the alkalinity of the blood is reduced by the formation of an acid. The substances, whatever they may be, are not excreted by the urine, and are, therefore, perhaps readily oxidised (Loewy). C. Lehmann has proved that, in rabbits, the acidification of the blood produced by muscular exertion plays an important part in the stimulation of the respiratory centre.]

4. Asphyxia.-If blood, abnormal as regards the amount and quality of its gases, continue to circulate in the medulla, or if the condition of the blood become still more abnormal, the respiratory centre is over-stimulated, and ultimately exhausted. The respirations are diminished both in number and depth, and they become feeble and gasping in character; ultimately the movements of the respiratory muscles cease, and the heart itself soon ceases to beat. This constitutes the condition of asphyxia, and if it be continued, death from suffocation takes place. (Langendorff asserts that in asphyxiated frogs the muscles and grey nervous substance have an acid reaction.) If the conditions causing the abnormal condition of the blood be removed, the asphyxia may be prevented under favourable circumstances, especially by using artificial respiration (\$134); the respiratory muscles begin to act and the heart begins to beat, so that the normal eupnoic stage is reached through the condition of dyspnœea. If the venous condition of the blood be produced slowly and very gradually, asphyxia may occur without there being any symptoms of dyspnœa, as happens when death takes place quietly and very gradually $(\$ 324,5)$.

Causes of Dуврnсеа.-(1) Direct limitation of the activity of the respiratory organs; diminution of the respiratory surface by inflammation, acute oedema ( $\$ 47)$, or collapse of the 
alveoli, occlusion of the capillaries of the alveoli, compression of the lungs, entrance of air into the pleura, obstruction or compression of the windpipe. (2) Obstruction to the entrance of the normal amount of air by strangulation, or enclosure in an insufficient space. (3) Enfeeblement of the circulation, so that the medulla oblongata does not receive a sufficient amount of blomel: in degeneration of the heart, valvular cardiac disease; and artificially by ligature of the carotid and verteliral arteries (Kussmaul and Tenner), or by preventing the free eftlux of venous liloul from the skull, or hy the injection of a large quantity of air or indifferent particles into the right heart. (4) Direct loss of blood, which acts by arresting the exchange of gases in the melulla ( $J$. Resmlhal). This is the cause of the "biting or snapping at the air" manifeste.1 l,y th. decapitated heads of young animals, e.g., kittens. [The phenomenon is well marked in the head of a tortoise separated from the body ( $W$. Stirling).]

If we study the rapilly fatal effects of these factors on the respiratory activity, we observe that at first the respirations become quicker and deeper, then after an attack of general convulsions. emling in expiratory spasm, there follows a stage of complete cessation of respiration. Before death takes place, there are usually a few "snapping" or gasping efforts at inspiration (Hogyes, Siym. Mayer-\$111).

Condition of the Blood-Gases. - As a ceneral rule, in the production of dyspncea, the want of $\mathrm{O}$ amd the excess of $\mathrm{C}(1)$, act simultaneously (I'flüger and Dohmen), but each of these alone may act as an efficient cause. According to Bernstein, blood containing a small amount of $O$ ants chicfly upon the inspiratory centre, and hlood rich in $\mathrm{CO}_{2}$ on the expiratory centre. (1) Dyspnaea, from want of 0 , occurs during respiration in a space of moderate size (\$ 133), in spaces where the tension of the air is diminished, and by breathing indifferent gases or those containing no free $\mathrm{O}$. When the blood is freely ventilated with $\mathrm{N}$ or $\mathrm{H}$, the amount of $\mathrm{CO}_{2}$ in the hlmol mav even he liminished, and ieath oceurs with all the signs of asphyxia (Pflüger). (2) Dyspnces, from the blood being overcharged with $\mathrm{CO}_{2}$, occurs by breathing air containing much $10 .,(\$ 133)$ A Ar containing much $\mathrm{CO}$. may cause lyspucea, even when the amount of $\mathrm{O}$ in the bluod is sreater than that in the atmosphere (Thiry). The blood may even contain more 0 than normal (Pflüger).

Heat Dyspnoa. - An increased temperature increases the activity of the respiratory centre $(\$ 214,11,3)$. This ocurs when blood warmer than natural flows through the brain, as Fick and follstein observed when they placed the exposed carotids in warm tubes, so as to heat the blool passing through them. In this case the heated blood acts directly upon the brain, the medulla, and the rereliral respiratory centres $(G a d)$. Direct cooling diminishes the excitability (Fridivin). When the temperature is increased, vigorous artificial respiration does not produce apnora, although the blool is highly arterialised (Ackcrmann). Emeties act in a similar manner (Hermann and Grimm).

Electrical stimulation of the medulla oblongata, after it is separated from the brain, discharges respiratory movements or increases those already present (Kronecker and Marckwali). Langendorff found that electrical, mechanical, or chemical (salts) stimulation usually (aused an expiratory effect, while stimulation of the cervical spinal cord (subordinate centre) gave an inspiratory effect. According to Laborde, a superficial lesion in the region of the ralamus scriptorius causes standstill of the respiration for a few minutes. If the peripheral end of the vagus be stimulated, so as to arrest the action of the heart, the respirations also cease after a few seconds. Arrest of the heart's action causes a temporary anæmia of the medulla, in consequence of which its excitability is lowered, so that the respirations cease for a time (Langendorff).

Action on the Centre.-The respiratory centre, besides being capable of being stimulated directly, may be influenced by the will, and also reflexly by stimulation of a number of afferent nerves.

1. By a voluntary impulse we may arrest the respiration for a short time, but only until the blood becomes so venous as to excite the centre to increased action. The number and depth of the respirations may be voluntarily increased for a long time, and we may also voluntarily change the rhythm of respiration.

2. The respiratory centre may be influenced reflexly both by fibres which excite it to increased action and by others which inhibit its action. (a) The exciting fibres lie in the pulmonary branches of the vagus, in the optic, auditory, and cutaneous nerves; normally their action overcomes the action of the inhibitory fibres. Thus, a cold bath deepens the respirations, and causes a moderate acceleration of the pulmonary ventilation (Speck).

Section of both vagi causes slower and deeper respiratory movements, owing to the cutting off of those impulses which under normal conditions pass from the lungs to excite the respiratory centre (p. 661). The amount of air taken in the 
$\mathrm{CO}_{2}$ given off, however, is unchanged, but the inspiratory efforts are more vigorous and not so purposive $(G a d)$. Weak tetanising currents applied to the central end of the vagus, cause acceleration of the respirations, while, at the same time, the efforts of the respiratory muscles may be increased, or diminished, or remain unchanged $(\mathrm{Gad})$. Strong tetanising currents cause standstill of the respiration in the inspiratory phase (Traube), or especially in fatigue of the nerves, in the expiratory phase (Budge, Burkart). Single induction shocks have no effect (Marckwald and Kronecker).

[Marckwald, while admitting that the respiratory centre is automatically active, as well as capable of being affected reflexly, comes to the conclusion, that, when the centre is separated from all nerve-channels by which afferent impulses can be conveyed to it, it is incapable of discharging rhythmical respiratory movements. He also asserts that the normal rhythmical respiration is a reflex act discharged chiefly through the vagi, and that the normal excitant of the respiratory centre is not dependent on the condition of the blood, either on the diminution of $\mathrm{O}$, or the increase of $\mathrm{CO}_{2}$. These results are opposed to the usually accepted view, and they are controverted by Loewy. Division of the medulla oblongata above the respiratory centre, so as to cut off all cerebral channels of communication, has very little effect on the respirations. If, after this, one, or both vagi be divided, there is-(1) an extraordinaryslowing of the respiration; the number of respirations may fall in the rabbit, from 20 to 2 or 4 per minute; (2) the rhythm is changed, in some cases the inspiration may be twice or thrice as long as the expiration, but, whatever the ratio of inspiration to expiration, the respiration is rhythmical ; (3) the volume of air respired is diminished (p. 664), but the volume for each respiration is deeper; $(4)$ the intra-thoracic pressure is increased, during inspiration, and during expiration it is the same as before the vagotomy.]

\begin{tabular}{|c|c|c|c|c|c|c|c|c|c|c|c|}
\hline \multicolumn{5}{|c|}{ Before Vagotomy. } & \multicolumn{7}{|c|}{ After Vagotomy. } \\
\hline 离 & 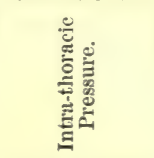 & 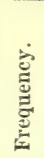 & 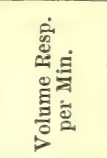 & 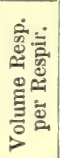 & 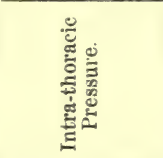 & 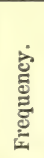 & 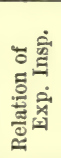 & 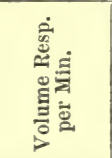 & 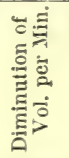 & 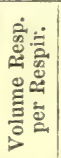 & 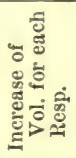 \\
\hline $\begin{array}{l}1 \\
2\end{array}$ & $\begin{array}{c}\text { mm. } \\
-30 \text { to }-40 \\
-22 \text { to }-24\end{array}$ & $\begin{array}{l}20 \\
32\end{array}$ & $\begin{array}{c}\text { c.cm. } \\
310-350 \\
530-540\end{array}$ & $\begin{array}{l}\text { c.cm. } \\
16 \\
16\end{array}$ & $\begin{array}{l}-60 \text { to }-70 \\
-50 \text { to }-60\end{array}$ & $\begin{array}{l}4 \\
2 \frac{1}{2}\end{array}$ & $\frac{1}{2}$ & $\begin{array}{c}\text { c.cm. } \\
130-140 \\
105-120\end{array}$ & $\begin{array}{l}\% \\
59 \\
79 \cdot 5\end{array}$ & $\begin{array}{l}33 \\
40\end{array}$ & $\begin{array}{l}\% \\
100 \\
150\end{array}$ \\
\hline
\end{tabular}

[The above table (from Loewy) shows the result. Loewy finds that, if the centre be separated from all centripetal channels, it still discharges respiratory movements, which are rhythmical, and he has shown that these rhythmical discharges are due to the condition of the blood.]

[If one lung be made atelectic, i.e., devoid of air, e.g., by plugging its bronchus with a sponge-tent, then the pulmonary fibres of the vagus from this lung are no longer excited during respiration, and their section has no effect on the respiration. Section of the vagus on the sound side, however, has the same consequence as double vagotomy (Loewy).]

Wedenski and Heidenhain find that a temporary, weak, electrical stimulus applied to the central end of the vagus, at the beginning of inspiration (rabbit), affects the depth of the succeeding inspirations, while a similar strong stimulus affects also the depth of the following expirations. If the stimulus be applied just at the commencement of expiration, stronger stimuli being required in this case, there is a diminution of the expiration and of the following inspiration. Continued tetanic stimulation of the vagus may cause decrease in the depth of the expirations, or at the same time alteration in the depth of the inspirations, without affecting the respiratory rhythm; when the stimulation is stronger, inspiration and expiration are diminished with or without alteration of the frequency, and with the strongest stimuli, respirations cease either in the inspiratory or expiratory phase.

(b) The inhibitory nerves which affect the respiratory centre run in the superior laryngeal nerve (Rosenthal), and also in the inferior (Pfiüger and Burkart, Hering, Breuer), to the respiratory centre (fig. 467 , inh).

According to Langendorff, direct electrical, mechanical, or chemical stimulation of the centre may arrest respiration, perhaps in consequence of the stimulus affecting the central ends of these inhibitory nerves where they enter the ganglia of the respiratory centre. During the reflex inhibition of the respiration in the expiratory phase, there is a suppression of the motor impulse in the inspiratory centre (Wegete).

Stimulation of the superior or inferior laryngeal nerves $(b)$ or their central ends 
causes slowing, and even arrest of the respiration (in expiration-Rosenthal). Arrest of the respiration in expiration is also caused by stimulation of the nasal (Hering und Kiotsikmer) and ophthalmic branches of the trigeminus (Christiani), of the olfactory, and glosso-pharyngeal (Marckwald). [Kratschmer found that tobacco-smoke blown into a rabbit's nostrils, or puffed through a hole in the trachea into the nose, by stimulating the nasal branch of the fifth nerve, arrested the respiration in the expiratory phase; while it had no effect when blown into the lungrs. Ammonia vapour applied to the nostrils arrests it in the same way. If ammonia vapour be blown into the lungs (the nasal cavity being protected from its action), the respiration may be accelerated, or deepened, or arrested occasionally in expiration, i.e., according to the fibres of the vagus acted on by the vapour in the lungs $(K n o l l)$.$] \quad Stimulation of the pulmonary branches of the vagus by breathing$ irritating gases ( $K_{n}$ oll $)$ causes standstill in expiration, although some other gases cause standstill in inspiration. Chemical stimulation of the trunk of the vagus, by dilute solutions of sodic carbonate,-causes expiratory inhibition of the respiration; and mechanical stimulation,-_rubbing with a glass rod,-inspiratory inhibition (Knill). The stimulation of sensory cutaneous nerves, especially of the chest and abdomen (as occurs on taking a cold douche), and stimulation of the splanchnics, cause standstill in expiration, the first cause often giving rise to temporary clonic contractions of the respiratory muscles. The respirations are often slowed to a very great extent by pressure unon the brain, [whether the pressure be due to a depressed fracture or effusion into the ventricles and subarachnoid space]. The respiration may be greatly oppressed and stertorous.

The amount of work done by the respiratory muscles is altered during the reflex slowing of the respiratory muscles, the work being increased during slow respiration, owing to the ineffectual inspiratory efforts ( $\mathrm{rad}$ ). The volume of the gases which passes through the lungs during a given time remains unchanger (Valentin), and the gaseous exchanges are not altered at first (Voit and Rauber).

Automatic Regulation.-Under normal circumstances, it would seem that the pulmonary branches of the vagus act upon the two respiratory centres, so as to set in action what has been termed the self-adjusting mechanism; thus, the inspiratory dilatation of the lungs stimulates mechanically the fibres which reflexly excite the exprirutory centres, while the diminution of the lungs during expiration excites the nerves which proceed to the inspircutory centre (Hering and Breuer, Head). [Thus, blowing into the lungs excites the act of expiration, and sucking air out of them excites inspiration.]

In this way we may explain the alternate play of inspiration and expiration. In deep narcosis, however, dilatation of the thorax in animals is followed first by cessation of the respiratory movements, and then by inspiration (P. Guttmann).

Discharge of the First Respiration.-The foetus is in an apnoic condition until birth, when the umbilical cord is cut. During intrauterine life, $\mathrm{O}$ is freely supplied to it by the activity of the placenta. All conditions which interfere with this due supply of $\mathrm{O}$, as compression of the umbilical vessels and prolonged labour pains, cause a decrease of the $\mathrm{O}$ and an increase of the $\mathrm{CO}_{2}$ in the blood, so that the condition of the foetal blood is so altered as to stimulate the respiratory centre, and thus the impulse is given for the discharge of the first respiratory movement (Schurertz). A fretus, still within the unopened fœtal membranes, may make respiratory movements (Vesalius, 1542). If the exchange of gases be interrupted to a sufficient extent, dyspncea and ultimately death of the foetus may occur. If, however, the venous condition of the mother's blood develops very slowly, as in cases of quiet slow death of the mother, the medulla oblongata of the foetus may gradually die without any respiratory movement being discharged $(\S 324,5)$.

According to this view, the respiratory movements are due to the direct action of the dyspnoeic blood upon the medulla oblongata. [The excitability of the respiratory centre is less 
in the foetus than in the newly born, and it increases from day to day after birth. Amongst the causes of the diminished excitability are the small amount of $\mathrm{O}$ in foetal blood, and the slow velocity of the circulation. If an inspiration is discharged in the foetus, it is at once inhibited by fluid passing into the nostrils and inhibiting the act reflexly. The chief cause of the first respiration after birth, is undoubtedly the increasing venosity of the blood, and also the disappearance of the above-named reflex inhibitory process.] Death of the mother acts like compression of the umbilical cord. In the former case, the maternal venous blood robs the foetal blood of its $\mathrm{O}$, so that death of the fotus occurs more rapidly (Zuntz). If the mother be rapidly poisoned with $\mathrm{CO}(\S 17)$, the foetus may live longer, as the CO-hæmoglobin of the maternal blood cannot take any $\mathrm{O}$ from the fœetal blood (\$ 16-Hogyes). In slow poisoning the CO passes into the foetal blood (Gréhant and Quinquand).

In many eases, especially in cases of very prolonged labour, the excitability of the respiratory centre may be so diminished, that after birth, the dyspnœic condition of the blood alone is not sufficient to excite respiration in a normal rhythmical manner. In such cases stimulation of the skin also acts, e.g., partly by the cooling produced by the evaporation of the amniotic fluid from the skin. When air has entered the lungs by the first respiratory movements, the air within the lungs also excites the pulmonary branches of the vagus (Pflüger), and thus the respiratory centre is stimulated reflexly to increased activity. According to v. Preuschen's observations, stimulation of the cutaneous nerves is more effective than that of the pulmonary branches of the vagus. In animals which have been rendered apnoic by free ventilation of their lungs, respiratory movements may be discharged by strong cutaneous stinuli, e.g., dashing on of cold water. The mechanical stimulation of the skin by friction or sharp blows, or the application of a cold douche, excites the respiratory centre. When the placental circulation is intact, cutaneous stimuli do not discharge respiratory movements (Zuntz and Cohnstein), (Artificial respiration, $\$ 134$ ).

[Action of Drugs on the Respiratory Centre.-Ammonia, salts of zinc and copper, strychnin, atropin, duboisin, apomorphin, emetin, the digitalis group, and heat increase the rapidity and depth of the respirations, while they become frequent and shallower after the use of alcohol, opium, chloral, chloroform, "physostigmin. The excitability of the centre is first increased and then diminished by caffein, nicotin, quinine, and sapomin (Brunton).]

369. CENTRE FOR THE INHIBITORY NERVES OF THE HEART(CARDIO-INHIBITORY).- The fibres of the vagus, when moderately stimulated, diminish the action of the heart; when strongly stimulated, however, they arrest its action and cause it to stand still in diastole $(\$ 352,7)$; they are supplied to the vagus through the spinal accessory nerve, and have their centre in the medulla oblongata (\$353).

[Gaskell has shown that stimulation of the vagus not only influences the rhythm of the heart's action, but modifies the other functions of the cardiac muscle. Stimulation of the vagus influences- $(a)$ the automatic rhythm, i.e., the rate at which the heart contracts automatically; $(b)$ the force of the contractions, more especially the auricles, although in some animals, e.g., the tortoise, the ventricles are not affected; $(c)$ the power of conduction, i.e., the capacity for conducting the muscular contractions. According to Gaskell, the vagus acts upon the rhythmical power of the muscular fibres of the heart.]

This centre may be excited directly in the medulla, and also reflexly, by stimulating certain afferent nerves.

Many observers assume that this centre is in a state of tonic excitement, i.e., that there is a continuous, uninterrupted, regulating, and inhibitory action of this centre upon the heart through the fibres of the vagus. According to Bernstein, this tonic excitement is caused reflexly through the abdominal and cervical sympathetic.

I. Direct Stimulation of the Centre.-This centre may be stimulated directly, by the same stimuli that act upon the respiratory centre. (1) Sudden ancemia of the oblongata, ligature of both carotids or both subclavians, or decapitating a rabbit, the vagi alone being left undivided, cause slowing and even temporary arrest of the action of the heart. (2) Sudden venous hypercemia acts in a similar manner, e.g., by ligaturing all the veins returning from the head. (3) Increased venosity of the blood, produced either by direct cessation of the respiraticns (rabbit), or by forcing into the lungs a quantity of air containing much $\mathrm{CO}_{2}$ (Traube). As the circulation in the placenta (the respiratory organ of the foetus) is interfered 
with during severe labour, this sufficiently explains the enfeeblement of the action of the heart which occurs during protracted labour; it is due to stimulation of the central end of the vagus by the dyspnoic blood (B.S. Schultze). (4) At the moment the respiratory centre is excited, and an inspiration occurs, there is a variation in the inhibitory activity of the cardiac centre (Donders, Pflüger, Frédéricq $-574, a .4)$. (5) The centre is excited by increased blood-pressure within the cerebral arteries.

II. The centre may be excited reflexly by-(1) Stimulation of sensory nerves (Livin). (2) Stimulation of the central end of one vagus, provided the other vagus is intact. (3) Stimulation of the sensory nerves of the intestines, by tapping upon the belly (Croltz's tapping experiment), whereby the action of the heart is arrested. Stimulation of the splanchnic directly (Asp and Ludvig), or of the abdominal or cervical sympathetic, produces the same result. Very strong stimulation of sensory nerves, however, arrests the above-named retlex effects upon the vagus (\$3 361,3$)$.

Tapping Experiment.-Goltz's experiment succeeds at once, by tapping the intestines of a frog directly, say, with the handle of a scalpel, especially if the intestine has been exposed to the air for a short time, so as to become inflamed (Turchanoff). Stimulation of the stomach of the dog causes slowing of the heart-beat (Sig. Hayer and Pribram). [M'William finds that the artion of the heart of the eel may be arrested reflexly with very great facility. The reflex inhibition is obtainel by slight stimulation of the gills (through the branchial nerves), the skin of the head and tail, and parietal peritoneum, by severe injury of almost any part of the animal, except the abdominal organs.]

[Effect of Swallowing Fluids. - Kronecker has shown that the act of swallowing interferes with or abolishes temporarily the cardio-inhibitory action of the vagus, so that the pulse-rate is greatly acceleraterl. Merely sipping a wine-glassful of water may raise the rate 30 per cent. Hence, sipping cold water acts as a powerful cardiac stimulant.]

Accorling to Hering, the excitalility of the cardio-inhibitory centre is diminished by vigorous artificial ventilation of the lungs with atmospheric air. At the same time, there is a considerable fall of the blood-pressure $(\$ 352,8,4)$. In man, a vigorous expiration, owing to the increased intra-pulmonary pressure, causes an acceleration of the heart-beat, which Sommerbrodt ascribes to a diminution of the activity of the vagi. At the same time the activity of the vaso-motor centre is diminished $(\S 60,2)$.

Stimulation of the trunk of the vagus from the centre downwards, along its whole course, and also of certain of its cardiac branches [inferior cardiac], causes
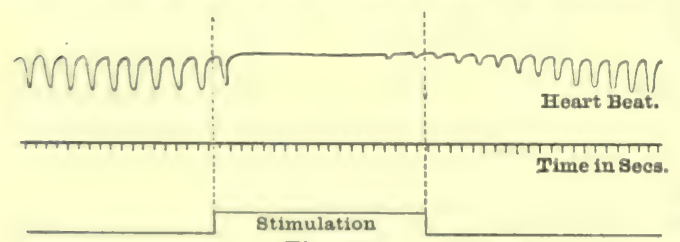

Fig. 468. the heart either to beat more slowly, or arrests its action in diastole. The result depends upon the strength of the stimulus employed; feeble stimuli slow the action of the heart, while strong stimuli arrest it in diastole. The frog's heart may be Beating of a frog's heart taken by means of a lever rest. arrested by stimulating the fibres ing on the heart. The lowest curve shows when the of the vagus upon the sinus vevagus was stimulated and the consequent arrest of the nosus [or by stimulating the
heart-beat (Stirling).

468]. If strong stimuli be applied, either to vagus in its course as in fig. for a long time, the part stimulated becomes fatigued and the heart beats more rapidly in spite of the continued stimulation. If a part of the nerve lying nearer the heart be stimulated, inhibition of the heart's action is brought about, as the stimulus acts upon a fresh portion of nerve. The following points have also been ascertained regarding the stimulation of the inhibitory
fibres :-

1. The experiments of Löwit on the frog's heart, confirmed by Heidenhain, showed that electrical and chemical stimulation of the vagus produce different results, as regards the extent of the ventricular systole, as well as the number of heart-beats; the contractions either become 
smaller, or less frequent, or they become smaller and less frequent simultaneously. Strong stimuli cause, in addition, well-marked relaxation of the heart-muscle during diastole.

2. In order to cause inhibition of the heart, a continuous stimulus is not necessary. A rhythmically interrupted moderate stimulus suffices (v. Bezold); 18 to 20 stimuli per second are required for mammals, and 2 to 3 per second for cold-blooded animals.

3. Donders, with Prahl and Niel, observed that arrest of the heart's action did not take place immediately the stimulus was applied to the vagus; but about $\frac{1}{6}$ of a second-period of latent stimulation-elapsed before the effect was produced on the heart.

4. If the heart be arrested by stimulation of the vagus, it can still contract, if it be excited directly, e.g., by pricking it with a needle, when it executes a single contraction. [This holds good only for some animals, e.g., frog, tortoise, birds and mammals. In fishes, only the ventricle responds to stimulation during marked inhibition; in the newt, only the bulbus arteriosus. In the newt's heart, the sinus, auricles, and ventricle are all inexcitable to direct stimulation during strong inhibition.]

5. According to A. B. Meyer, inhibitory fibres are present only in the right vagus in the turtle. It is usually stated that the right vagus is more effective than the left in other animals, e.g., rabbit (Masoin); but this is subject to many exceptions (Landois and Langendorff). [In the newt, the right vagus acts more readily on the ventricle than on the other parts of the heart; slight stimulation of the right vagus can arrest the ventricle, while the sinus and auricles go on beating.]

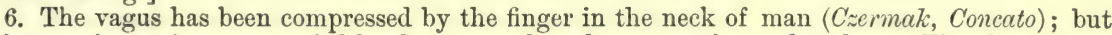
this experiment is accompanied by danger, and ought not to be undertaken. The electrotonic condition of the vagus is stated in $\S 335$, III.

7. Schiff found that stimulation of the vagus of the frog caused acceleration of the heart-beat, when he displaced the blood of the heart with saline solution. If blood-serum be supplied to the heart, the vagus regains its inhibitory action.

8. Many soda salts in a proper concentration arrest the inhibitory action of the vagus, while potash salts restore the inhibitory function of the vagi suspended by the soda salts. If, however, the soda or potash salts act too long upon the heart, they produce a condition in which, after the inhibitory function of the vagi is abolished, it is not again restored. The heart's action in this condition is usually arhythmical (Löwit).

9. If the intracardial pressure be greatly increased, so as to accelerate greatly the cardiac pulsations, the activity of the vagus is correspondingly diminished (.,$M$. Ludwig and Luchsinger).

[Differences in Animals. - Perhaps the most remarkable fact in connection with the influence of the vagus on the eel's heart and that of all other fishes examined, is, that vagus-stimulation causes the sinus and auricle to be entirely inexcitable to direct stimulation during strong inhibition. Nerve-stimulation has in this case the very peculiar effect of rendering the muscular tissue temporarily incapable of responding to even the strongest direct stimuli, e.g., powerful induction shocks. This would appear to be decisive evidence that the vagus acts on muscle directly, and not simply on automatic motor ganglia, as was held according to the old view (M'William).]

Poisons. - Muscarin stimulates the terminations of the vagus in the heart, and causes the heart to stand still in diastole (Schmiedeberg and Koppe). [See p. 85 for Gaskell's views.] If atropin be applied in solution to the heart, this action is set aside, and the heart begins to beat again. [Atropin abolishes completely the inhibitory action of the vagus on the heart. If it be injected into the jugular vein of a rabbit, the pulse-beats are increased 27 per cent., in the dog, they may be trebled, and in a man under its full influence the pulse-beats may rise from 70 to $\mathbf{1 5 0}$ or more. After atropin, it is impossible to arrest the action of the heart by stimulation of the vagus, and in the frog this cannot be done even by stimulation of the inhibitory centre in the heart itself, so that atropin must be regarded as paralysing the intracardiac terminations of the vagus.] Digitalin diminishes the number of heart-beats by stimulating the cardio-inhibitory centre (vagus) in the medulla. Large doses diminish the excitability of the vagus centre, and increase at the same time the accelerating cardiac ganglia, so that the heart-beats are thereby increased. In small doses, digitalin raises the bloodpressure by stimulating the vaso-motor centre and the elements of the vascular wall (Klug). Nicotin first excites the vagus, then rapidly paralyses it. Hydrocyanic acid has the same effect (Preyer). Atropin (v. Bezold) and curara (large dose-Cl. Bernard and Kölliker) paralyse the vagi, and so does a very low temperature or high fever.

370. CENTRE FOR THE ACCELERATING CARDIAC NERVES.Nervus Accelerans. - It is more than probable that a centre exists in the medulla oblongata, which sends accelerating fibres to the heart. These fibres pass from the medulla oblongata-but from which part thereof has not been exactly ascertained -through the spinal cord, and leave the cord through the rami communicantes of the lower cervical and upper six dorsal nerves (Stricker), to pass into the sympathetic 
nerve. Some of these fibres, issuing from the spinal cord, pass through the first thoracic sympathetic ganglion and the ring of Vieussens, to join the cardiac plexus (figs. 469, 470). [These fibres, proceeding from the spinal cord, frequently accompany the nerve running along the vertebral artery], and they constitute the Nervus accelerans curdis. [Fig. 470 shows the accelerator fibres passing through the ganglion stellatum of the cat to join the cardiac plexus.] If the vagi of an animal be divided, stimulation of the medulla oblongata, of the lower end of the divided cervical spinal cord,

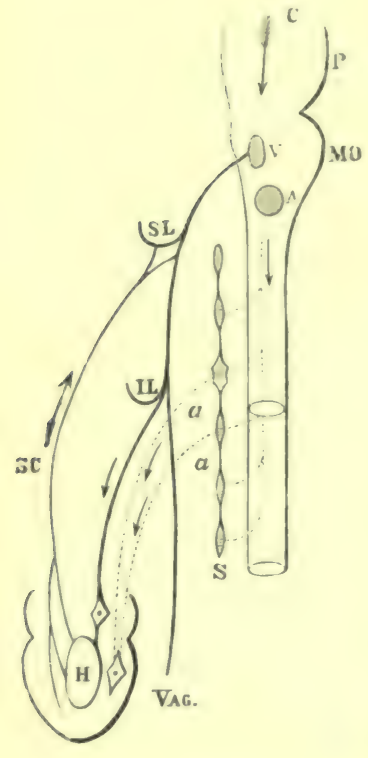

Fig. 469.

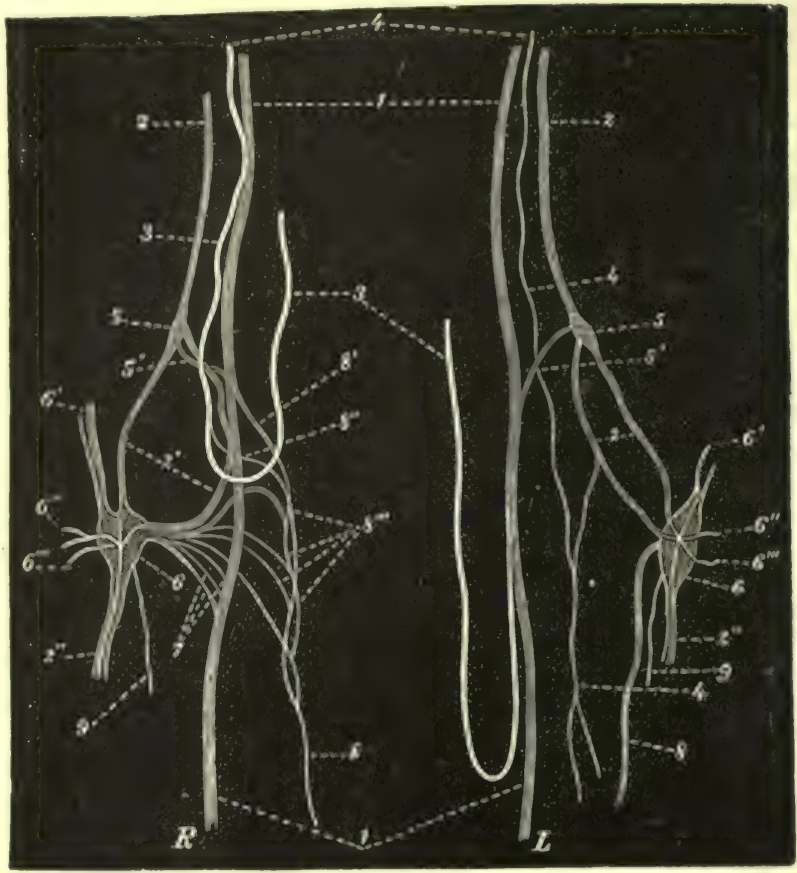

Fig. 470 .

Fig. 469. - Scheme of the course of the accelerans fibres. P, pons; MO, medulla oblongata ; C, spinal cord ; V, inhibitory centre for heart ; A, accelerans centre ; VAG., vagus ; SL, superior, IL, inferior laryngeal ; SC, superior, IC, inferior cardiac ; H, heart ; C, cerebral impulse; $\mathrm{s}$, cervical sympathetic ; $a, a$, accelerans fibres. Fig 470.-Cardiac plexus, and ganglion stellatum of the cat. $\mathrm{R}$, right, $\mathrm{L}$, left $\times 1 \frac{1}{2} ; 1$, vagus; $2^{\prime}$ cervical sympathetic, and in the annulus of Vieussens; 2, conmunicating branches from the middle cervical ganglion and the ganglion stellatum; $2^{\prime \prime}$, thoracic sympathetic; 3 , recurrent laryngeal ; 4, depressor nerve ; 5 , middle cervical ganglion ; $5^{\prime}$, communication between 5 and the vagus; 6 , ganglion stellatum (1st thoracic ganglion); 7 , communicating branches with the vagus; 8 , nervus accelerans ; $8,8^{\prime}, 8^{\prime \prime}$, roots of accelerans ; 9 , branch of the ganglion stellatum.

even of the lower cervical ganglion, or of the upper dorsal ganglion of the sympathetic (Gang. stellatum), causes acceleration of the heart-beats in the dog and rabbit, without the blood-pressure undergoing any change $(\mathrm{Cl}$. Bernard, v. Bezold, Cyon).

On stimulating the medulla oblongata or the cervical portion of the spinal cord, the vasomotor nerves are, of course, simultaneously excited. The consequence is that the blood-vessels, supplied by vaso-motor nerves from the spot which is stinulated, contract, and the bloodpressure is greatly increased. Again, a siniple increase of the blood-pressure accelerates the action of the heart ; this experiment does not prove directly the existence of accelerating fibres lying in the upper part of the spiual cord. If, however, the splanchnic nerves be divided beforehand (when, as they supply the largest vaso-motor area in the body, the result of their division is to cause a great fall of the blood-pressure), then on stimulating the above-named 
parts, after this operation, the heart-beats are still. increased in number, so that this increase cannot be due to the increased blood-pressure. Indirectly it may be shown, by dividing or extirpating all the nerves of the cardiac plexus, or at least all the nerves going to the heart, that stimulation of the medulla oblongata, or cervical part of the spinal cord, no longer causes an increased frequency of the heart's action to the same extent as before division of these nerves. The slightly increased frequency in this case is due to the increased bloodpressure.

The accelerating centre is certainly not continually in a state of tonic excitement, as section of the accelerans nerve does not cause slowing of the action of the heart; the same is true of destruction of the medulla oblongata or of the cervical spinal cord. In the latter case, the splanchnic nerves must be divided beforehand, to avoid the slowing effect on the action of the heart produced by the great fall of the blood-pressure consequent upon destruction of the cord, otherwise we might be apt to ascribe the result to the action of the accelerating centre, when it is in reality due to the diminished blood-pressure (Cyon).

According to the results of the older observers ( $v$. Bezold and others), some accelerating fibres run in the cervical sympathetic. A few fibres pass through the vagus to reach the heart $(\$ 352,7)$, and when they are stimulated, either the heartbeat is accelerated or the cardiac contractions strengthened (Heidenhain and Löwit), or the latter alone occurs (Pawlow). The inhibitory fibres of the vagus lose their excitability more readily than the accelerating fibres, but the vagus fibres are more excitable than those of the accelerans.

Tarchanoff has described some very rare cases of individuals who, by a merely voluntery effort, and while at rest, the respirations remaining unaffected, could nearly double the number of their pulse-beats.

Modifying Conditions. - When the peripheral end of the nervus accelerans is stimulated, a considerable time elapses before the effect upon the frequency of the heart takes place, i.e., it has a long latent period. Further, the acceleration thus produced disappears gradually. If the vagus and accelerans fibres be stimulated simultaneously, only the inhibitory action of the vagus is manifested. If, while the accelerans is being stimulated, the vagus be suddenly excited, there is a prompt diminution in the number of the heart-beats; and if the stimulation of the vagus is stopped, the accelerating effect of the accelerans is again rapidly manifested (C. Ludwig with Schmicdebery, Bowditch, Baxt). According to the experiments of Stricker and Wagner on dogs, with both vagi divided, a diminution of the number of the heart-beats occurred when both accelerantes were divided. This would indicate a tonic innervation of the latter nerves.

[Accelerans in the Frog.-Gaskell showed that stimulation of the vagus might produce two opposing effects; the one of the nature of inhibition, the other of augmentation. In the crocodile, the accelerans fibres leave the sympathetic chain at the large ganglion corresponding to the ganglion stellatum of the dog, and run along the vertebral artery up to the superior vena cava, and, after anastomosing with branches of the vagus, pass to the heart. "Stimulation of these fibres increases the rate of the cardiac rhythm, and augments the force of auricular contractions; while stimulation of the vagus slows the rhythm, and diminishes the strength of the auricular contractions." The strength of the ventricular contraction, both in the tortoise and crocodile, does not seem to be influenced by stimulation of the vagus, and probably also it is unaffected by the sympathetic. The so-called vagus of the frog, in reality, consists of pure vagus fibres and sympathetic fibres, and is in fact a vago-sympathetic. Gaskell finds that stimulation of the sympathetic, before it joins the combined ganglion of the sympathetic and vagus, produces purely augmentor or accelerating effects; while stimulation of the vagus, before it enters the ganglion, produces purely inhibitory effects. The two sets of fibres are quite distinct, so that in the frog, the sympathetic is a purely augmentor (accelerator), and the vagus a purely inhibitory nerve. Acceleration is merely one of the effects produced by stimulation of these nerves; hence, Gaskell suggests that they ought to be called "augmentor," or simply cardiac sympathetic nerves.]

[In his more recent researches Gaskell asserts that vagus stimulation produces first an inhibi- 
tory or depressing effect, but that it ultimately improves the condition of the heart as regards force, rate, or regularity - one or all of these. He regards it as a true anabolic nerve ( $\$ 342$, d). J

\section{VASO-MOTOR CENTRE AND VASO-MOTOR NERVES. - Vaso-motor} Centre.-The chief dominating or general centre, which supplies all the nonstriped muscles of the arterial system with motor nerves (vaso-motor, vaso-constrictor, vaso-hypertonic nerves), lies in the medulla oblongata, at a point which contains many ganglionic cells (Ludwig and Thiry). Those nerves which pass to the blood-ressels are known as vaso-motor nerves. The centre (which is 3 millimetres long and $1 \frac{1}{2}$ millimetre broad in the rabbit) reaches from the region of the upier part of the floor of the medulla oblongata to within 4 to $5 \mathrm{~mm}$. of the calamus scriptorius. Each half of the body has its own centre, placed $2 \frac{1}{2}$ millimetres from the middle line on its own side, in that part of the medulla oblongata which represents the upward continuation of the lateral columns of the spinal cord; according to Ludwig, Owsjannikow, and Dittmar, in the lower part of the superior olives. Stimulation of this central area causes contraction of all the arteries, and, in conserfuence, there is great increase of the arterial blood-pressure, resulting in swelling of the veins and heart. Paralysis of this centre causes relaxation and dilatation of all the arteries, and consequently there is an enormous fall of the blood-pressure. Inder ordinary circumstances, the vaso-motor centre is in a condition of muderute tonir excitement $(\$ 366)$. Just as in the case of the cardiac and respiratory centres, the vaso-motor centre may be excited directly and reflexly.

[Position-How ascertained.-As stimulation of the central end of a sensory nerve, the blood-pressure, even after removal of the cerebrum, it is evident that the centre is not in the cerebrum itself. For the effect of chloral, under the same conditions, see p. 67t. lis making a series of sections from above downwards, it is found that this reflex effect is not affected until a short distance above the medulla oblongata is reached. If more and more of the medulla oblongata be removed from above downwards, then the reflex rise of the blood-pressure becomes less and less, until, when the section is made 4 to $5 \mathrm{~mm}$. above the calamus scriptorius, the effect ceases altogether. This is taken to be the lower limit of the general vasomotor centre. The bilateral centre corresponds to some large multipolar nervecells, described by Clarke as the antero-lateral nucleus.]

I. Direct Stimulation of the Centre.-The amount and quality of the gases contained in the blood flowing through the medulla are of primary importance. In the condition of apnoea $(\$ 368,1)$, the centre seems to be very slightly excited, as the blood-pressure undergoes a considerable decrease. When the mixture of bloodgases is such as exists under normal circumstances, the centre is in a state of moderate excitement, and running parallel with the respiratory movements are variations in the excitement of the centre (Traube-Hering curves- $\$ 85$ ), these variations being indicated by the rise of the blood-pressure. When the blood is highly venous, produced either by asphyxia or by the inspiration of air containing a large amount of $\mathrm{CO}_{n}$, the centre is strongly excited, so that all the arteries of the body contract, while the venous system and the heart become distended with blood (Thiry). At the same time, the velocity of the blood-stream is increased (Heidenhain). The same result is produced by ligature of both the carotid and subclavian arteries, thus causing sudden anæmia of the medulla oblongata; and, no doubt, also by the sudden stagnation of the blood in venous hyperæmia.

Emptinese of the Arteries after Death. - The venosity of the blood which occurs after death always produces an energetic stimulation of the vaso-motor centre, in consequence of which the arteries are firmly contracted. The blood is thereby forced towards the cavillaries and veins, and thus is explained the "emptiness of the arteries after death."

Effect on Hæmorrhage. - Blood flows much more freely from large wounds, when the vasomotor centre is intact, than if it be destroyed (frog). As psychical excitement undoubtedly 
influences the vaso-motor centre, we may thus explain the influence of psychical excitement (speaking, \&c.) upon the cessation of hæmorrhage. If the hæmorrhage be severe, stimulation of the medulla oblongata, due to the anæmia, may ultimately cause constriction of the small arteries, and thus arrest the bleeding. Thus, surgeons are acquainted with the fact that dangerous hæmorrhage is often arrested as soon as unconsciousness, due to cerebral anæmia, occurs. If the heart be ligatured in a frog, all the blood is ultimately forced into the veins, and this result is also due to the anæmic stimulation of the oblongata (Goltz). In mammals, when the heart is ligatured, the equilibration of the blood-pressure between the arterial and venous systems takes place more slowly when the medulla oblongata is destroyed than when it is intact ( $v$. Bezold, Gscheidlen).

[Effect of Destruction of the Vaso-motor Centre.-If two frogs be pithed and their hearts exposed, and both be suspended, then the hearts of both will be found to beat rhythmically and fill with blood. Destroy the medulla oblongata and spinal cord of one of them, then immediately in this case, the heart, although continuing to beat with an altered rhythm, ceases to be filled with blood; it appears collapsed, pale, and bloodless. There is a great accumulation of the blood in the abdominal organs and veins, and it is not returned to the heart, so that the arteries are empty. This experiment of Goltz is held to show the existence of venous tonus depending on a cerebro-spinal centre. If a limb of this frog be amputated, there is little or no hæmorrhage, while in the other frog the hæmorrhage is severe. The bearing of this experiment on conditions of "shock" is evident.]

Action of Poisons.-.-Strychnin stimulates the centre directly, even in curarised dogs, and so do nicotin and Calabar bean.

Direct Electrical Stimulation. - On stimulating the centre directly in animals, it is found that single induction shocks only become effective when they succeed each other at the rate of 2 to 3 shocks per second. Thus there is a "summation" of the single shocks. The maximum contraction of the arteries, as expressed by the maximum blood-pressure, is reached when 10 to 12 strong, or 20 to 25 moderately strong shocks per second are applied (Kronecker and Nicolaides).

Course of the Vaso-motor Nerves. - From the vaso-motor centre fibres proceed directly through some of the cranial nerves ta their area of distribution; through the trigeminus partly to the interior of the eye ( $\$ 347, \mathrm{I} ., 2)$, through the lingual and hypoglossal to the tongue $(\S 347$, III., $4)$, through the vagus to a limited extent to the lungs $(\$ 352,8,2)$, and to the intestines $(\S 352,11)$.

All the other vaso-motor nerves descend in the lateral columns of the spinal cord $(\S 364,9)$; hence, stimulation of the lower cut end of the spinal cord causes contraction of the blood-vessels supplied by the nerves below the point of section (Pfiuger). In their course through the cord, these fibres form connections with the subordinate vaso-motor centres in the grey matter of the cord $(\$ 362,7)$, and then leave the cord either directly through the anterior roots of the spinal nerves to their areas of distribution, or pass through the rami communicantes into the sympathetic, and from them reach the blood-vessels to which they are distributed (§356) [see fig. 439].

The following is the arrangement of these nerves in the region of the head:- The cervical portion of the sympathetic supplies the great majority of the blood-vessels of the head (see Sympathetic, § $356, \mathrm{~A}, 3$ ). In some animals, the great auricular nerve supplies a few vasomotor fibres to its own area of distribution (Schiff, Loven, Moreau). The vaso-motor nerves to the upper extremities pass through the anterior roots of the middle dorsal nerves into the thoracic sympathetic, and upwards to the 1st thoracic ganglion, and from thence through the rami communicantes to the brachial plexus (Schiff, Cyon). The skin of the trunk receives its vaso-motor nerves through the dorsal and lumbar nerves. The vaso-motor nerves to the lower extremities pass through the nerves of the lumbar and sacral plexuses into the sympathetic, and from thence to the lower limbs (Pflïger, Schiff, Cl. Bernard). The lungs, in addition to a few fibres through the vagus, are supplied from the cervical spinal cord through the 1st thoracie ganglion (Brown-Séquard, Fick and Badoud, Lichtheim). The splanchnic is the greatest vasomotor nerve in the body, and supplies the abdominal viscera (\$356, B-v. Bezold, Ludwig and Cyon). The vaso-motor nerves of the liver $(\S 173,6)$, kidney $(\S 276)$, and spleen $(\S 103)$ have been referred to already. According to Stricker, most of the vaso-motor nerves leave the spinal cord between the 5th cervical and the 1st dorsal vertebræ. [Gaskell finds that in the dog (fig. 439) they begin to leave the cord at the 2nd dorsal nerve ( $\$ 366)$.]

As a general rule, the blood-vessels for the skin of the trunk and extremities are innervated from those nerves which give other fibres (e.g., sensory) to those regions. The different vascular areas behave differently with regard to the intensity of the action of the vaso-motor nerves. The most powerful vaso-motor nerves are those that act upon the blood-vessels of peripheral parts, e.g., the toes, the fingers, and ears; while those that act upon central parts seem to be less active (Lewaschew), e.g., on the pulmonic circulation (\$ 88).

II. Reflex Stimulation of the Centre.-There are fibres contained in the different afferent nerves, whose stimulation affects the vaso-motor centre. There are nerve-fibres whose stimulation excites the vaso-motor centre, thus causing a 
stronger contraction of the arteries, and consequently an increase of the arterial blood-pressure. These are called "pressor" fibres. Conversely, there are other fibres whose stimulation reflexly diminishes the excitability of the vaso-motor centre. These act as reflex inhibitory nerves on the centre, and are known as "depressor" fibres.

Pressor, or excito-vaso-motor nerves, have already been referred to in connection with the superior and inferior laryngeal nerves $(\$ 352,12, a)$, in the trigeminus, which, when stimulated directly (\$3.7), causes a pressor action, as well as when stimulating valours are blown into the nostrils (Hering and Kratschmer). [The rise of the blood-pressure in this case, however, is accompanied by a change in the character of the heart's beat and in the respirations. Rutherford has shown that in the rabbit the vapour of chloroform, ether, amyl nitrite, acetic acid, or ammonia held liefore the nose of a rabbit, greatly retards or even arrests the heart's action, and the same is true if the nostrils be closed by the hand. This arrest does not occur if the trachea be opened, and Rutherford regards the result as due not to the stimulation of the sensory fibres of the trigeminus, but to the state of the blood acting on the cardio-inhibitory nerve alyparatus.] Hubert and Roever found pressor fibres in the cervical symprathetic; S. Mayer and Pribram found that mechanical stimulation of the stomach, especially of its serosa, caused pressor effects $(\$ 352$, 12, r). According to Lovin, the first effect of stimulating every sensory nerve is a pressor action.

[If a dog be joisoned with curara, and the central end of one sciatic nerve be stimulated, there is a great and steady rise of the blood-pressure, chiefly owing to the contraction of the abdominal blood-vessels, and at the same time there is no change in the heart-beat. If, however, the animal be poisoned with chloral, there is a fall of the blood-pressure resembling a depressor effect.]

0. Nimmann found that weak, electrical stimulation of the skin caused at first contraction of the bloml-vessels, esperially of the mesentery, lungs, and the web, with simultaneous excitement of the carliar activity and acceleration of the circulation (frog). Strong stimuli, however, harl an oplosite effert, i.c., a depressor effect, with simultaneous decrease of the cardiac activity. Grutzner and Heidenhain found that contact with the skin caused a pressor effect, while painful impressions produced no effect. The application of heat and cold to the skin produces reflexly a change in the lumen of the blood-vessels and in the cardiac activity (Röhrig, Winter. nit: l'inching the skin causes contraction of the vessels of the pia mater of the rabbit i. Sikillir), and the same result was prodnced hy a warm bath, while cold dilated the vessels. These results are due partly to pressor and partly to depressor effects, but the chief cause of the dilatation of the bloul-vessels is the increased blood-pressure due to the cold constricting the cutaneous vesseis. Heat, of course, has the opposite effect. In man, most stimuli applied to sensory nerves produce an effect:- feeble cutaneous stimuli, tickling (even unpleasant odours, bitter or acil tastes, optical and acoustic stimuli) at the parts where they are applied, cause a fall of the cutancous temperature, and diminution of the volume of the corresponding limb, sometimes increase of the gencral hlood-pressure and change of the heart-beat. The opposite effects are jroduced by painful stimulation, the action of heat (and even by pleasant odours and sweet tastes). The former cause simultaneously dilatation of the cerebral vessels and increase the vascular contents of the skull, - the latter cause the opposite results (Istominow and Tarchanoff).

Depressor fibres, i.e., fibres whose stimulation diminishes the activity of the vaso-motor centre, are present in many nerves. They are specially numerous in the superiog curliuc branch of the vagus, which is known as the depressor nerve $(\$ 352,6)$. The trunk of the vagus below the latter also contains depressor fibres (v. Bezold), as well as the pulmonary fibres (dog). The latter also act as depressors, during strong expiratory efforts (\$74); while Hering found that inflating the lungs (to $50 \mathrm{~mm}$. $\mathrm{Hg}$ j)ressure) caused a fall of the blood-pressure (and also accelerated the heart beats- $\$ 369$, II.). Stimulation of the central end of sensory nerves, especially when it is intense and long-continued, causes dilatation of the bloodvessels in the area supplied by them (Lovèn). According to Latschenberger and Deahna, all sensory nerves contain both pressor and depressor fibres. 
If a rabbit be poisoned with curara, and the central end of the great auricular nerve be stimulated, there is a double effect-one local and the other general; the blood-vessels throughout the body, but especially in the splanchnic area, contract, so that there is a general rise of the blood-pressure, while the blood-vessels of the ear are dilated. If the central end of the tibial nerve be stimulated, there is a rise of the general blood-pressure, but a local dilatation of the saphena artery in the limb of that side (Lovèn). Again, the temperature of one hand and the condition of its blood-vessels influence that of the other. If one hand be dipped in cold water, the temperature of the other hand falls. Thus, pressor and depressor effects may be obtained from the same nerve. The vaso-motor centre, therefore, primarily regulates the condition of the blood-vessels, but through them it obtains its importance by regulating and controlling the blood supply according to the needs of an organ.]

The central artery of a rabbit's ear contracts regularly and rhythmically 3 to 5 times per minute. Schiff observed that stimulation of sensory nerves caused a dilatation of the artery, which was preceded by a slight temporary constriction.

Depressor effects are produced in the area of an artery on which direct pressure is made, as occurs, for example, when the sphygmograph is applied for a long time-the pulse-curves become larger, and there are signs of diminished arterial tension $(\$ 75)$.

Rhythmical Contraction of Arteries. - In the intact body slow alternating contraction and dilatation, without a uniform rhythm, have been observed in the arteries of the ear of the rabbit, the membrane of a bat's wing, and the web of a frog's foot. This arrangement, observed by Schiff, supplies more or less blood to the parts according to the action of external couditions. It has been called a "periodic regulatory vascular movement." This movement may be useful when a vessel is occluded, as after ligature, and may help to establish more rapidly the collateral circulation. Stefäni has shown that this occurs with more difficulty after section of the nerves.

Direct, local, applications may influence the lumen of the blood-vessels; cold and moderate electrical stimuli cause contraction; while, conversely, heat and strong mechanical or electrical stimuli cause dilatation, although with the last two there is usually a preliminary constriction.

Poisons.-Almost all the digitalis group of substances cause constriction; quinine and salicin constrict the splenic vessels. The other febrifuges dilate the vessels (Thomson). See p. 95 .

Effect on Temperature.-The vaso-motor nerves influence the temperature, not only of individual parts, but of the whole body.

1. Local Effects. - Section of a peripheral vaso-motor nerve, e.g., the cervical sympathetic, is followed by dilatation of the blood-vessels of the parts supplied by it (such as the ear of the rabbit), the intra-arterial pressure dilating the paralysed walls of the vessels. Much arterial blood, therefore, passes into and causes congestion and redness of the parts, or hyperæmia, while, at the same time, the temperature is increased. There is also increased transudation through the dilated capillaries within the dilated areas; the velocity of the blood-stream is of course diminished, and the blood-pressure increased. The pulse is also felt more easily, because the blood-vessels are dilated. Owing to the increase of the blood-stream, the blood may flow from the veins almost arterial (bright red) in its characters, and the pulse may even be propagated into the veins, so that the blood spouts from them $(C l$. Bernard $)$. Stimulation of the peripheral end of a vaso-motor nerve causes the opposite results - pallor, owing to contraction of the vessels, diminished transudation, and fall of the temperature on the surface. The smaller arteries may contract so much that their lumen is almost obliterated. Continued stimulation ultimately exhausts the nerve, and causes at the same time the phenomena of paralysis of the vascular wall.

Secondary Results. - The immediate results of paralysis of the vaso-motor nerves lead to other effects; the paralysis of the muscles of the blood-vessels must lead to congestion of the blood in the part; the blood moves more slowly, so that the parts in contact with the air cool more easily, and hence the first stage of increase of the temperature may be followed by a fall of the temperature. The ear of a rabbit with the sympathetic divided, after several weeks 
hecomes cooler than the ear on the sound one. If in man the motor muscular nerves, as well as the raso-motor fibres, are paralysed, then the paralysed limb becomes cooler, because the paralysed muscles no longer contract to aid in the production of heat (\$338), and also because the dilatation of the muscular arteries, which accompanies a muscular contraction, is absent. Should atruphy of the paralysed muscles set in, the blood-vessels also become smaller. Hence, paralysed limbis in man generally become cooler as time goes on. The primary effect, however, in a limb, $\epsilon_{0} y_{\text {. }}$, after section of the sciatic or lesion of the brachial plexus, is an increase of the temperature.

If, at the same time, the vaso-motor nerves of a large area of the skin be paralysed, e.\%, the lower half of the body after section of the spinal cord, then so much heat is given otf from the dilated blood-vessels that, either the warming of the skin lasts for a very short time and to a slight degree, or there may be cooling at once. Some observers (Tschetschichin, Naunyn, Quincke) observed a rise of the temperature after section of the cervical spinal cord, but Riegel did not observe this increase.

2. Effect on the Temperature of the Body.-Stimulation or paralysis of the vaso-motor nerves of a smull area has practically no effect on the general temperature of the body. If, however, the vaso-motor nerves of a considerable area of the skin be suddenly paralysed, then the temperature of the entire body falls, because more heat is given off from the dilated vessels than under normal circumstances. This occurs when the suinal cord is divided high up in the neck. The inhalation of a few drops of amyl nitrite, which dilates the blood-vessels of the skin, causes a fall of the temperature (Sassetzki and Manassën). Conversely, stimulation of the vaso-motor nerves of a large area increases the temperature, because the constricted vessels give off less heat. The temperature in fever may be partly explained in this way $(\$ 220,4)$.

The activity of the heart, i.e., the number and energy of the cardiac contractions, is influenced by the condition of the vaso-motor nerves. When a large vaso-motor area is paralysed, the blood-channels are dilated, so that the blood does not flow to the heart at the usual rate and in the usual amount, as the pressure is considerably diminished. Hence, the heart executes extremely small and feeble contractions. Stricker observed that the heart of a dog ceased to beat on extirpating the spinal cord from the 1st cervical to the Sth dorsal vertebra. Conversely, we know that stimulation of a large vaso-motor area, by constricting the bloodvessels, raises the arterial blood-pressure considerably. As the arterial pressure affects the pressure within the left ventricle, it may act as a mechanical stimulus to the cardiac wall, and increase the cardiac contractions both in number and strength. Hence, the circulation is accelerated (Heidenhain, Slavjansky).

Splanchnic.-By far the largest vaso-motor area in the body is that controlled by the splanchnic nerves, as they supply the blood-vessels of the abdomen ( $\$ 161)$; hence, stimulation of their peripheral ends is followed by a great rise of the blood-pressure. When they are divided, there is such a fall of the blood-pressure that other parts of the body become more or less anæmic, and the animal may even die from "being bled into its own belly," i.e., from what has been called "intravascular hæmorrhage." Animals whose portal vein is ligatured die for the same reason (C. Lulwig and Thiry) [see \$ 87]. The capacity of the vascular system, depending as it does in part upon the condition of the vaso-motor nerves, influences the bodyweight. Stimulation of certain vascular areas may cause the rapid excretion of water, and we may thus account in part for the diminution of the body-weight, which has been sometimes observed after an epileptic attack terminating with polyuria.

Trophic disturbances sometimes occur after affections of the vaso-motor nerves $(\S 342, \mathrm{I}, c)$. Paralysis of the vaso-motor nerves not only causes dilatation of the blood-vessels and local increase of the blood-pressure, but it may also cause increased transudation through the capillaries [\$203]. When the active contraction of the muscles is abolished, the blood-stream at the same time becomes slower, and in some cases, the skin becomes livid, owing to the venous congestion. There is a diminution of the normal transpiration, and the epidermis may be dry and preel off in scales. The growth of the hair and nails may be affected by the congestion of blood, and other tissues may also suffer.

Vaso motor Centres in the Spinal Cord.-Besides the dominating centre 
in the medulla oblongata, the blood-vessels are acted upon by local or subordinate vaso-motor centres in the grey matter of the spinal cord, as is proved by the following observations :---If the spinal cord of an animal be divided, then all the bloodvessels supplied by vaso-motor nerves below the point of section are paralysed, as the vaso-motor fibres proceed from the medulla oblongata. If the animal lives, the blood-vessels regain their tone and their former calibre, while the rhythmical movements of their muscular walls are ascribed to the subordinate centres in the lower part of the spinal cord (Lister, Goltz-\$362, 7).

The subordinate spinal centres may, further, be stimulated directly by dyspnoic blood, and also reflexly, in the rabbit and frog (Ustimowitsch). After destruction of the medulla oblongata, the arteries of the frog's web still contract reflexly when the sensory nerves of the hind leg are stimulated (Putnam, Nussbaum, Vulpian). In the dog, opposite the 3rd to 6th dorsal nerve is a spinal vaso-motor centre (origin of the splanchnic), which can be excited reflexly (Smirnow), and there is a similar one in the lower part of the spinal cord (Vulpian).

If the lower divided part of the cord be crushed, the blood-vessels again dilate, owing to the destruction of the subordinate centres. In animals which survive this operation, the vessels of the paralysed parts gradually recover their normal diameter and rhythmical movements. This effect is ascribed to ganglia, which are supposed to exist along the course of the vessels. [It is to be recollected that the existence of these peripheral nervous mechanisms has not been proved.] 'These ganglia [or peripheral nervous mechanisms] might be compared to the ganglia of the heart, and seem by themselves capable of sustaining the movements of the vascular wall. Even the blood-vessels of an excised kidney exhibit periodic variations of their calibre (C. Ludwig and Mosso). It is important to observe that the walls of the blood-vessels contract as soon as the blood becomes highly venous. Hence, the blood-vessels offer a greater resistance to the passage of venous than of arterial blood (C. Ludwig). Nevertheless, the blood-vessels, although they recover part of their tone and mobility, never do so completely.

The effects of direct mechanical, chemical, and electrical stimuli on blood-vessels may be due to their action on these peripheral nervous mechanisms. The arteries may contract so much as almost to disappear, but sometimes dilatation follows the primary stimulus.

Lewaschew found that limbs, in which the vaso-motor fibres had undergone degeneration, reacted like intact limbs to variations of temperature; heat relaxed the vessels, and cold constricted them. It is, however, doubtful if the variations of the vascular lumen depend upon the stimulation of the peripheral nervous mechanisms. Amyl nitrite and digitalis are supposed to act on those hypothetical mechanisms.

The pulsating veins in the bat's wing still continue to beat after section of all their nerves, which is in favour of the existence of local nervous mechanisms (Luchsinger, Schiff).

Influence of the Cerebrum.-The cerebrum influences the vaso-motor centre, as is proved by the sudden pallor that accompanies some psychical conditions, such as fright or terror. There is a centre in the grey matter of the cerebrum where stimulation causes cooling of the opposite side of the body.

Although there is one general vaso-motor centre in the medulla oblongata, which influences all the blood-vessels of the body, it is really a complex composite centre, consisting of a number of closely aggregated centres, each of which presides over a particular vascular area. We know something, e.g., of the hepatic (\$175) and renal centres $(\$ 276)$.

Many poisons excite the vaso-motor nerves, such as ergotin, tannic acid, copaiba, and cubebs ; others first excite, and then paralyse, e.g., chloral hydrate, morphia, laudanosin, veratrin, nicotin, Calabar bean, alcohol: others rapidly paralyse them, e.g., amyl nitrite, CO (§ 17), atropin, muscarin. The paralytic action of the poison is proved by the fact that, after section of the vagi and accelerantes, neither the pressor nor the depressor nerves, when stimulated, produce any effect. Many pathological infective agents affect the vaso-motor nerves.

The veins are also influenced by vaso-motor nerves, and so are the lymphatics, but we know very little about this condition.

Pathological. - The angio-neuroses, or nervous affections of blood-vessels, form a most important group of diseases. The parts primarily affected may be either the peripheral nervous 
mochanisms, the suhordinate centres in the cord, the dominating centre in the medulla, or the grey matter of the cerelirum. The effect may be direct or reflex. The dilatation of the vessels may also be due to stimulation of vaso-dilator nerves, and the physician must be careful to listinguish whether the result is due to paralysis of the vaso-constrictor nerves or stimulation of the vaso-dilator fibres.

Angio-neuroses of the skin occur in affections of the vaso-motor nerves, either as a diffuse redness or prillor: or there may be circumscribed affections. Sometimes, owing to the stimulation of inlivilual vasu-motor nerves, there are loul cutaneous arterio-spasms (Nothnagel). In certian a ute f.lirile attacks-after previous initial violent stimulation of the vaso-motor nerves, esperially during the cold stage of fever-there may be different forms of paralytic phenomena of the ctitaneous ressels. In some cases of epileprsy in man, Trousseau observed irregular, red, angin.pralytic patches (ataches cérébrales). Continued strong stimulation may lead to interruption of the circulation, which may result in gangrene of the skin and deeper-seated parts (I'ciss).

Hemicrania, due to unilateral spasm of the branches of the carotid on the head, is accompanied los severe headache (I) IJuis-liellmomd). The cervical sympathetic nerve is intensely stimulater a pale, collapsed, and cool side of the face, contraction of the temporal artery like a firm whip-cord, iliatation of the pupil, secretion of thick saliva, are sure signs of this affec. tion. This form may be followed by the opyosite condition of paralysis of the cervical sympathetic, where the efficts are reversed. Sometimes the two conditions may alternate.

Basedow's disease is a remarkable condition, in which the vaso-motor nerves are concerned; the lieart heats very rapilly (90 to $129-200$ heats yer minute), causing palpitation; there is swelling of the thrond gland (struma), and projection of the eyeballs (exophthalmos), with imperfectly co-rrilinatinl movements of the upjer eyelid, whereby the plane of vision is raised or lowereil. P'erhaps in this disease we have to deal with a simultaneous stimulation of the arelerans corlis $\$ 37(1)$, the motor fibres of Miiller's museles of the orbit and eyelids $(\$ 347, \mathrm{I}$.), as well as of the vaso-ililators of the thyroil gland. The disease may be due to direct stimulation of the sympathetic channels or their spinal origins, or it may be referred to some reflex cause. It has also ixeen explained, however, thus, that the exophthalmos and struma are the conseyucnce of vaso-motor paralysis, which results in enlargement of the blood-vessels, while the in reased carrliae action is a sign of the diminished or arrested inhibitory action of the vagus. .1l these phenomena may be caused, according to Filehne, by injury to the upper part of both restiform bodies in rablits.

Visceral Angio-neuroses. - The oceurrence of sudden hyperamia with transudations and ecchymoses in some thoracic or aldominal organs may have a neurotic basis. As already mentioned, injury to the pons, corpus striatum, and optic thalamus may give rise to hyperæmia, and erhymuses in the lungs, pleurie, intestines, and kidneys. According to Brown-Séquard, compression or section of one-half of the pons causes ecchymoses, especially in the lung of the "Inosite sile: he also observed ecelymoses in the renal capsule after injury of the lumbar portion of the spinal cord $(\$ 379)$.

The dependence of diabetes mellitus upon injury to the vaso-motor nerves is referred to in $\$ 175$; the action of the vaso-motor nerves on the secretion of urine in $\$ 276$; and fever in $\$ 220$.

372. VASO-DILATOR CENTRE AND NERVES.-Although a vaso-dilator centre has not been definitely proved to exist in the medulla, still its existence there has been surmised. Its action is opposite to that of the vaso-motor centre. The centre is certainly not in a continuous or tonic state of excitement. The vaso-dilator nerves behave in their functions similarly to the cardiac branches of the vagus; both, when stimulated, cause relaxation and rest (Schiff, $\mathrm{Cl}$. Bernard). Hence, these nerves have been called vasi-inhibitory, vaso-hypotonic, or vaso-dilator nerves. Iyspnceic blood stimulates this centre as well as the vaso-motor centre, so that the cutaneous vessels are dilated, while simultaneously the vessels of the internal organs are contracted and the organs anæmic, owing to the stimulation of their vaso-motor centre (I)astre and Morcut). Nicotin is a powerful excitant of the vaso-dilator nerves (Ostroumoff); it raises the temperature of the foot $(\operatorname{dog})$, and increases the formation of lymph (Rogorvicz).

[The existence of vaso-dilator nerves is assumed in accordance with such facts as the following:- If the chorda tympani be divided, there is no change in the blood-vessels of the sub-maxillary gland; but if its peripheral end be stimulated, in addition to other results (\$145), there is dilatation of the blood-vessels of the submaxillary gland, so that its veins discharge bright florid blood, while they spout like an artery. Similarly, if the nervi erigentes be divided, there is no effect on 
the blood-vessels of the penis $(\$ 362,4)$; but if their peripheral ends be stimulated with Faradic electricity, the sinuses of the corpora cavernosa dilate, become filled with blood, and erection takes place $(\$ 436)$. Other examples in muscle and elsewhere are referred to below.]

Course of the Vaso-dilator Nerves.-To some organs they pass as special nerves-to other parts of the body, however, they proceed along with the vaso-motor and other nerves. According to Dastre and Morat, the vaso-dilator nerves for the bucco-labial region (dog) pass out from the cord by the 1 st to the 3rd dorsal nerves, and go through the rami communicantes into the sympathetic, then to the superior cervical ganglion, and lastly through the carotid and intercarotid plexus into the trigeminus. [The fibres occur in the posterior segment of the ring of Vieussens, and if they be-stimulated there is dilatation of the vessels in the lip and cheek on that side (p. 624).] The maxillary branch of the trigeminus, however, also contains vasodilator fibres proper to itself (Laffont). In the grey matter of the cord, there is a special subordinate centre for the vaso-dilator fibres of the bucco-labial region. This centre may be acted on reflexly by stimulation of the vagus, especially its pulmonary branches, and even by stimulating the sciatic nerve. The ear receives its nerves from the 1st dorsal and lowest cervical ganglion, the upper limb from the thoracic portion, and the lower limb from the abdominal portion of the sympathetic. The vaso-dilator fibres run to the sub-maxillary and sub-lingual glands in the chorda tympani ( $\$ 349,4)$, while those for the posterior part of the tongue run in the glosso-pharyngeal nerve $(\$ 351,4-V u l p i a n)$. Perhaps the vagus contains those for the kidneys $(\S 276)$. Stimulation of the nervi erigentes proceeding from the sacral plexus causes dilatation of the arteries of the penis, together with congestion of the corpora cavernosa $(\$ 436)$ (Eckhard, Loven). Eckhard found that erection of the penis can be produced by stimulation of the spinal cord and of the pons as far as the peduncles, which may explain the phenomenon of priapism in connection with pathological irritations in these regions. The muscles receive the vaso-dilator fibres for their vessels through the trunks of the motor nerves. Stimulation of a motor nerve or the spinal cord causes not only contraction of the corresponding muscles, but also dilatation of their blood-vessels (§ 294, II._C. Ludwig and Sczelkow, Hafiz, Gaskell).- the dilatation of the vessels taking place even when the muscle is prevented from shortening. [Gaskell observed under the microscope, the dilatation produced by stimulation of the nerve to the mylohyoid muscle of the frog.] The vaso-dilators remain medullated up to their terminal ganglion (Gaskell).

The vaso-dilators (like the vaso-motors, p. 676) also have subordinate centres in the spinal cord; e.g., the fibres of the labio-buical region at the 1 st to 3 rd dorsal vertebræ. This centre may be influenced reflexly through the pulmonary fibres of the vagus, and also through the sciatic (Laffont, Smirnow). According to Holtz, a similar centre lies in the lowest part of the cord.

Goltz showed that, in the nerves to the limbs (e.g., in the sciatic nerve), the vaso-motor and vaso-dilator fibres lie side by side in the same nerve. If the peripheral end of this nerve be stimulated immediately after it is divided, the action of the vaso-constrictor fibres overcomes that of the dilators. If the peripheral end be stimulated 4 to 6 days after the section, when the vaso-constrictors have lost their excitability, the blood-vessels dilate under the action of the vaso-dilator fibres. Stimuli, which are applied at long intervals to the nerve, act especially on the vaso-dilator fibres; while tetanising stimuli xct on the vaso-motors. The latent period of the vaso-dilators is longer, and they are more easily exhausted than the vaso-motors (Bowditch and Warren). The sciatic nerve receives both kinds of fibres from the sympathetic. It is assumed that the peripheral nervous mechanisms in connection with the blood-vessels are influenced by both kinds of vascular nerves; the vaso-motors (constrictors) increase, while the vaso-dilators diminish the activity of these mechanisms or ganglia. [It is, however, possible to explain their effects by supposing that they act directly upon the muscular fibres of the blood-vessels, without the intervention of any nervous ganglionic structures.]

[Section of the spinal cord high up in the neck causes, of course, a great fall of the bloodpressure, owing to the division of the vaso-motor nerves. In the dog the pressure may fall to $30-40 \mathrm{~mm}$. Hg. After isolation of the cord, in rabbits alone, stimulation of the central end of a sensory nerve causes a rise of the blood-pressure; in dogs, however, under the same conditions, the blood-pressure falls. Dyspuœic blood also causes a rise of the blood-pressure, which is preceded by a fall (Ustimowitch). This reflex fall of the blood-pressure takes place after section of the splanchnies, and the nerves to the extremities, but it does not take place if the spinal cord be divided at the lumbar or lower dorsal region. If the cord be divided in the lower dorsal region, stimulation of the brachial plexus has no effect, while the fall occurs after stimulation of the central end of the sciatic. These experiments indicate that the vaso-dilator nerves which cause the fall of the blood-pressure arise in the lower part of the spinal cord (lumbar), and that they are probably contained in the visceral nerves and not in those for the extremities (Thayer and Pal).] 
In the muscles of the face, paralysed by extirpation of the facial nerve, stimulation of the ring of Vieussens causes pseudo-motor contractions of these muscles, just as stimulation of the chorda tympani causes such contractions in the paralysed tongue $(\$ 349,4)$, after section of the hypoglossal nerve (Rogowicz).

In analysing the vascular phenomena resulting from experiments on these nerves, we must He very careful to determine, whether the dilatation is the result of stimulation of the vasodilutms, or a consequence of paralysis of the vaso-constrictors. Psychical conditions act upon the ravo-lilator nerves-the blush of shame, which is not confined to the face, but may even expul over the whole skin, is probably due to stimulation of the vaso-dilator centre.

Influence on Temperature. - The vaso-lilator nerves obviously have a considerable influence (1in the trmpriuture of the body and on the heat of the individual parts of the body. Both vascular centres must act as important regulatory mechanisms for the radiation of heat through the cutaneous ressels $(\$ 214$, II.). P'robably they are kept in activity reflexly by sensory nerres. l histurbances in their function may lead to an abnormal accumulation of heat, as in fever $(\$ 220)$, or to abuormal cooling $(\$ 213,7)$. Some observers, however, assume the existence of an inirawanial "heat-regulating centre" (Tschetschichin, Naunyn, Quincke). According to Wood, s.paration of the medulla ollongata from the pons causes an increased radiation and a diminished froduction of heat, due to the cutting off of the influences from the heat-regulatiug centre $(\$ 37 \tau)$.

373. SPASM-CENTRE - SWEAT-CENTRE. - Spasm-Centre. - In the medulla oblongata, just where it joins the pons, there is a centre, whose stimulation causes generil sprisins. The centre may be excited by suddenly producing a highly venous condition of the blood ("asphyxia spasms"), in cases of drowning in mammals (but not in frogs), sudden aniemia of the medulla oblongata, either in conseluence of hamorrhage or ligature of both carotids and subclavians (Kussmaul and Tinner), and lastly, by sudden venous stagnation caused by compressing the veins comin from the head. In all these cases, the stimulation of the centre is due to the sudden intrrmption of the normal exchange of the !ases. When these factors act ynite gradually, death may take place without convulsions. Direct stimulation by means of chermicul substances (ammonia carbonate, potash, and soda salts, \&c.) applied to the medulla, quickly causes general convulsions (Pupellier). Intense direct merhenicul stimulation of the medulla, as by its sudden destruction, causes general convulsions.

Position. - Nothnagel attempted by direct stimulation to map out the position of the spasmrentre in ral,hits; it extends from the area above the ala cinerea upwards to the corpora quadrigemina. It is limited externally by the locus coruleus and the tuberculum acusticum. In the frog, it lies in the lower half of the 4 th ventricle (Houbl). The centre is affected in extensive reflex spasms $(\$ 364,6), c . y$. , in poisoning with strychnin and in hydrophobia.

Poisons.-Many inorganic and organic poisons, most cardiac poisons, nicotin, picrotoxin, ammonia $(\leqslant 2 \pi)$, and the conplounds of harium cause death after producing convulsions, by acting on the spasm-centre (Röber, Heubel, Böhm).

If the arteries going to the brain be ligatured so as to paralyse the medulla oblongata, then, on ligaturing the alulominal aorta, spasms of the lower limbs occur, owing to the anæmic stimulation of the motor ganglia of the spinal cord (Sigm. Mayer).

Pathological-Epilepsy. - Schrieeter van der Kolk found the blood-vessels of the oblongata dilated and increasel in cases of epilepsy. Brown-Séquard observed that injury to the central or peripheral nervous system (spinal cord, oblongata, peduncle, corpora quadrigemina, sciatic nerve) of guinea-pigs produced epilepsy, and this condition even became hereditary. Stimulation of the cheek or of the face "epileptic zone," on the same side as the injury (spinal cord), caused at once an attack of epilepsy; but when the peduncle was injured, the opposite side must be stimulated. Westphal made guinea-pigs epileptic by repeated light blows on the skull, and this condition also became hereditary. In these cases, there was effusion of blood in the medulla oblongata and upper part of the spinal cord ( $\$ 375$ and 378, I. $)$. Direct stimulation of the cerebrum also produces epileptic convulsions. Strong electrical stimulation of the motor areas of the cortex cerebri is often followed by an epileptic attack $(\$ 375)$. [It is no unfrequent occurrence while one is stimulating the motor areas of the cortex cerebri of a dog, to find the snimal exhibiting symptoms of local or general epilepsy.]

Sweat-Centre.--A dominating centre for the secretion of the sweat of the entire surface of the body ( $\$ 289$, II.) - with subordinate spinal centres $(\$ 362,8)$ nccurs in the medulla oblongata (Adamkiewicz, Marmé, Nawrocki). It is double, 
and in rare casses the excitability is unequal on the two sides, as is manifested by unilateral perspiration $(\$ 289,2)$.

Poisons.-Calabar bean, nicotin, picrotoxin, camphor, and ammonium acetate, cause a secretion of sweat by acting directly on the sweat-centre. Muscarin causes local stimulation of the peripheral sweat-fibres-it causes sweating of the hind limbs after section of the sciatic nerves. Atropin arrests the action of muscarin (Ott, Wood, Field, Nawrocki).

[Regeneration of the Spinal Cord.-In some animals, true nervous matter is reproduced after part of the spinal cord has been destroyed, at least this is so in tritons and lizards (H. Müller). In these animals, when the tail is removed, it is reproduced, and Miiller found that a part of the spinal cord corresponding to the new part of the tail is reproduced. Morphologically, the elements were the same, but the spinal nerves were not reproduced, while physiologically, the new nerve substance was not functionally active ; it corresponds, as it were, to a lower stage of development. According to Masius and Vanlair, an excised portion of the spinal cord of a frog is reproduced after six months; while Brown-Séquard maintains that re-union of the divided surfaces of the cord takes place in pigeons after six to fifteen months. A partial re-union is asserted to occur in dogs by Dentan, Naunyn, and Eichhorst, although Schieferdecker obtained only negative results, the divided ends being united only by connective-tissue (Schwalbe).]

374. PSYCHICAL FUNCTIONS OF THE BRAIN.--The hemispheres of the cerebrum are usually said to be the seat of all the psychical activities. Only when they are intact are the processes of thinking, feeling, and willing possible. After they are destroyed, the organism comes to be like a complicated machine, and its whole activity is only the expression of the external and internal stimuli which act upon it. The psychical activities appear to be located in both hemispheres, so that after destruction of a considerable part of one of them, the other seems to act in place of the part destroyed. [Objection has been taken to the term the "seat of " the will and intelligence, and undoubtedly it is more consistent with what we know, or rather do not know, to say, that the existence of volition and intelligence is dependent on the connection of the cerebral cortex with the rest of the brain.]

[That a certain condition of the cerebral hemispheres is necessary for the manifestation of the intellectual faculties, is admitted on all hands ; for, compression of the brain, e.g., by a depressed fracture of the skull, and sudden cessation of the supply of blood to the brain, abolish conscionsness. The intellectual faculties are affected by inflammation of the meninges involving the surface of the brain, the action of drugs affects the intellectual and other faculties; but while all this is admitted we cannot say preeisely upon what parts of the brain ideation depends. The pre-frontal area, or the convolutions in front of the ascending frontal supplied by the anterior cerebral artery, are sometimes regarded as the anatomical substratum of certain mental acts. At any rate, electrical stimulation of these parts is not followed by muscular motion, and, according to Ferrier, if this region be extirpated in the monkey, there is no motor or sensory disturbance in this animal ; it still exhibits emotional feeling, all its special senses remain, and the power of voluntary motion is retained; but, nevertheless, there is a decided alteration in the animal's character and behaviour, so that it exhibits considerable psychological alterations, and, according to Ferrier, "it has lost to all appearance the faculty of attention and intelligent observation."]

Observations on Man. - Cases in which considerable unilateral Iesions or destruction of one hemisphere have taken place, without the psychical activities appearing to suffer, sometimes occur. The following is a case communicated by Longet:-A boy, 16 years of age, had his parietal bone fractured by a stone falling on it, so that part of the protruding brain-matter had to be removed. On reapplying the bandages, more brain-matter had to be removed. After 18 days lie fell out of bed, and more brain-matter protruded, which was removed. On the 35th day he got intoxicated, tore off the bandages, and with them a part of the brain-matter. After his recovery, the boy still retained his intelligence, but he was hemiplegic. Even when both hemispheres are moderately destroyed, the intelligence appears to be intact; thus, Trousseau describes the case of an officer whose fore-brain was pierced transversely by a bullet. There was scarcely any appearance of his mental or bodily faculties being affected. In other cases, destruction of parts of the brain peculiarly alters the character. We must be extremely careful, however, in forming conclusions in all such cases. [In the celebrated "American crow-bar case " recorded by Bigelow, a young man was hit by a bar of iron $1 \frac{1}{4}$ inch in diameter, which traversed the anterior part of the left hemisphere, going clean out at the top of his head. This man lived for thirteen years without any permanent alterations of motor or sensory functions; but "the man's disposition and character were observed to have undergone a serious change. There were, however, some changes which might be referable to injury to the frontal region." In all cases it is most important to know both the exact site and the extent of the lesion. Ross 
points out that the characteristic features of lesions in the pre-frontal cortical region are afforded hy " usvehival disturbances, consisting of dementia, apathy, und somnolency."]

Imperfect development of the cerebrum. - Microcephalia and hydrocephalus yield every result between diminution of the psychical activities and idiocy. Extensive inflammation, degeneration, pressure, ancemia of the blood-vessels, and the actions of many poisons produce the same effect.

Flourens Doctrine.-Flourens assumed that the whole of the cerebrum is concerned in cery psychical process. From his experiments on pigeons, he concluded that, if a small part of the hemispheres remained intact, it was sufficient for the manifestation of the mental functions: just in proportion as the grey matter of the hemispheres is removed, all the functions of the cerebrum are enfeebled, and when all the grey matter is removed, all the fun tions are abolisherl. Accorling to this view, neither the diflerent faculties nor the different forceptions are localised in special areas. Goltz holds a somewhat similar view to that of Flourens, He assumes that if an uninjured part of the cerebrum remain, it can to a certain extent perform the functions of the parts that have been removed. This Vulpian has called the law of "functional substitution" (loi de suppléance).

The Phrenological doctrine of (iall $(+1828)$ and Spurzheim assumes that the different mental faculties are located in different parts of the brain, and it is assumed that a large development of a particular organ may be detected by examining the external configuration of the head (Cranioscopy).

Removal of the Cerebrum.-After the removal of both cerebral hemispheres, in most animals, every voluntary movement and all conscious impression and sensory perception entirely cease. On the other hand, the whole mechanical movements and the maintenance of the equilibrium of the movements are retained. The maintenance of the equilibrium depends upon the mid-brain, and is regulated by important reflex channels $(\$ 379)$.

sudlen cessation of the circulation in the hrain, e.g., by decapitation, is followed at once by cessation of the mental faculties. When Hayem and Barrier perfused the blood of a horse through the carotils of a decapitated dog's head, the head showed signs of consciousness, and will for 10 seconls, but not longer.

The mid-brain (corpora (juadrigemina) is connected not only with the grey matter of the spinal cord and medulla oblongata, the seat of extensive reflex mechanisms $(\$ 367)$, but it also receives tibres coming from the higher organs of sense, which also excite movements reflexly. The corpora quadrigemina are also supposed to contain a reflex inhibitory appraratus $(\$ 361,2)$. The joint action of all these parts makes the corpora quadrigemina one of the most important organs for the harmonious execution of movements, and this even in a higher degree than the medulia oblongata itself $(G o l t z)$. Animals with their corpora quadrigemina intact retain the equilibrium of their bodies under the most varied conditions, but they lose this power as soon as the mid-brain is destroyed $\left(G^{\prime}\right.$ oltz). Christiani locates the co-ordinating centre for the change of place and the maintenance of the equilibrium, in mammals, in front of the inspiratory centre in the 3rd ventricle $(\$ 368)$.

That impressions from the skin and sense-organs are concerned in the maintenance of the equilibrium, is proved by the following facts :- $\mathbf{\Lambda}$ frog without its cerebrum at once loses its power of balancing itself as soon as the skin is removed from its hind limbs. The action of impressions communicated through the eyes is proved by the difficulty or impossibility of maintaining the efuilibrium in nystagmus $(\$ 350)$, and by the vertigo which often accompanies jaralysis of the external ocular muscles. In yersons whose cutaneous sensibility is diminished, the eyes are the chief organs for the maintenance of the equilibrium; they fall over when the eyes are closed. [This is well illustrated in cases of locomotor ataxia (p. 647).]

Frog. - A frog with its cerebrum removed retains its power of maintaining its equilibrium. It can sit, spring, or execute complicated co-ordinated movements when appropriate stimuli are applied; when placed on its back, it immediately turns into its normal position on its belly; if stimulated it gives one or two springs, and then comes to rest; when thrown into water, it swims to the margin of the vessel, and it may crawl up the side, and sit passive upon the edge of the vessel. When incited to move, it exhibits the most complete harmony and unity in all its movements. Unless it is stimulated, it never makes independent, voluntary, purposive movements. It sits in the same place continually as if in 
sleep, it takes no food, it has no feelings of hunger and thirst, it shows no symptoms of fear, and ultimately, if left alone, it becomes desiccated like a mummy on the spot where it sits. [If the flanks of such a frog be stroked, it croaks with the utmost regularity according to the number of times it is stroked. Langendorff has shown that a frog croaks under the same circumstances, when both optic nerves are divided. It seems to be influenced by light; for, if an object be placed in front of it so as to throw a strong shadow, then on stimulating the frog it will spring not against the object, $a$, but in the direction, b (fig. 471). Steiner finds that if a glass plate be substituted for an opaque object like a book, the frog always jumps against this obstacle. Its balancing movements on a board are quite remarkable and acrobatic in character. If it be placed on a board, and the board gently inclined (fig. 472 ), it does not fall off, as a frog with only its spinal cord will do, but as the board is inclined, so as

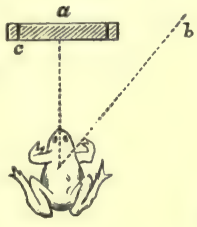

Fig. 471.

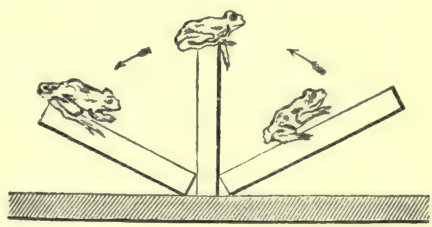

Fig. 472.

to alter the animal's centre of gravity, it slowly crawls up the board until its equilibrium is restored. If the board be sloped as in fig. 472 , it will crawl up until it sits on the edge, and if the board be still further tilted, the frog will move as indicated in the figure. It only does so, however, when the board is inclined, and it rests as soon as its centre of gravity is restored. It responds to every stimulus just like a complex machine, answering each stimulus with an appropriate action.]

A pigeon without its cerebral hemispheres behaves in a similar manner (fig. 473). Whien undisturbed it sits continuously, as if in sleep, but when stimulated, it shows complete harmony of all its movements; it can walk, fly, perch, and balance its body. The sensory nerves and those of special sensation conduct impulses to the brain; they only discharge reflex movements, but they do not excite conscious impressions. Hence, the bird starts when a pistol is fired close to its ear; it closes its eyes when it is brought near a flame, and the pupils contract; it turns away its head when the vapour of ammonia is applied to its nostrils. All these impressions are not per-

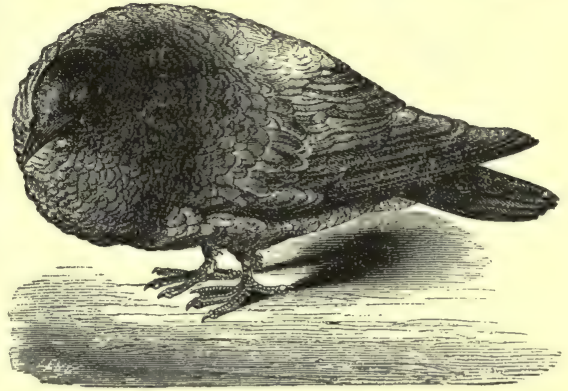

Fig. 473. ceived as conscious perceptions. The Pigeon with its cerebral hemispheres removed. perceptive faculties - the will and memory-are abolished; the animal never takes food or drinks spontaneously. But if food be placed at the back part of its throat it is swallowed [reflex act], and in this way the animal may be maintained alive for months (Flourens).

Fish appear to behave differently. A carp with its cerebrum removed (fig. 483, VI. 1) can see and may even select its food, and seems to execute its movements voluntarily (Steiner, Vulpian).

Mammals (rabbit), owing to the great loss of blood consequent on removal of the cerebrum, are not well suited for experiments of this kind. Immediately after the operation they show great signs of muscular weakness. When they recover, they present the same general phenomena; only when they are stimulated they run, as it were, blindfold against an obstacle. Vulpian observed a peculiar shriek or cry which such a rabbit makes under the circumstances. Sometimes even in man 
a peculiar cry is emitted in some cases of pressure or inflammation rendering the cerebral hemispheres inactive.

Observations on somnambulists show that in man, also, complete harmony of all movements may be retained, without the assistance of the will or conscious impressions and perceptions. As a matter of fact, many of our ordinary movements are accomplished without our being conscious of them. They take place under the guidance of the basal ganglia.

The degree of intelligence in the animal kingdom is in relation to the size of the cerebral lemispheres, in proportion to the mass of the other parts of the central nervous system. Taking the brain alone into consideration, we ohserve that those animals have the highest intelligence in which the cerebral hemispheres greatly exceed the mid-brain in weight. The mid-brain is represented by the optic lobes in the lower vertebrates, and by the corpora quadrigemina in the higher vertebrates. In fig. 483 , VI, represents the brain of a carp; V, of a frog; and $\mathrm{IV}$, of a pigron. In all these cases 1 indicates the cerebral hemispheres; 2 , the optic lubes; 3 , the cerebellum; and 4, the medulla oblongata. In the carp, the cerebral hemispheres are smaller than the optic lobes; in the frog, they exceed the latter in size. In the jigeon, the cerehrum begins to project backward over the cerebellum. The degree of intelligence increases in these animals in this proportion. In the dog's brain (fig. 483, II) the hemispheres completely cover the corpora quadrigemina, but the cerebellum still lies behind the cerebrum. In man the oceipital lobes of the cerebrum completely overlap the cerelvellum (fig. 479). [The projection of the occipital lobes over the cerebellum is due to the development of the frontal lobes pushing backwards the convolutions that lie behind them, and not entirely to increased development of the occipital lobes.]

Meynert's Theory.-According to Meynert, we may represent this relation in another way. As is known, filmes proceed downwarls from the cerebral hemispheres, through the crusta or hasis of the cerebral peduncle. These fibres are separated from the upper fibres or tegmentum of the perluncle by the locus niger, the tegmentum being connected with the corpora quadrigemina and the optic thalamus. The larger, therefore, the cerebral hemispheres, the more numerous will be the fibres proceeding from it. In fig. 461, II, is a transverse section of the posterior corpora quadrigemina, with the aqueduct of Sylvius and both cerebral peduncles of an adult man ; $p, p$, is the crusta of each peduncle, and above it lies the locus niger, $s$. Fig. 461, IV, shows the same parts in a monkey; III, in a $\log$; and $\mathrm{V}$, in a guinea-pig. The crusta diminishes in the above series. There is a corresponding diminution of the cerebral hemispheres, and, at the same time, in the intelligence of the corresponding animals.

Sulci and Gyri. - The degree of intelligence also depends upon the number and depth of the convolutions. In the lowest vertebrates (fish, frog, bird) the furrows or sulci are absent (fig. $461, \mathrm{IV}, \mathrm{V}, \mathrm{VI}$ ); in the rabbit there are two shallow furrows on each side (III). The dog has a completely furrowed cerebrum (I, II). Most remarkable is the complexity of the sulci and "onvolutions of the cerebrum of the elephant, one of the most intelligent of animals. Nevertheless, some very stupid animals, as the ox, have very complex convolutions.

The absolute weight of the brain cannot be taken as a guide to the intelligence. The clephant has absolutely the heaviest brain, but man has relatively the heaviest brain. [We uught also to take into account the complexity of the convolutions and the depth of the grey matter, its vascularity, and the extent of anastomoses between its nerve-cells. ]

Time an Element in all Psychical Processes. - Every psychical process requires a certain time for its occurrence-a certain time always elapses between the application of the stimulus and the conscious reaction.

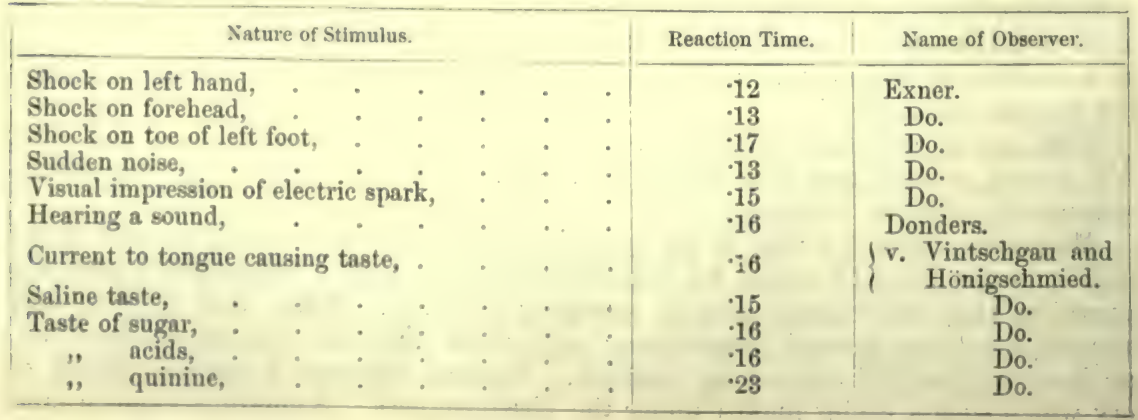


Reaction Time.-This time is known as "reaciion time," and is distinctly longer than the simple reflex time required for a reflex act. It can be measured by causing the person experimented on to indicate by means of an electrical signal the moment when the stimulus; is applied. The reaction time consists of the following events :-(1) The duration of perception, i.e., when we become conscious of the impression; $(2)$ the duration of the time required to direct the attention to the impression, i.e., the duration of apperception; and (3) the duration of the voluntary impulse, together with (4) the time required for conducting the impulse in the afferent nerves to the centre, and (5) the time for the impulse to travel outwards in the motor nerves. If the signal be made with the hand, then the reaction time for the impression of sound is 0.136 to 0.167 second ; for taste, 0.15 to 0.23 ; touch, 0.133 to 0.201 second (Horsch, v. Vintschgau and Hönigschmied); for olfactory impressions, which, of course, depend upon many conditions (the phase of respiration, current of air), 0.2 to 0.5 second. Intense stimulation, increased attention, practice, expectation, and knowledge of the kind of stimulus to be applied, all diminish the time. Tactile impressions are most rapidly perceived when they are applied to the most sensitive parts ( $v$. Vintschgau). The time is increased with very strong stimuli, and when objects difficult to be distinguished are applied ( $v$. Helmholtz and Baxt). The time required to direct the attention to a number consisting of 1 to 3 figures, Tigerstedt and Bergquist found to be 0.015 to 0.035 second. Alcohol and the anæsthetics alter the time ; according to their degree of action they shorten or lengthen it (Kraplin). In order that two shocks applied after each other be distinguished as two distinct impressions, a certain interval must elapse between the two shocks-for the ear, 0.002 to 0.0075 second ; for the eye, 0.044 to 0.47 second; for the finger, 0.277 second.

[The Dilemma. - When a person is experimented on, and he is not told whether the right or left side is to be stimulated, or what coloured disc may be presented to the eye, then the time to respond correctly is longer.]

[Drugs and other conditions affect the reaction time. Ether and chloroform lengthen it, while alcohol does the same, but the person imagines he really reacts quicker. Noises also lengthen it.]

In sleep and waking, we observe the periodicity of the active and passive conditions of the brain. During sleep, there is diminished excitability of the whole nervous system, which is only partly due to the fatigue of afferent nerves, but is largely due to the condition of the central nervous system. During sleep, we require to apply strong stimuli to produce reflex acts. In the deepest sleep the psychical or mental processes seem to be completely in abeyance, so that a person asleep might be compared to an animal with its cerebral hemispheres removed. Towards the approach of the period when a person is about to waken, psychical activity may manifest itself in the form of dreams, which differ, however, from normal mental processes. They consist either of impressions, where there is no objective cause (hallucinations), or of voluntary impulses which are not executed, or trains of thought where the reasoning and judging powers are disturbed. Often, especially near the time of waking, the actual stimuli may so act as to give rise to impressions which become mixed with the thoughts of a dream. The diminished activity of the heart $(\S 70,3, c)$, the respiration $(\$ 127,4)$, the gastric and intestinal movements $(\S 213,4)$, the formation of heat $(\S 216,4)$, and the secretions, point to a diminished excitability of the corresponding nerve-centres, and the diminished reflex excitability to a corresponding condition of the spinal cord. The pupils are contracted during sleep, the deeper the latter is; so that in the deepest sleep they do not become contracted on the application of light. The pupils dilate when sensory or auditory stimuli are applied, and the lighter the sleep, the more is this the case; they are widest at the moment of awaking (Plotke). [Hughlings Jackson finds that the retina is more anæmic than in the waking state.] During sleep, there seems to be a condition of increased action of certain sphincter muscles-those for contracting the pupil and closing the eyelids (Rosenbach). The soundness of the sleep may be determined by the intensity of the sound required to waken a person. Kohlschïtter found that at first sleep deepens very quickly, then more slowly, and the maximum is reached after one hour (according to Mönninghoff and Priesbergen after $1 \frac{3}{4}$ hour); it then rapidly lightens, until several hours before waking it is very light. External or internal stimuli may suddenly diminish the depth of the sleep, but this may be followed again by deep sleep. The deeper the sleep, the longer it lasts. [Durham asserts that the brain is anæmic, that the arteries and veins of the pia mater are contracted during sleep and the brain smaller; but is this cause or effect?]

The cause of sleep is the using up of the potential energy, especially in the central nervous system, which renders a restitution of energy necessary. Perhaps the accumulation of the decomposition-products of the nervous activity may also act as producers of sleep (? lactatesPreyer). Sleep cannot be kept up for above a certain time, nor can it be interrupted voluntarily. Many narcotics rapidly produce sleep. [The "diastolic phase of cerebral activity," as sleep has been called, is largely dependent on the absence of stimuli. We must suppose that there are two factors, one central, represented by the excitability of the cerebrum, which will vary under different conditions, and the other external, represented by the impulses reaching the cerebrum through the different sense-organs. We know that a tendency to sleep is favoured by removal of external stimuli, by shutting the eyes, retiring to a quiet place, \&c. The external sensory 
impressions, indeed, influence the whole metabolism. Strumpell describes the case of a boy whose sensory inlets were all paralysed except one eye and one ear, and when these inlets were closed the boy fell asleep, showing how intimately the waking condition is bound up with sensory afferent impulses reaching the cerebral centres.]

[Hypnotics, such as opium, morphia, KBr, chloral, are drugs which induce sleep.]

Hypnotism, or Animal Magnetism. - [Most important observations on this subject were made by Brail of Manchester, whose results are confirmed by many of the recent re-discoveries of Weinhold, Heidenhain, and others.] Heidenhain assumes that the cause of this condition is due to an inhibition of the ganglionic cells of the cerebrum, produced by continuous feeble stimulation of the face (slightly stroking the skin or electrical applications), or of the optic nerve (as by gazing stealily at a small brilliant object), or of the auditory nerve (by uniform sounls): while sulden and strong stimulation of the same nerves, especially blowing upon the face, abolishes the condition. Berger attributes great importance [as did Carpenter and Braid long agol to the psychological factor, whereby the attention was directed to a particular part of the buly. The facility with which different persons become hypnotic varies very greatly. When the hypuotic condition has been produced a number of times, its subsequent occurrence is farilitated, e.g., by merely pressiug upon the brow, by placing the body passively in a certain position, or ly stroking the skin. In some people the mere idea of the condition suffices. A hypuotised person is no longer able to open his eyelids when they are pressed together. This is fullowed by spasm of the apparatus for accommolation in the eye, the range of accommodation is diminished, and there may be deviation of the position of the eyeballs ; then follow phenomena of stimulation of the sympathetic in the oblongata; dilatation of the fissure of the eyelids and the pupil, exophthalmos, and increase of the respiration and pulse. At a certain stage, there may he a great increase in the sensitiveness of the functions of the sense-organs, and also of the muscular sensibility. Afterwards there may be analgesia of the part stroked, and loss of taste; the sense of temperature is lost less readily, and still later that of sight, of smell, and of hearing. Owing to the abolition or suspension of consciousness, stimuli applied to the sense-organs do not produce conscious impressions or perceptions. But stimuli applied to the sense-organs of a hypnotised person cause movements, which, however, are unconscious, although they simulate voluntary a.ts. In persons with greatly increased reflex excitability, voluntary movements may exite reflex spasms; the person may be unable to co-ordinate his organs for speech.

Types. - According to Griitzner, there are several forms of hypnotism :-(1) Passive sleep, where words are still understood, which occurs especially in girls; (2) owing to the inereased reflex exitability of the striped museles, certain groups of muscles may be contracted-a condition which may last for days, especially in strong people; at the same time ataxia may occur, and the muscles may fail to perform their functions (artificial katalepsy). During the stage of lethargy in hysterical persons, the tendon reflexes are often absent (Charcot and Richer); (3) untonomy ut call, i.e., the hymotised person-in most cases the consciousness is still retainedobeys a command, in his condition of light sleep. When the hand is grasped or the head stroked, he executes involuntary movements - runs about, dances, rides on a stool, and the like; (4) hullucinutions occur only in some individuals when they waken from a deep sleep, the hallucinations (usually consisting of the sensation of sparks of fire or odours) being very strong and wellpronounced; (5) imitation is rare, ordinary movements, such as walking, are easily imitated, the finer movements occur rarely. The "echo-speech" is produced by pressure upon the neck, speaking into the throat, or against the ablomen. Pressure over the right eyebrow often ushers in the speech. Colour-sensation is suspended by placing the warm hand on the eye, or by stroking the opposite side of the head $(\mathrm{Cohn})$. Stroking the limbs in the reverse direction grailually removes the rigility of the limbs and causes the person to waken. Blowing on a part does sn at once. Insane persons can be hypnotised. Disagreeable results follow only when the condition is induced too often and too continuously.

Hypnotism in Animals. - A hen remains in a fixed position when an object is suddenly placed before its eyes, or when a straw is placed over its beak, or when the head of the animal is pressed on the ground and a chalk line made before its beak (Kircher's experimentum mirabile, 1644). [Langley has hypnotised a crocodile.] Birds, rabbits, and frogs remain passive for a time after they have been gently stroked on the back. Crayfish stand on their head and claws (Czermak).

375. STRUCTURE OF THE CEREBRUM-MOTOR CORTICAL CENTRES. - [Cerebral Convolution. - A vertical section of a cerebral convolution consists of a thin layer of grey matter externally enclosing a white core (fig. 478). The cortex consists of cells and fibres embedded in a matrix, and to the nerve-cells nerve-fibres proceed from the white matter. The nerve-cells of the cortex vary in size, form, and distribution in the different layers and also in different convolutions. Taking such a convolution as the ascending frontal or motor-area type, we get the appearances shown in fig. 474. It is covered on its surface by the pia mater. (1) The most superficial layer is narrow, and consists of much neuroglin, a network of branched nervefibrils, a few scattered small multipolar nerve-cells, and a layer of very small medullated nervefibres: (2) a layer of close-set small, angular, or short pyramidal nerve-cells ; (3) the thickest layer or formation of the cornu ammonis, consisting of many layers of larger pyramidal cells, 


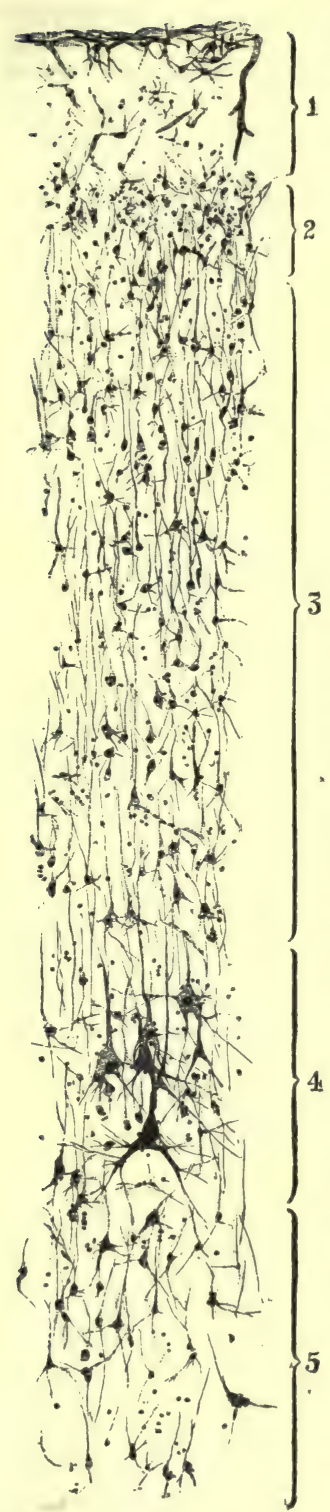

Fig. 474.

which are larger in the deeper than in the more superficial layers. They are not so closely packed together, as many granules lie between them. At the lowest part of this layer, the cells are larger than elsewhere, presenting some resemblance to the cells of the anterior cornu of the grey matter of the spinal cord. By some it is described as a special layer, and termed the ganglioncell layer. This layer is specially well marked in those convolutions which are described as containing motor centres. Amongst the large cells are a few small angular-looking cells, which become more numerous lower down, and from (4) a narrow layer of numerous small, branched, irregular, ganglionic cells-the "granular formation" of Meynert. In the motor areas mixed with these are large pyramidal cells, disposed in groups called "cell-clus-

3 ters." (5) A layer of spindle-shaped fusiform branched cells-the claustral formation of Meynert-lying for the most part parallel to the surface of the convolution. No layer is composed exclusively of one form of cell. The above represents the motor type. Then follows the white matter $(m)$, consisting of medullated nerve-fibres, which run in groups into the grey matter, where they lose their myelin. The fibres are somewhat smaller than in the other parts of the nervous system (diameter $\frac{1}{1000}$ inch), and between them lie a few nuclear elements.]

[In the sensory type, as in the occipital lobe (fig. 475), the first and second layers are not unlike the corresponding layers in the motor type, and the fusiform cells in the seventh layer also resemble the latter. The layer of pyramidal cells (3) is not so large, while its deeper part, sometimes called the "ganglion-cell layer," contains no large cells. (5) Between the two is (4) a layer with numerous angular granulelike bodies or cells, called the "granulelayer."]

5 [The hippocampus major contains, besides a layer of neuroglia and some white matter on the surface, a regular series of pyramidal cells, which give it a characteristic appearance. This is the part which varies most. It is to be remembered that the transition from one

Cortex of motor area type to the other takes place gradually.] of brain of monkey [Pyramidal Cells of the Cortex. $(x$ 150). 1, super- Each cell is more or less pyramidal in ficial layer; 2, small shape, giving off several processes- $(a)$ angular cells ; 3, py- an apical process, which is often very ramidal cells; 4, gan-long, and rưns towards the surface of glionic cells and cell- the cerebrum, where it is said to terclusters ; 5, fusiform minate in an ovoid corpuscle, closely cells (Ferrier, after Bevan resembling those in which the ultimate Lewis). branches of Purkinje's cells of the cere-

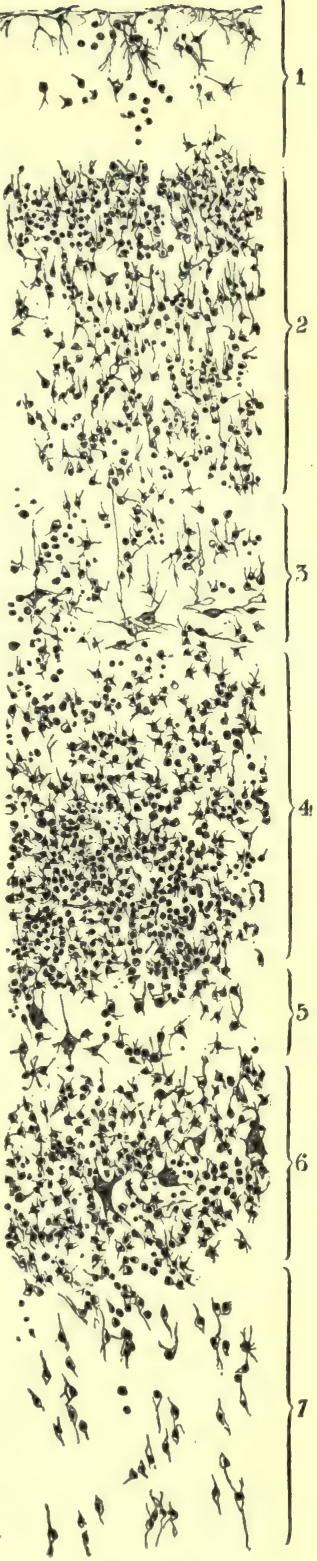

Fig. 475.

Cortex of occipital lobe. 1 , superficial layer; 2 , small angular cells ; 3,5 , pyramidal cells ; 4, granule layer; 6 , granules and ganglionic layer ; 7, spindle-cells (Ferrier, after Bevan Lewis). 
bellum end ; ( $($ ) the unbrauched median basilar process, which is an axial cylinder process, and becomes continuous with the axial cylinder of a nerve-fibre of the white matter. It ultimately becomes invested by myelin. (c) The lateral processes are given off chiefly near the base of

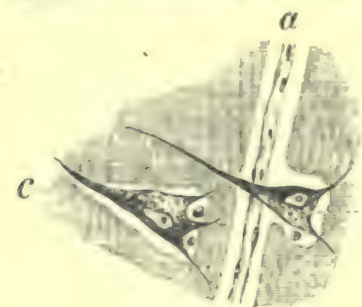

Fig. 476 . the cell, and they soon branch to form part of the ground plexus of fibrils which everywhere pervades the grey matter.]

Each cell is surrounded by a lymph-space, and so are the bloodvessels, in the latter case forming a perivascular space, which com6 municates with the pericellular lymph-space, as in fig. 476 .

[Nerve-fibres in the Cortex. The ordinary methods of hardening the brain do not enable us to detect Perivascular and pericellular the enormous number of medullymph-spaces. $a$, capillary lated nerve-fibres in the grey with a lymph-space communi- matter. By using Exner's osmic. cating with the vericellular acid method, or Weigert's or Pal's lymph-space $b$, round the cell method, we obtain such a result a lympli-space $c$, containing as is shown in fig. 477. Under the two lymull-corpuscles. $\times 150$. pia $(P)$ is a layer of connectivetissue $(a)$ devoid of nerve-fibres. Beneath it is a layer (b) occuyying about the half of the outer layer, which is almost entirely taken up by medullated uerve-fibres; most of these are fine, but a few of them are coarse, and run parallel to the surface and tangential to the arc of the outer contour of the convolution. Internal to this is a layer of medullated fibres (c), which cross each other in various directions ; while a similar network $(d)$ occurs in the small-celled layer. (2) In the layer of large pyramidal cells (3) there are bundles of medullated fibres, running radially $(e)$; but at the lower part of this layer there is a very dense network $(f)$, forming (in a Weigert's preparation) a dense, dark band, corresponding to the outer layer of Baillanger. In the layers marked $(g$ and $h$ ), which are partly in the third and partly in the fourth cortical layer, the radial arrangement is more marked and more compact, and the thick fibres are more numerous. In the middle is $(h)$ a narrow dense network corresponding to Baillanger's inuer layer. The lower part of the fourth layer, and the whole of the fifth, are occupied by $i$. It is to be remembered, that all the convolutions do not present exactly the same structure and arrangement (Obersteiner).]

[Variations.-The grey matter differs in different parts of the brain. In the grey matter of the cornu ammonis, the large pyramidal cells of (3) make up the chief mass; in the claustrum (4) is most abuniant. In the central convolutions (ascending frontal and parietal), according to Betz, Mierzejewski, and Bevan Lewis, very large pyramiclal cells are found in the lower part of the third layer. Similar cells have been found in the posterior extremities of the frontal convolutions in some auimals-the posterior parietal lobule, and para-eentral lobule, all of which have motor functions. In those convolutions which are regarded as subserving sensory functions, a somewhat different type prevails, e.g., the occipital gyri or annectant convolution (B. Lewis). The very large pyramidal cells are absent, while the granule layer exists as a well-marked layer between the layer of large pyramidal cells and the ganglion cell-layer (fig. 475).]

[Fuchs finds that there are no medullated fibres either in the cortex or medulla until the end of the first month of life. The medullated fibies appear in the uppermost layer at the fifth month, and in the second at the end of the first year, the radial bundles in the deeper layers at the second month. The uedullated fibres increase until the seventh or eighth year, when they have the same arrangement as in the adult.]

[Blood-Vessels. - The adventitia of the small cerebral vessels contains pigment and granular cells, filled with oil-granules. In the new-born child, the blood-vessels of the brain are beset with cells, filled with fatty granules. Perhaps the granules supply part of the material for the formation of the myelin sheath on the nerve-fibres. About the fifth year, the fat is replaced by a yellow pigment. In adults, yellow or brown glancing pigment-granules are found in the 
adventitia of the arteries. In the adventitia of the veins there is no pigment, but generally some fat. The grey matter is much more vascular than the white, and when injected, a section of a convolution presents the appearance shown in fig. 478. The nutritive arteries consist of - (a) the long medullary arteries (1) which pass from the pia mater through the grey matter into the central white matter or centrum ovale. They are terminal arteries, and do not communicate with each other in their course; thus, they supply independent vaseular areas; nor do they anastomose with any of the arteries derived from the ganglionic system of bloodvessels; 12 , to 15 of them are seen in a section of a convolution. $(b)$ The short cortical nutritive arteries $(2)$ are smaller and shorter than the foregoing. Although some of them enter the white matter, they chiefly supply the cortex, where they form an open meshed plexus in the first layer $(a)$, while in the next layer $(b)$ the plexus of capillaries is dense, the plexus again being wider in the inner layers $(c)$.]

[Central or Ganglionic Arteries.-From the trunks constituting the circle of Willis (fig. in $\S 381$ ), branches are given off, which pass upwards and enter the brain to supply the basal ganglia with blood. They are arranged in several groups, but they are all terminal, each one supplying its own area, nor do they anastomose with the arteries of the cortex.]

Cerebral Arteries. - From a practical point of view, the distribution of the blood-vessels of the brain is important. The artery of the Sylvian fissure supplies the motor areas of the brain in animals; in man, the precentral lobule is supplied by a branch of the anterior cerebral artery (Duret). The region of the third left frontal convolution, which is connected with the function of speech, is supplied by a special branch of the Sylvian artery. Those areas of the frontal lobes whose injury results in disturbance of the intelligence, are supplied by the anterior cerebral artery. Those regions of the cortex cerebri, whose injury, according to Ferrier, causes hemianæsthesia, are supplied by the posterior cerebral artery.

[In connection with the localisation of the centres in the cortex, it is important to be thoroughly acquainted with the arrangement of the cerebral convolutions. Each half of the outer cerebral surface is divided by certain fissures into five lobes-frontal, parietal, occipital, temporo-sphenoidal, and central, or island of Reil (fig. : 481). The

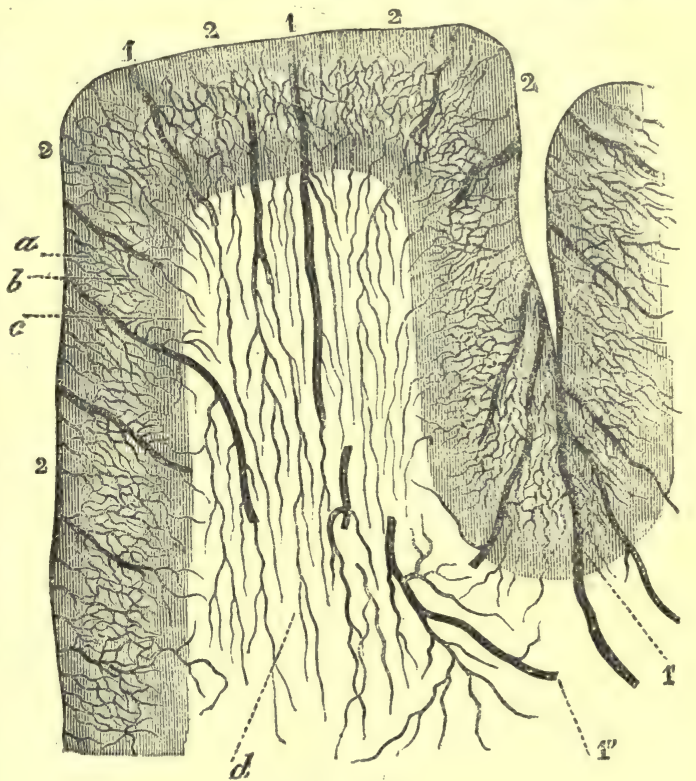

Fig. 478.

1,1 , medullary'arteries; and $1^{\prime}, 1^{\prime}$, in groups between the convolutions; 2,2 , arteries of the cortex cerebri ; $a$, large meshed plexus in first layer; $b$, closer plexus in middle layer; $c$, opener plexus in the grey matter next the white substance, with its vessels $(d)$.

frontal lobe (fig. 479) consists of three convolutions, with numerous secondary folds running nearly horizontal, named superior $\left(\mathrm{F}_{1}\right)$, middle $\left(\mathrm{F}_{2}\right)$, and inferior $\left(\mathrm{F}_{3}\right)$ frontal convolutions. Behind these is a large convolution, the ascending frontal (A), which ascends almost vertically, immediately behind these-separated from them, however, by the præcentral fissure $\left(f_{3}\right)$, and mapped off behind by the fissure of Rolando, or the central sulcus $(c)$.

[The parietal lobe (fig. 479, P) is limited in front by the fissure of Rolando, below in part by the Sylvian fissure, and behind by the parieto-occipital fissure. It consists of the ascending parietal (posterior central) convolution (fig. 479, B), which ascends just behind the fissure of Rolando, and parallel to the ascending frontal, with which it is continuous below; above, it becomes continuous with the superior parietal lobule $\left(\mathrm{P}_{1}\right)$, while the latter is separated from the inferior parietal lobule ("pli courbe") by the interparietal sulcus. The inferior parietal lobule consists of $(\alpha)$ a part arching over the upper end of the Sylvian fissure, the supra-marginal convolution $\left(\mathrm{P}_{2}\right)$, which is continuous with the superior temporo-sphenoidal convolution. Behind is $(b)$ the angular gyrus $\left(\mathrm{P}_{2}^{\prime}\right)$, which arches round the posterior end of the parallel fissure, and becomes connected with the middle temporo-sphenoidal convolution.]

[The temporo-sphenoidal or temporal lobe (fig. 479, T) consists of three horizontal convolu. 
tions-superior, middle, and inferior-the two former being separated by the parallel sulcus, while the whole lobe is mapped off from the frontal by the Sylvian fissure (S).]

[The occipital lobe (fig. $479, \mathrm{O}$ ) is small, forms the rounded posterior end of the cerebrum, and is sejarated from the parietal lobe by the paricto-occipital fissure, which fissure is bridged over at the lower part by the four anuectant gyri (plis de passage of Gratiolet). It has three convolutions superior $\left(\mathrm{O}_{1}\right)$, midule $\left(\mathrm{O}_{2}\right)$, and inferior $\left(\mathrm{O}_{3}\right)$ - on its outer surface.]

The central lobe or island of Reil, consists of five or six short, straight convolutions (gyri operti-fig. 481). radiating outwards and backwards from near the anterior perforated spot, and can only he seen when the margins of the Sylvian fissure are pulled asunder. The operculum, consisting of the extremities of the inferior frontal, ascending parietal, and frontal convolutions, lie outside it, cover it, and conceal it from view. ]

(U) the inner or mesial surface of the cerebrum are-the gyrus fornicatus (fig. 480, Gf), or convinlution of the corpus callosum, which ruus parallel to and bends round the anterior and

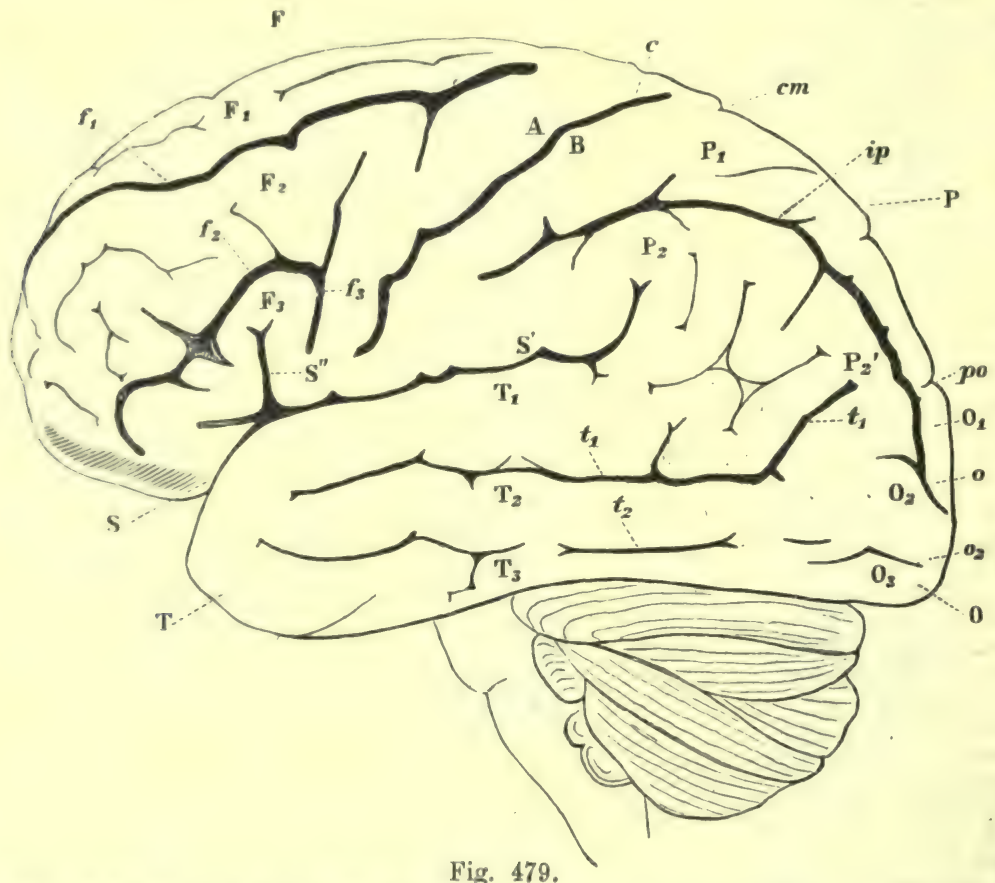

Left sile of the human brain (diagrammatic). F, frontal; $\mathrm{P}$, parietal; O, occipital; T, tempro-splienoidal lobe; S, fissure of Sylvius; $S^{\prime}$, horizontal, $\mathbf{S}^{\prime \prime}$, ascending ramus of $\mathbf{S}$; c, sulcus centralis, or fissure of Rolando; $\Lambda$, ascending frontal, and $B$, ascending parietal convolution; $\mathrm{F}_{1}$, superior, $\mathrm{F}_{2}$, middle, and $\mathrm{F}_{3}$, inferior frontal convolutions; $f_{1}$, superior, and $f_{2}$, inferior frontal fissures; $f_{3}$, sulcus precentralis; $\mathrm{P}$, superior parietal lobule ; $\mathrm{P}_{2}$, inferior parietal lolule, consisting of $\mathrm{P}_{2}$, supra-marginal gyrus, and $\mathrm{P}_{2}^{\prime}$, angular gyrus; ip, sulcus interparietalis; $\mathrm{cm}$, termination of calloso-marginal fissure ; $\mathrm{O}_{1}$, first, $\mathrm{O}_{2}$, second, $\mathrm{O}_{\mathrm{g}}$, third occipital convolutions; po, parietal-occipital fissure; 0 , transverse occipital fissure ; $o_{2}$, inferior longitudinal occipital fissure; $\mathrm{T}_{1}$, first, $\mathrm{T}_{2}$, second, $\mathrm{T}_{3}$, third temporosphenoidal convolutions; $t_{1}$, first, $t_{9}$, second temporo-sphenoidal fissures.

posterior extremities of the corpus callosum, terminating posteriorly in the gyrus uncinatus or gyrus hiplocampi (fig. 480, H), and ending anteriorly in a crooked extremity, the subiculum cornu ammonis (fig. 480, U). Above it is the calloso-marginal fissure (fig. $480, \mathrm{~cm}$ ), and ruming parallel to it is the marginal convolution (fig. 480), which lies between the latter fissure and the margin of the longitudinal fissure; it is, however, merely the mesial aspect of the frontal and parietal convolutions. The quadrate lobule or præcuneus lies (fig. 480, $\mathrm{Pi}$ ), between the posterior extrenity of the calloso-marginal fissure and the parieto-occipital fissure; it is merely the mesial aspect of the ascending parietal convolution. The parieto-occipital 
fissure terminates below in the calcarine fissure (fig. 480,oc), and the latter runs backwards in the occipital lobe dividing it into two branches, $o c^{\prime}, o c^{\prime \prime}$. Between the parieto-occipital and calcarine fissures lies the wedge-shaped lobule termed the cuneus (fig. 480, Oz). The calcarine fissure indicates on the surface the position of the calcar avis or hippocampus minor, in the posterior cornu of the lateral ventricle. The dentate fissure or sulcus hippocampi (fig. 480, $h$ ) marks the position of the elevation of the hippocampus major, or cornu ammonis, in the lateral ventricle. The temporo-sphenoidal lobe terminates anteriorly in the uncinate gyrus, while, running along the former and the occipital lobes, is the collateral fissure (occipito-temporal sulcus), which marks the position of the eminentia collateralis in the descending cornu of the lateral ventricle, while it also separates the superior from the inferior temporo-occipital convolutions $\left(\mathrm{T}_{4} \text { and } \mathrm{T}_{5}\right)_{\text {.] }}$

Motor Centres.-In 1870 Fritsch and Hitzig discovered a series of circumscribed regions on the surface of the cerebral convolutions, whose stimulation

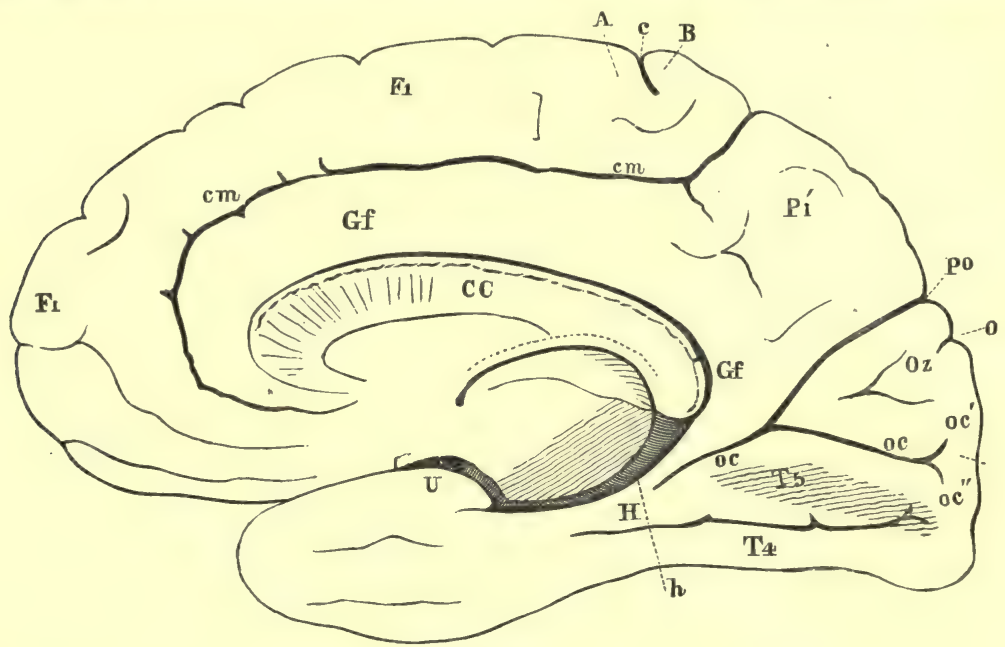

Fig. 480.

Median aspect of the right hemisphere. CC, corpus callosum divided longitudinally; Gf, gyrus fornicatus; $\mathrm{H}$, gyrus hippocampi ; $h$, suleus hippocampi; $\mathrm{U}$, uncinate gyrus ; $\mathrm{cm}$, calloso-marginal fissure; F, first frontal convolution; $c$, terminal portion of fissure of Rolando; A, ascending frontal ; B, ascending parietal convolution and paracentral lobule ; $\mathrm{P}_{1}^{\prime}$, præcuneus or quadrate lobule; $\mathrm{Oz}$, cuneus; $\mathrm{Po}$, parieto-occipital fissure; $o_{1}$, transverse occipital fissure ; $o c$, calcarine fissure; $o c^{\prime}$, superior, $o c^{\prime \prime}$, inferior ramus of the same ; $\mathrm{D}$, gyrus descendens; $\mathrm{T}_{4}$, gyrus occipito-temporalis lateralis (lobulus fusiformis); $\mathrm{T}_{5}$, gyrus occipito-temporalis medialis (lobulus lingualis).

by means of electricity causes co-ordinated movements in quite distinct groups of muscles of the opposite side of the body (fig. 483, I, II).

Methods-Stimulation. - The surface of the cerebrum is exposed in an animal (dog, monkey) by removing a part of the skull covering the so-called motor convolutions and dividing the dura mater. When the convolutions are fully exposed, a pair of blunt non-polarisable (\$328) needle electrodes are applied near each other to various parts of the cerebral surface. We may employ the closing or opening shock of a constant current, or the constant current may be rapidly interrupted, the current being of such a strength as to be distinctly perceived when it is applied to the tip of the tongue (Fritsch and Hitzig). Or, the induced current may be used, also of such a strength that it is readily felt when applied to the tip of the tongue (Ferrier, 1873). The cerebrum is completely insensible to severe operations made upon it.

The areas of the cerebral cortex, whose stimulation discharges the characteristic movements, are regarded by some as actual centres, because the reaction-time after stimulation of the centres and the duration of the muscular contraction are longer than when the subcortical fibres which lead towards the deeper parts of the brain are stimulated. Another circumstance favouring this view is, that the excitability 
of these areas is influenced by the stimulation of afferent nerves (Bubnoff und Heidenhrin). It may be that these centres are acted upon by voluntary impulses in the execution of voluntary movements. Hence, they have been called "psychomotor centres." [At auy rate, these areas have a definite relation to certain motor acts,

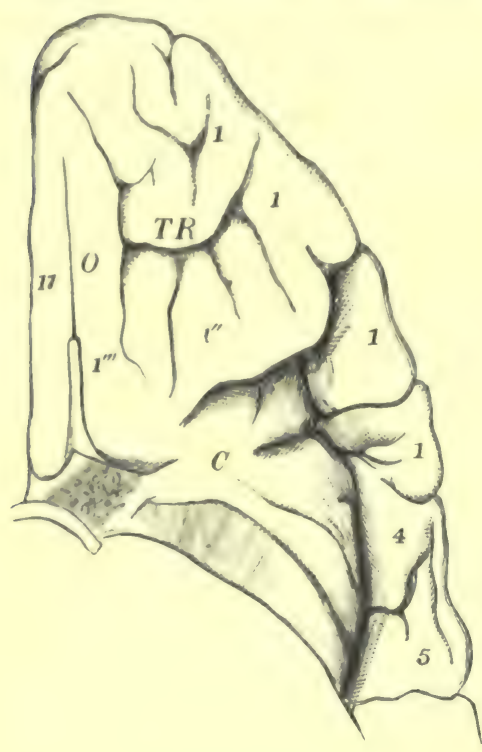

Fig. 481.

Orbital surface of the left frontal lobe and the island of Reil, the tip of the temporosphenoidal lobe removed to show the latter. 17 , convolution of the margin of the longitudinal fissure ; $O$, olfactory fissure, with the olfactory lobe removed; TR, triradiate fissure; $1^{\prime \prime}$ and $1^{\prime \prime \prime}$, convolutions on the orbital surface; $1,1,1,1$, under surface of the infero-frontal convolution; 4 , undersurface of the ascending frontal, and 5 of the ascending parietal convolutions; C, central lobe or island.

und perhaps it is well to speak of them as "areas of representation" of the function to which they are related.] The motor areas of the cerebrum (dog, cat, sheep) are characterised by the presence of specially large pyramidal cells (Betz, Merzejewsky, Bevan Lewis); while similar cells were found by Obersteiner in the areas marked 4 and 8 (fig. 483 ), and Betz found them in the ascending frontal convolution of man, in the third frontal convolution, and in the island of Reil. O. Soltmann found that stimulation of the motor areas in newly-born animals is without result, while only the deeper fibres of the corona radiata are excitable.

Modifying Conditions. - In the condition of deep narcosis produced by chloroform, ether, chloral, morphia, or in apnoea, the excitability of the centres is abolished (Schiff), whilst the subcortical conducting paths still retain their excitability (Bubnoff and Heidenhain). Small doses of these poisons and also of atropin at first increase the excitability of the centres. Moderate loss of blood excites them, while a great loss of blood diminishes and then abolishes the excitability (Munk and Orschansky). Slight inflammation increases, while cooling diminishes, the excitability. If the cortex cerebri be removed in animals, the excitability of the fibres of the corona radiata is completely abolished about the fourth day, just as in the case of a peripheral nerve separated from its centre (Albertoni, Dupuy, Franck and Pitres).

Stimulation of Subcortical Parts. - As the fibres of the corona radiata converge towards the centre of the hemisphere, it is evident that, after removal of the cortex, stimulation of these fibres in the deeper parts of the hemisphere is followed by the same motor results (Gliky and Eckhard). The stimulus is applied merely to a deeper part of the motor path. If the stimulus be applied to parts situated still more deeply, as for example to the internal cupsule, general contraction of the muscles on the opposite side is the result.

Time Relations of the Stimulation.-According to Franck and Pitres, the time which elapses between the moment of stimulation of the cortex and the resulting movement, after deducting the periorl of latent stimulation for the muscles, and the time necessary for the conduction of the impulse through the cord and nerves of the extremities, is 0.045 second. Heidenhain and Bulmoff found that, luring moderate morphia narcosis, when the stimulating current was increased in strength, the muscular contraction and the reaction-time became shorter. After removal of the cortex, the occurrence of the muscular contraction from the moment of stimulation of the white matter is diminished $\frac{1}{4}$ to $\frac{1}{3}$. The form of the muscular contraction is longer and more extended when the cortex, than when the subcortical paths, are stimulated. If the animal (dog) be in a state of high reflex excitability, these differences disappear ; in both cases the contraction follows very rapidly (Bubnoff and Heidenhain). If the stimulus he very strong the muscles of the same side may contract, but somewhat later than those of the opposite side. If the motor areas for the fore and hind limbs be stimulated simultaneously, the latter contract somewhat after the former.

Number of Stimuli. - If 40 stimuli per second be applied to a motor area, then the corre. sponding muscles yield 40 single contractions; while with 46 single stimuli per second there results a continued complete contraction (Franck and Pitres). In one and the same animal, 
the same number of stimuli is required to produce a continuous contraction, whether the cortical centre, the motor nerve, or even the muscle itself be stimulated. With very feeble stimuli, summation of stimuli takes place, for the muscular contraction only begins after several ineffective stimuli have been applied. [It is generally held that the rhythm of a contracting muscle is the same as the rhythm of the stimuli applied to its motor nerve, but Schäfer and Horsley contend that this holds good for rates of stimuli to about 10 or 12 per second. They find that the same is true for the cortex cerebri, corona radiata, and medulla spinalis, viz., that the muscular response does not vary with the rhythm, i.e., number of stimuli per sec.), but that the rhythm is constant-about 10 per sec. - and independent of the number of stimuli per sec., provided they are above 10 per sec. applied to these parts. Indeed, all voluntary contractions show a similar rate of undulation in the muscle-curve. Perhaps the rhythm of the efferent impulses is modified in the motor nerve-cells of the spinal cord.]

[The matter, as regards electrical stimulation of the cortex cerebri, resolves itself into this, that stimulation of certain cortical areas always causes contraction in

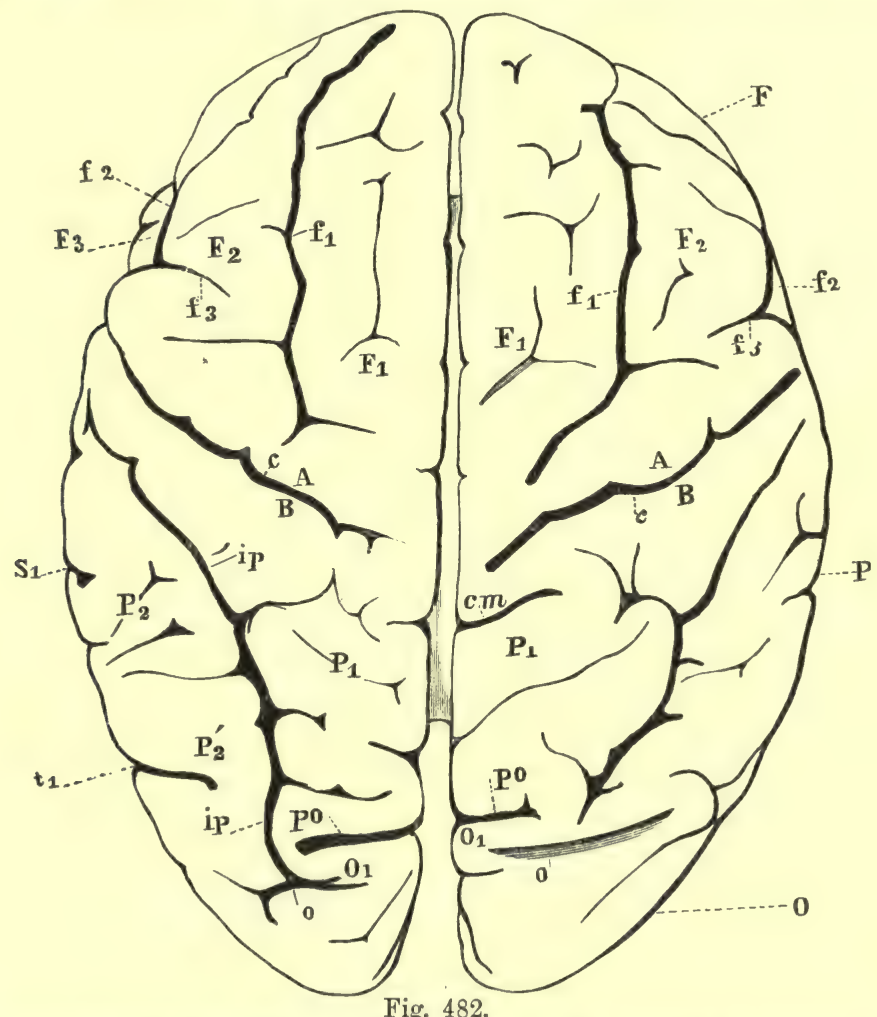

Fig. 482.

View of the brain from above (semi-diagrammatic). $\mathbf{S}_{1}$, end of ramus of the Sylvian fissure.

The other letters refer to the same parts as in fig. 479 .

definite muscles or groups of muscles, resulting in definite co-ordinated movements on the opposite side of the body ; the areas have been called "motor areas." They have been mapped out and ascertained in a large number of animals, and the question comes to be-Are there similar areas.in man ?]

Primary Fissures and Convolutions of the Dog's Brain.-The position of the motor centres in the dog's brain is indicated in fig. 483, I and II. The dog's brain is marked by two "primary fissures," viz., the sulcus cruciatus (S), which intersects the longitudinal fissure at a right angle at the junction of its anterior with its middle third. This fissure has been called the sulcus frontalis, or the fissura coronalis. The second primary fissure is the fossa Sylvii $(F)$. 
Four " primary convolutions," in addition, are arranged with reference to these primary fissures. The first primary convolution (I), in the form of a sharply curved knee, embraces the fossa
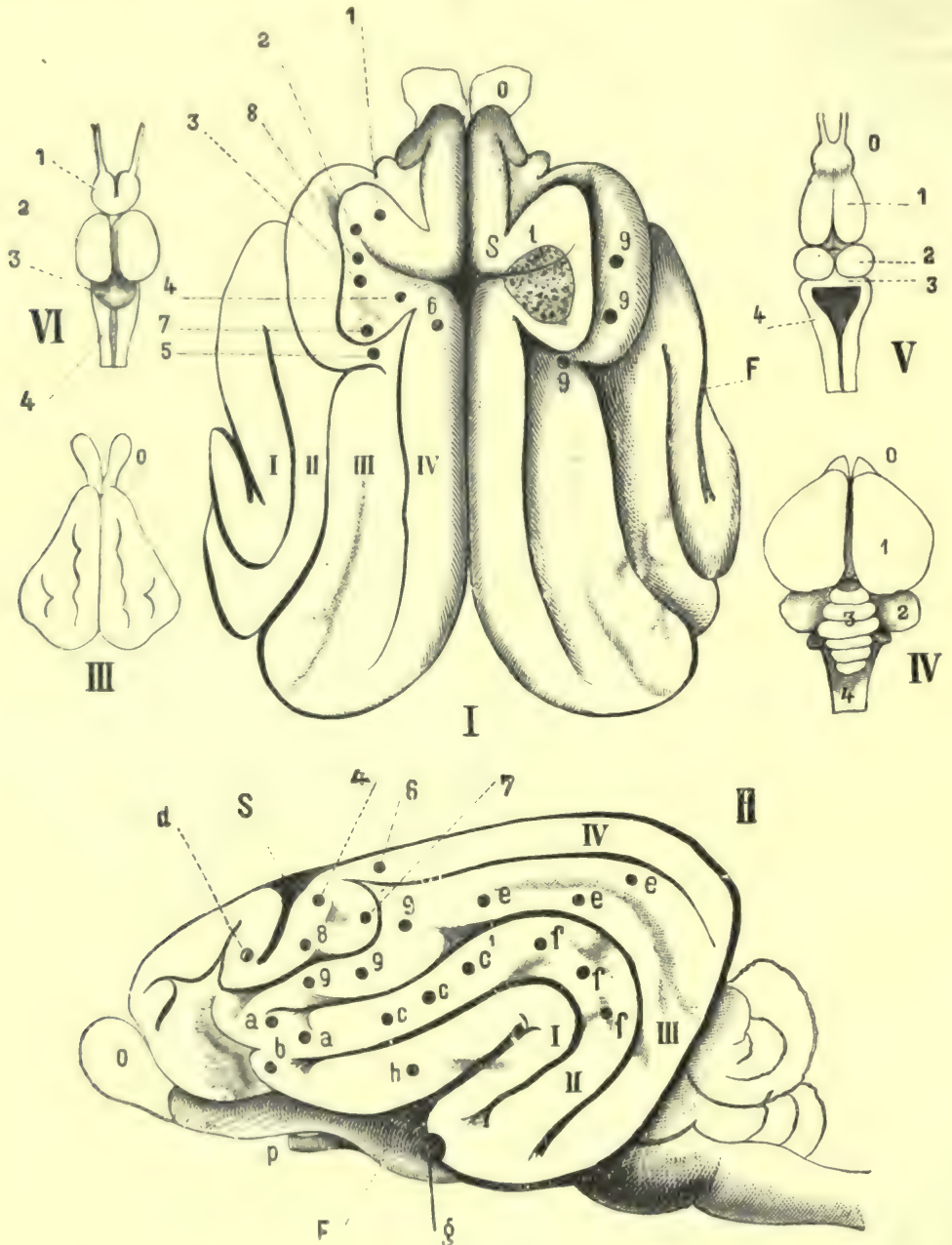

Fig. 483

I. Cirebrum of the dog from above; II, from the side; I, II, III, IV, the four primary convolutions, $-\mathrm{S}$, sulcus cruciatus; $\mathrm{F}$, Sylvian fossa ; 0 , olfactory lobe ; $p$, optic nerve; 1 , motor area for the muscles of the neck; 2 , extensors and abductors of the fore limb; 3 , flexors and rotators of the fore limb; 4, the muscles of the hind limb; 5, the facial muscles; 6 , lateral switching movements of the tail ; 7 , retraction and abduction of the fore limb ; 8, elevation of the shoulder and extension of fore limb (movements as in walking) ; 9,9 , orbicularis palpebrarum, zygomaticus, closure of the eyelids. II, $a, a$, retraction and elevation of the angle of the mouth; $b$, opening of the mouth and movements of the oral centre ; $c, c$, platysma; $d$, opening of the eye : I, $t$, thermic centre, according to Eulenburg and Landois. III, cerebrum of the rabbit from above; IV, cerebrum of the pigeon from above; V, cerebrum of the frog from above; VI, cerebrum of the carp from above-(in all these $o$ is the olfactory lobe; 1 , cerebrum; 2, optic lobe; 3 , cerebellum; 4, medulla oblongata).

Sylvii (F). The second convolution (II) runs nearly parallel to the first. The fourth primary 
convolution (IV) bounds the longitudinal fissure, and is separated from its fellow of the opposite side by the falx cerebri ; anteriorly it embraces the sulcus cruciatus (S), so that it is divided into two parts by this sulcus, a part, the gyrus præcruciatus or præfrontalis, lying in front of the sulcus, and the gyrus postcruciatus (postfrontalis) lying behind it. The third primary convolution (III) runs parallel to the fourth. Some authors count the convolutions from the longitudinal tissure outwards: In fig. 483, I and II, the motor arcas or centres are indicated by dots on the individual primary convolutions. We must remember, however, that the centres are not mere points, but that they vary in size from that of a pea upwards, according to the size of the animal. Motor areas have been mapped out in the brain of the monkey, rabbit, rat, bird, and frog.

Position of the Motor Centres (Dog).-Fritsch and Hitzig, in 1870, mapped out the following motor areas, whose position may be readily found on referring to fig. $483:-1$, is the centre for the muscles of the neck; 2 , for the extensors and adductors of the fore limb; 3 , for the flexion and rotation of the fore leg; 4, for the movements of the hind limb, which Luciani and Tamburini resolved into two antagonistic centres; 5 , for the muscles of the face, or the facial centre. In 1873 Ferrier discovered the following additional centres :- 6 , for the lateral switching movements of the tail; 7 , for the retraction and abduction of the fore limb; 8 , for the elevation of the shoulder and extension of the fore limb, as in walking; the area marked $9,9,9$, controls the movements of the orbicularis palpebrarum, and of the zygomaticus (closure of the eyelids), together with the upward movement of the eyeball and narrowing of the pupil. Stimulation of the areas $a, a$ (fig. II) is followed by retraction and elevation of the angle of the mouth, with partial opening of the mouth ; at $b$, Ferrier observed opening of the mouth with protrusion and retraction of the tongue, while the dog not unfrequently howled. $\mathrm{He}$ called this centre the "oral centre." Stimulation of $c c$ causes retraction of the angle of the mouth, owing to the action of the platysma, while $c^{\prime}$ causes elevation of the angle of the mouth and of one-half of the face, until the eye may be closed, just as in 9. Stimulation of $d$ is followed by opening of the eye and dilatation of the pupil, while the eyes and head are turned towards the other side. According to $\mathrm{H}$. Munk, the prefrontal region has an influence upon the attitude of the body (?). The perineal museles contract when the gyrus postcruciatus is stimulated. Stimulation of the gyrus præcruciatus on its anterior and sloping aspect causes movements in the pharynx and larynx.

The position of the individual motor areas may vary somewhat, and they may be slightly different on the two sides (Luciani and Tamburini).

Strong Stimuli.-If the stimulation be very strong, not only the muscles on the opposite side, but those on the same side, may contract. These latter movements belong to the class of associated movements, and are due to conduction through commissural fibres. Those muscles, which usually (muscles of mastication) or always (muscles of eye, larynx, and face) act together, appear to have a centre not only in the opposite but also in the hemisphere of the same side (Exner). [All observers have found that stimulation of the facial centre causes identical (associated) movements on both sides of the face, so that both sides of the face seem to be represented in each hemisphere. Schäfer and Horsley's experiments make it very probable that some other muscles, e.g., some of the trunk muscles, pectorals, and recti abdominis, are represented bilaterally in the hemispheres. This is an important point, in relation to recovery after the supposed destruction of a centre, and has an intimate bearing on the question of "Substitution," in reference to the restoration of nerve-function (p. 682).]

Mechanical stimulation, e.g., scraping the motor areas for the limbs, produces movements in these parts (Luciani).

Cerebral Epilepsy.-It is of great practical diagnostic importance to ascertain if stimulation of the motor areas in man, due to local diseases (inflammation, tumours, softening, degenerative irritation), causes movements. [Hughlings-Jackson has shown that local diseases of the cortex may cause spasmodic contractions in certain groups of muscles, a condition known as "Jacksonian Epilepsy," and he explains in this way the occurrence of unilateral local epileptiform spasms, which were observed by Ferrier and Landois to occur after inflammatory irritation.] Luciani observed these spasms in dogs, and sometimes they were so violent and general as to constitute an attack of epilepsy. This condition became hereditary, and the animals ultimately died from epilepsy (§ 373$)$. According to Eckhard, epileptic 
attacks are never produced by stimulation of the surface of the posterior convolutions.

Strong stimulation of the motor regions may give rise in dogs to a complete general convulsive epileptic attack, which usually begins with contractions of the groups of muscles specially related to the stimulated centre (Firrier, Eulenburg and Landois, Albertoni, Luciuni and Tamlurimi); then often passes to the corresponding limb of the opposite side (associated movements: and lastly, all the muscles of the body are thrown into tonic and then into clonic sprasms. The opposites side of the body has been observed to pass into spasm from below upward. after the contractions were developed in the other side. The spasmodic excitement jasses from centre to centre, an intermediate motor region never being passed over. After this comlition has unce been prolucel, the slightest stimulation may suffice to bring on a new (jil.pti. attack $(\$ 373$ ). During the attack, the cerebral circulation is accelerated. According in E. khari and Danillo, epileptic attacks eaunot be discharged from the posterior part of the .... linm hy means of weak currents. Stimulation of the subcortical white matter causes -pilipls, which, however, begins in the museles of the same side (Bubnoff and Heidenhain). These contractions are due to an escape of the electrical current, which thus reaches the medulla oblongata $(\$ 373)$.

If certain motor areas are extirpated, the epileptic attack is absent from the muscles contrulled by these areas (Lucucit). Separation of the motor cortical area by means of a horizontal sertion iluring an attack cuts short the latter $(M u n k)$. During an epilejtic attack it is possible (i) exise the motor area of one extremity, and thus exclude this limb from the attack whilst the rest of the body is convulsed.

Drug8. - The continued use of potussium bromide prevents the production of epilepsy on stimulating the cortical areas.

Chrmical Stimulation.-Substances such as occur in urine, e.g., kreatinin, kreatin, acid potassic phosphate, and sediment of urates, when sprinkled on the motor areas of the dog, cause pronounced eclampsic, clonic convulsions, which recur spontaneously, and are followed by deep coma. These symptoms are like those of uratmic poisoning. The sensory centres, especially that for vision, seem also to be affected by chemical stimulation (Landois).

Motor Centres in the Monkey. - Ferrier has mapped out a large number of centres on the outer surface of the brain in the monkey, and to each centre he has given a number. These numbers have been transferred to corresponding convolutions on the human brain, numbered accordingly. These areas are specially distributed on the convolutions around the fissure of Rolando, including in the monkey, the posterior extremities of the posterior and middle frontal convolutions, the ascending frontal, ascending parietal, and part of the parietal lobule.]

[Fig. 484 represents these areas transferred to the corresponding areas in man. (1) On the superior pratietal lobule (alvance of the opposite hind limb, as in walking). (2), (3), (4) Around the upuer extremity of the fissure of Rolando (complex movements of the opposite leg and arm, and of the trunk, as in swimming). (a), (b), (c), (d), On the ascending parietal or posterior central convolution (iulividual and combined movements of the fingers and wrist of the opposite hand or jrehensile movements). (5) Posterior end of the superior frontal convolution (extension forward of the opposite arm and hand). (6) Ulper part of the ascending frontal or anterior central convolution (supination and flexion of the opposite fore-arm). (7) Middle of the same convolution (retraction and elevation of the opposite angle of the inouth). (8) At the lower end of the same convolution (elevation of the ala nasi and upper lip, and depression of the lower lip on the opposite side). (9), (10) Broca's convolution (opening of the mouth with protrusion and retraction of the tongue-aphasic region). (11) Between 10 and the lower end of the ascending parietal convolution (retraction of the opposite angle of the mouth, the head turns towaris one sile). (12) l'osterior part of the superior and middle frontal convolutions (the eyes open widely, the pupils dilate, and the head and eyes turn towards the opposite side). (13), (13') Supra-marginal and angular gyrus (the eyes move towards the opposite side, and upwards or downwards - centre of vision). (14) Superior tenıporo-sphenoidal convolution (pricking of the opposite ear, pupils dilate, and the head and eyes turn to the opposite side-hearing centre).]

[Experiments on Monkeys.-Electrical stimulation of the anterior part of the frontal lobes yields negative results; but behind the anterior end of the sagittal limb of the precentral sulcus, there are lateral movements of the head and eyes. If the anterior third or fourth be removed, Schäfer and Horsley observed no motor 
paralysis nor any deficiency of general or special sensibility... Excitation of the external surface (motor area) led Ferrier to map out the areas named on p. 695 . Schäfer and Horsley's experiments agree with Ferrier's, and they map out the

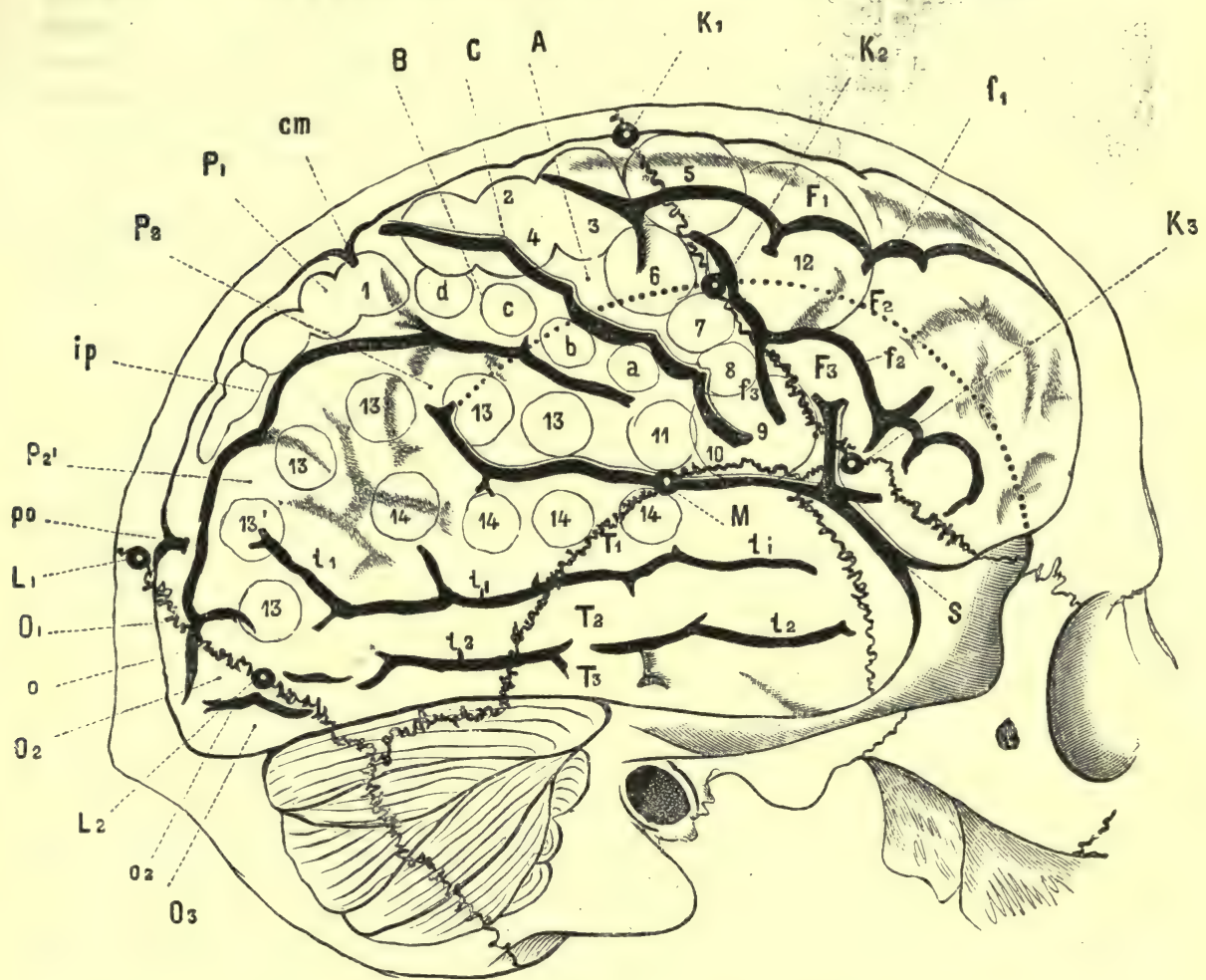

Fig. 484.

The brain with the chief convolutions (after Ecker). See also figs. 498, 499 in their relation to the skull. The numbers 1 to 14 , and the letters $a$ to $d$, indicate cortical areas (p. 696). $\mathrm{S}$, Sylvian fissure ; C, central sulcus, or fissure of Rolando ; A, anterior, and B, posterior central convolutions; $\mathrm{F}_{1}$, upper, $\mathbf{F}_{2}$, middle, and $\mathrm{F}_{3}$, lowest frontal convolutions ; $f_{1}$, superior, and $f_{2}$, inferior frontal fissure ; $f_{3}$, sulcus præcentralis; $\mathrm{P}_{1}$, superior, $\mathrm{P}_{2}$, inferior parietal lobe, with $\mathrm{P}_{2}$, gyrus supra-marginalis; $\mathrm{P}_{2}^{1}$, gyrus angularis; $i p$, sulcus interparietalis ; $\mathrm{cm}$, end of calloso-marginal fissure; $\mathrm{O}_{1}, \mathrm{O}_{2}, \mathrm{O}_{3}$, occipital convolutions ; $p o$, parieto-occipital fissure; $\mathrm{T}_{1}, \mathrm{~T}_{2}, \mathrm{~T}_{3}$, temporo-sphenoidal convolutions; $\mathrm{K}_{1}, \mathrm{~K}_{2}, \mathrm{~K}_{3}$, points in the coronal suture $; 4_{1}, 4_{2}$, in the lambdoidal suture.

motor area into a number of main areas, each of which is particularly concerned with the movement of a particular part or limb, and in some of which, centres concerned with more specialised movements may be marked out. The arm-area is roughly triangular (fig. 485), and "occupies most of the upper half of the ascending parietal and ascending frontal gyri, from a little beneath the level of the sagittal part of the precentral fissure below, nearly to the margin of the hemisphere above, together with the adjacent part of the frontal lobe below the small antero-posterior sulcus." It bends round and is continuous with a part of the marginal gyrus. The special movements of the arm are indicated in fig. 485.]

[The face-area gives rise not only to movements of the facial muscles, but also of the whole of the upper end of the alimentary tube. It comprises the whole of the 
ascending parietal and frontal convolutions below the arm-area, down to the fissure of Sylvius, and including the external surface of the operculum.]

[The head-area, or aroa for visual clirection, comprises part of the frontal lobe from the margin of the hemisphere to the face-area. In front it is bounded by the non-excitable part of the frontal lobe. Its stimulation gives the results obtained by Ferrier on stimulating his No. 12 centre. The leg-area is partly situate on the mesial surface, but it extends over to the external surface from the parietooccipital fissure nearly to the level of the anterior end of the small sulcus marked $\therefore$ The trunk-area scarcely extends over the margin to reach the external surface.

[ichafer and Horsley have extended Ferrier's researches, and shown that motor centres exist in the marginal convolution (fig. 486), which is excitable only in that portion corresponding in extent (antero-posteriorly) to the excitable portion

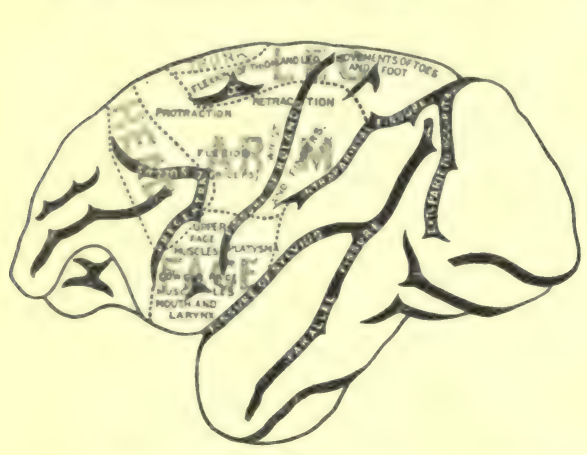

Fig. 485 .

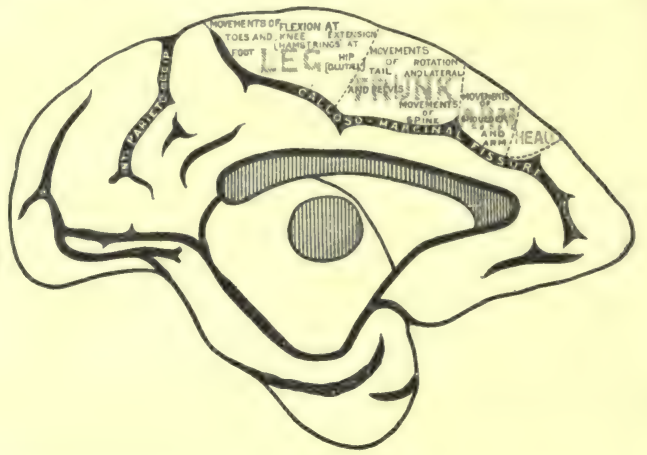

Fig. 486 .

Fig. 4A. - Diagram of the motor areas on the outer surface of a monkey's brain (Horsley and Sithitz). Fig. 486.-I)iagram of the motor areas on the marginal convolution of a monkey's brain'(Horsley and Schäfer).

of the outer surface of the hemisphere. Anteriorly it reaches forward to a line which is opposite the junction of the posterior and middle thirds of the superior frontal convolution (centre 12), while posteriorly it extends backwards opposite to the parictal lobule, including the paracentral lobule, which contains large multipolar lyramidal motor cells. The rest of the mesial surface is excitable. They find that the centres are arranged from before backwards in the following order:-(1) Movements of the head-this area is very small, and belongs to the large headarea on the external surface; (2) of the fore-arm and hand; $(3)$ of the arm at the shoulder; (4) of the upper dorsal part of the trunk; (6) of the leg at the hip ; (7) of the lower leg at the knee; (8) of the foot and toes.]

Excitation of the Area AS produces movements of the arm (fig. 489). These vary according to the spest stimulated, but towards the anterior part of the area, movements of the wrist and fore-arm, towards the posterior part movements of the arm and shoulder, are more frequently the result of the excitation. Excitation of $\mathbf{T r}$ produces movernents of the trunk, generally arching and rotation. Those movements which are called forth by stimulating the anterior part of the area are usually confined to the upper part of the trunk (thoracic region), and are often assoreiated with movements of the shoulder and arm; those called forth by stimulating the posterior part are movements of the abdominal and pelvic regions and of the tail, and are often associated with movements of the hip and leg. Excitation of the area $\mathbf{L}$ produces movements in the lower limb. These vary according to the part stimulated, extension of the hip beiug especially associated with excitation of the anterior part of the area, and contraction of the hamstrings with excitation of the middle part.]

[Do similar Centres exist in Man?-The results of clinical and pathological investigations show, that similar although not absolutely identical areas exist in man. The motor areas, or those which have a special relation to voluntary motion 
in man, exist in part on the convolutions bounding the fissure of Rolando, and occupy the "central" convolutions, i.e., the ascending frontal and ascending parietal convolutions along with the superior parietal lobule, and along the mesial surface of the hemisphere, the paracentral lobule and precuneus (fig. 488). In this region, the upper third of the ascending frontal and parietal convolutions along with the superior parietal are the leg area (fig. 488 , leg), the middle third of the ascending parietal and ascending frontal for the arm, and the upper part of the lowest third of these convolutions for the face, while the very lowest part of the ascending frontal convolution is the area for the movements of the lips $(\mathrm{L})$ and tongue (T). (Compare figs. 485, 490.) The last area, with the posterior extremity of the third left frontal convolution, is the centre for voluntary speech. We cannot say whether these "centres" are sharply mapped off from each other. In any case a very strong stimulation of one centre may involve an adjacent area. So far as is yet known, centres Nos. 5 and 12, as represented on the monkey's brainthose on the posterior extremity of the superior and middle frontal convolutions, (5) for extension forward of the arm and hand, and (12) for opening the eyes and turning the head towards the opposite side (as in surprise), are not represented in the human brain. So accurately have certain of these areas been located, that surgeons, in suitable cases, have been able to excise a tumour causing certain symptoms, with relief of those symptoms.]

[We may, therefore, assert as a general proposition that the muscles of one lateral half of the body are regulated by certain areas in the opposite cerebral hemisphere.]

[Gowers maintains that the motor region is not exclusively motor, but that destruction of this area also leads to some loss of sensation. Starr also asserts that perceptions occur in the grey matter of the cortex of the "central" region and parietal convolutions, and that the various sensory areas for the various parts of the body lie about, and coincide to some extent with, the motor various areas for similar parts, but the sensory area is more extensive than the motor area, extending into the parietal behind the motor area, which is confined to the ascending frontal and parietal convolutions.]

II. Method of Destruction or Ablation of Parts of the Cortex.-Much confusion in this matter has arisen from comparing the results obtained on animals of different species. [It seems quite certain that the results obtained in the dog are quite different from those in the monkey. The motor areas may be simply excised with a knife, or the surface of the brain may be washed away with a stream of water, as was done by Goltz in dogs.]

[In the dog, the areas which are described as motor may be removed either by the knife (Hermann) or by means of a stream of water so directed as to wash away the grey matter (Goltz). In both cases, although there was some paralysis on the opposite side of the body, this was but temporary, for the paralysis disappeared within a few days, the animals having very decided control over their muscles, although Goltz admits that certain acts, especially those which the dogs had been trained to execute, e.g., giving a paw, were executed "clumsily," indicating some failure of complete control, which Goltz ascribed to loss of tactile sensibility. Goltz thinks that the extent of the injury has more to do with the result than the locality. The restoration of motion was not due to the action of the corresponding centre of the opposite side, as destruction of this centre, although it produced the usual symptoms on the side which it governed, had no effect on the previous result (Carville and Duret).]

[In the monkey, there can be no doubt from the experiments of Ferrier that destruction of a motor centre, e.g., that for the arm, results in permanent paralysis of the arm of the opposite side, and if the centres for the arm and leg are destroyed, there is permanent hemiplegia of the opposite side. "In order that the hemiplegia or paraplegia produced by cortical ablation shall be complete, it is necessary to include the part of the marginal gyrus corresponding in longitudinal extent to the excitable areas of the external surface." The amount of paralysis produced by ablation of the marginal gyri alone is as great as that caused by removal of the much more extensive external areas; but the complexity of the muscular movements which are governed from these areas is much greater than in those governed from the marginal gyrus (Schäfer and Horsley). 1 
[In man, records of destructive lesions of the motor areas in whole or part have now accumulated to such an extent as to leave no doubt, that if there be, say, a destructive lesion of the middle third of the cortex of the ascending frontal and ascending parietal convolutions, there will be paralysis of the arm of the opposite side; and the same is true for the other centres.]

[In extirpation or ablation of the motor centres, again, much confusion has arisen from comparing the results obtained on different animals. In the dog, there is no permanent motor paralysis, in the monkey and man there is. The difference is this, that in the dog the lower centres, perhaps the basal ganglia, are able to suliserve the execution of those co-ordinated movements required for standing. progression, drc. As we proceed higher in the animal scale, the motor cortical centres assume more and more of the functions subserved by the basal ganglia in lower animals. There is, as it were, a gradual displacement of motor centres to the cortical region, as we ascend in the zoological scale.]

Differences in Animals. - The higher the development of the intelligence of animals, the more have their movements been learned, and the more have they gradually come to be controlled hy the will; in them the disturbance of the motor phenomena becomes more pronounced and persistent after destruction of the cortical psychomotor centres. Whilst in the lower vertelrates, including the birds, extirpation of the whole hemispheres does not materially interfere with movements, the co-ordinated reflex movements being sufficient-in dogs necasionally, but exceptionally, extirlation of several motor areas produces visible permanent disturlance of motor acts - and in monkeys and $\operatorname{man}(\$ 378)$, the paralytic phenomena may be intense and persistent.

Acquired Movements. - Among the movements performed by men are many which have been acipuired after much practice, and have been subjected to voluntary control, e.g., the movements of the hauls for many manual occupations. After a lesion of certain motor areas, such movements are rencipuired only very slowly and incompletely, or it may be not at all. [The interference with these finer acquired movements sometimes becomes very marked in lesions of the motor areas produced by hemorrhage, and in some cases of hemiplegia.] Those movements, however,: which are, as it were, innate [or as they are sometimes termed fundamental in opposition to acyuired], and are under the control of the will without much practice-such as the assoriated movements of the eyes, face, some of those of the limbs-are either rapidly restured after the lesion, or they appear to suffer hut slightly after a lesion of the cerebral cortex; the facial museles are never so completely paralysed as from a lesion of the trunk of the facial nerre : usually the eye can be closed in the former case. The movements necessary for sucking have been performed by hemicephalic infants.

Theoretical. - Hitzig ascribes the disturbance of movement, after the removal of the motor centres, to the loss of the "muscular sensibility." Schiff refers it to the loss of tactile sensibility. According to Ferrier, the tactile and sensory impressions are not appreciably diminished or altered. The descending degeneration of the pyramidal tracts in the lateral columns, according to Schiff, occurs after section of the posterior half of the cervical spinal cord, or even after section of the posterior frart of the lateral columns. After dividing the latter, and allowing secondary degeneration to take place, it is not possible to discharge movements by stimulating the cortex cerebri. [Schiff divided the posterior column of the cord, and found that stimulation of the opposite motor cortex failed to excite movements in the opposite fore limbs. He supprsed that this result was due to ascending degeneration. Horsley finds, however, that Schiff's results are due to transverse aseptic myelitis at the seat of operation, thus causing a "block" there in the motor tract.] The posterior columns, and their continuation upwards to the brain, are supposed to carry the impulses upwards to the cerebrum (ascending the limb of the reflex arc), where, after being modified in the centres, they are carried outwards by the pyramilal tracts (descending limb of the reflex arc). [Some hold that the posterior columns are directly connected with the cortical motor area, while others think that a sensory perceptive centre is interposed between the afferent and efferent impulses.] Between, but deeper in the brain, lie the centres for tactile sensibility. Landois and Eulenburg observed in a dog, from which the motor centres for the extremities had been removed on both sides, that the movements became completely ataxic, i.e., the animal could not execute such co-ordinated movements as walking, standing, \&c. Goltz regards the disturbances of movement after injury of the cortex as due to inhibition. Schiff maintains that when the cortex cerebri is stimulated we do not stimulate a cortical centre, but only the sensory channels of a reflex arc, the continuation of the posterior columns, so that on this supposition the movements resulting from stimulation of the motor points would be reflex movements. The centres lie deeper in the brain. This view is not generally entertained.

Modifying Conditions.-The excitability of the motor centres is capable of 
being considerably modified. Stimulation of sensory nerves diminishes it; thus, the curve of contraction of the muscles becomes lower and longer, while the reaction-time is lengthened simultaneously. Only when, owing to strong stimulation, the reflex muscular contractions are vigorous, the excitability of the cortical centres appears to be increased. Specially noteworthy is the fact that, in a certain stage of morphia-narcosis, a stimulus which is too feeble to discharge a contraction becomes effective at once, if immediately before the stimulus is applied to the cortical centre, the skin of certain cutaneous areas be subjected to gentle tactile stimulation. When strong pressure is applied to the foot, the contractions become tonic in their nature, so that all stimuli, which under normal conditions produce only temporary stimulation, now stimulate these centres continuously. If, during the tonic contraction, one gently strokes the back of the foot, blows on the face, gently taps the nose, or stimulates the sciatic nerve, suddenly relaxation of the muscles again occurs. These phenomena call to mind the analogous observations in hypnotised animals (\$374). Another very remarkable observation is, that when either owing to a reflex effect, or to strong electrical stimulation of a cortical centre, contraction of the corresponding muscles is produced, then feeble stimulation of the same centre, but also of other centres, suppresses the movement. Thus, we have the remarkable fact that, according to the strength of the stimulus applied to the motor apparatus, we can either produce movement or suppress a movement already in progress (Bubnoff and Heidenhain).

[Excision of the Thyroid affects the nerve-centres. After thyroidectomy (twenty-four hours) the tetanus obtained by stimulating the cortex is greatly changed. It ceases. when the stimulating current is shut off, as suddenly as that observed on stimulating the corona radiata. In more advanced cases, the tetanus is soon exhausted, and is often followed by clonic epileptoid spasms. In the latter stages, after thyroidectomy, there may be only a feeble tetanus, or none at all, on stimulating the motor areas, so great is the state of depression of function of these centres (Horsley).]

[Warner has directed attention to visible muscular movements apart from those studied in epilepsy, chorea, athetosis - and including attitude, gait, movements of the eyeballs, position of the hand, and posture in general, \&c. —as expressive of states of the brain and nerve-centres.]

376. SENSORY CORTICAL CENTRES.-[There must be some connection between the surface of the brain and the afferent channels through which sensory impulses pass inwards, and although the channels for sensory impulses are, perhaps, not so definitely localised as those for voluntary motion, still we know that sensory impulses for the opposite half of the body travel upwards through the posterior third of the posterior limb of the internal capsule (fig. 500, S), to radiate in all probability into the occipital and temporo-sphenoidal lobes. Parts of these convolutions are sometimes spoken of as "sensory centres" or "psycho-sensorial " areas.]

[The same methods have been applied to the investigation of these centres, viz., stimulation and extirpation. Stimulation, - Ferrier found that electrical stimulation of the angular gyrus (monkey) caused movements of the eyeballs towards the side, with sometimes associated movements of the head, but he regarded these as reflex movements, so that for this and other reasons he, in his earliest contributions, considered the angular gyrus and adjacent parts as the "centre for vision." On stimulating the first temporo-sphenoidal convolution, the monkey pricked the opposite ear, the pupils dilated, while the head and ears turned to the opposite side, it exhibited movements similar to those caused by a loud sound; these movements are also reflex phenomena, so that he located the "auditory centre" in this region, and on somewhat similar grounds. As the result of inferences from the stimulation and extirpation of other parts, he referred the centres for smell and taste to the tip of the temporo-sphenoidal lobe, and for touch to the hippocampus major, but all these statements have not been confirmed.]

[Goltz experimented on dogs by washing away the cortex cerebri, and found that when a sufficient amount of the grey matter is removed, and after recovery from the immediate effects of the operation, there is a peculiar defect of vision and other sensory defects, but so far Goltz has not found that there is any difference in this respect between removal of the anterior and posterior lobes of the dog's brain. The dog is not blind, as it can see and use its eyes to avoid obstacles, but it seemed as if the animal failed to recognise food or flesh as such, when placed before it; while exhibitions, which, before the operation, greatly excited the dog, ceased to do 
so. Goltz caused his servant to dress himself in a mummer's red coloured garb, which previously had greatly excited the dog, but after the operation the dog, although it was not blimd, was no longer excited thereby. Nor was it afterwards cowed by the appearance of a whip. After a time there was recovery to a certain extent if the animal was trained, whether by the deposition of new impressions, or by opening up new channels, or by the partial recovery of some parts of the grey matter not removed, it is impossible to say.]

[Munk has mappeel out the surface of the brain into a series of "sensory" or psycho-sensorial centres, hut he distinguishes between complete and total extirpation of these centres and the phenomena which follow these operations. ]

When these centres are partially disorganised, the mechanism of the sensory activity may remain intact, but "the conscious link is wanting." A dog with its centres thus destroyed, sees, hears, or smells, but it no longer knows what it sees, hears, or smells. These centres are in a certain sense the seat of experience that has been acquired through the organs of sense. Stimulation of these centres may give rise to movements, such as occur when sudden intense sensory impressions are produced. These movements, therefore, are to be regarded as reflex, partly as extensive co-ordinated reflex movements, and are in no way to be confounded with the movements which result from direct stimulation of the motor cortical centres. To this belongs dilatation of the pupil and the fissure of the eyelids, as well as lateral movements of the eyeball.

1. The "visual area," according to Munk, embraces the outer convex part of the occipital lobe of the dog's brain. [This area and its connections are represented

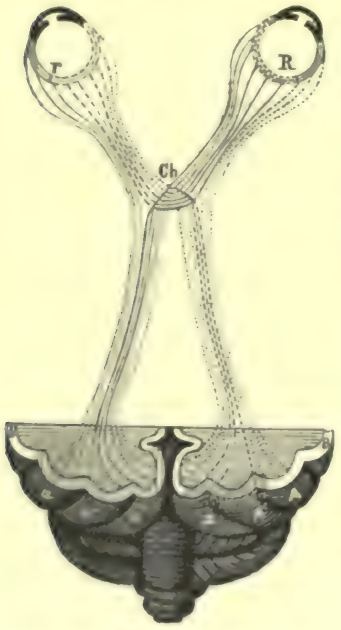

Fig. 487.

Course of the usycho-optic fibres (after Munk). in fig. 487. It is, therefore, in the area supplied by the posterior cerebral artery.] If the occipital lobes be completely destroyed, the dog remains permanently blind ("cortical or absolute blindness"). If, however, only the central circular area be destroyed, there is loss of the conscious visual sensation, which may be called "psychical blindness" (Munk) [a condition of visual defect like that observed by Goltz in the dog, in which the dog saw an object, e.g., its food, but failed to recognise it as such. There is a certain amount of recovery if the whole visual area be not removed. According to Schäfer, the visual area of the cerebral cortex in the monkey comprises the whole of the occipital lobe, and perhaps a part of the angular gyrus. He finds, with Munk, that removal of one occipital lobe is followed by hemianopia, i.e., blindness in the lateral half of each retina corresponding to the side operated on. The blindness passes off. Removal of both occipital lobes is said to produce total and permanent blindness, whereas destruction of the cortex of both angular gyri is not followed by any appreciable permanent defect of vision. Ferrier, however, does not accept these statements.]

[Ferrier and Yeo find that after operations conducted antiseptically, removal of both occipital lobes (monkeys) does not cause any recognisable disturbance of vision, or other bodily or mental derangement, provided the lesion does not extend beyond the parieto-occipital fissure. Nor does destruction of both angular gyri cause permanent loss of vision; such loss of vision lasts only three days, so that in Ferrier's original experiments, the animals lived for too short a time after the operation, to enable a just conclusion to be arrived at. Destruction of both angular gyri and occipital lobes causes total and permanent blindness in both eyes in monkeys, without any impairment of the other senses or motor power. This region Ferrier calls the "occipito-angular region." 
[Stimulation of the angular gyrus causes movements of the eyes to the opposite side, with closure of the eyelids and contraction of the pupil. The eyeballs were directed upwards or downwards according as the electrodes were applied to the anterior or posterior limb of the angular gyrus (Ferrier). Stimulation of the whole of the cortex of the occipital lobe, including its mesial and under surfaces, causes conjugate deviation of the eyes to the opposite side, the direction of movement varying with the position of the electrodes.]

Mauthner denies the existence of cortical blindness, and believes that, after destruction of the middle of the visual centre, the reason why the dog does not recognise the object with the opposite eye is because, owing to there being only indirect vision, there is no distinct impression on the retina. The position of the visual centre has been variously stated by different observers. According to Ferrier, in the dog it lies in the occipital part of the III primary convolution, near the spot marked $e, e, e$, in fig. 483 ; according to his newer researches, in the occipital lobe and gyrus angularis.

Connection with the Retina.-Munk asserts that in dogs both retinæ are connected with each visual cortical centre, and in such a manner that the greatest part of each retina is connected with the opposite cortical centre, and only by its most external lateral marginal part with the centre of the same side (fig. 487). If we imagine the surface of one retina to be projected upon the centres, then the most external margin of the first is connected with the centre of the same side, the inner margin of the retina with the inner area of the opposite centre, the upper margin with the anterior area, and the lower marginal part of the retina with the posterior area of the opposite side. The (shaded) middle of the centre eorresponds to the position of direct vision of the retina of the opposite side (compare $\$ 344$ ).

Stimulation of the visual centre in dogs causes movements of the eyes towards the other side, sometimes with similar movements of the head and contraction of the pupils. If one eye be excised, from new-born dogs, the opposite visual centre, after several months, is less developed (Munk). After extirpation of the visual centre in young dogs, the channels which connect it with the optic nerve undergo degeneration (Monakow) (§ 344).

In monkeys; the centre occupies the occipital lobe. Unilateral destruction causes temporary blindness of the halves of both retinæ, $i . \dot{e}$., hemianopia on the side of the injury. The visual centre in pigeons (fig. 483, IV, where 1 is placed) lies somewhat behind and internal to the highest curvature of the hemispheres (M'Kendrick, Ferrier, Musehold). The visual centre in the frog lies in the optic lobe (Blaschko).

[The visual path is along the optic nerve to the chiasma, where the fibres from the nasal half of each retina cross to the optic tract, some of the fibres perhaps becoming connected with the external corpora geniculata, and some with the pulvinar of the optic thalamus and corpora quadrigemina, while the great mass sweeps backwards to the occipital lobes as the optic expansion of Gratiolet. Destruction of this path behind the chiasma causes hemiopia or hemianopia, and certain diseases of the occipital cortex cause a similar result. Perhaps, however, there is another centre in the angular gyrus (and supra-marginal lobe), for in eases of word-blindness disease has been found in these regions. Sometimes flashes of light or the appearance of a ball of fire form the aura in epilepsy, and Hughlings Jackson thinks that discharging lesions of the right occipital lobe cause coloured vision more frequently than on the left.]

2. The centre for hearing, or "auditory area," lies in the dog, according to Ferrier, in the region of the second primary convolution at $f, f, f$ (fig. 483 , II), while in the monkey and man it is in the first temporal or temporo-sphenoidal gyrus (Ferrier's centre, No. 14). Munk locates it in the same region. Áccording to Munk, destruction of the entire region causes deafness of the opposite ear, while destruction of the middle shaded part alone causes "psychical deafness" ("Seelentaubheit"). Electrical irritation of the upper two-thirds of the superior temporal convolution is followed by a reaction which closely resembles that produced by a sudden fright, or that produced by a sudden unexpected noise. [There is a quick retraction of the opposite ear, i.e., "pricking" of the ear as if toward the supposed origin of the sound, combined generally with turning of the head and eyes to that side, and dilatation of the pupil.]. Ferrier locates the centre for hearing in the monkey in the superior temporo-sphenoidal convolution, and he finds that, when the centres on both sides are extirpated, the animal is absolutely deaf; it takes no cognisance of a pistol fired in its neighbourhood. [From his experiments on monkeys, 
Schifer denies absolutely the conclusions of the above-named experiments. Schïfer points out that it is not difficult to substantiate hearing in monkeys ; it is difficult to substantiate deafness, for quite normal monkeys will often fail to pay the least attention $t_{0}$ ) loud sounds. In six monkeys, Schaifer asserts that after more or less complete destruction of the superior temporal gyrus on both sides, hearing was not perceptilily affected. In one case, both temporal lobes were completely removed without any frermanent diminution in the acuteness of hearing. These results are olposed to the ordinary clinical teaching on this subject.] In man, injuries to the first and second temporo-sphenoidal convolutions on one side do not appear to cause complete deafness of one ear, as it seems that the sense of hearing for each ear is perhaps represented on both sides. Bilateral lesions of these convolutions in man cause complete deafness. Disease of these two convolutions is associated with word-deafness (p. 713). Wernicke cites the case of a person first affected with word-deafness, who afterwards became completely deaf; and after death, a bilateral lesion was found in the first temporo-sphenoidal convolution. These convolutions are supplied with blood by the middle cerebral or Sylvian artery.

[The auditory paths are from the auditory nuclei in the medulla oblongata through the jons, where they perhaps cross into the tegmentum, thence into the "sensory crossway," and onwards to the auditory centre.]

[Auditory Auræ. - Eiqually important with these effects of disease are the sensory impressions, or "auræ," which sometimes usher in an attack of epilepsy ; sometimes these auræ consist of soumls or noises, and in these cases the seat of the disease is often in the first temporo-sphenoidal convolution.]

[3. The olfactory centre has not been so definitely located as some of the others. There is strong presumptive evidence that it is situated in the hippocamplal region of the temporal lobe, at its lower extremity. This view is strengthened by the anatomical relations of this region to the olfactory tract and anterior commissure (Ferrier). M'Lane Hamilton has recorded a case of epilepsy ushered in by an aura of a disagreeable odour, in which there was atrophy of the grey matter of the right uncinate gyrus.]

[Olfactory Path. - Although the outer root of the olfactory tract runs direct to the uncinate gyrus, in hemiunusthesice resulting from injury to the "sensory crossway," smell is lost on the opprosite sile, while it is lost on the same side when the uncinate gyrus is involved. It may be that the impulses go first to their own side, and cross afterwards.]

[4. We do not know the centre for taste, and even the course of the nerve of taste is disputed. Ferrier places it close to that of smell.]

On stimulating the subiculum in monkeys, dogs, cats, and rabbits, he observed peculiar mnvements of the lips and partial closure of the nostrils on the same side (\$365). In man, suljective olfactory and gustatory perceptions are regarded as irritative phenomena, while loss of these sensory activities, often complicated with other cerebral phenomena, is regarded as a symptom of their paralysis.

[The gustatory path crosses in the posterior part of the posterior segment of the internal capsule. While Gowers adnits that the chorda tympani is the nerve of taste for the anterior twothirls of the tongue, he thinks that it reaches the facial nerve from the spheno-palatine ganglion through the Vidian nerve. He denies that the glosso-pharyngeal is concerned in taste, and "he believes that taste impressions reach the brain solely by the roots of the 5th uerve." $\mathrm{He}$ almits that the nerves of taste to the back part of the tongue may be distributed with the glisso-pharyngeal, reaching them through the otic ganglion by the small superficial petrosal and tympanic plexus.]

[5. Ferrier places the centre for tactile sensation in the hippocampal region, close to the distribution of part of the posterior cerebral artery; so far this has not been confirmed. The centre for the sensation of pain has not been defined; probably it is very diffuse. The limbic lobe, according to Broca, includes the hippocampal convolution and the gyrus fornicatus. Ferrier found that removal of the hippocampal region resulted in a diminution of the sensibility of the opposite side of the 
body. Horsley and Schäfer observed only a temporary hemianæsthesia, but they found that an extensive lesion of the gyrus fornicatus was followed by hemianæsthesia, more or less marked and persistent. From their experiments, these observers conclude that the limbic lobe " is largely, if not exclusively, concerned in the appreciation of sensations, painful and tactile."]

6. Munk is of opinion that the surface of the cerebrum in the region of the motor centres acts at the same time as "sensory areas" ("Fühlsphäre"), i.e., they serve as centres for the tactile and muscular sensations and those of the innervation of the opposite side. He asserts that after injury to these regions the corresponding functions are affected.

According to Bechterew, the centres for the perception of tactile impressions, those of innervation, of the muscular sense, and painful impressions are placed in the neighbourhood of the motor areas (dog) ; the first immediately behind and external to the motor areas, the others in the region close to the origin of the Sylvian fissure. [So far this agrees with the views of Starr (p. 699).]

Goltz, who first accurately described the disturbances of vision following upon injuries to the cortex in dogs, is opposed to the view of sensory localisation. He believes that each eye is connected with both hemispheres. He asserts that the disturbance of vision, after injury to the brain, consists merely in a diminished colour- and space-sense. The recovery of the visual perception of one eye after injury of one side of the cortex cerebri, he explains by supposing that this injury merely causes a temporary inhibition of the visual activity in the opposite eye, which disappears at a later period. Instead of psychical blinduess and deafness he speaks of a "cerebro-optical" and "cerebro-acoustical weakness."

377. THERMAL CORTICAL CENTRES. - Euleuberg and Landois discovered an area on the cortex cerebri, whose stimulation produced an undoubted effect upon the temperature and condition of the blood-vessels of the opposite extremities. This region (fig. 483, I, $t$ ) generally embraces the area in which, at the same time, the motor centres for the flexors and rotators of the fore limb (3), and for the muscles of the hind limb (4) are placed. The areas for the anterior. and posterior limbs are placed apart, that for the anterior limb lies somewhat more anteriorly, close to the lateral end of the crucial sulcus. Destruction of this region causes increase of the temperature of the opposite extremities; the temperature may vary considerably $\left(1.5^{\circ}\right.$ to $2^{\circ}$, and even rising to $13^{\circ}$ C.). This result has been confirmed by Hitzig, Bechterew, Wood, and others. This rise of the temperature is usually present for a considerable time after the injury, although it may undergo variations. Sometimes it may last three months, in other cases it gradually reaches the normal in two or three days. In well-marked cases, there is a diminution of the resistance of the wall of the femoral artery to pressure, and the pulse-curve is not so high (Reinke). Local electrical stimulation of the area causes a slight temporary cooling of the opposite extremities, which may be detected by the thermo-electric method. Stimulation by means of common salt acts in the same way, but in this case the phenomena of destruction of the centre soon appear. As yet, it has not been proved that there is a similar area for each half of the head. The cerebro-epileptic attacks $(\$ 375)$ increase the bodily temperature, partly owing to the increased production of heat by the muscles $(\S 302)$, partly owing to diminished radiation of heat through the cutaneous vessels, in consequence of stimulation of the thermal cortical nerves. The experiments led to no definite results when performed on rabbits. According to Wood, destruction of these centres occasions an increased production of heat that can be measured by calorimetric methods, while stimulation causes the opposite result.

These experiments explain how psychical stimulation of the cerebrum may have an effect upon the diameter of the blood-vessels and on the temperature, as evidenced by sudden paleness and congestion (\$378, III.).

[Heat Production. - Injury to the fore-brain has no effect on the temperature. If the brain of a rabbit be punctured through the large fontanelle, and the stylette be forced through the grey matter on the surface, white matter, and the median portion of the corpus striatum right to the base of the brain, there is a rapid rise of the temperature which may last several days. Injury to the grey cortex does not affect the temperature. After puncture of the corpus striatum, the highest temperature is reached only after twenty-four to seventy hours, but when the puncture reaches the base of the brain this result occurs in two to four hours. Electrical stimulation of these areas causes the same effect on the temperature. Direct injury to certaiu parts of the brain is followed by a rise of the temperature-or fever. See also, p. 329, for further evidence of the existence of thermal centres. There is at the same time an increase of the $\mathrm{O}$ taken in, the $\mathrm{CO}_{2}$ given off and a decided increase of the $\mathrm{N}$ given off, indicating an increase in the proteid metabolism, which points to an increased production of heat (Aronsohn and Sachs, Richet, $\left.W_{0 o d}\right)$.]

General and Theoretical._Goltz's View.-Goltz uses a different method to remove the cortex cerebri-he makes an opening in the skull of a dog, and by means of a stream of water washes away the desired amount of brain-matter. He describes, first of all, inhibitory phenomena, which are temporary and due to a temporary suppression of the activity of the nervous 
apparatus, which, however, is not injured anatomically; this may be explained in the same way as the suppression of reflexes ly strong stimulation of sensory werves $(\$ 361,3)$. In addition, there are the permanent phenomena, due to the disappearance of the activity of the nervous apparatus, which is removel by the operation. A dog, with a large mass of its cerebral cortex removed, may lie compared to an eating, complex, reflex machine. It behaves like an intensely stupill log, walks slowly, with its head hanging lown; its cutaneous sensibility is diminished in all its yualities-it is less sensitive to pressure on the skin; it takes less cognisance of variations of temperature, and does not comprehend how to feel; it can with difficulty ancommolate itself to the outer world, especially with regard to seeking out and taking its food. On the other hand, there is no paralysis of its muscles. The dog still sees, but it does not unlerstand what it loes see; it looks like a somnambulist, who avoids obstacles without whtaining a clear perception of their nature. It hears, as it can be wakened from sleep by a :all, hut it hears like a person just wakened from a leep sleep by a voice-such a person does nut at once olitain a distinct perception of the sound. The same is the case with the other wnses. It howls from hunger, and eats until its stomach is filled; it manifests no symptoms of sexual excitement.

Goltz supposes that every part of the brain is concerned in the functions of willing, feeling, jerception, and thinking. Every section is, independently of the others, connected by coniluting paths with all the voluntary museles, and, on the other hand, with all the sensory nerves of the hoiy. He regards it as possible that the indivilual lobes have different functions.

After removal of the anterior or frontal convolutions and the motor areas, there is at first unilateral motor and sensory paralysis and affection of vision. After some months, there remains wnly the loss of the muscular sense. If the operation be bilateral, the phenomena are more marked; there are innumerable purposeless associated movements, and the dogs become vi.ious. Marked anil permanent disturhance in the capacity to utilise the impressions from the sense-organs is not a necessary consequence of removal of the frontal convolutions.

Removal of the occipital lobes interferes most with vision. Bilateral removal makes the animal almost blind. The dog remains obedient and lively. There is no disturbance of motion or of the muscular sense.

Inhibitory Phenomena.-Injury to the brain also causes inhibitory phenomena, such as the disturban... of motion, the complete hemiplegia which is frequently observed after large miniateral injuries of the cortex rerehri; these are regarded by Goltz as inhibitory phenomena, lue to the injury acting on lower infra-cortical centres, whose action inhibits movement, but these movements are recovered as soon as the inhibitory action ceases.

378. TOPOGRAPHY OF THE CORTEX CEREBRI.-A short resumé of the arrangement of convolutions, according to Ecker, is given in $\$ 375$.

I. The cortical motor areas for the face and the limbs are grouped around the

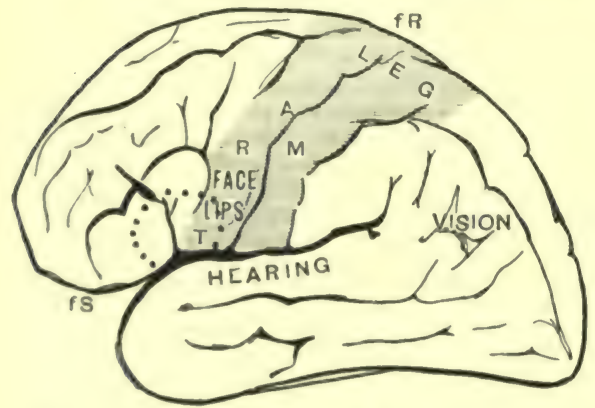

Fig. 488,

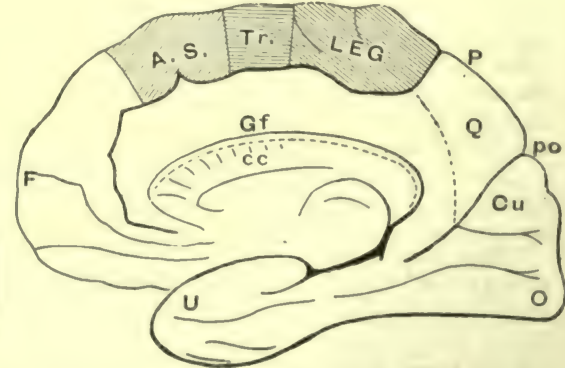

Fig. 489.

Fig. 488. - Motor areas in man shaded-outer surface of the left side of human brain. Dotted area, the aphasic region (modified from Gowers). Fig. 489. - Inner surface of right hemisphere. AS, area governing the movenients of the arm and shoulder; $\mathrm{Tr}$, of the trunk ; $l e g$, those of the leg; $G f$, gyrus fornicatus ; $C C$, corpus callosum; $U$, uncinate gyrus ; $O$,
occipital lobe.

fissure of Rolando, including the ascending frontal, ascending parietal, and part of the parietal lobule (fig. 488). The centre for the face occupies the lowest third of the ascending frontal convolution, and reaches also to the lowest fifth of the ascending parietal. The arm centre occupies the middle third of the ascending frontal and 
middle three-fifths of the ascending parietal convolutions, while the leg centre lies at the upper end of the sulcus and extends backwards into the parietal lobule (and perhaps on to the superior frontal convolution) (fig. 488). The leg centre is continued over on to the paracentral lobule, opposite the upper end of the fissure of Rolando, in the marginal convolution on the mesial aspect of the hemisphere (fig. 490), where the centres for the muscles of the trunk also exist (p. 698). The centre for speech is in the posterior part of the third left frontal convolution (fig. 488).

Blood Supply. - These convolutions are supplied with blood from 4 to 5 branches of the Sylvian artery, which may sometimes be plugged with an embolon. When a clot lodges in this artery, the branches to the basal ganglia may remain pervious, whilst the cortical branches may be plugged (Duret, Heubner) (\$381).

[Hemiplegia consists of motor paralysis of one-half of the body, although, as a rule, all the muscles are not paralysed to the same extent; in some there may be complete paralysis, i.e., they are entirely removed from voluntary control, while in others, there is merely impaired voluntary control. It may be caused by affections of the cortical areas or by lesion of the motor tracts above the medulla, and the

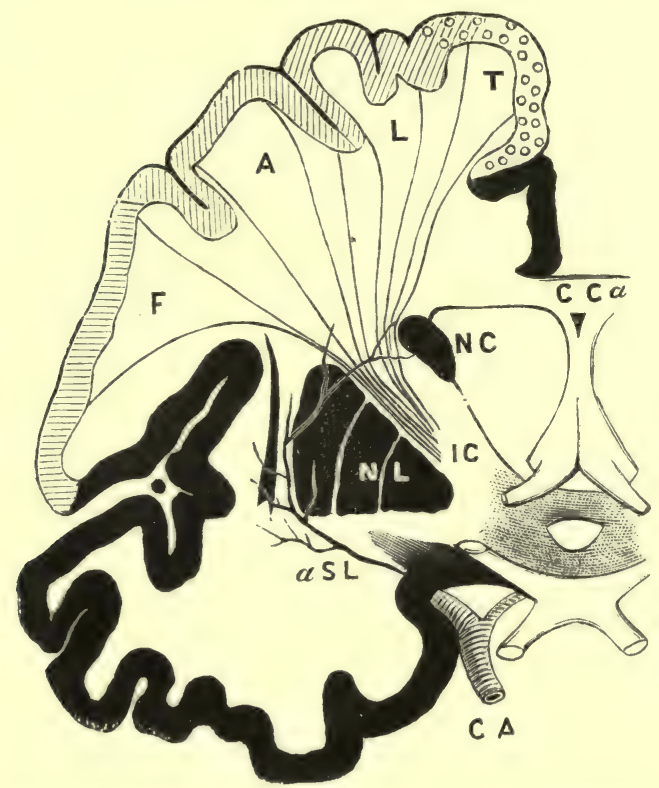

Fig. 490 . paralysis is always on the side opposite to the lesion, owing to the decussation of the motor paths in the medulla. If the case be a severe one, we have what Charcot terms hémiplégie centrale vulgaire, or "complete hemiplegia," due to lesion of the cortical centres for the face, arm, and leg. While the arm and leg are completely paralysed, the lower part of the face is

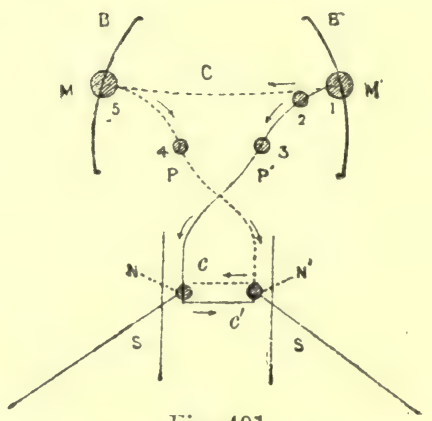

Fig. 491.

Fig. 490.-Transverse section of a cerebral hemisphere. CC 2 , corpus callosum; NC, caudate nucleus; NL, lenticular nucleus ; IC, internal capsule ; CA, internal carotid artery ; $a \mathrm{SL}$, lenticulo-striate artery; ("Artery of hæmorrhage"); F, A, L, T, position of motor areas governing the movements of the face, arm, leg, and trunk muscles of the opposite side (Horsley). Fig. 491. - Scheme of the innervation of bilaterally associated muscles (Ross).

more affected than the upper half, which is usually not much affected. All those movements under voluntary control, and especially those that have been learned, are abolished, whilst the associated and bilateral movements, which even animals can execute immediately after birth, remain more or less unaffected. Hence, the hand is more paralysed than the arm; this, again, than the leg; the lower facial branches more than the upper; the nerves of the trunk scarcely at all (Fervier). When an extraordinary effort is made, it will be found that there is sorne impairment of the power of the muscles of mastication and respiration, although the muscles on opposite sides act together (Gowers). The trunk-muscles, as a rule, are 
but slightly affected, or not at all, as their centre is elsewhere. There may be alterations of sensibility and of the reflexes.]

[Conduction through the whole of the pyramidal fibres coming from one hemisphere may be interrupted, and yet all the muscles on the opposite side of the body are not paralysed. The muscles which are comparatively unaffected are those associated in their action with the muscles of the oprosite side, $\epsilon . \%$, the respiratory muscles. Broadbent assumes that such muscles have a bilateral representation in the motor areas. Suppose in fig. $491, \mathrm{~B}, \mathrm{~B}^{\prime}$, to represent the cerebral cortex : M. M, motor centres in it: $\mathbf{N}, \mathrm{N}^{\prime}$, nerve nuclei in the spinal corl or medulla oblongata; P. P' the prramidal tracts pussing to spinal nuclei $N, N^{\prime} ; m, m^{\prime}$, nerves proceeding from the last. $1,2,3,4,5$, represent different lesions. In the case of muscles on opposite sides of the body, which act independently, e.g., those of the hand, this is all the mechanism, but in bilaterally assuriated musiles there is another mechanism, viz., commissural fibres between the nerve mullei, the one $c$ conducting from right to left, and $c^{\prime}$ from left to right. When there is an injury at 1 or 3 , impulses can still pass from the uninjured side $\mathbf{I l}$ to $\mathbf{N}^{\prime}$ and through $c^{\prime}$ to the musiles $m, m^{\prime}$. In this way, both muscles receive motor impulses from one hemisphere (Koss).]

Conjugate deviation of the eyes, with rotation of the head, is frequently present in the early jeriml of hemiplegia, although it usually disappears. When a person tums his head to one side, there is an associated movement of certain of the ocular muscles with those of the neck. The hear and eyes are usually turnerl to the side of the lesion ; this is termed "conjugate deviation," so that the power of voluntarily moving the eyes and head to the paralysed side is temporarily lost. The unopinsed muscles rotate the head and eyes to the sound side. If the lesion be in the posterior part of the pons, the deviation is to the paralysed side (Prérost).

[Subsequent Effects. - If there be a hamorrhage, say into these motor regions, or from the leutinulu-striate artery, so as to compress the pyranidal fibres in the knee and anterior twothirds of the posterior segment of the internal cajsule, then there is usually tonic or persistent contraction of the museles affected. These tonic spasms may accompany the hrmorrhage, or (wme on a few days after it, and set up' the condition of early rigidity. The contraction or spasm-if any -atecomplanying the hemorrhage, is due to direct irritation of the pyramidal filures, while that which comes on a few days later, and usually lasts a few weeks, is also due to irritation of these filures, probably produced by inflammatory action in and around the seat of the lesion. The affected limh is stiff and resists passive movement. After a few weeks, late rigidity sets in and is persistent, and it is characterised by structural changes in the pyramidal fath which leal to other results. There is secondary descending degeneration in the lyramidal tratts, which eauses "contracture" in the paralysed limbs, while at the same time, the deef or temlinous and periosteal reflexes (ankle-clonus, rectus-clonus, and the deep reflexes of the arm-tenduns, are exaggerated). The spastic rigidity is usually more marked in the arm than in the leg, and it generally affects the flexors more than the extensors, so that the upper arm is drawn close to the trunk, the elhow, arm, and fingers Hexed; in the leg, the extensors of the leg overome the peronei. Hitzig has pointed out that the contracture is less during sleep, and after rest. The muscles at first can be stretched by sustained pressure, but after months or years, structural changes occur in the muscles, ligaments, and tendons, and the limbs assume a jermanent and characteristic attitude.]

In heniplegic persons, the power of the umparalysed side is sometimes diminished, which is not sufficiently explained by the fact that some bundles of the pyramidal tracts remain on the same side (Brown Séquard, Charcot).

Acquired Movements. - Some movements performenl by man are learned only after much practica. and are only completely brought under the influence of the will after a time, such as the movments of the hand in learning a trade. Such movements are reacpuired only very slowly, or not at all, after injury to the motor areas in which they are represented. Those movements, however, which the boly performs without previous training, such as the associated movements of the eyeballs, the face, and some of those of the legs, are rapidly recovered after such an injury, or they suffer but little, if at all. Thus, the facial muscles seem never to be so completely paralysed after a lesion of the facial cortical centre, as in affections of the trunk of the facial nerve; the eye especially can he closed. Sucking movements have been observed in hemicephalous foetuses.

Degeneration of the Pyramidal Tracts.-After destruction of the cortical motor areas, descending degeneration of the cortico-motor paths, or "pyramilal tructs," takes place (\$365). Degenerative changes have been found to occur within the white matter under the cortex in the anterior two-thirds of the posterior segment of the internal capsule, [in the middle third of the crusta (figs. 492, *, 493, L], pons, in the anterior pyramids of the medulla oblongata (fig. 492), and thence they have been traced into the pyramidal paths (direct and crossed) of the spinal cord (Churcot, Singer). It is evident that lesions of these tracts at any part of their course must 
have the same result, viz., to produce hemiplegia. (For the subsequent effects, see p. 649.) In a case of congenital absence of the left fore-arm, Edinger found that the right central convolutions were less developed.

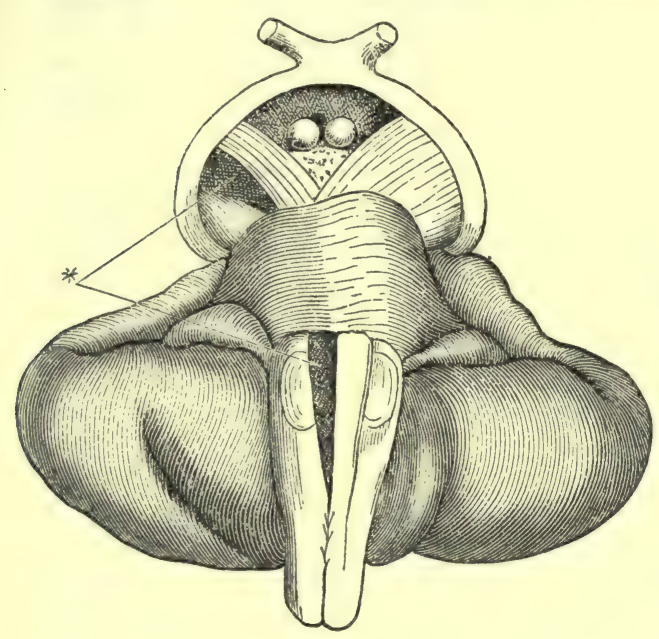

Fig. 492,

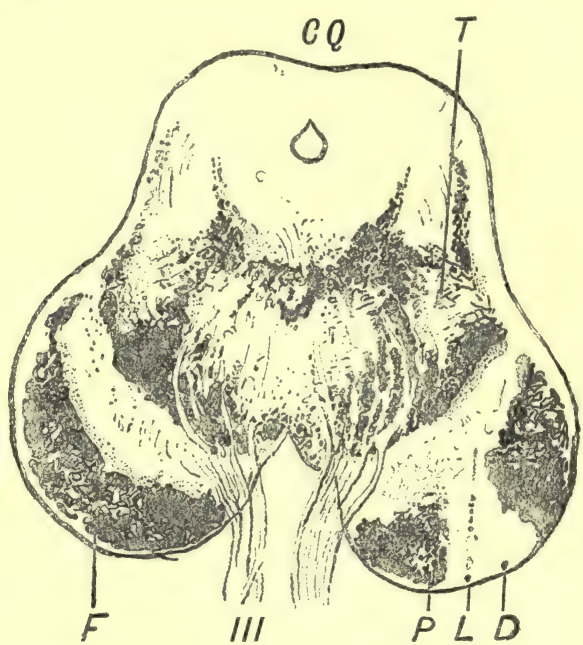

Fig. 493.

Fig. 492. - Secondary descending degeneration in midule third of right crus and medulla, after destruction of the cortical motor centres on the right side. Fig. 493.--Horizontal section of the cerebral peduncle in secondary degeneration of the pyramidal tracts, where the lesion was limited to the middle third of the posterior segment of the internal capsule. F, healthy crusta ; L, locus niger ; P, internal third of the crusta on the diseased side ; D, secondary degeneration in the middle third of the crusta; $\mathrm{CQ}$, corpora quadrigemina with the iter below them.

It is doubtful if the muscular sense is represented in the motor areas ; Nothnagel supposes it to be located in the temporal parietal lobes. It is to be noted, however, that in man there may be general loss of the muscular sense or of motor representations, and, on the other hand, a pure motor paralysis without loss of the former.

Ataxic motor conditions, similar to those that occur in animals (p. 700), take place in man, and are known as cerebral ataxia.

The position of the centres is given at p. 696 .

[But we may have localised lesions affecting one or more of the cortical motor areas ; these are called monoplegiæ. Cases in man are now sufficiently numerous to permit of accurate diagnosis.] Crural monoplegia [rare lesions recorded in the convolutions at the upper end of the fissure of Rolando, and the continuation of this area on to the paracentral lobule of the marginal convolution],- brachiocrural, more common, in the upper and middle thirds of the ascending frontal and ascending parietal convolutions-brachial, brachio-facial-facial, the last in the lowest part of the central convolutions.

Paralysis of the muscles of the neck and throat indicates a lesion of the central convolutions, and so does paralysis of the muscles of the eye. Lesions of the cortex always cause simultaneous movements of the head and eyeballs.

Irritation of the Motor Centres.-If the motor centres are irritated by pathological processes, such as hyperæmia, or inflammation in a syphilitic diathesismore rarely by tumours, tubercle, cysts, cicatrices, fragments of bone-there arise spasmodic movements in the corresponding muscle-groups. This condition of a sudden discharge of the grey matter resulting in local spasms is called "Jacksonian, or cerebral epilepsy."

[Convulsions and spasms may be discharged from motor cortical lesions, and 
these, whether they affect the general or localised areas, give rise to unilateral convolutions and monospasm respectively.

Monospasm. - A ciording to the seat of the spasm, it is called facial, brachial, crural monosjuism, de. Of course these spasms may affect several groups of muscles. Bartholow and s.iamanna have stimulated the exposed human brain successfully with electricity.

Cerebral Epilepsy. - Very powerful stimulation of one side may give rise to liliti,ill spasms, with loss of consciousness. In this case, impulses are conducted to the other hemisphere by commissural fibres $(\$ 379)$.

Morements of the Eye.- Nothing definite is known regarding the centre in the cortex for volumary combind merements of the evehalls in man. In paraly tic affections of the cortex and of the paths proceeling frum it, we occasionally find hot! eyes with a lateral deviation. If the faralytic affection lies in one cerebral hemisphere, the conjugate deviation of the eyeballs is iowards the souml side (1. 588). If it is situated in the conducting paths, after these have d.cussated, vi\%, in the pons, the eyes are turned towards the paralysed side (Prévost).

If the part he irritated so as to proiluce spasms in the opposite half of the body, of course the wes are turneal in the direction opposite to that in pure paralysis. Instead of the lateral deviation of the eveballs alrealy described, there is occasionally in cerebral paralysis merely a uembaing of the lateral recti muscles, so that during rest the eyes are not yet turned towards the soumd side, but they cannot be turned strongly towards the affected side (Leichtenstern, Hunnius). The (entre for the levator palpehre superioris appears to be placed in the angular gyrus (Grasset, Landuuzy).

II. The Centre for Speech.-The investigations of Bouilland [1825], Dax $[1 \times 36]$, Broca [1\&61], Kussnaul, Broadbent, and others have shown that the third left frontal convolution of the cerebrum (figs. $484, \mathrm{~F} 3$, and 488 ) is of essential importance for speech, while probably the island of Reil also is concerned. The island is deeply placed, and is seen on lifting up the overhanging part of the brain called the operculum, lying between the two branches of the Sylvian fissure (S). The motor centres for the organs of speech (lips, tongue) lie in this region, and here also the psychical processes in the act of speech are completed. In the great majority of mankind, the centre for speech is located in the left hemisphere. The fact that most men are right-handed also points to a finer construction of the motor ajyaratus for the upper extremity, which must also be located in the left hemisphere. Men, therefore, with pronounced right-handedness ("droiters") are evidently leftbrained ("gauchers du cerveau "-Broca). By far the greater number of mankind are "leit-brained spenkers" (Kussmaul); still there are exceptions. As a matter of fact, cases have been observed of left-hunded persons who lost their power of speech after a lesion of the right hemisphere $(O g l e)$. Investigations on the brains of remarkal,le men have shown that in them the third frontal convolution is more extensive and more complex than in men of a lower mental calibre. In deaf-mutes it is very simple; microcephales and monkeys possess only a rudimentary third frontal (Rüdinger).

The motor tract for speech prasses along the upper edge of the island' of Reil, then into the substance of the hemisplieres internal to the posterior elge of the knee of the internal capsule; from thence, through the rrusta of the left cerebral peduncle into the left half of the pons, where it crosses, then into the melulla oblongata, which is the place where all the motor nerves (trigeminus, facial, hypoglossal, vagus, and the respiratory nerves) concerned in speech arise. Total destruction of these paths, therefore, causes total aphasia; while partial destruction canses a greater or less disturlance of the mechanism of articulation, which has been called "anarthria" by Leyilen and Wernicke.

Conditions. - Three activities are required for speech-(1) the normal movement of the vocal apparatus (tongue, lips, mouth, and respiratory apparatus); (2) a kuowledge of the signs for objects and ideas (oral, written and imitative or mimetic signs); (3) the correct union of both.

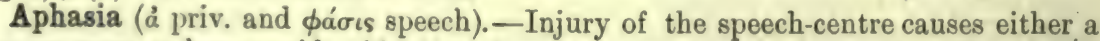
loss or more or less considerable disturbance of the power of speech. The loss of the power of speech is called " "pphasia." [Aphasia, as usually understood, means the partial or complete loss of the power of articulate speech from cerebral causes.] 
The following forms of aphasia may be distinguished :-

1. Ataxic aphasia (or the oro-lingual hemiparesis of Ferrier), i.e., the loss of speech owing to inability to execute the various movements of the mouth necessary for speech. Whenever such a person attempts to speak, he merely executes inco-ordinated grimaces and utters inarticulate sounds. [The muscles concerned in articulation, however, are not paralysed, but there is an absence of co-ordination of these nuscles due to disease of the cortical centre.] Hence, the patient cannot repeat what is said to him. Nevertheless, the psychical processes necessary for speech are completely retained, and all words are remembered; and hence, these persons can still give expression to their thoughts graphically or by writing. If, however, the finely adjusted movements necessary for writing are lost, owing to an affection of the centre for the hand, then there arises at the same time the condition of agraphia, or inability to execute those movements necessary for writing. Such a person, when he desires to express his ideas in writing, only succeeds in making a few unintelligible scrawls on the paper. Occasionally such patients suffer from loss of the power of imitation or the execution of particular movements of the limbs and body constituting pantomime speech or amimia (Kussmaul).

2. Amnesic Aphasia, or Loss of the Memory of Words. - Shonld the patient, however, hear the word, its significance recurs to him. 'The movements necessary for speech remain intact; hence, such a patient can at once repeat or write down what is said to him. Sometimes only certain kinds of words are forgotten, or it may be even only parts of certain words, so that only part of these words is spoken. [Nouns and proper names usually go first.] Cases of amnesic aphasia, or the mixed ataxic-amnesic form of disturbance of speech, point to a lesion of the third frontal convolution and of the island of Reil on the left side. Another form of amnesic aphasia consists in this, that the words remain in one's memory but do not come when they are wanted, i.e., the association between the idea and the proper word to give expression to it is inhibited (Kussmaul). It is common for old people to forget the names of persons or proper names; indeed, such a phenomenon is common within physiological limits, and it may ultimately pass into the pathological condition of amnesia senilis. Amongst the disturbances of speech of cerebral origin, Kussmaul reckons the following :-

3. Paraphasia, or the inability to connect rightly the ideas with the proper words to express these ideas, so that, instead of giving expression to the proper ideas, the sense may be inverted, or the form of words may be unintelligible. It is as if the person were continually making a "slip of the tongue."

4. Agrammatism and ataxaphasia, or the inability to form the words grammatically and to arrange them synthetically into sentences. Besides these, there is-

5. A pathological slow way of speaking (bradyphasia), or a pathological and stuttering way of reading (tumultus sermonis), both conditions being due to derangement of the cortex (Kussmaul). The disturbances of speech depending essentially upon affections of the peripheral, nerves, or of the muscles of the organs of the voice and speech, are already referred to in $\S 319,349$, and 354 .

[In word-blindness, the person cannot name a letter or a word, so that he cannot understand symbols, such as printed or written words, or it may be any familiar object, although he can

see quite well, while he can speak fluently and write correctly.]

[In word-deafness, the person hears other sounds and is not deaf, but he does not hear words.]

[The study of aphasia in its various forms is simplified by a study of the mode of acquisition of language by a child. The child hears spoken words and obtains auditory memories or impressions of

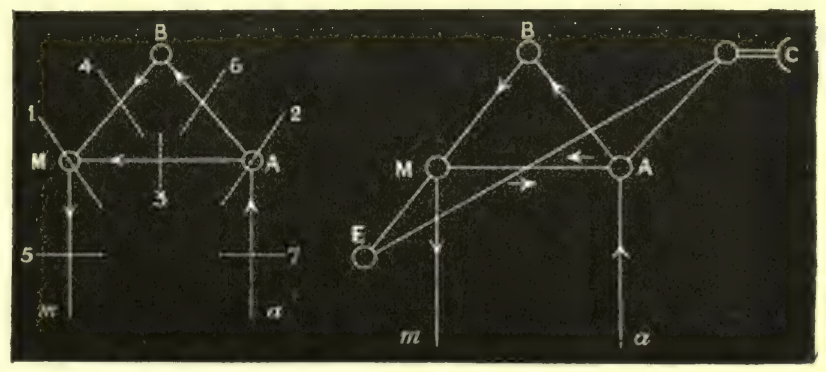

Fig. 494.

Fig. 495 .

Figs. 494, 495. - Schemes of aphasia. A, centre for auditory images; $\mathrm{M}$, for motor images; $\mathrm{B}$, perception centre ; Oc, eye ; $\mathrm{E}$, reading centre; 1 to 7 , lesions.(Lichtheim).

these sounds (called by Lichtheim "anditory word-representations"), and this must form the starting-point of language, and by and by it begins to co-ordinate its muscles to produce sounds imitative of these. Thus we have two centres, one for "auditory images" (fig. 494, A), and the other for "motor images" (fig. 494, M), and these two must be connected, thus establishing a reflex arc. There is a receptive and an emissive department as 
represented in the scheme. We must assume the existence of a higher centre (B), "in which concepts are elaboratel," where these sounds become intelligible. Politional language requires a connection between $\mathrm{B}$ and $\mathrm{M}$, as well as between $\mathrm{A}$ and $\mathrm{M}$. But we have also reading and writing. Suppose () to represent a centre for visual impressions (printed words or writing): these we can unlerstand through the connection between such visual impressions and auditory impressions, whereby a path is established through OA (fig. 495). In reading aloud, however, the oro-lingual museles must be co-ordinated, so we have the path OAM opened up. In writing, or copying written characters, the movements of the hand are special, and perhaps refuir. a special centre, or at least a special arrangement of the channels for impulses in the centre: the movements are learned under the guidance of ocular impressions, so we connect $\mathbf{O}$ and F. E being the centre guiling the movements in writing. As to volitional writing, the impulse fusses through $\mathrm{M}$ - but does it pass directly to E, or indirectly through A ? Lichtheim assumes that it goes lirect from II to $\mathbf{E}$. It is evilent that there are seven channels which mav lue interrupted, each one griving rise to a different form of aphasia (1 to 7$)$.]

[Lookel at from another point of view, either the ingoing $(a)$ or outgoing $(m)$ channels or centres, or the commissural fibres between both, may be affected. If the motor centre is affecteil. we have Wernicke's "motor aphasia"; if the sensory, his "sensory aphasia."]

In the must common form, or ataxic aphasia ( $\left.K^{\prime} u s s m a u l\right)$, which was that described by Broxa, or the "motor aphasia" of Wernicke, the lesion is in fig. 494, in M, i.e., in the motor, or what Ross calls the emissive department. In such a case, it is obvious that there will be loss of (1) volitional sprech, (2) repetition of worls, (3) reading aloud, (4) volitional writing, aul (5 writing to dictation; while there will exist $(a)$ understanding of spoken words, $(b)$ also

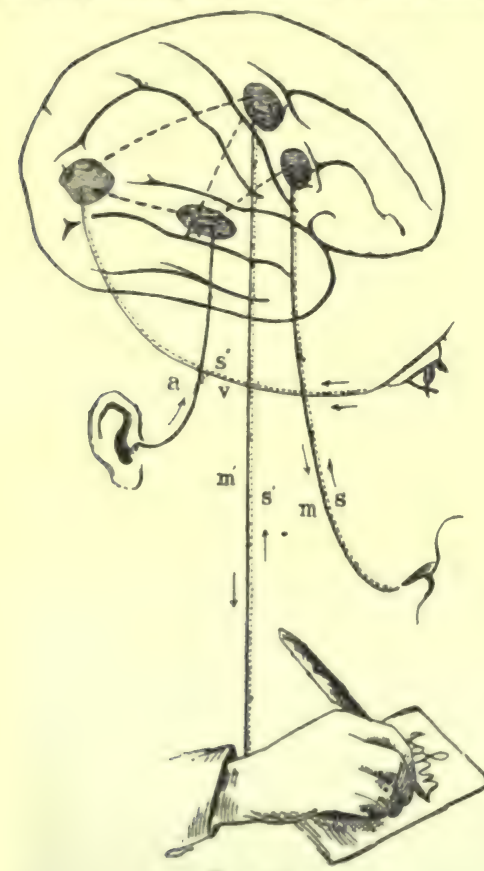

Fig. 496. of written words, $(c)$ and the faculty of copying. If the lesion be in A, we have the "sensorial aphasia", of Wernicke, i.e., in the acoustic word-centre; we find loss of (1) understanding of spoken language, (2) also of written language, (3) faculty of repeating words, (4) and of writing to dictation, (5) and of reading aloud; there will exist $(a)$ the faculty of writing, (b) of copying words, and (c) of volitional speech, but the volitional speech is imperfect, the wrong word being often used, so that there is the condition of I" paraphasia." If the connection between $\mathrm{A}$ and $\mathrm{M}$ be destroyed, other results will follow, and such cases of "commissural" aphasia have been described by Wernicke. If the interruption be between $B$ and $M$, we have a not uncommon variety of motor aphasia (4), where there is loss of (1) volitional speech, and (2) volitional writing, and there exist $(a)$ understanding of spoken language, (b) of written language, $(c)$ and the faculty of copying; but it differs from Broca's aphasia in that there also exists the faculty $(d)$ of repeating words, $(e)$ of writing to dictation, $(f)$, and of reading aloud. If the lesion is in $\mathrm{Mm}$ (5), the symptoms will be those of Broca's aphasia, but there will exist (1) the faculty of volitional writing, and (2) of writing to dictation. Many examples of this occur where patients have lost the faculty of speaking, but can express their thoughts in writing. In lesions of the path $\mathrm{AB}(6)$, there will be loss of (1) understanding of spoken language, and (2) of written language, and there will exist (a) volitional speech (but it will be paraphasic), (b) volitional writing (but it will have the characters of paragraphia), (c) the faculty of repeating worls, $(d)$ reacling aloud, $(c)$ writing to dictation, and $(f)$ power of copying words. The person will be quite unable to understand what he repeats, reads aloud, or copies.]

[Fig. 496 shows diagrammatically the conditions in motor and sensory aphasia. From the eye and car centripetal fibres ( $v$ and a) ascend to terminate in the visual (V) and auditory centres $(\Lambda)$, in the cortex, while afferent fibres $\left(s, s^{\prime}, s^{\prime \prime}\right)$, indicated by dotted lines, also pass from the articulations, muscles of the hand, and orbit to the cerebrum. The dotted lines on the surface of the cortex represent the association system of fibres which connects the centres with each other. The centres for vocal (V) and written expression (W) are connected by centrifugal fibres, $m$ and $m^{\prime}$, with the hand and larynx respectively (Ross).]

III. The thermal centre for the extremities is associated with the motor areas (\$377). Injury or degeneration of these areas causes inequality of the temperature on both sides (Bechterew). 
IV. The sensory regions are those areas in which conscious perceptions of the sensory impressions are accomplished. Perhaps they are the substratum of sensory perceptions, and of the memory of sensory impressions.

1. The visual centre, according to Munk, includes the occipital lobes (fig. 484, $\left.0^{1}, 0^{2}, 0^{3}\right)$, while, according to Ferrier, it also includes the angular gyrus. Huguenin observed, in a case of long-standing blindness, consecutive disappearance of the occipital convolutions on both sides of the parieto-occipital fissure, while Giovanardi, in a case of congenital absence of the eyes, observed atrophy of the occipital lobes, which were separated by a deep furrow from the rest of the brain. Stimulation of the centre gives rise to the phenomena of light and colour. Injury causes disturbance of vision, especially hemiopia of the same side ( $\$ 344-$ Westphal). When one centre is the seat of irritation, there is photopsia of the same halves of both eyes (Charcot). Stimulation of both centres causes the occurrence of the phenomena of light or colour, or visual hallucinations in the entire field of vision. Cases of injury to the brain, where the sensations of light and space are quite intact, and where the colour sense alone is abolished, seem to indicate that the colour sense centre must be specially localised in the visual centre (Samelsohn). After injury of certain parts, especially of the lower parietal lobe, "psychical blindness" may occur. A special form of this condition is known as "word-blindness" or alexia (Coecitas verbalis), which consists in this that the patient is no longer able to recognise ordinary written or printed characters (p. 711).

Charcot records an interesting case of psychical blindness. After a violent paroxysm of rage, an intelligent man suddenly lost the memory of visual impressions; all objects (persons, streets, houses) which were well known to him appeared to be quite strange, so that he did not even recognise himself in a mirror. Visual perceptions were entirely absent from his dreams.

Clinical observations on hemianopia (\$344) show that the field of vision of each eye is divided into a larger outer and a smaller inner portion, separated from each other by a vertical line passing through the macula lutea. Each right or left half of both visual fields is related to one hemisphere; both left halves are projected upon the left occipital lobes, and both right upon the right occipital lobes (fig. 487). Thus, in binocular vision, every picture (when not too small) must be seen in two halves; the left half by the left, the right half by the right hemisphere (Wernicke).

As a result of pathological stimulation of the visual centre, especially in the insane, visual spectres may be produced. Pick observed a case where the hallucinations were confiued to the right eye. Celebrated examples of ocular spectra occurred in Cardanus, Swedenborg, Nicolai, J. Kerner, and Hölderlin.

After degeneration of the cortical centre, the fibres which connect the occipital lobes with the external geniculate body, the anterior corpora quadrigemina, pulvinar, these structures themselves, and the origin of the optic tract undergo degeneration ( $v$. Monakow).

2. The auditory centre lies on both sides (crossed) in the temporo-sphenoidal lobes [according to Ferrier in the superior temporal convolution]; when it is completely removed, deafness results, while partial (left side) injury causes psychical deafness. [See p. 704 for contradictory results.] Amongst the phenomena caused by partial injury is surditas verbalis (word-deafness), which may occur alone or in conjunction with coecitas verbalis. Wernicke found in all cases of word-deafness softening of the first left temporo-sphenoidal convolution (p. 704). In left-handed persons, the centre lies perhaps in the right temporo-sphenoidal lobes (Westphal).

Clinical. - We may refer word-blindness and word-deafness to the aphataxic group of diseases, in so far as they resemble the amnesic form. A person word-blind or word-deaf resembles one who in early youth has learned a foreign tongue, which he has completely forgotten at a later period. He hears or reads the words and written characters; he can even repeat or write the words, but he has completely lost the significance of the signs. While an amnesic aphasic person has only lost the key to open his vocal treasure, in a person who is word-blind or worddeaf even this is gone. From a case of recovery it is known that to the patient the words sound like a confused noise. Huguenin found atrophy of the temporo-sphenoidal lobes after longcontinued deafness.

3. Gustatory and Olfactory Centre.-In the uncinate gyrus on the inner side of the temporo-sphenoidal lobe (especially on the inner side of that marked $U$ in 
fig. 480), Ferrier locates the joint centres for smell and taste. These two centres do not seem to be distinct locally from each other.

4. Tactile Areas. - According to Tripier and others, all the tactile cerebral fields from different parts of the body coincide with the motor cortical centres for these parts [compare p. 705].

Ocmionally, in epilepties, strong stimulation of the sensory centres, as expressed in the axesive suljuetive sensations, accompanies the spasmodic attacks (compare $\$ 393,12$ ). Such epileptifurm hallncinations, however, occur without spasms, and are accompanied only by disturbances of consciousness of very short duration (Berger).

Course of the Sensory Paths. - The nerve-fibres which conduct impulses from the sansory organs to the sensory cortical centres pass through the posterior third of the posterior limb of the internal copsule between the optic thalamus and the lenticular nucleus (fig. $500, \mathrm{~S}$ ). Hence, section of this part of the internal capsule causes hemianæsthesia of the cpposite half of the body (Charcot). In such a case, sensory functions are abolished-only the viscera retaining their sensibility. There may also be loss of hearing, smell, and taste,-and hemiopia (Bechterew).

Pathological. - In cases where there is more or less injury or degeneration of these paths, there is a corresponding greater or less pronounced loss of the pressure and temperature sense, of the cutaneous and muscular sensibility, of taste, smell, and hearing. The eye is rarely quite blind, but the sharpmess of vision is interfered with, the field of vision is narrowed, while the colour sense may be partially or completely lost. The eye on the same side may suffer to a slight extent.

V. Numerous cases of injury of the anterior frontal region, without interference with motor or sensory functions, have been collected by Charcot, Ferrier, and

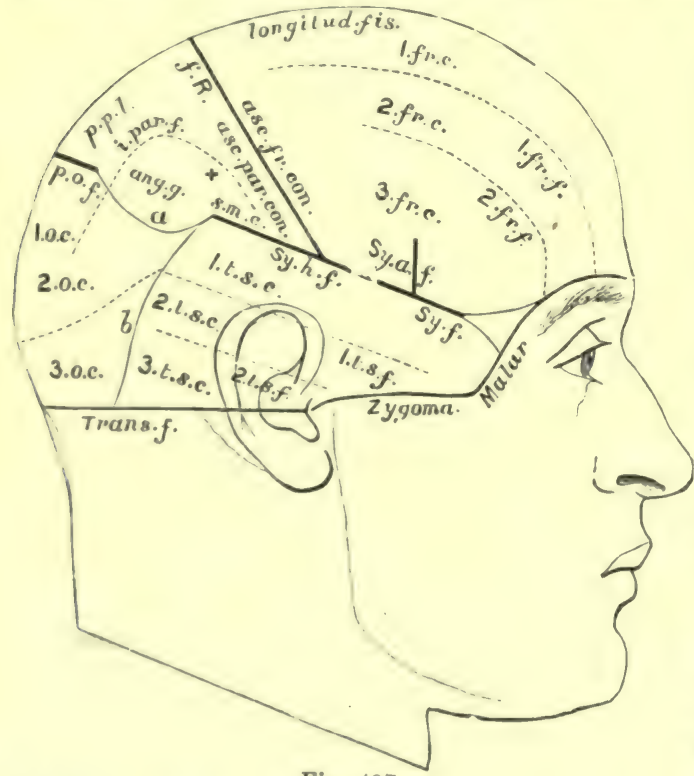

Fig. 497. others. On the other hand, enfeeblement of the intelligence and idiocy are often observed in acquired or congenital defects of the prefrontal region. In highly intellectualmen, Rüdinger found in addition a considerable development of the temporo-sphenoidal lobe. According to Flechsig, there is no doubt that the frontal lobes and the temporo-occipital zone are related to intellectual processes, more especially the "higher" of these.

Topography of the Brain.-The relations of the chief fissures and convolutions of the brain to the surface of the skull are given in fig. 484, the brain being represented after Fcker. [Turner and others have given minute directions for finding the position of the different convolutions by reference to the sutures and other prominent parts of the skull. The annexed diagram by R. W. Reid shows the relation of the convolutions to certain fixed

Relation of the fissures and convolutions to the surface of the sealp. +, most prominent part of the parietal eminence; $a$, convex line bounding parietal lobe below ; $b$, convex line bounding the temporo-sphenoidal lobe behind (R. W. Ricid).

ines (fig. 497).]

[The position of the fissure of Rolando, where its upper end joins the great longitudinal fissure, is obtained by measuring on the scalp $\mathrm{K}$ in the middle line the distance between the glabella and the external occipital protuberance, or the inion, which, in ordinary heals, varies from 11 to 13 inches (fig. 499). Measured from befure backwards, along this line, the distance from the glabella to the top of the fissure is $55^{\circ} 7$ 
per cent. of the length of the whole line. The direction of the fissure is downwards and forwards, and the long axis of the fissure forms, with the average mesial line, an angle of $67^{\circ}$, the angle opening forwards. Its average length is $3 \frac{3}{8}$ inches.]

[The fissure of Slyvius is found by drawing a line from the external angular process of the frontal bone backwards to the occipital protuberance, taking the nearest route between these two points. A point, $1 \frac{1}{8}$ inch backwards from the angular process along this line, marks the origin of the fissure; while a straight line drawn to the centre of the parietal eminence marks the course of its posterior limb. The parieto-occipital fissure will be two inches behind the upper end of the Rolandic fissure $(A . W$. Hare).

[Corpus Callosum. - It is usually stated that the corpus callosum connects the convolutions of one side of the brain with those on the other, i.e., that it is an inter. hemispherical commissure. D. J. Hamilton, however, is of opinion that it is not an inter-hemispheric commissure, but is due to cortical fibres coming from the cortex cerebri to be connected with the basal ganglia of the opposite side. On this view, the "corona radiata," as usually understood, consists only of the fibres which pass from the cerebral peduncle directly up to the cortex on the same side, and are contained in the posterior division and knee of the internal capsule. They correspond to the motor pyramidal tracts. Hamilton maintains that all the other fibres of the internal capsule pass into the crossed callosal tract, and, instead of running directly up to the cortex on the same side, cross in the corpus callosum to the cortex of the opposite side. Beevor, relying on the examination of the brain of monkeys, by Weigert's method, denies that any fibres of the corpus callosum pass into the external or internal capsules, and he supports the old view that the corpus callosum is a commissure between the two hemispheres.]

Erb observed a case of its almost complete destruction without any considerable effect on motility, co-ordination, sensibility, 'reflexes, senses, speech, or any marked impairment of intelligence.

379. BASAL GANGLIA

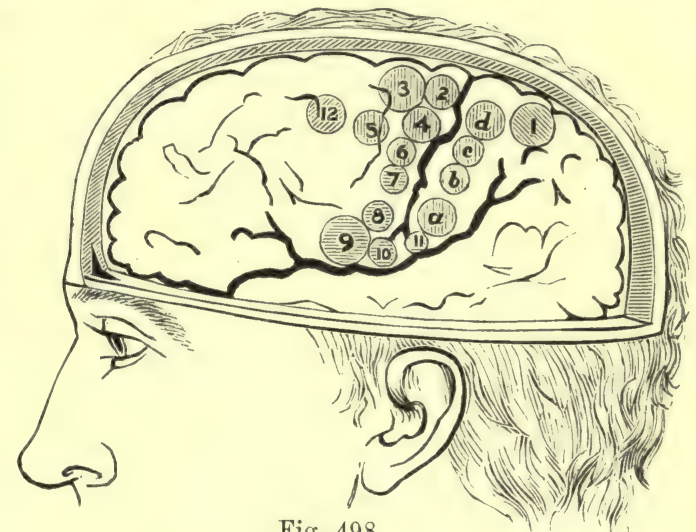

Fig. 498.

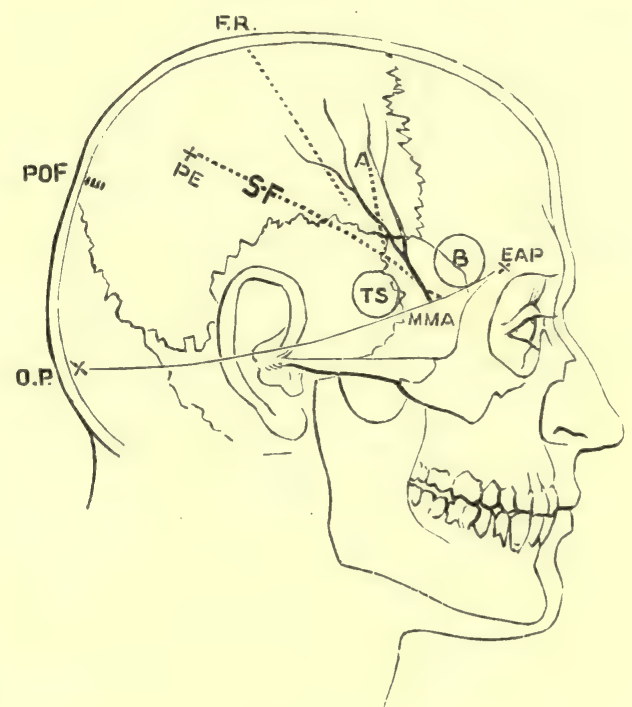

Fig. 499.

ig. 498. - The fissures of Rolando and Sylvius are marked as broad dark lines. The shaded circles mark approximately the motor areas. 1 , lower extremity; $2,3,4,5,6$, and $a, b, c, d$, upper extremity; $7,8,9,10,11$, orolingual muscles (A.W. Hare). Fig. 499.-Head, skull, and cerebral fissures. OPr, occipital protuberance ; EAP, external angular process; SF, Sylvian fissure; A, its ascending limb; FR, fissure of Rolando; $\mathrm{PE}$, parietal eminence; MMA, middle meningeal artery ; TS, tip of temporo-sphenoidal lobe; B, Broca's convolution; IF, inferior frontal sulcus; $\mathrm{POF}$, parieto-occipital fissure; IPF, intra-parietal sulcus ( $A$. W. Hare). 
ventricular portion, the lenticular nucleus. Between the head of the caudate nucleus internally, and the lenticular nucleus externally, lies the anterior division of the internal capsule. The fibres which pass between these ganglia do not seem to form connections with them. The expanded head of the caudate nucleus is in front, and lies inside and around the front, of the lenticular nucleus, with which and the anterior perforated space it is continuous; it sweeps backwards into a tailed extremity, which nearly surrounds the lenticular nucleus like a loop. The lenticular nucleus is biconvex in a horizontal section, but triangular and subdivided into three divisions when seen in a vertical section (fig. 501). The older observations on the corpora striata in man may be dismissed, as a distinction was not drawn between injury to its two parts on the one hand and the internal capsule on the other.]

[The caudate nucleus and lenticular nucleus in their development are co. ordinate with the development of the cortex cerebri. Electrical stimulation of these ganglia causes general muscular contractions in the opposite half of the body, which are due to simultanenus stimulation of the neighbouring cortico-muscular paths. The same result is obtained as if all the motor cortical centres were stimulated simultaneously.

(iliky did not observe movements on stimulating the corpus striatum in rabbits; it would seen that, in these animals the motor paths do not traverse these ganglia, but merely pass along. side of them.

[Lesions of the lenticular nucleus or of the caudate nucleus do not seem to give rise to any lermanent symptoms, provided the internal capsule be not injured.] Destruction of the internal capsule, however, causes paralysis of motion or sensibility, or both, on the opposite side of the body, according to the part of it which is injured. The corpus striatum is quite insensible to painful stimulation (Longet).

Pathological.-In man, a lesion, not too small, destroying the anterior part of the corpus striatum is followed by jermanent paralysis of the opposite side, provided the internal capsule is injured, but the paralysis gradually disappears, if the lenticular and caudate nucleus only are affected (compare $\$ 365)$. Sometimes there is dilatation of the blood-vessels in consequence of vaso-motor paralysis ( $\$ 37 \boldsymbol{i})$ if , the posterior part is injured (Nothnagcl); redness and a slightly increasel temperature of the paralysed extremities, at least for a certain time; swelling or wilema of the extremities; sweating; anomalies of the pulse detectable by the sphygmograph; decubitus acutus on the paralysed side ; abnormalities of the nails, hair, skin ; acute inflammations of joints, especially of the shoulder. Later, contracture or permanent contraction of the paralysed musrles takes place (Huguenin, Charcot). In some cases there is cutaneous anæsthesia, and oceasionally enfeeblement of the sense-organs of the paralysed side, and both when the posterior thinl or sensory crossway of the posterior section of the internal capsule is affected. Usually, however, hemiplegia and hemiancsthesia occur together.

Optic Thalamus.--Ferrier did not observe any movements on stimulating the optic thalami with electricity. As the pulvinar, or posterior extremity of the optic thalamus, is in part the origin of the optic nerve, and is also connected by fibres with the cortex cerebri, it is probably related to the sense of sight. Injury to its posterior third in man, results in disturbance of vision (Nothnagel). Ferrier surmises that the sensory fibres pass through the optic thalami on their way to the cortex, so that when they are destroyed, insensibility of the opposite half of the body is produced. Removal of the optic thalamus, or destruction of the part in the neighbourhood of the inspiratory centre in the wall of the third ventricle, influences the co-ordinated movements in the rabbit (Christiani).

We know very little definitely as to the functions of these organs. After injury to one thalamus, there has been observed enfeeblement or paralysis of the muscles of the opposite side, together with mouvements de manège; and sometimes hemianresthesia of the opposite side, with or without affections of the motor areas, have been recorded. Extirpation of certain cortical areas (rabbit) is followed by atrophy of certain parts of the thalamus (v. Monakove).

[Internal Capsule.-In connection with the functions of the basal ganglia, it is most important to remember their relation to the internal capsule. The corpus 
striatum consists of an intra-ventricular part, the caudate nucleus; and an extraventricular part, the lenticular mucleus. The lenticular nucleus consists of three parts, best seen in a vertical section (fig. 501, 1, 2, 3), with white matter between them, the striæ medullares. The anterior limb of the internal capsule sweeps

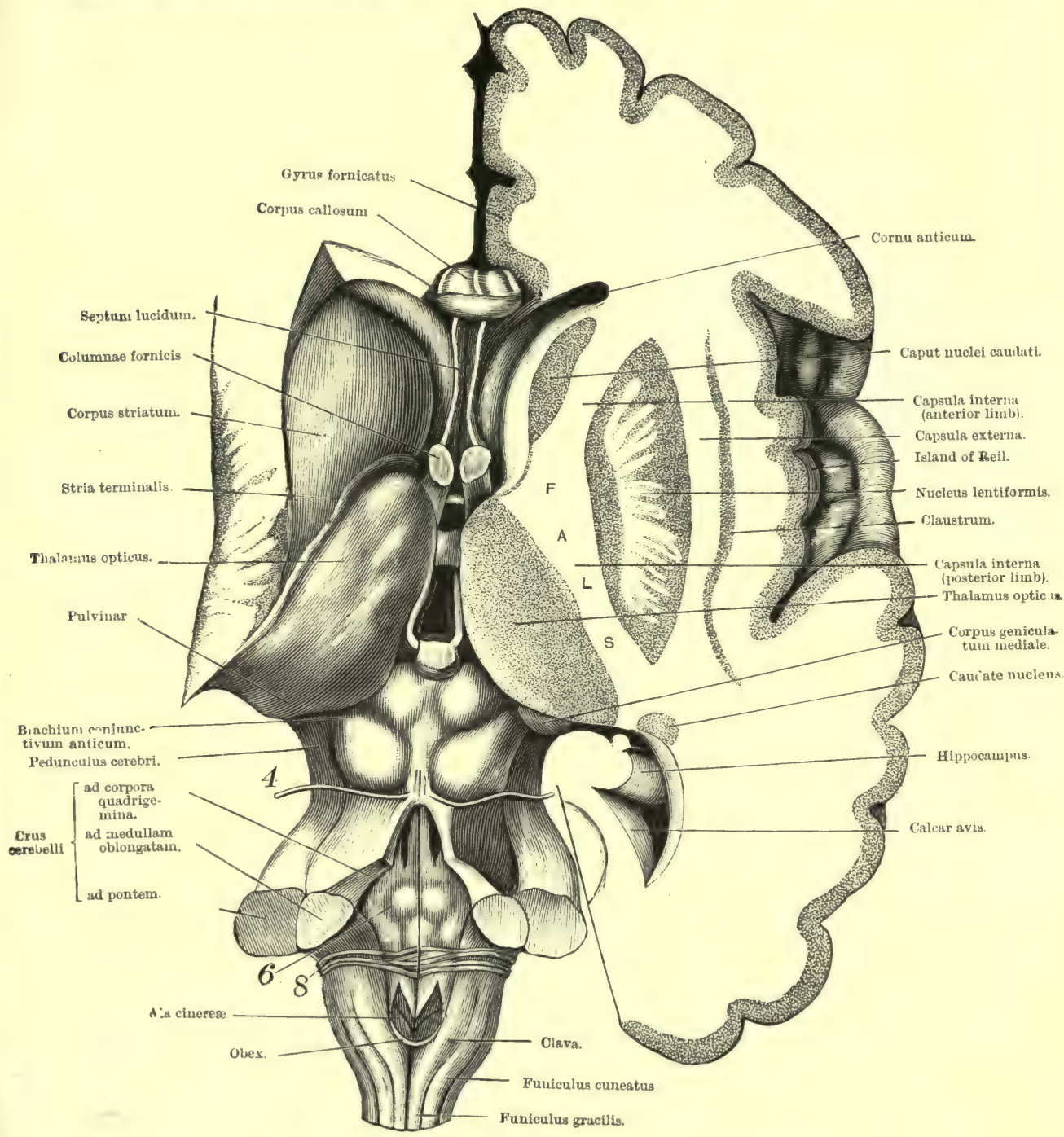

Fig. 500 .

Human brain, with the hemispheres removed by a horizontal incision on the right side. 4, trochlear ; 8 , acoustic nerve ; 6 , origin of the abducens ; F, A, L, position of the pyramidal (motor) fibres for the face, arm, and leg; $\mathrm{S}$, sensory fibres.

between the caudate and lenticular nucleus, while the posterior segment lies between the optic thalamus and the lenticular nucleus (fig. 500). External to the first division of the lenticular nucleus is the external capsule (figs. 500, 501), whose function is unknown. External to this is the claustrum, whose function is also 
unknown. It is evident that hemorrhage into or about the basal ganglia is apt to involve the fibres of the internal capsule. [When the lenticulo-striate artery, or as it is called the "artery of hæmorrhage," ruptures (tig. 490, aSL), it may not only destoy the lenticular nucleus, but the internal capsule will be compressed; and the same is the case with the lenticulo-optic artery-the external capsule will tend to force the blood inwards. We know that, in the posterior segment of the capsule, the volitional or pyramidal fibres lie in the following order from before backwards-those for the face (and tongue) in the knee, in the anterior third those for the arm and hand, and in the middle third for the leg, and perhaps behind these those for the trunk (fig. 500, F, A, L), so that a very small lesion in this region will affect a large number of these fibres, converging as they do like the lays of a fan from the motor cortical areas, where the arrangement of these centres is a sulero-inferior one (fig. 4R8), to become an antero-posterior one in the knee

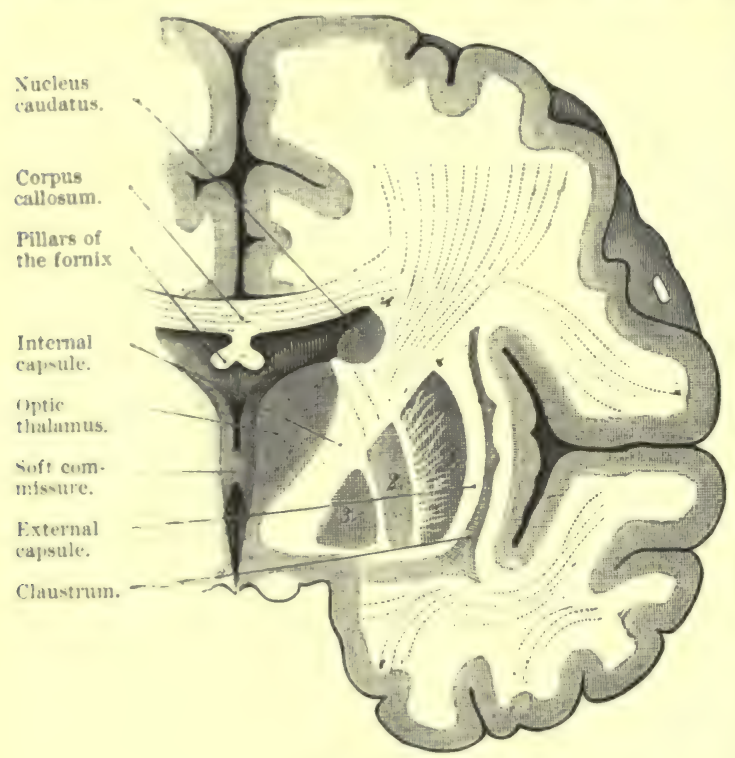
and posterior limb of the internal capsule (fig. 500). The posterior third of this limb is sensory and is the "sensory crossway."]

[Horsley points out that hæmorrhage from the lenticulostriate artery affects in order the muscles of the face, arm, leg, and trunk, while recovery is in the inverse order.]

[The crura cerebri (fig. $461, \mathrm{P})$, or cerebral peduncles, are two thick strands as they emerge above the pons, and as they are much larger than the pyramidal tracts, they must receive many fibres within the pons. A transverse section (fig. 502) shows that, on them posteriorly and connecting them, are the corpora quadrigemina (CQ). The crus proper is divided Frontal section through the right cerebral hemisphere in front of the soft commissure (posterior surface of the section). by the substantia nigra (SN) into a lower part, the crusta or basis, and an upper part, or tegmentum. The crusta is composed of ascending and descending nerve-fibres; but the tegmentum, in addition to many nerve-fibres, contains much grey matter with nerve-cells. Near the middle is the "red nucleus" or "tegmental nucleus" (RN). Outside this is the fillet (F), a well-defined bundle of nerve-filures running upwards from the pons. Above the nucleus, near the middle line, is the "posterior longitudinal bundle" (p. l. b.), which is triangular in section. Above the tegmentum lie many nerve-cells, the origin of the third nerve (III), and arranged around the iter is much grey matter.]

Injury to one cerebral peduncle causes, in the first place, violent pain and spasm of the opposite side, while the blood-vessels on that side contract, and the salivary glands secrete. These phenomena of irritation are followed by paralytic symptoms of the olpposite side, viz., anæsthesia $(\$ 365)$ and paresis, or incomplete voluntary control over the muscles, as well as paralysis of the vaso-motor nerves. In affections of the cerebral peduncle in man, we must remember the relation of 
the oculomotorius to it, as the latter is often paralysed on the same side [while the extremities, tongue, and half the face are paralysed on the opposite side from the lesion].

The middle third of the crusta of the cerebral peduncle (fig. 502) includes the direct pyramidal tracts $(\$ \$ 365,378)$. The fibres of the inner third connect the frontal lobes with the cerebellum through the superior cerebellar peduncles. In the outer third are fibres which connect the pons with the temporal and occipital cerebral lobes (Flechsig). The fibres which pass from the tegmentum in to the corona radiata conduct sensory impulses (Flechsig).

[The pons varolii contains ascending and descending fibres, as well as transverse ones, and, in addition, the continuation upwards of grey matter from the medulla, special masses of grey matter, and the nuclei of certain cranial nerves. Its appearance in section necessarily varies with the region where the section is made. Fig. 503 is a transverse section through part of the seventh nerve. The lower part shows the superficial (s.t.f.) and deep (d.t.f.) transverse fibres, with the pyramidal fibres $(\mathrm{Py})$ between them.]

Stimulation or section of the pons causes pain and spasms; after the section, there may be sensory, motor, and vaso-motor paralysis, together with forced movements.

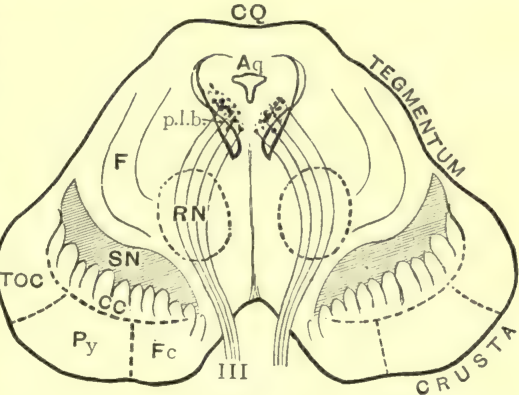

Fig. 502.

Scheme of transverse section of the cerebral peduncles. $\mathrm{CQ}$, corpora quadrigemina; $\mathrm{Aq}$, aqueduct; p.l.b., posterior longitudinal bundle; F, fillet or lemniscus; RN, red nucleus ; SN, substantia nigra; III, third nerve; Py, pyramidal tracts; FC, frontocerebellar; and TOC, temporo-occipital fibres of the crusta ; CC, caudate-cerebellar fibres in upper part of crusta (after Wernicke and Gowers).

For diagnostic purposes in man, it is important to observe if alternate hemiplegia be present.

[In lesions situated in the lower half of one side of the pons, there is facial paralysis on the

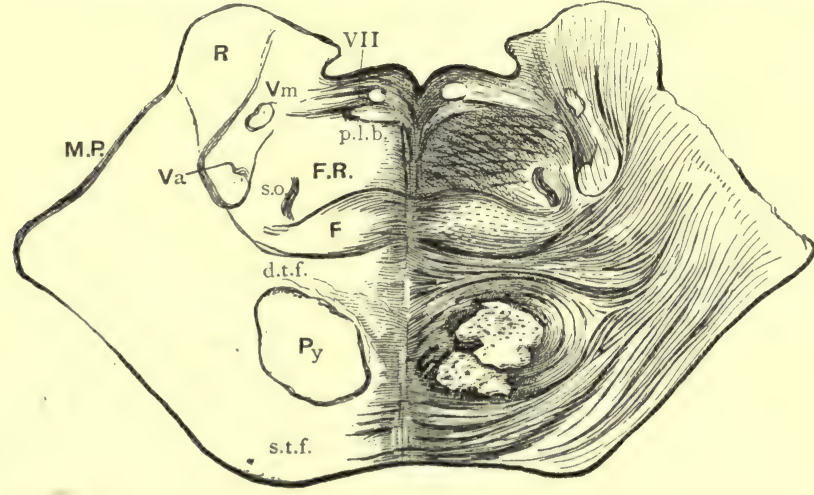

Fig. 503.

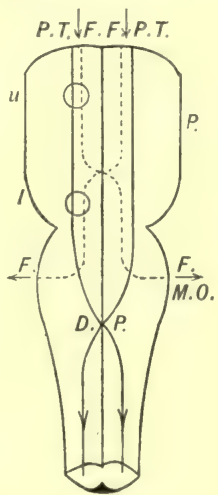

Fig. 504.

Fig. 503. - Transverse section of the pons through part of the seventh nerve. $\times 2$. F.R., formatio reticularis; VII, seventh nerve; Va, ascending root, and Vm, motor root of the fifth nerve; F, fillet; s.o., superior olive; s.l.b., superior longitudinal bundle; Py, pyramidal fibres ; R, restiform body ; M.P., middle peduncles of cerebellum ; d.t.f. and s.t.f., deep and transverse superficial fibres of the pons (after Wernicke). Fig. 504. - Scheme of the fibres in the pons ; PT, pyramidal tracts; F, facial fibres ; $u$, upper, $l$, lower lesion ; MO, medulla oblongata ; DP, decussation of pyramids.

same side as the lesion and paralysis (motor and sensory, and more or less complete) on the opposite side of the body - this is called alternate paralysis ; while, if the lesion be in the upper 
half of one side of the pons, the facial paralysis is on the same side as the paralysis of the body. But the parts supplied by the 5 th and 6 th nerves may also be involved. This is explained by fig. 504 , where the upret facial fibres cross in the pons. Sudden and extensive lesions of the pons are freyuently associated with hyperpyrexia, the temperature often rising rapidly within an hour, perhaps from the grey matter in the floor of the 4 th ventricle being affected; but whether it is due to some effect on a heat-regulating or heat-producing centre is uncertain. Tumours of considerable size may press on the pons without producing very marked symptoms, as tumours tend to push aside tissues, unless they be infiltrating in their character. Lesions of the transverse superficial fibres (middle cerebellar peduncles) often give rise to involuntary forced movenuents, there being a tendency to move to one side or the other.]

The Corpora Quadrigemina.-Destruction of these bodies on one side in mammals (or their homologues, the optic lobes in birds, amphibians, and fishes) rauses actual l, lininess, which may be on the same or the opposite side, according to the relation of the fibres crossing at the optic chiasma (\$ 344). Total destruction causes blindness of both eyes. At the same time, the reflex contraction of the jupil, due to stimulation of the retina with light, no longer takes place (Flourens), where the optic is the afferent and the oculomotorius the efferent nerve $(\$ 345)$. If the cerebral hemispheres alone be removed, the pupil still contracts to light, as well as after mechanical stimulation of the optic nerve (H. Mayo). Destruction of the corpora yuadricemina interferes with the complete harmony of the motor acts; disturbance of equilibrium and inco-ordination of movements occur (Serres). In frogs, Goltz observed not only awkward clumsy movements, but at the same time the animals have to a large extent lost the power of completely balancing the body (p. 6×3). A similar result was observed in pigeons (M'Kendrick) and rablits (Ferrier). Extirpation of the eyeball is followed by atrophy of the opposite anterior corpus quadrigeminum (Gudden).

Acording to Bechterew, the fibres of one optic tract pass through the anterior brachium (fig. 5 iii) into the anterior pair (nates) of the corpora quadrigemina; while those fibres which cross in the rhiasma (fig. 4.5 pass into the posterior pair (testes). According to this arrangement we have partial hlininess, arcorling as one or other pair of these bodies is destroyed.

In man, very little is known regarding the effects of disease of the corpora quadrigemina, interferene with the ocular museles being the most marked symptom; but the inco-ordination of movem.nt which has hen olserved may be due to pressure upon the superior cerebellar perluncle, while it is by no means certain that the defects of vision are directly lue to lesions of these bodies.]

Stimulation of the Corpora Quadrigemina. - The corpora quadrigemina react to electrical, chemical, and mechanical stimuli. The results of stimulation are very variously stated. Accorling to some observers, there is dilatation of the pupil on the same side; according to Ferrier, it may be the jupil on the opposite or on the same side. The stimulation may le conducterl from the corpora yualrigemina to the medulla oblongata, and to the origin of the sympathetic, for, after section of the sympathetic nerve in the neck, dilatation of the pupil no longer takes place. Acerrling to linoll, the contraction of the pupil observed by the older exprimenters occurs ouly when the adjoining optic tract is stimulated. Stimulation of the right anterior corpus quailriceminum causes deviation of both eyes to the left (and conversely); on continuing the stimulition, the head is turned to this side. On dividing the corpora quadrigemina by a vertical median incision, stimulation of one side causes the result to take place only on one sile (Alwmiit). Ferrier observed signs of pain on stimulating these organs in mammals. Carville and I)uret conclude from their experiments, that these organs are centres for the extensor movements of the trunk. Ferrier found, on stimulating one optic lobe in a pigeon, dilatation of the opjosite jupil, turning of the head towards the other side and backwards, movement of the opposite wing and leg; strong stimulation caused flapping movements of both wings. Danilewsky, Ferrier, and Lauder Brunton observed a rise of the blood-pressure and slowing of the heart-heat, together with deeper inspiration and expiration.

Bechterew ascribes all the phenomena, except those of vision itself, which accompany injury or stimulation of these bolies, to affections of deeper seated parts. He asserts that the corpora 'uluarigumina contain neither the centre for the movements of the pupils nor that for the combined movements of the eyeballs; not even the centre for maintaining the equilibrium of the boly. Stimulation of these boulies causes the animals to perform marked movements. Reflex phenomena, nystagmus, forcel movements, and unsteadiness of the gait only occur, however, when the deeper parts are injured.

Pathological. - Lesions of the anterior pair in man, according to the extent of the lesion, cause disturbance of vision, failure of the pupil to contract to light, and even blindness; there 
may be paralysis of the oculomotorii on both sides. Disease of the posterior pair may be associated with disturbances of co-ordination (Nothnagel).

Forced Movements.-It is evident from what has been said regarding the importance of the corpora quadrigemina for the harmonious execution of movements, that unilateral injury of such parts as are connected with them by conducting channels, must give rise to peculiar unilateral disturbance of the equilibrium, causing variations from the symmetrical movements of both sides of the body. These movements are called forced movements. To this class belong the "mouvements de manège," where the animal, instead of moving in a straight line, runs round in a circle; index movements, where the anterior part of the body is moved round the posterior part, which remains in its place, just like the movements of an index round its axis ; and rolling movements, when the animal rolls on its long axis. All these forms of movement may pass into each other, and they are, in fact, merely different varieties of the same kind of movement. The parts of the nervous system whose injury produces these movements are the corpus striatum, optic thalamus, cerebral peduncle, pons, middle cerebellar peduncles, and certain parts of the medulla oblongata. Eulenburg observed index movements in the rabbit, after injury to the surface of the brain, and Bechterew observed the same in dogs. Forced movements, together with nystagmus and rotation of the eyeballs, are caused by injury to the olives (Bechterew). The statements of observers vary as to the direction and kind of movement produced by injuring individual parts. The following observations have been made :- Section of the anterior part of the pons, and of the crura cerebelli causes index, or, it may be, rolling movernents towards the other side; section of the posterior part of the same regions causes rolling move ments towards the same side, while the same result is caused by a deeper puncture into the tuberculum acusticum, or into the restiform body. Section of one cerebral peduncle causes mouvements de manège, while the body is curved with the convexity towards the same side. The nearer to the pons the section is made, the smaller is the circle described; ultimately index movements occur. Injury to one optic thalamus produces results similar to puncture of the anterior part of the cerebral peduncle, because the latter is injured along with it at the same time. Injury to the anterior part of one optic thalamus causes the opposite kind of forced movement, viz., with the concavity of the body towards the injured side. Injury to the spinal portion of the medulla oblongata is followed by bending of the head and vertebral column, with the convexity towards the injured side, along with movements in a circle. When the anterior end of the calamus and the part above it are injured, the movements are towards the sound side.

Strabismus and Nystagmus.-Amongst the forced movements may be reckoned deviation of the eyeballs, strabismus or squinting, and involuntary oscillation of the eyeballs, constituting nystagmus. The latter condition occurs after superficial lesions of the restiform body, as well as of the floor of the 4 th ventricle. A unilateral, deep, transverse injury, from the apex of the calamus upwards as far as the tuberculum acusticum, causes the eye of the same side to squint downwards and forwards, that of the other side backwards and upwards. Section of both sides causes this condition to disappear (Schwahn). Hence, Eckhard assumes that the medulla oblongata is the seat of an apparatus controlling the movements of the eyes (Eckhard), which can be excited by sudden anæmia, e.g., ligature of the cephalic arteries in a rabbit.

In pathological degeneration of the olivary body of the medulla oblongata in man, Meschede observed intense rotatory movements towards the same side.

Theory. - In order to explain the oecurrence of forced movements, it is suggested that there is unilateral incomplete paralysis (Lafarque), so that the animal in its efforts to move onwards leaves the paralytic side slightly behind the other, and hence there is a variation from the syminetry of the movements. Brown-Séquard regards the matter in exactly an opposite light, viz., as due to stimulation from injury, causing an excessive activity of one-half of the body. 
Henle ascribes the movements to vertigo, or a feeling of giddiness caused by the injury. In all operations on the central nervous system, where the equilibrium is deeply affected, there is a consilerable increase in the number and depth of the respirations (Landuis).

Other Effects. - Some olsservers noticed variations of the blood-pressure and a change in the number of heart-beats after stimulation of the cortex cerebri, e.g., after electrical stimulation of the motur areas for the extremities (Bochefontaine). Balogh olserved acceleration of the pulse, on stimulating several points on the cortex cerebri of a dog, and from one point slowing of the pulse. Echhard stimulatel the surface of the brain in rabbits, and, as a rule, he observed that, as long as singl. crossed :unvements oceurred in the anterior extremities, there was no effect upon the heart, but that the heart became affected as soon as other movements occurred. This consists in slow strong pulse-beats, with oecasional weaker beats, while at the same time the blood-pressure is slirhtly increased (Berhefontuine). If the vagi be divided beforehand, the effect upon the pulse uisap'pears, while the increase of the blool-pressure remains. That psychical processes affect the action of the heart was known to Homer and Clırysipp. Bochefontaine and Lepine, on stimulat. ing several points, esprecially in the neighbourhood of the sulcus cruciatus in the dog, observed increased secretion of saliva, slowing of the movements of the stomach, peristalsis of the intestine, contraction of the spleen, of the uterus, of the bladder, and increased respirations. linfalini, on stimulating those parts of the cortex which cause movements of the jaw, observed seretion of gastric juice with increase of the temperature of the stomach. Schifl, BrownSiquard, Ebstein, Kilosterhalfen, anil others have observed that injury to the pons, corpus striatum, thalamus, cerebral perlumcle, and medulla oblongata often causes hyperamia and hremorrhage into the lung (accorling to Brown-Séquard, especially after injury to one side of the jons, which affects the opposite lung), under the pleura, in the stomach, intestine, and kilueys. Gastric bimorrhage is common after injury to the pons just where the cerebral perluncles join it. Sinilar phenomena have been observed in suan after apoplexy or cerebral Læmorrhage.

Sperially interesting is the cerebral ninilateral decubitus acutus or bed-sore, described by Charcot, which always occurs on the jaralysed side of the body, i.e., on the side oplosite to

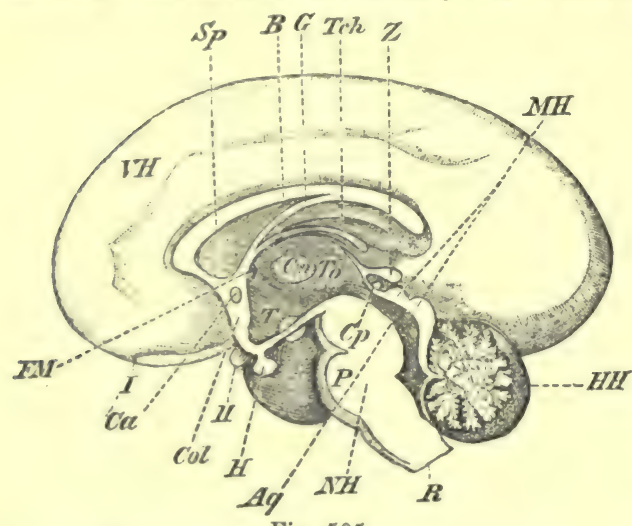

Fig. 505 .

Longitudinal section of an adult human brain. $A q$, ayueduct of Sylvius ; $B$, corpus callosum; $C c$, anterior commissure; Cm, middle corrmissure; Col, lamina terminalis ; $C p$, posterior commissure ; $F M$, foramen of Munro; $G$, fornix ; $H$, pituitary body; $H H$, cerebellum ; $M H$, corpors quadrigemina ; $M H$, medulla oblongata; $P$, pons Varolii ; $R$, spinal cord; $S p$, septum lucidum; $I$, infundibulum; $T$ ch, tela choroidea ; To, optic thalamus ; $V H$, cerebrum ; $Z$, pineal gland; $I$, olfactory lobe and nerve; $I I$, optic nerve. as a hollow outgrowth from the part of the embryonic brain connected with the third ventricle. It loses its cavity and its nervous tissue; is permeated by connective-tissue and blood-vessels; and is connected with the floor of the third ventricle by the infundibulum. The anterior lobe is developed as a tubular invagination of the stomodæum, i.e., from the ectoderm of the buccal cavity; but it soon loses its connection with this cavity as the upper end enlarges, and the stalk atrophies. In mammalia, the upper expanded end unites with the anterior lobe to form the pituitary body. For the effects of its removal, see $\$ 103, \mathrm{~V}$.] 
380. STRUCTURE AND FUNCTIONS OF THE CEREBELLUM. - [Structure. - On examining a vertical section of a cerebellar leaflet, we observe the following microscopic appearances : -Externally, is the pia mater with its blood-vessels (fig. 506, $\alpha$ ), which penetrate into the grey matter; within is the medulla, composed of white fibres. The grey matter consists of $\zeta$, a broad outer or molecular layer, largely composed of branched fibrils; and internal to it is $d$, the "granular," nuclear, or rust-coloured layer. On the boundary line between these two, is the layer of Purkinje's cells, $c$. The cells of Purkinje form a single layer of large multipolar

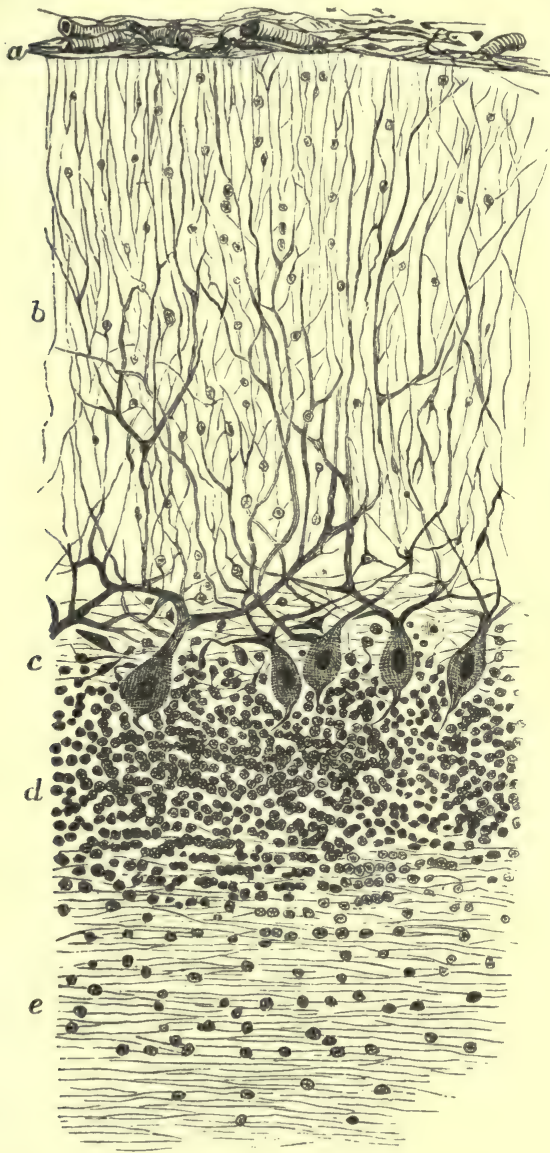

Fig. 506. flask-shaped nerve-cells, which have been compared to the branched antlers of a stag (fig. 507). From their outer surface is given off a process which rapidly divides, and gives rise to a large number of smaller processes running outwards in the outer grey layer. Some of these processes form part of the ground-plexus of fibrils in this layer: An unbranched axial cylinder process is sent inwards to the granular layers, where it becomes continuous with a nerve-fibre-every cell of Purkinje being continuous with a straight unbranched medullated nerve-fibre. The unbranched fibres run straight from the medulla through the granular layer, forming no connection with its granules. A second set of branched or anastomosing, often varicose, nerve-fibres, finer than the foregoing, pass from the medulla into the granular layer, where they form a network which is

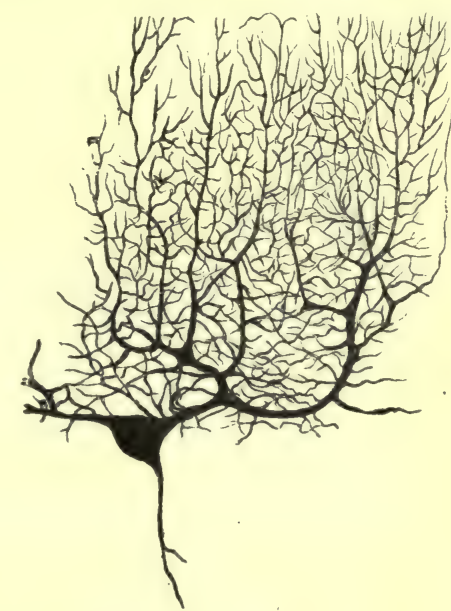

Fig. 507.

Fig. 506. - Vertical section of the cerebellum. $\alpha$, pia mater; $b$, external layer ; $c$, layer of Purkinje's cells : $d$, inner layer ; $e$, medullary white matter. Fig. 507.-Purkinje's cell, sublimate preparation. $\times 120$.

continued into the molecular layer. The granular layer is composed of closely packed granules of two kinds; one is stained by hæmatoxylin, and the other with eosin (Denissenko). The hæmatoxylin stained cells are most numerous; they consist of a nucleus surrounded by protoplasm, and are what were formerly called granules. The eosin-stained cells, which are also stained by nigrosin (Beevor), are interposed in the course of medullated nervefibres. The hrmatoxylin cells, called glia-cells by Beevor, have processes, and form a network throughout the granular layer, which also extends into the molecular layer. This network is regarded as the continuation of the modified myelin of the nerve-fibres, and it forms a capsule for the cells of Purkinje. The molecular layer consists of a ground substance, composed of a spongy network of fine fibrils, which seem to be of the nature of neuro-keratin, strengthened here and there by stronger fibres. In the meshes lies a homogeneous substance. 
Some of this substance is more condensed to form a limitans externa on the surface of tho cerebellum, while on the boundary line next the granular layer the branches of the glia-cells form a limitans interna, and between the two stretches the neuro-keratin network. Some small varicose nerve-fibres exist in this layer continuous with those in the granular layer. The branched provess of the cells of Purkinje is fibrillated, and the finer processes are composed also of fihrils, which are gradually distributed until they become isolated. It is suggested by Beevor that these fibrils bend at a right angle in a plane parallel to the surface, and rearrange themselves as fibres surrounded by a medullated sheath, and that these fibres run inwards through the molecular and granular layers-as the branched fibres-to the medulla.]

Function.-Injuries of the cerebellum cause disturbances in the harmony of the movements of the body. Most probably, the cerebellum is a great and important central organ for the finer co-ordination and integration of movements. The fact that it is connected with all the columns of the spinal cord and with all the central ganglionic masses, renders this very probable. The direct cerebellar tracts from the lateral column of the cord conduct sensory impressions to the cerebellum, and thus indicate the posture of the trunk. The cerebellum may affect the motor nerves of the cord through fibres which pass downward in the lateral columns of the cord from the restiform bodies (Flechsig). Injury of the cerebellum neither produces disturbance of the psychical activities, nor does it interfere with the will or consciousness. Injuries to the cerebellum itself do not give rise to pain.

According to Schiff, the cerebellum does not actually regulate the co-ordination of movements. According to him, there is a mechanism on both sides of the middle line, which increases all the complicated muscular movements--not only those for powerful contractions, but also the jeculiar fine movements which fix the limbs and joints. Luciani asserts that destruction of the cerebellum produces a condition of incomplete tonus, there being a want of energy to control the voluntary muscles. Each half of the organ acts on both halves of the body.

Injury or Removal of Cerebellum.-The immediate results produced by injury to or removal of the cerebellum have been admirably described by Flourens (fig. 508).

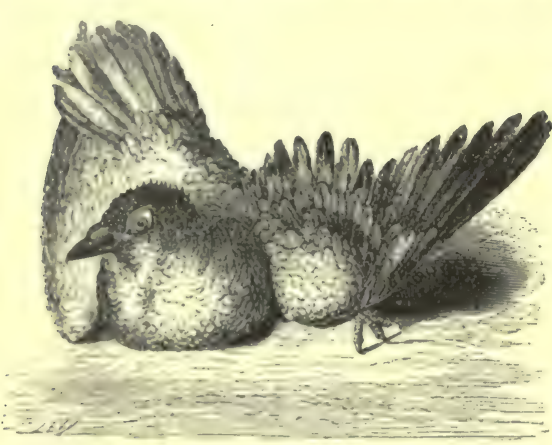

Fig. 508.

Pigeon with its cerebellum removed. On removing the most superficial layers in a pigeon, the animal merely showed signs of weakness and interference with the uniformity of its movements. On removing more of the cerebellum, the animal became greatly excited, and made violent irregular movements, which did not partake of the character of convulsions. The sensorium was unaffected, while vision and hearing were intact. Co-ordinated movements, such as walking, flying, springing, and turning, could be executed but imperfectly. After removal of the deepest layers, the power of executing the above-named movements was completely abolished. On placing the pigeon on its back, it could not get on its legs; at the same time it made coutinually the greatest exertions in its movements, but these were always inco-ordinated, and therefore without any satisfactory result. The will, intelligence, and perception remained intact ; the animal could see and hear, and sought to avoid obstacles placed in its way. It gradually exhausted itself in fruitless efforts to get on its legs, and ultimately remained in its abnormal position, quite exhausted. Flourens concluded from these experiments that the cerebellum is the centre for co-ordinating voluntary movements. Lussana and Morganti regard the cerebellum as the seat of the muscular sense.

[Extirpation in Mammals. - The dangers attending this operation are so great, that but few animals survive. Luciani, however, by using antiseptic and other precautions, has been able to operate so that complete cicatrisation was obtained, the animal (young bitch) being restored to health for a few months. The cerebellum alone was removed, but not its peduncles. As in 
all other similar operations, we must distinguish sharply the phenomena manifested during recovery from those after complete recovery. During the first period of six weeks, from the time of the operation until complete recovery, the symptoms are those of injury and irritation of the divided peduncles, along with those resulting from the removal of the organ. They are clonic contractions of the muscles of the fore limb, neck, and back, passing into tonic contractions when the animal attempts to move, and also weakness of the hind legs, so that all the normal voluntary movements are interfered with, i.e., inco-ordinated, although these symptoms may be explained by the injury to adjoining parts. There was no sensory disturbance or loss of the muscular sense, although closing the eyes rendered standing impossible. As recovery takes place, these symptoms disappear, and the animal enters on the second period, where the symptoms depending on the actual loss of the organ are pronounced. The contracture and psendo-paralytic weakness disappear, while there are alterations in the tone of the individual muscles, producing a sort of "cerebellar ataxy." The dog could swim in quite a normal manner, its power of equilibration was not interfered with, but acts requiring a greater development of muscular energy could not be properly executed. This period lasted four to five months. After this time its health gave way, there was otitis, conjunctivitis, articular and cutaneous inflammations, while a peculiar form of marasmus set in, the animal dying after eight months. In fishes also, the removal of the cerebellum does not affect their power of locomotion (Bandelot).]

Duration of the Phenomena.-After superficial lesions, or after a deep incision, the disturbances of co-ordination soon pass away (Flourens). If the injury affects the lowest third of the cerebellum, the motor disturbances remain permanently. Symmetrical lesions do not disturb co-ordination (Schiff). After removing the greater portion of the cerebellum in birds, Weir-Mitchell has observed that the original disturbances gradually disappear; and after months only slight weakness and a condition of rapid fatigue remain.

After ablation of the cerebellum, secondary degeneration occurs in the part of the pons around the pyramids, the lower olives, all the cerebellar peduncles and the direct cerebellar tract, usually on the same side (Flechsig). It is found also in individual fibres in all the cranial nerves and the anterior roots of the spinal nerves (Marchi).

In the dog, superficial injuries of the vermiform process, or of one-half of the organ, produce merely temporary disturbances; while deep injuries to the vermiform process, or removal of one hemisphere and a part of the vermiform process, cause permanent rigidity of the legs and shaking of the head; if the worm and both halves are destroyed, there follows permanent pronounced disturbance of co-ordination ( $v$. Mering). According to Baginsky, destruction of a large part of the vermiform process alone causes in mammals permanent disturbance of co-ordination. Ferrier found that a vertical section of the cerebellum in monkeys, produced only inconsiderable disturbances of equilibrium; after injury of the anterior part of the middle lobe, the animal often tumbles forward; while, when the posterior part is injured, it falls backward. After injury of the lateral lobe, the animal is drawn towards the affected side (Schiff, Vulpian, Ferrier, Hitzig). If the middle commissure be injured, the animal rolls violently on its long axis towards the injured side (Magendie). Paralysis never occurs after injuries of the cerebellum, nor is there ever disturbance of sensation or of the sense of touch. Luciani found that, in animals with the cerebellum extirpated, marasmus ultimately set in. In frogs, an important organ concerned with motion lies at the junction of the oblongata with the cerebellum (Eckhard). After it is removed the animal can no longer execute co-ordinated jumping movements, nor cau it crawl (Goltz).

[In man, the cerebellum is connected with the maintenance of the equilibrium. There may be a lesion of the hemispheres without any marked symptoms; but if the middle lobe be injured or pressed on by a tumour, there is usually a reeling or staggering gait, like that of a drunken man. Ross points out that, if the tumour affect the upper part of this lobe, the tendency is to fall backwards, and if in the lower part, to fall forwards or to revolve round a horizontal axis. Voniting is frequently persistent and well inarked, while there may be nystagmus and tonic retraction of the head.]

After injuries of the cerebellum, involuntary oscillations of the eyeballs or nystagmus, as well as squinting (Magendie, Hertwig), have been observed; while Ferrier observed movements of the eyeballs after electrical stimulation. According to Curschmann, Eckhard, and Schwann, this occurs only when the medulla oblongata is involved ( $\$ 379)$.

Effects of Electricity and Vertigo. - If an electrical current be passed through the head, by placing the electrodes in the mastoid fossæ behind both ears, with the + pole behind the right and the - pole behind the left ear, then on closing the current, there is severe vertigo, and the head and body lean to the + pole, while the objects around seem to be displaced to the left. If the eyes be closed, while the current is passing, the movements appear to be transferred to the person himself, so that he has a feeling of rotation to the left (Purkinje). At the moment 
the head leans towards the anode, the eyes turn in that direction, and often exhibit nystagmus. The electrical current probably stimulates the nerves of the ampulle, as we know that affections of these bolies cause vertigo $(\$ 350)$. The cerebellum has no relation to the sexual activities, as was maintainel by Gall. The contractions of the uterus observed by Valentin, Budge, and Spiegelber: after stimulation of the cerebellum, are as yet unexplained.

Pathological. - Lesions of one hemisphere may give rise to no symptoms ; but if the middle lobe is involverl, there is inco-ordination of movement, especially a tendency to fall, unsteady Gait, and pronounced vertigo. Irritative lesions of the middle peduncle cause complete gyrating movements of the horly around its axis, together with rotation of the eyes and head (Nothnagel).

381. PROTECTIVE APPARATUS OF THE BRAIN. - The Membranes. - The dura mater cerebralis is intimately united to the periosteum of the cavity of the skull, while the spinal Inra nater forms around the spinal cord a freely suspended long sac, fixed only on its anterior -urface. It is a fibrous membrane, consisting of firm bundles of comnective-tissue intermixed

v

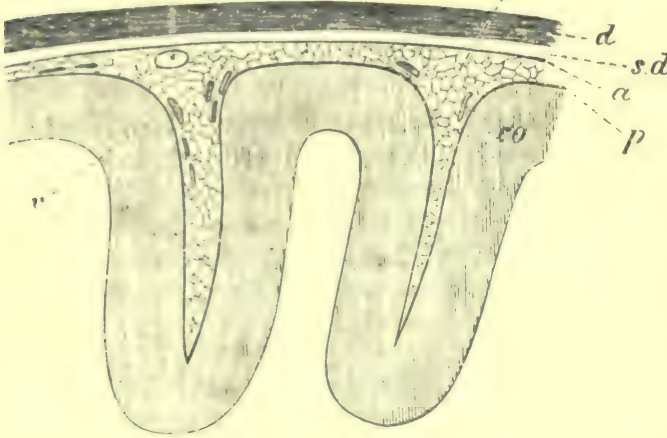

Fig. 509. with numerous elastic fibres, and provided with flattened connectivetissue corpuscles and Waldeyer's plasma cells. The smooth, inner surface is covered with a layer of endothelium. It is but slightly supplied with blood-vessels, although they are more numerous in the outer layers; the lymphatics are numerous, while nerves whose terminations are unknown give to the dura its exquisite sensibility to painful operations on it. Pacinian corpuscles have been found in the dura over the temporal bone. The lymphatic subdural space (Key and Retzius) lies between the dura and the arachnoid, and between the pia and arachnoid is the subarachnoid space (fig. 509). These two spaces do not

lertical section of the cortex cerebri and its membranes; communicate directly. The delicate $\times 2 \frac{1}{2}$. $c o$, cortex cerebri; $p$, intima pie dipping into the arachnoid, thin and partially persulci; " arachnoid, connected with $p$ by means of the forated, poor in blood-vessels and louse subarachnoid trabeculie in the subarachnoid space, without nerves, is covered on both $s a$; $v, v$, blood-vessels ; $d$, dura ; sd, subdural space. surfaces with squamous endothelium. Anly on the spinal cord is it separated from the pia, so that between the two lies the lymphatic subarachnoil space; over the brain, the two membranes are for the most part united together, "xcept the parts brilging over the sulci between adjacent convolutions. The arachnoid passes from convolution to convolution without dipping into the sulci, while the pia dips into each sulcus (fig. $509, a$ ). The ventricles of the brain communicate freely with the lymphatic subarachnoid space, but not with the subdural space. 'The pia consists of delicate bundles of connective-tissue without any admixture of elastic fibres; it is richly supplied with bloodvessels and lymphaties, aud carries nerves which accompany the blood-vessels into the substance of the brain. The lymphatics open into the subarachnoid space (\$ 196).

[Subarachnoid Fluid, or cerebro-spinal fluid, lies in the subarachnoid space, which is traversel by trabeculee of connective-tissue. Within the brain are a series of cavities called ventricles, which communicate one with another in a definite way. The fourth ventricle is lined by a layer of columnar epithelium, and covered in dorsally by a membrane and continuation of the pia mater, from the midllle of which there hangs into the roof of the fourth ventricle twn vascular processes composed of capillaries-the choroid plexuses of the fourth ventricle, which are comparalile to the larger plexuses of the lateral ventricles. In this membrane is the foramen of Magendie and two other smaller foramina, whereby the fluid in the subarachnoid slace communicates with that in the fourth ventricle; but the lymphaties of the nerve-sheaths can be injected from the subarachnoud space, so that there is direct continuity of the fluid in the ventricles of the brain with that in the subarachnoid space, perivascular spaces of the cerebral substance, and the perineural lymphatics of nerves. The average quantity is about 2 ounces, and if it be suddenly withdrawn, epilepsy or convulsions may be produced; or, if it be rapidly increased in amount, coma may be produced. The middle and posterior parts of the brain and the medulla oblongata do not rest directly on bone, but are separated by a distinct interval from their osseous case, an interval occupied by the cerebro-spinal fluid and traversed by trabecule, so that, as Hilton expresses it, this fluid forms a perfect water-bed for those parts, heing sustained by the venous circulation and the elasticity of the dura. It has important mechanical functions, protecting delicate parts of the brain from injury; by distributing 
vibratory impulses it insulates the nerve-roots, and has important relations to the quantity of blood in the brain and the cerebral circulation (Chemical Composition, \$198).]

[Spina bifida. - Sometimes the laminæ of the vertebræ in the lumbar or other region of the spinal column are imperfectly developed, in which case the membranes project through as a tumour distended by cerebro-spinal fluid and covered by skin. The effects of rapid tapping or compressing the sac are readily studied in such cases.]

The Pacchionian bodies, or granulations, are connective-tissue villi, which serve for the outflow of lymph from the subdural and subarachnoid spaces into the sinuses of the dura mater, especially the longitudinal sinus. The subarachnoid space also communicates with the spaces in the spongy bone of the skull, and with the veins of the skull and surface of the face (Kollmann). The subdural space also communicates with the lymphatic spaces in the dura, while the latter communicate directly with the veins of the dura. Both the subdural and subarachnoid lymphatic spaces communicate with the lymphatics of the nasal mucous membrane. The space outside the dura of the spinal cord is called the epidural space, and may be regarded as lymphatic in its nature; the pleural and peritoneal cavities may be fillecl from it; but it does not communicate with the cavity of the skull. The plexuses of blood-vessels are surrounded by undeveloped connective-tissue. The telæ choroider in the new-born are still covered with ciliated epithelium.

Movements of the Brain.--The pulsations of the large basal cerebral vessels communicate their pulsatile movements $(\$ 79,6)$ to the brain-the respiratory movements also affect it, so that the brain rises during expiration and sinks during inspiration. Lastly, there are slight alternating vascular elevations and depressions, occurring 2 to 6 times per minute, due to the periodic dilatation and contraction of the blood-vessels ( $\$ 371$ ). Psychical excitement influences these, and they are most regular during sleep. The movements are best seen especially where the membranes of the brain offer little resistance, e.g., over the fontanelles in children, and where the membranes have been exposed by trephining. The presence of the cerebrospinal fluid is most important for the occurrence of these movements, as it propagates the pressure uniformly, so that every systolic and expiratory dilatation of the blood-vessels is concentrated upon those parts of the cerebral membrane which do not offer any resistance (Donclers). When the fluid escapes, the movements may almost disappear.

Mental excitement increases the pulsations of the brain. At the moment of awaking, the amount of blood in the brain diminishes; sensory stimuli applied luring sleep, so that the sleeper does not awake, increase the amount of blood. As the arteries within the rigid skullcase change their volume with each pulse-beat, the veins (sinuses) exhibit at every beat a pulsatile variation in volume, the opposite of that occurring in the arteries (Mosso).

The Cerebral Blood-Vessels.--The blood-vessels of the pia, of course, are regulated by the vaso-motor nerves $(\$ 356, \mathrm{~A}, 3)$, and their calibre may also be influenced by the stimulation of more distant parts of the body ( $\$ 347)$. Donders trephined the skull so as to make a round hole, and filled it with a piece of glass, so that with a microscope he could observe changes in the calibre of the blood-vessels. Paralysis of the vaso-motor nerves and narcotics dilate the blood-vessels; they become greatly contracted at death (\$ 373, I.). The blood-vessels are dilated during cerebral activity ( $\$ 100, A)$, as well as during sleep. Increased pressure within the skull causes great derangement of the cerebral activity-laboured respiration $(\S 368, \mathrm{~B})$, unconsciousness even to coma, and paralytic phenomena-all of which may in part be referable to disturbances of the circulation. If all the cranial arteries be ligatured suddenly, there is immediate loss of consciousness, together with strong stimulation of the medulla oblongata and its centres, and death takes place rapidly with convulsions (compare \& 373).

By the free anastomosis which takes place at the base of the brain, forming the circle of Willis (fig. 510), the individual parts of the brain are preserved from want of blood, when one or other blood-vessels is compressed or ligatured. Within the brain, the arteries are distributed as "terminal " arteries, i.e., the terminal branches of any one artery end in their own area, and do not anastomose with those of adjoining areas (Cohnheim). On the other hand, the peripheral arteries (arteries of the corpus callosum, Sylvian fissure, and deep cerebral) which run externally on the brain, form free anastomoses (Tichomirow).

[The nutrient or ganglionic arteries for the central ganglia arise in groups from the circle of Willis, or from the first two centimetres of its trunks. The antero-median group (1) supplies the anterior part of the head of the candate nucleus. The postero-median (2) enter the posterior perforated space and supply the internal surface of the optic thalami and the walls of the third ventricle. The antero-lateral groups $(3,3)$ from the middle cerebral enter the anterior perforated space, supply the corpora striata, the anterior part of the optic thalamus, and the internal capsule. These branches are apt to rupture. The postero-lateral $(4,4)$ 
supply a large part of the optic thalami (Charcot). A line drawn at a distance of two centimetres outsicle the circle of Willis, encloses the ganglionic area. The cerebral convolutions are supplied by the large brauches of the circle of Willis. The anterior cerebral curves round the corpus callosum, and supplies the gyrus rectus and the supraorbital, the first and second frontal convolutions, the upper part of the ascending frontal, and the inner surface of the hemisphere as far as the quadrate lobule (fig. 510, I). The posterior cerebral goes to the region of the occipital lobe and the inferior aspect of the temporal lobe; the middle cerebral rr Sylvian artery divicles into four branches, which go to the posterior part of the frontal lobe,

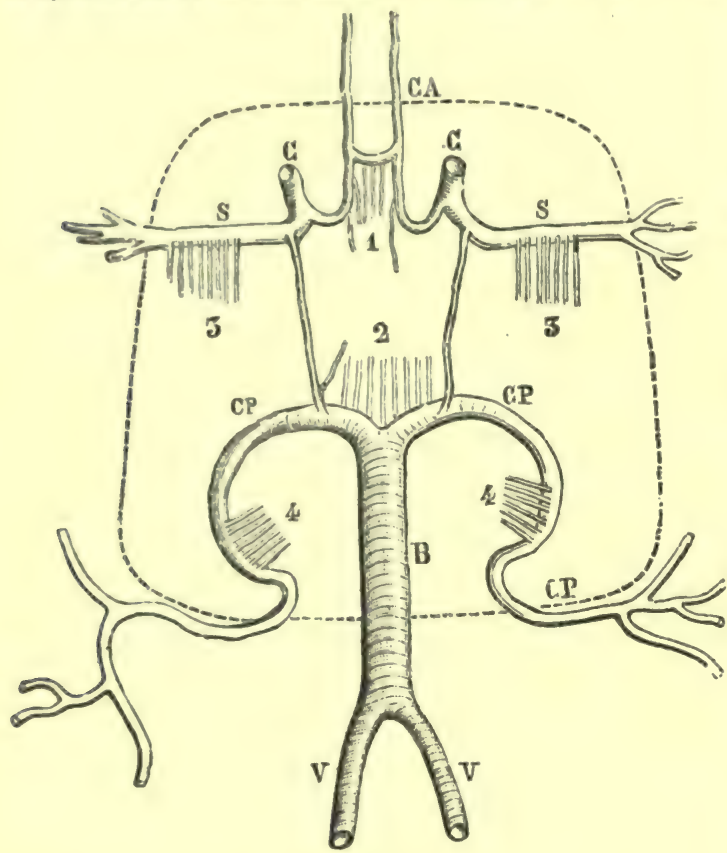

Fig. 510. ascending frontal, and to all the parietal lobes, i.e., chiefly to the motor areas (III), the angular gyrus, and to the first temporosphenoidal lobule. The terminal branches of these ganglionic arteries do not anastomose with the cortical system. Fig. 511 shows the ganglionic arteries pierciug the basal ganglia. $\mathrm{Ob}$ viously, when hæmorrhage of the lenticulo-striate artery or "artery of hæmorrhage" $(4,4)$ occurs, it will compress the lenticular nucleus, or tear it up, and may even injure the parts outside, such as the external capsule, claustrum ( $T$ ), and island of Reil (R), or those inside, e.g., the internal capsule.]

[Thus, the anterior cerebral supplies the prefrontal area and a small part of the motor area, that for the leg-centre in the paracentral lobule and upper end of the ascending frontal (and perhaps that for the trunk). The posterior cerebral supplies the centre for vision, and that connected with the course of the posterior part of the optic expansion, and also the sensory part of the internal capsule. The middle cerebral supplies the motor areas of the cortex, except part of the leg-centre and the basal ganglia, the auditory centre, and that for speech.]

[The cerebral circulation has the limit of the ganglionic area. many pecnliarities. The curves on the arteries serve to modify the effect of the cardiac shock,
the circle of Willis permits within limits a free circulation ; but, in as far as the skull is largely a rigid box, it was at one time taught that, as the brain substanee and its fluids were practically incompressible, it was impossible to alter the amount of blood in the brain. This is a mistake. The amount of blood undergoes an alteration in this 'way, that when more blood passes in, some cerebro-spinal fluid moves out, and vice vers $A$, so that there is an intimate relation between these fluils. In the developing skull, the cerebro-spinal fluid may accumulate in large amount within the ventricles, and greatly distend both them and the yielding skull-case from internal pressure, as in acute hydrocephalus. The peculiarities and independence of the cortical and ganglionic arteries have already been referred to. Plugging by means of a clot, vegetation, or wart, earried from the heart, is common in the left middle cerebral artery.-Why?-When the plug is washed away by the blood-stream, owing to the left carotid springing from the aorta nearly in line with the blood-current, the plug readily passes into the carotid and so into the left middle cerebral which is in line with the internal carotid. In such a case, the convolutions and parts supplied by it are suddenly deprived of blood with immediate and serious results.]

[The venous circulation is peculiar. The sinuses are really spaces between the layers of the tough dura mater, and partly bounded by bone. The blood moves in the longitudinal sinus from before backwards, but most of the cortical veins open into it in a forward direction, so that their stream is opposed to that in the sinus. Thus, the blood which enters the brain by ascending arteries reaches the sinuses by ascending veins, the reverse of what obtains elsewhere, 
in parts where ascending veins convey blood from descending arteries, whereby the hydrostatic pressure and gravity aid the circulation, but here gravitation is opposed to the flow of blood in the cerebral veins. This will help to explain the occurience of thrombosis in these vessels. Some of the veins on the surface communicate with intracranial veins, e.g., those of the nose, the facial through the ophthalmic, mastoid veins, and veins of the diploœ. Hence, morbid processes affecting the scalp (erysipelas), ear (caries), or face (carbuncle) may readily affect intracranial structures (Gowers).]

If a person who has been in bed for a long time, and whose blood is small in amount, be suddenly raised into the erect position, cerebral anæmia is not unfrequently produced, owing to hydrostatic causes. At the same time, there may be loss of consciousness and impairment of the senses. Liebermeister regards the thyroid gland as a collateral blood-reservoir which empties its blood towards the head during such changes of the position of the body. Perhaps this may explain the swelling of the thyroid as a compensatory act, when the heart beats violently, and the brain is surcharged with blood (\$§ 103, III., and 371). Very violent muscular exertion, as well as marked activity of other organs, causes a very considerable fall of the blood-pressure in the carotid.

Pressure on the Brain. - The brain and the fluid surrounding it are constantly subjected to a certain mean pressure, which must ultimately depend upon the blootl-pressure within the vascular system, The investigations of Naunyn and Schreiber on the cerebral pressure (or cerebro-spinal pressure) showed that the pressure must be slightly less than the pressure within the carotid, before the symptoms proper to pressure on the brain oceur. These are, sudden attacks of headache, with vertigo, or it may be loss of consciousness, vomiting, slowing of the pulse, slow and shallow respiration, convulsions-while the pressure of the cerebro

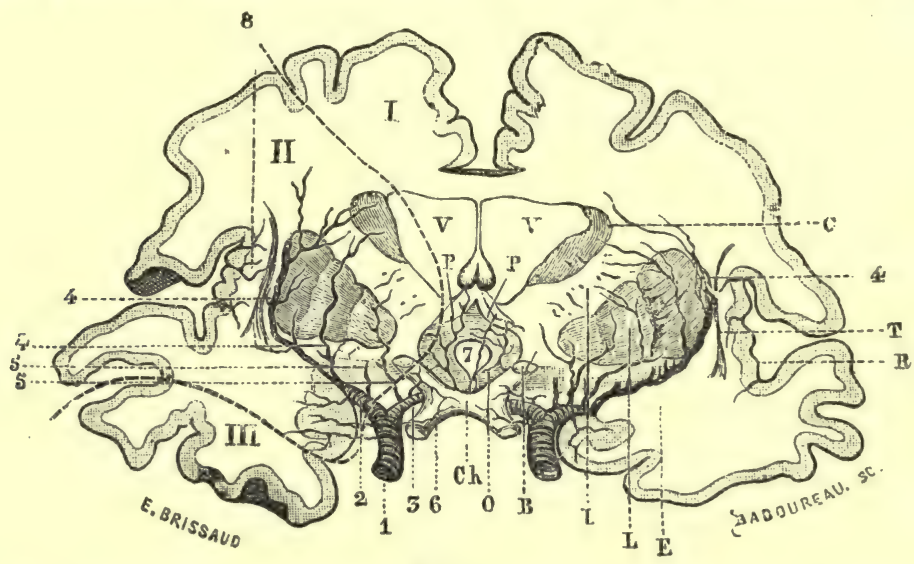

Fig. 511.

Transverse section of the cerebrum behind the optic chiasma. Arteries of the coryus striatum. $\mathrm{C} h$, optic chiasma; B, section of optic tract; L, lenticular nucleus; I, internal capsule ; C, caudate nucleus; E, external capsule; T, claustrum ; R, convolutions of the island of Reil; V, V, section of the lateral ventricles; P, P, pillars of the fornix; $O$, grey substance of the third ventricle. Vascular areas-I, anterior cerebral artery; II, Sylvian artery; III, posterior cerebral artery ; 1, internal carotid ; 2, Sylvian, 3, anterior cerebral artery ; 4, 4, lenticulo-striate arteries; 5, 5, lenticular arteries.

spinal fluid is increased. The cause of these phenomena lies in the anæmia of the brain. If the pressure is moderate, the above-named symptoms may remain latent; nevertheless, disturbances of the nutrition of the brain occur, with consecutive phenomena, such as persistent slight headache, feeling of vertigo, muscular weakness, and disturbances of vision (owing to neuro-retinitis with choked disc). Increase of the blood-pressure diminishes the symptoms, while diminution of the blood-pressure causes more pronounced phenomena of cerebro-spinal pressure. In the dog, pain begins with a pressure of 70 to $80 \mathrm{~mm}$. $\mathrm{Hg}$. Consciousness is abolished when the pressure is higher, and at 80 to $100 \mathrm{~mm}$. spasms take place. A pressure of 100 to $120 \mathrm{~mm}$. causes sloving of the pulse, owing to stimulation of the vagus at its origin ; the respirations are temporarily accelerated and then diminish. Long-continued severe compression always, sooner or later, ends fatally. The blood-pressure at first is increased, owing to reflex stimulation of the vaso-motor centre from the pressure stimulating the sensory nerves; ultimately, the blood-pressure falls, and the pulse becomes very slow. Irregular variations in the blood-pressure point to a direct central stimulation of the vaso-motor centre 
hy pressure. The application of continued slowly increasing pressure compresses the brain (Adamkieviez).

382. COMPARATIVE-HISTORICAL. - Comparative. - Nerves are absent in the protozoa. Neuro-muscular cells occur in the coelenterata, in the hydroida and meduse, and they are the first indieations of a nervous apparatus $(\$ 296)$. The umbrella of the medusa is covered with a plexus of nerve-fibrils, which at various parts along its margin is provided with small cellular thickenings corresponding to ganglia, and from these, nerve-fibres proceed to the sense organs. Many of the worms possess a nervous ring in the cephalic portion, and in those provided with an intestine a single or double nervous cord, in the form of a ring, surrounds the jharynx. Branches (often two) pass from this into the elongated body, and usually these carry ganglia corresponding to each ring of the body of the animal. In the leech, only one zangliated corl is present. In the echinodermata, a large nerve-ring surrounds the mouth; aml from it large nerves proceed, corresponding to the chief trunks of the water-vascular ystem. At the pints where the nerves are given off, the nervous ring is provided with the wi-eallem "ambulacral brains." The arthropoda are provided with a large cephalic ganglion Ilacenl alove the pharynx, from which nerves pass to the sense organs. Another ganglion lies on the under surface of the pharynx, and is connected with the former by commissures. The pharynx is thus embraced by a gangliated ring, and from it proceeds the abdominal gangliated doulile chain, along the ventral surface of the body, through the thorax and abdomen. Sometimes several ganglia unite to form a large compound ganglion, while, in other cases, each segment of the boly entains its own ganglia. In the mollusca, the cesophageal nervous ring is present, although the sanglionic masses vary much in position within it. A number of cmmpouml ganglia lie scattered in different parts of the body, and are united by nerves to the former. They represent the sympathetic system. In the cephalopoda, the oesophageal ring has almost no commissure, and a prart of the ganglionic matter is enclosed in a cartilaginous "apsule, aml is often spoken of as a "brain." Aidditional ganglia are found in the mantle, heart, ani stomach. In vertebrates, the nervous system invariably lies on the clorsal aspect of the londy. In the amphioxus, there is no separation into brain and spinal cord. (See $\$ 374$ and 375 .)

Historical.- $11 \mathrm{~km}$ ion (580 $\mathrm{B.}(\mathrm{C})$ ) placed the seat of consciousness in the brain; Galen 131 203 A.I. regarded it as the seat of the impulses for voluntary movements. Aristotle 3.4 1.,.) ascrilied the relatively largest brain to man; he stated that it was inexcitable to stimuli (insensible). One of the functions he ascribed to the brain was to cool the heat assemling from the heart. Herophilus (300 B.c.) gave the name calamus scriptorius; and he regardel the 4 th ventricle as the most important organ for the maintenance of life. Even in Homer there are repeated references to the dangers of injuries of the neck. Aretaeus and Cassius Felix $(97$ A.1.) were aware of the fact that lesion of one cerebral hemisphere caused faralysis on the opposite side of the body. Galen was acquainted with the path in the spinal cord connected with movement and sensation. Vesalius (1540) described the five ventricles of the hain. R. Colombo (1559) observed the movements of the brain isochronous with the action of the heart. A more careful description of these movements was given by Riolan (1618). Coiter (1573) discovered that an animal can live after removal of its cerebrum. About the millille of the 17th century, Wepfer discovered the hiemorrhagic nature of apoplexy. Schmeiler (1660) estimated the weight of the brain in different animals. Mistichelli (1709) and l'etit (1710) described the decussation of the fibres of the spinal cord below the pons. Gall discovered the jartial origin of the optic nerve from the anterior pair of the corpora yuadrigemina, and by dissecting the brain from below, he attempted to trace the course of the nerve-fibres to the convolutions (1810). Rolando described more accurately the form of the grey matter of the spinal cord. Carus (1814) discovered the central canal. The most compendious work on the brain was written by Burdach (1819-1826). The more recent observations are referred to in the text. 


\section{Physiology of the Sense Organs.}

383. INTRODUCTORY OBSERVATIONS.-Requisite Conditions.-The sense organs have the function of transferring to the sensorium impressions of the various phenomena of the external world; they are, in fact, the intermediate instruments of sensory perceptions. In order that this may occur, the following conditions must be fulfilled:-(1) The sense organ, provided with its specific endorgan, must be anatomically perfect, and capable of acting physiologically. A "specific stimulus" must be present, which under normal conditions acts upon the end-organ. (3) The sense organ must be connected with the cerebrum by means of a nerve, and the conduction through this path must be uninterrupted. (4) During the act of stimulation, the psychical activity (attention) must be directed to the process, and then the sensation results, e.g., of light or sound, through the sense organ. (5) Lastly, when, by a psychical act, the sensation is referred to the external cause, then there is a conscious sensory perception. Often, however, this relation is completed as an unconscious conclusion, as it is essentially a deduction from previous experience.

Stimuli. - With regard to the stimuli which are applied to the sensory apparatus, we distinguish :-(1) Adequate or homologous stimuli, $i . e$., stimuli for whose action the sense organs are specially adapted, such as the rods and cones of the retina for the vibrations of the ether. Thus, each sense organ has a specific form of stimulus best adapted to act upon it. This is what Johannes Miiller called the "law of specific energy." (2) There are many other forms of stimuli (mechanical, thermal, chemical, electrical, internal somatic) which act upon the sense organs, producing the flash of light beheld when the eye is struck; singing in the ears when there is congestion of the head. These heterologous stimuli act upon the nervous elements of the sensory apparatus along their entire course, from the end-organ to the cortex cerebri. The homologous stimuli, on the other hand, act only on the end-organ, i.c., light has no effect whatever upon the trunk of the exposed optic nerve.

Strength and Liminal Intensity.-Homologous stimuli act upon the sensory organs only within certain limits as to strength. Very feeble stimuli at first produce no effect. That strength of stimulus which is just sufficient to cause the first trace of a sensation is called by Fechner the "liminal intensity" of the sensation. As the strength of the stimulus increases, so also do the sensations, but the sensations increase equally when the strength of the stimulus increases in relative proportions. Thus, we have the same sensation of equal increase of light when, instead of 10 candles, 11, or instead of 100 candles, 110 are lighted-the proportion of increase in both cases is equal to one-tenth. As the logarithm of the numbers increases in an equal degree, when the numbers increase in the same relative proportion, the law may be expressed thus :- " The sensations do not increase with the absolute strength of the stimuli, but nearly as the logarithm of the strength of the stimulus." This is Fechner's "psycho-physical law," but its accuracy has recently been challenged by E. Hering. [It holds good only with regard to stimuli 
of medium strength.] If the specific stimulus be too intense, it gives rise to peculiar painful sensations, e.\%, a feeling of blindness or deafness, as the case may be. The sense organs respond to adequate stimuli, but only within certain limits of the stimulus, e.\%., the ear responds only to vibrating bodies emitting a certain range of vibrations per second; the retina responds only to the vibrations of the ether hetween red and violet, but not to the so-called heat vibrations or to the chemically active vibrations.

[It was Weher who worked out the relation between the intensity of stimuli and the changes in the quantity of the resulting sensations. He used the method of "least observable differ"nices. as apritiel to sensations of pressure and the measurements of lines by the eye. Hence, it is callel Weber's Law; but Fechner expanded it and assumed that all just observable differences are equally great, and so the law is sometimes called by his name.]

[Fechner's Law. - Expressed in another way, the result depends on (1) the strength of the stimulus, and (2) the degree of excitability. Supposing the latter to be constant, while the former is varied, it is fouml that if the stimulus be doubled, tripled, or quadrupled, the sensation increases only as the legarithm of the stimulus. Suppose the stimulus to be increased 10, 100. or 1000 times, then the sensation increases only as 1, 2, or 3. Just as there is a lower limit of creitation liminal intensity (or threshold), so there is an upper limit or maximum of rritution or height if sensibility, when any further increase produces no appreciable increase in the sensation. Thus, we do not notice any difference between the central and peripheral portion of the sun's disc, though the difference of light intensity is enormous (Sully). Between these two is the reinge of sensibility ( $W$ undt). There is always a constant ratio between the strength of the stimulus and the intensity of the sensation. The stronger the stimulus already applied, the stronger must be the increase of the stimulus in order to cause a perceptible increase of the sensation (Weber's Law). The neeessary increment is proportional to the intensity of the stimulus, and it varies for each sense organ. If a weight of 10 grammes be placed in the hand, it is found that 3.3 grammes must be added or removed before a difference in the sensation is pereeptible; if 100 grammes are held, $33 \cdot 3$ grammes must be added or removed to obtain a perceptible difference in the sensation. The magnitude of the fraction indicating the increment of stimulus necessary to obtain a perceptible difference of the sensation, is spoken of as the constant propsition or the discriminative sensibility. In the above case it is $1: 3$. The following table gives approximately the constant proportion for each sense :-
Tactile Sensation,
$1: 3$.
$1: 3$.
$1: 3 . \frac{1}{3}$
$1: 3$
Muscular Sensation, Visual
$6: 100 \cdot \frac{6}{100}$
Thermal

\section{Auditory ,"}

[The application of the law to temperature sensations is beset with great difficulties, while for taste and smell we do not know that it is really applicable. From an experimental point of view, it cannot be said to be proved, and its application is obviously somewhat restricted to certain sensations, and to these, only within a certain range. It certainly does not hold good for sensations of pressure, and muscular' sense, near the lower limits for these senses. "At hest it is only an approximately correct statement of what holds true of the relative intensity of certain sensations of light and hearing, and less exactly of pressure and the muscular sense, when these sensations are of moderate strength " (Ladd).]

The term after-sensation is applied to the following phenomenon, viz., that, as a rule, the sensation lasts longer than the stimulus producing it; thus, there is an after-sensation, after pressure is applied to the skin. Subjective sensations occur when stimuli due to internal somatic causes excite the nervous apparatus of the sense organ. The highest degrees of these, depending mostly upon pathological stimulation of the sensory cortical centres, are characterised as hallucinations, e.\%., when a delirious person imagines he sees figures or hears sounds which have no objective reality. In opposition to this condition, the term illusion is applied to modifications by the sensorium of sensations actually caused by external objects, e.g., when the rolling of a waggon is mistaken for thunder.

In a new-born child, the sense of touch is strongly developed, that of pain slightly, muscular sensations are undoubtedly present, while smell and taste are frequently confounded. Auditory stimuli are heard from the second day onwards, the stimulus of light immediately after birth, but a peripheral field of vision does not yet exist (Cuignet). Towards the fourth to fifth week, the movements of convergence and accommodation are noticable, while after four months, colours are distinguished. The various stimuli are not perceired simultaneously - a reflex inhibitory centre is not yet developed (Genemer). 


\section{The Visual Apparatus-The Eye.}

384. HISTOLOGICAL OBSERVATIONS. - In the following remarks it is assumed that the student is familiar with the anatomical structure of the eye :-

The cornea, for the sake of simplicity, is regarded as uniformly spherical, although, properly speaking, it differs slightly from this form. It is more like a vertical section of a somewhat oblique ellipsoid, which we must suppose to be formed by rotating an ellipse around its long axis (Brïcke). It is nearly of uniform thickness throughout, only in the infant it is slightly thicker in the centre, and in the adult slightly thinner. The cornea consists of the following layers :-
[1. Anterior stratified epithelium.
2. Anterior elastic lamina.
4. Posterior elastic lamina.
3. Substantia propria.
5. Single layer of epithelium.]

1. The anterior epithelium, stratified and nucleated, consists of many layers of cells (fig. 514, a). The deepest cells are more or less columnar, are arranged side by side, and are called supporting cells. The cells of the middle layers are more arched, and dip with finger-shaped processes into corresponding spaces between their neighbours. The most superficial cells are Hat, perfectly smooth, hard, keratin-containing squamous epithelium. 2. The epithelial layer rests upon the anterior elastic membrane (Bowman's elastic lamina), a structureless clear base-

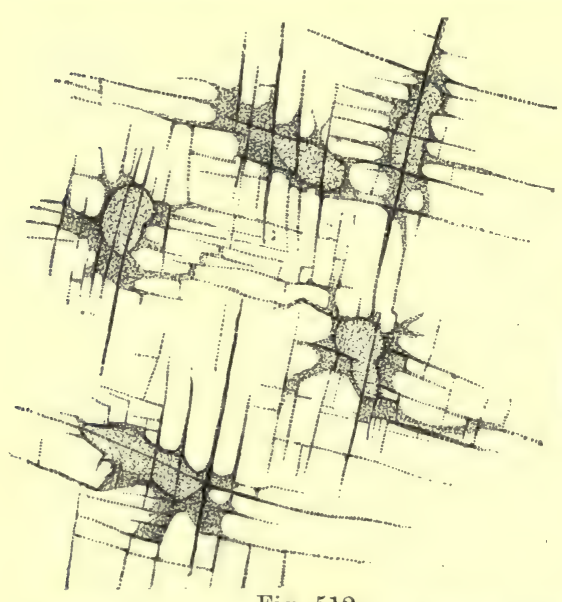

Fig. 512.

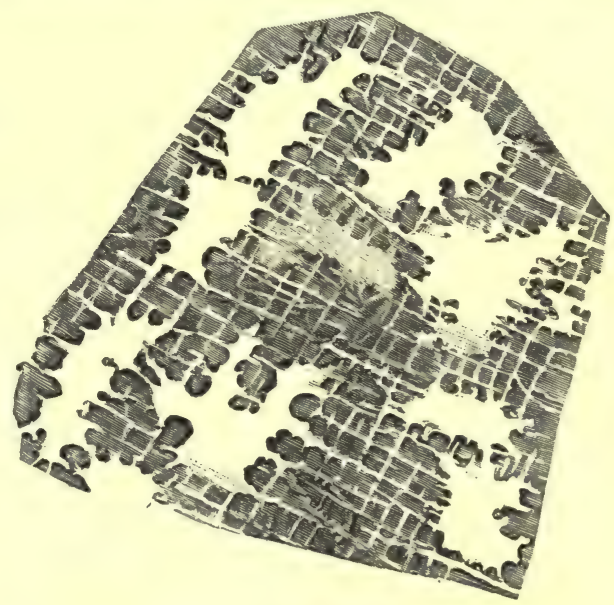

Fig. 513 ,

Fig. 512. - Cornea of the frog treated with chloride of gold, showing the corneal corpuscles stained, and a few nerve-fibrils. Fig. 513. - Cormea of the frog treated with silver nitrate; the ground substance is stained, while the spaces for the corneal corpuscles are left unstained.

ment-like membrane $(b)$, whose existence is denied by Brücke. 3. The substantia propria of the cornea consists of (chondrin-yielding) fibres composed of delicate fibrils of connective-tissue. The fibres are arranged in mat-like thin lamellæ $(l)$, more or less united together, and are placed in layers over each other. Towards the anterior elastic lamina, the fibres bend round and perforate the superficial lamellæ, thus serving as supporting fibres. [These perforating fibres are comparable to Sharpey's fibres in bone.] Between the lamellæ are a series of intercommunicating spaces lined by endothelium. These spaces are really lymph-spaces, and they communicate with the lymphatics of the conjunctiva. The fixed corneal corpuscles lie in these spaces $(c)$, and are provided with numerous processes, which anastomose with the processes of corpuscles lying between the lamellæe above and below, and on either side of them. Kühne observed that stimulation of the corneal nerves was followed by contraction of these cells $(\$ 201$, 7), while Kühne and Waldeyer maintain that they are connected with the corneal nerve-fibrils.

[The corneal corpuscles are looked upon as branched connective-tissue corpuscles lying in and not quite filling the branched spaces between the lamellæ. The processes anastomose freely with similar cells in the same plane, and to a less extent with the processes of cells in 
planes immediately above and below them. In a section stained with gold chloride, they present the appearance seen in fig. 512. In a vertical section of the cornea, they appear fusiform and parallel to the free surface of the cornea (fig. 514). If the cornea of a frog be pencilled with silver nitrate, the cement-substance between the lamella is blackened, and the branched cell-spaws remain clear, as in fig. 513. The one figure represents, as it were, the [ositive, and the other the negative image.]

Leucoertes also pass into these lymph-spaces or juice-canals. The importance of these lencuryt in inflammation is referred to in $\$ 200$. 4. The transparent, structureless, posterior elastic membrane $(d)$, the membrane of Descemet or Demours, is in many animals fibrillated, and shows vidence of stratification, while towards the margin of the cornea there are occasionally slight conical elevations. This membrane is very tough and very resistant (of grrat injurtance in inflammation). If it be removed, it rolls up towards the convex side. At its periphery it becomes continuous with the fibro-elastic reticulated ligamentum pectinatum irilis, whose trabecula are covered by epithelium. 5. The posterior single layer of epithelium

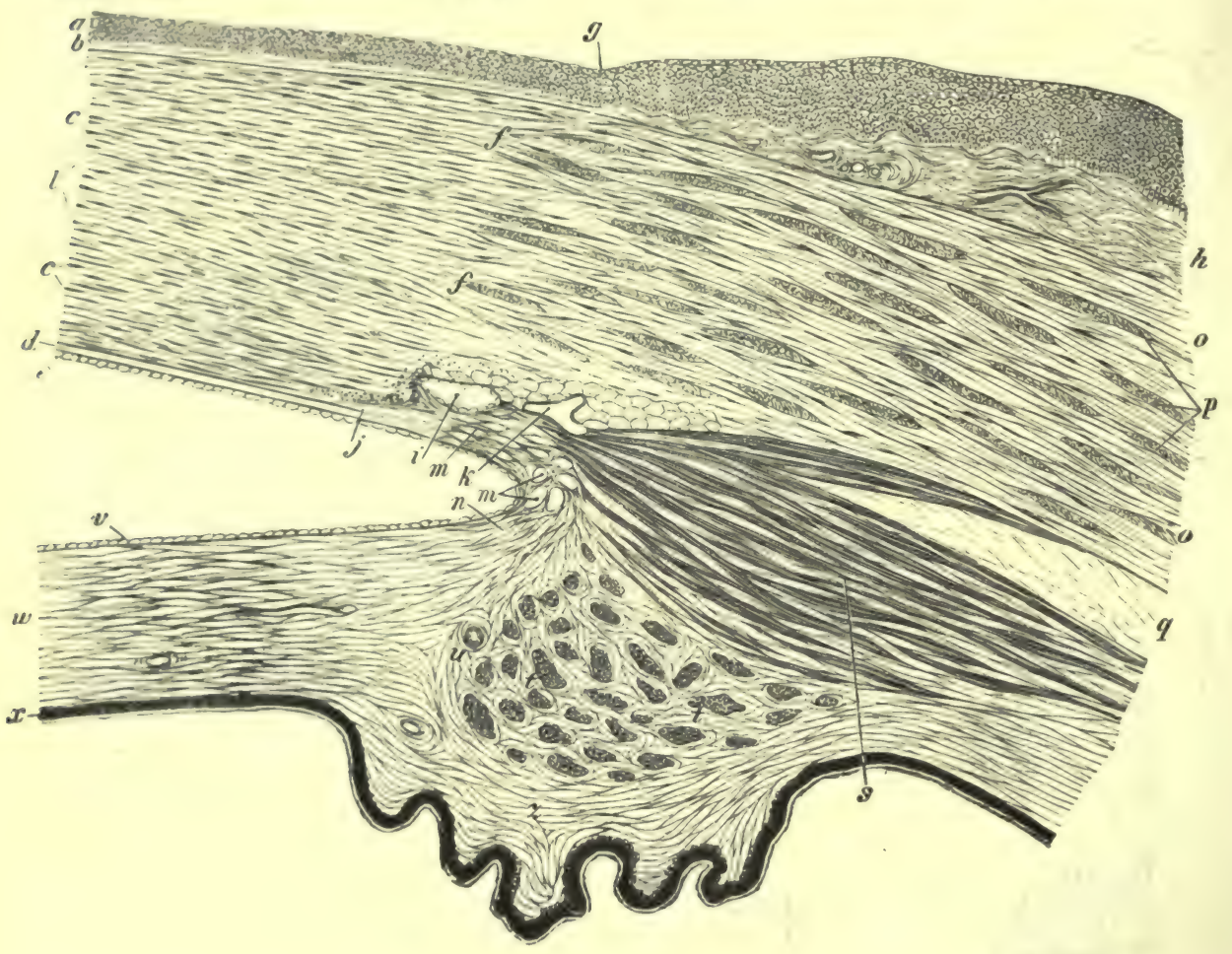

Fig. 514.

Antero-posterior section at the junction of the cornea with the sclerotic. $\alpha$, anterior corneal epitheliun; $b$, Bowman's lamina; $c$, corneal corpuseles; $l$, corneal lamellæ (the whole thickness lying between $b$ and $d$ is the substantia propria corneæ); $d$, Descemet's membrane $: e$, its epithelium ; $f$, junction of cornea with the sclerotic ; $g$, limbus conjunctivæ ; $h$, conjunctiva ; $i$, canal of Schlemm; $k$, Leber's venous plexus (is regarded by Leber as belonging to $i$ ); $m, n$, meshes in the tissue of the lig. iridis pectinatum ; $n$, attachment of the iris; $o$, longitudinal, $p$, circular (divided transversely) bundles of fibres of the sclerotic ; $q$, perichoroidal space ; $s$, meridional [radiating], $t$, equatorial (circular) bundles of the ciliary muscle ; $u$, transverse section of a ciliary artery; $v$, epithelium of the iris (a continuation of that on the posterior surface of the cornea); $w$, substance of the iris ; $x$, pigment of the iris; $z$, a ciliary process.

consists of flat, delicate, nucleated cells $(e)$, which are continued from the margin of the cornea on to the anterior surface of the iris $(v)$. Fine juice-canals exist in the spaces between the individual cells (v. Rccklinghuusen). These spaces communicate with a system of fine tubes under 
the epithelium, perforate Descemet's membrane, and thus communicate with the corneal spaces.

[Bowman's tubes are artificial productions, formed by forcing air or a coloured fluid between the lamellæ, when it passes between the bundles of fibrils, forming a series of tubes with dilatations on them and running at right angles to one another between the lamellæ.]

The nerves of the cornea, which are derived from the long and short ciliary nerves $(\$ 347)$, are partly sensory in function. They enter the cornea at its margin as medullated fibres, but the myelin soon disappears, while the axial cylinders split up into tibrils. [The axial cylinders branch and form a plexus between the lamellæ, especially near the anterior surface, the fundamental or ground plexus (fig. $515, n$ ). There are triangular nuclei at the nodal points, but they probably belong to the sheath of flattened cells which cover the larger branches. There is a finer and denser plexus of fibrils immediately under the anterior epithelium, sub-epithelial plexus, which is derived from the former, the fibrils arising in pencils or groups (fig. 516). Some fibrils perforate the anterior elastic lamina, rami perforantes, and pass between the anterior epithelial cells to form the intra-epithelial network (fig. $515, b, p$ ). Some observers suppose that they terminate in free, pointed, or bulbous ends. There is also a fine plexus of fibrils in the posterior layers of the cornea, near Desceniet's membrane. It gives off numerous fine fibrils, which come into intimate, if not direct, anatomical relation with the corneal corpuscles. The trophic fibres of the coinea (\$347) are, perhaps, those deeper branches which are connected with the còrneal corpuseles. ]

[Method._-These fibrils åre best revealed by staining a cornea with chloride of gold, which tinges them of a purplish line after exposure to light (Cohnheim).]

Blood-vessels occur only in the outer margin of the cornea (fig. $518, v$ ), and extend $2 \mathrm{~mm}$.

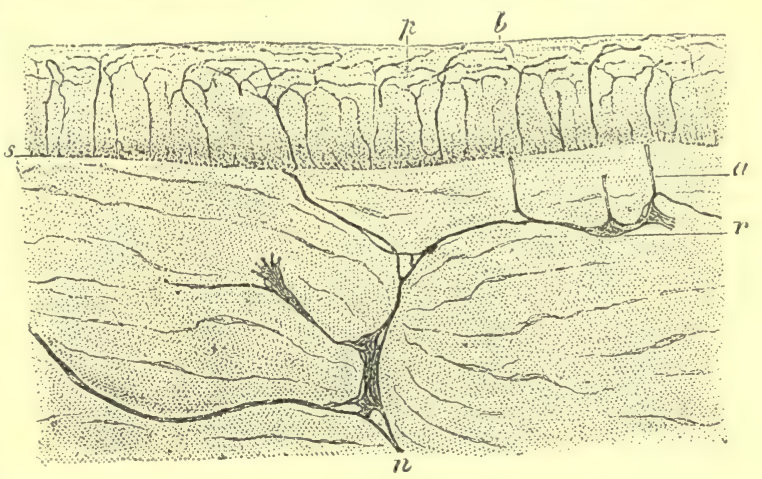

Fig. 515.

Vertical section of the cornea stained with gold chloride. $n$, nerve-fibrils ; $a$, perforating branch ; $r$, nucleus ; $p, b$, interepithelial termination of tibrils; $s$, anterior elastic lamina. over the cornea above, $1.5 \mathrm{~mm}$. below, and $1 \mathrm{~mm}$. laterally-the most external capillaries form arched loops, and thus turn on themselves. The cornea is nourished from the blood-vessels in its margin. Opacities of the cornea give rise to many forms of visual defects.

The sclerotic is a thick fibrous membrane, composed of, $p$, circular (equatorial) and, o, longitudinal (meridional) bundles of connective-tissue woven together (fig. 514). The spaces between the bundles contain colourless and pigmented connective-tissue corpuscles and also leucocytes. It is thickest posteriorly, thinner at the equator, while in front of this it again becomes thicker, owing to the insertion of the tendons of the straight muscles of the eyeball. It contains few blood-vessels, which form a wide-meshed eapillary plexus, immediately under its deep surface. Other vessels form an arterial ring around the entrance of the optic nerve. It rarely is quite spherical ; it rather resembles an ellipsoid, which we might imagine to be formed by the rotation of an ellipse around its short axis (short eyes) or around its long axis (long eyes). Above and below, the sclerotic overlaps like a fold the clear margin of the cornea; hence, when the cornea is viewed from before, it appears transversely elliptical, when seen from behind, it appears circular. Following the margin of the cornea, but lying still within the substance of the sclerotic, is the circular canal of Schlemm $(i)$, which communicates with other anastomosing veins, the venous plexus of Leber $(k)$. Schwalbe and Waldeyer regard Schlemm's canal as a lymphatic. Posteriorly, the sclerotic becomes continuous with the fibrous covering of the optic nerve derived from the dura mater. The selerotic is provided with nerves, which are said to terminate in the cells of the seleral substance (Helfreich).

The tunica uvea, or the uveal tract, is composed of the choroid, the ciliary part of the choroid, and the iris.

The choroid is composed of the following layers (fig. 517):-(1) Most internally is the transparent limiting membrane, $0 \cdot 7 \mu$ in thickness; but it is slightly thicker anteriorly. (2) The very vascular capillary network of the chorio-capillaris, or membrane of Ruysch, embedded in a homogeneous layer. Then follows-(3) a layer of a thick elastic network, covered on both surfaces by endothelium (Sattler). (4) The choroid proper consists of a layer with pigmented connective-tissue corpuscles, together with a thick elastic network, containing the numerous venous vessels as well as the arteries. The pigmented layers are known as the 
supra-choroidea, or lamina fusca, which surrounds the large lymphatic space lined with endothelium and called the perichoroidal space, $q$. In new-born infants, which according to Aristotle have the iris lark blue, the uveal tissue is devoid of pigment; in brunettes it is developed later, and in blondes not at all.

In the ciliary part of the choroid, the pigmented connective-tissue corpuscles are not so numeruns: The ciliary muscle (tensor choroidere, or muscle of accommodation) is placed in this regien. It arises (s), hy means of a branched, reticulated, connective-tissue origin, from the imu.r sicle of the junction of the cornea and sclerotic, near the canal of Schlemm, and passes lackwards to be inserted into the choroid. This constitutes the radiating fibres. Other fitres lying internal to these are arranged circularly, $t$, in bundles in the ciliary margin. Thene ircular filires are sometimes called Heinrich Miiller's muscle. The muscle consists of smooth muscular fibres, and is supplied by the oculomotorius $(\$ 345,3)$.

Th. iris consists of the following parts from before backwards : - a layer of epithelial cells $(v)$ continuons with those covering the posterior surface of the cornea, a layer of reticulated

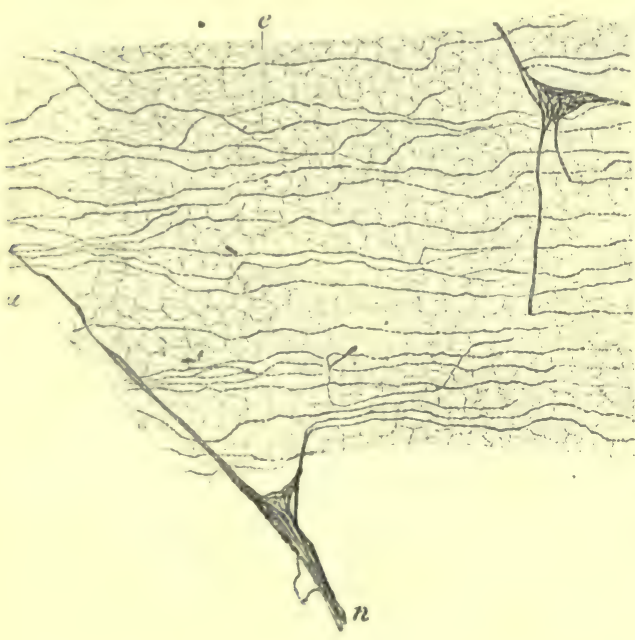

Fig. 516.

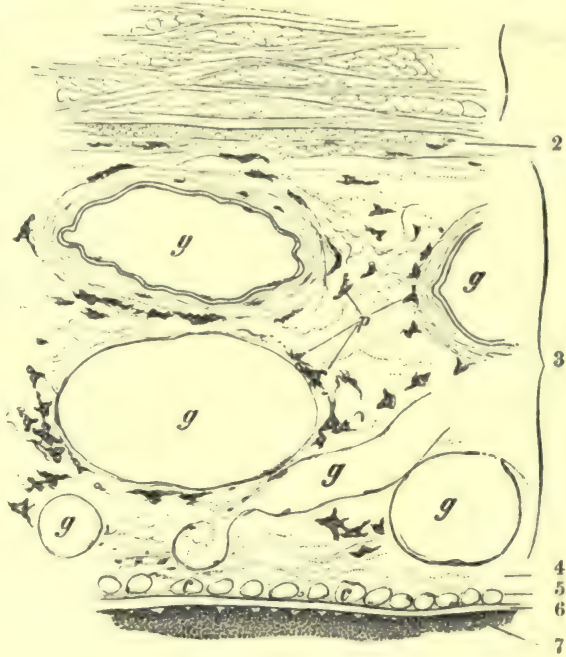

Fig. 517.

Fig. 516. - Nerve-plexus in the cornea after gold chloride. $n$, nerve; $a$, fibrils. Fig. 517.-Vertical section of the choroid and a part of the sclerotic. (1) sclerotic ; (2) lamina suprachoroilea ; (3) layer of large vessels ; (4) limiting layer ; (5) chorio-capillaris ; (6) hyaline membrane ; ( 7$)$ pigment epithelium ; $(g)$ large blood-vessels; $(p)$ pigment-cells; $(c)$ sections of carpillaries.

cumnective-tissue, the layer of blood-vessels, and lastly a posterior limiting membrane, which contains the ligmentary epithelium $(x)$ (Michel). In brunettes, the texture of the iris contains pigmented connective-tissue corpuscles. The iris in some animals is described as containing two muscles composed of smooth muscular fibres - one set constituting the sphincter pupillø (circular-fig. 533), which surrounds the pupil, and lies nearer the posterior than the anterior surface of the iris (\$392). Its nerve of supply is derived from the oculomotorius $(\$ 345,2)$. The other fibres constitute the dilator pupillw (radiating), which consists of a thinner layer of fibres arranged in a radiate manner. Some of the fibres reach to the margin of the pupil, while others bend into the sphincter. [The existence of a dilator pupillæ in man is denied (\$392).] At the outer margin of the iris, the radial bundles are arranged in anastomosing arches, and form a circular muscular layer ( $\mathrm{Merkel})$. The chief nerre of supply for the dilator fibres is the sympathetic $(\$ 347,3)$. Ganglia occur in the ciliary nerves in the choroid, [and they are found also in the iris]. Gerlach has recently applied the term ligamentum annulare bulli to that complex fibrous arrangement which surrounds the iris, and at the same time forms the point of union of the ciliary body, iris, ciliary muscle, sinus venosus iridis, and the line of junction of the cornea and sclerotic.

The choroidal vessels are of great importance in conuection with the nutrition of the eye. $\Lambda$ ccording to Leber, they are arranged as follows:-The arteries are-1. The short posterior ciliary, which are about twenty in number and perforate the sclerotic near the optic nerve 
(fig. 518, a, a). They terminate in the vascular network of the chorio-capillaris $(m$ ), which reaches as far as the ora serrata. 2. The long posterior ciliary; one of these lies on the nasal and the other on the temporal side, and they run $(b)$ to the ciliary part of the choroid, where they divide dichotomously, and penetrate into the iris, where they help to form the circulus arteriosus iridis major $(p)$. 3. The anterior ciliary $(c)$, which arise from the muscular branches, perforate the sclerotic anteriorly, and give branches to the ciliary part of the choroid and to the iris. About twelve branches run backwards $(0)$ from them to the chorio-capillaris.

Veins. - -1. The anterior ciliary veins (c) receive the blood from the anterior part of the uvea and carry it outwards. These branches are connected with Schlemm'scanal and Leber's venous plexus. They do not receive any blood from the iris. 2. The venous plexus of the ciliary processes $(r)$ receives the blood from the iris $(q)$, and passes backwards to the choroidal veins. 3. The large vasa vorticosa Stenonis $(h)$ perforate the sclerotic behind the equator of the bulb.

The inner margin of the iris rests upon the anterior surface of the lens ; the posterior chamber is small in adults, and in the new-born child it may be said scarcely to exist-it is so small. When. Berlin blue is injected into the anterior chamber of the eye, it generally passes into the anterior ciliary veins (Schwalbe). Even in living animals, carmin also behaves in a similar manner (Heisrath); hence, these observers conclude that there is a direct communication between the veins and the aqueous chamber, as these substances do not diffuse through membranes.

Internal to the choroid, lies the single layer of hexagonal cells $(0.0135$ to 0.02 $\mathrm{mm}$. in breadth) filled with crystalline pigment. This layer really belongs to the retina. It consists of a single layer of cells as far as the ora serrata-it is continued on to the ciliary processes and the posterior surface of the iris, where it forms several layers (fig. $514, x$ ). In albinos it is devoid of pigment ; on the other hand, the uppermost cells, which lie on the ridges of the ciliary processes, are always devoid of pigment. [The processes of these cells vary in length with the nature and kind of light acting on the retina ( $\$ 398)$.]

The retina externally is in contact with the layer of hexagonal pigment-cells $(P i)$, which in its development and functions really belongs to the retina. The cells are not flat, but they send pigmented processes

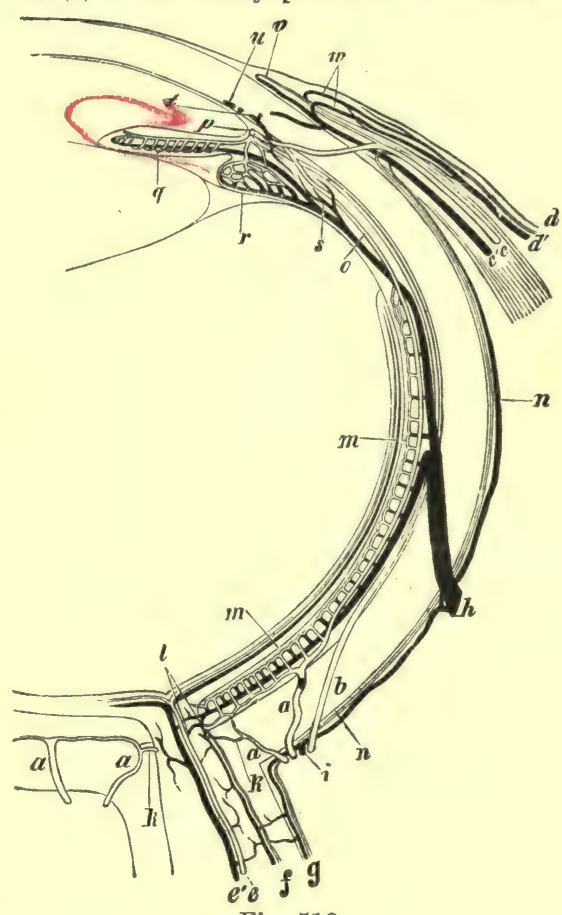

Fig. 518.

Diagram of the blood-vessels of the eye (horizontal view, veins black, arteries light, with a double contour). $\quad \alpha, a$, short posterior ciliary ; $b$, long posterior ciliary ; $c, c^{\prime}$, anterior ciliary artery and vein ; $d, d^{\prime}$, artery and vein of the conjunctiva ; $e, e^{\prime}$, central artery and vein of retina ; $f$, bloodvessels of the inner, and $g$, of the outer optic sheath; $h$, vorticose vein ; $i$, posterior short ciliary vein confined to the sclerotic ; $k$, branch of the posterior short ciliary artery to the optic nerve; $l$, anastomosis of the choroidal vessels with those of the optic ; $m$, chorio-capillaris ; $n$, episcleral branches; $o$, recurrent choroidal artery; $p$, great circular artery of iris (transverse section); $q$, blood-vessels of the iris ; $r$, ciliary process ; $s$, branch of a vorticose vein from the ciliary muscle; $t$, branch of the anterior ciliary vein to the ciliary muscle ; $u$, circular vein ; $v$, marginal loops of vessels on the cornea; $w$, anterior artery and vein of the conjunctiva.

In some animals (rabbit) the cells contain fatty granules and other substances. The cells are larger and darker at the orra serrata (Kiihne). The retina is composed of the following layers, proceeding from without inwards :-

[1. Layei of pigment-cells.

2. Rods and cones.

3. External limiting membrane.

4. Outer nuclear layer.

5. Outer molecular (granular or internuclear) layer.
6. Inner nuclear layer.

7. Inner molecular (granular) layer.

8. Layer of nerve-cells (ganglionic) layer.

9. Layer of nerve-fibres.

10. Internal, limiting membrane.] 
1. The hexagonal p:gment-cells already described. 2. The layer of rods and cones (St) or 1. The hexagonal pigment-cells already described. visual cells, or visual epithelium of Kiihme]
(figr. 520). These lie externally next the choroid, but they are absent at the entrance of the optie nerve. Then follows the external limiting membrane $(L e)$, which is perforated by the
lases of the rods and cones. 3. The external nuclear layer $(\ddot{a} u . K)$; this and all the succeeding lavers are called "brain layers" by Schwalbe. 4. The external granular (äu.gr), or internuclear layer, which is perforated by the filres which proceed inwards from the nuclei of 3 to reach 5 , the nuclei of the internal nuclear layer $(i n K)$. The nuclei of this layer, which are connected hy fibres with the rods and cones, are marked by transverse lines in the macula lutea (Krause, Denissenko). 6. The finely granular internal granular layer (in. $g r)$, through which the fibres proceeding from the inner nuclear layer cannot be traced. It would seem as if these fibres break up into the finest fibrils, into which also the branched processes of the ganglionic cells

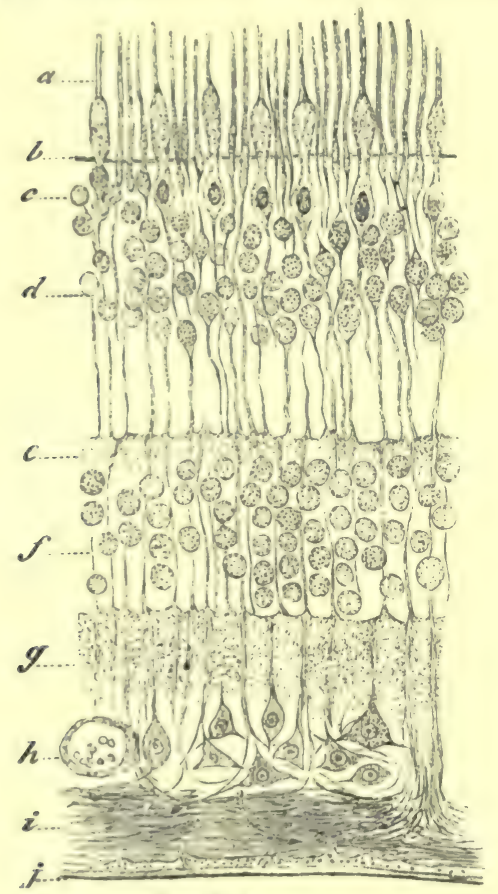

Fig. 519.

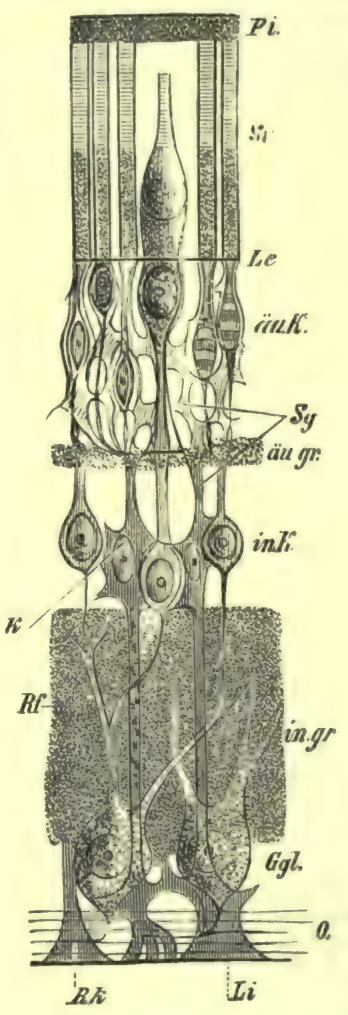

Fig. 520.

Fig. 519. - Vertical section of human retina. $a$, rods and cones ; $b$, ext., and $j$, int. limit. meml). ; c, ext., and $f$, int. nucl. layers ; $e$, ext., and $g$, int. gran. layers; $h$, blood-vessel anil nerve-cells; $i$, nerve-fibres. Fig. 520.-Layers of the retina. Pi, hexagonal pigmentcells; St, rouls and cones; $L e$, ext. limiting membrane ; äu.K, ext. nuclear layer ; äu.gr, ext. granular layer ; in $K$, int. nuclear ; in.gr, int. granular; $G g l$, ganglionic nerve-cells ; $o$, fibres of optic nerve; $L i$, int. limit. membrane ; $R k$, fibres of Miiller ; $K$, nuclei ; $S y$, spaces for the nervous elements.

of 7 , the ganglionic layer, extend. According to v. Vintschgau, the processes of the ganglionic cells are connected with the fibres. 8. The next, or fibrous layer, consists of the fibres of the optic nerve (o), and most internally is the internal limiting membrane $(L i)$. According to W. Krause, there are 400,000 broad, and as many narrow, optic fibres, so that for every fibre there are 7 cones, about 100 rods, and 7 pigment-cells. The optic fibres are absent from the macula lutea, where, however, there are numerous ganglionic cells. Between the two homogeneous limiting membranes ( $L e$ and $L i$ ) lies the connective-tissue substance of the retina. It contains the perforating fibres, or Müller's fibres, which run in a radiate manner between the two membranes, and hold the various layers of the retina together. They begin by a wing-shaped expansion at the internal limiting membrane $(R k)$, and in their course outwards contain nuclei $(k)$. They are abseut at the yellow spot. The supporting tissue forms a 
network in all the layers, holes being left for the nervous portions $(S g)$. The inner segments of the rods and cones are also surrounded by a sustentacular substance. As the retina passes forward to the ora serrata, it becomes thinner and thinner, gradually becoming richer in connective-tissue elements and poorer in nerve elements, until, in the ciliary part, only the cylindrical cells remain (fig. 519).

Macula Lutea and Fovea Centralis. - There are no rods in the fovea, while the cones are longer and narrower than in the other parts of the retina (fig. 521). The other layers also are thinner, especially at the macula lutea, but they become thicker towards the margins of the fovea, where the ganglionic layer consists of several rows of bipolar cells. The yellow tint is due to pigment lying between the layers composing the yellow spot.]

The blood-vessels of the retina lie in the inner layers near the inner granular layer. Only near the entrance of the optic nerve are they connected by fine branches with the choroidal vessels ; they are surrounded by perivascular lymph-spaces. The greatest number of capillaries runs in the layers external to the inner granular layer (Hesse). The fovea centralis is devoid of blood-ressels (Nettleship, Becker). Except in mammals, the eel (Denissenko), and some tortoises $(H$. Müller), the retina receives no blood-vessels. Destruction of the retina is followed by blindness.

[Retinal Epithelium. - The single layer of pigmentary cells containing granules of melanin sends processes downwards, like the hairs of a brush, between the rods and cones ( $\$ 398)$. Kiihne has shown that the nature and amount of light influence the condition of these processes

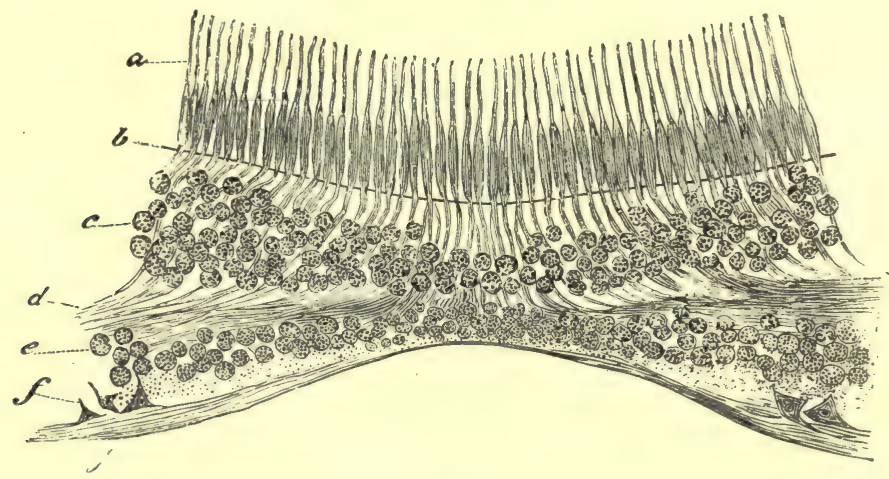

Fig. 521.

Section of the forea centralis. $a$, cones ; $b$ and $g$, int. and ext. linit. memb. ; $c$, ext., and $e$, nuclear layer ; $d$, fibres ; $f$, nerve-cells.

(fig. 563). The protoplasm of these cells in a frog kept for several hours in the dark, is retracted, and the pigment-granules lie chiefly in the body of the cell and in the processes near the cell. In a frog kept in bright daylight, the processes loaded with pigment penetrate downwards between the rods and cones as far as the external limiting membrane.]

Each rod and cone consists of an outer and an inner segment. During life, the outer segment contains a reddish pigment or the visual purple (Boll).

Visual purple [or rhodopsin] may be preserved by keeping the eye in darkness; but it is soon bleached by daylight, while it is again restored when the eye is placed in darkness. It can be extracted from the retina by means of a 2.5 per cent. solution of the bile acids, especially from eyes that have been kept in 10 per cent. solution of common salt (Ayres). The rods are 0.04 to $0.06 \mathrm{~mm}$. high and 0.0016 to $0.0018 \mathrm{~mm}$. broad, and exhibit longitudinal striation, produced by the presence of fine grooves; a fine fibril runs in their interior (Ritter). The external segment occasionally cleaves transversely into a number of fine transparent dises. [It is a very resistant structure, and in this respect resembles neuro-keratin.] Krause found an ellipsoidal body, the "rod ellipsoid," at the junction of the inner and outer segments of the rods. The cones are devoid of visual purple, but their outer segment is striated longitudinally, and it also readily breaks across into thin discs. Only cones are present in the macula lutea. In the neighbourhood of the yellow spot, each cone is surrounded by a ring of rods. The cones become less numerous towards the periphery of the retina. In nocturnal animals, such as the owl and bat, there are either no cones or imperfect ones. The retinæ of birds contain many cones, that of the tortoise only cones. The rods and cones rest on the sieve-like perforated external limiting membrane $(L e)$. Both send processes through the membrane, the cones to the larger and higher-placed nuclei, the rods to the nuclei, with transverse markings in the external nuclear layer. [The cones are particularly large in some fishes, e.g., the cod, while 
the skate has no cone's, but only rods. The same is the case in the shark and sturgeon, heilgehog, bat, and mole.]

[Distribution and Regeneration of Rhodopsin. - Keep a rabbit in the dark for some time, kill it, remove its eyeball, and examine its retina by the aid of monochromatic (sodium) light. The retipa will he purple-red in colour, all except the macula lutea and a small part at the ora serrata. The pigment is confined to the outer segments of the rods, It is absent in pigeons, hens, and one bat, although the last has only rods. It is found both in nocturnal and diurnal animals. Its colour is quickly bleached by light, and it fades rapidly at a temperature of $50^{\circ}$

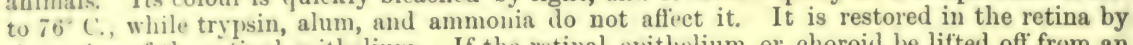
the action of the retinal epithelium. If the retinal epithelium or choroid be lifted off from an exciseal eye exposed to light, the purple is lestroyed; but if the eye be placed in darkness and the retinal epithelium replaced, the colour is restored.]

Chemistry of the retina. - The reaction of the retina, when quite fresh, is acid, and becomes alkaline in darkness. The rods and cones contain albumin, neuro-keratin, nuclein, and in the "ones are the pigmented oil globules, the so-called "chromophanes." The other layers contain the constituents of the grey matter of the brain.

[Cones. - There is no colouring matter in the outer segment of the cones, but in fishes, reptiles, and birds the imner segment contains a globular-coloured body often red and yellow, the jignent being held in solution by a fatty body. Kiihne has separated a green (chlorophane), a yellow (xanthophane), and a red (rhodophane) pigment. They all give a blue with iodine, and are bleached by light (Schwalbe).]

The crystalline lens is enclosed in a transparent capsule, thicker anteriorly than posteriorly, aud it is coverel on the inner surface of the anterior wall by a layer of low epithelium. Towards the margin of the lens, these cells elongate into nucleated fibres, which all bend round the

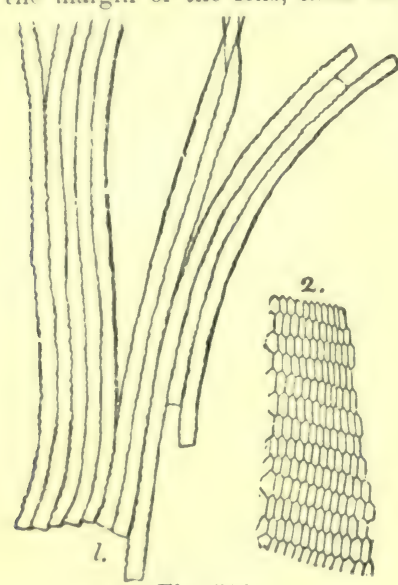

Fig. 522. margin of the lens, and on both sides of the lens abut with their ends against each of the triradiate figures. The lens fibres contain globulin enclosed in a kind of membrane. Owing to mutual pressure, they are hexagonal when seen in transverse section (fig. 522, 2), while in many animals, especially fishes, their margins are serrated [the teeth dovetail into each other]. For the sake of simplicity, we may regard the lens as a biconvex body with spherical surfaces, the posterior surface being more curved. As a matter of fact, the anterior part is part of an ellipsoid formed by rotation on its short axis. The posterior surface resembles the section of a paraboloid, i.e., we might regard it as formed by the rotation of a parabola on its axis (Brïcke). The outer layers of the lens have less refractive power than the more internal layers. The central part of the lens or nucleus is, at the same time, firmer, and more convex than the entire lens. The margin of the lens is always separated from the ciliary processes by an intermediate space.

[Chemistry. - The lens contains about two-thirds of its weight of water, while its chief solid is a globulis, called by Berzelius crystallin $\left(24^{\circ} 6\right.$ per cent.), with a little serumalbumin, salts, cholesterin, and fats.]

[Cataract. - Sometimes the lens becomes more or less

1, Fibres of the lens; 2, transverse sections of the lens fibres.

opaque, the opacity beginning either in the middle or outer parts of the lens. This is generally due to fatty degeneration of the fibres, cholesterin being deposited. An opaque, cataractous condition of the lens may be produced in frogs, by injecting a solution of some salts or sugar into the lymph-sacs; the result is that these salts absorb the water from the lens, and thus make it opaque. The cataract of diabetes is probably produced from the presence of grape-sugar in the blood.]

The zonule of Zinn, at the ora serrata, is applied as a folded membrane to the ciliary part of the uvea, so that the ciliary processes are pressed into its folds, and are united to it. It passes to the margins of the lens, where it is inserted by a series of folds into the anterior part of the capsule of the lens. Behind the zonule of Zinn, and reaching as far as the vitreous humour, is the canal of Petit. The zonule is a fibrous perforated membrane. According to Merkel, the canal of Petit is enclosed by very fine fibres, so that it is really not a canal but a complex com. municating system of spaces (Gerlach). Nevertheless, the zonule represents a stretched mem. brane, holding the lens in position, and may therefore be regarded as the suspensory ligament of the lens.

Opacity or cloudiness of the lens (grey cataract) hinders the passage of light into the eye. $\Delta$ phakia, or the absence of the leus (as after operations for cataract), may be remedied by a pair of strong convex spectacles. Of course, such an eye does not possess the power of accommorlation.

The vitreous humour, as far as the ora serrata, is bounded by the internal limiting membrane 
of the retina (Henle, Iwanoff). From here forwards lying between both, are the meridional fibres of the zonule, which are united with the surface of the vitreous and the ciliary processes. A part of the fibrous layer bends into the saucer-shaped depression, and bounds it. A canal, $2 \mathbf{~ m m}$. in diameter, runs from the optic papilla to the posterior surface of the capsule of the lens; it is called the hyaloid canal, and was formerly traversed by blood-vessels. The peripheral part of the vitreous humour is laminated like an onion, the middle is homogeneous ; in the former, especially in the foetus, are round fusiform or branched cells of the mucous tissue of the vitreous, while in the centre there are disintegrated remains of these cells (Iwanoff). The vitreous humour contains a very small percentage of solids, 1.5 per cent. of mucin, [and, according to Picard, there is 0.5 per cent. of urea, and about $\cdot 75$ of sodic chloride].

[Structure. - The vitreous humour consists essentially of mucous tissue, in whose meshes lies a very watery fluid, containing the organic and inorganic bodies in solution. According to

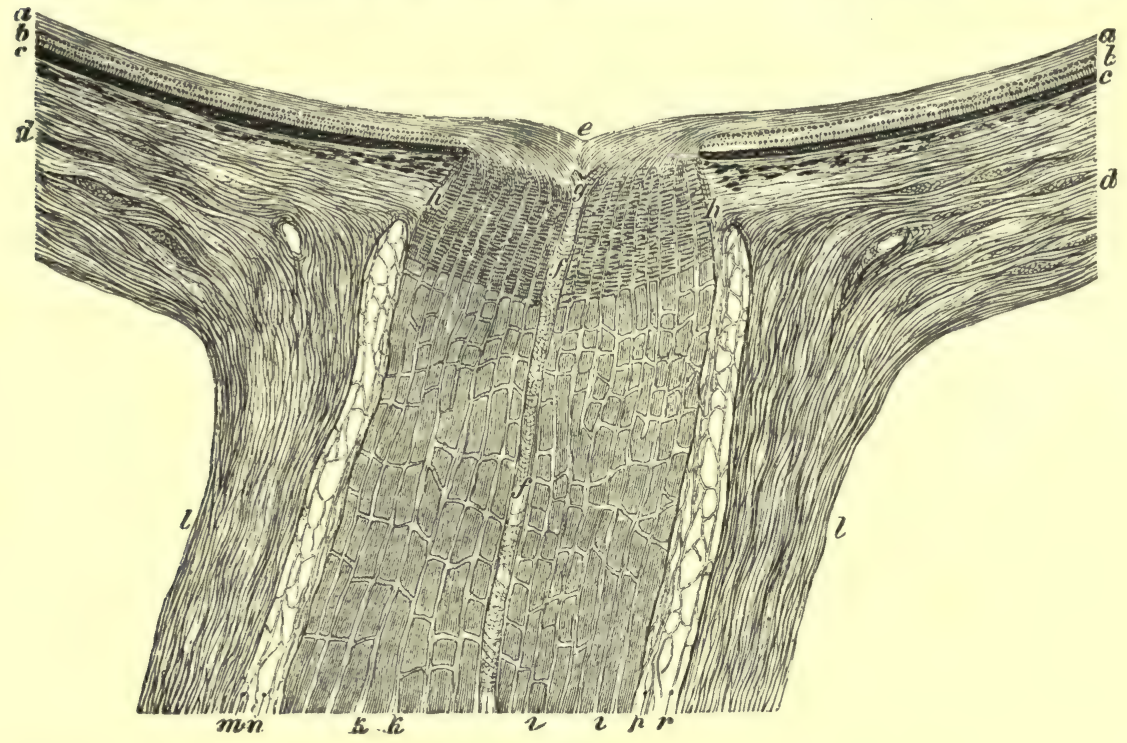

Fig. 523.

Ilorizontal section of the entrance of the optic nerve and the coats of the eye. $a$, inner, $b$, outer layers of the retina ; $c$, choroid ; $d$, sclerotic ; $e$, physiological cup ; $f$, central artery of retina in axial canal; $g$, its point of bifurcation ; $h$, lamina cribrosa $; l$, outer (dural) sheath ; $m$, outer (subdural) space; $n$, inner (subarachnoid) space; $r$, middle (arachnoid) sheath ; $p$, inner (pial) sheath ; $i$, bundles of nerve-fibres; $k$, longitudinal septa of connective-tissue.

Younan, the vitreous contains two types of cells-(1) amœboid cells of various shapes and sizes. They lie on the inner surface of the lining hyaloid membrane and the other mem. branes in the cortex of the vitreous; (2) large branching multipolar cells. The vitreous is permeated by a large number of transparent, clear, homogeneous hyaloid membranes, which are so disposed as to give rise to a concentric lamination. The canal of Stilling represents in the adult the situation of the hyaloid artery of the fotus. It can readily be injected by a coloured fluid.]

The lymphatics of the eye consist of an anterior and a posterior set. The anterior consist of the anterior and posterior chambers of the eye (aqueous), which communicate with the lymphatics of the iris, ciliary processes, cornea, and conjunctiva. The posterior consist of the perichoroidal space between the sclerotic and the choroid (Schwalbe). This space is connected by means of the perivascular lymphatics around the trunks of the vasa vorticosa, with the large lymph-space of Tenon, which lies between the sclerotic and Tenon's capsule. Posteriorly, this is continued into a lymph-channel, which invests the surface of the optic nerve; while anteriorly it communicates directly with the sub-conjunctival lymph-spaces of the eyeball. The optic nerve has three sheaths-(1) the dural ; (2) the arachnoid ; and (3) the pial sheath, derived from the corresponding membranes of the brain. Two lymph-spaces lie between these three sheaths-the subdural space between 1 and 2, and the subarachnoid space between 2 and 3 'fig. 509). Both spaces are lined by endothelium; and the fine trabeculæ passing from 
one wall to the other are similarly covered. According to Axel Key and Retzius, these lymphspaces communicate anteriorly with the perichoroidal space.

The aqueous humour closely resembles the cerebro-spinal fluid, and contains albumin and sugar; the former is increased, and the latter disappears after death. The same occurs in the vitreous. The allomin increases when the difference between the blood-pressure and the intraocular jiressure rises. Such variations of pressure, and also intense stimuli applied to the "ye, cause the proluction of fibrin in the anterior chamber (Jesner and Grïnagen).

Intraocular Pressure. - The cavity of the bulb is practically filled with watery fluids, which, during lifi, are constantly subjected to a certain pressure, the "intraocular pressure." Ultimately, this depends upon the blood-pressure within the arteries of the retina and uven, and must rise and fall with it. The pressure is determined by pressing upon the eyeball, and ascertaining whether it is tense, or soft and compressible. Just as in the case of the arterial fresiure, the intraocular pressure is influenced by many circumstances ; it is inereased at every puls-beat and at every expiration, while it is decreased during inspiration. The elastic tension of the sclerotic and cornea regulates the increase of the arterial pressure by acting like the airchamber in a fire-engine; thus, when more arterial blood is pumperl into the eyeball, more venous blood is also expelled. The constancy of the intraocular pressure is also influenced by the fact that, just as the aqueous humour is removed, it is secreted, or rather formed, as rapidly as it is alsorbed $(\$ 392)$. [Fick has invented an instrument for the direct measurement of the intraocular pressure, a small plate of known size is pressed against the eyeball, and the pressure exerted is registered by means of a spring and index.]

The secretion of the aqueous humour occurs pretty rapidly, as may be surmised from the fact, that hiemoglobin is found in the ayueous humour half an hour after dissolved blood (lamb's) is injected into the blood-vessels of a dog. It is rapidly reformed, after evacuation, through a wound in the cornea. According to Knies, the watery fluid within the eyeball is secreted, esperially from the chorio-capillaris, and reaches the suprachoroidal space, in the lymph-sheaths of the optic nerve, and partly through the network of the sclerotic. It saturates the retina, vitreous, lens, and for the most part passes through the zonula ciliaris into the posterior chamber, and through the pupil into the anterior chamber. The movements of the fluid within the eyeliall have been recently studied by Ehrlich, who used fluorescin, an indifferent substance, which, on being introduced into the body, passes into the fluids of the eyeball, and in a very dilute solution may be recognised by its green fluorescence in reflected light. From observations on the entrance of this substance into the eye, Schöler and Uhthoff regard the posterior surface of the iris and the ciliary body as the secretory organs for the aqueous humour. It passes through the pupil into the anterior chamber; some passes into the lens, and along the canal of l'etit into the vitreous humour (Pflüger). Section of the cervical sympathetic, and still more of the trigeminus, accelerates the secretion of the aqueous, but its amount is diminished. If the substance is dropped into the conjunctival sac, it percolates towards the centre of the cornea, and through the latter into the anterior chamber (Pflüger).

$A$ current passes forwards from the vitreous humour around the lens, and there is an outflow along the central artery of the retina backwards through the optic nerve to the cavity of the skull (fifford). The current in the spaces between the sheaths flows from the brain to the eye (Quincke).

The outflow of the arjueous humour, according to Leher and Heisrath, takes place chiefly letween the meshes of the ligamentum pectinatum iridis (fig. $514, m, m$ ), and the canal of Schlemm $(i, k)$, into the anterior circular veins (p. 737). A small part of the aqueous hurnour diffuses into the posterior layers of the cornea, to nourish it (Leber). None of the water is conducted from the eyeball by any special efferent lymphatics (Leber). Under normal circumstances, the pressure is nearly the same in the vitreous and aqueous chambers, but atropin seems to diminish the pressure in the former and to increase it in the latter, whilst Calabar bean has an opposite action $(A d$. Weber). Arrest of the outflow of the venous blood often increases the pressure in the vitreous, and diminishes that in the aqueous chamber. Compres. sion of the bulb from without causes more fluid to pass out of the eye temporarily than enters it. The diminution of the intraocular pressure is well-marked after section of the trigeminus, while it rises when this nerve is stimulated. The statements of observers regarding the effeet of the sympathetic nerve upon the pressure vary. Interruption to the venous outflow increases the pressure, while an imperfect supply of blood, the outflow being normal, diminishes the pres. sure. The innervation of the blood-vessels of the eye is referred to at $\$ 347$.

385. DIOPTRIC OBSERVATIONS. - The eye as an optical instrument is comparable to a camers obscura ; in both, an inverted diminished image of the objects of the external world is formed upon a background, the field of projection. Instead of the single lens of the camera, however, the eye has several refractive media placed behind each other-cornea, aqueons humour, lens (whose individual parts-capsule, cortical layers, and nucleus, all possess different refractive indices), and vitreous humour. Every two of these adjacent media are bounded by a "refractive surface," which may be regarded as spherical. The field of projection of the eye is the retina, which is coloured with the visual purple (Boll, Kühnc). As this substance is 
bleached chemically by the direct action of light, so that the pictures may be temporarily fixed upon the retina, the comparison of the eye with the camera of the photographer becomes more striking. In order that the passage of the rays of light through the media of the eye may be rightly understood, we must know the following factors:-(1) the refractive indices of all the media; (2) the form of the refractive surfaces; (3) the distance of the various media from each other and from the field of projection or retina.

Action of a converging lens.- We must know how a convex lens acts upon light. In a convex lens we distinguish the centre of curvature, i.e., the centre of both spherical surfaces (fig. 524, I, $m, m_{1}$ ). The line connecting both is called the chief axis ; the centre of this line is the optical centre of the lens (o). All rays which pass through the optical eentre of the lens pass through unbent, or without being refracted; they are called the chief or principal rays $\left(n, n_{1}\right)$ The following are the laws regulating the action of a convex lens upon rays of light:-

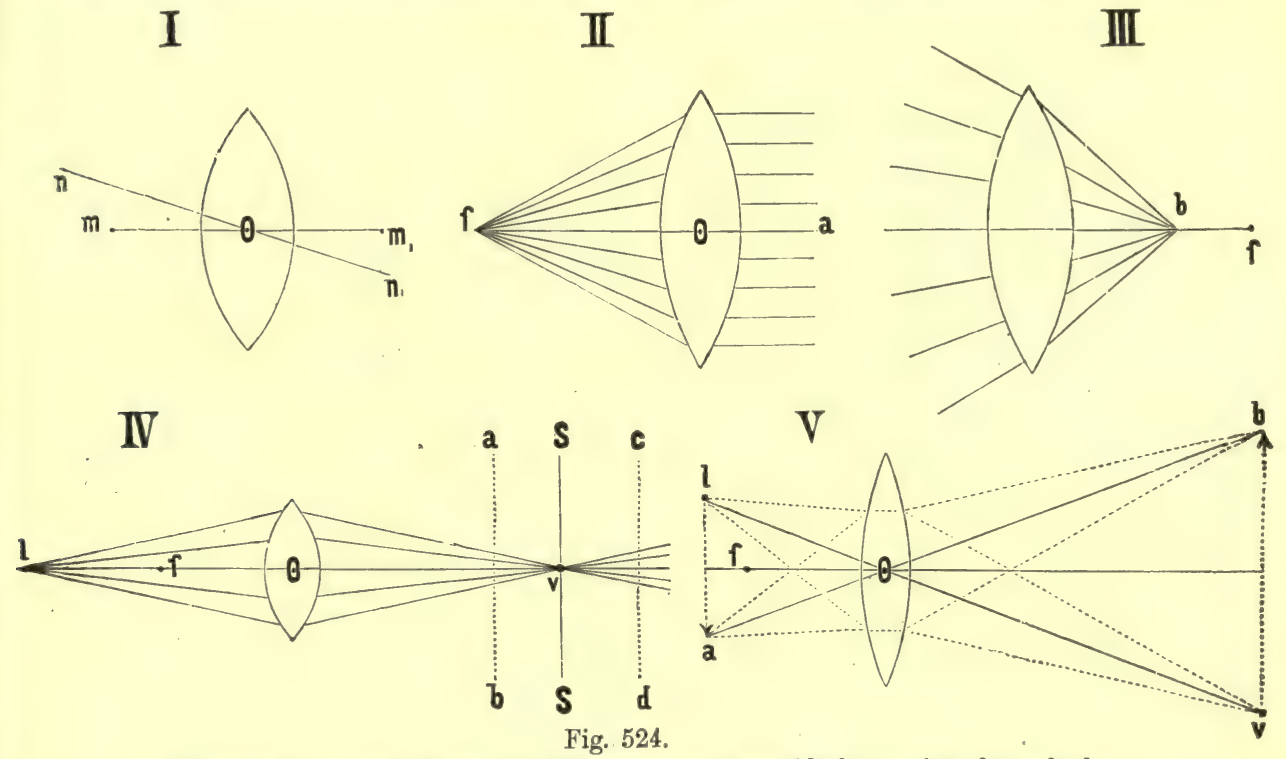

Figures illustrating the action of lenses upon rays of light passing through them.

1. Rays which fall upon the lens, parallel with the principal axis $(I I, f, a)$, are so refracted that they are collected on the other side of the lens, at a point called the focus or principal focus $(f)$. The distance of this point from the central point $(o)$ of the lens, is called the focal distance of the lens $(f, o)$. The converse of this condition is evident, viz., rays which diverge from a focus and reach the lens, pass through it to the other side, parallel with the principal axis, without again coming together.

2. Rays of light proceeding from a source of light $(\mathrm{IV}, l)$ in the prolonged principal axis, but beyond the focal point $(f)$, again converge to a point on the other side of the lens. The following cases may occur:-(a) When the distance of the light from the lens is equal to twice the focal distance, the focus or point of convergence lies at the same distance on the other side of the lens, i.e., twice the focal distance. (b) If the luminous point be moved nearer to the focus, then the focal point is moved farther away. (c) If the light is still farther from the lens than twice the focal distance, then the focal point comes correspondingly near to the lens.

3. Rays proceeding from a point of the chief axis (III, $b$ ) within the focal distance, pass out at the other side less divergent, but do not come to a focus again. Conversely, rays which are convergent, and pass through a collecting lens, have their focal point within the focal distance.

4. If the luminous point $(\mathrm{V}, a)$ is placed in the secondary ray $(a, b)$, the same laws obtain, provided the angle formed by the secondary ray with the principal axis is small.

Formation of images by convex lenses. - After what has been stated, regarding the position of the point of convergence of rays proceeding from a luminous point, the construction of the image of any object by a convex lens is easily accomplished. This is done simply by projecting images of the various parts of the object. Thus, evidently (in V), $b$ is the focal point of the object, $a$, while $v$ is the focal point of the object $l$. The picture is inverted. Collecting lenses form an inverted and real-image (i.e., upon a screen) only of such objects as are placed beyond the focal point of the lens. 
With regard to the size and distance of the image from the lens, there are the following cases :- $(a)$ If the object be placed at twice the focal distance from the lens, the image of the same is just the same size and at the same distance from the lens as the object is. (b) If the object be nearer than the focus, the image recedes and at the same time becomes larger. $(c)$ If

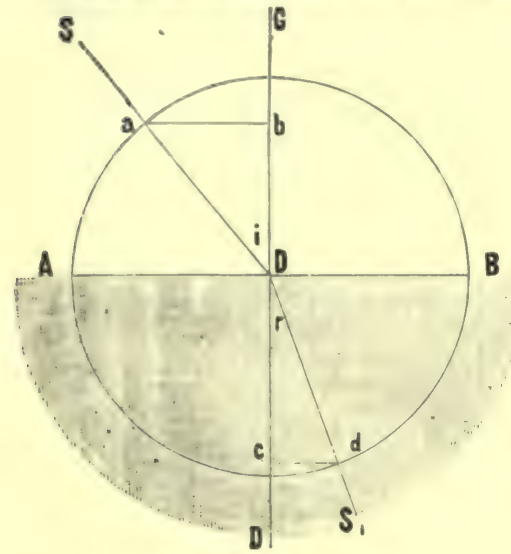

Fig. 525 .

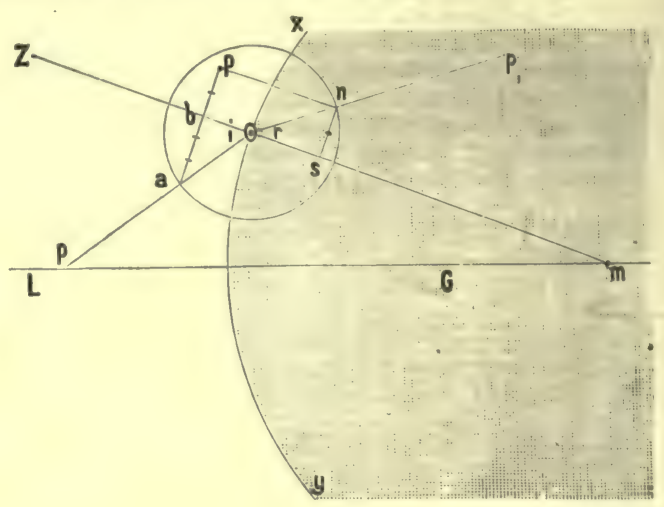

Fig. 526.

the ol,ject be farthes removed from the lens than twice the focal distance, then the image is nearer to the lens and at the same time becomes smaller.

Position of the focal point. - The distance of the focal point from the lens is readily calcu. lated according to the following formula :-Where $l=$ the distance of the luminous point, $b=$ the listance of the image, and $f=$ the focal distance of the lens: $\frac{1}{l}+\frac{1}{b}=\frac{1}{f}$, or $\frac{1}{b}=\frac{1}{f}-\frac{1}{l}$.

Example. -Let $l=24$ centimetres, $f=6 \mathrm{~cm}$. Then $\frac{1}{b}=\frac{1}{6}-\frac{1}{24}=\frac{1}{8}$; so that $b=8 \mathrm{~cm}$., i.e., the image is formed $8 \mathrm{~cm}$. behind the lens. Further, let $l=10 \mathrm{~cm}$., $f=5 \mathrm{~cm}$. (i.e., $l=2 f)$. Then $\frac{1}{b}=\frac{1}{5}-\frac{1}{10}=\frac{1}{10}$; so that $b=10, i$.c., the image is placed at twice the focal distance of the lens. Lastly, let $l=\infty$. Then $\frac{1}{b}=\frac{1}{f}-\frac{1}{\infty}$; so that $b=f$, i.e., the image of parallel rays coming from infinity lies in the focal point of the lens.

Refractive Indices. - $\mathrm{A}$ ray of light, which passes in a perpendicular direction from one medium into another medium of different density, passes through the latter without changing its course or being refracted. In fig. 525 , if $\mathrm{G} \mathrm{D}$, is $\perp \mathrm{A} \mathrm{B}$, then so is $\mathrm{D} \mathrm{D,} \perp$ A B ; for a plane surface A B is the horizontal, and G D the vertical line. If the surface be spherical, then the vertical line is the prolonged radius of this sphere. If, however, the ray of light fall obliquely npon the surface, it is "refracted," $i . e .$, it is bent out of its original course. The incident and the refracted ray nevertheless lie in one plane. When the oblique incident ray passes from a less dense inedium (c.g., air) into one more dense (e.g., water), the refracted or excident ray is bent tow:ards the perpendicular. If, conversely, it pass from a more dense to a less dense medium, it is bent away from the perpendicular. The angle $(i, \mathrm{G} \mathrm{D} \mathrm{S})$ which the incident ray (S D) forms with the perpendicular (G D) is called the angle of incidence, the angle former by the refracted ray $\left(\mathrm{D} \mathrm{S}_{1}\right)$ with the prolonged perpendicular $(\mathrm{D} \mathrm{D})$ is called the angle of refraction, $\mathrm{D} \mathrm{D} \mathrm{S}_{1}$ $(r)$. The refractive power is expressed as the refractive index. The term refractive index (n) means, that number which shows for a certain substance, how many times the sine of the angle of incidence is greater than the sine of the angle of refraction, when a ray of light passes from the air into that substance. Thus, $n=\sin . i: \sin . r=a b,: c d$. On comparing the refractive indices of two media, we always assume that the ray passes from air into the medium. On passing from the air into water, the ray of light is so refracted that the sine of the angle of incidence is to the sine of the angle of refraction, as $4: 3$; the refractive index $=\frac{4}{3}$ (or more exactly $=1 \cdot 336)$. With glass the proportion is $=3: 2 \quad=1 \cdot 535$-Snellius, 1620 ; Descartes $)$. The sine of the incident and refractive-angles are related as the velocity of light with both media.

The construction of the refracted ray, the refractive index being given, is simple:-Example -Suppose in fig. $526, \mathrm{~L}=$ the air, $\mathrm{G}=\mathrm{a}$ dense medium (glass) with a spherical surface, $x y$, and 
with its centre at $m ; p o=$ the oblique incident ray the $m Z$ is the perpendicular $<)=i$ the angle of incidence. The refractive index given is $\frac{3}{2}$; the object is to find the direction of the refracted way. From $o$ as centre describe a circle with a radius of any length; from $a$ draw a perpendicular, $a b$ to $m Z$; then $a b$ is the sine of the angle of incidence, $i$. Divide the line $\alpha b$ into three equal parts, and prolong it to the extent of two of these parts, viz., to $p$. Draw the line $p$ parallel to $m Z$. The line joining $o$ to $n$ is the direction of the refracted ray. On making a line, $n s$, perpendicular to $m Z, n s=b p$. Further, $n s=\operatorname{sine}<)=r$. So that $a b: s \|$ or : $b p)=3: 2$ or $\sin . i: \sin . r=\frac{3}{2}$.

Optical cardinal point of a simple collecting system. - Two refractive media (fig. 527, L and

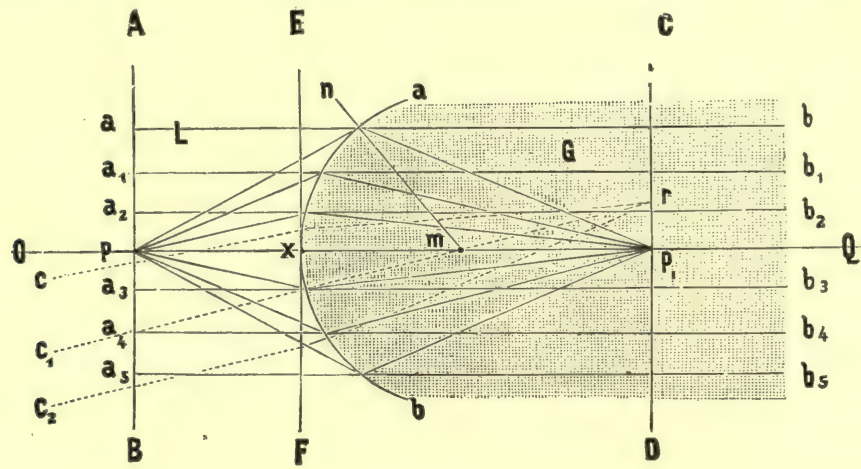

Fig. 527.

G), which are separated from each other by a spherical surface $(a, b)$ form a simple collecting system. It is easy to estimate the construction of an incident ray coming from the first medium $(\mathrm{L})$ and falling obliquely upon the surface $(\alpha, b)$ separating the two media, as well as to ascertain its direction in the second medium, $\mathrm{G}$, and also from the position of a luminous point in the first medium, to estimate the position of the corresponding focal point in the second medium. The factors required to be known are the following:-L (fig. 527) is the first, and $\mathrm{G}$ the second medium, $a, b=$ the spherical surface whose centre is $m$. Of course, all the radii drawn from $m$ to $a b(m x, m n)$ are perpendiculars, so that all rays falling in the direction of the radii must pass unrefracted through $m$. All rays of this sort are called rays or lines of direction ; $m$, as the point of intersection of all these, is called the nodal point. The line which connects $m$ with the vertex of the spherical surface, $x$, and which is prolonged in both directions, is called the optic axis, $\mathrm{O}$ Q. A plane $(\mathrm{E}, \mathrm{F})$ in $x$, perpendicular to $\mathrm{O} \mathrm{Q}$, is called the principal plane, and in it $x$ is the principal point. The following facts have been ascertained :-(1) All rays $\left(a\right.$ to $\left.\alpha_{5}\right)$, which in the first medium are parallel with each other and with the optic axis, and fall upon $a b$, are so refracted in the second medium that they are all again united in one point $\left(p_{1}\right)$ of the second medium. This is called the second principal focus. A plane in this point perpendicular to $\mathrm{O} Q$ is called the second focal plane (C D). (2) All rays ( $c$ to $c_{2}$ ), which in the first medium are parallel to each other, but not parallel to $\mathrm{O} Q$, reunite in a point of the second focal plane $(r)$, where the non-refracted directive ray $\left(c_{1}, m r\right)$ meets this. (In this case, the angle formed by the rays $c$ to $c_{2}$ with $\mathrm{C} Q$ must be very small.) The propositions 1 and 2 of course may be reversed; the divergent rays proceeding from $p$ towards $a b$ pass into the first medium parallel to each other, and also with the axis $\mathrm{C} Q\left(a\right.$ to $\left.a_{5}\right)$; and the rays proceeding from $r$ pass into the first medium parallel to each other, but not parallel to the axis $\mathrm{OQ}$ (as $c$ to $c_{2}$ ). (3) All rays, which in the second medium are parallel to each other $\left(b\right.$ to $\left.b_{5}\right)$ and with the axis $\mathrm{O} Q$, reunite in a point in the first medium $(p)$ called the first focal point; of course the converse of this is true. A plane in this point perpendicular to $\mathrm{O} Q$ is called the first focal plane (A, B). The radius of the refractive surface $(m, x)$ is equal to the difference of the distance of both focal points $\left(p\right.$ and $\left.p_{1}\right)$ from the principal focus $(x)$; thus $m x=$ $p_{1} x-p x$. From these comparatively simple propositions it is easy to determine the following points :-

1. The construction of the refracted ray.-Let A be the first (fig. 528); B, the second medium; $c d$, the spherical surface separating the two; $a b$, the optical axis; $k$, the nodal point ; $p$, the first and $p_{1}$ the second principal focus ; C, D, the second focal plane. Suppose $x y$ to represent the direction of the incident ray, what is the construction of the refracted ray 
in the second medium? Prolong the unrefracted ray, $\mathrm{P}, k, \mathrm{Q}$ parallel to $x, y$, then $y, \mathrm{Q}$ is the lirection of the refracted ray (according to 2 ).

2. Construction of the image for a given object. - In fig. $529, \mathrm{~B}, c, d, a, b, k, p$, and $p_{1}$,

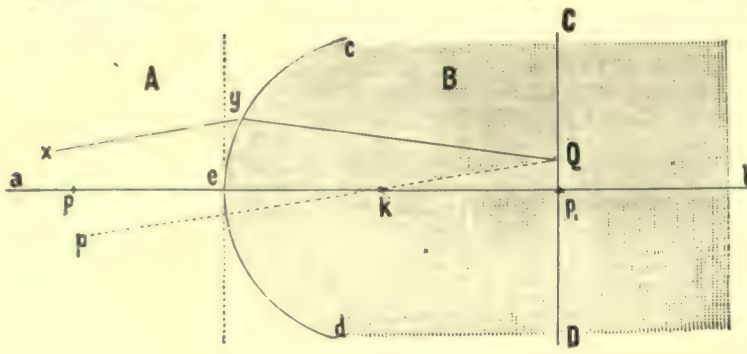
C, D are as before. Suppose a luminous point $(o)$ in the first medium, what is the position of the image in the second medium? Prolong the nnrefracted ray $(0, k, \mathbf{P})$, and draw the ray $(o, x)$ parallel to the axis $\mathbf{b}_{(a, b)}$. The parallel rays $(a, e$ and $o, x$ ) reunite in $p$ (according to proposition 1). Prolong $x, p_{1}$ until it intersects the ray $(o, P)$, then the image of $o$ is at $\mathrm{P}$, the rays of light ( $o x$ and $o k$ ) proceeding from the lumi-

Fig. 528. nous point $(0)$ reunite in $P$.

Construction of the refracted ray and the image in several refractive media. - If several refractive media be placed behind each other, we must proceed from medium to medium with

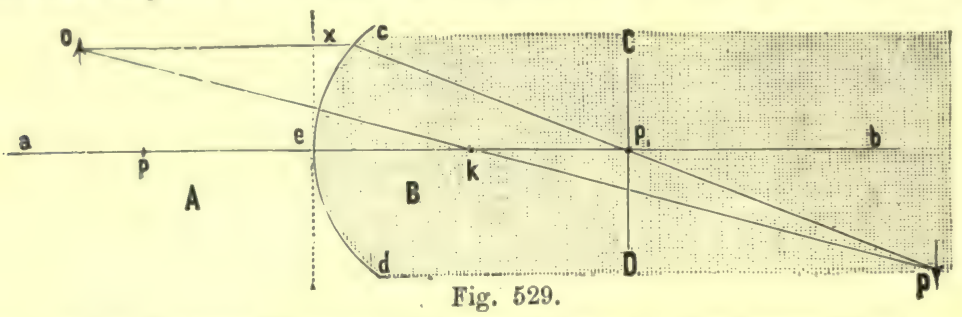

the sann mithouls as above described. This would be very tedious, especially when dealing with small objects. Gauss (1840) calculated that in such cases the method of construction is
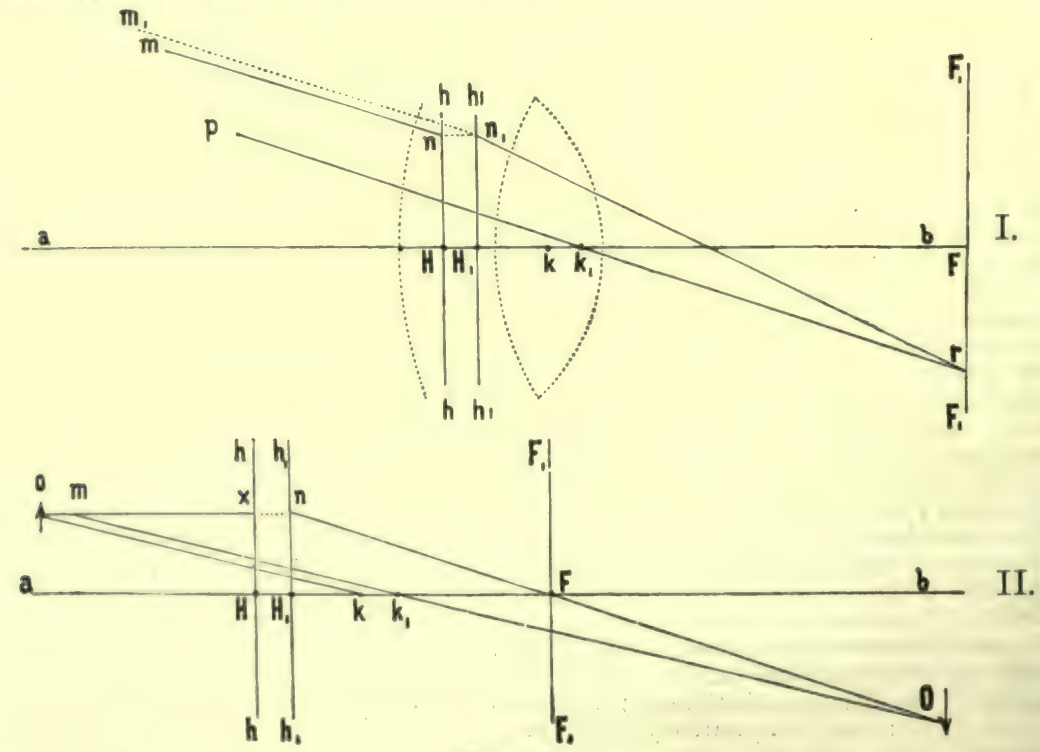

Fig. 530,

very simple. If the several media are "centred," i.c., if all have the same optic axis, then the refractive indices of such a centred system may be represented by two equal, strong, refractive surfaces at a certain distance. The rays falling upon the first surface are not refracted by it, 
but are essentially projected forwards parallel with themselves to the second surface. Refraction takes place first at the second surface, just as if only one refractive surface was present. In order to make the calculation, we must know the refractive indices of the media, the radii of the refractive surfaces, and the distance of the refractive surfaces from each other.

Construction of the refracted ray is accomplished as follows :-Let $a b$ represent the optical axis (fig. $530, \mathrm{I}$.) ; H, the first focal point determined by calculation ; $h h$, the principal plane : $\mathrm{H}$, the second focal point; $h_{1}, h_{1}$, the second principal plane; $k$, the first, and $k_{1}$ the second nodal point; $\mathrm{F}$, the second focal point ; and $\mathrm{F}_{1}, \mathrm{~F}_{1}$, the second focal plane. Make the ray of direction $p k_{1}$ parallel to $m_{1}, n_{1}$. According to proposition 2, $p, k_{1}$ and $m_{1}, n_{1}$ must meet in a point of the plane $\mathrm{F}_{1} \mathrm{~F}_{1}$. As $p k_{1}$ passes through unrefracted, the ray from $n_{1}$ must fall at $r$; $n_{1} r$ is, therefore, the direction of the refracted ray.

Construction of the focal point.-Let $o$ be a luminous point (fig. 530, II.), what is the position of its image in the last medium? Prolong from $o$ the ray of direction $o k$, and make $o, x$ parallel to $a b$. Both rays are prolonged in a parallel direction to the second focal plane. The ray parallel to $a b$ goes through $\mathrm{F} ; m, k_{1}$ as the ray of direction passes through unrefracted. 0 , where $n, F$, and $m k_{1}$ intersect each other, is the position of the image of $o$.

386. DIOPTRICS-RETINAL IMAGE-OPHTHALMOMETER-Position of the cardinal points. - The eye surrounded with air on the anterior surface of the cornea, represents a concentric system of refractive media with spherical separating surfaces. In order to ascertain the course of the rays through the various media of the eye, we must know the position of both principal foci of both nodal points as well as the two principal focal points. Gauss, Listing, and v. Helmholtz have calculated the position of these points. In order to make this calculation, we require to know the refractive indices of the media of the eye, the radii of the refractive surfaces, and the distance of the latter from each other. These will be referred to afterwards. (1) The first principal point is $2 \cdot 1746 \mathrm{~mm}$; and (2) the second principal point is $2.5724 \mathrm{~mm}$. behind the anterior surface of the cornea. (3) The first nodal point, $0.7580 \mathrm{~mm}$. ; and (4) the second nodal point, $0.3602 \mathrm{~mm}$. in front of the posterior surface of the lens. (5) The second principal focus, $14.6470 \mathrm{~mm}$. behind the posterior surface of the lens; and (6) the first principal focus, 12.8326 in front of the anterior surface of the cornea.

Listing's reduced eye.-The distance between the two principal points, or the two nodal points, is so small (only $0.4 \mathrm{~mm}$.), that practically, without introducing any

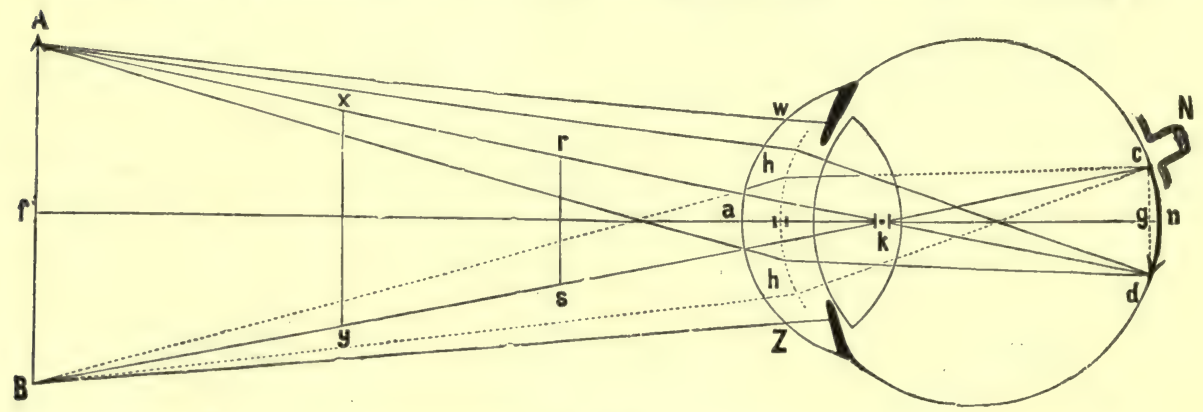

Fig. 531.

great error in the construction, we may assume one mean nodal or principal point lying between the two nodal or principal points. By this simple procedure we gain one refractive surface for all the media of the eye, and only one nodal point, through which all the rays of direction from without must pass without being refracted. This schematic simplified eye is called "the reduced eye" of Listing.

Formation of the retinal image. - Thus, the construction of the image on the retina becomes very simple. In distinct vision, the inverted image is formed on the retina. Let A B represent an object placed vertically in front of the eye (fig. 531). A pencil of rays passes from $\mathrm{A}$ into the eye; the ray of direction, $\mathrm{A} d$, 
passes without refraction through the nodal point, $k$. Further, as the focal point for the luminous point, $\mathrm{A}$, is upon the retina, all the rays proceeding from $\mathrm{A}$ must reunite in $d$. The same is true of the rays proceeding from $\mathrm{B}$, and, of course, for rays sent out from an intermediate point of the body, A B. The retinal image is, as it were; a mosaic, composed of innumerable foci of the object. As all the rays of direction must pass through the common nodal point, $k$, this is also called the "point of intersection of the visual rays."

The invertenl image on the retina is easily seen in the excised eye of an albino rabbit, by holding ul any cliject in front of the cornea and observing the inverted image through the transparent coats of the eyeball.

The size of the retinal image may also be calculated, provided we know the size of the ohject, and its distance from the cornea. As the two triangles, A B $k$ and $c d k$ are similar, A $B: c d=f k: k g$, so that $c d=(\mathrm{A} \mathrm{B}, k g): f k$. All these values are known, viz., $k g=15 \cdot 16$ mm.: further, $f k=a k \times a, f$, where $a f$ is measured clirectly, and $a k=7 \cdot 44 \mathrm{~mm}$. The size of $\mathrm{A} B$ is measured directly.

The angle, $A k B$, is called the visual angle, and of course it is equal to the angle $c k d$. It is evident that the nearer objects, $x y$, and $r s$, must have the same visual angle. Hence, all the three objects, A B, $x y$, and $r s$, give a retinal image of the same size. Such objects, whose ends when united with the nodal point form a visual angle of the same size, and consequently form retinal images of the same size, have the same " apparent size."

In order to determine the optical cardinal points by calculation, after the method of Gauss, we must know the following factors:-

1. The refractive indices: for the cornea, $1 \cdot 377$; aqueous humour, 1.377 ; lens, 1.454 (as the mean value of all the layers); vitreous humour, 1.336 ; air being taken as 1 , and water $1 \cdot 335$.

2. The radii of the spherical refractive surfaces: of the cornea, $7.7 \mathrm{~mm}$; of the anterior surface of the lens, 10.3 ; of the posterior, $6.1 \mathrm{~mm}$.

3. The distance of the refractive surfaces: from the vertex of the cornea to the anterior surface of the lens, $3.4 \mathrm{~mm}$.; from the latter to the posterior surface of the lens (axis of the lens), $4 \mathrm{~mm}$. ; dianeter of the vitreous humour, $14.6 \mathrm{~mm}$. The total length of the optic axis is $22.0 \mathrm{~mm}$.

[Kuhne's Artificial Eye.-The formation of an inverted image, and the other points in the dioptrics of the eye can be studied most effectively on Kiihne's artificial eye, the course of the rays of light being visible in water tinged with eosine.]

Ophthalmometer. - This is an instrument to enable us to measure the radii of the refractive media of the eye. As the normal curvature cannot be accurately measured on the dead eye, owing to the rapid collapse of the ocular tunics, we have recourse to the process of Kohlrausch, for calculating the radii of the refractive surfaces from the size of the reflected images in the living eye. The size of a luminous body is to the size of its reflected image, as the distance of both to half the rulius of the convex mirror. Hence, it is necessary to measure the size of the re-
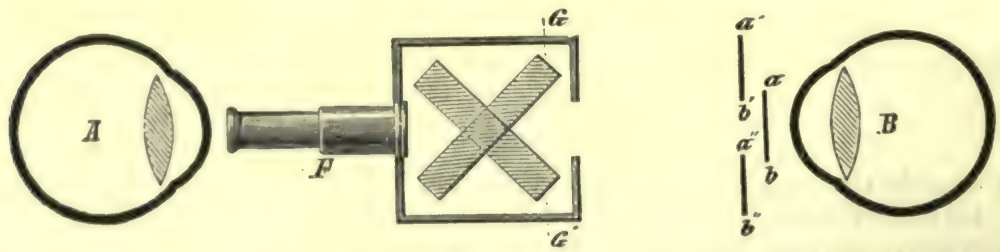

Fig. 532.

Scheme of the ophthalmometer of Helmholtz.

flected image. This is done by means of the ophthalmometer of Helmholtz (fig. 532). The apparatus is constructed on the following principle:--If we observe an object through a glass plate placed obliquely, the object appears to be displaced laterally; the displacement becomes greater, the more obliquely the plate is moved. Suppose the observer, $A$, to look through the telescope, F, which has the plate, G, placed obliquely in front of the upper half of its objective, he sees the corneal reflected image, $a b$, of the eye, $\mathrm{B}$, and the 
image appears to be displaced laterally, viz., to $a^{\prime} b^{\prime}$. If a second plate, $\mathrm{G}$, be placed in front of the lower half of the telescope, but placed in the opposite direction, so that both plates, corresponding to the middle line of the objective, intersect at an angle, then the observer sees the reflected image, $a b$, displaced laterally to $a^{\prime \prime} b^{\prime \prime}$. As both glass plates rotate round their point of intersection, the position of both is so selected, that both reflected images just touch each other with their inner margins, (so that $b^{\prime}$ abuts closely upon $a^{\prime \prime}$ ). The size of the reflected image can be determined from the size of the angle formed by both plates, but we must take into calculation the thickness of the glass plates and their refractive indices. The size of the corneal image, and also that in the lens, may be ascertained in the passive eye, and also in the eye accommodated for a near object, and the length of the radius of the curved surface may be calculated therefrom (Helmholtz and others).

Fluorescence. - All the media of the eye, even the retina, are slightly fluorescent; the lens most, the virteous humour least ( $v$. Helmholtz).

Erect Vision. - As the retinal image is inverted, we must explain how we see objects upright. By a psychical act, the impulses from any point of the retina are again referred to the exterior, in the direction through the nodal point; thus the stimulation of the point $d$ is referred to A, that of $c$ to B (fig. 531). The reference of the image to the external world happens thus, that all points appear to lie in a surface floating in front of the eye, which is called the field of vision. The field of vision is the inverted surface of the retina projected externally ; hence, the field of vision appears erect again, as the inverted retinal image is again projected externally but inverted (fig. 531).

That the stimulation of any point is again projected in an inverse direction through the nodal point, is proved by the simple experiment, that pressure upon the outer aspect of the eyeball is projected or referred to the inner aspect of the field of vision. The entoptical phenomena of the retina are similarly projected externally and inverted; so that, e.g., the entrance of the optic nerve is referred externally to the yellow spot (see $\$ 393)$. All sensations from the retina are projected externally.

387. ACCOMMODATION OF THE EYE.-According to No. 2. (p. 743), the rays of light proceeding from a luminous point, e.g., a flame, and acted upon by a collecting (convex) lens, are brought to a focus or focal point, which has always a definite relation to the luminous object. If a projection-surface or screen be placed at this distance from the lens, a real and inverted image of the object is obtained upon the screen. If the screen be placed nearer to the lens (fig. 524, IV, $a, b$ ), or farther away from it $(c, d)$, no distinct image of the object is formed, but diffusion circles are obtained; because, in the former case, the rays have not united, and in the latter, because the rays, after uniting, have crossed each other and become divergent. If the luminous point be brought nearer to, or removed farther from, the lens, in order to obtain a distinct image, in every case, the screen must be brought nearer, or removed from the lens, to keep the same distance between the lens and the screen. If, however, the screen be fixed permanently, whilst the distance between the luminous point and the lens varies, a distinct image can only be obtained upon the screen, provided the lens, as the luminous point approaches it, becomes more convex, i.e., refracts the rays of light more strongly - conversely, when the distance between the luminous point and the lens becomes greater, the lens must become less curved, i.e., refract less strongly.

In the eye, the projection surface or screen is represented by the retina, which is permanently fixed at a certain distance; but the eye has the power of forming distinct images of near and distant objects upon the retina, so that the refractive power, i.e., the form of the crystalline lens in the eye, must undergo a change in curvature corresponding in every case to the distance of the object. [It is important to remember, that we cannot see a near object and a distant one with equal distinctness at the same time, and hence arises the necessity for accommodation.]

Accommodation. - By the term "accommodation of the eye," is understood that property of the eye, whereby it forms distinct images of distant as well as near objects upon the retina. This power depends upon the fact, that the crystalline lens alters its curvature, becoming more convex (thicker), or less curved (flatter), according to the distance of the object. When the lens is absent from the eyeball, accommodation is impossible (Th. Young, Donders-p. 740):

During rest [or negative accommodation], or when the eye is passive, it is accommodated for the greatest distance, i.e., images of objects placed at an infinite distance (e.g., the moon) are formed upon the retina. In this case, rays coming 
from such a distance are practically parallel, and when they enter the eye, are in the passice normul eye (emmetropic) brought to a focus on the retina. When

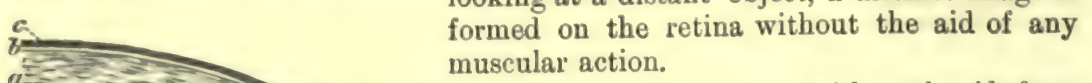

That distant objects are seen without the aid of any muscular action is shown by the following cousiderations :-(1) With the normal, or emmetropic eye, we can see distant objects clearly and distinctly, without experiencing any feeling of

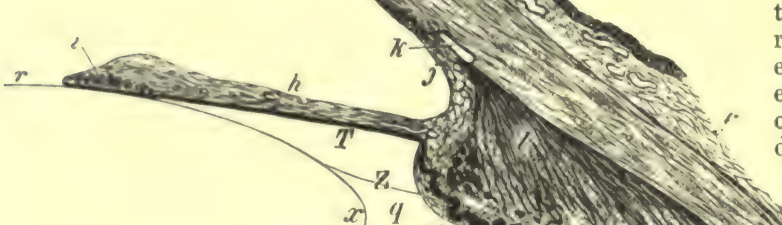
effort. On opening the eyelids aftera long period of rest, the objects at a distance are at once dis-

Anterior yualrant of a horizontal section of the eyeball, cornea, and lens. $a$, substantia propria of the cornea; $b$, Bowman's elastic membrane; $c$, anterior corneal epithelium ; $d$, Descement's membrane; $e$, its epithelium $; f$, conjunctiva ; $g$, sclerotic $; h$, iris ; $i$, sphincter iridis ; $j$, ligamentum pectinatum iridis, with the adjoining vacaolated tissue ; $k$, canal of Schleum; $l$, longitudinal, $m$, circular nuscular fibres of the ciliary muscle ; $n$, ciliary process ; $o$, ciliary part of the retina ; $q$, canal of Petit, with $Z$, zonule of Zinn in front of it ; and $p$, the posterior layer of the hyaloid membrane; $r$, anterior, $s$, posterior part of the capsule of the lens; $t$, choroid ; $u$, perichoroidal space; $\mathrm{T}$, pigment epithelium of the iris ; $x$, margin of the lens.

tinctly visille in the field of vision. (2) If, in consequence of paralysis of the mechanism of accommodation (e.(1., through paralysis of the oculomotor nerve- $-\$ 345,7)$, the eye is unable to focus images of objects placed at different distances, still distinct images are obtained of distant objects. Thus, paralysis of the mechanism of accommodation is always accompanied by inability to focus a near oljject, never a distant object. A temporary paralysis occurs with the same results when a solution of atropin or duboisin is dropped into the eye, and also in poisoning with these drugs (\$392).

When the eye is accommodated for a near object, [positive accommodation], the lens is thicker, its anterior surface is more curved (convex), and projects farther into the anterior chamber of the eye (Cramer, 1851, v. Helmholtz, 1853). The mechanism producing this result is the following :-During rest, the lens is kept somewhat flattened against the vitreous humour lying behind it, by the tension of the stretched zonule of Zinn, which is attached round the margin of the lens (fig. 533, Z). When the muscle of accommodation, the ciliary muscle $(l, m)$, contracts, it pulls forward the margin of the choroid, so that the zonule of Zinn in intimate relation with it is relaxed. [When we accommodate for a near object, the ciliary muscle contracts, pulls forward the choroid, relaxes the zonule of Zinn, and this in turn diminishes the tension of the anterior part of the capsule of the lens.] The lens assumes a more curved form, in virtue of its elasticity, so that it becomes more convex as soon as the tension of the zonule of Zinn, which keeps it flattened, is diminished (fig. 534). As the posterior surface of the lens lies in the saucer-shaped unyielding depression of the vitreous humour, the anterior surface of the lens in becoming more convex must necessarily protrude more forwards.

Nerves.-According to Hensen and Völckers, the origin of the nerves of accom- 
modation lies in the most anterior root-bundles of the oculomotorius. Stimulation of the posterior part of the floor of the third ventricle causes accommodation ; if a part lying slightly posterior to this be stimulated, contraction of the pupil occurs. On stimulating the limit between the third ventricle and the aqueduct, there results

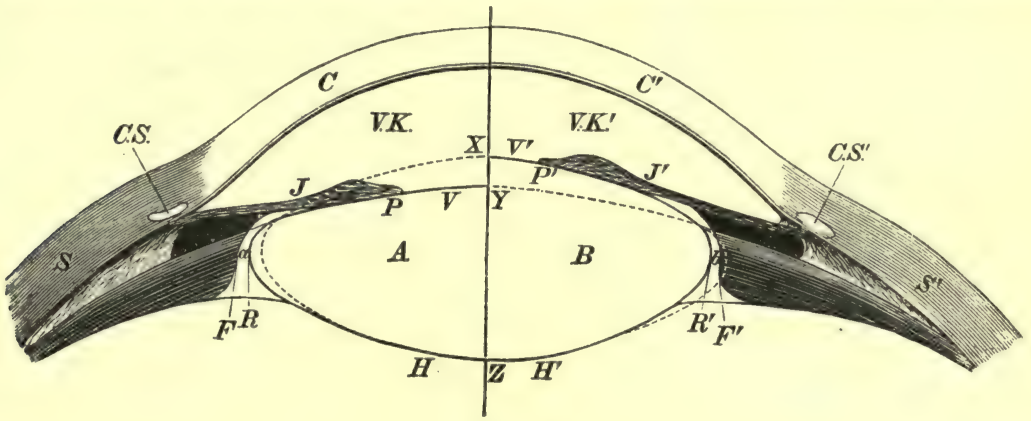

Fig. 534.

Scheme of accommodation for near and distant objects. The right side of the figure represents the condition of the lens during accommodation for a near object, and the left side when the eye is at rest. The letters indicate the same parts on both sides ; those on the right side are marked with a dash ; $A$, left, $B$, right half of the lens ; $C$, cornea ; $S$, sclerotic ; C.S., canal of Schlemm; $V . K$., anterior chamber; $J$, iris ; $P$, margin of the pupil ; $V$, anterior surface ; $H$, posterior surface of the lens; $R$, margin of the lens ; $F$, margin of the ciliary processes ; $a$ and $b$, space between the two former ; the line $Z, X$, indicates the thickness of the lens during accommodation for a near object; $Z, Y$, the thickness of the lens when the eye is passive.

contraction of the internal rectus muscle, while stimulation of the other parts around the iter causes contraction of the superior rectus, levator palpebræ, rectus inferior, and inferior oblique muscles.

Proofs. - That the lens undergoes an alteration in its curvature, luring accommodation, is proved by the following facts:-

1. Purkinje-Sanson's Images. - If a lighted candle be held at one side of the eye, or if light be allowed to fall on the eye through two triangular holes, placed above each other and cut in a piece of cardboard, in the latter case the observer will see three pairs of reflected images [in the former, three images]. The brightest and most distinct image (or pair of images) is erect and is produced by the anterior surface of the cornea (fig. $535, a)$. The second image (or pair of images) is also erect. It is the largest, but it is not so bright $(b)$, and it is reflected by the anterior surface of the lens. (The size of a reflected image from a convex mirror is greater, the longer the radius of curvature of the reflecting surface.) The latter image lies $8 \mathrm{~mm}$. behind the plane of the pupil. The third image (or pair of images) is of medium size and medium brightness-it is inverted and lies nearly in the plane of the pupil (c). The posterior capsule of the lens, which reflects the last image, acts like a concave mirror. If a luminous object be
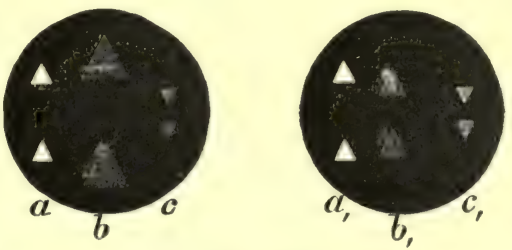

Fig. 535.

Sanson-Purkinje's images. $a, b, c$, during negative, and $\alpha_{l}, b_{l}, c_{t}$, positive accommodation.

placed at a distance from a concave mirror, its inverted, diminished, real image lies close to the focus towards the side of the object. If the images be studied when the observed eye is passive, i.e., in the phase of negative accommodation, on asking the person experimented upon to accommodate his eye for a near object, at once a change in the relative position and size of some of the images is apparent. The middle pair of images reflected by the anterior surface of the lens diminish in size and approach each other $(b)$, which depends upon the fact that the anterior surface of the lens has become more convex. At the same time, the image (or pair of images) comes nearer to the image formed by the cornea $\left(a_{1}\right.$ and $\left.c_{l}\right)$ as the anterior surface of the lens lies nearer to the cornea. The other images (or pairs of images) neither change their size nor posi- 
tion. Helmholtz, with the aid of the ophthalmometer, has measured the diminution of the ralius of curvature of the anterior surface of the lens during accommodation for a near object.

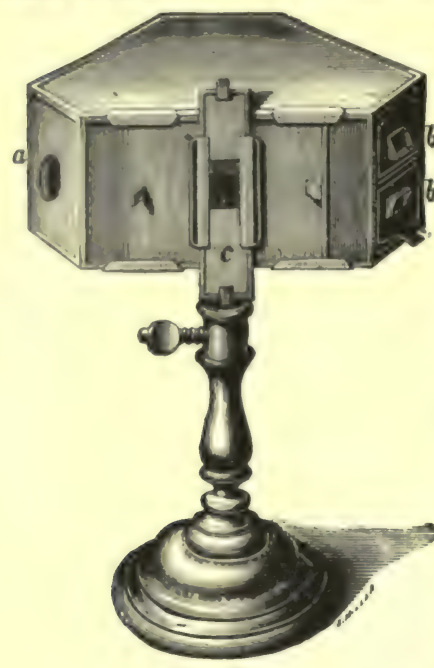

Fig. 536.

Phakoscope. - These images may be readily shown by means of the phakoscope of v. Helmholtz (fig. 536). It consists of a triangular box with its angles cut off and blackened inside. The observer's eye is placed at $a$, while on the opposite side of the box are two prisms, $b, b^{1}$; the observed eye is placed at the side of the box opposite to $\mathrm{C}$. When a candle is held in front of the prisms, $b$ and $b^{1}$, three pairs of images are seen in the observed eye. Ask the person to accommodate for a distant object, and note the position of the images. On pushing up the slide $\mathrm{C}$ with a pin attached to it, and asking him to accommodate for the pin, i.e., for a near object, the position and size of the middle images chiefly will be seen to alter as described above.]

2. In consequence of the increased curvature of the lens during accommodation for a near object, the refractive indices within the eye must undergo a change. According to $\mathrm{v}$. Helmholtz the annexed measurements obtain in negative and positive accommodation respectively.

3. Lateral View of the Pupil.-If the passive eye be looked at from the side, we observe only a small black strip of the pupil, which becomes broader as soon as the person experimented on accommodates for a near object, as the whole pupil is pushed more forwards.

4. Focal Line. - If light be admitted through the cornea into the anterior chamber, the "focal line" formed by the concave surface of the cornea falls upon the iris.

l'hakoscope of Helmholtz.

If the experiment be made upon a jerson whose eye is accommodated for a distant object, so that the line lies near the margin of the pupil, it gradually recedes towards the scleral margin

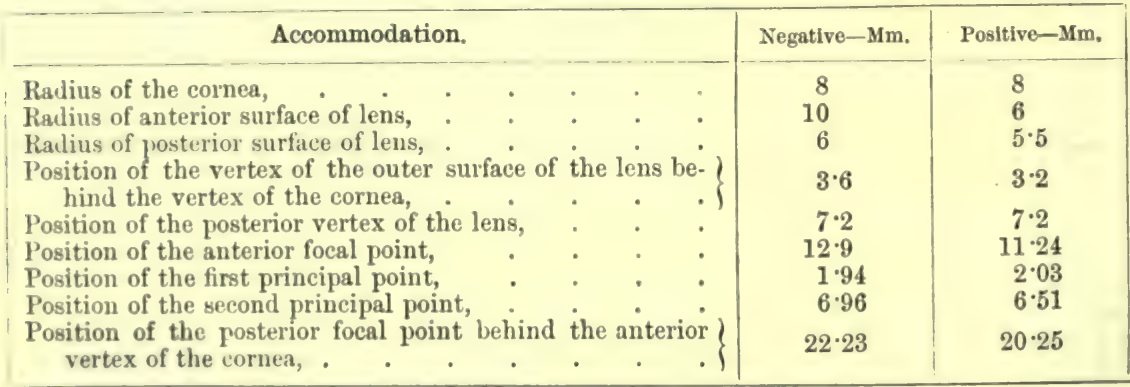

of the iris, as soon as the person accommodates for a near ubject, because the iris becomes more oblique as its inner margin is pushed forward.

5. Change in Size of Pupil. - On accommodating for a near object, the pupil contracts, while in accommodation for a distant object, it dilates (Descartes, 1637). The contraction takes place slightly after the accommodation (Donders). This phenomenon may be regarded as an associated movement, as both the ciliary muscle and the sphincter pupillæ are supplied by the oculomotorius $(\$ 345,2,3)$. A reference to fig. 533 shows that the latter also directly supports the ciliary muscle; as the inner margin of the iris passes inwards (towards $r$ ), its tension tends to be propagated to the ciliary margin of the choroid, which also must pass inwards. The riliary processes are made tense, chiefly by the ciliary muscle (tensor choroidæ). Accommodation can still be perforned, even though the iris be absent or cleft.

6. Internal Rotation of the Eye.-On rotating the eyeball inwards, accommodation for a near object is performed involuntarily. As rotation of both eyeballs inwards takes place when the axes of vision are directed to a near object, it is evident that this must be accompanied involuntarily by an accommodation of the eye for a near object.

7. Time for Accommodation.-A person can accommodate from a near to a distant object (which depends upon relaxation of the ciliary muscle) inuch more rapidly than conversely, from a distant to a near object ( $V$ icrordt, $A b b y$ ). The process of accommodation requires a longer time, the nearer the object is brought to the eye (Vierordt, Vülchers and Hensen). The time 
necessary for the image reflected from the anterior surface of the lens to change its place during accommodation, is less than that required for subjective accommodation (Aubert and Angelucci).

8. Line of Accommodation.-When the eye is placed in a certain position during accommodation, we may see not one point alone distinctly, but a whole series of points behind each other: Czermak called the line in which these points lie the line of accommodation. The more the eye is accommodated for a distant object, the longer does this line become. All objects placed at a greater distance from the eye than 60 to 70 metres appear equally distinct to the eye. The line becomes shorter the more we accommodate for a near object-i.e., when we accommodate as much as possible for a near object, a second point can only be seen indistinctly at a short distance behind the object looked at.

9. The nerves concerned in the mechanism of accommodation are referred to under Oculo. motorius ( $\$ 345$, and again in $\S 704$ ).

Scheiner's Experiment.-The experiment which bears the name of Scheiner (1619) serves to illustrate the refractive action of the lens during accommodation for a near object, as well as for a distant object. Make two small pin-holes $(\mathrm{S}, d)$ in a piece of cardboard (fig. 537, $\mathrm{K}, \mathrm{K}_{1}$ ), the holes being nearer to each other than the diameter of the pupil. On looking through these holes, $\mathrm{S}, d$, at two needles $(p$ and $r$ ) placed behind each other, then on accommodating for the near needle $(p)$, the far needle $(r)$ becomes double and inverted. On accommodating for the near needle $(p)$, of course the rays proceeding from it fall upon the retina at the focus $\left(p_{1}\right)$; while the rays coming from the far needle $(r)$ have already united and crossed in the vitreous humour, whence they diverge more and more and form two pictures $\left(r_{1}, r_{u}\right)$ on the retina. If the right hole in the cardboard $(d)$ be closed, the left picture on the retina $\left(r_{11}\right)$ of the double images of the far needle disappears. An analogous result is obtained on accommodating for the far needle (R). The near needle $(\mathrm{P})$ gives a double image $\left(\mathrm{P}, \mathrm{P}_{4}\right)$, because the rays from it have not yet come to a focus. On closing the right hole $(d)$, the right double image $(\mathrm{P})$ disappears $($ Porterfield). When the eye of the observer is accommodated for the near needle, on closing one aperture the double image of the distant point disappears on that side ;

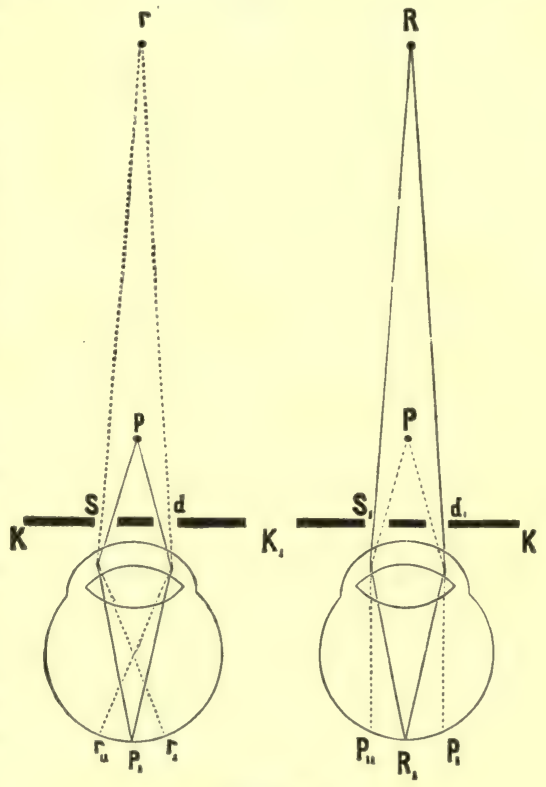

Fig. 537.

Scheiner's experiment. but if the eye is accommodated for the distant needle, on closing one hole the crossed image of the near needle disappears.

388. REFRACTIVE POWER OF THE EYE-ANOMALIES OF REFRACTION.-The limits of distinct vision vary very greatly in different eyes. We distinguish the far point [p.r., punctum remotum] and the near point [p.p., punctum proximum]; the former indicates the distance to which an object may be removed from the eye, and may still be seen distinctly ; the latter, the distance to which any object may be brought to the eye, and may still be seen distinctly. The distance between these two points is called the range of accommodatior. The types of eyeball are characterised as follows-

1. The normal or emmetropic eye is so arranged when at rest that parallel rays (fig. 538, r, r) coming from the most distant objects can be focussed on the retina 
$\left(r_{1}\right)$. The far point, therefore, is $=\infty$ (infinity). When accommodating as much as possible for a near object, whereby the convexity of the lens is increased (fig. $538, a)$, rays from a luminous point placed at a distance of 5 inches are still focussed
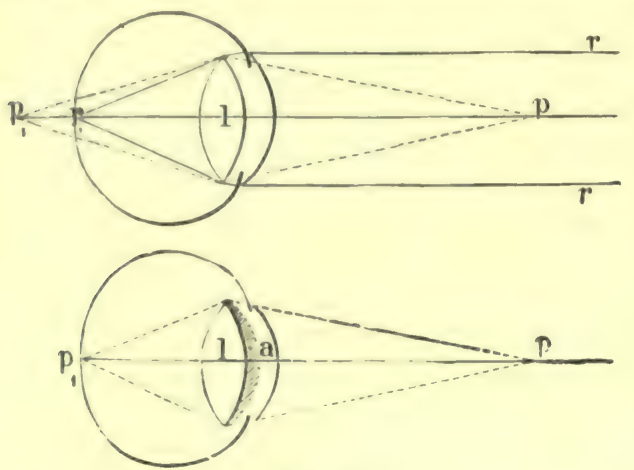

Fig. 538.

Condition of refraction in the normal passive eye and during accommodation. on the retina, i.e., the near point is $=5$ inches $(1$ inch $=27 \mathrm{~mm}$.). The range of accommodation, or ["the range of distinct vision"], therefore, is from 5 inches (10-12 $\mathrm{cm}$.) to $\infty$.

2. The short-sighted, myopic eye (or long eye) cannot, when at rest, bring parallel rays from infinity to a focus on the retina (fig. 539). These rays decussate within the vitreous humour (at $\mathrm{O}$ ), and after crossing form diffusion circles upon the retina. The object must be removed from the passive eye to a distance of 60 to 120 inches (to $f^{*}$ ), in order that the rays may be focussed on the retina. The passive myopir eye, therefore, can only focus divergent rays upon the retina. The iil. print, therefore, lies abnormally near. With an intense effort at accommodation,

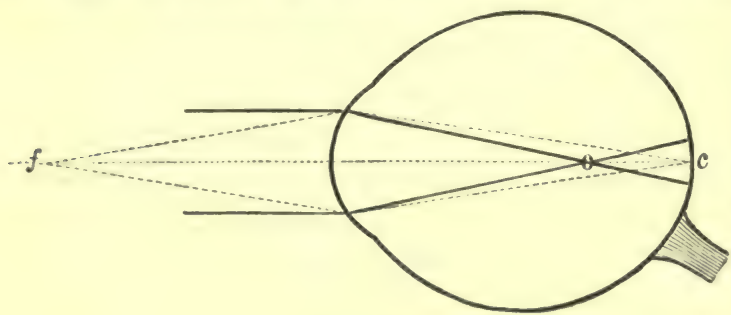

Fig. 539 .

Myopic eye. objects at a distance of 4 to 2 inches, or even less, from the eye may be seen distinctly. The near point, therefore, lies abnormally near; the range of accommodation is diminished.

Short-sightedness, or myopia, usually depends upon congenital, and frequently hereditary, elongation of the eyeball. This anomaly of the refractive media is easily corrected by using a

diverging lens (concave), which makes parallel rays divergent, so that they can then be brought to a focus on the retina. It is remarkable that most children are myopie when they are born. This myopia, however, depends upon a too-curved condition of the cornea and lens, and on the lens being ton near to the cornea. As the eye grows, this short-sightedness disappears,

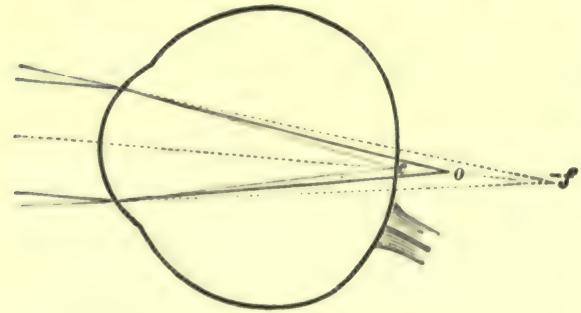

Fig. 540 .

Hypermetropie eye. The cause of myopia in children is ascribed to the continued activity of the ciliary musele in reading, writing, \&c., or the continued convergence of the eyeballs, whereby the external pressure upon the eyeball is increased.

3. The long-sighted, hypermetropic eye, hyperoptic (flat eye) when at rest, can only cause convergent rays to come to a focus on the retina (fig. 540). Distinct images can only be formed when the rays proceeding from objects are rendered convergent by means of a convex lens, as parallel rays would come to a focus behind the retina (at $f$ ). All rays proceeding from natural objects are either divergent, or at most nearly parallel, never convergent. Hence, 
a long-sighted person, when the eye is passive, i.e., is negatively accommodated, cannot see distinctly without a convex lens. When the ciliary muscle contracts, slightly convergent, parallel, and even slightly divergent rays may be focussed, according to the increasing degree of the accommodation. The far point of the eye is negative, the near point abnormally distant (over 8 to 80 inches), while the range of accommodation is infinitely great.

The cause of hypermetropia is abnormal shortness of the eye, which is generally due to imperfect development in all directions. It is corrected by using a convex lens.

[Defective Accommodation.-In the presbyopic eye, or long-sighted eye of old people, the near point is farther away than normal, but the far point is still unaffected. In such cases, the person cannot see a near object distinctly, unless it be held at a considerable distance from the eye. It is due to a defect in the mechanism of accommodation, the lens becoming somewhat flatter, less elastic, and denser with old age, while the ciliary muscle becomes weaker. In hypermetropia, on the contrary, the mechanism of accommodation may be perfect, yet from the shape of the eye the person cannot focus on his retina the rays of light from a near object. In presbyopia the range of distinct vision is diminished. The defect is remedied by weak convex glasses. The defect usually begins about forty-five years of age.]

Estimation of the Far Point-Snellen's Types. - In order to determine the far point of an eye, gradually bring nearer to the eye objects which form a visual angle of 5 minutes (e.g., Snellen's small type letters, or the medium type, 4 to 8 , of Jaeger), until they can be seen distinctly. The distance from the eye indicates the far point. In order to determine the far point of a myopic person, place at 20 inches distant from the eye the same objects which give a visual angle of 5 minutes, and ascertain the concave lens which will enable the person to see the objects distinctly. To estimate the near point, bring small objects (e.g., the finest print) nearer and nearer to the eye, until it finally becomes indistinct. The distance at which one call still see distinctly indicates the far point.

Optometer. - The optometer may also be used to determine the near and far points. A small object, e.g., a needle, is so arranged as to be movahle along a scale, along which the eye to be investigated can look as a person looks along the sight of a rifle. The needle is moved as near as possible, and then removed as far as possible, in each case as long as it is seen distinctly. The distance of the near and far point and the range of accommodation can be read otf directly upon the scale (Gräfe).

389. FORCE OF ACCOMMODATION. - Force.-The range of accommodation, which is easily determined experimentally, does not by itself determine the proper power or force of accommodation. The measure of the latter depends upon the mechanical woik done by the muscle of accommodation, or the ciliary muscle. Of course this camot be directly determined in the eye itself. Hence, this force is measured by the optical effect, which results in consequence of the change in the shape of the lens, brought about by the energy of the contracting muscle.

In the normal eye, during the passive condition, the rays coming from infinity, and therefore parallel (which are dotted in fig. 541), are focussed upon the retina at $f$. If rays coming from a distance of 5 inches (p. 756) are to be focussed, the whole available energy of the ciliary muscle must be brought into play to allow the lens to become more convex, so that the rays may be brought to a focus at $f$. The energy of accommodation, therefore, produces an optical effect in as far as it increases the convexity of the anterior surface of the passive lens (A), by the amount indicated by B. Practically, we may regard the

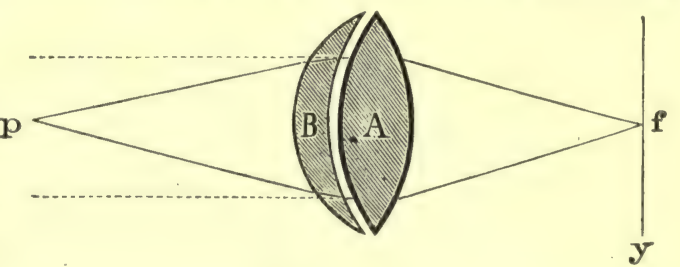

Fig. 541. matter as if a new convex lens (B) were added to the existing convex lens (A). What, therefore, must be the focal distance of the lens (B), in order that rays coming from the near point (5 inches) may be focussed upon the retina at $f$ ? Evidently the lens B must make the diverging rays coming from $p$, parallel, and then A can focus them at $f$. Convex lenses cause those rays proceeding from their focal points to pass out at the other side as parallel rays (\$385, I.). Hence, in our case, the lens must have a focal distance of 5 inches. The normal eye, therefore, with the far point $=\infty$, and the near point $=5$ inches, has a power of accommodation equal to a lens of 5 inches 
focal distance. When the lens by the energy of accommodation is rendered more powerfully refractive, the increase (B) can readily be eliminated by placing before the eye a concave lens which possesses exactly the opposite optical effect of the increase of accommodation(B). Hence, it is possible to indicate the power (force) of accommodation of the eye by a lens of a definite focal distance, i.e., by the optical effect produced by the latter. Therefore, according to Donders, the measure of the force of accommodation of the eye is the reciprocal value of the focal distance of a concave lens, which, when placed before the accommodated eye, so refracts the rays of light coming from the near point $(p)$ as if they came from the far point.

Example. - We may calculate the force of the accommodation according to the following formula : $-\frac{1}{x}=\frac{1}{p}-\frac{1}{r}, i . c$, the force of accommodation, expressed as the dioptric value of a lens (of $x$ inch focal distance), is equal to the difference of the reciprocal values of the distances of the near point $(p)$ and of the far point $(r)$ of the eye. In the cmmetropic eye, as already mentioned, $p=5, r=\infty$. Its force of accommodation is therefore $\frac{1}{x}=\frac{1}{p}-\frac{1}{8}$, so that $x=5$, i.e., it is equal to a lins of 5 inches focal distance. In a myopic eye, $p=4, r=12$, so that $\frac{1}{x}=\frac{1}{4}-\frac{1}{12}$, i.e., $x=$ 6. In another myopic eye, with $p=4$ and $r=20$, then $x=5$, which is a normal force of accommolation. Hence, it is evident that two different eyes, possessing a very different range of accommodation, may nevertheless have the same force of accommodation. Example. - The one eye has $p=4, r=\infty$, the other, $p=2, r=4$. In both cases, $\frac{1}{x}=\frac{1}{4}$, so that the force of accommodation of both eyes is equal to the dioptric value of a lens of 4 inches focal distance. Conversely, two eyes may have the same range of accommodation, and yet their force of accommodation be very unequal. Example.-The one eye has $p=3, r=6$; the other $p=6, r=9$. Both, therefore, have a range of accommodativn of 3 inches. For these, the force of accommodation, $\frac{1}{x}=\frac{1}{3}-\frac{1}{6}, x=6$; and $\frac{1}{x}=\frac{1}{6}-\frac{1}{9}, x=18$.

Relation of range to force of accommodation. - The general law is, that, the ranges of accommodation of two eyes being equally great, then their forces of accommodation are equal, proviled that their near points are the same. If the ranges of accommodation for both eyes are equally great, but their near points unequal, then the forces of accommodation are also unequal - the latter being greater in the eyes with the smallest near point. This is due to the fact that every difference of distance near a lens has a much greater effect upon the image as compared with differences in the distance far from a lens. The emmetropic eye can see distinctly oljects at 60 to 70 metres, and even to infuity, without acconmodation.

While $p$ and $r$ may be directly estimated in the emmetropic and myopic eyes, this is impossible with the hypernetropic (long-sighted) eye. The far point in the latter is negative ; indeed, in very pronouncel hypermetropia even the near point may be negative. The far point may be estimated by making the hypermetropic eye practically a normal eye by using suitable convex lenses. The relative near point may then be determined by means of the lens.

Even from the 15th year onwards, the power of accommodation is generally diminished for near objects-perhaps this is due to a diminution of the elasticity of the lens (Donders).

390. SPECTACLES. - The focal distance of concave (diverging), as well as convex (converg. ing) spectacles, depends upon the refractive inclex of the glass (usually $3: 2$ ), and on the length of the radius of curvature. If the curvature of both sides of the lens is the same (biconcave or bieonvex), then, with the ordinary refractive index of glass, the focal distance is the same as the radius of curvature. If one surface of the lens is plane, then the focal distance is twice as great as the radius of the spherical surface. Spectacles are arranged according to their focal dishance in inches, but a lens of shorter focal distance than 1 inch is generally not used. They may also be arranged according to their refractive power. In this case, the refractive power of 9 lens of 1 inch focus is taken as the unit. A lens of 2 inches focus refracts light only half as much as the unit measure of 1 inch focus; a lens of 3 inches focus refracts $\frac{1}{3}$ as strongly, \&c. This is the case both with convex and concave lenses, the latter, of course, having a negative focal distance; thus, "concave $-\frac{1}{3}$," indicates that a concave lens diverges the rays of light one-eighth as strongly as the concave lens of 1 inch (negative) focal distance.

Choice of Spectacles. - Having determined the near point in a myopic eye, of course we require to render parallel the divergent rays coming from the far point, just as if they came from infinity. This is done by selecting a concave lens of the focal distance of the far point. The greatest distance is the far point of the emmetropic eye. Suppose a myopic eye with a far point of 6 inches, then such a person requires a concave lens of 6 inches focus to enable him to see distinctly at the greatest distance. Thus, in a myopic eye, the distance of the far point from the eye is directly equal to the focus of the (weakest) concave lens, which enables one to see distinctly objects at the greatest distance. These lenses generally have the same number as the spectacles required to correct the defect. Example.,A myopic eye with a far point of 
8 inches requires a concuve lens of 8 inches focus, i.e., the concave spectacles No. 8. For the hypermetropic (long-sighted) eye, the focal distance of the strongest convex lens, which enables the hypermetropic eye to see the most distant objects distinctly, is at the same time the distance of the far point from the eye. Example. - A hypermetropic eye which can see the most distant objects with the aid of a convex lens of 12 inches focus has a far point of 12 ; the proper spectacles are convex No. 12.

[Diopter or Dioptric.-The focal length of a lens used to be expressed in inches ; and as the unit was taken as 1 inch, necessarily all weaker lenses were expresser in fractions of an inch. In the method advocated by Donders, the standard is a lens of a focal distance of 1 metre (39.370 English inches, about 40 inches), and this unit is called a dioptric. Thus, the standard is a weak lens, so that the stronger lenses are multiples of this. Hence, a lens of 2 dioptrics= one of about 20 inches focus ; 10 dioptries $=4$ inches focus ; and so on. The lenses are numbered from No. 1, i.e., 1 dioptric onwards. It is convenient to use signs instead of the words convex and concave. For convex the sign plus + is used, and for concave the sign minus - . Thus a $+4^{\circ} 0$ means a convex lens of 4 dioptries, and $a-4^{\circ} 0=a$ concave lens of $4^{\circ}$ dioptrics.]

In all cases of myopia or hypermetropia, the person ought to wear the proper spectacles. In a myopic eye, when the far point is still more than 5 inches, the patient ought always to wear spectacles ; but generally the working distance, e.g., for reading, writing, and for handicrafts, is about 12 inches from the eye. If the person desires to do finer work (etehing, drawing), requiring the object to be brought nearer to the eye, so as to obtain a larger image upon the retina, then he should either remove the spectacles altogether or use a weaker pair.

The hypermetropic person ought to wear his convex spectacles when looking at a near olject, and especially when the illumination is feeble, because then, owing to the dilatation of the pupil, the diffusion circles of the eye tend to become very pronounced. It is advisable to wear at first convex spectacles, which are slightly too strong. Cylindrical lenses are referred to under Astigmatisin. Spectacles provided with dull-coloured or blue glasses are used to protect the retina when the light is too intense. Stenopaic spectacles are narrow diaphragms placed in the front of the eye, which cause it to move in a definite direction in order to see through the opening of the diaphragm.

391. CHROMATIC AND SPHERICAL ABERRATION, ASTIGMATISM. -Chromatic Aberration.-All the rays of white light, which undergo refraction, are at the same time broken up by dispersion into a bundle of rays which, when they are received on a screen, form a spectrum. This is due to the fact that the different colours of the spectrum possess different degrees of refrangibility. The violet rays are refracted most strongly; the red rays least.

A white point on a black ground does not form a sharp simple image on the retina, but many coloured points appear after each other. If the eye is accommodated so strongly as to focus the violet rays to a sharp image, then all the other colours must form concentric diffusion circles, which become larger towards the red. In the centre of all the circles, where all the colours of the spectrum are superposed, a white point is produced by their mixture, while around it are placed the coloured circles. The distance of the focus of the red rays from that of the violet in the eye $=0.58$ to $0.62 \mathrm{~mm}$. The focal distance for red is, according to v. Helmholtz, for the reduced eye, $20.524 \mathrm{~mm}$.; for violet, $20.140 \mathrm{~mm}$. Thus, the near and far points for violet light are nearer each other than in the case of red light; white objects, therefore, appear reddish when beyond the far point, but when nearer than the near point they are violet. Hence, the eye must accommodate more strongly for red rays than for violet, so that we judge red objects to be nearer us than violet objects placed at an equal distance (Brücke).

Monochromatic, or Spherical Aberration.-Apart from the decomposition or dispersion of white light into its components - the rays of white light, proceeding from a point if transmitted through refractive spherical surfaces - we find that, before the rays are again brought to a focus, the marginal rays are more strongly refracted than those passing through the central parts of the lens. Hence, there is not one focus but many. In the eye this defect is naturally corrected by the iris, which, acting as a diaphragm, cuts off the marginal rays (fig. 531), especially when the lens is most convex, when the pupil also is most contracted. In addition, the margin of the lens has less refractive power than the central substance ; lastly, the margins of the refractive spherical surfaces of the eye are less curved towards their margins than the parts lying nearer to the optical axis. Compare the form of the cornea (p. 733) and the lens (p. 740).

Imperfect Centring of the Refractive Surfaces.--The sharp projection of an image is somewhat interfered with by the fact that the refractive surfaces are not exactly centred (Brïcke). Thus, the vertex of the cornea is not exactly in the termination of the optic axis ; the vertices of both surfaces of the lens, and even the different layers of the lens itself, are not exactly in the optic axis. The variations, however, and the disturbances produced thereby are very small indeed. 
Regular Astigmatism. - When the curvature of the refractive surfaces of the eye is unequally great in its different merilians, of course the rays of light cannot he united or focussed in one noint. Generally, in such eases, the cornea is more curved in its rertical meridian and least in the horizontal (as is shown by ophthalmometric measurements, p. 748). The rays passing through the vertical meridian come to a focus, first, in a horizontal focal line; while the rays entering horizontally unite afterwards in a vertical line. There is thus no common focus for the light rays in the eye ; hence the name "astigmatism." The lens also is unequally curved in its merilians, but it is the reverse of the cornea; consequently, a part of the inequality of the curvature of the cornea is thereby compensated, and only a part of it affects the rays of light. The emmetropic eye has a viy sliyht degree of this inequality (normal astigmatism). If $t w n$ very tine lines of equal thickness be drawn on white paper, so as to intersect each other at right angles, it will be found that, in order to see the horizontal line quite sharply, the paper must ie brought slightly nearer to the eye, than when we focus the vertical line. When the inefuality of curvature of the meridians is considerable, of course exact vision is no longer irossible.

[Fig. 542 shows the effect of an astigmatic surface on the rays of light. Let $a b c d$ be such a surfiue, and suplose diverging rays to proceed from $t$. The rays passing through $c d$ come to

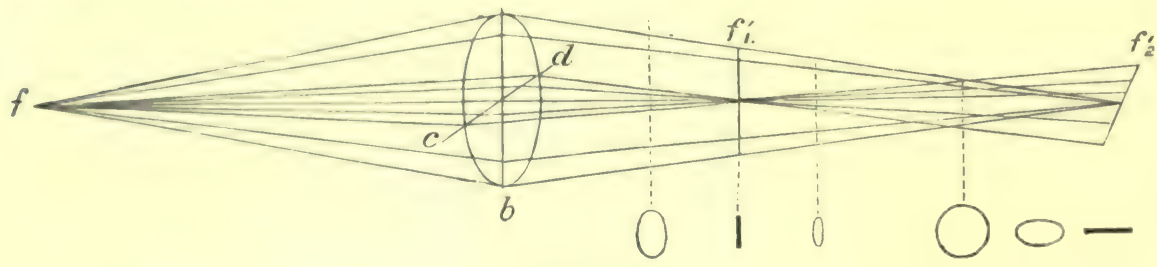

Fig. 542 .

Action of an astigmatic surface on a cone of light (Frost).

a fw.us at $f_{1}$, while thos passing through the vertical meridian are focussed at $f_{y}$ The outline of the cone of rays between $a b c d$ and $f_{2}$ varies, as shown in the figure. At a certain part it is oval, with its axis rertical, at another the long axis of the oval is horizontal, while at other places it is circular, or the rays are focussed in a horizontal or vertical line.]

Correction. - This condition is corrected by a cylindrical lens, i.e., a lens so cut as to be without (urvature in one direction, while in the other direction (vertical to the former) it is curved. The lens is placed in front of the eye, so that the direction of its curvature coincides with the direction of least curvature of the eye ( $v$. Helmholt $\approx$, Knapp, Donders).

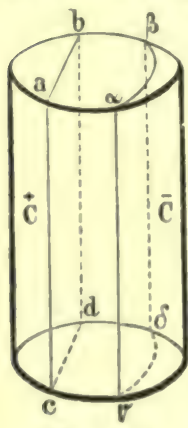

Fig. 543.

Cylindrical

glasses for a stigmatism. The section $\mathrm{C} a b c d$ of the cylindrical lens (fig. 543) represents a plano-convex, the section $\mathrm{C} \alpha \boldsymbol{\beta} \gamma \delta$, a concavo-convex lens.

[Test. - Draw two lines of equal thickness at right angles to each other. An astigmatic person camnot see both lines with equal distinctness at the same time, one line will appear thicker than the other. Or, a series of lines radiating from a centre may be used (astigmatic clock) when that line which is parallel to the astigmatic merilian will be seen most distinctly; while, with the vertical meridian most curved, it would be the vertical line.]

Irregular Astigmatism.--Owing to the radiate arrangement of the fibres in the interior of the crystalline lens, and in consequence of the unequal course of the fibres within the different parts of one and the same meridian of the lens, the rays of light passing through one meridian of the lens, cannot all be brought to one focus. Hence, we do not obtain a distinct sharp image of distant luminous points, such as stars or street lamps, but we see a radiate jaggeel figure provided with rays. The same obtains on holding a piece of cardboard with a small hole in it towards the light, at a distance from the eye slightly greater than the far point. Slight degrees of this irregular astigmatism are normal, but when it is highly developed it greatly interferes with vision, by forming screial foci of an object instead of one (Polyopia monocnlaris). Of course this condition cannot obtain in an eye devoid of a lens.

392. IRIS.-Functions.-1. The iris acts like a diaphragm in an optical apparatus by cutting off the marginal rays, which, if they entered the eye, would cause spherical alverration, and thus produce indistinct vision (fig. 531).

2. As the pupil contracts strongly in a bright light, and dilates when the light is feeble, it regulates the amount of light entering the eye; thus, fewer rays enter the eye when the light is strong than when it is feeble. 
3. To a certain extent it supports the action of the ciliary muscle.

Muscles and Nerves.-The iris is usually described as being provided with two sets of muscular fibres-the sphincter, which immediately surrounds the pupil and is supplied by the oculomotorius $(\S 345,2)$, and the dilator pupillæ (p. 736), supplied chiefly by the cervical sympathetic ( $\$ 356, \mathrm{~A}, 1)$, and the trigeminus $(\S 347,3)$. Both muscles stand in an antagonistic relation to each other $(\S 345)$, the pupil dilates moderately after section or paralysis of the oculomotorius, owing to the contraction of the dilator fibres which are supplied by the cervical sympathetic; conversely, the pupil contracts when the sympathetic is divided or extirpated (Petit, 1727). When both nerves are stimulated simultaneously, the pupil contracts, so that the excitability of the oculomotorius overcomes the sympathetic.

[The existence of a dilator pupillæ muscle is not universally recognised, and in fact some observers doubt its existence. The muscular nature of the radial fibres in the posterior limiting membrane of the iris is denied by Grïnhagen, while Koganei regards these as in no case muscular, and the dilating fibres as represented by fibres radiating from the iris. These fibres are well-developed in birds and the otter, exist in traces in the rabbit, and are absent in man. Gaskell points out that in this case the size of the pupil must in part depend on the elasticity of the radial fibres of the iris, while the dilator nerve-fibres must act on the sphincter fibres, causing them to relax. Gaskell groups the sphincter of the iris with those muscles "supplied by two nerves of opposite character, the one motor, the other inhibitory." The dilatation of the pupil caused by stimulation of the cervical sympathetic is usually explained by the hypothesis that this nerve contains motor fibres, which act on the dilator fibres. Griinhagen thought that it might be due chiefly to the constriction of the blood-vessels of the iris : Gaskell suggests that the nerve acts on the sphincter muscle, and is the inhibitory 1rerve of that muscle, dilatation taking place because the sphincter is normally in a condition of tonic contraction, and also because the posterior limiting membrane is elastic.]

Nerves, - Arnstein and A. Meyer have studied the mode of termination of the nerve-fibres in the iris. All the medullated nerve-fibres lose their white sheaths after a time; most of the fibres (motor) in the region of the sphincter consist of naked bundles of fibrils. A network of very delicate sensory nerves lies under the anterior epithelium. Numerous fibrils pass to the capillaries and arteries as raso-motor nerves. [Many ganglionic cells are intercalated in the course of the fibres.]

Movements of the iris occur under the following conditions :-

1. Action of light on the retina causes, (according to its intensity and amount), a corresponding contraction of the pupil ; the same effect is produced by stimulation of the optic nerve itself (Herbert Mayo, +1852 ). This movement is a reflex act, [the afferent nerve being the optic and the efferent the oculomotorius; the impulse is transferred from the former to the latter in a centre situated somewhere below the corpora quadrigemina (fig. 544, C)]. The older observers locate the centre in the corpora quadrigemina, the recent observers in the medulla oblongata (p. 660). Both pupils always react, although only one retina be stimulated ; generally under normal circumstances both contract to the same extent (Donders), owing to the intercentral communication [coupling] of the two pupillo-constricting centres. [This is called consensual contraction of the pupil.] After section of the optic nerve the pupil dilates, and subsequent section of the oculomotorius no longer produces any further dilatation (Knoll).

2. The centre for the dilator fibres of the pupil (pupillo-dilating centre) is excited by dyspnoic blood $(\$ 367,8)$. If the dyspnœa ultimately passes into asphyxia, the dilatation of the pupil diminishes. Of course, if the peripheral dilating fibres $(\S 247,3)\left[e_{.} g\right.$. , the sympathetic nerve in the neck] be previously divided, this effect cannot take place, as the dyspnœic blood acts on the centre and not on the nerve-fibres.

3. The centre, as well as the subordinate "cilio-spinal region" of the spinal cord $(\S 362,1)$, is also capable of being excited reflexly ; painful stimulation of sensory nerves, in addition to causing protrusion of the eyeballs $(\S 347)$, a fact proved in the case of persons subjected to torture, produces dilatation of the pupils (Arndt, Bernard, Westphal, Luchsinger); while a similar effect is caused by labour pains, a loud call in the ear, stimulation of the nerves of the sexual organs, and even by slight tactile impressions (Fod and Schiff). According to Bechterew, the foregoing results are due to inhibition of the light-reflex in the sense expressed in $\$ 361,3$.

4. The condition of the blood-vessels of the iris influences the size of the pupil; all conditions causing injection or congestion of these vessels contract the pupil, all conditions diminishing them dilate it. The pupil, therefore, is contracted by forced expirution, which prevents the return of venous blood from the head, momentarily by every pulse-beat, owing to the diastolic filling of the arteries; diminution of the intraocular pressure, e.g., after puncture of the anterior 
chamber, because, owing to the diminished intraocular pressure, there is less resistance to the passage of blood into the blool-vessels of the iris (Hensen and Völckers); paralysis of the vasomotor fibres of the iris $(\$ 347,2)$. Conversely, the pupil is dilated by conditions the reverse of those already mentioneil, and also by strong muscular evertion, whereby blood flows freely into the dilated inuseular blood-vessels; also, when death takes place. The condition of the filling of the blood-vessels also explains the fact, that the pupil dilated with atropin becomes smaller when a part of the sympathetic in the upper cervical ganglion, carrying the vaso-motor fibres of the iris, is excised : also, that after extirpation of this ganglion, atropin always causes a less diminution of the pupil on this side. The fact that when the pupil is already dilated by stimulation of the sympathetic, it is further dilated by atropin, is due to a diminished injection of the hlool-vessels of the iris. If an animal with its pupils dilated with atropin be rapidly h.mi, the fupils contract, owing to the antmic stimulation of the origin of the oculomotorius (.). The diutation of the pupils observed in cases of neuralgia of the trigeminus, is partly ilue to the stimulation of the dilating fibres, partly to the stimulation of the vaso-motor fibres of the iris $(\$ 347,2)$.

5. Contraction of the pupil occurs as an associated movement, luring accommodation for a near oljewt (1. 752,5), and when the cyctulls are rotated inuards, which is the case during siof [1. 65:5!. Conversely, intense movements of the iris, caused by variations in the brightness of lazzling illumination, e.\%, of the electrie light, are followed by disturbing associated movenuents of the ciliary muscle (Ljulinsky). In eertain movements discharged from the medulla ol,longata (forced respiration, chewing, swallowing, vomiting), dilatation of the pupil oceurs as a kind of associated movement.

[Argyll Robertson Pupil. - In this condition the pupil does not contract to light, although it contrats when the eye is accommolated for a near olject, vision usually being normal. The

\section{A}

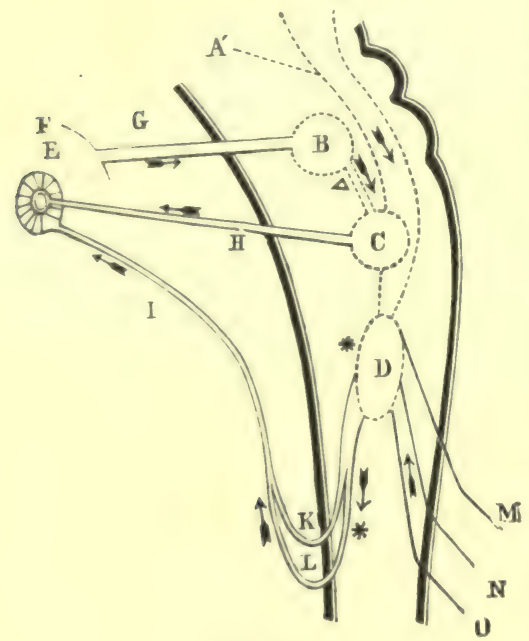

Fig. 544 . lesion is situated in those structures connecting the afferent and efferent fibres at their central ends (at $\Delta$ in fig. 544), i.e., the connection between the corpora quadrigemina and the oculomotorius. It is most frequently found in locomotor ataxia, although it also occurs in progressive paralysis of the insane.]

Direct stimulation at the margin of the cornea causes dilatation of the pupil $(\boldsymbol{E} . H$. Weber $)$; in fact, direct stimulation of circumscribed areas of the margin of the iris causes partial contraction of the dilator fibres (Bernstein and Dogiel). Stimulation near the centre of the cornea contracts the pupil (E. H. Weber). In addition, we must assume that the iris itself contains elements that influence the diameter of the pupil (Sig. Mayer and Pribram).

Our knowledge of the action of poisons on the iris is still very obscure. Those substances which dilate the pupil are called mydriatics, e.g., atropin, homatropin, duboisin, daturin, and hyoscyamin. They act chiefly by paralysing the oculomotorius. But, in addition, there must be also an effect upon the dilating fibres, for after complete paralysis (section) of the oculomotorins, the moderate dilatation of the pupil thereby Nicheme of the nerves of the iris. B, centrum produced $(\$ 345,5)$ is still further increased by optici ; C, oculomotor centre; D, dilator centre (spinal); E, iris; G, optic nerve; $H$, oculomotor' (sphincter) roots; I, sym. pathetic (dilator); $\mathrm{k}, \mathrm{L}$, anterior roots; $\mathrm{M}, \mathrm{N}, \mathrm{O}$, posterior roots; $\Delta$, seat of lesion, causing pupillary immobility; * probable seat of lesion, causing myosis. pupil, owing to stimulation of the pupilloconstrictor fibres; cnormous doses cause moderate dilatation of the pupil in consequence of paralysis of the dilating as well as of the constricting nerve-fibres. Atropin acts after destruction of the ciliary [ophthalmic] ganglion (Hensen and $V$ ïlckers) [and division of all the nerves except the optic], and in the excised eye (De Ruyter), [so that atropin is a local mydriatic. In moderate doses it paralyses the nervous terminations of the 3rd nerve (but not in birds whose iris contains striped muscle), and in larger doses it also paralyses the muscular fibres]. [Cocaine, or cucaine, is obtained from the leaves of Erythroxylon coca. When applied locally it acts as a powerful local anesthetic, and hence it is very useful for operations about the muco-cutaneous orifices. A 4 per cent. solution dropped into the eye produces complete insensibility of the cornea in a few minutes. It causes dilatation of the pupils, though they react to light and to the movements of accommodation. It also causes teniporary paralysis of 
accommodation, a sensation of heaviness and coldness of the eyeball, enlargement of the palpebral fissure, constriction of the small peripheral vessels, and slight lachrymation.]

Myotics are those substances which contract the pupil :-Physostigmin (=Eserin, the alka. loid of Calabar bean), nicotin, pilocarpin, muscarin, morphia, according to some observers (Grünhagen) cause stimulation of the oculomotorius, while others say they paralyse the sympathetic. As these substances cause spasm of the ciliary muscle, it is supposed that the first of these has an analogous action on the sphincter. It is probable that they paralyse the dilator fibres and stimulate the oculomotor fibres. [Amongst local myotics, i.e., those which act on the eye, some act on the muscular fibres of the iris, e.g., physostigmin or eserin, while others act on the peripheral terminations of the 3rd nerve, e.g., pilocarpin, muscarin. Muscarin causes very great contraction of the pupil from spasm of the circular fibres, due to its action on the 3rd nerve; eserin, on the other hand, although contracting the pupil, also affects the dilator fibres. The contraction of the pupil due to opium is central in its cause.]

If the one pupil be contracted or dilated by these substances, the other pupil, conversely, is dilated or contracted, owing to the change in the amount of light admitted into the eye into which the poison has been introduced. The anæsthetics (ether, chloroform, alcohol, \&c.), when they begin to cause stupor, contract the pupil, and when their action is intense they dilate it (Dogiel). Chloroform, during the stage when it causes excitement (preceding the narcosis), stimulates the centre for the dilatation of the pupil; after a time this centre is paralysed, so that the pupil no longer dilates on the application of external stimuli. Thereafter the pupilloconstrictor centre is stimulated, whereby the pupil may be contracted to the size of a pin's head ; ultimately this centre is paralysed, and the pupil becomes dilated.

Time for Movements of Iris. - The reflex dilatation of the pupil oscurs slightly later than the reflex contraction, the time in the two cases being 0.5 and 0.3 second respectively, after stimulation by light ( $v$. Vintschgau). A certain time always elapses, until the iris, corresponding to the strength of the stimulus of light exciting the retina, "adapts" itself to produce a suitable size of the pupil (Aubert). Contraction of the pupil occurs very rapidly after stimulation of the oculomotorius in birds; in rabbits 0.89 second elapses after stimulation of the sympathetic, until the dilatation begins ( $A r l t)$.

Excised Eye. - Light causes contraction of the pupil in the excised eye of amphibians and fishes (Amold). Even the iris of the eel, when cut out and placed in normal saline solution, contracts to light (Arnold), the green and blue rays being most active. Increase of the temperature causes mydriasis in the excised eye of the frog or eel, while cooling causes myosis $(\boldsymbol{H}$. Müller).

[Size of the Pupil. - Jonathan Hutchinson recommends a pupilometer, consisting of a metal plate perforated with a series of holes of different sizes. The smallest hole measures about $\frac{1}{3}$ of a line, and the largest is $4 \frac{1}{2}$ lines. The plate is placed just below the patient's eye, and the hole is selected which corresponds with the size of the pupil.]

[Gorham's Pupil Photometer.-This ingenious instrument may be used as a pupilometer, and also as a photometer. It consists of a piece of bronzed tubing $1.9 \mathrm{in}$. long and $1.5 \mathrm{in}$. diameter (figs. 545 and 546). One end is closed by a disc or cap, which is pierced in its radii by

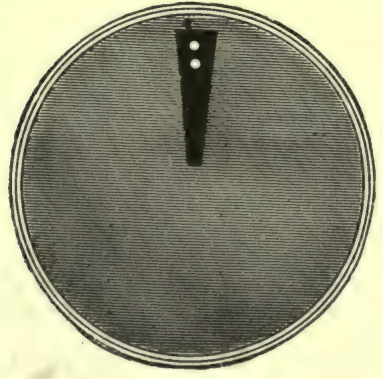

Fig. 545.

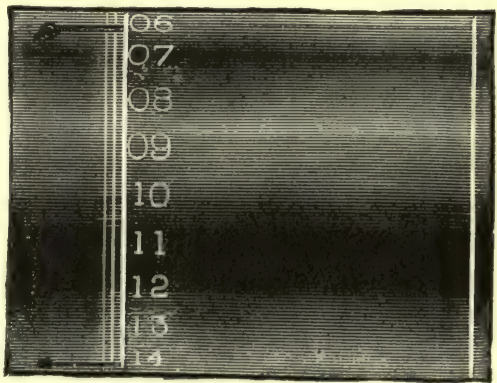

Fig. 546.

Gorham's pupil photometer. Fig. 545 shows the disc with a slot and two holes. Fig. 546 gives a side view with the diameter of the pupil marked on it. The upper end is closed by the disc, while the lower end is open.

a series of holes at distances varying from $05 \mathrm{in}$. to $28 \mathrm{in}$. There is a slot in the cap which allows one pair of holes to be visible at a time, while on the cylinder is engraved the linear distance of each pair of holes. In using the instrument as a pupilometer, look through the open end of the tube (the bottom in fig. 546), with both eyes open, towards a sheet of white paper or the sky, when two dics of light will be seen. Then revolve the lid or cap slowly until the two white dics just touch one another at their edges. The decimal fraction opposite the two 
apertures seen on the scale outside indicates the diameter of the pupil in 100ths of an inch. When using it as a photometer, it is assumed that the size of the pupil gives an index of the intensity of the amount of light which influences the diameter of the pupil.]

Intraocular Pressure. - The movements of the iris are always accompanied by variations of the intracular pressure. The muscles of the iris affect the intraocular pressure, in that the dilatation of the pupil increases it, while contraction of the pupil diminishes it. The increased or diminishel tension can be felt when two fingers are pressed on the eyeball. Stimulation of the ssmuathetic increases, while its section diminishes the pressure. Action of Drugs. - Atropin dropinel into the eye, after producing a short temporary diminution of the tension, increases it ; eserin, after a prinary increase, causes a diminution of the pressure (Graser and Hölzke).

393. ENTOPTICAL PHENOMENA-Entoptical phenomena depend upon the perception of objects present within the eyeball itself.

1. Shadows are formed upon the retina by different opraque bodies. In order to see them in on's own eye, froceed thus :-By means of a strong convex lens project a small image of a flame IIjn a paper screen, prick a small oprening through the image of the flame, and place one eye at the other side of the screen, so that the illuminated puncture lies in the anterior focus of the we, $i .$, , about $13 \mathrm{~mm}$, in front of the cornea. As the rays proceeding from this point pass jarallel through the media of the eye, a diffuse bright field of vision, surrounded by the black margins of the iris, is obtained. All dark boclies which lie in the course of the rays of light

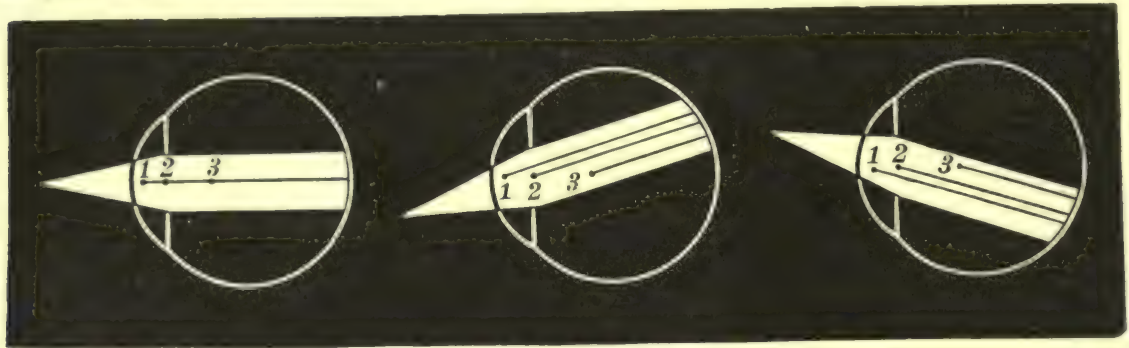

Fig. 547 .

Entoptical Shadows.

throw a shadow upon the retina, and appear as specks. There are various forms of these shadows (fig. 547):-

(a) The spectrum mucro-lacrimale, especially upon the margin of the eyelids, depending ujon prarticles of mucus, fat globules from the Meibomian glands, dust mixed with tears, causing cloudy or drop-like retinal shadows, which are removed by winking.

(b) Folds in the cornea. - If the cornea be pressed laterally with the finger, wrinkled shadows, due to temporary wrinkles in the cornea, are produced.

(c) Lens' shadows. - Bead-like or dark specks, bright and star-like figures, the former due to deposits on and in the lens, the latter to the radiate structure of the lens.

(d) Muscæ volitantes (1)cchales, 1690), like strings of beads, circles, groups of balls or pale stripes, depend upon opaque particles (cells, disintegrating cells, granular fibres) in the vitreous humour. They move about when the eye is moved rapidly. Listing (1845) showed that one may determine pretty accurately the position of these objects. Whilst making the observation upon one's own eyes, raise or uepress the source of light; those shadows which are caused by bodies on a level with the pupil retain their relative positions in the bright fields of vision. Shadows which appear to move in the same direction as the source of light are caused by bodies which lie in front of the plane of the pupil-those, however, which appear to move in the opposite direction depend upon objects behind the plane of the pupil.

2. Purkinje's figtre (1819) depends upon the blood-vessels within the retina, which cast a shadow upon the most external layer of the retina, viz, upon the rods and cones, these being the parts acted upon by light. In ordinary vision we do not observe these shadows. According to v. Helmholtz, this is due to the fact that the sensibility of the shaded parts of the retina is greater, and their excitability is less exhausted, than all the other parts of the retina. As soon, however, as we change the position of the shadow of the blood-vessels, instead of being directly behind, so that the blood-vessels come to lie more laterally and behind them, i.e., upon places which do not receive shadows from the blood-vessels when the rays of light pass through the eye in the ordinary way, then the figure of the blood-vessels becomes apparent at once. All that is necessary is to cause the light to enter the eyeball obliquely. Method. - (1) This may be done by passing an intense light through the sclerotic, e.g., by throwing upon the sclerotic a small, bright, luminous image from a source of light. On moving the source of light, the 
figure of the blood-vessels moves in the same direction. (2) Look directly upwards to the sky, wink with the upper eyelid drooping, so that for a moment, corresponding to the act of winking, rays of light enter obliquely the lowest vart of the pupils. (3) Look through a small aperture towards a bright sky, and move the aperture rapidly to and fro, so that from both sides of the blood-vessels shadows fall rapidly upon the nearest series of rods and cones. (4) In a darkened room look straight ahead, and move a light to and fro close under the eyes. Occasionally, whilst performing this experiment, one may see the macula lutea as a non-vascular shaded depression, and, owing to the inversion of the objects, it lies on the inner side of the entrance of the optic nerve.

3. Movements of the blood-corpuscles in the retinal capillaries.--On looking, without accommodating the eye, towards a large bright surface, or through a dark blue glass towards the sun, we see bright spots, like points, forming longer or shorter chains, moving in tortuous paths. The phenomenon is, perhaps, caused by the red blood-corpuscles (in the capillaries posterior to the external granular layer) acting as small light-collecting concave dises, concentrating the light falling upon them from bright surfaces, and throwing it upon the rods of the retina. Each corpuscle must be in a special position; should it rotate, the phenomenon disappears. Vierordt, who projected the movement upon a screen, calculated, from the velocity of their motion, the velocity of the blood-stream in the retinal capillaries as equal to 0.5 to $0.75 \mathrm{~mm}$. in a second, which corresponds very closely with the results obtained directly in other capillaries by E. H. Weber and Volkmann $(\S 90,4)$. When the carotids are compressed, the movement is slower on freeing them from the compression; during short forced expirations the movement is accelerated (Landois).

4. The entoptical pulse $(\S 79,2)$ depends upon the pulsating arteries irritating mechanically the rods lying outside them.

5. Pressure Phosphenes.-Pressure applied to the eye canses a series of phenomena:- $(\alpha)$ Partial pressure upon the eyeball causes the so-called illuminated "pressure-picture" or phosphene, which was known to Aristotle. As the impression upon the retina is referred to something outside the eye, the phosphene is always perceived on the side of the field of vision opposite to where the pressure, affects the retina, e.g., pressure upon the outer surface of the eyeball causes the flash of light to appear on the inner side. If the retina is not well lighted, the phosphene appears luminous; if the retina is well lighted, it appears as a dark speck, within which the visual perception is momentarily abolished. (b) If a uniform pressure be applied to the eyeball continuously from before backwards, as Purkinje pointed out, after some time there appear in the field of vision very sparkling variable figures, which perform a wonderful fantastic play, and often resemble the sparkling effects obtained in a kaleidoscope $(v$. Helmholtz), and are probably comparable to the feeling of formication produced by pressure upon sensory nerves ("sleeping of the limbs"). (c) By applying equable and continued pressure, Steinbach and Purkinje observed a network with moving contents of a bluish-silvery colour, which seemed to correspond to the retinal voins. Vierordt and Laiblin observed the branching of the blood-vessels of the choroid as a red network upon a black ground. (d) According to Houdin, we may detect the position of the yellow spot by pressure upon the eyeball.

6. The entrance of the optic nerve may be detected on moving the eyes rapidly backwards, and especially inwards, as a fiery ring or semicircle about the size of a pea. Probably, owing to the movement of the retina, the entrance of the optic nerve is stimulated mechanically by the rapid bending. Purkinje and others observed that the ring remained persistent on turning the eye strongly inwards. If the retina be brightly illuminated, the ring appears dark, and when the freld of vision is coloured, the ring has a different tint. If Purkinje's figure be produced at the same time, one may observe that the vascular trunk proceeds from this ring-a proof that the ring corresponds to the entrance of the optic nerve (Landois).

7. Accommodation Spot.-On accommodating the eye strongly towards a white surface, there appears in the middle a small, bright, trembling shimmer, and in its centre a coarse brown speck, about the size of a pea, is seen (Purkinje). If pressure be applied externally to the eyeball, this speck becomes more distinct. After having once observed the phenomenon, occasionally on pressing laterally upon the opened eye we may see it as a bright speck in the field of vision-another proof that the intraocular pressure is increased during accommodation.

8. Mechanical Optical Stimulation.-On dividing the optic nerve in man, as in extirpation of the eyeball, a flash of light is observed at the moment of section by the person operated on. The section of the nerve-fibres themselves is painless, but section of the sheaths is painful.

9. The accommodation phosphene is the occurrence of a fiery ring at the periphery of the field of vision, seen on suddenly bringing the eyes to rest after accommodating for a long time in the dark (Purkinje). The sudden tension of the zonule of Zinn resulting from the relaxation causes a mechanical stretching of the outermost part of the margin of the retina, or it may be of a part of the retina behind this. Purkinje observed the phenomenon after suddenly relaxing the pressure on the eye.

10. Electrical Phenomena.-Electrical currents, when applied to the eye, cause a strong flash of light over the whole field of vision. One pole of the battery may be placed on the under eyelid and the other on the neck. The flash at closing [making] the current is strongest 
with an ascending current, that with opening [breaking] the current with a descending current. If a uniform continuous ascending current be transmitted through the closed eyes, the dark dise of the elevation at the entrance of the optic nerve appears in a whitish-violet field of vision; with a elescending current, the field of vision is reddish and dark, in which the position of the optic nerve appears light blue ( $v$. Helmholtz). If external colours are looked at simultaneously, these colours blenil to form a violet or yellow with the colours looked at (Schelske). During the passage of the ascending current we see external objects indistinctly and smaller when the eves are open ; while with the descending current they are larger and more distinct (Ritter). Simetimes the position of the macula lutea appears dark on a bright ground, or the reverse, areording to the direction of the current. If the current be opened [broken] the phenomena are reversed $(\$ 335)$, and the eye soon returns to rest.

11. The yellow spot appears sometimes as a dark circle when there is a uniform blue illumimation. In a strong light the position of the yellow spot is surrounded by a bright area, twice ur thrice as large, called "Löwe's ring." [Clerk-Maxwell's Experiment.-On looking through a solution of chrome-alum in a bottle or ressel with parallel glass sides, we observe an oval purplish spot in the greenish colour of the alum. 'This is due to the pigment of the yellow spot.]

Haidinger's Brushes. - On directing the eye towards a souree of polarised light, "Haidinger's polariserl brushes "appear at the point of fixation. They are seen on looking through a Nicol's frism at a bright cloul ( $v$. Helmholtz). They are bright and bluish on a surface, bounded by two neighbouring hyperbola on a white field; the dark hundle separating them is smallest in the centre and yellow. Of the various colours of homogeneous light, blue alone shows the brushes (Stokics). Accorling to v. Helmholtz the seat of the phenomenon is the yellow spot, and is tue to the yellow-coloured elements of the yellow spot being slightly doubly refractive, while at one part they absorb more, at another less, of the rays entering the eye.

12. Lastly, there are the visual sensations depending on internal causes, e.g., increased bounding of the bloot through the retina, as during violent coughing, increased intraocular pressure. Stimulation of the risual areas $(\$ 378, I V$.) may produce spectra, which Cardanus (1550), Goethe, Nicolai, and Johannes Miiller could produce voluntarily.

394. ILLUMINATION OF THE EYE.-OPHTHALMOSCOPE.-The light which enters the eye is partly absorbed by the black uveal pigment, and partly agrain reflected from the eye, and always in the same direction in which the rays entered the eye. By placing oneself in front of the eye of another person, of course the head, being an opaque body, cuts off a large number of rays. Owing to the position of the head, no rays of light can enter

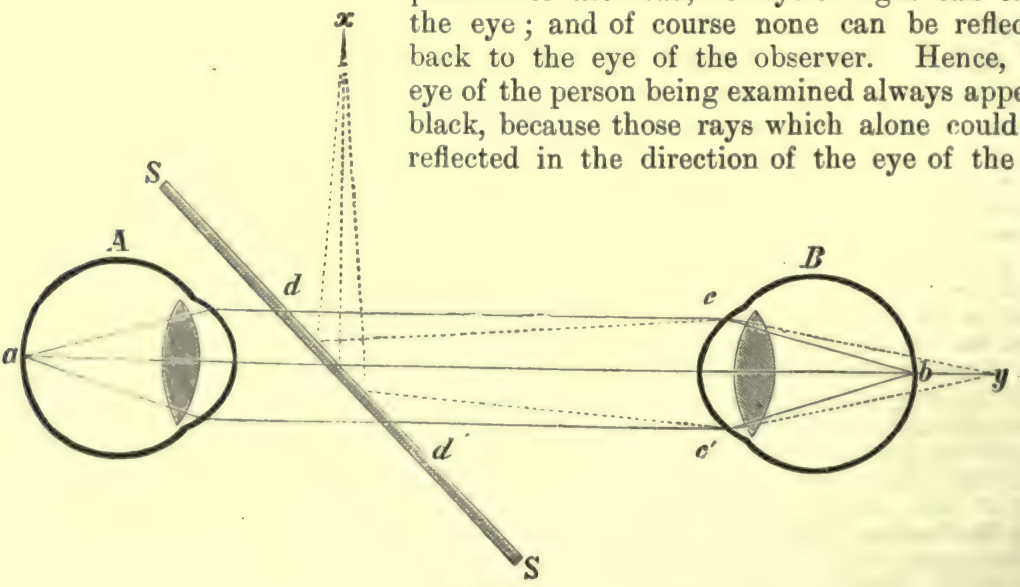

Fig. 548.

Arrangement for examining the eye of $\mathrm{B}$. A, eye of observer ; $x$, source of light; $\mathrm{S}, \mathrm{S}$, plate of glass directed obliquely, reflecting light into $\mathrm{B}$.

server are cut off. As soon, however, as we succeed in causing rays of light to enter the eye at the same time and in the same direction in which we observe the eye of another person, the fundus of the eye appears brightly illuminated.

The following simple arrangement is sufficient for the purposes (fig. 548) :-Let B be the eye 
of the patient, $\mathbf{A}$ that of the observer, and let a flame be placed at $x$. The rays of light proceeding from $x$ impinge upon the obliquely placed plate of glass $(\mathbf{S}, \mathbf{S})$, and are reflected in the direction of the dotted lines into the eye (B). The fundus of the eye appears in this position to be brightly illuminated in diffusion circles around $b$. As the observer (A) can see through

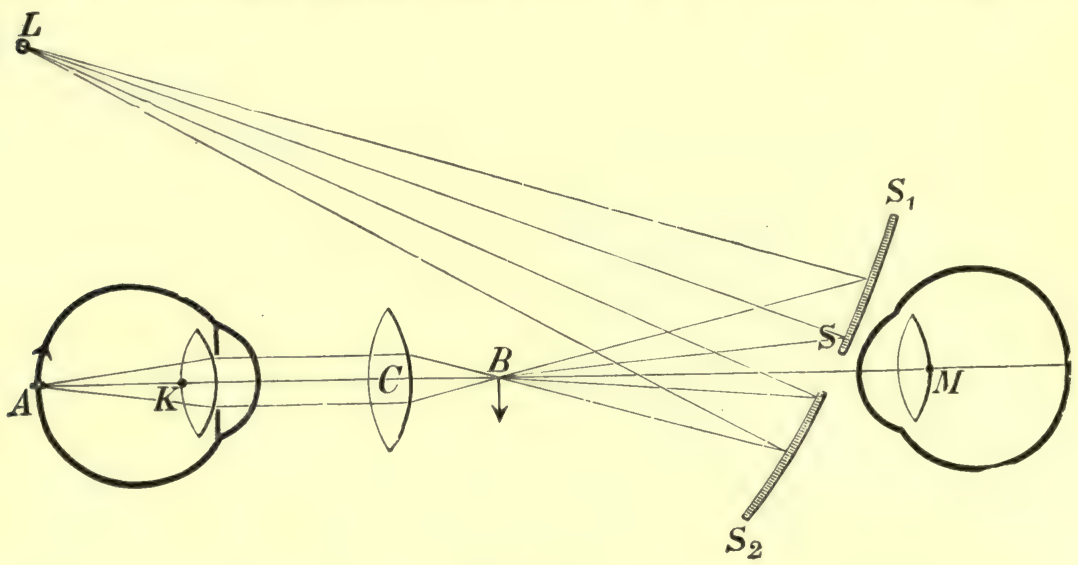

Fig. 549.

the obliquely placed glass plate $(\mathbf{S}, \mathbf{S})$, and in the same direction as the reflected rays $(x, y)$, he sees the retina around $b$ brightly illuminated.

In order that this method be made available for practical purposes, we must, of course, be able to distinguish the details, such as the blood-vessels of the fundus of the eye, the macula

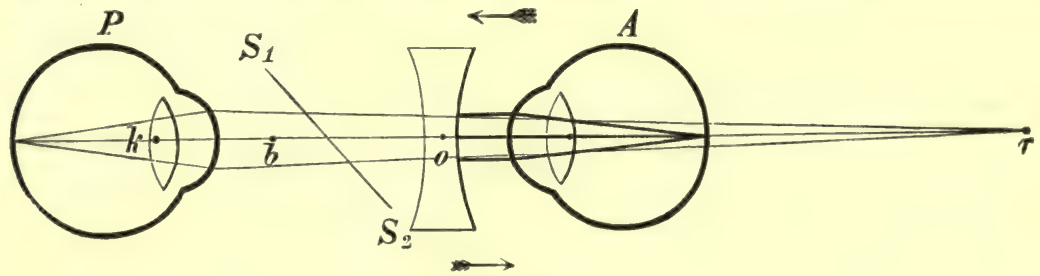

Fig. 550.

lutea, the entrance of the optic nerve, abnormalities of the retina, and the choroidal pigment, \&c. The following considerations show us how to proceed in order to accomplish this. As already mentioned, and as fig. 531 shows, a small inverted image is formed on the retina $(c, d)$ when we look at an object $(\AA, B)$; conversely, according to the same dioptric law, an enlarged inverted real image of a small distinct area of the retina $(c, d$-depending on the distance for which the eye was accommodated) must be formed outside the eye (A, B).

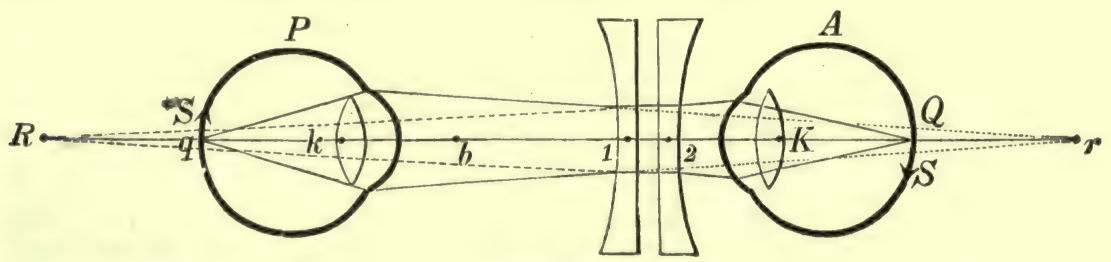

Fig. 551.

If the fundus of this eye be sufficiently illuminated, this aerial image will be correspondingly bright.

In order to see the individual parts of the retinal picture more distinctly, the observer must accommodate his own eye for the position of this image. In such circumstances the eye of the observer would be too near the observed eye. His eye when so accommodated is removed from 
the eye of the patient by his own visual distance, and by the visual distance of the patient. As this distance is considerable, the individual small details of the fundus cannot be seen distinctly. Further, owing to the contraction of the pupil of the patient, only a small area of the fundus can be seen, and this only under a small visual angle, quite apart from the fact that it is often impossible to accommolate for the real image of the fundus of the patient.

Hence, the eye of the observer must be brought nearer to the eye of the patient. This may be done in two ways:-(1) Either by placing in front of the eye of the patient a strong convex lens (of 1 to 3 inclies focus-fig. $549, \mathrm{C}$ ). This causes the retinal image to be nearer to the eye (at 13), owing to the strong lens refracting the rays of light. The observer (M) can come nearer to the eye, and can still accommodate for the image of the fundus of the eye. (2) Or a concave lens is jlaced inmediately in front of the eye of the patient (fig. 550, o). The rays of light emerging from the eye of the patient $(\mathrm{P})$ are either made parallel by the concave lens $(0)$, and are lirought to a focus on the retina of the emmetropic observer (A); or, if the lens causes the rays to diverge (fig. 551), an erect, virtual image is formed at a distance behind the eye of the jatient (at R). In these cases, also, the observer can go much nearer to the eye of the patient.

The ophthalmoscope invented by v. Helmholtz enables us to examine the whole of the fundus of the eye.

[Direct Method. - Use a concave mirror of 20 centimetres focal distance, with a central open. ing. Reflect a beam of light into the patient's eye, where the rays cross in the vitreous and

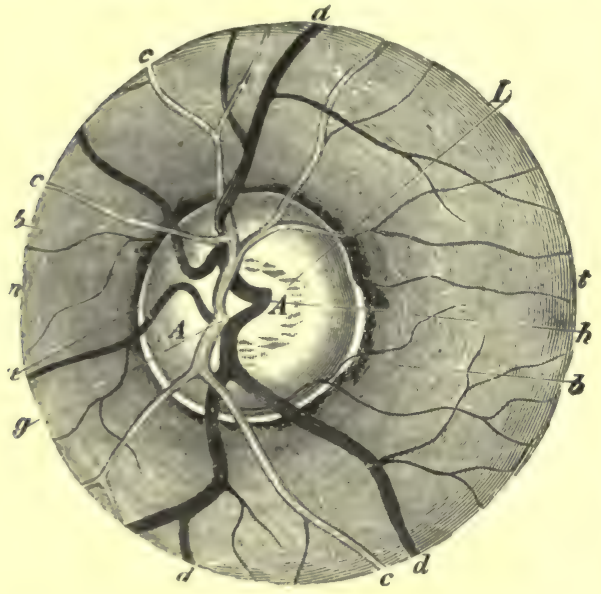

Fig. 552 .

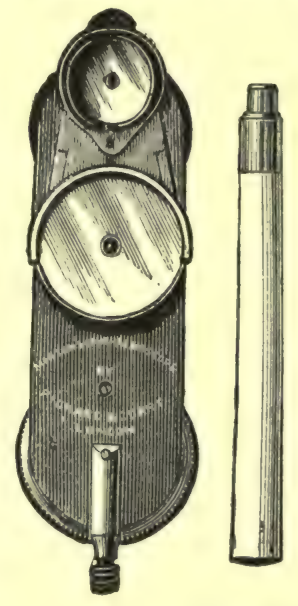

Fig. 553.

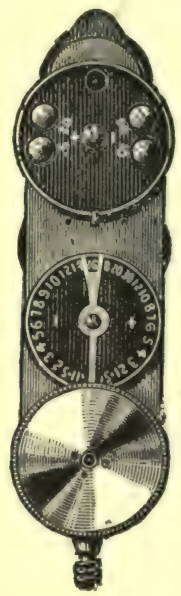

Fig. 552. - The entrance of the optic nerve with the adjacent parts of the fundus of the normal eye. $a$, ring of connective-tissue ; $b$, choroidal ring; $c$, arteries ; $d$, veins ; $g$, division of the central artery; $h$, division of the central vein; L, lamina cribrosa ; $t$, temporal (outer) side; $n$, nasal (inner) side. Fig. 553.- Morton's ophthalmoscope.

illuminate the fundus of the eye. These rays again pass out of the eye and reach the observer's eye through the central hole in the mirror. If the observer be emmetropic they come to a focus on his retina. In this way all the parts of the retina are seen in their normal position, but enlargerl. Hence, it is sometimes called the examination of the upright image. The eye of the patient and observer must be at rest, i.e., be negatively accommodated, while the mirror must be brought as near as possible to the eye of the patient.]

[Indirect Method, by which a more general view of the fundus is obtained. Throw the light into the patient's eye by an ophthalmoscopic mirror as above, but held at a distance of about 50 cm. (10 inches) from the patient's eye. Hold a biconvex lens of 14 dioptrics focal length vertically letween the mirror and the patient's eye (fig. 549), the observer looking through the hole of the mirror. What he does see is an inverted aerial image at B. Only a small part of the fundus oculi can be seen at one time.]

[The ophthalmoscope, besides being used for examining the interior of the eyeball, is of the utmost use in determining the existence and amount of anomalies of refraction in the refractive media. For this purpose an ophthalmoseope requires to be provided with plus and minus lenses, which can be readily brought before the eye of the observer. This is readily done by an ingenious mechanism devised by Couper, and made use of in the handy students' ophthalmoscope of Morton (fig. 553). The lenses are moved by a driving-wheel on the left figure, 
while at the same time is indicated at a certain aperture the lens presented at the sight hole. The instrument is also provided with a movable arrangement carrying a concave mirror at either end. One of these mirrors is 10 inches in focus, and is used for indirect examination and retinoscopy, while the other is of 3 inches focus for direct examination, and is fixed at an angle of $25^{\circ}$.]

[Retinoscopy.-The ophthalmoscope is used also for this purpose. A beam of light is reflected into the eye by the ophthalmoscopic mirror, and the play of light and shade on the fundus oculi observed. A study of this is important in determining anomalies of refraction. For the method, the student is referred to a text-book on "Diseases of the Eye."]

[Artificial Eye. - The student may practise the use of the ophthalmoscope on an artificial eye, such as that of Frost (fig. 554) or Perrin.]

Illumination. - In order to illuminate the interior of the eye, v. Helmholtz used several plates of glass, placed behind each other, in the position of S, S, in fig. 548. Afterwards he used a plane or concave mirror of 7 inches focus (fig. 549), with a hole in the centre. Fig. 552 shows the appearance of the fundus of the eye, as seen with the ophthalmoscope. In albinos the fundus of the eye appears red, because light passes into the eye through the sclerotic and

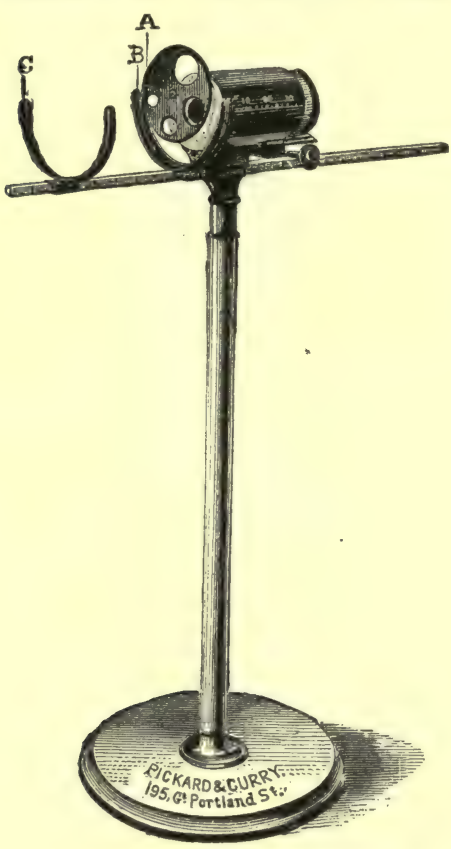

Fig. 554.

Frost's artificial eye.

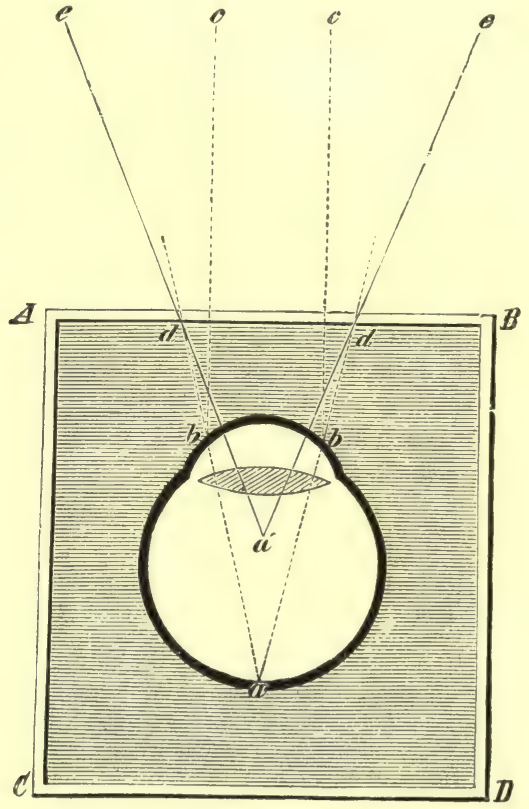

Fig. 555 .

Action of the orthoscope.

uvea, which are devoid of pigment. If a diaphragm be placed over the eye, so that the pupil alone is free, the eye appears black (Donders).

Tapetum. - In many animals the eyes have a bright green lustre. These eyes have a special layer, the tapetum, or the membrana versicolor of Fielding; in carnivora it consists of cells, in herbivora of fibres, placed between the capillaries of the choroid and the stroma of the uvea. These structures exhibit interference-colours and reflect much light, so that the coloured lustre appears in the eye.

Oblique illumination is used with advantage for investigating the anterior chamber. A bright beam of light, condensed by a convex lens, is thrown laterally upon the cornea into the eye, and so directed upon the point to be investigated as to illuminate it. A point so illuminated, e.g., a part of the iris, may be examined from a distance by means of a lens, or even by a microscope (Liebreich).

The Orthoscope. - Czermak constructed this instrument, in which the eye is placed under water (fig. 555). It consists of a small glass trough with one of its walls removed. The margins of the open side are pressed firmly against the region of the eye. The eye and its surroundings form, as it were, the sixth side of the trough, which is filled with water, so that the 
cornea is bathed therewith. As the refractive index of water is almost the same as the refractive index of the media of the eye, the rays of light pass into the eye in a straight direction without being refracted. Hence, objects in the anterior chamber can be seen directly, as if they were not within the eye at all. Another advantage is that the objects can be brought nearer to the eye of the observer. The rays of light emerging from the point $(a)$ of the fundus, if the eve were surrouuded by air, would leave the eye as the parallel lines, $b, c, b, c$. Under water, these rays, $a, b$, contiuue in the direction $a, b$, as far as $b, d$, where they emerge from the water, and are bent from the perpendicular to $d, e, d, e$. The eye of the observer, looking in the direction $c$, $d$, sees the point, $a$, nearer, viz., in the direction $e, d, a^{\prime}$, lying at $\alpha$.

395. ACTIVITY OF THE RETINA IN VISION.-I. Blind Spot.-The rods and cones alone are the parts of the retina sensitive to light, they alone are excited by the vibrations of the ether. This is confirmed by Mariotte's experiment (1688), which proves that the entrance of the optic nerve, where rods and cones are absent, is devoid of visual sensibility. Hence it is spoken of as the "blind 8)ot."

Mariotte's Experiment.-Make two marks, about 3 inches apart, upon paper (fig. 556). Jook at the cross with the right eye, keeping the left eye closed, and hold the paper about a foot from the eye, when both the cross and the circle will

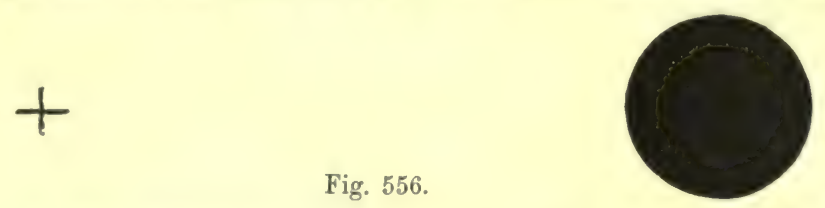

be seen. Gradually approximate the paper to the eye, keeping the open eye steadily fixed on the cross; at a certain moment the circle will disappear, and on bringing the paper nearer to the eye it will reappear. The moment when the circle disappears is when its image falls upon the entrance of the optic nerve.]

Position and Size.-The entrance of the optic nerve lies about $3.5 \mathrm{~mm}$. internal to the visual axis of the eyeball, in the retina. Its diameter is $1.8 \mathrm{~mm}$. The apparent diameter of the hlind spot in the field of vision is in a horizontal direction $6^{\circ} 56^{\prime}$ - this lies $12^{\circ} 35^{\prime}$ to $18^{\circ} 55^{\prime}$ horizontally from the fixed point. Eleven full moons placed side by side would disappear on the surface, and so would a human face at a distance of over 2 metres.

Proofs. - The following facts prove that the entrance of the optic nerve is insensible to light:-(1) Donders projected, by means of a mirror, the small image of a flame upon the entrance of the optic nerve of another person, and the person had no sensation of light. But a sensation of light was experienced, when the image of the flame was projected upon the neighbouring parts of the retina. (2) On combining with Mariotte's experinent the experiment which causes entoptical phenomena at the entrance of the optic nerve, this coincides with the blind spot ( 8393,6 and 7$)$.

Form of Blind Spot. - In order to determine the form and apparent size of the blind spot in one's own eye, fix the head at about 25 centimetres from a surface of white paper ; select a small point on the latter and keep the eye directed towards it; then, starting from the position of the blind spot, move a white feather in all directions over the paper; whenever the tin of the feather becomes visible, make a mark at this spot. The blind spot inay be mapped out in this way. It has an irregular, elliptical forn from which processes proceed, due to the equally non-sensitive origins of the large blood-vessels of the retina (Hueck). (Mariotte concluded from his experiment that the choroid, which is perforated by the optic nerve, is the membrane sensitive to light, as the nerves are nowhere absent from the retina.)

The blind spot causes no appreciable gap in the field of vision. - As this area is not excited by light, a black spot cannot appear in the field of vision, for the sensation of black implies the presence of retinal elements, which, however, are absent from the blind spot. The circumstance, however, that in spite of the existence of an inexcitable spot during vision, no part of the field of vision appears to be unoccupied, is due to a psychical action. The unoccupied area of the field of vision, corresponding to the blind spot, is filled in according to probability by a psychical process $(E, H . W e b e r)$. Hence, when a white point disappears from a black surface, the whole surface appears to us black; a white surface, from which a black point falls on the blind spot, appears quite white; a page of print, grey throughout, \&c. According to the probabilities, certain parts are supplied-parts of a circle, the middle parts of a long line, the central part of a cross. Such images, however, as cannot be constructed 
according to the probabilities, are not perfected, e.g., the end of a line or a human face. In other cases the condition known as "contraction" of the field of vision tends to fill up the gap. This will be evident on looking at the nine adjoining letters, so that $e$ disappears; we no longer see the three letters on each side of it in straight lines, but $b, f, h, d$ are turned in towards $e$. The adjoining parts of the field of vision seem to extend over and around the blind spot, and thus help to compensate for the blind spot.

II. Optic Fibres Inexcitable to Light.- The layer of the fibres of the optic nerve in the retina is not sensitive to light. This is proved by the fact that, in the fovea centralis, which is the area of most acute vision, there are no nerve-fibres. Further, Purkinje's figure proves that, as the arteries of the
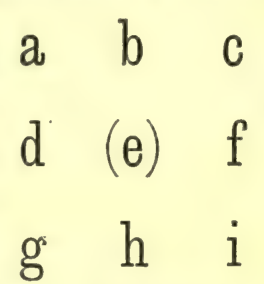
retina lie behind the optic fibres, the latter cannot be concerned in the perception of the former.

III. Rods and Cones.-The outer segments of the rods and cones have rounded outlines, and are packed close together; but natural spaces must exist betweeu them, corresponding to the spaces that must exist between groups of bodies with a circular outline. These parts are insensible to light, so that a retinal image is composed like a mosaic of round stones. The diameter of a cone in the yellow spot is 2 to $2.5 \mu(M$. Schultze). If two images of two small points, placed very near each other, fall upon the retina, they will still be distinguished as distinct images, provided that both images fall upon two different cones. The two images on the retina need only be $3-4-5 \cdot 4 \mu$ apart, in order that each may be seen separately, for then the images still fall upon two adjoining cones. If the distance be diminished so very much that both images fall upon one cone, or one upon one cone and the other upon the intermediate or cement substance, then only one image is perceived. The images must be further apart in the peripheral portion of the retina in order that they may be separately distinguished.

As the rounded end-surfaces of the cones do not lie exactly under each other, but are so arranged that one series of circles is adapted to the interstices of the following series, this explains why fine dark lines lying near each other appear to have alternating twists upon them, as the images of these must fall upon the cones, at one time to the right, at another to the left.

IV. The fovea centralis is the region of most acute vision, where only cones are jresent, and where they are very numerous and closely packed (fig. 521). The cones are less numerous in the peripheral areas of the retina, and consequently vision is much less acute in these regions. We may therefore conclude that the cones are more important for vision than the rods. When we wish to see an object distinctly, we involuntarily turn our eyes so that the retinal image falls upon the fovea centralis. In doing this, we are said to "fix" our eyes upon an object. The line drawn from the fovea to the object is called the axis of vision (fig. 557, $S r$ ). It forms an angle of only $3.5-7^{\circ}$ with the "optical axis" $(O A)$, which unites the centres of the spherical surfaces of the refractive media of the eye. The point of intersection, of course, lies in the nodal point $(K n)$ of the lens (p. 770). The term "direct vision" is applied to vision when the direction of the axis of vision is in line with the object, [i.e., when the image of the object falls directly on the fovea centralis.]

"Indirect vision" occurs when the rays of light from an object fall upon the peripheral parts of the retina. Indirect vision is much less acute than the direct.

To test the acuity of direct vision, draw two fine parallel lines close to each other, and gradually remove them more and more from the eye, until both appear almost to unite and form one line. The size of the retinal image may be ascertained by determining the distance of the two lines from each other, and the distance of the lines from the eye, or, from the corresponding visual angle, which is generally from 60 to 90 seconds.

Perimetry. - In order to test indirect vision, we may use the perimeter of Aubert and Förster. The eye is placed opposite a fixed point, from which a semicircle proceeds, so that the eye lies in the centre of it. As the semicircle rotates round the fixed point, on rotating the. 
former we can circumscribe the surface of a hemisphere, in the centre of which the eye is placed. Proceeding from the fired point, objects are placed upon semicircles, and are gradually pushed more and more towards the periphery of the field of vision, until the object becomes indistinct, and finally disappears. The process of testing is continued by placing the arc successively in the different meridians of the field of vision.

[M 'Hardy's perimeter is a very convenient form (fig. 558). It consists of two uprights (C and D), which are fixed to the opposite ends of a flat hasal plate (A). C carries an arrangement for supprorting the patient's head, while $\mathrm{D}$ carries the automatic arrangement for the perimetric

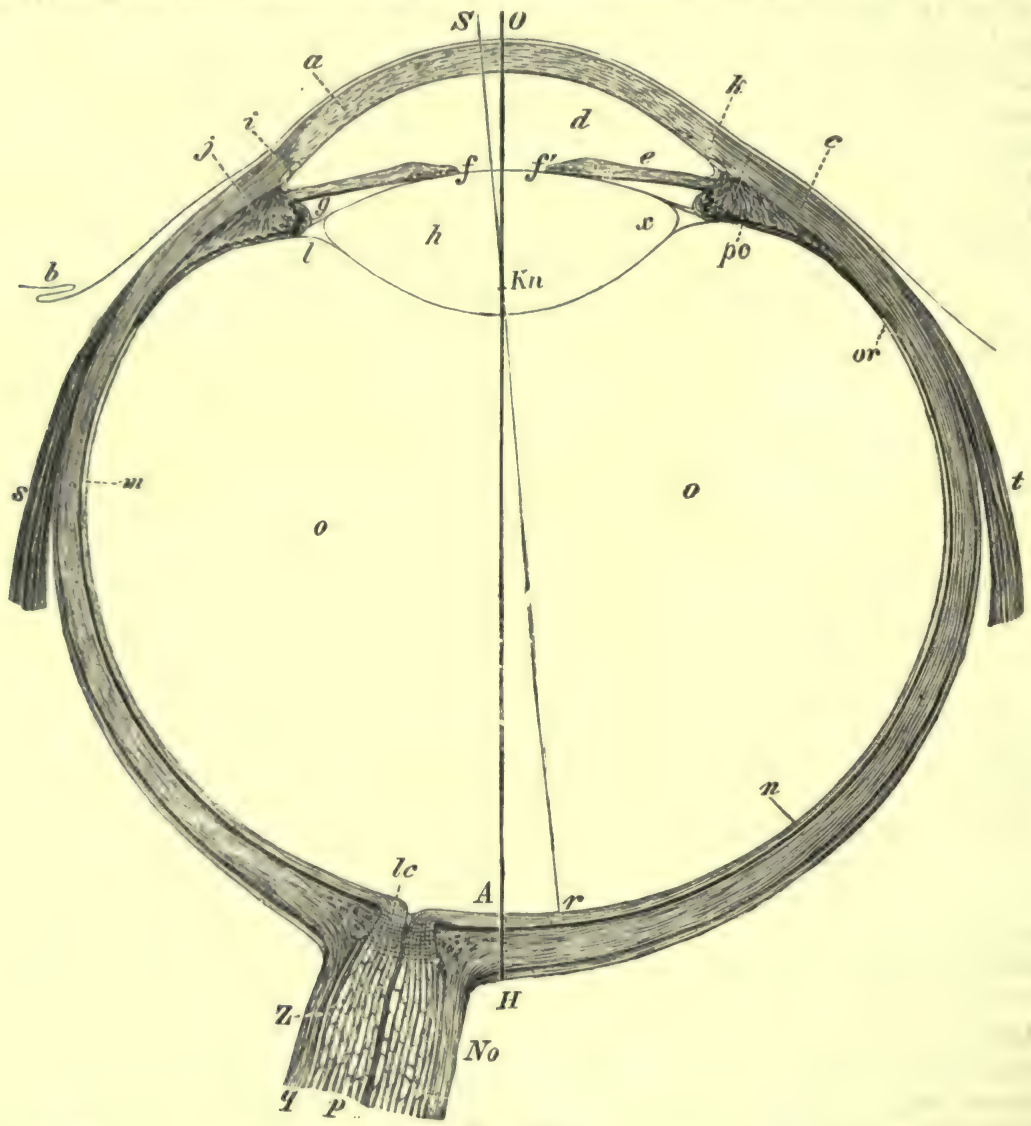

Fig. 557.

Horizontal section of the right eye. $a$, cornea ; $b$, conjunctiva ; $c$, sclerotic; $d$, anterior chamber containing the aqueous humour ; $e$, iris ; $f f^{\prime}$, pupil; $g$, posterior chamber ; $l$, Petit's canal; $j$, ciliary muscle ; $k$, corneo-scleral limit; $i$, canal of Schlemm ; $m$, choroid; $n$, retina; 0 , vitreous humour; $N o$, optic nerve; $q$, nerve-sheaths ; $p$, nerve-fibres ; $l c$, lamina cribrosa. The line $O A$ indicates the optic axis; $S r$ the axis of vision; $r$, the position of the fovea centralis.

record. Both of these can be raised or depressed by the screws ( $G$ and $b$ ). The patient's chin rests on the chin-rest $(\mathrm{E})$, while in the mouth is placed Landolt's biting fixation $(\mathrm{L})$, which is detachable. The position of the head can be altered by sliding $\mathrm{F}$ on $\mathrm{L}$, which can be fixed in any position by the serew $(0)$. The porcelain button $(I)$ just below the patient's eye $(l)$ is connected with the adjustment of the "fixation point." The automatic recording apparatus consists of a revolving quadrant $(h, h)$, which describes a hemisphere round a horizontal axis passing through the centre of the hollow male axle, turning in the female end of $\alpha$, which is supported by D. The quadrant can be fixed at any point by $g$. On the front concave surface of the quadrant is fixed a circular white piece of ivory, representing the "fixation point," fron 
which a needle projects, and which is the zero of the instrument. A carriage $(i)$, in which the test objects are placed, can be moved in the concave face of the quadrant by means of the milled head $(j)$, which moves the carriage by means of a tooth and pinion wheel.]

[When the milled head $(j)$ is turned, it moves the carriage and two slides ( $k$ and $l$ ), the two slides moving in the ratio of 2 to 1 . The rate of the carriage is so adjusted that it travels ten times faster than $l$, and five times faster than $k$. The pointer $(p)$ is connected with these slides, so that it moves when they move, and records its movements by piercing the record chart, which is fixed in the double-faced frame $(e)$. The frame for the record chart is hinged near $c$ to the upright (D). The frame, when upright, comes so near the pointer that the latter can pierce a chart placed in the frame. The patient is directed to look at the "fixation point," which is merely a small ivory button placed in the imaginary axis of the hemisphere on the

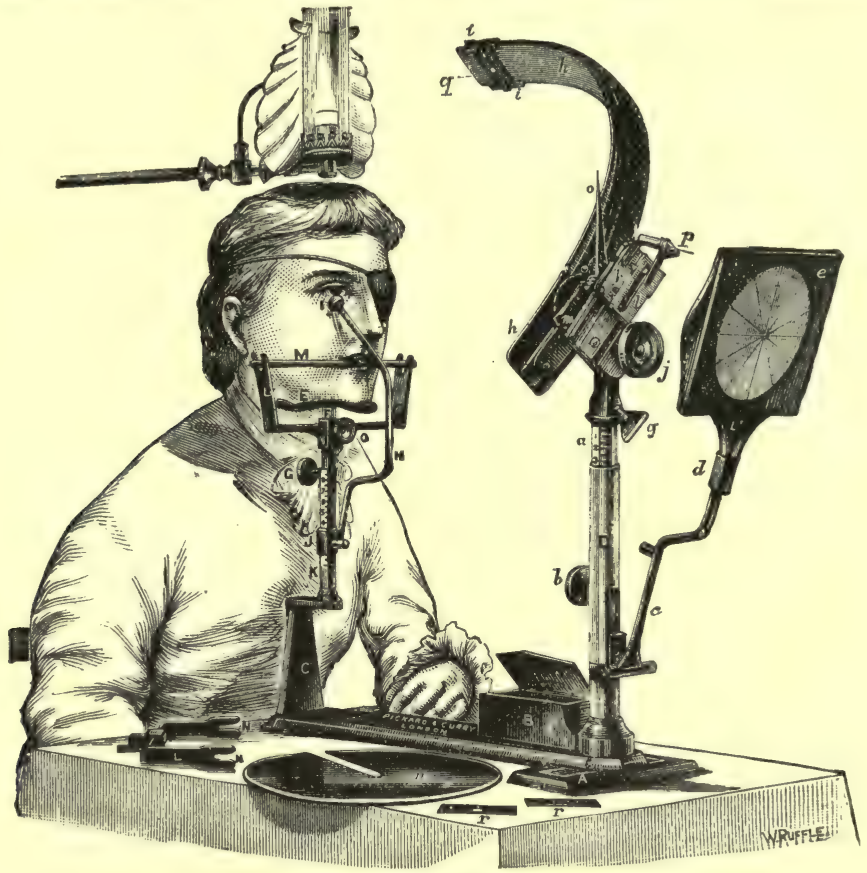

Fig. 558.

$\mathbf{M}$ ['Hardy's perimeter. I, porcelain button; M, bit; $\mathbf{E}$, for fixing the head; $g, h$, quadrant ; $o$, fixation point; $p$, pointer for piercing the record chart held in the frame $(e)$ which moves on $c ; \mathrm{D}$, upright supporting the quadrant and the automatic arrangement of slides $(k$ and l), which are moved by $j$.

front of the centre of the concave surface of the quadrant; the projecting needle-point $(0)$ indicates its position. This is the zero of the quadrant, and on each side of it the quadrant is divided into $90^{\circ}$.]

[In testing the field of vision, place the carriage so as to cover zero, adjust the eye for the fixation point, and look steadily at it, when, if all is right, the pointer $(p)$ ought to pierce the centre of the chart. Move the carriage along the quadrant by $j$ until it disappears from the field of vision, and when it does so the pointer is made to pierce the chart. Make another observation in another direction by altering the position of the quadrant, and go on doing so until a complete record is obtained of the field of vision. Test the other eye in the same way. The colour-field may be tested by using coloured papers in the carriage.]

[Priestley Smith's perimeter (fig. 559). - The wooden knob on the left of the figure is placed under the eye of the patient, who stares at the fixed point in the axis of the quadrant, which can be moved in any meridian. The test object is a square piece of white paper, which is moved along the quadrant. The chart is placed on the posterior surface of the hand-wheel and moves with it, so that the meridians of the chart move with the quadrant. There is a scale behind the hand-wheel corresponding with the circles on the chart, so that the observer can prick off his observations directly.] 
[Scotoma is the term applied to dimness or blindness in certain parts of the field of vision,

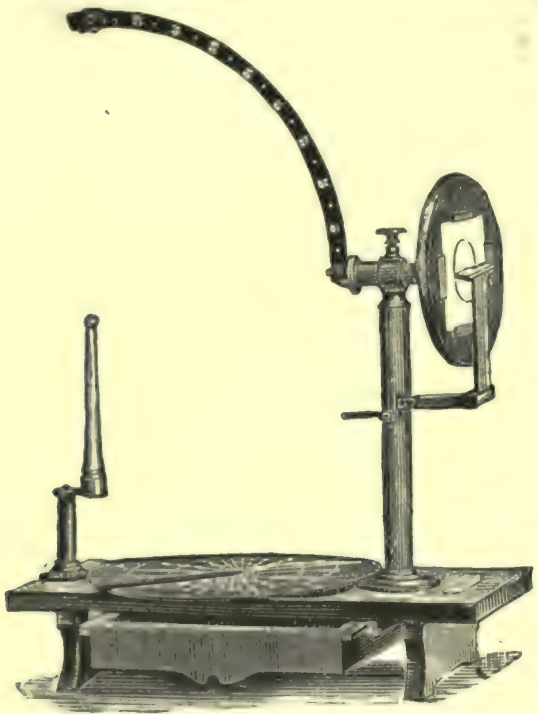
which may be central, marginal, or in patches. ]

The capacity for distinguishing colours diminishes more rapidly at the periphery of the retina, than that for distinguishing differences in the brightness or intensity of light. In fact, the periphery of the retina is slightly red blind. The diminution is greater in the vertical meridian of the eye than in the horizontal, and it dinin. ishes with the distance from the fixation point (Aubert and Förster). These observers also state that, during accommodation for a distant object, the diminution of the capacity to distinguish brightness and colour towards the periphery of the lens, occurs more rapidly than with near vision. The excitability of the retina for colours and brightness is greater at a point equally distant from the fovea centralis on the temporal than on the nasal side of the eye (Schön).

Perimetric Chart. - If the arc of the perimeter (fig. 559) be divided into 90 degrees, beginning at the fixation point (central point), and proceeding to L and M (fig. 560); and if a series of concentric circles be inscribed on this, with the point of fixation as their centre, we can construct a topographical chart of the visual capaeity of the normal or healthy eye from the data obtained by the examination of the retina.

Fig. 560 is an example; the thick lines indi-

Fig. 559.-Priestley Smith's perimeter. cate a diseaserl eye the corresponding thin lines a healthy eye. The continuous line indicates

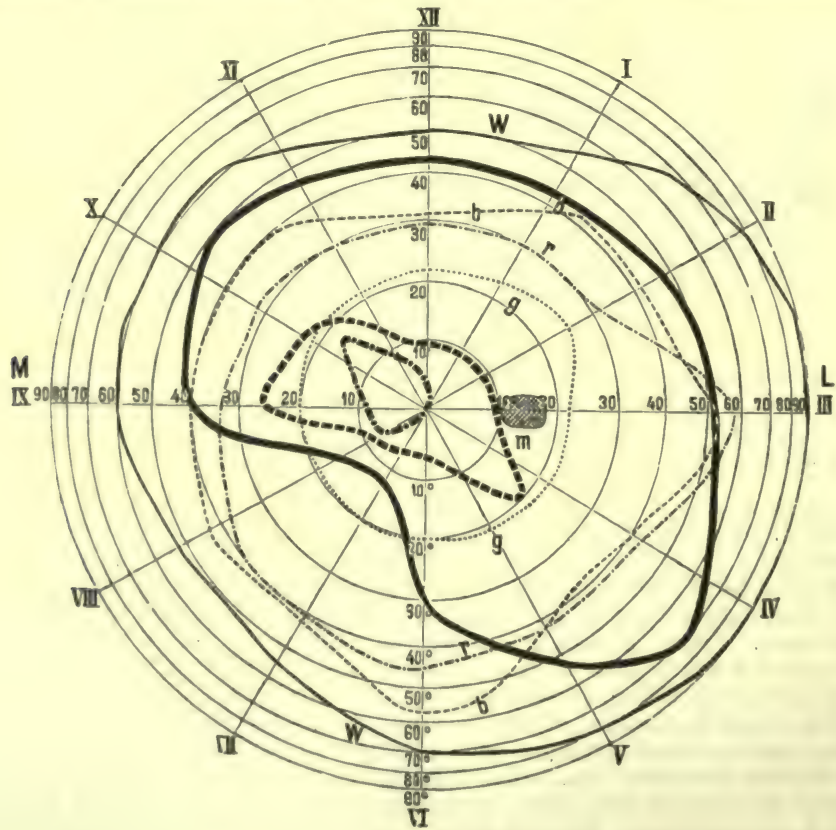

Fig. 560.

Perimetric chart of a healthy and a diseased eye.

the limits for the perception of white; the interrupted line that for blue; the punctuated and 
interrupted line that for red; $m$ is the blind spot. In the normal eye the limits for the perception of colours are as under:-

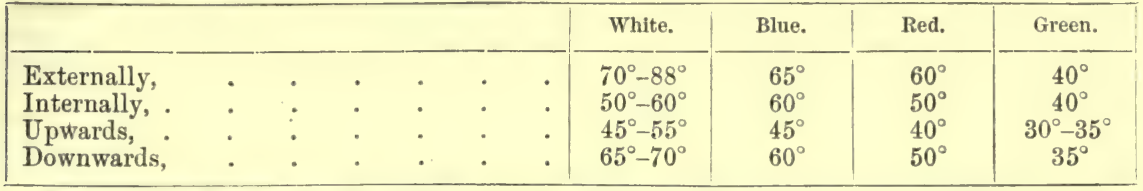

V. Specific Energy.-The rods and cones alone are endowed with what Johannes Müller called "specific energy," i.e., they alone are set into activity by the ethereal vibrations, to produce those impulses which result in vision. Mechanical and electrical stimuli, however, when applied to any part of the course of the nervous apparatus, produce visual phenomena. Mechanical stimuli are more intense stimuli than light rays, as is shown by performing the dark pressure figure with the eyes open $(\$ 393,5, a)$, whereby the circulation in the retina is interfered with ; in the region of pressure, we cannot see external objects which affect the retina uniformly and continuously.

VI. The duration of the retinal stimulation must be exceedingly short, as the electrical spark lasts only 0.000000868 second; still, as a general rule, a shorter time is required, the larger and brighter the object looked at. Alternate stimulation with light, 17 to 18 times per minute, is perceived most intensely (Brïcke). Further, an increase or diminution of 0.01 part of the intensity of the light is perceptible (\$383). A shorter time is required to perceive yellow than is required for violet and red (Vierordt). The retina becomes more sensitive to light, after a person has been kept in the dark for a long time, and also after repose during the night. If light be allowed to act on the eyes for a long time, and especially if it be intense, it causes fatigue of the retina, which begins sooner in the centre than in the periphery of the organ (Aubert). At first the fatigue comes on rapidly and afterwards develops more slowly-it is most marked in the morning (A. Fick). The periphery of the retina is specially characterised by its capacity for distinguishing movements (Exner).

VII. Visual Purple.-The mode of the action of light upon the end-organs of the retina has already been referred to (p. 739) in connection with the "visual purple" or rhodopsin (Boll, Kühne). Kühne showed that, by illuminating the retina, actual pictures (e.g., the image of a window) could be produced on the retina, but they gradually disappeared. From this point of view we might regard the retina as comparable, to a certain extent, to the sensitive plate of a photographic apparatus.

Optogram. - The visual purple is formed by the pigment-epithelium of the retina. Perhaps we might compare the process to a kind of secretion. The visual purple may be restored in a retina by laying the latter upon living choroidal epithelium. The pigment disappears from the mammalian retina by the action of light 60 times more rapidly than from the retina of the frog. In a rabbit's eye, whose pupil was dilated with atropin, Ewald and Kuihne obtained a sharp picture or optogram of a bright object placed at a distance of $24 \mathrm{~cm}$. from the eye-the image was "fixed" by a 4 per cent. solution of alum. Visual purple withstands all the oxidising reagents; zinc chloride, acetic acid, and corrosive sublimate change it into a yellow substance -it becomes white only through the action of light; the dark heat-rays are without effect, while it is decomposed above a temperature of $52^{\circ} \mathrm{C}$. [As visual purple is absent from the cones, and cones only are present in the fovea centralis, we cannot explain vision by optograms formed by the visual purple.]

VIII. Destruction of the rods and cones of the retina causes corresponding dark spots in the field of vision.

396. PERCEPTION OF COLOURS. - Physical. - The vibrations of the light-ether are perceived by the retina only within distinct linits. If a beam of white light, e.g., from the sun, be transmitted through a prism, the light rays are refracted and dispersed, and a "prismatic spectrum" is obtained (fig. 17). White light contains rays of very different wave-lengths or periods of vibration. The dark heat-rays, whose wave-length is $0.00194^{\circ} \mathrm{mm}$., are refracted least (Fizeau). They do not act upon the retina, and are therefore invisible. They act, however, 
npon sensory nerves. Abont 90 per cent. of these rays is absorbed by the media of the eye (Brilele and Knobleuch). From Franenhofer's line, A, onwards, the oscillations of the lightether excite the retina in the following order:-Red with 481 billions of vibrations per second, nange with 532, yellono with 563 , green with 607 , blue with 653 , indigo with 676 , and violet with 764 billion vibrations per second. The sensation of colour therefore depends on the number of vibrations of the light-ether, just as the pitch of a note depends on the number of vilirations of the sounding body (Newton, 1704; Hartley, 1772). Beyond the violet lie the chemically active [actinic] rays of the spectrum. After cutting out all the spectrum, including the violet rays, $r$. Helmholtz succeeded in seeing the ultra-violet rays, which had a feeble greyish-line colour. The heat-rays in the coloured part of the spectrum are transmitted by the meilia of the eve in the same way as through water. The existence of the ultra-violet rays is best ascertained by the phenomenon of fluorescence. Von Helmholtz, on illuminating a solution of sulphate of quinine with the ultra-violet rays, saw a bluish-white light proceeding from all parts of the solution which were acted on by the ultra-violet rays. As the media of the eye themselves exhibit fluorescence ( 2 . Helmholtz), they must increase the power of the retina to distin¿nish these rays. The ultra-violet rays are not largely absorbed by the media of the eye (Brückc).

In order that a colour be perceived, it is essential that a certain amount of light fall upon the retina. 13lue, when at the lowest degree of brightness, gives a colour sensation with an amount of light which is sixteen times less than that required for red (I)olroulosky).

Intensity of the Impression of Light. - While light of different periods of vibration applied to the eye excites the different sensations of colour, the amplitude of the vibrations (height of the waves) letermines the intensity of the impression of light; just as the loudness of a note leprends on the amplitude of the vibrations of the sounding body. The sun's light contains all the rays which excite the sensation of colour in us, and when all these rays fall simultaneously npon the retina we experience the sensation of white. If the colours of the spectrum obtained by means of a prism be remited, white light is again obtained. If no vibrations of the lightwher reach the retina, every sensation of light and colour is absent, but we can scarcely apply the term black to this conclition. It is rather the absence of sensation, such as, for example, is the ease when a heam of light falls on the skin of the back. This does not give the sensation of black, but rather that of no sensation of light.

Simple and Mixed Colours. - We distinguish simple colours, e.g., those of the spectrum. In order to perceive these, the retina must be excited (set into vibration) by a distinct number of oscillations (see above). Further, we distinguish "mixed colours," whose sensations are produced when the retina is excited by two or more simple colours, simultaneously or rapidly alternating. The most complex mixed colour is white, which is composed of a mixture of all the simple colours of the spectrum.

The "complementary colours" are important. Any two colours which together give the sensation of white are complementary to each other. The "contrast colours" are mentioned here merely to complete the list. They are closely related to the complementary colours. Any two colours which, when mixed, supplement the generally prevailing tone of the light, are contrast colours. When the sky is blue, the two contrast colours must be bluish-white: with bright gaslight they must be yellowish-white, and in pure white light of course all the complementary are the same as the contrast colours (Briicke).

Methods of Mixing Colours. - 1. Two solar spectra are projected upon a screen, and the spectra are so arranged as to cause any one part of one spectrum to cover any part of the other. 2. look obliquely through a vertically arranged glass plate at a colour placed behind it. Another colour is placed in front of the glass plate, so that its image is also reflected into the eye of the observer ; thus, the light of one colour transmitted through the glass plate and the reflecterl light from the other colour reach the eye simultaneously. [Lambert's Method.-This is easily done by Lambert's method. Use coloured wafers and a slip of glass; place a red wafer on a sheet of black paper, and about 3 inches behind it another blue one. Hold the plate of glass midway and vertically between them, and so incline the glass that, while looking.through it at the red wafer, a reflected image of the blue one will be projected into the eye in the same clirection as that of the rel image, when we have the sensation of purple.]

3. A rotatory disc, with sectors of various colours, is rapidly rotated in front of the eyes. On rapidly rotating the coloured disc, the impressions produced by the individual colours are united to produce a mixed colour. If the rotating dise, which yields, let us suppose, white, on mixing the colours of the spectrum, be reflected in a rapidly rotating mirror, then the individual components of the white reappear.

4. Place in front of each of the small holes in the cardboard used for Scheiner's experiment 
(fig. 537) two differently coloured pieces of glass ; the coloured rays of light passing through the holes unite on the retina, and produce a mixed colour (Czermak).

Complementary Colours. - Investigation shows that the following colours of the spectrum are complementary, i.e., every pair gives rise to white :-

$$
\begin{aligned}
& \text { Red and greenish-blue, } \\
& \text { Yellow and indigo-blue, }
\end{aligned}
$$

Orange and Cyan-blue,

Greenish-yellow and violet,

while green has the compound complementary colour, purple ( $v$. Helmholtz).

The mixed colours may be determined from the following table. At the top of the vertical and horizontal columns are placed the simple colours; the mixed colours occur where they

\begin{tabular}{|c|c|c|c|c|c|c|c|}
\hline & Violet. & Indigo. & Cyan-blue. & $\begin{array}{l}\text { Bluish- } \\
\text { green. }\end{array}$ & Green. & $\begin{array}{l}\text { Greenish- } \\
\text { yellow. }\end{array}$ & Yellow. \\
\hline Red & Purple & Dk.-rose & Wh.-rose & White & Wh.-yellow & Gold-yellow & Orange \\
\hline Orange & Dk.-rose & Wh.-rose & White & Wh.-yellow & Yellow & Yellow & $\ldots$ \\
\hline Yellow & Wh.-rose & White & Wh.-green & Wh.-yellow & Gr.-yellow & .. & $\ldots$ \\
\hline Gr.-yellow & White & Wh.-green & Wh.-green & Green. & $\ldots$ & $\ldots$ & $\ldots$ \\
\hline Green & White-blue & Water-blue & Bl.-green & $\ldots$ & ... & ... & $\ldots$ \\
\hline Bluish-green & Water-blue & Water-blue & $\ldots$ & $\ldots$ & $\ldots$ & $\ldots$ & $\ldots$ \\
\hline Cyan-blue & Indigo & $\ldots$ & ... & ... & $\cdots$ & $\ldots$ & ... \\
\hline
\end{tabular}
intersect the corresponding vertical and horizontal columns (Dk. = dark ; wh. $=$ whitish) :-

The following results have been obtained from observations on the mixture of colours :-

1. If two simple, but non-complementary, spectral colours be mixed with each other, they give rise to a colour sensation, which may be represented by a colour lying in the spectrum between both, and mixed with a certain quantity of white. Hence we may produce every impression of mixed colours by a colour of the spectrum + white (Grassman).

2. The less white the colours contain, the more "saturated" they are said to be; the more white they contain, the more unsaturated do they appear. The saturation of a colour diminishes with the intensity of the illumination.

Geometrical Colour Table. - Since the time of Newton, attempts have been made to construct a so-called "geometrical colour table," which will enable any mixed colour to be readily found. Fig. 561 shows such a colour table; white is placed in the middle, and from it to every point in the curve, - which is marked with the names of the colours, - suppose each colour to be so placed that, proceeding from white, the colours are arranged, beginning with the brightest tone, always followed by the most saturated tone, until the pure saturated spectral colour lies in the point of the curve marked with the name of the colour. The mixed colour, purple, is placed between violet and red. In order to determine from this table the mixed colour of any two spectral colours, unite the points of these colours by a straight line. Suppose weights corresponding to the units of intensity of these colours, to be placed on both points of the curve indicating colours, then the position of the centre of gravity of both in the line connecting the colours indicates the posi-

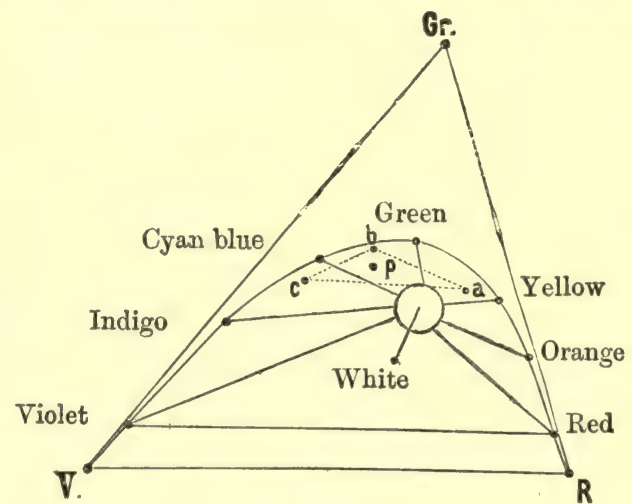

Fig. 561.

Geometrical colour cone or table.

tion of the mixed colour in the table. The mixed colour of two spectral colours always lies in the colour table in the straight line connecting the two colour points. Further, the impression of the mixed colour corresponds to an intermediate spectral colour mixed with white. The complementary colour of any spectral colour is found at once by making a line from the point of this colour through white, until it intersects the opposite margin of the colour table; the point of intersection indicates the complementary colour. If pure white be produced by mixing two complementary colours, the colour lying nearest white on the connecting line must be specially strong, as then only would the centre of gravity of the lines uniting both colours lie in the point marked white. 
By means of the enlour table we may ascertain the mixed colour of three or more colours. For example, it is required to find the mixed colour resulting from the union of the point, $\alpha$ (pale yellow), $b$ (fairly saturated bluish-green), and $c$ (fairly saturated blue). On the three points place weights corresponding to their intensities, and ascertain the centre of gravity of the weight, $a, b, c$; it will lie at $p$. It is obvious, however, that the impression of this mixed colour, whitish green-blue, can be proluced by green-blue + white, so that $p$ may be also the centre of gravity of two weights, which lie in the line connecting white and green-blue.

We may describe a triangle, $\mathrm{V}, \mathrm{Gr}, \mathrm{R}$, about the colour table so as to enclose it completely. The three fundamental or primary colours lie in the angles of this triangle, red, green, violet. It is criclent that 'ach of the coloured impressions, i.e., any point of the colour table, may be determinel by placing weights corresponding to the intensity of the primary colours at the angles of the triangle, so that the joint of the colour table, or what is the same thing, the desirel mixed colour, is the centre of gravity of the triangle with its angles weighted as above. The intensity of the three primary colours, in order to produce the mixed colour, must be represented in the same proportion as the weights.

Theories.- Various theories have been proposed to account for colour sensation.

1. Accorling to one theory, colour sensation is producel by one kind of element present in the retina, being excited in different ways by liglit of different colours (oscillations of the lightether of different wave-lengths, number of vibrations, and refractive indices).

2. Young-Helmholtz Theory.-The theory of Thomas Young (1807) and v. Helmholtz (1852) assumes that three different kinds of nerve-elements, corresponding to the three primary colours, are present in the retina. Stimulation of the first kind causes the sensation of red, of the second green, and of the third violet.

The elements sensitive to red are most strongly excited by light with the longest wavelength, the reel rays; those for green by melium wave-lengths, green rays; those for violet by the rays of shortest wave-length, violet rays. Further, it is assumed, in order to explain a number of phenomena, that irry! colour of the spectrum excites all the kinds of fibres, some of

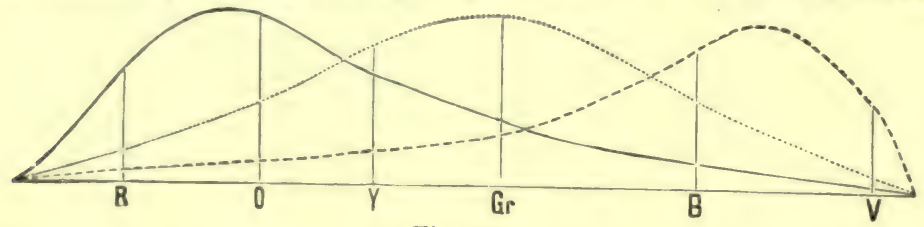

Fig. 562.

them firbly, others strongly. Suppose in fig. 562 the colours of the spectrum are arranged in their natural order from red to violet horizontally, then the three curves raised upon the abscissa might inlicate the strength of the stimulation of the three kinds of retinal elements. The continuous curve corresponds to the rays producing the sensation of red, the dotted line that of green, and the broken line that of violet. Pure real light, as indicated by the height of the ordinates in $\mathrm{R}$, strongly excites the elements sensitive to red, and feebly the other two kinds of terminations, resulting in the sensation of rad. Simple yellow excites moderately the elements for rel and green, and feebly those for violet = sensation of yellow. Simple green excites strongly the elements for green, but much more feebly the two other kinds=sensation of gren. Simple blue excites to a moderate extent the elements for green and violet; more fecbly those for red=sensation of blue. Simple violet excites strongly the corresponding elements, feebly the others = sensation of violet. Stimulation of any two elements excites the impression of a mixed colour; while, if all of them be excited in a nearly equal degree, the sensation of white is produced. As a matter of fact, the Young-Helmholtz theory gives a simple explanation of the phenomena of the physiological doctrine of colour. It has been attempiteil to make the results obtained by exanination of the structure of the retina accord with this view. According to Max Schultze, the cones alone are end-organs connected with the perreption of colour. The presence of longitudinal striation in their outer segments is regarded as constituting them multiple terminal end-organs. Our power of colour sensation, 8) far as it depends on the retina, would, on this view of the matter, bear a relation to the number of cones. The degree of colour sensation is most developed in the macula lutea, which contains only cones, and diminishes as the distance from the point increases, while it is absent in the peripheral parts of the retina. The rods of the retina are said to be concerned only with the eajacity to distinguish between quantitative sensations of light.

3. Hering's Theory.-Ew. Hering, in order to explain the sensation of light, proceeds from the axiom stated under 1, p. 775. What we are conscious of, and call a visual sensation, is the physical expression for the metabolism in the visual substance ("Schsubstanz"), i.e., in those nerve-masses which are excited in the process of vision. Like every other corporeal 
matter, this substance during the activity of the metabolic prosess undergoes decomposition or "disassimilation"; while during rest it must be again renewed, or "assimilate" new material. Hering assumes that for the perception of white and black, two different qualities of the chemical processes take place in the visual substance, so that the sensation of white corresponds to the disassimilation (decomposition), and that of black to the assimilation (restitution) of the visual substance. According to this view, the different degrees of distinctness or intensity with which these two sensations appear, occur in the several transitions between pure white and deep black; or, the proportions in which they appear to be mixed (grey), correspond to the intensity of these two psycho-physical processes. Thus, the consumption and restitution of matter in the visual substance are the primary processes in the sensation of white and black. In the production of the sensation of white, the consumption of the visual substance is caused by the vibrating ethereal waves acting as the discharging force or stimulus, while the degree of the sensation of whiteness is proportional to the quantity of the matter consumed. The process of restitution discharges the sensation of black; the more rapidly it occurs, the stronger is the sensation of black. The consumption of the visual substance at one place causes a greater restitution in the adjoining parts. Both processes influence each other simultaneously and conjointly. [In the production of a visual sensation, it is important to remember that the condition of one part of the retina influences contemporaneously the condition of adjoining parts of the retina, i.e., "the sensation which arises through the stimulation of any given point of the retina, is also a function of the state of other immediately contiguous points."] This explains physiologically the phenomenon of contrast of which the old view could give only a psychical interpretation (p. 782).

Similarly, colour sensation is regarded as a sensation of decomposition (disassimilation) and of restitution (assimilation); in addition to white, red and yellow are the expression of decomposition; while green and blue represent the sensation of restitution. Thus, the visual substance is subject to three different ways of chemical change or metabolism. We may explain in this way the coloured phenomena of contrast and the complementary after-images. The sensation of black-white may occur simultaneously with all colours; hence, every colour sensation is accompanied by that of dark or bright, so that we cannot have an absolutely pure colour. There are three different constituents of the visual substance ; that connected with the sensation of black-white (colourless), that with blue-yellow, and that with red-green. All the rays of the visible spectrum act in disassimilating the black-white substance, but the different rays act in different degrees. The blue-yellow or the red-green substances, on the other hand, are disassimilated only by certain rays, some rays causing assimilation, whilst others are inactive. Mixed light appears colourless when it causes an equally strong disassimilation and assimilation in the blue-yellow and in the red-green substance, so that the two processes mutually antagonise each other, and the action on the black-white substance appears pure. Two objective kinds of light, which together yield white, are not to be regarded as complementary, but as antagonistic, kinds of light, as they do not supplement each other to produce white, but on!y allow this to appear pure, because, being antagonistic, they mutually prevent each other's action.

The imperfection of the Young-Helmholtz theory of colour sensation is that it recognises only one kind of excitability, excitement, and fatigue (corresponding to Hering's disassimilation), and that it ignores the antagonistic relation of certain light rays to the eye. It does not regard white as consisting of complementary light rays, which neutralise each other by their action on the coloured visual substance, but as uniting to form white (Hering).

[While it suffices to explain a great many of the phenomena of light and colour, e.g., the mixing of colours and complementary colours, it does not satisfactorily explain contrast or colour-blindness. Fick admits that it does not explain the following important fact :-Every ray of light, while exciting a colour sensation if it falls on a sufficient area of the posterior polar part of the eyeball, provided it acts on an extremely limited part of the retina, even if it be coloured light, produces a whitish impression. This is exactly the opposite of what we should expect, viz., the smaller the area of retina acted on, the more readily should the particular nerve-ending be excited and a pure colour sensation result.]

In applying this theory to colour-blindness ( $\$ 397)$, we must assume that those who are red-blind want the red-green visual substance; there are but two partial spectra in their solar spectrum, the black-white and the yellow-blue. The position of green appears to such an one to be colourless; the rays of the red part of the spectrum are visible, so far as the sensation of yellow and white produced by these rays is strong enough to excite the retina. Hering divides his spectrum into a yellow and a blue half. A violet-blind person wants the yellow-blue visual substance ; in his spectrum there are only two partial spectra, the black-white and the red-green. In cases of complete colour-blindness, the yellow-blue and red-green substances are absent. Hence, such a person has only the sensation of bright and dark. The 
sensibility to light and the length of the spectrum are retained; the brightest part in this case, as in the normal eye, is in the yellow (Hering).

\section{COLOUR-BLINDNESS AND ITS PRACTICAL IMPORTANCE-} Canses. - By the term colour-blindness (dyschromatopsy) is meant a pathological condition in which some individuals are unable to distinguish certain colours. Huddart (17ii) was acyuainted with the condition, but it was first accurately described by l)alton (1794), who himself was red-blind. The term colour-blindness was given to it by Brewster.

The supurerters of the loung-Helmholtz theory assume that, corresponding to the paralysis of the three colour-perceiving elements of the retina, there are the following kinds of colourhininess :-

\section{Red-blindness, 2. Green-blindness, 3. Violet-blindness,}

The highest degree being termed complete colour-blindness.

The supperters of E. Hering's theory of colour sensation distinguish the following kinds :-

1. Complete Colour-blindness (Achromatopsy). - The spectrum appears achromatic; the position of the greenish-yellow is the brightest, while it is darker on both sides of it. A coloured pirture aprears like a photograph or an engraving. Occasionally the different degrees of lifht intensity are perceived in one shade of colour, e.g., yellow, which cannot be compared with any wther colour. O. Berker and v. Hippel observed cases of unilateral congenital com. Wlete colour-llindness, whilst the other eye was normal for colour-perception.

2. Blue-yellow Blindness. - The spectrum is dichromatic, and consists only of red and green. The hlue-violet end of the spectrum is usually greatly shortened. In pure cases only the red and rreen are eorrectly distinguished (Mauthner's erythrochloropy), but not the other colours. Unilateral cases have been observed.

3. Red-green Blindness. - The sprectrum is also dichromatic. Yellow and blue are correctly distingnishenl; violet anl blue are both taken for blue. The sensations for red and green are ahisent altugether. There are several forms of this- $(\alpha)$ Green-blindness, or the red-green hindurss, with undiminisherl spectrum (Mauthner's xanthokyanopy), in which bright-green and dark-rul are confounded. In the spectrum yellow abuts directly on blue, or between the two, at must. there is a strip of srey. The maximum of brightness is in the yellow. It is often milateral and often hereditary. (li) Red-blindness (or the red-green blindness with undiminishel sjectrum, also called Daltonism), in which bright-red and dark-green are confounded. The spertrum consists of yellow and blue, but the yellow lies in the orange. The red end of the sjectrum is uncoloured, or even dark. 'The greatest brightness, as well as the limit between yellow and blue, lies more towards the right.

4. Incomplete colour-blindness, or a diminished colour sense, indicates the condition in which the acuteness of colour perception is diminished, so that the colours can be detected only in large olijects, or only when they are near, and when they are mixed with white they no longer arjuear as such. A certain degree of this form is frequent, in as far as many persons are unable to distinguish greenish-blue from bluish-green.

Acquired colour-blindness occurs in cliseases of the retina and atrophy of the optic nerve in commencing tabes, in some forms of cerebral disease (p. 713), and intoxications. At first green-blinduess ocenrs, which is soon followed hy red-blindness. The peripheral zone of the retina suffers sooner than the central area. In hysterical persons there may be intermittent attacks of colour-hlindness (Charcut); and the same occurs in hypnotised persons (p. 686).

H. Cohn found that, on heating the eyeball of some colour-blind persons, the colour-blindness tisappeared temporarily. Oceasionally in persons without a lens red vision is present, and is ilue to unkunwn causes. Percentage.-Holmgren found that 2.7 per cent. of persons were colour-hlind, most being red and green blind, and very few violet blind.

Limits of Normal Colour-blindness. - The investigations on the power of colour perception in the numul retina are best carried out by means of Aubert-Förster's perimeter, or that of MI'Harily (\$395). It is found that our colour perception is complete only in the middle of the fivel of vision. Around this is a middle zone, in which only blue and yellow are perceived, in which, therefore, there is red hlindness. Outside this zone, there is a peripheral girdle, where there is complete colour-blindness $(\$ 395)$. Hence a red-blind person is distinguished from a person with normal vision, in that the central area of the normal field of vision is absent in the former, this being rather included in the middle zone. The field of vision of a green-blind person differs from that of a person with normal vision, in that his peripheral zone corresponds to the intermediate and peripheral zones of the normal eye. The violet-blind person is distinguished by the complete absence of the normal peripheral zone. The incomplete colourblindness of these two kinds is characterised by a uniformly diminished central field. [When very intense colours are used, such as those of the solar spectrum, the retina can distinguish them quite up to its margin (Landolt).] 
In poisoning with santonin, violet-blindness (yellow vision) occurs in consequence of the paralysis of the violet perceptive retinal elements, which not unfrequently is preceded by stimulation of these elements, resulting in violet vision, i.e., objects seem to be coloured violet (Hiifner). Such is the explanation of this phenomenon given by Holmgren. Max Schultze, however, referred the yellow vision, i.e., seeing objects yellow, to an increase of the yellow pigment in the macula lutea.

When coloured objects are very small, and illuminated only for a short time, the normal eye first fails to perceive red (Aubert); hence, it appears that a stronger stimulus is required to excite the sensation of red. Brücke found that very rapidly intermittent white light is perceived as green, because the short duration of the stimulation fails to excite the elements of the retina connected with the sensation of red.

[The practical importance of colour-blindness was pointed out by George Wilson, and again more recently by Holmgren.] No person should be employed in the marine or railway service until he bas been properly certified as able to distinguish red from green.

Methods of Testing Colour-blindness. - Following Seebeck, Holmgren used small skeins of coloured wools as the simplest material, in red, orange, yellow, greenish-yellow, green, greenishblue, blue, violet, purple, rose, brown, grey. There are five finely graduated shades of each of the above colours. When testing a person, select only one skein-e.g., a bright red or rosefrom the mass of coloured wools placed in front of him, and place it aside, asking him to seek out those skeins which he supposes are nearest to it in colour.

Macé and Nacati have measured the acuteness of vision by illuminating a small object with different parts of the spectrum. They compared the observations on red and green-blind persons with their own results, and found that a red-blind person perceives green light as much brighter than it appears to a normal person. The green-blind had an excessive sensibility for red and violet. It appears that what the colour-blind lose in perceptive power for one colour they gain for another. They have also a keen sense for variations in brightness.

398. STIMULATION OF THE RETINA.-As with every other nervous apparatus, a certain but determinable time elapses after the rays of light fall upon the eye before the action of the light takes place, whether the light acts so as to produce a conscious impression, or produces merely a reflex effect npon the pupil. The strength of the impression produced depends partly and chiefly upon the excitability of the retina and the other nervous structures. If the light acts for a long time with equal intensity, the excitation, after having reached its culminating point, rapidly diminishes again, at first more rapidly, and afterwards more and more slowly.

[When the retina is stimulated by light, there is (1) an effect on the rhodopsin (p. 740). (2) The electro-motive force is diminished (\$332). (3) The processes of the hexagonal pigment-cells of the retina dipping between the rods and cones are affected; thus they are retracted in darkness, and protruded in the light (fig. 563). (4) Engelmann has shown that the length and shape of the cones vary with the action of light. The cones are retracted in darkness and protruded under the influence of light (fig. 563). This alteration in the shape of the cones takes place even if the light acts on the skin, and not on the eyeball at all.]

After-Images. - If the light acts on the eye for some time so as to excite the retina,

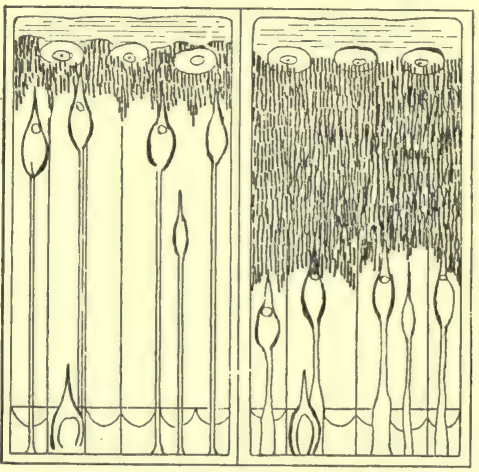

1. $\quad$ Fig. 563.2.

The cones of the retina and pigment-cells (of the frog) as affected by light and darkness : 1. after two days in darkness; 2 . after ten minutes in daylight. and if it be suddenly withheld, the retina still remains for some time in an excited condition, which is more intense and lasts longer, the stronger and the longer the light may have been applied, and the more excitable the condition of the retina. Thus, after every visual perception, especially if it is very distinct and bright, there remains a so-called "after-image." We distinguish a "positive afterimage," which is an image of similar brightness, and a similar colour. 
"That the impression of any picture remains for some time upon the eye is a physiological phenomenon; when such an impression can be seen for a long time, it becomes pathoIngieal. The weaker the eye is, the longer the image remains upon it. The retina does not revover itself so yuickly, and we may regard the action as a kind of paralysis. This is not to the wonlered at in the case of clazzling pictures. After looking at the sun, the image may remain on the retina for several days. A similar result sometimes occurs with pictures which are not lazzling. Busch records that the impression of an engraving, with all its details, remained on his eye for 17 minutes " (Gocthe).

Experiments and Apparatus for Positive After-Images. - 1. When a burning stick is rapidly rotated, it appears as a fiery circle.

2 . The phanakistoscope (I'luteu $)$ or the stroboscopic discs (Stampfer). Upon a disc or cvlinler, a series of objects is so depicted that successive drawings represent individual factors uf one continuous movement. On looking through an opening at such a disc rotated rapidly, we see pictures of the different phases moving so fuickly that each rapidly follows the one in frunt of it. As the impression of the one picture remains until the following one takes its flac\%, it has the appearance as if the successive phases of the movement were continuous, and ine and the same figure. The apparatus under the name of zoetrope, which is extensively used as a toy, is generally stated to have been invented in 1832. It was described by Cardanus in 15.51. It may be used to represent certain movements, e.g., of the spermatozoa and ciliary motion. the novements of the heart and those of locomotion.

3. The colour top contuins on the sectors of its lisc the colours which are to be mixed. As the culuur of each sector leaves a condition of excitation for the whole duration of a revolution, all the colours must be perceived simultaneously, i.e., as a mixed colour.

[llusions of Motion. - Silvanus P. Thompson points out that if a series of concentric circles in hlack and white he made on paper, and the sheet on which the circles are drawn be moved with a motiun, as if one were rinsing out a pail, but with a very minute radius, then all the .ircles aplear to lotate with the same angular velocity as that imparted. Professor Thompson has contrived other forms of this illusion, in the form of strobic discs.]

Negative After-Images.-Occasionally, when the stimulation of the retina is strong and very intense, a "negative," instead of a positive after-image, appears. In a negative after-image, the bright parts of the object appear dark, and the coloured parts in corresponding contrast colours (p. 774).

Examples of Negative After-Images. - After looking for a long time at a dazzlingly-illuminated white winlow, on closing the eyes we have the impression of a bright cross, or crosses, as the case may be, with dark panes.

Negative coloured after-images are beautifully shown by Nörrenberg's apparatus. Look stralily at a coloured surface, e.g., a yellow board with a small blue square attached to the centre of its surface. A white screen is allowed to fall suddenly in front of the board-the white surface now has a bluish appearance, with a yellow square in its centre.

The usual explanation of dark negative after-images is that the retinal elements are fatigued by the light, so that for some time they become less excitable, and consequently light is but feebly pereived in the corresponding areas of the retina; hence, darkness prevails.

Hering explains the dark after-images as due to a process of assimilation in the black-white visual substance. In explaining coloured after-images, the Young-Helmholtz theory assumes that, under the action of the light waves, e.g., red, the retinal elements connected with the perception of this colour are paralysed. On now looking suddenly on a white surface, the mixture of all the colours appears as white minus red, i.e., the white appears green. In bright daylight the contrast colour lies very near the complementary colour. According to Hering, the contrast after-image is explained by the assimilation of the corresponding coloured visual substance, in this case, of the "resl-green" ( $\$ 397)$. From the commencement of a momentary illumination until the appearance of an after-image, 0.344 sec. elapses $(v$. Vintschgau and Lustig).

Not unfrequently, after intense stimulation of the retina, positive and negative after-images alternate with each other until they gradually fuse. After looking at the dark-red setting sun we see alternate discs of red and green.

The phenomena of contrast undergo some modification in the peripheral areas of the retina, owing to the partial colour-blindness which occurs in these areas (Adamück and Woinow).

Irradiation is the term applied to certain phenomena where we form a false estimate of visual impressions, owing to inexact accommodation. If, from inexact accommodation, the margins of the object are projected upon the retina in diffusion circles, the mind tends to add the undefined margin to those parts of the visual 
image which are most prominent in the image itself. What is bright appears larger and overcomes what is dark, while an object, without reference to brightness or colour, has the same relation to its background (fig. 564). When the accommodation is quite accurate, the phenomenon of irradiation is not

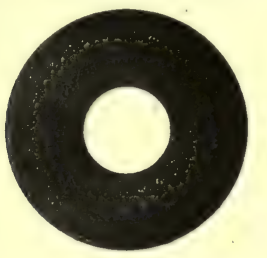

Fig. 564.

For irradiation.

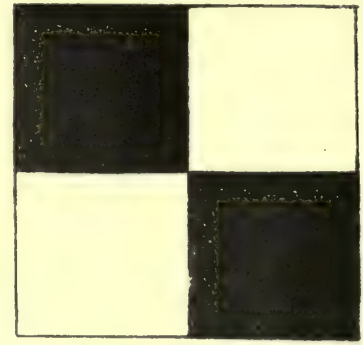

Fig. 565.

For irradiation.

present. [On looking at fig. 565 from a distance, the white squares appear larger and as if they were united by a white band.]

"A dark object appears smaller than a bright one of the same size. On looking at the same time from a certain distance at two circles of the same size, a white one on a black background, and a black on a white background, we estimate the latter to be about one-fifth less than the former (fig. 564). On making the black circle one-fifth larger they will appear equal. Tycho de Brahe remarks that the moon, when in conjunction (dark), appears to be one-fifth smaller than in opposition (full, bright). The first lunar crescent appears to belong to a larger disc than the dark one adjoining it, which can occasionally be distinguished at the time of the new light. Black clothes make persons appear to be much smaller than light clothes. A light seen behind a margin gives the appearance of a cut in the margin. A ruler, behind which is placed a lighted candle, appears to the observer to have a notch in it. The sun, when rising and setting, appears to make a depression in the horizon" (Goethe).

[Contrast.-The fundamental phenomena are such as these, that a bright object looks brighter surrounded by objects darker than itself ; and darker with surroundings brighter than itself. There may be contrasts either with bright or dark objects or with coloured ones.]

Simultaneous Contrast. - By this term is meant a phenomenon like the following :-When bright and dark parts are present in a picture at the same time, the bright (white) parts always appear to be more intensely bright the less white there is near them, or, what is the same thing, the darker the surroundings, and, conversely, they appear less bright the more white tints that are present near them. A similar phenomenon occurs with coloured pictures. A colour in a picture appears to us to be more intense the less of this colour there is in the adjoining parts, that is, the more the surroundings resemble the tints of the contrast colour. Simultaneous contrast arises from simultaneous impressions occurring in two adjoining and different parts of the retina.

Examples of Contrast for Bright and Dark.-1. Look at a white network on a black ground ; the parts where the white lines intersect appear darker, because there is least black near them.

2. Look at a point of a small strip of dark grey paper in front of a dark black background. Push a large piece of white paper between the strip and the background; the strip on the white ground now appears to be much darker than before. On again removing the white paper, the strip at once again appears bright (Hering).

3. Look with both eyes towards a greyish-white surface, e.g., the ceiling of a room. After gazing for some time, place in front of the eye a paper tube eight inches long, and an inch to an inch and a quarter in diameter, blackened in the inside. The part of the ceiling seen through the tube appears as a round white spot (Landois).

Examples for Colours.-1. Place a piece of grey paper on a red, yellow, or blue ground; the contrast colours appear at once, viz., green; blue, or yellow. The phenomenon is made still more distinct by covering the whole with transparent tracing paper (Herm. Meyer). Under similar circumstances, printed matter on a coloured ground appears in its complementary colour (W. v. Bezold). 
2. An air-bubble in the strongly tinged field of vision of a thick microscopical preparation appears with an intense contrast colour (Landois).

3. Paste funr green sectors upon a rotatory white disc, leave a ring round the centre of the dise uncovered liy green, and cover it with a black strip. On rotating such a dise the black frart appears red and not grey (Bricke).

4. Look with both eyes towards a greyish-white surface, and place in front of one eye a tube about the length and breadth of a finger, comprosed of transparent oiled paper, gummed together to such thickness as will permit light to pass through its walls. The part of the surface seen through the tule appears in its contrast colour. The experiment also shows the contrast in the intensity of the illumination (Lamlois). A white piece of paper, with a round black spot in its centre, when looked at through a blue glass appears blue with a black spot. If a white spot of the same size on a black ground be placed in front, so that it is reflected in the glass plate and just covers the black spot, it shows the contrast colour yellow (Ragona Scina).

5. The coloured shadows also belong to the group of simultaneous contrasts. "Two conditions are necessary for the production of coloured shadows-firstly, that the light gives some kint of a colour to the white surface; second, that the shadow is illuminated, to a certain extent, by another light. During the twilight, place a shert lighted candle on a white surface, between it and the fading daylight hold a pencil vertieally, so that the shadow thrown by the "an!le is illuminated, hut not abolished, by the feeble daylight; the shadow appears of a beautiful llue. 'The blue shadow is easily seen, but it requires a little attention to observe that the white paper acts like a reddish-yellow surface, wherely the blue colour apparent to the eye is improved. One of the most beautiful cases of coloured shadows is seen in connection with the full moon. The light of the candle and that of the moon can be completely equalised. Both shadows can be obtained of equal strength and distinctness, so that both colours are completely balanced. Place the plate opposite the light of the moon, the lighted candle a little to one side at a suitable distance. In front of the plate hold an opaque body, when a double shalow aplears, the one thrown by the moon and lighted by the candle being bright reddishyelluw; and, conversely, the one thrown by the candle and lighted by the moon appears of a beautiful blue. Where the two shadows come together and unite is black" (Goethe).

6. "Take a plate of green glass of considerable thickness and hold it so as to get the bars of a window reflected in it, the hars will be seen double, the image formed by the under surface of the glass being green, while the inage coming from the under surface of the glass, and which ought really to be colourless, appears to be purple. The experiment may be performed with a vessel filled with water, with a mirror at its base. With pure water colourless images are obtained, while by colouring the water coloured images are produced" (Goethe).

Explanation of Contrast. - Some of these phenomena may be explained as due to an error of judgment. During the simultaneous action of several impressions, the judgment errs, so that when an effect ocrurs at one place, this acts to the slightest extent in the neighbouring parts. When, therefore, brightness acts upon a part of the retina, the judgment ascribes the smallest possible action of the brightness to the adjoining parts of the retina. It is the same with colours. It is far more probable that the phenomena are to be referred to actual physiological processes (Hering). Partial stimulation with light affects not only the part so acted on, but also the surrounding area of the retina (p. 782); the part directly excited undergoing increased disassimilation, the (indirectly stimulated) adjoining area undergoing increased assimilation; the increase of the latter is greatest in the immediate neighbourhood of the illuminated portion, and rapidly diminishes as the distance from it increases. By the increase of the assimilation in those parts not acted on by the image of the object, this is prevented, so that the diffused light is perceived. The increase of the assimilation in the immediate neighbourhood of the illuminated spot is greatest, so that the perception of this relatively stronger different light is largely rendered impossible (Hering).

[Helmholtz thus ascribed the phenomena of contrast to psychical conditions, i.e., errors of judgment, but this explauation is certainly not complete. A far more satisfactory solution of the problem is that of Hering, that stimulation of one part of the retina affects the condition of adjoining parts. If a white disc on a black background be looked at for a time, and then the eyes be closed, a negative after-image of the disc appears, but it is darker and blacker than the visual area, and it has a light area around, brightest close to the disc, i.e., the adjacent part of the retina is affected. This Hering has called successive light induction. ]

Successive Contrast. - Look for a long time at a dark or bright object, or at a coloured (e.g., red) one, and then allow the effect of the contrast to occur on the retina, i.e., with reference to the above, bright and lark, or the contrast colour green, then these become very intense. This phenomenon has also been called "successive contrast." In this case the negative afterimage obviously plays a part.

[Some drugs cause subjective visual sensations, but these do so by acting on the brain, e.g., alcohol, as in delirium tremens, cannabis indica, sodic salicylate, and large doses of digitalis (Brunton).] 
eyeball is capable of extensive and free movement on the correspondingly excavated fatty pad of the orbit, just like the head of a long bone in the corresponding socket of a freely movable arthroidal joint. The movements of the eyeball, however, are limited by certain conditions, by the mode in which the eye-muscles are attached to it. Thus, when one muscle contracts, its antagonistic muscle acts like a bridle, and so limits the movement; the movements are also limited by the insertion of the optic nerve. The soft elastic pad of the orbit on which the eyeball rests is itself subject to be moved forward or backward, so that the eyeball also must participate in these movements.

Protrusion of the eyeball takes place-1. By congestion of the blood-vessels, especially of the veins in the orbit, such as occurs when the overflow of the venous blood from the head is interfered with, as in cases of hanging. 2. By contraction of the smooth muscular fibres in Tenon's capsule, in the spheno-maxillary fissure, and in the eyelids (\$ 404), which are inmervated by the cervical sympathetic nerve. 3. By voluntary forced opening of the palpebral fissure, whereby the pressure of the eyelids acting on the eyeball is diminished. 4. By the action of the oblique muscles, which act by pulling the eyeball inwards and forwards. If the superior oblique be contracted when the eyelids are forcibly opened, the eyeball may be protruded about $1 \mathrm{~mm}$. When protrusion of the eyeball occurs pathologically (as in 1 and 2), the condition is called exophthalmos.

Retraction of the eyeball is the opposite condition, and is caused-1. By closing the eyelids forcibly. 2. By an empty condition of the retrobulbar blood-vessels, diminished succulence, or disappearance of the tissue of the orbit. 3. Section of the cervical sympathetic in dogs causes the eyeball to sink somewhat in the orbit. The smooth muscular fibres of Tenon's capsule are perhaps antagonistic in their action to the four recti when acting together, and thus prevent the eyeball from being drawn too far backwards. Many animals have a special retractor bulbi muscle, e.g., amphibians, reptiles, and many mammals; the ruminants have four.

The movements of the eyes are almost always accompanied by similar movements of the head, chiefly on looking upwards, less so on looking laterally, and least of all when looking downwards.

The difficult investigations on the movements of the eyeballs have been carried out, especially by Listing, Meissner, Helmholtz, Donders, A. Fick, and E. Hering.

Axes.-All the movements of the eyeball take place round its point of rotation (fig. 566, 0), which lies $1.77 \mathrm{~mm}$. behind the centre of the visual axis, or $10.957 \mathrm{~mm}$. from the vertex of the cornea (Donders). In order to determine more carefully the movements of the eyeball, it is necessary to have certain definite data:-1. The visual axis $\left(S, S_{1}\right)$, or the antero-posterior axis of the eyeball, unites the point of rotation with the fovea centralis, and is continued straight forwards to the vertex of the cornea. 2. The transverse, or horizontal axis $\left(Q, Q_{1}\right)$, is the straight line connecting the points of rotation of both eyes and its extension outwards. Of course, it is at right angles to 1.3 . The vertical axis passes vertically through the point of rotation at right angles to 1 and 2 . These three axes form a co-ordinate system. We must imagine that in the orbit there is a fixed determinate axial system, whose point of intersection corresponds with the point of rotation of the eyeball. When the eye is at rest (primary position), the three axes of the eyeball completely coincide with the three axes of the co-ordinate system in the orbit. When the eyeball however is moved, two or more axes are displaced from this, so that they must form angles with the fixed orbital system.

Planes of Separation.-In order to be more exact, and also partly for further estimations, let us suppose three planes passing through the eyeball, and that their position is secured by any two axes. 1. The horizontal plane of separation divides the eyeball into an upper and lower half ; it is determined by the visual transverse axis. In its course through the retina it forms the horizontal line of separation of the latter; the coats of the eyeball itself cut it in their horizontal meridian. 2. The vertical plane divides the eyeball into an inner and outer half; it is determined by the visual and vertical axes. It cuts the retina in the vertical line of separation of the latter and the periphery of the bulb in the vertical meridian of the eyeball. 3. The equatorial plane divides the eyeball into an anterior and posterior half; its position is determined by the vertical and transverse axes, and it cuts the sclerotic in the equator of the eyeball. The horizontal and vertical lines of separation of the retina, which intersect in the fovea centralis, divide the retina into four quadrants.

In order to define more precisely the movements of the eyeball, v. Helmholtz has introduced the following terms :- $-\mathrm{He}$ calls the straight line which connects the point of rotation of the eye with the fixed point in the outer world, the visual line ("Blicklinie"), while a plane passing through these lines in both eyes he called the visual plane; the ground line of this plane is the line uniting the two points of rotation, viz., the transverse axis of the eyeball. Suppose a 
sagittal section (antero-posterior) to be made through the head, so as to divide the latter into a right and left half, then this plane would halve the ground line of the visual plane, and when prolonged forward would intersect the visual plane in the median line. The visual point of the eye can be (1) raised or lowered-the field which it traverses being called the visual field ("Blickfeld"); it is part of a spherical surface with the point of rotation of the eye in its centre. Proceeding from the primary position of both eyes, which is characterised by both visual lines heing parallel with each other and horizontal, then the elevation of the visual plane can be determined liy the angle which this forms with the plane of the primary position. This angle is called the angle of elcration-it is positive when the visual plane is raised (to the foreheail), and negative when it is lowered (chinwards). (2) From the primary position, the visual line can be turned laterally in the visual plane. The extent of this lateral deviation is measured by the angle of lateral rotation, i.c., by the angle which the visual line forms with the median line of the visual plane; it is said to be positive when the posterior part of the visual line is turued to the right, negative when to the left. The following are the positions of the "yeball :-

1. Primary position [or "position of rest"], in which both the lines of vision are parallel with each other, and the visual planes are horizontal. The three axes of the eyeball coincide with the three fixed axes of the co-ordinate system in the orbit.

2. Secondary positions are due to movements of the eye from the primary position. There are two different varieties- $-(a)$ where the visual lines are parallel, but are directed upwards or dowmvards. The transverse axis of both eyes remains the same as in the primary position; the deviations of the other two axes expressed by the amount of the angle of elevation of the ine of vision. (b) The second variety of the secondary position is produced by the convergence or divergence of the lines of vision. In this variety the vertical axis, round which the lateral rotation takes jlace, remains as in the primary position; the other axes form angles; the amount of the deviation is expressed by the "angle of lateral rotation." The eye, when in the primary position, can be rotated from this position $42^{\circ}$ outwards, $45^{\circ}$ inwards, $34^{\circ}$ upwards, and $57^{\circ}$ downwards (Schuurmann).

3. Tertiary position is the position brought about by the movements of the eye, in which the lines of vision are convergent, and are at the same time inclined upwards or downwards.

[Listing's Law is that which expresses the movements of the eyeball. When the eyeball moves from the primary position, or position of rest, the angle of rotation of the eye in the second position is the same as if the eye were turned about a fixed axis perpendicular to both the first and the second positions of the visual line (Helmholtz).]

All the three axes of the eye are no longer coincident with the axes in the primary position. The exact direction of the visual lines is determined by the amount of the angle of lateral rotation and the angle of elevation. There is still another important point. The eyeball is always rotated at the same time round the line of vision aud round its axis (Volkmann, Hering). As the iris rotates round the visual line like a wheel round its axis, this rotation is called "circular rotation" ("Ruddrehung") of the eye, which is always connected with the tertiary positions: Even oblique movements may be regarded as composed of - (1) a rotation round the vertical axis, and (2) round the transverse axis; or it may be referred to rotation round a single constant axis placed between the above-named axes, passing through the point of rotation of the eyeball, and at right angles to the secondary and primary direction of the visual axis (line of vision)-(Listing). The amount of circular rotation is measured by the angle which the horizontal separation line of the retina forms with the horizontal separation of the retina of the eye in the primary position. This angle is said to be positive, when the eye itself rotates in the same direction as the hand of a watch observed by the same eye, i.e., when the upper end of the vertical line of separation of the retina is turned to the right.

According to Donders, the angle of rotation increases with the angle of elevation and the angle of lateral rotation-it may exceed $10^{\circ}$. With equally great elevation or depression of the visual plane, the rotation is greater, the greater the elevation or depression of the line of vision. 
On looking upvards in the tertiary position, the upper ends of the vertical lines of separation of the retina diverge; on looking downwards they converge. If the visual plane be raised, the eye, when it deviates laterally to the right, makes a circular rotation to the left. Whell the visual plane is depressed, on deviating the eye to the right or left, there is a corresponding circular rotation to the right or left. Or we may express the result thus:-When the angle of elevation and the angle of deviation have the same sign $(+$ or -$)$, then the rotation of the eyeball is negative; when, however, the signs are unequal, the rotation is positive. In order to make the circular rotation visible in one's own eye, accommodate one eye for a surface divided by vertical and horizontal lines until a positive after-image is produced, and then rapidly rotate the eye into the third position. The lines of the after-image then form angles with the lines of the background. As the position of the vertical meridian of the eye is important from a practical point of view, it is necessary to note that, in the primary and secondary positions of the eyes, the vertical meridian retains its vertical position. On looking to the left and up-

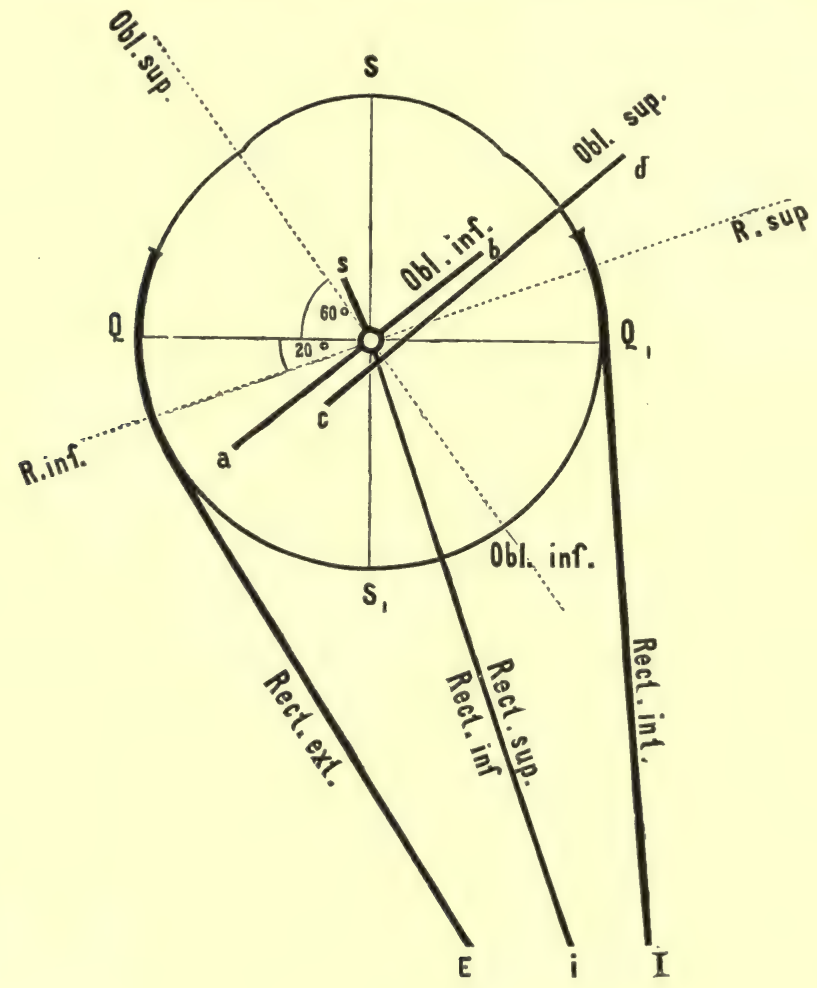

Fig. 566.

Scheme of the action of the ocular muscles.

wards, or to the right and downwards, the vertical meridians of both eyes are turned to the left; conversely, they are turned to the right on looking to the left and downwards, or to the right and upwards.

In the secondary positions of the eye, rotation of the axis of the eye never occurs (Listing). Very slight rolling of the eyes occurs, however, when the head is inclined towards the shoulder, and in the direction opposite to that of the head, it is about $1^{\circ}$ for every $10^{\circ}$ of inclination of the head (Slkrebitak).

Ocular Muscles. - The movements of the eyeball are accomplished by means of the four straight and two oblique ocular muscles. In order to understand the action of each of these muscles, we must know the plane of traction of the muscles 
and the axis of rotation of the eyeball. The plane of traction is found by the plane lying in the middle of the origin and insertion of the muscle and the point of rotation of the eyeball. The axis of rotation is always at right angles to the plane of traction in the point of rotation of the eyeball.

1. The rectus internus $(\mathrm{I})$ and externus $(\mathrm{E})$ rotate the eye almost exactly inwards and outwards (fig. 566). The plane of traction lies in the plane of the paper; Q, $\mathrm{E}$, is the direction of the traction of the external rectus, $Q_{1}, I$, that of the internal. The axis of rotation is in the point of rotation, $\mathrm{O}$, at right angles to the plane of the pajer, so that it coincides with the vertical axis of the eyeball. 2. The axis of rotation of the $\mathbf{R}$. superior and inferior (the dotted line, R. sup., $R$. inf.), lies in the horizontal plane of separation of the eye, but it forms an angle of about $20^{\circ}$ with the transverse axis $\left(Q, Q_{1}\right)$; the direction of the traction for both muscles is indicated by the line, $s, i$. Jiy the action of these muscles, the cornea is turned upwards and slightly inwards, or downwards and slightly inwards. 3. The axis of rotation of both oblique muscles (the dotted lines, Obl. sup. and Obl. inf.) also lies in the horizontal plane of separation of the eyeball, and it forms an angle of $60^{\circ}$ with the transverse axis. The direction of the traction of the inferior oblique gives the line, $a, l$; that of the superior, the line, $c, d$. The action of these muscles, therefore, is in the one case to rotate the cornea outwards and upwards, and in the other outwards and downwards. These actions, of course, only obtain when the eyes are in the primary position-in every other position the axis of rotation of each muscle changes.

When the eyes are at rest, the muscles are in equilibrium. Owing to the power of the internal recti, the visual axes converge and would meet, if prolonged 40 centimetres in front of the eye. In the movements of the eyeball, one, two, or three muscles may be concerned. One muscle acts only when the eye is moved directly outwards or inwards, especially the internal and external rectus. Two muscles act when the eyeball is moved directly upwards (superior rectus and inferior oblique) or downwards (inferior rectus and superior oblique). Three muscles are in action when the eyeballs take a diagonal direction, especially for inwards and upurards, by the internal and the superior rectus and inferior oblique; for inwards and domenerls, the internal and inferior rectus and superior oblique; for outwards and dounmerls, the external and inferior rectus and superior oblique; for outwards and unuerds, the external and superior rectus and inferior oblique.

[The following table shows the action of the muscles of the eyeball :-

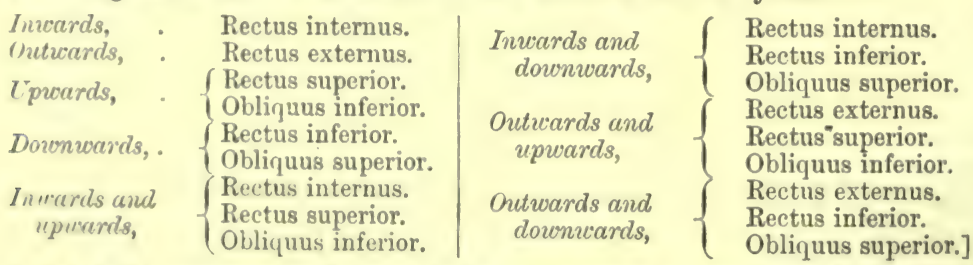

Ruete imitated the movements of the eyeballs by means of a model, which he called the ophthalmotrope.

The size of the eyeball and its length diminish with age. The mobility is less in the vertical than in the lateral clirection, and less upwards than downwards. The normal and myopic eye can be moved more outwards, and the long-sighted eye more inwards, the external and internal recti act most when the eye is moved outwards, the obliqui when it is rotated inwards. An eye can be turned inwards to a greater extent when the other eye at the same time is turned nutwards than when the other is turned inwards. During near vision, the right eye can be turned less to the right, and the left to the left, than during distant vision (Hering).

Simultaneous Ocular Movements. - Both eyes are always moved simultaneously. Even when one eye is quite blind, the ocular muscles move when the whole eyeball is excited. When the head is straight, the movements always take place so that 
both visual planes (visual axes) lie in the same plane. In front both visual axes can diverge only to a trifling extent, while they can converge considerably. If individual ocular muscles are paralysed, the position of the visual axis in the same place is disturbed, and squinting results, so that the patient no longer can direct both visual axes simultaneously to the same point, but be directs the one eye after the other. Even nystagmus (p. 721) occurs in both eyes simultaneously, and in the same direction. The innate simultaneous movement of both eyes is spoken of as an associated movement (Joh. Müller). E. Hering showed that in all ocular movements there is a uniformity of the innervation as well. Even during such movements, in which one eye apparently is at rest, there is a movement, due to the action of two antagonistic forces, the movements resulting in a slight to and fro motion of the eyeball.

The motor nerves of the ocular muscles are the oculomotorius $(\S 345)$, the trochlearis $(\$ 346)$, and the abducens $(\$ 348)$. The centre lies in the corpora quadrigemina, and below it ( $\$ 379)$, and partly in the medulla oblongata (\$379).

400. BINOCULAR VISION.-Advantages.-Vision with both eyes affords the following advantages:-(1) The field of vision of both eyes is considerably larger than that of one eye. (2) The perception of depth is rendered easier, as the retinal images are obtained from two different points. (3) A more exact estimate of the distance and size of an object can be formed, in consequence of the perception of the degree of convergence of both eyes. (4) The correction of certain errors in the one eye is rendered possible by the other.

When the position of the head is fixed, we can easily form a conception as to the form of the entire field of vision if we close one eye and direct the open eye inwards. We observe that it is pear-shaped, broad above and smaller below, the silhouette, or profile of the nose, causes the depression between the upper and lower part of the field.

401. IDENTICAL POINTS-HOROPTER-Identical Points.-If we imagine the retinæ of both eyes to be a pair of hollow saucers placed one within the other, so that the yellow spots of both eyes coincide, and also the similar quadrants of the retinæ, then all those points of both retinæ which coincide or cover each other are called "identical" or "corresponding points" of the retina. The two meridians which separate the quadrants coinciding with each other are called the "lines of separation." Physiologically, the identical points are characterised by the fact that, when they are both simultaneously excited by light, the excitement proceeding from them is, by a psychical act, referred to one and the same point of the field of vision, lying, of course, in a direction through the nodal point of each eye. Stimulation of both identical points causes only one image in the field of vision. Hence all those objects of the external world, whose rays of light pass through the nodal points to fall upon identical points of the retina, are seen singly, because their images from both eyes are referred to the same point of the field of vision, so that they cover each other: All other objects whose images do not fall upon identical points of the retina cause double vision, or diplopia.

Proofs.-If we look at a linear object with the points $1,2,3$, then the corresponding retinal images are 1, 2, 3 and 1, 2, 3, which are obviously identical points of the retinæ (fig. 567). If, while looking at this line, there be a point, $A$, nearer the eyes, or $B$, further from them, then, on focussing for $1,2,3$, neither the rays $(\mathbf{A}, a, \mathbf{A}, a)$ coming from $\mathbf{A}$, nor those $(\mathrm{B}, b, \mathrm{~B}, b)$ from B, fall upon identical points; hence $\mathrm{A}$ and $\mathrm{B}$ appear double.

Make a point $(e . g ., 2)$ with ink on paper; of course the image will fall upon both fover centrales of the retinæ $(2,2)$, which of course are identical points. Now press laterally upon one eye, so as to displace it slightly, then two points at once appear, because the image of the point no longer falls upon the fovea centralis of the displaced eye, but on an adjoining non-identical part of the retina. When we squint voluntarily all objects appear double.

The vertical surfaces of separation of the retina do not exactly coincide with the vertical meridians. There is a certain amount of divergence $\left(0^{\circ} 5^{\circ}-3^{\circ}\right)$, less above, which varies in different individuals, and it may be in the same individual at different times (Hering). The horizontal lines of separation, however, coincide. Images which fall upon the vertical lines of 
separation appear to be vertical to those on the horizontal lines, although they are not actually 80. Hence, the vertical lines of separation are the apparent vertical meridians. Some observers regard the ilentical points of the retina as an acquired arrangement ; others regard it as normally innate. Persons who have had a squint from their birth see singly; in these cases, the identical prints must be differently disposed.

The horopter represents all those points of the outer world from which rays of

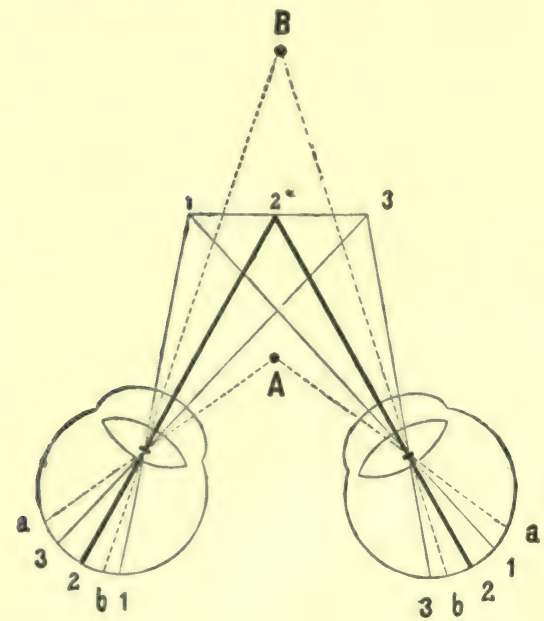

Fig. $56 \pi$.

Scheme of identical and non-identical points of the retina.

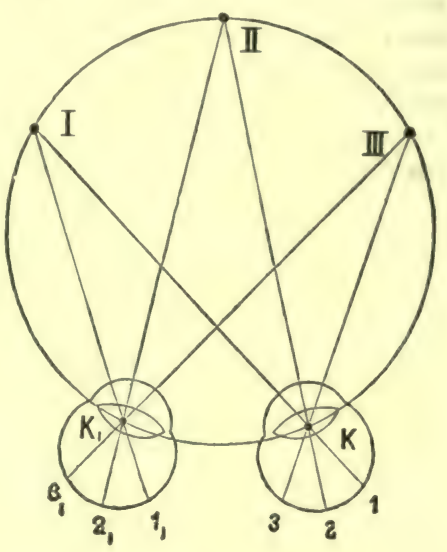

Fig. 568.

Horopter for the secondary position, with convergence of the visual axes.

light passing into both eyes fall upon identical points of the retina, the eyes being in a certain position. It varies with the different positions of the eyes.

1. In the primary position of both eyes with the visual axes parallel, the rays of direction proceeding from two identical points of the two retinæ are parallel and intersect only at infinity. Hence for the primary position the horopter is a plane in infinity.

2. In the secondary position of the eyes with converging visual axes, the horopter for the transverse lines of separation is a circle which passes through the nodal points of both eyes (fig. $568, K, K$ ), and through the fixed points I, II, III. The horopter of the vertical lines of separation is in this position vertical to the plane of vision.

3. In the symmetrical tertiary position, in which the horizontal and vertical lines of separation form an angle, the horopter of the vertical lines of separation is a straight line inclined towards the horizon. There is no horopter for the identical points of the horizontal lines of separation, as the lines of direction prolonged from the identical points of these points do not intersect.

4. In the unsymmetrical tertiary position (with rolling) of the eyes, in which the fixed point lies at unequal distances from both nodal points, the horopter is a curve of a complex form.

All objects, the rays proceeding from which fall upon non-identical points oi the retina, alpear double. We can distinguish direct or crossed double images, according as the rays prolonged from the non-identical points of the retina intersect in front of or behind the fixed point.

Experiment. - Hold two fingers-the one behind the other-before both eyes. Accommo. date for the far one and then the near one appears double, and when we accommodate for the near one the far one appears double. If, when accommodating for the near one, the right eye be closed, the left (crossed) image of the far finger disappears. On accommodating for the far finger and closing the right eye, the right (direct) double image of the near finger disappears.

Double images are referred to the proper distance from the eyes, just as single images are. 
Neglect of Double Images.-Notwithstanding the very large number of double images which must be formed during vision, they do not disturb vision. As a general rule they are "neglected," so that the attention must, as a rule, be directed to them before they are perceived. This condition is favoured thus:-

1. The attention is always directed to the point of the field of vision which is accommodated for at the time. The image of this part is projected on to both yellow spots, which are identical points of the retina.

2. The form and colour of objects on the lateral parts of the retina are not perceived so sharply.

3. The eyes are always accommodated for those points which are looked at. Hence, indistinct images with diffusion circles are always formed by those objects which yield double images, so that they can be more readily neglected.

4. Many double images lie so close together that the greater part of them, when the images are large, covers the other.

5. By practice images which do not exactly coincide may be united.

402. STEREOSCOPIC VISION.-On looking at an object, both eyes do not yield exactly similar images of that object-the images are slightly different, because the two eyes look at the object from two different points of view. With the right eye we can see more of the side of the body directed towards it, and the same is the case with the left eye. Notwithstanding this inequality, the two images are united. How two different images are combined is best understood by analysing the stereoscopic images.

Let, in fig. 569, $\mathrm{L}$ and $\mathrm{R}$ represent two such images as are obtained with the left and right eyes. These images, when seen with a stereoscope, look like a truncated pyramid, which projects towards the eye of the observer, as the points indicated

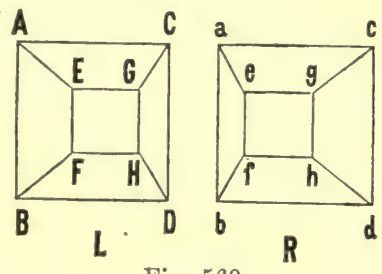

Fig. 569.

Two stereoscopic drawings. by the same signs cover each other. On measuring the distance of the points, which coincide or cover each other in both figures, we find that the distances $\mathrm{A}, a, \mathrm{~B}, b, \mathrm{C}, c, \mathrm{D}, d$ are equally great, and at the same time are the widest of all the points of both figures; the distances $\mathrm{E}, e$, $\mathrm{F}, f, \mathrm{G}, g, \mathrm{H}, h$ are also equal, but are smaller than the former. On looking at the coinciding lines ( $\mathrm{A}, \mathrm{E}, a, e$, and $\mathrm{B}, \mathrm{F}, b, f)$, we observe that all the points of this line which lie nearer to $\mathrm{A} a$ and $\mathrm{B} b$ are further apart than those lying nearer $\mathrm{E} e$ and $\mathrm{F} f$.

Comparing these results with the stereoscopic image, we have the following laws for stereoscopic vision:-1. All those points of two stereoscopic images, and of course of two retinal images of an object, which in both images are equally distant from each other, appear on the same plane. 2. All points which are nearer to each other, compared with the distance of other points, appear to be nearer to the observer. 3. Conversely, all points which lie further apart from each other appear perspectively in the background.

The cause of this phenomenon lies in the fact that, "in vision with both eyes we constantly refer the position of the individual images in the direction of the visual axis to where they both intersect."

Proofs. - The following stereoscopic experiment proves this (fig. 570):-Take both images of two pairs of points $(\alpha, b$, and $\alpha, \beta)$, which are at unequal distances from each other on the surface of the paper. By means of small stereoscopic prisms cause them to coincide, then the combined point, $\mathrm{A}$ of $a$, and $\alpha$ appears at a distance on the plane of the paper, while the other point, B, produced by the superposition of $b$ and $\boldsymbol{\beta}$, floats in the air before the observer. Fig. 570 shows how this occurs. The following experiment shows the same result:-Draw two figures, which are to be superposed similar to the lines $\mathrm{B}, \mathrm{A}, \mathrm{A}, \mathrm{E}, b, a$, and $a, e$, in fig. 569 . In the lines $\mathbf{B}, \mathbf{A}$, and $b, a$, all the points which are to be superposed lie equally distant from each other, while, on the contrary, all the points in $\mathbf{A}, \mathbf{E}$, and $a$, $e$, which lie nearer $\mathbf{E}$ and $e$, are constantly nearer to each other. When looked at with a stereoscope, the superposed verticals, $\mathrm{A}, e$, and $\mathrm{B}, b$, lie in the plane of the paper, while the superposed lines, $\mathrm{A}, \alpha$, and $\mathrm{E}$, $e$, project obliquely towards the observer from the plane of the paper. From these two fundamental experiments we may analyse all pairs of stereoscopic pictures. Thus, in fig. 569, if we exchange the two pictures, so that $\mathrm{R}$ lies in the place of $\mathrm{L}$, then we must obtain the impression of a truncated hollow pyramid. 
Two stereoscopic pictures, which are so constructed that the one contains the body from the front and above, and the other it from the front and below (suppose in fig. 569 the lines A B, and $a b$, were the ground lines), can never be superposed by means of the stereoscope.

This process has been explained in another way. Of the two figures, $R$ and $\mathrm{L}$ (fig. 569), only A B C D, and $a b c d$, fall upon identical points of the retina, hence these alone can be superposed; or, when there is a different convergence of the visual axis, only E F G H, and ef $g h$, can be superposed for the same reason. Supyose the square ground surfaces of the figures are first superposed, in order to explain the stereoscopic impression, it is further assumed that both eyes, after superposition of the ground squares, are rapidly moved towards the apex of the

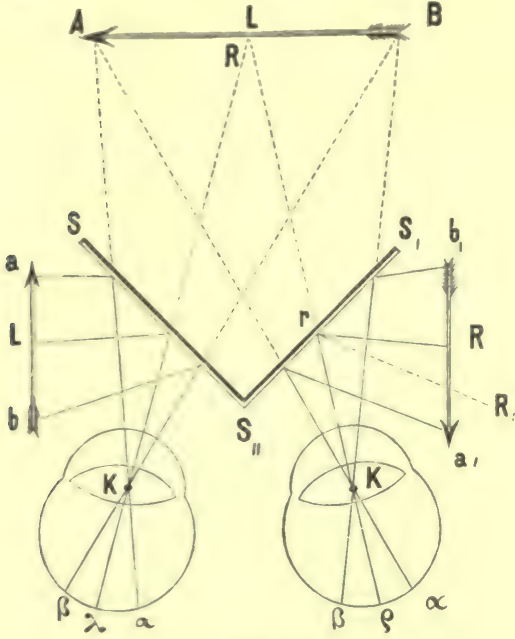

Fig. 570.

6. Wheatstone's stereoscope.

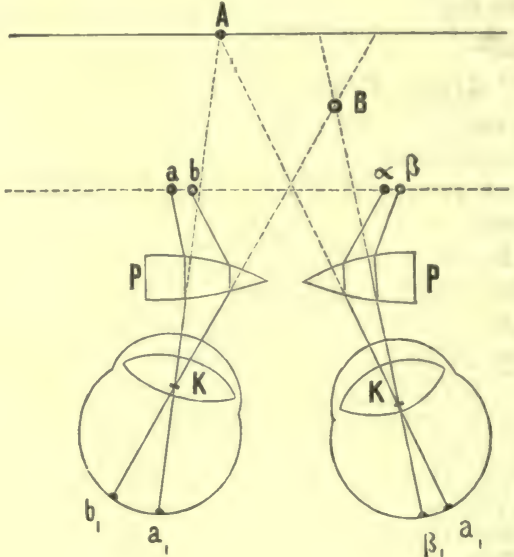

Fig. 571.

Scheme of Brewster's stereoscope.

pyramid. As the axis of the eyes must thereby converge more and more, the apex of the pyramid appears to project; as all points which require the convergence of the eyes for their vision appear to us to be nearer (see below). Thus, all corresponding parts of both figures would be brought, one after the other, upon identical points of the retina by the movements of the eyes (Briucke).

It has been urged against this view that, the duration of an electrical spark suffices for stereoscopic vision (Dove) - a time which is quite insufficient for the movements of the eyes. Although this may be true for many figures, yet in the correct combination of complex or extraordinary figures, these movements of the visual axes are not excluded, and in many individuals they are distinctly advantageous. Not only the actual movements necessary for this act, but the sensations derived from the muscles are also concerned.

When two figures are momentarily combined to form a stereoscopic picture, there being no movement of the eyes, clearly many points in the stereoscopic pictures are superposed which, strictly speaking, do not fall upon identical points of the retina. Hence we cannot characterise the identical points of the retina as coinciding mathematically ; but from a physiological point of view we must regard such points as identical, which, as a rule, by simultaneous stinulation, give rise to a single image. The mind obviously plays a part in this combination of images. There is a certain psychical tendency to fuse the double images on the retinæ into one image, in accordance with the fact that we, from experience, recognise the existence of a single object. If the differences between two stereoscopic pictures 
be too great, so that parts of the retina too wide apart are excited thereby, or when new lines are present in a picture, and do not admit of a stereoscopic effect, or disturb the combination, then the stereoscopic effect ceases.

The stereoscope is an instrument by means of which two somewhat similar pictures drawn in perspective may be superposed so that they appear single. Wheatstone (1838) obtained this result by means of two mirrors placed at an angle (fig 570); Brewster (1843) by two prisms (fig. 571). The construction and mode of action are obvious from the illustrations.

Some pairs of two such pictures may be combined, without a stereoscope, by directing the visual axis of each eye to the picture held opposite to it.

Two completely identical pictures, i.e., in which all corresponding points have exactly the same relation to each other as the same sides of two copies of a book, appear quite flat under the stereoscope; as soon, however, as in one of them one or more points alters its relation to the corresponding points, this point either projects or recedes from the plane.

Telestereoscope.-When objects, placed at a great distance, are looked at, e.g., the most distant part of a landscape, they appear to us to be flat, as in a picture, and do not stand out, because the slight differences of position of our eyes in the head are not to be compared with the great distance. In order to obtain a stereoscopic view of such objects, v. Helmholtz constructed the telestereoscope (fig. 572), an apparatus which by means of two parallel mirrors, places, as it were, the point of view of both eyes wider apart. Of the mirrors, $L$ and $R$ each projects its image of the landscape upon $l$ and $r$, to which both eyes, $\mathrm{O}, o$, are directed. According to the distance between $\mathrm{L}$ and $\mathrm{R}$ the eyes, $\mathrm{O}, 0$, as it were, are displaced to $\mathrm{O}, 0$. The distant landscape appears like a stereoscopic view. In order to see distant parts more clearly and nearer, a double telescope or opera-glass may be placed in front of the eyes.

Take two corresponding stereoscopic pictures, with the surfaces black in one case and light in the other. Draw two truncated pyramids like fig. 569, make one figure exactly like $\mathrm{L}$, i.e.,

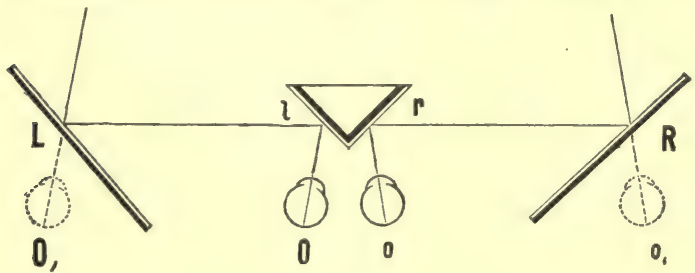

Fig. 572.

Telestereoscope of v. Helmholtz.

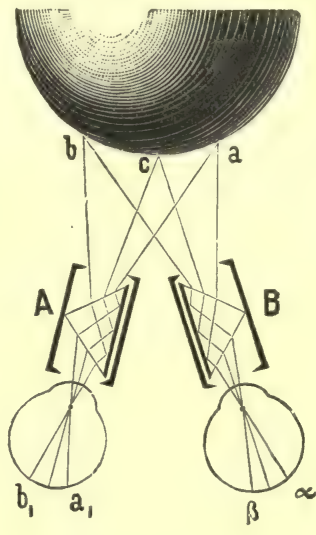

Fig. 573.

Wheatstone's Pseudoscope.

with a white surface and black lines, and the other with white lines and a black surface, then under the stereoscope such objects glance. The cause of the glancing condition is that the glancing body at a certain distance reflects bright light into one eye and not into the other, because a ray reflected at an angle cannot enter both eyes simultaneously (Dove).

Wheatstone's Pseudoscope consists of two right-angled prisms (fig. 573, A and B) enclosed in a tube, through which we can look in a direction parallel with the surfaces of the hypothenuses. If a spherical surface be looked at with this instrument, the image formed in each eye is inverted laterally. The right eye sees the view usually obtained by the left eye, and conversely; the shadow which the body in the light throws upon a light ground is reversed. Hence the ball appears hollow.

Struggle of the Fields of Vision. - The stereoscope is also useful for the following purpose :In vision with both eyes, both eyes are almost never active simultaneously and to the same extent; both undergo variations, so that first the impression on the one retina and then that on the other is stronger. If two different surfaces be placed in a stereoscope, then, especially when they are luminous, these two alternate in the general field of vision, according as one or other eye is active (Panum). Take two surfaces with lines ruled on them, so that when the surfaces are superposed the lines will cross each other, then either the one or the other system of lines is more prominent (Panum). The same is true with coloured stereoscopic figures, so that there is a contest or struggle of the coloured fields of vision.

403. ESTIMATION OF SIZE AND DISTANCE.-Size.-We estimate the size of an object-apart from all other factors-from the size of the retinal image ; thus the moon is estimated to be larger than the stars. If, while looking at a 
distant landscape, a fly should suddenly pass across our field of vision, near to our eye, then the image of the fly, owing to the relatively great size of the retinal image, may give one the impression of an object as large as a bird. If, owing to defective accommodation, the image gives rise to diffusion circles, the size may appear to be even greater. But objects of very unequal size give equally large retinal images, especially if they are placed at such a distance that they form the same visual angle (fig. 531); so that in estimating the actual size of an object, as opposed to the apparent size determined by the visual angle, the estimate of rlistence is of the greatest importance.

As to the distance of an object, we obtain some information from the feeling of accommodation, as a greater effort of the muscle of accommodation is required for exact vision of a near object than for seeing a distant one. But, as with two objects at uneyual distances giving retinal images of the same size, we know from experience that that object is smaller which is near, then that object is estimated to be the smaller for which, during vision, we must accommodate more strongly.

In this way we explain the following : $-A$ person beginning to use a microscope always observes with his eves accommodlated for a near object, while one used to the microscope looks through it without accommodating. Hence beginners always estimate microscopic objects as too small, and on making a drawing of them it is too small. If we produce an after-image in one eye, it at once appears smaller on accommodating for a near object, and again becomes larger during negative accommolation. If we look with one eye at a small body placed as near is frossilile to the eye, then a hody lying behind it, but seen only indirectly, appears smaller.

Angle of Convergence of Visual Axes.-In estimating the size of an object, and taking into account our estimate of its distance, we also obtain much more important information from the degree of convergence of the visual axes. We refer the position of an object, viewed with both eyes, to the point where both visual axes intersect. The angle formed by the two visual axes at this point is called the "angle of convergence of the visual axes" ("Gesiclitswinkel"). The larger, therefore, the visual angle, the size of the retinal image remaining the same-we judge the olject to be nearer. The nearer the object is, it may be the smaller, in order

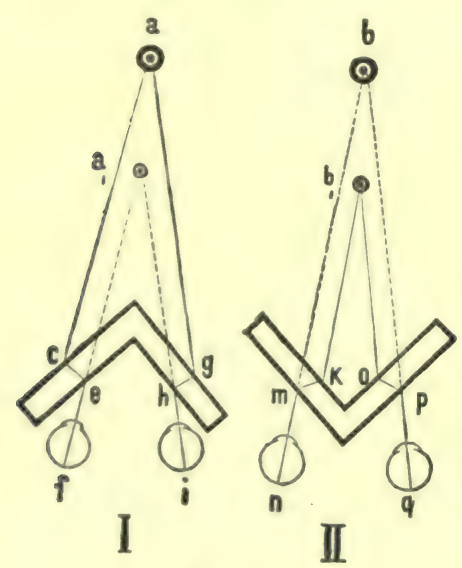

Fig. 574.

Rollett's glass plate apparatus. to form a "visual angle" of the same size, such as a distant large object would give. Hence, we conclude, that with the same apparent size (equally large visual angle, or retinal images of the same size) we judge that object to be smallest which gives the greatest convergence of the visual axes during binocular vision. As to the muscular exertion necessary for this purpose, we obtain information from the muscular sense of the ocular muscles.

Experiments and Proofs - The chess-board phenomenon of H. Meyer.-1. If we look at a uniform chess-board-like pattern (tapestry or carpet), then, when the visual axes are directed directly forwards, the spaces on the pattern appear of a certain size. If, now, we look at a nearer object, we may cause the visual axes to cross, when the pattern apparently moves towards the plane of the fixed point, so that the crossed double images are superposed, and the pattern at once appears smaller.

2. Rollett looks at an object through two thick prisms of glass placed at an angle. The plates are at one time so placed that the apex of the angle is directed towards the observer (fig. 574, II), at another in the reverse position (I). If both eyes, $f$ and $i$, are to see the object $a$, in I, then as the glass plates so displace the rays, $a, c$, and $a, g$, as to make them parallel with the direction of these rays, viz., $e, f$, and $h, i$, then the eyes must convrrge more than when they are turned directly towards $a$. Hence the object appears nearer and smaller, as at $a$. In II, the rays, $b_{1} k$, and $b_{1} o$, from the nearer object $b_{1}$, fall upon 
the glass plates. In order to see $b_{1}$, the eyes $(n$ and $q$ ) must diverge more, so that $b$ appears more distant and larger.

3. In looking through Wheatstone's reflecting stereoscope (fig. 571), it is obvious that the more the two images approach the observer, the more must the observer converge his visual axes, because the angles of incidence and reflexion are greater. Hence the compound picture now appears to him to be smaller. If the centre of the image, $R$, recedes to $R_{1}$, then of course the angle, $\mathrm{S}_{11}, r_{\rho}$, is equal to $\mathrm{S}_{1}, r \mathrm{R}_{1}$, and the same on the left side.

4. In using the telestereoscope, the two eyes are, as it were, separated from each other, then of course in looking at objects at a certain distance the convergence of the visual axes must be greater than in normal vision. Hence, objects in a landscape appear as in a small model. But as we are accustomed to infer that such small objects are at a great distance, hence the objects themselves appear to recede in the distance.

Estimation of Distance.-When the retinal images are of the same size, we estimate the distance to be greater the less the effort of accommodation, and conversely. In binocular vision, when the retinal images are of the same size, we infer that that object is most distant for which the optic axes are least converged, and conversely. Thus, the estimation of size and distance go hand in hand, in great part at least, and the correct estimation of the distance also gives us a correct estimate of the size of objects (Descartes). A further aid to the estimation of distance is the observation of the apparent displacement of objects, on moving our head or body. In the latter, especially, lateral objects appear to change their position toward the background, the nearer they are to us. Hence, when travelling in a train, in which case the change of position of the objects occurs very rapidly, the objects themselves are regarded as nearer, and also smaller (Dove). Lastly, those objects appear to us to be nearest which are most distinct in the field of vision.

Example. - A light in a dark landscape, and a dazzling crown of snow on a hill, appear to be near to us; looked at from the top of a high mountain, the silver glancing curved course of a river not unfrequently appears as if it were raised from the plane.

False Estimates of Size and Direction.-1. A line divided by intermediate points appears longer than one not so divided. Hence the heavens do not appear to us as a hollow sphere, but as curved like an ellipse; and for the last reason the disc of the setting sun is estimated to be larger than the sun when it is in the zenith. 2. If we move a circle slowly to and fro behind a slit, it appears as a horizontal ellipse ; if we move it rapidly, it appears as a vertical ellipse. 3 . If a very fine line be drawn obliquely across a vertical thick black line, then the direction of the fine line beyond the thick one appears to be different from its original direction. 4. Zöllner's Lines. - Draw three parallel horizontal lines 1 centimetre apart, and through the upper and lower ones draw short oblique parallel lines in the direction from above and the left to below and the right; throngh the middle line draw similar oblique lines, but in the opposite direction, then the three horizontal-lines no longer appear to be parallel. [Fig. 575 shows a modification of this. The lines are actually parallel, although some of them appear to converge and others to diverge.] If we look in a dark room at a bright vertical line, and then bend the

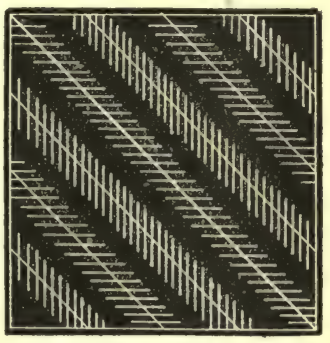

Fig. 575.

Zöllner's Lines. head towards the shoulder, the line appears to be bent in the opposite direction (Aubert).

404. PROTECTIVE ORGANS OF THE EYE.-I. The eyelids are represented in section in fig. 576. The tarsus is in reality not a cartilage, but merely a rigid plate of connective-tissue, in which the Meibomian glands are imbedded; acinous sebaceous glands moisten the edges of the eyelids with fatty matter. At the basal margin of the tarsus, especially of the upper one, close to the reflection of the conjunctiva, open the acino-tubular glands of Krause. The conjunctiva covers the anterior surface of the bulb as far as the margin of the cornea, over which the epithelium alone is continued. On the posterior surface of the eyelid, the conjunctiva is partly provided with papillæ. It is covered by stratified prismatic epithelium. Coiled glands occur in ruminants just outside the margin of the cornea, while outside this, towards the outer angle of the eye in the pig, there are simple glandular sacs. Waldeyer describes modified sweat glands in the tarsal margins in man. Small lymphatic sacs in the conjunctiva are called trachoma glands. Krause found end-bulbs in the conjunctiva bulbi (\$ 424). The blood-vessels in the conjunctiva communicate with the juice-canals in the cornea and sclerotic (p. 737). 
The secretion of the conjunctiva, besides some mucus, consists of tears, which may be as abundant as that formed in the lachrymal glands.

Closure of the eyelids is effected by the orbicularis palpebrarum (facial nerve,

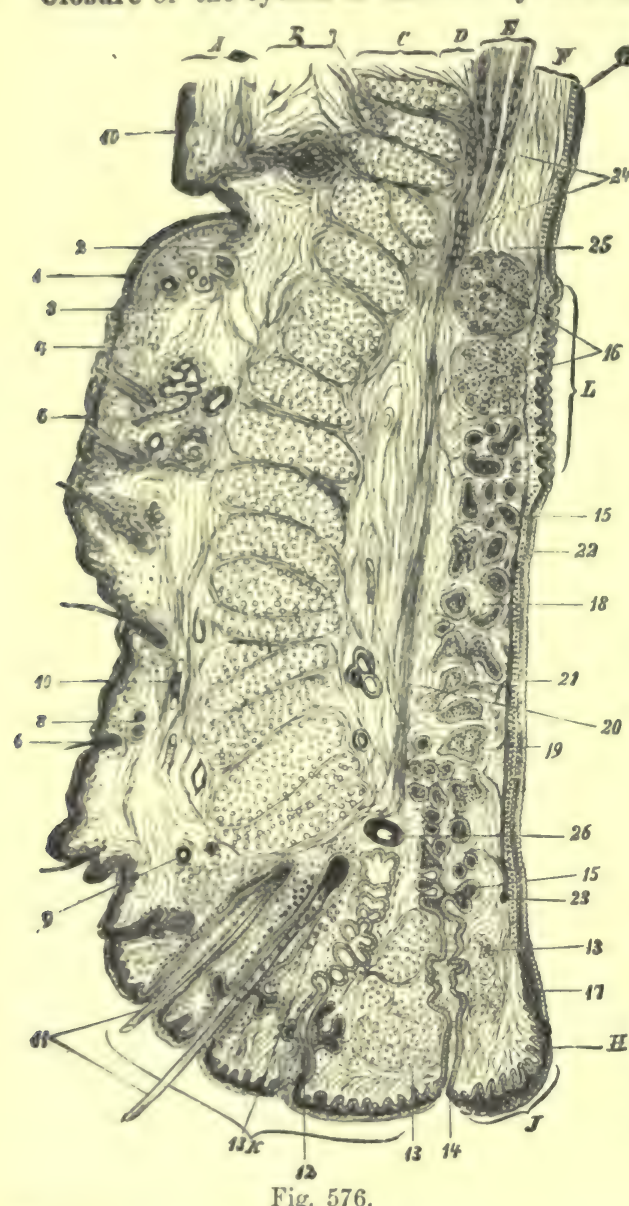

Fig. 576.

$\S 349)$, whereby the upper: lid falls in virtue of its own weight. This muscle contracts-(1) voluntarily; (2) involuntarily (single contractions); (3) reflexly by stimulation of all the sensory fibres of the trigeminus distributed to the bulb and its immediate neighbourhood (\$347), also by intense stimulation of the retina by light; (4) continued involuntary closure occurs during sleep.

Opening of the eyelids is brought about by the passive descent of the lower one, and the active elevation of the upper eyelid by the levator palpebræ superioris (§ 345). The smooth muscular fibres of the eyelids also aid (p. 593). In looking downwards, the lower eyelid is pulled downwards by bands of connective-tissue which run from the inferior rectus to the inferior tarsal cartilage (Schwalbe).

II. The lachrymal apparatus consists of the lachrymal glands, which in structure closely resemble the parotid, their acini being lined by low cylindrical granular epithelium. Four to five larger, and eight to ten smaller excretory ducts conduct the tears above the outer angle of the lid into the fornix conjunctivæ. The tear ducts, beginning at the puncta lachrymalis, are composed of connective and elastic-tissue, and are lined by stratified squamous epithelium. Striped muscle accompanies the duct, and by its contraction keeps the duct open. Toldt found no sphincter surrounding the Vertical section through the upper eyelid. $A$, cutis ; puncta lachrymalia, while Gerlach found 1 , epidermis ; 2 , chorium ; $B$ and 3 , subcutaneous an incomplete circular musculature. The connective-tissue ; $C$ and 7 , orbicularis muscle; $D$, loose sub-muscular connective-tissue ; $E$, insertion of H. Miller's muscle ; $F$, tarsus ; $G$, conjunctiva ; $J$, inner, $K$, onter edge of the lid; 4 , pigment-cells ; 5 , sweat glands; 6 , hair follicles; 8 and 23 , sections of nerves; 9, arteries; 10 , veins; 11 , cilia; 12 , modified sweat glands; 13, circular muscle of Riolan ; 14, Meibomian gland; 15 , section of an acinus of the same; 16, posterior tarsal glands; 18 and 19 , tissue of the tarsus ; 20 , pretargal or sub-muscular connective-tissue; 21 and 22 , conjunctiva, with its epithelium ; 24, fat ; 25 , loosely woven posterior end of the tarsus; 26, section of a palpebral artery. connective-tissue covering of the tear sac and canal is united with the adjoining periosteum. The thin mucous membrane, which contains much adenoid tissue and lymph-cells, is lined by a single layer of ciliated cylindrical epithelium, which below passes into the stratified form. The opening of the duct is often provided with a valve-like fold (Hasner's valve).

The conduction of the tears occurs between the lids and the bulb by means of capillarity, the closure of the eyelids aiding the process. The Meibomian secretion prevents the overflow of the tears [just as greasing the 
edge of a glass vessel prevents the water in it from overflowing]. The tears are conducted from the puncta through the duct, chiefly by a siphon action. Horner's muscle (also known to Duvernoy, 1678) likewise aids, as every time the eyelids are closed it pulls upon the posterior wall of the sac, and thus dilates the latter, so that it aspirates tears into it (Henke).

E. H. Weber and Hasner ascribe the aspiration of the tears to the diminution of the amount of air in the nasal cavities during inspiration. Arlt asserts that the tear sac is compressed by the contraction of the orbicularis muscle, so that the tears must be forced towards the nose. Lastly, Stellwag supposes that when the eyelids are closed the tears are simply pressed into the puncta, while Gad denies that there is any kind of pumping mechanism in the nasal canal. Landois points out that the tear ducts are surrounded by a plexus of veins, which according to their state of distension may influence the size of these tubes.

The secretion of tears takes place only by direct stimulation of the lachrymal nerve ( $\$ 347$, I., 2), subcutaneous malar (\$ 347, II., 2), and cervical sympathetic ( $356, \mathrm{~A}, 6)$, which have been called secretory nerves. Secretion may also be excited reflexly (p. 591) by stimulation of the nasal mucous membrane only on the same side (Herzenstein). The ordinary secretion in the waking condition is really a reflex secretion produced by the stimulation of the anterior surface of the bulb by the air, or by the evaporation of tears. A very bright light also causes a reflex secretion of tears, the optic being the afferent nerve. The centre in the rabbit does not extend forward beyond the origin of the fifth nerve, but it extends downwards to the fifth vertebra (Eckhard). During sleep all these factors are absent, and there is no secretion. Histological changes.-Reichel found that in the active gland (after injection of pilocarpin) the secretory cells became granular, turbid, and smaller, while the outlines of the cells became less distinct, and the nuclei spheroidal. In the resting gland, the cells are bright and slightly granular with irregular nuclei. Intense stimulation by light acting on the optic nerve causes a reflex secretion of tears. The flow of tears accompanying certain violent emotions, and even hearty laughing, is still unexplained. During coughing and vomiting the secretion of tears is increased partly reflexly, and partly by the outflow being prevented by the expiratory pressure.

Function.-The tears moisten the bulb, protect it from drying, and float away small particles, being aided in this by the closure of the eyelids. Atropin diminishes the tears (Mogaard).

Composition.-The tears are alkaline, saline to taste, and represent a "serous" secretion. Water 98.1 to $99 ; 1.46$ organic substances $(0.1$ albumin and mucin, 0.1 epithelium); 0.4 to 0.8 salts (especially $\mathrm{NaCl}$ )

[Action of Drugs.-Essential volatile oils and eserin increase the secretion of tears, atropin arrests it, while eserin antagonises the effect of atropin and causes an increased secretion.]

405. COMPARATIVE-HISTORICAL. - Comparative. - The simplest form of visual apparatus is represented by aggregations of pigment-cells in the outer coverings of the body, which are in connection with the termination of afferent nerves. The pigment absorbs the rays of light, and in virtue of the light-ether discharges kinetic energy, which excites the terminations of the nervous apparatus. Collections of pigment-cells, with nerve-fibres attached, and provided with a clear refractive body, occur on the margin of the bell of the higher medusæ, while the lower forms have only aggregations of pigment on the bases of their tentacles. Also, in many lower worms there are pigment spots near the brain. In others, the pigment lies as a covering round the terminations of the nerves, which occur as "crystalline rods" or "crystalline spheres." In parasitic worms, the visual apparatus is absent. In star-fishes, the eyes are at the tips of the arms, and consist of a spherical crystal organ surrounded with pigment, with a nerve going to it. In all other echinodermata there are only accumulations of pigment. Amongst the annulosa there are several grades of visual apparatus-(1) Without a cornea, there may be only one crystal sphere (nervous end-organ) near the brain, as in the young of the crab; or there may be several crystal spheres forming a compound eye, as in the lower crabs. (2) With a cornea, consisting of a lenticular body formed from the chitin of the outer integument, the eye itself may be simple, merely consisting of one crystal rod, or it may be compound. The compound eye consists of only one large lenticular cornea, common to all the crystal rods, as in the spiders; or each crystal rod has a special lenticular cornea for itself. The numerous rods 
surroundel by pigment are closely packed together, and are arranged upon a curved surface, so that their free enils also form a part of a sphere. The chitinous investment of the head is facetted, and forms a small corneal lens on the free end of each rod. According to one view, each facette, with the lens and the crystal sphere, is a special eye, and just as man has two eyes so insects have several hundred. Each eye sees the picture of the outer world in toto. This view is supported by the following experiment of van Leeuwenhoek:-If the cornea be sliced off, earli facette thereof gives a special image of an object. If a cross be made on the mirror of a microscopre, while a piece of the facetted cornea is placed as an object upon the stage, then we see an image of the cross in each facette of the cornea. Thus for each rod (crystal sphere) there would be a sprecial image. Each corneal facette, however, forms only a part of the image of the outer world, so that we must regard the image as composed like a mosaic. Amongst mollusca, the fixed brachiofoda have two jigment spots near the brain, but only in their larval condition; while the mussel has, under similar conditions, pigment spots with a refractive body. The alult mussel however, has pigment spots (ocelli) only in the margin of the mantel, but some molluscs have stalked and highly developed eyes. Some of the lower snails have no eyes, some have pigment spots on the head, while the garden snail has stalked eyes provided with a cornea, an oftic nerve with retina and pigment, and even a lens and vitreous body. Amongst cepha. lopoda, the nautilus has no cornea or lens, so that the sea-water flows freely into the orbits. Dthers have a lens and no cornea, while some have an opening in the cornea (Loligo, Sepia, Octopus). All the other parts of the eye are well developed. Amongst vertebrata, Amphioxus has no eyes. They exist in a degenerated condition in Proteus and the mammal Spalax. In many fishes, amphibians, and reptiles, the eye is covered by a piece of transparent skin. [Pineal or Epiphysial Eye. - Some lizarls, e.g., Hatteria, have a rudimentary median eye in the median line of the head, and lodged in the parietal foramen. It is developed from the pineal body, and its lens is formed from the optic cup, so that light falls upon the retina without jenctrating the fibres of the optic nerve. Thus, it is an invertebrate type of eye, where the retina and lens are developed from epidermal structures, while in the vertebrate eye, the retina is leveloped from the cerebrum.] Some hag-fishes, the crocodile, and birds have eyelids, and a nictitating membrane at the inner angle of the eye. Connected with it is the Harderian gland. In mammals the nictitating membrane is represented only by the plica semilunaris. There is no lachrymal apparatus in fishes. The tears of snakes remain under the watch-glasslike eutis with which the eyes are covered. The sclerotic often contains cartilage which may ossify. A vascular organ, the processus falciformis, passes from the middle of the choroid into the interior of the vitreous body in osseous fishes, its anterior extremity being termed the campanula Halleri. Similarly, there is the pecten in birds, but it is provided with muscular fibres. In birds the cornea is surrounded by a bony ring. The whale has an enormously thick sclerotic. In aquatic animals, the lens is nearly spherical. The muscles of the iris and churoid are transversely striped in birds and reptiles. The retinal rods in all vertebrates are lirected from before backwards, while the analogous elements (crystal rods and spheres) in invertebrata are directed from behind forward.

Historical. - The Hippocratic School were acquainted with the optic nerve and lens. Aristotle (384 B.C. ) mentions that section of the optic nerve causes blindness-he was acquainted with after-images, short and long sight. Herophilus (307 B.c.) discovered the retina, and the ciliary frocesses received their name in his school. Galen (131-203 A.D.) described the six muscles of the eyeball, the puncta lachrymalia, and tear duct. Aerengar (1521) was aware of the fatty matter at the edge of the eyelids. Stephanus (1545) and Casseri (1609) described the Meibomian glauls, which were afterwards redescribed by Meibom (1666). Fallopius described the vitreous membraue and the ciliary ligament. Plater (1583) mentions that the posterior surface of the lens is more curved. Aldrovandi observed the remainder of the pupillary membrane (1599). ()bservations were made at the time of Vesalius (1540) on the refractive action of the lens. Leonardo da Vinci compared the eye to a camera obscura. Maurolykos compared the action of the lens to that of a lens of glass, but it was Kepler (1611) who first showed the true refractive index of the lens and the formation of the retinal image, but he thought that during accommodation the retina moved forward and backward. The Jesuit, Scheiner $(+1650)$, mentions, however, that the lens becomes more convex by the ciliary processes, and he assumed the existence of muscular fibres in the uvea. He referred long and short sight to the curvature of the lens, and he first showed the retinal image in an excised eye. With regard to the use of spectacles there is a reference in Pliny. It is said that at the beginning of the 14 th century the Florentine, Salvino d'Armato degli Armati di Fir (†1317), and the monk, Alessandro de Spina (†1313), invented spectacles. Kepler (1611) and Descartes (1637) described their action. Mayo $(† 1852)$ described the 3 rd nerve as the constrictor nerve of the pupil. Zinn contributed considerably to our knowledge of the structure of the eye. Ruysch described muscular fibres in the iris, and Monro described the sphincter of the pupil (1794). Jacob described the bacillary layer of the retina-Scemmering (1791) the yellow spot. Brewster and Chossat (1819) tested the refractive indices of the optical media. Purkinje (1819) studied subjective vision. 


\section{Hearing.}

406. THE ORGAN OF HEARING.-Stimulation of the Auditory Nerve. - The normal manner in which the auditory nerve is excited, is by means of sonorous vibrations, which set in motion the end-organs of the acoustic nerve, which lie in! the endolymph of the labyrinth of the inner ear, on membranous expansions of the cochlea and semicircular canals. Hence, the sonorous vibrations are first transmitted to the fluid in the labyrinth, and this, in turn, is thrown into waves, which set the endorgans into vibration. Thus, the excitement of the auditory nerves is brought about by the mechanical stimulation of the wave-motion of the lymph of the labyrinth.

The fluid or lymph of the labyrinth is surrounded by the exceedingly hard osseous mass of the temporal bone (fig. 577). Only at one small roundish and slightly triangular area, the fenestra rotunda $(r)$, the fluid is bounded by a delicate yielding membrane, which is in contact with the air in the middle ear or tympanum

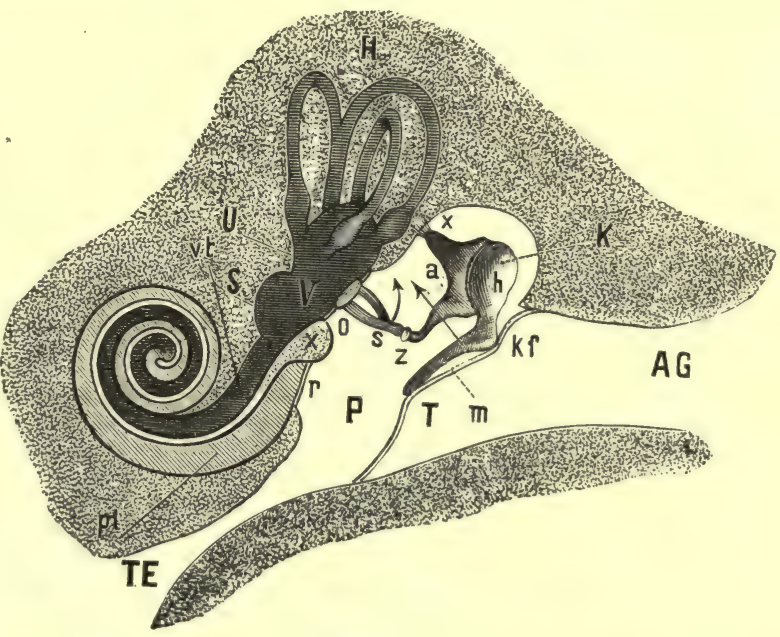

Fig. 577.

Scheme of the organ of hearing. AG, external auditory meatus ; T, tympanic membrane; $K$, malleus with its head $(h)$, short process $(k f)$, and handle $(m) ; a$, incus, its short process $(x)$, and its long process united to the stapes $(s)$ by means of the Sylvian ossicle $(z)$; P, middle ear; $o$, fenestra ovalis ; $x$, fonestra rotunda ; $x$, beginning of the lamina spiralis of the cochlea; $p t$, scala tympani, and $v t$, scala vestibuli; $\mathrm{V}$, vestibule; $\mathrm{S}$, saccule; $\mathrm{U}$, utricle; H, semicircular canals; TE, Eustachian tube. The long arrow indicates the line of traction of the tensor tympani; the short curved one, that of the stapedius,

(P). Not far from the fenestra rotunda is the fenestra ovalis $(0)$, in which the base of the stapes $(s)$ is fixed by means of a yielding membranous ring. The outer surface of this also is in contact with the air in the middle ear. As the perilymph of the inner ear is in contact at these two places with a yielding boundary, it is clear that the lymph itself may exhibit oscillatory movements, as it must follow the movements of the yielding boundaries. 
The sonorous vibrations may set the perilymph in vibration in three different ways :-

1. Conduction through the Bones of the Head.-This occurs especially when the vibrating solid body is applied directly to some part of the head, e.g., a tuningfork placed on the head, the sound being propagated most intensely in the direction of the prolongation of the handle of the instrument-also when the sound is conducted to the head by means of fluid, as when the head is ducked under water. Vibrations of the air, however, are practically not transferred directly to the bones of the heal, as is shown by the fact that we are deaf when the ears are stopped.

The soft parts of the heal, which lie immediately upon bone, conduct sound best, and of the projerting lart, the hest conductor is the cartilaginous portion of the external ear. But even under the most favourable cireminstance, conduction through the bones of the head is far less effective than the comluction of the sound-waves through the external auditory meatus. If a tuning-fork be made to vibrate between the teeth until we no longer hear it, its tone may still le heard on hringing it near the ear (kinne). The conduction through the bones is favoured when the uscillations are not transferred from the bones to the tympanic membrane, and are thus transferrel to the air, in the outer ear. Hence, we hear the sound of a tining-fork applied to the head better when the ears are stopped, as this prevents the propagation of the sound. waves through the air in the outer ear. If, in a deaf person, the conduction is still normal through the cranial bones, then the cause of the deafness is not in the nervous part of the ear, but in the external sound-conducting part of the apparatus.

2. Normal hearing takes place through the external auditory meatus. The enormous vibrations of the air first set the tympanic membrane in vibration (fig. $577, \mathrm{~T})$; this moves the malleus $(h)$, whose long process is inserted into it; the malleus moves the incus $(\alpha)$, and this the stapes $(s)$, which transfers the movements of its plate to the perilymph of the labyrinth.

3. Direct Conduction to the Fenestra. - In man, in consequence of occasional disease of the millile ear, whereby the tympanic inembrane and auditory ossicles may be destroyed, the auditory apparatus may be excited, although only in a very feeble manner, by the vibrations of the air being directly transferred to the nembrane of the fenestra rotunda $(r)$, and the parts r. losing the fenestra ovalis $(0)$. The membrane of the fenestra rotunda may vibrate alone, even when the oval window is closed with a rigid body ( $W$ eber.Liel).

407. PHYSICAL INTRODUCTION. - Sound is produced by the vibration of elastic bodies capable of vibration. Alternate condensation and rarefaction of the surrounding air are thus produced ; or, in other words, sound-waves in which the particles vibrate longitudinally or in the direction of the propiagation of the sound are excited. Around the point of origin of the semnd, these condensations and rarefactions occur in equal concentric circles, which conduct the somul vibrations to our outer ear. The vibrations of the sounding body are so called "stationary vilurations," i.e., all the particles of the vibrating body are always in the same phase of movement, in that they pass into movement simultaneously, they reach the maximum of movement simultaneously, e.g., in the particles of a sounding vibrating metal rod. Sound is produced by the stationary vibrations of elastic bodies; it is propagated by progressive wave-motion of elastic media, generally the air. The wave-length of a tone, i.e., the distance of one maximum of condensation to the next one in the air, is proportional to the duration of the vibration of the body, whose vibrations produce the sound-waves.

If $\lambda$ is the wave-length of a tone, $t$ in second the durations of a vibration of the body prorlucing the wave, then $\lambda=n t$, where $n=340.88$ metres, which is the rate per second of propagation of sound-waves in the air. The rapidity of the transmission of sound-waves in water $=1435$ metres per second, i.e., nearly four times as rapid as in air; while, in solids rapable of vilbration, it is propagated from seven to eighteen times faster than in the air. Sound-waves are conducted best through the same medium; when they have to pass through several media they are always weakened.

Reflection of the sound-waves occurs when they impinge upon a solid obstacle, in which case the angle of reflection is always equal to the angle of incidence.

Wave Movements. - We distinguish-I. Progressive wave movements which occur in two forms-(1) As longitudinal waves, in which the individual particles of the vibrating body vilurate around their centre of gravity in the direction of the propagation of the wave; examples are the waves in water and air. This movement causes an accumulation of the particles at certain places, c.g., on the crests of the waves in water-waves, while at other places they are diminished. This kind of wave is called a wave of condensation and rarefaction. (2) If, however, each particle in the progressive wave moves vertically up and down, i.e., transversely 
to the direction of the propagation of the wave, then we have the simple transverse waves, or progressive waves, in which there is no condensation or rarefaction in the direction of propagation, as each particle is merely displaced laterally. An example of this is the progressive waves in a rope.

II. Stationary Flexion Waves.-When all the particles of an elastic vibrating body so oscillate that all of them are always in the same phase of movement as the limbs of a vibrating tuning-fork or a plucked string, then this kind of movement is described as stationary flexion waves. As bodies, whose expansion in the direction of oscillation is very slight, vibrate to and fro in the stationary flexion wave, so we see that the small parts of the auditory apparatus (tympanic membrane, ossicles, lymph of the labyrinth) oscillate in stationary flexion waves.

408. EAR MUSCLES-EXTERNAL AUDITORY MEATUS.-When the external ear is absent, little or no impairment of the hearing is observed; hence, the physiological functions of these organs are but slight. Boerhaave thought that the elevations and depressions of the outer ear might be connected with the reflection of the sound-waves. Numerous sound-waves, however, must be again reflected outwards; and those waves which reach the deep part of the concha are said to be reflected towards the tragus, to be reflected by it into the external auditory meatus. According to Schneider, when the depressions in the ear are filled up with wax, hearing is impaired; other observers, however, have found the hearing to be unaffected. Mach points out that the dimensions of the external ear are proportionally too small to act as reflecting organs for the wave-lengths of noises.

Muscles of the External Ear. - (1) The whole ear is moved by the retrahentes, attrahens, and. attollens. (2) The form of the ear may be altered by the tragicus, antitragicus, helicis major and minor internally; and by the transversus and obliquus auriculæ externally. Persons who can move their ears do not find that the hearing is influenced during the movement. The $\mathrm{Mm}$. helicis major and minor are regarded as elevators of the helix, the transversus and obliquus auriculæ as dilators of the concha; the tragicus and antitragicus as constrictors of the meatus. In animals, the external ear and the action of its muscles have a marked effect upon hearing. The muscles point the ear in the direction of the sound, while other muscles contract or dilate the space within the external ear. In many diving animals, the meatus can be closed by a kind of valve.

The external meatus is 3 to $3.25 \mathrm{~cm}$. long [ $1 \frac{1}{8}$ to $1 \frac{1}{4}$ inch], 8 to $9 \mathrm{~mm}$. high, and 6 to 8 $\mathrm{mm}$. broad at its outer opening (fig. 578). It is the conductor of the sound-waves to the tympanic membrane, so that almost all the sound-waves first impinge upon its wall, and are then reflected towards the tympanic membrane. To see well down into the meatus, we must pull the auricle upwards and backwards. Occlusion of the meatus, especially by a plug of inspissated wax (\$287), of course interferes with the hearing, [and when it presses on the membrana tympani may give rise to severe vertigo].

\section{TYMPANIC MEMBRANE.-The} tympanic membrane, which is tolerably laxly fixed in a special osseous cleft, with a thickened

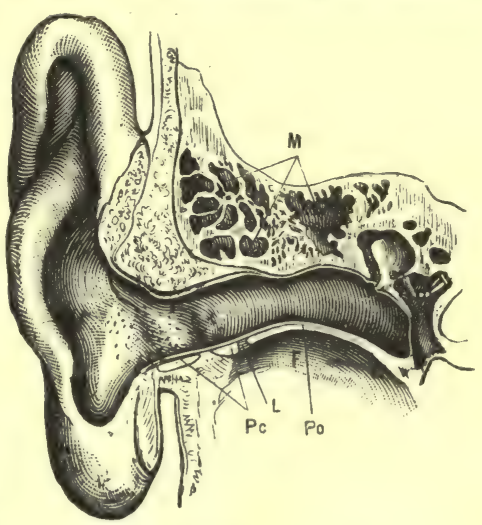

Fig. 578.

The external auditory meatus and the tympanic cavity. M, osseous spaces in the temporal bone; $\mathrm{P} c$, cartilaginous part of the meatus; L, membranous union between both ; F, auricular surface for the condyle of the lower jaw. margin, is an elastic, unyielding, and almost non-extensible membrane of about $0 \cdot 1$ $\mathrm{mm}$. in thickness, and with a superficial area of 50 square millimetres (fig. 581). It is elliptical in form, its greater diameter being 9.5 to $10 \mathrm{~mm}$., and its lesser 8 $\mathrm{mm}$., and it is fixed in the floor of the external meatus obliquely, at an angle of $40^{\circ}$, being directed from above and outwards, downwards and inwards. Both tympanic membranes converge anteriorly, so that if both were prolonged they would meet to form an angle of $130^{\circ}$ to $135^{\circ}$. The oblique position enables a larger surface to be presented than would be obtained if it were stretched vertically, so that more sound-waves can fall vertically upon it. The membrane is not stretched flat, but a little under its centre (umbilicus) it is drawn slightly inwards by the 
handle of the malleus, which is attached to it; while the short process of the malleus slightly bulges out the membrane near its upper margin (figs. 577, 584).

Structure. - The tympanic membrane consists of three layers :-(1) The membrana propria is a fibrous membrane with radial fibres on its outer surface, and circularly arranged fibres on

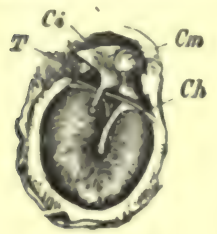

Fig. 579.

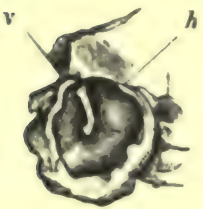

Fig. 581.

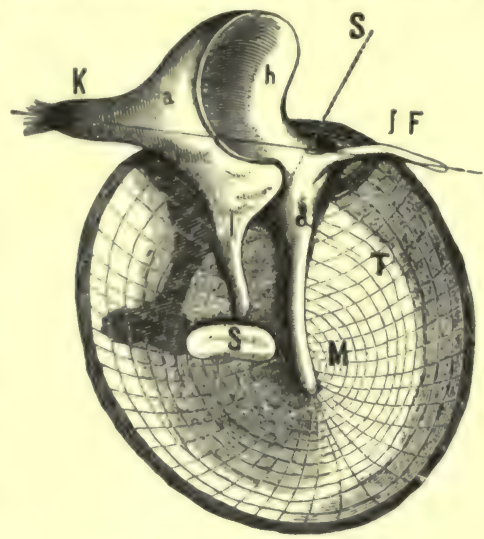

Fig. 580 . its inner aspect. (2) The surface directed towards the meatus is covered with a thin and semi-transparent part of the cutis. (3) The side towards the tympanum is covered with a delicate mucous membrane, with simple squamous epithelium. Numerous nerves and lymph-vessels, as well as inner and outer bloodvessels, occur in the mem. brane.

[The middle layer, or substantia propria, is fixed to a ring of bone, which is deficient above. It is filled up by a layer composed of the mucous and cutaneous layers called

Fic. 579. - Tympanic membrane with the auditory ossicles (left) the membrana flaccida, or seen from within. $C^{\prime} i$, incus; $C m$, malleus; $C h$, chorda tymjani ; $T$, jouch-like depression (after Urbantschitsch). Fig. 580 . - Trmanic membrane and the auditory ossicles (left) seen from within, i.c., from the tympanic cavity. M, manubrium or handle of the malleus; $\mathrm{T}$, insertion of the tensor tympani ; $h$, head: $l \mathrm{~F}$, long process of the malleus; $a$, incus, with the short $(\mathrm{K})$ and the long $(l)$ process; $\mathrm{S}$, plate of the stapes; $A x, A x$, is the common axis of rotation of the auditory ossicles ; $s$, the pinion-wheel arrangement between the malleus and incus. Fig. 581.-Tympanic membrane of a new-born child seeu from without, with the handle of the malleus visible on it. $\Lambda t$, tympanic ring with its anterior $(v)$ and posterior $(h)$ ends.

\section{Shrapnell's membrane.]}

[Examination.-When examining the outer ear and membrana tympani, pull the auricle upwards and backwards. The membrana tympani is examined by means of an ear speculum (fig. 582). The speculum is placed in the ear, and light is reflected into it by means of a concave mirror, perforated in

the centre, and having a focal distance of four or five inches. It is convenient to have the mirror fixed to a band placed round the head, as in the case of the laryngoscopic reflector (fig. 359). It is important to remember that the membrane is placed obliquely, so that the posterior and upper parts are nearer the surface. The membrane in health is greyish in colour

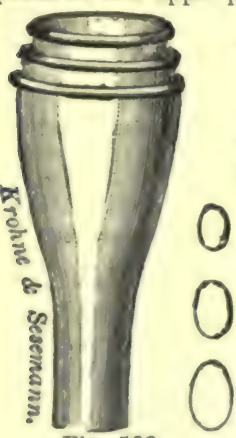

Fig. 582.

Ear specula of various sizes. and transparent, so that the handle of the malleus is seen running from above downwards and lackwards, while at the anterior and inferior part there is a cone of light with its apex directed inwards.]

Function.-The tympanic membrane catches up the soundwaves which penetrate into the external meatus, and is set into vibration by them, the vibrations corresponding in number and amplitude to the vibrating movements of the air. Politzer connected the auditory ossicles fixed to the tympanic membrane of a duck with a recording apparatus, and could thus register the vibrations produced by sounding any particular tone. Owing to its small dimensions, the tympanic membrane can vibrate in toto, to and fro in the direction of the sound-waves corresponding to the condensations and rarefactions of the vibrating air, and therefore executes transverse vibrations, for which it is specially adapted, owing to the relatively slight resistance.

Fundamental Note.- Stretched strings and membranes are generally only thrown into actual and considerable sympathetic vibration when they are affected by tones which correspond with their own fundamental tone, or 
whose number of vibrations is some multiple of the number of vibrations of the same, as the octave. When other tones act on them, they exhibit only inconsiderable sympathetic vibration. If a membrane be stretched over a funnel or cylinder, and if a nodule of sealing wax attached to a silk thread be made just to touch the centre of the membrane, then the sealing wax remains nearly at rest when tones or sounds are made in the neighbourhood; as soon, however, as the fundamental or proper tone of this arrangement is sounded, the nodule is propelled by the strong vibrations of the membrane.

If we apply this to the tympanic membrane, then it also should exhibit very great vibrations when its own fundamental note is sounded, but only slight vibrations when other tones are produced. This, however, would produce great inequality in the audible sounds. There is an arrangement of the membrane whereby this is prevented. (1) Great resistance is offered to the vibrations of the tympanic membrane, owing to its union with the auditory ossicles. These act as a damping apparatus, which provides, as in damped membranes generally, that the tympanic membrane shall not exhibit excessive sympathetic vibrations for its own fundamental note. But the damping also makes the sympathetic vibrations less for all the other tones. In this way, all vibrations of the tympanic membrane are modified; especially, however, is the excessive vibration diminished during the sounding of its fundamental tone. The membrane is at the same time rendered more capable of responding to the vibrations of different wave-lengths. The damping also prevents after-vibrations. (2) Corresponding to the small mass of the tympanic membrane, its sympathetic vibrations must also be small. Nevertheless, these slight elongations are quite sufficient to convey the sonorous movements to the most delicate end-organs of the auditory nerve; in fact, there are arrangements in the tympanum which still further diminish the vibrations of the tympanic membrane.

As v. Helmholtz has shown, the strong sympathetic vibrations of the tympanic membrane are not completely set aside by this damping arrangement. The painful sensations produced by some tones are, perhaps, due to the sympathetic vibration of the membrana tympani. According to Kessel, certain parts of the membrane vibrate to certain tones; the shortest radial fibres at the upper part of the anterior and upper segment vibrate with the highest tones, the longest fibres at the posterior segment with the deepest tones. At the upper part of the posterior segment noises are transmitted.

According to Fick, the tympanic membrane, besides possessing the property of taking up all vibrations with nearly equal intensity, has also the properties of a resonance apparatus; i.e., it causes a summation of the energy of successive vibrations. This is due to the funnel-shape of the membrane, and to the radial, rigid insertion of the handle of the malleus.

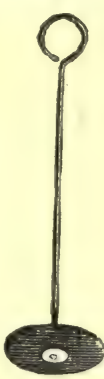

Fig. 583.

Pathological. - Thickenings or inequalities of the tympanic membrane inter- Toynbee's artififere with the acuteness of hearing, owing to the diminished capacity for vibra- cial membrana tion thereby produced. Holes in and loss of its substance act similarly. In tympani. extensive destruction, an artificial tympanum is placed in the external meatus, and its vibrations, to a certain extent, replace those of the lost membrane (Toynbee). [Fig. 583 shows an artificial tympanic membrane.]

410. AUDITORY OSSICLES AND THEIR MUSCLES.-The auditory ossicles have a double function.-(1) By means of the "chain" which they form, they transfer the vibrations of the tympanic membrane to the perilymph of the labyrinth. (2) They also afford points of attachment for the muscles of the middle ear, which can alter the tension of the membrana tympani, and the pressure on the lymph of the labyrinth.

Mechanism.-The form and position of the ossicles are given in figures 584 and 585. They form a jointed chain which connects the tympanic membrane, M, by means of the malleus, $h$, incus, $a$, and stapes, $\mathrm{S}$, with the perilymph of the labyrinth. The mode of movement of the ossicles is of special importance. The handle of the malleus is firmly united to the fibres of the tympanic membrane (fig. 585, n). 
Besides this, the malleus is fixed by ligaments which preseribe the direction of its movements. Two ligaments - the lig. mallei anticum (passing from the processus Folianus) and the posticum (from a small crest on the neck)-together form a common axial band ( $v$. Helmholtz), which acts in the direction from behind forwards, i.e., parallel to the surface of the tympanic membrane. The neck of the malleus lies between the insertions of both ligaments. The united ligament determines the

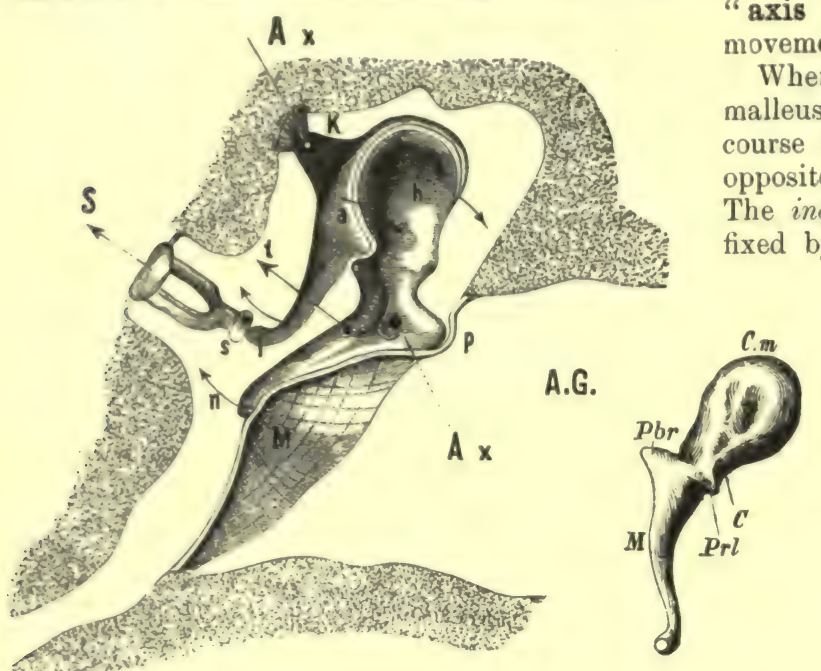

Fig. 585. "axis of rotation" of the vement of the malleus.

When the handle of the malleus is drawn inwards, of course its head moves in the opposite direction, or outwards. The incus, $a$, is only partially fixed by a ligament, which at-

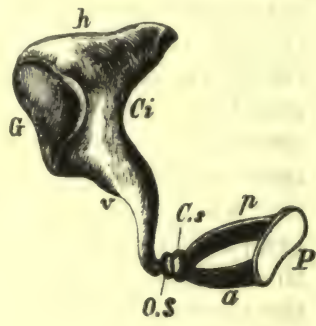

Fig. 584.

Fig. 584. - The aulitory ossicles (right). C.m, head; $C$, neck; $P b r$, short process ; Prl, long jrocess : $M$, handle of the malleus; $C i$, body ; $G$, articular surface ; $h$, short, and $v$, long process of the incus; $O . S$, so-called lenticular ossicle ; $C . s$, head ; $\alpha$, anterior, and $p$, posterior limb; $P$, plate of the stapes. Fig. 585. - Tympanum and auditory ossicles (left) magnified. A.G., external meatus ; II, membrana tympani, which is attached to the handle of the malleus, $n$, and near it the short process, $p ; h$, head of the malleus ; $a$, incus; $k$, its short process with its ligament; $l$, long process; $s$, Sylvian ossicle ; $\mathbf{S}$, stapes ; $\mathbf{A} x, \mathbf{\Lambda} x$, is the axis of rotation of the ossicles, it is shown in perspective, and must be imagined to penetrate the plane of the paper; $t$, line of traction of the tensor tympani. The other arrows indicate the movement of the ossicles when the tensor contracts.

taches its short process to the wall of the tympanic cavity, in front of the entrance to the mastoid cells, $k$. The not very tense articulation joining it to the head of the malleus, $h$, which lies with its saddle-shaped articular surface in the hollow of the incus, is important. The lower margin of the incus (fig 584, S) acts like a tooth of a cog-wheel. Thus, when the handle of the malleus moves inwards to the tympanic cavity, the incus, and its long process, $b$, which is parallel to the handle of the malleus, also pass inwards. The incus forms almost a right angle with the stapes, $\mathrm{S}$, through the intervention of the Sylvian ossicle, s. If, however, as by condensation of the air in the tympanum, the membrana tympani and the handle of the malleus move outwards, the long process of the incus does not make a similar movement, as the malleus moves away from this margin of the incus. Hence, the stapes is not liable to be torn from its socket. The malleus and incus form an angular lever, which moves round a common axis (fig. 581 and fig. 585, $\mathrm{A} x, \mathrm{~A} x$ ). In the inward movement, the malleus follows the incus, as if both formed one piece. The common axis (fig. 580) is not, however, the axial ligament of the malleus, but it is formed anteriorly by the processus Folianus, $l F$, directed forwards, and posteriorly by the short process of the incus directed backwards. The rotation of both ossicles around this axis occurs in a plane vertical to the plane 
of the membrana tympani. During the rotation, of course the parts above this axis (head of the malleus and upper part of the body of the incus) take a direction opposite to the parts lying below it (the handle of the malleus and the long process of the incus), as is indicated in fig. 585 by the direction of the arrows. The movement of the handle of the malleus must follow that of the membrana tympani, and vice vers $\hat{A}$, while the movement of the stapes is connected with the movement of the long process of the incus. As the long process of the incus is only twothirds of the length of the handle of the malleus (figs. 577, 581, 585), of course the excursion of the tip of the former, and with it of the stapes, must be correspondingly less than the movement of the tip of the handle of the malleus; while, on the other hand, the force of the movement of the tip of the handle of the malleus, corresponding to the diminution of the excursion, will be increased.

Mode of Vibration. - Thus, the movement of the membrana tympani inwards causes a less extensive but a more powerful movement of the foot of the stapes against the perilymph of the labyrinth. V. Helmholtz and Politzer calculated the extent of the movement to be $0.07 \mathrm{~mm}$. The mode in which the vibrations of the membrana tympani are conveyed to the lymph of the labyrinth, through the chain of ossicles, is quite analogous to the mechanism of these parts already described. Long delicate glass threads have been fixed to these ossicles, and their movements were thus graphically recorded on a smoked surface (Politzer, Hensen). Or, strongly refractive particles are fixed to the ossicles, while the beam of light reflected from them can be examined by means of a microscope (Buck, v. Helmholtz). All the experiments showed that the transference of the sound-waves is accomplished by means of the mechanism " of the angular lever, composed of the auditory ossicles already described. As the vibrations of the membrana tympani are conveyed to the handle of the malleus, they are weakened to about one-fourth of their original strength (Politzer). [The membrana tympani is many times (30) larger than the fenestra ovalis, and the relation in size might be represented by a funnel. The arm of the malleal end of the lever where the power acts is $9 \frac{1}{2} \mathrm{~mm}$. long, while the short or stapedial arm is $6 \frac{1}{2} \mathrm{~mm}$., so that the latter moves less than the former, but what is lost in extent is gained in force.]

[Methods. - Politzer attached small, very light levers to each of the ossicles, and inscribed their movements on a revolving cylinder. An organ-pipe was sounded, and when the levers were of the same length, the malleus made the greatest excursion and the stapes the least. Buck attached starch grains to the ossicles, illuminated them, and observed the movements of the refractive starch granules by means of a microscope provided with a micrometer.]

[The ossicles move en masse, and not in the way of propagating molecular vibrations.] As the excursions of the ossicles during sonorous vibrations are, however, only nominal, there is practically no change in the position of the joints with each vibration. The latter will only occur when extensive movements take place by means of the muscles.

The muscles of the auditory ossicles alter the position and tension of the membrana tympani, as well as the pressure of the lymph of the labyrinth. The tensor tympani, which lies in an osseous groove above the Eustachian tube, has its tendon deflected round an osseous projection [processus cochleariformis], which lies external to it, almost at right angles to the groove above it, and is inserted immediately above the axes of rotation of the malleus (fig. 580, M). When the muscle contracts in the

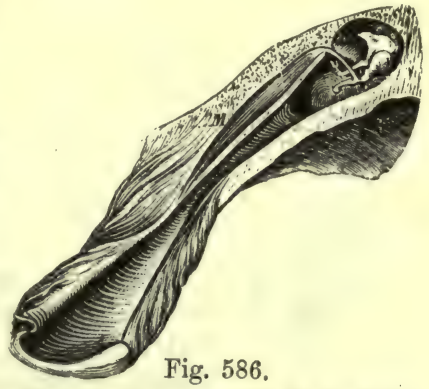

Tensor tympani-the Eustachian tube (left).

direction of the arrow, $t$, then the handle of the malleus $(n)$ pulls the membrana tympani (M) inwards and tightens it (fig. 585). This also causes a movement 
of the incus and stajes (S) which must be pressed more deeply into the fenestra ovalis as already described. When the muscle relaxes, then owing to the elasticity of the rotated axial ligament and the tense membrana tympani itself, the position of equilibrium is again restored. The motor nerve of this muscle arises from the trigeminus, and passes through the otic ganglion (p. 597). C. Ludwig and Politzer observed that stimulation of the fifth nerve within the cranium [dog] caused the above mentioned movement.

Use of the tension. - The tension of the membrana tympani caused by the tensor trmuani has a double function (Joh. Mïller) - 1. The tense membrane offers very great resistance to sympathetic vibrations when the sound-waves are very intense, as it is a physical fact that stretched membranes are more difficult to throw into sympathetic vibrations the tenser they are. Thus, the tension so far protects the auditory organ, as it prevents too intense vibrations applied to the membrana tympani from reaching the terminations of the nerves. 2. The tension of the membrana tympani must vary according to the degree of contraction of the tensor. Thus, the membrana for the time being has a different fundamental tone, and is thereby capable of vibrating to the correspondingly higher tone, it, as it were, being in a certain sense accommodated for.

Comparison with Iris. - The membrana tympani has been compared with the iris. Both menbranes prevent by contraction-narrowing of the pupil and tension of the membrana tvmpani-the too intense action of the specifie stimulus from causing too great stimulation, and linth wilipt the sensory apparatus for the action of moderate or weak stimuli. This movement in loth nuembranes is buotught alont reftedy, in the ear through the $\mathbf{N}$. acusticus, which causes a reflex stimulation of the motor fibres for the tensor tympani.

Effect of Tension.- That increased tension of the membrana tympani renders it less sensitive to sonud-waves is easily proved, thus :-Close the mouth and nose, and make either a forced expiration, so that the air is forced into the Eustachian tube, which bulges out the membrana tyminani, or inspire forcilly, wherely the air in the tympanum is diminished, so that the nembrania bulges inwards. In both cases, hearing is interfered with, as long as the increased tension lasts. If a funnel with a small lateral opening, and whose wide end is covered by a membrane, be placeed in the external meatus, hearing becomes less distinct when the membrane is stretched (Juh. Muiller). If air be blown into the external auditory meatus, both tensores tymprani contract, and in consequence of this the hearing of the other ear is temporarily affected (rellé).

Normally, the tensor tympani is excited reftexly. The muscle is not directly and by itself subjeet to the control of the will. According to L. Fick, the following phenomenon is due to an "assoriated movement" of the tensor:-When he pressed his jaws firmly against each other, the heart in his ear a piping, singing tone, while a capillary tube, which was fixed air-tight into the meatus, had a drop of water which was in it rapidly drawn inwards. During this experiment, a jersun with normal hearing hears all musical tones as if they were louder, while all the highest non-musieal tones are enferblesl (Lucue). When yauning, v. Helmholtz and Politzer found that hearing was enfeebled for certain tones.

Contraction of the tensor.- Hensen showed that the contraction of the tensor tympani during hearing is not a continued contraction, but what might be termed a "twitch." A twitch takes place at the beginning of the act of hearing, which favours the perception of the sound, as the membrana tympani thus set in motion

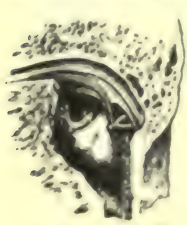

Fig. 587.

Right stapredius muscle. vibrates more readily to higher tones than when it is at rest. On exposing the tympanum in cats and dogs, it was found that this contraction or twitch occurs only at the beginning of the sound, and that it soon ceases, although the sound may continue.

Action of the Stapedius. - The muscle arises within the eminentia pyramidalis, and is inserted into the head of the stapes and Sylvian ossicle (fig. 583); when it draws upon the head of the stapes, as indicated in fig. 577 , by the small curved arrow, it must place the bone obliquely, whereby the posterior end of the plate of the stapes is pressed somewhat deeper inwards into the fenestra ovalis, while the anterior is, as it were, displaced somewhat outwards. The stapes is thereby more fixed, as the fibrous mass [annular ligament] which surrounds the 
fenestra ovalis and keeps the stapes in its place becomes more tense. The activity of this muscle, therefore, prevents too intense shocks, which may be communicated from the incus to the stapes, from being conveyed to the perilymph. It is supplied by the facial nerve $(\$ 349,3)$.

The stapedius in many persons executes an associated movement, when the eyelids are forcibly closed (\$349). Some persons can cause it to contract reflexly by scratching the skin in front of the meatus, or by gently stroking the outer margin of the orbit (Henle). It seems to be excited reflexly in many diseases of the ear when the tympanum is being syringed.

Other Views. - According to Lucae, when the stapes is displaced obliquely, its head forces the long process of the incus, and also the membrana tympani, outwards, so that it is regarded as an antagonist of the tensor tympani. Politzer observed that the pressure within the labyrinth fell, when he stimulated the muscle. According to Toynbee, the stapedius acts as a lever and moves the stapes slightly out of the fenestra ovalis, thus making it more free to move, so that it is more capable of vibrating. Henle supposes that the stapedius is more concerned in fixing than in moving the stapes, and that it comes into action when there is danger of too great movement being communicated to the stapes from the incus. Landois agrees with this opinion, and compares the stapedius with the orbicularis palpebrarum, both being protective muscles.

Pathological. - Immobility of the auditory ossicles, either by adhesions or anchyloses, causing diminished vibrations, interferes with hearing; while the same result occurs when the stapes is firmly anchylosed into the fenestra ovalis. The tendon of the tensor tympani has beeu divided in cases of contracture of the muscles. For paralysis of the tensor, see p. 598, and for the stapedius, p. 603.

411. EUSTACHIAN TUBE-TYMPANUM.-The Eustachian tube [4 centimetres in length, $1 \frac{5}{8}$ in.] is the ventilating tube of the tympanic cavity. It keeps the tension of the air within the tympanum the same as that within the pharynx and outer air (figs. 577, 586). Only when the tension of the air is the same outside and inside the tympanum, is the normal vibration of the membrana tympani possible. The tube is generally closed, as the surfaces of the mucous membrane lining it come into apposition. During swallowing, however, the tube is opened, owing to the traction of the fibres of the tensor veli palatini [spheno-salpingostaphylinus sive abductor tubae ( $v$ Tröltsch), sive dilator tubae (Rüdinger)] inserted into the membrano-cartilaginous part of the tube (Toynbee, Politzer). (Compare $\$ 139,2$.) When the tube is closed, the vibrations of the membrana tympani are transferred in a more undiminished condition to the auditory ossicles than when it is open, whereby part of the vibrating air is forced through the tube (Mach and Kessel). If, however, the tympanic cavity is closed permanently, the air within it becomes so rarefied ( $\S 139)$ that the membrana tympani, owing to the abnormally low tension, becomes drawn inwards, thus causing difficulty of hearing. As the tube is lined by ciliated epithelium it carries outwards to the pharynx the secretions of the tympanum (p. 452).

Noise in the Tube.-A sharp hissing noise is heard in the tube during swallowing, when we swallow slowly and at the same time contract the tensor tympani, due to the separation of the adhesive surfaces of its lining membrane. Another person may hear this noise by using a stethoscope or his ear.

In Valsalva's experiment ( $\S 60)$, as soon as the pressure of the air reaches 10 to $40 \mathrm{~mm}$. $\mathrm{Hg}$, air enters the tube. The sound is heard first, and then we feel the increased tension of the tympanic membrane, owing to the entrance of air into the tympanum. During forced inspiration, when the nose and mouth are closed, air is sucked out, while the tympanum is ultimately drawn inwards.

The M. levator veli palatini, as it passes under the base of the opening of the tube into the pharynx, forms the levator-eminence or cushion (fig. 354, W). Hence, when this muscle contracts and its belly thickens, as at the commencement of the act of deglutition and during phonation, the lower wall of the pharyngeal opening is raised, and the opening thereby narrowed (Lucae). The contraction of the tensor, occurring during the later part of the act of deglutition, dilates the tube.

Other Views. - According to Riidinger, the tube is always open, although only by a very narrow passage in the upper part of the canal, while the canal is dilated during swallowing. According to Cleland, the tube is generally open, and is closed during swallowing.

[Practical Importance. - The tympanic cavity forms an osseous box, and therefore a protective organ for the auditory ossicles and their muscles, while the 
increased air space, obtained by its communication with the mastoid cells, permits free vibration of the membrana tympani. The six sides of the tympanum have important practical relations. It is about half an inch in height, and one or two lines in breadth, i.e., from without inwards. Its roof is separated from the cavity of the brain by a very thin piece of bone, which is sometimes defective, so that encephalitis nay follow an abscess of the middle ear. The outer wall is formed by the membrana tympani, while on the inner wall are the fenestra ovalis and rotunda, the ridge of the aqueductus Fallopii, the promontory, and the pyramid. The floor consists of a thin plate of bone, which roofs in the jugular fossa and separates it from the jugular vein. Fractures of the base of the skull may rupture the carotid artery or internal jugular vein; hence, hxmorrhage from the ears is a bad symptom in these cases. Caries of the ear may extend to other organs. The anterior wall is in close relation with the carotid artery, while the posterior communicates with the mastoid cells, so that fluids from the middle ear sometimes escape through the mastoid cells.]

That the air in the tymuanum ean communicate its vibrations to the membrane of the fenestra rotumda is true $(1.797,3$ ), but normally this is so slight, when compared with the conduction through the auditory ossicles, that it scarcely need be taken into account.

Structure.-'The tube and tympanum are lined by a common mueous membrane, covered by (iliater epithelium, while the membrana is lined by a layer of squamous epithelium. Mucous

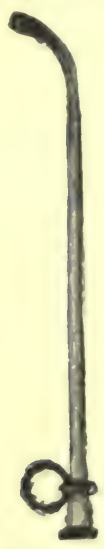

Fig. 588.

Eustachian catheter. glands were found by Troiltsch and Wendt in the mucous membrane. ['The efithelium covering the ossicles and tensor tympani is not ciliated.]

Pathological. - The tube is often occluded, owing to chronic catarrh and narrowing from cicatrices, hypertrophy of the mucous membrane, or the presence of tumours. The deafness thereby produced may often be cured by catheterising the tube from the nose (fig. 588). Effusions into or suppuration within the tympanum of course paralyse the soundconducting mechanism, while inflammation often causes subsequent affections of the plexus tympanicus. If the temporal bone be destroyed

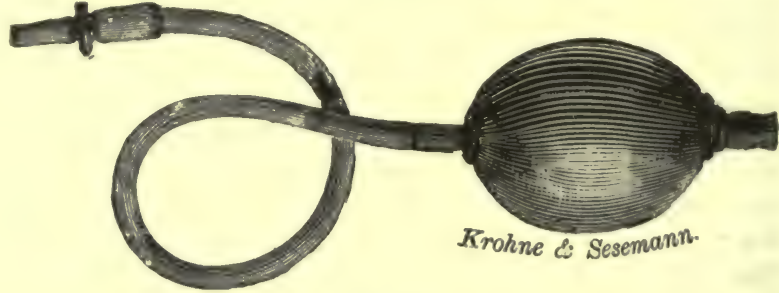

Fig. 589.

Polit\%er's ear bag.

by progressive caries within the tympanum, inflammation of the neighbouring cerebral struc. tures may occur and cause death.

[Methods. - Not unfrequently the aurist is called upon to dilate the Eustachian tube, which in certain cases requires the use of a Eustachian catheter introduced into the tube along the floor of the nose (fig. 588). At other times he requires to fill the tympanic cavity with air, which is easily done by means of a Politzer's bag (fig. 589). The nozzle is introduced into one nostril, while the other nostril is closed, and the patient is directed to swallow, while at the same moment the surgeon compresses the bag, and the patient's mouth being closed, air is forced through the open Eustachian tube into the middle ear. Sometimes a small curved narrow manometer, containing a drop of coloured water, is placed in the outer ear (Politzer). Normally, when the patient swallows, the fluid ought to move in the tube.]

412. CONDUCTION OF SOUND IN THE LABYRINTH.-The vibrations of the foot of the stapes in the fenestra ovalis give rise to waves in the perilymph within the inner ear or labyrinth. These waves are so-called "flexion waves" i.e., the perilymph moves in mass before the impulse of the base of the stapes. This is only possible from the existence of a yielding membrane-that filling the fenestra rotunda, and sometimes called the membrana secundaria, which during rest bulges 
inwards to the scala tympani, and can be bulged outwards towards the tympanic cavity by the impulse communicated to it by the movement of the perilymph (fig. 577, r). The flexion waves must correspond in number and intensity to the vibrations of the auditory ossicles, and must also excite the free terminations of the auditory nerve, which float free in the endolymph.

As the endolymph of the saccule and utricle lying in the vestibule receives the first impulse, and as these communicate anteriorly with the cochlea, and posteriorly with the semicircular canals, consequently the motion of the perilymph must be propagated through these canals. To reach the cochlea, the movement passes from the saccule (lying in the fovea hemispherica) along the scala vestibuli to the helicotrema, where it passes into the scala tympani, where it reaches the membrane of the fenestra rotunda, and causes it to bulge outwards. From the utricle (lying in the fovea hemielliptica), in a similar manner the movement is propagated through the semicircular canals. Politzer observed that the endolymph in the superior semicircular canal rose when he caused contraction of the tensor tympani by stimulating the trigeminus, just as the base of the stapes must be forced against the perilymph with every vibration of the membrana tympani.

[Practical.-It is well to view the organ of hearing as consisting of two mechanisms :-

1. The sound-conducting apparatus.

2. The sound-perceiving apparatus.

The former includes the outer ear, with its auricle and external meatus; the middle ear and the parts which bound it, or open into it. The latter consists of the inner ear with the expansion of the auditory nerve in the labyrinth, the nerve itself, and the sound-perceiving and interpreting centre or centres in the brain (p. 703).]

[Testing the Sound-conduction.-In any case of deafness, it is essential to estimate the degree of deafness by the methods stated at $p .798$, and it is well to do so both for such sounds as those of a watch and conversation. We have next to determine whether the sound-conducting or the sound-perceiving apparatus is affected. If a person is deaf to sounds transmitted through the air, on applying a sounding tuning-fork to the middle line of the head or teeth, if it be heard distinctly, then the sound-perceiving apparatus is intact, and we have to look for the cause of deafness in the outer or middle ear. In a healthy person, the sound of the tuningfork is heard of equal intensity in both ears. In this case the sound is conducted directly to the labyrinth by the cranial bones. In cases of disease of the soundconducting mechanism, the sound of the tuning-fork is heard loudest in the deafer ear. Ed. Weber pointed out that, if one ear be stopped and a vibrating tuningfork placed on the head, the sound is referred to the plugged ear, where it is heard loudest. It is assumed that when the ear is plugged, the sound-waves transmitted by the cranial bones are prevented from escaping (Mach). If, on the contrary, the sound be heard loudest in the good ear, then in all probability there is some affection of the sound-perceiving apparatus or labyrinth, although there are exceptions to this statement, especially in elderly people. Another plan is to connect two telephones with an induction machine, provided with a vibrating Neef's hammer. The sounds of the vibrations of the latter are reproduced in the telephones, and if they be placed to the ears, then the healthy ears hear only one sound, which is referred to the middle line, and usually to the back of the bead. In diseased conditions this is altered-it is referred to one side or the other.] 
413. LABYRINTH AND AUDITORY NERVE. - Scheme. -The vestibule (fig. 591, III) contains two separate saes; one of them, the saccule, $s$ (round sac or S. hemisphæricus), com. municates with the ductus cochlearis, $\mathrm{C} c$, of the cochlea; the other, the utricle, $\mathrm{U}$ (elliptical sac, or sacculus hemiellipticus), communicates with the semicircular canals, Cs, Cs.

The cochlea consists of $2 \frac{1}{2}$ turns of a tube disposed round a central column or modiolus. The tube is diviled into two compartments by a horizontal septum, partly osseous aud partly memlirannus, the lamina spiralis ossea and membranacea (fig. 595; fig. 591, I). The lower compartment is the scala tympani, and is separated from the cavity of the tympanum by the membrane of the fenestra rotunda.

The upper compartment is the scala vestibuli, which communicates with the vestibule of the labyrinth (fig. 591, I). These two compartments communicate directly by a small opening at the apex of the cochlea, a sickle-shaped edge ["hamulus"] of the lamina spiralis bounding the helicotrema (fig. 577). The scala vestibuli is divided by Reissner's membrane (fig. 591, I),

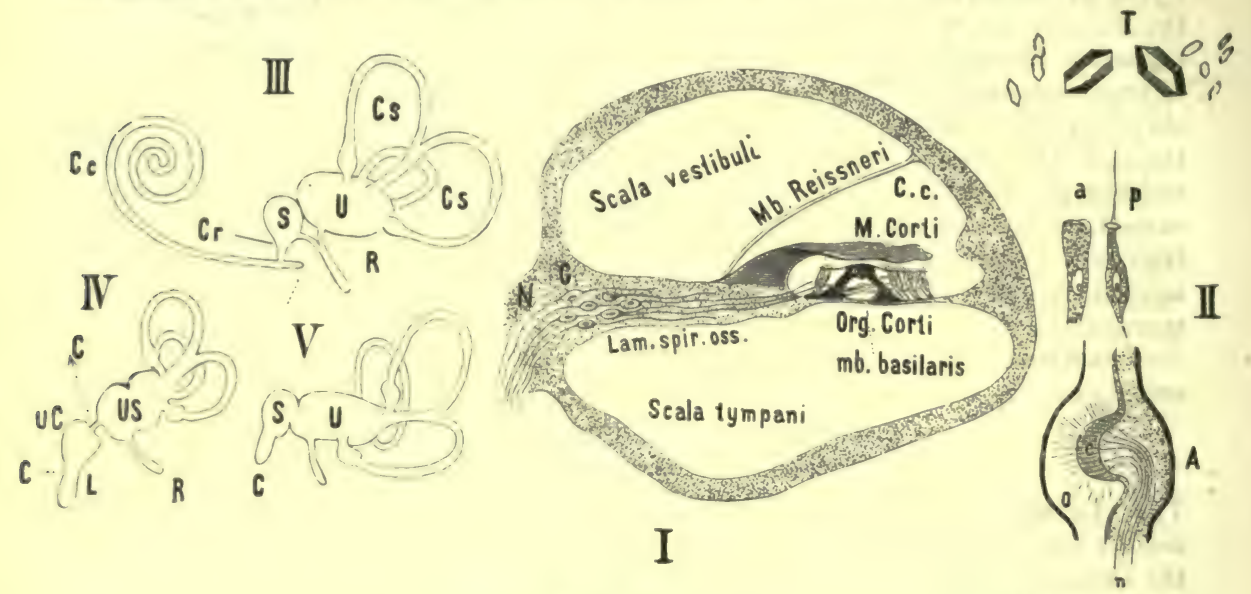

Fig. 591.

I, transverse section of a turn of the cochlea ; II, A, ampulla of a semicircular canal with the crista acustica ; $a, p$, auditory cells; $p$, provided with a fine hair ; T, otoliths ; III, scheme of the human labyrinth; IV, scheme of a bird's labyrinth; V, scheme of a fish's labyrinth.

which arises near the outer part of the lamina spiralis ossea, and runs obliquely outwards to the wall of the cochlea so as to cut off a small triangular canal, the ductus or canalis cochlearis, or scala medis, $\mathrm{C}_{c}$, whose floor is formed for the most part by the lamina spiralis membranacea, and on which the end-organ of the auditory nerve-Corti's organ-is placed. The lower end of the canalis cochlearis is blind, III, and divided towards the saccule, with which it communicates by means of the small canalis reuniens, $\mathrm{Cr}$ (Honsen). The utricle (fig. 591, III, U) cornmunicates with the three semicircular canals, $\mathrm{Cs}, \mathrm{Cs}$-each by means of an ampulla, within which lie the terminations of the ampullary nerves, but as the posterior and the superior canals unite, there is only one common ampulla for them. The membranous semicircular canals lie within the osseous canals, perilymph lying between the two. Perilymph also fills the scala vestibuli and tympani, so that all the spaces within the labyrinth are filled by fluid, while the spaces themselves are lined by short cylindrical epithelium.

The system of spaces, filled by endolymph, is the only part containing the nervous end-organs for hearing. All these spaces communicate with each other; the semicircular canals directly with the ntricle, the ductus cochlearis with the saccule through the canalis reuniens; and lastly, the saccule and utricle through the "saccus endolymphaticus," which springs by an isolated limb from each sac; the limbs then unite, as in the letter $Y$, and pass through the osserous aqueductus vestibuli to end blindly in the dura mater of the brain (fig. III, R-Bötcher, Retzins). The aqueductus cochlex is another narrow passage, which begins in the scala tympani, immediately in front of the fenestra rotunda, and opens close to the fossa jugularis. It forms a direct means of communication between the perilymph of the cochlea and the subarachnoid space.

Semicircular Canals and Vestibular Sacs. - The membranous semicircular canals do not fill the corresponding osseous canals completely, but are separated from them by a pretty wide space, which is filleil with perilymph (fig. 592). At the concave margin they are fixed by con- 
nective-tissue to the osseous walls. The ampullæ, however, completely fill the corresponding osseous dilatations. The canals and ampullæ consist externally of an outer, vascular, connective-tissue layer, on which there rests a well-marked hyaline layer, bearing a single layer of flattened epithelium.

Crista Acustica. - The vestibular branch of the auditory nerve sends a branch to each ampulla and to the saccule and utricle (fig. 593). In the ampullæ (fig. 591, II, A), the nerve (c) ter-

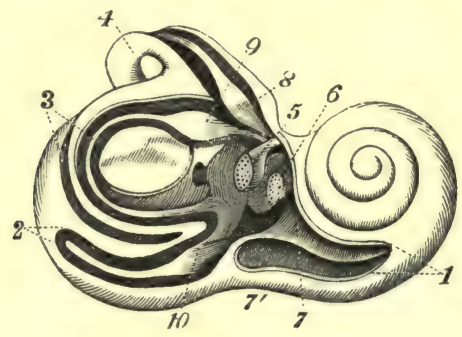

Fig. 592.

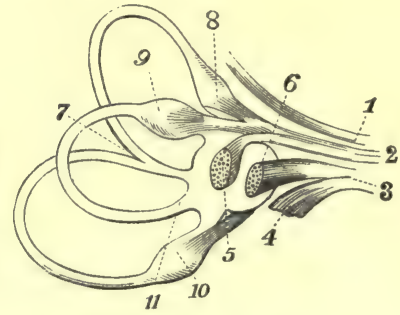

Fig. 593.

The interior of the right labyrinth with its membranous canals and nerves. In fig. 592, the outer wall of the bony labyrinth is removed to show the membranous parts within-1, commencement of the spiral tube of the cochlea; 2, posterior semicircular canal, partly opened; 3 , horizontal; 4, superior canal; 5 , utricle; 6 , saccule; 7, lamina spiralis ; 7 , scala tympani; 8 , ampulla of the superior membranous canal; 9 , of the horizontal; 10 , of the posterior canal. Fig. 593 shows the membranous labyrinth and nerves detached-1, facial nerve in the internal auditory meatus; 2 , anterior division of the auditory nerve giving branches to 5,8 , and 9 , the utricle and the ampullæ of the superior and horizontal canals ; 3 , posterior division of the auditory nerve, giving branches to the saccule, 6 , and posterior ampulla, 10, and cochlea, $4 ; 7$, united part of the posterior and superior canals: 11, posterior extremity of the horizontal canal.

minates in connection with the crista acustica, which is a yeilow elevation projecting into the equator of the ampulla. The medullated nerve-fibres, $n$, form a plexus in the connective-tissue layer, lose their myelin as they pass to the hyaline basement membrane, and each ends in a cell provided with a rigid hair $(o, p) 90 \mu$ in length, so that the crista is largely covered with these hair-cells, but between them are supporting cells like cylindrical epithelium $(\alpha)$, and not unfrequently containing granules of yellow pigment. The hairs or "auditory hairs" (M. Schultze) are composed of many fine fibres (Retzius). An excessively fine membrane (membrana tectoria) covers the hairs (Pritchard, Lang).

Maculæ Acusticæ.-The nerve-terminations in the maculæ acustice of the saccule and utricle are exactly the same as in the ampullæ, only the free surface of their membrana tectoria is sprinkled with small white chalk-like crystals or otoliths (II, T), composed of calcic carbonate, which are sometimes amorphous and partly in the form of arragonite, lying fixed in the viscid endolymph. The non-medullated axis-cylinders of the saccular nerves enter directly into the substance of the hair-cells. The terminations of the nerves have been investigated, chiefly in fishes, in the rays.

[Fig. 594 is a vertical section of a macula acustica of the rabbit. The medullated nerves $(n)$ lose their myelin at the external limiting membrane, become non-medullated, pierce this membrane, and form a basal plexus $(p b)$ between $(i)$ the epithelial cells, and finally terminate in the sensory ciliated cells $(r)$. The epithelium itself consists of basal cells $(c b)$, fusiform or supporting cells $(f)$, and the ciliated neuro-epithelium $(r)$, each cell being provided with a cilium, which perforates the external limiting membrane $(a)$. There is thus a remarkable likeness to the olfactory epithelium.]

Cochlea. - The terminations of the cochlear branch of the audi-

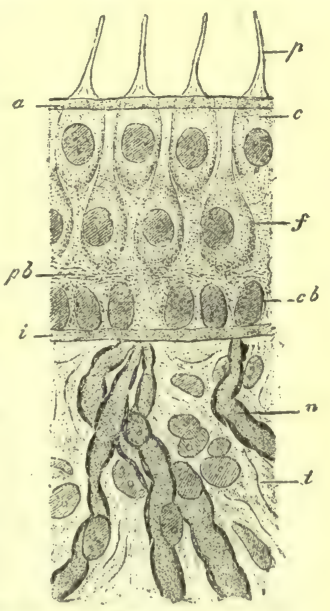

Fig. 594.

Vertical section of the macula acustica of a rabbit. tory nerve lie in connection with Corti's organ, which is placed in the canalis or ductus cochlearis (fig. 591, I, C $c$, and III, C $c$, and fig. 595), the small triangular chamber [or scala media, ] cut off from the scala vestibuli by the membrane of Reissner. Corti's organ is placed on the lamina spiralis membranacea, and consists of a supporting apparatus composed of the so-called Corti's arches, each of which consists of two Corti's rods $(z, y)$, which lie upon each other like 
the beams of a house. But every two rods do not form an arch, as there are always three inner

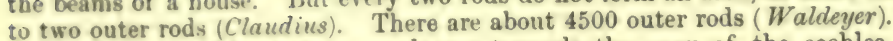

The ductus cochlearis becomes larger towards the apex of the cochlea, and the rods also become longer : the inner ones are $30 \mu$ long in the first turn, and $34 \mu$ in the upper, the outer rods $47 \mu$ and $69 \mu$ respectively. The span of the arches also increases (Hensen). [The

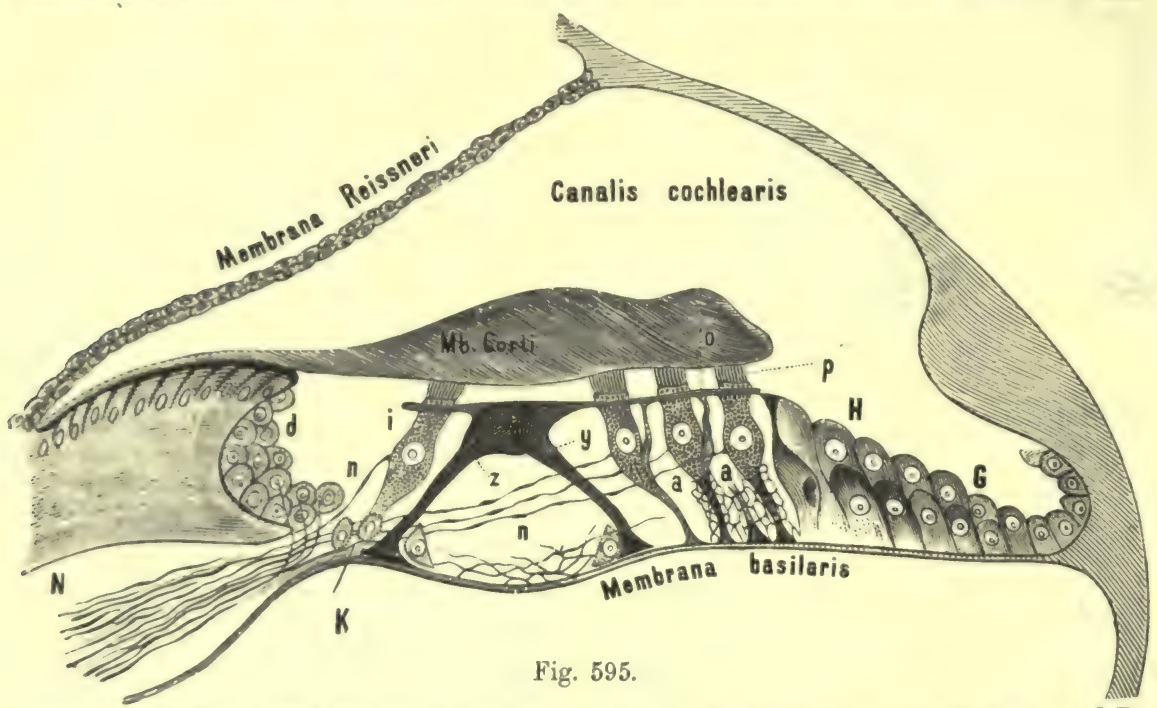

Scheme of the ductus cochlearis and the organ of éorti. N, cochlear nerve; K, inner, and P, outer hair-cells; n, nerve-fibrils terminating in $\mathrm{P} ; a, a$, supporting cells; $d$, cells in the suleus spiralis; $z$, inner rod of Corti; $\mathrm{Mb}$. Corti, membrane of Corti, or the membrana tectoria; $\theta$, the membrana reticularis; $\mathrm{H}, \mathrm{G}$, cells filling up the space near the outer wall.

arches leave a triangular tunnel beneath them.] The proper end-organs of the cochlear nerve are the cylindrical "hair-cells" (Kijlliker) previously observed by Corti, which are from 16,400 to 20,000 in number (Hensen, Waldeyer). There is one row of inner cells (i), which rests on a layer of small granular cells ( $\mathrm{K}$ ) (Böttcher, Waldeyer); the outer cells $(a, a)$ number 12,000 in man (Retzius), and rest upon the basement membrane, being disposed;in three or even four rows. Between the outer hair-cells there are other cellular structures, which are either regarled as special cells (Deiter's cells), or are regardecl merely as processes of the haircells (Luvdouskiy). [The cochlear branch of the auditory nerve enters the modiolus, and runs upwards in the osseous channels there provided for it, and as it does so gives branches to the lamina spiralis, where they run between the osseous plates which form the lamina.] The fibres ( $\mathbf{N}$ ) come out of the lamina spiralis after traversing the ganglionic cells in their course (figs. 591, 595, I, G), and end by tine varicose fibrils in the hair-cells (fig. 595) (Waldeyer, Gottstein, Lavdowsky, Retzins).

Membrana Reticularis. - Corti's rods and the hair-cells are covered by a special membrane (o), the membrana reticularis of Külliker. The upper ends of the hair-cells, however, project through holes in this membrane, which consists of a kind of a cement-substance holding these parts together (Lavdousky). [Springing from the outer end of the lamina spiralis, or crista spiralis, is the membrana tectoria, sometimes called the membrane of Corti. It is a' welldefined structure, often fibrillated in appearance, and extends outwards over the organ of Corti.] Waldeyer regards it as a damping apparatus for this organ (fig. 595, Mb. Corti).

[Basilar Membrane. - Its breadth increases from the base to the apex of the cochlea. This fact is important in connection with the theory of the perception of tone. It is supposed that high notes are appreciated by structures in connection with the former, and low notes by the upper parts of the basilar membrane. In one case, recorded by Moos and Steinbrugge, a patient heard low notes only in the right ear, and after death it was found that the auditory nerve in the first turn of the cochlea was atrophied.]

Intra-Labyrinthine Pressure.- The lymph within the labyrinth is under a certain pressure. Every diminution of the pressure of the air in the tympanum is accompanied by a corresponding diminution of the intra-labyrinthine pressure, while conversely every increase of pressure is accompanied by an increase of the lymph-pressure (F. Bezold). 
The perilymph of the inner ear flows away chiefly through the aqueductus cochleæ, in the circumference of the foramen jungulare, into the peripheral lymphatic system, which also takes up the cerebro-spinal fluid of the subarachnoid space, while a small part drains away to the sub-dural space through the internal auditory meatus. The endolymph flows through the arachnoid sheath of the N. acusticus into the subarachnoid space (C. Hasse).

414. AUDITORY PERCEPTIONS.-Every normal ear is able to distinguish musical tones and noises. Physical experiments prove that tones are produced when a vibrating elastic body executes periodic movements, i.e., when the sounding body executes the same movement in equal intervals of time, as the vibrations of a string which has been plucked. A noise is produced by non-periodic movements, i.e., when the sounding body executes unequal movements in equal intervals of time. [The non-periodic movements clash together on the ear, and produce dissonance; as when we strike the key-board of a piano at random.] This is readily proved by means of the siren. Suppose that there are forty holes in the rotatory disc of this instrument, placed at exactly the same distance from each other-on rotating the disc and directing a current of air against it, obviously with every rotation the air will be rarefied and condensed exactly forty times. Every two condensations and rarefactions are separated from each other by an equal interval of time. This arrangement yields a characteristic musical tone or note. If a similar disc with holes perforated in it at unequal distances be used, on air being forced against it, a whirring non-musical noise is produced, because the movements of the sounding body (the condensations and rarefactions of the air) are non-periodic. [The double siren of $\mathrm{v}$. Helmholtz is an improved instrument for showing the same facts.]

The normal ear also distinguishes in every tone three distinct factors :-

[(1) Intensity or force ; (2) Pitch ; (3) Quality, timbre or "klang."]

1. The intensity of a tone depends upon the greater or lesser amplitude of the vibrations of the sounding body. It is well known that a vibrating string emits a feebler sound when its excursions are smaller. (The intensity of a sound corresponds to the degree of illumination or brightness in the case of the eye.)

2. The pitch depends upon the number of vibrations which occur in a given time [or the length of time occupied by a single vibration]. This is proved by means of the siren. If the rotating disc have a series of forty holes at equal intervals, and another series of eighty equidistant from each other, on blowing a stream of air against the rotating disc we hear two sounds of unequal pitch, one being the octave of the other. (The perception of pitch corresponds to the sensation of colour in the case of the eye.)

3. The quality or timbre ("Klangfarbe") is peculiar to different sonorous bodies. [It is the peculiarity of a musical tone by which we are enabled to distinguish it as coming from a particular instrument, or from the human voice. Thus, the same note struck on a piano and sounded on a violin differs in quality or timbre.] It depends upon the peculiar form of the vibration, or the form of the wave of the sonorous body. (There is no analagous sensation in the case of light.)

I. Perception of Pitch. - By means of the organ of hearing, we can determine that different tones have a different pitch. In the so-called musical seale, or gamut, this difference is very marked to a normal ear. But in the scale there are again four tones, which, when they are sounded together, cause in a normal ear the sensation of an agreeable sound, which once heard can readily be reproduced. This is the tone of the so-called accord, Triad, or Common Chord, consisting of the 1st, 3rd, and 5th tones of the scale, to which the 8th tone or octave is added. We have next to determine the pitch of the tones of the chord, and then that of the other tones of the scale. The siren is used for the fundamental experiment, from which the others can easily be calculated. Four concentric circles are drawn upon the rotatory disc of the siren; the inner circle contains 40 holes, the second 50, the third 60, and the outer 80 -all the holes being at equal distances from each other. If the dise be rotated, and air forced against each 
series of holes in turn, we distinguish successively the four tones of the accord (major chord with its octave); when all the four series are blown upon simultaneously, we hear in complete purity the major chord itself. The relative number of the holes in the four series indicates in the simplest manner the relative pitch of the tones of the major chord. While one revolution of the dise is necessary to proluce the fundimental ground-tone (key-note or tonic) with 40 condensations and rarefactions of the air-in order to produce the octave, we must have donble the number of condensations and rarefactions during one revolution in the same time. Thus, the relation of the number of vibrations of the Ground-tone or Tonic to the Octave next above it, is $1: 2$. In the second series we have 50 holes, which causes the pitch of the third; hence, the relation of the Ground-tone to the Third in this case is $40: 50$, or $1: 1 \frac{1}{4}=\frac{5}{4}, i \cdot e$, for very vibration of the Ground-tone there are $\frac{5}{4}$ vibrations in the Third. In the third series are 60 holes, which, when blown upon, yiell the fifth; hence, the ratio of the Ground-tone to the Fifth in our disc is $40: 60$, or $1: 1 \frac{1}{2}=\frac{3}{2}$. In the same way we can estimate the pitch of the rourth tone, and we find that the number of vibrations of the First, Third, Fifth, and Octave are to each other as $1: 1: \frac{s}{2}: 2$.

The minor chord is quite as characteristic to a normal ear as the major. It is distinguished e'ssentially from the latter by its Third being half a tone lower. We can easily imitate it by the siren, as the Minur Third consists of a number of vibrations which stand to the Groundtone as $6: 5$, i.c., if 5 vibrations occur in a given time in the Ground-tone, then 6 occur in the Minor Third; its vibration number, therefore, is $\frac{6}{5}$.

From these relations of the Major and Minor common chords, we may calculate the relative tones in the scale, and we must remember that the Octave of a tone always yields the fullest and most complete harmony. It is evident that as the Major Third, and Minor Third, and the Fifth harmonise with the fundamental Ground-tone or key-note, they must also harmonise with the Oetave of the key-note. We obtain from the Major Third with the uumber of vibrations $\frac{A}{4}$, the Minor Sixth $\frac{8}{5}$, from the Minor Third with $\frac{6}{5}$, the Major Sixth $=\left(\frac{6}{10}=\right) \frac{8}{6}$; from the Fifth with $\frac{7}{2}$, the Fourth $=\frac{3}{4}$. These relations are known as the "Inversions of the intervals." These relations of the tones are, collectively, the consonant intervals of the scale. The dissonant stages, or discords, of the scale can be obtained as follows:- Suppose that we have the Groundtone or key-note $C$, with the number of vibrations $=1$, the Third $E=\frac{5}{4}$, the Fifth $G=\frac{3}{2}$, and the Octave $=2$, we then derive from the Fifth or Dominant G a Major chord-this is G, B, D'. The relative number of vibrations of these 3 tones is the same as in the Major chord of $\mathrm{C}, \mathrm{C}, \mathrm{E}$, (i. Hence, the number of vibrations of $\mathrm{G}: \mathrm{B}$ is as $\mathrm{C}: \mathrm{E}$. When we substitute the values we obtain $\frac{3}{2}: \mathrm{B}=1: \frac{5}{4}-$ i.e., $\mathrm{B}=\frac{1 \mathrm{~s}}{8}$. But $\mathrm{D}: \mathrm{B}=\mathrm{G}: \mathrm{E}$; so that $\mathrm{D}: \frac{15}{8}=\frac{5}{2}: \frac{5}{4}, i . e_{.}, \mathrm{D}=\frac{18}{8}$, or an octave lower, we have $\mathrm{D}=\frac{2}{8}$. Deduce from $\mathrm{F}$ (subdominant) a Major chord, F, A, C. The relation of $\mathrm{A}: \mathrm{C}=\mathrm{E}: \mathrm{G}$, or $\mathrm{A}: 2=\frac{5}{4}: \frac{3}{2}, i . c ., \mathrm{A}=\frac{5}{3}$. Lastly, $\mathrm{F}: \mathrm{A}=\mathrm{C}: \mathrm{E}$, or $\mathrm{F}: \frac{5}{3}=1: \frac{5}{4}$, i.c., $\mathrm{F}=\frac{4}{\mathrm{~s}}$. So that all the tones of the scale have the following number of vibrations:- I., $\mathrm{C}=1 ; \mathrm{II}, \mathrm{D}=\frac{9}{9}$; III., $\mathrm{E}=\frac{5}{2} ; \mathrm{IV} ., \mathrm{F}=\frac{4}{3} ; \mathrm{V} ., \mathrm{G}=\frac{3}{2} ; \mathrm{VI} . ; \mathrm{A}=\frac{5}{3} ;$ VII., $\mathrm{B}=\frac{15}{8} ; \mathrm{VIII} ., \mathrm{C}=2$.

Conventional Estimate of Pitch.-Conventionally, the pitch or concert-pitch of the note, $a$, is taken at 440 vibrations in the second (Scheibler, 1834), although in France it is taken at 435 vibrations per second. From this we can estimate the absolute number of vibrations for the tones of the scale :- $\mathrm{C}=33, \mathrm{D}=37 \cdot 125, \mathrm{E}=41 \cdot 25, \mathrm{~F}=44, \mathrm{G}=49 \cdot 5, \mathrm{~A}=55, \mathrm{~B}=61 \cdot 875$ vibrations. The number of vibrations of the next highest octave is found at once by multiplying these numbers by 2 .

Musical Notes, - The lowest notes used in music are the double-bass, E, with $41 \cdot 25$ vibrations. rianoforte $\mathrm{C}$ with 33 , grand piano $\mathrm{A}$ with $27 \cdot 5$ and organ $\mathrm{C}$ with $16 \cdot 5$. 'The highest

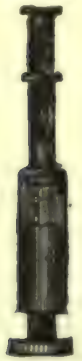
notes in music are the pianoforte $c^{v}$ with 4224 , and $d^{v}$ on the piccolo-flute, with 4752 vibrations per second.

Limits of Auditory Perception.-According to Preyer, the limit of the perception of the lowest andible tone lies between 16 and 23 vibrations per second, and $\mathrm{e}^{\text {vill }}$ with 40,960 vibrations as the highest audible tone; 80 that this embraces about $11 \frac{1}{2}$ octaves.

[Audibility of Shrill Notes. - This varies very greatly in different persons ( $W$ olInston). There is a remarkable falling off of the power as age advances (Galton). For testing this Galton uses a small whistle made of a brass tube, with a diameter of less than soth of an inch (fig. 596). A plug is fitted at the lower end to lengthen or shorten the tube, whereby the pitch of the note is altered. Amongst animals Galton finds none superior to cats in the power of hearing shrill sounds, and he

Fig. 596. attributes this "to differentiation by natural selection amongst these animals until Falton's they have the power of hearing all the high notes made by mice and other little Whistle, creatures they have to catch."]

Variations in Auditory Perception.-It is rare to find that tones produced by more than 35,000 vibrations per second are heard. When the tensor tympani is contracted, the pereeption may be increased for tones 3000 to 5000 vibrations higher, but rarely more. Pathologically, the perception for high notes may be abnormally acute-(1) When the tension of the 
sound-conducting apparatus generally is increased. (2) By elimination of the sound-conducting apparatus of the middle ear, which offers greater or less resistance to the propagation of very high notes, as perforation of the membrane tympani, or loss of the incus and malleus. In these cases, the stapes is directly set in vibration by the sound-waves, when tones up to 80,000 vibrations have been perceived. Diminished tension of the sound-conducting apparatus causes diminution of the perception for high tones (Blake).

A smaller number of vibrations than 16 per second (as in the organ) are no longer heard as a tone, but as single dull impulses. The tones that are produced beyond the highest audible note, as by stroking small tuning-forks with a violin bow, are also no longer heard as tones, but they cause a painful cutting kind of impression in the ear. In the musical scale the range is, approximately, from $\mathrm{C}$ of the first octave with 16.5 vibrations to e, the eighth octave.

Comparison of Ear and Eye.-In comparing the perception of the eye with that of the ear, we see at once that the range of accommodation of the ear is much greater. Red has 456 billions of vibrations per second, while the visible violet has but 667 , so that the eye only takes cognisance of vibrations which do not form even one octave.

Lowest Audible Tone.-As to the smallest number of successive vibrations which the ear can perceive as a sensation of tone, Savart and Pfaundler considered that two would suffice. If, however, we exclude in our experiments the possibility of the occurrence of overtones, 4 to 8 (Mach) or even 16 to 20 vibrations ( $F$. Auerbach, Kohlrausch) are necessary to produce a characteristic tone.

When tones succeed each other rapidly, they are still perceived as distinct, when at least 0.1 second intervenes between two successive tones ( $v$. Helmholtz); if they follow each other more rapidly, they fuse with each other, although a short-time interval is sufficient for many musical tones.

By the term, "fineness of the ear," or, as we say, a "good ear," is meant the capacity of distinguishing from each other, as different, two tones of nearly the same number of vibrations. This power can be greatly increased by practice, so that musicians can distinguish tones that differ in pitch by only $\frac{1}{300}$, or even $\frac{1}{1200}$, of their vibrations.

With regard to the time-sense, it is found that beats are more precisely perceived by the ear than by the other sense-organs (Höring, Mach).

Pathological. - According to Lucae, there are some ears that are better adapted for hearing low notes and others for high notes. Both conditions are disadvantageous for hearing speech. Those who hear low notes best hear the highest consonants imperfectly. The low notes are heard abnormally loud in rheumatic facial paralysis, while the high tones are heard abnormally loud in cases of loss of the membrana tympani, incus, and malleus. The stapedius is in full action, whereby the highest tones are heard louder at the expense of the lower notes. Many persons with normal hearing hear a tone higher with one ear than with the other. This condition is called diplacusis binauralis. In rare cases, sudden loss of the perception of certain tones has been observed, e.g., the bass-deafness of Moos. In a case described by Magnus, the tones d', b', were not heard ( $\$ 316$ ).

II. Perception of the Intensity of Tone.-The intensity of a tone depends upon the amplitude of the vibrations of the sounding body. The intensity of the tone is proportional to the square of the amplitude of vibration of the sounding body, i.e., with 2,3 , or 4 times the amplitude the intensity of the tone is 4, 9, 16 times as strong. As sonorous vibrations are communicated to our ears by the wave-movements of the air, it is evident that the tones must become less and less intense the further we are from the source of the sound. The intensity of the sound is inversely proportional to the square of the distance of the source of the sound from the ear.

Tests. - 1. Place a watch horizontally near the ear, and test how close it may be brought to the ear, and also how far it ntay be removed, and still its sounds be heard. Measure the distance. 2. Itard uses a small hammer suspended like a pendulum, and allowed to fall upon a hard surface. 3. Balls of different weights are allowed to fall from varying heights upon a plate. In this case the intensity of the sound is proportional to the product of the weight of the ball into the height it falls.

As to the limits of the perception of the intensity of a tone, it is found that a spherule weighing $1 \mathrm{milligram}$, and falling from a height of $1 \mathrm{~mm}$. upon a glass plate, is heard at a distance of 5 centimetres (Schafhäult).

415. PERCEPTION OF QUALITY_VOWELS. - By the term quality ("Klangfarbe"), musical colour or timbre, is understood a peculiar character of the tone, by which it can be distinguished apart from its pitch and intensity. Thus, a flute, horn, violin, and the human voice may all sound the same note with equal intensity, and yet all the four are distinguished 
at once by their specific quality. Wherein lies the essence ("Wesen ") of tone-colour ? The investigations of $\mathrm{v}$. Helmholtz have proved that, amongst mechanisms which produce tones, only those that produce pendulum-like vibrations, i.c., the to-and-fro vibrations of a metallic rod with one end fixed, and tuning-forks, execute simple pendulum-like vibrations. This can be shown by making a tuning-fork write off its vibrations on a recording surface, when a completely uniform wave-line, with equal elevations and depressions is noted. The term "tone" is restricterl to those sounds, hardly ever occurring in nature, which are due to simple pendulum-like vibrations.

Other investigations have shown that the tones of musical instruments and of the human voice, all of which hare a characteristic quality of their own, are composed of many single simple tones. Amongst these one is characterised by its intensity, and at the same time it iletermines the pitch of the whole conpround musical "tone-picture." This is called the fundamental tone or key-note. The other weaker tones which, as it were, spring from and are wingled with this, vary in different instruments both in intensity and number. They are "upper tones," and their vibrations are always some multiple-2, 3, 4, 5 ... times-of

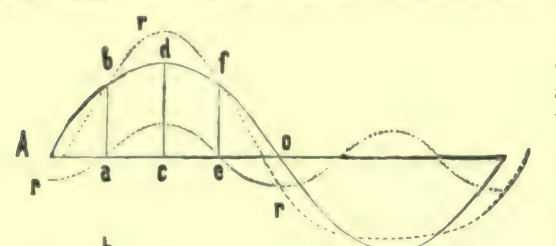
the fundamental tone or key-note. In general, we say that all those outbursts of sound which embrace numerous strong upper tones, especially of high pitch, in addition to the fundamental tone, are characterised by a sharp, piercing, and rough quality, such as emanates from a trumpet or clarionet, and that conversely the quality is characterised by mildness and softness when the overtones are few, feeble, and

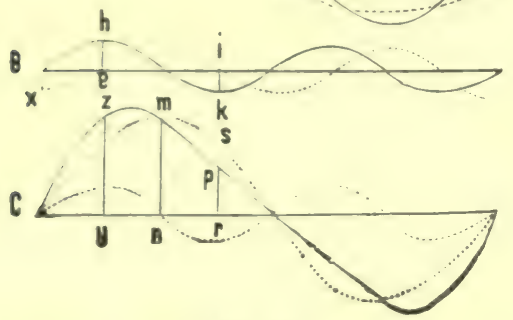

! Fig. 597. low, e.g., such as are produced by the flute. It requires a well-trained musical ear to distinguish, in an instrumental burst, the overtones apart from the fundamental tone. But this is very easily done with the aid of resonators (fig. 600). These consist of spherical or funnelshaped hollow bodies, made of brass or some other substance, which, by means of a short tube, can be placed in the outer ear. If a resonator be placed in the ear, we can hear the feeblest overtone of the same number of vibrations as the fundamental tone. Thus, musical instruments are distinguished by the number,

Curves of a musical tone obtained by com- intensity, and pitch of the overtones which they jounding the curve of a fundamental tone produce. A vibrating metallic rod and a tuningwith that of its overtones. fork have no overtones; they only give the fundamental tone. As already mentioned, the term simple tone is applied to sounds due to sinple jenclulum-like vibrations, while a sound composed of a fundamental tone and overtones is called a "klang" or compound musical tone.

Vibration Curve of a Musical Tone. - When we remember that a musical tone or clang consists of a funlamental tone, and a number of overtones of a certain intensity, which determine its quality, then we ought to be able to construct geometrically the vibration curve of the inusical tone. Let A represent the vibration curve of the fundamental tone, and B that of the first moderately weak overtone (fig. 597). The combination of these two curves is obtained simply by computing the height of the ordinates, whereby the ordinates of the overtone curve, lying above the abscissa or horizontal line, are added to the fundamental tone curve, while those of the ordinates below the line are subtracted from it. Thus we obtain the curve $\mathrm{C}$, which is not a simple pendulum-like curve, but one which corresponds to an unsteaciy movement. A new curve of the second overtone may be added to $\mathrm{C}$, and so on. The result of all these combinations is that the vibration curves corresponding to the compound musical tones are unsteady periodic eurves. All these curves must, of course, vary with the number and pitch of the compounded overtone curves.

Displacement of the Phases. - The form of the vibration of one and the same musical tone may vary very greatly if, in compounding the curves $A$ and $B$, the curve $B$ is only slightly displaced laterally. If $B$ is lisplaced so that the hollow of the wave $r$ falls under $A$, the addition of both curves yields the curve $r, r, r$, with small elevations and broad valleys. If $B$ le displaced still further, until the elevation of the wave, $h$, coincides with A, we obtain still another form, so that by displacement of the phases of the wave-motions of the compounded simple pendulum-like vibrations, we obtain numerous different forms of the same musical tone. The displacement of the phases, however, has no effect on the ear.

The general result of these observations, and those of Fourier, is that the quality of a musical tone deprends unon the characteristic form of the vibratory movement.

Analysis of Vowels. - The human voice represents a reed instrument with vibrating elastic 
membranes, the vocal cords (\$312). In uttering the various vowels the mouth assumes a characteristic form, so that its cavity has a certain fundamental tone peculiar to itself. Thus, to the fundamental tone of a certain pitch produced within the larynx, there are added certain overtones, which communicate to the laryngeal tone the vocal or vowel quality. Hence, a vowel is the timbre or quality of a musical tone which is produced in the larynx. The quality depends upon the number, intensity, and pitch of the overtones, and the latter, again, depend on the configuration of the "vocal cavity" in uttering the different vowels (\$317).

Suppose a person to sing the vowels one after the other on a special note, e.g., b b, we can, with the aid of resonators determine the overtones, and in what intensity they are mixed with the fundamental tone, $\mathrm{b} b$, to give the characteristic quality. According to v. Helmholtz, when we sound the vowels on $\mathrm{b} b$, for each of the three vowels, one overtone is specially characteristic for $\mathbf{A}-\mathbf{b}^{\|} \mathbf{b}$; for $\mathbf{O}-\mathbf{b}^{\prime} b$; for $\mathrm{U}-\mathrm{f}$. The other vowels and the diphthongs have each two specially characteristic overtones, because in these oases the mouth is so shaped that the posterior larger cavity, and also the anterior narrower part, each yields a special tone $(\$ 316$, I. and $E)$. These two overtones are for $E-B \| ! b$ and $f^{\prime}$; for $I-d^{\text {iv }}$ and $f$; for $A-g \mid \|$ and

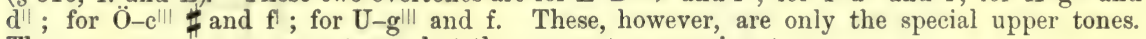
There are many more upper tones, but they are not so prominent.

Artificial Vowels. - Just as it is possible to analyse a vowel into its fundamental tone and its upper tones, it is possible to compound tones to produce the rowels by simultaneously sounding the fundamental tone and the corresponding upper tones. (1) A vowel is proluced simply by singing loudly a vowel, e.g., A, upon a certain note against the free strings of an open piano, whilst by the pedal the damper is kept raised. As soon as we stop singing, the characteristic vowel is sounded by the strings of the piano. The voice sets into sympathetic vibration all those strings whose overtones (in addition to the fundamental tone) occur in the vocal compound tone, so that they vibrate for a time after the voice ceases ( $v$. Helmholtz). (2) The vowel apparatus devised by $v$. Helmholtz consists of numerous tuning-forks, which are kept vibrating by means of electro-magnets. The lowest tuning-fork gives the fundamental tone, $\mathrm{B} b$, and the others the overtones. A resonator is placed in front of each tuning-fork, and the distance between the two can be varied at pleasure. The resonators can be opened and closed by a lid passing in front of their openings. When the resonator is closed, we cannot hear the tone emitted by the tuning-fork placed in front of it; but when one or more resonators are opened the tone is heard distinctly, and it is louder the more the resonator is opened. By means of a series of keys, like the keys of a pianoforte, we can rapidly open and close the resonators at will, and thus combine various overtones with the fundamental tone so as to produce vowels with different qualities. V. Helmholtz makes the following compositions:$\mathrm{U}=\mathrm{B} b$ with $\mathrm{b} b$ weak and $\mathrm{f}^{\prime} ; \mathrm{O}=$ damped $\mathrm{B} b$ with $\mathrm{b}^{\prime} \mathrm{b}$ strong and weaker $\mathrm{b} b^{\prime}, \mathrm{f}^{\prime}, \mathrm{d}^{\prime \prime} ; \mathrm{A}=\mathrm{b} b$ (fundamental tone) with moderately strong $b^{\prime} b$ and $f^{\prime \prime}$, and strong $b^{\|} b$ and $d^{\prime \prime} ; A=b \quad b$ (fundamental tone) with $\mathrm{b} b$ and $\mathrm{f}^{\prime} \mid$ somewhat stronger than for $\mathrm{A}$, $d$ strong, $b$ and $f \|$ as strong as possible; $E=b b$ (as fundamental tone) moderately strong, with $b \mid b$ and $f$ moderate also, and $f^{\prime \prime}, a^{\| l \mid} b$, and $b \| l \mid b$, as strong as possible; I could not be produced.

In Appunn's apparatus, the fundamental tone and the overtones are produced by means of organ pipes, whose notes can be combined to produce the vowels, but it is not so good as the tuning-forks, since the organ pipes do not yield simple tones, but nevertheless some of the vowels can be admirably reproduced with this apparatus.

Edison's Phonograph. - If we utter the vowels against a delicate membrane stretched over the end of a hollow cylinder, and if a writing style be fixed to the centre of the membrane, and the style be so arranged that it ean write or record its movements on a piece of soft tinfoii arranged on a revolving apparatus, then the vowel curve is stamped as it were upon the tinfoil. If the style now be made to touch the tinfoil while the latter is moved, then the style is movedit moves the membrane, and we hear distinctly by resonance the vowel sound reproduced.

[Koenig's Manometric Flames. - By means of this apparatus the quality of the vowel sounds is easily shown. It consists of a small wooden capsule, A, divided into two compartments by a piece of thin sheet india-rubber. Ordinary gas passes into the chamber on one side of the membrane, through the stop-cock, and it is lighted at a small burner. To the other compartment is attached a wider tube with a mouthpiece. The whole is fixed on a stand, and near it is placed a four-sided rotating mirror, $\mathbf{M}$, as suggested by Wheatstone (fig. 598). On speaking or singing a vowel into the mouthpiece, and rotating the mirror, a toothed or zigzag flamepicture is obtained in the mirror. The form of the flame-picture is characteristic for each vowel, and varies of course with the pitch.] [Fig. 599 shows the form of the flame-picture obtained in the rotating mirror when the vowels, oU, $0, A$, are sung at a pitch of $u t_{1}, s o l_{1}$, and ut $t_{2 .}$ This series shows how they differ in quality.]

[Koenig has also invented the apparatus for analysing any compound tone whose fundamental tone is $\mathrm{UT}_{2}$ (fig. 600). It consists of a series of resonators, from $\mathrm{UT}_{2}$ to $\mathrm{UT}_{5}$, fixed in an iron frame. Each resonator is connected with its special flame, which is pictured in a long narrow, square rotating mirror. If a tuning-fork $\mathrm{UT}_{2}$ be sounded, only the flame $\mathrm{UT}_{2}$ is affected, and so on with each tuning-fork of the harmonic series. Suppose a compound note containing the fundamental tone $\mathrm{UT}_{2}$, and its harmonics be sounded, then the flame of $\mathrm{UT}_{2}$, and those of 
the other harmonies in the note are also affecterl, so that the tone can be analysed optically. The same may be done with the vowels.]

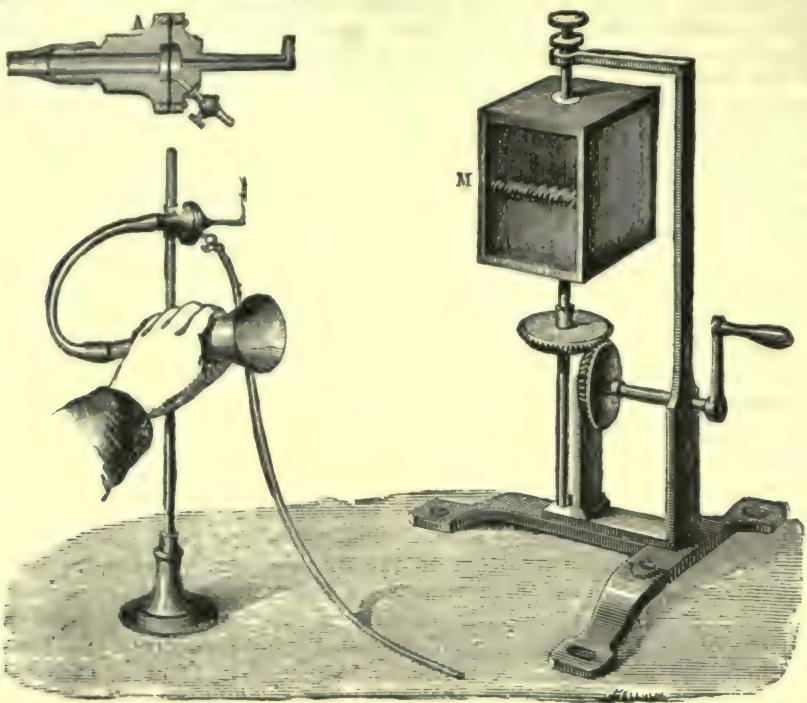

Fig. 598.

Koenig's manometric capsule (A) and mirror (M)-(Koenig).

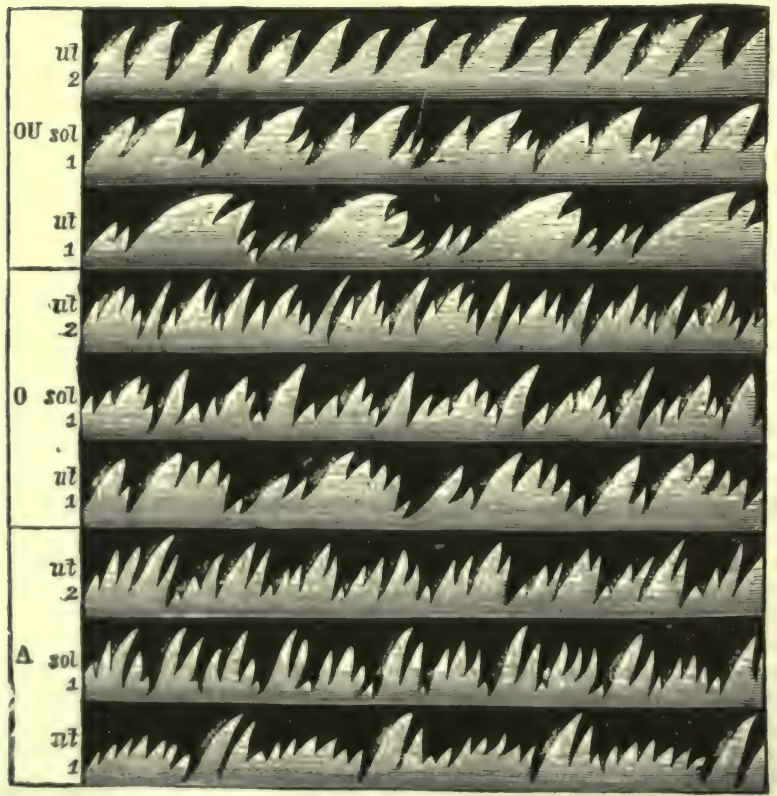

Fig. 599.

Flame-pictures of the vowels ov, 0 , and $\mathbf{A}$ (Koenig).

416. LABYRINTH DURING HEARING.-If we ask what rôle the ear plays in the perception of the quality of sounds, then we must assume that, just as with 
the help of resonators a musical note can be resolved into its fundamental tone and overtones, so the ear is capable of performing such an analysis. The ear resolves the complicated wave-forms of musical tones into their components. These com. ponents it perceives as tones harmonious with each other; with marked attention each is perceived singly, so that the ear distinguishes as different tone-colours only different combinations of these simple tone-sensations. The resolution of complex vibrations, due to quality, into simple pendulum-like vibrations is a characteristic function of the ear. What apparatus in the ear is capable of doing this? If we sing vigorously-e.g., the musical vowel A on a definite note, say $b$-against the strings of an open pianoforte while the damper is raised, then we cause all those strings, and only those, to vibrate sympathetically, which are contained in the vowel so sung. We must, therefore, assume that an analogous sympathetic apparatus occurs in the ear, which is tuned, as it were, for different pitches, and which will vibrate sympathetically like the strings of a pianoforte. "If we could so connect every string of a piano with a nerve-fibre that the nervefibre would be excited and perceived as often as the string vibrated, then, as is actually the case in the ear, every musical note which affected the instrument would excite a series of sensations exactly corresponding to the pendulum-like vibrations into which the original movements of the air can be resolved ; and thus the existence

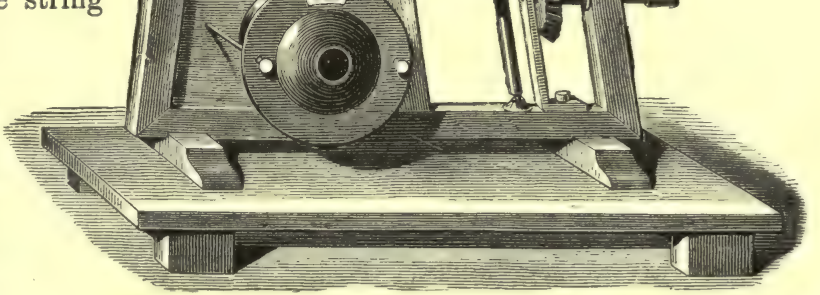

Fig. 600 .

Koenig's apparatus for analysing a compound tone with the fundamental tone $\mathrm{UT}_{2}$.

of each individual overtone would be exactly perceived, as is actually the case with the ear. The perception of tones of different pitch, would under these circumstances depend upon different nerve-fibres, and hence would occur quite independently of each other. Microscopic investigation shows that there are somewhat similar structures in the ear. The free ends of all the nerve-fibres are connected with small elastic particles which we must assume are set into sympathetic vibration by the sound-waves" (v. Helmholtz).

Resolution by the Cochlea - Formerly v. Holmholtz considered the rods of Corti to be the apparatus that vibrated and stimulated the terminations of the nerves. But, as birds and amphibians, which certainly can distinguish musical notes, have $n o$ rods (Hasse), the stretched radial fibres of the membrana basilaris, on which the organ of Corti is placed, and which are shortest in the first turn of the cochlea, becoming longer towards the apex of the cochlea, are now regarded as the vibrating 
threads (Hensen). Thus, a string-like fibre of the membrana basilaris, which is capable of vibrating, corresponds to every possible simple tone. According to Hensen, the hairs of the labyrinth, which are of unequal length, may serve this purpose. Destruction of the apex of the cochlea causes deafness to deeper tones (Buyinsky).

[Hensen's Experiments. - That the hairs in connection with the hair-cells vibrate to a particular note is also rendered probable by the experiments of Hensen on the crustacean Mysis. He found that certain of the minute hairs (auditory hairs) in the auditory organ of this animal, situate at the base of the antennæ, vibrated when certain tones were sounded on a keyed horn. The movements of the hairs were observed by a low-power microscope. In mammals, however, there is a difficulty, as the hairs attached to the cells appear to be all about the same length. We must not forget that the perception of sound is a mental act.]

This assumption also explains the perception of noises.

of noises in the strictly physical sense, it is assumed that they, like single impulses, are perceived by the aid of the saccules and the ampullæ.

It is assumed that the saccules and the ampullæ are concerned in the general perception of hearing, i.e., of shocks communicated to the auditory nerve (by impulses and noises); while by the cochlea we estimate the pitch and depth of the vibrations, and musical character of the vibrations produced by tones.

The relation of the semicircular canals to the equilibrium of the body is referred to in $\$ 350$.

417. SIMULTANEOUS ACTION OF TWO TONES-HARMONY-BEATS -DISCORDS - DIFFERENTIAL TONES. - When two tones of different pitch fall upon the ear simultaneously, they cause different sensations according to the difference in pitch.

1. Consonance. - If the number of vibrations of the two tones is in the ratio of simple multiples, as $1: 2: 3: 4$, so that when the low notes make one vibration the higher one makes $2: 3$ or 4 ... then we experience a sensation of complete harmony or concord.

2. Interference.-If, however, the two tones do not stand to each other in the relation of simple multiples, then when both tones are sounded simultaneously interference takes place. The hollows of the one sound-wave can no longer coincide with the hollows of the other, and the crests with the crests, but, corresponding to the difference of number of vibrations of both curves, sometimes a wave-crest must coincide with a wave-hollow. Hence, when wave-crest meets wave-crest, there must he an increase in the strength of the tone, and when a hollow coincides with a crest, the sound must be weakened. Thus we obtain the impression of those variations in tone intensity which have been called " beats."

The number of vibrations is of course always efunal to the difference of the number of vibrations of both tones. The beats are perceived most distinctly when two organ tones of low pitch are soumlerl together in unison, but slightly out of tune. Suppose we take two organ Iipes with 33 vibrations per secoml, and so alter one pipe that it gives 34 vibrations per second, then mo distinct heat will be heard every second. The beats are heard more frequently the greater the difference between the number of vibrations of the two tones.

Successive Beats. - The beats, however, produce very different impressions upon the ear according to the rapidity with which they succeed each other.

1. Isolated Beats. - When they occur at long intervals, we may perceive them as completely isolated, but single intensifications of the sound with subsequent enfeeblement, so that they give rise to the impression of isolated beats.

2. Dissonance.-When the beats occur more rapidly they cause a continuous disagreeable whirring impression, which is spoken of as dissonance, or an unharmonious sensation. The greatest degree of unpleasant painful dissonance occurs when there are 33 beats per second. 
3. Harmony. - If the beats take place more rapidly than 33 times per second, the sensation of dissonance gradually diminishes, and it does so the more rapidly the beats occur. The sensation passes gradually from moderately inharmonious relations (which in music have to be resolved by certain laws) towards consonance or harmony. The tone relations are successively the Second, Seventh, Minor Third, Minor Sixth, Major Third, Major Sixth, Fourth, and Fifth.

4. Action of the Musical Tones ("Klënge").-Two musical "klangs," or compound tones, falling on the ear simultaneously, produce a result similar to that of two simple tones; but in this case we have to deal not only with the two fundamental tones; but also with the overtones. Hence the degree of dissonance of two musical tones is the more pronounced the more the fundamental tones and the overtones (and the "differential" tones) produce beats which number about 33 per second.

5. Differential Tones.-Lastly two "klangs," or" two simple musical tones sounding simultaneously, may give rise to new tones when they are uniformly and simultaneously sounding in corresponding intensity. We can hear, if we listen attentively, a third new tone, whose number of vibrations corresponds to the difference between the two primary tones, and hence it is called a "differential tone."

Summational Tones. - It was formerly supposed that new tones could arise from the summation or addition of their number of vibrations, but it has been shown that these tones are in reality differential tones of a high order (Appunn, Preyer).

\section{PERCEPTION OF SOUND - OBJECTIVE AND SUBJECTIVE AUDITION-AFTER-SENSATION.-Objective and Auditory Perceptions.-} When the stimulation of the terminations of the nerves of the labyrinth is referred to the outer world, then we have objective auditory perceptions. Such stimulations are only referred to the outer world as are conveyed to the membrana tympani by vibrations of the air, as is shown by the fact that if the head be immersed in water, and the auditory meatuses be filled thereby, we hear all the vibrations as if they occurred within our head itself $(E d$. Weber), and the same is the case with our own voice, as well as with the sound-waves conducted through the bones of the head, when both ears are firmly plugged.

Perception of Direction.-As to the perception of the direction whence sound comes, we obtain some information from the relation of both meatuses to the source of the sound, especially if we turn the head in the supposed direction of the sound. We distinguish more easily the direction from which noises mixed with musical tones come than that of tones (Rayleigh). When both ears are stimulated equally, we refer the source of the sound to the middle line anteriorly, but when one ear is stimulated more strongly than the other, we refer the source of the sound more to one side (Kessel). The position of the ear-muscles, which perhaps act like an ear-funnel, is important. According to Ed. Weber, it is more difficult to determine the direction of sound when the ears are firmly fixed to the side of the head. Further, if we place the hollow of both hands in front of the ear, so as to form an open cavity behind them, we are apt to suppose that a sounding body placed in front is behind us. The semicircular canals are said also to be concerned, as sound coming from a certain direction must always excite one canal more than the others. Thus, the left horizontal canal is most stimulated by horizontal soundwaves coming from the left (Preyer). Other observers assert that the membrana tympani localises the sound, as only certain parts of it are affected by the soundwaves.

The distance of a sound is judged of partly by the intensity or loudness of the sound, such as we have learned to estimate from sound at a known distance. But still we are subject to many misconceptions in this respect. 
Amongst subjective auditory sensations are the after-vibrations, especially of intense and continued musical tones: the tinnitus aurium (p. 606), which often accompanies abnormal movements of the bloul in the ear, may be due to a nechanical stimulation of the auditory fibres, perhaps by the blood-stream (Brenner).

[Drugs. - Cannabis iudica seems to act on the hearing centre, giving rise to subjective sounds ; the hearing is rendered more acute by strychnin; while quinine and sodic salicylate in large doses cause ringing in the ears (Brunton).]

Entotical perceptions, which are due to causes within the ear itself, are such as hearing the pul. lients in the surroumding arteries, and the rushing sound of the blood, which is especially strung "hen there is increased resonance of the car (as when the meatus or tympanum is closed, (1) when fluil acrumulates in the latter), during increased cardiac action, or in hyperæsthesia of the atulitory nerve (Brenne). Sometimes there is a cracking noise in the maxillary artivulation, the noise produced by traction of the muscles on the Eustachian tube (\$ 411), and when air is forcel into the latter, or when the membrana tympani is forced outwards or in wards $(\$ 350)$.

Fatigue. - The ear after a time becomes fatiguel, either for oue tone or for a series of tones whi.h have acted on it, while the perceptive activity is not affected for other tones. Complete recovery, however, takes place in a few seconds (Urbantschitsch).

Auditory After-Sensations. - (1) Those that correspond to positice after-sensations, where the after...nsation is so clusely connected with the original tone that both appear to be continuous. (:) There are some after-sensations, where a pause intervenes between the end of the objective and the begiming of the suljective tone (Thuntschitsch). (3) There seems also to be a form corresponding to mgative after-images.

In sume prersons, the pereeption of a tout is accompanied by the occurrence of subjective colours, or the sensation of light, $e . y$, the soumd of a trumpet, accompanied by the sensation of yellow. Jore seldom visual sensations of this kind are observed when the nerves of taste, smell, or

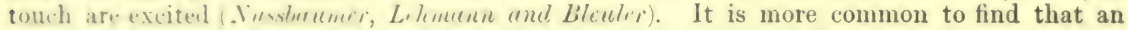
intense sharp souml is accomplanied ly an associated sensation of the sensory nerves. Thus many pople exprerience a cold shudeler when a slate pencil is drawn in a peculiar manuer across a slate.

[Colour Associations. - Colour is in some jersons instantaneously associated with sound, and Gialton remalks that it is rather common in children, although in an ill-developed degree, and the tendency sems to he very herelitary. Sometimes a particular colour is associated with a particular letter, vowel sounds particularly evoking colours. Galton has given coloured representations of these colour associations, and he points out their relation to what he calls number-forms, or the association of certain forms with certain numbers.]

An aulitury impulse communicated to one ear at the same time often causes an increase in the auditory function of the other ear, in consequence of the stimulation of the auditory centres of both sides (Urbantschitsch, Eitelbery).

Other Stimuli. - The aulitory aplraratus, besides heing excited by sound-waves, is also afficted ly heterologous stimuli. It is stimulated mechanically by a sudden blow on the ear. The effects of electricity and pathological conditions are referred to in $\$ 350$.

419. COMPARATIVE-HISTORICAL. - The lowest fishes, the cyclostomata (Petromyzon), have a sarcule proviled with auditory hairs containing otoliths, and communicating with two semicireular canals, while the myxinuids have only one semicircular canal. Most of the other fishes, however, have a utricle communieating with three semicircular canals. In the carp, prolongations of the labyrinth communicate with the swimming-bladder. In amphibia, the structure of the labyrinth is sumewhat like that in fishes, but the cochlea is not typically developred. Most amphibia, except the frog, are devoid of a membrana tympani. Only the fenestra ovalis (not the rotunda) exists, and it is connected in the frog by three ossicles with the freely-exposed membrana tympani. Amongst reptiles the appendix to the saccule, corresponding to the cochlea, begins to be prominent. In the tortoise it is saccular, but in the crocodile it is longer, and somewhat curved and dilated at the end. In all reptiles the fenestra rotumla is developed, whereby the cochlea is connected with the labyrinth. In crocodiles and birds, the cochlea is divided into a scala vestibuli and $\mathrm{S}$. tympani. Snakes are devoid of a tympanic cavity. In birds both saccules (fig. 591, IV, U S') are united (Hasse), the canal of the cochlea ( $\mathrm{C}$ ), which is connected by means of a fine tube (C) with the snceule, is larger, and shows indications of a spiral arrangement, and has a flask-like blind end, the lagena $(\mathrm{L})$. The auditory ossicles in reptiles and birds are reduced to one column-like rod, corresponding to the stayes, and called the columella. The lowest mammals (Echidna) have structures very like those of birds, while the higher mammals have the same type as in man (fig. 591, 1II). The Eustachian tube is always open in the whale.

Amongst invertebrata, the auditory organ is very simple in medusie and mollusca. It is merely a bladder filled with fluid, with the anditory nerves provided with the ganglia in its walls. Hair-cells occur in the interior, provided with one or more otoliths. Hensen observed that in some of the annulosa, when sound was conducted into the water, some of the auditory 
bristles vibrated, being adapted for special tones. In cephalopoda, we distinguish the first differentiation into a membranous and cartilaginous labyrinth.

Historical. - Empedocles (473 B.c.) referred auditory impressions to the cochlea. The Hippocratic School was acquainted with the tympanum, and Aristotle (384 B.c.) with the Eustachian tube. Vesalius (1561) described the tensor tympani ; Cardanus (1560) the conduction through the bones of the head; while Fallopius (1561) described the vestibule, the semicircular canals, chorda tympani, the two fenestræ, the cochlea, and the aqueduct. Eustachius $(+1570)$ described the modiolus, the lamina spiralis of the cochlea, the Eustachian tube, as well as the muscles of the ear ; Plater the ampullæ (1583); Casseri (1600) the lamina spiralis membranacea. Sylvius (1667) discovered the ossicle called by his name; Vesling (1641) the stapedius. Mersenne (1618) was acquainted with overtones; Gassendus (1658) experimented on the conduction of sound. Acoustics were greatly advanced by the work of Chladni (1802). The most recent and largest work on the ear in vertebrates is by G. Retzius (1881-84).

\section{The Sense of Smell.}

420. STRUCTURE OF THE ORGAN OF SMELL. - Regio Olfactoria.-The area of the distribution of the olfactory nerve is the regio olfactoria, which embraces the upper part of the septum, the upper, and part of the mildle $(\mathrm{Cm})$ turbinated bone (fig. 601, $\mathrm{Cs}$ ). All the remainder of the nasal cavity is called the regio respiratoria. These two regions are distinguished as follows :-(1) The regio olfactoria has a thicker mucous membrane. (2) It is covered by a single layer of cylindrical epithelium, the cells being often branched at their lower ends, and contain a yellow or brownish-red pigment (figs. 602,603, E). (3) It is coloured by this pigment, and is thereby distinguished from the uncoloured regio respiratoria, which is covered

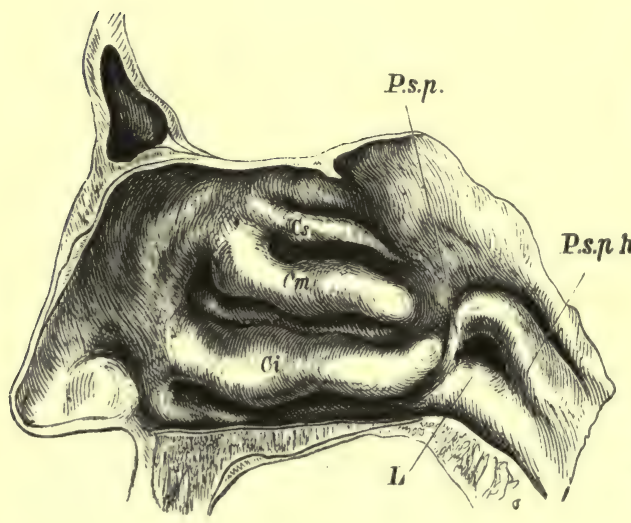

Fig. 601.

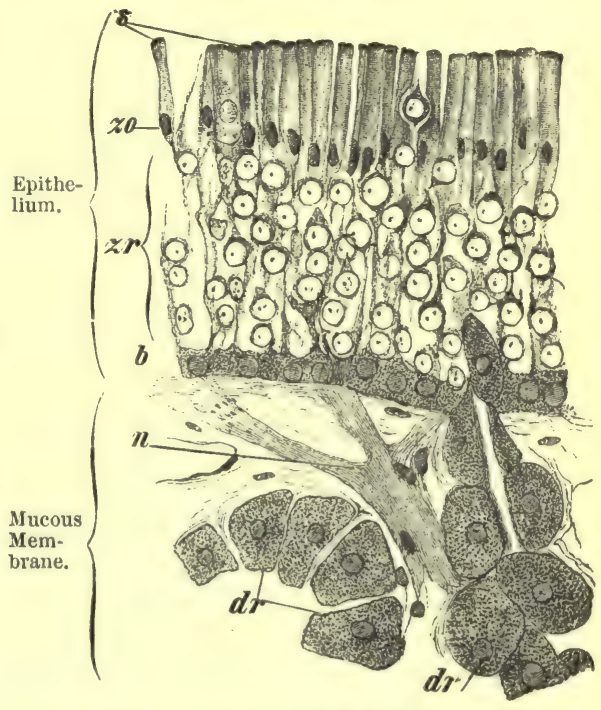

Fig. 602.

Fig. 601.-Nasal and pharyngo-nasal cavities. $L$, levator elevation ; P.s.p., plica salphingopalatina ; $\mathrm{Cs}, \mathrm{Cm}$, Ci, the three turbinated bones (Urbantschitsch). Fig. 602.-Vertical section of the olfactory region (rabbit), $\times 560 . s$, disc; $z 0$, zone of oval, and $z r$ of spherical nuclei ; $b$, basal cells ; $d r$, part of a Bowman's gland ; $n$, branch of the olfactory nerve.

by ciliated epithelium. (4) It contains peculiar tubular glands (Bowman's glands), described as "mixed glands" by Paulsen ( $\$ 142$ ), while the rest of the mucous membrane contains numerous acinous serous glands (Heidenhain); but in man the latter are said to be mixed glands (Stöhr) (fig. 602). Lymph-follicles lie in the mucous membrane, and from them numerous leucocytes pass out on to free surface $(S t o ̈ h r)$. (5) Lastly, the regio olfactoria embraces the 
end-organs of the olfactory nerve. The long narrow olfactory cells (fig. 603, N) are distributed between the ordinary cylindrical epithelium (E) covering the regio olfactoria. The body of the cell is spindle-shaped, with a large nucleus containing nucleoli, and it sends

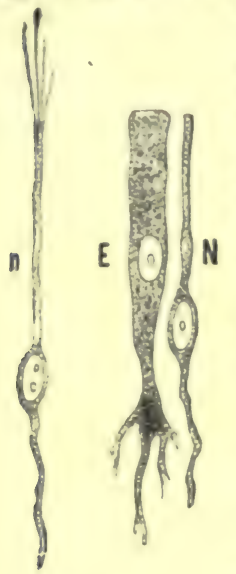

Fig. 603 . upwards between the cylindrical cells a narrow $(0.9$ to $1.8 \mu)$ smooth rod, quite up to the free surface of the mucous membrane. In the frog (n) the free end carries delicate projecting hairs or bristles. In the deeper part of the mucous membrane, the olfactory cells pass into, and become continuous with, varicose fine nerve-fibres, which pass into the olfactory nerve (\$321, I., 1). According to C. K. Hoffmann and Exuer, after section of the olfactory nerve, the specific olfactory end-organs become ehanged into cylindrical epithelium (frog), and in warm-blooded animals they undergo fatty degeneration, even on the 15th day. V. Brunn found a homogeneous limiting membrane, which had holes in it for transmitting the processes of the olfactory cells only.

[The respiratory part of the nasal mucous membrane is lined by ciliated epithelium stratified like that in the trachea and resting on a basement membrane. Below this there are many lymph-corpuscles and aggregations of alenoid tissue.]

[The organ of Jacobson is present in all mammals, and consists of two narrow tubes protected by cartilage, and placed in the lower and anterior part of the nasal septum. Each tube terminates blindly behind, but anteriorly it opens into the nasal furrow or into the naso-palatine canal (dog). The wall next the middle line is covered by olfactory epithelium, and receives olfactory nerves (rabbit, guinea-pig), and it contains glands similar to those of the olfactory region; the outer wall is covered by columnar epithelium ciliated in some animals (Klein).]

X. olfactory eells (hu. man); $i$, from the frog : E, epithelimm of the regio olfac. toria.

421. OLFACTORY SENSATIONS.-Olfactory sensations are produced by the action of gaseous, odorous substances, being brought into direct contact with the olfactory cells, during the act of breathing. The current of air is divided by the anterior projection of the lowest turbinated bone, so that a part above the latter is conducted to the regio olfactoria. Odorous bodies taken into the mouth and then expired through the posterior nares are said not to be smelt (Bidder). [This is certainly not true, as has been proved by Aronsohn.]

[It is usually stated that only odorous particles suspenderl in air excite the sensation of smell. This is certain $\tilde{y}_{\mathrm{y}}$ not the whole truth-otherwise, how do aquatic animals, like fish, smell? Moreover the mucous membrane is always moist, and in some cases where there is a profuse secretion from the olfactory mucous membrane, there is no impairment of the sense of smell.]

During inspiration, the air streams along close to the septum, while little of it passes through the nasal passages, especially the superior (Poulsen and Exner). [The expired air takes almost the same course as the inspired air.]

'The first moment of contact between the odorous body and the olfactory mucous membrane apjears to be the time when the sensation takes place, as, when we wish to obtain a more exact perception, we sniff several times, i.e., a series of rapid inspirations are taken, the mouth being kept closed. During sniffing, the air within the nasal cavities is rarefied, and as air rushes in to equilibrate the pressure, the air, laden with odorous particles, streams over the olfactory region. Odorous fluids are said not to give rise to the sensation of smell when they are brought into direct contact with the olfactory mucous membrane, as by pouring eau de Cologne into the nostrils (Tourtual, 1827; E. H. Weber, 1847). [Aronsohn has, however, shown that these experiments are not accurate, for one can smell eau de Cologne, clove oil, dc., when a mixture of these bodies with 73 per cent. $\mathrm{NaCl}$ is applied to the olfactory mucous membrane; the most suitable medium is 73 per cent. $\mathrm{NaCl}$ and its temperature $40-43^{\circ}$ C.] Even water alone temporarily affects the cells. We know practically nothing about the nature of the action of odorous bodies, but many odorous vapours have a considerable power of absorbing heat ( $T y n d a l l$ ). [Odorous bodies diminish the number of respirations (Gourewitsch).]

The intensity of the sensation depends on -1 . The size of the olfactory surface, as animals with a very keen sense of smell are found to have complex turbinated 
bones covered by the olfactory mucous membrane. 2. The cencentration of the odorous mixture of the air. Still, some substances may be attenuated enormously (e.g., musk to the two-millionth of a milligramme), and still be smelt. 3. The frequency of the conduction of the vapour to the olfactory cells (sniffing).

[The acuteness of the sense of smell is greatly improved by practice. A boy named James Mitchell, who was deaf, dumb, and blind, used his sense of smell, like a dog, to distinguish persons and things.]

[As in the case of sight and hearing, it has been sought to connect the quality of taste and smell with the kind of vibrating stimulus. Ramsay showed that many facts pointed to the dependence of smell upon the vibratory motion of odorous particles; thus, many gases and vapours of low specific gravity-i.e., with a very rapid vibration of their molecules-are perfectly odourless, while such substances as the alcohols and fatty acids, alike in chemical and physical properties, can excite generic smells, the higher members of the group being more powerful in this respect than the lower ones. Taking the elements as arranged in a "Natural Classification" by Mendelejeff, Haycraft has shown that elements in the same group are capable of producing similar or related tastes, and the same seems to be true for smell (Haycraft).]

We can smell the following substances in the following proportions:-Bromine $\frac{1}{3 \frac{1}{0} \overline{0} \overline{0}}$, sulphuretted hydrogen $\frac{1}{5000 \sigma}$ milligramme in $1 \mathrm{c.cm}$. of air (Valentin); also $\frac{1}{4600 \overline{0}}$ gramme of chlorphenol, and $\frac{1}{46000 \overline{0 \sigma}}$ of a milligramme of mereaptan (E. Fischer and Penzold $t$ ).

Electrical stimuli give rise to olfactory sensations. [Althaus found that electrical stimulation of the olfactory mucous membrane gave rise to the sensation of the smell of phosphorus, and Aronsohn found that he smelt on making the current when the cathode-and on breaking the current when the anode-was in the nose.

The variations are referred to in $\$ 343$. If the two nostrils are filled with different odorous substances there is no mixture of the odours, but we smell sonetimes the one and sometimes the other (Valentin). [Some substances appear to affect some regions of the olfactory membrane, while others affect othęr parts.] The sense of smell, however, is very soon blunted, or even paralysed. [It can be blunted or fatigued in a few minutes; but after it is completely fatigued it can recover in a minute.] Morphia, when mixed with a little sugar and taken as snuff, paralyses the olfactory apparatus, while strychnin makes it more sensitive (Lichtenfels and Fröhlich).

The sensory nerves of the nasal mucous membrane (\$ 347, II.) [i.e., those supplied from the fifth cranial nerve] are stimulated by irritating vapours, and may even cause pain, $e, g$., ammonia and acetic acid. In a very diluted condition they may even act on the olfactory nerves. The nose is useful as a sentinel for guarding against the introduction of disagreeable odours and foods. The sense of smell is aided by the sense of taste, and conversely.

[Flavour depends on the sense of smell, and, to test it, use substances, solid or fluid, with an aroma or bouquet, such as wine or roast beef.]

[Method of Testing. - In doing so, avoid the use of pungent substances like ammonia, which excite the fifth nerve. Use some of the essential volatile oils, such as cloves, bergamot, and the fretid gum resins, or musk and camphor. Electrical stimuli are not available. Action of Drugs, \& 343.]

Comparative. - In the lowest vertebrata, pits, or depressions provided with an olfactory nerve, represent the simplest olfactory organ. Amphioxus and the cyclostomata have only one olfactory pit ; all other vertebrates have two. In some animals (frog) the nose communicates with the mouth by ducts. The olfactory nerve is absent in the whale.

Historical. - Rufus Ephesius (97 A.D.) described the passage of the olfactory nerve through the ethmoid bone. Rudius (1600) dissected the body of a man with congenital anosmia, in whom the olfactory nerves were absent. Majendie originally supposed that the nasal branch of the fifth was the nerve of smell, a view successfully combated by Eschricht.

\section{The Sense of Taste.}

422. STRUCTURE OF THE GUSTATORY ORGANS.-Gustatory Region. -There is considerable difference of opinion as to what regions of the mouth are endowed with taste:-(1) The root of the tongue in the neighbourhood of the circumvallate papillæ, the area of distribution of the glosso-pharyngeal nerve, is undoubtedly endowed with taste ( $\$ 351)$. (2) The tip and margins of the tongue are gustatory, but there are very considerable variations. (3) The lateral part of the soft palate and the glosso-palatine arch are endowed with taste from the glosso-pharyn- 
geal nerve. (4) It is uncertain whether the hard palate and the entrance to the larynx are endowed with taste (Drielsma). The middle of the tongue is not gustatory.

[Tongue-Mucous membrane. - The structure of the tongue, as a muscular organ covered with mueous membrane, has already been described (\$155). The dorsal surface of the tongue, in front of the blind foramen, is beset with elevations of the mucous membrane, which extend to its tip and borders. These elevations, or papillm, are of three kinds;--filiform, fungiform, and circumvallate. They consist of elevations of the mucous membrane, visible to the naked eye, and covered by stratified squamous epithelium, while the central core of connective-tissue contains bloul-and lymph-vessels and nerves. The filiform papillæo occur over the whole tongue, and are smallest and most numerous. They are conical eminences covered by stratified squamous

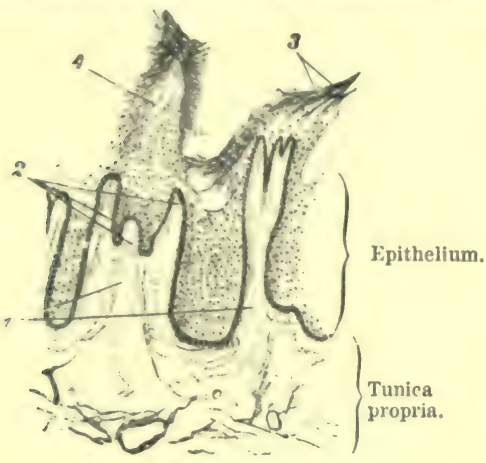

Fig. 604.

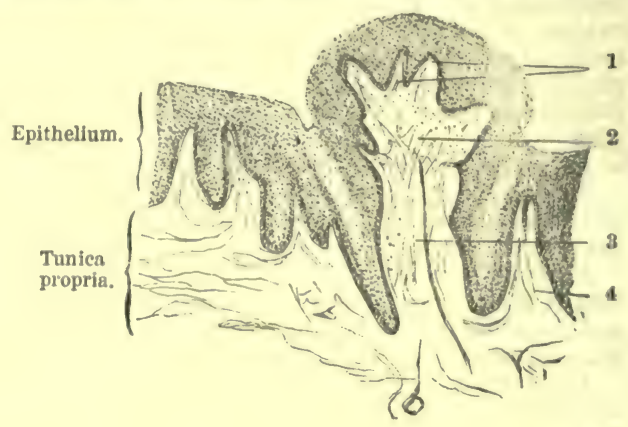

Fig. 605 .

Fig. 604.-Longitudinal section of the dorsum of the human tongne. 1, section of two filiform papillie, with secondary papillix (2); 3 , double, 4, single process of epithelium with loose epithelial scales. $\times 30$. Fig. 605. - Longitudinal section of the human tongue. 1, secondary papille on 2 , the fungiform papilla ; 3 , base of $2 ; 4$, small filiform papilla. $\times 30$.

"pithelium, and often beset with secondary papillæ (fig. 604). The fungiform papillse occur chiefly over the middle and front part of the tongue, and are not so numerous as the last. They are club-shaped, with a narrow base, and broad expanded rounded head. They also have secondary papillie. They are generally brighter red than the others (fig. 605). The circum. vallate papillø, 8 to 12 in number, diverge from the foramen cæecum at the back part of the

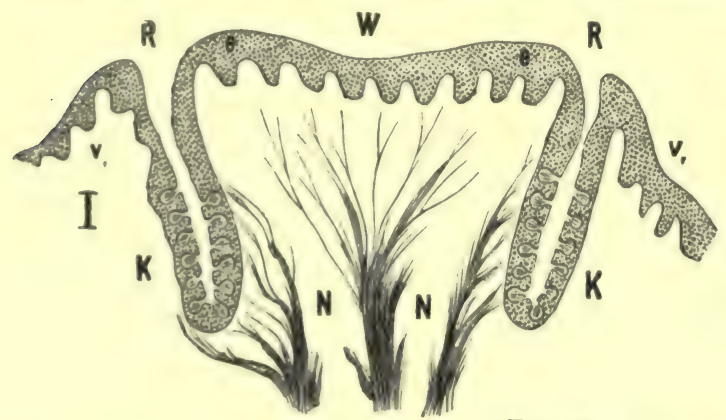

Fig. 606 .

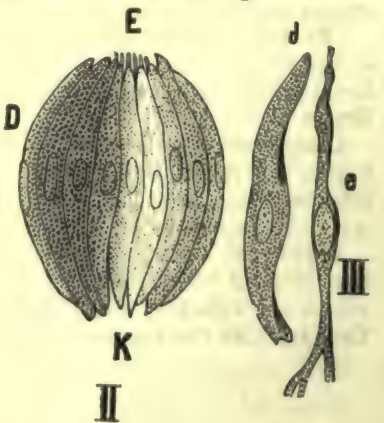

I, Tranverse section of a circumvallate papilla; $\mathrm{W}$, the papilla ; $v_{1}, v_{1}$, the wall in section ; $\mathrm{R}, \mathrm{R}$, the circular slit or fossa ; $\mathrm{K}, \mathrm{K}$, the taste-bulbs in position; $\mathrm{N}, \mathrm{N}$, the nerves. II, Isolated taste-bulb; D, supporting or protective cells; $\mathrm{K}$, under end ; $\mathrm{E}$, free end, open, with the projecting apices of the taste-cells. III, Isolated protective cell $(d)$ with a taste-
cell $(e)$.

tongue in two rows in the form of a wide $\mathrm{V}$, the open angle of the $\mathrm{V}$ being directed forwards. They are large, with a broad expanded top, and are lodged in a depression of the mucous membrane, being surrounded by a wall of mucous membrane, and separated from it by a circular treuch, into the base of which gland-ducts often open. They have numerous secondary papilla, and in them are taste-buds, fig. 606, I.] 
Taste-bulbs. - The end-organs of the gustatory nerves are the taste-bulbs or taste-buds discovered by Schwalbe and Lovèn (1867). They occur on the lateral surfaces of the circumvallate papillæ (fig. 606, I), and upon the opposite side, $\mathrm{K}$, of the fossa or capillary slit, $\mathrm{R}$, $\mathrm{R}$, which surrounds the central eminence or papilla; they occur more rarely on the surface. They also occur on the fungiform papillæ, in the papillæ of the soft palate and uvula (A. Hoffmann), on the under surface of the epiglottis, the upper part of the posterior surface of the epiglottis, and the inner side of the arytenoid cartilages (Verson, Davis), and on the vocal cords (Simanowsky). Many buds or bulbs disappear in old age.

[In the rabbit and some other animals, there is a folded laminated organ on each side of the posterior part of the tongue, called the papilla foliata; the folds have on each side of them numerous taste-buds (fig. 607).]

Structure of the taste-bulbs. - They are $81 \mu$ high and $33 \mu$ thick, barrel-shaped, and embedded in the thick stratified squamous epithelium of the tongue. Each bulb consists of a series of lancet-shaped, bent, nucleated, outer supporting or protective cells, arranged like the staves of a barrel (fig. 606, II, D, insolated in III, a). They are so arranged as to leave a small opening, or the "gustatory pore," at the free end of the bulb. Surrounded by these cells, and lying in the axis of the bud, are 1 to 10 gustatory cells (II, E), some of which are provided with a delicate process (III, e) at their free ends, while their lower fixed ends send out basal processes, which become continuous with the terminations of the nerves of taste, which have become non-medullated. After section of the glosso-pharyngeal, the taste-buds degenerate, while the protective cells become changed into ordinary epithelial cells within four months (v. Vintschgau and Hönigschmied). Very similar structures were found by Leydig in the skin of freshwater fishes. The glands of the tongue and their secretory fibres from the 9 th cranial nerve are referred to in $\S 141$ (Drasch).

423. GUSTATORY SENSATIONS. - Varieties. - There are four different gustatory qualities, the sensations of 1 . Sweet; 2 . Bitter; 3. Acid ; 4. Saline. Acid and saline substances at the same time also stimulate the sensory nerves of the tongue, but when greatly diluted, they only excite the
end-organs of the specific nerves of taste. Perhaps there are special nerve-fibres for each different gustatory quality ( $v$. Vintschgau).

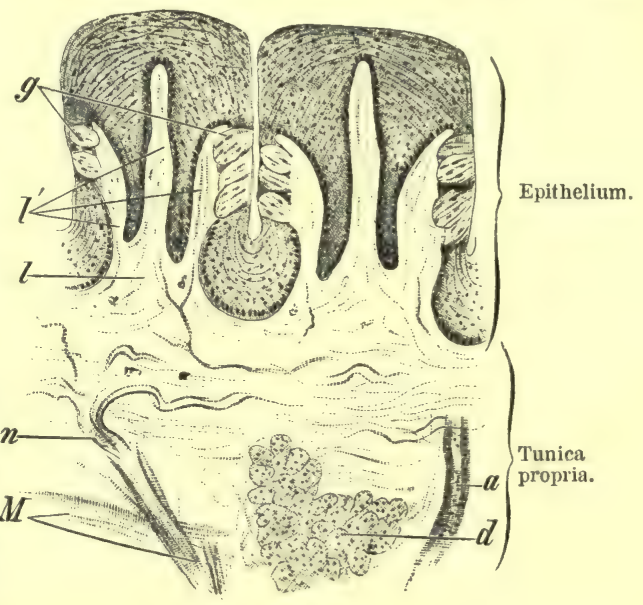

Fig. 607 .

Vertical section of two septa of the papilla foliata (rabbit). $\times 80$. Each septum, $l$, has secondary septa, $l^{\prime} ; g$, taste-buds ; $n$, medullated nerve ; $d$, serous gland, and part of its duct, $\alpha ; M$, muscular fibres of the tongue.

Conditions.- Sapid substances, in order that they may be tasted, require the following conditions:-They must be dissolved in the fluid of the month, especially substances that are solid or gaseous. The intensity of the gustatory sensation depends on :-1. The size of the surface acted on. Sensation is favoured by rubbing in the substance between the papillæ, in fact, this is illustrated in the rubbing movements of the tongue during mastication (\$354). 2. The concentration of the sapid substance is of great importance. Valentin found that the following series of substances ceased to be tasted in the order here stated, as they were gradually diluted-syrup, sugar, common salt, aloes, quinine, sulphuric acid. Quinine cau be diluted 20 times more than common salt and still be tasted (Camerer). 3 . The time which elapses between the application of the sapid substance and the production of the sensation varies with different substances. Saline substances are tasted most rapidly (after 0.17 second, according to $v$. Vintschgau), then sweet, acid and bitter (quinine after 0.258 second, v. Vintschgau). This even occurs with a mixture of these substances (Schirmer). The last-named substances produce the most 
persistent "after-taste." 4. The delicacy of the sense of taste is partly congenital, but it can be greatly improved by practice. If a person continues to taste the same sapid substance, or a nearly related one, or even any very intensely sapid substance, the gustatory sense is soon affected, and it becomes impossible to give a correct judgment as to the taste of the sapid body. 5. Taste is greatly aided by the sense of smell, and in fact we often confound taste with smell ; thus, ether, chloroform, musk, and assafatida only affect the organ of smell. [The combined action of taste and smell in some cases gives rise to flavour (p. 823).] The eye even may aid the determination, as in the experiment where in rapidly tasting red and white wine one after the other, when the eyes are cavered, we soon become unable to distinguish between the one and the other. 6. The most advantageous temperature for taste is between $10^{\circ}$ to $35^{\circ} \mathrm{C}$. ; hot and cold water temporarily paralyse taste.

Ice placed on the tongue suppresses, sometimes entirely, the whole gustatory apparatus; cocain alone, bitter tastes, and water containing 2 per cent. of $\mathrm{H}_{2} \mathrm{SO}_{4}$, excite afterwards a sweet taste (Aducoo and Mosso).

Electrical Current. - The constant current, when applied to the tongue, excites, both during its passage and when it is opened or closed, a sensation of acidity at the + pole, and at the - pole an alkaline taste, or, more correctly, a harsh burning sensation (Sulzer, 1752). This is not due to the action of the electrolytes of the fluid in the mouth, for even when the tongue is moistened with an acid fluid the alkaline sensation is experienced at the - pole (Volta). We cannot, however, set aside the supposition that perhaps electrolytes, or decomposition-products, may he formed in the deeper parts and excite the gustatory fibres. Rapidly interrupted currents do not excite taste (Grïnhagen). V. Vintschgau, who has only incomplete taste on the tip of the tongue, finds that when the tip of the tongue is traversed by an electrical current, there is never a gustatory sensation, but always a distinct tactile one. In experiments on Honigschmied, who is possessed of normal taste in the tip of the tongue, there was often a metallic or acid taste at the +pole on the tip of the tongue, while at the-pole taste was often alsent, and when it was jresent it was almost always alkaline, and acid only exceptionally. After interrupting the current there was a metallic after-taste with both directions of the current.

[Testing Taste.--Direct the person to put out his tongue and close his eyes, and after drying the tongue apply the sapid substance by means of a glass rod or a small brush. Try to confine the stimulus as much as possible to one place, and after each experiment rinse the mouth with water. A wine-taster chews an olive to "clean the palate," as he says. For testing litter taste use a solution of quinine or quassia ; for sueet, sugar, [or the intensely sweet substance "saccharine" obtained from coal tar]; saline, common salt; and acid, dilute citric or acetic acid. The galvanic current may also be used.]

Pathological. - Diseases of the tongue, as well as dryness of the mouth caused by interference with the salivary secretion, interfere with the sense of taste. Subjective gustatory impressions are common amongst the insane, and are due to some central cause, perhaps to irritation of the centre for taste $(\$ 378, \mathrm{IV}, 3)$. After poisoning with santonin, a bitter taste is experienced, while after the subcutaneous injection of morphia, there is a bitter and acid taste. The terms hypergensia, hypogeusia, and ageusia are applied to the increase, diminution, and abolition of the sense of taste. Many tactile impressions on the tongue are frequently confounded with gustatory sensations, e.g., the so-called biting, cooling, prickling, sandy, mealy, astringent, and harsh tastes.

Comparative.-About 1760 taste-bulbs occur on the circumvallate papillæ of the ox. The term papilla foliata is applied to a large folded gustatory organ placed laterally on the side of the tongue (fig. 607), especially of the rabbit (Rapp, 1832), which in man is represented by analogous organs, composed of longitudinal folds, lying in the fimbrix linguæ on each side of the posterior part of the tongue (Krause, $v$. Wyss). Taste-bulbs are absent in reptiles and birds. They are numerous in the gill-slits of the tadpole $(F . E$. Schultze), while the tongue of the frog is covered with epithelium resembling gustatory cells (Billroth, Axel Key). The goblet-shaped organs in the skin of fishes and tadpoles have a structure similar to the taste-bulbs, and may perhaps have the same function. There are taste-bulbs in the mouth of the carp and ray.

Historical.-Bellini regarded the papillæ as the organs of taste (1711). Richerand, Mayo, and Fodera thought that the lingual was the only nerve of taste, but Majendie proved that, after it was divided, the posterior part of the tongue was still endowed with taste. Panizza (1834) described the glosso-pharyngeal as the nerve of taste, the gustatory as the nerve of touch, and the hypoglossal as the motor nerve of the tougue. 


\section{The Sense of Touch.}

424. TERMINATIONS OF SENSORY NERVES. - 1. The touch-corpuscles of Wagner and Meissner lie in the papillm of the cutis vera $(\$ 283)$, and are most numerous in the palm of the hand and the sole of the foot, especially in the fingers and toes, there being about 21 to every square millimetre of skin, or 108 to 400 of the papillæ containing blood-vessels. They are less abundant on the back of the hand and foot, mamma, lips, and tip of the tongue, rare on the glans clitoridis, and occur singly and scattered on the volar side of the fore-arm, even in the anthropoid apes. They are oval or elliptical bodies, 40-200 $\mu$ long $\left[\frac{1}{30}\right.$ in. $]$, and $60-70 \mu$ broad [ $\frac{1}{500}$ to $\left.\frac{1}{300} \mathrm{in}.\right]$, and are covered externally by layers of connective-tissue arranged transversely in layers, and within is a granular mass with elongated striped nuclei (figs. 608,

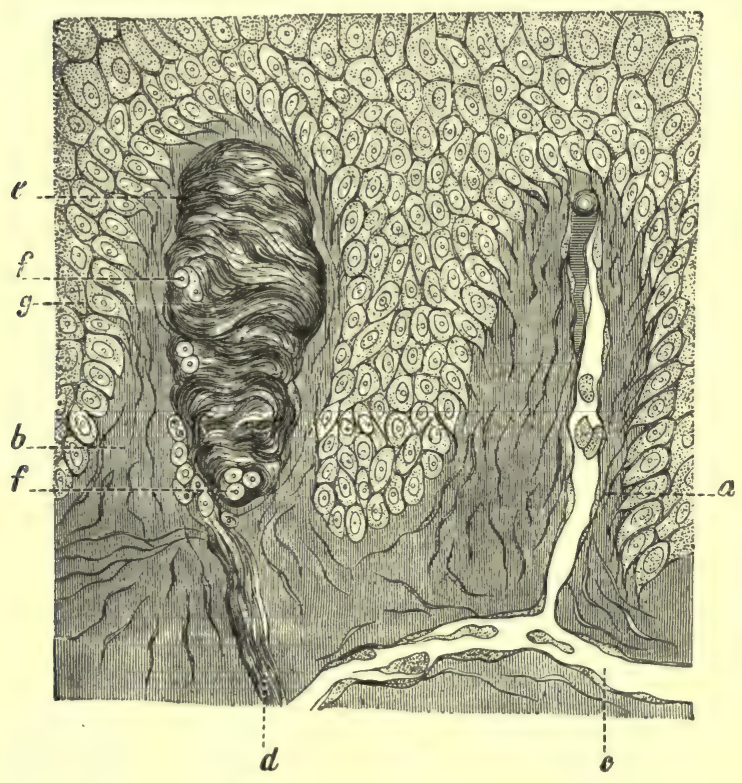

Fig. 608 . $609, e)$. One to three medullated nerve-fibres pass to the lower end of each corpuscle, and surround it in a spiral manner two or three times; the fibres then lose their myelin, and, after dividing into 4 to 6 fibrils, branch within the corpuscle. The exart

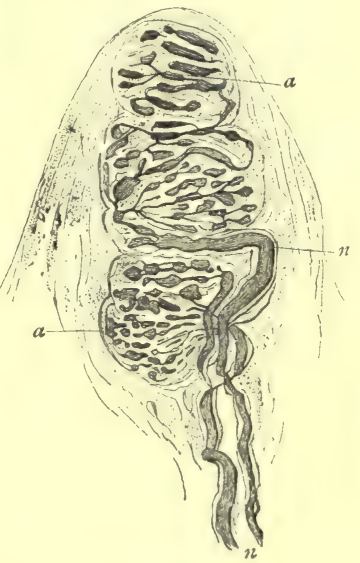

Fig. 609.

Fig. 608.-Vertical section of the skin of the palm of the hand. $a$, blood-vessels ; $b$, papilla of the cutis vera ; $c$, capillary ; $d$, nerve-fibre passing to a touch-corpuscle; $f$, nervefibre divided transversely ; $e$, Wagner's touch-corpuscle ; $g$, cells of the Malpighian layer of the skin. Fig. 609. - Wagner's touch-corpuscle from the palm, treated with gold chloride; $n$, nerve-fibres; $a, \alpha$, groups of glomeruli.

mode of termination of the fibrils is not known. Some observers suppose that the transverse fibrillation is due to the coils or windings of the nerve-fibrils; while according to others, the inner part consists of numerous flattened cells lying one over the other, between which the pale terminal fibres end either in swellings or with disc-like expansions, such as occur in Merkel's corpuseles.

[These do not contain a soft core such as exists in Pacini's corpuscles. The corpuscles appear to consist of connective-tissue with imperfect septa passing into the interior from the fibrous capsule. After the nerve-fibre enters it loses its myelin, and then branches, while the branches anastomose and follow a spiral course within the corpuscle, finally to terminate in slight enlargements. According to Thin, there are simple and compound corpuscles, depending on the number of nerve-fibres entering them.]

Kollmann deseribes three special tactile areas in the hand :-(1) The tips of the fingers with 24 touch-corpuscles in a length of $10 \mathrm{~mm}$.; (2) the three eminences lying on the palm behind the slits between the fingers, with 5.4-2.7 touch-corpuscles in the same length; and (3) the ball of the thumb and little finger with $3 \cdot 1-3 \cdot 5$ touch-corpuscles. The first two areas also contain many of the corpuscles of Vater or Pacini, while in the latter these corpuscles are fewer and scattered. In the other parts of the hand the nervous end-organs are much less developed. 
2. Vater's (1741) or Pacini's corpuscles are oval bodies (fig. 610), 1-2 mm. long, lying in the subcutaneous tissue on the nerves of the fingers and toes (600-1400), in the neighbourhood of joints and muscles, the sympathetic abdominal plexuses, near the aorta and coccygeal gland on the dorsum of the penis and clitoris, and in the mesocolon [ant mesentery] of the cat. [They also occur in the course of the intercostal and periosteal nerves, and Stirling has seen them in the capsule of lymphatic glands. They are attached to the nerves of the hand and feet, and are so large as to be visible to the naked eye, both in these regions and between the layers of the mesentery of the cat. They are whitish or somewhat transparent, with a white line in the centre (cat); in man, they are $\frac{1}{15}$ to $\frac{1}{10}$ inch long, and $\frac{1}{25}$ to $\frac{2}{20}$ inch broad, and are attached by a stalk or pedicle (fig. $610, a$ ) to the nerve.] They consist of numerous nncleated connective-tissue capsules or lamellæ lined by endothelium, separated from each other by fluid, and lying one within the other like the coats of an onion, while in the axis is a

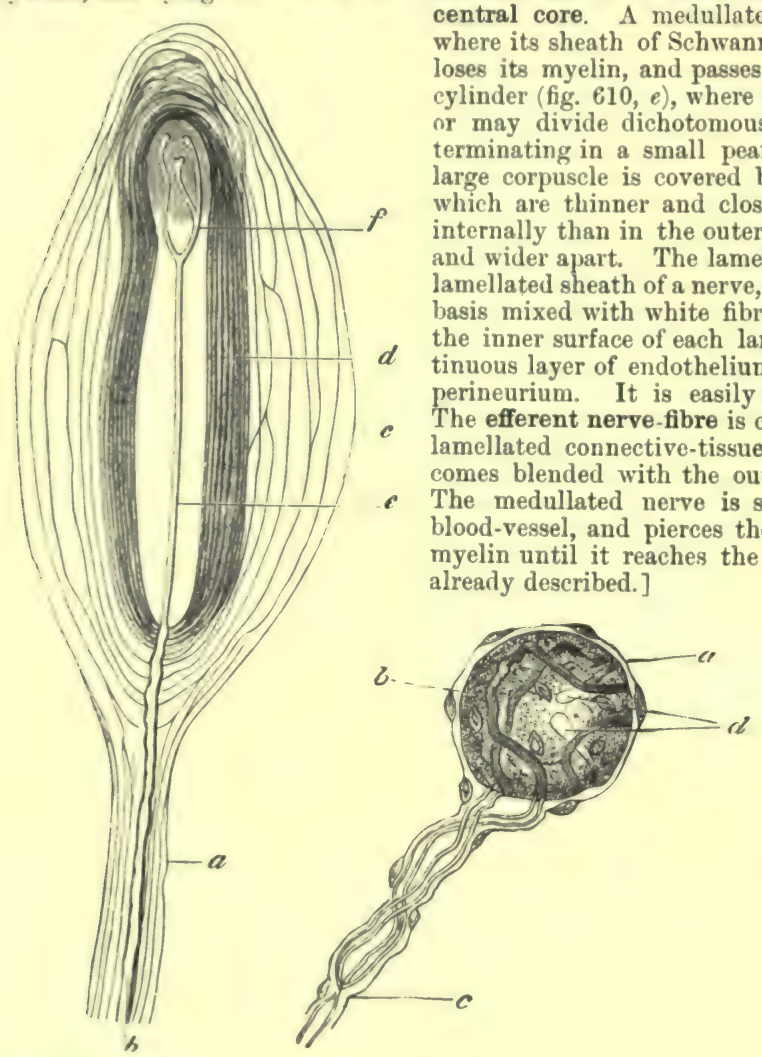

Fig. 610.

Fig. 611.

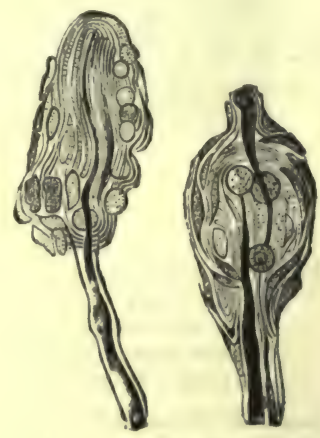

Fig. 612 .

Fig. 610. - Vater's or Pacini's corpuscle. $a$, stalk; $b$, nerve-fibre entering it; $c, d$, connectivetissue envelope; $e$, axis-cylinder, with its end divided at $f$. Fig. 611. - End-bulb from human conjunctiva. $a$, nucleated capsule; $b$, core ; $c$, fibre entering and branching, terminating in core at $d$. Fig. 612. - Tactile corpuscles, clitoris of rabbit.

3. Kranse's end-bulbs very probably occur as a regular mode of nerve-termination in the cutis and mucous membranes of all mammuls (fig. 611). They are elongated, oval, or round borlies, 0.075 to $0.14 \mathrm{~mm}$. long, and have been found in the deeper layers of the conjunctiva bulbi, floor of the mouth, margins of the lips, nasal mucous membrane, epiglottis, fungiform and circumvallate papillæ, glans penis and clitoris, volar surface of the toes of the guinea-pig, ear and bolly of the monse, and in the wing of the bat. [In the calf, the "cylindrical end. bulbs" are oval, with a nerve-fibre terminating within them. The sheath of Henle becomes continuous with the nucleated capsule, while the axial cylinder, devoid of its myelin, is continued into the soft core. In man the end-bulhs are "spheroidal," and consist of a nucleated 
connective-tissue capsule continuous with Henle's sheath of the nerve, and enclosing many cells, amongst which the axis-cylinder which enters the bulb branches and terminates.] The spheroidal end-bulbs occur in man, in the nasal mucous membrane, conjunctiva, mouth, epiglottis, and the mucous folds of the rectum. According to Waldeyer and Longworth, the nerve-fibrils terminate in the cells within the capsule. These cells are said to be comparable to Merkel's tactile cells ( Waldeyer).

The genital corpuscles of Krause, which occur in the skin and mucous membrane of the glans penis, clitoris, and vagina, appear to be end-bulbs more or less fused together (fig. 612).

The articulation nerve-corpuscles occur in the synovial mucous membrane of the joints of the fingers. They are larger than the end-bulbs, and have numerous oval nuclei externally, while one to four nerve-fibres enter them.

4. Tactile or touch-corpuscles of Merkel, sometimes also called the corpuscles of Grandry, occur in the beak and tongue of the duck and goose, in the epidermis of man and mammals, and in the outer root-sheath of tactile hairs or feelers (fig. 613). They are small bodies composed of a capsule enclosing two, three, or more large, granular, somewhat flattened nucleated and nucleolated cells, piled one on the other in a vertical row like a row of cheeses. Each corpuscle receives at one side a medullated nerve-fibre which loses its myelin, and branches, to terminate, according to some observers ( $\mathrm{Merkel})$, in the cells themselves, and according to others (Ranvier, Izquierdo, Hesse) in the protoplasmic transparent substance or disc lying between the cells. [This intercellular dise is the "disc tactil" of Ranvier, or the "Tastplatte" of Hesse.] When there is a great aggregation of these cells, large structures are formed which appear to form a kind of transition between these and touchcorpuscles. [According to Klein, the terminal fibrils end neither in the touchcells nor tactile dise, but in minute

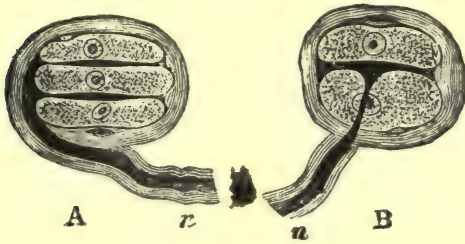

Fig. 613.

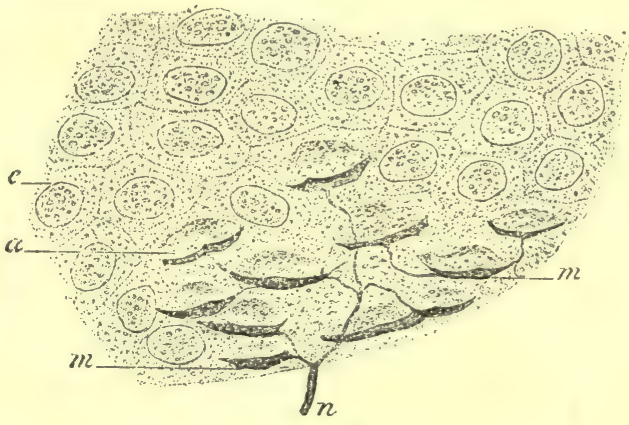

Fig. 614 .

Fig. 613.-Tactile corpuscles from the duck's tongue. A, composed of three cells with two interposed discs, with axis-cylinder, $n$, passing into them. $\mathrm{B}$, two tactile cells and one disc. Fig. 614. - Bouchon epidermique from the groin of a guinea-pig, after the action of gold chloride. $n$, nerve-fibre ; $a$, tactile cells ; $m$, tactile dises ; $c$, epithelial ceils.

swellings in the interstitial substance between the touch-cells, in a manner very similar to that occurring in the end-bulbs.]

[According to Merkel, tactile cells, either isolated or in groups, but in the latter case never forming an independent end-organ, occur in the deeper layers of the epidermis of man and mammals and also in the papillæ. They consist of round or flask-shaped cells, with the lower pointed neck of the flask continuous with the axis-cylinder of a nerve-fibre. They are regarded by Merkel as the simplest form of a tactile end-organ, but their existence is doubted by some observers.]

Amougst animals there are many other forms of sensory end-organs. [Herbst's corpuscles occur in the mucous membrane of the tongue of the duck, and resemble small Vater's corpuscles, but their lamellæ are thinner and nearer each other, while the axis-cylinder within the central core is bordered on each side by a row of nuclei.] In the nose of the mole there is a peculiar end-organ (Eimer), while there are "end-capsules" in the penis of the hedgehog and the tongue of the elephant, and "nerve-rings" in the ears of the mouse.

5. [Other Modes of Ending of Sensory Nerves. - Some sensory nerves terminate not by means of special end-organs, but their axis-cylinder splits up into fibrils to form a nervous network, from which fine fibrils are given off to terminate in the tissue in which the nerve ends. These fibrils, as in the cornea ( $\$ 384$ ), terminate by means of free ends between the epithelium on the anterior surface of the cornea, and some observers state that the free ends are provided with small enlargements ("boutons terminals") (fig. 614, a). These enlargements or "tactile cells" occur in the groin of the guinea-pig and mole. A similar mode of termination oecurs between the cells of the epidermis in man and mammals (fig. 293).]

6. Tendons, especially at their junction with muscles, have special end-organs (Sachs, Rollett, Golgi), which assume various forms; it may be a network of primitive nerve-fibrils, or flattened 
and-flakes or plates in the sterno-radial muscle of the frog, or elongated oval end-bulbs, not unlike the end.bulbs of the conjunctiva, or small simple Pacinian corpuscles.]

Prus found ganglion cells more frequently in the subcutaneous tissue than in the corium, and they appreared to have some relation to the blood-vessels and sweat-glands.

425. SENSORY AND TACTILE SENSATIONS. - In the sensory nervetrunks there are two functionally different kinds of nerve-fibres:-(1) Those which administer to reinful impressions, which are sensory nerves in the narrower sense of the word ; and (2) those which administer to tactile impressions and may therefore be called tintile nerves. The sensations of temperature and pressure are also reckoned as belonging to the tactile group. It is extremely probable that the sensory and tactile nerves have different end-organs and fibres, and that they have also special jerceptive nerve-centres in the brain, although this is not definitely proved. This view, however, is supported by the following facts :-

1. That sensory and tactile impressions cannot be discharged at the same time from all the parts which are endowed with sensibility. Tactile sensations, including pressure and temperature, are only discharged from the coverings of the skin, the mouth, the entrance to and floor of the nose, the pharynx, the lower end of the rectum and genito-urinary orifices; feeble indistinct sensations of temperature are felt in the esophagus. Tactile sensations are absent from all internal viscera, as has been proved in man in cases of gastric, intestinal, and urinary fistulæ. Pain alone can be discharged from these organs. 2. The conduction chanuels of the tactile and sensory nerves lie in different parts of the spinal cord ( $\$ 364,1$ and 5). This renders probable the assumption that their central and peripheral ends also are different. 3. Very probably the reflex acts discharged by both kinds of nerve fibres - the tactile and pathic-are controlled, or even inhibited, by special central nerve-organs (\$361-?). 4. Under pathological conditions, and under the action of narcotics, the one sensation may be suppressed while the other is retained $(\S 364,5)$.

Sensory Stimuli.-In order to discharge a painful impression from sensory nerves, relatively strony stimuli are required. The stimuli may be mechanical, hemical, electrical, thermal, and somatic, the last being due to inflammation or anomalies of nutrition and the like.

Peripheral Reference of the Sensations.-These nerves are excitable along their entire course, and so is their central termination, so that pain may be produced by stimulating them in any part of their course, but this pain, according to the "law of peripheral perception," is always referred to the periphery.

The tactile nerves can only discharge a tactile impression or sensation of contact when moderately strong mechanical pressure is exerted, while thermal stimuli are required to produce a temperature sensation, and in both cases, the results are obtained only when the appropriate stimuli are applied to the end-organs. If pressure or cold be applied to the course of a nerve-trunk, e.g., to the ulna at the inner surface of the elbow-joint, we are conscious of painful sensations, but never of those of temperature, referable to the peripheral terminations of the nerves in the inner fingers. All strong stimuli disturb normal tactile sensations by over-stimulation, and hence cause pain.

The law of the specific energy of nerves leads us to assume that the cutaneous nerves contain different kinds of nerve-fibres with different kinds of end-organs, which subserve different kinds of impressions, e.g., pressure, temperature, and pain. Blix and Goldscheider have found such differences. Electrical stimulation causes different sensations according to the part of the skin where it is applied; at one spot, pain only is produced, at another a sensation of cold, at a third a sensation of heat, and at a fourth, a sensation of pressure. At every temperature point or spot, there is insensibility for pain or pressure. The "pressure-points" or pressure-spots lie much closer together, and are more numerous than the temperature-points. There are special "pain-spots" and even "tickling-spots." These spots are 
arranged in a linear chain, which usually radiates from the hair-follicles. The "tickling-spots" coincide with the pressure and pain-spots. The feeling of tickling corresponds to the feeblest stimulation of a nerve-fibre, and pain to the strongest. The pain-spots can be isolated by means of a needle, or electrically, especially in the cutaneous furrows, in which the pressure-sense is absent.

Goldscheider removed from his own body small pieces of skin, in which he had previously ascertained the presence of these "spots," and then investigated the excised skin microscopically. At each such spot he found a rich supply of nerves; at the pressure-spots, there were no touch-corpuscles.

[By means of the skin, impressions are supplied also to the brain, whereby we become conscious of the amount and direction of a body moved in contact with the skin. Indeed, the discriminative sensibility is more acute for motion than for touch ; but the liability to error in judging of the distance and direction is great $(\mathrm{Hall})$.]

[Very complex sensations are obtained by means of the combined action of the skin and muscles, e.g., those known as "feelings of double contact." These sensations are of the greatest advantage in acquiring the use of instruments and tools. If we touch an object with a rod, we seem to feel the object at the point of the rod, and not in the hand where the cutaneous nerves are actually stimulated. With a walking stick, we feel the ground at the end of the stick. Touch the tips of the hair, or a tooth, and the sensation is referred to the tips of the hair in the one case, and the crown of the tooth in the other ( $\operatorname{Lad} d)$.]

426. SENSE OF LOCALITY.-We are not only able to distinguish differences of pressure or temperature by our sensory nerves, but we are able to distinguish the part which has been touched. This capacity is spoken of as the sense of space or locality.

Methods of Testing. - 1. Place the two blunted points of a pair of compasses (fig. 615) upon the part of the skin to be investigated, and determine the smallest distance at which the two points are felt only as one impression. Sieveking's æsthesiometer may be used instead (fig. 616); one of the points is movable along a graduated rod, while the other is fixed. 2. The distance between the points of the instrument being kept the same, touch several parts of the skin, and ask if the person feels the impression of the points coming nearing to or going wider apart. 3. Touch a part of the skin with a blunt instrument, and observe if the spot touched is correctly indicated by the patient. 4. Separate the points of two pairs of

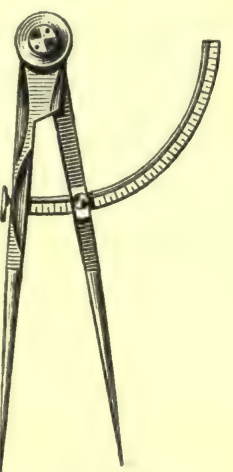

Fig. 615 .

Esthesiometer. compasses unequally, and place their points upon different parts of the skin, and ask the person to state when the points of both appear to be equally far anart. A distance of 4 lines on the

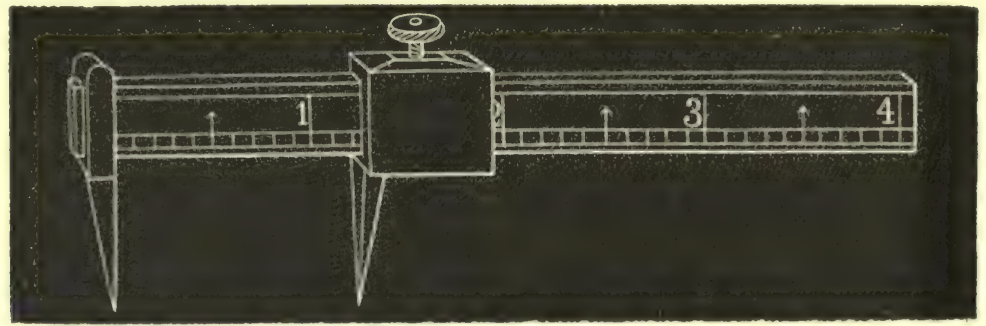

Fig 616.

Æsthesiometer of Sieveking

forehead appears to be equal to a distance of 2.4 lines on the uppes lip. This is Fechner's "methods of equivalents."

The following results have been obtained. The sense of locality of a part of the skin is more acute under the following conditions :-

1. The greater the number of tactile nerves in the corresponding part of the skin.

2. The greater the mobility of the part, so that it increases in the extremities towards the fingers and toes. The sense of locality is always very acute in parts of the body that are very rapidly moved (Vierordt). 
3. The sensibility of the limbs is finer in the transverse axis than in the long axis of the limb, to the extent of $\frac{1}{8}$ th on the flexor surface of the upper limb, and $\frac{1}{4}$ th on the extensor surface.

4. The mode of application of the points of the asthesiometer:- $(a)$ According as they.are applied one after the other, instead of simultaneously, or as they are considerably warmer or colder than the skin (Klug), a person may distinguish a less distance between the points. (b) If we begin with the points wide apart and approximate them, then we can distinguish a less distance than when we proceed from imperceptible distances to larger ones. (c) If the one point is warm and the other cold, on exceeding the next distance we feel two impressions, but we cannot rightly judge of their relative positions (Czermak).

5. Exercise greatly improves the sense of locality; hence the extraordinary acuteness of this sense in the blind, and the improvement always occurs on both sides of the body (Volkmann).

[Fr. (ialton finds that the reputed increased acuteness of the other senses in the case of the blind is not so great as is generally alleged. He tested a large number of boys at an educational blind asylum, with the result that the performances of the blind boys were by no means superior to those of other boys. He points out, however, that "the guidance of the blind depends mainly on the multitude of collateral indications, to which they give much heed, and not in their superiority in any one of them."]

6. Moistening the skin with indifferent fluids increases the acuteness. If, however, the skin between two points, which are still felt as two distinct objects, be slightly tickled, or be traversed by an imperceptible electrical current, the impressions become fused ('suslou' $u$ ). The sense of locality is rendered more acute at the cathode when a constant current is used (Suslowa), and when the skin is congested by stimulation (Klinkenberg), and also by slight stretching of the skin (Schmey); further, by baths of carbonic acid ( $v$. Basch and $v$. Dietl), or warm common salt, and temporarily by the use of caffein (Rumpf).

7. Anxmiu, produced by elevating the limbs, or venous hyperamia (by compressing the veins), blunts the sense, and so does too frequent testing of the sense of locality, by producing fatigue. The sense is also blunted by cold applied to the skin, the influence of the anode, strong stretching of the skin, as over the abdomen during pregnancy, previous exertion of the muscles under the part of the skin tested, and some poisons, e.\%, atropin, daturin, morphin, strychnin, alcohol, potassium bromide, cannabin, and chloral hydrate.

Tip of tongue,

Third phalanx of finger, volar surface,

Red part of the lip,

Secoul phalanx of finger, volar surface,

First phalanx of finger, volar surface, .

Third phalanx of finger, dorsal surface,

Tip of nose,

Head of metacarpal boue, volar,

Ball of thumb,

Ball of little finger, $\cdot 6 \cdot 5-7$

Centre of palm,

Dorsum and side of tongue, whit. of the lips, metacarpal part of the thumb,

Third phalanx of the great toe, plantar surface,

Second phalaux of the fingers, dorsal surface,

Back,
Millimetres.

$1 \cdot 1[1 \cdot 1]$

$2 \cdot-2 \cdot 3[1 \cdot 7]$

$4 \cdot 5[3 \cdot 9]$

$4 \cdot-4 \cdot 5[3 \cdot 9]$

$5 \cdot-5 \cdot 5$

$6 \cdot 8[4 \cdot 5]$

$6 \cdot 8[4 \cdot 5]$

$5 \cdot-6 \cdot 8[4 \cdot 5]$

$6 \cdot 5-7 \cdot$
$5 \cdot 5-6 \cdot$

$8 \cdot-9$.

$9 \cdot[6 \cdot 8]$

$11 \cdot 3[6 \cdot 8]$

$11 \cdot 3$ [9.]

$11 \cdot 3[9$.
Eyelid,

Centre of hard palate,

Lower third of the fore-arm, volar surface, . $15^{\circ}$

In front of the zygoma, . . $15 \cdot 8$ [11.3]

Plantar surface of the great toe, . $15 \cdot 8\left[9{ }^{\circ}\right]$

Inner surface of the lip,

Behind the zygoma,

Forehead,

Occiput,

Back of the hand,

Under the chin,

Vertex,

Knee,

Sacrum, gluteal region,

Fore-arm and leg,

Neck,

Back at the fifth dorsal vertebra, lower dorsal and lumbar region,

Middle of the neck,

Upper arm, thigh, and centre of the back, 
Smallest Appreciable Distance.-The preceding statement gives the smallest distance, in millimetres, at which two points of a pair of compasses can still be distinguished as double by an adult. The corresponding numbers for a boy twelve years of age are given within brackets.

musions of the sense of locality occur very frequently; the most marked are :-(1) A uniform movement over a cutaneous surface appears to be quicker in those places which have the finest sense of locality. (2) If we merely touch the skin with the two points of an æsthesiometer, then they feel as if they were wider apart than when the two points are moved along the skin (Fechner). (3) A sphere, when touched with short rods, feels larger than when long rods are used (Tourtual). (4) When the fingers of one hand are crossed, a small pebble or sphere placed between them feels double (Aristotle's experiment). [When a pebble is rolled between the crossed index and middle finger (fig. $617, \mathrm{~B}$ ), it feels as if two balls were present, but with the fingers uncrossed single.] (5) When pieces of skin are transplanted, e.g., from the forehead, to form a nose, the person operated on feels, often for a long time, the new nasal part as if it were his forehead.

Theoretical. - Numerous experiments were made by E. H. Weber, Lotze, Meissner, Czermak, and others to explain the phenomena of the sense of space. Weber's theory goes upon the assumption, that one and the same nerve-fibre proceeding from the brain to the skin can only take up one kind of impression, and administer thereto. He called the part of the skin to which each single nerve-fibre is distributed a "circle of sensation." When two stimuli act simultaneously upon the tactile end-organ, then a double sensation is felt, when one or more circles of sensation lie between the tywo points stimulated. This explanation, based upon anatomical considerations, does not explain how it is that, with practice, the circles of sensation become smaller, and also how it is that only one sensation occurs, when both points

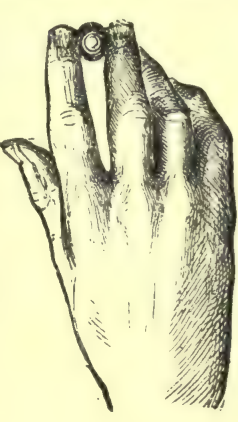

A.

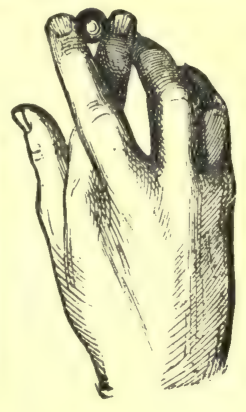

B.

Fig. 617 .

Aristotle's experiment.

of the instruments are so applied, that both points, although further apart than the diameter of a circle of sensation, at one time lie upon two adjoining circles, at another between two others with another circle intercalated between them.

Wundt's Theory. - In accordance with the conclusions of Lotze, Wundt proceeds from a psycho-physiological basis, that every part of the skin with tactile sensibility always conveys to the brain the locality of the sensation. Every cutaneous area, therefore, gives to the tactile sensation a "local colour" or quality, which is spoken of as the local sign. He assumes that this local colour diminishes from point to point of the skin. This gradation is very sudden in those parts of the skin where the sense of space is very acute, but occurs very gradually where the sense of space is more obtuse. Separate impressions unite into a common one, as soon as the gradation of the local colour becomes imperceptible. By practice and attention differences of sensation are experienced, which ordinarily are not observed, so that he explains the diminution of the circles of sensation by practice. The circle of sensation is an area of the skin, within which the local colour of the sensation changes so little that two separate impressions fuse into one.

427. PRESSURE SENSE-By the sense of pressure we obtain a knowledge of the amount of

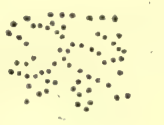

$\alpha$

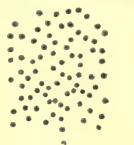

b

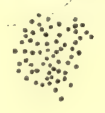

c

Fig. 618 .

Pressure-spots. $\quad a$, middle of the sole of the foot; $b$, skin of zygoma; $c$, skin of the back.

weight or pressure which is being exercised at the time on the different parts of the skin.

A specific end-apparatus arranged in a punctated manner is connected with the pressure sense (iig. 618). These points or spots are called "pressure-spots" or "pressure-points" (Blix), and are endowed with varying degrees of sensibility; at some places (back, thigh) they are distinguished by a markedly pronounced aftersensation. The arrangement of the pressure-spots follows the type of the arrangement of the temperature-spots. The pressure-spots have usually another direction than that of hot and cold spots, as a rule, they are denser. The minimal distance at which two pressure-spots, when simultaneously stimulated, are felt as double, is 
-on the back, 4 to $6 \mathrm{~mm}$; breast, 0.8 ; abdomen, 1.5 to 2 ; cheek, 0.4 to 0.6 ; upper arm, 0.6 to 0.8 ; fore-arm, 0.5 ; back of the hand, 0.3 to 0.5 ; palm, 0.1 to 0.5 ; leg, 0.8 to 2 ; back of foot, 0.8 to 1 ; sole of foot, 0.8 to $1 \mathrm{~mm}$.

Methods. - 1. Place, on the part of the skin to be investigated, different weights, one after the other, and ascertain what perceptions they give rise to, and the sense of the difference of pressure to which they give rise. We must be careful to exclude differences of temperature and prevent the displacement of the weights-the weights must always be placed on the same spot, and the skin should be covered beforehand with a disc, while the muscular sense must be eliminated (\$430). [This is done by supporting the hand or part of the skin which is being tested, so that the action of all the muscles is excluded.] 2. A process is attached to a balance and made to touch the skin, while by placing weights in the scale-pan or removing them, we

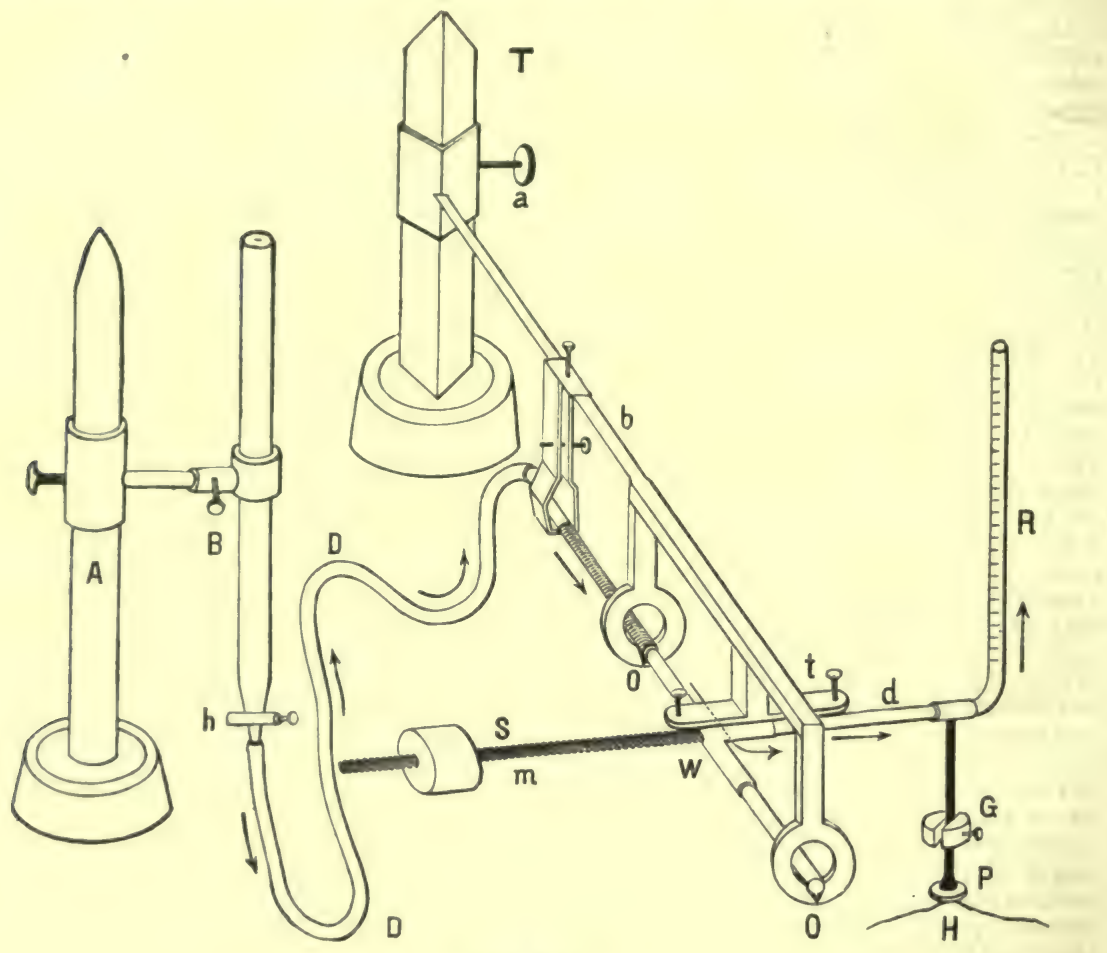

Fig. 619 .

Landois' mercurial balance for testing the pressure sense.

test what differences in weight the person experimented on is able to distinguish (Dohrn). $\mathbf{3}$ In order to avoid the necessity of changing the weights, A. Eulenburg invented his barrs. thesiometer, which is constructed on the same principle as a spiral spring paper-clip or balance. There is a small button which rests on the skin and is depressed by the spring. An index shows at once the pressure in grammes, and the instrument is so arranged that the presssure can be very easily varied. 4. Goltz uses a pulsating elastic tube, in which he can produce waves of different height. He tested how high the latter must be before they are experienced as pulse-waves, when the tube is placed upon the skin. 5. Landois uses a mercurial balance (fig. 619). The beam of a balance (W) moves upon two knife-edges $(\mathrm{O}, \mathrm{O})$, and is carried on the on which arm $(b)$ of a heavy support $(\mathrm{T})$. One arm of the beam is provided with a screw $(m)$ calibrated weight $(G)$, and which Below this is the pressure-pad $(P)$, which can be loaded as desired by a adjoining burette (B) held be placed upon the part of the skin to be tested $(\mathbf{H})$. From an the arrows, to one part of the balauce and into the tube (R). On the stop-cock $(h)$ being 
closed, whenever pressure is exerted on the tube $(\mathrm{D}, \mathrm{D})$, the mercury rises through $d$ into $\mathrm{R}$, and increases the pressure on $\mathrm{P}$. We measure the weight of the mercury corresponding to each division of the tube $(R)$. This instrument enables rapid variations of the weight to be made without giving rise to any shock. In estimating both the pressure sense and temperature sense, it is best to proceed on the principle of "the least perceptible difference," i.e., the different pressures or temperatures are graduated, either beginning with great differences, or proceeding from the smallest difference, and determining the limit at which the person can distinguish a difference in the sensation.

Results.-1. The smallest perceptible pressure, when applied to different parts of the skin, varies very greatly according to the locality. The greatest acuteness of sensibility is on the forehead, temples, and the back of the hand and fore-arm, which perceive a pressure of 0.002 grm.; the fingers first feel with a weight of 0.005 to $0.015 \mathrm{grm}$. ; the chin, abdomen, and nose with 0.04 to $0.05 \mathrm{grm}$.; the finger nail 1 grm. (Kammler and Aubert).

The greater the sensibility of the skin, the more rapidly can stimuli succeed each other, and still be perceived as single impressions ; 52 stimuli per second may be applied to the volar side of the upper arm, 61 on the back of the hand, 70 to the tips of the fingers, and still be $\mathrm{fel}^{4}$ singly (Bloch).

2. Intermittent variations of pressure, as in Goltz's tube, are felt more acutely by the tips of the fingers than with the forehead.

3. Differences between two weights are perceived by the tips of the fingers when the ratio is $29: 30$ (in the fore-arm as $18 \cdot 2: 20$ ), provided the weights are not too light or too heavy. In passing from the use of very light to heavy weights, the acuteness or fineness of the perception of difference increases at once, but with heavier weights, the power of distinguishing differences rapidly diminishes again (E. Hering, Biedermann). This observation is at variance with the psycho-physical law of Fechner $(\S 383)$.

4. A. Eulenburg found the following gradations in the fineness of the pressure sense :-The forehead, lips, dorsum of the cheeks, and temples appreciate differences of $\frac{1}{40}$ to $\frac{1}{30}(200: 205$ to $300: 310$ grm.). The dorsal surface of the last phalanx of the fingers, the fore-arm, hand, 1st and 2nd phalanx, the volar surface of the hand, fore-arm, and upper arm, distinguish differences of $\frac{1}{10}$ to $\frac{1}{20}$ (200:220 to $220: 210$ grm.). The anterior surface of the leg and thigh are similar to the forearm. Then follow the dorsum of the foot and toes, the sole of the foot, and the posterior surface of the leg and thigh. Dohrn determined the smallest additional weight, which, when added to $1 \mathrm{grm}$. already resting on the skin, was appreciated as a difference, and he found that for the 3rd phalanx of the finger it was 0.499 grm.; back of the foot, 0.5 grm.; 2nd phalanx, 0.771 grm.; 1st phalanx, 0.02 grm.; leg, 1 grm. ; back of the hand, 1.156 grm.; palm, 1.018 grm. ; patella, 1.5 grm.; fore-arm, 1.99 grm.; umbilicus, 3.5 grms. ; and the back, 3.8 grms.

5. Too long time must not elapse between the application of two successive weights, but 100 seconds may elapse when the difference between the weights is $4: 5$ (E. H. Weber).

6. The sensation of an after-pressure is very marked, especially if the weight $\mathrm{i}$ considerable and has been applied for a length of time. But even light weights, when applied, must be separated by an interval of at least $\frac{1}{480}$ to $\frac{1}{6} \frac{1}{10}$ second, in order to be perceived. When they are applied at shorter intervals, the sensations become fused. When Valentin pressed the tips of his fingers against a wheel provided with blunt teeth he felt the impression of a smooth margin, when the teeth were applied to the skin at the intervals above mentioned; when the wheel was rotated more slowly, each tooth gave rise to a distinct impression. Vibrations of strings are distinguished as such when the number of vibrations is 1506 to 1552 per second (v. Wittich and Grünhagen).

7. It is remarkable that pressure produced by the uniform compression of a part of the body, e.g., by dipping a finger or arm in mercury, is not felt as such ; the 
sensation is felt only at the limit of the fluid, on the volar surface of the finger, at the limit of the surface of the mercury.

428. TEMPERATURE SENSE.-The temperature sense makes us aquainted with the variations of the heat of the skin.

A spiecific mol-apparatus arranged in a punctated manner is connected with the temperature sense.

These "temperature-spots" are arranged in a linear manner or in chains, which are usually slightly curved (figs. 620,621). They generally radiate from certain points of the skin, usually the hair-roots. The chain of the "cold-spots " usually does not coincide with those of the "hot-spots," although the point from which they radiate may be the same. Frequently, these punctated lines are not complete, but they may be indicated by scattered points, between which, not unfrequently, points or spots for other qualities of sensation may be intercalated. Near the hairs there are almost always temperature-spots. In parts of the skin, where the temperature sensibility is slight, the temperature-points are present only near the hairs.

The sensation of cold occurs at once, while the sensation of heat develops gradually. Mechanical and electrical stimulation also excite the sensation of

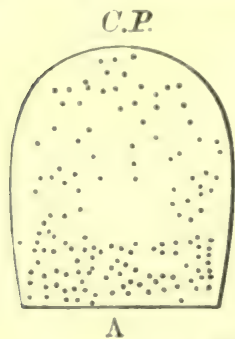

Fig. 620.

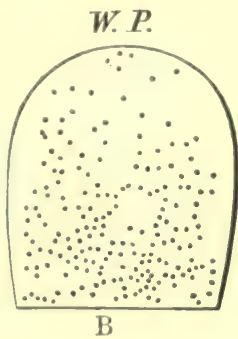

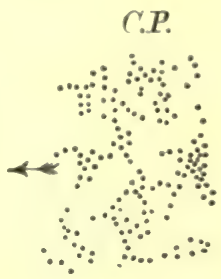

C

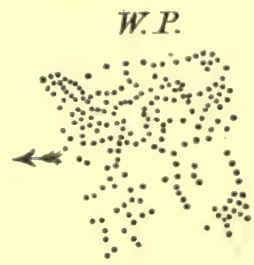

D

Fig. 621 .

Fig. 621.-A, cold-spots, B, hot-spots, from the volar surface of the terminal phalanx of the index-finger to the margins of the nail. Fig. 622 . - C, cold-spots, and D, warm-spots of the radial half of the dorsal surface of the wrist. The arrow indicates the direction in which the hair points.

temperature. A gentle touch of the temperature-spots is not perceived; these points seem to he anæsthetic towards pressure and pain. As a general rule, the cold-spots are more abundant over the whole body-there are more of them in a given area-while the hot-spots may be quite absent. The hot-spots are, as a rule, perceived as double at a greater distance apart than the cold-spots. The minimal distance on the forehead is $0.8 \mathrm{~mm}$. for the cold-spots and 4 to $5 \mathrm{~mm}$. for the warm-spots; on the breast the corresponding numbers are 2 and 4 to 5 ; back, 1.5 to 2 and 4 to 6 ; back of hand, 2 to 3 and 3 to 4 ; palm, 0.8 to 2 ; thigh and leg, 2 to 3 and 3 to $4 \mathrm{~mm}$.

Method.-To test the hot-and cold-spots, use a hot or cold metallic rod ; at the cold-spots, when they are lightly touched, only the sensation of cold will be felt, and a corresponding effect with a hot rod at the hot-spots. Both spots are insensible to objects of the same temperature as the skin.

According to E. Hering, what determines the sensation of temperature is the temperature of the thermic end-apparatus itself, i.e., its zero-temperature. As often as the temperature of a cutaneous area is above its zero-temperature, we feel it as warm; in the opposite case, cold. The one or the other sensation is more marked, the more the one or other temperature varies from the zero-temperature. The zerotemperature can undergo changes within considerable limits, owing to external conditions.

Methods. - To the surface of the skin objects of the same size and with the same thermal conductivity are applied successively at different temperatures:-1. Nothnagel uses small 
wooden cups with a metallic base, and filled with warm and cold water, the temperature being registered by a thermometer placed in the cups. [2. Clinically, two test-tubes filled with cold and warm water, or two spoons, the one hot and the other cold, may be used.]

Results.-1. As a general rule, the feeling of cold is produced when a body applied to the skin robs it of heat; and, conversely, we have a sensation of warmth when heat is communicated to the skin.

2. The greater the thermal conductivity of the substance touching the skin, the more intense is the feeling of heat or cold (\$218).

3. At a temperature of $15.5^{\circ}-35^{\circ} \mathrm{C}$., we distinguish distinctly differences of temperature of $0 \cdot 2^{\circ}-0 \cdot 16^{\circ} \mathrm{R}$. with the tips of the fingers $(E . H$. Weber). Tem-
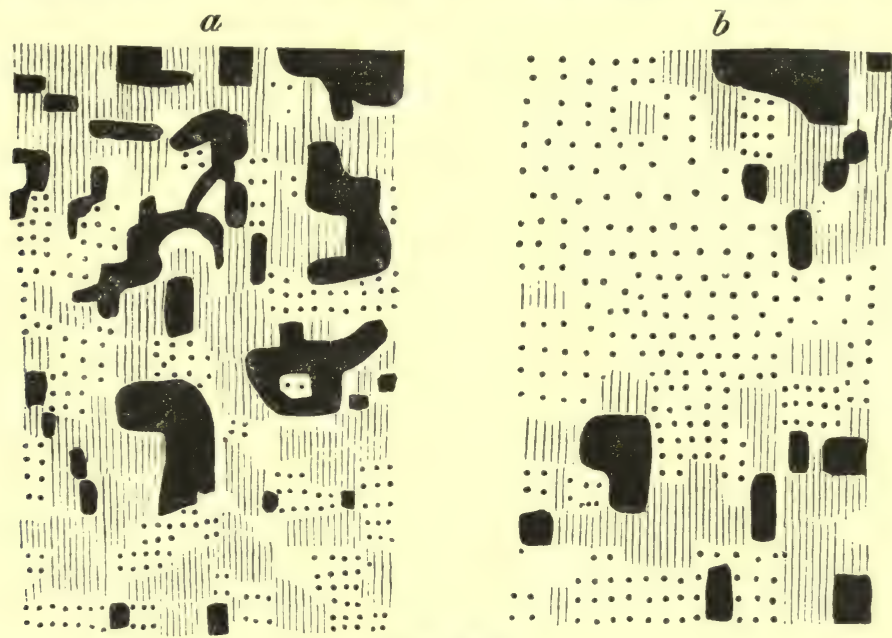

Fig. 622.

Cold- and hot-spots from the same part of the anterior surface of the fore-arm. $\alpha$, cold-spots ; $b$, hot-spots. The dark parts are the most sensitive, the hatched the medium, the dotted the feebly, and the vacant spaces the non-sensitive.

peratures just below that of the blood $\left(33^{\circ}-27^{\circ}\right.$ C. - Nothnagel $)$ are distinguished most distinctly by the most sensitive parts, even to differences of $0.05^{\circ} \mathrm{C}$. (Linder$\operatorname{man} n)$. Differences of temperature are less easily made out when dealing with temperatures of $33^{\circ}-39^{\circ} \mathrm{C}$, as well as between $14^{\circ}-27^{\circ} \mathrm{C}$. A temperature of $55^{\circ} \mathrm{C}$., and also one a few degrees above zero $\left(2.8^{\circ} \mathrm{C}\right.$.), cause distinct pain in addition to the sensation of temperature.

4. The sensibility for cold is generally greater than for warmth, - that of the left hand is greater than the right (Goldscheider). The different parts of the skin also vary in the acuteness of their thermal sense, and in the following order :-Tip of the tongue, eyelids, cheeks, lips, neck, and body. The perceptible minimum Nothnagel found to be $0.4^{\circ}$ on the breast; $0.9^{\circ}$ on the back; $0.3^{\circ}$, back of the hand; $0.4^{\circ}$, palm; $0.2^{\circ}$, arm; $0.4^{\circ}$, back of the foot; $0.5^{\circ}$, thigh; $0.6^{\circ}$, leg; $0.4^{\circ}-0.2^{\circ}$, cheek; $0.4^{\circ}-0.3^{\circ}$ C., temple. The thermal sense is less acute in the middle line, e.g., the nose, than on each side of it (E. H. Weber). Fig. 622 shows that in one and the same portion of skin, the cold- and hot-spots are differently located, i.e., their different topography.

If the mucous membrane of the mouth be pencilled with a 10 per cent. solution of cocain; the sensibility for heat is abolished; the cooling sensation of menthol depends upon its stimulation of the cold nerves; $\mathrm{CO}_{2}$ applied to the skin excites the heat nerves (Goldscheider).

5. Differences of temperature are most easily perceived when the same part of the skin is affected successively by objects of different temperature. If, however, two 
different temperatures act simultaneously and side by side, the impressions are apt to become fused, especially when the two areas are very near each other.

6. Practice improves the temperature sense; congestion of venous blood in the skin diminishes it ; diminution of the amount of blood in the skin improves it $(M$. Alstery). When lurge areas of the skin are touched, the perception of differences is more acute than with small areas. Rapid variations of the temperature produce more intense sensations than gradual changes of temperature. Fatigue occurs soon.

Illusions are very common :- 1 . The sensations of heat and cold sometimes alternate in a paraloxical manner. When the skin is dipped first into water at $10^{\circ} \mathrm{C}$. we feel cold, and if it Fe then lipreel at once into water at $16^{\circ} \mathrm{C}$., we have at first a feeling of warmth, but soon again of cold. 2. The same temperature applied to a large surface of the skin is estimated to be greater than when it is appilied to a small area, $c . g$., the whole hand when placed in water at $2.95 \mathrm{C}$. feels warmer than when a finger is dipped into water at $32^{\circ} \mathbf{C}$. 3. Cold weights are judged to be heavier than warm ones.

Pathological. - Tactile sensibility is only seldom increased (hyperpselaphesia), but great sensibility to differenees of temperature is manifested by areas of the skin whose epidernis is fartly removed or altered by vesicants or herpes zoster, and the same occurs in some cases of Incomotor ataxia ; while the sense of locality is rendered more acute in the two former cases and in erysipelas. An abnormal condition of the sense of locality was described by Brown-Séquard, where three points were felt when only two were applied, and two when one was applied to the skin. Lanlois finds that in himself pricking the skin of the sternum over the angle of Ludovirus is always accompanied by a sensation in the knee. [Some persons, when cold water is applied to the scalp, have a sensation referable to the skin of the loins (Stirling).] A remarkable variation of the sense of locality occurs in moderate poisoning with morphia, where the person feels himself abnormally large or greatly diminished. In degeneration of the posterior columns of the cord, Obersteiner observed that the patient was unable to say whether his right or left side was touched ("allochiria"). Ferrier observed a case where a stimulus applied to the right side was referred to the left, and vice versa.

Diminution and paralysis of the tactile sense (Hypopselaphesia and Apselaphesia) occur either in conjunetion with simultaneous injury to the sensory nerves, or alone. It is rare to find that on of the qualities of the tartile sense is lost, e.g., either the tactile sense or the sense of temperature-a condition which has been called "partial tactile paralysis." Limbs which are "sleeping" feel heat and not cold (Herzen).

429. COMMON SENSATION-PAIN.-By the term common sensation we understand jleasant or unpleasant sensations in those parts of our bodies which are endowed with sensibility, and which are not referable to external objects, and whose characters are difficult to describe, and cannot be compared with other sensations. Each sensation is, as it were, a peculiar one. To this belong pain, hunger, thirst, malaise, fatigue; horror, vertigo, tickling, well-being, illness, the respiratory feeling of free or impeded breathing.

Pain may occur wherever sensory nerves are distributed, and it is invariably caused by a stronger stimulus than normal being applied to sensory nerves. Every kind of stimulation, mechanical, thermal, chemical, electrical, as well as somatic (inflammation or disturbances of nutrition), may excite pain. The last appears to be especially active, as many tissues become extremely painful during inflammation (e.g., muscles and bones), while they are comparatively insensible to cutting. Pain may be produced by stimulating a sensory nerve in any part of its course, from its centre to the periphery, but the sensation is invariably referred to the peripheral end of the nerve. This is the law of the peripheral reference of sensations. Hence, stimulation of a nerve, as in the scar of an amputated limb, may give rise to a sensation of pain which is referred to the parts already removed. Too violent stimulation of a sensory nerve in its course may render it incapable of conducting impressions, so that peripheral impressions are no longer perceived. If a sufficient stimulus to produce pain be then applied to the central part of the nerve, such an impression is still referred to the peripheral end of the nerve. Thus we explain the paradoxical anæsthesia dolorosa. In connection with painful impressions, the patient is often unable to localise them exactly. This is most easily done when a small injury (prick of a needle) is made on a peripheral part. When, however, the 
stimulation occurs in the course of the nerve, or in the centre, or in nerves whose peripheral ends are not accessible, as in the intestines, pain (as belly-ache), which cannot easily be localised, is the result.

Irradiation.-During violent pain there is not unfrequently irradiation of the pain $(\$ 364,5)$, whereby localisation is impossible. It is rare for pain to remain continuous and uniform; more generally there are exacerbations and diminutions of the intensity, and sometimes periodic intensification, as in some neuralgias.

The intensity of the pain depends especially upon the excitability of the sensory nerves. There are considerable individual variations in this respect, some nerves, e.g., the trigeminus and splanchnic, being very sensitive. The larger the number of fibres affected the more severe the pain. The duration is also of importance, in as far as the same stimulation, when long continued, may become unbearable. We speak of piercing, cutting, boring, burning, throbbing, pressing, gnawing, dull, and other kinds of pain, but we are quite unacquainted with the conditions on which such different sensations depend. Painful impressions are abolished by anæsthetics and narcotics, such as ether, chloroform, morphia, \&c. (\$364, 5).

Methods of Testing.-To test the cutaneous sensibility, we usually employ the constant or induced electrical current. Determine first the minimum sensibility, i.e., the strength of the current which excites the first trace of sensation, and also the minimum of pain, i.e., the feeblest strength of the current which first causes distinct impressions of pain. The electrodes consist of thin metallic needles, and are placed 1 to $2 \mathrm{~cm}$. apart.

Pathological.--When the excitability of the nerves which administer to painful sensations is increased, a slight touch of the skin, nay, even a breath of cold air, may excite the most violent pain, constituting cutaneous hyperalgia, especially in inflammatory or exanthematic conditions of the skin. The term cutaneous paralgia is applied to certain anomalous, disagreeable, or painful sensations which are frequently referred to the skin-itching, creeping, formication, cold, and burning. In cerebro-spinal meningitis, sometimes a prick in the sole of the foot produces a double sensation of pain and a double reflex contraction. Perhaps this condition may be explained by supposing that in a part of the nerve the condition is delayed $(\S 337,2)$. In neuralgia there is severe pain, occurring in paroxysms, with violent exacerbations and pain shooting into other parts (p. 598). Very frequently excessive pain is produced by pressure on the nerve where it makes its exit from a foramen or traverses a fascia.

Valleix's Points Douloureux (1841). - The skin itself to which the sensory nerve runs, especially at first, may be very sensitive; and when the neuralgia is of long duration the sensibility may be diminished even to the condition of analgesia ( $T$ ürck); in the latter case there may be pronounced anæsthesia dolorosa (p. 838).

Diminution or paralysis of the sense of pain (hypalgia and analgia) may be due to affections of the ends of the nerves, or of their course, or central terminations.

Metalloscopy.-In hysterical patients suffering from hemianæsthesia, it is found that the feeling of the paralysed side is restored, when small metallic plates or larger pieces of different metals are applied to the affected parts (Burcq, Charcot). At the same time that the affected part recovers its sensibility the opposite limb or side becomes anæsthetic. This condition has been spoken of as transference of sensibility. The phenomenon is not due to galvanic currents developed by the metals; but it may be, perhaps, explained by the fact that, under physiological conditions, and in a healthy person, every increase of the sensibility on one side of the body, produced by the application of warm metallic plates or bandages, is followed by a diminution of the sensibility of the opposite side. Conversely, it is found that when one side of the body is rendered less sensitive by the application of cold plates, the homologous part of the other side becomes more sensitive (Rumpf).

430. MUSCULAR SENSE.-Muscular Sensibility.-The sensory nerves of the muscles (\$292) always convey to us impressions as to the activity or nonactivity of these organs, and in the former case, these impressions enable us to judge of the degree of contraction. It also informs us of the amount of the contraction to be employed to overcome resistance. Obviously, the muscular sense must be largely supported and aided by the sense of pressure, and conversely. E. H. Weber showed, however, that the muscle sense is finer than the pressure sense, as by it we can distinguish weights in the ratio of $39: 40$, while the pressure sense only enables us to distinguish those in the ratio of $29: 30$. In some cases there has been observed total cutaneous insensibility, while the 
muscular sense was retained completely. A frog deprived of its skin can spring without any apparent disturbance. The muscular sense is also greatly aided by the sensibility of the joints, bones, and fasciæ. Many muscles, e.g., those of respiration, have only slight muscular sensibility, while it seems to be absent normally in the heart and non-striped muscle.

[The muscular sense stands midway between special and common sensations, and by it we obtain a knowledge of the condition of our muscles, and to what extent they are contracted; also the position of the various parts of our bodies and the resistance offered by external objects. Thus, sensations accompanying muscular movement are two-fold-(a) the movements in the unopposed muscles, as the movements of the limbs in space; and (b) those of resistance where there is opposition to the movement, as in lifting a weight. In the latter case the sensations due to innervation are important, and of course in such cases we have also to take into account the sensations obtained from mere pressure upon the skin. Our sensations derived from muscular movements depend on the direction and duration of the movements. On the sensations thus conveyed to the sensorium, we form judgments as to the direction of a point in space, as well as of the distance between two points in space. This is very marked in the case of the ocular muscles. It is also evident that the muscular sense is intimately related to, and often combined with, the exercise of the sensation of touch and sight (Sully).]

Methods of Testing. - Weights are wrapped in a towel and suspended to the part to be testel. The patient estimates the weight ly raising and lowering it. The electro-muscular scnsitritity also may be proved thus: cause the muscles to contract by means of induction shocks, and observe the sensation therely produced. [Direct the patient to place his feet together while stanling, and then close his eyes. A healthy person can stand quite steady, but in one with the muscular sense implaired, as in locomotor ataxia, the patient may move to and fro, or even fall (p. 647). Agrain, a person with his muscular sense impaired may not be able to touch accurately and at once some prart of his body, when his eyes are closed.]

$\Lambda$ healthy person perceives a weight of 1 gramme applied to his upper arm; when a weight of 15 grms. is alplieul, he perceives an addition of $1 \mathrm{grm}$. If the original weight be $50 \mathrm{grms}$., he will detect the addition of 2 grms.; if the original weight be 100 grms., he will detect 3 grms. The weight detectable by the individual finger varies. With the leg, when the weight is apylied at the knee, the individual may detect 30 to 40 grms.; but sometimes only a greater weight. Often one can detect a difference of 10 to 20 , or 30 to $70 \mathrm{grms}$.

Section of a sensory nerve causes disturbance of the fine graduation of movement (p. 619). Meynert supposes that the cerebral centre for muscular sensibility lies in the motor cortical centres, the muscles being connected by motor and sensory paths with the ganglionic cells in these centres.

Too severe muscular exercise causes the sensation of fatigue, oppression, and weight in the limbs (\$ 304).

Pathological,-Ahnormal increase of the muscular sense is rare (muscular hyperalgia and hypreresthesiat), as in anxictas tibiarum, a painful condition of unrest which leads to a continual change in the prosition of the limbs. In cramp there is intense pain, due to stimulation of the sensory nerves of the muscle, and the same is the case in inflammation. Diminution of the muscular sensibility occurs in some choreic and ataxic persons $(\$ 364,5)$. In locomotor ataxia the muscular sense of the upyer extremities may be normal or weakened, while it is usually considerably diminished in the legs. [The muscular sense is said to be increased in the hypnotic condition, and in somnambulists.] 


\section{Reproduction and Development.}

431. FORMS OF REPRODUCTION,-I. Abiogenesis (Generatio aequivoca, sive spontanea, spontaneous generation). - It was formerly assumed that, under certain circumstances, nonliving matter derived from the decomposition of organic materials became changed spontaneously into living beings. While Aristotle ascribed this mode of origin to insects, the recent observers who advocate this form of generation restrict its action solely to the lowest organisms. Experimental evidence is distinctly against spontaneous generation. If organised matter be heated to a very high temperature in sealed tubes, and be thus deprived of all living organisms or their spores, there is no generation of any organism. Hence, the dictum "Omne vivum ex ovo" (Harvey, or, ex vivo). Some highly organised invertebrate animals (Gordius, Anguillula, Tardigrada, and Rotatoria) may be dried, and even heated to $140^{\circ} \mathrm{C}$, , and yet regain their vital activities on being moistened (Anabiosis).

II. Division or fission occurs in many protozoa (amœba, infusoria). The organism, just as is the case with cells, divides, the nucleus when present taking an active part in the process, so that two nuclei and two masses of protoplasm forming two organisms are produced. The Ophidiasters amongst the echinoderms divide spontaneously, and they are said to throw off an arm which may develop into a complete animal. According to Trembley (1744), the hydra may be divided into pieces, and each piece gives rise to a new individual [although under normal circumstances the hydra gives off buds, and is provided with generative organs].

[Division of Cells.-Although a cell is defined as a "nucleated mass of living proto-
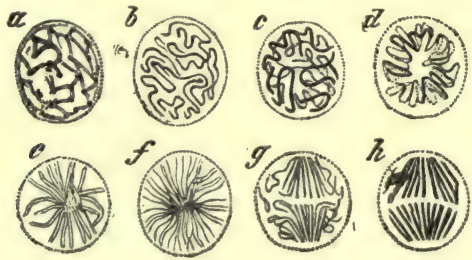

Fig. 623.

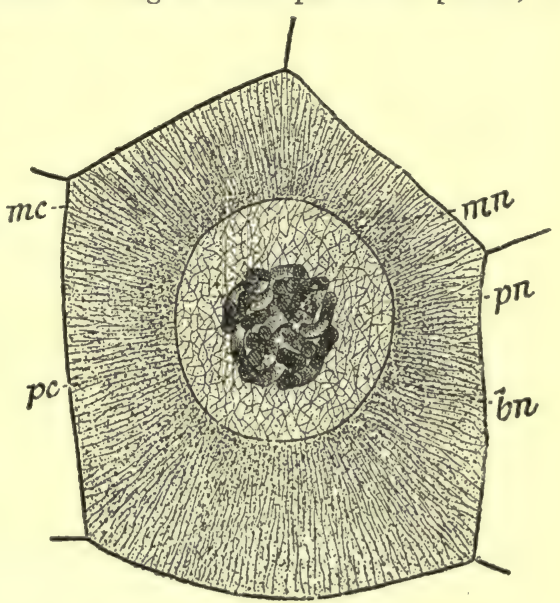

Fig. 624.

Fig. 623. - Changes in a cell nucleus during karyokinesis. Fig. 624.-Typical nucleated cell of the intestinal epitheliun of a flesh-maggot. $m c$, membrane of cell; $m n$, membrane of nucleus ; $p c$, cellular protoplasm, with the radiating reticulum, and the enchylema enclosed. in its meshes ; $p n$, plasma of nucleus ; $b n$, nuclear filament showing numerous twists.

plasm," recent researches have shown that, from a histological as well as from a chemical point of view, a cell is really a very complex structure. The apparently homogeneous cell-substance is traversed by a fine plexus of fibrils, with a homogeneous substance in its meshes, while a similar network of fibrils exists within the nucleus itself (fig. 623).]

[The nucleus of a typical cell is a spherical vesicle, consisting of a membrane containing what is called "achromatin," because it is not readily stained by staining reagents. Flemming has also called it nuclear fluid, or intermediate substance. The achromatin substance is permeated 
by a delicate reticular network, or plexus of fibrils, which has been called "chromatin," "nucleoplasm," "karyoplasma," and "karyomiton." The network stains readily with pigments, hence the name "chromatin" given to it by Flemming. The nodal points of the network give a dotted or granular appearance to the nucleus, especially when it is examined with a low power. The nuclear membrane also consists of chromatin (fig. 624). In the meshes of the network lie nucleoli, which seem to differ in constitution, and perhaps in function. According to Flemming, there are principal and accessory nucleoli in some nuclei. In Carnoy's nomenclature the several parts are spoken of as a fine reticulum of fibrils, enclosing in its meshes a fluid-the cuchylema - which contains various particles in suspension.]

[Direct Cell-Division. - A cell may divide directly, as it were, by simple cleavage, and in the process the nucleus usually divides before the cell protoplasm. The nucleus becomes constricted in the centre, has an hour-glass shape, and soon divides into two.]
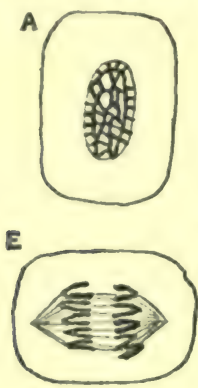

B
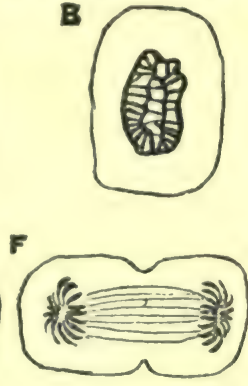
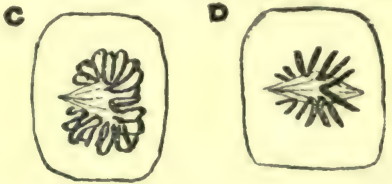

H

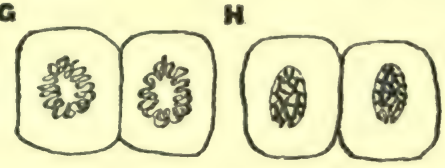

Fig. 625 .

Mitosis. A, nuclear reticulum, resting state ; B, preparing for division; C, wreath stage ; D, monaster stage ; $\mathrm{E}$, barrel stage; $\mathrm{F}$, diaster stage ; $\mathrm{G}$, daughter wreath stage; $\mathrm{H}$, daughter cells, passing to resting stage.

[Indirect Cell-Division.-Recent observations, confirmed by a great number of investigators, conclusively prove that the process of division in cells is a very complicated one, the changes in the nucleus being very remarkable. The terms karyokinesis, mitosis, or indirect division have been applied to this process. Figs. 623,625 show the changes that take place in the nucleus. The chromatin or intranuclear network $(a, \mathrm{~B})$ passes into a convolution of fibrils, while the nuclear envelope becomes less distinct, the fibrils at the same time becoming thicker and forming loops, which gradually arrange themselves around a centre $(c$ and $d)$ in the form of a wreath, rosette, or spirem (C). The fibres curve round both at the periphery and the centre and form loops; but when their peripheral connections are severed or dissolved, we obtain a star-shaped form or aster (D), composed of single loops radiating from the centre (e). The loops divide in the direction of their length; their number is doubled, but they are thinner. By this further subdivision, the whole is composed of fine radiating fibrils $(f)$, which gradually arrange themselves around two poles, or new centres, to form the barrel-form or pithode (E); the two groups of loops then separate still further, and arrange themselves so as to form a diaster, or double star $(g)$, the two gronps being separated by a substance called the equatorial plate. Each of the groups of fibrils becomes more elongated, and forms a nuclear spindle, which indicates the position of a new nucleus. The protoplasm separates into two parts. In each of these parts the chromatin rearranges itself into an irregular coil, and the whole is called dispirem $(\mathrm{G})$, and when division is complete, the chromatin filaments assume the form seen in a resting nucleus. This whole complex process may be accomplished in 1 to 4 hours. The separate groups of fibrils again become convoluted, each group gets a nuclear membrane, while the cell protoplasm divides, and two daughter nuclei are obtained from the original cell.]

The following scheme represents some of the more important changes:-
1. Network.
Mother nucleus.
2. Convolution.
3. Wreath or Spirem.
4. Aster,

|

\section{Equatorial grouping of chromatin.}

Daughter nuclei.
8. Network.
7. Convolution.
6. Dispirem.
5. Diaster.

III. Budding or gemmation occurs in a well-marked form among the polyps and in some infusorians (Vorticella). $\Lambda$ bud is given off by the parent, and gradually comes more and more to resemble the latter. The hud either remains permanently attached to the parent, so that a complex organism is produced, in which the digestive organs communicate with each other directly, or in some cases there may be a "colony" with a common nervous system, such as the 
polyzoa. In some composite animals (siphonophora) the different polyps perform different functions. Some have a digestive, others a motor, and a third a generative function, so that there is a physiological division of labour. Buds which are given off from the parent are formed internally in the rhizopoda. In some animals (polyps, infusoria), which can reproduce them. selves by buds or division, there is also the formation of male and female elements of generation, so that they have a sexual and a non-sexual mode of reproduction.

IV. Conjugation is a form of reproduction which leads up to the sexual form. It occurs in the unicellular Gregarinæ. The anterior end of one such organism unites with the posterior end of another; both become encysted, and form one passive spherical body. The conjoined structures form an amorphous mass, from which numerous globular bodies are formed, and in each of which numerous oblong structures-the pseudo-navicelli-are developed. These bodies become, or give rise to an amoboid structure, which forms a nucleus and an envelope, and becomes transformed into a gregarina.

Sexual reproduction requires the formation of the embryo from the conjunction of the male and female reproductive elements, the sperm-cell and the germ-cell. These products may be formed either in one individual (hermaphroditism, as in the flat worms and gasteropods), or in two separate organisms (male or female). Sexual reproduction embraces the following varieties :-

V. Metamorphosis is that form of sexual reproduction in which the embryo from an early period undergoes a series of marked changes of external form, e.g., the chrysalis stage, and the pupa stage, and in none of these stages is reproduction possible. Lastly, the final sexually developed form (the imago stage in butterflies) is produced, which forms the sexual products whose union gives rise to organisms which repeat the same cycle of changes. Metamorphosis occurs extensively amongst the insects; some of them have several stages (holo-metabolic), and others have few stages (hemi-metabolic). It also occurs in some arthropoda, and worms, e.g., trichina; the sexual form of the

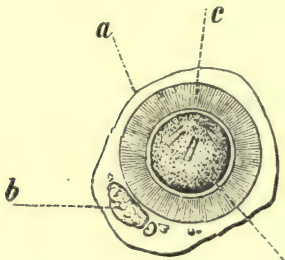

Fig. 626.

A ripe egg taken from the uterus of Tænia solium. $a$, Albuminous envelope; $b$, remains of the yelk; $c$, covering of the embryo; $d$, embryo with embryonal hooklets.

\section{sexually mature.]} animal occurs in the intestine, the numerous larvoe wander into the muscles, where they become encysted, and form undeveloped sexual organs, constituting the pupa stage of the muscular trichina. When the encysted form is eaten by anotheranimal, the sexual organs come into activity, a new brood is formed, and the cycle is repeated. Metamorphosis also occurs in the frog and in petromyzon. [This is really a condition in which the embryo undergoes marked changes of form before it becomes

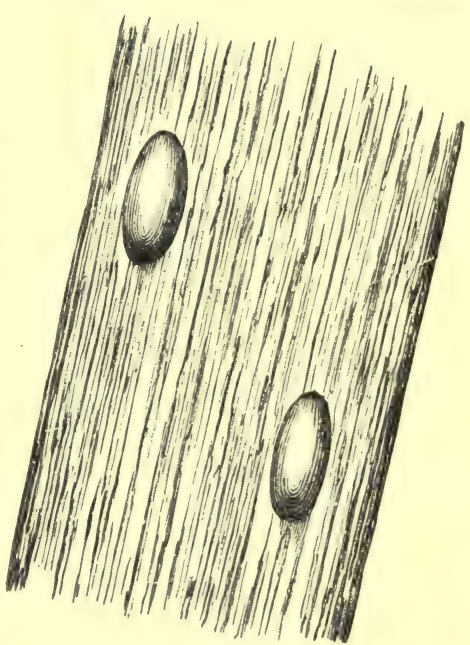

Fig. 627.

Encapsuled cysticercus from Tænia solium embedded in a human sartorius. Natural size.

VI. Alternation of Generations (Steenstrup). - In this variety some of the members of the cycle can produce new beings non-sexually, while in the final stage reproduction is always sexual. From a medical point of view, the life-history of the tape-worm or Tænia is most important. The segments of the tape-worm are called proglottides (fig. 631), and each segment is hermaphrodite, with testes, vas deferens, penis, ovary, \&c., and numerous ova. The segments are evacuated with the fæces. The eggs are fertilised after they are shed (fig. 626), and from them is developed an elliptical embryo, provided with six hooklets, which is swallowed by another animal, the host. These embryos bore their way into the tissues of the host, where they undergo development, forming the encysted stage (Cysticercus (fig. 627), Coenurus, or Echinococcus (fig. 630). The encysted capsule may contain one (cysticercus) or many (coenurus) sessile heads of the tænia. In order to undergo further development, the cysticercus must be eaten alive by another animal, when the head or scolex fixes itself by the hooklets and suckers to the intestine of its new host (fig. 629), where it begins to bud and produce a series of new segments between the head and the last-formed segment, and thus the cycle is repeated.

The most important flat-worms are :-Tænia solium, in man; the Cysticercus cellulosæ (fig. 628 ), in the pig, when it constitutes the measle in pork; Tænia mediocanellata (fig. 631), the 
encysted stage, in the $0 x$; Trnia coenurus, in the dog's intestine; the encysted stage, or Coenurus cerebralis, in the brain of the sheep, where it gives rise to the condition of "staggers"; Twonia echinococcus, in the dog's intestine; the embryos or scolices occur in the liver of man as "hydatids."

The medusie also exhibit alternation of generations, and so do some insects, especially the plant liee or aphides.

VII. Parthenogenesis $($ Owen, $v$. Sicbold). - In this variety, in addition to sexual reproduction, new inliviluals may be produced without sexual union. The non-sexually produced brood is always of one sex, as in the bees. $\mathrm{A}$ beehive contains a queen, the workers, and the drones or males. During the nuptial flight, the queen is impregnated by the males, and the seminal fluid is stored up in the receptaculum seminis of the queen, and it

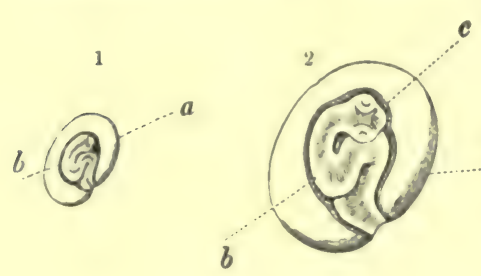

Fig. 628 .

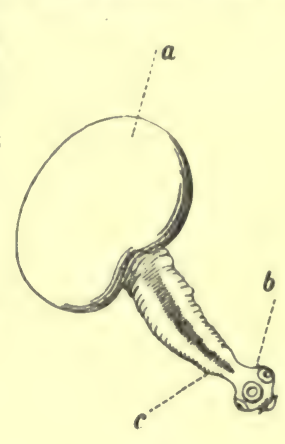

Fig. 629 .

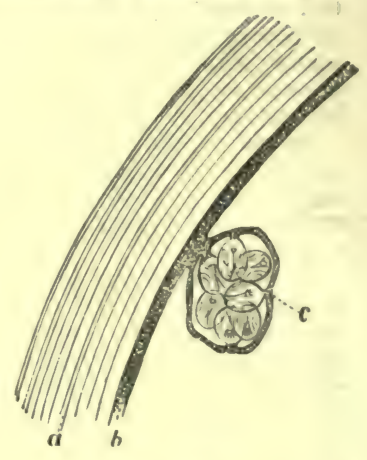

Fig. 630 .

Fig. 628.-Cysticerci from Tænia solium removed from their capsule. 1, natural size; 2, magnifiel. $a$, embryo-sac; $b$, cavity produced by budding of the embryo-sac; $c$, suctorial discs and hooklets. Fig. 629.-Cysticercus of Tænia solium, with its head and segments protruded. $a$, caudal-sac; $b$, head of the tape-worm, with discs and hooklets (scolex); c, neck. Fig. 630.-Part of an Echinococcus capsule, with developing buds. $a$, sheath ; $b$, parenchymatous layer ; $c$, germinating capsule filled with scolices.

appears that the queen may voluntarily permit the contact of this fluid with the ova or withhold it. All fertilised eggs give rise to female, and all unfertilised ones to male bees.

VIII. Sexual reproduction without any intermediate stages occurs in, besides man, mammals, birds, reptiles, and most fishes.

432. TESTIS - SEMINAL FLUID.-[Testis.-In the testis or male reproductive organ, the seminal fluid which contains the male element or spermatozoa is formed. The framework of the gland consists of a thick strong white fibrous covering, the tunica albuginea, composed chiefly of white interlacing fibrous tissue. Externally, this layer is covered by the visceral

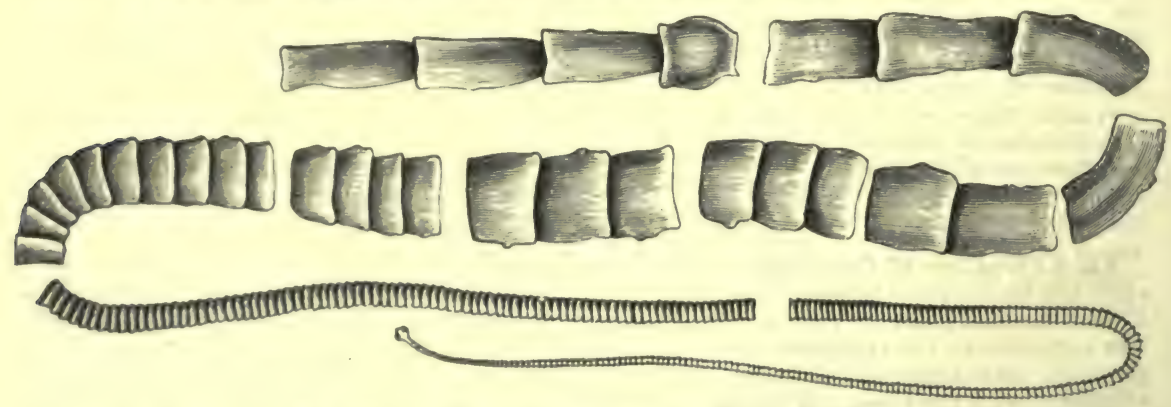

Fig. 631.-Trnia mediocanellata. Natural size.

layer of the serous membrane, or the tunica vaginalis, which invests the testis and epididymis. The tunica albuginea is prolonged for some distance as a vertical septum into the posterior part of the testis, to form the mediastinum testis or corpus Highmori. Septa or trabeculæmore or less complete-stretch from the under surface of the T. albuginea towards the medias. tinum, so that the organ is subdivided thereby into a number of compartments or lobules, with their bases directed outwards and their apices towards the mediastinum. From these, finer sustentacular fibres pass into the compartments to support the structures lying in these compartments.] 
[Arrangement of Tubules.-Each compartment contains several seminal tubules, long convoluted tubules $\left(\frac{1}{100}\right.$ in. in diam.) which rarely branch except at their outer end; they are about 2 feet in length and exceed 800 in number. These tubules run towards the mediastinum, those in one compartment uniting at an acute angle with each other, to form a smaller number of narrower straight tubules-tubuli recti (fig. 632). These straight tubules open into a network of tubules in the mediastinum to form the rete testis, a dense network of tubules of irregular diameter (fig. 632). From this network there proceed 12 to 15 wider ducts,-the vasa efferentia - which after emerging from the testis are at first straight, but soon become convoluted-and form a series of conical eminences-the coni vasculosiwhich together form the head of the epididymis. These tubes gradually unite with each other and form the body and globus minor of the epi. didymis, which, when unravelled, is a tube about 20 feet long terminating in the vas deferens ( 2 feet long), which is the excretory duct of the testis.]

[Structure of a Tubule. - The seminal tubules consist of a thick well-marked basement membrane, composed of flattened nucleated cells arranged like membranes (fig. 637). These tubes are lined by several layers of more or less cubical cells ; there is an outer row of such cells next the basement membrane, and often showing a dividing large nucleus. Internal to these are several layers of inner large clear cells, with nuclei often dividing, so that they form many daughter cells which lie internal to them and next the lumen. From these daughter cells are formed the spermatozoa, and they constitute the spermatoblasts. These several layers of cells leave a distinct lumen. The tubuli recti are narrow in diameter, and lined by a single layer of squamous or flattened epithelium (fig. 633). The rete testis consists merely of channels in the fibrous stroma without a distinct membrana propria, but lined by flattened epithelium. The vasa efferentia and coni vasculosi have circular smooth muscular fibres in their walls, and are lined by a layer of columnar ciliated epithelium with striated protoplasm. At the bases of these cells in some parts is a layer of smaller granular cells. These tubules form the epididymis, whose tubules have the same structure (fig. 634). In the sheep, pigment cells are often found in the basement membrane. The vas

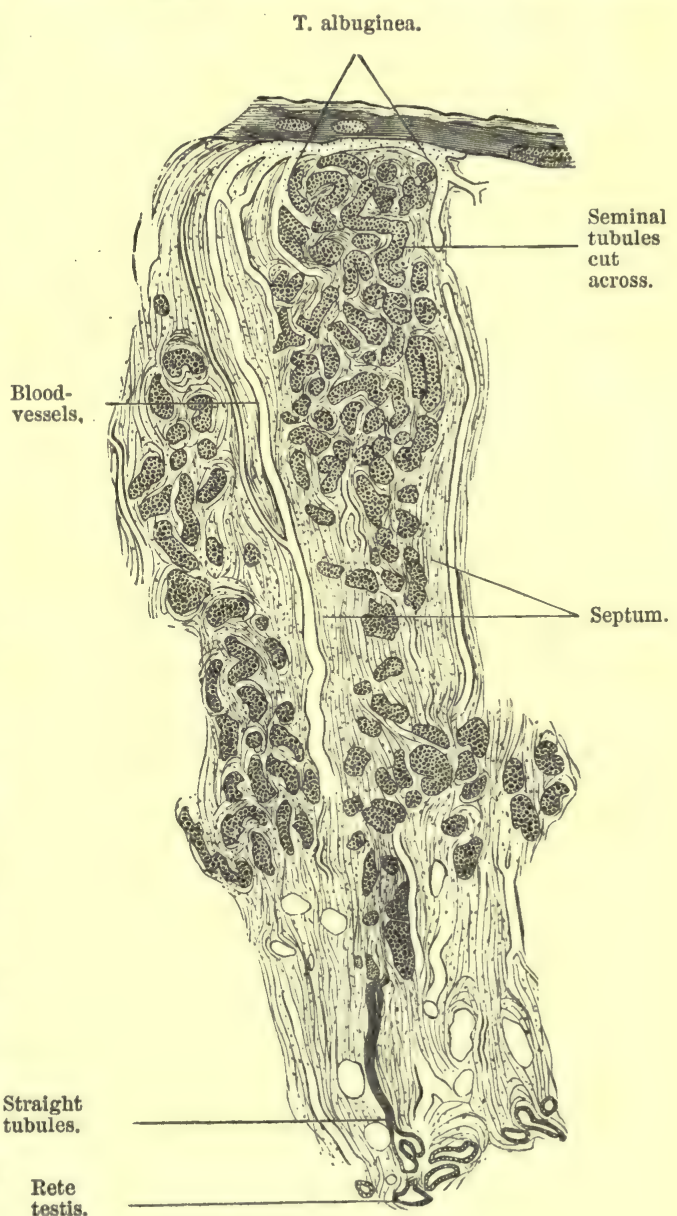

Fig. 632 .

Transverse section of the testis (low power view).

deferens is lined by several layers of columnar epithelinm resting on a dense layer of fibrous tissue-the mucosa. Outside this is the muscular coat, a thick layer of non-striped muscle composed of a thick inner circular, and thick outer longitudinal layer, a thin submucous coat connecting the muscular and mucous coats together; outside all is the fibrous adventitia.]

[The interstitial tissue (fig. 632), supporting the seminal tubules, is laminated and covered by endothelial plates, with slits or spaces between the lamellæ, which form the origin of the lymphatics. These lymph-spaces are easily injected by the puncture method. In fact, if Berlin blue be forced into the testis, the lymphatics of the testis and spermatic cord are readily filled with the injection. In some animals (boar), and to a less extent in man, dog, there are 
also fairly large polyhedral interstitial cells, often with a large nucleus and sometimes pigmented. They represent the residue of the epithelial cells of the Wolffian bodies (Klein), or, according to Waldeyer, they are plasma cells. The blood-vessels are numerous, and form a dense plexus outside the basement membrane of the seminal tubules.]

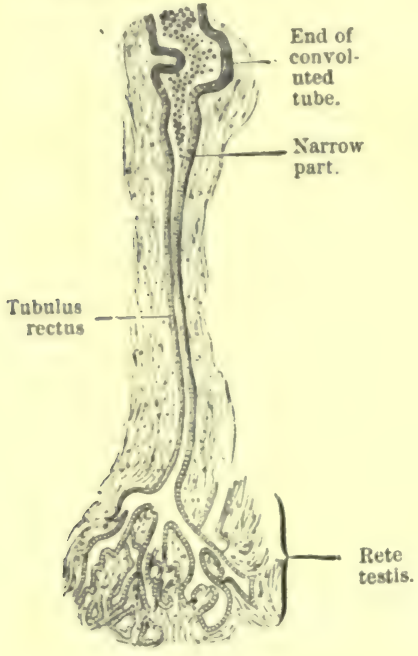

Fig. 633.

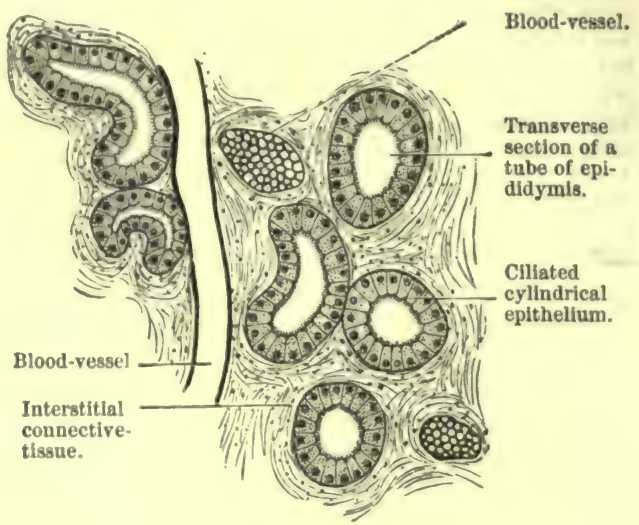

Fig. 634 .

Fig. 633. - Convoluted seminal tubule opening into a narrow straight tubule. Fig. 634.Transverse section of the tubules of the epididymis.

Chemical Composition. - The seminal fluid, as discharged from the urethra, is mixed with the secretion of the glands of the vas deferens, Cowper's glands, and those of the prostate, and with the fluid of the vesiculæ seminales. Its reaction is neutral or alkaline, and it contains 82 per cent. of water, serum-albumin, alkalialbuminate, nuclein, lecithin, cholesterin, fats (protamin ?), phosphorised fat, salts (2 per cent.), especially phosphates of the alkalies and earths, together with sulphates, carbonates, and chlorides. The odorous body, whose nature is unknown, was called "spermatin" by Vauquelin.

Seminal Fluid.-The sticky, whitish-yellow seminal fluid, largely composed of a mixture of the secretions of the above-named glands, when exposed to the air, becomes more fluid, and on adding water it becomes gelatinous, and from it separate whitish transparent flakes. When long exposed, it forms rhomboidal crystals, which, according to Schreiner, consist of phosphatic salts with an organic base $\left(\mathrm{C}_{2} \mathrm{H}_{5} \mathrm{~N}\right)$. These crystals (fig. 635 ) are said to be derived from the prostatic fluid, and are identical with the so-called Charcot's crystals (fig. 149, $c$, and § 138). The prostatic fluid is thin, milky, amphoteric, or of slightly acid reaction, and is possessed of the seminal olour. The phosphoric acid necessary for the formation of the crystals is obtained from the seminal fluid. A somewhat similar odour occurs in the albumin of eggs not quite fresh. The non-poisonous ptomain, cadaverin (pentamethyldiamin of Ladenburg), isolated by Brieger from dead bodies, has a similar odour. 'The secretion of the vesiculæ seminales of the guinea-pig contains much fibrinogen (p. 376).

The spermatozoa are $50 \mu$ long, and consist of a flattened pear-shaped head (fig. 636,1 and $2, k$ ), which is followed by a rod-shaped middle piece, $m$ (Schweigger-Seidel), and a long tail-like prolongation or cilium, $f$. The spermatozoon is propelled forwards by the to-and-fro movements of the tail at the rate of 0.05 to $0.5 \mathrm{~mm}$. per second; the movement is most rapid immediately after the fluid is shed, but it gradually becomes feebler.

Finer Structure. - The observations of Jensen have shown that the middle piece and head are still more complex, although this is not the case in human spermatozoa and those 
of the bull (G. Retzius). These consist of a flattened, long, narrow, transparent, protoplasmic mass, with a fibre composed of many delicate threads in both margins. At the tip of the tail both fibres unite into one. The fibre of the one margin is generally straight the other is thrown into wave-like folds, or winds in a spiral manner round the other ( $W$. Krause, Gibbes). G. Retzius describes a special terminal filament (fig. 636, e). An axial thread surrounded by an envelope of protoplasm, traverses the middle piece and the tail (Eimer, v. Braun). [Leydig showed that in the salamander there is a delicate membrane attached to the tail, and Gibbes has described a spiral thread attached to the head (newt) and connected with the middle piece by a hyaline membrane. $]$

Motion of the Spermatozoa.-[After the discharge of the seminal fluid, the spermatozoa exhibit spontaneous movements for many hours or days.] The movements are due to the lashing movements of the tail, which moves in a circle or rotates on its long axis, the impulse to movement proceeding from the protoplasm of the middle piece and the tail, which seem to be capable of moving when they are detached (Eimer). These movements are comparable to those that occur in cilia (\$292), and there are transition forms

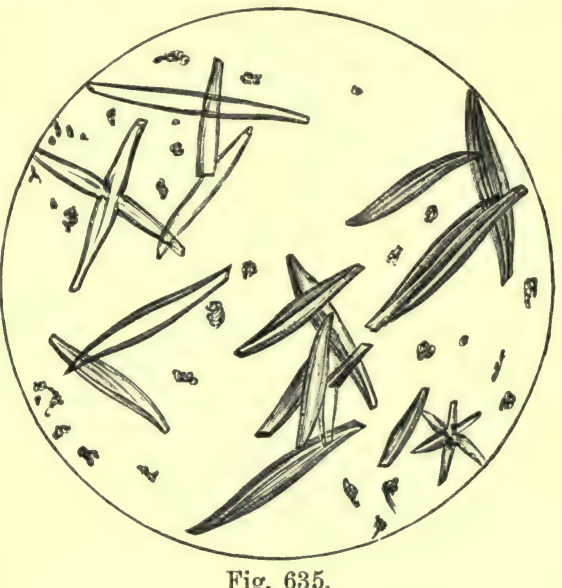

Fig. 635 .

Crystals from spermatic fluid.

between ciliary and amœboid movements, as in the Monera. Reagents.-Within the testis they do not exhibit movement, as the fluid is not sufficiently dilute to permit them to move. Their movements are specially lively in the normal secretion of the female sexual organs (Bischoff), and they move pretty freely, and for a long time, in all normal animal secretions except saliva. Their movements are paralysed by water, alcohol, ether, chloroform, creosote, gum, dextrin, vegetable mucin, syrup of grape-sugar, or very alkaline or acid uterine or vaginal mucus (Donné), acids and metallic salts, and a too high or too low temperature. The narcotics, as long as they are chemically indifferent, behave as indifferent fluids, and so do medium solutions of urea, sugar, albumin, common salt, glycerin, amygdalin, \&c.; but if these be too dilute or too concentrated, they alter the amount of water in the spermatozoa and paralyse them. The quiescence produced bytwater may be set aside by dilute alkalies (Virchow), as with cilia (p. 452). Engelmann finds that minute traces of acids, alcohol, and ether excite movements. The spermatozoa of the frog may be frozen four times in succession without killing them. They bear a heat of $43.75^{\circ} \mathrm{C}$, and they will live for 70 days when placed in the abdominal cavity of another frog (Mantegazza).

Resistance.-Owing to the large amounts of earthy salts which they contain, when dried upon a microscopical slide, they still retain their form (Valentin). Their form is not destroyed by nitric, sulphuric, hydrochloric, or boiling acetic acid, or by caustic alkalies; solutions of $\mathrm{NaCl}$ and saltpetre (10 to 15 per cent.) change them into amorphous masses. Their organic basis resembles the semi-solid albumin of epithelium.

Seminal fluid, besides spermatozoa, also contains seminal cells, a few epithelial cells from the seminal passages, numerous lecithin granules, stratified amyloid bodies (inconstant), granular yellow pigment, especially in old age, leucocytes, and sperma crystals (Fürbinger).

Development of Spermatozoa. - The walls of the seminal tubules, $n$, which are made up of spindle-shaped cells, are lined by a nucleated, protoplasmic layer (fig. $637, I, b$, and IV, $h$ ), from which there project into the lumen of the tube long (0.053 mm.) column-like prolongations (I, $c$, and II, III, IV), which break up at their free end into several round or oval lobules (II.) - the spermatoblasts (v. Ebner) ; these consist of soft finely granular protoplasm, and usually have an oval nucleus in their lower part. During development, each lobule of the spermatoblast elongates into a tail (IV, $r$ ), while the deeper part forms the head and middle pieces of the future spermatozoon (IV, $k$ ). At this stage the spermatoblast is like a greatly enlarged, irregular, cylindrical epithelial cell. When development is complete, the head and middle piece are detached (III, $t$ ), and ultimately the remaining part of the spermatoblast undergoes fatty degeneration. Not unfrequently in spermatozoa we may observe a small mass of protoplasm adhering to the 
tail and the middle piece (III, $t$ ). Between the spermatoblasts are numerous round amceboid cells devoid of an envelope, and connected to each other by processes.
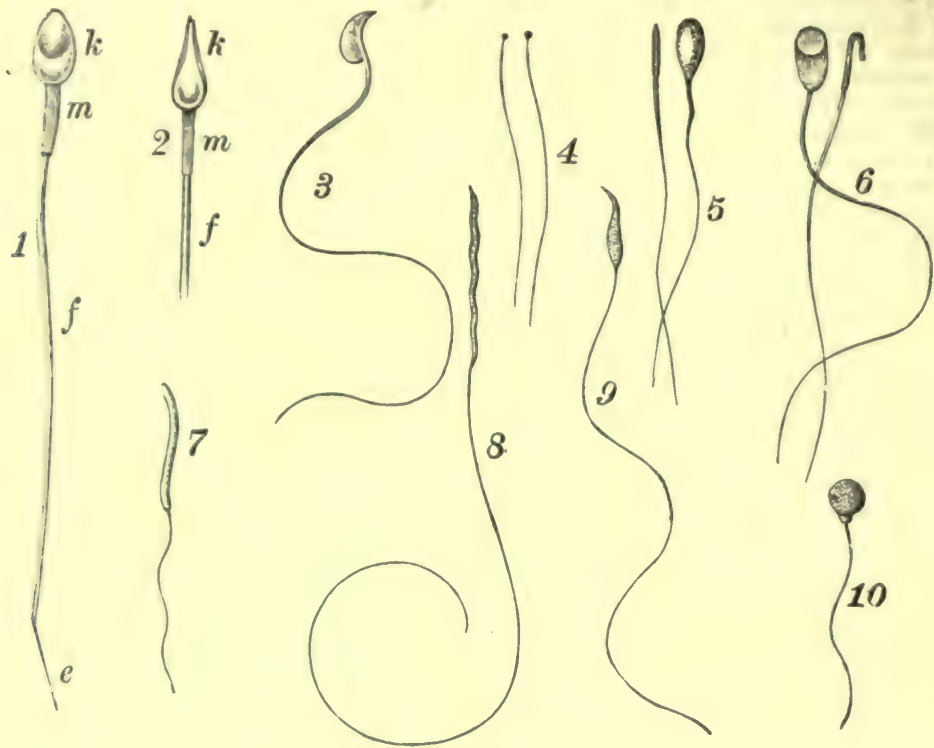

Fig. 636.

Sivermatozoa. 1, human $(\times 600)$, the head seen from the side ; 2 , on edge ; $k$, head ; $m$, middle piece ; $f$, tail ; $c$, terminal filament; 3 , from the mouse; 4 , bothriocephalus latus ; 5 , deer; 6 , mole; 7 , green woodpecker; 8 , black swan; 9 , from a cross between a goldfinch (II) and a canary $(\hat{\mathrm{F}}) ; 10$, from cobitis.

They seem to secrete the fluid part of the semen, and they may therefore be called seminal cells (I, s, II, III, IV, $p$ ). A spermatozoon, therefore, is a detached

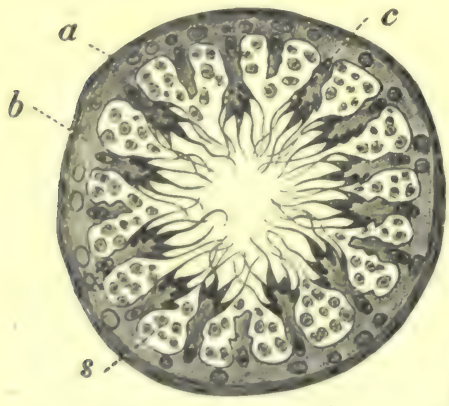

$I$

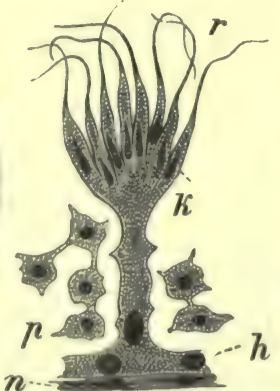

IV

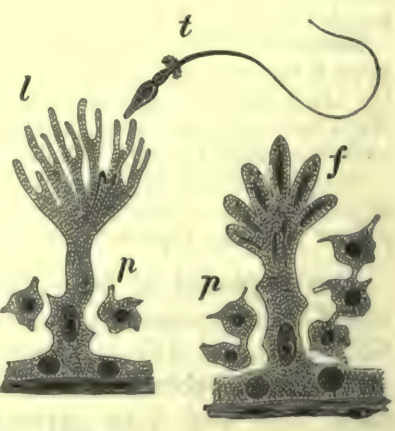

III

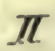

Fig. 637.

Semi-diagrammatic spermatogenesis : I, transverse section of a seminal tubule- $a$, membrane ; $b$, protoplasmic inner lining ; $c$, spermatoblast ; $s$, seminal cells. II, Unripe spermatoblast $-f$, rounded clavate lobules ; $p$, seminal cells. IV, spermatoblast, with ripe spermatozoa (k) not yet detached; tail, $r$; $n$, wall of the seminal tubule; $h$, its protoplasnic layer. III. spermatoblast with a spermatozoon free, $t$.

independently mobile cilium of an enlarged epithelial celt. Some observers adhere 
to the view that the spermatozoa are, in part at least, formed within round cells, by a process of endogenous development.

According to Benda and v. Ebner, the spermatoblasts are formed by the coalescence (copulation) of a group of seminal cells with the lower part of the foot-plate and stalk of the spermatoblasts. Each seminal cell forms from its nucleus the head, and from its protoplasm the tail of a spermatozoon. For the complete formation of these parts, there must be a coalescence of the seminal cells with the spermatoblasts.

Shape.-The spermatozoa of most animals are like cilia with larger or smaller heads. The head is elliptical (mammals), or pear-shaped (mammals), or cylindrical (birds, amphibians, fish), or cork-screw (singing birds, paludina), or merely like hairs (insects-fig. 636). Immobile seminal cells, quite different from the ordinary forms, occur in myriapoda and the oyster.

433. THE OVARY - OVUM UTERUS.-[Structure of the Ovary. - The ovary consists of a connectivetissue framework, with blood-vessels,

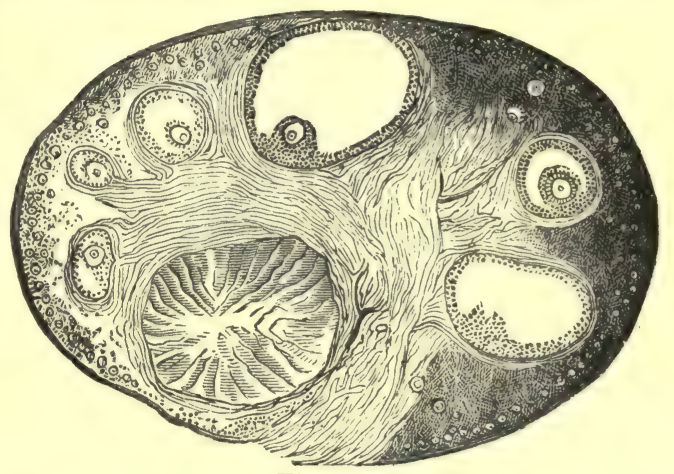

Fig. 638 .

Section of a cat's ovary. The place of attachment or hilum is below. On the left is a corpus luteum. nerves, lymphatics, and numerous non-striped muscular fibres. The ova are embedded in this matrix (fig. 638). The surface of the ovary is covered with a layer of columnar epithelium (fig. $639, e$ ), the remains of the germ-epithelium. The most superficial layer is called the albuginea; it does not contain any ova. Below it is the cortical layer of Schrön, which contains the smallest Graafian follicles ( $\frac{1}{100}$ inch - fig. 638), while deeper down are the larger follicles ( $\frac{1}{30}$ to $\frac{1}{100}$ inch). There are 40,000 to 70,000 follicles in the ovary of a female infant. Each ovum lies within its follicle or Graafian vesicle.]

Structure of an 0vum.The human ovum (C. E. $v$. Baer, 1827) is 0.18 to $0.2 \mathrm{~mm}$. $\left[\frac{1}{120}\right.$ in. $]$ in diameter, and is a spherical cellular body with a thick, solid, elastic envelope, the zona pellucida, with radiating striæ (fig. 640). The zona pellucida encloses the cell-contents represented by the protoplasmic, granular, contractile vitellus or yelk, which in turn contains the eccentrically placed spherical nucleus or germinal vesicle

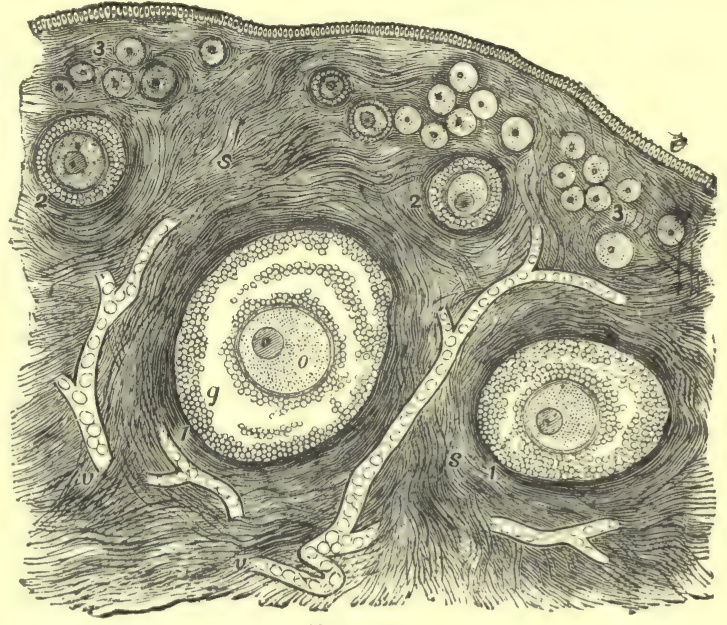

Fig. 639.

Section of an ovary. $e$, germ-epithelium; 1 , large sized follicles; 2,2 , middle sized, and 3,3 , smaller sized follicles; $o$, ovum within a Graafian follicle; $v, v$, blood-vessels of the stroma; $g$, cells of the membrana granulosa. (40-50 $\mu$-Purkinje, 1825; Coste, 1834). The germinal vesicle contains the nucleolus or germinal spot $(5-\tau \mu-R$. Wagner, 1835). The chemical composition is given in $\$ 232$.

[0vum. Cell.

Zona pellucida corresponds to the Cell-wall.

$\begin{array}{llll}\text { Vitellus } & , & \text { Cell-contents. } \\ \text { Germinal vesicle } & , ", & \text { Nucleus. } \\ \text { Germinal spot } & \text { Nucleolus.] }\end{array}$

[This arrangement shows the corresponding parts in a cell and the ovum, and in fact tire ovum represents a typical cell.] 
The zona pellucida (figs. $640,641, \mathrm{~V}, \mathrm{Z}$ ), to which cells the Graafian follicles are often adherent, is a cuticular membrane formed secondarily by the follicle (Pflïger). According to Van Beneden, it is lined by a thin membrane next the vitellus, and he regards the thin membrane as the original cell-membrane of the ovum. The fine radiating striæ in the zona are said to be

Cells of discus proligerus.

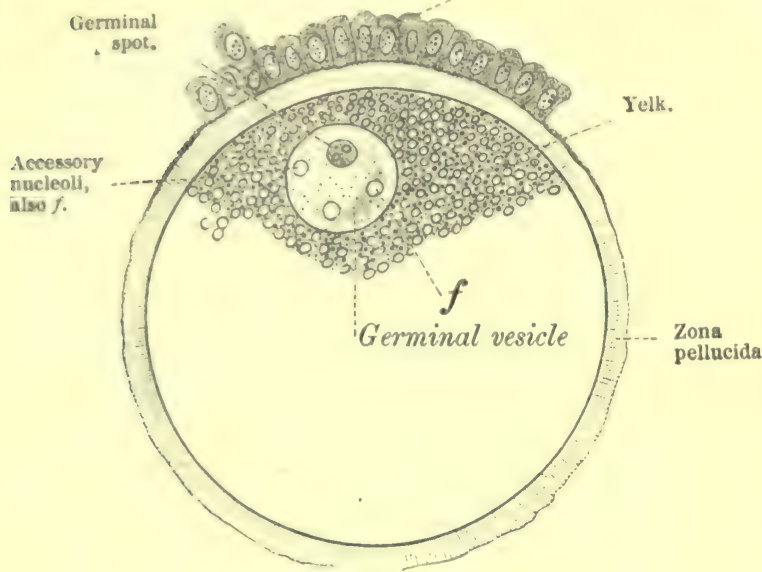

Fig. 640 .

Ripe ovum of rabbit. due to the existence of numerous canals (Kölliker, $v$. Sehlen). It is still undecided whether there is a special micropyle or hole for the entrance of the spermatoza.

A micropyle has been observed in some ova (holothurians, many fishes, mussels). The ova of some animals (many insects, e.g., the flea) have porous canals in some part of their zona, and these serve both for the entrance of the spermatozoa and for the respiratory exchanges in the ovum.

The development of the ova takes place in the following manner:-The surface of the ovary is covered with a layer of cylindrical epitheliumthe so-called "germ-epithelium"- and between these cells lie somewhat spherical "primordial ova" (fig. 641, I, a, a). The epithelium covering the surface dips into the ovary at various places to form "ovarian tubes" (fig. 683). These tubes, from and in which the ova are developed (Waldeyer), become deeper and deeper, and they contain, in their interior, large single spherical cells with a nucleus and a nucleolus, and other smaller and more numerous cells lining the tube. The large cells are the cells (primordial ova) that are to develop into ova, while the smaller cells are the epithelium of the tube, and are direct continuations of the cylindrical epithelium on the surface of the ovary. The upper extremities of the tubes become closed, while the tube itself is divided into a number of rounded compartments-snared off, as it were, by the ingrowth of the ovarian stroma (I, c). Each compartment so snared off usually contains one, or at most two, ova (IV, o, o), and becomes developed into a Graafian follicle. The embryonic follicle enlarges, and fluid appears within it ; while its lateral small cells become changed into the epithelium lining the Graafian follicle itself, or those of the membrana granulosa. The cells of the membrana granulosa form an elevation at one part-the discus proligerus -by which the ovum is attached to the membrana granulosa. The follicles are at first only $0.03 \mathrm{~mm}$. in diameter, but they become larger, especially at puberty. [The smaller ova are near the surface of the ovary, the larger ones deeper in its substance (fig. 639).] When a Graafian follicle with its ovum is about to ripen (IV), it sinks or passes downwards into the substance of the ovary, and enlarges at the same time by the accumulation of fluid-the liquor folliculi-between the tunica and membrana granulosa. It is covered by a vascular outer membranethe theca folliculi-which is lined by the epithelium constituting the membrana granulosa (IV, g). When a Graafian follicle is about to burst, it again rises to the surface of the ovary, and attains a diameter of 1.0 to $1.5 \mathrm{~mm}$., and is now ready to burst and discharge its ovum. [The tissue between the enlarged Graafian follicle and the surface of the ovary gradually becomes thinner and thinner and less vascular, and at last gives way, when the ovum is discharged and caught by the 
fimbriated extremity of the Fallopian tube embracing the ovary, so that the ovum is shed into the Fallopian tube itself.] Only a small number of the Graafian follicles undergo development normally, by far the greatest number atrophy and never ripen. (The study of the development of the ova and ovary was advanced particularly by Martin Barry, Pfliuger, Billroth, Schrön, His, Waldeyer, Kölliker, Koster, Lindgren, Schulin, Foulis, Balfour, and others.)

According to Waldeyer, the mammalian ovum is not a simple cell, but a compound structure. The original primitive ovum is, according to him, formed only of the germinal vesicle and germinal spot, with the surrounding membranous clear part of the vitellus (fig. 641, III). The remainder of the vitellus is developed by the transformation of granulosa cells, which also form the zona pellucida.

Holoblastic and Meroblastic 0va. - The ova of frogs and cyclostomata have the same type as mammalian ova; they are called holoblastic ova, because all their contents go to form cells which take part in the formation of the embryo. In contrast with these, the birds, the monotremes alone amongst the mammals (Caldwell), the reptiles and the other fishes have meroblastic

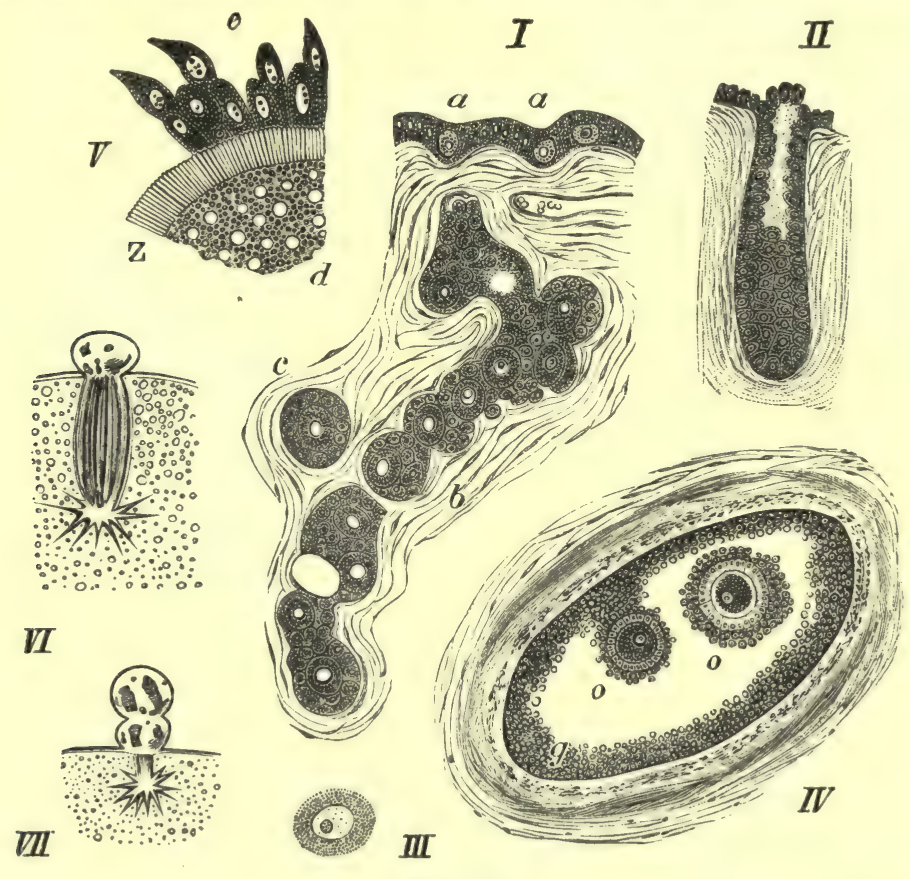

Fig. 641.

I, An ovarian tube in process of development (new-born girl). $\alpha, \alpha$, young ova between the epithelial cells on the surface of the ovary; $b$, the ovarian tube with ova and epithelial cells ; $c$, a small follicle cut off and enclosing an ovum. II, Open ovarian tube from a bitch. III, Isolated primordial ovum (human). IV, Older follicle with two ova $(0,0)$ and the tunica granulosa $(g)$ of a bitch. V, Part of the surface of a ripe ovum of a rabbit $\_z$, zona pellucida; $d$, vitellus; $e$, adherent cells of the membrana granulosa. VI, First polar globule formed. VII, Formation of the second polar globule $(\vec{F} \circ)$.

ova (Reichert). The latter, in addition to the white or formative yelk, which corresponds to the yelk of the holoblastic eggs, and gives rise to the embryonic cells, contains the food-yelk (yellow in birds), which during development is a reserve store of food for the developing embryo.

Hen's Egg.-The small, white, round, finely granular speck, the cicatricula, blastoderm, or tread, which is $2 \cdot 5-3.5 \mathrm{~nm}$. broad and $0.28-0.37$ thick, lying upon the surface of the yellow yelk, corresponds to the contents of the mammalian ovum, and is, therefore, the formative yelk. In the cicatricula lie the germinal vesicle and spot (fig. 642). From the tread in which lie the 
characteristic white yelk elements, processes pass into the yellow yelk. A part passes as an exceedingly thin layer round the yelk, or cortical protoplasm. [The cicatricula in an unincubated egg is always uppermost whatever the position of the egg, provided the contents can rotate freely, and this is due to the lighter specific gravity of that part of the yelk in connection with the cicatricula. In a fecundated egg the cicatricula has a white margin (the area opaca), surrounding a clear transparent area, the beginning of the area pellucida, containing an opaque

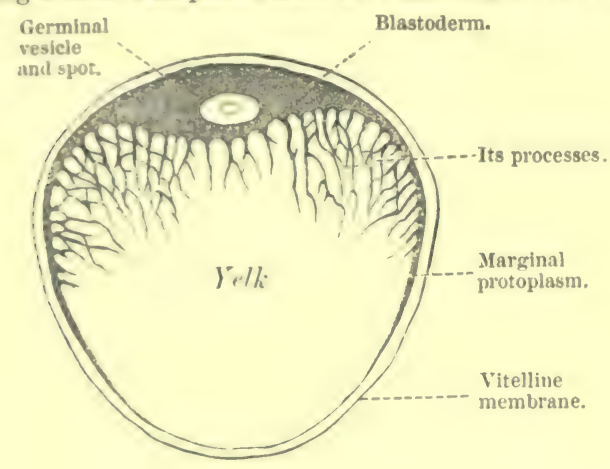

Fig. 642.

Scheme of a meroblastic egg.

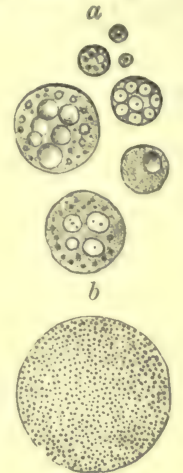

Fig. 643 .

$a$, White; $b$, yellow yelk granules. spot in its centre. If an exg he boiled very hard and a section made of the yelk, it will be found to consist of alternating lityers of white and yellow yelk. The outermost layer is a thin layer of white yelk, which is slightly thicker at the margin of the cicatricula. Within the centre of the velk in a llask-shaped mass of white yelk, the neek of the flask being connected with the white yelk outside. This flask-shaped mass does not become so hard on being boiled, and its upper expamial end is known as the "nucleus of Pander." The great mass of the yelk is made uy, however, of yellow yelk.] Microscopically, the yellow yelk consists of soft yellow spheres, of from $23-100 \mu$ in diameter, and they are often polyhedral from mutual pressure (fig. $643,1, \%$. [They are very delicate and non-nucleated, but fillerl with fine granules, which are, perhap's, proteid in their nature, as they are insoluble in ether and alcohol. They are developed by the proliferation of the granulosa cells of the Graafian follicle, which also seem ultimately to form the granulo-fibrous luuble envelope or the vitelline membrane (Eimer). The whole yelk of the hen's egg is regarded by some observers as equivalent to the mammalian ovum plus the

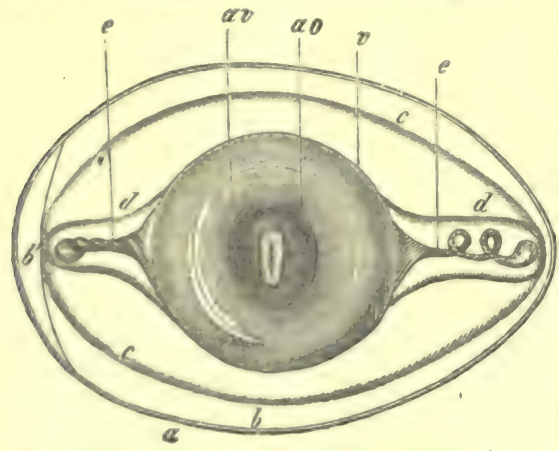

Fig. 644 . corpus luteum. Microscopically, the white yelk consists of small vesicles $(5-75 \mu)$ containing a refractive substance and larger spheres containing several smaller spherules (fig. $643, a$ ). The whole yelk is enveloped by the vitelline membrane, which is transparent, but possesses a fine fibrous structure, and it seems to be allied to elastic tissue.]

When the yelk is fully developed within the Graafian follicles of the hen's ovarium, the follicle bursts and discharges the yelk, which passes into the oviduct, where in its passage it rotates, owing to the direction of the folds of the mucous membrane of the oviduct. The numerous glands of the oviduct secrete the albumin, or white of the egg, which is deposited in layers around the yelk in its passage along the duct, and forms at the anterior and posterior chalazae. [The chalazae are two twisted cords

Fowl's egg after thirty hours incubation. shell; $b$, shell-membrane ; $b^{\prime}$, air-chamber; composed of twisted layers of the outer denser $c$, boundary between outer and middle portion part of the albumin. They extend from the

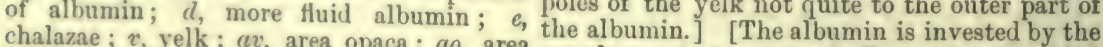
vasculosa, and in its centre is the embryo. is composed of two layers-an outer thicker and an inner thinner one (fig. 644). Over the greater part of the albumin these two layers are united, but at the broad end of the hen's egg they tend to separate, and air passing through 
the porous shell separates them more and more as the fluid of the egg evaporates. This airspace is not found in fresh-laid eggs.] The layers consist of spontaneously coagulated keratinlike fibres arranged in a spiral manner around the albumin (Lindvall and Hamarsten). [External to this is the test, or shell, which consists of an organic matrix impregnated with lime salts.] The shell consists of albumin impregnated with lime salts, which form a very porous mortar. [The shell is porous, and its inner layer is perforated by vertical canals, through which the respiratory exchange of the gases can take place.] In the eggs of some birds there is an outer structureless, porous, slimy, or fatty cuticula. The shell is secreted in the lower part of the oviduct. The shell is partly used up for the development of the bones of the chick (Prout, Gruve, although this is denied by Polt and Preyer). The pigment which often occurs in many layers of the surface of the eggs of some birds appears to be a derivative of hæmogloblin and biliverdin.

Chemical Composition. - The yellow yelk is alkaline, and coloured yellow owing to the presence of lutein, which contains iron. It contains several proteids [including a globulin body called vitellin (p. 376)], a body resembling nuclein, lecithin, vitellin, glycerin-phosphoric acid, cholesterin, olein, palmitin, dextrose, potassic chloride, iron, earthy phosphates, fluoric and silicic acids. The presence of cerebrin, glycogen, and starch is uncertain. [Dareste states that starch is present.]

[The albumin of egg contains-water, 86 per cent.; proteids, 12 ; fat and extractives, 1.5; saline matter, including sodic and potassic chlorides, phosphates, and sulphates, 5 per cent. ]

[The uterus, a thick hollow muscular organ, is covered externally by a serous coat, and lined internally by a mucous membrane, while between the two is the thick muscular coat composed of smooth muscular fibres arranged in a great number of layers and in different directions. The mucous membrane of the body of the uterus in the unimpreguated condition hasnofolds, while the muscularis mucosæ is very well developed, and forms a great part of uterine muscular wall. The mucous membrane is lined by a single layer of columnar ciliated epithelium. A vertical section shows the mucous membrane to contain 'numerous tubular glands (fig. 645 ) - the uterine glands - which branch towards their lower ends. They have a membrana propria, and are lined by a single layer of ciliated epithelium, a small lumen being left in the centre. The utricular glands are not formed during intra-uterine life (Turner), nor are there any glands in the human uterus at birth (G. J. Engelmann). There are. numerous slit-like lymphatic spaces in the mucous membrane (Leopold), which communicate with well-marked lymphatic vessels existing in this and the other layers of the organ. In the cervix, the mucous membrane is folded, presenting in the virgin the appearance known as the arbor vitæ. The external surface of the vaginal part of the neck is covered by stratified squamous epithelium, like the vagina.]

[The Fallopian tubes are really the ducts of the ovaries (fig. 646). They

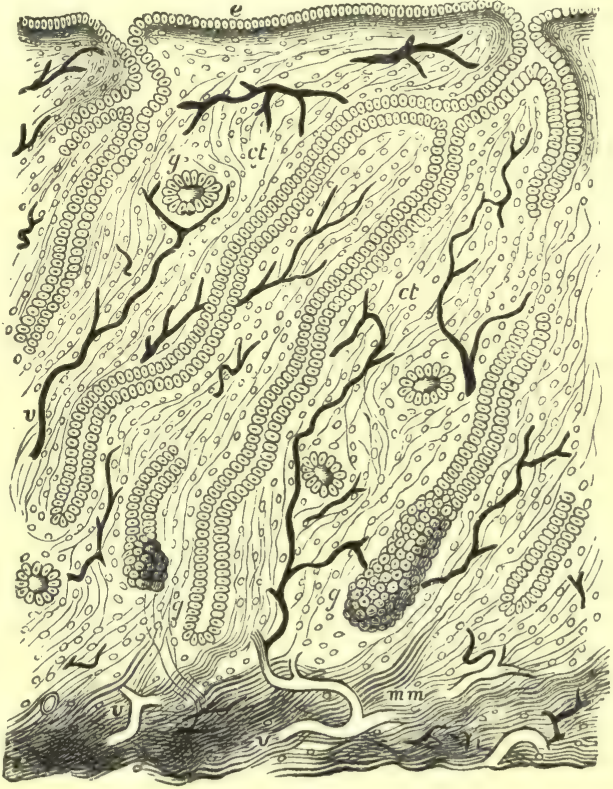

Fig. 645 .

Vertical section of the mucous membrane of the human uterus. e, columnar epithelium, the cilia absent; $g g$, utricular glands ; $c t$, intra-glandular connectivetissue ; $v v$, blood-vessels ; $m m$, muscularis mucosæ. consist of a serous, museular (an external, longitudinal, and an internal circular) layer of nonstriped muscle, and a mucous layer thrown into many folds and lined by a single layer of ciliated columnar epithelium, but no glands (fig. 647).

434. PUBERTY.-The term puberty is applied to the period at which a human being becomes capable of procreating, which occurs from the 13 th to 15 th years in the female, and the 14 th to 16 th in the male. In warm climates, puberty may occur in girls even at 8 years of age. Towards the 40 th to 50 th year, the procreative faculty ceases in the female with the cessation of the menses; this con- 
stitutes the menopause or grand climacteric, whilst in man the formation of seminal fluid has been observed up to old age. From the period of puberty

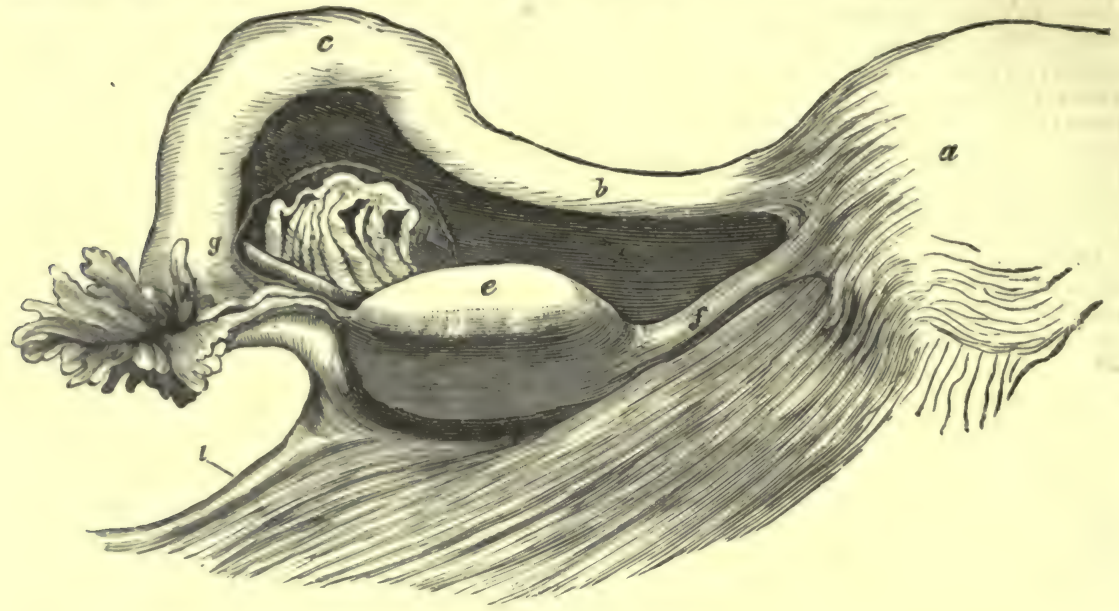

Fig. 646.

Left broad ligament, Fallopian tube, ovary, and parovarium. $a$, uterus ; $b$, isthmus of Fallopian tube; $c$, ampulla ; $y$, fimbriated end of the tube, with the parovarium to its right; $c$, ovary ; $f$, ovarian ligament.

nnwards, the sexual appetite occurs, and the ripe ova are discharged from the nvary. [But ova are discharged even before puberty or menstruation has occurred.]

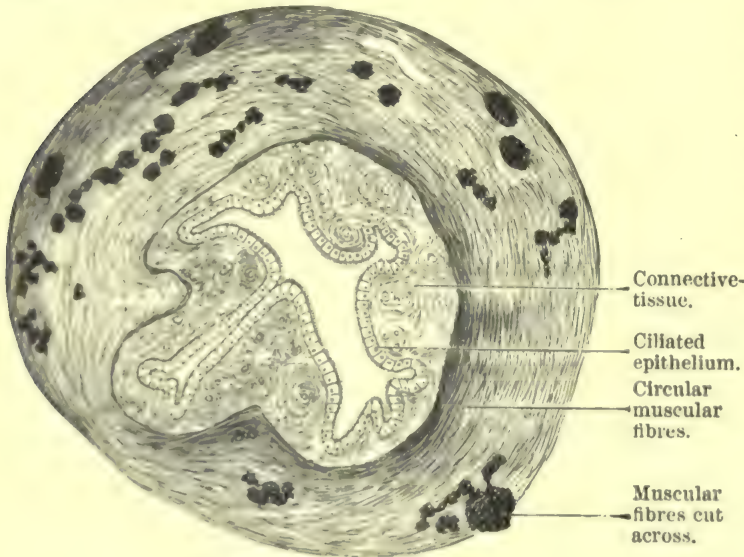

Fig. 647 .

Transverse section of the Fallopian tube.

At puberty, the internal and external generative organs and their annexes become more vascular and undergo development; the pelvis of the female assumes the characteristic female shape. For the changes in the mammæ see $\S 230$. At the same time hair is developed on the pubes and axilla, and in the male on the face, while the sebaceous glands become larger and more active.

Other changes occur, especially in the larynx. In the boy the larynx elongates in its antero-posterior diameter, the thyroil, or Adam's apule, becomes more prominent, while the vocal cords lengthen, so that the voice is hoarse, or lusky, or "breaks," the voice being lowered at least an octave. In the female the larynx becomes longer, while the comprass of the voice is increased. The vital capacity (\$ 108), corresponding to the increase in the size of the chest, undergoes a considerable increase ; the whole form and expression assume the characteristic sexual appearance, while the psychical energies also receive an impulse.

435. MENSTRUATION. - External Signs. - At regular intervals of time, of $27 \frac{1}{3}-28$ days in a mature female, there is a rupture of one or more ripe Graafian follicles, while at the same time there is a discharge of blood from the 
external genitals. This is known as the process of menstruation (or menses, catamenia, or periods). Most women menstruate during the first quarter of the moon, and only a few at new and full moon $(S t r o h l)$. In mammals, the analogous condition is spoken of as the period of heat [or the "rut" in deer]. There is a slightly bloody discharge from the external genitals in carnivora, the mare, and cow (Aristotle), while apes in their wild condition have a well-marked menstrual discharge (Neubert). [Observations on cases where abdominal section has been performed have shown that the Graafian follicles mature and burst at any time (Lawson Tait, Leopold).]

The onset of menstruation is usually heralded by constitutional and local phenomenathere is an increased feeling of congestion in the internal generative organs, pain in the back and loins, tension in the region of the uterus and ovaries, which are sensitive to pressure fatigue in the limbs, alteruate feeling of heat and cold, and even a slight increase of the temperature of the skin (Kersch). There may be retardation of the process of digestion and variations in the evacuation of the freces and urine, and in the secretion of sweat. The discharge is slimy at first, and then becomes bloody, lasting three to four days; the blood is venous, and shows little tendency to coagulate, provided it is mixed with much alkaline mucus from the genital passages; but, if the hæmorrhage be free, the blood may be clotted. The quantity of blood is 100 to 200 grms. [The blood contains many white blood-corpuscles and epithelial cells.] After cessation of the discharge of blood there is a moderate amount of mucus given off.

The characteristic internal phenomena which accompany menstruation are:-(1) The changes in the uterine mucous membrane; and (2) the rupture of the Graafian follicle.

1. Changes in the uterine mucous membrane.-The uterine mucous membrane is the chief source of the blood. The ciliated epithelium of the congested, swollen, and folded, soft, thick ( 3 to $6 \mathrm{~mm}$.) mucous membrane is shed. The orifices of the numerous mucous glands of the mucous membrane are distinct, the glands enlarge, and the cells undergo fatty degeneration, and so do the tissue and the blood-vessels lying between the glands. The tissue contains more leucocytes than normal. This fatty degeneration and the excretion of the degenerated tissue occur, however, only in the superficial layers of the mucosa, whose

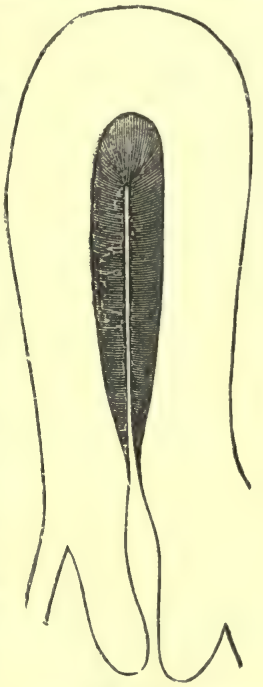

Fig. 648 .

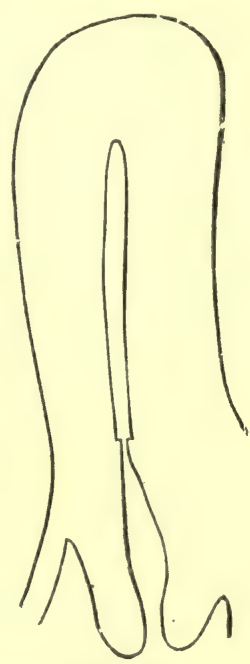

Fig. 649 .

Fig. 648.-Diagram of the uterus just before menstruation. The shaded portion represents the mucous membrane. Fig. 649.Uterus when menstruation has just ceased, showing the cavity of the body deprived of mucous membrane (J. Williams). blood-vessels, when torn across, yield the blood. The deeper layers remain intact, and from them, after menstruation is over, the new mucous membrane is developed (Kundrat and G.J. Engelmann). [Leopold denies the existence of this fatty degeneration. According to Williams, the entire mucous membrane is removed at each menstrual period, and it is regenerated from the muscular coat (fig. 649). The mucous membrane of the cervix remains free from these changes.]

2. Ovulation.-The second important internal phenomenon is ovulation, in which process the ovary becomes more vascular-the ripe follicle is turgid with fluid, and in part projects above the surface of the ovary. The follicle ultimately bursts, its membranes and the epithelium covering of the ovary are torn or give way under the pressure, the bursting being accompanied by the discharge of a small amount of blood. At the same time, the congested, turgid, and erected fimbriated extremity of the Fallopian tube is applied to the ovary, so that the 
discharged ovum, with its adherent granulosa cells, and the liquor folliculi, are caught by the funnel-shaped extremity of the tube (fig. 646). The ovum, when discharged, is carried towards the uterus by the ciliated epithelium $(\$ 433)$ of the tube, and perhaps also partly by the contraction of its muscular coat. Ducalliez and Kiiss found that, by fully injecting the blood-vessels, they could imitate the erection of the Fallopian tube. Rouget points out that the non-striped muscle of the broad ligaments may cause constriction of the vessels, and thus secure the necessary injection of the blood-vessels of the Fallopian tube.

Pfliger s Theory. - There are two theories as to the connection between ovulation or the discharge of an ovum and the escape of blood from the uterine mucous membrane. Pfliger renaris the hlooly discharge from the superficial layers of the uterine mucous membrane as a physiologieal preparation of "freshening" of the tissue (in the surgical sense), by which it will h. prepared to receive the ovmu when the latter reaches the uterus, so that union can take place hetween the orum and the freshly-exposed surface of the mucous membrane, and thus the ovum will receive nourishment from a new surface.

Reichert's Theory.- This view is opposed to that of Reichert, Engelmann, Williams, and wethers. According to Reichert's theory, before an ovum is discharged at all there is a sympathet $i$, change in the uterine nucous membrane, whereby it becomes more vascular, more -pengy, and swollen up. The mucous membrane so altered is spoken of as the membrana deciituin menstrualis, and from its nature it is in a proper condition to receive, retain, and murish a fertilisel ovum which may come into contact with it. If the ovum, however, be not frrtilisul, am escape from the genital passages, then the uterine mucous membrane degenerates, and blood is shed as above described. According to this view, the hremorrhage from the

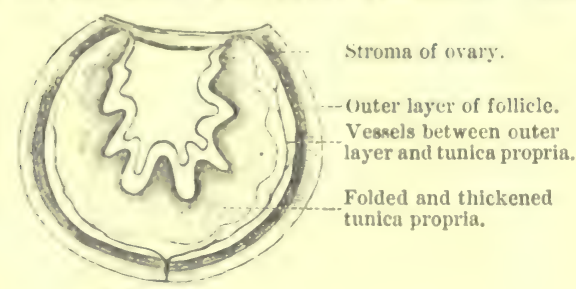

Fig. 650 .

Fresh corpus liteum.

tion without ovulation, and ovulation without menstruation.

Corpus Luteum. - When a Graatian follicle bursts, it discharges its contents and collapses ; in the interior are the remains of the membrana granulosa and a small effusion of blood, which

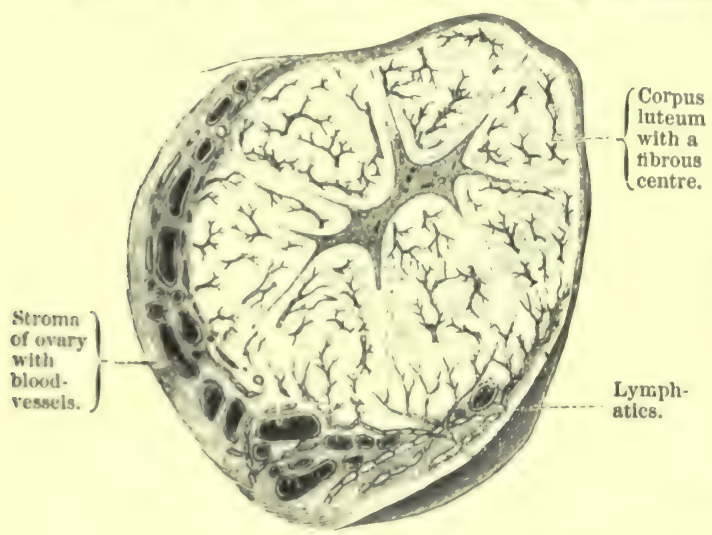

Fig. 651. soon coagulates. The small rupture soon heals, after the serum is absorbed. The vascular wall of the follicle swells up. Villous prolongations or granulations of young connective-tissue, rich in capillaries and cells, grow into the interior of the follicle (fig. 651). Colourless blood-corpuscles also wander into the interior. At the same time the cells of the granulosa proliferate, and form several layers of cells, which ultimately,

Corpus luteum of $\operatorname{cow}\left(\times 1 \frac{1}{3}\right)$.
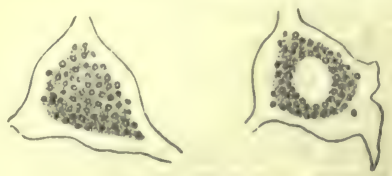

Fig. 652 .

Lutein cells from the corpus luteum of cow. after the disappearance of a number of blood-vessels, undergo fatty degeneration, lutein, and fatty matter being formed, and it is this mass which gives the corpus luteum its yellow colour (fig. 
652). The capsule becomes more and more fused with the ovarian stroma. If pregnancy does not take place after the menstruation, then the fatty matter is rapidly absorbed, and the effused blood is changed into hæmatoidin $(\$ 20)$ and other derivatives of hæmoglobin, while there is a gradual shrivelling of the whole mass, which is complete in about four weeks, only a very small remainder being left. Such a corpus luteum, i.e., one not accompanied by pregnancy, is called a false corpus luteum. If, however, pregnancy occurs, then the corpus luteum, instead of shrivelling, grows and becomes a large body, especially at the third and fourth month, the walls are thicker, the colour deeper, so that the corpus luteum at the period of delivery may be 6 to $10 \mathrm{~mm}$. in diameter, and its remains may be found in the ovary for a very long time thereafter (fig. 651). This form is sometimes spoken of as a true corpus luteum. [We cannot draw a sharp distinction between these two forms.] Only a very small number of the ova in the ovary undergo development and are discharged; by far the greater number degenerate (Slavjansky).

436. PENIS-ERECTION.-Penis.-[The penis is composed of the two long cylindrical corpora cavernosa, the corpus spongiosum, which lies between and below them, and surrounds the urethra; these are held together by fibrous and muscular sheaths, and are composed of erectile tissue.] Our knowledge of the distribution of the blood within the penis is chiefly due to C. Langer's researches. The albuginea of the corpus spongiosum consists of tendinous connective-tissue, containing thickly-woven elastic tissue and smooth muscular fibres, which together form a solid fibrous envelope, from which numerous interlacing trabeculæ pass into the interior, so that the corpus spongiosum comes to resemble a sponge. The anastomosing spaces bounded by these trabeculæ form a series of inter-communicating venous spaces or sinuses filled with blood and lined by a layer of endothelium constituting erectile tissue (fig. 653). The largest sinuses lie in the lower and external part of the corpus cavernosum, while they are less numerous and smaller in the upper part. The small arteries arise from the A. profunda penis, which runs along the septum, and pass to the trabeculæ after following a very sinuous course. At the outer part of the corpus spongiosum, some of the small arteries become directly continuous with the larger venous sinuses; some of them, however, terminate in capillaries both in the outer part and within the corpus spongiosum, the capil. laries ultimately terminating in the venous sinuses. The helicine arteries of the penis described by Joh. Müller are merely much twisted arteries. The deep veins of the penis arise by fine veinlets within the body of the organ,

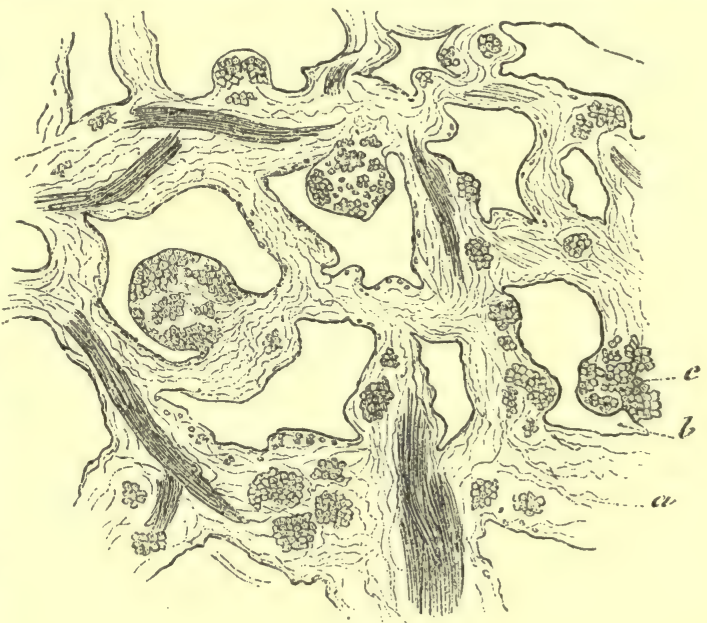

Fig. 653. while the veins proceeding from

Erectile tissue. $a$, trabeculæ of connective-tissue with elastic fibres and smooth muscle $(c) ; b$, venous spaces.

the cavernous spaces pass to the dorsum of the penis to form the vena dorsalis penis. As these vessels have to traverse the meshes of the vascular network in the cortex of the corpora cavernosa penis, it is evident that, when the network is congested by being filled with blood, it must compress the outgoing venous trunks. The corpus cavernosum urethræ consists for the most part of an external layer of closely packed anastomosing veins, which surround the longitudinally directed blood-vessels of the urethra.

In the dog, all the arteries of the penis run at first towards the surface, where they divide into penicilli. The veins arise from the capillary loops in the papillæ, and they empty their blood into the cavernous spaces. Only a small part of the blood passes to the cavernous spaces through the internal capillaries and veins, but arterial blood never flows directly into these spaces (M. v. Frey).

Mechanism of Erection.-Erection is due to the overfilling of the bloodvessels of the penis with blood, whereby the volume of the organ is increased four or five times, while, at the same time, there are also a higher temperature, increased blood-pressure (to $\frac{1}{6}$ of that in the carotid-Eckhard), with at first a pulsatile movement, increased consistence, and erection of the organ. 
Regner de Graaf obtained complete erection of the penis by forcibly injecting its bloodvessels (1668).

The preliminary phenomena consist in a considerable increase of the arterial blood-supply, the arteries being dilated and pulsating strongly. The arteries are controlled by the nervi erigentes. The nervi erigentes [called by Gaskell the pelvic sulanchnics (fig. 439] arise chiefly from the second (more rarely the third) sacral nerves (dog), and have ganglionic cells in their course (Loven, Nikolsky). These nerves contain vaso-dilator fibres, which can be excited in part reflexly from the sensory nerves of the penis, the transference centre being in the centre for erection in the spinal cord $(\$ 372,4)$. Sensory impressions produced by voluntary movements of the genital apparatus (by the ischio- and bulbo-cavernosi

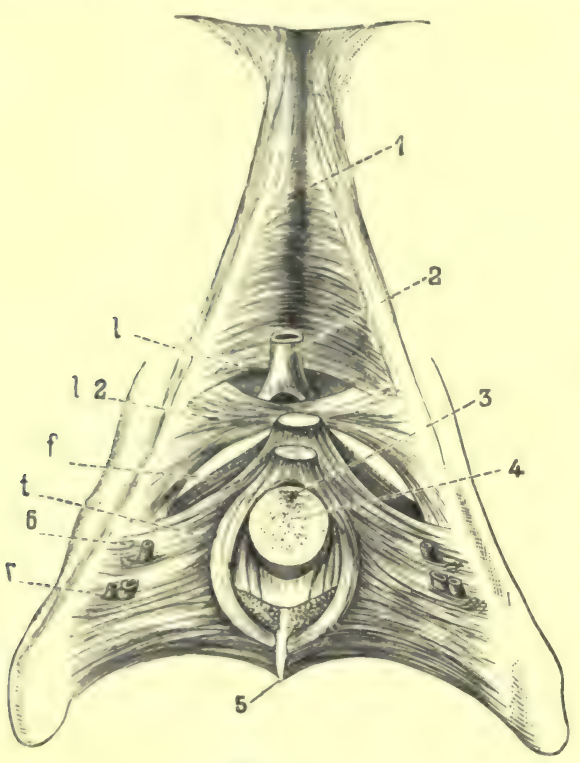

Fig. 654.

Anterior wall of the jelvis with the urogenital septum seen from the front. The corpus cavernosum (4) with the urethra (3) is cut across below its exit from the pelvis. 1, symphysis pubis; 2, dorsal vein of the penis; 5 , part of the bulbocavernosus; $t$, deep transversus perinei with its fascia $(f) ; 6$, vena profunda penis ; 7 , artery and vein of the bulbo-cavernosus. and cremaster muscles) can also discharge this reflex; while the thought of sexual impulses, referable to the penis, tends to induce erection. The nervi erigentes also supply the longitudinal fibres of the rectum (Fellner).

The centre for erection in the spinal cord $(\$ 362,2)$ is, however, controlled by the dominating vasodilator centre in the medulla oblongata ( $\$ 372)$, and the two centres are connected by fibres within the cord; hence stimulation of the upper part of the cord, as by asphyxiated blood $(\$ 362,5)$ or muscarin, may also be followed by erection(Nikolsky). [The seminal fluid is frequently found discharged in persons who have been hanged.]

The psychical activity of the cerebrum has a decided influence on the genital vaso-dilator nerves. Just as the psychical disturbance which accompanies anger or shame is followed by dilatation of the blood-vessels of the head, owing to stimulation of the vaso-dilator fibres, so when the attention is directed to the sexual centres there is an action upon the nervi erigentes. This action of the brain is more comprehensible, since we know that the diameter of the blood-vessels is affected by the cortex cerebri ( $\$ 377)$. The fibres probably pass from the cerebrum through the peduncles of the cerebrum and the pons; as a matter of fact, if these parts be stimulated erection may take place $(\S 362,4)$ (Eckhard).

When the impulse to erection is obtained by the increased supply of arterial blood, the full completion of the act is brought about by the activity of the following transversely striped muscles:-(1) The ischio-cavernosus arises from the coccyx, and by its tendinous union surrounds the root of the penis (fig. 172). When it contracts, it compresses the root of the penis from above and laterally, so that the outflow of blood from the penis is hindered. It has no action on the dorsal vein of the penis, as this vessel lies in a groove on the dorsum of the penis, and is therefore protected from compression by the tendon. (2) The deep transversus 
perinei is perforated by the venæ profundæ penis, which come from the corpora cavernosa, so that when it contracts it must compress these veins between the tense horizontal fibres (fig. 654,6). The deep veins of the penis join the common pudendal vein and the plexus Santorini. (3) Lastly, the bulbo-cavernosus is concerned in the hardening of the urethral corpus spongiosum, as it compresses the bulb of the urethra (figs. 654,5, 172). All these muscles are partly under the control of the will, whereby the erection may be increased. Normally, however, their contraction is excited reflexly by stimulation of the sensory nerves of the penis $(\$ 362,4)$.

The congestion of blood is not complete, else, in pathological cases, continuous erection, as in satyriasis, would give rise to gangrene. The accumulation of the blood in the penis is favoured by the fact that the origins of the veins of the penis lie in the corpus cavernosum, which, when it enlarges, must compress them. There are also trabecular smooth muscular fibres, which compress the large venous plexus of Santorini.

That erection is a complex motor act depending on the nervous system, is proved by an experiment of Hausmann, who found that section of the nerves of the penis prevented erection in a stallion. The imperfect erection which occurs in the female is confined to the corpora cavernosa clitoridis and the bulbi vestibuli. During erection, the passage from the urethra to the bladder is closed, partly by the swelling of the caput gallinagiuis, and partly by the action of the sphincter urethræ, which is connected with the deep transversus perinei.

\section{EJACULATION-RECEPTION OF THE SEMEN.-In connection} with the ejaculation of the seminal fluid, we must distinguish two different factors -(1) its passage from the testicles to the vesiculæ seminales; (2) the act of ejaculation itself. The former is caused by the newly secreted fluid forcing on that in front of it, by the action of the ciliated epithelium (which lines the epididymis to the beginning of the vas deferens), and also by the peristaltic movements of the smooth muscular fibres of the vas deferens. Ejaculation, however, requires strong peristaltic contractions of the vasa deferentia and the vesiculæ seminales, which are brought about by the reflex stimulation of the ejaculation centre in the spinal cord $(\S 362,5)$. As soon as the seminal fluid reaches the urethra, there is a rhythmical contraction of the bulbo-cavernosus muscle (produced by the mechanical dilatation of the urethra), whereby the fluid is forcibly ejected from the urethra. Both vasa deferentia and vesiculæ do not always eject their contents into the urethra simultaneously. With moderate excitement the contents of only one may be discharged. The ischio-cavernosus and deep transversus perinei contract at the same time as the bulbo-cavernosus, although the former have no effect on the act of ejaculation. In the female also, under normal circumstances, at the height of the sexual excitement there is a reflex movement corresponding to ejaculation. It consists of a movement analogous to that in man. At first there is a reflex peristaltic movement of the Fallopian tube and uterus, proceeding from the end of the tube towards the vagina, and produced reflexly by the stimulation of the genital nerves. Dembo observed that stimulation of the anterior upper wall of the vagina in animals caused a gradual contraction of the uterus. By this movement, corresponding to that of the vasa deferentia in man, a certain amount of the mucus normally lining the uterus is forced into the vagina.

This is followed by the rhythmical contraction of the sphincter cunni (analogous to the bulbo-cavernosus), also of the ischio-cavernosus, and deep transversus perinei. The uterus is erected by the powerful contraction of its muscular fibres and round ligaments, while at the same time it descends towards the vagina, its cavity is more and more diminished, and its mucous contents are forced out. When the uterus relaxes after the stage of excitement, it aspirates into its cavity the seminal fluid injected into the vestibule (Aristotle, Bischoff).

But the suction of the greatly excited uterus is not necessary for the reception of the semen (Aristotle). The spermatozon may wriggle by their own movements from the vagina into the orifice of the uterus (Kristeller). The cases of pregnancy where from some pathological causes 
(partial closure of the vagina or rulva), the penis has not passed into the vagina during coition, prove that the spermatozoa can traverse the whole length of the vagina, and pass into the uterus.

\section{FERTILISATION OF THE OVUM. - The ovum is fertilised by one spermatozoon passing into it.}

Swanmerdam $(+1685)$ proved that contact of the semen with the ovum was necessary for fertilisation. Spallanzani (1768) proved that the fertilising agent was the spermatozoa, and not the clear filtered fluid part of the semen, and that the spermatozoa, even after being enormously diluted, were still capable of action. Martin Barry (1850) was the first to observe the entrance of a sjermatozoon into the ovum of the rabbit. This occurs pretty rapidly, by a boring movement through the vitelline membrane (Leuckhart). The entrance is effected either through the porous canals or the micropyle (Keber, p. 850).

Theories. $-\mathrm{As}$ to the manner in which the spermatozoon affects the ovum, there are great differences of opinion. Aristotle compared it to an action like that of rennet on milk ; Bischoff, to that of yeast on a fermentable mass, i.e., to a catalytic action. These theories, however, are quite unsatisfactory, as we know that the unfertilised ova of the hen, rabbit (Hensen), pig (Kisichin), salpa (Küppfer) (but not the frog-Pffüger) can undergo the initial stages of development as far as the stage of cleavage, and the star-fishes even as far as the larval form (Greef).

Place of Fertilisation.-The place where fertilisation occurs is either the ovary, as indicated by the occurrence of abdominal pregnancy, or the Fallopian tube, and the numerous recesses in the latter afford a good temporary nidus for the spermatozoa. This view is supported by the occurrence of tubal pregnancy. Thus, the spernatozoa must be able to pass through the Fallopian tube to the ovary, which is probably brought about chiefly by the movements proper to the spermatozoa themselves. It is uncertain whether the peristaltic movements of the uterus and Fallopian tube are concerned in this process ; certainly ciliary movement is not concerned, as the cilia of the Fallopian tube act from above downwards. When once the ovum has passed unfertilised into the uterus, it is not fertilised in the uterus. It is assumed that the ovum reaches the uterus within 2 to 3 weeks (in the bitch, 8 to 14 days).

Twins occur in 1 in 87 preguancies, but oftener in warm climates; triplets, $1: 7600$; four at a birth, $1: 330,000$. More than six at a time have not been observed. The average number of pregnancies in a woman is $4 \frac{1}{2}$.

Superfecundation. - By this term is understood the fertilisation of two ova at the same menstruction, by two different acts of coition. Thus, a mare may throw a foal and a mule, after being covered first by a stallion and then by an ass. A white and a black child have been born as twins by a woman.

Superfœtation is when a second impregnation takes place at a later period of pregnancy, as in the second or third month. This, however, is only possible in a double uterus, or when menstruation persists until the time of the second impregnation. It is said to occur frequently in the hare.

Hybrids are produced when there is a cross between different species (horse, ass, zebra-dog, jackal, wolf-goat, ibex-goat, sheep-species of llama-camel, dromedary-tiger, lion-species of pheasant-goose, swan-carp, crucian-species of butterflies). Most hybrids are sterile, especially as regards the formation of properly formed spermatozoa; while the hybrid females are for the most part fertile with the male of both parents, e.g., the mule; but the characters of the offspring tend to return to those of the species of the parents. Very few hybrids are fertile when crossed by hybrids. In many species of frogs the absence of hybrids is accounted for by the mechanical obstacles to fertilisation of the ova.

Tubal Migration of the 0vum.-Under exceptional circumstances, the ovum discharged from a ruptured Graafian follicle passes into the Fallopian tube of the other side, as is proved by the occurrence of tubal pregnancy and pregnancy of an abnormal rudimentary horn of the uterus, in which case the true corpus luteum is found on the other side of the ovary. This is spoken of as "external migration" (Kussmaul, Leopold). This observation coincides with experiment, as granular fluids, e.g., China-ink, when injected into the peritoneal cavity, pass into both Fallopian tubes, and are carried by the ciliated epithelium to the uterus (Pinner). In animals, with a double uterus with two orifices, the ova may migrate through 
the os of the one into the other uterus, a condition which is spoken of as "internal migration."

439. IMPREGNATION-CLEAVAGE-IAYERS OF THE EMBRYO.Maturation of the 0vum.-In birds and mammals, important changes occur in the ovum before impregnation. The germinal vesicle comes to the surface and disappears from view, while the germinal spot also disappears. In place of the germinal vesicle, a spindle-shaped body appears. The granular elements of the protoplasmic vitellus arrange themselves around each of the two poles of the spindle, in the form of a star, the double-star, or diaster of Fol-nuclear spindle (figs. 655, 656). When this takes place, the peripheral pole of the nucleus or altered germinal vesicle, along with some of the cellular substance of the ovum, protrudes upon the surface of the vitellus, where they are nipped off from the ovum in the form of small corpuscles just like an excretory product (fig. 657). These bodies, which are not made use of in the further development and growth of the ovum, are called polar or directing globules (Fol, Bütschli, O. Hertwig), although the elimination of small bodies from the yelk was known to Dumortier [1837], Bischoff, P. J. van Beneden, Fritz Müller [1848], Rathke, and others. The remaining part of the germinal vesicle stays within the vitellus and travels back towards the centre of the ovum, to form the female proneucleus (O. Hertwig, Fol, Selenka, $E$. van Beneden). [Before, however, the altered germinal vesicle travels downwards again into the substance of the ovum, it divides again as before, and from it is given off the second polar globule, and then the remainder of the germinal vesicle

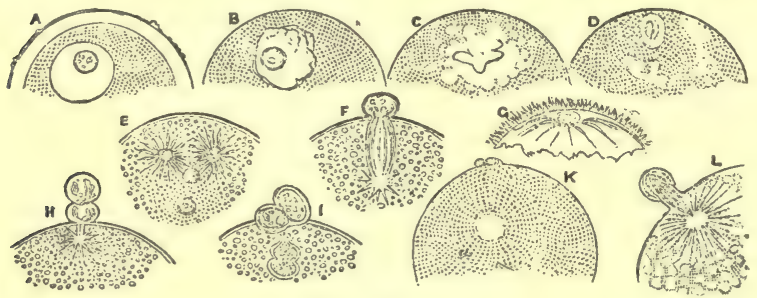

Fig. 655 .

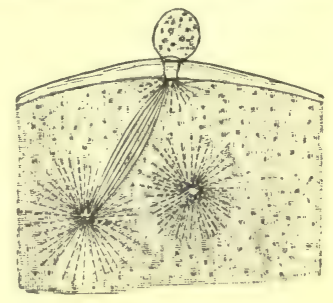

Fig. 656.

Fig. 655. - Formation of polar globules in a star-fish (Asterias glacialis). A, ripe ovum with excentric germinal vesicle and spot; B-E, gradual metamorphosis of germinal vesicle and spot, as seen in the living egg, into two asters ; F, formation of first polar globule, and withdrawal of the remaining part of the nuclear spindle within the ovum; G, surface view of living ovum with view of first polar globule; $\mathrm{H}$, formation of second polar globule; I, a later stage, showing the remaining internal part of the spindle in the form of two clear vesicles; $\mathrm{K}$, ovum with two polar globules and radial striæ around the female pronucleus ; L, extrusion of polar globule. (Geddes: A-K, after Fol; L, after O. Hertviig.) Fig. 656.-Egg of Scorpaena scrofa. The germinal vesicle is extruding a polar globule, and withdrawing towards the centre of the ovum. Near it is the male pronucleus.

forms the female proneucleus (fig. 655). At the same time the vitellus shrinks somewhat within the vitelline membrane.]

Impregnation.-As a rule, only one spermatozoon penetrates the ovum, and as it does so, it moves towards the female pronucleus, while its head becomes surrounded with a star; it then loses its head and eilium, or tail, the latter only serving as a motor organ, while the remaining middle piece swells up to form a second new nucleus, the male pronucleus ( Fol, Selenka). According to Flemming, it is the anterior part of the head, and according to Rein and Eberth, it is the head which is so changed. Thereafter, the male and female pronucleus unite, undergoing amœboid movements at the same time, to form the new nucleus of the fertitised ovum. The female pronucleus receives the male pronucleus in a little depression on its surface. Thereafter the yelk assumes a radiate appearance (Rein). 
[The union of the representatives of the male and female elements forms the first embryonic segmentution sphere or blastosphere, which divides into two cells, and

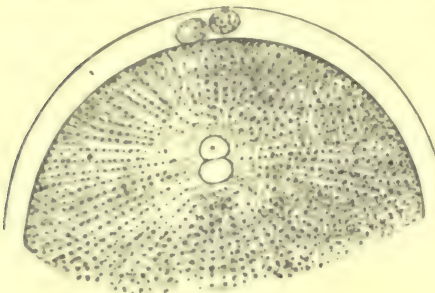

Fig. $65 \%$.

these again into four, and so on (fig. 658).]

In Echinoderms, O. Hertwig and Fol observed that several embryos were formed when, under abnormal conditions, several spermatozoa penetrated an ovum. The male pronuclei, formed from the several spermatozoa, then fused each with a fragment of the female pronucleus. Under similar circumstances, Born observed in amphibians abnormal cleavage, but no further de. velopment.

Cleavage of the Yelk. - In an ovum so fertilised the yelk contracts somewhat around the newly-formed nucleus, so that it becomes slightly Fog of a Star-fish (Asteracanthion) with separated from the vitelline membrane, and for two extruded I rolar globules. Male the first time the nucleus and the yelk divides and female pronuclei near each other. into two nucleated spheres. This process is sploken of as a complete cleavage or fission (fig. 658). Each of these two cells arain divides into two, and the process is repeated, so that $4,8,16,32$, and so on,

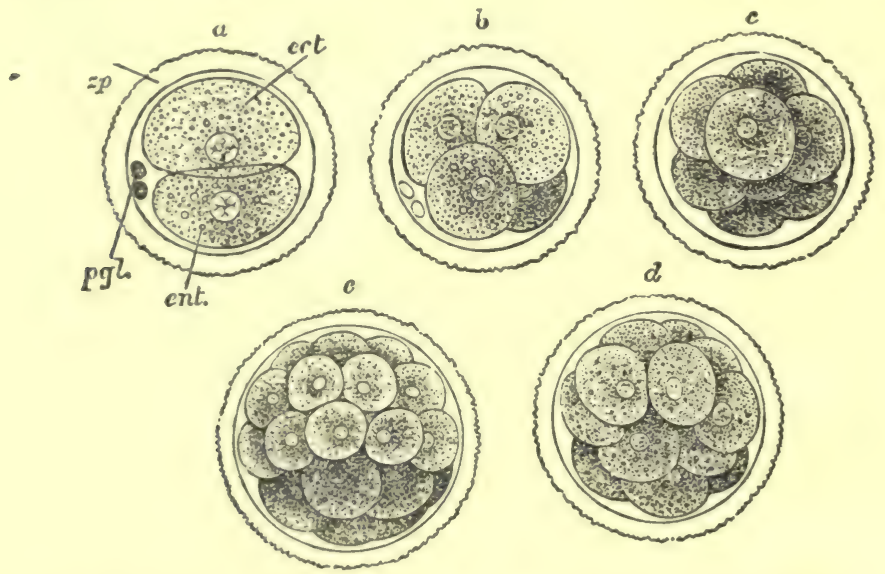

Fig. 658.

Sigmentation of a rabbit's ovum. $a$, two-celled stage; $b$, four-celled stage; $c$, eight-celled stage ; de, many blastomeres showing the more rapid division of the outer-layer cells, and the gradual enclosure of the inner-layer cells ; ect, outer-layer cells ; ent, inner-layer cells ; $p g l$, polar globules; $z p$, zona pellucida.

spheres are formed (fig. 659). This constitutes the cleavage of the yelk, and the process goes on until the whole yelk is subdivided into numerous small, nucleated
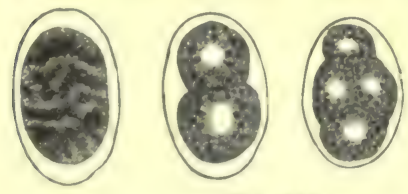

Fig. 659 .

Cleavage of the yelk of the egg of Anchylo. stomum duodenale.

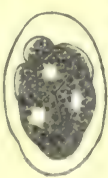

spheres, the "mulberry mass" or "seg. mentation spheres " or "morula," or the protoplasmic primordial spheres (20 to 25 $\mu$ ) which are devoid of an envelope. [Each cell divides by a process of karyokinesis. According to Van Beneden, the segmentation begins in 1-2 hours after the union of the pronuclei, and the process is complete in about 75 hours. These primi-

tive cells, from which all the tissues of the future embryo are formed, are called blastomeres.] 
Variation of Lines of Cleavage.-According to the observations of Pfliger, the ova of the frog can be made to undergo cleavage in very different directions, according to the angle between the axis of the egg and the line of gravitation. This of course we can alter as we please, by placing the eggs at any angle to the line of gravitation. By the axis of the ovum is meant a line connecting the centre of the black surface and the middle of the white part, which, in the fertilised ovum, is always vertical. In such cases of abnormal cleavage the deposition of the organs takes place from other constituents of the egg than those from which they are formed under normal conditions. Under normal circumstances, according to Roux, the first line of cleavage in the frog is in the same direction as the central nervous system. The second intersects the first at a right angle, so as to divide the mass of ovum into two unequal parts, the larger of which forms the anterior part of the embryo.

Blastoderm.-During this time the ovum is enlarging by absorption of fluid into its interior. All the cells, from mutual pressure against each other, become polyhedral, and are so arranged as to form a cellular envelope or bladder, the blastoderm or germinal membrane, which lies on the internal surface of the vitelline membrane (De Graaf, v. Baer, Bischoff, Coste). A small part of the cells not used in the formation of the blastoderm is found on some part of the latter. [In the ovum of the bird, where there is only partial segmentation, the blastoderm is a small round body resting on the surface of the yelk, under the vitelline membrane, so that it does not completely surround the yelk, or a hollow cavity, as

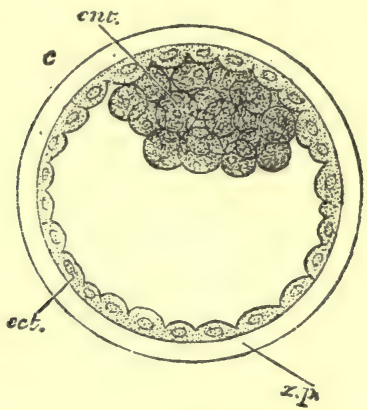

Fig. 660 .

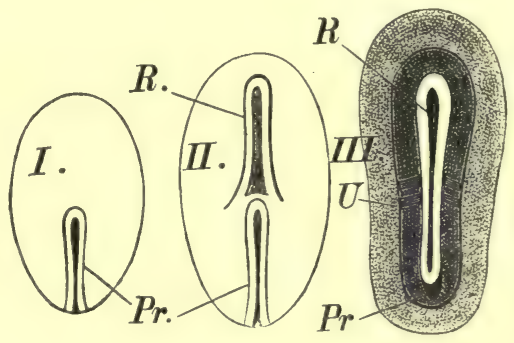

Fig. 661 .

Fig. 660.-Blastodermic vesicle of a rabbit. ect, ectoderm, or outer layer of cells ; ent, inner layer of cells. Fig. $661 .-P r$, prinitive streak ; $R$, medullary groove ; $U$, first protovertebra.

in mammals. In mammals, this cavity is called the segmentation cavity.] The hollow sphere, composed of cells, is called the blastodermic vesicle by Reichert (fig. 660), and in the human embryo it is formed at the 10th to 12 th day, in the rabbit at the 4 th, the guinea-pig at the $3 \frac{1}{2}$, the cat 7 th, dog 11 th, fox 14 th, ruminantia at the 10th to 12 th day, and the deer at the 60 th day.

When the blastoderm grows to $2 \mathrm{~mm}$. (rabbit), whereby the vitelline membrane is distended to a very thin delicate membrane, then at one part of it there appears the germinal area, the area germinativa, or the embryonal shield (Coste, Kölliker), as a round white spot, in which the blastoderm, owing to the proliferation of its cells, becomes double. The upper layer is called the ectoderm or epiblast, and in some animals it consists of several layers of cells, while the lower layer is the endoderm or hypoblast. The hypoblast continues to grow at its edges, so that it ultimately forms a completely closed sac, on which the epiblast is applied concentrically. The embryonal area soon becomes more pear-shaped, and afterwards biscuit-shaped. At the same time the surface of the zona pellucida develops numerous small, hollow, structureless villi, and is called the primitive chorion.

At the posterior part, or narrow end, of the embryonic shield, the primitive streak (fig. 661, I, $P^{r}$ ) appears at first as an elongated opaque circular thickening, and later as a longer streak or groove, the primitive groove. [The opacity is due 
to the fact that there are several layers of cells in this region (fig. 662). In a transverse section through the primitive streak, three layers of cells are seen. They form part of the middle layer or mesoblast, and are originally derived from the hypoblast. These cells fuse with those of the epiblast. The remainder of the hypoblastic cells retain their steliate character.] At the same time a new layer of

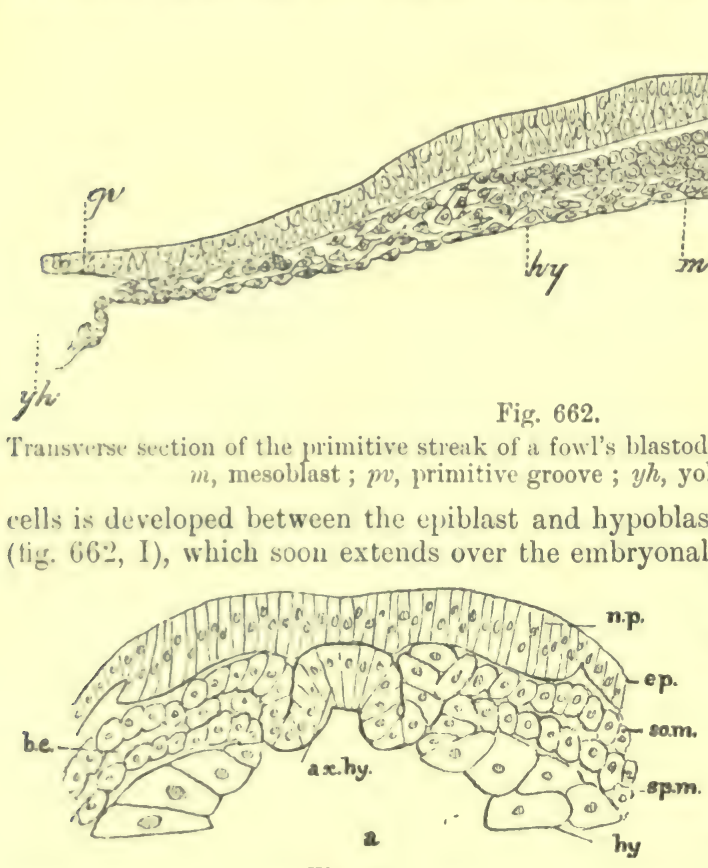

Fig. 662 .

Transverse section of the primitive streak of a fowl's blastoderm. $c p$, epiblast; hy, hypoblast ; $m$, mesoblast ; $w$, primitive groove ; $y h$, yoke of germinal wall.

cells is developed between the epiblast and hypoblast, the mesoderm or mesoblast (fig. 66:- I), which soon extends over the embryonal area, and into the blastoderm.

Fig. 663 .

Transverse section of an embyro newt. $a$, mesenteron; ux. hy, axial hypol,last, forming the notochord; $b c$, cerlon or herly-cavity; ep, epiblast; hy, digestive hypoblast; sim, somatic nesoblast ; spm, splanchnic mesoblast ; $n p$, neural plate.
[There has been much discussion as to the origin of the mesoblast, but in vertebrates it seems to be originally developed from the hypoblast. Fig. 663 shows a portion of the hypoblast in its axial part, in process of forming the notochord, which is described as mesoblastic.] Blood-vessels are formed within the mesoblast, and are distributed over the blastoderm to form the area vasculosa.

Medullary Groove. - A longitudinal groove, the medullary groove, is formed at the anterior part of the embryonal shield, but it gradually extends posteriorly, embracing the anterior part of the primitive streak with its divided posterior end, while the primitive streak itself gradually becomes relatively and absolutely smaller and less distinct, until it disappears altogether (fig. 661, I, and II, $\mathrm{Pr}$ ).

The position of the embryo is indicated by the central part becoming more transparent, - the area pellucida, - which is surrounded by a more opaque partthe area opaca. [The area opaca rests directly upon the white yelk in the fowl, and it takes no share in the formation of the embryo, but gives rise to structures which are temporary, and are connected with the nutrition of the embryo. The embryo is formed in the area pellucida alone.]

From the epiblast [neuro-epidermal layer] are developed the central nervous system and epidermal tissues, including the epithelium of the sense-organs.

From the mesoblast are formed most of the organs of the body [including the vascular, muscular, and skeletal systems, and, according to some, the connectivetissue. It also gives rise to the generative glands and excretory organs].

From the hypoblast epithelio-glandular layer [which is the secretory layer], arise 
the intestinal epithelium, and that of the glands which open into intestine. The notochord is also formed from its axial portion. [The mouth and anus being formed by an inpushing of the epiblast, are lined by epiblast, and are sometimes called the stomodæum and proctodæum respectively.]

[Structure of the Blastoderm (fig. 664). - Originally it is composed of only two layers, and in a vertical section of it the epiblast consists of a single row of nucleated granular cells, arranged side by side, with their long axes placed vertically. The hypoblast consists of larger cells than the foregoing, although they vary in size. They are spherical and very granular, so that no nucleus is visible in them. The cells form a kind of network, and occur in more than one layer, espe-

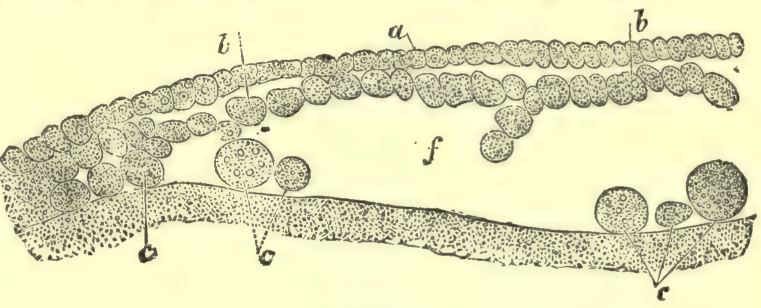

Fig. 664 .

Vertical section of part of the unincubated blastoderm of a hen. $a$, epiblast; $b$, hypoblast; $c$, formative cells resting on white yelk ; $f$, archenteron.

cially at the periphery. It rests on white yelk, and under it are large spherical refractive cells, spoken of as formative cells $(c)$.]

The cells of the epiblast, and especially those of the hypoblast, nourish themselves by the direct absorption and incorporation of the constituents of the yelk into themselves. The amoboid movements of these cells play a part in the process of absorption. "The absorbed particles are changed, or, as it were, digested within the cells, and the product used in the processes of growth and development (Kollmann).

440. STRUCTURES FORMED FROM THE EPIBLAST.-Laminæ Dorsales.-The medullary groove upon the epiblast (also called outer, serous, sensorial, corneal, or animal layer) becomes deeper (fig. 665, II). The two longitudinal elevations or laminæ dorsales consist of a thickening of the epiblast, and grow up over the medullary groove, to meet each other and coalesce by their free edges in the middle line posteriorly. Thus, the open groove is changed into a closed tube - the medullary or neural tube (III). The cells next the lumen of the tube ultimately become the ciliated epithelium lining the central canal of the spinal cord, while the other cells of the nipped-off portion of the epiblast form the ganglionic part of the central nervous system and its processes.

Primary Cerebral Vesicles. - [The laminæ dorsales unite first in the region of the neck of the embryo, and soon this is followed by the union of those over the future head.] The medullary tube is not of uniform diameter, for at the anterior end it becomes dilated and mapped out by constrictions into the primary vesicles of the brain, which at first are arranged, one behind the other, in the following order, each one being smaller than the one in front of it:- the fore-brain (representing the structures from which the cerebral hemispheres are developed); the mid-brain (corpora quadrigemina); the hind-brain (cerebellum); and the after-brain (medulla oblongata), which is gradually continued into the spinal cord (IV and V). The posterior part of the medullary tube has a dilatation at the lumbar enlargement. In birds, the medullary groove remains open in this situation to form a lozenge-shaped dilatation, the sinus rhomboidalis.

Cranial Flexures.- The anterior part of the medullary tube curves on itself, especially at the junction of the spinal cord and oblongata, between the mid-brain and hind-brain, and again almost at right angles between the fore-brain and midbrain. [Thus, a displacement of the primary vesicles is produced, and the head of the future embryo is mapped off.] At first all the cerebral vesicles are devoid of convolutions and sulci. On each side of the fore-brain there grows out a stalked 
hollow vesicle $(\mathrm{VI})$, the primary optic vesicle. The remainder of the epiblast forms the epidermal covering of the body. At an early period we can distinguish the stratum corneum and the Malpighian layer of the skin (\$283); from the former are developed the hairs, nails, feathers, dic.

Partial Cleavage. - Only a partial cleavage takes place in the eggs of birds and in meroblastic sva, i. ., only the uchite yelk in the neighbourhood of the cicatricula divides into nume. rous segmentation spiheres (Coste, 1848). The cells arrange themselves in two layers lying one
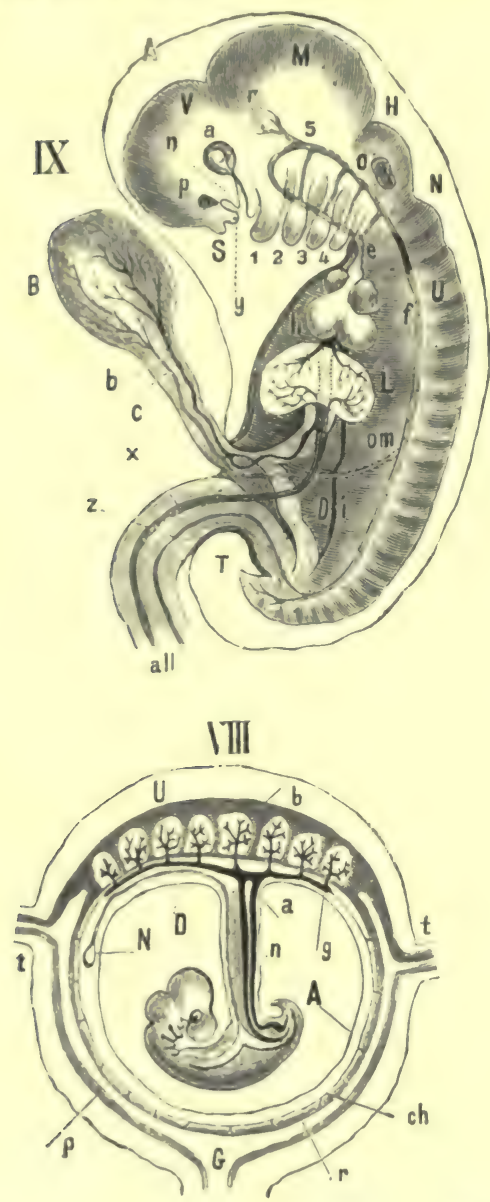
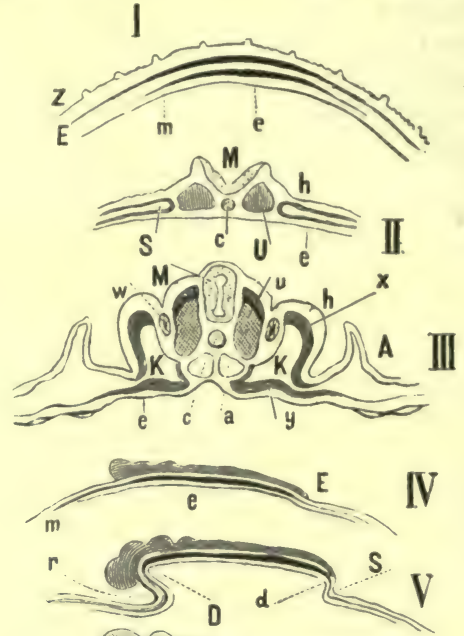

VI

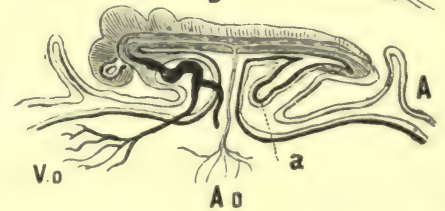

Ao

Fig. 665 .

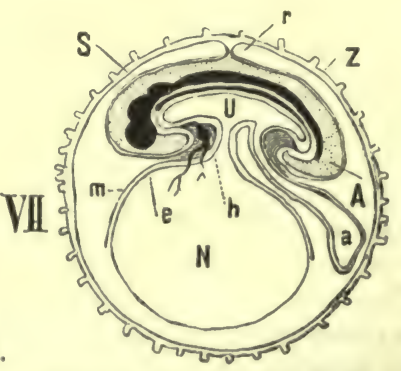

1, The three layers of the blastoderm of a mammalian ovum-Z, zona pellucida; $\mathbf{E}$, epiblast ; $m$, mesoblast ; $e$, hypoblast. II, Section of an embryo, with six protovertebræ at the 1st day - II, medullary groove; $h$, somatopleure; U, protovertebra ; $c$, chorda dorsalis; $\mathbf{S}$, the lateral plates divided into two ; $e$, hypoblast. III, Section of an embryo chick at the 2nd day in the region behind the heart-M, medullary groove; $h$, outer part of somatopleure ; $u$, protovertebra ; $c$, chorda ; $w$, Wolffian duct; $\mathrm{K}$, colom ; $x$, inner part of somatopleure; $y$, inver part of splanchnopleure ; $\mathbf{A}$, amniotic fold ; $a$, aorta ; $e$, hypoblast. IV, Scheme of a longitudinal section of an early embryo. V, Scheme of the formation of the head-and tail-folds $-r$, head-fold ; D, anterior extremity of the future intestinal tract; $\mathrm{S}$, tail-fold, first rudiment of the cavity of the rectum. VI, Scheme of a longitudinal section through an embryo after the formation of the head-and tail-folds-A o, omphalo-mesenteric arteries; 
V o, omphalo-mesenteric veins; $a$, position of the allantois; A, amniotic fold. VII, Scheme of a longitudinal section through a human ovum $-\mathrm{Z}$, zona pellucida; S, serous cavity ; $r$, union of the amniotic folds ; $\mathrm{A}$, cavity of the amnion; $a$, allantois ; $\mathbf{N}$, umbilical vesicle ; $m$, mesoblast; $h$, heart ; $U$, primitive intestine. VIII, Schematic transverse section of the pregnant uterus during the formation of the placenta ; $U$, muscular wall of the uterus ; $p$, uterine mucous membrane, or decidua vera ; $b$, maternal part of the placenta, or decidua serotina; $r$, decidua reflexa ; $c h$, chorion ; A, amnion ; $n$, umbilicai cord ; $a$, allantois, with the urachus; $\mathrm{N}$, umbilical vesicle, with $\mathrm{D}$, the omphalo-mesenteric duct; $t t$, openings of the Fallopian tubes; G, canal of the cervix uteri. IX, Scheme of a human embryo, with the visceral arches still persistent-A, amnion; V, fore-brain ; $\mathbf{M}$, mid-brain ; $\mathrm{H}$, hind-brain ; $\mathrm{N}$, after-brain ; $\mathrm{U}$, primitive vertebræ ; $a$, eye ; $p$, nasal pits; $\mathbf{S}$, frontal process ; $y$, internal nasal process ; $n$, external nasal process ; $r$, superior maxillary process of the 1st visceral arch ; 1, 2, 3, and 4, the four visceral arches, with the visceral clefts between them; 0 , auditory vesicle ; $h$, heart, with $e$, primitive aorta, which divides into five aortic arches ; $f$, descending aorta ; om, omphalo-mesenteric artery; $b$, the omphalo-mesenteric arteries on the umbilical vesicle ; $c$, omphalo-mesenteric vein ; $\mathrm{L}$, liver, with venæ advehentes and revehentes; D, intestine; $i$, inferior cava; T, coceyx ; all, allantois, with $z$, one umbilical artery, and $x$, an umbilical vein.

over the other. The upper layer or epiblast is the larger, and contains small pale cells ; the lower layer, or hypoblast, which at first is not a continuous layer, ultimately forms a continuous layer, but its periphery is smaller than the upper layer, while its cells are larger and more granular.

Between the epiblast and hypoblast there is formed, from the primitive streak as a product of cell-proliferation, the mesoblast, which is said by Kölliker to be due to the division of the cells of the epiblast. It gradually extends in a peripheral direction between the two other layers. All the three layers grow at their periphery. In the mesoblast blood-vessels are developed. All the three layers, as they grow, come ultimately to enclose the yelk, so that their margins come together at the opposite pole of the yelk.

\section{STRUCTURES FORM- ED FROM THE MESOBLAST} AND HYPOBLAST. - The meso-

blast (vascular layer or middle layer) forms immediately under the medullary groove, a cylindrical cellular cord, the chorda dorsalis, or notochord, which is thicker at the tail than at the cephalic end (fig. 665, II, III, c). It is present in all vertebrata, and also in the larval form of the ascidians, but in the latter it disappears in the adult form (Kowalewsky). In man it is relatively small. It forms the basis of the bodies of the vertebræ, and around it, as a central core, the substance of the bodies of the vertebræe is deposited, so that they are strung on it, as it were, like beads on a

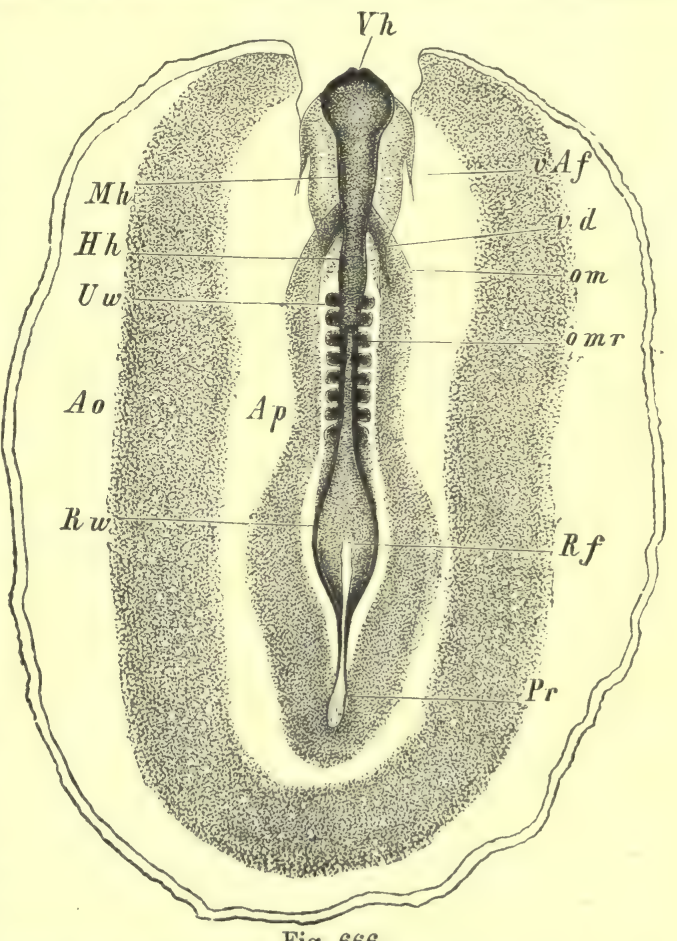

Fig. 666 .

Embryo fowl of the 2nd day, $\times 50$. Ao, area opaca ; $A p$, area pellucida ; $H h$, hind-brain ; $M h$, mid-brain ; $V$, fore-brain ; om, omphalo-mesenteric veins ; omr, point where the closure of the neural groove is travelling backwards with the protovertebræ; $V w$, muscle-plates; $R f$, posterior part of widely-open neural groove ; $R w$, neural ridge ; $v A f$, anterior amniotic fold. string. After it is formed, it becomes surrounded by a double sheath-like covering (Gegenbaur, Kölliker). 
The recent observations of L. Gerlach and Strahl show that the chorda dorsalis is derived from the hypoblast (fig. 663). It does not contain chondrin or glutin, but albumin (Retzius).

Protovertebræ. - The cells of the mesoblast, on each side of the chorda, arrange themselves into cubical masses, always disposed in pairs behind each other, the protovertebræ (fig. $665, U$ and $u$ ). The first pair correspond to the atlas. At a later period each protovertebra shows a marginal cellular area and a nuclear area (fig. 665). Only part of it goes to form a future vertebra. The part of the mesoblast lying external to the protovertebra, the lateral plates (fig. 665, II, s), splits into two layers, an upper one and a lower one, which, however, are united by a median plate at the protovertebræ. The space between the two layers of the mesoblast is called the pleuro-peritoneal cavity, or the cœlom of Haeckel (III, K). The upper layer of the lateral plate becomes united to the epiblast, and forms the cutaneo-muscular plate of German authors, or the somatopleure (fig. 665, III, $r$; fig. $667, s()$, while the inner one unites with the hypoblast to form the intestinal plate of German authors, or the splanchnopleure (fig. 665, III, y fig. $i 6 i, s p)$. On the surfaces of these plates, which are directed towards each other, the endothelium lining the pleuro-peritoneal cavity is developed. On the surface

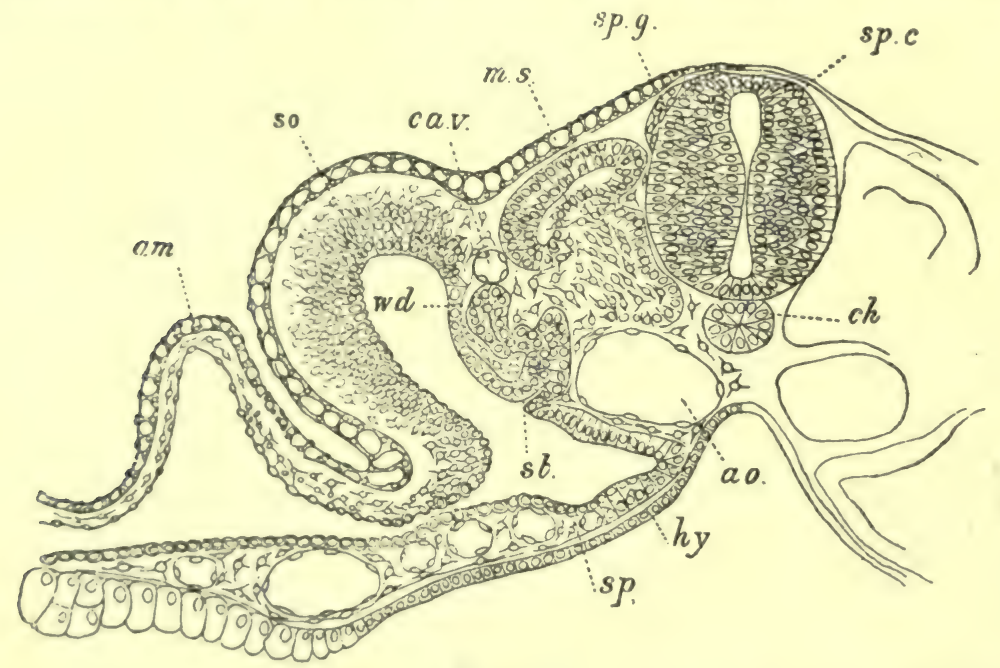

Fig. 667 .

Transverse section of an embryo duck. am, ammion; $\iota 0$, aorta ; $c a . v$, cardinal vein ; $c h$, notochord ; hy, hypohlast; $m s$, muscle-plate ; so, somatopleure; $s p$, splanchnopleure ; sp.e, spinal cord ; sp. $y$, spinal ganglion; st, segnental tube; $w d$, Wolffian (segmental) duct.

of the merlian plate, directed towards the coelom, some cylindrical cells, the "germ-epithelium" of Waldeyer, remain, which form the ovarian tubes and the ova (\$ 438).

According to Remak, the skin, the muscles of the trunk, and the blood-vessels, and according to His, only the musculature of the trunk, are derived from the somatopleure. Both observers agree that the splanchnopleure furnishes the musculature of the intestinal tract.

Parablastic and Archiblastic Cells.-According to His, the blood-vessels, blood, and connective-tissue are not developed from true mesoblastic cells, but he asserts that for this purpose certain cells wander in from the margins of the blasto derm between the epiblast and hypoblast, these cells being derived from outside the position of the embryo, from the elements of the white yelk. His calls these structures parablastic, in olposition to the archiblastic, which belong to the three layers of the embryo. Waldeyer also adheres to the parablastic structure of blood and 
connective-tissue, but he assumes that the material from which the latter is formed is continuous protoplasm, and of equal value with the elements of the blastoderm.

The hypoblast does not undergo any change at this time; it applies itself to the inner layer of the mesoblast, as a single layer of cells, to form the splanchnopleure.

442. FORMATION OF EMBRYO, HEART, PRIMITIVE CIRCULATION. -Head-and Tail-Folds. - Up to this time the embryo lies with its three layers in the plane of the layers themselves. The cephalic end of the future embryo is first raised above the level of this plane (fig. 665, V). In front of, and under the head, there is an inflection or tucking-in of the layers, which is spoken of as the head-fold $(\mathrm{V}, r)$. [It gradually travels backwards, so that the embryo is raised above the level of its surroundings.] The raised cephalic end is hollow, and it communicates with the space in the interior of the umbilical vesicle. The cavity in the head is spoken of as the head-gut or fore-gut $(V, D)$. The formation of the fore-gut, by the elevation of the head from the plane of the three layers, occurs on the second day in the chick, and in the dog on the 22 nd day. The tail-fold is formed in precisely the same way, in the chick on the 3rd day, and in the dog on the 22nd day. The tail-fold, S, also is hollow, and the space within it is the hind-gut, $d$. Thus, the body of the embryo is supported or rests on a hollow stalk, which at first is wide, and communicates with the cavity of the umbilical vesicle. This duct or communication is called the omphalo-mesenteric duct, or the vitello-intestinal or vitelline duct. The saccular vesicle attached to it in mammals is called the umbilical vesicle (VII, N), while the analogous much larger sac in birds, which contains the yellow nutritive yelk, is called the yelk-sac. The omphalo-mesenteric or vitelline duct in course of time becomes narrower, and is ultimately obliterated in the chick on the 5th day. The point where it is continuous with the abdominal wall is the abdominal umbilicus, and where it is inserted into the primitive intestine, the intestinal umbilicus.

[Sometimes part of the vitelline duct remains attached to the intestine, and may prove dangerous by becoming so displaced as to constrict a loop of intestine, and thus cause strangulation of the gut.]

Heart.-Before this process of constriction is complete, some cells are mapped off from that part of the splanchnopleure which lies immediately under the headgut ; this indicates the position of the heart, which appears in the chick at the end of the first day, as a small, bright red, rhythmically contracting point, the punctum

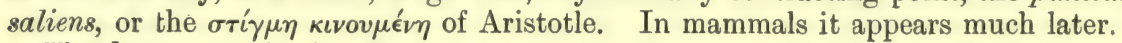

The heart, VI, begins first as a mass of cells, some of which in the centre disappear to form a central cavity, so that the whole looks like a pale hollow bud (originally a pair) of the splanchnopleure. The central cavity soon dilates; it grows, and becomes suspended in the cœlom by a duplicature like a mesentery (mesocardium), while the space which it occupies is spoken of as the fovea cardica. The heart now assumes an elongated tubular form, with its aortic portion directed forwards, and its venous end backward; it then undergoes a slight $f$-shaped curve (fig. 675,1 ). From the middle of the 2nd day, the heart begins to beat in the chick, at the rate of about 40 beats per minute. [It is very important to note that at first, although the heart beats rhythmically, it does not contain any nervecells.]

From the anterior end of the heart, there proceeds from the bulbus aortæ, the aorta which passes forward and divides into two primitive aortæ, which then curve and pass backwards under the cerebral vesicles, and run in front of the protovertebræ. Opposite the omphalo-mesenteric duct, each primitive aorta in the ehick sends off one, in mammals several (dog 4 to 5), omphalo-mesenteric arteries (VI, A, o), which spread out to form a vascular network within the mesoblast of the 
umbilical vesicle. From this network, there arise the omphalo-mesenteric veins, which run backwards on the vitelline duct, and end by two trunks in the venous end of the tubular heart. In the chick, these veins arise from the sinus terminalis of the future vena terminalis of the area vasculosa. Thus, the first or primitive circulation is a closed system, and functionally it is concerned in carrying nutriment and oxygen to the embryo. In the bird, the latter is supplied through the porous shell, and the former is supplied up to the end of incubation by the yelk. In mammals, both are supplied by the blood-vessels of the uterine mucous membrane to the ovum. In lirds, owing to the absorption of the contents of the yelk-sac, the vascular area steadily diminishes, until ultimately, towards the end of the period of incubation, the shrivelled yelk-sac slips into the abdominal cavity. In mammals, the circulation on the umbilical vesicle, i.e., through the omphalo-mesenteric vessels, soon diminishes, while the umbilical vesicle itself shrivels to a small appendix, and the second circulation is formed to replace the omphalo-mesenteric system. The first hlood-ressels are formed in the chick, in the area vasculosa, outside the position of the embryo, at the last quarter of the first day, before any part of the heart is visible. The blood-vessels begin in vaso-formative cells [constituting the "bloodislands" of Pander]. At first they are solid, but they soon become hollow (§ 7, A).

A narrow-mesherl plexus of 7ymplatirs is formed in the area vasculosa of the chick (His), and it communicates with the amniotic cavity (A. Biulye).

443. FORMATION OF THE BODY.-Body-Wall-(1) The cœlom, or pleuro-peritoneal cavity, becomes larger and larger, while at the same time, the lifference between the body-wall and the wall of the intestine becomes more pronounced. The latter becomes more separated from the protovertebræ, as the middle plate begins to be elongated to form a mesentery. The body-wall, or somatopleure, composed of the epiblast and the outer layer of the cleft mesoblast, becomes thickened by the ingrowth into it of the muscular layer from the musclejlate, and the position of the bones and the spinal nerves from the protovertebræ. These grow between the epiblast and the outer layer of the mesoblast (Remak). [The somatopleure, or parietal lamina, from each side grows forward and towards the middle line, where they meet to form the body-wall, while at the same time, the splanchnopleure, or visceral lamina, on each side also grow and meet in the middle line, and when they do so, they enclose the intestine. Thus, there is one tube within the other, and the space between is the pleuro-peritoneal cavity.]

(2) Vertebral Column.-A dorsally placed structure, called the muscle-plate (fig. $667, m s$.$) , is differentiated from each of the protovertebræ; the remainder of$ the protovertebra, the protovertebra proper, coalesces with that on the other side, so that both completely surround the chorda, to form the membrana reuniens inferior, in the chick on the $3 \mathrm{rd}$, and in the rabbit on the 10 th day, while, at the same time, they close over the medullary tube dorsally, in the chick at the 4th day, to form the membrana reuniens superior (Reichert). Thus, there is a union of the masses of the protovertebra in front of the medullary tube, which encloses the chorda, and represents the basis of the bodics of all the vertebræ, whilst the membrana reuniens superior, pushed between the muscle-plates and the epiblast on the one hand and the medullary tube on the other, represents the position of the entire vertebral lamince as well as the intervertebral ligaments between them. In some rare cases the membrana reuniens superior is not developed, so that the medullary tube is covered only by the epiblast (epidermis), either throughout its entire extent, or at certain parts. This constitutes the condition of spina bifida, or, when it occurs in the head, hemicephalia. The vertebral column at this membranous stage is in the same condition as the vertebral column of the cyclostomata (Petromyzon). The membranes of the spinul cord, the spinal ganglia, and spinal nerves are formed from the membrana reuniens superior. 
Lastly, parts of the somatopleures also grow towards the middle line of the back, and insinuate themselves between the muscle-plate and the epiblast; thus, the dorsal skin is formed (Remak).

In the membranous vertebral column, there are formed the several cartilaginous vertebræ, the one behind the other, in man at the 6th to 7 th week, although at first they do not form closed vertebral arches; the latter are closed in man about the 4th month. Each cartilaginous vertebra, however, is not formed from a pair of protovertebræ, i.e., the 6th cervical vertebra from the 6th pair of protovertebræ, but there is a new subdivision of the vertebral column, so that the lower half of the preceding protovertebra and the upper half of the succeeding protovertebra unite to form the final vertebra. While the bodies are becoming cartilaginous the chorda becomes smaller, but it still remains larger in the intervertebral discs. The body of the first vertebra or atlas unites with that of the axis to form its odontoid process, and in addition it forms the arcus anterior atlantis and the transverse ligament (Hasse). The chorda can be followed upwards through the ligamentum suspensorium dentis as far as the posterior part of the sphenoid bone.

The histogenetic formation of cartilage from the indifferent formative cells takes place by division and growth of the cells, until they ultimately form clear nucleated sacs. The cement substance is probably formed by the onter parts of the cells (parietal substance) uniting and secreting the intercellular substance. It is supposed by some that the latter contains fine canals, which connect the protoplasm of the adjoining cells.

Visceral Clefts and Arches.-Each side of the cervical region contains four slit-like openings - the visceral clefts or branchial openings (Rathlie); in the chick, the upper three are formed at the 3rd, and the fourth on the 4th day. Above the slits are thickenings of the lateral wall, which constitute the visceral or branchial arches (fig. 671). The clefts are formed by a perforation from the fore-gut, but this, perhaps, does not always occur in the chick, mammal, and man (His), and they are lined by the cells of the hypoblast. On each side in each visceral arch, i.e., above and below each cleft, there runs an aortic arch, five on each side (fig. 665, IX). These aortic arches persist in fishes. In man, all the slits close, except the uppermost one, from which the auditory meatus, the tympanic cavity, and the Eustachian tube are developed. The four visceral arches are for the most part made use of later for other formations (p. 879).

Primitive Mouth and Anus.-Immediately under the fore-brain, in the middle line, is a thin spot, where there is at first a small depression, and ultimately a rupture, forming the primitive oral aperture, which represents both the mouth and the nose. Similarly, there is a depression at the caudal end, and the depression ultimately deepens, thus communicating with the hind-gut to form the anus. When the latter part of the process is incomplete, there is atresia ani, or imperforate anus. Several processes are given off from the primitive intestine, including the hypoblast and its muscular layers, to form the lungs, the liver, the pancreas, the cæcum (in birds), and the allantois.

The extremities appear at the sides of the body as short unjointed stumps or projections at the 3rd cr 4th week in the human embryo.

444. AMNION AND ALLANTOIS.-Amnion.-During the elevation of the embryo from its surroundings, immediately in front of the head (at the end of the 2nd day in the chick), there rises up a fold consisting of the epiblast and the outer layer of the mesoblast, which gradually extends to form a sort of hood over the cephalic end of the embryo (fig. 665 , VI, A). In the same way, but somewhat later, a fold rises at the caudal end, and between both along the lateral borders similar elevations occur, the lateral folds (fig. 665, III, A). All these folds grow over the back of the embryo to meet over the middle line posteriorly, where they unite at the $3 \mathrm{rd}$ day, in the chick, to form the amniotic sac. Thus, a cavity which 
becomes filled with fluid-the amniotic fluid-is developed around the embryo [so that the embryo really floats in the fluid of the amniotic sac]. In mammals also, the amnion is developed very early, just as in birds (fig. 665, VII, A). From the middle of pregnancy onwards, the amnion is applied directly to the chorion; and united to it by a gelatinous layer of tissue, the tunica media of Bischoff.

Amniotic Fluid.-The amnion, and the allantois as well, are formed only in mammals, birds, and reptiles, which have hence been called amniota, while the lower vertebrates, which are livoil of an amnion, are called anamnia. Composition. - The amniotic fluid is a clear, serens, alkaline fluid, specific gravity 1007 to 1011 , containing, besides enithelium, lanugo hairs, $\frac{1}{2}$ to 2 per cent. of fixed solids. Amongst the latter are albumin ( $\frac{1}{10}$ to $\frac{1}{3}$ per cent.), mucin, glolulin, a vitelline-like hody, some grape-sugar, urea, ammonium carbonate, very probalily derived from the decomposition of urea, sometimes lactic acid and kreatinin, calcic sulphate and phosphate, and common salt. About the middle of pregnancy, it amounts to about $1-1 \cdot 5$ kilo. $[2 \cdot 2-3 \cdot 3$ lbs. $]$, and at the end about 0.5 kilo. The amniotic fluid is of fotal origin, as is shown by its occurrence in lirds, and is, perhaps, a transudation through the fietal membranes. In mammals, the urine of the fotus forms part of it during the second half of pregnancy (Giuserour). In the pathological condition of hydramnion, the blood-vessels of the uterine mucous membrane secrete a watery fluid. The fluid preserves the foetus, and also the ressels of the fietal membranes from mechanical injuries; it permits the limbs to move freely, and protects them from growing together; and, lastly, it is important for dilating the os uteri during labour. The annion is capable of contraction at the 7 th day in the chick; and this is due to the smooth muscular fibres which are developed in the cutaneous plate in its mesoblastic portion, but nerves have not been found.

Allantois.-From the anterior surface of the caudal end of the embryo, there grows out a small double projection, which becomes hollowed out to form a sac projecting into the cavity of the colom or pleuro-peritoneal cavity (fig. 665); it constitutes the allantois, and is formed in the chick before the 5th day, and in man, during the 2nd week. Being a true projection from the hind-gut, the allantois has two layers, one from the hypoblast, and the other from the muscular layer, so that it is an off'shoot from the splanchnopleure. From both sides, there pass on to the allantois the umbilical arteries from the hypogastric arteries, and they ramify on the surface of the sac. The allantois grows, like a urinary bladder gradually being distended, in front of the hind-gut in the pleuro-peritoneal cavity towards the umbilicus; and lastly, it grows out of the umbilicus, and projects beyond it alongside the omphalo-mesenteric or vitelline duct, its vessels growing with it (fig. 665 , VII, a); but, after this stage, it behaves differently in birds and mammals.

In birds, after the allantois passes out of the umbilicus, it undergoes great development, so that within a short time it lines the whole of the interior of the shell as a highly vascular and saccular membrane. Its arteries are at first branches of the primitive aortæ, but with the development of the posterior extremities they appear as branches of the hypogastric arteries. 'Two allantoidal, or umbilical veins, proceed from the numerous capillaries of the allantois. They pass backwarl through the umbilicus, and at first unite with the omphalo-mesenteric veins to join the venous end of the heart. In birds this circulation on the allantois, or second circulation, is respiratory in function, as its vessels serve for the exchange of gases through the porous shell. The circulation gradually assumes the respiratory functions of the umbilical vesicle, as the latter gradually becomes smaller and smaller, and ceases to be a sufficient respiratory organ. Towards the end of the period of incubation the chick may breathe and ery within the shell (Aristotle) - a proof that the respiratory funetion of the allantois is partly taken over by the lungs. The allantois is also the excretory organ of the urinary constituents. Into its cavity in mammals the ducts of the primitive kidneys, or the IVolffian ducts, open, but in hirds and reptiles, which possess a cloaca, these open into the posterior wall of the cloaca. The primitive kidneys, or Wolffian bodies, consist of many glomeruli, and empty their secretion through the Wolffian ducts into the allantois (in birds into the cloaca), and the secretion passes through the allantois, per the umbilicus, into the peripheral part of the urinary sac. Remak found ammonium and sodium urate, allantoin, grape-sugar, and salts in the contents of the allantois. From the 8th day onwards, the allantois of the chick is contractile (Vulpian), owing to the presence of smooth fibres derived from the splanchnopleure. Lymphatics accompany the branches of the arteries $(A . B u d g e)$.

Allantois in Mammals. - In mammals and man, the relation of the allantois is 
somewhat different. The first part or its origin forms the urinary bladder, and from the vertex of the latter there proceeds through the umbilicus a tube, the urachus, which is open at first (fig. 665, VIII, a). The blind part of the sac of the allantois outside the abdomen is in some animals filled with a fluid like urine. In man, however, this sac disappears during the 2 nd month, so that there remains only the vessels which lie in the muscular part of the allantois. In some animals, however, the allantois grows larger, does not shrivel, but obtains through the urachus from the bladder an alkaline turbid fluid, which contains some albumin, sugar, urea, and allantoin. The relations of the umbilical vessels will be described in connection with the fœtal membranes.

\section{FETAL MEMBRANES, PLACENTA, FETAL CIRCULATION.-} Decidua. - When a fecundated ovum reaches the uterus, it becomes surrounded by a special covering, which William Hunter (1775) described as the membrana decidua, because it was shed at birth. We distinguish the decidua vera (fig. 665 , VIII, $p$ ), which is merely the thickened, very vascular, softened, more spongy, and somewhat altered mucous membrane of the uterus. [Sometimes in a diseased condition, as in dysmenorrhœa, the superficial layer of the uterine mucous membrane is thrown off nearly en masse in a triangular form (fig. 668). This serves to show the shape of the decidua, which is that of the uterus.] When the ovum reaches the uterus, it is caught in a crypt or fold of the decidua, and from the latter there grow up elevations around the ovum; but these elevations are thin, and soon meet over the back of the ovum to form the decidua reflexa (fig. 665, VIII, $r)$. At the 2nd to 3rd month, there is still a space in the uterus outside the reflexa; in the 4th month, the whole cavity is filled by the ovum. At one part the ovum lies directly upon the d. vera [and that part is spoken of as the

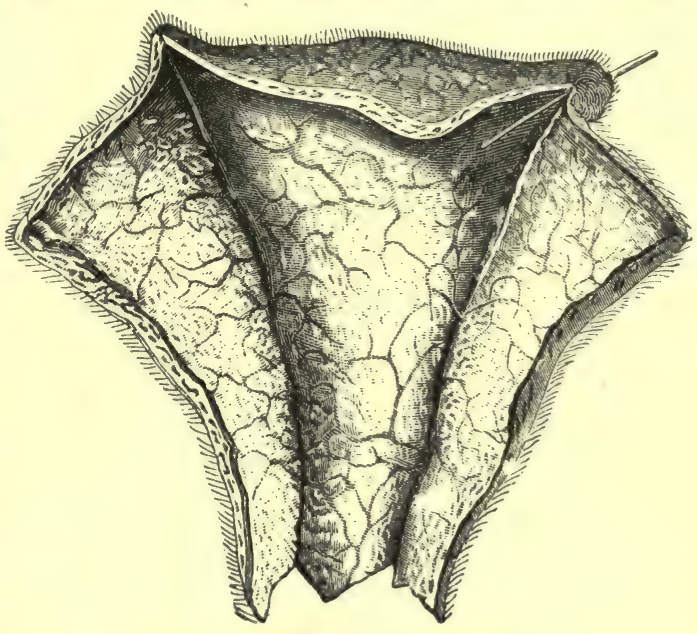

Fig. 668 .

A dysmenorrhoeal membrane laid open. decidua serotina], but by far the greatest part of the surface of the ovum is in contact with the reflexa. In the region of the $d$. serotina the placenta is ultimately formed.

Structure of the Decidua Vera.--The d. vera at the 3rd month is 4 to $7 \mathrm{~mm}$. thick, and at the 4 th only 1 to $3 \mathrm{~mm}$., and it no longer has any epithelium; but it is very vascular, and is possessed of lymphatics around the glands and blood-vessels (Leopold), and in its loose substance are large round cells (decidua cells-Kölliker), which in the deeper parts become changed into fibre cells-there are also lymphoid cells. The uterine glands, which become greatly developed at the commencement of pregnancy, at the 3rd to the 4th month form non-cellular, wide, bulging tubes, which become indistinct in the later months, and in which the epithelium disappears more and more. The $d$. reflexa, nuch thinner than the vera from the middle of pregnancy, is devoid of epithelium, and is without vessels and glands. Tuwards the end of pregnancy both deciduæ unite.

The ovum, covered at first with small hollow villi, is surrounded by the decidua. From the formation of the amnion it follows that, after it is closed, a completely closed sac passes away from the embryo to lie next the primitive chorion. This membrane is the "serous covering" of v. Baer (fig. 665, VII, s), or the false 
amnion. It becomes closely applied to the inner surface of the chorion, and extends even into its villi. The allantois proceeding from the umbilicus comes to lie directly in contact with the foetal membrane; its sac disappears about the 2nd month in man, but its vascular layer grows rapidly and lines the whole of the inner surface of the chorion, where it is found on the 18th day (Coste). From the 4 th week the blood-vessels, along with a covering of connective-tissue, branch and penetrate into the hollow cavities of the villi, and completely fill them. At this time the prinitive chorion disappears. Thus, we have a stage of general vascularisation of the chorion. In the place of the derivative of the zona pellucida we lave the vascular villi of the allantois, which are covered by the epiblastic cells derived from the false amnion. This stage lasts only until the 3rd month, when the chorionic villi disappear all over that part of the surface of the ovum which is in contact with the decidua reflexa. On the other hand, the villi of the chorion, where they lie in direct contact with the decidua serotina, become larger and more lranched. Thus, there is distinguished the chorion laeve and c. frondosum.

The thorion laeve, which consists of a connective-tissue matrix covered externally by several lavers of cells, has a few isolated villi at wide intervals. Between the chorion and the amnion is a felatinous substance (membrana intermedia) or undeveloped connective-tissue.

Placenta. - The large villi of the chorion frondosum penetrate into the tissue of the decidua serotina of the uterine mucous membrane. [It was formerly supposed that the chorionic villi entered the mouths of the uterine glands, but the researches of Ercolani and Turner have shown that, although the uterine glands enlarge during the early months of utero-gestation, the villi do not enter the glands. The villi enter the crypts of the uterine mucous membrane. The glands of the inner layer of the decidua serotina soon disappear.] As the villi grow into the decidua

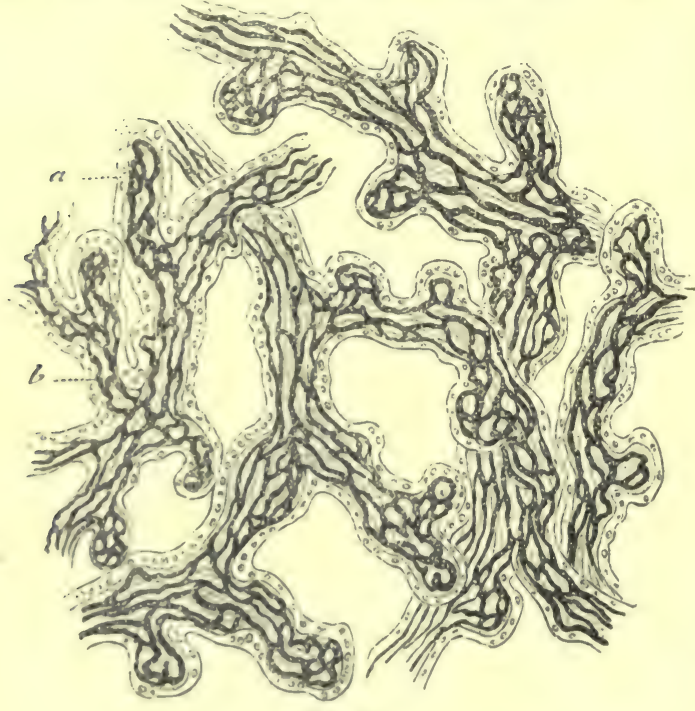

Fig. 669.

Human placental villi. Blood-vessels black. serotina, they push against the walls of the large bloodvessels, which are similar to capillaries in structure, so that the villi come to be bathed by the blood of the mother in the uterine sinuses, or they float in the colossal decidual capillaries (fig. 665, VII, b). The villi do not float naked in the maternal blood, but they are covered by a layer of cells derived from the decidua. Some villi, with bulbous ends, unite firmly with the tissue of the uterine part of the placenta to form a firm bond of connection. [The placenta is formed by the mutual intergrowth of the chorionic villi and the decidua serotina.] Thus, it consists of a fœtal part, including all the villi, and a maternal or uterine part, which is the very vascular decidua serotina. At the time of birth, both parts are so firmly united that they cannot be separated. Around the margin of the placenta is a large venous vessel, the marginal sinus of the placenta. [Friedländer found the uterine sinuses below the placental site blocked by giant cells after the 8 th month of pregnancy. Leopold confirms this, and found the same in the serotinal veins.] 
Functions. - The placenta is the nutritive, excretory, and respiratory organ of the fotus ( $\S 368)$; the latter receives its necessary pabulum by endosmosis from the maternal sinuses through the coverings and vascular wall of the villi in which the foetal blood circulates. [The placenta also contains glycogen.]

[Structure.-A piece of fresh placenta teased in normal saline solution, shows the villi provided with lateral offshoots, and consisting of a connective-tissue framework, containing a capillary network with arteries and veins, while the villi themselves are covered by a layer of somewhat cubical epithelium (fig. 669).]

Uterine Milk.-Between the villi of the placenta there is a clear fluid, which contains numerous small, albuminous globules, and this fluid, which is abundant in the cow, is spoken of as the uterine milk. It seems to be formed by the breaking up of the decidual cells. It has been supposed to be nutritive in function. [The maternal placenta, therefore, seems to be a secreting structure, while the foetal part has an absorbing function. The uterine milk has been analysed by Gamgee, who found that it contained fatty, albuminous, and saline constituents, while sugar and casein were absent.]

The investigations of Walter show, that after poisoning pregnant animals with strychnin, morphia, veratrin, curara, and ergotin, these substances are not found in the fotus, although many other chemical substances pass into it.

[Savory found that strychnin injected into a fotus in utero caused tetanic convulsions in the mother (bitch), while syphilis may be communicated from the father to the mother through the medium of the foetus (Hutchinson). A. Harvey's record of observations on the crossing of breeds of animals-chiefly of horses and allied species-show that materials can pass from the fœetus to the mother.]

On looking at a placenta, it is seen that its villi are distributed on large areas separated from each other by depressions. This complex arrangement might be compared with the cotyledons of some animals.

The position of the placenta is, as a rule, on the anterior or posterior wall of the uterus, more rarely on the fundus uteri, or laterally from the opening of the Fallopian tube, or over the internal orifice of the cervix, the last constituting the condition of placenta praevia, which is a very dangerous form of placental insertion, as the placenta has to be ruptured before birth can take place, so that the mother often dies from hæmorrhage. The umbilical cord may be inserted in the centre of the placenta (insertio centralis), or more towards the margin (ins. marginalis), or the chord may be fixed to the chorion laeve. Sometimes, though rarely, there are small subsidiary placentre $(p l$. succenturiata), in addition to the large one. When the placenta consists of two halves, it is called duplex or bipartite, a condition said by Hyrtl to be constant in the apes of the old world.

Structure of the Cord.-The umbilical cord ( 48 to $60 \mathrm{~cm}$. [20 to 24 inches] long, 11 to $13 \mathrm{~mm}$. thick) is covered by a sheath from the amnion. The blood-vessels make about forty spiral turns, and they begin to appear about the 2nd month. [The cause of the twisting is not well understood, but Virchow has shown that capillaries pass from the skin for a short distance on the cord, and they do so unequally, and it may be that this may aid in the production of the torsion.] It contains two strongly muscular and contractile arteries, and one umbilical vein. The two arteries anastomose in the placenta ( Hyrtl). In addition, the cord contains the continuation of the urachus, the hypoblastic portion of the allantois (fig. 665, VIII, a), which remains until the 2nd montb, but afterwards is much shrivelled. The omphalo-mesenteric duct of the umbilical vesicle $(\mathrm{N})$ is reduced to a threadlike stalk (fig. 665, VIII, D). Wharton's jelly surrounds the umbilical bloodvessels. Wharton's jelly is a gelatinous-like connective-tissue, consisting of branched corpuscles, lymphoid cells, some connective-tissue fibrils, and even elastic fibres. It yields mucin. It is traversed by numerous juice-canals lined by endothelial cells, but other blood- and lymphatic-vessels are absent. Nerves occur $3-8-11 \mathrm{~cm}$. from the umbilicus (Schott, Valentin).

The fœtal circulation, which is established after the development of the allantois, has the following course (fig. 670):-The blood of the foetus passes from the hypogastric arteries through the two umbilical arteries, through the umbilical cord to the placenta, where the arteries split up into capillaries. The blood is 
returned from the placenta by the umbilical vein, although the colour of the blood cannot be distinguished from the venous or impure blood in the umbilical arteries. The umbilical vein (fig. $673,3, u$ ) returns to the umbilicus, passes upwards under the margin of the liver, gives a branch to the vena porte $(a)$, and runs as the

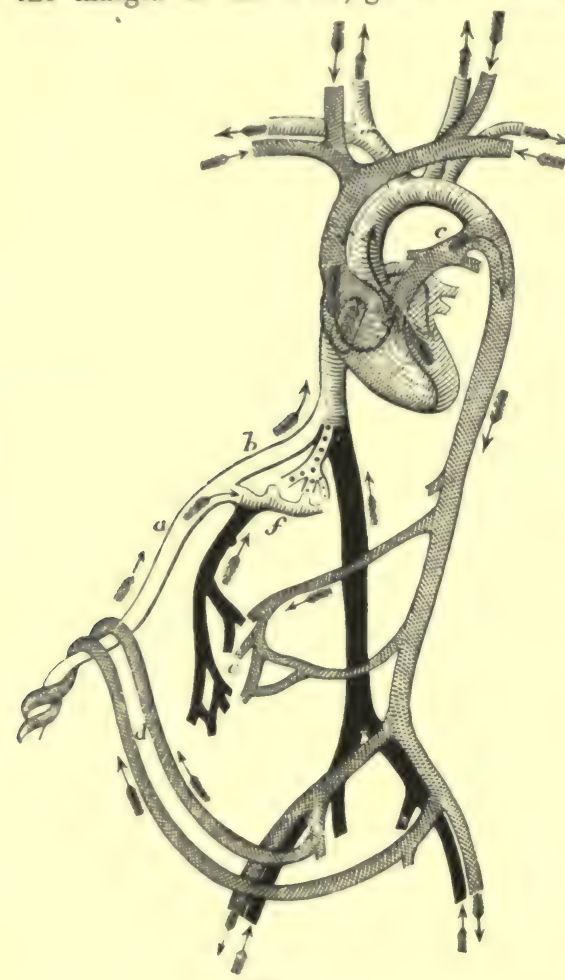

Fig. 670

Course of the fotal circulation (Cleland). ductus venosus into the inferior vena cava, which carries the blood into the right auricle. Directed by the Eustachian valve and the tubercle of Lower (fig. $675,6, t L$ ), the great mass of the blood passes through the foramen ovale into the left auricle, owing to the presence of the valve of the foramen ovale. From the left auricle it passes into the left ventricle, aorta, and hypogastric arteries, to the umbilical arteries. The blood of the superior vena cava of the fotus passes from the right auricle into the right ventricle (fig. $675,6, C s$ ). From the right ventricle it passes into the pulmonary artery (fig. 675, 7, p), and through the ductus arteriosus of. Botalli $(B)$ into the aorta. There are, therefore, two streams of blood in the right auricle which cross each other, the descending one from the head through the superior vena cava, passing in front of the transverse one from the inferior vena cava to the foramen ovale.] Only a small amount of the blood passes through the as yet small branches of the pulmonary artery to the lungs (fig. 675 , $7,1,2)$. The course of the blood makes it evident that the head and upper limbs of the foetus are nourished by purer blood than the remainder of the trunk, which is supplied with blood mixed with the blood of the superior vena cava. After birth, the umbilical arteries are obliterated, and become the lateral ligaments of the bladder, while their lower parts remain as the superior vesical arteries. The umbilical vein is obliterated, and remains as the ligamentum teres, or round ligament of the liver, and so is the ductus venosus Arantii. Lastly, the foramen ovule is closed, and the ductus arteriosus is obliterated, the latter forming the lig. arteriosus.

The condition of the membranes where there are more foetuses than one :-(1) With twins there are two completely separated ova, with two placentæ and two deciduæ reflexæ. (2) Two completely separated ova may have only one reflexa, whereby the placentre grow together, while their blood-vessels remain distinct. The chorion is actually double, but cannot be separated into two lamellæ at the point of union. (3) One reflexa, one chorion, one placenta, two umbilical cords, and two amuia. The vessels anastomose in the placenta. In this case there is one ovum with a double yelk, or with two germinal vesicles in one yelk. (4) As in (3), but only one amnion, caused by the formation of two embryos in the same blastoderm of the same germinal vesicle.

Formation of the footal membranes. - The oldest mammals have no placenta or umbilical vessels; these are the Mammalia implacentalia, including the monetremata and marsupials. The second group includes the Mammalia placentalia. Amongst these (a) the non-deciduata possess only chorionic villi supplied by the umbilical vessels, which project into the depressions of the uterine mucous membrane, and from which they are pulled out at birth (Pl. diffusa, e.g., 
pachydermata, cetacea, solidungula, camelidæ). In the ruminants, the villi are arranged in groups or cotyledons, which grow into the uterine mucous membrane, from which they are pulled out at birth. (b) In the deciduata, there is such a firm union between the chorionic villi with the uterine mucous membrane, that the uterine part of the placenta comes away with the fotal part at birth. In this case the placenta is either zonary (carnivora, pinnipedia, elephant) or discoid (apes, insectivora, edentata, rodentia).

446. CHRONOLOGY OF HUMAN DEVELOPMENT.-Development during the 1st Month. -At the 12-13th day the ovum is saccular $(5.5 \mathrm{~mm}$. and $3 \mathrm{~mm}$. in diameter); there is simply the blastodermic vesicle, with the blastoderm at one part, consisting of two layers; the zona pellucida beset with sinall villi (Reichert). At the 15th-16th day the ovum $(5-6 \mathrm{~mm}$.) is covered with simple cylindrical villi. The zona pellucida consists of embryonic counectivetissue covered with a layer of flattened epithelium. The primitive groove and the laminx dorsales appear. Then follows the stage when the allantois is first formed. At the 15th-18th day Coste investigated an ovum. It was $13 \cdot 2 \mathrm{~mm}$. long, with small branched villi; the embryo itself was $2.2 \mathrm{~mm}$. long, of a curved form, and with a moderately enlarged cephalic end. The amnion, umbilical vesicle with a wide vitelline duct, and the allantois were developed, the last already united to the false amnion. The S-shaped heart lies in the cardiac cavity, shows a cavity and a bulbus aortæ, but neither auricles nor ventricles. The visceral arches and clefts are indicated, but they are not perforated. The omphalo-mesenteric vessels forming the first circulation on the umbilical vesicle are developed, the duct (vitelline) is still quite open, and two primitive aortæ run in front of the protovertebræ. The allantois attached to the foetal membranes is provided with blood-vessels. The two omphalo-mesenteric veins unite with the two umbilical veins, and pass to the venous end of the heart. The mouth is in process of formation. The limbs and sense-organs absent; the Wolffian bodies probably present.

At the 20th day all the visceral arches are formed, and the clefts are perforated. The mid. brain forms the highest part of the brain, while the two auricles appear in the heart. The connection with the umbilical vesicle is still moderately wide. The embryo is $2 \cdot 6-3 \cdot 3-4 \mathrm{~mm}$. long, while the head is turned to one side (His). At a slightly later period the temporal and cervical flexures take place, and the hemispheres appear more prominently ; the vitelline duct is narrowed, the position of the liver is indicated, while the limbs are still absent (His).

At the 21 st day the ovum is $13 \mathrm{~mm}$. long and the embryo $4-4.5 \mathrm{~mm}$.; the umbilical vesicle $2 \cdot 2 \mathrm{~mm}$., and the intestine almost closed. Three branchial clefts, Wolffian bodies laid down, and the first appearance of the limbs, three cerebral vesicles, auditory capsules present $(\boldsymbol{R}$. Wagner). Coste also observed, in addition, the nasal pits, eye, the opening for the mouth, with the frontal and superior maxillary processes, the heart with two ventricles and two auricles.

End of the 1st Month. - The embryos of 25-28 days are characterised by the distinctly stalked condition of the umbilical vesicle and the distinct presence of limbs. Size of the ovum, $17 \cdot 6 \mathrm{~mm}$.; embryo, $13 \mathrm{~mm}$.; umbilical vesicle, $5 \cdot 5 \mathrm{~mm}$., with blood-vessels.

2nd Month. - The embryos of 28-35 days are more elongated, and all the branchial clefts are closed except the first. The allantois has now only three vessels, as the right umbilical vein is obliterated. At the 5th week the nasal pits are united with the angle of the mouth by furrows, which close to form canals at the 6th week (Toldt). At 35-42 days the nasal and oral orifices are separated, the face is flat, the limbs show three divisions, the toes are not so sharply defined as the fingers. The outer ear appears as a low projection at the 7 th week. The Wolffian bodies are much reduced in size. Length of body at 7 th to 8 th week, 1.6-4.1 $\mathrm{cm}$.

End of the 2nd Month.-Ovum, $6 \frac{1}{2} \mathrm{~cm}$; villi, $1.3 \mathrm{~mm}$. long; the circulation on the umbilical vesicle has disappeared ; embryo, $26 \mathrm{~mm}$. long, and weighs 4 grammes. Eyelids and nose present, umbilical cord $8 \mathrm{~mm}$. long, abdominal cavity closed, ossification beginning in the lower jaw, clavicle, ribs, bodies of the vertebræ; sex indistinct, kidneys laid down.

3rd Month. -Ovum as large as a goose's egg, beginning of the placenta, embryo 7-9. cm., weighing 20 grammes, and is now spoken of as a footus. External ear well formed, umbilical cord $7 \mathrm{~cm}$. long. Beginning of the difference between the sexes in the external genitals, umbilicus in the lower fourth of the linea alba.

4th Month, - Fœetus, $17 \mathrm{~cm}$. long, weighing 120 grammes, sex distinct, hair and nails begin. ning to be formed, placenta weighs 80 grammes, umbilical cord $19 \mathrm{~cm}$. long, umbilicus above the lowest fourth of the linea alba, contractions or movements of the limbs, meconium in the intestine, skin with blood-vessels shining through it, eyelids closed.

5th Month. -Fotus, length of body, $9 \cdot 7-14 \cdot 7 \mathrm{~cm}$., total length 18 to $28 \mathrm{~cm}$., weighing 284 grammes; hair on the head and lanugo distinct; skin still somewhat red and thin, and covered with vernix caseosa $(\S 287,2)$, is less transparent ; weight of placenta, 178 grammes ; umbilical cord, $31 \mathrm{~cm}$. long.

6th Month. - Foetus, length of body, 15-18.7, total length, 29-37 cm., weighing 634 grammes ; lanugo more abundant; vernix more abundant; testicles in the abdomen ; pupillary membrane and eyelashes present; meconium in the large intestine.

7th Month. - Foetus, length of body, 18-22.8, total length, $35-38 \mathrm{~cm}$., weighing 1218 
grammes, the descent of the testicles hegins-one testicle in the inguinal canal, the eyes open, the pupillary memlirane often absorbed at its centre in the 28th week. In the brain other fissures are formed besides the primary ones. The foetus is capable of living independently. At the leginning of this month there is a centre of ossification in the os calcis.

8th Month. - Fuctus, length of body, 24-27.8, total length $42 \mathrm{~cm}$., weighing 1.5 to 2 kilos. $(3 \cdot 3$ to $4.4 \mathrm{lbs}$. , hair of the head abundant, $1 \cdot 3 \mathrm{~cm}$. long, nails with a small margin, umbilicus helow the midale of the linea allos, one testicle in the scrotum.

9th Month. - Fietus, length of louly, 30-37, total length, 47-67 cm., weighing 2234 grammes, and is not distinguishable from the child at the full period.

Footus at the Full Period. - Length of hody, $51 \mathrm{~cm}$. [20 inches], weight, 3 \& kilos. [7 lbs.], lanuro present only on the shoulders, skin white. The nails of the fingers project beyond the tips of the fingers, umbilicus slightly below the middle of the linea alba. The centre of ossification in the lower epiphysis of the femur is 4 to $8 \mathrm{~mm}$. broad.

\section{Period of Gestation or Incubation (Schenk).}

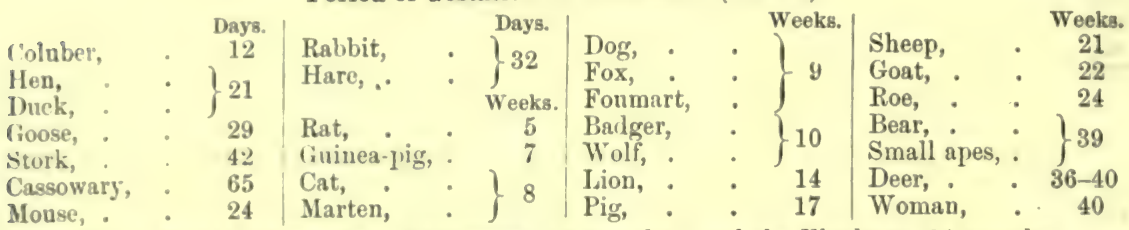

Horse, Canel, 13 months; Rhinoceros, 18 months; and the Elephant, 24 months.

Limitation of the supply of $\mathrm{O}$ to eggs, during incubation, leads to the formation of dwarf chicks.

447. FORMATION OF THE OSSEOUS SYSTEM. - Vertebral Column. - The ossification of the vertebræ begins at the 8 th to the 9th week, and first of all there is a centre in each verte. bral arch, then a centre is formed in the body behind the chorda, which, however, is composed of two closely apposed centres. At the 5th month the osseous matter has reached the surface, the chorla within the body of the vertebra is compressed; the three parts unite in the 1st year. The atlas has one centre in the anterior arch and two in the posterior; they unite at the 3rd year. The epistrophens has a centre at the 1st year. The three points of the sacral vertehra unite or anchylose between the 2nd and the 6th year, and all the vertebræ (sacral) become united to form one body between the 18th and 25th years. Each of the four coccygeal vertebræ has a centre from the 1st to 10 th year. The vertehre in later years produce 1 to 2 centres in wach process; 1 to 2 centres in each transverse process; 1 in the mamillary process of the lumbar vertebrie; and 1 in euch articular process (8 to 15 years). Of the upper and under surfaces of the looly of a vertebra each forms an epiphysial thin osseous plate, which may still be visible at the 20th year. Grouls of the cells of the chorda are still to be found within the intervertebral dises. As long as the coccygeal vertebre, the odontoid process, and the base of the skull are cartilaginous, they still contain the remains of the chorda ( $H$. Müller). The eoocygeal vertebre form the tail, and they originally project in man like a tail (fig. 665, IX, T), which is ultimately covered over by the growth of the soft parts (His).

The ribs bul out from the protovertebrie, and are represented on each vertebra. The thoracic ribs become cartilaginous in the 2 nd month and grow forwards into the wall of the chest, whereby the seven upper ones are united by a median portion (Rathke), which represents the position of one-half of the sternum, and when the two halves meet in the middle line the sternum is formed. When this does not occur we have the condition of the cleft sternum. At the 6th month there is a centre of ossification in the manubrium, then 4 to 13 in pairs in the body, and 1 in the ensiform process. Each rib has a centre of ossification in its body at the 2nd month, and at the 8 th to 14 th one in the tubercle and another in the head. These anchylose at the 14 th to 25 th year. Sometimes cervical ribs are present in man, and they are largely developed in birds.

The skull. - The chorda extends forwards into the axial part of the base to the sphenoid bone. The skull at first is membranous, or the primordial cranium; at the 2 nd month the basal portion becomes cartilaginous, including the occipital bone, except the upper half, the anterior and posterior part and wings of the sphenoid bone, the petrous part and mastoid process of the temporal bone, the ethmoid with the nasal septum, and the cartilaginous part of the nose. The other parts of the skull remain membranous, so that there is a cartilaginous and membranous primordial cranium.

I. The occipital bone has a centre of ossification in the basilar part at the 3rd month, and one in the condyloid part and another in the fossa cerebelli, while there are two centres in the membranous cerebral fosse. The four centres of the body unite during intra-uterine life. All the other parts unite at the 1 st to 2 nd year.

II. The post-sphenoid. - From the 3rd month it has two centres in the sella turcica, two in the suleus caroticus, two in both great wings, which also form the lamina externa of the ptery- 
goid process, while the non-cartilaginous and previously formed inner lamina arises from the superior maxillary process of the first branchial arch. During the first half of foetal life these centres unite as far as the great wings; the dorsum sellæ and the clinoid process, as far as the synchondrosis spheno-occipitalis, are still cartilaginous, but they ossify at the 13 th year.

III. The pre-sphenoid at the 8th month has two centres in the small wings and two in the body. At the 6th month they unite, but cartilage is still found within them even at the 13th year.

IV. The ethmoid has a centre in the labyrinth at the 5 th month, then in the 1 st year a centre in the central lamina. They unite about the 5 th or 6 th year.

V. Amongst the membranous bones are the inner lamina of the pterygoid process (one centre), the upper half of the tabular plate of the occipital (two points), the parietal bone (one centre in the parietal eminence), the frontal bone (one double centre in the frontal eminence), three small centres in the nasal spine, spina trochlearis and zygomatic process, nasal (one centre), the edges of the parietal bones (one centre), the tympanic ring (one centre), the lachrymal, vomer, and intermaxillary bone.

The facial bones are intimately related to the transformations of the branchial arches and branchial clefts (fig. 671). The median end of the first branchial arch projects inwards from each side towards the large oral aperture. It has two processes, the superior maxillary process which grows more laterally towards the side of the mouth, and the inferior maxillary process, which surrounds the lower margin of the mouth (fig. 665, IX). From above downwards there grows as an elongation of the basis cranii the frontal process $(s)$, a broad process with a point $(y)$ at its lower and outer angle, the inner nasal process. The frontal and the superior maxillary processes $(r)$ unite with each other in such a way that the former projects between the two latter. At the same time there is anchylosed with the superior maxillary process the small external nasal process $(n)$, a prolongation of the lateral part of the skull, and lying above the superior maxillary process. Between the latter and the outer nasal process is a slit leading to the eye $(\alpha)$. Thus the mouth is cut off from the nasal apertures which lie above it.' But the separation is continued also within the mouth; the superior maxillary process produces the upper jaw, the nasal process, and the intermaxillary process (Goethe) - the latter is present in man, but is united to the upper jaw. The intermaxillary bone, which in many animals remains as a separate bone (os incisivum), carries the incisor teeth. At the 9th week the hard palate is closed, and on it rests the septum of the nose, descending vertically from the frontal process. 'The lower jaw is formed from the inferior maxillary process. At the circumference of the oral aperture the lips and the alveolar walls are formed. The tongue is formed behiud the point of the union of the second and third branchial arches (His); while,

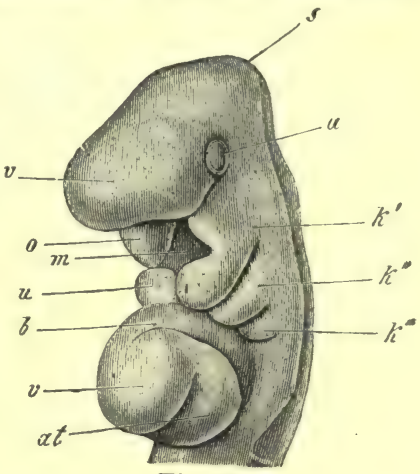

Fig. 671.

Head of embryo rabbit of 10 days $(\times 12) . \quad a$, eye ; at, atrium or primitive auricle of heart; $b$, aortic bulb; $K^{\prime}, K^{\prime \prime}, K^{\prime \prime \prime}$, first (mandibular), second (hyoid), third (1st branchial) visceral arch; $m$, mouth; $s$, superior, and $u$, inferior maxillary process ; $s$, mid-brain ; $v$, part of head and fore-brain; $v$, ventricle of heart.

according to Born, it is formed by an intermediate part between the inferior maxillary processes.

These transformations may be interrupted. If the frontal process remains separate from the

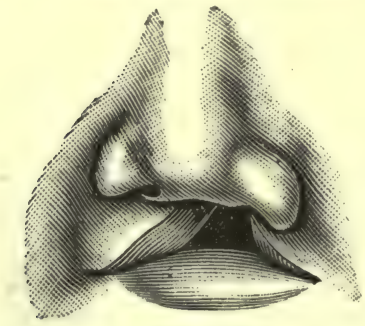

Fig. 672.

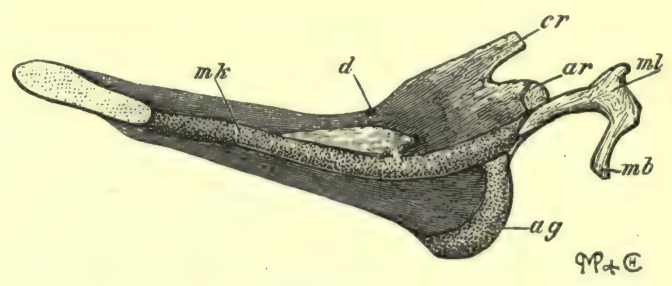

Fig. 673.

Fig. 672.-Hare lip on the left side. Fig. 673. - Inner view of the lower jaw of an embryo pig 3 inches long $\left(\times 3 \frac{1}{2}\right)$. mk, Meckel's cartilage; $d$, dentary bone ; $c r$, coronoid process ; $a r$, articular process (condyle); $a g$, angular process ; $m l$, malleus ; $m b$, manubrium.

superior maxillary processes, then the mouth is not separated from the nose. This separation may occur only in the soft parts, constituting hare-lip (fig. 672); or it may involve the hard 
palate, constituting cleft palate. Both conditions may occur on one or both sides. From the justerior jart of the first branchial arch are formed the malleus (ossified at the 4 th month), and Meckel's cartilage (fig. 673), which proceeds from the latter behind the tympanic ring as a long cartilaginous jrocess, extending along the inner side of the lower jaw, almost to its midule. It disappears after the 6 th month; still its posterior part forms the internal lateral ligament of the maxillary articulation. Near where it leaves the malleus is the processus Folii (Baumïller). A part of its median end ossifies, and unites with the lower jaw. The lower jaw is laid down in membrane from the first branchial arch, while the angle and condyle are formed from a rartilaginous process. The union of both bones to form the chin occurs at the 1st year. From the sujwrior maxillary process are formed the inner lamella of the pterygoid process, the palatine process of the upler jaw, and the palatine bone at the end of the 2 nd month, and lastly the inalar bove.

The second arch [hyoul], arising from the temporal hone, and running parallel with the first arch, rives rise to the stapes (although accorling to Salensky, this is derived from the first ar.hl, the eminentia pyramidulis, with the stapedius muscle, the incus, the styloid process of the temporal hone, the (formerly cartilaginous) stylohyoid ligament, the smaller cornu of the hyoil bone, and lastly the glosso-palatine arch (His).

The third arch (thyro-hyvil) forms the greater cornu and body of the hyoid bone and the pharyngo-palatine arch ( $\mathrm{His}$ ).

The fourth arch gives rise to the thyroid cartilage ( $\mathrm{His}$ ).

Branchial Clefts. - The first hranchial or visceral is represented by the external auditory matus, the tympanic cavity, and the Eustachian tube; all the other clefts close. Should one ir other of the elefts remain open, a condition that is sometimes hereditary in some families, a cervical fistula results, and it may he formed either from without or within. Sometimes only a lilind diverticulum remains. Branchiogenic tumours and cysts depend upon the branchial arches (R. Vulkmann).

Relation of Branchial Clefts to Nerves. - It is important to note that the clefts in front of the mouth (pre-oral), and those behind it (post-oral), have a relation to certain nerves. The linhrmmal slit between the frontal and nasal processes is supplied by the first division of the trim minus. The nasal slit between the superior maxillary process and the nasal process is suplienl hy the bifureation of the thirl nerve. The oval cleft, between the superior maxillary froresses and the mandibular arch, is supplied by the second and third divisions of the trigeminus. The first post-oral or tympanic-Eustachian cleft, between the mandibular arch (1st) and the hyoil arch, is supplied by the portio lura. The next cleft is supplied by the glosso. pharyngcal, and the succeeding clefts by branches of the vagus.]

The thymus and thyroid glands are formed as paired diverticula from the epithelium covering the liranchial arches. The epithelium of the last two clefts does not disapyear (pig), but jroliferates and pushes inwards cylindrical processes, which develop into two epithelial vesicles, the paired commencement of the thyroid glands. These vesicles have at first a central slit, whirh communicates with the pharynx ( $\mathrm{W}$ ijlfler $)$. According to His, the thyroid gland appears as an epithelial vesicle in the region of the $2 \mathrm{nd}$ pair of visceral arches in front of the tonguein man at the 4 th week. Solid buds, which ultimately become hollow, are given off from the "avity in the centre of the embryonic thyroid gland. The two glands ultimately unite together. The only epithelial part of the thymus which remains is the so-called concentric corpuscles 1. 154). According to Born, this gland is a diverticulum from the 3rd cleft, while His ascribes its origin to the 4 th and 5 th aortic arches in man at the 4 th week. The carotid gland is of epithelial origin, being a variety of the thyroid (Stieda).

The Extremities.- The origin and course of the nerves of the brachial plexus (p. 616) show that the upper ertremity was originally placed much nearer to the cranium, while the position of the prostrrior jair corresponds to the last lumbar and the $3 \mathrm{rd}$ or 4 th sacral vertebre (His).

The clavicle, according to Bruch, is not a membrane bone, but is formed in cartilage like the furculum of birds (feycubaur). At the 2 nd month it is four times as large as the upper limb; it is the first bone to ossify at the 7 th week. At puberty a sternal epiphysis is formed. Episterual bones must be referred to the clavicles (Göttc). Ruge regards pieces of cartilages existing between the clavicle and the sternum as the analogues of the episternum of animals. The clavicle is absent in many mammals (carnivora); it is very large in flying animals, and in the rabbit is half membranous. The furculum of birds represents the united clavicles.

The scapula at first is united with the clavicle (Rathke, Götte), and at the end of the 2nd month it has a median centre of ossification, which rapidly extends. Morphologically, the accessory centre in the coracoid process is interesting; the latter also forms the upper part of the articular surface. In birds the corresponding structure forms the coracoid bone, and is united with the sternum; while in man only a membranous band stretches from the tip of the coracoid process to the sternum. The long, basal, osseous strip corresponds to the supra. scapular bone of many animals. The other centres of ossification are-one in the lower angle, two or three in the acromion, one in the articular surface, and an inconstant one in the spine. Complete consolidation occurs at puberty.

The humerus ossifies at the 8 th to the 9 th week in its shaft. The other centres are-one in 
the upper epiphysis, and one in the capitellum (1st year); one in the great tuberosity and one in the small tuberosity (2nd year); two in the condyles (5th to 10 th year); one in the trochlea (12th year). The epiphyses unite with the shaft at the 16th to 20 th year.

The radius ossifies in the shaft at the 3rd month. The other centres are-one in the lower epiphysis (5th year), one in the upper (6th year), and an inconstant one in the tuberosity, and one in the styloid process. They unite at puberty.

The ulna also ossifies in the shaft at the 3rd month. There is a centre in the lower end (6th year), two in the olecranon (11th to 14 th year), and an inconstant one in the coronoid process, and one in the styloid process. They consolidate at puberty.

The carpus is arranged in mammals in two rows. The first row contains three bones-the radial, intermediate, and ulnar bones. In man these are represented by the scaphoid, semilunar, and cuneiform bones; the pisiform is only a sesamoid bone in the tendon of the flexor carpi ulnaris. The second row really consists of as many bones as there are digits (e.g., salamander). In man the common position of the 4 th and 5 th fingers is represented by the unciform bone. Morphologically, it is interesting to observe that an os centrale, corresponding to the os carpale centrale of reptiles, amphibians, and some mammals, is formed at first, but disappears at the 3rd month, or unites with the scaphoid. Only in very rare cases is it persistent. All the carpal bones are cartilaginous at birth. They ossify as follows:-Os magnum, unciform (1st year), cuneiform (3rd year), trapezium, semilunar (5th year), scaphoid (6th year), trapezoid (7th year), and pisiform (12th year).

The metacarpal bones have a centre in their diaphyses at the end of the 3rd month, and so have the phalanges. All the phalanges and the first bone of the thumb have their cartilaginous epiphyses at the central end, and the other metacarpal bones at the peripheral end, so that the first bone of the thumb is to be regarded as a phalanx. The epiphyses of the metacarpal bones ossify at the 2 nd, and those of the phalanges at the 3rd year. They consolidate at puberty.

The innominate bone, when cartilaginous, consists of two parts-the pubis and the ischium (Rosenberg). Ossification begins with three centres-one in the ilium (3rd to 4 th month), one in the descending ramus of the ischium ( 4 th to 5th month), one in the horizontal ramus of the pubis (5th to 7 th month). Between the 6th to the 14th year, three centres are formed where the bodies of the three bones meet in the acetabulum, another in the superficies auricularis, and one in the symphysis. Other accessory centres are:-One in the anterior inferior spine, the crests of the ilium, the tuberosity and the spine of the

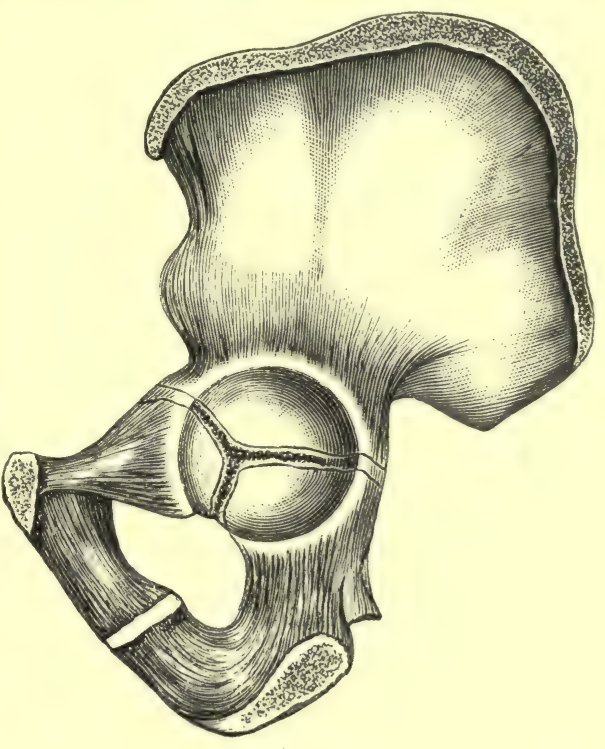

Fig. 674.

Centres of ossification of the innominate bone. ischium, the tuberculum pubis, eminentia iliopectinea, and floor of the acetabulum. At first the descending ramus of the pubis and the ascending ramus of the ischium unite at the $7 \mathrm{th}$ to 8 th year; the Y-shaped suture in the acetabulum remains until puberty (fig. 674).

The femur has its middle centre at the end of the 2nd month. At birth, there is a centre in the lower epiphysis; slightly later in the head. In addition, there is one in the great trochanter (3rd to 11 th year), one in the lesser trochanter (13th to 14th year), two in the condyles (4th to 8 th year); all unite about the time of puberty. The patella is a sesamoid bone in the tendon of the quadriceps femoris. It is cartilaginous at the 2 nd month, and ossifies from the 1st to the 3rd year.

The tarsus generally resembles the carpus. The os calcis ossifies at the beginning of the 7 th month, the astragalus at the beginning of the 8 th month, the cuboid at the end of the 10 th, the scaphoid ( 1 st to 5 th year), the I. and II. cuneiform ( 3 rd year), and the III. cuneiform ( 4 th year). An accessory centre is formed in the heel of the calcaneum at the 5 th to 10th year, which consolidates at puberty.

The metatarsal bones are formed like the metacarpals, only later.

[Histogenesis of Bone. - The great majority of our bones are laid down in cartilage, or are preceded by a cartilaginous stage, including the bones of the limbs, backbone, base of the skull, sternum, and ribs. These consist of solid masses of hyaline cartilage covered by a membrane, 
which is identical with and ultimately becomes the periosteum. The formation of bone, when preceded by cartilage, is called endochondral bone. Some bones, such as the tabular bones of the vault of the cranium, the facial bones, and part of the lower jaw, are not preceded by cartilage. In the latter there is merely a membrane present, while from and in it the future bone is formed. It becomes the future periosteum as well. This is called the intra-membranous or periosteal mode of formation.]

[Endochondral Formation.-(1) The cartilage has the shape of the future bone only in miniature, and it is covered with periosteum. In the cartilage an opaque spot or centre of ossification appears, due to the deposition of lime-salts in its matrix. The cartilage cells moliferate in this area, but the first bone is formed under the periosteum in the shaft, so that an osseous case like a muff surrounds the cartilage. This bone is formed by the subleriostual osteoblasts. (2) Blool-vessels, accompanied by osteoblasts and connective-tissue, "row into the cartilage from the osteogenic layer of the periosteum (periostcal processes of Firchow), so that the cartilage becomes channelled and cascular. As these channels extend they open into the already enlarged cartilage lacunx, alsorption of the matrix taking place, while nther parts of the cartilaginous matrix hecome ealcified. Thus a series of cavities, bounded by alcifiel cartilage-the primary medullary cavities-are formed. They contain the primary or curtiluif marvic, consisting of blool-vessels, osteoblasts, and osteoclasts, carried in from the osteocenic layer of the periosteum, and of course the cartilage cells that have been liberated from their faruna. (3) The osteoblasts are now in the interior of the cartilage, where they dispose themselves on the calcified cartilage, and secrete or form around them an osseous matrix, thus enclosing the calcified cartilage, while the ostroblasts themselves become embedded in the products of their own activity and remain as cone-corpuscles. Bone therefore is at first spongy bone, and as the primary meilullary spaces gradually become filled up by new osseous matter it becomes denser, while the calcified cartilage is gradually absorbed. It is to be remembered that, pari passu with the deposition of the new lione, bone and cartilage are being absorbed by the osteoclasts.]

Chemical Composition of Bone.-Dry bone contains $\frac{1}{3}$ of organic matter or ossein, from which relatin can be extracted by prolonged boiling; and about $\frac{2}{3}$ mineral matter, which consists of neutral calcic phosphate, $5 \frac{1}{7}$ per cent.; calcic carbonate, 7 per cent.; magnesic phosphate, 1 to 2 per cent. : calci, fluoride, 1 per cent., with traces of chlorine; and water, about 23 per cent The marrow contains fluid fat, albumin, hypoxanthin, cholesterin, and extractives. The red marrow contains more iron, corresponding to its larger proportion of hæmoglobin (Nasse).

[The medullary cavity of a long bone is occupied by yellow marrow, which contains about 96 jer cent. of fat. The red marrow occurs in the ends of long bones, in the flat bones of the skull, and in some short bones. It contains very little fat, and is really lymphoid in its characters, being, in fact, a blood-forming tissue (p. 12).]

Growth of Bones. - Long bones grow in thickness by the deposition of new bone from the periosteum, the osteoblasts becoming embedded in the osseous matrix to form the bone-corpuscles. Some of the fibres of the connective-tissue, which are caught up, as it were, in the process, remain as Sharpey's fibres, which are calcified fibres of white fibrous tissue, bolting together the jeripheric lamellie. [Miiller and Schäfer have shown that there are also fibres in the peripheric lamelle, comparable to yellow elastic fibres; they branch, stain deeply with magenta, and are best developed in the bones of birds.]

[At the same time that bone is being deposited on the surface, it is being absorbed in the marrow cavity by the action of the osteoclasts, so that a metallic ring placed round a bone in a young animal ultimately comes to lie in the medullary cavity (Duhamel). The growth in length takes place by the continual growth and ossification of the epiphysial cartilage. The rartilage is gradually absorbel from below, but it proliferates at the same time, so that what is lost in one direction is more than made up in the other (J. Hunter).

When the growth of bone is at an end, the epiphysis becomes united to the diaphysis, the epiphysial cartilage itself becoming ossified. It is not definitely proved whether there is an interstitial expansion or growth of the true osseous substance itself, as maintained by Wolff $(\$ 244,9)$.

[Howship's Lacunø.-The osteoclasts or myeloplaxes are large multinuclear giant-cells, which erode bone. They can be seen in great numbers lying in small depressions corresponding to them-Howship's lacuuæ-on the fang of a temporary tooth, when it is being absorbed. They are readily seen in a microscopical section of spongy bones with the soft parts preserved.]

The form of a bone is influenced by external conditions. The bones are stronger the greater the activity of the muscles acting on them. If pressure acting normally upon a bone be removed, the bone develops in the direction of least resistance, and becomes thicker in that direction. Bone develops more slowly on the side of the greatest external pressure, and it is curved by unilateral pressure (Lesshaft).

448. DEVELOPMENT OF THE VASCULAR SYSTEM,-Heart. - [The heart appears as a solid mass of cells in the splanchnopleure, at the front end of the embryo, immediately under the "fore-gut." Very soon a cavity appears in this mass of cells; some of the latter float free in the fluid, while the cellular wall begins to pulsate rhythmically. This hollow cellular 
structure elongates into a tube, which very soon assumes a shape somewhat like an $\mathrm{S}$ (fig. 675,1)], and there are indications of its being subdivided into $(a)$ an upper aortic part with the bulbus arteriosus ; $(b)$ a middle or ventricular part; and $(v)$, a lower venous or auricular part. The heart then curves on itself in the form of a horse-shoe $(2)$, so that the venous end $(A)$ comes to lie above and slightly behind the arterial end. On the right and left side, respectively, of the venous part is a blind hollow outgrowth, which forms the large auricle on each side $\left(3, o_{0} o_{1}\right)$. The flexure of the body of the heart corresponding to the great curvature $(2, V)$ is divided into two large compartments (3), the division being indicated by a slight depression on the surface. The large truncus venosus $(4, v)$, which joins with the middle of the posterior wall of the auricular part, is composed of the superior and inferior venæ cavæ. This common trunk is absorbed at a later period into the enlarging auricle, and thus arise the separate terminations of the superior and inferior venæ cavæ. In man, the heart soon comes to lie in a special cavity, which in part is bounded by a portion of the diaphragm (His). At the 4th to 5 th week, the heart begins to be divided into a right and a left half. Corresponding to the position of the vertical ventricular furrow, a septum grows upwards vertically in the interior of the heart, and divides
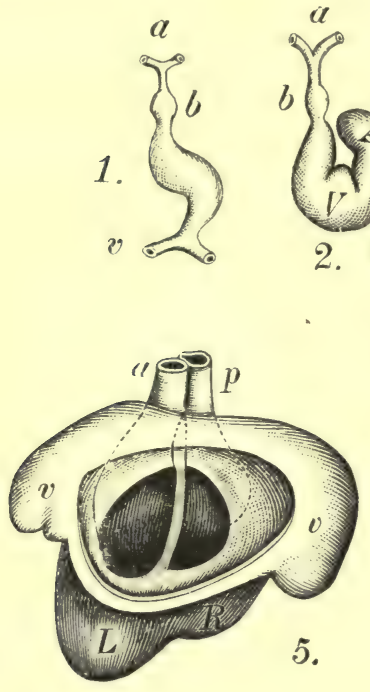

2.
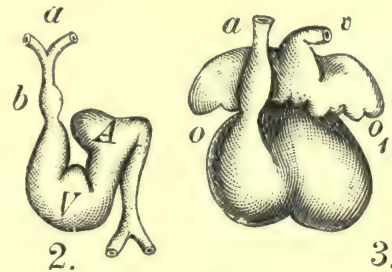

3.

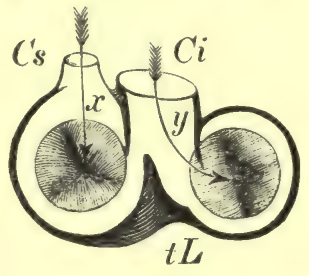

6.

Fig. 675 .
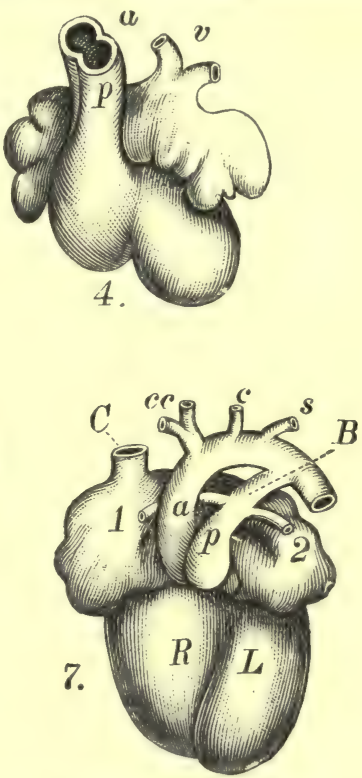

Development of the heart. 1, Early appearance of the heart- $a$, aortic part, with the bulbus, $b ; v$, venous end. 2, Horse-shoe shaped curving of the heart-a, aortic end, with the bulbus, $b ; V$, ventricle; $A$, auricular part. 3 , Formation of the auricular appendages, $o, o_{1}$, and the external furrow in the ventricle. 4, Commencing division of the aorta, $p$, into two tubes, $a .5$, View from behind of the opened auricle, $v, v$, into the $L$, and $R$, ventricles, and between the two latter the projecting ventricular septum, while the aorta (a) and pulmonary artery $(p)$ open into their respective ventricles. 6, Relation of the orifices of the superior $(C s)$ and inferior vena cava $(C i)$ to the auricle (schematic view from above $-x$, direction of the blood of the superior vena cava into the right auricle; $y$, that of the inferior cava to the left auricle ; $t L$, tubercle of Lower. 7 , Heart of the ripe foetus $-R$, right, $L$, left ventricle ; $a$, aorta, with the innominate, $c, c$, carotid, $c$, and left subclavian artery, $s ; B$, ductus arteriosus ; $p$, pulmonary artery, with the small branches 1 and 2 , to the lungs.

the ventricular part into a right and left ventricle $(5, R, L)$. There is a constriction in the heart, between the auricular and ventricular portions, forming the canalis auricularis. It contains a communication between the auricle and both ventricles, lying between an anterior and posterior projecting lip of endothelium, from which the auriculo-ventricular valves are formed ( $F$. Schmidt). The ventricular septum grows upwards towards the canalis auricularis, and is complete at the 8 th week. Thus, the large undivided auricle communicates by a right and left auriculo-ventricular opening with the corresponding ventricle (5). At the same time two septa $(4, p a)$ appear in the interior of the truncus arteriosus $(4, p)$, which ultimately meet, and thus divide this tube into two tubes $(5, a p)$, the latter forming the aorta and pulmonary 
artery, and are disposed towards each other like the tubes in a double-barrelled gun. The septum grows downwards until it meets the ventricular septum (5), so that the right ventricle comes to be connected with the pulmonary artery, and the left with the aorta. The division of the truncus arteriosus, however, takes place only in the first part of its course. The division does not take place above, so that the pulmonary artery and aorta unite in one common trunk alveve. 'This communication between the pulmonary artery and the aorta is the ductus arteriosus Botalli $(7, B)$.

In the auricle a septum grows from the front and behind, ending internally with a concave mariu. The vena cava smperior $(6, C s)$ terminates to the right of this fold, so that its blood will tend to go towards the right ventricle, in the direction of the arrow in $6, x$. The cava inferior, on the other hand $\left(\hat{b}, \hat{C}_{i} i\right)$, opens directly opposite the fold. On the left of its orifice the valve of the foramen ovale is formed by a fold growing towards the auricular fold, so that the blood-current from the inferior vena cava goes only to the left, in the direction of the arrow, $y$; on the right of the orifice of the cava, and opposite the fold, is the Eustachian valve, which, in conjunction with the tubercle of Lower $(t L)$, directs the stream from the inferior vena cava to the left into the left auricle, through the pervious foramen ovale. Compare the futal circulation (1. 876). After birth, the valve of the foramen ovale closes that aperture, while the ductus arteriosus also becomes impervious, so that the blood of the pulmonary artery is forced to so through the pulmonary branches proceeding to the expanding lungs. Sometimes the foramen ovale remains pervious, giving rise to serious symptoms after a time, and constituting morbus ceruleus.

Arteries. - With the formation of the branchial arches and clefts, the number of aortic arches on each side becomes increased to 5 (fig. 676 ), which run above and below each branchial cleft,
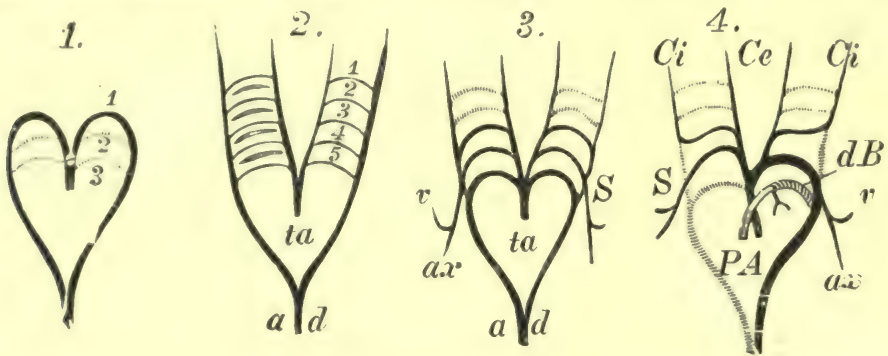

Fig. 676 .

The aortic arches. 1. The first position of the 1, 2, and 3 arches. 2. 5 aortic arches; $t a$, common aortic trunk; ad, descending aorta. 3. Disappearance of the upper two arches on each side $-S$, subclavian artery; $v$, vertebral artery; $a x$, axillary artery. 4. Transition to the final stage $-P$, pulmonary artery ; $A$, aorta ; $d B$, ductus arteriosus (Botalli) ; $S$, right subclavian, united with the right common carotid, which divides into the internal $(C i)$ and external carotid $(C e)$; ax, axillary ; $v$, vertebral artery.

in a branchial arch, and then all reunite behind in a common descending trunk $(2, a d)$ (Rathkc). 'These blood-vessels remain only in animals that breathe by gills. In man, the upper two arches disappear completely (3). When the truncus arteriosus divides into the pulmonary artery and the aorta $(4, P, A)$, the lowest arch on the left side, with its origin, forms the julmonary artery (4), and it springs from the right side of the heart. Of these the left lowest arch forms the ductus arteriosus $(l B)$, and from the commencement of the latter proceed the pulmonary branches of the pulmonary artery. Of the remaining arches which are united with the aorta, the left middle one (i.e., the fourth left) forms the permanent aortic arch into which the ductus arteriosus opens, while the right one (fourth) forms the subclavian artery; the third arch forms on each side the origin of the carotids $(C i, C e)$. The arteries of the first and second circulations have been referred to already (p. 870). When the unbilical vesicle, with its primary circulation, diminishes, only one omphalo-mesenteric artery is present, which gives a branch to the intestine. At a later period, the omphalo-mesenteric arteries atrophy, while the artery to the intestine-the superior mesenteric-becomes the largest of all, it being originally derived from one of the omphalo-mesenteric arteries.

Veins of the Body. - The veins first formed in the body of the embryo itself are the two cardinal veins; on each side an anterior (fig. $677, \mathrm{I}, c s$ ), and a posterior (ci), which proceed towards the heart and unite on each side to form a large trunk, the duct of Cuvier $(D C)$, which passes into the venous part of the heart. The anterior cardinal veins give off the subclavian veins $(b b)$ and the comunon jugular veins, which divide into the external $(I c)$ and internal (Ji) jugular veins. In addition, there is a transverse ancestonnosing branch passing obliquely from the left (where it divides) to the right, which joins their trunk lower down. In the final 
arrangement (II) this anastomosis $(A s)$ becomes very large to form the left innominate vein, while with the growth of the arms the subclavian veins increase $(b b)$; and lastly, the calibre of the jugular vein changes, the internal jugular $(J i)$ becoming very large, and the external jugular (Ie) smaller. In some animals, e.g., the dog and rabbit, the large embryonic size is retained. The part of the left superior cardinal vein, from the anastomosis downwards to the left duct of Cuvier, disappears. The posterior cardinal veins divide in the pelvis into the hypogastric $(I, h)$ and external iliac $(f, f)$. The inferior cava at first is very small $(I, V c)$, divides at the entrance to the pelvis, and on each side goes into the point of division of the cardinal veins. There is also a transverse ascending anastomosis between the right and left cardinal veins. For the final arrangement, the cava inferior (II, Ci) dilates, and with it the hypogastric and external iliac vein on each side. The right cardinal vein remains very small ( ${ }^{\text {ena }}$ azygos, $A z$ ), and also the lower part from the left one to the transverse anastomosis. The latter itself also remains very small (Vena hemiazygos, $H z$ ). On the other hand, the upper part above the anastomosis to the duct of Cuvier disappears. Lastly, the common large venous trunk is so absorbed into the wall of the auricle $(V)$ that both venæ cavæ have each a separate orifice (p. 876). The embryonic condition of the veins persists in fishes.

Veins of the First and Second Circulation, and Portal System.-The two omphalo-mesenteric veins $\left(o m, o m_{1}\right)$ open into the posterior or venous end of the tubular heart (fig. $678,1, H$ ). The right vein, however, disappears very soon. As soon as the allantois is formed, the two nmbilical veins join the truncus venosus $\left(1, u, u_{1}\right)$. At first the omphalo-mesenteric veins are larger than the umbilical

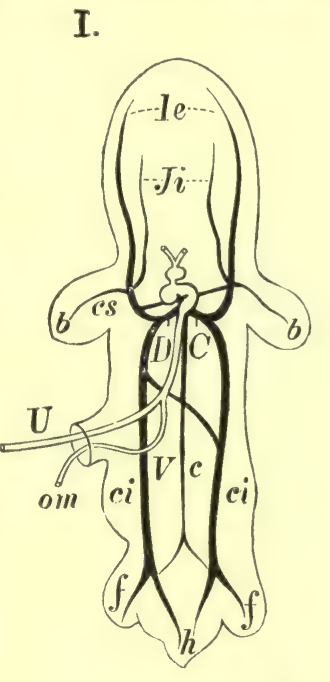

Fig. 677.

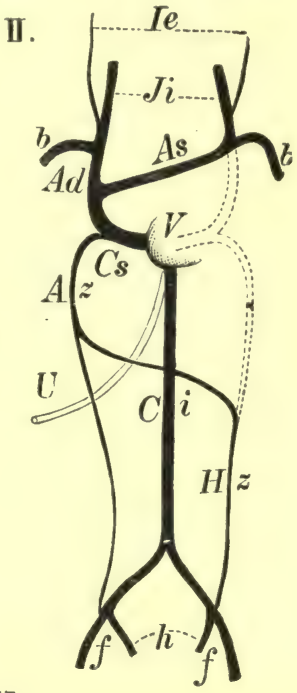

I, First appearance of the veins of the embryo. II, Their transformations to form the final arrangement.

veins; at a later period this is reversed, and the right umbilical vein disappears. As soon as veins are formed within the body proper of the embryo, the inferior cava also opens into the

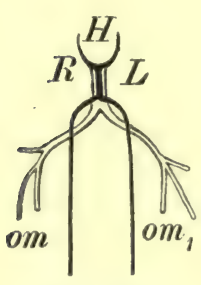

$u_{1}$

1.

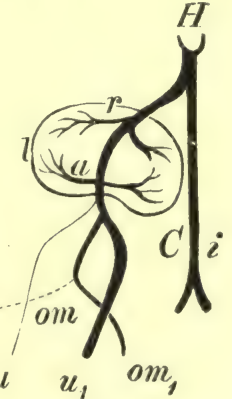

2.

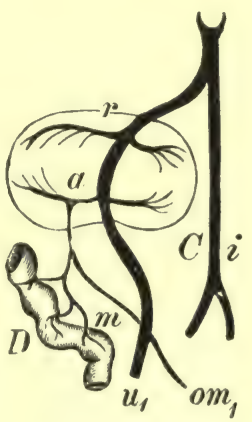

3.

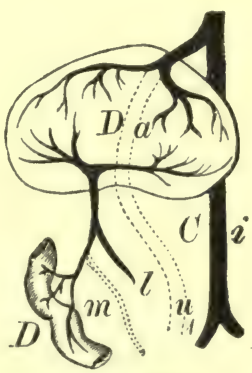

4. $\mathrm{om}$,

Fig. 678.

Development of the veins and portal system. $H$, heart; $R, L$, right and left side of the body; om, right omphalo-mesenteric vein ; om, left; $u$, right umbilical vein; $u_{1}$, left ; $C i$, vena cava inferior ; $a$, venæ advehentes; $r$, venæ revehentes; $D$, intestine; $m$, mesenteric vein ; $4, l$, splenic vein ; $2, l$, liver.

truncus venosus $(2, C i)$. Gradually, the umbilical vein $\left(2, u_{1}\right)$ becomes the chief trunk, while the small omphalo-mesenteric $\left(2, \mathrm{om}_{1}\right)$ carries little blood.

Portal System. - The umbilical and omphalo-mesenteric veins pass in part directly under the 
liver to reach the heart. They send branches-carrying arterial blood-to the liver, and the latter grows round these vessels. These brauches are the venæ advehentes (2 and $3, a)$. The hlool eirculating through the liver from the venæe advehentes is returned by other veins, the vense revehentes ( 2 and $3, r$ ), which reunite at the blunt margin of the liver with the chief trunk of the umbilical vein. The umbilical vein $\left(3, u_{1}\right)$ and the omphalo-mesenteric vein $\left(3, o m_{1}\right)$ anastomose in the liver. When the intestine develops $(3, D)$, the mesenteric vein ( $m$ ) oprens into the omphalo-mesenteric vein, and the splenic vein as well $(4, l)$, when the spleen is formed. At a later period, when the omphalo-mesenteric vein $\left(4, o m_{1}\right)$ disappears, the vein from the intestine now becomes the common trunk of the previously united vessels. It unites in the liver with the umbilical vein to form the trunk of the vena porta: When, after birth, the umbilical vein disappears $\left(4, u_{1}\right)$, the mesenteric alone remains as the portal vein. As the ductus venosus is obliterated, the portal vein must send its blood through the liver, and thus the portal circulation is completed.

449. FORMATION OF THE INTESTINAL CANAL, - The primitive intestine, or gut, consists of a straight tulve proceeding from the head to the tail. 'The vitelline duct is inserted at that point, which at a later period corresponds to the lower part of the ileum. At the 4th week the tulre makes a slight lend toward the umbilicus (fig. 679, I). As already mentioned,

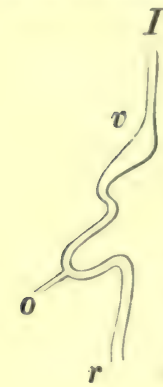

$I$

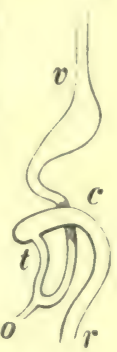

III

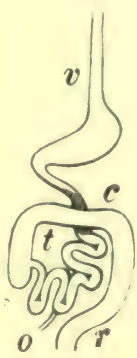

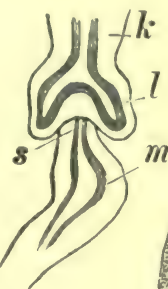

A

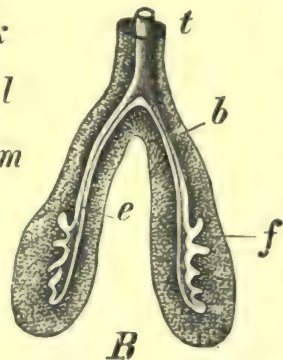

Fig. 680 .

Fig. 679.

Fig. 679. - Development of the intestine. $v$, stomach; $o$, insertion of the vitelline duct ; $t$, small intestine ; $c$, colon ; $r$, rectum. Fig. 680. -Formation of the lungs. $\Lambda$, Diverticula of the lungs as double sacs- $k$, mesol,lastic layer ; l, hypoblastic layer; $m$, stomach; $s$, nesophagus. B, Further hranching of the lungs $-t$, trachea ; $b, e$, bronchi ; $f$, projecting vesicles. the vitelline duct is obliterated, remaining only for a time as a thread attached to the intestine, being still visible at the $3 \mathrm{rd}$ month. Sometimes it remainsasa short blind tube communicating with the intestine. This is the so-called "true intestinal diverticulum"; occasionally a cord - the obliterated omphalo-mesenteric vessels-passes from it to the umbilicus. In very rare cases, the duct may remain open as far as the um.

lilicus, forming a congenital fistula of the ileum, or it may give rise to eystic formations ( $M$. loth). In a human foetus at the 4 th week, His distinguished the eavity of the mouth, pharynx, resophagns, stomach, duorlenum, mesenterial intestine, and the hind-gut, with the cloaca. The intestine then forms the first coil (fig. 679, II) by rotating on itself at the intestinal umbilicus, so that the lower part of the intestine lying next the knee-like lend comes to lie above, while the upper part lies below. From the lower part of this loop, there proceed the coils of the small intestine (III, $t$ ), which gradually grow longer. From the upper limb of the loop, which also elongates, the large intestine is formed; first the descending colon, then by elongation the transverse colon, and lastly the ascending colon.

Gland6. - By diverticula, or protrusions from the intestine, the various glands are formed. The cells of the hypoblast proliferate and take part in the process, as they form the secretory cells of the glands, while the mesoblastic part of the splanchnopleure forms the membranes of the glands, giving them their shape. The diverticula are as follows :-

1. The salivary glands, which grow out from the oral cavity at first as simple solid buds, but afterwards become hollow and hranched. [The salivary glands are developed from the epiblast lining the mouth (stomodreum).]

2. The lungs, which arise as two separate hollow buds (fig. $680, \mathrm{~A}, 2$ ), and ultimately have only one common duct, are protrusions from the oesophagus. The upper part of the united tracheal tube forms the larynx. The epiglottis and the thyroid cartilage originate from the part which forms the tongue (Ganghofner). The two hollow spheres grow and ramify like branched tubular glands with hollow processes $(\mathrm{B}, f)$. In the first period of development, there is no essential difference between the epithelium of the bronchi and that of the primitive airvesicles (Sticila). The spleen and suprarenal capsules, however, are not developed in this way. The former arises in a fold of the mesogastrium at the 2nd month (His); the latter are origin ally larger than the kidneys. 
3. The pancreas arises in the same way as the salivary glands, but is not visible at the 4 th week (His).

4. The liver begins very early, and appears as a diverticulum, with two hollow primitive hepatic ducts, which branch and form bile-ducts. At their periphery they penetrate between the solid masses of cells-the liver-cellswhich are derived from the hypoblast. At the 2nd month the liver is a large organ, and secretes at the 3rd month (\$182).

5. In birds two small blind sacs are formed from the hind-gut.

6. The fœtal respiratory organ, the allantois, is treated of specially (\$ 444).

Peritoneum and Mesentery. The inner surface of the coelom, or body-cavity, the surface of the intestine, and its mesentery are covered by a serous coat-the peritoneum. At first the simple intestine is contained in a fold, or duplicature of the peritoneum ; on the stomach, which is merely at first a spindle-shaped dilatation of the tube placed vertically, it is called mesogastrium. Afterwards, the stomach turns on its side, so that the left surface is directed forwards and the right backwards. Thus, the insertion of the mesogastrium, which originally was directed backwards (to

$\boldsymbol{I}$

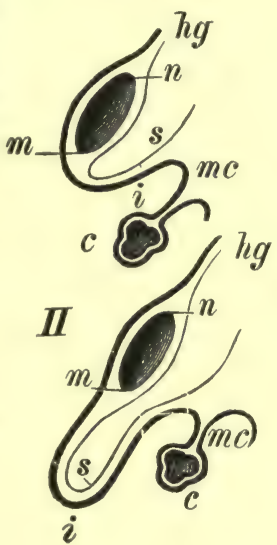

III

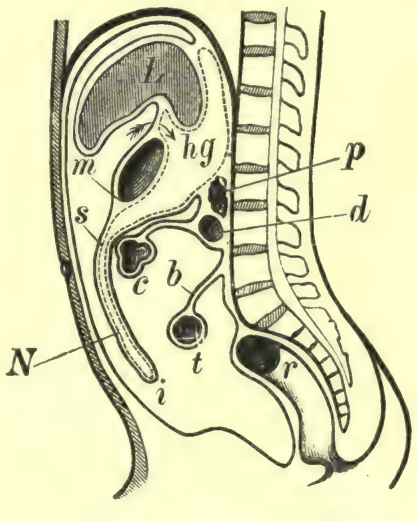

Fig. 681.

Formation of the omentum. I and II. - hg, gastro-hepatic ligament; $m$, great, $n$, lesser curvature of the stomach ; $s$, posterior, and $i$, anterior fold or plate of the omentum ; $m c$, mesocolon; $c$, colon. III. $-L$, liver ; $t$, small intestine; $b$, mesentery $; p$, pancreas ; $d$, duodenum ; $r$, rectum ; $N$, great omentum.

the vertebral column), is directed to the left; the line of insertion forming the region of the

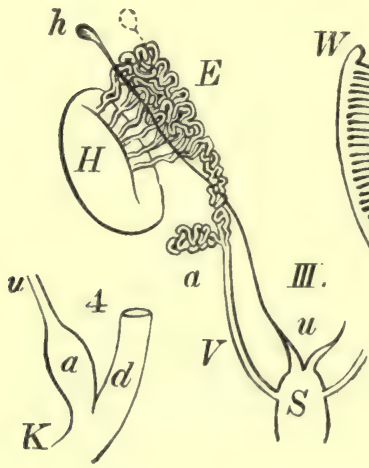

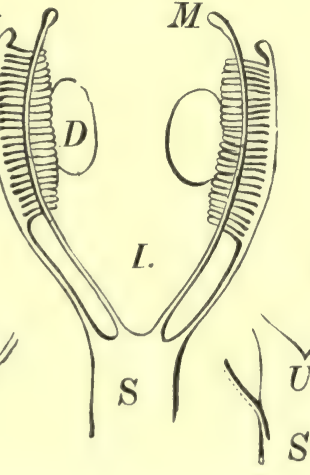

Fig. 682 .

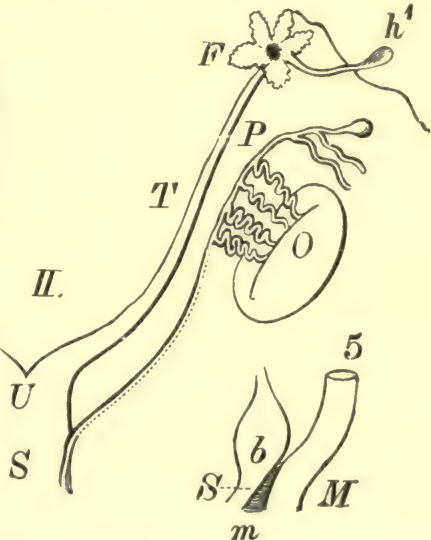

$m$

Development of the internal generative organs. I., Undifferentiated condition-D, reproductive gland, lying on the tubules of the Wolffian body ; W, Wolffian duct; M, Müllerian duct; $\mathrm{S}$, uro-genital sinus. II., Transformations in the female-F, fimbria, with the hydatid, $h^{1}$; T, Fallopian tube ; U, uterus ; S, uro-genital sinus ; O, ovary ; P, parovariun.. III., Transformations in the male- $\mathrm{H}$, testis; $\mathrm{E}$, epididymis, with the hydatid, $h ; a$, vas aberrans; $\mathrm{V}$, vas deferens; $\mathrm{S}$, uro-genital sinus; $u$, male uterus. $4, d$, hind-gut ; $a$, allantois ; $u$, urachus ; $\mathrm{K}$, cloaca. 5 , M, rectum ; $m$, perineum ; $b$, position of the bladder; S, uro-genital sinus.

great curvature, which becomes still more curved. From the great curvature, the mesogastrium becomes elongated like a pouch (fig. 681 , I and II, $s, i$ ), constituting the omental sac, which 
extends so far downwards as to pass over the transverse colon and the loops of the small intestine (III,, $\mathrm{V}$ ). As the mesogastrium originally consists of two plates, of course the omentum must consist of four plates. At the 4 th month, the posterior surface of the omental sac unites with the surface of the transverse colon (Joh. Müller).

450. URINARY AND GENERATIVE ORGANS. - Urinary apparatus, - The first indication of this apparatus occurs in the chick at the 2nd day and in the rabbit at the 9 th, as the ducts of the primitive kidneys or Wolffian ducts (fig. 682, 1, W), which are formed from some cells mapred off from the lateral plate above and to the side of the protovertebre, and extending from the fifth to the last vertebra. The ducts are solid at first, but soon become hollow, and from their cavities there extend laterally a series of small tubes, which in the chick communirate freely with the peritoneal cavity (Kölliker). Into one end of each of these tubes grows a tuft of blood-vessels forming a structure resembling the glomeruli of the kidney. The tubes longate, form convolutions, and increase in number. The upper end of the Wolffian duct is "losed at first, its lower end, which lies in a projecting fold - the plica urogenitalis of Waldeyer -in the peritoneal cavity, opens into the uro-genital sinus. Close above the orifice of the Wolffian duct appears the ureter as the duct of the kidney. The duct elongates, and branches at its upper end. Each canal at its end is like a stalked caoutchouc sac (Toldt), and into it there grow the already formed glomeruli. The duct of the kidney opens independently into the uro-genital sinus, and forms the ureter. The part where the branching of the duct stops forms the pelvis of the kidney, and the branches themselves the renal tubules. Toldt found

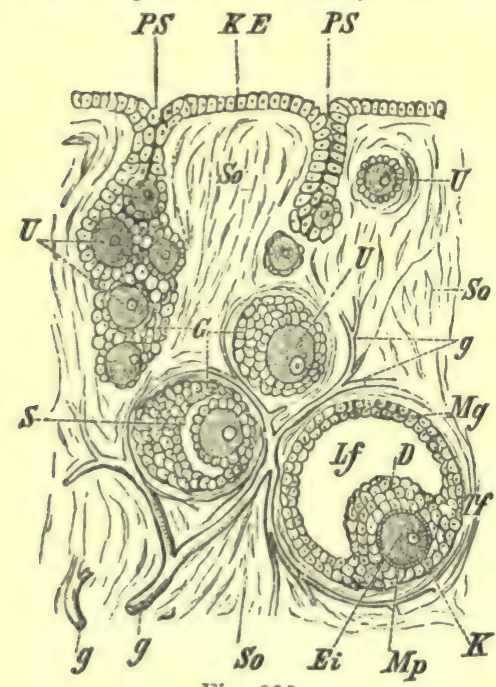

Fig. 683.

Section of mammalian ovary showing de. velopment of ova and their follicles. $E i$, Ripe ovum; $G$, follicular cells of germinal epithelium; $g$, blood-vessels ; $K$, germinal vesicle and spot; $K E$, germinal epithelium; $L f$, liquor folli. culi ; $M g$, membrana granulosa ; $M p$, zona pellucida; $P S$, ingrowths from germinal epithelium, ovarian tubes, by means of which some of the nests retain their connection with the epithelium; $\mathbb{S}$, eavity which appears within the Graafian follicle; So, stroma of ovary; Tf, Theca folliculi or ovi-capsule; $U$, primative ova.

uterus or vesicula prostatica (III, $u$ )-the

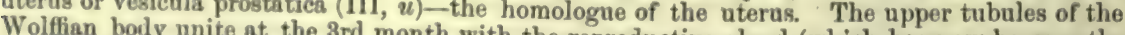
body of the ty unite at the 3rd month with the reproductive gland (which has now become the body of the testis), and become the coni vasenlosi of the epididymis, which are lined by eiliated epithelium (E); the remainder of the Wolffian body disappears. Some detached tubules form Malpighian corpuscles in the human kidney at the 2nd month, and Henle's loops at the 4th. The first appearance of the urinary bladder is at the 4 th week (His), and is more distinct at the 2nd month, as the dilated first part of the allantois (fig. 682). The upper part of the allantois remains as the obliterated urachus, in the middle vesicle ligament.

Internal Reproductive Organs. - In front of and internal to the Wolffian bodies, there arises in the mesoblast the elongated reproductive gland, germridge, or mass of germ-epithelium (fig. 682, I, D), which in both sexes is originally alike (fig. $683, \mathrm{~K}, \mathrm{E}$ ). In addition, there is formed a canal or duct parallel to the Wolffian duct (W), which also opens into the uro-genital sinus; this is Muller's duct (M). The elevation of the future reproductive gland is covered originally by germ-epithelium (Waldeyer). The upper end of the Miillerian duct opens free into the abdominal cavity, while the lower ends of both ducts unite for a distance. Some of the germinal cells covering the surface of the future ovary enlarge to form ova, and sink into the stroma to form ova embedded in their Graatian follicles (\$ 433) (fig. 683). In the female, the Miillerian ducts form the Fal. lopian tube (II, T), and the lower united ends the uterus.

In the male, the germ-epithelium is not so tall. According to Waldeyer, there are two kinds of tubes in the Wolffian bodies, and some of these penetrate the position of the reproductive gland. These tubes, which are connected with the Wolffian ducts, hecome the seminiferous tubules (v. Wittich), and the Wolffian duct itself becomes the vas deferens, with the vesiculæ seminales. According to some other observers, however, tubes which become the seminiferous tubules, are developed within the reproductive gland itself, and these tubes lined with their germ-epithelium ultimately form a connection with the Wolffian ducts.

The Muillerian ducts, which are really the ducts of the reproductive glands, disappear in man, all except the lowest part, which becomes the male 
the vasa aberrantia $(a)$ of the testicle (Kobelt). The hydatid of Morgagni $(h)$, at the head of the epididymis, according to Luschka and others, is a part of the epididymis-Fleischl regards it as the rudiment of the male ovary. The organ of the Giraldès is part of the Wolffian body. The Wolffian duct itself becomes the vas deferens (V) from which the vesiculæ seminales are developed. The two Wolffian and two Müllerian ducts, as they enter the pelvis, unite to form a common cord-the genital cord.

In the female, the tubes of the Wolffian bodies disappear, all except a few tubules, lined with ciliated epithelium, constituting the parovarium, or organ of Rosenmiiller (fig. 646), and a part

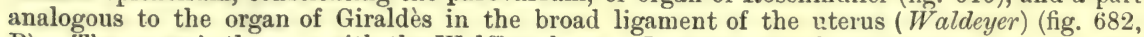
P). The same is the case with the Wolffian ducts. In some animals (ruminants, pig, cat, and fox) they remain permanently as the ducts of Gaertner.

The Miillerian duct is frayed out at its upper end to form the fimbrix of the Fallopian tube, and it is often provided with a hydatid $\left(h^{1}\right)$. That part of the uro-genital sinus into which the four ducts open grows above into a hollow sphere, which forms the vagina (Ruthke). According to Thiersch and Leuckart, however, the two Miillerian ducts unite at their lower ends to form the united uterus (U) and vagina, while their free upper ends form the Fallopian tubes $(\mathrm{T})$. The Miillerian ducts at first open into the posterior part of the urinary bladder below the ureters (uro-genital sinus, S), while ultimately this part of the bladder becomes so elongated posteriorly that the vagina (the united Muillerian ducts) and the urethra are united below and deeply within the vestibule of the vagina. It the 3 rd to the 4 th month, the uterus and vagina are not separate from each other, but at the 5 th to the 6 th month the uterus is defined from the vagina.

The testicles lie originally in the lumbar region of the abdominal cavity (fig. $684, \mathrm{~V}, t$ ), and are carried by a fold of the peritoneum-the mesorchium $(m)$. From the hilum of the testicle a cord, the gubernaculum testis, runs through the inguinal canal into the base of the scrotum. At the same time a septum-like process is developed independently from the peritoneum to the base of the scrotum $(p v)$. The testicle passes through the inguinal canal into the scrotum, but the mechanism and the cause of the descent are not accurately ascertained.-[Descent of testis, § 446.]

The ovaries also descend somewhat. The round ligament of the uterus corresponds to the gubernaculum testis. A process of the peritoneum passes in the female into the inguinal canal as Nuck's canal. It is rare to find the ovaries descending into the labia majora.

[The origin of the urinary and generative organs is undoubtedly associated with the development of the Wolffian bodies. The researches of Semper and Balfour on elasmobranch fishes show that the process is a very complex one. There is a mass of cells on each side of the vertebral column, which is divided into three parts, the first called the pronephros, or head-kidney of Balfour and Sedgwick, the middle one, the mesonephros or Wolffian body, and the posterior one or metanephros, which is formed after the other two, gives origin to the permanent kidney in the arnniota. The Miillerian duct is connected with the pronephros, the Wolffian duct with the mesonephros, and the ureter with the metanephros.]

[The following table, modified from Quain, shows the destiny of these structures :-

$$
\text { Müllerian Ducts (Ducts of the Pronephros). }
$$

Female.

Fallopian tubes. Hydatid.

Uterus and vagina.

\section{Wolffian Bodies (Mesonephros).}

Parovarium.

Paroöphoron.

Round ligament of the uterus.

\section{WolfFiAN Ducts.}

Chief tube of parovarium.

Ducts of Gaertner.
Male.

Hydatid of Morgagni.

Male uterus.

Vasa efferentia, Coni vasculosi.

Organ of Giraldès, Vasa aberrantia.

Gubernaculum testis.

Convoluted tube of epididymis.

Vas deferens and vesiculæ seminales.

\section{Metanephros. \\ Kidney. Ureter.]}

The external genitals are at first not distinguishable in the two sexes (fig. 684, $I$ ). At the 4 th week, there is merely an orifice at the posterior extremity of the trunk, representing both the anus and the opening of the urachus, and forming a cloaca (fig. 682, 4, K). In front of this an elevation-the genital eminence - appears about the 6 th week, and on each side of the orifice a large cutaneous elevation $(I I, w)$. At the end of the 2 nd month, there is a groove on the under surface of the genital eminence, leading back to the cloaca, and with distinct walls bounding it $(I I, r)$. At the middle of the 3rd month, the cloacal opening is divided by the growth of the 
perineum, between the urachus (now become the urinary bladder) (fig. 682, 5, b) and the rectum (M).

In the male, the genital eminence enlarges, its groove deepens from the opening of the blaclder onwards to the apex of the elevation at the 10th week. The two edges unite to enclose

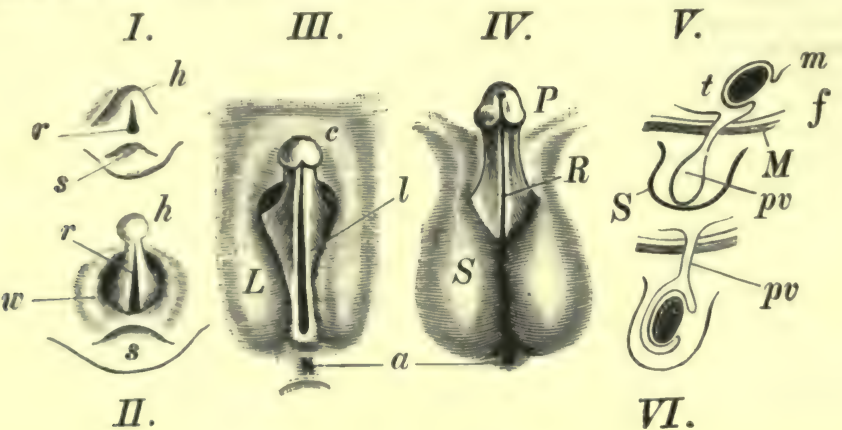

Fig. 684.

Development of the external genitals. $I$. and $I I$. - Genital eminence; $r$, genital groove ; $s$, coceyx ; $u$, cutaneous elevations. $I V .-P$, penis ; $R$, raphe penis ; $S$, scrotum. III.c, clitoris; I, labia minora; $L$, labia majora ; $a$, anus. $V$. and $V I$. -Descent of the testicle; $t$, testis ; $m$, mesorchium ; $p v$, processus vaginalis of the peritoneum; $M$, abdominal wall ; $S$, scrotum.

the groove which hecomes the urethra. When this does not take place, hypospadias occurs. At the 4 th month the glans, and at the 6 th the prepuce, are formed. The large cutaneous folds meet in the middle line or raphe to form the scrotum.

In the female, the undifferentiated condition remains to a certain extent permanent. The small genital eminence remains as the clitoris, the margins of its furrow become the nymphæ, the cutaneous elevations remain separate to form the labia majora. The uro-genital sinus

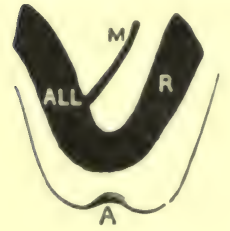

Fig. 685.

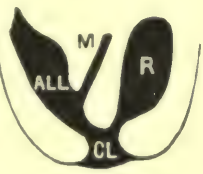

Fig. 686 .

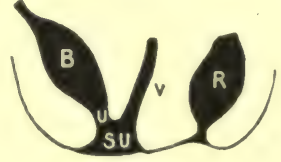

Fig. 687.

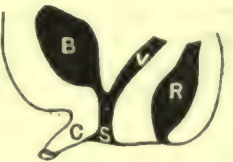

Fig. 688.

Fig. 685.-R, rectum continuous with the allantois (ALL-bladder); M, duct of Miuller (vagina); A, depression of skin below genital eminence, growing inwards to form the vulva. Fig. 686.-The depression has become continuous with the rectum and allantois to form the cloaca (CL). Fig. 687.-The cloaca is becoming divided into uro-genital sinus (SU) and anus by the downward growth of the perineal septum. The ducts of Miiller are united to form the vagina (V). Fig. 688. - Perineum completely formed.

remains short as the vestibule of the vagina, while in man, by the closing of the genital groove, it has a long additional tube, the urethra. [The accompanying illustrations, after Schroeder, show the changes of the external organs of generation in the female. In the early period (6th week), the hind-gut (fig. 685, R), allantois (ALL), and the Miillerian ducts (M) communicate, but not with the exterior. About the 10th week a depression or inflection of the skin takes place, genital cleft, until it meets the hind-gnt and allantois, whereby the cloaca (fig. 686, CL) is formed. The cloaca is then divided into an anterior part, the uro-genital sinus, into which the Miillerian ducts open, and a posterior part, the anus. There is a downward growth of the tissue between the hind-gut and the allantois to form the perineum (fig. 687). The uro-genital sinus then contracts at its upper part to form the short urethra, its lower part remaining as the vestibule (fig. $688, \mathrm{SV}^{\mathrm{V}}$ ), while the vagina has been formed by the union of the lower parts of the two Miillerian ducts. The bladder (B) is the expanded lower end of the stalk of the allantois.]

The causes of the difference of sex are by no means well known. From a statistical analysis of 80,000 cases, the influence of the age of the parents has been shown by Hofacker and 
Sadler. If the husband is younger than the wife, there are as many boys as girls ; if both are of the same age, there are 1029 boys to 1000 girls ; if the husband is older, 1057 boys to 1000 girls. In insects, food has a most important influence. Pfliger's investigations on frogs show that all external conditions during development are without effect on the determination of the sex, so that the latter would seem to be determined before impregnation.

451. FORMATION OF THE CENTRAL NERVOUS SYSTEM. - Fore-brain.-At each side of the fore-brain, or anterior cerebral vesicle, which is covered externally by epiblast and internally by the ependyma, there grows out a large stalked hollow vesicle, the rudiment of the cerebral hemispheres. The relatively wide opening in the stalk, or communication, ultimately becomes very small, and is the foramen of Monro. The middle part between the two cerebral vesicles remains small, and is the 'tween or interbrain with the $3 \mathrm{rd}$ ventricle in its interior. It elongates at the second month towards the base of the brain as a funnel-shaped projection, to form the tuber cinereum with the infundibulum. The thalami optici, projecting and enlarging from the sides of the 3rd ventricle, narrow the foramen of Monro to a semilunar slit. At the base of the brain are formed, in the 2nd month, the corpora albicantia, at the 3rd the chiasma; while within the 3rd ven. tricle the commissures are formed. The hypophysis, belonging to the mid-brain, is a diverticulum of the nasal mucous membrane, extending $\mathrm{T}$ through the base of the skull towards the hollow infundibulum, which grows to meet it (fig. 505, $T)$. There is, as it were, a tendency to the union of the cavity of the fore-gut with the medullary tube. In the amphioxus (Kowalewsky), goose (Gasser), and lizard (Strahl) the medullary tube communicated originally with the hind-

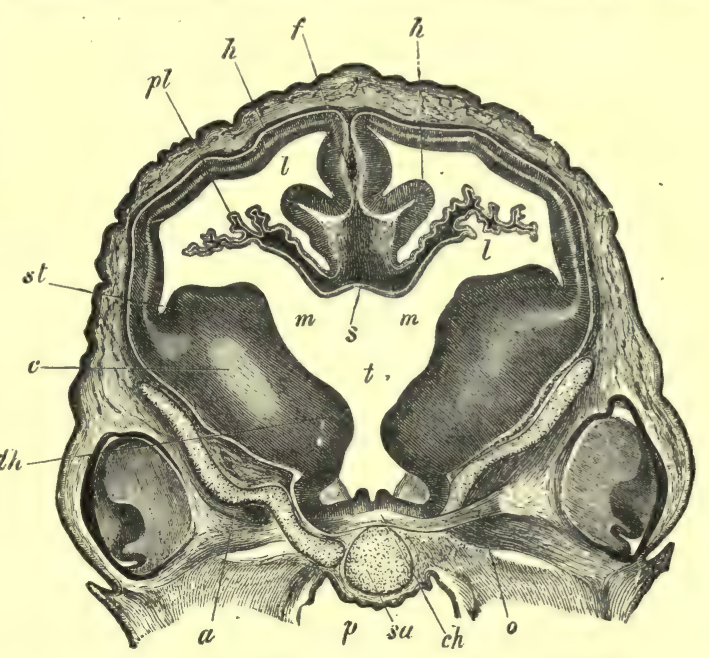

Fig. 689.

Transverse section of the brain of an embryo sheep $2.7 \mathrm{~cm}$. long ; $\times 10 . \quad a$, cartilage of orbito-sphenoid ; $c$, peduncular fibres ; $c h$, optic chiasm; $f$, median cerebral fissure; $h$, cerebral hemispheres, with a convolution upon their inner wall, projecting into the lateral ventricle, $l ; m$, foramen of Monro; $o$, optic nerve; $p$, pharynx; $p l$, lateral plexus; $s$, termination of the median fissure, which forms the roof of the third ventricle; $s a$, body of the anterior sphenoid; st, corpus striatum ; $t$, third ventricle ; th, anterior deep portion of the optic thalamus (Kölliker). gut by the canalis myeloentericus.

The choroid plexus, which grows into the ventricles of the hemispheres through the foramen of Monro, is a vascular development of the ependyma. At the 4 th month, the conarium (pineal gland) is formed, and at this time the corpora quadrigemina cover the hemispheres. The corpora striata begin to be developed in the cerebral (lateral) ventricle at the 2 nd month, while the cornu ammonis is formed at the 4 th month. [The external walls and floor of the primitively simple central hemispheres become much thickened, the thickenings in the floor constitute the corpora striata, which protrude into the lateral ventricles, their position being indicated on the surface of the brain by the Sylvian fissure. As they extend backwards, they become connected with the optic thalami (fig. $689, s t, t h$ ). The corpora striata are connected together by the anterior commissure. From the inner wall of each hemisphere, there grow into each lateral ventricle two projections; the upper one forms the hippocampus major or cornu ammonis (fig. $689, h$ ), while the lower one becomes folded, remains thin, receives numerous blood-vessels from the falx cerebri, and forms the choroid plexus (fig. 689, $p l$ ).] At the 3rd month the Sylvian fissure is formed, and the basis of the island of Reil. The permanent cerebral convolutions are formed from the 7 th month onwards.

The mid-brain, or middle cerebral vesicle, is gradually covered over by the backward growth of the hemispheres; its cavity forms the aqueduct of Sylvius (fig. 690). Depressions appear on the surface of the vesicle to divide it into four, the corpora quadrigemina, in birds into two, the corpora bigemina (fig. $690, b g$ ), the longitudinal depression being formed at the 3rd, and the transverse one at the 7 th month. The cerebral peduncle is formed by a thickening in the base of this vesicle. 
Iu the hind.brain are found the cerebellar hemispheres, which grow backwards to meet in the middle line. The vermis is formed at the 7 th month. The cerebellum covers in the part of the medullary tube lying below it, which is not closed, as far as the calamus. The pons arises in the floor of the hind-brain at the 3rd month.

The spindle-shaped narrow after-brain forms the medulla oblongata, with the opening of the medullary tube in its upper part.

[The following table, from Quain, shows the destiny of each cerebral vesicle :-

\begin{tabular}{|c|c|c|c|}
\hline & & $\begin{array}{c}\text { 1. Prosencephaton, } \\
\text { (fore-brain) }\end{array}$ & ix, lateral \\
\hline cosese, & . . & $\begin{array}{l}\text { 2. Thalamencephalon, } \\
\text { (inter or 'tween brain) }\end{array}$ & $\begin{array}{l}\text { eal gland, pitui- } \\
\text { cerebri, aqueduct } \\
\text { erve. }\end{array}$ \\
\hline $\begin{array}{l}\text { Middle } \\
\text { Vexicl }\end{array}$ & $\begin{array}{c}\text { Primary } \\
\cdot \cdot \cdot \text {. }\end{array}$ & $\left\{\begin{array}{r}\text { 3. Mescneephalon, } \\
\text { (mid-brain) }\end{array}\right.$ & $\begin{array}{l}\text { quadrigemina, cruri cerebri, } \\
\text { ct of Sylvius, optic nerve } \\
\text { arily). }\end{array}$ \\
\hline $\begin{array}{r}\text { Poster } \\
\text { Vesi }\end{array}$ & Prim & $\begin{array}{l}\text { 4. Epencephalon, : } \\
\text { (hind-brain) } \\
\text { 5. Metencephalon, } \\
\text { (after-brain) }\end{array}$. & $\begin{array}{l}\text { Cerebellum, pons, anterior part of } \\
\text { the fourth ventricle. } \\
\text { Medulla oblongata, fourth ventricle, } \\
\text { auditory nerve. }\end{array}$ \\
\hline
\end{tabular}

Spinal Cord.-.The spinal cord is developed from the medullary tube behind the medulla ohlongata, first the grey matter around the canal, while the white matter is added afterwards

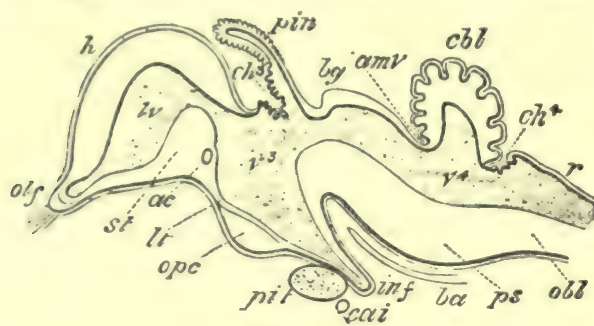

Fig. 690 .

Diagram of an embryonic fowl's brain. $a c$, anterior commissure; amv, anterior medullary velum, and below it the aqueduct of Sylvius and the cerebral peduncles; $b a$, basilar artery ; $b g$, corpora bigemina; cai, internal carotid artery ; cbl, cerebellum ; $c h^{3}, c h^{4}$, choroid plexuses of the third and fourth ventricles; $h$, cerebral hemispheres; inf, infundibulum; $l t$, lamina terminalis; $l i$, lateral ventricle; $o b l$, medulla oblongata ; olf, olfactory lobe and nerve; opc, optic commissure; pin, pineal gland ; pit, pituitary bolly ; $p s$, pons Varolii ; $r$, floor of fourth ventricle; st, corpus striatum ; $v^{3}$, third ventricle; $i^{t}$, fourth ventricle (Quain, "fter Mihalkovies). outside this. The ganglionic cells increase by division in amphibians (Lominsky). At first the spinal cord reaches to the coccyx. In the adult, the spinal cord reaches only to the 1 st or 2 nd lumbar vertebræ, so that it does not elongate so much as the vertebræ can. It is a question how far this want of harmony in the development of these two structures may lead to disturbances of sensibility or paralysis of the lower limbs in children. The first muscles are formed in the lack at the 2nd month; at the 4th month they are red. The spinal ganglia are formed from a special strip of epiblastic cells. They are seen at the 4th week, and so are the anterior spinal roots and some of the trunks of the spinal nerves, while the posterior roots are still absent. At this period the ganglia of the 5 th, 7 th, 8 th, 9 th, and 10th nerves and part of their origins are present, while the 1st, 2nd, 3rd, and 12th nerves and the sympathetic are not yet far differentiated (His). The peripheral nerves grow out from the ganglia of the spinal cord (first the motor and afterwards the sensory nerves), and penetrate into the other parts of the body (His). At first they are devoid of myelin.

452. THE SENSE ORGANS. - Eye.-The primary optic vesicle grows out from the forebrain towarls the onter covering of the head or epiblast, and soon becomes folded in on itself (4th week), so that the stalked optic vesicle is shaped like an egg-cup (fig. 691, I). The cavity in the interior of this cup is called the secondary optic vesicle. The inflected part becomes the rwina $(\mathrm{IV}, r)$, while the prosterior part becomes the choroidal epithelium $(\mathbf{I V}, p)$. The stalk heenmes the optic nerve. At the under surface of the depression there is a slit-the choroidal fissure-which permits some of the mesoblast to gain access to the interior of the eye. This slit forms the coloboma (II); it is prolonged backward on the stalk, and contains the central artery of the retina. The margins of the coloboma afterwards unite completely with each other, but in some rare conditions this does not take place, in which case we have to deal with a coloboma of the choroid or retina, as the case may be. In the bird the embryonio coloboma slit does not close up, but a vascular process of the mesoblast dips into it, and passes into the eye to form the pecten (p. 796). The same is the case in fishes, where there is a large vascular process of the nieso- and epiblast forming the processus falciformis (p. 796).

The depression or inflection of the optic vesicle is due to the downgrowth into it of a 
thickening of the epiblast (I, L). It is hollow, and as it grows inwards ultimately becomes spherical and separated from the epiblast to form the crystalline lens, so that the lens is epiblastic in its origin, while the capsule of the lens is a cuticular structure formed from the epiblast. That part of the epiblast which covers the vesicle in front of the lens ultimately becomes the stratified epithelium of the cornea. The layer of pigment of the invaginated optic vesicle is applied to the ciliary body, and the posterior surface of the iris, when the latter is formed. The cornea is formed at the 6 th week. The substance of the choroid, sclerotic, and cornea is formed around the position of the eye from the mesoblast $(m)$. The capsule of the lens is at first completely surrounded by a vascular membrane-the membrana capsulo-pupillaris. Afterwards, the lens passes more posteriorly into the eye-the anterior part of the capsulopupillary membrane, however, remains in the anterior part of the eye, while towards it grows
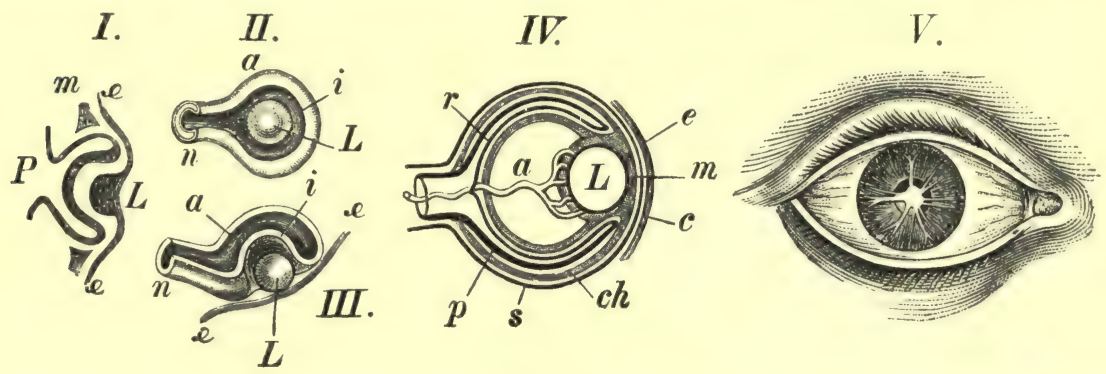

Fig. 691.

Development of the eye. I., Inflexion of the sac of the lens (L) into the primary optic vesicle

(P) $-e$, epidermis ; $m$, mesoblast. II., The inflexion seen from below-n, optic nerve ; $e$, the outer, $i$, the inner layer of the inflected vesicle; L, lens. III., Longitudinal section of II. IV., Further development- $e$, corneal epithelium; $c$, cornea; $m$, membrana capsulo-pupillaris ; L, lens ; $a$, central artery of the retina; $s$, sclerotic ; $c h$, choroid ; $p$, pigment layer of the retina ; $r$, retina. V., Persistent remains of the pupillary membrane.

the margin of the iris (7th week), so that the pupil is closed by this part of the vascular capsule, membrana pupillaris. The blood-vessels of the iris are continuous with those of the pupillary membrane ; those of the posterior capsule of the lens give off the hyaloid artery, a continuation of the central artery of the retina; its veins pass into those of the iris and choroid. The vitreous humour at the 4 th week is represented by a cellular mass between the lens and the
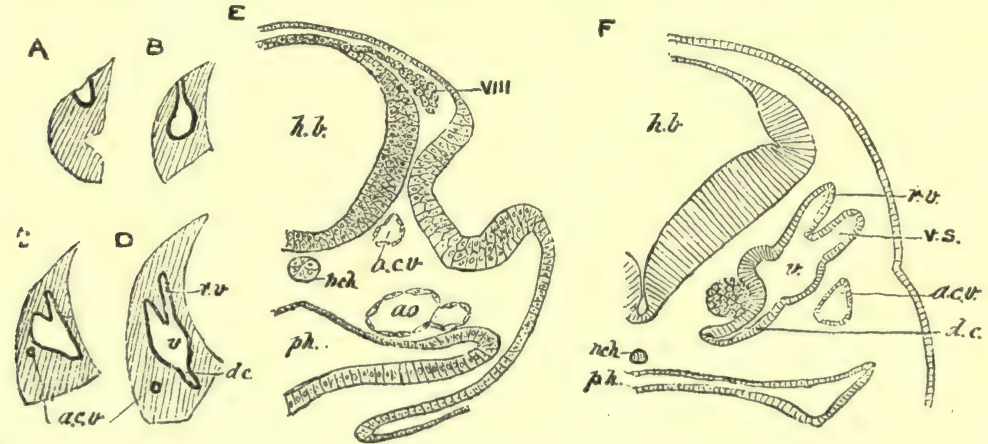

Fig. 692.

Early stages in the development of the vertebrate ear. A-D, Early stages in the chick (Reissner). E, Transverse section through the auditory pit of a 50 hours' chick (Marshall). F, Transverse section through the hind-brain of a foctal sheep. acv, anterior cardinal (jugular) vein ; $a m$, amnion; $\alpha o$, aortic arch ; $c e$, cochlea ; $r v$, recessus (aqueductus) vestibuli ; $v$, vestibultum ; vc, vertical semicircular canal ; viöi, auditory nerve.

retina. The pupillary membrane disappears at the 7 th month. It may remain throughout life (V).

Organ of Smell. - On the under surface and lateral limit of the fore-brain, the epiblast forms a groove or pit with thickened epithelium, which forms a depression towards the brain, but 
always remains as a pit or depression; this is the olfactory or nasal pit, to which the olfactory nerve afterwards senus its branches. For the formation of the nose, see p. 879 .

Organ of Hearing. - On both sides of the after-brain or posterior brain vesicle, above the first visceral or hyoil arch, there is a depression or pit formed in the epiblast, which gradually extends deeprer towards the brain - this is the labyrinth pit or auditory sac, which soon becomes flask-8haped (fig. 692, A, B).

[The stalk, which originally connected the cavity of the sac with the surface, persists as the aqueductus vestibuli; and its blind swollen distal extremity as the saccus endolymphaticus, or reressus vestibuli (Houldon, fig. $692, r, v)$.] The pit is ultimately completely cut off from the epililast, just like the lens, and is now called the vesicle of the labyrinth or primary auditory vesicle. Its related portion forms the utricle, from which, at the 2 nd month, the semicircular canals and the cochlea are developed (fig. 692, D). The union with the brain wcurs later, along with the development of the auditory nerve. The first visceral cleft remains as an irregular prassage from the Eustachian tuhe to the external auditory meatus. The outer car appears at the 7 th week.

Organ of Taste. - The gustatory papillie are developed in the later period of intra-uterine life, and several days before birth the taste-buds appear (Fr. Hermann).

453. BIRTH. - With the growth of the ovum, the uterus becomes more distended, its walls more muscular and more vascular, although the uterine walls are not thicker at the end of pregnancy. Toward the end of gestation the cervical canal is intact until labour begins, or at any rate it is but slightly opened up at its upper part. - Ifter a period of 280 days of gestation, "labour" begins, whereby the contents of the uterus are discharged. The labour pains occur rhythmically and periodically, leing sellarated from each other by intervals free from pain. Each pain begins gradually, reaches a maximum, and then slowly declines. With each pain the heat of the uterus increases $(\$ 303)$, while the heart-beat of the fotus becomes slower and feebler, which is due to stimulation of the vagus in the medulla oblongata $(\$ 369$, 3).

[At the full time the membranes and placenta line the uterus. The membranes consist, from within outwards, of amnion, chorion, decidua reflexa, and decidua vera. The fundi of the uterine glands persist in the deep part of the decidua vera, and thus form a spongy layer, the part above this being the compact layer in the deep part of the placenta, e.\%., near the uterine wall, we have also the fundi of the uterine gland persisting in the decidua serotina. When the placenta and membranes are expelled after birth, the line of separation takes place in the part of the membranes and placenta where the fundi of the glands persist. After labour is completely finished, the uterus is lined by the remains of the spongy layer of the decidua vera and serotina, e.g., is lined by a layer which contains the fundi of the uterine glands. The new mucous membrane is regenerated by the growth of the epithelium and connective-tissue in this part. The membranes expelled are made up of amnion, chorion, deciduæ reflexæ, and the compact layer of the decidua vera.]

The uterine movements during labour proceed in a peristaltic manner from the Fallopian tube to the cervix, and occupy 20 to 30 seconds. In the curve registered by these movements there is usually a more steep ascent than descent.

[Power in Ordinary Labour. - Sometimes the ovum is expelled whole, the membranes containing the liquor amnii remaining unruptured. Poppel has pointed out that the force which ruptures the hag of membranes is sufficient to complete delivery, so that, as Matthews Duncan remarks, the strength of the membranes gives us a means of ascertaining the power of labour in the easiest class of natural labours. Matthews Duncan, from experiments on the pressure required to rupture the membranes, concludes that the great majority of labours are completed by a propelling force not exceeding $40 \mathrm{lbs}$.]

Polaillon estimates the pressure exerted by the uterus upon the foetus at each pain to be $\mathbf{1 5 4}$ kilos. [338.8 lbs.], so that, according to this calculation, the uterus at each pain performs 8820 kilogrammetres of work $(\$ 301)$. [This estimate is certainly far too high.]

After-Birth. - After the foetus is expelled, the placenta remains behind; but it is soon expelled by the contractions of the uterus. During the contraction of the uterus to expel the placenta, a not inconsiderable amount of the placental blood is forced into the child (\$ $(\$ 0)$. [ It is more probable that the child aspirates the blood from the foetus portion of the placenta. This can be seen in late ligature of the cord. The child may thus gain two ounces of blood.] 
After a time the placenta, the membranes, and the decidua-constituting the after-birth-are expelled.

Influence of Nerves on the Uterus, - 1 . Stimulation of the hypogastric plexus causes contraction of the uterus. The fibres arise from the spinal cord, from the last dorsal, and upper three or four lumbar nerves, run into the sympathetic, aud then reach the hypogastric plexus (Frankenhäuser). 2. Stimulation of the nervi erigentes, which are derived from the sacral plexus, causes movement ( $v$. Basch and Hofmann). 3. Stimulation of the lumbar and sacral parts of the cord causes powerful movements (Spiegelberg). There is a centre for the act of parturition in the lumbar region of the cord $(\$ 362,6)$. The uterus, like the intestine, probably contains independent or parenchymatous nerve-centres (Körner), which can be excited by suspension of the respiration, and by anæmia (by compressing the aorta, or rapid hæmorrhage). Decrease of the bodily temperature diminishes, while an increase of the temperature increases the movement, which, however, ceases during high fever (Fromme). The experiments made by Rein upon bitches show that, if all the nerves going to the uterus be divided, practically all the functions connected with conception, pregnancy, and parturition can take place, even although the uterus is separated from all its cerebro-spinal connections. Hence, we must look to the presence of some automatic ganglia in the uterus itself. According to Dembo, there is a centre in the anterior wall of the vagina of the rabbit. According to Jastreboff, the vagina of the rabbit contracts rhythmically. Sclerotic acid greatly excites the uterine contractions $(v$. Swiecicki), so does anæmia (Kronecker and Jastreboff'). 4. The uterus contracts reflexly on stimulating the central end of the sciatic nerve ( $v$. Basch and Hofmann), the central end of the branchial plexus (Schlesinger), and the nipple (Scanzoni). 5. The uterus is supplied by vasomotor nerves (hypogastric plexus), which come from the splanchnic; and also by vaso-dilator fibres, the latter through the nervi erigentes. The vaso-motor nerves are affected reflexly by stimulation of the sciatic nerve ( $v$. Basch and Hofmann).

[In the rabbit the vagina and uterine cornua exhibit regular movements of a "peristaltic" nature. These exist apart from any extraneous stimulus, and are probably a vital property of the tissue. They can be demonstrated in animals a few weeks old, and have been recorded continuously for many hours.* Frequently they are more vigorous six hours after than at the beginning, showing that they are not due to the irritation of the operation necessary to demonstrate them.

Their rate and extent vary. In young animals they are frequent ( 1 to 4 per minute), but irregular in character. In nulliparous adults they are less frequent and somerwhat more regular. During pregnancy they increase greatly in extent, and their rate becomes 1 in 120 to 130 seconds. These characters are retained after pregnancy for many months at least. They are diminished or abolished by chloroform narcosis, are scarcely affected by ether. Water at $100^{\circ}$ to $120^{\circ} \mathrm{F}$. produces a persistent contraction accompanied by blanching of the tissue. Similar effects are produced by dilute acetic acid (Milne Murray).]

Lochia.-After birth the whole mucous membrane (decidua) is shed; its inner surface, therefore, represents a large wounded surface, on which a new mucous membrane is developed. The discharge given off after birth constitutes the lochia.

Involution of the Uterus.-After birth the thick muscular mass decreases in size, some of its fibres undergoing fatty degeneration. Within the lumen of the blood-vessels of the uterus itself, there begins in the interna of these vessels a proliferation of the connective-tissue elements, whereby within a few months the bloodvessels so affected become completely occluded. The smooth muscular fibres of the middle coat of the arteries undergo fatty degeneration. The relatively large vascular spaces in the region of the placenta are filled by blood-clots, which are ultimately traversed by outgrowths of the connective-tissue of the vascular walls.

Milk-Fever.-After birth, there is a peculiar action on the vaso-motor system, constituting milk-fever, while at the 2 nd to 3 rd day there is a more copious supply of blood to the mammary gland for the secretion of milk ( $\$ 231$ ). [After birth the pulse becomes slow and remains so in a normal puerperium. The so-called milk-fever is not found in cases where strict cleanliness is observed during the labour and puerperium.] For the cause of the first respiration in the child, see p. 666 .

454. COMPARATIVE-HISTORICAL. - A sketch of the development of man must necessarily have some reference to the general scheme of development in the Animal Kingdom. The question as to how the numerous forms of animal life at present existing on the globe have arisen has been answered in several ways. It has been asserted that each species has retained its characters unchanged from the beginning, so that we speak of the "constancy of species." This view, developed by Linnæus, Cuvier, Agassiz, and others, is opposed by that supported 
by Lamarck 1809, or the doctrine of the "Unity of the Animal Kingdom," corresponding to the ancient view of Empedocles, that all species of animals were derived by variations from a few fundamental forms; that at first there were only a few lower forms from which the numerous species were leveloped-a view supported by Geoffrey St Hilaire and Goethe. After a long periol this view was restated and elucidated in the most brilliant and most fruitful manner by Charles Darwin in his "Origin of Species" (1859) and other works. He attempted to show how modifications may be brought about by uniform and varying conditions acting for a long time. Amongst created beings each one struggles with its neighbour, so that there is a real "struggle for existence." Many qualities, such as vigour, rapidity, colour, reproductive activity, \&c., are hereditary, so that in this way by "natural selection" there may be a gradual improvement, and therewith a gradual change of the species. In addition, organisms can, within certain limits, accommodate themselves to their surroundings or environment. Thus certain useful organs or parts may undergo development, while inactive or useless parts may, undergen retrogression, and form "rudimentary organs." "This process of " natural selection," causing gradual changes in the form of organisms, finds its counterpart in "artificial selection" amougst plants and animals. Breeders of animals, for example, by selecting the proper crosses, can within a relatively short time produce very material alterations in the form and characters of the animals which they breed, the changes being more pronounced than many of those that separate well-defined species. But, just as with artificial selection, there is sometimes a sudden "reversion" to a former type, so in the development of species by natural selection there is sometimes a condition of atavism. Obviously, a wide distribution of one species in different climates must increase the liability to change, as very different conditions of environment come into play. Thus, the migration of organisms may gradually lead to a change of spereies.

Biological Law. - Without discussing the development of different organisms, we may refer to the "jundumenful biological lau;" of Haeckel, viz., "that the ontogeny is a short repetition of the phylugeny," [ontogeny being the history of the rlevelopment of single beings, or of the individual from the ovum onwards, while phylogeny is the history of the development of a whole stock of organisms, from the lowest forms of the series upwards] (p. xxxv). When applied to man, this law asserts that the individual stages in the course of the development of the human embryo, e.y., its existence as a unicellular ovum, as a group of cells after complete cleavage, as a blastolermic vesicle, as an organism without a body-cavity, \&c.; that these stanes of development indicate or represent so many animal forms, through which the human species in the course of untold ages has been gradually evolved. The individual stages which the human race has passed in this process of evolution are rapidly rehearsed in its embryonic development. This conception has not passed without challenge. In any case, the comparison of the human development and its individual organs with the corresponding perfect organs of lower vertebrates is of great importance. Thus, a mammal during the development of its organs is originally possesserl of the tubular heart, the branchial clefts, the undeveloped brain, the cartilagiuous chorda dorsalis, and many arrangements of the vascular system, \&c), which are permanent throughout the life of the lowest vertebrates. These incomplete stages are perfected in the ascending classes of vertebrates. Still, there are many difficulties to contend with in establishing both the evolution hypothesis of Darwin and the biological law of Haeckel.

Historical. - Although the impetus to the study of the history of development has been most stimulated in recent times, the ancient philosophers held distinct but very varied views on the question of development. Passing over the views of Pythagoras (550 B.C.) and Anaxagoras (500 B.c.), Empredocles (473 B.c.) taught that the embryo was nourished through the umbilicus; while he named the chorion and amnion. Hippocrates observed incubated eggs from day to day, noticed that the allantois protruded through the umbilicus, and observed that the chick escaped from the egg on the 20 th day. He taught that a 7 months' fotus was viable, and explained the possibility of superfuetation from the horns of the uterus. The writings of Aristotle (born 384 B.C.) contain many references to development, and many of them are already reforred to in the text. He taught that the embryo receives its vascular supply through the umbilical vessels, and that the placenta sucked the blood from the vascular uterus like the rootlets of a tree absorbing moisture. He distinguished the polycotyledonary from the diffuse placenta; and he reforred the former to animals without a complete row of teeth in both jaws. In the incubated egg of the chick he distinguished the blood-vessels of the umbilical vesicle, which carried food from the cavity of the latter, and also the allantois. He also observed that the head of the chick lay on its right leg, and that the umbilical sac was ultimately absorbed into the body. The formation of double monsters he ascribed to the union of two germs or two embryos lying near each other. During generation the female produces the matter, the male the principle which gives it form and motion. There are also numerous references to reproduction in the lower animals. Erasistratus ( 304 B.c.) described the embryo as arising by new formations in the ovum-Epigenesis, - while his contemporary, Herophilus, found that the pregnant uterus was closed. He was aware of the glandular nature of the prostate, and named the vesiculse seminalis and the epididymus. Galen (131-203 A.D.) was acyuainted with the existence of the foramen ovale, and the course of the blood in the foetus 
through it, and through the ductus arteriosus. He was also aware of the physiological relation between the breast and the blood-vessels of the uterus, and he described how the uterus con. tracted on pressure being applied to it. In the Talmud it is stated that an animal with its uterus extirpated may live, that the pubes separates during birth, and there is a record of a case of Cæsarian section, the child being saved. Sylvius described the value of the foramen ovale; Vesalius (1540) the ovarian follicles; Eustachius $(+1570)$ the ductus arteriosus (Botalli) and the branches of the umbilical vein to the liver. Arantius investigated the duct which bears his name, and he asserted that the umbilical arteries do not anastomose with the maternal vessels in the placenta. In Libavius (1597) it is stated that the child may cry in utero. Riolan (1618) was aware of the existence of the corpus Highmorianum testis. Pavius (1657) investigated the position of the testes in the lumbar region of the fotus. Harvey (1633) stated the fundamental axiom, "Omne vivum ex ovo." Fabricius ab Aquapendente (1600) collected the materials known for the history of the development of the chick. Regner de Graaf described more carefully the follicles which bear his name, and he found a mammalian ovum in the Fallopian tube Swammerdam $(\dagger 1685$ ) discovered metamorphosis, and he dissected a butterfly from the chrysalis before the Grand Duke of 'Tuscany. He described the cleavage of the frog's egg. Malpighi $(+1694)$ gave a good description of the development of the chick with illustrations. Hartsoecker (1730) asserted that the spermatozoa pass into the ovum. The first half of the 18th century was occupied with a discussion as to whether the ovum or the sperm was the more important for the new formation (the Ovulists and Spermatists); and also as to whether the foetus was formed or developed within the ovum (Epigenesis), or if it merely increased in growth. The question of spontaneous generation has been frequently investigated since the time of Needham in $\mathbf{1 7 4 5}$.

New Epoch. - A new epoch began with Caspar Fried. Wolff (1759), who was the first to teach that the embryo was formed from layers, and that the tissues were composed of smaller parts (corresponding to the cells of the present period). He observed exactly the formation of the intestine. William Hunter (1775) described the membranes of the pregnant uterus. Sœmmering (1799) described the formation of the external human configuration, and Oken and Kieser that of the intestines. - Oken and Goethe taught that the skull was composer of vertebræ. Tiedemann described the formation of the brain, and Meckel that of monsters. The basis for the study of the development of an animal from the layers of the embryo was laid by the researches of Pander (1817), Carl Ernst v. Baer (1828-1834), Remak, and many other observers; and Schwann was the first to trace the development of all the tissues from the ovum. [Schleiden enunciated the cell-theory with reference to the minute structure of vegetable tissues, while Schwann applied the theory to the structure of animal tissues. Amongst those whose names are most prominent in connection with the evolution of this theory are Martin Barry, von Mohl, Leydig, Remak, Goodsir, Virchow, Beale, Max Schultze, Briicke, and a host of recent observers.] 


\section{APPENDIX A.}

\section{General Bibliography,}

SYSTEMATIC WORKS AND TEXT-BOOKS.-A. v. Haller, Elementa physiologiæ corporis humani, 1757-1766, 8 vols., Auctarium, 1780.-F. Magendie, Précis élémentaire de physiologie, 1816, 2nd ed., 1825. - Johannes Müller, Handbuch der Physiologie des Menschen, 2nd ed., 1858-1861 (translated by W. Baly).-Donders, Phys. d. Mensch., pt. i., Leip., 1856. -C. Ludwig, Lehrbuch der Physiologie des Menschen, 2nd ed., 1858-1861. - Otto Funke, Lehrbuch ler Physiologie, 7th ed., by A. (irinhagen, 1884.-G. Valentin, Lehrbuch der Physiologie, 1844 (translated by Brinton, 1853). - Moleschott, Phys. d. Nahrungsmittel, 2nd ed., Giessen, 1859. - F. A. Longet, Traite de physiologie, 2nd ed., 1860-1861.-Joh. Ranke, Grundziige der Physiologie, 4th ell., 1881.-E. Brücke, Vorlesungen iiber Physiologie, 3rd ed., 1885.-I. Hermann, Grundriss der Physiologie, 8th ed., 1885 (translated and enlarged by $\mathbf{A}$. Gamgee, 2nd English ed., 1878).-W. Wundt, Lehrbuch der Physiologie, 4th ed., 1878, and Grundziige 1. physiol. Psycholog., 3rd ed., Leip., 1887.-M. Foster, Text-book of Physiology, 4th ed., 1883. - H. Milne-Edwards, Leçons sur la physiologie et l'anatomie comparée, 14 vols., 18571880. - G. Colin, Traité de Physiologie comparée des animaux, Paris, 1871-1873.-Bernard, Leç. de Pathol. expér., Paris, 1872. - Marshall, Phys. (Diagrams and Text), 1875. - Stricker, Vorles. ii. allg. u. exp. Path., Wien, 1878. --Munk, Physiologie d. Menschen u. d. Säugethiere, Berlin, 2nd ed., 1888. - Schmidt-Mulheim, Grundriss d. spec. Physiologie d. Haussäugethiere, Leipzig, 1879.-Vierordt, Grundriss d. Physiologie d. Menschen, 5th ed., Tübingen, 1857.Todd and Bowman's Cyclopredia of Anat. and Phys.-Hermann, Expt. Toxicologie, 1874.-W. Rutherford, A Text-book of Physiology, pt. i., Ėlinburgh, 1880.-W. B. Carpenter, Princip. of Phys., 8th ed., edited by Power, Loudon, 1876.-J. Beclard, Traité élém. de Phys., Paris, 1880. - Cohnheim, Vorlesungen ii. allgem. Pathologie, Berlin, 1880. - Huxley's Elements, 1885. - H. Beaunis, Nouveaux éléments de Physiologie humaine, 2nd ed., 1881.-Flint, Textbook, New York, 1876 ; and Phys. of Man, 1866-1873.-Kirkes, Handbook of Physiology, 11 th ed., 1884.-Dalton, Text-book, 1882.-J. G. M'Kendrick, Text-book of Physiology, Glus(gow, 1888. - Samuel, Handh. d. allg. Path., Stutt., 1879. - The works of Herbert Spencer and G. H. Lewe8. - E. D. Mapother, Manual of Physiology, 3rd ed., rewritten by J. F. Knott, Dublin, 1882. - A. Fick, Compendium d. Phys., 1882. - Steiner, Physiologie, 4th ed., Leipzig, 1888. - Nuel and Frédéricq. Elém. de Phys., Gand, 1883.-Preyer, Elemente der allgemeinen Physiologie, 1883.--T. Lauder-Brunton, Pharmacology, Therapeutics, and Materia Medica, 1887. - H. Power, Elements of Physiol., London, 1884.-Wundt, Phys. méd., 1878.-Daniell, Text-book of the Principles of Physies, 1884.-Fick, Med. Physik., 2nd ed., 1884. - M'Gregor Robertson, P'hysiological Physics, London, 1885. - Draper, Med. Physics, 1885. - Yeo, Manual of Physiology, 2nd ed., London, 1887.- L. v. Thanhoffer, Grundziige d. vergl. Physiologie u. Histologie, Stuttgart, 1885. - Ziegler, Text-book of Path. Anat. (trans. by D. Macalister), 1883-1884. - P. H. Pye-Smith, Syllabus of Lectures on Physiology, London, 1885. - Chapman, Treatise on Human Phys., Philad., 1887.-Klein, Micro-organisms and Disease, 1884.- Magnin and Steinberg, Bacteria, 1884. - Woodhead and Hare, Mycology, 1885. - Crookshank, Bacteriology, 1886. - Davis, Text-hook of Biol., London, 1888. - Vines, Physiology of Plants. Albertoni and Stefani, Manuale di Fisiol. umana, 1888. - Ellenberger, Lehrb. d. vergleich. Histol. u. Physiol. d. Hausthiere, Berlin, 1887. - Landois and Stirling, Text-book, 3rd ed., 1888.

YEARLY REPORTS, BIBLIOGRAPHICAL WORKS.-1834-1837: "Jahresberichte iiber die Fortschritte der Physiologie," by Joh. Muiller, in his Archiv.-1838-1846 : by Th. I. Bischoff, cbenda.-1836-1843? in "Repertorium fuir Anatomie und Physiologie," by G. 
Valentin, 8 vols. - 1856-1871 : in "Zeitschrift für rationelle Medicin," by G. Meissner, and continued since 1872 under the title- "Jahresberichte iiber die Fortschritte der Anatomie und Physiologie," by F. Hofmann, and G. Schwalbe, Leipzig. -1841-1865 : Jahresbericht iiber die Fortschritte der gesammten Medicin, by Canstatt, continued by Virchow and Hirsch. - 1822 1849 : Froriep's Notizen, 101 vols. (References and Bibliography).-Centralblatt für die medicinischen Wissenschaften, Berlin; yearly since 1863.-Biologisches Centralblatt, Erlangen, since 1881.-1817-1818: Isis, by Oken,--Catalogue of Scientific Papers compiled and published by the Royal Society of London, 1800-1873, 8 vols.-Engelmann, 1700-1846 : Bibliotheca historico-naturalis (Titles of Books on Comparative Physiology). - Jahrbuch der gesammten Medicin, by Schmidt, since 1826. - Bibliotheca anatomica qua seripta ad anatomen et physiologiam facientia a rerum initus recensentur auctore Alberto von Haller, 2 vols. (important for the older literature up to 1776). - Yearly Reports on Physiology, in Journal of Auat. and Phys., by Rutherford, Gamgee, and Stirling; also Monthly Reports in London Med. Record, since its commencement in 1873. - Index medicus. - Neurologisches Centralblatt. - Med. Bibliographie by A. Würzburg, since 1886. - Fortschritt d. Med.

HISTORICAL. - Kurt Sprengel, Versuch einer pragmatischen Geschichte der Arzneykunde, 3rd ed., 1821.-W. Hamilton, Hist. of Med. Surg. and Anat., 1831.-Bostock's Syst. of Phys., 3rd ed., 1836.-J. C. Poggendorf, Geschichte der exacten Wissenschaft, 1863.-J. Goodsir, Titles of Papers on Anat. and Phys., 1849-1852, Edin., 1853.-Meyer, Gesch. d. Botanik, Königs., 1854-1857.-H. Haeser, Lehrbuch der Geschichte der Medicin, Jena, 1875. - Julius Sachs, Geschichte der Botanik seit 16. Jahrh. bis 1860 ; 1875. - Bouchut, Hist. de la méd., Paris, 1873. - Fournié, Applic. de la scien. a la méd., Paris, 1873. - Willis's William Harvey, 1878 ; and his Servetus and Calvin, London, 1877. Biographisches Lexikon, Vienna, 1884.

ENCYCLOP EDIAS. - R. Wagner, Handwörterbuch der Physiologie, 4 vols., 1842-1853.R. B. Todd, The Cyclopædia of Anatomy and Physiology, 1836-1852.-Pierer and N. Choulant, Anatomisch-physiologisches Realwörterbuch, 8 vols., 1816-1829.-L. Hermann, Handbuch der Physiologie, 1879-1884. Real-Encyclop. d. gesam. Med., edited by Eulenberg. Wien, 1888.

PRACTICAL WORK IN THE LABORATORY.-R. Gscheidlen, Physiologische Methodik, 1876 (not yet completed).-E. Cyon, Methodik der physiologischen Experimente u. Vivisektionen, with Atlas, 1876 (only one part issued).-Ott, The Actions of Medicines, Phil., 1878. Claude Bernard and Huette, Précis iconographique de médecine operatoire et d'anatomie chirurgicale, with 113 plates, 1873; also Lecons de physiologie operatoire (edited by Duval), Paris, 1879. - Sanderson, Foster, Klein, and Brunton, Handbook for the Physiological Laboratory (Text and Atlas). The French edition contains additional matter.-Rutherford, Ontlines of Pract. Hist., 1876. - Meade-Smith, Trans. of Hermann's Toxicol._-J. Burdon-Sanderson, Practical Exercises in Physiology, London, 1882.-Foster and Langley, Pract. Phys., London, 1884. - B. Stewart and Gee, Pract. Physics. - Vierordt, Anat. Physiol. u. Physik. J)aten u. Tabellen, Jena, 1888.-Müller-Pouillet, Lehrb. d. Physik., 8th ed., Braunschweig.-Wüllner, Lehrb. d. exp. Physik.-Livon, Nanuel de Vivisect., Paris, 1882.- Harris and Power, Manual for the Phys. Lab., 5th ed., 1888. - Straus-Durckheim, Anat. descrip. coinp. du. chat, Paris, 1845.-W. Krause, Die Anatomie des Kaninchens, Leipzig, 2nd ed., 1883.-A. Ecker, Die Anatomie des Frosches, 1864-1882, 2nd ed., pt. i., 1888. - Biolog. Memoirs, edited by BurdonSanderson. - Stirling, Outlines of Pract. Physiol., Lond., 1888.

SPECIAL LABORATORY REPORTS.-Ludwig and his pupils, Arbeiten ans der physiologischen Anstalt zu Leipzig, since 1866.-Burdon-Sanderson and Schäfer, Collected papers from the Physiological Laboratory of University College, London, 1876-1885. - Gamgee, Studies froin the Physiological Laboratory of Owens College, Manchester, 1877-78.--Traube, Beitr. z. Path. u. Phys., Berlin, 1871.-J. Czermak, Gesammelte Schriften, 1879.-Marey, Physiologie expérimentale, Travaux du laboratoire, Paris, 1875. - L. Ranvier, Laboratoire d'histologie du Collège de France, Paris, since 1875. - Lovén, Physiol. Mittheil., Stockholm, 1882-84. - W. Kühne, Untersuchungen des physiologischen Instituts der Universität Heidelbery, since 1877.- R. Heidenhain, Studien des physiologischen Instituts zu Breslau, 1861-68. -.-Stricker, Studien aus dem Institute für experimentelle Pathologie, Vienna.-John Reid, Physiological and Anatomical Researches, Edinburgh, 1848.-Rollett's Untersuch. a. d. Inst. zu Gratz, since 1870. - Schenk, Mitth. a. d. embryol. Inst. z. Wien, 1877-. - Preyer, Sammlung phys. Abhandl., Jena, 1877-.--Von Wittich, Mitth. a. d. Königsb. Phys. Lab., 1878.Rossbach, Pharmacol. Unters. Wuirzb., 1873--Fick, Arb. a. d. Wuirzburger Hochschule, Würzburg, 1872.--Hoppe-Seyler, Med.-chem. Unters., 1866-71.-Laborde, Travaux de Lab. de Phys. de la Faculté de Méd., Paris, 1885. Studies from the Biol. Lab. of Owens College, pt. i., 1886. - Tigerstedt, Mitth. v. d. phys. Lab. in Stockholm, 1888.

JOURNALS, PERIODICALS.-Arehiv für die Physiologie, by J. C. Reil and Autenrieth. 12 vols., Halle, 1796-1815. Continued as-Deutsches Archiv fiir die Physiologie, by J. F. Meckel, 8 vols., Halle, 1815-1823. Continued as-Archiv fïr Anatomie und Physiologie, by J. F. Meckel, 6 vols., Leipzig, 1826-1832. Continued as-Archiv für Anatomie und wissen- 
schaftliche Medicin, by Johannes Müller, 25 vols., Berlin, 3834-1858. Continued under the same tille by-C. B. Reichert and E. du Bois-Reymond, 1859-1876. When it was divided into -Zeitschrift firr Anatomie und Entwickelungsgeschichte, by W. His and Braune, and Archiv fiir Physiologie, by E. du Bois-Reymond, until 1877. Is continued as-Archiv fuir Anatomie und Physiologie by W. His, W. Braune, and E. du Bois-Reymond.-Archiv für die gesammte l'hysiologie des Menschen und der Thiere, by E. F. W. Pflüger, Bonn, since 1868. - Zeitschrift fur Biologie, elited from 1865 by Buhl, Pettenkofer, Voit, and Radlkofer; from 1875 by the first three, and since 1580 by Pettenkofer and Voit, presently by Voit and Kühne.--Journal de Physiologie exprerimentale et pathologique, by F. Magendie, 11 vols., Paris, 1821-1831. Zeitschrift fur die organische Physik, by C. F. Heusinger, 4 vols., Eisenach, 1827-1828.\%eitschrift fuir Physiologie, by F. Tiedemann and Treviranus, 5 vols., 1824-1833. —Journal de l'anatomie et de la physiologie normales et pathologiques de l'homme et des animaux, by Ch. Robin anil Pouchet, since 1864.- Archives de physiologie normale et pathologique, by BrownSequard, Charcot, Vulpian, Paris, since 1868. - Journal of Anatomy and Physiology, edited by Humphry. Turner, and M'Kendrick, since 1867.- Journal of Physiology, edited by M. Foster, since 1875.--Archives Italiennes de Biologie, by C. Emery and A. Mosso, since 1881.-Annales des sciences naturelles, l'aris, since 1824. - Archives de Zoologie expérimentale et générale by Lacaze-Duthiers, Paris, since 1872. - Archives de Biologie, by Ed, van Beneden, and Ch. van Bambeke, since 1880.-Physiolog. Centralblatt, by Exner and Gad, since 1887.-Zeitschrift fir wissenschaftliche Zoologie, by C. T. von Siebold and A. von Kölliker, Leipzig, since 1849. Arrhir fiir pathologisehe Anatomie und Physiologie und fuir klinische Medicin, by $\mathbf{R}$. Virchow, Berlin, since 1847.-Zeit. f. wissensch. Mikroscop., Behrens, Braunschweig. -Arehiv frir Naturgeschichte, by Wiegmann; continued by Erichson and Troschel, Berlin, since 1835.Untersuchungen zur Naturlehre des Menschen und der Thiere, by Jac. Moleschott, since 1857. -Zeitschrift fir rationelle Medicin, Henle, Pfeufer, and Meissner. -Sitzungsberichte der Akademie der Wissenschaften (Math. Nat.Wiss. Classe), Vienna.-Philosophical Transactions, Londor.-Proceedings of the Royal Society, London.-Transactions of the Royal Society, Edinburgh.-Proceelings of the Royal Society, Edinburgh.-Quarterly Microscopical Journal, London. -Monthly Microscopical Journal, London.-Journal of the Royal Microscopical Society, London._-Comptes rendus, Paris.-Anatomisches Anzeiger.-Index Medicus. - Cohn, Beiträge zur Physiologie der Pflanzen, Breslau, 1872.-The Philosophical Magazine, Eilinburgh, London, and Cambridge.-Boston Medical and Surgical Journal.Vurhandlungen der physikal.-medicinischen Gesellsehaft zu Wiirzburg.-Archives of Medicine, editecl by $\mathbf{L}$. Beale, London, 1856.-Annals and Magazine of Natural History.-Annales (Mémoires) Archives du Muséum d'histoire naturelle, Paris.-Jenaische Zeitschrift für Naturwissenschaft. - Mémoires de l'Academie des Sciences de l'Institut de France, Paris. Morphologisches Jahrbuch, by C. Gegenbaur, since 1876. - Nova Acta Academiæ LeopoldinoCaroline.-Zoologischer Anzeiger, by V. Carus, since 1878.-Abhandlungen and Monatsherichte der k. preussischen A kademie der Wissenschaft zu Berlin. - Archiv fur experimentelle Pathologie und Pharmakologie, hy Naunyn and Schreiber, Leipzig, since 1873.--Deutsehes Archiv fuir klin. Medicin, by v. Ziemsen and Zenker, Leipzig. -Journal de Pharmacie et de Chimie, Paris. - A rehiv für Psychiatrie und Nervenkrankheiten, by Gudden and others, Berlin, since 1868. - Archiv fuir wissenschaftliche und praktische Thierheilkunde by Roloff, Berlin, 1874. - Archives générales de médecine, P'aris. - Brain, since 1879, edited by de Watteville.Archives de Neurologie, by Charcot, Paris, 1875. - Zeit. f. Hygiene, by Koch and Flügge.The various Medical Journals, including the Lancet and British Medical Journal, Edinburgh Medical Journal, London Medical Record, New York Med. Record, Practitioner, Medical Chronicle.-Arch. for Otology, N. Y.-Arch. for Ophthal., N. Y.-Internat. Jour. of Med. Sciences, Edin.-Revue de Med., Paris.-Zeit. f, Klin. Med., Berlin.-Intern. Monatssch. f. Anat. u. Physiol.-Asclepiad.-Nature.

HISTOLOGY. - Henle, Handbuch der systematischen Anatomie des Menschen, 3rd ed., 18661883. - Rutherford, Outlines of Pract. Histol., 1876. - W. Krause, Allgemeine und Mikroskopische Anatomie, Hannover, 1876. - F. Leydig, Lehrbuch der Histologie des Menschen und der Thiere, Hamm, 1857; and his Untersuchungen, 1883; and his Zell u. Gewebe, Bonn, 1885. - V. Mihalkovics, General Anatomy (Hungarian), 1881. - L. Ranvier, Traité technique d'histologie, Paris, 1875-1888. - G. Schwalbe, Lehrbuch der Neurologie, Erlangen, 1881; Lehrb. d. Anat. d. Siunesorgane, - S. Stricker, Handbook of Histology (translated by the New Sydenham Society), 1871-1873. - Archiv fuir mikroskopische Anatomie, Bonn; edited formerly by Max Schultze, and presently by Waldeyer and La Valette.-Quarterly Microscopical Journal, London. - Monthly Microscopical Journal, London. - Journal of the Royal Microscopical Society, London. - Schwann, Mikrosk. Untersuch., 1838 (translated by the Sydenham Society, 1847). W. Kuhne, Das Protoplasma, Leipzig, 1864.-Max Schultze, Das Protoplasma, Leipzig, 1863.R. Virchow, Die Cellular Pathologie (translated by Chance), 1860. - L . Beale, The Structure of the Elementary Tissues, London, 1881. - A. Kolliker, A Manual of Human Microscopic Anatomy, London, 1860; and his Icones Histolog., Leip., 1864.-J. Goodsir, Anatomical and Pathological Observations, edited by W. Turner, Edinburgh._-Quain's Anatomy, 9th ed., edited 
by A. Thomson, Schäfer, and Thane, London, 1882. - Rindfleisch, A Manual of Pathological Anatomy (translated by R. Baxter), London, 1873.-C. Toldt, Lehrbuch der Gewebelehre, Stuttgart, 3rd ed., 1888.-E. Klein and E. Noble-Smith, Atlas of Histology, London, 1872. H. Frey, Handbuch der Histologie und Histochemie des Menschen, Leipzig, 1876, Grundziige, 1885, and Das Mikroskop., 8th ed., 1886. -Fol, Lehr. d. vergleich. mikros. Anatomie, Leip., 1885. -Behrens, Tabellen z. Gebrauch b. mik. Arbeiten, Braunschweig, 1887. - Fearnley, Pract. Histol., 1887.-Brass, Kurzes Lehrb. d. Histol., Leip., 1888.-Beale, How to Work with the Micros., Lond., 1880. - W. Stirling, Histological Memoranda, Aberdeen, 1880.-E. A. Schäfer, Practical Histol., 1877 ; and Essentials of Histol., 1887.-W. Stirling, Text-book of Practical Histology, London, 1881.-Heitzmann, Microse. Morphology, 1882,-Purser, Man. of Hist., Dublin. - E. Klein, Elements of Hist., London, 1883.-W. Flemming, Zellsubst. u. Zelltheil., Leipzig, 1882. - Cadiat, Traité d'anat. gén., Paris, 1879.-Bizzozero, Hand. d. klin. Mikroskop., Erlang., 2nd ed., 1888.-Carnoy, Gilson and Denys, Biol. Cellul., Louvain, 1884-88.-Friedländer, Mik. Technik., 3rd ed., Berlin, 1888. -Gierke, Farberei z. mik. Zwvecken., Braun., 1885. - Frommann, Unters. u. thier. u. pflanz. Zellen, Jena, 1884.-Wiedersheim, Lehrb. d. vergl. Anat., Jena, 1888. - S. L. Schenk, Grundriss der Histologie d. Menschen, Vienna, 1885. —Orth, Cursus d. norm. Histol., 4th ed., 1886.-S. Mayer, Histolog. Taschenbuch, Prag., 1887. -Stöhr, Lehrb. d. Histol., Jena, 1888. - Lee and Henneguy, Traité de meth. de l'Anat., Paris, 1888.

PHYSIOLOGICAL CHEMISTRY.-Hoppe-Seyler, Physiologische Chemie, Berlin, 18771879. -Lehmann, Lehrb. d. phys. Chem., 3rd ed., Leipzig, 1853; and Handbuch, 1859.-J. König, Chemie der menschlichen Nahrung und Genussmittel, 2nd ed., Berlin, 1883.--Leo. Liebermann, Grundziige der Chemie des Menschen, Stuttgart, 1880.-Robin and Verdeil, 'Traité de chim. anat. et phys. (with Atlas), Paris, 1853.-J. Moleschott, Physiologie der Nahrungsmittel, 2nd ed., Giessen, 1859.-E. Smith, Foods, 1873.-A. Wynter Blyth, Foods, 1887.-Gorup-Besanez, Anleitung zur Zoo-chemischen Analyse, 1871.-Gautier, Chimie appliqué à la Physiologie, 1874.--Lehmann's Phys. Chem. (translated by Cavendish Soc., 1851-54), with Atlas of O. Funke's plates. - Kingzett, Animal Chem., 1878. - Thudichum, Ann. of Chem. Med., 1879.-A. Gamgee, Physiological Chemistry of the Animal Body, vol. i., 1880. - Hoppe-Seyler, Medicinische-Chemische Untersuchungen, Berlin.-Zeitschrift füir physiologische Chemie, by Hoppe-Seyler, Strassburg, since 1877. -Watts' Dictionary of Chemistry, second supplement, London, 1875. - Ralfe, Clinical Chemistry, London, 1880 ; and Clinical Chem., 1883. - Wurtz, Traité de chim. biol., Paris, 1880.-T. C. Charles, Physiological and Pathological Chemistry, London, 1884.-Parkes' Hygiene, 7th ed.-Flügge, Lehrb. d. hygien. Untersuchung., Leip., 1881.-Maly's Jahresb. ii. Thierchemie since 1870.-Landolt, Das opt. Drehungsvermog. org. Subst. Braun., 1879. - Articles in Hermann's Handbuch d. Physiologie, 1879-1884, and the various Text-books on Organic Chemistry.-Roscoe and Schorlemmer, (Organic) Chem., 1884.-Nowak, Lehrbuch d. Hygiene, Wien.-Beilstein, Handb. d. org. Chem., Hamb. and Leip., 2nd ed., 1885. - Ladenburg, Handb. d. Chemie, Breslau, 1883.Rosenthal, Vorles. ii. iffent. u. priv. Gesundheitspflege, Erlangen, 1887. - Kossel, Leitfaden f. med.-chem. Curse, 2nd ed., Berlin, 1888. 
APPENDIX B.

\section{COMPARISON OF THE METRICAL WITH THE COMMON MEASURES. \\ By Dr Warren De la Rue.}

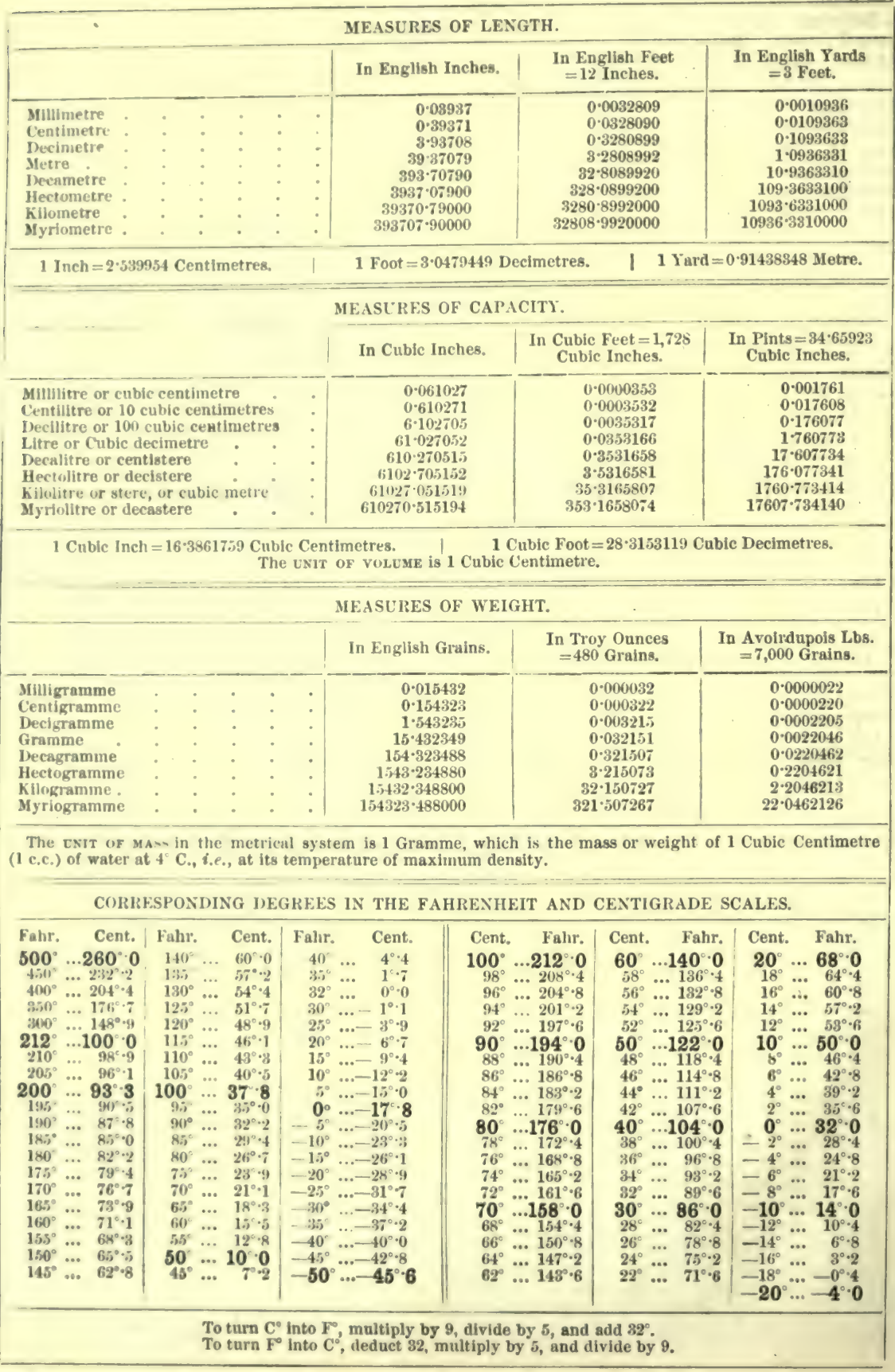




\section{N D E X.}

Abdominal muscles in respiration, 173.

Abdominal reflex, 641.

Abducens, 599.

Aberration, chromatic, 757 . ,, spherical, 757.

Abiogenesis, 841 .

Absolute blindness, 702 .

Absorption by fluids, 39 .

Absorption ofby solids, 39 .

Carbohydrates, 298.

Colouring matter, 299.

Digested food, 295.

Effusions, 312 .

Fat soaps, 299.

Forces of, 295.

Grape-sugar, 298.

Influence of nerves on, 301.

Inorganic substances, 297 .

Nutrient enemata, 301.

Organs of, 290.

Oxygen, 188, 191.

Peptones, 298.

Small particles, 300.

Solutions, 297.

Sugars, 298.

Unchanged proteids, 299.

Absorption spectra, 21.

Accelerans nerve, 669. ,$\quad$ in frog, 671 .

Accommodation of eye, 749 .

$\begin{array}{ll}, & \text { defective, } 755 . \\ , & \text { force of, } 755 . \\ , & \text { line of, } 753 . \\ , & \text { nerves of, } 753 . \\ \text { phosphene, } 763 . & \text { range of, } 756 . \\ , & \text { spot, } 763 .\end{array}$

Accord," 811.

time for, $75^{\circ}$.

Acetic acid, 381.

Aceton, 405, 415 .

Acetylene, 25.

Achromatin, 841.

Achromatopsy, 778.

Achroodextrin, 218.

Acid-albumin, 377.

Acid-hæmatin, 25.

Acids, free, 374.

Acoustic nerve, 603 .

Acquired movements, 708.

Acrylic acid series, 381.

Action currents, 554 .

Active insufficiency, 502.

Addison's disease, 156, 446 .

Adelomorphous cells, 241.

Adenin, 385.

Adenoid tissue, 304.
Adipocere, 365.

Adventitia, 95.

Egophony, 181.

Aërobes, 280.

Asthesiometer, 831.

Esthesodic substance, 645 .

Afferent nerves, 583.

After-birth, 894.

After-images, 779 .

After-sensation, 732.

Ageusia, 826 .

Agoraphobia, 606.

Agrammatism, 711.

Agraphia, 711 .

Ague, 153.

Air, changes in respiration, 187.

, collection of, 184 .

," composition of, 186.

", diffusion of, 190.

, expired, 187.

, impurities in, 200.

," quantity exchanged, 188.

Air-cells, 161.

Albumimeter, 410.

Albuminoids, 378.

Albumin of egg, 853 .

Albumins, 374.

Albuminuria, 408.

Albumoses, animal, 377.

Alcohol, 352. vegetable, 377 .

Alcohols, 382

Alcoholic drinks, 353.

Alcool au tiers, 10.

Aleurone grains, 377.

Alexia, 713.

Alkali-albumin, 377.

Alkali-hæmatin, 26.

Alkaline fermentation, 408.

Alkaloids, 353.

Allantoin, $355,403$.

Allantois, 871.

Allochiria, 838.

Allorhythmia, 107.

Alloxan, 400.

Almén's test, 412.

Alternate hemiplegia, 655 .

Alternation of generations, 843.

Amaurosis, 587.

Amblyopia, 587.

American crow-bar case, 681.

Amido-acids, 384.

Amido-acetic acid, 267, 384.

Amido-caproic acid, 255.

Amimia, 711.

Amines, 384.

Ammoniæmia, 430.

Amnesia, 711.
Amnion, 871.

Amniota, 872 .

Amniotic fluid, 872.

Amoeboid movement, 15, 451 .

Ampère's rule, 543.

Amphiarthroses, 499.

Ampho-peptone, 248.

Amphoric breathing, 180.

Amygdalin, 312.

Amyloid substance, $37 \%$.

Amylopsin, 254.

Amylum, 383.

Anabiosis, 841 .

Anacrotism, 109.

Anæmia, 17, 48.

, metabolism in

, pernicious, 17.

Anærobes, 280.

Anæsthesia dolorosa, 838.

Anæsthetic leprosy, 582.

Anæsthetics, 839.

Anabolic nerves, 583.

Anabolism, 341.

Anakusis, 604.

Analgesia, 647.

Analgia, 839 .

Anamnia, 872.

Anarthria, 710.

Anasarca, 313.

Anelectrotonus, 566.

Aneurism, 114, 115.

Angiograph, 100.

Angiometer, 108.

Angioneuroses, 678.

Anidrosis, 448.

Animals, characters of, xlii.

Animal foods, 343 .

, magnetism, 686 .

Anions, 545.

Anisotropous substance, 455 .

Ankle clonus, 643.

Anode, 545.

Anosmia, 584.

Antagonistic muscles, 502.

Anthracometer, 184.

Anthracosis, 164.

Anti-albumin, 248.

Antiar, 312.

Anti-emetics, 233.

Antihydrotics, 446.

Antipeptone, 248.

Antiperistalsis, 233.

Anti-pyretics, 336.

Anti-sialics, 215.

Aortic valves, 54.

insufficiency of,

Aperistalsis, 237. 110.

Apex-beat, 61, 69 .

Aphakia, 740 . 
Aphasia, 710.

Aphonia, 5\%2.

Apnuea, 663.

Appunn's apparatus, 815.

tpselaphesia, 838.

ๆฺueous humour, 7:20.

Arachnoid mater, 726.

Archiblastic cells, 868 .

Area opnes, 864

" pellucida, 864 .

vasoulosa, 870 .

Argyll Robertson juyil, 760.

Arhythmia cordis, 57.

Aristotle's experiment, 833.

Aromatic acids, 382.

oxyacisls, 385 .

Arrector pili muscle, 441.

Arterial tension, 105.

Arteries, 93.

" blood-pressure in, 122. central, 689 .

development of, 884 . emptiness of, 672 .

rhythmical contraction of, 675 .

sounds in, 140 .

structure of, 93.

tension in, 122.

termination in veins, 137

Arteriogram, 101.

Arthroidal joints, 499.

Articular cartilage, 498.

Articulation nerve-corpuscles, 829.

Artificial cold-blooded condition, 339.

Artificial ey€, 748

, digention, 250 .

, gastric juice, 247.

, pancreatic juice, 255.

"respiration, 198.

, Marshall Hall's method, 199.

, Sylvester's method, 199.

selection, 896 .

Aspartic acid, 256, 385 .

Asphyxia, 196, 663 .

,. artificial respiration in, 198.

Aspirates, 542. recovery from, 198

Aspiration of heart, 129.

" thoracic, 129 "ventricles, 58

Assimilation, 341.

Associated movement, 760,787 .

Astatic needles, 544.

Asteatosis, 449.

Asthma nervosum, 614. ," dyspepticum, 614.

Astigmatism, 757.

$$
\text { ", correction of, } 758 .
$$

"̈ism, 896 .

test for, 758 .

Ataxaphasia, 911.

Ataxia, 619, 700, 709.

Ataxic tabes, 647.

Atelectasis, 182, 199.

Atmospheric pressure, 203.

$$
\text { , diminution of, } 204 .
$$$$
\text { ", increase of, } 204 \text {. }
$$

Atresia ani, 871.

Atrophy, 504

"' of thin, 475 .

of the face, 598 .

Atropin, 475.

in eye, 588,760 .

Attention, time for, 685 .

Aurlible tone, lowest, 813.

Aulitory after-sensations, 820 .

$$
\text { , area, } 703 .
$$

, auræe, 704.

, centre, 703 .

," delusions, 604 .

". meatus, 799

" nerve, 797.

, ossicles, 801

," paths, 704

," perceptions, 811.

sac, 894.

Auerbach's plexus, 237, 294.

Augmentor nerves, 671 .

Auricles of heart, $50,52,57$.

, development of, 883 .

Auscultation of heart, 75 . of lungs, 179.

Automatic excitement, 625 .

Autonomy, 686 .

Auxocardia, 86.

Avidity, 245.

Axis of vision, 769 .

Bacilius, 49, 279.

, acidi lactici, 280

, anthracis, 49.

") butyricus, 280.

, subtilis, 281.

, tubercle and others, 200.

Bacterium, 49, 279, 284.

$$
\text { , aceti, } 280 .
$$

coli, 285.

, foetidum, 449 .

lactis, 284.

," synxanthum, 348 .

Ball and socket joints, 499.

Bantingism, 366.

Baræsthesiometer, 834 .

Basal development, 891 .

, ganglia, 650, 715 .

Basedow's disease, 155, 678.

Bases, 374.

Basilar membrane, 810 .

Bass-deafness, 813 .

Batteries, galvanic, 545.

" Bunsen's, 545 .

, Daniell's, 545 .

, Grennet's, 546.

, Grove's, 545.

, Lechlanche's, 546 .

Beats, 818

$$
\text { Smee's, } 546 .
$$

," isolated, 818.

successive, 818 .

Bed-sores, 542.

Beef-tea, 350.

Beer, 354

Bell's law, 617.

, deductions from, 619.

Bell's paralysis, 602 .

Benzoic acid, 402

Bert's experiment, 574.

Bidder's ganglion, 78 .

Bile, 267.

," acids, 267.
Bile, composition of, 270 .

," crystallised, 267.

", ducts, 261.

, , ligature of, 262.

," effects of drugs on, 273.

, excretion of, 271.

, fate of, 275 .

,. functions of, 274

, gases of, 269.

, passage of drugs into, 272

" pigments, 268.

," pressure, 272.

, reabsorption of, 272

," secretion of, 270.

, spectrum of, 269 .

test for $267,268$.

Biliary fistula, 271.

Bilicyanin, 269.

Bilifuscin, 269.

Biliprasin, 269.

Bilirubin, 268.

Biliverdin, 268.

Binocular vision, 787 .

Biological law, 896.

Biology, xxxv.

Biot's respiration, 172 .

Birth, 894.

Biuret reaction, 376 .

Blastoderm, 851, 862.

Blastomere, 862.

Blastosphere, 862.

Blepharospasm, 603.

Blind spot, 768 .

Blood, 1.

, abnormal, 46.

,, analysis, 29.

, arterial, 45.

, carbon dioxide in, 44.

, clot, 29.

, coagulation, 31

, colour, 1.

, colouring matter, 18 .

, composition of, 20 .

,. defibrinated, 29.

, distribution of, 144 .

", electrical condition of, 579.

extractives, 39 .

fats in, 38 .

, fibrin in, 17, 30 .

", gases in, 39 .

", granules of, 17

, islands, 10,870 .

, lake-coloured, 8.

," loss of, 48 .

". microseopic examination, 3.

nitrogen in, 45.

odour, 2.

organisms in, 49.

oxygen in, 42 .

ozoue in, 43.

plasma, 29.

plates, 16.

portal vein, 45 .

proteids of, 37.

quantity, 45.

reaction, 1 .

salts in, 39 .

serum, 29.

specific gravity, 2.

taste, 2.

temperature, 3. 
Blood, transfusion of, 46 . variations in, 46 . venous, 45 .

" water in, 39.

Blood-channels, intercellular, 95.

Blood-corpuscles-stroma, 6. , abnormal changes, 17.

, action of reagents on, 6 , 7,15 .

, amoboid movements, 15.

change of form, 7 .

", chemical composition, 18.

circulation, 137.

colour, 6.

colourless, 13.

conservation of, 8 .

crenation, 6 .

decay, 12 .

diapedesis, 15, 139.

effect of drugs, 15.

effect of reagents, 6 .

form, 3,9 .

Gower's method, 5 .

histology of, 6.

human, red, 3 . white, 13, 29.

intracellular origin, 11 .

Malassez's method, 4.

nucleated, 17.

number, $4,17$.

of newt, 13.

origin, 10.

parasites of, 18.

pathological changes, 17 .

proteids of, 28.

rouleaux of, 6 .

size, $3,9,17$.

staining of, $\tau$.

stroma, 6.

transfusion of, 46 .

weight, 3 .

white, 13

Blood-current, 137.

, in capillaries, 137.

," velocity of, 134 .

Blood-gases, 39.

" estimation of, $\mathrm{O}, \mathrm{CO}_{2}$, and N, 42.

extraction, 40.

gas-pumps for, 40.

quantity, 42.

Blood-glands, 148.

Blood-islands, 10, 870 .

Blood-plasma, 29.

Blood-pressure, 119. arterial, 122.

capillary, 128.

estimation of, 119.

in pulmonary artery, 130.

in veins, 128 .

relation to pulse, 127 .

variations of, 122,127 .

Blood-vessels, 92. action of drugs on, 95 .

cohesion of, 97.

elasticity of, 96 .

lymphatics, 95 .

pathology of, 97.

properties of, 95, 96.

structure of, 92.
Blue pus, 449.

," sweat, 449.

Body, vibrations of, 116 .

Body-wall, formation of, 870 .

Bone, chemical composition of, 882.

, callus of, 371.

, development of, 881 .

, effect of madder on, 371.

, fracture of, 371.

, growth of, 882.

, histogenesis of, 881.

, red marrow, 12.

Bones, mechanism of, 498 .

Böttger's test, 220.

Boutons terminals, 829.

Bowman's tubes, 735 .

,, glands, 821.

Box pulse-measurer, 97.

Bradyphasia, 711.

Brain, 649.

, arteries of, 728.

blood-vessels of, 727 .

," general scheme of, 649 .

," impulses, course of, 633 .

," in invertebrata, 730 .

," membranes of, 726 .

," motor centres of, 691 .

,, movements of, 727 .

," of dog, 693 .

, pressure on, 729.

, protective apparatus of, 726.

, psychical functions of, 681.

, pulse in, 115.

, pyramidal tracts of, 653 , 708.

topography of, 706,714 .

," weight of, 649 .

Branchial arches, 871 .

Brandy, 354 clefts, 871,880 .

Bread, 351.

Brenner's formula, 603.

Broca's convolution, 710.

Bromidrosis, 449.

Bronchial breathing, 179, 180. fremitus, 180 .

Bronchiole, 161

Bronchophony, 181.

Bronchus extra-pulmonary, 166. ,$\quad$ intra-pulmonary, 161. ," small, 161.

Bronzed skin, 156 .

Brownian movement, 217.

Bruit, 140.

, de diable, 141.

Brunner's glands, 276, 293.

Buchanan's experiments, 33.

Bulbar paralysis, 661 .

Bulbus arteriosus, 883.

Butter, 346.

Butyric acid, 280, 381.

Cachexia, 154.

Caffein, 353.

Calabar bean on eyé, 588 .

Calcic phosphate, 373.

Calculi, biliary, 269, 287.

, salivary, 216, 285.

Callus, 371 .
Calorimeter, 315.

Canal of cochlea, 808.

, hyaloid, 741 .

,, Nuck, 889.

, of spinal cord, 626 .

," of Stilling, 741.

,Petit, 740 .

", Schlemm, 735.

semicircular, 808 .

Canalis cochlearis, 808.

, reuniens, 808 . reunie
ries, 93.

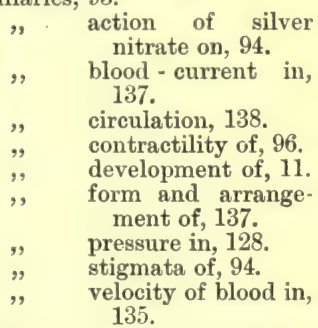

Capillary electrometer, 554 .

Capsule, external, 717.

, Glisson's, 259.

internal, 716 .

," of Tenon, 741 .

Carbohydrates, 382. fermentation of,
280 .

Carbolic acid urine, 404.

Carbon dioxide, conditions affecting, 188 .

estimation of, 183.

," excretion of, 188, 192.

, in air, 186.

, in blood, 44

,$\quad$ in expired air, 187.

, where formed, 194.

Carbonic oxide-hæmoglobin, 24.

, oxide, 24.

, poisoning by, 24 .

Cardiac cycle, 57.

, dulness, 76 .

", ganglia, 76 .

,, hypertrophy, 6\%.

,, impulse, 61,69 .

," movements, 66 .

, murmurs, 74 .

, nerves, 76 .

,$\quad$ nutritive fluids, 779

,2 plexus, 76 .

," poisons, 85 .

, revolution, 57 .

, sounds, 71 .

Cardinal points, 747 .

Cardiogram, 61.

Cardiograph, 61.

Cardio-inhibitory centre, 125, 667.

nerves, 667.

Cardio-pneumatic movement, 86.

Caricin, 256.

Carnin, 349.

Carotid gland, 77, 157.

Cartilage, .498, 871 .

Casein, 346, 377.

Catacrotic pulse, 102.

Cataphoric action, $\mathbf{5 4 8 .}$

Cataract, 740. 
Cathartios, 239.

Cathelectrotonus, 565.

Cathode, 545.

Caudate nucleus, 715.

Cavernous formations, 95 .

Cells, division of, 841 .

Cellulose, 218.

Cement, 225.

, action of silver nitrate on, 95 .

substance, 95 .

('entre, accelerans, 669.

ano-spinal, 644 .

auditory, 713 .

cardio-inhibitory, 667 .

cilio-spinal, 643.

closure of eyelids, 659 . coughing, 660 .

dilator of pupil, 643 , 660

ejaculation, 644 .

erection, 644,858

for coughing, 660 .

for defacation, 644 .

for mastication and sucking, 660 .

for saliva, 660 .

gustatory, 713 .

heat regulating, 680 .

micturition, 644 .

olfactory, 713 .

parturition, 644 .

pupil, 660 .

respiratory, 661.

sensory, 701.

sneezing, 660 .

spasm, 680 .

speech, 710 .

swallowing, 660 .

sweat, 644,680 .

vaso-dilator, 644,678 .

vaso-motor, 644,672 .

vesico-spinal, 644 .

visual, 713

vomiting, 660 .

Centre of gravity, 505 .

Centrifugal nerves, 581.

Centripetal nerves, 583.

Centro-acinar cells, 252.

Cereals, 351.

Cerebellum, 723.

Action of electricity on, 725 .

Connections of, 652 .

Function of, 724 .

Pathology of, 725 .

Removal of, 724 .

Structure of, 728 .

Cerebral arteries, 689, 728.

,, epilepsy, 695, 709 .

fissures, dog, 693.

inspiratory centre, 662.

motor centres, 706 . sensory centres, 713 . vesicles, 865 .

Cerebrin, 380, 581.

Cerebro-spinal fluid, 307.

Cerebrum, 649.

blood-vessels of, 688,
$689,727$.
convolutions of, 689.
epilepsy of, 695.
excision of centres,
700.

Cerebrum, Flourens? doctrine, 682.

, functions of, 681.

", Goltz's theory of, 705 .

imperfect development of, 682 .

lobes of, 689 .

motor regions of, 694.

movements of, 727 .

removal of, 682 .

sensory centres, 701 .

structure of, 686 .

sulci and gyri of, 689 .

thermal centres of, 705 .

weight of, 649 .

Cerumen, 445.

Cervical sympathetic, section of, 6:2:3.

Chalazx, 852.

Charcot's crystals, 203.

Cheese, 348.

lisease, 582 .

Chemical affinity, xl.

Chess-board phenomenon, 792.

Chest, dimensions of, 176 .

Cheyne-Stokes' phenomenon, 172 .

Chiasma, 585.

Chitin, 380.

Chloasma, 446.

Chloral, $6 \pi 4$.

Chlorophane, 740.

Chlorosis, 17.

Chocolate, 353.

Cholæmia, 272.

Cholalic acid, 267, 382.

Cholesteræmia, 274.

Cholesterin, 28, 269, 275, 532.

Choletelin, 269.

Cholin, 531.

Choloidinic acid, 268.

Choluria, 413.

Chondrin, 379.

Chondrogen, 379.

Chorda dorsalis, $86 \pi$.

Chorda tympani, 600, 678 .

Chordæ tendiniæ, 59.

Chorion læve, 874. , frondosum, 874. ,, primitive, 873 .

Choroid, 735.

Choroidal fissure, 892.

Christison's formula, 393.

Chromatic aberration, 757 .

Chromatin, 842.

Chromatophores, 450.

Chromatopsia, 587.

Chromidrosis, 449.

Chromophanes, 740 .

Chronograph, 481.

Chyle, 307.

, movement of, 310 .

," vessels, 301.

Chylous urine, 418.

Chyme, 247.

Cicatricula, 851.

Cilia, 451.

" conditions for movement, 452.

, effect of reagents on, 452 .
Cilia, functions of, 452.

Ciliary ganglion, 591.

, motion, 451. , force of, 452. muscle, 736,750 . nerves, 591.

Ciliated epithelium, 451.

Cilio-spinal region, 643 .

Circle of Willis, 728.

Circulating albumin, 356.

Circulation, capillary, 137.

$$
\begin{array}{ll}
, & \text { duration of, } 136 . \\
\text {, } & \text { first, } 870 . \\
\text { foetal, 875. } & \text { portal, 50. } \\
, & \text { pulmonary, } 50 . \\
, & \text { schemata of, } 118 . \\
, & \text { second, 870. } \\
, & \text { systemic, 50. }
\end{array}
$$

Circumpolarisation, 221.

Circumvallate papillæ, 824 .

Claustrum, 717.

Cleft sternum, 71.

, palate, 880 .

Clerk-Maxwell's 764.

Clevage of yelk, 862 .

,$\quad$ lines of, 863 .

, partial, 866.

Climacteric, 854.

Clitoris, 890.

Closing, continued contraction, 569.

Closing shock, 549 .

Clothing, 331.

Coagulable fluids, 37.

Coagulated proteids, 377 .

Coagulation experiments, 35 .

Coagulation of blood, 30, 31, 33 . , theories of, 33,34 ,

Cocaine, 760 . 36.

Coccygeal gland, 77, 157 .

Cochlea, 808.

Cocoa, 353.

Coecitas verbalis, 703.

Coelom, 868.

Coffee, 353.

Cold-blooded animals, 318.

Cold on the body, 337 .

", uses of, 339 .

Cold-spots, 836.

Collagen, 379.

Colloids, 296.

Coloboma, 892.

Colostrum, 347.

Colour associations, 820.

Colour-blindness, 778.

, acquired, 778.

"testing, 779 .

Colour sensation, 774 .

, Hering's theory, 776.

"Y Young-Hemholtz theory, 776 .

Coloured shadows, 782.

Colourless corpuscles, 13.

Colour top, 780.

Colours, complementary, 774.

") contrast, 780 .

, geometrical table, 775 .

," methods of mixing, 775 .

, mixed, 77.

, simple, 774 . 
Columella, 820 .

Columns of the cord, 626 .

Coma, diabetic, 266.

Comedo, 449.

Common sensation, 838 .

Comparative -

Absorption, 314.

Circulation, 157.

Digestion, 288 .

Hearing, 820 .

Heat, 340 .

Kidney and urine, 437.

Metabolism, 385 .

Motor organs, 508.

Nerve centres, 730 .

Nerves and electrophysiology, 579.

Peripheral nerves, 624 .

Reproduction and develop. ment, 895 .

Respiration, 205.

Sight, 795.

Skin, 449.

Smell, 823 .

Taste, 826 .

Voice and Speech, 523.

Compensation, 554 .

Complemental air, 166.

Complementary colours, 774.

Compound eye, 795 .

Concretions, 284.

Condensed milk, 348 .

Condiments, 352.

Conduction in the cord, 633, 646.

Conductivity, 573.

Conglutin, 378.

Congo red, 243.

Conjugate deviation, 588, 708.

Conjugation, 842.

Connective-tissue spaces, 302.

Consonance, 818.

Consonants, 521.

Constant current, action of, 482.

Constant elements-

Bunsen's, 545.

Daniell's, 545 .

Grennet, 546.

Grove's, 545 .

Leclanché's, 546.

Smee's, 546.

Constipation, 287.

Contraction, cardiac, 86.

, fibrillar, 476 .

, initial, 487.

, muscular (see Myogram).

, $\quad$ of blood-vessels, 95 .

," remainder, 480 .

, $\quad$ rhythmical, 474

, secondary, 558,559

, without metals, 556 .

Contracture, 480.

Contrast, 780 .

colours, 774, 780 .

Convergent lens, action of, 743 .

Cornea, 733.

Coronary vessels, 55.

, effects of ligature of, 56.

Corpora quadrigemina, 650 , 652,720 .

Corpulence, 366.
Corpus callosum, 715

"luteum, 856.

, spongiosum, 887.

, striatum, 650,715 .

Corresponding points, 787 .

Cortical blindness, 702 .

Corti's organ, 808.

Cotyledons, 877 .

Coughing, 182.

, centre for, 660 .

Cracked pot sound, 179 .

Cramp, 840.

Cranial flexures, 865 .

,, nerves, 584.

Cranioscopy, 682

Creamometer, 347.

Cremasteric reflex, 641 .

Crepitation, 180.

Crescents of Gianuzzi, 209.

Crista acustica, 809.

Crossed reflexes, 638.

Crura cerebri, 718 .

Crusta, 718.

", petrosa, 225 .

, phlogistica, 30

Crying, 183 .

Crystallin, 376, 740 .

Crystalline lens, 740. spheres, 795 .

Crystallised bile, 267.

Crystalloids, 297.

Cubic space, 200.

Curara, action of, 471, 474, 674 .

Cutaneous respiration, 193. trophic affections, 581.

Cuticular membrane, 225.

Cyanogen, 25.

Cylindrical lenses, 757

Cynuric acid, 403.

Cyrtometer, 177.

Cysticercus, 843.

Cystin, 416.

Cytozoon, 7 .

Daltonism, 778 .

Damping apparatus, 801.

Darby's fluid meat, 249 .

Death of a nerve, 541 .

Débove's membrane, 161.

Decidua reflexa, 873 .

,, serotina, 873.

, vera, 873 .

Decubitus acutus, 582, 722 .

Decussation of pyramids, 657 .

Defæcation, 235. , centre for, 644 .

Degeneration, fatty, 366, 539 .

Deglutition, 227. traumatic, 539 .

Deiter's cells, 810 .

Delomorphous cells, 241.

Demarcation current, 554 .

Demodex folliculorum, 445 .

Denis's plasmine, 33 .

Dentine, 224.

Dentition, 226.

Depressor fibres, 610, 613, 674 .

, nerve, 124, 610.

Deutero-albumose, 248.

Development, chronology of, 877.
Dextrin, 383.

Dextrose, 383.

Diabetes mellitus, 264.

Diabetic coma, 266.

Dialysis, 297.

Diapedesis, 139.

Diaphanometer, 348.

Diaphoretics, $4 \mathbf{4} 6$.

Diaphragm, 173.

Diarrhcea, 288.

Diastatic action, $218,254,278$, 380.

Diastole, 57.

Dichroism, 18.

Dicrotic pulse, 106.

, wave, 103.

Diet, adequate, 359 .

,, effect of age on, 360 .

, effect of work on, 360 .

" flesh, 362.

,, flesh and fat, 364 .

,, of carbohydrates, 363 .

, quality of, 356 .

, quantity, 356.

Difference theory, 563 .

Differential rheotom, 560 .

Diffusion, 295. tones, 819 .

, circles, 749 .

, of gases, 39 .

Digestion during fever, 286.

, in plants, 289.

Digestion, 206. artificial, 250, 255.

Digestive apparatus, 221.

Dilatation of pupil, centre for, 644.

Dilator pupillæ, 759 .

Dilemma, 685.

Dioptric, 757.

observations, 742 .

Diphthongia, 523.

Diphthongs, 521.

Diplacusis, 813.

Diplopia, 588, 787.

Direct vision, 769 .

Direction, 819.

Discharging forces, 470 .

Discus proligerus, 850 .

Disdiaclasts, 462.

Dissociation, 193.

Dissonance, 818 .

Distance, estimation of, 793 .

, false estimate of, 793 .

smallest appreciable, 833.

Diuretics, 422.

Division of cells, 841 .

Double conduction in nerve, 573.

Double contact, feeling of, 831 .

Double images, neglect of, 789 .

Dreams, 685 .

Drepanidium, 7 .

Dromograph, 133.

Dropsy, 312.

Duct of Cuvier, 884.

," Gaertner, 889.

Ductus arteriosus, 876 .

" venosus, 876 .

Dura mater, 726.

Dust particles, 200.

Dys-albumose, 248 
Dyschromatopsy, 778.

Dyslysin, 268.

Dysperistalsis, 237.

Dyspncen, 171, 196, 663.

Ear, 797.

", conduction in, 798 .

", development of, 894 .

", external, 799.

"fatigue of, 820 .

, fineness of, 813 .

". labyrinth of, 893 .

" mestus of, 799 .

". Ossicles of, 801 .

", speculum, 800 .

"tympanum of, 799

Karthy phosphates, 406.

Eccentric hypertrophy, 60 .

Echo speech, 686.

Ectoderm, 863.

Ectopia cordis, 67.

Efferent nerves, 581.

Effusions, 312.

Egg albumin, 376, 411.

Figgs, 349.

Ejaculation, centre for, 644 .

Elastic after-affect, $96,492$.

,, elevations, 105.

.. tension, 130.

" tubes, 92

Elasticity of blood-vessels, 96 .

$$
\begin{array}{ll}
\text { ") lens, } 750 . \\
\text { lungs, } 165 . \\
\text { muscle, } 491 .
\end{array}
$$

Elastin, 379 .

Electrical charge of body, 579 .

$$
\begin{array}{ll}
, \quad \text { fishes, } 579 . \\
, \quad \text { nerves, } 573 . \\
\text {, } & \text { organs, } 580 .
\end{array}
$$

Electrical currents of muscle, 554,557 eye, 561 .

glands, 561 .

heart, 558 .

mucous membranes, 561.

nerve, 554, 557.

plants, 565 .

skin, 561 .

Electricity, therapeutical uses, 574 .

klectrodes, non-polarisable, 547.

Electrolysis, 545 other forms, 574

Electrometer, 554 .

Electro-motive force, 541 .

Electro-physiology, 541 .

Electro-therapeutics, 574.

Electrotonus, 561 .

currents in, 561.
in conductors, 562.
in inhibitory
n $\quad$ nerves, 567.
,$\quad$ in motor nerves,
$561,565$.
in muscle, 567.
in sensory nerves,
567.

Eleidin, 438. 567.

Elementary granules of blood, 17.

Embryo formation of, 869.

Emetics, 232.
Emmetropic eye, 750, 753.

Emotions, expression of, 523 .

Emulsification, 256.

Emulsin, 312.

Emulsion, 256.

Emydin, 377.

Enamel, 225.

Enamel-organ, 225.

Enchylema, 842.

End-arteries, 148.

,, bulbs, 828 .

, organs, 581.

,' plate, 458.

Endocardial pressure, 67.

Endocardium, 54.

Endoderm, 863.

Endolymph, 808 .

Endomysium, 453.

Endoneurium, 530.

Endosmometer, 296.

Endosmosis, 295.

Endosmotic equivalent, 296.

Enemata, 301.

Energy, conservation of, xli. ") potential, xli.

Eneuresis nocturna, 437.

Entoptical phenomena, 762. pulse, 763 .

Entotical perceptions, 820 .

Enzym, 379.

Epiblast, 863

Epicardium, 51.

Epidermal appendages, 369 .

Epididymis, 845.

Epidural space, 727.

Epigenesis, 869.

Epiglottis, 229.

$$
, \text { injury to, } 229 \text {. }
$$

Epilepsy, 680, 695.

Epineurium, 529.

Epiphysis cerebri, 722 .

Epithelium, ciliated, 159, 451.

Eponychium, 440.

Equator, 561.

Equilibrium, 603, 647, 682 .

Erectile tissue, 857 .

Erection, centre for, 857 .

Erect vision, 749 . of penis, 857 .

Errhines, 183.

Erythrochlorophy, 778.

Erythro-dextrin, 218. granulose, 383

Esbach's method, 410.

Eserine, 761 .

Ether, xxxvi.

Eudiometer, 40.

Eukalyn, 334.

Euperistalsis, 237.

Eupnoen, 663.

Eustachian catheter, 806

" tube, 805.

Excitability, action of poisons on, 646 .

Excitable points of a nerve, 541 .

Excito-motor nerves, 583.

Excretin, 282.

Excretion of freal matter, 233.

Exophthalmos, 783.

Expectorants, 202.

Experimentum mirabile, 686.

Expiration, 169.

Expiratory muscles, 173.
Explosives, 521.

Extensor tetanus, 637 .

External capsule, 717. genitals, 890 .

Extra-current, 549 .

Extrapolar region, 565.

Extremities, development of, 871.

Exudation, 313.

Eye, 733.

, accommodation of, 749 .

,, artificial, 748 .

,2 astigmatism, 758.

, chromatic aberration of, 757.

, compound, 793 .

, development of, 892.

, effect of electrical currents. 763.

, emmetropic, 750 .

, entoptical phenomena, 762 .

epiphysial, 796.

excised, 761.

fundus of, 766 .

hypermetropic, 754 .

, illumination of, 764 .

movements of, 782 .

, muscles of, 782 .

myopic, 754 .

," pineal, 796.

, presbyopic, 755 .

, protective organs of, 793 .

, refractive power of, 753 .

, structure of, 733 .

Eyeballs, axis of, 783 .

", movements of, 785 .

, muscles of, 785 .

, planes of, 783 .

, positions of, 784 .

,. protrusion of, 783 .

," retraction of, 783 .

, simultaneous movements of, 780 .

Eye-currents, 561.

Eyelids, 793.

Facial nerve, 599.

Fæcal matter, 283.

Fainting, 61 .

Fallopian tubes, 853.

Fall-rhetom, 563.

Falsetto voice, 578 .

Faradic current, 549.

Faradisation in paralysis, 577.

Far point, 753 .

Fascia, lymphaties of, 310 .

Fatigue of muscle, 495 .

"s stuffs, 496 .

, decomposition of, 256.

," metabolism of, 363.

, origin of, 364 .

Fat-splitting ferment, 256.

Fatty acids, 381.

, degeneration, 366.

Febrifuges, 336.

Fechner's law, 732.

Fehling's solution, 220, 414.

Fermentation, 354 .

Ferments, 380 . 
Ferments, fate of, 279. , organised, 380.

, unorganised, 379 .

Fertilisation of ovum, 860 .

Fever, 335.

" after transfusion, 147.

Fibres of Tomes, 224.

Fibrillar contraction, 476.

Fibrin, 17, 30, 31.

Fibrin-factors, 35.

Fibrin-ferment, 35 .

Fibrinogen, 34, 376.

Fibrinoplastin, 34.

Fibroin, 378 .

Field of vision, 749 . contest of, 791 .

Filaria sanguinis, 418.

Filiform papillæ, 824 .

Filtration, 297.

First respiration, discharge of, 666.

, effects of, on thorax, 182.

Fish extract, $356^{\circ}$.

Fission, 841.

Fistula, biliary, 271.

, gastric, 247.

, intestinal, 277.

," pancreatic, 253.

, pyloric, 245.

", Thiry's, 277.

, Vella's, 277.

Flame spectra, 21.

Flavour, 823.

Fleischl's law of contraction, 568.

Flesh", 349. hæmometer, 20 .

Flight, 509

Floor-space, 201.

Flourens' doctrine, 682.

Fluid vein, 140.

Fluids, flow of, 89 .

, introduction of, 222.

Fluorescence, 774 .

Fluorescin, 742

$$
\text { of eye, } 749 \text {. }
$$

Focal distance, 743 .

,, line, 752 .

, point, 744 .

Foetal circulation, 875 . membranes, 873 .

Follicles, solitary, 293.

Fontana's markings, 532.

Fontanelle, pulse in, 115 .

Foods, isodynamic, 316 .

, plastic, 356 .

", quantity, 356, 358 .

, respiratory, 356 .

, utilisation of, 350 .

, vegetable, 350 .

Foramen ovale, 876 of Magendie, 726.

Force of accommodation, 755 .

Forced movements, 721 .

Forces, xxxvii.

Fore-gut, 869 .

Formatio reticularis, 658 .

Formative cells, 865 .

Fovea cardica, 869 . centralis, 739, 769 .

Fractional heat coagulation, 38.

Free acid, formation of, 245.

Fremitus, 180.
Friction sounds, 180.

Frog current, 557.

Fromann's lines, 529.

Fruits, 352.

Fundamental note, 814 .

Fundus glands, 241.

Fungi, 279.

Fungiform papillæ, 824.

Gaertner, ducts of, 889 .

Galactorrhoea, 345.

Galactose, 383.

Gallop, 508.

Gall stones, 287.

Galton's whistle, 812.

Galvanic battery, 545 . , excitability, 578 .

Galvano-cautery, 579

Galvanometer, 544. ,$\quad$ reflecting, 547.

Galvano-puncture, 579. , tonus, 536.

Gamgee's method, 35 .

Ganglionic arteries, 689.

Gangrene, 583.

Gargling, 183.

Gaseous exchanges, 188.

Gases, absorption of, 39 .

, diffusion of, 39 .

,extraction of, 40 .

, in blood, 39.

, in lymph, 195.

, in stomach, 252.

, indifferent, 200.

, irrespirable, 200.

, poisonous, 199

respired, 188.

Gaskell's clamp, 80.

Gas-pump, 41.

Gasserian ganglion, 591.

Gas-sphygmoscope, 101.

Gastric digestion, 247.

, conditions affecting, 250 .

". fistula, 247. ", pathological variations,

Gastric giddiness, 606.

Gastric juice, 241.

," action of drugs on, 246.

,. action on tissues, 251 .

, actions of, 247.

,, artificial, 2.

Gaule's experiment, 7 .

Gelatin, 251, 379.

Gelatin $v$, albumin, 362 .

Gemmation, 842 .

Genital cord, 889.

, corpuscles, 829 .

, eminence, 889 .

Genu valgum, 503 .

,, varum, 503.

Geometrical colour-table, 775.

Gerlach's theory, 629 .

Germ-epithelium, 849, 868.

Germinal area, 863. membrane, 863

Germinating cells, 304 .

Germs, 200.

Gestation, period of, 878 .

Giddiness, 601, 605 .

Ginglymus, 498.

Giraldès, organ of, 889 .

Girdle sensation, 648 .
Gizzard, 231.

Glance, 791 .

Glands, albuminous, 206.

Bowman's, 821.

Brunner's, 276, 293.

buccal, 206.

carotid, 77, 157.

ceruminous, 445 .

changes in, 209.

coccygeal, $77,157$.

Ebner's, 206.

fundus, 241.

Harderian, 796.

lachrymal, 794.

Lieberkühn's, 277, 293.

lingual, 206.

lymph, 304 .

mammary, 343.

Meibomian, 793

mixed, 209.

Moll's, 443.

mucous, 206.

Nuhn's, 206.

parotid, 214.

peptic, 241.

Peyer's, 294.

pyloric, 241.

salivary, 206.

sebaceous, 442.

serous, 206.

solitary, 293.

sublingual, 214

submaxillary, 209.

sweat, 442.

uterine, 853 .

Weber's, 206.

Glaucoma, 594.

Gliadin, 378.

Glisson's capsule, 259.

Globin, 377.

Globulins, 376.

Globuloses, 248.

Glomerulus, 387.

Glosso-pharyngeal nerve, 606 .

Glossoplegia, 615 .

Glossy skin, 582.

Glottis, 512 .

Glucose, 264, 383, 413 . , tests for, 220,414 .

Glucosides, 380.

Glutamic acid, 385.

Gluteal reflex, 641.

Gluten, 378.

Glycerin, 381, 382.

method, 219.

Glycerin-phosphoric acid, 382.

Glycin, 384.

Glycocholic acid, 267.

Glycogen, 262, 383.

Glycolic acid, 382 .

Glycosuria, 264, 413.

Gmelin-Heintz' reaction, 269.

Goblet cells, 291. .

Goitre, 154.

Goll's column, 633 .

Goltz's balancing experiments, 583.

, croaking experiment, 638.

embrace experiment, 638.

œsophagus experiments, 229. 
Gorham's pupil photometer, 761.

Gout, 48.

Graafian folliole, 849

Gracilis experiment, 574 .

Grandry's corpuscles, 829 .

Granules; elementary, 17.

Granulose, 218.

Grape-sugar, 383, 413 absorption of, 298. estimation of, 221 injection of, 264. in urine, 413. tests for, 220. volumetric analy sis, 41 .

(iravitation, xxxvii.

(ireat suricular nerve, 6,5 .

Green-blindness, 778 .

ireen vegetables, 352 .

(irowth, 37.).

fruanidin, 482.

(iuanin, 439, 453.

fiuarana, 353.

Gubernaculum testis, 889.

Gium, 384.

Gustatory centre, 704, 713. fibres, 600 .

region, 823 .

sensations, 825 .

"'

(iymnotus, 580 .

(iyri, 649 .

Hay's reaction, 268, 413 .

Hrmacytometer, 5.

Hamadynamometer, 119.

Hitmatin, 25, 26.

Hamatoblasts, 17.

Hamatohidrosis, 449.

Hematoidin, 27.

Hzmatoma aurium, 583.

Hæmatoporphyrin, 26.

Hzmaturia, 411.

Hzmautography, 101.

Hrmin and its tests, 26, 412

Hamochromogen, 26.

Hamocyanin, 38.

Hamocytolysis, 7.

Hamocy tometer, 5 .

Hamocytotrypsis, 7.

Hrmodromometer, 133.

Hæmodynamometer, 119.

Hamoglobin, 18.

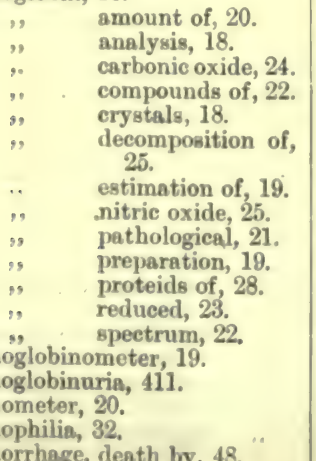

Hæmorrhage, effect on, 672 .

Hæmorrhagic diathesis, 32.

Hæmotachometer, 133.

Haidinger's brushes, 764 .

Hair, 440.

, cells, 810.

follicle, 441.

Halisterisis, 503.

Hallucinations, 732 .

Hammarsten, 34 .

Hammarsten on blood, 34.

Harderian gland, 796.

Hare-lip, 879.

Harmony, 818.

Harrison's groove, 171.

Hassall's corpuscles, 154 .

Hawking, 183.

Hay's test, 268.

Head-fold, 869 .

Head-gut, 869.

Hearing, 797 .

Heart, 51 .

, accelerated action, 67.

, action of fluids on, 81 .

" action of gases, 85 .

, action of poisons on, 83, 85.

apex, 82 .

apex beat, 61 .

, arrangement of fibres, 51.

, aspiration of, 129 .

, auricular systole, 57.

, automatic centres, 78 .

, automatic regulation, 55 .

" blood-vessels of, 56 .

, changes in shape, 65 . chordæ tendiniæ, 59.

cutting experiments, 69 . development of, 869, 882 .

diastole, 57.

duration of movements, 74.

endocardium, 54.

examination of, 75 .

frog's, 77

, ganglia of, 76 .

, hypertrophy of, 60 .

, impulse of, 61

, innervation of, 76 .

, movements of, 57 .

, muscular fibres, 53 .

, myocardium, 51.

nerves, 76 .

nutritive fluids, 79 .

, palpitation of, 60 .

pause of, 59,66 .

" pericardium, 54 .

". Purkinje's fibres, 55.

, regulation of, 55 .

section of, 80 .

sounds of, 71 .

stairease beats of, 81,84 .

systole, 57.

valves of, 54 .

weight, 55 .

work of, 136

Heat, xxxix.

\section{balance of, 331 .}

calorimeter, 324

centres, $329,705$.

conductivity, 325 .

dyspncea, 171, 664 .
Heat, employment of, 337.

estimation of, 324 .

excretion of, 380 .

income and expenditure, 332.

, in inflamed parts, 339.

, in muscle, 493 .

latent, 315 .

,, production, 317.

regulating centre, 329 .

,, relation to work, 333.

, sources of, 315 .

,' specific, 324 .

stiffening, 468.

, storage of, 334

units, $x l, 316$.

variations in production, 333.

Helicotrema, 808.

Heller's test, 202, 409.

blood-test, 412 .

Helmholtz's modification, 549 .

Hemeralopia, 587.

Hemialbumin, 248.

Hemialbumose, 247.

Hemianresthesia, 714

Hemianopsia, 586.

Hemicrania, 678.

Hemiopia, 586.

Hemipeptone, 248.

Hemiplegia, 707.

Hemisystole, 71.

Henle's loop, 387.

Hen's egg, 851.

Hensen's experiments, 818.

Hepatic cells, 260.

". chemical composition of, 262. zones, 260 .

Hepatogenic icterus, 273.

Herbst's corpuscles, 829 .

Hermann's theory of tissue currents, 563 .

Herpes, 582.

Hetero-albumose, 248.

, -xanthin, 401

Heterologous stimuli, 731 .

Hewson's experiments, 33.

Hiccough, 183.

Hippocampus, 687.

Hippuric acid, 402.

Hippus, 588.

Histo-hrematin, 156.

Historical-

Absorption, 314.

Circulation, 158.

Digestion, 289.

Hearing, 820.

Heat, 340.

Kidney and urine, 437.

Metabolism, 385.

Nerves and electro-physiology, 579.

Nerve-centres, 730.

Peripheral nerves, 624.

Reproduction and development, 896.

Respiration, 205.

Sight, 795 .

Skin, 449 .

Simell, 823.

Taste, 826 . 
Historical-

Voice and speech, 523.

Hoarseness, 523.

Holoblastic ova, 851 .

Homoiothermal animals, 318 .

Homologous stimuli, 731 .

Horopter, 788.

Hot-spots, 836.

Howship's lacunæ, 882.

Humour, aqueous, 720.

Hunger and starvation, 360.

Hyaloid canal, 741.

Hybernation, 339 .

Hybrids, 860.

Hydatids, 844.

Hydræmia, 48.

Hydramnion, 871.

Hydrobilirubin, 269.

Hydrocele, 34.

Hydrocephalus, 728.

Hydrochinon, 404.

Hydrochloric acid, 243.

Hydrocyanic acid, 25.

Hydrogen in body, 373 .

Hydrolytic ferments, 379 .

Hydronephrosis, 436 .

Hydrostatic test, 165.

Hydroxylbenzol, 404.

Hyo-cholalic acid, 267.

Hypakusis, 604.

Hypaigia, 839.

Hyperæsthesia, 646.

Hyperakusis, 604.

Hyperalgia, 604.

Hyperdicrotism, 106.

Hypergeusia, 826 .

Hyperglobulie, 47.

Hyperidrosis, 448.

Hyperkinesia, 646.

Hypermetropia, 754.

Hyperoptic, 754 .

Hyperosmia, 584.

Hyperpselaphesia, 838.

Hypertrophy of heart, 60,70 .

Hypnotism, 686 . of muscle, 504 .

Hypoblast, 863 .

Hypogeusia, 826.

Hypoglossal nerve, 615 .

Hypophysis cerebri, 156, 722.

Hypopselaphesia, 838 .

Hyposmia, 584.

Hypospadias, 890.

Hypoxanthin, 385.

Iehthidin, 377 .

Icterus, 273.

Identical points, 787 .

Ileo-colic valve, 233 .

Ileus, 233.

Illumination of eye, 764 .

Illusion, 732 .

Images, formation of, 747 .

Imbibition currents, 564 .

Impregnation, 861.

Impulse, cardiac, 61.

Impulses in brain, course of, 653.

Inanition, 360 .

Incisures, 528.

Income, 358.

Indican, 404.

Indifferent point, 554 .
Indigo blue, 404.

Indigo-carmine test, 414.

Indigogen, 404.

Indirect vision, 769 .

Indol, 255, 282.

Induction, 549 .

Inductorium, 551.

Inferior maxillary nerve, 595.

Inhibition, nature of, 640 .

Inhibition of reflexes, 639 .

Inhibitory action of brain, 706 .

,$\quad$ nerves, 583.
,$\quad$ for heart, 667.
,$\quad$ for intestine, 238.

Inion, 714.

Initial contraction, 487.

Inosinic acid, 385.

Inosit, 384.

Insectivorous plants, 289.

Inspiration, 169.

$$
\begin{array}{ll}
, & \text { centre for, } 662 . \\
, & \text { muscles of, } 172 .
\end{array}
$$

Intelligence, degree of, 684 .

Intercellular blood-channels, 95 .

Intercentral nerves, 583 .

Intercostal muscles, 174.

Interference, 818.

Interglobular spaces, 225.

Interlobular vein, 259.

Internal capsule, 716 .

reproductive 888.

respiration, 159, 194.

Intestinal fistula, 277 .

,, gases, 279.

" $\quad$, actions of, 278. " paresis, 238.

Intestine, 233

, artificial circulation, 238.

, development of, 886.

, effect of drugs on, 239.

" fermentation processes in, 279.

,, large, 283, 294.

, movements of, 233.

small, 290.

Intralabyrinthine pressure, 810 .

Intralobular vein, 259.

Intraocular pressure, 594, 742, 762.

Intrathoracic pressure, 181.

Intravascular hæmorrhage, 676 .

Inulin, 384.

Inunction, 449 .

Invertin, 281.

Invert sugar, 281.

Inverted image, 747.

Ions, 545 .

Iris, 736.

, action of poisons on, 760 .

, blood-vessels of, 737 .

," functions of, 758 .

," movements of, 759 .

, muscles of, 759 .

", nerves of, 759 .

Irradiation, 780 .

Ischuria, 436.
Island of Reil, 690.

Isodynamic foods, 316 .

Isolated beats, 818 .

Isometrical act, 487.

Isotropous, 476.

Jacksonian epilepsy, 695, 709.

Jacobson's organ, 822 .

Jaeger's types, 755 .

Jaundice, 272.

Jaw-jerk, 643.

Joints, 498.

, arthrodial, 499

,. ball and socket, 499.

, ginglymus, 498.

,, mechanism of, 498 .

,, rigid, 499.

, screw-hinge, 493.

Juice canals, 302.

Karyokinesis, 842 .

Karyomiton, 842.

Karyoplasma, 842.

Katabolic metabolism, 341.

Katalepsy, 686 .

Kations, 545 .

Keratin, 378.

Keratitis, 602 .

Keys-

Capillary contact, 553 .

Friction, 552.

Plug, 552.

Kidney, 386.

,' blood of, $426^{\circ}$.

," chemistry of, 426 .

, conditions affecting, 427.

, reabsorption in, 424.

,$\quad$ structure of, 386.

, volume of, 428

Kinæsodic substance, 645.

Kinetic energy, 315.

Klang, 811.

$$
\text { theory, } 604 \text {. }
$$

Knee phenomenon, 643.

, -jerk, 643.

, reflex, 643 .

Konig's monometric flames, 815 .

Koumiss, 348.

Krause's end-bulbs, 828.

Kreatin, 385 .

Kreatinin, 385, 401.

,, properties, 401.

, quantity, 401.

, test, 401 .

Kresol, 385.

Kryptophanic acid, 405.

Kühne's artificial eye, 74 .

, experiments, 556, 573.

, pancreas powder, 256.

Kymograph, 119.

$\begin{array}{cl}\text {," } & \text { Fick's, 121. } \\ \text { ", } & \text { Hering's, 121. } \\ & \text { Ludwig's, } 119 .\end{array}$

Kyphosis, 503.

Labials, 522.

Labour, power of, 894.

Labyrinth, 806.

Lachrymal apparatus, 794.

Lacteals, 290, 302. 
Lactic acid, $243,346,382$.

Lactometer, 347. ferment, 251 .

Lactoprotein, 346.

Lactoscope, 348.

Iactose, 383.

Levulose, 281, 383.

Lagophthalmus, 588 .

lambert's method, 774 .

Lamina dorsales, 86.).

Lamina spiralis, 80x.

Language, 711 .

Lanoline, 445.

Lanugo, 442.

Lapping, 22:2.

Ianlacein, $37 \pi$.

Iarge intestine, 283, 294.

$$
\text { absorption }
$$
283.

Laryngoscope, 515.

I arynx, cartilages of, 510 . during respiration, 517 . experiments on, 518 .

illumination of, 515 . mucous membrane of, 514.

muscles of, 511 .

view of, 516 . vocal cords, 510

latent heat, 315 .

, period, 480 .

Iateral plates, 868 .

Iaughing, 183 .

Law of conservation of energy, xli.

contraction, 568

, isolated conduction, 573.

, peripheral perception, 830 .

, specific energy, 731 .

Leaping, 507.

Lecithin, 28, 381, 531.

Legumin, 351 .

Lens, chemistry of, 740 .

" crystalline, 740 .

,9 development of, 893.

Lenticular nucleus, 716 .

Leptothrix epidermalis, 449. buccslis, 217.

Leucic acid, 382.

Leucin, 255.

Leucocytes, 13.

Leucoderma, 582.

Leucomaines, 249.

Leukæmia, 18

Levers, 501.

Lichenin, 384.

Lieben's test, 405 .

Lieberkühn's glands, 277 .

$$
\text { , jelly, } 377 .
$$

Liebermann's reaction, 376 .

Liebig's extract, 350 .

Life, xli.

Limbic lobe, 704.

Limb plexus, 620.

Liminal intensity, 731 .

Line of accommodation, 753 .

Ling's system, 503.

Lingual nerve, 596.

Lipaemin, 47.

Liquor anguinis, 29.

Listing's reducerl eye, 747.

Liver, 258. law, 784 .
Liver, action of drugs on cells, 261.

" chemical composition, 262.

," cirrhosis of, 262

,, development of, 887 .

" fat in, 264

". functions of, 266.

,. glycogen in, 263.

, influence on metabolism, 271.

, pathology of, 262

," pulse in, 143 .

," regeneration of, 262 .

, structure of, 258.

Lobes of brain, 689 .

Locality, sense of, 830 .

Locomotor ataxia, 647 . illusions of, 833 .

Lordosis, 503.

Loss by skin, 194 .

Loss of weight, 361 .

Löwe's ring, 764 .

Lungs, 159.

", chemical composition of, 165.

, development of, 886 .

" elastic tension of, 87 , $130,166$.

examination of, 177 .

excision of, 165 .

limits of, 177.

physical properties, 165 . structure of, 161. tonus, 165.

Lunule, 440.

Lutein, 856.

Luxus consumption, 356.

Lymph, 306.

" movement of, 310 .

, gases of, 195, 308.

Lymphatics, 301.

$$
\text { ,, } \quad \text { of eye, } 741 .
$$

Lymph-corpuscles, 305.

, origin and decay of, 309 .

Lymph-follicles, 304

," glands, 304.

,. hearts, 311

Macropia, 588 .

Macula lutea, 739 .

Maculæ acusticæ, 809 .

Madder, feeding with, 371.

Magnetisation, 549 .

Magneto-induction, 550 .

Major chord, 812.

Malapterurus, 579.

Malt, 354.

Maltose, 218, 383.

Mammary glands, 343.

, changes in, 343 .

") development of, 344 .

" structure of, 343 .

Manometer, 119.

$$
\begin{array}{ll}
, & \text { frog, } 82 . \\
\text { ", } & \text { maximum, } 59 . \\
\text { minimum, } 59 .
\end{array}
$$

Manometric flames, 815 .

Marey's tambotur, 67.

Margarin, 438.

Marginal convolutions, 690.
Mariotte's experiment, 768 .

Massage, 503.

Mastication, 223.$$
\text { " }
$$
muscles of, 223.

Mate, 353. nerves of, 223.

Matter, xxxvi.

Maturation of ovum, 861.

Meat soup, 350.

Meckel's cartilage, 880 .

Meconi ganglion

Medulla oblongata, 655 .

Functions of, 359 .

Grey matter of, 657 .

Reflex centres in, 659 .

Structure of, 655 .

Medullary groove, 864 . ," tube, 865 .

Meibomian glands, 793 .

Meiocardia, 86 .

Meissner's plexus, 230, 236, 294.

Melanæmia, 18.

Melanin, 381

Melitose, 383

Mellitæmia, 47.

Mellituria, 47.

Membrana decidua menstrualis, 873.

,, flaccida, 800 .

,, reticularis, 810 .

,, reuniens, 870 .

,, secundaria, 806 .

," tectoria, 810 .

,, tympani, 799 .

Membranes of brain, 726 .

Menière's disease, 605 .

Menopause, 854.

Menstruation, 854 .

Mercurial balance, 834 .

Merkel's cells, 829 .

Meroblastic ova, 851 .

Mesentery, development of, 887.

Mesoblast, 864.

Mesonephros, 889 .

Metabolic equilibrium, 355. phenornena, 341.

Metabolism, 341.

$$
\begin{aligned}
& , \quad \text { in anæmia, } 48 . \\
& \text { on flesh and other } \\
& \text { diets, } 362 .
\end{aligned}
$$

Metakresol, 404.

Metalbumin, 376.

Metallic tinkling, 180.

Metalloscopy, 839 .

Metamorphosis, 843.

Metanephros, 889 .

Meteorism, 238.

Methæmoglobin, 23.

Methylamine, 384.

Meynert's projection systems, 650 .

Micrococci, 41. theory, 684 .

Micrococcus urere, 408.

Microcytes, 17.

Micropyle, 850 .

Microscope, 137.

Micro-organisms, 284.

Micro-spectroscope, 21.

Micturition, 434 . centre for, 644

Migration of ovum, 860 . 
Milk, 345.

" action of drugs on, 347.

" coagulation of, 346 .

", colostrum, 343.

,, composition of, 344 .

", curdling ferment, 243, 346.

, digestion of, 250.

, fever, 345,895 .

", globules of, 345 .

, peptonised, 258.

" plasma, 345.

" preparations of, 348 .

", substitutes for, 347 .

", sugar, 346 .

tests for, 347

Millon's reagent, 375 .

Mimetic spasm, 603.

Mimicry, 523.

Minor chord, 812.

Mitosis, 842.

Mixed colours, 774 .

Modiolus, 808.

Molecular basis of chyle, 306 .

Molecules, xxxvi.

Molisch's test, 222.

Monoplegia, 709 .

Monospasm, 710.

Moore's test, 220.

Moreau's experiment, 278.

Mormyrus, 579.

Morphology, xxxv.

Morula, 862.

Motion, illusions of, 780 .

Motor areas, 706.

Motor centres, dog, 691, 695.

, excision of, 699 .

" in man, 698 .

, in monkey, 696.

", nerves, 581 .

, paths, 653 .

" points on the surface, 575.

Mouth, 206

,, glands of, 206.

Mouvements de manège, 721.

Movements of the eye, 782 .

$$
\begin{aligned}
& \text { acquired, } 700 . \\
& \quad \text { forced, } 721 . \\
& \text { inco-ordinated, } 619 .
\end{aligned}
$$

Mucedin, 378.

Mucigen, 291.

Mucin, 267, 378.

Mucous membrane currents, 561.

Mucous tissue, 741 .

Mucus, effect of drugs on, 201. formation of, $201,267$.

Mulberry mass, 862 .

Mulder's test, 220.

Müller's ducts, 888 . " experiment, 88, 112.

, fibres, 738 . valve, 184

Multiplicator, 543.

Murexide test, 400 .

Murmurs, cardiac, 74. venous, 141.

Muscre volitantes, 762.

Muscarin, 669.

Muscle, 453.

" action of two stimuli on, 484 .
Muscle, action of veratrin, 483. active changes in, 475 . arrangement of, 500 .

atrophic proliferation of, 504 .

blood-vessels of, 457 .

cardiac, 51, 460 .

changes during contraction, 475 .

chemical composition, 462.

current, 547.

curve of, 479 .

degenerations of, 504 .

development of, 640 .

effect of acids on, 469 .

effect of cold on, 469 .

effect of distilled water on, 469 .

effect of exercise on 503.

effect of heat on, 468 .

elasticity of, 491 .

electric currents of, 554 .

excitability of, 470 .

extractives of, 465 .

fatigue of, 495 .

ferments, 463.

fibrillæ, 455 .

formation of heat in, 493.

gases in, 464.

glycogen in, 463,465 .

hypertrophy of, 504 .

involuntary, 453 .

lymphatics of, 458 .

metabolism of, 464 .

myosin of, 463 .

nerves of, 458 .

nutrition of, 503.

of heart, 51,460 .

perimysium of, 453 .

physical characters, 462.

plasma of, 463 .

plate, 870 .

polarised light on, 462 .

reaction, 462 .

recovery of, 497 .

red and pale, 460.

relation to tendons, 457.

rhythmical contraction, 474.

rigor mortis of, 466 . rods, 456 .

sensibility, 459,493 .

serum of, 463 .

smooth, $453,460$.

sound of, 495 .

spectrum of, 460 .

staircase of, 485 .

stimuli of, 473 .

structure of striped, 453.

tetanus, 485 .

tonus, 493,644 .

uses of, 500 .

volume of, 475 .

voluntary, 453 .

work of, 489 .

Muscle-current, 554 .
Muscular contraction (see Myogram), rate of, 487 .

Muscular energy, 466. exercise, 189. sense, 839 .

work, 465 .

" " laws of, 489.

Mutes, 521.

Mydriasis, 588.

Mydriatics, 760 .

Myelin forms, 527.

Myocardium, 51 .

Myogram, 479.

" effect of constant current on, 482.

effect of fatigue on, 482.

effect of poisons on, 482.

effect of veratrin on, 483.

effect of weights on, 482.

method of studying, 477.

stages of, 479 .

Myograph, Helmholtz's, 477.

pendulum, 477.

" Pfliger's, 478.

" simple, 478 .

"spring, 478.

Myohæmatin, 381, 460.

Myopia, 754 .

Myoryctes Weismanni, 462.

Myosin, 376, 463.

Myosis, 588 .

Myotics, 761 .

Myxoedema, 155, 582.

Nails, 440

Narcotics, 839.

Nasal breathing, 182.

, timbre, 521.

Nasmyth's membrane, 225.

Native albumins, 376 .

Natural selection, 896 .

Near point, 753 .

Neef's hammer, 551.

Negative accommodation, 749 .

,, after-images, 780 .

,, pressure, 297.

, variation, 557, 559.

Nephrozymose, 405.

Nerve-cells, 525, 530 .

" hipolar, 530 .

," multipolar, 530, 628.

,2 of cerebrum, 687 .

, Purkinje's, 723.

," with a spiral fibre, 530 .

Nerve centres, general functions, 625 .

Nerve-current, 554.

Nerve-fibres, 525.

, action of nitrate of silver on, 529 .

" chemical properties of, 531.

classification of, 581 .

death of, 541 .

degeneration of, 537.

development of, 530 .

division of, 529. 
Nerve-fibres, effect of a constant Nervus trigeminus, 590. current on, 535. , trochlearis, 589.

"electrical current of, 554 .

electrical stimuli, 535 .

excitability of, 533 .

fatigue of, 537 .

incisures of, 528 .

mechanical properties of, 532.

medullated, 525 .

metabolism of, 532 .

nutrition of, 538 .

Ranvier's nodes, 52S.

reaction of, 532 .

recovery of, 537 .

regeneration of, 537,540 .

sheaths of, 529 .

stimuli of, 533 .

structure of, 525 .

suture of, 540 .

terminations of, $82 \pi$.

to glands, 212 .

traumatic degeneration of, 538 .

,. trophic centres of, 539

4. unequal excitability of, 536.

, union of, 540 .

, unipolar stimulation, 537.

Nerve-impulse, rate of, 570 .

method of measuring, 571

modifying conditions, 570.

Nerve-motion, 573.

Nerve-inuscle preparation, 555 .

Nerves, 581.

afferent, 583 .

anabolic, 583 .

centrifugal, 581.

centripetal, 583.

cranial, 584.

electrical, 573 .

intercentral, 583 .

katabolic, 583.

motor, 581.

secretory, 581.

sensory, 583.

special sense, 583.

spinal, 615 .

trophic, 581.

union of, 540 .

vaso-dilator, 678 .

vaso-motor, 672.

visceral, 583 .

Nerve-stretching, 533.

Nervi nervorum, 530.

Nervous system, 725. development of, 891.

Nervus abducens, 599 . accelerans, 669 .

accessorius, 611 .

acusticus, 603 .

depressor, 610 .

erigens, 614, 679, 859 .

facialis, 599 .

gloseopharyngeus, 606.

hypoglossus, 615 .

oculomotorius, 587.

olfactorius, 584 .

opticus, 584.

sympathicus, 620 .

Nyctalopia, 587.
" trochlearis,

Neubauer's test, 220.

Neuralgia, 598, 631 .

Neural tube, 865 .

Neurasthenia gastrica, 286.

Neurin, 531.

Neuro-epithelium, 738.

Neuro-keratin, 528.

Neuro-muscular cells, 473.

New-born child, digestion of, 246. pulse, 107.

" $\quad$ sulse, 372 .

", temperature, 326 .

,$\quad$ urine of, 393.

, weight, 372 .

Nictitating membrane, 796 .

Nitrites, 24.

, on pulse, 105.

Nitrogen in air, 186.

,$\quad$ in blood, 44.

Noud vital, 661.

Noises, 811.

Nose, development of, 879 .

,, structure, 821.

Notochord, 867 .

Nuclear spindle, 861.

Nuclein, 378.

Nucleus of Pander, 852.

Number-forms, 820.

Nussbaum's experiments, 423.

Nutrient enemata, 310.

Nystagmus, 605, 721.

Oatmeal, 351.

Octave, 812.

Oculomotorius, 587.

Odontoblasts, 224.

Edema, 313.

,, cachectic, 313.

" pulmonary, 182.

Esophagus, 229, 230.

Ohm's law, 542.

Oleic acid, 381.

Oligæmia, 48.

Olivary body, 656 .

Olfactory centre, 704, 713.

, nerve, 584 . sensations, 822

Omphalo-mesenteric duct, 869 .

Ona", ", vessels, 870 .

Onamatopoesy, 523.

Oncometer, 428 .

Ontogeny, 896.

Opening shock, 549.

Ophthalmia neuro - paralytica, 594.

intermittens, 594.

Ophthalmic nerve, 591 .

Ophthalmometer, 748 .

Ophthalmoscope, 764 .

Optic nerve, 584, 763.

, radiation, 585 .

" thalamus, 716.

" tract, 584 .

," vesicle, 866 .
Optical cardinal points, 745 .

Optogram, 773.

Optometer, 755 .

Ordinates,' 121.

Organic albumin, 356.

" compounds, 374.

, reflexes, 643 .

Ortho-kresol, 404.

Orthopnoea, 171.

Orthoscope, 767.

Osmasome, 350.

Ossein, 379.

Osseous system, formation of, 878.

Osteoblasts, 882.

Osteoclasts, 882 .

Osteomalacia, 503.

Otic ganglion, 597.

Ovarian tubes, 850 .

Ovary, 849.

Overcrowding, 201.

Ovulation, 855.

Ovu', 849. theories of, 856 .

," development of, 850 .

, discharge of, 855 .

, fertilisation of, 860 .

,, impregnation of, 861 .

, maturation of, 861 .

, migration of, 860 .

, structure of, 849.

Oxalic acid, 382, 401.

Oxalưria, 401. series, 382.

Oxaluric acid, 401.

Oxy-acids, 405.

Oxyakoia, 603.

Oxygen in blood, 42.

," estimation of, 42, 184 .

, forms of, 44 .

, in body, 373 .

Oxyhæmoglobin, 22.

Ozone in blood, 43 .

Pacehionian bodies, 727 .

Pacini's corpuscles, 828.

Pain, 838.

,$\quad$ irradiation of, 839 .

," points, 830 .

Painful impressions, conduction of, 647.

Palmitic acid, 381.

Palpitation, 60.

Pancreas, 252.

, changes in, 253.

," development of, 887.

, fistula of, 253.

," juice of, 254.

, paralytic secretion, 258.

, powder, 256.

, salt, 257.

Pancreatic secretion, 253.

, actions of, 254 .

, artificial juice, 255.

" action of nerves on, 257. action of poisons on, 258. composition, 254

", extracts, 257.

Panophthalmia, 59.

Pansphygmograph, 61. 
Papain, 256.

Papilla foliata, 825.

Papillæ of tongue, 824 .

Parablastic cells, 868 .

Paradoxical contraction, 562.

Paraglobulin, 37.

Parakresol, 385, 404.

Paralbumin, 376.

Paralgia, 839.

Paralytic secretion of saliva, 214.

Paramylum, 384 pancreatic juice, 258 .

Para-peptone, 247.

Paraphasia, 711.

Paraxanthin, 385, 401.

Parelectronomy, 563.

Paridrosis, 449.

Paroöphoron, 889.

Parotid gland, 207, 214.

Parovarium, 889.

Parthenogenesis, 844.

Partial pressure, 40. reflexes, 636 .

Particles, xxxvi

Parturition, centre for, 644.

Passive insufficiency, 502.

Patellar reflex, 642 .

Pavy's test, 220.

Pecten, 796, 892.

Pectoral fremitus, 181.

Pedunculi cerebri, 718.

Penis, erection of, 857 .

Pepsin, 242.

Pepsinogen, 244

Peptic glands, 241. changes in, 244.

Peptogenic substances, 246.

Peptone, 247, 249.

, absorption of, 298.

, forming ferment, 212 .

,$\quad$ injection of, $32,299$.

, metabolism of, 363 .

, tests for, 249

Peptonised foods, 258 .

Peptonuria, 410.

Percussion of heart, 76 .

$$
\text { ,, lungs, } 178 .
$$$$
\text { sounds, } 178
$$
wave, 102.

Perforating ulcer of the foot, 583.

Pericardium, 54.

Perilymmp, 808 .

$$
\text { fluid of, } 307 .
$$

Perimeter, Aubert and Forster, 769.

$\begin{array}{ll}\text {, } & \text { M'Hardy's, } 770 . \\ \text {, } & \text { Priestley Smith's, }\end{array}$ 772.

Perimetric chart, 770 .

Perimetry, 769.

Perimysium, 51, 453 .

Perineurium, 529.

Periodontal membrane, 225.

Peristaltic movement, 233. action of blood on, 237. action of nerves on, 238.

Peritoneum, development of, 887.

Perivascular spaces, 688.
Pernicious anæmia, 17.

Pettenkofer's test, 268. apparatus, 185.

Peyer's glands, 294.

Pflüger's law, 567. , law of reflexes, 637 .

Phagocytes, 15.

Phakoscope, 752.

Phänakistoscope, 780 .

Phases, displacement of, 814 .

Phenol, 282, 404.

Phenylsulphonic acid, 404.

Phlebogram, 142.

Phloro-glucin-vanilin, 243.

Phonation, 513.

Phonograph, 815.

Phonometry, 179 .

Phosphenes, 763.

Phosphoric acid, 406

Photo-hæmatachometer, 133.

Photophobia, 603.

Photopsia, 587.

Phrenograph, 169.

Phrenology, 682

Phylogeny, 896.

Physostigmin, 761.

Phytalbumose, 377.

Phytomycetes, 417.

Pia mater, 626.

Picric acid test, 410 .

Picro-saccharimeter, 415 .

Pigment cells, 452.

Pineal eye, 722 .

,' gland, 722.

Pitch, 811.

Pituitary body, 712 .

Placenta, 874.

Placental bruit, 141.

Plantar reflex, 642 .

Plants, characters of, xlii. , digestion by, 289 .

", electrical currents in, 565 .

Plasma cells, 726. ,2 of blood, $29,37$.

," of invertebrates, 38 .

, of lymph, 307.

," of milk, 345 .

of muscle, 462

Plasmine, 33.

Plethora, 47.

Plethysmography, 144.

Pleura, 163.

Pleuro-peritoneal cavity, 868 .

Pleximeter, 177.

Pneumatic cabinet, 112.

Pneumatogram, 170.

Pneumatometer, 182.

Pneumograph, 86, 169.

Pneumonia after section of vagi, 611.

Pneumothorax, 166.

Poikilothermal animals, 318.

Poiseuille's space, 137.

Poisons, heart, 85.

Polar globules, 861.

Polarisation, galvanic, 545. internal, 548.

Polarising after-currents, 562.

Politzer's ear-bag, 806.

Polyæmia, 46.

apocoptica, 46.

aquosa, 47 .
Polyæmia hyperalbuminosa, 47. , polycythæmica, 47 . , serosa, 47.

Polyopia monocularis, 758.

Pons Varolii, 719.

Porret's phenomenon, 548.

Portal canals, 259.

, circulation, 50 .

" system, development of, 885 .

, vein in liver, 259.

Positive accommodation, 749 .

,, after-images, 779 .

Potash salts, 373.

Potassium sulphocyanide, 216, 405.

Potatoes, 351.

Presbyopia, 754.

Pressor fibres, 674.

Pressure, arterial, 122.

, atmospheric, 203.

, intra-labyrinthine, 810.

of blood, 119

", phosphenes, 763 .

,$\quad$ points, 830,833 .

, respiratory, 181 .

,$\quad$ sense of, 833 .

Presystolic sound, 74.

Prickle cells, 438.

Primitive anus, 871 .

, aorta, 869 .

, chorion, 863,873

, circulation, 870 .

, groove, 863 .

, kidneys, 888

mouth, 871.

, streak, 863.

Primordial cranium, 878.

$$
\text { , ova, } 850 \text {. }
$$

Principal focus, 743.

Proctodæum, 865.

Proglottis, 843.

Progressive muscular atrophy, 504.

Pronephros, 889.

Pronucleus, male, 861 .

Propepsin, 244

female, 861 .

Propeptone, 247.

Protagon, 380.

Proteids, 374 .

, coagulated, 377.

," gastric digestion of, 247.

fermentation of, 281. metabolism of, 362 .

pancreatic digestion of, 255 .

reactions of, 375 .

". reactions of, 375

roteolytic ferments, 379 .

Proteoses, 248.

Protistæ, xxxv, xliv.

Proto-albumose, 248.

Protovertebræ, 868.

Pseudo-hypertrophic paralysis, 504.

Pseudo-motor action, 601, 603.

Pseudoscope, 791.

Pseudo-stomata, 162.

Psychical activities, 681 .

, blindness, 702 . 
Psychical deafness, 703. processes, time of, 684 .

Psycho-physical law, 731.

Ptomaines, 249.

Ptosis, 588.

Ptyalin, 219.

Ptyalism, 215.

Puberty, 853.

Pulmonary artery, pressure in, 130.

vessels, 162.

I'ulmonary cedema, 182.

Pulp of tooth, 225. of spleen, 148 .

l'ulse, 97 .

.. anacrotic, $10 \%$

capillary, 118.

catacrotic, $10^{\circ}$.

characters of, 102.

conditions affecting, 107 .

curve, 101.

dicrotic, 106

entoptical, 115 .

hyperdicrotic, 106.

in animals, 107.

in jugular vein, 142

in liver, 143.

influence of pressure on, $11 \%$.

influence of respiration on, 110.

instruments for investigating, 97 .

monocrotic, 106.

of various arteries, 108

" paradoxical, 112.

,. pathological, 115

rate, 107,127 .

recurrent, 110

tracing, 102.

trigeminal, 108.

variations in, 108 .

venous, 142 .

wave, 113.

Pulses, 351

Pulsus alternans, 108.

", bigeminus, 108

, caprizans, 106.

". dicrotus, 106.

,, intercurrens, 108.

myurus, 108

Pumping mechanisms, 310 .

Pupil, 752.

,$\quad$ action of poisons on, 760 .

"Argyll Robertson, 660.

,, functions of, 758 .

, movements of, 759 .

,. photometer, 761.

, size of, 761 .

Pupilometer, 761.

Purgatives, 239.

Purkinje, cells of, 723 .

, fibres of, 55, 460.

,, figure, 762.

"Sanson's images, 751.

Pus-corpuscles, 139.

Putrefaction, pancreatic, 255.

Putrefactive processes, 283.

Pyloric glands, 241.

Pyramidal cells, 687 .

changes in, 244 fistula, 245 .

". tracts, 633.
Pyramidal tracts, degenerat on of, 708.

Pyrokatechin, 382, 405.

Pyuria, 416.

Quality of a note, 811, 813.

Quantity of blood, 45 . of food, 357 .

,, of gases, 188.

kales, dry, 180. moist, $] 80$

Rami communicantes, 620 .

Range of accommodation, 756 .

Ranvier's nodes, 528.

Raynaud's disease, 583 .

Reaction impulse, 63.

Reaction of degeneration, 578 .

Reaction time, 573, 685 .

Recoil wave, 103.

Rectum, 238.

Recurrent pulse, 110.

, sensibility, 617.

Red-blindness, 778.

Reduced eye of Listing, 747 .

Reducing agents, 43.

Retluctions in intestine, 283.

Retlex acts, examples of, 636 .

, inhibition of, 639 .

, law of, 637 .

movements, 636

Reflex movements, theory of, 641 .

,, nerves, 583.

,. organic, 643 .

,, spasms, 636 .

, tactile, 647

, time, 639 .

, tonus, 645 .

Retlexes, crossed, 638.

$$
\begin{array}{ll}
, & \text { deep, } 642 . \\
, & \text { spinal, } 635 . \\
, & \text { organic, } 643 .
\end{array}
$$

Refracted ray, 744 .

Refractive indices, 744 .

Regeneration of tissues, 369 . of nerve, 540 .

Regio olfactoria, 821 . respiratoria, 821 .

Regnault's apparatus, 184.

Reissner's membrane, 808 .

Relative proportions of diet, 357.

Remak's ganglion, 77 .

Renal plexus, 427 .

Rennet, 251, 346.

Reproduction, forms of, 841 .

Requisites in a proper diet, 357 .

Reserve air, 166.

Residual air, 166.

Resistance, 89.

Resonance organs, 510 .

Resonants, 522.

Resonators, 817.

Resorcin, 404.

Respiration, 159.

,$\quad \begin{aligned} & \text { amphoric, } 180 . \\ & \text { artificial, } 198 .\end{aligned}$
Biot's, 172.
bronchial, 180.
centre for, 661.
chemistry of, 183.
cog.wheel, 180.

Respiration, cutaneous, 193.

,$\quad$ first, 666 .

, forced, 172.

, foreign gases, 199.

, in a closed space, 196.

in animals, 168.

internal, 194.

mechanism of, 165 .

muscles of, 172 .

nasal, 182 .

number of, 167.

pathological, 171.

periodic, 172.

pressure during, 181.

pressure on heart, 86.

sounds of, 179 .

time of, 168 .

type, 170 .

variations of, 167 .

vesicular, 179 .

Respiratory apparatus, 159.

Andral and Gavarret, 184 .

centre, 661 .

mechanism of, 165.

v. Pettenkofer, 185.

quotient, 186.

Regnault and Reiset, 185 .

Scharling, 185.

undulations, 124.

Restiform bodies, 655 .

Rete mirabile, 51 .

Retina, 737.

$$
\text { activity in vision, } 768 \text {. }
$$

blood-vessels of, 739 .

capillaries, movements in, 763 .

chemistry of, 740 .

epithelium of, 737 .

rods and cones of, 738 , 769.

stimulation of, 779 .

structure of, 737 .

visual purple of, 739 .

Retinal image, formation, of 747 .

size of, 748 .

Retinoscopy, 767

Reversion, 896.

Rheocord, 542.

Rheometer, 132.

Rheophores, 575.

Rheoscopic limb, 556.

Rheostat, 543 .

Rheotom, 560 .

Rhinoscopy, 517.

Rhodophane, 740 .

Rhodopsin, 739 .

Ribs, 174 .

Ricket's, 503.

Rigor mortis, 466.

Ritter's opening tetanus, 567. tetanus, 567.

Ritter-Valli law, 541.

Rods and cones, 738 .

Rods of Corti, 809 .

Rosenthal's modification, 472.

Rotatory disc for colours, 774.

Rudimentary organs, 896. 
Rumination, 288.

Running, 505.

Saceharomycetes, 354 .

Saccharose, 383.

Saccule, 808 .

Saftcanälchen, 302.

Saline cathartica, 239.

Saliva, action of nerves on, 212.

, action of poisons on, 213.

, action on starch, 218 .

," chorda, 212.

", composition of, 217.

, facial, 212

, functions of, 216 .

mixed, 217.

new-born child, 218.

" paralytic secretion, 214

, parotid, 216

," pathological, 285.

,, ptyalin, 216, 219.

,$\quad$ reflex secretion of, 214.

,$\quad$ sublingual, 217.

, submaxillary, 216.

, sympathetic, 212.

, theory of secretion, 216.

Salivary corpuscles, 217.

Salivary glands, 207.

, changes in, 209.

," development of, 886 .

, extirpation of, 215 .

Salts, 373 . nerves of, 211.

Sanson-Purkinje's images, 751.

Saponification, 256.

Sarcina ventriculi, 287.

Sarcoglia, 459 .

Sarcolactic acid, 463.

Sarcolemma, 455 .

Sarcolytes, 640.

Sarcoplasts, 640.

Sarcous elements, 455.

Sarkin, 401.

Sarkosin, 385.

Saviotti's canals, 253.

Scheiner's experiment, 753.

Schiff's test, 400 .

Schizomycetes, 49, 279.

Schmidt's researches, 34 .

Schreger's lines, 225.

Schwann's sheath, 528.

Sclerotic, 735.

Scoliosis, 503.

Scotoma, 772.

Screw-hinge joint, 499.

Scrotum, formation of, 890 .

Scurvy, 48.

Scyllit, 384

Sebaceous glands, 442. secretion, 444.

Seborrhoea, 449.

Secondary circulation, 870.

contraction, 558.
decompositions, 545.
degeneration, 633.

, tetanus, 559 .

Secretion currents, 561 .

Secretory nerves, 581 .

Sectional area, 134.

Segmentation sphere, 862 .

Self-stimulation of muscle, 556 .

Semen, composition of, 846 . ejaculation of, 859 .
Semen, reception of, 859 .

Semicircular canals, 604, 808 .

Sensation, 731 .

Sense organs, 731 .

,, development of, 892 .

Sensory areas, 701 .

, crossway, 654, 718 .

,, paths, 644 .

, sensations, 830 .

Serin, 385 .

Serous cavities, 303.

Serum of blood, 30 .

Serum-albumin, 37, 376.

Serum-globulin, 37, 376.

Setschenow's centres, 639.

Sex, difference of, 890 .

Shadows, lens, 762. , coloured, 782.

Sharpey's fibres, 882.

Short-sightedness, 754 .

Shunt, 548.

Sialogogues, 215.

Sighing, 183.

Silver lines, 94.

Simple colours, 778.

Simultaneous contrast, 780 .

Sinuses, 95.

Sitting, 505.

Size, 372 .

, estimation of, 791.

, increase in, 372.

, false estimate of, 793 .

Skatol, 255, 282, 405 .

Skin, absorption by, 449 .

, chorium of, 437.

, currents of, 557 .

, epidermis, 437.

, functions of, 444 .

, galvanic conduction of, 449.

, glands of, 442

," historical, 450 .

,, pigments, 446 .

,, protective covering, 443.

respiratory organ, 193 , 444.

, structure of, 437.

, varnishing the, 444.

Skin currents, 061 .

Sleep, 655 .

Small intestine, 290.

$\begin{array}{cc}, & \text { absorption by, } \\ 297 . & \\ & \text { structure of } \\ & 290 .\end{array}$

Smegma, 445.

Smell, sense of, 821 .

Sneezing, 183.

Snellen's types, 754 .

Sniffing: 822.

Snoring, 183.

Sodic chloride, 373.

salts, 373 .

Solitary follicles, $293,295$.

Somatopleure, 868.

Somnambulism, 684 .

Sorbin, 384.

Sound, 798.

, cardiac, 67.

", conduction to ear, 798, 807.

direction of, 819.

distance of, 819 .
Sound, perception of, 819 .

,, reflection of, 798 .

Sounds, cardiac, 71 .

, cracked pot, 180 .

,, respiratory, 179 .

,, tympanitic, 179 .

, vesicular, 179.

Spasm centre, 680 .

Spasmus nictitans, 603.

Specific energy, 731, 773.

Spectacles, 756 .

Spectra, absorption, 21.

", flame, 21.

,. ocular, 764 .

Spectroscope, 21.

Spectrum mucro-lacrimale, 762.

, of bile, 269.

, of blood, 22.

, of muscle, 460 .

Speech, 519.

, centre for, 710

, pathological variations, 522.

Spermatin, 846

Spermatozoa, 846.

Spermatoblasts, 847 .

Spheno-palatine ganglion, 595.

Spherical aberration, 757 .

Sphincters, 502.

Sphincter ani, 233.

$$
\begin{aligned}
& \text {, } \quad \text { pupillæ, } 736 . \\
& \text { urethræ, } 433 .
\end{aligned}
$$

Sphygmogram, 102.

Sphygmograph, 97.

$$
\begin{array}{ll}
, & \text { Dudgeon's, } 99 . \\
, & \text { Ludwig's, 99. } \\
,, & \text { Marey's, 98. }
\end{array}
$$

Sphygmometer, 97.

Sphygmomanometer, 122.

Sphygmoscope, 101.

Sphygmotonometer, 97.

Spina bifida, 727, 870 .

Spinal accessory nerve, 614.

Spinal cord, 626. action of blood and poisons on, 646 .

blood-vessels of, 632 .

centres, 643 .

conducting paths in, 633 .

conducting system of, 633,646 .

degeneration of, 636 .

development of, 892 .

excitability of, 645 .

Flechsig's systems, 633 .

functions of, 632 .

ganglion, 616 .

Gerlach's theory, 629 .

nerves, 615 .

tneuroglia of, 631.

nutritive centres in, 635 .

reflexes, 635 .

regeneration of, 681 .

secondary degeneration of, 636 .

segment of, 635 .

structure of, 626 .

time of development, 635 .

transverse section of, 648.

unilateral section of, 649 .

vaso-motor centres in, 676. 
Spinal oord, Woroschiloff's observations, 627.

Spinal nerves, 615 .

1, anterior roots of, 619

posterior roots of, 620 .

Spiral joints, 499.

Spirillum, 49, 279 .

spirochreta, $49,279$.

Spirometer, 167.

Splanchnic nerve, 238.

Splanchnopleure, 868.

Spleen, 148.

, action of drugg on, 153.

," chemical composition, 150.

contraction of, 151 .

extirpation of, 150 .

functions of, 150 .

influence of nerves on, 152.

oncograph, 151.

regeneration of, 150 .

structure, 148.

tumours of, 153.

spongin, 379.

Spontaneous generation, 841 .

Sipores, 281.

sipring kymograph, 121.

Spring myograph, 479.

springing, 505.

Sputum, abnormal, 201.

Squint, 588 . normal, 200.

Staircase, 485 .

Stammering, 523.

Standing, 504.

Stannius's experiment, 79.

Stapedius, 804.

Nitarch, 383.

Starvation, 360.

Stasis, 139.

Statical theory of Goltz, 604.

Stationary vibrations, 799 .

Steapsin, 256.

Stenopaic spectacles, 757.

Stenosis, 70 .

Stenson's experiment, 468.

Stercobilin, 269, 284.

Stercorin, 284.

Stereoscope, 791.

Stereoscopic vision, 789 .

Sternutatories, 183.

Stethograph, 169.

Sitigmata, 94.

Stilling, canal of, 741 .

Stimuli, 470 .

, adequate, 731.

heterologous, 731.

hoinologous, 731 . muscular, 473.

itoffwechsel, xliv.

stomach, 239. cancer of, 286. catarrh of, 286. changes in glands, 244. gases in, 252. glands of, 241. movements of, 230 . structure of, 239 .

Stornata, 139, 303.

Stomorlieum, 865.

Storage albumin, 356.

Strabismus, 721 .
Strangury, 437.

Strassburg's test, 413.

Strixe medullares, 717.

Strobic dises, 780.

Stroboscopic discs, 780.

Stroma-fibrin and plasma-fibrin, 37.

Stromuhr, 132 .

Struggle for existence, 896.

Struma, 678.

Strychnin, action of, 637 .

Stuttering, 528.

Subarachnoid space, 726. fluid, 726 .

Subdural space, 726 .

Subjective sensations, 732 .

Sublingual gland, 214.

Submaxillary ganglion, 598 .

$\begin{array}{ll},, & \text { atropin on, } 213 . \\ , & \text { gland, 209. } \\ ,, & \text { saliva, 216. }\end{array}$

Substantia gelatinosa, 628 .

Successive beats, 818 . , contrast, 782 .

, light-induction, 782.

Succinic acid, 405 .

Succus entericus, 278. action of drugs on, 278 .

Suction, 222

Sudorifics, 446.

Sugars, 382 .

, estimation of, 161.

, tests for, 220 .

Sulphindigotate of soda, 423 .

Summation of stimuli, 485, 637.

Summational tones, 819 .

Superfecundation, 860 .

Superficial reflexes, 641 .

Superfoetation, 860 .

Superior maxillary nerve, 594.

Supplemental air, 166.

Supra-renal capsules, 156.

Surditas verbalis, 713 .

Sutures, 499.

Swallowing fluids, 668 .

Sweat, 445 .

" chemical composition, 445.

, conditions influencing secretion, 446.

, excretion of substances by, 446 .

,. glands, 443 .

insensible, 445 .

,. nerves, 447.

", pathological variations of, 448 .

Sweat centre, 447, 680 .

Swimming, 509 . pinal, 680 .

Sympathetic ganglion, 621 .

$\begin{array}{ll}" & \text { nerve, } 620 . \\ & \text { section of, } 623,675 . \\ \text { stimulation of, } 624,\end{array}$

Symphyses, 499. 675 .

Synchondroses, 499 .

Syncope, 61.

Synergetic muscles, 502.

Synovia, 498.

Syntonin, 247, 377.

Systole, cardiac, 51.
Tabes, 647 .

Taches cerebrales, 678 .

Tactile areas, 714.

", corpuscles, 829.

, sensations, 829 .

, sensations, conduction of, 646 .

Tænia, 843 .

Tail-fold, 869 .

Talipes calcaneus, 503.

,$\quad$ equinus, 503 .

, varus, 503.

Tambour, Marey's, 99.

Tapetum, 767.

Tapping experiment, 668 .

Taste, centre for, 704 .

,, organ of, 823 .

, testing, 826.

Taste-bulbs, 825 .

Taurin, 385 .

Taurocholic acid, 247.

Tea, 353.

,. effects of, 220 .

Tears, 794.

Tegmentum, 718

Telestereoscope, 791 .

Telolemma, 459.

Temperature of animals, 319 .

accommodation for, 334 .

artificial increase of, 336 .

estimation of, 319 .

febrile, 335 .

". how influenoed, 322.

lowering of, 338 .

" post-mortem, 337 .

,' regulation of, 328 .

topography of, 321 .

, variations of, 325 .

Temperature-sense, 836 .

, illusions of, 838.

Tendon, 457.

, nerves of, 462,829 .

reflexes, 642 .

Tensor choroideæ, 750 .

tympani, 803 .

Terminal arteries, 137.

Testicle, descent of, 889 .

Testis, 844.

Tetanomotor, 534

Tetanus, 485, 486, 536. secondary, 539 .

Tetronerythrin, 38 .

Theobromin, 353 .

Thermal centre, 329 . nerves, 329 .

Thermo-electric methods, 320 .

$$
\text { ,n needles, } 321 .
$$

Thermogenesis, 317.

Thermometer, 319 . clinical, 319.

" maximal and
" metastatic, 320 .

Thermometry, 319.

Thirst, 356 .

Thiry's fistula, 277.

Thomsen's disease, 484.

Thoracometer, 176. 
Thrombosis, 33.

Thymus, 153.

Thyroid, 154 .

development of, 880 .

Tidăl air, 166.

development of, 880 .

wave, 102.

Timbre, 519, 521, 811.

Time in psychical processes, 684 .

Time-sense, 813.

Tinnitus, 606.

Tissue-formers, 356.

metabolism of, 367.

regeneration of,

Tizzoni's reaction, 264 .

Tobin's tubes, 201.

Tomes, fibres of, 224.

Tone-inductorium, 487.

Tones, 814.

Tongue, glands of, 206.

,$\quad$ movements of, 227.

,, nerves of, 227.

taste-bulbs of, 825 .

Tonometer, 82.

Tonsils, 207.

Tonus, 644 .

Tooth, 223.

,' action of drugs on, 226.

", chemistry of, 225 .

" development of, 225 .

, eruption of, 226 .

," permanent, 226.

, pulp of, 224 .

, structure of, 223.

, temporary, 226.

Topography, cerebral, 706, 714.

Toricelli's theorem, 89 .

Torpedo, 579

Torticollis, 615 .

Touch corpuscles, 827.

Touch, sense of, 827 .

Trachea, 159

Transfusion, 145.

of blood, 145 . of other fluids, 148 .

Transitional epithelium, 432.

Transplantation of tissues, 371 .

Transudations, 313.

Trapezius, spasm of, 615 .

Traube-Hering curves, 126.

Traumatic degeneration of nerves, 539.

Trehalose, 383.

Trichina, 843.

Trigeminus, 590 .

\begin{tabular}{|c|c|}
\hline & $\begin{array}{c}\text { ganglia of, } 591,595 \text {, } \\
597,598 .\end{array}$ \\
\hline & $\begin{array}{l}\text { inferior maxillary } \\
\text { branch, } 595 \text {. }\end{array}$ \\
\hline & neuralgia of, 598 . \\
\hline & ophthalmic branch, \\
\hline & paralysis of, 599 . \\
\hline & $\begin{array}{l}\text { pathological, } 598 \text {. } \\
\text { section of, } 593,599 \text {. }\end{array}$ \\
\hline & superior maxillary \\
\hline & $\begin{array}{l}\text { branch, } 594 . \\
\text { trophic functions } \\
\text { of, } 593 \text {. }\end{array}$ \\
\hline
\end{tabular}

Triple phosphate, 408.

Trismus, 598.
Trochlearis, 589.

Trommer's test, 221.

Tropæolin, 242.

Trophic centres, 539.

, fibres, 539 .

," nerves, 539, 582.

Trophoneuroses, 582 .

Trotting, 508 .

Trypsin, 255.

Trypsinogen, 255.

Tryptone, 254.

Tube casts, 417.

Tubes, capillary, 91.

, division of, 91 .

, elastic, 92 .

" movements of fluids in, 92.

rigid, 92.

Tumultus sermonis, 711.

Tunicin, 384.

Turacin, 381.

Türck's method, 641 .

Twins, 860 .

Twitch, 479.

Tympanic membrane, 799 . artificial, 801.

Tyrosin, 255, 385, 416.

U1cer of foot, perforating, 583 .

Umbilical arteries, 872 .

$$
\begin{array}{ll}
, & \text { cord, } 875 . \\
, & \text { veins, } 872 . \\
, & \text { vesicle, } 869 .
\end{array}
$$

Unipolar induction, 550 .

Upper tones, 814. timulation, 537 .

Urachus, 888 .

Uræmia, 430 .

Urates, 399 .

Urea, 395 .

, antecedents of, 396.

,, compounds of, 397.

, decomposition of, 395 .

, effect of exercise on, 396.

ferment, 408 .

, formation of, 396,425 .

nitrate of, 397

occurrence of, 396 .

, oxalate of, 397.

, pathological, 396.

, phosphate of, 397.

,, preparation of, 397.

,$\quad$ properties of, 395 .

, qualitative estimation of, 397.

, quantitative estimation of, 397.

, quantity of, 395 .

,, relation of, to muscular work, 396 .

Ureameter, 397.

Ureter, ligature of, 424.

", pressure in, 412.

"structure and functions of, 431 .

Uric acid, 399.

, diathesis, 431.

, formation of, 426 .

,, occurrence, 399 .

, properties of, 399.

," qualitative estimation, 400 .

, quantitative estimation of, 400.
Uric acid, quantity, 399.

,, solubility, 399 .

,. tests for, 400 .

Urinary bladder, 433.

, calculi, 419.

, closure of, 433

, deposits, 416 .

, development of, 873 .

, organs, 386

Urine, 392 .

pressure in, 436 .

, accumulation of, 434.

aceton in, 415 .

acid fermentation, 408 .

acidity, 394 .

albumin in, 408.

alkaline fermentation, 408.

alkaloids in, 431.

amount of solids, 393 .

bile in, 413 .

blood in, 411

calculi, 419 .

changes of in bladder, 436.

characters of, 392.

colour, 393.

colouring matters of, 403 .

consistence, 394 .

cystin in, 416 .

deposits in, 416 .

dextrin in, 415 .

effect of blood-pressure on, 421.

egg-albumin in, 411.

electrical condition 579.

excretion of pigments by, 424.

fermentations of, 407 .

ferments in, 405 .

fluorescence, 393 .

fungi in, 417.

gases in, 407 .

globulin in, 410 .

hemi-albumose, 411.

incontinence of, 437 .

influence of nerves on, 427.

inorganic constituents, 405.

inosit in, 415 .

leucin in, 416.

milk-sugar in, 415 .

movement of, 432 .

mucin in, 393, 411.

mucus in, $393,411$.

organisms in, 417.

passage of substances into, 426.

peptone in, 410 .

phosphoric acid in, 406.

physical characters of, 392.

pigments of, 403.

propeptone in, 410 .

quantity, 392.

reaction, 394 .

retention of, 436 .

secretion of, 420 .

silicic acid in, 407.

sodic chloride in, 405.

solids of, 393 . 
Vrine, specific gravity, 392.

" spontaneous changes in, 407.

sugar in, 413.

sulphuric acid in, 406.

, taste of, 394 .

,. test for albumin in, 409.

," tube casts in, 417 .

tyrosin in, 416.

Urinometer, 393.

Urobilin, 26, 403.

Urochrome, 403.

Uroerythrin, 403.

Vro-genital sinus, 890 .

Iromelanin, 403.

Urorubin, 403.

Urostealith, 419.

Vterine milk, 875 .

I'terus, 853.

, development of, 890 .

", involution of, 895 .

," nerves of, 895 .

I'tricle, 808.

ITvea, 735 .

Vugotomy, 665 .

Vagus, 606. cardiac branches, 611.

, depressor nerve of, 124, 610.

effect of section, 611 .

on heart, 126.

pathological, 613.

pneumonia after section, bill.

reflex effects of, 613 .

" stimulation of, 127,668 .

" unequal excitability of, 613.

Valleix's points douloureux, 839.

Valsalva's experiment, 88, 111.

Valve, illeo-colic, 233. pyloric, 230.

Valves of heart, 54 .

, disease of, 70 .

, injury to, 60 .

" of veins, 94 .

". sounds of, 142 .

Valvula conniventes, 290.

Varicose fibres, 526.

Varix, 129.

Varnishing the skin, 339.

Vas deferens, 845.

Vasa vasorum, 95.

Vascular system, development of, 882 .

Vaso-dilator centre, 678 . "nerves, 678 .

Vaso-formative cells, 11.

Vaso-motor centre, 672.

" destruction of, 673 .

"nerves, $67^{\circ}$.

"spinal, 676.

Vaso-motor nerves, course of, 673.

Vater's corpuscles, 828.

Vegetable albumin, 377.

$$
\text { " cosein, } 377 .
$$

", foods, 350 .
Vegetable, preserved, 352.

Veins, 94. cardinal, 884.

", development of, 884 .

, movement of blood in, 139.

murmurs in, 141.

", pressure in, 128.

," pulse in, $\mathbf{1 4 2}$.

, structure of, 94 .

, tonus of, 673 .

," valves in, 94, 140 .

,. valvular sounds in, 142 .

,, varicose, 129.

, velocity of blood in, 139.

Vella's fistula, 277.

Velocity of blood-stream, 90 .

Venous blood, 45.

Ventilation, 200.

Ventricles, 53, 66.

\begin{tabular}{|c|c|}
\hline ", & aspiration of, 58 . \\
\hline , & brain, 726 . \\
\hline , & $\begin{array}{l}\text { capacity of, 118, } 135 \text {. } \\
\text { fibres of } 53 .\end{array}$ \\
\hline ", & impulse of, 62 . \\
\hline$"$ & $\begin{array}{l}\text { negative pressure in, } \\
59 .\end{array}$ \\
\hline & systole of, 59,66 . \\
\hline
\end{tabular}

Veratrin, 483.

Vernix caseosa, 445.

Vertebræ, mobility of, 504.

Vertebral column, 870 .

Vertigo, 605.

Vestibular sacs, 808.

Vibrations of body, 116.

Vibratives, 522.

Vibrio, 49.

Villus, intestinal, 291.

,, absorption by, 300 .

,. chorionic, 873 .

," contractility of, 292.

placental, 874

Violet-blindness, 778 .

Visceral arches, 871 . clefts, 871.

Vision, binocular, 787 .

, stereoscopic, 789 .

Visual angle, 748 .

, apperatus, 731.

," centre, 702, 713.

, purple, 739, 773.

Vital capacity, 166.

Vitellin, 376.

Vitelline duct, 869.

Vitreous humour, 740.

Vocal cords, 509

, conditions influencing the,

Voice, 509. 518 .

, falsetto, 518 .

, in animals, 523.

, pathological variations of, 522 .

physies of, 510 .

", pitch of, 510

, production of, 519

, range of, 419 .

Volume pulse, 145.

Volumetric method, 398.
Vomiting, 231.

Vowels, 520

centre for, 232,660 .

, analysis of, 520,814 .

, artificial, 815 .

, formation of, 520 .

" Konig's apparatus for, 817.

Wagner's corpuseles, 827 .

Waking, 685.

Walking, 505.

Wallerian law of degeneration, 538.

Wandering cells, 303.

Warm-blooded animals, 318.

Washed blood-clot, 33 .

Water, 341, 373.

" absorbed by skin, 449.

, absorption of, $29 \%$.

", exhaled by skin, 194, 425.

, exhaled from lungs, 188.

, hardness of, 342 .

," impurities, 342.

, in urine, 392.

, vapour of, in air, 187.

Wave-pulse, 102.

, propagation of, 111.

Wave-motion, 92.

Wave-movements, 798 .

Waves, in elastic tubes, 113.

Weber's paradox, 493.

, law, 732.

Weigert's method, 529 .

Weight, 372.

Weyl's test, 401.

Wharton's jelly, 875 .

Whispering, 519.

White of egg, 375.

Wine, 354.

Wolftian bodies, 888 . ducts, 888 .

Word-blindness, 711 .

Word-deafness, 704, 711.

Work, 489.

, unit of, xxxviii.

Xanthin, 401.

Xanthokyanopy, 778.

Xanthophane, 740.

Xanthoproteic reaction, 375

Xerosis, 594.

Yawning, 183.

Yeast, 380.

Yelk, 852.

" cleavage of, 862 .

, sac, 869.

Yellow-spot, 764.

Young-Helmholtz theory, 776.

Zero-temperature, 836.

Zimmermann, particles of, 17.

Zinn, zonule of, 740 .

Zoetrope, 780.

Zöllner's lines, 793.

Zona pellucida, 849 .

Zoogluea, 280.

Zymogen, 255. 


\title{
BY JOHN THORBURN, M.D., F.R.C.P.,
}

LATE PROFESSOR OF OBSTETRIC MEDICINE, OWENS COLLEGE AND VICTORIA UNIVERSITY, MANCHESTER; OBSTKTRIC PHYSICIAN TO THE MANCHESTER ROYAL INFIRMARY.

Royal 8vo, with Chromo-Lithograph and over 200 Illustrations, Cloth. 21s.

\section{THE DISEASES OF WOMEN}

\author{
(A PRACTICAL TREATISE ON). \\ Prepared with Special Reference to the Wants of the General Practitioner and \\ Advanced Student.
}

\section{OPINIONS OF THE PRESS.}

"A task of extraordinary difficulty ... handled with great JUDICIOUSNESS and HIGH LITERARY skill. ${ }^{-}$Professor Thorburn has done his duty. he lets his readers understand all sides of a question, and never fails to inculcate the highest principles. INSTRUCTIVE, and in every way worthy of its author."-British Medical Journal.

"As a Manual for Students and Practitioners, Dr ThORBurn's Is ALL THAT Could BE DEsIRED. Knowledge is power, and that power is trebled when JUDGMENT AND COMNON-SENSE KEEP PACE WITH KNOWLEDGE. We can say no less of Dr Thorburn's book."-Glasgow Medical Journal.

"Free from prejudices, candid and careful, exhibiting sound judgment and discretion, WE KNOW OF NO BOOK which affords to Students and Practitioners a letter digest of the subject than DR THORBURN's "DISEASES OF WOMEN."-Birmingham Medical Reviev.

\section{WORKS BY A. WYNTER BLYTH, M.R.C.S., F.C.S., PUBLIC ANALYST FOR THE COUNTY OF DEVON, AND MEDICAL OFFICRR OF HEALTH FOR ST MARYLEBONE. New EDition. Revised and partly Rewritten.}

\section{FOODS: THEIR COMPOSITION AND ANALYSIS.}

In Crown 8vo, Cloth, with Elaborate Tables, Folding Litho-Plate, and Photographic Frontispiece. THIRD EDITION. Price 16s.

GENERAL CONTENTS.

History of Adulteration-Legislation, Past and Present-Apparatus useful to the FoodAnalyst - "Asl " - Sugar-Confectionery-Honey-Treacle-Jams and Preserved FruitsStarches - Wheaten-Flour-Bread-Oats-Barley-Rye-Rice-Maize-Millet-Potatoe-Peas -Chinese Peas - Lentils - Beans-Milk-Cream-Butter-Cheese-Tea-Coffee-Cocoa and Chocolate - Alcohol - Brandy - Rum - Whisky - Gin - Arrack — Liqueurs - Beer-Wine Vinegar-Lemon and Lime Juice-Mustard-Pepper-Sweet and Bitter Almond-AnnattoOlive Oil-Water. Appendix: Text of English and American Adulteration Acts.

"Thoroughly practical. . . . Should be in the hands of every medical practitioner."-Lancet.

"An admirable digest of the most recent state of kuowledge. . . . Interesting even to lay readers." -Chemical Neros.

"STANDS UNRIVALLED for completeness of information."-Sanitary Record.

* * The new Edition contains many Notable Additions, especially on the subject of MILK and its relation to FEVER EPIDEMICS, the PURITY of WATER-SUPPLY, the New MARGARINE ACT, \&c. \&c.

\section{POISONS: THEIR EFFECTS AND DETECTION.} With Tables and Illustrations. SECOND EDITION. Price 16s. GENERAL CONTENTS.

Historical Introduction-Statistics-General Methods of Procedure-Life Tests-Special Apparatus-Classification: I.-Organic PoIsons: $(a$.$) Sulphuric, Hydrochloric, and Nitric$ Acids, Potash, Soda, Ammonia, \&c. ; (b.) Petroleum, Benzene, Camphor, Alcohols, Chloroform, Carbolic Acid, Prussic Acid, Phosphorus, \&c. ; (c.) Hemlock, Nicotine, Opium, Strychnine, Aconite, Atropine, Digitalis, \&c.; (d.) Poisons derived from Animal Substances; $\left(e_{.}\right)$The Oxalic Acid Group. II.-Inorganic Poisons : Arsenic, Antimony, Lead, Copper, Bismuth, Silver, Mercury, Zinc, Nickel, Iron, Chromium, Alkaline Earths, \&c. Appendix: (A.) Examination of Blood and Blood-Spots; (B.) Hints for Emergencies.

"One of the best and most comprehensive works on the subject."-Saturday Review.

"A sound and Practical Manual of Toxicology, which cannot be too warmly recommended. . . . One of its chief merits is that it discusses substances which have been overlooked." - Chemical Nev8.

London: CHARLes GRIFFiN \& CO., Exeter Street, Strand. 


\section{By PROF. HADDON.}

In Royal 8vo, with 190 Illustrations. $18 \mathrm{~s}$.

\section{E M B R Y O L O G Y}

(AN INTRODUCTION TO THE STUDY OF).

Jfor the Use of itudents.

BY ALFRED C. HADDON, M.A., M.R.I.A., Professon of Zoology in the Royal College of Science, Dublin.

OPINIUNS OF THE PRESS.

"The pullication of this volume supplies a long-felt want."-Nature.

"AN EX(ELLENT RÉtuE OF' RECENT RESEARCH, well adapted for 'self-study.' (iive remarkal)ly gool accumts (including all recent work) of the development of the heart and other organs. . . . The book is handsomely got up."-Lancet.

"Profesor Haddon has the real seientific spirit for work of this kind. . . . The develejment of the virious organs ably demonstrated. . . Forms a handsome volume."-British Medical Joumal.

\section{By J. R. AINSWORTH DAVIS.}

In Large Crown 8vo, with 158 Illustrations. 12s. 6d.

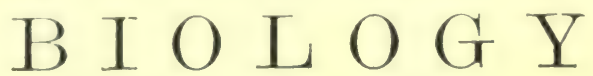

(A TEXT-BOOK OF).

Comprising Vegetalle and Animal Norphology and Physiology,

BY J. R. A. DAVIS, B.A.,

Lecturer on Biology, University College, Aberystwyth.

"As at general Work of Reference, Mr Davis's Manual will be highly serviceable to Medical Men."-British Medical Journal.

"Th. volume is literally packed with information."-Glasgow Medical Journal.

\section{By Drs PORTER and GODWIN.}

Thinv Entrox. Revised and enlarged. Foolscap 8vo, Roan, with 152 Illustrations and Folding-plate. 7s. 6d. Postage $3 d$.

\section{THE SURGEON'S POCKET-BOOK.}

\section{specially adapted to tbe public Medical services.}

By Surgeon-Major J. H. PORTER.

Revised and in great part rewritten

BY BRIGADE-SURGEON C. H. T. GODWIN, Assistunt-Professor of Military Surgery in the Army Medical School.

"Every Medical Officer is recommended to have the 'Surgeon's Pocket-Book,' by Surgeon-Major Porter, accessiljle to refresh his memory and fortify his judgment."Précis of Field-Service Medical Arrangements for Afghan War.

"The present editor-Brigade-Surgeon Godwin-has introduced so much that is new and practical, that we can recommend this 'Surgeon's Pocket-Book' as an INvaluable. GOIDE to all engaged, or likely to be engaged, in Field Medical Service."-Lancet.

"A complete vade mecum to guide the military surgeon in the field."-British Medical Journal.

“A capital little book . . . of the greatest practical value. . . . A surgeon with this Manual in his pocket becomes a man of resource at once."-Westminster Review. 
By PROFESSOR T. M'CALL ANDERSON, M.D.

Now ready, with two Coloured Lithographs, Steel Plate, and munerous Wiondents. Royal 8vo, Handsome Cloth, 25 s.

\section{DISEASES OF THE SKIN}

(A TREATISE ON),

With Special Reference to Diagnosis and Treatment, ixcuudis; ax Analysis of 11,000 Consecutive Cases.

Br T. M'CALL ANDERSON, M.D., Professor of Clinical Medicine, University of Glasyon.

The want of a manual embodying the most recent advances in the treatment of cutaneous affections has made itself much felt of late years. Professor M'Call Anderson's Treatise, therefore, affording, as it does, a complete résumé of the best modern practice, will be doubly welcome. It is written-not from the standpoint of the University Professor-but from that of one who, during upwards of a quarter of a century, has been actively engaged both in private and in hospital practice, with unusual opportunities for studying this class of disease, hence the PRACTICAL and CLINICAL directions given are of great value.

Speaking of the practical aspects of Dr ANDERSON's work, the British Medical Journal says:- "Skin diseases are, as is well known, obstinate and troublesome, and the knowledge that there are ADDITIONAI, RESOURCES besides those in ordinary use will give confidence to many a puzzled medical man, and enable him to encourage a doubting patient. Almost ANY PAGE MIGHT BE USED TO ILLUSTRATE THE FULNESS OF THE WORK IN THIS RESPECT. . . . The chapter on Eczema, that universal and most troublesome ailment, describes, in a comprehensive spirit, and with the greatest accuracy of detail, the various methods of treatment. Dr Anderson writes with the authority of a man who has tried the remedies which he discusses, and the information and advice which he gives cannot fail to prove extremely valuable."

\section{Opinions of the Press.}

\footnotetext{
"Beyond doubt, the MosT IMPORTANT WORK on Skin Diseases that has appeared in England for many years. - . Conspicuous for the AMOUNT AND EXCELLENCE of the CLINICAL AND PRACTICAL information which it contains."-British Medical Journal.

"Professor M'Call Anderson has produced a work likely to prove very acceptable to the busy practitioner. The sections on treatment are very full. For example, EczEms has 110 pages given to it, and 73 of these pages are devoted to treatment."-Lancet.
}

CHARLES GRIFFIN \& CO., Exeter Street, Strand, London. 
By Drs MEYER and FERGUS.

Now Reculy, with Three Coloured Plates and numerous Illustrations.

Royal 8vo, Handsome Cloth, $25 s$.

\title{
DISEASES OF THE EYE
}

(A PRACTICAL TREATISE ON),

\author{
BY EDOUARD MEYER, \\ Prof. ì REcole Pratique de la Fuculté de Médecine de Paris, \\ Chev. of the Ley. of Honour, dic.
}

\begin{abstract}
Translated from the Third French Edition, with Additions as contained in the Fourth German Edition, By F. FERGUS, M.B., Ophthalmic Surgeon, Glasgow Infirmary.
\end{abstract}

The particular features that will most commend Dr Meyer's work (1) English readers are-its concIseness, its HeLPFULNESs in explanation, and the PRACTICALITY of its rlirections. The best proof of its worth may, perhajs, be seen in the fact that it has now gone through three French and four German editions, and has been translated into most European langruages-Italian, Spanish, Russian, and Polish-and even into Japanese.

\section{Opinions of the Press.}

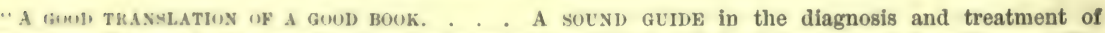
the various disenses of the eye that are likely to fall under the notice of the general Practitioner. The l'aper, Type, and Chromo-Lithographs are all that could be desired. . . . We know of no work in which the IISFisks and INFurMitiss of the LIIS are more fully treated. Numerous figures illustrate almost every defect remediable by operation."-Practitioner.

"A VERY тKUSTWORTHY GUIDE in all respects. . . . THOROUGHLY PRACTICAL. Excellently translated, and very well got up. Type, Woodcuts, and ('hromo-Lithographs are alike excellent."-Lancet.

"Any Student will find this work of GREAT ValuE. . . . The chapter on Cataract is excellent. The Illustrations describing the various plastic operations are specially helpful."-Brit. Med. Journal.

"An nxcelient translation of a standard French Text-Book. . . We can cordially recommend Dr Meyer's work. It is essentially a PRAcTICAL WORK. The Publishers have done their part in the

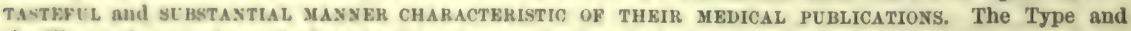
the Illustrations are in marked contrast to most medical works."-Ophthalmic Review. 



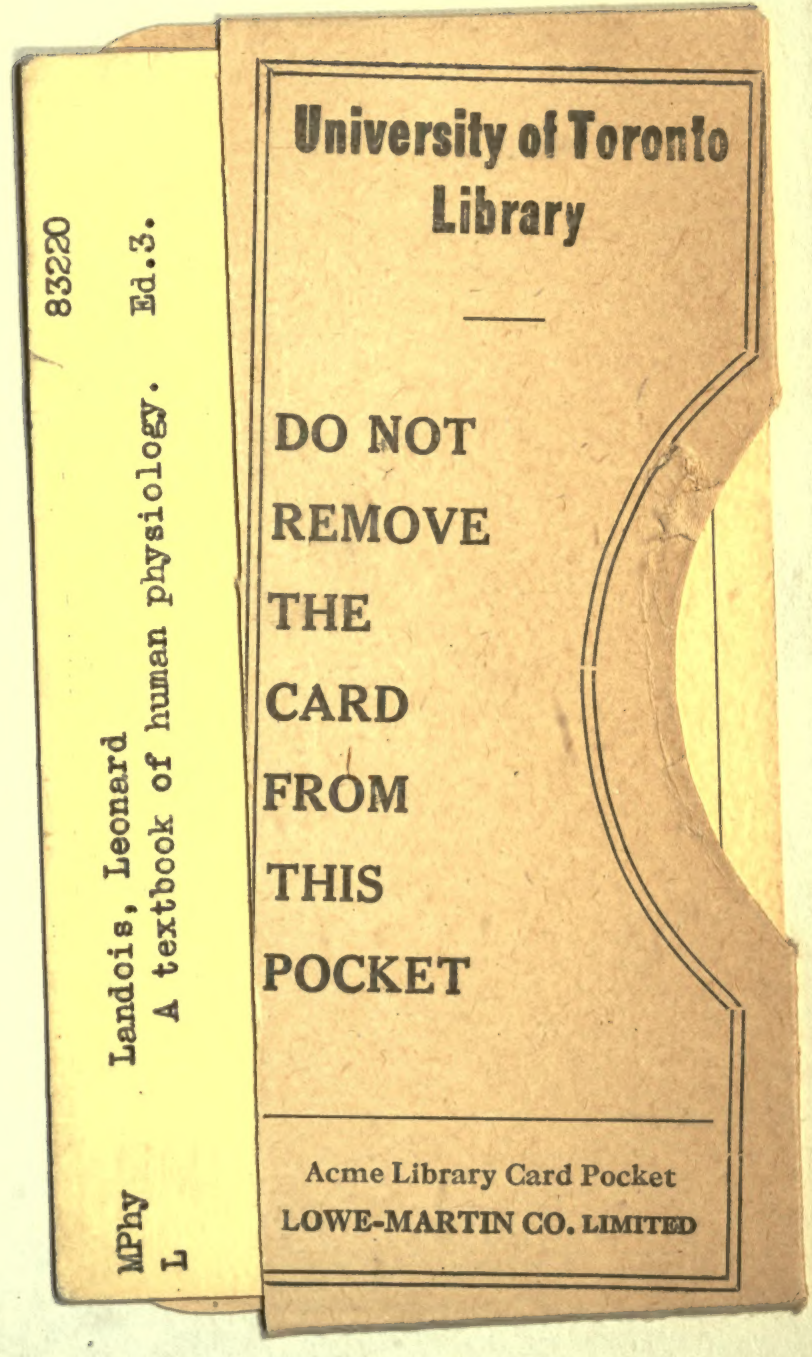


